\title{
Abstracts from the 35th Annual Meeting of the Society of General Internal Medicine
}

\section{ABSTRACTS OF SUBMISSIONS ACCEPTED FOR PRESENTATION}

\section{SCIENTIFIC ABSTRACTS}

2011 VETERANS' HEALTH ADMINISTRATION EMERGENCY SERVICES FOR WOMEN (ESW) SURVEY Kristina M. Cordasco ${ }^{1,2}$; Laurie C. Zephyrin ${ }^{3,4}$; Ismelda Canelo ${ }^{1}$; Chad Kessler ${ }^{5,6}$; Merri Mallard $^{3}$; Lisa V. Rubenstein ${ }^{1,2}$; Elizabeth M. Yano ${ }^{1,7}$. ${ }^{1}$ VA Greater Los Angeles Healthcare System, Los Angeles, CA; ${ }^{2}$ The David Geffin School of Medicine at The University of California, Los Angeles (UCLA), Los Angeles, CA; ${ }^{3}$ The Veterans' Health Administration, Washington, DC; ${ }^{4}$ VA New York Harbor Healthcare System, New York, NY; ${ }^{5}$ Jesse Brown VA Hospital, Chicago, IL; ${ }^{6}$ The University of Illinois - Chicago, Chicago, IL; ${ }^{7}$ UCLA School of Public Health, Los Angeles, CA. (Control ID \#1337288)

BACKGROUND: More women are using Veterans' Health Administration (VHA) Emergency Departments (EDs). Women Veterans presenting to VHA EDs may have different needs, and therefore require different resources and processes of care, than their male counterparts. VHA EDs' capacities to meet these needs have not been previously assessed. In a research-operations partnership, we surveyed VHA ED capabilities relevant to caring for women Veterans.

METHODS: We surveyed all 120 VHA ED directors between May 24th and June 30th, 2011. We report here primarily on results for gynecologic and sexual assault care and pregnancy testing. Because VHA EDs are known to vary, we compared female-specific capabilities (resources and processes of care) to gender neutral capabilities to ground our analyses. We also assessed capabilities stratified by number of ED encounters by women, total ED encounters per year, facility complexity, and facility location in large versus small or non-metropolitan areas.

RESULTS: All VHA EDs (100\%) completed the survey. Thirty-five percent of VHA EDs have emergent gynecology consultations available at all times compared to $77 \%$ having cardiology and $74 \%$ urology. An additional $28 \%$ of VHA EDs have emergent gynecology consultations available some of the time. Emergency psychiatric consultation for sexual assault is available in $86 \%$ of EDs at all times, and $77 \%$ are able to arrange follow-up mental health services within 48 hours for this problem. Most (92\%) VHA EDs transfer sexual assault victims to other institutions for physical and evidentiary evaluation and treatment. EDs commonly use point-of-care testing (i.e., immediate testing in the ED) for troponin (58\%) but not for pregnancy $(8 \%)$. Similarly, standing ordersets are common for electrocardiogram $(88 \%)$ and fingerstick glucose (75\%) but less common for pregnancy tests $(40 \%)$. Nurse triage note templates have a designated space for last menstrual period in $33 \%$ of the EDs. Basic female-specific supplies, such as speculums and gynecologic examination tables, are available in most, but not all VHA EDs ( $98 \%$ and $88 \%$, respectively). Emergency contraception is available in $87 \%$ of VHA EDs and Rho(D) immune globulin in 53\%. Seventy-two percent of VHA EDs have pelvic ultrasounds available to ED patients, 39\% have this available at all times. VHA EDs with fewer encounters by women, located in small or non-metropolitan areas, and part of less complex VHA healthcare systems have less capabilities for caring for women.

CONCLUSIONS: While many VHA EDs have capabilities for femalespecific care, gaps remain, especially in those with fewer women Veteran encounters. Lack of point of care capability to carry out pregnancy tests, for example, may result in delays in emergency gynecologic and radiologic care to women. Such gaps must be further assessed and addressed if VHA is to provide comprehensive care to women. These data will be used as the basis for developing recommendations for ensuring quality care to women in VHA EDs. 
A BRIEF CURRICULAR INTERVENTION TO IMPROVE SCREENING AND BRIEF INTERVENTIONS FOR SUBSTANCE ABUSE IN THE PRIMARY CARE SETTING DOES NOT IMPROVE PERFORMANCE ON A STANDARDIZED PATIENT ASSESSMENT Maria A. Wamsley ${ }^{1}$; Steven L. Batki ${ }^{2,3}$; Miranda Dunlop ${ }^{1}$; Katherine Julian $^{1}$; Elinore McCance-Katz ${ }^{2}$; Patricia S. O'Sullivan ${ }^{1}$; Jason Satterfield ${ }^{1}$. IUniversity of California, San Francisco, San Francisco, CA; 2 University of California, San Francisco, San Francisco, CA; ${ }^{3}$ San Francisco Veterans Administration Medical Center, San Francisco, CA. (Control ID \#1340097)

BACKGROUND: The need for screening and brief intervention for substance use disorders (SUDs) in primary care settings is widely recognized. Barriers include inadequate provider skills and lack of confidence. The optimal format for curricular interventions to address barriers remains unclear. We implemented a 3 hour Screening, Brief Intervention and Referral to Treatment (SBIRT) curriculum for internal medicine (IM) residents using a Team-based Learning (TBL) format. The TBL format was selected for its adherence to educational principles and active learning. The objective of our study was to evaluate the impact of the curriculum on resident SBIRT skills, knowledge and confidence using a standardized patient (SP) assessment. METHODS: This study took place at an academic residency training program. 54 PGY2 and PGY3 IM residents participated. 26 residents participated in the SP assessment prior to the curriculum (control group) and 29 participated in the SP assessment after the curriculum (experimental group). The SP assessment consisted of 3 twenty-minute encounters with a patient with at-risk alcohol use, alcohol dependence or prescription opioid misuse. SPs evaluated residents on history(HX), information sharing(IS), and patient-physician interaction(PPI) and a single-item to assess overall satisfaction(OS) with the resident. Individual and mean scores for each case were calculated for each of the domains(HX,IS,PPI,OS) and a mean summary score for each domain was calculated for all cases. After the SP assessment, residents completed surveys about their satisfaction with the SP assessment and confidence in performing SBIRT. Residents were surveyed about their satisfaction with the TBL curriculum. T-tests were performed to detect differences between experimental and control groups. RESULTS: There were no statistically significant differences between control and experimental groups on the mean scores for each case in the four domains (HX,IS, PPI,OS) or in the mean summary scores (HX,IS,PPI,OS) for all cases combined. For HX items, residents received 54\% of the total points possible (experimental) vs $61 \%$ (control; $\mathrm{p}=.05$ ) and for IS, residents received overall scores of $72 \%$ (experimental) vs $67 \%$ (control; $p=.78$ ). PPI and OS for both groups were rated as good to very good. HX and IS scores were lowest for the prescription opioid misuse case. Resident confidence in screening for drugs [mean $=3.93$ vs $3.45(1=$ strongly disagree, $5=$ strongly agree), $\mathrm{p}=.013$ ] and alcohol (mean $=4.24$ vs $3.66, \mathrm{p}=.002$ ), assessing stage of behavioral change (mean $=4.21$ vs $3.34, p<.001$ ) and making treatment plans for patients with SUDs (mean $=3.90$ vs. $2.90, \mathrm{p}<.001$ ) was significantly higher in the experimental group. Residents in the experimental group were more likely to say they would recommend the SP assessment to a colleague (mean $=3.41$ vs $2.83, \mathrm{p}=.04$ ). Both groups were close to neutral on whether they would recommend the TBL training to a colleague.

CONCLUSIONS: A 3 hour TBL session improved resident confidence in their SBIRT skills, but did not improve SBIRT skills as measured by a SP assessment. Resident SBIRT skills in both the experimental and control groups showed room for improvement in all areas (HX,IS,PPI, OS) with a particular need for improvement in handling prescription opioid misuse. Overall satisfaction with SP assessment and the TBL curriculum was positive. A more intensive curriculum with opportunities to practice skills and receive feedback over time may be required to improve behavioral skills.

A BRIEF STRUCTURED PEER-TO-PEER FEEDBACK INTERVENTION TO IMPROVE THE QUALITY OF RESIDENT DISCHARGE SUMMARIES Krishan Soni; Mia Lozada; Michelle Schneidermann. San Francisco General Hospital, University of California San Francisco (UCSF), San Francisco, CA. (Control ID \#1315178)

BACKGROUND: High quality communication is required for safe hospital discharges and is accomplished primarily through the discharge summary.
Clear, concise, and meaningful correspondence between providers is essential and yet, first year medical residents are generally charged with the responsibility of completing discharge summaries with little or no training. While prior studies have shown improvement in discharge summary quality after introducing a didactic curriculum with direct feedback, we are not aware of any curricula that take advantage of peer-to-peer feedback.

METHODS: Based on literature review and an internal needs assessment of problems with discharge summaries, we developed an educational curriculum consisting of (1) a didactic session illustrating the quality gaps in discharge summaries at our institution, and best practices for improvement, followed by (2) a one hour session dedicated to directed peer-to-peer feedback for interns to evaluate their own summaries. A discharge summary evaluation tool, focusing on 5 elements of the discharge summary was developed to facilitate peer assessment of discharge summaries. During the peer-to-peer feedback session, interns completed a brief pre-test regarding their confidence with discharge summaries. They exchanged their own discharge summary with a partner and reviewed/evaluated them with the standardized rubric. The larger group congregated and summarized the strengths and weaknesses of their own discharge summaries and focused on areas they would work on in the future, and then completed a brief post-test to assess the experience with the peer-to-peer feedback session.

RESULTS: 58 learners participated in this curriculum and completed the pre and post test evaluation form. $29(50 \%)$ of the respondents were medical interns, 13 $(22 \%)$ were prelim interns from other departments and, $16(28 \%)$ were students. While $69 \%$ of respondents thought that completing a discharge summary solidifies their understanding of a patient's hospital course, only $22 \%$ were satisfied with the quality of their discharge summary. In the post test analysis, $98 \%$ of residents agreed or strongly agreed that it is useful to develop and improve skills in writing $\mathrm{D} / \mathrm{C}$ summaries. $81 \%$ of residents thought the peer-topeer feedback session was comfortable, and $85 \%$ thought that it was helpful. CONCLUSIONS: We developed a unique educational innovation involving a peer-to-peer feedback session for PGY1 residents to assess the quality of their own discharge summaries. The peer-to-peer evaluation component was a powerful tool for allowing interns to identify their own strengths and weaknesses in their ability to write concise and accurate discharge summaries. Additionally, the group generated best practices and specific approaches to change their own practice immediately after this session. Having residents provide feedback and teaching to their peers can be a high yield mechanism for promoting learning and retention compared to traditional didactic sessions. This peer-to-peer feedback session could easily be repeated in most academic clinical settings to improve the quality of discharge summaries written by residents.

A COMPARISON OF EVISITS AND OFFICE VISITS FOR SINUSITIS AND URINARY TRACT INFECTIONS AT FOUR PRIMARY CARE PRACTICES Ateev Mehrotra ${ }^{1}$; Suzanne J. Paone ${ }^{2}$; Cynthia Carbine ${ }^{2}$; G. D. Martich $^{2}$; Grant Shevchik ${ }^{2}$. ${ }^{1}$ University of Pittsburgh, Pittsburgh, PA; ${ }^{2}$ UPMC, Pittsburgh, PA. (Control ID \#1339525)

BACKGROUND: There is growing recognition that many physician-patient encounters do not require face-to-face contact. The availability of secure internet portals and personal health records creates the opportunity for online care or structured "eVisits." An increasing number of health systems provide eVisits and more health plans reimburse for eVisits (identified via CPT code 99444). While there is growing use of eVisits, little is known about their clinical effectiveness. To fill this gap in knowledge, we compared the care between eVisits and office visits. To complete an eVisit in our healthcare system, patients log onto their secure personal health record and go through a structured questionnaire with branching logic that reviews their symptoms. This information is sent to their primary care physician who reviews this information and the patient's chart and answers on average in $\sim 4$ hours. The physician response is via the secure portal, prescriptions are sent electronically to the pharmacy, and the care provider's note is automatically put into the healthcare system's electronic health record (EHR). Four commercial health plans in the region reimburse eVisits and for the eVisit a patient pays the typical office visit co-payment.

METHODS: In the EHR for the University of Pittsburgh Medical Center, we used diagnosis codes to identify all eVisits and office visits for urinary tract 
infections (UTI) and sinusitis at four primary care practices that occurred between $1 / 1 / 10$ and 5/31/11. We focused on UTI and sinusitis, because these were common reasons for eVisits and there are published practice guidelines on management. The four practices chosen were the first to adopt eVisits within the system (they are now offered at all practices) and have the highest number of eVisits per month. We abstracted the necessary information (e.g. problem list, prescriptions, tests ordered, follow-up care) from the EHR. RESULTS: We identified 5595 sinusitis visits ( $9 \%$ of which were eVisits) and 3341 UTI visits ( $3 \%$ eVisits). eVisit users were on average only slightly younger ( $>65$ yo, eVisits $6 \%$ vs. $13 \%$ office visits) and employed by self-report ( $75 \%$ vs. $62 \%$ ). Surprisingly, there was no statistically significant difference in socio-economic status between eVisit and office visit patients (e.g. median household income in zip code $<\$ 30,000,12 \%$ vs. $10 \%$ ). At eVisits, physicians were less likely to order any relevant testing for the condition (e.g. urine culture or urinalysis, $6.5 \%$ UTI eVisits vs. $53 \%$ UTI office visits). At eVisit physicians were more likely to prescribe antibiotics (e.g. visits with antibiotic prescription, $99 \%$ sinusitis eVisits vs. $93 \%$ sinusitis office visits). A similar number of patients had any follow-up in the subsequent 3 weeks for the condition (e.g. 9.9\% UTI eVisits vs. $11.8 \%$ UTI office visits).

CONCLUSIONS: At these four primary care practices, eVisits accounted for almost 7\% visits for sinusitis and UTI. eVisits attracted patients who were slightly younger and employed. In an eVisit, physicians are more likely to prescribe antibiotics and are less likely to order testing. There was no difference in the need for follow-up. Recognizing we cannot account for differences in diagnosis and severity of illness between the two groups of patients, these results will be helpful to physicians and health systems that are considering eVisits.

A NATIONAL ASSESSMENT OF HEALTH PROFESSIONAL TRAINING AND PIPELINE PROGRAMS IN COMMUNITY HEALTH CENTERS Jaya Aysola ${ }^{1}$; LeRoi Hicks ${ }^{2}{ }^{1}$ Harvard Medical School and Brigham and Women's Hospital, Boston, MA; ${ }^{2}$ University of Massachusetts Memorial Medical Center, Worcester, MA. (Control ID \#1338538)

BACKGROUND: Training and pipeline programs within community health centers (CHCs) are cited as potential solutions to the shortage and maldistribution of primary care providers, yet the impact of such programs is not well understood. We conducted a nationally representative survey of $\mathrm{CHCs}$ to examine the effects of such programs on recruitment and retention of primary care providers in shortage areas.

METHODS: We partnered with the National Association of Community Health Centers to develop and pilot a self-administered survey instrument to evaluate the effects of health professional training activities at community health centers. We fielded the online survey to executive directors at a total of 976 federally qualified health centers with an email address in the 2009 Uniform Data System (UDS). Data collection was from March to June 2010, with 390 CHCs $(40 \%)$ responding. Survey data was then linked to UDS, which provides a range of information on the operations and performance of all community health centers receiving federal funding. Our four predictors were the presence or absence of 1) health professional training programs (providers and/or students) 2) Area Health Education Center programs (AHEC) 3) Student/Resident Experiences and Rotations in Community Health (SEARCH) and 4) Career Ladder/Advancement programs. Our two primary outcomes were self-reported difficulty in primary care provider recruitment and retention in the past 5 years, with difficulty measured by a scaled response (no/minimal, some, great). We ran both univariate and multivariable ordinal logistic regression models evaluating the associations between our four predictors and outcomes of interest. In our multivariable models, we adjusted for our predictors and several CHC level characteristics, including urban versus rural location, hospital affiliation, and receipt of Title VII funding.

RESULTS: Of the $390 \mathrm{CHCs}, 53 \%$ were rural, $34.5 \%$ reported hospital/ medical school affiliation, $89.6 \%$ reported having health professional training programs, $48.5 \%$ reported AHEC programs, $29.7 \%$ reported SEARCH programs, and $54 \%$ reported Career Ladder programs. The majority of CHCs reported "some" difficulty in both recruiting and retaining primary care providers in the last 5 years, with $32.6 \%$ reporting "great" difficulty in recruiting providers and $16.2 \%$ reporting "great" difficulty with retaining providers. Multivariable analyses demonstrated urban compared to rural health centers were more likely to report no/minimal difficulty in provider recruitment (OR 1.82; 95\% CI: 1.14, 2.90; $\mathrm{p}=.01$ ) and retention (OR 1.94; 95\% CI: 1.21 , $3.12 ; \mathrm{p}=.006$ ). In adjusted analyses, only one of our four predictors, having career ladder programs, was independently associated with both recruitment and retention of primary care providers. Centers with career ladder programs were more likely to report no/minimal difficulty with both recruitment (OR 1.93; 95\% CI: $1.21,3.08 ; \mathrm{p}=, 006$ ) and retention (OR 2.17; 95\% CI: 1.35, 3.49; $\mathrm{p}=.001$ ) compared to health centers without those programs.

CONCLUSIONS: Our findings suggest that career ladder/advancement programs have a significant effect on recruitment and retention of primary care providers in shortage areas. Our study underscores the need for further evaluation of health professional training and pipeline programs in order to expand models that demonstrate effectiveness in improving the primary care workforce in shortage areas.

\section{A NEW MODEL OF RETENTION FOR HEALTH EDUCATION/ BEHAVIORAL INTERVENTIONS: URBAN AFRICAN-AMERICANS IN THE DIABETES EMPOWERMENT PROGRAM Katie Raffel ${ }^{1}$; Anna Goddu ${ }^{2,3}$; Monica Peek ${ }^{2,3}$. ${ }^{1}$ University of Chicago Pritzker School of Medicine, Chicago, IL; ${ }^{2}$ University of Chicago Medical Center, Chicago, IL; ${ }^{3}$ Diabetes Research and Training Center, Chicago, IL. (Control ID \#1333585)}

BACKGROUND: Culturally-tailored diabetes education can improve diabetes self-management and self-efficacy among African-Americans, but attrition is often a barrier to program effectiveness. Attrition from basic diabetes education ranges from $4-57 \%$ and is associated with higher $\mathrm{HbA1c}$, blood pressure and BMI as well as increased complications. Despite the importance of retention in health outcomes, research describing minority retention in health education/behavioral interventions is lacking. The Diabetes Empowerment Program combines diabetes education and patient/provider communication training and is culturally-tailored for African-Americans. This program not only improved diabetes self-management but also had notably high retention rates. Despite the intensity of the ten-week program, $70 \%$ of the 50 participants attended $\geqq 80 \%$ of the classes. We sought to investigate the program's successful retention in order to address significant gaps in the literature describing minority retention in health education.

METHODS: We conducted four focus groups $(n=31)$ and seven in-depth interviews $(n=7)$ with former participants of the Diabetes Empowerment Program. Interviews and focus groups were conducted by trained interviewers with experience discussing health and communication. Using a structured topic guide, interviewers asked participants to identify and discuss factors influencing their attendance. Each interview/focus group was audiotaped, transcribed verbatim and analyzed using Atlas.ti software. Coding was conducted using an iterative process; each transcription was independently coded by two members of the research team.

RESULTS: Participants discussed multiple program characteristics contributing to successful retention, from which we identified the following key themes: 1 . Relevant information: The curriculum was culturally-tailored and practical, incorporating participants' traditional diets and community resources and reinforcing information with practical skills training (e.g. touring grocery stores, understanding lab results). 2. Program leadership: Educators were experienced with the local population and in culturally-tailored patient activation. Instructors' willingness to listen and their caring disposition were consistently cited. 3. Social support: Drawing on the African-American tradition of testifying, participants were encouraged to share their stories. The opportunity to be heard and to hear others' experiences was described as relieving, encouraging and educational. 4. Principles of the African-American Helping Tradition: Many participants explained that by sharing their new knowledge and experiences, they hoped to help their peers prevent and manage diabetes. CONCLUSIONS: Based on these results, we suggest a new conceptual model of retention for African-Americans in health education/behavioral interventions. Our model is different from frameworks describing retention in clinical trials, which emphasize contact, scheduling and logistics of research visits. Our results support prioritizing culturally-tailored program design, qualified and openly caring educators and interpersonal support among participants. Our recommended strategies are low-cost and can contribute importantly to interventions in low-resource settings. This understanding of 
program factors that improve retention among urban African-Americans with diabetes may be applicable to other health education/behavioral interventions and may help to reduce disparities in disease control and outcomes.

\section{A PROTOCOL FOR ELICITING CLINICAL DECISION SUPPORT OBJECTIVES FOR MEDICAL SPECIALTIES: DEVELOPMENT AND PILOT DEMONSTRATION Douglas S. Bell ${ }^{2}$; Justin W. Timbie ${ }^{1}$; Cheryl L. Damberg ${ }^{2}$; Eric Schneider ${ }^{3}$; $\overline{\text { Amber L. Smith }}^{2}$. ${ }^{1}$ RAND, Arlington, VA; ${ }^{2}$ RAND, Santa Monica, CA; ${ }^{3}$ RAND, Boston, MA. (Control ID \#1339532)}

BACKGROUND: A process for developing meaningful requirements for clinical decision support (CDS) within incentive programs does not exist. We developed a systematic process that combines evidence review with expert opinion to elicit clinically meaningful objectives for CDS from specialists by: 1) prioritizing the clinical performance gaps within each specialty and 2) evaluating CDS opportunities that might address the prioritized gaps.

METHODS: Our conceptual framework for deriving CDS objectives involved specifying CDS "targets" that comprised high priority performance gaps for which CDS would be effective and feasible in addressing the gap. We used a two-stage modified Delphi expert panel process to elicit and prioritize the set of CDS targets. In the first stage, experts rated the importance of performance gaps, beginning with a candidate list generated through an environmental scan and supplemented through nominations by panelists. In the second stage, for each of the highest-priority performance gaps, panelists rated the extent to which specific CDS opportunities (existing CDS tools or feasible CDS concepts) would be effective in ameliorating the gap and would be compatible with clinical workflows of the specialty. Each stage consisted of an initial rating (using a web-based program), followed by a moderated discussion of ratings via webmeeting, and a final rating immediately following the discussion. We convened four expert panels representing one medical specialty (oncology), one surgical specialty (orthopedic surgery), one non-surgical procedural specialty (interventional cardiology), and one primary care specialty (pediatrics). All panels except pediatrics focused on a pre-determined, narrow set of clinical conditions. A total of 54 experts completed all ratings.

RESULTS: The oncology panel considered 15 of the 22 performance gaps to be high priority and thus potential targets for CDS. Results for the other panels were as follows: 6 of 28 (orthopedics); 11 of 23 (interventional cardiology); and 11 of 28 (pediatrics). Of the high priority performance gaps, many were based on panelists' own clinical experience rather than existing performance measures. After considering whether effective and compatible CDS opportunities existed for each performance gap, the final set of high priority CDS targets was reduced to 14 (oncology); 4 (interventional cardiology); and 3 (orthopedics and pediatrics). Order sets were the most common form of CDS found to be effective and compatible (30\% of all opportunities), followed by alerts and reminders $(26 \%)$, and documentation templates $(19 \%)$.

CONCLUSIONS: The protocol successfully elicited high-priority performance gaps having the greatest opportunities for implementation of CDS within four clinically disparate specialties. The staged approach and the use of webmeetings minimized participant burden. Most specialties readily identified high-priority performance gaps, but only the oncology panel rated a wide range of CDS opportunities highly. The protocol could be replicated across many specialties to produce lists of high-priority CDS targets that could serve as measurable CDS objectives for use in EHR policy actions such as the "meaningful use" requirements of the Medicare and Medicaid EHR Incentive Programs. Framing objectives as performance gap 'targets' along with specific examples of effective and workable CDS creates enforceable objectives without limiting innovation by developers.

A RANDOMIZED CONTROLLED TRIAL EVALUATING THE EFFECT OF FACILITATED SMALL GROUP SESSIONS ON PHYSICIAN QUALITY OF LIFE, BURNOUT, AND MEANING FROM WORK Colin P. West $^{1,2}$; Liselotte Dyrbye ${ }^{1}$; Jeff A. Sloan ${ }^{2}$; Tait Shanafelt ${ }^{1} .{ }^{1}$ Mayo Clinic, Rochester, MN; ${ }^{2}$ Mayo Clinic, Rochester, MN. (Control ID \#1339963)

BACKGROUND: Despite the recognized prevalence of burnout, low job satisfaction, and poor quality of life among physicians, few studies have evaluated interventions to address these issues. Outcome measures from these studies are scarce, and application of validated instruments has been uncommon. In addition, prior studies have been largely observational and limited by volunteer bias. To address these limitations, we conducted a randomized controlled trial of an organizational small group facilitated intervention designed to positively impact physician well-being and job satisfaction, with additional comparison with a cohort of non-study participants.

METHODS: We conducted a randomized controlled trial of a 9-month intervention based on biweekly small group facilitated sessions. Each session lasted 1 hour, and both intervention and control participants received 1 hour of protected time every two weeks during the study, funded by their health care employer. The 17 covered topics included work-life balance, medical mistakes, meaning in work, and resiliency, among other topics relating to the physician experience. Participants completed surveys at baseline and then quarterly for 1 year. Surveys included linear analog self assessment of overall quality of life (QOL), the Empowerment at Work Scale, which includes an assessment of meaning derived from work, and the Maslach Burnout Inventory. The trial groups were compared using generalized estimating equations for repeated measures. In addition, the two study groups were compared with non-study participants from the Mayo Clinic Department of Medicine (DOM) on results from annual well-being surveys in 2010 and 2011 occurring simultaneous with the baseline and 1-year study surveys.

RESULTS: $\mathrm{N}=37$ participants were randomized to each arm of the study, with $n=34$ respondents to each survey in each arm. $\mathrm{N}=493 \mathrm{DOM}$ faculty comprised the non-study comparison group, with 340 respondents. Overall, data were provided by 408 of 567 (72.0\%) DOM faculty. At baseline, no differences were observed between the 3 study groups for any well-being variable. Results at one year are shown in the Table. Compared to non-study participants, those in the intervention arm improved on all 5 variables. The intervention arm also yielded superior average results for each of the 5 outcomes relative to the control arm, although these differences did not reach statistical significance.

CONCLUSIONS: Relative to non-study faculty, study participants receiving a small amount of protected time (one hour every other week) experienced substantial reductions in burnout with larger reductions for those in the facilitated small group curriculum. Those in the facilitated small group curriculum also experienced improvement in QOL and meaning from work. These findings are consistent with prior uncontrolled studies of interventions to promote physician well-being. However, further study is needed to define the optimal approach to delivery of these interventions.

Absolute Change in Rates of Poor QOL, Burnout, and High Meaning from Work from Baseline to Year 1.

\begin{tabular}{llll}
\hline \hline Outcome & $\begin{array}{l}\text { Intervention } \\
(\mathrm{n}=34)\end{array}$ & $\begin{array}{l}\text { Control } \\
(\mathrm{n}=34)^{*}\end{array}$ & $\begin{array}{l}\text { Non-Study Faculty } \\
(\mathrm{n}=340)^{* *}\end{array}$ \\
Poor QOL & $-15.2 \%$ & $+0.6 \%$ & $-7.3 \%$ \\
High Emotional Exhaustion & $-20.4 \%$ & $-5.3 \%$ & $+4.3 \%$ \\
High Depersonalization & $-13.3 \%$ & $-8.3 \%$ & $+2.5 \%$ \\
Overall Burnout & $-25.8 \%$ & $-13.8 \%$ & $+4.9 \%$ \\
High Meaning from Work & $+6.3 \%$ & $-6.3 \%$ & $-13.4 \%$
\end{tabular}

*All $\mathrm{p}$ values comparing intervention versus control $>0.05 * *$ All $\mathrm{p}$ values comparing all 3 groups $<0.01$

A RANDOMIZED CONTROLLED TRIAL OF FINANCIAL INCENTIVES TO PROMOTE WEIGHT LOSS AMONG OBESE EMPLOYEES Jeffrey T. Kullgren ${ }^{1}$; George Loewenstein ${ }^{2}$; Andrea B. Troxel ${ }^{3}$; Laurie Norton ${ }^{1}$; Lisa Wesby ${ }^{3}$; Jingsan $\mathrm{Zhu}^{3}$; Yuanyuan Tao ${ }^{3}$; Kevin G. Volpp ${ }^{1}$. ${ }^{1}$ Philadelphia VA Medical Center and University of Pennsylvania, Philadelphia, PA; ${ }^{2}$ Carnegie Mellon University, Pittsburgh, PA; ${ }^{3}$ University of Pennsylvania, Philadelphia, PA. (Control ID \#1339878)

BACKGROUND: Obesity is a leading cause of chronic disease and leads to substantial economic costs in the workplace. Consequently, employers are 
increasingly offering different types of financial incentives to motivate weight loss among obese employees. However, there is little evidence on how to optimally design incentives to promote weight loss in this setting. The objective of this study is to test the relative effectiveness of two novel financial incentive approaches in promoting weight loss among obese employees.

METHODS: We developed a partnership with the Children's Hospital of Philadelphia to recruit 105 employees with a body mass index between 30 and $40 \mathrm{~kg} / \mathrm{m} 2$ who were interested in losing weight. Participants were given a weight loss goal of 1 pound per week for 24 weeks, provided with access to a website to track their progress, and randomized to one of 3 groups: (1) monthly weigh-ins alone (control group), (2) \$100 per month for being at or below their monthly target weight (individual arm), or (3) $\$ 500$ per month split between groups of 5 participants, who received a larger share when they were at or below their monthly target weight but other group members were not (group arm). The primary outcome is weight loss at 24 weeks. Secondary outcomes include weight loss in a subsequent 12 week observation period without incentives to assess sustainability (i.e., weight loss over the full 36 weeks) and intervention period changes in eating behaviors, physical activity, and participation in weight-related wellness programs. We report here baseline participant characteristics, primary outcome results, and secondary outcome results available through December 2011. Complete data on sustainability will be available by February 2012 .

RESULTS: Participants have a mean age of 45.3 years [standard error (SE) 1.0] and a mean household income of $\$ 94,952$ (SE 3,757). Most are female (88.6\%), White $(62.9 \%)$ or African American (29.5\%), and have at least a college degree (59.0\%). Group arm participants lost more weight (mean 10.7 pounds, SE 1.8) than participants in the control (mean 1.1 pounds, SE 2.0, $\mathrm{P}=$ 0.0004 ) and individual (mean 3.7 pounds, $\mathrm{SE} 1.9, \mathrm{P}=0.0079$ ) arms. Group arm participants also experienced a greater increase in cognitive restraint around eating [mean 19.9 (measured on a 0 to 100 scale), SE 4.4] than control (mean 4.6, SE 2.8, $\mathrm{P}=0.0017$ ) and individual (mean 7.3, SE 2.5, $\mathrm{P}=0.0087$ ) arm participants. There were no significant differences across arms in changes in emotional eating, uncontrolled eating, physical activity, and weight-related wellness program participation.

CONCLUSIONS: A monthly financial incentive that involved competition within a group was significantly more effective than an individual incentive in promoting weight loss and greater cognitive restraint around eating among obese employees at 24 weeks. Between now and February 2012 we will assess the sustainability of these initial effects. Evidence on the relative effectiveness of different financial incentives will help employers maximize the impact of incentives designed to help obese employees lose weight and modify weight-related behaviors.

A RANDOMIZED CONTROLLED TRIAL OF THE FEASIBILITY OF A PROTECTED SLEEP PERIOD FOR MEDICAL INTERNS IN THE HOSPITAL FOR PROLONGED DUTY Kevin G. Volpp ${ }^{1,2}$; Laurie Norton ${ }^{1,2}$; Judy A. Shea ${ }^{3,1}$; Jingsan $\mathrm{Zhu}^{1,3}$; $\overline{\text { Dylan }}$ Small $^{4,1}$; Mathias Basner ${ }^{5}$; Adrian Ecker ${ }^{5}$; David Dinges ${ }^{5}$; Daniel Mollicone ${ }^{6}$; Cristina Novak ${ }^{1,2}$; Lisa Bellini ${ }^{3}$. ${ }^{1}$ University of Pennsylvania, Philadelphia, PA; ${ }^{2}$ Philadelphia VA Medical Center, Philadelphia, PA; ${ }^{3}$ University of Pennsylvania, Philadelphia, PA; ${ }^{4}$ University of Pennsylvania, Philadelphia, PA; ${ }^{5}$ University of Pennsylvania, Philadelphia, PA; ${ }^{6}$ Pulsar Informatics, Philadelphia, PA. (Control ID \#1340423)

BACKGROUND: In 2008 an Institute of Medicine Report recommended protected sleep periods for medicine trainees on extended overnight shifts, a position reinforced in recent ACGME requirements. We evaluated the feasibility and consequences of mandatory naps during extended duty. METHODS: 4-week blocks at the Philadelphia VA Medical Center (PVAMC) medicine service and at the Hospital of the University of Pennsylvania (HUP) oncology service were randomly assigned to either a standard intern schedule (extended duty overnight shifts of up to 30 hours), or 'mandatory naps' (interns given protected time from 00:30-05:30 to sign out their cell phones and sleep). Study participants were asked to wear wrist actiwatches, complete sleep diaries, and perform daily Psychomotor Vigilance Tests (PVT-B) to measure behavioral alertness.

RESULTS: On $98.3 \%$ of intern on call nights, cell phones were signed out to residents as designed. Mean sleep time during the protected period at
PVAMC was 2.6 hours in the intervention months compared with 1.6 in the control months (p-value $<0.0001$ ). At HUP, mean sleep time was 2.8 hours in the intervention months and 1.9 hours in the control ( $p$-value <0.0001). Interns with mandatory naps were less likely to have on call nights with no sleep at both sites (PVAMC: $12.5 \%$ vs. $26.9 \%$, p-value $<0.0001$; HUP: $11.1 \%$ vs. $17.1 \%$, p-value 0.04 ). In contrast $20.7 \%$ of interns during intervention months slept 4-5 hours during the protected period compared with $6.6 \%$ during control months at PVAMC (p-value $<0.001$ ) and at HUP, $27.6 \%$ vs. $8.0 \%$, (p-value $<0.001$ ). At both HUP and PVAMC, response speed on the PVT-B was significantly faster after on-call nights in the intervention relative to the control group (PVAMC: 4.07 vs. 3.84 per second (s-1), p-value 0.021 ; HUP: 4.04 vs. $3.89 \mathrm{~s}-1$, p-value 0.016 ), and the number of lapses of attention was lower, albeit not significantly at HUP (PVAMC: 3.88 vs. 5.72, p-value 0.031 ; HUP: 3.95 vs. 4.73 , p-value 0.059 ).

CONCLUSIONS: This study provides the first evidence that a mandatory program of "protected time for sleep on extended duty shifts" is feasible with high rates of adherence and that it can produce a significant increase in mean hours slept and an increase in behavioral alertness on mornings after overnight shifts. While there is evidence that obtaining nap sleep (relative to no sleep) during prolonged duty helps reduce fatigue, it remains to be determined whether the gain in sleep time and alertness afforded by a mandatory protected nap reduces fatigue-related errors and accidents involving residents. Protected nap periods are feasible and improve alertness, suggesting they may provide a reasonable alternative to mandated shorter shifts.

A RANDOMIZED, DOUBLE-BLIND, PLACEBO-CONTROLLED SWITCH STUDY OF THE SAFETY, TOLERABILITY, AND EFFICACY OF MILNACIPRAN IN FIBROMYALGIA PATIENTS WHO INADEQUATELY RESPOND TO DULOXETINE Allan Spera $^{2}$; Lucinda Bateman ${ }^{1}$; Robert H. Palmer ${ }^{2}$; Joel M. Trugman ${ }^{2}$; Jennifer $\operatorname{Lin}^{2} .{ }^{1}$ The Fatigue Consultation Clinic, Salt Lake City, UT; ${ }^{2}$ Forest Research Institute, Jersey City, NJ. (Control ID \#1330962)

BACKGROUND: Fibromyalgia (FM) is characterized by a multitude of symptoms that include chronic widespread pain, fatigue, stiffness, impaired physical functioning, sleep disturbances, cognitive dysfunction, and depressed mood. Since individual patient responses among analgesics often differ, switching therapies with the objective of achieving greater benefit or avoiding certain side effects is common in clinical practice. Milnacipran and duloxetine are both serotonin/norepinephrine reuptake inhibitors approved in the United States for the management of FM. Although these medications are in the same pharmacologic class, their properties differ, which may result in different responses and adverse-effect profiles in some FM patients. This study evaluates the safety, tolerability, and efficacy of milnacipran following a direct switch from duloxetine in FM patients who clinically experience an inadequate response to duloxetine.

METHODS: Patients were required to have been taking duloxetine $60 \mathrm{mg} /$ day for $\geq 4$ weeks at the time of enrollment into this study. Following a 2-week open-label period with duloxetine $60 \mathrm{mg} /$ day, patients with VAS pain scores $\geq 40$ and $\leq 90 \mathrm{~mm}$ (range, $0-100 \mathrm{~mm}$ ) and dissatisfied with duloxetine were randomized $4: 1$ to milnacipran $100 \mathrm{mg} /$ day $(n=86)$ or placebo $(n=21)$ for 10 weeks. The purpose of the small placebo group was to minimize expectation bias rather than provide a comparator arm, since patients would be discontinuing a treatment that may have been partially efficacious. Patients randomized to milnacipran were directly switched with no tapering or titration periods. The primary efficacy outcome was Patient Global Impression of Change (PGIC) responder status, with PGIC responders defined as patients rating themselves as "much improved" or "very much improved" at Week 10. The secondary efficacy outcome was the change from baseline in 1-week recall VAS pain. Additional outcomes included changes from baseline in Fibromyalgia Impact Questionnaire Revised (FIQR), which measures overall FM severity, and Multiple Ability Self-Report Questionnaire (MASQ), which measures self-reported cognitive functioning. Missing data were imputed by the last observation carried forward approach.

RESULTS: In the group of patients switched from duloxetine to milnacipran, $32.9 \%$ were PGIC responders and the mean reduction from baseline in VAS pain was $12.3 \mathrm{~mm}$. In the small group of patients switched to placebo, $23.8 \%$ 
were PGIC responders and the mean decrease in VAS pain was only $1.3 \mathrm{~mm}$. Patients switched to milnacipran had a mean improvement in FIQR total scores of 7.8 points, representing a $14 \%$ improvement (patients switched to placebo improved by only 1.4 points). Patients switched to milnacipran also showed improved cognitive functioning, with a mean improvement in MASQ total score of 2.4 points, while the mean score worsened from baseline by 3.2 points in patients switched to placebo. The most common treatment-emergent adverse events were nausea and dizziness; these occurred in $21 \%$ and $15 \%$ of patients switched to milnacipran, and in $29 \%$ and $5 \%$ of patients switched to placebo, respectively.

CONCLUSIONS: These results indicate that members of the same pharmacologic class may have different clinical response profiles and that directly switching FM patients from duloxetine to milnacipran is safe and well-tolerated, and may improve pain and other FM symptoms in patients who inadequately respond to duloxetine.

A REVIEW OF HOMELESSNESS AMONG DISCHARGE DELAYS: MAKING THE CASE FOR MEDICAL RESPITE CARE FOR INDIVIDUALS EXPERIENCING HOMELESSNESS Jacob Feigal ${ }^{1}$; Carolyn Bramante ${ }^{1}$; John Song ${ }^{2,1}$; Curtis Nordgaard ${ }^{1}$; Brian Park ${ }^{\text {T. }}{ }^{1}$ University of Minnesota Medical School, Minneapolis, MN; ${ }^{2}$ University of Minnesota Academic Health Center, Minneapolis, MN. (Control ID \#1338108)

BACKGROUND: Homelessness is associated with higher morbidity, mortality, and hospital costs. Medical respite is an emerging care management option that decreases hospital costs and readmissions by combining a 24-hour supportive shelter setting with specialized services for homeless patients with health care needs after hospital discharge. Future efforts to establish respite care centers would benefit from better understanding the health care needs of its target population. Additionally, little research has examined discharge delays of housed versus homeless patients. This study examined medical needs and discharge delays of individuals experiencing homelessness in Minneapolis, Minnesota following discharge from inpatient hospitalization to determine whether respite care could facilitate more expedient and appropriate discharges.

METHODS: The study was conducted at Hennepin County Medical Center (HCMC), a 477-bed regional safety net hospital that provides care for much of Minneapolis' homeless population. We performed a retrospective cohort analysis of admissions between January 1 and June 30, 2009 at HCMC. Data were abstracted from charts defined by HCMC as "discharge delays for external reasons," an operational definition used by the hospital's Utilization Management department to assess eligibility for continued inpatient stay. Using the inpatient electronic medical record, as well as electronic records from Utilization Management, patients were classified as "housed," "not housed," or "unknown" based on the federal definition of homelessness. Demographics, reason for admission, interventions, diagnoses, reason for delay, length of delay, and readmissions within 90 days were also abstracted. Analyses were then conducted to evaluate the relationship between housing status and discharge delay.

RESULTS: A total of 304 charts included hospitalizations during the study period that were affected by discharge delays; 93 of which belonged to homeless patients. The median number of delay days was significantly longer for homeless than housed patients $(\mathrm{p}<0.001)$. The charts of 107 patients $(43.3 \%)$ included psychiatric diagnoses on admission. The median number of delay days remained significantly longer for homeless patients after controlling for psychiatric diagnoses $(\mathrm{p}=0.012)$. For homeless patients, the most common reason for discharge delay was awaiting group home placement (35.6\%) while $9.9 \%$ of delays were due to inability to find shelter placement. Other reasons included awaiting healthcare coverage, medication management, and follow-up requirements. Homeless individuals were less likely to have either public or private insurance than housed patients $(\mathrm{p}=0.011)$.

CONCLUSIONS: This study found that homeless patients had longer discharge delays than housed patients after inpatient hospitalizations for both medical and psychiatric reasons. The most common reason for discharge delays of homeless patients was awaiting a group-home bed. Another common reason for delay was inability to find shelter placement. Respite care facilities could potentially expedite discharge in these situations and other situations identified. Respite care could also improve the healing and recovery of individuals experiencing homelessness, a group that faces many challenges to good health.

A STORY OF CHANGE: THE INFLUENCE OF NARRATIVE ON
AFRICAN-AMERICAN PATIENTS WITH DIABETES Anna Goddu'; AFRICAN-AMERICAN PATIENTS WITH DIABETES Anna Goddu
Katie Raffel ${ }^{3}$; ; Monica Peek ${ }^{1,2}$. ${ }^{1}$ University of Chicago, Chicago, IL; ${ }^{2}$ University of Chicago, Chicago, IL; ${ }^{3}$ University of Chicago, Chicago, IL. (Control ID \#1338420)

BACKGROUND: Identifying culturally-tailored methods to improve diabetes self-care and shared decision-making (SDM) are key areas of research that can improve the health of racial/ethnic minorities. Narrative in the form of storytelling, entertainment, and role-play has shown promise as a means of facilitating behavior change, yet recent models describing its influence have not been fully explicated, and little is known about its effect in diabetes self-care or SDM

METHODS: The Diabetes Empowerment Program (DEP) is a culturallytailored intervention to improve self-care and SDM among AfricanAmericans; it has improved patients' self-efficacy, self-care behaviors and diabetes control. The DEP incorporates several narrative elements: personal testimonials, role-play, and a film with SDM vignettes. Our study had two aims: 1) To understand how program narrative elements may have affected diabetes-related behavior change, and 2) Whether our results would validate Larkey and Hecht's conceptual model, which describes narrative elements as mediated by transportation (i.e. engagement), identification (e.g. with characters), and social proliferation (rehearsal, discussion, support) to influence health behavior change. We conducted four focus groups and seven in-depth interviews with former DEP patients. Using a structured topic guide, trained interviewers asked participants to describe their experience with DEP's narrative elements. Each interview/focus group was audio-taped and transcribed verbatim. Transcripts were independently coded by two researchers using an iterative process and analyzed using Atlas.ti software. RESULTS: 31 patients participated in the focus groups and 7 were interviewed in-depth. $76 \%$ were female, and the mean age was 58 . The mean income was $<\$ 25 \mathrm{k}$, and the mean number of years with diabetes was 8 . Participants reported many narrative elements that influenced their behavior change, from which we identified three central themes. The program's narrative elements: 1) facilitated skills training in diabetes self-care and SDM ("when we role played...it broke down my shell. She was teaching us stepby-step"); 2) generated relevant teachable moments, making the material more applicable and memorable ("somebody might say...'when I started doing this eating properly...my [sugars] went down'... Okay, I wonder if I did that will mine go down"); 3) built strong social support among participants that facilitated program retention and behavioral change ("instead of me pushing away from [the education]...it's an inspiration because you hear what others go through and we get a chance to share"). These themes align closely with Larkey and Hecht's three social proliferation mediators: rehearsal/reinforcement, discussion/diffusion, and reciprocal support. Participants also described transportation ("it had really brought me back") and identification ("I saw me there"), but these were less prominent themes.

CONCLUSIONS: Our study suggests that the use of narrative can facilitate behavior change among African-Americans with diabetes. The social mediation of narratives - the discussion and rehearsal of stories, and the support that results from sharing them - may be particularly relevant for this population, especially given their salience to existing African-American cultural traditions of shared knowledge creation, the use oral testimonials and the "helping tradition."

A SURVEY OF PRIMARY CARE CLINICIANS ON USE OF DECISION AIDS AND CONFIDENCE IN SHARED DECISION MAKING SKILLS Leigh H. Simmons ${ }^{1}$; Lauren Leavitt ${ }^{2,1}$; Christine Greipp ${ }^{3,1}$; Karen R. Sepucha ${ }^{2}$. ${ }^{1}$ Massachusetts General Hospital, Boston, MA; ${ }^{2}$ Massachusetts General Hospital, Boston, MA; ${ }^{3}$ Massachusetts General Hospital, Boston, MA. (Control ID \#1339540)

BACKGROUND: Since 2005, primary care clinicians at Massachusetts General Hospital in Boston, MA, have been able to "prescribe" decision 
aids (DAs) to their patients through the electronic medical record. There are 35 video and paper-based DAs available that cover a range of medical conditions such as prostate cancer screening and treatment of knee osteoarthritis. The use of the DAs has increased over time, however, some providers use them often and others never use them. Clinicians were surveyed regarding their perceptions about the use of decision aids and shared decision making in routine clinical practice.

METHODS: A survey was distributed to 361 primary care clinical staff across 15 primary care practices through a survey website. Participants also had the option to receive the survey on paper, via fax. Up to three reminder emails were sent to non-responders. The survey closed after 3 weeks. The survey included 20 questions that examined use of decision aids, perceived barriers to use, and a self-assessment of two key shared decision making skills: risk communication and elicitation of patients' goals and preferences. RESULTS: 179 of 361 clinicians (50\%) invited to complete the survey responded. Respondents from all 15 primary care sites submitted surveys. The majority of respondents were women (66\%) and physicians $(85 \%)$, with $51 \%$ attending physicians, $34 \%$ resident physicians, and $14 \%$ nurse practitioners. About half of respondents (53\%) indicated that they had personally prescribed at least one decision aid ("users") and 47\% indicated that they had never prescribed a decision aid ("non-users"). Users were more likely to have watched a video decision aid compared to non-users $(63 \%$ vs. $23 \%, p<0.001)$. Users were more likely to feel that the programs definitely helped provide better care for patients $(72 \%$ vs. $34 \%, \mathrm{p}<0.001)$ and less likely to feel it was difficult to provide decision aids to patients $(23 \%$ vs. $53 \%, p<0.001)$ compared to non-users. The most frequent barriers to use of decision aids were not remembering to prescribe $(63 \%)$ and having few patients who were eligible for the available programs $(21 \%)$. Very few non-users $(2.3 \%)$ mentioned concern about increasing visit length as a barrier to using DAs, and only $10 \%$ of users felt that using the programs increased the length of their visits. Only $19 \%$ of respondents stated that they felt very confident in their ability to communicate risks of treatments and probabilities of outcomes to their patients, and $38 \%$ felt very comfortable eliciting patients' goals and preferences.

CONCLUSIONS: Many primary care clinicians at Massachusetts General Hospital have experience using decision aids with patients. Potential facilitators for increasing use of decision aids were identified, including provider viewing of the programs to increase familiarity with the content and automation of the prescription process to reduce reliance on clinicians remembering to prescribe programs. The finding that few primary care clinicians felt very comfortable and confident in their shared decision making skills is an impetus for enhancing training in these areas.

\section{A SYSTEM DYNAMIC MODEL OF STROKE REHABILITATION ADHERENCE: HOW MUCH SHOULD WE SPEND TO IMPROVE? David Matchar ${ }^{1,3}$; Gerald $\mathrm{Koh}^{2}$; John Ansah ${ }^{1}$; Sean Love ${ }^{1} .{ }^{1}$ Duke-National University of Singapore Graduate Medical School, Singapore, Singapore; ${ }^{2}$ National University of Singapore, Singapore, Singapore; ${ }^{3}$ Duke University Medical Center, Durham, NC. (Control ID \#1340283)}

BACKGROUND: Despite evidence that rehabilitation following stroke is effective in reducing morbidity and mortality, rates of use of rehabilitation services is low; in Singapore, a major issue is adherence with recommendations, with approximately 80 percent of referred individuals not attending stroke rehabilitation. Evidence that rehabilitation can reduce long-term disability and attendant costs in general has not provided a compelling policy case for funding a program to improve attractiveness of rehabilitation services. Our objective was to use simulation modeling to provide a vivid demonstration of the potential benefit of various policies to improve uptake of stroke rehabilitation, including a dynamic policy of linking funding to projected cost-effectiveness.

METHODS: A stock and flow model representing the potential sequences of events following a stroke-including rehabilitation, improvement, or another serious event-was constructed. The model focused on a major factor in reducing rehabilitation adherence: the attractiveness of services (e.g., convenience and cost). The model provides an estimate of the costeffectiveness of rehabilitation at different rates of utilization, accounting for the cost of raising levels of utilization. On this a prospective policy is imposed: to enhance the attractiveness of rehabilitation services through increased levels of government support up to the point at which marginal cost-effectiveness exceeds a prescribed threshold. Based on different hypothetical production functions for service uptake (i.e., relationships between added cost and increased use of rehabilitation service), we examine the value of the policy in terms of social cost-effectiveness. Estimates of model inputs were based on extant population surveys and estimates from the research team.

RESULTS: The model suggests that the proposed policy of improving the attractiveness of rehabilitation services could be constructed in such a way that would allow spending for enhancing rehabilitation services to expand only to the threshold of an acceptable level of health value for money. Depending on the nature of the true production function, and the desire for rapid service expansion and the tolerance for overshoot, a robust policy is feasible.

CONCLUSIONS: Substantial underuse of stroke rehabilitation services provides an excellent opportunity to search for policies that will increase uptake. Modeling helps to provide an evidence-based approach on how to improve uptake of rehabilitation services and quality of life for post-stroke patients.

A YEAR IN REVIEW: IMPLEMENTATION OF A REFUGEE CLINIC AT PENN CENTER FOR PRIMARY CARE Rachael Truchil; Joseph M. Garland; Matthew H. Rusk. University of Pennsylvania, Philadelphia, PA. (Control ID \#1339689)

BACKGROUND: A partnership between a Philadelphia-area refugee resettlement agency and Penn Center for Primary Care, the site of an internal medicine resident clinic, was developed in October 2009. From that partnership a Refugee Clinic was created that opened in October 2010. Now one year after the clinic has been operating we have reviewed our progress and evaluated the clinic's compliance with national guidelines for refugee screening so as to improve the clinic in moving forward.

METHODS: A retrospective audit was performed using our electronic medical record and results were compiled and analyzed using Excel.

RESULTS: Seventy-five patients were seen during the course of the year, including refugees from five ethnic groups: Bhutanese Lhotshampa, Chin and Karen from Burma, Eritrean, and Russian patients. As per CDC guidelines, our goal is to see all patients within 30 days of arrival in the US. Over the past year we met this goal with $78.7 \%$ of the patients we saw, however during the past six months, we met this goal with $97.4 \%$ of patients suggesting significant improvement. All patients received a screening complete blood count, QuantiFERON Gold testing for tuberculosis (TB) infection, and hepatitis B serologies. Eosinophilia was common, with 13 patients (17.3\%) having an absolute eosinophil count greater than $450.94 \%$ of patients were screened for Strongyloides with three patients testing positive and all received treatment. Serologic testing for Schistosoma infection was positive in 4 of 11 patients tested. One third of patients had positive QuantiFERON Gold testing without symptoms or evidence on chest radiograph of active TB. Rates of immunization for our patients were high with greater than $93 \%$ of patients having received MMR, Td or Tdap, and Hepatitis B vaccination (when indicated). Varicella immunity was assessed by history and serology in $96 \%$ of patients; all 6 of the non-immune patients were vaccinated. $64 \%$ of patients received the seasonal influenza vaccine, $75 \%$ of eligible patients received the pneumococcal vaccine, and 16 patients received HPV vaccination. We have also focused attention on making sure the patients seen in clinic received routine age-appropriate preventive health screening. $80.6 \%$ of female patients received a Pap test and a lipid profile was checked in $57.3 \%$ of patients. Several older patients have required screening colonoscopy, DEXA scans, and mammography. We did not start referring patients for these tests until midway through the year and therefore do not have estimates of number of patients receiving screening.

CONCLUSIONS: Our compliance with CDC guidelines was high but still could be improved upon. In moving forward, we hope to improve upon our rates of routine preventive health screening and have higher rates of vaccination. We also hope to improve treatment rates for latent tuberculosis infection. Finally, we hope to create protocols for the work-up of commonly encountered problems, including eosinophilia, thrombocytopenia, and abdom- 
inal pain. We also plan to evaluate the educational value of resident participation in the clinic.

\section{A BROAD ASSESSMENT OF THE ORAL CASE PRESENTATION FROM THE PERSPECTIVES OF INTERNAL MEDICINE FACUL- TY AND 4TH YEAR MEDICAL STUDENTS Reza Sedighi Manesh ${ }^{1}$; Michael Elnicki ${ }^{2} .{ }^{1}$ University of Pittsburgh, Pittsburgh, PA; ${ }^{2} \mathrm{UPMC}$, Pittsburgh, PA. (Control ID \#1333678)}

BACKGROUND: The oral case presentation is recognized as a primary mode in which physician-physician communication occurs. It is recognized as an educational goal for trainees by the Clerkship Directors in Internal Medicine (CDIM), the Association of American Medical Colleges (AAMC), and the Accreditation Council for Graduate Medical Educators (ACGME). Nevertheless, many medical schools lack formal teaching of oral presentation skills to medical students. Further, senior trainees lack proper oral presentation skills in the inpatient and outpatient settings. There are limited data regarding how medical students learn skills necessary to present patients and whether teaching of such skills are lacking in medical education curriculum. We had 3 major goals: to further understand the teaching and learning of oral presentation skills, to gain the student's perspective in regard to various aspects of the oral case presentation and compare it to that of faculty, and to assess whether students and faculty believe there is a need for formal education in oral presentation skills.

METHODS: We designed and administered a faculty-specific-survey to 44 internal medicine faculty members of the University of Pittsburgh School of Medicine (UPSOM) and a student-specific-survey to 156 fourth year medical students at UPSOM. Both surveys consisted of 10 questions: two free response questions, three or four multiple-choice questions, and four or five 5-point Likert response questions. The questions addressed various aspects of the oral case presentation: ideal time of a presentation in minutes, importance of various attributes of an oral case presentation, and need for formal education. Also, questions addressed the methods of learning and teaching of oral presentation skills by students and faculty. We generated descriptive statistics, used chi-square to compare categorical data, and compared means by t-tests. RESULTS: The survey response rate of faculty and students were $68 \%$ $(29 / 44)$ and $76.2 \%(119 / 156)$, respectively. Faculty and students primarily learned "by observing senior students and/or faculty" ( $96.7 \%$ vs $96.6 \%$, respectively, $\mathrm{p} \geq 0.1$ ). Faculty and students less commonly learned from "textbooks" (17.6\% vs $10 \%$, respectively, $\mathrm{p} \geq 0.1)$ and "journal articles" (7.6\% vs $17.6 \%$, respectively, $\mathrm{p} \geq 0.1)$. Seven faculty members $(23 \%)$ taught by "Lecture" and "Walk Rounds," 16 (53\%) taught by "Walk Rounds", 1 taught by "Lecture", and $6(20 \%)$ taught by "Other" methods. Mean response regarding the ideal length of oral presentation of a new patient in minutes by faculty and students were $6.4 \pm 3$. Mean response of a "need for formal education" of oral presentation skills by faculty and students were $4 \pm 1$ and $4.2 \pm 0.9$ ( $p \geq 0.1$ ), respectively. Forty-six percent of faculty and $38.7 \%$ of students believe there is an absolute need (5/5 Likert) for formal education of oral case presentation skills.

CONCLUSIONS: Our study addressed each of the aforementioned goals: (1) students primarily learn oral presentation skills from observing senior trainees, and faculty primarily teach students such skills during walk rounds. (2) Students and faculty members' opinions regarding length of an ideal presentation, importance of various attributes of a presentation, and contribution of students' presentation skills towards final grade on medicine clerkship were similar. (3) The majority of students and faculty "absolutely" (5/5 Likert) believe there is a need for formal education in oral presentation skills.

\section{A COMPARISON OF PATIENTS' AND PHYSICIANS' PERSPEC-} TIVES ON PRESCRIPTION MEDICATION COSTS Leslie Ramirez; Toshiko Uchida; Kenzie A. Cameron; Charlie Zei; Ariane M. Garrett; Anne Henson; Eric D. Christoff; Ami Desai; Erik Orelind; Michael Zielinski; David W. Baker. Northwestern University Feinberg School of Medicine, Chicago, IL. (Control ID \#1327007)

BACKGROUND: Patients are shouldering a larger and larger portion of the costs of their medical care. In particular, out-of-pocket costs for prescription medications have increased substantially in recent years. In an earlier study in our patient population $55 \%$ of patients reported being concerned about the costs of their prescription medications. The physicians in our Practice-Based Research Network (PBRN) also expressed concern about patients' ability to pay for their medications. The goals of this study included: 1) Comparing patients' and physicians' perspectives on the magnitude of the problem of prescription medication costs, and 2) Comparing patients' and physicians' perspectives on the roles of the physician and patient in addressing prescription medication costs.

METHODS: This study was conducted by the PBRN at Northwestern University Feinberg School of Medicine known as the Research and Education for Academic Achievement (REACH) Network. We surveyed patients and physicians at 9 of the academic and private practice internal medicine sites in the REACH Network. During the study period, every patient who came for an appointment was given a paper survey to complete after the visit regarding prescription medication costs. Physicians were sent similar surveys by email with paper copies delivered in person to those who failed to complete the email survey. The surveys included both Likerttype and yes/no items about patients' and physicians' attitudes regarding prescription medication costs. This study was a subset of a larger REACH study regarding prescription medication costs.

RESULTS: Three hundred nine (response rate of 26.2\%) patients and 66 (response rate of $88.0 \%$ ) physicians responded to the surveys. Among patients, $56.1 \%$ agreed/strongly agreed that they were concerned about the costs of their prescription medications. In comparison, physicians estimated that $60.5 \%$ of their patients were concerned about medication costs. In addition, $25.4 \%$ of patients agreed/strongly agreed that they have difficulty paying, while physicians estimated that $28.1 \%$ of their patients have difficulty paying for prescription medications. In contrast, $62.9 \%$ of patients compared to $87.9 \%$ of physicians agreed/strongly agreed that the physician should discuss prescription costs with patients. Finally, $85.8 \%$ of patients agreed/strongly agreed that they feel comfortable talking with their doctor about prescription costs, whereas $93.9 \%$ of physicians stated they agreed/strongly agreed that they feel comfortable talking with patients about the costs of their prescription medications.

CONCLUSIONS: When compared to patients' self-report, physicians in this study were remarkably close in estimating the proportion of patients who were concerned about the costs and had difficulty paying for their prescription medications. In contrast, many fewer patients than physicians felt it was the physicians' responsibility to discuss prescription medication costs. Also, in a clinical encounter, patients reported feeling less comfortable discussing medication costs than did physicians. Educational materials and outreach to patients are needed to encourage patients to discuss their concerns about medication costs with their physicians.

A NOVEL CONCEPTUAL MODEL FOR CLINICAL DECISION COMPLEXITY Donna M. Zulman ${ }^{2,1}$; Susana B. Martins'; Steven Asch $^{2,1}$; Mary K. Goldstein ${ }^{3,1}$. ${ }^{1}$ Stanford University, Stanford, CA; ${ }^{2}$ VA Palo Alto Health Care System, Menlo Park, CA; ${ }^{3}$ VA Palo Alto Health Care System, Palo Alto, CA. (Control ID \#1336944)

BACKGROUND: Patient complexity is frequently cited as impeding adherence to chronic disease management guidelines. While it is generally assumed that complex patients generate complex management decisions, this relationship has not been rigorously studied. Our objective is to develop a novel conceptual model for clinical decision complexity, and use data from a clinical decision support (CDS) system to illustrate the model's dimensions in the setting of hypertension management.

METHODS: We used a cognitive engineering approach to develop a conceptual model with two major dimensions of clinical decision complexity: Component complexity (the number of information cues associated with a decision) and coordinative complexity (the level of interaction between components) (Figure). We illustrate the dimensions of this model in the setting of hypertension management decisions, using electronic medical record data from a Veterans Affairs hypertension CDS system (ATHENA-HTN).

RESULTS: In an analysis of a preliminary sample of 4006 patients with hypertension, common contributors to decision component complexity were older age $(67 \%$ were $>=65)$, blood pressure above target $(35 \%),>=3$ prescribed anti-hypertensives $(23 \%)$, diastolic blood pressure $<60 \mathrm{mmHg}$ 
$(10 \%)$, and a history of an adverse reaction to an anti-hypertensive $(8 \%)$ Coordinative complexity arose when multiple, interacting components were present (e.g. among 1417 individuals with blood pressure above target, 17\% were over the age of 65 and were on $>=3$ anti-hypertensives). Coordinative complexity also arose due to the presence of comorbidities with potential disease-disease or medication-disease interactions (mean 4, SD 2.4). For example, $295(7 \%)$ individuals had a condition that constituted a compelling indication for adding a specific anti-hypertensive medication, but also had a condition that constituted a relative contraindication to that same medication (e.g. a patient with heart failure and COPD had a compelling indication and a relative contraindication for a cardioselective beta-blocker, respectively).

CONCLUSIONS: We successfully applied this novel model for decision complexity to hypertension management decisions. Further investigation should examine how best to quantify the dimensions of component and coordinative complexity in order to develop CDS systems that reduce both dimensions. Distinguishing between patient and decision complexity could inform cognitive service reimbursement policies and the development of quality metrics.

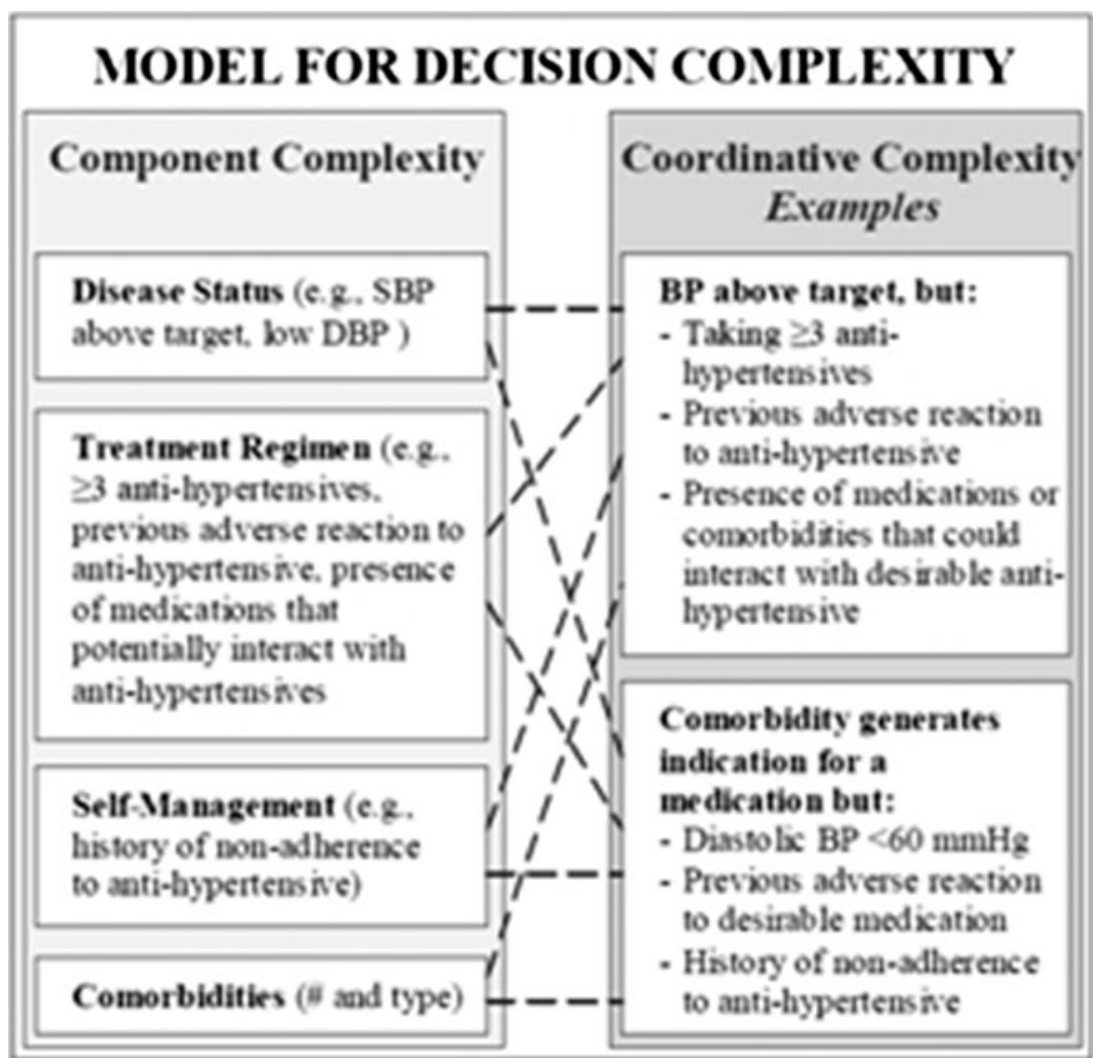

A NOVEL METHOD TO TEACH INTERNAL MEDICINE RESIDENTS BEHAVIORAL COUNSELING SKILLS: THE PREVENTIVE MEDICINE EDUCATION PARTNERSHIP (PEP) CURRICULUM. Jennifer Rockfeld; Jennifer Neuman; Anju Dayal; Jenny J. Lin. Mount Sinai School of Medicine, New York, NY. (Control ID \#1339067)

BACKGROUND: Most physicians understand the benefits of diet and exercise in the management of many chronic diseases, including obesity, hypertension and diabetes. Despite this awareness, many cite time constraints and lack of training as barriers to providing adequate patient guidance. To address this issue, we developed an evidence-based nutrition and exercise curriculum and created a group visit model for Internal Medicine residents to develop their behavioral counseling skills outside of the usual constraints of a clinic visit.

METHODS: All third year Internal Medicine residents participated in the Preventive Medicine Education Partnership (PEP) curriculum as part of their ambulatory four-week block. Each month, three to six residents underwent a one-hour lecture based on the New York City Department of Health "Primary Care Nutrition 101" curriculum as well as three brief lessons on evidencebased exercise recommendations, small group teaching skills and health care literacy. The residents then developed a one-hour lesson plan for their patients focusing on a low salt diet, a low fat diet, or portion control. They were encouraged to make the session both interactive and appropriate for a low literacy audience. The residents recruited patients from their clinic to participate in three weekly sessions focusing on the topics above. A 30minute walk and establishment of individual goals followed each session. Residents' attitudes toward and confidence in their ability to provide nutrition and exercise counseling were assessed before and after the intervention using an adaptation of a validated survey on a 4-point Likert scale. They also answered qualitative questions at the end of the block to assess whether the curriculum contributed to their education as a whole. All of the residents' responses were anonymous, and student's t-test was used to compare pre and post-intervention answers

RESULTS: Thirty-five third-year residents received the PEP curriculum. Of these, $31(88 \%)$ and $27(77 \%)$ completed the pre and post surveys, respectively. Before and after participation in the PEP program, all residents agreed that all overweight, hypertensive and diabetic patients should be counseled on diet and exercise. The vast majority agreed that it was their responsibility to counsel on diet (97\% pre, $93 \%$ post) and exercise $(94 \%$ pre, $93 \%$ post). Time constraints and other medical issues of higher priority were cited as barriers to providing adequate counseling. Following participation in the PEP curriculum, residents were more comfortable identifying what is important when providing dietary counseling (mean 2 vs $2.5, \mathrm{p}<0.0046$ ). 
They felt more prepared to follow evidence-based guidelines in counseling their patients about diet (mean 2.1 vs $2.8, \mathrm{p}<0.0001$ ) and exercise (mean 2.2 vs $2.6, \mathrm{p}<0.0207)$ and also were more confident in their ability to improve patients' diets (mean 2.3 vs $2.7, \mathrm{p}<0.0054$ ). All of the residents believed the curriculum should be continued as part of their ambulatory experience.

CONCLUSIONS: The PEP curriculum improved residents' confidence in their ability to provide evidence based, effective nutrition and exercise counseling. Our findings suggest that a nutrition and exercise curriculum paired with a group visit model is an innovative and potentially successful method to teach residents relevant skills while minimizing the time constraints of the typical clinic environment.

A OBSERVATIONAL STUDY TO MEASURE THE AMOUNT OF TIME NEEDED TO ADMIT A NEW GENERAL MEDICINE PATIENT Kathlyn E. Fletcher ${ }^{1}$; Alexis M. Visotcky ${ }^{2}$; Jason M. Slagle ${ }^{3}$; Matthew Weinger ${ }^{4,5}$; Sergey Tarima ${ }^{2}$; Marilyn Schapira ${ }^{5}$. ${ }^{1}$ Milwaukee VAMC/Medical College of Wisconsin, Milwaukee, WI; ${ }^{2}$ Medical College of Wisconsin, Milwaukee, WI; ${ }^{3}$ Vanderbilt University, Nashville, TN ${ }^{4}$ Tennessee Valley VAMC, Nashville, TN; ${ }^{5}$ University of Pennsylvania/ Philadelphia VAMC, Philadelphia, PA. (Control ID \#1324279)

BACKGROUND: Workload is often conceptualized as the numbers of patients for whom a given physician is responsible. However, little information exists about how much work individual patients actually contribute to the workload of a physician. We conducted this study to determine the amount of time internal medicine interns spend on new patient admissions

METHODS: We conducted a prospective time-motion study on general internal medicine wards at a single tertiary care VAMC, with IRB approval. We consented internal medicine interns and patients admitted by those consented interns. Inclusion criterion for the patients was being admitted to a consented intern. Exclusion criteria were being 1) unable to give consent, 2) admitted after $1 \mathrm{AM}$; and 3) admitted directly to the ICU. We trained observers to shadow interns during an on call period. We used specialized software on laptop computers to continuously record the tasks performed by interns. We also recorded work performed by the interns for consented patients. We calculated the total time spent on individual patients at 4,6 and 8 hours after admission. We also calculated the amount of time spent on different types of work (e.g., direct patient care) for each patient. We recorded demographic information for the patients and interns and collected other workload data for the interns such as the number of patients crosscovered and the number of patients on the team at the start of the day. We evaluated the relationship between time spent on patients and possible predictors of time spent by using Pearson's correlations.

RESULTS: Twenty-five of $36(69 \%)$ interns and 26 of $43(60 \%)$ of patients agreed to participate, although 1 patient left AMA prior to data being collected. Mean age of interns was 28.6 (SD 2.4) and mean age of patients was 62.5 (SD 14.2); $98 \%$ of the patients were men. Interns spent a mean of 69 \pm 31 minutes with each new admission in the first 4 hours after admission, 89 \pm 41 minutes after 6 hours and $107 \pm 87$ minutes at 8 hours. In the first 4 hours, interns spent a mean of $32.0 \pm 19.9$ minutes $(47 \%)$ in documentation tasks, $16.5 \pm 13.3$ minutes $(25 \%)$ communicating with other healthcare professionals, and $15.90 \pm 15.03$ minutes $(22 \%)$ at the bedside of each new patient. The remainder was spent in other activities such as teaching about the patient (2 minutes or $2 \%$ ). Care for patients occurred episodically, with a mean of 36 tasks devoted to each patient in the first 4 hours of admission. We examined correlations between the number of hours spent on individual patients and other workload parameters. We found that the amount of time that interns predicted that they would spend on the patient was negatively correlated with amount of time they actually spent $(\mathrm{r}=-0.43, \mathrm{p}=0.03)$. The number of months an intern had been in training was also negatively correlated with the amount of time actually spent $(\mathrm{r}=-0.38, \mathrm{p}=0.06)$. Team census, intern census and number of patients cross-covered were not significantly correlated with time spent on individual patients.

CONCLUSIONS: We have demonstrated that it is possible to measure the amount of time interns spend on new admissions. These admissions take a significant amount of time. This work is fragmented, and predominantly composed of indirect patient care. Less experienced interns spend more time than more experienced interns, which reinforces the need for graduated responsibility of patient care. A PATIENT-CENTERED TEAM APPROACH TO HOME-BASED
PRIMARY CARE: A MODEL FOR MULTIDISCIPLINARY PRI-
MARY CARE AT THE MOUNT SINAI VISITING DOCTORS PROGRAM Cameron R. Hernandez; Meng Zhang; Silvia Chavez; Katherine Ornstein; Theresa Soriano. Mount Sinai Medical Center, New York, NY. (Control ID \#1340699)

BACKGROUND: There are at least three million homebound adults in the United States, comprising a costly population at high risk for fragmented care and hospitalization. Mount Sinai Visiting Doctors Program (MSVD), the largest academic home-based primary care program in the U.S., cares for over 1000 homebound patients in Manhattan annually. Despite the program's growth, there continues to be a greater need in the community for primary care services for the homebound than the program is able to meet at its current capacity and structure. In October 2009, MSVD launched a project designed to extend our capacity to care for the most vulnerable homebound patients. Utilizing a patient-centered care team consisting three physicians (two full time equivalents), a social worker, a nurse practitioner, and an administrative assistant, the goal was to extend primary and palliative care services to $50 \%$ more homebound individuals per primary care provider while maintaining high quality care.

METHODS: We evaluated the cost-effectiveness of the new multidisciplinary team approach compared to our existing physician-centered model. Additionally, we compared hospitalization rates in 2010, patient satisfaction, and provider burnout for patients receiving team versus standard care. RESULTS: The interdisciplinary team was able to care for 393 patients from $10 / 1 / 09$ to $11 / 15 / 11$ which was a $40 \%$ increase in each physician's panel size. The wait list to enter MSVD was decreased from over 100 to 40 patients. Wait time until entry into MSVD was decreased from over 12 weeks to 5 weeks. One hundred percent of team patients were seen by one of the team provider post-hospital discharge. Eighty percent of new team patients were evaluated by a social worker. While there was no difference in the number of hospitalization admissions per 1000 patient-days (3.65 team vs. 3.75 nonteam) or repeat admissions ( $10 \%$ team vs. $9 \%$ non-team) for 2010 , the team providers reported less feelings of burnout than non-team providers. Team patients were as satisfied as non-team patients. Team patients had greater recognition of social work and nursing staff. There was $40 \%$ increased monthly revenue for one team physician FTE versus one non-team physician FTE. Finally, team patients were cared for at a $15 \%$ reduced cost per patient compared to patients receiving standard non-team care at MSVD.

CONCLUSIONS: Although we did not find reduced hospitalizations through the use of the team approach, we did find that the program expanded care capacity at no additional cost with no negative impact on quality of care for patients. Home-based primary care programs should continue to explore multidisciplinary approaches to patient care which may be more cost-effective and result in improved patient experience and provider satisfaction, thereby reducing unnecessary healthcare costs.

A POSITIVE DEVIANT ANALYSIS OF SELF-MANAGEMENT STRATEGIES AMONG AFRICAN-AMERICAN AND LATINO PATIENTS WITH DIABETES Ian Huntington ${ }^{1}$; Ashley Harris ${ }^{2}$; LeRoi Hicks ${ }^{3}$. ${ }^{1}$ Brigham \& Women's Hosp, Boston, MA; ${ }^{2}$ Brigham \& Women's Hospital, Jamaica Plain, MA; ${ }^{3}$ University of Massachusetts Medical Center and UMass Medical School, Worcester, MA. (Control ID \#1340000)

BACKGROUND: Positive deviance is a research method that seeks to identify those people who have achieved an unexpected good outcome despite high risk, and then uncover the individual psychological, environmental, and behavioral factors that are contributing to that success. To our knowledge, this method has yet to be utilized among high-risk diabetes patients. This study focused on those patients with diabetes who had a history of poor disease control but had then made improvements in self-management.

METHODS: We examined African-American and Latino patients with Medicaid insurance receiving care at a hospital based outpatient residency 
clinic with either residents or faculty as their primary care physicians. "Positive deviants" (PDs) were defined as having had at least two HbAlc measurements greater than $9 \%$ at least 3 months apart and then at least 3 months of HbAlc measurements $<7.5 \%$, including the most recent value. We excluded patients who were only on oral agents at the time of poor control, on dialysis, had dementia or had bariatric surgery. Semi-structured interviews were conducted with subjects by co-authors in English (IH) and Spanish (AH). Interview transcripts were recorded, transcribed, and in the case of Spanish language interviews, translated into English for analysis. After coding, we utilized NVivo9 qualitative software to search for themes that occurred frequently in a single interview or across interviews and resulted in categorical nodes, and then grouped them into larger themes. Several interviews were coded by both $\mathrm{IH}$ and $\mathrm{AH}$ to insure consistency of coding.

RESULTS: Of a total of 490 patients with a $\mathrm{HbAlc}>9 \%$ from $2003-2010$, we identified 55 potential subjects who met the above criteria for periods of both poor and improved control. Of these, 14 patients $(25 \%)$ met further inclusion criteria for being PDs and seven consented for an interview. Two year after data collection, six of the PDs had had HbAlc levels $>7.5 \%$. A common theme for the period of poor control was social stressors, including ill parents, a dysfunctional marriage, and homelessness. These stressors caused patients to both deny that they had diabetes and to neglect medications, exercise or dietary self-management. Transitions to improved control were in several cases centered on diabetes health related events which occurred both to the PD and within their families, causing the PDs to take their disease more seriously. For other subjects, it was addressing the significant stressors in their lives. All subjects discussed the sequelae of diabetes (dialysis, vision changes, amputations), and patients were more likely mention these effects if they had family members with that complication. Specific strategies of change included walking more after the transition and diet changes that included removing ill-advised foods from the home and strategies for reducing consumption.

CONCLUSIONS: We found that among traditionally high-risk groups for diabetes complications, the family context of diabetes emerged as an important contributor to the process of change through providing examples of the complications of diabetes and supporting behavioral change; this familial context should be explored by health care providers. Providers should also establish programs to assist high-risk patients with the social factors that may take priority for patients over disease self-management. The difficulty of sustained change is underscored by the rising $\mathrm{HgAlc}$ in six of the seven subjects.

\section{A PROFILE OF POORLY CONTROLLED DIABETICS IN SOUTH} FLORIDA: THE MIAMI HEALTHY HEART INITIATIVE Olveen Carrasquillo; Elizabeth Patberg; Sonjia Kenya; Yisel Alonzo. University of MIami, Miami, FL. (Control ID \#1339706)

BACKGROUND: Given the growing epidemic of diabetes, particularly among Latino populations, there is an impetus to define and implement culturally sensitive interventions aimed at helping to improve health status and the quality of diabetes care in this group. There is increasing consensus that Community Health Workers (CHW's) can have an essential role in narrowing such diabetes and cardiovascular health disparities. However, evidence of their effectiveness from rigorous randomized controlled clinical trails is limited.

METHODS: The Miami Healthy Heart Initiative is clinical trial of poorly controlled Latino diabetic patients randomized to usual care or a one year CHW intervention. Patients are identified from our local public hospital clinic using automated EMR queries followed by opt-out letters and recruitment phone calls. Our primary outcomes are changes from baseline in systolic blood pressure, LDL and HgA1C. Additional outcomes include self-reported medication adherence (Morisky), medication intensification, self-efficacy and self-reported dietary intake (BRFSS) and physical activity (IPAQ). Other measures include acculturation (Marin), health literacy (SAHLSA), and depression (PHQ-9).

RESULTS: To date we have sent recruitment letters to 540 potential patients. Of the 294 subjects that we have been able to contact and whom on screening were study eligible, $79 \%$ have agreed to participate. So far we have randomized 178 of our planned 360 patients. Mean age is $56 \mathrm{yrs} \pm 7$ and approximately $30 \%$ of our sample is Cuban. The rest of the the study subjects comprise a variety of other Latino ethnicities with no other group representing $>15 \%$ of the sample. Enrolled participants have a mean SBP of $132 \mathrm{mmHg} \pm 18$, LDL $99 \mathrm{mg} / \mathrm{dl} \pm 41$, and $\mathrm{HbAlc}$ of $9.0 \% . \pm 1.7$. Most subjects are obese (mean BMI $32.4 \pm 7.7$ ), $44 \%$ have low acculturation, but only $12 \%$ have low health literacy. Nearly all $(70 \%)$ have inadequate medication adherence and $48 \%$ reported low diabetes related self-efficacy. Although, we are still not adequately powered to make planned statistical comparisons some emerging trends are already evident. For example, the least acculturated Latinos are trending to have better diabetes control than those most acculturated $(8.8 \pm 1.7$ vs $9.4 \pm 1.9)$. Diabetes self-efficacy also seems to be related to poorer glycemic control $(8.5 \pm 1.5$ vs $9.4 \pm 1.7)$. In the intervention group, each of our three CHWs is handling a caseload of approximately 30 patients. CHWs have made 8.1 home visits and 14.2 phone calls per patient. Patients have participated in a mean of 2.1 group educational sessions.

CONCLUSIONS: As compared to other communities, poorly controlled Latinos diabetics in MHHI are very ethnically diverse and have relatively high health literacy but similar to others low acculturation status. MMHI is already generating important cross-sectional data of Latino diabetics in Miami. Preliminary findings suggest that existing measures of self-efficacy are valid in this South Florida Latino population. We are also finding evidence of the Latino paradox in our cohort.

\section{A QUALITATIVE INVESTIGATION OF THE DECISION TO INITIATE DO NOT HOSPITALIZE ORDERS AMONGST HEALTH CARE PROXIES OF NURSING HOME RESIDENTS WITH DEMENTIA Elizabeth A. Mann ${ }^{1}$; Wanda Colon-Cartagena ${ }^{2}$; Sarah L. Goff $^{2}$; Sandra Bellantonio ${ }^{2}$; Michael Rothberg $2 .{ }^{1}$ University of Pennsylvania, Philadelphia, PA; ${ }^{2}$ Baystate Medical Center/Tufts University School of Medicine, Springfield, MA. (Control ID \#1337596)}

BACKGROUND: Approximately 5 million patients with advanced dementia reside in nursing homes in the United States. Advanced dementia is a fatal illness, and routine hospitalization does not necessarily positively impact patients' quality of life or health outcomes. Despite this, do not hospitalize $(\mathrm{DNH})$ orders are rare. The demographics of patients with DNH orders have been studied, but little is known about the reasons why some health care proxies choose to initiate DNH orders. The objective of this study was to explore how health care proxies make this decision.

METHODS: In this qualitative study, in-depth, in person semi-structured interviews were held with health care proxies (HCPs) of elderly nursing home residents with dementia. An interview guide was developed and tested with nursing home staff. All nursing home residents aged $\geq 65$ years with an activated HCP were considered eligible. Nursing home staff mailed a letter to all HCPs informing them of the study and allowing them to opt out. HCPs were then contacted and invited to come to the nursing home for an interview. The interviews were audio-taped and transcribed verbatim. Applying grounded theory, transcripts were coded in an iterative process with revision of the interview guide as indicated to prompt for emerging themes. Codes were then organized into pertinent themes. Demographic and clinical characteristics of the nursing home residents were collected from the Minimum Data Set and patient charts.

RESULTS: Of the 31 eligible HCPs, 9 declined to be interviewed, 5 could not be reached and 1 was unable to come for an interview because the resident was hospitalized. The remaining $16 \mathrm{HCPs}$ participated in approximately 1 hour long interviews. Eight participants had transfer limitations in place, and in most cases had a stipulation allowing for hospitalization in the case of an acute injury. Only 1 participant had an absolute DNH order. The subject of DNH orders elicited a wide variety of responses. Not all HCPs were familiar with the orders; of those that were, their opinions towards the orders were generally strongly positive or negative. All participants, regardless of their opinion of DNH orders, reflected on the challenges of health care decisionmaking. Major themes that arose included factors that facilitate initiating DNH orders and factors that present barriers to initiating DNH orders. Facilitators included HCP experience in the health care industry, an accurate understanding of the resident's prognosis, and a desire to limit resident 
distress during hospital transfers. Barriers included a perceived lack of physician involvement in decision-making, a limited understanding of the resident's prognosis and a limited understanding of the orders. Other salient themes included misunderstandings about the role of HCPs in decisionmaking and the personal nature of the decision to limit care.

CONCLUSIONS: In this qualitative study of HCP decision-making, we found a number of barriers to initiating $\mathrm{DNH}$ orders, some of which appear to be modifiable. Randomized trials are necessary to explore whether interventions to overcome these barriers can increase the number of HCPs who choose to initiate DNH orders.

A RANDOMIZED TRIAL OF PHONE-BASED MOTIVATIONAL INTERVIEWING ON ADHERENCE TO CLOPIDOGREL AFTER A CORONARY STENT AMONG BLACK AND HISPANIC SUBJECTS. Ana M. Palacio ${ }^{1}$; Leonardo Tamariz ${ }^{1}$; Hua $\mathrm{Li}^{1}$; Desiree Garay ${ }^{1}$; Claudia Uribe ${ }^{2}$; Leslie Hazel-Fernandez ${ }^{2}$; Olveen Carrasquillo ${ }^{1} .{ }^{1}$ University of Miami, Miami, FL; ${ }^{2}$ Competetive Health Analytics, Miami, FL. (Control ID \#1336675)

BACKGROUND: Racial/ethnic minorities who receive coronary stents have lower medication adherence to antiplatelet agents. Motivational interviewing (MI) has been effective at inducing behavior change among patients with cardiovascular risk factors. The aim of this study is to compare the efficacy of phone-delivered MI to an educational video at improving medication adherence to clopidogrel among insured minorities. METHODS: We conducted a randomized trial of Black and Hispanic patients enrolled in a health benefits plan who had recently received a coronary stent. Study patients were randomly assigned to either a telephone based MI intervention or a culturally tailored educational video. The MI intervention consisted of 4 phone-based encounters by trained nurses over 9 months each lasting about 20 minutes made from a centralized location. Outcomes variables collected at baseline and 12-month using surveys and administrative data included self -reported adherence (Morisky score), self reported forgetfulness and carelessness when taking antiplatelet medications and self reported completion of 12 month of therapy (only in 12 month survey). We used ttest and chi-square methods to compare outcomes between groups and used logistic regression to model predictors of self-reported forgetfulness taking medications.

RESULTS: We recruited 339 minorities ( $58 \%$ Hispanics and $42 \%$ Black) from 21 different states who had received a new coronary stent. Patients had a mean age of $69.5 \pm 8.8,52 \%$ were males, $78 \%$ had an income less than $\$ 30,000 / \mathrm{yr}$ and only $22 \%$ had greater than high school education. At 12 months, as compared to the video group, patients in the MI group had significantly better self reported adherence (lower Morisky score), and were less likely to report being forgetful or careless about taking antiplatelet medications (Table 1). Other important predictors of self-reported adherence at 12 months were low health literacy, black race, and depression $(\mathrm{p}<0.01)$. Adjusting for these factors as well as socio-demographic characteristics, co-morbidities and baseline Morisky score at baseline did not significantly change our findings.

CONCLUSIONS: Among blacks and Hispanics patients, phone-based motivational interviewing resulted in higher self-reported adherenceto clopidogrel after a coronary stent versus a mailed video.A centralized phone-based MI seems to be a promising cost-effective strategy which may help prevent stent re-occlusion among a large geographically diverse sample of minority patients.Analyses of medication adherence using claims data (medication possession ratio) will be completed by May 2012 .

\begin{tabular}{llll}
\hline \hline Outcome & $\begin{array}{l}\text { Motivational } \\
\text { interviewing } \\
\text { group(n=169) }\end{array}$ & $\begin{array}{l}\text { Video } \\
\text { group(n=170) }\end{array}$ & p-value \\
$\begin{array}{l}\text { Not completing 12 months } \\
\text { antiplatelet therapy, \% }\end{array}$ & 9 & 17 & $<0.01$ \\
$\begin{array}{l}\text { Mean Morisky score } \\
\begin{array}{l}\text { Forgetting to take } \\
\text { antiplatelet therapy, \% }\end{array}\end{array}$ & $0.18 \pm 0.44$ & $0.46 \pm 0.79$ & $<0.01$ \\
$\begin{array}{l}\text { Being careless about } \\
\text { taking antiplatelet }\end{array}$ & 7 & 24 & $<0.01$ \\
therapy, \% & & 16 & $<0.01$ \\
\hline
\end{tabular}

A SYSTEMATIC REVIEW OF LONGITUDINAL POPULATIONBASED STUDIES ON THE PREDICTORS OF SMOKING CESSATION IN ADOLESCENT AND YOUNG ADULT SMOKERS Semanur Cengelli, ${ }^{1,2}$; Jennifer L. O'Loughlin ${ }^{2,3}$; Beatrice Lauzon ${ }^{2,3}$; Jacques Cornuz ${ }^{1,4}$. ${ }^{1}$ University of Lausanne, Lausanne, Switzerland; ${ }^{2}$ Centre de Recherche du CHUM, Montreal, QC, Canada; ${ }^{3}$ University of Montreal, Montreal, QC, Canada; ${ }^{4}$ University of Lausanne, Lausanne, Switzerland. (Control ID \#1335312)

BACKGROUND: Tobacco use causes more than five million deaths worldwide annually. In Canada in 2009, the prevalence of smoking was $13 \%$ among adolescents aged $15-19$ years, and $23 \%$ among young adults aged 20-24 years. Many young smokers express the desire to quit, but most have a great deal of difficulty doing so. Empirical reviews have generally concluded that smoking cessation programs in youth have limited efficacy. In order to provide a solid knowledge base for future tobacco control interventions, the determinants of self-initiated cessation of duration of at least 6 months in youth need to be better understood.

METHODS: A systematic search of the PubMed and EMBASE databases using smoking, tobacco, cessation, quit and stop as keywords was performed. Limits included articles related to humans, in English, published between January 1984 and August 2010, and study population aged 10-29 years. A total of 4502 titles and 871 abstracts were reviewed independently by 2 and 3 reviewers, respectively. Nine articles were retained for data abstraction. The number of studies that reported a statistically significant association between each determinant investigated and cessation were tabulated, from among all studies that assessed the determinant.

RESULTS: Three of the nine studies retained defined smoking cessation as abstinence of at least 6 months and six studies defined it as abstinence of 12 months. Despite heterogeneity in methods across studies, five factors robustly predicted quitting across studies in which the factor was investigated: not having friends who smoke, not having intentions to smoke in the future, resisting peer pressure to smoke, being older at first use of cigarette and having negative beliefs about smoking. Additional factors are significant in some studies but not others or only once assessed.

CONCLUSIONS: The literature on longitudinal predictors of cessation in adolescent and young adult smokers is not well developed. Cessation interventions for this population will remain less than optimally effective until there is a solid evidence base on which to develop interventions.

A1C VARIABILITY AND THE RISK OF DEVELOPING NEW DIABETES FOR THE HEALTHY ADULTS IN JAPAN Osamu Takahashi $^{1,2}$; Daiki Kobayashi ${ }^{1}$; Gautam A. Deshpande ${ }^{2}$; Sachiko $\frac{\mathrm{Ohde}^{2} \text {; }}{\text { Th }}$ Tsuguya Fukui ${ }^{1,2}$; Paul Glasziou ${ }^{3}$. ${ }^{1}$ St.Luke's International Hospital, Tokyo, Japan; ${ }^{2}$ St.Luke's Life Science Institute, Tokyo, Japan; ${ }^{3}$ Bond University, Gold Coast, QLD, Australia. (Control ID \#1340192)

BACKGROUND: Recently, the ADA proposed a shift to A1C for the initial diagnosis of diabetes. However, the association of A1C's variability with developing new diabetes has been little studied. We aimed to evaluate the effect of A1C variability on the risk of developing new diabetes in healthy adults in Japan.

METHODS: Population-based, cohort study from 2005 to 2008 in Tokyo, Japan of healthy adults not taking diabetes medication and with a $\mathrm{HbAlc}$ lower than $6.5 \%$ at baseline. Based on annual measurement of serum $\mathrm{HbAlc}$ we calculated the annual visit-to-visit variability, and used this as a predictor of new onset diabetes in a multivariate logistic regression.

RESULTS: At baseline, 14,587 people $(50 \%$ female) with a mean age of 51 years old (SD: 12 years, range: 23 to 92 ), a mean fasting plasma glucose (FPG) level of $98.4 \mathrm{mg} / \mathrm{dl}(\mathrm{SD}: 9.3 \mathrm{mg} / \mathrm{dl})$ and a mean $\mathrm{HbAlc}$ level of $5.3 \%$ (SD: $0.4 \%$ ) had annual check-ups over 4 years. After adjusting for the other potential risk factors new diabetes was predicted by the A1C variability (odds ratio (OR): 10.3 for highest (>=0.16\%)) versus the lowest quantile $(<0.08 \%), 95 \% \mathrm{CI}: 5.9-18.0)$ and by the baseline A1C (OR: 55.2 for A1C of 6.0 - $6.4 \%$ versus A1C of $<5.0 \%$, 95\% CI: 13.2 - 230). FPG (OR: 1.1, 95\%CI: $1.1-1.2$ ) and Smoker (OR: 1.8, 95\%CO: 1.3 - 2.6) weakly but also significantly related to develop the new diabetes. For predicting the development of diabetes, the 
combination of the level of AIC at baseline and the variability (Area Under the Curve (AUC) for the ROC $=0.94$ ) was superior to the level of $\mathrm{A} 1 \mathrm{C}$ at baseline alone $(\mathrm{AUC}=0.89)$. (Figure)

CONCLUSIONS: Visit-to-visit variability in A1C independently added to the baseline $\mathrm{A} 1 \mathrm{C}$ in predicting the risk of developing new diabetes for the healthy adults.

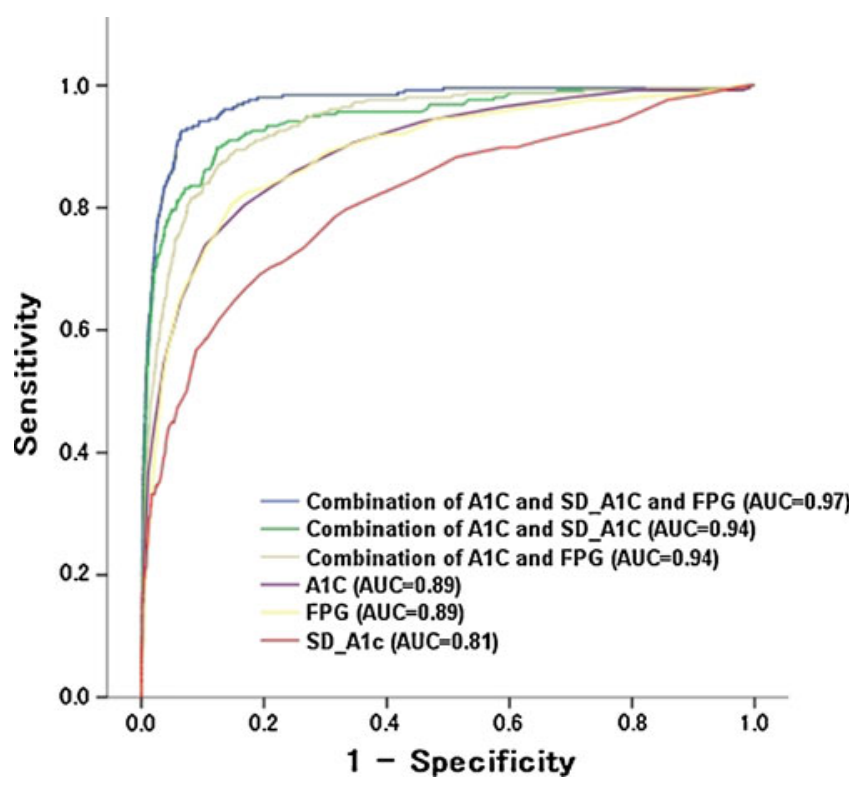

ARROW PLOTS: AN INNOVATIVE METHOD OF PLOTTING SURROGATE AND CLINICAL OUTCOMES IN META-ANALYSES KoKo Aung ${ }^{1,2}$; Fanglong Dong ${ }^{4}$; Robert G. Badgett ${ }^{3,4}$. ${ }^{1}$ University of Texas $\overline{\text { Health }}$ Science Center at San Antonio, San Antonio, TX; ${ }^{2}$ University of Texas School of Public Health, Houston, TX; ${ }^{3}$ University of Kansas School of Medicine, Wichita, KS; ${ }^{4}$ University of Kansas School of Medicine, Wichita, KS. (Control ID \#1334315)

BACKGROUND: Meta-analyses of health care interventions usually study dichotomous patient-centered clinical outcomes such as mortality. Clinicians titrate medical therapy to a surrogate outcome that is a continuous biomarker. Forest plots in meta-analyses do not, however, show well the relation between a surrogate outcome, baseline risk, and a clinical outcome. The objective of this study is to develop a visual display of quantitative information from meta-analyses to illustrate the link between a purported biomarker, baseline risk, and clinical outcome. METHODS: Differences in outcomes of randomized controlled trials are plotted on two dimensions for two independent variables: changes in a clinical outcome and changes in a surrogate outcome. Arrows begin with the results of the control groups, reflecting baseline risk, and end with the results of the intervention groups. Bayesian meta-analysis is performed and impact of therapy is described with judicial analogies. We illustrate by reanalyzing 3 recent meta-analyses, intensive lowering of LDL-cholesterol (CTT 2010), intensive insulin therapy in hospitalized patients (Kansagara 2011), and intensive glucose lowering treatment in type 2 diabetes (Boussageon 2011) RESULTS: The change in the y-coordinate of each arrow indicates relative risk reduction. The slopes of the arrows represent the relation between change in the biomarker and change in the clinical outcome. For intensive lowering of LDL-cholesterol (Figure), arrows are homogeneous suggesting a consistent association between biomarker and clinical outcome. For diabetes in hospitalized patients, arrows are heterogeneous suggesting inconsistent association between biomarker and outcome. However, two visually identifiable regions suggest association between biomarker and outcome in subpopulations. Intervention may improve clinical outcome in subpopulations with very high baseline glucose or very high baseline risk of mortality. For intensive glucose lowering in type 2 diabetes, arrows are heterogeneous without forming any identifiable homogeneous regions suggesting inconsistent association between biomarker and outcome.

CONCLUSIONS: This data visualization is innovative because two independent variables are dimensioned and Bayesian analyses of regions facilitate interpretation. Arrow plots allow assessment of biomarkers as indicators of therapy response and influence of baseline risk. Further research should develop a statistical measure for homogeneity of slopes of arrows and a method of identifying the thresholds of biomarker and baseline risks that separate benefit from harm.

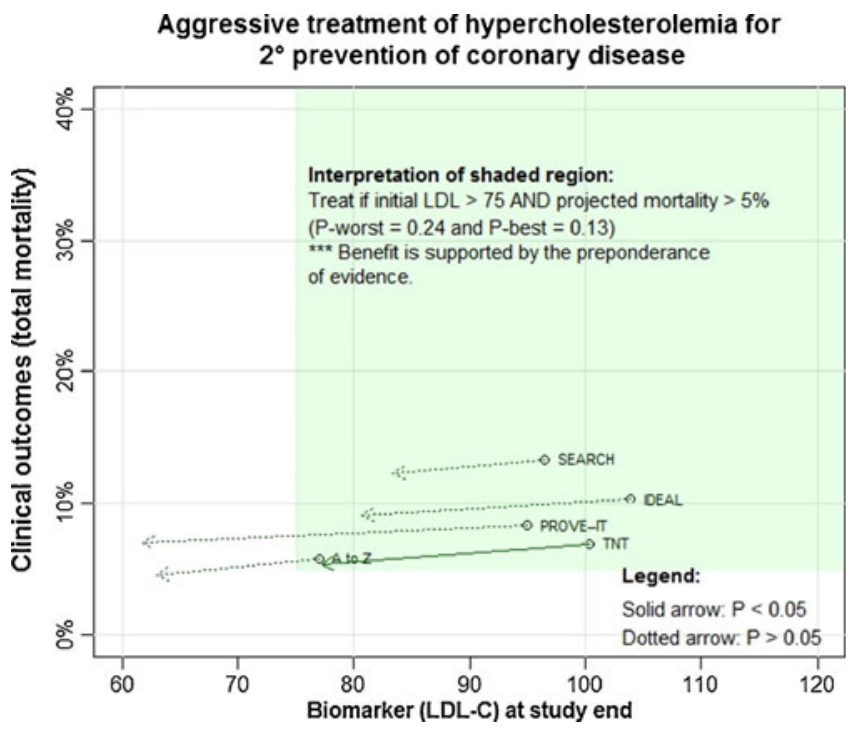

Figure

ACCEPTANCE OF COMBIVENT RESPIMAT INHALATION SPRAY IN COPD PATIENTS Gary T. Ferguson ${ }^{1}$; Mo Ghafouri ${ }^{2}$; Luyan $\mathrm{Dai}^{2}$; Leonard J. Dunn ${ }^{3}$. ${ }^{1}$ Pulmonary Research Institute of Southeast Michigan, Livonia, MI; ${ }^{2}$ Boehringer-Ingelheim Pharmaceuticals, Inc, Ridgefield, CT; ${ }^{3}$ Clinical Research of West Florida, Inc, Clearwater, FL. (Control ID \#1325478)

BACKGROUND: Chronic obstructive pulmonary disease (COPD) medications are preferentially delivered via oral inhalers. Combivent (CVT; ipratropium+albuterol) is currently only available as a metered-dose inhaler (CVT-MDI) that uses a chlorofluorocarbon (CFC) propellant. The Respimat inhaler is a novel, soft-mist inhaler that is CFC propellant-free. In association with the phase-out of CFCs worldwide, the Combivent Respimat Inhalation Spray (CVT-R) has recently been approved for patients with COPD in the USA, and will replace the phasing out MDI device. This study evaluated patient satisfaction+long-term safety of CVT$\mathrm{R}$ vs CVT-MDI vs the free combination of ipratropium (Atrovent) HFA and albuterol HFA metered dose inhalers $(\mathrm{I}+\mathrm{A})$.

METHODS: The study was a Phase III, 1-year, 3-treatment, open-label, randomized, active-controlled, parallel-group study. After initial screening, patients received CVT-MDI during a $3 \neg-4$ week baseline period, followed by randomization to 1 of 3 treatments: CVT-R, CVT-MDI, or I+A for 48 weeks. Patients were provided with albuterol MDI rescue medication as needed. Study participants were males and females with COPD, aged $\geq 40$ years, who were current or ex-smokers (smoking history, $\geq 10$ pack-years). Treatment compliance was assessed using a patient Daily Diary Card. The primary endpoint was the Performance Domain Score (PDS) evaluated using a modified Patient Satisfaction and Preference Questionnaire (PASAPQ) at Visit 2 (Day 0; before randomization) and at treatment Visits 3-7 (Weeks 348). Adverse events (AEs) were recorded at each visit.

RESULTS: 688 patients were enrolled at 55 US sites and 470 were randomized to open-label treatment following baseline treatment with CVTMDI. Baseline demographics were similar for all treatment groups. Overall, 
$58.7 \%$ were male, $93.5 \%$ were white, $52.0 \%$ were current smokers, and $63.0 \%$ used pulmonary medication at the time of informed consent. After 48 weeks of treatment, a statistically significantly higher PASAPQ PDS was observed for the CVT-R group vs the CVT-MDI and I+A groups $(\mathrm{P}<0.0001)$. Patients also indicated that they liked the actuation indicator component of the Respimat device. Improvements in lung function were similar for all groups from Day 1 through Week 48. Time to first COPD exacerbation (TTFE) hazard was slightly longer in the CVT-R group compared to other treatment groups, and the cumulative risk of COPD exacerbation was comparable. There was no significant difference among groups for TTFE leading to hospitalization and exposure-adjusted event rates. Overall, rescue medication use between the groups was not significantly different. Patients in the CVT-R group withdrew from the study in fewer numbers than patients in the $\mathrm{I}+\mathrm{A}$ group $(\mathrm{P}=0.0059)$. All patients who received at least 1 dose of study medication were included in the safety analysis. Overall, $78.1 \%$ of patients completed the study and $72.0 \%$ reported an AE; the percentage of patients with serious AEs was similar in the 3 groups.

CONCLUSIONS: The environmentally friendly CVT-R was statistically superior vs CVT-MDI and I+A with regard to patient satisfaction, and fewer patients dropped out while receiving CVT-R. All 3 treatments showed a similar safety profile. A relative improvement in TTFE with CVT-R suggests that effective drug delivery and/or patient acceptance of the Respimat inhaler may benefit adherence and, therefore, clinical outcomes in patients with COPD.

\section{ACCESS MATTERS: IMPROVED DETECTION OF PREMA- LIGNANT POLYPS WITH A SCREENING COLONOSCOPY PROGRAM FOR THE UNINSURED Damian Casadesus; Orlando Penaloza; Delaram Moazami; Armen Simonian; Daniel Goldsmith. Capital Health Regional Medical Center, Trenton, NJ. (Control ID \#1309505)}

BACKGROUND: Colonoscopy is an effective screening modality for the early detection of colonic polyps and cancer, but screening rates are low particularly among minorities and the uninsured. In 2008, Capital Health obtained a clinical grant from the American Cancer Society to perform screening colonoscopies for patients without insurance who were established at the hospital's primary care clinic. The aim of this study is to evaluate the grant program with respect to endoscopic findings and changes in the demographics of patients undergoing colonoscopy.

METHODS: A retrospective review was performed of all patients registered for colonoscopy at the endoscopy suite of Capital Health Regional Medical Center. A pre- and post-implementation analysis was designed to compare 3 groups: 1) all colonoscopies performed in the twenty four months prior to the program's start (pre-program group) 2) all screening colonoscopies performed on uninsured patients in the program during twenty four months period (program group) and 3) all other colonoscopies performed in the same suite during the time of the program (parallel group). A descriptive analysis of demographics, ethnicity and insurance status was performed. The clinical endpoints included rate of polyp detection by screening and the histological description of polyps detected.

RESULTS: There were a total of 3596 colonoscopies performed during this 48 month period, $86(2.3 \%)$ of them were excluded because the data was incomplete. The pre-program group had 1624 colonoscopies, 296 of which were screening colonoscopies, the program group had 339 screening colonoscopies, and the parallel group had 1547 colonoscopies, 292 of which were screening colonoscopies. Implementation of the grant program resulted in more than double the screening colonoscopies performed as compared to the pre-program period (296 vs 631). There was no change in the number of the reimbursed procedures performed in the same suite after initiation of the grant program. The proportion of minority patients undergoing a screening procedure was significantly increased compared to the pre-program group $(89 / 296$ vs $280 / 339 ; \mathrm{p}<0.001)$ and parallel group $(136 / 292$ vs $280 / 339 ; \mathrm{p}<0.001)$. This increase was largely accounted for by a higher proportion of Hispanic and African American patients. The number of patients with polyps was higher in the program group compared with the preprogram and parallel group $(42 \%, 36.8 \%$, and $33.9 \%$, respectively) The total number of polyps and the number of premalignant polyps detected was higher in the program group probably related with the increased number of procedures during the two years period; however, the rate of detection of premalignant polyps did not change among the groups.

CONCLUSIONS: Retrospective analysis of a grant-funded program offering screening colonoscopies to uninsured patients demonstrated a higher rate of screening for an underserved population consisting largely of minority patients. Furthermore, the program resulted in detection of a higher number of premalignant polyps that might otherwise have been undetected. Our experience suggests that targeting health care disparities by insurance status can increase access to preventive services and detect a high number of premalignant lesions.

ACCESS TO PREVENTIVE COUNSELING SERVICES: HEALTH CENTERS VERSUS OTHER PHYSICIAN OFFICES Leiyu Shi ${ }^{2}$; Lydie A. Lebrun ${ }^{1}$; Quyen Ngo-Metzger ${ }^{1}$. ${ }^{1}$ U.S. Department of Health and Human Services, Rockville, MD; ${ }^{2}$ Johns Hopkins Bloomberg School of Public Health, Baltimore, MD. (Control ID \#1324096)

BACKGROUND: Vulnerable populations seek primary and preventive care from a range of health settings such as health centers ( $\mathrm{HCs})$ and physician offices (POs), which include private solo or group practices, free standing clinic/urgicenters, health maintenance organizations, and faculty practice plans. These settings vary in their mission, financial resources, staffing, and patient demographics, and thus, may also differ in the extent to which they are able to operate like a medical home for vulnerable populations. Given the well-known relationship between preventive care and health outcomes, it is important to identify healthcare providers or settings that best promote preventive care for vulnerable populations. The purpose of this study was to compare access to preventive counseling services by $\mathrm{HC}$ patients versus patients seen at POs. Racial/ethnic and insurance-related disparities in accessing preventive counseling services were also assessed.

METHODS: Data came from the 2008 National Ambulatory Medical Care Survey (NAMCS), a nationally representative annual survey, which reports on 28,741 visits to non-federal, office-based physicians. In 2008, the NAMCS also included an oversampling of $104 \mathrm{HCs}$ to provide more reliable data on $\mathrm{HC}$ patient visits. Key study variables included: 1) Measures of preventive counseling services: a. health education services for all patients; b. disease management services for patients with a variety of chronic conditions such as asthma, depression, diabetes, hyperlipidemia, hypertension, and obesity; c. asthma education for patients with asthma, d) tobacco use education for patients who smoke, and d. weight control education for patients who are overweight. 2) Measures of health care settings (HCs and POs), taking specific provider and community characteristics into account (e.g., \% population below poverty level in patient's zip code, $\%$ adults with bachelors degree or higher in patient's zip code, urban-rural classification). 3) Measures of individual sociodemographics. Individual characteristics such as age, gender, race/ethnicity, education, income, health status, and presence and type of insurance were used as covariates.

RESULTS: Patients seen at HCs were more likely to be racial/ethnic minorities (65\%) and have Medicaid (46\%) or no insurance (11\%) compared to patients seen at POs (26\% minorities, $10 \%$ Medicaid, $4 \%$ uninsured). About $40 \%$ of $\mathrm{HC}$ patients received health education and counseling services compared to $36 \%$ at POs. After controlling for patient sociodemographic and health status characteristics and physician practice characteristics, logistic regressions showed that $\mathrm{HC}$ patients were as likely to receive preventive counseling service as patients seen in POs (health education: odds ratio 0.92, CI 0.64-1.33; disease management: odds ratio 0.95, CI 0.34-2.65; asthma education: odds ratio 1.34 , CI $0.47-3.81$ ). Furthermore, no adverse racial/ ethnic and insurance-related disparities were noted.

CONCLUSIONS: Patients seen at HCs reported comparable access to preventive counseling services compared to patients seen at POs. No significant racial/ethnic and insurance-related disparities were noted in accessing certain preventive counseling services. As safety-net providers for uninsured and vulnerable populations, HCs provide high levels of accessibility to preventive care services and contribute to overcoming healthcare disparities. 
ACUTE RENAL FAILURE POST TWO STAGE ARTHROPLASTY USING ANTIBIOTIC LADEN SPACERS: A SYSTEMATIC REVIEW. Fahd Syed ${ }^{1}$; Madhumati Rao ${ }^{2} .{ }^{1}$ Mount Auburn Hospital, Cambridge, MA; ${ }^{2}$ Tufts Medical Center, Boston, MA. (Control ID \#1334962)

BACKGROUND: Two stage arthroplasty is currently the treatment of choice for infected hip and knee joint prostheses. This technique is associated with more than a $90 \%$ success rate in terms of eradication of joint space infection (1-3). A number of complications including acute renal failure have been reported following spacer implantation. However, the data on these complications is often limited. Acute renal failure has been reported with the use of vancomycin, gentamicin as well as tobramycin in antibiotic spacers, and has been reported to occur as late as 5 months post implantation(4-8). However,in this instance again, the data regarding this complication are limited. Our aim was to do a systematic review of literature to assess the number of patients who develop acute renal failure post antibiotic spacer implantation.

METHODS: Our inclusion eligibility criteria were original studies, both observational and clinical trials as well as case series or case reports and systematic review articles dealing with 2 stage revision arthroplasty (knee and hip), antibiotic loaded spacers, and antibiotic elution from spacers, and post operative complications/systemic complications, in particular acute kidney injury after two stage revision using antibiotic loaded spacers. Abstracts and manuscripts were reviewed by two independent investigators to determine eligibility. Reference lists from all reviewed articles from 1989 to 2011, including relevant narrative reviews were assessed to complete the literature search. Data about materials, methods, and results of each original article included was extracted into a specific form. The materials extracted were compared to each other and conflicting data were re-checked from the original papers and corrected after discussion.

RESULTS: 7 out of a total of 297 (2.4\%) patients in whom renal function was followed up developed acute renal failure. 5 of these were those who had hip spacers and 2 had knee spacers. Different antibiotics and dosages were present in the spacers so it is difficult to establish a relationship between the antibiotic concentration in the spacers and renal dysfunction. In addition to these studies there were 5 cases reported in 4 case reports that had AKI. 2 patients had non oliguric and 1 had oliguric renal failure. 3 of these patients required dialysis. Of these patients 3 had knee spacers and 2 had hip spacers. CONCLUSIONS: Infection itself is a risk factor for the development of renal impairment, which might suggest that patients with infected prostheses are at higher risk, although to date there is little published on this in the literature. With an ageing population it could be expected that the number of patients developing AKI after undergoing hip surgery will increase, the identification of patients at risk of development of AKI will have important prognostic implications. In addition, given that there is no standardization of antibiotic content of the cement used in joint spacers, the study of systemic absorption of the antibiotics used will be important in determining the optimal proportion of antibiotics needed in spacer cement.

\section{ADDRESSING DISPARITIES IN COLORECTAL CANCER} SCREENING AT SAN FRANCISCO GENERAL HOSPITAL Claire Horton; Joyce Viloria; Ellen Chen; Hali Hammer. ucsf, San Francisco,

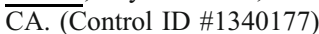

BACKGROUND: Colorectal cancer [CRC] is the third most common cancer and second leading cause of cancer death in the U.S.1 Approximately 18,000 lives could be saved each year, and $60 \%$ of CRC deaths prevented if all U.S. adults over 50 were screened.2 Unfortunately, national rates of $\mathrm{CRC}$ screening rates are low [55\%], and significant disparities exist for racial minorities. At San Francisco General Hospital, the Family Health Center [FHC] and the General Medicine Clinic [GMC] are on-campus clinics providing general adult primary care. Given limited resources within our safety net system, CRC screening is offered through annual FOBTs in primary care, with referral to colonoscopy for abnormal results. In 2010, 48\% of our patients completed CRC screening. 1 U.S. Preventive Task Force.org, accessed December 7, 2010. 2 Cancer statistics, 2007. CA Cancer J Clin. 2007.
METHODS: Beginning in January of 2010, GMC and FHC developed a project to identify and decrease disparities in CRC screening. We performed focus groups to a assess specific barriers to CRC screening in patients with disproportionately low CRC screening rates. Our primary intervention was to develop a panel management system for CRC screening for our two clinics. Two staff members were trained in reviewing registry reports, counseling patients to complete FOBT tests, and providing logistical support for test completion. We also trained staff in colorectal cancer screening counseling, posted screening rates prominently throughout clinic, and involved resident providers in outreach efforts.

RESULTS: The racial/ethnic groups with the lowest screening rates were African American and White patients. Marginal housing status, substance abuse, and psychiatric comorbidity were correlated with lower rates of screening. Focus groups identified the following major barriers to performing FOBT testing: confusion about $\mathrm{CRC}$ and who was at-risk; inadequate clinic educational efforts; logistical barriers to screening for homeless patients; and cultural beliefs among African-Americans about cancer detection and treatment. To date, the project has resulted in significant increases in CRC screening for all racial/ethnic groups $(10 \%$ relative improvement over baseline) with a narrowing of screening disparities among racial/ethnic groups. CONCLUSIONS: In two urban safety-net clinics, the lowest rates of CRC screening were seen in Whites, African-Americans, and patients with psychsocial comorbidities. Focus groups helped identify barriers to screening in at-risk groups, and outreach efforts have resulted in improvement for all racial/ethnic groups and a narrowing of healthcare disparities. Next steps include increasing targeted outreach efforts for at-risk groups.

\section{ADHERENCE TO GUIDELINES FOR LOW BACK PAIN IMAG-} ING: COMPARISON OF A TEACHING AND NON-TEACHING CLINIC Claudia P. Taramona Espinoza; Melissa Skupin; Dora Montezuma; Zahrae Sandouk; Sean Drake. Henry Ford Hospital, Detroit, MI. (Control ID \#1311087)

BACKGROUND: Acute low back pain (LBP) has a prevalence of $80 \%$ and is the fifth most common reason for clinic visits in the United States. Its evaluation depends mainly on the clinical picture. Numerous studies show that clinicians continue to order routine imaging, despite evidence that it is not beneficial, increases radiation exposure and raises healthcare costs. The American College of Radiology (ACR) published guidelines in 2008 for indications to image based on certain red flags. The objectives of our study were to assess adherence to the ACR guidelines and to compare a teaching and non-teaching clinic.

METHODS: We conducted a retrospective descriptive study. Inclusion criteria were: patients older than 18 with acute $(<6$ weeks $)$ musculoskeletal LBP, seen in the clinics from March 17, 2008 to June 30, 2011. Exclusion criteria were: non-musculoskeletal LBP, previous LBP work-up, acute on chronic and chronic LBP and patients without a clinic note. A list of medical record numbers and clinic visit dates was retrieved using the ICD9 billing code of LBP for both an urban teaching and urban non-teaching clinics. Office notes were reviewed. We documented if imaging was ordered and type of imaging, and compared it to the ACR guidelines. Data was analyzed using a chi-square test was used to compare categorical data, and two-sample independent t-tests for continuous data.

RESULTS: We reviewed 1,776 charts (teaching clinic: 954; nonteaching clinic: 822). Patients were excluded for the following reasons: 467 were non-musculoskeletal; 734 were not acute; 149 were previously evaluated; 15 lacked documentation. 404 patients met inclusion criteria: 203 from the teaching clinic; 201 from the non-teaching clinic. The teaching clinic adhered to guidelines $67 \%$ of the time, versus $35.3 \%$ adherence in the non-teaching clinic $(p<0.001)$. Management was inconsistent with guidelines $23.1 \%$ of the time in the teaching clinic, versus $35.8 \%$ of the time in the non-teaching clinic $(p=0.005)$. For patients who met the ACR criteria but were not imaged, $30.6 \%$ were from the non-teaching clinic and $69.4 \%$ were from the teaching clinic $(\mathrm{p}<0.01)$. For patients who did not meet the imaging criteria, but were still imaged, most were from the non-teaching clinic $(73.5 \%$ non-teaching, $26.5 \%$ resident clinic). The most common red flag was age $>70$, and it was also the most commonly missed red flag. 
CONCLUSIONS: Overall, we found that the teaching clinic adhered to guidelines more than the non-teaching clinic. For patients who were managed inappropriately, the teaching clinic more frequently spared imaging when it was indicated, while the non-teaching clinic more frequently ordered imaging inconsistently with the guidelines. There are several possible explanations for our results. Studies have found that residents are more receptive to using guidelines than supervisors, because they lack experience and research guidelines more often. Supervisors may trust their experience over clinical guidelines. In general, adherence to hospital guidelines is often low to moderate $(40-60 \%)$. In institutions where residents are not independent decision makers, any implementation plan should combine strategies aimed at both residents and staff physicians. The mindset that more testing means better care must be abandoned in favor of a more evidence-based approach.

ADJUSTING FOR HOSPITALIZED PATIENTS' CLINICAL RISK OF URINARY TRACT INFECTIONS: A NECESSARY STEP IN PAY-FOR-PERFORMANCE WHEN COMPARING BY HOSPITALACQUIRED CONDITION RATES. Jennifer Meddings; Heidi Reichert; Mary A.M. Rogers; Laurence F. McMahon. University of Michigan, Ann Arbor, MI. (Control ID \#1338627)

BACKGROUND: Hospitals no longer receive more pay to treat certain hospital-acquired conditions (HACs), regardless of a patient's risk to develop HACs. Reduced pay for all admissions will occur in 2015 for hospitals with the highest quartile of risk-adjusted HAC rates. Our objective was to develop models to predict a hospitalized patient's risk of developing a HAC (using the example of a urinary tract infection, UTI) in order to risk-adjust a hospital's performance by its patients' inherent risks of the HAC.
METHODS: Using claims data from the Healthcare Cost and Utilization Project State Inpatient Dataset for 1,792,288 adults discharged from 297 acute care California hospitals in 2009 without a principal diagnosis of UTI, we developed models to predict a patient's risk of developing a UTI as a secondary diagnosis using patient characteristics at admission supported by clinical literature as risk factors, such as age, gender and comorbidities by CMS's hierarchical condition categories. We assessed how hospitals' observed UTI rates would be modified by risk-adjustment.

RESULTS: Unadjusted hospital rates (Graph A) of UTIs ranged from 0.8$30.5 \%$ (mean $12 \%$ ). Using a logistic regression model with patient characteristics (c-statistic of 0.77 ), age, gender and age-gender interactions contributed the most to the model's predictiveness (generating a c-statistic of 0.66 versus a model with no predictors). Compared to patients admitted for medical diagnoses, patients discharged with surgical diagnosis-related groups had fewer UTIs coded as secondary diagnoses (OR 0.30 for scheduled surgery admits, 0.92 for unscheduled surgery admits). Other conditions at admission associated with significantly higher UTI rates included decubitus ulcers (OR 2.5), ventilator dependence (2.5), shock (2.8), or a urologic or gynecologic diagnosis (1.90). Significant risk factors for UTI with OR 1.11.7 included diabetes, renal failure, malnutrition, hip fracture, chronic liver disease, dementia, and other serious neurologic disease. Observed-toexpected ratios for hospitals (Graph B) ranged from 0.35-1.98. Of 74 hospitals in highest quartile of unadjusted UTI rates (Graph A), 23 were reassigned to better quartiles using observed-to-expected ratios (Graph B). CONCLUSIONS: With risk-adjustment by patient characteristics at admission in claims data, 1 in 3 hospitals identified as poor performers by UTI rates would be reassigned as better performers. Age and gender were the most critical patient characteristics for adjustment. It is unclear if lower rates of UTIs coded for surgical patients indicate fewer UTIs occurred versus a coding bias of not listing UTIs when other diagnoses justify equal payment.

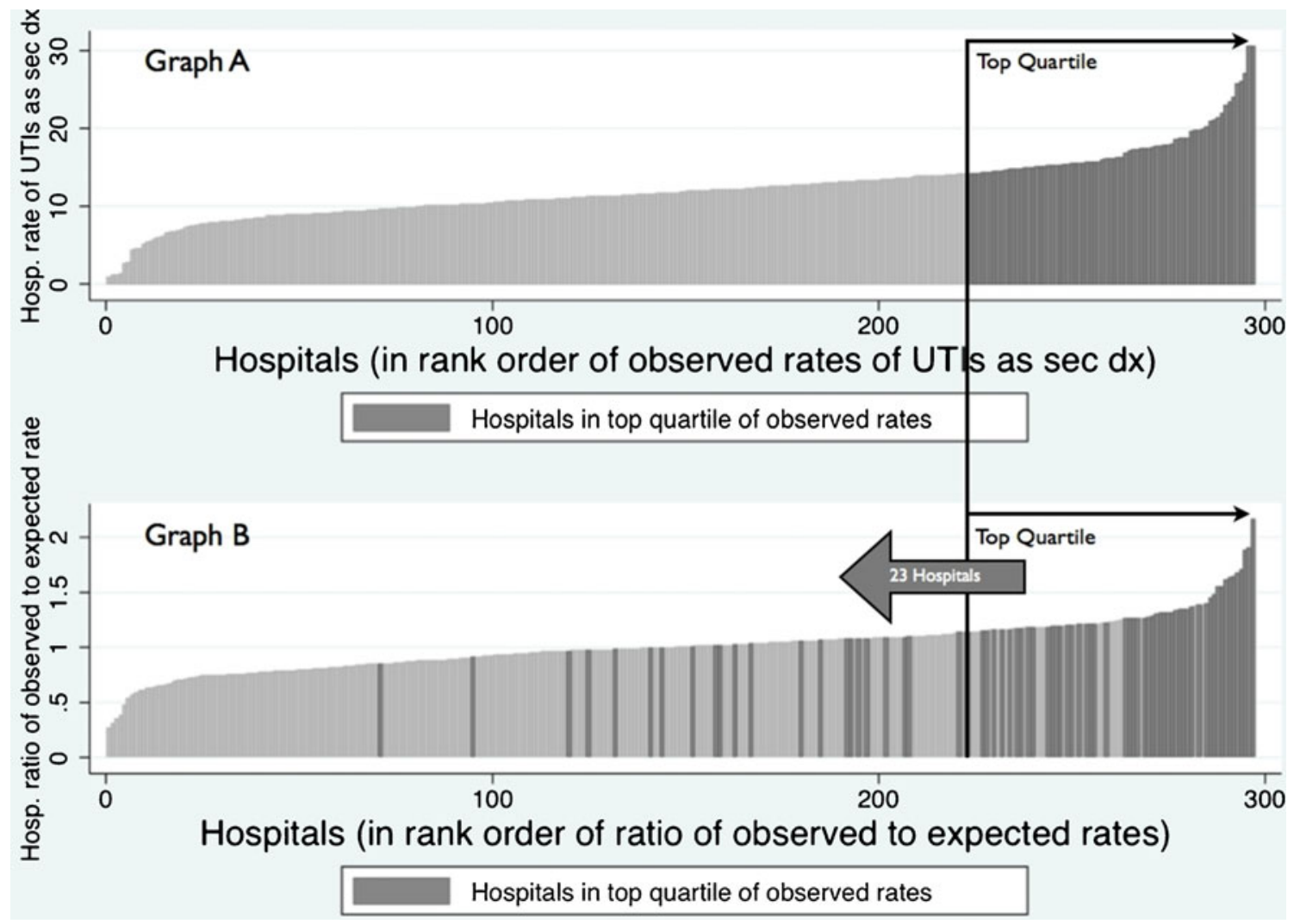


ALCOHOL USE AFTER TOBACCO CESSATION: IMMEDIATE CONSEQUENCES Kristin M. Berg ${ }^{1}$; Megan Piper $^{2}$; Michael Fiore $^{2}$; Timothy Baker ${ }^{2}$; Douglas E. Jorenby ${ }^{2} .{ }^{1}$ University of Wisconsin Hospitals and Clinics, Madison, WI; ${ }^{2}$ University of Wisconsin School of Medicine and Public Health, Madison, WI. (Control ID \#1333712)

BACKGROUND: While tobacco use has declined in American society, $20.6 \%$ percent of adults continue to use tobacco. Upwards of $51 \%$ percent of American adults consume some alcohol, and studies have shown that use of one substance primes the use of the other. There have been several studies on tobacco cessation and its long-term relationship with alcohol use, but little is known about the immediate consequences of tobacco cessation.

METHODS: The Wisconsin Smokers' Health Study is a large clinical trial of 1504 participants, designed to compare smoking cessation pharmacotherapies with each other and placebo in a double-blind, randomized controlled trial. Including participants drinking at least 0.5 drinks daily on average, mean alcohol use pre- and post- quit were compared using student's $\mathrm{T}$ test for statistical significance. Average daily alcohol use was also compared between groups to determine trends over the 28 day pericessation period ( 2 weeks pre- and 2 weeks post- quit).

RESULTS: Alcohol use in general increases leading up to the planned tobacco quit date. Second, as participants attempt to quit tobacco, they decrease their mean alcohol use by approximately 0.5 drinks daily post-quit $(\mathrm{p}<0.05)$, with two notable exceptions. Males who do not use cessation pharmacotherapies to assist in their efforts not only demonstrate a lack of change in mean alcohol consumption, but appear to increase alcohol use within 48-72 hours post-tobacco cessation when evaluating daily alcohol trends. Moreover, their alcohol use declines if they relapse to smoking. Females using bupropion as their cessation pharmacotherapy appear to likewise demonstrate no change in their mean alcohol use post-quit, but they do not have the same post-cessation increase in alcohol use as do the males in the placebo group. Third, those participants maintaining abstinence seem to delay and reduce the amount of alcohol consumed in the immediate postquit period when evaluating daily trends.

CONCLUSIONS: In general, alcohol use significantly decreases in the postcessation period, possibly indicative of an overall attempt at improved health. However, fluctuations in alcohol use trends in various treatment groups could indicate underlying factors influencing success rates in this immediate pericessation period. These intriguing trends give healthcare professionals an opportunity for counseling and intervention to improve outcomes in their patients seeking tobacco cessation.

\section{AN ANALYSIS TO DETERMINE DRIVERS OF SATISFACTION AND DISSATISFACTION IN THE PATIENT EXPERIENCE OF INPATIENT CONTINUITY OF CARE Ryan Thompson; Elizabeth Immen; Andrea Litvak; Cathleen Ward; Catherine Desroches; David Torchiana; Timothy Ferris. Massachusetts General Hospital, Boston, MA. (Control ID \#1340377)}

BACKGROUND: The highly specialized nature of tertiary hospital care increasingly requires multiple specialized physicians caring for a single patient. At the same time, ensuring that patient information, management plan, and trusting relationships are shared among the care team remains an essential characteristic of patient-centered care. Given these competing realities, we undertook an effort to improve continuity of care for hospitalized patients, starting with the development of a system to measure patients' perception of their care continuity. We describe here our measurement approach, descriptive results, and the aspects of continuity that correlate with both satisfaction and dissatisfaction.

METHODS: We designed our survey to assess a patient's perception of continuity at three time points during an inpatient episode of care - ED, ward, and post-discharge. We derived sixteen continuity measurement concepts from the three continuity domains (information, management plan, and relationships) defined in the literature. We then developed and tested 34 Likert-type survey items for 3 distinct surveys (ED, ward, post-discharge); each measurement concept is measured by one to three survey items. Using a random number generator for enrollment, we surveyed consenting patients in-person in the ED and ward, and by telephone post-discharge. We excluded patients who were clinically or psychologically unstable, or who lacked suitable proxy. The postdischarge survey was not administered to patients discharged to a post-acute facility. Using survey responses re-coded as binary variables based on whether or not the subject selected the "top box" option, we used regression models to control for age, gender, and insurance type. We calculated measurement concept scores by averaging the top box percentages for survey items associated with each concept. Satisfaction and dissatisfaction with care continuity were assessed by combining multiple survey items which assessed general satisfaction with care.

RESULTS: We enrolled 206 patients through the ED and 834 directly admitted patients between November 2010 and May 2011. Overall, 949 patients completed the inpatient survey ( $87 \%$ of eligible), and 644 patients completed the discharge survey ( $69 \%$ of eligible). Overall unadjusted top box scores with department low-high ranges were $62 \%$ for continuity of information $(42 \%, 86 \%), 75 \%$ for management $(68 \%, 90 \%)$, and $71 \%$ for relationships $(58 \%, 78 \%)$. We found that shared decision-making between patient and providers $(\mathrm{p}=0.01)$, and patient understanding of provider roles $(p=0.01)$ were associated with greater satisfaction in our patients' experience of continuity. Drivers of dissatisfaction were lack of awareness of provider coverage and transitions $(\mathrm{p}=0.01)$ and not seeing their longitudinal MGH physician(s) while in the hospital $(\mathrm{p}=0.01)$. Two concepts were associated with both satisfaction and dissatisfaction - effective communication about test results and progress in the hospital $(\mathrm{p}=0.01 ; \mathrm{p}=0.01)$ and provider responsiveness to patient needs $(\mathrm{p}=0.01 ; \mathrm{p}=0.05$ ).

CONCLUSIONS: We demonstrated a reliable approach to measuring patient perception of the continuity of their inpatient care. We found significant departmental variability in all three domains of continuity. Our analysis suggests efforts to improve continuity should focus on specific areas most associated with general satisfaction with care.

AN ASSESSMENT OF ELIGIBILITY AND ENROLLMENT INTO CANCER CLINICAL TRIALS AMONG A DISADVANTAGED URBAN POPULATION Tracy Battaglia ${ }^{2}$; Naomi Y. Ko ${ }^{1}$; Sarah E. Caron $^{2}$; Julie C. Fu ${ }^{3}$; Kathleen T. Finn ${ }^{1}$; Sandy Allten ${ }^{1}$; Nectaria Vassilakis ${ }^{1}$; Lisa Stober ${ }^{1}$; Lisa A. Kachnic ${ }^{4}$. ${ }^{1}$ Boston University School of Medicine, Boston, MA; ${ }^{2}$ Boston University School of Medicine, Boston, MA; ${ }^{3}$ Boston University School of Medicine, Boston, MA; ${ }^{4}$ Boston University School of Medicine, Boston, MA. (Control ID \#1337453)

BACKGROUND: Enrollment rates onto cancer clinical trials among adult population remains approximately $2-11 \%$ with the majority being whites from high socioeconomic status and educational backgrounds. In order to address the unequal burden of cancer among underserved populations, it is imperative to accrue underrepresented populations onto cancer clinical trials, however, the barriers to minority enrollment must first be better understood. We aim to examine the eligibility and enrollment criteria for disadvantaged populations in cancer clinical trials as the initial step towards increasing minority accrual. METHODS: Boston Medical Center, a Commission on Cancer Accredited and recently designated National Cancer Institute Minority Based Community Clinical Oncology Program, is the largest Safety Net medical institution in New England serving the city's most vulnerable cancer patients. Using the hospital cancer registry to identify incident cases in calendar year 2010 (n= 1,228 ), we conducted a retrospective review of the electronic medical record (EMR) to identify rates of screening for available cancer clinical trials. Hospital protocol dictates that each patient identified as eligible for an existing cancer clinical trial has documentation in their EMR of screening from a clinical trials nurse. This screening note documents eligibility for enrollment and reasons for ineligibility/non-enrollment. Socio-demographic information was obtained from the cancer registry. Descriptive statistics were used to report our main outcomes: screening rate, eligibility and accrual rate (of those screened), and reasons for not enrolling.

RESULTS: Among 1,228 newly diagnosed cancer patients in calendar year 2010 , the mean age was 59 years; $50 \%$ female; $47 \%$ White, $30 \%$ Black, $11 \%$ Hispanic, $8 \%$ Other and 4\% Asian. 99 clinical trials were available for accrual during time period. Overall, we found documentation of screenings for specific cancer trials performed for 543 individual patients, resulting in a $44 \%$ screening rate. Each patient may have been screened for multiple trials 
(mean 1.6 screens per patient, range 1 to 7 screens) for a total of 854 screenings across all patients. No trial was available for 155 screenings, and 122 screens were excluded due to pending status; among the remaining 577 screenings, we found only $11 \%(65 / 577)$ were deemed eligible for the specific clinical trial. The most commonly noted reasons for not meeting trial-specific eligibility criteria were: disease stage and/or type (33\%), prior treatment $(27 \%)$ and patient co-morbidities $(21 \%)$. Administrative reasons for ineligibility were mostly $(78 \%)$ due to sponsor level issues, such as "trial on hold." Of the 65 patients who were deemed eligible for clinical trials, $46 \%$ (n $=31$ ) were enrolled, of which $53 \%$ were White, $43 \%$ Black and $4 \%$ Hispanic. Only $5 \%$ declined enrollment when deemed eligible. The most commonly noted reason for declining enrollment was aversion to extra treatment.

CONCLUSIONS: Less than half of all newly diagnosed patients had evidence of screening for clinical trial enrollment. The main reason for low screening is lack of available cancer clinical trials. When specific trials are available, only $11 \%$ were found to be eligible; the most common reason for ineligibility was disease characteristics and patient co-morbidities. When eligible, a racially representative sample was enrolled. Our findings suggest the need to identify and open clinical trials that are more relevant to our diverse and co-morbid patient population.

AN AUTOMATED TELEPHONE NUTRITION SUPPORT SYSTEM FOR SPANISH-SPEAKING PATIENTS WITH DIABETES Raman R. Khanna $^{1}$; Pamela J. Stoddard ${ }^{2}$; Mariana Villagran-Flores ${ }^{3}$; Paul Bayard ${ }^{3}$; Joan Thompson ${ }^{3}$; Ralph Gonzales ${ }^{1}$. ${ }^{1}$ University of California, San Francisco, San Francisco, CA; ${ }^{2}$ Santa Clara Valley Medical Center, San Jose, CA; ${ }^{3} \mathrm{La}$ Clinica de la Raza, Oakland, CA. (Control ID \#1336012)

BACKGROUND: Automated telephone-based nutrition support (ATNS) provided through interactive voice response survey software is capable of providing tailored counseling at varying times of day and to limited English proficiency patients, and at a very low cost; but its efficacy is uncertain. Based on a very promising pilot study of ATNS conducted among a medically underserved population living in Cuernavaca, Mexico, we conducted a randomized trial of this system in a predominantly Spanish-speaking safety net population with type 2 diabetes in the United States.

METHODS: We worked with physicians, dietitians and medical care coordinators at a large community health center in Oakland, California to design an ATNS system which, using Telesage ${ }^{\mathrm{TM}}$ software, called patients approximately twice a week. The ATNS system asked patients to respond (using their telephone key pads) with the number of each of 15 low and high glycemic index foods they had eaten in the previous 24 hours. It then added the number of high glycemic index portions and provided either reinforcement of the current diet or encouragement to choose low glycemic index substitutes depending on the patient's total. After designing the intervention, we recruited patients with uncontrolled type 2 diabetes (hemoglobin A1c $\geq 8.0$ ) in a randomized, blinded fashion to receive diet and exercise counseling followed by ATNS phone calls either immediately (intervention) or after a period of 3 months (control). We recorded hemoglobin $\mathrm{A} 1 \mathrm{c}$ at baseline and at 3 months; the betweengroup difference in change over 3 months was our primary endpoint. We also recorded other anthropometric and laboratory values at baseline and at 3 months.

RESULTS: We recruited 71 patients between August 2010 and January 2011 and were able to follow up $44(62 \%)$ in that period, with 20 in the intervention group and 24 in the control group. There were no differences between the intervention and control groups at baseline or between patients who did and did not follow up. All patients at baseline had poorly controlled disease, with a median A1c of 8.9 despite taking a median of 2 diabetes medications. Patients in the intervention group received a median of 24 calls (approximately 1.8/week) during the 3 months of follow up; however, they only completed a median of 10 calls (less than $1 /$ week). The median change in A1c from baseline to 3 months was -0.2 ; in the intervention group it was -0.3 and in the control group it was $-0.1 ; \mathrm{p}=0.83$ by the Wilcoxon Rank-Sum test. There were no significant differences between the intervention and the control groups in change in blood pressure, body mass index, waist circumference, or cholesterol.
CONCLUSIONS: The ATNS system was easy to set up and cheap to maintain, but despite promising results in the pilot program in Mexico it did not lead to improved Hemoglobin A1c or other metabolic parameters in our USbased study population. Patients completed less than half of the calls that were placed to them by the ATNS system. More work is required to define whether ATNS is effective or appropriate in other populations and at other levels of automated counseling intensity.

AN EDUCATIONAL INTERVENTION TO IMPROVE DISCHARGE SUMMARIES IN A COMMUNITY HOSPITAL Nicole Gill-Duncan ${ }^{1}$; Philippe Leveille ${ }^{1}$; Dean Luu ${ }^{1}$; Julie M. Pearson ${ }^{2}$; Julie Kanevsky ${ }^{1}$; Daniel Giaccio ${ }^{1}$. ${ }^{1}$ Lutheran Medical Center, Brooklyn, NY; ${ }^{2}$ Lutheran Medical Center, Brooklyn, NY. (Control ID \#1330276)

BACKGROUND: The quality of discharge summaries significantly affects the ability of primary care providers to adequately care for patients after hospital discharge. As the pressure on hospitals to decrease length of stay rises, so too does the percentage of patients discharged with significant test results still pending. Relaying information about the hospital course and pending test results to the primary care provider is crucial to ensure patient safety.

METHODS: We developed an assessment tool for rating the quality of discharge summaries. It incorporated ratings of the narrative of the history of present illness and hospital course, as well as scoring the presence of important elements that affect post-hospital care, such as primary and secondary diagnoses, medication reconciliation, and appropriate follow-up. Our educational intervention consisted of two parts. The first was a morning conference teaching residents how to write an effective discharge summary. The second was a noon conference simulation exercise in which a long, detailed description of a hospital course was provided, and residents were asked to write discharge summaries in real time using the guidelines provided in the morning conference. These discharge summaries were then anonymously submitted, and a selection of the best and worst examples were reviewed with the group. Discharge summaries dictated before and after the educational intervention were then compared using the assessment tool. Two members of the research team rated each discharge summary. The interrater reliability was moderate (Cronbach alpha $=0.50$ ). The average absolute difference between the reviewers' scores was $21 \%$. Discharge summaries with an absolute difference greater than $30 \%$ (fourth quartile) were reviewed by a third reviewer and then all three scores were averaged. Paired t-tests were used to assess the differences in final weighted averages between pre-/ post-test scores.

RESULTS: A total of 104 discharge summaries were assessed from 26 residents. The weighted average score for discharge summaries significantly increased from $52 \%$ to $67 \%$ after the educational intervention ( $p<0.01$ ). $70 \%$ of the post-intervention discharge summaries were rated as being effective overall (agree or strongly agree), compared to $47 \%$ of the pre-intervention discharge summaries. The elements of the discharge summaries that were rated the poorest pertained to medication reconciliation; more than $90 \%$ of the summaries from both the pre- and post-intervention groups were missing information on medications that were changed or discontinued.

CONCLUSIONS: Our educational intervention improved the quality of hospital discharge summaries written by Internal Medicine residents. Medication reconciliation was rated the poorest. Computer-generated discharge summary forms with specific fields for medication reconciliation may help to improve discharge summaries.

AN EVALUATION OF CONTINUITY CLINIC RE-DESIGN IN AN INTERNAL MEDICINE RESIDENCY PROGRAM Mark L. Wieland ${ }^{1}$; Andrew J. Halvorsen ${ }^{2}$; Rajeev Chaudhry ${ }^{1}$; Furman S. McDonald ${ }^{3}$; Kris G. Thomas ${ }^{1} .{ }^{1}$ Mayo Clinic, Rochester, MN; ${ }^{2}$ Mayo Clinic, Rochester, MN; ${ }^{3}$ Mayo Clinic, Rochester, MN. (Control ID \#1336566)

BACKGROUND: There have been recent calls for improved internal medicine outpatient training. Charged by participation in the ACGME Education Innovation Project, the Mayo Clinic Internal Medicine Residency Program implemented a continuity clinic re-design in the 2010-11 academic 
year. Changes included 1) separation of the inpatient and outpatient experiences, 2) development of outpatient care teams, and 3) additional rotations with concentrated continuity clinic exposure. In order to link these structural processes to outcomes, we assessed the impact of clinic re-design on clinical and educational outcomes.

METHODS: 96 residents in our Primary Care Internal Medicine site participated in this study. The pre-intervention study interval was July '09-June ' 10 ; the post-intervention interval was July "10-June '11. Continuity of care was assessed from the perspective of the resident (proportion of visits in which residents see their own patients). Quality of care was assessed through an existing primary care database for diabetes, hypertension and preventive service measures. Patient satisfaction was measured with the ABIM Patient Assessment Module. Residents assessed perceived safety and quality of the care environment through a 13-item survey administered four times per year. Educational outcomes were assessed through an existing electronic evaluation database across multiple domains. We used this database to measure resident satisfaction with clinic, resident performance in clinic, and faculty rating of the clinic experience. Attendance at teaching conferences was tracked via electronic card swipe. Outcomes were assessed for each variable using generalized estimating equations.

RESULTS: Clinical outcomes before and after re-design are depicted in Table 1 . Perceived safety and quality of the clinic was higher in the postintervention year ( $\mathrm{p}$-value range 0.026 to $<0.0001$ ). Mean attendance at teaching conferences was higher in the post-intervention year (56.7 vs. 63.1, $\mathrm{p}=<.001$ ). There was no significant difference between study intervals for the remaining educational outcomes.

CONCLUSIONS: Continuity clinic re-design through separation of the inpatient-outpatient experiences and additional structural changes was associated with increased resident panel size but a reduction in continuity of care with little change in other clinical parameters. Perceived safety and quality in the outpatient setting improved. Attendance at teaching conferences improved while the remaining education outcomes were unchanged. These data provide important information for iterative residency re-design to optimize clinical care, patient continuity, and educational experiences.

Table 1. Clinical outcomes before and after continuity clinic re-design

\begin{tabular}{|c|c|c|c|}
\hline Domain & $\begin{array}{l}\text { Pre- } \\
\text { Intervention }\end{array}$ & $\begin{array}{l}\text { Post- } \\
\text { Intervention }\end{array}$ & $\begin{array}{l}\text { p- } \\
\text { value }\end{array}$ \\
\hline Panel Size $($ mean \pm SE) & $120.0 \pm 1.0$ & $137.6 \pm 0.9$ & $<.001$ \\
\hline $\begin{array}{l}\text { Patient Visits (mean } \pm \\
\text { SE) }\end{array}$ & $216.0 \pm 3.8$ & $228.5 \pm 5.4$ & 0.06 \\
\hline \multicolumn{4}{|l|}{$\begin{array}{l}\text { Continuity of Care } \\
\text { (\% of all resident visits) }\end{array}$} \\
\hline $\begin{array}{l}\text { Individual (seeing } \\
\text { own panel) }\end{array}$ & $62.9 \% \pm 1.3 \%$ & $48.1 \% \pm 1.1 \%$ & $<.001$ \\
\hline $\begin{array}{l}\text { Team (seeing own or } \\
\text { team member's panel) }\end{array}$ & $67.0 \% \pm 1.2 \%$ & $63.5 \% \pm 0.8 \%$ & 0.001 \\
\hline $\begin{array}{l}\text { Diabetes Care } \\
(\% \text { with } \mathrm{HgbA} 1 \mathrm{C}<8+ \\
\mathrm{LDL}<100+\mathrm{BP}<140 / 90, \\
1567 \text { eligible })\end{array}$ & $40.2 \% \pm 1.8 \%$ & $42.2 \% \pm 2.0 \%$ & 0.40 \\
\hline $\begin{array}{l}\text { Microalbumin within one } \\
\text { year (1567 eligible) }\end{array}$ & $64.6 \% \pm 1.8 \%$ & $70.2 \% \pm 1.6 \%$ & 0.01 \\
\hline $\begin{array}{l}\text { Hypertension Care } \\
(\% \text { with } \mathrm{BP}<140 / 90, \\
4030 \text { eligible })\end{array}$ & $71.1 \pm 1.1 \%$ & $72.1 \pm 0.9 \%$ & 0.40 \\
\hline $\begin{array}{l}\text { Preventive Services } \\
\text { (\% up to date) }\end{array}$ & & & \\
\hline $\begin{array}{l}\text { Mammography } \\
\text { (4648 eligible) }\end{array}$ & $55.9 \% \pm 1.1 \%$ & $49.2 \% \pm 1.3 \%$ & $<.001$ \\
\hline $\begin{array}{l}\text { Cervical cancer screening } \\
\text { (9535 eligible) }\end{array}$ & $68.3 \% \pm 0.8 \%$ & $67.2 \% \pm 0.7 \%$ & 0.12 \\
\hline $\begin{array}{l}\text { Bone densitometry } \\
\text { (1063 eligible) }\end{array}$ & $60.1 \% \pm 2.2 \%$ & $60.0 \% \pm 2.1 \%$ & 0.93 \\
\hline $\begin{array}{l}\text { Lipid screening } \\
\text { (11253 eligible) }\end{array}$ & $76.5 \% \pm 0.7 \%$ & $76.8 \% \pm 0.6 \%$ & 0.67 \\
\hline $\begin{array}{l}\text { PatientSatisfaction } \\
\text { (mean } \pm \text { SE, 5-point scale) }\end{array}$ & $4.69 \pm .02$ & $4.70 \pm .02$ & 0.62 \\
\hline
\end{tabular}

AN EXPLORATORY ANALYSIS OF SUCCESSFUL PEER COACHES: OUR ASSUMPTIONS ABOUT IMPORTANT CHARACTERISTICS MAY NOT BE CORRECT Elizabeth Rogers; Tom Bodenheimer; Danielle Hessler; David Thom. University of $\mathrm{Cal}$ ifornia San Francisco, San Francisco, CA. (Control ID \#1339936)

BACKGROUND: The training of lay personnel to provide self-management support is proving effective for chronic diseases such as diabetes. However, little has been published about what, if any, characteristics of lay personnel are associated with effective coaching. We sought to understand what characteristics are associated with successful diabetes peer coaches in an urban low-income U.S. population.

METHODS: We carried out a prospective cohort study of diabetic patients from six urban health centers serving a low-income population who participated in a randomized controlled trial to assess the impact of peer health coaching on patient change in glycosylated hemoglobin (HbA1c). The cohort included 25 well-controlled diabetic patients who were trained as peer coaches and 123 poorly controlled diabetic patients who were assigned to coaching. All spoke English or Spanish. We defined level of peer coaching success to be the degree of improvement in patient $\mathrm{HbAlc}$ from baseline to 6 months. We used linear regression, to account for clustering by coach, to assess for the association between patient change in $\mathrm{HbAlc}$ and baseline coach characteristics of age, gender, number of years with diabetes, body mass index (BMI), HbAlc level, and scores on two previously validated questionnaires composed of Likert-scale items Perceived Diabetes Self-Management Scale (PDSMS) and Diabetes Distress Scale (DDS). We also used linear regression to evaluate the association between patient-coach concordance of age, gender, ethnicity, and educational level and patient change in $\mathrm{HbAlc}$ over 6 months. All dyads were language-concordant.

RESULTS: Average patient change in $\mathrm{HbA} 1 \mathrm{c}$ by coach over the 6-month intervention ranged from an increase in $\mathrm{HbAlc}$ of $0.7 \%$ to a decrease of $2.7 \%$. Higher coach BMI was associated with a greater reduction in patient HbAlc $(\mathrm{p}=0.01)$. For example, patients of coaches in the highest BMI tertile had a mean reduction in $\mathrm{HbA} 1 \mathrm{c}$ of $1.6 \%$ while those with coaches in the lowest BMI tertile reduced their $\mathrm{HbA} 1 \mathrm{c}$ by only $0.6 \%$. A lower coach PDSMS score, indicating lower perceived self-management, and a higher DDS score, indicating higher levels of diabetes-associated distress, were both associated with a greater decrease in patient $\mathrm{HbA1c}(\mathrm{p}=0.04$ for both associations). Coach age, gender, number of years with diabetes, and baseline HbAlc level did not predict more successful coaching. Patient-coach concordance by gender ( $60 \%$ concordant), ethnicity ( $63 \%$ concordant), and educational level (60\% concordant) were not associated with change in patient $\mathrm{HbA1C}$, but age concordance ( $59 \%$ concordant), defined as being no more than 10 years apart in age, was associated with a trend toward greater decrease in patient $\mathrm{HbAlc}(\mathrm{p}=0.09)$.

CONCLUSIONS: Contrary to our hypothesis, coaches with lower BMI, higher perceived self-management of diabetes, and lower diabetes distress were not more successful in reducing the $\mathrm{HbAlc}$ levels of the patients they coached, and in fact they may be less successful. One could hypothesize that those coaches struggling with their own disease management and lifestyle changes may be more open to training and make more empathetic patient connections to prompt improvement in a patient's self-management. Coach age, gender, years with diabetes, and $\mathrm{HbAlc}$ were not important for successful coaching, but patient-coach age concordance may be. These findings can help guide diabetes peer coach selection in future interventions.

AN UNDERGRADUATE MEDICAL STUDENT GERIATRICS HOME VISIT, REVISITED DURING INTERNSHIP Gerald D. Denton; Paul Hemmer; Janice Hanson. Uniformed Services University,

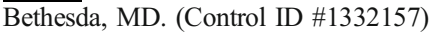

BACKGROUND: Home visits, especially with elderly patients, have been resurgent in medical education, and, when paired with self-reflection and narrative writing, have at least a short-term influence on attitudes towards caring for the elderly. Anecdotal reports indicate durability of at least the 
memory of the home visit during medical school experience, but little research supports this.

METHODS: This study had two parts. First, all medical students at the Uniformed Services University (USU) completed a critique on their mandatory Geriatrics Home Visit (GHV) during academic year 2008-9. Second, 18-30 months after their GHV, during the second half of their PGY-1 intern year (2010-11), an electronic survey was provided to these physicians and their colleagues who were graduates of other medical schools. The survey included questions about demographics, home visits during medical school, current attitudes towards care of the elderly, and knowledge of geriatrics. Means and proportion of responses above and below the neutral point for likertscaled questions were calculated. Qualitative responses were analyzed using constant comparative method. The IRB at USU reviewed this project and granted exempt status.

RESULTS: For the post-GHV critique analysis, 137 of 168 critiques $(81.5 \%)$ were available for review. The GHV was acceptable to students and changed the way they thought about caring for elderly patients $(59 \%$ agreed or strongly agreed; mean Likert scale response 3.4/5). Most students felt the GHV was a good way to learn geriatric medicine (56\% agreed or strongly agreed; mean Likert scale response 3.4/5). For the PGY-1 intern survey, 14 of the 17 sites where USU graduates were training agreed to participate. $60 / 133$ $(45 \%)$ of the USU graduates at those sites responded. 124/501(24.7\%) of non-USU graduates responded. Overall, $61 \%$ performed a home visit during medical school. $88 \%$ of PGY-1 interns who performed a home visit as a medical student remembered their patient, and those PGY-1 interns had better attitudinal scores towards care of the elderly $(83.1 \%$ vs $58.5 \%$; $=0.003$, student's t-test). Knowledge scores were similar between PGY-1 interns who did and did not perform a GHV. However, PGY-1 interns who performed a home visit as a medical student had better attitudinal scores towards care of the elderly than PGY-1 interns who did not $(84.3 \%$ vs $74.9 \%$; $p=0.01$, students t-test). Qualitative analysis added depth and understanding to the results of both analyses.

CONCLUSIONS: A geriatrics home visit program is an acceptable curricular addition to medical students and is memorable for 18-30 months after the experience. While a geriatric home visit program may not provide a lasting impact on knowledge of geriatrics, PGY-1 intern attitudes towards care of the elderly may be improved. Given the aging of the population and the need for positive attitudes towards care of the elderly, medical schools should consider geriatrics home visits as an addition to their curriculum.

\section{AN ASSESSMENT OF INTERNAL MEDICINE RESIDENT AND MEDICAL STUDENT KNOWLEDGE OF ADDICTION MEDICINE Angel Brown; Victor Kolade; Neha Patel; Lisa Staton. University of Tennessee College of Medicine Chattanooga, Chattanooga, TN. (Control ID \#1312234)}

BACKGROUND: Approximately 30 million Americans suffer with addiction, and nearly 7 million people misuse prescription medications. Further, addiction complicates the management of co-morbid conditions. There are too few addiction specialists in the United States and generalists are not well versed in addiction disorders; as a result, many patients are not treated adequately. In many medical schools and residency programs, lectures regarding alcohol and drug addiction are limited. The objective of our study was to assess and compare baseline knowledge among medical students and residents in a community-based internal medicine residency program and evaluate the impact of an addiction medicine curriculum on knowledge of substance abuse disorders. We also compared knowledge of medical students and residents from US medical schools to knowledge of international medical school graduates (IMGs).

METHODS: A pretest was administered via the internet and in person to determine baseline knowledge of the subject. Study subjects included internal medicine and transitional year residents, as well as medical students who were enrolled in an internal medicine program at the time of the lecture series. Participants were given four structured sessions, one each week, on the topics of addiction, opioids, alcohol, benzodiazepines and illicit stimulants. An expert panel discussion was also convened. After the completion of the symposium the participants were instructed to complete a posttest online to assess if learning had occurred. ANOVA was used to compare means. Paired t-test was used to compare before and after scores.

RESULTS: Thirty-six (36) of $44(81.8 \%)$ medical students and medicine residents completed the pretest: internal medicine residents fared the best with an average of $65.4 \%$, while third year internal medicine residents scored an average of $59.2 \%$. Fourth year medical students scored $64 \%$. Second year medicine residents averaged $62.3 \%$ and third year medical students averaged $62.5 \%$. For all medical students, the average score was $63.3 \%$; for residents, the average score was $62.5 \%$. United States graduates averaged $65.0 \%$ correct while the average for international graduates was $58.6 \%$. The differences between groups were not statistically significant. Of the 36 participants, $20(55.6 \%)$ completed both surveys. Posttest scores, average $68.75 \%$, were higher than pretest scores, which averaged $61.75 \% ; \mathrm{p}=0.003$. Among IMGs, the average score rose from $57.5 \%$ (pretest: range $35-75 \%$ ) to $72.5 \%$ after the seminars (range 50-85\%); all 6 participants had higher posttest scores than pretest scores. Among the US trainees, the average score rose was $63.6 \%$ on the pretest (range $55-70 \%$ ) and $67.1 \%$ on the posttest (range $45-85 \%)$; 8 of 14 (57.1\%) participants had higher posttest than pretest scores.

CONCLUSIONS: There is room for improvement in the knowledge base of medical students and residents concerning addiction medicine. Knowledge gains can be demonstrated after structured sessions. Similar studies in larger resident populations may elucidate specific components of the curriculum that are most valuable.

AN ASSESSMENT OF NON-AMERICAN INTERNS' CLINICAL AND LIFE SKILLS: PRELIMINARY DATA Josh Baru; Suja Mathew; Brian P. Lucas; Benjamin Mba. Cook County Hospital, Chicago, IL. (Control ID \#1339984)

BACKGROUND: Each year, approximately $25 \%$ of medical residency positions are filled by international medical graduates (IMGs). When they begin their training, $80 \%$ of these graduates are non-US citizens. Several studies note that non-American IMGs experience unique problems relative to their co-residents such as loneliness, social isolation, concerns related to family members left behind in home countries, a decrease in social status, lack of financial resources and worries about visas/immigration issues. Previous studies have described orientation programs for IMGs, but these have generally focused on acculturation into the healthcare system. In this preliminary study, we explored non-American IMGs' comfort and skills in American life as well as the healthcare system.

METHODS: The study group was first-year residents ("interns") in the internal medicine residency program at Cook County Hospital $(\mathrm{CCH})$ in Chicago, IL. We also included participants from a pilot study performed at Rush University Medical Center (RUMC). We surveyed the participants within the first 2 months of the 2011 academic year. Surveys were distributed electronically and manually at mandatory teaching conferences. The survey assessed 3 domains: life skills, clinical skills, and communication skills. The survey instrument was created by the research group and was based on consensus opinion and a literature review. All questions were rated on a 7-point Likert scale. We generated summary scores for each intern within each domain and transformed these sums into domainspecific $\mathrm{z}$-scores; $1 \mathrm{z}$-score unit is equal to 1 standard deviation (SD). We separated participants into American citizens and non-American citizens. We compared the scores for the groups using Student's t-test.

RESULTS: 32 (68\%) CCH residents completed the survey. 10 residents from the RUMC program were included. 13 participants were American. We found no significant differences in the scores for the clinical or communication skills domains. There were significant differences in life skills as US citizens scored 1.2 SD higher in life skills than non-US citizens $(p=0.0001)$. Specific examples are illustrative. Whereas all 13 American interns strongly agreed with the statement "I am comfortable managing a checking/banking account", only $9(31 \%)$ of the non-Americans strongly agreed with it. Similarly, only 8 (28\%) non-Americans strongly agreed with the statement "I know how to access emergency assistance from local police or fire personnel" while $12(92 \%)$ Americans strongly agreed with it.

CONCLUSIONS: Our early data of internal medicine residents in 2 urban programs suggests that there are significant differences between non- 
American interns' life skills upon entering residency in the United States. This data suggests specific goals for orientation programs for IMGs, particularly those that are citizens of other countries. In future work we plan to use factor analysis to refine our instrument. We also plan a citywide sample of internal medicine interns to obtain adequate statistical power to stratify our analysis by potential confounders such as age, sex, and country of origin.

AN ASSESSMENT OF UNDOCUMENTED IMMIGRANTS ATTITUDES TOWARDS ORGAN DONATION Josh Baru ${ }^{1,2}$; Carmen Martinez ${ }^{1} .{ }^{1}$ Cook County Hospital, Chicago, $\overline{\mathrm{IL} ;{ }^{2} \mathrm{Rush}}$ University Medical Center, Chicago, IL. (Control ID \#1312760)

BACKGROUND: There are approximately 12 million undocumented immigrants living in the United States; 7 million of these are uninsured. Undocumented immigrants can donate organs into the organ pool and, in compliance with the JCAHO and Medicare "required request" policies, must be asked about their wishes regarding organ donation if they are imminently dying. While they are asked to donate organs, to receive organs they would need to independently cover all expenses. This is an exceedingly rare occurrence. This conflict potentially violates ethical principles of justice and informed consent. The purpose of this study was to determine if undocumented immigrants are aware of this discrepancy and whether they consider it relevant to their decision to donate.

METHODS: The setting is a 500-bed, university-affiliated, safety-net hospital in Chicago, IL. We used a convenience sample of patients older than 18 years old who were admitted to the general medicine wards. Participants were interviewed at the bedside on their first hospital day. Participants were eligible for the study if they spoke English or Spanish, self-identified as undocumented immigrants on the initial screen, and were not aware that they had a condition which would preclude organ donation (e.g., cancer, HIV, or chronic hepatitis) They were excluded if they were incarcerated or cognitively impaired. After the initial screen, study investigators asked participants about willingness to donate. We asked participants if they were insured and then informed them that, if not, they would have to pay for an organ transplant. We then asked participants to guess the costs of solid organ transplantation (heart, liver, and kidney) and informed them of the actual costs. Investigators then reassessed participants' willingness to donate and opinions about the importance of this information. Basic demographic characteristics were collected.

RESULTS: 81 patients were screened. 54 were eligible and completed the survey. Spanish was the primary language for all participants. See Table for demographic data. 14 participants $(26 \%$; $95 \%$ confidence interval [CI], 14 $38 \%$ ) did not know that they would have to pay for an organ if they didn't have health insurance. The cohort grossly underestimated the cost of transplants. Median estimated cost (MEC) for heart transplant was $\$ 95 \mathrm{~K}$ (Interquartile range [IQR] \$45 K-200 K); actual cost is $\$ 620 \mathrm{~K}$. MEC for liver transplant was $\$ 50 \mathrm{~K}$ (IQR \$35 K-150 K); actual cost is $\$ 525 \mathrm{~K}$. MEC for kidney transplant was $\$ 50 \mathrm{~K}$ (IQR $\$ 30 \mathrm{~K}-170 \mathrm{~K}$ ); actual cost is $\$ 250 \mathrm{~K}$. Before the intervention, 40 participants $(74 \%$; $95 \%$ CI, $62-86 \%)$ were willing to donate their organs. After the intervention, 42 participants (78\%; 95\% CI, 66-89\%) were willing to donate. 46 participants $(85 \%$; $95 \%$ CI, $73-93 \%)$ felt that this was information that their doctor should tell them.

CONCLUSIONS: This study of a group of undocumented, Spanishspeaking immigrants at a safety-net hospital suggests that being practically unable to receive a transplant does not lead most to opt out of the organ donor pool. Nonetheless, this information is considered important to this group.

Table 1 Demographic characteristics of study population

\begin{tabular}{ll}
\hline \hline Characteristics & N (\%) \\
Country of Origin Mexican Non-Mexican & $51(94) 3(6)$ \\
Education $\leq$ High school & $48(89)$ \\
Related to US Citizen Yes No & $34(63) 20(37)$ \\
Children $\geq 1$ & $37(87)$ \\
Uninsured & $50(93)$ \\
\hline
\end{tabular}

AN ASSOCIATION BETWEEN TIMING OF ADMISSION, MEDICAL TEAM WORKLOAD, AND 30-DAY READMISSION RATE. Yelena Averbukh; William Southern. Montefiore Medical Center, Bronx, NY. (Control ID \#1297088)

BACKGROUND: The "July effect" refers to a drop in the quality of medical care due to trainees' changeover at the beginning of academic year in July. Greater teaching team workload is associated with worse patient outcomes, but it is unknown if the association is the same in the early part of the academic year and the later months. To address this we examined the associations between medical team workload, timing of admission during academic year, and 30-day readmission rate.

METHODS: In this retrospective observational study we examined all admissions to the medicine teaching service of an urban academic medical center from 3/1/09 to 6/30/10. Admissions were divided into two groups based on the admission date during either first quarter of the academic year, or during the rest of the year. The two patient groups were compared with respect to demographic characteristics, co-morbidities (Charlson score), severity of illness (Laboratory-based Acute Physiology Score, LAPS), and number of prior admissions. Admissions were further divided into groups as follows: those assigned to "busy" teams (total admissions $>49$ ) and those assigned to "less busy" teams (total admissions $<=49$ ). The primary outcome was 30-day readmission rate. Multivariate logistic regression models were constructed to determine the independent association between teaching team workload and readmission rates, stratified by time of year of admission, after adjustment for demographic and clinical characteristics.

RESULTS: Of 12,118 admissions examined, 2,352 (19.4\%) were admitted during the first quarter of the academic year (July - September) and 9,766 $(80.6 \%)$ were admitted during the rest of the year (October - June). Patients admitted during the first quarter were similar to the patients admitted during the rest of the year with respect to age, gender, race/ethnicity, insurance, and clinical characteristics (LAPS and Charlson score). Patients admitted to busier teams in the first quartile had similar 30-day readmission rate $(15.26 \%$ vs. $14.93 \%$, p-value 0.82 ), but greater 30 -day readmission rate during the rest of academic year $(17.3 \%$ vs. $14 \%$, p-value 0.04$)$. After multivariate adjustment for age, LAPS, Charlson score, 90-day prior admissions, patients admitted to more-busy vs. less-busy teams in the first quartile had similar 30day readmission rate $(\mathrm{OR}=1.03(0.82-1.30))$. During the rest of the academic year admission to a busier team was associated with increased risk of readmission $(\mathrm{OR}=1.16(1.03-1.30))$.

CONCLUSIONS: During the first quarter of the academic year admission to a busier team is not associated with increased odd of 30-day readmission. In contrast, during the rest of academic year admission to busier teams is associated with $16 \%$ increased odds of 30 -day readmission

Odds of 30-day readmission if admitted to More vs. Less busy team, stratified by time of year

\begin{tabular}{lll}
\hline \hline & Univariate & Multivariate* \\
1st Quarter (Jul - Sep) & $1.03(0.82-1.29)$ & $1.03(0.82-1.30)$ \\
2nd -4th Quarters (Oct - Jun) & $1.27(1.14-1.41)$ & $1.16(1.03-1.30)$
\end{tabular}

* Adjusted for age, LAPS, Charlson score, 90-day prior admissions

AN EDUCATIONAL INTERVENTION TO IMPROVE OPIATE PRESCRIBING PRACTICES IN RESIDENT CLINIC Erin Snyder ${ }^{1,2}$; Analia Castiglioni ${ }^{1,2}$; Carlos Estrada ${ }^{1,2}$; Zhiying You $^{3}$; Stefan $\mathrm{Kertesz}^{3,2}$; Joseph E. Schumacher ${ }^{3}$. ${ }^{1}$ University of Alabama at Birmingham, Birmingham, AL; ${ }^{2}$ Birmingham VA Medical Center, Birmingham, AL; ${ }^{3}$ University of Alabama at Birmingham, Birmingham, AL. (Control ID \#1339197)

BACKGROUND: Prescription of chronic opiates is common in resident continuity clinics. Based on prior research data documenting low comfort and lack of key knowledge among internal medicine residents and faculty, we implemented a multi-pronged educational intervention intended to improve comfort, self efficacy, and knowledge of chronic opiate prescribing. METHODS: Our intervention consisted of development and delivery of 1) educational posters to each resident clinic site including standard recom- 
mendations and a guide to urine drug test interpretation, 2) pocket cards to all residents with similar information and 2) two large group lectures. Internal medicine residents and teaching faculty at a single academic medical center were surveyed pre- and post-intervention at intervals 18 months apart (each a convenience sample of persons attending a required educational activity). Using identical questions each time, the survey assessed: a) experience with opiate management (5 items, 4-point Likert), b) comfort with both opiate management (11 items) and tobacco/alcohol counseling (4 items, Likert scale), c) self-efficacy (comparing current practice to ideal practice) and d) knowledge related to typical opiate prescription management (anticipation of correct drug screen results (10 items), and 15 true/false (T/F) questions). Mean scores for the pre-intervention and post-intervention samples were compared, adjusting for gender and training level.

RESULTS: The pre-intervention sample consisted of 48 participants $(76 \%$ residents, $24 \%$ faculty) while the post-intervention consisted of $39(64 \%$ residents, 36\% faculty). Pre- to post, overall comfort with opiate management decreased slightly from 32.2 (out of a possible 45, higher scores corresponding to higher comfort) to $29.9(\mathrm{p}=0.09)$. There was no significant change in selfefficacy (Pre: 27.4 (out of possible 40) vs Post: $28.8(\mathrm{p}=0.13$ ). Prior to our intervention, correct anticipation of standard urine drug screen results was poor, scoring 5.8 (out of 10). Incorrect responses were especially prevalent for fentanyl, demerol, and oxycodone (on this last item, only $10 \%$ responded correctly). Post-intervention, knowledge increased to 6.8 ( $\mathrm{p}<0.01$ unadjusted, and $p=0.09$ adjusting for gender and training level). Correct anticipation of oxycodone results increased to $41 \%(\mathrm{p}<0.001)$. Knowledge rating on $\mathrm{T} / \mathrm{F}$ questions increased from 12.1 (out of 15 ) to 13.3 post-intervention ( $\mathrm{p}=.002$, adjusting for gender and training level).

CONCLUSIONS: A relatively modest intervention consisting of lectures, posters, and pocket cards helped to close key knowledge gaps, but did not improve comfort. This discomfort may be appropriate, however, as our intervention may have alerted learners to previously unrecognized levels of complexity in chronic opiate prescribing.

\section{ANTIBIOTIC PRESCRIBING BY TELEPHONE IN PRIMARY} CARE Edward Ewen ${ }^{1}$; Vincent J. Willey ${ }^{2}$; Kanchala Vichaichanakul ${ }^{2}$; Tanmay $\mathrm{Khole}^{2}$; Pranav Dabeer ${ }^{2}$; Nikhil Loka ${ }^{2}$; William F. McGhan ${ }^{2}$; Marci Drees ${ }^{1}$. ${ }^{1}$ Christiana Care Health System, Newark, DE; ${ }^{2}$ University of the Sciences in Philadelphia, Philadelphia, PA. (Control ID \#1333660)

BACKGROUND: Overuse of antibiotics in outpatient settings has been well documented despite numerous randomized controlled trials finding no meaningful clinical benefit of antibiotic treatment for illnesses such as upper respiratory infections (URIs). Because telephone calls between patient and provider are rarely billed, little is known about antibiotic prescribing by telephone and the extent to which this practice contributes to antibiotic overuse is unknown. This study examines the patterns of use and reasons for telephonic antibiotic prescribing in a group of primary care practices.

METHODS: Antibiotic prescription data and telephone notes from 18 primary care practices, including pediatric, internal and family medicine, and women's health, were retrieved from a large, Mid-Atlantic healthcare system outpatient electronic medical record for calendar year 2008. All telephone notes associated with antibiotic prescriptions were reviewed manually and characterized according to antibiotic class, infection type, evidence supporting treatment (empiric or directed by culture/laboratories/imaging), treatment context (new, ongoing, or recurring infection), and temporal relationship to office visits. Antibiotics for chronic use were excluded and practices were categorized as teaching or private. Descriptive statistics were compared using chi-square and Mann-Whitney U tests.

RESULTS: Of 64,105 patients cared for in these practices in 2008, 1790 antibiotics were prescribed by telephone during 1736 calls, for an overall rate of 2.8 per 100 patient-years. The rate of telephonic antibiotic prescribing was greatest in internal and family medicine practices (3.3/ $100 \mathrm{pt}$-yrs vs $0.6 / 100 \mathrm{pt}$-yrs for pediatrics and $0.4 / 100 \mathrm{pt}$-yrs for women's health). In addition, private practices prescribed telephonically at more than twice the rate of teaching practices $(3.3$ vs. 1.4 per 100 pt-yrs, $\mathrm{p}<0.001)$. The majority of infections were URIs $(43.7 \%)$ and genito-urinary tract infections $(32.6 \%)$. These infections were considered new occurrences in
$65.7 \%$ and recurrent infections in $13.7 \%$. Treatment was directed by cultures, labs, or imaging results in only $13 \%$ of prescriptions. Newer macrolides were most commonly prescribed $(23.5 \%)$ followed closely by quinolones $(23.1 \%)$. When examining only URIs, broad spectrum antibiotics comprised $74.1 \%$ of those prescribed. A total of $796(44.5 \%)$ prescriptions were associated with an office visit within 14 days prior to the telephone call. Patients recently seen in office visits were younger ( 43.7 [SD 21.5] vs 50.6 [SD 19.9] years, $\mathrm{p}<0.001$, more frequently male $(25.8 \%$ vs $20.9 \%, \mathrm{p}=0.018)$, more often African-American $(18.0 \%$ vs $12.2 \%$, p < 0.001 ), more likely to receive a newer quinolone (moxifloxacin or levofloxacin, $13.3 \%$ vs $7.4 \%, \mathrm{p}<0.001$ ), and more often received directed therapy $(21.4 \%$ vs. $6.2 \%, p<0.001)$ compared to those managed only by telephone.

CONCLUSIONS: Antibiotic prescribing by telephone is relatively common in primary care and significant differences exist in the prescribing rate by teaching and practice type. The majority of prescriptions are generated for the empiric treatment of new onset URIs using broad spectrum antibiotics, and more than half of these patients were not seen in the office within the prior 2 weeks. The frequency and prescribing patterns associated with telephone antibiotic prescribing in this population support the case for further study of its impact on antibiotic resistance.

ANTICOAGULATION MANAGEMENT: DOES FACE-TO-FACE DELIVERED CARE IMPROVE PERCENT TIME INR IN RANGE OVER TELEPHONE-BASED CARE FOR PATIENTS ON CHRONIC WARFARIN THERAPY? Payam B. Bokhoor ${ }^{1}$; Lei Xuan²; Eve Glazier ${ }^{3}$; Shannon M. Ruiz ${ }^{3}$; Brandon Koretz; Jason Fish ${ }^{2,3}$. ${ }^{1} \mathrm{UC}$ Davis, Sacramento, CA; ${ }^{2}$ UT Southwestern, Dallas, TX; ${ }^{3}$ UCLA, Los Angeles, CA. (Control ID \#1318201)

BACKGROUND: Successful anticoagulant management requires careful monitoring of the international normalized ratio (INR) for those patients on warfarin, with several studies indicating patients have better outcomes when managed by an "anticoagulation management service" (AMS). Yet, the literature is limited in delineating which patients might benefit most from the different types of AMS configurations. We sought to identify if patients switched from a telephone-based AMS to a face-to-face AMS improved their percent time INR in range as well as to identify those benefiting the most from the switch.

METHODS: Using a retrospective random effects model, we evaluated 209 patients at an academic institution who were transitioned from a telephone-based AMS using venipuncture (non-POCT) to a face-to-face AMS using fingerstick point-of-care testing (POCT). We analyzed 18 months of data: 9 months before and after the switch. To identify any elements of secular trend, we analyzed 132 patients to be switched later over the same 18 months, managed only by non-POCT. Exclusion criteria included patients off of warfarin or hospitalized for greater than one month, or who had two or more consecutive months without data. The primary endpoint was percent time of INR in range (TIR) evaluated as a continuous variable. The independent variable was AMS configuration (POCT versus non-POCT), evaluated as a categorical variable. Additional variables included age, gender, and anticoagulation indication. We also included an interaction term involving diagnosis of atrial fibrillation/atrial flutter (afib) and AMS configuration, evaluated as a categorical variable.

RESULTS: There were 231 eligible patients in the intervention group, of which 22 were excluded ( 4 off of warfarin and 18 with missing data) with a final sample size of 209. The average age was 72.9 (SD 15.7), with $52.6 \%$ being female. Half of the patients had afib, with the remaining patients with DVT, PE or CVA (38.8\%), Cardiac Valve Replacement $(5.7 \%)$, coagulation defect $(3.4 \%)$, CAD or PAD $(1.4 \%)$, or Pulmonary HTN $(0.5 \%)$. The average unadjusted TIR was $72.6 \%$. We observed a mean $3.3 \%(95 \%$ CI: $1.37,5.24 ; \mathrm{p}=0.001)$ increase in TIR in the intervention group after their transition to POCT AMS, controlling for age, gender, and warfarin indication. Using a random effects model with the interaction term, we found that those patients with afib had a mean increase in TIR of $1.07 \%$ (Interaction Term $\beta=-4.49 ; 95 \%$ CI $-8.36,-0.62 ; \mathrm{p}=0.02$ ) compared to those patients without afib who had a mean increase of $5.56 \%(95 \% \mathrm{CI}$ : $2.81,8.30 ; \mathrm{p}<0.001)$, controlling for age, gender and warfarin indication. 
In the control group, using the same methodology, there was no significant change in TIR during the study period $0.53 \%$ (95\% CI: $-0.24,3.10 ; \mathrm{p}=$ 0.68 ). No other variables were significant.

CONCLUSIONS: The POCT AMS led to a greater TIR than the nonPOCT AMS. Our research also indicated patients on warfarin for a diagnosis other than afib benefited the most from a POCT AMS, and secular trend did not appear to account for the differences. Further research is needed to better understand which specific components of the POCT AMS led to the improved TIR and why certain patients, typically thought of as complex patients, achieved a greater TIR.

ARE ANGIOTENSIN-CONVERTING ENZYME INHIBITORS (ACEI) AND ANGIOTENSIN-II RECEPTOR BLOCKERS (ARB) A RISK FACTOR FOR CIN? - A HOSPITAL-BASED STUDY Anil Nalubotula; Daniel Gutteridge; Siva K. Talluri; Pramod Kalagara; Daniel Tismal; Vanessa Pauig; Bhavana Siddegowda Bangalore; Marcello F. Schmidt; Radhika Kakarala. McLaren Regional Medical Center, Flint, MI. (Control ID \#1333666)

BACKGROUND: Contrast media-Induced nephropathy (CIN) is a major complication in hospitalized patients undergoing procedures using contrast media. CIN is the third most common cause of acute kidney injury (AKI) following hypo-perfusion and nephrotoxic medications, responsible for $11 \%$ of hospital-acquired AKI. The incidence of CIN in the general population $(<2 \%)$ increases to $\geq 20-30 \%$ in high-risk patients. Chronic therapy with ACE-I/ARBs has been proposed as a risk factor in some studies but considered protective in others. CIN risk due to ACE-I/ARBs therapy has not been prospectively studied in critically ill and hospitalized patients. The goal of our study is to determine the association of ACE-I/ARB use on CIN incidence in hospitalized patients who had undergone coronary angiogram with or without PCI.

METHODS: A retrospective analysis was performed in patients who underwent emergent/non-emergent cardiac catheterization during 2009 at a US community hospital. Patients were excluded if they met any of following criteria: (i) end stage renal disease; (ii) acute kidney injury preceding cardiac catheterization; (iii) hyperkalemia (K>5.5 meq/l); (iv) nephrotoxic agent administration within 3 days before the procedure; (v) pregnancy and (vi) prior cardiac catheterization within one month. CIN was defined as a $25 \%$ increase from baseline creatinine within $48 \mathrm{~h}$. Patients were sub-classified based on Mehran CIN score. The association of ACE-I/ARBs with CIN development was evaluated.

RESULTS: Two hundred forty-six patients who underwent cardiac catheterization met the inclusion criteria (55\% men, 35\% diabetic, $43 \%$ anemic, $19 \%$ CHF). Of the 246 patients, $50 \%(n=123)$ were taking ACE-I/ARBs. PCI was done in $42 \%$ of cardiac catheterization patients $(n=100)$. The median Mehran CIN risk score was similar between ACE-I/ARBs (6.5) and non- ACE-I/ARBs groups (5.0) $(\mathrm{P}=0.24)$. There was no significant difference in the incidence of CIN between the two groups (OR: $1.451 ; 95 \%$ CI: $0.677-3.107 ; \mathrm{P}=0.339$ ). A sub-group analysis of 100 patients who underwent PCI revealed CIN development in a total of 8 patients. Of these $8,75 \%(n=6)$ were using ACE-I/ARBs. There was also no statistically significant difference in the incidence of CIN in patients who were using ACE-I/ARBs compared to nonACE-I/ARBs group (OR: 3.5; CI: 0.68 - 18.63; $\mathrm{P}=0.131$ ).

CONCLUSIONS: We did not find an association between ACE-I/ARB use and CIN. Nevertheless, in patients who underwent PCI, the risk of developing CIN while using ACE-I/ARBs was 3.5 times higher, though not reaching statistical significance. Further randomized controlled trials with more power are required especially in view of the conflicting evidence.

ARE INCOMING INTERNS' KNOWLEDGE LEVELS ABOUT CORE CONTINUOUS QUALITY IMPROVEMENT CONCEPTS IMPROVING? Anne Tomolo ${ }^{1}$; Mamta K. Singh ${ }^{2}$; Renee H. Lawrence ${ }^{2}$. ${ }^{1}$ Atlanta VA Medical Center, Atlanta, GA; ${ }^{2}$ Cleveland VA Medical Center, Cleveland, OH. (Control ID \#1314457)

BACKGROUND: The importance of continuous quality improvement (CQI) for providers' skill sets and healthcare systems' toolsets is well-recognized and there is a move to shift such curriculum "downstream" so that many medical schools offer training experiences related to CQI. However, few studies have evaluated whether exposure and knowledge levels have changed over time. This information is important in terms of shaping residency training related to CQI. We addressed these questions by using data collected on two cohorts of Internal Medicine interns at the beginning of a Practice Based Learning Improvement curriculum in 2005 and 2011.

METHODS: At the beginning of a four-week ambulatory rotation curriculum, interns' completed a questionnaire assessing knowledge of core concepts related to CQI in 2005 and 2011. Specifically, learners completed a question about previous experience and answered open-ended (describe or define) questions about the following core concepts: change concept, creating a cause effect diagram, elements of the improvement model, common cause and special cause variation, and why the distinction in types of variation is important. A previously developed coding system was used to score the responses and create a total knowledge score (maximum of 51 points).The coding system scores key variables needed for ideal responses to evaluate short answer definitions for core knowledge concepts (30 variables). The coding system has demonstrated good inter-coder reliability (percent agreement $>85 \%$; Lin's concordance for total knowledge was 0.98 ), and face, construct and discriminative validity.

RESULTS: Previous experience was significantly related to cohort year: Of the 34 interns in 2005, 73.5\% $(\mathrm{n}=25)$ indicated absolutely no experiences with CQI. In contrast, of the 33 interns (missing data for one) in 2011 , only $15.2 \%(n=5)$ indicated no previous experience and $51.5 \%(\mathrm{n}=17)$ indicated having attended a lecture or teaching session on CQI. However, level of knowledge for the cohorts was not significantly different and was low with the majority of both cohorts scoring zero $(70.6 \%, n=24 / 34$ in 2005 and $73.5 \%, n=25 / 34$ in 2011).

CONCLUSIONS: Despite increasing emphasis upon the inclusion of CQI education in undergraduate medical education these findings suggest that intern baseline knowledge in some core principles of CQI has not changed over six years. Further defining and standardizing core CQI skills sets needed upon graduation from medical school would be helpful. In addition, residency programs may benefit from incorporating a curriculum that includes discussion of CQI core principles early in the training program, particularly prior to participation in Practice-Based Learning and Improvement curriculum that requires the application of these core concepts.

\section{ARE MEDICAL INTERNS MISLED BY DIRECT-TO-CONSUMER PRESCRIPTION DRUG ADVERTISING? A PILOT CASE STUDY Huai Cheng. University of Virginia, Charlottesville, VA. (Control ID \#1336251)}

BACKGROUND: Direct-to-consumer prescription advertising is epidemic and costly. The claimed benefit and effectiveness of the drug in the advertising is often misleading and not evidence-based. Health care providers have similar exposure to such advertising and may be also influenced to prescribe the drug. Despite critical appraisal of the evidence as part of evidence-based medicine work shops or lectures for medical trainees, how much trainees learn in critcal appraisal of direct-to-consumer prescription advertising has not been well studied. The purpose of this pilot study was to test whether medical interns could correctly recognize the wrongly claimed benefit from a drug advertising figure by a drug company.

METHODS: The study subjects were medical interns who rotated in a 4 week geriatric rotation at a univeristy teaching hospital. During the rotation, they were required to participate in a 4 week evidence-based medicine workshop. The learning objectives of the workshop were provided to medical interns in the begining of the workshop. One was to show them a drug advertising figure and to ask them the following question: "Does the combination therapy of drug A and drug B (real drug name with figure was provided to the class) improve cognitive function based on the figure?" They were asked to choose one of 4 answeres: yes, probably yes, no, probably no. After the medical interns answered the question, the author (H Cheng) explained the scale of cognitive function assessment in the figure. They were asked to answer the same question again. The title of this figure was "Improved cognitive benefits with drug A+drug B". The scale on the $\mathrm{X}$-axis was the duration ranged from 4 to 24 weeks. The scale 
$(0-4)$ on the Y-axis appeared impressive with $4 \mathrm{p}$ values ranged from 0.03 to $<0.001$. The problem was that the cognitive function scale (Severe Impairment Battery) ranged from 1-100, not shown in the figure. Decriptive statistics were used for data analysis.

RESULTS: $44 \%$ (72/165) of medical interns from 2007 to 2010 participated in this pilot study. $29 \%$ (21/72) said "yes", agreeing that the combination therapy of drug A and drug B improves cognitive function; $22 \%$ (16/72) said "probably yes"; $17 \%$ (12/75) said "no"; and 32\% (23/72) said "probably no". Everybody said "no" once I explained that the scale of the cognitive function assessment actually ranged from 1-100. They all agreed that the changes of 1-3 in the scale of 1-100 was minimal and the drug advertising figure was misleading.

CONCLUSIONS: This pilot study has shown that the incorrectly claimed drug benefit from a drug advertising figure was not recognized by about half medical interns at an univeristy teaching hospital. Whether this could change their prescription behavivor needs to be further studied. Critical appraisal of direct-to consumer prescription drug advertising should be taught to medical interns and perhaps other trainees.

\section{ARE PRIMARY CARE PHYSICIANS READY TO CARE FOR} CANCER SURVIVORS? Jeong H. Oh ${ }^{1}$; Lewis Foxhall ${ }^{2}$; Karen BasenEngquist ${ }^{3}$; Maria Suarez-Almazor ${ }^{1}$. ${ }^{1}$ UT MD Anderson Cancer Center, Houston, TX; ${ }^{2} \mathrm{UT}$ MD Anderson Cancer Center, Houston, TX; ${ }^{3} \mathrm{UT}$ MD Anderson Cancer Center, Houston, TX. (Control ID \#1309614)

BACKGROUND: Cancer survivorship has sharply risen since the advent of widespread screening practices and the recent advances in treatment options. Chemotherapy, one of the cornerstones in winning the battle against cancer, has lasting health effects that require medical attention. The purpose of this study was to describe primary care physicians' perception of their own knowledge and comfort level ascertaining and managing long-term and late (lasting) effects of chemotherapy in cancer survivors, and their preferences for further Continuing Medical Education (CME) in this area.

METHODS: We surveyed a random sample of office-based primary care physicians (PCPs), practicing in Texas with a self-administered crosssectional fax/mail-based survey instrument. Physicians' demographics, cancer diagnosis of survivors, familiarity with lasting effects of chemotherapy, barriers to optimal care, preferences for further CME, and communication patterns with oncologists were obtained. Each item and sub-scale on the survey was summarized using standard descriptive and chi-square statistics, and two-sided precision (95\% confidence interval) for a one sample proportion was calculated when appropriate.

RESULTS: Most responses were received by fax (61.2\%) and from 128 participants the mean age was 49.8 years, $68.3 \%$ were males, $49.2 \%$ were general internists and $50.8 \%$ were family physicians, and the mean time since their last post-graduate training was 19.4 years. PCPs estimated that cancer survivors constituted $20.6 \%$ of all patients seen in the 4 week period prior to the survey, $64.0 \%$ of the respondents reported they were unfamiliar with the lasting effects of chemotherapy, $75.0 \%$ were uncomfortable with screening practices, and $73.7 \%$ were uncomfortable with management strategies of these complications. "Complexity of chemotherapy agents and their lasting effects" was the most significant barrier reported, and "Lack of time during patient encounters" the least significant barrier. Only $17.6 \%$ responded that they had ever been offered any CME opportunity on screening and management of lasting effects of chemotherapy, however $85.6 \%$ stated that they would attend such CME if offered. "Lecture format in conferences" (63.4\%) and "Printed educational materials by mail" $(44.6 \%)$ were the preferred methods of CME. Most physicians (81.6\%) reported that it would be helpful to receive advice/guidelines from oncologists.

CONCLUSIONS: Our study suggest that most primary care physicians felt they were unfamiliar with lasting effects of chemotherapy, and reported lack of opportunities for training. These findings underscore the need for additional medical education in this area so that gains achieved in cancer survival are not offset by loss in quality of life, comorbidities, and premature mortality from the same treatment used to save patients' lives.

ARE PROGRAM DIRECTORS READY FOR ACGME MILESTONES? Pamela P. Reynolds; Megan Madaras. University of Virginia, Charlottesville, VA. (Control ID \#1336313)
BACKGROUND: Supporting a national effort to teach professionalism along the continuum, in 2002 the Accreditation Council on Graduate Medical Education (ACGME) launched its initiative to require program directors (PDs) to focus on 6 core competencies as part of residency training. In 2010 the ACGME announced its Milestones Project, designed to require PDs to document residents' mastery of these competencies prior to graduation. Efforts are now underway to develop curricula and tools that assess residents' competencies, including professionalism. This project was designed to determine if PDs at the University of Virginia (UVa) are prepared to implement the Milestones requirements on professionalism.

METHODS: Ten PDs, representing clinical disciplines, were interviewed from January through July 2011 by one of two investigators. A structured interview was followed that asked 9 questions. PDs were asked to define professionalism, describe ACGME requirements for professionalism and discipline specific RRC requirements, whether they have a professionalism curriculum, the importance of role modeling versus didactic teaching on professionalism, whether they formally assess professional behaviors, and agreement or disagreement with behaviors included in a 15-item Medical Professionalism Behavior Assessment Tool (MPB). All interviews were transcribed. The two investigators read all interviews, and analyzed them for specific responses to questions, and common themes across the interviews. RESULTS: All 10 PDs provided a definition of professionalism that included ethical behavior and professional responsibilities. Two (of 10) PDs could state the ACGME's specific requirements for professionalism. Two (of 10) PDs could describe additional RRC requirements for professionalism. Three (of 10) PDs had formal curricula on professionalism that included seminars, lectures, workshops, and journal club case discussions; one also required an advocacy project. All relied on a GME lecture series to provide some of the content of professionalism. All PDs thought role modeling was essential to teaching professionalism, but all thought it was insufficient without structured education on the expectations of professional conduct and formal training on ethics and related issues. Seven (of 10) PDs used 360 evaluations in formative and/or summative evaluations of professionalism. None used a specific professionalism assessment tool. Three (of 10) PDs expressed concern that bad role modeling occurred and all articulated the need for faculty development prior to use of a professionalism assessment tool. All 10 PDs affirmed that the behaviors included in the MPB were appropriate for assessment of residents' professionalism.

CONCLUSIONS: PDs at UVa primarily rely on role modeling for teaching professionalism. All PDs believe a curriculum with instruction on the expectations of professionalism is necessary, and that case-based seminars highlighting ethics, conflicts of interest, legal issues in medicine, cultural sensitivity and professional standards would be a valuable addition to residency training. No PD currently provides instruction on the "hidden curriculum" or the "resident as role model". One PD includes skills-based training on cultural sensitivity. While all of the UVa PDs articulate the critical importance of professionalism, most appear unprepared to formally assess residents' professionalism beyond previously described clinical competence.

ARE WE ALL EQUAL? COMPARING STUDENTS PURSUING GENERAL INTERNAL MEDICINE TO THOSE PURSUING OTHER PRIMARY CARE SPECIALTIES Martha Grayson ${ }^{1,4}$; Dale A. Newton ${ }^{2}$; Lori Foster Thompson ${ }^{3} .{ }^{1}$ Albert Einstein College of Medicine, Bronx, NY; ${ }^{2}$ Brody School of Medicine, Greenville, NC; ${ }^{3}$ North Carolina State University, Raleigh, NC; ${ }^{4}$ New York Medical College, Valhalla, NY. (Control ID \#1323953)

BACKGROUND: Research examining the factors relevant to medical students' career choice commonly combines primary care specialties into one group. While this approach facilitates comparisons between those pursuing primary care and non-primary care specialties, it may mask important differences within the primary care family. The objective of this study was to determine whether students pursuing general Internal Medicine (GIM) differ from their peers interested in Family Practice (FP) and general Pediatrics (PEDS) with respect to anticipated income and the self-reported values driving their career specialty decisions.

METHODS: Fourth-year (M4) students at New York Medical College (NYMC) and Brody School of Medicine at East Carolina University 
(ECU) were surveyed annually immediately prior to graduation. This study included 18 consecutive years of M4 data from the students graduating between 1993 and 2010 (response rate $=77 \%$ ). Respondents were asked to indicate their future specialty plans. Those intending to pursue GIM $(\mathrm{N}=$ 296), FP ( $N=358)$, and PEDS ( $N=247)$ were included in the analyses, for a total sample size of $\mathrm{N}=901$ ( $57 \%$ female). Respondents reported their annual expected income five years after completion of residency training. Beginning in 1998 , respondents $(\mathrm{N}=640)$ were also asked to used a 1 (no influence) to 4 (major influence) scale to rate the degree to which their career decisions were affected by 29 considerations, reflected in items such as "allows me to be viewed as a medical authority." Prior factor analysis found that these 29 items clustered into 7 career values: Comprehensive Patient Care, Prestige, Lifestyle, Helping Others, Working with the Poor, Research, and Income. ANCOVA analyses followed by post hoc tests were conducted to examine whether those pursuing GIM placed higher or lower weight on each factor when compared to those pursuing FP and PEDS. We statistically controlled for any effects of school (NYMC, ECU). Differences in anticipated income were also examined.

RESULTS: For three outcome measures, GIM respondents stood out as notably distinct, placing significantly higher value on Prestige and Research and significantly lower value on Helping Others than did both FP and PEDS $(p<.05)$ respondents. For other outcome measures, GIM tended to cluster with one of the other two primary care specialties, though the particular specialty with which GIM clustered depended on the factor at hand. GIM ratings were similar to PEDS but significantly (p $<.05)$ lower than FP with respect to the importance students placed on Comprehensive Patient Care, Lifestyle, and Working with the Poor when selecting a career specialty. However, in other cases, GIM looked similar to FP, with both GIM and FP diverging significantly from PEDS. Specifically, students aspiring to GIM and FP placed a higher importance on income and they anticipated significantly higher incomes compared to those intending to pursue PEDS $(\mathrm{p}<.05)$.

CONCLUSIONS: Important differences in income expectations and career values exist between students pursuing GIM and those pursuing other primary care specialties. Although researchers often lump primary care specialties into a common category, this study indicates that a more nuanced view would be appropriate. Awareness of these differences may improve career counseling by medical school faculty and residency program directors in the three primary care fields and ultimately lead to enhanced career satisfaction.

ARE PHYSICIANS ADHERING TO US PUBLIC HEALTH SERVICE SMOKING CESSATION GUIDELINES? Shibani M. Pokras ${ }^{1}$; Richard Chapman'; Aaron Galaznik ${ }^{2}$. ${ }^{1}$ IMS Consulting Group, Alexandria, VA; ${ }^{2}$ Pfizer Global Pharmaceuticals, New York, NY. (Control ID \#1280040)

BACKGROUND: Recent US Public Health Service (PHS) guidelines urge clinicians to use the "5 A's" model to treat tobacco dependence. Clinicians should ask and document patients tobacco use at every visit, advise users to quit, assess willingness to quit, assist by offering medication and counseling, and arrange for follow-up. The guidelines urge providers to recommend a combination of counseling and medication, instead of either alone, as there is a strong association between the number of sessions of counseling, when combined with medication, and the likelihood of successfully quitting. We assessed providers' agreement with this recommendation and consistency in adherence to the 5A's model among patients seen in the last week

METHODS: Our convenience sample consisted of physicians who hosted smoking cessation workshops designed to support patients preparing to quit and ZIP-code matched control physicians who did not participate (using the AMA physician masterfile). Hosts and controls were faxed a post-workshop survey evaluating self-reported adherence to PHS guidelines among patients seen in the past week.

RESULTS: Of 215 host $(\mathrm{H})$ and 2,215 control (C) physicians surveyed, 113 $(52.6 \%)$ and $240(10.8 \%)$ responded, respectively. Mean age overall was $51.5 \mathrm{y}, 24 \%$ were female. Hosts were more likely to be non-smokers, to have fewer years practicing, and to have more patients who smoked, and less likely to be solo practitioners ( $\mathrm{p}<0.05$ for each). Overall, $83.6 \%$ of providers agreed that pharmacotherapy plus multimodal support was a more effective method to quit smoking than either strategy alone. Significantly more hosts agreed with this statement vs. controls ( $87.6 \%$ vs. $81.7 \%, \mathrm{p}=0.0055)$. The percentage of physicians who reported completing the following action in $>80 \%$ of patients seen in the last week were: actively solicited smoking status (ask), $34.6 \%$ (H:36.3\%, C:33.8\%); documented discussion of smoking status and treatment strategies (ask), 29.2\% (H:32.7\%, C:27.5\%); initiated the discussion regarding smoking cessation (advise, assess), 27.2\% (H:31.9\%; C:25.0\%); suggested patient consider quitting (assist) supported by (a) counseling: $22.9 \%$ (H:34.5\%, C:17.5\%), (b) medication: 30.3\% (H:39.8\%, C:25.8\%), (c) support: $23.8 \%$ (H:33.6\%, C:19.2\%); arranged follow-up, 9.6\% (H:14.2\%, C:7.5\%). Significantly more hosts reported completing each action vs. controls ( $\mathrm{p}<0.05$ for each).

CONCLUSIONS: The majority of providers agreed that guideline-recommended combination treatment (medication and counseling) is more effective than either treatment alone, but they were not consistent in recommending each of these components to patients and most frequently recommended pharmacotherapy. Highest adherence to the 5A's model was seen in soliciting smoking status and lowest adherence in arranging for smoking-related follow-up visits. Physicians who had recently hosted smoking cessation workshops reported better adherence to guidelines vs. controls, although no definitive conclusions can be drawn between the groups due to baseline differences and self-selection to participate in these workshops. There was significant room for improvement in adherence to the 5A's model even among hosts of smoking cessation clinics, suggesting the need for greater awareness and training on guidelines to providers.

\section{AS IF HIV WEREN'T ENOUGH: PERSPECTIVES ON MANAGING MEDICAL COMORBIDITIES IN PEOPLE WITH HIV Anne Monroe;} Tashi L. Rowe; Richard D. Moore; Geetanjali Chander. Johns Hopkins University, Baltimore, MD. (Control ID \#1340425)

BACKGROUND: Since the introduction of antiretroviral therapy (ART), HIV-positive patients have experienced a dramatic decline in AIDS-related mortality but increasing morbidity and mortality from cardiovascular disease (CVD). An aging population with HIV faces medical comorbidities such as diabetes mellitus (DM) and hypertension (HTN), which must be wellcontrolled to decrease CVD risk and optimize care. A high level of medication adherence is required for disease control in HIV as well as DM and HTN. The purpose of this study was to explore perceptions of living with HIV complicated by DM or HTN and to elicit barriers to and facilitators of medication adherence.

METHODS: We conducted 6 focus groups, each consisting of 5 or 6 individuals with HIV and DM or HTN on treatment for both conditions. The participants were identified by purposive sampling from the Johns Hopkins HIV Clinic in Baltimore, Maryland. The discussions were audiorecorded and transcribed. Two investigators independently coded transcripts for thematic content using editing style analysis.

RESULTS: There were 35 participants in the sample, of whom $54 \%$ were male and $94 \%$ were black. Mean age was 50.8 years (standard deviation 5.1 ) and median body mass index was $28.6 \mathrm{~kg} / \mathrm{m} 2$ (IQR 24.3, 35.8). The median duration of HIV was 13 years (IQR 5, 18), and most participants had HTN and HIV (65.7\%) or DM, HTN, and HIV (25.7\%). The thematic domains identified were: 1) perceptions of HIV versus other conditions, 2) attitudes towards medications, and 3) acceptance of HIV and integration of multiple diagnoses into daily life. For perceptions of HIV versus other conditions, many participants identified comorbid conditions as a greater threat than HIV and expressed frustration at not being able to control comorbid conditions: "Well you know what? It was told to me [by my doctor] HIV's not going to kill me. High blood pressure is going to kill me . . . that's what I think about the most." For attitudes towards medications, participants discussed perceived ineffectiveness of medications for comorbid conditions and lack of clinical consequences of missed doses: “. . . with the high blood pressure medicine ... my doctor [says], 'take your high blood pressure [medicine],' but then [he says], 'you ain't taking it', 'cause my pressure be up. I say, 'Well, let me take it in front of you and then, and then when I come back you will see . . it still will be high."' For acceptance of HIV and integration of multiple diagnoses into daily life, subthemes included: the importance of social support in medication adherence and the use of various reminder systems. For example, 
one participant stated, “. . . my family and my friends check with me to make sure that I'm doing what I'm supposed to be doing, keeping my appointments, taking my insulin, taking my HIV drugs, and everything else." CONCLUSIONS: Achieving control of medical comorbidities is an important component of comprehensive HIV care, with particular relevance for patients whose HIV is well-controlled. In contrast with HIV, medication adherence alone may not be sufficient to achieve disease control in DM and HTN. Rather, educational-behavioral interventions to decrease CVD risk in HIV-positive patients must include lifestyle modification and integration of disease management into patients' daily lives with social support to achieve treatment goals.

ASKING FOR WHAT SHE NEEDS? PREGNANCY TESTING OR EMERGENCY CONTRACEPTION E. Bimla Schwarz ${ }^{1,2}$; Sara M. Parisi $^{1}$; Erin Baldauf ${ }^{1}$; Rachel B. Rapkin ${ }^{2}$; Glenn M. Updike ${ }^{2}{ }^{1}$ University of Pittsburgh, Pittsburgh, PA; ${ }^{2}$ University of Pittsburgh, Pittsburgh, PA. (Control ID \#1326256)

BACKGROUND: Emergency contraceptive (EC) pills are safe and effective in preventing pregnancy when taken up to 5 days after unprotected sex. Although dedicated EC products have been available in the US since 1998, EC is used relatively rarely and unintended pregnancy remains common in the US. We assessed how often women seeking pregnancy testing might have benefited from EC and explored characteristics associated with women asking for EC when it was needed.

METHODS: We surveyed women aged 15-45 years who sought EC or walkin pregnancy testing from a Title $\mathrm{X}$ family planning clinic between January 2011 and December 2011. Women were eligible to complete surveys if they had a negative pregnancy test and did not want to be become pregnant at the time of their visit. Surveys were conducted as part of a larger study on use of highly effective reversible contraceptives. Respondents were asked what had prompted their visit to the clinic, how many days since they last had unprotected sex, and how many times they had used EC in the past. They were also asked about their reproductive history and sociodemographic characteristics. We calculated the proportion of women seeking pregnancy testing who might have benefited from same-day use of EC and identified patient characteristics associated with seeking EC when its use was indicated.

RESULTS: Two hundred and thirteen women who visited the study clinic completed the survey questions of interest (a response rate of $30 \%$ ). Respondents were $22 \pm 5$ years of age; $70 \%$ were black, $17 \%$ were white and $13 \%$ self-identified as other; $59 \%$ had previously been pregnant; $40 \%$ had been tested for sexually transmitted infection (STI) in the prior 3 months. Twenty-eight percent $(n=59)$ of respondents were seeking EC and $72 \%(n=$ 154) were seeking pregnancy testing. Of those seeking pregnancy testing, $49 \%$ might have benefited from same-day use of EC, as they reported a contraceptive emergency (i.e. unprotected sex or sex where the contraceptive method may have failed) within the past 5 days; an additional $4 \%$ said that they "didn't know" how many days it had been since they had unprotected intercourse and might have also benefitted from same-day use of EC. Of those seeking EC, $98 \%$ were eligible for same-day use of EC; only one woman $(2 \%)$ reported it had been more than 5 days since she had unprotected sex. Among women who may have benefited from same-day use of EC, those who asked for EC differed from those who asked only for pregnancy testing in a number of ways. Women who requested EC were older ((mean+SD): $24.3+5.2$ vs. $21.4+4.3$ years, $\mathrm{p}<0.01)$, more likely to have experienced an unwanted pregnancy $(55 \%$ vs. $34 \%, \mathrm{p}=0.03)$, more likely to have had an abortion $(46 \%$ vs. $19 \%, \mathrm{p}<0.01)$ and more likely to have ever used EC in the past ( $68 \%$ vs. $39 \%, \mathrm{p}<0.01)$. In logistic regression models adjusted for these factors as well as other demographics, previous use of EC was the strongest predictor of whether or not a woman who could have benefited from EC actually asked for it (OR:4.17, 95\%CI:1.42-12.24). CONCLUSIONS: A significant portion of women seeking pregnancy tests may benefit from information about and same-day access to EC. Clinicians should ensure that all women seeking pregnancy testing are asked whether they want to be pregnant and how long it has been since unprotected sex. All women at risk of unintended pregnancy should receive timely access to $\mathrm{EC}$.
ASSESSING ORGANIZATIONAL READINESS FOR QUALITY IMPROVEMENT IN A GENERAL INTERNAL MEDICINE FACULTY PRACTICE C. E. Lu; Abigail E. Wilkes; Lisa M. Vinci; Monica Peek; Marshall Chin. University of Chicago, Chicago, IL. (Control ID \#1339953)

BACKGROUND: Many change management experts have suggested that the failure to establish sufficient organizational readiness for change accounts for half of all unsuccessful organizational change efforts. However, few studies have explicitly examined what role readiness for change plays within the context of implementing a particular quality improvement intervention. This study examines the organizational readiness for implementing a provider feedback intervention in order to determine whether provider attitudes towards quality improvement may impact their participation in quality improvement interventions.

METHODS: In this study, we define organizational readiness for change as encompassing three domains: perceived need for change, perceived value of the specific quality improvement intervention, and perceived capacity for change. The quality improvement intervention assessed was a roster of providers' diabetes patients with select follow-up actions to intensify their care. These rosters were given to academic faculty within the department of general internal medicine that provided care at a primary care clinic within the academic medical center. Organizational readiness for the intervention was assessed using a post-intervention survey and semi-structured interview. Likert scaled questions were analyzed using a Fisher's exact test. Qualitative survey questions and interview responses were analyzed using template analysis.

RESULTS: Most (69\%) physicians agreed or strongly agreed that they were effective at helping their patients with diabetes. Less than half of the physicians surveyed (41\%) agreed or strongly agreed that the feedback intervention provided them with useful information. Providers were more likely to review the feedback roster if they agreed that the rosters were helpful $(\mathrm{p}=0.05)$ and if they agreed that they were effective at helping their patients with diabetes $(\mathrm{p}=0.03)$. While we found that many physicians surveyed $(43 \%)$ found their clinic load to be "heavy" or "very heavy" indicating lower capacity for change, this did not seem to correlate with lower tendency to rate the rosters as helpful or review the rosters. Furthermore, while most physicians felt they were effective and the clinic as a whole did well in providing their diabetic patients with appropriate care, most physicians were also able to articulate specific changes needed to improve diabetes care.

CONCLUSIONS: In this study, provider readiness for a particular quality improvement intervention appears to affect their tendency to participate in the intervention. Other factors, such as their personal confidence in their abilities, may also affect their tendency to participate; however, at least for this intervention, provider confidence in their own abilities had the opposite effect expected. Further investigation is needed to determine the role of provider confidence in participation in quality improvement interventions. Further studies are also needed to determine whether such methods could be used at other clinics with other quality improvement interventions, and to further refine methods of assessing readiness for change.

ASSESSING BARRIERS TO UPTAKE OF AVAILABLE SERVICES AND DIABETES EDUCATION AMONGST PATIENTS WITH DIABETES: A QUALITATIVE STUDY IN DELHI, INDIA. Farah N. Khan. UAB, Birmingham, AL. (Control ID \#1339125)

BACKGROUND: Diabetes is a growing problem in India, yet little is known about the awareness levels and barriers to care amongst patients with diabetes. The purpose of this qualitative study was to investigate patients' knowledge related to diabetes and its complications as well as barriers to care.

METHODS: Semi-structured interviews were conducted with patients with diabetes seen at Dr. Shroff's Charity Eye Hospital (SCEH) in Delhi. The moderator's guide incorporated constructs from the Health Belief Model. Interviews were recorded and transcribed. Two independent reviewers used a combined inductive-deductive approach to identify themes. 
RESULTS: 23 participants were interviewed; the mean age was 59 , nearly half were men $(42 \%)$, most were married $(83 \%)$, and $48 \%$ had less than 10 years of education. Participants had general knowledge about the severity of diabetes, but did not necessarily perceive personal susceptibility to the complications of diabetes. Cues to action were typically symptom-related. Most patients recognized exercise and diet as key components of diabetes management, but self-efficacy for these behaviors was low. Often patients had difficulty identifying their diabetes related needs based on limited knowledge; when identified, limited education and financial constraints were the main barriers reported.

CONCLUSIONS: This study suggests there is a need for accessible diabetes education programs in India. Further research is needed to elucidate specific barriers to uptake of available healthcare services amongst patients with diabetes in India.

ASSESSING THE NEEDS OF CALIFORNIA HOSPITALS TO IMPROVE THE REPORTING OF PATIENT RACE, ETHNICITY, AND PRIMARY SPOKEN LANGUAGE David Zingmond ${ }^{1}$; Punam Parikh ${ }^{\text {; }}$; Zahabiya H. Chithiwala ${ }^{1}$; Scarlett Lin-Gomez; Daphne Y. Lichtensztajn ${ }^{2}$; Ninez A. Ponce; ${ }^{3}$ Romana Hasnain-Wynia ${ }^{4}$; Cheryl Mercado ${ }^{1} \cdot{ }^{1}$ UCLA, School of Medicine, Los Angeles, CA; ${ }^{2}$ Cancer Prevention Institute of California, Fremont, CA; ${ }^{3}$ UCLA School of Public Health, Los Angeles, CA; ${ }^{4}$ Northwestern University Feinberg School of Medicine, Chicago, IL. (Control ID \#1338778)

BACKGROUND: Disparities in health care are documented to be significantly higher among racial/ethnic minority populations. Although California mandates that hospitals collect and report patient race, ethnicity, and primary spoken language, a lack of specific guidelines and standardized practices on what, how, and when these data should be collected has contributed to inconsistent and incomplete data. Implementing standardized methods to collect race, ethnicity, and language data is one strategy to improve quality and elucidate disparities in care, and is recommended by the Institute of Medicine. As part of a multi-step quality improvement program, we performed a baseline assessment of how hospitals collect these data in California.

METHODS: A baseline needs assessment hospital survey was developed that explored information on policies and practices regarding patient data collection, including types of data collected, data sources, and the use of a standardized form. The survey also asked about barriers encountered in the collection of these data, data auditing practices, and strategies for improving collection of data on race, ethnicity, language, and place of birth within hospitals. We identified all general acute care hospitals submitting inpatient data to the California Office of Statewide Health Planning and Development (OSHPD), the state agency that is mandated to collect patient-level data. Hospitals were contacted and asked to identify 1 to 2 people appropriate to complete this survey. A long form baseline survey was sent via U.S. post and electronically to these individuals, hospital chief administrators, and hospital registrars. A short form survey was sent to non-respondents. Respondents received a $\$ 10$ gift card for their participation.

RESULTS: The survey was sent to 367 general acute care hospitals statewide. Fifty-six percent $(n=205)$ hospitals completed the survey. Respondents included admissions/registration, quality improvement, patient services and fiscal personnel, and hospital CEOs. Participating hospitals were generally urban/ suburban, privately-owned facilities. Nearly all hospitals reported collecting race/ethnicity (97\%) and spoken language (97\%), while only $60 \%$ collected place of birth. Five percent of hospitals did not collect any type of patient race or ethnicity information. The majority of hospitals reported using standardized forms to collect race/ethnicity ( $82 \%$ ) and spoken language (79\%), while only $48 \%$ did so for place of birth. Seventy-five percent of hospitals reported auditing patient registration information for completeness. Strategies with the most hospital support to improve the quality and completeness of patient information included collecting data at a patient's first visit, offering routine staff training, incorporating questions into existing admissions forms, the development and enforcement of hospital policies regarding data collection, and the availability of a frequently asked questions and answers document to staff.

CONCLUSIONS: Hospitals in California are collecting information on patient race, ethnicity, and language as mandated, but variation in data collection exists. Hospitals endorse many reasonable approaches for standard- ization, but lack information on the accuracy of their data. Future work must focus on methods to assess and improve the collection of these data.

ASSESSING THE ACCURACY AND COMPLETENESS OF RESIDENT SIGOUTS FOR IMPROVING PATIENT HANDOFFS Nadia K. Ali; Salma Baksh; Kristin Chapman; Nancy Law; Sarun Thomas; Ahad Lodhi. Crozer Chester Medical Center, Upland, PA. (Control ID \#1330691)

BACKGROUND: A direct consequence of the enactment of the duty-hour standards by Accreditation Council on Graduate Medical Education in 2003 followed by further modifications in 2010 has been an increase in the number of hand-offs taking place. Breakdown in communication has been cited as the leading root cause of sentinel events by The Joint Commission. A transition task force consisting of interns, residents, chief resident and faculty mentor was formed to evaluate the accuracy and completeness of the written signouts created by interns at a teaching community hospital. METHODS: The transition task force used the 'ANTICIPATE' checklist proposed by Vidyarthi et al. as a framework for assessing the accuracy and completeness of the signout. They identified 28 variables that were grouped into 9 categories: demographic data, admitting complaint and diagnosis, medications \& allergies, assessment \& plan, baseline vital signs and mental status, recent procedures and significant events, follow up plan, primary team contact information and organization of content. Signouts were obtained from 17 interns (77\%) on 4 randomly selected weekdays over a course of 2 weeks. The collected data was corroborated by cross-checking the patient charts. Data was recorded and analyzed using descriptive statistics.

RESULTS: The average intern score for accurate and complete signout was around $60 \%$ (16.77 \pm 3.05 of 28 points). The categories which achieved accurate completion score of over $80 \%$ included organization of content ( $88 \pm$ 1) and documentation of recent procedures and significant events $(92 \pm 1)$. The categories that were accurately documented less than $50 \%$ included assessment and plan $(21 \% \pm 0)$ and baseline vital signs and mental status $(2 \% \pm 3)$. Medication and allergies received an average score of $56 \%$ and Primary team contact information received an average score of $51 \%$.

CONCLUSIONS: The study indicates that there is substantial variability in the accuracy and completeness of the interns' signouts. Multiple categories of critical significance are deficient indicating the need for standardization of signouts as well as provision of handoffs training to all interns and residents. This study also provides an approach to evaluate the accuracy and completion of signouts for physicians in training. The next step for the transition task force is to create a standardized template and conduct a handoffs workshop for interns and residents.

Table 1: Percentage completion for the different sign-out assessment categories

\begin{tabular}{lll}
\hline \hline$\#$ & Assessment Categories & $\begin{array}{l}\text { Accurately completed } \\
\text { (Percentage } \pm \text { Standard } \\
\text { Deviation) }\end{array}$ \\
1 & Demographic Data & $62 \pm 29$ \\
2 & Admitting Complaint \& Diagnosis & $64 \pm 30$ \\
3 & Medications \& Allergies & $56 \pm 49$ \\
4 & Assessment \& Plan & $21 \pm 0$ \\
5 & Baseline vital signs and mental status & $2 \pm 3$ \\
6 & Recent procedures \& significant events & $92 \pm 1$ \\
7 & Follow-up plan & $56 \pm 46$ \\
8 & Primary Team Contact information & $51 \pm 8$ \\
9 & Organization of content & $88 \pm 1$ \\
\hline
\end{tabular}

ASSESSING THE EFFECTIVENESS OF A PATIENT- AND PHYSICIAN-TARGETED INTERVENTION TO ADDRESS PRESCRIPTION MEDICATION COSTS Toshiko Uchida; Charlie Zei; Kenzie A. Cameron; Ariane M. Garrett; Anne Henson; Michael Zielinski; Leslie Ramirez; Erik Orelind; Ami Desai; Eric D. Christoff; David W. Baker. Northwestern University Feinberg School of Medicine, Chicago, IL. (Control ID \#1327032) 
BACKGROUND: Patients are being required to pay a larger percentage of rising healthcare costs each year. A recent survey conducted by the REACH Practice-Based Research Network affiliated with Northwestern University Feinberg School of Medicine revealed that patients have significant concerns about the costs of their prescription medications, but they seldom discuss ways to reduce these costs with their physicians. In response to these findings, we designed a bundled intervention consisting of an educational brochure targeted to patients and a brief (20-minute) educational seminar delivered to physicians, both focusing on strategies to help patients reduce their prescription medication costs. The current study sought to assess the effectiveness of this patient- and provider-targeted intervention for: 1) increasing the frequency of discussions between patients and physicians about prescription medication costs; and 2) increasing patient use of strategies to help reduce prescription medication costs.

METHODS: We surveyed separate samples of patients at 9 academic and private practice internal medicine sites before and after implementing the bundled intervention. During the study period, every patient who came for an appointment was given a paper survey to complete after the visit. The survey included items about patients' concerns about prescription costs and attitudes about discussing prescription medication costs with their physicians. A subset of patients agreed to complete a follow-up questionnaire via phone or web survey approximately two weeks later. These follow-up items related to patients' use of various strategies to help decrease prescription medication costs.

RESULTS: Three hundred nine (response rate $26.2 \%$ ) patients completed the pre-intervention survey, and 284 patients (response rate 23.4\%) completed the post-intervention survey. One hundred ninety-six $(68.8 \%)$ and $177(67.3 \%)$ of pre- and post-intervention survey respondents completed the follow-up survey, respectively. We observed no difference in the frequency of discussions about prescription medication costs between the pre- and post-tests. However, among patients who did discuss prescription medication costs with their physicians, a significantly larger proportion reported that they (instead of their physician) initiated the discussion in the post-intervention group ( $70 \%$ ) compared to the pre-intervention group ( $42 \%)$ $(p=0.04)$. For follow-up questions related to use of various strategies to help reduce costs (e.g., using mail order pharmacies, using pharmacy discount cards, etc.), only use of websites to comparison shop for prescription medications increased (pre $2 \%$, post $16 \% ; \mathrm{p}=0.04$ ).

CONCLUSIONS: Combined use of a patient-targeted brochure and a physician-targeted seminar designed to increase patient-provider discussions about prescription medication costs was effective in increasing patient-initiated discussions about such costs. This bundled intervention was also effective in increasing patient use of websites to compare prescription medication costs. The intervention was not effective for increasing patient use of more traditional strategies, such as using mailorder pharmacies or pharmacy discount cards.

ASSESSMENT OF TYPE 2 DIABETES RISK FACTORS AVAILABLE IN THE ELECTRONIC MEDICAL RECORD BY USING ASSOCIATION RULE MINING Jane W. Njeru; Gyorgy J. Simon; M. Regina Castro; Stephen S. Cha; Peter W. Li; Pedro J. Caraballo. Mayo Clinic, Rochester, MN. (Control ID \#1336961)

BACKGROUND: The prevalence of diabetes continues to grow worldwide. Identifying individuals at risk is at the core of any prevention efforts. Multiple diabetes prediction models have been developed using clinical and/ or laboratory factors. However, most models are rarely used due to several barriers. New models with novel techniques could provide solutions to these barriers. Association rules mining can discover relationships between unrelated data, and when applied to large databases could help identify new risk factors. In this project we seek to validate the clinical relevance of risk factors selected by association rules mining and compare them against those from previously published models.

METHODS: We used a cohort of 53,393 adults Olmsted County, MN, residents who visited Mayo Clinic between 1999-2004. They all have a glucose value in the electronic medical record (EMR) and no previous diagnosis of diabetes or glucose $\geq 126 \mathrm{mg} / \mathrm{dl}$. We collected 29 baseline characteristics available as structured data in the EMR including vital signs, medications, diagnoses, and laboratory values. We assessed 5-year incidence of diabetes; 2,349 subjects developed diabetes during the follow up period. We used association rule mining to discover rules that associate risk factors (alone or in combination) with increased risk of diabetes, adjusted for age and timeto-event. This adjustment was performed using the Cox proportional hazard model. For each rule, we calculated its relative risk and statistical significance (Wilcox and Poisson tests). We compared our model with risk factors described in a 2011 systematic review by Collins et al. These risk factors were selected using statistical models other than association rule mining.

RESULTS: The association rule mining identified 331 rules with statistically significant increased risk of diabetes (risk factors alone or in combination). Only 16 of the 29 baseline characteristics were included in at least one rule as risk factors. These factors were glucose $(\mathrm{n}=207), \mathrm{BMI}(\mathrm{n}=170)$, triglycerides $(n=154)$, hypertension $(n=139)$, HDL cholesterol $(n=88)$, statins $(n=83)$, ACEI-ARB $(n=71)$, aspirin $(n=69)$, systolic BP $(n=50)$, cholesterol $(n=46)$, beta blockers $(n=37)$, diuretics $(n=23)$, diastolic BP $(n=11)$, tobacco $(n=10)$, ischemic heart disease $(n=7)$ and fibrates $(n=5)$. Each rule had between 1 and 6 risk factors. The relative risk was calculated for each rule and was between 1.34 (beta blockers use) and 3.87 (BMI+HDL+glucose+triglycerides+aspirin+hypertension). Collins et al. reviewed 39 studies with 43 prediction models for incident and prevalent diabetes. These models used a median cohort of 2,562 and a median of 14 candidate risk factors (range 4 to 64). The final models included a median of 6 risk factors (range 2 to 11). The most common risk factors included age, family history of diabetes, BMI, hypertension, waist circumference, gender, ethnicity, glucose level, smoking status, physical activity, systolic blood pressure, triglycerides, HDL, CVD, and HTN and CAD medications. All 16 risk factors in our model have been included in previous models although their definitions may differ; i.e. HTN medications vs. specific class of medications.

CONCLUSIONS: Our novel risk model derived using a large cohort, EMR data, and association rules mining identifies clinically relevant risk factors to predict type 2 diabetes. Further research is needed to better assess this model and its implementation in clinical practice.

ASSOCIATION BETWEEN FOOD INSUFFICIENCY AND MORTALITY: JOINT EFFECT WITH INCOME ON ALL-CAUSE AND CAUSE-SPECIFIC MORTALITY Jennifer Zhu ${ }^{1}$; Ankit Parikh ${ }^{2}$; Stuart R. Lipsitz ${ }^{3}$; Sundar Natarajan ${ }^{4} .{ }^{1}$ NYU School of Medicine, New York, NY; ${ }^{2}$ NYU School of Medicine, New York, NY; ${ }^{3}$ Brigham and Womens Hospital, Boston, MA; ${ }^{4}$ VA New York Harbor and NYU School of Medicine, New York, NY. (Control ID \#1338680)

BACKGROUND: Food insufficiency is the state in which people have an inadequate intake of food due to a lack of resources. It is associated with chronic diseases and poor health outcomes. However, the association between food insufficiency and mortality is not known.

METHODS: Since income may modify the effect of food insufficiency, participants were categorized as: food sufficient middle-income, food insufficient middle-income, food sufficient low-income, and food insufficient low-income. We evaluated the relationship between food insufficiency and mortality among US adults in the Third National Health and Nutrition Examination Survey. Age and sex-adjusted Kaplan-Meier curves characterized survival over time, and a log-rank test was used to test if survival differences existed between groups. The effect of the 4-level food insufficiency income variable on mortality was evaluated using age and sex-adjusted Cox models and multivariate Cox models that also adjusted for race-ethnicity, education, diet, physical activity, smoking, diabetes, obesity, hyperlipidemia, and hypertension. To further elucidate the effect of each variable in the causal pathway on the relationship between the food sufficiencyincome variable and mortality, Cox models sequentially incorporated age, sex, race, and risk factors. Low-income food sufficient adults were the referent group in all Cox models.

RESULTS: Of the 13,722 adults surveyed, $62.2 \%$ were food sufficient middle-income, $1.1 \%$ food insufficient middle-income, $32.8 \%$ food sufficient low-income, and 3.8\% food insufficient low-income. At 18 years, food 
sufficient low-income adults had a higher survival rate compared to food insufficient low-income adults in age and sex-adjusted Kaplan-Meier curves ( $72 \%$ vs. $68 \%, \mathrm{p}<.001)$. In multivariate Cox models, low-income food insufficient adults had higher all-cause mortality (HR 1.31, CI 1.05-1.63, $\mathrm{p}=.02$ ) and cancer mortality (HR 1.72, CI 1.07-2.78, $\mathrm{p}=.03$ ). Cardiovascular mortality did not reach statistical significance (HR 1.26, CI 0.80-2.00, $\mathrm{p}=.32$ ). In Cox models that excluded variables in the putative causal pathway to mortality, the multivariate HR in low-income food insufficient adults was 1.46 for all-cause mortality (CI 1.19-1.79, $\mathrm{p}<.001), 1.95$ for cancer mortality (CI 1.14-3.33, $\mathrm{p}=.02$ ), and 1.50 for cardiovascular mortality (CI 1.01-2.22, $\mathrm{p}=.046$ ). In sequential models for all-cause mortality, socioeconomic variables and demographic characteristics had modest effects on the relationship between the food insufficient low-income group and mortality (HR changed from 1.91 to 1.83). However, adding lifestyle and risk factors changed the HR from 1.83 to 1.40 . While these sequential adjustments attenuated the effect on mortality, the association between food insufficiency and all-cause mortality, as well as cancer mortality, remained significant even after full adjustment.

CONCLUSIONS: Low-income adults who are food insufficient have higher all-cause and cancer mortality than low-income adults who are food sufficient. Cardiovascular mortality is also higher after variables associated with the putative causal pathway to mortality are excluded. Food insufficiency is at the highest level it has ever been since the U.S. Department of Agriculture started collecting data in 1995. Screening for food insufficiency may provide a cost-effective way for policy makers and health care providers to identify people at high risk of negative health outcomes and intervene by directing people to the appropriate resources.

ASSOCIATION BETWEEN HEALTH LITERACY, PATIENT
ACTIVATION, AND GLYCEMIC CONTROL AMONG DIABETIC
VETERANS WITH MULTIMORBIDITY LeChauncy D. Woodard;
Cassie R. Landrum; Degang Wang; Laura A. Petersen; Aanand D. Naik.
Michael E. DeBakey VA Medical Center HSR\&D Center of Excellence
and Baylor College of Medicine, Houston, TX. (Control ID \#1336197)

BACKGROUND: Diabetes is a highly prevalent condition that often coexists with other chronic illnesses. Although the benefits of glycemic control have been widely documented, diabetic patients often have suboptimal control. Prior studies have shown that higher patient activation is associated with better self management and that health literacy is associated with the confidence to make health-related decisions. Both patient activation and health literacy may be particularly important for patients with diabetes, as it is largely a self-managed condition that requires patient involvement in most aspects of care. We examined the interaction of self-reported health literacy and patient activation on glycemic control among diabetic patients with coexisting cardiovascular disease

METHODS: We administered a survey that included previously validated items on patient activation (Patient Activation Measure) and health literacy (Three-item Health Literacy Measure) to 387 diabetic patients with coexisting hypertension and ischemic heart disease (IHD). We used VA administrative data to screen patients receiving care at a large, urban VA Medical Center from November-December 2010 for our eligibility criteria. We included patients who had 2 outpatient ICD-9 diagnosis codes or 1 inpatient code indicating the study conditions. We also identified patients with IHD using relevant cardiovascular procedure codes. After identifying eligible patients from administrative data, we confirmed the target diagnoses with a medical record review before administering the surveys. Among those who completed surveys, we reviewed medical records to obtain evidence of diabetes control. We used logistic regression to examine the association between achievement of guideline-recommended $\mathrm{HbAlc}$ thresholds (

RESULTS: 195 individuals (50.4\%) returned surveys. Participants were $71 \%$ White, $25 \%$ Black, $4 \%$ Other, $99 \%$ male, and had a mean age of 68.1 years. $87 \%$ of patients had $\mathrm{HbA} 1 \mathrm{c}$ levels

CONCLUSIONS: We found that both patient activation and health literacy are associated with achievement of guideline-recommended $\mathrm{HbA1c}$ control. Our results suggest that patient activation may play an influential role in achieving the less-stringent $\mathrm{HbAlc}$ threshold (
ASSOCIATION BETWEEN PHYSICIANS' BELIEFS AND WHETHER THE OPTION OF COMFORT CARE IS OFFERED FOR PATIENTS WITH ADVANCED CRITICAL ILLNESS Yael Schenker $^{1}$; Greer A. Tiver ${ }^{1}$; Seo Yeon $\mathrm{Hong}^{3}$; Douglas B. White ${ }^{2}$. ${ }^{1}$ University of Pittsburgh, Pittsburgh, PA; ${ }^{2}$ University of Pittsburgh, Pittsburgh, PA; ${ }^{3}$ University of Pittsburgh, Pittsburgh, PA. (Control ID $\# 1310165)$

BACKGROUND: For patients with advanced critical illness, treatment focused on comfort is an accepted alternative to treatment focused on lifeprolongation. Little is known about whether and how physicians present this option to surrogates. The objectives of this study were to assess how comfort care is presented to surrogates and whether physicians' beliefs about whether life support should be withdrawn are associated with the presentation of comfort care.

METHODS: We conducted a mixed-methods study of audio-recorded physician-family conferences in 5 ICUs at two academic hospitals from January, 2006 through August, 2008. Participants included 169 family members and 54 physicians who participated in 72 conferences about treatment decisions for patients at high risk of death or severe functional impairment. Transcripts of audio-recorded conferences were coded to identify 1) the presentation of comfort care by the physician, 2) discussion of the risks and benefits of comfort care, and 3) treatment options discussed when comfort care was not offered. Physicians completed a questionnaire indicating the strength of their belief that life support should be withdrawn. We used hierarchical logistic regression to assess the association between the physician's belief that life support should be withdrawn and the presentation of comfort care as an option.

RESULTS: Audio-recorded conferences took place an average of 10 (SD 12) days after admission to the ICU. The inpatient mortality rate was $72 \%$. Using a broad definition of comfort-oriented treatment, comfort care was presented as an option in 56\% [95\% CI, 44\%-67\%] of conferences. Few risks and benefits of comfort care were described (mean number of risks per conference was 0.2 (SD 0.5), range 0-2; mean number of benefits 0.9 (SD 1.0), range 0-4). Of the 32 conferences in which comfort care was not discussed, $78 \%$ included only discussion of continued unlimited intensive care, $9 \%$ included only discussion of limited intensive care, and $13 \%$ included discussion of unlimited intensive care and placing some limit on intensive care. In many conferences, intensive care was described as the default option. In clustered multivariate models adjusting for APACHE II score and number of days in the ICU, the only independent predictor of offering comfort care as an option was the strength of the physician's belief that life support should be foregone (OR 1.38 [1.14-1.66], $\mathrm{p}=.01$ ).

CONCLUSIONS: Among a cohort of critically ill patients with a high mortality rate, comfort care was not discussed as an option in nearly half of clinician-family conferences, and the more strongly the physician believed that life support should be continued, the lower the odds that comfort care was offered. These data suggest a previously unrecognized problem with decision making for incapacitated patients with advanced illness: the possibility that physicians' personal values may consciously or unconsciously influence whether widely-accepted (and often preferred) end-of-life treatment options are offered. Clinicians should be aware of this potential bias. Researchers should develop and test interventions to ensure that surrogates are able to consider the range of medically-permissible treatment options, including comfort care, when making decisions for loved ones with far advanced illness.

\section{ASSOCIATION BETWEEN RESIDENT ACGME CORE COMPETENCIES AND OUTPATIENT LABORATORY UTILIZATION Jessica R. Singer $^{1}$; Nancy Chang ${ }^{1}$; Rafael A. Lantigua ${ }^{1}$; Steven Shea ${ }^{1,2}$. ${ }^{\mathrm{T}}$ New York Presbyterian Hospital - Columbia University Medical Center, New York, NY; ${ }^{2}$ Columbia University, New York, NY. (Control ID \#1317386)}

BACKGROUND: There is significant variation in laboratory utilization by physicians. This variation matters because there are potential negative consequences for patients, provider organizations and the health care system from under- and over-utilization. Previous studies 
have demonstrated that part of the variation in laboratory utilization can be explained by patient-specific factors, physician demographic factors and physician behaviors. Our interest is specifically in the behavioral factors which are potentially modifiable during the formative years of residency training. Previous literature in this area has been limited to attending physician laboratory utilization, self report of behaviors, and focus on inpatient utilization. We aim to determine whether there is an association between Internal Medicine resident physician behaviors, including Accreditation Council for Graduate Medical Education (ACGME) competencies, and laboratory utilization in the outpatient setting.

METHODS: Participants included all 39 first-year Internal Medicine residents at New York Presbyterian Hospital - Columbia University Medical Center (NYPH-CUMC) during the academic year 7/2010-6/ 2011 who had their outpatient continuity practice at the Association of Internal Medicine (AIM) practice. The 4 first-year residents who had their outpatient clinic at other sites were excluded. The AIM practice is a joint resident physician and faculty group practice serving the publicly insured Northern Manhattan community. Laboratory utilization was measured as the total number of outpatient labs ordered by each resident during the academic year adjusted for the resident's total number of patient visits that year. Resident behaviors and competencies were measured by supervising inpatient attendings using standardized monthly electronic evaluations of patient care, medical knowledge, practice-based learning and improvement, interpersonal and communication skills, professionalism, systems-based practice, teaching and teamwork and overall clinical competency. Evaluation scores were scaled from 1 to 9. Spearman rank correlation coefficients were calculated between the outcome and independent variables.

RESULTS: Participants were $46 \%$ female. The distribution of laboratory utilization was right skewed with an average of 3.2 (IQR 2.4-4.0) labs ordered per patient visit. The Spearman rank correlation coefficients demonstrated inverse associations between laboratory utilization and patient care $(\mathrm{r}=-0.13 \mathrm{p}=0.40)$, medical knowledge $(\mathrm{r}=-0.18 \mathrm{p}=0.27)$, practice-based learning and improvement $(\mathrm{r}=-0.13 \mathrm{p}=0.42)$, interpersonal and communication skills $(\mathrm{r}=-0.19 \mathrm{p}=0.24)$, professionalism $(\mathrm{r}=-0.25 \mathrm{p}=$ $0.11)$, systems-based practice $(\mathrm{r}=-0.15 \mathrm{p}=0.35)$, teaching and teamwork $(\mathrm{r}=$ $0.30 \mathrm{p}=0.06)$, and overall clinical competency $(\mathrm{r}=-0.22 \mathrm{p}=0.18)$. Correlation coefficients between resident competencies ranged from 0.79 to 0.92 (all $\mathrm{p}$ values $<0.001$ ) indicating that there is significant overlap in the competencies as evaluated by attending physicians.

CONCLUSIONS: Better performance on attending rated competencies and behaviors is associated with decreased laboratory utilization in first year residents. Further studies will (1) investigate these relationships in larger sample sizes including second and third year residents to have more power to assess significant associations, and (2) specifically assess whether patterns of utilization on both ends of the spectrum are inappropriate.

\section{ASSOCIATION BETWEEN THE COMMERCIAL CHARACTER- ISTICS OF PSYCHOTROPIC DRUGS AND THE PROBABILI- TY OF THEIR OFF-LABEL USE G. Caleb Alexander, ${ }^{4,5}$; Chris Graziul $^{1}$; Robert Gibbons ${ }^{2,3}$. ${ }^{1}$ University of Chicago, Chicago, IL; ${ }^{2}$ University of Chicago, Chicago, IL; ${ }^{3}$ University of Chicago, Chicago, IL; ${ }^{4}$ University of Chicago, Chicago, IL; ${ }^{5}$ University of Chicago, Chicago, IL. (Control ID \#1326157)}

BACKGROUND: Off-label prescribing, or the use of a medicine for nonFDA approved indications, is especially common for psychotropic therapies and often lacks scientific support. We quantified the association between four commercial characteristics of prescription medicines product age, therapeutic class age, drug volume, and promotional expenditures - and off-label use of antidepressants, antipsychotics and mood stabilizers from 1998 through 2009.

METHODS: We linked data from the IMS Health National Disease and Therapeutic Index ${ }^{\mathrm{TM}}$, a nationally representative audit of office-base physicians, with data from FDA@gov and the drug compendium DrugDex ${ }^{\mathrm{TM}}$, to derive information regarding off-label use. Our primary outcome was the probability that a drug was used for non-FDA approved indications during a given calendar year. We used mixed-effects regression models with random intercepts for each drug, adding measures of commercial characteristics as fixed effects within this model.

RESULTS: We examined agents that represented 981 million [M] drug mentions over the time period examined; this was comprised of $143 \mathrm{M}$ mentions for antipsychotics, $532 \mathrm{M}$ mentions for antidepressants and $306 \mathrm{M}$ mentions for mood stabilizers. From 1998 through 2009, mood stabilizers were the most likely to be used off-label (average annual off-label use of class members ranged from $46 \%-59 \%$ ) and anti-depressants were least likely (25\%-29\%). There was a positive association between the annual proportion of all uses of a therapy that occurred off-label and drug volume (incidence rate ratio [IRR] $1.25,95 \%$ confidence intervals [CI] 1.20-1.31), although the strength of this association differed across the therapeutic classes examined. For each order of magnitude increase in volume of therapy, the fraction of off-label use increased by $42 \%$ for antipsychotics, $26 \%$ for mood stabilizers, and $18 \%$ for anti-depressants. By contrast, there was no statistically significant association between product age (IRR 0.99, CI 0.97-1.00) or class age (IRR 1.01, CI 0.99-1.03) and the probability of off-label use. There was a small, statistically significant association between promotional expenditures and less off-label use (IRR 0.97, CI 0.96-0.98). These associations were similar when examining scientifically unsupported rather than all off-label use.

CONCLUSIONS: Our findings suggest that psychotropic drug volume, rather than product age or therapeutic class age, should be scrutinized to identify settings where the public health impact of unsupported off-label prescribing may be particularly important.

\section{ASSOCIATION OF PATIENT CHARACTERISTICS AND PHYSICIAN} SPECIALTY WITH PROSTATE SPECIFIC ANTIGEN TEST FOLLOWUP Lipika Samal ${ }^{1,2}$; Adam Wright ${ }^{1,2}$; Francine L. Maloney ${ }^{2}$; Julie Fiskio ${ }^{2}$; Stuart R. Lipsitz ${ }^{1,2}$; Gianna Zuccott ${ }^{1,3}$. ${ }^{1}$ Brigham and Women's Hospital, Boston, MA; ${ }^{2}$ Partners HealthCare, Boston, MA; ${ }^{3} \mathrm{CRICO} /$ Risk Management Foundation, Boston, MA. (Control ID \#1336410)

BACKGROUND: Prostate cancer disparities exist, but it is unknown whether the disparities are attributable to differences in initial follow-up of abnormal prostate specific antigen (PSA) tests. Few studies have examined the process of PSA follow-up using data from clinical information systems. METHODS: We queried our clinical information system for PSA test results on adult patients performed at Brigham and Women's Hospital from June 30, 2008 - June 29, 2009. We determined whether abnormal results were followed by either: 1) repeat PSA test, 2) problem list diagnosis (e.g., benign prostatic hyperplasia), 3) a visit with Urology, 4) a visit with Oncology, 5) a procedure such as a biopsy or operation, 6) death, or 7) documentation of patient refusal. Patients with problem list documentation of pre-existing conditions predisposing them to elevated PSA (e.g., prostate cancer) were excluded. Chart review was performed for all failures to followup. Data analysis examined the association of patient characteristics and physician specialty with follow-up. We used regression analysis of survival data based on the Cox proportional hazards model to adjust for potential confounders and clustering by patient.

RESULTS: Over a one-year period, we found 1,166 abnormal PSA test results, $97 \%$ of which had a follow-up within 1,026 days. Follow-up actions were a repeat PSA test in $27 \%$ of cases and a visit in $53 \%$ of cases. The median index PSA value was 6.0 (maximum value 11,016). Higher index PSA value $(\mathrm{p}=0.001)$ and younger patient age were significantly associated with follow-up $(\mathrm{p}=0.004)$, but race, language preference, and marital status were not. Physician specialty was significantly associated with follow-up. As compared to PSA tests ordered by Internal Medicine, PSA tests ordered by Radiation Oncology were followed-up in a more timely manner $(\mathrm{p}=0.002$, see Figure line 5 as compared to line 1) and after adjustment for patient age, index PSA value, and clustering by patient, this difference remained significant $(\mathrm{p}=$ $0.002)$. Follow-up of PSA tests ordered by Transplant Surgery $(\mathrm{p}<0.001)$ and tests without a physician's name on the order $(\mathrm{p}=0.03)$ was less timely than for those tests ordered by Internal Medicine in unadjusted analyses, and the difference between Transplant Surgery and Internal Medicine persisted after adjustment $(\mathrm{p}<0.001)$. 
CONCLUSIONS: We have found that physician specialty is related to timeliness of follow-up in a large sample of PSA tests. In future, queries of clinical information systems may help us to create systems-level solutions to improve timeliness of abnormal test result follow-up.

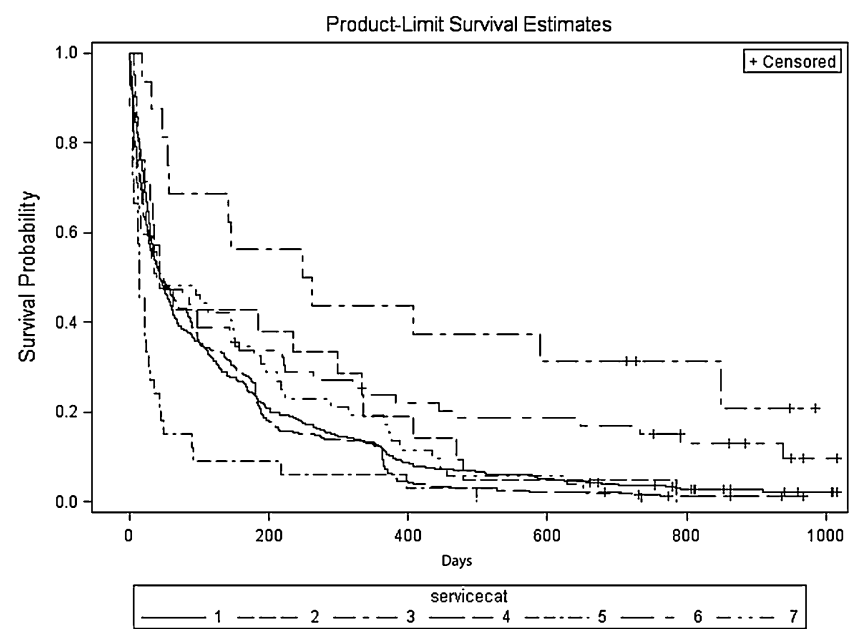

Servicecat: 1) Internal Medicine, 2) Cardiology, 3) Transplant Surgery, 4) Urology, 5) Radiation Oncology, 6) No name, 7) Other specialty

ASSOCIATIONS BETWEEN ACTIVE TRANSPORT AND CARDIOVASCULAR DISEASE RISK FACTORS IN US ADULTS Gregg L. Furie. ${ }^{1}$ Yale School of Medicine, New Haven, CT; ${ }^{2}$ Department of Veterans Affairs, West Haven, CT. (Control ID \#1334562)

BACKGROUND: Despite the known cardiovascular health benefits of exercise, many US adults do not obtain recommended amounts of physical activity. Active transport - defined as walking or biking for transportation could be a valuable source of physical activity, particularly for individuals with low levels of work or recreational physical activity. However, little is known about the health benefits of active transport in the US adult population, and, therefore, whether recommending walking and biking for transportation would be a clinically useful disease prevention strategy. Thus, we investigated the association between active transport and cardiovascular disease risk factors among US adults.

METHODS: We used cross-sectional data from the 2007-2008 and 20092010 cycles of the National Health and Nutrition Examination Survey (NHANES). Adults aged $\geq 20$ years who were not pregnant and reported unimpaired mobility were classified according to their weekly amount of active transport: no active transport $(0 \mathrm{~min} / \mathrm{wk})$, low active transport (1$149 \mathrm{~min} / \mathrm{wk}$ ), or high active transport ( $\geq 150 \mathrm{~min} / \mathrm{wk}$ ). Multivariable linear and logistic regression analyses were performed to examine the associations between level of active transport and physical activity-related cardiovascular disease risk factors, including body mass index (BMI), abdominal waist circumference, hypertension, diabetes, and abnormal high-density lipoprotein (HDL) level. Analyses were weighted to account for the complex survey design and adjusted for sociodemographic characteristics, smoking status, and minutes per week of combined work and recreational physical activity.

RESULTS: Of 9,933 adults included in the final sample, $75 \%$ engaged in no active transport, $11 \%$ engaged in low active transport, and $14 \%$ engaged in high active transport. Overall, there were significant inverse associations between level of active transport and BMI, abdominal waist circumference, hypertension, and diabetes. After adjusting for covariates, when compared with no active transport, individuals engaging in low active transport and high active transport had $0.9 \mathrm{~kg} / \mathrm{m}^{2}(95 \%$ confidence interval $[\mathrm{CI}]=0.5-1.4)$ and $1.2 \mathrm{~kg} / \mathrm{m}^{2}(95 \% \mathrm{CI}=0.8-1.7)$ lower mean BMIs, respectively, and $2.2 \mathrm{~cm}$ $(95 \% \mathrm{CI}=1.2-3.2)$ and $3.1 \mathrm{~cm}(95 \% \mathrm{CI}=1.0-4.3)$ smaller mean waist circumferences, respectively. Odds of hypertension were $24 \%$ lower (adjusted odds ratio $[\mathrm{OR}]=0.76,95 \% \mathrm{CI}=0.61-0.94)$ and $31 \%$ lower $(\mathrm{OR}=0.69,95 \%$
$\mathrm{CI}=0.58-0.83$ ) among those with low and high levels of active transport. High levels of active transport were associated with $31 \%$ lower (OR $0.69,95 \% \mathrm{CI}=$ 0.54-0.88) odds of diabetes. Active transport was not associated with abnormal HDL level.

CONCLUSIONS: Active transport is associated with lower BMI, smaller waist circumference, and lower odds of hypertension and diabetes in US adults. This association is consistent with the known relationship between physical activity and cardiovascular disease risk factors and supports the utility of recommending active transport as an alternative form of physical activity for patients.

ATTITUDES AND PREFERENCES TOWARD THE PROVISION OF MEDICATION ABORTION IN AN URBAN ACADEMIC INTERNAL MEDICINE PRACTICE Cameron S. Page ${ }^{1}$; Marji Gold ${ }^{3}$; Sarah Stumbar ${ }^{2} .{ }^{1}$ Beth Israel Medical Center, New York, NY; ${ }^{2}$ Stony Brook University School of Medicine, Stony Brook, NY; ${ }^{3}$ Albert Einstein College of Medicine, Bronx, NY. (Control ID \#1276165)

BACKGROUND: Mifepristone (formerly known as RU-486) became commercially available for early abortion in the US in 2000. This offered Internal Medicine doctors the opportunity to greatly expand abortion access for their patients. A decade later, almost $70 \%$ of pregnancy terminations still occur in specialist clinics, with less than $1 \%$ of abortions performed by generalists. Our goal was to determine whether patient preference is a reason for the limited uptake of medication abortion among Internal Medicine physicians.

METHODS: The study took place at an urban primary care center with Internal Medicine (IM) and $\mathrm{Ob} / \mathrm{Gyn}$ clinics. Between December 2008 and July 2009, we approached women in the waiting room of the IM clinic, and invited them to be anonymously interviewed. All consecutive women aged $18-45$ who presented to the clinic during research sessions were offered participation in the study. A semistructured 43-item questionnaire was used to inquire about demographic characteristics, pregnancy history, contraceptive practices, and abortion preferences. Support for medication abortion was assessed with a binary yes/no question, followed by the open-ended question, "Why do you think this clinic should or should not offer medication abortion?" Responses to the open-ended question were coded into one of nine categories. The importance of abortion services was assessed with a question that had three possible responses: very important, somewhat important, and not important.

RESULTS: Of 102 women who met inclusion criteria, 90 completed the survey, yielding a response rate of $88 \%$. $49 \%$ were Hispanic, $51 \%$ black. The average age was $33.82 \%$ of respondents reported having vaginal intercourse with a man in the past three months. $22.2 \%$ were at risk of unintended pregnancy. $47 \%$ had had at least one abortion in the past. None of the 77 pregnancy terminations reported were medication abortions. 59\% responded Yes to the question, "Do you think this clinic should offer medication abortions?" and $66 \%$ stated that it was "very important" or "somewhat important" to offer this service. Among women who did not support medication abortion, the most common reason given was "abortion is morally wrong" $(71 \%)$. Among women who would consider having an abortion in the future, $87 \%$ stated that they would be interested in receiving medication abortion from their primary care doctor.

CONCLUSIONS: To our knowledge, this is the first study to examine the abortion preferences of Internal Medicine patients specifically. We found rates of sexual intercourse, unintended pregnancy, and abortion use consistent with national trends. Among our respondents who would consider abortion, a wide majority would like to receive it from their primary care doctor. Our data suggest that patient preference is not a reason for limited uptake of medication abortion among Internal Medicine doctors. Alternate factors, such as physician comfort, should be explored. These data are limited by the small sample size, and a larger study should be done to assess whether these findings are generalizable. The provision of medication abortion by Internal Medicine physicians has the potential to greatly expand abortion access for women 
ATTITUDES REGARDING COLORECTAL CANCER SCREENING TESTS AMONG HISPANIC/LATINO AND NON-HISPANIC BLACK PATIENTS: HOW DO THEY COMPARE? Kenzie A. Cameron'; Vanessa Ramirez-Zohfeld ${ }^{1}$; Alfred W. Rademaker ${ }^{2}$; M. Rosario Ferreira ${ }^{1,4}$; Nancy C. Dolan ${ }^{1,3}$; Dachao Liu ${ }^{2} .{ }^{1}$ Northwestern University Feinberg School of Medicine, Chicago, IL; ${ }^{2}$ Northwestern University Feinberg School of Medicine, Chicago, IL; ${ }^{3}$ Northwestern Medical Faculty Foundation, Chicago, IL; ${ }^{4}$ Jesse Brown VAMC, Chicago, IL. (Control ID \#1338868)

BACKGROUND: Research has explored attitudes toward colorectal cancer (CRC) screening among minority populations; however, such studies often focus exclusively on one racial and/or ethnic population at a time. We sought to explore attitudes toward CRC screening among Hispanic/Latino (H/L) and Non-Hispanic Black (NHB) patients seeking care within the same Federally Qualified Health Center (FQHC) system in a large, urban area.

METHODS: Data were extracted from a larger, ongoing randomizedcontrolled study to promote colorectal cancer screening. English and Spanish-speaking patients seeking care at one of six FQHCs participated in an interviewer- administered survey that included five attitude questions about stool tests (FOBT and FIT); five related questions were asked regarding attitudes toward colonoscopy. Participants responded on a Likert scale $(1=$ strongly disagree to $4=$ strongly agree). Independent sample t-tests were used to examine differences in attitude scores among $\mathrm{H} / \mathrm{L}$ and $\mathrm{NHB}$ participants.

RESULTS: Among 186 patients, $48.92 \%$ identified as $\mathrm{H} / \mathrm{L}$ and $51.08 \%$ as NHB with a mean age of 58.23 years $(\mathrm{SD}=6.52) ; 71.50 \%$ were female. The groups differed in their attitudes toward the CRC screening tests on only three of the 10 items. Although neither group perceived stool tests as particularly painful, $\mathrm{H} / \mathrm{L}$ participants reported perceiving a stool test as more painful $(\mathrm{M}=1.94, \mathrm{SD}=1.08)$ than NHB $(\mathrm{M}=1.53, \mathrm{SD}=0.80 ; \mathrm{p}<0.01)$. $\mathrm{H} / \mathrm{L}$ participants also perceived a colonoscopy as easier to do $(\mathrm{M}=3.23, \mathrm{SD}=$ $0.94)$, but as more embarrassing $(\mathrm{M}=2.68, \mathrm{SD}=1.16)$ than $\mathrm{NHB}$ (ease: $\mathrm{M}=$ $2.76, \mathrm{SD}=1.15 ; \mathrm{p}<.01 ;$ embarrassment: $\mathrm{M}=2.28, \mathrm{SD}=1.17 ; \mathrm{p}<.05)$. No other differences emerged (see Table 1).

CONCLUSIONS: Overall, attitudes toward both stool tests and colonoscopy among the two groups were fairly similar. If the discrepant attitudes reported here are found across multiple samples, these subtle, yet significant differences can inform future prevention efforts. Although a significant difference was seen regarding perceptions of ease of colonoscopy, we are unable to ascertain how participants defined "easy to do" (e.g., in terms of preparation, access, etc.); making interpretation of this difference difficult. These findings may help to better understand attitudes toward potential screening tests across underserved minority groups and consequently help provide optimal CRC screening counseling in FQHC settings.

\begin{tabular}{|c|c|c|c|}
\hline Attitude Item & $\begin{array}{l}\text { Hispanic/ } \\
\text { Latino M (SD) }\end{array}$ & $\begin{array}{l}\text { Non-Hispanic } \\
\text { Black M (SD) }\end{array}$ & $\begin{array}{l}\text { Statistical } \\
\text { Significance }\end{array}$ \\
\hline $\begin{array}{l}\text { A stool test would } \\
\text { be easy to do. }\end{array}$ & $3.61(0.63)$ & $3.65(0.67)$ & NS \\
\hline $\begin{array}{l}\text { A stool test would } \\
\text { be messy. }\end{array}$ & $2.82(1.09)$ & $2.55(1.14)$ & NS \\
\hline $\begin{array}{l}\text { A stool test would } \\
\text { be painful. }\end{array}$ & $1.94(1.08)$ & $1.53(0.80)$ & $<0.01$ \\
\hline $\begin{array}{l}\text { A stool test would } \\
\text { be embarrassing. }\end{array}$ & $2.29(1.17)$ & $2.00(1.13)$ & NS \\
\hline $\begin{array}{l}\text { A stool test would } \\
\text { be scary. }\end{array}$ & $2.08(1.16)$ & $1.96(1.15)$ & NS \\
\hline $\begin{array}{l}\text { A colonoscopy would } \\
\text { be easy to do. }\end{array}$ & $3.23(0.94)$ & $2.76(1.15)$ & $<0.01$ \\
\hline $\begin{array}{l}\text { A colonoscopy would } \\
\text { be messy. }\end{array}$ & $2.36(1.18)$ & $2.38(1.08)$ & NS \\
\hline $\begin{array}{l}\text { A colonoscopy would } \\
\text { be painful. }\end{array}$ & $2.83(1.08)$ & $2.74(1.09)$ & NS \\
\hline $\begin{array}{l}\text { A colonoscopy would } \\
\text { be embarrassing. }\end{array}$ & $2.68(1.16)$ & $2.28(1.17)$ & $<0.05$ \\
\hline $\begin{array}{l}\text { A colonoscopy would } \\
\text { be scary. }\end{array}$ & $2.81(1.13)$ & 2.85 & NS \\
\hline
\end{tabular}

$1=$ Strongly disagree; 2 =slightly disagree; 3 =slightly agree; $4=$ strongly agree $\mathrm{NS}=$ not statistically significant
AUTO-IMMUNITY, SUBCLINICAL HYPOTHYROIDISM AND THE RISK OF CORONARY HEART DISEASE AND MORTALITY Tinh-Hai Collet ${ }^{1}$; Drahomir Aujesky ${ }^{2}$; Eric Vittinghoff ${ }^{3}$; Douglas Bauer ${ }^{4,3}$; Jacobijn Gussekloo ${ }^{5}$; Anne R.Cappola ${ }^{6}$; Wendy P. den Elzen ${ }^{5}$; José Sgarbi $^{7,8}$; Jacques Cornuz ; Alexandra P. Bremner'; Rui M.B. Maciel ${ }^{7}$; Henry Völzke ${ }^{10}$; John P. Walsh ${ }^{11,12}$; Nicolas Rodondi ${ }^{2,1}$. ${ }^{1}$ University of Lausanne, Lausanne, Switzerland; ${ }^{2}$ Inselspital, University of Bern, Bern, Switzerland; ${ }^{3}$ University of California, San Francisco, CA; ${ }^{4}$ University of California, San Francisco, CA; ${ }^{5}$ Leiden University Medical Center, Leiden, Netherlands; ${ }^{6}$ University of Pennsylvania School of Medicine, Philadelphia, PA; ${ }^{7}$ Federal University of Sao Paulo, Sao Paulo, Brazil; ${ }^{8}$ Faculdade de Medicina de Marília, Marília, Brazil; ${ }^{9}$ The University of Western Australia, Crawley, WA, Australia; ${ }^{10}$ University of Greifswald, Greifswald, Germany; ${ }^{11}$ The University of Western Australia, Crawley, WA, Australia; ${ }^{12}$ Sir Charles Gairdner Hospital, Nedlands, WA, Australia. (Control ID \#1318985)

BACKGROUND: Our recent participant-level meta analysis found that subclinical hypothyroidism (defined as elevated TSH but normal thyroxine levels) was modestly associated with coronary heart disease (CHD) mortality and CHD events, particularly for those with TSH $>10 \mathrm{mIU} / \mathrm{L}$. Thyroid autoantibodies predict the risk of progression from subclinical to overt hypothyroidism, and several guidelines recommend measuring thyroid auto-antibodies to better identify patients who should receive thyroxine replacement. However, it is unknown whether thyroid auto-antibodies predict CHD outcomes associated with subclinical hypothyroidism. We aimed to compare the risks of CHD mortality and CHD events associated with subclinical hypothyroidism in adults with positive vs. negative thyroid auto-antibodies.

METHODS: We searched MEDLINE and EMBASE without language restrictions, and reference lists of retrieved articles to find prospective cohort studies with baseline assessment of thyroid function and autoantibodies that followed subsequent CHD mortality and CHD events. Individual data on 9,063 participants with 109,017 person-years of followup between 1981 and 2005 were supplied from 4 prospective cohorts in Europe, Australia and Brazil. Data on CHD events were available in 4,976 participants from 2 cohorts. Euthyroidism was defined as a TSH 0.45-4.49 $\mathrm{mIU} / \mathrm{L}$ and subclinical hypothyroidism as TSH between 4.5 and $19.9 \mathrm{mIU} /$ $\mathrm{L}$ with normal thyroxin levels. We used study-specific cutoff values for thyroxin and anti-thyroid peroxidase antibodies (anti-TPO).

RESULTS: Among 9,063 adults (54\% of women), 8,418 were euthyroid and 645 had subclinical hypothyroidism $(7.1 \%)$, of whom $278(43.1 \%)$ had positive anti-TPO. During follow-up, 643 participants died of CHD and 1514 had CHD events. In age and gender-adjusted analyses compared to euthyroid individuals, risks of CHD mortality were similar among those with anti-TPO positive subclinical hypothyroidism ( $\mathrm{HR}=1.23,95 \% \mathrm{CI} 0.87-1.74)$ and those with anti-TPO negative subclinical hypothyroidism ( $\mathrm{HR}=1.82,95 \% \mathrm{CI} 0.74-$ $4.48, \mathrm{p}$ for interaction 0.43 ). The risks of CHD events were also similar among subclinical hypothyroid subjects with and without anti-TPO antibodies $(\mathrm{HR}=1.23,95 \%$ CI $0.70-2.18$ vs. $\mathrm{HR}=1.32,95 \%$ CI $0.78-2.23$, $\mathrm{p}$ for interaction 0.86). Risks of CHD mortality and CHD events increased with higher TSH levels, but did not differ between positive and negative anti-TPO. CONCLUSIONS: Risks of CHD mortality and CHD events associated with subclinical hypothyroidism did not differ according to anti-TPO status. Thyroid autoimmunity does not add prognostic information for subsequent CHD outcomes among adults with subclinical hypothyroidism. The role of the measurement of anti-TPO to target thyroxine replacement should be reevaluated.

BARRIERS AND FACILITATORS TO RECOMMENDED BLOOD PRESSURE MEASUREMENT TECHNIQUE IN COMMUNITY-BASED PRIMARY CARE CLINICS Romsai T. Boonyasai ${ }^{1}$; Jill A. Marsteller ${ }^{2,1}$; Cheryl A. Anderson ${ }^{2,1}$; Jeanne B. Charleston ${ }^{1,2}$; Katherine Dietz ${ }^{1}$; Sarah J. Flynnn'; Crystal D. Salcido ${ }^{3}$, Gary Noronha, ${ }^{4,1}$; Lisa A. Cooper ${ }^{1,2}$. ${ }^{1} \mathrm{JHU} / \mathrm{APL}$, Baltimore, MD; ${ }^{2}$ Bloomberg School of Public Health, Baltimore, MD; ${ }^{3}$ Albert Einstein College of Medicine, Bronx, NY; ${ }^{4}$ Johns Hopkins Community Physicians, Baltimore, MD. (Control ID \#1339987)

BACKGROUND: Blood pressure (BP) measurement in clinical settings often deviates from evidence-based standards, which may lead to suboptimal 
hypertension treatment. Prior studies have examined barriers to accurate BP assessment, but few have examined what interventions would be practical for clinical settings.

METHODS: This exploratory study was conducted as part of a pragmatic trial aimed at improving hypertension management at 6 urban, communitybased primary care clinics. Data were collected in two stages: 1) prior to introducing a standardized BP measurement protocol and automated BP measurement device to intervention sites, we conducted 1-hour semistructured focus groups with primary care physicians (PCPs, $n=37$ in 6 groups) and medical assistants (MAs, $n=62$ in 6 groups) to identify potential barriers to performing recommended techniques. 2) After modifying the intervention based on focus group feedback and introducing it to the clinics, we collected written and photographic observations during scheduled followup visits. During these visits we identified implementation issues and engaged in collaborative, solution-oriented problem-solving with clinic staff. We uploaded the transcribed focus group recordings, written diaries from follow-up visits, and photographs to NVIVO for coding and analysis. We used grounded theory to identify principal themes.

RESULTS: Qualitative analysis revealed four barrier themes. First PCPs and MAs expressed concern that performing the recommended BP measurement techniques would interfere with workflow. Second, they identified team dynamics as important barriers to performing recommended procedures. PCPs expressed reluctance to cede BP measurement to MAs, citing concern with reliability of MA-obtained readings and the importance of PCPs personally measuring $\mathrm{BP}$ as a means to enhance patient-provider alliance. MAs cited comfort in having PCPs provide "back-up" for potentially inaccurate measurements. Third, they identified site-specific challenges in the equipment and physical environment, including rooms that lack tables on which to support patients' arms during BP measurement and inadequate size of $\mathrm{BP}$ cuffs for morbidly obese patients. Fourth, they identified site-specific challenges from clinic organization, including limited staffing at lunchtime and patient stacking at the beginning of clinic sessions. Shared problemsolving with clinic staff identified four facilitators to adoption of recommended techniques. First, we provided tools to support recommended practices, including detachable arm boards that allow patients to support their arms on the BP measurement device. Second we reorganized MA workflow. In one example, MAs prepared vaccines while patients rest prior to $\mathrm{BP}$ measurement. Third, we reorganized duties within PCP-MA teams, and in doing so created efficiencies that allowed for the extra time needed to perform the recommended techniques. Finally, we created patient education materials to explain the process changes and enlist patient support for the intervention. CONCLUSIONS: This work suggests that workflow, team dynamics, physical environment, and clinic organization all pose major barriers to performing guideline-based BP measurement techniques. Collaborative problem solving generated 4 categories of feasible solutions to overcoming these barriers. Future studies could examine if the facilitators to intervention adoption can be disseminated to generalizable settings.

BARRIERS AND FACILITATORS TO IMPLEMENTING A MULTIMODAL INTERVENTION TO INCREASE HIV TESTING Barbara Bokhour $^{1,2}$; Stephen R. Henry ${ }^{3}$; Hemen Saifu'; Gemmae M. Fix ${ }^{1,2}$; Michael $\overline{\text { Fletcher }}^{3}$; Matthew B. Goetz ${ }^{3}$; Herschel Knapp ${ }^{3}$; Jane Burgess ${ }^{3}$; Steven Asch ${ }^{4}{ }^{1}$ VA New England Healthcare System, Bedford, MA; ${ }^{2}$ VA New England Healthcare System, Bedford, MA; ${ }^{3}$ Greater Los Angeles Healtcare System, Los Angeles, CA; ${ }^{4}$ Palo Alto VAMC, Palo Alto, CA. (Control ID \#1340651)

BACKGROUND: HIV testing offers significant benefits yet, many patients remain untested. In the Department of Veterans Affairs, increasing HIV testing is a major initiative, requiring novel approaches to implementing this evidence-based practice. This study examined barriers and facilitators to implementing a multimodal intervention to increase HIV testing.

METHODS: We implemented an HIV testing intervention using social marketing, electronic medical rcord clinical reminders (CR's) and performance feedback to facilitate testing at $16 \mathrm{VA}$ medical centers in three regional VA hospital networks. We conducted qualitative formative evaluation at each site, using the PARiHS framework, to understand the context, evidence and facilitation of implementation. Prior to the implementation, we conducted 50 interviews with HIV lead clinicians, primary care lead clinicians, nurse managers and social workers to identify perceived barriers and facilitators to HIV testing. We then conducted 41 follow-up interviews four to six months afterwards to identify which perceived barriers had been addressed by the intervention, which remained problematic, and which interventional factors facilitated an increase in HIV testing rates.

RESULTS: Pre-implementation, providers indicated that lack of resources; clinical reminder overload; an onerous written informed consent processes; stigma associated with talking about HIV; concerns about how to communicate positive test results; and concerns about how best to link individuals to care were significant barriers. Concerns for patient welfare, professional responsibility, viewing CRs as helpful and organizational support were key to facilitating testing. Notably, issues of HIV stigma were more prominent in the southern region, with providers referring to trying to discuss HIV testing in the 'Bible Belt' as particularly difficult. Providers noted a need for education for providers and patients about the benefits of HIV testing. Simplifying the consent and testing process was also viewed as critical to increasing testing. Post-implementation interviews indicated that several of these perceived barriers had been addressed; however others endured including concerns about HIV stigma and management of patients who test positive.

CONCLUSIONS: Providers found the multi-modal intervention effective in addressing many pre-implementation concerns, including the potential burden of an additional clinical reminder. Several barriers remained and need to be addressed in the future. Addressing organizational and attitudinal barriers can facilitate increasing HIV testing rates in the VA through interventions incorporating clinical reminders, provider education and performance feedback. This study indicates that interventions to implement HIV testing can be successful when utilizing proven quality improvement techniques, addressing providers' perceptions of the evidence and taking into consideration the local context of implementation. Attending to concerns about persistent stigma associated with HIV may further help this effort.

BARRIERS TO ADOPTION AND USE OF A NATIONWIDE WEB-BASED PERSONAL HEALTH RECORD AND PATIENT PORTAL: A QUALITATIVE STUDY OF VA'S MYHEALTHEVET Steven R. Simon ${ }^{1,2}$; Gemmae Fix ${ }^{3,6}$; Cliona Archambeault ${ }^{5}$; Max D. Stewart $^{1,2}$; Keith Mclnnes ${ }^{3,6}$; Timothy P. Hogan ${ }^{7,4}$; Rebecca Grochow ${ }^{1,8}$. ${ }^{1}$ VA Boston Healthcare System, Boston, MA; ${ }^{2}$ Brigham and Women's Hospital, Boston, MA; ${ }^{3}$ VA Medical Center ENRM, Bedford, MA; ${ }^{4}$ University of Massachusetts Medical School, Worcester, MA; ${ }^{5}$ VA Boston Healthcare System, Boston, MA; ${ }^{6}$ Boston University School of Public Health, Boston, MA; ${ }^{7}$ VA Medical Center ENRM, Bedford, MA; ${ }^{8}$ Boston University School of Medicine, Boston, MA. (Control ID \#1338859)

BACKGROUND: My HealtheVet (MHV), the VA's personal health record and web-portal, allows patients to track health parameters, view and download elements of their electronic health record, communicate with their providers, and access health education materials. However, low adoption rates prevent this technology from transforming care delivery. Only $3 \%$ of VA patients have completed in-person authentication (IPA), necessary for access to key features on the portal. VA primary care clinics are a logical site to engage patients in IPA; however, little is known about how best to intervene. With an overarching goal of developing an intervention to improve adoption and use of MHV, we undertook a qualitative study to identify the barriers to adoption and use of MHV and to characterize current approaches used in primary care to promote enrollment.

METHODS: Two researchers observed 3 primary care clinics for a total of 10 hours. We interviewed 4 primary care clinicians and 10 individuals with national roles in MHV ("key informants"). We conducted 4 patient focus groups - 2 among Veterans $(\mathrm{N}=11)$ previously enrolled in MHV and 2 among Veterans $(\mathrm{N}=12)$ who had not enrolled - to characterize barriers to MHV adoption and use. We recorded detailed field notes after every encounter, documenting methodological details about data collection; descriptive details about interactions, observations and content; and analytic information such as interpretive ideas linking information across interviews and amongst the types of data collection. We iteratively coded data using an emergent, thematic strategy. 
RESULTS: We observed high variability in primary care clinic processes surrounding MHV enrollment, including: presence and involvement of clinical champions; standardization of MHV documentation; and descriptions of MHV. Veterans in all 4 focus groups had Internet access and an interest in MHV; those who had not yet enrolled lacked clarity on what, exactly, MHV is; its relevancy to their health care; and the steps needed to achieve full enrollment. Additionally, many Veterans had encountered problems while trying to enroll in the portal, such as a closed enrollment office, logon errors, and difficulties navigating the user interface. Some primary care providers saw MHV as a "nuisance" and irrelevant for many of their patients; these clinicians were concerned about time constraints and felt someone else should be helping Veterans to enroll in MHV. In contrast, other providers saw MHV as a way to enhance their practice by facilitating conversations with patients outside of the usual, cumbersome phone system; these clinicians encouraged their patients to enroll in and use the portal. Key informants emphasized the need to publicize the benefits of the patient portal to both Veterans and their clinicians.

CONCLUSIONS: Taken together, the findings from this multi-modal qualitative study reveal a broad spectrum of awareness and understanding of MHV and its potential to influence health care. Discussions with Veterans and clinicians support key informants' perceptions that both Veterans and staff will benefit from education and outreach about MHV. Multiple lines of evidence indicate that primary care-based efforts to enroll Veterans in MHV need standardization and improved visibility. These findings will form the basis for the development of primary-care based strategies to foster Veterans' adoption and use of the patient portal.

BARRIERS TO POST-HOSPITALIZATION FOLLOW-UP: UNDERSTANDING THE PATIENT'S PERSPECTIVE Caroline DeFilippo; Lello Tesema; Ramiro Jervis. Mount SInai Medical Center, New York, NY. (Control ID \#1324675)

BACKGROUND: Timely access to primary care follow-up is an important factor in preventing hospital readmissions. Patients that lack primary care follow-up within four weeks of discharge are more likely to be readmitted. Moreover, resident clinics are often understaffed and have poor continuity of care which may further aggravate poor post hospitalization follow up. As a significant proportion of patients discharged from a hospitalist service have follow up primary care in a resident clinic, our institution undertook a survey of this important patient population. Few studies have looked at the patient perspective to primary care follow up, and none have looked at this in a resident run clinic. While some studies have addressed risk factors associated with poor primary care follow-up, none to our knowledge have addressed the patient's views of the role of primary care physician (PCP) after hospitalization.

METHODS: A qualitative and quantitative survey tool was administered to hospitalized patients known to an outpatient primary care clinic staffed primarily by housestaff affiliated with a major medical center. Patients had to have been to the clinic within the past 18 months for a primary care appointment, have an assigned primary care physician, speak English or Spanish and lack cognitive difficulties. Patients consented to have their medical record reviewed and were followed after discharge to see if they attending post-hospitalization appointments.

RESULTS: A total of 50 patients were surveyed over 5 months. The majority of patients felt it was important to follow-up with a physician after hospitalization; however $45 \%$ felt they only needed to see a specialist, not their PCP. Qualitative analysis identified the following roles of the PCP: referrals, medications, coordination of care, general check-up and less often follow-up after hospitalization. In addition, patients expressed specific concerns about their clinic experience including lack of continuity with their PCP. Preliminary data suggest that $25 \%$ of these patients did not have followup appointments with their PCP upon discharge and an additional $25 \%$ of patients had appointments and did not attend. The re-hospitalization rate was $20 \%$ at 30 days and $28 \%$ at 90 days.

CONCLUSIONS: Patients at a large urban tertiary medical center who receive their primary care in a resident run clinic appreciate the importance of post discharge follow up, but a large percentage value sub specialty follow up over primary care. A large number of patients are discharged without appropriate follow-up care. Future analysis will determine if there is an association between perceived role of the PCP and hospital readmission rates.

BARRIERS TO UTILIZATION OF FREE SPECIALTY CARE IN AN UNINSURED POPULATION Catherine Handy ${ }^{1}$; Sai $\mathrm{Ma}^{2}$; Lauren Block $^{3}$; Desiree M. de la Torre ${ }^{4}$; Anne Langley ${ }^{4}$; Barbara G. Cook ${ }^{4} .{ }^{1}$ Johns Hopkins School of Medicine, Baltimore, MD; ${ }^{2}$ Johns Hopkins Bloomberg School of Public Health, Baltimore, MD; ${ }^{3}$ Johns Hopkins School of Medicine, Baltimore, MD; ${ }^{4}$ Johns Hopkins Medicine, Baltimore, MD. (Control ID \#1321184)

BACKGROUND: Uninsured individuals face many barriers to accessing specialty care. The Access Partnership (TAP) was launched in 2009 by an urban, academic medical center to provide and coordinate free specialty care to qualified uninsured patients. Patients were referred by their primary provider and asked to pay a $\$ 20$ program entry fee (waived for financial hardship). In the first year, 333 eligible adult patients were referred yet 104 $(31 \%)$ did not enroll. We sought to understand the barriers to utilization of care within the TAP program to find ways to improve access to specialty care for this population.

METHODS: A cross sectional, qualitative study was done through telephone surveys. Open ended responses were analyzed using word count analysis and categorized as 'program specific', 'patient specific,' or both. RESULTS: Eighteen (17\%) of non-enrollees were surveyed yielding 23 unique responses. The most commonly cited barriers to joining the program were insufficient program follow up (6 responses) and financial barriers related to the program entry fee ( 4 responses). Seven responses were related to program-specific barriers and were within the capacity of the program to change. These included insufficient program follow up (6 responses) and unclear eligibility (1 response). Six responses were related to patient-specific barriers. These including work conflicts (2 responses), poor health status and forgetting and not wanting or needing services (1 response each). Ten responses were related to a combination; barriers not within the current design of the program but potentially within the capacity of the program to change. These barriers included financial barriers posed by the entry fee ( 4 responses), transportation/mobility issues ( 2 responses), not understanding the reason for referral (2 responses) and choosing other services (2 responses).

CONCLUSIONS: While lowering costs is necessary to improve access to specialty care for underserved patients, it is insufficient. Improving follow up and communication from the program can be used to reduce the program specific barriers. Future work should focus on identifying patients who are less likely to utilize specialty care and improve communication and resource coordination to encourage these patients to follow through with recommended care.

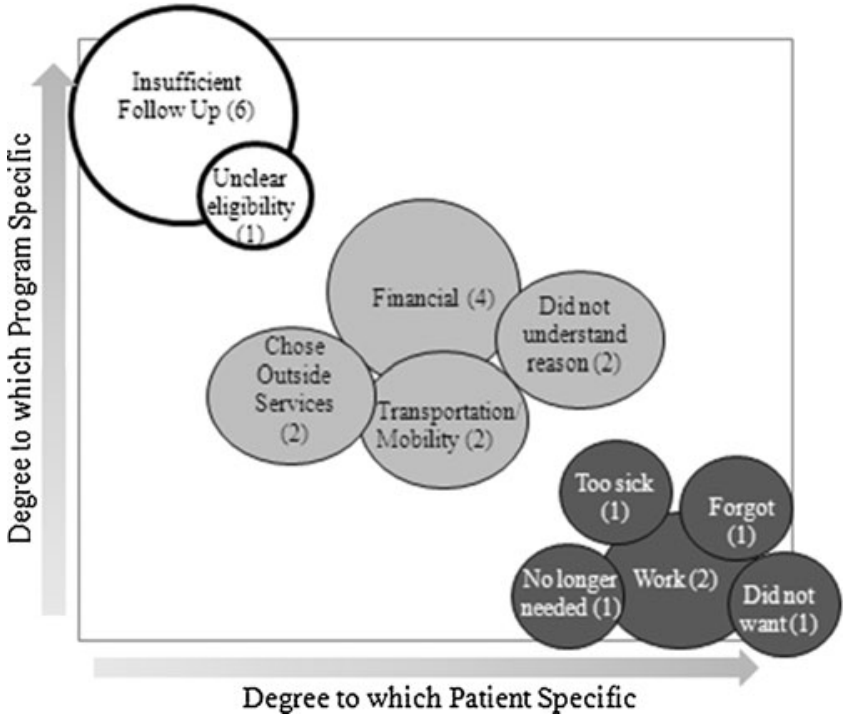


Bubble plot showing degree to which barriers to utilization of care were program specific, patient specific or a combination. Size of bubble reflects number of responses obtained (listed in parentheses).

BETTER MEASURES OF QUALITY FOR PATIENTS HOSPITALIZED WITH ACUTE MYOCARDIAL INFARCTION OR PNEUMONIA Lena M. Chen ${ }^{1,2}$; John D. Birkmeyer ${ }^{2}$; Douglas Staiger ${ }^{3}$; Wenying Zhang'; Justin B. Dimick ${ }^{2} .{ }^{1}$ VA Center for Clinical Management Research, Ann Arbor, MI; ${ }^{2}$ University of Michigan, Ann Arbor, MI; ${ }^{3}$ Dartmouth University, Hanover, NH. (Control ID \#1315612)

BACKGROUND: The Centers for Medicare and Medicaid and other large payers have launched numerous initiatives aimed at improving the quality of inpatient medical care. Whether efforts such as public reporting on hospital quality will improve clinical outcomes by steering patients towards higher quality hospitals is uncertain, however. Existing quality measures are generally sub-optimal in identifying hospitals with superior (or inferior) performance. In surgery, composite measures of quality have been found to better predict future outcomes than individual measures. Therefore we assessed how well composite measures of quality predict future hospital mortality for acute myocardial infarction (AMI) and pneumonia (PNA).

METHODS: Using publicly reported performance measures from Hospital Compare and national Medicare data, we examined the ability of two different measures of quality to predict future hospital mortality rates for patients 65 years of age or older with a primary diagnosis of AMI $(n=644,967)$ or PNA $(n=1,184,119)$. For each condition, our quality measures were: 1) mortality rates as reported on Hospital Compare, and 2) a composite measure that was empirically weighted and reliability adjusted, and incorporated volume and mortality. We used each measure separately to place hospitals into quintiles of performance, based on July 2005 to June 2008 data. We then examined the association between hospital rank in 2005-2008 and hospital mortality rates in calendar year 2009 .

RESULTS: For AMI and PNA, a composite measure better predicted future hospital mortality rates, compared to Hospital Compare measures. For example, if an AMI patient had chosen a hospital in 2009 using rankings based on the 2005-2008 composite (Hospital Compare) measure, he or she would have had 0.64 (0.72) times the odds of dying in 30 days (CI for odds of dying at best vs. worst hospital quintile based on composite: 0.61-0.66; CI for odds of dying at best vs. worst hospital quintile based on Hospital Compare: 0.690.74). If a patient with PNA had chosen a hospital in 2009 using the rankings based on the 2005-2008 composite (Hospital Compare) measure, he or she would have had $0.64(0.71)$ times the odds of dying in 30 days (CI for odds of dying at best vs. worst hospital quintile based on composite: 0.62-0.66; CI for odds of dying at best vs. worst hospital quintile based on Hospital Compare: 0.68-0.73).

CONCLUSIONS: Composite measures of quality for AMI and PNA are better at predicting future mortality than current measures on Hospital Compare. Incorporating composite measures into public reporting may provide better guidance to patients about which hospitals to choose for their care.

BISPHOSPHONATES - RISKY BUSINESS? Chi-Na $\mathrm{Pak}^{1}$; Jennifer Burkham ${ }^{1,2}$. ${ }^{1}$ Santa Clara Valley Medical Center, San Jose, CA $;{ }^{2}$ Stanford University Hospital, Palo Alto, CA. (Control ID \#1311844)

BACKGROUND: Bisphosphonates account for an estimated $80 \%$ of all prescribed medications for osteoporosis, but certain serious conditions have recently been linked to its long-term use. In addition to severe bone and muscle pains, there have been reports of atypical subtrochanteric and diaphyseal femur fractures, osteonecrosis of the jaw, atrial fibrillation, and esophageal cancer associated with its chronic use. Additionally, alendronate therapy in postmenopausal women with osteopenia who have no history of clinical fractures or other risk factors for fractures has not been found to be cost-effective and could potentially be harmful. Given these recent findings, guidelines for the management of osteoporosis is under active review, particularly regarding the appropriate use of bisphosphonates. In an effort to gauge how well the current guidelines are being followed in clinical practice, we studied the percent of patients in our county hospital system, Santa Clara Valley Health and Hospital System in San Jose, California, who are being inappropriately treated with bisphosphonates.

METHODS: Using existing registries of DEXA reports and prescribed medications from the past five years in the Santa Clara Valley Health and Hospital System, an electronic registry was used to pool all patients who have had both a DEXA scan as well as a prescription for bisphosphonates, which came out to 1874 patients. A random number generator was used to select a sample population of 200 from the cohort of 1874. From the sample population, each patient's DEXA report was reviewed to see if their $\mathrm{T}$ score met diagnostic criteria for osteoporosis, signifying indication for bisphosphonate treatment. If their $\mathrm{T}$ score was normal or osteopenic by $\mathrm{T}$ score, a chart review was then conducted to look for other indications for bisphosphonate treatment such as fragility fracture found on imaging, documented past fragility fractures, documentation of outside hospital DEXA scans showing osteoporosis, or other documented very high risk factors such as chronic steroid or immunosuppressant use. If any of these other factors were found, then treatment was also considered indicated. If not, then it was determined that bisphosphonate treatment was not clinically indicated. Given our sample size in this descriptive analysis, a confidence level of $95 \%$ was used to calculate the confidence interval of 5.38

RESULTS: We found that $68.5 \%$ of patients on bisphosphonates who have had a DEXA met osteoporosis criteria by their T-score and indicated treatment. There were $10 \%$ of patients on bisphosphonates who have had a DEXA who did not meet criteria by DEXA but had other indications for treatment upon chart review. Overall, $78.5 \%$ of the population were appropriately treated. The percent of people on bisphosphonates who lacked a clear indication for the drug was $21.5 \%$ [16.12, 26.88\%]. Of this subset, $11.6 \%$ were diagnosed with osteoporosis without meeting clinical criteria on chart review and $39.5 \%$ were actually diagnosed with osteopenia but still treated with bisphosphonates.

CONCLUSIONS: These results show that many patients are on bisphophonates without documented indication. This suggests a possible gap between knowledge of osteoporosis treatment guidelines and application in clinical practice. It is important to keep providers up to date on changes to osteoporosis treatment guidelines since bisphosphonate use may have more risk implications than previously thought, particularly in those with normal bone density or osteopenia.

BOSTON PATIENT NAVIGATION RESEARCH PROGRAM: THE IMPACT OF NAVIGATION ON TIME TO DIAGNOSTIC RESOLUTION AFTER ABNORMAL CANCER SCREENING Tracy Battaglia ${ }^{1}$; Sharon $\mathrm{Bak}^{1}$; Timothy Heeren ${ }^{3}$; Clara $\mathrm{Chen}^{3}$; Richard Kalish $^{2}$; Stephen Tringale ${ }^{5}$; James O. Taylor ${ }^{4}$; Barbara Lottero ${ }^{7}$; Patrick Egan $^{6}$; Nisha Thakrar ${ }^{2}$; Karen Freund ${ }^{1} .{ }^{1}$ Boston University School of Medicine, Boston, MA; ${ }^{2}$ South Boston Community Health Center, Boston, MA; ${ }^{3}$ Boston University School of Public Health, Boston, MA; ${ }^{4}$ East Boston Community Health Center, Boston, MA; ${ }^{5}$ Codman Square Health Center, Boston, MA; ${ }^{6}$ Dorchester House Multi-Service Center, Boston, MA; ${ }^{7}$ Greater Roslindale Medical \& Dental Center, Boston, MA. (Control ID \#1317072)

BACKGROUND: There is a need for controlled research studies to assess the impact of patient navigation on cancer outcomes in a vulnerable population. The objective of this study was to evaluate whether patient navigation decreased time to diagnosis for subjects with a breast or cervical cancer screening abnormality.

METHODS: The Boston PNRP program was designed as a clinical effectiveness study of patient navigation as a new standard of care. Boston Patient Navigation Research Program collected baseline data (2004-2005) and intervention data (2007-2008) at 6 community health center sites (CHCs) on all women with cervical or breast cancer screening abnormalities. During the intervention period $3 \mathrm{CHCs}$ were assigned breast 
navigators and 3 were assigned cervical navigators, and served as a control site for the other abnormalities. Kaplan-Meier survival curves and proportional hazards regression examined the effect of navigation on time to definitive diagnosis, adjusting for clustering by clinic and adjusting for age, race, language, and insurance. Hazard ratios greater than 1.0 indicate a decrease in time to diagnosis.

RESULTS: We enrolled $\mathrm{N}=997$ subjects in the baseline period and $\mathrm{N}=$ 3,041 subjects during the intervention period ( $n=1,499$ navigated, $n=1,542$ control). 30\% were African African, 28\% were Hispanic and 34\% were white; $32 \%$ had no insurance, $38 \%$ were publically insured. Among those with a breast screening abnormality, there was a significant decrease in time to diagnosis for navigated subjects who resolved after 60 days (aHR 1.4, 95\% CI: 1.1-1.9) compared with controls, but no differences for those who resolved before 60 days (aHR1.04, $.83-1.3$ ). Among those with a cervical screening abnormality, there was a significant decrease in time to diagnosis for all navigated subjects when compared with controls (aHR 1.5, 95\% CI: 1.4-1.9).

CONCLUSIONS: This clinical effectiveness study documents a benefit of patient navigation on time to diagnosis among a racially/ethnically diverse inner city population cared for in CHCs.

BOTULINUM A TOXIN FOR HEADACHES, A META-ANALYSIS Jeffrey L. Jackson ${ }^{1}$; Akira Kuriyama ${ }^{2}$; Yasuaki Hayashino ${ }^{2} .{ }^{1}$ Zablocki VAMC, Milwaukee, WI; ${ }^{2}$ Kyoto University, Kyoto, Japan. (Control ID \#1337567)

BACKGROUND: Our study objective was to assess the efficacy of botulinum toxin for the prophylactic treatment of headaches in adults.

METHODS: We searched Medline, Embase and Cochrane, including RCTs comparing botulinum to placebo or other interventions among adults. Data was abstracted and quality was assessed independently by two reviewers with good inter-rater reliability. We pooled continuous outcomes as standardized mean differences (SMD), also known as effect sizes, using random effects models. By convention, effects less than 0.2 are considered trivial, 0.2-0.5 small, 0.5-0.8 modest and 0.8-1.0 large.

RESULTS: Our search produced 249 articles. Among 31 included trials, 27 compared botulinum to placebo and 4 to other medications (amitriptyline, prednisone, topiramate, valproate). Among placebo controlled trials, 13 evaluated episodic migraine headaches $(<15$ headaches/month), 8 chronic tension-type headaches ( $\geq 15$ days per month), 1 trial evaluated a mixed population of patients with episodic or chronic tension and 5 studied chronic daily headaches (migraine or tension-type headaches occurring more than 14 days per month). Botulinum was more effective than placebo for chronic daily headaches (SMD: $-0.33,95 \%$ CI: -0.56 to $-0.11, \mathrm{Q}=8.24,5$ studies, $\mathrm{I} 2=51.5 \%$ ) and no more effective for episodic migraine (SMD: $-0.08,95 \%$ CI: -0.22 to $0.05, \mathrm{Q}=30.09,13$ studies, $\mathrm{I} 2=$ $60.1 \%, \mathrm{p}=0.03$ ) or chronic tension-type headaches (SMD: $-0.32,95 \% \mathrm{CI}$ : -0.69 to $0.05, \mathrm{Q}=27.42,8$ studies, $\mathrm{I} 2=74.5 \%, \mathrm{p}<0.005$, Figure 2 ). The single trial with a combination of episodic and chronic tension-type headaches also found no benefit (SMD: $-0.11,95 \%$ CI:-0.97 to 0.74 ). Among fewer trials, botulinum was no better than placebo in producing $50 \%$ headache improvement for migraine (5 studies, RR: $0.97,95 \% \mathrm{CI}$ : $0.86-1.10, \mathrm{Q}=2.77, \mathrm{I} 2=0.0 \%$ ), chronic tension-type headaches ( 1 study, RR: $1.11,95 \%$ CI: $0.87-1.43)$ or chronic daily headaches (2 studies, RR: $1.17,95 \%$ CI: $0.93-1.47, \mathrm{Q}=0.76 . \mathrm{I} 2=0.0 \%$ ). Poor quality, small, single trials found botulinum equivalent to topiramate (SMD: 0.20 , 95\% CI: -0.36 to 0.76 ) or amitriptyline (SMD: $0.29,95 \%$ CI: -0.17 to 0.76 ) for chronic migraine and valproate for a trial that included both episodic $(n=45)$ and chronic $(n=14)$ migraines (SMD: $-0.20,95 \%$ CI: -0.91 to 0.31 ). All trials used doses generally inadequate for headache prophylaxis. Botulinum was better than prednisone in a single trial of chronic tension-type headaches (SMD: $-2.19,95 \%$ CI: -3.32 to -1.06 ). CONCLUSIONS: Botulinum is effective for "chronic daily headaches" though ineffective for episodic or chronic tension-type headaches or episodic migraines. The size of the effect is smaller than those found in systematic reviews of tricyclic antidepressants, beta-blockers and anticonvulsants. The direct comparisons found no differences though all were low quality, small and used inadequate doses of prophylactic medications.
While FDA approved for chronic migraine headaches, there are no clinical trials investigating this specific type of headache-instead trials evaluated "chronic daily headache" which is a mixture of patients experiencing chronic migraine $(68 \%)$ or chronic tension $(32 \%)$ headaches. Given that botulinum is ineffective for chronic tension headaches, it's efficacy for chronic migraines may be underestimated in these studies. Further research is needed to determine the relative efficacy of botulinum compared to other available agents and to confirm its effectiveness for chronic migraine headaches.

BREAST CANCER PATIENTS' RECALL OF RECEIVING PATIENT ASSISTANCE SERVICES Jenny J. Lin; Kezhen Fei; Rebeca Franco; Nina A. Bickell. Mount Sinai, New York, NY. (Control ID \#1339549)

BACKGROUND: Accuracy of recall is highly variable and recall bias may be more pronounced with the passage of time. As surveys are used to assess utilization and effectiveness of interventions, it is critical to assess factors that affect patient recall, particularly if future funding and services are determined by patients' recall of service utilization.

METHODS: We compared newly-diagnosed breast cancer patients' recall of receiving patient assistance services at 2 weeks and at 6 months after needs assessment was performed in a patient-assistance randomized controlled trial. All patients received English or Spanish written information about breast cancer treatment and resources; those in the intervention group received information about assistance programs targeted to their specific needs and created an action plan to contact the program. 374 women with new early-stage breast cancer operated at $8 \mathrm{NYC}$ hospitals participating in the RCT were eligible to complete the 6 month follow-up survey. To date, 333 completed the follow-up (89\%); 32 completing the baseline survey were unreachable, 7 refused, and 2 were deceased; 164 were assigned to the intervention group. Scales of social support were calibrated to a 100 point scale; SF12 measured physical and emotional health.

RESULTS: Of the 333 women, $210(63 \%)$ had informational needs. At 2 weeks, 96\% (202/210) reported receiving breast cancer information but at 6 months, only $69 \%(140 / 210)$ recalled receiving informational material. Of the 140 who recalled getting informational material, $80 \%$ had their informational needs met. $100 \%$ of women who had their informational needs met, recalled getting informational material, compared to $31 \%$ of those who did not get their informational needs met $(\mathrm{p}<0.0001)$. Age, race, income, education, adjuvant treatment, physical health status and social support did not affect recall. Of the 109 women in the intervention group with psychosocial or practical needs who were told of relevant assistance programs, $67(61 \%)$ contacted the patient assistance program specified in their action plan at 2 weeks. However, at 6 months, only 25/67 (37\%) recalled contacting a program. Women who did not recall contacting a program at 6 months were less likely to have had their psychosocial or practical needs met ( $13 \%$ vs $78 \%$; $\mathrm{p}<0.0001)$. Age, income, race, education, language spoken, social support, adjuvant treatment and physical or emotional health status did not affect recall of programs contacted.

CONCLUSIONS: Nearly all women who receive information about breast cancer and treatment recall getting this information at 2 weeks but this proportion drops substantially by 6 months. Of the women with underlying psychosocial or practical needs, more than half report contacting patient assistance programs within 2 weeks, but by 6 months, only a third of these women recall contacting a program. Women's recall of receipt of patient assistance is strongly related to having had their needs met. Care should be taken when using patient surveys to evaluate the impact and utilization of patient assistance programs due to poor levels of recall.

BREAST CANCER TREATMENT DECISION-MAKING: ARE WE ASKING TOO MUCH OF PATIENTS? Nina A. Bickell; Jennifer C. Livaudais; Rebeca Franco; Kezhen Fei. Mount Sinai School of Medicine, New York, NY. (Control ID \#1336437)

BACKGROUND: Physicians are mandated to offer treatment choices to breast cancer patients yet not all patients may feel comfortable with the 
responsibility that entails. Prior studies show that breast cancer patients who are not given enough responsibility for treatment decision-making have poorer treatment knowledge and report worse quality of care. In contrast, few studies explore potential adverse consequences for breast cancer patients who feel they are given too much responsibility for treatment decision-making.

METHODS: We sought to describe and compare women by degree of responsibility they felt they had for making decisions about their breast cancer treatment. Our sample includes women with early-stage breast cancer treated surgically at $8 \mathrm{NYC}$ hospitals, recruited for an RCT of patient assistance to improve receipt of adjuvant treatment. In multivariable analyses, we explored the association between treatment decision-making responsibility and a) baseline knowledge of treatment benefit (of surgical and other discussed treatments) and b) regret of treatment decisions after 6 months.

RESULTS: Of 368 women aged 28-89y enrolled at baseline, $72 \%$ reported a "reasonable amount", $21 \%$ "too much", and 7\% "not enough" responsibility for treatment decision-making. The majority in the first group were White $(53 \%)$, earned $>\$ 150,000 /$ year (33\%), completed college (53\%) and had no health literacy problems $(50 \%)$. In contrast, the majority with "not enough" responsibility were Black $(42 \%)$, earned $<\$ 50,000 /$ year $(58 \%)$, did not finish high school (39\%), and had health literacy problems (68\%). The majority with "too much" responsibility were Hispanic (68\%), earned $<\$ 15,000 /$ year $(54 \%)$, did not finish high school (37\%), and also had health literacy problems $(62 \%)$. Only $29 \%$ of women had knowledge of treatment benefits and $40 \%$ experienced decision regret at 6 months. Multivariable analysis found that women with "too much" vs. a "reasonable amount" of responsibility had less treatment knowledge ([OR] $0.46[95 \% \mathrm{CI}] 0.21,0.99$; model $\mathrm{c}=0.7301 ; \mathrm{p}<$ $0.001)$ and more decision regret at 6 months ([OR] $3.16[\mathrm{CI}] 1.59,6.29$; model $\mathrm{c}=0.7821 ; \mathrm{p}<0.001)$. The few women with "not enough" responsibility also had less treatment knowledge, and more decision regret, though results were not statistically significant in this small group ([OR] $0.74[\mathrm{CI}] 0.23,2.37$ and [OR] $3.26[\mathrm{CI}]$ 0.93, 11.4).

CONCLUSIONS: Overall, treatment knowledge was low and decision regret common in this group of breast cancer patients. Having "not enough" or having "too much" responsibility for treatment decisions adversely impacted treatment knowledge and decision regret. Health literacy problems were common in both groups, suggesting that physicians find alternative ways to communicate with low health literacy patients, and consider using decision-aid tools to enable all patients to obtain adequate treatment knowledge, achieve desired levels of responsibility and reduce decision regret.

BRIEF TOOLS FOR ASSESSING DIETARY QUALITY IN PRIMARY CARE: A PILOT STUDY Melanie Jay ${ }^{1}$; Christopher Still²; Jamie Seiler ${ }^{2}$; Nora Henderson ${ }^{1}$; Stella Savarimuthu ${ }^{1}$; Iryna Lobach ${ }^{1}$; Colleen Gillespie ${ }^{1}$; Adina Kalet ${ }^{1} .{ }^{1}$ New York University School of Medicine, New York, NY; ${ }^{2}$ Geisinger Health System, Danville, PA. (Control ID \#1336195)

BACKGROUND: Several chronic diseases and conditions including diabetes, hyperlipidemia, obesity and hypertension can be prevented by a healthful diet or managed in part with dietary change. Promoting these behavior changes in primary practice, however, is hampered by the lack of clinically useful, brief, simple, and validated tools for assessing diet and physical activity. The purpose of this pilot study was to identify useful screening questions for targeting dietary change by asking primary care patients to report on the quality of their diets and dietary behaviors and comparing results with the gold standard data from a standardized 24-hour dietary recall.

METHODS: We recruited adult female patients while they waited to see their primary care physician in a public inner city clinic. After the PCP visit, patients were given a questionnaire (self-administered) asking them to report on dietary behaviors and physical activity (30 items) and to rate the quality of their diets (six items) on a four-point Likert-type scale (strongly disagree to strongly agree). Within 1-month of the visit questionnaire, over a period of two weeks, patients were contacted by phone and asked to provide detailed information in three, 24-hour dietary recalls. The final sample for these analyses was 28 patients with both visit questionnaire and 24-hour recall data. Wilcoxon tests were used to assess associations between visit questionnaire items and the 24-hour recalls.

RESULTS: 49 patients completed the surveys, and all were contacted by telephone. 21 patients either could not be reached, declined to complete 24hour recalls, or only completed 1 or 2 recalls and were excluded. Among 28 participants who completed $24-\mathrm{hr}$ recall and dietary questionnaire, $35.7 \%$ were obese and $60.7 \%$ were Hispanic. The mean number of kcal consumed was $1218 \mathrm{kcal}$. Compared to those who disagreed, patients who agreed with statement "I generally eat a healthy diet" had fewer kcal (1073 vs. $1465, \mathrm{p}=$ $0.06)$, lower percent saturated fat ( 7.39 vs. $10.79, p=0.02)$, lower added sugar ( 27.87 vs. $45.99, \mathrm{p}=0.048)$, and higher percent calories from protein ( $16.88 \mathrm{vs.}$ 21.93). Patients who agreed with the statement "I usually control portions, eat less of what is on my plate" had lower kcal (1122 vs. 1595, $\mathrm{p}=0.06)$ and lower saturated fat $(10.47$ vs. $17.7, p=0.06)$. Patients who agreed with the statement "I usually pay attention to fat in my diet" and "I eat plenty of fruits and vegetables each day" also trended towards having healthier diets as measured by the 24 -hour dietary recalls. In contrast, the statements "I do not eat too much food", "I eat enough fruit", and "I don't eat too many calories" were not associated with healthier diets.

CONCLUSIONS: In this pilot study, we identified four questions that prospectively identified people with healthier dietary behaviors, as validated the gold standard of the 24-hour recall, and therefore may facilitate dietary counseling and targeted interventions. Future studies are needed to clarify these questions' operating characteristics and validate them in larger, more diverse patient populations.

\section{BURDEN OF MEDICALLY-RELATED LEGAL NEEDS AMONG} PATIENTS SEEKING CANCER CARE SERVICES AT AN URBAN SAFETY NET HOSPITAL Tracy Battaglia ${ }^{1}$; Rebecca Gupta-Lawrence ${ }^{2}$; Sarah E. Caron ${ }^{1}$; Megan Eldridge ${ }^{3}$; Jessica Schiller ${ }^{2}$; Tibrine da Fonseca ${ }^{2}$; Samantha Morton ${ }^{2}$; Jennifer Rosen ${ }^{4}$. ${ }^{1}$ Boston University School of Medicine, Boston, MA; ${ }^{2}$ Boston Medical Center, Boston, MA; ${ }^{3}$ Boston Medical Center, Boston, MA; ${ }^{4}$ Boston University School of Medicine, Boston, MA. (Control ID \#1336439)

BACKGROUND: It is well known that underserved populations in the United States bear a disproportionate burden of cancer. It has also been shown that a host of community-specific social, cultural, behavioral and systems barriers to accessing timely quality cancer care exist for these same low-income, racial and ethnic minority populations. Many of these barriers may be legal in nature, requiring solutions residing in laws and their enforcement, an area in which medical providers have little training and also lack resources to address. The burden of legal barriers to accessing cancer care is unknown amongst vulnerable populations.

METHODS: We conducted a cross-sectional survey of women presenting for ambulatory cancer care services at an inner-city safety net hospital to assess legal needs, defined as adverse social circumstances with legal remedies that reside in laws, regulations or policies. We included patients seeking care in three ambulatory cancer practice sites to capture the needs of patients across the entire cancer care spectrum, from screening through diagnosis and treatment. Clinical sites included: a breast health specialty practice where patients are referred for screening and diagnostic services, a medical oncology clinic and a same-day surgery clinic for cancer patients in active treatment. Eligible participants were age 18 and older and able to speak and read English. The Research Assistant-administered survey contained questions regarding 25 specific legal barriers to medical care across the following domains: Income Supports, Housing and Utilities, Employment/Education, Legal Status (Immigration), and Personal \& Family Stability and Safety. Descriptive statistics were used to quantify the reported presence of medically-related legal barriers and bivariate associations using T-tests and Chi-square analyses were utilized to compare those with and without reported legal barriers. 
RESULTS: Among 104 respondents, 70\% were female, $65 \%$ were NonWhite (48\% Black), $43 \%$ had income $<\$ 20,000$ annually and only $34 \%$ had private insurance. Overall, eighty $(77 \%)$ reported concerns with one or more legal needs in the past month. Compared to those with no reported legal needs, those with reported legal needs were more likely to be Black $(54 \%$ v. $29 \%, \mathrm{p}=0.05)$, single, low-income ( $48 \%$ v. $29 \%, \mathrm{p}=0.03)$, and not own their own home $(24 \%$ v. $63 \%, \mathrm{p}=0.01)$. The most common legal needs reported were related to income supports (69\%), housing and utilities (51\%) and employment/education (32\%). Only 35\% discussed these legal needs with their health care provider, and among those who did, only $20 \%$ utilized resources to address their need.

CONCLUSIONS: This study is the first to provide a comprehensive assessment of the medically-related legal needs of a vulnerable population seeking care across the cancer care spectrum. We have documented a high prevalence of reported legal needs, which are largely unmet in the current healthcare system. Our findings support the need for innovations in cancer care to address legal barriers to care.

CPOE-RELATED MEDICATION ERRORS: ANALYSIS OF 10,000 ERROR REPORT NARRATIVES AND VULNERABILITY TESTING OF CURRENT SYSTEMS Gordon D. Schiff ${ }^{1,3}$; Andrew C. Seger $^{1,2}$; Mary Amato $^{2}$; Diana L. Whitney ${ }^{1}$; Jennifer Boehne ${ }^{1}$; Ali Rashidee $^{5}$; Robert B. Elson ${ }^{6}$; Ross Koppel ${ }^{4}$; Adam Wright ${ }^{1,3}$; David W. Bates ${ }^{1,3}$. ${ }^{1}$ Brigham and Women's Hospital, Boston, MA; ${ }^{2}$ Massachusetts College of Pharmacy \& Health Sciences, Boston, MA; ${ }^{3}$ Center for Patient Safety Research and Practice, Boston, MA; ${ }^{4}$ University of Pennsylvania, Philadelphia, PA; ${ }^{5}$ Quantros, Inc, Milpitas, CA; ${ }^{6}$ MetroHealth Center for Health Care Research and Policy, Cleveland, OH. (Control ID \#1338737)

BACKGROUND: Although CPOE has been shown to reduce medication errors, like any new technology it also has potential for introducing new errors. A recent IOM report on HIT safety urges studying problems to analyze their causes and implement prevention strategies. Since 1998 the USP MEDMARX medication error reporting system has collected nearly 2 million reports; in 2003 a coded field was added for CPOE as a contributing cause of the error. As these reports had not been studied previously, we obtained and analyzed the structured and narrative details of these CPOE-related error reports.

METHODS: A team of general internists and pharmacists analyzed MEDMARX CPOE-related error reports using 2 approaches: a) in-depth quantitative and qualitative review of report content, particularly narrative descriptions, and b) by testing vulnerability of leading current CPOE systems to actual error cases by attempting to enter these erroneous orders. We used qualitative analytic software to create a new taxonomy of CPOE errors categorized by type, cause, and prevention strategies. Representative cases were selected to construct "use case" scenarios based on frequency, severity, generalizability, testability, and correctability.

RESULTS: Of 1.6 million reported errors, 63,040 were reported as CPOErelated. We reviewed and coded 10,000 (15.8\%) reports and derived a taxonomy of 73 codes describing error causes, 112 codes describing error effects, and 76 codes describing potential prevention strategies. Leading error causes included multiple electronic systems (1166 cases), problematic use of abbreviations (489), failure to follow procedures/protocol (468), profiling failure/issues (420), lack of computer training/system knowledge (301), hybrid (electronic \& paper) systems (202), entry/typing errors (199), medication reconciliation issues (177), and alerts ignored/overridden (143). Leading error effects included missing/incorrect sig (2078), missing/wrong quantity ordered (872), wrong dose or strength (849), wrong schedule (548), duplicate orders (474), wrong formulation/dosage form (360), overdose/potential overdose (355), wrong drug (295), and comments field with conflicting information (254). Testing vulnerability of selected CPOE systems to 21 selected prototypical cases found that of 307 attempted erroneous orders, 174 (57\%) could be relatively easily replicated (entered easily or w/ minor workarounds) with no warning or blocking of potentially dangerous orders. Observations of typical users (mostly medical residents) documented multiple instances of error-prone ordering and ignoring of warnings.

CONCLUSIONS: Medication error narrative reports are a rich source of descriptions and insights into medication errors, especially those related to CPOE. Review of 10,000 CPOE-related errors provided the foundation for a new taxonomy of errors, and subsequent vulnerability testing revealed that the majority of errors tested could be replicated in current CPOE systems. Selected insights from this analysis include potential for CPOE propagating errors that are perpetuated in recurring orders, potential for facilitation of adjacency/pull down errors, poor integration of CPOE across multiple systems, widespread evidence of alert fatigue/ ignoring with repeated examples of prescribers overriding true positive alerts, and evidence that prescribers often have difficulty entering desired orders leading to potentially dangerous workarounds, in particular description of their intent in free text comments.

CAN TELEPHONE SMOKING CESSATION PROGRAMS REACH AND TREAT HOMELESS SMOKERS? Erin Rogers ${ }^{1,2}$; Scott Sherman ${ }^{1,2}$. ${ }^{1}$ VA NY Harbor Healthcare System, New $\overline{\text { York, NY; }}{ }^{2}$ New York University School of Medicine, New York, NY. (Control ID \#1336007)

BACKGROUND: Telephone smoking cessation counseling is effective in the general population, but it is not clear how feasible telephone cessation programs are for smokers with housing problems. We compared the feasibility and acceptability of telephone tobacco cessation treatment among non-homeless veterans, homeless veterans residing in long-term VA housing (HUD-VASH), and homeless veterans not residing in HUDVASH housing.

METHODS: These data were collected as part of a six-site VA trial evaluating the implementation of a telephone care coordination program for smokers who use VA mental health services. Mental health providers referred patients to the program. We collected housing status on all referred patients via medical record review at the time of referral. We categorized referred patients as "non-homeless" if they did not have any medical record documentation of homelessness in the year prior to their referral. We categorized patients as "HUD-VASH" if there was documentation that they were enrolled in the VA's HUD-VASH housing program at the time of referral. We categorized patients as "homeless" if they had medical record documentation of homelessness in the year prior to their referral and they were not enrolled in the VA's HUD-VASH program at the time of referral. All referred patients were contacted by phone to offer enrollment in the treatment program. Participants who enrolled were offered self-help materials, smoking cessation medications, and proactive multi-call telephone counseling. We used chi-square analyses to examine the ability of the program to reach and engage non-homeless, HUD-VASH, and homeless patients into treatment.

RESULTS: This report describes the first 1074 patients referred to the program. 912 patients were classified as non-homeless, 87 as HUDVASH, and 75 as homeless. Non-homeless patients and HUD-VASH patients were equally likely to be reached by phone to offer treatment ( $79 \%$ versus $74 \%$; OR $=1.32, \mathrm{CI}=.80-2.18$ ), but homeless patients were significantly less likely to be reached than non-homeless patients $(61 \%$ versus $79 \%$; OR $=.43, \mathrm{CI}=.26-.71)$. Among patients who were reached by phone, there was no difference in treatment enrollment between non-homeless and HUD-VASH patients (67\% versus $64 \%$; $\mathrm{OR}=1.15, \mathrm{CI}=.68-1.97)$ or between non-homeless and homeless patients $(67 \%$ versus $61 \%$; $\mathrm{OR}=1.32, \mathrm{CI}=.72-2.44)$. Among patients who enrolled in treatment, there was no difference in odds of starting telephone counseling between non-homeless and HUD-VASH patients $(69 \%$ versus $68 \%, \mathrm{OR}=1.04, \mathrm{CI}=.35-1.53)$. However, homeless patients were significantly less likely to begin telephone counseling than non-homeless patients $(46 \%$ versus $69 \%, \mathrm{OR}=.39, \mathrm{CI}=.18-.84)$. CONCLUSIONS: Telephone program reach and treatment uptake was good in all three patient groups. Veterans in long-term VA housing showed no differences in their ability to use a telephone tobacco cessation program compared to non-homeless patients. Homeless patients were less likely to be reached by the telephone program to offer treatment, although just as likely to enroll when they were reached. Homeless patients who enrolled in treatment were less likely to begin telephone counseling after enrolling. Future research should identify ways to increase the ability of homeless patients to use telephone tobacco cessation programs. 
CAN SERUM COBALAMIN SENSIBLY BE DROPPED FROM THE "ANEMIA PANEL" IN MICROCYTIC INPATIENTS? Sindhu L. Joseph; Kapil Yadav; John Erikson Yap; Swarna Rai; Pramoda Koduru; Brian P. Lucas. John H. Stroger Jr. Hospital of Cook County, Chicago, IL. (Control ID \#1338655)

BACKGROUND: Cobalamin (vitamin B12) deficiency is a cause of macrocytic anemia. Yet serum cobalamin levels are often ordered in the diagnostic evaluation of microcytic anemia. This is despite both the low reported prevalence of cobalamin deficiency among patients with microcytic anemia and the fact that such patients must have a combined disorder: cobalamin deficiency plus one known to cause microcytosis. We hypothesized, therefore, that in a large cohort of inpatients with anemia who had cobalamin levels ordered the incidence of cobalamin deficiency would be lower among patients with microcytosis when compared to patients without microcytosis (normo- or macrocytosis). In addition, we hypothesized that patients with microcytic anemia and cobalamin deficiency would have an elevated red cell distribution width (RDW), suggesting a second disorder causing microcytosis

METHODS: Our cohort was individual hospitalizations of general medicine patients discharged from Cook County Hospital from July 2009 through June 2010. From this cohort, we identified patients who had a cobalamin level drawn during their hospital stay and who were anemic (hemoglobin less than $12 \mathrm{mg} / \mathrm{dl}$ for women and less than $13.5 \mathrm{mg} / \mathrm{dl}$ in men) at the time that the cobalamin level was ordered. Trained physician investigators systematically reviewed electronic medical charts to identify the etiology of anemia and determine whether cobalamin deficiency was present. Patients were considered cobalamin deficient if they fulfilled at least 1 out of 3 objective criteria: a serum cobalamin level less than $200 \mathrm{pg} / \mathrm{ml}$, a methylmalonic acid level greater than $376 \mathrm{nmol} / \mathrm{l}$, or receipt of cobalamin replacement therapy.

RESULTS: Among consecutive hospitalizations with anemia during the yearlong study period, 1888 ( $21 \%$ of 9157$)$ had a serum cobalamin level ordered when the patient was anemic and 344 (18\% of 1888) when the patient was also microcytic. Cobalamin deficiency was present in $5 \%$ of these 1888 hospitalizations overall. This proportion did not vary by whether or not patients were microcytic (chi-squared test, $\mathrm{P}$ value $=0.9$ ). Of the 16 patients with both microcytic anemia and cobalamin deficiency, all had elevated RDWs (greater than $14.5 \%$ ), suggesting combined disorders. Indeed 12 out of the 16 patients had concurrent iron deficiency anemia. However, the proportion of patients with an elevated RDW was nearly as high $(97 \%)$ among microcytic anemia patients who did not have cobalamin deficiency (Fisher exact test, $\mathrm{P}$ value $=1.0$ ).

CONCLUSIONS: Among anemic inpatients that had serum cobalamin levels drawn, the proportion diagnosed with cobalamin deficiency was low and associated neither with patients' MCVs nor RDWs. Despite the selection biases inherent in our methods, our findings suggest that general medicine inpatient physicians should continue to test for cobalamin deficiency when they suspect it as a cause of anemia regardless of whether or not microcytosis is present or the red cell population is disperse in size.

CARE GUIDES: USING TRAINED LAYPERSONS TO IMPROVE CHRONIC DISEASE CARE. A RANDOMIZED CONTROLLED TRIAL. Richard Adair ${ }^{1}$; Jon Christianson ${ }^{2}$; Douglas R. Wholey ${ }^{2}$; Katie M. White ${ }^{2}$; Heather R. Britt ${ }^{1}$; Suhna R. Lee ${ }^{2} .{ }^{1}$ Allina Hospitals and Clinics, Minneapolis, MN; ${ }^{2}$ University of Minnesota, Minneapolis, MN. (Control ID \#1316299)

BACKGROUND: Can we improve care for chronic disease patients while keeping costs down? We tested whether trained laypersons located in clinic waiting rooms, where they could interact face-to-face with patients and providers and build ongoing relationships, could improve care.

METHODS: We conducted a randomized controlled trial in 5 primary care clinics diverse in location, patients, and providers. We hired 12 "care guides" (title chosen by patients). Eligibility for this position was two years of college plus strong social skills and a desire to help others; average salary was $\$ 16 / \mathrm{h}$. Most were recent college graduates working in non-medical fields; none had clinical training. The care guides received two weeks' training that included medical ethics and confidentiality, motivational interviewing, the electronic health record (EHR), and basic information about diabetes, hypertension, and heart failure. They were located in small office cubicles in clinic waiting rooms. Their sole task was to help patients who had these diseases and their primary care physicians (PCPs) work together to reach each patient's evidence-based care goals, as defined by national experts. PCPs were asked to refer patients who "needed help" to care guides for enrollment. Patients who agreed to participate $(87 \%)$ were given written information about their diseasespecific care goals, then randomized in a 2:1 ratio to work with a care guide and their PCP to meet these goals (CG patients), or to work with their PCP in the usual way (UC patients). The end point was percent of care goals unmet at baseline and one year later. Care guides and patients could determine the manner (in person, by telephone) and frequency of their interactions; care guides sent PCPs quarterly EHR progress reports and met informally with them as needed.

RESULTS: A total of 2125 patients were enrolled, 1423 randomized to CG and 702 randomized to UC. Care guides served an average panel of 120 patients/full time position. Most patients had more than one diagnosis (1365 diabetes, 1723 hypertension, 122 heart failure). The groups were well matched for diagnoses, age, sex, insurance status, race, educational attainment, and goals met at baseline (75\%). We report interim (6-month) results. In the 1365 patients with diabetes, unmet goals declined $18 \%$ in CG patients $(\mathrm{p}<.001)$ and $1 \%$ in UC patients, with a significant difference between the two groups $(\mathrm{p}<.001)$. In the 705 patients with hypertension without diabetes, unmet blood pressure goals declined $45 \%$ in CG patients $(\mathrm{p}<.001)$ but $51 \%$ in UC patients $(\mathrm{p}<.001)$, with no significant difference between groups. For all randomized patients, unmet goals declined $24 \%$ in CG patients $(\mathrm{p}<.001)$ and $17 \%$ in UC patients $(\mathrm{p}<.001)$, with $\mathrm{p}=.065$ for a difference between groups. Data collection for the randomized patients will be carried out for 1 year. CG and UC patients will be compared with 13,647 propensity-score matched patients in similar clinics without care guides, for a subset of goals.

CONCLUSIONS: Trained laypersons integrated into the primary care delivery process can improve care for chronic disease patients, especially those with diabetes, at a relatively low cost.

CESSATION OF ANTIBIOTICS THROUGH USE OF PROCALCITONIN BIOMARKER LEVEL? Quang V. Ton; Sudhir R. Dudekonda; Benjamin Hohmuth. Englewood Hospital Medical Center, Englewood, NJ. (Control ID \#1309812)

BACKGROUND: Studies have suggested that serum procalcitonin biomarker levels improve the ability to differentiate between bacterial and nonbacterial infections. Previous trials based in the European health care setting have shown a reduction in antibiotic use through clinical algorithms based on procalcitonin levels. In the United States, procalcitonin biomarker has only been recently made available. We conducted a study to evaluate the relationship of procalcitonin biomarker and subsequent antibiotic administration. We hypothesized that antibiotics will not be discontinued when the procalcitonin level discourages use of antibiotics.

METHODS: We retrospectively reviewed electronic medical records of all patients that had a procalcitonin biomarker level during the first nine months of availability between July 2010 and March 2011 in a single center community hospital. Patient were included if they had a procalcitonin level measured in the emergency department or inpatient setting. Patients were divided into two groups based on procalcitonin levels: discouraged or encouraged use of antibiotics. The discouraged use of antibiotics group included patients who had a procalcitonin level of less than $0.25 \mathrm{ug} / \mathrm{L}(\mathrm{n}=242)$; while the encouraged use of antibiotics group included patients who had a procalcitonin level of greater than $0.25 \mathrm{ug} / \mathrm{L}(\mathrm{n}=419)$. The subsequent cessation or continuation of antibiotics was then documented. The specific antibiotic, length of use, and admitting diagnosis were also recorded. Statistical analyses were performed using chi-square analysis for nominal data and unpaired t test (two sided). RESULTS: In the discouraged use of antibiotics group, 78 procalcitonin levels $(32.2 \%)$ had antibiotics discontinued, while in 164 levels $(67.8 \%)$ antibiotics were continued. In the encouraged use of antibiotics group, antibiotics were discontinued in 59 levels (14.1\%) and continued in 360 levels $(85.9 \%)$. Clinical adherence with procalcitonin guided therapy was shown in 438 instances $(66.3 \%)$ while non-adherence was in 223 instances $(33.7 \%)$ in accordance with procalcitonin levels $(\mathrm{p}<0.0001)$. Average length of antibiotic use was 4.8 days in the discouraged use of antibiotics group and was 5.9 days in the encouraged use of antibiotics group $(p<0.001)$. 
CONCLUSIONS: Recent reports from Europe suggest the potential for reduction in antibiotic exposure using clinical algorithms for antibiotic therapy decisions in patients with suspected bacterial infections. Our results suggest that antibiotics are being continued despite procalcitonin levels that should prompt discontinuation of antibiotics. Possible explanations for our findings include lack of procalcitonin algorithms and studies in the United States health care setting. This is one of the first studies to examine clinical adherence in terms of procalcitonin levels and antibiotics in the United States. Further US studies perhaps are needed to alleviate clinician concerns in regards to practice and population differences.

CHANGES IN PATIENT EXPERIENCE IN A PAYMENT-LINKED PATIENT CENTERED MEDICAL HOME DEMONSTRATION PROJECT Asaf Bitton ${ }^{1,2}$; Gordon Schiff ${ }^{1}$; Tony $\mathrm{Yu}^{1}$; Daniel Henderson ${ }^{1,4}$; Stuart R. Lipsitz ${ }^{1}$; Steven R. Simon ${ }^{1,5}$; Lydia A. Flier ${ }^{1}$; Carol Keohane ${ }^{1}$; David W. Bates ${ }^{1,3}$. ${ }^{1}$ Brigham and Women's Hospital, Boston, MA; ${ }^{2}$ Harvard Medical School, Boston, MA; ${ }^{3}$ Harvard School of Public Health, Boston, MA; ${ }^{4}$ University of Connecticut School of Medicine, Farmington, CT; ${ }^{5}$ VA Boston HealthCare System, Jamaica Plain, MA. (Control ID \#1338719)

BACKGROUND: Patient-centered medical homes (PCMH) pilots are garnering attention for their potential to improve primary care delivery. Linking practice transformation to payment reform may help accelerate this change process, but previous research on the short-term impact of transformation on patient experience has shown mixed results. We examined the impact of a payerinitiated, payment-linked PCMH demonstration on patient experience.

METHODS: As part of a multi-modal evaluation, we analyzed data from the first 2 years (2009 and 2010) of a PCMH demonstration initiated by a non-profit commercial insurer in New York. The pilot tested facilitated primary care practice transformation to team-based care linked with comprehensive payment reform in 3 practices with 11,500 health plan patients. The practices received risk-adjusted monthly payments, initial transformation stipends, and significant performance bonus opportunities. Patient experience was surveyed by mail via the Clinical Group-Consumer Assessment of Health Plans (CG-CAHPS) survey, a wellvalidated measure for outpatient care. We focused on 4 domains: care when needed, physician communication, office staff helpfulness, and global physician rating. Each domain is measured on a 0-100 scale. The survey collects information on patient age, sex, race, education, duration of physician relationship, number of visits in prior year, and self-reported health status. We conducted bivariate analyses comparing patients of $\mathrm{PCMH}$ practices to non-PCMH practices, examining means of the domains. We constructed multivariable regression models to assess adjusted differences over time between the PCMH and nonPCMH patients, adjusting for the a priori covariates listed above and accounting for physician-level clustering effects using generalized estimating equations.

RESULTS: In 2009 and 2010, 44,626 patients responded to the CG-CAHPS survey, 1689 of whom were linked to a PCMH pilot. Over $47 \%$ of the sample was from 2009. Compared to non-PCMH, PCMH patients were older, more likely to be white, and had higher education, according to bivariate analysis. They were significantly less likely to report seeing their physician for more than 5 years, and reported fewer visits per year. In unadjusted analyses, patient experience mean measures were significantly improved in global physician rating in both the PCMH and non-PCMH group. In the two year model adjusted for the significant covariates above, gender, and health status, $\mathrm{PCMH}$ ratings in office staff helpfulness improved (84.6 to 85.7), while adjusted means in the non-PCMH group decreased (89.7 to 89.4) (difference in differences $=1.4(\mathrm{SE} 0.7), \mathrm{P}=0.045)$. Similar trends toward improvement in the PCMH patients existed in the other domains, though without statistical significance (care when needed: 1.4 (SE 0.9), $\mathrm{P}=0.14$; physician communication: 1.2 ( $\mathrm{SE} 0.8), \mathrm{P}=0.11$; global rating of physician $0.9(\mathrm{SE} 0.8), \mathrm{P}=0.24)$. CONCLUSIONS: In the first two years of a large PCMH pilot demonstration linked to payment reform, patient experience improved significantly in the office staff interaction domain, and trended toward improvement in other domains. These results are consistent with the pilot's transformation focus on team-based care and improving patient interactions and goal-setting. Aligned payment reform efforts to promote and reward this type of transformation may be a valuable tool for primary care delivery reform. Further research is needed to assess whether the changes are sustainable and replicable.
CHANGES IN USE AND GEOGRAPHIC VARIATION IN PERCUTANEOUS CORONARY INTERVENTION (PCI) FOR STABLE ANGINA FOLLOWING PUBLICATION OF THE COURAGE TRIAL Arun Mohan ${ }^{1}$; Yu-Chu Shen ${ }^{2}$; David Howard ${ }^{3}$. ${ }^{1}$ Emory University School of Medicine, Atlanta, GA; ${ }^{2}$ Naval Postgraduate School, Monterrey, CA; ${ }^{3}$ Rollins School of Public Health, Atlanta, GA. (Control ID \#1339681)

BACKGROUND: Prior research demonstrates substantial geographic variation in clinical practice that is not associated with health status or patient outcomes. Lack of clarity regarding the best approach to a clinical condition is often cited as a cause of such variation. The COURAGE trial, published in 2007, was a highly publicized, multi-center randomized, controlled trial that found that optimized medical therapy alone was as effective as percutaneous coronary intervention (PCI) plus optimized medical therapy for patients with stable angina. We examine whether overall use and geographic variation in use of PCI for stable angina declined following publication of COURAGE.

METHODS: We measured trends in PCI volume in Arizona, California, Florida, Massachusetts, Maryland, and New Jersey using the State Inpatient Discharge databases. These capture a near $100 \%$ sample of all inpatient discharges, including discharges for non-elderly patients. We supplemented these data with regional characteristics from the Dartmouth Atlas of Healthcare such as HRR-level inpatient spending for decedents in the last 6 months of life (a generally-accepted measure of regional practice patterns), hospital characteristics, physician availability, and availability of PCI. We examined geographic variation in hospital referral region (HRR)-level PCI volume for stable angina pre- (2006) and post- (2008) publication of the COURAGE trial and compared those to contemporaneous changes in PCI volume for two "control" conditions not studied in the COURAGE trial, acute myocardial infarction and unstable angina (AMI/UA). Geographic variation was measured in two ways: 1) weighted coefficient of variation (CV), which is the ratio of the standard deviation of the prevalence rates to the mean rate among the HRR, weighted by the population in each HRR, and 2) interquartile ratio (IQR), the ratio of the highest-use HRR to the lowest-use HRR. Multivariate linear and logistic regression was used to examine the impact of age, sex, prevalence of $\mathrm{AMI} / \mathrm{UA}$ (measure of health status), and non-clinical factors on the use of PCI for stable agina. 72 hospital referral regions which represented 290,950 PCI for stable angina and 350,398 PCI for AMI/UA were included in the analysis.

RESULTS: Following the publication of the COURAGE trial PCI volume for stable angina declined $25 \%$ (mean $=1.2$ per 1,000 residents to $0.82, \mathrm{SD}=0.68$ to $0.48, \mathrm{p}=0.002$ ) and decreased $9 \%$ for $\mathrm{AMI} / \mathrm{UA}$ (mean $=1.28$ to $1.14, \mathrm{SD}=$ 0.72 to $0.66, p=0.22)$. Both the $\mathrm{CV}(0.62$ to 0.63 for stable angina and 0.58 to 0.58 for UA/AMI) and the IQR (2.5 to 2.5 for stable angina and 2.3 to 2.5 for $\mathrm{AMI} / \mathrm{UA}$ ) were unchanged. In multivariable regression models, rate of AMI/ UA and spending for decedents in the last 6 months of life were found to be significantly associated with changes in PCI for stable angina $(p<0.05)$. Other non-clinical factors were not associated with changes in PCI volume for either stable angina or AMI/UA.

CONCLUSIONS: While use of PCI for stable angina declined after the publication of the COURAGE trial, there was substantial geographic variation in use of PCI for stable angina that existed prior to its publication that did not change following it. Although non-clinical factors explain this finding in part, even this large, well conducted trial with clear implications for the optimal treatment of PCI did not have the desired effect of reducing geographic variation in healthcare utilization for this condition.

CHARACTERISTICS OF CHRONIC KIDNEY DISEASE PATIENTS IN THE TEXAS PANHANDLE Sudha P. Chennasamudram ${ }^{1}$; Tetyana L. Vasylyeva ${ }^{1}$; Georges Maliha ${ }^{2}$; Giron A. Milton ${ }^{5}$; Sharma Prabhakar ${ }^{4}$; Roger D. Smalligan ${ }^{2} .{ }^{1}$ Texas Tech University Health Sciences Center, Amarillo, TX; ${ }^{2}$ Texas Tech University Health Sciences Center, Amarillo, TX; ${ }^{3}$ Amarillo Medical Specialists, LLP, Amarillo, TX; ${ }^{4}$ Texas Tech University health Sciences center, Lubbock, TX. (Control ID \#1332270)

BACKGROUND: Chronic kidney disease (CKD) is a growing healthcare problem. It is estimated that over twenty-six million people are affected with CKD in the United States. In addition, about 500,000 patients have end stage renal disease (ESRD) and are on chronic dialysis. The state of 
Texas ranks second highest in the prevalence of CKD and costs associated with it in the nation. The goal of this study was to identify the stage at which patients nearing ESRD were referred to nephrologists in the Panhandle region of Texas and to compare these patients with national data available from the Centers for Disease Control and Prevention (CDC). METHODS: A retrospective chart review of CKD patients attending local nephrology clinics in Amarillo, TX was performed (there are no university or public nephrology clinics in the area). Demographic data including age, gender and ethnicity; laboratory values such as serum-creatinine, eGFR, urinary protein excretion, hemoglobin, lipid panel, serum protein and hemoglobin A1c were recorded. Etiology, risk factors for $\mathrm{CKD}$, duration of $\mathrm{CKD}$, medication profile and family history were also collected. Data was compared to the national data from the $\mathrm{CDC}$.

RESULTS: A total of 394 sequential charts were reviewed; 192 men and 192 women. The average age of the patients was 68 ( $8 \%$ 20-44yo, 26\% 45-64yo, $66 \%$ over $65 \mathrm{yo}$ ). With regard to etiology of CKD mentioned at initial visit to the nephrologist, the majority had HTN and/or DM. The average HbAlc among diabetic patients was 11. Other mentioned etiologies/comorbidities in approximate decreasing frequency included acute renal failure, coronary artery disease/CHF, polycystic kidney disease, SLE, glomerulonephritis, recurrent infection and obstructive uropathy. Most patients were referred to the nephrology clinic at CKD stage 3 (GFR 30-59).

CONCLUSIONS: Patients in this study had similar causes of CKD in comparison with national CDC data (CDC data: $35 \%$ of CKD due to DM, $20 \%$ due to HTN). The age distribution of this cohort of Panhandle patients with CKD was significantly older than that in the national database $(66 \%$ vs $45 \%$ (CDC) over 65 yo; $26 \%$ vs $12 \%$ age $45-64$; and $8 \%$ vs $4 \%$ under 45). The high average HbAlc among patients in the cohort showed relatively poor glucose control among diabetics. Research has shown that earlier referral to nephrology can slow the progression to ESRD in patients with DM and HTN. It is important to encourage primary care physicians to screen for CKD and aggressively treat the most common contributors to CKD in the USA: DM and HTN. In addition, earlier referral to nephrology may aid in reducing the rate of progression and high associated costs of ESRD in the Panhandle of Texas and the USA in general.

CHARACTERISTICS OF ISOLATED AND SERIAL RE-HOSPITALIZATIONS SUGGEST A NEED FOR DIFFERENT TYPES OF PATIENT-CENTERED STRATEGIES Romsai T. Boonyasai; Huy Do; Jennifer E. Bracey; Regina Landis; Scott Wright. JHU/APL, Baltimore, MD. (Control ID \#1339935)

BACKGROUND: Early readmission is considered to be an indicator of healthcare quality as well as of utilization. Characterizing sub-groups among readmitted patients can guide quality improvement efforts.

METHODS: We conducted an IRB-approved mixed-methods study of readmitted adults at a 365 bed teaching hospital. 1) We prospectively screened all admissions to identify patients who had been discharged from the hospitalist service within 30 days. 2) A trained research assistant reviewed the index admission and readmission records to collect demographic and health information. 3) Patients were then interviewed using a brief structured questionnaire to ask why they returned to the hospital, whether they felt ready for the index discharge, and whether they had unmet needs after the index discharge. Patients were also asked to rate the patient-centeredness of the index discharge using the Care Transitions Measure and to explain the reason for their rating. 4) We asked hospitalists who had cared for a patient to comment on the preventability of the readmission. 5) We followed patients for 6 months after the index discharge to categorize the episode as an isolated readmission (IR, 1 readmission $/ 6 \mathrm{mos}$ ) or part of serial readmissions (SR, $>1$ readmission/6 mos). All abstracted data and interview responses were transcribed and coded. We used a grounded theory approach to identify principal themes. RESULTS: We identified 82 readmissions involving 76 patients. Median time to readmission was 14.5 days (range $0-30$ days). In the 6 months after index discharge, $23(30.3 \%)$ were readmitted once and $53(69.7 \%)$ were readmitted $>1$ time (range 2-12 times). Only (23.7\%) patients rated their index discharge as not patient-centered. Emerging themes related to lack of patient-centeredness include perceptions of poor communication, unmet expectations (for discharge and for service), and non-improvement of clinical condition. These themes were expressed by both IR and SR patients. Hospitalists provided comments for 66 (80.5\%) encounters involving 64 patients. Emerging themes related to the etiology and preventability of readmission include new medical problem, suboptimal quality of care (e.g., fall at skilled nursing facility), care coordination issues (e.g., lack of PCP), disease progression, patient non-adherence, psychiatric illness, and substance abuse. IR patients appeared to be readmitted for suboptimal quality of care and care coordination issues, whereas SR patients were readmitted for disease progression, psychiatric illness and substance abuse.

CONCLUSIONS: Isolated readmissions may be reduced by improving care coordination and quality of care. Communication and expectation management can improve patient-centeredness of discharge processes. Serial rehospitalizations often result from advanced physiologic or psychiatric disease that only complex systems-wide interventions can improve. Hospitals should apply different targeted interventions for IR and SR populations.

CHARACTERISTICS OF PATIENTS SEEN BY AN INPATIENT SUBSTANCE USE DISORDERS CONSULTATION SERVICE Alvanzo Anika; Patti R. Burgee; Diane Moses; Donnie T. Missouri. Johns Hopkins University School of Medicine, Baltimore, MD. (Control ID \#1340533)

BACKGROUND: Substance use disorders are common, with an estimated lifetime prevalence of $30 \%$ for alcohol and $10 \%$ for drug use disorders, respectively. Further, it has been reported that up to $1 / 5$ of hospitalized patients may have a current substance use disorder. The aim of this study is to characterize patients seen by an inpatient Substance Use Disorders Consultation Service. METHODS: Participants were patients seen by the Substance Use Disorders Consultation Service (SUDS) at an urban, mid-Atlantic academic medical center. The SUDS provides consultation services for patients admitted with medical problems related to or exacerbated by use of alcohol and other drugs. Services include provision of recommendations for management of substance withdrawal syndromes, patient education and brief behavioral counseling, and facilitation of linkage to hospital and community-based alcohol and drug treatment programs. Data on patient demographics and substance use was collected at time of SUDS assessment and frequencies and means and standard errors were used to describe the population. Chi square analyses were used for bivariate comparisons of substance use by sex. Between January and June of 2011, there were 564 referrals for SUDS evaluation, representing 477 unique patients. Data is presented for 422 patients with complete substance use information from their first referral to the SUDS.

RESULTS: Patients had a mean age of 49.8 years (SD 10.66) were predominantly men $(69.5 \%)$ and a majority were either uninsured or receiving Medicaid (57.8\%). One quarter of the patients reported polysubstance use, with a range of one to four substances reported. Alcohol was the most common substance reported $(60.2 \%)$, followed by opiates $(26.8 \%)$, cocaine $(25.6 \%)$, marijuana (6.9\%) and sedative hypnotics $(2.1 \%)$. Men were more likely than women to report alcohol use ( $71.5 \%$ vs. $60.6 \%, p=.029)$, while more women reported cocaine use $(32.0 \%$ vs. $22.7 \%, \mathrm{p}=.043)$ when compared to men. There were no sex differences in polysubstance use.

CONCLUSIONS: Patients referred for inpatient Substance Use Disorders consultation were more likely to be middle aged men reporting use of a single substance, primarily alcohol. Further research should examine the impact of the SUDS on hospital length of stay, substance abuse treatment entry, and 30-day hospital readmission.

CHARACTERIZING LEADERSHIP IN THE MEDICAL HOME TRANSFORMATION: A QUALITATIVE STUDY OF CLINICIAN-LED CHANGE Daniel Henderson ${ }^{1,3}$; Asaf Bitton ${ }^{1,2}$; Greg Schwartz ${ }^{1}$; Elizabeth Stewart $^{4}$; Carol Keohane ${ }^{1}$; David W. Bates ${ }^{1,5}$; Gordon Schiff ${ }^{1}{ }^{1}$ Brigham and Women's Hospital, Boston, MA; ${ }^{2}$ Harvard Medical School, Boston, MA; ${ }^{3}$ University of Connecticut School of Medicine, Farmington, CT; ${ }^{4}$ National Research Network, Kansas City, MO; ${ }^{5}$ Harvard School of Public Health, Boston, MA. (Control ID \#1333093) 
BACKGROUND: The Patient-Centered Medical Home has emerged as a vehicle for reshaping and reviving primary care, offering hope for providers, patients, and a health care system struggling to control costs. Implementing the model broadly presents substantial challenges, both for the health care system, and the individual clinicians undertaking major change. We sought to understand how physicians lead in this setting. We examined leadership behaviors within physician-led PCMH transformations in three primary care practices. Our aim was to benchmark features with a validated model of transformational leadership, and to explore the association between leadership approaches and initial successes.

METHODS: Pursuing a qualitative comparative approach, the research team evaluated three primary care practices $12-18$ months into PCMH transformation. Over two days, we observed facilities and operations, conducted structured interviews with staff, and collected artifacts from the sites. These descriptive and qualitative data were compiled, thematically coded, and analyzed. Drawing evidence from this qualitative data pool we classified practice-level approaches to transformation based on a validated framework, the Eight Step Process for Leading Change developed by John Kotter (below). We also identified indicators of success in transformation, and obtained payer-derived scores rating practices on a composite of quality, efficiency, and patient satisfaction. RESULTS: The categorization along the Kotter framework identified many differences in leadership approaches between practices (see table). In addition, practices $\mathrm{B}$ and $\mathrm{C}$ showed clearer evidence of sustained success than practice A. Site B staff demonstrated satisfaction with success and described individual and team self-efficacy toward a vision. Practice $\mathrm{C}$ staff described successes, but focused on the leadership of a single physician. Site A informants tentatively described success, but with negative spillover effects or reversals. Practice B had the highest initial scores but these declined after the transformation began, eventually rebounding partially. By contrast, practice $\mathrm{C}$ started with lower scores, but improved rapidly to equal practice B's. Practice A showed modest improvement of continually lower scores. All three had difficulty sustaining their gains.

CONCLUSIONS: This qualitative study identified clear differences between three primary care practices undergoing PCMH adoption on the basis of their fit to a validated model of transformational leadership. The degree to which they matched the framework was associated with measures of better performance for a successful PCMH practice, and these associations were proportionate and plausible, if not entirely specific. These results suggest that leadership may be an important co-factor in the success of PCMH adoption, and deserves continuing study in transforming primary care practices.

Table: Transformation resemblance to framework

\begin{tabular}{llll}
\hline \hline & Site & Site & Site \\
& A & B & C \\
Create a sense of urgency & I & + & + \\
Form a powerful guiding coalition & I & + & - \\
Create a vision & - & + & + \\
Communicate a vision & I & + & I \\
Empower others to act on the vision & - & + & - \\
Plan and create short term wins & - & I & I \\
Consolidate improvements and produce more & - & + & I \\
change & & & \\
Institutionalize new approaches & - & I & I \\
\hline
\end{tabular}

(- clearly inconsistent, I intermediate or indeterminate, + clearly consistent):

CHARACTERIZING HEALTHCARE UTILIZATION AMONG OLDER ADULT LATINOS WITH A HISTORY OF DEPRESSION AND ACCESS TO HEALTHCARE Adriana Izquierdo ${ }^{1}$; Catherine Sarkisian ${ }^{2,3}$; Jeanne Miranda ${ }^{4}$. ${ }^{1}$ University of California Los Angeles, Los Angeles, CA; ${ }^{2}$ University of California Los Angeles, Los Angeles, CA; ${ }^{3}$ VA Greater Los Angeles Healthcare System GRECC, Los Angeles, CA; ${ }^{4}$ University of California Los Angeles, Los Angeles, CA. (Control ID \#1335124)

BACKGROUND: Effective treatments for depression in older adults are available. Older adult Latinos, however, are less likely to be treated for depression than older non-Latino whites. Using the largest qualitative dataset collected to date on older Latinos with a history of depression and healthcare access, we aim to characterize how and why this unique population uses healthcare services and treatments for depression management.

METHODS: We used data from the qualitative sub-study at 10-year followup of participants in the Partners in Care (PIC) study, a RCT of QI programs for depression among people with depression and healthcare access. For the qualitative sub-study, all PIC Latino participants $(n=205)$ were invited to complete 3 semi-structured qualitative interviews over 12 months. We evaluated Latino sub-study participants age $\geq 50$. We developed a descriptive framework to model the pathways along which a patient may proceed as $\mathrm{s} / \mathrm{he}$ engages in health care services/treatments for depression management. This framework allowed us to identify participants who moved further along the healthcare utilization pathway. To facilitate data management and analysis, we constructed a data matrix (Microsoft Excel 2007) based on the framework; each column represents a step along the healthcare utilization pathway outlined by the framework, each row an individual transcript. For every health care encounter described, we identified meaningful quotations using standard qualitative content-analysis methods and input them into the data matrix. We identified recurring concepts representing contextual factors and participants' behaviors and attitudes associated with use, or lack of use, of mental healthcare services/treatments, which we developed into codes. We organized coded quotations into themes, and analyzed the themes for their association with participants' progression along the healthcare utilization pathway.

RESULTS: Ninety-five older adult Latinos ( $77 \%$ response) completed at least one qualitative interview ( $75 \%$ female, age range 50-88 years, 265 interviews). Certain themes (e.g., patient activation, patient awareness of depression as a disease, positive past experiences with depression care, perceived provider traits (e.g., empathy, willingness to listen), repeated provider-led outcomes assessment, patient-provider ethnic/gender concordance) facilitated care and were more common in responses among depressed older Latinos who proceeded further along the healthcare utilization pathway. Other themes (e.g., mistrust of provider due to a negative experience and/or general beliefs, absent outcomes tracking) inhibited care and were more likely to be present in responses of those participants who disconnected from care. Some themes (e.g., beliefs about depression, attitudes towards medications, other medical problems, social relationships, media) were associated with variable progression along healthcare utilization pathway.

CONCLUSIONS: Our findings highlight that, even with a history of depression and healthcare access, depressed older Latinos engage in \&/or disconnect from using depression services/treatments for myriad reasons at different points in the healthcare utilization process. By describing the reasons associated with patient utilization of and disengagement from mental healthcare services and treatments, our study may help inform the development of future interventions to improve depression care for older depressed Latinos and reduce mental health disparities.

CHECKBOOK: IMPROVING COST AWARENESS THROUGH AUDIT AND FEEDBACK Jason Post; Darcy Reed; Andrew J. Halvorsen; Furman S. McDonald. Mayo Clinic, Rochester, MN. (Control ID \#1336601)

BACKGROUND: Rising healthcare costs have created an urgent need to improve physicians' education regarding cost and value in healthcare. The ACGME core competency of systems-based practice encompasses cost awareness and risk-benefit analysis, but there has recently been a call to expand this to create a new, seventh core competency of high-value, costconscious care. We set out to determine if audit and feedback would improve resident attitude and knowledge regarding costs of commonly ordered tests. METHODS: We developed an electronic audit and feedback tool called "Checkbook" which provides data on costs and charges for patients cared for at Mayo Clinic-Rochester. This tool allows modeling of healthcare costs and charges by adding or deleting tests and services to the "bill". We conducted a pre-post analysis of the effect of Checkbook on residents' knowledge and attitudes of healthcare costs. First, we asked 48 first and 48 third year residents to estimate charges for commonly ordered tests and services, and surveyed their attitudes regarding cost. Residents then used Checkbook to examine 
billing data from three hospitalized patients for whom they personally provided care. Residents reflected upon which, if any, tests or services may have been avoidable. Following the Checkbook exercise, residents completed an identical post-test of knowledge and attitudes. Pre and post study results were compared using paired t-tests.

RESULTS: Forty-three PGY-1 (89.5\%) and 40 PGY-3 (83.3\%) residents completed the Checkbook exercise. Post-test data showed improvement in estimates of charge as measured by percentage error for commonly ordered tests and services including electrolyte panel (mean percentage error $83.1 \%$ vs $13.4 \%, \mathrm{p}<0.0001)$, serum calcium $(17.2 \%$ vs $12.0 \%, \mathrm{p}=0.006)$, chest $\mathrm{x}$-ray (324.6\% vs $128.9 \%, \mathrm{p}=0.003)$, ECG $(38.5 \%$ vs $9.5 \%, \mathrm{p}=0.001)$, and a onenight hospital stay $(35.0 \%$ vs. $3.6 \%, p=0.01)$. Less commonly ordered tests such as abdominal CT and head MRI did not show an improvement in accuracy of charge estimate. After using Checkbook, both PGY-1 and PGY-3 residents were more likely to agree that they knew the costs of common tests (PGY-1: $2.7 \%$ vs. $27 \%, p=0.003$; PGY-3: $9.7 \%$ vs. $41.9 \%, p=0.002$ ) and that they had received adequate education regarding cost of care (PGY-1: $2.7 \%$ vs. $51.4 \%$, $\mathrm{p}=<0.001$; PGY-3: $0 \%$ vs. $32.3 \%, \mathrm{p}=0.002$ ). PGY-1 residents were more likely to agree that cost influenced their ordering decisions $(37.8 \%$ vs $67.6 \%, \mathrm{p}=$ 0.005 ), that their supervising physicians encouraged them to consider cost when ordering tests $(24.3 \% \mathrm{vs} 46 \%, \mathrm{p}=0.01)$, and that they had adequate access to the costs of care that they provide ( $5.4 \%$ vs $54.1 \%, \mathrm{p}<0.001$ ).

CONCLUSIONS: This study shows that chart audit and feedback with the aid of a cost/charge calculator reflection tool improves residents' knowledge and attitudes regarding costs of care. Previous studies have shown improvement in knowledge of costs using different methods, but to our knowledge this study is the first to demonstrate this using audit and feedback with actual patient cost and charge data. Reflecting upon actual costs of care is an important component of curricula teaching high-value, cost-conscious care.

CHEST RADIOGRAPHS AT PRESENTATION AND CLINICAL OUTCOMES IN A COHORT OF ADULTS HOSPITALIZED WITH COMMUNITY ACQUIRED PNEUMONIA Melissa Simpson ${ }^{1}$; Joseph Mazza $^{1}$; John Schmelzer ${ }^{1}$; Satye Varre ${ }^{1}$; Liang $\overline{\text {Hong}^{2} \text {; Steven H. Yale }}{ }^{1}$. ${ }^{1}$ Marshfield Clininc Research Foundation, Marshfield, WI; ${ }^{2}$ Marshfield CLinic Research Foundation, Marshfield, WI. (Control ID \#1336832)
BACKGROUND: Community acquired pneumonia (CAP) is a leading cause of morbidity, mortality, and health care expenditures. Although decreasing in incidence, CAP still accounts for an estimated 28 hospitalizations per 100,000, across all age groups. Diagnosis is symptom based with supporting laboratory and radiographic data. Although chest radiographs are routine, evidence suggests they lack sensitivity for diagnosing CAP. The purpose of this study was to examine the association between chest radiograph findings at the time of presentation and clinical outcomes in a retrospective cohort of 1,126 adults hospitalized for CAP at St. Joseph's Hospital (SJH), Marshfield, WI from January 1, 1992 to December 31, 2007.

METHODS: Electronic screening using International Classification of Diseases, 9th Revision (ICD-9) codes 480.0-487.9 identified patients with manual confirmation and chart abstraction. One hundred fifty nine patients had clinical symptoms of CAP but no pulmonary parenchymal abnormalities (chest radiograph negative); 967 patients had CAP and chest radiographs showing pulmonary opacities. Logistic regression was used to analyze associations between chest radiographs and the following clinical outcomes: length of hospitalization, in-hospital mortality, inhospital complications (excluding death), 30 day readmission, and 90 day all-cause mortality (table).

RESULTS: CAP and chest radiographs showing pulmonary opacities. Chest radiograph negative patients were older $(\mathrm{p}<0.0001)$, had higher blood pressure (systolic $\mathrm{p}=0.001$, diastolic $\mathrm{p}=0.02$ ), had a body temperature outside $96.8-100.4^{\circ} \mathrm{F}(\mathrm{p}=0.04)$, and were more likely to be nursing home residents $(p=0.02)$. Chest radiograph positive status was associated with an approximately 5 fold increase in the odds of experiencing an inhospital complication. Chest radiograph results were not associated other outcomes. Among chest radiograph negative patients, $82(52 \%)$ had a repeat radiograph and 38 (24\%) developed changes.

CONCLUSIONS: This study suggests that chest radiographs have limited prognostic utility for hospitalized patients and should not guide treatment decisions. The association between in-hospital complications and a positive chest radiograph is puzzling given that chest radiograph results were not associated with any other outcomes. Further research should examine other imaging modalities for their utility in prognostication. In our study of CAP, chest radiograph negative patients were older, perhaps contributing to delayed and/or attenuated immune response.

Unadjusted and adjusted odds ratios for the association between chest radgiograph results and clinical outcomes in a cohort of adults hospitalized for community acquired pneumonia

\begin{tabular}{|c|c|c|c|c|c|c|c|c|}
\hline \multirow[t]{2}{*}{$\overline{\text { Outcome }}$} & \multirow{2}{*}{$\begin{array}{l}\text { Chest radiograph } \\
\text { negative } \\
n=159\end{array}$} & \multirow{2}{*}{$\begin{array}{l}\text { Chest radiograph } \\
\text { positive } \\
n=967\end{array}$} & \multicolumn{3}{|c|}{$\overline{\text { Unadjusted }}$} & \multicolumn{3}{|c|}{ Adjusted } \\
\hline & & & OR & $95 \% \mathrm{CI}$ & p-value & OR & $95 \% \mathrm{CI}$ & p-value \\
\hline Mean ( \pm sd) length of stay (days) & $5.8(5.2)$ & $5.5(5.0)$ & 0.98 & $0.73-1.31$ & 0.89 & 1.09 & $0.81-1.46$ & 0.57 \\
\hline $\mathrm{n}(\%)$ with in-hospital complication(s) & $5(3)$ & $122(13)$ & 4.67 & $1.70-12.88$ & 0.003 & 5.18 & $1.87-14.33$ & 0.002 \\
\hline $\mathrm{n}(\%)$ died in hospital & $10(6)$ & $38(4)$ & 0.61 & $0.30-1.25$ & 0.18 & 0.58 & $0.27-1.22$ & 0.15 \\
\hline $\mathrm{n}(\%)$ readmitted within 30 days of discharge & $11(7)$ & $77(8)$ & 1.17 & $0.61-2.25$ & 0.64 & 0.79 & $0.47-1.34$ & 0.38 \\
\hline $\mathrm{n}(\%)$ died within 90 days of discharge & 21(13) & $101(10)$ & 0.77 & $0.46-1.27$ & 0.3 & 1.22 & $0.63-2.35$ & 0.56 \\
\hline
\end{tabular}

CHRONIC DISEASE RISK FACTORS AND ANTICIPATED BEHAVIOR CHANGE FROM GENETIC RISK TESTING IN YOUNG ADULTS Jason L. Vassy ${ }^{1,2}$; Karen Donelan ${ }^{5,2}$; Marie-France Hivert ${ }^{3}$; Kelsey E. O'Brien ${ }^{1}$; Richard W. Grant ${ }^{4} .{ }^{1}$ Massachusetts General Hospital, Boston, MA; ${ }^{2}$ Harvard Medical School, Boston, MA; $;{ }^{3}$ Université de Sherbrooke, Sherbrooke, QC, Canada; ${ }^{4}$ Kaiser Permanente Division of Research, Oakland, CA; ${ }^{5}$ Massachusetts General Hospital, Boston, MA. (Control ID \#1326402)

BACKGROUND: Preventive behaviors in young adulthood may decrease the lifetime morbidity from heart disease (HD), type 2 diabetes (T2D), and stroke. However, adherence to diet and exercise guidelines in young adults remains low. Genetic susceptibility testing may identify young adults at increased risk prior to onset of phenotypic risk factors like hypertension and hypercholesterolemia. It is not known whether such testing would motivate behavior change.
METHODS: We conducted a nationally representative Internet survey of young adults aged 25 to 30 years to test the hypothesis that young adults would be motivated by high-risk genetic results to adopt behavior changes. Survey participants were recruited through random digit dialing or addressbased sampling. We measured 6 baseline risk factors for $\mathrm{HD}, \mathrm{T} 2 \mathrm{D}$, and stroke: non-white race/ethnicity, smoking, poor diet, low physical activity, family history, and obesity. We used a 3-point scale (not at all/somewhat/very) to measure respondents' interest in undergoing a genetic susceptibility test for the 3 diseases and a 5-point scale (from "Much less likely" to "Much more likely") to ask whether a hypothetical high-risk genetic result would change their likelihood of improving their physical activity and diet. For those anticipating an improvement, we asked whether this change would occur now vs. 5 years or more from now. We used weighted $\chi 2$ and t-tests to account for sampling design.

RESULTS: Of 521 survey respondents (completion rate 54\%), 40\% were nonwhite, and $30 \%$ were obese. Two-thirds expressed interest in genetic testing. Respondents generally reported that high-risk genetic results would increase their 
likelihood of improving physical activity and diet (median response "Somewhat more likely" for both). Of the 6 baseline risk factors, non-white race/ethnicity and current obesity were both associated with greater anticipated likelihood of increasing physical activity $(\mathrm{P}<0.05$ for both), whereas less-than-average current physical activity was associated with a lower likelihood of improving $\operatorname{diet}(\mathrm{P}=0.03)$ in response to high-risk genetic test results. Of the respondents anticipating improvement in physical activity $(n=419)$ or diet $(n=411)$, no risk factor was associated with a greater likelihood of making that change now versus $\geq 5$ years from now. Indeed, poorer baseline physical activity was associated with delaying physical activity and diet changes ( $\mathrm{P}<0.05$ for both behaviors), and poorer baseline diet was associated with delaying dietary changes $(\mathrm{P}=0.02)$, compared to better current physical activity and dietary patterns.

CONCLUSIONS: Testing young adults for genetic susceptibility to future lifestyle-related chronic diseases has the potential to motivate behavior changes. However, those with worse baseline health behaviors were least likely to change behavior while still young. These results suggest that to be effective, such testing may need to be coupled with interventions to support behavior change.

CLINICAL SIGNIFICANCE OF RESTING HEART RATE AND INFLAMMATION IN OLDER ADULTS David Nanchen ${ }^{1,2}$; Jacobijn Gussekloo $^{3}$; Simon P. Mooijaart ${ }^{1,4}$; Rudi G. Westendorp ${ }^{1,5}$; J. Wouter Jukema $^{6}$; Peter W. Macfarlane ${ }^{7}$; Naveed Sattar ${ }^{7,8}$; David J. Stott ${ }^{7,8}$; Jacques Cornuz ${ }^{2}$; Nicolas Rodondi ${ }^{9}$; Anton J. de Craen ${ }^{1,5}$. ${ }^{1}$ Leiden University Medical Center, Leiden, Netherlands; ${ }^{2}$ University of Lausanne, Lausanne, Switzerland; ${ }^{3}$ Leiden University Medical Center, Leiden, Netherlands; ${ }^{4}$ Institute for Evidence-Based Medicine in Old Age, Leiden, Netherlands; ${ }^{5}$ Leiden University Medical Center, Leiden, Netherlands; ${ }^{6}$ Leiden University Medical Center, Leiden, Netherlands; ${ }^{7}$ University of Glasgow, Glagsgow, United Kingdom; ${ }^{8}$ University of Glasgow, Glagsgow, United Kingdom; ${ }^{9}$ University of Bern, Bern, Switzerland. (Control ID \#1329461)

BACKGROUND: Traditional cardiovascular risk factors have a lower predictive capacity in older people. Thus, resting heart rate might be a promising cardiovascular risk marker in older adults, but previous results have not led to any clinical utility partly because incertitude remains about the causal role of heart rate in the development of atherosclerosis, or other clinical morbidities such as heart failure, or infection. Particularly, the interrelation between heart rate and inflammation might play a key role in older adults. We aimed to assess the clinical significance of resting heart rate along with inflammation on heart failure, atrial fibrillation and cardiovascular and non-cardiovascular mortality and morbidities in older adults.

METHODS: We studied 4084 older adults aged 70-82 years with known cardiovascular risk factors or previous cardiovascular disease, without preexisting heart failure or beta-blockers in the PROSPER study. Over a 3.2-year follow-up period, we compared (non-) cardiovascular mortality and morbidities according to gender-adjusted tertiles of resting heart rate. Inflammation was measured in all participants with C-reactive protein (CRP).

RESULTS: In men (women) mean resting heart rate was 55 (60) beat per minute (bpm) for the first tertile, 66 (70) bpm for the second tertile, and 81 (83) bpm for the third tertile, respectively. An elevated resting heart rate (highest vs lowest tertile) was associated with incident heart failure hospitalization (multivariate adjusted hazard ratio [HR] 1.75, 95\% confidence interval [CI], $1.19-2.59, \mathrm{p}$ for trend across categories $=0.001$ ), but not with nonfatal myocardial infarction, stroke or atrial fibrillation. Incident nonfatal infections were also more common (highest vs lowest tertile), with a HR of $1.90,95 \%$ CI, 1.33-2.71, $\mathrm{p}$ for trend $<0.001$. Both non-cardiovascular mortality (HR 1.70, 95\% CI, 1.24-2.34, p for trend $<0.001$ ) and cardiovascular mortality (HR 1.73, 95\% CI 1.22-2.44, $\mathrm{p}$ for trend $=0.001$ ) were increased in older adults in the highest tertile of heart rate. Further adjustments for inflammation assessed by CRP decreased associations with heart failure to $1.72,95 \% \mathrm{CI}, 1.16-2.55, \mathrm{p}$ for trend $=0.001$, and with cardiovascular mortality to $1.60,95 \% \mathrm{CI}, 1.12-2.28$, $\mathrm{p}$ for trend $=0.005$ (Figure).

CONCLUSIONS: In older adults, an elevated resting heart rate predicted first incident heart failure and infection event, but not nonfatal cardiovascular event. Inflammation seems to play only a minor explicative role in the association between resting heart rate and heart failure and cardiovascular mortality in older adults. The specific role of heart rate reduction in cardiovascular prevention management needs further evaluation in older adults.
Figure: Association of heart rate with cardiovascular mortality and heart failure hospitalization, with respect to heart rate tertiles (T1, T2, T3).

\section{Cardiovascular mortality}

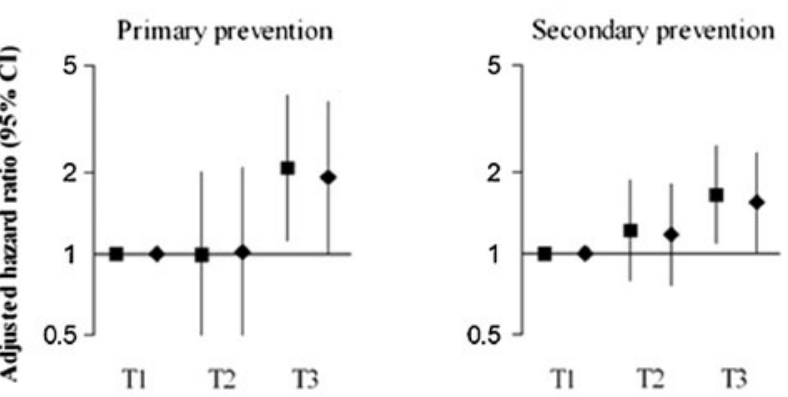

\section{Heart failure hospitalization} Primary/secondary prevention includes older adults without/with cardiovascular disease or diabetes.

Squares (model 1) represent hazard ratios adjusted for age, smoking status, hypertension, body mass index and creatinine. Diamonds (model 2) represents hazards ratios additionally adjusted for $\log$ CRP.
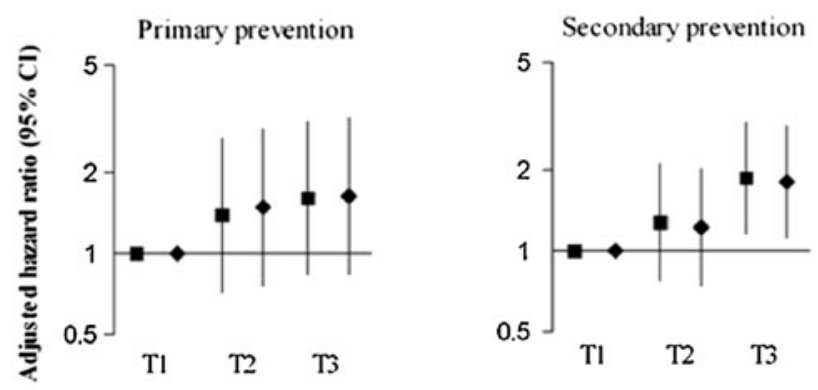

- Model 1 Model 2 
CLINICIAN-EDUCATORS ARE MORE BURNED OUT AS CLINICIANS THAN AS EDUCATORS: IMPLICATIONS FOR TEACHING (AND PRACTICE). Anne Dembitzer ${ }^{1,2}$; Colleen Gillespie ${ }^{2}$; Kathleen Hanley $^{2}$; Ruth Crowe ${ }^{2}$; Sondra Zabar ${ }^{2}$; Nina Yeboah ${ }^{2}$; Audrey Grask $^{2}$; Joseph Nicholson ${ }^{2}$; Adina Kalet ${ }^{2}$; Mark D. Schwartz ${ }^{1,2}$. ${ }^{1}$ NY Harbor VA, New York, NY; ${ }^{2}$ NYU School of Medicine, New York, NY. (Control ID \#1335960)

BACKGROUND: Clinician-educators are the primary teachers of medical students and residents, yet most have not had formal training in medical education. Physician burnout is common and is associated with job turnover and absenteeism, poor morale, and reduced efficiency, and may lead to poorer patient care. As part of a faculty development program (FDP) designed to improve mentoring and teaching skills, we sought to determine the degree of burnout among clinician-educators in an urban, underserved setting, how burnout differed for clinician and educator roles, and the impact of burnout on teaching.

METHODS: 30 clinician-educators (CEs) involved in a FDP completed a survey that included the clinician and educator versions of the Maslach burnout inventory. These scales each assess three features of burnout: Emotional Exhaustion, Depersonalization, and Personal Accomplishment, each classified as low, moderate, or high. They rated their perceived competence in five teaching domains (establishing a learning climate, control of session, assessment/evaluation, instructional skills, and giving feedback). They reported on practice characteristics, career fit, job satisfaction, and commitment to lifelong learning (Jefferson Lifelong Learning Scale). Standardized learners assessed clinician-educators' teaching skills in the same five domains in a threestation Objective Structured Teaching Examination (OSTE). Scores were calculated as percentage of items within each domain rated as Done Well (vs. Not or Partly Done).

RESULTS: More than half of the clinician-educator faculty were internists, the rest were pediatricians or family medicine physicians. They spent an average of $24 \%$ (SD $11 \%$ ) of their time teaching or precepting, $55 \%$ of their time providing outpatient care (SD 22\%), and $20 \%$ of their time providing inpatient care (SD 20\%). Burnout scores by clinician and educator roles are shown below (Table). Clinician-educators with high "emotional exhaustion" as clinicians reported lower overall confidence in teaching than did those with only low or moderate emotional exhaustion (one-way ANOVA $\mathrm{F}=9.91, \mathrm{p}=.001$, pairwise comparisons $\mathrm{p}<.05$ ), and this pattern was upheld in the five specific teaching domains as well. Those who reported "high" depersonalization as clinicians were less confident in teaching than were those with only low or moderate scores (one-way ANOVA $\mathrm{F}=4.51, \mathrm{p}=.020$, pairwise comparisons $\mathrm{p}<.05$ ). Commitment to lifelong learning was lower for those with high clinician-related emotional exhaustion $(\mathrm{F}=$ 7.91, $\mathrm{p}=.002)$ and high depersonalization $(\mathrm{F}=1.967, \mathrm{p}=.05)$, compared to that of those with low or moderate clinician burnout in these areas. Teaching skills in the OSTE did not significantly differ by clinician or educator burnout.

CONCLUSIONS: This sample of clinician-educators in urban, underserved settings had a higher rate of burnout as clinicians than as educators. Burnout as a clinician may impact one's effectiveness as an educator since clinically burned-out faculty had less confidence in their teaching skills and fewer life-long learning habits. This study suggests the need to further investigate how clinician-educators balance the demands and effects of their dual roles.

Burnout scores by clinician and educator roles

\begin{tabular}{|c|c|c|c|}
\hline $\begin{array}{l}\text { Maslach burnout } \\
\text { inventory } \\
\text { (scored low, medium, } \\
\text { high) }\end{array}$ & $\begin{array}{l}\text { Emotional } \\
\text { exhaustion } \\
\% \text { high }\end{array}$ & $\begin{array}{l}\text { Depersonalization } \\
\% \text { high }\end{array}$ & $\begin{array}{l}\text { Personal } \\
\text { accomplishmen } \\
\% \text { low }\end{array}$ \\
\hline Clinician & 53 & 23 & 20 \\
\hline Educator & 10 & 0 & 23 \\
\hline $\begin{array}{l}\text { Significance (Wilcoxon } \\
\text { Signed Ranks Test) }\end{array}$ & $\mathrm{p}<.001$ & $\mathrm{p}=.001$ & $\mathrm{p}=.803$ \\
\hline
\end{tabular}

CO-MORBIDITY AND COMPLEXITY OF CARE, A WEIGHTY MATTER: FOUR CO-MORBIDITY INDEXES AND PREDICTION OF DEATH, AMBULATORY CARE VISITS, POLYPHARMACY, AND THE PRIMARY CARE CHECKLIST Lillian Min ${ }^{1,2}$; Neil Wenger $^{4,5}$; Caroline Blaum ${ }^{1,2}$; Chris Cigolle ${ }^{1,2}$; Eve A. Kerr ${ }^{3,1}{ }^{1}$ University of Michigan, Ann Arbor, MI; ${ }^{2}$ VA Healthcare Systems, Ann Arbor, MI; ${ }^{3}$ VA Healthcare Systems, Ann Arbor, MI; ${ }^{4}$ UCLA, University of California, Los Angeles, CA; ${ }^{5}$ RAND Health, Santa Monica, CA. (Control ID \#1334704)

BACKGROUND: Understanding clinical complexity of older ambulatory care patients due to their co-morbidities is crucial to providing high-quality ambulatory care. Co-morbidity indexes measure conditions associated with specific outcomes, assigning greater weights for co-morbidities with the strongest associations with death (Charlson Comorbidity Index, CCI) and expenditures (Medicare Hierarchical Condition Categories, HCC). We previously reported how an unweighted count of 9 conditions was associated with quality of care (ACOVE simple count, ACOVE$\mathrm{SC}$ ). We also developed the Geriatric Complexity of Care (GCC) score, 32 medical, psychosocial and geriatric conditions further sub-classified into severity categories (total 117 categories) common in geriatric ambulatory care. Using an expert panel, the 117 categories were weighted according to the difficulty of providing high-quality primary care for geriatric patients with those conditions. We hypothesized that the GCC (calculated as the total of the difficulty weights for each patient's co-morbidities) would predict external (i.e., non-comorbidity) measures of primary care complexity: polypharmacy, primary care visits, and number of ACOVE QIs eligible (the care processes a clinician or medical home would be responsible for).

METHODS: Sample: We reviewed 1 year of ambulatory medical records for 644 patients (age $>=75$, screened for symptoms of $1+$ geriatric condition) in the Assessing the Care of Vulnerable Elders-2 (ACOVE-2) study to obtain the 117 GCC co-morbidity categories. Meaures: We calculated 4 co-morbidity indexes for each patient using weights from the CCI, HCC (ambulatory care sample), ACOVE-SC, and the GCC. For CCI and HCC, we omitted weights for age and gender because multivariable models included these co-variables. Analysis: Using logistic and linear regression, we compared the unadjusted model fit (Area under the Receiver Operating Curve [AUC] and R-squared [R2]) and adjusted effect size (predicted values for top minus bottom quintile, adjusted for age, gender, and functional status) for predicting: (1) 3-year mortality, (2) primary care and specialty ambulatory visits over 1 year, and (3) complexity of care (polypharmacy [14+ longterm medications] and the "primary care checklist", calculated as the number of ACOVE QIs each patient was eligible for over 1 year)

RESULTS: Of the 4 co-morbidity indexes, the HCC-weighted score predicted the most variation in mortality ( $\mathrm{AUC}=69.1 \%$ ) and specialty visits ( $\mathrm{R} 2=9.1 \%$ ) but the $\mathrm{GCC}$ was the best predictor of primary care visits $(\mathrm{R} 2=13.6 \%)$, polypharmacy $(\mathrm{AUC}=78 \%)$, and the primary care checklist $(\mathrm{R} 2=30.5 \%$ ). The most complex (top quintile of GCC) patients received more ambulatory medical care ( 7.0 vs 3.7 primary care visits, 6.2 vs 2.4 specialist visits), polypharmacy ( $14.3 \%$ vs $0 \%$ had $14+$ medications) and longer ambulatory care checklists (33 vs 25 QIs) compared to the lowest quintile. The effect of GCC on complexity of care outcomes was independent of age, gender, and functional status, even when other indices (CCI, HCC, and ACOVE-SC) were entered into the multivariable models. CONCLUSIONS: Geriatric complexity of care reflects health care needs not represented by simpler and routinely-collected co-morbidity indexes. Future planning of systems with the capacity to provide high quality geriatric ambulatory care should consider targeting complex patients with extra clinic visits and resources (e.g., pharmacy to review additional medication burden).

COMMUNITY HEALTH CENTER PROVIDERS' SPANISH LANGUAGE ABILITY AND KNOWLEDGE OF LATINO CULTURE Arshiya A. Baig ${ }^{1}$; Cara A. Locklin ${ }^{2}$; Amanda Campbell ${ }^{3}$; Cynthia T. Schaefer $^{4}$; Loretta J. Heuer ${ }^{5}$; Sang Mee Lee ${ }^{6}$; Marla C. Solomon ${ }^{7}$; Michael T. Quinn ${ }^{1}$; J. Martin Vargas ${ }^{8}$; Deborah L. Burnet ${ }^{1}$; Marshall Chin ${ }^{1}$. ${ }^{1}$ University of Chicago, Chicago, IL; ${ }^{2}$ University of Illinois at Chicago, Chicago, IL; ${ }^{3}$ MidWest Clinicians' Network, Lansing, MI; ${ }^{4}$ University of Evansville, Evansville, IN; ${ }^{5}$ University of North Dakota, Fargo, ND; ${ }^{6}$ University of Chicago, Chicago, IL; ${ }^{7}$ University of Illinois at Chicago, Chicago, IL; ${ }^{8}$ Community Action Partnership of Western Nebraska, Gering, NE. (Control ID \#1332311) 
BACKGROUND: Many Latino patients with diabetes receive care at community health centers. However, little is known regarding health center providers' Spanish language ability and knowledge of Latino culture.

METHODS: We collaborated with the MidWest Clinicians' Network and conducted a survey of providers who manage or treat patients with diabetes from member health centers. Providers included advanced practice nurses (APN), certified diabetes educators, certified medical assistants (MA), dietitians, health educators, licensed practical nurses (LPN), physicians, physician's assistants (PA), registered nurses (RN), and social workers. Participants self-reported their ability to speak, understand, read and write Spanish. Providers also rated their knowledge of Latino culture across 8 different domains: role of family, religious beliefs, folk remedies, traditional diet modifications, variations among different Latino cultures, patient-doctor interactions, health barriers for seasonal workers, and culturally tailored care. The composite language ability (Cronbach's alpha 0.94) and cultural knowledge scores (Cronbach's alpha 0.96) were divided into terciles and then dichotomized as "high" and "moderate/low." Participants self-reported ever having received cultural competency training. Participants also reported their access to on-site interpretation services (defined as available $\leq 50 \%$ or $>50 \%$ of the time) and cultural competency training (available or not). We assessed overall frequency of language and cultural competency scores and also evaluated differences across provider types using chi-square tests. Availability of interpreter services and cultural competency training were assessed for the providers with low language and cultural knowledge scores.

RESULTS: We received responses from 621 providers $(47 \%$ adjusted response rate) from 87 health center sites across 10 states. Twenty-nine percent of providers reported never having received cultural competency training. Overall, 12\% had high Spanish language scores and 20\% had high cultural knowledge scores. Across providers, $8 \%$ of APNs, $4 \%$ of LPNs, $10 \%$ of MAs, $14 \%$ of physicians, $16 \%$ of PAs, and $2 \%$ of RNs reported having high language scores $(\mathrm{p}=0.10)$. In terms of cultural knowledge, $23 \%$ of APNs, $8 \%$ of LPNs, $10 \%$ of MAs, $19 \%$ of physicians, $24 \%$ of PAs, and $17 \%$ of RNs had high cultural knowledge scores $(\mathrm{p}=0.27)$. Of the providers who had low Spanish language scores, $69 \%$ had access to interpreter services on-site the majority of the time. Of the providers who had low cultural competency scores, $45 \%$ had access to cultural competency training on-site.

CONCLUSIONS: Spanish language ability and cultural knowledge scores were low across all types of providers. Strengthening access to interpreter services and providing cultural competency training may be promising strategies to support health center providers who manage Latino patients.

COMORBID CONDITIONS IN COPD Daniel A. Belletti ${ }^{1}$; Christopher Zacker ${ }^{1}$; Jennifer Wogen ${ }^{2} .{ }^{1}$ Novartis Pharmaceuticals, East Hanover, NJ; ${ }^{2}$ Medmentis Consulting, Towaco, NJ. (Control ID \#1339649)

BACKGROUND: COPD is associated with other risk factors and co-morbid conditions which may complicate COPD management. Primary care treatment patterns of COPD remain largely undocumented despite research suggesting that the majority $(63 \%)$ of patients with COPD in the US are managed by primary care physicians (PCPs). As PCPs deliver care to many patients with COPD, our study objective was to understand treatment patterns related to 5 specific comorbid conditions addressed in the 2007 Global Initiative for Chronic Obstructive Lung Disease (GOLD) Guidelines.

METHODS: This cross-sectional study was performed via retrospective chart review at 11 primary care sites, using a random sample of from 50 150 patients/site aged 40 through 89 years with a diagnosis of $\operatorname{COPD}(\mathrm{N}=$ 1517). Data extracted from patient medical records included patient demographics and clinical information (spirometry testing and documented COPD stage, respiratory medications, and co-morbid conditions). Appropriate evaluation or treatment of comorbid conditions was assessed, including glycemic control in diabetic patients, blood pressure (BP) control in hypertensive patients, bone mineral density (BMD) testing for at-risk patients, and depression screening/treatment for all patients.

RESULTS: Mean patient age was $67.2(\mathrm{SD}+11.3)$ years, $54 \%$ were female, $62 \%$ were Caucasian, and $34 \%$ were current smokers. Mean duration of COPD was 48 months (range $1-612$ ) . Only $27 \%$ of patients had a current FEV1\% result documented. $20 \%$ were classified as mild (stage I), $13 \%$ moderate (stage II), $12 \%$ stage III (severe), 3.4\% very severe (stage IV). The average number of comorbid conditions was $2.9(+/-1.6)$; $66 \%$ had hypertension, $27 \%$ diabetes, $26 \%$ depression/ anxiety, Comorbid conditions were most prevalent in the severe and very severe categories.(Table 1) Mean number of all medications was $8.1(+/-6.7)$, mirroring the greater number of comorbid conditions present with more severe stages of COPD. (Table 1) Of the patients with the 5 comorbid conditions of interest, about $49 \%$ had all of the benchmarks of treatment/evaluation (Figure 1).

CONCLUSIONS: Comorbid conditions are frequently associated with COPD. Concurrent medication use is also higher in those with co-morbid conditions. Comprehensive management of the patient with COPD is complicated by this confluence of conditions, poly- medication use and associated care. Addressing comorbid conditions in COPD remains a critical component of care for primary care.

Avg. \# of Medications and Comorbid Conditions by Severity

\begin{tabular}{lll}
\hline \hline COPD Stage & Avg. \# All Medications & Avg. \# Comorbidities \\
Mild & 5.8 & 2.6 \\
Moderate & 7.8 & 2.8 \\
Severe & 10.4 & 3.3 \\
Very Severe & 9.8 & 3.0 \\
Unkown/Missing & 7.7 & 3.0 \\
\hline
\end{tabular}

COMPARATIVE ANALYSIS OF PRINT AND MULTIMEDIA HEALTH MATERIALS: A SYSTEMATIC REVIEW OF THE LITERATURE Elizabeth A. Wilson ${ }^{1}$; Gregory Makoul ${ }^{2,3}$; Elizabeth A. Bojarski ${ }^{1}$; Katherine R. Waite ${ }^{1}$; David N. Rapp ${ }^{4}$; David W. Baker ${ }^{1}$; Michael S. Wolf ${ }^{1,4}$. ${ }^{1}$ Northwestern University, Chicago, IL; ${ }^{2}$ University of Connecticut School of Medicine, Farmington, CT; ${ }^{3}$ Saint Francis Hospital and Medical Center, Hartford, CT; ${ }^{4}$ Northwestern University, Evanston, IL. (Control ID \#1338447)

BACKGROUND: Although the prevalence of multimedia educational tools has increased within the context of healthcare, there is little guidance as to what modalities of presentation are most effective. We completed a systematic literature review to evaluate the existing empirical evidence regarding the relative effectiveness of multimedia versus print as modes of dissemination for patient education materials and to examine the development of these materials. METHODS: To examine the impact of type of medium on patient outcomes including preference, comprehension, behavior, and anxiety, we completed a structured literature review utilizing Medline, PsychInfo, and the Cumulative Index to the Nursing and Allied Health Literature (CINAHL), supplemented by reference mining.

RESULTS: Of 738 studies screened, 31 effectively compared equivalent forms of multimedia and print materials. Studies offered 58 opportunities for assessing the effect of medium on various outcomes. In the majority of instances (34 occurrences, 59\%), no difference was noted between multimedia and print on patient outcomes. Multimedia was associated with improvements in outcome in 19 instances $(33 \%)$ and print in $5(9 \%)$. For material development, six studies $(19 \%)$ assessed readability of materials and $4(13 \%)$ involved patients in tool development.

CONCLUSIONS: Multimedia appears to be a promising medium for patient education; however, the majority of studies found that print and multimedia performed equally well in practice. Few studies involved patients in material development or assessed the readability of patient education tools. As such, future research should focus on comparing message-equivalent tools and assessing their effect on behavioral outcomes. Materials should be developed with consideration of universal cognitive constraints and patients input during the development process.

COMPARATIVE EFFECTIVENESS OF HOSPITAL-BASED EDUCATIONAL INTERVENTIONS FOR PATIENTS WITH COPD OR ASTHMA Valerie G. Press ${ }^{1}$; Vineet Arora $^{2}$; Lisa M. Shah ${ }^{3}$; Stephanie Lewis ${ }^{4}$; Jeffery Charbeneau ${ }^{4}$; Edward Naureckas ${ }^{5}$; Jerry Krishnan ${ }^{4}$. ${ }^{1}$ University of Chicago Medical Center, Chicago, IL; ${ }^{2}$ University of Chicago Medical Center, Chicago, IL; ${ }^{3}$ Johns Hopkins University, Baltimore, MD; ${ }^{4}$ University of Illinois at Chicago, Chicago, IL; ${ }^{5}$ University of Chicago Medical Center, Chicago, IL. (Control ID \#1311106) 
BACKGROUND: There has been an increasing focus on avoiding preventable readmissions through patient coaching and education. This is particularly salient for inpatients with Chronic Obstructive Pulmonary Disease (COPD), as it is the 3 rd leading cause of 30-day hospital readmissions. However, little is known about how to to improve inpatient self-management skills, especially for medication use, for patients with obstructive lung disease (e.g., COPD, asthma). The primary objective of the study was to test the comparative effectiveness of two interventions that provide hospital-based education on effective inhaler technique for hospitalized patients with COPD or asthma.

METHODS: Adult inpatients with COPD or asthma were randomized to receive either Teach-to-Goal (TTG) or Brief Intervention (BI) education. Participants receiving TTG were provided with a demonstration on inhaler technique and verbal and written instructions and then asked to redemonstrate their technique (i.e., "teachback"); this cycle continued until they demonstrated mastery ( $\leq$ two rounds). BI participants received verbal and written instructions. Use of metered dose inhaler (MDI) and Diskus ${ }^{\circledR}$ devices was assessed using detailed checklists. Misuse was defined as $<75 \%$ of steps correct. Self-reported inhaler technique confidence was measured using a 5-point Likert scale. Follow-up data were collected at 30days post-discharge (phone interviews). Overall health-related events (ED visits, hospitalizations and/or deaths), post-discharge were assessed. Chisquared, Fisher's exact, and t-tests were performed using STATA 11.

RESULTS: Participants were enrolled and randomized to TTG $(n=24)$ or BI $(\mathrm{n}=26)$. The mean age was 54 years, and the majority had COPD $(60 \%)$, were female $(68 \%)$ and were African-American $(78 \%)$. This was a high-risk population with over half $(58 \%)$ having $\geq 1$ hospitalization in the past year for COPD or asthma, and nearly half (44\%) having had a near-fatal event (ICU admission and/or intubation). While the majority of participants reported being confident with their inhaler technique (MDI 70\%, Diskus ${ }^{\circledR}$ 94\%), most misused their inhalers pre-intervention (MDI $62 \%$, Diskus ${ }^{\circledR} 78 \%$ ). The proportion who misused MDIs post-intervention decreased significantly for both TTG and BI groups $(\mathrm{p}<0.05)$. Further, there was a significantly greater reduction in the prevalence of misuse in the TTG group vs the BI group $(50 \%$ vs. $30 \%, p=0.01$ ). There was also a nearly significant decrease in misuse for Diskus in the TTG vs. BI group $(\mathrm{p}=0.05)$. Thirty-nine $(78 \%)$ participants had 30-day follow-up data; there were 3 deaths and 36 completed follow-up interviews (77\% BI, 79\% TTG). There were significantly more health-related events in the BI group vs. TTG at 30-days post discharge $(\mathrm{p}=0.02)$.

CONCLUSIONS: Our study demonstrates that providing hospital-based instructions on inhaler technique for patients hospitalized with COPD or asthma can decrease prevalence of inhaler misuse prior to hospital discharge. Further, we demonstrate that TTG may be a superior strategy for improving inhaler technique, and may lead to improved clinical outcomes compared to BI. Finally, high-risk inpatients over-estimate their inhaler technique, emphasizing the need for hospital-based interventions to correct inhaler misuse. Larger, multi-institution comparative studies are needed to evaluate the effects of TTG vs. BI for different patient subgroups (e.g., level of health literacy), the durability of the hospital-based education and associated clinical outcomes.

COMPARATIVE OVERDOSE DEATH RATES BETWEEN ILLICIT AND PRESCRIBED SUBSTANCES Susan Calcaterra ${ }^{1}$; Ingrid A. Binswanger ${ }^{2}$. ${ }^{1}$ University of Colorado, Denver, CO; ${ }^{2}$ University of Colorado, Denver, CO. (Control ID \#1334470)

BACKGROUND: Overdose death rates in the United States (US) vary significantly by drug type. Cocaine and heroin were historically considered leading causes of overdose death in the US. In 2000, prescription opioid overdose deaths surpassed cocaine overdose deaths and are rising at an alarming rate. This trend has been attributed to the widespread use of pain scales, "pain as the 5th vital sign," and heavy prescribing for non-malignant pain. We aimed to describe the death rate from prescription opioid pain relievers as compared to the death rates of other commonly abuse substances, and describe the demographic characteristics of overdose deaths by substance. METHODS: Using the CDC Wonder Database, we reviewed all cocaine (T40.5), heroin (T40.1), psychostimulant (i.e. methamphetamine, T43.6), and prescription opioid poisoning (overdose) deaths (T40.2-T40.4) among 15-64 year olds in the US from 1999-2008. We calculated age-adjusted death rates (number of deaths $/ 100,000$ person- years [p-y] and $95 \%$ confidence interval $[\mathrm{CI}]$ ) for those who died using substance specific ICD10 codes listed among their causes of death. We calculated death rates by age, gender, and race/ethnicity. We identified the most common underlying causes of death among those who died. Lastly, we categorized all deaths by autopsy status during 2003-2008 to help support the validity of the listed causes of death.

RESULTS: By 2008, prescription opioid overdose deaths were more common than cocaine, heroin, and psychostimulant overdose deaths combined. Deaths increased significantly for heroin, psychostimulants, and prescription opioids from 1999 to 2008. Prescription opioid overdose deaths increased more than three-fold from 1999 to 2008 (2.24/100,000 p-y [95\% CI 2.17-2.31] to 7.34/ 100,000 p-y [ $95 \%$ CI 7.22-7.45]). Psychostimulant overdose deaths doubled from 1999 to $2008(0.37 / 1000$ p-y [95\% CI $0.34-0.39$ ] to $0.78 / 100,000$ p-y [95\% CI 0.74-0.82]). Cocaine overdose deaths peaked in $2006(4.29 / 100,000 \mathrm{p}-$ y [95\% CI 4.20-4.38]), but declined in 2008 (2.84/100,000 p-y [95\% CI 2.77 2.92]). Overdose death rates were higher overall in men than woman, and peak death rates were frequently observed among 35-44 year olds. African Americans had the highest death rate due to cocaine (8.00/100,000 p-y [95\% CI 7.88-8.11]), American Indians/Alaska Natives had the highest death rate due to psychostimulants $(1.45 / 100,000$ p-y [95\% CI 1.26-1.64]) and prescription opioids $(6.42 / 100,000$ p-y [95\% CI 6.03-6.82]), closely followed by nonHispanic whites, $(6.17 / 100,000$ p-y [95\% CI 6.12-6.21]). After combining cocaine, heroin, prescription opioid, and psychostimulant overdose deaths, "accidental poisonings" (X40-49) was the most frequently listed cause of death. Autopsies were performed on $80 \%$ of overdose deaths.

CONCLUSIONS: While overall overdose death rates due to heroin, cocaine, and psychostimulants have risen since 1999, overdose deaths due to prescription opioids have increased more than three-fold in the last decade. Practice-based interventions to reduce the rising risk of overdose deaths should be tested as a method to decrease the death rates of all substances, not only prescription opioids.

COMPARATIVE EFFECTIVENESS OF ENDOVASCULAR VERSUS OPEN REPAIR OF RUPTURED ABDOMINAL AORTIC ANEURYSM IN THE MEDICARE POPULATION Samuel T. Edwards ${ }^{1}$; Marc Schermerhorn'; A. James O'Malley ${ }^{3}$; Rodney P. Bensley ${ }^{2}$; Rob Hurks $^{2}$; Philip Cotterill ${ }^{4}$; Bruce E. Landon ${ }^{1,3}$. ${ }^{1}$ Beth Israel Deaconess Medical Center, Boston, MA; ${ }^{2}$ Beth Israel Deaconess Medical Center, Boston, MA; ${ }^{3}$ Harvard Medical School, Boston, MA; ${ }^{4}$ Center for Medicare and Medicaid Services, Bethesda, MD. (Control ID \#1339713)

BACKGROUND: Ruptured abdominal aortic aneurysm (rAAA) is the 13th leading cause of death in the United States and causes up to 9000 deaths annually. The traditional treatment for patients who survive to hospital presentation is open aortic repair (OAR), but perioperative mortality remains over $40 \%$. Endovascular aortic repair (EVAR) has become the dominant mode of treatment for intact AAA, and is becoming increasingly common in the repair of rAAA. In this retrospective cohort study, we sought to compare the perioperative and long-term mortality and perioperative complications of patients who received endovascular versus open repair for rAAA in Medicare patients.

METHODS: We examined Medicare beneficiaries 67 years of age or older who were admitted to a US hospital with a primary diagnosis of ruptured abdominal aortic aneurysm (ICD-9 441.3) between 2001 and 2008. We excluded patients with concurrent diagnoses of non-ruptured abdominal aortic aneurysm, thoracic aneurysm, thoracoabdominal aneurysm and aortic dissection and patients with a prior diagnosis of ruptured AAA, or aortic surgery. Treatment was determined using ICD9-CM procedure codes for open aortic repair and endovascular repair. Outcomes consisted of perioperative and long-term mortality, and perioperative complications including myocardial infarction, pneumonia, acute renal failure, reoperation/conversion to open repair, wound dehiscence, embolectomy and tracheostomy. We also examined discharge disposition, and length of hospital stay. Propensity score models were used to create matched cohorts of patients equally likely to receive endovascular or open repair.

RESULTS: Our cohort consisted of 10998 patients with 1126 receiving EVAR and 9872 receiving OAR. Patients receiving EVAR tended to be older ( 78.2 v. 77.2 years, $\mathrm{p}<0.001)$, and were more likely to have a preexisting diagnosis of AAA $(25.6 \%$ v. $16.4 \%, \mathrm{p}<0.01)$. Patients receiving OAR were more likely to require urgent admission $(80.5 \%$ v. $63.2 \%, p<$ 
$0.01)$, and less likely be transferred between hospitals before surgery $(2.4 \%$ v. $6.0 \%, \mathrm{p}<0.01)$. Propensity matching yielded 1099 patient pairs. Perioperative mortality was $33.7 \%$ for EVAR patients and $47.7 \%$ for OAR patients and mortality benefit of EVAR persisted to five years. EVAR was associated with a larger mortality benefit with increased age. Patients treated with open repairs suffered a higher rate of most post-operative complications including post operative pneumonia $(28.5 \%$ vs. $35.9 \%$, $\mathrm{p}=0.0002)$, acute renal failure $(33.4 \%$ vs. $45.4 \%, \mathrm{p}<.0001)$, embolectomy $(3.6 \%$ vs. $6.3 \%, \mathrm{p}<0.01)$, tracheostomy $(4.6 \%$ vs. $9.9 \%, \mathrm{p}<0.01)$ wound dehiscence $(2.5 \%$ vs. $4.6 \%, \mathrm{p}<0.01)$, mesenteric ischemia (7.6\% vs. $14.7 \%, \mathrm{p}<0.01)$, bowel obstruction $(12.74 \%$ vs. $17.02 \%, \mathrm{p}<$ $0.01)$, and colon resection $(4.37 \%$ vs $8.36 \%, \mathrm{p}<0.01)$. Conversion to open repair occurred in $4.9 \%$ of patients treated with EVAR. Mean length of stay was shorter for EVAR patients (10.8 days vs. 18.1 days, $\mathrm{p}<0.01$ ), and EVAR patients were more likely to be discharged home $(62.8 \%$ vs. $40.7 \%, \mathrm{p}<0.01)$.

CONCLUSIONS: Compared to open repair of ruptured AAA, endovascular repair is associated with lower peri-operative and long-term mortality, fewer in-hospital complications, shorter length of stay and more frequent discharge home. Long-term survival benefit of EVAR persisted to five years, and older patients had a larger survival benefit. Increased use of EVAR for rAAA may lead to decreased mortality for treated ruptured AAA.

COMPARING PHYSICIAN VERSUS PATIENT PERCEPTION OF PHYSICIAN HOSPITAL DISCHARGE COMMUNICATION: A PRELIMINARY STUDY Michael Maniaci; Nancy Dawson. Mayo Clinic in Florida, Jacksonville, FL. (Control ID \#1313111)

BACKGROUND: Physician discharge instructions are critical to patient care because they are the link transitioning the hospital care plan to the home. We hypothesized that physician perception of discharge instructions communication is better than patient perception. The results will be used to shape future resident didactics on discharge communication.

METHODS: This observational, survey-based study was done in a 330bed adult teaching hospital. Surveys were mailed to 100 patients discharged home and 49 internal medicine physicians responsible for those patients' care. Each physician had between 1 and 4 of their patients surveyed. Patients and physicians received anonymous 5-item questionnaires concerning physician communication at discharge. Patient surveys inquired about their physician's communication at the specific physician encounter, while physician surveys asked about the physician's overall self-perception of discharge communication skills. Physician responses were separated by physician training level

RESULTS: Completed questionnaires were returned by 59 patients and 40 physicians (26 residents, 14 attendings). All physicians reported a noticeably better perception of communication than patients than the patients: $83 \%$ vs. $61 \%(\mathrm{P}=0.027)$ for spending adequate time reviewing the discharge plan, $98 \%$ vs. $80 \%(\mathrm{P}=0.013)$ for speaking slowly enough to understand, $100 \%$ vs. $68 \%(\mathrm{P}<0.001)$ for using wording that can be easily understood, and $85 \%$ vs. $59 \%(\mathrm{P}=0.008)$ for taking the time to answer questions before discharge. Patients believed more than physicians that further training on patient communication would be beneficial, but this was not statistically significant $(41 \%$ vs $25 \% ; \mathrm{P}=0.13)$. Attending physicians perceived having more time to review the discharge plan $(\mathrm{P}=0.025)$ and answer questions before discharge $(\mathrm{P}=0.018)$. Significantly more first-year residents reported that further communication training would be beneficial $(\mathrm{P}=0.003)$.

CONCLUSIONS: Patient and physician perceptions of adequate discharge communication in the health care setting differ greatly. While young trainees recognize the constraints of time placed on physician patient interaction, as experience increases, this recognition decreases. Although all resident levels had a poorer perception than their patients, first-year residents were more perceptive of their communication flaws and more likely to consider further education on effective communication. Based on these preliminary findings, programs should strongly consider didactics for all residents focusing on the basic fundamentals of physician-patient communication.
COMPARING THE PERFORMANCE OF THE S-TOFHLA AND NVS IN A RACIALLY/ETHNIC DIVERSE STUDY POPULATION Vanessa Ramirez-Zohfeld $^{1}$; Alfred W. Rademaker ${ }^{2}$; Nancy C. Dolan ${ }^{1,3}$; M. Rosario Ferreira ${ }^{1,4}$; Milton "Mickey" Eder'; Dachao Liu' ${ }^{5}$; Michael S. Wolf ${ }^{1}$; Kenzie A. Cameron ${ }^{1} .{ }^{1}$ Northwestern University Feinberg School of Medicine, Chicago, IL; ${ }^{2}$ Northwestern University Feinberg School of Medicine, Chicago, IL; ${ }^{3}$ Northwestern Medical Faculty Foundation, Chicago, IL; ${ }^{4}$ Jesse Brown VAMC, Chicago, IL; ${ }^{5}$ ACCESS Community Health Network, Chicago, IL. (Control ID \#1332071)

BACKGROUND: Numerous assessment tools are used to estimate health literacy in healthcare settings, including the Short Test of Functional Health Literacy in Adults (S-TOFHLA) and the Newest Vital Sign (NVS). However, there is a dearth of data on the performance of the NVS when administered within a diverse population and in a language other than English. We compared the performance of the S-TOFHLA with the NVS among both English and Spanish-speaking patients.

METHODS: Data was extracted from a larger, ongoing randomizedcontrolled study to promote colorectal cancer screening. Participating English and Spanish-speaking patients seeking care at one of six federally qualified health centers serving predominately low-income patients were administered a pre-test which included the S-TOFHLA. After their physician visit they were administered a post test, which included the NVS. Scores on the S-TOFHLA range from $0-100 ; 0-53$ indicates inadequate health literacy, 54-66 marginal health literacy and 67-100 adequate health literacy. Scores on the NVS range from 0-6; 0-1 indicates limited literacy likely, 2-3 limited literacy possible and 4-6 almost always indicates adequate literacy. Spearman correlation coefficients were used to examine the relationship between scores on the S-TOFHLA and NVS. Weighted kappa statistics were used to find the strength of agreement between the scoring categories of the S-TOFHLA and the NVS.

RESULTS: Among 146 patients, $27.40 \%$ were men and $72.60 \%$ were women with a mean age of 57.79 years $(\mathrm{sd}=5.97)$. Hispanic/Latinos comprised $47.26 \%$ of study patients while $52.74 \%$ identified as non-Hispanic. Fifty-six percent of patients completed the literacy tests in English while 44\% completed them in Spanish. Overall, the correlation between the S-TOFHLA and the NVS was $0.73(\mathrm{p}<0.0001)$. There was a stronger correlation for those completing the test in Spanish $(\mathrm{r}=0.83, \mathrm{p}<0.0001)$ compared to those completing the tests in English $(\mathrm{r}=0.55, \mathrm{p}<0.0001)$. An agreement analysis revealed moderate agreement between the three S-TOFHLA literacy categories and the three NVS categories (kappa $=0.36,95 \%$ CI 0.26 - 0.46). Among English test takers, the agreement across the three categories was lower (kappa $=0.20,95 \%$ CI $0.11-0.30$ ) than for those completing it in Spanish (kappa $=$ $0.54,95 \%$ CI $0.37-0.71$ ). Of 77 patients classified as having adequate health literacy by the S-TOFHLA, $35.06 \%$ were classified by the NVS as having a high likelihood of limited literacy and $32.47 \%$ were classified as having limited literacy possible. Of 60 English test takers classified as having adequate health literacy by the S-TOFHLA, $40.00 \%$ were classified by the NVS as likely having limited health literacy and $28.33 \%$ were classified as having limited literacy possible. Of 17 Spanish test takers who were classified as having adequate health literacy by the S-TOFHLA, 17.65\% were classified by the NVS as having a high likelihood of limited literacy and $47.06 \%$ were classified as having limited literacy possible.

CONCLUSIONS: Overall, there is moderate agreement between the STOFHLA and the NVS literacy categories; however, the NVS regularly classifies respondents as having more limited literacy than the S-TOFHLA. Agreement is better between the two measures when the tests are taken in Spanish.

COMPARISON OF COMORBIDITY RISK ADJUSTMENT METHODS IN A NATIONAL COHORT OF VETERANS WITH DIABETES Cheryl ${ }_{\text {P. Lynch }}^{1,2}$; Mulugeta Gebregziabher ${ }^{1,2} ;$ R. Neal Axon ${ }^{1,2} ;$ Kelly J. Hunt ${ }^{1,2}$; Carrae Echols ${ }^{2}$; Leonard E. Egede ${ }^{1,2}$. ${ }^{1}$ Ralph H Johnson Veterans Affairs Medical Center, Charleston, SC; ${ }^{2}$ Medical University of South Carolina, Charleston, SC. (Control ID \#1340373)

BACKGROUND: Adjustment for disease burden or comorbidity risk is essential in health outcomes research. Outcomes models that use administrative data sets, which are generally not collected for research 
purposes, may be biased if they do not properly account for comorbidity. Some researchers adjust for comorbidity using an ICD-9-CM adaptation of the Charlson Comorbidity Index (Deyo-Charlson), Elixhauser or their categorical form, while others include diagnostic indicators of individual comorbidities in their model (Quan approach). However, it is unclear which is optimal for risk adjustment. Therefore, we examined these three approaches to determine the most optimal comorbidity risk adjustment with applications to data from a national, longitudinal cohort of veterans with diabetes.

METHODS: We investigated Deyo-Charlson, Elixhauser and Quan's comorbidity measures using three modeling approaches: as a continuous predictor, as a categorical form $(0=$ none, $1=$ one, $2=$ two, $3=$ three or more $)$ and as a list of all diagnostic indicators of disease. We considered three types of outcomes (binary, continuous, time to event). Generalized linear mixed models (GLMM) were fitted for glycemic control, assessed by HbA1c (binary and continuous) and Cox regression was used for modeling mortality with comorbidity as the main covariate. We examined area under the receiver operating characteristics curve (AUC) and goodness-of-fit criteria to determine the optimal comorbidity modeling approach. AIC values were standardized by the total sample size used to fit each model. RESULTS: Application of comorbidity measures to a national cohort of 892,223 veterans with diabetes (followed from 2002 to 2006) showed that modeling comorbidity, irrespective of index, in a categorical form ( $\mathrm{AIC}=8.77$, $\mathrm{R} 2=0.42, \mathrm{AUC}=0.95$ ) leads to greater risk adjustment than modeling it as a continuous predictor $(\mathrm{AIC}=8.79, \mathrm{R} 2=0.41, \mathrm{AUC}=0.92)$. In $\mathrm{GLMM}$ with continuous $\mathrm{HbA1c}$, a list of diagnostic indicators of disease (Quan approach) consistently exhibited the best goodness-of-fit. In GLMM with binary $\mathrm{HbAlc}$, a better fit was observed when a list of diagnostic indicators $(\mathrm{AIC}=12.5, \mathrm{R} 2=$ $0.95, \mathrm{AUC}=0.61)$ or four categories $(\mathrm{AIC}=12.4, \mathrm{R} 2=0.61, \mathrm{AUC}=0.95)$ modeled comorbidity compared to including a score $(\mathrm{AIC}=12.9, \mathrm{R} 2=0.61$, AUC $=0.95$ ). Results were similar for mortality outcome.

CONCLUSIONS: How we model comorbidity leads to different levels of risk adjustment irrespective of the type of comorbidity index used. A robust approach seems to categorize the count of diagnostic indicators from ICD-9 codes. Researchers need to consider alternative scenarios of modeling previously validated comorbidity scores when adjusting for risk in outcomes research, especially with more widespread use of ICD-10 codes.

COMPARISON OF TAILORED VERSUS MAINSTREAM PRIMARY CARE DELIVERY FOR HOMELESS PERSONS: A NEW, VALIDATED PATIENT ASSESSMENT TOOL Stefan Kertesz ${ }^{1,2}$; David E. Pollio ${ }^{2}$; Cheryl Holt ${ }^{4}$; Jocelyn L. Steward ${ }^{2}$; Adam Gordon ${ }^{6}$; Theresa W. Kim ${ }^{3}$; Erin Stringfellow ${ }^{3}$; Erika Austin ${ }^{1}$; Joya Golden ${ }^{5}$; Nancy Johnson'; Lori L. Davis ${ }^{2} .{ }^{1}$ Birmingham VAMC, Homewood, AL; ${ }^{2} \mathrm{U}$. Alabama, Birmingham, AL; ${ }^{3}$ Boston Health Care for the Homeless, Boston, MA; ${ }^{4} \mathrm{U}$. Maryland, Baltimore, MD; ${ }^{5} \mathrm{VA}$ Greater Los Angeles, Los Angeles, CA; ${ }^{6}$ VA Pittsburgh Health Care System, Pittsburgh, PA. (Control ID \#1332698)
BACKGROUND: Tailoring primary care (PC) service delivery for homeless patients may result in more positive experiences with care. Some organizations tailor services for the homeless through co-located medical, mental, \& social services, homeless-dedicated medical staff, a homeless mission focus, outreach to streets and shelters, or including formerly homeless persons in leadership. As no consumer assessment surveys were specifically designed for homeless patients, we developed a Primary Care Quality survey for the Homeless (PCQ-H) and compared PCQ-H scores from sites that differed in the degree of homeless-specific service tailoring.

METHODS: Based on 60 interviews with homeless patients and expert clinicians, the PCQ-H survey was designed to assess 11 areas (access, coordination, cooperation, evidence-based care, accountability, patient control, continuity, shared knowledge, respect, substance abuse/mental illness, homeless-specific needs). A multidisciplinary team crafted 78 items (Likert, 1-4) and administered them to random samples of clients from a highly tailored 25-year old Health Care for the Homeless Program (HT: $n=182$ ), 2 mainstream VA sites with no (Main 1, n=150) or some (Main 2, $\mathrm{n}=101$ ) tailoring, and a new tailored VA program (VA-HT, $n=41$ ). We used confirmatory factor analysis, item-scale and inter-scale correlations to select 50 items for the present analysis. To assess the effect of service tailoring on patient assessment of care, we first compared scores across sites adjusting for demographics, economic status, chronic and street homelessness, alcohol/drug use, psychiatric symptoms, medical status, and general health. Since ratings of care tended to the favorable end of the Likert scale, we also created a binary indicator of more than the median number of unfavorable or negative responses to items on each scale (e.g. agreeing that "I have had to walk out because getting care was too much trouble") and compared the proportion of negative responses across site.

RESULTS: We found 4 overarching scales: Patient-Clinician Relationship (PCR), Access/Coordination (A/C), Perceived Cooperation (COOP) and Homeless-Specific Needs (HSN). There was good model fit $(\mathrm{RMSEA}=0.069 ; \mathrm{CFI}=0.957$ ), internal reliability (all alphas $>0.76$ ), and convergence with Safran's Primary Care Assessment Survey (PCAS) (r's 0.26-0.31, all $\mathrm{p}<.001$ ). In adjusted analyses, the highly tailored program exceeded the 2 VA sites for PCR $(\mathrm{p}<.01)$, COOP $(\mathrm{p}<.01)$, and HSN $(\mathrm{p}<.05)$. Unfavorable response patterns were 10 $25 \%$ more prevalent (in absolute terms) for the 2 mainstream VA sites for PCR, COOP and HSN, compared to both tailored sites (all $\mathrm{p}<.05$ in raw \& adjusted analyses)

CONCLUSIONS: The PCQ-H appears to provide a valid measure of homeless patients' satisfaction with primary care. Homeless patients rate their relationship to their provider, cooperation among caregivers, and care for homeless-specific needs more highly when primary care services are highly tailored. Whether such results are attainable across diverse settings remains to be seen.

Table: Primary Care Service Design and Patient-Rated Quality Scores (PCQ-H)

\begin{tabular}{|c|c|c|c|c|}
\hline & \multicolumn{4}{|r|}{ Highly } \\
\hline & Mainstream & Mainstream & & Tailored \\
\hline & VA 1 & VA 2 & Tailored VA & Non-VA \\
\hline Subscale & $N=150$ & $N=101$ & $(n=41)(+)$ & $(n=182)$ \\
\hline Patient-Clinician Relationship $\S$ & 3.23 & $3.36^{*}$ & 3.37 & $3.44^{* *}$ \\
\hline Cooperation & 2.75 & 2.82 & 2.82 & $3.05^{* *}$ \\
\hline Access/Coordination & 3.15 & 3.22 & 3.18 & 3.26 \\
\hline Homeless-Specific Needs $(p=.06)$ & 3.14 & 3.19 & 3.23 & $3.30 * *$ \\
\hline
\end{tabular}

$\S$ indicates $p<0.05$ in 4-site comparison. For comparison to Mainstream $1, *(p<.05)$ and

$* *(p<.01)$. All models fully adjusted. $+: 1 / 2$ of planned surveys complete 
COMPARISON OF ELECTRONIC CARESETS TO GUIDE VENOUS THROMBOEMBOLISM RISK ASSESSMENT Larissa Verda; William Trick; Ashima Sahni; Abhimanyu Saini; Samrat Khanna; Monica Muppidi; Shane Borkowsky; Krishna Das. John H Stroger Jr Hospital of Cook County, Chicago, IL. (Control ID \#1331870)

BACKGROUND: Hospital acquired venous thromboembolism (VTE) is a significant cause of mortality in hospitalized patients. National quality organizations and expert panels recommend a VTE risk assessment and risk-based prophylaxis for every inpatient. Point scoring systems used in risk stratification have been associated with errors in risk stratification and choice of VTE prophylaxis. Thus, the optimal tool for assessing VTE risk remains unknown. The aim of this study was to compare electronic caresets that differed regarding the extent of clinical guidance provided at the time of VTE risk assessment.

METHODS: We performed a cross-sectional study at a 464-bed public teaching hospital. We integrated two separate risk assessment tools into VTE prophylaxis electronic caresets within admission orders. One assessment tool provided detailed guidance on VTE risk factors (RF-G), the other provided no specific guidance (No-G); both of them directed clinicians to categorize the patient's VTE risk into three levels (low, moderate/high, very high). Providers were allowed to exit the careset without completing the risk assessment in order to facilitate the admission process. Patients admitted to the medicine service were randomly assigned to one of three inpatient care teams (i.e., firms). We randomly assigned one VTE risk assessment tool to each firm; thus, two firms were exposed to the RF-G tool, and the other firm used the No-G tool. Medical patients were randomly selected and their charts abstracted by four expert housestaff reviewers trained through literature review, case discussion and participation in guideline development; they exhibited excellent agreement in determining VTE risk $(\kappa=0.8)$. Reviewers assessed VTE risk blinded to the teams' determination and then recorded the primary teams' VTE risk assessment. We compared the level of agreement between the reviewers vs. the RF-G and No-G groups.

RESULTS: We reviewed 344 patient admissions. AVTE risk assessment tool was completed by the housestaff for 135 patients $(39 \%)$. Physicians using the RF-G tool arm were more likely to complete the VTE prophylaxis risk assessment careset than those using the No-G tool $(44 \%$ vs $30 \%, P<0.01)$. By expert reviewer assessment, the distribution of risk levels between the RF-G and No-G groups were similar. However, the No-G clinical teams were more likely to assign patients to low or very high risk categories compared to the RF$\mathrm{G}$ determinations (Table); the distributions among the three categories were significantly different $(\mathrm{P}<0.01)$. Agreement between the expert review and clinical team assessments was significantly higher for the RF-G arm compared to the No-G arm $(90 \%$ vs $63 \%, P<0.05)$. Overall, in 14 of 19 cases of disagreement, the clinical team underestimated VTE risk compared to experts. CONCLUSIONS: Clinical determination of VTE risk improved when an assessment tool at the point of care provided risk factor guidance within a venous thromboembolism prophylaxis careset. Although only $40 \%$ of physicians completed the electronic risk assessment tool, they were more likely to complete it when risk factor guidance was included.

\begin{tabular}{lllll}
\hline \hline & \multicolumn{2}{l}{ Risk-factor guidance } & \multicolumn{2}{l}{ No guidance (No-G) } \\
& \multicolumn{2}{l}{ RF-G) N=102 } & N=33 & \\
& Team N & Reviewer N & Team N & Reviewer N \\
& $(\%)$ & $(\%)$ & $(\%)$ & $(\%)$ \\
Low risk & $7(7)$ & $3(3)$ & $7(21)$ & $0(0)$ \\
$\begin{array}{l}\text { Moderate/high } \\
\text { risk }\end{array}$ & $91(89)$ & $95(93)$ & $22(67)$ & $31(94)$ \\
Very high risk & $4(4)$ & $4(4)$ & $4(12)$ & $2(6)$ \\
\hline
\end{tabular}

CONTINUITY CLINIC MAKEOVER: AN AMBULATORY EDUCATION NEEDS ASSESSMENT FOR RESIDENTS PRACTICING IN AN URBAN SAFETY-NET CLINIC Ryan Laponis; Claire Horton; Katherine Julian; Sharad Jain; Reena Gupta. University of California San Francisco, San Francisco, CA. (Control ID \#1340074)

BACKGROUND: Educating residents in resource-poor primary care clinics that care for a large volume of vulnerable patients can provide many positive experiences, but also present barriers to high-quality resident education. While some barriers (high patient volume, low support staff ratios) may be unavoidable, others could be addressed by clinicianeducators to enhance the educational experience for resident providers. Using mixed method inquiry, we sought to identify areas needing improvement to meet resident educational goals.

METHODS: We surveyed all second and third year internal medicine residents (primary care and categorical) whose continuity clinic was based at the San Francisco General Hospital General Medicine Clinic during the 2010-2011 academic year using the Veterans Health Administration Learners' Perceptions Survey-a 76-item instrument with established reliability and validity that measures satisfaction on a Likert-like scale (1 $=$ poor, $5=$ excellent $)$ in 6 domains: faculty interactions (14 items); the learning (16), working (12), clinical (14), and physical (6) environments; and personal/professional experience (14). We further explored the resident experience of items with a mean score $<4$ by conducting four resident focus groups (primary care and categorical) led by the ambulatory chief resident and captured responses by transcription. We used iterative thematic analysis to identify emergent themes.

RESULTS: 16 of $32(50 \%)$ residents participated in the survey. Of the 76 survey items, 35 averaged a score below $4: 14 \%$ of items in the faculty interactions domain, $56 \%$ in the learning environment, $50 \%$ in the working environment, $71 \%$ in the clinical environment, $50 \%$ in the physical environment and $36 \%$ in personal/professional experience. 15 of 32 (47\%) residents participated in one of four focus groups. Exploration of the transcripts yielded 4 themes: 1 . Need for longitudinal clinical mentorship ("For my most complicated patients, I need a 'go to' [preceptor] ...sometimes, its really complicated and re-presenting [to a different attending every visit]...just doesn't work); 2 . Increased direct observation and feedback ("[I would like] a much more structured set up where [the same preceptor] just walks in and watches us[monthly]"); 3. More time for panel management ("We are learning things on our own in a time constrained environment and the only time you can do it is when you are in clinic...it is not realistic to do panel management while you are on an inpatient rotation"); 4. Learning clinic efficiency and time management skills ("[I] Feel like I never have enough time [to get through clinic] and when I graduate, I will have to see even more patients [per clinic session]")

CONCLUSIONS: Residents identify multiple domains in which their safety-net continuity clinic experience can be educationally enhanced. Interventions that increase longitudinal clinical precepting, enhance direct observation and feedback, and teach panel management skills may improve resident satisfaction with continuity clinic experiences and increase retention in primary care careers

CONTROLLED TRIAL OF A COMBINED DECISION AID+ PATIENT NAVIGATOR INTERVENTION TO INCREASE COLORECTAL CANCER SCREENING AMONG MEDICAID PATIENTS IN NORTH CAROLINA Lucia A. Leone ${ }^{1}$; Daniel S. Reuland $^{2,3}$; Carmen L. Lewis ${ }^{2,3}$; Mary Ingle; Brian Erman ${ }^{3}$; Annette DuBard ${ }^{4}$; Michael Pignone ${ }^{2,3}$. ${ }^{1}$ University of North Carolina, Chapel Hill, $\mathrm{NC} ;{ }^{2}$ University of North Carolina, Chapel Hill, NC; ${ }^{3}$ University of North Carolina, Chapel Hill, NC; ${ }^{4} \mathrm{NC}$ Community Care Networks, Raleigh, NC; ${ }^{5}$ Community Care of the Lower Cape Fear, Wilmington, NC. (Control ID \#1324456)

BACKGROUND: Screening rates for colorectal cancer (CRC) among Medicaid patients are lower than those for the general population despite insurance coverage for tests. The goal of this study was to test, in a controlled trial, an intervention to improve CRC screening rates consisting of a mailed decision aid followed by telephone support from an offsite, payer-based patient navigator.

METHODS: The study was conducted in cooperation with Community Care of North Carolina (CCNC), the quality improvement organization for Medicaid in NC. We selected one of fourteen CCNC regions for our study. Within this region, we selected six practices for the intervention and matched them with six control practices. Practices were selected to allow for a mix with regards to size, geographic location (urban vs. rural) and 
presence of residents. Eligible patients at each practice were age 50 and older, not up-to-date with CRC screening based on Medicaid claims data, and currently covered by Medicaid, but not Medicare. A CCNC patient outreach coordinator was trained to serve as a patient navigator for the study. Training included an overview of CRC screening plus motivational interviewing techniques focused on helping patients overcome screening barriers. We mailed a packet containing a decision aid DVD, written survey, and a letter from the patient's physician encouraging screening to all eligible patients in intervention practices. The patient navigator called patients beginning one month after the packet was mailed and attempted to contact each person at least 3 times. Patients who had not returned the mailed survey, had the option to complete it over the phone prior to receiving counseling from the navigator. Our primary outcome was completion of a CRC screening test within six months of enrollment as determined by Medicaid claims data. We compared screening test completion rates among intervention participants with those from the matched control practices, using intent to treat analysis. Other study outcomes were measures of intervention reach based on navigator logs and survey data.

RESULTS: Based on claims data, we identified 240 eligible patients who were not up-to-date with $\mathrm{CRC}$ screening from intervention practices (overall screening rate among age-eligible patients was 35.6\%) and 174 eligible patients from control practices (overall screening rate of $46.0 \%$ ). At six months, 9.2\% $(n=22)$ of intervention patients had received CRC screening during the intervention period based on claims, compared to $7.5 \%(n=13)$ of control patients. The rate difference was 1.7 percentage points (95\% CI: -3.6, 7.0) After excluding anyone who declined participation or self-reported as ineligible, 207 patients remained in the intervention group at follow-up. Of those, $26.6 \%(\mathrm{n}=55)$ completed the decision aid survey and $40 \%(n=22)$ of survey completers reported watching some or all of decision aid. The patient navigator discussed CRC screening with $25.6 \%(\mathrm{n}=53)$ of the 207 intervention patients at least once by phone.

CONCLUSIONS: A mailed decision aid plus phone-based patient navigator intervention had limited reach and was not able to increase CRC screening among older Medicaid patients compared with control. Higher-intensity interventions, such as practice-based navigators, may be needed to better reach challenging patients and improve screening rates among vulnerable populations such as this one.

COPING SKILLS ASSOCIATED WITH INTERNAL MEDICINE RESIDENT BURNOUT: A MULTI-CENTER SURVEY STUDY Jessica Cohen ${ }^{1}$; Ravi K. Gopal ${ }^{2}$; Jonathan Ripp ${ }^{1} .{ }^{1}$ Mount Sinai School of Medicine, New York, NY; ${ }^{2}$ University of Colorado, Denver, CO. (Control ID \#1334764)

BACKGROUND: Job burnout is common among internal medicine (IM) residents and may lead to depression, poor academic performance and selfreported suboptimal patient care. Potential predictors of burnout include excessive work hours, loan debt burden and some personality traits. Little is known about the relationship between coping techniques and job burnout in IM residents. This study attempts to determine whether an association exists between coping mechanisms and IM resident burnout.

METHODS: Two academic medical centers, the Mount Sinai School of Medicine and the University of Colorado, administered a survey to IM residents once late in the academic year between April 2010 and June 2011. Likert scale survey measures included job burnout, personality traits and coping mechanisms. Burnout was defined using the most commonly identified definition of the Maslach Burnout Inventory, a previously validated instrument. The validated Brief COPE scale was used to assess both adaptive and maladaptive coping responses to stressful conditions. Combining both centers into one cohort, we conducted chi-square tests to identify statistically significant differences in proportions $(\mathrm{P}<0.05)$ of burnout across survey items.

RESULTS: Of 259 eligible residents from both institutions, 154 (59\%) completed the survey. Sixty-six percent (101/154) of these residents met criteria for burnout. Residents with burnout more frequently used ("a lot") self-distraction $(25$ v. $3 ; 25 \%$ v. $6 \% ; \mathrm{P}=0.034)$ and self-blame ( 15 v. 5 ; $15 \%$ v. $9 \% ; \mathrm{P}=0.050$ ) as coping mechanisms when compared with residents who were burnout free. Residents with burnout also more commonly used ("at all") behavioral disengagement ( 25 v. $3 ; 25 \%$ v. $6 \% ; \mathrm{P}=0.051)$ and venting $(71$ v. $30 ; 71 \%$ V. $57 \% ; \mathrm{P}=0.012)$ as coping mechanisms when compared with residents who were burnout free. There were no significant associations between resident burnout prevalence and other coping mechanisms such as humor, religion, substance use, support network, planning, acceptance or denial.

CONCLUSIONS: In keeping with previous studies of IM residents, we found a high prevalence of burnout late in the academic year. The correlations we observed between burnout and coping mechanisms suggest that burnt out residents use different techniques to manage stressful conditions. Further investigation utilizing a longitudinal design to measure burnout incidence can make use of our findings to identify whether these potentially adverse coping strategies predict the development of burnout. A better understanding of the relationship between burnout and coping may help identify those at risk and inform potential future interventions.

\section{CORRELATES OF SELF-REPORTED HYPERTENSION IN} PATIENTS WITH AIDS IN THE ERA OF HIGHLY ACTIVE ANTI-RETROVIRAL THERAPY (HAART) Katherine Krauskopf ${ }^{1}$; Mark L. Van Natta ${ }^{2}$; Alex Federman ${ }^{1}$; Andrea D. Branch ${ }^{1}$; Curtis L. Meinert ${ }^{2}$; Douglas A. Jabs ${ }^{3,1}$. ${ }^{1}$ Mount Sinai School of Medicine, New York, NY; ${ }^{2}$ The Johns Hopkins University Bloomberg School of Public Health, Baltimore, MD; ${ }^{3}$ Mount Sinai School of Medicine, New York, NY. (Control ID \#1336248)

BACKGROUND: Cardiovascular (CV) disease is among the most common non-AIDS comorbidities developed by patients with HIV on treatment. While multiple factors contribute to its development in this population (including chronic inflammation and HAART), hypertension (HTN) represents a significant, modifiable risk factor. It is therefore important to identify distinct clinical predictors and risk factors for HTN in this population. This study evaluated risk factors for prevalent and incident HTN in a large cohort of HIV-infected individuals, including retinal vascular measurements that are associated with incident HTN in the general population.

METHODS: We analyzed standardized interview data from the Longitudinal Study of Ocular Complications of AIDS (LSOCA). LSOCA is a large, prospective study beginning in 1998 of AIDS patients $\geq 13$ years of age attending 19 AIDS ophthalmology centers for a routine examination. Outcomes were self-reported HTN at study entry and 3- or 6-month follow-up. Predictors of HTN included traditional risk factors, AIDS duration, severity and disease control, and HIV treatment history. Secondary analysis was performed in LSOCA participants without an ocular opportunistic infection (OOI) to determine associations between central retinal vascular measurements (central retinal artery equivalent [CRAE] and central retinal vein (central retinal vein equivalent [CRVE]) and HTN. The unadjusted associations of risk factors with HTN were assessed using the t-test or the chi-square test. Multiple logistic regression was used to assess adjusted associations of predictors with prevalent self-reported HTN. Cox regression was used to assess associations of predictors with incident selfreported HTN during follow-up among those without self-reported HTN at enrollment. All models were adjusted for socio-demographics, components of metabolic syndrome (hyperlipidemia and diabetes), and hepatitis $\mathrm{C}$ coinfection.

RESULTS: Of the 2,359 participants, $80 \%$ were male, $60 \% 40-59$ years old, $36 \%$ Black, and $14 \%$ Hispanic. Prevalence of self-reported HTN was $20 \%$ ( $95 \%$ confidence interval [CI]: 18-21). In adjusted analysis, prevalent HTN was associated with Black race (odds ratio [OR]: 1.99, 95\% CI: 1.53-2.61], older age [OR: 1.07 per year, $95 \%$ CI: 1.06-1.09], and higher weight [OR 1.25 per $10 \mathrm{~kg}$, 95\% CI 1.15-1.36), longer time since AIDS diagnosis (OR 1.04 per year, $95 \%$ CI 1.00-1.07) and higher CD4+ T cell counts (OR 1.09 per 100 cells $/ \mathrm{uL}, 95 \%$ CI 1.03-1.16). HTN incidence was $26 / 1000$ person years (PYs) $(95 \%$ CI $22-$ 29). Traditional risk factors were also associated with an increased risk for HTN; as were shorter time since AIDS diagnosis (RR 0.95 per year, $95 \% \mathrm{CI}$ 0.91-0.99) and lower peak HIV viral load (RR 0.86 per log copies $/ \mathrm{ml}, 95 \%$ CI $0.75-0.99)$. In secondary adjusted analysis, prevalent HTN was associated with narrower CRAE (OR 2.24 1st vs. 4th quartile, $95 \% \mathrm{CI}$ ), as was risk for HTN (RR 5.131 st vs. 4th quartile, $95 \%$ CI 2.58-10.20). 
CONCLUSIONS: In this large, prospective study of participants with AIDS, hypertension was relatively prevalent. Risk factors for and clinical indicators of hypertension in this population mirror those in the general population. The results of this study highlight the importance of screening for hypertension as a modifiable CV risk factor among primary care patients with AIDS, including via retinal microvascular evaluation.

COST-EFFECTIVENESS OF PNEUMOCOCCAL CONJUGATE VACCINATION STRATEGIES IN US ADULTS AGED 65 AND OLDER Kenneth J. Smith; Angela Wateska; Mary Patricia Nowalk; Mahlon Raymund; Richard K. Zimmerman. University of Pittsburgh, Pittsburgh, PA. (Control ID \#1310720)

BACKGROUND: The 13-valent pneumococcal conjugate vaccine (PCV13) awaits US adult licensure. Its potential role in adults aged $\geq 65$ years is unclear, particularly since it could prevent nonbacteremic pneumococcal pneumonia, which the presently recommended 23-valent pneumococcal polysaccharide vaccine (PPSV23) has not been consistently shown to do.

METHODS: Using a Markov model, we estimated the cost-effectiveness of vaccination strategies using PCV13 and PPSV23 alone or in combination in 65- and 75-year-old cohorts. For the base case, we assumed no prior vaccination; we also examined prior vaccination and hyporesponsiveness to repeated vaccination scenarios. We estimated age- and comorbidity-specific pneumococcal disease rates, indirect effects on adults from childhood vaccination with PCV13, and costs using CDC Active Bacterial Core surveillance data and US national databases. An expert panel estimated vaccine-related protection. We took a societal perspective and discounted outcomes 3\%/yr. One-way and probabilistic sensitivity analyses were performed to test the robustness of results.

RESULTS: The table summarizes incremental cost-effectiveness ratios (ICER) for non-dominated strategies. PPSV23 strategies were dominated (more costly, less effective) by PCV13 strategies. Results were most sensitive to varying vaccine effectiveness estimates. Probabilistic sensitivity analyses, where parameter values were simultaneously varied, supported baseline results. When we assumed prior vaccination and $80 \%$ effectiveness of subsequent vaccinations, base case ICERs increased by $37-$ $78 \%$ for single dose strategies and $29-35 \%$ for multiple dose strategies. In addition, PCV13 strategies are less favored if greater indirect effects due to childhood vaccination occur.

CONCLUSIONS: Single dose PCV13 strategies may be worth considering for elderly patients. Multiple dose strategies are more expensive, particularly when prior vaccination and the possibility of hyporesponsiveness with repeated vaccination are considered.

Results Table

\begin{tabular}{lll}
\hline \hline $\begin{array}{l}\text { Cohort } \\
\text { age }\end{array}$ & Strategy & ICER (per QALY) \\
65 & PCV13: 65 PCV13: 65, 80 PCV13: & $\$ 11,300 \$ 83,000$ \\
& 65, 75 PCV13: 65, 75,85 & $\$ 263,000 \$ 272,000$ \\
75 & PCV13: 75 PCV13: 7585 & $\$ 62,800 \$ 278,000$ \\
\hline
\end{tabular}

ICER $=$ incremental cost-effectiveness ratio $\mathrm{QALY}=$ quality adjusted life year

COST-EFFECTIVENESS OF PROCALCITONIN-GUIDED ANTIBIOTIC USE IN COMMUNITY ACQUIRED PNEUMONIA Kenneth J. Smith; Richard K. Zimmerman; Angela Wateska; Mary Patricia Nowalk; Mahlon Raymund; Michael J. Fine. University of Pittsburgh, Pittsburgh, PA. (Control ID \#1315469)

BACKGROUND: Although prior randomized trials have demonstrated that procalcitonin (PCT) guided antibiotic therapy effectively reduces antibiotic treatment rates and duration for patients with community acquired pneumonia (CAP), the cost implications of PCT protocols remain unclear.
METHODS: We used a decision model examining hypothetical patient cohorts to estimate the cost-effectiveness of PCT protocols vs. usual care in low-risk patients hospitalized for CAP, taking a third-party payer perspective over the duration of that hospitalization. Since studies show no outcome differences between PCT and usual care strategies, we assumed no length of stay (LOS) or quality of life utility differences in the base case, biasing against PCT use, but relaxed these assumptions in sensitivity analyses. Two PCT protocols were evaluated: 1) PCT levels only at hospital admission, affecting only the decision to begin antibiotic therapy; and 2) PCT levels drawn at admission and on days 3,5, and 7 as indicated, affecting both prescribing and therapy duration decisions. In clinical trials, PCT protocols decreased absolute antibiotic prescription risk (mean 9.9\%) and therapy duration (mean 4.5 days) in CAP patients. Medicare reimbursement for PCT is $\$ 38.36$. These and other parameter values were varied in sensitivity analyses.

RESULTS: PCT protocols performed only on admission were cost saving compared to usual care if the total per patient cost (including administration) of the antibiotic regimen was $>\$ 387$ in usual care patients. If PCT was drawn on admission and every two days thereafter while on antibiotic, PCT protocols were cost saving if antibiotic costs were $>\$ 23 /$ day; the Figure shows a 2-way sensitivity analysis, varying both antibiotic costs and decreased antibiotic duration. Either PCT protocol was cost saving if total hospital costs decreased $>\$ 119$ (or LOS decreased $>0.18$ days) through their use. If PCT use improved quality of life utility through decreased antibiotic side effects or shorter hospitalization, PCT protocols cost $<\$ 100,000 /$ QALY if they gained $\geq 0.0012$ QALYS ( $\sim 10.5$ hours) when oral azithromycin (regimen cost \$39) was used, with even more favorable costeffectiveness ratios when more expensive antibiotic regimens were evaluated. Results favoring PCT use were otherwise robust in sensitivity analyses.

CONCLUSIONS: PCT regimens for low-risk patients hospitalized with CAP are likely to be either cost saving or cost-effective compared to usual care in an analysis biased against PCT use. Further pragmatic trials of PCTguided therapy and other evidence-based antibiotic decision rules in CAP are warranted.

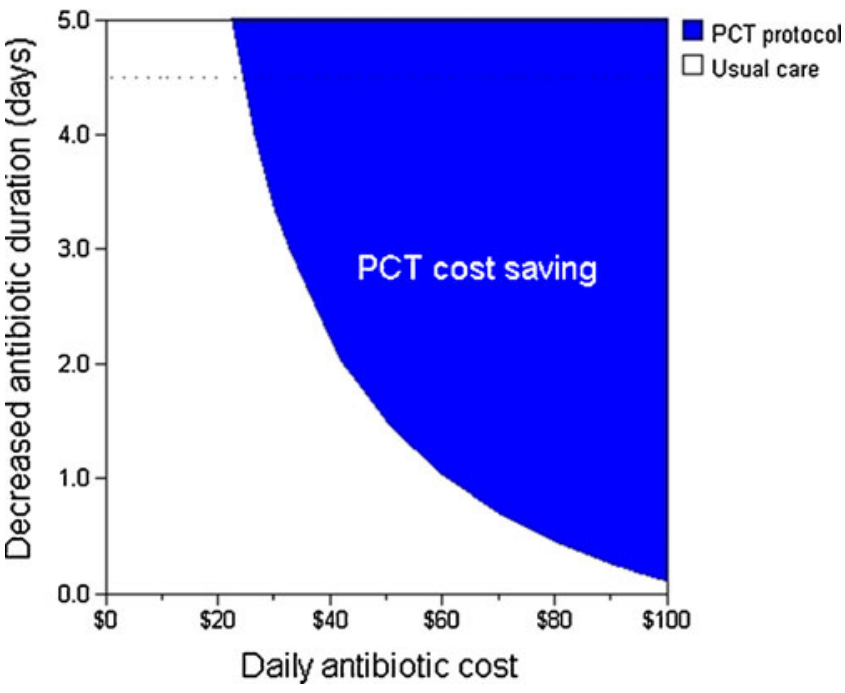

2-Way Sensitivity Analysis

CREATING A POSITIVE LEARNING ENVIRONMENT FOR FEMALE MEDICAL STUDENTS: RESULTS FROM A LONGITUDINAL QUALITATIVE STUDY Palav Babaria ${ }^{1,2}$; Sakena Abedin $^{2}$; David Berg ${ }^{2}$; Marcella Nunez-Smith ${ }^{2}$. ${ }^{1}$ University of California, San Francisco, San Francisco, CA; ${ }^{2}$ Yale University, New Haven, CT. (Control ID \#1339999)

BACKGROUND: Continued high rates of sexual harassment and gender discrimination (SH/GD) amongst female medical students have important implications on medical education and learning. Harassment and belittlement of both male and female students have been associated with 
decreased confidence and self-worth, but little is known about how SH/ GD specifically affect the clinical learning environment for third-year female medical students and how these experiences evolve over time. METHODS: A qualitative longitudinal study consisting of in-depth interviews with 12 third-year female medical students after each clinical clerkship. Participants were purposefully selected from a single New England medical school to represent a range of ages, ethnicities, and prior life experiences. Using a grounded theory approach, a diverse coding team analyzed the transcripts to identify recurring themes. The coding team met regularly to review all coded transcripts and resolve any discrepancies. Data was organized using the scientific software, ATLAS.ti 5.0 (Berlin). RESULTS: Four themes emerged, with illustrative quotes, that characterized the effect of gender on the learning environment of participants (1) Gendered experiences affected participants' comfort with the medical team, often leading to awkwardness and avoidance; (2) Experiences that were gender-neutral or gender-positive fostered learning and question-asking; (3) Participants often tolerated sexualized or gendered encounters in order to facilitate learning; (4) Over time, participants' repeated gendered interactions led to fatigue and disengagement from clinical learning.

CONCLUSIONS: Gendered experiences significantly shape the learning environment for third year female medical students, resulting in positive learning environments with increased comfort, engagement and questionasking or negative learning environments with silence, fatigue and disengagement. Educators should attempt to identify these factors and foster improved clinical learning environments for female medical students.

CROSSING THE DIGITAL LINE: MEDICAL TRAINEE AND ATTENDING PHYSICIAN RELATIONSHIPS VIA ONLINE SOCIAL NETWORKS Chapy Venkatesan ${ }^{1}$; Henry Tran ${ }^{2}$; Shirley Kalwaney ${ }^{1} .{ }^{1}$ Inova Fairfax Hospital, Falls Church, VA; ${ }^{2}$ New York University Langone Medicine Center, New York, NY. (Control ID \#1309489)

BACKGROUND: Online social networks such as Facebooktm are very commonly used by medical trainees. However, few studies have examined the content, perceptions, and potential interpersonal issues that may arise between residents and attending physicians via online social networks. The authors sought to quantify and describe the online interactions between medical trainees and attending physicians.

METHODS: From February to April 2010, residents at four academic institutions were asked to complete a 15-question, anonymous web-based survey assessing their online social network behaviors and relationships. Approximately $25 \%$ of respondents were categorical internal medicine residents. Respondents were asked to describe specifically the number, status, and history of online friendships with attending physicians.

RESULTS: 130 respondents $(90.3 \%)$ reported using an online social network. Among these users, $76(64.4 \%)$ reported a friendship with at least one attending physician (mean 7.7 friendships). Respondents did not uniformly use security filters to obscure information or photographs. 45 respondents $(46.9 \%)$ felt social pressure to accept a request of friendship from an attending. 3 respondents $(2.8 \%)$ reported online interactions with attending physicians that "crossed the line".

CONCLUSIONS: Online friendships between residents and attending physicians via social networks are common. A small percentage of residents report pressure to accept online friendships and experienced negative interactions with attending physicians. National accrediting bodies, GME departments and residency programs need to provide guidance and possibly create policies in order to ensure that the potential problems of these interactions are mitigated.

CUMULATIVE CHANGES IN USE OF CHRONIC MEDICATIONS: A NEW MEASURE OF PRESCRIBING INTENSITY Khoa D. Lam ${ }^{1}$ Yinghui $\mathrm{Miao}^{2}$; Michael A. Steinman ${ }^{1,2}$. ${ }^{1}$ University of California San Francisco, San Francisco, CA; ${ }^{2}$ San Francisco VA Medical Center, San Francisco, CA. (Control ID \#1337247)

BACKGROUND: Polypharmacy is a major concern in older adults, yet a simple cross-sectional count of medications does not capture the potential complications (and benefits) that occur when medications are started, stopped, and changed over time. This study explored a novel measure of longitudinal changes in use of chronic medications as a marker of prescribing intensity in elderly veterans.

METHODS: Using a combination of Veteran Affairs (VA) outpatient pharmacy data and VA and Medicare outpatient and inpatient claims data from October 1, 2006 through September 30, 2008, we identified veterans age 65 years or greater who predominantly used VA services and received at least one chronic medication from VA at the beginning of a 1-year study period. Changes in use of chronic medications (drugs supplied for periods of 1 month or longer) were defined as additions (new medications started), discontinuations (medications present at baseline which were no longer filled), disruptions (interruption of refills for $>=6$ months), intraclass substitution (medication replaced with another drug from the same class) and dose changes. Negative binomial models were used to assess predictors of the rate of medication changes.

RESULTS: Among 834,299 veterans, the mean age was $76(+/-7)$ years. These patients had 5.7 (+/- 2.6) chronic conditions. At baseline, subjects were taking a mean of $4.9(+/-2.9)$ medications, and one year later were taking an identical number of medications. However, over this one-year period patients had a mean of $5.3(+/-5.1)$ changes in their chronic medications, including an average of 1.7 additions, 1.5 discontinuations, 0.6 disruptions, 1.3 dose changes, and 0.2 intra-class substitutions. Fourteen percent of patients had 10 or more changes in their chronic medications over one year. On multivariate analyses, older patients had fewer medication changes (IRR 0.96 for each 10 -year increase in age, $95 \%$ CI 0.95-0.96). In contrast, medication changes were more frequent in patients taking more medications at baseline (IRR 1.11 for each additional medication, 95\% CI 1.11-1.11) and in patients having more outpatient visits (IRR 1.07 for each additional visit, 95\% CI 1.07-1.07). The frequency of medication changes varied substantially between VA's 21 regions. After adjusting for characteristics of patients, medical use, and visit frequency, there was a $27 \%$ difference in the rate of medication changes between the regions with the lowest and highest rates of change. CONCLUSIONS: Changes in chronic medications are frequent in elderly veterans. Cumulative measurement of medication changes captures important information about drug regimen complexity and potential problems that are not captured by simple drug counts at discrete time points.

CYSTATIN C-BASED GLOMERULAR FILTRATION RATE: AN IMPROVED PREDICTOR OF MORTALITY Alexandra Milin ${ }^{1}$; Ankit Parikh $^{1}$; Stuart R. Lipsitz ${ }^{2}$; Sundar Natarajan ${ }^{3}$. ${ }^{\top}$ New York University School of Medicine, New York, NY; ${ }^{2}$ Brigham and Women's Hospital, Harvard Medical School, Boston, MA; ${ }^{3}$ Veterans Administration New York Harbor Healthcare System, New York, NY. (Control ID \#1338733)

BACKGROUND: Creatinine (cr) is widely used to track kidney function. However, its utility is limited by partial secretion in renal tubules and dependence on age, gender, race, and muscle mass. Cystatin C (cys) is produced by nucleated cells and is neither secreted nor reabsorbed in nephrons. It is also influenced much less by the aforementioned characteristics. Consequently, it is a reliable marker of renal function and possibly mortality. Yet, it is not known whether clinically relevant glomerular filtration rate (GFR) thresholds, using cys or cr, are superior predictors of mortality in U.S. adults. Identifying the more discriminating marker will allow providers to employ the finest modality to detect and treat kidney disease.

METHODS: GFR was calculated using the standard of care Modification of Diet in Renal Disease cr equation and the new Chronic Kidney Disease Epidemiology Collaboration cys equation. Using the Third National Health and Nutrition Examination Survey linked mortality data, with follow-up until 2006, we evaluated the relationship between GFR and all-cause mortality using life table analyses and Cox proportional hazards models that incorporated survey weights, strata, and clusters, while adjusting for potential confounders.

RESULTS: Cys and cr measurements were obtained from 7,205 adults. GFR was dichotomized as normal $(\geq 60 \mathrm{~mL} / \mathrm{min} / 1.73 \mathrm{~m} 2)$ or low $(<60 \mathrm{~mL} /$ 
$\min / 1.73 \mathrm{~m} 2) ; 74.55 \%$ had normal GFR based on cr and cys (NGFRCYCR), $2.58 \%$ had low GFR by cr only (LGFR-CR), $12.20 \%$ had low GFR by cys only (LGFR-CY), and $10.67 \%$ had low GFR by cr and cys (LGFR-CYCR). At 18 years (figure), the proportion surviving was $36.38 \%$ in LGFR-CYCR, $44.96 \%$ in LGFR-CY, $67.24 \%$ in LGFR-CR, and $68.52 \%$ in NGFR-CYCR. When directly compared to LGFR-CR, LGFR-CY was better at predicting all-cause mortality [(hazard ratio (HR) 1.53, 95\% confidence interval (CI) 1.09-2.15]; LGFR-CYCR was the best (HR 2.34, 95\%, CI 1.65-3.32). Further, among adults with normal cr-based GFR, the multivariate-adjusted mortality HR for each unit increase in cys was 7.79 (CI 5.67-10.69); for participants with a low cr-based GFR, the HR was 1.62 (CI 1.49-1.78). Finally, the population attributable risk was $5.03 \%(\mathrm{CI}$ $3.72-6.43$ ) in LGFR-CY, 5.86\% (CI 4.58-7.20) in LGFR-CYCR, and $0.84 \%$ (CI $0.02-1.79$ ) in LGFR-CR

CONCLUSIONS: Using established clinical thresholds, cys-based GFR is superior to cr-based GFR for predicting all-cause mortality and should be the preferred marker or used in combination with creatinine to assess risk. Even when stratified by cr-based GFR, cys is related to mortality in a leveldependent fashion, with a stronger effect among those with normal crbased GFR. Finally, cys-based GFR has a greater population impact than cr-based assessments.

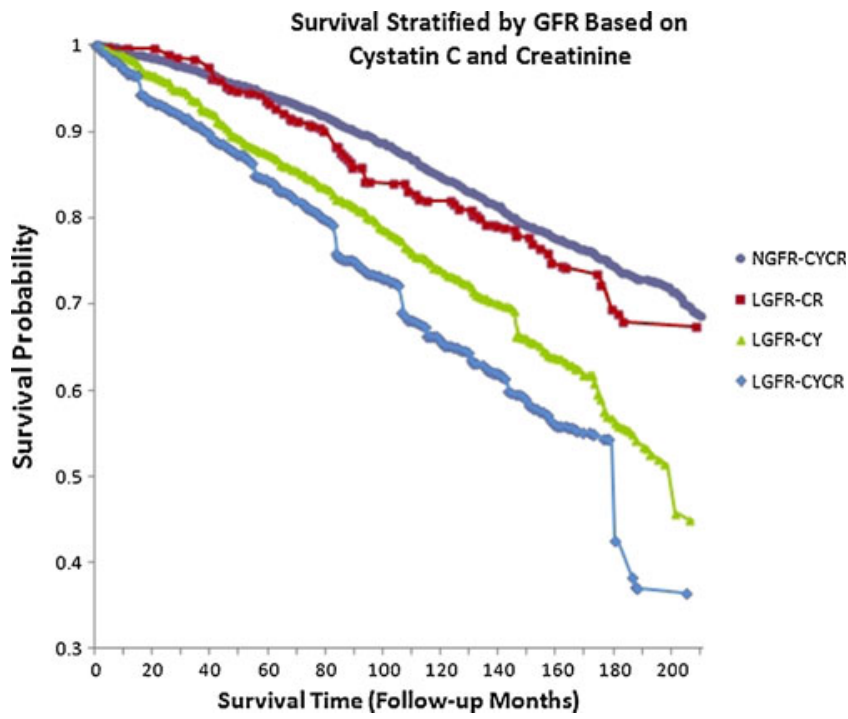

DOES IMPROVED CONTINUITY OF PRIMARY CARE IMPROVE CLINICIAN-PATIENT COMMUNICATION IN VA? David A. $\mathrm{Katz}^{1,2}$; Kimberly $\mathrm{McCoy}^{2}$; Mary Vaughan Sarrazin ${ }^{2,1}$. ${ }^{1}$ University of $\overline{\text { Iowa }}$ Carver College of Medicine, Iowa City, IA; ${ }^{2}$ VA Medical Center, Iowa City, IA. (Control ID \#1326883)

BACKGROUND: Implementation of the Patient Aligned Care Team (PACT) model within the VA aims to improve care coordination and access to first-contact care, but its effects on continuity of care (COC) and on interpersonal dimensions of care are unknown. The aim of this study is to evaluate the association between longitudinal $\mathrm{COC}$ with the same primary care provider (PCP) and patients' ratings of physician-patient communication during the ongoing PACT initiative.

METHODS: We conducted a retrospective cohort study of 4393 VA outpatients (Region 23) who satisfied the following criteria: 1) were assigned to a PCP and had at least 3 primary care visits to physicians or physician extenders during FY2009-10), and 2) completed the Survey of Healthcare Experiences of Patients (SHEP) following a primary care visit in FY2011. Data from the 2009 Patient Care Management Module were linked to VA outpatient datasets and clinic stop codes were used to identify primary care visits; telephone contacts, home-based contacts, or contacts with a non-PCP were excluded. Three measures of longitudinal COC, Usual Provider of Continuity (UPC), Modified Modified Continuity Index (MMCI), and Known Provider Continuity (K index), were calculated for each eligible VISN 23 primary care patient (on a scale of $0-1$, where 1 is perfect continuity). We grouped COC values into four categories: 1.0 (excellent), 0.75-0.99 (high intermediate), 0.50-0.74 (low intermediate), and $<0.50$ (poor). Quality of communication was measured using the 4item CAHPS-HP communication subscale in 3717 patients with complete data. To identify excellent care, we used an "all-or-none" scoring strategy: when all items within a scale were rated "always," the subscale score was assigned a value of 1 (otherwise 0). Patients were also asked to rate whether they participated in shared decision making $(\mathrm{SDM})(\mathrm{N}=1948$ respondents with a treatment choice). Multivariable random effects logistic regression models were used to predict interpersonal communication and shared decision making during FY2011, after controlling for demographics, disability status, chronic medical and psychiatric conditions (Elixhauser comorbidities plus generalized anxiety disorder and post-traumatic stress disorder), number of primary care clinic visits, usual site of care (modeled as a random effect), and PCP participation in a PACT Learning Collaborative

RESULTS: $51,19,18$, and $12 \%$ of outpatients had excellent $(\mathrm{UPC}=1)$, high-intermediate $(\mathrm{UPC}=0.75-0.99)$, low-intermediate $(\mathrm{UPC}=0.50-0.74)$, and low $(\mathrm{UPC}<0.50)$ continuity, respectively. In bivariate analysis, 61,59 , 55 , and $53 \%$ of patients in each continuity category rated their patientprovider communication as excellent, respectively $(\mathrm{p}=.0002$ by CochranMantel-Haenszel test for trend). In multivariable models, only low UPC was associated with a decreased odds of excellent communication (adjusted $\mathrm{OR}=0.80,95 \% \mathrm{CI}=0.64-1.0$ ). Similar results were obtained with MMCI and K-index, which account for dispersion of visits across providers. There was no significant association between low UPC and SDM (adjusted $\mathrm{OR}=0.89,95 \% \mathrm{CI}=0.66-1.21$ ), but patients with low MMCI tended to be less likely to rate SDM as excellent (adjusted $\mathrm{OR}=$ $0.71,95 \% \mathrm{CI}=0.50-1.01$ )

CONCLUSIONS: Reduced PCP continuity may significantly decrease the quality of patient-provider communication in VA primary care. Innovative models of care that emphasize team-based care need to be monitored for unintended reductions in longitudinal continuity with the patient's PCP.

DAILY DIARY-RECORDED, BUT NOT GLOBAL SELFREPORTED, ADHERENCE TO WEIGHT MONITORING IS ASSOCIATED WITH REDUCED HEART FAILURE HOSPITALIZATION Christine D. Jones ${ }^{1}$; George M. Holmes ${ }^{2,5}$; Darren A. DeWalt ${ }^{3,5}$; Brian Erman'; Victoria Hawk ${ }^{5}$; Kimberly Broucksou ${ }^{5}$; Crystal $\mathrm{Cene}^{3,5}$; Jia-Rong $\mathrm{Wu}^{4}$; David W. Baker ${ }^{7}$; Dean Schillinger ${ }^{6}$; Bernice $\mathrm{Ruo}^{7}$; Kirsten Bibbins-Domingo ${ }^{6}$; Aurelia Macabasco-O'Connell ${ }^{8}$; Michael Pignone ${ }^{3,5}$. ${ }^{1}$ University of North Carolina at Chapel Hill, Chapel Hill, NC; ${ }^{2}$ University of North Carolina at Chapel Hill, Chapel Hill, NC; ${ }^{3}$ University of North Carolina at Chapel Hill, Chapel Hill, NC; ${ }^{4}$ University of North Carolina at Chapel Hill, Chapel Hill, NC; ${ }^{5}$ University of North Carolina at Chapel Hill, Chapel Hill, NC; ${ }^{6}$ San Francisco General Hospital, University of California San Francisco, San Francisco, CA; ${ }^{7}$ Northwestern University, Chicago, IL; ${ }^{8}$ University of California Los Angeles, Los Angeles, CA. (Control ID \#1314646)

BACKGROUND: Heart failure (HF) self-care programs can improve outcomes, but suboptimal adherence to program components may limit effectiveness. Accurately and feasibly measuring adherence can be challenging. We sought to examine the relationship between adherence to daily weight monitoring and HF hospitalization using both self-reported and diary-recorded measures of adherence.

METHODS: We conducted a prospective cohort study among participants who underwent intensive HF self-care training as part of a 4-site randomized trial. All participants received in-person training followed by ongoing telephone-based support and education over 1 year. All partic- 
ipants were given digital scales and instructed to weigh themselves daily, record weights in a diary, and mail diaries back to a clinical educator monthly. In addition, participants were instructed about appropriate responses to significant weight changes, including contacting their provider. Self-reported weight monitoring adherence was assessed at 12 months in all participants with the question: "How often do you weigh yourself?" Patients who indicated weighing daily or more than once daily were categorized as optimally adherent; those who indicated weighing less frequently than daily were categorized as sub-optimally adherent. Diaryrecorded weight monitoring adherence was based on return of weight diaries and dichotomized into $\geq 80 \%$ and $<80 \%$ adherence to daily weight monitoring over the entire follow-up period to reflect optimal and suboptimal adherence, respectively. We evaluated the outcome of HF hospitalization over 1 year; HF hospitalization was determined by a masked adjudication committee. We used negative binomial regression to examine the relationship between self-reported and then diary-recorded weight monitoring adherence with the incidence of HF hospitalization, adjusted for study site, NYHA class, subjective SES, age, gender, race, literacy, HF related quality of life, systolic dysfunction, chronic kidney disease (GFR $<60 \mathrm{~mL} / \mathrm{min}$ ), coronary heart disease, beta-blocker use, and ACE inhibitor or ARB use.

RESULTS: Among 303 participants, we identified 74 HF hospitalizations over 12 months. Participants' mean age was 61 years, $52 \%$ were male, $32 \%$ had NYHA Class III or IV at enrollment, and $39 \%$ were African American. At 12 months, $83 \%$ of participants who completed a survey (241 of 290) self-reported weighing themselves at least daily and $37 \%$ of participants (112 of 303) recorded and returned $\geq 80 \%$ of daily weights. We found that patients with daily or greater self-reported weight monitoring adherence had an incidence rate ratio (IRR) of $1.69(0.46,6.21)$ for HF hospitalizations compared to those reporting less than daily weight monitoring; patients with $\geq$ $80 \%$ diary-based weight monitoring adherence had an IRR of 0.21 (95\% CI 0.11, 0.37) for HF hospitalizations compared to those with< $80 \%$ adherence. Sensitivity analyses lowering adherence thresholds yielded little change in results.

CONCLUSIONS: Daily diary-recorded, but not global self-reported, adherence to weight monitoring was associated with fewer HF hospitalizations. The multiple steps involved in diary-recorded weight monitoring adherence (weighing, recording, and mailing diaries) may identify more rigorous adherence to HF self-management skills. Self-care training programs should incorporate objective measures of weight monitoring adherence to ensure optimal clinical outcomes.

DAILY OPIOID USE, BUT NOT RECENT OVERDOSE, PREDICTS PERCEIVED HEALTH STATUS IN HOSPITALIZED OPIOID DEPENDENT PATIENTS Lidia Meshesha ${ }^{1}$; Judith Tsui ${ }^{1}$; Jane M. Liebschutz; Denise Crooks ${ }^{1}$; Bradley J. Anderson ${ }^{2}$; Debra S. Herman ${ }^{2}$; Michael D. Stein ${ }^{2} .{ }^{1}$ Boston University Medical Center, Boston, MA; ${ }^{2}$ Butler Hospital, Providence, RI. (Control ID \#1322637)

BACKGROUND: Patients entering addiction treatment generally report low perceived health, suggesting health status' contribution to motivation for treatment. However, perceived health status, and its predictors, among those not seeking treatment has not been studied. This study examined the association between drug addiction severity and perceived health status in non-treatment seeking persons with opioid dependence admitted to a general medical hospital.

METHODS: Baseline data from 113 subjects in a randomized clinical trial of opioid dependent persons hospitalized for medical or surgical indications. Subjects met DSM-IV criteria for opioid dependence and were not currently engaged in addiction treatment. The primary outcome, perceived health status of good, very good, or excellent, was determined by responses to a single question from SF-12 asking subjects to rate their general health, it was then compared to responses of fair or poor health. Primary predictors of interest were past 1 month frequency of opioid use (daily v. non-daily) and past 6-month accidental overdose. Multivariable logistic regression models assessed associations between perceived health status and substance use related covariates, adjusting for age, gender, race/ethnicity and alcohol use.

RESULTS: Participants were $72 \%$ male, had a mean age of 40 years $(\mathrm{SD} \pm 12$ ), $40 \%$ Caucasian, 32\% African-American and 22\% Hispanic. Forty-four percent $(44 \%)$ of patients reported good or better health. In the past 30 days, $88 \%$ reported any use of heroin, and $45 \%$ endorsed daily opioid use. Individuals who used opioids daily were significantly less likely to report good or better health status $(\mathrm{OR}=.30, \mathrm{p}=.01 ; 95 \%$ CI: $0.12 ; 0.75$ ) (Table 1). Recent accidental overdose was not significantly associated with health status $(\mathrm{OR}=2.3, \mathrm{p}=.16 ; 95 \% \mathrm{CI}$ : $0.71 ; 7.54)$. Indeed, among the $20 \%$ who reported accidental overdose in the past 6 months $60 \%$ indicated having good or better health. There were no significant associations between recent cocaine use or injection drug use and perceived health status.

CONCLUSIONS: Non-treatment seeking opioid dependent patients' perceived health status was predicted by daily opioid use but not by recent accidental overdose. Since perceptions of poor health status appear to motivate individuals to engage in treatment, changing perceptions of overdose as a potentially lethal health risk among opioid dependent persons merits further exploration.

Logistic Regression Model Predicting Good or Better than Good Health Status $(\mathrm{n}=113)$

\begin{tabular}{lll}
\hline \hline Predictor & OR $(95 \% \mathrm{CI})$ & $\mathrm{z}(\mathrm{p}=)$ \\
Daily Opioid Use & $0.30^{*}(0.12 ; 0.75)$ & $-2.59(.01)$ \\
Past 30-Day Cocaine Use & $1.78(0.76 ; 4.19)$ & $1.32(.19)$ \\
Past 30-Day IDU & $0.53(0.17 ; 1.60)$ & $-1.12(.26)$ \\
Accidental OD Prior 6-Mo. & $2.31(0.71 ; 7.54)$ & $1.39(.17)$ \\
\hline
\end{tabular}

* Adjusted for age, gender, race and alcohol use

DEAFNES AMONG PHYSICIANS AND TRAINEES: A NATIONAL SURVEY. Christopher Moreland ${ }^{1}$; Darin Latimore ${ }^{2}$; Ananda $\mathrm{Sen}^{3}$; Nora Arato $^{3}$; Philip Zazove ${ }^{5}$. The University of Texas HSC - San Antonio, San Antonio, TX; ${ }^{2}$ The University of California, Davis, Sacramento, CA; ${ }^{3}$ The University of Michigan, Ann Arbor, MI. (Control ID \#1339453)

BACKGROUND: While the deaf and hard of hearing (DHoH) community, a linguistically diverse population, forms a medically underserved minority in the United States (US), many of this community's members are seeking healthcare professional training, including as physicians, and are entering the healthcare workforce in increasing numbers, with their training facilitated in part by legal support (e.g., from the Americans with Disabilities Act of 1990, or ADA) and technological advances (e.g., electronic stethoscopes connected to hearing aids). Yet little is known about them or the accommodations they receive under the ADA, how these impact their practice, or the patients they serve. The authors designed this study to describe $\mathrm{DHoH}$ physicians' and trainees' characteristics and accommodations used, as well as their current or anticipated future patient characteristics.

METHODS: The authors created an online survey by focus group and expert review. Eligibility criteria for respondents included being a $\mathrm{DHoH}$ medical student, resident physician, or practicing physician. Multi-pronged snowball sampling identified 86 potential participants via physicians' professional networks, the American Association on Medical Colleges Group on Student Affairs, and medical schools directly; 56 of these individuals agreed to participate in the survey, which was administered July-September 2010. The primary, descriptive measure was the specific accommodations used by survey respondents, as well as frequency of and situations for use. Secondary measures included accommodation satisfaction, career satisfaction, sense of institutional support of accommodations, 
likelihood of recommending medicine as a career, and the current/ anticipated $\mathrm{DHoH}$ patient population size.

RESULTS: The survey obtained a $65 \%$ response rate. Nearly half were trainees (medical students or residents), with a mean age of 37 years; almost all were comfortable with spoken English, while nearly one-third used signed communication. Specific accommodations (e.g., interpreters, captioning) varied widely, but $93 \%$ of respondents reported at least one accommodation, while $73 \%$ reported using more than one form of accommodation. 64\% of physicians and $68 \%$ of trainees reported their accommodations met their needs well, although some spent up to 10 hours weekly arranging accommodations. Of practicing physicians, $76 \%$ reported primary-care specialties; $31 \%$ of trainees planned to enter primary-care specialties. Over $20 \%$ of trainees anticipated working with $\mathrm{DHoH}$ patients, whereas $10 \%$ of practicing physicians did. For practicing physicians, accommodation satisfaction was positively associated with career satisfaction and recommending medicine as a career.

CONCLUSIONS: DHoH physicians and trainees appear satisfied with frequent, multi-modal accommodations from employers and educational institutions. Our results can assist such organizations in planning accommodation provisions. Because $\mathrm{DHoH}$ physicians and trainees appear interested in serving $\mathrm{DHoH}$ patients as generalist physicians, recruiting and training DHoH physicians has implications for the care of this underserved population as well as the composition of a subset of the US generalist healthcare workforce.

DECREASING DISPARITIES IN BREAST CANCER SCREENING IN REFUGEE WOMEN USING CULTURALLY TAILORED PATIENT NAVIGATION Sanja Percac-Lima ${ }^{1,3}$; Barbara Bond ${ }^{4}$; Jeffrey Ashburner ${ }^{2}$; Sarah $\mathrm{Oo}^{1,3}$; Steven Atlas ${ }^{2} .{ }^{1}$ Massachussetts General Hospital, Chelsea, MA; ${ }^{2}$ Massachusets General Hospital, Boston, MA; ${ }^{3}$ Massachusets General Hospital, Boston, MA; ${ }^{4}$ Massachusets General Hospital, Boston, MA. (Control ID \#1333593)

BACKGROUND: Refugee and recent immigrant women have low breast cancer screening rates. Patient navigation can improve breast cancer screening in low income, ethnic/racial minorities, but little information is available for refugee women. The objective of this study was to evaluate the effect of a culturally tailored patient navigator program on decreasing disparities in breast cancer screening in refugee women from Africa, the Middle East and Bosnia.

METHODS: Since April 2009, all women who self-identified as speaking Arabic, Somali or Serbo-Croatian (Bosnian) and were eligible for breast cancer screening at an urban community health center, were enrolled in a patient navigator program. Patient navigators were women from the same community who spoke the same language as the patients they served. Patient navigators educated women about breast cancer screening, explored their barriers to screening, and tailored interventions to individual women to help them complete mammography screening. We compared breast cancer screening rates in refugee women to English and Spanish speaking patients receiving care at the same health center over a four year period, starting 1 year prior to the navigator program (2008), using Chi-square tests.

RESULTS: Over the 4 year period, there were on average 147 refugee women eligible for breast cancer screening. Among these women, $19 \%$ were Somali speaking, 25\% were Arabic speaking, and 56\% were SerboCroatian speaking. Over the same period, there were on average 1555 English and 1494 Spanish speaking women eligible for breast cancer screening at the same health center. In 2008, prior to the start of the navigator program for refugee women, there were marked disparities in mammography screening rates: $35.5 \%$ for refugee women compared to $58.3 \%$ for English and $62.4 \%$ for Spanish speaking patients $(\mathrm{p}<0.001)$. Over the three years of the patient navigator program, mammography rates increased among all patients, showing an increase of $3.4 \%$ for English, and $5.4 \%$ for Spanish speaking women and $29.3 \%$ for refugee women. As of October 31, 2011, mammography screening rates were similar in all groups, with $64.8 \%$ of refugee women screened compared to $61.7 \%$ in English and $67.8 \%$ in Spanish speaking patients (both $\mathrm{p}=$ 0.47) (Table 1).
CONCLUSIONS: A culturally tailored patient navigator program can decrease disparities in breast cancer screening that are present in women refugees from Somalia, the Middle East, and Bosnia.

Mammography rates in Refugee women compared to English /Spanish speaking patients

\begin{tabular}{|c|c|c|c|c|c|}
\hline Year & Refugee & English & $\begin{array}{l}\mathrm{p}- \\
\text { value }\end{array}$ & Spanish & $\begin{array}{l}\mathrm{P}- \\
\text { value }\end{array}$ \\
\hline 2008 & $\begin{array}{l}49 / 138 \\
(35.5 \%)\end{array}$ & $\begin{array}{l}960 / 1647 \\
(58.3 \%)\end{array}$ & $<0.001$ & $\begin{array}{l}942 / 1510 \\
(62.4 \%)\end{array}$ & $<0.001$ \\
\hline 2009 & $\begin{array}{l}81 / 151 \\
(53.6 \%)\end{array}$ & $\begin{array}{l}963 / 1599 \\
(60.2 \%)\end{array}$ & 0.12 & $\begin{array}{l}1006 / 1536 \\
(65.5 \%)\end{array}$ & 0.004 \\
\hline 2010 & $\begin{array}{l}85 / 156 \\
(54.5 \%)\end{array}$ & $\begin{array}{l}936 / 1560 \\
(60.0 \%)\end{array}$ & 0.18 & $\begin{array}{l}1047 / 1533 \\
(68.3 \%)\end{array}$ & 0.001 \\
\hline $2011^{*}$ & $\begin{array}{l}92 / 142 \\
(64.8 \%)\end{array}$ & $\begin{array}{l}872 / 1414 \\
(61.7 \%)\end{array}$ & 0.47 & $\begin{array}{l}946 / 1396 \\
(67.8 \%)\end{array}$ & 0.47 \\
\hline
\end{tabular}

* Through 10/31/2011

DECREASING DISPARITIES IN COLORECTAL CANCER PREVENTION USING A CULTURALLY TAILORED PATIENT NAVIGATOR PROGRAM Sanja Percac-Lima ${ }^{1,3}$; Lenny Lopez ${ }^{2,4}$; Jeffrey M. Ashburner ${ }^{2}$; Alexander Green $^{2}$; Steven J. Atlas ${ }^{2}$. ${ }^{1}$ Massachussetts General Hospital, Chelsea, MA; ${ }^{2}$ Massachusetts General Hospital, Boston, MA; ${ }^{3}$ Massachussetts General Hospital, Chelsea, MA; ${ }^{4}$ Massachusetts General Hospital, Boston, MA. (Control ID \#1338780)

BACKGROUND: Despite evidence that reductions in colorectal cancer (CRC) morbidity and mortality can be achieved through early detection and treatment, CRC screening rates are relatively low, particularly in low-income and minority patients. In 2007, a culturally-tailored, multi-faceted CRC screening navigator program was implemented at an urban community health center (CHC) with the aim of increasing $\mathrm{CRC}$ screening. We sought to evaluate the impact of the CRC screening patient navigator program on disparities in $\mathrm{CRC}$ prevention during the four years of the program.

METHODS: All patients due for CRC screening at the CHC were offered patient navigation $(\mathrm{PN})$. $\mathrm{CRC}$ screening rates in patients getting care at the $\mathrm{CHC}$ with $\mathrm{PN}$ were compared with the $\mathrm{CRC}$ screening rates in patients receiving care in the other practices within the same academic primary care network during 2006- 2010 using chi-square tests. CRC screening rates for non-English speaking and Latino patients in these two settings were analyzed separately. To determine whether the rate of increase in CRC screening was greater at the site with PN compared to other practices over the four years, we ran logistic regression models which adjusted for age, gender, race, language, insurance status, and calendar year among all patients. Similar models were also performed comparing nonEnglish speaking patients and Latino patients at the site with PN compared to those at the non-PN clinical sites. Adjusted slopes were calculated by including an interaction term between primary care site and calendar year.

RESULTS: In 2006, before the CRC screening PN program was established, $49.2 \%$ of all eligible patients at the $\mathrm{CHC}$ were up to date for CRC screening compared with $62.5 \%$ of patients cared for in other practices. In 2010 the CRC screening rate at the $\mathrm{CHC}$ was $69.2 \%$, compared with $73.6 \%(\mathrm{p}<0.001)$ in practices without $\mathrm{PN}$. Non-English speaking patients from the $\mathrm{CHC}$ had similar CRC screening rates in 2006 compared to other practices $(44.3 \%$ vs. $44.7 \%, \mathrm{p}=$ $0.79)$, but higher screening rates in $2010(70.6 \%$ vs. $58.6 \%, \mathrm{p}<0.001)$. Latino patients at the $\mathrm{CHC}$ had lower CRC screening rates in 2006 compared to other practices $(47.5 \%$ vs. $52.1 \%, \mathrm{p}=0.02)$, but had higher screening rates in $2010(73.5 \%$ vs. $67.3 \%, \mathrm{p}<0.001)$. Among all patients, the adjusted rate of increase was higher in patients from the $\mathrm{CHC}$ compared to other practices $(5.0 \%$ vs. $3.4 \%$ per year, difference $=1.6 \%, \mathrm{p}<0.001)$. Additionally, the adjusted rate of increase was higher in patients from the CHC compared to other practices for non-English speaking patients $(6.4 \%$ vs. $3.6 \%$ per year, difference $=2.8 \%, p<0.001)$ and Latino patients $(6.3 \%$ vs. $3.8 \%$ per year, difference $=2.5 \%, \mathrm{p}<0.001)$. CONCLUSIONS: Culturally-tailored CRC screening patient navigator programs can not only increase the overall colorectal screening rates, but can significantly decrease disparities in CRC prevention in vulnerable patients. Long term support of patient navigator programs is a promising method for reducing cancer screening disparities. 
DEFINING PROFESSIONAL ROLES IN AN INTERDISCIPLINARY ENVIRONMENT: THE VACHS CENTER OF EXCELLENCE IN PRIMARY CARE EDUCATION Rebecca Brienza ${ }^{1,2}$; Emily M. Meyer ${ }^{1,3}$. ${ }^{1}$ VA Connecticut Healthcare System, West Haven, CT; ${ }^{2}$ Yale University School of Medicine, New Haven, CT; ${ }^{3}$ Yale University School of Medicine, New Haven, CT. (Control ID \#1331785)

BACKGROUND: The Veterans Affairs Connecticut Healthcare System (VACHS) was one of five sites to receive funding to develop a Center of Excellence (COE) in Primary Care Education. The goal of the COE is to build a transformative model of health care professions education. COE teams at VACHS include medicine residents and nurse practitioner fellows who provide shared team care for a panel of patients over 8-week immersion blocks and throughout the year. Through a curriculum of interprofessional clinical care months, health policy seminars, facilitation, and shared decision making training, the COE offers trainees an innovative approach to post-graduate medical education. The goal of this project was to elicit trainees' attitudes towards this approach to training prior to entering the COE.

METHODS: A qualitative methodologist conducted semi-structured interviews with COE trainees between September and December 2011 (n $=9$ ). Interviews were audiotaped, transcribed verbatim, and de-identified. The transcripts were topic-analyzed and iteratively coded using the qualitative data analysis software program, Atlas.ti. Using the constant comparative method of Glaser and Strauss' Grounded Theory, textual data underwent three rounds of reduction. Any coding ambiguities were resolved by project staff prior to embarking on the next phase of analysis. Through a process of conceptual mapping and code refinement, major themes and domains of experience were identified and documented.

RESULTS: COE trainees are eager to learn and care for patients in an interprofessional team environment: "There's a high demand for physicians and it is important to bring in other people from different perspectives, other professions like PAs, NPs... it's really necessary to optimize patient care." Despite this, resident trainees were unfamiliar with the roles and scope of practice of their nurse practitioner trainee colleagues. Several residents could not articulate what a nurse practitioner actually does ("I think that, I mean, we just know that they are there and we don't really know what they do. I mean that's the truth"). Nurse practitioner fellows, on the other hand, saw their own role in accordance with their physician peers' scope of practice: ("I think that nurse practitioners are immediately more open to the various things that affect a person's health in a much broader realm"). Every participant agreed that a transformation from silo to team models of health professional training was necessary to meet the needs of the current health care system and improve patient outcomes.

CONCLUSIONS: The VACHS COE offers health care professional trainees an opportunity to learn and care for patients in a team based interdisciplinary environment. This model is in contrast to the typical "silo model" of post-graduate health professional education. Our findings suggest an initial lack of interprofessional understanding between medical and nursing models. However, the philosophical differences, potential challenges, and role ambiguities noted by trainees demonstrate the need for this type of training. It is imperative that trainees talk openly about their roles, relative contributions, strengths, and weaknesses early on in their professional development. Only then can they begin to work together to provide excellent patient-centered care and optimize patient safety and clinical outcomes.

DEMENTIA IS INDEPENDENTLY ASSOCIATED WITH A HIGHER 30-DAY HOSPITAL READMISSION RATE Rebekah Gardner $^{1,6}$; Lori A. Daiello ${ }^{2,3}$; Gary Epstein-Lubow ${ }^{4,5}$; Kristen Butterfield ${ }^{6}$; Stefan Gravenstein ${ }^{1,6}$. ${ }^{1}$ Alpert Medical School of Brown University, Providence, RI; ${ }^{2}$ Alpert Medical School of Brown University, Providence, RI; ${ }^{3}$ Rhode Island Hospital, Providence, RI; ${ }^{4}$ Alpert Medical School of Brown University, Providence, RI; ${ }^{5}$ Butler Hospital, Providence, RI; ${ }^{6}$ Healthcentric Advisors, Providence, RI. (Control ID \#1339460)

BACKGROUND: Individuals with dementia have high healthcare utilization and are at increased risk for hospitalization, but it is unknown if their 30-day readmission rates are higher than in people without dementia. Dementia is frequently co-morbid with the discharge diagnoses targeted by CMS for improvement in 30-day readmission rates, and as our population ages, understanding the role of dementia will be increasingly important. Our objective was to investigate the association between a diagnosis of dementia and the risk of hospital readmission within 30 days of discharge in a cohort of Medicare beneficiaries.

METHODS: We performed a retrospective cohort study to examine Medicare Fee for Service beneficiaries who were admitted to any Rhode Island hospital between January 2009 and December 2009. Index and subsequent hospital admissions were identified through Medicare hospital claims data. A diagnosis of dementia was identified by either 1) an ICD-9 code for dementia from Medicare Part A or Part B claims or 2) documentation of 2 or more prescriptions for medications used to treat dementia from Medicare Part D pharmacy claims. The odds of 30-day readmission were calculated using conditional logistic regression, controlling for age, gender, race, antipsychotic use, number of comorbidities, dual eligible status, length of stay of index admission, number of admissions in the previous year, and hospital of index admission.

RESULTS: From a cohort of 25,839 hospitalized patients, we identified 3,908 patients with dementia. Patients with a dementia diagnosis were older (mean age (SD), 81.0 (11.7) vs. $72.4(14.5)$ years; $\mathrm{p}<0.0001)$, were more likely to be female $(62.4 \%$ vs. $55.0 \% ; \mathrm{p}<0.0001)$, and had more comorbid illnesses $(\geq 3$ comorbidities; $28.1 \%$ vs. $25.3 \%$; $<<0.001)$ than those without a dementia diagnosis. Nearly $20 \%$ of the cohort $(\mathrm{N}=5,133)$ was readmitted within 30 days of the index hospitalization. Compared to Medicare beneficiaries without a dementia diagnosis, those with dementia were more likely to be readmitted within 30 days, even after adjusting for potential confounders (adjusted odds ratio, $1.18 ; 95 \% \mathrm{CI}, 1.08-1.29$ ).

CONCLUSIONS: Medicare Fee for Service beneficiaries with a dementia diagnosis are at increased risk for 30-day hospital readmission. Much of the recent research aimed at decreasing 30-day readmission rates excludes patients with cognitive impairment or focuses on self-management and patient activation, which may not be as effective in patients with dementia. Given the high healthcare costs and negative health outcomes associated with acute hospitalization in people with dementia, our findings have important implications for quality improvement efforts to decrease readmission rates in elderly, hospitalized patients.

DEMOGRAPHIC ANALYSIS OF CLINICA ESPERANZA, A STUDENT-RUN FREE CLINIC IN MEMPHIS, TN Sumeet S. Vaikunth; Whitney Cesari; Mukta Panda; J. B. Lewis. University of

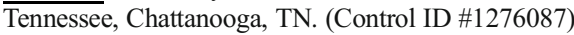

BACKGROUND: Census data show that Memphis's Hispanic population increased from $3.0 \%$ to $6.5 \%$ from 2000 to 2010 . To serve the growing uninsured Hispanic population, medical students with faculty sponsor Alicia McClary, $\mathrm{PhD}$, at the University of Tennessee Health Science Center (UTHSC) began Clinica Esperanza in 2005. The clinic runs Wednesday evenings at Christ Community Health Center on Broad Avenue. This study aims to identify the clinic's patient demographics and health issues.

METHODS: A retrospective chart review of patient files from 2005 through 2010 was undertaken, as approved by UTHSC's Institutional Review Board. RESULTS: From 2005 through 2010, Clinica Esperanza fielded 2,551 patient visits, comprised of 951 patients, 609 females and 342 males. Mean age was 34 years. $60 \%$ of patients presented once, while $13 \%$ followed up for 1 year, $9 \%$ for 2 years, $6 \%$ for 3, $6 \%$ for 4 and $4 \%$ for 5 . "Pap smear," "abdominal pain" and "follow-up lab results" ranked as the three most frequent presenting issues, respectively. Women's health accounted for $19 \%$ of patient visits, followed by HEENT $(14.5 \%)$, health maintenance $(12 \%)$, gastrointestinal $(9.5 \%)$ and musculoskeletal (9.2\%) complaints. 65\% reported length of time in Memphis$14 \%$ had lived in Memphis less than 1 year, $8 \%$ for 1 year, $13 \%$ for 2 years, $13 \%$ for 3 years, $12 \%$ for 4 years, $10 \%$ for 5 years and $32 \%$ for more than 5 years. $72 \%$ reported marital status- $61 \%$ were married and $36 \%$ were single. $73 \%$ reported country of origin- $70 \%$ were from Mexico, $11 \%$ from Honduras, $8 \%$ from Guatemala and 5\% from El Salvador. 50\% reported occupation- $31 \%$ worked in construction/ landscaping, 28\% in factories/ warehouses and $16 \%$ in cleaning/housekeeping. $24 \%$ reported educational attainment- $19 \%$ attended elementary school, $27 \%$ middle school, $49 \%$ high school and $6 \%$ college.

CONCLUSIONS: Based on these demographics of the clinic's population, improvements in health care services can be made, including better continuity, 
emphasis on gynecologic services and provider education in medical Spanish. Thus a medical Spanish elective has been created, and the clinic has been open for the entirety of 2011. Future plans include on site pharmacy, smoother referrals, and new clinics on UTHSC's other campuses

DEPRESSION AND INCREASED ASTHMA MORBIDITY IN THE ELDERLY: POOR ADHERENCE MAY BE A KEY FACTOR Katherine Krauskopf $^{1}$; Anastasia Sofianou ${ }^{1}$; Melissa Martynenko ${ }^{1}$; Mita Goel ${ }^{2}$; Michael S. Wolf ${ }^{2}$; Ethan $\mathrm{Halm}^{3}$; Howard Leventhal ${ }^{4}$; Jonathan Feldman ${ }^{5}$; Alex Federman ${ }^{1}$; Juan P. Wisnivesky ${ }^{1} .{ }^{1}$ Mount Sinai School of Medicine, New York, NY; ${ }^{2}$ Northwestern University, Chicago, IL; ${ }^{3}$ UT Southwestern, Dallas, TX; ${ }^{4}$ Rutgers, The State University of New Jersey, New Brunswick, NJ; ${ }^{5}$ Yeshiva University, Albert Einstein School of Medicine, New York, NY. (Control ID \#1338700)

BACKGROUND: Asthma is a growing cause of morbidity and mortality for elderly urban Americans. The impact of depression, a common comorbidity in the elderly, on asthma morbidity is unknown. We sought to determine the association between depression and asthma outcomes in a cohort of older urban asthmatics and to assess if this relationship would be mediated, in part, by adherence.

METHODS: Asthmatics $\geq 60$ years of age were recruited from two hospitalbased primary care clinics in New York City and Chicago for this prospective cohort study $(\mathrm{n}=317)$. Data was obtained through standardized English or Spanish interviews. Depression was evaluated using the Patient Health Questionnaire (PHQ-9); patients were classified as depressed if they had a PHQ9 score of $\geq 10$. Outcomes included asthma control (Asthma Control Questionnaire), quality of life (Asthma Quality of Life Questionnaire), medication adherence (Medication Adherence Report Scale), and asthma-related acute resource utilization at baseline. Multiple regression adjusting for sociodemographics (age, sex, race, income) asthma history (years with asthma, past intubation) and comorbid conditions (diabetes, hypertension, congestive heart failure) was used to evaluate the association of depression with these outcomes. RESULTS: The mean age of subjects was $67.4 \pm 6.6$ years; $83 \%$ were female, $33 \%$ were Black and $30 \%$ Hispanic. A third of all subjects were college graduates. Overall, $17 \%$ had symptoms of depression. In unadjusted analyses, depressed subjects were more likely to have inpatient resource utilization (odds ratio $[\mathrm{OR}] 2.03,95 \%$ confidence interval $[\mathrm{CI}] 1.04-3.99)$, worse asthma control (mean difference: $1.01 \pm 0.16, \mathrm{p}<0.0001$ ) and lower asthma-related quality of life (mean difference: $-1.4 \pm 1.8, \mathrm{p}<0.0001$ ). The presence of depressive symptoms was also associated with poorer medication adherence (OR $0.23,95 \%$ CI $0.10-0.52$ ). Similarly, in adjusted analyses, depressed subjects were more likely to have worse asthma control (mean difference: $0.87 \pm 0.17, \mathrm{p}<0.0001)$ and lower asthma-related quality of life (mean difference: $-1.22 \pm 0.18, \mathrm{p}<0.0001$ ). Poorer asthma medication adherence (OR $0.18,95 \%$ CI $0.06-0.51)$ was also associated with depression in adjusted analysis.

CONCLUSIONS: Depression is a risk factor for increased asthma morbidity and poorer quality of life in elderly patients. Our results indicate that medication adherence may mediate, in part, these findings. Further studies examining self-management behaviors and self-efficacy in this population might elucidate the mechanism underlying this association and suggest possible targets for effective interventions.

DEPRESSIVE SYMPTOMS ARE RELATED TO PERCEIVED BUT NOT OBJECTIVE SEVERITY OF HEART FAILURE Bruce L. Rollman; Bea Herbeck Belnap; Fanyin He; Sati Mazumdar; Charles F. Reynolds. University of Pittsburgh, Pittsburgh, PA. (Control ID \#1340613)

BACKGROUND: Heart failure (HF) affects over 5.7 million Americans with over 660,000 newly diagnosed cases and 277,000 deaths annually, and is the only major cardiovascular disease whose mortality rate has remained essentially unchanged over the past decade despite advancements in care. One potential cause of these persistently poor outcomes is depression which is present in 16$38 \%$ of hospitalized patients with HF, and strongly linked to poorer adherence with HF treatment regimens and increased risk of cardiovascular morbidity and mortality. Physicians may have difficulty recognizing depression in HF patients as the symptoms of these conditions can overlap (e.g., fatigue, disordered sleep). Patients may therefore be harmed if initiation of effective depression treatment is delayed pending adjustments in HF pharmacotherapy and additional tests ordered to "treat" symptoms that are, in fact, due to the underlying depressive episode. Therefore, we examined the relationship between mood symptoms and with objective and subjective measures of HF severity to guide physicians caring for these patients and to highlight the impact of co-morbid depression.

METHODS: Over a 16-month period ending 4/09, two trained study nurses screened and enrolled 471 patients from 4 Pittsburgh-area hospitals with: (a) systolic HF (left ventricular ejection fraction (EF) $<40 \%$ confirmed objectively by cardiac catheterization, echocardiogram, or MUGA); and (b) New York Heart Association (NYHA) functional class II-IV symptoms by subjective patient self-report prior to discharge. They collected sociodemographic and clinical information by patient self-report and chart review, and administered the PHQ-9 to assess the 9 cardinal symptoms of depression described in DSMIV. We used scatterplots and Pearson correlation coefficients to determine the association of EF with: (a) total PHQ-9 score; (b) somatic subscale (psychomotor, sleep, appetite, fatigue); and (c) cognitive subscale (mood, anhedonia, wothlessness, concentration, suicidal ideation) symptoms of depression. We also used Fischer's exact test to calculate a P-value for the association of NYHA class with: (a) total PHQ-9 score; and (b) EF.

RESULTS: The 471 enroled patients had a mean age of 66 (range 23-93) and $65 \%$ were male, $85 \%$ White, $41 \%$ diabetic, and they had a mean EF of $26 \%$ (SD: 7.6) and mean PHQ-9 score of 9.6 (SD: 5.2). EF was neither associated with total PHQ-9 score nor with the somatic or cognitive subscales (all Rsquared $<0.0002$ ). Moreover, while EF was similar across perceived severity level of HF symptoms as categorized by NYHA class, greater perceived HF severity was associated with higher mean PHQ-9 score (Table).

CONCLUSIONS: Severity of depressive symptoms is associated with perceived but not objective severity of HF. Patients whose HF symptoms are out-of-proportion to the objective severity of their disease and those whose symptoms fail to respond to appropriate medical therapy for $\mathrm{HF}$ should be screened for depression. Physicians caring for HF patients expressing mood symptoms should not delay initiation of appropriate therapy for depression as effective treatment can reduce symptom amplification and improve health-related quality of life.

\begin{tabular}{lllll}
\hline \hline & \multicolumn{5}{l}{ NYHA } \\
& Class & & \\
& II (N= & III (N=178) & IV (N= & P \\
& $183)$ & & $110)$ & \\
EF, mean (SD) & $26.4(7.5)$ & $25.0(7.3)$ & $25.7(8.3)$ & 0.25 \\
PHQ-9 score, mean & $7.7(5.1)$ & $9.8(4.8)$ & $12.6(4.7)$ & $<0.0001$ \\
(SD) & & & &
\end{tabular}

DESIGN AND EVALUATION OF A MULTI-DISCIPLINARY WEB-BASED HANDOFF TOOL Jeffrey L. Schnipper ${ }^{1,2}$; Andrew S. Karson $^{3,2}$; Susan K. Morash ${ }^{4}$; Brett Glotzbecker $^{6,2}$; Marsha J. Milone ${ }^{7}$; Deborah S. Yolin Raley ${ }^{6}$; Nyryan V. Nolido ${ }^{1}$; Jan Horsky ${ }^{8,1}$; Laura Leinen $^{8}$; Ishir Bhan ${ }^{5,2}$; Christian Dankers ${ }^{3,2}$; Kendra L. Church ${ }^{6}$; Jacquelyn A. Minahan ${ }^{1}$; Catherine Yoon ${ }^{1} .{ }^{1}$ Brigham and Women's Hospital, Boston, MA; ${ }^{2}$ Harvard Medical School, Boston, MA; ${ }^{3}$ Massachusetts General Hospital, Boston, MA; ${ }^{4}$ Massachusetts General Hospital, Boston, MA; ${ }^{5}$ Massachusetts General Hospital, Boston, MA; ${ }^{6}$ Brigham and Women's Hospital, Boston, MA; ${ }^{7}$ Brigham and Women's Hospital, Boston, MA; ${ }^{8}$ Partners Healthcare, Boston, MA. (Control ID \#1336148)

BACKGROUND: Failures in communication among healthcare personnel during intra-hospital handoffs in care are known threats to patient safety. In August, 2009, our healthcare system held a multi-stakeholder summit on handoffs, developed consensus around the need for a system-wide electronic handoff tool, and recommended a pilot study to develop and evaluate this technology.

METHODS: We adapted a web-based, multi-disciplinary handoff tool used by a single residency program. Enhancements to the existing tool included: 1) implementation at a second hospital in our system; 2) support for simultaneous handoffs by nurses, residents/PAs, and attendings with shared information among the different roles; 3) custom structured templates for each user group; and 4) the ability to create progress notes and multiple sign-out forms from the same core data. The tool was refined and tested on a general medicine teaching service at one hospital and a hematologic malignancy PA service at the other. 
For 3 months pre-intervention and 4 months post-implementation, we surveyed receivers of handoffs regarding continuity of care and evaluated signout content using explicit criteria. We also conducted formal usability testing using simulated cases. We conducted principal components analysis to derive categories from the survey questions and create composite scores for each category.

RESULTS: We received survey responses from 315 clinicians (66\% response rate). In a pre-post analysis, 2 of 5 composite scores improved: perceived negative impact of handoff on clinical information and decision-making (composite score 14.7 pre, 10.2 post, $\mathrm{p}=0.01$ ), and negative subjective rating of handoff quality and accuracy (28.4 vs. 25.8, p=0.01). Among survey questions to nurses, 10 improved, including an increase in how well handoffs prepared them for things that might go wrong ( 47.3 vs. $65.2, \mathrm{p}=0.01)$. In the explicit review of written sign-outs, inclusion of 5 data elements (e.g., \% tasks with if/then statements) increased, but decreases were noted in other data elements. Usability testing revealed a tension between desire for a clinical narrative and the use of structured template fields.

CONCLUSIONS: A multi-disciplinary, web-based sign-out tool was able to increase subjective measures of sign-out quality and impact on clinical decision-making, particularly among nurses. Much of the improvement may have come from the ability to produce both a progress note and sign-out with one tool, which led to more frequent updating of sign-outs and greater faith in their accuracy. The use of customized "templated" fields was inconsistent and suggests that these should be minimized to those most necessary for continuity of care. Greater improvements in care may require further enhancements in usability of the tool, training in use of the tool, and education in best practices in handoffs in care.

DETECTING AND MEASURING DEPRIVATION IN PRIMARY CARE: DEVELOPMENT, VALIDITY AND RELIABILITY OF A SELF-REPORTED QUESTIONNAIRE - THE DIPCARE-Q Paul Vaucher $^{1,2}$; Thomas Bischoff ${ }^{3}$; Esther-Amélie Diserens ${ }^{1}$; Lilli Herzig ${ }^{3}$; Giovanna Meystre-Agustoni ${ }^{4}$; Francesco Panese ${ }^{5}$; Bernard Favrat ${ }^{1}$; Catherine Sass ${ }^{6}$; Patrick Bodenmann ${ }^{1} .{ }^{1}$ University of Lausanne, Lausanne, Switzerland; ${ }^{2}$ University of Geneva, Geneva, Switzerland; ${ }^{3}$ University of Lausanne, Lausanne, Switzerland; ${ }^{4}$ University of Lausanne, Epalinges, Switzerland; ${ }^{5}$ University of Lausanne, Lausanne, Switzerland; ${ }^{6}$ Centres d'Examens de Santé, St-Etienne, France. (Control ID \#1317320)
BACKGROUND: General practitioners play a central role in taking deprivation into consideration when caring for patients in primary care. Social environment and the subjective social and individual conditions of life affect health by enhancing stress and diminishing material means to fight against affections. Validated questions to identify deprivation in primary-care practices are still lacking. For both clinical and research purposes, this study therefore aims to develop and validate a standardized instrument measuring both material and social deprivation at an individual level.

METHODS: The Deprivation in Primary Care Questionnaire (DiPCare-Q) was developed using qualitative and quantitative approaches between March 2008 and April 2011. A systematic review identified 199 questions related to deprivation. Using judgmental item quality, these were reduced to 38 questions. Two focus groups (primary-care physicians, and primary-care researchers), structured interviews (10 laymen), and think aloud interviews (eight cleaning staff) assured face validity. Content validity was evaluated by six experts in the field of social medicine, and 17 primary-care physicians. Item response theory analysis was then used to derive the DiPCare-Q index using data obtained from a random sample of 200 patients. Patients completed the questionnaire a second time over the phone three days later to enable us to assess reliability. For construct and criterion validity, the final 16 questions were administered to a random sample of 1,898 patients attending one of 47 different private primary-care practices along with questions on subjective social status (subjective SES ladder), education, income, and welfare status.

RESULTS: Deprivation was defined in three distinct dimensions; material deprivation (eight items), social deprivation (five items) and health deprivation (three items). Item consistency was high in both the derivation $(\mathrm{KR} 20=0.827)$ and the validation set $(\mathrm{KR} 20=0.778)$. The DiPCare-Q index was reliable (ICC $=$ 0.847), and items were relevant (Table). The DiPCare-Q index was correlated to patients' estimation of their position on the subjective SES ladder $\left(r_{\mathrm{s}}=0.539\right)$. This position was correlated to both material and social deprivation independently suggesting two separate mechanisms enhancing the feeling of deprivation.

CONCLUSIONS: The DiPCare-Q is a rapid, reliable and validated instrument useful for measuring both material and social deprivation in primary care. Compared to commonly used socio-economic determinants, these questions are better social indicators of patients' perceptions of themselves. Questions from the DiPCare-Q are easy to use when investigating patients' social history and could improve clinicians' ability to detect underlying social distress related to deprivation.

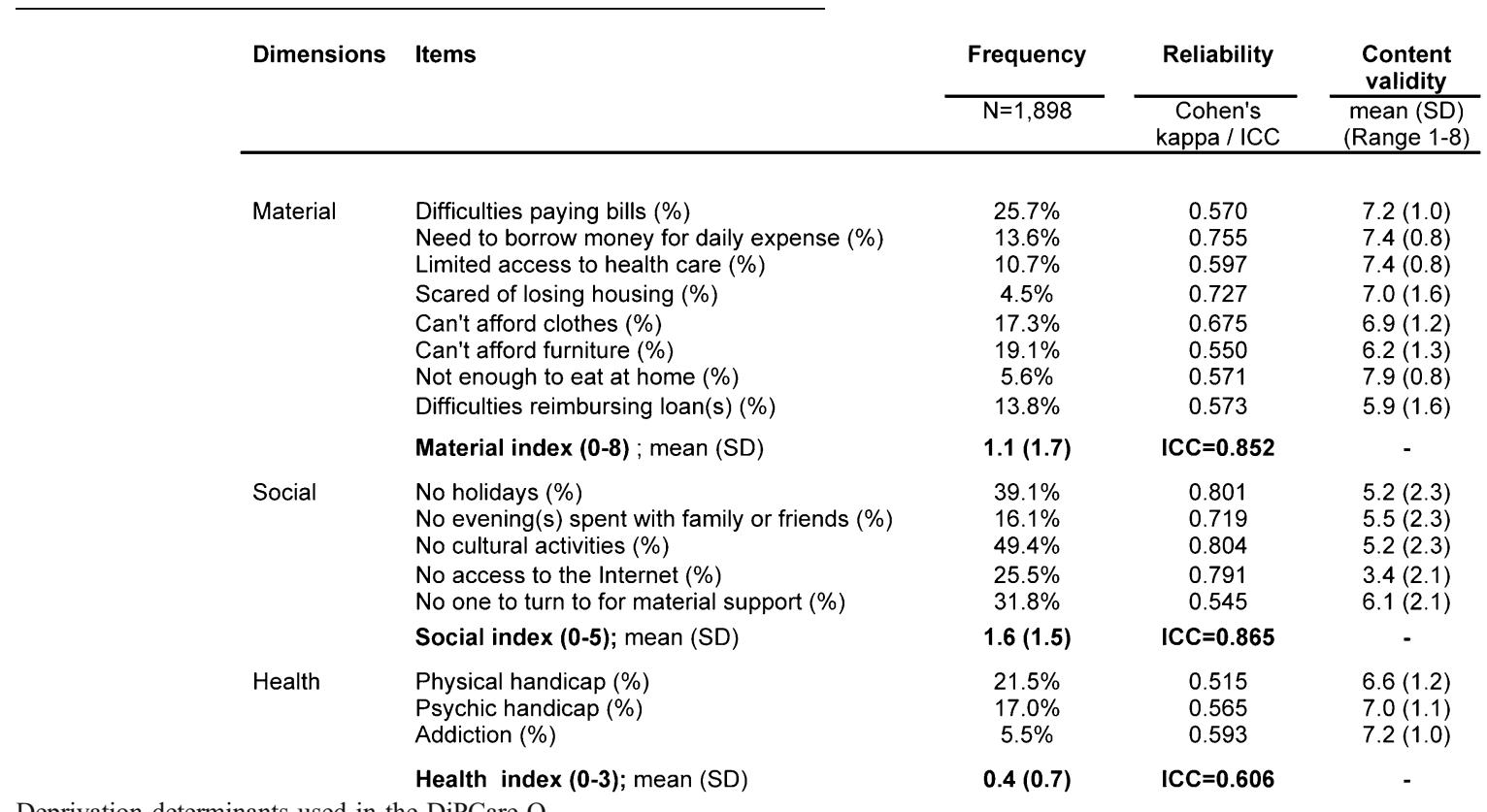


DETERMINANTS OF READINESS FOR PRIMARY CARE-MENTAL HEALTH INTEGRATION (PC-MHI) IN THE VA HEALTH CARE SYSTEM Evelyn Chang ${ }^{1}$; Danielle Rose ${ }^{2}$; Elizabeth M. Yano ${ }^{2}$; Kenneth Wells ${ }^{3,4}$; Martin Lee ${ }^{2}$; Lisa V. Rubenstein ${ }^{2,4}$. ${ }^{1}$ VA- Greater Los Angeles, Los Angeles, CA; ${ }^{2}$ VA HSR\&D Center of Excellence for the Study of Healthcare Provider Behavior, North Hills, CA; ${ }^{3}$ UCLA, Los Angeles, CA; ${ }^{4}$ RAND, Santa Monica, CA. (Control ID \#1311610)

BACKGROUND: Substantial evidence demonstrates the value of primary care-mental health integration (PC-MHI) to improve patient outcomes. While several evidence-based models exist, the readiness of practices to adopt them has not been well-studied. In 2006, the VA endorsed three different PC-MHI approaches: (1) co-located collaborative care, where mental health services are offered in PC, (2) a care manager-based depression collaborative care intervention called TIDES, and (3) a computer-assisted screening intervention for depression/alcohol/anxiety called Behavioral Health Laboratory (BHL).

METHODS: We examined variations in the adoption rates for these PCMHI approaches in relationship to organizational readiness factors in VA clinics. In this cross-sectional study of the 2007 VA Clinical Practice Organization Survey $(n=225)$, we assessed adequacy of resources, motivation for change, staff attributes, and organizational climate for each clinic. The survey included the level of implementation for each PC-MHI approach. We performed bivariate analyses for each readiness variable by each PC-MHI approach. We then constructed separate multivariate models for PC-MHI approaches with sufficient sample size.

RESULTS: By 2007, 107 (47.5\%) clinics had implemented co-located clinics, $39(17.3 \%)$ had implemented TIDES, and $17(7.6 \%)$ had implemented BHL. In bivariate analyses, PC clinics with psychologists or psychiatrists, greater financial sufficiency and greater spatial sufficiency were significantly more likely to implement BHL. Multivariate analyses largely confirmed bivariate RESULTS: clinics with established processes for quality improvement (OR 2.30, 95\% CI [1.36, 3.87], $\mathrm{p}=0.002$ ) or a depression clinician champion (OR 2.36, [1.14, 4.88], $\mathrm{p}=0.02$ ) were more likely to adopt co-location. Clinics with greater IT sufficiency (OR 1.61, $[0.97,2.67], \mathrm{p}=0.07)$ or located in VA regional networks that had endorsed TIDES (OR 8.42, [3.69, 19.26], $\mathrm{p}<0.001$ ) were more likely to adopt TIDES.

CONCLUSIONS: Adoption of each PC-MHI approach was associated with different readiness factors. Better understanding of local readiness factors may be used to improve the match between PC-MHI approaches and local site resources.

DEVELOPING FEMALE ACADEMIC LEADERS: OUTCOMES OF THE VA WOMEN'S HEALTH FELLOWSHIPS 1995-2011. Sarah A. Tilstra $^{1,2}$; Melissa McNeil ${ }^{1,2}$; Doris Rubio ${ }^{2} .{ }^{1}$ UPMC/VAPHS, Pittsburgh, $\overline{\mathrm{PA} ;{ }^{2}}$ University of Pittsburgh, Pittsburgh, PA. (Control ID \#1322148)

BACKGROUND: While some progress has been made in allocating research grants and administrative positions to women the medical community remains dissatisfied with the low proportion of female academic leaders. The VA has been a pioneer in developing academic leaders in women's health, many of whom are women, by instituting the VA Women's Health Fellowships (VAWHF). These programs have never been evaluated to determine if these fellowships are producing female academic leaders. The objectives of this study are to describe the career outcomes of female graduates of the VAWHF in terms of academic productivity, leadership roles, and clinical practices in women's health.

METHODS: In this cross-sectional survey study, all graduates of the VAWHF from 1995-2011 identified by the VA central office were eligible for participation. A 60 -item survey consisting of multiple choice, short answer and Likert scale questions was developed to assess employment and academic advancement. Contact information was obtained for 80 graduates from current program directors, PubMed and the internet. Graduates received a letter or email inviting them to participate in this survey, which were completed online using REDCap electronic data capture tools hosted at the University of Pittsburgh. Preliminary results were analyzed with descriptive statistics.

RESULTS: Twenty-five (31\%) graduates have responded to date. Average age was $40 \mathrm{yrs}$, average time post-fellowship was $8 \mathrm{yrs}, 96 \%$ were female, and $64 \%$ have obtained an advanced degree during fellowship (MS in Research
(44\%), Public Health (19\%), Education (19\%), or Epidemiology (13\%)); one pursued a $\mathrm{PhD}$. Most graduates were trained in internal medicine $(80 \%)$. For female graduates, $79 \%$ currently hold academic positions with $47 \%$ on a tenure track. Eighty percent have held a position in a teaching setting. Clinician educators represent $38 \%$ of graduates, $38 \%$ are clinician researchers, $13 \%$ are clinicians, $5 \%$ are administrators, and 5\% fill other academic roles. Seventyfive percent practice women's health and of these, $85 \%$ teach women's health to trainees. Graduates show a high rate of productivity for academic activities including successful grant funding $(88 \%)$, published peer reviewed article $(76 \%)$, presented at national meeting $(96 \%)$, curricular development/evaluation (64\%), and received teaching/research award (56\%). All academic graduates $<5$ yrs in training are assistant professors. Associate professors comprise $10 \%$ of graduates $5-10 \mathrm{yrs}$ in training. Of graduates $>10 \mathrm{yrs}$ in training, only $25 \%$ have achieved associate professor status without any obtaining professorship. Sixty percent of graduates have obtained administrative/leadership roles in primary medical education $(31 \%)$, residency/fellowship (20\%), division/section (27\%), and clinical settings (27\%).

CONCLUSIONS: The VAWHF Program has been successful in training leaders in women's health while producing exceptional female role models in academic medicine. These graduates are likely to stay in academics, practice and teach women's health, and have a high rate of productivity and achievement of leadership positions. However, the rate of academic promotion is slow. This may be due to the phenomenon of late-peaking academic careers for female graduates due to family rearing or part-time work, which were not assessed in this study. The unique elements of the VAWHF that have made it successful bear further scrutiny and may serve as a template for future development of female leaders in academic medicine.

DEVELOPING A COMMUNITY OF PRACTICE USING AN HIV TREATERS' MEETING David Feldstein ${ }^{1}$; Marge Sutinen ${ }^{1}$; Julie Yendrek $^{1}$; Barbara E. Cuene ${ }^{2}$; Raymond G. Bachhuber ${ }^{1}$; Peter L. Havens $^{3,2}$; Iram Nadeem ${ }^{3}$; James M. Sosman ${ }^{1}$. ${ }^{1}$ University of Wisconsin School of Medicine and Public Health, Madison, WI; ${ }^{2}$ Children's Hospital of Wisconsin, Milwaukee, WI; ${ }^{3}$ Medical College of Wisconsin, Milwaukee, WI. (Control ID \#1320004)

BACKGROUND: The development of new therapies and longer life expectancies has led to increased complexity in HIV infection management. Low-volume HIV providers find it difficult to keep up with changes in care and often feel isolated. The HIV Treaters' Meeting is a monthly, 1hour, case based conference. The Meeting links five sites throughout Wisconsin via videoconference. Two cases are discussed at each meeting. Clinicians present cases for discussion and seek input from attendees on treatment decisions. Our objective was to evaluate the HIV Treaters' Meeting to determine its impact on patient care and as a community of practice.

METHODS: The evaluation consisted of three components: 1) presenter pre and post-meeting questionnaires; 2) attendee session evaluation questionnaires; 3) a web-based questionnaire. Case presenters received pre-meeting questionnaires asking them to identify specific patient questions they wanted answered. Six weeks after the Treaters' Meeting they were sent a post-meeting questionnaire and reported if their patient questions were answered satisfactorily and the effect of the meeting on their patient's treatment. Attendees completed session evaluation questionnaires between March 2010 and May 2011 at the end of each Treaters' Meeting. Attendees rated aspects of the meeting on a 5-point Likert scale and provided free text answers describing what they would do differently as a result of the meeting. The web-based questionnaire was sent to all 132 people who attended a Treaters' Meeting between September 2009 and November 2010. For the evaluation, all categorical answers and Likert scales were analyzed using descriptive statistics and all free text answers were analyzed using content analysis.

RESULTS: Twenty three cases were presented at 13 Treaters' Meetings. Eighteen presenters $(78 \%)$ answered the pre-meeting questionnaire and eleven (48\%) answered both pre and post-meeting questionnaires. The 11 presenters had 29 of their 30 patient care questions answered to their satisfaction. The meeting changed the treatment plan for two patients and confirmed the treatment plan for the nine others. Meeting attendees 
completed 523 session evaluation questionnaires. The average overall rating for the meetings was 1.68 (SD 0.69) $(1=$ excellent to $5=$ poor). Major themes of the effect on attendees' practice include changes in screening or testing, changes in patient counseling, changes in therapy, increased collaboration for patient care and system changes. Fifty-six people (42\%) completed the web-based survey. Forty seven (84\%) of those completing the survey reported the meeting introduced them to new experts in HIV care. Forty four $(78 \%)$ reported that they would contact experts from the meetings with clinical questions. Twenty six (47\%) had contact with other meeting attendees outside of the meetings with the majority for patient care and patient resources. Forty seven (84\%) felt that the Treaters' Meetings have improved the patient care they provide. One respondent described how the meeting made them aware of a problem their system had with collecting HIV RNA samples that led to a system wide process change.

CONCLUSIONS: The HIV Treaters' Meeting has successfully established a community of practice with attendees who share a passion for HIV care and their patients receive improved care because of the interaction. Attendees interact outside of the meeting providing a venue to support low volume HIV treaters. We will identify ways to expand the program in the future.

DEVELOPING AN INTERDISCIPLINARY PRIMARY CARE TRAINING PROGRAM: PRE-PROGRAM ASSESSMENT OF PERCEPTIONS OF INTERNAL MEDICINE RESIDENTS AND NURSE PRACTITIONER STUDENTS Theodore Long ${ }^{1,2}$; Ali $\mathrm{Khan}^{1,2}$; Rebecca Brienza ${ }^{1,2}$. ${ }^{1}$ Yale University, New Haven, CT; ${ }^{2} \mathrm{VA}$ Connecticut Healthcare System, West Haven, CT. (Control ID \#1310517)

BACKGROUND: In 2010, the Veterans' Administration (VA) Office of Academic Affiliations funded five sites to establish Centers of Excellence (COE) in Primary Care Education. These programs were charged with transforming health-care professional training by developing new interprofessional educational training models. During the development of our COE, we sought to understand internal medicine resident and nurse practitioner perceptions about their current training as well as level of interest in participating in this new model.

METHODS: We developed two surveys focused on eliciting perspectives of internal medicine residents and nurse practitioner students about current education models. The resident survey included Likert scale questions about the trainee's primary care experience, in addition to a qualitative section about interdisciplinary education and level of trainee interest in participating in our program. The survey was sent to residents of the Yale Traditional Internal Medicine Residency Program ( $\sim 60$ residents), with 30 surveys being returned. Means were calculated from the Likert scale questions, and the qualitative data was evaluated for common themes. We administered a similar survey to a random sample of nurse practitioner students at Fairfield University School of Nursing (approximately 30 students) with 14 surveys being returned.

RESULTS: Among the residents who responded to the survey, the mean score on a scale of one to five ( $1=$ not at all confident, $5=$ very confident $)$ regarding their level of confidence about their primary care education was 4.0. The mean score regarding perception of preparedness for practicing team based ambulatory care was 3.8. Residents most commonly indicated that the need for inter-professional training is a current reality and a future direction of primary care. The qualitative data revealed a common theme that the primary care ambulatory experience could be most improved by including more continuous experience in the ambulatory clinic setting. Among the nurse practitioner students, the mean response of confidence in providing outpatient care at time of graduation was 3.14. The majority of nurse practitioner students $(71 \%)$ reported that they would be interested in completing a one year post-graduate fellowship in primary care.

CONCLUSIONS: The majority of medical residents indicated that primary care education would be improved through increased experience in the ambulatory setting, and that interdisciplinary training and care is important to the future direction of primary care. The majority of nurse practitioner students indicated that they were modestly confident about providing independent outpatient care at the time of graduation, but the majority demonstrated an interest in completing a primary care post-graduate fellowship including interdisciplinary education and team care. These surveys were utilized to inform the development of the VACHS COE in Primary Care Education.
DEVELOPMENT AND DISSEMINATION OF NEW PHYSICIAN DECLARATION IN POST HIPPOCRATIC ERA. Seiji Bito; Shinji Matsumura. NHO Tokyo Medical Ceter, Tokyo, Japan. (Control ID \#1315848)

BACKGROUND: "Medical Professionalism in the New Millennium: A Physician Charter" precisely describes the attitudes and behavior expected of modern physicians and has been accepted in many countries as a new set of standards. We aimed at the development of new physician declaration that support the everyday activities of physicians in modern society.

METHODS: Research Design: Action Research Development process: The development process involved the following steps. STEP 1. First, we established web page to facilitate development of the declaration document. We conducted open brainstorming using "Twitter" for recruitments of candidate declarations. Every tweet should be started with "I will" and had hashtag "ishisengen" (means "physician declaration" in Japanese). STEP 2. The development committee were consist of 7 physicians and 4 non MD advisers. After discussions regarding the basic concepts underlying the declaration, the 11 members selected statements they individually felt should be either included in the final draft, consolidated with other declarative statements, or omitted from the final prospective declaration. STEP 3. A publicly advertised World Café session was held, in which the content was carefully examined and prospective items were selected. STEP 4. Core members selected final candidate statements based on the results of the World Café session. STEP 5. After the prospective items were displayed on the homepage and "Facebook" fanpage, a public vote was held using the Facebook voting function. STEP 6. By incorporating the results of the vote, the core members and advisers established a final declarative statement. To disseminate the declaration after it was finalized, medical professionals who approved of actions based on the declaration were able to register on the homepage as followers by interfacing with the profile function on Facebook.

RESULTS: In STEP 1, 235 initial statements were collected and classified into 4 broad categories and 12 subcategories. In STEP 2, the 63 items that received scores of 5 or more points were shortlisted. The breakdown showed that 36 items received 10 or more points and 27 items received 5-9 points. These items were classified into 5 categories: (1) questioning one's motives, (2) confronting risk and uncertainty, (3) considering the interests of the patient, (4) doing one's best while being aware of one's limitations, and (5) accepting others and conducting introspection. The 12 items included in the new physician's declaration were ultimately established as a result of the processes in STEPS 3-6. These items included the following: "I, will not forget that medical practice may harm the patient at any time. If unfortunately an event of a serious side effect occurs to the patient, I will continue to sincerely confront the sorrow of the patient and the family."'"I, will say "thank you" in voice, when patients and colleagues had helped out. Also when a colleague close by is about to break down, I will ask "what happened?" and listen." One month after the declaration was made public, 625 Japanese medical professionals became followers of the declaration.

CONCLUSIONS: This declaration for a new generation of physicians encompasses doctors' relationships with patients, potential harm during treatment, and diverse outcomes. The process of creating the declaration prompted the interest, involvement, and understanding of many medical professionals.

DEVELOPMENT AND IMPLEMENTATION OF A PATIENT ADVISORY GROUP IN A STUDENT-RUN FREE CLINIC Devora Aharon; Yasmin S. Meah; Linda Wang; Temitope P. Awosogba; Matthew Spinelli; Laura Belland; David C. Thomas. Mount Sinai School of Medicine, New York, NY. (Control ID \#1333736)

BACKGROUND: Clinics that aim to provide patient-centered care should offer venues to elicit patient feedback and increase patient investment in selfcare. The time and resource limitations of a student-run free clinic, however, may hinder the ability to provide a patient-driven clinical experience. To increase patient involvement in our clinic operations, the East Harlem Health Outreach Partnership (EHHOP) established a Patient Advisory Group (PAG) consisting of patients invested in improving the quality of care at EHHOP. Composed of three meetings per year, the goals of the PAG are as follows: 1) to 
improve EHHOP's patient-centered services, 2) to empower patients to take a more active role in their clinic experience, 3 ) to foster relationships among fellow patients, and 4) to cultivate dialogue and promote collaboration between patients and students.

METHODS: All patients who visited clinic in the weeks prior to each PAG meeting were invited to participate, and about 30 patients expressed interest in participating. The first meeting took place in May 2011, the second in November 2011, and the third meeting will take place in January 2012. 5 patients and 3 student moderators participated in the first session, and 8 patients and 4 student moderators were present at the second. Both sessions were conducted in English and Spanish and audio taped. Conversation was participant-driven, with student-moderators facilitating discussion through open-ended questions.

RESULTS: Feedback revealed key themes around wait-times, navigation of referrals, and patient-clinician relationships. Participants offered creative, realistic solutions to streamline clinic flow and improve patients' understanding of their treatment plans and referrals. Participants also agreed to serve as patient-liaisons who will publicize the results of the PAG to fellow patients and encourage their participation in future feedback sessions. A number of patient complaints addressed issues that studentclinicians were already working to improve, indicating that patients and clinicians concurred on areas in need of improvement. Changes already implemented in response to PAG feedback include streamlining the phone system; creating a welcome guide for patients; utilizing downtime in clinic to conduct benefit screening and patient education; and instituting patientnavigators to assist patients through a complex referrals process.

CONCLUSIONS: A Patient Advisory Group can provide a means for clinics to generate insightful feedback and suggestions from patients about clinic operations, policies and quality-improvement measures. Furthermore, it has the potential to strengthen patient-clinician partnerships, create patient-patient partnerships and promote patients' investment in their own care. Finally, the institution of a PAG at a student-run free clinic helps train students to foster a patient-centered approach in a healthcare delivery system.

DEVELOPMENT AND VALIDATION OF A PREDICTIVE MODEL TO IDENTIFY PATIENTS WHO PHYSICIANS DEFINE AS COMPLEX Clemens S. Hong; Yuchiao Chang; Richard W. Grant; Jeffrey M. Ashburner; Timothy Ferris; Steven J. Atlas. Massachusetts General Hospital, Brookline, MA. (Control ID \#1340103)

BACKGROUND: Health system redesign efforts increasingly focus on patients with complex health needs. The ability to prospectively identify patients defined as complex by their primary care physician (PCP) using available electronic data sources may allow health systems to better allocate resources and tailor programs to improve patient care and physician experience. We previously characterized physician-defined complexity and described associated factors. Here we develop and validate a predictive model for physician-defined complex patients.

METHODS: 40 PCPs from 12 primary care practices in the Massachusetts General Hospital Practice-based Research Network reviewed a random sample of 120 of their own patients. After excluding patients for whom they were not responsible, PCPs identified 1126 of their 4302 patients as complex. We randomly split this PCP-reviewed cohort into two subsets: $2 / 3$ for model development and the remaining $1 / 3$ for model validation. We generated 1000 bootstrap samples from the development subset. For each sample, we ran separate logistic regression models with backward elimination (significance $=$ 0.05 ) to identify predictors for physician-defined complexity, stratifying our models by patient age (age $<60$, age $\geq 60$ ). We included only variables chosen in greater than half of bootstrap samples. We evaluated model discrimination using c statistics and calculated test characteristics. We obtained all predictive variables from an electronic data repository.

RESULTS: Among patients under 60 years old, the odds of being identified as complex increased with age (OR 1.04 [1.03-1.06]), Charlson score (OR 1.52 [1.11-2.06]), number of prescribed medication (OR 1.08 [1.06-1.10]) and number of no shows in 3 years (OR 1.24 [1.13-1.36]), Hemoglobin A1c >9 (OR 9.43 [2.86-31.11]), Medicare (OR 5.07 [2.70-9.51]) or Medicaid (OR 1.76 [1.18-2.62]) insurance, depression (OR 1.73 [1.25-2.38]) or alcohol related diagnosis (OR 2.80 [1.39-5.65]), and billing codes for MRI (OR 1.54 [1.07-2.22]). Among patients 60 years or older, the odds of being identified as complex increased with age (OR 1.04 [1.02-1.06]), number of prescribed medications (OR 1.08 [1.05-1.10]) and number of no shows in 3 years (OR 1.34 [1.13-1.59]), Medicaid insurance (OR 2.66 [1.14-6.20], diabetes (OR 1.96 [1.26-3.05]), atrial fibrillation (OR 2.19 [1.25-3.83]), and billing codes for psychotherapy (OR 3.31 [1.68-6.52]) or a complex patient visit (OR 1.54 [1.11-2.15]). C statistics from the development cohort were 0.82 for age $<60$ and 0.79 for age $\geq 60$ and 0.83 and 0.77 in the validation cohort, respectively. We defined complexity when predicted probability was $\geq 0.45$ for age $<60$ and $\geq 0.59$ for age $\geq 60$ to achieve highest accuracy, and this resulted in an overall accuracy of $82 \%$. Table 1 shows model test characteristics in the to subsets.

CONCLUSIONS: We were able to develop a robust general model to predict PCP-defined patient complexity. Age, markers of chronic disease and mental illness, Medicaid insurance, and no show visits were common predictors of complexity in both age groups. Applying this type of predictive model to populations of patients may help health systems effectively identify complex patients for resource allocation and interventions to improve primary care quality and physician experience.

Model Test Characteristic for Development and Validation Datasets

\begin{tabular}{lllllll}
\hline \hline Dataset & $\begin{array}{l}\text { Prevalence of } \\
\text { Complexity }\end{array}$ & Accuracy & Sensitivity & Specificity & $\begin{array}{l}\text { Positive Predictive } \\
\text { Value }\end{array}$ & $\begin{array}{l}\text { Negative Predictive } \\
\text { Value }\end{array}$ \\
Developmental & 0.16 & 0.82 & 0.45 & 0.95 & 0.76 & 0.83 \\
Validation & 0.15 & 0.81 & 0.43 & 0.95 & 0.74 & 0.82 \\
\hline
\end{tabular}

DEVELOPMENT OF A POTASSIUM REPLETION PROTOCOL TO DECREASE INTERRUPTIONS DURING ICU TRANSFER OF CARE Lyudmila Shvets ${ }^{1}$; Wajahat Khan ${ }^{1}$; Mona $\mathrm{Ali}^{2}$; Michael P. Carson ${ }^{1} .{ }^{1}$ Jersey Shore University Medical Center, Neptune, NJ; ${ }^{2}$ Jersey Shore University Medical Center, Neptune, NJ. (Control ID \#1312273)

BACKGROUND: Resident work hour limitations have highlighted the importance of avoiding interruptions during transitions of care, especially in the intensive care unit (ICU). Calls regarding abnormal potassium $(\mathrm{K}+)$ results are a common cause of such interruptions in our ICU. We implemented an automated $\mathrm{K}+$ repletion protocol for ICU patients with mild hyperkalemia and collected data to a) determine if it could decrease the number of phone calls made by nurses and received by residents, b) monitor and assess the response to the repletion dictated by the protocol, and c) determine whether protocol use changed the average time to first $\mathrm{K}+$ dose.

METHODS: ICU nurses and residents completed surveys regarding the current potassium replacement system, and for a week tracked the relative number of calls/pages made regarding $\mathrm{K}+$ repletion. A written order set was developed, approved by the ICU committee and piloted 
for a month. The nurses used the automated protocol to direct $\mathrm{K}+$ repletion only for patients whose eGFR was $>=50 \mathrm{cc} / \mathrm{min}$ AND initial morning $\mathrm{K}+$ was between 3.3 and $3.9 \mathrm{meq} / \mathrm{L}$ (Protocol Used Group). For those with an eGFR $<50$ OR an initial $\mathrm{K}+<=3.2$, the nurses called/paged the residents as usual (Standard Care). The protocol was only used once per day. The following were recorded each day: initial $\mathrm{K}+$ levels, creatinine, eGFR, $\mathrm{K}+$ supplement dose, time to administration, repeat $\mathrm{K}+$ values, and medications. $\mathrm{K}+$ repletion was separately tracked for those with eGFR $<50 \mathrm{cc} / \mathrm{min}$.

RESULTS: Prior to the pilot program, residents received an average of 7 pages/day from nurses during morning sign-out rounds regarding potassium repletion orders. The median time to the first $\mathrm{K}+$ dose was longer for the Standard Care patients, and was over 9 hours for 4 (Table), but the difference between the mean time to repletion was not significantly different (Wilcoxn-Rank Sum $p=0.13$ ). 14 additional patients with an eGFR < 50 (range 12-44) treated by Standard Care were tracked: the average morning $\mathrm{K}$ + was $3.5 \mathrm{meq} / \mathrm{L}$, the average repletion dose was 43 meq of $\mathrm{KCl}$, and the average next morning $\mathrm{K}+$ value was $3.7 \mathrm{meq} / \mathrm{L}$, similar to the patients with an eGFR $>=50$. The nurses and residents thought the protocol was an effective tool.

CONCLUSIONS: The pilot protocol was well received by the staff, did not cause hyperkalemia, prevented long delays in repletion, and prevented 2-3 interruptions/day during the morning transition of care when the pilot was implemented. Those with an eGFR $<50$ received similar repletion doses without developing hyperkalemia. Patients on the protocol received less total repletion because it was only implemented for those with a $\mathrm{K}+>=3.3 \mathrm{meq} / \mathrm{L}$. The protocol is now in place as part of our standard, computerized, ICU order set and except for those with critically low $\mathrm{K}+$, it is being used to address the first daily $\mathrm{K}+$ regardless of eGFR. As $88 \%$ of patients had a $\mathrm{K}+>=3.3 \mathrm{meq} / \mathrm{L}$, it has the potential to prevent at least 5-6 interruptions per day.

\begin{tabular}{llll}
\hline \hline & $\begin{array}{l}\text { Protocol } \\
\text { Used }(\mathrm{n}=52)\end{array}$ & $\begin{array}{l}\text { Standard } \\
\text { Care }(\mathrm{n}=31)\end{array}$ & $\begin{array}{l}\text { Entire } \\
\text { Cohort } \\
71.5\end{array}$ \\
$\begin{array}{l}\text { Mean Age (years) } \\
\begin{array}{l}\text { Mean Morning Potassium } \\
\text { (meq/L) }\end{array}\end{array}$ & 31 & 73 & 3.5 \\
$\begin{array}{l}\text { Potassium administered via } \\
\quad \text { Protocol (meq) }\end{array}$ & 28 & & 28 \\
$\begin{array}{l}\text { Potassium administered via } \\
\text { Other Route (meq) }\end{array}$ & 30 & 60 & 25 \\
$\begin{array}{l}\text { Range of Time For K+Repletion } \\
\text { (Hours:Minutes) }\end{array}$ & $00: 41$ to & $00: 44$ to & \\
$\begin{array}{l}\text { Mean Time to Administration of } \\
\text { First Potassium Dose Once }\end{array}$ & 136 & $16: 45$ & \\
$\quad \begin{array}{l}\text { Result } \\
\text { Available in the Computer } \\
\text { (Minutes) }\end{array}$ & & 220 & \\
$\begin{array}{l}\text { Median Time to Administration } \\
\text { of First Potassium Dose Once }\end{array}$ & 109 & 141 & \\
$\quad$ Result Available in the & & & \\
$\quad$ Computer(Minutes) & & 3.8 & \\
Next Morning K+Level (meq/L) & 3.8 & & \\
\hline
\end{tabular}

DEVELOPMENT OF A QUALITY MEASURE SET FOR CHRONIC KIDNEY DISEASE CARE IN PRIMARY CARE PRACTICE Cara Litvin $^{1}$; Steven M. Ornstein ${ }^{2} .{ }^{1}$ Medical University of South Carolina, Charleston, SC; ${ }^{2}$ Medical University of South Carolina, Charleston, SC. (Control ID \#1336225)

BACKGROUND: Chronic kidney disease (CKD) is commonly managed by primary care physicians. Although there are existing practice guidelines for the management of patients with CKD, many of these are based on expert opinion and/or focus on patients with later stages of disease usually managed by nephrologists. Early interventions may reduce progression of renal disease and risk for cardiovascular disease, yet a standard set of CKD quality measures for primary care physicians is lacking. The purpose of this study was to develop a primary care CKD quality measure set as the first step of a quality improvement project.

METHODS: This study was conducted in 2011 within PPRNet, a national primary care practice based research network whose members use a common electronic health record and pool data for quality improvement and research purposes. Ten practices from 9 states with 52 providers volunteered to participate. Eight were family medicine practices, one an internal medicine practice and one a family medicine residency. An extensive review of current CKD practice guidelines, published quality measures and existing literature was performed to generate a preliminary list of CKD quality measures. Using a measure development process based on the RAND Appropriateness method, face validity and reliability of these measures was determined through an online survey. Respondents were asked to rate each potential measure against a 1 to 9 integer scale: "Is this a useful measure of quality?" with 1 being the lowest quality and 9 being the highest quality. Respondents were also invited to provide comments on each measure. Measures with an overall median value of 8-9 were considered face valid and reliable.

RESULTS: Eighteen measures were developed and included in the preliminary CKD survey. Thirty-two out of $52(61.5 \%)$ providers completed the survey. The median score for seven of these measures was '7,' for nine measures ' 8 ,' and for two measures '9.' The 11 measures with a median score of 8-9 include: 1)Estimated Glomerular Filtration Rate (eGFR) for patients at risk for CKD or with known CKD; 2)Screening for proteinuria in all at-risk or known CKD patients; 3)Blood pressure (BP) measurement for CKD patients; 4) Most recent $\mathrm{BP}<140 / 90$ for all CKD patients; 5)Prescription for ACEinhibitor or angiotensin receptor blocker for patients with $\mathrm{CKD}$, hypertension and proteinuria; 6)Lipid measurement for all CKD patients; 7)Complete blood count in Stage 3-5 CKD; 8)Avoidance of non-steroidal anti-inflammatory drugs in Stage 3-5 CKD; 9)Avoidance of most sulfonylureas in Stage 3-5 CKD; 10)eGFR every 6 months in Stage 3-5 CKD; 11)Referral to nephrologist for eGFR $<30$. Measures eliminated from the preliminary set included diagnosis of CKD for patients with eGFR $<60$, statin therapy and lipid control, measurement of bone metabolism (calcium, phosphorus, parathyroid hormone) for stage 3-5 CKD, avoidance of nitrofurantoin and bisphosphonates in patients with reduced eGFR, and eGFR every 3 months in stage 4 CKD. Comments on these measures included concern about their level of evidence and the preference to have nephrologists handle management of bone metabolism and monitoring of later CKD stages. CONCLUSIONS: Using a consensus process, a set of primary care CKD quality measures rated as valid and reliable has been developed. Future steps based on these measures include an initial assessment of CKD management followed by a quality improvement project.

DEVELOPMENT OF A COMPREHENSIVE MOBILE SYSTEM FOR OUTREACH MEDICAL RESEARCH DATA COLLECTION AND MANAGEMENT: THE DOC-R PROJECT (DATA OUTREACH COLLECTOR FOR RESEARCH) Nicolas Senn ${ }^{1}$; Romain Thérisod ${ }^{2}$; Constant Barthel ${ }^{1}$; Jacques Cornuz; Markus Jaton ${ }^{2}$. ${ }^{1}$ University of Lausanne, Lausanne, Switzerland; ${ }^{2}$ University of Applied Sciences of Western Switzerland, Yverdon, Switzerland. (Control ID \#1321909)

BACKGROUND: In clinical research, data are often collected through paper questionnaires, with high potential of missing data, time consuming data entry and cleaning. With the rapid emergence of electronic technologies (Smartphone), new systems have been developed to achieve more efficiently the collection of data. However, most technologies are often not designed for medical research, expensive, requiring high level of computer expertise or incompatible with the confidentiality of medical research. A high degree of mobility is also mandatory when research is performed in primary care or community medicine. A school of engineering and a university primary care research institution combined their efforts to develop the DOC-R system (Data Outreach Collector for 
Research), a comprehensive, mobile system able to manage data acquisition in a primary care.

METHODS: The objectives of the DOC-R project are: 1) to develop an integrated system allowing medical researchers to perform all steps of data collection: generate electronic questionnaires, organize mobile data collection through GSM devices, and access database directly in a format suitable for analysis 2) to provide the researcher with a system that is owned by himself 3 ) to respect confidentiality of data following medical research standards. The DOC-R system comprises: 1) a server based on LAMP technology (Linux Apache MySQL PHP) requiring no specific framework 2) EditQuest, a Java/Swing-based software developed within our institution to generate offline XML format questionnaire 3) ad hoc Android application for mobile device (tablets/Smartphone).

RESULTS: To assess if DOC-R was operational, 2 researchers in primary care were asked to set up a simple survey with a minimal training (based on simple didactic material). First tests showed that the entire system was operational and the different steps displayed in figure 1 could be achieved by naïve users. The Android mobile client application uses caching and delayed write techniques in order to achieve full functionality even in absence of any network access; thus, the user is kept largely unaware from any technical contingencies, with the exception of battery life. Typical answer size is less than 1000 bytes/questionnaire, which grants an optimal efficiency, even in poor network quality conditions.

CONCLUSIONS: This work established the proof of principle that the integrated mobile data collection system DOC-R is operational and able to provide researchers with a simple, reliable and efficient tool to manage all critical steps of outreach data collection. Further developments are already going on to test its feasibility in real life research settings. DOC-R will be available for testing and demonstration.

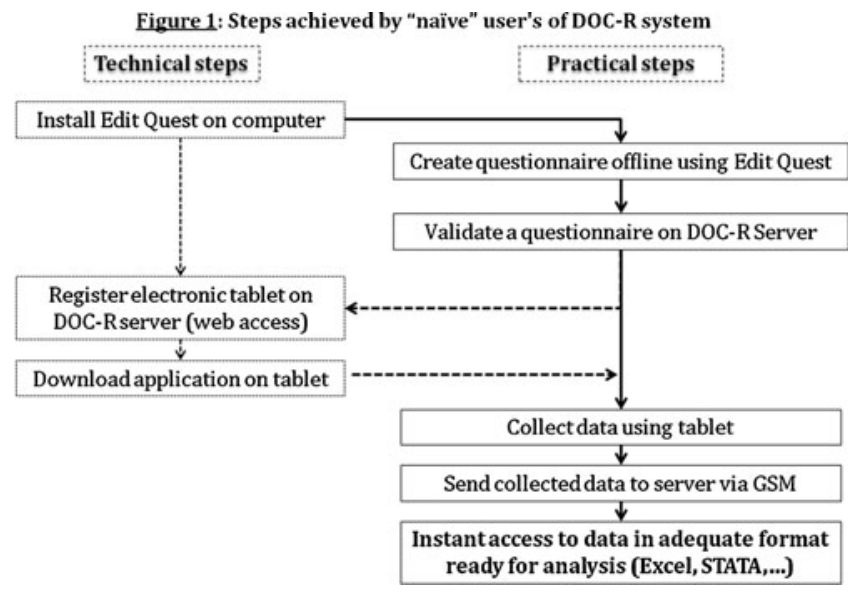

DIAGNOSTIC UNCERTAINTY AND ANTIBIOTIC PRESCRIBING FOR ACUTE COUGH IN PRIMARY CARE Lauren E. Whaley; Alexandra C. Businger; Patrick P. Dempsey; Jeffrey A. Linder. Brigham and Women's Hospital, Boston, MA. (Control ID \#1338072)

BACKGROUND: Despite guidelines stating that clinicians should not prescribe antibiotics for acute cough/acute bronchitis, nationwide rates of antibiotic prescribing for acute bronchitis are over $70 \%$. To inform solutions to reduce inappropriate antibiotic prescribing, we evaluated clinician diagnoses and antibiotic prescribing for acute cough in a primary care practice.

METHODS: We identified all acute cough visits in one primary care practice between March 1, 2011 and December 20, 2011. We defined acute cough as a cough lasting 21 days or less in an adult aged 18-64 without chronic lung disease. We excluded patients who had a visit within the previous 30 days. From acute cough visit notes in the electronic health record, we abstracted information about patient demographics, symptoms, signs, diagnoses, and prescribed medications. We also assessed whether the diagnosis was antibioticappropriate and whether clinicians expressed uncertainty regarding the diagnosis - using words like "possible" or balancing two different diagnoses, like "URI versus sinusitis."

RESULTS: During the study period, we excluded 17,182 of $33,215(52 \%)$ visits to the practice due to age, the presence of chronic lung disease, and having made a visit in the previous 30 days. We reviewed 16,033 visits from 10,487 patients, of which 601 ( $2 \%$ of all visits) met our definition of acute cough. Of acute cough visits, patients were $73 \%$ women and their mean age was 44 years old. The mean duration of cough at the time of visit was 8 days. A total of 160 different clinicians saw the acute cough patients. The most common non-cough symptoms were nasal congestion $(57 \%)$, sore throat $(49 \%)$, and phlegm (37\%). Clinicians documented a lung abnormality in $12 \%$ of visits. The most common cough-related diagnoses were upper respiratory infection (43\%), acute bronchitis (9\%), sinusitis (9\%), and pneumonia (9\%). Overall, clinicians prescribed antibiotics to $23 \%$ (140) of patients. Nonantibiotic-appropriate diagnoses accounted for $25 \%$ of antibiotic prescribing. Clinicians prescribed antibiotics to $25 \%$ of patients diagnosed with acute bronchitis. Overall, clinicians expressed diagnostic uncertainty in $112(19 \%)$ visits. Clinicians expressed diagnostic uncertainty more often for antibiotic-appropriate diagnoses than for non-antibiotic-appropriate diagnoses $(47 \%$ versus $8 \% ; \mathrm{p}<0.001)$. Likewise, clinicians expressed diagnostic uncertainty more often when prescribing antibiotics than when not prescribing antibiotics $(34 \%$ versus $14 \% ; \mathrm{p}<0.001)$.

CONCLUSIONS: The antibiotic prescribing rate for acute cough in this practice was lower than national rates. Non-antibiotic-appropriate diagnoses accounted for a quarter of antibiotic prescribing. Diagnostic uncertainty was strongly associated with antibiotic prescribing. To further reduce antibiotic prescribing for acute cough, future efforts should focus on eliminating antibiotic prescribing for non-antibiotic-appropriate diagnoses and improving diagnostic certainty.

DIFFERENCES IN PATIENT PERCEPTIONS OF THE QUALITY OF COMMUNICATION AMONG STAFF AND RESIDENT PRIMARY CARE PHYSICIANS Jeffrey M. Ashburner; Charlotte E. Ward; Yuchiao Chang; Blair W. Fosburgh; Steven J. Atlas. Massachusetts General Hospital, Boston, MA. (Control ID \#1337504)

BACKGROUND: Physicians are increasingly evaluated based on measures of performance. The Consumer Assessment of Healthcare Providers and Systems (CAHPS) survey is a standardized instrument which asks patients to report on and evaluate their experience with health care. We examined how staff primary care physicians (PCPs) compared to resident PCPs on items related to communication skills from the CAHPS patient experience of care survey. 
METHODS: We studied 209 staff PCPs and 245 resident PCPs in 13 primary care practices within the Massachusetts General Hospital practicebased research network with completed CAHPS surveys between August 2008 and June 2011. For each of 6 individual questions within the communication domain of the survey, staff and resident PCPs were compared by "top-box" score, or the proportion of surveys where "Always" was chosen, using chi-square tests. A communication composite score was calculated for each survey as the proportion of all questions where the top-box was chosen, and requiring that at least 4 of the 6 questions were answered. We compared unadjusted composite scores among staff and resident physicians in linear regression models accounting for clustering by PCP. Adjusted scores were also compared after controlling for patient age, gender, insurance status, race, language, education, Charlson score, and date of survey completion.

RESULTS: CAHPS data were available for 209 staff PCPs (median 84 surveys per PCP) and 245 resident PCPs (median 4 surveys per PCP). Patients of staff PCPs were older (59.6 vs. 53.2 years), more likely to be female $(61.5 \%$ vs. $54.5 \%)$, white $(83.5 \%$ vs. $68.7 \%)$, speak English $(94.3 \%$ vs. $86.8 \%)$, graduate college $(54.7 \%$ vs. $41.0 \%)$, have private insurance $(56.0 \%$ vs. $53.1 \%)$, and have higher mean Charlson scores (1.33 vs. 1.24 , $\mathrm{p}=0.02$ ) compared to patients of resident PCPs (all $p<0.001$ unless noted). For individual items, staff PCPs had higher top-box scores for explaining things in an easily understood way $(87.3 \%$ vs. $82.1 \%)$, giving easy to understand instructions $(86.3 \%$ vs. $82.4 \%)$, knowing the patient's medical history ( $80.8 \%$ vs. $72.7 \%)$, and respecting patient's comments $(91.2 \%$ vs. $87.9 \%$ ) (all $\mathrm{p}<0.001$ ). Resident PCPs had higher top-box scores for spending enough time with the patient $(84.1 \%$ vs. $81.7 \%, p=0.04)$. Scores were similar for listening carefully to the patient $(86.3 \%$ vs. $87.7 \%$ in Resident PCPs, $p=0.17$ ). Mean communication composite scores were higher for patients seen by staff PCPs compared to resident PCPs $(85.8 \%$ vs. $82.4 \%$, difference: $3.4 \%, \mathrm{p}<0.001)$. After adjusting for patient characteristics, staff PCP mean scores remained higher than resident PCPs ( $85.9 \%$ vs. $83.0 \%$, difference: $2.9 \%, \mathrm{p}=0.004)$.

CONCLUSIONS: Staff PCPs scored higher in most communicationrelated measures of patient experience of care, including a composite measure of communication survey items. Staff PCPs had average scores higher than the overall CAHPS 75 th percentile $(84 \%)$ for the communication composite, while resident PCP average scores fell below this threshold. Adjusting for differences in the patient characteristics of those seen by staff or resident PCPs did not substantially change mean composite scores. Residency programs should provide feedback on patient experience of care survey data to residents as part of training on quality assessment and improvement. Future studies should address whether specific training interventions can decrease these differences among staff and resident PCPs.

DIFFERENCES IN PRESCRIBING PRACTICES OF ANALGESICS TO TREAT MODERATE TO SEVERE ACUTE PAIN AMONG PHYSICIAN SPECIALTIES Aarti $\mathrm{Patel}^{2}$; Bill McCarberg'; Carmela Benson $^{2}$; Samir Mody ${ }^{2}$; Wing $\mathrm{Chow}^{2}$; Gary Vorsanger'; Myoung $\mathrm{Kim}^{2}$. ${ }^{1}$ Kaiser Permanente, San Diego, CA; ${ }^{2}$ Janssen Scientific Affairs, LLC, Raritan, NJ. (Control ID \#1333223)

BACKGROUND: Patients have reported inadequate control of their moderate to severe acute pain. Additionally, opioid-related side effects can be a significant barrier to adequate treatment of such pain. The objective of this survey was to gain insight into the differences among physician specialties in the management of acute pain in their practices, including prescribing practices of analgesics for the treatment of moderate to severe acute pain and management of opioid-related side effects, among physician specialties through the Physicians Partnering Against Pain (P3) Survey.

METHODS: The P3 survey, the largest survey of pain management practices in the U.S., was a nationwide study of U.S. physicians and their patients with severe to moderate acute pain. Physicians completed a brief questionnaire upon enrollment and were surveyed about the management of acute pain in their practice: volume of patients presenting in a typical week, frequency of prescribing opioid analgesics, the percentage of patients that return for a follow-up visit after treatment, reasons patients discontinue treatment, frequency of prescribing or recommending treatment for opioid-related gastrointestinal (GI) side effects, and frequency of patients taking opioid analgesics that also take additional treatments to manage GI side effects.

RESULTS: A total of 5,982 physicians were surveyed about the management of moderate to severe acute pain in their practice of which $52 \%$ were primary care physicians (included family practitioners, general practitioners, and internists), $23 \%$ other specialists (included rheumatologists and orthopedic surgeons), and 25\% pain specialists. Of the 1,042 physicians surveyed that responded seeing $>60$ patients with moderate to severe acute pain per week, $17 \%$ were primary care physicians (PCPs), 23\% were other specialists, and $61 \%$ were pain specialists. PCPs and other specialists were less likely than pain specialists to prescribe opioid analgesics to their patients with moderate to severe acute pain $(25.8 \%, 29.5 \%$ and $44.8 \%$, respectively) but were slightly more likely to have $75 \%$ or more of their patients with moderate to severe acute pain return for a follow-up visit $(37.7 \%, 35.1 \%$ and $27.3 \%)$. Of the 3,226 physicians surveyed that ranked unacceptable gastrointestinal side effects as primary or secondary reasons for discontinuing opioid analgesic treatment in patients with moderate to severe acute pain, 53.2\% were PCPs compared with $21.7 \%$ other specialists and $25.1 \%$ pain specialists. Despite this, there was little difference among PCPs, other specialists and pain specialists in those that recommend or prescribe treatments to manage opioid-related side effects, such as nausea, vomiting and constipation $(38.3 \%, 23.1 \%$ and $38.5 \%$, respectively).

CONCLUSIONS: The P3 Study confirms the challenge of pain management: balancing efficacy and tolerability of opioid treatments. Differences among PCPs, pain specialists and other specialists exist in pain management for patients with moderate to severe acute pain. Compared to pain specialists and other specialities, PCPs were less likely to prescribe opioid analgesics and were more likely to discontinue treatment due to unacceptable GI side effects.

DISCONTINUATION OF DIABETES MEDICATIONS DIFFERS BY SOCIOECONOMIC STATUS FOLLOWING LAPAROSCOPIC BANDING, BUT NOT GASTRIC BYPASS Kristina H. Lewis ${ }^{1}$; Matthew W. Gillman ${ }^{1}$; David E. Arterburn ${ }^{2}$; Claire F. Canning ${ }^{3}$; Fang Zhang $^{3}$; Dennis Ross-Degnan ${ }^{3}$; James F. Wharam ${ }^{3}$. ${ }^{1}$ Harvard Medical School/ Harvard Pilgrim Health Care Institute, Boston, MA; ${ }^{2}$ Group Health Center for Health Studies, Seattle, WA; ${ }^{3}$ Harvard Medical School/Harvard Pilgrim Health Care Institute, Boston, MA. (Control ID \#1333552)

BACKGROUND: Few long-term studies compare outcomes after laparoscopic (lap) banding and gastric bypass, and no national studies have determined the more effective surgery among low socioeconomic status (SES) patients. Gastric bypass causes rapid resolution of diabetes (DM) largely independent of patient behavior, whereas lap banding requires some weight loss and behavior change to resolve DM. We hypothesized that SES would not modify rates of DM resolution after bypass, but that lower SES patients would do worse after lap banding due to poorer adherence to necessary post-op lifestyle changes.

METHODS: Using CPT and ICD-9 codes from claims to a national commercial insurer, we identified 14,434 patients age 18-64 y who underwent lap band or gastric bypass during 2001-08. Of these, we studied 3,393 patients (17\% lap band, 37\% lap bypass, $46 \%$ open bypass) who were dispensed one or more DM meds in the $6 \mathrm{~m}$ before surgery, and who had at least $1 \mathrm{y}$ follow-up after surgery. From a linked consumer credit database, we obtained individual data on race/ethnicity, household income, net worth, and education. Our outcome was discontinuation of DM meds, i.e, no dispensing for a period of at least $6 \mathrm{~m}$ after surgery. We used survival analysis and Cox proportional hazards models to assess differences in discontinuation rates by surgical type and by educational level (HS or less v. some college or greater), income ( $\leq$ or $>$ $\$ 20 \mathrm{~K})$ and net worth $(\leq$ or $>\$ 25 \mathrm{~K})$ within surgical type.

RESULTS: Mean age was 46.8 y; $77 \%$ were white and $75 \%$ were female. $41 \%$ had a HS education or less, $10 \%$ had incomes $<\$ 20 \mathrm{~K}$, and $18.9 \%$ had 
net worth $<\$ 25 \mathrm{~K}$. Mean (SD) follow-up time after surgery was 2.6 (1.5) y. Baseline age, sex, race/ethnicity, and SES factors were similar between the surgical groups. After adjusting for education, income, and race/ethnicity, lap band patients were less likely to discontinue DM meds than bypass patients (HR 0.55, 95\% CI 0.49-0.61). Among bypass patients, however, DM med discontinuation did not differ by education (HR 1.00, 95\% CI 0.91-1.08, Figure 1), or income. However, lap band patients with post-HS education discontinued DM meds at a slower rate than those with HS education or less (HR 0.80,
95\%CI 0.65-0.98, Figure 2), a finding that was unaltered after adjusting for race (HR $0.80,95 \%$ CI $0.65-0.99$ ). Findings were similar, but NS, when stratifying by income (HR $0.75,95 \%$ CI $0.54-$ 1.04 ) or by net worth (HR $0.78,95 \%$ CI $0.55-1.11$ ).

CONCLUSIONS: As expected, patients discontinued DM meds at a lower rate after lap band than after bypass, and SES factors did not modify discontinuation rates after bypass. However, contrary to our hypothesis, higher (not lower) SES was associated with reduced discontinuation rates after lap band.

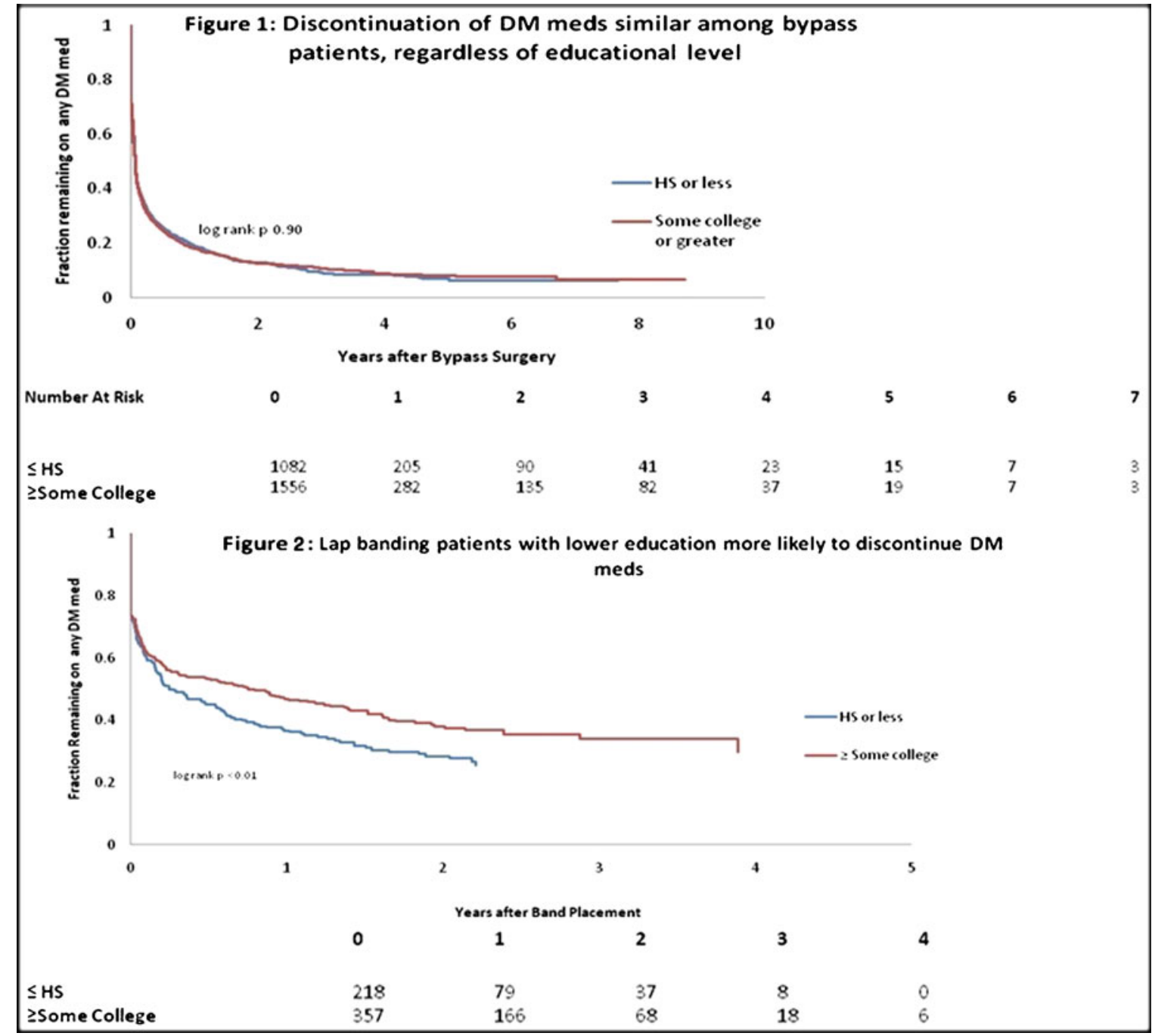

DISCOUNT FOOD STORES IN HIGH DISADVANTAGE LOS ANGELES COUNTY NEIGHBORHOODS EXPLAIN MOST INDIVIDUAL DIFFERENCE IN BODY MASS INDEX (BMI) Peter Capone-Newton ${ }^{3,2}$; Arleen Brown ${ }^{1}$; Paul M. Ong ${ }^{2}$. ${ }^{1}$ University of California Los Angeles, Los Angeles, CA; ${ }^{2}$ University of California Los Angeles, Los Angeles, CA; ${ }^{3}$ University of California Los Angeles, Los Angeles, CA. (Control ID \#1340106)

BACKGROUND: Poor diet and physical inactivity is the second leading cause of mortality in the US after smoking. Cross-sectional, ecologic studies have associated specific obesogenic food environments (OFE examples: smaller distance to fast food restaurants, higher counts of fast food per population, larger distance to grocery stores, lower counts of grocery stores per population) to higher rates of poor diet or higher body mass index (BMI). These OFEs are more prevalent in some low-income and racial/ethnic minority neighborhoods potentially contributing to widening health disparities. Recent analyses of two longitudinal cohorts (CARDIA; Framingham Offspring Cohort), found no associations between ecologic measures of OFEs and poor diet or BMI, possibly because they do not capture the characteristics of the OFEs associated with poor diet or BMI. We assessed the hypothesis that current ecologic OFE measures do not capture the link between food environments and BMI because they ignore variability in food store types and ignore actual distance traveled to purchase food. Populations defined by store type or distance may better describe the potential causal link.

METHODS: The Los Angeles Family and Neighborhood Survey (LAFANS) is a longitudinal cohort of 2619 households in Los Angeles County. In 2001-2002, households were asked where they shopped for groceries, (store name and location), self-reported BMI and details of household structure and resources. A six-category food environment 
measure based on store name and frequency was developed: highfrequency (HF) English-language named stores ("major chain"), discount stores ("less, "value", etc. in the name), HF Spanish-language stores, Englishlanguage specialty stores, multi-purpose or bulk purchase stores, other HF stores, and other low frequency stores of any language. We analyzed associations of this food environment measure with self-reported BMI, controlling for individual and household characteristics.

RESULTS: Of all LAFANS households, 2297 (88\%) reported both BMI and a valid store name. In Los Angeles County, $37 \%$ of households shop at the nearest grocery store, the remaining bypass an average of 13 stores, and $13 \%$ shop in their home census tract. The median distance to store is 1.12 mi (IQR 1.32), and the distribution of shopping by concentric radii of $<1$, $1-2.99$ and 3 to $4.99 \mathrm{~km}$, is $28 \%, 46 \%, 16 \%$. Adjusting for individual, household and neighborhood characteristics, discount store shoppers have substantially higher BMI than the referent group, major chain store shoppers in low disadvantage neighborhoods (BMI difference 1.40 points, $95 \%$ CI $0.62-2.18, p=0.004$ ), equivalent to a weight difference of $8.4 \mathrm{lbs}$. for an individual of median height and weight (5'5",160 lbs.).

CONCLUSIONS: Distinguishing between store types may better describe the causal link between individuals, stores and BMI than ecologic measures. In L.A. County, discount stores, found almost exclusively in high disadvantage and racial/ethnic minority neighborhoods are associated with individual differences in BMI. Further research should assess whether the association between discount stores and BMI is related to unmeasured elements of store content or individual characteristics. Current policy efforts focused on modifying small markets or building major chain stores in high disadvantage neighborhoods may inadequately address food environment based racial/ethnic and income based health disparities in BMI.

DISCRIMINATION BASED ON CRIMINAL RECORD AND ACCESS TO HEALTHCARE Joseph Frank ${ }^{1}$; Emily Wang ${ }^{2}$; Marcella Nunez-Smith ${ }^{2}$; Megan Comfort ${ }^{3}$. 'Brigham and Women's Hospital, Boston, MA; ${ }^{2}$ Yale School of Medicine, New Haven, CT; ${ }^{3}$ RTI International, San Francisco, CA. (Control ID \#1334729)

BACKGROUND: Incarceration is a common experience among lowincome populations and is associated with poor access to healthcare. The experience of healthcare discrimination attributed to a criminal record may play a role, as it does in employment and housing sectors. Therefore, we sought to examine the association between reported discrimination attributed to having a criminal record and access to healthcare.

METHODS: We used data from the Relate Project (R01MH0787443), a cross-sectional survey of 172 male-female couples recruited based on a recent release from state prison of the male partner. The independent variable was self-reported lifetime history of discrimination based on criminal record history by doctors, nurses, psychiatrists and others in "helping jobs". We stratified by gender as inclusion criteria and therefore incarceration history differed markedly by gender. Among the 172 male respondents, we examined the association of patient-reported discrimination with three outcome variables measuring healthcare access: 1) lifetime history of a "general check-up", 2) history of an Emergency Department (ED) visit since age 18, and 3 ) history of a medical visit while incarcerated. We used chi-square tests and multivariable logistic regression models, adjusting for age, race, insurance status and number of times incarcerated. Chi-square tests were used to compare characteristics by reported discrimination history among the smaller group of 85 female respondents surveyed regarding discrimination based on criminal record.

RESULTS: Among 172 male respondents, 73 (42\%) reported a history of healthcare discrimination attributed to criminal record. The group reporting healthcare discrimination was significantly older (mean, 42 vs. 39), had a more extensive incarceration history (median, 15.5 yrs vs. 8.5 yrs) and was more likely to report prior drug or alcohol treatment ( $82 \%$ vs. $67 \%$ ). Among all male respondents, $146(84 \%)$ reported a history of a "general check-up", $148(86 \%)$ reported an ED visit since age 18 and $117(68 \%)$ reported a physician visit while incarcerated. In adjusted analyses, the group reporting discrimination was less likely to report a history of a "general check-up" (OR 0.48, 95\% CI 0.19-1.22), more likely to report an
ED visit (OR $1.76,95 \%$ CI $0.67-4.63$ ) and significantly more likely to report a medical visit while incarcerated (OR 2.31, 95\% CI 1.11-4.78). Among female respondents, 20 (24\%) reported discrimination based on criminal history. The group reporting discrimination had a more extensive incarceration history (median, 2.5 yrs vs. $76 \mathrm{~d}$ ), was more likely to report a history of depression ( $65 \%$ vs. $34 \%$ ), anxiety ( $65 \%$ vs. $31 \%)$, drug or alcohol treatment (90\% vs. $55 \%)$ and current intravenous drug use $(55 \%$ vs. $17 \%$ ). Reported discrimination by female respondents was not significantly associated with study outcomes.

CONCLUSIONS: To our knowledge, this is the first description of patient-reported discrimination by healthcare providers attributed to criminal record. Reported discrimination was associated with duration of incarceration and contact with correctional healthcare. Trends toward decreased utilization of primary care and increased use of ED services, though not significant in this small sample, suggest that the experience of discrimination based on criminal record may act as a barrier to healthcare access. Efforts to improve access for ex-prisoners should seek to better understand and mitigate experiences of discrimination.

DISCRIMINATION AND MEDICATION ADHERENCE IN HYPERTENSIVE AFRICAN AMERICANS: THE ROLE OF STRESS AND DEPRESSION Jessica M. Forsyth; Antoinette Schoenthaler; Joseph Ravenell; Gbenga Ogedegbe. NYU School of Medicine, New York, NY. (Control ID \#1338663)

BACKGROUND: Poor adherence to antihypertensive medication among African Americans is recognized as a major contributor to racial disparities in blood pressure control. Research has shown that psychosocial factors such as stress and depression are associated with poor medication adherence. Perceived racial discrimination is an important psychosocial factor that has been associated with poor health outcomes in African Americans; its effect on medication adherence among hypertensive patients remains untested. In this study, we examined the influence of discrimination on medication adherence among hypertensive African American patients, and the mediating role of stress and depressive symptoms on this relationship.

METHODS: Participants were 740 patients enrolled in the Counseling African American To Control Hypertension (CAATCH) trial. CAATCH was a cluster-randomized trial designed to evaluate the effectiveness of a multilevel intervention, targeted at physicians and patients, on blood pressure control among hypertensive African Americans followed in 30 underserved community health centers. Analysis for this substudy utilized baseline measurements from CAATCH participants with complete data. The outcome variable was medication adherence assessed with the Morisky scale; higher scores indicate poor adherence. The predictor variable, perceived racial discrimination was assessed with the lifetime scale of the Schedule of Racist Events; higher scores indicate more frequent exposure to perceived discrimination. The mediator variables were stress, assessed with the Perceived Stress Scale, and depressive symptoms, assessed with the PHQ-9, with higher scores indicating more stress and depressive symptoms, respectively. Mediation was tested using the Baron and Kenny analytic framework examining four regression pathways. Age, income and education level were controlled for in all analyses. The Sobel test was used to determine if the mediating effect was significant.

RESULTS: Most patients were low-income and had a high school education, with a mean age of 57 years. In pathway 1 , discrimination was associated with poor medication adherence $(\beta=.086, p=.019)$. In pathway 2 , discrimination was associated with increased stress $(\beta=.197, p$ $=.000)$ and depressive symptoms $(\beta=.363, \mathrm{p}=.000)$. In pathway 3 , stress $(\beta=.236, p=.000)$ and depressive symptoms $(\beta=.190, p=.000)$ were associated with poor medication adherence. In pathway 4 , when stress and depressive symptoms were added to pathway 1, the relationship between discrimination and medication adherence became non-significant, indicating a mediating relationship $(\beta=.008, \mathrm{p}=.832)$. The Sobel test confirmed that the mediation was significant.

CONCLUSIONS: This study provides evidence that exposure to discrimination is associated with poorer medication adherence among low-income hypertensive African Americans, and that this relationship is partially explained by the negative effects of discrimination on perceived stress and depressive symptoms. Further assessment of situations where 
African American patients report high exposure to discrimination in combination with higher levels of perceived stress and depressive symptoms could provide a key starting point in addressing the lower rates of adherence in this patient population.

DISCUSSIONS BETWEEN PREMENOPAUSAL WOMEN AND THEIR PHYSICIANS REGARDING CALCIUM, VITAMIN D, AND OSTEOPOROSIS Kenzie A. Cameron ${ }^{1}$; Karin B. Ulstrup ${ }^{2,1}$; Charlie Zei ${ }^{1}$; Anne Henson ${ }^{1} .{ }^{1}$ Northwestern University, Chicago, IL; ${ }^{2}$ Chicago Lake Shore Medical Associates, Chicago, IL. (Control ID \#1332733)

BACKGROUND: More than 40 million adults in the United States have or are at risk of developing osteoporosis. Although the risk of osteoporosis in women increases significantly after menopause, by maintaining strong bones throughout life with proper calcium and vitamin D intake, premenopausal women may decrease their risk of osteoporosis. It is not known how frequently physicians are discussing osteoporosis and/or calcium and vitamin D supplementation with female premenopausal patients.

METHODS: Female premenopausal (18-49 years old) patients were approached by a research assistant in the waiting rooms of an internal medicine practice or an obstetrics and gynecology practice in Chicago, IL. Those who consented were asked to complete a self-administered survey about calcium, vitamin D, and osteoporosis and were called 1-2 weeks later to complete a follow-up phone interview.

RESULTS: Three hundred and forty-five participants completed the initial self-administered survey; these results report on the 198 participants who also completed the follow-up phone interview. Participants' mean age was 31.3 years (SD 6.5); the majority of participants were white $(77.8 \%), 10.6 \%$ were African American, 5.1\% Hispanic/Latino, and 6.1\% Asian/Pacific Islander. $45.5 \%$ reported taking a calcium supplement and $46.0 \%$ reported taking a vitamin D supplement. However, only $31.5 \%$ and $36.3 \%$ of those who reported taking calcium and vitamin D supplements, respectively, reported having started supplementation based upon a physician recommendation. Only $17.2 \%$ reported ever having discussed osteoporosis with their physician, with $41.2 \%$ of those 34 individuals having done so at their most recent visit. Participants reported that physicians brought up the topic of osteoporosis $47.1 \%$ of the time. In comparison, $35.4 \%$, and $28.8 \%$ reported ever having discussed calcium and vitamin D intake, respectively, with their physician, with $45.7 \%$ and $63.2 \%$ having done so at their most recent visit. Physicians brought up these topics $74.3 \%$ and $75.4 \%$ of the time. Women reported obtaining information about calcium and vitamin D from a range of 1 - 5 sources (mean 1.8 different sources), including: the internet (46.0\%); family and friends (43.9\%); the doctor's office (34.8\%); newspaper, books, magazines (31.6\%); and television $(17.7 \%)$.

CONCLUSIONS: Physicians appear to be more likely to raise the topics of calcium and vitamin D intake than they are to raise the topic of osteoporosis with their premenopausal female patients; however, even discussions of calcium intake are reported by fewer than $40 \%$ of surveyed patients. Women are receiving information about calcium and vitamin D from multiple sources, including the Internet and family and friends. The accuracy of the information from these sources is unknown and worthy of further study, particularly as almost $50 \%$ of the surveyed women indicate they are taking calcium and/or vitamin D supplementation. The majority of women who did start calcium and vitamin $\mathrm{D}$ did not do so based upon a conversation with their physician. We are unable to assess the context in which calcium and vitamin D supplementation is being discussed, but it appears as though such discussions are often occurring in the absence of any dialogue regarding osteoporosis. Physicians may wish to consider explicitly stating the connection between calcium, vitamin $\mathrm{D}$ and osteoporosis in discussions with their patients.

DISPARITIES IN PROCESS OF CARE FOR PATIENTS WITH WITH CIRRHOSIS IN A PUBLIC HOSPITAL CLINIC VERSUS A FACULTY PRACTICE Seth N. Sclair; Paul Martin; Frank Czul; Olveen Carrasquillo. University of MIami, Miami, FL. (Control ID \#1339094)

BACKGROUND: Vulnerable populations are at higher risk of death from liver disease and receipt of services at facilities disproportionately serving such groups is a major contributor to health care disparities. We sought to examine differences in process of care among cirrhotic patients at two health care facilities in our Miami community. One is the hepatology clinic of a public hospital ( $\mathrm{PH}$ ) and the other is a private faculty practice (FP). Academic hepatologists provide and/or oversee care by trainees and midlevel providers at both sites.

METHODS: We used claims data to identify 596 cirrhotic patients seen at each practice over a 6 month period. We selected 210 consecutive records for review of which 153 met inclusion criteria $(\mathrm{FP}=74, \mathrm{PH}=79)$. We conducted a structured retrospective chart review of electronic records (physician notes, labs and procedures) to examine adherence to cirrhosis practice guidelines by the American Association for the Study of Liver Disease. These include hepatitis A \& B vaccinations, surveillance for hepatocellular carcinoma (HCC) and esophageal varices (EV), antibiotic prophylaxis for spontaneous peritonitis (SBP), and referral for liver transplant (LT) evaluation. Chi square analysis was used to test for statistically significant differences.

RESULTS: Patients at both sites were similar in age, gender, number of visits, and cirrhosis etiology. $72 \%$ of $\mathrm{PH}$ patients were uninsured/Medicaid versus $8 \%$ of FP patients. PH patients were more likely than FP patients to have met vaccination guidelines (proven immunity or completion of vaccination series); $81 \%$ vs $46 \%$ and $76 \%$ vs $29 \%$ for hepatitis $A$ and $B$, respectively $(\mathrm{p}<.01$ for both). PH patients were more likely to have received annual HCC screening by serum AFP and imaging, $90 \%$ vs $70 \%$ $(\mathrm{p}<.01)$. However, patients at the FP practice were more likely than $\mathrm{PH}$ patients to have had endoscopic screening for EV, 97\% vs $87 \%(\mathrm{p}<.05)$. Further, excluding patients with a prior index variceal bleed, $71 \%$ of FP patients underwent primary EV screening within 6 months of establishing care vs $43 \%$ of $\mathrm{PH}$ patients $(\mathrm{p}<.01)$. Among those with varices, B blocker utilization was similar, but $\mathrm{PH}$ patients tended to have less prophylactic endoscopic banding therapy. Documentation of and scores for Model for End Stage Liver Disease (MELD) were similar at both sites, but FP patients were more likely than $\mathrm{PH}$ patients to have had a documented discussion about LT with their provider or to have been referred for LT evaluation; $81 \%$ vs $53 \%$, and $42 \%$ vs $20 \%,(p<.01$ for both). The number of patients with an indication for antibiotic prophylaxis for SBP was small (14 at FP and 13 at PH), however, the proportion of $\mathrm{PH}$ patients $(1 / 13)$ receiving antibiotics was lower than FP patients $(6 / 14)$

CONCLUSIONS: We found very large disparities in the adherence to cirrhosis practice guidelines across sites. However the direction of the observed disparities was not uniform with the safety net site having higher adherence rates than the faculty practice in several process of care measures. In general, PH patients had higher adherence to less service intense measures such as vaccinations, imaging and blood testing. In contrast, FP patients had higher adherence to more service intense measures, such as endoscopy and transplant evaluation. Providers noted difficulties in arranging elective endoscopies for indigent patients in $\mathrm{PH}$ and insurance reimbursement policies for vaccinations of FP patients as barriers to care.

DO IMMIGRANTS SUBSIDIZE THE HEALTH CARE OF THE USBORN THROUGH MEDICARE? Leah Zallman ${ }^{1,2}$; Steffie Woolhandler ${ }^{3,1}$; David Himmelstein ${ }^{3,1}$; David Bor ${ }^{1,2}$; Danny McCormick ${ }^{1,2}$. ${ }^{1}$ Cambridge Health Alliance, Cambridge, MA; ${ }^{2}$ Harvard Medical School, Boston, MA; ${ }^{3}$ City University of New York School of Public Health, New York, NY. (Control ID \#1331397)

BACKGROUND: The Medicare Trustees forecast that the Hospital Insurance Trust Fund (HITF) will be exhausted by 2024; the HITF funds Medicare Part A (primarily hospitalizations, skilled nursing, and hospice) and partially funds Part C (Medicare Advantage). Immigrants contribute to the HITF through taxes but may withdraw less than the US-born because they are younger and because they are often ineligible to receive Medicare benefits (i.e. they are undocumented or have legally resided in the US for $<$ five years).

METHODS: We calculated HITF contributions from and withdrawals by immigrants and the US-born. Using US Census Bureau data from the 2008 Current Population Survey (which represents data from 2007) we calculated the proportion of Medicare tax collections accounted for by immigrants and by US-born individuals. To calculate payroll contributions, we multiplied wage and salary earnings by $2.9 \%$ (the rate of payroll taxes that fund Medicare). To calculate taxes paid on social security benefits, we 
calculated the taxable portion of social security benefits that funds Medicare according to taxation laws and multiplied this number by the federal income tax rate. We made a small adjustment to account for miscellaneous sources of HITF revenue (interest, premiums and other) as reported by the 2008 Medicare Trustees Report (which reflects 2007 events). For this correction, we assumed that immigrant's share of contributions to miscellaneous revenue was the same as their share of total payroll taxes. We tabulated HITF withdrawals using nationally representative data from the 2007 Medical Expenditure Panel Survey (MEPS). We first calculated the proportion of hospitalization, home health care, and Medicare Advantage expenditures made on behalf of immigrants and US-born individuals. In order to account for the known underrepresentation of high expenditure Medicare hospitalizations in the MEPS, we used a weighting correction proposed by Zuvekas and Olin (2009). We applied this corrected proportion to total HITF expenditures as reported in the 2008 Medicare Trustees Report. This procedure corrected for underestimates of home health care, Medicare Advantage, and inpatient expenses in the MEPS as well as skilled nursing facility, administration, and other expenses. Finally, for each group, we calculated the overall and per capita subsidies defined as the difference between contributions and withdrawals from the HITF. We used the same process to calculate subsidies provided by citizen and non-citizen immigrants.

RESULTS: In 2007, immigrants made up $12.2 \%$ of the U.S. population. They contributed $\$ 30.8$ billion to the HITF and withdrew $\$ 19.1$ billion (net subsidy $=\$ 11.6$ billion). US-born individuals contributed $\$ 192.9$ billion and withdrew $\$ 184.0$ billion (net subsidy $=\$ 9.0$ billion). On average, immigrants provided $\$ 305$ per capita in subsidies, nearly nine times the per capita subsidy of $\$ 35$ provided by US-born individuals. All of the net subsidy was accounted for by non-citizen immigrants who contributed \$15.3 billion and withdrew \$2.6 billion (net subsidy $=\$ 12.7$ billion or $\$ 645$ per capita).

CONCLUSIONS: In 2007, immigrants withdrew only two dollars worth of care from the HITF for every three dollars they contributed; non citizen immigrants withdrew only one dollar for every six dollars they contributed. Despite the wide perception that immigrants, in particular non-citizen immigrants, consume healthcare resources, we find evidence that non-citizen immigrants heavily subsidize Medicare.

DO INSURED YOUNG ADULTS SEE THEIR PRIMARY CARE DOCTOR MORE? INSURANCE COVERAGE AND PRIMARY CARE ACCESS AMONG ADULTS AGES 27-32 Jennifer H. Chuang ${ }^{1}$; Karen Soren ${ }^{1,2}$; John Santelli ${ }^{1,2}$. ${ }^{1}$ Columbia University Medical Center, New York, NY; ${ }^{2}$ Columbia University, New York, NY. (Control ID \#1339516)
BACKGROUND: After maturing out of pediatrics, young adults face challenges of inadequate health insurance coverage and access to ageappropriate primary care. Prior to 2011, adults ages 18-26 were the age group most likely to be uninsured in the United States. Passage of the Affordable Care Act in 2010 allowed coverage under parents' health insurance plans up to age 26. Surveys in early 2011 show that adults 27-35 are now the group most at risk of being uninsured in the United States. This study examines whether insured adults ages 27-32 in 2007-2008 were more likely to have routine check-ups compared to those without health insurance coverage.

METHODS: Cross-sectional analysis of Wave IV of the National Longitudinal Study of Adolescent Health collected in 2007-2008 of 13,105 adults ages 27-32 in the United States. Primary predictor was health insurance coverage in the previous 12 months. 12 months of insurance was considered full coverage, 1-11 months as partial coverage, and 0 months as no coverage. Outcome variable of interest was having had a routine check-up in the previous 12 months. Chi-squared analysis examined covariate relationships of tobacco use, binge drinking, illicit drug use, low household income, contraception, having a sexually transmitted disease, exercise, diagnosis of mental illness, and diagnosis of a chronic health condition. Multivariable logistic regression was performed to analyze the association between health insurance coverage and having a routine check-up in the previous 12 months, adjusting for the above covariates.

RESULTS: 11,243 subjects ages $27-32$ were included in the analysis after excluding respondents with missing values. $71.7 \%$ had full health insurance coverage for the previous 12 months, $14.8 \%$ had partial coverage, and $13.5 \%$ had no coverage. $63.0 \%$ of respondents had a routine check-up within the previous 12 months and $37.0 \%$ had not had a check-up in over 12 months. $40.8 \%$ of respondents had one or more chronic health conditions. Chi-squared analysis demonstrated significant associations between insurance status and routine check-ups with the covariates described above in the methodology. Results of the multivariable logistic regression model are shown in the table. CONCLUSIONS: This study demonstrates that before implementation of the Affordable Care Act, over one-quarter of adults ages 27-32 in this sample had partial or no insurance coverage in 2007-2008. Adults in this age group had greater odds of accessing routine check-ups if they had full or partial insurance coverage. With the passage of the Affordable Care Act, young adults may now be covered under their parents' health insurance plan up to age 26. This study suggests that adults ages 27-32 are more likely to have routine check-ups if they have health insurance coverage, but this group remains at risk for insufficient health insurance coverage. Future studies performed after the implementation of the Affordable Care Act may reveal further information regarding insurance coverage and primary care access among this age group.

Relationship between health insurance coverage and routine check-up

\begin{tabular}{|c|c|c|c|c|c|c|c|c|c|}
\hline & \multicolumn{3}{|l|}{$\overline{\text { Total }}$} & \multicolumn{3}{|c|}{ Males } & \multicolumn{3}{|c|}{$\overline{\text { Females }}$} \\
\hline & OR & $95 \% \mathrm{CI}$ & p-value & OR & $95 \% \mathrm{CI}$ & p-value & OR & $95 \% \mathrm{CI}$ & p-value \\
\hline Full vs. partial and no coverage & 2.2 & $2.0-2.4$ & $<0.0001$ & 2.0 & $1.8-2.3$ & $<0.0001$ & 2.4 & $2.1-2.8$ & $<0.0001$ \\
\hline Full and partial vs. no coverage & 3.6 & $3.2-4.1$ & $<0.0001$ & 3.0 & $2.5-3.5$ & $<0.0001$ & 4.0 & $3.4-4.8$ & $<0.0001$ \\
\hline Full vs. no coverage & 3.9 & $3.4-4.4$ & $<0.0001$ & 3.1 & $2.6-3.7$ & $<0.0001$ & 4.5 & $3.7-5.4$ & $<0.0001$ \\
\hline
\end{tabular}

Full coverage $=12$ months insurance; Partial coverage $=1-11$ months insurance; No coverage $=0$ months insurance

DO PATIENT ACTIVATING INTERVENTIONS IMPROVE QUALITY OF LIFE FOR ADULTS WITH TYPE 2 DIABETES? Alida M. Gertz $^{1}$; Carl V. Tyler ${ }^{2}$; Apoorva K. Chandar ${ }^{4}$; Adam T. Perzynski ${ }^{3}$; Corinna Falck-Ytter ${ }^{3}$; Donna Windish ${ }^{5}$; Paulette A. Sage ${ }^{3}$; Shari Bolen ${ }^{3}$. ${ }^{1}$ Johns Hopkins Hospital, Baltimore, MD; ${ }^{2}$ Cleveland Clinic, Cleveland, $\mathrm{OH} ;{ }^{3}$ Metro Health Medical Center, Cleveland, OH; ${ }^{4}$ Case Western Reserve University, Cleveland, OH; ${ }^{5}$ Yale, New Haven, CT. (Control ID \#1339037)

BACKGROUND: Studies of self-management interventions effects on quality of life (QOL) in adult patients with type 2 diabetes have had mixed results. Comprehensive information regarding the impact of patient activating interventions on QOL is critical since patients may be more inclined to participate in interventions that improve their QOL. We therefore conducted a systematic review of patient activation interventions (defined as interventions targeting knowledge, beliefs, and skills for self management) effects on QOL in adults with type 2 diabetes.

METHODS: We searched the MEDLINE, EMBASE, CINAHL and Cochrane Central Register of Controlled Trials databases from inception for original English-language articles. Our search strategy combined terms for Type 2 diabetes, randomized controlled trials, and self-management interventions. We selected original studies in adults 
with type 2 diabetes that assessed the impact of patient-activating interventions on QOL. Reviewers extracted data for each article using standardized protocols. Data from these articles was analyzed and examined to compare the different types of patient activation interventions and to determine their respective effects on QOL. Patient activation interventions were grouped according to which of the 7 AADE (American Association of Diabetes Educators) behaviors were targeted (Healthy Eating, Being Active, Monitoring, Taking Medication, Problem Solving, Reducing Risks and Healthy Coping).

RESULTS: Out of 10,219 citations, 30 articles reported a validated QOL measure as either a primary or secondary outcome. On average, the studies reported enrolling 193 participants (range 25 to 2570). The majority of studies were published after $2000(\mathrm{~N}=26)$, and most occurred in the U.S. $(\mathrm{N}=$ 16). The mean time of follow-up was 11.9 months. The $\mathrm{SF}-36(\mathrm{~N}=10)$ and DQOL (Diabetes Quality of Life; N=7) were the two most common instruments used to measure QOL out of the 10 types of instruments used. Eighteen studies (60\%) found significant improvements in QOL in intervention groups compared to control groups. The most frequently targeted AADE behaviors among studies that reported significant improvements in QOL were healthy eating $(\mathrm{N}=13)$ and being active $(\mathrm{N}=12)$. Dietitian-led interventions were the most commonly used $(\mathrm{N}=5)$ among studies that reported positive outcomes. Problem solving $(\mathrm{N}=9)$ and didactic education $(\mathrm{N}=9)$ were the most commonly cited in studies with positive outcomes, whereas studies without positive outcomes cited didactic education $(\mathrm{N}=6)$ and individual care plans $(\mathrm{N}=6)$ most frequently. Of studies that used non-diabetes specific QOL measurement tools $(\mathrm{N}=20)$, only $45 \%(\mathrm{~N}=9)$ demonstrated positive QOL outcome, whereas $64 \%(\mathrm{~N}=7)$ of the studies that used diabetes specific QOL measurement tools $(\mathrm{N}=11)$ demonstrated positive $\mathrm{QOL}$ outcomes.

CONCLUSIONS: The majority of patient activation interventions improve QOL in adults with type 2 diabetes, in particular when assessed with diabetes specific QOL instruments. Future interventions targeting improvements in QOL should consider using patient activation interventions.

\section{DOCTOR SHOPPING BY OVERWEIGHT AND OBESE PATIENTS} IS ASSOCIATED WITH INCREASED HEALTHCARE UTILIZATION Kimberly Gudzune ${ }^{1}$; Sara N. Bleich ${ }^{1}$; Thomas M. Richards ${ }^{1}$; Krista Hodges ; Jonathan Weiner ${ }^{1}$; Jeanne Clark ${ }^{1} .{ }^{1}$ Johns Hopkins, Baltimore, MD; ${ }^{2}$ Healthways Hawaii LLC, Honolulu, HI. (Control ID \#1320754)

BACKGROUND: Negative interactions with healthcare providers may lead patients to switch physicians or "doctor shop." We hypothesized that overweight and obese patients would be more likely to doctor shop, and as a result, have increased rates of emergency department (ED) visits and hospitalizations as compared to normal weight non-shoppers.

METHODS: We combined claims data from a single health plan with information from beneficiaries' health risk assessments. The primary outcome was "doctor shopping," which we defined as having claims for visits with $\geq 5$ different primary care physicians (PCPs) during a 24-month period. The independent variable was NIH standard categories of weight by BMI. We performed multivariate logistic regression to evaluate the association between weight categories and doctor shopping. We conducted multivariate zero-inflated negative binominal regression to evaluate the association between weight-doctor shopping categories with counts of ED visits and hospitalizations. All regression models were adjusted for age, sex, survey year, mental health diagnosis, and co-morbid disease severity.

RESULTS: Of the 20,726 beneficiaries, the mean BMI was $26.3 \mathrm{~kg} / \mathrm{m} 2$ (SD 5.1), mean age was 44.4 years (SD 11.1) and 53\% were female. As compared to normal weight beneficiaries, overweight beneficiaries had $23 \%$ greater adjusted odds of doctor shopping (OR 1.23, 95\%CI 1.04-1.46, $\mathrm{p}=0.02)$ and obese beneficiaries had $52 \%$ greater adjusted odds of doctor shopping (OR 1.52, 95\%CI 1.26-1.82, $\mathrm{p}<0.01$ ). As compared to normal weight non-shoppers, overweight and obese shoppers had significantly higher rates of ED visits (IRR $1.85,95 \%$ CI $1.37-2.45, \mathrm{p}<0.01$; IRR $1.83,95 \% \mathrm{CI}$ $1.34-2.50, \mathrm{p}<0.01$, respectively), but not hospitalizations (IRR $1.72,95 \% \mathrm{CI}$ $0.82-3.62, p=0.15$; IRR $1.92,95 \%$ CI 0.93-3.96, $\mathrm{p}=0.08$, respectively).

CONCLUSIONS: Frequently changing PCPs may impair continuity of care for overweight and obese patients and result in increased healthcare utilization.
DOES PCP SUPPORT CONTRIBUTE TO WEIGHT MAINTENANCE EFFORTS FOLLOWING LIFESTYLE INTERVENTION? Molly B. Conroy $^{1}$; Kathleen Spadaro ${ }^{2}$; Kathleen Sward ${ }^{1}$; Irina Karpov' ${ }^{1}$, Dana L. Tudorascu ${ }^{1}$; Wishwa N. Kapoor ${ }^{1}$. ${ }^{1}$ University of Pittsburgh, Pittsburgh, PA; ${ }^{2}$ Chatham University, Pittsburgh, PA. (Control ID \#1327113)

BACKGROUND: Unfortunately, weight regain and return to sedentary behavior are very common after lifestyle interventions. Little is known about specific strategies and behaviors that may be used to maintain weight and physical activity (PA) following a lifestyle intervention, and in particular, the contribution of primary care provider (PCP) support to such efforts.

METHODS: Data were gathered from the Healthy Bodies, Healthy Hearts Study, in which 99 inactive women aged 45-65 with BMI $\geq 30$ were recruited from 3 primary care clinics associated with the University of Pittsburgh. Participants were randomized to a 12-week, in-person activity intervention program (IP) or to a self-guided, at-home (AH) intervention. At the one-year follow-up assessment, participants were weighed by a research assistant and completed a survey assessing maintenance strategies used in the past 6 months. Physical activity levels were measured with the one-month version of the Modifiable Activity Questionnaire. Strategies were categorized as selfmonitoring, group/commercial support, behavioral skills, and professional support, with the last category including PCP support. PCP support items were general discussion, encouragement, more frequent visits, diet counseling, PA counseling, referral to health educator, and referral to commercial program. Chi-square tests were used to determine differences in frequency of strategies by intervention group. Wilcoxon tests were used to compare change in weight and PA by use of strategies.

RESULTS: Data was available from 83 women ( $84 \%$ of sample). Mean (SD) age was $53.6(5.0)$ years at baseline and $34 \%$ were black. Mean baseline weight was $92.6(18.6) \mathrm{kg}$. Mean weight loss at one year was -1.3 (5.5) $\mathrm{kg}$. Participants reported using a high number of maintenance strategies in the past 6 months: $65 \%$ reported self-monitoring, $69 \%$ reported group/commercial support, $98 \%$ reported behavioral skills and $72 \%$ reported professional support. $64 \%$ of participants reported discussion with PCP. Most (54\%) participants received encouragement from PCP, but fewer received specific dietary $(40 \%)$ or physical activity $(38 \%)$ counseling. $24 \%$ were referred by PCP to health educator, but few were asked to come for more frequent visits $(11 \%)$ or referred to a commercial program (4\%). PCP support did not differ by intervention arm and was not related to weight or PA change outcomes at one year.

CONCLUSIONS: Women who had recently participated in a lifestyle intervention reported frequent use of maintenance strategies at one year after randomization, including PCP support. However, PCP support was not associated with weight or physical activity outcomes, consistent with literature suggesting low- to moderate-intensity counseling has limited efficacy in weight management. It remains to be seen whether more intensive PCP support could be effective in maintenance efforts.

DOES PATIENT NAVIGATION IMPROVE TIME TO DIAGNOSTIC RESOLUTION OF ABNORMAL MAMMOGRAMS AMONG DEPRESSED VULNERABLE WOMEN? Andrea Kronman ${ }^{1}$; Ignacio DeLaCruz ${ }^{1}$; Karen Freund ${ }^{1,3}$; Sharon Bak ${ }^{1}$; Cynthia Schoettler ${ }^{1}$; Timothy Heeren $^{2}$; Tracy Battaglia ${ }^{1} .{ }^{1}$ Boston University School of Medicine, Boston, MA; ${ }^{2}$ Boston University School of Public Health, Boston, MA; ${ }^{3}$ TUFTS University, Boston, MA. (Control ID \#1338261)

BACKGROUND: Depression is more common in women, lower socioeconomic groups, certain racial/ethnic minorities, and is associated with higher breast cancer mortality. Patient navigation has been shown to improve access to timely cancer care in a patient-centered manner, by identifying and helping to overcome barriers to healthcare in vulnerable populations. Our objective is to determine if patient navigation improves time to diagnostic resolution after abnormal mammograms for vulnerable women who are depressed.

METHODS: The Boston University Patient Navigation Research Program provided navigation for all patients who had an abnormal screening mammogram in 3 inner-city community health center (CHC) 2007-2008, while another $3 \mathrm{CHCs}$ served as control sites and received usual care. A woman was categorized as "depressed" if depression was recorded in her 
medical record during the 12 months preceding the screening test abnormality. All women were followed for 12 months to determine if they completed diagnostic evaluation. Demographic characteristics of depressed and non-depressed women were compared through chi-square tests. Differences between the time to resolution of depressed women in the control group and depressed women in the navigated group were tested using Cox Proportional time to event analysis, and described with hazard ratios and $95 \%$ confidence intervals.

RESULTS: Among 1299 women with abnormal mammograms, $17 \%$ were depressed, $12 \%$ had anxiety. Most of the women were non-White: $28 \%$ Hispanic, 35\% Black, and 11\% Vietnamese; English was the primary language in $58 \%$, and less than one third had private health care insurance. Depression was positively associated with white race and Hispanic ethnicity $(\mathrm{P}<0.01)$, English and Spanish language $(\mathrm{P}<0.001)$, older age $(\mathrm{P}=0.03)$, anxiety $(\mathrm{P}<$ $0.001)$, and public health insurance status $(\mathrm{P}<0.001)$; and negatively associated with no insurance status $(\mathrm{P}<0.001)$. Among depressed women $(\mathrm{N}=221)$, there was no significant difference in time to resolution between navigated and control patients $(\mathrm{HR}=0.96(0.73,1.3) \mathrm{p}=0.8)$.

CONCLUSIONS: Depression is common in vulnerable women needing diagnostic testing for abnormal cancer screening. In this preliminary analysis among women with depression, patient navigation did not significantly improve time to diagnostic resolution after abnormal breast cancer screening, even after adjusting for demographics and insurance status. These results suggest that patient navigators may need additional training to accommodate the needs of depressed patients in order to impact health outcomes. This analysis was limited by the small sample size, which limited adjusting for all relevant covariates. Future analyses will use a larger sample size, and adjust for variations within $\mathrm{CHCs}$, interactions with anxiety, time-dependent covariates, and depression treatments.

DOES SEVERITY OF MENTAL HEALTH SYMPTOMS AFFECT QUIT RATES IN SMOKING CESSATION TELEPHONE COUNSELING? Juan E. Arevalo ${ }^{1}$; Nicholas Lanzieri ${ }^{1}$; Erin Rogers ${ }^{1,2}$; Scott Sherman ${ }^{1} .{ }^{1}$ New York University, New York, NY; ${ }^{2}$ VA New York Harbor Healthcare System, New York, NY. (Control ID \#1331057)

BACKGROUND: Proactive telephone-based smoking cessation counseling is an emerging approach to helping mental health patients quit smoking, and it is unclear whether all patients benefit equally. We examined whether mental health patients enrolled in a telephone smoking cessation program with higher Global Assessment of Functioning (GAF) scores had better quit rates at 6 month follow-up compared to mental health patients with lower GAF scores.

METHODS: We analyzed preliminary data from a multi-site VA study implementing a telephone care coordination program for smokers with mental illness. VA providers referred smoking patients to the program via a CPRS consult, and 366 referred patients enrolled in the program. We randomized participants to receive counseling from either a state Quitline or a VA counselor. We collected patient GAF scores through the CPRS system for those enrolled in counseling using the most recent GAF score received prior to their program enrollment date. Based upon the GAF's definition of functionality and of what constituted serious impairment of functioning, we categorized GAF scores above 50 as "high functioning" and scores 50 and below as "low functioning." Patients completed a phone survey at 6 months that assessed their 30-day point prevalence abstinence.

RESULTS: GAF scores were available for $103 / 180$ patients who had enrolled in counseling and completed the 6 month follow-up. Of those patients, $70(68 \%)$ had high GAF scores and $33(32 \%)$ had low GAF scores. At 6-month follow-up, 33/103 (32\%) had quit smoking. There was no difference in abstinence rates between the high and low functioning GAF groups $(33 \%$ versus $30 \%, \mathrm{OR}=1.13,95 \% \mathrm{CI}=0.46-2.75)$. The GAF score was completed on average 125 days before the smoking cessation referral, and there was no significant difference in the interval between the high GAF and low GAF groups (99 vs. 181 days; $\mathrm{p}>.05$ ).

CONCLUSIONS: The telephone smoking cessation program produced excellent long-term abstinence rates and was equally effective for persons with mental illness who have high and low GAF scores. Telephone cessation counseling is effective for mental health patients regardless of their GAF scores. Mental health providers should not use severity of mental health symptoms in determining who would be appropriate for tobacco cessation treatment.

\section{DOES TRANSITION TO A PATIENT CENTERED MEDICAL HOME} EFFECT TEAMWORK AND BURNOUT AT RESIDENCY AND NONRESIDENCY CLINICS SIMILARLY? Erin J. Goss' ${ }^{1}$; Jason Fletcher'; Claudia Lechuga ${ }^{2}$; Paul Meissner ${ }^{2}$; David Lounsbury ${ }^{2}$; Arthur Blank ${ }^{2}$; Diane $\mathrm{McKee}^{2} .{ }^{1}$ Montefiore Medical Center, Bronx, NY; ${ }^{2}$ Albert Einstein College of Medicine, Bronx, NY. (Control ID \#1339561)

BACKGROUND: Patient Centered Medical Home (PCMH) pilot projects have shown that practice transformation, especially to team-based care, is challenging. Projects have described the experience of providers and staff at both independent and residency clinics, however we found no studies comparing outcomes of practice transformation among clinics with and without residents. We observed previously that staff at resident clinics within our institution report lower levels of teamwork than non-teaching clinics. Currently two pilot clinics are one-year into PCMH transition. Our objective is to evaluate change over time in perceived teamwork and burnout among staff at pilot clinics relative to comparison clinics, and at teaching relative to nonteaching clinics.

METHODS: Two pilot ambulatory clinics (1 with residents), and 4 comparison clinics ( 2 with residents), are included in this study. Over the past year, $\mathrm{PCMH}$ pilot clinics have received support from a PCMH facilitator, added support staff, redistributed tasks, and instituted team huddles. Staff (including clinical and clerical staff) completed a survey at baseline and year 1, which included the Healthcare Team Vitality Instrument (HTVI) and Burnout Self Report - a 15-item questionnaire where higher scores indicate more burnout. Both are rated on a 1-5 Likert scale and the ten-question HTVI was adapted to the outpatient setting. Using validated methods, factor scores were calculated for support structures, engagement and empowerment, patient care transitions, and team communication. Results from the Burnout Self Report and HTVI factors were compared between baseline and year 1 at pilot and comparison clinics using $t$ tests. The Maslach Burnout Inventory - General Survey, added at year 1, was analyzed within 3 subscales of professional efficacy, exhaustion, and cynicism. Results from resident and nonresident clinics, as well as between pilot and comparison clinics were compared using $t$ tests.

RESULTS: To date, 196 employees responded to the year 1 survey ( 76 from pilot clinics; 117 from comparison clinics), compared to 335 responses to the baseline survey. At year 1, resident clinics have significantly higher levels of exhaustion ( 13.7 vs. $8.4 ; \mathrm{p}<0.001)$ and cynicism ( 8.9 vs $5.0 ; \mathrm{p}<0.001)$ on the Maslach than non-teaching clinics; there were no differences in the professional efficacy subscale $(\mathrm{p}>0.05)$. No significant differences were observed between pilot and comparison clinics on Maslach subscales. Compared to baseline, intervention clinics did not significantly improve in regards to mean scores on the Burnout Self Report (40 vs 37.5) or HTVI factors of support structures (3.70 vs. 3.56), empowerment (3.18 vs 3.2), care transitions (3.18 vs 3.17), or team communication ( 4.01 vs 3.82; $p>0.05$ for all). Similarly there were no improvements at comparison clinics $(\mathrm{p}>0.05)$. CONCLUSIONS: While resident clinics demonstrate higher levels of burnout, neither pilot clinic showed improvement in teamwork or burnout at 1 year. These results, although disappointing, generate further questions. Early survey respondents may differ from clinic staff as a whole and skew our results. Negative effects from practice disruption may have obscured the benefits from interventions intended to improve teamwork within the PCMH model. Alternatively, interventions at our pilot clinics may truly not improve staff perceptions of teamwork at one year. Further analysis is needed to determine the barriers to teamwork at teaching and non-teaching clinics.

DOES A BITE CAUSE CANCER? MISPERCEPTIONS OF BREAST CANCER ETIOLOGY AMONG LOW-INCOME URBAN WOMEN. Erin N. Marcus ${ }^{1}$; Noella Dietz ${ }^{2} .{ }^{1}$ University of Miami Miller School of Medicine, Sylvester Comprehensive Cancer Center, Miami, FL; ${ }^{2}$ University of Miami Miller School of Medicine, Miami, FL. (Control ID \#1337724)

BACKGROUND: Adequate patient understanding is an important component of shared decision making (SDM) in the screening, diagnosis and 
treatment of breast cancer. Health beliefs about the causes of cancer may influence women's perceptions of their personal cancer risk and ability to participate effectively in SDM. The objective of this qualitative study is to describe the beliefs of a cohort of urban low-income women regarding the causes of breast cancer.

METHODS: A convenience sample of 34 women was recruited in the community to participate in 4 focus groups, each of which was led by an AfricanAmerican female moderator. All of the women had undergone mammogram screening within the past 2 years. None had ever been diagnosed with cancer. The overarching purpose of the focus groups was to explore the women's experiences learning of their mammogram results and elicit their preferences for how they would like to learn of their results. Focus group discussions were audiotaped and transcribed. Two investigators separately performed a thematic analysis of the transcripts using an immersion and crystallization approach.

RESULTS: 36 women participated in the study. $85 \%(n=29)$ self-identified as black or African-American. $39 \%(n=14)$ qualified for Medicaid; $36 \%(n=$ 13) reported that they lacked insurance. $56 \%(n=20)$ reported an annual income of less than $\$ 10,000.38 \%(n=13)$ reported that they needed help reading health materials, a marker of low health literacy. A prominent theme that emerged in the discussion was an uncertainty of why women develop breast cancer. Many participants stated that they believed that certain foods, infections, bites, trauma, large breast size, and sexual activity could cause breast cancer. Even though all the women had undergone mammography screening, many voiced skepticism about mammography's effectiveness in women with no obvious symptoms of breast cancer.

CONCLUSIONS: Despite widespread educational campaigns about mammography, misperceptions about the causes of breast cancer still exist among low-income urban women. The effect of these misperceptions on screening and treatment behavior is unclear. Nonetheless, these beliefs may serve as a barrier to successful SDM regarding cancer screening, diagnosis, and treatment. Clinicians should be cognizant of possible misperceptions among their patients when discussing breast cancer with them. Educational media about breast cancer should seek to dispel such beliefs. Additional research is needed to elucidate the role of these beliefs in shaping patient behavior about breast cancer screening and treatment.

DOES DOCUMENTATION OF CHRONIC KIDNEY DISEASE ON A PATIENT'S PROBLEM LIST CORRELATE WITH HIGHER QUALITY CARE? Lipika Samal ${ }^{1}$; Adam Wright ${ }^{1}$; David W. Bates ${ }^{1,2}$. ${ }^{1}$ Brigham and Women's Hospital, Boston, MA; ${ }^{2}$ Harvard Medical School, Boston, MA. (Control ID \#1319137)

BACKGROUND: Most electronic health records allow for maintenance of a discrete clinical problem list. Such problem lists can be used for patient care, clinical decision support, quality measurement and research, but problem list accuracy is variable. Chronic kidney disease (CKD) is asymptomatic, but carries high morbidity and mortality in late stages. We hypothesized that problem list documentation of CKD may be associated with higher quality care because it is an explicit marker of recognition of the disease and because it prompts stage-appropriate monitoring and treatment for CKD. We assessed the relationship between problem list documentation and quality of care.

METHODS: We obtained problem list, medication list, and laboratory data on adult patients seen in 12 Brigham and Women's Hospital-affiliated primary care practices. We included 3,149 patients with stages 3 or $4 \mathrm{CKD}$ (defined as two past estimated glomerular filtration rates (eGFR) $15-60 \mathrm{~mL} / \mathrm{min} / 1.73 \mathrm{~m} 2$ ) who also had at least one primary care visit during 2009. We examined the association of demographic characteristics and comorbidities with problem list documentation using the chi-squared test. We assessed the association of problem list documentation with five outcomes: 1) annual serum eGFR test, 2) annual urine protein test, 3) an ACE/ARB prescription, 4) mean systolic blood pressure, and 5) blood pressure control $<130 / 80 \mathrm{mmHg}$. We used multivariable logistic regression to adjust for confounders.

RESULTS: Only 496/3149 patients had CKD on the problem list (16\%). Problem list documentation was more likely in patients with lower mean eGFR ( 38 vs. $48 \mathrm{~mL} / \mathrm{min} / 1.73 \mathrm{~m} 2)$, male gender ( $53 \%$ vs. $32 \%$ ), black race ( $29 \%$ vs. $22 \%)$, hispanic ethnicity $(10 \%$ vs. $8 \%)$, diabetes $(40 \%$ vs. $25 \%)$, or hypertension ( $78 \%$ vs. $65 \%)$. We performed a multivariate analysis of race/ ethnicity and problem list documentation and found that there was no significant association after controlling for diabetes and hypertension. Problem list documention was significantly positively associated with annual serum eGFR measurement before ( $98 \%$ vs. $93 \%, \mathrm{p}<0.0001)$ and after adjustment for eGFR, gender, race, diabetes and hypertension ( $98 \%$ vs. $95 \%, \mathrm{p}=0.03)$, annual urine protein measurement before $(48 \%$ vs. $29 \%, \mathrm{p}<0.0001)$ and after adjustment $(49 \%$ vs. $42 \% \mathrm{p}=0.04)$, as well as ACE/ARB prescription before $(75 \%$ vs. $64 \%, \mathrm{p}<0.0001)$ and after adjustment $(82 \%$ vs. $77 \%, \mathrm{p}=0.02)$. Problem list documentation was not associated with mean blood pressure or blood pressure control in unadjusted or adjusted analyses.

CONCLUSIONS: Problem list documentation was poor for chronic kidney disease. Severity of disease, demographic characteristics, and comorbidities were associated with problem list documentation. We found that patients with $\mathrm{CKD}$ on the problem list were more likely to undergo monitoring of serum eGFR and to receive ACE/ARB, but they were not more likely to have blood pressure under control. In the future, clinical decision support may improve recognition of CKD, for example by automatically flagging patients with sustained low eGFR, and may also improve stage-appropriate monitoring and treatment for CKD.

DOES IMPROVING THE EDUCATIONAL ENVIRONMENT REDUCE ADOLESCENT SUBSTANCE USE AND EXPOSURE TO SUBSTANCE-USING PEERS? RESULTS FROM A NATURAL EXPERIMENT. Mitchell D. Wong ${ }^{1}$; David Kennedy ${ }^{2}$; Martin F. Shapiro ${ }^{1}$; Richard Buddin ${ }^{2}$; Paul Chung ${ }^{3}$; Rebecca Dudovitz ${ }^{3}$; Arleen Brown ${ }^{1}$; Karen Coller ${ }^{1} .{ }^{1}$ David Geffen School of Medicine at UCLA, Los Angeles, CA; ${ }^{2}$ RAND, Los Angeles, CA; ${ }^{3}$ David Geffen School of Medicine at UCLA, Los Angeles, CA. (Control ID \#1340138)

BACKGROUND: The link between wealth and health is well documented in observational studies, but few randomized trials have been conducted to improve health through interventions targeting socioeconomic status. Despite the obvious challenges of trying to improve socioeconomic conditions, our nation's public schools may be one of the few social institutions with the potential to create large, long-term reductions in poverty and socioeconomic disparities. Encouragingly, innovative educational programs have recently demonstrated remarkable results in improving educational achievement among low-income minorities, suggesting that reducing poverty through education may be more possible than commonly believed. This study examines whether adolescents randomly selected to attend an academically successful charter high school report lower substance use, and less exposure to substance-using peers, compared to control school students attending poorly performing public high schools. METHODS: This study takes advantage of a natural experiment in Los Angeles in which students applied to attend one of the three high-performing charter high schools and were randomly selected via a lottery admissions process. The charter schools in our study, which are located in very low income neighborhoods in Los Angeles, have graduation rates around 90\% compared to $30-50 \%$ in nearby public schools. Analyses compare 498 students selected to attend a charter school (Experimental Group) vs. 437 who were not selected (Control Group). Students were excluded from analyses if they went to other charter or private schools. Intent-to-treat analyses were used to compare the two groups on their personal social network characteristics (based on naming 20 network members and then answering follow-up questions about each network member to identify same-aged peers and substance use by network members), as well as their own past month alcohol, cigarette, and drug use. RESULTS: Students in the Experimental and Control Groups attended schools with an average California standardized test score of 751 and 685, respectively (Academic Performance Index score 0-1000 scale with higher scores indicating better performance). The two groups were similar in terms of race/ethnicity, gender, language preference, and parental education and income. The Experimental Group reported a greater number of same-aged peers in their network who drank (6.10 vs. 5.50, p<.001) and used drugs (3.83 vs. $3.27, \mathrm{p}<.001)$ compared to the Control Group. However, there were no group differences with respect to students' 30-day rates of smoking cigarettes $(11 \%$ vs. $9 \%, \mathrm{p}=0.31)$, alcohol use ( $33 \%$ vs. $34 \%, \mathrm{p}=0.93)$, and marijuana use ( $21 \%$ vs. $21 \%, p=0.91$ ). An important caveat to these findings is that the Control Group was more likely to refuse participation $(27.7 \%$ vs. $18.1 \%)$ and difficult to find (20.2\% vs. 9.6\%) compared to the Experimental Group.

CONCLUSIONS: Although being in more academically rigorous and successful school environment was associated with greater exposure to 
peer network alcohol and drug use, students in this environment were not more likely to engage in substance use. Although limited by differential consent and participation rates, results from this study suggest that improving an adolescent's educational environment might buffer students from pro-drug network influences.

DOES PRIMARY CARE MANAGEMENT OF CHRONIC KIDNEY DISEASE DIFFER FROM CO-MANAGEMENT WITH NEPHROLOGY? Lipika Samal; Adam Wright; Joseph V. Bonventre; Jeffrey A. Linder. Brigham and Women's Hospital, Boston, MA. (Control ID \#1319268)

BACKGROUND: Primary care physicians (PCPs) commonly manage early stage chronic kidney disease (CKD). We sought to determine whether patients managed solely by PCPs receive better or worse quality care than patients co-managed with nephrologists.

METHODS: We obtained data on 3,149 adults with stages 3 or 4 CKD (defined as two estimated glomerular filtration rates [eGFR] $15-60 \mathrm{~mL} / \mathrm{min}$ $1.73 \mathrm{~m} 2$ measured three months apart) with at least one visit to a PCP in the Brigham and Women's Primary Care Practice-Based Research Network practice during 2009. We identified patients managed solely by a PCP and patients who had at least one visit with a nephrologist and compared number of PCP visits, demographic characteristics, eGFR value, and co-morbidities between the two groups. We assessed the relationship of nephrology comanagement with five quality measures: 1 ) annual serum eGFR test, 2) annual urine protein test, 3) ACE/ARB prescription, 4) mean systolic blood pressure, and 5) blood pressure control $(<130 / 80 \mathrm{mmHg})$. For each measure, we used multivariable logistic or linear regression to adjust for confounding factors and we present adjusted percentages and means.

RESULTS: Fourteen percent of patients (453/2923) adults with stage 3 and 4 CKD were co-managed with a nephrologist. Solo PCP management was associated with older patient age ( 75 vs. 68 years), higher eGFR ( 48 vs. $35 \mathrm{~mL}$ $\mathrm{min} / 1.73 \mathrm{~m} 2$ ), fewer PCP visits (3.9 vs. 4.8$)$, female gender ( $66 \%$ vs. $54 \%$ ), white race $(68 \%$ vs. $52 \%)$, less diabetes ( $25 \%$ vs. $43 \%)$, and less hypertension ( $66 \%$ vs. $74 \%$; all comparisons, $\mathrm{p}<0.01)$. PCP solo management was significantly associated with less annual serum eGFR testing $(93 \%$ vs. $100 \%$, $\mathrm{p}<0.0001$ ), a relationship that persisted after adjustment for number of PCP visits, age, gender, race, eGFR, diabetes, and hypertension ( $96 \%$ vs. $100 \%$, $\mathrm{p}=$ 0.002). Solo PCP management was associated with less annual urine protein testing before $(25 \%$ vs. $74 \%, \mathrm{p}<0.0001)$ and after adjustment $(35 \%$ vs. $80 \%$, p < $0.0001)$. Solo PCP management was associated with fewer patients given an ACE/ARB prescription $(63 \%$ vs. $77 \%, p<0.0001)$, but not after multivariable adjustment $(74 \%$ vs. $78 \%, p=0.13)$. Solo PCP versus nephrology comanagement was not associated with a difference in mean systolic blood pressure in unadjusted analysis ( $133 \mathrm{mmHg}$ vs. $132 \mathrm{mmHg}, \mathrm{p}=0.32)$, but after adjustment the systolic blood pressure of co-managed patients was significantly lower $(131 \mathrm{mmHg}$ vs. $129 \mathrm{mmHg}, \mathrm{p}=0.04)$. Solo PCP versus nephrology comanagement was not associated with likelihood of BP control $<130 / 80 \mathrm{mmHg}$ in crude $(43 \%$ vs. $47 \%, \mathrm{p}=0.09)$ or adjusted analyses $(49 \%$ vs. $53 \%, \mathrm{p}=0.17)$. CONCLUSIONS: Though only $14 \%$ of patients were co-managed with nephrology, quality of care for primary care patients with CKD was generally better with nephrology co-management. Specifically, nephrology co-management was associated with better stage-appropriate monitoring and marginally lower mean blood pressure, but not a higher rate of blood pressure control. To improve the quality of care for CKD patients, we should develop and evaluate strategies - for example, education, feedback, or clinical decision support - to facilitate referral and co-management with nephrology as well as improve stage-appropriate monitoring and treatment in primary care.

DOES PROVIDING ACCESS TO SPECIALTY CARE TO AN UNINSURED POPULATION REDUCE ED UTILIZATION? Lauren Block ${ }^{1}$; Sai $\mathrm{Ma}^{2}$; Matthew Emerson ${ }^{3}$; Anne Langley ${ }^{3}$; Desiree M. de la Torre ${ }^{3}$; Gary Noronha ${ }^{4} .{ }^{1}$ Johns Hopkins University School of Medicine, Baltimore, MD; ${ }^{2}$ Johns Hopkins Bloomberg School of Public Health, Baltimore, MD; ${ }^{3}$ Johns Hopkins Medicine, Baltimore, MD; ${ }^{4} \mathrm{Johns}$ Hopkins Community Physicians, Baltimore, MD. (Control ID \#1321075)

BACKGROUND: Nationally, the annual number of emergency department (ED) visits increased 23\% between 1997 and 2007. Higher utilization rates of ED services by the uninsured has been identified as a possible contributor to this increase. We hypothesized that care coordination and access to specialty care services provided by The Access Partnership (TAP) program would reduce ED utilization among uninsured patients with a usual source of primary care. We further hypothesized that while rates of inpatient admission would not change significantly, TAP patients would have fewer "non-necessary" ED visits that did not result in admission.

METHODS: We conducted a retrospective cohort study to examine rates of monthly ED utilization and inpatient admission among TAP patients and a comparison group of eligible patients who were referred to the program but did not enroll. Administrative data on ED visits and inpatient hospitalization was used to examine monthly ED utilization and inpatient admission rates for both groups before referral to TAP and subsequent to referral, within a study period 2007-2011. Difference-in-differences estimation and multivariate linear regression were used to compare TAP and non-TAP patients, while adjusting for demographic and socioeconomic factors.

RESULTS: During the first 21 months of the program, 673 patients were referred to TAP. 374 patients joined the program (the treatment group), and the other 299 patients did not join the program (the comparison group). Rates of ED visits per month increased in both groups, from 0.039 to 0.051 (difference post-pre $=0.012$ ) visits per month among TAP patients and 0.057 to 0.083 (difference post-pre $=0.026$ ) visits per month among non-TAP patients. Difference-in-difference of monthly ED use comparing TAP with non-TAP patients was $0.014(p=0.15)$. Among heavy users with at least one visit per three months, ED utilization declined among TAP patients (difference post-pre $=-0.012$ ) and increased among non-TAP patients (difference $=$ $0.022, \mathrm{p}=0.16$ ). Among the subgroup of ED visits that did not result in inpatient admissions, the number of visits per month was relatively constant among TAP patients (difference post-pre $=0.006$ ), whereas the rate increased among non-TAP patients (difference $=0.023$ ). Difference-in-difference comparing TAP with non-TAP patients was $0.017(\mathrm{p}=0.05)$. Non-TAP status was a moderate predictor of ED visits not leading to admission, after controlling for age, gender, and zip code $(\mathrm{t}=2.01, \mathrm{p}=0.045)$.

CONCLUSIONS: Our evaluation finds some promising evidence that a program combining access to specialty care services with care coordination may help shift utilization patterns relative to a comparison group of patients who did not enroll in the program. A longer study period may help obtain stronger indications of decreased ED use.

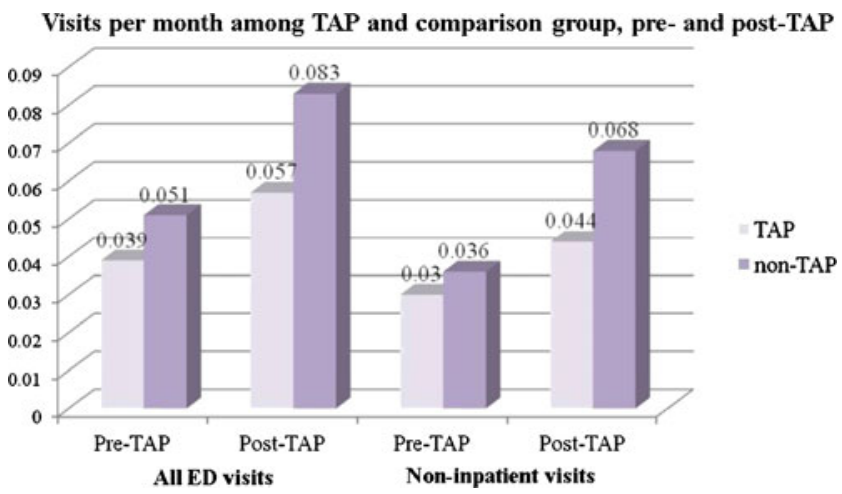

DOES THE KNOWLEDGE OF PATIENT READMISSIONS AFFECT HOSPITALISTS' ATTITUDES OR BEHAVIOR? Jennifer E. Bracey ${ }^{1,4}$; Scott Wright ${ }^{2,4}$; Regina Landis ${ }^{1}$; Romsai T. Boonyasai ${ }^{3,4}$. ${ }^{1}$ Johns Hopkins Bayview Medical Center, Baltimore, MD; ${ }^{2}$ Johns Hopkins Bayview Medical Center, Baltimore, MD; ${ }^{3}$ Johns Hopkins University School of Medicine, Baltimore, MD; ${ }^{4}$ Johns Hopkins University School of Medicine, Baltimore, MD. (Control ID \#1338968)

BACKGROUND: Hospitalists are often not aware when a patient for whom they have provided care is rehospitalized. Yet, there may be much to learn from readmissions, both in terms of clinical care and systems improvement. To address this concern, we conducted an intervention to inform hospitalists when their patients were readmitted. We hypothesized 
that doing so would improve hospitalists' clinical care and communication behaviors.

METHODS: This IRB approved intervention was conducted at a 365 bed teaching hospital. Over a 12 week period, all hospitalists involved with a patient's care (admission, in-house follow-up care, or discharge) were electronically notified if the patient was rehospitalized within 30 days of discharge from the hospitalist service. The electronic notification linked to a website where a research assistant had uploaded clinical summaries and patients' responses to a bedside interview conducted during their rehospitalization. We evaluated this intervention by surveying participants' attitudes and self-reported behaviors before and after the intervention. All responses were collected on a 5-point Likert scale. For analytic purposes, we dichotomized responses to compare the 2 highest responses ("always" and "often" or "strongly agree" and "agree") with the remaining responses. Comparisons were analyzed using Chi-square tests.

RESULTS: During the study period, 194 notifications involving 82 rehospitalized patients were sent. Hospitalists viewed 134 of these notifications $(68 \%)$. Twenty-seven hospitalists $(100 \%)$ at our institution completed the pre- and postintervention surveys. Thirteen (48\%) were male and $20(74 \%)$ had worked within the hospitalist group for $>1$ year. Results from the survey are shown in Table I. CONCLUSIONS: Self-reported care coordination practices, including communication with patients, PCP's, and nurses improved following this intervention. However, hospitalists' attitudes and behaviors related to giving and receiving feedback about their clinical practice did not change. Yet, hospitalists could learn from one another. Future provider notification systems of patient readmissions should foster the sharing of best practices and feedback amongst colleagues.

Table I

Variable

Usually or always contact the primary care provider (PCP) after patient's discharge Usually or always ask patient to repeat what you $3(11) \quad 7(27) \quad 0.04$
discussed at discharge

Usually or always discuss discharge plan with $\quad 14$ (52) 20 (77) $\quad 0.05$ patient's nurse

Usually or always communicate with

readmitting provider

Usually or always ask for feedback from

$11(41) \quad 5(19) \quad 0.01$

colleague about the medical care you provided

Usually or always give feedback to colleague

about the medical care they provided

A lot or complete comfort in giving feedback to 8 (29) $\quad 6(23) \quad 0.13$

colleague

A lot or complete comfort in asking for feedback 12 (45) 13 (50) 0.09

from colleague

A lot or complete confidence that nursing will $\quad 10$ (37) 14 (54) 0.05 complete assigned task
DUCTAL CARCINOMA IN SITU: KNOWLEDGE OF ASSOCIATED RISKS AMONG LATINAS AND NON-LATINA WHITES Leah Karliner $^{1,2}$; Anna M. Napoles ${ }^{1,2}$; Celia Kaplan ${ }^{1,2} .{ }^{1}$ UCSF, San Francisco, CA; ${ }^{2}$ UCSF, San Franicsco, CA. (Control ID \#1334627)

BACKGROUND: Since the advent of mammography screening for breast cancer, ductal carcinoma in situ (DCIS) has become a common diagnosis, accounting for almost one-third of breast cancers. While DCIS is not lifethreatening, it can progress to invasive disease if left untreated and does confer a higher risk of future breast cancer. Several treatment courses are possible and understanding the disease is crucial for women to deciding the optimal treatment course. Among women with DCIS, we investigated their knowledge of DCIS and whether knowledge differed by language and ethnicity.

METHODS: Telephone interviews of California Latina and non-Latina White women diagnosed with DCIS between 2002-2005. We examined participant's knowledge of DCIS with four true/false statements about DCIS. The four statements were: "This type of breast problem is not itself life-threatening", "Women with this type of breast problem have more chances of developing breast cancer in the future", "If untreated, this type of breast problem can become invasive cancer", and "The chances of dying from the breast problem are the same for women who have a mastectomy and for those who have a lumpectomy with radiation". We modeled the odds of giving a correct answer for each question by ethnicity-language (Latina English-speakers, Latina Spanish-speakers, white English-speakers) and surgical treatment type (lumpectomy or mastectomy), adjusting for family history of breast cancer, educational attainment, age, insurance, geographic region in California, time since diagnosis to interview, and having sought out a second-opinion.

RESULTS: Of 710 participants, $52 \%(\mathrm{n}=368)$ were white English-speakers, $21 \%(n=152)$ Latina English-speakers, and 27\% ( $n=190)$ Latina Spanishspeakers. Overall, $67 \%$ had undergone lumpectomy and 33\% mastectomy. Less than half $(41 \%)$ of participants were aware that DCIS is not lifethreatening and only $32 \%$ knew that mortality risk is the same for mastectomy and lumpectomy plus radiation; whereas two-thirds (67\%) were aware that DCIS confers increased risk of future breast cancer, and almost all (92\%) knew that it could become invasive if not treated. In adjusted analyses, compared to White English-speakers, both Latina English- and Spanish-speakers had significantly lower odds of knowing that their DCIS was not life-threatening. In contrast, compared to white English-speakers, Latina Spanish-speakers had more than two-fold higher odds of knowing that DCIS increases risk of future breast cancer, but English-speaking Latinas were no different from Englishspeaking whites. Surgical treatment type was not associated with knowledge. CONCLUSIONS: Our data suggest that physicians diagnosing and treating women with DCIS are more successful at conveying the risks conferred by DCIS than the nuances of the difference between DCIS and invasive cancer. This uneven communication is most marked for Spanish-speaking Latinas. Efforts are needed to create culturally and linguistic standardized information for DCIS patients.

\begin{tabular}{|l|l|l|l|l|}
\hline & $\begin{array}{l}\text { Not Itself Life- } \\
\text { Threatening }\end{array}$ & $\begin{array}{l}\text { More Chances of } \\
\text { Future Breast } \\
\text { Cancer }\end{array}$ & $\begin{array}{l}\text { If Untreated, } \\
\text { Can Become } \\
\text { Invasive }\end{array}$ & $\begin{array}{l}\text { Recurrence same } \\
\text { for mastectomy } \\
\text { and lumpectomy + } \\
\text { radiation } \\
\text { OR }(95 \% \mathrm{Cl})\end{array}$ \\
\hline OR $(95 \% \mathrm{Cl})$ & OR $(95 \% \mathrm{Cl})$ & & \\
\hline $\begin{array}{l}\text { Ethnicity- } \\
\text { Language }\end{array}$ & Reference & Reference & Reference & Reference \\
White English & $\mathbf{0 . 6 ( 0 . 4 - 0 . 9 )}$ & $1.0(0.6-1.4)$ & $0.9(0.4-1.8)$ & $1.1(0.7-1.7)$ \\
Latina English & $\mathbf{0 . 6 ( 1 . 6 - 4 . 4 )}$ & $0.7(0.3-1.6)$ & $1.2(0.7-1.9)$ \\
Latina Spanish & $\mathbf{0 . 5 ( 0 . 3 - 0 . 9 )}$ & $\mathbf{2 . 6})$ & \\
\hline
\end{tabular}

DUPLICATE FEDERAL EXPENDITURES FOR DUAL ENROLLEES IN MEDICARE ADVANTAGE PLANS AND THE VETERANS AFFAIRS HEALTH CARE SYSTEM Amal N. Trivedi ${ }^{1,2}$; Regina C. Grebla $^{2}$; Lan Jiang ${ }^{2}$; Jean Yoon ${ }^{3}$; Vincent $\overline{\text { Mor }^{1,2} \text {; Kenneth Kizer }}{ }^{4} .{ }^{1}$ Alpert Medical School of Brown University, Providence, RI; ${ }^{2}$ Providence VA Medical Center, Providence, RI; ${ }^{3}$ Palo Alto VA Medical Center, Palo Alto, CA; ${ }^{4}$ UC Davis, Sacramento, CA. (Control ID \#1339259)
BACKGROUND: When eligible adults simultaneously enroll in a Medicare Advantage (MA) plan and the Veterans Affairs health care system (VA), MA plans receive federal subsidies to insure veterans who receive health services from another taxpayer-funded health system. Using national data from 2004 to 2009 , we quantified the prevalence of dual enrollment in VA and MA, the concurrent use of medical and surgical services in each setting, and the costs of Medicare-covered services incurred by the VA to care for MA enrollees. 
METHODS: We merged VA and MA administrative data to derive the national population of veterans with at least one month of simultaneous enrollment in an MA plan during the study period. To estimate the cost of VA services, we used VA HERC average cost methods and the VA's direct payments for third party and pharmaceutical care. We inflationadjusted all cost estimates using the CPI-U. We determined the demographic characteristics and use of services among: (1) exclusive VA users, (2) VA - MA dual users, (3) exclusive MA users, and (4) non-users. We used Chi-square and ANOVA tests to determine whether characteristics differed among these groups. For each dual user, we calculated the proportionate reliance on VA inpatient and outpatient care. A sensitivity analysis excluding $9 \%$ of beneficiaries who enrolled in their MA plan after January or exited their MA plan prior to December yielded similar findings.

RESULTS: The number of persons concurrently enrolled in the VA and Medicare Advantage increased from 485,651 in 2004 to 924,792 in 2009. Over $60 \%$ of dual enrollees used VA health care services each year. In $2009,11 \%$ of all VA enrollees and $8 \%$ of all MA enrollees were dually enrolled. The total inflation-adjusted cost of VA care for MA enrollees was $\$ 12.4$ billion over 6 years, increasing from $\$ 1.0$ billion to $\$ 3.2$ billion. Among VA users enrolled in MA plans, the mean annual non-drug spending increased from $\$ 3,151$ in 2004 to $\$ 4,703$ in 2009. Among dual enrollees, $10 \%$ exclusively used the VA for outpatient and acute inpatient services, $38 \%$ exclusively used the MA plan, $48 \%$ used both the VA and MA, and 3\% received no services. Compared to dual users and exclusive MA users, exclusive VA users were more likely to be black, reside in the south, and have more intensive use of outpatient visits and acute hospital care ( $p<0.001$ for each comparison). The VA financed $45 \%$ of outpatient visits, $19 \%$ of acute medical admissions, $24 \%$ of acute medical hospital days, $11 \%$ of acute surgical admissions, and $10 \%$ of acute surgical inpatient days. In 2009, the VA submitted collection requests totaling $\$ 52.3$ million on behalf of care provided to MA enrollees. Of these requests, the VA collected $\$ 9.4$ million from MA plans, representing $0.3 \%$ of the total cost of care for this population.

CONCLUSIONS: From 2004 to 2009, the VA spent a substantial and rapidly increasing amount of funds to care for enrollees in MA plans. Because the federal government simultaneously pays MA plans to provide comprehensive care, policymakers should consider measures to identify and eliminate these duplicative expenditures.

DUTY HOURS 2.0: EFFECT OF THE ACGME 16-HOUR RULE ON QUALITY AND EFFICIENCY OF CARE Neesha N. Choma; Eduard E. Vasilevskis; Kelly C. Sponsler; Jacob Hathaway; Daniel G. Stover; Cecelia Theobald; Jennifer Green; Joshua Denny; Shea Polancich; Neeraja B. Peterson; Sunil Kripalani. Vanderbilt University Medical Center, Nashville, TN. (Control ID \#1324596)

BACKGROUND: Effective July 1, 2011, the Accreditation Council for Graduate Medical Education reduced interns' maximum consecutive hours of duty from 30 to 16. Vanderbilt's Internal Medicine Residency Program instituted 16-hour duty limits for all residents in April, 2011. We compared the quality and efficiency of care delivered to non-intensive care unit (ICU) medical inpatients under the 30 -hour and 16-hour duty limits.

METHODS: We defined two cohorts of patients admitted to and discharged from six internal medicine teaching services at Vanderbilt University Hospital, Nashville, TN, between July 1 and September 30, 2010 (30-hour cohort) and July 1 and September 30, 2011 (16-hour cohort). Data were extracted from the Vanderbilt Enterprise Data Warehouse (EDW) and the Rapid Response Team (RRT) Database. The EDW is a relational electronic data repository of clinical and administrative information and the RRT database is a manually maintained database of demographic and RRT/code event specific information. We compared the cohorts on indices of hospital continuity (number of handovers per week); efficiency (adjusted length of stay [LOS]); and the quality and safety of care delivery (30-day readmissions to the same facility, observed to expected mortality, in-hospital rapid response and code events, escalations of care to an ICU, and adverse events). Adverse events were defined by the number of Agency for Healthcare Research and Quality (AHRQ) patient safety indicators and University HealthSystem Consortium (UHC) complications. Nonparametric statistical tests were conducted to compare the quality and efficiency outcomes in the two groups.

RESULTS: The 30-hour cohort included 987 patients and the 16-hour cohort included 903 patients. The 30-hour and 16-hour groups were similar in terms of age (median 53 vs. 54 years), gender (51\% vs. $50 \%$ male), and payor mix ( $78 \%$ vs. $77 \%$ Medicare or commercial insurance). The median all patient refined diagnosis related group (APR-DRG) risk of mortality was 2 (interquartile range [IQR] 1,3) in the 30-hour cohort and 3 (IQR 2,3 ) in the 16-hour cohort. The structural changes made to accommodate the 16-hour duty rule more than doubled the total number of weekly handovers across the six medicine teams (41 to 95). Adjusted LOS in days did not differ significantly ( 4.34 for 30 -hour vs. 4.65 for 16 -hour, $\mathrm{p}=0.33$ ). No differences were seen in AHRQ patient safety indicators (5 vs. 1 per 1000 patients, $p=0.13$ ); UHC complications (34 vs.30 per 1000 patients, $\mathrm{p}=0.58)$; observed to expected mortality ( 0.23 vs. $0.3, \mathrm{p}=0.9)$; or all-cause 30 -day readmission $(19.55 \%$ vs. $19.05 \%, p=0.75)$. While there was no significant difference in the number of rapid response team calls ( 26 vs. 38 per 1000 patients, $p=0.18)$ and codes $(1$ vs. 3 per 1000 patients, $p=0.28$ ), there was an increase in the number of escalations of care to an ICU ( 9 vs. 23 per 1000 patients, $p=0.01)$ in the 16-hour cohort.

CONCLUSIONS: Despite the shortened resident duty hours and increased number of total handovers of care, we observed no statistically significant difference in the efficiency or quality of care provided to non-ICU medical inpatients. The finding of increased care escalations to an ICU needs to be further monitored from both a quality and utilization perspective. This may be due to temporal trends towards higher acuity of patients as reflected by APR-DRG risk of mortality or may represent a more proactive escalation of care.

E-LEARNING AND DELIBERATE PRACTICE FOR ORAL CASE PRESENTATION SKILLS: A RANDOMIZED TRIAL Heather L. Heiman ${ }^{1,2}$; Toshiko Uchida ${ }^{1,2}$; Craig Adams ${ }^{4}$; John Butter ${ }^{1,2}$; Elaine Cohen $^{1}$; Stephen D. Persell ${ }^{1}$; Paul Pribaz ${ }^{4}$; William C. McGaghie ${ }^{3}$; Gary J. Martin ${ }^{1}$. ${ }^{1}$ Northwestern University Feinberg School of Medicine, Chicago, IL; ${ }^{2}$ Northwestern University Feinberg School of Medicine, Chicago, IL; ${ }^{3}$ Northwestern University Feinberg School of Medicine, Chicago, IL; ${ }^{4}$ Northwestern University Feinberg School of Medicine, Chicago, IL. (Control ID \#1338669)

BACKGROUND: The oral case presentation is an essential clinical skill. A competent presentation allows for efficient transfer of information between providers, and it permits evaluation of students' clinical reasoning. Medical students express anxiety about their oral presentation skills. They seek a rule-based structure to use in developing their presentations. We therefore aimed to develop and rigorously evaluate an oral presentations curriculum using on-line learning and deliberate practice to better prepare second year medical students for clinical clerkships.

METHODS: We developed a web-based, interactive oral presentation curriculum emphasizing conciseness and clinical reasoning. The curriculum consists of two components: 1) an on-line, interactive module followed by 2) deliberate practice of case presentations with detailed checklist-based feedback from a fourth-year medical student coach. To permit standardized feedback and assessment, we created a set of video cases with accompanying written physical exam reports for students to use as the basis for their presentations. Chief concerns for all cases were common primary care symptoms. Using a waitlist control design, groups of second year students were randomly assigned to receive the curriculum in December 2010 (intervention group) or in April 2011 (waitlist control group). Medical students completed presentation assessments at baseline, at a midpoint when half had taken the curriculum, and at a final point when all students had taken the curriculum. Performance of a class of students who did not receive the curriculum was also examined as a historical comparison.

RESULTS: One hundred thirty-two second-year medical students (67 in the intervention group and 65 in the waitlist control group) were evaluated 
at the three time points. At the midpoint evaluation, mean scores of the intervention students improved from $60.2 \%$ to $70.1 \%$, while scores of the waitlist control students improved from $61.8 \%$ to $64.5 \%$ ( $p<0.01$ for between-group difference in improvement). At the final evaluation, the mean scores for the intervention and waitlist control students rose to $77.8 \%$ and $78.4 \%$, respectively, as compared to $68.1 \%$ for the 142 untrained comparison students $(\mathrm{p}<0.0001$ compared to all curriculum students). Inter-rater reliability across the content portions of the checklists was substantial at 0.73 (range $0.61-0.81$ ).

CONCLUSIONS: This study demonstrates that a curriculum of online learning followed by deliberate practice improved performance on a reliable assessment of oral presentation skills. The curriculum is sustainable, potentially exportable, and can be used to ensure that early medical students demonstrate competence in the case presentation before moving to the clinical environment. .

EARLY VS. LATE ACCESS TO PALLIATIVE CARE CONSULTATION: DOES IT MAKE A DIFFERENCE? Jessi Humphreys; Stephanie Harman. Stanford University, Stanford, CA. (Control ID \#1333817)

BACKGROUND: Palliative care services in the United States are increasing in their prevalence but continue to vary in their implementation, with different approaches to palliative care team composition, referral policies and patient access to services. Stanford Hospital's Palliative Care Service currently depends on referrals from inpatient attending physicians, with no current policies or triggers guiding when patients are referred to palliative care. As a result, there is potential for large variability in the time similar patients may wait before being referred to palliative care. For patients who receive a palliative care consult, it is important to understand whether the timing of referrals has an impact on patient outcomes, as this can help optimize care and guide hospital policies. While some studies have found lower quality of care associated with later referrals to hospice, it is currently unknown what impact the timing of inpatient palliative care consultation has on patient outcomes.

METHODS: A retrospective analysis was done on patients with pre-existing oncologic diagnoses who received a palliative care consultation $(n=1249)$ since the establishment of Stanford Hospital's Palliative Care Service. Multiple linear \& logistic regression analyses were applied to data to look at the impact of timing of referral on patient outcomes.

RESULTS: Those oncologic patients referred to palliative care in the first week following admission had shorter lengths of stay, a greater desire to limit aggressive interventions and lower in-hospital mortality as compared to patients referred after one week. Groups were similar in terms of DNR status, level of sickness as measured by number of recent inpatient admissions, and demographic variables, except later referrals tended to be younger and were more likely to be white. Groups differed significantly in time-to-consultation and length-of-stay following consultation. Regression analyses, adjusted for demographic variables, DNR status and sickness revealed that for each day palliative care consultation was delayed, length-of-stay following the consult was associated with an increase of 0.22 days $(\mathrm{P}<0.001)$. Waiting one week or more to refer a patient was associated with an overall increased length-of-stay of 2.76 days $(\mathrm{P}<$ $0.001)$, which increased to 5.27 days $(\mathrm{P}<0.001)$ when patients who died in-hospital were removed from the data, suggesting in-hospital mortality was not independently driving the trend. Waiting one week or more to refer was associated with increased odds of a patient dying in the hospital (vs. being discharged alive) by a factor of 3.15 ( $\mathrm{P}<$ 0.001). Additionally, if a patient was referred in the first week, the odds of the patient deciding to limit aggressive interventions increased significantly (OR:1.7; $\mathrm{P}<0.001)$.

CONCLUSIONS: Recent studies have shown that decreased hospital length-of-stay for advanced cancer patients has been linked to increased patient quality of life, decreased hospital-acquired infection rates and decreased patient and hospital cost. If palliative care referral timing has the capacity to impact length-of-stay, and allow for potentially improved care and patient quality of life at lower cost, this argues for the design and implementation of hospital policies that encourage early referral to palliative care for advanced cancer patients.
EDUCATOR-ASSESSED SALT REDUCTION KNOWLEDGE, BUT NOT SELF-REPORTED SALT REDUCTION IS ASSOCIATED WITH REDUCED HEART FAILURE HOSPITALIZATION Christine D. Jones ${ }^{1}$; George M. Holmes ${ }^{2,4}$; Darren A. DeWalt ${ }^{3,4}$; Brian $\overline{\text { Erman }^{4} \text {; }}$ Victoria Hawk $^{4}$; Kimberly Broucksou ${ }^{4}$; Crystal Cene ${ }^{3,4}$; Jia-Rong $\mathrm{Wu}^{5}$; David W. Baker ${ }^{6}$; Dean Schillinger ${ }^{7}$; Bernice Ruo ${ }^{6}$; Kirsten BibbinsDomingo $^{7}$; Aurelia Macabasco-O'Connell ${ }^{8}$; Michael Pignone ${ }^{3,4}$. ${ }^{1}$ University of North Carolina at Chapel Hill, Chapel Hill, NC; ${ }^{2}$ University of North Carolina at Chapel Hill, Chapel Hill, NC; ${ }^{3}$ University of North Carolina at Chapel Hill, Chapel Hill, NC; ${ }^{4}$ University of North Carolina at Chapel Hill, Chapel Hill, NC; ${ }^{5}$ University of North Carolina at Chapel Hill, Chapel Hill, NC; ${ }^{6}$ Northwestern University, Chicago, IL; ${ }^{7}$ San Francisco General Hospital, University of California San Francisco, San Fracisco, $\mathrm{CA} ;{ }^{8}$ University of California Los Angeles, Los Angeles, CA. (Control ID $\# 1334412$ )

BACKGROUND: Patients participating in HF self-care programs are often educated about the importance of salt reduction. Yet, whether salt reduction knowledge or self-reported salt reduction is associated with HFrelated outcomes is uncertain. We sought to measure the relationship between both educator-assessed salt reduction knowledge and self-reported salt intake reduction with HF hospitalizations.

METHODS: We conducted a prospective cohort study among participants who underwent intensive HF self-care training as part of a 4-site randomized trial. All participants received a 40-minute in-person training session followed by one year of ongoing telephone-based support and education on topics including salt reduction. Educator-assessed salt reduction knowledge was evaluated by a clinical educator through structured telephone assessment. Patients were considered to have achieved mastery of salt reduction knowledge once they correctly answered a series of nine questions on two separate attempts that included recognizing the effects of salt on the body, reading sodium content on food labels, and identifying foods high and low in salt. Self-reported salt intake reduction was assessed at 12 months using the following question: "“"How successful have you been at sticking to your plans to eat foods that are low in salt?" Patients who indicated that they were "very" successful were categorized as having salt reduction success; those who indicated that they were "somewhat" or "not at all" successful were categorized as not having salt reduction success. We evaluated the outcome of HF hospitalization over 1 year; HF hospitalizations were determined by a masked adjudication committee. We used negative binomial regression to examine the relationship between self-reported and then educator-assessed mastery of salt reduction with the incidence of $\mathrm{HF}$ hospitalization, adjusted for study site, NYHA class, subjective SES, age, gender, race, literacy, HF related quality of life, systolic dysfunction, chronic kidney disease (GFR $<60 \mathrm{~mL} / \mathrm{min}$ ), hypertension, coronary heart disease, betablocker use, and ACE inhibitor or ARB use.

RESULTS: Among 303 participants over one year, we identified $74 \mathrm{HF}$ hospitalizations. Participants' mean age was 61 years, $52 \%$ were male, $32 \%$ had NYHA Class III or IV at enrollment, and $39 \%$ were African American. At 12 months, $42 \%$ of participants who completed a survey (122 of 291) selfreported success with salt intake reduction and $76 \%$ of participants ( 230 of 303 ) had achieved educator-assessed mastery of salt reduction knowledge. We found that participants who attained educator-assessed mastery of salt reduction knowledge had an incidence rate ratio (IRR) of $0.31(0.21,0.46)$ for HF hospitalizations compared to those who did not attain mastery. Participants who reported success with salt intake reduction had an IRR of $1.11(0.38,3.22)$ for HF hospitalizations compared with those who did not report success with salt. CONCLUSIONS: We found that educator-assessed mastery of salt reduction knowledge, but not self-reported success with salt intake reduction, was associated with fewer HF hospitalizations. Mastery of how to reduce salt intake may be an important goal for self-management training programs.

EFFECT OF ELIMINATING THE MEDICARE PART D COVERAGE GAP ON HEALTH OUTCOMES OF DIABETES AND CARDIOVASCULAR DISEASE Amal N. Trivedi ${ }^{1,2}$; Chang $\mathrm{Liu}^{1}$; Vincent Mor ${ }^{1,2}$; Alan Zaslavsky ${ }^{3,2}$; Lewis Kazis ${ }^{4}$; John Z. Ayanian ${ }^{3}$. ${ }^{1}$ Alpert Medical School of Brown University, Providence, RI; ${ }^{2}$ Providence VA Medical Center, Providence, RI; ${ }^{3}$ Harvard Medical School, Boston, MA; ${ }^{4}$ Boston University, Boston, MA. (Control ID \#1332271) 
BACKGROUND: Although the Affordable Care Act will phase out the Medicare Part D coverage gap over ten years at a projected total cost of $\$ 43$ billion, there is little empirical evidence about the health consequences of closing this coverage gap. Using a difference-in-difference design, we examined whether enrollees age 65 and older in Medicare Advantage (MA) plans that eliminated the Part D coverage gap experienced better health outcomes compared with concurrent trends for seniors in matched control plans that did not change drug benefits.

METHODS: We identified 15 "case" MA plans with continuous participation in Medicare from 2006-9 that eliminated the coverage gap for at least $90 \%$ of enrollees in either 2007 or 2008 . We matched each case plan to at least one control plan with continuous Medicare participation from 2006-9 that retained the coverage gap over this time period. Control plans $(n=28)$ were located in the same state or a neighboring state with the same tax-status and model type. Among enrollees ages 65-75, key outcomes derived from HEDIS measures were control of LDL cholesterol $<100 \mathrm{mg} / \mathrm{dl}$ among enrollees with diabetes and coronary heart disease, $\mathrm{HbA} 1 \mathrm{c}<9.0 \%$ among enrollees with diabetes, and blood pressure $<140 / 90 \mathrm{~mm} \mathrm{Hg}$ among enrollees with hypertension. We estimated generalized linear regression models for each outcome with indicators for whether the plan was a case or control, time (the year before or year after the addition of gap coverage), and their interaction. The models also adjusted for age, sex, race, area-level income, area-level education, plan fixed effects, and multiple observations from enrollees using GEE. Observations from control plans were weighted to reflect the sample size of their matched case plans.

RESULTS: The sample included 33,862 observations in case plans and 55,123 in control plans. The characteristics of enrollees in case plans were: mean age 71.6 years (SD 4.2 years), $51 \%$ female $87 \%$ white, $9 \%$ black, $3 \%$ other, $8 \%$ below poverty and $34 \%$ with college attendance. Characteristics of enrollees in control plans were: mean age 71.6 years (SD 4.4 years), $51 \%$ female $86 \%$ white, $12 \%$ black, $3 \%$ other, $9 \%$ below poverty and $30 \%$ with college attendance. None of these characteristics changed by more than 1 percentage point or 0.1 years between the year before and year after the coverage gap was eliminated. Trends in outcomes and adjusted estimates are shown in the Table and indicate no significant differences in the clinical outcomes.

CONCLUSIONS: We found no evidence that eliminating the coverage gap resulted in significantly improved control of blood pressure, cholesterol, and glucose for seniors with Part D drug coverage.

Table. Change in Alc. Cholesterol. and Blood Pressure Control in Case Plans that Added Gap Coverage and Control Plans that Did Not Provide Gap Coverage

\begin{tabular}{|c|c|c|c|c|c|c|c|c|}
\hline \multirow[t]{2}{*}{ Oufcome } & \multicolumn{3}{|c|}{ Case Plans } & \multicolumn{3}{|c|}{ Control Plans } & S.S & Adj. $\Delta-1 \%$ nts \\
\hline & $\begin{array}{l}\text { Yr before } \\
\text { adding gap } \\
\text { coversge. \% }\end{array}$ & $\begin{array}{l}\text { Yr after } \\
\text { adding } \\
\text { gap } \\
\text { coverage. } \\
\text { o. }\end{array}$ & $\begin{array}{l}\mathrm{A} .0 \\
\mathrm{pts}\end{array}$ & $\begin{array}{l}\text { Ye before } \\
\text { case plan } \\
\text { added gap } \\
\text { coverage. } \\
\text { \% }\end{array}$ & $\begin{array}{l}\text { Yr sfter } \\
\text { case plan } \\
\text { solded gap } \\
\text { coverage. } \\
\%_{6}\end{array}$ & 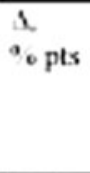 & & \\
\hline$\overline{A 10=90^{\circ}}$ & 790 & 80.7 & 1.7 & 81.4 & 81.3 & 0.1 & 1.8 & $\begin{array}{l}2.0 \\
(-2.0,6,1)\end{array}$ \\
\hline $\begin{array}{l}\text { L.DL }=100 \mathrm{mg} \text { dl } \\
\text { disabetes) }\end{array}$ & 526 & 55.1 & 2.5 & 52.9 & 57.9 & 50 & .2 .5 & $\begin{array}{l}.2 .3 \\
(-6.5,2.0)\end{array}$ \\
\hline $\begin{array}{l}\text { LDL } 100 \mathrm{mg} \text { dl } \\
\text { CHD) }\end{array}$ & 589 & 56.7 & -22 & 61.6 & 66.2 & 46 & -6.8 & $\begin{array}{l}-6.8 \\
(-11.7,-1.9)\end{array}$ \\
\hline $\begin{array}{l}\text { Blood pressure } \\
140.90 \mathrm{mmHs}\end{array}$ & 59.8 & 62.5 & 2.7 & 53.6 & 60.4 & 2.8 & .0 .1 & $\begin{array}{l}-1) .2 \\
(-3.6,3.2)\end{array}$ \\
\hline
\end{tabular}

EFFECT OF TRANSFORMATION OF THE VETERANS AFFAIRS HEALTH CARE SYSTEM ON THE QUALITY OF HYPERTENSION CARE Rebecca Brienza ${ }^{1,2}$; Daren Anderson ${ }^{4}$; Joseph L. Goulet ${ }^{1,5}$; Emily M. Meyer ${ }^{1,3}$; Aldo Peixoto ${ }^{1,2}$. ${ }^{1}$ VA Connecticut Healthcare System, West Haven, CT; ${ }^{2}$ Yale School of Medicine, New Haven, CT; ${ }^{3}$ Yale School of Medicine, New Haven, CT; ${ }^{4}$ Community Health Center, Inc., Middletown, CT; ${ }^{5}$ Yale School of Medicine, New Haven, CT. (Control ID \#1337903)

BACKGROUND: Over the past decade, Veterans Health Administration (VA) has achieved substantial improvement in hypertension control. This accomplishment exceeds national rates and coincides with a broad, national VA redesign and quality improvement effort. We hypothesized that the improvement in blood pressure control is the result of an improvement in clinician "treatment intensification" or patient medication adherence brought about by a broad series of reforms in the measuring and monitoring of clinical performance.

METHODS: We measured patient adherence to antihypertensive treatment and treatment intensification rates between 2000 and 2008 using VA pharmacy data from the local facility. Hypertension control was defined as a blood pressure of $<140 / 90$ or $<130 / 80$ among Veterans with both hypertension and diabetes (ICD-9 250.xx). Adherence was determined by calculating the total days of medication dispensed from a VA pharmacy during the year for each patient with a diagnosis of hypertension. Treatment intensification was defined as a binary variable (yes/no) indicating an increase in the dose of an existing anti-hypertensive medication or the addition of a new anti- hypertensive medication after a clinic visit in which the patient had an above goal blood pressure reading. We modeled intensifications and adherence using linear mixed effects regression models that included fixed effects for time and random effects for provider and facility.

RESULTS: Between 1999 and 2008, 52,215 Veterans had a diagnosis of hypertension and at least one primary care visit: $97.0 \%$ were male, and $31.4 \%$ had diabetes. The mean age was 74 years. Over time, hypertension control rates increased from $50 \%$ to $78 \%$. Patients with diabetes saw a similar rate of improvement, increasing from $28 \%$ to $54 \%$. Average adherence rates improved significantly from a low of $60 \%$ in 2000 to a high of $68 \%$ in $2007(\mathrm{p}<0.0001)$. Adherence was significantly higher for older Veterans, males, diabetics and those with $1+$ visits. During the observation period, 37,712 Veterans with hypertension had 150,812 visits with a high BP event. Of these, 77,780 (51.5\%) events had subsequent treatment intensification. Results from the analyses of yearly changes that accounted for clustering were similar in that treatment intensification rates were relatively stable. Provider-specific intensification rates increased, from an average low of $55 \%$ in 2000 to a high of $61 \%$ in $2003(p=0.0068)$. However, intensification rates declined after $2003(\mathrm{p}=0.0034)$. Diabetics were less likely to receive intensification but trends over time were similar between these groups.

CONCLUSIONS: The improvement in hypertension control over the past ten years at the VA provides an important opportunity to study systems redesign and to learn more about quality improvement. Our results suggest that rates of Veteran medication adherence to anti-hypertensive medications have improved from year to year from 2000-2008. Measures utilized 
in this study could be applied to other chronic diseases such as diabetes, obstructive lung disease, or pain management where both adherence and treatment intensification are also critically related to clinical outcomes. The results have broad applicability for other VA and non-VA practice settings.

\section{EFFECT OF A MODIFIED MEDITERRANEAN DIET AND THER- APEUTIC LIFE-STYLE CHANGE ON BODY MASS INDEX(BMI) AND FAT MASS IN A COMMUNITY MEDICAL PRACTICE Reshmi Siddique $^{1}$; Rania Siddique ${ }^{1}$; Iftekhar Mahmud ${ }^{1}$; Anthony Can- non $^{1}$; Mahmood Siddique ${ }^{1,2}$. ${ }^{1}$ Sleep and Wellness Medical Associates, LLC, Hamilton, NJ; ${ }^{2}$ Robert Wood Johnson Medical School, New Brunswick, NJ. (Control ID \#1282637)}

BACKGROUND: Although randomized studies have shown that Mediterranean-style, low glycemic load diets may treat metabolic syndrome and reduce obesity, real world cohort studies are needed to increase external validity of such therapies. A modified Mediterranean diet, along with therapeutic life style changes and medical foods were incorporated as part of a comprehensive program in a community medical practice. The objective of this study was to assess the impact of this program on Body Mass Index (BMI) and fat mass in obese and overweight subjects.

METHODS: Eighteen obese subjects, aged 35 to 70 were recruited to participate in this program, with a follow-up of 6 months. Participants were instructed to follow a modified Mediterranean style diet, including a list of allowable phytochemically-rich foods that had low glycemic load. Whole grains were limited to 1 serving per day (75-100 calories). Patients were asked to consume from this list until satisfied. Additionally, they were asked to consume a phytochemical (soy protein, phytosterols, proanthocyanidins and rho iso-alpha acids) enriched powdered medical beverage of 2 servings a day. Life-style modifications of incorporating exercise was also a part of this program. Data on BMI and fat mass measurements were collected at baseline and 6 months for analysis. Means were calculated and $\mathrm{T}$ tests were used to determine statistical significance.

RESULTS: The mean age was 54.4. Males comprised $53 \%$ of the subjects. Across all patients, BMI decreased from 38.9 to 36.7 ( $\mathrm{p}<0.2382$ ). Fat mass decreased from 41.8 to 40.6 . For males, BMI decreased from 36.7 to 34.3. $(\mathrm{p}<0.2367)$. Male fat mass decreased from 35.7 to 35.1. For females, BMI decreased from 41.9 to 38.7. $(\mathrm{p}<0.4740)$. Female fat mass decreased from $49.5 \%$ to $47.6 \%(\mathrm{p}<0.1650)$.

CONCLUSIONS: The improvement in BMI and fat mass in obese subjects demonstrated the clinical relevance of this program in a community practice setting. Further studies with larger sample sizes are needed to confirm such findings.

EFFECT OF A PAY-FOR-PERFORMANCE INCENTIVE TO INCREASE TOBACCO USE DOCUMENTATION IN AN ELECTRONIC HEALTH RECORD Gina R. Kruse ${ }^{1,2}$; Yuchiao Chang ${ }^{3,2}$; Jennifer H. Kelley ${ }^{4,5}$; Jeffrey A. Linder $^{6,2}$; Nancy A. Rigotti ${ }^{1,2}$. ${ }^{1}$ Massachusetts General Hospital, Boston, MA; ${ }^{2}$ Harvard Medical School, Boston, MA; ${ }^{3}$ Massachusetts General Hospital, Boston, MA; ${ }^{4}$ Massachusetts General Hospital, Boston, MA; ${ }^{5}$ Partners Health Care, Boston, MA; ${ }^{6}$ Brigham and Womens Hospital, Boston, MA. (Control ID \#1339349)

BACKGROUND: Documentation of tobacco use status is a 'Meaningful Use' standard for electronic health records (EHR) and is necessary to permit the use of chronic disease and population management strategies to reduce tobacco use. However, achieving high rates of tobacco use documentation is a challenge for many systems. Starting on January 1, 2010, 3 commercial insurers for a large Massachusetts integrated health care delivery system offered a pay-for-performance incentive (P4P) to practices achieving $80 \%$ documentation for eligible patients. Eligibility was defined as having (1) an outpatient visit in 2010, (2) a high-risk chronic condition (hypertension, diabetes, or coronary heart disease) and (3) a participating insurer. To help reach the goal, a tobacco use documentation reminder was added to the EHR for all patients. We studied whether the P4P incentive and the EHR reminder increased documentation for eligible and ineligible patients.

METHODS: Among adult $(\geq 18$ yo) patients who visited outpatient practices, we measured the change in the proportion of patients with tobacco use documented over the year before (2009) and the year after (2010) $\mathrm{P} 4 \mathrm{P}$ implementation. To calculate this change, we measured documentation on December 31 of 2008, 2009, and 2010. We compared P4P-eligible patients to a subset of similar but ineligible patients; these patients had a qualifying highrisk condition and a commercial insurer but the insurer was not in the P4P program. We used a logistic regression model with generalized estimating equations techniques to compare documentation before and after P4P implementation by P4P eligibility, adjusting for patient factors (age, gender, race/ethnicity, English-speaking, visits/year) and provider factors (age, gender) and accounting for provider-level clustering.

RESULTS: Over 460,000 adults visited an outpatient provider each year in 2009 and $2010 ; 3 \%(\mathrm{n}=16,364)$ met P4P eligibility criteria in $2009,5 \%(\mathrm{n}=$ $21,063)$ were P4P-eligible in 2010 and $1 \%(n=3,370$ in 2009 and $n=4,005$ in 2010) were in the ineligible subset with a chronic condition but non-P4P commercial insurance. In the year after P4P implementation (2010), tobacco use documentation accelerated for all patients; documentation rates in 2008 , 2009 , and 2010 were $55 \%, 57 \%$, and $70 \%$, respectively. The increase was even more rapid among both the P4P-eligible group $(61 \%, 63 \%$, and $80 \%)$ and the subset of ineligible patients $(61 \%, 63 \%$, and $77 \%)$. In a multivariable model, documentation improved after P4P implementation compared to before implementation in both the P4P-eligible (AOR, 2.3; 95\% CI, 1.9 to 3.0 ) and the P4P-ineligible patients (AOR, 1.9; 95\% CI, 1.5 to 2.5 ). The documentation rate was significantly higher for P4P-eligible vs. ineligible patients (AOR, 1.3; $95 \% \mathrm{CI}, 1.2$ to $1.5, \mathrm{p}=0.009$ ).

CONCLUSIONS: A targeted P4P incentive from insurers plus a nontargeted EHR reminder significantly accelerated tobacco use documentation in a large health care delivery system. The improvement was greatest among adults targeted by the P4P incentive, but documentation also improved significantly for ineligible patients. The improvement among ineligible patients may be attributable to the EHR reminder provided for all patients or to spillover in documentation behavior stimulated by the $\mathrm{P} 4 \mathrm{P}$ incentive. The performance incentive and EHR enhancement were effective quality improvement tools that will facilitate the health care system's use of population management strategies to reduce tobacco use.

EFFECT OF A ROTATION IN A PRIMARY CARE BASED HEPATITIS C CLINIC ON RESIDENT KNOWLEDGE OF HEPATITIS C MANAGEMENT Shelly-Ann Fluker; Lesley Miller; Leslie Davis-Singletary; Michael Fost. Emory University School of Medicine, Atlanta, GA. (Control ID \#1334372)

BACKGROUND: Hepatitis $\mathrm{C}$ virus (HCV) infection is the most common chronic blood-borne disease in the United States and chronic hepatitis $\mathrm{C}$ $(\mathrm{CHC})$ is a leading cause of cirrhosis and death from liver disease. Most patients are diagnosed with $\mathrm{CHC}$ by generalists, yet studies reveal that generalists have significant knowledge deficits regarding the natural history and treatment of $\mathrm{CHC}$. Given a projected shortage in available specialist care for $\mathrm{CHC}$, generalists' ability to care for $\mathrm{CHC}$ will become increasingly important. Internal medicine (IM) residents represent the next generation of practicing internists but studies show that residents also have suboptimal knowledge about screening and management of HCV infection. To our knowledge no study has documented a successful strategy to improve IM resident knowledge about CHC. At Grady Memorial Hospital, which serves a predominantly low-income, African American population, general internists have provided management and treatment of $\mathrm{CHC}$ in the "Liver Clinic" since 2002. We hypothesized that a resident rotation of 3 - 4 sessions in the Liver Clinic would lead to improved and sustained knowledge about management and treatment of HCV infection. The objective of our study was to evaluate IM residents' knowledge of hepatitis $\mathrm{C}$ management and treatment using a self-administered questionnaire at three time points: just prior to, just after, and 6 months after the rotation. METHODS: IM residents assigned to the Liver Clinic were recruited at the beginning of the rotation and completed informed consent. Residents then completed a 23 item self-administered questionnaire (pre-test) that evaluated their knowledge of management of HCV infection. Subjects completed an identical questionnaire at the conclusion of the rotation (posttest), and 6 months after their rotation (late post-test). The questionnaires were graded by the investigators (who were blinded to the subject) to yield an overall score that was compared at the three time points. 
RESULTS: Thirty two residents were enrolled in the study from March 2010 to October 2011. All residents completed the pre-test. As of December 2011, $69 \%$ and $28 \%$ of the residents had completed the post-test and late post-test respectively. The average score on the pre-test was $71 \%$. The average improvement in score from the pre-test to the post-test was 14.6 points which represented an increase of $21.91 \%(\mathrm{n}=22, \mathrm{p}$-value $=0.00000002)$. The average improvement in score from the pre-test to the late post-test was 11.12, which represented a $17.5 \%$ increase from the baseline $(n=9$, $p$-value $=0.009158)$.

CONCLUSIONS: IM resident knowledge of management and treatment of $\mathrm{HCV}$ infection, as tested by a self-administered questionnaire, significantly improved after a rotation in a primary care based hepatitis $\mathrm{C}$ clinic. Six months after completing the rotation resident knowledge showed a sustained increase in knowledge of $17.5 \%$ over baseline. Other studies that have evaluated resident knowledge after other types of IM specialty rotations have noted similar gains in knowledge. These findings have several implications, including: 1) Our primary care based hepatitis $C$ clinic can serve not only as a model for provision of hepatitis $\mathrm{C}$ care for urban patients but also as a venue for resident education about hepatitis $\mathrm{C}$;2) Our study adds to the small body of literature that describes methods of evaluating and validating the educational experiences that residents get on specialized outpatient clinical rotations.

EFFECT OF PHYSICIAN FOLLOW-UP AND SPECIALTY DIFFERENCES ON 30-DAY READMISSION FOR HEART FAILURE PATIENTS David Jang; Haiyong Xu; Jose Escarce; Michael Ong. UCLA, Los Angeles, CA. (Control ID \#1340492)

BACKGROUND: One-fifth of Medicare patients are re-hospitalized within 30 days after discharge, with heart failure being the most common diagnosis for the initial hospitalization. On an institutional level, hospitals with lower rates of physician follow-up within seven days after discharge have higher rates of 30-day readmission. We sought to determine on an individual level whether heart failure patients who had an outpatient physician visit within 30 days after discharge had a lower rate of 30-day readmission than patients without such a visit. We also examined whether the medical specialty of the physician seen at the first outpatient visit had an effect on the rate of 30-day readmission.

METHODS: We examined 1265 Medicare Advantage patients discharged with a principal diagnosis of heart failure from an academic medical center's affiliated hospitals between June 2005 and July 2010. We then examined hospitalizations not preceded by another hospitalization within 30 days. We examined inpatient and outpatient data to determine if a rehospitalization occurred within 30 days and if an outpatient visit occurred within 30 days of these index hospitalizations. Outpatient visits occurring after a rehospitalization were not included. The academic medical center's medical staff rosters were used to determine the identity and specialty of physicians who provided the initial post-discharge outpatient care. We examined the relationship between 30-day readmissions and outpatient visits within 30 days using bivariable and multi-variable analyses.

RESULTS: Of heart failure patients who saw a physician within 30 days after discharge, $17.0 \%$ had a 30 -day readmission, compared to $29.8 \%$ of patients who did not see a physician. Compared to a reference group of patients who had no outpatient visit, the adjusted odds ratio for 30-day readmission for patients who first saw a cardiologist was 0.33 . The odd ratios for patients seen by primary care and other specialty physicians were 0.48 and 0.49 , respectively.

CONCLUSIONS: This study suggests that patients discharged for heart failure should have physician follow-up within 30 days after discharge to reduce the risk of 30-day readmission. Follow-up with a cardiologist provides for the greatest reduction in readmission risk.

EFFECT OF THE ACGME 16-HOUR RULE ON INTERNAL MEDICINE INTERN EDUCATIONAL OPPORTUNITIES Daniel G. Stover; Cecelia N. Theobald; Jacob Hathaway; Neesha N. Choma; Neeraja B. Peterson; Jennifer Green; Joshua Denny. Vanderbilt University Medical Center, Nashville, TN. (Control ID \#1329844)

BACKGROUND: The most recent regulations regarding resident duty hours became effective in July, 2011 and included restriction of shifts to a maximum of 16 hours for interns in all specialties. There is little objective data regarding the impact of these new duty hour limits on resident education. We hypothesized that the duty hour changes would not have a significant impact on intern educational opportunities.

METHODS: Vanderbilt University Medical Center is a large tertiary care hospital in Nashville, TN with 47 interns in 2010 and 50 interns in 2011. We evaluated intern educational experience at our university hospital training site over the first six 4-week blocks of the 2010 and 2011 academic years. All inpatient services went from a 30-hour maximum shift length (2010) to a 16hour maximum shift length (2011). Using KnowledgeMap Portfolio, a concept-based curriculum management program that captures all traineeauthored notes written in the electronic medical record, we evaluated several objective metrics: number of notes written (history and physical or daily progress note), number of unique patients seen, numbers of five common procedures (arterial line placement, central line placement, thoracentesis, paracentesis, and lumbar puncture), and attendance at two academic conferences. All analyses were limited to inpatient experiences and restricted to intern experiences. We also compared average weekly intern duty hours.

RESULTS: When comparing the first six blocks of 2011 to 2010, interns cared for more unique patients (mean 140 vs. 118 patients per intern; $\mathrm{p}=0.005$ ) and wrote more history and physicals (mean 87 vs. $73 ; p=0.005$ ). There was no difference in the number of daily progress notes $(289 \mathrm{vs.} 286, \mathrm{p}=0.92)$ and overall notes ( 376 vs. $360, p=0.51$ ). There was no difference in the median number of procedures performed ( $4 \mathrm{vs} .3$ per intern $\mathrm{p}=0.71$ ) or exposure to any individual procedure. Attendance was higher at the weekly noon chief resident conference $(68 \%$ vs. $60 \% \mathrm{p}<0.0001)$, but unchanged at morning report conferences $(79 \%$ vs. $78 \%, \mathrm{p}=0.49)$. There was no difference in average weekly duty hours per intern (69.4 vs. 68.2 hours/week; $p=0.293$ ).

CONCLUSIONS: Using four objective metrics of educational opportunities - notes written, unique patients seen, procedures performed, and attendance at academic conferences - we demonstrate that educational opportunities for interns were not decreased after implementation of 16hour shifts. In fact, our data suggests that interns cared for more unique patients, did more initial patient evaluations (history and physicals), and had higher attendance at a weekly educational conference. It will be important to evaluate the impact of these increased opportunities on medical knowledge and decision-making over the course of residency training. To our knowledge, this is the first study to objectively evaluate the impact of the 16-hour rule change on intern education.

EFFECTIVENESS AND SAFETY OF PATIENT ACTIVATING INTERVENTIONS FOR ADULTS WITH TYPE 2 DIABETES: A SYSTEMATIC REVIEW AND METAREGRESSION Shari Bolen ${ }^{1,6}$; Adam T. Perzynski ${ }^{1}$; Donna Windish ${ }^{3}$; Carl V. Tyler ${ }^{2}$; $\overline{\text { Corinna Falck- }}$ Ytter $^{1}$; Alida M. Gertz ${ }^{4}$; Apoorva K. Chandar ${ }^{6}$; Paulette A. Sage ${ }^{5}$; Steven Lewis ${ }^{1} .{ }^{1}$ MetroHealth/Case Western Reserve University, Cleveland, OH; ${ }^{2}$ Cleveland Clinic, Cleveland, OH; ${ }^{3}$ Yale University, New Haven, CT; ${ }^{4}$ Johns Hopkins University, Baltimore, MD; ${ }^{5}$ Case Western Reserve University, Cleveland, $\mathrm{OH} ;{ }^{6}$ Case Western Reserve University, Cleveland, OH. (Control ID \#1334407)

BACKGROUND: Given the multitude of recent publications on self management interventions for adults with type 2 diabetes, health care providers and patients alike could benefit from a comprehensive overview of potential interventions. We conducted a systematic review of studies which targeted improvements in patient activation (defined as targeting knowledge, beliefs, and skills for self management) in adults with type 2 diabetes.

METHODS: We searched the MEDLINE, EMBASE, CINAHL and Cochrane Central Register of Controlled Trials databases from inception for original English-language articles. Our search strategy combined terms for Type 2 diabetes, randomized controlled trials, and self management interventions. We selected original studies in adults with type 2 diabetes that assessed intermediate outcomes (HbAlc, LDL cholesterol, weight, and systolic blood pressure), long term clinical outcomes (e.g. cardiovascular morbidity) and serious adverse events (e.g. hypoglycemia) of patient activating interventions. Reviewers extracted data for each article using standardized protocols. We conducted meta-analyses when there were at least 20 trials, using a random-effects model to derive pooled estimates. We also conducted metaregression to identify which intervention characteristics had a greater impact on $\mathrm{HbAlc}$. 
RESULTS: Out of 10,219 citations, 100 articles were included in the review. Too few studies $(\mathrm{N}<10$ for each outcome) with too few events reported on the safety and long-term clinical outcomes; therefore, we were unable to draw firm conclusions on these outcomes. Patient activation interventions improved all intermediate outcomes including $\mathrm{HbA} 1 \mathrm{c}$ (pooled estimate $-0.3 \%, 95 \% \mathrm{CI}-0.4$ to $-0.2 \%$ ), weight (pooled estimate $-2.4 \mathrm{lbs}, 95 \% \mathrm{CI}-4.3$ to $-0.6 \mathrm{lbs}$ ), LDL cholesterol (pooled estimate $-2.9 \mathrm{mg} / \mathrm{dL}, 95 \% \mathrm{CI}-5.6$ to $-0.3 \mathrm{mg} / \mathrm{dL}$ ), and SBP (pooled estimate $-3.0 \mathrm{mmHg}$, $95 \% \mathrm{CI}-4.3$ to $-1.8 \mathrm{mmHg}$ ). No publication bias was found and no single study influenced the results except for the outcome of weight. Metaregression for $\mathrm{HbAlc}$ revealed that interventions focused on the AADE (American Association of Diabetes Educators) self management behaviors of problem solving skills, reducing risks of complications, and exercise had larger between group differences in HbA1c after adjusting for study quality, mean baseline HbA1c, study followup, the other AADE self management behaviors, and other aspects of the intervention $(p<0.02)$. The intensity and location of the intervention did not account for significant heterogeneity in the metaregression for $\mathrm{HbAlc}$.

CONCLUSIONS: The combined improvements of patient activation interventions on multiple intermediate outcomes can have a strong impact on health in adults with type 2 diabetes. Despite the variety of interventions, few intervention characteristics besides the 7 AADE behaviors accounted for the heterogeneity of the results in $\mathrm{HbAlc}$. Problem solving skills, reducing risks, and exercise may be particularly important components of interventions to more effectively lower HbAlc.

\section{EFFECTIVENESS OF HEALTH PROMOTION OUTREACH FOR} DIABETES CARE: A RANDOMIZED-CONTROLLED TRIAL AT THE UNIVERSITY OF COLORADO Ingrid Lobo; Mitra A. Razzaghi; L Miriam Dickinson; William G. LeBlanc; Crystal Reingardt; Trina C. Mizrahi; Stephen Ross. University of Colorado, Denver School of Medicine, Aurora, CO. (Control ID \#1327160)

BACKGROUND: The chronic care model recommends using clinical information systems and delivery system redesign to improve management of diseases like diabetes. A novel care delivery system (the "Center for Health Promotion", CHP) has shown promise in advancing guidelineconcordant preventive care. This study was conducted to assess whether CHP could improve diabetes management.

METHODS: A randomized-controlled trial enrolled patients from February 1, 2009 to March 31, 2010 from primary care practices in an academic medical center. Inclusion criteria included (1) an ICD-9 code of 250.XX on two separate administrative billing claims records (2) practice visit in the past 18 months, and (3) lack of concordance to one or more of the following criteria: $\mathrm{HgAlC}<7 \%$, LDL-C $<100 \mathrm{mg} / \mathrm{dL}$, systolic blood pressure $<130 \mathrm{mmHg}$, diastolic blood pressure $<80 \mathrm{mmHg}$, and urine microalbumin assessment in the last year. Eligible patients were identified weekly and were sequentially assigned to the intervention or control group in a 3:1 ratio using a predetermined randomization scheme. Tailored outreach interventions included scheduling a primary care visit, lab work, Endocrinology consultation, diabetes education, and a retinal examination. Using generalized linear mixed models and data obtained in the processes of care, we assessed whether the two groups differed in changes in guideline concordance (for $\mathrm{HgA1C}$, LDL-C, blood pressure, urine microalbumin, and a composite measure) over the 12 month period following enrollment. We also assessed for differential changes in continuous measures.

RESULTS: 3243 patients were assessed for eligibility; 2800 were randomized (2195 intervention, 605 control). 54\% of patients were female and the mean age was 60.9. While continuous measures improved in both groups, the composite measure of quality actually declined in both groups, and the observed changes did not differ between the intervention and control groups (Table 1).

CONCLUSIONS: CHP outreach did not result in superior process or outcome measures of diabetes quality relative to usual care. The primary limitation of this analysis is that observations were collected during the course of care rather than systematically, which could reduce the power to detect intervention effects. Further analysis may identify why the composite measure of quality declined in spite of outreach, whether outreach reduced clinical inertia, and whether outreach could be targeted to subgroups of patients with diabetes who are more likely to benefit.

Table 1: Continuous Variables

\begin{tabular}{lllllllll}
\hline Measure & \multicolumn{2}{l}{$\begin{array}{l}\text { Baseline Value } \\
\text { (adjusted) }\end{array}$} & $\begin{array}{l}\text { FollowUp } \\
\text { Value } \\
\text { (adjusted) }\end{array}$ & \multicolumn{2}{l}{$\begin{array}{l}\text { Change in } \\
\text { adjusted } \\
\text { values }\end{array}$} & $\begin{array}{l}\text { Difference } \\
\text { in change }\end{array}$ & $\begin{array}{l}p \text {-value of } \\
\text { difference } \\
\text { in change }\end{array}$ \\
\hline $\begin{array}{l}\text { Composite } \\
\text { Quality }\end{array}$ & 1.45 & 1.59 & 1.07 & 1.20 & -0.38 & -0.39 & -0.01 & 0.85 \\
$\begin{array}{l}\text { Measure } \\
(0-4)\end{array}$ & & & & & & & & \\
\hline $\begin{array}{l}\text { HgA1C } \\
(\%)\end{array}$ & 8.03 & 7.79 & 7.89 & 7.72 & -0.14 & -0.06 & 0.08 & 0.36 \\
$\begin{array}{l}\text { SBP } \\
\text { (mmHg) }\end{array}$ & 132.04 & 130.89 & 130.99 & 130.28 & -1.05 & -0.61 & 0.44 & 0.58 \\
$\begin{array}{l}\mathrm{DBP} \\
(\mathrm{mmHg})\end{array}$ & 76.59 & 75.83 & 76.00 & 75.61 & -0.59 & -0.22 & 0.37 & 0.44 \\
$\begin{array}{l}\mathrm{LDL} \\
(\mathrm{mg} / \mathrm{dl})\end{array}$ & 90.30 & 90.08 & 85.53 & 86.42 & -4.77 & -3.67 & 1.11 & 0.58 \\
\hline
\end{tabular}

*adjusted for age, sex, marital status, ethnicity, race, and insurance

${ }^{* *} \mathrm{C}=\mathrm{C}$ ontrol group, $\mathrm{I}=$ Intervention group

EFFECTIVENESS, PATIENT PREFERENCE AND COMPLIANCE OF SPLIT-DOSE POLYETHYLENE GLYCOL (PEG) COMPARED WITH STANDARD DOSE PEG IN HOSPITALIZED PATIENTS AT A TERTIARY CARE HOSPITAL. A PILOT STUDY Ali Raza; Kashif Ahmed; Jonathan Kushner. The University of Cincinnati, Cincinnati, OH. (Control ID \#1313013)

BACKGROUND: The importance of good quality bowel preparation cannot be overstated. The likelihood of missing a small lesion is higher in patients with poor bowel preparation. Split-dose PEG is shown to be better than standard PEG in the outpatient setting. The aim of this study is to evaluate if similar results can be accomplished in hospitalized patients, thus decreasing the need of re-scoping and prolonging hospital stay.

METHODS: In this on-going prospective, single blind study, we have enrolled 22 hospitalized patients who required colonoscopy for their care. Patients were randomized to receive split-dose PEG (2 liters the evening prior to colonoscopy and 2 liters the morning of colonoscopy) or standard PEG (4 liters the evening prior to colonoscopy, ending before morning). Both groups were placed on the same dietary restrictions (clear liquids the day prior to colonoscopy and nothing to eat or drink except PEG after midnight). Patients with bowel obstruction, intractable nausea and vomiting before PEG administration, or massive gastrointestinal bleed were excluded from the 
study. Patients with a past experience of a different PEG dosing schedule were asked to answer a question comparing the ease of current and past experience. An independent operator, who was blind to the nature of the PEG regimen received, evaluated each patient's colonoscopic findings using an Ottawa score. Patient's ability to finish the PEG was also recorded.

RESULTS: Twelve of 22 patients were males. Median age of the sample was 50 years (Range 23 to 81 years; mean, + SD 54+17 years). Thirteen patients received standard PEG-ELS bowel regimen while 9 received the split-dose regimen. Six (42\%) of 13 patients receiving standard regimen could finish it, compared to 7 (78\%) of 9 patients receiving the split-dose regimen (Fisher's exact test $\mathrm{p}$-value 0.184 , odds ratio 4.08). Mean Ottawa score for standard regimen was significantly higher than the split-dose regimen $(8.33$ and 2.22 respectively; p-value 0.023). Four patients in the split-dose regimen group had a standard regimen in the past. All of these patients $(100 \%)$ preferred the splitdose regimen over the standard regimen. No patient in standard PEG-ELS group ever received a split-dose PEG-ELS regimen in the past.

CONCLUSIONS: Split-dose PEG was significantly better than standard PEG in terms of quality of bowel preparation. Patients having the split-dose bowel preparation were four times more likely to finish the whole solution compared with patients having standard PEG. All patients in the split-dose PEG arm who had standard bowel preparation in the past, found split-dose preferable. Split-dose may reduce the length of hospital stay in patients requiring colonoscopy by improving quality and adherence to the regimen.

EFFECTS OF HEALTH-PROMOTING COUNSELING ON HYPERTENSIVE PATIENTS' WILLINGNESS TO CHANGE Courtney Moore; Maithili Shenoy; Angela Zanardelli; Kenneth D. Dorsette; Diane L. Levine. WSUSOM, Detroit, MI. (Control ID \#1324669)

BACKGROUND: Per the Seventh Report of the Joint National Committee on Prevention, Detection, Evaluation, and Treatment of High Blood Pressure (JNC 7) about 50 million Americans and 2 billion people globally are hypertensive [systolic blood pressure (SBP) $>140$ or diastolic blood pressure (DBP) of $>90$ ]. Current recommendations outline the importance of counseling to adopt a health-promoting lifestyle beginning at the pre-hypertension stage. However, there is little data exploring a relationship, if any, between counseling and their willingness towards lifestyle changes. It is unclear if time spent counseling, age, history or stage of hypertension impact willingness to change. METHODS: We conducted a cross sectional study utilizing a four-stage counseling station at which patients received blood pressure screening and either 5 or 10 minutes of hypertension, cardiovascular health, nutrition and stress management counseling. (Table 1) At each station we identified behaviors that were less than optimal for blood pressure (BP) control and, through counseling, provided the patient with more health-promoting alternatives. At the conclusion of the four stations patients were asked if they felt more willing to change their behaviors to integrate health-promoting choices following counseling.

RESULTS: Mean age of our sample was $54 \pm 12$ years with $49 \%$ males. $44 \%$ reported past history of hypertension. 11\% had normal BP at exam, $50 \%$ had BP $<139 / 89,30 \%$ had BP $<159 / 99,8 \%$ had BP $>160 / 100$. 55\% were counseled for up to 5 minutes, $45 \%$ were counseled for 5 to 10 minutes. $70 \%$ reported willingness to change after being counseled. People who were willing to change based on counseling were significantly younger ( $49.7 \pm 9.9$ vs. $61.1 \pm$ $11.3, \mathrm{p}<0.001)$. Multivariate logistic regression analysis revealed that age was the only significant predictor of willingness to change with counseling (OR 0.9, $95 \%$ CI $0.86-0.95, \mathrm{p}<0.001)$ after adjustment for potential confounders. People aged $\geq 50$ years were 0.25 times less likely to change as compared to people aged $<50$ years (CI 0.09-0.67, p 0.006). Patients with $\mathrm{BP}<120 / 80$ were most receptive to counseling $(92.3 \%$ vs $63.7 \%$, p 0.039). Willingness to change was independent of sex, history or stage of hypertension and time spent counseling. CONCLUSIONS: $70 \%$ patients were willing to change after counseling. However, spending more time being counseled did not improve willingness to change. Younger patients were more willing to modify health behaviors with counseling. Patients with normal blood pressures were more receptive to counseling than their hypertensive counterpart which indicates the need for health-promoting lifestyle counseling before the development of hypertension. Further studies are required to determine if longer sessions are needed or if repeated counseling sessions make a difference. Additionally, integration of age-specific strategies for older patients needs to be explored.

\begin{tabular}{|c|c|c|}
\hline STATION & VARIABLE ASSESSED & HEALTHPROMOTING COUNSELING OFFERED \\
\hline Blood Pressure Reading & 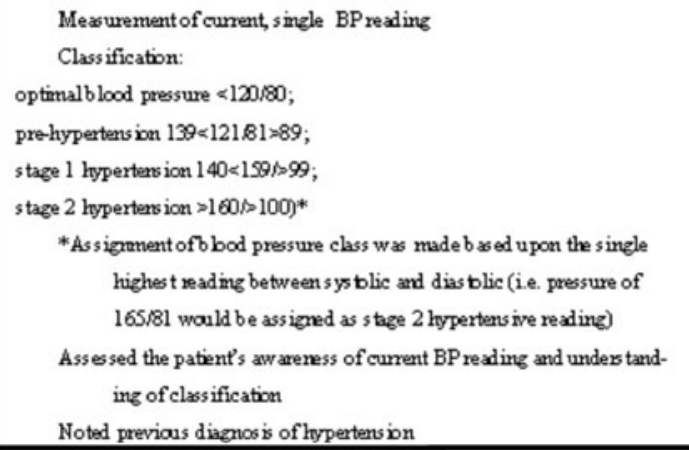 & Educated patient on ilealb bod pressure meacurements. \\
\hline $\begin{array}{l}\text { Cardiovascular Heatth } \\
\text { Screening }\end{array}$ & $\begin{array}{l}\text { Assessed the frequency of moderate exercise in minutes perday and } \\
\text { week }\end{array}$ & $\begin{array}{l}\text { Length of ourent moderate exercise regimenwas sub tracted fiom ounent } \\
\text { recormendation b amive at patient's deficit from optimal } \\
\text { Patient were provided with b ility and situational appropriate sugges tions b } \\
\text { help them attain oal minutes }\end{array}$ \\
\hline Stress Management & $\begin{array}{l}\text { Dis cussed individual resporse b s tress, coping mechanis ms, support } \\
\text { system, while paying particular attention to ron-health promoting } \\
\text { behavios such } ₫ \text { alooblol consumption, smoking, and poor eating } \\
\text { habit }\end{array}$ & $\begin{array}{l}\text { Health-promoting s tress mamagements trategies were explained, such a } \\
\text { meditation, smoking oess ation, and limiting aloolol intaloe to cunent } \\
\text { recormendation* } \\
\text { *Less than } 2 \text { drink equivalent/day for men, less than } 1 \text { drink equivalent/day } \\
\text { forwomen }\end{array}$ \\
\hline Nutrition Counseling & $\begin{array}{l}\text { Assessed the patient's cunenteating hab it, foousing on intake of DASH } \\
\text { sugges ted foods (fnits and vegetbles) and DASH limited iterm } \\
\text { (such } ₫ \text { sodinm) }\end{array}$ & $\begin{array}{l}\text { DASH diet regimens stressed, healthy altemative lis t and sugges tions } \\
\text { tailored b patient's tas tes were provided }\end{array}$ \\
\hline
\end{tabular}

Table 1 
EFFECTS OF MEDICAID DRUG POLICIES ON PRESCRIPTION FILLS FOR PEOPLE DIAGNOSED WITH MENTAL ILLNESS IN COLORADO AND OREGON Judy T. Zerzan ${ }^{1,2}$; Dan Hartung ${ }^{6}$; Nancy E. Morden ${ }^{5}$; Traci Yamashita ${ }^{2}$; Suhong Tong ${ }^{4}$; Anne Libby ${ }^{3} .{ }^{1}$ State of Colorado, Denver, CO; ${ }^{2}$ University of Colorado School of Medicine, Denver, CO; ${ }^{3}$ University of Colorado School of Pharmacy, Denver, CO; ${ }^{4}$ University of Colorado School of Public Health, Denver, CO; ${ }^{5}$ Dartmouth University, Hannover, NH; ${ }^{6}$ OHSU College of Pharmacy, Portland, OR. (Control ID \#1339756)

BACKGROUND: Colorado and Oregon State Medicaid Programs implemented a series of drug policy interventions on prescriptions for sleep aids. Insomnia is a common symptom in individuals with mental illnesses and affects both function and mental illness treatment outcomes. These two states were concerned about policy impact on people diagnosed with severe and persistent mental illness (SMI), and people with less severe mental health diagnoses (non-SMI). Colorado Medicaid implemented cost sharing in July 2003 and added sedative hypnotics to a preferred drug list (PDL) in April 2008; Oregon required cost sharing in Jan 2003 and PDL for these drugs in October 2007. Medicaid officials hypothesized the policies reduced utilization of sedative hypnotics and possibly been associated with substitution to low dose atypical antipsychotics and first generation antihistamines.

METHODS: State prescription fill claims, associated medical claims and enrollment data were obtained from Colorado and Oregon Medicaid from 2002-2009. Prescription rates were calculated in monthly time series as prescription fills per 1,000 person months. Interrupted time series was implemented with shift factors at each policy implementation date. Policy effects were measured as mean change in fill rate at implementation and as pre- vs post-policy slope changes. Policy impact on expenditures and medical services utilization are underway.

RESULTS: During this time period Colorado Medicaid covered 129,848 individuals using any mental health drug of which $14.7 \%$ had SMI and $57.7 \%$ non-SMI. For Oregon Medicaid 222,479 individuals were covered with $17.5 \%$ SMI and $66.3 \%$ non-SMI. Among both SMI and non-SMI patients in Colorado, we observed increases in sedative hypnotic fill rates at implementation of cost sharing (SMI .55 p=.05; non-SMI 1.46, $\mathrm{p}=.01$ ) Post-cost sharing antipsychotic fill rate increases per month were attenuated increasing by .15 per month compared to the .58 monthly increase prior to cost sharing $(\mathrm{p}=.03)$. Sedative hypnotic fill rates declined after implementation of PDL with corresponding increases in atypicals and antihistamines. However, significant increases were only observed among SMI and non-SMI patients using antihistamines (SMI .35, p $=.04 ;$ non-SMI $.43, \mathrm{p}=.05$ ). In Oregon, we observed reduction in sedative hypnotic fills at PDL implementation for SMI $(-.34, \mathrm{p}<.001)$ and non-SMI $(-.47, \mathrm{p}=.03)$ with corresponding decrease in pre vs post PA antipsychotic fills among non-SMI $(\mathrm{p}<.001)$ and decrease for SMI $(\mathrm{p}=.05)$. The impact on use of clinical services and total costs are currently being analyzed.

CONCLUSIONS: Drug policies that create hurdles for prescribers change prescription use and may impact sleep and clinical symptoms in people with SMI and non-SMI. The policy intent was to encourage providers to be more mindful of prescribing sedatives. We interpret these findings as evidence that Medicaid drug policies influence prescription behavior by providers and SMI patients may be more affected by changes in policy. Policymakers are aware of unintended consequences of policy actions and researchers can assist them in using observational data.

EFFICACY AND TOLERABILITY OF ARMODAFINIL IN HEALTHCARE WORKERS WITH EXCESSIVE SLEEPINESS ASSOCIATED WITH SHIFT WORK DISORDER Mary G. Umlauf ${ }^{1}$; Richard K. Bogan ${ }^{2}$; Ronghua Yang ${ }^{3} .{ }^{1}$ University of Alabama, Hoover, AL; ${ }^{2}$ SleepMed, Inc, Columbia, SC; ${ }^{3}$ Teva Pharmaceuticals Ltd., Frazer, PA. (Control ID \#1334491)

BACKGROUND: Individuals, such as healthcare workers, who typically work permanent or rotating night shifts are susceptible to developing excessive sleepiness and, ultimately, shift work disorder (SWD). Excessive sleepiness in shift workers may lead to increased workplace accidents and mistakes. The wakefulness-promoting agent armodafinil has been shown to significantly improve clinical condition and wakefulness during the night shift and overall functioning in patients with SWD. Specifically, this study examined the late hours of the night shift. This post-hoc analysis examined efficacy and tolerability of armodafinil in healthcare workers with SWD.

METHODS: Patients in this 6-week, randomized, double-blind study were clinically diagnosed with SWD (DSM-IV and ICSD-2 criteria), worked at least five 6- to 12-hour night shifts per month (between 10:00 PM and 8:00 AM), with Global Assessment of Functioning (GAF) score $<70$, and late-in-shift sleepiness (between 4:00 AM and 8:00 AM) represented by a mean Karolinska Sleepiness Scale (KSS) score $>6$. Following randomization, patients received $150 \mathrm{mg}$ armodafinil or placebo 30-60 minutes before beginning their night shift. Efficacy assessments were change in Clinical Global Impression-Change (CGI-C) related to excessive sleepiness late in the shift (including the commute home), GAF, late-in-shift KSS, and modified Sheehan Disability Scale (SDS-M) from baseline to final visit. The SDS-M was modified to determine the effect of shift work on work, family, and social life. Final visit data included last observation carried forward.

RESULTS: Of the 383 patients enrolled in the original study, 56 (15\%) were healthcare practitioners and $37(10 \%)$ were healthcare support staff. After pooling both healthcare worker populations, 47 patients received armodafinil and 46 received placebo. In contrast to what was previously observed for the general study population, the proportion of patients in this analysis with an improvement in late-in-shift CGI-C from baseline was not significantly greater in the armodafinil group versus the placebo group at final visit ( $67 \%$ vs. $51 \%$; $\mathrm{p}=0.0978$ ). However, the proportion of patients with late-in-shift CGI-C improvement from baseline was significantly greater in armodafinil patients who completed the 6 -week study $(72 \%$ vs. $49 \%$; $=0.0350)$. Significant improvements in the GAF, late-in-shift KSS, and SDS-M were observed at final visit and for Week 6 completers. Headache and nausea were the most common adverse events.

CONCLUSIONS: Consistent with earlier findings, these results demonstrate that armodafinil significantly improved late-in-shift clinical condition after 6 weeks of treatment. Armodafinil also significantly improved overall functioning and late-in-shift wakefulness and reduced patient disability score. Similar to the overall study population, headache and nausea were the most common adverse events. This study was funded by Cephalon, Inc, now a part of Teva Pharmaceuticals Ltd.

ELECTRONIC HEALTH RECORD ADOPTION IN FEDERALLY FUNDED HEALTH CENTERS Michael Wittie ${ }^{1}$; Lydie A. Lebrun ${ }^{1}$; Leiyu Shi ${ }^{2}$; Heather Ngai ${ }^{1}$; Quyen Ngo-Metzger $^{1}{ }^{1}$ U.S. Department of Health and Human Services, Rockville, MD; ${ }^{2}$ Johns Hopkins Bloomberg School of Public Health, Baltimore, MD. (Control ID \#1324046)

BACKGROUND: Health Information Technology (HIT) has been widely promoted for its potential to improve the quality, safety, efficiency, and costeffectiveness of health care in the US, but there is concern that a 'digital divide' in adoption could cause or exacerbate disparities in access to quality care. Health centers, supported by the Health Resources and Services Administration (HRSA), provide comprehensive primary care and supportive services to vulnerable populations to increase access and reduce disparities, and must report data each year to the Uniform Data System (UDS). The UDS collects information on their operations, clinical quality, and electronic health record (EHR) adoption and use. We analyzed UDS data to determine health centers' use of EHRs and the possibility of a digital divide.

METHODS: Organization-level data from the 2010 UDS on 1124 health centers, which served 19.5 million patients in 2010, were used to examine the relationships between EHR adoption and a variety of patient, provider, and organizational characteristics. Relationships between EHR adoption and geography, patient characteristics, size, funding, and duration of operation were assessed using $\chi 2$ or $t$ tests.

RESULTS: In $2010,65 \%$ of health centers reported having an EHR in use, including $50 \%$ of health centers using EHR at all of their sites. The data also reveal that health centers were using many advanced EHR functionalities, including computerized provider order entry for lab tests ( $85 \%$ of EHR users) and electronic entry of prescriptions 
( $94 \%$ of EHR users). $80.4 \%$ of centers had the capability to provide clinical summaries, and $63.5 \%$ had the capability to provide patients with an electronic copy of their health information. There was considerable geographic variation in adoption rates, with the US Department of Health and Human Services' Region X (Washington, Alaska, Idaho, Oregon) being the highest at $81.4 \%$ and Region IX (Nevada, American Samoa, Arizona, California, Guam, Hawaii, Northern Mariana Islands) the lowest at $46.2 \%$. State adoption rates ranged from $0 \%$ to $100 \%$. However, there were no indications of disparities or a digital divide in adoption based on centers' urbanicity, HRSA funding stream, or payer mix.

CONCLUSIONS: The 2010 UDS represents the first and to date only opportunity to examine EHR adoption in the full universe of health centers. Findings reveal that a large proportion of centers are using EHRs. In addition, there was no evidence of a digital divide among centers, indicating that EHRs are being implemented in keeping with their mission to reduce disparities in access to quality care among vulnerable patient populations. Federally funded health centers are adopting EHR apace with other ambulatory providers, and without the disparities seen in those settings or evidence of a digital divide in HIT. Health centers appear on track to make the quality transformations which are critical to improving health outcomes, population health, and the efficiency of care enabled by the Meaningful Use of EHRs. Additional data from the upcoming 2011 UDS will shed additional light on health centers' progress towards Meaningful Use and further narrowing of the digital divide for HIT.

ELECTRONIC HEALTH RECORD TOOL REDUCES ANTIBIOTIC USE: THE INTEGRATED CLINICAL PREDICTION RULES (ICPR) TRIAL Devin Mann ${ }^{1,3}$; Joseph Kannry ${ }^{3}$; Juan P. Wisnivesky ${ }^{3}$; James Stulman $^{3}$; Lauren $\mathrm{McCullagh}^{2}$; Anastasia Sofianou ${ }^{3}$; Alice $\mathrm{Li}^{3}$; Diego Chiluisa $^{3}$; Megan Knaus ${ }^{2}$; Daniel Edonyabo ${ }^{3}$; Thomas McGinn ${ }^{2} .{ }^{1}$ Boston University, Boston, MA; ${ }^{2}$ North Shore-LIJ/Hofstra, Manhasset, NY; ${ }^{3}$ Mount Sinai, New York, NY. (Control ID \#1338921)

BACKGROUND: Clinical decision supports (CDS) have been developed as platforms within electronic health records (EHRs) to help introduce evidence-based medicine (EBM) into routine care. Clinical prediction rules (CPRs) are frontline decision aids that combine evidence with real-time patient history, exam, and laboratory data. Despite being well-validated EBM tools, CPRs have been underutilized in practice. Previous studies of CDS for upper respiratory infections (URIs) have demonstrated negative results with utilization rates as low as $6 \%$. We have developed an Integrated Clinical Prediction Rules Clinical Decision Support system (iCPR) that incorporates two well-validated URI CPRs (Walsh Streptococcal Pharyngitis and Heckerling Pneumonia CPRs) into the most widely used commercial outpatient EHR system (EpicCare ${ }^{\mathrm{TM}}$ ). Our study is a randomized controlled trial of the effectiveness of the iCPR tool in changing provider antibiotics and diagnostic test ordering behaviors for URI's within an urban ambulatory primary care practice that uses a large commercial EHR system. METHODS: The study setting was a large ambulatory academic primary care practice. All primary care providers (140 residents and faculty) were recruited for participation. Consenting providers were randomized in a 1:1 fashion into intervention or control. After a brief training, intervention providers had the iCPR tool activated in their EHR profile. The intervention consisted of an optional EHR embedded CDS that triggered from specific complaints, diagnoses, and/or orders relevant to strep or pneumonia placed at the point-of-care. The interface then guided risk stratification and facilitated antibiotic orders, notes, supportive therapies, and patient instructions. The primary outcome was the difference in antibiotics ordered for step or pneumonia during encounters between intervention and control providers after one year. Secondary outcomes included differences in diagnostic test ordering between groups and use of each component of the $\mathrm{iCPR}$ tool among intervention providers. Generalized estimating equations were used to test for differences in antibiotic utilization among groups accounting for clustering by provider. RESULTS: Over 1 year, enrolled providers conducted 1007 (586 intervention, 421 control) encounters that triggered the iCPR tool, representing $3 \%$ of all their encounters during this period. More than half (63\%) of the encounters seen by providers enrolled in the intervention arm launched the risk stratification tool and 58\% utilized the bundled iCPR interface associated ordering option. We observed a $40 \%$ reduction in the likelihood of antibiotic ordering in intervention versus control encounters (30\% vs. 39\%, OR: $0.6[0.5-$ $0.9], p=.01$ ). Chest $\mathrm{x}$-rays for pneumonia were ordered in $20 \%$ of control encounters and $21 \%$ of intervention encounters (OR: 0.9 [0.5-1.6], $\mathrm{p}=.70$ ) while rapid strep tests were $30 \%$ less likely in the intervention arm (29\% vs. $40 \%$, OR: 0.7 [0.5-1.0], $\mathrm{p}=.05$ ). Broad spectrum antibiotics were ordered less frequently amongst intervention encounters ( $71 \%$ vs. $77 \%$, OR: 0.75 [0.44-1.31], $\mathrm{p}=0.31$ ). CONCLUSIONS: The iCPR randomized control study demonstrated significant reductions in antibiotic and some diagnostic test ordering. Moreover, the tool was frequently used by providers; a significant improvement over previous CDS studies for URIs. These data suggest that EHR embedded CPRs have the potential to enhance the implementation of EBM in primary care and improve quality.

\section{ELECTRONIC HEALTH RECORD-BASED PATIENT IDENTIFI-} CATION AND INDIVIDUALIZED MAILED OUTREACH FOR PRIMARY CARDIOVASCULAR DISEASE PREVENTION: A CLUSTER RANDOMIZED TRIAL Stephen D. Persell ${ }^{1}$; Donald M. Lloyd-Jones $^{2}$; Elisha M. Friesema ${ }^{1}$; Andrew J. Cooper ${ }^{1}$; David W. Baker ${ }^{1}$. ${ }^{1}$ Northwestern University, Chicago, IL; ${ }^{2}$ Northwestern University, Chicago, IL. (Control ID \#1336645)

BACKGROUND: Many individuals at higher risk for cardiovascular disease (CVD) don't get beneficial treatments. Prior interventions using personalized risk information to promote prevention did not test the clinicwide effectiveness of these approaches.

METHODS: We compared the effectiveness of a strategy that included EHR-based identification of patients with increased CVD risk and individualized mailed outreach delivered at the level of the practice. We performed a 9-month cluster-randomized trial, clustered by primary care physician. Internists at a practice affiliated with an academic medical center were eligible. Physicians were enrolled. Their eligible patients were included with a waiver of consent. Patients were included in the intention-to-treat analysis if: age was 40 to 79 years, they were not prescribed lipid lowering medication, they had no CVD or diabetes mellitus, and had at least two clinic visits in the past 24 months. LDL cholesterol had to have been done in the past 5 years and: $\mathrm{LDL}$ was $\geq 100 \mathrm{mg} / \mathrm{dl}$ and Framingham Risk Score (FRS) was $>20 \%$, LDL $\geq 130 \mathrm{mg} / \mathrm{dl}$ and FRS was 10 to $20 \%$, or $\mathrm{LDL} \geq 160 \mathrm{mg} / \mathrm{dl}$ and FRS was 5 to $<10 \%$. The FRS was calculated from EHR data retrieved electronically. Physicians could indicate patients they did not wish to have the intervention. Patients were mailed individualized CVD risk depicted in written and graphic formats and describing benefits of using a statin (and treating hypertension or quitting smoking when relevant). The control group received usual care. The primary outcome was occurrence of a LDL-cholesterol level that was repeated and was at least $30 \mathrm{mg} / \mathrm{dl}$ lower than prior. Secondary outcomes included: lipid lowering drug prescribing; aspirin prescribing (among those not prescribed at baseline); change in blood pressure, increase in number of antihypertensive drugs prescribed (among patients who had uncontrolled hypertension at baseline); and documentation of quitting smoking (among smokers). The study was powered to detect a $10 \%$ increase in primary outcome assuming control rate $\leq 5 \%$. Analyses used generalized linear mixed models with physicians as random effects. Clinicaltrials.gov identifier: NCT01286311.

RESULTS: 14 physicians with 218 patients were randomized to intervention, and 15 physicians with 217 patients to control. Characteristics were similar. There was no difference in the primary outcome, LDL-cholesterol level repeated and at least $30 \mathrm{mg} / \mathrm{dl}$ lower at 9 months $(11.0 \%$ intervention, $11.1 \%$ control, OR 1.0, 95\% CI 0.53-1.86). Few patients returned for repeat lipid testing during the study period after receiving a new prescription. Intervention group patients were twice as likely to receive a prescription for lipid lowering medication $(11.9 \%$, vs. $6.0 \%$, OR 2.1 , CI $1.03-4.4, \mathrm{p}=0.041)$. Among the subgroup with uncontrolled hypertension with follow up blood pressure, decline in systolic blood pressure was non-significantly greater in the intervention group $(-13.3 \mathrm{mmHg}$ vs. $-10.4 \mathrm{mmHg}, \mathrm{p}=0.18)$. More intervention patients with uncontrolled hypertension had an increase in the number of antihypertensive drugs prescribed, $11.8 \%$ vs. $4.7 \%$ (also non-significant, $\mathrm{p}=$ $0.15)$. Other outcomes did not differ. 
CONCLUSIONS: In this effectiveness trial, individualized mailed CVD risk messages increased the frequency of new lipid lowering drug prescriptions but not the primary study outcome. The duration of follow up may have been insufficient to demonstrate the effects of increased prescribing on lipid levels.

ELEVATED SERUM COBALAMIN: SHOULD WE BE PAYING MORE ATTENTION? Sindhu L. Joseph; Geetanjali K. Dang; Vishnu Vandana Palle; Arvind Rangarajan Murali; Geeta Kutty; Brian P. Lucas. John H. Stroger Jr. Hospital of Cook County, Chicago, IL. (Control ID \#1326823)

BACKGROUND: Roughly $15 \%$ of serum cobalamin (vitamin B12) levels are elevated. Studies suggest an association of high serum cobalamin with liver disease and hematologic malignancies. These studies were based on small patient populations. We hypothesized that there is a higher association of liver disease and cancer with elevated serum cobalamin levels than previously understood. We therefore conducted a retrospective chart review of patients with elevated cobalamin levels to determine the frequency of liver disease and cancer.

METHODS: Our cohort was general medicine patients discharged from Cook County Hospital from July 2009 through June 2010 who had at least one cobalamin level drawn between their admission and when our review began in July 2011. From this cohort we identified patients who had a serum cobalamin greater than or equal to $900 \mathrm{ng} / \mathrm{dL}$. Among these patients, we selected a $50 \%$ random sample. Trained investigators then abstracted data from electronic medical records with standardized data collection instruments to determine whether or not patients had a diagnosis of liver disease or cancer

RESULTS: Of 10,003 inpatients, 3055 patients had at least one cobalamin result drawn during the 2 year study period. Among these patients, $25 \%$ (778 out of 3055) had an elevated level. $61 \%$ of our random sample of 778 patients with high cobalamin (237 out of 388 patients) had either liver disease or cancer. At the time of our review, 151 patients were not diagnosed with either liver disease or cancer. Among these patients, 26 had a disease likely to be associated with elevated cobalamin; these included systemic lupus erythematosus $(n=2)$, alcohol abuse $(n=3)$, and heart failure $(n=21) .125$ patients, however, had no clear cause of elevated cobalamin by the end of the study period.

CONCLUSIONS: We found that a quarter of our patients hospitalized on the general medicine wards have an elevated serum cobalamin. More than half of these patients have liver disease or cancer, a higher proportion than previously recognized. This suggests that elevated serum cobalamin levels should not be ignored and may provide some diagnostic value. We plan to follow up this early data with a matched case-control study comparing the prevalence of liver disease or cancer among patients with elevated serum cobalamin to those with normal serum cobalamin.

\section{EMERGENCY DEPARTMENT UTILIZATION AMONG RECENTLY} RELEASED EX-PRISONERS Joseph Frank; Christina Andrews; Traci C. Green; Aaron M. Samuels; Trong T. Trinh; Peter D. Friedmann. Alpert Medical School of Brown University, Providence, RI. (Control ID \#1320137)

BACKGROUND: The population of ex-prisoners returning to their communities is large. Morbidity and mortality is increased during the period following release. Understanding utilization of emergency services by this population may inform interventions to reduce adverse outcomes. We sought to examine Emergency Department utilization among a cohort of recently released ex-prisoners.

METHODS: We linked data on all Emergency Department (ED) visits in a single large hospital system in Rhode Island occurring between 2007-2009 with data from the Rhode Island Department of Corrections (333,369 ED visits with 5,147 visits by recent ex-prisoners). We used primary ICD-9 visit diagnoses to identify visits related to substance use and mental health as defined by the New York University ED Algorithm and ambulatory care sensitive conditions per Agency for Healthcare Research and Quality criteria. We reported the proportion of ED visits for each of these conditions among exprisoners and the general population. Fixed effects logistic regression modeling estimated the association between ex-prisoner status and use of the ED for these conditions, controlling for age, gender, race/ethnicity, visit year and location and ZIP code level unemployment and population of individuals' place of residence.

RESULTS: ED visits by ex-prisoners were more likely to be made by men $(85 \%$ vs. $48 \%, \mathrm{p}<0.001)$ and by Blacks $(26 \%$ vs. $16 \%, \mathrm{p}<0.001)$ compared to the Rhode Island general population. Visits by ex-prisoners were more likely to have a substance use diagnosis $(16 \%$ vs. $4 \%, \mathrm{p}<0.001)$ or a mental health diagnosis $(6 \%$ vs. $4 \%, \mathrm{p}<0.001)$. Unadjusted rates of ambulatory sensitive condition diagnoses were equivalent between groups ( $14 \%$ vs. $14 \%, p=0.79)$. After controlling for patient and community level factors, ex-prisoners' ED visits were significantly more likely to be due to a substance use diagnosis (OR 1.93; 95\% CI 1.77, 2.11), a mental health diagnosis (AOR 1.43; 95\% CI 1.27-1.61) or an ambulatory care sensitive conditions (AOR 1.09; $95 \%$ CI 1.003-1.181)

CONCLUSIONS: Among ex-prisoners in Rhode Island, recent release from prison was independently associated with increased Emergency Department visits related to mental health, substance use and ambulatory care sensitive conditions. Future work should determine whether greater access to outpatient services during the transition from prison might reduce ex-prisoners' utilization of emergency services.

EMERGENCY DEPARTMENT AND HOSPITAL UTILIZATION AMONG THE HOMEBOUND ELDERLY Ania Wajnberg ${ }^{1}$; Kristofer L. Smith ${ }^{2}$; David Russell ${ }^{3}$; Joseph S. Ross ${ }^{4}$; John Doucette ${ }^{2}$; Anastasia Sofianou ${ }^{1}$; Alex Federman ${ }^{1} .{ }^{1}$ Mount Sinai School of Medicine, New York, NY; ${ }^{2}$ North Shore University Hospital, Manhasset, NY; ${ }^{3}$ Visiting Nurse Service of New York, New York, NY; ${ }^{4}$ Yale School of Medicine, New Haven, CT. (Control ID \#1331186)

BACKGROUND: Over three million community-dwelling seniors have functional impairments that limit their access to office-based physician care. Although these patients are likely to be high utilizers of healthcare services, little is known about their patterns of acute care use.

METHODS: English and Spanish speaking homebound adults over 65 years of age were recruited into two home-based care programs, the Mount Sinai Visiting Doctors (MSVD) program or the Visiting Nurse Services of New York Long-term Home Health Care Program (VNS). Homebound status was defined as leaving the home infrequently and requiring assistance when leaving the home. All patients were able to provide written consent or had a proxy to provide consent. Healthcare utilization was assessed by asking patients/proxies to report how many Emergency Department (ED) visits and hospital admissions they had three months before and after MSVD or VNS enrollment. Univariate tests were performed to determine overall and within group change in ED visits and hospital admissions before and after enrollment. Multivariate Poisson regression was used to examine differences in utilization rates between the MSVD and VNS groups, controlling for pre-enrollment utilization and demographics.

RESULTS: To date, we have collected complete baseline, pre enrollment and post enrollment data on 73 patients. At baseline, 31 (43\%) were personally interviewed and $42(57 \%)$ required proxies/surrogate interviews due to cognitive impairment. Mean age was 82 years $(\mathrm{SD}=9.5), 61(84 \%)$ were female, $26(36 \%)$ were Latino and $21(29 \%)$ were African-American. Most were poorly educated and poor: $29(43 \%)$ had not graduated from high school and $50(75 \%)$ reported an income $<\$ 1350$ /month. $61(84 \%)$ lived with family, friends or a paid caregiver and among those who had a home attendant/aide (94\%), $60(95 \%)$ required $>5$ days/week assistance. The majority of patients $(66 \%)$ rated their baseline General Health (SF-1) as poor or fair. In the three months prior to enrollment, 33 (45\%) reported at least one ED visit and 29 $(39 \%)$ had at least one hospitalization. Of the patients who reported $\geq 1$ hospital 
admission in the three months prior to enrollment, $86 \%$ had no admissions during their first three months in the program $(\mathrm{p}<0.001)$; and $78.8 \%$ of those who had $\geq 1 \mathrm{ED}$ visit 3 months prior to baseline had 0 visits after enrollment $(\mathrm{p}<0.001)$. In regression analysis, baseline utilization, proxy interviews and increased ADL dependencies were predictive of increased hospital admissions.

CONCLUSIONS: Homebound older adults in this study have substantial physical and cognitive limitations and experience high rates of ED visits and hospitalizations. At three months after enrollment into physician or nursing led home-based care programs, our data indicates a $30 \%$ decrease in $\mathrm{ED} / \mathrm{admission}$ utilization.

ENHANCING OUT-OF-HOME INFORMAL CAREGIVING THROUGH HEALTH INFORMATION TECHNOLOGY Donna M. Zulman $^{1,2}$; John D. Piette ${ }^{3,4}$; Steven Asch ${ }^{1,2}$; Ann-Marie Rosland ${ }^{3,4}$. ${ }^{7}$ Stanford University, Stanford, CA; ${ }^{2}$ VA Palo Alto Health Care System, Menlo Park, CA; ${ }^{3}$ University of Michigan, Ann Arbor, MI; ${ }^{4}$ Ann Arbor VA, Ann Arbor, MI. (Control ID \#1337076)

BACKGROUND: Rapidly advancing health information technology (HIT), such as electronic personal health records and mobile health applications, offer opportunities to actively engage patients' family members and friends in their health care. This study aimed to understand HIT use by out-of-home caregivers (individuals who provide care for a family member or friend living in a different household), and to identify barriers to such use.

METHODS: We conducted an Internet-based survey of a nationallyrepresentative sample of individuals who previously reported a willingness to care for a family member or friend with a chronic illness living outside of their household. Respondents reported their use of computers, the Internet, and e-mail to help care recipients manage their health and health care, for example by finding health information online, sending messages to health care providers, tracking personal health record information, or filling medications. We also asked caregivers to describe barriers to HIT use. Using bivariate statistics, we examined differences in HIT use across caregiver subgroups defined by sociodemographic characteristics.

RESULTS: Among the 452 current or potential caregivers who completed the survey (response rate $75 \%$ ), the mean age was 48 years (SD 15;28\%> 60 years), $62 \%$ were female, $41 \%$ were Black or Hispanic, $33 \%$ had at most a high school education, and $42 \%$ had an annual income $<\$ 50,000$. The majority of care recipients were either the respondent's parent (35\%) or a sibling (34\%). All caregivers had access to the Internet, either through independent means (77\%), or as compensation for their participation in the research panel, and $64 \%$ reported that their care recipient also used the Internet. Only $30 \%$ of caregivers reported using HIT to help manage a care recipient's health, most commonly to find health information online $(n=93,21 \%)$. In bivariate analyses, non-Hispanic Blacks were more likely than non-Hispanic Whites to use HIT for caregiving (OR 1.68, $\mathrm{p}=0.04$ ), but technology use did not vary significantly by caregiver age, sex, education, or income. Among technology non-users, $69 \%$ reported that - if it would help their care recipient improve his or her health - they would be likely to use HIT in the future, for example to find health information (63\%), track personal health or health care information $(45-50 \%)$, or send messages to health care providers $(42 \%)$. The most commonly cited barriers to using technology for caregiving were lack of familiarity with relevant programs or websites $(24 \%)$ and health system privacy rules that limit caregivers' access to care recipients' personal health information $(24 \%)$

CONCLUSIONS: In this diverse sample of current and potential outof-home caregivers, there was strong interest in using HIT for caregiving activities, although fewer than one-third of out-of-home caregivers currently do so. Electronic personal health records and other mobile health technologies should incorporate and promote features that enable long-distant caregiver engagement, such as shared access to patient health information, and applications that offer education and tools for caregivers.
ENROLLMENT AND SATISFACTION WITH COMMUNICATION USING AN ELECTRONIC PERSONAL HEALTH RECORD Joan Neuner $^{2}$; Megan F. Fedders ${ }^{1}$; Marilyn Schapira ${ }^{3}$. ${ }^{1}$ Medical College of Wisconsin, Milwaukee, WI; ${ }^{2}$ Medical College of Wisconsin, Milwaukee, WI; ${ }^{3}$ University of Pennsylvania, Philadelphia, PA. (Control ID \#1332677)

BACKGROUND: Electronic personal health records (PHRs) have the potential to improve patient involvement in care and communication with physicians, particularly with regard to medications. Little is known, however, about their actual use or effects, particularly with older patients who use the internet less overall.

METHODS: We examined enrollment in and satisfaction with a commercially available personal health record (EpicCare) linked to the electronic medical record of a multispecialty academic group practice and seven affiliated community primary care clinics. The PHR allows patients to make appointments, email providers, and review major medical record content (problem lists of diagnoses, medications, laboratory and radiology results, and immunizations). PHR medication lists included doses and frequency, and diagnosis and laboratory information were linked to a commercial library of patient educational materials. Enrollment and use of the PHR was examined electronically, and satisfaction with communication was examined through an anonymous survey sent electronically a single time to all active PHR users in early 2011 (response rate, 20.1\%). Survey items included general satisfaction, satisfaction with specific elements of the EMR, desire for additional elements, and communication measures from the Consumer Assessment of Healthcare Providers and Systems (CAHPS) clinician and group surveys. Subjects were asked to respond to the CAHPS questions for only one of their providers. Most items used four-point Likert scales (eg very dissatisfied to very satisfied, never to always).

RESULTS: PHR utilization: Between $10-30 \%$ of adults in each of the practices enrolled in the personal health record in its first two years. $49.8 \%$ of enrollees accessed the PHR in the month prior to the survey, with each user averaging 4.6 visits. $4 \%$ of those visits included access to patient educational materials. Survey RESULTS: $30.3 \%$ of the 2,989 survey respondents were age $56-65$ and $21.4 \%$ were over $65.80 \%$ reported having a primary care provider and $81 \%$ a specialty care provider at our organization. $96.5 \%$ were satisfied with the PHR $(66.5 \%$ very satisfied and $30.0 \%$ satisfied), and $97.7 \%$ would recommend it to a friend/family member. There were no statistically significant differences by age $+/-65$ in these responses. Few patients $(6.6 \%)$ used another PHR, and more patients were interested in additional education information for the PHR in future $(75.1 \%)$ than were interested in integration with a separate web site to hold information from many healthcare providers (52.1\%). 86.6\% of respondents agreed that the PHR improved communication with the care team. In response to questions from CAHPS about communication overall (ie not just with the PHR), $78.7 \%$ reported that their provider always gave easy to understand instructions about how to take their medicines, and $69.4 \%$ that he/she always explained the side effects of medications in a way that was easy to understand.

CONCLUSIONS: Our results show patients frequently use and are highly satisfied with a PHR linked to an EMR. Patients also reported improved communication with providers with the PHR. A substantial minority reported communication quality issues even in areas like medication information that optimal future PHR design and use could help address.

ESTIMATING THE STAFFING INFRASTRUCTURE FOR A PATIENT-CENTERED MEDICAL HOME Mitesh Patel ${ }^{1}$; Martin Arron ${ }^{2}$; Thomas Sinsky ${ }^{3}$; Eric Green ${ }^{4}$; David W. Baker'; Judith Bowen ${ }^{6}$; Susan Day ${ }^{1} .{ }^{1}$ Hospital of the University of Pennsylvania, Philadelphia, PA; ${ }^{2}$ Beth Israel Medical Center, New York, NY; ${ }^{3}$ Medical Associates Clinic, Dubuque, IA; ${ }^{4}$ Mercy Catholic Medical Center, Darby, PA; ${ }^{5}$ Feinberg School of Medicine at Northwestern, Chicago, IL; ${ }^{6}$ Oregan Health and Science University, Portland, OR. (Control ID \#1334413) 
BACKGROUND: The patient-centered medical home (PCMH) offers an innovative method of delivering primary care by utilizing a team-based approach where members work collaboratively at the top of their scope of practice. The staffing infrastructure required for a PCMH is not well established

METHODS: The objective of this study was to define the personnel infrastructure, staffing ratio, and associated costs needed to implement a PCMH within an academic clinical practice that includes internal medicine trainees. In February 2011, with support from the Josiah Macy Jr. Foundation, the Society of General Internal Medicine convened an Education Summit to discuss implications of implementing such a model. Based on this discussion, we designed a study that had two phases. First, we completed a thematic review of the PCMH literature to elucidate the roles and training of team members required for implementation. Second, we used a social networking approach to determine a convenience sampling of primary care clinical practices that were known to have transitioned to a PCMH practice model or in process of implementing this model. Nine leaders of primary care health systems agreed to be interviewed. Practices included academic medical centers, non-profit and for-profit institutions, and government-owned health systems. Interview results were aggregated and ranges in staffing at each position were calculated. A model incorporating an estimate of staffing at each level was developed based on published literature standards and data ranges from the interviews. To translate this increased staffing estimate into additional resources required, we calculated the incremental cost of staffing a PCMH compared to benchmark MGMA data.

RESULTS: In addition to physician providers, literature review indicated the importance of including the following members/augmented functions in the staffing of a PCMH: nurse practitioner/physician assistant, MA/ Tech/LPN, health coaches, nurse case manager, pharmacist, social worker, nutritionist, data analyst/population health manager, clerical staff. Interviews revealed that panel sizes for attending physicians varied significantly based on the PCMH setting. Panel sizes ranged from 625-2500 patients for full-time practicing physicians compared to 30-60 patients for PGY-1 residents and 100-125 patients for PGY-3 residents. Per physician FTE, we estimate that 3.95 FTEs should be allocated to PCMH staffing, compared to the base-case MGMA model of 2.68 FTE. The incremental cost perpatient-per month (pppm), including population health, is estimated to be $\$ 4.90$. Annually this rate and staffing ratio totals about $\$ 126,346$ for staffing per physician FTE.

CONCLUSIONS: Implementing a patient-centered medical home requires an evaluation of the practice's current staffing and functional roles, along with a determination of the needs of its patient population. Experience from PCMH practices suggests that in order to achieve the goals of improved outcomes and better care, at reasonable cost, additional staff with specific expertise and training will be required. Further investigation on the value added with increased staffing, and opportunities for funding is needed if the full potential of the PCMH-model of care is to be realized within an academic clinical practice.

EVALUATING ALLOPURINOL THERAPY AND SERUM URIC ACID LEVELS IN MEDICARE BENEFICIARIES WITH GOUT Melea Ward ${ }^{1}$; Anthony M. Louder ${ }^{1}$; Keith Szymanski ${ }^{2}$; Leonardo Tamariz ${ }^{3}$. ${ }^{\mathrm{T}}$ Competitive Health Analytics, Inc, Louisville, KY; ${ }^{2}$ Takeda Pharmaceuticals America Inc., Deerfield, IL; ${ }^{3}$ University of Miami, Miami, FL. (Control ID \#1285856)

BACKGROUND: Higher serum uric acid levels in gout patients have been associated with an increased frequency and risk of gout flares and greater subsequent healthcare costs. Despite the wide availability of allopurinol, achieving a therapeutic serum uric acid (sUA) level remains problematic for clinicians and patients. The objectives of this study included identifying predictors of an sUA response to allopurinol and investigating the associated healthcare costs.

METHODS: A retrospective cohort study of a large health benefits company was conducted among Medicare Advantage Prescription Drug (MAPD) plan patients with gout newly initiated on allopurinol between 1/ $1 / 08$ and $12 / 31 / 10$. Patients were separated into two cohorts defined by their
sUA response to allopurinol ( $\mathrm{sUA}<6 \mathrm{mg} / \mathrm{dL}$ or $\mathrm{sUA}>6 \mathrm{mg} / \mathrm{dL}$ ). Mean allopurinol adherence, as measured by proportion of days covered (PDC), was reported at 12 months follow-up. Multivariate logistic regression was used to determine factors associated with allopurinol response. A generalized linear model was developed to assess the association between allopurinol response and total healthcare costs.

RESULTS: Of the 2,703 patients initiated on allopurinol, $57 \%$ had a baseline sUA and $33 \%$ had sUA $\leq 6 \mathrm{mg} / \mathrm{dL}$ in the follow-up period. Higher adherence was associated with achieving an sUA $<6 \mathrm{mg} / \mathrm{dL}$ compared to $>$ $6 \mathrm{mg} / \mathrm{dL}$ ( $\mathrm{PDC}=0.74$ and 0.59 , respectively, $\mathrm{p}<.0001$ ). Predictors of sUA $<$ $6 \mathrm{mg} / \mathrm{dL}$ included female sex, higher allopurinol PDC, and allopurinol dose $>100 \mathrm{mg}$ /day (OR:1.74, CI:1.41-2.13; OR:12.28, CI:8.25-18.28; OR:5.84, CI:4.73-7.20, respectively). Hispanic ethnicity, higher baseline sUA, renal impairment (Stage 3 vs. Stage 1), colchicine use, and NSAID use were associated with lower odds of responding to allopurinol therapy (OR:0.32, CI:0.14-0.76; OR:0.60, CI:0.56-0.66; OR:0.41, CI:0.30-0.55; OR:0.79, CI:0.65-0.97; OR:0.68, CI:0.55-0.85, respectively). There were no significant differences in total healthcare costs between the two cohorts.

CONCLUSIONS: A large percentage of patients initiated on allopurinol did not have adequate sUA monitoring, and did not achieve an $\mathrm{sUA}<6 \mathrm{mg} / \mathrm{dL}$. Drivers of a therapeutic response included increased adherence and dose escalation. This study demonstrates that ample opportunity exists for clinicians and patients to improve sUA monitoring and treatment adherence when initiating urate lowering therapy.

EVALUATING FAILURES IN WEIGHT AND DIABETES MANAGEMENT IN AN HIV COHORT Barbara S. Taylor ${ }^{1,3}$; L. Sergio Garduno $^{1}$; Margit B. Gerardi ${ }^{5,1}$; Elizabeth Walter ${ }^{1}$; Delia Bullock ${ }^{1}$; Barbara J. Turner ${ }^{2,4}$. ${ }^{1}$ University of Texas Health Science Center San Antonio, San Antonio, TX; ${ }^{2}$ University of Texas Health Science Center San Antonio, San Antonio, TX; ${ }^{3}$ University of Texas School of Public Health, San Antonio, TX; ${ }^{4}$ University Health System, San Antonio, TX; ${ }^{5}$ University of Texas Health Science Center San Antonio, San Antonio, TX. (Control ID $\# 1334930)$

BACKGROUND: HIV-infected (HIV+) people in the U.S. die from nonHIV-related causes more than HIV-specific complications. Diabetes (DM) is a growing threat with several studies reporting a greater risk of DM for $\mathrm{HIV}+$ than non-HIV+persons. Limiting weight gain and controlling DM should be considered clinical care metrics for HIV providers. In an HIV clinic serving South Texas, we evaluated prevalence and predictors of: a) clinically significant weight gain and b) poor DM control. We hypothesized that less frequent HIV clinic visits and lack of comanagement with non-HIV providers would be associated with these poor outcomes.

METHODS: Of 1938 HIV + patients with $\geq 2$ HIV clinic visits in 200710,1464 had $\geq 12$ mos observation, and 1292 had a body mass index (BMI) recorded at $\geq 2$ visits. We excluded $36 \quad(3 \%)$ with $\mathrm{BMI}<$ $18.5 \mathrm{~kg} / \mathrm{m} 2$. From first to last BMI in the most recent $6-12 \mathrm{mos}$ [median $336 \mathrm{~d}$ intraquartile range (IQR) 228, 367], we calculated \% change in BMI with $\geq 3 \%$ increase considered clinically significant per non-HIV cohorts. DM was defined from ICD9 codes at $\geq 2$ visits or hemoglobin A1c (HbA1c) $\geq 6.5 \%$. HIV clinic visits in the most recent $12 \mathrm{mos}$ was analyzed in quartiles, then dichotomized to high/low intensity at the median [6, IQR:4, 8]. Co-management was defined as any primary care or diabetes specialist visit in the same $12 \mathrm{mos}$. Insurance categories were based on type recorded in the majority of visits in 2007-10. HIV disease status was assessed by CD4+ count and viral load. The two study outcomes were: a) $\geq 3 \%$ increase in $\mathrm{BMI} /$ year and b), among those with $\mathrm{DM}$, a most recent $\mathrm{HbA1c}$ $\geq 8.0 \%$. The key predictor was HIV care intensity in logistic regression models that adjust for demographics, insurance, HIV disease status, and baseline BMI.

RESULTS: In $1256 \mathrm{HIV+subjects,} \mathrm{median} \mathrm{age} \mathrm{was} \mathrm{42y} \mathrm{[IQR:35,} \mathrm{48].} \mathrm{Most}$ were men $(77 \%)$ and Hispanic $(55 \%)$; fewer were white $(27 \%)$ or black $(14 \%)$. Most common HIV risks were men having sex with men $(43 \%)$ and heterosexual sex (22\%). Predominant insurance types were: Medicare (29\%), a county indigent medical assistance plan (CareLink, 25\%), Medicaid (13\%), 
and self pay (10\%). Median CD4+ cell count was 411 cells/mm3 [IQR: 255 , 581 ]; $64 \%$ had virologic control, and $90 \%$ on antiretrovirals. Baseline BMIs were: $36 \%$ normal $(18.5$ to $<25 \mathrm{~kg} / \mathrm{m} 2), 36 \%$ overweight $(25$ to $<30 \mathrm{~kg} / \mathrm{m} 2)$, and $28 \%$ obese ( $\geq 30 \mathrm{~kg} / \mathrm{m} 2)$. DM was diagnosed in $184(15 \%)$. Only $3 \%$ were co-managed.Thirty percent had $\geq 3 \% / \mathrm{yr}$ gain in BMI and $17 \%$ of diabetics had $\mathrm{HbAlc} \geq 8 \%$. A $\geq 3 \%$ gain was not associated with frequent HIV visits [adjusted odds ratio (AOR) $0.96 ; 95 \% \mathrm{CI}: 0.71,1.30$ ] but AORs were increased for: Medicaid or CareLink [AOR 1.53;95\%CI:1.07, 2.18] and self pay [AOR $1.65 ; 95 \%$ CI: $1.04,2.63]$ versus Medicare. Obese persons had lower odds of $\geq 3 \%$ gain [AOR $0.54 ; 95 \% \mathrm{CI}: 0.37,0.79$ ]. The only significant predictor of poor diabetes control was less frequent $(<6 / \mathrm{yr}) \mathrm{HIV}$ visits [AOR 4.87; 95\%CI:1.72, 13.7].

CONCLUSIONS: The $28 \%$ prevalence of obesity in this largely Hispanic HIV + cohort is far higher than in other HIV+cohorts (14-17\%). Thirty percent had $\geq 3 \%$ BMI gain in one yr compared with NHANES data for a national U.S. sample reporting mean 3.4-5.2\% weight gain over $10 \mathrm{yrs}$. Co-management was rare. Lower HIV clinic visit frequency $(<6 / \mathrm{yr})$ was not predictive of weight gain but was associated with a fourfold greater odds of poor DM control. HIV providers should make obesity management a priority at visits and patients with poor DM control should be followed more closely. Comanagement may also help improve these outcomes.

EVALUATION OF COPD MEDICATION USE IN THE PRIMARY CARE SETTING Daniel A. Belletti ${ }^{1}$; Christopher Zacker $^{1}$; Jennifer Wogen ${ }^{2}$. ${ }^{1}$ Novartis Pharmaceuticals, East Hanover, NJ; ${ }^{2}$ MedMentis Consulting, Towaco, NJ. (Control ID \#1339300)

BACKGROUND: The Global Initiative for Chronic Obstructive Lung Disease (GOLD) guidelines recommend the use of short-acting bronchodilators only in Stage I (mild) COPD, with the use of inhaled corticosteroids (ICS) recommended only for symptomatic patients with Stage III (severe) or IV (very severe) COPD. While ICS use may be appropriate for those patients with COPD and asthma, prior research has suggested that ICS may be over-utilized in COPD treatment, particularly among those with less severe disease. Our study objective was to describe treatment patterns in the primary care setting related to respiratory medication use among COPD patients with and without comorbid asthma.

METHODS: This cross-sectional study was performed via retrospective chart review by investigators at 11 primary care sites, using a random sample of from 50-150 adult patients, aged 40 through 89 years with a diagnosis of COPD, per site. (total $\mathrm{N}=1517$ ). Data extracted from patient primary care medical records included patient demographics, clinical information (spirometry testing, respiratory medications, co-morbid conditions, and risk reduction measures). Medication use was evaluated, focusing on ICS use by severity and comorbid asthma.

RESULTS: Mean patient age was $67.2(\mathrm{SD}+11.3)$ years, 54\% were female, and $62 \%$ were Caucasian. $18.7 \%$ of patients had co-morbid asthma, . Mean duration of COPD was about 4.8 years. Only $27 \%$ of patients had a current FEV1\% result documented in their chart. More than half $(52 \%)$ of patients did not have a documented COPD stage; $20 \%$ were classified as mild (stage I), $13 \%$ moderate (stage II), $12 \%$ stage III (severe), $3.4 \%$ very severe (stage IV). Medication class distribution is shown in figure 1 . Mean number of respiratory medications was 1.8 , and $23 \%$ were on no respiratory medications (mostly stage I). $14.8 \%$ were on monotherapy and $62.8 \%$ were on polytherapy. Overall, $51 \%$ of patients were prescribed inhaled corticosteroids (ICS); of patients without asthma, 39\% were prescribed ICS, including a combined $28.7 \%$ in the mild and severe stages.

CONCLUSIONS: While almost 1 in 4 patients with COPD are not prescribed respiratory medications, more than $25 \%$ of patients with mild/ moderate COPD are prescribed early ICS use (mild or moderate stages). These individual and concurrent scenarios suggest the use of potentially inappropriate therapeutic strategies which may limit COPD treatment options as disease severity progresses. Our findings highlight a need for educational initiatives geared at increasing primary care providers' knowledge of COPD guideline-recommended therapeutic management strategies.

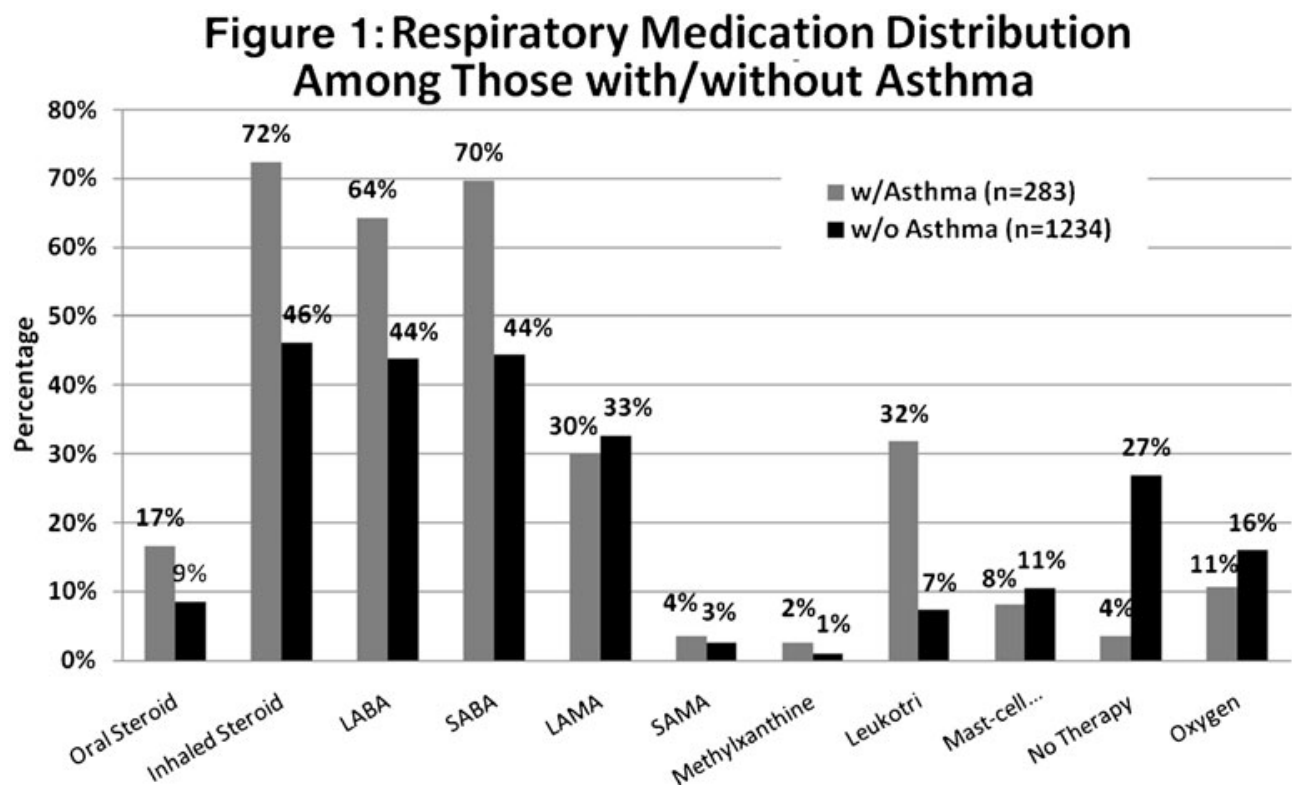

EVALUATION OF INITIAL EMPIRIC ANTIBIOTIC THERAPY IN A COMMUNITY HOSPITAL FOR PATIENTS DIAGNOSED WITH SEPSIS AND ITS IMPACT ON HOSPITAL LENGTH OF STAY. John Kim; Stephen RIso; Saleha Butt; Arismendy Nunez; Carlo Palarca; Simi Philip; Samuil Rafailov; Kell Julliard; Naser Yazigi. Lutheran Medical Center, Brooklyn, NY. (Control ID \#1332621)
BACKGROUND: Infection-related hospital admissions are highlighted in recent years for their associated higher mortality, morbidity and high financial burden. Recent studies have suggested incidence of initial inappropriate antibiotic therapy to be significantly high leading to higher mortality and morbidity and longer length of stay. Goal of this retrospective analytic cohort study was to evaluate the role of the initial 
antibiotic selected based on patient illness, residence and suspected etiology and then comparing mortality, morbidity, ICU admission and length of stay in patients who received appropriate versus inappropriate antibiotics.

METHODS: Patients with sepsis of infectious etiology were selected and all cultures taken within the first 72 hours were examined for microbiological pathogen and subsequent susceptibility. Antibiotics selected were classified as appropriate versus inappropriate by the senior author. Patients were subclassified by severity of disease based on APACHE II and Charlson score and were tracked through hospital stay. ICU admission, total length of stay, mortality and morbidity and were then compared in appropriately versus inappropriately treated groups.

RESULTS: Of the 152 patients meeting study criteria, 35 (23\%) were deemed to have received inappropriate antibiotics in the ED. Those receiving appropriate vs. inappropriate antibiotics did not differ in hospital length of stay, ICU length of stay, Apache II score, Charlson Index, gender, residence before admission, or ICU admission. In-hospital mortality of those treated appropriately $(21 \%)$ was significantly lower than those treated inappropriately $(54 \%)(\mathrm{p}<0.001)$. Of 59 patients with sensitivity information, sensitivity of $96 \%$ of the 49 patients treated appropriately was present, and was present in $20 \%$ of 10 patients treated inappropriately $(\mathrm{p}<0.001)$. CONCLUSIONS: This study suggests that inappropriate antibiotic administration in the ED is associated with higher mortality, even though the appropriate and inappropriate groups were similar in disease severity. Because mortality would be expected to shorten both hospital and ICU lengths of stay, this could explain the equivalence of these lengths of stay in the inappropriately and appropriately treated groups. The implication that the timeliness and appropriateness of antibiotics can influence both mortality and length of stay indicates the importance of this topic for future research and clinical practice.

EVALUATION OF A CURRICULAR INNOVATION IN OBESITY ASSESSMENT AND MANAGEMENT Pardha Devaki; Diane L. Levine. Wayne state university, Detroit, MI. (Control ID \#1339078)

BACKGROUND: Obesity substantially increases the risk of morbidity from illnesses such as Diabetes Mellitus, coronary artery disease, dyslipidemia, and hypertension. Evidence shows that patients who receive weight loss advice from physicians show increased effort at weight loss. Educational intervention aimed at improving the confidence and frequency of dietary counseling by the resident physicians was shown to be effective and also resulted in changing dietary behaviors of patients. At Wayne State University (WSU)/Detroit Medical Center we evaluated the effectiveness of an obesity curriculum by measuring improvement in care of paper based obese patients by Internal Medicine residents.

METHODS: Residents attending a conference were provided with a primary-care patient (Scenario \#1) and asked to develop a diagnostic and management plan. Residents then received two one-hour educational sessions (obesity curriculum) in obesity evaluation and management. Within two months residents were provided with a second primary-care patient scenario (Scenario \#2) providing a nearly identical patient and again asked to develop a diagnostic and management plan. Residents did not know the focus of the study and were told that purpose of the scenarios was to evaluate their primary care management. Patient plans were analyzed based on NHLBI obesity guidelines with a focus on if and how obesity evaluation and management was addressed. Data from the first and second scenarios were compared. Data was analyzed using Fisher's exact test. The study was exempted by the investigational review board at WSU. RESULTS: Fifty-six residents completed the first case scenario. Twentyfive completed the second scenario. A statistically significant decrease in inappropriate drug prescribing practice was observed; 11 of 56 residents prescribed statins for hyperlipidemia for the first case (ten year risk $<10 \%$ ) compared to 0 of 25 after the curriculum $(\mathrm{p}<0.01)$. Approximately $50 \%$ of residents diagnosed obesity. However, there was no increase in obesity history obtained, BMI measurement, waist circumference, or diagnosis of obesity. An increase in referrals to nutrition services was noted $(\mathrm{p}<0.02)$.
All residents recommended some component of lifestyle change even those who did not diagnose obesity.

CONCLUSIONS: Residents have poor knowledge of obesity evaluation and management. A two-hour educational curriculum impacted inappropriate statin utilization but was insufficient to improve knowledge and overall management of obesity. Residency programs need to identify effective strategies to improve care of obese patients by the resident physicians.

Table: Summary of pre and post intervention data

\begin{tabular}{llll}
\hline \hline & $\begin{array}{l}\text { Pre- } \\
\text { intervention }\end{array}$ & $\begin{array}{l}\text { Post- } \\
\text { intervention }\end{array}$ & $\begin{array}{l}\mathrm{p}- \\
\text { value }\end{array}$ \\
BMI - calculated & $15 / 56$ & $8 / 25$ & 0.79 \\
BMI - calculated correct value & $14 / 56$ & $6 / 25$ & 1.0 \\
$\begin{array}{l}\text { Diagnosis of obesity } \\
\text { Used BMI for diagnosis of }\end{array}$ & $26 / 56$ & $12 / 25$ & 1.0 \\
obesity & $14 / 56$ & $6 / 25$ & 1.0 \\
Waist circumference & $2 / 56$ & $0 / 25$ & \\
Dietary assessment & $6 / 56$ & $6 / 25$ & 1.0 \\
Additional dietary history & $7 / 56$ & $1 / 25$ & 0.17 \\
Body weight history & $4 / 56$ & $0 / 25$ & 0.42 \\
Some type of dietary strategy & $56 / 56$ & $25 / 25$ & 0.3 \\
Drug therapy & $11 / 56$ & $0 / 25$ & 1.0 \\
Referral & $5 / 56$ & $8 / 25$ & $<0.01^{*}$ \\
& & & $<0.02^{*}$ \\
\hline
\end{tabular}

*statistically significant

EVALUATION OF A PATIENT FINANCIAL REWARDS PROGRAM TO ENCOURAGE PREVENTIVE CARE Ateev Mehrotra ${ }^{1}$; Ruopeng $\mathrm{An}^{2}$; Deepak Patel ${ }^{3}$; Roland Sturm ${ }^{2} .{ }^{1}$ University of Pittsburgh, Pittsburgh, PA; ${ }^{2}$ RAND, Santa Monica, PA; ${ }^{3}$ DiscoveryHealth, Durban, South Africa. (Control ID \#1339627)

BACKGROUND: The Affordable Care Act increased coverage for preventive health services, but research has shown that removing out-ofpocket costs will only have a limited impact on the discrepancy between recommended and actual use. Patient incentives have been promoted as a mechanism to increase receipt of preventive care. When making the choice to receive preventive care, patients must tradeoff current and future costs/ benefits. In theory, incentive programs can help by increasing short term benefits. An unusually innovative preventive care incentive program has been pioneered by Discovery, a private health insurance company operating in South Africa, the United States, the UK, and China. In their voluntary program, which now includes over one million people, the receipt of preventive care services "earns" an enrollee points and the points can then be used for gifts such as discounted travel and retail goods such as movie tickets or cell phones. These types of incentive programs have significant potential to improve preventive care, but there have been no empirical evaluations of their impact. We assessed the impact of the incentive program on receipt of preventive care services among 3.3 million Discovery health members in South Africa of whom 1.5 million were ever enrolled in the incentive program. Of note, South Africans with private health insurance are similar to the US population in terms of education and income.

METHODS: We analyzed the receipt of 9 preventive care services (e.g. mammogram, Pap smear, HIV test) among all members of Discovery (those in the incentive program and those that are not) over the years 2005-10. To try and address the selection bias inherent in an elective program, we used a difference-in-difference analysis where we compared the change in an individual's receipt of preventive care services before and after they enter the program compared to similar patients who are in not in the incentive program. We used a logistic model with random effects for each individual with controls for year and type of health plan product. 
RESULTS: Compared to those who never joined the incentive program, those who joined the incentive program were younger (e.g. $\geq 60 \mathrm{yo}, 5 \%$ vs. $20 \%$ ) and healthier (no chronic illnesses, $68 \%$ vs. $62 \%$ ). In our models, being part of the incentive program was associated with a statistically higher odds of receiving all 9 preventive care services (e.g. Odd ratio for Cholesterol testing 1.5, Glucose testing 1.2, HIV test 1.9, Mammogram 1.3, Pap smear 1.3). However, receipt of preventive care among those in the incentive program was still low. For example, among members of incentive program eligible for the care, receipt of Pap smear was only $24 \%$. CONCLUSIONS: Voluntary participation in a patient incentive program was associated with a higher likelihood of receiving preventive care. However, receipt of preventive care among those in the program was still much lower than ideal.

EVALUATION OF A POCKET-SIZED ULTRASOUND DEVICE AS AN AID TO THE PHYSICAL EXAMINATION James Colbert ${ }^{1,2}$; Jason Ojeda ${ }^{1,2}$; Maria Yialamas ${ }^{1,2}$; Joel T. Katz ${ }^{1,2}$; Graham T. McMahon ${ }^{1,2}$; Carol B. Benson ${ }^{3,2}$; Peter Doubilet ${ }^{3,2}$. ${ }^{1}$ Brigham and Women's Hospital, Boston, MA; ${ }^{2}$ Harvard Medical School, Boston, MA; ${ }^{3}$ Brigham and Women's Hospital, Boston, MA. (Control ID \#1342364)

BACKGROUND: Advances in technology have allowed for miniaturization and decreased price of ultrasound devices such that individual ownership and daily use is now practical. These pocket-sized ultrasound devices are powerful tools and have been found to perform as well as more traditional high-end portable systems when used by experienced clinicians. It remains to be seen, however, if such devices will improve the diagnostic accuracy of internal medicine residents in a broader range of physical findings with minimal training in the performance and interpretation of ultrasonography.

METHODS: We hypothesize that the use of pocket-sized ultrasound devices will improve the diagnostic accuracy of internal medicine residents. Physical findings being studied: pleural effusion, hepatomegaly, cirrhotic liver, splenomegaly, ascites, aortic stenosis, mitral regurgitation, right atrial pressure, abdominal aortic aneurysm, deep vein thrombosis. Study Design: 40 residents will be randomized into two groups. One group will receive training on pocket-sized ultrasound and will then have 4 weeks to practice with the devices. The other 20 residents will receive a training session on physical exam skills. After 4 weeks all of the residents will undergo an evaluation to determine if use of the pocket-sized ultrasound allows them to identify a greater percentage of physical findings.

RESULTS: Our study is still in process and the results are not yet final, however we will be able to report the results at the SGIM conference in May, 2012.

CONCLUSIONS: Primary Aim: - Determine the diagnostic accuracy of residents using a handheld ultrasound device compared with residents using more traditional physical exam techniques for the physical findings mentioned above. Secondary Aims: - Determine Medical Residents comfort with the traditional physical exam versus a pocket-sized ultrasound - Evaluate the perceived utility of the physical exam versus ultrasound for various findings among medical residents - Evaluate the perceived ability of residents to integrate ultrasound examination into their daily patient care routines - Determine the diagnostic abilities of residents using the physical exam for various physical findings Determine the ability of residents to diagnose various physical findings using ultrasound.

EVALUATION OF A WEB-BASED RISK ASSESSMENT TOOL IN THE PRIMARY CARE SETTING Heather J. Baer ${ }^{1,2}$; Louise I. Schneider $^{1,2}$; Graham A. Colditz ${ }^{3}$; Hank Dart $^{5}$; Analisa Andry ${ }^{1}$; Endel J. Orav $^{1,2}$; Jennifer Haas ${ }^{1,2}$; George Getty ${ }^{4}$; Elizabeth Whittemore ${ }^{1}$; Deborah H. Williams ${ }^{1}$; David W. Bates ${ }^{1,2}$. ${ }^{1}$ Brigham and Women's Hospital, Boston, MA; ${ }^{2}$ Harvard Medical School, Boston, MA; ${ }^{3}$ Washington University, St.Louis, MO; ${ }^{4}$ Partners HealthCare, Boston, MA. (Control ID \#1321789)
BACKGROUND: Primary care clinicians play an important role in identifying individuals at increased risk of cancer and other chronic diseases, but they have limited time and often lack adequate systems for collecting and synthesizing risk factor information. We conducted a pilot study to evaluate the feasibility and effectiveness of using a brief, web-based risk assessment tool in the primary care setting.

METHODS: We adapted a web-based risk assessment tool to collect information from patients on family history and lifestyle factors and estimate their risk of cancer, heart disease, diabetes, and stroke. We then conducted a pilot study of this tool in several primary care practices within a large, academic medical institution. Three practices were assigned to the intervention group, and two practices were assigned to the control group. Eligible patients in the intervention practices were asked to complete the tool on a laptop computer in the waiting room before an annual visit. Patient-entered information on family history of cancer was sent to their electronic health record (EHR) for their primary care clinician to view during the visit. If the clinician accepted it, it populated existing fields in the EHR and could trigger reminders about colon and breast cancer screening. Patients also received a summary report with their estimated risk of each condition and tailored recommendations for prevention. Eligible control group patients received usual care. We examined the percentage of patients who had new information on family history of cancer entered into their EHR within 30 days after the visit and the percent who received moderate or high risk reminders for colon and breast cancer screening. Logistic regression models with generalized estimating equations were used to compute odds ratios (ORs) and 95\% confidence intervals (CIs), accounting for clustering and adjusting for potential confounders.

RESULTS: There were 9647 eligible patients in the intervention group and 5848 eligible patients in the control group. Among eligible patients in the intervention group, 996 (10.3\% of eligible) completed the web-based risk assessment tool at their visit. Among eligible patients in the intervention group, $2.0 \%$ had new information on family history of cancer entered into the EHR within 30 days after the visit, compared to $0.6 \%$ of eligible patients in the control group (adjusted $\mathrm{OR}=4.3,95 \%$ CI: $1.2-15.7$, $\mathrm{P}=0.03$ ). Of the 996 eligible patients in the intervention group who completed the tool, $10.6 \%$ had new information on family history of cancer entered into the EHR, compared to $0.8 \%$ of 637 eligible patients in the control group who would have been most likely to complete the tool, based on a propensity score model (adjusted OR $=15.9,95 \%$ CI: 3.5-72.1, $\mathrm{P}=0.0003$ ). There were no significant differences in the percent of patients who received moderate or high risk reminders for colon or breast cancer screening in the two groups.

CONCLUSIONS: It is feasible to use a brief, web-based risk assessment tool to collect information on family history and lifestyle factors from patients in the primary care setting. Use of this tool was associated with increased documentation of family history of cancer in the EHR, although the percentage of patients with new family history information was very low in both groups. Further research is needed to determine how risk assessment tools can be better integrated with practice workflow and how they affect screening and health behaviors.

EXCESSIVE SLEEPINESS AND ITS IMPACT ON SHIFT WORKERS: AN INTERNET SURVEY OF SHIFT WORKERS AND PATIENTS WITH SHIFT WORK DISORDER Lauren Sylvester; Sharon Paik. Ipsos, Norwalk, CT. (Control ID \#1333577)

BACKGROUND: Shift work disorder (SWD) is a circadian rhythm sleep disorder characterized by excessive sleepiness and/or insomnia in people who work permanent or rotating shifts. It is estimated that $45 \%$ of shift workers (SWs) have excessive sleepiness and up to $23 \%$ of SWs have SWD, although the exact prevalence is unknown due to under recognition by both patients and physicians. This market research examined how shift work impacted quality of life in SWs 
with excessive sleepiness and compared the similarities and differences between patients with diagnosed SWD and those SWs without a formal diagnosis of SWD.

METHODS: An online survey was administered to SWs with or without a self-reported diagnosis of SWD. Participation in the SWs survey required $\geq 21$ hours per week working shifts in the previous 2 weeks, a diagnosis of SWD or $\geq 10$ score on the Epworth Sleepiness Scale (ESS), and $\geq 5$ score on any of the subscales of the Sheehan Disability Scale (SDS). Significant differences between respondents diagnosed with SWD and without a SWD diagnosis were tested at a 95\% confidence interval.

RESULTS: The survey was completed by 260 respondents (103 with diagnosed SWD and 157 without a SWD diagnosis). Diagnosed and undiagnosed respondents demonstrated similar ESS (13.7 vs. 13.6) and Karolinska Sleepiness Scale (KSS) (6.0 vs. 5.5) scores suggesting similar levels of sleepiness in these groups. SDS social life and family life scores were similar between the two groups although diagnosed respondents had a greater SDS work disability score compared with undiagnosed respondents (6.7 vs. $5.5 ; \mathrm{p}<0.0001)$. Significantly more respondents diagnosed with SWD had chronic fatigue syndrome, restless leg syndrome, decreased sexual desire, and insomnia compared to undiagnosed respondents $(\mathrm{p} \leq$ 0.0161 ). Quality of life was more impaired in diagnosed patients in terms of ability to drive safely, propensity for accidents, work performance, and anxiety ( $\mathrm{p} \leq 0.039$ vs. undiagnosed). However, other quality of life measures such as alertness, irritability, motivation, ability to get sufficient sleep, and energy level were impaired in a similar proportion of respondents in both groups. Work-related accidents $(16 \%$ vs. $5 \% ; \mathrm{p}=$ $0.0076)$ and injuries at work $(17 \%$ vs. $7 \% ; p=0.0233)$ were also reported by significantly more diagnosed respondents than undiagnosed. Many respondents used caffeine and $57 \%$ of diagnosed respondents received prescription medication to treat symptoms such as excessive sleepiness and/or insomnia. The use of behavioral modifications was significantly more common among diagnosed respondents. However, there was not a significant difference in overall use of non-prescription treatments with the exception of "natural/ herbal remedies/vitamins" for wakefulness.

CONCLUSIONS: Individuals with diagnosed SWD demonstrated impairment in many quality of life measures and increased work-related accidents and injuries and use of non-prescription/behavioral remedies compared to undiagnosed respondents. Still, many measures of quality of life and prescription drug usage were similar between groups. This study was funded by Cephalon, Inc, now a part of Teva Pharmaceuticals Ltd.

EXERCISE REDUCES BLOOD PRESSURE IN DIABETICS WITH PERIPHERAL ARTERIAL DISEASE Philip Twumasi-Ankrah; Tracie Collins. KU School of Medicine - Wichita, Wichita, MN. (Control ID $\# 1338252$ )

BACKGROUND: Patients with diabetes mellitus and peripheral arterial disease (PAD - defined by an ankle-brachial index - an objective measure of lower limb blood flow - <0.9) are at high risk for systemic ischemic events (i.e., myocardial infarctions and ischemic cerebrovascular accidents) in addition to critical limb ischemia. A major risk factor for such adverse events is hypertension. Routine exercise is known to reduce blood pressure in sedentary populations but less is known about its impact in persons with diabetes mellitus and PAD. We sought to determine the association of exercise behaviors and blood pressure in persons with diabetes mellitus and PAD.

METHODS: We conducted a secondary analysis of a clinical trial in which we enrolled participants with diabetes mellitus and PAD to a six-month home-based walking intervention versus control. Participants completed baseline, three-month, and six-month assessments of exercise behaviors, as captured by the Stanford Patient Education Research Center Exercise Behaviors Instrument (higher scores indicate more frequent use of exercise), and treadmill walking distance. Linear regression was used to assess the relationship of the independent variables of exercise behaviors and walking distance with the dependent variables of systolic and diastolic blood pressure measurements for all three time points.

RESULTS: We analyzed data for 145 participants (mean age 66.5 years \pm 10.1 years). Forty-five (31\%) were women, 119 (82\%) had a known diagnosis of hypertension, and $132(91 \%)$ had a body mass index (BMI)> $=25$. At baseline, mean systolic blood pressure was 138 (SD 18.95), mean diastolic blood pressure was 70.53(SD 12.51), and mean exercise behavior score was 18 (SD 16.5), which corresponds to 30-60 minutes of exercise per week. Mean maximal walking distance was 0.255 meters (SD 0.15). At baseline, for each unit increase in exercise behavior score, participants had only a $0.01 \mathrm{mmHg}$ decrease in systolic blood pressure. At three-months, resting systolic blood pressure decreased by $6.64 \mathrm{mmHg}$ for every unit increase in the exercise behavior score (adjusted for multiple comparisons, $\mathrm{P}=0.0145$, as compared to baseline). At six-months, resting systolic blood pressure decreased by $8.87 \mathrm{mmHg}$ for every unit increase in the exercise behavior score (adjusted for multiple comparisons, $\mathrm{P}=0.0026$, as compared to baseline). These findings were more pronounced among participants with a BMI $>=25$. There were no statistically significant associations of exercise behavior scores with resting diastolic blood pressure. Comparing six-months to baseline, each unit increase in mean maximal walking distance was associated with a $6.1 \mathrm{mmHg}$ decrease in systolic blood pressure $(\mathrm{P}=0.007)$.

CONCLUSIONS: Greater levels of exercise are associated with lower resting systolic blood pressure and this association is more pronounced with time and in persons who are obese. These findings highlight the need for routine exercise to reduce systolic blood pressure among high risk groups including obese persons with diabetes mellitus and PAD. Further research is needed to identify which forms of exercise are most efficacious to reduce blood pressure in persons with multiple risk factors for adverse systemic events.

EXPANDING PRIMARY CARE RESIDENCY TRAINING MEDICAL CARE IN UNDERSERVED COMMUNITIES Kelly White; Karen Chacko. University of Colorado SOM, Aurora, CO. (Control ID \#1332138)

BACKGROUND: There is a national need for primary care doctors in rural and underserved areas of the United States. Our primary care internal medicine training program selects for students demonstrating an interest in practicing in these areas. Historically, we have offered month long preceptorships in rural and urban underserved settings for both educational value and evidence that exposure to both rural and underserved populations leads to increased probability of career choice in these areas (reference here). Despite high interest in doing such rotations, few residents previously chose rural preceptorships mainly due to financial and logistical barriers. Our goal was to couple the interest and the exposure to primary care in rural and underserved sites in hopes of increasing the number of primary care residency graduates who choose careers in rural and underserved areas.

METHODS: Potential rural and underserved teaching sites were identified. Site visits were completed which included faculty development focusing on enhancing teaching skills in the clinic setting and competency-based evaluation process. Primary care residents were educated regarding rural sites and rural health career opportunities including loan repayment options. Resident preceptorship choices were recorded.

RESULTS: The grant began in April of 2009. Site visits were completed shortly afterwards. Resident education was also completed shortly after funding. Career choice at graduation is actively being tracked. (See Chart-1)

CONCLUSIONS: Offering established rural and underserved preceptorship sites to internal medicine primary care track trainees increases their likelihood of doing such a preceptorship. It is our hope that this will also lead to an increase number of our graduates choosing careers in such sites, and our early data would suggest this is true. This model can be implemented at other programs around the country as we work together to realign the physician supply and patient demand for services in our country. We will continue to track the career choices of our graduates both 
in the short and long-term to better understand how training exposure affects career choice after graduation.

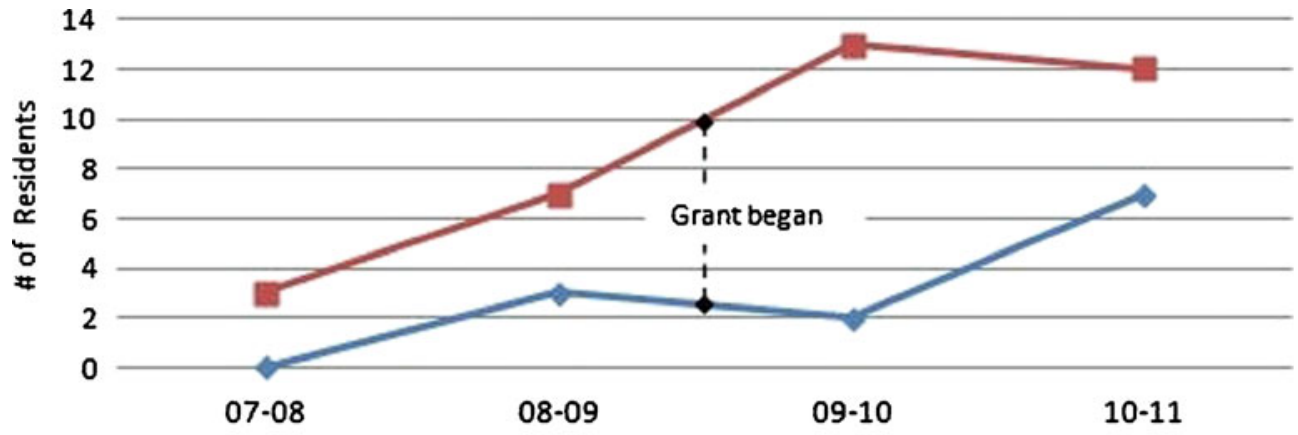

\section{Chart-1}

- Rural Preceptorship

EXPECTATIONS FOR MEDICAL STUDENT WORK HOURS IN INPATIENT CLINICAL CLERKSHIPS Rebecca A. Mazurkiewicz ${ }^{1}$; Erica Friedman ${ }^{2}$; Reena Karani ${ }^{2}$; Jenny J. Lin ${ }^{1}$. ${ }^{1}$ Mount Sinai School of Medicine, New York, NY; ${ }^{2}$ Mount Sinai School of Medicine, New York, NY. (Control ID \#1321077)

BACKGROUND: Each medical school must develop mission-based competencies and curricula to attain them; however, no standards exist regarding the specific skills that medical students should learn, or how much time should be allotted to learn them, during the clinical undergraduate medical experience. As time spent on activities may imply which associated skills are acquired, we aimed to determine how third-year medical students allocate their time in the hospital during clerkships. We also assessed and compared how students and clerkship directors (CDs) reported time should be allocated to specific activities to best achieve clerkship goals.

METHODS: From January 2011 to June 2011, Mount Sinai School of Medicine third-year students from the Class of 2012 were given an anonymous survey midway through an inpatient rotation during their Internal MedicineGeriatrics or Surgery clerkships. This survey asked students to estimate the number of hours they spent on common clerkship activities on an average noncall day during the rotation. These activities included direct patient care (e.g. admissions, pre-rounding, post-OR checks, etc.), rounding, coordinating care (e.g. calling consults, speaking with ancillary staff, etc.), participating in procedures or in OR, attending didactics and conferences, writing notes, studying and researching, idle time, and other. Students were also asked to indicate the amount of time they felt they should have spent on those activities in order to achieve the clerkship competencies. CDs $(n=4)$ were given an analogous survey asking them to estimate the amount of time their students are currently spending on common clerkship activities and how much time they should have spent on those activities. Mann-Whitney U-tests were performed comparing results between students and CDs.

RESULTS: All 4 CDs (100\%) and 104 of 110 eligible students (95\%) completed surveys. For the Internal Medicine-Geriatrics clerkship, CDs reported that their students are spending significantly more time rounding ( 3.25 hours vs. 2 hours, $p=0.007$ ) and studying ( 2 hours vs. 0.75 hours, $p=$ 0.04) than students reported they were. For the Surgery clerkship, CDs perceived that their students are spending significantly more time writing notes ( 1 hour vs. 0 hours, $\mathrm{p}=0.02$ ) and studying ( 3 hours vs. 0.5 hours, $\mathrm{p}=$ 0.02 ) and significantly less idle time ( 0 hours vs. 1 hour, $p=0.04)$ than students reported they were. CDs felt students should spend more time rounding ( 3 hours vs. 2 hours, $\mathrm{p}=0.004$ ) during the medicine clerkship and more time on educational activities ( 2 hours vs. 1 hour, $p=0.02$ ), studying ( 3.5 hours vs. 1 hour, $\mathrm{p}=0.03$ ), and total time in hospital ( 16.5 hours vs. 12 hours, $p=0.04$ ) during the surgery clerkship than students felt they should. CONCLUSIONS: There is discordance between how students and CDs report students allocate their time in the hospital during clerkships. In addition, there is discrepancy between students' and CDs' reports regarding the amount of time that should be allotted to certain educational activities in order to achieve clerkship objectives. These differences may be due to external mediators; however, CDs should ensure clear communication with students to promote their understanding of which activities will prepare them for exams and internship.

EXPECTATIONS FOR STUDENT ORAL CASE PRESENTATIONS: DO INTERNAL MEDICINE AND PEDIATRIC COURSE DIRECTORS SEE EYE TO EYE Eric Green ${ }^{1}$; Linda O. Lewin ${ }^{2}$; Steven Durning $^{7}$; Linda DeCherrie ${ }^{3}$; Mark J. Fagan ${ }^{4}$; Bradley A. Sharpe ${ }^{5}$; Warren Hershman ${ }^{6}{ }^{1}$ Mercy Catholic Medical Center, Darby, PA; ${ }^{2}$ University of Maryland, Baltimore, MD; ${ }^{3}$ Mount Sinai School of Medicine, New York, NY; ${ }^{4}$ Alpert Medical School of Brown University, Providence, RI; ${ }^{5}$ University of California San Francisco, San Francisco, CA; ${ }^{6}$ Boston University School of Medicine, Boston, MA; ${ }^{7}$ Uniformed Services University of Health Sciences, Bethesda, MD. (Control ID \#1322482)

BACKGROUND: There are no universally accepted standards for oral case presentations (OCP) by medical students. We hypothesized that pediatric and internal medicine medical educators would value the same attributes of the OCP.

METHODS: We included questions regarding 22 attributes of the OCP done by a third year student on an annual survey of pediatric medical education leaders conducted by the Counsel of Medical Student Education in Pediatrics (COMSEP) in 2010. Respondents rated the relative importance of each of these attributes using a Likert scale ranging from 1 (not important) to 5 (very important). 18 of these questions were identical to the questions posed to members of the Clerkship Directors in Internal Medicine (CDIM) in 2007. We also asked both groups about the expected length of the OCP. We used chi squared analysis and t-tests for comparisons.

RESULTS: COMSEP received 157 complete replies from 334 surveys (response rate $47 \%$ ) with at least 1 respondent from $71 \%$ of all U.S. allopathic medical schools, while CDIM received 82 of 110 surveys (response rate $=75 \%$ ). Eight attributes of the oral case presentation were rate as very important by more than $50 \%$ of internists and pediatricians (see table). The only statistical difference seen between internists and pediatricians was in the prioritized problem list, rated as very important by $76 \%$ of internists and $63 \%$ of pediatricians $(\mathrm{p}=.04)$. The only pediatric-specific attribute with $>50 \%$ rating as very important was inclusion of growth parameters. Pediatricians, compared to internists, believed OCPs should be slightly shorter ( $6.8 \pm 3.3$ vs $8.2 \pm 4.2 \mathrm{~min}, \mathrm{p}=.006)$.

CONCLUSIONS: North American undergraduate medical education leaders in internal medicine and pediatrics share common expectations for oral case presentations. They both expect the OCP to include a comprehensive report of the history, along with a targeted and reasoned 
physical exam, assessment, and plan. Pediatricians' expectations only differ from internists' in the expected length of the presentation and inclusion of a single pediatric-specific attribute. Our findings suggest that the OCP, like history taking and physical exam, has interdisciplinary rather than discipline-specific standards. The identified shared attributes can be used to develop interdepartmental instruction in, and assessment of, OCPs.

Percentage of Respondents Rating Attribute as Very Important

\begin{tabular}{|c|c|c|c|}
\hline Attribute & $\begin{array}{l}\text { Medicine } \\
(\mathrm{n}=81)\end{array}$ & $\begin{array}{l}\text { Pediatrics } \\
(\mathrm{n}=147)\end{array}$ & $\begin{array}{l}\mathrm{P} \\
\text { value }\end{array}$ \\
\hline Organized According to Standards & 85 & 77 & .13 \\
\hline Chief Complaint & 80 & 76 & .41 \\
\hline Accurately describe symptoms & 82 & 72 & .13 \\
\hline Includes all pertinent facts & 65 & 67 & .85 \\
\hline $\begin{array}{l}\text { Sequence of events leading to } \\
\text { hospitalization }\end{array}$ & 64 & 64 & .97 \\
\hline $\begin{array}{l}\text { Detailed assessment and plan for the } \\
\text { most important problems }\end{array}$ & 76 & 63 & .04 \\
\hline $\begin{array}{l}\text { Targeted physical examination germane } \\
\text { to the patient's complaint }\end{array}$ & 62 & 62 & .99 \\
\hline Prioritized problem list & 67 & 61 & .45 \\
\hline Growth history & not asked & 56 & \\
\hline $\begin{array}{l}\text { Structured to guide the listener to } \\
\text { conclusion (e.g } \backslash \text { "makes a casel") }\end{array}$ & 43 & 36 & .31 \\
\hline $\begin{array}{l}\text { Comprehensive assessment and plan } \\
\text { for all problems }\end{array}$ & 12 & 25 & .02 \\
\hline Clearly Spoken & 30 & 23 & .24 \\
\hline Complete physical exam & 10 & 17 & .77 \\
\hline Excludes all not pertinent facts & 18 & 14 & .45 \\
\hline Uses Precise terminology & 24 & 14 & .09 \\
\hline Detailed social history & 15 & 13 & .74 \\
\hline Developmental history & not asked & 12 & \\
\hline Complete review of systems & 5 & 12 & .09 \\
\hline Impact of the illness on the patient & 18 & 10 & .07 \\
\hline Complete family history & 7 & 10 & .55 \\
\hline Vaccination history & not asked & 7 & \\
\hline
\end{tabular}

EXPECTATIONS OF IPAD USE IN AN INTERNAL MEDICINE RESIDENCY PROGRAM: IS IT WORTH THE "HYPE"? Nancy Luo $^{1}$; Bhakti Patel ${ }^{2}$; Christopher G. Chapman ${ }^{1}$; James Woodruff ${ }^{1}$; Vineet Arora ${ }^{1}{ }^{1}$ University of Chicago Medical Center, Chicago, IL; ${ }^{2}$ University of Chicago Medical Center, Chicago, IL. (Control ID \#1339342)

BACKGROUND: Hospitals and residency programs are increasingly looking to mobile computing to enhance physician efficiency and improve patient experience. While anecdotal reports highlight its benefits, introduction of any new technology in the workplace could result in an "inflated peak of expectations" according to the Gartner Hype Cycle. Few studies examine this phenomenon. The aim of the study is to compare expectations of use of an iPad among residents in a teaching hospital with perceptions after deployment.

METHODS: One-hundred and fifteen internal medicine residents were given Apple iPadsTM (Cupertino, CA) with access to the electronic medical record (EMR) [EpicTM (Verona, WI)] via Citrix client in October of 2010. Residents were surveyed in the month prior and four months after deployment of iPads to assess the potential impact on their workflow and efficiency. Questions used a five-point Likert scale to assess excitement, anticipated and actual use, as well as barriers to use in terms of portability and user interface. Data were merged in Excel, and Pearson's chi squared tests were performed to assess for differences before and after iPad deployment.

RESULTS: Ninety-nine percent (114/115) of residents completed a matched pre- and post-deployment survey. Most residents [78\%] reported using the iPad during the call day. After deployment of the iPad, fewer residents reported spending time looking for a computer (16\% post vs $65 \%$ pre, $\mathrm{p}<0.0001)$ and waiting for a computer to log into the EMR $(30 \%$ post vs $63 \%$ pre, $p<0.0001)$. Interestingly, while most residents before deployment believed that the iPad would improve attendance at educa- tional conferences, patient care, and efficiency on the wards, there were fewer residents who 'strongly agreed' with each of these statements after deployment of the iPads $(17 \%$ post vs $44 \%$ pre, $\mathrm{p}<0.0001$ for education; $15 \%$ post vs $34 \%$ pre, $\mathrm{p}=0.009$ for patient care; $24 \%$ post vs $41 \%$ pre, $\mathrm{p}=$ 0.034 for efficiency). The biggest barriers to why residents did not use their iPad more were: difficult to type (47\%), cumbersome to carry $(42 \%)$, wireless access point (e.g. "hotspot") connection delay (37\%), and slow user interface of the EMR on the iPad (32\%). Surprisingly, compared to before deployment, more residents reported after deployment of the iPad that they preferred pen and paper to organize their thoughts $(67 \%$ post vs $39 \%$ pre, $\mathrm{p}<0.001$ ). In spite of this, $84 \%$ of residents thought the iPad was a good investment for the residency program, and over half of residents (58\%) reported that patients commented on the iPad in a positive way. After using the iPads for 4 months, more residents agreed with the statement "[the $\mathrm{iPad}]$ has changed my life" $(16 \%$ post vs $6 \%$ pre, $\mathrm{p}=0.02)$. CONCLUSIONS: While the use of mobile computing devices like the iPad is associated with improved efficiency among internal medicine residents and is generally well-received by residents and patients, the high initial expectations of the iPad highlight the danger of implementing new technologies. Here, we show inflated expectations specifically in the areas of education, patient care, and efficiency, in addition to a stronger preference for pen and paper after the iPad. For those considering deployment of mobile devices, it is important to consider how to manage initial expectations to avoid the 'trough of disillusionment' and maximize the adoption of new technologies.

EXPERIENCES OF REPORTED HEALTHCARE DISCRIMINATION: INSIGHTS FROM FOCUS GROUPS WITH SPANISH-LANGUAGE SPEAKING PATIENTS. Gladys Rodriguez; Rosana Gonzalez-Colaso; Marcella Nunez Smith. Yale School of Medicine, New Haven, CT. (Control ID \#1339692)

BACKGROUND: Healthcare discrimination is emerging as an important correlate of outcomes for racial/ethnic minority patients. However, Hispanic/Latino (H/L) populations have been largely underrepresented within this body of research and the influence of English language proficiency is rarely disaggregated. Building upon a previously conducted qualitative study and embedded within a parent study (2R21 CA134980-01A2) we sought to gain a better understanding of reported healthcare discrimination in this population and capture emerging novel themes.

METHODS: We conducted four online English- and Spanish-language focus groups with self-identified $\mathrm{H} / \mathrm{L}$ participants $(\mathrm{n}=5-6$ participants). Focus groups were defined by participant English language proficiency (proficient or less than proficient) and geographic location (Connecticut or Texas). A moderated online forum was established within the parent study's webpage; participants confidentially posted and replied to comments at their convenience over a 3-day period. Focus groups began with the broad question "How does your ability to speak English influence your interactions in health care settings, if at all?" Two additional focus groups are currently in progress.

RESULTS: Five novel themes capturing unique perspectives not previously described in the prevailing literature emerged from preliminary analysis: 1) Patients defined a range of healthcare discrimination experiences that they attributed to English language proficiency; 2) Regardless of their level of English proficiency, participants viewed their role within the healthcare system as advocates for high quality care; 3 ) Participants detailed unexpected standards to assess interactions with individual providers regarding healthcare discrimination; 4) Participants prioritized several key components in the provider-patient interaction above ethnic or language concordance; 5) Participants valued information sharing regardless of the type of clinical situation or setting.

CONCLUSIONS: We were surprised to find data from the focus groups that ran counter to many prevailing ideas regarding Spanish-speaking patient populations. This study provides support that neither $\mathrm{H} / \mathrm{L}$ patients nor LEP Spanish-speaking patients are monolithic. A broad diversity of knowledge and expectations exist within this population that can inform patient-provider interactions and reduce the experience of healthcare discrimination. 
EXPLORING CHALLENGES AND PREFERENCES AT HOSPITAL DISCHARGE IN A LOW HEALTH LITERACY POPULATION Cristina M. Gonzalez ${ }^{1,2}$; Pajtesa Kukaj ${ }^{1}{ }^{1}$ Albert Einstein College of Medicine, Bronx, NY; ${ }^{2}$ Montefiore Medical Center, Bronx, NY. (Control ID \#1340186)

BACKGROUND: Health literacy is a set of skills, including the ability to perform basic reading and numerical tasks, required to function in the health environment. Low health literacy (LHL) contributes to health disparities. Patients with LHL have a poorer understanding of prescription medication names, indications for use, instructions and more unreconciled medications. Evidence suggests that poor communication plays a role in many medication errors after hospital discharge, and is exacerbated by LHL. The objective of this study was to ascertain the challenges and preferences of patients at risk for LHL when providers are communicating with them about their medications at the time of hospital discharge.

METHODS: Patients were selected by convenience sampling from the medicine service at a university hospital in Bronx, NY. Informed consent was obtained and semi-structured interviews (in English or Spanish) were conducted regarding current or previous hospital discharge. Two investigators independently reviewed the transcribed data to generate a codebook using grounded theory. A third researcher reviewed any data where disagreement in coding arose. Saturation of themes was reached after 14 interviews.

RESULTS: Three major themes emerged from analysis of the interview data: challenges to asking questions, patient's preferences for delivery of medication instructions, and the effect of trust on patient's perceptions of the discharge process. Most patients acknowledged that at times they wanted to ask questions and did not. Frequently a barrier to question asking could not be identified, with patients merely stating: "It didn't occur to me to ask." Patients preferred instructions to contain pictures of pills. Most patients organized their medications with a pillbox and identified pills by color; in contrast to the delivery of medication instructions by name, dosage and indication. They preferred explicit, simple language, with careful attention to any dangers. "Don't take the medicine you got at home because you could get real sick. I remember that one. It's important." "She just kept emphasizing what he shouldn't have." Low trust in the medical system challenged patient's ability to solve problems after discharge. Patients with regular follow-up stated they would call their doctor or pharmacist if they had a problem. Without a trusted provider, problem solving suffered. "If I can't get it [medication] that day, I'll have to wait the next day, sometimes two days." Patients worried when they could not get their medications. Regarding pill color changes, "I really don't know what I would do" was a common response. "I wanted to know what I'm taking and why...it never happened. Then I refused to take the medication." Some mistrust stemmed from a misconception of standard residency program practice. "So many different doctors and they come and ask different questions so many times."
"They don't know too much." "You have a young doctor, they don't know what they're doing. So they [patients] won't tell them."

CONCLUSIONS: The results of this analysis suggest providers need a mechanism to uncover patient's questions and that medication instructions are not currently delivered in a way to minimize post-discharge medication errors. Additionally, efforts to enhance trust may increase patient problem solving abilities after discharge. Further research is needed to develop and evaluate methods for patient-centered discharge planning that account for low health literacy.

EXTREME HYPERFERRITINEMIA IN HOSPITALIZED PATIENTS: CAUSES AND PROGNOSTIC SIGNIFICANCE Jay Lipshitz; Yair Keilson; Nelli Fromer; Summer R. Branda; Oscar Lahoud; Yiqing Xu; Alan B. Astrow; William B. Solomon. Maimonides Medical Center, Brooklyn, NY. (Control ID \#1336714)

BACKGROUND: Extreme hyperferritinemia (serum ferrtin $>1000 \mathrm{mcg}$ / dL) commonly occurs in states of iron overload where it serves as a marker for toxic levels of tissue iron. Very high serum ferritin values may also occur in conditions of acute or chronic inflammation. There is little information regarding the causes and significance of extreme hyperferritinemia in hospitalized patients.

METHODS: We reviewed the records of all adult inpatients with serum ferritin values greater than $1000 \mathrm{mcg} / \mathrm{dL}$ over a 6-month period at our institution to determine the causes of extreme hyperferritinemia and markers of disease severity. Markers for severity included ICU admission, length of stay and in-hospital mortality. We compared the results in our patient cohort to those in all hospitalized adults for the same 6-month period to assess whether there may be an association between extreme hyperferritinemia and adverse outcomes.

RESULTS: Ninety-two patients with a serum ferritin value greater than $1000 \mathrm{mcg} / \mathrm{dL}$ were identified. In the vast majority of cases extreme hyperferritinemia could be attributed to one of four causes, infection (42.4\%), hepatitis (16.3\%), malignancy (15.2\%) and renal insufficiency $(10.9 \%)$. Iron overload accounted for a small minority (5.4\%). Patients with extreme hyperferritinemia had worse outcomes in all clinical measures. Mortality was $10.9 \%$ compared with $2.7 \%$ for all adult inpatients. $18.5 \%$ of patients with extreme hyperferritinemia had an ICU visit during hospitalization compared with $7.4 \%$ for all patients. Median length of stay was 10 days for the patients with extreme hyperferritinemia compared with 3 days for all patients.

CONCLUSIONS: Extreme hyperferritinemia in hospitalized patients can usually be attributed to infection, malignancy, hepatitis or renal insufficiency. A serum ferritin level greater than $1000 \mathrm{mcg} / \mathrm{dL}$ may be associated with adverse outcomes in hospitalized patients.
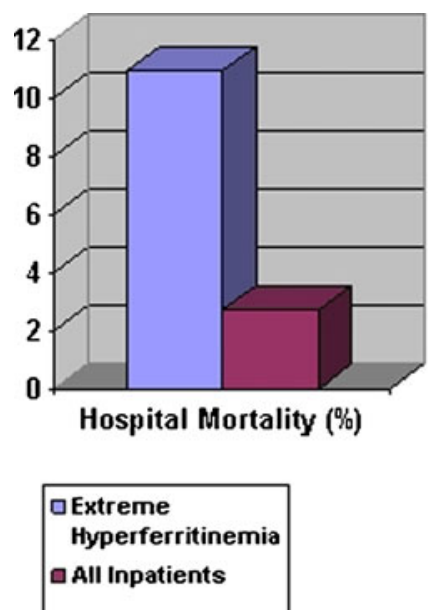

Figure 1: Markers of disease severity for patients with extreme hyperferritinemia compared with all adult inpatients
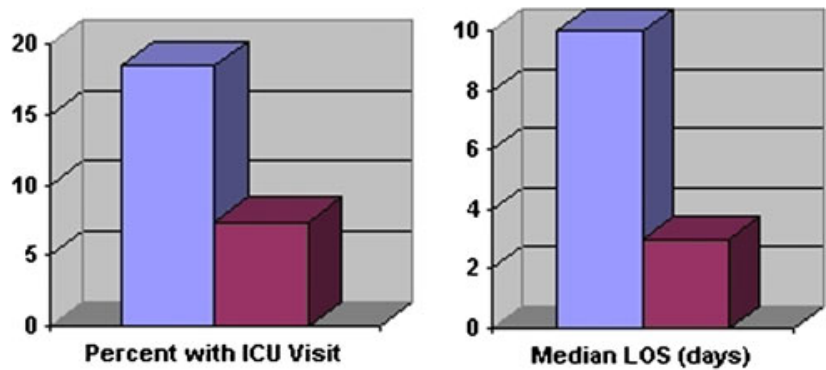

Median LOS (days) 
FACTORS CONTRIBUTING TO IMPROVED HYPERTENSION MANAGEMENT AT THE VA Rebecca Brienza ${ }^{1,2}$; Emily M. Meyer ${ }^{1,3}$; Daren Anderson; Aldo Peixoto ${ }^{1,2}$. ${ }^{1}$ VA Connecticut Healthcare System, West Haven, CT; ${ }^{2}$ Yale University School of Medicine, New Haven, CT; ${ }^{3}$ Yale University School of Medicine, New Haven, CT; ${ }^{4}$ Community Health Center, Inc., Middletown, CT. (Control ID \#1331509)

BACKGROUND: Every $10 \mathrm{mmHg}$ reduction in blood pressure results in a $35 \%$ reduction in rates of stroke. The Veterans Health Administration (VHA) has achieved substantial improvement in hypertension control over the past decade, far exceeding national rates. While this improvement coincides with a broad, national VHA redesign and quality improvement efforts, it is unknown how VHA achieved these superior results in blood pressure control. Therefore, we hypothesized that the improvement in blood pressure control is the result of an improvement in clinician "treatment intensification" brought about by a broad series of reforms in the measuring and monitoring of clinical performance.

METHODS: Our study used a mixed methods approach to evaluate hypertension treatment from 1998 to 2008. This abstract will focus on our qualitative results. We conducted four focus groups with clinicians, pharmacists, and health care leaders using a semi-structured data collection protocol. All sessions were digitally recorded and transcribed verbatim. The transcripts were topic-analyzed and iteratively coded by an independent investigator with extensive training in qualitative data analysis. We did not utilize an existing theoretical model to frame analysis; rather we implemented the constant comparative method of Glaser and Strauss' Grounded Theory. Through a series of open coding, the development of data tables, conceptual mapping and iterative code refinement, hierarchical themes and associated sub-themes were generated.

RESULTS: Our qualitative data suggest that providers are taking a more active role in hypertension management through enhanced VHA system redesign (e.g. electronic health records, clinical reminders, and performance reports), interdisciplinary collaboration and prescribing optimal pharmacological therapies and doses. According to one provider participant, "while seeing patients in the primary care clinic, it [electronic reminders] just reinforces the importance of blood pressure control." Participants also noted several barriers to improved blood pressure management. Many Veterans still struggle with challenges in proper medication adherence due to polypharmacy and low health literacy. Failure to refill prescriptions regularly, self-discontinuation due to side effects, and reluctance to take multiple medications were also noted. Our participants observed that adherence was especially problematic among women; this is possibly the result of multiple mental health comorbidities. Providers suspect that some of the challenges associated with adherence are attenuated when men have a supportive spouse or family caregiver to assist them

CONCLUSIONS: This study aimed to understand why blood pressure control improved at the Department of Veterans Affairs (VA) over the past decade. Focus group participants indicated that systems improvements and an interdisciplinary team approach to blood pressure management played major roles. They also felt that a general attitude shift towards aggressive monitoring and treatment occurred during the last ten years. Challenges to medication adherence in women Veterans with psychiatric comorbidities and the powerful role of supportive caregivers to maintaining a treatment regimen were highlighted. Future research should investigate the impact of systems redesign for other chronic conditions on Veteran health care outcomes and the unique social needs and challenges to medication adherence for Veterans receiving primary care at VHA.

FACTORS INFLUENCING ADHERENCE TO AN URBAN PUBLIC HOSPITAL WEIGHT MANAGEMENT PROGRAM Himali Weerahandi; Lisa Parikh; Gaëlle C. Pierre; Brian Diskin; Elenore Patterson; Albert Ahn; Camila Deza; Colleen Gillespie; Michelle McMacken. NYU School of Medicine, New York, NY. (Control ID \#1333574)

BACKGROUND: Much research has been devoted to identifying factors that predict weight loss and weight loss maintenance. Studies of low-income and minority populations, who are disproportionately affected by obesity, have shown that increased social support, increased nutritional knowledge, and attendance at structured weight loss programs have been linked to increased weight loss. Previous research from our own urban weight management clinic demonstrated that the number of revisits to clinic was the most significant predictor of weight loss and that approximately half of the patients did not return for a second visit. There are limited data on factors influencing adherence to structured weight loss programs in a minority, low-income population. Our study sought to identify predictors of revisits to our clinic. METHODS: We retrospectively reviewed electronic medical records of all patients seen in our weight management clinic between January 2006 and July 2010. The following data were collected for each patient: age at first visit, gender, provider-documented race, referral source (primary care provider vs other), presence of family/friend support (patient self-report, indicated as yes/ no) diagnosis of axis 1 psychiatric disorder, use of medications associated with weight gain, language concordance with weight management clinic provider, weight lost at second visit, total weight lost, and total number of revisits.

RESULTS: Data were collected for 318 patients seen during the study period. Of these, 146 had documented information on age, gender, family/ friend support, language concordance with provider, diagnosis of axis 1 psychiatric disorder, referral source, and use of medications associated with weight gain. These were the factors included in the regression analysis. Two patients were identified as outliers (standardized residuals greater than 3 ), and excluded from the analysis. Of those included, $46 \%$ were identified as Hispanic, $15 \%$ as black, $3 \%$ as white, $2 \%$ as Asian, and $1 \%$ as another racial group; race/ethnicity data were missing for 33\%. Also, $79 \%$ were female, $29 \%$ carried an axis 1 psychiatric diagnosis, 34\% were on medications associated with weight gain (primarily insulin, thiazolidinediones, sulfonylureas, and antipsychotics), $78 \%$ were referred to the clinic by their primary care provider, $76 \%$ had language concordance with the weight management clinic provider, and 59\% reported support from family/friends. The average age at the first visit was 46 . When controlling for age, gender, and use of medications causing weight gain, age at first visit was negatively related to the total number of revisits $(\beta=-.22, p<.01)$ - older patients had fewer revisits. Presence of family/friend support was also negatively related $(\beta=-.29, p<.001)$. Use of medications associated with weight gain was positively related to total number of revisits $(\beta=.25$, $\mathrm{p}<.01$ ). No other variables were significantly related to revisits.

CONCLUSIONS: In our urban, safety-net hospital weight management clinic, older patients and those who reported receiving support from family and friends were less likely to adhere to return visits; in contrast, patients taking medications associated with weight gain were more likely to follow up. No other variables were associated with adherence. Future studies should further refine the reasons for attrition in weight management programs, as the number of return visits seems to correlate with success in weight loss.

FACTORS ASSOCIATED WITH DEPRESSIVE SYMPTOMS AT 6 WEEKS POSTPARTUM AMONG WOMEN WITH RECENT GESTATIONAL DIABETES MELLITUS Jacinda M. Nicklas ${ }^{1,2}$; Laura J. Miller ${ }^{3}$; Chloe A. Zera ${ }^{4}$; Sue E. Levkoff ${ }^{3,6}$; Ellen W. Seely ${ }^{2}$. ${ }^{1}$ Beth Israel Deaconess Medical Center, Boston, MA; ${ }^{2}$ Brigham and Women's Hospital, Boston, MA; ${ }^{3}$ Brigham and Women's Hospital, Boston, MA; ${ }^{4}$ Brigham and Women's Hospital, Boston, MA; ${ }^{5}$ Brigham and Women's Hospital, Boston, MA; ${ }^{6}$ University of South Carolina, Columbia, SC. (Control ID \#1339498)

BACKGROUND: Postpartum depression affects $10-15 \%$ of new mothers, and can impair both self-care and infant care. Identifying women at increased risk for postpartum depression may help guide prevention efforts. Little is known about predictors for postpartum depression among women with a history of gestational diabetes (GDM). We sought to identify factors associated with symptoms of postpartum depression at 6 weeks postpartum among women with recent GDM.

METHODS: We administered the Edinburgh Postnatal Depression Scale (EPDS) at 6 weeks postpartum to women whose most recent pregnancy was complicated by GDM (confirmed by laboratory data or chart diagnosis). An EPDS score $\geqq 9$ indicated depressive symptoms. We measured weight and height, measured thyroid stimulating hormone, and administered a questionnaire to collect demographic data as well as information about breastfeeding and sleep. We obtained pre-pregnancy weight by self-report, and calculated 
pre-pregnancy BMI using measured height (BMI categorized as $<25,25-30$, $>30$ ). We performed a chart review to obtain data about medical history, including insulin use during pregnancy. We used backward selection to fit multivariable logistic regression models to identify factors associated with depressive symptoms.

RESULTS: Our study included 74 women (mean age 33 years \pm 5 ; $51 \%$ Caucasian, 32\% African-American, 12\% Asian, 5\% Other; 20\% Hispanic; mean pre-pregnancy BMI $30 \mathrm{~kg} / \mathrm{m}^{2} \pm 6$ ). Measured weight at 6 weeks postpartum was $184 \mathrm{lbs} \pm 40$. Fifty-four percent reported annual household incomes $>\$ 75,000$, while $25 \%$ reported incomes of $<\$ 25,000$ annually. No cases of hypothyroidism were identified. Thirty-five percent of women studied had depressive symptoms at 6 weeks postpartum. When controlling for income level, higher pre-pregnancy BMI category (OR 2.5, 95\% CI 1.26.0) was associated with depressive symptoms. Use of insulin during pregnancy, perceived lack of sleep, breastfeeding status, and race/ethnicity were not retained in the model. Among those women with weights recorded within 5 days of delivery ( $N=63)$, gestational weight gain was $28 \mathrm{lbs} \pm 16$. In this group, gestational weight gain was also associated with depressive symptoms (OR 1.3, 95\% CI 1.1-1.6 per $5 \mathrm{lb}$ increment of weight gain).

CONCLUSIONS: Higher pre-pregnancy BMI was associated with postpartum depressive symptoms among women with recent GDM in this study. Gestational weight gain may also be associated with depressive symptoms in this population. If confirmed in larger studies, pre-pregnancy BMI and gestational weight gain may identify women with GDM at increased risk for postpartum depression.

FACTORS THAT SUSTAIN HUMANISM IN TEACHERS OF INTERNAL MEDICINE RESIDENTS Carol Chou; Katherine Kellom; Judy A. Shea. University of Pennsylvania, Philadelphia, PA. (Control ID \#1339628)

BACKGROUND: The humanistic care of patients has been shown to improve patient satisfaction and patients' trust in their doctors. Humanism is often taught by example; teaching faculty are thus charged with modeling humanistic behavior to learners. Residents are vulnerable to declining empathy during their training and therefore can especially benefit from humanistic teachers. However, with many demands and pressures on teaching faculty, it can be hard to sustain humanism, much less to model humanistic behavior to learners. The goal of this study was to identify factors that exemplary humanistic teachers believe help them to maintain their caring attitudes.

METHODS: A survey was administered to all internal medicine residents at the University of Pennsylvania in June 2011, asking them to nominate up to three teaching attending physicians who served as excellent role models for the humanistic care of patients. Faculty members who received the most nominations were invited to participate in a one-on-one interview to identify factors that sustain humanism in their teaching and patient care. Interviews were conducted until themes reached saturation. The interviews were transcribed anonymously and coded with NVivo software by an independent coder; codes were verified by one of the investigators (CC). RESULTS: 119 of the 150 internal medicine residents at the University of Pennsylvania responded to the survey (71\% participation). 92 of the 591 internal medicine teaching faculty $(15.5 \%)$ received one or more votes. The range of votes per faculty member was 1 to 21 . The top sixteen nominees, with votes ranging from 5 to 21 , were invited to be interviewed; response rate was $100 \%$. Demographics describing interviewees are as follows: mean age was 43.2 years. Nine interviewees were women $(56 \%)$ and seven $(43.7 \%)$ were general internists. Other specialties included pulmonary/critical care $(25 \%)$, hematology/oncology (12.5\%), infectious disease (12.5\%), and GI (6.25\%). Factors that sustain humanism for these interviewees include: engaging in ongoing self-reflection $(68.7 \%)$; deriving sustenance for humanism from the patient interaction itself, including a desire to find a connection with the patient (62.5\%); being humble to receiving feedback and striving to improve one's practice (50\%); and being conscious of living up to a standard of being a "good doctor," as well as treating others as one would want to be treated (37.5\%). 15 of the 16 interviewees identified role models within medicine that had positively influenced them; the sixteenth cited negative role models in medicine that were a reminder of how not to act. 8/16 (50\%) had had personal experiences with illness or close family members with illness which inform their current humanistic tendencies.
CONCLUSIONS: These findings have implications for producing future generations of humanistic internal medicine physicians. Identifying teachable factors that help to sustain exemplary humanistic physicians may allow us to replicate those factors and create an environment in which those factors are fostered. Faculty development programs based on these factors may improve the teaching and delivery of humanistic care.

FACULTY DEVELOPMENT UTILIZING EDUCATIONAL VIDEOBASED SCENARIOS AND EVALUATIVE INSTRUMENT FOR HANDOFF COMMUNICATION Saba Berhie ${ }^{1}$; Vineet Arora ${ }^{2,1}$; Leora I. Horwitz ${ }^{3}$; Mark Saathoff ${ }^{2,1}$; Paul G. Staisiunas ${ }^{2}$; Jeanne M. Farnan ${ }^{2,1}$. ${ }^{1}$ University of Chicago, Chicago, IL; ${ }^{2}$ University of Chicago, Chicago, IL; ${ }^{3}$ Yale, New Haven, CT. (Control ID \#1333816)

BACKGROUND: The most recent iteration of the Accreditation Council for Graduate Medical Education (ACGME) duty hour regulations, released in July 2011, has further limited PGY-1 shift duration to 16 hours. Explicit language in these regulations also mandates handoff education for trainees and for residency training programs to assess handoff quality. However, there is a lack of validated tools for the assessment of handoff quality and to utilize for trainee education.

METHODS: Faculty were recruited via email to participate in a workshop on handoff education and evaluation to both pilot test the videos and for instrument validation. Six video-based scenarios were developed which highlight varying levels of performance in the domains of communication skills, professionalism and setting. Each video permuted one domain of performance while holding the others constant. Scripts were based upon real-time clinical observations. Videos ranged in length from 3-5 minutes. Videos were shown in a random order and faculty were instructed to use the Hand-off CEX to rate the performance; debrief occurred immediately after to identify barriers and facilitators to the displayed behaviors. The Handoff CEX was developed is a paper-based instrument in which individuals are rated in six domains on a nine point scale (unsatisfactory[1] to superior [9]) with qualitative anchors defining each level of performance. Descriptive statistics and two tests of reliability, Cronbach's alpha and Kendall's coefficient of concordance, were performed. Two tests of validity were performed: a test of trend across ordered groups and a Two-Way ANOVA to examine for rater bias.

RESULTS: 172 of a possible 189 (91\%) handoff observations were captured. Reliability testing revealed a Cronbach's alpha of $0.77(0.8=$ optimal) and Kendall's coefficient of concordance of 0.68 to $0.77(>0.6=$ high reliability). Faculty were able to reliably distinguish the different levels of performance in professionalism and setting, but had greater difficulty distinguishing between satisfactory and superior communication. Two-way ANOVA revealed no evidence of rater bias. Faculty participants commented on face validity of video scenarios, specifically those portraying setting and communication skills. In addition, robust discussion resulted in identifying the barriers and facilitators to the behaviors demonstrated in the video.

CONCLUSIONS: Video-based scenarios, utilized to highlight differing levels of performance, with focused debrief are an effective way to observe specific domains and behaviors in handoff communication. In addition, the Hand-off CEX is a reliable and valid tool to assess varying levels of videos depicting handoff performance.

FACULTY-LIBRARIAN COLLABORATION ON AN EVIDENCEBASED MEDICINE BLOCK CURRICULUM IN INTERNAL MEDICINE RESIDENCY Roy L. Kao ${ }^{1}$; Ellen M. Justice ${ }^{2}$; Sharon EasterbyGannett $^{2}$; Ene Belleh ${ }^{2}$; Daniel J. Elliott ${ }^{1}$. ${ }^{1}$ Christiana Care Health System, Newark, DE; ${ }^{2}$ Christiana Care Health System, Newark, DE. (Control ID $\# 1324925)$

BACKGROUND: As a component of the practice-based learning competency of the Accreditation Council for Graduate Medical Education (ACGME), Internal Medicine residents must demonstrate the ability to practice evidence-based medicine (EBM). Previous EBM efforts at our institution were focused on critical appraisal of articles but lacked 
comprehensive development of knowledge and skills in the four steps of EBM. These steps include formulating an answerable question, finding the best evidence, appraising the results for validity and usefulness, and then applying these findings to clinical practice and evaluating performance. Our objective was to create a curriculum to increase residents' knowledge, skills, and comfort levels in EBM.

METHODS: We developed a 2-week, dedicated EBM block rotation for first and second year internal medicine residents. Internal Medicine faculty and medical librarians jointly taught the course. The curriculum was organized around the four steps of EBM and included limited didactics with an emphasis on hands-on sessions to teach literature searching and critical appraisal within a small group setting. At the conclusion of the rotation each resident presented a critically appraised topic at a residency program core lecture. We assessed EBM knowledge and skills with the validated Fresno test at the beginning and end of the rotation. Residents similarly rated comfort with EBM resources on a Likert scale. Pre- and post-test scores and confidence survey results were compared using the paired t-test.

RESULTS: In 2011, eleven residents completed the curriculum and the test and survey. Residents' performance on the 212-point Fresno Test improved from a mean score of 114.3 on the pre-test to 154.6 on the post-test (mean difference 40.3, p<0.001). There was also significant improvement in the subsections addressing formation of questions (mean difference 3.7, p=0.01), creating search strategies (mean difference $4.3, \mathrm{p}=0.004$ ), appraising an article's relevance (mean difference 6.2, $\mathrm{p}=0.006$ ), and calculating and understanding biostatistics (mean difference 18.9, $\mathrm{p}<0.0001$ ). Confidence ratings on a 4-point Likert scale significantly improved in defining a question; searching and using MeSH terms, advanced limits, and subheadings in Ovid within a reasonable amount of time; and finally conducting a literature review and presenting it to a wider audience (all $\mathrm{p}<0.001$ ). Residents also rated their comfort with EBM databases significantly higher $(\mathrm{p}<0.001)$.

CONCLUSIONS: Our findings demonstrate that a comprehensive, dedicated evidence-based medicine block rotation utilizing a facultylibrarian partnership can be effective in improving residents' knowledge, skills, and comfort levels in the four steps of EBM.

\section{FALLING THROUGH THE CRACKS: STATIN USAGE BASED} ON POPULATION CHARACTERISTICS IN AMBULATORY CARE Anurag Mehrotra; Arun K. Muthusamy; Kavyashri Kodlipet Jagadeesh; Khalid Zakaria; Sarwan Kumar; Palaniappan Manickam. Crittenton Medical Center / Wayne State University, Rochester Hills, MI. (Control ID \#1340265)

BACKGROUND: Statin therapy is recommended for secondary prevention in all patients with known cardiovascular disease or the risk equivalent. Knowledge about the possible differences in the use of statin among high risk population in US is vital in order to stimulate a positive change in the health of society. Our objective is to assess the association between statin use and patient demographics in high risk population in ambulatory setting.

METHODS: We conducted a retrospective cross sectional analysis on the limited access dataset of National Ambulatory Medical Care Survey from the year 2007 to 2009. Patients less than 18 years of age were excluded from our analysis. High risk patients were defined by history of smoking, diabetes mellitus, hypertension, hyperlipidemia and coronary artery disease. Multivariate logistic regression model was used to compute the adjusted odds ratio to compare the demographic characteristics with and without statin use in this high risk population. All statistical analysis was performed using SAS 9.2 version.

RESULTS: There were a total of 45, 391 high risk patient visits in United States from 2007 to 2009 . Statin use was documented in only $20 \%$ of the total visits. Compared to the younger patients (age 18 - 45 years), middle- aged (age 45-64 years) and elderly ( $>65$ yrs) patients were more likely to receive statin [OR $6.2395 \%$ CI (5.16 to 7.51)]. Men were more likely to receive statin [OR $1.38 ; 95 \% \mathrm{CI}(1.27$ to 1.51$)$ ] compared to women in high risk population. The use of statin was more likely in the Medicare patients [OR 1.13; $95 \%$ CI (1.01 to 1.29$)$ ] and the community health centers [OR $1.36 ; 95 \% \mathrm{CI}$ (1.16 to 1.61$)$ ] compared to private practices. High risk patients visiting internists were more likely to be prescribed statin compared to family practitioners [OR $1.21 ; 95 \%$ CI (1.05 to 1.39)]. Among different races, statin use was less likely in African Americans [OR 0.89; $95 \%$ CI ( 0.81 to 0.97$)$ than Caucasians. Despite the similar prevalence, statin use was less likely in lower socio-economic strata [OR $0.86 ; 95 \%$ CI $(0.75$ to 0.98$)$ ] compared to patients of higher socioeconomic status. No differences were noted in the statin use based on the region and ethnicity.

CONCLUSIONS: Our findings indicate consistent association between patient 's demographic characteristics and statin use in high risk population. Future efforts should concentrate on enhancing more statin use among African Americans and uninsured high risk population, with a goal of reducing adverse events from cardiovascular and cerebrovascular incidents.

FAMILY HISTORY OF DIABETES IS LINKED TO MORE AWARENESS, LESS FATALISM, BUT NO DIFFERENCES IN PHYSICIAN COUNSELING BEHAVIOR AMONG MINORITIES WITH HIGH DIABETES RISK. Kezhen Fei; Ashley Fox; Euny C. Lee; Carol Horowitz. Mount Sinai School of Medicine, New York, NY. (Control ID \#1338299)

BACKGROUND: Family history is powerful risk factor for diabetes, but the non-modifiable quality of this risk factor may lead patients to be fatalistic about their ability to prevent diabetes onset. Alternatively, having a family history of diabetes could make patients more aware of diabetes risk factors and increase the likelihood of modifying their behaviors to prevent diabetes. Physicians may also counsel patients differently based on their family history, which may also affect patients' risk perceptions. The objective of this study was to assess overweight adults' perceived risk for diabetes and their physicians' roles in defining preventive strategies as part of a community-academic partnership in East Harlem.

METHODS: Using a community-based participatory research approach, we recruited overweight adult residents of East Harlem (measured BMI of $\geq 25$ ) with no known history of diabetes, and surveyed their family history of diabetes, and dietary attitudes and behaviors. Respondents were asked to list the three most powerful risk factors diabetes and a series of questions regarding their ability to control diabetes. In addition, participants were asked about advice that they had received from their primary care providers regarding their weight, diet and exercise and ways to minimize diabetes risk. Bivariate analysis and multivariate models were run to assess the relationship between family history of diabetes and knowledge of perceived risk factors for diabetes, fatalism and providers counseling regarding diabetes risk factors.

RESULTS: The final cohort was predominately low-income $(47 \%<$ $\$ 15,000)$, female (84\%) and Latino (74\%). Fifty-six percent had a family history of diabetes. Respondents listed unhealthy eating (67\%), being overweight $(47 \%)$, being physically inactive $(38 \%)$ and having a family history of diabetes $(32 \%)$ as the most powerful risk factors for diabetes. Nearly a quarter could not name any risk factor for diabetes. Those with a family history were more likely to identify family history as a risk factor for diabetes ( $35 \%$ vs. $28 \%$; $=0.04)$. Those with a family history were less likely to agree with the statement, "If you are going to get diabetes, there is not much you can do about it" ( $56 \%$ vs. $47 \% ; \mathrm{p}=0.02$ ). Among the $76 \%$ of participants with a regular doctor, two thirds had been informed by their physicians that they needed lose weight, be more active, and eat healthier. Multivariate models suggested that physicians did not counsel patients differently whether or not they had a family history of diabetes.

CONCLUSIONS: In a cohort of overweight participants who have not received a diagnosis of pre-diabetes or diabetes, those with a family history of diabetes were more likely to accurately report family history as a risk factor. Although the relationship could have gone either way, individuals with a family history were less fatalistic about their ability to prevent diabetes onset. Nevertheless, physicians did not adjust their counseling based on whether an individual had a family history of diabetes. Clinicians should consider eliciting this history and determining whether tailored counseling for those with a diabetes family history will better engage this high risk group in diabetes prevention activities. 
FAMILY ACCOMPANIMENT TO ROUTINE MEDICAL VISITS IS ASSOCIATED WITH BETTER SELF-CARE BEHAVIOR IN HEART FAILURE PATIENTS Crystal Cene; Laura B. Haymore; Diane Dolan-Soto; Feng-Chang Lin; Michael Pignone; Darren A. DeWalt; Christine D. Jones; Jia-Rong Wu; Giselle Corbie-Smith. University of North Carolina, Chapel Hill, NC. (Control ID \#1335013)

BACKGROUND: Family involvement is one dimension of patientcentered care and a tenet of the chronic illness care model. Family accompaniment to routine medical visits may be associated with better inter-personal processes of care, but findings are inconsistent. In order to better understand the pathways by which families exert their influence within routine medical visit and their impact on health outcomes, we examined the association between more (vs. less) frequent family accompaniment to medical visits and Heart Failure (HF) self-care behaviors.

METHODS: We conducted a cross-sectional survey of communitydwelling patients with systolic or diastolic HF seen in a university internal medicine or cardiology clinic. We separately assessed the following HF self-care behaviors: self-care maintenance (i.e., engaging in behaviors to help maintain physiologic stability, such as medication adherence and frequent weighing) and self-care management (i.e., decision-making in response to symptoms, such as recognizing signs of fluid overload and adjusting diuretic dose) using the well-validated Self-Care of Heart Failure Index (SCHFI). SCHFI scores range from 0-100 (higher scores represent better self-care) and scores $\geq 70$ are considered "adequate" self-care. Family accompaniment was assessed with the question, "how often does one of your family members or friends come in the exam room with you for your doctor's visit" (scored from 1-5 ranging from never to every visit). We dichotomized this variable as "never/rarely" vs. "some/most/every visit." We used logistic regression to examine associations between family accompaniment and adequate self-care maintenance and management, adjusting for age, race, gender, household living status, educational level, and self-rated health.

RESULTS: Of the $150 \mathrm{HF}$ patients (mean age $61 \mathrm{yrs} ; 51 \%$ female; $44 \%$ African Americans), $62 \%$ of patients were accompanied to some/most/ every visit. Mean HF self-maintenance and management scores were 69.9 (SD 13.9) and 56.8 (SD 24.2), respectively; $52 \%$ had "adequate" self-care maintenance and $32 \%$ had "adequate" self-care management. In multivariate analysis, more frequent family accompaniment to visits was associated with adequate (vs. inadequate) self-care maintenance (OR 2.3; 95\% CI 1.1$5.0 ; \mathrm{p}=0.03$ ) and adequate (vs. inadequate) self-care management (OR 5.0; $95 \%$ CI $1.7-14.8 ; \mathrm{p}<0.01$ ). These results were qualitatively similar when we analyzed family accompaniment as a continuous variable and when we analyzed self-care maintenance and self-care management as continuous variables.

CONCLUSIONS: More frequent family accompaniment to medical visits is strongly associated with behaviors to maintain physiologic stability and with decision-making related to symptom management in HF patients. Future studies should examine mechanisms (e.g. patient-provider communication) that might explain these associations. Clinicians should encourage patients to have family members attend clinic visits as it may further enhance self-care processes.

FEASIBILITY AND ACCEPTABILITY OF A SYSTEMATIC SPECIALIZED SMOKING CESSATION INTERVENTION TO SMOKERS HOSPITALIZED FOR AN ACUTE CORONARY SYNDROME. Reto Auer ${ }^{1,2}$; Baris Gencer $^{2}$; Rodrigo Tango ${ }^{3}$; Pierre-Frédéric Keller ${ }^{4}$; David Carballo ${ }^{4}$; Jean-Paul Humair ${ }^{3}$; Jacques Cornuz ${ }^{2}$; Nicolas Rodondi ${ }^{5}$. ${ }^{1}$ UCSF, San Francisco, CA; ${ }^{2}$ University of Lausanne, Lausanne, Switzerland; ${ }^{3}$ University Hospital of Geneva, Geneva, Switzerland; ${ }^{4}$ University Hospital of Geneva, Geneva, Switzerland; ${ }^{5}$ University of Bern, Bern, Switzerland. (Control ID \#1340142)

BACKGROUND: Smoking cessation interventions initiated in hospital increase smoking cessation rates among smokers hospitalized for an acute coronary syndrome (ACS). Hospitals offering specialized smoking cessation services currently do not provide systematic interventions to patients admitted for an ACS in Switzerland. Clinicians in charge of patients may decide to request a specialized smoking cessation consultation according to their perception of patients' needs. The shift from a cognitive to a motivational approach in behavior change and the effectiveness of smoking cessation interventions for inpatients suggest that these should be offered to all smokers, regardless of their motivation to quit. Our study aimed to determine the feasibility and acceptability of a proactive, systematic, smoking cessation intervention using motivational interviewing for all smokers hospitalized for an ACS.

METHODS: We designed a before-after study where we prospectively included smoking patients hospitalized with the main diagnosis of ACS in two university hospitals in Switzerland. In the observation phase, clinicians in charge of patients could request a specialized smoking cessation intervention through a simple phone call. In the intervention phase, we actively screened and included in the study all smokers hospitalized for an ACS who agreed to participate. A trained resident physician systematically offered to all subjects a smoking cessation intervention that included prescription of nicotine replacement therapy and counseling tailored to the clinical situation and the patient's stage of behavioral change. In the intervention phase, discharged patients also received four telephone counseling sessions over two months. To assess feasibility and acceptability, we measured the change in smoking cessation interventions rates between both phases and the participation rate in the intervention phase. RESULTS: In the observational phase (September 2009 to October 2010), $24 \%(\mathrm{~N}=58 / 233)$ of ACS smokers received a specialized smoking cessation intervention based on the evaluation of their HCP. In the intervention phase (November 2010 to October 2011) using a systematic approach, 93\% $(\mathrm{N}=166 / 178)$ of ACS smokers received a specialized smoking cessation intervention $(\mathrm{p}<0.001)$. In the intervention phase, less than $2 \%(\mathrm{~N}=3 / 178)$ of smokers refused discussions about their smoking habits and $5 \%(\mathrm{~N}=9 / 178)$ were discharged before any smoking cessation counseling could take place. The duration of smoking cessation intervention ranged from 2 to 120 minutes (mean: 52 minutes). $90 \%$ of patients accepted the ambulatory telephone counseling. Smoking cessation rates at one year were $40.1 \%(\mathrm{~N}=59$ / $147)$ in the observation phase and $71.4 \%(\mathrm{~N}=60 / 84)$ at 2 months in the intervention phase.

CONCLUSIONS: A proactive strategy offering a specialized smoking cessation intervention based on motivational interviewing to all smoking patients hospitalized for an ACS is well accepted and significantly increases the proportion of patients receiving an intervention as compared to a reactive strategy dependent on the request of health care providers. Further research should assess the impact on long-term smoking abstinence and cardiovascular events.

FEASIBILITY AND VALIDATION OF A SIGN-OUT EVALUATION TOOL. Leora I. Horwitz ${ }^{1}$; David A. Rand ${ }^{2}$; Paul G. Staisiunas ${ }^{3}$; Jeanne M. Farnan ${ }^{3}$, Vineet Arora ${ }^{3}{ }^{1}$ Yale University, New Haven, CT; ${ }^{2}$ Hahnemann University Hospital, Philadelphia, PA; ${ }^{3}$ University of Chicago, Chicago, IL. (Control ID \#1338984)

BACKGROUND: Residency programs must now ensure and monitor effective, structured hand-over processes, and ensure that residents are competent in communicating with team members in the hand-over process ("sign-out"). There is a lack of validated sign-out assessment tools.

METHODS: We developed two sign-out evaluation tools based on the mini-CEX format: one for the sign-out provider and one for the signout recipient. The provider tool included domains for setting, organization, communication, content, judgment, professionalism, and overall competency. The recipient tool included the same domains with the exception of content, which was omitted. Each domain was scored 1-9 where 1-3 was unsatisfactory, 4-6 was satisfactory and 7-9 was superior. Extremes were anchored by descriptors. We tested the tool in house staff day to night shift changes at Yale and in hospitalist day to night changes at University of Chicago. Each handoff was evaluated by a peer (participant in the hand-off) and by at least one external observer. One consistent external observer scored all handoffs 
at each institution. In addition, many handoffs at Yale included supervising residents as additional external observers. Mean scores were compared with t tests.

RESULTS: A total of 149 sign-out sessions involving 98 subjects were observed, yielding 343 sign-out CEX evaluations of sign-out providers and 330 of sign-out recipients. 34 unique subjects were evaluated at the University of Chicago and 64 unique subjects were evaluated at Yale. A total of 468 evaluations $(69.5 \%)$ assessed trainees or mid-level providers (sub-interns, interns, residents and nurse practitioners); the remainder assessed hospitalist attendings. Evaluators used the full spectrum of unsatisfactory (1-3) to superior (7-9) scores. For sign-out providers, mean scores were lowest for content (6.9) and highest for professionalism (7.5). Scores for recipients were higher than scores for providers at borderline significance for most domains: setting $(p=0.05)$, organization $(p=0.07)$, judgment $(p=0.05)$ and overall $(p=0.01)$. Hospitalists and trainees received similar scores for providing sign-out except that attendings received significantly higher ratings for judgment than trainees (mean score 7.6 vs. 7.2, $\mathrm{p}=0.03$ ). Conversely, attendings received significantly lower scores than trainees for judgment $(7.2$ vs. $7.6, p=0.01)$ and organization $(7.1$ vs. $7.5, p=$ 0.03 ) when receiving sign-out. Overall, external evaluators gave significantly lower scores than peer evaluators in every domain for both sign-out providers and recipients $(\mathrm{p}<0.0001$ for every domain using paired $\mathrm{t}$ test). External and peer evaluator scores were well correlated, with Spearman correlation coefficients ranging 0.33-0.39 for provider sub-domains and 0.47 for the overall provider score. Weighted kappas for interobserver reliability of provider unsatisfactory/satisfactory/superior scores were in the low-moderate range: setting 0.22 (0.09-0.35); organization 0.35 (0.21-0.48); communication 0.32 (0.20-0.44); content 0.29 (0.18-0.41); judgment 0.27 (0.14-0.40); professionalism $0.42(0.28-0.55)$ and overall $0.40(0.27-0.53)$. Kappas for recipient scores were lower, ranging $0.14-0.33$.

CONCLUSIONS: An evaluation tool for providers of sign-out is feasible and moderately reliable. Peers provide significantly higher scores than external observers, suggesting difficulty in providing negative feedback to peers. The sign-out CEX is best used by an external observer such as an attending.

FEASIBILITY OF PORTABLE TECHNOLOGY TO DIAGNOSE SLEEP APNEA IN UNDERSERVED COMMUNITIES Jillian E. Nickerson ${ }^{1}$; Ana C. Krieger ${ }^{2}$; Ellen P. Simon ${ }^{3}$; Sandra Talavera ${ }^{4}$; Euny C. Lee ${ }^{1}$; Michael Nedelman ${ }^{1}$; Carol Horowitz ${ }^{1}$. ${ }^{1}$ Mount Sinai School of Medicine, New York, NY; ${ }^{2}$ Weill Cornell Medical College, New York, NY; ${ }^{3}$ Union Settlement Association, New York, NY; ${ }^{4}$ Senior Health Partners, A Healthfirst Company, New York, NY. (Control ID \#1339585)

BACKGROUND: Despite high prevalence of risk factors such as obesity and diabetes, Latinos and Blacks are under-diagnosed and under-treated for obstructive sleep apnea (OSA), partly due to decreased access to costly traditional overnight studies. One in four adults have pre-diabetes. Untreated OSA may speed their development of diabetes, both because compensatory mechanisms to compensate for apnea may exacerbate hyperglycemia, and because the associated fatigue may thwart weight loss. Yet, little is known about the prevalence of OSA among pre-diabetics. In addition, portable OSA monitoring devices may improve access to diagnosis among high-risk, low-income populations, but no data exist on portable testing among vulnerable populations. A community-academic partnership in East Harlem, the epicenter of diabetes in New York City, aimed to assess the feasibility and acceptability of portable OSA diagnosis and explore the prevalence in OSA in a pre-diabetic, minority population. METHODS: We recruited a convenience sample of participants who completed a diabetes prevention randomized controlled trial. All participants wore an ApneaLinkPlus ${ }^{\mathbb{R}}$ portable monitor overnight and afterwards engaged in open-ended interviews about their experience using the equipment and underlying perceptions about OSA. Those with moderate or severe OSA ( $>15 \mathrm{AHI}$ per hour) received auto-titrating CPAP machines and returned at 30 days for follow-up. Measurements of health were collected in conjunction with the parent study. We analyzed interview transcripts and calculated means and standard deviations for continuous variables, proportions for categorical variables and group comparisons using chi-square and student's t-test in SPSS v17.

RESULTS: We tested 52 pre-diabetic, overweight adults. Most were Latino $(64 \%)$, or Black $(31 \%)$, uninsured $(31 \%)$, low-income $(50 \%$ earned $<\$ 15,000$ per year), and undereducated $(28 \%$ with $<$ high school diploma). Use of at-home sleep monitors was feasible in this population: of the 121 approached, $58 \%$ agreed to testing, and $100 \%$ of those given home monitors returned them promptly. No participants required OSA test in overnight lab, as the home diagnoses were conclusive. Participants found monitors comfortable and convenient $(87 \%$ would recommend the test). Nearly half $(48 \%)$ had OSA $(>5$ AHI events/hour); $13 \%$ had moderate to severe OSA ( $>15$ events/ hour) requiring treatment with CPAP. Screening with the commonly used, verbally administered Epworth Sleepiness Scale did not accurately predict which participants had OSA compared to those diagnosed by the ApneaLink Plus $(\mathrm{p}=.506)$. Mean systolic blood pressure (BP) was significantly higher in those with OSA ( 119 vs $109 \mathrm{mmHg}, \mathrm{P}=0.01$ ), but $\mathrm{BMI}$, cholesterol and depressive symptoms were not statistically different between those with and without OSA We interviewed all 18 participants with moderate to severe OSA and found that most had a misconception that OSA would cause them to die in their sleep. However, they were appropriately concerned that OSA could cause progression to diabetes, and thought weight loss could prevent or improve OSA.

CONCLUSIONS: At-home sleep monitors represent a feasible and acceptable method for diagnosing OSA in high-risk minorities. The prevalence of mild-severe OSA in this population is much higher than the US average (48\% compared $3-28 \%$ ) indicating need to increase accessibility to OSA diagnosis in this population.

FEEDBACK ABOUT FACULTY FEEDBACK: DO STUDENTS AND FACULTY AGREE ON WHAT IS USEFUL? Neil Mehta; Alan L. Hull; J. H. Isaacson; Amy S. Nowacki; Pavel Ermakov. Cleveland Clinic Lerner College of Medicine of Case Western Reserve University, Cleveland, OH. (Control ID \#1340475)

BACKGROUND: Students need useful performance feedback to improve skills and behaviors. Many institutions, provide faculty development to help faculty provide useful feedback, faculty get little information about the usefulness of the assessments they provide. Our institution has a web-based formative feedback system where faculty provide written narrative feedback to students on observed patient encounters. The objective of our study was to compare student and "expert" perceptions of the usefulness of these assessments. This is the first step in a plan to provide faculty regular feedback on the usefulness of the student assessments they provide.

METHODS: Students can rate the written faculty comments as useful or not (or no comments) by answering the question "This feedback identifies specific behaviors/skills I did well and/or I can improve." Data were collected for 1 month. We randomly selected 100 assessments received and rated by students during 7 core disciplines. Two "experts" also independently rated the blinded comments as useful or not. We analyzed the agreement between the 2 "experts" and between the experts' consensus and the students. Iif either or both experts rated the comments as useful, the expert consensus was considered useful.

RESULTS: A total of 65 students received 945 assessment forms from 389 faculty members during this 1 month period. The 100 randomly selected assessments were completed by 74 different faculty for 27 unique students. A total of 75 forms were used in the analysis (25 excluded because students did not provide a usefulness rating). Students rated $89 \%$ of faculty feedback comments as useful $(\mathrm{N}=67)$ while the "experts" rated $68 \%$ of faculty feedback comments as useful $(\mathrm{N}=51)$. Experts had good to excellent agreement (Kappa $=.69$, see Table 1$)$. Students and the combined "expert" assessment showed poor agreement overall (Kappa $=.28$, see Table 2). When students rated a form as not useful, experts agreed with them $63 \%(5 / 8)$ of the time. When students rated a form as useful, experts agreed $81 \%(54 / 67)$ of the time. 
CONCLUSIONS: While students identified more of the feedback as useful compared to the "experts", there was good agreement ( $81 \%$ of cases) between the two. There was less agreement between the two when the students rated the forms as not useful. One explanation is that students rate the usefulness of the feedback in the context of their learning experience. They may also interpret the comments in light of any verbal feedback they might have received during the patient encounter. It is also possible that students were reluctant to identify faculty comments as not useful. We believe that student generated assessments of faculty comments have potential to provide strong reinforcement for faculty to provide useful, behaviorally-based, feedback to students and help target faculty development.

table 2

\begin{tabular}{lllll}
\hline \hline & & student & & \\
\multirow{4}{*}{ experts } & not useful & useful & \\
& not useful & 5 & 13 & 18 \\
& Useful & 3 & 54 & 57 \\
& & 8 & 67 & 75 \\
\hline
\end{tabular}

\begin{tabular}{|c|c|c|c|c|}
\hline & & Expe & ert2 & \\
\hline & Frequency & $\mid \begin{array}{l}\text { Not Usef } \\
\text { ul }\end{array}$ & Useful & Total \\
\hline & Not Useful & 18 & 4 & 22 \\
\hline ert & Useful & 6 & 47 & 53 \\
\hline & Total & 24 & 51 & 75 \\
\hline
\end{tabular}

FINANCIAL CONSIDERATIONS IN RESIDENTS' DECISIONS ABOUT END-OF-LIFE CARE Neil J. Farber; Lisa J. Wastila. University of California, San Diego, La Jolla, CA. (Control ID \#1319231)

BACKGROUND: One of the most contentious issues in end-of-life care is that of financial concerns in decisions made on the behalf of patients by patients' surrogates. While physicians emphasize the emotional cost involved, some patients emphasize the financial costs, including the fear that the patient's family would suffer financially if the patient died. Since there have been no studies which examine physicians' decisions to withdraw life-sustaining treatment based on the influence of surrogates' financial gain from such decisions we studied internal medicine residents regarding their attitudes about withdrawing life-sustaining treatment when financial considerations are involved.

METHODS: A survey was developed which was pre-tested for face and content validity among 40 general internal medicine physicians. The survey contained 8 scenarios in which a patient who was terminally ill and lacked capacity had a decision to make regarding withdrawal of the ventilator. The life sustaining treatment had been deemed by the ethics committee to be medically futile. Nested variables included agreement or disagreement between the surrogate and patient, decision to withdraw or continue the ventilator, and financial gain or no financial gain for the surrogate based on the decision. Residents were asked how likely they were to withdraw the ventilator in each scenario based on a 4 point Likert scale. The survey was administered to all internal medicine residents at UCSD. The differences between scenarios in which there was the presence or absence of each of the three nested variables was analyzed via $\mathrm{T}$ tests.

RESULTS: Residents were more likely to withdraw the ventilator when requested to do so, than when it was requested to be continued, despite the scenario indicating futility of care $(p<0.001)$. They were also more likely to withdraw the ventilator when there was agreement in the decision between the surrogate and the patient $(\mathrm{p}<0.001)$. Residents were more likely to withdraw or withhold the ventilator when financial benefits were not an issue for the spouse, than when the spouse was likely to accrue such benefits $(p=0.032)$. This difference was largely due to the scenario in which there was disagreement between the surrogate and patient but in which the ventilator was requested to be removed, where residents were significantly more likely to remove the ventilator when financial benefits were not to be accrued by the surrogate $(p=0.02)$.

CONCLUSIONS: Internal Medicine residents make some decisions about whether to withdraw life sustaining treatment based on financial considerations for the patient's surrogate. Residents also make such decisions based on the wishes of the surrogate and the agreement or disagreement of the patient and surrogate, despite determination of futility by and ethics committee. There needs to be ongoing communication with residents and education about end-of life decisions in terminally ill patients where conflicts may exist between the surrogates and patients and between the surrogates and physicians.

FOLLOW-UP PROVIDER VISITS AFTER HOSPITALIZATIONS: WHO YA GONNA CALL? Claire F. Snyder ${ }^{1}$; Kevin D. Frick ${ }^{2}$; Robert J. Herbert $^{2}$; Amanda L. Blackford ${ }^{3}$; Bridget A. Neville ${ }^{4}$; Antonio C. Wolff ${ }^{3}$; Michael A. Carducci ${ }^{3}$; Craig C. Earle ${ }^{5}$. ${ }^{1}$ Johns Hopkins School of Medicine, Baltimore, MD; ${ }^{2}$ Johns Hopkins Bloomberg School of Public Health, Baltimore, MD; ${ }^{3}$ Sidney Kimmel Comprehensive Cancer Center at Johns Hopkins, Baltimore, MD; ${ }^{4}$ Consultant, Boston, MA; ${ }^{5}$ Institute for Clinical Evaluative Sciences, Toronto, ON, Canada. (Control ID \#1315642)

BACKGROUND: Patients who are hospitalized for conditions such as stroke, diabetes, and depression require follow-up visits after discharge. However, it is not always clear whether these follow-up visits should be with the patient's primary care provider (PCP) or a specialist. This issue may be exacerbated for cancer survivors who frequently have visits with a variety of primary care and specialty care providers. This analysis examined the provider specialties visited by cancer survivors following hospitalizations for non-cancer medical problems.

METHODS: We used the Surveillance, Epidemiology and End Results (SEER)-Medicare linked database which combines the clinical cancer data from the SEER registry system with Medicare claims. The sample included survivors of loco-regional breast, colorectal, or prostate cancer who were diagnosed in 2004, age $>=66$ at diagnosis, continuously enrolled in fee-for-service Medicare, and survived $>=3$ years from diagnosis. The study time period was the transition from cancer treatment to survivorship, defined as days 366-1095 from the cancer diagnosis. We examined the provider specialties visited during the 2 or 4 weeks following discharge (based on published quality indicators) for the following conditions: depression, diabetes, malignant/severe hypertension, gastrointestinal bleeding, transient ischemic attack, cerebrovascular accident, unstable angina, acute myocardial infarction, and congestive heart failure. Provider specialty was determined using the American Medical Association Masterfile and was grouped into PCP, oncology specialist, or other specialist. We analyzed the data descriptively to examine the proportion of patients with visits to each provider type to determine the specialties from whom patients were most likely to seek care.

RESULTS: The total sample included 8661 cancer cases (53\% prostate, $22 \%$ breast, $26 \%$ colorectal). The mean age was $75,65 \%$ male, $85 \%$ white. The table summarizes the number of patients who were hospitalized for each condition and the number (\%) of patients with visits to PCPs, oncology specialists, and other specialists during the time period specified by the published quality indicator. With the exception of patients with unstable angina $(39 \%)$, patients were most likely to have visits with a PCP $(67 \%-85 \%)$. Patients were equally likely to see other specialists as PCPs following hospitalization for acute myocardial infarction $(67 \%)$ and more likely to visit other specialists following hospitalization for unstable angina $(85 \%)$. Visits to oncology specialists following hospitalization for any of the conditions were rare $(<22 \%)$.

CONCLUSIONS: Patients were most likely to visit a PCP during the period immediately following hospitalization. This finding is particularly noteworthy given our sample of cancer survivors, many of whom seek care from a complex array of physician specialties. These results support the critical role PCPs play in managing patients' overall health. 
Providers Visited After Hospitalization

\begin{tabular}{|c|c|c|c|c|}
\hline Reason for & Number & $\mathrm{N}(\%)$ & N (\%) with & $\mathrm{N}(\%)$ with \\
\hline Hospitalization (Time & Eligible & with & Oncology & Other \\
\hline Period for Follow-Up & & $\mathrm{PCP}$ & Specialist & Specialist \\
\hline Visit) & & Visits & Visits & Visits \\
\hline Depression ( 2 weeks) & 72 & $56(78)$ & $6(8)$ & $26(36)$ \\
\hline Diabetes ( 4 weeks) & 299 & $\begin{array}{l}211 \\
(71)\end{array}$ & $52(17)$ & $142(48)$ \\
\hline $\begin{array}{l}\text { Malignant/Severe } \\
\text { Hypertension ( } 4 \\
\text { weeks) }\end{array}$ & 13 & $11(85)$ & $0(0)$ & $5(39)$ \\
\hline $\begin{array}{l}\text { Gastrointestinal } \\
\text { Bleeding ( } 4 \text { weeks) }\end{array}$ & 63 & $48(76)$ & $10(16)$ & $22(35)$ \\
\hline $\begin{array}{l}\text { Transient Ischemic } \\
\text { Attack (4 weeks) }\end{array}$ & 32 & $24(75)$ & $5(16)$ & $19(59)$ \\
\hline $\begin{array}{l}\text { Cerebrovascular } \\
\text { Accident ( } 4 \text { weeks) }\end{array}$ & 117 & $90(77)$ & $8(7)$ & $52(44)$ \\
\hline $\begin{array}{l}\text { Unstable Angina (4 } \\
\text { weeks) }\end{array}$ & 33 & $13(39)$ & $4(12)$ & $28(85)$ \\
\hline $\begin{array}{l}\text { Acute Myocardial } \\
\text { Infarction ( } 4 \text { weeks) }\end{array}$ & 52 & $35(67)$ & $11(21)$ & $35(67)$ \\
\hline $\begin{array}{l}\text { Congestive Heart } \\
\text { Failure (4 weeks) }\end{array}$ & 236 & $\begin{array}{l}164 \\
(70)\end{array}$ & $27(11)$ & $119(50)$ \\
\hline
\end{tabular}

FOOD INSECURITY IN RELATION TO CHANGES IN SELFEFFICACY, NUTRITION, AND HEMOGLOBIN A1C DURING A DIABETES EDUCATIONAL INTERVENTION Courtney R. Lyles $^{1}$; Michael S. Wolf ${ }^{2}$; Allison Dahlke ${ }^{2}$; Terry Davis ${ }^{3}$; Laura Curtis $^{2}$; Darren A. DeWalt ${ }^{4}$; Dean Schillinger ${ }^{1}$; Hilary Seligman ${ }^{1} .{ }^{1}$ University of California San Francisco, San Francisco, CA; ${ }^{2}$ Northwestern University Feinberg School of Medicine, Chicago, IL; ${ }^{3}$ Louisiana State University Health Sciences Center - Shreveport, Shreveport, LA; ${ }^{4}$ University of North Carolina Chapel Hill School of Medicine, Chapel Hill, NC. (Control ID \#1336988)

BACKGROUND: Food insecurity refers to being at risk of going hungry because of the inability to afford food. It is a way in which poverty may predispose individuals to poorer diabetes management, as patients may shift their dietary intake toward inexpensive, calorically- dense foods. While previous, cross-sectional analyses have shown an association between food insecurity and worse glycemic control and diabetes self-management, this study uses longitudinal data to assess food insecurity in relation to changes in self-efficacy, nutritional intake, and glycemic control. We hypothesized food insecure individuals would have worse outcomes over time.

METHODS: The dataset is from the Missouri Health Literacy and Diabetes Communication Initiative, conducted in 2008-2009. We enrolled 621 patients with diabetes from urban, suburban, and rural safety net sites into a trial evaluating a low-literacy diabetes guide for self-management support. Two thirds received a defined intervention designed to engage patients in setting feasible diabetes action plans, and remaining patients received usual diabetes care. In this study, we conduct a secondary analysis of baseline food insecurity (6-item scale dichotomized into food secure vs. food insecure) in relation to several outcomes over 1 year. We compared unadjusted differences of diet and diabetes self-efficacy (scales scored 1-5), fruit and vegetable consumption (\# per day), and glycemic control (A1c) by food insecurity status at each time point using two-sided t-tests. Adjusted differences by food insecurity over time were examined using generalized estimating equations, clustering on individual and controlling for time, age, gender, race, income, and intervention arm, as well as an interaction between time and food insecurity.

RESULTS: $35 \%(n=214)$ of the diabetes sample reported being food insecure. These participants were younger, with less income, and were more likely to be current smokers and unemployed. At baseline, food insecure individuals had higher A1c as well as lower diet and overall diabetes self-efficacy and fruit and vegetable consumption compared to food secure individuals (Table). Food insecure individuals had significantly greater improvements in A1c (reduced on average by $0.39 \%$ ), diet self-efficacy (increased by 0.23 ), and diabetes selfefficacy (increased by 0.26 ) over time (interaction terms: $\mathrm{p}<0.05$ ). This improvement for food insecure individuals resulted in no significant difference in A1c between the groups at follow-up.

CONCLUSIONS: As expected, individuals experiencing food insecurity were more likely to begin this study with poorer measures of self-efficacy, nutritional intake, and glycemic control. However, contrary to our hypotheses, food insecure patients made significant improvements on A1c and self-efficacy over time. This finding may suggest that food insecure patients are particularly sensitive to diabetes self-management support.

Outcomes of Interest by Food Insecurity

\begin{tabular}{llllllll}
\hline \hline & \multicolumn{2}{l}{ Unadjusted Baseline } & & \multicolumn{2}{c}{ Unadjusted Follow-up } & & \multicolumn{2}{c}{$\begin{array}{l}\text { Adjusted Longitudinal Model } \\
\text { Comparing Insecure to Secure } \dagger\end{array}$} \\
& & & & & & \\
Mean \# fruit/day & $1.00( \pm 0.7)$ & $0.78( \pm 0.7)$ & $0.001^{*}$ & $1.09( \pm 0.8)$ & $1.00( \pm 0.9)$ & 0.27 & $-0.003(-0.17,0.16)$ \\
Mean \# veg/day & $2.03( \pm 1.1)$ & $1.75( \pm 0.9)$ & $0.01^{*}$ & $2.10( \pm 1.2)$ & $1.76( \pm 0.9)$ & $0.002^{*}$ & $-0.25^{*}(-0.45,-0.05)$ \\
Mean diet self-efficacy & $3.49( \pm 0.8)$ & $3.09( \pm 1.0)$ & $<.001^{*}$ & $3.55( \pm 0.8)$ & $3.41( \pm 0.8)$ & 0.07 & $-0.17^{*}(-0.33,-0.01)$ \\
Mean diabetes self-efficacy & $3.57( \pm 0.4)$ & $3.33( \pm 0.5)$ & $<.001^{*}$ & $3.70( \pm 0.4)$ & $3.60( \pm 0.4)$ & $0.01^{*}$ & $-0.09^{*}(-0.16,-0.01)$ \\
Mean Alc & $7.8( \pm 1.6)$ & $8.4( \pm 1.9)$ & $0.002^{*}$ & $7.8( \pm 1.6)$ & $8.1( \pm 1.8)$ & 0.12 & $0.22(-0.17,0.60)$ \\
\hline
\end{tabular}

$\dagger$ Adjusted for age, gender, income, race, intervention arm, time, and interaction between food insecurity and time. $*$ p $<0.05$ Note: Self-efficacy and nutrition outcomes: $\mathrm{n}=455$; A1c: $\mathrm{n}=343$

FORMATIVE EVALUATION OF A PRIMARY CARE SHARED PANEL MANAGEMENT PROGRAM: THE FIRST YEAR'S EXPERIENCES Tara F. Bishop ${ }^{1}$; Melinda A. Chen ${ }^{1}$; Amanda S. Parsons ${ }^{2}$; Daniel I. Gottlieb ${ }^{2}$; Priya K. Desai ${ }^{2}$; Sarah C. Shih' ; Lawrence P. Casalino ${ }^{1} .{ }^{1}$ Weill Cornell Medical College, New York, NY; ${ }^{2}$ Primary Care Information Project, New York City Department of Health and Mental Hygeine, New York, NY. (Control ID \#1326122)
BACKGROUND: Outreach to patients who are overdue for recommended chronic disease care is difficult for small practices. These practices often lack the time and resources to utilize their electronic health records' (EHR) disease registries to arrange follow-up appointments. We describe the motivations for, barriers to, facilitators of, and experiences with the first year of a publicly initiated shared panel management program for small primary care practices in underserved areas. 
METHODS: We conducted a formative evaluation of the New York City Department of Health and Mental Hygiene's Primary Care Information Project's (PCIP) shared panel management program using individual interviews with administrators, shared prevention outreach specialists (POSs), physicians, and practice staff. We used the constant comparative method to identify major domains and themes from interviews.

RESULTS: Prior to the program, none of the physicians interviewed regularly used the EHR's registry function to track patients. Physicians and POSs generally perceived the program as useful. Barriers to program implementation were technical problems, lack of practice workspace, POS training, and difficulty recruiting practices. Facilitators of implementation included having POSs onsite at each practice, creating a portable training manual, and engaging providers in the process.

CONCLUSIONS: This program demonstrates that it is possible for a public agency to provide shared technical and personnel resources to enable small primary care practices in underserved areas to promote patient follow-up. With pressures from health care reform and new payment models to improve care coordination, programs like this could serve as a model enabling small primary care practices to improve patient follow-up.

FREQUENT USERS OF EMERGENCY DEPARTMENTS PRESENT VERY HIGH PREVALENCE OF MENTAL HEALTH AND SUBSTANCE USE DISORDERS, WHICH ARE LARGELY UNDERDIAGNOSED BY CLINICIANS Francis $\mathrm{Vu}^{1}$; Jean-Bernard Daeppen ${ }^{2}$; Olivier Hugli ${ }^{3}$; Katia Iglesias ${ }^{4}$; Stephanie Stucki ${ }^{4}$; Patrick Bodenmann ${ }^{1}$. ${ }^{1}$ Department of Ambulatory Care and Community Medicine, Lausanne, Switzerland; ${ }^{2}$ Lausanne University Hospital, Lausanne, Switzerland; ${ }^{3}$ Lausanne University Hospital, Lausanne, Switzerland; ${ }^{4}$ Lausanne University Hospital, Lausanne, Switzerland. (Control ID \#1330179)

BACKGROUND: The objective of this study was to determine if mental health and substance use diagnoses were equally detected in frequent users (FUs) compared to infrequent users (IUs) of emergency departments (EDs).

METHODS: In a sample of 399 adult patients ( $\geq 18$ years old) admitted to a teaching hospital ED, we compared the mental health and substance use disorders diagnoses established clinically and consigned in the medical files by the ED physicians to data obtained in face-to-face research interviews using the Primary Care Evaluation of Mental Disorders (PRIME-MD) and the Alcohol, Smoking and Involvement Screening Test (ASSIST). Between November 2009 and June 2010, 226 FUs (>4 visits within a year) who attended the ED were included, and 173 IUs ( $\leq 4$ visits within a year) were randomly selected from a pool of identified patients to comprise the comparison group.

RESULTS: For mental health disorders identified by the PRIME-MD, FUs were more likely than IUs to have an anxiety ( 34 vs. $16 \%$, Chi2(1) $=16.74$, $\mathrm{p}<0.001$ ), depressive $(47$ vs. $25 \%$, Chi2 $(1)=19.11, \mathrm{p}<0.001)$ or posttraumatic stress (PTSD) disorder (11 vs. $5 \%$, Chi2 $(1)=4.87, \mathrm{p}=0.027)$. Only $3 /$ 76 FUs $(4 \%)$ with an anxiety disorder, $16 / 104$ FUs $(15 \%)$ with a depressive disorder and none of the 24 FUs with PTSD were detected by the ED medical staff. None of the 27 IUs with an anxiety disorder, 6/43 IUs (14\%) with a depressive disorder and none of the 8 IUs with PTSD were detected. For substance use disorders identified by the ASSIST, FUs were more at risk than IUs for alcohol $(24$ vs. $7 \%$, Chi2 $(1)=21.12, \mathrm{p}<0.001)$ and drug abuse/dependence ( 36 vs. $25 \%$, Chi2 $(1)=5.52, \mathrm{p}=0.019$ ). Of the FUs, $14 / 54(26 \%)$ using alcohol and $8 / 81(10 \%)$ using drugs were detected by the ED physicians. Of the IUs, 5/12 (41\%) using alcohol and none of the 43 using drugs were detected. Overall, there was no significant difference in the rate of detection of mental health and substance use disorders between FUs and IUs (Fisher's Exact Test: anxiety, $p=$ 0.567 ; depression, $\mathrm{p}=1.000$; PTSD, $\mathrm{p}=1.000$; alcohol, $\mathrm{p}=0.517$; and drugs, $\mathrm{p}=0.053$ ).
CONCLUSIONS: While the prevalence of mental health and substance use disorders was higher among FUs, the rates of detection were not significantly different for FUs vs. IUs. However, it may be that drug disorders among FUs were more likely to be detected.

FUNCTIONAL IMPAIRMENT AND DISABILITY DURING THE LAST TWO YEARS OF LIFE Alexander K. Smith; Yinghui Miao; W. John Boscardin; Kenneth Covinsky. University of California, San Francisco, San Francisco, CA. (Control ID \#1324289)

BACKGROUND: We know little about the national prevalence of disability at varying points across the last years of life.

METHODS: Data are from participants ages $50+$ who died in the Health and Retirement Study (HRS) between the years 1995 and 2008. Each participant was interviewed once in the last 24 months of life. We used the HRS interview closest to death to reconstruct national estimates of the monthly prevalence of functional impairment and disability in the two years prior to death. Measures of functional impairment and disability included: IADL difficulty (Difficulty with cooking, shopping, using telephone, taking medications, managing money); ADL dependence (requires assistance with dressing, bathing, eating, transferring, walking across the room, and toileting). We estimated the predicted probability of functional impairment or disability by age at death and gender, adjusting for race or ethnicity, educational level, net worth, and proxy status.

RESULTS: There were 7624 decedents (mean age at death $80,52 \% \%$ women, $84 \%$ White, $10 \%$ African-American, 4\% Latino, 27\% proxy interview). The unadjusted prevalence of all forms of functional impairment rose linearly over the last two years of life (Table). The predicted prevalence of disability prior to death rose with advancing age. After adjustment for gender, race/ethnicity, educational level, net worth, and proxy status, the predicted prevalence of any ADL dependency for elders ages 50-69 12 months prior to death was $24 \%$ (95\% CI 18-29), compared to $29 \%$ (24-34) for ages 70 to 79 , $37 \%(31-44)$ for ages 80 to 89 , and $47 \%(41-54)$ for ages 90 and older ( $\mathrm{p}$ for trend $<.0001$ ). Adjusting for age and the above factors, the predicted prevalence of any ADL disability was more common in women 12 months before death $(38 \%, 95 \%$ CI 33-44) then men $(29 \%$, 24-34) $(\mathrm{p}<.0001)$

CONCLUSIONS: Those who live to an older age are more likely to experience a protracted period of disability prior to death. Nearly half of all elders who live to their tenth decade will be dependent in ADL a year prior to death. Independent of age, women are at greater risk then men.

Prevalence of Disability in the Months Before Death

\begin{tabular}{llll}
\hline \hline Domain & $\begin{array}{l}\text { 24 months before } \\
\text { death }(95 \% \mathrm{CI})\end{array}$ & $\begin{array}{l}12 \text { months } \\
\text { before death }\end{array}$ & $\begin{array}{l}\text { Last month } \\
\text { of life } \\
73 \%(61-\end{array}$ \\
$\begin{array}{l}\text { Prevalence of any } \\
\text { IADL difficulty }\end{array}$ & $34 \%(27-41)$ & $50 \%(44-56)$ & $\begin{array}{l}72 \% \\
82)\end{array}$ \\
$\begin{array}{l}\text { Prevalence of any } \\
\text { ADL dependence }\end{array}$ & $21 \%(16-27)$ & $35 \%(29-41)$ & $\begin{array}{l}56 \%(47- \\
65)\end{array}$ \\
\hline
\end{tabular}

GENDER, ETHNIC AND RACIAL DIFFERENCES IN PRIMARY PREVENTION OF STROKE IN YOUNG ADULTS Arun K. Muthusamy; Diane L. Levine. Detroit Medical Center / Wayne State University, Detroit, MI. (Control ID \#1276251)

BACKGROUND: There has been an increase in the incidence of stroke in young adults. From 1994 to 2004 stroke increased from $4.5 \%$ to $7.3 \%$ in adults younger between ages 18 and 45 years old. Primary prevention of stroke in this 
age group is particularly important to prevent years of disability. Furthermore, life style modification alone has been shown to lower risk of a first stroke by $80 \%$. Our aim is to assess how frequently primary care physicians provide counseling to address modifiable risk factors such as smoking, hypertension, obesity, diabetes mellitus and hyperlipidemia in young adults and whether such rates differ by patient gender, ethnicity and race.

METHODS: The National Ambulatory Medical Care Survey is a limited access dataset that provides visit-based data on documented preventive counseling services in primary care physician offices. All patients of age 18 to 45 years were included in the analysis. The likelihood of being counseled on three health promotion topics (i.e., diet, exercise, and weight reduction) and two risk reduction topics (i.e., tobacco use/ exposure, medication prescription for hypertension, diabetes mellitus, obesity and hyperlipidemia) were measured. Multivariate survey logistic regression model was used to investigate the discrepancies based on patient demographic characteristics.

RESULTS: There were a total of 10,580 patient visits between 18 to 45 years in 2008 and 2009. The majority of these visits were to Family Medicine $(45.8 \%)$ and Internal Medicine (20.5\%) practices and only $2.5 \%$ visits were to Neurology. Smoking (21.6\%) was the most prevalent risk factor, followed by obesity $(17.1 \%)$, hypertension $(13.3 \%)$, hyperlipidemia $(8.6 \%)$ and diabetes $(7 \%)$. Women were more likely to receive prescription medications for obesity and statins than men. Men were more likely to receive diet counseling in diabetes mellitus [OR 1.60, 95\% CI (1.04 to 2.45)], hypertension [OR 1.47 $95 \% \mathrm{CI}$ (1.07 to 2.02$)$ ] and hyperlipidemia [OR $1.97,95 \% \mathrm{CI}$ (1.33 to 2.91$)$ ] than women. Men with hyperlipidemia were more likely to receive exercise counseling [OR $1.75,95 \%$ CI (1.12 to 2.72)] compared to women. Hispanics were more obese and were more likely to receive diet counseling compared to non-Hispanics. Hispanics were also less likely to receive tobacco cessation counseling [OR 2.40, 95\% CI (1.07 to 5.39)]. Among hypertensives, Hispanics were more likely to receive diet counseling than non-Hispanics. There were no statistically significant differences between Caucasians and African-Americans in preventive counseling.

CONCLUSIONS: Our results indicate that less than one third of the atrisk patient population received any preventive health counseling during outpatient office visits. In addition, there were significant differences in health promotion counseling based on gender and ethnicity. Efforts should be undertaken by primary care physicians to improve counseling rates for patients with risk factors for stroke and to bridge the differences across the gender and race.

GENDER, RACIAL AND ETHNIC DISPARITIES IN THE UTILITY OF ELECTRONIC HEALTH RECORD IN AMBULATORY CARE Arun K. Muthusamy; Diane L. Levine. Detroit Medical Center / Wayne State University, Detroit, MI. (Control ID \#1276235)

BACKGROUND: Health Information Technology (HIT) has been recognized as an important tool to improve overall health care. Significant emphasis has been made on the use of Electronic Health Records (EHRs), a component of HIT, to improve the quality of ambulatory care. However, data on the role of the EHR in improving outcomes in this setting is scant. Our aim was to assess the association between EHR use and the quality of ambulatory care delivered. A secondary aim was to determine if use of an EHR evaluated the racial, gender and ethnic differences as implemented in a nationally representative survey.

METHODS: The National Ambulatory Medical Care Survey is a limited access dataset that provides visit-based patient data from 2008 to 2009 in outpatient physician offices. We analyzed the utility of EHR use to the guideline-concordant care using 16 previously validated quality indicators by Institute of Medicine (IOM). Multivariable logistic regression model was used to examine the racial and gender differences across the strata adjusted for other risk factors.

RESULTS: There were a total of 61,022 patient visits in 2008 and 2009 which represents a weighted frequency of an estimated 1.7 billion annual visits in the United States. EHRs were used in $49.5 \%$ of the weighted frequency visits. Use of an EHR was associated with improvement in two of $16 \mathrm{IOM}$ indicators (diet/nutrition counseling and exercise advice in high risk adults). In women, EHR use was associated with higher rates of ACE inhibitor use in congestive heart failure $[42 \%$ Vs $35 \%(\mathrm{P}=0.02)]$ and aspirin use in coronary artery disease $[76 \%$ Vs $67 \%(\mathrm{P}=0.003)]$. Among men, smoking cessation counseling $[22 \% \mathrm{Vs} 18.6 \%(\mathrm{P}=0.03)]$ was associated with use of an EHR as compared to non-EHR visits. There was no difference in use of inappropriate medication prescription in elderly [93.5\% Vs $94.1 \%$ $(\mathrm{P}=0.04)]$ by EHR use. Caucasian patients seeing physicians who used an EHR had higher rates of statin use than those seeing a physician who did not use an EHR [46\% vs 42\%; $(\mathrm{P}=0.01)]$. There was no difference in concordant guideline care in statin use among African American patients seeing a physician who used an EHR [44.6 Vs 43.7; $(\mathrm{P}=0.98)]$. In Hispanics, EHR use was associated with higher rates of anti-thrombotic therapy in atrial fibrillation $[91 \% \mathrm{Vs} 64 \%$ $(\mathrm{P}=<0.0001)]$, aspirin in coronary artery disease $[69 \%$ Vs $53 \%(\mathrm{P}=$ $0.03)]$ and TMP-SMZ in urinary tract infection $[98 \% \mathrm{Vs} 88 \%(\mathrm{P}=<$ 0.0001)] as compared to non-EHR use. There was no difference in concordant guideline care in among non-Hispanic patients seeing a physician who used an EHR in these three areas.

CONCLUSIONS: Our findings indicate that EHR use had significant but limited impact on a panel of quality indicators and that in African Americans and non-Hispanics an EHR did not result in better care as measured by IOM indicators. Use of an EHR does not alone fulfill the promise of improving quality. Additional research is needed to improve quality of care.

GENERAL INTERNIST CONCEPTUALIZATION OF THE COMPLEX PATIENT: A QUALITATIVE STUDY Danielle F. Loeb ${ }^{1}$; Ingrid A. Binswanger ${ }^{1,2}$; Carey Candrian ${ }^{3}$; Elizabeth A. Bayliss ${ }^{4}{ }^{1}$ University of Colorado Denver, Aurora, CO; ${ }^{2}$ Denver Health Medical Center, Denver, CO; ${ }^{3}$ University of Lugano, Lugano, Switzerland; ${ }^{4}$ Kaiser Permanente, Denver, CO. (Control ID \#1337469)

BACKGROUND: While improving care for complex patients has become a national research and policy priority, the definition of patient complexity requires further elucidation. Definitions of patient complexity generally include patient demographic and psychosocial factors that may affect disease outcomes as well the state of having multiple chronic diseases. However, since demographic and psychosocial factors can be difficult to quantify, most measures of complexity focus on the number and types of chronic illnesses. We used in-depth open-ended interviews to explore how primary care physicians (PCPs) conceptualize patient complexity.

METHODS: We used personal emails to recruit 15 physicians from 2 university clinics and 3 community health clinics. We used systematic nonprobabilistic sampling to achieve an even distribution with respect to gender, years in practice, and type of practice. The in-person interviews focused on PCP experiences with complex patients in general, with additional focus on specific patient-level factors that contribute to patient complexity. PCPs brought de-identified notes from 3 patients they considered complex. Interview transcripts were coded and analyzed utilizing a participatory general inductive approach.

RESULTS: PCPs identified four types of complexity: 1) medical complexity in which patients had chronic conditions that complicated treatment of other chronic conditions; 2) mental illness with medical illness; 3) socio-economic challenges; and 4) cognitive challenges that interfered with patients' ability to manage their medical conditions (Table 1). PCPs expressed the belief that truly complex patients had additional contributing factors beyond chronic medical illness, as expressed by one PCP: "I think for me, a complex patient is a patient who makes me think outside of the exam room...." (p. 13) Or more specifically, "I don't think it is a number of conditions. I think it is somebody that... I have difficulty controlling their symptoms for whatever reason... because of psychosocial factors, economic factors, transportation difficulties." (p. 14)

CONCLUSIONS: In this qualitative study we were able to gain a better understanding of PCPs' conceptualization of complex patients. They conceived patients as "highly complex" if they had medical illnesses, mental illnesses, socioeconomic challenges, and/or cognitive deficiencies that complicated care for chronic medical illnesses. Although challenging to quantify, this study points to the importance of taking socio-economic, 
psychological, and cognitive factors into account when measuring complexity.

Table 1. Primary Care Physicians' Categorization of Complex patients

\begin{tabular}{|c|c|}
\hline Theme & Illustrative Quote \\
\hline $\begin{array}{l}\text { Medically complex with } \\
\text { discordant conditions }\end{array}$ & $\begin{array}{l}\text { "[H]e brings up that highly complex thing. [H]e has cirrhosis in his } \\
\text { transplant liver due to hepatitis C infection... he has a hernia in his } \\
\text { abdomen that he can't have operated on ... Because of that hernia } \\
\text { he has chronic abdominal wounds...that don't heal well despite } \\
\text { ongoing would care efforts...those are about } 4 \text { different things that } \\
\text { really complicate each other in his case." (p. 1) }\end{array}$ \\
\hline $\begin{array}{l}\text { Mental illness complicating } \\
\text { medical illness }\end{array}$ & $\begin{array}{l}\text { "I have to say that the patients who also have medical co- } \\
\text { morbidities, I think the more difficult thing, the severe depression, } \\
\text { the not terribly responsive to treatment depression, or the } \\
\text { depression that sort of colored by their frustration by their medical } \\
\text { co-morbidities. That I think really affects the management of their } \\
\text { medical problems because of the hopelessness and that affects } \\
\text { adherence and other things." (p. 8) }\end{array}$ \\
\hline $\begin{array}{l}\text { Socio-economic issues } \\
\text { complicating medical illness }\end{array}$ & $\begin{array}{l}\text { "[S]he has a whole lot of medical illnesses going on and was } \\
\text { diagnosed with cancer while I was taking care of her and then lost } \\
\text { her insurance.... And so now we have a really hard time getting her } \\
\text { any of the care she used to get before she lost her insurance. So } \\
\text { that is really frustrating." (p. 6) }\end{array}$ \\
\hline $\begin{array}{l}\text { Cognitive issues or poor health } \\
\text { literacy complicating medical } \\
\text { illness }\end{array}$ & $\begin{array}{l}\text { "[P]articularly challenging are issues regarding developmental } \\
\text { delay. And how that interferes with some patient's abilities to } \\
\text { understand their medical problems." (p. 8) }\end{array}$ \\
\hline
\end{tabular}

Table 1

GEOGRAPHIC AND FACILITY CHARACTERISTICS ASSOCIATED WITH DIALYSIS FACILITY QUALITY Milda R. Saunders. ${ }^{1}$ University of Chicago Medical Center, Chicago, IL; ${ }^{2}$ University of Chicago Medical Center, Chicago, IL. (Control ID \#1340052)

BACKGROUND: In 2012, the Centers for Medicare and Medicaid Services (CMS) began mandatory pay-for-performance for dialysis facilities. Dialysis facilities are an important unit of analysis and site of intervention because they affect clinical outcomes for patients with end-stage renal disease through the quality of the medical care provided. We examined whether dialysis facility characteristics, neighborhood demographics, and region are associated with CMS dialysis facility quality measures.

METHODS: We linked US census data to the Center for Medicare and Medicaid Services (CMS) Dialysis Compare File which contains information for facility outcomes for all CMS certified dialysis facilities in 2009 (n =5616). We used three CMS measures of quality: 1) patient survival, facilities actual survival rates compared to predicted, after controlling for demographic and clinical characteristics; 2) adequate anemia management, proportion of patients with $\mathrm{Hgb}$ between 10 and $12 \mathrm{~g} / \mathrm{dL}$; and 3) dialysis adequacy, proportion of patients with a urea reduction rate $>65 \%$. We then used linear and logistic regression to characterize the association between dialysis facility quality and dialysis facility characteristics (profit status, size, length of operation, chain), neighborhood demographics (percent African American and percent of population below poverty), and region.

RESULTS: In adjusted analysis, worse than expected survival was associated with for-profit status (OR 2.22, 95\% CI 1.63, 3.02), increasing length of operation (OR 1.03, 95\% CI 1.02, 1.04), increasing proportion of African Americans (OR 3.27, 95\% CI 2.10, 5.09), and increasing proportion of poverty in the neighborhood (OR 7.19, 95\% CI 2.46, 20.98). Compared to facilities in the Midwest, dialysis facilities in the South had a worse than expected patient survival (OR 2.18, 95\% CI 1.63, $2.90)$. Greater dialysis adequacy is associated with for-profit status $(\beta=1.26)$, being part of a chain $(\beta=1.85)$, and having a greater number of stations $(\beta=$ $0.113)$, all $p<0.05$. Facilities in the South $(\beta=1.59)$ and Northeast $(\beta=1.66)$ also had greater dialysis adequacy, all $\mathrm{p}<0.05$. Lower dialysis adequacy is associated with dialysis facilities with an increasing proportion of African Americans in the neighborhood $(\beta=-3.14)$. Better anemia management within a dialysis facility is associated with having a greater number of stations $(\beta=$ $0.065, \mathrm{p}<0.05$ ). Worse anemia management is associated with being part of a chain $(\beta=-5.02)$, having an increased proportion of African Americans in the neighborhood $(\beta=-3.36)$, and dialysis facilities in the South $(\beta=-2.22)$, all $\mathrm{p}<$ 0.05 .

CONCLUSIONS: Neither region nor dialysis facility characteristics such as profit status, size, length of operation, or being part of a chain 
have a consistent relationship across all three measures of dialysis facility quality. Having an increasing proportion of African Americans in the neighborhood is consistently associated with worse dialysis facility outcomes, even after controlling for neighborhood poverty. Quality improvement efforts, while important overall, are particularly needed in minority communities.

GROUP MEDICAL VISITS VERSUS USUAL CARE ON SATISFACTION AND PATIENT-CENTERED CARE EXPERIENCES: RESULTS FROM A THREE-YEAR STUDY. Leonie Heyworth ${ }^{1}$; Ronen Rozenblum ${ }^{1}$; Mark Meterko ${ }^{3}$; Debra Prescott ${ }^{4}$; Zeev Neuwirth ${ }^{4}$; Steven R. Simon ${ }^{2,1}$. ${ }^{1}$ Brigham and Women's Hospital, Boston, MA; ${ }^{2}$ VA Boston Health Care System, Boston, MA; ${ }^{3}$ VA Boston Health Care System, Boston, MA; ${ }^{4}$ Atrius Health, Boston, MA. (Control ID \#1339480)

BACKGROUND: Group medical visits (GMV) aim to improve access, efficiency and outcomes. As patient-centered medical homes emerge, GMV are an increasingly common approach to patient-care delivery. However, little is known about the impact of GMV on patient satisfaction or other measures of patient-centered care.

METHODS: In a large multispecialty group practice, we identified 36539 adults who had at least one internal medicine office visit from 2008-2010, including 981 who had taken part in a GMV and 35558 who received usual care (UC). Each 90-minute GMV comprised approximately 10 patientparticipants and a care team consisting of a primary care internist, a "behaviorist" (e.g., a psychologist, social worker or nurse practitioner) and documentation specialist. UC participants experienced a routine one-on-one clinician-patient encounter. Mail surveys were sent to both groups one week following their visits and were completed by $40 \%$ of GMV patients and $37 \%$ of UC patients. We used chi-squared analysis to compare GMV and UC patients' overall satisfaction, the primary endpoint, and responses to specific items measuring elements of the patient-centered medical home. To control for baseline characteristics of participants, we dichotomized overall satisfaction and employed multivariable logistic regression to compare overall satisfaction between GMV and UC participants.

RESULTS: Compared with UC patients, GMV participants were more likely to be over age 65 years $(p<0.001)$, male $(p<0.001)$, white $(p<0.001)$ and have a chronic illness (hypertension, diabetes, heart disease, each $\mathrm{p}<0.001$ ). Overall, $70 \%$ of GMV participants and $71 \%$ of UC participants were very satisfied with their visit $(\mathrm{p}=0.76)$. Compared with UC patients, GMV participants were more likely to report enhanced access to care $(\mathrm{p}<0.001)$, whole-person orientation of their care provider $(p<0.001)$, and team-based care $(p<0.001)$. There was no difference in perceptions of care coordination $(p=0.66)$. After adjusting for baseline patient characteristics, overall patient satisfaction for GMV and UC participants was similar (adjusted odds ratio $0.9,95 \%$ confidence interval 0.7 1.1). Of GMV participants, $76 \%$ reported that they were likely or very likely to schedule another GMV.

CONCLUSIONS: Overall patient satisfaction was similar among participants of GMV and UC, although potential selection bias limits inference. GMV participants were significantly more likely to report that their care embodied five elements of the patient-centered medical home. Organizations and providers adopting the medical-home model should consider group visits as a way to enhance patient-centered care.

GUATEMALA INTERNAL MEDICINE PHYSICIANS' KNOWLEDGE OF NON-COMMUNICABLE DISEASE CLINICAL PREVENTIVE SERVICES. Juan E. Corral ${ }^{1}$; Erwin E. Argueta ${ }^{1}$; Akshay Ganju ${ }^{2}$; Lauren Arnold $^{2}$; Joaquin Barnoya ${ }^{1,2}$. ${ }^{1}$ Cardiovascular Unit of Guatemala, Guatemala, Guatemala; ${ }^{2}$ Washington University in St. Louis, School of Medicine, St. Louis, MO. (Control ID \#1294700)

BACKGROUND: Non-communicable diseases (NCDs) represent a considerable toll in Guatemala accounting for $47 \%$ of all deaths. Clinical preventive services (e.g. smoking cessation counseling and colonoscopy) are key to reducing the burden of NCDs. Internal medicine staff are fundamental to providing these services, and their knowledge and practices are a cornerstone of NCDs control and prevention. On February 2011, the Guatemalan Ministry of Health released their NCDs Prevention and Treatment Guidelines. Designed to be implemented in rural health posts and centers, the guidelines are based on reports and guidelines from international associations (e.g. Alliance for Cervical Cancer Prevention) and other countries (e.g. Mexico) rather than on cost-effectiveness analyses from Guatemala.

METHODS: We conducted a cross-sectional survey of internal medicine departments in teaching hospitals nationwide. Four trained surveyors invited interns, residents, and attendings to participate. The self-administered anonymous survey included demographic data, percentage of time devoted to outpatient care, preventive service recommendations, perceived availability and barriers to providing each service, and opinion on who should be responsible for preventive services guidelines and training. Answers regarding recommendation practices were compared with US Preventive Services Task Force (USPSTF) guidelines due to the scope and limitations of Guatemalan guidelines. USPSTF classifies preventive services as A (strongly recommend), $\mathrm{B}$ (recommend), $\mathrm{C}$ (recommend against routine use but can provide on individual basis), D (recommend against), or I (insufficient evidence recommend for or against). Services were considered cost-effective if they require less than $\$ 35,000$ per quality adjusted life-year saved. Data analysis was done with STATA/SE 11.2.

RESULTS: Of 443 physicians invited to participate, 394 completed the survey (88.94\% response rate). Recommended services (grade A or B) were offered as frequently as non-recommended services (grade D or I) (51.09\% vs. 50.39\%, p=0.9). Among A and B services, physicians did not prioritize those considered cost-effective. Only colorectal $(\mathrm{p}<0.001)$ and prostate $(\mathrm{p}=0.006)$ cancer screening recommendation practices differed by level of training. Tobacco cessation interventions (grade A) had the highest recommendation rates $(99.20 \%)$ and screening for colorectal cancer (grade A) had the lowest recommendation rates $(55.75 \%)$. Almost two-thirds of physicians recommended screening for coronary heart disease with an EKG (grade D). Regarding services availability, hypertension screening was perceived as the most available service $(93.57 \%$ indicated it is available at their hospital) and tobacco cessation pharmacotherapy as the least available $(4.83 \%)$. Furthermore, the most frequent barriers to providing services were lack of time $(46.38 \%)$ and inadequate patient's resources $(31.34 \%)$. When asked about who should provide training in preventive services, $42 \%$ considered the Ministry of Health responsible, followed by Schools of Medicine (30\%), and the Guatemalan College of Physicians (14\%)

CONCLUSIONS: Internal medicine physicians in Guatemala are inappropriately trained on preventive services for NCDs control and prevention and do not prioritize recommendations based on costeffectiveness. These data should prove useful to strengthen preventive medicine education and implement an evidence-based national screening program.

HIV CONSPIRACY BELIEFS AMONG RACIAL/ETHNIC MINORITIES IN CHICAGO: IMPLICATIONS FOR HIV TESTING AND PARTICIPATION IN RESEARCH Elizabeth Jacobs ${ }^{1}$; Ryan Westergaard ${ }^{1}$; Mary Catherine Beach ${ }^{2}$; Somnath Saha ${ }^{3}$. ${ }^{1}$ University of Wisconsin School of Medicine \& Public Health, Madison, WI; ${ }^{2}$ Johns Hopkins University, Baltimore, MD; ${ }^{3}$ Oregon Health \& Science University, Portland, OR. (Control ID \#1340669)

BACKGROUND: Conspiracy beliefs about the origin of HIV and the government's role in HIV research have been reported among AfricanAmericans in the United States. We hypothesized that endorsing such beliefs would be associated with decreased willingness to undergo physician-initiated screening for HIV infection or to participate in HIV research.

METHODS: We conducted a cross-sectional, computer-adapted survey of a convenience sample of African American $(n=208)$, white $(n=198)$, and 
Latino or Hispanic ( $\mathrm{n}=195)$ adults shopping at one of 12 supermarkets located within a socioeconomically diverse group of Chicago neighborhoods. Using a 5-point Likert scale, participants rated their level of agreement with 6 statements reflecting HIV conspiracy beliefs (Figure). Respondents then indicated whether they would agree to be tested for HIV infection if it was recommended by a doctor and rated their willingness to participate in an HIV vaccine study. Ordinal logistic regression models were used to assess the influence of holding conspiracy beliefs on willingness to undergo HIV testing or participate in research while adjusting for race/ethnic group, gender, age, family income and education level.

RESULTS: African-American and Hispanic/Latino respondents were more likely to agree or strongly agree with at least one HIV conspiracy belief compared to white/Caucasian respondents $(60.4 \%$ and $59.0 \%$ vs. $38.8 \%$, respectively, $\mathrm{p}<0.001)$. African-Americans were significantly more likely to report they would accept HIV testing if recommended by a doctor $(82.9 \%)$ than whites $(73.9 \%, \mathrm{p}<0.05)$, and expressed greater willingness to participate in an HIV vaccine study (58.9\% "very willing" or "probably willing") than whites $(39.3 \%)$ or Hispanic/Latino respondents $(49.5 \%, \mathrm{p}<0.001$ for trend). In adjusted models, level of agreement with HIV conspiracy beliefs showed no association with either report of HIV test acceptance or willingness to participate in HIV research. Of the variables analyzed, racial/ ethnic group was the only significant predictor of HIV test acceptance: African-Americans had 75\% increased odds of reporting they would accept an HIV test compared to whites (adjusted OR= $1.75,95 \% \mathrm{CI}=1.0-3.1)$.

CONCLUSIONS: HIV conspiracy beliefs remain common and are disproportionately held by racial/ethnic minorities. Endorsing such beliefs, however, does not appear to influence reported willingness to undergo HIV testing in health care settings or to participate in HIV research.

Figure. Percent agreement with HIV conspiracy beliefs, by racial/ethnic group

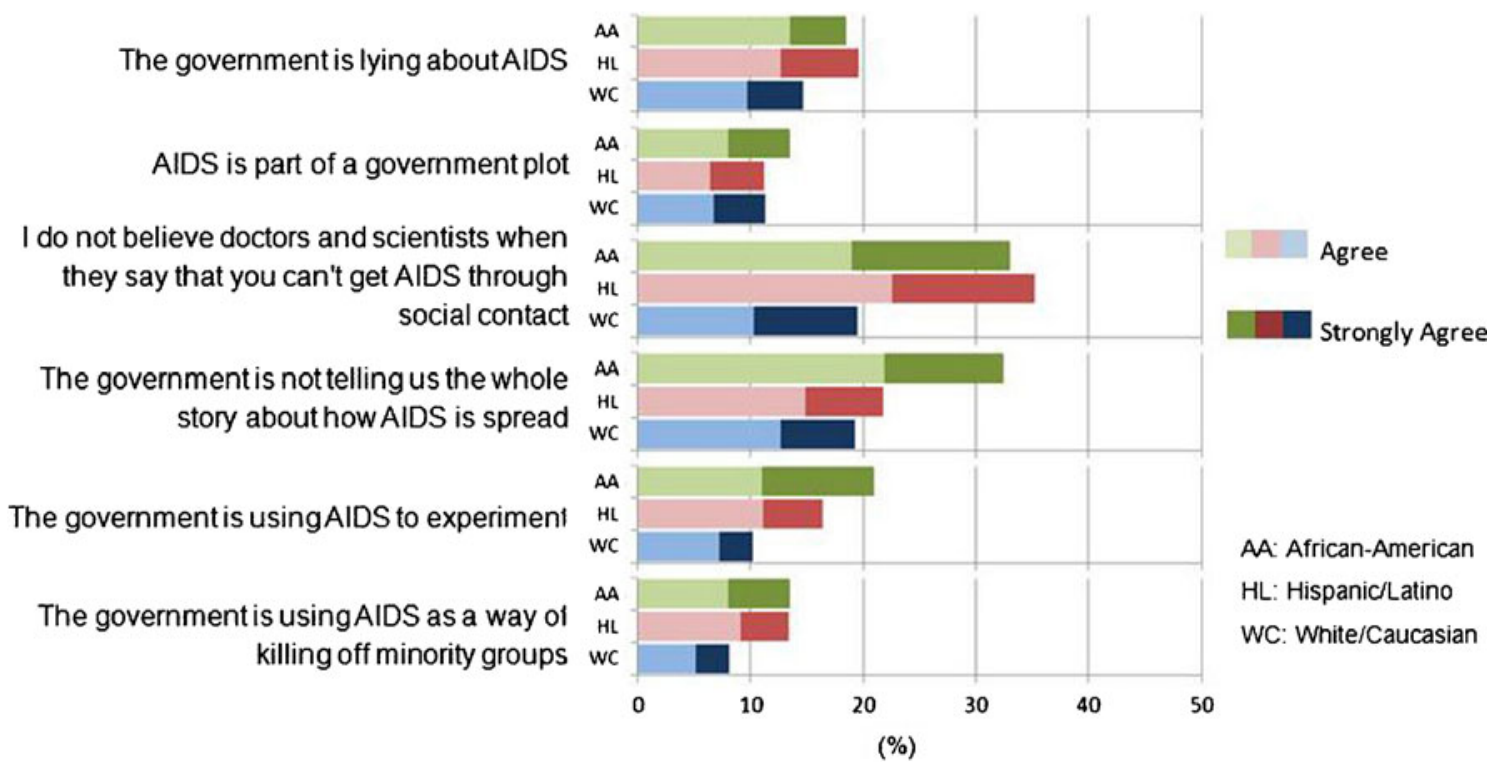

HIV STATUS IS AN INDEPENDENT PREDICTOR OF RECEIVING OPIOID ANALGESICS E. J. Edelman ${ }^{1,2}$; Kirsha S. Gordon ${ }^{3,4}$; William Becker ${ }^{2,3}$; Joseph L. Goulet ${ }^{3}$; Melissa Skanderson ${ }^{3}$; Julie R. Gaither $^{5}$; Jennifer B. Braden ${ }^{6}$; Adam Gordon ${ }^{7}$; Robert Kerns ${ }^{3}$; Amy C. Justice $^{3,2}$; David A. Fiellin ${ }^{2} .{ }^{1}$ Yale University School of Medicine, New Haven, CT; ${ }^{2}$ Yale University School of Medicine, New Haven, CT; ${ }^{3}$ VA Connecticut Healthcare System, West Haven, CT; ${ }^{4}$ Columbia University School of Public Health, New York, NY; ${ }^{5}$ Yale University School of Public Health, New Haven, CT; ${ }^{6}$ University of Washington, Seattle, WA; ${ }^{7}$ University of Pittsburgh and VA Pittsburgh Healthcare System, Pittsburgh, PA. (Control ID \#1339823)

BACKGROUND: Opioid analgesics are increasingly prescribed. HIVinfected patients may be particularly vulnerable to the toxicities and risks associated with opioid analgesics, yet limited data about receipt of opioids comparing HIV-infected and uninfected patients exist.

METHODS: We conducted a cross-sectional analysis of the Veterans Aging Cohort Study - Virtual Cohort (VACS-VC), using data from October 1, 2005 to September 30, 2006 of 40,594 HIV-infected and 81,188 age/ race/ethnicity/site-matched uninfected patients. We excluded patients who had an ambiguous HIV status; a cancer diagnosis other than nonepithelial skin cancers; no inpatient or outpatient visit in FY2006; or unclear opioid pharmacy data. Clinical characteristics, including pain diagnoses, and alcohol and substance use disorders, were determined based on ICD-9 codes. Using pharmacy data, we defined: opioid receipt as at least one outpatient prescription for an opioid; high dose opioid therapy as an average daily dose of at least $120 \mathrm{mg}$ of morphine equivalents; and long-term therapy as 90 consecutive days of opioids. Descriptive statistics for all variables by HIV status and opioid receipt were performed. We constructed multivariate models, adjusting for demographics, site of care based on urbanicity, Hepatitis C virus (HCV) status, pain diagnosis (acute, chronic), serious mental illness, and alcohol and substance use disorder, to determine factors independently associated with opioid receipt.

RESULTS: Our sample $(\mathrm{N}=78,748)$ was $97 \%$ male, with a mean age of 46 years, diverse (39\% white, $48 \%$ black, $8 \%$ Hispanic), with $86 \%$ receiving care in urban settings. Thirty-one percent of HIV-infected and $28 \%$ of uninfected patients $(\mathrm{p}<0.001)$ received any opioids. Among patients who received opioids, HIV-infected patients, were less likely to have a pain diagnosis $(60.7 \%$ vs. $73.8 \%)$, but more likely to have depression $(8.7 \%$ vs. $7.6 \%)$, Hepatitis $\mathrm{C}$ infection $(40.8 \%$ vs. $19.1 \%)$, and an alcohol (13.0\% vs. $11.2 \%)$ or substance use disorder $(17.3 \%$ vs. $10.4 \%)$ than uninfected patients. Median average daily morphine equivalent dose was $20.0 \mathrm{mg}$ (Interquartile range (IQR) 
13.5, 36.0) among HIV-infected patients and $20.6 \mathrm{mg}$ (IQR 14.6, $36.8)$ among uninfected patients, with $6.1 \%$ vs. $4.9 \% \quad(\mathrm{p}<0.001)$ receiving high doses. Median number of days of opioids supplied was 44 days (IQR 14, 189) among HIV-infected and 60 days (IRQ 17, 212 ) among uninfected patients, with $38 \%$ vs. $42 \%$ receiving longterm opioid therapy. HIV status was associated with receipt of opioids unadjusted (OR 1.17, 95\% CI 1.13, 1.21) and adjusted (AOR 1.40, $95 \%$ CI $1.35,1.46$ ) analyses. HCV infection, pain (acute or chronic), PTSD, and depression were positively associated, while non-White race/non-Hispanic ethnicity, schizophrenia, and alcohol abuse were negatively associated with receipt of opioids.

CONCLUSIONS: Though HIV-infected patients may be particularly vulnerable to the toxicities and risks associated with opioid analgesics, they are $40 \%$ more likely than uninfected patients to receive opioids after controlling for pain and other factors.

HIV KNOWLEDGE AND TESTING AMONG PREGNANT WOMEN IN RURAL MOZAMBIQUE: VALIDATION OF THE HIV KNOWLEDGE SCALE-27 (HK-27) Shannon Skinner ${ }^{1}$; Carolyn Audet $^{1}$; Sérgio Roques ${ }^{2}$; Troy Moon ${ }^{2,1}$; Sten Vermund ${ }^{1}$; Russell L. Rothman ${ }^{1}$; Philip J. Ciampa ${ }^{1}{ }^{1}$ Vanderbilt University School of Medicine, Nashville, TN; ${ }^{2}$ Friends in Global Health, Zambézia, Mozambique. (Control ID \#1337858)

BACKGROUND: The prevalence of HIV infection in Mozambique was $14 \%$ among women in 2009 , yet it is estimated only $18 \%$ of women have comprehensive HIV knowledge. Limited knowledge may have negative implications for testing uptake and treatment adherence, but research has been limited by the lack of a comprehensive, validated instrument to measure HIV knowledge in Mozambique.

METHODS: We adapted items from existing measures of HIV knowledge (HIV-KQ-45, BSS, DHSAIDS) and added items about HIV treatment. The adapted scale (the HK-27) was translated into Portuguese and Echuabo and orally administered to women seeking prenatal care at two clinics in rural Mozambique. The HK-27 consists of 27 items that assess knowledge across several HIV content domains; for each item, respondents could agree, disagree, or state that they were uncertain. A summary score is based on the percent correct (range 0-100\%). Sociodemographic characteristics and HIV testing were determined by self-report. HIV status was abstracted from medical records. Analyses were stratified by survey language. KuderRichardson (KR-20) coefficients estimated internal reliability. Construct validity was established by testing bivariate associations between HK-27 score and sociodemographic characteristics chosen $a$ priori. The association between knowledge and HIV test utilization was estimated by multivariable logistic regression.

RESULTS: Participants $(\mathrm{N}=348)$ had a median age (IQR) of 24 (2028); 188 spoke Portuguese, and 160 spoke Echuabo. Over half (57.6\%) had no formal education. Mean HK-27 scores were higher for Portuguese-speaking participants (68.3, SD 18.6) than Echuabospeaking participants (42.2, SD 22.6). Internal reliability was strong (KR-20>0.8) for scales in both languages. Higher HK-27 scores were significantly $(p \leq 0.05)$ correlated with more education, more media items in the home, and maternal work outside of the home (Table 1). Eighty-five percent of women reported past HIV testing. Women with higher HIV knowledge had higher odds of past HIV testing, even after adjusting for study language, site, travel time to the clinic, and maternal work in the multivariable model (aOR 5.0, 95\% CI:1.2-22.1, $\mathrm{p}=0.03$ ).

CONCLUSIONS: HK-27 is a reliable and valid measure of HIV knowledge of Portuguese and Echuabo-speaking women seeking prenatal care in rural Mozambique. HIV knowledge was higher than in previous estimates, though gaps remain especially for non-Portuguese speakers. HIV knowledge was associated with HIV testing in this cross-sectional sample, though the study design limits its ability to prove causation. Further work should explore the relationship among HIV knowledge and health-seeking behaviors in low income settings.
Table 1: Correlation among HIV knowledge and sociodemographic data

\begin{tabular}{|c|c|c|c|c|c|c|}
\hline \multirow{2}{*}{$\begin{array}{l}\text { Characteristic } \\
\text { Continuous } \\
\text { Variables }\end{array}$} & \multicolumn{3}{|c|}{$\begin{array}{l}\text { Portuguese-Speaking } \\
\text { Participants }\end{array}$} & \multicolumn{3}{|c|}{$\begin{array}{l}\text { Echuabo-Speaking } \\
\text { Participants }\end{array}$} \\
\hline & $\mathbf{N}$ & $\begin{array}{l}\text { Correlation } \\
\text { with HK- } \\
27(\rho)\end{array}$ & $\mathbf{P}$ & $\mathbf{N}$ & $\begin{array}{l}\text { Correlation } \\
\text { with HK- } \\
27(\rho)\end{array}$ & $\mathbf{P}$ \\
\hline Age & 188 & 0.05 & 0.49 & 160 & -0.02 & 0.78 \\
\hline $\begin{array}{l}\text { Years of } \\
\text { education }\end{array}$ & 188 & 0.68 & $<0.001$ & 159 & 0.19 & 0.02 \\
\hline $\begin{array}{l}\text { Number } \\
\text { of children }\end{array}$ & 187 & -0.18 & 0.01 & 160 & 0.00 & 0.90 \\
\hline $\begin{array}{l}\text { Media items } \\
\text { owned }\end{array}$ & 182 & 0.28 & $<0.001$ & 159 & 0.17 & 0.04 \\
\hline $\begin{array}{l}\text { Categorical } \\
\text { Variables }\end{array}$ & $\mathbf{N}$ & $\begin{array}{l}\text { Mean HK- } \\
27 \text { Score } \\
\text { (SD) }\end{array}$ & $\mathbf{P}$ & $\mathbf{N}$ & $\begin{array}{l}\text { Mean HK- } \\
27 \text { Score } \\
\text { (SD) }\end{array}$ & $\mathbf{P}$ \\
\hline $\begin{array}{l}\text { HIV test } \\
\text { obtained }\end{array}$ & 188 & & 160 & & & \\
\hline Yes & 156 & $69.7(18.3)$ & 0.05 & 141 & $43.5(22.6)$ & 0.04 \\
\hline No & 32 & $62.5(19.3)$ & & 19 & $32.4(20.7)$ & \\
\hline Maternal work & 188 & & 160 & & & \\
\hline $\begin{array}{l}\text { Domestic } \\
\text { work }\end{array}$ & 99 & $65.4(20.4)$ & 0.01 & 14 & $64.0(11.1)$ & 0.02 \\
\hline Agriculture & 53 & $65.8(15.2)$ & & 143 & $39.6(22.3)$ & \\
\hline Business & 15 & $74.3(15.4)$ & & 2 & $63.0(0.10)$ & \\
\hline Teacher & 21 & $84.8(7.0)$ & & 1 & $59.3(-)$ & \\
\hline
\end{tabular}

HOSPITALIZATION: A MISSED OPPORTUNITY TO ASSESS FOR OSTEOPOROSIS Eileen Hennrikus; Christopher Weber; Nida Rizvi; Edward Fox. Penn State Milton Hershey Medical Center, Hershey, PA. (Control ID \#1338639)

BACKGROUND: More than 1.5 million fractures result from osteoporosis annually in the United States at a cost of fifteen billion dollars. Less than $25 \%$ of these patients receive treatment for osteoporosis. We hypothesize that hospitalization offers an opportunity to screen patients utilizing the very simple Osteoporosis SelfAssessment Tool (OST).

METHODS: From $2007-2010,790$ patients, >age 50, were identified by ICD-9 diagnoses for fragility fractures. Patients were excluded if they: had a high impact, or pathologic fracture, not consistent with a fragility fracture, could not be contacted, received pre and post-fracture care at another facility, suffered dementia, or were deceased. 320 patients with insufficiency fractures remained and responded to a phone survey. They were asked about a prior diagnosis of osteoporosis and the administration of a Dexa scan, Calcium and Vitamin D. Medical records were reviewed for all hospitalizations five years prior to the patient's fracture. Data collected included the principle diagnosis, age, weight and Bone Mineral Density (BMD) scores among other variables. The OST was utilized to calculate a fracture risk assessment score based on weight and age. The Spearman correlation test was used to test correlation between the OST score and Hip and Lumbar T-scores.

RESULTS: 127 of the 320 patients $(40 \%)$ were hospitalized within the past 5 years. These 127 patients were admitted 312 times with a mean of 2.5 hospitalizations per person. The top 5 reasons for hospitalization included: Cardiac, Infectious, Musculoskeletal, Gastrointestinal and Neurologic/Psychiatric. $78 \%$ of patients were female. The mean age was 74 years. $45 \%$ had a prior diagnosis of osteoporosis. Of the 127 patients, only 45 (39\%) had BMD tested prior to their fragility fracture. The mean lumbar T-score was -1.71 and the mean hip T-score was -2.17 .87 patients $(69 \%)$ had an OST $<$ 2.0. The mean OST score was 1.47 (an OST value $<2.0$ indicates osteoporosis risk). Although the OST score did not correlate with the lumbar T-score, it did correlate moderately with the hip T-score 0.46 (95\% CI 0.25-0.67). 
CONCLUSIONS: Forty percent of patients $>50$ years old who sustained an osteoporotic fracture had been hospitalized during the previous 5 years. Of these, less than half had been screened with a DEXA scan. $69 \%$ of these patients had an OST score $<2.0$. The OST score is easy to calculate and can easily be incorporated as a screening tool for hospitalized patients. Although there is only a moderate correlation with the hip T-score value, the OST score has potential to function as the initial hospital screening tool by virtue of its simplicity, thereby making it possible to routinely screen patients in the hospital.

HAS QUALITY IMPROVEMENT RESULTED IN THE REDUCTION OF INAPPROPRIATE CARE? Minal Kale ${ }^{1}$; Alex Federman ${ }^{1}$; Salomeh Keyhani ${ }^{2} .{ }^{1}$ Mount Sinai School of Medicine, New York, NY; ${ }^{2}$ University of California, San Francisco, CA. (Control ID \#1330375)

BACKGROUND: In the past decade there has been a major focus on improving the quality of care. However, efforts to improve the quality of healthcare have largely concentrated on developing publicly reportable underuse measures. Overuse and misuse of health care services are also important aspects of the quality of healthcare. We compared changes in the quality of ambulatory care in the US between 1998/1999 and 2008/2009 using measures of underuse, misuse, and overuse of health care services.

METHODS: We performed a cross-sectional analysis of the National Ambulatory Medical Care Survey (NAMCS) and the outpatient department component of the National Hospital Ambulatory Medical Care Survey (NHAMCS), which are nationally representative annual surveys of visits to non-federally funded ambulatory care practices. We identified a total of twenty-three quality indicators using a combination of current quality measures and guideline recommendations. Each indicator was chosen because it pertained to outpatient quality of care and could be reliably calculated using information in the 1998, 1999, 2008, and 2009 NAMCS and NHAMCS. We categorized our quality indicators into underuse (9), overuse (12), and misuse (2). We estimated the rates of underuse, overuse, and misuse and their $95 \%$ confidence intervals, accounting for the complex sampling design of the NAMCS and NHAMCS

RESULTS: We observed a statistically significant improvement in six out of nine underuse quality indicators. In the ten year interval under consideration, there was an improvement in the use of antithrombotic therapy for atrial fibrillation $(45.9 \%$ to $71.9 \%, \mathrm{p}<.01)$, the use of aspirin in coronary artery disease (CAD) $(28.4 \%$ to $64.5 \%, \mathrm{p}<.01)$, the use of beta blockers in congestive heart failure (CHF) $(20.6 \%$ to $59.7 \%, \mathrm{p}<.01)$ and beta blockers in $\operatorname{CAD}(28.1 \%$ to $55.2 \%, \mathrm{p}<.01)$. There were also improvements in the use of statins in CAD $(26.8 \%$ to $58.6 \%, \mathrm{p}<.01)$ and diabetes $(12.1 \%$ to $36.2 \%, \mathrm{p}<.01)$. We observed an improvement in only two of 12 overuse quality indicators, one indicator became worse and 9 did not change. There was a statistically significant decrease in the overuse of cervical cancer screening in visits for females older than $65,(3.1$ to $2.2 \%$, $\mathrm{p}=.02$ ) and a statistically significant decrease in the overuse of antibiotics in asthma exacerbations $(22.3 \%$ to $6.8 \%, \mathrm{p}<.01)$. However, there was an increase in the overuse of prostate cancer screening in men older than 74 , from $3.5 \%$ to $5.7 \%(p=.03)$. There were no changes in the remaining nine overuse measures: laboratory screening tests and ECG testing in general medical exams, antibiotics for upper respiratory infections and acute bronchitis, mammography for women older than 74, pap tests in women younger than 21, and imaging in acute back pain. Out of the two misuse indicators, there was one significant improvement. The proportion of patients with a urinary tract infection who were prescribed an inappropriate antibiotic decreased from $24.9 \%$ to $2.7 \%(\mathrm{p}<.01)$. There was no change in the proportion of elderly patients who were prescribed inappropriate medications.

CONCLUSIONS: Quality improvement efforts have resulted in the reduction in the underuse of appropriate ambulatory care. However despite significant policy attention focused on reducing waste in the US health care system, we found little improvement in the delivery of inappropriate ambulatory care in the past decade.
HEALTH INFORMATION EXCHANGE REDUCES HOSPITAL ADMISSIONS FOR CHEST PAIN James E. Bailey ${ }^{1}$; Xinhua $\mathrm{Yu}^{2}$; Ryan D. Ward ${ }^{1}$; Guy L. Reed ${ }^{1} .{ }^{1}$ University of Tennessee Health Science Center, Memphis, TN; ${ }^{2}$ University of Memphis, Memphis, TN. (Control ID \#1339000)

BACKGROUND: Presentation to the emergency department (ED) for chest pain can result in potentially avoidable hospital admission and/or cardiac evaluation. By providing useful patient data to emergency staff, health information exchange (HIE) may reduce unnecessary hospital admissions, cardiac testing, and associated costs. This study seeks to determine whether HIE reduces hospitalizations and cardiac catheterizations in patients presenting to the ED with chest pain.

METHODS: Cross-sectional analyses of 21,257 individual patientvisits for 12,385 adults diagnosed with chest pain without active cardiac conditions presenting to any Memphis Metropolitan Area (MMSA) ED 2 or more times between 8/1/2007 and 7/31/2009. Outcome measures included hospital admission and cardiac catheterization.

RESULTS: The majority of the repeat patient-visits for chest pain were for males $(58.3 \%)$, Medicare patients $(32.5 \%)$ with median age of 54 (IQR 42 - 69) and median of 1 previous ED visit for chest pain (IQR 1 - 2; range 1 48). HIE data was accessed for $6.5 \%$ of visits for chest pain. For $80.2 \%$ of visits troponin was obtained, 7,964 (37.5\%) resulted in hospital admission, and $1,134(5.3 \%)$ resulted in cardiac catheterization. After controlling for demographic factors, comorbidity, hospital system, and previous visits, HIE use was associated with decreased odds of hospital admission (OR 0.77 , CI 0.65 - 0.91) but was not associated with reduced odds of cardiac catheterization (OR 1.08, CI 0.81 - 1.46).

CONCLUSIONS: HIE use in the ED is associated with decreased hospital admission for patients seen for chest pain. Rates of cardiac catheterization were appropriately low overall for this population and HIE did not impact use of cardiac catheterization. Low HIE use rates by providers in the ED limited the effectiveness of HIE in reducing potentially avoidable hospitalizations. We estimate that over 1,150 hospitalizations could be potentially prevented annually in the MMSA if providers used HIE for all ED chest pain visits. Assuming a similar reduction in admissions as seen in Memphis, we estimate that $100 \%$ provider HIE adherence nationwide could avoid over 297,000 chest pain hospitalizations yielding potential cost savings between $\$ 1.3 \mathrm{~B}$ and $\$ 3.2 \mathrm{~B}$ annually. Further studies are needed to assess best methods to increase HIE use.

HEALTH LITERACY AND COGNITIVE DECLINE IN OLDER ADULTS Shwetha S. Sequeira ${ }^{1}$; Rebecca Silliman ${ }^{1}$; Timothy Bickmore ${ }^{2}$; Lori Henault ${ }^{1}$; Michael Winter ${ }^{1}$; Debbie M. Cheng ${ }^{1}$; Kerrie Nelson ${ }^{1}$; Laura Eggermont $^{3}$; Michael Paasche-Orlow ${ }^{1} .{ }^{1}$ Boston University Medical Center, Boston, MA; ${ }^{2}$ Northeastern University, Boston, MA; ${ }^{3} \mathrm{VU}$ University, Amsterdam, Netherlands. (Control ID \#1321298)

BACKGROUND: Limited health literacy is associated with a range of neurocognitive measures including executive function and verbal fluency. We hypothesized that people with limited health literacy would have greater decline in executive function and verbal fluency over time than people with higher health literacy.

METHODS: Participants were recruited from three urban ambulatory care practices for a randomized controlled trial of an intervention to promote walking. Enrollment criteria were age $\geq 65$, community dwelling, and permission from their primary care physician to participate. Subjects were excluded if they screened positive for cognitive impairment ( $\leq 2$ on the Mini-Cog) or depression (PHQ-9 $\geq 15$ ). The study is a secondary analysis of information collected on health literacy, demographics, education status, co-morbidities and neurocognitive measures. Health literacy, the main independent variable, was assessed with the Short Test of Functional Health Literacy in Adults, categorized as limited (0-22) and higher (23-36). Executive function was assessed using the Trails Making Test (TMT): the time taken to connect a series of numbers (TMT-A) and alternating numbers 
and letters (TMT-B) is recorded in seconds and scored as time for TMT-B minus time for TMT-A (TMT B-A). A faster time reflects better executive function. Verbal fluency was assessed using the FAS test, where the score is the average number of words starting with F, A and $\mathrm{S}$ said in one minute for each and the Naming test, where the score is the number of animals and vegetables named in one minute. A higher score indicates better verbal fluency. The primary outcomes were the change in each neurocognitive measure between enrollment and 12 months. The association between health literacy and neurocognitive measures at baseline was modeled with multivariate linear regression adjusting for clinic location, age, sex, race, number of comorbidities, PHQ-9 score, and education. In addition, baseline neurocognitive scores and randomization group were added to model change from baseline to 12 months.

RESULTS: Of 263 participants, 226 completed baseline and 12 month visits and were included in the analysis. Mean (sd) age at enrollment was 71.4(5.4); $63 \%$ were Black; $63 \%$ female and $37 \%$ had limited health literacy. At baseline, those with limited health literacy were 74 seconds slower in TMT B-A $(159.3$ vs 85.6, p<0.0001), scored 12 fewer words in FAS ( 22 vs $34, \mathrm{p}<0.0001)$ and 6 fewer words in Naming ( 24.1 vs $29.8, \mathrm{p}<0.0001)$ compared to higher literacy participants. In adjusted models, those with limited health literacy were 49 seconds slower for TMT B-A $(\mathrm{p}<0.0001)$, scored 8 fewer words in FAS $(\mathrm{p}<$ $0.0001)$ and 2 fewer words in Naming $(\mathrm{p}=0.06)$ at enrollment. In the adjusted models of change from baseline, TMT B-A was 24 seconds longer $(\mathrm{p}=0.01)$ for those with limited health literacy compared with the higher health literacy group, but there were no significant differences in change from baseline on Naming and FAS scores by health literacy status.

CONCLUSIONS: As we explore mechanisms of cognitive decline it is important to understand the role of health literacy. Interventions aimed at delaying cognitive decline should be evaluated in relation to health literacy.

HEALTH PROMOTION IN AFRICAN AMERICAN CHURCHES: WHO SAYS THEY WILL ATTEND? Adebowale Odulana ${ }^{1}$; Mimi $\mathrm{Kim}^{1}$; Malika Roman Isler ${ }^{1}$; Melissa Green ${ }^{1}$; Yhenneko Taylor ${ }^{2}$; Paul Godley ${ }^{1}$; Giselle Corbie-Smith ${ }^{1} .{ }^{1} \mathrm{UNC}$ at Chapel Hill School of Medicine, Chapel Hill, NC; ${ }^{2} \mathrm{UNC}$ Charlotte College of Human and Health Services, Charlotte, NC. (Control ID \#1339215)

BACKGROUND: While churches have been identified as an important partner for improving health within the African American community, there is little literature describing who and how congregants are reached by health promotion activities. Increasing emphasis on community-academic partnerships to address health disparities creates a need to understand the expectations and interests of those engaged by outreach activities. Identifying individual attributes and contextual characteristics associated with willingness to attend health promotion programs allows faith based health collaborations to better target members to improve health. We examined how church characteristics and congregants' beliefs and interests in faithbased health promotion are associated with their willingness to attend church-based health promotion activities.

METHODS: We surveyed adult members $(n=1204)$ of 11 predominately African American churches in regions with significant health disparities in North Carolina. We collected data on demographics, health concerns, lifestyle habits, health beliefs, and health ministry characteristics and these independent variables were categorized into four domains: demographics (age, sex, education, health status), behavioral (church attendance, respondent food choices, and physical activity), cognitive(church based health promotion belief, bible based healthy living interest, healthy living resource interest), or environmental (family health, church travel distance, church health ministry activity, church members' food choices). We used a dichotomous outcome; interest in attending programs offered by the health ministry (yes vs. no). . We constructed a series of domain specific models and a full model and used logistic generalized estimating equations to adjust for clustering of the data.

RESULTS: Of the 1204 congregants, $72 \%$ were female, $57 \%$ age $\geq 50$, $84 \%$ had $\geq$ a high school education, and $77 \%$ had a chronic health condition. In bivariate analyses, congregants who wanted to learn more about resources for healthy living (OR 6.67, 95\% CI 4.37-10.19) and who believed the church is responsible for health promotion (OR 5.15, CI 3.33-7.96) had the highest odds of willingness to attend. These cognitive characteristics remained significant in domain-specific models. Additionally, in the behavioral and environmental domains respectively, factors with the highest odds of willingness to attend were reporting making healthy food choices (OR 2.53, CI 1.77-3.61), and having fellow church members who make healthy food choices (OR 2.90, CI 1.70-4.93). In models adjusting for all four domains simultaneously, cognitive factors were most highly associated with willingness to attend, while no demographic characteristics remained significant.

CONCLUSIONS: Congregants' belief in the church's role for health promotion and their desire to learn about healthy behaviors highlight the role of the black church as a partner in addressing health disparities and the need to capitalize on this expectation through stronger partnerships between medical and faith communities. Additionally in a time of limited resources, these findings help churches and investigators more efficiently target the concerns of those who are most willing to attend.

HEALTH STATUS AND HEALTH CARE EXPERIENCES AMONG HOMELESS PATIENTS IN FEDERALLY SUPPORTED HEALTH CENTERS: FINDINGS FROM THE 2009 PATIENT SURVEY Travis P. Baggett ${ }^{2}$; Lydie A. Lebrun ${ }^{1}$; Darlene Jenkins ${ }^{3}$; Alek Sripipatana ${ }^{1}$; Ravi Sharma ${ }^{1}$; Arthur S. Hayashi ${ }^{1}$; Charles Daly ${ }^{1}$; Quyen Ngo-Metzger ${ }^{1}{ }^{1}$ U.S. Department of Health and Human Services, Rockville, MD; ${ }^{2}$ Harvard Medical School, Boston, MA; ${ }^{3}$ National Health Care for the Homeless Council, Nashville, TN. (Control ID \#1324072)

BACKGROUND: The Health Resources and Services Administration (HRSA) provides federal funding to health centers to provide health services to homeless persons as well as other low-income, minority, and uninsured or publicly insured individuals. In 2010, health centers provided primary health care, as well as mental health services and substance abuse treatment, to over 1 million homeless patients and individuals who were formerly homeless or at risk of homelessness. The purpose of this study was to examine the health status and health care experiences of homeless patients in health centers, and compare them with their housed counterparts.

METHODS: Nationally representative data came from the 2009 Health Center Patient Survey conducted by HRSA. Computer-assisted personal interviews were conducted with health center patients. Crosssectional analyses were limited to adults $(n=2,735)$. We compared sociodemographic characteristics, health conditions, access to health care, and utilization of services among homeless and non-homeless patients. Health status and medical conditions included self-reported fair/poor health status, any activity restrictions in the past 3 months, food insufficiency, obesity, various chronic conditions, vision impairment, oral health problems, mental health problems, and substance use. Access and utilization measures included unmet needs for medical care, usual source of care, emergency department (ED) visits, hospitalizations, and receipt of preventive services. We employed multiple logistic regression models to examine the independent effect of homelessness on health care access and utilization.

RESULTS: Homeless patients were mostly male (57\%), not married (93\%), and non-Hispanic White (36\%) or African American (37\%). Homeless patients had worse health status-lifetime burden of chronic conditions, mental health problems, and substance use problemscompared with housed respondents. For instance, $42 \%$ of homeless patients had 2 or more chronic conditions, compared with $33 \%$ of non-homeless patients $(\mathrm{p}<0.01)$. Psychological distress in the past month was more prevalent among homeless patients than nonhomeless patients $(68 \%$ vs. $41 \%, \mathrm{p}<0.001)$. About $60 \%$ of homeless patients were current smokers, compared with $30 \%$ of non-homeless patients $(\mathrm{p}<0.001)$. In addition, twice as many homeless patients reported binge drinking than non-homeless patients $(40 \%$ vs. $20 \%, p<$ 
0.001 ), and $14 \%$ of homeless patients had ever injected drugs compared with $3 \%$ of non-homeless patients $(\mathrm{p}<0.001)$. In adjusted analyses, homeless patients were twice as likely as housed patients to have an $\mathrm{ED}$ visit in the past year $(\mathrm{OR}=2.00,95 \% \mathrm{CI}$ : $1.37-2.92)$, and twice as likely to have unmet medical care needs $(\mathrm{OR}=1.98,95 \% \mathrm{CI}$ : 1.24-3.16). Among homeless patients, having a usual source of care was associated with $50 \%$ lower odds of an $\mathrm{ED}$ visit $(\mathrm{OR}=0.51,95 \%$ CI: $0.29-0.90)$.

CONCLUSIONS: There is an ongoing need to focus on the health issues which disproportionately affect homeless populations, and federally supported health centers play a key role in providing quality primary care to these patients. Addressing the primary and preventive care needs of homeless populations may help to curb ED visits and unmet medical needs.

\section{HEALTH-RELATED QUALITY OF LIFE IN INSURED, OLDER ADULTS WITH DIABETES DIFFERS ONLY MARGINALLY BY RACE OR ETHNICITY: RESULTS FROM THE DIABETES \& AGING STUDY Neda Laiteerapong ${ }^{1}$; Andrew J. Karter ${ }^{2}$; Jennifer Y. $\mathrm{Liu}^{2}$; Howard H. Moffet ${ }^{2}$; Priya John ; Marshall Chin ${ }^{1}$; Elbert Huang ${ }^{1}$. ${ }^{1}$ University of Chicago, Chicago, IL; ${ }^{2}$ Kaiser Permanente Northern California, Oakland, CA. (Control ID \#1332363)}

BACKGROUND: Racial and ethnic disparities exist in the quality of diabetes care and rates of diabetes-related complications in adults, and a previous study reported racial/ethnic differences in HRQL in older rural adults with diabetes. Since race/ethnic differences in access to care may confound the relationship between race/ethnicity and HRQL, we explored this relationship among older adults with diabetes who are members of an integrated health care delivery system with uniform access to care.

METHODS: We studied a race/ethnic stratified, random sample of adults $\geq 60$ years with diabetes, enrolled in Kaiser Permanente Northern California's Diabetes Registry, who self-reported HRQL (based on SF8TM Health Survey) in the DISTANCE (Diabetes Study of Northern California) survey. Race/ethnicity was classified as self-reported nonHispanic White ("White"), non-Hispanic Black ("Black"), Hispanic, Asian (non-Filipino), Filipino, Multi-racial, and other race. Responses to the HRQL assessment were transformed into physical and mental HRQL scores (both ranges: 0-100, and means: 50) using published algorithms. We constructed a series of weighted linear regressions models to estimate the associations between race/ethnicity and HRQL, and evaluate whether they were explained by potential mediating factors: Model 1: race/ethnicity adjusting for demographics (age and sex); Model 2: Model 1+marital status, education, income, birthplace and English proficiency; Model 3: Model $2+$ health behaviors (alcohol/smoking history, physical activity); Model 4: Model 3+geriatric conditions (fall history, underweight, chronic pain, depression, incontinence), Model 5: Model 4+diabetes complications (congestive heart failure, myocardial infarction, stroke, end-stage renal disease, amputation, blindness, foot ulcer, and neuropathy). Least square means of the HRQL scores are reported with the p-values comparing the HRQL scores between each race/ethnicity and Whites (reference group).

RESULTS: There were 6,317 eligible survey respondents with mean age of 67 years and diabetes duration of 12 years. The sample size adequately supported race/ethnic contrasts. Physical HRQL changed marginally across Models 1 to 5 for all races; thus we present only Model 1 results. Whites (Model 1: 42.9; 95\% Confidence Interval (CI), 42.6-43.2, $\mathrm{p}<.001$ ) had only a marginally lower physical HRQL score than Blacks (44.2; CI 43.345.1, $\mathrm{p}<.001$ ), Latinos (45.1; CI, 44.2-46.0, $\mathrm{p}<.001$ ), Asians (48.1; CI, $46.8-49.3, \mathrm{p}<.001)$, Filipinos $(48.3 ; 95 \% \mathrm{CI}, 44.9-47.9, \mathrm{p}<.001)$, and respondents reporting other race $(46.1 ; \mathrm{CI} 43.9-48.5, \mathrm{p}=.03)$. Physical HRQL did not differ significantly between Whites and Multi-racials. Mental HRQL differed only minimally by race/ethnicity. In Models 1, 2, and 3, only Asians (e.g. Model 1: 52.7; 51.6-53.7, $\mathrm{p}=.01$ ) reported slightly higher mental HRQL than Whites (51.0; CI, 50.7-51.3). These differences were not present in Models 4 or 5. In Models 4 and 5, respondents reporting other race (e.g. Model 4: 47.3; CI, 45.4-49.3) had slightly lower mental HRQL than Whites (50.2; CI, 49.6-50.8).

CONCLUSIONS: In an integrated health care delivery system affording uniform access to health care, health-related quality of life in older adults with diabetes differs only marginally by race/ethnicity. Moreover, these differences were not clinically relevant or explained by a wide range of potentially explanatory factors.

HEPATITIS B SURFACE ANTIBODY TITERS AS A MARKER OF IMMUNITY IN HEMODIALYSIS (HD) PATIENTS: A SINGLE CENTER PROSPECTIVE QUALITY IMPROVEMENT STUDY Amit P. Ladani ${ }^{1}$; Raghavesh Pullalarevu ${ }^{1}$; Prabhat Singh $^{1}$; Saba Akhtar ${ }^{1}$; Derek Evans ${ }^{4}$; Maureen Lawlor ${ }^{3}$; Mohamed H. Yassin ${ }^{2} .{ }^{1}$ UPMC MERCY, Pittsburgh, PA; ${ }^{2}$ UPMC Mercy, Pittsburgh, PA; ${ }^{3}$ UPMC Mercy, Pittsburgh, PA; ${ }^{4}$ UPMC Mercy, Pittsburgh, PA. (Control ID \#1326328)

BACKGROUND: Hepatitis B virus (HBV) is a DNA virus that is capable of causing significant liver disease including acute hepatitis, chronic hepatitis, cirrhosis and hepatocellular carcinoma. HBV is responsible for $80 \%$ of hepatocellular carcinoma cases worldwide. The burden of HBV in HD patients has markedly decreased. The main factors include: introduction of HBV vaccination, routine screening of blood products for $\mathrm{HBV}$, the use of erythropoietin and effective Infection Control measures. It is the standard of care to confirm that all ESRD patients are vaccinated for HBV prior to starting HD. This response is measured by monitoring protective antibody titer (HBs $\mathrm{Ab})$. A titer above 10 is considered protective. Despite all the efforts to vaccinate $\mathrm{HD}$ patients, their response to $\mathrm{HBV}$ vaccine is lower and less sustained than healthy individuals. The $\mathrm{CDC}$ recommends post vaccination testing only in certain population, which includes HD patients. HBV is an efficiently transmitted blood borne pathogen. HBV can stay alive on surfaces, which make HBV more capable of producing outbreaks especially in HD units. Inpatient $\mathrm{HD}$ units receive higher percentage of acute $\mathrm{HD}$ patients that could lead to HBV outbreaks if a new HBV positive patient is introduced to the HD unit.

METHODS: The goal of the study was to estimate the base-line protection against $\mathrm{HBV}$ in $\mathrm{HD}$ patients. It was a quality improvement initiative to improve protection against $\mathrm{HBV}$ in $\mathrm{HD}$ patients. All HD patients at our inpatient unit were included in the study and got HBV serology monitored during their HD session. The following serum markers were monitored: $\mathrm{HBsAg}, \mathrm{HBsAb}$ titer, $\mathrm{HBcAb}$ total. Vaccination was offered to all patients with no evidence of protective $\mathrm{HBsAg}($ titer $<10)$. The schedule of $\mathrm{HBV}$ vaccination was based on CDC recommendations. Outpatient HD units were notified with our recommendations regarding further vaccination needs. The study was done over a period of two months and included $60 \mathrm{HD}$ patients.

RESULTS: Sixty patients were included in this study. Forty two (70\%) patients had received prior vaccinations, either complete or incomplete and eighteen $(30 \%)$ patients had no history of prior vaccination. Seventeen $(40 \%)$ patients who had prior vaccination had low HbsAb titers and hence were vulnerable. All patients who were vulnerable [thirty five $(57 \%)$ patients] were offered vaccination. Twelve patients received HBV vaccination shots during their inpatient stay. Rest of the vulnerable patients could not be vaccinated due to factors like death, vaccination refusal and discharges before vaccine administration.

CONCLUSIONS: Although the rates of HBV infection have decreased markedly in the general population as well as in HD patients, it still poses a great potential for producing outbreaks particularly in inpatients HD units. Monitoring $\mathrm{HBV}$ serology ( $\mathrm{HBsAg}, \mathrm{HBs} \mathrm{Ab}, \mathrm{HBc} \mathrm{Ab}$ ) on routine basis is essential to prevent such potential outbreaks. Monitoring HBs Ag alone is not sufficient and has to be coupled with other serology to assure adequate protection for this particularly vulnerable population.

HETEROGENEITY AND VARIATION IN BODY MASS INDEX IN THE FRAMINGHAM HEART STUDY OFFSPRING COHORT OVER 37 YEARS Jason P. Block ${ }^{1}$; S. V. Subramanian ${ }^{3}$; Nicholas A. Christakis ${ }^{2}$; A. James $\mathrm{O}^{\prime}$ Malley ${ }^{2}$ ' Harvard Pilgrim Health Care Institute/Harvard Medical School, Boston, MA; ${ }^{2}$ Harvard Medical School, Boston, MA; ${ }^{3}$ Harvard School of Public Health, Boston, MA. (Control ID \#1339547)

BACKGROUND: The rapid trajectory of average weight in the US has implicated changing environmental or neighborhood factors as possible 
contributing factors. Using longitudinal data from the Framingham Heart Study (FHS) Offspring Cohort over 37 years, we examined trends in BMI accounting for residential mobility to determine variation in BMI at the individual and neighborhood levels over time.

METHODS: Subjects were examined up to eight times through 2008. We excluded observations with missing body mass index, smoking status, alcohol intake or census tract of residence and when a subject was living in a nursing home or the age of a subject was less than 21 years. The final sample size was 4,148 subjects with 27,133 observations. We used gender-stratified, crossclassified multilevel models to account for time-varying attributes of individuals and residential neighborhoods (census tracts) with measured BMI as the outcome, controlling for individual demographics and behaviors and neighborhood poverty. We included a random slope for linear time at the individual level to account for heterogeneity in BMI trajectories between individuals.

RESULTS: Mean BMI increased from $24.0 \mathrm{~kg} / \mathrm{m} 2$ at Wave 1 to 27.7 at Wave 8 for women and from 26.6 to 29.0 for men. The proportion of the total BMI variance explained by the individual level, the intraclass correlation coefficient (ICC), was high, 0.88 for women and 0.86 for women, meaning that the individual level explained $88 \%$ and $86 \%$ of all variation in BMI. Inclusion of the individual-level covariates did not appreciably change the ICCs, explaining less than $10 \%$ of the individual-level variance for women and none of the variance for men. Neighborhood-level variance contributed minimally to BMI variance. Large individual-level random slopes demonstrated that the variance in BMI increased significantly over time for both men and women (Figure 1). Covariates that were positively associated with BMI for men and women were increasing age, alcohol consumption, a high school education, being married, and being employed. Smoking was negatively associated. Neighborhood poverty was not significantly associated with BMI. CONCLUSIONS: Unmeasured individual factors explained almost all of the variation in BMI over nearly 40 years, and BMI variance increased substantially over time. The limited relationship of the neighborhood of residence to BMI variation over time suggests that neighborhood-level interventions may have an overall limited impact on weight.

Siandard Deviarion in Body Mass Index for Men and Women, 1971.2008

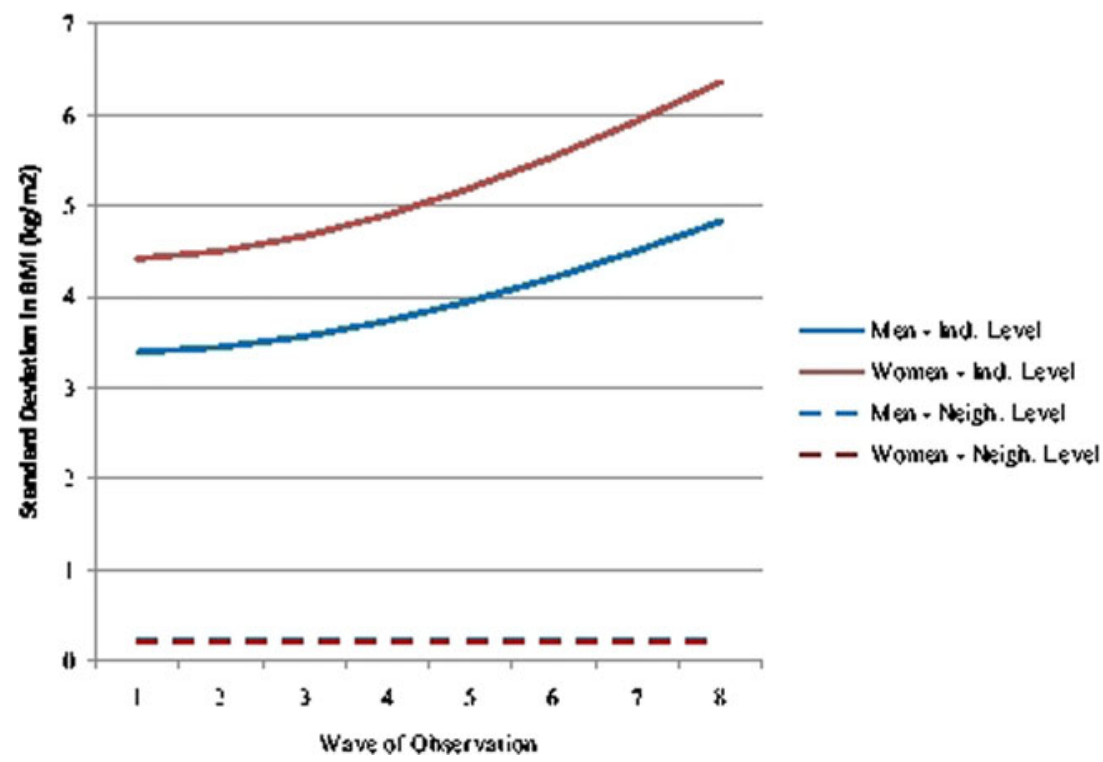

HIGH PREVALENCE OF PATIENTS $<65$ YEARS OLD WITH COMPLEX BEHAVIORAL AND MEDICAL PROBLEMS AMONG HOSPITAL READMISSIONS - A PILOT STUDY Brent C. Williams; Laura Haley; Donna Fox. University of Michigan, Ann Arbor, MI. (Control ID \#1319638)

BACKGROUND: Most studies examining the characteristics of patients readmitted to hospitals in the United States have focused on patients $>65$ years of age. Relatively little is known about the prevalence and characteristics of patients $<65$ among hospital readmissions. We sought to compare the prevalence and reasons for readmission among patients $>65$ ("older") and $<65$ ("younger") years old readmitted to medical services at an academic medical center.

METHODS: At the University of Michigan discharge summaries for all Medicare patients not already under care management (e.g., dialysis and transplant patients); and all patients with capitated public insurance (Medicaid managed care, county health insurance, and uninsured) discharged from UM medical services are reviewed by 5 care managers (social workers or nurses) through the Complex Care Management Program (CCMP), who call all patients post-discharge, and provide care management to high-risk patients. Patients: Consecutive case series of all 108 patients discharged from the hospital and reviewed by the CCMP over a 3 -month period from $6 / 15 / 2010$ to $9 / 14 / 2010$ who had been admitted $<30$ days following a hospital discharge. In addition to demographic and clinical information from the medical record, responses by care managers (social workers or nurses) to standardized questions completed after the initial post-discharge call to the patient regarding the reason for readmission were recorded. Simple descriptive statistics were applied using either patients or readmissions as the unit of analysis. P values were calculated sparingly due to small sample size.

RESULTS: The 108 readmissions were experienced by 69 patients, the majority of whom $(65 \%)$ were $<65$ years old. 16 patients accounted for 55 $(51 \%)$ of readmissions. Younger patients disproportionately accounted for multiple readmissions; with all 5 patients with $5-6$ readmissions $<65(\mathrm{p}=0.15)$. Equal proportions of patients older and younger than 65 experienced at least one readmission for the same diagnosis as the immediately previous discharge (67\% in each group), whereas younger patients were more likely to have had at least one readmission due to missed follow up appointments (27\% vs. $12 \%$; p $=.2)$ or to have a new diagnosis as reason for at least one admission $(40 \%$ vs. $33 \% \mathrm{p}=\mathrm{NS})$. Using readmissions as the unit of analysis, $77(71 \%)$ were among younger patients. Among readmissions a similar but stronger pattern of reasons for readmission was observed as for individual patients, with $73 \%$ of readmissions among younger vs. $64 \%$ among older patients due to the same diagnosis as the previous admission, and $34 \%$ vs. $3 \%$ due to missed follow-up appointments $(\mathrm{p}<0.001)$.

CONCLUSIONS: In a population of discharged medical patients not already in disease-based care management, younger patients were highly prevalent, and more likely to be readmitted for behavioral reasons as compared to older patients. Future studies and current debates over the size and scope of programs to decrease readmissions should examine whether 
age split at 65 (a marker for insurance and disability status) provides a readily available marker for differences in patient characteristics, clinical needs, and most appropriate interventions. One example is the current debate in the State of Michigan over proposed program and finance reform for 'dual eligibles' (patients on Medicare and Medicaid), which frequently make no distinction among patients above and below 65 years of age.

\section{HIGH PREVALENCE OF ENGLISH-ONLY LABELED MEDICINE BOTTLES FOR SPANISH-ONLY SPEAKING ELDERS: IS IT TIME TO CHANGE LABELS? Huai Cheng. University of Virginia, Charlottes- ville, VA. (Control ID \#1314569)}

BACKGROUND: It is very important for patients to understand what medications they are taking and how to take them. Without this understanding, medication errors, adverse drug events, and nonadherence are more likely to occur. It is known that medication bottle labeling is often challenging to understand, not only due to low literacy but also due to the lack of standardized medication labeling. Minority older patients, especially those who have multiple co-existing conditions, are on multiple medicaitons, and are non-English speaking, are vulnerable to medication errors, adverse drug events, and nonadherence. While it is obvious that that giving Spanish-labeled medications to Spanish-speaking patients makes sense, how often this occurs has not been studied. In this study, we determined the language of medicine bottle labels among a group of Spanish-only speaking elderly patients.

METHODS: The study subjects were Spanish-only speaking older patients (65 years old and above) who were seen consecutively at a hospital-based ambulatory clinic in New York City between 06/2008 and $06 / 2009$. This clinic was surrounded by a large low income Spanishspeaking population; was staffed by a Spanish-speaking clerk, a nursing assistant, and a nurse practioner; and was designed as a medication review and reconciliation clinic for minority elderly patients. These patients were usually refered by hospitalists and other subspecialty physicians. Pharmacies were located in Broadway and nearby. At each visit, patients were requested to bring all their medication bottles (prescribed and over the counter). Each medication bottle label was examined by the author with help from Spanish-speaking staff or translator and recorded as Englishlabel, Spanish-label, or both. The age, gender, comorbility, and the number of medications were also recorded. Decriptive statistics was used for data analysis. IRB approved this study.

RESULTS: 78 older patients were identified as Spanish-only speaking. The majority of patients $(73 \%, 57 / 78)$ were women. The mean age was 77 years (range 65-96), the mean number of co-existing conditions per patient was 4 (range 1-10), and the mean of number of medications per patient was 6 (range 2-20). A total of 506 medication bottle labels from 78 patients were examined and recorded. $44 \%(222 / 506)$ of medication bottles from 39 patients were labeled in English, 45\% medication bottles (230/ 506) from 35 patients in Spanish, and 11\% medication bottles $(54 / 506)$ from 4 patients in both.

CONCLUSIONS: There was a high prevalence of English-only labeled medication bottles among older Spanish-only speaking patients at an ambulatory clinic in New York City. The association of English-only medication labels with medication errors, adverse drug events, and nonadherence in this vulerable population needs to be further examined. A larger, systematic survey is warranted to further define the extent of the problem and to identify strategies to improve medication labeling.

HIGH PREVALENCE OF HEALTH CARE FORGONE FOR ECONOMIC REASONS IN A COUNTRY WITH UNIVERSAL HEALTH-INSURANCE COVERAGE: A POPULATION-BASED STUDY Idris Guessous; Jean-Michel T. Gaspoz; Jean-Marc Theler; Hans Wolff. Geneva University Hospitals, Geneva 14, Switzerland. (Control ID \#1336496)

BACKGROUND: Forgoing health care for economic reasons may worsen chronic diseases and increase the risk of complications and hospitalization.
Little is known about the importance and trends of health care forgone for economic reasons in Switzerland. We estimated the extent and the 4-year evolution of health care forgone for economic reasons in Switzerland and identified associated factors.

METHODS: A population-based cross-sectional survey (2007-2010) of a representative sample aged 35-74 years in the Canton of Geneva, Switzerland. Health care forgone, income level categories $(1 \mathrm{CHF} \approx 1$ \$, Jan 2012), education, job position, civil status, children dependence, insurance status (franchise, premium subvention) and cardiovascular comorbidities and risk factors were collected using standardized questionnaires and measures. Prevalences with $95 \% \mathrm{CI}$ intervals were calculated and Cochran-Armitage test used to test trends. Logistic regression models were used to test associations between forgoing health care for economic reasons and study variables.

RESULTS: 2601 subjects $(50.3 \%$ women, mean age 51.7 years) were included in the analyses. $13.8 \%(358 / 2243)(95 \% \mathrm{CI} 12.4-15.1)$ forgone health care for economic reasons. This prevalence varied from $3.7 \%$ $(\geq 13,000 \mathrm{CHF}$ monthly income category) to $30.9 \%$ ( $<3,000 \mathrm{CHF}$ monthly income category). In subjects with monthly income $<3,000 \mathrm{CHF}$, forgoing health care increased from $22.5 \%$ in $2007 / 8$ to $32.7 \%$ in 2009 and $34.7 \%$ in 2010. It remained stable in other income categories. In multivariate analyses, forgoing health care for economic reasons was positively associated with decreasing income category $(<3,000 \mathrm{CHF}$ vs $\geq 13,000 \mathrm{CHF}$ $\mathrm{OR}=7.59$, $\mathrm{P}$ trend $<0.0001)$, female gender $(\mathrm{OR}=1.32)$, smoking $(\mathrm{OR}=$ 1.58), having dependent children at home $(\mathrm{OR}=1.50)$, being divorced not in couple $(\mathrm{OR}=1.53)$, increasing franchise insurance category $(\mathrm{OR}=1.27)$, and receiving premium subvention $(\mathrm{OR}=1.41)$. Having a complementary health insurance decreased the risk of forgoing health care for economic reasons $(\mathrm{OR}=0.45)$. Among those who renounced $(\mathrm{N}=358), 75.1 \%$ renounced dental care, $39.9 \%$ physician consultation $(25.4 \%$ specialist, $14.5 \%$ general practitioner), $25.7 \%$ health devices, $12.1 \%$ medication, and $5.8 \%$ surgery. These prevalences remained stable across survey years.

CONCLUSIONS: In a Swiss region with universal health-insurance coverage, the prevalence of forgoing health care for economic reasons was high and highly dependent of socioeconomic status. In 2010, more than $30 \%$ of the lowest income group forgone health care for economical reasons in the previous year. This important and increasing lack of socioeconomic equity for health care in Switzerland needs to be addressed.

HOSPITAL CHARACTERISTICS ASSOCIATED WITH HIGH RATES OF IMAGING UTILIZATION Jason S. Mathias; Joe Feinglass; David W. Baker. Northwestern University Feinberg School of Medicine, Chicago, IL. (Control ID \#1339770)

BACKGROUND: The Hospital Outpatient Quality Reporting Program (HOQR) publicly reports U.S. hospitals' performance on imaging use measures for four tests that may be problematic if overused: magnetic resonance imaging for low back pain without antecedent therapy (MRI), follow-up imaging after screening mammography (MAM), and abdominal and thoracic computed tomography performed with and without contrast (A-CT and T-CT). In this study, we sought to characterize performance on these measures, determine whether performance was consistent across measures, and identify hospital characteristics associated with high outlier performance. METHODS: Variations in performance were examined with descriptive statistics. Correlation across measures was assessed using Spearman rankorder tests. We linked $2008 \mathrm{HOQR}$ data to the 2009 American Hospital Association Survey and examined associations between hospital characteristics and high outlier status using multivariable logistic regression. The dependent variable was high-outlier performance on an HOQR imaging use measure, defined as a performance score in the top decile among reporting hospitals. Independent variables included HOQR-reported volume and AHAreported for-profit ownership, rural setting, and non-teaching status. All hospital characteristics were entered as dichotomous variables except for imaging volume (i.e., the $\mathrm{N}$ in the measure denominator), for which we created quartiles and used the middle $50 \%$ as the reference group. In addition, models included the proportion of all discharged patients with Medicare or Medicaid insurance as a covariate, entered as a continuous variable.

RESULTS: Hospital performance varied widely. Performance across measures was weakly correlated $($ rho $<0.10)$ except A-CT and T-CT 
(rho $=0.42, \mathrm{p}<0.0001)$. Imaging volume was inversely associated with high-outlier performance. Compared to hospitals with measure-specific imaging volume in the 25 th-75th percentile, adjusted ORs [95\% CI] for high-outlier performance among $<25$ th percentile volume hospitals were MRI 4.22 [3.04-5.84], MAM 1.72 [1.31-2.26], A-CT 1.38 [1.05$1.80]$, T-CT 1.66 [1.28-2.16]. Rural location was associated with increased likelihood of high-outlier performance on all measures except MAM (adjusted OR [95\% CI] MRI: 1.42 [1.21-1.68], MAM: .80 [.69-.92], A-CT: 1.46 [1.28-1.66], T-CT: 1.32 [1.16-1.51]). Forprofit ownership was significantly associated with increased likelihood of high-outlier status on both MAM (aOR 1.47 [1.10-1.98]) and T-CT (aOR 1.71 [1.28-2.27]). Non-teaching status was significantly associated with high-outlier status only on T-CT (aOR 1.69 [1.11-2.58]).

CONCLUSIONS: Wide variation in performance suggests low-value imaging use is common. Inconsistent performance across measures will make it difficult for patients to identify and avoid hospitals with high use of all types of imaging, because few such hospitals exist. Although publicly reporting data may drive some hospitals to change imaging practices to avoid the stigma associated with public identification as a high outlier, the types of hospitals that are more likely to be high outliers may be unable or unwilling to support efforts to reduce imaging use that, if successful, could decrease hospital revenue. The effect of publicly-reporting hospital performance on these measures may be limited. It may be necessary to institute stronger policies to influence hospitals that continue to have very high use of these imaging tests.

HOSPITAL REPORT CARDS FOR HOSPITAL-ACQUIRED PRESSURE ULCERS: HOW DOES HOSPITAL PERFORMANCE BY CLAIMS DATA COMPARE TO PERFORMANCE BY STANDARDIZED SURVEILLANCE EXAMS FOR HOSPITAL-ACQUIRED PRESSURE ULCER RATES? Jennifer Meddings; Heidi Reichert; Laurence F. McMahon. University of Michigan, Ann Arbor, MI. (Control ID \#1318710)

BACKGROUND: Since March 2011, hospital rates of hospital-acquired complications (e.g., pressure ulcers) from administrative discharge data (i.e., claims data) are publicly reported online. In 2015, these claims-data generated complication rates will be used by Medicare to identify the top quartile of poor performing hospitals, to penalize by $1 \%$ less pay for all admissions. Concern exists regarding the accuracy and validity of complication rates as reported in claims data to compare hospitals. We evaluated whether hospitals in the top quartile of poor performance for hospital-acquired pressure ulcer (HAPU) rates according to claims data would be scored similarly when compared by HAPU rates from quarterly hospital-wide patient surveillance exams.

METHODS: Using claims data from the Healthcare Cost and Utilization Project (HCUP) State Inpatient Dataset for all adults discharged from 185 acute care California hospitals in 2007, we generated hospital rates as a percentage of discharges with at least one HAPU. We assessed how hospital ranking according to HAPU rates from claims data compared to these hospitals' ranking according to HAPU rates from quarterly prevalence surveillance exams, as conducted by trained examiners in 2007 and reported by hospitals to the California Nursing Outcomes (CalNOC) Pressure Ulcer Prevalence Study and CalHospitalCompare.org. RESULTS: According to HCUP data, $6378(0.4 \%)$ of admissions had a HAPU diagnosis; 1258 (19.7\%) of these admissions also had a pressure ulcer diagnosis listed as present-on-admission. $886(0.06 \%)$ had a pressure ulcer with unspecified status at admission. Hospital HAPU rates ranged from 0 to $1.7 \%$ (mean $0.3 \%$ ) of discharges according to HCUP data, and from 0 to $9.4 \%$ (mean 3.3\%) according to the CalNOC dataset. Of the 46 hospitals identified in the top quartile (Graph A) of poor performing hospitals according to claims data, only $20(43 \%)$ also were identified in the top quartile of poor performing hospitals according to CalNOC prevalence data (Graph B).

CONCLUSIONS: Overall, hospital rates of hospital-acquired pressure ulcers were much higher according to quarterly prevalence exams compared with claims data. Relative hospital performance regarding HAPU rates varied significantly from claims data compared with prevalence surveillance exams, with less than half of hospitals identified in the top quartile as poor performing hospitals by claims data also being in the top quartile according to prevalence exam data. These findings imply that claims data may not be an accurate or valid datasource for comparing hospitals for public reporting or financial penalties for hospital-acquired pressure ulcers.

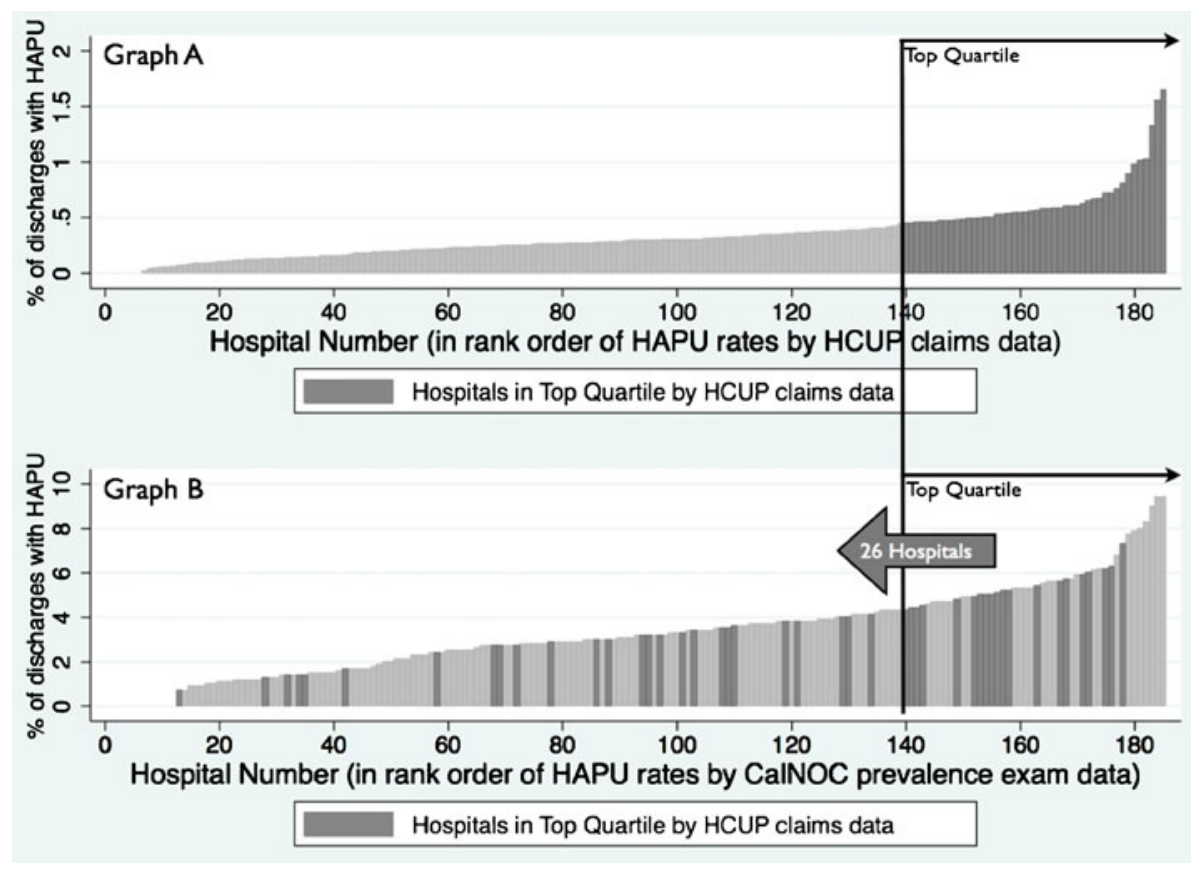

Hospital Ranks by HAPU rates according to HCUP data (Graph A) and CalNOC data (Graph B) 
HOSPITAL VARIATION IN USE OF POST-OPERATIVE MEDICINE CONSUlTATIONS Lena M. Chen ${ }^{1,2}$; Mousumi Banerjee ${ }^{2}$; John D. Birkmeyer ${ }^{2}$. $V A$ Ann Arbor Healthcare System, Ann Arbor, MI;

${ }^{2}$ University of Michigan, Ann Arbor, MI. (Control ID \#1311052)

BACKGROUND: Medicare payments around episodes of inpatient surgery vary as much as $40-70 \%$ across hospitals. Although such variation is attributable to multiple factors, differential use of physician services is one key component. In this context, we explored the use of medicine consultations after inpatient surgery, factors associated with increased utilization, and variations in practice patterns across hospitals.

METHODS: We used national Medicare data to identify elderly patients undergoing colectomy or total hip replacement (THR) between January 2005 and November 2007. Hierarchical logistic regression was used to examine factors associated with medicine consult use. A random effect was incorporated to account for variation due to unmeasured hospital factors. To better understand reasons for variations in medical consultation rates among hospitals, we compared use in low risk patients $(<75$ yo with $\leq 1$ co- morbid condition) versus high risk patients ( $\geq 80$ yo with $\geq 2$ co-morbid conditions)

RESULTS: Our cohort included 105,810 $(244,693)$ patients undergoing colectomy (THR) at 3,957 $(3,447)$ hospitals. In this sample, $49.2 \%(47.8 \%)$ of colectomy (THR) patients received $\geq 1$ general medicine consult. Other common consults were cardiology $(21.7 \%)$ and oncology $(22.0 \%)$ after colectomy, and physical medicine and rehabilitation (8.6\%) after THR. Factors associated with a greater likelihood of receiving a medicine consult included older age, more co-morbidities, and being treated at a nonteaching hospital (all p-values $<0.05$ ). The use of medical consultations varied widely across hospitals (Figure). Although high risk patients received substantially more consultations, low risk patients were primarily responsible for wide variation in consultation rates across hospitals.

CONCLUSIONS: Post-operative medical consultations are common, but their use varies widely across hospitals. The greatest variation was seen among low risk patients. It is unclear whether this represents an opportunity for hospitals to improve efficiency, or whether these practice patterns benefit patients.

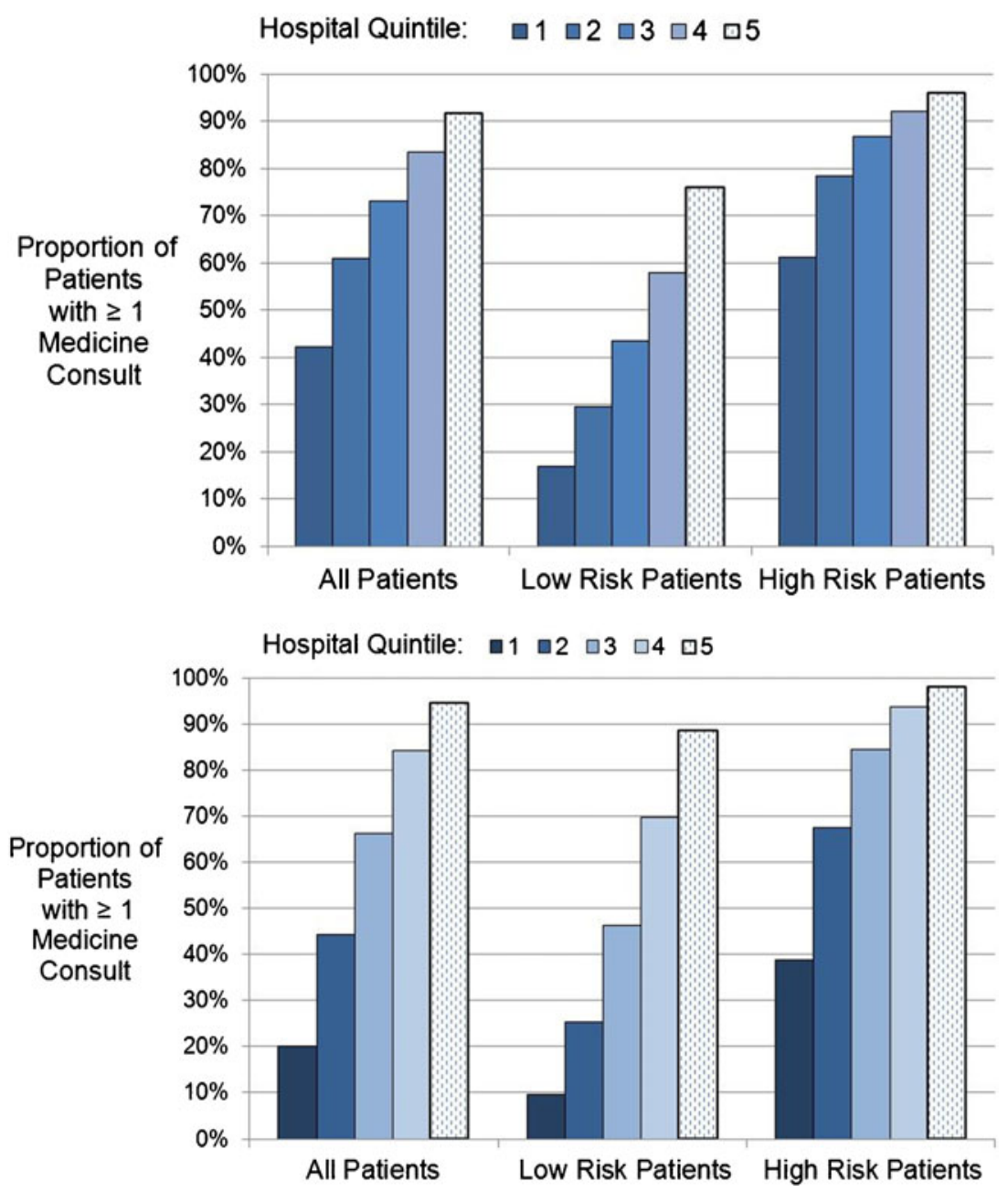

Variation in Hospital Use of Medicine Consults after Colectomy (top panel) and Total Hip Replacement (bottom panel)

HOSPITALIST STAFFING AND PATIENT SATISFACTION IN THE NATIONAL MEDICARE POPULATION Lena M. Chen ${ }^{1,2}$; John D. Birkmeyer ${ }^{2}$; Sanjay Saint ${ }^{1,2}$; Ashish K. Jha ${ }^{3,4}$. ${ }^{1}$ VA Ann Arbor Healthcare System, Ann Arbor, MI; ${ }^{2}$ University of Michigan, Ann Arbor, MI; ${ }^{3}$ VA Boston Healthcare System, Boston, MA; ${ }^{4}$ Harvard School of Public Health, Boston, MA. (Control ID \#1314918)
BACKGROUND: Starting in October 2012, Medicare will base part of its hospital payments on how hospitals perform on patient experience metrics. Given the inpatient expertise of hospitalists and their $24 / 7$ presence, increasing hospitalist staffing may be one approach to enhancing patient satisfaction. METHODS: Using 2009 national Medicare data we examined the association between hospitalist staffing and patient satisfaction scores. To determine 
hospitalist staffing, we estimated the proportion of medicine inpatients cared for by hospitalists at each included hospital. We used a well-validated approach that classifies general internists with at least $90 \%$ of their evaluation and management (E\&M) billings from inpatient care to be a hospitalist. Patient experience metrics were derived from the Hospital Consumer Assessment of Providers and Systems (HCAHPS) survey (Table). In multivariable regression, we adjusted for potential confounders, including percent Medicaid patients, hospital size and teaching status, location, presence of a medical intensive care unit, and nurse staffing.

RESULTS: Our cohort included 132,814 patients' index medicine admissions at 2,843 hospitals in 2009 . Overall, $44 \%$ of general medicine admissions were cared for by hospitalists. At $17 \%$ of hospitals no medicine admissions were cared for by hospitalists; at $4 \%$ of hospitals all medicine admissions were cared for by hospitalists. In multivariable analyses, compared to hospitals with low hospitalist staffing, hospitals with high hospitalist staffing had modestly higher patient satisfaction scores across most dimensions of care (Table). For example, among hospitals in the highest tertile of hospitalist staffing, $80.4 \%$ of patients reported being satisfied with discharge compared to $77.6 \%$ in the lowest tertile (p-value $<0.001$ ).

CONCLUSIONS: Hospitals with higher levels of hospitalist staffing have modestly higher performance on patient experience scores across most dimensions of care, including satisfaction with discharge planning. Understanding why hospitals with more hospitalists have higher patient satisfaction is important for clinical leaders hoping to improve performance on these metrics.

Hospital-level Association Between Hospitalist Staffing and Patient Satisfaction, Adjusted

\begin{tabular}{|c|c|c|c|c|}
\hline \multirow[b]{2}{*}{$\begin{array}{l}\text { Dimension } \\
\text { of Care }\end{array}$} & \multicolumn{4}{|c|}{ Patient Satisfaction Score } \\
\hline & $\begin{array}{l}\text { Hospitals in } \\
\text { Highest Tertile } \\
\text { of Hospitalist } \\
\text { Staffing }\end{array}$ & $\begin{array}{l}\text { Hospitals in } \\
\text { Lowest Tertile } \\
\text { of Hospitalist } \\
\text { Staffing }\end{array}$ & $\begin{array}{l}\text { Difference } \\
\text { (Highest minus } \\
\text { Lowest Tertile) }\end{array}$ & P-Value \\
\hline Would Recommend & 68.6 & 64.8 & 3.8 & $<0.001$ \\
\hline Overall Satisfaction & 65.6 & 62.7 & 2.9 & $<0.001$ \\
\hline Discharge & 80.4 & 77.6 & 2.8 & $<0.001$ \\
\hline Nursing Services & 62.8 & 61.4 & 1.4 & $<0.001$ \\
\hline $\begin{array}{l}\text { Communication } \\
\text { with Nurses }\end{array}$ & 75.4 & 74.1 & 1.3 & $<0.001$ \\
\hline Pain Control & 68.8 & 67.7 & 1.2 & $<0.001$ \\
\hline Quiet & 59.4 & 58.3 & 1.1 & 0.0012 \\
\hline $\begin{array}{l}\text { Communication } \\
\text { about Medications }\end{array}$ & 58.3 & 57.4 & 0.9 & $<0.001$ \\
\hline Clean & 68.5 & 68.0 & 0.6 & 0.004 \\
\hline $\begin{array}{l}\text { Communication } \\
\text { with Doctors }\end{array}$ & 80.4 & 80.5 & 0.0 & 0.91 \\
\hline
\end{tabular}

NOTE: Numbers are rounded, so the numbers in the difference column do not always equal rounded patient satisfaction scores for hospitals in the highest tertile of hospitalist staffing minus rounded patient satisfaction scores for hospitals in the lowest tertile of hospitalist staffing.

HOSPITALS WITH LOWER RATES OF PERIOPERATIVE BLOOD CLOTS BY CLAIMS DATA DO NOT REPORT BETTER ADHERENCE TO PROPHYLAXIS MEASURES BY MEDICAL RECORD REVIEW. Jennifer Meddings ${ }^{1}$; Heidi Reichert ${ }^{1}$; Mary A.M. Rogers ${ }^{1}$; Jack Iwashyna ${ }^{1,2}$; Laurence F. McMahon ${ }^{1} .{ }^{1}$ University of Michigan, Ann Arbor, MI; ${ }^{2}$ Ann Arbor VA HSR\&D Center for Excellence, Ann Arbor, MI. (Control ID \#1334934)

BACKGROUND: Discharge data (i.e., claims data) have been used since 2008 to identify hospital-acquired complications (HACs) as non-payable diagnoses, and more recently to compare hospitals by HAC rates as measures of patient safety. Previously, hospitals were compared only by adherence to certain HAC prevention measures using medical record reviews, as self-reported on-line by HospitalCompare. Using the example of hospital-acquired blood clots (venous thromboembolism, HA-VTE), we evaluated whether hospitals that report higher adherence to perioperative VTE prophylaxis were more likely to have lower rates of HA-VTE during an admission for knee or hip replacement as recorded in claims data.

METHODS: Using claims data from the Healthcare Cost and Utilization Project State Inpatient Dataset for adults discharged from 177 acute care California hospitals in 2009 that performed knee and hip replacements, we generated hospital-specific rates of HA-VTE among patients undergoing knee or hip replacements. We linked each hospital's VTE rates from claims data with the hospital's adherence to perioperative VTE prophylaxis for Medicare patients as reported on HospitalCompare, using a composite measure averaging the SCIP-VTE1 and SCIP-VTE2 measures (correlation $r=0.97, p$ $<0.001$ ) which assess prophylaxis ordered from admit until $24 \mathrm{~h}$ post-op. Using zero-inflated negative binomial regression, we tested the hypothesis that a hospital's rate of HA-VTE during admissions for hip and knee replacement would be significantly and inversely associated with the hospital's adherence to VTE prophylaxis.

RESULTS: Hospital rates of HA-VTEs during admissions for knee and hip replacements ranged from $0 \%$ for $110(62 \%)$ hospitals to $13 \%$, with a mean of $1.2 \%$. Hospitals with zero HA-VTE events in claims data (noted by black dots in Figure) reported VTE prophylaxis from $37-100 \%$ (mean $84 \%$ ). For hospitals that reported at least one of these VTE events (gray dots), prophylaxis ranged from 59-100\% (mean 90\%). Overall, a $1 \%$ increase in composite SCIP-VTE adherence score was associated with an increase in VTE rate by a factor of $1.02, \mathrm{p}=0.04$.

CONCLUSIONS: Overall, hospital rates of hospital-acquired VTE events during admissions for hip and knee replacements are low by claims data, with more than half of hospitals reporting zero cases. Unexpectedly, hospitals with lower VTE event rates did not report higher adherence to perioperative VTE prophylaxis. These findings suggest important limitations in comparing hospitals by HA-VTEs from claims data, which may be an incomplete record of HA-VTE events and does not adjust for individual patient risks for VTE

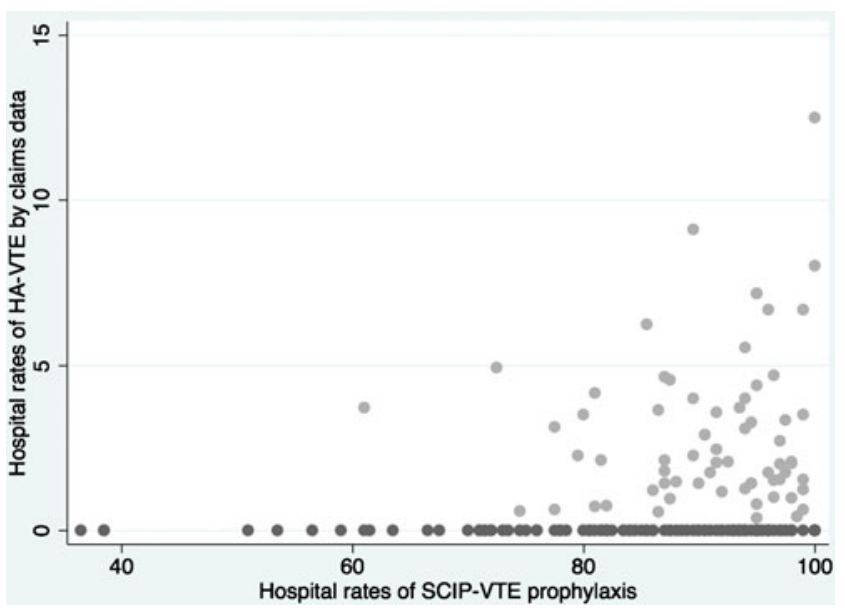

HOW MUCH DOES PATIENT PANEL COMPOSITION INFLUENCE PHYSICIAN PERFORMANCE RANKING ON PATIENT EXPERIENCE OF CARE QUALITY MEASURES? Charlotte E. Ward; Clemens S. Hong; Jeffrey M. Ashburner; Yuchiao Chang; Steven J. Atlas. Massachusetts General Hospital, Boston, MA. (Control ID \#1338082)

BACKGROUND: Quality assessment for primary care physicians (PCPs) and practices increasingly includes assessment of patient experience of care through surveys, such as the Consumer Assessment of Healthcare Providers and Systems (CAHPS) survey. Previous research suggests that adjustment for differences in patient panel composition impacts CAHPS outcomes. We compared patient experience of care rankings among PCPs within a large academic primary care network with and without adjustment for differences in patient panel composition.

METHODS: We analyzed 15,612 adult patients who completed 18,874 CAHPS surveys following visits to any of 13 Massachusetts General 
Hospital practices between August, 2008 and June, 2011. We calculated CAHPS composite measures for access, communication and office staff by taking the average 'Top Box' score (the percentage of patients that answered 'Always' to each question within the composite). We ranked 167 PCPs based on unadjusted CAHPS composite measures and re-ranked them after adjusting for patient gender, age, language, education, race, insurance, Charlson score, and no-show appointments using linear regression models. We also categorized PCPs into tertiles based on their rankings for each composite and compared patient panel characteristics between PCPs in the top and bottom tertiles.

RESULTS: PCP panels varied by gender $(0-80.7 \%$ male), age (41.381.3 years), language spoken $(18.2-100 \%$ English), education $(7.8-86.4 \%$ college or above), race (0- $93.9 \%$ minority), insurance ( $0-37.9 \%$ Medicaid/ uninsured), Charlson score (9.2-71\% Charlson score $\geq 2)$, and no-shows over the prior year $(0-38.7 \% \geq 1$ no-show). Patient characteristics varied significantly between top and bottom tertile PCPs. Top tertile PCPs for the 'Access' composite cared for more males than bottom tertile PCPs ( $46.0 \%$ vs. $30.5 \%$, P $<0.001)$. PCPs in the top tertile for the 'Communication' composite cared for fewer minority $(10.2 \%$ vs. $19.5 \%, \mathrm{P}<0.001)$ and non-English-speaking patients $(1.7 \%$ vs. $7.5 \%, \mathrm{P}=0.01)$, and more patients with a Charlson score $\geq$ $2(41.2 \%$ vs. $36.1 \%, \mathrm{P}=0.05)$. PCPs in the top tertile for the 'Office Staff' composite cared for more males $(45.1 \%$ vs. $28.6 \%, \mathrm{P}<0.001)$, and patients with a Charlson score $\geq 2(45.9 \%$ vs. $33.3 \%, \mathrm{P}<0.001)$, and had patients that were older $(64.2$ years vs. 58.0 years, $\mathrm{P}<0.001)$. Adjusting for patient panel composition among individual PCPs resulted in a relative mean change in PCP ranking of 2.7 percentiles for 'Communication', 7.6 percentiles for 'Access', and 12.6 percentiles for 'Office Staff'. Within 'Communication', $6 \%$ of PCPs were reclassified into different tertiles, while $10.8 \%$ of PCPs were reclassified within 'Access', and $18 \%$ of PCPs were reclassified within 'Office Staff'.

CONCLUSIONS: Among PCPs practicing within the same primary care network, PCP panels with greater proportions of minority, female, nonEnglish speaking, and younger patients with fewer comorbidities were associated with lower relative patient experience of care rankings. Adjusting for differences in patient panel composition had a differential impact on PCP rankings with greater impact seen with the access and office staff composites compared to the communication composite. As the use of patient experience measures increases, including for physician reporting and pay-for-performance, we must be aware of the potential effect of patient panel composition on physician ranking for different patient experience composites.

HOW NOT TO GET LOST IN TRANSLATION: ASSESSING BARRIERS TO INTERPRETER UTILIZATION Alissa Detz ${ }^{1}$; Hemal K. Kanzaria ${ }^{2}$; Sara L. Swenson ${ }^{1}$. ${ }^{1}$ California Pacific Medical Center, San Francisco, CA; ${ }^{2}$ University of California, San Francisco; San Francisco General Hospital, San Francisco, CA. (Control ID \#1340127)

BACKGROUND: Using professional interpreters for patients with limited English proficiency (LEP) improves health outcomes. Despite the importance of language services and a national mandate to provide professional interpreters for LEP patients, this resource is often unavailable or underutilized. Understanding barriers at both the individual and system level is key to improving access to professional interpreters. We sought to identify utilization patterns and barriers to the use of professional interpreters at a community-based teaching hospital.

METHODS: California Pacific Medical Center (CPMC) is a communitybased teaching hospital in San Francisco. Approximately 10\% of CPMC patients are LEP. In-person professional interpreters provide services to four hospital campuses in the most commonly spoken languages during business hours with limited weekend availability. Professional interpreters are available by phone 24 hours a day. We solicited a convenience sample of 74 internal medicine residents and attending physicians to assess perceptions of interpreter services and obstacles to interpreter use in the hospital setting. We conducted a 34-item anonymous online questionnaire. Respondents quantified their frequency and practice patterns of interpreter use, time parameters of interpreter-aided encounters, use of ad hoc interpreters, and desire for education regarding interpreters and LEP patients. We limited our analysis to descriptive statistics.
RESULTS: Our response rate was $68 \%$, with $71 \%$ of residents and $48 \%$ of attending physicians completing surveys. Only $55 \%$ of physicians reported using any interpreter (professional or ad hoc) for the majority of encounters with LEP patients. Use of interpreters varied with the type of clinical encounter. Physicians were more likely to use professional interpreters to obtain consent for procedures ( $66 \%$ vs. $33 \%$ ) and for code status discussions ( $72 \%$ vs. $26 \%$ ). Ad hoc interpreters were more frequently used for daily rounds ( $43 \%$ vs. $19 \%$ ). When asked about their most recent encounter using an interpreter, physicians reported a median delay of 10 minutes (range 0-30 minutes), and 32\% felt their patient's care was postponed. Seventy-four percent of physicians reported that it was "somewhat" or "very challenging" to arrange a professional interpreter, and $75 \%$ cited at least one instance of not using a professional interpreter, but wishing they had. Constraints of time and availability posed significant barriers, and physicians often cited the availability of ad hoc interpreters as a reason for not obtaining professional language services. Only $11 \%$ cited "lack of comfort working with interpreters" as an important barrier, despite reporting little formal training on working with interpreters.

CONCLUSIONS: We found low utilization of interpreters in the hospital setting, even for "high stakes" encounters such as admissions, discharges, and code status discussions. Time constraints and availability were the most important barriers to using professional interpreters. Availability of ad hoc interpreters may also detract from widespread use of professional language services. We aim to use these results to design targeted systemsbased changes. Onsite interpreters dedicated to one hospital campus, computer alerts to signal language preference to staff, and improved technology for utilization of remote interpreters could make using professional interpreters more efficient and feasible in the inpatient setting.

HYDROCORTISONE IN ACYCLOVIR 5\%/ HYDROCORTISONE 1\% CREAM DOES NOT ADVERSELY AFFECT HEALING TIME IN IMMUNOCOMPROMISED PATIENTS WITH RECURRENT HERPES SIMPLEX LABIALIS Jason T. Olin ${ }^{2}$; Stephen K. Tyring ${ }^{1}$; Tina $\mathrm{Lin}^{2}$. ${ }^{1}$ University of Texas Medical School at Houston, Houston, TX; ${ }^{2}$ Valeant Pharmaceuticals North America LLC, Bridgewater, NJ. (Control ID \#1336336)

BACKGROUND: Acyclovir (AC) 5\%/ hydrocortisone 1\% cream (AHC) has been shown to be a well-tolerated and efficacious treatment option for patients with recurrent herpes simplex labialis (HSL); however, the effects of hydrocortisone in immunocompromised patients are unknown, and could be associated with inhibited immune function and increased healing time.

METHODS: In this randomized (2:1), double-blind study, immunocompromised adults (CD4+ T-cell count $100-500 / \mathrm{mm}^{3}$ ) with recurrent HSL were assigned to a self-initiated treatment with topical AHC or AC (in AHC vehicle) 5 times per day for 5 days after the first sign of a recurrence. Patients were tracked for up to 1 year. The primary outcome measure was episode duration; a non-inferiority analysis was used to assess whether healing time was at least twice as long in the AHC group compared with the AC group.

RESULTS: A total of 201 participants were randomized (136 AHC; 65 AC); 77 and 30 participants, respectively, experienced an HSL recurrence and initiated treatment. The mean episode duration was 6.7 days in both treatment groups; median duration was 6.4 days in the AHC group and 6.6 days in the $\mathrm{AC}$ group. Adverse events (AEs), experienced by $6(8 \%)$ participants in the AHC group and $5(17 \%)$ in the $\mathrm{AC}$ group, were consistent with an immunocompromised study population. No participants discontinued the trial due to AEs.

CONCLUSIONS: These data suggest that AHC cream is a safe and welltolerated treatment option in immunocompromised patients with recurrent HSL, with efficacy and tolerability comparable to that of AC cream.

IBCD: EFFECTIVENESS AND SUSTAINABILITY OF A CHECKLIST TO IMPROVE QUALITY OF CARE FOR HOSPITALIZED GENERAL MEDICAL PATIENTS Anthony V. Aspesi ${ }^{1}$; Greg Kauffmann ${ }^{1}$; Andrew M. Davis ${ }^{1}$; Elizabeth Schulwolf ${ }^{2}$; Valerie G. Press ${ }^{1}$; Vineet Arora ${ }^{1} .{ }^{1}$ University of Chicago, Chicago, IL; ${ }^{2}$ Loyola University, Chicago, IL. (Control ID \#1326430) 
BACKGROUND: Although checklists have shown significant promise as a tool to improve care in surgery and intensive care units, they have been underutilized in hospitalized medical patients. The objectives of this study were to ascertain whether there were sustained improvements in processes of care addressing four hospital conditions [(I) pneumococcal immunization, (B) pressure ulcers (bedsores), (C) catheter-associated urinary tract infections (UTIs), and (D) deep venous thrombosis (DVT)] for hospitalized general medical patients during the IBCD checklist intervention.

METHODS: The IBCD checklist was integrated into the established routine of post-call morning rounds for new admissions. Checklists prompted teams to offer Pneumovax $\subseteq$ vaccine for indicated patients (I), perform a skin exam for patients high risk for bed sores (B), remove Foley catheters from patients without an indication (C), and administer pharmacologic DVT prophylaxis when indicated (D). Charts were reviewed to ascertain if documentation on the checklist resulted in care intended.

RESULTS: Seventy percent $(46 / 66)$ of medical teams during July 2010 - March 2011 used the IBCD checklist with 1168 (52.5\%) patients. While participation varied by month, the variation was not statistically significant $(X 2=8.37, \mathrm{p}=0.40)$. Overall, the IBCD checklist prompted 301 actions, and overall adherence to these four domains

\section{IMMUNIZATIONS}

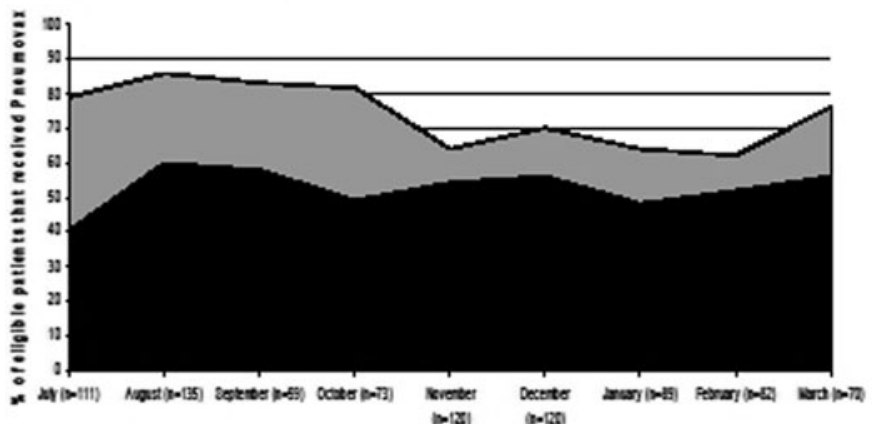

\section{CATHETERS}

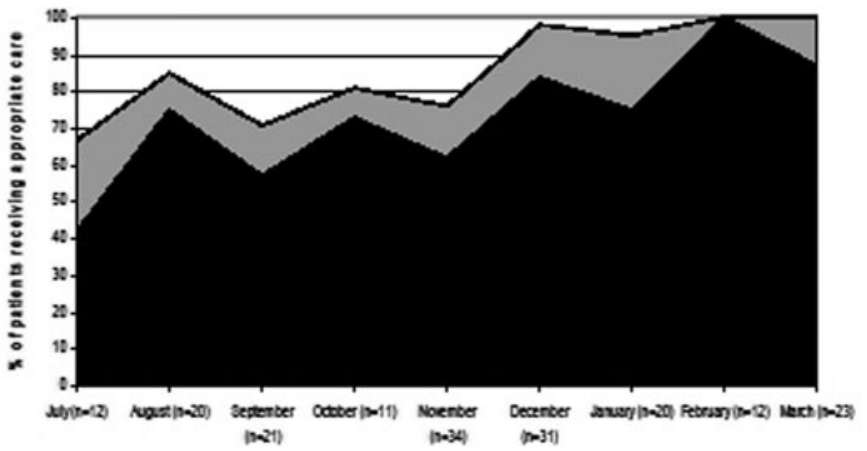

increased from $68 \%$ on admission to $82 \%$ after checklist use ( $\mathrm{p}<$ 0.001). During the IBCD checklist intervention, average adherence rates for immunizations increased from $52 \%$ on admission to $74 \%$ after checklist use $(\mathrm{p}<0.001)$. For pressure ulcers, average adherence increased from $44 \%$ to $62 \% \quad(\mathrm{p}<0.001)$. For catheters, average adherence increased from $73 \%$ to $86 \%(\mathrm{p}<0.001)$. While DVT prophylaxis was high on admission prior to checklist use $(93 \%)$, the checklist was associated with near universal prophylaxis $(96 \%, \mathrm{p}<$ 0.01 ). A statistically significant improvement on adherence to quality measures on admission, a learning effect, was observed for bedsores (0.034) and catheters (0.01) suggesting more residents completed the quality measure on admission before use of the checklist.

CONCLUSIONS: A checklist can be a useful tool to improve adherence to four key quality indicators in general medicine. Improvements were greatest for appropriate use of catheters. The observed learning effects indicate that the checklist intervention can be effective in helping teams incorporate quality measures into their routine of care. Future work will include chart review of a historical control group, examining the use of a new checklist adapted for our electronic health record, and studying the effect of the checklist on patient outcomes.

\section{BEDSORES}

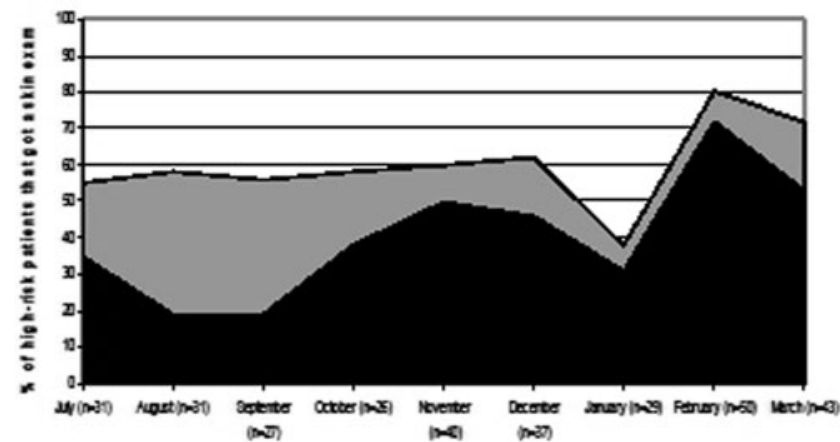

DVT

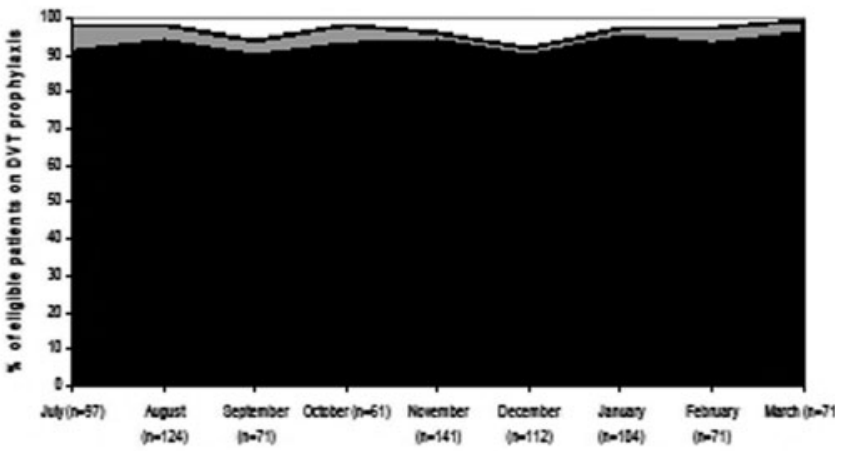

Figure 1: Adherence to four process measures over the intervention period of July 2010 March 2011. Average overall adherence raised from $68 \%$ to $82 \%$ (p 0.001$)$ for 1135 patients.

- Completed on admission - Completed after checklist
IDENTIFYING KEY BARRIERS TO MEDICATION ADHERENCE IN SURVIVORS OF STROKES AND TIAS Ian Kronish ${ }^{1}$; Michael Diefenbach $^{2}$; L. Alison Phillips ${ }^{3}$; Kezhen $\mathrm{Fei}^{2}$; Carol Horowitz $2 .{ }^{1}$ Columbia University Medical Center, New York, NY; ${ }^{2}$ Mount Sinai Medical Center, New York, NY; ${ }^{3}$ George Washington University, Washington, DC. (Control ID \#1323578)
BACKGROUND: Stroke is the 3 rd leading cause of death and most common cause of severe disability in US adults. Adherence to antihypertensive, antiplatelet, and statin medications is essential to reducing the risk of recurrent stroke. Despite the importance of medications, about 1 in 3 stroke survivors has suboptimal medication adherence. We aimed to identify key barriers to medication adherence 
in survivors of stroke or TIA. As stroke disproportionately affects patients from low income and minority groups, we sought to assess barriers in stroke/TIA survivors from these groups.

METHODS: We surveyed 510 participants who were recruited into a stroke prevention intervention targeted at stroke survivors in underserved communities in New York City. Participants were eligible if they were $\geq 40 \mathrm{yrs}$ and had a stroke or TIA in the past 5 years. Medication adherence was measured at baseline using the 8 -item Morisky questionnaire (score 0 to $5=$ non-adherent; 6 to $8=$ adherent). Potential barriers to adherence were assessed at baseline by interviewing participants using previously validated instruments (e.g., Beliefs about Medicines Questionnaire). Our selection of 9 potential barriers (see Table) was guided by Osterberg and Blaschke's model postulating that barriers emerge from suboptimal patient-doctor and patient-health system interactions. Hierarchical multivariate logistic regression was then used to calculate the adjusted ORs of barriers to adherence. Models were additionally controlled for age, race/ethnicity, income, and years since most recent stroke/TIA.

RESULTS: The mean age of participants was 63 yrs, $61 \%$ were women, $14 \%$ were White, $23 \%$ preferred Spanish, and 55\% had income $<\$ 15,000 / y r$. Thirty-nine percent had poor adherence to medications. Both the cluster of variables representing patient-doctor $(\Delta$ Nagelkerke $\mathrm{R}$ square $0.09, \mathrm{p}<.001)$ and patient-health system interactions $(\Delta$ Nagelkerke $\mathrm{R}$ square $0.03, \mathrm{p}=.04$ ) were significantly associated with poor adherence beyond sociodemographic factors. More specifically, in the fully adjusted model (Table), high concerns about medications [OR $2.7,95 \%$ CI 1.7 to 4.4 ) and perceived discrimination by the health care system [OR 1.9, 95\% CI 1.2 to 3.2] were associated with increased odds of poor medication adherence.

CONCLUSIONS: In this low-income, minority population where medication adherence is particularly important, poor adherence was common Key barriers to medication adherence were unfavorable beliefs about medications and perceived discrimination by the health care system. Thus, special efforts are needed to assess adherence in this population. Interventions to modify medication beliefs and overcome perceptions of discrimination may improve adherence and outcomes in stroke/TIA survivors.

\begin{tabular}{|c|c|c|c|c|}
\hline $\begin{array}{l}\text { Potential Barriers of } \\
\text { Medication Adherence } \\
\text { Patient-Doctor Barriers }\end{array}$ & $\begin{array}{l}\text { Model 1 OR } \\
(95 \% \mathrm{CI})\end{array}$ & $\begin{array}{l}\text { P- } \\
\text { Value }\end{array}$ & $\begin{array}{l}\text { Model } 2 \text { OR } \\
(95 \% \mathrm{CI})\end{array}$ & $\begin{array}{l}\text { P- } \\
\text { Value }\end{array}$ \\
\hline $\begin{array}{l}\text { High Concerns About } \\
\text { Medications }\end{array}$ & $2.8(1.8-4.4)$ & $<.001$ & $2.7(1.7-4.4)$ & $<.001$ \\
\hline $\begin{array}{l}\text { Low Perceived Necessity of } \\
\text { Medications }\end{array}$ & $1.6(0.7-3.7)$ & .26 & $1.7(0.7-3.8)$ & .26 \\
\hline $\begin{array}{l}\text { Low Knowledge of Risk } \\
\text { Factors for Stroke }\end{array}$ & $0.7(0.6-1.8)$ & .74 & $1.1(0.6-1.8)$ & .78 \\
\hline $\begin{array}{l}\text { Low Trust in Personal } \\
\text { Doctor }\end{array}$ & $1.2(0.6-2.5)$ & .57 & $0.9(0.4-1.9)$ & .78 \\
\hline $\begin{array}{l}\text { Problems Communicating } \\
\text { with Doctor Due to } \\
\text { Language }\end{array}$ & $1.6(0.9-3.1)$ & .13 & $1.4(0.7-2.7)$ & .31 \\
\hline \multicolumn{5}{|c|}{ Patient-Health System Barriers } \\
\hline $\begin{array}{l}\text { Perceived Discrimination } \\
\text { Due to Race, } \\
\text { Ethnicity, Education, or } \\
\text { Income }\end{array}$ & . & - & $1.9(1.2-3.2)$ & .01 \\
\hline $\begin{array}{l}\text { Problems Affording } \\
\text { Medications }\end{array}$ & - & - & $0.9(0.5-1.5)$ & .64 \\
\hline $\begin{array}{l}\text { Difficulty Accessing Health } \\
\text { Care }\end{array}$ & - & - & $1.4(0.7-2.9)$ & .32 \\
\hline $\begin{array}{l}\text { Poor Continuity of Health } \\
\text { Care }\end{array}$ & - & - & $1.3(0.7-2.4)$ & .41 \\
\hline
\end{tabular}

Nagelkerke R square Model $1=0.15$; Model $2=0.18$
IDENTIFYING OVERUSED PROCEDURES WITH CLAIMS DATA Jodi B. Segal ${ }^{1,2}$; John Bridges ${ }^{2}$; Hsien-yen Chang ${ }^{2}$; Eva Chang ${ }^{2}$; Najlla Nassery'; Jonathan Weiner ${ }^{2}$; Kitty Chan ${ }^{2} .{ }^{1}$ Johns Hopkins University School of Medicine, Baltimore, MD; ${ }^{2}$ Bloomberg School of Public Health of the Johns Hopkins University, Baltimore, MD. (Control ID \#1319820)

BACKGROUND: The spending on healthcare in the United States may surpass $20 \%$ of the Gross Domestic Product by the year 2020. This is unsustainable and was a driving force for the passage of the Patient Protection and Affordable Care Act of 2010. Provisions in this law encourage adoption of medical practices with proven effectiveness with the expectation that this will restrain spending. Many healthcare practices do not have widely-proven effectiveness and are rightly considered as overused practices. Overuse is defined as a service used in the absence of a clear medical basis for its use, when the risk of harm exceeds its likely benefit; or more expansively, when the added costs do not provide proportional benefits. As a step in our developing an index of overuse, we identified therapeutic and diagnostic procedures that have been called overused procedures, and quantified them, by region, using claims data. METHODS: We identified key organizations with interests in quality improvement and/or cost-containment. These included professional societies, federal agencies, and non-profit organizations. We reviewed relevant publications that described the medical procedures classified as overused by these organizations, which typically used group process methods to identify them. We took the initial unrestricted list and classified the procedures according to the feasibility of determining overuse of the procedure with only administrative claims data. For each procedure deemed feasible, we developed an algorithm to be used in claims data for determining usage of the procedure. We applied the algorithms to a 5\% Medicare data set from 2008, and tabulated counts for each Health Referral Area (HRA) as defined by the Dartmouth Group. Our population was 1,379,521 enrollees in Medicare 65 years of age and older who were not enrolled in a managed Medicaid plan in 2008, and who had continuous enrollment during that year. These enrollees are a representative sample of Medicare-insured individuals across the U.S.

RESULTS: We identified guidelines or practice recommendations from a consortium of North American cardiology societies, the American Academy of Orthopedic Surgeons, the Agency for Healthcare Quality and Research, the National Quality Forum, the National Health Service (United Kingdom), the National Guidelines Clearinghouse, and the Institute of Medicine Board on Health Care Services. We identified 337 procedures considered to be overused by these organizations. We graded 100 of these as possibly feasible for operationalizing with claims data and selected 32 representing different clinical areas for further development. The volume of use of these 32 procedures varied importantly across Health Referral Areas, even with casemix adjustment, and the magnitude of this variation differed across procedures. CONCLUSIONS: Overused procedures can be quantified with claims data. We found unexplained variation in usage of these procedures. Future research will include the development of tools for use with claims data for identifying regions or health systems with global overuse practices, and for evaluating the outcomes of interventions to reduce overuse. These tools will also facilitate exploration of determinants of overuse, including systematic processes that drive overuse that could respond to intervention.

IDENTIFYING RACIAL/ETHNIC DISPARITIES IN DIABETES MANAGEMENT IN FEDERALLY SUPPORTED HEALTH CENTER PATIENTS Alek Sripipatana; Lydie A. Lebrun; Quyen Ngo-Metzger. U.S. Department of Health and Human Services, Rockville, MD. (Control ID \#1324011)

BACKGROUND: Racial/ethnic minorities have higher risks for cardiovascular disease morbidity and mortality compared with whites. This excess burden of morbidity and mortality among minorities may be attributable to uncontrolled diabetes and subsequent complications. Health Centers care for over 19 million patients, $93 \%$ of whom are below $200 \%$ poverty, and $63 \%$ of 
whom are racial/ethnic minorities. Given their patient population, Health Centers have the opportunity to reduce diabetes-related health disparities among America's most vulnerable populations.

METHODS: Cross-sectional analyses of a 2009 nationally-representative sample of federally-supported Health Center (HC) patients. For patients 18 years and older with diabetes $(n=848)$, we estimated logistic regression models to predict the likelihood of: 1) Process measures including: a) eye exam in past year, b) foot exam in past year, and c) hemoglobin A1C test at least twice in past year; and 2) Intermediate outcome measures including: a) frequency of high blood glucose and b) diabetes-related hospitalization or ED visit in the past 2 years. Our models statistically controlled for patient characteristics including age, gender, type of diabetes medication, diabetes care support, health care access, and transportation.

RESULTS: Among Health Center patients 18 years and older with diabetes, there were no racial/ethnic disparities found in: receipt of eye exam ( $\mathrm{p}=0.816)$, receipt of A1C testing $(\mathrm{p}=0.637)$, high blood glucose levels $(\mathrm{p}=0.569)$, and diabetes-related hospitalization or ED visit $(\mathrm{p}=$ 0.252), after controlling for confounders. In fact, Hispanic/Latino (OR: $1.58,95 \%$ CI: 1.01, 2.47) and non-Hispanic African Americans (OR: 2.16, $95 \%$ CI: $1.39,3.38$ ) were more likely than non-Hispanic whites to have a foot exam in the past year. Having health insurance and a usual source of care increased the likelihood of having an eye exam (ORs: $2.52,95 \% \mathrm{CI}$ : $1.85,3.44 ; 1.65,95 \%$ CI: $1.08,2.51$ ) and foot exam (ORs: $1.78,95 \%$ CI: $1.25,2.54 ; 2.25,95 \%$ CI: 1.32, 3.85). Reporting high blood glucose "Sometimes/Most of time/Always" was associated with increasing age. Men were more likely to have a diabetes-related ED visit or hospitalization (OR: 1.61, 95\% CI: 1.08, 2.40).

CONCLUSIONS: Health Centers are part of larger federal efforts to eliminate racial/ethnic health disparities, like the National Partnership for Action to End Health Disparities. The finding that racial/ethnic minority patients did not appear to be disadvantaged in the diabetes measures being studied indicate that Health Centers are making progress in this effort. In addition, health care access (having insurance and a usual source of care) may increase preventive health care use (eye and foot exams) which may ultimately reduce diabetic retinopathy and amputations across all racial/ethnic groups. Targeted and early interventions directed at men and older adults may help reduce the complications associated with diabetes.

IDIOPATHIC GRANULOMATOUS MASTITIS: NON-SURGICAL MANAGEMENT WITH STEROID THERAPY IN A CASE SERIES OF 49 WOMEN Tanu Pandey; Pamela Ganschow; Leah Bressler; Elizabeth Marcus. John H Stroger Hospital of Cook County, Chicago, IL. (Control ID \#1339893)

BACKGROUND: Idiopathic granulomatous mastitis (IGM) is a rare benign breast disease of unclear etiology. Only a few hundred cases have been reported as case reports and small case series. Clinical features are similar to infectious mastitis and it may radiologically resemble breast cancer. Treatment options remain controversial and recent studies have advocated surgical treatment with wide excision or mastectomy as well as oral corticosteroids. We present one of the largest case series of women with IGM. The aim of the study was to describe the demographic characteristics, clinical features, associated conditions, and the outcomes of initial treatment focused on the use of non-surgical therapies.

METHODS: We conducted a prospective observational study in the breast clinics of a large safety net hospital in Chicago by enrolling all women with biopsy proven granulomatous mastitis between 2006 and 2010. Demographic, clinical, laboratory, and radiological data were obtained from electronic and paper records. Secondary causes of breast granulomas were excluded. Treatments prescribed included oral steroids, observation or surgical excision. The primary end points were the number of women who achieved complete resolution of disease with non-surgical treatment and the time to resolution. Resolution was defined by clinical examination and not self-report. IRB approval and patient consent were obtained.
RESULTS: 49 women were diagnosed with IGM during this period and all were enrolled into the study. The mean age was 35 years (range 24-67). 39(80\%) women were Hispanic whereas one third of overall referrals to the breast clinics are Hispanic. 34(70\%) were born in Mexico. 39(80\%) women presented with a painful breast mass with overlying erythema. $29(59 \%)$ women were initially prescribed antibiotics for presumed infectious mastitis to which they had minimal response. 44(90\%) women were prescribed oral steroids, $2(3 \%)$ women had surgical excision and 3(6\%) remained under observation only. Of the 44 women who received oral steroids, $35(80 \%)$ had complete resolution of disease, $6(14 \%)$ were lost to follow up, $2(5 \%)$ remained on steroid treatment at the time of submission and 1 had surgical excision after failure of steroid treatment. Excluding one woman who was non-adherent to her treatment, the mean time to complete resolution on steroids was 194 days (range 45-581) with 20(59\%) resolving within 6 months. Weight gain and epigastric discomfort were the most common side effects from steroid treatment during the study period.

CONCLUSIONS: Idiopathic granulomatous mastitis is a rare benign breast disease for which surgery, including mastectomy, has been widely used for treatment but may be unnecessary. In our case series, surgery-sparing treatments (primarily with oral steroids) led to resolution in over $90 \%$ of women suggesting that most patients with this disease may be successfully managed with conservative therapy alone. In addition, the predilection of IGM among young Hispanic women of childbearing age in our study supports similar findings from other studies and may suggest a common genetic, environmental, immunological or infectious etiology, which warrants further multidisciplinary investigation.

\section{IMMUNE RISKS FOR PERSISTENT DEBILITY AFTER MOLD} EXPOSURE \& RESPONSE TO ANTIFUNGAL BIOFILM THERAPY Irene H. Grant; James F. Rini. New York Medical College, Valhalla, NY. (Control ID \#1340240)

BACKGROUND: Tissue barriers \& cell-mediated immunity(CMI) defend against fungal penetration. Stachybotrys'(S) products irreversibly damage tissues.Culture \& microscopy routinely fail to detect Aspergillus-Penicillium (AP) invasion in the severely immunocompromised. Non-invading fungal biofilms support mold proliferation, persistence \& elaboration of myriad inflammatory, toxic, anticoagulant or immunosuppressive products. While only Amphotericin (Ampho) eradicates mold biofilms, it cannot cross tissue planes. Since toxin-producing biofilms may cause persistent debility long after exposure,biofilm-directed antifungal therapy (Rx) was studied. Objectives:(1) Correlate clinical spectrum after mold exposure with immune risks, antibody \& mycotoxin detection, (2) Determine efficacy of antifungal biofilm Rx.

METHODS: Prospectively monitored 47 ill mold-exposed patients (16 M,31 F,13-86 yrs.),for immune risks, fungal antibodies, urine mycotoxins (MT's) \& response to antifungal Rx (nasal lavage with oral "swish \& swallow" Ampho +/- systemic azoles or capsofungin).

RESULTS: Of 47 patients with documented exposure to S \&/or AP, 55\% had severe persistent symptoms (sxs), 28\% disabling \& 11\% life-threatening. Those exposed to both S\&AP were at greater risk for more sxs. Prolonged debility was associated with exposure intensity, impaired CMI, \& multiple MT excretion. $11 \%$ had impaired CMI risks: vitamin $\mathrm{D}$, protein or zinc deficiencies, steroids,neutropenia. A.fumigatus $+\operatorname{IgG}$ titers in $25 / 26(96 \%)$; S.chartarum + IgG in $21 / 28(75 \%),+\operatorname{IgA}$ in $2 \&$ IgE undetectable in $28 / 28$. MT excretion in 28/32 (88\%): Ochratoxin 27, Tricothecenes(T's) 21, Aflatoxin 11; 19 excreting multiple MT's were severely debilitated. All 21 excreting T's had chronic fatigue syndrome (CFS), fibromyalgia (FM) \& persistent pain. Rx response was striking: $44 / 47$ (94\%) unequivocally improved: $25 \%$ completely; $28 \%$ residual disability; $55 \%$ relapsed with $\mathrm{Rx}$ interruption, most improving on restart. Flares were common on initiation, waning over time. Sxs improved in all categories, most in 2-3 wks; some 1-3 mo. Many with impaired CMI or markers for penetration (adenopathy, nodules, calcification) required prolonged(> 2 yrs), relapsing with interruptions. Most refusing to continue RX steadily deteriorated. 18 relapsed on mold re-exposure.

CONCLUSIONS: Mold exposure with impaired immunity \&/or Stachybotrys-exposure can result in persistent debility \& MT excretion. Biofilmfocused antifungal +/- systemic Rx dramatically reverses chronic, 
unexplained debility in over $90 \%$ (bleeding, CFS, FM, sinusitis, vertigo, choking, GERD, IBS, tremors, ataxia, paresis, seizure, delerium, sleep apnea). Better methods to detect mold \& controlled clinical trials for antifungal $\mathrm{Rx}$ are needed.

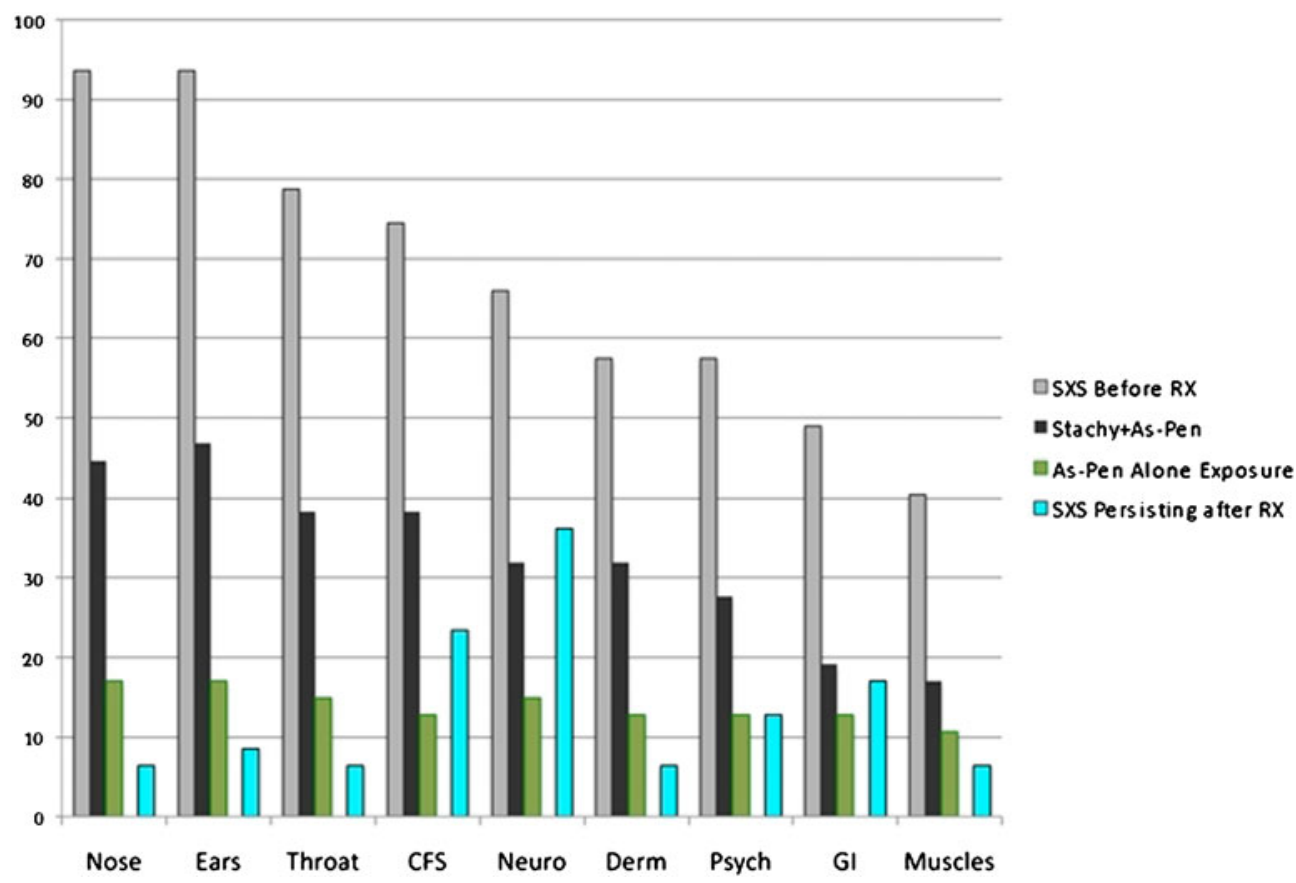

IMPACT OF HEALTH DISPARITIES EDUCATION ON MEDICAL STUDENTS' CULTURAL COMPETENCE April S. Fitzgerald ${ }^{1}$; Mary Catherine Beach ${ }^{1}$; Somnath Saha ${ }^{2} .{ }^{1}$ Johns Hopkins University, Baltimore, MD; ${ }^{2}$ Oregon Health \& Science University, Portland, OR. (Control ID $\# 1340803$ )

BACKGROUND: In response to mounting evidence that physicians may contribute to widespread disparities in the quality of health and health care, medical educators have worked to determine the most effective methods of teaching about health disparities and increasing the cultural competence of physicians-in-training. Although many studies have demonstrated a positive impact of these educational efforts, few if any have examined the impact of health disparities education on the cultural competence of medical students.

METHODS: The Health Care Disparities course is the first course taught to first year medical students at Johns Hopkins University, occupying 3 full days in the week following Orientation, and modeled after a health disparities course developed, taught and evaluated at University of Chicago. The goals of the course are to improve 1-awareness of the effect of social needs and demands on care of patients, 2-knowledge of existence of health care disparities and the demographic influences on health care quality and effectiveness, 3-selfawareness of personal biases in their approach to health care delivery, and 4cross-cultural communication skills. The course contains short lectures, small group work with video presentation and active learning exercises, community tours and service experiences. One-hundred and twenty students are in the Class of 2015; of those, 119 students completed the health disparities course in August 2011. We evaluated the effectiveness of the course using a previously validated instrument measuring 5 domains of physician cultural competence: Cultural Awareness, Perceived Cultural Self-Efficacy, Awareness of Racial Disparities, Valuing Diverse Perspectives, Support for System-Level Cultural Accommodations. Each domain includes 5-8 individual items, rated on a 5-point scale (strongly agree - strongly disagree). Resulting scores for each domain range from 1 (lowest cultural competence) to 5 (highest cultural competence). We used ttests to measure differences before and after the course in students' selfassessment of their cultural competence across all five remaining domains.
RESULTS: Most students completed a pre- course cultural competency selfassessment $(\mathrm{n}=103)$ and all completed the post-course self-assessment $(\mathrm{n}=$ 119). Students demonstrated statistically significant improvements in all five domains measured (See Table).

CONCLUSIONS: Our health disparities course was effective in improving the cultural competence of medical students. To the extent that greater cultural competence is associated with more equitable health care delivery, health disparities education in medical school may help reduce the physician contribution to racial and ethnic disparities in health care.

Table. Student Self-Rated Cultural Competence Before and After Health Disparities Curriculum

\begin{tabular}{lllll}
\hline Domain & $\begin{array}{l}\text { Cronbach's } \\
\text { Alpha }\end{array}$ & $\begin{array}{l}\text { Mean } \\
(\mathrm{SD}) \\
\text { Pre-Test } \\
\mathrm{N}=103\end{array}$ & $\begin{array}{l}\text { Mean } \\
(\mathrm{SD}) \\
\text { Pre-Test } \\
\mathrm{N}=119\end{array}$ & $\begin{array}{l}\mathrm{p} \text { - } \\
\text { value }\end{array}$ \\
& & 2.66 & 3.20 & $<0.001$ \\
\hline $\begin{array}{l}\text { Cultural } \\
\text { Awareness }\end{array}$ & 0.82 & $(0.84)$ & $(0.73)$ & \\
\hline $\begin{array}{l}\text { Perceived } \\
\text { Cultural Self- }\end{array}$ & 0.71 & 4.14 & 4.29 & 0.031 \\
$\begin{array}{l}\text { Efficacy } \\
\begin{array}{l}\text { Awareness of } \\
\text { Racial Disparities }\end{array}\end{array}$ & 0.83 & $(0.52)$ & $(0.51)$ & \\
\hline $\begin{array}{l}\text { Valuing Diverse } \\
\text { Perspectives }\end{array}$ & 0.64 & 3.56 & 4.08 & $<0.001$ \\
$\begin{array}{l}\text { Support for CLAS } \\
\text { Standards }\end{array}$ & 0.71 & $(0.91)$ & $(0.72)$ & \\
\hline
\end{tabular}

IMPACT OF HEALTH INSURANCE, EDUCATION AND INCOME STATUS ON CANCER SCREENING RATES IN MINORITY POPULATIONS: 2001 - 2010 Raxitkumar Jinjuvadia; Prateek Lohia; Theresa E. Vettese. Wayne State University/Detroit Medical Center, Detroit, MI. (Control ID \#1339654) 
BACKGROUND: Despite the beneficial effect of cancer screening methods, utilization of these resources remains low among minority population. Our aim was to evaluate impact of medical insurance, education level and income status on colorectal cancer (CRC), breast cancer (BC) and cervical cancer (CC) screening among minority populations using Behavioral Risk Factor Surveillance System (BRFSS) 2001 to 2010.

METHODS: 184,092 and 451,075 individuals participated in BRFSS in 2001 and 2010 respectively, including those from minority populations (African American, Asian, Native Hawaiian, American Indian). CRC screening was defined as having FOBT in last 1 year or colonoscopy in last 10 years in individuals $>50$ years. BC screening was defined as mammogram in last 2 years in women $>40$ years. Individual was considered to have $\mathrm{CC}$ screening if she had Pap smear in last 3 years. Education status was categorized as less than high school, and high school or more. Participants in an annual income group $>\$ 25,000$ were compared with those in a lower income group. Cancer screening rates were evaluated for years 2001 to 2010 with respect to medical insurance, education and income status. Logistic regression analysis was used to calculate adjusted odds ratios (aOR) for receiving cancer screening for the year 2010. All analyses were performed using SAS 9.2.

RESULTS: In 2010, after implementing eligibility criteria, the study cohort for CRC, BC and CC included 25426, 22302 and 22083 participants respectively.
Overall screening rate for $\mathrm{CRC}, \mathrm{BC}$ and $\mathrm{CC}$ for minority population in year 2010 was $62.2 \%, 77.5 \%$ and $83.7 \%$ respectively. Individuals with medical insurance had a significantly higher screening rate compared to those without, for all cancer screenings. (CRC- $65.9 \%$ vs $37.7 \%$; BC- $81.2 \%$ vs $56.1 \%$; CC$86.2 \%$ vs $72.9 \%$, p values $<0.001$ ). Though not as prominent as insurance status, a significant difference was noted with respect to a higher education level (CRC-64.3\% vs $52.6 \%$; BC-78\% vs $74.5 \%$; CC- $84.6 \%$ vs $77.2 \%$ ). Participants with $>\$ 25,000$ income had higher screening rate (CRC- $68.4 \%$ vs $55.6 \%$; BC$81.7 \%$ vs $72.8 \%$; CC- $87.8 \%$ vs $78.7 \%$ ). After adjusting for age, sex, race, BMI, smoking, alcohol and cost factors; insurance, education and income status were found to be independent predictors of CRC and CC screening. The aOR for insurance, education and income status were 2.09 (1.92-2.27), 1.52 (1.41- 1.64) and 1.66 (1.56-1.76) for $\mathrm{CRC}$; and 2.37 (2.17-2.59), 1.15 (1.03-1.28) and 1.72 (1.59-1.87) for CC screening respectively. Only insurance $(2.66,2.45-2.89)$ and income $(1.57,1.46-1.69)$ status were found to independent predictors of $\mathrm{BC}$ screening, but not education $(1.05,0.95-1.15)$. Similar results were noted for other years.

CONCLUSIONS: Among minority populations, health insurance status seems to be the most important socioeconomic factor affecting cancer screening rate, along with education and income status. More targeted efforts are required to improve screening rate among this population.

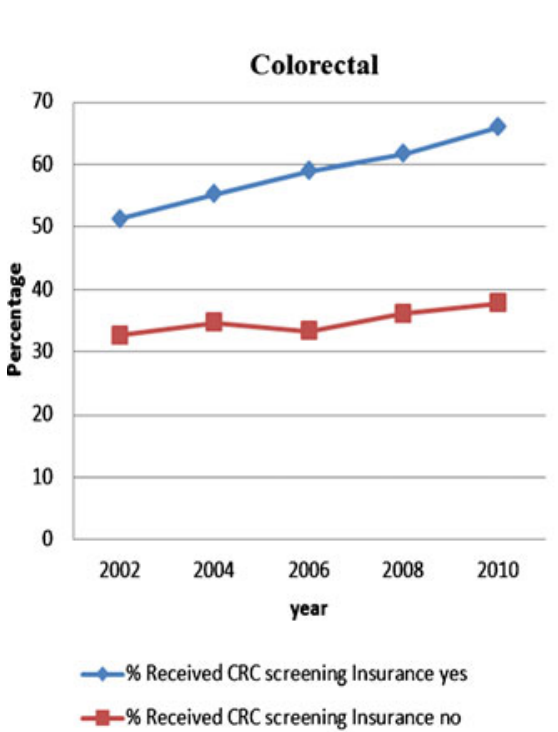

\section{Health Insurance and cancer screening rates}

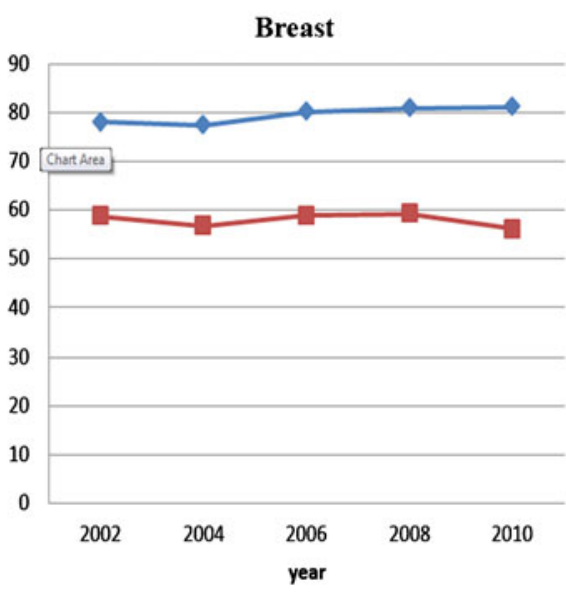

$\neg \leftarrow \%$ Received breast cancer screening Insurance yes

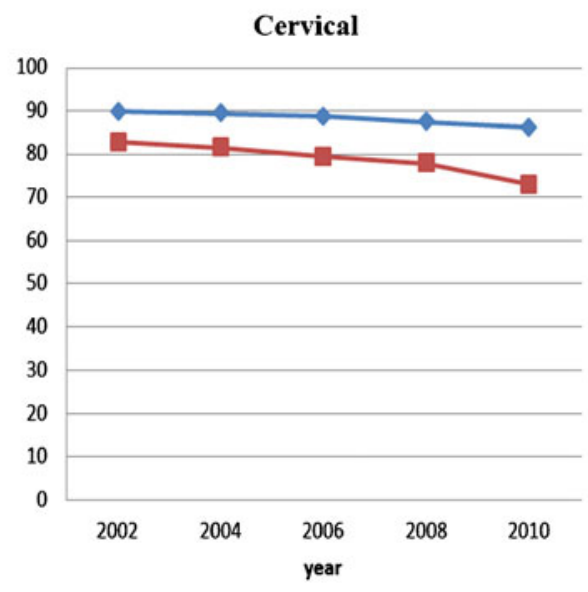

$\multimap \%$ Received cervical cancer screening Insurance yes

$-2=\%$ Received cervical cancer screening Insurance no
IMPACT OF INTERN WORKLOAD AND DISCONTINUITY OF CARE ON 30-DAY READMISSION Stephanie Mueller; Jacques Donze; Robert Burke; Jeffrey L. Schnipper. Brigham and Women's Hospital, Boston, MA. (Control ID \#1332280)

BACKGROUND: Recent modifications to Accreditation Council for Graduate Medical Education (ACGME) duty hour restrictions have resulted in greater variation of intern workload and increased transitions of care by housestaff. We evaluated the association between intern workload and discontinuity of patient care on probability of 30-day readmission.

METHODS: We performed a retrospective cohort study at an academic medical center in Boston, MA. Data were obtained from administrative sources and electronic medical records. Patients were eligible for inclusion if discharged from the general medicine service to home or a rehabilitation facility between July, 2009 and June, 2010. The outcome of interest was 30day readmission to any of three hospitals within the healthcare system. Intern workload predictors included: (1) Number of admissions completed by intern on day of patient discharge, dichotomized into $>2 \mathrm{vs} \leq 2$ to account for on-call vs non call days, (2) Number of discharges completed by intern on day of patient discharge, and (3) Intern census (i.e., total patients cared for) on day of patient discharge. Discontinuity of care predictor was defined as the number of different physicians writing orders on the patient during hospitalization, which was categorized into quartiles of $<8$ physicians (reference), $8-11,11-15$, and $>$ 15 . We performed logistic regression to examine the association of predictors with 30-day readmission, accounting for clustering by intern, and controlling for patients' demographics, healthcare utilization in past year, length of stay, comorbidities, source of admission, discharge destination, number of medications on discharge, weekday vs weekend discharge, and nurse workload on day of patient discharge (i.e., number of patients cared for on the nursing unit).

RESULTS: Of the 10,731 patient discharges, 2398 (22\%) were readmitted within 30 days. Intern workload predictors demonstrated a mean of 0.7 patient admissions, 1.7 patient discharges, and mean census of 4 patients on day of patient discharge. Of the 10,731 discharges, 1098 (10.2\%) occurred on intern call days (admissions $>2$ ). Discontinuity of care predictor showed the mean number of physicians writing orders on a patient during hospitalization was 12.2. Results of multivariate analysis demonstrated no association between number of intern admissions or intern census and odds of 30-day readmission. However, with every increase in number of intern discharges on day of patient discharge, the odds of 30-day readmission increased by 5\% (adjusted OR 1.05 [1.00, 1.11], $\mathrm{p}<0.05$ ). Increasing number of physicians writing orders on a patient during hospitalization was not significantly associated with increased odds of 30-day readmission on adjusted analysis. 
CONCLUSIONS: We found that increased number of patients an intern discharges on day of patient discharge is associated with increased odds of 30 -day readmission. We also found that only $10 \%$ of patients are discharged on intern call days, less than expected given a traditional 4day call schedule. Our findings suggest that workload associated with multiple patient discharges may adversely impact patient outcomes, and that interns potentially self-regulate workload on call days by decreasing the number of patient discharges. Future research should further examine workload associated with patient discharge and potential mechanisms of mitigating negative consequences.

IMPACT OF MASSACHUSETTS HEALTH CARE REFORM ON THE RISK OF HOSPITALIZATION FOR CARDIOVASCULAR AMBULATORY CARE SENSITIVE CONDITIONS. Danny McCormick $^{1}$; Amresh D. Hanchate ${ }^{2,3}$; Nancy R. Kressin ${ }^{2,3}$; Mengyun Lin ${ }^{3}$; Meredith D'Amore ${ }^{3}$; Karen E. Lasser ${ }^{3}$. ${ }^{1}$ Harvard Medical School / Cambridge Health Alliance, Cambridge, MA; ${ }^{2} \mathrm{VA}$ Boston Healthcare System, Boston, MA; ${ }^{3}$ Boston Medical Center/Boston University School of Medicine, Boston, MA. (Control ID \#1337863)

BACKGROUND: The 2006 Massachusetts (MA) health reform increased the number of insured residents, particularly among racial/ethnic minorities. Yet, little is known about whether this insurance expansion translated into improvements in access to medical care for MA residents as a whole or for the state's racial and ethnic minorities. Ambulatory care sensitive conditions (ACSCs) such as congestive heart failure (CHF), hypertension and angina, are a set of medical conditions for which good outpatient care can potentially prevent the need for hospitalization. Decreased admission rates for these conditions are validated measures of improved access to outpatient care. We therefore evaluated whether such rates declined for residents of MA following health reform and whether racial and ethnic disparities in admission rates declined.

METHODS: Using complete data on acute care hospital admissions in MA and in two states that did not implement comprehensive health care reform, New York and Pennsylvania, we identified all hospital admissions for cardiovascular ACSCs (CHF, angina and hypertension) during the 21 months preceding and following health reform implementation (7/1/ 2006 - 12/31/2007). Using US census population data we calculated preand post-reform age- and sex-standardized admission rates for the 3 ACSCs combined among patients 18-64 (those affected by reform). Treating MA as the intervention cohort, and NY and PA together as the control cohort we used multivariate Poisson regression models to conduct "difference-in-difference" analyses to estimate post-reform changes in admission rates in MA adjusted for contemporaneous changes occurring in control states. The models were also adjusted for age, gender and race. Using this approach, we also assessed whether health reform was associated with decreases in admission rates for racial and ethnic minorities compared with whites.

RESULTS: There were 84,286 hospital admissions for CHF, angina and hypertension combined in the pre and post reform periods in MA and 535,726 during the same time periods in control states. The hospital admission rate (number/100 k population) for ACSCs declined in MA (128.7 to 121.7 [5.4\%]) and in control states (232.2 to 208.4 [10.2\%] from the pre to post reform period. When adjusted for secular trends in control states, age, gender and race, however, there was a $6.2 \%$ (95\% CI, 2.9-9.6) increase in admissions in MA. The admission rate among blacks in MA increased (477.5 to 511.5 [7.1\%]) but in control states increased less $(683.3$ to $693.3[1.5 \%])$. The admission rate among Hispanics declined in both MA (295.3 to $267.2[9.5 \%])$ and control states (278.1 to 254.4 [8.5\%]). The admission rate among whites declined in both MA (102.9 to 92.1 [10.5\%]) and control states (150.5 to 121.3 [19.4\%]). After adjustment, there was no significant change in the admission rate for blacks compared with whites in MA $(+3.7 \%[95 \% \mathrm{CI},-11.1-4.5])$ or for Hispanics compared with whites in MA $(+9.3 \%[95 \% \mathrm{CI},-18.2-0.6])$.

CONCLUSIONS: Hospital admissions for cardiovascular ACSCs did not decline in MA as a whole, or for minorities relative to whites, in comparison with 2 large control states that did not implement health reform. Additional insurance or health care system reforms in MA may be needed to decrease potentially avoidable hospitalizations and improve access to care.
IMPACT OF MASSACHUSETTS HEALTH REFORM ON RACIAL AND ETHNIC DISPARITIES IN USE OF INPATIENT CARDIOVASCULAR SURGERIES Amresh D. Hanchate ${ }^{1,2}$; Xin Zheng ${ }^{2}$; Karen E. Lasser ${ }^{2}$; Danny McCormick ${ }^{3}$; Meredith $\mathrm{D}^{\prime} \mathrm{A} m o r{ }^{2}$; Alok Kapoor ${ }^{2}$; Nancy R. Kressin ${ }^{1,2}$. ${ }^{1}$ VA Boston Healthcare System, Boston, MA; ${ }^{2}$ Boston Medical Center/Boston University School of Medicine, Boston, MA; ${ }^{3}$ Cambridge Health Alliance, Cambridge, MA. (Control ID \#1336492)

BACKGROUND: The 2006 Massachusetts (MA) health reform increased insurance coverage to near-universal levels, but its impact on access to care or disparities in access is unclear. We examined post-reform change in racial/ ethnic differences in use of two cardiovascular $(\mathrm{CV})$ procedures, percutaneous transluminal coronary angioplasty (PTCA) and coronary artery bypass graft $(\mathrm{CABG})$, for which disparities in use have been noted in numerous past studies. As use of these procedures is sensitive to outpatient referral, impact of reform on procedure use can serve as a marker for impact on access to care. We hypothesized that reform will result in increased use of procedures, and that the increase in use will be greater among minorities. We contrasted the results with those for two comparable orthopedic procedures (total knee replacement and total hip replacement)

METHODS: Using the comprehensive MA Hospital Inpatient Data of all discharges in all non-Federal hospitals from 1/1/2004 to 9/30/2009, we obtained state-level counts of individual procedures stratified into 30 cohorts by age (40-44, 45-49, 50-54, 55-59 \& 60-64), sex and race/ethnicity (Whites, Blacks and Hispanics) for each quarter. To separate the impact of MA reform from secular changes, we used corresponding data from New York (SPARCS Inpatient Data) to capture changes unrelated to MA reform. To convert procedure counts into rates (\# procedures/10,000 population), we obtained Census data to create a measure of population at risk for each cohort. Treating a quarter as the unit of time, the study period comprised of 10 pre-reform, 6 transition and 7 post-reform quarters. Applying a difference-in-difference specification, we used segmented time series Poisson regression models to estimate the impact of MA reform on the procedure rates for Blacks and Hispanics relative to those for Whites.

RESULTS: Reflecting national trends, there was a secular decrease in MA in the use of PTCA and CABG (-8.0\% per year for each) throughout the study period. For the pre-reform period procedure rates in MA were: $\mathrm{PTCA}=31.5$ and $\mathrm{CABG}=7.7$ per 10,000 population. In $\mathrm{MA}$, compared to Whites, prereform rates of both procedures were lower among Blacks (Incidence Rate Ratio [IRR]: $\mathrm{PTCA}=0.79 \& \mathrm{CABG}=0.73$; all $\mathrm{p}$ values $<0.001$ ) but similar among Hispanics. For the post-reform period, the procedure rate in MA was unchanged for PTCA but was $11 \%$ higher $(95 \% \mathrm{CI}=[2 \%, 20 \%])$ for CABG. In MA, health reform was associated with $14 \%$ higher post-reform rates of PTCA $+\mathrm{CABG}$ (combined) use $(95 \% \mathrm{CI}=[3 \%, 27 \%])$ among Hispanics than that among Whites; no significant impact was noted for Blacks $(2 \%$; $95 \% \mathrm{CI}=[-$ $9 \%, 15 \%])$. In contrast, the orthopedic procedures experienced secular increases in use. In MA, reform was associated with higher post-reform use of both procedures (combined) among Blacks $(15 \%, 95 \% \mathrm{CI}=[4 \%, 28 \%])$ and Hispanics $(36 \%, 95 \% \mathrm{CI}=[17 \%, 58 \%])$ than that among Whites.

CONCLUSIONS: MA health reform may have increased the use of CV and orthopedic procedures among minorities, thereby indicating improved access to care. While orthopedic procedure use increased among Blacks and Hispanics, the increase for $\mathrm{CV}$ procedures was smaller in magnitude and limited to Hispanics. The weaker impact on CV procedures may be indicative of less pent-up demand or greater acuity of conditions targeted relative to orthopedic procedures.

IMPACT OF MEDICAL AND PSYCHIATRIC COMORBIDITY BURDEN AND MORTALITY IN VETERANS WITH TYPE 2 DIABETES Cheryl P. Lynch ${ }^{1,2}$; Mulugeta Gebregziabher ${ }^{1,2}$; Kelly J. Hunt $^{1,2}$; Carrae Echols ${ }^{1}$; Gregory Gilbert ${ }^{1}$; Leonard E. Egede ${ }^{1,2}$. ${ }^{1}$ Ralph H Johnson Veterans Affairs Medical Center, Charleston, SC; ${ }^{2}$ Medical University of South Carolina, Charleston, SC. (Control ID \#1340363)

BACKGROUND: Greater disease burden earmarks affected individuals for premature disability, hospitalizations and death. Among people with diabetes, common medical and psychiatric conditions such as obesity and depression greatly increase morbidity and mortality risks. Therefore, we examined the 
relationship of medical versus psychiatric comorbidity burden and mortality among veterans with type 2 diabetes.

METHODS: A national cohort of veterans with type 2 diabetes was created by linking multiple patient and administrative files from 2 large VA databases. The main outcome measure was time to death, defined in months between the date of study entry and the date of death (or study end or censored). The predictor was comorbidity burden measured by count of medical and psychiatric conditions. Cox regression methods were used to model the association between time to death and comorbidity burden. Both unadjusted hazard ratios (HR) and adjusted HR were computed with their corresponding $95 \%$ confidence intervals.

RESULTS: The study cohort comprised a total of 629,563 veterans, nearly all male, with type 2 diabetes followed up over a 4-year period. The overall mortality rate was $22.2 \%$. Obesity (67\%) and hypertension (59\%) were the most common medical comorbidities. In comparing those with no comorbidities to those with one, two and three or more additional medical comorbidities the unadjusted HR were $1.16(1.14,1.18), 1.60(1.57,1.63)$ and 2.47 $(2.42,2.51)$, respectively. After adjusting for all covariates, the corresponding HR were $1.01(0.99,1.03), 1.34(1.32,1.37)$ and $1.99(1.96,2.03)$, respectively. Having three or more comorbidities in addition to diabetes is associated with a two-fold risk of death compared to those having only diabetes. Similarly, having one or two psychiatric comorbidities in addition to diabetes was associated with $1.26(1.25,1.28)$ and $1.46(1.41,1.50)$ fold risk of death, respectively. Looking at both comorbidity groups together, having three, four or five comorbidities was associated with $1.86(1.82,1.89), 1.98(1.93,2.02)$ and $1.99(1.92,2.06)$ fold risk of death, respectively.

CONCLUSIONS: Risk of death doubled for veterans with diabetes having 3 or more additional medical comorbidities. In addition, those having two psychiatric comorbidities had nearly $50 \%$ greater odds of death. These findings amplify the crucial need for healthcare providers to co-treat or integrate care management plans for diabetes-related medical and psychiatric comorbidities in veterans.

\section{IMPACT OF PRIOR ANTIBIOTIC USE ON OUTCOMES FOR} PATIENTS HOSPITALIZED WITH PNEUMONIA Eric Mortensen ${ }^{1}$; Ata Rahman ${ }^{2}$; Antonio Anuzeto ${ }^{2} .{ }^{1}$ VANTHCS, Dallas, TX; ${ }^{2}$ UTHSCSA, San Antonio, TX. (Control ID \#1336324)

BACKGROUND: Despite the growing number of antibiotic-resistant pathogens, the effect of prior use of antibiotics on pneumonia-related outcomes is unclear. Prior studies examining the effect of use of antibiotics prior to hospitalization for pneumonia have lead to divergent findings. Therefore, the purpose of our study was to examine the association between prior outpatient antibiotic use and clinical outcomes for those subsequently hospitalized with pneumonia.

METHODS: We identified patients $>65$ years of age admitted to a Department of Veterans Affairs hospital in fiscal years 2002 - 2007 with a discharge diagnosis of pneumonia. We examined the impact of prior antibiotic use within 90 days on 30-day mortality, intensive care unit (ICU) admission, use of mechanical ventilation, and use of vasopressor therapy. RESULTS: Our cohort was comprised of 50,119 patients with a mean age of 75.0 years (standard deviation 6.8 years) and $98.2 \%$ of subjects were male. In this cohort, $29 \%$ received antibiotics within 90-days of admission. Mortality at 30-days was similar for the prior antibiotic vs. no prior antibiotic groups $(13.9 \%$ vs. $14.2 \%, p=0.4)$. Patients that did not receive antibiotics prior to hospitalization had higher rates of ICU admissions $(14.2 \%$ vs. $13.2 \%, \mathrm{p}=0.005)$, but not use of mechanical ventilation $(6.6 \%$ vs. $6.3 \%, \mathrm{p}=0.2)$. Vasopressor use was similar $(4.4 \%$ vs. $4.6 \%, \mathrm{p}=0.4)$. Rates of staphylococcus aureus and pseudomonas infections were higher for those who received beta-lactams or fluoroquinolones vs. non-users. In a multilevel regression model, after adjusting for other potential confounders, prior receipt of beta-lactams, but not other types of antibiotics, was associated with increased risk for 30-day mortality (OR $1.23,95 \%$ CI $1.05-1.45$ )

CONCLUSIONS: A clinically important proportion of patents in our cohort were found to have antibiotic use prior to hospitalization for pneumonia, and the prior use of beta-lactams was associated with increased risk of mortality. Patients treated as outpatients for pneumonia should be treated with non-beta lactam antibiotics.
IMPACT OF VENDOR COMPUTERIZED PHYSICIAN ORDER ENTRY ON ADVERSE DRUG EVENTS IN PATIENTS WITH RENAL IMPAIRMENT Alexander A. Leung ${ }^{1}$; Gordon Schiff'; Carol Keohane $^{1}$; Mary Amato ${ }^{1,2}$; Steven R. Simon ${ }^{1}$; Bismarck Cadet $^{3}$; Michael Coffey $^{4}$; Nathan Kaufman ${ }^{4}$; Eyal Zimlichman ${ }^{1}$; Diane L. Seger ${ }^{1}$; Catherine Yoon ${ }^{1}$; David W. Bates ${ }^{1} .{ }^{1}$ Brigham and Women's Hospital, Boston, MA; ${ }^{2}$ Massachusetts College of Pharmacy and Health Sciences, Boston, MA; ${ }^{3}$ New England Medical Specialists, Boston, MA; ${ }^{4}$ Partners Community Healthcare, Inc., Boston, MA. (Control ID \#1326705)

BACKGROUND: Adverse drug events (ADE) are common among hospitalized patients with renal impairment. Computerized physician order entry (CPOE) systems with clinical decision support (CDS) may help prevent many ADEs by providing timely laboratory information, recommending renally-adjusted doses, and offering a knowledge base to assist with prescribing. However, decision support for renal disease varies widely among current vendor systems. Given the uncertain benefits of CPOE, especially with the wide range of associated CDS, we sought to determine the impact of these systems on the rates of ADEs among patients with kidney disease in the community hospital setting, where mainly vendor-developed applications are used.

METHODS: We conducted a before-and-after quasi-experimental study from January 2005 to September 2010 at five Massachusetts community hospitals to evaluate the impact of CPOE implementation on ADE rates. Three distinct levels of CDS were studied: basic CPOE only; rudimentary CDS with laboratory display; and, advanced CDS with suggested renal dosing and automated corollary laboratory orders for monitoring. We sampled a total of 1,590 patients with renal impairment (defined as an admission creatinine $\geq 1.5 \mathrm{mg} / \mathrm{dL}$ ) who were prescribed a renally-cleared and/or nephrotoxic drug. Charts were reviewed for orders, medication lists, laboratory reports, admission histories, notes, discharge summaries, and flow sheets. The primary outcome was the rate of preventable ADEs. Secondary outcomes were the rates of potential ADEs and the average lengths of stay. The occurrence of each outcome was determined according to hospital site and rates were calculated. To account for hospital effects in the analysis, we fit a fixed-effects model using Poisson regression.

RESULTS: There was a $45 \%$ decrease in the rate of preventable ADEs following implementation ( $8.0 / 100$ vs. $4.4 / 100$ admissions; $p<0.01)$, and the impact was related to the level of decision support $(\mathrm{p}=0.03$ and 0.02 for pairwise comparisons between advanced CDS vs. rudimentary CDS and basic CPOE, respectively). Basic CPOE was not associated with any significant benefit (4.6/100 vs. $4.3 / 100$ admissions; $p=0.87)$, and there was a decrease in preventable ADEs with rudimentary CDS, which did not meet statistical significance $(9.1 / 100$ vs. $6.4 / 100$ admissions; $p=0.22)$. However, substantial reduction was seen with advanced CDS (12.4/100 vs. 0/100 admissions; $p=$ $0.01)$. Despite these benefits, a significant increase in potential ADEs was found for all systems $(55.5 / 100$ vs. $136.8 / 100$ admissions; $p<0.01)$. There was a significant decrease in median length of stay following implementation at all sites ( 5.0 vs. 4.0 days; $\mathrm{p}<0.01$ ).

CONCLUSIONS: Vendor-developed CPOE with appropriate CDS can reduce the occurrence of preventable ADEs, and was associated with a decreased average length of stay. Our findings support the use of vendor CPOE systems as a means to reduce drug-related injury and harm. The potential ADE rate could be reduced by making refinements to the vendor applications and their associated decision support.

IMPACT OF A PATIENT ACTIVATION TOOL TO IMPROVE AGENDA SETTING IN CHRONIC DISEASE ENCOUNTERS: A RANDOMIZED CONTROLLED TRIAL Patrick G. O'Malley ${ }^{1,2}$; Dorothy Becher $^{1,2}$; Janice Hanson ${ }^{4,1}$; Jacqueline LeeHoffman ${ }^{2}$; Chengqing $\mathrm{Li}^{2}$; John J. Ominski ${ }^{2}$; Jeffrey L. Jackson ${ }^{3,1}$. ${ }^{1}$ Uniformed Services University, Bethesda, MD; ${ }^{2}$ Walter Reed National Military Medical Center, Bethesda, MD; ${ }^{3}$ Medical College of Wisconsin, Milwaukee, WI; ${ }^{4}$ University of Colorado School of Medicine, Aurora, CO. (Control ID \#1321978)

BACKGROUND: Patients with chronic illness often have a complicated visit agenda. Their visit interactions tend to be poorly organized resulting in miscommunication and potentially poor health outcomes. We hypothesized that a brief intervention before the visit could help 
patients organize their thoughts in order to improve communication, satisfaction with the encounter, and health-related outcomes.

METHODS: Randomized Controlled Trial. We enrolled a consecutive sample of 120 consenting participants aged 40-80yo with hypertension, at least 2 additional chronic conditions (excluding dementia), who were scheduled to see their internist for a routine appointment. Of 11 participating providers, each had 8-12 patients randomized (using a computer generated random sequence with allocation concealment) to a previsit intervention tool or usual care. Intervention: Using patient and physician focus groups, the tool was systematically designed to prompt the patient to reflect on, articulate, and prioritize their visit goals. The tool was administered at least 20 minutes prior to the visit. The primary outcomes were change in 4 and 12 week blood pressure (BP) readings and hypertension medication adherence using validated measurement processes. Analyses were perfomed by intention to treat. Covariates included patient functional status, health literacy, locus of control, and physician psychosocial attitudes and mindfulness.

RESULTS: Of the 106 patients who attended and completed their visit, the demographics were: mean age, 67; 53\% female; 55\% AfricanAmerican (AA); $88 \%$ were on $\geq 5$ medications; and only $8 \%$ had "poor" health literacy. The physician profile was: 48 yo, $55 \% \mathrm{~F}, 27 \%$ AA, 19 yrs since graduation. At baseline, patient characteristics, comorbidities, and biometrics were equally distributed and comparable among the study arms indicating effective randomization. Immediately after the visit, there was no difference in patient satisfaction or trust. At 4 and 12 weeks follow-up, there was no difference between groups on BP, medication adherence (by pill count or Morisky score) or trust (see Table). Intervention patients were more satisfied with physicians' explanations but this only approached statistical significance $(78.6 \%$ vs $68.9 \%$ fully satisfied; $\mathrm{P}=0.07$ )

CONCLUSIONS: A single, simple pre-visit tool designed to prompt patient initiated agenda setting and articulation of visit goals does not impact BP control, adherence, patient satisfaction, or trust in chronic illness patients with hypertension. More robust patient and physician interventions are likely needed to optimize agenda setting and communication in ways that improve patient-centered outcomes.

\begin{tabular}{|c|c|c|c|c|c|c|c|}
\hline & $\begin{array}{l}\text { Baseline - } \\
\text { Visit }\end{array}$ & Post & 4 Weeks & & 12 Weeks & & $\begin{array}{l}\mathrm{P} \\
\text { Valu }\end{array}$ \\
\hline & Activated & Usual & Activated & Usual & Activated & Usual & \\
\hline $\begin{array}{l}\text { Systolic BP } \\
\text { (mm Hg) }\end{array}$ & 139.0 & $\frac{\overline{C a r e}}{136.0}$ & 133.0 & $\frac{\overline{\text { Care }}}{130.5}$ & 129.2 & $\frac{\overline{\text { Care }}}{128.6}$ & NS \\
\hline $\begin{array}{l}\text { Diastolic } \\
\text { BP } \\
(\mathrm{mm} \mathrm{Hg})\end{array}$ & 81.5 & 80.2 & 78.1 & 77.0 & 76.8 & 76.3 & NS \\
\hline $\begin{array}{l}\text { Pill Count } \\
\text { (\% } \\
\text { Adherence) }\end{array}$ & 51.7 & 42.8 & 45.8 & 49.5 & 49.3 & 51.1 & NS \\
\hline $\begin{array}{l}\text { Morisky } \\
\text { Score }\end{array}$ & 4.5 & 4.8 & 5.0 & 5.1 & 4.9 & 5.1 & NS \\
\hline $\begin{array}{l}\text { Satisfaction } \\
(\%)\end{array}$ & 78.6 & 73.8 & & & & & NS \\
\hline $\begin{array}{l}\text { Trust } \\
\text { Score }\end{array}$ & 11.3 & 12.8 & 12.3 & 12.7 & 11.9 & 13.6 & NS \\
\hline
\end{tabular}

$B P$ (avg of 3 measurements, 5-min apart); Morisky (6-item; motivation + knowledge); Satisfaction (\% fully satisfied, RAND); Trust (8-item PCAS); $P$-values were for comparisons of change in continuous values, and for differences in \% fully satisfied.

IMPACT OF A TRAINING PROGRAM ON SUPERVISORS' ABILITY TO PROVIDE FEEDBACK ON RESIDENTS' COMMUNICATION SKILLS Noelle Junod Perron ${ }^{1}$; Mathieu Nendaz; Johanna Sommer ${ }^{2}$; Martine Louis Simonet ${ }^{1}$; Anne Gut ${ }^{2}$; Anne Baroffio ${ }^{2}$; Diana Dolmans ${ }^{3}$; Cees Van der Vleuten ${ }^{3}$. ${ }^{1}$ Geneva University Hospitals, Geneva, Switzerland; ${ }^{2}$ Geneva Medical School, Geneva, Switzerland; ${ }^{3}$ School of Health Professions Education, Maastricht, Netherlands. (Control ID \#1334027)
BACKGROUND: Lack of clinical supervisors' training in teaching skills is often cited as a main obstacle to post-graduate teaching of communication skills (CS). We report the results of a training program for clinical supervisors on how to provide feedback on residents' communication skills.

METHODS: We designed a pretest-posttest controlled study in which clinical supervisors working in the service of general internal medicine and the division of primary care at the Geneva University Hospitals, Switzerland, were invited to attend a program in teaching CS including small group training sessions with simulated patients and 2 individual supervision sessions over a period of 6 to 9 months. Controls were recruited among supervisors of a similar service not taking part in the training program. Before and after the training program, supervisors were videotaped while giving feedback on residents' communication skills bu using an OSTE-video (objective structured teaching encounter). The feedback given by the supervisors was analysed using a 20 -item feedback rating instrument.

RESULTS: Twenty nine 29 clinical supervisors took part into the training program and 20 were included in the control group. Apart from age and training experience, the two groups were similar in terms of sociodemographic data and self-perceived knowledge in CS and teaching experiences. After training, a higher percentage of trained participants demonstrated statistically significant improvement (differences in mean scores before and after intervention using Wilcoxon signed rank test) in the following items: exploring residents' feelings/needs (intervention: $p r e=2.92$ and post $=$ 4.16, $\mathrm{p}=0.01$; control: $\mathrm{pre}=2.90$ and $\mathrm{post}=3.35, \mathrm{p}=0.87$ ), stimulating residents' self-assessment (intervention: $p r e=2.24$ and post $=3.68, p<0.001$; control pre $=1.90$ and post $=2.45, \mathrm{p}=0.04)$, limiting feedback to a few items (intervention: pre $=3.40$ and post $=4.24, p<0.001$; control: pre $=3.70$ and post $=3.60, \mathrm{p}=0.87$ ), making the resident active in finding solutions (intervention: pre $=2.40$ and post $=4.04, p<0.001 ;$ control: pre $=2.90$ and post $=2.85, p=0.78$ ), checking understanding(intervention: $\mathrm{pre}=1.36$ and post $=4.28, \mathrm{p}<0.001$; control: pre $=1.40$ and post $=1.35, \mathrm{p}=0.80$ ). Improvement was higher among inpatient clinical supervisors than among outpatient clinical supervisors.

CONCLUSIONS: These preliminary results suggest that a training program is effective in increasing clinical supervisors' feedback skills on residents' communication skills.

IMPACT OF INSTALLING AN OFFICE BASED AUTOMATED POINT OF CARE MEDICATION DELIVERY SYSTEM ON OUTCOMES AMONG DIABETIC PATIENTS ON CHOLESTEROL LOWERING MEDICATIONS Ana M. Palacio ${ }^{1}$; Leonardo Tamariz ${ }^{1}$; Jessica $\mathrm{Chen}^{2}$; Hua $\mathrm{Li}^{1}$; Olveen Carrasquillo ${ }^{1}{ }^{1}$ University of Miami, Miami, FL $;{ }^{2}$ Chen Medical Associates, Miami, FL. (Control ID \#1339234)

BACKGROUND: To date most patients receive their medications from traditional or mail order pharmacies. Automated point of care medication delivery system (POCMDS) are a relatively new innovation allowing patients to receive their medications at the time of their visit with their health care provider. Literature on the impact of POCMDS on outcomes is sparse. The aim of this study is to evaluate the impact of a POCMDS implementation in medium size practice on adherence, costs and lipid control among a group of diabetic patients.

METHODS: Between 2008 and 2010, the POCMDS was implemented in a network of five South Florida capitated private practice clinics located predominantly in underserved areas. The POCMDS formulary included mostly generic medications provided to patients by their physicians during the clinic visit free of charge. When possible, patients were switched from name brand to generic medications as part of this process. To evaluate the impact of POCMDS on outcomes we conducted a pre-post analysis in a cohort of 308 diabetics who had 12 months of follow-up before and after the POCMDS was installed. Eligible patients had to have an ICD-9 codes for diabetes in the pre-evaluation period, drug code for a statin medication prior to the POCMDS period and at least one LDL measurement within a year before and after the POCMDS. Race, co- morbidities and LDL were obtained from the electronic medical record. Medication refills and costs were collected from claims data prior to POCMDS and subsequently from the POCMDS prescription records. As a measure of adherence, we used the medication possession ratio (MPR) calculated as the percentage of time 
the subject had a statin available during the 12 months before and after the intervention.

RESULTS: We analyzed data on 238 Black and 70 White subjects. With respect to medication adherence, the medication possession ratio nearly doubled among both whites and blacks $(\mathrm{p}<.01$, Table 1). Our costs analyses show a significant reduction in per patient statin related costs from an average of $\$ 120$ before POCMDS to $\$ 90$ after the enrollment on POCMDS $(\mathrm{p}=0.04)$. However, while both groups experienced significant improvements in the MPR, we did not observe a significant improvement in the average LDL.

CONCLUSIONS: Among diabetic patients in this capitated system a POCMDS implementation was associated with significant improvements in the medication possession ratio and lower statin medication drug costs. Lack of change on LDL levels could be related to the transition from more potent brand statins to less potent generic ones or relatively low LDL levels at baseline. Our cost data excludes the savings experienced by patients whom are no longer are charged drug co-payments. Ongoing analyses are examining data on diabetes and blood pressure control.

\begin{tabular}{lllll}
\hline \hline Race & Number & MPR before POCMDS & MPR after POCMDS & p-value \\
White & 70 & $0.49 \pm 0.28$ & $0.80 \pm 0.22$ & $<0.01$ \\
Black & 238 & $0.40 \pm 0.26$ & $0.84 \pm 0.19$ & $<0.01$ \\
& & LDL before POCMDS & LDL after POCMS & \\
White & 70 & $95.6 \pm 31.8$ & $92.1 \pm 28.0$ & 0.51 \\
Black & 238 & $96.0 \pm 29.6$ & $96.5 \pm 32.4$ & 0.72 \\
\hline
\end{tabular}

IMPACT OF THE ELECTRONIC MEDICAL RECORD (EMR) ON RESIDENTS' MEDICAL EDUCATION Diane L. Levine; Satyam Patel; Ahmad Muneer. Wayne State University/Detroit Medical Center, Detroit, MI. (Control ID \#1334914)

BACKGROUND: With the 2009 American Recovery and Reinvestment Act, new funding for Health Information Technology will result increased adoption of EMR. A previous study showed that interns spent 5\% more time entering orders with computerized physician order entry (CPOE) than before implementation of CPOE. In 2009 our institution implemented an EMR with level-five capabilities (Healthcare Information and Management Systems Society classification). In 2010, the Department of Medicine mandated full use of the EMR for all patient documentation. Anecdotal evidence suggested that this resulted in residents spending excessive amounts of time on the EMR. The impact of use of a level-five EMR on graduate education is unknown. We conducted a survey-study to determine the impact of the EMR on residents' daily activities, time spent on the EMR, and on residents' reading and education.

METHODS: A survey consisting twenty-eight multiple-choice questions was developed. An email with an information sheet and an online encrypted survey link was sent to trainees at the Detroit Medical Center/ Wayne State University (WSU). Participation in the survey was voluntary and anonymous. The study was exempted by the Institution Review Board at WSU.

RESULTS: A total 138 residents completed the survey; response rates were highest from the Departments of Medicine (49), Pediatrics (26), and Emergency Medicine (23). Trainees spent considerable time on the EMR during duty hours. The majority (95\%) spent more than one hour and almost $73 \%$ spent more than two hours. In contrast, $56 \%$ of residents spent less than two hours in direct patient care and $45 \%$ spent less than 90 minutes. Residents spent additional time on the EMR after duty-hours with $42 \%$ spending more than one hour. Primary-care residents spent significant time on the EMR after duty hours as compared to non primary-care residents $(\mathrm{p}=0.036)$. Residents who spent more time on the EMR during the work were more likely to spend time on the EMR after the work and less likely to spend time reading; $52 \%$ of residents spending more than two hours on the EMR during the work-day spent more than 60 minutes on the EMR after work as compared to $15.8 \%$ of residents spending less than two hours $(p<0.001) .66 \%$ of residents spending more than two hours on the EMR during work spent less than 30 minutes reading as compared to $44.7 \%$ of residents spending less than two hours ( $p=$ 0.023 ). $81 \%$ of residents spending more than one hour on the EMR after work spent less than 45 minutes for reading as compared to $63.8 \%$ of residents spending less than one hour $(\mathrm{p}=0.027)$. Residents spending more than two hours on the EMR perceived that use of the EMR decreased efficiency ( $p=$ 0.021 ) and did not contribute to education as compared to those spending less than two hours $(\mathrm{p}=0.028)$.

CONCLUSIONS: Residents spent considerable time on the EMR impacting time for reading and other activities. It is unclear whether time spent on the EMR after duty-hours should be included in duty-hour computations. The impact of the EMR on resident education needs to be further studied.

IMPACT OF USING ADMINISTRATIVE VS. CHART-ABSTRACTED DATA ON CALCULATIONS OF FINANCIAL INCENTIVES FOR HEALTH CARE PROVIDERS IN A PAY-FOR-PERFORMANCE PROGRAM Laura A. Petersen; Tracy Urech; Kate Simpson; LeChauncy D. Woodard; Salim S. Virani; Mark Kuebeler; Meghan Lutschg. Michael E. DeBakey VA Medical Center HSR\&D Center of Excellence and Baylor College of Medicine, Houston, TX. (Control ID \#1338522)

BACKGROUND: Pay-for-performance programs often rely on claims and other administrative data sources, but it is not clear whether these data accurately reflect information contained in patients' medical records. To evaluate the impact of using administrative data on the calculation of financial incentives in a study of pay for performance, we compared the calculation of financial incentives based upon administrative data with calculations using data collected via chart review to reward use of guideline-recommended antihypertensive medications.

METHODS: Trained abstractors collected data from VA medical records reflecting care provided to hypertensive patients between April and July 2009. The same data elements were collected from administrative data, including patients' comorbidities that impact drug regimens, current antihypertensive medications, and medication allergies or contraindications. Two clinicians, an internist and a cardiologist, independently reviewed procedure and diagnosis codes (ICD-9-CM and CPT) for conditions identified as compelling indications by JNC 7 guidelines for hypertension treatment: diabetes mellitus, chronic kidney disease, unstable angina, myocardial infarction, ischemic heart disease, and nephropathy. We also reviewed diagnoses codes to identify contraindications to certain therapies, e.g., angioedema. When possible, we supplemented diagnoses and procedure codes with laboratory data to identify certain conditions (e.g., diabetes). We identified clinically relevant allergy information and vital sign data from the VA Corporate Data Warehouse (one of the administrative data sources). Using these data, we determined whether or not a patient's antihypertensive medication regimen was consistent with JNC 7 guidelines.

RESULTS: 2834 of 2840 patients (99.8\%) were eligible for evaluation in the administrative data. Comparing the chart abstracted and administrative data, $84.4 \%$ of patients had the same comorbidity history for the 6 conditions examined. Agreement was highest for diabetes (kappa $=0.92)$ and lowest for unstable angina $(\mathrm{kappa}=0.38)$. In the medical record data, $72.3 \%$ of patients received guideline-recommended medications compared to only $62.7 \%$ in the administrative data. Over half $(55.6 \%)$ were identified in both sources $(\mathrm{kappa}=0.46)$. Among the patients with the same comorbidity history in both data sources $(n=2391)$, chart abstracted data showed that $71.9 \%$ received appropriate medications while administrative data identified $63.1 \%$ of patients (kappa $=0.51$ ). Overall, incentive earnings decreased by an average of $\$ 28.37(\mathrm{SD}=\$ 33.68)(11.6 \%[\mathrm{SD}=9.9 \%])$ for the 85 health care personnel when we calculated payments using only administrative data. Compared to the payments providers received in the study, seventy-nine $(92.9 \%)$ providers would have received less money, three $(3.5 \%)$ would have received the same amount, and three $(3.5 \%)$ would have earned more money.

CONCLUSIONS: We found moderate agreement for use of guidelinerecommended antihypertensive medications when we compared abstracted data from medical records to data in VA administrative sources. Overall, incentive payments decreased by an average of $11.6 \%$ when evaluating providers' performance using only administrative data. Given the resources and time needed to abstract medical record data, quality initiatives implementing pay for performance should consider using administrative and claims data to evaluate provider performance. 
IMPACT ON LENGTH OF STAY AND COSTS IN PATIENTS ADMITTED TO AN OBSERVATION UNIT WITH CHEST PAIN, SYNCOPE AND ABDOMINAL PAIN Aziz Ansari; Elizabeth Schulwolf. Loyola University Medical Center, Maywood, IL. (Control ID \#1339798)

BACKGROUND: Hospitals are focusing more on patient admission status as inpatient or observation due to changes in reimbursement and increased scrutiny from regulatory agencies. Observation units allow for more efficient and appropriate care for patients admitted under observation status. Implementation of these units may decrease resource utilization, length of stay (LOS) and costs.

METHODS: We compared data of patients admitted under observation status either to the general floor in Quarter 4 of 2009 or to the observation unit in Quarter 4 of 2010. The data was obtained from our administrative database and included primary diagnosis, LOS and total charges. We excluded patients who were classified as inpatient at any time during the admission, regardless of the patients' length of stay. All patients were cared for by a hospitalist. Patient cohorts were compared overall and then by primary diagnosis: chest pain, syncope and abdominal pain. Continuous variables were compared using the Student's t-test. Dichotomous variables were compared using the chi-square test. All data was analyzed using Stata 11, College Station, TX and Microsoft Excel.

RESULTS: The overall mean LOS significantly decreased in the OBS unit (19.04 hours vs. 29.55 hours, $\mathrm{P}<0.001$ ). Overall mean total charges also significantly decreased $(\$ 7,230$ vs. $\$ 8,709, \mathrm{P}<0.001)$. The percentage of patients staying beyond 24 hours was reduced from $42.9 \%$ baseline to $21.9 \%$ post implementation of the OBS unit $(\mathrm{P}<0.001)$. In chest pain patients, significant decreases were seen in LOS (17.92 hours vs. 26.71 hours, $\mathrm{P}<0.001)$ and total charges $(\$ 7,157$ vs. $\$ 8,920, \mathrm{P}<0.001)$. Significantly fewer patients were hospitalized for more than 24 hours $(19.5 \%$ vs. $35.7 \%, \mathrm{P}<0.001)$. In syncope patients, LOS for patients admitted to the OBS unit significantly decreased (23.00 hours vs. 43.44 hours, $\mathrm{P}<0.05$ ). There was no significant difference in total charges but a significantly lower proportion were hospitalized for more than 24 hours ( $28.7 \%$ vs. $66.6 \%, \mathrm{P}=0.003)$. In abdominal pain patients, no statistically significant differences were seen any in of the metrics. Readmission rates were negligible both pre and post implementation.

CONCLUSIONS: There was an improvement in overall LOS, total charges and percentage of patients staying longer than 24 hours. In subgroup analyses, patients admitted with chest pain or syncope had a significant reduction in all metrics. The lack of effect in abdominal pain patients is likely due to small sample size and variability in clinical presentation and evaluation needs. Our comparison shows that implementation of an observation unit improves key metrics in hospital efficiency without affecting readmission rates. Additional studies are needed to assess other metrics such as patient satisfaction, ER throughput of patients admitted to the observation unit, effects of incorporating case managers into the triaging process to determine admission classification, and the use of standardized clinical protocols.

IMPLEMENTATION OF A PHARMACIST-MANAGED TELEPHONIC HOSPITAL DISCHARGE FOLLOW-UP PROGRAM Sarah L. Anderson ${ }^{1,3}$; Joel C. Marrs ${ }^{1,3}$; Joseph P. Vande Griend $^{1}$; Rebecca Hanratty ${ }^{2,4}$. ${ }^{1}$ University of Colorado Skaggs School of Pharmacy and Pharmaceutical Sciences, Aurora, CO; ${ }^{2}$ Denver Health Medical Center, Denver, CO; ${ }^{3}$ Denver Health Medical Center, Denver, CO; ${ }^{4}$ University of Colorado School of Medicine, Denver, CO. (Control ID \#1340091)

BACKGROUND: The early post-hospital discharge time period can be wrought with patient confusion and potential for medication errors, potentially resulting in patient harm and/or unnecessary 30-day readmissions. Low income and minority patients may be at higher risk for hospital readmission. Because of their intricate knowledge of medications, pharmacists are well-positioned to assist patients during this transitional period with medication-related questions and problems and prioritization of post-hospital discharge follow-up appointments. In this study we conducted a retrospective observational cohort analysis to determine the rates of attendance at post-hospital discharge follow-up appointments and 30- day readmissions in patients who were successfully contacted and intervened upon by a Clinical Pharmacy Specialist via telephone within 48 to 96 hours post-discharge compared to rates in those patients unable to be contacted.

METHODS: Adult patients who were discharged from a safety-net hospital between July 1, 2010 and June 30, 2011 and were included in the hospital discharge follow-up quality improvement initiative at a community health center for underserved populations were included in this retrospective observational cohort study. Patients were categorized into one of two cohorts: 1) those who were successfully contacted and 2) those who received a voice message or were unable to be contacted.

RESULTS: Clinical Pharmacy Specialists attempted to contact 470 patients within 48 to 96 hours of hospital discharge. Of those, 207 were successfully contacted and intervened upon (Group 1), 112 were left voice messages and 151 were unable to be contacted (Group 2). Of those patients with scheduled follow-up appointments at time of discharge, those in Group 1 were more likely to attend their follow-up appointments (104 attended, 40 unattended) than those in Group 2 (92 attended, 63 unattended; $p=0.03$ ). Similarly, patients who did not have scheduled follow-up were more likely to schedule and attend a visit with their Primary Care Physician within 30 days of discharge if they were in Group 1 (36 attended, 27 unattended) compared to those in Group 2 (28 attended, 84 unattended; $\mathrm{p}<0.0001$ ). Patients who were contacted had lower rates of 30 -day readmission (25 readmissions [12\%]) compared to those who were not $(53$ readmissions $[20 \%] ; \mathrm{p}=0.03)$. The reduction in readmissions represents a potential savings of over $\$ 300,000$.

CONCLUSIONS: Clinical pharmacists in the ambulatory care setting are well-positioned to improve patient transitions of care from the inpatient to outpatient setting based on their extensive medication knowledge and ties to the patients' clinic care in a county health system. Telephonic intervention by a Clinical Pharmacy Specialist in the early post-discharge time period increased patient attendance at post-hospital discharge follow-up appointments and resulted in a lower 30-day hospital readmission rate compared to patients who were left voice messages or were unable to be contacted. Because such advantages have both positive clinical and financial impact, institutions should consider allocating resources for pharmacist-managed post-hospital discharge follow-up services.

IMPLEMENTATION OF AN ELECTRONIC HEALTH-RECORDBASED TOBACCO CARE MANAGEMENT SYSTEM FOR IMPROVING TREATMENT IN PRIMARY CARE Gina R. Kruse ${ }^{1,2}$; Jennifer H. Kelley ${ }^{4,1}$; Jeffrey A. Linder ${ }^{3,2}$; Elyse R. Park ${ }^{6,5}$; Nancy A. Rigotti ${ }^{4,2}$. ${ }^{1}$ Massachusetts General Hospital, Boston, MA; ${ }^{2}$ Harvard Medical School, Boston, MA; ${ }^{3}$ Brigham and Womens Hospital, Boston, MA; ${ }^{4}$ Massachusetts General Hospital, Boston, MA; ${ }^{5}$ Massachusetts General Hospital, Boston, MA; ${ }^{6}$ Massachusetts General Hospital, Boston, MA. (Control ID \#1312192)

BACKGROUND: Effective treatments for tobacco cessation are underused in primary care. Using principles of chronic disease management, we designed and implemented a novel Tobacco Care Management system to improve the delivery of tobacco treatment at primary care visits. It used the electronic health record (EHR) and team-based care coordination to link smokers to treatment without increasing the burden on primary care providers (PCPs). We assessed the system's feasibility and acceptability to patients and to PCPs.

METHODS: At 2 community health centers affiliated with a Boston, MA, health care system, we added a 1-click referral functionality to the EHR. It gave PCPs an easy way to refer smokers to a centrally-located tobacco treatment coordinator (TTC). The TTC called smokers referred from both health centers to counsel and connect them to local specialty tobacco services and the state quitline. The TTC reported back to PCPs after each referral and was available for their inquiries. We evaluated the implementation of the system over 18 months with a mixed-methods design. Using TTC and EHR records, we measured PCPs' utilization of the referral function and the proportion of smokers connected to treatment. At 2 semistructured focus groups $(n=24)$, we elicited PCP's reasons for using the 
system, barriers to use, expectations of the referral, and experience with realtime feedback. Two coders independently conducted content analyses of focus group transcripts.

RESULTS: From 2/1/10-7/31/11, 33 (92\%) of 36 PCPs used the functionality, generating 466 referrals for 422 unique patients $(15 \%$ of 2,894 total smokers seen in the health centers during the study period, 42 patients were referred $\geq 2$ times). The health centers differed in the race/ethnicity and insurance status of smokers, but within each center there was no difference between smokers who were and were not referred. The TTC reached 246 smokers $(58 \%$ of 422 referred smokers) by telephone and connected 133 (32\% of those referred) to further treatment. At 1 center, the clinic leader spontaneously sent PCPs monthly feedback about their utilization compared to their peers. This center generated $79 \%$ of the referrals, and in focus groups PCPs identified this feedback as a motivator for using the system. Other themes that emerged were appreciation of (1) the simplicity of the 1click function, (2) the easy access to up-to-date tobacco resources, and (3) a time-efficient means of addressing smoking in addition to their own efforts. PCPs were discouraged when the TTC was unable to reach the smokers they had referred and wanted information about the quit rates of referred smokers. They collectively supported continuation of the system.

CONCLUSIONS: A novel EHR-based Tobacco Care Management system was adopted by PCPs, especially those who received regular performance feedback, and successfully connected one-third of referred smokers to tobacco treatment resources. It is a promising, time-efficient model for improving tobacco treatment in primary care. The integrated design with a system-wide care coordinator has the potential for adoption by patient-centered medical homes and is scalable to multiple clinics within a healthcare system.

\section{IMPROVEMENTS IN DIABETES QUALITY OVER TIME ARE ASSOCIATED WITH DIFFERENCES IN PAYER MIX, TEACHING STATUS, AND THE PRESENCE OF DIABETES PERSONNEL IN A LARGE URBAN AMBULATORY NETWORK Calie Santana; James Grigg; Yuming Ning. Montefiore Medical Center, Bronx, NY. (Control ID} \#1337375)

BACKGROUND: Although policies like Accountable Care Organizations encourage integrated networks, the performance of these networks in diabetes care for vulnerable populations has not been studied. Previously, we studied an established, 20-site integrated network in NY. We found significant cross-sectional differences among sites, associated with site payer mix and presence of residents (teaching), but not presence of diabetes personnel like nutritionists and care managers. We now sought to measure whether these site characteristics are associated with improvements in diabetes quality over time. We hypothesized that commercial insurance- predominant and teaching sites would improve over time, while those with diabetes personnel would not.

METHODS: Our study population included adults with diabetes (by ICD9, HbA1c $\geq 6.5$, or problem list) seen in both 2008 and 2010 ( $\geq$ twice/year). Diabetes quality outcomes were: (1) $\mathrm{HbAlc} \leq 8 \%$, (2) LDL $\leq 100$, (3) microalbumin checked once, (4) blood pressure (BP) $\leq$ $130 / 80$, and (5) BP $\leq 140 / 90$ based on the last value for 2008 and 2010 . Payer mix was the payer for $60 \%$ of patients (commercial or government-sponsored). Presence of residents, and diabetes personnel was gathered from a survey of site medical directors. Payer mix and teaching status were combined (commercial/nonteaching, $n=10$; government/teaching, $\mathrm{n}=5$; and government/nonteaching, $\mathrm{n}=5$ ) due to the relationship between payer and being seen at a teaching site. Four sites had nutritionists and 11 had care managers at least half-time. We measured the association between each independent variable and our main dependent variable (site percentage of each outcome met in 2010 minus the 2008 percentage) using a 2-level mixed effects logistic regression with sites as random effects. This technique accounts for clustering of patients within sites when using patient-level outcomes and site-level independent variables. Models were adjusted for patient age, sex, race/ethnicity and insurance.
RESULTS: Our analysis included 13,001 patients. A significant association was found between improvement in 2 of 5 outcomes and payer mix/teaching status. Commercial/nonteaching and government/teaching sites were significantly more likely to improve over time than government/non-teaching sites in microalbumin checks (OR 1.89 and $1.41, \mathrm{p}<0.001)$ and $\mathrm{BP} \leq 130 / 80$ (OR 1.75 and $1.40, \mathrm{p}<0.005)$. There was no improvement in the other measures of diabetes quality. The association of diabetes quality and the presence of nutritionists or of care managers followed an identical pattern, with significant improvements in microalbumin checks and BP control.

CONCLUSIONS: Contrary to our previous findings, we found that both site characteristics like payer mix and teaching status, and diabetes personnel are associated with significant improvements in diabetes quality over time. Since personnel are a modifiable site characteristic, adding them might help alleviate differences in diabetes quality over time in our ambulatory network. We still observe that sites where government insurance predominates and no residents perform worse than other sites, even in this centrally managed network. Additional patient-level factors (e.g.adherence) and site resources (improvement activities) likely still play a role in diabetes quality. Although the promise of system integration is driving policy changes, we must continue to carefully identify and promote workforce and other changes beyond integration that can promote quality in vulnerable populations.

IMPROVING COMPLIANCE WITH CDC RECOMMENDATIONS REGARDING PRENATAL VITAMIN ADVICE IN AN INTERNAL MEDICINE RESIDENT-RUN CONTINUITY CLINIC Taral Jobanputra; Christina Gulotta; Sunil Asnani; Michael P. Carson. Jersey Shore University Medical Center, Neptune, NJ. (Control ID \#1331613)

BACKGROUND: Prenatal vitamins (PNV) with $400 \mathrm{mcg}$ folic acid can decrease the rate of neural tube defects when taken before pregnancy. $50 \%$ of the pregnancies in the U.S are unplanned, so women of childbearing age should be advised to use PNV. We determined the historical rate of PNV usage or recommendations in our medical clinic, then developed a tool to increase those rates. The primary outcomes were the rates at which the tool was used and documentation of advice regarding use of PNV or Family Planning.

METHODS: Retrospective chart review of women aged 18-45 seen in the continuity clinic in 2008. Hysterectomy (TAH), tubal ligation (TL), IUD, and oral contraceptive use (OCP) status were noted. A checkbox prompt was added to the progress note: "Is Prenatal Vitamin Indicated? Yes/No". The form was introduced and the staff oriented in December 2010. Women with TAH or TL were considered to be "surgically sterilized". Chart review was repeated in May 2011.

RESULTS: PRE-INTERVENTION: 77 charts were reviewed, 9\% were on PNV. $10 \%$ of the 59 women who did not have a prior TAH or TL took a PNV. Discussions regarding pregnancy (FIGURE) were documented in $17 \%$ of the total cohort, and $22 \%$ of the women who did not have TAH/TL. No notes had documentation regarding advice to use PNV. POSTINTERVENTION: 42 of $92(46 \%)$ notes had the PNV prompt checked. FIGURE: The rate of documentation regarding PNV use (Striped Columns) rose from "zero" to $12 \%$ among the total cohort (chi2 $\mathrm{p}=0.0017$ ), and by $16 \%$ for those surgically sterilized. The Black Columns show Family Planning discussions. Documentation regarding PNV use OR Family Planning (White Columns) was 25\% for the entire cohort, and 29\% among those not surgically sterilized. Advice regarding PNV/Family planning was documented for $23 \%$ of those with the box checked, and $26 \%$ of those without it. PNV use was $9 \%$. The second review was not timed to detect an increase in PNV usage.

CONCLUSIONS: The simple prompt on the progress notes was associated with a significant increase in the rate of documentation regarding PNV advice, regardless of "box check" status, and a more modest $7-8 \%$ increase regarding use of PNV OR Family Planning. The first clinical goal was to change behavior by increasing awareness among all women of childbearing age, not just those on birth control. Provider awareness increased, but only $46 \%$ of the PNV prompts were checked. This information will be fed back to the medical staff, and consideration given to a systems based approach such as adding additional prompts to the "Plan" section of the progress notes, in order 
to increase compliance with this evidence based intervention with proven benefit.

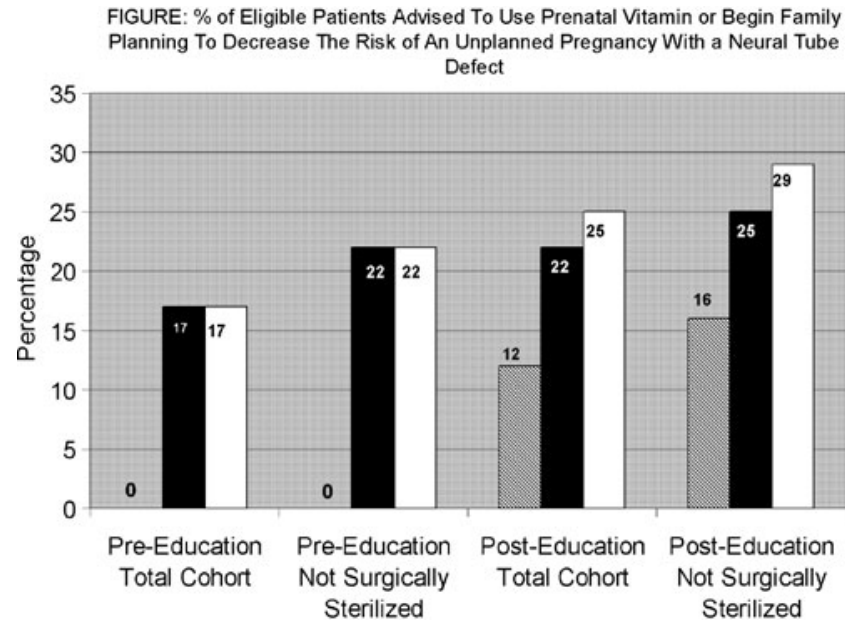

IMPROVING PARTNER NOTIFICATION: A QUALITATIVE STUDY OF MEDICAL CASE MANAGERS, DISEASE INTERVENTION SPECIALISTS AND MEN WHO HAVE SEX WITH MEN E. J. Edelman $^{1,2}$; Christopher A. Cole ${ }^{3}$; Wanda Richardson ${ }^{4}$; Nicholas Boshnack ${ }^{3}$; Marjorie S. Rosentha $1{ }^{1,5}$. ${ }^{1}$ Yale University, New Haven, CT; ${ }^{2}$ Yale University, New Haven, CT; ${ }^{3}$ AIDS Project New Haven, New Haven, CT; ${ }^{4}$ CT Department of Public Health, Hartford, CT; ${ }^{5}$ Yale University, New Haven, CT. (Control ID \#1339674)

BACKGROUND: Approximately $21 \%$ of HIV infected individuals are unaware of their status. Partner notification, or contact tracing, is the strategy whereby partners are notified of a potential exposure to a particular disease, including HIV. Notably, the rate of newly diagnosed infections among tested partners ranges from $10-30 \%$. Partner notification, however, is limited by suboptimal referral rates and difficulty eliciting partner names and contact information, particularly from men who have sex with men (MSM). To identify opportunities to improve partner notification for MSM, we explored the perspectives and experiences of medical case managers, state public health authorities responsible for partner notification known as Disease Intervention Specialists (DIS), and MSM.

METHODS: In partnership with the Connecticut Department of Public Health and AIDS Project New Haven, we used a community-based participatory research approach to conduct a qualitative study of the perspectives and experiences of partner notification. The in-depth interviews and focus group were audio-recorded and professionally transcribed. Our multi-disciplinary, academic/community-based research team analyzed the transcripts using grounded theory and the constant comparative method. We determined sample size at the point we reached thematic saturation.

RESULTS: We conducted 21 in-depth interviews (seven DIS and 17 MSM) and one focus group (14 medical case managers). We identified four domains which hinder the implementation of partner notification for MSM, including: 1.) client-based, such as concerns about confidentiality; shame about both HIV and sexuality; and fears of partner rejection; 2.) relationship-based, such as need to have a trusting relationship before discussing notification; sense of need to manage crisis intervention vs. prevention; 3 .) structurally-based, such as availability of clinic appointments for follow-up testing; and 4.) knowledgebased, such as lack of knowledge about the referral process among health care providers and lack of knowledge online social networking among state public health authorities.

CONCLUSIONS: Through a community-based participatory research process, we found multiple opportunities for developing interventions that may improve partner notification for HIV among MSM. Through our multi-disciplinary team, we plan to translate our findings into systematic changes. As the National HIV/AIDS Strategy and routine HIV testing becomes implemented, health care providers, including Generalists, will have the opportunity to lead and participate in these changes.
IMPROVING A MEDICAL STUDENT EBM CURRICULUM WITH A WEB-BASED EDUCATIONAL PRESCRIPTION David Feldstein $^{1}$; Craig A. Umscheid ${ }^{3}$; Matthew Maenner ${ }^{2}$; Mark A. Albanese ${ }^{2}$.

${ }^{1}$ University of Wisconsin School of Medicine and Public Health, Madison, WI; ${ }^{2}$ University of Wisconsin - Madison, Madison, WI; ${ }^{3}$ University of Pennsylvania Medical School, Philadelphia, PA. (Control ID \#1320177)

BACKGROUND: The AAMC requires that medical students apply the principles of Evidence-based Medicine (EBM) to patient care as part of the Learning Objectives for Medical Student Education. Integrating EBM teaching into clinical care improves learners' skills. The objective of this study is to evaluate the feasibility and impact of integrating a web-based, EBM educational prescription (EP) into clinical clerkships.

METHODS: The study was performed over one year at the University of Pennsylvania School of Medicine. Third year medical students completed EPs on questions arising from clinical care on their Internal Medicine, Family Medicine and Pediatrics clerkships. The web-based EP was developed to guide learners through the steps of EBM to answer patient care questions including describing a clinical question, documenting a search strategy, analyzing the quality of evidence found, reporting the results, and describing how to apply the evidence to their individual patients. EP results were evaluated using a web-based form by physician fellows in a Masters of Clinical Epidemiology program. EPs were graded on: 1) question formation; 2) searching; 3) evaluation of evidence; 4) application to patient; and 5) overall competence. Each area was scored on a scale of 1 (not yet competent) to 9 (superior) using an integrated grading rubric. Time to complete and grade EPs, and whether the EP changed patient care were recorded with each EP. Students and fellows completed end of study questionnaires about their attitudes toward the EP and barriers to its use. Student change in EP scores over time was evaluated using a mixed-model regression controlling for within-person effects.

RESULTS: 166 students completed 541 EPs (mean 3.3 EPs). The majority of EPs $(73 \%)$ involved a question about therapy. Twenty three fellows graded 523 of the EPs (mean 22.7 EPs). Students' average overall competency score was 7.36 (SD 1.57). The change in overall score over one year was 1.26 points $(95 \% \mathrm{CI}: 0.79-1.73)$. Students took a median of 90 minutes (IQR 60-120) to complete EPs and fellows took a median of 20 minutes (IQR 15-30) to grade EPs. The EPs changed patients' treatment plans or would have changed the treatment plan if they had the information sooner $19 \%$ of the time. Seventy three students $(44 \%)$ and $86 \%$ of fellows completed end of study surveys. Forty one (56\%) students reported they received adequate instruction on using the EP and $30(41 \%)$ described the $\mathrm{EP}$ as a valuable experience. Students' most frequently cited barriers to using the EP included time (34\%), personal attitude toward EBM (27\%), and comfort evaluating the quality of evidence (21\%). Fellows' only commonly cited barrier to grading was time $(79 \%)$.

CONCLUSIONS: This represents the first study of the EP in medical students, which was previously evaluated in Internal Medicine residents. The EP was easily incorporated into medical student clerkships. Performing EPs effected patient care and student scores increased significantly over the year. However, students reported inadequate instruction on how to perform EPs and the majority did not feel that it was valuable. Time and student attitudes were major barriers. Increased student training and further assessment of barriers are necessary to improve the students' experience. We will expand the results of this study to include analysis of the validity of the EP as an evaluation tool in students, as well as the impact of the EP on EBM skills.

IMPROVING THE EFFICIENCY OF BLOOD PRESSURE TREATMENT WITH BENEFIT-BASED DECISION-MAKING Jeremy Sussman ${ }^{1,2}$; Sandeep Vijan ${ }^{2,3}$; Rodney A. Hayward ${ }^{1,3}$. ${ }^{1}$ University of Michigan, Ann Arbor, MI; ${ }^{2}$ Ann Arbor Veterans Affairs Hospital, Ann Arbor, MI; ${ }^{3}$ University of Michigan, Ann Arbor, MI. (Control ID \#1339911)

BACKGROUND: Current guidelines for blood pressure (BP) medication use, such as the Joint National Committee (JNC 7), focus on 
achieving BP targets with limited attention to the effectiveness of medications in reducing cardiovascular (CV) events. Since many factors beyond BP, such as cardiac risk, influence the chance that $\mathrm{BP}$ treatment will prevent a $\mathrm{CV}$ event, we hypothesized that $\mathrm{BP}$ treatment could be made more efficient. We developed a BP treatment strategy based on an individualized estimate of the expected benefit of advancing BP treatment (tailored treatment) and compared it with JNC 7 guidelines

METHODS: We developed a data-driven Monte Carlo simulation model to estimate the clinical implications of each strategy. Model inputs were drawn from nationally representative CVD risk factor data, randomized studies of blood pressure reduction, and CVD outcome data. We used a society perspective to assess the benefits of each strategy - the JNC 7 guidelines and the tailored treatment strategy that we developed on a representative population of U.S. adults aged 30 to 75 years with no history of $\mathrm{CV}$ disease (primary prevention). Our primary outcome measure was lifetime change in quality-adjusted life-years (QALYs) for 5 years of treatment by the JNC7 vs. the tailored treatment regimens. We also examined the implications of these different strategies on representative individuals.

RESULTS: Compared with the standard JNC 7 regimen, the tailored treatment approach is more efficient. Using JNC 7 guidelines $42 \%$ of adults aged 30-74 would receive BP medications (mean number of BP medications per patient treated $=2.27$ ) compared to $38 \%$ treated with a mean of 2.24 BP medications for tailored treatment. In spite of treating fewer people and using fewer BP medications, tailored treatment prevented 300,000 more major CV events than following JNC 7, an almost $20 \%$ gain in relative effectiveness. Treatment by JNC 7 guidelines would save 260 QALYs per 1000 persons treated, while tailored treatment would save 300 QALYs per 1000 persons treated. Tailored treatment had greater efficiency due to treating higher $\mathrm{CV}$ risk patients more intensively and lower $\mathrm{CV}$ risk patients less intensively, particularly people with known cardiac risk factors, such as smokers. The greater efficiency of tailored treatment was generally robust to broad variations in model assumptions in our sensitivity analyses.

CONCLUSIONS: Compared to the traditional treat-to-target approach to hypertension therapy, tailored treatment has the potential to prevent more CVD events while limiting polypharmacy, treatment side effects, and costs. Prevention of CVD can be made more efficient and effective by basing BP treatment decisions on a patient's estimated $\mathrm{CV}$ event reduction, rather than purely on a patient's BP level.

\section{IMPROVING THE STATE OF QUALITY MEASUREMENT:} LESSONS FROM "DOWN UNDER" Sangeeta Ahluwalia. Veterans Administration Greater Los Angeles, Los Angeles, CA. (Control ID \#1326627)

BACKGROUND: Quality problems in today's healthcare system are widely manifest, and a major thrust of recent reform has been to develop and implement better signals of quality to guide purchasers and users of care. However, how should quality indicators be optimally implemented to guide improvement? How can signals of quality be appropriately linked with resources for improvement to maximize their impact? We evaluated these questions across multiple levels of the healthcare system to better understand the individual and organizational factors that facilitate or hinder quality measure implementation and to inform policy, programmatic, and clinical efforts in the Australian and United States healthcare systems to improve the quality of care.

METHODS: We conducted semi-structured in-depth interviews with 35 clinicians, senior government officials, and agency managers across the scope and levels of the cancer care system in Australia and representing a wide range of organizations $(n=27)$ with responsibility for care delivery and policy-making in the cancer field within national and regional government, area health services, practice management organizations, hospital systems and individual practice units. Interviews were audiotaped, transcribed and analyzed according to qualitative content analysis methods.
RESULTS: We identified several factors associated with quality measure implementation. Identified barriers included: the complexity of managing multiple sources of information regarding care quality; difficulties in coordinating quality measurement efforts across practice units; difficulty coordinating patient-provider activities necessary for quality measurement such as follow-up, tracking, and monitoring, and the lack of clinician support for quality measurement activities. Identified facilitators included: having a robust informatics infrastructure, especially care registries and point-of-care decision support; utilizing regional provider-led quality improvement groups to track performance and train clinicians within individual practice units, and employing various policy levers, such as linking accreditation to quality improvement efforts, shifting reimbursement for quality improvement from the practice level to the individual clinician, and providing financial incentives for having relevant policies and processes in place.

CONCLUSIONS: Several immediate opportunities for improving quality measurement efforts exist. Working with clinicians from the outset to develop and implement quality measures can assure buy-in and a clinically meaningful measurement process. A robust informatics system is key to supporting provider efforts to improve quality, by providing clinical decision support, facilitating tracking and monitoring activities, managing multiple sources of information, and coordinating patient-provider interactions. Finally, financial incentives targeted towards specific efforts supportive of quality measurement, such as having a care registry in place, or towards specific clinicians, rather than broadly disseminated, can foster more sustainable and enduring quality improvement efforts.

INADEQUATE UNDERSTANDING OF CODE STATUS IMPROVED BY CASE-BASED LEARNING Aroonsiri Sangarlangkarn"; Margaret Drickamer ${ }^{2} .{ }^{1}$ Yale School of Medicine, New Haven, CT; ${ }^{2}$ Yale School of Medicine, New Haven, CT. (Control ID \#1310190)

BACKGROUND: Multiple studies demonstrate continued deficiencies in physician's education on end-of-life care. However, there is limited literature on whether healthcare providers have an adequate understanding of DNR/ DNI, which is essential in end-of-life care and discussion. Our study evaluates the understanding of DNR/DNI among physicians in training and the efficacy of case-based learning in code status education.

METHODS: From September-October 2011, we surveyed medical students and residents at noon conferences during internal medicine rotations at Yale School of Medicine, before and after a course on challenging end-of-life cases that provide clinical applications of DNR/ DNI. Constructed using the same standards as the nationally utilized Yale Office-Based Medicine Curriculum, the course focused on DNI/not DNR patients, reversibility of code status, and futility of care. The exact Mcnemar test was used to evaluate changes in responses.

RESULTS: Results from 44 surveys are shown in attached image. Figure 1 demonstrated that a significant number of participants would provide contraindicated interventions to coding DNR patients. Figure 2 demonstrated that while none of the participants would intubate DNI patients, many failed to offer manual lung inflation to DNI patients in respiratory distress. After the course, participants were 9 times more likely to correctly forego amiodarone in coding DNR patients $(p=$ 0.02 ), and 11 times more likely to correctly offer ambu-bag in DNI patients $(p=0.01)$. When asked if participants possess adequate understanding of DNR/DNI on a scale of 1 (disagree) to 5 (agree), there was a 0.93 point increase after the course (before $=3.26$, after $=$ $4.19, \mathrm{p}=0.00$ ). While $7 \%$ of the participants feel neutral, $93 \%$ would recommend the course to others.

CONCLUSIONS: Surveys showed that many participants would provide contraindicated interventions to coding DNR patients while failing to offer appropriate interventions to DNI patients. After the course, more participants reported having an adequate understanding of DNR/DNI. A significant number correctly withheld antiarrhythmics in coding DNR patients and appropriately offered manual lung inflation to DNI patients. Our study showed that physicians in training may benefit from code status education, particularly case-based 
learning which may improve their understanding of DNR/DNI and the quality of end-of-life discussion.
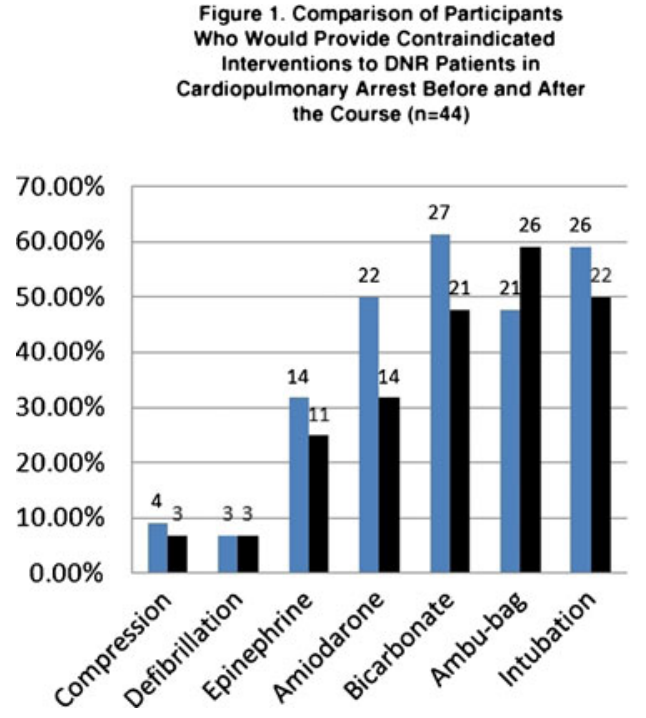

INCARCERATION HISTORY AND CHRONIC DISEASE MANAGEMENT AMONG NEW YORK CITY ADULTS Joseph Frank $^{1}$; Emily Wang ${ }^{2}$; Heather J. Baer ${ }^{1}$; LeRoi Hicks ${ }^{3} .{ }^{1}$ Brigham and Women's Hospital, Boston, MA; ${ }^{2}$ Yale School of Medicine, New Haven, CT; ${ }^{3}$ UMass Memorial Medical Center, Worcester, MA. (Control ID \#1334523)

BACKGROUND: Individuals with a history of incarceration have disproportionately higher rates of chronic disease and lower rates of community health care. A better understanding of chronic disease management among ex-prisoners is important given the imminent expansion of health insurance coverage in this population.

METHODS: We used data from the New York City Health and Nutrition Examination Survey (NYC HANES), a population-based, cross-sectional survey of adults $\geq 20$ years old. Among respondents with at least one of three common chronic disease diagnoses (diabetes (DM), hypertension (HTN) and hypercholesterolemia), we examined the association between self-reported prior incarceration and three disease management outcomes: 1) disease control (defined as HgbAlc $<7 \%$, blood pressure $<140 / 90$ and $\mathrm{LDL}<160 \mathrm{mg} / \mathrm{dL}$ ), 2) healthcare utilization (self-reported number of healthcare visits in the prior year) and 3) access to primary care (selfreport of either a regular site of primary care vs. no routine place of care or the Emergency Department as the site of routine care). We used a multivariable binomial regression model to determine the odds of uncontrolled disease for each participant given their total number of diagnoses. We used multivariable logistic regression models to determine the odds of the utilization and access outcomes. All models adjusted for age, gender, race/ethnicity, insurance status, obesity, disability, tobacco, alcohol and drug use. Results were considered significant at $\mathrm{p}<0.05$.

RESULTS: Among 1701 survey respondents, 755 (44.4\%) had at least one of three chronic disease diagnoses. $65(8.6 \%)$ reported a history of incarceration. Individuals with a history of incarceration were significantly more likely to be male, Black, disabled, smokers and to report heavy alcohol and recent drug use. The proportion of respondents who were unaware of a chronic disease diagnosis (i.e. were diagnosed by survey testing) or had an untreated diagnosis did not differ significantly between the groups. $20.9 \%$ of all individuals with a chronic disease reported no routine place of care, $13.4 \%$ reported no healthcare visits in the prior year and $41.3 \%$ had at least one uncontrolled chronic disease. After adjustment, participants with a history of incarceration were significantly more likely to report having no routine place of care (OR 2.2, 95\% CI 1.0-4.7) when
Figure 2. Comparison of Participants Who Would provide the Following Interventions to DNA patients in Respiratory Distress Before and After the Course $(n=44)$

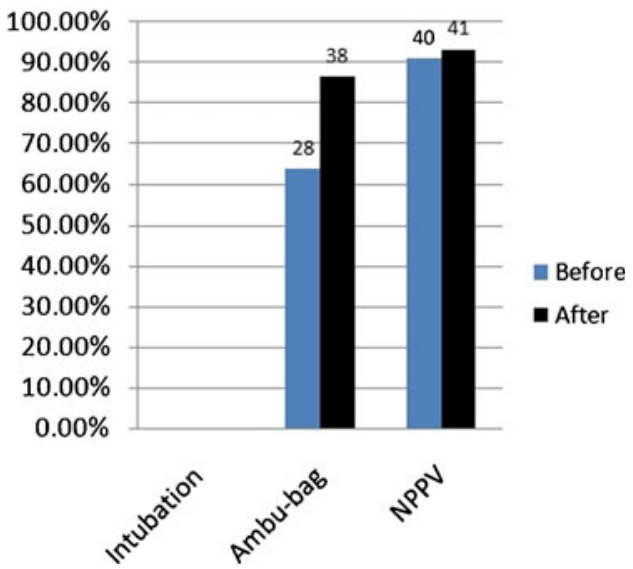

compared to participants without prior incarceration. Incarceration history was not significantly associated with rates of uncontrolled chronic disease diagnoses (OR 1.3, 95\% CI 0.7-2.3) or with rates of no healthcare utilization in the prior year (OR 1.21, 95\% CI 0.4-3.4).

CONCLUSIONS: Our analysis of a population-based survey found that history of incarceration was significantly associated with poor access to primary care but not chronic disease control or rates of recent healthcare utilization. These findings suggest that individuals with a history of incarceration are more likely to receive their medical care in non-primary care settings such as the Emergency Department or correctional facilities. Efforts to capitalize on expanding insurance coverage among ex-prisoners must consider current care-seeking behaviors.

INCIDENCE OF VENOUS THROMBOEMBOLISM (VTE) AFTER BARIATRIC SURGERY: A POPULATION-BASED COHORT STUDY David A. Froehling; Paul Daniels; Karen F. Mauck; Scott Litin; Maria L. Collazo-Clavell; Tanya M. Petterson; Michael G. Sarr; John A. Heit. Mayo Clinic, Rochester, MN. (Control ID \#1314877)

BACKGROUND: Bariatric surgery is considered a high VTE-risk operation, but the incidence of VTE after bariatric surgery is uncertain. In order to better estimate the incidence of symptomatic VTE after bariatric surgery, we carried out a retrospective populationbased cohort study.

METHODS: Using the resources of the Rochester Epidemiology Project and a Mayo bariatric surgical database, we identified all Olmsted County, MN residents who underwent bariatric surgery and all residents with incident VTE over the 19-year period, 1987-2005; all discrepancies were resolved by direct review of the patient operative report. Using the dates of bariatric surgery and VTE events, we estimated the cumulative incidence of VTE after bariatric surgery using the Kaplan-Meier estimator with censoring at date of death or last follow-up, or 12/31/2005, whichever came first. The log-rank test and Cox proportional hazards model were used to test patient age at bariatric surgery and sex as potential predictors of VTE after bariatric surgery.

RESULTS: 404 residents (mean age of 43.8 years at first bariatric operation, $\mathrm{SD}=10.1$ years, $80.5 \%$ women) underwent 410 bariatric operations. Unfractionated heparin (5000 units SubQ BID or TID) and external pneumatic compression for the duration of the postoperative 
hospitalization were used routinely for thromboprophylaxis. Nine patients developed VTE within six months (seven within one month) after bariatric surgery. Three patients had only pulmonary emboli, two patients had both pulmonary emboli and deep vein thrombosis, and four patients had only deep vein thrombosis within six months after surgery. The cumulative incidence of VTE at 7, 30, 90, and 180 days after bariatric surgery was $0.3 \%, 1.8 \%, 2.1 \%$, and $2.3 \%$, respectively (180-day $95 \%$ CI: $0.8 \%, 3.8 \%$ ). Seven VTE events occurred after hospital discharge. Patient age (but not sex) was a predictor of postoperative VTE (hazard ratio $=2.10$ per 10 -year increase in age; $95 \%$ CI: $1.16,3.83 ; \mathrm{p}=0.015)$.

CONCLUSIONS: Compared to a mean $2.5 \%$ and $1.8 \%$ three-month cumulative incidence of VTE after total hip or knee replacement surgery, bariatric surgery is a high VTE-risk operation, especially among older patients. Because most VTE events occurred after hospital discharge, a trial of extended out-of-hospital thromboprophylaxis is warranted.

INCIDENCE OF POST-SURGICAL INFECTIONS AFTER LOWRISK, SHORT-STAY SURGERIES PERFORMED IN AMBULATORY AND INPATIENT SETTINGS Claudia Steiner ${ }^{1}$; Pamela L. Owens ${ }^{3}$; Marguerite L. Barrett ${ }^{2}$. ${ }^{1}$ Agency for Healthcare Research and Quality, Rockville, MD; ${ }^{2}$ M.L. Barrett, Inc, Del Mar, CA $;{ }^{3}$ Consultant to AHRQ, Rockville, MD. (Control ID \#1339423)

BACKGROUND: Healthcare-associated infections rank among the leading causes of death in the U.S. and are a growing public health concern. Limited information is available on post-surgical infections following short-stay surgeries. The objective of this study was to determine the incidence of post-surgical infections across a spectrum of low-risk procedures routinely performed in ambulatory surgery centers (ASCs) and short-stay (less than two days) hospitalizations.

METHODS: Surgical procedures across body systems, general and gender-specific (e.g., cholecystectomy, hernia repair, anterior cruciate ligament (ACL) repair, hysterectomy, and transurethral prostatectomy (TURP)) were selected. The study included patients from eight states (CA, FL, GA, HI, MO, NE, NY, TN) that participate in the Healthcare Cost and Utilization Project (HCUP). Surgeries were performed in hospital-owned ASCs or community hospitals between February and November 2009 in which the patient was discharged with no indication of infection. State Inpatient, Ambulatory Surgery, and Emergency Department Databases with verified, synthetic person identifiers were used to identify surgeries and track infections. Postsurgical 14- and 30-day rates per 1,000 surgeries were calculated for each surgery and setting. We differentiated the rates for directlyrelated surgical site infections (SSIs), such as wound infections, indirectly-related infections such as pneumonia, and post-surgical hospital care for any cause.

RESULTS: The rate of directly-related SSIs within 14 days per 1,000 ASC surgeries varied from 1.3 (laproscopic inguinal or femoral hernia repair) to 9.7 (laproscopic incisional or abdominal hernia repair), while indirectlyrelated SSIs within 14 days ranged from 1.1 (ACL repair) to 21.3 (TURP). The average directly-related rate increased from 4.9 to 7.8 when the time frame was extended to 30 days; and the indirectly-related rate increased from 6.4 to 9.6. The average rate of all-cause, post-surgical hospital care per 1,000 ASC surgeries was 58.2 (14 days) and 88.8 (30 days) and included visits primarily for pain. Surgeries performed as a short-stay inpatient often resulted in the same or higher infection rates as those in an ASC.

CONCLUSIONS: This study provides baseline estimates of postsurgical infections following low-risk, short stay surgeries performed in ambulatory and inpatient settings. Although there is a range of rates across type of surgery, infection, and setting, the rates are relatively low, and much lower that the any-cause post-surgical hospital care. Inpatient hospitals with strict infection control standards did not demonstrate lower infection rates than ASCs. Further investigation is needed to determine whether inpatient cases were more complicated, and therefore, at higher risk of infection.
Post-Surgical Infection Rates per 1,000 ASC Surgeries

\begin{tabular}{lllll}
\hline \hline Surgical Procedure & $\begin{array}{l}\text { Directly- } \\
\text { related SSI } \\
\text { rate in first } \\
14 \text { days }\end{array}$ & $\begin{array}{l}\text { Directly- } \\
\text { related SSI } \\
\text { rate } 30 \\
\text { days }\end{array}$ & $\begin{array}{l}\text { Indirectly- } \\
\text { related } \\
\text { infection rate } \\
\text { in first 14 days }\end{array}$ & $\begin{array}{l}\text { Indirectly- } \\
\text { related } \\
\text { infection rate } \\
\text { in 30 days }\end{array}$ \\
$\begin{array}{l}\text { Cholecystectomy - } \\
\text { Laparoscopic }\end{array}$ & 4.3 & 5.9 & 5.2 & 7.3 \\
$\begin{array}{c}\text { Hernia - Inguinal/ } \\
\text { Femoral - Open }\end{array}$ & 2.8 & 4.0 & 3.2 & 4.7 \\
$\begin{array}{c}\text { Hernia - Inguinal/ } \\
\text { Femoral - Laparoscopic }\end{array}$ & 1.3 & 3.2 & 3.0 & 4.2 \\
$\begin{array}{c}\text { Hernia - Incisional/ } \\
\text { Abdominal - Open }\end{array}$ & 7.8 & 16.7 & 4.9 & 7.7 \\
$\begin{array}{l}\text { Hernia - Incisional/ } \\
\text { Abdominal - }\end{array}$ & 9.7 & 12.1 & 12.1 & 16.0 \\
$\begin{array}{l}\text { Laparoscopic } \\
\text { ACL Repair }\end{array}$ & 3.7 & 6.8 & 1.1 & \\
$\begin{array}{l}\text { Spine Surgery } \\
\text { Hysterectomy }\end{array}$ & 4.0 & 8.1 & 3.7 & 6.1 \\
TURP & 7.3 & 9.4 & 9.0 & 13.7 \\
\hline
\end{tabular}

Source: Agency for Healthcare Research and Quality, Healthcare Cost and Utilization Project.

INCREASE IN PREVALENCE OF NON-MEDICAL USE OF PRESCRIPTION OPIOIDS IN THE U.S., 2000-2009 William Becker; David A. Fiellin. Yale University School of Medicine, New Haven, CT. (Control ID \#1339656)

BACKGROUND: Opioid prescribing increased markedly in the U.S. from 2000-2009, concurrent with leading healthcare organizations' emphasis on assessment and treatment of pain and aggressive product marketing by pharmaceutical companies. This increase included a shift towards Drug Enforcement Agency (DEA) schedule II opioids, which are of higher potency and may increase risk for adverse consequences including dependence and overdose. We sought to describe changes in the prevalence of non-medical use of prescription opioids and prescription opioid abuse and/or dependence over the 10 years from 2000-2009.

METHODS: We analyzed data from the 2000 through 2009 annual waves of the National Survey on Drug Use and Health (NSDUH). For each of the ten years, we calculated the following demographic and clinical features among those who reported non-medical use of prescription opioids: mean age, proportion female, proportion non-white and proportion with selfreported good, very good or excellent health compared to fair or poor health. Next, for each of the ten years, we calculated the following outcomes related to prescription opioids: prevalence of past-year nonmedical use and past-year abuse and/or dependence, mean age of first nonmedical use, and prevalence of lifetime non-medical use of opioid classes (e.g. DEA schedule II) and individual opioids. We then compared sequential year-to-year pairs of demographic and clinical features and outcomes related to prescription opioids, using chi-square tests to compare proportions and t-tests to compare means.

RESULTS: The full analytic sample consisted of 557,282 individuals with approximately 55,000 individuals per year. From 2000 until 2009, the prevalence of past-year non-medical use of prescription opioids increased from $2.8 \%$ to $4.8 \%(\mathrm{p}<0.0001)$. Among individuals with non-medical use of prescription opioids, mean age increased from 22.4 to 24.2 years $(\mathrm{p}<.0001)$. The proportion female decreased from $49.6 \%$ to $43.7 \%(\mathrm{p}=.02)$ while the proportion non-white and the proportion reporting good, very good or excellent health did not change significantly across the 10 years. The prevalence of past-year prescription opioid abuse and/or dependence increased from $0.3 \%$ to $0.7 \%(\mathrm{p}<.0001)$. Mean age of first use did not change. The prevalence of lifetime non-medical use of a DEA schedule II opioid increased from $42.3 \%$ to $48.3 \%(p<0.0001)$. The proportion of these respondents with any lifetime non-medical use of propoxyphene, codeine; hydrocodone; oxycodone; and controlled release oxycodone all increased. 
CONCLUSIONS: Non-medical use of prescription opioids, lifetime nonmedical use of DEA schedule II opioids and prescription opioid abuse and/ or dependence increased during 2000-2009, a period of increased prescribing of these medications.

INCREASING HIV TESTING IN PRIMARY CARE: OVERCOMING BARRIERS AND LESSONS LEARNED Elana R. Sydney; Penelope Demas; Jason Leider. Jacobi Medical Center, Bronx, NY, NY. (Control ID \#1320336)

BACKGROUND: Failure to diagnose HIV in a timely manner contributes to increased morbidity, mortality and high rates of transmission. CDC data support routine HIV testing in the primary care setting. According to the CDC, approximately 250,000 people are unaware that they are HIV infected. It is estimated that these unaware adults account disproportionately for $54 \%$ of new sexual transmissions. Clearly routine screening is crucial to the reduction of transmission. Despite these data, primary care physicians have reported multiple barriers to HIV testing: insufficient time, competing priorities, perceived burden of consenting requirements. Our goal was to test a model that would increase HIV screening in a busy medical clinic.

METHODS: The study site was an urban medical clinic with 300 patient visits per day. This pilot study had two phases conducted over four months during one weekly clinic session. In Phase I (4 weeks), all patients were offered testing by patient care assistants (PCA's) regardless of risk stratification. If the patient consented, they were brought to the HIV counselor prior to being seen by their physician and received pre and post test counseling. If patients did not consent, they were given a printed version of the 7 points of counseling mandated by NY State. The provider then met with the patient and assessed whether the patient was now willing to consent. In Phase II (11 weeks), all elements were the same except a pre-test counseling video replaced the written material. Patients watched a vignette where a patient interacted with their doctor and learned of the importance of HIV testing. The primary study end points were number of patients consenting to HIV testing in the pilot, and in the clinic as a whole. In addition, the role of PCA's, physicians, and educational materials (written vs. video) in obtaining consent was analyzed.

RESULTS: In phase I, a total of 32 patients were approached and 19 agreed to be tested $(59 \%)$. Men $=19 \%$ and Women $=81 \%$, Mean age $=56$, Hispanic $43 \%$, AA $34 \%$, Caucasian $12 \%$, other $11 \%$. The PCA's consented $68 \%$ of the patients. An additional 6 patients, $32 \%$ that initially refused the PCA, consented to the MD after reading the 7 points. In phase II, a total of 66 patients were approached and 32 agreed to be tested (48\%). Men $=28 \%$ and Women $=72 \%$, Mean age $=58$, Hispanic $62 \%$, AA $27 \%$, other $11 \%$. Of the 32 patients that consented, 17 (53\%) consented to the PCA while an additional $15(47 \%)$ that refused the PCA, consented to the MD after watching the video. In total, 51 patients agreed to HIV testing out of 98 patients approached. The PCA consented 28 (55\%), while the MD was able to consent an additional 23 (45\%) of the patients. Moreover, of the 70 patients who refused the PCA, the MD was able to change the mind of 22 patients $(30 \%)$. Baseline testing rates in the ambulatory clinic were approximately 80 per month prior to the pilot study. At the end of the 4 months, testing rate of the patients rose $200 \%$ ( 275 tests per month). The pilot study increased awareness of HIV screening and led to an overall increase in the referral rate throughout the clinic.

CONCLUSIONS: Widespread screening in a busy urban clinic can be accomplished. Barriers to HIV testing can be improved by patient education through the addition of HIV screening videos, written material, and by assisting physicans in the consenting and counseling process with ancillary staff. The patient physician relationship is powerful in persuading patients to consent to HIV testing.

INFLUENCE OF HEALTH LITERACY ON DIABETES MELLITUS OUTCOMES Daniel Goldsmith; Mushtaq Anis; Katia Dieguez Otero; Saba A. Hasan. Capital Health Regional Medical Center, Trenton, NJ. (Control ID \#1337421)
BACKGROUND: Health Literacy is a measure of patients' ability to read, comprehend, and act on medical information. Poor health literacy is common among diabetic patients and those with other chronic diseases, especially in racial and ethnic minorities and elderly persons. Little is known about the extent to which health literacy affects clinical health outcomes. This study intends to examine the association between health literacy and clinical outcomes among patients with diabetes mellitus.

METHODS: This is a single-center observational study of 48 English and Spanish speaking adult patients, with either type 1 or type 2 diabetes mellitus. We identified our study group from the Internal Medicine Residency Program's continuity clinic patients. In 2009, a program of focused diabetic visits was instituted. At that time, patients' baseline health literacy was assessed using the Rapid Estimate of Adult Literacy in Medicine (REALM) and Short Assessment of Health Literacy for Spanish Adults (SAHLSA-50), and scored as either adequate or inadequate. Measures of diabetes control and other health risk parameters were collected retrospectively from these patients' charts. The patients were then closely followed in focused diabetic visits, emphasizing patient education and counseling. Health literacy was then re-assessed along with diabetic parameters including glycohemoglobin, LDL, urine microalbumin, blood pressure and BMI, and compared to data from the baseline assessment. Results were evaluated using the Pearson Chi-square test.

RESULTS: The patients were $44 \%$ female and $56 \%$ male, and $45.8 \%$ Hispanic, 27\% African-American, $20.8 \%$ Caucasian, and 6.2\% Asian. In the first assessment, $64.5 \%$ of patients had inadequate health literacy, but in the second assessment, $35.5 \%$ had inadequate health literacy, indicating that $31.2 \%$ of patient improved their health literacy from inadequate to adequate. $35.5 \%$ of patients remained inadequate, and $33.3 \%$ were adequate in both assessments. In the group that improved health literacy, rates of achieving goal parameters improved with glycohemoglobin $(\mathrm{p}<$ $0.003), \operatorname{LDL}(\mathrm{p}<0.003)$, urine microalbumin $(\mathrm{p}<0.021)$, and blood pressure $(p<0.012)$. No improvements were observed in any parameter in the groups where health literacy remained unchanged. Also, BMI was unchanged in all groups.

CONCLUSIONS: Among our primary care patients with type 1 and 2 diabetes mellitus, improved control of diabetes and related parameters was associated with whether health literacy had improved over the study time. No improvement was seen in those whose health literacy remained static, even when that literacy was measured as adequate at both assessments. Our experience suggests that focused diabetic visits should be coupled with educational efforts to improve overall health literacy in order to maximize benefit.

INFLUENCE OF GENDER ROLE ATTITUDES IN SMOKING AND DRINKING AMONG GIRLS FROM JUJUY, ARGENTINA Raul Mejia. UCSF, San Francisco, CA. (Control ID \#1324365)

BACKGROUND: Gender role is influenced by culture, temporal factors, traditions, expectations and assumptions. Ideas about gender roles and femininity may have a role in explaining differences in tobacco and alcohol consumption in adolescents. Objective: To evaluate the effect of gender role attitudes in smoking and drinking among mostly Indigenous girls from northwest Argentina.

METHODS: Self-reported data were obtained from a 2006 survey of tenth grade female students attending a random sample of 27 urban and rural schools in Jujuy Province. Questions about tobacco smoking and alcohol consumption were adapted from global youth surveys. We also constructed a scale to assess adolescents' perception of traditional or egalitarian sex roles based on a comprehensive literature review. In formative work, the questionnaire was reviewed by a panel of experts in gender studies and administered to 10 adolescents during in-depth interviews to evaluate comprehension and face validity. The final scale consisted of 10 items with 5-point response options of agreement-disagreement scale where $1=$ strongly agree to $5=$ strongly disagree. Factor analysis of the item data suggested a 5 item factor with a Cronbach alpha of 0.72 . These items asked about women staying at home to care for children, men working to earn a living for the family, women accepting men's decision if they disagree, women working outside the home leading to more crime by youth and the women belonging at home and not in a job. Responses 
to the items that minimize sexual stereotypes reflected non-traditional/ egalitarian gender roles. In this study we hypothesized those girls who ascribe to more egalitarian gender roles have higher risk of ever or current smoking or drinking than those who ascribe to more traditional roles.

RESULTS: In 2006, 2,133 girls aged 13-18 responded, 71\% identified as Indigenous, $22 \%$ as mixed indigenous/European, and $7 \%$ as European. Nearly $60 \%$ were ever smokers (ever tried or experimented with cigarette smoking), $32 \%$ were current smokers (in past 30 days), $58 \%$ were ever drinkers, $27 \%$ have drunk in the previous month, and $13 \%$ were heavy drinkers (had 5 or more drinks in past month). A logistic regression model was generated for each outcome using the gender role scale as the main predictor and adjusting for known confounders from these data (having any friends who smoke, having a job, repeating a grade in school, living with both parents, living with someone who smokes at home, depressive symptoms in previous year, worked in tobacco growing and/or selling, and smoking media literacy). Gender role was positively associated with significantly increased odds of ever smoking and ever drinking, drinking in the last month and heavy drinking.

CONCLUSIONS: Girls in Jujuy who ascribe to more egalitarian gender roles have greater chances of smoking or drinking than those who ascribe to more traditional ones

\begin{tabular}{lllll}
\hline \hline Outcome & $\begin{array}{l}\text { Odds Ratio } \\
\text { (OR) }\end{array}$ & $\begin{array}{l}\text { OR Lower } \\
\text { CL }\end{array}$ & $\begin{array}{l}\text { OR Upper } \\
\text { CL }\end{array}$ & P \\
$\begin{array}{l}\text { Ever smoker (even a } \\
\text { puff) }\end{array}$ & 1.25 & 1.09 & 1.44 & 0.001 \\
$\begin{array}{l}\text { Current cigarette smoker } \\
\text { (past 30 days) }\end{array}$ & 1.17 & 0.99 & 1.39 & 0.056 \\
$\begin{array}{l}\text { Ever drink alcohol } \\
\begin{array}{l}\text { Drink an alcoholic } \\
\text { beverage in past }\end{array}\end{array}$ & 1.24 & 1.10 & 1.40 & $<0.001$ \\
$\begin{array}{c}\text { month? } \\
\begin{array}{c}\text { Heavy drinking ( } \geq 5 \\
\text { drinks in past month) }\end{array}\end{array}$ & 1.15 & 1.07 & 1.37 & 0.002 \\
\hline
\end{tabular}

\section{INNOVATIVE CARE DELIVERY MODEL TO ADDRESS HEALTH} DISPARITY IN UNDERSERVED POPULATION Nia S. Mitchell ${ }^{1,4}$; David L. Washington ${ }^{5}$ J James O. Hill ${ }^{2,3}$. ${ }^{1}$ University of Colorado, Aurora, $\mathrm{CO} ;{ }^{2}$ University of Colorado, Aurora, CO; ${ }^{3}$ University of Colorado, Aurora, CO; ${ }^{4}$ University of Colorado, Aurora, $\mathrm{CO} ;{ }^{5} \mathrm{Center}$ for African American Health, Denver, CO. (Control ID \#1340007)

BACKGROUND: African Americans are more likely to be overweight or obese compared to other racial/ethnic groups, and are subsequently more likely to be diagnosed with weight-related co-morbidities such as hypertension, diabetes, and cardiovascular disease. Based on the chronic care model, obesity may be effectively managed in a setting that combines self-management, patient education, and outreach utilizing the infrastructure of an existing community organization. The objective of this study is to describe the implementation and effectiveness of a community-based participatory research project that recruited African American subjects through a community organization to join a weight loss program and determine weight change after 12 weeks.

METHODS: The Senior Wellness Initiative and TOPS Collaboration for Health (SWITCH) study is a prospective analysis of the weight change of the individuals who were recruited through a community organization to join a weight loss program. Participants were recruited through the Senior Wellness Initiative (SWI), a community program designed to help older African Americans maintain their independence. SWI participants aged 50 and older with $\mathrm{BMI} \geq 25$ were eligible to participate. Take Off Pounds Sensibly (TOPS) is a national, peer-led weight loss program that has been shown to help participants lose $5 \%$ of their initial weight, which is clinically significant. Its program consists of weekly peer-led meetings where there is a private weigh-in followed by educational programming on nutrition, physical activity, and behavior modification. TOPS chapters were started at three SWI sites-a senior center, a senior residence for independent living, and a church. Informational sessions were held at each site, and participants were encouraged to invite others to attend. Weight change in $\mathrm{kg}$ was calculated as the average difference from the initial date of participation to week 12. For participants who were in the program for less than 12 weeks, their last weight measurement was carried forward. Weight change was also calculated as the percentage change from initial weight. Weight was subsequently categorized as weight loss or gain of 0 to $5 \%$ and $\geq 5 \%$.

RESULTS: Sixty-six people attended the informational sessions, and 50 people joined the three TOPS chapters. The average age of participants was 69.5 years $(\mathrm{SD}=8.2)$. Their average starting weight was $91.7 \mathrm{~kg}(\mathrm{SD}=17.5)$ and the average baseline BMI was $34.7 \mathrm{~kg} / \mathrm{m} 2 \quad(\mathrm{SD}=6.4)$. The average weight change for all participants was $1.8 \mathrm{~kg}(\mathrm{SD}=2.5)$, equal to $2.0 \%$ $(\mathrm{SD}=2.7)$ of initial weight. Almost $82 \%$ of participants lost weight during the 12 week period $-10 \%$ of all participants lost $5 \%$ or more and $71 \%$ lost between 0 and $5 \%$ of their initial weight.

CONCLUSIONS: This study suggests that integrating community organizations with effective self-management tools can help address health disparities. Using the infrastructure of a community organization, recruitment goals of a study in the African American community were met with only three informational sessions. Ten percent of the individuals who participated in the SWITCH program lost a clinically significant amount of weight, while a majority of participants either maintained their weight or lost a modest amount.

INSIGHTS FROM THE POWER PRACTICE-BASED WEIGHT LOSS TRIAL: A FOCUS GROUP STUDY ON THE PCP'S ROLE IN WEIGHT MANAGEMENT Wendy L. Bennett; Kimberly Gudzune; Lawrence J. Appel; Jeanne Clark. The Johns Hopkins University, Baltimore, MD. (Control ID \#1339591)

BACKGROUND: Despite the obesity epidemic, primary care providers (PCPs) infrequently engage in weight counseling due to lack of time and knowledge. The Practice-based Opportunities for Weight Reduction (POWER) Trial at Hopkins was a 3 arm, randomized trial that tested two behavioral weight loss interventions implemented as part of routine medical practice compared to usual care. POWER used external counselors to provide weight loss counseling, but still engaged the PCP unlike current commercial programs. This trial provided a unique opportunity to understand what PCPs perceived their role to be when a patient is enrolled in an external weight management program.

METHODS: During the summer of 2010, we conducted five focus groups of community-based PCPs who had at least 4 patients enrolled in POWER. Focus groups were moderated using a semi-structured guided, audio recorded and transcribed verbatim. Two investigators independently coded transcripts for thematic content using editing style analysis.

RESULTS: Of the 30 eligible PCPs, 26 participated in 1 of 5 focus groups. Mean years in practice were 16.4 (SD 11.7) and 77\% practiced general internal medicine. We identified 5 themes (with illustrative quotations) related to PCPs' role during the POWER weight management program. 1) Refer patients into the program and provide endorsement: "I really pushed the patients that I had...'cause I felt like it was something that I could do for them, whereas, normally, I don't have a lot because...nutrition's not covered...So this was something free and easy that I could encourage them to get involved in." 2) Have limited role in management: "She did great. And she did it with the help of the coach, and not from any input from me." 3) Provide accountability for patients: "You can drop out of Weight Watchers and nobody will know or... care...but he's going back to his primary care physician. [Y]ou are adding a layer of accountability with the patient, which I think could potentially be important." 4) "Cheerlead" for patient's successes during interval visits: "And just trying to encourage them, to say, 'If you're heading in the right direction that's better than going backwards." "5) Maintain trust and support through the long-term relationship with patients despite ups and downs: "I think one key may be just having a primary care doctor that you know for a while instead of switching...it helps to know someone for a while and to get into the head of your patient a little bit and get to have a comfort level with them."

CONCLUSIONS: PCPs with patients in the POWER trial described themes related to their role in patients' weight management, including program referral and endorsement, yet continuing long-term relationships 
with patients, and "cheerleading" for their successes. Practice based weight loss programs have great potential to partner with PCPs to build upon the existing the patient-provider relationship to improve patient accountability and sustain behavior change.

INTEGRATED ANALYSIS OF EFFICACY OF A ONCE-DAILY GASTRORETENTIVE FORMULATION OF GABAPENTIN IN PATIENTS WITH POSTHERPETIC NEURALGIA WHO ARE AT LEAST 75 YEARS OLD Daniel Kantor ${ }^{3}$; John T. Mathis ${ }^{1}$; Richard L. Rauck $^{2}$; Gordon Irving ${ }^{4}$; Michael Sweeney ${ }^{1}$; Geertrui F. Vanhove ${ }^{1}$. ${ }^{1}$ Depomed Inc, Menlo Park, CA; ${ }^{2}$ Carolinas Pain Institute, Winston-Salem, NC; ${ }^{3}$ Neurologique Foundation, Ponte Vedra, FL; ${ }^{4}$ Swedish Pain Center, Seattle, WA. (Control ID \#1332499)

BACKGROUND: Gabapentin is a first-line treatment for postherpetic neuralgia (PHN). However, due to gabapentin's short elimination halflife and its absorption by a saturable transporter with limited distribution in the proximal small bowel, gabapentin needs to be dosed TID. Gabapentin TID is associated with a high incidence of somnolence and dizziness that may prevent efficacious dosages from being reached. Recently, a once-daily gastroretentive formulation of gabapentin (G-QD) was approved for the treatment of PHN. Upon contact with gastric fluid, G-QD tablets swell to a size that promotes retention in the fed stomach. This prolonged retention (approximately 8 to 10 hours) allows gabapentin to be gradually released to the site of absorption, minimizing the chance of saturating transporter uptake, and resulting in greater absorption. This permits once-daily dosing and may result in less somnolence and dizziness, which is especially important for older individuals, who are at risk for falls.

METHODS: Integrated efficacy analyses were performed on two placebocontrolled, Phase 3 studies in patients with PHN. The analysis included 333 randomized to receive $1800 \mathrm{mg}$ G-QD gastroretentive gabapentin and 340 to receive placebo, both taken with the evening meal. The primary efficacy assessment was the change in "average pain for the past 24 hours" as assessed by the Numeric Pain Rating Scale (NPRS), with scores recorded every morning from Baseline to Week 10. Subgroup analyses were performed on patients 75 and older (the very elderly), for whom PHN is more common.

RESULTS: A total of 179 patients (94, G-QD; 85, placebo) were 75 and older. The mean absolute last observation carried forward (LOCF) change in NPRS score was significantly greater with G-QD than with placebo (2.2 vs. $-1.4 ; \mathrm{p}=0.032$ ). A greater proportion of G-QD patients achieved a $\geq 30 \%$ response compared with placebo ( $52 \%$ vs. $29 \%$; $p=0.002)$. At Week 10 , significantly more G-QD patients felt "Very Much or "Much" improved compared with placebo $(36 \%$ vs. $19 \%$; $p=0.001)$ and the mean absolute LOCF change in SIS was significantly greater with G-QD than with placebo $(-2.4$ vs. $-1.3 ; \mathrm{p}=0.0044)$.

CONCLUSIONS: G-QD can be an effective treatment option for PHN in the very elderly and had a similar efficacy profile in the subgroup $\geq 75$ compared to the overall efficacy population.

INTENSITY OF MESSAGING NECESSARY TO ENCOURAGE PATIENTS TO ACCESS THE PHR: PRELIMINARY RESULTS FROM THE SMART-PHR STUDY Rachel Hess ${ }^{1}$; Gary Fischer ${ }^{1}$; Melissa Weimer ${ }^{1}$; Sunday Clark ${ }^{2}$; Caroline Zieth $^{1}$; XinXin Dong ${ }^{1}$; Mark S. Roberts ${ }^{1} .{ }^{1}$ University of Pittsburgh, Pittsburgh, PA; ${ }^{2}$ Weill Cornell Medical College, New York, NY. (Control ID \#1339903)

BACKGROUND: Care for individuals with chronic disease, such as cardiovascular disease, in the United States is not optimal. Personal Health Records (PHR) provide an opportunity to more actively involve patients in their own care. We have developed and are testing an active PHR that pushes information regarding prevention gaps, (diabetes foot exam, dilated retinal exam, A1C testing, creatinine testing, lipid testing, and influenza vaccination) to patients with high cardiovascular risk. We describe our preliminary findings regarding the intensity of messaging necessary before the patient accesses the PHR.
METHODS: Beginning in July, 2010, patients with high cardiovascular risk were recruited from 64 primary care practices in Western Pennsylvania and enrolled in a randomized controlled trial comparing the active PHR in which patients are alerted to prevention gaps to the traditional passive PHR in which patients were not alerted to prevention gaps. Participants randomized to the active PHR receive an initial email alert regarding prevention gaps. If the PHR is not accessed, an additional email alert is sent followed by a mailed letter informing them of the prevention gaps. The cycle repeats every two months. We describe the numbers of messages sent, the percent of individuals who accessed the PHR after receiving an alert, and the closure of prevention gaps between message cycles.

RESULTS: Of the 1169 people enrolled, 559 are randomized to the active PHR and form the basis of the data described here. On average, subjects in the active arm are 60.9 year old, $48 \%$ are female, $11 \%$ are non-white. Among these 1169 participants, 1069 initial alerts regarding prevention gaps were delivered in any cycle. The PHR was accessed $582(54 \%)$ times after the initial alert and $725(68 \%)$ times after the initial alert and one reminder. The PHR-access rate was $71 \%$ in the first cycle and decreased slightly to $62 \%$ in the second cycle and $64 \%$ in the third cycle. Sixty-two percent of prevention gaps open at the beginning of a cycle were closed by the beginning of the subsequent cycle.

CONCLUSIONS: Among participants with high cardiovascular risk enrolled from a large number of primary care practices throughout Western Pennsylvania, a low intensity of messaging prompts a significant proportion of patients to access their PHR regarding prevention gaps. Further work will examine if the active PHR results in a reduction in prevention gaps compared to the traditional, passive PHR.

INTEREST IN QUITTING SMOKING AND SELF-PERCEPTION OF SMOKING-RELATED DISEASE AMONG DAILY AND NONDAILY CIGARETTE SMOKERS AT AN ACUTE CARE CLINIC: A UNIQUE OPPORTUNITY. Yinchong E. Mak; Pamela M. Ling; Ralph Gonzales. UCSF, San Francisco, CA. (Control ID \#1310447)

BACKGROUND: Acute care clinics have assumed a prominent place in the US health care delivery system by providing same-day walk-in medical attention to patients with limited access to primary care. Because acute care clinics serve a disproportionate number of patients who do not have a primary care provider, this setting also represents an opportunity to deliver important preventive health services such as smoking cessation counseling. We examined the prevalence of smoking, patterns of use, self-perception of smoking-related disease, and interest in quitting among patients at an acute care clinic in California.

METHODS: The study was conducted April - September 2011 in an academic-affiliated walk-in acute care clinic that provides care to 75 100 adults daily. As part of routine clinical care, patients completed a computerized check-in process that included questions about demographics, chief complaint, past medical history, social history, smoking history, interest in smoking cessation, and if they believed their reason for visit was caused or made worse by smoking. The 364 patients who self-reported smoking on at least 1 of the past 30 days were classified into three groups: daily smokers (smoked 30 out of the previous 30 days), regular non-daily smokers (smoked 20-29 days in the past month), and occasional non-daily smokers (smoked 1-19 days in the past month). Comparisons were conducted between daily and non-daily smokers using chi-square test for categorical variables, and t-test for continuous variables. Multivariable logistic regression analysis was used to identify factors independently associated with interest in quitting (defined as smokers reporting they "already quit in past month", "are trying to quit right now", or "will quit in the next month").

RESULTS: The prevalence of smoking within the last 30 days was $13.1 \%$ among patients completing the computerized check-in system. The majority of smokers were non-daily smokers $(65 \%)$ and did not identify 
a primary care doctor $(67 \%)$. Of the non-daily smokers, $68 \%$ were occasional smokers. Overall, $30 \%$ of daily, $53 \%$ of regular, and $73 \%$ of occasional smokers were interested in quitting $(\mathrm{P}<0.0001)$. Multivariable logistic regression analysis showed a stepwise relationship between smoking frequency and interest in quitting, with occasional non-daily smokers (OR 6.80, 95\% CI 4.03-11.48) and regular non-daily smokers (OR $2.80,95 \%$ CI $1.54-5.10$ ) being significantly more likely to be interested in quitting compared to daily smokers. Believing that the reason for visit may be caused or made worse by smoking was also independently associated with patients' interest in quitting (OR 4.24, 95\% CI 1.05-17.06). Age, gender, race/ethnicity, insurance status, having a primary care doctor, alcohol and recreational drug consumption, blood pressure, level of nicotine dependence, and the number of cigarettes smoked per smoking day were not independently associated with interest in quitting.

CONCLUSIONS: Acute care clinics present an important opportunity to screen for tobacco use and conduct brief interventions for smoking cessation - particularly for occasional non-daily smokers - as smokers in this setting have a high interest in quitting and these patients frequently do not have an established primary care provider. Helping patients to understand when their symptoms or reason for visit are related to smoking may be one strategy to motivate smoking cessation and transform acute care visits into true "teachable moments."

INTERNAL MEDICINE RESIDENT SENIOR COMMUNITY SERVICE LEARNING EXPERIENCE Rachel K. Miller ${ }^{1}$; Karen M. Goldstein $^{2}$; Jennine Groce-Martin ${ }^{1}$; Gala True ${ }^{1}$;erry Johnson ${ }^{1}$. ${ }^{1}$ University of Pennsylvania, Philadelphia, PA; ${ }^{2}$ Durham VA Medical Center, Durham, NC. (Control ID \#1339633)

BACKGROUND: We developed, piloted, and evaluated a program to provide a service-learning and community-based, interactive experience focused on Geriatric Medicine and ACGME core competencies to medical residents. Service-based learning promotes core competencies by enriching learners' experiences, promoting understanding of community resources and cultural norms, providing help to service agencies and communities, and developing leadership and professional skills.

METHODS: We developed and piloted a service-based community learning program with 20 Internal Medicine residents of the University of Pennsylvania. The program, which consisted of a half-day morning session, was embedded in a local senior center or senior housing facility. A community outreach coordinator of the Division of Geriatrics orchestrated program implementation, assisted in site selection, and facilitated communication between the project leader and key contacts at each site. At the site, residents toured the facility, learned about the mission and activities of the site, and gave a supervised presentation or "brown-bag" review for the seniors on a Geriatrics topic. A tenminute presentation on local senior resources ended the experience.

RESULTS: Resident evaluation of the community based learning and service experience was administered electronically via an IRB-approved survey using the internal medicine evaluation system. Of 19 evaluations received, 84\% (16) of the internal medicine residents found the tour of the facility informative; $42 \%$ (8) of the residents agreed or strongly agreed that the service they performed expanded their knowledge of senior health issues; $68 \%$ (13) of the residents agreed or strongly agreed that this interaction with the seniors helped them learn more about communicating with older patients. Themes from openended questions included increased knowledge in senior community resources and facilities, enjoyment of interactions and learning from seniors outside a hospital setting.

CONCLUSIONS: Overall, this pilot program of service-based community learning provided residents with insight into the lived environment and community resources available to seniors while increasing awareness of the importance of effective communication with seniors. Future directions include having all trainees perform an aging presentation, expansion of topics, and obtaining evaluations from the seniors at the sites.

INTERPERSONAL TRUST, RACE/ETHNICITY, AND ACCESS TO HEALTHCARE Anthony Nguyen ${ }^{1}$; Paul Rathouz ${ }^{2}$; Elizabeth Jacobs ${ }^{1}$. ${ }^{1}$ University of Wisconsin School of Medicine and Public Health, Madison, WI; ${ }^{2}$ University of Wisconsin School of Medicine \& Public Health, Madison, WI. (Control ID \#1338126)
BACKGROUND: Interpersonal trust between a patient and their physician is important to delivering effective medical care. Compared to Non-Hispanic white populations, interpersonal trust has been shown to be lower in racial/ethnic minority groups and may contribute to health disparities. Our objective was to examine the relationship between race/ ethnicity and interpersonal trust, and if healthcare access modified this relationship.

METHODS: To achieve a sample of individuals with a range of socioeconomic status and healthcare experiences, we conducted a crosssectional, computer-adapted survey study of a convenience sample of African American $(\mathrm{n}=138)$, white $(\mathrm{n}=154)$, and Mexican American $(\mathrm{n}=$ 144) adults shopping in a diverse group of Chicago supermarkets who indicated they had a personal doctor. We used the validated 11-item Hall Trust Scale to measure interpersonal trust. The scores were normally distributed and we used tertiles indicating "low", "middle", and "high" interpersonal trust in a multivariate logistic regression analysis. We first examined the relationship between race/ethnicity and interpersonal trust. Then we added 4 measures of healthcare access (individually and then together): "In the last 2 years, how hard has it been for you to get health services you have needed? ("Hard" vs. "Not Very Hard"); "The last time you were sick or needed medical attention, how quickly could you get an appointment to see a doctor or health professional?" ( $<5$ days vs. $>6$ days); "Is there anytime during the past 2 years when you did not seek medical care because it was too expensive or health insurance did not cover it?" and "Has there been a time that you did you not follow the doctor's advice or treatment plan because it cost too much?" (Yes vs. No, for both items). Analyses were adjusted as follows Model $1=$ gender, marital status, age, race/ethnicity; Model 2=Model 1+employment status, highest level of education, family income; Model $3=$ Model $2+$ healthcare setting, number of doctor visits within 12 months, and insurance status.

RESULTS: The majority of respondents were female, married, had a family income $>\$ 16,000$, not currently working for pay, and had a high school degree or less. The mean age was 41 years. The Hall measure had high reliability $(\alpha=0.91)$. Before adjustment, there was no significant difference in interpersonal trust between the $3 \mathrm{racial} / \mathrm{ethnic}$ groups $(\mathrm{P}=.41)$ and this relationship did not change after adjustment. Before adjustment, all 4 access barriers were statistically significant predictors $(\mathrm{P}<.05)$ of lower trust. After adjustment, only difficulty getting care in the last 2 years, longer wait time to last doctor's appointment, and not following physician advice/treatment plan because of cost were significantly $(\mathrm{P}<.05)$ associated with lower trust. Results did not vary significantly across the adjusted Models.

CONCLUSIONS: We found that patterns of interpersonal trust did not differ significantly across racial/ethnic groups and that systemic factors appear to be important determinants of interpersonal trust regardless of race/ethnicity and other patient characteristics. Healthcare reform may enhance physician trust through improved access and reduced cost

INTERVENTION THRESHOLDS: AN ESSENTIAL ELEMENT OF ADVANCE CARE PLANNING Karen G. Scandrett; Brian T. Joyce; Linda Emanuel. Northwestern University, Chicago, IL. (Control ID \#1309507)

BACKGROUND: Advance care planning (ACP) can refer to designation of a surrogate decision maker, documentation of physician orders, or a living will. Each of these approaches results from discussion with the patient about care preferences, and each, despite limitations, contributes important information to the decision-making process in real time. We present empirical evidence for another element of ACP: the individual's intervention threshold. The intervention threshold is intuitively understood by clinicians and lay public, but has not been thoroughly described, measured, or analyzed.

METHODS: We used a mixed-methods approach to develop the concept of the intervention threshold. From a population of chronically ill general medicine outpatients, we recruited 52 subjects for a 20 -minute, structured telephone interview assessing knowledge, attitudes, and prior ACP activities. Next, respondents were presented with four scenarios representing a spectrum of life-limiting medical situations, and a list of 11 interventions. For each scenario, they were asked if they would accept or decline each intervention. Descriptive statistics were generated via excel, and preferences for each intervention by scenario were plotted. The 
presence of intervention thresholds was evaluated by visual inspection of the data, and patterns were identified in an iterative process. Disagreements in data interpretation were resolved through consensus.

RESULTS: Complete data were obtained from 52 patients, aged 2181 years (mean 64.5, SD 13.0), 34.6\% of whom were male. Although $92.3 \%$ of respondents declined at least one intervention, only $17.3 \%$ reported a prior discussion with a physician. Five threshold patterns were identified: wanting all interventions, wanting no interventions, wanting interventions if there is potential for recovery, wanting interventions in select baseline states, and wanting only the least invasive interventions in all scenarios.

CONCLUSIONS: These data provide evidence for individual, intervention thresholds that may be identified using a scenario-driven process. Factors contributing to the threshold included the possibility of recovery, the baseline health state, and the invasiveness of the medical procedure. The intervention threshold, and the values represented by each dimension, may provide guidance for future surrogate decision-makers. Further research is needed to determine effective ways to identify, measure, and represent an individual's intervention threshold, to facilitate informed decision making during future incapacity.

INTERVENTION REDUCES INPATIENT ORDERING OF CARDIAC ENZYMES AT JOHNS HOPKINS BAYVIEW MEDICAL CENTER Marc Larochelle; Amy Knight; Jeff Trost. Johns Hopkins Bayview Medical Center, Baltimore, MD. (Control ID \#1333625)

BACKGROUND: Inpatient diagnostic testing is overused. Modifying physician ordering behavior has the potential to reduce costs and improve patient care. Physicians for Responsible Ordering (PRO), a multidepartmental, physician-led committee was organized to understand and improve physician ordering of diagnostic tests at Johns Hopkins Bayview Medical Center. As an initial target, we set out to influence cardiac enzyme test ordering.

METHODS: Based on a review of clinical evidence and guidelines, and discussions with cardiologists, criteria for appropriate ordering of cardiac enzymes for the diagnosis of acute coronary syndromes (ACS) were identified. At an academic hospital in Baltimore, we conducted an assessment of an inpatient intervention that promoted appropriate cardiac enzyme ordering. Presentations were made to faculty and housestaff to outline the project objectives and communicate the guidelines. A quick reference card summarizing appropriate ordering was developed and disseminated in September 2011. Changes to the computerized provider order entry system were made in October 2011 to facilitate adherence to the guideline. Cross-sectional analysis was performed on all inpatients admitted to JHBMC in November 2010 (pre-intervention) and November 2011 (post-intervention). Primary outcome was mean number of orders per patient for total creatine kinase (CK), CK-MB, and troponin I. Secondary outcomes were total charges for CK, CK-MB, and troponin I. The number of patients with a primary diagnosis of ACS (ICD-9 codes $410 \mathrm{x}$ or $411 \mathrm{x}$ ) was also tracked in the pre- and post-intervention groups. Subgroup analysis was conducted for patients admitted to a medicine service as medicine services admit the majority of patients with possible ACS and are more likely to order cardiac enzymes.

RESULTS: Orders were compared for 1,881 patients in the pre period and 1,865 patients in the post period. Mean age was 49 years in both groups, and $52 \%$ of patients were female in both groups (both $\mathrm{p}>0.05$ ). There was no difference in the incidence of ACS between groups, with $20(1.06 \%)$ patients in the pre period and $21(1.13 \%)$ patients in the post period $(\mathrm{p}=0.85)$. The mean numbers of tests per patient for the pre and post groups respectively were: 1.23 and 0.26 for total $\mathrm{CK}(\mathrm{p}<0.0001) ; 1.08$ and 0.14 for CK-MB ( $<<$ $0.0001)$; and 1.06 and 0.74 for troponin I $(\mathrm{p}<0.0001) .774(41 \%)$ of the pre group and $781(42 \%)$ of the post group were admitted to a medicine service. In this medicine subgroup, the mean numbers of tests per patient for the pre and post groups respectively were: 2.41 and 0.42 for Total CK ( $<<0.0001) ; 2.15$ and 0.22 for CK-MB $(\mathrm{p}<0.0001)$; and 2.05 and 1.45 for troponin $\mathrm{I}(\mathrm{p}<$ 0.0001). The total number of cardiac lab tests (total CK, CK-MB, and troponin I) declined from 6,329 during the pre period to 2,125 during the post period, a $66 \%$ reduction. This change corresponds to a decrease in charges of $\$ 109,946$ for the one month of this analysis.
CONCLUSIONS: The multi-faceted intervention changed physician behaviors to be consistent with guidelines. The new practice patterns resulted in substantive savings without affecting the incidence of ACS. This approach is scalable to other diagnostic tests and institutions and represents an opportunity to improve care quality and reduce costs nationwide.

INTERVENTIONS TO IMPROVE MINORITY HEALTH AND REDUCE RACIAL AND ETHNIC DISPARITIES IN CARE FOR CERVICAL CANCER: A SYSTEMATIC REVIEW Amanda R. Clarke $^{1}$; Susan Glick ${ }^{3}$; Anita Blanchard ${ }^{2}$; Amy K. Whitaker ${ }^{2}{ }^{1}$ University of Chicago, Chicago, IL; ${ }^{2}$ University of Chicago, Chicago, IL; ${ }^{3}$ University of Chicago, Chicago, IL. (Control ID \#1322071)

BACKGROUND: Cervical cancer is a preventable illness yet women in the United States continue to develop the disease and to die from it. This burden is not shared equally among women of different races and ethnicities. Interventions to maximize the prevention, screening, diagnosis or treatment of cervical cancer have the potential to reduce racial and ethnic disparities, yet there exists no comprehensive review of the literature to determine which interventions are effective. Our objective was to systematically review the intervention research literature to characterize interventions with the potential to improve the prevention, screening, diagnosis, or treatment of cervical cancer among racial and ethnic minority women.

METHODS: We performed electronic searches in the Medline, Cochrane Register of Controlled Trials, CINAHL, PsycINFO and Cochrane Systematic Reviews databases for original articles published in English between the inception of the databases and 2010. Medical Subject Heading $(\mathrm{MeSH})$ terms related to cervical cancer or interventions were combined with terms for minority populations. We included studies with at least one intervention designed to improve cervical cancer prevention, screening, diagnosis or treatment. Additionally, included studies linked participants to the healthcare system; presented data for US racial and/or ethnic minority populations; and measured medical outcomes. We reviewed the included articles to determine the intervention(s), study design, sample size and constituency, length of follow up, limitations and outcomes. Study quality was assessed using a modified version of the Downs and Black (DB) checklist, designed to assess the methodological quality of health care interventions. This checklist has a maximum score of 29; studies with scores at or above 15 were determined to be of good or higher quality.

RESULTS: Thirty-one studies met inclusion criteria. DB quality scores ranged from nine to 23 , with a median score of 14 . Although 25 studies described interventions to increase cervical cancer screening, only 11 of these earned DB scores of 15 or above. Of the six studies to improve the diagnosis of premalignant lesions of the cervix, 3 were of good or higher quality. No studies examined interventions to prevent or to treat cervical cancer. The most common interventions studied were patient education, patient navigation or a combination of the two. Seven higher quality studies involved direct contact with patients via telephone or in-person (with or without an indirect component). Of these, 6 showed positive results, although one of these did not show an effect for the minority groups included. Four higher quality studies described interventions reaching patients indirectly only, via mail or media; 2 were ineffective and 2 resulted in a negative outcome. Two studies with both direct and indirect contact showed a significantly better response for the direct contact group.

CONCLUSIONS: Although high quality data is limited, interventions that target patients directly via telephone or in-person show promise for improving cervical cancer screening and diagnosis among racial and ethnic minorities. Telephone counseling in particular appears effective for increasing diagnosis of premalignant disease of the cervix. More high quality studies are needed to explore ways to improve the prevention, screening, diagnosis, and treatment of cervical cancer for minority women.

INVESTIGATING OLDER ADULT MULTIMORBIDITY: A LATENT CLASS FACTOR MODEL OF CHRONIC DISEASES AND GERIATRIC CONDITIONS Christine Cigolle ${ }^{1,2}$; Jersey Liang ${ }^{1}$; Jinkyung $\mathrm{Ha}^{1}$; Lillian Min ${ }^{1,2}$; Tanya Gure ${ }^{1}$; Pearl Lee ${ }^{1,2}$; Caroline Blaum ${ }^{1,2}$. ${ }^{1}$ University of Michigan, Ann Arbor, MI; ${ }^{2}$ VA Ann Arbor Healthcare System, Ann Arbor, MI. (Control ID \#1336881) 
BACKGROUND: The traditional disease model is limited as an approach to multimorbidity in older adults. Their health status may be more fully conceptualized and described in terms of their combinations of chronic diseases and geriatric conditions. Latent class factor analysis enables the examination of the structure of underlying patterns of diseases and conditions. We hypothesized that (1) geriatric conditions constitute a critical component in the complex health status of older adults and (2) latent classes of chronic diseases and geriatric conditions predict disability and mortality in older adults. METHODS: We analyzed the 2004-2008 waves of the Health and Retirement Study, a nationally-representative longitudinal health interview survey. Our study sample included adults $>=65$ years old $(n=11,113$, representing 37.1 million). Independent variables included 4 geriatric conditions (dementia, DEM; falls, FALL; incontinence, UI; sensory impairment, SI) and 5 chronic diseases (diabetes, DM; hypertension, HTN; heart disease, HEART; stroke, CVA; musculoskeletal conditions, MSK). Outcome variables included new dependency in activities of daily living (ADL) and instrumental activities of daily living (IADL) and mortality. We employed latent class factor modeling to examine classes of older adult health status, using the Akaike information criterion, the Bayesian information criterion, the likelihood ratio test for goodness of fit, and misclassification error to select the model that best fit the data.

RESULTS: Using latent class factor analysis, older adult morbidity proved to sort best by two factors: first, cardiovascular disease (low and high burden groups), and, second, geriatric conditions (low, intermediate, and high burden groups). Jointly, these two factors resulted in six classes, as depicted in the profile plot (Figure). These classes were characterized by distinctive 2-year and 4-year outcomes (Table).

CONCLUSIONS: Confirming our hypotheses, latent class factor modeling demonstrated the importance of geriatric conditions to the complex health status of older adults. Further, the classes of diseases and conditions predicted disability and mortality at two- and four-years. This approach provides new insight into multimorbidity in the older adult population and can be a tool to more completely characterize complexity in clinical settings.

\begin{tabular}{|c|c|c|c|c|c|c|}
\hline \multirow[t]{3}{*}{ Outcomes } & \multicolumn{6}{|l|}{ Classes } \\
\hline & Class & Class & Class & Class & Class & Class \\
\hline & $1-1$ & $1-2$ & $1-3$ & $2-1$ & $2-2$ & $2-3$ \\
\hline New ADL & 3.0 & 7.7 & 42.0 & 7.8 & 10.0 & 26.3 \\
\hline $\begin{array}{l}\text { Dependency at Two } \\
\text { Years }(\%)\end{array}$ & & & & & & \\
\hline $\begin{array}{l}\text { New IADL } \\
\text { Dependency at Two } \\
\text { Years }(\%)\end{array}$ & 5.1 & 13.9 & 60.2 & 11.5 & 15.3 & 53.2 \\
\hline $\begin{array}{l}\text { Mortality at Two } \\
\text { Years }(\%)\end{array}$ & 4.8 & 13.7 & 44.4 & 6.4 & 13.0 & 35.5 \\
\hline $\begin{array}{l}\text { Mortality at Four } \\
\text { Years (\%) }\end{array}$ & 10.3 & 25.7 & 73.3 & 13.8 & 24.2 & 59.8 \\
\hline
\end{tabular}

Note: $\mathrm{p}<0.01$ for each outcome.

Figure: Profile Plot for Latent Class Factor Model

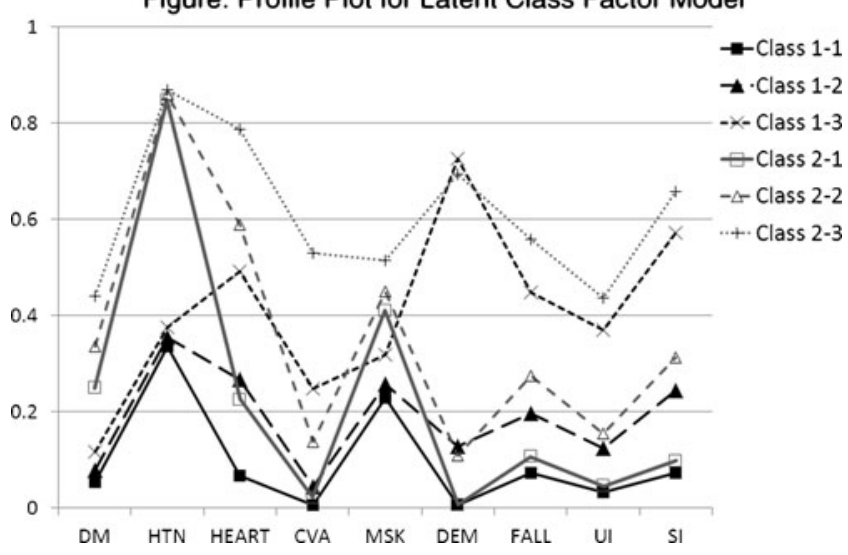

IS CHADS2 SCORE PREDICTIVE OF LA THROMBUS IN A PATIENT WITH ATRIAL FIBRILLATION? Siddesh V. Besur; Rohit Bishnoi; Muni Krishna Theertham; Anil Nalubotula; Vidya Sagar Kollu; Mahesh Borhade; Siva K. Talluri. McLaren Regional Medical Center, FLINT, MI. (Control ID \#1318901)

BACKGROUND: Atrial fibrillation (AF) is associated with an increased risk of systemic thromboembolic events due to Left Atrial (LA) thrombus. The presence of LA thrombus is excluded by Transesophageal echocardiography (TEE) before cardioversion. While the CHADS2 score predicts chronic thromboembolic risk in patients with $\mathrm{AF}$, it is unknown if it can also predict LA thrombus obviating the need for TEE. We aim to study if CHADS2 score predicts the LA thrombus in patients with AF.

METHODS: We retrospectively studied consecutive patients with AF who underwent TEE at a Midwestern tertiary care medical center during years 2005-2009. All patients with AF aged 18-100 years were included. Patients with rheumatic valvular heart disease, mitral stenosis and those requiring valve replacement were excluded. We identified patients with $\mathrm{AF}$ who have undergone TEE by using ICD-9 and CPT codes respectively. The TEE reports were reviewed for the presence of LA thrombus. The information collected included demographic data,comorbid factors (history of diabetes meleitus, hypertension,congestive heart failure,prior stroke or transient ischemic attack, cancer, hypercoagulable state,aspirin use, warfarin use and most recent INR). We calculated CHADS2 scores for each patient by assigning 1 point for age more than 75 years, hypertension, diabetes, heart failure, and 2 points for prior stroke or transient ischemic attack. The association between CHADS2 and LA thrombus were analysed using Fisher's exact test.

RESULTS: We identified 173 patients with AF who underwent TEE. We included 145 patients after applying the inclusion and exclusion criteria. LA thrombus was identified in $9(6.2 \%)$ patients. Patients with LA thrombus were predominantly female $(56 \%)$, aged less than 75 years $(67 \%)$ and had hypertension (89\%). Higher percentage of patients with CHADS2 score $\geq 2$ developed LA thrombus compared to those with CHADS2 score $<1$ ( $8.6 \%$ vs $1.9 \%, \mathrm{p}=0.16$ ).

CONCLUSIONS: We found a high incidence of LA thrombus in patients with higher CHADS2 score as compared to those with lower CHADS2 score, though it was statistically not significant.

IS EPIDEMIOLOGY LOSING IMPORTANCE IN AN ERA OF MOLECULAR TARGET SCIENCE AND PERSONALIZED MEDICINE? Prantesh Jain ${ }^{1}$; Harsha Poola ${ }^{1}$; Sushma Bharadwaj ${ }^{1}$; Sonia Sandhu ${ }^{2}$; Shweta Gupta ${ }^{2,1}$; Susan McDunn ${ }^{2,1}$. ${ }^{1}$ John H Stroger Jr Hospital of Cook County, Chicago, IL; ${ }^{2}$ John H Stroger Jr Hospital of Cook County, Chicago, IL. (Control ID \#1340182)

BACKGROUND: Epidemiology is an essential science, which over time has proved its worth by discovery of various causative agents of many diseases, including certain cancers. The role of human papilloma virus in the causation of cervical, anal and head \& neck cancers is very well established. In breast cancer epidemiological factors help identify patients who may have a worse prognosis. Such factors then in turn promote the development of agents designed to overcome the process. Gynecological and obstetric history is important in a patient with breast cancer but is often forgotten about. We undertook this study as a short clinical research project to see the documentation of this history in patients with breast cancer.

METHODS: We retrospectively reviewed the charts of 211 consecutive women with breast cancer since January 2009 identified from the tumor registry of our inner city community-based teaching hospital. Patients who were seen in a general medicine or sub-specialty clinic (breast clinic, breast surgery, medical oncology, gynecology) at least once were included in the study. The charts with no pathology report, no clinical notes or incomplete notes were excluded. Patients whose charts had only emergency room or Medical ICU visits were also excluded. The charts were screened for age of onset of cancer, menarche, menopause, age at first pregnancy, gravidity and parity of the woman. Patients with any of the above data not reported were identified and a note was made of the number of different licensed physicians who had seen the patient in a non-emergent setting at least once. 
RESULTS: A total of 211 charts were screened out of which 12 were excluded based on the above criteria. A total of 199 patients were included in the study. 104 patients (52.3\%) patients lacked the complete gynecological history. An average 7 physicians each saw these patients in a nonemergent setting with a range from 1 to 37 physicians. The median number of physicians seeing these patients was 5. A number of these patients lacking complete data were also seen by medical students, who also did not chart the detailed gynecologic history. The medical student number was not included in the physician count

CONCLUSIONS: In the era of great advancement in cancer care with targeted therapy and personalized medicine around the corner, we believe physicians may be losing the importance of epidemiology, which actually forms the basis of many of the treatment developments in the field of oncology. Our study is just one small example of the same in the most common cancer in women in the United States. We believe this knowledge gap should be identified and attempts be made to further promote the importance of collecting epidemiological data at the medical student curriculum and resident training level so that clinicians can have a more solid base, especially ones who would one day be clinical or translational researchers.

IS WEIGHT GAIN BENEFICIAL FOR HIV INFECTED INDIVIDUALS INITIATING COMBINATION ANTIRETROVIRAL TREATMENT (CART) REGARDLESS OF INITIAL BODY MASS INDEX? Mary Logeais ${ }^{1}$; Janet P. Tate ${ }^{2,1}$; Amy C. Justice ${ }^{2,1}$. ${ }^{1}$ Yale University School of Medicine, New Haven, CT; ${ }^{2}$ VA Connecticut Healthcare System, West Haven, CT. (Control ID \#1339321)

BACKGROUND: Since the advent of combination anti-retroviral therapy (cART), patients are living longer and increasingly experiencing comorbid disease associated with obesity. Most individuals initiating cART are now normal weight or overweight. We compare 12-month trends in weight among uninfected and HIV infected individuals initiating cART and ask whether benefits associated with weight gain depend upon weight status at cART initiation.

METHODS: We analyzed data from the Veterans Aging Cohort Study (VACS), a longitudinal prospective multi-site observational study of HIV infected and uninfected veterans across 128 nation-wide Veterans Administration (VA) sites. We identified 4,732 HAART initiators between the years of 2000 and 2008 who were alive and in follow-up at one year, as well as 18,769 HIV negative comparators. Changes in weight were examined over a 12-month period. Patients were stratified into those who gained weight $(>5 \mathrm{lb}$. increase in weight), those who lost weight $(>5 \mathrm{lb}$. decrease in weight), and those who stayed the same (weight change within $5 \mathrm{lbs}$.). Our outcomes were the VACS Risk Index (a validated prognostic index comprising age, CD4, viral load, hemoglobin, FIB4, GFR and hepatitis infection) and all cause mortality.

RESULTS: Fifty-one percent of HIV positive patients gained $>5 \mathrm{lbs}$. in the first year after cART initiation compared to $32 \%$ of uninfected patients in the same period $(\mathrm{p}<.0001)$. Mean one year weight change was $6.6 \mathrm{lbs}$. greater in HIV infected compared to uninfected veterans $(\mathrm{p}>0.05)$. Among the $43 \%$ HIV + who started out overweight (BMI $>25), 72 \%$ gained $>10$ pounds and $36 \%$ gained $>20$ pounds. HIV positive patients who gained weight had lower baseline CD4 counts, higher viral loads, and experienced greater improvements in their VACS index. Among HIV positive veterans, weight gain was associated with improved survival across all starting BMI categories. For every five pounds of weight gained, mortality decreased by $4 \%(\mathrm{p}<0.0001$, HR 0.96 , CI $0.93-0.98)$. Half of this association was explained by VACS Index Score.

CONCLUSIONS: In the first year of cART, HIV infected individuals gain weight well beyond that observed among demographically and behaviorally similar uninfected veterans and, compared to HIV infected individuals who maintain weight, weight gain is associated with improved clinical biomarkers and overall survival regardless of baseline weight. Despite real weight gain after cART among normal and overweight individuals, we find no support for weight loss or maintenance interventions in this population.

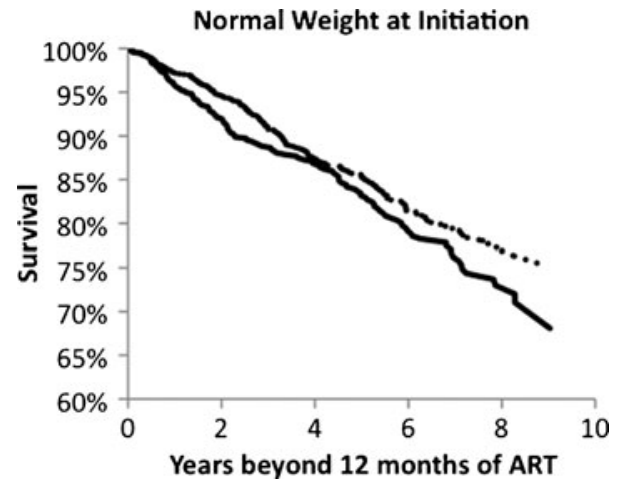

Dashed $=>5$ lbs weight gain Solid $=$ no change in weight

IS HONESTY THE BEST POLICY? THE IMPACT OF ROUTINE CONFLICT OF INTEREST DISCLOSURE BY PRE-CLINICAL LECTURERS ON MEDICAL STUDENT ATTITUDES TOWARD THE PHARMACEUTICAL AND DEVICE INDUSTRIES. Azalea Kim ${ }^{2}$; Lawrence A. Mumm ${ }^{2}$; Deborah Korenstein ${ }^{1}$. ${ }^{1}$ Mount Sinai School of Medicine, New York, NY; ${ }^{2}$ Mount Sinai School of Medicine, New York, NY. (Control ID \#1334000)

BACKGROUND: Pharmaceutical industry influence pervades medical education. Disclosure has been recommended to manage potential conflicts of interest (COI), but data on the impact of disclosure is limited. In September 2010, Mount Sinai School of Medicine mandated disclosure of relevant COI by lecturers to pre-clinical medical students. Our objective was to determine the impact of routine exposure to COI disclosure on preclinical medical students' attitudes toward industry relationships and disclosure.

METHODS: The intervention included brief lectures introducing COI concepts and the disclosure policy for year 1 and 2 (pre-clinical) students followed by routine COI disclosure. Disclosures were made during lectures (verbally or slide-based) and were available in an on-line database. We administered a survey to all pre-clinical students prior to the intervention and again at the end of the academic year. The survey was adapted from a published survey and used a 4-point Likert scale to assess attitudes toward the appropriateness of industry gifts to physicians, industry-sponsored education and industry/faculty relationships and the role of COI disclosure. We performed 2 analyses, first comparing student attitudes before and after disclosure exposure and second utilizing quasi-experimental design to compare post-exposure first year (intervention) students to pre-exposure second year (control) students, both having completed 1 medical school year. We dichotomized responses as strongly agree/agree or disagree/ strongly disagree and used Fischer's exact test to determine the significance of between-group differences.

RESULTS: Most lecturers (77.4\%) completed disclosures; $92 \%$ had no COI. Survey response rates at the beginning and end of the academic year were $66.2 \%$ and $60.9 \%$. Demographic data were similar in the 2 classes. Exposure to COI disclosure was associated in both analyses with significant $(\mathrm{p}<0.05)$ increases in student beliefs that schools should limit industry meetings with students $(56.5 \%$ vs $71.9 \%$, pre-post; $54.3 \%$ vs. $73.5 \%$ control-intervention) and educators (39.8\% vs $56.5 \%$ pre-post; $37.6 \%$ vs $53.0 \%$ control-intervention), a decrease in student belief that industry should fund medical school programs $(61.5 \%$ vs $44.2 \%$ pre-post; $61.1 \%$ vs $44.9 \%$ control-intervention), and a near-significant increase in the belief that receiving gifts/food from industry increases a physicians' prescribing of that company's products $(70.1 \%$ vs $79.4 \%$ pre-post, $\mathrm{p}=0.05$; $68.5 \%$ vs $84.5 \%$ control-intervention, $\mathrm{p}=0.01$ ). There were no changes in student attitudes toward industry educational products, acceptability of gifts/lunches, or the impact of industry relationships on educational content. There was a near-significant increase post-intervention in students strongly agreeing with the statement "The content of lectures...is evidencebased and unbiased regardless of any relationship the lecturer may have 
with private industry" $(8.9 \%$ vs $19.5 \%, \mathrm{p}=0.05)$. There was no change in attitudes toward disclosure though nearly all students (97.0-98.9\%) favored disclosure in all surveys.

CONCLUSIONS: Routine COI disclosure to medical students lead students to favor more limitations in some industry interactions, but did not impact perceptions of the impact of industry relationships on education content or of disclosure itself. Our study suggests that COI disclosure may make students less aware of potential bias in lectures, though further investigation is needed.

IS INSURANCE STATUS ASSOCIATED WITH THE TRANSFER OF HOSPITALIZED PATIENTS? Janel Hanmer; Peter Cram. University of Iowa, Iowa City, IA. (Control ID \#1340802)

BACKGROUND: Little is know about factors associated with the transfer of hospitalized patients to other acute care hospitals.

METHODS: We used 2008 all-payor State Inpatient Data to identify all patients age 18 to 64 discharged from seven states with any of nine common principal diagnoses (pneumonia, acute myocardial infarction, congestive heart failure, cerebrovascular accident, chronic obstructive pulmonary disease, diabetes, hip fracture, skin infection, or gastrointestinal bleed). We excluded patients who were admitted in transfer from another acute care hospital, died during hospitalization, or left against medical advice. The outcome of interest was whether a given patient was transferred to another acute care hospital. The independent variable of interest was patient insurance status categorized as Medicare, Medicaid, private insurance, or self-pay. Univariate, bivariate, and multivariate logistic regression methods were used to examine the association between patient insurance coverage and inter-hospital transfers while accounting for an array of patient factors (age, sex, race, comorbid conditions) and hospital factors (teaching status, community hospital status, bed size, and hospital ownership).

RESULTS: We identified 499,562 discharges across 9 diagnoses (range of 8276 - 78633 patients per diagnosis). In total, 3.5\% discharges were transferred to another acute care hospital (range of $0.9 \%$ for skin infections to $17 \%$ for acute myocardial infarction). Overall, $23 \%$ of patients were insured by Medicare, $22 \%$ by Medicaid, $41 \%$ by private insurance, and $14 \%$ were uninsured. In bivariate analysis, uninsured patients were more likely to be transferred compared to other insurance categories for 6 of our 9 diagnoses. In multivariate analyses, uninsured patients were significantly more likely to be transferred when compared to privately insured patients for 9 of our conditions even after adjusting for patient and hospital factors. Patients with Medicaid were significantly more likely to be transferred when compared to privately insured patients for 3 of our conditions after adjusting for patient and hospital factors. We also observed that smaller hospitals $(<100$ beds $)$ were less likely to transfer patients for all of our conditions (when compared with larger hospitals).

CONCLUSIONS: Uninsured patients were significantly more likely to be transferred than patients with private insurance, Medicare, or Medicaid. This raises important concerns about potential treatment delays for acutely ill uninsured patients and potential violations of federal Emergency Medical Treatment and Active Labor Act Laws.

\section{IS REDUCTION IN SMOKING A MEANINGFUL OUTCOME IN} MENTAL HEALTH PATIENTS? RESULTS FOR A TELEPHONE TREATMENT STUDY Alfredo Axtmayer; Erin Rogers; Scott Sherman. Department of Veterans Affairs, New York, NY. (Control ID \#1335644)

BACKGROUND: Providers and patients are encouraged by reduction in the number of cigarettes smoked, but it is unclear this represents a meaningful outcome. A proactive telephone smoking cessation counseling study in smokers with mental illness examined whether a reduction in number of cigarettes smoked per day (CPD) from baseline to 2 months associated with sustained reduction or abstinence at 6 months.

METHODS: Participants (421) from a multi-site VA study evaluating telephone care for VA smokers with mental illness received smoking cessation education, were offered cessation medications and received telephone counseling from the VA or State Quitline. Participants completed a structured assessment upon enrollment, and 2 and 6 months postenrollment. Chi-square analyses examined whether persons who were abstinent or had reduced their CPD by at least $50 \%$ by 2 months were more likely to be abstinent or have continued their reduction in smoking at 6 months.

RESULTS: 104 participants completed baseline, 2 month and 6 month surveys. $49 \%(50 / 104)$ achieved at least a $50 \%$ reduction in CPD by 2 months, $44 \%(22 / 50)$ were abstinent. 50\% (26/50) of patients who achieved at least $50 \%$ reduction in CPD by 2 months remained at the reduced number of CPD or decreased their CPD more by 6 months. Patients who reduced CPD by $50 \%$ by 2 months were more likely to be abstinent at 6 months compared to patients who had not achieved $50 \%$ reduction in CPD by 2 months ( $44 \%$ vs. $19 \%$, OR $=3.46,95 \%$ CI 1.43-8.38). Patients who were abstinent by 2 months were more likely to be abstinent at 6 months compared to patients who were not abstinent by 2 months $(81 \%$ vs. $17 \%$, OR $=21.9,95 \%$ CI $6.4-74.5)$.

CONCLUSIONS: Data support short-term harm reduction as a model for long-term abstinence in a mental health population. Referral to smoking cessation counseling and initial harm-reduction steps should be encouraged for patients not ready or able to immediately quit. All smokers, no matter their stage of change, should be offered effective cessation treatment. Although smoking reduction is a meaningful outcome for mental health patients, short-term abstinence should remain a primary goal of cessation treatment, as it is significantly associated with long-term abstinence.

KICKING THE TIRES OF CPOE SYSTEMS: TESTING VULNERABILITY TO REPORTED CPOE-RELATED MEDICATION ERRORS Gordon D. Schiff ${ }^{1,3}$; Andrew C. Seger ${ }^{1,2}$; Mary Amato ${ }^{2}$; Diana L. Whitney ${ }^{1}$; Jennifer Boehne ${ }^{1}$; Ali Rashidee ${ }^{5}$; Robert B. Elson ${ }^{6}$; Ross Koppel ${ }^{4}$; David W. Bates ${ }^{1,3}$. ${ }^{1}$ Brigham and Women's Hospital, Boston, MA; ${ }^{2}$ Massachusetts College of Pharmacy \& Health Sciences, Boston, MA; ${ }^{3}$ Center for Patient Safety Research and Practice, Boston, MA; ${ }^{4}$ University of Pennsylvania, Philadelphia, PA; ${ }^{5}$ Quantros, Inc., Milpitas, CA; ${ }^{6}$ MetroHealth Center for Health Care Research and Policy, Cleveland, OH. (Control ID \#1338870)

BACKGROUND: A recent IOM report on HIT and Safety highlighted the need for enhanced EMR safety. The Leapfrog Group performed tests of CPOE capabilities and found significant safety gaps. As part of an in-depth review of CPOE-related medication errors reported to MEDMARX, a medication error reporting database, we extracted recurrent errors and created test scenarios designed to evaluate the vulnerability of leading CPOE systems to these reported errors. We examined ways current commercial and "home grown" systems were vulnerable to these erroneous/dangerous orders.

METHODS: MEDMARX collected medication error reports with a coded field designating CPOE as a cause of error. We analyzed a $15 \%$ random sample of 63,040 reports and constructed a taxonomy of error types, causes, and prevention strategies. We flagged representative cases and created 21 scenarios based on error frequency, severity, generalizability, testability, and correctability. These included erroneous/problematic orders related to: wrong units, overdoses, drug allergies, omission errors, wrong frequency, and drug-disease contraindications. Additionally, we developed 3 correct but "complex" test orders (e.g. prednisone tapers, alternate-day dosing) that often led to problemprone workarounds. We identified test sites and obtained permission to enter orders on test patients in a sample of leading vendor and homegrown $\mathrm{CPOE}$ systems. We recruited typical users (mostly medical residents), and instructed them to enter the erroneous orders. Outcomes (whether orders were successfully entered) and behaviors (of MDs and CPOE systems) were recorded by a pharmacist and RA observer who rated the ease (or difficulty) of entering the orders using a Likert scale with accompanying operational definitions.

RESULTS: Testing the vulnerability of selected CPOE systems to 21 selected prototypical cases found that of 307 attempted erroneous (incorrect) orders, $174(57 \%)$ were able to be easily replicated (entered easily or $\mathrm{w} /$ minor workarounds) with no warning/blocking of such potentially dangerous drug orders. In total $>80 \%$ of attempted erroneous orders were able to be "successfully" entered. Of 57 attempted "complex" orders, prescribers had moderate or significant difficulty entering $30(53 \%)$ orders. $30 \%$ of orders generated specific warnings related to the erroneous order. Of these, $61 \%$ were passive alerts, often ignored, another $28 \%$ 
required workarounds but could still be entered. Failures included no warnings for a 100 -fold levothyroxine overdose in $84 \%$, erroneous orders for pioglitazone accepted for CHF patients in $74 \%$, and orders for insulin 60 "ml" (rather than "units") going through in 79\%. In thinking aloud test subjects articulated multiple frustrations and insights about CPOE systems and practices.

CONCLUSIONS: Testing reported CPOE errors on current systems revealed a significant and disturbing rate of "successful" entry of potentially dangerous medication orders. Scenarios harvested from actual reported cases were useful in probing systems and physicians' ordering behaviors. We uncovered remarkable variability between inpatient and outpatient systems as well as different implementations of same systems at different sites. Ethnographic study of physician ordering demonstrated frustrations with specific potentially correctable features, patterns of overriding/ignoring warnings, frustrations with complex orders, and tolerance for suboptimal orders based on assumptions that potential errors would be corrected by pharmacists downstream.

LEARNING MORE ABOUT THE FEEDBACK PROCESS Laura K. Snydman; Daniel Chandler; Kimberly Dowdell; Joseph Rencic. Tufts Medical Center, Boston, MA. (Control ID \#1339385)

BACKGROUND: It is generally assumed that feedback is a good thing. Guidelines on feedback techniques to enhance learning have been published. While many models exist on how to deliver effective feedback, there is little research about the feedback process in the setting of clinical teaching. This is most likely due to the complexity of the feedback process itself and the challenge in measuring learner outcomes in the clinical setting. The objective of this study was to analyze the content and nature of the feedback session involving attending physician observers and residents after direct observation of teaching on work rounds.

METHODS: All post-graduate year 2 internal medicine residents at one academic medical center participated in a randomized controlled trial involving direct observation and feedback of work rounds. Residents were randomized to a feedback group or control group. Each resident was observed on two occasions 3 days apart. After the first observation, residents in the feedback group received verbal feedback from a trained attending observer using a standardized process created by the authors (LKS, DC, JR) using expert opinion and existing literature. Residents in the control group did not receive feedback after the first observation. All residents received feedback after the second observation. The feedback sessions were audiotaped to allow for qualitative analysis. One author (LKS) coded comments into dominant and supporting themes using the Stanford Faculty Development Program's (SFDP) clinical teaching framework.

RESULTS: Twenty-five internal medicine residents participated. The feedback sessions ranged in length from 7-35 min, mean 18 min, median 15 min. Residents' self-assessments of their teaching were vague $29 \%$ of the time and nearly half of the time they stated only things they did well $(47 \%)$. When asked to identify behaviors they did well versus behaviors they could improve upon, $45 \%$ of the statements were in the SFDP categories of Understanding and Retention (UR) and Control of Session (CS) respectively. Most of the time residents' self-assessments (reinforcing and corrective) did not correlate with the attending assessments (85-90\%). Residents in the feedback group did not provide more detailed self-assessments after the 2nd observation despite having participated in the same standardized feedback process 3 days prior. The majority of attending reinforcing and corrective feedback was in the categories UR and Learning Climate (LC). When asked to recall the attending's 3 pieces of reinforcing feedback and 3 pieces of corrective feedback given a few minutes earlier, $33 \%$ and $83 \%$ were remembered respectively. Residents in the feedback group were better at remembering the reinforcing feedback after the 2nd observation (52\%).

CONCLUSIONS: Our data indicate that corrective feedback is more likely to be remembered than reinforcing feedback, though nearly a fifth of the corrective feedback was not remembered minutes after it was discussed. While educators discuss the importance of providing feedback to learners as a means of enhancing skills or correcting mistakes, if the feedback is not remembered, is there still utility in giving it? What can be done to enhance their memory? These questions deserve further study.
LEARNING OUTCOMES DESCRIBED IN MEDICAL EDUCATION COMPETENCY-BASED FRAMEWORK TO PREPARE STUDENTS TO TAKE INITIATIVE ROLES FOR HEALTH PROMOTION: INTERNATIONAL COMPARISON AND FUTURE CHALLENGES Yuko Takeda ${ }^{1}$; Katsuya Takemura ${ }^{2}$; Ann Wylie ${ }^{1} .{ }^{1}$ King's College London School of Medicine, London, United Kingdom; ${ }^{2}$ University of the Ryukyus Hospital, Nishihara, Japan. (Control ID \#1340280)

BACKGROUND: The emphasis on learning outcomes that equip medical graduates with the competencies required to provide optimal care for patients and society, has led various regulatory bodies and accrediting agencies to develop a set of standards within the competency-based framework. Physicians need to understand and respond appropriately to the complex relationships of individual behaviour and health determinants, including environmental and social conditions. Learning outcomes to prepare students for such competencies should be stated in the medical education framework

METHODS: In order to identify a set of learning outcomes, we reviewed the medical education frameworks in the UK (Tomorrow's Doctors 2009), the USA (Standards for Accreditation of Medical Education Programmes leading to the M.D. Degree), Canada (The CanMEDS 2005 Physician Competency Framework), Australia (Assessment and Accreditation of Medical Schools), and Japan (Model Core Curricula 2010), as well as World Federation for Medical Education (The WFME Global Standards for Quality Improvement). The learning outcomes and competencies in each framework were carefully reviewed twice by the authors. When any of the following words were contained or reflected in a sentence it was extracted: prerequisites for health; determinants of health; political, economic, social, cultural, environmental, behavioural; equality, equity; intersectoral approach (interprofessional collaboration). We also conducted a literature review using six databases: MEDLINE (via Ovid), EMBASE (via Ovid), Web of Sciences, IBSS, CINAHL and ERIC, to find practical examples of curricula/programmes designed to achieve the outcomes. In order to locate educational programmes focusing on the disciplines of health promotion described in the Ottawa Charter, [determinant of health] (or [health determinant]), [inequity], and [disparity] were used.

RESULTS: The term "health promotion" is a standard term used in medical education quidelines in the UK, the USA, Canada and WFME without specification. However, in various frameworks, there are statements regarding relevant competencies; recognising advocacy roles and social responsibility of physicians; understanding cultural factors and diversity; describing principles of health policy; discussing social justice and social equity/inequity; and identifying social determinants of health. After conducting the literature review, five main themes were identified in the educational programmes: "social construction of health", "knowledge and skills to promote healthier lifestyle", "determinants of health", "social responsibility" and "professionalism". The majority of these programmes have been provided in communities that have become part of the recent trend for global health programs conducted in low-income countries. Examples of community-based activities were community needs assessment, community service, and collaboration with community (community mobilisation). It has been pointed out that medical schools bear responsibility to provide students adequate knowledge and practical skills to prepare them for both the clinical and ethical challenges of working in socially disadvantaged and medically underserved community.

CONCLUSIONS: In competency-based frameworks, there has been an emphasis on preparing graduating students to identify the social determinants of health in order to become aware of their potential roles as clinicians. How to achieve these has presented challenges yet to be significantly addressed.

LESSONS IN RECRUITMENT OF OLDER ADULTS USING ANTIDEPRESSANTS Elizabeth M. Haney; Melanie Abrahamson. Oregon Health \& Science University, Portland, OR. (Control ID \#1340291)

BACKGROUND: We recruited two groups of older adults to participate in a prospective study of bone turnover: men and women who were starting selective serotonin reuptake inhibitors (SSRIs) and a comparison group who had not taken SSRIs in at least 1 year. Our intention was to follow 150 participants ( 75 users and 75 non-users) at 6 clinic visits over 1 year. We were interested in patients who had started SSRIs within the past 30 days. 
METHODS: Initial recruitment began in the Internal Medicine clinic and was expanded to include other primary care clinics within the university health system (Family Medicine and Women's Health clinics). Subsequently, a local health maintenance organization was also added. Both health systems had electronic medical records that were used for recruitment; the two differed on whether direct contact with patients was allowable without consent of the PCP.

RESULTS: To achieve our desired enrollment, we made early changes to our recruitment strategy and expanded our clinic sites. Multiple comorbidities and health concerns were prominent and some people reported being to sick to participate. Some participants had difficulty scheduling around work either because of their own job or a family member's job. To combat these issues we offered transportation, when this was the main barrier to participation; home visits once someone had completed the baseline assessment, in order to gather as much longitudinal data as possible; increased the monetary incentive for participation; allowed verbal responses to our surveys instead of just written; and attempted to coordinate study visits with other appointments in the university health system when applicable. We successfully utilized the electronic medical record, under both organizations' IRBs for identification of potential participants. This greatly reduced the burden on research staff and primary care providers. In total we recruited and enrolled 94 users and 106 SSRI users in order to assure $>150$ participants at the end of the study. We identified 1274 SSRI users and 1060 non-user controls through the electronic medical records in order to enroll this number. Of the 200 men and women enrolled, 166 completed the 12 month study (a 17\% drop out rate). SSRI users were more difficult to retain than controls: $78 \%$ of users completed the 12 month study vs $86 \%$ non-users. SSRI users completed a lower percentage of their total possible study visits than non-users ( $85 \%$ vs. $91 \%)$. Anecdotally, women were easier to recruit than men; they seemed to perceive osteoporosis as a personally important problem, may have more flexible work schedules, and have higher rates of SSRI use.

CONCLUSIONS: Several lessons about recruitment of older adults taking antidepressant medications may be generalizable to other studies wishing to include an older frail population with and without mental health concerns. Health issues, care giving and care giver schedules, coordination with other appointments, and transportation to the study site are all important factors in recruitment of older adults. Those with mental health concerns require additional attention and extra recruitment effort.

LIMITED AVAILABILITY OF INFLAMMATORY BOWEL DISEASE CLINICAL TRIAL RESULTS ON CLINICALTRIALS.GOV Zackary Berger; Rachel Blair; Eric Bass; Lisa M. Wilson; Susan Huftless. Johns Hopkins University School of Medicine, Baltimore, MD. (Control ID \#1330731)

BACKGROUND: To reduce selective reporting of trial results, the International Committee of Medical Journal Editors required in 2005 that all clinical trials be registered in publicly accessible databases. In 2007, the US Food and Drug Administration (FDA) required reporting of trial results on ClinicalTrials.gov within a year of trial completion. Inflammatory bowel disease (IBD) is a condition for which multiple drugs have been studied in randomized controlled trials (RCTs) funded by industry and non-industry sources. To ascertain availability of IBD trial results and compliance with the FDA requirement, and to determine the relationship between funding source and such reporting, we conducted a systematic review of RCTs of IBD treatments.

METHODS: We searched ClinicalTrials.gov in July 2011 for Phase II and III clinical trials in IBD, ulcerative colitis, and Crohn's disease. Two reviewers independently reviewed RCTs that tested biologics, immunomodulators, steroids, or aminosalicylates. We excluded trials that did not conduct interclass or intraclass medication comparisons or were not placebo-controlled. We extracted data on intervention, study result availability, and funding source (industry, government, academic, or other). We specified if results were provided via a link to a peer reviewed publication, a table of results in ClinicalTrials.Gov, or on an outside website (ClinicalStudyResults.org). To identify publications not directly listed on ClinicalTrials.gov, we searched PubMed, EMBASE, and Cochrane databases for IBD RCTs conducted after 1997 using the same criteria as was used for the registry entries. We then matched trial registry entries to their corresponding publications using NCT number, study acronym, intervention, and participants enrolled. To examine compliance with FDA mandates, we analyzed the subset of trials initiated or ongoing as of September 2007 and completed by July 2010 .

RESULTS: Of 513 trials identified in ClinicalTrials.gov, 133 trials met inclusion criteria. $50(38 \%)$ of the included trials had available results. Of the 67 trials completed by July 2010, 35 (52\%) had available results. 30 (45\%) had corresponding peer reviewed publications, but only $19(28 \%)$ directly provided links to peer reviewed publications containing trial results on ClinicalTrials.gov. 39\% (23/53) of exclusively industry funded trials had corresponding peer reviewed publications, compared with $75 \%(3 / 4)$ of exclusively non-industry funded trials. Only $18 \%$ of trials required to report results on ClinicalTrials.gov complied with the 2007 FDA mandate ( $21 \%$ of exclusively industry funded trials and $0 \%$ of exclusively nonindustry funded trials).

CONCLUSIONS: Availability of trial results for Phase II and III IBD RCTs is limited, and few trial sponsors are complying with 2007 FDA requirements to post trial results within a year of trial completion. The FDA may need to increase monitoring and enforcement of its requirement in order for ClinicalTrials.gov to effectively decrease the impact of publication bias on the availability of trial results in IBD and other conditions.

LONG-TERM EFFICACY AND TOLERABILITY OF MILNACIPRAN FOR THE MANAGEMENT OF FIBROMYALGIA: RESULTS FROM AN OPEN-LABEL, FLEXIBLE-DOSING STUDY FOLLOWED BY A RANDOMIZED, DOUBLE-BLIND, PLACEBOCONTROLLED DISCONTINUATION STUDY Allan Spera ${ }^{3}$; Daniel J. Clauw ${ }^{1}$; Lesley M. Arnold ${ }^{2}$; Yimin $\mathrm{Ma}^{3}$. ${ }^{1}$ University of Michigan, Ann Arbor, MI; ${ }^{2}$ University of Cincinnati College of Medicine, Cincinnati, OH; ${ }^{3}$ Forest Research Institute, Jersey City, NJ. (Control ID \#1338386)

BACKGROUND: Fibromyalgia (FM) is a chronic widespread pain disorder often accompanied by symptoms including fatigue, stiffness, impaired physical function, and cognitive dysfunction. Due to the persistent nature of this disorder, medications that can manage the symptoms of FM over a long-term period are important. Milnacipran is a dual serotonin and norepinephrine reuptake inhibitor approved for the management of FM. The efficacy of milnacipran in treating the symptoms of FM has been demonstrated in several 3- to 6-month placebo-controlled trials and in 6- to 9-month extension studies. The current studies evaluate the efficacy and tolerability of milnacipran in patients with FM during a 3year, open-label (OL) study and a subsequent randomized, double-blind, placebo-controlled discontinuation study.

METHODS: Patients completing previous milnacipran studies were eligible to participate in a 3-year, OL study (Study 1). Study 1 comprised a 2-week washout period, a 2-week dose-escalation period to $100 \mathrm{mg} /$ day, an 8 -week stable-dose period at $100 \mathrm{mg} /$ day, and a flexible-dose period (50-200 mg/day) for up to 3.25 years. Key efficacy outcomes included weekly-recall VAS pain and the Patient Global Impression of Change (PGIC). Patients completing Study 1 were eligible to enroll in a double-blind, placebo-controlled discontinuation study (Study 2). Patients enrolling in Study 2 continued OL milnacipran treatment for 4 weeks at the dose received in Study 1. After the 4week OL period, patients achieving a $\geq 50 \%$ reduction in VAS pain score from pre-milnacipran exposure and receiving $\geq 100 \mathrm{mg}$ /day were considered responders and randomized to milnacipran or placebo for 12 weeks. The primary outcome in Study 2 was the time to loss of therapeutic response, defined as a worsening in VAS pain score to $\leq 30 \%$ reduction from premilnacipran exposure or worsening of FM requiring an alternative treatment. RESULTS: In Study $1(\mathrm{~N}=1227)$, the group of patients reaching the 3-year visit $(n=217)$ demonstrated a $23.9 \mathrm{~mm}$ improvement in weekly recall VAS pain score. Additionally, $70.3 \%$ of patients at the final visit rated themselves "much improved" or "very much improved" from Study 1 baseline on the PGIC scale. The most common treatment-emergent adverse events (TEAEs) during Study 1 were nausea, headache, hypertension, and sinusitis. AEs led to study discontinuation in $20.5 \%$ of patients, most commonly due to nausea $(2.7 \%)$. Of those continuing into Study $2(\mathrm{~N}=358)$, a total of 150 patients met the responder criteria ( $50 \%$ pain improvement and dosage $\geq 100 \mathrm{mg} /$ day) and were included in the intent-to-treat analysis population. At the end of Study 2, $64 \%$ of the patients switched to placebo $(n=50)$ experienced a loss of 
therapeutic response vs $35 \%$ of patients who continued milnacipran $(n=100)$. The time to loss of response was significantly shorter for patients switched to placebo than for patients who continued milnacipran $(\mathrm{P}=.0004)$. There were no discontinuations due to AEs in patients switched to placebo; 2 patients continuing milnacipran discontinued due to AEs.

CONCLUSIONS: These findings provide support for sustained long-term efficacy (in some cases exceeding 3 years of continuous usage) and tolerability of milnacipran in the treatment of FM. The loss of therapeutic response upon discontinuation of long-term milnacipran treatment provides further evidence of the continuing efficacy of milnacipran in FM patients.

LOW RATE OF COLORECTAL CANCER SCREENING AMONG A COHORT OF HIV-INFECTED PATIENTS Greer Burkholder; Ashutosh Tamhane; Lauren E. Appell; Michael J. Mugavero; James H. Willig; Michael S. Saag. University of Alabama at Birmingham, Birmingham, AL. (Control ID \#1322079)

BACKGROUND: Given the dramatic improvement in survival among HIV-infected patients due to potent combination antiretroviral therapy (ART), we can expect increasing incidence of colorectal cancer (CRC) in this aging population. Prospective studies comparing CRC screening in HIV-infected patients at average risk with uninfected controls have found higher prevalence of neoplastic lesions and more advanced disease among those diagnosed with CRC. HIV primary care guidelines recommend screening colonoscopy beginning at age 50 in HIVinfected patients at average risk. However, there are few studies on rates and determinants of screening in this population. This study evaluated time to CRC screening from age 50 in HIV-infected patients and examined associations between socio-demographic, clinical, and psychosocial variables and likelihood of screening.

METHODS: A retrospective follow-up cohort study was conducted at the UAB 1917 HIV Clinic. Patients with $\geq 1$ primary HIV provider visit at age 50 between January 1, 2003 and December 31, 2010 were included. Patients with history of CRC, colonic polyps or inflammatory bowel disease prior to age 49 were excluded. Patients receiving diagnostic colonoscopy between ages 40 and 48 were also excluded, as they were not "at risk" for screening by age 49 . Patients screened between age 49 and 50 were considered screened at time 0 (age 50).

RESULTS: Of 1,869 patients, 242 met eligibility criteria (age range 50.2-57.8 years; $78.5 \%$ male; $38.4 \%$ African American; $86.0 \%$ insured). $77.7 \%$ of patients received primary care from their HIV provider. Overall, $83(34.2 \%)$ of patients ever received CRC screening. Median time to screening from age 50 was 1.2 years (IQR 0.2-2.4), with a maximum of 5.5 years. Among screened patients, $12(14.5 \%)$ had neoplastic polyps. Among unscreened patients $(\mathrm{n}=159), 29$ were referred but never received CRC screening, 6 declined referral, and in 33 patients the provider documented the need for CRC screening but did not refer the patient; there was no documentation of the need for CRC screening in the remaining 91 patients. In a multivariable Cox proportional hazards model, only male gender was significantly associated with screening $(\mathrm{HR}=2.2,95 \% \mathrm{CI}: 1.1-4.3, \mathrm{p}=0.02)$ while adjusted for: African-American race $(H R=1.0 ; p=0.93)$, public insurance $(H R=1.6, p=$ 0.23 ), private insurance $(\mathrm{HR}=1.6, \mathrm{p}=0.23)$, history of smoking $(\mathrm{HR}=0.7 ; \mathrm{p}=$ $0.12)$, CD4 count $<200$ cells $/ \mathrm{mm}^{3}(\mathrm{HR}=0.8 ; \mathrm{p}=0.58)$, having an outside general medicine provider $(\mathrm{HR}=1.6 ; \mathrm{p}=0.07)$, and history of $\mathrm{CRC}$ in a first degree relative $(\mathrm{HR}=1.1 ; \mathrm{p}=0.86)$.

CONCLUSIONS: Only modest delays were observed among patients receiving CRC screening in this cohort of HIV-infected patients. However, despite the majority of patients having insurance, the overall rate of screening was low, and lower among women. Although frequency of neoplastic polyps was lower than in other studies of HIV-infected patients, it was substantial enough to raise serious concerns for missed lesions in our unscreened patients. Barriers to $\mathrm{CRC}$ screening appeared to occur at multiple points along the continuum from HIV provider visits to screening. Further research using qualitative methods is urgently needed to determine patient and provider factors along this pathway that contribute to low screening rates, in order to allow for the development of effective interventions.
LOW SES IS ASSOCIATED WITH INCREASED RISK FOR HYPOGLYCEMIA IN TYPE 2 DIABETES PATIENTS: RESULTS FROM THE DIABETES STUDY OF NORTHERN CALIFORNIA (DISTANCE) Seth Berkowitz ${ }^{1}$; Andrew J. Karter ${ }^{2}$; Jennifer Y. Liu' ${ }^{2}$ (Dean Schillinger ; Nancy E. Adler ${ }^{1}$; Howard H. Moffet ${ }^{2}$; Urmimala Sarkar ${ }^{1}$. ${ }^{1}$ UCSF, San Francisco, CA; ${ }^{2}$ Kaiser Permanente Northern California, Oakland, CA. (Control ID \#1312930)

BACKGROUND: Minimizing hyperglycemia is a clinical priority and quality goal in diabetes care. Hyperglycemia-lowering therapies can improve diabetes-related outcomes, but may also increase the risk of hypoglycemia. Past European studies have reported greater risk of hypoglycemia among those with low socioeconomic status (SES), but this research was conducted prior to the focus on more intensive glucose lowering. Therefore, we sought to determine if low income, a marker of low SES, was associated with greater risk for hypoglycemia in a modern U.S. cohort of adults with diabetes.

METHODS: We conducted a cross-sectional analysis of the DISTANCE study, a survey follow-up cohort comprised of adult diabetes patients enrolled in the Kaiser Permanente Northern California diabetes registry. DISTANCE included an ethnically stratified random sample, and the baseline survey (response rate 62\%) was conducted in English, Spanish, Mandarin, Cantonese, and Tagalog. The outcome of interest was patient report of 1 or more episodes of significant hypoglycemia, those resulting in unconsciousness or requiring outside assistance, in the past 12 months. The exposure of interest was household income. We specified unadjusted (bivariate) and multivariate logistic regression analysis, controlling for sociodemographic variables such as age, gender, race/ethnicity, education, and health literacy, and clinical variables such as medication use (insulin, secretagogues, metformin, or mixed), HbA1c, duration of diabetes, chronic kidney disease, self blood glucose monitoring, CVA, and dementia. Models accounted for the complex sampling design (non-proportional sampling fractions) through expansion weights, for survey response bias using Horvitz-Thompson weights, and for item non-response with multiple imputation.

RESULTS: 14,357 patients were included. The mean age of our sample was 58 years (SD 10 years), and was $49 \%$ female. The sample was $23 \%$ Asian, $22 \%$ white (non-Latino/a), 18\% Latino/a, 17\% African-American, and 20\% mixed/other. The mean HbA1c was 7.6 (SD 1.6). 17\% had household income below $\$ 25,000$ annually, and $33 \%$ had income $>\$ 65,000$. Hypoglycemia was common, with $11 \%$ of patients reporting significant hypoglycemia in the past year. In bivariate analysis, low SES was associated with nearly twice the frequency of hypoglycemia, with $16 \%$ of those with incomes $<\$ 25,000$ reporting hypoglycemia, compared to only $8.8 \%$ of those with incomes $>$ $\$ 65,000$ (Cochran-Armitage test for trend $\mathrm{Z}$ score 7.82, $\mathrm{p}<0.0001$ ). In our multivariate logistic regression analysis, hypoglycemia remained significantly associated with low SES. The effect was significant for both the poorest (incomes $<\$ 15,000$, OR hypoglycemia $1.51,95 \%$ CI 1.11-2.05, $\mathrm{p}=0.008$ ) and the second poorest (incomes $\$ 15,000-24,999$, OR $1.41,95 \%$ CI $1.01-1.99, \mathrm{p}=$ 0.045 ) cohorts (reference group income $>\$ 65,000$ ).

CONCLUSIONS: Low income is associated with substantially increased hypoglycemia risk. This finding persisted even after adjustment for potentially confounding and mediating risk factors. Clinicians should be aware of this increased risk when caring for vulnerable patients and prioritize treatment strategies which minimize hypoglycemia. This has important implications for diabetes clinical guidelines and quality metrics, which must balance the risk of hypoglycemia against the benefits of diabetes treatment. More work is needed to understand why lower income is linked with greater risk of hypoglycemia and whether this disparity is modifiable.

LOW INCIDENCE OF ADVERSE EVENTS FOLLOWING VARENICLINE PRESCRIPTION AMONG OPIATE DEPENDENT PATIENTS WITH COMORBID PSYCHIATRIC AND MEDICAL ILLNESS Shadi Nahvi ${ }^{1}$; Bryan $\mathrm{Wu}^{1}$; Kimber P. Richter ${ }^{2}$; Steven L. Bernstein ${ }^{3}$; Julia H. Arnsten ${ }^{1}{ }^{1}$ Albert Einstein College of Medicine, Bronx, NY; ${ }^{2}$ University of Kansas, Kansas City, KS; ${ }^{3}$ Yale University, New Haven, CT. (Control ID \#1340381)

BACKGROUND: Substance abuse treatment patients smoke at four times the prevalence of the general population and suffer high rates of tobacco- 
related disease and mortality. Varenicline is more efficacious for smoking cessation in non-substance abusers than bupropion and nicotine replacement therapy, but has potential psychiatric and cardiovascular risks. Little data exist on the safety or effectiveness of varenicline among smokers with mental health and substance abuse issues.

METHODS: In this retrospective observational study, we reviewed all patient charts in two urban methadone clinics that provide on-site primary care. Subjects included all smokers prescribed varenicline between May 2006 and December 2009. We evaluated adverse events, including events prompting treatment discontinuation, psychiatric symptoms, and cardiovascular events in the six months following varenicline prescription. We also assessed varenicline treatment course, smoking cessation, and the association between treatment course and smoking cessation.

RESULTS: We reviewed 718 patient charts and identified 581 smokers $(80.9 \%)$. Seventy smokers $(12 \%)$ were prescribed a total of 82 courses of varenicline treatment. There was six months of clinical follow up for $100 \%$ of patients following each of the 82 varenicline treatment courses; patients had a median of six [Interquartile range (IQR) 3-9] medical visits and 10 (IQR 7-13) substance abuse counselor visits in the six months following each varenicline prescription. The mean age of patients prescribed varenicline was 50 years and $46 \%$ were female. Cardiovascular risk factors and psychiatric illness were prevalent: $20 \%$ had diabetes, $51 \%$ had hypertension, $23 \%$ had hyperlipidemia, $53 \%$ had preexisting depression, $30 \%$ had preexisting anxiety, $8.6 \%$ had preexisting bipolar disease, and $10 \%$ had preexisting psychotic disorders. Among 82 varenicline courses, nine (11\%) were discontinued due to adverse effects; two discontinuations were due to depressive symptoms. One patient initiated new psychiatric treatment within six months of initiating varenicline, but did not discontinue varenicline. There were no reports of suicidal ideation, no reports of agitation prompting clinical intervention, and no psychiatric hospitalizations. There were also no incident cardiac or vascular events within 6 months of varenicline prescription. Only 16 patients $(22.9 \%)$ received the recommended 12 week treatment course; those with $a \geq$ 12 week treatment course were significantly more likely to report tobacco abstinence at follow up than those with a $<12$ week treatment course $(29.4 \%$ v $2.8 \%, \mathrm{p}=0.01)$.

CONCLUSIONS: Smokers in treatment for opiate dependence, who have high rates of psychiatric and cardiovascular comorbidity, experience adverse events from varenicline infrequently. Treatment course duration is significantly associated with cessation. Interventions are needed to optimize use of smoking cessation treatment among opiate dependent smokers.

\section{LOWER RATES OF NEPHROTOXICITY AND OTHER ADVERSE EFFECTS WITH LIPOSOMAL AMPHOTERICIN B VS. LIPID COMPLEX AMPHOTERICIN B IN HOSPITALIZED NEUTRO- PENIC PATIENTS Paresh Chaudhari ${ }^{1}$; Rolin Wade ${ }^{2}$; Jaime L. Natoli ${ }^{2}$; Robert Taylor ${ }^{2}$; Brian Nathanson ${ }^{3}$; David Horn ${ }^{4} .{ }^{1}$ Astellas Pharma US, Inc., Deerfield, IL; ${ }^{2}$ Cerner LifeSciences, Beverly Hills, CA; ${ }^{3}$ OptiStatim, LLC, Longmeadow, MA; ${ }^{4}$ David Horn, LLC, Doylestown, PA. (Control ID \#1275580)}

BACKGROUND: In previous clinical studies, liposomal amphotericin B (L-AMB) and amphotericin B lipid complex (ABLC) were significantly less nephrotoxic compared to conventional amphotericin $\mathrm{B}$ deoxycholate. Additionally, some trials have suggested differences in nephrotoxicity between L-AMB and ABLC. We examined rates of nephrotoxicity and other adverse effects among adult inpatients administered L-AMB or ABLC with neutropenia for therapy of invasive fungal infections (IFIs).

METHODS: The Health Facts $\left.{ }^{(}\right)$database (Cerner Corp, Kansas City, MO) was used to extract information for analysis regarding neutropenic hospitalized patients aged $>=18$ y receiving L-AMB or ABLC from 1/1/016/30/10 from 141 participating US hospitals. Patients had a positive blood culture or diagnosis of Aspergillus, Candida, and/or Cryptococcus and at least one absolute neutrophil count $<1000$ cells $/ \mathrm{mm} 3$ prior to L-AMB or ABLC administration. Renal function was evaluated by changes in serum creatinine ( $\mathrm{SCr}$ ) and nephrotoxicity was defined as a post $\mathrm{SCr}>100 \%$ increase from baseline and an absolute level $>1.2 \mathrm{mg} / \mathrm{dL}$. Univariate inference tests and multivariate analyses (MVA) utilizing generalized linear models were used to assess differences between groups.

RESULTS: 63 L-AMB and 116 ABLC patients were identified for analysis. The L-AMB and ABLC groups were similar in gender $(67 \%$ vs $57 \%, \mathrm{p}=$ $0.202)$ and mean (SD) age 48.4 (22.2) vs 55.8 (18.1) ( $\mathrm{p}=0.171)$. The most common diagnosis was myeloid leukemia seen in $17.5 \%$ of L-AMB and $20.7 \%$ of ABLC patients $(\mathrm{p}=0.603)$. Organ system dysfunction (respiratory, hematologic, hepatic, cardiovascular or renal) within $48 \mathrm{~h}$ of admission was seen in $77.8 \%$ of L-AMB and $67.2 \%$ of ABLC patients ( $p=0.138$ ). Comorbid conditions were generally similar between the two cohorts with the mean (SD) Charleston Comorbidity Score for L-AMB of 2.52 (1.99) and for ABLC of $2.97(2.09)(\mathrm{p}=0.164)$. Mean (SD) baseline $\mathrm{SCr}$ was $1.2(0.9) \mathrm{mg} / \mathrm{dL}$ for L-AMB and $1.1(0.7) \mathrm{mg} / \mathrm{dL}$ for ABLC $(\mathrm{p}=0.411)$. For those with sufficient data, mean (SD) pre-to-post \% increase in $\mathrm{SCr}$ was 58.1 (84.8) for L-AMB $(n=52)$ and $120.66(159.1)$ for ABLC $(n=106)(p=0.004)$, while $14.3 \%$ of L-AMB and $31.9 \%$ of ABLC patients met the definition of nephrotoxicity $(p=0.01)$. In MVA, ABLC patients were 2.97 times more likely to have nephrotoxicity than patients receiving L-AMB (95\% CI, 1.22 $7.25, \mathrm{p}=0.016$ ). While not statistically significantly different, hypokalemia was more frequent for $\operatorname{ABLC}(69.1 \%, \mathrm{n}=97)$ vs. L-AMB $(62.5 \%, \mathrm{n}=48, \mathrm{p}=0.429)$ as was hypomagnesemia (L-AMB $36.8 \%, n=38)$ vs. ABLC $(41.2 \%, n=85)(p=$ $0.650)$, and infusion-related reactions requiring treatment were more frequent in $\operatorname{ABLC}(23.3 \%)$ vs. L-AMB $(14.3 \%, \mathrm{p}=0.152)$.

CONCLUSIONS: In this retrospective study of neutropenic hospitalized inpatients comparing the use of L-AMB to ABLC for therapy of IFIs, LAMB demonstrated a significantly lower rate of nephrotoxicity. When an amphotericin B agent is required, the consequences of nephrotoxicity should be strongly considered before selecting a particular agent.

M.O.L.E.S (MULTIMEDIA ON-LINE EDUCATION IN SKIN CONDITIONS) OFFERED AS SELF-DIRECTED TEACHING MODULE RESULTS IN LOW RATES OF USE AMONG INTERNAL MEDICINE ATTENDINGS Carrie Mahowald; Oluwatumininu Johnson; Ross Radusky; Miguel Sanchez. NYU, New York, NY. (Control ID \#1337805)

BACKGROUND: A study in 2004 by Antic, et al. in Dermatology studied the diagnostic accuracy of dermatologic conditions in 1290 patients by General Internists. Only $51.1 \%$ of these conditions were accurately diagnosed and a significant amount of referrals from Generalists are deemed unnecessary by receiving Dermatologists. We identified the top ten dermatologic conditions that both Dermatologists and Internists in our NYU community believe that Internists should be able to diagnose and manage. We built a multi-media online teaching tool targeting these conditions to aid in improving patient care and preventing unnecessary referrals. Despite great initial enthusiasm from Internists to have access to the modules we have seen a very low rate of completion in the first six months of availability.

METHODS: We developed a self-directed multi-media on-line teaching tool based on data collected using an IRB approved structured survey method of facilitated focus groups (twenty Internists and Dermatology Residents and Attendings, associated with NYU School of Medicine). The top ten dermatologic conditions generated from these focus groups that generalists should be able to diagnose and manage included: general skin care, skin exam and basic nomenclature, drug eruptions, suspicious moles, dermatitis, alopecia, acne, lower extremity lesions (stasis dermatitis, cellulitis, vasculitis), common outpatient procedures, and fungal infections. The modules were announced in person and via email to invite participation of Internists. Email reminders were sent monthly for 6 months to those requesting access. We monitored module use and pre- and post-test scores.

RESULTS: Despite initially greatly expressed enthusiasm of Internists to have access to these modules, very few attempted or completed them. Of the 43 Internists who requested access to the on-line modules, only 9 attempted all 10 modules, and only 1 completed 8 modules. Eight completed module 1 , six completed module 2 , two completed modules 3 , 4 and 5 , one completed modules 6,7 , and 8 , and zero completed modules 9 or 10 . The majority of attempts and completions occurred within the first 
month of their release. This drop off in participation demonstrates a participant cascade effect.

CONCLUSIONS: The low rate of interested Attnendings attempting the modules might be a reflection of lack of time and sustained motivation. Incentives such as CME credits, gift cards, protected learning time, and more frequent email reminders may ameliorate this participant cascade effect. Surveying the Attendings about the obstacles to attempting the modules might prove very helpful in evaluating the usability of our teaching tool. Because of the low rate of completion so far, thorough assessment of the module's effectiveness is difficult at this time. We are hopeful that through the aforementioned incentives and surveys, better participation rates shall occur.

MEDLEAD: DEVELOPMENT AND IMPLEMENTATION OF A HEALTH PROFESSIONAL LEADERSHIP AND POLICY CURRICULUM Ali Khan ${ }^{1,2}$; Theodore Long ${ }^{1,2}$; Rebecca Brienza ${ }^{2,1}$. ${ }^{1}$ Yale School of Medicine, New Haven, CT; ${ }^{2}$ VA Connecticut Healthcare System, West Haven, CT. (Control ID \#1310845)

BACKGROUND: Increasing attention has been placed to the need for greater integration of health policy and team-based improvement in medical education. In 2010, the Veterans Administration Office of Academic Affiliations established five Centers of Excellence (CoE) in Primary Care Education. The program represents a new frontier in the transformation of American medicine: educational paradigms that foster new inter-professional care models while emphasizing innovation, continuous improvement and team leadership. In creating leaders in primary care delivery, however, attention must be paid to another key domain: grounding in translational health policy with a focus on the skill set necessary to succeed as agents of change. Via deliberate, structured curricula in health policy and public leadership, the VA Connecticut $\mathrm{CoE}$ aims to endow trainees with the tools necessary for effectual action across sectors - while building a national model for training in medicine and public service.

METHODS: We developed a survey assessing two major components: content assessment of trainees' knowledge of public policy and leadership concepts and attitude assessment of trainees' comfort with application of those concepts, focused on the Batalden medical education domains and measured on five-point Likert type scales. The survey was administered to matched groups of $\mathrm{CoE}$ and non-CoE resident physician/nurse practitioner fellow trainees prior to participation in the CoE's MedLEAD curriculum, a learner-driven educational paradigm focused on domestic health policy, leadership and public sector engagement. Championed, developed and taught by $\mathrm{CoE}$ senior residents, curricula include novel assessment models to both determine the impact of MedLEAD training and flexibly accommodate learners of varying experience to allow for rapid advancement in skill acquisition. Interval means were calculated and analyzed for statistical significance.

RESULTS: Baseline knowledge of and comfort with core health policy and leadership concepts was low among $\mathrm{CoE}$ trainee respondents. Most respondents $(66 \%)$ rated their prior involvement in public policy and leadership experiences as minimal to non-existent $(1=$ non-existent, $5=$ extensive, mean $=2.3$ ); similar proportions indicated that the integration of health policy and leadership development training within their medical education was lacking, with mean Likert ratings of disagreement to neutrality (2.6) as to whether prior training in these areas was appropriate. Respondents uniformly $(100 \%)$ expressed neutrality to discomfort (mean 2.2 ) with their ability to effect cultural, structural or policy change across a variety of sectors (public/private/non-governmental).

CONCLUSIONS: Findings among trainees mirror the growing consensus in American medicine: training in health policy and leadership is necessary (and frequently absent) within medical education. Policy and leadership curricula that exist, however, overwhelmingly emphasize either international or private sector action. MedLEAD's public sector focus thus represents a unique curricular model in American medical education. More research is needed to measure MedLEAD's impact on its intended core outcomes: acquisition and retention of knowledge of core policy and leadership concepts, confidence in newly acquired knowledge - and, uniquely, the application of those concepts in everyday practice (as measured by skills, behaviors and clinical outcomes).
MALPRACTICE RISK AND RATES OF IMAGING USE AT US HOSPITALS Jason S. Mathias; Joe Feinglass; David W. Baker. Northwestern University Feinberg School of Medicine, Chicago, IL. (Control ID \#1339819)

BACKGROUND: Physicians consistently report that fear of malpractice claims encourages them to order diagnostic tests, even when tests are of low-value. The Centers for Medicare and Medicaid Services has identified four imaging tests that may be of low value if overused, and U.S. hospitals' rates are publicly reported in the Hospital Outpatient Quality Reporting Program (HOQR): magnetic resonance imaging for low back pain (MRI), follow-up imaging after screening mammography (MAM), and abdominal and thoracic computed tomography with and without contrast (A-CT and T-CT). We sought to determine if high-outlier (i.e., top decile) performance on the HOQR imaging use measures was associated with three measures of local malpractice risk: 1) average county malpractice insurance premiums, 2) state claims risk per physician, and 3) caps on non-economic damages. METHODS: The dependent variable was high-outlier performance on an HOQR imaging use measure, defined as a performance score in the top decile among reporting hospitals. We used logistic regression to examine the association between high-outlier performance and three measures of regional malpractice environment 1) a county level average of internal medicine, general surgery, and obstetrics and gynecology annual malpractice premiums derived from the Medical Liability Monitor 2008, 2) state claims risk per physician (2008 claims per physician times average payment per claim), and 3) states with tort law reforms capping noneconomic damages as of 2008. The premium and claims risk data were divided into tertiles and entered as categorical variables with the lowest tertile as the reference group. Caps on non-economic damages were coded as present or absent. All analyses were adjusted for hospital's HOQRreported measure specific imaging volume and 2009 American Hospital Association Survey reported geographic setting, ownership status, teaching status, and percentage of discharges with Medicare or Medicaid insurance. RESULTS: When compared to hospitals in areas in the lowest tertile for malpractice premiums, those in the highest tertile were more likely to be highoutliers on MAM (adjust OR 1.98 [1.43-2.74]) and less-likely to be highoutliers on MRI (OR .56 [.37-.84]). Similarly, when compared to hospitals in areas with lowest tertile claims risk per physician, those with highest tertile claims risk per physician were more likely to be high-outliers on MAM (OR 1.76 [1.32-2.35] and less likely to be high-outliers on MRI (OR .61 [.41-.91]). Neither premiums nor claims risk were significantly associated with highoutlier status on A-CT or T-CT. There was no consistent relationship between the presence of caps on non-economic damages and high-outlier status (adjusted OR [95\% CI] MRI: .73 [.53-.99]; MAM: .99 [.77-1.27]; A-CT: 1.62 [1.27-2.06]; T-CT: 1.39 [1.08-1.78]).

CONCLUSIONS: Higher local malpractice risk (e.g., higher premiums, higher claims risk) was associated with increased likelihood of high-outlier status on MAM but not MRI, A-CT, or T-CT. The relationship between caps on non-economic damages and high-outlier status was inconsistent. Interventions focused solely on alleviating malpractice risk may decrease overuse of specific imaging studies. However, to meaningfully reduce overuse of all imaging studies, interventions will need to simultaneously address other factors that may encourage imaging overuse.

MANAGEMENT OF CARDIOVASCULAR RISK FACTORS IN DIABETIC PATIENTS: COMPLIANCE TO GUIDELINES IN THE UNITED STATES. Bhavana Siddegowda Bangalore ${ }^{1}$; Siva K. Talluri ${ }^{1}$; Jyosthna Talluri ${ }^{1}$; Punnaiah Marella ${ }^{2}$; Siddesh Besur ${ }^{1}{ }^{1}$ Mclaren Regional Medical Center, Flint, MI; ${ }^{2}$ Banner Estrella Medical Center, phoenix, AZ. (Control ID \#1320393)

BACKGROUND: Individuals with diabetes have twice the risk of myocardial infarction and stroke as the general population. Current guidelines recommend treatment of all modifiable cardiovascular disease risk factors (CVD-RF) in diabetic patients. Although the incidence of CVD events in patients with diabetes has declined over the past decade, many studies have shown that CVD-RF control has been inadequate. Our study 
objective is to examine compliance to American Diabetic Association (ADA) guidelines for CVD-RF modification in the United States (US). METHODS: National Health and Nutrition Examination Survey (NHANES) is conducted by the US National Center for Health Statistics. It collects participants' demographic, socioeconomic, dietary and health-related information. Its complex design allows nationally representative random sampling of US patients. We conducted a secondary data analysis of a retrospective cohort of adult diabetic patients included in the 2008 NHANES. We excluded those not seeing a doctor for their diabetes. The primary outcome was to measure the proportion of US diabetic patients that met ADA diabetes care guidelines on control of blood sugar, blood pressure, cholesterol and albuminuria and secondarily examine gender differences. We defined as diabetic those individuals told by their doctor to have diabetes. We described their demographic characteristics. We calculated the proportion meeting diabetes care guidelines. We compared compliance to guidelines among men and women using Chi-square test. We analyzed the data with SPSS complex samples 19.0 software (SPSS Inc, Chicago, IL). Our study was reviewed and exempted by the Institutional Review Board.

RESULTS: There were 759 adults with diabetes in our sample (projected to 18.5 million in the US). We excluded 182 who were not seen by a doctor for diabetes (leaving a projected US population of 14.6 million). The goal of hemoglobin A1C $<7$ was met in $49 \%$ (95\% CI $39 \%-59 \%$ ), LDL was $<100$ in $62 \%(95 \%$ CI $54 \%-69 \%)$, triglycerides $<150$ in $54 \%(95 \%$ CI $46 \%-62 \%)$, blood pressure $<130 / 80$ in $51 \%$ (95\% CI $41 \%-62 \%)$, HDL goal of $>40$ in men and $>50$ in women was met in $60 \%(95 \%$ CI $50 \%-69 \%)$, urine albumin creatinine ratio $<30$ in $59 \%$ (95\% CI 51\%-66\%). Majority were non-smokers $65 \%$ (95\% CI $51 \%-66 \%$ ). Men were more likely to meet guideline goal achievement only for LDL, odds ratio 2.7 (95\% CI 1.4-5.3, P =0.006), and HDL, odds ratio 2.18 (95\% CI 1.3-3.5, $\mathrm{P}=0.003)$. No other gender differences were appreciated.

CONCLUSIONS: Treatment goals for CVD-RF were met in slightly more than half of diabetic patients. Men are more likely to meet LDL and HDL goals compared to women. The proportion not meeting goals of care was similar to the 2002 NHANES published results except for an improvement in control of HDL and triglycerides in 2008. We did not have information about medication compliance, which plays an important role in reaching treatment goals. We need to focus on strategies to optimize CVD-RF modification and compliance to ADA diabetes care guidelines.

MANAGEMENT OF IMPLANTABLE DEFIBRILLATORS IN HOSPICE PATIENTS-NEED FOR A POLICY? Deirdre R. Pachman ${ }^{1}$; Abigale L. Ottenberg ${ }^{2}$; Nou Chang ${ }^{3}$; Katlyn E. Cook ${ }^{3,2}$; Paul S. Mueller ${ }^{3,2}$; Keith M. Swetz ${ }^{3,4}$. ${ }^{1}$ Mayo Clinic, Rochester, MN; ${ }^{2}$ Mayo Clinic, Rochester, $\mathrm{MN} ;{ }^{3}$ Mayo Clinic, Rochester, MN; ${ }^{4}$ Mayo Clinic, Rochester, MN. (Control ID \#1326201)

BACKGROUND: Implantable cardioverter-defibrillators (ICDs) are common as indications for device implantation expand. However, shocks from ICDs can be painful and distressing, and may negatively affect quality of life in patients at end of life. Recent studies have demonstrated that patients enrolled in hospice may experience shocks at end of life yet many hospice programs do not have policies addressing ICD deactivation. In this study, we explored the experiences of regional hospice programs with patients with ICDs, the final goal was to assist programs in policy development. METHODS: Forty-nine hospice programs were identified in the referral area of our academic medical center (i.e., Minnesota, Iowa, and Wisconsin). An email with a link to a survey was sent to each program. The survey was comprised of 23 closed and open-ended questions and participants were given 4 weeks to respond. The survey collected data on organizational characteristics, professional experiences with ICD management, and the existence and function of policies related to the care of patents with ICDs. Descriptive statistics were used.

RESULTS: Twelve hospice programs responded to the survey. All programs admitted patients with active ICDs but only $25 \%$ had questions on the intake form that inquired about the presence of an ICD. The discussion and decision to deactivate the ICD caused distress for $50-75 \%$ of patients and families. In addition, $58 \%$ of programs reported that the discussion caused distress for the hospice staff and this was more common in programs without a policy. Only five programs specifically trained staff in having conversations with patients and their caregivers about ICD deactivation. The most common method of ICD deactivation was in the patient's home by an industry employed allied professional and the majority of programs did not have a magnet available for emergency ICD deactivation. Some programs $(25 \%)$ reported that patients were shocked at end of life and $50 \%$ of programs had policies. All six programs without policies perceived they would benefit from a policy. A sample policy was developed with input from experts in the field of palliative medicine, cardiology, and ethics. This policy will be provided to interested hospice programs.

CONCLUSIONS: ICD shocks can be a source of pain, suffering, and anxiety for patients at end of life. However, the discussion of ICD deactivation can also cause distress for patients, family, and hospice staff. A formal policy to address ICD management in hospice patients may reduce the stress and discomforts associated with ICD deactivation discussions by providing direction, ensuring consistency, and promoting communication, as well as prevent unwanted shocks at end of life.

Management of ICDs in Hospice Programs

\begin{tabular}{ll}
\hline \hline $\begin{array}{l}\text { Program Responses } \\
\text { Programs with a formal policy addressing ICD deactivation }\end{array}$ & $\begin{array}{l}\mathrm{N}=12 \\
6(50 \%)\end{array}$ \\
$\begin{array}{l}\text { Staff specifically trained in conversations with patients/ } \\
\text { caregivers }\end{array}$ & $5(42 \%)$ \\
about ICD deactivation & $6(50 \%)$ \\
Discussion to deactivate ICD caused distress for patients & $9(75 \%)$ \\
Discussion to deactivate ICD caused distress for family & $7(58 \%)$ \\
Discussion to deactivate ICD caused distress for hospice staff & $3(25 \%)$ \\
Patient shocked by ICD at end of life in past 5 years & $4(33 \%)$ \\
Magnet available for emergency ICD deactivation &
\end{tabular}

MANAGEMENT OF SUBSTANCE ABUSE AND DEPENDENCE IN U.S. AMBULATORY CARE, 2001-2009 Joseph Frank; John Z. Ayanian; Jeffrey A. Linder. Brigham and Women's Hospital, Boston, MA. (Control ID \#1334748)

BACKGROUND: Psychosocial therapy has long been a mainstay in the treatment of substance abuse and dependence but effective pharmacologic options have increased over the past decade. We sought to examine predictors and trends in the provision of pharmacotherapy and psychosocial therapy for the treatment of substance abuse and dependence in U.S. ambulatory care.

METHODS: We conducted a serial cross-sectional analysis of the 2001-2009 National Ambulatory Medical Care Survey ( $\mathrm{N}=252,450$ visits) and the National Hospital Ambulatory Medical Care Survey ( $N=302,440$ visits). We identified visits related to alcohol or drug abuse or dependence $(\mathrm{N}=9741)$ by diagnosis codes (ICD-9) or reason for visit codes. Among these 9741 visits, we identified visits in which either of the two main modalities of treatment of these conditions was provided. We defined pharmacotherapy as the prescription of methadone, buprenorphine, acamprosate, naltrexone or disulfiram. We defined psychosocial therapy as the provision of either psychotherapy or other mental health counseling. We examined treatment trends across 3 -year time periods using chi-square trend tests. We used multivariable logistic regression to identify predictors of each treatment modality, adjusting for time period and available patient, visit, and practice characteristics. We used SUDAAN software to account for the complex survey design.

RESULTS: Of an estimated 46.8 million visits for substance abuse and dependence in the US between 2001 and $2009,19 \%$ of patients were $>55$ years old, $58 \%$ were male, $81 \%$ were White and $39 \%$ had a primary payment source of Medicaid or Medicare. Total visits increased from 12.4 million in 20012003 to 19.3 million in 2007-2009 ( $p=.02$ ). Physicians prescribed pharmacotherapy in 6.3 million (13\%) visits, psychosocial therapy in 25 million $(55 \%)$ visits, and both therapies in 4.2 million (9\%) visits; neither therapy was recorded in 18.7 million (40\%) visits. Methadone and buprenorphine accounted for $77 \%$ of pharmacotherapy visits. Rates of pharmacotherapy were $5 \%$ (643,000 visits) in 2001-2003, 12\% (1.7 million visits) in 2004-2006 and $20 \%$ (3.9 million visits) in 2007-2009 ( $<<.001)$. Independent predictors of pharmacotherapy included age $>55$ years old (OR 0.3 compared to age $<35$ years old; $95 \%$ CI $0.2-0.6$ ), Psychiatry clinic visits (OR 0.4 compared to Internal Medicine/Family Practice clinic visits; 95\% CI 0.2-0.8) and 
Substance Abuse clinic visits (OR 0.4 compared to Internal Medicine/Family Practice clinic visits; 95\% CI 0.2-0.9). Rates of psychosocial therapy were unchanged over time $(59 \%$ in $2001-2003,52 \%$ in $2004-2006$, and $56 \%$ in 2007-2009, $\mathrm{p}=.28)$. Independent predictors of psychosocial therapy included age $>55$ (OR 0.6 compared to age $<35 ; 95 \%$ CI $0.4-0.9$ ), Psychiatry visits (OR 14.5 compared to Internal Medicine/Family Practice clinic visits; 95\% CI 924 ) and Substance Abuse clinic visits (OR 3.4 compared to Internal Medicine/ Family Practice clinic visits; 95\% CI 1.8-6.2).

CONCLUSIONS: Total ambulatory visits involving substance abuse or dependence have increased over time, and visits involving pharmacotherapy have accounted for an increasing share. Adoption within Internal Medicine and Family Practice specialties appears to have driven this growth while psychosocial therapy remained more common in Psychiatry and Substance Abuse visits. Greater use of both pharmacotherapy and psychosocial is needed as $40 \%$ of visits with a diagnosis of substance abuse or dependence did not involve treatment.

MANAGING CHRONIC PAIN WITH OPIOID MEDICATIONS: PATIENT VOICES Alicia A. Bergman ${ }^{1}$; Jessica Coffing ${ }^{1}$; Marianne S. Matthias $^{1,2}$; Erin E. Krebs ${ }^{1,2}$. ${ }^{1}$ Roudebush VAMC, Indianapolis, IN; ${ }^{2}$ Indiana University School of Medicine, Indianapolis, IN. (Control ID \#1342335)

BACKGROUND: Over the past two decades opioid prescribing for chronic pain has increased dramatically, along with rates of opioid abuse and opioid-related overdose deaths. National guidelines stress that health care providers should monitor for opioid effectiveness, adverse effects, adherence, and signs of misuse, addiction, or diversion among patients on opioid therapy. Some monitoring strategies, such as contracts and urine screening, have potentially punitive undertones and may have unintended alienating or stigmatizing effects. The goal of this qualitative study was to develop a better understanding of the experiences, perceptions, and challenges patients with chronic pain face when communicating with their primary care physicians about opioid therapy and monitoring.

METHODS: Semi-structured interviews were conducted with 24 patients from a federally funded hospital in the Midwest. Purposive and snowball sampling techniques were first used to identify 14 primary care physicians, who then provided lists from which patients were randomly identified. All of the patients were receiving long-term opioids for chronic pain.

RESULTS: Qualitative thematic analysis of the interviews revealed three major themes: 1) avoiding opioids; 2) wanting pain to be acknowledged; and 3) treating patients as individuals. Wanting to avoid opioids was one of the most common themes and was overwhelmingly driven by patient fears of addiction, although other reasons included side effects, as well as how opioids limit daily functioning and cover up underlying symptoms. Patients discussed their initial discomfort with starting opioids and their desires to taper off of them or stop them completely. As one patient described, "You prescribe pain medicine, you just started a junkie." Another patient recalled, "each one of them [opioids] I took myself off of....like, I'm not gonna become a dope addict, you know, because of pain." The second theme of wanting pain to be acknowledged deals to a large extent with how the patients often did not feel as though the physicians wanted to hear about their experience of pain or talk about it during the appointment. As one patient said, "I mean they don't really ask you how, you know, 'what's your problems'... she [current doctor] never really wanted to listen to what I tell her. And I'm like, 'well, I'm trying to explain to you that I'm hurting, you know.' It's like, 'what do you want me to do?' That's her words, 'what do you want me to do?"” The third theme was related to patients wanting their physicians to treat them as individuals, as opposed to being stereotyped (e.g., assuming patients are addicts based on "that one bad apple" as put by one patient), or lumped into broad categories when it comes to prescribing. As stated by another patient, "everything needs to be on a... one-to-one, on a personal basis, no matter what it is."

CONCLUSIONS: Gaining in-depth knowledge about patients' struggles with opioid therapy is an important step in creating interventions that help both patients and providers with chronic pain management. Important implications for clinical practice from this study include specific strategies for how primary care physicians can approach patients.
MANY HOSPITALIZATIONS THAT ORIGINATE FROM NURSING HOMES ARE POTENTIALLY PREVENTABLE Rebekah Gardner $^{1,4}$; Orna Intrator ${ }^{3}$; David Gifford ${ }^{2,1}$; Stefan Gravenstein ${ }^{1,4}$. ${ }^{1}$ Alpert $\overline{M e}$ dical School of Brown University, Providence, RI; ${ }^{2}$ American Health Care Association, Washington, DC; ${ }^{3}$ Brown University, Providence, RI; ${ }^{4}$ Healthcentric Advisors, Providence, RI. (Control ID \#1334907)

BACKGROUND: Quality initiatives are increasingly focused on preventing unnecessary hospital admissions, as hospitalizations are burdensome for patients, costly to the healthcare system, and may indicate an opportunity to improve care. Ambulatory care sensitive conditions have been used by researchers to measure potentially preventable hospitalizations in an outpatient population. These outpatient conditions may not apply to the generally frailer and more medically complex nursing home residents, who reside in an environment offering 24-hour observation and care. We sought to develop a model that identified potentially preventable hospitalizations from the nursing home setting in order to examine regional variation and to highlight structural issues that could be targeted for improvement.

METHODS: We convened an expert panel to review diagnoses in a revised version of the ambulatory care sensitive conditions and to identify which diagnoses best identified potentially preventable hospitalizations of nursing home patients. Diagnoses from "preventable" hospitalizations are those conditions that are more likely to be avoided or managed in the nursing home. The Residential History File (based on fee-for-service Medicare claims and eligibility files and minimum data set [MDS] assessments) was used to identify hospitalizations originating from a nursing home in 2009. The closest MDS prior to the hospitalization was used for demographic and clinical characteristics. Residents were stratified based on length of nursing home stay (0-7 days, 8-30 days, 31-90 days, and long-stayers over 90 days), and the prevalence of potentially preventable nursing home hospitalizations (PPNH) for each group was examined. Variation in the rate of PPNH was explored at the state and regional levels.

RESULTS: Twenty six PPNH categories with ICD-9 codes were identified. There were 563,036 nursing home residents who had at least one hospitalization, and a total of 808,937 hospitalizations. Almost $60 \%$ of all hospitalizations were among nursing home residents who had been in the facility for at least 90 days. Hospitalized nursing home residents were more often female $(64.5 \%)$ and $39.4 \%$ were 85 years or older. Among all hospitalizations, $25.8 \%$ were classified as potentially preventable in residents who had been in the nursing home for fewer than 7 days, $29.3 \%$ in residents who had been in the nursing home for $8-30$ days, $31.7 \%$ in residents there for 31-90 days, and 34.8\% in residents there for $>90$ days. Most potentially preventable conditions were more prevalent among long-term residents, including COPD, glycemic abnormalities, pneumonia, and urinary tract infections. However, CHF, diarrhea, and delirium/confusion were more common diagnoses among short-stay residents. There was substantial variation in rates of PPNH by state (ranging from $27.0 \%$ in California to $44.7 \%$ in South Dakota).

CONCLUSIONS: Adapting ambulatory care sensitive conditions for the nursing home setting revealed a high number of hospitalizations from nursing homes that are potentially preventable. The regional variation both in the number of hospitalizations and the diagnoses leading to those admissions indicates an opportunity for improvement. These measures can be used to test quality improvement interventions in this setting as well as to highlight concerns about national long-term care infrastructure and policy.

MASSACHUSETTS HEALTH REFORM DID NOT REDUCE 30DAY READMISSIONS FOR ACUTE MYOCARDIAL INFARCTION AMONG MASSACHUSETTS RESIDENTS OVERALL OR AMONG RACIAL/ETHNIC MINORITIES. Karen E. Lasser ${ }^{1,4}$; Chieh $\mathrm{Chu}^{1}$; Danny McCormick ${ }^{2}$; Nancy R. Kressin ${ }^{3,1}$; Meredith D'Amore ${ }^{1}$; Howard Cabral ${ }^{4}$; Alok Kapoor ${ }^{1}$; Adam Rose ${ }^{5,1}$; Amresh Hanchate ${ }^{3,1}$. ${ }^{1}$ Boston Medical Center/Boston University School of Medicine, Boston, MA; ${ }^{2}$ Cambridge Health Alliance/Harvard Medical School, Cambridge, MA; ${ }^{3}$ VA Boston Healthcare System, Boston, MA; ${ }^{4}$ Boston University School of Public Health, Boston, MA; ${ }^{5}$ Bedford VA Medical Center, Bedford, MA. (Control ID \#1310972) 
BACKGROUND: Following Massachusetts (MA) health reform, the percent of uninsured residents fell from $8.4 \%$ to $3.4 \%$. Prior studies have not examined the effect of this policy change on 30-day readmission rates for acute myocardial infarction (AMI). We hypothesized that these admissions, which are sensitive to access to outpatient care, would decline in MA following reform, particularly among minorities historically disadvantaged in terms of insurance coverage.

METHODS: We analyzed 2004-2009 inpatient discharge data from MA and New York ([NY], which did not undergo health reform). We compared 30-day readmission rates (for any cause) after a hospitalization for AMI overall, and among racial/ethnic subgroups, for patients age 18-64 (those affected by health reform) in the 21 months prior to and following MA reform. We used chi-square tests to compare unadjusted 30-day readmissions for AMI between groups. Treating MA adults as the intervention cohort, and NY adults as the control cohort, we used logistic regression to conduct a difference-in-difference analysis that estimates odds of readmission in the post-reform period vs. the pre-reform period in MA adjusted for secular changes unrelated to reform. We performed this analysis for the entire sample and also stratified by whites, blacks, and Hispanics. The model was also adjusted for age, gender, and Charlson Comorbidity Score. In order to assess differences in white vs. minority disparities over time between the pre and post reform periods in MA relative to NY, we used logistic regression to conduct a difference-in-difference-in-differences analysis.

RESULTS: There were 50,720 admissions for AMI in NY and MA over the study period. In MA, pre-reform and post-reform readmission rates were $11.5 \%$ and $10.5 \%$. In NY, they were $12.8 \%$ and $12.3 \%$ respectively. The postreform decrease in MA was not significantly different than that in NY (difference-in-difference adjusted OR [AOR] 1.0, 95\% confidence interval [CI], 0.9-1.1). In MA, blacks had higher readmission rates than whites both pre-reform $(16.9 \%$ vs. $11.3 \%$; $=0.003)$ and post-reform $(16.1 \%$ vs. $10.2 \%$; $\mathrm{p}=0.0006)$. In NY, blacks also had higher readmission rates than whites prereform $(18.8 \%$ vs. $11.4 \%$; $<<0.0001)$ and post-reform $(17.2 \%$ vs. $11.2 \%$; $<<$ $0.0001)$. Prior to reform in MA, Hispanics had significantly higher readmission rates than whites $(16.5 \%$ vs. $11.3 \%, \mathrm{p}=0.005)$; post-reform there was no significant difference in readmission rates $(12.8 \%$ vs. $10.2 \%, \mathrm{p}=0.1)$. In NY, Hispanics had significantly higher readmission rates than whites, both pre- and post-reform $(15.9 \%$ vs. $11.4 \%, \mathrm{p}<0.0001$ and $16.3 \%$ vs. $11.2 \%, \mathrm{p}<$ 0.0001 , respectively). Difference-in-difference estimates stratified by race/ ethnicity indicated that the post-reform changes in readmission rates in MA and NY were not significantly different among whites, blacks, and Hispanics. In difference-in-difference-in-differences analyses, there was no significant change in the presence of disparities between whites and blacks or between whites and Hispanics in NY vs. MA pre- and post-reform.

CONCLUSIONS: A major coverage expansion in MA was not associated with a reduction in 30-day readmissions for AMI overall or a reduction in racial and ethnic disparities in this outcome. Other interventions may be needed to further reduce 30-day readmissions for AMI overall, and to decrease disparities in such readmissions.

\section{MAXIMIZING TEACHING EFFECTIVENESS: PREDICTORS OF LEARNER SATISFACTION WITH TEACHING ON ROUNDS Chad} Stickrath $^{1,2}$; Megan Griffiths ${ }^{2}$; Allan V. Prochazka ${ }^{1,2}$; Eva M. Aagaard ${ }^{2}$; $\overline{\text { Melver Anderson }}{ }^{1,2}$; Melissa N. Deloughry ${ }^{2} .{ }^{1}$ Denver VA Medical Center, Denver, CO; ${ }^{2}$ University of Colorado Denver, Aurora, CO. (Control ID \#1339996)

BACKGROUND: Traditionally, inpatient academic internal medicine teams have employed "attending rounds" to fulfill their patient care and teaching duties. As a part of a study to assess the current characteristics and impressions of internal medicine attending rounds, we investigated the predictors of learner satisfaction with attending teaching on rounds.

METHODS: We conducted a cross-sectional study of attending ward rounds on the general inpatient medicine services at four teaching hospitals affiliated with a large public medical school. Trained, independent observers accompanied general internal medicine teams on attending rounds to observe, time, and record the activities of these rounds, including the location, participants, patient care activities, communication activities, and teaching activities. A single observer followed each team, observing one post-call day and one non-post-call day. Observations did not occur on the first day of the month and were otherwise completed on random days during the month. After observing the entire episode of attending rounds for the day, observers then invited attendings and trainees to complete a 12item self-administered questionnaire. Items on the questionnaire utilized a 4-point Likert scale to assess: overall teaching effectiveness, attending input to patient care plans, effective teaching of history-taking or physical exam skills, the opportunity for trainees to direct the learning, medical topic teaching, helpful references being made to the medical literature, areas for future learning, the attending providing useful feedback, the creation of a safe learning environment, and whether the observed rounds were representative of a normal day. We used Chi Square testing and logistic regression to assess predictors of learner satisfaction with attending teaching.

RESULTS: We observed 63 rounding sessions involving 605 patients and 206 trainees (34\% residents, $27 \%$ interns, $25 \%$ third-year medical students, $7 \%$ sub-interns). Rounds lasted on average 123 minutes $( \pm 42)$. Trainee satisfaction with teaching on rounds was high overall $(45 \%$ strongly agreed and $50 \%$ agreed that effective teaching occurred on rounds that day). Perceptions about the quality of teaching was not influenced by trainee level or the duration of rounds ( $\mathrm{p}=\mathrm{NS}$ for all comparisons). In logistic regression, the independent predictors of high satisfaction with attending teaching were the attending adding valuable patient care information (OR 7.44), effectively teaching physical exam skills on rounds (OR 2.01), being given the opportunity to identify topics for learning (OR 2.48), helpful references to the literature being given (OR 1.83), and history taking skills being taught effectively (OR 1.76). Provision of feedback and a safe learning environment were not independently predictive of teaching satisfaction.

CONCLUSIONS: We found that trainee satisfaction with attending teaching related to discrete activities that emphasize clinical skills (history/physical exam), discussion of medical literature and providing information that is felt to be valuable for patient care. Interventions to improve the quality of attending rounds teaching should consider focusing on these key elements.

MEASURING SURVIVAL-TO-DISCHARGE RATES FOR IN-HOSPITAL CARDIAC ARREST WITH THE USE OF THE LUCAS-CPR CARDIOPULMONARY RESUSCITATION (CPR) ASSIST DEVICE Daniel Gutteridge; Siva K. Talluri; Bhavana Siddegowda Bangalore; Pramod K. Kalagara. McLaren Regional Medical Center, Flint, MI. (Control ID \#1339491)

BACKGROUND: Cardiopulmonary resuscitation gained popularity during the 1960's. The national in-hospital survival-to-discharge rates are 15$20 \%$, similar to those observed two decades earlier. The recent addition of cardiopulmonary resuscitation (CPR) assist devices may have the potential to improve the current survival-to-discharge rates. The LUCAS-CPR device, an active compression/decompression CPR assist tool, provides chest compressions at an optimal rate and depth in an uninterrupted manner. This eliminates human error due to physical fatigue by both limiting interruptions and inadequate compressions that can impair adequate circulation. The LUCAS-CPR creates higher coronary perfusion pressures with a lower incidence of rib fractures. Early studies in the out-of-hospital setting showed no increase in survivalto-admission rates with the adoption of CPR assist devises. We found no studies reviewing in-hospital use of assist devices. Our objective is to compare survival-to-discharge rates of in-hospital cardiac arrests using the LUCASCPR assist device to those that did not employ the device at our facility.

METHODS: This is a retrospective analysis of hospitalized patients who experienced a CPR-related event over a 21-month period (20102011) in a US community hospital. Patients were excluded if "Code Sheets" were incomplete or Advanced Cardiac Life Support (ACLS) was stopped within 5 minutes due to by family request or advance directives noted in the chart. The population studied was characterized 
according to age, sex, race, cardiac rhythm, co-morbid conditions and the use of LUCAS-CPR at the time of CPR initiation. We also looked at the individual patient's All Patient Refined-Diagnosis Related Group (APR-DRG) morbidity and mortality risk, a system wide insurance tool that stratifies risk to evaluate severity of illness of hospitalized patients based on current condition and procedures.

RESULTS: One hundred nineteen patients had in-hospital CPR-related events with "Code Sheets". Thirty met exclusion criteria leaving a study population of 89 . The mean age (SD) of study population is 71 (13.5). It was predominantly men $57.3 \% \quad(\mathrm{~N}=51)$ and Caucasian $70.8 \%(\mathrm{~N}=63)$. Cardiac rhythm at the time of cardiac arrest was predominantly Pulseless Electrical Activity (PEA) $50.6 \%(\mathrm{~N}=45)$ followed by asystole $20.2 \%(\mathrm{~N}=18)$. Survival-to-discharge rate in the study population was $25 \%(\mathrm{~N}=22)$. LUCAS-CPR machine was used in $57.3 \%(\mathrm{~N}=51)$ of patients. The mean age (SD) of patients in LUCAS-CPR group was 70 (13.2) and non-LUCAS-CPR group was 71 (14.1). Cardiac rhythm at the time of arrest was similar in LUCAS-CPR group, PEA 49\% ( $\mathrm{N}=25)$ and asystole $21.6 \%(\mathrm{~N}=11)$ and non- LUCAS-CPR group PEA 52.6\% $(\mathrm{N}=20)$ and asystole $18.4 \%$ $(\mathrm{N}=7)$. We found that there was no statistically significant difference in survival-to-discharge rates between patients in the LUCAS-CPR and non-LUCAS-CPR groups. Small sample size may have prevented us from achieving statistical significance (odds ratio $1.42,95 \%$ CI $0.53-3.83, \mathrm{P}=0.53$ ). Survival to discharge rate in patients in the non LUCAS CPR group was $21.2 \%$ and in the LUCAS-CPR group was $27.6 \%$. Absolute risk reduction was $6.4 \%$ and number need to treat was 15.6 .

CONCLUSIONS: Though not statistically significant the rate of survival was 1.4 times higher in the LUCAS-CPR group. Due to our limited sample size, a large multi-center study is required to further explore these findings.

MEDICAL INTENSIVE CARE UNIT ADMITTING PATTERNS IN A NATIONAL COHORT Lena M. Chen ${ }^{1,2}$; Anne E. Sales ${ }^{1}$; Edward H. Kennedy ${ }^{1}$; TImothy Hofer ${ }^{1,2}$. ${ }^{T}$ VA Ann Arbor Healthcare System, Ann Arbor, MI; ${ }^{2}$ University of Michigan, Ann Arbor, MI. (Control ID \#1314351)

BACKGROUND: Critical care makes up nearly $1 \%$ of the US gross domestic product, but there is wide hospital variation in critical care resource use. It is possible that some hospitals have higher ICU admission rates because of patient factors beyond their control such as admitting diagnosis. Therefore, we sought to describe the diagnosisspecific association between severity of illness and ICU admission rates.

METHODS: We created a retrospective cohort of the first non-surgical admission of patients admitted from the Emergency Department or Outpatient Clinic to 120 Veterans Affairs (VA) acute care hospitals from July 2009 to June 2010. Our primary predictor was severity of illness, which was defined as 30day predicted mortality on admission, and estimated using the validated VAICU severity score. We constructed separate multilevel models (random intercept and slope) predicting ICU admission rates for each of the nine most common admitting diagnoses. Our models did not adjust for any covariates beyond severity and admitting diagnosis.

RESULTS: The 278,335 patients in our cohort had a median 30-day predicted mortality of $1.5 \%$. The ICU admission rate for a median severity patient varied from $3.8 \%$ for pneumonia to $46.5 \%$ for acute myocardial infarction. The odds ratio for the change in ICU admission rate for a one standard deviation change in severity of illness varied from 1.08 for chest pain to 2.39 for gastrointestinal hemorrhage. Compared to non-cardiac diagnoses (i.e., sepsis, chronic obstructive pulmonary disease, gastrointestinal hemorrhage, and pneumonia), ICU admission rates for cardiac diagnoses were not as strongly associated with changes in predicted mortality (Figure).

CONCLUSIONS: Our results quantify the disparate way in which the ICU is used across diagnoses, and suggest that measures of ICU utilization that aggregate across diagnoses will not work well as global measures of efficiency. Our findings also raises the larger question of whether current diagnosis-specific patterns of ICU utliization represent the best use of an expensive resource.
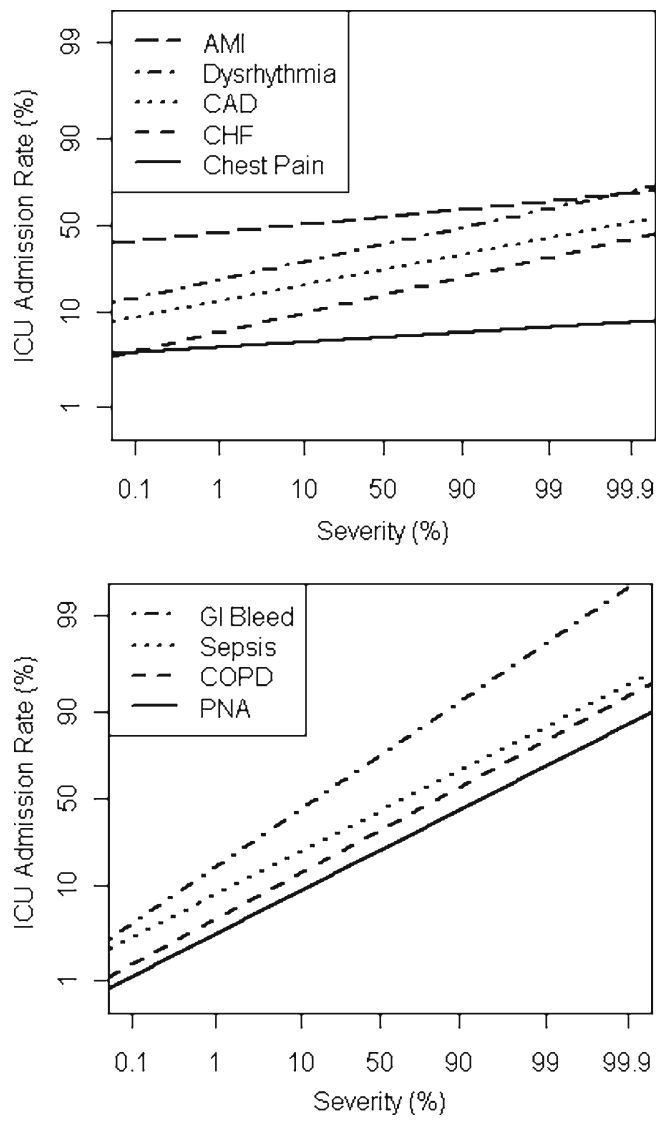

MEDICAL HOME RECOGNITION AND DIABETES QUALITY OF CARE IN COMMUNITY HEALTH CENTERS Robin Clarke'; Chihong Tseng ${ }^{1}$; Robert Brook ${ }^{1,2}$; Arleen Brown ${ }^{1} .{ }^{1}$ UCLA, Santa Monica, CA; ${ }^{2}$ RAND Corporation, Santa Monica, CA. (Control ID \#1310231)

BACKGROUND: The Affordable Care Act increased funding to community health centers (CHCs) to double the number of newly insured, low-income patients for whom they provide ambulatory care services. The Center for Medicare and Medicaid Innovation and Health Resources and Services Administration (HRSA) have launched programs incentivizing $\mathrm{CHCs}$ to transform into patient-centered medical homes (PCMH). These programs measure a PCMH by the assessment tool developed by the National Committee for Quality Assurance (NCQA). At this time, little is known about if and how CHC performance on the NCQA tool is associated with the quality of chronic disease care provided by these clinics.

METHODS: CHCs which were members of a Los Angeles County consortium, employed at least one physician and provided health services were invited to participate. Participating organizations completed the NCQA's 2008 PCMH tool. A NCQA level was assigned to each organization based on a 100 -point scale and number of "must-pass" elements passed. Patient sociodemographic information and diabetes clinical data were collected for 50 randomly selected adult diabetic patients from each organization. National Quality Forum diabetes care processes (e.g. Hemoglobin A1c in last 12 months) and intermediate outcomes (e.g Alc $<8 \%$ ) were collected. CHC structural characteristics about each organization were extracted from a public database. Random intercept mixed effects models were conducted using NCQA performance as the independent variable and adjusting for patient- and clinic-level characteristics and clustering within clinics. Predicted probabilities and relative risks were created for each diabetes outcome comparing clinics at higher levels of NCQA recognition to those at the lowest level. 
RESULTS: Of the 46 organizations within the consortium, 40 were eligible, and $30(75 \%)$ participated in the study. The structural characteristics of the participating and non-participating organizations were not substantially different. Each of the organizations exceeded the threshold to be recognized by the NCQA as a medical home. Eight (27\%) earned the highest Level 3 recognition, three (10\%) received Level 2, and $19(63 \%)$ received Level 1. Forty-five patient observations were dropped because of missing values creating a final sample of 1455 patients. For each care process and intermediate outcome, there was a distribution with organizations providing diabetes care below and above established standards from other $\mathrm{CHC}$ samples. The relative risks for each of the diabetes care processes and intermediate outcomes comparing the Levels 2 and 3 NCQA level clinics to the Level 1 clinics were non-significant. Sensitivity analyses using score on the 100-point scale and on each of the tool's 9 domains also produced no significant relationship between NCQA performance and diabetes care.

CONCLUSIONS: These analyses indicate that a diabetic patient has a similar probability of receiving a screening test or having a risk factor controlled in CHCs eligible for the highest and lowest NCQA recognition levels. Our findings raise the possibility that implementing the NCQA medical home components may be necessary but not sufficient for improving quality of diabetes care in CHCs. The implications are that the HRSA and Innovation Center programs should consider expanding PCMH transformation beyond just the NCQA tool in order to shape the primary care delivered by $\mathrm{CHCs}$ to millions of newly insured Americans.

\section{MEDICATION DISCREPANCIES IN INTEGRATED ELECTRON-} IC HEALTH RECORDS Amy Linsky ${ }^{1,2}$; Steven R. Simon ${ }^{1,3} \cdot{ }^{1} \mathrm{VA}$ Boston Healthcare System, Boston, MA; ${ }^{2}$ Boston University School of Medicine, Boston, MA; ${ }^{3}$ Brigham and Women's Hospital, Boston, MA. (Control ID \#1314930)

BACKGROUND: Medication reconciliation has emerged as a process to reduce medication-related errors. Medication discrepancies are often used as a proxy for potential errors. Electronic health records (EHRs) have been suggested as a way to reduce the presence of these discrepancies, but there is no evidence that EHRs or electronic medication reconciliation processes are associated with fewer discrepancies. Given that the Veterans Affairs (VA) healthcare system has an established EHR integrated with pharmacy dispensing, we sought to determine the prevalence of medication discrepancies, the medications involved and the factors associated with them.

METHODS: We analyzed data from a convenience sample of patients $(\mathrm{N}=105)$ seen in ambulatory care clinics at VA Boston, 3/10-7/11, by fourth-year medical students. Students performed medication reconciliation to a computer-generated medication list ("List") that included all medications and non-durable medical supplies dispensed at VA Boston (i.e., locally-dispensed), from other VA facilities (i.e., remotelydispensed) and non-VA sources. The main outcomes were the presence of non-mutually exclusive types of discrepancies, including commissions (medications present on List but not taken per patient report), omissions (medications not present on List but taken per patient report), duplications (present more than once) or alterations in dose or frequency. Characteristics included patient age ( $\geq 65$ vs. $<65$ years), sex, number of items on List, care provided at other VA facilities (yes vs. no) and presence of non-locally dispensed medications (yes vs. no). Single predictor and multivariable logistic regression models estimated the associations of patient and system factors with the presence of each type of discrepancy.

RESULTS: We analyzed 104 medication reconciliations. This Veteran cohort was predominantly male $(95 \%)$; $59(57 \%)$ were age 65 years or older. The median number of medications was 8 (IQR 5-13). Sixteen patients $(15 \%)$ had evidence of care in other VA systems, and $50(48 \%)$ had documentation of non-locally dispensed medications. Sixty-two $(60 \%)$ patients had at least one medication discrepancy. Prevalence of commissions, omissions, duplications and alterations in dose or frequency were $36 \%, 27 \%, 11 \%$ and $19 \%$, respectively. In unadjusted analyses, each additional medication was associated with higher likelihood of commissions (OR 1.1; 95\% CI 1.1-1.2) and duplications (OR 1.2; $95 \%$ CI 1.1-1.4) and with lower likelihood of omissions (OR $0.9 ; 95 \%$ CI $0.8-1.0)$. Non-locally dispensed medications was associ- ated with increased odds of duplications (OR 5.7; 95\% CI 1.2-27.9). In adjusted analyses, an increasing number of medications remained associated with commissions (OR 1.2; 95\% CI 1.1-1.3) and omissions (OR $0.9 ; 95 \%$ CI $0.8-1.0$ ). The involved medications differed by type of discrepancy, but non-opioid analgesics and herbal therapies were commonly seen in errors of commission and omission.

CONCLUSIONS: In a system with a well-established EHR that is directly linked to pharmacy dispensing, medication discrepancies occurred in $60 \%$ of patients visiting ambulatory clinics. Patients with greater number of medications were more likely to have errors of commission and duplication, but were less likely to have errors of omission. Our findings highlight that relying on EHRs alone will not ensure an accurate medication list and stress the need to review medication taking thoroughly with patients to capitalize on the full potential of EHRs.

MEETING PERFORMANCE STANDARDS FOR ANTICOAGULATION EDUCATION: WHAT SHOULD BE TAUGHT? Christopher Moreland ${ }^{1}$; Richard L. Kravitz ${ }^{2}$; Debora Paterniti ${ }^{2}$; Chin-Shang $\mathrm{Li}^{2}$; Tzu-Chun Lin ${ }^{2}$; Richard H. White ${ }^{2} .{ }^{1}$ The University of Texas HSC - San Antonio, San Antonio, TX; ${ }^{2}$ University of California, Davis, Sacramento, CA. (Control ID \#1339651)

BACKGROUND: The Joint Commission (JC) Venous Thromboembolism quality measure outlines four criteria for the education of patients starting warfarin on discharge. However, these criteria do not specify educational content regarding patient recognition of potentially dangerous warfarin-related scenarios, nor has any study investigated this potentially critical element of warfarin education. The authors designed this study to investigate how well patients assess the risks and consequences of potential warfarin-related safety threats, as well as what high-risk scenarios are not recognized as dangerous. METHODS: From a population of adult patients on long-term warfarin therapy through an anticoagulation clinic at an academic medical center, we randomly selected 480 for a telephone-based survey, with $38.3 \%$ participation. Warfarin knowledge questions were drawn from a previous survey; warfarin-associated risk scenarios were developed via focus interviews. Expert anticoagulation pharmacists categorized each scenario as urgent, moderately urgent, or not urgent; survey participants did the same. The initial measure was percentage of correct responses to knowledge questions. Main measures were accuracy, over-estimation, and under-estimation when categorizing clinical scenarios' urgency, as well as identification of scenarios for which patients' characterization most diverged from pharmacists'.

RESULTS: 184 participants completed the survey. The mean knowledge score was $69 \%$ (SD 0.20). Overall classification accuracy of situational urgency was $59 \%$ (95\% CI 57.3-60.3\%). Respondents over-estimated nonurgent-severity situations $23 \%$ of the time (95\% CI $20.8-24.7 \%$ ), while underestimating urgent-severity situations $21 \%$ of the time (95\% CI $19.0-23.9 \%)$. Four situations were underestimated by $>20 \%$ : acute vision loss, head trauma, prescription of a new medication, and missing a warfarin dose.

CONCLUSIONS: Despite fair factual knowledge of warfarin, participants did not appear to recognize well the clinical severity of warfarin-associated scenarios. In addition to JC measure requirements, warfarin education programs should incorporate patient-centered strategies to teach recognition of high-risk situations that compromise patient safety, including stroke symptoms, head trauma, and medication management.

MENTAL HEALTH STATUS AND PHYSICAL FUNCTION OF COMBAT-INJURED MILITARY PERSONNEL AT HOSPITAL DISCHARGE Valerie Lawrence ${ }^{1,2}$; Polly H. Noel ${ }^{1,2}$; Linda H. Yoder ${ }^{3,4}$; John E. Cornelli ${ }^{2,1}$; Anthony E. Johnson; Joseph R. Hsu ${ }^{4,5}$; Steven E. Wolf ${ }^{6} .{ }^{1}$ South Texas Veterans Health Care System, San Antonio, TX; ${ }^{2}$ University of Texas Health Science Center, San Antonio, TX; ${ }^{3}$ University of Texas at Austin, Austin, TX; ${ }^{4}$ United States Army Institute for Surgical Research, Fort Sam Houston, TX; ${ }^{5}$ San Antonio Military Medical Center, Fort Sam Houston, TX; ${ }^{6}$ University of Texas Southwestern Medical Center, Dallas, TX. (Control ID \#1326553)

BACKGROUND: Much is unknown regarding mental health and physical function outcomes among service members injured in Iraq and Afghani- 
stan. The San Antonio Military Medical Center (SAMMC) provides combat trauma care to all active duty personnel whose home base is in the Great Plains Regional Medical Command, which encompasses 17 states. We hypothesized that combat-injured service members would have significant residual mental and physical impairment when well enough for discharge from inpatient care at SAMMC.

METHODS: Joint VA-DoD prospective cohort study of consecutively consenting combat-wounded service members $\geq 18$ years old, hospitalized $\geq 72$ hours at SAMMC after medical evacuation from Iraq or Afghanistan, surveyed at the time of discharge from inpatient care, using a battery of psychosocial and functional status measures, and annually thereafter for 4 years.

RESULTS: Of 70 combat-injured enrolled, 67 were men (95.7\%). Other demographic characteristics were: age range $19-45$ years old with a mean (sd) age of 27.8 (6.2) and 54 (77\%) nonHispanic white, 10 (14.3\%) Hispanic, and 6 (8.6\%) African-American or other. Mean ADL and IADL scores were 10.7 (2.7) and 14.4 (3.5). Mean pain score (McGill Short Form Pain Questionnaire) was 3.8 (2.1). For health-related quality of life, mean SF12V PCS and MCS scores were 29.03 (7.4) and 54.6 (10.0), respectively. Regarding PTSD and depression, mean PCL-M (military) and CES-D scores were 32.2 (12.2) and 18.1 (6.1) respectively. Based on National Center for PTSD cutoff scores for those serving in Iraq or Afghanistan, $48(66 \%)$ had positive PTSD screens and $44(63 \%)$ met criteria for diagnosis. Using a CES-D score of $\geq 16,44(63 \%)$ met criteria for clinical depression. CONCLUSIONS: After recovering from combat injury sufficiently to be discharged from inpatient care, this cohort had some impairment in ADL and IADL but still scored better than the U.S. average on the PCS. Despite scoring at about the U.S. average on the MCS, a high proportion $(>60 \%)$ met criteria for clinical depression and screening and diagnostic criteria for PTSD. These data will be important for examining recovery trajectories over the four-year follow-up period for the cohort. The findings suggest that primary care providers will need to coordinate care for mental health and physical rehabilitation, in addition to medical comorbidities, for substantial numbers of combat-injured as they continue recovery as outpatients and after leaving the military.

MENTORING IN ACADEMIC MEDICINE: THE PERSPECTIVE OF THE MENTOR Rebecca E. Selling ${ }^{1}$; Scott De La Cruz ${ }^{2}$; Steven R. Lowenstein $^{3}$; Traci Yamashita ${ }^{1}$; Eva M. Aagaard ${ }^{2}{ }^{1}$ University of Colorado School of Medicine, Aurora, CO; ${ }^{2}$ University of Colorado School of Medicine, Aurora, CO; ${ }^{3}$ University of Colorado School of Medicine, Aurora, CO. (Control ID \#1340036)

BACKGROUND: Mentoring is an important predictor of career success and productivity in academic medicine. Opinions regarding mentoring have previously been studied predominantly from the perspective of the protégé.

METHODS: We conducted a cross-sectional online survey of all faculty members at the University of Colorado School of Medicine in April, 2010. The survey included 28 items and assessed faculty attitudes toward mentoring, ways in which mentorship was valued by the department and school, barriers to mentoring and interest in mentor-training programs. Data analysis included basic descriptive statistics (frequencies, means, standard deviations, medians, interquartile ranges) and proportions with $95 \%$ confidence intervals.

RESULTS: The response rate was $24 \%$; the final sample included 499 faculty members. Fifty-two percent of participants were female; with respect to academic rank, $23 \%$ were professors, $28 \%$ were associate professors, $36 \%$ were assistant professors, and $13 \%$ were instructors. Thirty-eight percent of participants spent $\geq 50 \%$ professional time on research. Forty-four percent of participants spent $\geq 50 \%$ professional time on clinical practices. Seventy-nine percent $(95 \%$ confidence interval $[\mathrm{CI}]$ $75.8-82.2)$ of faculty reported acting as a mentor. Mentors had a median of 4 (IQR 3,8) protégés. The average time spent mentoring was $5.4 \pm 5.4$ hours per week. Ninety-seven percent $(95 \%$ CI $95-98)$ of participants felt mentoring was an important part of their job and 95\% (95\% CI 93-96) felt the School of Medicine should do more to encourage mentoring. Acknowledgement in annual performance reviews was the most common way in which mentorship was rewarded $(57 \%, 95 \%$ CI 53-61); a monetary stipend was the least common $(8 \%, 95 \%$ CI $6-10)$. Inadequate time $(90 \%$,
$95 \%$ CI 88-92) and the importance of other responsibilities $(87 \%, 95 \% \mathrm{CI}$ 84-90) were identified as the greatest barriers to mentoring, but two-thirds of survey participants $(67 \% ; 95 \%$ CI $63-71)$ also identified a lack of adequate training as a barrier. A similar proportion of faculty $(68 \% ; 95 \%$ CI 64-72) were interested in a mentor-training program. The preferred format for such training was a workshop $(82 \%, 95 \%$ CI $78-86)$. Participants reported the greatest interest in learning how to mentor with regard to career planning $(86 \%, 95 \%$ CI $83-89)$ and conflict resolution skills $(75 \%, 95 \%$ CI $71-79)$.

CONCLUSIONS: This study contributes the perspective of the mentor to the current literature regarding mentoring in academic medicine. Recognition of the time and effort required for this important role is necessary. Moreover, most faculty are interested in training to improve their skills as mentors.

METHADONE DOSE-RELATED INCREASE IN QTC WITHOUT CLINICALLY SIGNIFICANT PROLONGATION OR ARRHYTHMIA Karran A. Phillips ${ }^{1}$; David H. Epstein ${ }^{1}$; Dave Reamer ${ }^{1}$; Gavin Bart ${ }^{2}$; Kenzie L. Preston ${ }^{1}{ }^{1}$ NIDA/NIH, Baltimore, MD; ${ }^{2}$ University of Minnesota, Minneapolis, MN. (Control ID \#1340383)

BACKGROUND: Methadone is an effective treatment for opioid dependence. However, some studies suggest that methadone may increase QTc intervals, possibly leading to cardiac arrhythmias and death. We prospectively determined the impact of methadone dose on QTc prolongation in methadone-maintained participants.

METHODS: Methadone-maintained outpatients underwent electrocardiograms (ECGs) in triplicate at baseline prior to first dose and at 4-6 week intervals for 30 weeks ( 7 time points). Automated measurements for QTe were averaged for each triplicate. We defined QTc prolongation as $>450 \mathrm{~ms}$ for males and $>470 \mathrm{~ms}$ for females. Participants who provided ECGs at baseline and at least one other time point were included in this analysis.

RESULTS: The 93 participants provided 1683 ECGs (561 triplicates). Mean age was 38.4 (SD 8.2) years; 55\% were African American, and $74 \%$ male. Baseline positive urines were $98 \%$ for heroin, $82 \%$ for cocaine, and $4 \%$ for benzodiazepines. Baseline ECG findings and self-report suggested a low prevalence of structural heart disease, hypertension, and hyperlipidemia. Use of QTc-affecting medications and electrolyte abnormalities were infrequent. At baseline, mean QTc was 410.2 (SD 16.6) ms, with only 1 participant meeting criteria for QTc prolongation. During the study, only $33(7.5 \%)$ of the 561 ECG triplicates met criteria for QTc prolongation. Controlling for age and sex, mean QTc was higher at each time point than at baseline (all $\mathrm{p}<0.001$ ), but no mean increase occurred after week 6 . Controlling for study week, age, and sex, only mean methadone dose (range $70-190 \mathrm{mg}$ ) in the 5 days prior to ECG was associated with QTc prolongation $(\mathrm{F}(1,338)=19.58, \mathrm{p}<0.001)$. In all $561 \mathrm{ECG}$ triplicates obtained, only 7 (in 5 participants) had an increase in QTc interval of $>60 \mathrm{~ms}$ or $>15 \%$ from baseline or $>500 \mathrm{~ms}$. Multiple logistic regressions showed no significant effect of dose on these outcomes. There were no adverse clinical outcomes associated with QTc prolongation in this sample.

CONCLUSIONS: Our findings, and the lack of evidence that QTc monitoring is an effective risk stratification tool for sudden cardiac death, suggest that it is premature to recommend QTc risk evaluation in this population

\section{METHODS TO IMPROVE INFORMED CONSENT PROCEDURES FOR RESEARCH SUBJECTS WITH LOW LITERACY: A SYSTEM- ATIC REVIEW Leonardo Tamariz; Ana M. Palacio; Mauricio Robert;} Erin N. Marcus. University of Miami, Miami, FL. (Control ID \#1336856)

BACKGROUND: Research subjects do not adequately understand information presented to them during the informed consent (IC) process. Inadequate health literacy may exacerbate the limited understanding of the informed consent. The purpose of this study is to evaluate the evidence supporting interventions to improve comprehension of the IC process in low literacy research subjects. METHODS: A MEDLINE database search (1966 to November 2011) supplemented by manual searches of bibliographies of key relevant articles 
was performed. We selected all studies in which an intervention was tested to improve comprehension of informed consent and the intervention was evaluated in low literacy populations. The main outcome evaluated was comprehension measured using written test or verbal comprehension.

RESULTS: Our search strategy yielded 58 studies, of which only 4 met our eligibility criteria. The four studies included 593 research participants. The table summarizes the results of each study. The studies predominantly included populations that were older (median age 61 range 49-64), ethnic minority, and with literacy level of 8th grade or below. Only one study had a randomized design. The specific intervention differed in each study. Two of the studies included the teach-back method or teach to goal method and achieved the highest level of comprehension. An intervention that involved changing the readability level of the informed consent document resulted in the lowest comprehension among study subjects.

CONCLUSIONS: The evidence supporting interventions to improve the informed consent process in low literacy populations is extremely limited. Efforts to improve understanding through the use of multimedia and enhanced consent forms have had only limited success. As in the field of health education having a study team member spend more time talking one-on-one to study participants is the most effective available way of improving research participants understanding. Additional research is needed because of the lack of randomized controlled trials.

\begin{tabular}{|c|c|c|c|c|c|}
\hline$\overline{\text { Source }}$ & Intervention & $\begin{array}{l}\text { Population } \\
\text { and literacy }\end{array}$ & Sample size & $\begin{array}{l}\text { Comprehension } \\
\text { score in control arm }\end{array}$ & $\begin{array}{l}\text { Comprehension score } \\
\text { in intervention arm }\end{array}$ \\
\hline Bickmore, 2009 & $\begin{array}{l}\text { Computer agent with } \\
\text { touch screen interaction }\end{array}$ & $\begin{array}{l}\text { Older minority with } \\
\text { 8th grade or lower level }\end{array}$ & 13 & 30 & 25 \\
\hline Kripalani, 2008 & Teach back method & $\begin{array}{l}\text { Older minority with CAD and }<8 \text { th } \\
\text { grade level }\end{array}$ & 284 & NA & 31 \\
\hline Sudore, 2006 & Teach to goal method & Older minority with TOHFLA <23 & 45 & NA & 98 \\
\hline Young, 1990 & $\begin{array}{l}\text { Changing IC to } 6 \text { th } \\
\text { grade reading level }\end{array}$ & Middle age & 251 & NA & 13 \\
\hline
\end{tabular}

NA: Not controlled arm studies IC: informed consent

MISSED OPPORTUNITIES FOR EFFECTIVE PATIENT EDUCATION AND COUNSELING: WHAT THE UNANNOUNCED STANDARDIZED PATIENT EXPERIENCE CAN TELL US Colleen Gillespie; Nina Yeboah; Angela Burgess; Kathleen Hanley; David Stevens; Andrew B. Wallach; Sondra Zabar. New York University School of Medicine, New York, NY. (Control ID \#1337759)

BACKGROUND: Patient education and counseling skills are critical to patient safety and outcomes, especially for achieving behavior change and managing chronic conditions. The goal of this study was to explore the nature and quality of patient education and counseling skills through indepth qualitative analysis of resident physician interactions with Unannounced Standardized Patients (USPs) - trained actors integrated incognito into practice. We sought to describe, from the ground up, variation in how resident physicians educate and counsel patients, focusing on aspects that might not be captured through commonly used checklists based on the ASK-TELL-ASK model of patient education.

METHODS: Highly trained USPs portrayed two clinical cases, one involving asthma medication education and the other a routine visit requiring both education about a common condition and general preventive recommendations. They were scheduled as new patients and seen by Internal Medicine Residents in two busy, urban primary care clinics. Residents were aware they would see USPs in clinic, but did not know when. USPs completed a comprehensive checklist that assessed communication skills, including those patient education and counseling skills associated with the ASKTELL-ASK model (assess understanding, provide clear explanations, check understanding), as well as other core clinical skills, after each visit and used a concealed digital recorder to audiotape visits. The 18/ 37 audible encounters comprise the analysis sample; Asthma case $=$ 8 and Routine Visit case $=10$. Average visit length was 26 minutes (range 12 - 37 minutes). Case portrayal was assessed and found to be consistent. Tapes were transcribed and entered into Atlas TI, a qualitative analysis software program, to facilitate coding and analysis. Major themes were identified by listening to the audio while reading the transcripts so as to include tone, emotion, and other verbal cues. RESULTS: The qualitative data validated core aspects of the checklist assessment, namely that the clarity of explanations and degree of summarizing/reviewed varied, and that evaluation of patient under- standing was rare. Further analysis revealed that checklists may miss some significant aspects of the interaction -critical moments when patients could be more fully educated and engaged. These missed opportunities fell into four categories: 1) failing to orient patients (e.g., not setting an agenda or explaining reasons for actions; mean $=4.2$ times/visit); 2) failing to engage patients in behavior change (e.g., not reinforcing connections between symptoms and behavior change; postponing decisions until later visits; not making direct recommendations; mean $=5.1$ times $/$ visit ); 3) failing to engage patients in the treatment plan (e.g., not explaining reasons for medication, how to take, what to expect, when to follow-up and why; mean=2.9 times/ visit); and 4) failing to help patients navigate the system to obtain recommended services (mean $=1.9$ times/visit).

CONCLUSIONS: While checklists capture critical positive aspects of education and counseling practice during the patient-physician encounter, in-depth qualitative exploration of USP interactions suggests the need to include assessment of missed opportunities to educate and activate patients. These missed opportunities may be essential to achieving intended outcomes of care with enhanced efficiency and therefore not only merit further study but are important targets for education and training.

MISSED OPPORTUNITIES FOR TREATMENT OF UNCONTROLLED HYPERTENSION AT PHYSICIAN OFFICE VISITS IN THE UNITED STATES, 2005-2009 Raman R. Khanna ${ }^{1}$; Ronald G. Victor $^{2}$; Farzaneh Pour Ansari ${ }^{3}$; Premere Knowles ${ }^{2}$; Kirsten BibbinsDomingo ${ }^{1}$; Eric Vittinghoff ${ }^{4}$; Pamela G. Coxson ${ }^{1}$; Larissa Thomas ${ }^{1}$; Martin F. Shapiro ${ }^{5}$; Mark J. Pletcher ${ }^{1,4}$. ${ }^{1}$ University of California, San Francisco, San Francisco, CA; ${ }^{2}$ Cedars-Sinai Medical Center, Los Angeles, CA; ${ }^{3}$ University of California, San Francisco, San Francisco, CA; ${ }^{4}$ University of California, San Francisco, San Francisco, CA; ${ }^{5}$ University of California, Los Angeles, Los Angeles, CA. (Control ID \#1333898)

BACKGROUND: National guidelines recommend prescribing new blood pressure medication for all untreated patients with an established diagnosis of hypertension and at least Stage I-level blood pressure elevation (systolic blood pressure 140-159 and/or diastolic blood pressure 90-99). Some recent guidelines also recommend prescribing new blood pressure 
medication (as compared to escalating medication dose) for patients who, despite treatment, have Stage II-level blood pressure elevation (systolic blood pressure $\geq 160$ or diastolic blood pressure $\geq 100$ ). It is unclear how often U.S. physicians adhere to these guidelines at each office visit. METHODS: We analyzed the National Ambulatory Medical Care Survey (NAMCS), a multistage, nationally representative, detailed survey of all office visits to non-federally employed physicians in the U.S. from 2005-2009 to determine the likelihood of new blood pressure medication prescribing for patients with uncontrolled hypertension at each office visit in the U.S. The NAMCS does not record medication dose so were not able to assess blood pressure medication dose escalation. We restricted our analysis to a visit population of non-pregnant adults with an established hypertension diagnosis and a visit blood pressure above the JNC 7 goal (systolic $\geq 140 \mathrm{~mm} \mathrm{Hg}$ and/or diastolic $\geq 90 \mathrm{~mm} \mathrm{Hg}$ ) who were seeing a family/general practitioner, internist, or cardiologist. In this population, we analyzed predictors of new blood pressure medication prescribing, including demographic, clinical, and physician characteristics using multivariable regression. All analyses were sample weighted to account for the complex survey design.

RESULTS: From 2005-2009, NAMCS recorded 8,071 observations meeting the above-specified restrictions, which represent approximately 58 million annual U.S. office visits. New blood pressure medication was prescribed to only $27.5 \%$ of untreated patients, $18.2 \%$ of treated patients with Stage II level blood pressure elevation, and $17.5 \%$ of all patients with uncontrolled blood pressure. Prescribing was more likely with higher visit blood pressure (adjusted odds ratio $[\mathrm{aOR}]=1.27,95 \%$ confidence interval [CI] 1.20-1.34, and 1.44, 95\% CI 1.25-1.65, for each $10 \mathrm{~mm} \mathrm{Hg}$ of systolic and diastolic blood pressure, respectively), when patients were previously untreated $(\mathrm{aOR}=0.42$ for one current medication, $95 \%$ confidence interval [CI] $0.34-0.52$; aOR $=0.18$ for two or more current medications, $95 \% \mathrm{Cl}$ $0.14-0.22)$, and when patients identified blood pressure as a visit reason $(\mathrm{aOR}=2.58,95 \%$ CI $2.16-3.10)$. New medication prescribing only exceeded $50 \%$ in untreated patients with Stage II-level blood pressure elevation who identified blood pressure as a visit reason. New medication prescribing did not increase from 2005-2009 ( $\mathrm{p}=0.40$ for trend).

CONCLUSIONS: Missed opportunities to improve blood pressure control by prescribing new blood pressure medication for patients with uncontrolled hypertension are common during outpatient physician office visits in the U.S.
MODEL OF ADHERENCE TO BLOOD PRESSURE MEDICATIONS Jeffrey L. Jackson ${ }^{1}$; Patrick G. O'Malley ${ }^{2}$; Janice Hanson ${ }^{2}$. ${ }^{1}$ Zablocki VAMC, Milwaukee, WI; ${ }^{2}$ Walter Reed Army Medical Center, Bethesda, MD. (Control ID \#1336718)

BACKGROUND: Hypertension is a common medical problem. Aherence to blood pressure medications is low, generally less than $50 \%$. Better understanding of factors contributing to hypertension control could improve interventions to improve medication adherence.

METHODS: We enrolled a consecutive sample of consenting participants aged 40-80 y.o. who had hypertension, at least 2 additional chronic medical conditions, and were scheduled to see their internist for a routine appointment. Surveys assessed depression (PHQ-9), somatization (PHQ-15) and functional status (MOS SF-6). Post-visit surveys assess satisfaction (Rand-9). Adherence was assessed at 1 and 3 months using pill counts and was defined as greater than $80 \%$. Models were developed using structural equation modeling and reported direct and indirect effects using standardized estimates, also known as effect sizes.

RESULTS: Among 106 participants, the average age was 66, 53\% were women, $56 \%$ African-American and $88 \%$ were on $\geq 5$ medications. Average adherence at baseline, 1 and 3 months was: $47 \%, 49 \%$ and $50 \%$ respectively. The models for both 1 month and 3 month adherence were identical. Variables that directly increased adherence included baseline blood pressure (ES: $0.18,95 \%$ CI: 0-0.37), Patient satisfaction $(0.24,95 \%$ CI: 0.05 to 0.42$)$ and patient functional status $(0.18,95 \%$ CI: $0-0.38)$. Patient characteristics that indirectly decreased adherence (through effects on functional status) included worse depression scores (ES: - $0.62,95 \% \mathrm{CI}$ : -1.3 to 0.0$)$, somatization $(-0.1895 \% \mathrm{CI}$ : -0.42 to 0.00$)$ and number of comorbid conditions $(-0.40,95 \%$ CI: -0.97 to 0.0$)$. The total number of medications was not related to hypertensive medication adherence (ESL $-0.86,95 \%$ CI: -2.3 to 0.61 ). Our model was well fitted (Chi2: 0.58)

CONCLUSIONS: There are a number of patient characteristics that modulate adherence to hypertensive medications. Being depressed, having a greater number of comorbid conditions, higher somatization and poor functional status decrease adherence, while higher blood pressure readings and satisfaction with their provider increase adherence. Among these factors, depression and number of comorbid medical conditions had the greatest impact.

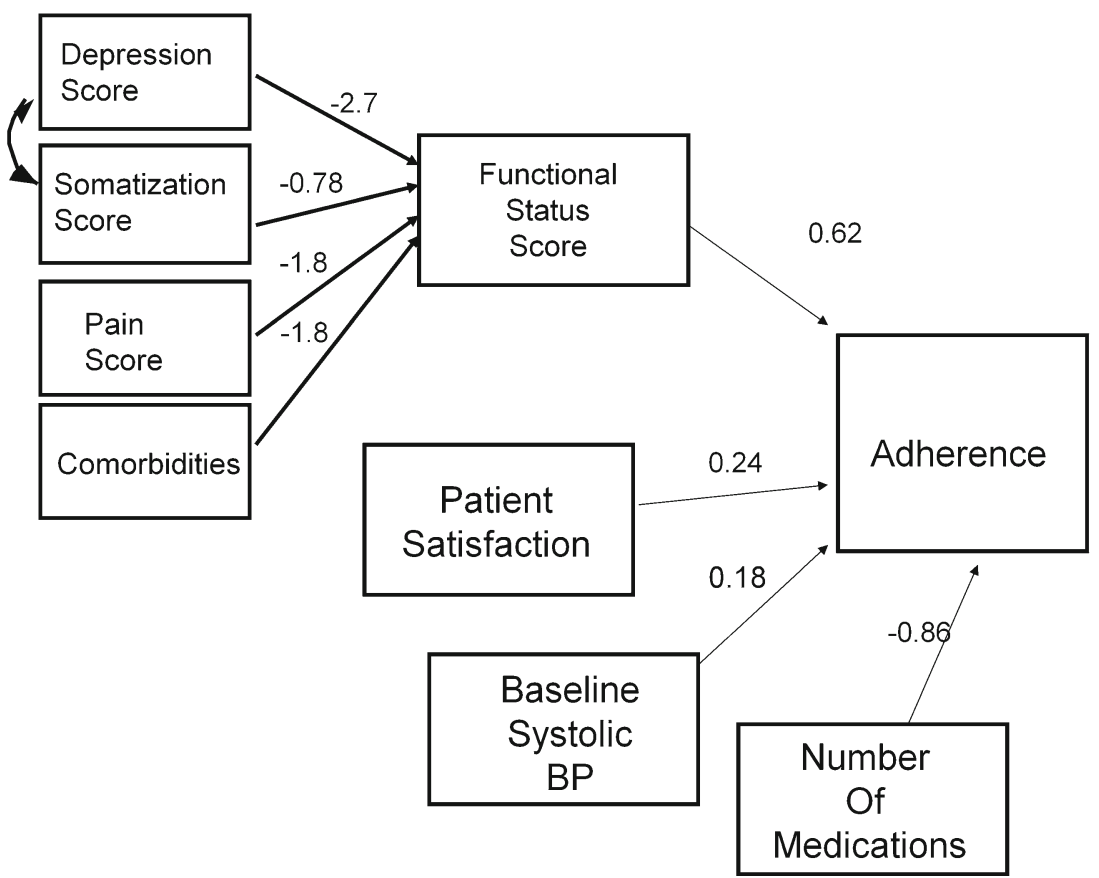


MONO AND DUAL RENIN-ANGIOTENSIN-ALDOSTERONE-SYSTEM (RAAS) INHIBITION STRATEGIES IN TYPE 2 DIABETES MELLITUS: SAFETY AND EFFICACY EXPERIENCES OF AN ENDOCRINOLOGY PRACTICE Michael P. Kane ${ }^{1,2}$; Alecia M. Heh ${ }^{1,2}$; Robert S. Busch' ${ }^{2}$ Gary Bakst ${ }^{2}$; Jill M. Abelseth ${ }^{2}$; Robert A. Hamilton'; Daniel A. Belletti ${ }^{3}$. ${ }^{1}$ Albany College of Pharmacy and Health Sciences, Albany, NY; ${ }^{2}$ The Endocrine Group, LLP, Albany, NY; ${ }^{3}$ Novartis Pharmaceuticals, East Hanover, NJ. (Control ID \#1279236)

BACKGROUND: Blockade of the RAAS has been shown to lower BP, decrease proteinuria and slow the decline in GFR in patients with CKD, and decrease morbidity and mortality in patients with chronic heart failure. While fairly standard practice, the utility of dual RAAS inhibition has not been studied in real world practice. We report our experience of various dual RAAS inhibition therapies in hypertensive, type 2 diabetes mellitus (T2DM) patients with proteinuria.

METHODS: This is a cross-sectional observational study comparing two cohorts of hypertensive, diabetes patients of a private endocrinology practice. Patients were identified via a computerized text search of patient electronic medical records (EMR) using generic and brand names of ACEI's, ARB's and direct renin inhibitors (DRI) available in the United States in April, 2011. Inclusion criteria: age between 18-89 years, documented diagnosis of HTN and T2DM for at least one year, history of proteinuria, at least one year of care with the practice, and stable RAAS agent therapy for a minimum of six months. Exclusion criteria: pregnancy, type 1 diabetes, and patients with ESRD. Mono and dual therapies were compared to each other in regards to efficacy (BP and urinary creatinine/ albumin ratio), and safety (serum potassium, BUN, serum creatinine, and mortality) at 6 and 12 months after initiation of therapy. A last observation carried forward (LOCF) analysis was performed; statistical analysis utilized the Kruskal-Wallis one-way nonparametric analysis of variance.

RESULTS: EMR search identified 866 patient records, including 242 patients who met all study criteria. 53 patients received ACEI or ARB monotherapy, 67 patients received ACEI/ARB combination therapy, 51 patients received ACEIDRI therapy, and 71 patients received ARB-DRI therapy. Average baseline patient age was 63 years $( \pm 12), 63.6 \%$ were men, $89.3 \%$ were Caucasian, mean diabetes duration was 10 years $( \pm 7)$, and average $\mathrm{BMI}$ and $\mathrm{A1C}$ values were $35.3 \mathrm{~kg} / \mathrm{m} 2( \pm 7.7)$ and $7.59 \%( \pm 1.57)$, respectively. Significant baseline differences between groups included a shorter mean duration of diabetes, lower weight, and significantly less mean and median albuminuria at baseline in the monotherapy group compared to dual RAAS inhibition groups, and a lower baseline serum potassium and higher diastolic blood pressure in the monotherapy group compared to the DRI groups. There were no significant baseline differences between groups in BUN, serum creatinine, SBP, or A1C. LOCF analysis demonstrate similar overall efficacy in BP and albuminuria reduction, though the ACEI/DRI group had a greater reduction in albuminuria compared to the monotherapy group, but not compared to other dual therapy groups. LOCF analysis demonstrated similar overall safety between the groups (i.e., no significant differences in subsequent BUN, SCr, or K values). While baseline serum potassium was statistically different between monotherapy and DRI-based dual RAAS therapies $(4.39+0.42$ vs. $4.58+0.44)$ the LOCF values were equivalent and the changes were not significantly different $(0.21+0.42$ for mono; $0.16+0.41$ for ACE/ARB; $0.11+0.47$ for DRI based dual therapy) between groups. There were no significant differences between the groups in mortality.

CONCLUSIONS: Dual RAAS blockade using ACEI/ARB or DRI-based dual RAAS inhibition was well tolerated, demonstrating similar safety and efficacy compared to monotherapy in hypertensive patients with T2DM and proteinuria who required additional therapy.

MORE THAN MEETS THE EYE: MEASURING THE DUAL RISKS OF LOW HEALTH LITERACY AND POOR VISION FOR HOSPITALIZED GENERAL MEDICINE PATIENTS Madeleine I. $\underline{\text { Shapiro }}^{1}$; Vineet Arora $^{2}$; Ainoa M. Mayo ${ }^{3}$; David Meltzer'; Valerie G. Press $^{3}{ }^{1}$ University of Chicago, Chicago, IL; ${ }^{2}$ University of Chicago Medical Center, Chicago, IL; ${ }^{3}$ University of Chicago Medical Center, Chicago, IL. (Control ID \#1310958)
BACKGROUND: As our nation focuses on how to reduce healthcare costs, there is an increasing focus on improving care transitions by empowering patients. In fact, SGIM along with 5 other professional medical societies has adopted a set of care transition principles which include communication with patients and their caregivers about not only who their medical home is, but also focused education on treatment plans and follow-up expectations. While we should aim for a "universal precaution" approach when communicating with our patients, it is important to recognize that in resource-limited hospital settings, identification of vulnerable, high-risk (e.g. low health literacy [HL]) patients may be necessary to ensure adequate education for safe care transitions. In order to identify these at-risk patients, an effective clinical screening tool is needed. Chew et al have validated a questionnaire to detect low HL among outpatients. Our objective is to evaluate the validity of the Chew tool for hospitalized patients.

METHODS: General medicine inpatients were enrolled from an ongoing study of resource allocation and quality of care at our hospital. Eligible patients (cognitively intact, English-speaking) completed the Chew tool: (q1) "How often do you have problems learning about your medical condition because of difficulty understanding written information?"; (q2) "How confident are you filling out medical forms by yourself?"; (q3) "How often do you have someone help you read hospital materials?" Participants responded on a Likert scale from 0-4 and were considered "atrisk" for poor HL if they answered "sometimes, often, or always" (q1, 3) or "somewhat, a little bit, or not at all" (q2). To validate the tool, we administered the REALM-R to participants' if their vision was sufficient (Snellen screening chart)

RESULTS: To date we have enrolled 216 participants. The mean age is 53; the majority were female (54\%) and African-American (82\%). About $1 / 2$ have $\leq$ high-school degree. Of the 122 participants who completed both HL tools (Chew and REALM-R), 39 (32\%) were considered "at-risk" based on the Chew screening tool, while $56(46 \%)$ were considered to have inadequate HL based on the REALM-R ( $\mathrm{p}=0.02)$. The Chew tool had a sensitivity of $45 \%$. Of note, about one-third $(n=57)$ of participants approached for the REALM-R ( $\mathrm{n}=179)$ were unable to complete it due to insufficient vision; $51 \%$ of these participants did not have their glasses with them in the hospital. Participants with insufficient vision $(32 / 57,56 \%)$ were more likely to be 'at risk' for poor HL on the Chew tool compared to those with sufficient vision $(39 / 122,32 \% ; p=0.002)$.

CONCLUSIONS: We demonstrate that in a low-income, primarily AfricanAmerican urban hospitalized population, two health literacy tools found differing prevalence of low HL. Our data suggest that the Chew screening tool may have low sensitivity among hospitalized and/or African-American patients; if upon completion of the study these findings remain, further evaluation of clinically relevant tools is needed. Additionally, the prevalence of poor vision $(\sim 1 / 3$ of participants) in this population is non-trivial and may be an underrecognized risk factor for hospitalized patients. Internists should take into account the implications of the dual risks of poor $\mathrm{HL}$ and poor vision: up to $63 \%$ of patients may have difficulty reading, and/or understanding written material provided in the hospital setting, which may impede patient empowerment and high-quality care transitions.

MOTIVATION, SELF-EFFICACY, AND PERCEIVED AUTONOMY SUPPORT IN PATIENTS ENROLLED IN A CLINIC-BASED BEHAVIORAL WEIGHT LOSS PROGRAM Stephanie A. Rose'; Shu Shen ${ }^{2}$; Kelly H. Webber ${ }^{3}$. ${ }^{1}$ University of Kentucky, Lexington, KY; ${ }^{2}$ University of Kentucky, Lexington, KY; ${ }^{3}$ University of Kentucky, Lexington, KY. (Control ID \#1336374)

BACKGROUND: Level and type of motivation can be predictive of completion and success in weight loss programs. The type of motivation (autonomous and controlled), level of self-efficacy, and perceived level of autonomy support in patients enrolled in a clinical weight loss program were assessed. The relationships between the measured variables were also explored.

METHODS: We performed a quantitative survey of patients at the start of a 12-week clinic-based medically-managed weight loss program based on the Diabetes Prevention Program between January and November 2011. 
The survey included questions from the Treatment Self-Regulation Questionnaire (TSRQ), the Health Care Climate Questionnaire for maintaining a healthy diet (HCCQ), the Perceived Competence Scale (PCS), as well as questions regarding obesity screening and treatment practices by the patient's primary care physician (PCP).

RESULTS: 28 overweight and obese patients (body mass index (BMI) $\geq 25 \mathrm{~kg}$ / $\mathrm{m}^{2}$ ) agreed to participate. $85 \%$ reported having seen their PCP at least once in the past year. All patients reported that their PCP had ever told them that they needed to lose weight. $64 \%$ felt that their PCP was able to help them with weight loss, but only $36 \%$ felt it was their PCP's responsibility to help them with weight loss. The mean level of autonomous motivation (AM) was 6.04 (SD 0.65 ) and the mean level of controlled motivation (CM) was 2.76 (SD 1.24). The mean level of self-efficacy was 4.75 (SD 1.46). The mean level of perceived PCP support of AM was 4.73 (SD 1.74). There was a positive correlation between self-efficacy and AM ( $\mathrm{r}=0.27)$, and a negative correlation between self-efficacy and $\mathrm{CM}(\mathrm{r}=-0.15)$. There was a small negative correlation between PCP support of AM and AM ( $\mathrm{r}=-0.05)$, and a negative correlation between perceived PCP support of AM and CM $(\mathrm{r}=-0.41)$.

CONCLUSIONS: Patients enrolled in a weight loss program reported higher mean levels of AM versus CM. This is similar to previous weight loss programs. Enrolled patients reported high levels of self-efficacy and perceived health care provider autonomy support. There was a positive correlation between self-efficacy and AM and a negative correlation between self-efficacy and CM. There was not a positive correlation between perceived AM support and $\mathrm{AM}$, though there was a negative correlation between perceived $\mathrm{AM}$ support and CM. Future goals include linking these surveys to patient demographics, BMI change, and responses to post-intervention data, comparison of surveys of patients not enrolled in the weight loss program, and implementation of an intervention that improves health care provider support of autonomy.

\section{MOTIVATIONAL INTERVIEWING BY HIV CARE PROVIDERS} IS ASSOCIATED WITH PATIENT COMMITMENT TO REDUCE UNSAFE SEXUAL BEHAVIOR. Tabor E. Flickinger ${ }^{1}$ Somnath $\mathrm{Saha}^{2}$; Todd Korthuis ${ }^{2}$; Ira Wilson ${ }^{3}$; Victoria L. Sharp ${ }^{4}$; Jonathan A. Cohn ${ }^{5}$; Gary S. Rose ${ }^{6}$; Stephen Berry ${ }^{1}$; Michael B. Laws ${ }^{3}$; Mary Catherine Beach ${ }^{1}$. ${ }^{1}$ Johns Hopkins University School of Medicine, Baltimore, MD; ${ }^{2}$ Oregon Health and Science University, Portland, OR; ${ }^{3}$ Brown University, Providence, RI; ${ }^{4}$ St. Luke's Roosevelt, New York, NY; ${ }^{5}$ Wayne State University, Detroit, MI; ${ }^{6}$ Massachusetts School of Professional Psychology, Boston, MA. (Control ID \#1337733)

BACKGROUND: Commitment to behavior change, when expressed by patients during clinical encounters, is associated with better patient outcomes. Motivational interviewing (MI) is a counseling style with the potential to elicit this commitment. The extent to which HIV providers use MI when counseling patients about safe sex, and whether its use is associated with patient commitment to safer sex practices, is unknown. We hypothesized that more MI-adherent provider counseling would be associated with patient expressions of commitment to safer sex.

METHODS: Routine follow-up visits between $426 \mathrm{HIV}$-infected patients and 45 healthcare providers collected as part of the Enhancing Communication and HIV Outcomes (ECHO) Study were audio-recorded, transcribed and searched for sexual risk counseling. Our study outcome was the presence of expressed patient commitment to reduce high-risk sexual behavior, coded using the Client Language Assessment in Motivational Interviewing. The independent variable was the extent to which providers used communication behaviors consistent with MI, coded by the Motivational Interviewing Treatment Integrity (MITI). Using the MITI, we calculated an overall summary score reflecting the balance of MI adherent minus non-adherent provider talk. We used logistic regression analysis, with generalized estimating equations accounting for clustering of patients within providers, to investigate whether more provider MI-adherence was associated with patient commitment to sexual behavior change.

RESULTS: Of the 426 total audio-recorded encounters, 27 contained provider counseling regarding unsafe sexual practices. Six of the 27 dialogues included patient commitment talk to reduce unsafe sexual practices. The most common provider behaviors within the 27 dialogues were giving information (e.g. "The higher the viral load, the more likely the risk of infection" $n=114$ utterances), and asking closed questions (e.g. "Do you always use protection?" $\mathrm{n}=95$ ). The most common MI-adherent behaviors were reflections (e.g. "You always use condoms then" $n=56$ ) and patient affirmation (e.g. "That is a very smart decision" $n=19$ ). Less common MI-adherent behaviors were emphasizing patient control (e.g. "You're the one who would say yes or no" $n=7$ ) and support (e.g. "You know we are here for you" $n=1$ ). The most common MInonadherent behaviors were advising without permission (e.g. "If you're gonna have sex with him, just use a condom" $n=16$ ), directing the patient ("Don't give it to anybody" $n=12$ ) and confronting the patient ("It's not okay for you to have sex without a condom" $n=6$ ). The summary score of MI balance was higher in dialogues in which patients expressed commitment than in dialogues in which they did not (mean 4.0 vs. 1.2). The odds of expressed patient commitment were higher when providers used more MI adherent than non-adherent behaviors, OR: 1.17 (1.01-1.38).

CONCLUSIONS: Patients were more likely to express commitment to safer sex during clinical encounters in which their healthcare providers used communication behaviors consistent with the principles of motivational interviewing. With 1.2 million HIV-infected individuals in the United States, and $84 \%$ of new HIV infections caused by sexual transmission, MI holds promise as one strategy to help reduce the spread of HIV. More research is urgently needed to determine the most effective communication strategies to influence patient sexual risk behavior.

MOTIVATIONS OF RESIDENT TIME SPENT IN CLINICAL AND EDUCATIONAL ACTIVITIES AT HOME: IMPLICATIONS OF NEW DUTY HOURS SIGLIN, S; CORK, D; DEANO, R; GIANGRECO, D; APPANAGARI, A; DOLL, J; DEKOSKY, A.; POTTS, S; VARKEY, A; ARORA, A. Scott Siglin; David Cork; Roderick Deano; Vineet Arora. University of Chicago Medical Center, Chicago, IL. (Control ID \#1338847)

BACKGROUND: The ACGME required implementation of resident duty hour restrictions across the U.S. in July 2011 and time spent performing patient care activities while out of the clinical setting is now being counted as part of the maximum allowed hours. While prior studies show that many residents access electronic health records (EHR) from home to complete clinical tasks, no study has yet examined what compels residents to complete clinical tasks from home after leaving the hospital. The aim of this study is to ascertain the motivation among Internal Medicine housestaff from three teaching hospitals to perform clinical work outside of the monitored duty hours.

METHODS: An anonymous two-page survey was created to assess the frequency by which residents perform clinical activities from home via telephone, internet, or remote access of EHR (checking labs, reviewing records, placing orders, communicating with ward teams, or managing clinic patients). Residents were also surveyed regarding their motivation to perform clinical duties from home on a 5-point agreement Likert scale. Paper surveys were distributed to Internal Medicine residents at mandatory housestaff meetings at three Midwestern teaching hospitals in Spring 2011. The surveys were entered into an Excel database and statistical analysis was performed by use of STATA 10.0. Chi square tests were utilized to assess differences by site or by residency training year.

RESULTS: 156 of residents responded with an overall response rate of $77 \%$, Site responses varied from $68 \%$ to $86 \%(\mathrm{p}=0.02)$. Residents reported accessing the electronic health record from home for checking labs for inpatients (86\%) and outpatients (73\%), ordering labs for inpatients (71\%) and outpatients $(56 \%)$. Communicating via phone or text with a co-resident was common $(84 \%)$, and $74 \%$ reported contacting the cross-cover team from outside the hospital. Nearly a third (29\%) of residents reported coming to the hospital to complete patient care work on their designated day off. $39 \%$ came to work on their day off for educational purposes. The most common reason residents reported working from home was to monitor a patient's progress $(81 \%)$ and to complete unfinished work (65\%). Most residents $(88 \%)$ believe the ability and skill-set to work from home is useful for future independent practice. There were differences between PGY classes, where interns were 
more likely to believe their program expects them to access the EMR at home for inpatient and outpatient care (32\% of interns compared to $25 \%$ of residents agreed). Almost half (45\%) believe outside hospital clinical activity should also be more closely and formally monitored by the ACGME. Significant site differences were noted regarding resident motivation and the program's expectation of residents to manage clinics from home, to complete unfinished work from home, and the desire for formal ACGME monitoring. The majority of housestaff (40\%) believed that accessing EMR from home did not interfere with their personal life versus those that did $(26 \%)$

CONCLUSIONS: In spite of residency duty hour restrictions, many residents report accessing the EHR from home to advance care, primarily to monitor a patient's progress and to complete unfinished work. As most residents believe this is a useful practice skill, it is important for residency programs to recognize this out of hospital activity and develop curricula and policies for remote use of electronic health records.

NATIONAL TRENDS IN ORAL ANTICOAGULANT USE IN THE UNITED STATES, 2007-2011 Rachel Kornfield ${ }^{1}$; Kate Kirley ${ }^{2}$; Dima M. Qato $^{1}$; Randall S. Stafford ${ }^{3}$; G. Caleb Alexander ${ }^{1,4}$. ${ }^{1}$ University of Chicago, Chicago, IL; ${ }^{2}$ University of Chicago, Chicago, IL; ${ }^{3}$ Stanford University, Palo Alto, CA; ${ }^{4}$ University of Chicago, Chicago, IL. (Control ID \#1322519)

BACKGROUND: Oral direct thrombin inhibitors offer a new means of preventing thromboembolism in ambulatory settings, yet little is known regarding their adoption in clinical practice. We described trends in oral anticoagulation for the prevention of thromboembolism in the United States.

METHODS: We used the IMS Health National Disease and Therapeutic Index, a serial cross-sectional nationally representative audit of officebased providers, to quantify patterns of oral anticoagulant use among all subjects and stratified by clinical indication. Our main outcome measure was an office visit where oral anticoagulation was used (treatment visit). We also quantified oral anticoagulant expenditures using the IMS Health National Prescription Audit.

RESULTS: Between 2007 and 2011, warfarin treatment visits declined from approximately 2.1 million [M] quarterly visits to approximately 1.6 M visits. Since its market release in October 2010, dabigatran use increased from $0.062 \mathrm{M}$ quarterly visits (2010Q4) to $0.231 \mathrm{M}$ visits (2011Q3), reflecting its increasing share of oral anticoagulant visits from $3.1 \%$ to $12.3 \%$. The majority of oral anticoagulant treatment visits occurred with patients aged 65-84 years, but dabigatran use was even more focused within this age group than warfarin. The proportion of dabigatran use that occurred among individuals 85 years or older decreased from $22 \%$ to $3 \%$ over the four calendar quarters of available data. In contrast to warfarin, the majority of dabigatran visits have been for atrial fibrillation, though this proportion decreased from $92 \%$ (2010Q4) to $71 \%$ (2011Q3), with concomitant increases in dabigatran's off-label use. The most common off-label uses of dabigatran were for coronary artery disease, hypertensive heart disease and venous thromboembolism. Among atrial fibrillation visits, warfarin use decreased from $55.8 \%$ of visits $(2010 \mathrm{Q} 4)$ to $45.4 \%$ (2011Q3), while dabigatran use increased from $4.0 \%$ to $11.9 \%$. Of atrial fibrillation visits, the fraction without any oral anticoagulant use has remained unchanged at approximately $40 \%$ since the introduction of dabigatran. Prior to the availability of dabigatran, the majority of visits reporting oral anticoagulant use were with physicians practicing in internal medicine (30\%), cardiology (34\%), and family practice (19\%), with fewer visits accounted for by physicians affiliated with osteopathy (5\%), oncology $(3 \%)$, or other specialties $(8 \%)$. By contrast, most dabigatran visits during the four calendar quarters of available data were accounted for by cardiologists $(54 \%)$, with fewer visits associated with internal medicine $(29 \%)$, family practice $(12 \%)$ or other clinical fields $(6 \%)$. Expenditures related to dabigatran increased rapidly from $\$ 16 \mathrm{M}$ in $2010 \mathrm{Q} 4$ to $\$ 141 \mathrm{M}$ in 2011Q3, approaching expenditures on warfarin $(\$ 144 \mathrm{M})$ in $2011 \mathrm{Q} 3$.

CONCLUSIONS: Although representing a minority of all oral anticoagulant visits, dabigatran has been rapidly adopted into ambulatory practice in the United States, primarily for treatment of atrial fibrillation, but increasingly for off-label indications. We did not find evidence that it has increased overall atrial fibrillation treatment rates.

NEUROBIOLOGICAL CHANGES ACCOMPANY PATIENT-CENTERED INTERVIEWING Robert C. Smith; Issidoros Sarinopoulos; Seungcheol Lee; Chelsea Gordon; Lu Wang; Ashley Hesson; Francesca C. Dwamena. Michigan State University, East Lansing, MI. (Control ID \#1324573)

BACKGROUND: Patient-centered interviewing (PCI) enhances the provider-patient relationship (PPR) and health outcomes, but the biological basis for this is unknown. Recent fMRI investigations report neurobiological changes during other dyadic interactions, for example, reduced threatrelated neural activation in anterior insula in the presence of a strong marital relationship. In the first fMRI study of PCI/PPR we are aware of, we hypothesized that an evidence-based, behaviorally-defined PCI method, compared to isolated clinician-centered interviewing $(\mathrm{CCI})$, would be associated with attenuated brain activation in the pain processing regions in anterior insula, the region that instantiates the emotional component of pain and where pain-related activation can be attenuated by supportive figures.

METHODS: We recruited 9 right-handed females between 45 and 60 years of age from a primary care clinic. One of the authors (RCS) conducted the evidence-based PCI method in 5 randomly selected subjects or a standard, disease-focused CCI in the other 4 subjects for 20-25 minutes. One blinded, independent rater subsequently rated videotapes of the interviewer's success in achieving the 5 steps and 21 substeps in the PCI method. Another independent rater (AH) completed interactional sociolinguistic analyses of the interviews. Patients also completed a well-validated Satisfaction with the PPR questionnaire. Patients then underwent fMRI scans while we intermittently applied aversive vs. imperceptible stimulation to their left hand. Pain trials started with a red arrow cue and were followed by a red dot and aversive stimulation. Non-pain trials started with a blue arrow cue and were followed by a blue dot and imperceptible stimulation. Monitoring segments comprised blocks of three trials during which patients saw either the picture of the consulting doctor (PCI or CCI) or the picture of an unknown doctor. We analyzed single-subject fMRI time-series data to yield contrast maps between pain and non-pain conditions.

RESULTS: The satisfaction questionnaire and the rater successfully differentiated PCI and CCI (both $\mathrm{p}<.01$ ). The linguistic analysis showed significantly more physician space-providing moves in the PCI than $\mathrm{CCI}(\mathrm{p}<$ $0.05)$ and fewer physician questions in the PCI than CCI $(\mathrm{p}<0.05)$. Qualitatively, several markers indicated co-constructed, accommodative discourse in the PCI. We then confirmed that the anticipation and response to aversive stimulation elicited increased activation in pain related areas of interest in bilateral anterior insula $(\mathrm{p}<.05$, corrected). We then submitted extracted percent-signal change values from these regions to repeated-measures GLM analyses, Consultation 2 (PCI, CCI) x Doctor 2 (Consulting, Unknown) $\mathrm{x}$ Hemisphere 2 (Right, Left) x Period 2 (Anticipation, Experience). The type of intervention (PCI vs. CCI) predicted pain-related activation, as indicated by a statistically significant consultation $\mathrm{x}$ doctor interaction. Follow-up pairwise ttests revealed significantly decreased activation during the experience of pain associated with monitoring by the positive PPR doctor compared to the unknown doctor. Based on convention, all analyses were one tailed $(p<0.05)$. CONCLUSIONS: These findings represent an initial step in identifying the neural mechanisms underlying the previously observed positive health outcomes associated with PCI and a positive PPR.

NEW DRUGS: UNSAFE AT ANY SPEED? LENGTH OF TIME TO BLACK BOX WARNINGS AND WITHDRAWALS FROM 1975 TO 2010. Cassie Frank ${ }^{1}$; David Bor ${ }^{1}$; Steffie Woolhandler ${ }^{1}$; David Himmelstein ${ }^{1}$; Karen $\overline{\text { E. Lasser }}{ }^{2}$. ${ }^{1}$ Cambridge Health Alliance, Cambridge, MA; ${ }^{2}$ Boston Medical Center, Boston, MA. (Control ID \#1333853)

BACKGROUND: Black box warning labels are an important signal of drug safety problems, and a principle way that the FDA communicates such problems to doctors and the general public. An earlier study of drugs approved between 1975 and 1999 found that serious safety warnings often emerged years after drug approval. We extended this study to drugs approved through 
2009 to assess the safety of newly approved medications and the effects of regulatory efforts that have abbreviated the FDA approval process.

METHODS: We compiled a list of all drugs that the FDA approved from 1975-2009 and designated as new molecular entities (NMEs). NMEs contain an active ingredient that has not previously been approved for marketing in the United States in any form. We excluded over-the-counter medications, diagnostic agents, and biologics. We collected data from three sources: the Physician's Desk Reference (PDR), PDR.net, and drugs@FDA.gov. We determined if drugs were withdrawn for safety reasons from information on the FDA website and the Center for Drug Evaluation and Research (CDER) Report to the Nation 2005. First, we searched the 2010 PDR and PDR.net to establish if a drug currently has a black box warning. We then looked through earlier editions of the PDR from 2000-2010 and at drug label information on drugs@FDA.gov to determine the date the black box warning first appeared. If a black box warning was present in the PDR or FDA-approved label when the drug first appeared, we excluded it. We used the date the drug was first approved to approximate the date the drug was first marketed, and we counted an event as the date a drug received a black box warning or was withdrawn for safety reasons. For drugs that had more than one black box warning, we counted each new warning as a separate event. We performed survival analyses to generate Kaplan-Meier plots to determine how much time passed between drug approval and withdrawal or receipt of a black box warning.

RESULTS: The FDA approved 745 NMEs between $1975-2009 ; 117$ $(15.7 \%)$ drugs received new black box warning and $26(3.5 \%)$ drugs were withdrawn from the market for safety reasons. In Kaplan-Meier Analyses, the estimated probability of acquiring a new black box warning or being withdrawn from the market over 35 years was $44 \%$.

CONCLUSIONS: Despite efforts to ensure drug safety prior to drug approval, many new drugs receive serious new warnings or are withdrawn from the market many years after they are first approved. New drugs should be used with caution, and older drugs with a more established safety profile should be used preferentially.

NO DETECTABLE EFFECT OF MARIJUANA USE ON HEALTH OR HEALTHCARE UTILIZATION AMONG PATIENTS WITH ANY ILLICIT DRUG USE IDENTIFIED BY SCREENING IN PRIMARY CARE. Daniel Fuster ${ }^{1}$; Debbie M. Cheng ${ }^{1,2}$; Donald Allensworth-Davis ${ }^{3}$; Tibor P. Palfai ${ }^{4}$; Jeffrey H. Samet ${ }^{1}$; Richard Saitz ${ }^{1}$. ${ }^{1}$ Boston Medical Center/ Boston University School of Medicine, Boston, MA; ${ }^{2}$ Boston University School of Public Health, Boston, MA; ${ }^{3}$ Boston University School of Public Health, Boston, MA; ${ }^{4}$ Boston University, Boston, MA. (Control ID \#1326904)

BACKGROUND: Federal programs encourage screening for drug use, including marijuana, in general health settings. Marijuana is the illicit drug most commonly used by patients in primary care identified by screening, and when identified in patients using other drugs, questions arise regarding how to address it. Although use can be associated with adverse health effects, the impact of marijuana on health is not extensive. Therefore, we assessed the association between marijuana use and health and healthcare utilization among patients with any illicit drug use identified by screening in primary care.

METHODS: We analyzed data from patients in an urban primary care clinic who, when screened, reported any past 3-month drug use (marijuana, opioids, cocaine, others). We assessed comorbidity with the Charlson comorbidity index (Deyo modification) using ICD-9 codes obtained from the electronic medical record. By interview, we determined health status with the Euroquol [index from 0 (worst) to 100 (best possible health)] and healthcare utilization (past 3-month emergency department use and hospitalization). Recent marijuana use (past 3 months) was the main independent variable and defined as any vs. no use. Separate multivariable models adjusting for age, sex, and any recent (past 3 months) other type of substance use (separate indicators for heavy episodic drinking; cocaine use; opioid use [prescription opioids or heroin] and any other drug use [sedatives, amphetamines, hallucinogens and inhalants]) were used to assess the association between recent marijuana use and the four outcomes of interest: any comorbidity, health status, any emergency department use, and any hospitalization.
RESULTS: Participants $(\mathrm{n}=554)$ were $69 \%$ male and mean age was 41 years. All participants reported recent drug use: marijuana $84 \%$, heavy episodic drinking $44 \%$, cocaine use $26 \%$, opioid use $23 \%$, other drug use $8 \% ; 57 \%$ reported use of marijuana only, $8 \%$ cocaine use only and $4 \%$ reported opioid use only. The median Charlson comorbidity index value was 0 (range $0-7,37 \%$ with values $\geq 1$ ); the mean Euroquol score was $70 ; 36 \%$ had recent emergency department use and $14 \%$ a recent hospitalization. In analyses adjusted for age, sex and use of any other drugs, recent marijuana use was not significantly associated with emergency department use (adjusted odds ratio (AOR) $0.71,95 \%$ confidence interval (CI) $0.42,1.22$ ), hospitalization (AOR $1.16,95 \%$ CI $0.59,2.28$ ), health status (adjusted mean Euroquol score 66 versus $65, \mathrm{p}=0.86$ ) or Charlson index $\geq 1$ (AOR 0.64, 95\% CI $0.37,1.13)$. A multivariable analysis with recent marijuana use defined in 3 categories (daily use, less than daily use, vs. no use) yielded similar results.

CONCLUSIONS: Among adults in primary care who reported any recent illicit drug use, we were unable to detect an effect of marijuana use on health or on emergency department or hospital utilization. Addressing marijuana use among patients who use illicit drugs may not yield shortterm benefits as captured by health status, comorbidity and utilization measures. Future studies should assess the longitudinal impact of marijuana use on these and other measures and include patients who do not use other drugs.

NON-VISIT CLINICAL BURDEN OF EFFORT AMONG 82 ACADEMIC GENERAL INTERNISTS Liselotte Dyrbye; Colin P. West; Tim Burriss; Tait Shanafelt. Mayo Clinic, Rochester, MN. (Control ID \#1339411)

BACKGROUND: Primary care doctors are struggling to provide access to America's aging population. This problem is exacerbated by a declining supply of internists and - although welcomed - an expansion of insured patients who are seeking care. In response, some practices may expand hours and shorten visits lengths to accommodate more patients. Such practice redesign efforts, however, do not take into account the clinical workload that occurs outside the office visit. Previous small (4-33 physicians) observational or self-reported time studies over short intervals suggest physicians spend 8-15 hours per week on uncompensated work. In this study we electronically measured actual tasks commonly performed by general internists beyond interviewing and examining patients (e.g. prescriptions, notes, orders, etc.). The objective of the study was to evaluate the work burden of non-reimbursable practice related activity among general internists.

METHODS: Board-certified general internists at a large academic medical center who spent at least $20 \%$ of their work efforts in the outpatient setting were included. All use an electronic health record. From electronic databases we obtained the exact number of: prescriptions written and signed; orders placed; electronic clinical notes authored, edited, and signed; electronic patient care messages reviewed and responded to; outpatient visits; and clinic days for each physician for an entire calendar year (2010). In addition, we collected the number of minutes each physician spent dictating. We calculated basic summary statistics for each workload variable. The study was IRB approved.

RESULTS: Among the $82(71 \%)$ academic internists who devoted at least $20 \%$ to the outpatient practice, $50(61 \%)$ were male. In 2011 each of these physicians wrote a mean of 3,108 prescriptions, placed 7,627 orders, authored 1,967 clinical notes, responded to 681 electronic patient care messages, and spent 88 hours dictating. Mean totals per outpatient visit and per clinic day for each workload variable are shown in Table 1.

CONCLUSIONS: Outpatient visits are associated with substantial numbers of non-reimbursable tasks that must be completed by physicians. Efforts to increase the number of outpatients seen by internists must address this workload as it may form an under recognized limit to how many patients can be seen in a workday. 
Non-reimbursable practice related activity completed by 82 academic general internist in 2011

\begin{tabular}{lll}
\hline \hline & $\begin{array}{l}\text { Mean total per outpatient } \\
\text { visit }\end{array}$ & $\begin{array}{l}\text { Mean per clinic } \\
\text { day }\end{array}$ \\
$\begin{array}{l}\text { Prescriptions written } \\
\text { and signed }\end{array}$ & 2.84 & 30.80 \\
$\begin{array}{l}\text { Orders placed * } \\
\begin{array}{l}\text { Clinical notes } \\
\text { reviewed, }\end{array}\end{array}$ & 7.41 & 69.94 \\
$\begin{array}{l}\text { edited, and signed } \\
\text { Electronic messages }\end{array}$ & 0.86 & 19.11 \\
Minutes of dictation & 5.02 & \\
\hline
\end{tabular}

* includes imaging, laboratory studies, and consultations

NOVEL "TOBACCO" PRODUCT USE AND ASSOCIATION WITH SMOKING CESSATION: A NATIONAL STUDY Pamela M. Ling ${ }^{1,2}$; Lyudmila Popova ${ }^{2} .{ }^{1}$ University of California San Francisco, San Francisco, CA; ${ }^{2}$ University of California San Francisco, San Francisco, CA. (Control ID \#1340098)

BACKGROUND: Since 2006, the major US cigarette manufacturers acquired smokeless tobacco companies and have begun selling smokeless tobacco products bearing cigarette brand names (e.g. Marlboro snus, Camel snus). At the same time, electronic cigarettes (e-cigarettes) have begun to be aggressively promoted on the internet as a smoking cessation device or as a way to use nicotine in smokefree environments. There is a dearth of clinical data on effect of novel tobacco product use on smoking cessation, and these products are not approved for this purpose. We examined rates of trial and current use of novel alternative tobacco products among smokers and its association with smoking cessation behavior.

METHODS: A nationally representative probability-based sample of 1,836 current or recently former (quit within last two years) smokers completed an online cross sectional survey in November 2011. Current (past 30 day) and ever use of traditional smokeless tobacco (loose leaf, moist snuff), novel smokeless tobacco (snus, dissolvable), hookah, and electronic cigarettes was compared among former smokers ( $23.3 \%$ of sample), smokers who tried to quit in the past year $(59.9 \%)$, and smokers who did not try to quit $(16.8 \%)$. Comparisons between groups were made using chi-square tests for categorical variables, and multivariate logistic regression was used to evaluate the association between current alternative tobacco product use and quit attempts, controlling for age, sex, race/ethnicity and education.

RESULTS: Overall, $44 \%$ of smokers had tried one of the novel/alternative tobacco products, and $30 \%$ of smokers expressed interest in using novel tobacco products to try to reduce their health risk, cut down, or quit smoking. Electronic cigarettes had been tried most frequently $(20.4 \%$ of respondents had ever used, with $40 \%$ of ever users reporting current past month use), and dissolvable tobacco products had been tried least frequently $(2.8 \%$ ever used). Males used traditional smokeless and snus tobacco products more frequently than women $(p<.001)$; women used ecigarettes more frequently $(\mathrm{p}<0.05)$, and use of hookah, and dissolvable tobacco did not differ by gender. E-cigarettes had been used significantly more frequently by smokers who tried to quit smoking but failed $(22.5 \%)$, than successful quitters $(17.6 \%)$ or smokers who had not tried to quit smoking $(15.2 \%)(\mathrm{p}=0.006)$. Ever using an alternative tobacco product was associated with having made a cigarette quit attempt in the past year $(\mathrm{OR}=1.47,95 \% \mathrm{CI}[1.20,1.80])$, as was current use of any product $(\mathrm{OR}=$ $1.30[1.06,1.59])$. Ever using an alternative tobacco product was not independently associated with successful smoking cessation.

CONCLUSIONS: Many smokers in the USA have tried novel alternative tobacco products, and e-cigarettes were tried more frequently than both the traditional and newer smokeless tobacco products like snus. New smokeless tobacco products and e-cigarettes appear to appeal more to women than traditional smokeless tobacco. Alternative tobacco product use is associated with attempting to quit smoking, but not having quit successfully. Clinicians should be aware of novel tobacco products, particularly e-cigarettes, and should screen for use among smokers, as this may indicate stronger interest in smoking cessation and an opportunity to encourage evidence-based cessation treatments.

NUMERACY VERSUS READING SKILLS: UNPACKING HEALTH LITERACY Elizabeth A. Wilson ${ }^{1}$; Allison Dahlke ${ }^{1}$; Laura Curtis ${ }^{1}$; Lee Lindquist ${ }^{1}$; Alex Federman ${ }^{5}$; Michael S. Wolf ${ }^{1,2}$. ${ }^{1}$ Northwestern University, Chicago, IL; ${ }^{2}$ Northwestern University, Evanston, IL; ${ }^{3}$ Mount Sinai, New York, NY. (Control ID \#1338290)

BACKGROUND: Recent work in the field of health literacy has led to the development of a variety of distinct measures of literacy, including assessments that focus specifically on reading skills or on numeracy exclusively. We sought to investigate the relationship between reading and numeracy as well as examine the relative impacts of each on comprehension, retention, and problem solving for health-related information.

METHODS: Three-hundred-four primary care patients aged 55-74 completed a series of literacy assessments, three of which examined reading skills: the Rapid Estimate of Adult Learning in Medicine (REALM), the American Nelson Adult Reading Test (AMNART), and the reading section of the Test of Functional Health Literacy in Adults (TOFHLA), and three of which examined numeracy skills: the Lipkus Numeracy Scale, the Newest Vital Signs (NVS), and the numeracy portion of the TOFHLA. To assess the relationship between each measure, scores for all were correlated. Additionally, participants were presented with a number of different hypothetical health scenarios and asked to complete a series of health tasks about each to gauge comprehension, reasoning, and retention for the information contained in each scenario. To examine the relative impact of reading and numeracy skills on health performance, we performed a series of regression analyses to determine whether factor scores for reading skills and numeracy separately, as well as simultaneously, impacted health-task performance (scored on a scale of 1-100).

RESULTS: Scores on all assessments of numeracy and reading skills were highly correlated $(0.39 \leq \mathrm{r} \leq 0.69, \mathrm{p}<0.001)$. After adjusting for age, gender, race, education, and comorbidity, in regression analyses, when reading ability was entered into a model it was found to significantly predict health-task performance $(\beta, 10.4 ; \mathrm{CI}, 9.0-11.8, \mathrm{p}<0.001)$, and $64 \%$ of the variance was explained. When numeracy was entered into a separate model, it also significantly predicted performance $(\beta, 11.3 ; \mathrm{CI}, 9.7-12.8, \mathrm{p}<0.001)$, and again $64 \%$ of variance was explained. Finally, in a model including both reading and numeracy concurrently, both factor scores significantly predicted performance $(\beta, 7.1 ; \mathrm{CI}, 5.7-8.6, \mathrm{p}<0.001 ; \beta, 7.3 ; \mathrm{CI}, 5.7-8.9 ; \mathrm{p}<0.001$ for reading and numeracy, respectively). In this final model, $72 \%$ of the variance was explained, providing a significantly greater goodness of fit than was found for either model that included either reading or numeracy alone $(\mathrm{p}<0.001)$.

CONCLUSIONS: Reading and numeracy skills are highly associated, and each in isolation explains a large and comparable amount of variance in performance related to common health-based tasks. Additionally, consideration of both skills together provides an even clearer predictor for health-task success. As such, health literacy likely encompasses a broader set of abilities than either reading or numeracy alone, and rather entails cognitive processing at large. Designers of health-related interventions should consider universal cognitive constraints in information processing when creating interventions to mitigate the impacts of low health literacy.

NUTRITIONAL STATUS AND LONG-TERM SURVIVAL OF THE ELDERLY INITIATED ARTIFICIAL NUTRITION: FROM JAPAN ASSESSMENT STUDY ON PROCEDURES AND OUTCOMES OF ARTIFICIAL NUTRITION (JAPOAN) COHORT Seiji Bito ${ }^{1}$; Tetsuo Yamamoto $^{2}$; Harumi Tominaga ${ }^{3}{ }^{1} \mathrm{NHO}$ Tokyo Medical Ceter, Tokyo, Japan; ${ }^{2} \mathrm{NHO}$ Yonago Medical Center, Yonago, Japan; ${ }^{3} \mathrm{NHO}$ Kure Medical Center, Kure, Japan. (Control ID \#1333813)

BACKGROUND: Clinical choice of artificial nutrition therapy for the elderly requires careful consideration because artificial nutrition routes have both merits and demerits. The objective of this study is to compare the effect of different artificial nutrition methods on long-term survival and short-term nutritional condition for the benefit of the elderly who need long-term artificial nutrition support. 
METHODS: [Study Design] Multi center prospective cohort study. [Setting] Sixty-two National Hospital Organization (NHO) facilities in Japan. [Patients] Patients 60 years old or over who are admitted to each NHO facility and judged to be needed to take artificial nutrition therapy with an aim to maintain/ improve nutrition and who actually went under the artificial therapy, except postoperative management purpose. [Intervention variable] At the start of administration of artificial nutrition, patients were enrolled in either nutritional route group of total parenteral nutrition group (TPN), nasal tube feeding group (EN_N) or percutaneous endoscopic gastrostomy (PEG) nutrition group (EN_G). Nutritional routes were selected based on the clinical judgment, not experimentally. [Outcome measures] Observation started with the start of the artificial nutrition, and survival periods until the death were recorded. Serum albumin was also observed and recorded at 2 weeks and 4 weeks after the intervention.

RESULTS: A total of 548 patients were enrolled. 524 out of them, 152 patients, 183 patients and 189 patients were classified into TPN group, EN_N group and EN_G group, respectively. Slight but significant difference was observed in age at the time of registration, gender, performance status and Body Mass Index among three groups. Mean serum albumin level was $2.8 \mathrm{mg} / \mathrm{dl}, 3.1 \mathrm{mg} / \mathrm{dl}$ and $3.0 \mathrm{mg} / \mathrm{dl}$ in TPN group, EN_N group and NE_G group, respectively $(\mathrm{P}<0.0001)$. More than $80 \%$ of patients in both EN N group and NE G group were suffering from neurological disease as the primary disease, while $37 \%$ in TPN group $(\mathrm{p}<0.0001)$. Adjusted means of the serum albumin level at 2 weeks $/ 4$ weeks later were $2.5 \mathrm{mg} / \mathrm{dl}, 2.5 \mathrm{mg} / \mathrm{dl} ; 2.8 \mathrm{mg} / \mathrm{d}, 3.0 \mathrm{mg} / \mathrm{dl}$; and $2.8 \mathrm{mg} / \mathrm{d}, 3.0 \mathrm{mg} / \mathrm{dl}$ in TPN group, EN N group, and EN G group, respectively $(\mathrm{p}<0.0001)$. Estimated mean survival time $[95 \% \mathrm{CI}]$ from the intervention to the death was 361 days [297-426 days], 489 days [436543 days], 596 days [538-655 days] in TPN group, EN N group, and NE G group, respectively. As compared to EN G group, the relative hazard ratio $[95 \% \mathrm{CI}]$ against the survival time in $\bar{C}$ ox proportional-hazards model in EN_N group and TPN group was 1.2 [0.9-1.7] and 1.8 [1.3-2.5], respectively. CONCLUSIONS: [Limitations] Adjustments for center in multicenter study is needed. The distribution of the primary disease in TPN group differs from the other two groups. [Generalization] The result of our study supports the hypothesis, that is, enteral nutrition is superior, as compared to intravenous nutrition, in short-term retention of nutritional status as well as long-term survival for patients who need artificial nutrition continuously. It was indicated that the favorable effect of PEG nutrition was not significant comparing to nasal nutrition.

OFF THE HAMSTER WHEEL? QUALITATIVE EVALUATION OF A PAYMENT-LINKED PATIENT CENTERED MEDICAL HOME (PCMH) PILOT Gordon Schiff ${ }^{1}$; Asaf Bitton ${ }^{1,2}$; Elizabeth Stewart ${ }^{4}$; Greg Schwartz ${ }^{1}$; Carol Keohane ${ }^{1}$; Lydia A. Flier ${ }^{1}$; Daniel Henderson ${ }^{1,3}$; David W. Bates ${ }^{1,5}$. ${ }^{1}$ Brigham and Women's Hospital, Boston, MA; ${ }^{2}$ Harvard Medical School, Boston, MA; ${ }^{3}$ University of Connecticut School of Medicine, Farmington, CT; ${ }^{4}$ American Academy of Family Physicians, Farmington, CT; ${ }^{5}$ Harvard School of Public Health, Boston, MA. (Control ID \#1338014)

BACKGROUND: Deepening our understanding of ways to improve primary care represents a high priority for building a quality, caring, and cost-effective health system. Multiple Patient-Centered Medical Home (PCMH) initiatives are being implemented throughout the U.S, but few have combined comprehensive payment reform with PCMH transformation. As part of a larger evaluation of 2 unique PCMH initiatives centered around practice change and payment restructuring (Goroll, JGIM 2006), we conducted a qualitative evaluation of initial participating practices to better understand context, mechanisms, and impacts of these pilot efforts.

METHODS: We used a qualitative comparative case study approach to explore underlying dynamics of transformation efforts at 5 practices participating in a PCMH effort linked with payment reform. Sites were evaluated after engaging in transformation activities for 12-18 months. Data collection included review of practice-generated descriptive and quantitative data. Intensive 4-6 hour site visits by a team of 3 general internists included structured interviews, observations, and artifact review, and were followed by analysis of dictated transcripts guided by an experienced qualitative researcher to identify themes and insights.

RESULTS: The qualitative review generated detailed components of each practice's transformation efforts, as well as a grounded taxonomy of themes and insights. Data comparing the genesis, rationale for change, organizational structure, change approach, team care design, EHR deployment, practice-wide changes, clinician point-of-care changes, quality improvement measures, specifics of payment plan, helped to identify 8 recurrent but often contrasting themes among the study sites. They included 1) unique historical motivation and contexts, 2) wide variations in changes implemented, 3) varied role and value of change catalysts (consultants, trainers, and lean methods), 4) ubiquitous but multiple challenges in harnessing health information technology (HIT), 5) varying definitions and deployment of teams and teamwork, 6) centrality of compensation reorganization to organizational leadership in contrast with stated physician indifference, 7) challenges in pace of change if too rapid (change fatigue) or slow (disillusionment), 8) surprise confounders incidentally uncovered including unreported concurrent insurer restructuring toward high deductable plan. Practices differed in their emphasis on re-engineering the clinical encounters, vs. directing efforts at redesign of population management and intra-visit care level.

CONCLUSIONS: In a payment-linked PCMH transformation pilot, we noted widespread activation of clinician and staff experimentation with care delivery innovation, in addition to other adaptive responses that should inform further PCMH transformation activities and research. The issues raised included evidence of uncertain replicability; questions about comparability and standardization; challenges around HIT deployment, particularly related to obtaining ED and hospitalization information; ambiguous interpretations of teamwork varying from redesigned staffing to joint project activities; disconnect between leaders' and practitioners' understanding and commitment to payment reform; and outlier practices and practitioners. Interpretation and generalizability of metrics collected from this and other PCMH experiments will need to weigh the specifics and themes emerging from the on-the-ground realities of change implementation and outcomes.

ON THE WRONG SIDE OF THE DIGITAL DIVIDE: ACCESS, INTEREST, AND ATTITUDES TOWARD INTERNET-BASED COMMUNICATION FOR HEALTH AMONG PATIENTS IN THE SAFETY NET Urmimala Sarkar; Adam Schickedanz; David Huang; Andrea Lopez; Tom Bodenheimer. University of California, San Francisco, San Francisco, CA. (Control ID \#1326469)

BACKGROUND: Communication between patients and their health care team via email or a patient portal with secure web messaging has been shown to increase patient satisfaction, enhance provider productivity, and improve health outcomes. We sought to characterize access to and interest in web-based communication in a diverse group of patients from a large urban safety net clinic network.

METHODS: A cross-sectional, survey of English, Spanish, and Cantonese-speaking adults attending an ambulatory clinic visit in the San Francisco Community Health Network, a large group of urban community and hospital-based clinics. The primary outcomes were the level of patients' use of web-based communication and interest in web-based communication for health care related. Self-reported sociodemographic characteristics (age, gender, language, race/ethnicity, educational level) were collected.

RESULTS: Participants were generally representative of the overall clinic network patient population. Among the participants, 54\% were female, $79 \%$ were under 60 years old, and $68 \%$ had income less than $\$ 20,000$ per year. Eighty-one percent did not identify as white, only $55 \%$ were primarily English speaking, and 25\% reported poor English proficiency or no English proficiency at all. $60 \%$ of our study participants used email regularly, and $59 \%$ of those who used email accessed it in their home. Demographic characteristics associated with 
greater access to and use of email included younger age (especially age less than 40 years old), income over $\$ 20,000$ per year, being housed, some college education, white race, speaking primarily Spanish or English, and higher English proficiency. Cantonese language and Asian descent were negatively associated with email use. Seventeen percent of patients were already using email to communicate with their medical providers. Eighty-two percent agreed that email would improve the clinical communication with their doctor or nurse.

CONCLUSIONS: Our data show racial and ethnic disparities in email use and interest in electronic health-related communication among patients in the safety net. We found an unmet demand for technology for health communication among patients in the safety net. Furthermore, most of patients have access to the web, and most agreed it would likely improve clinical communication and efficiency. Usability testing and tailoring of these technologies in vulnerable populations will be critical to ensure that patients in disadvantaged populations benefit equally. Moreover, patients in resource-poor communities, especially those with limited health literacy and lower educational attainment, may require training in the use of these technologies to achieve the greatest benefit.

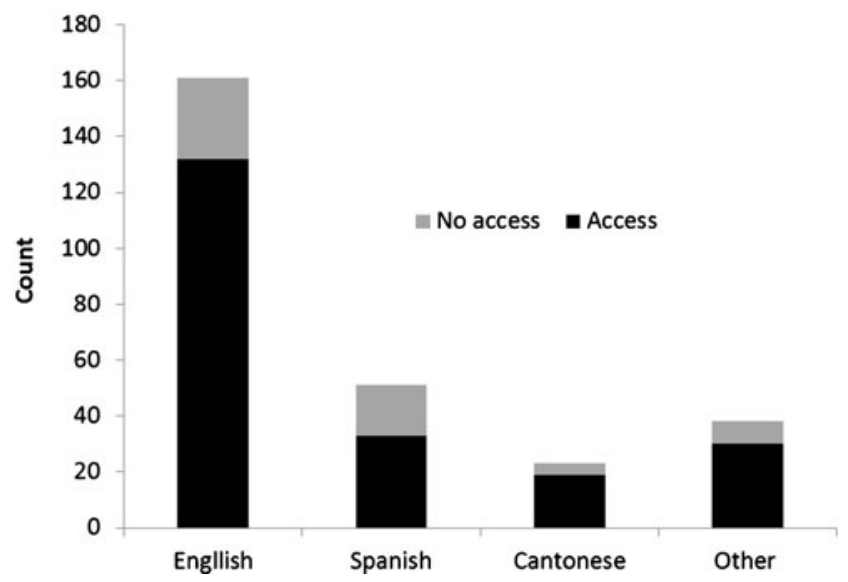

Interest by Access and Language

ONE YEAR AFTER: LUNG RESECTION SURGERY IMPROVES FUNCTIONAL OUTCOMES IN PATIENTS WITH EARLY STAGE LUNG CANCER. Samuel Cykert ${ }^{1}$; Franklin McGuire ${ }^{2}$; Paul Walker ${ }^{3}$; Giselle Corbie-Smith ${ }^{\text {T }}$ 'University of North Carolina School of Medicine, Chapel Hill, NC; ${ }^{2}$ University of South Carolina School of Medicine, Columbia, SC; ${ }^{3}$ Brody School of Medicine, East Carolina University, Greenville, NC. (Control ID \#1339607)

BACKGROUND: Many patients and their doctors decide against lung cancer surgery because of worries that lung resection could lead to significant debility or death. We recently found that surgical acceptance goes down markedly if patients believe that quality of life will be worse with surgical treatment one year after diagnosis. We performed a prospective cohort study designed to identify reasons for decisions against lung cancer surgery and Black-White disparities in surgical treatment. In this report we examined outcomes one year after diagnosis in the surgery and non-surgery groups related to important pre-operative variables including age, socioeconomic status, and race.

METHODS: Using pulmonary, oncology, thoracic surgery, and generalist practices in 5 communities, we enrolled 437 newly diagnosed patients with early stage, non-small cell lung cancer.
Inclusion criteria for patients were: at least 18 years old, have a tissue diagnosis or $>60 \%$ probability of non-small cell lung cancer using Bayesian methods, and be limited to Stage I or II disease by clinical and radiological testing. After being informed of the diagnosis of probable or definite lung cancer, but before the establishment of a treatment plan, patients were administered a 100-item survey that included a Short Form 12 Functional Assessment. Chart reviews were performed 4 months after enrollment to assess comorbidities, pulmonary function tests, and receipt of surgery. At one year follow-up, chart audits, follow up calls, and death certificate checks were used to identify survivors at which point the SF-12 was re-administered. To account for death and debility, we constructed a combination variable representing death or an SF-12 physical component score $<30$ one year after diagnosis. Descriptive statistics were compiled. Bivariate and multivariate analyses were performed. The independent variables examined included age, gender, marital status, SES, race, comorbid illness, having a regular source of care, and receipt of surgical treatment.

RESULTS: All deaths were identified and confirmed. Counting deaths, death-debility information at one year was available for 358 of 386 patients who remained eligible for lung cancer surgery based on diagnostic stage and absence of absolute contraindications. Of those patients enrolled, $29 \%$ were AA, $90 \%$ had health insurance, and the median age was 66 years. Sixty-six patients died within one year while 72 recorded a physical component score $<30$ yielding a combined death-debility rate of $39 \%$. Predictors of the one-year outcome of death or debility were 2 or more significant comorbid illnesses (OR 2.6, 95\% CI 1.3-5.2) and age $>66$ years (OR 1.9, 1.1-3.0). Surgical treatment significantly reduced the risk of the death or debility outcome (OR $0.53,0.33-0.86$ ). Despite an $11 \%$ decrement in lung cancer surgery rates for African-Americans, race was not an independent predictor of functional outcome.

CONCLUSIONS: Surgical treatment, even when controlling for comorbid illness and age, is associated with improved functional outcome at one year. Any patient perceptions that lung resection surgery leads to debility or death compared to no surgery need to be recognized and systematically refuted. More granular predictors for elderly patients with severe comorbidities need to be explored.

OPIOID ANALGESIC MISUSE IN A COMMUNITY-BASED COHORT OF HIV-INFECTED INDIGENT ADULTS Maya Vijayaraghavan $^{1}$; Joanne Penko ${ }^{2}$; David Guzman ${ }^{2}$; David Bangsberg ${ }^{3}$; Christine Miaskowski ${ }^{2}$; Margot Kushel ${ }^{2}$. ${ }^{1}$ University of California, San Diego, San Diego, CA $;{ }^{2}$ University of California, San Francisco, San Francisco, CA; ${ }^{3}$ Harvard School of Medicine, Boston, MA. (Control ID \#1320815)

BACKGROUND: Rising rates of opioid analgesic misuse and overdose have made the practice of prescribing opioid analgesics for chronic noncancer pain $(\mathrm{CNCP})$ controversial. Individuals with $\mathrm{CNCP}$ and cooccurring mental illness and substance use disorders are at higher risk for misuse than those without. In a longitudinal study of a community-based cohort of HIV-infected indigent adults, we examined rates of opioid analgesic misuse, the source of misused opioid analgesics, and determined factors associated with misuse.

METHODS: At a community-based field site, we interviewed participants every three months over two years about demographics, pain, treatment for pain, depression and illicit substance use (cocaine, heroin and methamphetamines). Using Audio Computer Assisted SelfInterviewing (ACASI) technology, participants self-reported opioid analgesic misuse (using opioid analgesics to get high, altering the route, selling or stealing, exchanging opioid analgesics for sex or illicit drugs, or attempting to forge a prescription). We determined lifetime (at baseline) and past 90-day rates of opioid analgesic misuse and the source of these medications. Using generalized estimating equations (GEE), we determined factors associated with opioid analgesic misuse. 
RESULTS: The mean age of the participants $(\mathrm{N}=296)$ was $49.4,41.2 \%$ were African American, and $82.1 \%$ had a lifetime history of homelessness. Of the 296 participants, $48.9 \%$ reported severe pain in the past week, $27.4 \%$ reported moderate to severe depression, and $34.8 \%$ used illicit substance in the past 90 days. Almost half (47.9\%) reported misuse in their lifetime; at baseline, $17.9 \%$ reported misuse in the past 90 days. A high proportion of participants reported receiving the misused opioid analgesic from a health care provider (HCP) $(37.9 \%$ for getting high, $69.2 \%$ for selling opioid analgesics, $45.8 \%$ for exchanging opioid analgesics for sex or illicit drugs, and $31.3 \%$ for altering the route). In GEE models, staying in a shelter or street (Adjusted odds ratio (AOR) 1.8, 95\% CI 1.1-2.8), men who have sex with men (MSM) (AOR 1.6, 95\% CI 1.0-2.6), current smoking (AOR 2.1, 95\% CI 1.3-3.5), illicit drug use (AOR 2.0, 95\% 1.42.8), moderate to severe depression (AOR 1.5, 95\% CI 1.0-2.1), and having severe pain (AOR 1.8, 95\% CI 1.1-2.8) were associated with misuse.

CONCLUSIONS: In this high-risk cohort, participants reported high rates of opioid analgesic misuse. Approximately half of the time, they reported obtaining the misused analgesics from a HCP. Consistent with previous studies, we found that illicit substance use, mental illness, current smoking, and severe pain were associated with misuse. We identified novel risk factors including current homelessness and MSM. Given the high rates of misuse using a prescribed opioid analgesic, HCP need to develop strategies for close assessment of the risk/benefit profile prior to deciding to prescribe these medications. This should be accompanied with careful monitoring for efficacy and misuse behaviors if prescribed, and a willingness to discontinue opioid analgesics when goals of treatment are unmet or when problematic behaviors develop.

ORGANIZATIONAL BARRIERS TO CHANGE AND REDUCING DISPARITIES IN HYPERTENSION CONTROL IN AN URBAN PRIMARY CARE NETWORK Brooke Cunningham ${ }^{1}$; Max J. Romano ${ }^{2}$; Jill A. Marsteller ${ }^{3}$; Lisa A. Cooper ${ }^{1,5}$. ' Johns Hopkins School of Medicine, Baltimore, MD; ${ }^{2}$ Johns Hopkins School of Medicine, Baltimore, MD; ${ }^{3}$ Johns Hopkins University, Baltimore, MD. (Control ID \#1339317)

BACKGROUND: National organizations and leaders in healthcare have affirmed that addressing disparities and achieving equity in healthcare delivery should be central objectives of quality improvement. Organizational culture affects the sucess of quality improvement efforts, but is relatively understudied. We hypothesize that organizational factors are associated with disparities in hypertension control between blacks and whites and will influence the uptake and success of interventions to improve hypertension control.

METHODS: The Multi-Method System Quality Improvement to Reduce Hypertension Disparities is a pragmatic trial of three system level interventions to reduce racial disparities in hypertension, set in six urban primary care clinics within the same primary care network in Baltimore. Semi-structured interviews were conducted with 25 senior and practice level managers. The interview guide focused on the process of identifying and implementing change within the organization, challenges with blood pressure management, and initiatives around health care disparities and cultural competency. Interviews lasted approximately an hour, were audio-recorded, and were transcribed. Two independent reviewers used the constant comparative method to code the transcripts for themes. Disagreements were adjudicated by a third reviewer.

RESULTS: Respondents identified several barriers to implementing and sustaining change in the organization. Barriers, such as time constraints, staffing, data quality, competing priorities, poor patient adherence and provider resistance, made organizational change and improving blood pressure difficult. Most respondents felt the best ways to improve blood pressure control were through interventions that targeted patients, such as reduced drug costs and improved outreach, rather than interventions directed at clinicians or staff.
Change initiated at the practice level tended to focus on the logistics of day to day work or was made in response to senior management objectives. Respondents report that there has been little explicit discussion of or activities related to health care disparities within the organization.

CONCLUSIONS: Primary care clinicians and staff face significant challenges in making changes to reduce racial disparities in hypertension. Interventions that improve workflow, target patients, or help practices meet senior management objectives are more likely to be implemented. For success in implementation and dissemination, organizational leaders must make the reduction of disparities in hypertension care a management priority.

OUTCOMES ASSOCIATED WITH EXPOSURE TO HIGH RISK MEDICATIONS IN ELDERLY WITH CHRONIC PAIN KoKo Aung ${ }^{1}$; Barbara J. Turner ${ }^{1}$; Mary Jo Pugh ${ }^{2,3}$. ${ }^{1}$ University of Texas Health Science Center at San Antonio, San Antonio, TX; ${ }^{2}$ University of Texas Health Science Center at San Antonio, San Antonio, TX; ${ }^{3}$ South Texas Veterans Affairs Medical Center, San Antonio, TX. (Control ID \#1340819)

BACKGROUND: Preventing adverse drug events (ADEs) in seniors is a patient safety priority. Exposure to high-risk medications for the elderly (HRME) is one of the HEDIS quality measures. Seniors with chronic pain may be at high risk for ADEs related to HRMEs such as opioids. Objective: To assess the association between incident HRME among seniors with chronic pain and a subsequent emergency room or hospital care indicating ADE.

METHODS: This retrospective cohort study was conducted among veterans aged $\geq 65$ in FY 2006 who received VA care from FY200406 , and had a chronic pain-associated diagnosis, defined by ICD-9 codes for conditions associated with persistent pain in the elderly by the American Geriatrics Society. Incident exposure to HRME was identified from pharmacy data. Medical records were searched for emergency room or inpatient encounters for diagnoses consistent with an ADE (likely ADE-related acute care) including: syncope, falls/ fractures and other conditions that may be associated with specific drug classes during one year following the date of initial exposure. Hierarchical logistic regression models examined association between likely ADE-related acute care and incident HRME exposure, controlling for demographic characteristics, chronic disease states, indicators of disease burden and prior history of these outcome events in the previous year.

RESULTS: Among 1,780,787 eligible veterans, 523,361 were diagnosed with chronic pain and, of these, $17.6 \%$ had incident HRME exposure compared with $4.5 \%$ in the overall cohort $(\mathrm{p}<0.001)$. Incident exposure to opioid analgesics significantly increased the odds of likely ADE-related acute care (adjusted odds ratio (AOR) $1.58 ; 95 \%$ CI, 1.08-2.30) within one year. The odds of likely ADErelated acute care after incident exposure to other HRME were also significantly increased for: AOR 1.93; 95\% CI, 1.67-2.24 (antihistamines), AOR 1.99; 95\% CI, 1.29-3.07 (psychotropics), and AOR 2.28; 95\% CI, 1.74-2.84 (muscle relaxants).

CONCLUSIONS: Incident exposure to HRME among older veterans with chronic pain is significantly higher than the overall population and is associated with adverse outcomes such as syncope, falls and fractures. Further research is needed to develop intervention measures to reduce exposure to these high risk drugs.

OVERACTIVE BLADDER MEDICATIONS: COMPARATIVE PERSISTENCE AND ADHERENCE OF PATIENTS WITH AND WITHOUT DIABETES Gabriel Haas ${ }^{1}$; Stephen Johnston ${ }^{2}$; Stephen Janning ${ }^{3}$; Kathleen Wilson ${ }^{2}$; David Smith ${ }^{2}$; GinaMarie Reckard ${ }^{1}$; Shun-Ping Quan ${ }^{1}$; Stan Bukofzer ${ }^{1} .{ }^{1}$ Astellas Pharma Global Development, Inc., Deerfield, IL; ${ }^{2}$ Thomson Reuters, Washington, DC; ${ }^{3}$ GlaxoSmithKline, Research Triangle Park, NC. (Control ID \#1325991) 
BACKGROUND: Overactive bladder (OAB) affects $\sim 33$ million adults in the US. A recent study showed $22.5 \%$ patients with diabetes had $\mathrm{OAB}$, which was more prevalent in individuals aged $>50$ years with diabetes for $>10$ years versus those with diabetes duration $\leq 10$ years and age $\leq 50$ years. Poor adherence and persistence to medication can lead to symptom relapse and high retreatment rates, yet $80-90 \%$ of patients discontinue their OAB medications within the first year of treatment, and only $30 \%$ of patients have an adherence rate $>80 \%$ at the end of first year. The objective of the current study was to evaluate and compare persistence and adherence to $\mathrm{OAB}$ medication in a subpopulation of patients with diabetes, versus those without the condition.

METHODS: Administrative claims and encounter records of patients $\geq 18$ years old who initiated at least one $\mathrm{OAB}$ medication (darifenacin, oxybutynin, solifenacin, tolterodine, trospium) between 1/1/2005 and 6/30/2008 were analyzed retrospectively using Thomson Reuters MarketScan ${ }^{\circledR}$ Research Databases. The date of the initial OAB medication claim was defined as the index date. A 12-month preindex baseline period was used to classify patients into diabetes (ICD-9-CM code 250.xx) and non-diabetes cohorts and measure demographics and clinical characteristics; patients with an $\mathrm{OAB}$ medication prescription during the baseline period were excluded. Patients in each cohort were directly matched 1:1 based on index year, age, sex, and geographic region. Primary outcomes included $\mathrm{OAB}$ medication adherence, refill of a second $\mathrm{OAB}$ medication prescription, and time to non-persistence with $\mathrm{OAB}$ medications (from the index date until a gap in $\mathrm{OAB}$ medication of $\geq 45$ days), each assessed over a 12-month post-index evaluation period. Multiple logistic regression was used to separately compare the outcomes of $\geq 80 \%$ adherence to $\mathrm{OAB}$ medications and refilling a second $\mathrm{OAB}$ medication prescription. The Cox proportional hazards model was used to compare the outcome of time to non-persistence with $\mathrm{OAB}$ medications. All models were adjusted for patient demographics and clinical characteristics.

RESULTS: The matched diabetes and non-diabetes cohorts each comprised 36,560 patients. In both cohorts, the mean age was $\sim 69$ years and $59.8 \%$ were female. Patients in the diabetes cohort had poorer baseline health and were more likely to have various comorbidities than patients in the non-diabetes cohort. Additionally, those in the diabetes cohort were more likely to have had more frequent encounters with the healthcare system compared with patients in the non-diabetes cohort. The diabetes cohort had $21.5 \%$ higher odds of $\geq 80 \%$ adherence to $\mathrm{OAB}$ medications, $16.6 \%$ higher odds of filling a second $\mathrm{OAB}$ medication prescription, and $10.3 \%$ lower hazard of non-persistence with $\mathrm{OAB}$ medications compared with the non-diabetes cohort; these differences were statistically significant.

CONCLUSIONS: This study showed that patients with diabetes were more likely to persist and adhere to OAB medications and had higher odds of filling a second $\mathrm{OAB}$ medication prescription than patients without diabetes. This finding may be important when considering treatment in diabetic patients with coexisting $\mathrm{OAB}$ and may advance the understanding of factors that contribute to improved persistence and adherence with $\mathrm{OAB}$ medications; further studies are needed to identify other contributory factors, such as disease severity, patient behaviour and response to $\mathrm{OAB}$ medication.

OVERWEIGHT/OBESITY AND WEIGHT-RELATED TREATMENT AMONG RACIALLY AND ETHNICALLY DIVERSE PATIENTS IN FEDERALLY SUPPORTED HEALTH CENTERS Lydie A. Lebrun; Joya Chowdhury; Alek Sripipatana; Suma Nair; Naomi Tomoyasu; Quyen Ngo-Metzger. U.S. Department of Health and Human Services, Rockville, MD. (Control ID \#1324032)
BACKGROUND: The Health Resources and Services Administration provides funding to health centers to improve access to primary care for about 20 million medically underserved patients, including racial/ethnic minorities, the uninsured, and low-income populations. The prevalence of overweight/obesity is a growing public health problem in the U.S., and health centers are well-positioned to serve as "first responders" to the obesity epidemic among vulnerable populations. We sought to determine the prevalence of overweight/ obesity, weight-loss attempts, and weight-related counseling among adult health center patients, and to investigate whether racial/ethnic disparities existed for these measures. We also examined whether certain sociodemographic and health-related characteristics were associated with the outcomes of interest.

METHODS: Nationally representative data came from the 2009 Health Center Patient Survey. Analyses were limited to adults ( $\mathrm{n}=$ 3,949 ). We examined several weight-related measures, including body mass index (BMI), self-perceived weight, and weight-loss attempts. We also included treatment measures relevant to overweight/obese patients: being told by provider of a weight problem, receipt of weight management counseling, nutritionist referrals, weight-loss prescriptions, and cholesterol checks. We conducted bivariate analyses to determine the distribution of sociodemographic, health, and weightrelated measures for the total sample, and also by race/ethnicity. We ran logistic regressions to determine which sociodemographic and health-related factors were associated with weight-loss attempts in past year and with being told by a provider of a weight problem.

RESULTS: Overall, $76 \%$ of the adult health center patients were overweight or obese (BMI $\geq 25.0 \mathrm{~kg} / \mathrm{m} 2)$. However, only $55 \%$ of overweight patients, and $87 \%$ of obese patients correctly perceived themselves as overweight. There were no racial/ethnic differences in BMI categories or self-perceptions of weight. About $60 \%$ of overweight/obese patients reported trying to lose weight in the past year; among these, $46 \%$ tried to do so through both diet change and exercise, while the remainder used either one or the other strategy. There were no racial/ethnic disparities in several weight-related treatment measures among overweight/obese patients, including weight management counseling, weight-loss prescriptions, and cholesterol checks. However, a larger proportion of Hispanic/Latino and African American patients received nutritionist referrals compared with non-Hispanic White patients $(61 \%, 53 \%, 26 \%$, respectively, $\mathrm{p}<0.05)$. In adjusted regressions, overweight/obese patients had higher odds of a weight-loss attempt if they perceived themselves as overweight $(\mathrm{OR}=3.30,95 \% \mathrm{CI}$ : 1.88-5.77), were female $(\mathrm{OR}=1.95,95 \% \mathrm{CI}: 1.05-3.64)$, or were African American $(\mathrm{OR}=3.34$, 95\% CI: $1.29-8.66)$ or Hispanic/Latino $(\mathrm{OR}=2.14,95 \%$ CI: $1.13-4.06)$. Overweight/obese patients had higher odds of being told by a provider that they had a weight problem if they were Hispanic/Latino $(\mathrm{OR}=2.56$, $95 \%$ CI: $1.21-5.38$ ) or if they had two or more chronic conditions $(\mathrm{OR}=2.77,95 \%$ CI: $1.28-6.01)$.

CONCLUSIONS: Health centers are uniquely positioned to address the obesity epidemic among a vulnerable segment of the population. Efforts to promote weight counseling during primary care visits, and patient education on healthy weight, diet, and physical activity have the potential to reduce the burden of obesity and its sequellae among health center patients.

PET-NEGATIVE AT 2, 3 OR 4 CYCLES OF ABVD IN HODGKIN'S LYMPHOMA IS STILL GOOD. George Yaghmour; Mirna Farhat; Bertha Sanchez Valdivieso; Nalini Janakiraman. henry ford health system, Detroit, MI. (Control ID \#1340172)

BACKGROUND: Our objective is to assess the prognostic value of anytime negative PET scan in the course of first line treatment in Hodgkin's lymphoma (HL) patients receiving ABVD (Adriamycin, bleomycin, vinblastine, and dacarbazine) chemotherapy 
METHODS: Thirty two patients with newly diagnosed HL were retrospectively included in the study. All underwent standard ABVD therapy. One, two, and three year survival was compared between patients with negative results after 2,3 or 4 cycles vs. positive results after 4 cycles. A two-sided log-rank test was used to test whether or not there was a significant difference in overall survival between the two groups

RESULTS: Thirty two patients were evaluated. 13 females and 19 males. Median age at diagnosis was 32.2 years. 25 patients achieved a negative PET status and 7 were persistently positive after the 4th cycle of ABVD. Ten patients received PET scan at 2 cycles ( 7 negative, 3 positive), 6 patients had PET scan at 3 cycles ( all 6 negative), and 16 patients had their first PET scan at 4 cycles (12 negative, 7 positive). When all 25 patients with negative PET by 4 cycles were compared to those with persistently positive PET scan at completion of 4 cycles, 1 year, 2 year, and 3-year survival were all higher in patients that had negative PET results. Overall survival was also significantly higher in patients with a negative result when compared to patients that had persistent positive results $(\mathrm{p}=0.0043)$ (see table)
CONCLUSIONS: The favorable prognosis of Hodgkin's disease patients achieving PET negative status after 2 cycles of chemotherapy is well recognized. We tried to extend this to up to 4 cycles and found that $t$ hey still do better than patients that are PET positive after 4 cycles. The numbers are too small for subset analysis looking at the influence of other prognostic factors. 1 year, 2 year, and 3 year survival were all higher in patients that had a negative PET results when compared to patients that had a persistent positive PET result. Overall survival was statistically significantly higher in patients with a negative result when compared to patients that had persistent positive results $(\mathrm{p}=0.0043)$.

Overall Survival in Hodgkin Lymphoma Patients after PET 4 cycle Detection

\begin{tabular}{|c|c|c|c|c|c|c|}
\hline $\begin{array}{l}\text { PET } \\
\text { Results }\end{array}$ & $\mathrm{N}$ & N Deaths $(\%)$ & $\begin{array}{l}1 \text { year } \\
\text { Survival }\end{array}$ & $\begin{array}{l}2 \text { year } \\
\text { Survival }\end{array}$ & $\begin{array}{l}3 \text { year } \\
\text { Survival }\end{array}$ & \\
\hline oga & 25 & $(2 / 25$ & 0.952 & 0.902 & 0.902 & 0.004 \\
\hline ositive & 7 & (4/7) $57.1 \%$ & 0.571 & 0.429 & 0.429 & \\
\hline
\end{tabular}

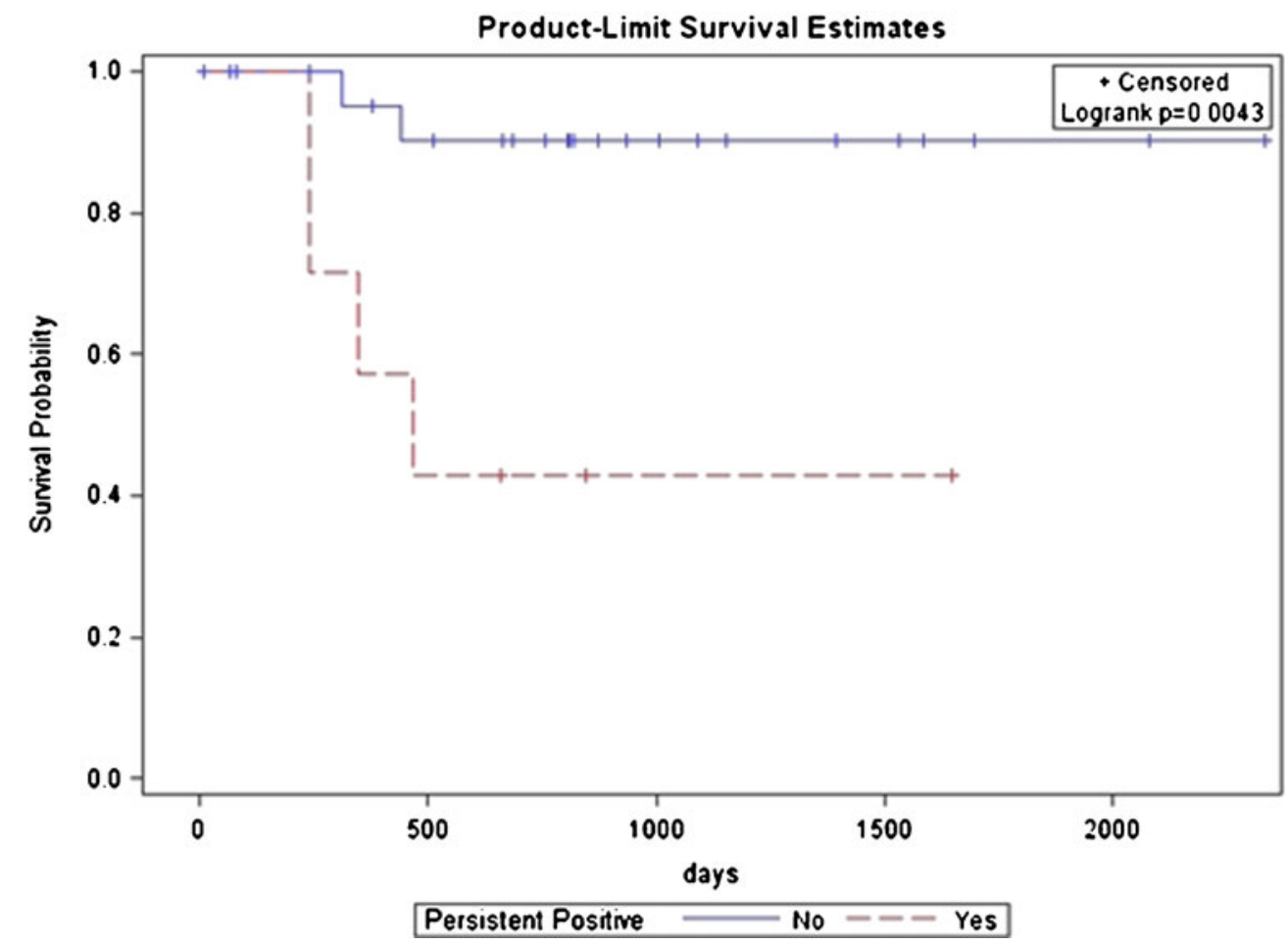

1 year, 2 year, and 3 year survival were all higher in patients that had a negative PET results when compared to patients that had a persistent positive PET result. Overall survival was statistically significantly higher in patients with a negative result when compared to patients that had persistent positive results ( $\mathrm{p}=$ $0.0043)$.

PAID MALPRACTICE CLAIMS FOR ADVERSE EVENTS RESOLVED BY JUDGMENT AND SETTLEMENT Tara F. Bishop; Jessica B. Rubin. Weill Cornell Medical College, New York, NY. (Control ID \#1320015)
BACKGROUND: Defensive medicine is commonly cited as a driver of overuse of health care services. Although previous research has shown that most malpractice cases are settled out of court, physicians, policymakers, and the public likely derive their perceptions of 
malpractice from cases that are judged in court because these are the cases that are often reported on by the lay and medical press. We sought to understand what characteristics predict whether a malpractice suit will be settled out of court or judged in court and how outcomes differ by the type of resolution.

METHODS: We performed a retrospective trend analysis and crosssectional comparison of malpractice claims paid on behalf of physicians using the National Practitioner's Data Bank (NPDB) from 2005-2009. We evaluated trends in the number and proportion of paid claims, and mean payment amount by resolution type. We used multivariable logistic regression to identify which patient, physician, and claim characteristics were most associated with each resolution type. Finally, we examined the effect of resolution type on mean payment amount and time to claim resolution.

RESULTS: Between 2005 and 2009, there were 58,667 claims paid on behalf of physicians. Of these paid claims, 56,850 (96.9\%) were settled outside of court, and 1,817 (3.1\%) were judged in court. There was no significant change in the proportion of suits resolved by settlement versus judgment over the five-year period $(\mathrm{p}=0.828)$; nor was there a significant change in the mean payment amount in either resolution group (settlement, $\mathrm{p}=0.9366$; judgment, $\mathrm{p}=0.3577$ ). Claims in which the physicians had no prior non-malpractice reports (adjusted odds ratio $[\mathrm{aOR}]=0.82,95 \%$ confidence intervals $[\mathrm{CI}] 0.70-0.96)$, that were paid by a state malpractice program $(\mathrm{aOR}=1.6595 \% \mathrm{CI} 1.39-1.97)$ for adverse events to a fetus (aOR $=3.39,95 \%$ CI 1.92-5.99), and for surgical error $(\mathrm{aOR}=1.44,95 \% \mathrm{CI} 1.26$ 1.65) were more likely to be judged in court. Mean payment amount $(\$ 592,283$ vs. $\$ 317,447, \mathrm{p}<0.001)$ and time to decision $(6.50$ years vs. 4.93 years, $\mathrm{p}<0.0001$ ) were significantly higher in judged claims compared with settled claims.

CONCLUSIONS: Although only a very small percentage of malpractice claims are judged in court, a number of physician, patient, and event characteristics are associated with judged cases. For example, cases on behalf of fetuses and for surgical errors are more likely to be judged in court. These differences may shape perceptions of which physicians are at greatest risk for malpractice and which errors are most common. Similarly, higher payment amounts in judged cases likely shape perceptions of the cost of medical malpractice to our health care system.

PAIN ADVERSELY AFFECTS RESPONSE TO A COLLABORATIVE CARE INTERVENTION FOR ANXIETY DISORDERS IN PRIMARY CARE Natalia Morone; Debra K. Weiner; Bea Herbeck Belnap; Fanyin He; Sati Mazumdar; Bruce L. Rollman. University of PIttsburgh, Pittsburgh, PA. (Control ID \#1341010)

BACKGROUND: Primary care patients with Panic Disorder (PD) and Generalized Anxiety Disorder (GAD) experience poorer than expected clinical outcomes despite the availability of efficacious pharmacologic and non-pharmacologic treatments. A barrier to recovery from PD/GAD may be the co-occurrence of pain. To evaluate whether pain intensity interfered with recovery from PD and/or GAD we studied primary care patients who had participated in a clinical trial of collaborative care for anxiety disorders. Our objective was to describe the prevalence of pain in this population and to determine the impact of pain on treatment response for $\mathrm{PD}$ and/or GAD.

METHODS: We performed a secondary data analysis on a randomized, controlled effectiveness trial comparing a telephone-delivered collaborative care intervention for primary care patients with severe PD and/or GAD to their doctor's "usual" care. Patients had to have a diagnosis of PD and/or GAD and a severe level of anxiety symptoms, defined as a Panic Disorder Severity Scale (PDSS) score $\geq 14$ or a Hamilton Anxiety Rating Scale (HRS-A) score $\geq 20$. Outcomes measures were collected over 12-months and included pain and anxiety symptoms. Pain was assessed using the Bodily Pain scale of the SF-36. Anxiety symptoms were measured with the HRS-A, PDSS and Generalized Anxiety Disorder Severity Scale (GADSS). The 124 patients randomized to the collaborative care intervention were analyzed. Participants were divided into two pain intensity groups based on their response to the SF-36 Bodily Pain scale (none or mild pain vs. at least moderate pain). Descriptive statistics were used to summarize demographic characteristics. Chi-squared tests were performed to test the proportions of patients achieving a $50 \%$ decline from baseline levels in the HRS-A, GADSS and PDSS at 12 months. To test the report of pain over time and the effects of baseline pain on anxiety outcomes a repeated measures mixed effect model was run.

RESULTS: The sample was predominantly white and well-educated. Patients with pain were significantly more likely to be older, single, and unemployed. At baseline, patients with pain were significantly more likely to endorse more anxiety symptoms on the HRS-A ( 30.1 vs. $25.4, \mathrm{P}<.001)$. They were also more likely to endorse significantly more pain on the SF36 Bodily Pain scale $(32.5$ vs. $50.4, \mathrm{P}<.0001)$. Patients with pain were significantly more likely to be on an NSAID or an opioid, and carry a chronic pain diagnosis, but there was no difference between groups in anxiolytic medication use at baseline. Among the patients with severe anxiety symptoms $65 \%(80 / 124)$ endorsed experiencing at least moderate pain in the previous month. A significantly lesser number of patients achieved response at 12-months on the HRS-A and GADSS in the pain group compared to the no pain group $(\mathrm{P}=.01$ and $\mathrm{P}=.04$, respectively). The PDSS did not show a significant difference between groups.

CONCLUSIONS: Coexisting pain was common in a sample of primary care patients with severe $\mathrm{PD} / \mathrm{GAD}$ and appeared to negatively affect response to anxiety treatment. It is important to remember that these conditions are commonly comorbid, and response to anxiety treatment may be hampered by the presence of pain.

PALLIATIVE CARE: PREPARING TO IMPLEMENT A BROADENED TRAINING CURRICULUM FOR INTERNAL MEDICINE RESIDENTS Sanaz Kashan ${ }^{1}$; Ghassan Bachuwa ${ }^{1}$; Kay Taylor ${ }^{2} .{ }^{1}$ Hurley Medical Center, Flint, MI; ${ }^{2}$ urley Research Center, Flint, MI. (Control ID \#1309416)

BACKGROUND: Chronic diseases including cancer, heart disease, lung disease, and diabetes account for three-quarters of our nation's health care expenses. Additionally there are 35 million Americans aged 65 and older which account for greater than one-third of outpatient visits and hospitalizations. Caring for those with chronic diseases and older patients involves providing not only effective preventive and curative therapies, but increasingly appropriate palliative care as well. The successful palliative care curriculum will need to use a multifaceted approach, incorporating a variety of intentional strategies to address the multiple competencies required. But when examining residency training program curriculum in this key area-most descriptions tend to focus on a single 'strategy' (e.g., reflection with use of individual case histories, a hospice rotation, reading materials, etc.). Our curriculum study team developed an expanded curriculum that would utilize multiple approaches to learning (including the aforementioned strategies as well as a noon conference lecture, a computer module and role-playing sessions). But it was important for us to learn what aspects of palliative care were 'weakest' for the resident physicians in order to help our teaching faculty to concentrate on those areas most in need of improvement.

METHODS: We developed a 40 -item survey with a true-false and multiple-choice option format. There were 35 knowledge items and 5 attitude items. Knowledge questions targeted such areas as palliative care definitions, goals, benefits, and roles. Attitude items targeted perceptions of competency as well as an assessment of the current palliative care curriculum. The questionnaire was distributed via Survey Monkey to all 1 st and 2nd year Internal Medicine resident physicians. (An exemption from full-board review was granted by the hospital's Institutional Review Board.)

RESULTS: Resident physicians had a strong understanding of the broad goals of palliative care - as well as the importance of working as a team with other health care professionals to support the patient and family. But there were some significant knowledge deficits about advance directivesincluding misperceptions about its impact on patient requests for 'all care possible'. Regarding self-evaluations of competency, only a small percentage (17\%) rated their overall knowledge level as being 'strong' or 'very strong'. And less than one-third expressed that they were 'very comfortable' or 'comfortable' in addressing the issue of palliative care with the patient and/or family. Finally, the majority $(60 \%)$ expressed the need to enhance the current palliative care curriculum. 
CONCLUSIONS: The intent of the planned expanded curriculum is to strengthen our residents' knowledge, attitudes and skills related to the provision of palliative care. We propose to implement a varied curriculum with both knowledge and experience-based activities. The pre-test survey allowed us to identify which palliative care issues would require the greatest attention. It also permitted us to establish the residents' current learner status such that we can later conduct a posttest to assess changes in knowledge, attitudes and skills. Palliative care is a growing field that represents an integral part of the foundational care that physicians offer to their patients. There is a need for more rigorous curricular evaluation.

PANEL MANAGEMENT IN PRIMARY CARE: WHAT PRIMARY CARE PROVIDERS COULD LEARN FROM NURSE CARE MANAGERS Colleen Gillespie; Jaclyn Fox; Alfredo Axtmayer; Anne Dembitzer; Joseph Leung; Scott Sherman; Mark Schwartz. New York University School of Medicine, New York, NY. (Control ID \#1332213)

BACKGROUND: Panel management strategies are generally viewed as a central element of Patient Centered Medical Home models and yet, there is little information on how to prepare health care professionals to actually manage the health of their patient panels. As part of an initiative to study the implementation and efficacy of panel management within the Patient Aligned Care Team (PACT) model in two VA primary care clinics, we report on a baseline survey of Primary Care Providers (PCPs) and Nurse Care Managers (RNs) designed to better understand how prepared these professionals feel to manage their patient panel, their attitudes toward panel management more generally, and their use of panel management strategies in routine practice. METHODS: Surveys were sent to the 46 PCPs (MDs with 1 NP and 1 PA) and 18 RN Care Managers at two urban VA Primary Care Clinics. 39 responses have been received thus far (61\% response rate, $57 \%$ for PCPs and $72 \%$ for RN Care Managers). Items assessed include perceived sufficiency of training and education in panel management (5-point, disagree/agree scale), panel management self-efficacy (4 items including: ability to recognize groups of patients with distinct needs, to use data to identify panel needs, and to implement specific strategies for targeting patients with poor outcomes; 10point Likert scale), attitudes toward panel management ( 2 items: influence of individual team member on panel outcomes and the importance of panel management to patient health; 5-point, disagree/agree scale), and routine use of panel management strategies (1 item, 5-point, disagree/agree scale).

RESULTS: Overall, only $23 \%$ of survey respondents reported that they had sufficient training and education in panel management (23\% of PCPs and $23 \%$ of RNs). Mean self-efficacy for four panel management skills was 6.73 (SD 2.51) on a 10-pt scale, $5=$ confident and $10=$ completely confident (PCP mean $=6.70, \mathrm{SD}=2.5$ and $\mathrm{RN}$ mean $=6.74, \mathrm{SD}=2.56, \mathrm{p}$ $=.96) .71 \%$ somewhat or strongly agreed that as an individual they had an influence on panel outcomes and $86 \%$ somewhat or strongly agreed that panel management is critical for improving patient health. Only $20 \%$, however, reported routinely using panel management strategies. All the RN Care Managers (13/13) agreed that panel management is critical compared with $77 \%$ of PCPs (20/26) (Chi Sq p=.040) and $38 \%$ of RN Care Managers (4/13) compared with 15\% (4/26) of PCPs (Fishers Exact $\mathrm{p}=.047)$ reported routinely using panel management strategies.

CONCLUSIONS: While primary care health professionals endorsed the importance and impact of panel management, they did not feel fully prepared or confident in managing patient panels. More RN Care Managers, perhaps due to their new role within the patient-centered home model currently being implemented throughout VA primary care, endorsed the importance of and routine use of panel management than PCPs in these settings. The small, single system sample limits generalizability but our findings suggest that RN Care Managers may be well suited to promote panel management within their teams and to educate team members, including PCPs, on how to effectively manage their panels of patients.

PANEL MANAGEMENT IN PRIMARY CARE: WHAT PRIMARY CARE PROVIDERS COULD LEARN FROM NURSE CARE MANAGERS Colleen Gillespie; Jaclyn Fox; Alfredo Axtmayer; Anne Dembitzer; Joseph Leung; Scott Sherman; Mark Schwartz. New York University School of Medicine, New York, NY. (Control ID \#1332213)
BACKGROUND: Panel management strategies are generally viewed as a central element of Patient Centered Medical Home models and yet, there is little information on how to prepare health care professionals to actually manage the health of their patient panels. As part of an initiative to study the implementation and efficacy of panel management within the Patient Aligned Care Team (PACT) model in two VA primary care clinics, we report on a baseline survey of Primary Care Providers (PCPs) and Nurse Care Managers (RNs) designed to better understand how prepared these professionals feel to manage their patient panel, their attitudes toward panel management more generally, and their use of panel management strategies in routine practice.

METHODS: Surveys were sent to the 46 PCPs (MDs with $1 \mathrm{NP}$ and 1 PA) and 18 RN Care Managers at two urban VA Primary Care Clinics. 39 responses have been received thus far $(61 \%$ response rate, $57 \%$ for PCPs and $72 \%$ for $\mathrm{RN}$ Care Managers). Items assessed include perceived sufficiency of training and education in panel management (5-point, disagree/agree scale), panel management self-efficacy (4 items including: ability to recognize groups of patients with distinct needs, to use data to identify panel needs, and to implement specific strategies for targeting patients with poor outcomes; 10-point Likert scale), attitudes toward panel management ( 2 items: influence of individual team member on panel outcomes and the importance of panel management to patient health; 5point, disagree/agree scale), and routine use of panel management strategies (1 item, 5-point, disagree/agree scale).

RESULTS: Overall, only $23 \%$ of survey respondents reported that they had sufficient training and education in panel management (23\% of PCPs and $23 \%$ of RNs). Mean self-efficacy for four panel management skills was 6.73 (SD 2.51) on a 10-pt scale, $5=$ confident and $10=$ completely confident ( $\mathrm{PCP}$ mean $=6.70, \mathrm{SD}=2.5$ and $\mathrm{RN}$ mean $=6.74, \mathrm{SD}=2.56, \mathrm{p}=.96$ ). $71 \%$ somewhat or strongly agreed that as an individual they had an influence on panel outcomes and $86 \%$ somewhat or strongly agreed that panel management is critical for improving patient health. Only $20 \%$, however, reported routinely using panel management strategies. All the RN Care Managers (13/13) agreed that panel management is critical compared with $77 \%$ of PCPs (20/26) (Chi Sq p $=.040)$ and $38 \%$ of RN Care Managers $(4 / 13)$ compared with $15 \%(4 / 26)$ of PCPs (Fishers Exact $\mathrm{p}=.047)$ reported routinely using panel management strategies.

CONCLUSIONS: While primary care health professionals endorsed the importance and impact of panel management, they did not feel fully prepared or confident in managing patient panels. More RN Care Managers, perhaps due to their new role within the patient-centered home model currently being implemented throughout VA primary care, endorsed the importance of and routine use of panel management than PCPs in these settings. The small, single system sample limits generalizability but our findings suggest that RN Care Managers may be well suited to promote panel management within their teams and to educate team members, including PCPs, on how to effectively manage their panels of patients.

PATIENT ACTIVATION AND IMPROVED OUTCOMES IN HIVINFECTED PATIENTS Todd Korthuis ${ }^{4,5}$; Rebecca Marshall ${ }^{1}$; Mary Catherine Beach ${ }^{2}$; Somnath Saha ${ }^{5,4}$; Tomi Mori ${ }^{5}$; Mark O. Loveless ${ }^{5}$; Judith H. Hibbard ${ }^{5,6}$; Jonathan A. Cohn ${ }^{7}$; Victoria L. Sharp ${ }^{8}$. ${ }^{1}$ Oregon Health \& Science University, Portland, OR; ${ }^{2}$ Johns Hopkins University School of Medicine, Baltimore, MD; ${ }^{3}$ Portland VA Medical Center, Portland, OR; ${ }^{4}$ Oregon Health \& Science University, Portland, OR; ${ }^{5}$ Oregon Health \& Science University, Portland, OR; ${ }^{6}$ University of Oregon, Eugene, OR; ${ }^{7}$ Wayne State University, Detroit, MI; ${ }^{8}$ St. Luke'sRoosevelt Hospital Center, New York, NY. (Control ID \#1332545)

BACKGROUND: Patient activation, the knowledge, skill, and confidence to manage one's own healthcare, is associated with improved health behaviors that may improve outcomes, but has not been studied in HIV primary care, where healthy behaviors such as medication adherence are essential to treatment success. The objective of this study was to determine 1) patient characteristics associated with patient activation and 2) associations between patient activation and HIV outcomes.

METHODS: The design was a cross-sectional survey conducted in 4 HIV clinics in Baltimore, Detroit, New York, and Portland. Participants were 433 HIV-infected patients, age 18 or greater receiving care from 45 HIV 
providers. Patient activation was measured using the 13-item Patient Activation Measure (PAM) (possible range 0-100). HIV outcomes included CD4 cell count $>200$ cells $/ \mathrm{mL}^{3}$ and HIV-1 RNA $<400$ copies $/ \mathrm{mL}$ (viral suppression), abstracted from medical records, and patient-reported adherence (100\% antiretrovirals taken as prescribed).

RESULTS: Overall, patient activation was high (mean PAM=72.3 [SD 16.5, range $34.7-100]$ ). Activation was lower among those without vs. with a high school degree ( 68.0 vs. $74.0, \mathrm{p}<.001)$, and greater depression (77.6 lowest, 70.2 middle, 68.1 highest tertile, $\mathrm{p}<.001$ ). In multivariable models, every 10-point increase in PAM was associated with greater odds of CD4 count $>200$ cells $/ \mathrm{mL}^{3}$ (aOR 1.22 [95\% CI 1.02, 1.46]), adherence (aOR 1.32 [95\% CI 1.07, 1.62]), and HIV-1 RNA suppression (aOR 1.18 [95\% CI 1.00, 1.38]). The association between PAM and viral suppression was mediated through adherence.

CONCLUSIONS: Higher patient activation was associated with more favorable HIV outcomes. Interventions to improve patient activation should be developed and tested for their ability to improve HIV outcomes.

\section{PATIENT IDENTIFICATION OF THEIR "MOST" AND "LEAST"} IMPORTANT MEDICATION Amy Linsky ${ }^{1,2}$; Steven R. Simon ${ }^{1,3} .{ }^{1}$ VA Boston Healthcare System, Boston, MA; ${ }^{2}$ Boston University School of Medicine, Boston, MA; ${ }^{3}$ Brigham and Women's Hospital, Boston, MA. (Control ID \#1314956)

BACKGROUND: Medication adherence is associated with better outcomes, but patients adhere to only half of prescribed medications. Static and dynamic factors influence medication-taking behaviors, and nonadherence suggests that patients place priorities on specific medications. It is unknown whether patients explicitly can and will identify and express to their providers one of their medications as "most important" or "least important." Our objective was to determine the frequency with which any medication was explicitly identified as most important or least important and the types of medications chosen by Veteran patients.

METHODS: We analyzed data from a convenience sample of patients ( $\mathrm{N}$ $=105$ ) seen in ambulatory care clinics at VA Boston, 3/10-7/11, by fourthyear medical students. Prior to the visit, patients independently answered two questions: "Which one of your medicines, if any, do you think is the most important? (if none, please write 'none')" and "Which one of your medicines, if any, do you think is the least important? (if none, please write 'none')." Reconciliation was made to a computer-generated medication list ("List") that included all medications and non-durable medical supplies dispensed at VA Boston (i.e., locally-dispensed), from other VA facilities and non-VA sources. Medications were classified according to the VA Medication Class codes. Additional factors included patient age ( $>65$ vs. $<65$ years), sex, number of items on List, care provided at other VA facilities (yes vs. no) and presence of non-locally dispensed medications (yes vs. no). We describe the patterns of responses and the medications chosen by Veteran patients. Associations with patient and system factors were tested with chi-square.

RESULTS: We analyzed 104 medication reconciliations. This Veteran cohort was predominantly male $(95 \%) ; 59(57 \%)$ were age 65 years or older. The median number of medications on the list was 8 (IQR 5-13). Sixteen patients $(15 \%)$ had evidence of care in VA systems outside of Boston, and $50(48 \%)$ had documentation of non-locally dispensed medications. In response to the most important, 41 (39\%) chose one specific medication; $26(25 \%)$ chose more than one medication or chose medications to treat a diagnosis; $21(20 \%)$ wrote "none" and $16(15 \%)$ responded with "n/a," "not sure," a nonsensical answer or left it blank. There was no association between choosing one medication and any measured factor. The three most commonly chosen classes of "most important" medications were beta blockers $(\mathrm{n}=8), \mathrm{ACE}$ inhibitors $(\mathrm{n}=7)$ and anticoagulants $(n=5)$. In response to the least important, $31(30 \%)$ chose one specific medication; $2(2 \%)$ chose more than one medication or chose medications to treat a diagnosis; $51(49 \%)$ wrote "none" and $20(19 \%)$ responded with "n/a," "not sure," or left it blank. The three most commonly chosen classes of "least important" medications were vitamins $(n=12)$, nonopioid analgesics (aspirin) $(n=4)$ and antilipemic agents $(n=2)$.
CONCLUSIONS: Approximately one in three Veteran patients did not identify a most important medication, and more than two in three patients did not identify a least important medication. This may reflect poor understanding of the question, limited knowledge of medications and their indications, or unwillingness to state preferences to health care providers. Further understanding of how patients prioritize their medications can guide interventions to enhance adherence to beneficial medications and withdrawal of less essential medicines.

PATIENT NAVIGATION TO IMPROVE COLPOSCOPY FOLLOW UP AFTER AN ABNORMAL PAP SMEAR AMONG LATINA WOMEN Carly Benner ${ }^{1}$; Bruce Chabner ${ }^{1,2}$; Raymond Lui ${ }^{1,3}$; Leslie Aldrich $^{5}$; Sarah $\mathrm{Oo}^{4,5}$; Nessa Rodgers ${ }^{5}$; Sanja Percac-Lima ${ }^{1,4}$. ${ }^{1}$ Harvard Medical School, Boston, MA; ${ }^{2}$ Massachusetts General Hospital, Boston, MA; ${ }^{3}$ Massachusetts General Hospital, Boston, MA; ${ }^{4}$ Chelsea HealthCare Center, Massachusetts General Hospital, Boston, MA; ${ }^{5}$ Center for Community Health Improvement, Massachusetts General Hospital, Boston, MA. (Control ID \#1333761)

BACKGROUND: Cervical cancer disproportionately affects Latina women in the United States and around the world. Patient navigation can improve compliance with cancer prevention, diagnosis and treatment in underserved populations. The aim of this study was to evaluate the impact of patient navigation on colposcopy clinic attendance rates, time to colposcopy follow up after an abnormal Pap smear, and grade of cervical abnormality at colposcopy among Latina women.

METHODS: Eligible women self-identified as Latina, had an abnormal Pap smear requiring colposcopy follow up, and were receiving care at a health center providing cervical patient navigation within an academic primary care network. The usual care group consisted of Latinas with abnormal Pap smears referred to the same colposcopy clinic from practices in the same network without navigation. Primary outcomes were the percentage of missed colposcopy appointments, time to colposcopy after an abnormal Pap smear, and grade of cervical abnormality at colposcopy. Data were collected using electronic medical records from January 1, 2004 to April 15, 2011. Comparisons were made with means and associated t-tests for continuous variables and chi-squared tests for categorical variables. Difference-indifference analysis was used to compare group trends over time, with the study period divided into two halves: 2004-2007 and 2008-2011. Grades of cervical abnormality were given numerical values: $1=$ no dysplasia, $2=$ mild dysplasia, $3=$ moderate dysplasia, $4=$ severe dysplasia, and $5=$ carcinoma in situ.

RESULTS: Of the 786 women in the study, $533(67.8 \%)$ were navigator program participants and $253(32.2 \%)$ received usual care. The mean age of each group was approximately 35 years $(\mathrm{p}=0.370)$. More comparison group women had private insurance compared to navigated women $(34 \%$ vs. $24 \%, p=0.006$ ). In the navigated group $47.6 \%$ had less than a high school education compared to $22.8 \%$ in the comparison group $(\mathrm{p}<0.001)$. Navigated women had a lower percentage of missed colposcopy appointments over time, with the average falling from $19.8 \%$ (95\% CI 17.5, 22.1) to $15.7 \%(95 \%$ CI $13.1,18.4, p=0.024)$. The usual care group no show rate did not change significantly, going from $18.6 \%(95 \%$ CI $14.8,22.5)$ to $20.6 \%$ (95\% CI 17.0, 24.2, $\mathrm{p}=0.454)$. The colposcopy clinic no show rate over the course of the study period was significantly lower for navigated women $(\mathrm{p}<0.001)$. Time to colposcopy follow up for navigated women decreased from 127 days $(95 \%$ CI 115,140$)$ to 120 days $(95 \%$ CI 108 , $131, \mathrm{p}=0.393)$. The comparison group follow up time did not change significantly, increasing from 116 days $(95 \%$ CI 97,134$)$ to 122 days $(95 \%$ CI 93, 151, $\mathrm{p}=0.717$ ). Comparison of group trends over the entire study period showed a significant difference in time to follow up $(p=0.010)$. The grade of cervical abnormality in navigated women decreased between the first and second study periods from a numerical value of $2.03(95 \% \mathrm{CI}$ $1.84,2.22)$ to $1.83(95 \% \mathrm{CI} 1.69,1.90, \mathrm{p}=0.035)$. In the non-navigated group there was an increase from $1.83(95 \%$ CI $1.66,2.0)$ to $1.92(95 \%$ CI $1.66,2.17, \mathrm{p}=0.573)$. Comparison of group trends over the full study period showed a significant decrease in the severity of cervical abnormality for the navigated group $(\mathrm{p}<0.001)$. 
CONCLUSIONS: Culturally-tailored patient navigator programs for cervical care can improve compliance with colposcopy appointments, shorten the time to colposcopy follow up, and decrease severity of cervical abnormalities over time among Latina women.

PATIENT NAVIGATION TO PROMOTE SMOKING CESSATION IN PRIMARY CARE: BASELINE RESULTS FROM A PILOT RANDOMIZED CONTROLLED TRIAL Karen E. Lasser ${ }^{1,2}$; Karey S. Kenst ${ }^{1}$; Lisa Quintiliani ${ }^{1}$; Renda S. Wiener ${ }^{3}$; Jennifer Murillo ${ }^{1}$; Deborah J. Bowen ${ }^{2} .{ }^{1}$ Boston Medical Center/Boston University School of Medicine, Boston, MA; ${ }^{2}$ Boston University School of Public Health, Boston, MA; ${ }^{3}$ Boston Medical Center/Boston University School of Medicine, Boston, MA. (Control ID \#1317420)

BACKGROUND: In primary care, few interventions have connected poor/minority smokers to smoking cessation treatments. Patient navigation is an evidence-based method for improving connectedness to the health care system, is well-suited to underserved populations, and holds promise for engaging patients in smoking cessation treatment.

METHODS: In a pilot randomized controlled trial (RCT) of the feasibility/ acceptability of navigation to link patients to smoking cessation treatment, we included patients age $>=18$ who smoked cigarettes in the past week, had a visit with a primary care provider (PCP), had a telephone, and spoke English. We excluded patients with a transient residence, who had cognitive impairment or severe illness; or who were using evidence-based smoking cessation treatment. Patients were recruited from 5 primary care practices at an urban safety-net hospital. Intervention patients received educational material followed by phone calls or in-person meetings with a navigator; control patients received only educational materials. The navigator was a community health worker trained in motivational interviewing, and in identifying/addressing barriers to engaging in smoking cessation treatment. The educational materials were print-based brochures about smoking risks, smoking cessation treatments, and resources to quit smoking such as quit lines and hospital-based smoking cessation groups. The navigator assisted with appointment scheduling and linked patients to resources to help with social needs such as housing. The primary outcome is engagement in smoking cessation treatment at 3 months; baseline data are presented below.

RESULTS: To date, 21 patients enrolled in the study. About half (48\%) were female; most (75\%) were non-white, with an average age of 46 years. One-fifth of patients were married or living with a partner; all reported that their partner smoked. Three-fourths had completed at least a high school education, and most $(81 \%)$ were unemployed. The majority $(81 \%)$ had a household income under $\$ 30,000$. Participants smoked a mean of 13 cigarettes per day, and had smoked for a mean of 29 years. Most patients (62\%) were in the precontemplation stage with respect to quitting smoking, and $29 \%$ had attempted to quit in the past year. Two-thirds had asked their PCP about ways to quit smoking, and two-thirds had been advised by their PCP to quit. $43 \%$ reported feeling "supported" or "very supported" by their PCP to quit. Only $43 \%$ of patients had heard of a smoker's quitline, and only $11 \%$ had ever been referred by a doctor to a quitline. Most (81\%) were aware of local programs to promote smoking cessation, but only $24 \%$ had ever been referred by their PCP, and $18 \%$ had attended such programs. $43 \%$ of patients agreed with the statement "I am suspicious" of nicotine replacement products, and $40 \%$ felt that they did not need these products to quit smoking.

CONCLUSIONS: Baseline data indicate that patients reported low levels of motivation to quit smoking, low awareness of quitlines, and infrequent referrals to quitlines from their PCP. Patient navigators could play a role not only in motivating patients to quit, but in educating patients about the safety/efficacy of nicotine replacement, and linking patients to evidencebased cessation.

PATIENT OPINION OF HOSPITAL CARE IN ENGLAND: ANALYSIS OF REVIEWS FROM THE NHS CHOICES WEBSITE Tar Lagu $^{1,2}$; Sarah L. Goff ${ }^{1,2}$; Nicholas S. Hannon ${ }^{1}$; Amy S. Shatz ${ }^{1}$; Peter K. Lindenauer ${ }^{1,2}$. ${ }^{1}$ Baystate Medical Center, Springfield, MA; ${ }^{2}$ Tufts University School of Medicine, Boston, MA. (Control ID \#1331588)
BACKGROUND: The increasing number of websites allowing patients to write reviews about health care providers suggests that health care consumers are often as interested in the subjective judgments of their peers as they are in more formal measures of quality. In the United Kingdom, the National Health Service (NHS) encourages patients to provide feedback to hospitals on their quality reporting website, Choices. We used mixed qualitative and quantitative methods to analyze the content of hospital reviews on Choices.

METHODS: We identified all English hospitals listed on Choices that provided medical care. We excluded dental, homeopathic, and psychiatric hospitals and further limited the sample to hospitals that had at least 10 reviews. Of 264 eligible hospitals, we used random number generation to select 20 hospitals for analysis and included the most recent 10 reviews from each (for a total of 200 reviews). Applying directed qualitative content analysis methods, four research team members (two physicians and two research assistants) developed an a priori codebook and coded small sets of up to 20 reviews to check for coding consistency, discuss emerging themes, and update the codebook as needed. Two team members then independently coded the remaining reviews. Reviews were analyzed until no new themes emerged in 100 sequential reviews. We calculated the frequency of recurring themes for descriptive purposes.

RESULTS: Major themes that emerged from the directed qualitative content analysis included positive and negative comments about staff, facilities, and technical aspects of care. Of the 200 reviews, $166(83 \%)$ contained positive content. One hundred forty nine reviews (74\%) contained positive comments about staff (e.g., "Nurses and doctors were professional, friendly, caring and polite at all times."), 55 reviews $(27 \%)$ commented positively about the facility (e.g., "The ward was exceptionally clean"), and 73 reviews (36\%) commented positively about technical aspects of care (e.g., "The bypass was expertly done."). A majority of reviews $(75 \%)$ contained negative content. Patients made negative comments about staff in $74 \%$ of reviews (e.g., "Doctors need improvement in bedside manner."). Sixty-one reviews (30\%) contained negative comments about technical aspects of care (e.g., "After two and a half weeks no correct diagnosis was made."), and 73 reviews (36\%) made negative comments about the facility (e.g., "Parking is an absolute nightmare."). Hospitals responded to reviews $55 \%$ of the time. Of these responses, $36 \%$ contained information about how the hospital would change policies in response to feedback (e.g., "We plan to expand our vegetarian menu.").

CONCLUSIONS: This content analysis of hospital reviews in England demonstrated similar domains to those covered in existing satisfaction surveys, such as the Hospital Consumer Assessment of Healthcare Providers and Systems (HCAHPS). Patients did provide feedback on technical aspects of care, but this was less frequent than comments regarding staff attributes. Unlike surveys such as HCAHPS, however, Choices enables all patients, not just a randomly selected subset, to share their experiences with other patients and with the hospital. The high response rate from hospitals demonstrates the potential for this type of feedback to be an effective tool for hospital care quality improvement.

PATIENT PERCEPTIONS OF HAVING 1ST AND 2ND YEAR MEDICAL STUDENTS INVOLVED IN THEIR CARE J. H. Isaacson ${ }^{1}$; Daniel Neides ${ }^{2}$; Mark Mayer ${ }^{1} .{ }^{1}$ Cleveland Clinic, Cleveland, $\mathrm{OH}$; ${ }^{2}$ Cleveland Clinic, Cleveland, OH. (Control ID \#1334771)

BACKGROUND: Many medical schools are developing earlier clinical experience in the ambulatory setting. Understanding patient perceptions of having students involved in their care is important to assess the impact of moving patient-based teaching of clinical skills earlier in the curriculum. Most literature regarding patient acceptance/satisfaction with medical student involvement in ambulatory settings has focused on $3 \mathrm{rd} / 4$ th year students. At our institution, each student is assigned a longitudinal primary care preceptor with whom they have 43 clinic sessions over the first 2 years. During these sessions student see 3 or 4 patients and practice their interviewing and physical examination skills. Preceptors see additional patients without students during these sessions. The objectives of our study 
were to answer the following questions and compare responses for patients seen by a 1 st or 2 nd year medical student and preceptor to those for patients seen by the preceptor alone. 1. How satisfied are patients when their visit includes a student? 2. How satisfied are patients with the time spent with their physician when their visit includes a student? 3. What is the patient perception of quality of care when their visit includes a student? 4. Do patients perceive any added value when their visit includes a student? 5. Do patients who see students want to have students involved in their care at future visits?

METHODS: We developed an anonymous survey that included demographic information and questions related to the 5 study objectives listed above. All patients answered questions related to overall visit satisfaction, adequacy of time spent with their physician and perceived quality of care. Patients seen by students answered questions related to the added value of a having a student involved in their care and interest in seeing a student again in the future. The survey was given to the patients of 23 faculty preceptors at 2 locations. During half-days when preceptors had 1st or 2nd year medical students in clinic all patients were asked to complete the survey after the visit. We compared responses for patients seen alone by the preceptor and patients seen by the preceptor and a student.

RESULTS: A total of 315 patients returned surveys, 202 of who saw a student with the preceptor and 113 of who saw only the preceptor. The percentage of patients "very satisfied" with their visit was high in both groups (83\% student, 91\% preceptor only; $\mathrm{p}=.07)$ and patients in both groups felt they had enough time with their physician $(98 \%$ student, $96 \%$ preceptor only; $\mathrm{p}=.41$ ). Most patients rated the quality of their visit as "excellent" (92\% students, 98\% preceptor only; $p=.047)$. Of the 205 patients who saw students, $35 \%$ would want to see a student again, $50 \%$ had no preference and $15 \%$ would prefer to see the preceptor alone at a future visit. Forty three percent thought having a student added value to their visit.

CONCLUSIONS: Our study suggests that patient satisfaction with their care was preserved and largely unaffected by the involvement of a 1st or 2nd year medical student in an outpatient primary care setting. Most patients would be willing to have medical students involved in their care again and many patients felt having a student added value to their visit. These results suggest that $1 \mathrm{st}$ and 2nd year students can be successfully integrated into an ambulatory primary care practice for early clinical experience.

PATIENT AND PHYSICIAN CONCORDANCE ON PERCEIVED DEGREE OF SHARED DECISION MAKING OF THE SAME MEDICAL ENCOUNTER Patrick G. O'Malley ${ }^{1,2}$; Janice Hanson ${ }^{4,1}$; Dorothy Becher ${ }^{1,2}$; Jeffrey L. Jackson ${ }^{3,1}$. ${ }^{1}$ Uniformed Services University, Bethesda, MD; ${ }^{2}$ Walter Reed National Military Medical Center, Bethesda, MD; ${ }^{3}$ Medical College of Wisconsin, Milwaukee, WI; ${ }^{4}$ University of Colorado School of Medicine, Aurora, CO. (Control ID \#1322301)

BACKGROUND: Patients with chronic illness often have a significant decision burden with complex trade-offs, necessitating a sophisticated process of shared decision making with their physician. Given the variation in decision making style among patients and physicians, we sought to determine how well patients and their physicians agree on the degree to which shared decision making is occurring. We hypothesized that patients and physicians could reliably agree on the relative degree of shared making within a shared interaction.

METHODS: We enrolled a consecutive sample of 120 consenting participants aged 40-80 y.o. who had hypertension, $\geq 2$ additional chronic medical conditions (excluding dementia), and were scheduled for a routine Internal Medicine appointment with their primary provider. Immediately prior to the visit, patients were surveyed to self-assess, on a 20-point visual analog scale, their preferred decision making style along a spectrum ranging from "doctor-dominant" to "shared" to "patient-dominant" decision making, for new and ongoing problems. The scale included behavioral descriptors along the spectrum in order to anchor one's choice. Immediately after the visit, both physicians and patients were independently asked to rate the style that best characterized their encounter using the same scale, while blinded to each other's ratings. Agreement between patient and physician post-visit ratings of the encounter, and between patients' pre-visit self-assessed style and their post-visit rating of what actually happened, were measured using the intraclass correlation coefficient (ICC).

RESULTS: Of the 120 patients who consented to participate, 106 attended and completed their visit: $53 \% \mathrm{~F}, 55 \%$ African-American, mean age 67 years; $8 \%$ with "poor" health literacy. Of the 11 physicians, 6 were female, 3 were AA, the mean age was 48 years, and the mean time since medical school was 19 years. Collapsed into 3 categories, patient preferences for decision-making were similar by type of problem, whether new or ongoing (See Table). Immediately after the visit, there was no agreement between patients and physicians on the degree of shared decision making that occurred during the visit $(\mathrm{ICC}=0.06, \mathrm{P}=0.37$ ). Agreement between the patients' rating of the actual encounter and their preferred style of decision making was moderate for both new (ICC $=0.54$, $\mathrm{P}<0.001$ ) and ongoing ( $\mathrm{ICC}=0.66, \mathrm{P}<0.001)$ problems.

CONCLUSIONS: In this cohort of mostly older, literate patients with multiple chronic problems who mostly prefer a shared style of decision making, there was no agreement between patients' and physicians' ratings regarding the degree to which shared decision making actually occurred in a routine visit. Patients' preferred style of decision making only moderately correlated with their assessment of what actually happened in the encounter. This indicates a gap in communication interactions which poses a substantial barrier to optimal shared decision-making.

\begin{tabular}{llllll}
\hline \hline & $\begin{array}{l}\text { Spectrum of Decision } \\
\text { Making } \\
\text { Control }\end{array}$ & & & \\
& Doctor & Shared & Patient & \\
Pre-Visit Patient Preference & $20.2 \%$ & $65.4 \%$ & $14.4 \%$ & \\
New Problem & $20.2 \%$ & $61.5 \%$ & $18.3 \%$ & ICC 0.06 \\
$\begin{array}{l}\text { Ongoing Problem } \\
\text { Post-Visit Rating of Encounter } \\
\text { By Patient }\end{array}$ & $23.3 \%$ & $68.0 \%$ & $8.7 \%$ & \\
By Doctor & $37.5 \%$ & $44.2 \%$ & $18.3 \%$ & \\
\hline
\end{tabular}

PATIENT AND PROVIDER ATTITUDES TOWARD OBESITY CARE IN THE PRIMARY CARE SETTING Stephanie A. Rose'; Arnold Stromberg ${ }^{2}$; Joseph Conigliaro ${ }^{3} .{ }^{1}$ University of Kentucky, Lexington, $\mathrm{KY} ;{ }^{2}$ University of Kentucky, Lexington, KY; ${ }^{3}$ New York University, New York City, NY. (Control ID \#1336230)

BACKGROUND: Despite published guidelines, physicians are not routinely screening and counseling for obesity. We assessed prevalence of and patient factors associated with obesity care. We hypothesized that obesity care varies widely in clinical practice and does not meet guideline standards.

METHODS: We developed a quantitative survey of primary care providers (PCPs) and adult ( $\geq 18$ years of age) patients (pts) at rural and urban primary care settings in Kentucky. PCPs were mailed paper surveys and reminder cards with a web link. Pts were invited to fill out a paper survey at their appointment time. We excluded non adult-care PCPs, and pts who reported not having seen their doctor or who were pregnant in the previous year.

RESULTS: Independent surveys were sent and $147 \mathrm{PCP}$ and $132 \mathrm{pt}$ surveys met criteria for analysis. PCPs were $93 \%$ primary care and $47 \%$ female. PCP practices included $25 \%$ urban, $38 \%$ rural, $31 \%$ academic, $26 \%$ community, and $22 \%$ private. Pts were $68 \%$ white, $70 \%$ female, median age of 52 , and $77 \%$ insured. $24 \%$ were normal weight (NW), $27 \%$ overweight (OW), and $49 \%$ obese (OB). $80 \%$ reported an obesity-related condition $(64 \% \mathrm{NW}, 75 \% \mathrm{OW}, 91 \% \mathrm{OB}, \mathrm{p}=0.024) .76 \%$ self-perceived the need to lose weight ( $32 \% \mathrm{NW}, 75 \%$ OW, $98 \%$ OB, $\mathrm{p}<0.001) .100 \%$ of PCPs reported discussing weight loss with their patients, while $44 \%$ of pts reported ever having been told by their PCP that they needed to lose weight $(4 \% \mathrm{NW}, 25 \% \mathrm{OW}, 75 \% \mathrm{OB}, \mathrm{p}<0.0001) .99 \%$ of PCPs reported measuring pt weight, while $96 \%$ of pts reported their PCP had ever 
measured their weight. $93 \%$ of PCPs and $41 \%$ of pts $(35 \%$ NW, $40 \%$ OW, $44 \%$ OB, $p=0.74$ ) felt it was the PCP's responsibility to help with weight loss. $16 \%$ of pts reported their PCP had ever helped them lose weight $(8 \%$ NW, $19 \%$ OW, $18 \%$ OB, $p=0.41$ ). Pts who reported their PCP told them they needed to lose weight were more likely to have tried to lose weight than those whose PCPs had not $(98 \%$ versus $53 \%$, p $<0.0001)$

CONCLUSIONS: PCPs report more patient weight loss counseling than reported by patients. Pts report a positive correlation between weight loss advice and weight loss attempt. PCPs appear to be missing opportunities for guideline-concordant obesity care. Future goals include focus groups to better understand ideas for improvement of obesity care.

PATIENT FACTORS ASSOCIATED WITH EXPERIENCES OF DISCRIMINATION IN HEALTH CARE IN A PROSPECTIVE COHORT AFTER HOSPITALIZATION WITH HEART FAILURE Howard S. Gordon $^{1,2}$; Erica Bauer ${ }^{3}$; Marvella Ford ${ }^{4}$. ${ }^{1}$ Jesse Brown VA Medical

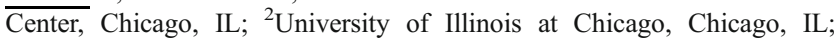
${ }^{3}$ Hines VA Hospital, Hines, IL; ${ }^{4}$ Medical University of South Carolina, Charleston, SC. (Control ID \#1341536)

BACKGROUND: Few studies have examined experiences of discrimination (EOD) in healthcare among diverse patients with severe chronic medical conditions and among patients in VA Medical Centers. Patients with chronic conditions who are frequent users of healthcare or are Veterans may have different EOD than others.

METHODS: We conducted a prospective observational cohort study of patients hospitalized for an exacerbation of heart failure at 2 VA Medical Centers (geographically located in Northern and Southern US). At up to two outpatient follow-up visits in the 6-months after discharge, we examined the association of reported EOD with patient demographics, visit outcomes, and adherence to physicians' recommendations for 162 patients; $61.7 \%$ Black $(\mathrm{N}=100)$ and $38.3 \%$ White $(\mathrm{N}=62)$. Patients with dementia and terminal illness were excluded. Patients completed questionnaires to collect demographics, functional status, trust in physician and healthcare system, heart failure related quality of life and functional status. All patients completed a previously validated self-report measure of EOD in healthcare and daily life. Analyses comparing discrimination with potential covariates used chi-square, t-tests, and correlations, as appropriate. Repeated measures analyses were conducted to examine the association of EOD in health care with trust, health status, and adherence measured after each visit.

RESULTS: There were no statistically significant differences for reported EOD in health care in black compared with white patients $(14.0 \%$ vs. $11.3 \% ; \mathrm{P}=0.62)$ or for ethnicity, gender, age, income, education, or geographical site $(\mathrm{P}>0.20)$. However, compared to white patients, black patients were significantly more likely to report EOD in every other area of daily life measured: school, employment, work, housing, store and restaurant services, financial services, public settings, and law enforcement $(\mathrm{P}<0.01)$. In repeated measures analyses examining all patients, those who reported EOD in health care also reported less trust of the VA health care system $(\mathrm{P}=0.02)$ and lower self-efficacy to communicate with their physician $(\mathrm{P}=0.04)$, but no statistically significant differences $(\mathrm{P}>0.05)$ were reported for trust in physician, heart failure specific health status, and adherence to physicians' recommendations.

CONCLUSIONS: In this cohort of heart failure patients, there was no significant racial difference in EOD in health care. EOD also did not significantly differ by geographical location of the VA hospital in the northern and southern US. Patients who indicated that they had EOD in health care indicated less trust in the VA system and lower selfefficacy to communicate with their physician. Possible limitations of our findings include an association with social desirability and that EOD in health care is measured with a single item. Nonetheless, our findings may also be explained by the fact that all participants were Veterans and were chronically ill. Unique characteristics of Veterans as a special population include Veterans shared sense of identity, which may have been just as strong as or stronger than identification with a particular racial group. Although patients reported being discriminated against in other settings, they reported adherence to their doctors' recommendations, which is consistent with veteran culture of following the orders of those who are in a perceived higher position of authority.

PATIENT NAVIGATION FOR FORMER PRISON INMATES: A PILOT RANDOMIZED CONTROLLED TRIAL Ingrid A. Binswanger ${ }^{1,2}$; Elizabeth Whitley ${ }^{2}$; Paul-Ryan $\mathrm{Haffey}^{2}$; Shane Mueller ${ }^{1}$; Sung-joon Min ${ }^{1} .{ }^{1}$ University of Colorado School of Medicine, Aurora, CO; ${ }^{2}$ Denver Health Medical Center, Denver, CO. (Control ID \#1338755)

BACKGROUND: Many states are releasing prison inmates early to address budget constraints, but prior research has demonstrated high mortality rates during the transition from prison to the community. Little is known about how to prevent poor health outcomes in former inmates. The objective of this study was to test the feasibility of a randomized controlled trial (RCT) of patient navigation to reduce barriers to healthcare and hospitalizations during the transition from prison to the community.

METHODS: Forty former prison inmates 18 and older were recruited into an RCT within 15 days of release from prison. Eligible individuals had a history of a drug related offense or endorsed 3/7 symptoms of substance dependence; could understand study procedures in English; did not plan to leave the area for 6 months; and were not living in a locked halfway house. Recruitment took place at a re-entry center, a program for returning prison inmates sponsored by the prison system. Participants were randomized to 3 months of patient navigation with facilitated enrollment into an indigent care discount program (intervention) or facilitated enrollment into an indigent care discount program alone (control). Structured interviews were conducted at baseline and 3 months which addressed the number of barriers to healthcare and the number of emergency department/ urgent care visits and hospitalizations. Outcomes were measured as a change in number of self-reported barriers to care and change in the rate of health service use per 100 person days from baseline to 3 months.

RESULTS: We recruited 40 participants in 2.5 months. There were no significant differences between intervention and control participants in days since release, age, race/ethnicity, and educational attainment. Participants were enrolled an average of 7 days after release. The mean age was 42 . Overall, $18 \%$ were women and $30 \%$ reported being Latino. In terms of race, $58 \%$ were white, $20 \%$ were African American, 5\% were American Indian, and 18\% did not report a race. At 3 months, 21(52.5\%) had completed the follow-up interview. Fourteen (35\%) participants were not available to be interviewed due to re-arrest, $3(7.5 \%)$ had absconded from the criminal justice system, $1(2.5 \%)$ could not be located, and $1(2.5 \%)$ had withdrawn. The mean number of reported barriers to care was reduced at 3 months in both groups (intervention: $-1.8 \pm 2.7$; control: $-1.1 \pm 2.4$ ). The change in rate of emergency department/urgent care visits per 100 person-days from baseline was $1.1 \pm 0.9$ among intervention participants and $0.5 \pm$ 0.5 among control participants. The change in rate of hospitalization per 100 person-days from baseline was $0.1 \pm 0.3$ in intervention participants and $0.8 \pm 1.5$ in control participants.

CONCLUSIONS: Our intervention feasibility work suggests that recruitment of former inmates into RCT of patient navigation was highly feasible, but follow-up was limited by re-arrests and individuals who absconded from the criminal justice system. Preliminary results suggest a trend towards lower hospitalization rates among navigation participants at 3 months, but that the rate of emergency room or urgent care visits was not improved, perhaps due to the high use of these services to access routine care. Given high recidivism, an adequately powered RCT should allow for follow-up in prison and repeated episodes of navigation at re-release. Future research will examine the effect of patient navigation on poor health outcomes and costs of care in former prison inmates. 
PATIENT PERCEPTIONS OF POST-DISCHARGE EDUCATION AND SUPPORT. Leora I. Horwitz ${ }^{1}$; John Moriarty ${ }^{1}$; Boback Ziaeian ${ }^{2}$; Sandhya V. Kanade $\overline{2}^{2}$ Grace Y. Jenq ${ }^{1}$; Christine Chen $^{2} .{ }^{1}$ Yale University, New Haven, CT; ${ }^{2}$ Yale New Haven Hospital, New Haven, CT. (Control ID \#1338865)

BACKGROUND: Patient education at the time of hospital discharge is a crucial component of transitional care. However, the degree to which patients recall receiving and understanding discharge information is uncertain. Furthermore, the degree to which hospitals adequately assess and meet post-discharge needs from the patient perspective is uncertain. METHODS: A prospective cohort of patients admitted to the medical service at Yale New Haven Hospital with pneumonia, heart failure or acute myocardial infarction and discharged home was enrolled from May 1, 2009 to April 4, 2010. The hospital has a higher than average readmission rate for pneumonia and heart failure. Patients were interviewed within one week of discharge by telephone. The interview consisted of approximately 50 questions, addressing diagnosis, discharge instructions, communication with primary physicians, follow-up appointments, medications and patient education. Where available, we used standardized, validated questions, including the CTM-3 and HCAHPS for assessment of patient satisfaction with discharge education. The CTM-3 includes three questions (the hospital staff took my preferences and those of my family or caregiver into account in deciding what my healthcare needs would be when I left the hospital, when I left the hospital I had a good understanding of the things I was responsible for in managing my health, and when I left the hospital I clearly understood the purpose for taking each of my medications).

RESULTS: The study cohort included 395 patients. Included patients had a mean age of 77 years; $54 \%$ were male. A total of $39 \%$ had heart failure, $24 \%$ had pneumonia and $52 \%$ had acute coronary syndrome. $14 \%$ had more than one diagnosis. One third of patients reported receiving less than one day's advance notice of discharge, and 246 (66.1\%) reported that staff asked if they would have the support they needed at home prior to discharge. After arriving home, $42(10.9 \%)$ reported that they would have liked the hospital to provide them with additional services. A total of 330 $(86.4 \%)$ of patients reported that they were told who to call if symptoms got worse after discharge, but only $201(51.0 \%)$ reported receiving a scheduled follow-up appointment prior to discharge. Overall, 354 (90.3\%) of patients reported receiving written discharge instructions prior to discharge. A total of $325(87.4 \%)$ found them easy to read, $319(86.0 \%)$ found them easy to understand, and $306(82.5 \%)$ reported being able to ask questions about them. $330(83.5 \%)$ reported that the instructions contained information about what symptoms or health problems to look out for after they left the hospital - identical to the publicly reported hospital-wide result on this HCAHPS measure (83\%) and slightly above the national average of $82 \%$. The mean CTM-3 score was 77.2 (SD 18.3). A total of $91(21.7 \%)$ patients would have liked a pharmacist to be involved in discharge education; only $14(3.3 \%)$ recalled actually speaking to one.

CONCLUSIONS: Patients at this hospital with above average readmission rates reported high levels of satisfaction with patient education and discharge instructions. By contrast, they reported receiving little time to prepare for discharge and inconsistent post-discharge follow-up. A substantial minority reported inadequate assessment of home needs. Our findings suggest that a successful discharge requires more than a focus on high quality patient education.

PATIENT RECALL AFTER MISUSE OF A BLOODLETTING DEVICE IN AN AMBULATORY CARE SETTING Philippe Staeger ${ }^{1}$; Giorgio Zanetti ${ }^{2}$; Françoise Ninane ${ }^{1}$; Lucia Mazzolai ${ }^{2}$; Darius Moradpour ${ }^{2}$; Eric Masserey ${ }^{3}$; Laurence Senn ${ }^{2}$; Jean-Blaise Wasserfallen ${ }^{2}$. ${ }^{1}$ Department of Ambulatory Care and Community Medicine, Lausanne, Switzerland; ${ }^{2}$ Lausanne University Hospital, Lausanne, Switzerland; ${ }^{3}$ Department of Public Health, Lausanne, Switzerland. (Control ID \#1323660)

BACKGROUND: Single- and multi-patients devices have been used indistinctly by mistake in our ambulatory care setting, combining primary care and anticoagulation clinics. Transmission of blood-borne pathogens may occur despite needle change between patients because of blood contamination of the tip of the device. Regarding to that risk, a recalling procedure of all concerned patients was implemented. The objective was to assess barriers, efficacy and relevance of recalling patients after misuse of a single-patient bloodletting device on several patients.

METHODS: A task force was set up, composed of representatives from the medical and nursing direction of the hospital, physicians and nurses from the ambulatory care setting, and specialists in infection control and public health. The patients potentially exposed to blood contact were identified, and a process for internal and external communication singled out. A procedure to recall the patients and organize the consultation, blood analyses and communication of results was established, including ways to deal with the reactions from both medias and patients. Recalled patients underwent serology testing for HBV (HBsAg, total anti-HBc), HCV (total anti-HCV), and HIV (anti-HIV1/2, p24 Ag). Possible transmissions were investigated by mapping visits to the clinics, and by comparing viral sequences in the suspected source and secondary cases.

RESULTS: A total of 280 patients were considered for recall. Of these, 263 patients $(94 \%)$ responded to the invitation to be tested, and seventeen patients $(6 \%)$ could not be tested $(9$ patients had died in the interval, with no suspicion of blood-borne infection; 5 patients refused to be tested; 2 patients could not be contacted; and 1 patient lived in a country where testing was not possible). From these 263 tested patients, 218 (83\%) had negative test results; 37 healed hepatitis B ; 5 hepatitis C (of which 4 were already known); and 3 both infections ( 2 already known). Only 1 was HIV positive (already known). For the newly discovered hepatitis C, contamination was considered very unlikely after genetic sequencing. For the newly discovered both infection case, the sequence of events excluded contamination through misuse of the bloodletting device. The recall process included 330 letters, 287 consultations, 1 year management and hotline availability, and 1'500 working hours for the nursing, administration and medical staff. All tested patients accepted that such an error could occur and submitted to testing and communication of results without difficulty. It implied however an important investment in time and energy from the clinicians and laboratories involved in the process.

CONCLUSIONS: Recalling patients after an error in a process of care is possible though difficult to carry out. Patients had a surprisingly good acceptance of the whole process and understanding that such an error could occur. Given the low yield of positive results, the usefulness of such a procedure in this kind of situation should be carefully assessed before launching it.

PATIENT SHARING AND THE COSTS OF CARE Craig E. Pollack ${ }^{1}$; Gary E. Weissman ${ }^{3}$; Klaus W. Lemke ${ }^{2}$; Peter S. Hussey ${ }^{4}$; Jonathan Weiner ${ }^{2}$. ${ }^{1}$ Johns Hopkins University School of Medicine, Baltimore, MD; ${ }^{2}$ Johns Hopkins Bloomberg School of Public Health, Baltimore, MD; ${ }^{3}$ Hospital of the University of Pennsylvania, Philadelphia, PA; ${ }^{4}$ RAND Corporation, Arlington, VA. (Control ID \#1333559)

BACKGROUND: Improving care coordination is a national priority and key focus of health care reforms. However, the success of these policies will be hindered by the lack of available care coordination measures that may be used with existing claims data. Prior research has found that doctors whose patient panels frequently overlap with one another in claims data (i.e. have many 'shared' patients) were more likely to communicate with and refer to one another. This may, in turn, facilitate care coordination. We tested whether patients with higher rates of patient sharing among his/her physicians tended to have lower costs of care and lower likelihoods of hospitalization.

METHODS: We performed a cohort study using 2009 data from 5 private insurers. Plans ranged in size from 460,000 to 890,000 members and represented all four US Census regions. We examined two clinical conditions for which care coordination has been postulated to reduce costs: congestive heart failure (CHF) and diabetes. For 9,596 patients with congestive heart failure (CHF) and 22,765 with diabetes, we calculated the amount of 'patient sharing' among his or her providers. The numerator in this measure is the total number of instances of patient sharing over a year 
among a patient's doctors. The denominator is the total number of pairs of doctors for that individual patient. We used multivariable models to test whether patient sharing — at the patient-level—was associated with costs and rates of hospitalization after adjusting for case-mix.

RESULTS: The average total annual health care cost for patients with CHF was $\$ 29,456$, and $\$ 16,508$ for those with diabetes. In risk adjusted analyses, the highest tertile of patient sharing was associated with lower total costs compared to the lowest tertile $(\$ 3,310$ lower for CHF and $\$ 2,121$ lower for diabetes, $\mathrm{p}<0.001$ ). Lower inpatient costs and rates of hospitalization were found for patients with CHF and diabetes with the highest amounts of patient sharing among his/her providers, and, for those with diabetes, lower outpatient costs were found for the highest patient sharing. Pharmacy costs were not significantly associated with patient sharing.

CONCLUSIONS: Patients treated by physicians who collaborate more frequently have lower costs. The degree of shared patients among a patient's providers may be an important measure that can be used to evaluate aspects of care coordination and track the performance of health care systems.

PATIENT-PROVIDER COMMUNICATION ABOUT RISK FACTORS FOR DIABETES COMPLICATIONS DURING BETWEENVISIT ENCOUNTERS Courtney R. Lyles ${ }^{1}$; Lou Grothaus ${ }^{2}$; Urmimala Sarkar'; James Ralston ${ }^{2}$. ${ }^{1}$ University of California San Francisco, San Francisco, CA; ${ }^{2}$ Group Health Cooperative, Seattle, WA. (Control ID \#1336722)

BACKGROUND: Secure messaging (SM) and phone encounters are becoming widespread to increase patient access to providers between visits. Although these encounters have the potential to improve the care of chronic conditions, we know little about their content in relation to traditional in-person visits. Within a diabetes patient population, we examined discussions about risk factors for diabetes complications, exploring patterns among all encounter types. We hypothesized that those with better control would report more risk factor discussions.

METHODS: We surveyed adult English-speaking patients with diabetes receiving care at Group Health Cooperative in Seattle, WA in 2009. All had $\geq 1$ in-person visit with their primary care provider in the previous year. Since 2003, all patients have been able to exchange SMs and have phone encounters with their providers, as well as have access to an electronic medical record with additional features (e.g., refilling medications, requesting in-person appointments). Patients reported on discussions with providers about risk factors for diabetes complications; automated data was obtained on A1c, BP, and LDL to determine which patients were in clinical control at the same time (A1c $\geq 7 \%$, $\mathrm{BP} \geq 130 / 80 \mathrm{mmHg}$, and $\mathrm{LDL} \geq 100 \mathrm{mg} / \mathrm{dL}$ ). We extracted data on outpatient encounters (in-person, phone, and SM encounters) and examined unadjusted associations of risk factor discussions by encounter type and clinical control. RESULTS: 592 patients responded to the survey (response rate 65\%). These analyses are limited to the $85 \%$ who responded to the risk factor discussion items: $51 \%$ were age $\geq 65,50 \%$ were male, $35 \%$ had $\geq$ college education, and $67 \%$ were white. In addition, $67 \%$ had $\mathrm{A} 1 \mathrm{c} \geq 7.0,57 \%$ had $\mathrm{BP} \geq 130 / 80$ and $27 \%$ had $\mathrm{LDL} \geq 100$, and $77 \%$ had $\geq 1$ between-visit encounter (63\% phone, $41 \% \mathrm{SM}$ ). Discussions of glycemic control with providers were reported by $89 \%$ of patients during in-person visits and $42 \%$ during between-visit encounters, compared to $82 \% \& 17 \%$ for BP and $76 \% \& 20 \%$ for LDL (all $\mathrm{p}<0.001$ ). During between-visit encounters, patients in poor control were more likely to have risk factor discussions (see table). During in-person visits, those with elevated BP were more likely to discuss BP.

CONCLUSIONS: In this system, diabetes patients reported significantly fewer risk factor discussions during between-visit encounters compared to inperson visits. Access to SM and phone visits may have fulfilled acute care needs of patients with diabetes, as opposed to shifting practices toward proactive chronic care management. Those with poorer risk factor control, however, reported more risk factor communication during between-visit encounters, suggesting some patients and providers are using between-visit encounters to help address unmet chronic care need. Additional information about all discussion content across encounters is needed to better understand these patterns. These findings are relevant for systems implementing phone and SM encounters, especially those transitioning to medical home models.

Visit type, clinical control, and patient-reported risk factor discussions

\begin{tabular}{|c|c|c|c|c|}
\hline & $\begin{array}{l}\text { Clinical } \\
\text { control }\end{array}$ & $\mathrm{N}$ & $\begin{array}{l}\text { \% reporting } \\
\text { risk factor } \\
\text { communication }\end{array}$ & $\begin{array}{l}\text { OR }(95 \% \mathrm{CI}) \\
\text { of } \\
\text { communication: } \\
\text { out of control vs. } \\
\text { in control }\end{array}$ \\
\hline \multirow{4}{*}{$\begin{array}{l}\text { In-person } \\
\text { visits }\end{array}$} & A1c in control & 163 & 9189 & $0.84(0.45,1.59)$ \\
\hline & $\begin{array}{l}\text { A1c } \\
\text { out of control }\end{array}$ & 327 & & \\
\hline & $\begin{array}{l}\text { BP in control } \\
\text { BP } \\
\text { out of control }\end{array}$ & $\begin{array}{l}214 \\
287\end{array}$ & 7885 & $\begin{array}{l}1.59 *(1.01, \\
2.50)\end{array}$ \\
\hline & $\begin{array}{l}\text { LDL in } \\
\text { control } \\
\text { LDL out of } \\
\text { control }\end{array}$ & $\begin{array}{l}324 \\
120\end{array}$ & 7681 & $1.33(0.79,2.27)$ \\
\hline \multirow[t]{3}{*}{$\begin{array}{l}\text { Between- } \\
\text { visit } \\
\text { encounters }\end{array}$} & $\begin{array}{l}\text { A1c in control } \\
\text { A1c out of } \\
\text { control }\end{array}$ & $\begin{array}{l}136 \\
244\end{array}$ & 3545 & $\begin{array}{l}1.56 *(1.01, \\
2.38)\end{array}$ \\
\hline & $\begin{array}{l}\text { BP in control } \\
\text { BP } \\
\text { out of control }\end{array}$ & $\begin{array}{l}167 \\
221\end{array}$ & 1221 & $\begin{array}{l}1.92 *(1.09, \\
3,45)\end{array}$ \\
\hline & $\begin{array}{l}\text { LDL in } \\
\text { control } \\
\text { LDL out of } \\
\text { control }\end{array}$ & $\begin{array}{l}247 \\
102\end{array}$ & 1725 & $\begin{array}{l}1.67 \dagger(0.96, \\
2.94)\end{array}$ \\
\hline
\end{tabular}

$* \mathrm{p}<0.05 \dagger \mathrm{p}<0.10$

PATIENTS' ESTIMATION OF EXPECTED LENGTH OF HOSPITALIZATION PREDICTS 30-DAY READMISSION RATES Dennis G. Gibson; Stephanie Pezzo. University of South Florida, Tampa, FL. (Control ID \#1276840)

BACKGROUND: Previous research has indicated that many hospitalized patients have a poor understanding of their plan of care. We hypothesize that patients are poor predictors of their expected length of stay, and that this may be related to 30 -day readmission rates.

METHODS: Sixty patients were interviewed about their expected hospital length of stay. The patients' physician and nurse completed the same interview. A chart review was performed thirty days after the study period to determine the actual discharge date and whether the patient was readmitted to our hospital. Fisher's exact test was used to assess for association between accuracy and readmission.

RESULTS: Physician, nurse, and patient accuracy in predicting length of stay was poor $(50 \%, 29.3 \%$, and $34.5 \%$ respectively). Although physician and nurse predictions were not associated with readmission rate, patients who were inaccurate about their length of stay had a significantly higher 30-day readmission rate $(\mathrm{p}=0.0105)$.

CONCLUSIONS: These results stress the importance of communication to patients regarding their expected discharge date.

PATIENTS' RETENTION OF HEPATITIS B KNOWLEDGE AFTER EDUCATION SESSIONS AT STUDENT-RUN SCREENING AND VACCINATION CLINICS Cindy Lai $^{1}$; David Ouyang ${ }^{1}$; Leslie Sheu ${ }^{1}$; Neal Yuan ${ }^{1}$; Gary Lau ${ }^{2}$; Cheng Chen ${ }^{3}{ }^{1}$ UCSF, San Francisco, CA; ${ }^{2}$ UCSF, San Francisco, CA; ${ }^{3}$ Keck School of Medicine of USC, Los Angeles, CA. (Control ID \#1339765)

BACKGROUND: Although hepatitis B virus (HBV) infection disproportionately affects the Asian Pacific Islander population, low HBV awareness and knowledge persist in this community, which may negatively impact preventive practices and early disease detection. Results are conflicting on whether community education can lead to 
long-term knowledge retention. The effectiveness of student-run education sessions on increasing patients' long-term knowledge retention has not been explored.

METHODS: From September to December 2011, we assessed patients' retention of HBV knowledge at two monthly student-run HBV screening and vaccination clinics affiliated with our urban health sciences campus and the Department of Public Health. One-on-one patient education was provided by trained first- and second-year medical, nursing, and pharmacy students using a script and aided by undergraduate interpreters. Using a 16-question true/false survey adapted from a previously validated tool for assessing hepatitis $\mathrm{C}$ knowledge, we evaluated patient knowledge of HBV risk factors, transmission modes, and management at three time points: before education (baseline), at the end of the initial visit, and at a one-month follow-up visit. Paired and unpaired T-tests were used to compare scores over time and a generalized linear model was used to assess associations between performance and socioeconomic factors.

RESULTS: Seventy-nine patients completed the first survey with a mean score of 8.72 out of $16(\mathrm{SD}=2.44) ; 78(99 \%)$ completed the second survey (mean score $10.99(\mathrm{SD}=2.37))$ and $64(81 \%)$ completed the third survey (mean score $10.91 \quad(\mathrm{SD}=2.56)$ ). There was a statistically significant difference in scores between the first and second tests $(\mathrm{p}<0.001)$ and the first and third tests $(p<0.001)$ but no significant difference between the second and third tests $(\mathrm{p}=0.85)$. A similar result was obtained when including only patients who completed all three tests (paired T-test, $\mathrm{p}<$ $0.001, \mathrm{n}=46,95 \% \mathrm{CI}$ of difference between first and third test: 1.28-2.76). Demographics provided by $36(46 \%)$ patients revealed that patients were older (mean age $53.2, \mathrm{SD}=18.2$ ), predominantly female $(68.6 \%$ ), of lower SES $(46.7 \%$ income less than $\$ 20,000)$, and had limited English proficiency (58.3\% monolingual East Asian language speakers). Patients had on average resided in the US for 22 years. A generalized linear model revealed no difference in performance by age $(p=0.15)$, gender $(p=$ $0.24)$, income $(\mathrm{p}=0.20)$, primary language $(\mathrm{p}=0.79)$, or years of residence in the United States $(\mathrm{p}=0.81)$. Patients demonstrated greater retention of knowledge related to positive risk factors of HBV transmission compared to knowledge of Hepatitis B management and treatment $(13.3 \%$ vs. $1.9 \%$ improvement).

CONCLUSIONS: Our study suggests that one-on-one education for patients led by trained health professional students at student-run clinics can be used to impart HBV health knowledge that is retained at one month after initial education. Retention appears to be independent of demographic or socioeconomic indicators. Patients may retain certain types of knowledge, such as information on positive risk factors for disease transmission, better than other types. Whether such knowledge retention ultimately translates into changed health behaviors remains unconfirmed. Nevertheless, these results warrant further consideration of student-led educational sessions at student-run clinics as a promising community health education model.

PATIENTS' CHOICES FOR LIFESTYLE CHANGE VERSUS MEDICATION USE TO REDUCE ELEVATED CVD RISK Stacey L. Sheridan $^{1,2}$; Lindy B. Draeger ${ }^{2}$; Michael Pignone ${ }^{1}$; Thomas C. Keyserling ${ }^{1,2}$. ${ }^{1}$ University of North Carolina at Chapel Hill, Chapel Hill, NC; ${ }^{2}$ University of North Carolina at Chapel Hill, Chapel Hill, NC. (Control ID \#1337795)

BACKGROUND: Coronary heart disease (CHD) is the leading cause of death in the United States. Although lifestyle change and medication use can substantially reduce CHD risk, little is known about patients' preferences for lifestyle versus medication to reduce elevated CHD risk or about what affects their preferences.

METHODS: We developed two versions (web-based and counselor-based) of a combined lifestyle and medication intervention to reduce CHD risk and compared their effects in a randomized trial conducted at five socioeconomically diverse clinics in a practice-based research network. Both versions included the same content: a web-based decision aid and 7 structured counseling sessions tailored to participants' baseline risk factors and treatment preferences. After completing baseline surveys and viewing the decision aid (which educated patients on their CHD risk and risk reducing options), participants reported their preferences for lifestyle, medication, or both to lower their CHD risk. Here we examine those preferences and whether they vary by patient characteristics.

RESULTS: We enrolled a consecutive sample of 340 participants with 10year Framingham CHD risk $>10 \%$. Mean age was $63.51 \%$ were female, $26 \%$ were African-American and $72 \%$ white. $14 \%$ read at less than a $7-8$ th grade reading level. $87 \%$ had a prescription drug plan. When offered a choice about how to reduce their CHD risk, 92\% (277/296) of participants with unhealthy diet chose to change their diet; $61 \%$ (204/243) with low physical activity chose to exercise more; $68 \%(55 / 81)$ who smoked chose to stop smoking; $30 \%$ (36/116) with high blood pressure chose to take medicine; 31\% (69/229) with abnormal cholesterol chose to take medicine; and $59 \%(38 / 112)$ who were eligible for aspirin chose to take it. $80 \%$ of individuals chose more than one intervention to lower their risk, with the most popular combinations including diet and exercise (36\%), diet and meds $(10 \%)$, and diet, exercise and meds (12\%). In unadjusted analysis, choices for lifestyle and medication interventions were consistent by gender, CHD risk level, health literacy level, race, and prescription drug plan with two notable exceptions: whites were less likely than AfricanAmericans to chose to take cholesterol medicines (25\% versus $49 \%, \mathrm{p}<$ 0.001 ), and those without a prescription drug plan were less likely to chose to quit smoking than those with a prescription drug plan ( $47 \%$ versus $74 \%$, $\mathrm{p}=0.03$ ).

CONCLUSIONS: When offered a choice, a majority of participants preferred to change their lifestyle to lower their CHD risk and to intervene on multiple risk factors at once. Preferences varied little by the patient characteristics we examined. More work is needed to understand whether patients make choices that optimize long term risk reduction.

PATIENT'S PATTERNS AND PREFERENCES OF USE AND CONTENT OF SOCIAL MEDIA Gurjeet Singh; Balaji Ramasamy; Satyam Patel; Gaurav Bhalla; Diane L. Levine. Wayne State University/ Detroit Medical Center, Detroit, MI. (Control ID \#1336667)

BACKGROUND: The use of various social media sites (such as Facebook, Twitter, and MySpace) as well as short messaging system (SMS) text messaging have seen dramatic increases in usage, particularly among adolescents and young adults. There is also an increased usage of social media by medical professionals and medical organizations. Social media offers healthcare organizations (HCOs) an abundance of potential benefits, including enhanced community outreach, improved patient satisfaction and management. As use of electronic media (social media, internet, email, or text messaging) continues to evolve, it is unclear which media type patients might prefer for their healthcare needs. Exploring these preferences could potentially help in designing more tailored and effective interventions. This study was done with the objective to assess patient's usage pattern of social media and to evaluate patient's perceptions and preferences of the use and content of social media for healthcare and medical purposes.

METHODS: We developed a survey instrument comprising 20 questions to assess patient perceptions of social media use. An informational sheet was provided. No personal identifying information was collected. All patients attending internal medicine clinics at Wayne State University (WSU) /Detroit Medical Center (DMC) were eligible to participate in the online survey. Patients willing to participate took the survey posted on Google documents using iPads or laptops. Participation in the study was completely anonymous and voluntary. The survey was conducted over a four week period from December 2011-January 2012. The study was exempted by the WSU institutional review board.

RESULTS: A total of 503 patients completed the survey. The majority of patients were 40-59 years old (47.5\%), were African American (84.3\%), female $(61.2 \%)$ and unmarried $(68.4 \%)$ with children $(73.4 \%)$. Nearly seventy percent had Medicare or Medicaid. Most (72\%) had chronic medical conditions. Although $30.4 \%$ of our patients have never used internet to access social media sites, almost an equal proportion $(29.4 \%$ of patients) use the internet to access such sites multiple times in a day. The remaining patients $(40.2 \%)$ access the internet occasionally. Patients using social media sites use home internet $(37.6 \%)$ or mobile phone $(29.0 \%)$ to access internet; the majority $(55.1 \%)$ do not use smart phones. The two most favored social media sites included Facebook and Google. The 
majority of patients have access to email and mobile texts. Most of our patients $(77.3 \%)$ do not follow any health-related webpage and do not trust medical information provided on such websites $(47.5 \%)$ and nearly all $(92.2 \%)$ do not share their medical concerns on social media sites. Almost half of patients $(45.1 \%)$ wants to be friends with their physicians on social media sites, and would like physicians to interact with patients utilizing Facebook or email, to help them better understand medial issues $(90.5 \%)$ and to provide them with information regarding appointment schedules and follow-up.

CONCLUSIONS: There is an untapped potential for use of social media to improve patients' healthcare delivery. Physicians and healthcare providers should utilize these tools for improving patient care.

PATTERN OF SEXUAL HEALTH ISSUES DOCUMENTED IN THE FIRST 6 MONTHS OF CARE OF RECENT COMBAT VETERANS Drew Helmer ${ }^{1}$; Gregory R. Beaulieu ${ }^{2,3}$; Cheryl Houlette ${ }^{4}$; Michael $\mathrm{Kauth}^{2,3}$. 'VA-NJHCS, East Orange, NJ; ${ }^{2}$ MEDVAMC, Houston, TX; ${ }^{3}$ Baylor College of Medicine, Houston, TX; ${ }^{4}$ MEDVAMC, Houston, TX. (Control ID \#1338739)

BACKGROUND: Despite the importance of sexual health and the known, common risk factors for sexual dysfunction in Veterans of Operations Enduring Freedom and Iraqi Freedom (OEF/OIF Veterans) (e.g., depression, PTSD, psychoactive medications), the sexual function of and best practices for addressing sexual health concerns in this group are not well described. We examined patient medical records to characterize and quantify the documented sexual health issues and related care to establish the importance and possible means of improving sexual health for these patients.

METHODS: We reviewed the medical records of consecutive patients presenting for an initial evaluation at the post-deployment clinic of a large, urban, academic, tertiary care VA medical center in late 2009 The clinic provides an integrated primary care, mental health, and case management assessment in a half-day visit. We abstracted information from the primary care and mental health clinician notes, templated clinical reminder screenings, and pharmacy report documented at the initial visit through the first 6 months of care. All text possibly related to sexual health issues was abstracted, as were screening results for PTSD, depression, alcohol use, military sexual trauma, and traumatic brain injury. Demographic information was also recorded. Sexual health issue text was coded and categorized. We noted whether each documented sexual health issue was an element of a standard note template or not. Medications prescribed by a VA provider in the study period were recorded by generic name and categorized into drug classes.

RESULTS: The sample $(\mathrm{n}=106)$ was primarily male $(\mathrm{n}=94(84 \%))$, white $(\mathrm{n}=51(48 \%))$ or black $(\mathrm{n}=28(26 \%))$, and non-Hispanic $(\mathrm{n}=106(100 \%))$ Mean age was 29.7 years (SD 7.1). Screening results indicated possible depression in 24 patients (23\%), PTSD in $52(49 \%)$, alcohol misuse in 43 (41\%), and TBI in 31 (29\%). Military sexual trauma was not report by any patients. A selective serotonin reuptake inhibitor or serotonin-norepinephrine reuptake inhibitor was prescribed to $35(33 \%)$ patients. A sexual health issue was documented in 26 patients $(25 \%)$ in the first 6 months of VA care. Of 30 sexual health issues documented, $18(60 \%)$ were recorded at a follow up visit. There were 8 categories of sexual health issues recorded. The most common templated sexual health issues were low libido and history of sexually transmitted disease (STD). The most common nontemplated issues were low libido, erectile dysfunction, and acute or resolved STD. At the initial visit, only $25 \%$ of sexual health issues were recorded as non-templated text; in the 6 month follow up period the proportion of non-templated issues rose to $56 \%$. Vardenafil was prescribed to 6 men $(6 \%)$.

CONCLUSIONS: Sexual health issues were common among VA careseeking OEF/OIF Veterans. The range of sexual health issues was broad and the frequency of documentation, nature of documentation (templated vs. non-templated), and type of issue differed between initial and follow up visits. More systematic use of a sexual health template might promote faster and more comprehensive assessment of sexual health in this population.
PAYING THE PRICE: THE DEARTH OF PUBLICALLY AVAILABLE PRISON HEALTHCARE COST DATA Brie Williams ${ }^{1,2}$; Cyrus Ahalt ${ }^{1} .{ }^{1}$ University of California San Francisco, San Francisco, CA; ${ }^{2}$ San Francisco VA Medical Center, San Francisco, CA. (Control ID \#1339155)

BACKGROUND: The U.S. prison population is aging rapidly. Over $10 \%$ of prisoners are age 50 or older and older prisoners often have poor health, adverse health outcomes and high medical costs. In 2001, state prisons spent $\$ 3.2$ billion on medical care for prisoners. By 2010, 4 state prison systems, with $26 \%$ of all prisoners, required the same amount of health spending. Prior medical research using the Dartmouth Atlas of Health Care has shown that easily accessible, publically available data can serve as a powerful tool to compare outcomes across health care systems and identify the drivers of healthcare costs. Given that recent dramatic increases in the cost of incarceration are associated primarily with prison healthcare and aging prisoners, we sought to assemble a publically available data compendium of correctional healthcare cost by age and expenditure category in 50 state prison systems.

METHODS: We reviewed all current publically available statistics, annual reports and special reports from the Departments of Corrections of all 50 states. Through content analysis, we identified those prison systems that make prison healthcare cost data publically available and those that provide cost data by age group and / or expenditure category. We then identified opportunities to improve the availability of relevant data.

RESULTS: All 50 state Departments of Correction issue publically available annual statistical reports and $90 \%$ make available some prison healthcare cost information. Yet none provide a systematic breakdown of healthcare costs for prisoners by age or age group and only 5 states $(10 \%)$ report healthcare costs by expenditure categories such as personnel, prescription medication, or outside medical care. Though no state Departments of Correction report age-stratified healthcare data in their annual reports, 4 states (8\%) have estimated healthcare costs for "older" versus "younger" prisoners on a per person per day basis in special reports. In these special reports, the average ratio of prison healthcare cost for older versus younger prisoners was 3.8:1. Finally, states significantly vary in the age cutoff used to define "older" prisoners. One state $(2 \%)$ did not report any age groups above age 40 yrs, 16 $(32 \%)$ used an age cutoff of $50 \mathrm{yrs}, 8(16 \%)$ used a cutoff of $55 \mathrm{yrs}, 2$ (4\%) used a cutoff of 60 yrs or older, and 23 states (46\%) did not clearly define the age of "older prisoners".

CONCLUSIONS: Despite both the aging of U.S. prisoners and an associated rise in prison healthcare costs, no state reports publically available data on prison healthcare costs by age and expenditure category. This absence of data represents a significant missed opportunity to engage in serious, cost-reducing correctional health policy reform as would be made possible by a data source like the Dartmouth Atlas. To provide researchers, public officials, and policy-makers with a vital tool to address the budgetary and healthcare crises in American corrections, we recommend four simple changes to data collection and dissemination by state Departments of Corrections: (1) determine a uniform age for "older prisoners" as indicated by experts in health and aging in the criminal justice system; (2) adjust data collection tools to report age-stratified health expenditures; (3) expand data to include health expenditures by category; and (4) disseminate data in the annual statistical reports currently issued by all 50 states.

PERCEIVED CONTROL \& SLEEP IN HOSPITALIZED ADULTS: A SOUND HYPOTHESIS? Marie Adachi; Paul G. Staisiunas; Kristen Knutson; David Meltzer; Eve Van Cauter; Vineet Arora. University of Chicago, Chicago, IL. (Control ID \#1320135)

BACKGROUND: Although sleep is important for recovery from acute illness, it is often hampered by noise in hospitals. Interestingly, some patients are more vulnerable to noise disruptions, which is now a publicly reported quality measure by Medicare for hospitals. Perceived control over sleep, or the belief of personal ability to bring about a health outcome related to sleep, could be responsible for this variation. High perceived control is associated with fewer hospitalizations and lower mortality rates in older patients, warranting its study in the context of sleep. The aim of 
this study was to assess the association between perceived control over sleep and inpatient sleep time after controlling for noise levels.

METHODS: All non-institutionalized patients over age 50, who were ambulatory on admission and admitted to a general medicine ward were eligible. Patients with prior sleep disorders, in the hospital greater than 72 hours, with prior ICU stay or in respiratory isolation were excluded. Perceived control over sleep was measured using the Sleep Self-Efficacy Scale (SSE) which is a 9 item scale which ranges from 9 to 45 and asks patients to report their confidence ranging from 1 (Not Confident) to 5 (Very Confident) of one's ability to carry out activities related to sleep (i.e. lie in bed, feeling mentally relaxed). Baseline sleep habits were assessed using the Epworth Sleepiness Scale. Patients were also asked daily about whether their sleep was disrupted by noise the night before. Sleep in hospital was measured nightly using wrist actigraphy (Actiwatch). Noise level was measured nightly in $\mathrm{dB}$ using bedside Larson Davis sound meters. Descriptive statistics and multivariate linear regression were used to discern the association between perceived control and sleep duration controlling for noise and clustered by subject.

RESULTS: From April 2010 to Aug 2011, 76 patients (61\%) were enrolled whose mean age was $67+/-12$ years. Two thirds were African American and $57 \%$ were female. Over two-thirds of subjects $(67 \%)$ were characterized as "average" or "above average" sleepers at baseline with Epworth Sleepiness Scale scores $\leq 9$. Mean in-hospital sleep time was $329 \pm 123$ mins, which was significantly shorter than the selfreported sleep duration of $393 \pm 119$ mins prior to admission $(\mathrm{p}<$ $0.0001)$. Roughly half $(46 \%)$ of patients complained of noise. Noise levels in the loudest tertile rooms ranged from $37 \mathrm{~dB}$ (conversation) to 94db (chainsaw). Median sleep self-efficacy was 35 (IQR 26-41). In unadjusted analyses, patients with above median SSE had almost and hour (58 min) more sleep (95\% CI [15-100], $\mathrm{p}=0.009)$. This effect remained significant after controlling for noise level and routine demographics. Of note, the loudest noise levels were associated with nearly an hour less sleep (-52 minutes). Patients with high SSE also had $70 \%$ lower odds of reporting noise-disrupted sleep [OR $0.31(0.13$, $0.73), \mathrm{p}<0.05]$ in both adjusted and unadjusted analyses. Sensitivity analysis using raw SSE scores showed similar findings.

CONCLUSIONS: Controlling for noise levels, high Sleep Self-Efficacy among hospitalized older patients is associated with longer sleep duration and fewer complaints of noise. In addition to noise control, hospitals should consider interventions (coaching, empowerment) to boost perceived control over sleep to improve the sleep of hospitalized adults.

\section{PERCEPTION OF CHEMOTHERAPY INFLUENCING TREAT-} MENT DECISIONS IN WOMEN WITH HORMONE-POSITIVE BREAST CANCER Tracy A. Proverbs-Singh ${ }^{1}$; Kezhen $\mathrm{Fei}^{2}$; Rebeca Franco $^{2}$; Nina A. Bickell ${ }^{1,2}$. ${ }^{1}$ The Mount Sinai Hospital, New York, NY; ${ }^{2}$ The Mount Sinai Hospital, New York, NY. (Control ID \#1339684)

BACKGROUND: Women with hormone receptor positive $(\mathrm{HR}+)(>1 \mathrm{~cm})$ breast cancer are a heterogeneous group for whom systemic chemotherapy can provide benefit for some, but not for all. Unnecessary chemotherapy can adversely affect patient quality of life and inflict undue cost and morbidity. Gene array analysis, to predict recurrence risk, can reduce unnecessary adjuvant chemotherapy; however, not all $\mathrm{HR}+$ patients receive this test. While tumor biology effects chemotherapy decision making, patient perception of cancer recurrence may also affect treatment decisions. Women who perceive a greater risk of recurrence may feel more obligated to undergo chemotherapy in addition to hormonal therapy. We undertook this study to determine whether women with $\mathrm{HR}+$ tumors $(>1 \mathrm{~cm})$ who undergo chemotherapy, have stronger concerns about cancer recurrence and stronger beliefs in the efficacy of chemotherapy than women who do not get chemotherapy.

METHODS: Women participating in a Breast Cancer Patient Assistance Trial in 2006-2010 were surveyed shortly after their definitive surgery about their cancer knowledge, beliefs and attitudes about breast cancer and its treatment; charts were abstracted 6 months later to measure treatments received. 211 women had $\mathrm{HR}+>1 \mathrm{~cm}$ tumors. Nottingham Prognostic indicator (NPI), which uses stage, tumor size and lymph node involvement was calculated. Chi-square and logistic regression analyses examined associations between receipt of chemotherapy and tumor stage, NPI, physician recommendation for chemotherapy, patient demographics and beliefs about chemotherapy making women live longer, reducing recurrence and concern about unwanted side effects.

RESULTS: $130 / 211$ (62\%) women with HR+tumors received chemotherapy and 197/211 (93\%) received hormonal therapy. Only 46/211 (22\%) had gene array analysis performed, and of these, all those with high risk recurrence scores $(7 / 46[15 \%]), 9 / 15(60 \%)$ with intermediate risk and 1/24 (4\%) low risk got chemotherapy. On average, women with $\mathrm{HR}+$ tumors treated with chemotherapy vs those without chemotherapy were younger (mean 52.5 yrs vs 62.3 yrs, $\mathrm{p}<0.0001$ ), had higher stage cancer (stage II: $65 \%$ vs $23 \%$, $\mathrm{p}<$ 0.0001 ), worse NPI (poor prognosis: $15 \%$ vs $2 \%$ \& moderate prognosis: $62 \%$ vs $30 \%$; $<<0.0001$ ), physician recommended chemotherapy ( $92 \%$ vs $34 \%$, p $<$ 0.0001 ) and patient concern that without chemotherapy their cancer would recur (very worried $70 \%$ vs $15 \% ; \mathrm{p}<0.0001$ ). Multivariate analysis found that physician recommendation and poor or moderate NPI were most strongly associated with receiving chemotherapy $[\mathrm{RR}=3.2$, (95\% CI: $1.8-5.6), 1.7$, (95\%CI:1.3-2.2); 1.6, (95\% CI:1.2-2.0)], respectively, followed by women's worry that without chemotherapy cancer would recur $(\mathrm{RR}=1.3 ; 95 \% \mathrm{CI}$ : $1.0-$ $1.6)$, and belief that chemotherapy allows women to live longer $(\mathrm{RR}=1.1$; $95 \%$ CI: $1.0-1.2$ ). Older age was associated with less use of chemotherapy $(\mathrm{RR}=0.99 ; 95 \% \mathrm{CI}: 0.978-0.995)$.

CONCLUSIONS: Physician recommendation and disease severity greatly influence the utilization of chemotherapy among women with HR+breast cancer. Decisions are also strongly affected by their concerns about recurrence and beliefs about chemotherapy's effectiveness. The study is limited in the small proportion of patients undergoing gene array analysis to help inform decision-making. Patients may benefit from education to augment their ability to make informed decisions about disease recurrence and risk of adverse outcomes from chemotherapy.

PERCEPTIONS AND BARRIERS TO USAGE OF GENERIC MEDICATIONS IN A RURAL AFRICAN-AMERICAN POPULATION Keri Sewell ${ }^{1}$; Susan J. Andreae ${ }^{2}$; Elizabeth Luke ${ }^{1}$; Monika Safford ${ }^{2}$. ${ }^{1} \mathrm{UAB}$, Birmingham, AL; ${ }^{2} \mathrm{UAB}$, Birmingham, AL. (Control ID \#1334886)

BACKGROUND: Usage of generic medications in chronic diseases has been shown to have many benefits, such as similar efficacy for lower price, leading to increased adherence. However, there is significant underuse of generic medicines in African-American communities as well as in communities with low socioeconomic status and low health literacy, such as those found in the rural Southeastern United States. Prior studies have found that such communities have lower trust of generics, particularly for chronic or serious diseases, and increased reluctance to switch from brand to generic. No studies, however, have focused on perceptions of rural, Southern African Americans toward generic medication use.

METHODS: To gain insight into causes of low usage of generic medications among Africans Americans in the rural South, we conducted focus groups in Alabama's underserved and low-income Black Belt area. Inclusion criteria included age of 18 years or older, residence in Alabama's Black Belt region, African-American race, and current use of a daily medication for chronic disease. A total of 30 community members participated in four focus groups. After transcription of the focus group recordings, analysis was performed using NVivo 9. Two authors independently identified themes and then reached a consensus on themes before coding all transcripts. The focus group members were primarily middle-aged women, with over half having only a high school education or less. Most were not employed, and one-fourth had no health insurance.

RESULTS: The general themes that emerged included perceived differences in efficacy and side effects of generic medications versus brand medications; the perception that generics were not "real" medicine; willingness to take generics for minor but not serious illnesses; mistrust in doctors and the health system that affects medication adherence; and the perception that, while generics cost less, people of limited means had to "settle" for less.

CONCLUSIONS: Our focus group data showed barriers to generic medication use in disadvantaged communities that include both misinformation about the safety and efficacy of generic medications as well as deeper feelings of mistrust and abuse by the medical system. While education about generics may rectify some of the misinformation, other views, such as mistrust of the health system and the belief 
that poor people must "settle" for inferior therapies by taking generics, may be more challenging to overcome. Both policy makers and physicians should consider these perspectives when working to increase generic drug usage in these populations.

PERCEPTIONS OF PHYSICAL ACTIVITY AMONG SOMALI MEN Ahmed A. Mohamed ${ }^{1}$; Jennifer A. Weis ${ }^{1}$; Irene G. Sia ${ }^{2}$; Mark L.

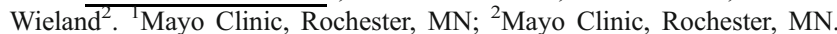
(Control ID \#1308494)

BACKGROUND: Immigrant and refugee populations arrive to the US healthier than the general population, but their cardiovascular risk profiles approximate and exceed those of the US average with increasing duration of residence. One critical factor contributing to these declines is adoption of a more sedentary lifestyle. Somali immigrants and refugees are the fastest growing subset of African migration to the US, yet little is known about their perceptions of physical activity in a new country. While preliminary work has described perceptions among Somali women, we report the first exploration (to our knowledge) of physical activity perceptions among Somali men. Community-based participatory research (CBPR) has been successful in targeting health issues among immigrant and refugee populations, and it is an intuitively appropriate approach for addressing health behaviors in a sociocultural context. The Rochester Healthy Community Partnership (RHCP) is an established CBPR partnership where community and academic members work together through all phases of research. Research question formation, recruitment, study implementation and data analysis for this study were all derived and executed through the RHCP infrastructure.

METHODS: An RHCP community partner recruited Somali men for three age-stratified focus groups to elicit perceived facilitators and barriers to physical activity. Moderators and note takers for the focus groups were Somali men trained in focus groups moderation. Three in-depth interviews were conducted to expand and/or clarify themes that emerged from the focus groups. All focus groups and interviews were digitally recorded, transcribed and translated. The Health Beliefs Model informed focus group question development and data analysis. Team-based, thematic inductive analysis was conducted with transcripts and field notes from the sessions with the help of NVIVO-9 software.

RESULTS: A total of twenty Somali men participated in the focus groups and interviews. Participants demonstrated significant knowledge of physical activity principles, but identified the following barriers to adhering to these principles: loss of walking culture in the US, embarrassment about standard exercise outfit, fear of harassment, competing priorities, cost, transportation for elders, and winter weather. Perceived facilitators to physical activity included a wealth of knowledge about how to be healthy, success stories as inspiration, and community cohesion.

CONCLUSIONS: Perceived barriers and facilitators to physical activity identified in this study may be used as cues to action for CBPR partnerships and public health agencies to derive interventions aimed at maintaining a healthy weight and cardiovascular risk profile among Somali immigrant and refugee men.

PERCEPTIONS OF READMITTED PATIENTS ON THE TRANSITION FROM HOSPITAL TO HOME Shreya Kangovi ${ }^{1,2}$; David Grande $^{3,4}$; Daniel A. Ryan ${ }^{2}$; Patricia Meehan ${ }^{5}$; Nandita Mitra ${ }^{6}$; Richard Shannon ${ }^{3}$; Judith A. Long ${ }^{1,3}$. ${ }^{1}$ Philadelphia Veterans Affairs Medical Center, Philadelphia, PA; ${ }^{2}$ Robert Wood Johnson Foundation Clinical Scholars Program, Philadelphia, PA; ${ }^{3}$ University of Pennsylvania Perelman School of Medicine, Philadelphia, PA; ${ }^{4}$ University of Pennsylvania, Philadelphia, PA; ${ }^{5}$ University of Pennsylvania Health System, Philadelphia, PA; ${ }^{6}$ University of Pennsylvania, Philadelphia, PA. (Control ID \#1336091)

BACKGROUND: Policymakers and hospital leaders are focused on reducing the risk of hospital readmissions. However, these efforts have had mixed success and have not fully considered the patient perspective on the root causes of readmission. The objective of this study was to describe challenges that patients believe contributed to their need for readmission and to determine whether these challenges vary by socioeconomic status.
METHODS: A six-item survey instrument was administered to 1,084 inpatients selected for inclusion if their hospitalization was an unplanned 30-day readmission from home. Surveys were administered at an urban tertiary care academic medical center and an affiliated urban community hospital, both located in West Philadelphia.

RESULTS: $45.5 \%$ of readmitted patients reported experiencing challenges during the transition from hospital to home which contributed to readmission. The most common transition challenges encountered by readmitted patients include lack of preparedness for discharge $(11.8 \%)$, difficulty performing activities of daily living (10.6\%), difficulty accessing $(5.0 \%)$ and adhering to $(5.7 \%)$ discharge medications and lack of social support (4.7\%). Readmitted patients of low socioeconomic status (SES) were significantly more likely than high SES patients to attribute readmission to difficulty understanding and executing discharge instructions (3.6\% vs. $1.3 \%$, $\mathrm{p}=0.01)$, difficulty accessing ( $7.2 \%$ vs. $4.1 \%, \mathrm{p}=0.04)$ and adhering to $(8.5 \%$ vs. $4.6 \%, \mathrm{p}=0.01)$ discharge medications, lack of social support $(7.5 \%$ vs. $3.6 \%, \mathrm{p}<0.01)$, substance abuse $(3.9 \%$ vs. $0.6 \%, \mathrm{p}<0.01)$ and lack of basic resources such as food, transportation or telephone (3.6\% vs. $1.3 \%, \mathrm{p}=0.01)$. CONCLUSIONS: Patients face modifiable transition challenges which they believe contributes to illness relapse and readmission. Interventions which are designed to address these challenges and tailored for patient characteristics such as SES may better address the root causes of readmission.

Transition Challenges by Socioeconomic Status

\begin{tabular}{|c|c|c|c|}
\hline & $\begin{array}{l}\text { Low SES (Uninsured/ } \\
\text { Medicaid/Dual } \\
\text { Eligible) } \\
\mathrm{n}=307\end{array}$ & $\begin{array}{l}\text { High SES } \\
\text { (Commercial/ } \\
\text { Medicare) } \\
\mathrm{n}=777\end{array}$ & $\mathrm{p}^{*}$ \\
\hline Unprepared for DC & $13.7 \%$ & $11.1 \%$ & 0.23 \\
\hline $\begin{array}{l}\text { Understanding DC } \\
\text { instructions }\end{array}$ & $3.6 \%$ & $1.3 \%$ & 0.01 \\
\hline $\begin{array}{l}\text { Executing DC } \\
\text { instructions }\end{array}$ & $5.2 \%$ & $2.4 \%$ & 0.02 \\
\hline $\begin{array}{l}\text { Activities of daily } \\
\text { living }\end{array}$ & $10.4 \%$ & $10.7 \%$ & 0.90 \\
\hline Medication access & $7.2 \%$ & $4.1 \%$ & 0.04 \\
\hline $\begin{array}{l}\text { Medication } \\
\text { adherence }\end{array}$ & $8.5 \%$ & $4.6 \%$ & 0.01 \\
\hline $\begin{array}{l}\text { Lack of social } \\
\text { support }\end{array}$ & $7.5 \%$ & $3.6 \%$ & $<0.01$ \\
\hline $\begin{array}{l}\text { Lack of food, } \\
\text { transportation, } \\
\text { telephone }\end{array}$ & $3.6 \%$ & $1.3 \%$ & 0.01 \\
\hline Substance abuse & $3.9 \%$ & $0.6 \%$ & $<0.01$ \\
\hline Symptoms only & $15.3 \%$ & $18.8 \%$ & 0.18 \\
\hline
\end{tabular}

* $\chi 2$ for categorical variables **Abbreviations: DC: Discharge

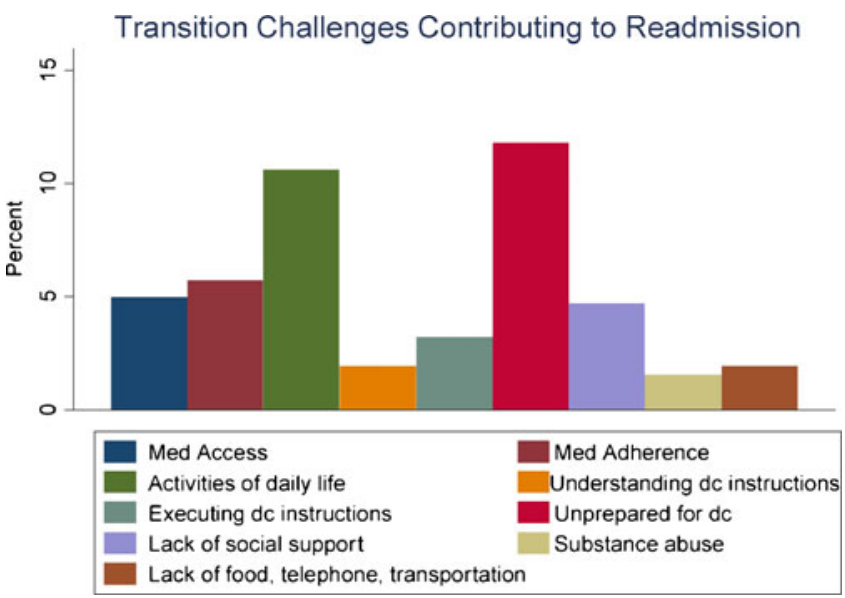

Reported Issues Contributing to Readmission 
PERCEPTIONS OF VULNERABLE PATIENTS ABOUT CLINICIAN COMPUTER USE IN A SAFETY NET CLINIC Neda Ratanawongsa ${ }^{1,2}$; Jennifer L. Barton ${ }^{1,2}$; Dean Schillinger ${ }^{1,2}$; Jennifer E. Hettema ${ }^{3}$; Paula J. Lum ${ }^{1} .{ }^{1}$ UCSF, San Francisco, CA; ${ }^{2}$ UCSF, San Francisco, CA; ${ }^{3}$ University of Virginia, Charlottesville, VA. (Control ID \#1317074)

BACKGROUND: Safety net clinics disproportionately serve patients with limited English proficiency (LEP) and limited health literacy (LHL), whose barriers to communication put them at risk of poorer health outcomes. Electronic health records (EHRs) are increasingly present in patient encounters due to Health Information Technology for Economic and Clinical Health Act incentives. Prior research suggests that EHR use may facilitate and inhibit clinician-patient communication, but little research exists regarding impact on LEP and LHL patients. We conducted a crosssectional survey to elicit patient attitudes toward EHR use in a safety net clinic preparing to transition to fully functional EHR.

METHODS: The county's current EHR allows clinicians to type unstructured notes, prescribe medications, review test results, track healthcare maintenance, and review hospitalization data; the EHR lacks computerized order entry, guideline-based alerts, and most subspecialty and inpatient notes. In JuneAugust 2011, we conducted a cross-sectional, anonymous, self-administered survey of English-, Spanish-, and Cantonese-speaking adults receiving primary care at an internal medicine clinic at a large academically-affiliated county hospital in San Francisco. Using daily convenience sampling, bilingual research assistants recruited patients in the waiting room and assisted patients who could not read or complete questionnaires. The questionnaire used items with closed-ended and LIkert scale responses to assess perceptions of EHR computer use. We analyzed proportions selecting responses and used multivariate logistic regression adjusting for age, gender, and race to examine the association between language / educational attainment and perceptions of high clinician EHR use.

RESULTS: Among 345 respondents, 31\% were Latino, 29\% Asian, 17\% African-American, and $18 \%$ Caucasian. The average age was 54.5 (SD 12.6 , range $21-88)$, and $55 \%$ were women. A quarter $(25 \%)$ had 8 th grade or less educational attainment, $22 \%$ were Spanish-speaking, and $18 \%$ were Cantonese-speaking. Thinking about a typical visit, 57\% of patients reported that their providers spent little or no time on the computer, $25 \%$ half the time, $12 \%$ most of the time, and $7 \%$ all the time. Most patients agreed or strongly agreed that the computer helped their providers understand the patients' health issues $(88 \%)$, remember the patients' concerns $(85 \%)$, or take better care of them ( $82 \%)$. However, $22 \%$ agreed or strongly agreed that their providers listened less carefully because of the computer, and $15 \%$ agreed or strongly agreed that their providers looked at them less because of the computer. These perceptions were not significantly associated with patient background. Patients with 8th grade or less educational attainment (OR 2.3,95\% CI 1.0-5.1, $\mathrm{p}=0.04)$ and Cantonese-speaking patients (AOR 3.8, 95\% CI 1.2- 12.4, p=0.03) had higher odds of reporting that their providers used the computer half or more of the time during a typical visit.

CONCLUSIONS: In this study, most patients in a safety net clinic perceived advantages to clinician computer use, but lower educational attainment and Cantonese language were associated with higher odds of perceiving high clinician computer use. Future studies using mixed methods approaches are needed to explore how patient-clinician-computer interactions differ by language and literacy and potentially affect care for vulnerable patients.

PERSONAL INFANT-FEEDING INTENTIONS AND BEHAVIOR OF PHYSICIANS IN INTERNAL MEDICINE Maryam Sattari ${ }^{1}$; Janet R. Serwint ${ }^{2}$; Dan Neal ${ }^{1}$; David Levine ${ }^{2}$. ${ }^{1}$ University of Florida College of Medicine, Gainesville, FL; ${ }^{2}$ Johns Hopkins University School of Medicine, Baltimore, MD. (Control ID \#1343128)

BACKGROUND: Physicians' breastfeeding advice has been shown to influence patients' breastfeeding initiation and continuation. A strong predictor of female physicians' breastfeeding advocacy is their personal breastfeeding experience. We sought to explore infant-feeding intentions and behavior of physicians in Internal Medicine (IM) or one of its subspecialties.
METHODS: Data was extracted from our database on breastfeeding of physician mothers, mainly affiliated with 2 academic medical centers in Baltimore, Maryland and Gainesville, Florida. Criteria for inclusion were (1) being a female physician, (2) current or previous training in IM, and (3) having had at least one biologic child. All physicians meeting above criteria were included regardless of stage of career or infant-feeding methods.

RESULTS: Thirty four physicians met eligibility criteria, of whom 26 had completed training and 8 were still in training. Maternal age ranged from 28 to 50 years (mean 36.7). These mothers had 67 children overall. While $71 \%$ of mothers intended to breastfeed for 12 months, only $52 \%$ of infants were breastfed up to 12 months. Breastfeeding rates were $96 \%, 77 \%$, and $52 \%$ at hospital discharge, 6 months, and 12 months respectively.

CONCLUSIONS: Breastfeeding continuation rates were higher than those reported in previous physician studies that had either only included certain specialties such as Obstetrics-Gynecology or had a lower proportion of participants form IM. This finding raises the question of impact of maternal specialty on breastfeeding duration and warrants further investigation. The discrepancy between actual and intended breastfeeding duration suggests importance of work-related factors in breastfeeding behavior of physician mothers in IM, which will be discussed further.

PHOTOGRAPHIC EVIDENCE FOR THE EFFICACY OF PIMECROLIMUS CREAM 1\% IN PEDIATRIC AND ADULT PATIENTS Jason T. Olin ${ }^{2}$; Vincent Falanga ${ }^{1} .{ }^{1}$ Roger Williams Medical Center, Providence, RI; ${ }^{2}$ Valeant Pharmaceuticals North America LLC, Bridgewater, NJ. (Control ID \#1336236)

BACKGROUND: Pimecrolimus cream 1\% (PIM), a topical calcineurin inhibitor, is indicated for the short-term and intermittent long-term treatment of mild-to-moderate atopic dermatitis (AD) in patients aged $\geq 2$ years. This study was designed to photographically document the effects of PIM in pediatric ( $\geq 2$ years of age) and adult patients with mildto-moderate $\mathrm{AD}$.

METHODS: This Phase 4, open-label study was designed to document the observed treatment response of twice-daily PIM over 6 weeks in patients aged $\geq 2$ years with mild-to-moderate $\mathrm{AD}$ who were affected at $\geq 5 \%$ of total body surface area, in 2-5 target areas. Efficacy measures included head/neck and overall Investigator's Global Assessment (IGA) and Eczema Area and Severity Index (EASI); safety measures included adverse events (AEs) and serious AEs (SAEs). Since this study was not designed for hypothesis testing regarding efficacy or safety, only photographic documentation and descriptive statistics were performed.

RESULTS: Of the 41 subjects who received $\geq 1$ dose of study medication (safety population), 38 had at least 1 post-baseline efficacy measurement (intent-to-treat population). More than half of the subjects (53.7\%) were 212 years of age; $9.8 \%$ were aged $13-17$ years, and $36.6 \%$ were aged $\geq 18$ years. At Day 43, approximately half of the subjects demonstrated improvement in head/neck and overall IGA scores $(50.0 \%$ and $52.6 \%$, respectively) with $63.2 \%$ (95\% confidence interval $=46.0-78.2)$ and $31.6 \%$ (17.5-48.7), respectively, attaining IGA scores of $\leq 1$, which was defined as treatment success. With each visit, mean EASI scores were successively reduced for a final reduction from Baseline of $52.8 \% \quad(\mathrm{SD}=36.2)$. Photographic evidence obtained during the study illustrates the results obtained for these secondary efficacy endpoints (photograph examples will be part of the presentation). Over half $(56.1 \%)$ of the patients experienced $\geq 1 \mathrm{AE}$ with the most frequently reported $(\geq 5 \%)$ being nasopharyngitis $(24.4 \%)$ and headache (7.3\%). All but $1 \mathrm{AE}$ (thermal burn SAE) were mild or moderate in intensity and most (approximately $75 \%$ ) were considered to be unrelated to treatment. Of the AEs considered to be related to treatment, all but 1 (rash AE) were administration site reactions. No deaths were reported. Two subjects withdrew due to an AE: 1 with moderate rash that was considered to be related to treatment, 1 with a mild molluscum contagiosum infection that was not considered treatment related.

CONCLUSIONS: In this study of pediatric and adult patients with mildto-moderate AD, PIM-treated patients showed improvement in signs and symptoms of $\mathrm{AD}$, as assessed by IGA and EASI and confirmed by photographic evidence. PIM was well tolerated in this patient population, with treatment-related AEs being predominately mild or moderate in severity and primarily involving application site reactions. 
PHYSICAL ACTIVITY, OPIOID MEDICATION USE, AND INTEREST IN YOGA IN AN URBAN COMMUNITY HEALTH CENTER Claudia Campos; Nancy M. Denizard-Thompson; Carolyn R. Pedley; David P. Miller; James L. Wofford. Wake Forest University, WinstonSalem, NC. (Control ID \#1320836)

BACKGROUND: The practice of yoga can decrease pain and enhance physical and psychological function. Yoga practice has been most popular among white, middle-aged educated females, and little is known about attitudes toward yoga among disadvantaged and minority populations. To explore the feasibilty of offering a yoga program at an urban community health center clinic, we surveyed patients to determine their attitudes towards yoga and whether their attitude was affected by current exercise habits or opioid medication use.

METHODS: We provided a written survey to all patients registering for an appointment in the adult medicine clinic during a one-week period in August 2011. The self-administered survey posed five questions (yes/no, short answer) related to current exercise patterns, perceived medical contraindications to exercise, previous experience with yoga, and willingness to enroll in a yoga program. One investigator (CC) reviewed the medical charts of surveyed patients to examine the use of and indication for opioid medications.

RESULTS: 213 patients returned survey cards during the designated 1week study period (response rate 38\%). Survey respondents were predominantly African-American (65\%) versus white $(29 \%)$ or Hispanic $(6 \%)$, and female $(69 \%)$, and the mean age was 50.4 years $(+13.2) .22 \%$ $(47 / 213)$ of respondents reported a sedentary lifestyle, and "walking" was the most common physical activity reported $(37 \%, 78 / 213)$ followed by "work" $(10 \%) .25 \%(52 / 213)$ of respondents were taking prescription opioid medications. The majority of clinical indications $(75 \%, 39 / 52)$ were for musculoskeletal complaints, with back pain being the leading cause for use of opioids $(25 \%, 13 / 52)$. Only $8 \%(18 / 213)$ of respondents reported a medical contraindication to exercise, most commonly lower back pain $(72 \%, 13 / 18)$. Most patients $(69 \%, 148 / 213)$ were willing to participate in a yoga program, $27 \%$ refused and $4 \%$ were undecided. Past yoga participants were no more willing to participate versus patients with no prior yoga experience $(74 \%, 26 / 35$ vs $68 \%, 20 / 177$, chi-square $p=.18)$. African American respondents were more yoga-willing than whites, or hispanics $(74 \%, 59 \%$, and $61 \%$ respectively), but there was little difference by age group (age $>50$ versus younger) or by gender. Opioid users were less likely to indicate interest in yoga than those who did not use opioids $(60 \%, 32 / 53$ vs $73 \%, 113 / 155$, chi-square $p=.036$ ).

CONCLUSIONS: The prevalence of sedentary lifestyle was high among survey respondents at this urban community health center. Over two-thirds of respondents were willing to participate in yoga, and African-Americans appear to be particularly receptive. The majority of opioid users expressed interest in yoga. Implementing a yoga program in this community health center clinic is supported by a high level of patient interest and is a potential strategy for improving pain management and encouraging healthy lifestyles.

PHYSICIAN ANXIETY DUE TO UNCERTAINTY AND THE USE OF RACE IN MEDICAL DECISION MAKING Brooke Cunningham ${ }^{1}$ Sherrill L. Sellers' ${ }^{2}$, Vence L. Bonham ${ }^{3}$; Lisa A. Cooper ${ }^{1}$. 'Johns Hopkins School of Medicine, Baltimore, MD; ${ }^{2}$ Miami University, Oxford, OH; ${ }^{3}$ National Human Genome Research Institute, Bethesda, MD. (Control ID \#1340393)

BACKGROUND: Physicians must manage uncertainty from ambiguous clinical presentations, incomplete information, multiple diagnosis and treatment possibilities, and poor communication between physicians and patients. Studies show that uncertainty leads to higher rates of repeat tests and healthcare costs. The 2003 Institute of Medicine report, Unequal Treatment, hypothesized that clinical uncertainty may promote the activation of prejudice and stereotypes. When there is uncertainty, a physician may treat the patient according to prior beliefs about the group to which the patient belongs, and by doing so, may poorly match care to the patient's needs. This study evaluates whether anxiety due to clinical uncertainty is associated with a higher propensity, among general internists, to use race as a heuristic in clinical decision-making.
METHODS: The Health Professionals' Genetics Education Needs Exploration (HP GENE) survey is a national web and mail survey developed to describe physician knowledge and clinical application of genetics and genomics. The survey was sent to a random sample of 1,738 practicing general internists Apr-Dec 2010. We measured anxiety due to clinical uncertainty (ACU) using a previously validated 5-item Likert scale by Gerrity et al. (1990, 1995). Bonham and Sellers Racial Attributes in Clinical Evaluation scale (RACE) is a new 7-item Likert scale (Cronbach's alpha 0.86). Two items relate to the use of race in determining genetic risk; 2 relate to medication choice and dose; and 3 relate to other aspects of clinical practice, such as initiating screening, how aggressive to be in treatment, and self-reported frequency of considering race. We used multivariate linear regression to assess the association of ACU with RACE, first coding ACU as a continuous predictor variable and then recoding it as a categorical variable (low, low-moderate, moderate, and high anxiety)

RESULTS: Responses were obtained from 787 (45\%) of the sample. Mean age was 48.6 years. $65 \%$ were male; $67 \%$ white, $20 \%$ Asian, $6 \%$ black, $8 \%$ another race; $30 \%$ foreign-born; and $75 \%$ US medical graduates (USMGs). The mean score on the anxiety due to clinical uncertainty scale (ACU) was $19.9(\mathrm{SD}=5.6)$. Women $(\beta=1.4, \mathrm{p}=.002)$, Asians $(\beta=2.7, \mathrm{p}<.0001)$ and those who do more clinical work $(\beta=0.59, p=0.005$, for every additional day of clinic per week) reported higher levels of ACU. USMGs $(\beta=-2.9, p<.0001)$ and those who have been out of residency longer $(\beta=-0.08, p=.002$, for every additional year) had lower ACU. Mean score on the RACE scale was 20.5 (SD =5.6). After adjusting for race, sex, years since residency, location of medical school, genetics coursework in medical school, fellowship training, clinical days per week, and the racial make-up of patient panels, physicians with higher levels of ACU had higher levels of RACE ( $\beta=0.08, p<.030$, for each onepoint increase in ACU). Using ACU categorically, those in the highest quartile of ACU scored 1.6 points higher $(p=.010)$ on RACE than those in the lowest quartile of ACU.

CONCLUSIONS: This is the first study to our knowledge to empirically demonstrate an association between anxiety due to clinical uncertainty (ACU) and the use of race in clinical decision making. This suggests that more attention needs to be paid to the factors that contribute to physician $\mathrm{ACU}$, such as poor patient-physician communication, and the potential impact of ACU on healthcare disparities.

PHYSICIAN AWARENESS AND USE OF RESOURCES TO REDUCE PRESCRIPTION MEDICATION COSTS FOR PATIENTS Toshiko Uchida; Kenzie A. Cameron; Charlie Zei; Anne Henson; Ariane M. Garrett; Eric D. Christoff; Ami Desai; Erik Orelind; Leslie Ramirez; Michael Zielinski; David W. Baker. Northwestern University Feinberg School of Medicine, Chicago, IL. (Control ID \#1326908)

BACKGROUND: Patients are concerned about costs of prescription medications, yet in the literature only a minority report discussing concerns with their physicians. Physicians report insufficient time and the lack of specific solutions to offer patients as barriers to initiating such discussions. This study assessed the effectiveness of a brief educational intervention to equip providers with resources and strategies to help their patients reduce medication costs.

METHODS: Physicians at 9 academic and private practice internal medicine sites in the Research and Education for Academic Achievement (REACH) Practice Based Research Network affiliated with the Northwestern University Feinberg School of Medicine were asked to complete an online pre-test; those who did not were provided a paper copy. Participants then attended a 20-minute educational session led by 2 experienced physicians focused on identifying resources and strategies to help patients reduce medication costs. Several weeks later, physicians were sent a posttest survey (on-line, with paper copy follow up). Both surveys included Likert-type and yes/no items about physicians' comfort with and perceptions of their responsibility in discussing medication costs. The surveys also inquired about physicians' knowledge and behaviors regarding patients' prescription medication costs.

RESULTS: Sixty-six out of 75 physicians completed the pre-test (88.0\%); 46 attended the training session $(61.3 \%)$, and 58 completed the post-test $(77.3 \%)$. These results report on the 40 physicians who attended the training session and 
completed both pre- and post-tests. Participants were $55.3 \%$ female, mean age $43.4(\mathrm{sd}=6.9)$ and had been practicing a mean of 13.25 years $(\mathrm{sd}=7.25)$. On a scale of $1=$ strongly disagree to $5=$ strongly agree, physicians felt very comfortable talking with their patients about prescription medication costs [pre-test $\mathrm{M}=4.25(\mathrm{sd}=0.87)$; post-test $\mathrm{M}=4.35(\mathrm{sd}=0.66), \mathrm{p}=\mathrm{ns}$ ]; felt that they should discuss medication costs with patients [4.08 (0.03); $4.25(0.67), \mathrm{p}=\mathrm{ns}]$; and disagreed that there was sufficient time to assist patients in lowering prescription medication costs $[2.15(0.89) ; 2.38(0.95), \mathrm{p}=0.06]$. Following the intervention physicians were significantly more likely to agree that there are ways to help lower prescription medication costs for patients [4.03 (0.48); 4.24 $(0.58), \mathrm{p}<0.01]$ and that they were knowledgeable about available resources [3.00 (0.88); $3.63(0.74), \mathrm{p}<0.01]$. At pre-test, physicians reported that they had switched a patient to a generic $(100 \%)$, discontinued medications due to cost $(80 \%)$, reviewed patients' formularies $(90 \%)$, prescribed a higher dose to allow for pill splitting $(90 \%)$, prescribed a 90 -day supply $(100 \%)$, and encouraged patients to apply to patient assistance programs (93\%), to comparison shop at multiple pharmacies $(93 \%)$ and to use a mail-order pharmacy $(95 \%)$ to lower prescription medication costs; no differences were seen at post-test. At post-test, significantly more providers reported encouraging patients to use a pharmacy discount card (pre-test $48 \%$; post-test $60 \%$, p $<0.05)$ and to visit websites to comparison shop $(30 \% ; 55 \%, \mathrm{p}<0.01)$.

CONCLUSIONS: Although many physicians are already engaging in behaviors to assist patients in lowering costs of prescription medications, a brief physician intervention appears to be effective in increasing physicians' knowledge of additional resources to help their patients save money on prescription medications.

PHYSICIANS' WILLINGNESS TO PAY FOR MEDICAL MEETINGS IN A HEALTH CARE SYSTEM COMBINING FEE FOR SERVICE AND UNIVERSAL COVERAGE. Shahzia Lambat Emery; Reto Auer; Nicolas Senn; Isabella Locatelli; Jacques Cornuz. Department of ambulatory care and community medicine, Lausanne, Switzerland. (Control ID \#1323885)

BACKGROUND: Sponsoring of physicians meetings and conferences by life science companies (e.g., pharmaceutical and health technology companies) has led to reduced participation fees for physicians but questions potential drawbacks including conflict of interest, as well as direct and indirect commercial products promotion. In many countries, ongoing discussions are proposing to ban such sponsoring which may increase physician's participation fees. We aimed to evaluate factors associated with physicians willingness to pay (WTP) for medical meetings and physicians support of a binding legislation in Switzerland, a country with a health care system combining fee for service and universal coverage. We also questioned Swiss physicians' opinion on alternative financing options such as the creation of a general fund set up by life science companies and centrally administered by an independent body.

METHODS: We sent an anonymous web-based questionnaire to the 447 general practitioners of one state of the French-speaking part of Switzerland, identified through their affiliation to the Swiss medical association. The questionnaire evaluated physician's WTP for congresses, their opinion on the introduction of a binding legislation and alternative financing options, their perception of a bias in prescription practices induced by commercial support, their frequency of exchange with pharmaceutical sales representatives and other relevant socioeconomic factors. We built a multivariate predictor logistic regression model to identify the determinants of WTP.

RESULTS: Of the 115 physicians answering (response rate of $26 \%$ ), about a half $(48 \%)$ of physicians were willing to pay more than what they currently pay for their congresses, $79 \%$ disagreed that commercial support introduced a bias in their prescription practices and $54 \%$ disagreed that it introduced a bias in their colleagues' prescription practices. Two thirds $(76 \%)$ of physicians did not support the introduction of a binding legislation prohibiting the sponsoring of congresses by life science companies and $53 \%$ were in favor of creating a general fund set up by life science companies and centrally administered by an independent body. Based on the multivariate logistic regression, the perception of a bias in peers prescription practices $(\mathrm{OR}=7.47,95 \% \mathrm{CI} 1.65-38.18)$ and group practice structure $(\mathrm{OR}=4.62,95 \%$ CI $1.34-22.29)$ were significantly associated with an increase in WTP.
CONCLUSIONS: Despite a low participation rate, our results suggest that perception of the influence of bias in peers prescription practices and group practice structure are predictors of an increase in physicians WTP for congresses. Most responders did not support the introduction of a binding legislation prohibiting the sponsoring of congresses by life science companies. For decision makers willing to regulate the mutual dependence of physicians and life science companies, an independent body that would centrally administer a general fund set up by life science companies to various congresses might be better received by physicians than a legislation banning the sponsoring of physicians congresses by life science companies.

POPULATION-BASED PREVALENCE OF HEALTH-RELATED INTERNET USE IN SENIORS AND ITS IMPACT ON HEALTH Joseph Finkelstein; Eunme Cha; Jeremy Barron. Johns Hopkins University, Baltimore, MD. (Control ID \#1318164)

BACKGROUND: Older adults are the fastest growing age group in the US. Despite the increasing trend of using the internet for obtaining health information, data on population-based prevalence of health-related internet (HRI) use in seniors are not readily available. The goal of this project is to determine the population-based prevalence of HRI usage in seniors, and to assess its predictors and impact on overall health status in seniors.

METHODS: In 2009, five questions were used in the National Health Interview Survey (NHIS) to collect information on HRI usage in the US population. The survey participants were asked if they accessed the Internet to look up health information, to learn about health topics in chat groups, to refill prescriptions, to schedule appointments, or to communicate with their provider. In our study, a positive answer to any of these 5 questions qualified a respondent to be assigned to the 'HRI user' group. The prevalence of HRI usage was stratified by age groups (60-64, 65-69, 70-74 and 75+ years old), gender, race and different chronic conditions. Further, two logistic regression models were performed to analyze the predictors of HRI use and its impact on overall health status. Independent variables included gender, race, income, education, marital status, and presence of any chronic health condition.

RESULTS: There were a total of 7,543 seniors with age 60+ in 2009 Sample Adult file. The final dataset included 6,076 subjects after excluding the missing frequencies of other variables. In this population, a decreasing trend of HRI use was observed with age. The population-based prevalence of HRI usage was $54 \%$ in $60-64,46 \%$ in $65-69,35 \%$ in $70-74$, and $18 \%$ in $75+$ age groups. Except for Asians, women were more active HRI users than men in all racial groups. The highest usage was reported from the White females in 60-64 age group (62\%), and the lowest was reported from the African American males in $75+$ age group $(1.2 \%)$. In all HRI users, the most prevalent chronic condition was hypertension (54\%) followed by arthritis (48\%). Only $4 \%$ reported to have stroke and 3\% had emphysema. Younger age groups ('60-64' OR 5.3, p<.0001; '65-69' OR 3.8, $\mathrm{p}<.0001$ ), females (OR 1.2, $\mathrm{p}=0.02$ ), being married (OR 1.2, $\mathrm{p}=0.02$ ), higher education (" $>12$ years' OR $9.5, \mathrm{p}<.0001)$, higher income level ('poverty income ratio $>=2$ ' OR 2.9, $\mathrm{p}<.0001$ ), and presence of a chronic condition (OR 1.4, $\mathrm{p}=0.008$ ) predicted HRI use. After adjusting for sociodemographic variables (age, gender, race, marital status, education, income level, presence of chronic conditions, physical activity, body mass index, smoking, and drinking), the seniors who were HRI users in the last 12 months were 1.4 times (CI 1.1-1.7, $\mathrm{P}=0.007$ ) more likely to report that they had better health compared to the last year.

CONCLUSIONS: About half of seniors are using internet for health related purposes in their sixties and internet use among older adults will likely continue to rise in the coming years. Socio-demographic factors and the nature of chronic condition may also have affected the HRI usage behavior. Furthermore, because the older adults who were HRI users were more likely to report overall improvement in health status compared to a previous year, the internet appears to be a potentially powerful means for assisting seniors with their health concerns.

POST-TRAUMATIC STRESS DISORDER (PTSD) IS ASSOCIATED WITH LOWER MEDICATION ADHERENCE IN US VETERANS Ian Kronish ${ }^{1}$; Yongmei Li ${ }^{2}$; Donald Edmondson ${ }^{1}$; Beth Cohen ${ }^{2} .{ }^{1}$ Columbia University Medical Center, New York, NY; ${ }^{2}$ University of California, San Francisco, San Francisco, CA. (Control ID \#1320452) 
BACKGROUND: Individuals with PTSD have worse prognosis from comorbid chronic medical diseases. The mechanisms explaining this association remain poorly understood. Medication adherence represents one of the most essential health behaviors for preventing complications from chronic disease. Accordingly, we tested whether PTSD was associated with worse medication adherence in a cohort of US veterans.

METHODS: The Mind Your Heart Study includes 747 patients recruited from 2 VA medical centers between 2008 and 2010. PTSD was assessed with the gold-standard Clinician Administered PTSD Scale. Medication adherence was assessed by asking participants how often they "forgot to take" and "decided to skip" their medications ["never", "once/mo", "2-3 times/mo", "once/wk", "several times/wk", "nearly every day"] and by asking how often they overall "took their medication as prescribed" ["all the time", "nearly all the time", "most of the time", "half the time", "less than half the time"]. Chi-square and t-tests were used to compare patients with and without PTSD. Ordinal logistic regression was used to examine the association of PTSD with each of the 3 adherence variables including all possible adherence response categories. Models were adjusted for covariates that differed significantly by PTSD status and that have been associated with adherence in prior studies. In bivariate analyses, participants who forgot or decided to skip medications once/wk or more and participants who took medications half the time or less were categorized as non-adherent.

RESULTS: The mean age of participants was 58 yrs, $58 \%$ were White, and $32 \%$ had income $<\$ 20,000 /$ yr. Thirty-nine percent had PTSD. Compared to patients without PTSD, those with PTSD were more likely $(\mathrm{p}<.05)$ to be female ( $10 \%$ vs $3 \%)$, to have depression $(82 \%$ vs $37 \%)$, hypertension $(59 \%$ vs $47 \%)$, COPD ( $23 \%$ vs $16 \%)$, diabetes ( $22 \%$ vs $16 \%)$, and prior MI $(14 \%$ vs 9\%). In bivariate analyses, participants with PTSD were more likely to forget $(\mathrm{p}<.001)$ and skip medications $(\mathrm{p}<.001)$ and had a trend toward lower overall adherence $(\mathrm{p}=0.11)$. In adjusted ordinal logistic regression analyses, participants with PTSD had increased odds of skipping medications (OR $1.8,95 \% \mathrm{CI}$ $1.2-2.7, \mathrm{p}=.002)$ and of having overall worse adherence (OR 1.5, 95\% $\mathrm{CI} 1.0-$ $2.1, \mathrm{p}=.03$ ), and had a trend toward increased odds of forgetting medications (OR 1.4, 95\% CI 1.0-1.9, $\mathrm{p}=.06$ ).

CONCLUSIONS: PTSD was associated with a greater prevalence of chronic illnesses and with worse adherence to medications. There was a stronger association between PTSD and intentionally skipping medications as compared to unintentionally forgetting medications. Clinicians should carefully assess adherence to medications in patients with PTSD. Future studies should explore the relationship between PTSD and beliefs about medications.

\section{DTSD no PTSD}

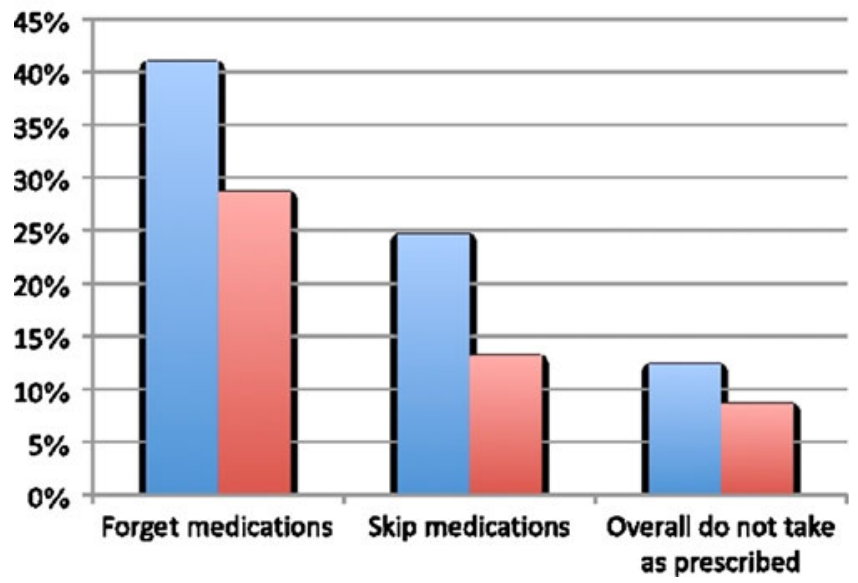

POTENTIAL HARM OF NON-STEROIDAL ANTI-INFLAMMATORY DRUGS TO FEBRILE ELDERLY PATIENTS: SECONDARY ANALYSIS FROM ELECTRONIC MEDICAL RECORD DATABASE Yasuhiro Yamada; Seiji Bito. Tokyo medical center, Meguro-ku, Japan. (Control ID \#1340154)

BACKGROUND: The elderly is often fragile and has an unstable hemodynamics. Administration of Non-steroidal anti-inflammatory drugs
(NSAIDs) has potential harm to the febrile elderly people. However, there are few empirical evidences that examined a risk of topical use of NSAIDs on vital signs of febrile elderly, compared with acetaminophen use or no drug use. We examined whether use of antipyretic have influence of blood pressure changes of the elderly.

METHODS: We examined secondary data analysis from electronic medical record database in two municipal hospitals in Japan to evaluate blood pressure changes of elderly (over 75 years) inpatients with fever above 38 degrees from October 2010 to April 2011. We set three cohorts for exposure variation; A1 using NSAIDs suppository, A2 using internal NSAIDs, B using acetaminophen or no drug. Clinical outcomes were blood pressure reductions, defined as over $50 \mathrm{mmHg}$ reduction of systolic blood pressure and under systolic blood pressure $100 \mathrm{mmHg}$.

RESULTS: There are 1609 inpatients, 230 in group A1, 195 in group A2, 1189 in group B. Blood pressure reductions occurred in 94 patients $(42 \%)$ in A1, 64 patients $(32.8 \%)$ in A2, $331(27.8 \%)$ in B. There was a significant difference between NSAIDs suppository and acetaminophen or no drug (Odds ratio 1.79, $\mathrm{P}<0.001$ ), while internal NSAIDs did not have the significant difference compared with acetaminophen or no drug (Odds ratio $1.27 \mathrm{P}=0.15$ )

CONCLUSIONS: Inadvertent using of NSADs may be harmful on hemodynamic of the elderly. When NSAIDs are administered to febrile elderly patients, their vital signs should be monitored carefully.

POTENTIALLY AVOIDABLE 30-DAY HOSPITAL READMISSIONS IN MEDICINE PATIENTS: DERIVATION AND VALIDATION OF A PREDICTION MODEL Jacques Donze ${ }^{1}$; Drahomir Aujesky ${ }^{2}$; Jeffrey L. Schnipper ${ }^{1}{ }^{1}$ Brigham and Women's Hospital, Boston, MA; ${ }^{2}$ University Hospital of Bern, Bern, Switzerland. (Control ID \#1336481)

BACKGROUND: Hospital readmission prediction models are useful to target post-discharge interventions to patients who might benefit the most. Most existing risk prediction models perform poorly, do not differentiate between avoidable and unavoidable readmissions, or rely on information not commonly available prior to discharge. To help clinicians target transitional care interventions most effectively, we derived and internally validated a prediction model for potentially avoidable 30-day hospital readmissions in medical patients using readily available administrative and clinical data.

METHODS: This retrospective cohort study included 10,731 admissions to the medical services of Brigham and Women's Hospital $(\mathrm{BWH})$ during the 2009-2010 academic year. The outcome was an index admission followed by a potentially avoidable 30-day readmission to any service at BWH or to two other hospitals in the Partners Healthcare system, which together account for over $80 \%$ of readmissions. Potentially Avoidable Readmissions (PAR) were differentiated from non-avoidable readmissions using a validated computerized algorithm based on administrative data (SQLape ${ }^{\mathbb{R}}$ ) commonly used in Switzerland to benchmark and compare hospitals. Admissions were randomly assigned to derivation (2/3) and validation (1/3) sets. Baseline demographic data, previous health care utilization, co-morbid conditions, and laboratory testing were used as predictors in a logistic regression model with 30-day PAR as the dependent variable.

RESULTS: There were 2,398 (22\%) admissions followed by a 30-day readmission, of which $1,101(10 \%)$ were identified as PAR. The prediction score identified 6 independent factors, which we refer to as the PAR ScOrE: any ICD-9 coded Procedure, number of Admissions in the previous 12 months $(0,1$ to 5 , or $>5$ ), Renal failure (glomerular filtration rate of $0-29,30-59$, or $\geq 60 \mathrm{ml} / \mathrm{min}$ ) at the time of discharge, low Sodium level $(<135 \mathrm{mmol} / \mathrm{l})$ at the time of discharge, discharge from an Oncology service, and Elective admission (Table). We then developed a scoring system to stratify risk of potentially avoidable 30 day readmission into 4 categories. With this prediction model, lowrisk patients having $0-1$ points ( $13 \%$ of patients) had a $3 \%$ risk of PAR, while high-risk patients having $\geq 7$ points ( $15 \%$ of patients) had a $27 \%$ risk of PAR. The PAR ScOrE had a good discriminatory power (C-statistic 0.72 and 0.70 ) and had good calibration (HosmerLemeshow goodness-of-fit statistic $\mathrm{P}=0.67$ and $\mathrm{P}=0.72$ ) in the derivation set and validation set, respectively. 
CONCLUSIONS: This simple prediction model accurately identifies the risk of potentially avoidable 30-day readmission in medical patients. While it still needs external validation, this score has potential to easily identify patients in need of more intensive transitional care interventions to prevent avoidable hospital readmissions.

Table 1. PAR ScOrE for 30-day potentially avoidable readmissions

\begin{tabular}{lll}
\hline \hline Attribute & Value & Points \\
Procedure during hospital stay (any ICD-9 coded & yes & 2 \\
procedure) & & \\
Number of hospital Admission(s) during the previous year & 0 & 0 \\
& $1-5$ & 2 \\
& $>5$ & 3 \\
Renal failure, glomerular filtration rate in ml/min at & $<30$ & 2 \\
discharge & $30-$ & 1 \\
& 59 & \\
& $\geq 60$ & 0 \\
Low Sodium level ( $<135$ mmol/1) at discharge & yes & 1 \\
Discharge from an Oncology service & yes & 3 \\
Elective admission & yes & 1 \\
\hline
\end{tabular}

PREDICTING RISK OF HOSPITALIZATION OR DEATH AMONG PATIENTS RECEIVING PRIMARY CARE WITHIN THE VETERANS HEALTH ADMINISTRATION Stephan D. Fihn ${ }^{1,2}$; Li Wang ${ }^{1}$; Brian Porter ${ }^{2}$; Charles Maynard ${ }^{1}$; Christopher L. Bryson ${ }^{1}$; Haili Sun'; Elliott Lowy ${ }^{1}$; Indra Gupta ${ }^{1}$; Mary McDonell ${ }^{1}$; Kathleen Frisbee ${ }^{1}$; Christopher Nielson ${ }^{1}$; Shawn Loftus ${ }^{1} .{ }^{1}$ Department of Veterans Affairs, Seattle, WA; ${ }^{2}$ University of Washington, Seattle, WA. (Control ID $\# 1338954)$

BACKGROUND: Statistical models to accurately identify patients at highest risk of hospitalization or death have been difficult to construct. The objective of the study was to use the clinical and administrative data sources available within VHA to produce models with better performance than those reported. METHODS: We identified a total of 4,505,501 patients who were receiving primary care from the Veterans Health Administration within 1 year prior to the index date, October 1, 2009, and tracked outcomes of hospitalization and death during the subsequent year. Using multinomial regression, we constructed statistical models to predict hospitalization or death occurring during the subsequent 90 days and 1 year. Predictors were selected from variables related to socio-demographics, medical conditions, vital signs, use of health care services, medications and laboratory tests. For each endpoint we sorted patients into 20 equal-sized risk strata based on quantiles of predicted probability for the endpoint in ascending order. RESULTS: The C-statistics for 90-day and 1-year outcomes were 0.840 (95\% CI $0.839,0.841)$ and $0.814(0.813,0.815)$, respectively for hospitalization; $0.860(0.858,0.861)$ and $0.847(0.846,0.848)$ respectively for death without hospitalizations; and $0.816(0.815,0.817)$ and $0.790(0.789,0.790)$ respectively for either event. Among patients who were in the 20th risk stratum, the 90-day event rates for hospitalization, death without hospitalization and either event were $20.1 \%, 6.3 \%$ and $23.2 \%$ respectively, as contrasted with the population averages of $2.7 \%, 0.7 \%$ and $3.4 \%$ respectively. The 1 -year event rates in the 20th risk stratum were $44.9 \%, 18.9 \%$ and $51.8 \%$ respectively, compared with the population average of $8.3 \%, 2.6 \%$ and $10.9 \%$ respectively.

CONCLUSIONS: These models incorporating demographic, administrative and clinical variables from electronic health records were able to accurately stratify the patient population according to the estimated risks with greater accuracy than those previously reported. The models have clinical implications to identify patients for whom selected interventions might reduce the risk for adverse outcomes.

PREDICTORS OF MAJOR CARDIAC ADVERSE EVENTS IN PATIENTS WITH CARBON MONOXIDE POISONING Osama Amro; Ahmed Ibrahim; Shadi Mayasy; Keyvan Ravakhah; Joseph Sopko; Srinivas Merugu; Robert Steele. St.Vincent Charity Medical Center, Cleveland, OH. (Control ID \#1311916)
BACKGROUND: Carbon monoxide (CO) is the most common cause of death from poisoning in the United States. Cardiovascular complications and their contribution to acute outcomes are yet to be defined.

METHODS: We retrospectively reviewed all the cardiovascular manifestations of CO poisoning in patients presented to St Vincent Charity Medical Center, the regional center for $\mathrm{CO}$ poisoning treatment between the periods of January 2009 to September 2011. Patient's demographics, comorbidities, electrocardiograms (EKG), laboratory results and echocardiograms were identified from chart review and retrieved for analysis.

RESULTS: We identified 47 patients, mean age 49.3 years and majority were male $(72 \%)$. The mean hospital stay was 2.6 days. The average carboxyhemoglobin $(\mathrm{COHb})$ level was $24.9 \%$. 20\% of cases were intentional poisoning, 19\% were unconscious on presentation (UOP) and $20 \%$ were intubated. Cardiovascular risk factors in these patients were DM $9 \%$, HTN $36 \%$, dyslipidemia $6 \%$, smoking $32 \%$ and family history of premature coronary artery disease $4 \%$. Obesity was present in $19 \%$ with $4 \%$ having documented history of CAD and $14 \%$ with cocaine abuse prior to admission. Troponin I was found to be greater than $0.2 \mathrm{ng} / \mathrm{mL}$ in $37 \%$ of patients and more than $1 \mathrm{ng} / \mathrm{mL}$ in $17 \%$. Ischemic EKG changes were found in $4 \%$ of patients. Corrected QT interval was prolonged (>440 msec) in $68 \%$ of patients. Echocardiogram was done in $9 \%$ of patients and $4 \%$ had depressed left ventricular ejection fraction which recovered after hyperbaric oxygen treatment. $92 \%$ of patients received treatment with hyperbaric oxygen (HBO). Two patients died by anoxic brain injury that was unrelated to their cardiac status. There was no correlation between $\mathrm{COHb}$ level and troponin or QTc ( $\mathrm{r}=-0.2$ and 0.02 respectively). UOP and intubation were the main predictors of significant myocardial injury with $55.5 \%$ of UOP group $(\mathrm{P}=0.002)$ and $62.5 \%$ of intubated patients having a troponin $\mathrm{I}>1 \mathrm{ng} / \mathrm{Ml}(\mathrm{P}=0.001)$. HTN was the only predictor of significant QTc prolongation with $88 \%$ of hypertensive group having a QTc $>440 \mathrm{msec}(\mathrm{P}=0.016)$.

CONCLUSIONS: Myocardial damage in patients presenting with $\mathrm{CO}$ poisoning is common and manifested by increased troponins and prolonged QTc. There is no correlation between $\mathrm{COHb}$ level and myocardial injury. In these patients, UOP and intubation are the only two significant predictors of troponins elevation while HTN is associated with more QTc prolongation. This damage is usually transient and is reversed with urgent HBO therapy. Our study although limited by being a retrospective study clearly adds to our understanding of predictors for carbon monoxide morbidity and helps prioritize medical care.

PREDICTORS OF PATIENTS WHO LEAVE AGAINST MEDICAL ADVICE FROM THE EMERGENCY DEPARTMENTS Mauli Mehta; Arun K. Muthusamy; Diane L. Levine. Detroit Medical Center / Wayne State University, Detroit, MI. (Control ID \#1333592)

BACKGROUND: Discharge against medical advice (AMA) from emergency departments (ED) continues to be a prevalent $(0.1-2.7 \%)$ and frustrating problem, yet the literature is limited to medical record reviews and retrospective analysis from single institutions. Data and guidelines for physicians on how to effectively manage and intervene with these patients are scant. In addition, multiple studies have shown that hospitalized patients that leave AMA are at increased risk of adverse medical outcomes and re-admission. Our objective is to determine the patient characteristics and clinical conditions associated with leaving AMA from ED across America.

METHODS: The National Hospital Discharge Survey is a limited access dataset that includes visit-based data during ED admissions. Patients of all ages from year 2007 to 2009 were included in the analysis. Patient demographic characteristics were compared. A multivariate survey logistic regression model was used to investigate the discrepancies in patient demographic characteristics and the reasons for visit between those who were discharged and who left AMA.

RESULTS: A total of 104,566 ED visits were documented, of which $1.1 \%$ (1135) were AMA. Patients who left AMA were significantly more likely to be male (Odds ratio[OR] 1.22; 95\% confidence interval[CI] 1.09-1.37), Hispanic (OR 1.26; 95\%CI 1.02-1.56) and from metropolitan areas (OR 1; reference). The positive predictors for leaving AMA are ages 18 to 65 (OR 1.89; 95\% CI 
1.53-2.34), annual income less than $\$ 52,388$ (OR 1.31; $95 \%$ CI 1.06-1.61) and pain described as none (OR 1.42; 95\%CI 1.08-1.87). Patients from the South (OR 1; reference), West (OR 0.88; 95\%CI 0.82-1.16) and Midwest (OR 0.97; $95 \%$ CI $0.75-1.04)$ regions had higher rates of leaving AMA than the Northeast (OR 0.79; 95\%CI 0.68-0.94). The day of the week did not influence AMA rates; but, rates of leaving AMA were higher during the months of January through July except for April and June (OR 1.39; 95\%CI 1.01-0.91). Compared to patients with private insurance (OR 1; reference), patients with Medicare (OR 1.59; 95\%CI 1.04-2.45), Medicaid (OR 1.66; 95\%CI 1.25 2.21) and self-pay (OR 1.78; 95\%CI 1.31-2.43) had higher rates of leaving AMA. Although most patients arrived to the hospital via personal transportation $(79.7 \%)$, a specific mode of arrival to ED was not statistically correlated with leaving AMA. African American race (OR 1.01; 95\%CI 0.85-1.21) and wait time to see MD/DO/PA/NP did not correlate with leaving AMA (OR $0.90 ; 95 \%$ CI $0.77-1.06)$. Patients with prior ED visits within a year had higher likelihood to leaving AMA (OR 1.49; 95\%CI 1.24-1.79). Of the top 10 presenting complaints to the $\mathrm{ED}$, AMA patients were significantly more likely to present with chest pain $(13.8 \%, \mathrm{p}<0.0001)$, musculoskeletal pain $(15.5 \%, \mathrm{p}<$ $0.0001)$, convulsions $(2.1 \%, \mathrm{p}=0.0004)$, shortness of breath $(6.2 \%, \mathrm{p}=0.0016)$, fever $(2.3 \%, \mathrm{p}=0.004)$, vertigo/dizziness $(3.9 \%, \mathrm{p}=0.02)$, gastrointestinal complication $(17.7 \%, \mathrm{p}=0.03)$ and MVA $(3.9 \%, \mathrm{p}=0.048)$. In our study, alcoholism $(1.5 \%, \mathrm{p}=0.16)$ and headache $(6.3 \%, \mathrm{p}=0.34)$ was not significantly associated with AMA

CONCLUSIONS: Predictors for patients who leave AMA from the ED can be identified at admission and include: males, Hispanics, in no pain, previous ED visits, not having private insurance and lower annual income. Efforts should be made to recognize patients at risk and intervene early to prevent adverse medical outcomes.

PREDICTORS OF SUBJECTIVE WORKLOAD IN ON-CALL INTERNS Kathlyn E. Fletcher ${ }^{1}$; Alexis M. Visotcky ${ }^{2}$; Jason M. Slagle ${ }^{3}$; Sergey Tarima ${ }^{2}$; Matthew Weinger, ${ }^{4,3}$; Marilyn Schapira ${ }^{5}$. ${ }^{1}$ Milwaukee VAMC/Medical College of Wisconsin, Milwaukee, WI; ${ }^{2}$ Medical College of Wisconsin, Milwaukee, WI; ${ }^{3}$ Vanderbilt University, Nashville, TN; ${ }^{4}$ Tennessee Valley VAMC, Nashville, TN; ${ }^{5}$ Philadelphia VAMC/University of Pennsylvania, Philadelphia, PA. (Control ID \#1334976)

BACKGROUND: Physician workload has been linked to patient care errors and adverse events. However, methods for measuring workload vary significantly from study to study. In this study, we sought to determine the relationship between intern self-rated workload during an admitting period and possible predictors of that workload.

METHODS: This was a prospective observational pilot study. Subjects were internal medicine interns rotating on general medicine wards at a VAMC. We trained observers to shadow interns during an on-call period. The observers shadowed the interns and continuously recorded the tasks the interns performed using laptop computers with specially designed software. The observer was also prompted to have the intern rate their self-rated workload on a validated $6-20$ scale $(6=$ low workload activity, i.e. using the internet). These prompts occurred randomly every 30-90 minutes, regardless of what activity the intern was performing. The observers also rated their own perceptions of the interns' workload before they asked the interns to rate themselves. We also collected demographic information and objective workload information such as the number of patients cross-covered by the intern and how many patients each intern had at the start of the day. Data was analyzed using SAS. We tested the univariate relationship between intern self-rated workload and various possible predictors using Pearson correlations, and we conducting multivariable modeling to predict intern self-reported workload using generalized estimating equation with intern as a random effect.

RESULTS: We recruited 25 (out of 36) interns to participate $(69 \%)$ Our sample had 14 women (56\%). The average age was 28.6 (SD2.4). Mean number of months in training was 4 (SD3.7). Interns started the day with a mean of 2.6 (SD1.6) patients and cross-covered a mean of 27.2 (SD12.1). The mean intern self-rated workload was 12 (SD 2.4), with a range of 8-16. Mean observer-rated workload was 11.6 (SD1.8). The observer and intern self-rated workload levels were highly correlated $(\mathrm{r}=0.92, \mathrm{p}<0.001)$. Mean intern self-rated workload while performing direct patient care tasks (such as taking a history) was 12.5 (SD 3.0). Intern self-reported workload while performing indirect patient care tasks (such as documentation) was 12.1 (SD 3.5). There was no significant difference between these workload values. In univariate analyses, there was no correlation between intern selfreported workload and months in training $(\mathrm{r}=-0.14, \mathrm{p}=0.5)$, intern census at the start of the day $(r=0.21, p=0.31)$, or the number of patients cross-covered by the intern $(\mathrm{r}=0.18, \mathrm{p}=0.44)$. In multivariable modeling including physician demographics, only physician age was significantly correlated with workload (beta $=0.46, \mathrm{p}<0.05$ ). However, number of patients at the start of the day (beta $=0.53, \mathrm{p}=0.18$ ) and number of patients cross-covered (beta $=0.05, \mathrm{p}=0.15$ ) trended toward significance.

CONCLUSIONS: Prior studies of physician workload have used physician census as the main proxy for workload. Our study suggests that physician age may also be important. In addition, other aspects of intern work such as cross-cover load may be important determinants of overall subjective workload. In order to understand how best to structure inpatient work to achieve maximum patient safety, it will be necessary to further explore the relationship between intern characteristics, the work done by interns and the impact of those on workload.

PREDICTORS OF WEIGHT LOSS IN AN URBAN, SAFETY-NET HOSPITAL WEIGHT MANAGEMENT PROGRAM. Himali Weerahandi; Elenore Patterson; Albert Ahn; Camila Deza; Lisa Parikh; Gaëlle C. Pierre; Colleen Gillespie; Michelle McMacken. New York University School of Medicine, New York, NY. (Control ID \#1334620)

BACKGROUND: More than one-third of U.S. adults are obese; minorities and populations with low socioeconomic status are disproportionately affected. These underserved populations face limited resources for weight management programs, and are not typically represented in large weight loss studies. Moreover, there is a paucity of data on variables that predict successful weight loss in any population.

METHODS: We conducted a retrospective chart review of all patients seen in an urban, safety-net hospital weight management clinic between January 2006 and July 2010. We examined the following variables: age, gender, initial weight, presence of an axis I psychiatric diagnosis, use of medications that may cause weight gain, prior enrollment in other weight management programs, source of referral, and number of revisits. The primary outcome measure was percentage weight change; we categorized patients into two groups: weight loss (defined as having lost $\geq 5 \%$ of initial body weight) or no weight loss. We used a hierarchical logistic regression analysis to examine which variables predicted weight loss.

RESULTS: Of the 323 patients enrolled during this time period, 161 had an initial weight and at least two total weights recorded. Of these, $80 \%$ were women and 32\% carried an axis I psychiatric diagnosis; the mean age was 48 . At baseline, $39 \%$ of patients were taking medications associated with weight gain (primarily insulin, thiazolidinediones, sulfonylureas, and antipsychotics). Approximately $11 \%$ had previously been enrolled in a weight loss program, and $72 \%$ were referred by their primary physician. Fifty-five patients (34\%) lost $\geq$ $5 \%$ of their initial body weight (weight loss group). Of all variables examined in descriptive, correlation, and regression analyses, only the number of revisits was significantly different between the weight loss and no weight loss groups $(\mathrm{p} \leq$ 0.01 ). The weight loss group averaged 6.62 visits (SD 6.96), versus 3.66 visits (SD 4.93) in the no weight loss group. A Pearson correlation of 0.24 was observed between number of revisits and percentage weight loss $(\mathrm{p}<0.01)$. Hierarchical logistic regression analysis revealed a positive association between number of revisits and weight loss with an odds ratio of 1.11 (95\% CI 1.041.19). No other variables were significantly associated with weight loss.

CONCLUSIONS: Among patients with at least one return visit, one-third achieved clinically significant weight loss in our urban, publicly funded, hospital-based weight management program. Patients who attended more visits were more likely to lose weight. Further research should focus on effective strategies for preventing attrition from weight loss programs. 
PRELIMINARY OUTCOMES OF GENETIC RISK TESTING IN PRIMARY CARE FOR COMMON DNA VARIANTS ASSOCIATED WITH TYPE 2 DIABETES Alex H. Cho ${ }^{7,1}$; Ley A. Killeya-Jones ${ }^{2}$; Sunil Suchindran $^{5}$; Julianne M. O'Daniel ${ }^{3}$; Kensaku Kawamoto ${ }^{4}$; Susanne Haga $^{5}$; Joseph E. Lucas ${ }^{5}$; Gloria M. Trujillo ${ }^{6}$; Scott Joy ${ }^{7,1}$; Geoffrey S. Ginsburg ${ }^{7,5}$. ${ }^{1}$ Duke University, Durham, NC; ${ }^{2}$ UNC-Chapel Hill, Chapel Hill, NC; ${ }^{3}$ Illumina, San Diego, CA; ${ }^{4}$ University of Utah, Salt Lake City, UT; ${ }^{5}$ Duke University, Durham, NC; ${ }^{6}$ Duke University, Durham, NC; ${ }^{7}$ Duke University, Durham, NC. (Control ID \#1340442)

BACKGROUND: Prevention of type 2 diabetes requires patient engagement and behavior change. Offering genetic risk testing for common DNA variants linked to type 2 diabetes has been proposed as a possible strategy for bolstering engagement. Little data exist about the uptake of such testing by patients in clinical settings where available; the impact on clinical and behavioral outcomes; and possible unanticipated negative consequences. Here we report three-month outcomes of a study of genetic risk testing for type 2 diabetes in primary care.

METHODS: Adult patients in two primary care clinics were offered DNA testing for four single nucleotide polymorphisms (SNPs) associated with type 2 diabetes risk. Interested patients were randomized to disclosure of results plus standard risk assessment $(\mathrm{SRA}+\mathrm{G})$, versus SRA alone with an option to receive genetic risk results at the end of the study. Those declining genetic testing were offered SRA without testing in a third study arm. Baseline measurements and surveys assessed weight, fasting glucose and insulin, family history, health behaviors, attitudes, and demographics. Participants were asked to return for risk counseling with a physician extender already working in the clinic. At three months they were asked to complete a survey including self-reported weight, health behaviors, and attitudes; and at 12 months to return for a final visit to repeat measurements and surveys done at baseline. For this preliminary analysis, linear-mixed models for longitudinal data were used to test for difference in weight change after three months between the SRA $+\mathrm{G}$ and SRA-only arms.

RESULTS: $30 \%$ of 1424 patients approached consented to participate. 391 of 409 eligible patients enrolled ( $96 \%$ ) wanted genetic risk testing. Only 18 patients entered a third, "no-test" arm. Mean age was $49.9 \pm 13.3$ years; $70 \%$ were female. $58 \%$ of participants self-identified as White, $29 \%$ African American, and $13 \%$ some other race. $37 \%$ did not complete college. There was a high prevalence of prediabetes, obesity, and positive family history. Nearly all participants (92\%) returned for counseling. Mean baseline weight was $190 \mathrm{lbs}$ in both the SRA $+\mathrm{G}$ and SRA-only arms. After three months, it was $185 \mathrm{lbs}$ in the SRA $+\mathrm{G}$ arm, and 186 in the SRA-only one $(\mathrm{p}=0.37)$. Subgroup analysis of overweight/obese participants found mean weight change of $-1.5 \%$ (SD 3.0\%) in the SRA $+\mathrm{G}$ arm; $-1.6 \%$ (SD 3.0\%) in the SRA-only arm, and $-1.1 \%$ (SD 3.4\%) in the third "no-test" arm. Additional analysis of overweight/obese participants without family history of diabetes found mean weight change of $-1.8 \%$ (SD $3.0 \%$ ) in the SRA $+\mathrm{G}$ arm and $-1.4 \%$ (SD $2.8 \%$ ) in the SRA-only one. In both subgroup analyses, differences did not achieve statistical significance.

CONCLUSIONS: Interest in genetic risk testing for type 2 diabetes was high in a heterogeneous sample of primary care patients. The high prevalence of risk factors and return rate for counseling may suggest interest in confirmatory or explanatory information regarding personal risk for diabetes. After three months, each of the three arms showed a small decrease in mean weight. There was no difference - positive or negative - in weight change between participants randomized to comprehensive type 2 diabetes risk assessment including disclosure of genetic risk testing results, and those receiving risk counseling based on conventional risk factors alone. Analyses of full 12-month and secondary outcomes are still ongoing.

PREMENOPAUSAL WOMEN, CALCIUM, AND VITAMIN D: HOW MUCH DO WE KNOW? Kenzie A. Cameron ${ }^{1}$; Anne Henson ${ }^{1}$; Charlie Zei ${ }^{1}$; Karin B. Ulstrup ${ }^{2,1}$. ${ }^{1}$ Northwestern University, Chicago, IL; ${ }^{2}$ Chicago Lake Shore Medical Associates, Chicago, IL. (Control ID \#1332709)

BACKGROUND: Calcium and vitamin D are two essential nutrients long known for their role in bone health; dietary intake alone seldom provides a sufficient amount of either nutrient. Women may be able to lower their risk of osteoporosis by adhering to the daily intake guidelines of $1000 \mathrm{mg}$ of calcium and $600 \mathrm{IU}$ of vitamin D set by the Institute of Medicine. The goal of this study was to assess premenopausal women's attitudes and knowledge regarding calcium, vitamin D and osteoporosis, as they are not well known.

METHODS: Premenopausal (18-49 years old) women presenting at either an internal medicine or an obstetrics and gynecology practice were approached, consented, and asked to complete a self-administered survey assessing knowledge and attitudes about calcium, vitamin D, and osteoporosis. Participants responded "true," "false," or "don't know" to knowledge items ("don't know" was later coded as "incorrect") and responded on a Likert scale ( 1 =strongly disagree to $5=$ strongly agree) for attitude items.

RESULTS: Three hundred forty-five participants completed the survey, mean age $31.7(\mathrm{sd}=6.7)$. The majority of participants $(75.1 \%)$ were white, $11.3 \%$ were African American, 6.1\% Hispanic/Latino and 7.0\% Asian/Pacific Islander. Women correctly answered a mean of $5.52(\mathrm{sd}=2.05)$ out of 9 knowledge items (range 0 - 9); with the vast majority $(94.5 \%)$ knowing that "osteoporosis leads to an increased risk of bone fractures." Fewer women (57.4\%) knew that "one in four women over the age of 60 will develop osteoporosis." Even fewer women recognized that two glasses of milk per day would not provide an adequate calcium intake (26.1\%). Reponses to the attitude items indicated that women perceive osteoporosis as a serious disease $(\mathrm{M}=4.53, \mathrm{sd}=0.75)$, but do not perceive themselves to be particularly at risk for developing osteoporosis $(\mathrm{M}=$ 3.56, $\mathrm{sd}=1.10$ ). $49.0 \%$ and $48.0 \%$ of participants report taking calcium or vitamin D supplements, respectively. Further, $33.6 \%$ and $40.9 \%$ of women report they believe that are not getting enough calcium or vitamin $\mathrm{D}$ from their diet, respectively, and $20.9 \%$ and $31.0 \%$ report they are unsure if they are receiving enough from their diet. When asked to identify the recommended daily intake of calcium, $77.7 \%$ indicated they did not know; and only $6.1 \%$ were able to provide the accurate answer of $1000 \mathrm{mg}$. When asked about the recommended daily dose of vitamin $\mathrm{D}, 80.0 \%$ responded they did not know; only five participants (1.4\%) accurately answered $600 \mathrm{IU}$.

CONCLUSIONS: Many women recognize that they are likely receiving insufficient amount of either calcium or vitamin D from their diet, yet only about half are supplementing their dietary intake. Although women are aware of potential severity of osteoporosis, they are less likely to perceive themselves to be at risk. Therefore, it is unlikely to be sufficient to educate premenopausal women about the need to take calcium and vitamin D supplementation. Rather, educational efforts must also provide information to allow women to better understand their risk of osteoporosis and be able to accurately assess their current dietary intake of calcium and vitamin D so they can better understand the need to discuss supplementation with their physician.

PREPARING INTERNAL MEDICINE RESIDENTS FOR EFFECTIVE PRACTICE IN THE PATIENT CENTERED MEDICAL HOME: IDENTIFYING EDUCATIONAL NEEDS AND PERCEIVED SKILLS Margaret Horlick, ${ }^{2,1}$ Jaclyn Fox ${ }^{2,1}$; Colleen Gillespie ${ }^{1} .{ }^{1} \mathrm{NYU}$ School of Medicine, New York, NY; ${ }^{2}$ NY Harbor VA Health Care System, New York, NY. (Control ID \#1340117)

BACKGROUND: Little is known about how best to prepare physicians, and particularly resident physicians, to work within Patient Centered Medical Home models of primary practice. This study explores changes in resident physicians' perceived medical home skills prior to and one year after implementation of a medical home in their continuity clinic and compares these skills to residents from the same program whose continuity clinic site had not yet implemented the medical home model.

METHODS: Prior to implementation of the medical home at one of two continuity clinic sites in the NYU Internal Medicine residency program, we assessed, via survey, residents' perceptions of their patient-centered medical home skills. One year after implementation of the medical home in the resident clinic of the VA clinic, which included an introductory conference and workshop and regular team meetings while on the ambulatory care block, we repeated the survey to assess change in perceived medical home skills and included a question about perceptions 
of the sufficiency of the education and training they had received. We also compared VA clinic residents' perceived skills with a sample of residents whose continuity clinic (a public safety net hospital's outpatient clinic) had not yet transformed into a medical home model. Perceived skills were assessed in 5 areas: practice-based learning and improvement (PBLI), system-based practice (SBP), panel management, effective primary care practice, and teamwork. Cronbach's alpha $>.70$ for all domains. Change from pre- to post-medical home was compared using paired sample t-tests and comparisons between medical home and non-medical home sites used independent sample t-tests.

RESULTS: Results 61\% (31/51) of the VA clinic-based residents completed the pre-survey and 10/51 have completed the one-year postsurvey to date. 11/60 city clinic-based residents (medical home not yet implemented) have thus far completed the survey. Among the residents in the medical home model $(n=10), 60 \%$ strongly and $40 \%$ somewhat agree that they have received sufficient training and education for the new model of care. Residents' perceived skills show improvement from pre- to postmedical home in $4 / 5$ domains with significant changes in SBP (pre mean= 2.40, SD .72; post mean $=3.45, \mathrm{SD} .49 ; \mathrm{p}<.05$ ) and PBLI (pre mean 2.35, SD .81; post mean 3.00 , SD .53; $<<.05$ ). Residents whose continuity clinic implemented the medical home model $(n=10)$ reported feeling more competent than residents whose continuity clinic had not $(n=11)$ in $4 / 5$ domains, 3 significantly: SBP (mean 2.60 , SD .88 vs 3.45 , SD $.50 ; \mathrm{p}$ $=.016$ ); PBLI (mean 2.10, SD .66 vs. 3.00, SD .53; $\mathrm{p}=.004$ ); and primary care practice (mean 2.33 , SD .70 vs. 3.33 , SD .67; $\mathrm{p}=.007$ ). Across all assessments, residents rated their panel management skills lowest.

CONCLUSIONS: Results suggest residents need more education/training in panel management but that they perceive the training they've received to practice in the medical home model to be at least adequate. In our small sample of residents exposed to the medical home model, we saw significant increases in some skills (SBP and PBLI) but not in others and that residents exposed to the medical home model in their continuity clinic reported greater competence than those not yet exposed. Results suggest a need for targeted education to fully prepare residents to practice in the medical home model and highlight the importance of having opportunities to practice within new models of care.

PREPARING HEALTHCARE PROVIDERS FOR DIVERSITY : CURRENT SITUATION REGARDING CROSS-CULTURAL CARE IN A SWISS UNIVERSITY HOSPITAL. Patrick Bodenmann ${ }^{2}$; Sophie Paroz ${ }^{1}$; Florence Faucherre ${ }^{3}$; Orest Weber ${ }^{3}$; Esther-Amélie Diserens ${ }^{3}$. ${ }^{1}$ Lausanne University Hospital, Lausanne, Switzerland; ${ }^{2}$ Lausanne University, Lausanne, Switzerland; ${ }^{3}$ Lausanne University Hospital, Lausanne, Switzerland. (Control ID \#1335406)

BACKGROUND: Switzerland is a multicultural country with a growing immigrant population. Around one third of Lausanne University Hospital patients are not Swiss citizens and part of them - asylum seekers, undocumented migrants, foreign-language speaking residents - are characterized by a poor access to care and health status. Improving the ability of the providers to address linguistic and cultural barriers is a key issue for Swiss healthcare institutions. Within a national initiative encouraging centers of competences in cross-cultural care, Lausanne University Hospital assessed the distribution of cross-cultural competences between the hospital departments as well as between medical doctors and nurses. METHODS: A self-administered questionnaire was used to explore 1) expert resources (knowledge, access), 2) education (access, self-perceived preparedness), 3) practices (specific issues, self-assessed skillfulness) and 4) opinions (hospital policy) regarding care of immigrants patients. The questionnaire consisted of 64 items, some of which were adapted from a US validated scale, whereas others were derived from a similar survey conducted at Geneva University Hospital. A mail survey was sent in November 2010 to every resident, chief resident and nurse of a sample of 11 hospital departments : the 5 departments with at least one internal crosscultural resource (clinical team or training program) and 6 departments chosen randomly within the departments without such resources. The first group of departments (primary care, psychiatry, gynecology, pediatrics, emergency room) was categorized as «sensitized» to cross-cultural care, the second group (internal medicine, neurology, rheumatology, urology, radiooncology) as «non sensitized».

RESULTS: A total of 371 out of 885 eligible healthcare providers participated (response rate $41.2 \%$ ). Respondents included $59.7 \%$ of healthcare providers from sensitized departments and $33.6 \%$ of medical doctors. Healthcare providers from sensitized departments had a significantly better knowledge and access to the hospital expert resources than providers from non-sensitized departments, they attended more crosscultural trainings and rated themselves more prepared to care for culturally diverse populations. Medical doctors had a significantly better knowledge and access to the expert resources than nurses, they attended more trainings and rated themselves more competent at most intercultural tasks. However no statistically significant difference of opinion appeared regarding the hospital diversity policy, neither between departments nor between professions : the necessity for hospitals to adapt to linguistic barriers (speaking or writing) was fairly accepted $(66.4 \%$ and $71.9 \%)$ while the necessity to adapt to cultural barriers was poorly accepted (16.2\%).

CONCLUSIONS: Despite a remarkable homogeneity of opinions regarding hospital diversity management, cross-cultural competencies appear to be unequally distributed among hospital departments and between medical doctors and nurses. Addressing diversity is a challenge that must not be limited to part of the medical specialties nor to part of the healthcare professions. In parallel to the development of expert resources in specific departments, hospitals have to make sure that cross-cultural care is integrated in every nurse's and physician's education cursus and that expert resources are known and available for every provider in all medical departments.

PRESCRIBING OPIATES IN RESIDENT CLINIC: CLINIC DIRECTOR AND RESIDENT PERSPECTIVES ON PROBLEMS AND POTENTIAL SOLUTIONS Alison R. Landrey ${ }^{2,1}$; Rachel Swigris ${ }^{1}$; Adam Abraham ${ }^{1} .{ }^{1}$ University of Colorado Hospital, Denver, CO; ${ }^{2}$ University of Colorado, Denver, CO. (Control ID \#1334312)

BACKGROUND: Use of opiate therapy for chronic nonmalignant pain (CNMP) and incidence of opiate overdose are increasing. Management of patients on opiate therapy for CNMP in resident training practices presents unique challenges and may negatively affect residents' continuity experience. Practice innovation to deliver safer and more patient-centered care for this population is vitally needed. We surveyed residency clinic directors and internal medicine residents to elicit perspectives on a) their experience managing CNMP in resident clinic b) problems they've identified, and c) potential solutions to these problems.

METHODS: We developed and emailed an electronic survey to a list-serve of US internal medicine residency clinic directors. We also modified and distributed a paper survey to internal medicine residents with continuity clinics associated with the University of Colorado. Both surveys asked questions on experiences managing CNMP in resident clinic and asked respondents to rate a variety of potential problems and solutions via 1-5 Likert scales. We additionally asked residents their opinion of a planned nursepharmacist run clinic for co-management of patients on opiates for CNMP.

RESULTS: $40 / 75$ clinic directors and 27/48 residents returned the surveys. The majority of clinic directors and residents perceived managing patients with CNMP in resident clinic as more or much more difficult than managing diabetes ( $92 \%$ and $96 \%$ respectively). Clinic directors identified both continuity of care and lack of resident knowledge of CNMP management as the biggest barriers to providing adequate care for these patients. Residents identified coexisting psychiatric illness $(4.2 / 5$ on the Likert scale), inability to address problems other than pain (4.4/5), and lack of continuity of care (4th out of 14 potential problems) as the largest challenges. A large majority ( $85 \%$ ) of residents reported that managing CNMP negatively or very negatively contributed to their continuity experience. Clinic directors felt that enhanced resident training and access to EMR systems that document red flags would most improve their ability 
to provide adequate care to patients with CNMP. Residents felt that a proposed nurse/pharmacist-run pain management clinic would be helpful and allow them to spend more time on medical issues other than pain.

CONCLUSIONS: The vast majority of residents and residency clinic directors perceive managing CNMP in residency clinic as more difficult than managing the complex chronic disease, diabetes. Furthermore, residents report a negative impact on their continuity clinic experience. Solutions to assist clinicians in training clinics to safely and effectively manage CNMP are urgently needed. Although clinic directors and residents have somewhat different perspectives on the greatest challenges, both identified continuity of care as a pressing issue. There was positive feedback from residents regarding a proposed nurse/pharmacist-run clinic for CNMP management. Based on our responses, to be most effective, this type of clinic should be established in a way that enhances continuity of care for patients and allows residents to focus on other medical issues with their patients.

PRESCRIPTION CAPS: RECENT USE BY STATE MEDICAID PROGRAMS AND IMPACT ON MEDICATION USE Daniel A. Lieberman $^{1,2}$; Niteesh K. Choudhry ${ }^{1,2}$; Jerry Avorn ${ }^{1,2}$; Michael Fischer $^{1,2}$. ${ }^{T}$ Brigham and Women's Hospital, Boston, MA; ${ }^{2}$ Harvard Medical School, Boston, MA. (Control ID \#1334283)

BACKGROUND: Prior studies have shown that Medicaid caps limiting the number of prescriptions covered lead to worse medical outcomes. Nevertheless, states continue to employ these policies. We examined prescription cap use by Medicaid programs from 2002 to 2010 and the impact of these policies on prescription use.

METHODS: We collected prescription cap policies for Medicaid fee-forservice plans from 2002 to 2010 for all states. We obtained policy data from reference publications, state websites, and direct contact with states. We classified prescription caps as applying to overall or branded prescriptions. We identified the number of states employing caps, the range in cap levels, and the number of times caps were implemented, removed, or changed. We defined essential medications for the treatment of chronic medical conditions based on prior work (Tamblyn et al., 2001). Using state-level aggregate Medicaid drug use data from CMS, we calculated the ratio of essential to total prescription use for 2 outcomes: number of prescriptions and prescription spending. We calculated these outcomes in 2002 and 2010 for (1) states implementing caps between 2003 and 2009 (2) states without caps during the study period and (3) states with caps throughout the study period. To determine the impact of cap implementation, we calculated the change in essential medication ratios between 2002 and 2010 for states implementing caps and states without caps. We compared the changes between those groups using a Student's T-test. For comparison, we present data for states with caps throughout the study period. RESULTS: We were unable to obtain policy data from Arizona. For several states, we obtained only partial information. 24 states had prescription caps during the study period. From 2002 to 2010, the number of states with caps increased from 11 to 20 . In 2002, 10 states had overall caps and 1 state had a brand cap. In 2010, 12 states had overall caps, 4 had brand caps, and 4 had overall and brand caps. Overall caps ranged from 3 to 15 prescriptions per month; brand caps ranged from 2 to 5 per month. During the study period states implemented 17 caps ( 8 overall, 9 brand) and removed 5 caps ( 3 general, 2 brand). States changed cap levels 12 times, 8 becoming more restrictive, 3 becoming less restrictive, and 1 unclear due to policy complexity. Table 1 presents ratios of essential to total medication use in 2002 and 2010 for each group of states and the change between 2002 and 2010 ratios. The essential medication ratio decreased from 2002 to 2010 . Compared to states without caps, states that implemented caps had a smaller decrease in the essential medications ratio for prescriptions $(\mathrm{p}<0.05)$ but not expenditures $(\mathrm{p}>0.4)$.

CONCLUSIONS: An increasing number of states are using prescription caps to reduce Medicaid costs. Our data suggest that implementing caps leads to a relative increase in the percentage of prescriptions for essential medications but not a significant change in the proportion of expenditures for essential medications. Given past research on the negative impact of caps, patient level evaluation is needed to understand how these recent cap policies affect patient outcomes.
Table 1. Ratio of essential to overall medication utilization $2002 \& 2010$

\begin{tabular}{lllllll}
\hline \hline & \multicolumn{3}{c}{ Prescriptions } & \multicolumn{4}{c}{ Expenditures } \\
& 2002 & 2010 & Change & 2002 & 2010 & Change \\
Cap implemented $(\mathrm{n}=9)$ & 0.343 & 0.278 & 0.066 & 0.388 & 0.354 & 0.034 \\
No cap ( $\mathrm{n}=26)$ & 0.358 & 0.260 & 0.098 & 0.390 & 0.331 & 0.059 \\
Cap throughout $(\mathrm{n}=6)$ & 0.318 & 0.241 & 0.077 & 0.376 & 0.333 & 0.043 \\
\hline
\end{tabular}

PREVALENCE OF HYPONATREMIA IN SMALL CELL LUNG CARCINOMA.

Jasleen K. Pannu ${ }^{1}$; Sriranjini C. Ramaswamy ${ }^{1}$; Nirmal S. Sharma ${ }^{2}$ A Anu Shrestha ${ }^{5}$; Hardeep Rai ${ }^{1}$; Abhijit Duggal ${ }^{4}$. ${ }^{1}$ Western Reserve Care System/ NEOUCOM, Youngstown, OH; ${ }^{2}$ Jacobi Medical Center, New York, NY; ${ }^{3}$ Cleveland Clinic, Cleveland, OH; ${ }^{4}$ Sunnybrook Health Sciences Center, Toronto, ON, Canada. (Control ID \#1340179)

BACKGROUND: Hyponatremia has been found to be the most common electrolyte abnormality in patients with advanced cancers. Degree of hyponatremia has been associated with poor outcomes in patients with cirrhosis, congestive heart failure, pneumonia and meningitis. Recent studies have recognized hyponatremia as an indicator of poor prognosis in advanced cancers and small cell carcinoma of lung. Both plasma sodium and urate are predictive of survival in limited stage small cell lung carcinomas and patients with lung cancer who do not fully regain normal values of sodium have poorer survival as compared to those who do. Few retrospective studies have shown hyponatremia to be a significant prognostic factor in lung cancer. The objective of our study is to study the demographic distribution and prevalence of hyponatremia in limited and extensive stage small cell carcinoma of lung. We also attempted to determine if the degree of hyponatremia was more prevalent in a certain subset of small cell lung carcinoma patients.

METHODS: We collected data for a retrospective cohort study from electronic data base of a community teaching hospital on patients diagnosed with small cell lung cancer from 1995 to 2010. Demographic data and clinical variables including age, sex, race, degree of hyponatremia (mild, $130-135 \mathrm{mmol} / \mathrm{L}$ or moderate/ severe, less than $130 \mathrm{mmol} / \mathrm{L}$ ), stage of small cell lung carcinoma (limited vs extensive) and co-morbidities such as renal failure, hypothyroidism and lung infiltrates were accounted for. Patients were staged into limited and extensive disease in accordance with Veterans Administration Lung Group 2 stage classification scheme. Patients aged less than 18 years and those with incomplete data on staging and serum sodium were excluded. Descriptive statistics for all variables and differences in means for the continuous variables and chi-square test for the categorical variables comparing degree of hyponatremia with these variables were performed.

RESULTS: Over a period of 15 years 80 patients with small cell carcinoma of lung and hyponatremia were included in our study. $65.63 \%$ of patients were male and $95 \%$ were Caucasian. Those with extensive disease constituted $48 \%$ and median duration from diagnosis of hyponatremia to death was 171 days. $41 \%$ patients had mild hyponatremia. $50.63 \%$ patients also had accompanying lung infiltrates, $17 \%$ had renal failure and $8 \%$ had concomitant hypothyroidism. Mild hyponatremia was present in $65.63 \%$ patients with limited disease whereas $58.33 \%$ of patients with extensive disease had moderate to severe hyponatremia $(\mathrm{p}<0.0425)$.

CONCLUSIONS: The results of our study show that moderate/severe degree of hyponatremia is more prevalent in extensive stage small cell carcinoma of lung whereas patients with limited stage disease have mild hyponatremia.

PREVALENCE AND CHARACTERISTICS OF PHYSICIANS WHO RECEIVE COMPENSATION FOR QUALITY OF CARE OR PATIENT SATISFACTION Kira L. Ryskina ${ }^{1}$; Tara F. Bishop ${ }^{2,3} .{ }^{1}$ New York Presbyterian Hospital - Weill Cornell, New York, NY; ${ }^{2}$ Weill Cornell Medical College, New York, NY; ${ }^{3}$ Weill Cornell Medical College, New York, NY. (Control ID \#1317991) 
BACKGROUND: Over the past decade, public and private payers initiated numerous incentive programs compensating physicians for quality of care and/ or patient satisfaction. Little is known about the prevalence of physician compensation for quality or satisfaction on a national level. The objective of this study was to estimate the national prevalence of physicians who are compensated for quality or satisfaction, to identify physician and practice characteristics associated with compensation for quality or satisfaction, and to determine whether physicians who are compensated for quality or satisfaction have more structural and process elements to improve quality of care.

METHODS: We performed a trend analysis and two cross-sectional analyses using the 2006-2008 National Ambulatory Medical Care Survey (NAMCS). NAMCS is a national survey of non-federal, non-hospital-based physicians who see patients in the ambulatory setting. Each physician is weighted to allow for national estimates of physician and practice characteristics. For the trend analysis, we used linear regression to determine whether the percentage of physicians who received compensation for quality and/or satisfaction changed from 2006 to 2008 . We then used multivariable logistic regression to identify physician and practice characteristics associated with physician compensation for quality or satisfaction. Finally, we used the Pearson $\chi 2$ test to identify which structural and process elements were associated with physician compensation for quality or satisfaction.

RESULTS: From 2006 to 2008, 3,813 eligible physicians completed the NAMCS induction survey. The proportion of physicians who received some compensation for quality increased from $17.2 \%$ in 2006 to $24.9 \%$ in 2008 ( $p=$ 0.002 ). Similarly, the proportion of physicians who received some compensation for patient satisfaction increased from $15.8 \%$ in 2006 to $21.4 \%$ in 2008 ( $p=$ 0.02 ). A substantial percentage of physicians did not know whether they received compensation for quality or patient satisfaction $(17.3 \%$ and $16.9 \%$, respectively). Physicians who received some compensation for quality were more likely to be an employee than an owner (adjusted $\mathrm{p}=0.001$ ), in an urban area (adjusted $\mathrm{p}=0.04$ ), in a primary care vs. surgical or medical specialty (adjusted $\mathrm{p}=0.0001$ and $\mathrm{p}=0.01$ ), and practicing in an HMO (adjusted $\mathrm{p}=$ 0.0001). Physician compensation for quality or satisfaction were significant predictors of the practice having an electronic medical record and evening or weekend office hours ( $\mathrm{p}=0.001$ and $\mathrm{p}=0.0001$, respectively).

CONCLUSIONS: A minority of physicians received compensation for quality and/or satisfaction in 2006 to 2008 although the proportion who did increased over that time period. We found that about one in six physicians did not know whether they received compensation for quality or patient satisfaction. Our findings suggest that on a national scale, pay-for-performance programs may not be effective because physicians are either not enrolled in or unaware of these programs.

PREVALENCE AND CHARACTERISTICS OF HOSPITALOWNED AMBULATORY PRACTICES IN THE U.S. Tara F Bishop; Jayme Mendelsohn; Lawrence P. Casalino. Weill Cornell Medical College, New York, NY. (Control ID \#1338777)

BACKGROUND: A number of articles in the medical literature and lay press report that hospitals are increasingly buying physician practices. Competitive and revenue pressures, as well as national policies such as bundled payments and incentives to create accountable care organizations (ACOs) may be propelling this change. Little is known about the characteristics of practices that are owned by hospitals and patient-mix of hospital versus physicianowned practices. We used data from a national survey of physicians to estimate the prevalence of hospital ownership, to identify physician and practice characteristics associated with hospital ownership, and to examine differences in infrastructure, patient access, and patient case-mix in hospital versus physician-owned practices.

METHODS: We used data from the 2005 through 2009 National Ambulatory Medical Care Survey (NAMCS) which is a national survey of physicians who see patients in the ambulatory setting. The sample does not contain information on physicians in hospital outpatient departments or in federally funded practices (e.g. Veteran's Affairs practices). We performed several analyses: a trend analysis to estimate the prevalence and change in prevalence of hospital-owned practices from 2005 to 2009, a cross sectional analysis of 2009 data that used multivariable logistic regression to identify which physician and practice characteristics predict hospital ownership, and a second cross-sectional analysis that used the Pearson X2 to examine differences in practice infrastructure, patient access, and patient case-mix.

RESULTS: From 2005 to 2009, the percentage of physicians practicing in hospital owned practices was low and did not increase over the time period ( $7.4 \%$ in 2005 to $5.6 \%$ in $2009, p=0.117$ ). Compared with physicianowned practices, physicians in hospital-owned practices were less likely to be surgical specialists than primary care physicians $(23.6 \%$ in physicianowned vs. $16.0 \%$ in hospital-owned, adjusted odds ratio $[\mathrm{aOR}]=0.51,95 \%$ confidence intervals [CI] $0.32-0.81$ ), more likely to be group practices (60.8\% vs.87.2\%, aOR $=4.43,95 \%$ CI $2.68-7.30)$, and more likely to be in rural locations $(10.4 \%$ vs. $17.9 \%$, aOR $=1.85,95 \%$ CI $1.21-2.85)$. Hospital owned practices were more likely to have an on-site laboratory $(41.9 \%$ for physician-owned vs. $64.3 \%$ for hospital-owned, $\mathrm{p}<0.001)$, electronic medical records $(18.9 \%$ vs. $22.6 \%, \mathrm{p}=0.003)$, accept Medicaid patients $(68.8 \%$ vs. $87.1 \%, p<0.001)$, and set aside time for same day appointments $(58.7 \%$ vs. $73.6 \%, \mathrm{p}=0.040)$. Patients who were seen by physicians in hospital-owned practices were more likely to be white $(74.3 \%$ vs. $77.2 \%$, p $=0.02)$ and black $(9.3 \%$ vs. $11.7 \%, p=0.02)$ than Hispanic $(11.4 \%$ vs. $6.9 \%, \mathrm{p}=0.02)$ and were more likely to have Medicaid $(10.9 \%$ vs. $17.8 \%$, $\mathrm{p}<0.001)$

CONCLUSIONS: We found that a minority of practices were owned by hospitals from 2005 to 2009 and the percentage did not increase over the time period. Hospital-owned practices had more processes to provide high quality care. Patients may benefit from improved processes of care if, in fact, there is an emerging trend for hospitals to own practices.

PREVALENCE AND CHARACTERISTICS OF HYPERTENSION AT A MEDICAL STUDENT-RUN FREE CLINIC Ognjen Katan ${ }^{1}$; Julie E. Risinger ${ }^{2}$; Thomas G. McLeod ${ }^{3}$; Mark L. Wieland ${ }^{3}$. ${ }^{\mathrm{I}}$ Mayo Clinic, Rochester, MN; ${ }^{2}$ University of Minnesota - Rochester, Rochester, MN; ${ }^{3}$ Mayo Clinic, Rochester, MN. (Control ID \#1339464)

BACKGROUND: Hypertension is a leading cause of morbidity and mortality throughout the world, but it is vastly under-diagnosed and undertreated. Medically underserved populations, the socioeconomically vulnerable, and racial/ethnic minorities are most likely to suffer from uncontrolled hypertension. Free clinics serve as safety nets for many of these patients, yet little is known about hypertension prevalence and characteristics among patients seen in these settings. Therefore, we explored hypertension and uncontrolled hypertension prevalence, treatment, and risk factors in patients who are seen by medical student providers at a free clinic.

METHODS: The REACH clinic is a medical student-run, free clinic that serves uninsured adults residing in Olmsted County, MN. Medical students participate in a longitudinal clinical experience at REACH under the supervision of faculty preceptors. We retrospectively analyzed all consecutive medical records of REACH clinic patients who were seen between January 2010 and December 2011. Data collection included hypertension diagnosis, most recent blood pressure, antihypertensive medications, comorbidities (diabetes, coronary artery disease, hyperlipidemia, tobacco use, alcohol use) and demographics (age, gender, ethnicity, and primary language spoken). Uncontrolled hypertension was defined as a systolic blood pressure $\geq 140$ or a diastolic blood pressure $\geq 90$. Demographics and prevalence of hypertension prevalence and uncontrolled hypertension were calculated through standard descriptive statistics. Associations between a diagnosis of hypertension or uncontrolled hypertension and study variables were assessed through weighted contingency tables with comparison of proportions through Pearson's chi squared test or Fisher's exact test as appropriate; level of significance was set at $<0.05$.

RESULTS: A total of 448 separate patients were seen by medical students at the REACH clinic during the study interval, of whom $393(88 \%)$ had a research authorization on file. Mean patient age was $46 \pm 13$ years; $232(59 \%)$ were Caucasian, 45 (11\%) African immigrant, $41(10 \%)$ Asian, $30(8 \%)$ Hispanic, and 22 (6\%) African American. 157 (40\%) carried a diagnosis of 
hypertension, 88 (56\%) of whom had uncontrolled hypertension. $129(82 \%)$ were prescribed at least one antihypertensive medication in the past. Diagnosis of hypertension was significantly associated with male gender $(p=.0373)$, Asian ethnicity $(\mathrm{p}=.03)$, diabetes $(\mathrm{p}<.0001)$, hyperlipidemia $(\mathrm{p}<.0001)$, coronary artery disease $(\mathrm{p}=.0007)$, and alcohol consumption $(\mathrm{p}=.02)$. A diagnosis of uncontrolled hypertension was associated with male gender $(\mathrm{p}$ $=.03$ ) and hyperlipidemia $(\mathrm{p}=.0008)$. Tobacco use and speaking primarily a foreign language were not associated with previous hypertension diagnosis or with uncontrolled hypertension.

CONCLUSIONS: Among uninsured patients who receive healthcare at a student-run, free clinic, a high percentage of this relatively young patient population carry a diagnosis of hypertension. Despite the fact that most patients have been prescribed antihypertensive medications in the past, the majority of these patients had uncontrolled hypertension. These results speak to the need for qualitative data to elucidate barriers to hypertension management among these vulnerable patient populations. Furthermore, structural interventions are needed to improve longitudinal hypertension management among patients who rely on student-run free clinics for their healthcare.

PREVALENCE AND CORRELATES FOR NONMEDICAL USE OF PRESCRIPTION OPIOIDS AMONG URBAN AND RURAL RESIDENTS Karen Wang ${ }^{1,2}$; William Becker ${ }^{2}$; David A. Fiellin ${ }^{2} .{ }^{1}$ Yale School of Medicine, New Haven, CT; ${ }^{2}$ Yale School of Medicine, New Haven, CT. (Control ID \#1333768)

BACKGROUND: Nonmedical use of prescription opioids and its complications are increasing in the United States. Concurrently, there has been an increase in unintentional overdose deaths related to prescription opioids, in particular within rural areas. Little is known about the differences in prevalence and correlates of nonmedical use of prescription opioids among urban and rural residents.

METHODS: We analyzed data from 2008-2009 National Survey on Drug Use and Health. We examined prevalence, type of opioid, and correlates of nonmedical use of prescription opioids among residents in large and small metropolitan (urban) compared with nonmetropolitan (rural) counties. We then examined bivariate and multivariate associations between nonmedical use of prescription opioids and sociodemographic and clinical characteristics, including age of first use of cigarettes, alcohol and illicit drugs and current (past year) use of these substances, stratified by urban and rural counties.

RESULTS: Among our study population $(\mathrm{n}=75964)$, prevalence of nonmedical use of prescriptions opioids was similar among residents in urban and rural counties $(4.7 \%$ vs. $4.3 \%, \mathrm{p}=0.15)$. Rural residents with nonmedical use of prescription opioids were more likely than urban residents with nonmedical use to be white, have an income under $\$ 20,000$ per year, report nicotine and stimulant use and less likely to have a high school education, be employed, report a good health status, or alcohol use $(\mathrm{p}<.05$ for all comparisons). Among those with nonmedical use of prescription opioids, rural residents were also more likely than urban residents to use acetaminophen with propoxyphene $(61.1 \%$ vs. $55.8 \%, \mathrm{p}=$ $0.02)$, methadone $(14.8 \%$ vs. $9.1 \%, p=0.003)$ and acetaminophen with codeine $(3.5 \%$ vs. $1.9 \%, \mathrm{p}=0.05)$. Among urban and rural residents, those with severe mental illness, age of first use of illicit drugs before the age of 18 , nicotine use, and nonmedical of other prescription drugs were more likely to report nonmedical use of prescription opioids. Among urban residents only, those whose age of first use of illicit drugs between age of 18-25 (AOR 1.53, CI 1.11-2.11) and those with alcohol use (AOR 1.60, CI $1.20-2.13$ ) were more likely to report nonmedical prescription use. Black and Hispanic urban residents were less likely to use prescription opioids nonmedically compared to white urban residents (Black AOR 0.62, CI 0.50-0.76; Hispanic AOR 0.68, CI 0.54-0.87). Rural residents reporting good health status were less likely to use prescription opioids nonmedically than residents reporting poor health status (AOR 0.62, CI 0.39-1.00).

CONCLUSIONS: Specific opioids and correlates of nonmedical use of prescription opioids differ between urban and rural counties. As characteristics differ by level of rurality, prevention and treatment interventions to address these problems may need to be tailored for specific communities.
PREVALENCE OF COMPLETE IMMUNIZATION STATUS AMONG NASHVILLE HIGH SCHOOL SENIORATHLETES Ashley R. Karpinos ${ }^{1,2}$; Katie H. Rizzone ${ }^{2}$; Sarah P. Cribbs ${ }^{3,4}$; Christianne Roumie ${ }^{1,2}$. ${ }^{\mathrm{T}}$ Tennessee Valley Healthcare System VA, Nashville, TN; ${ }^{2}$ Vanderbilt University Medical Center, Nashville, TN; ${ }^{3}$ The Ohio State University, Columbus, OH; ${ }^{4}$ Nationwide Children's Hospital, Columbus, OH. (Control ID \#1315027)

BACKGROUND: The pre-participation physical evaluation, which does not address immunizations, often serves as the only preventive healthcare visit for athletes. Our aim was to determine the proportion of high school senior athletes who received all recommended immunizations. Our hypothesis was that females would be less likely than males to be up-to-date on immunizations given the new human papillomavirus (HPV) vaccine recommendations.

METHODS: We conducted a cross-sectional evaluation of the immunization status of high school senior athletes in metropolitan Nashville. Surveys assessing immunization status and healthcare utilization were distributed to senior athletes' parents. The primary outcome was parent report of athletes being up-to-date on immunizations for tetanus (in the past 10 years), meningococcal (1 dose), and seasonal influenza (in the past 12 months). For females, the primary outcome also included having completed the 3-dose HPV vaccination series. We used Pearson's chi-squared to compare attainment of the primary outcome. We conducted a logistic regression model comparing immunization status among males and females adjusting for race, provider seen for pre-participation physical evaluation, and parental income.

RESULTS: 462 surveys were distributed, 170 (37\%) were returned, and 162 (95\%) were complete for analysis. There were 104 males and 58 females; median age was 17 vs. $17(\mathrm{p}=0.044)$. Males were more likely than females to have up-to-date immunizations ( $16 \%$ vs. $3 \%, \mathrm{p}=0.020)$. When we excluded HPV, there was no difference between males and females (16\% vs. $16 \%, \mathrm{p}=$ 0.982). In a multivariable logistic regression model, the odds of having received all recommended immunizations was 0.13 (95\% CI $0.03-0.68)$ for females vs. males. Athletes seen in sports medicine for their pre-participation physical evaluation were just as likely to have up-to-date immunizations as athletes seen in primary care (OR 0.53 ; 95\% CI 0.13-2.2). Our study population had a higher prevalence of tetanus vaccination, but a lower prevalence of other vaccinations than adolescents in Tennessee (figure 1).

CONCLUSIONS: The proportion of high school senior athletes with upto-date immunizations was low, and females were less likely than males to have recommended immunizations. There was no difference in immunization status among those who received their most recent pre-participation evaluation from sports medicine or primary care physicians. Policy changes recommending a review of immunizations at the pre-participation evaluation would benefit high school athletes.

\section{Study Sample $\square$ TN (3) $\square$ USA (4)}

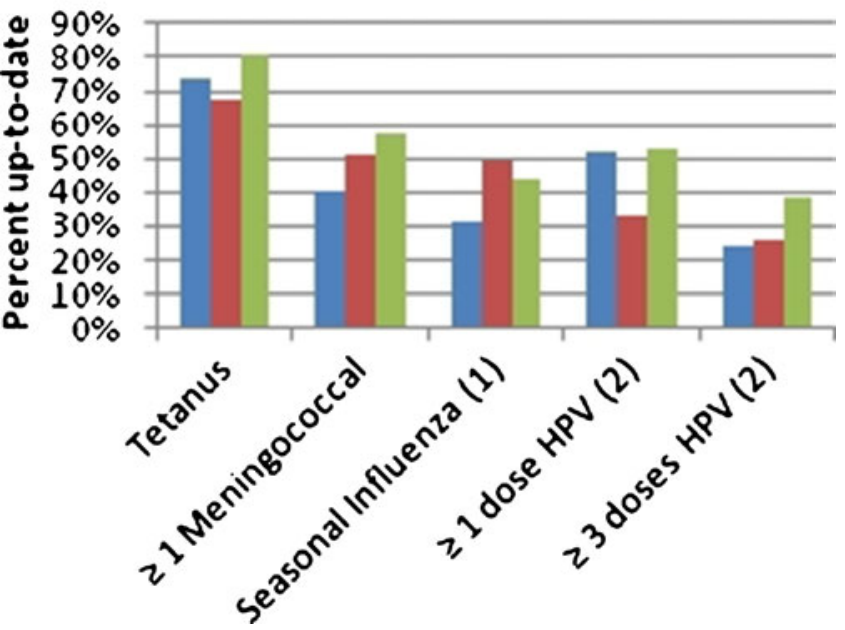

Figure 1. Immunization Status. ${ }^{1}$ Among 6 mo - 17 yr in '09-'10 influenza season ${ }^{2}$ Among females only ${ }^{3}$ Among $13-17 \mathrm{yr}$ in ' $10{ }^{4}$ Among $17 \mathrm{yr}$ in ' 10 
PREVALENCE OF THROMBO-EMBOLIC DISEASE IN END STAGE LIVER DISEASE PATIENTS IN AN URBAN SAFETYNET HOSPITAL 2005-2010. Maria G. Frank ${ }^{1,2}$; Angela Keniston ${ }^{1}$ ${ }^{1}$ Denver Health Hospital Authority, Denver, CO; ${ }^{2}$ University of Colorado, School of Medicine, Aurora, CO. (Control ID \#1310275)

BACKGROUND: The reported incidence of Thromboembolic disease (TED) in End Stage Liver Disease (ESLD) patients ranges from $0.5 \%$ to $4.7 \%$ in published series between 1995 and 2009. We hypothesize that ESLD patients receive less Deep Venous Thrombosis (DVT) prophylaxis than the non- ESLD group, hence they develop higher rate of TED.

METHODS: Data from Denver Health Data Warehouse were queried for all inpatient encounters discharged from Medicine between January 1, 2005 and December 31, 2010. Data were excluded for correctional care patients, patients known to be pregnant, and patients younger than 18 or older than 89. All encounters for all patients meeting inclusion criteria have been retained.

RESULTS: A total of 1448 encounters with primary or secondary diagnosis of ESLD (including chronic liver disease and cirrhosis, hepatic encephalopathy, portal hypertension, hepatorenal syndrome, and other sequelae of chronic liver disease) and 5 with simultaneous diagnosis of TED $(0.34 \%)$ were identified; in contrast with 43,821 non-ESLD discharges and 1,313 with simultaneous diagnosis of TED (2.9\%). When the discharge diagnosis of superficial thrombophlebitis was added to the TED group, numbers increased to $11(0.759 \%)$ in the ESLD group and $1,541(3.5 \%)$ in the non-ESLD group. Forty five percent of ESLD patients received DVT prophylaxis during hospital stay, $100 \%$ of TED patients were in this group.

CONCLUSIONS: Even though pathophysiology of coagulation in ESLD seems to suggest a pro-coagulant imbalance as recently reported by Tripodi, et.al; there is no clinical evidence of higher risk for developing thrombotic episodes in the Denver Health population. There have been 5 retrospective cohort studies to delineate the risk for TED in this group of patients. The largest, by Muhammad, et.al, reviewed 449,798 nationwide discharges and found rates of TED in ESLD of $1.8 \%$ and $2.4 \%$; the latter was true if patients were positive for HCV. Their percentage of TED for all-cause-discharge-diagnosis was 3.7\%. Another retrospective cohort study by Gulley, et.al, analyzed 963 discharges and reported an association of TED and ESLD of $1.8 \%$ in contrast to $3.2 \%$ for overall discharge diagnosis. However the above mentioned studies included superficial thrombophlebitis as one of the TED diagnosis. Northrup, et.al, described a TED frequency of $0.5 \%$ out of 21,000 ESLD patients. We report a rate of TED associated with ESLD lower than most series in English literature. We also found that only half of ESLD patients received DVT prophylaxis in contrast to $65 \%$ of the non-ESLD group. While DVT prophylaxis in ESLD patients appears to be a safe practice, there is no obvious benefit from it in Denver Health's population. Further clinical studies are necessary to develop DVT prophylaxis guidelines for this unique subset of patients.

PREVALENCE OF MORBIDITY POST-DISCHARGE IN AN OLDER PATIENT POPULATION. Leora I. Horwitz; Sandhya V. $\mathrm{Kanade}^{2}$; Christine $\mathrm{Chen}^{2}$; Boback Ziaeian ${ }^{2}$; John Moriarty ${ }^{1}$; Grace Y. Jenq ${ }^{1} .{ }^{1}$ Yale University, New Haven, CT; ${ }^{2}$ Yale New Haven Hospital, New Haven, CT. (Control ID \#1338911)

BACKGROUND: As length of stay has shortened in recent years, patients are being discharged earlier in their clinical course. Older patients in particular, who may take longer to recover from acute illness than younger patients, may experience morbidity post-discharge. Yet the overall experience of older patients post-discharge remains uncertain. The object of this study was to quantify the symptom burden of older patients after discharge home from an acute hospitalization.

METHODS: A prospective cohort of patients admitted to the medical service at Yale New Haven Hospital with pneumonia, heart failure or acute myocardial infarction and discharged home was enrolled from May 1, 2009 to April 4, 2010. The hospital has a higher than average readmission rate for pneumonia and heart failure. Patients were interviewed one month post- discharge by telephone. The interview consisted of 21 questions. Each participant was asked about the presence of 13 specific symptoms and had the opportunity to describe others. For each symptom, we determined whether it began post-discharge, whether the patient discussed it with a doctor, and whether it required a physician or hospital visit. Missing visit data was treated as negative (no physician or hospital visit).

RESULTS: The study cohort included 395 patients; 344 (87.1\%) were reached for the one month interview. Included patients had a mean age of 77 years; $54 \%$ were male. A total of $39 \%$ had heart failure, $24 \%$ had pneumonia and $52 \%$ had acute coronary syndrome. $14 \%$ of patients had more than one condition. Patients experienced a mean of 1.6 symptoms (SD 1.6) in the post-discharge period; $67.7 \%$ of patients experienced at least one symptom. A total of $36 \%$ of symptoms began only after hospitalization. Prevalence of symptoms ranged from $2 \%$ for fever to $32 \%$ for shortness of breath (see Table). More than half of diarrhea, rash, falls, nausea/vomiting, skin breakdown and fever began after discharge. At least $22 \%$ of symptoms required a visit to the physician or the hospital. A total of $17.3 \%$ of patients were readmitted within 30 days of discharge.

CONCLUSIONS: A substantial fraction of post-discharge morbidity begins in the post-discharge period. The frequency of diarrhea, rash, nausea and falls among new symptoms raise the possibility of medicationrelated adverse events and hospital-acquired infections in addition to chronic disease burden. Many of these symptoms require physician or hospital visits but may not be addressed in discharge instructions focused on symptoms related to hospitalization. Post-discharge care should include specific attention to common symptoms post-discharge and to potential medication adverse effects.

Post-discharge symptom burden

\begin{tabular}{lllll}
\hline \hline Symptom & $\begin{array}{l}\text { Prevalence } \\
\mathrm{n}(\% \text { of } \\
\text { patients) }\end{array}$ & $\begin{array}{l}\text { Began post- } \\
\text { discharge } \\
\mathrm{n}(\% \text { of } \\
\text { symptoms) }\end{array}$ & $\begin{array}{l}\text { Discussed } \\
\text { with doctor } \\
\text { n (\% of } \\
\text { symptoms) }\end{array}$ & $\begin{array}{l}\text { Required } \\
\text { physician or } \\
\text { hospital visit } \\
\text { n (\% of } \\
\text { symptoms) }\end{array}$ \\
Shortness of breath & $111(32.4)$ & $26(22 \%)$ & $86(75 \%)$ & $36(32 \%)$ \\
Polyuria & $77(22.6 \%)$ & $20(27 \%)$ & $39(53 \%)$ & $10(13 \%)$ \\
Edema & $71(20.9 \%)$ & $14(19 \%)$ & $54(76 \%)$ & $20(28 \%)$ \\
Pain & $70(20.5 \%)$ & $29(39 \%)$ & $56(76 \%)$ & $27(39 \%)$ \\
Cough & $67(19.7 \%)$ & $18(28 \%)$ & $33(54 \%)$ & $4(6 \%)$ \\
Diarrhea & $23(6.7 \%)$ & $17(74 \%)$ & $12(55 \%)$ & $2(9 \%)$ \\
Rash & $18(5.4 \%)$ & $12(71 \%)$ & $10(63 \%)$ & $4(22 \%)$ \\
Falls & $18(5.3 \%)$ & $16(80 \%)$ & $12(60 \%)$ & $4(22 \%)$ \\
Inability to eat & $17(5.0 \%)$ & $6(35 \%)$ & $14(88 \%)$ & $2(12 \%)$ \\
Nausea/ vomiting & $16(4.6 \%)$ & $10(59 \%)$ & $8(53 \%)$ & $0(0 \%)$ \\
Skin breakdown & $8(2.9 \%)$ & $6(67 \%)$ & $5(63 \%)$ & $2(25 \%)$ \\
Dysuria & $10(2.9 \%)$ & $5(50 \%)$ & $8(80 \%)$ & $2(20 \%)$ \\
Fever & $8(2.4 \%)$ & $5(71 \%)$ & $5(83 \%)$ & $4(50 \%)$ \\
Other & $18(5.3 \%)$ & $8(57 \%)$ & $8(53 \%)$ & $3(17 \%)$ \\
\hline
\end{tabular}

PREVENIR ES MEJOR QUE LAMENTAR: A CULTURALLY TAILORED COLORECTAL CANCER SCREENING INTERVENTION FOR LATINOS Rene Salazar; Monica Lopez; Juliette Hong; Eliseo Perez-Stable. UCSF, San Francisco, CA. (Control ID \#1324966)

BACKGROUND: Rates of colorectal cancer (CRC) screening are lower in racial and ethnic minorities, including Latinos. The goal of this study was to assess the impact of a culturally tailored CRC educational brochure and DVD on CRC cancer behavior among predominantly Spanishspeaking Latinos in a primary care setting.

METHODS: We conducted a randomized controlled trial of 204 Latinos age 50-79 recruited from academic and community primary care clinics in San Francisco CA. Participants were assigned to the intervention, which included a mailed bilingual CRC educational brochure and a Spanish-language DVD versus usual care. Usual care is defined by standard US Prevention Task Force recommendations within the clinical practice. Participants completed a baseline telephone survey and follow up survey 6-9 months later. Demographic data, knowledge of CRC screening and screening tests, facilitators and barriers to screening and self-reported rates of CRC screening were assessed. Descriptive 
statistics were computed for all demographic and dependent variables, including means and standard deviations for continuous data and frequency distributions for each of the categorical variables. Chi-square tests and t-tests were computed for assess differences in covariate distributions across groups. Multivariate analyses were performed to determine the independent impact of the intervention on rates of CRC screening. The primary outcome was the impact of the intervention on any CRC screening.

RESULTS: 190 Latinos completed the baseline and follow up survey. The majority of respondents were female $(69.5 \%)$ and the average age of study participants was 66 years (SD 9.2) Baseline survey revealed similar rates of self reported fecal occult blood testing between the intervention and control group $(58.5 \%$ vs. $55.3 \%, \mathrm{p}<0.65)$. At follow up, self reported rates of fecal occult blood testing (FOBT) were greater in the intervention group compared to the control group $(73.7 \%$ vs. $60.4 \%, p=0.05)$. Rates of colonoscopy at baseline were $58.2 \%$ in the intervention group and $71.4 \%$ in the control group $(\mathrm{p}<0.13)$ Rates of colonoscopy at follow up were similar between the intervention and control group $(73.5 \%$ vs $73.9 \%, \mathrm{p}<0.38)$. Multivariate analysis revealed that the intervention strongly improved rates of any CRC screening among participants (OR 2.23; 95\% CI 1.02-4.87). Those with prior knowledge of screening were more likely to report CRC screening (OR 6.65; $95 \%$ CI 2.8-15.6). Those who reported good health were less likely to have had any screening (OR $0.44 ; 95 \%$ CI $0.21-0.90$ ), as were those with fatalistic attitudes measured by a 4-item scale (OR 0.27 ; 95 CI 0.13-0.58).

CONCLUSIONS: Exposure to a culturally tailored brochure and DVD increased rates of self-reported CRC screening in Latinos.

PREVENTABLE FACTORS FOR REDUCING 30-DAY READMISSIONS IN PATIENTS WITH CARDIAC DISEASE IN A GENERAL INTERNAL MEDICINE CLINIC Ning Tang; Leah Karliner. UCSF, San Francisco, CA. (Control ID \#1339590)

BACKGROUND: Reducing hospital readmissions through improved care transitions is a national health care priority, and in October 2012, the Centers for Medicare and Medicaid Services will enact financial penalties on hospitals with higher than average risk-adjusted readmissions for patients with heart failure and acute myocardial infarction. Little is known about how primary care practices can target patients with heart disease and develop interventions that improve patient care and reduce hospital admission. We set out to understand in our own primary care population with congestive heart failure (CHF) and coronary artery disease (CAD) the reasons for readmission and whether those readmissions were preventable.

METHODS: A retrospective chart review of Division of General Internal Medicine (DGIM) clinic patients with CHF and/or CAD readmitted to University of California, San Francisco (UCSF) Medical Center within 30 days of initial hospital discharge between May 1, 2010 and April 30, 2011 was conducted. A standardized chart review tool, developed by the investigators, was used to review all inpatient and outpatient records. Study investigators developed criteria for determining if a readmission was potentially preventable and applied the criteria set to all reviewed cases.

RESULTS: During the study period, there were 386 hospital discharges for 183 DGIM patients with CHF or CAD; 46 patients had one or more readmissions within 30 days of hospital discharge, totaling 87 index admission-readmission pairs. The majority of patients were discharged from the medicine $(45 \%)$ or cardiology $(47 \%)$ service on their index admission, with length of stay largely less than 1 week (43\% 1-2 days and 43\% 3-7 days). Mean time between index and readmission was 12 days (range 1-29). Half (49\%) of the readmissions were directly related to the index readmission. In $60 \%$ of the index hospitalizations, a follow-up appointment in DGIM was scheduled. For patients who arrived to their outpatient appointments, compared to those who did not, mean time to readmission was 16 vs. 8 days. Almost half $(48 \%)$ of readmissions were classified as potentially preventable $(20 \%$ preventable with system actions alone, $18 \%$ preventable with patient actions alone, and $10 \%$ preventable with system and patient actions combined). The system-related preventable factors included: no timely follow-up appointment, inability to obtain prescriptions, inadequate teaching on discharge medications and disease management, lack of inpatient physical therapy consult for patients with poor functional status, lack of social work and case management involvement in discharge planning, and inadequate communication between inpatient and outpatient providers.

CONCLUSIONS: The potential to reduce preventable readmissions in a general internal medicine clinic population with cardiac disease is substantial. Interventions would require close collaboration between inpatient services and outpatient clinics to ensure appropriate discharge planning for patients with poor functional status, adequate communication between inpatient and outpatient providers, timely outpatient follow-up, and assistance for patients to obtain new prescriptions prior to hospital discharge.

PREVENTIVE CANCER SCREENING PRACTICES IN HIV POSITIVE PATIENTS Florence Momplaisiri'; Karam Mounzer ${ }^{2}$; Judith A. Long ${ }^{1}$. ${ }^{1}$ University of Pennsylvania, Philadelphia, PA; ${ }^{2}$ University of Pennsylvania, Philadelphia, PA. (Control ID \#1320210)

BACKGROUND: With the wide spread use of antiretroviral therapy, patients with HIV are living longer and are at risk of developing non-AIDS Defining Malignancies. In this study we evaluate the rates of routine colorectal and breast cancer screening in patients with and without HIV and identify factors associated with cancer screening.

METHODS: Design: We performed a cross-sectional survey of patients willing to complete the study questionnaire in waiting rooms of three outpatient HIV clinics and one general internal medicine clinic in Philadelphia, Pennsylvania. The survey asked about basic demographics; their colorectal and breast cancer screening history; cancer risk; and presence of other chronic diseases. The HIV positive patients were given additional questions about their HIV disease and their HIV providers. HIV clinics were categorized as integrated (providing HIV and primary care) and non-integrated (providing only HIV care). Study population: Women with and without HIV age 40 and older and men with and without HIV age 50 and older who agreed to complete the survey. Outcome: Survey respondents were considered current for their colorectal cancer (CRC) screening if they reported having at least one colonoscopy during the past 10 years, a flexible sigmoidoscopy during the past five years, or fecal occult blood testing during the last year regardless of the reason for the test. Women were on target on their breast cancer screening if they reported having at least one mammogram during the past year regardless of the indication for the study.

RESULTS: 762 complete surveys were collected. 401 respondents were HIV positive. Patients with HIV were younger (mean age 54 versus $62, \mathrm{p}<$ $0.001)$, mostly male $(53.6 \%$ versus $30.5 \%, \mathrm{p}<0.001)$ black, non-Hispanic $(62.8 \%$ versus $56.5 \%, \mathrm{p}=0.006)$ and low-income $(62.8 \%$ versus $27.4 \%, \mathrm{p}<$ $0.001)$. Co-morbidity counts were similar across both groups $(0.76)$. Patients with HIV were less likely to be up-to-date with their routine cancer screening $(45.6 \%$ versus $65 \%, \mathrm{p}<0.001$ for CRC screening and $20.4 \%$ versus $62.3 \% \mathrm{p}<0.001$ for breast cancer screening). After adjusting for demographic and clinical factors, the odds of up to date CRC screening were no longer significantly different between patients with and without HIV (OR 0.6; 95\% CI 0.3-1.2); however, HIV positive women remained significantly less likely to be current with breast cancer screening (OR 0.1 ; $95 \%$ CI $0.0-0.2$ ). HIV positive patients enrolled in the integrated and the non-integrated care clinics differed significantly: whites, males, high income and highly educated patients were more likely to attend the nonintegrated care clinic. There was a trend toward decreased CRC screening and increased breast cancer screening in integrated care clinics but this finding did not reach statistical significance.

CONCLUSIONS: Routine cancer screening needs to be improved in HIV positive patients. The integrated care clinic provides care to more disadvantaged HIV patients and is not associated with higher cancer screening rates. This could be secondary to non-adherence. It is also possible that patients in the integrated care clinic encounter competing priorities between HIV care and primary care during their visits in contrast to patients in the non-integrated care clinic. 
PREVIOUSLY UNDETECTED CHRONIC KIDNEY DISEASE FOUND TO BE COMMON IN PRIMARY CARE CLINICS IN THE TEXAS PANHANDLE Sudha P. Chennasamudram ${ }^{1}$; Tetyana L. Vasylyeva $^{1}$; Rodney Young ${ }^{2}$; Sharma Prabhakar ${ }^{3}$; Roger D. Smalligan ${ }^{4}$. ${ }^{1}$ Texas Tech University Health Sciences Center, Amarillo, TX; ${ }^{2}$ Texas Tech University Health Sciences Center, Amarillo, TX; ${ }^{3}$ Texas Tech University Health Sciences Center, Lubbock, TX; ${ }^{4}$ Texas Tech University Health Sciences Center, Amarillo, TX. (Control ID \#1338366)

BACKGROUND: Patients with chronic kidney disease (CKD) typically have multiple health problems including underlying hypertension (HTN) and/or diabetes (DM), cardiovascular disease (CVD), anemia and mineral bone diseases. These multiple comorbidities often require a team effort by various clinicians with the primary care physician (PCP) providing overall guidance and coordination of care. Early identification and prevention of progression of CKD is of great importance due to the aging of the US population. It is estimated that over twenty-six million people are affected with CKD in the United States. The state of Texas ranks second highest in the prevalence of CKD and costs associated with it in the nation. The purpose of this study was to identify the presence of CKD in a cohort of patients in the Panhandle region of Texas and offer quality of care suggestions to the primary care physician taking care of those patients.

METHODS: Patients arriving to either the Texas Tech University Health Sciences Center (TTUHSC, Amarillo) Internal Medicine or Family Medicine clinic who did not have documented renal disease were offered to participate in the study. After obtaining informed consent, data was collected including race, age, gender, presence or absence of CKD risk factors (HTN, DM, CVD, obesity) and a basic metabolic panel with estimated glomerular filtration rate (eGFR) was obtained if one was not in the record within the last 6 months. Rates and stages of CKD were determined based on eGFR by CKD EPI formula and a patient's PCP was advised of any important clinical findings along with a suggested course of action if indicated.

RESULTS: A total of 170 patients with no known renal problems were enrolled in the study after obtaining an informed consent. The average age of the participants was 62. Sixty-four percent were women and 36\% were men. Seventy-six percent were white, $7.6 \%$ Hispanic and $16.4 \%$ of other races. eGFR was above 90 in 23\%, CKD stage 2 (eGFR 60-89) in 48\%, stage 3 (30 - 59) in $27 \%$, stage $4 / 5$ (below 30 ) in $2 \%$. Risk factors for CKD among the participants included HTN (58\%), DM (10\%), and CVD (6\%). Among patients with DM, $70 \%$ had been tested for microalbuminuria during the past year.

CONCLUSIONS: This pilot study revealed a remarkably high number of patients who visited their PCP with no previously known renal disease to have early stages of CKD ( $77 \%$ had stage 2 or higher). Indeed there was a high percentage of participants with significant risk factors which would be predictive of CKD and should have alerted clinicians to screen for the condition. Similarly, age is associated with higher rates of CKD and the average age of participants was over 60 . This study reminds PCPs of the need to screen patients for CKD if they have the mentioned risk factors of DM, HTN, CAD or obesity. This will allow tighter control of these comorbid conditions by the PCP to delay CKD progression and early referral to a nephrologist for comanagement in more advanced cases. Identifying CKD earlier will also allow clinicians to be more vigilant while prescribing potentially nephrotoxic medications or ordering contrast involved imaging studies. Important limitations of this study include its small size and disproportionate sex distribution of subjects.

PRICING TRANSPARENCY: CAN U.S. HOSPITALS PROVIDE PRICE ESTIMATES FOR A COMMON ELECTIVE SURGICAL PROCEDURE? Jaime A. Rosenthal ${ }^{1}$; Xin $\mathrm{Lu}^{1}$; Peter Cram ${ }^{1,2} .{ }^{1}$ University of Iowa Carver College of Medicine, Iowa City, IA; ${ }^{2}$ Iowa City VA Medical Center, Iowa City, IA. (Control ID \#1333564)

BACKGROUND: Many proposals for healthcare reform incentivize patients to play a more active role in selecting providers on the basis of quality and price. While data on hospital and physician quality are increasingly available, the availability of pricing data for medical services is less certain. Our objective was to examine our ability to obtain pricing data for a common elective surgical procedure - total hip arthroplasty (THA).

METHODS: We used Medicare Part A data to identify all 4,058 US hospitals performing THA in 2008. We randomly selected two hospitals from each state as well as 21 top-ranked Honor Role Orthopaedic Hospitals identified in the 2011-2012 US News rankings. We contacted each hospital by telephone between May-August 2011. Using a standardized script a member of our study team contacted representatives at each hospital. The essence of the script was that the caller's grandmother required hip replacement surgery, had no comorbid medical conditions, and did not have insurance. The script also specified that the price quote being requested should include 4 days in the hospital and all bundled hospital fees. The caller explained that she was seeking the lowest total cost for the procedure (hospital fee plus physician fee), would be comparing prices among competing quotes, and that her grandmother would be able to pay for the procedure "out-of-pocket." When we encountered hospitals that could provide the hospital fee only, we selected a random orthopaedic surgery practice affiliated with the hospital to obtain the physician fee. This study was deemed exempt by the University of Iowa IRB. RESULTS: Of the 100 randomly selected hospitals, only $10(10 \%)$ were able to provide a single price (hospital plus physician fee); only $6(6 \%)$ were able to provide a single price on the first telephone call. An additional 54 hospitals (54\%) could provide a total price by contacting the hospital and orthopaedic surgery practice separately. There were 22 hospitals $(22 \%)$ that could provide either the hospital or physician price, but not both; 14 hospitals (14\%) could provide neither the hospital nor physician prices. The mean total price from the 64 randomly selected hospitals that were able to provide both the hospital and physician fees was $\$ 41,622$ (range $\$ 11,100$ to $\$ 125,798$ ); 8 had prices less than $\$ 25,000$ and 15 had prices more than $\$ 50,000$. Focusing on the 21 Honor Role Hospitals, 9 (43\%) could provide a single price (both hospital and physician fees) and 4 (19\%) provided the price on the first call; the mean price was $\$ 53,140$ (range $\$ 12,500$ to $\$ 105,000$ ). One top-ranked hospital offered a price of less than $\$ 25,000$, and 8 offered prices exceeding $\$ 50,000$.

CONCLUSIONS: We found it difficult to obtain price information for THA and wide variation in the prices that were quoted. Our results demonstrate that many healthcare providers can not provide reasonable price quotes and may not even have reasonable internal estimates of prices.

PRIMARY CARE CLINICIANS' PERCEPTIONS ABOUT ANTIBIOTIC PRESCRIBING FOR ACUTE BRONCHITIS: A QUALITATIVE STUDY Jeffrey A. Linder; Patrick P. Dempsey; Alexandra C. Businger; Lauren E. Whaley. Brigham and Women's Hospital, Boston, MA. (Control ID \#1326241)

BACKGROUND: Despite guidelines stating that antibiotics are not indicated for acute bronchitis, clinicians nationwide prescribe antibiotics at $70 \%$ of visits. We sought to identify and understand contemporary primary care clinician perceptions about antibiotic prescribing for acute bronchitis.

METHODS: We conducted a qualitative analysis of semi-structured, in-depth interviews with 13 primary care clinicians (12 physicians, 1 nurse practitioner) from 3 primary care practices in the Greater Boston Area. Interview questions addressed attitudes towards acute bronchitis guidelines, reasons for prescribing antibiotics, and potential solutions to decrease inappropriate antibiotic prescribing. Each interview was recorded, transcribed verbatim, and analyzed according to a standard comprehensive qualitative analysis method using structural then thematic coding.

RESULTS: Clinicians had a mean age of 43 years and 9 of the 13 respondents $(69 \%)$ were women. Clinicians universally agreed with guidelines that state antibiotics are not indicated for acute bronchitis. The reasons cited for prescribing antibiotics included direct patient demand (12 clinicians), saving time (4), diagnostic uncertainty (4), and validating patients' effort to come to clinic (3). Seven clinicians stated that patient demand for antibiotics was high. Five cited patient 
demand as a reason for bending the guidelines. Clinicians cited other contributors to antibiotic prescribing such as a lack of accountability or feedback about prescribing (7) and financial incentives that encourage antibiotic prescribing (3). In addition, clinicians cited having patients from cultures where antibiotics are over-the-counter and frequently used (2). Furthermore, clinicians perceived that patients are now used to getting antibiotics, and patients felt they needed antibiotics in order to get better. However, 6 clinicians indicated patient demand has decreased over the last 5 years. Six clinicians felt other clinicians believed antibiotics were either the right treatment, harmless, or that acute bronchitis was not viral. To decrease antibiotic prescribing for acute bronchitis, clinicians recommended better pre-visit triage by nurses or physicians (6); and ongoing patient education (4). Clinicians also recommended patient handouts and other educational materials (5), non-antibiotic prescriptions, coaching patients about the potential harms of antibiotics, providing work and school excuse letters, and other actions that validate patients' concerns. For system solutions, clinicians endorsed quality reports and clinical decision support as potentially helpful (13).

CONCLUSIONS: Clinicians agreed with guidelines that antibiotics are not indicated for acute bronchitis. Clinicians continued to cite patient demand as the main reason for antibiotic prescribing, although clinicians felt patient demand may have lessened in the past few years. To decrease inappropriate antibiotic prescribing for acute bronchitis, clinicians endorsed the use of pre-visit triage, educational materials, quality reports, and clinical decision support.

PRIMARY CARE PHYSICIAN BELIEFS, BEHAVIORS AND PREPAREDNESS IN THE MANAGEMENT OF DEMENTIA: RESULTS OF A NATIONWIDE SURVEY Elizabeth Cogbill ${ }^{1,2}$; Sherry Baker ${ }^{2}$; Jeffrey L. Jackson ${ }^{1,2}$; Marilyn Schapira ${ }^{3}{ }^{1}$ Clement J Zablocki VA Medical Center, Milwaukee, WI; ${ }^{2}$ Medical College of Wisconsin, Milwaukee, WI; ${ }^{3}$ University of Pennsylvania, Philadelphia, PA. (Control ID \#1336779)

BACKGROUND: Dementia is common and increasing in prevalence. Dementia has a highly variable clinical course and its management is complex due to many factors. Treatment options are limited to cholinesterase inhibitors. In the coming years dementia management will fall primarily to primary care physicians. Our study seeks to 1) describe primary care physician (PCP) beliefs about the effectiveness of cholinesterase inhibitors in the treatment of dementia, 2) describe cholinesterase use in clinical practice by PCP self-report, and 3) describe PCP perceptions of their preparedness to manage various aspects of dementia in light of the projected increase in prevalence.

METHODS: We administered a 4 page survey 800 internal medicine, family practice and geriatric medicine physicians. We excluded physicians in training, DOs, physicians employed by government, and retired. Physician and practice characteristics were collected. To assess PCP beliefs about the effectiveness of cholinesterase inhibitors we asked "Please rate the extent to which you agree with the following statement...Cholinesterase inhibitors are effective in treating cognitive/functional decline in dementia." To describe PCP cholinesterase use in clinical practice by self-report survey respondents answered one clinical vignette describing a patient newly diagnosed with dementia. To assess PCP perception of preparedness in the management of dementia survey respondents were asked to rate the extent to which they "feel prepared to manage dementia in the following areas: initiation of prescription of medications for dementia, diagnosis of dementia, discussion of risks and benefits of treatment, and managing side effects of medications." Univariate and bivariate relationships in the data were examined. To test for significant differences between the groups, 2-sample t-tests for continuous variables and $\mathrm{X} 2$ tests for categorical variables were used.

RESULTS: Of the 800 sampled physicians, 354 completed the survey. The majority of respondents were white and male. Sixteen point four percent of PCPs strongly agreed that cholinesterase inhibitors are effective in treating cognitive decline, and $13.3 \%$ felt that cholinesterase inhibitors are effective in treating functional decline. Eighty six percent of PCPs felt that every patient newly diagnosed with dementia should be offered treatment with a cholinesterase inhibitor, and $77 \%$ of PCPs indicated that they would offer and initiate treatment themselves in a straightforward case of newly diagnosed dementia. Less than half of PCPs felt very prepared to diagnose dementia, initiate medications, manage side effects, and discuss the risks and benefits of medication treatment with a patient newly diagnosed with dementia. Comments by PCPs included over 300 unique comments such as "Dementia care will suffer as the primary care base continues to erode" and "Primary care needs clearer diagnostic guidelines or an algorithm."

CONCLUSIONS: Dementia is a common and complex disease with a highly variable clinical course that makes its management challenging for PCPs. The results of our survey indicate that the majority of PCPs do not feel prepared to manage many aspects of dementia. The majority of PCPs do not feel that cholinesterase inhibitors are effective in the treatment of the cognitive or functional decline in dementia. Lastly, PCPs do not all approach the management of dementia in the same way.

PRIMARY CARE PHYSICIANS' CURRENT PRACTICES REGARDING ADULT VACCINATION AND USE OF IMMUNIZATION INFORMATION SYSTEMS Laura Hurley ${ }^{1,2}$; Lori Crane ${ }^{2}$; Erin D. Kennedy ${ }^{3}$; Sean T. O'Leary ${ }^{2}$; Brenda Beaty ${ }^{2}$; Shannon Stokley ${ }^{3}$; Mandy A. Allison ${ }^{4,2}$; Michaela Brtnikova ${ }^{2}$; Andrea Clinger ${ }^{2}$; Allison Kempe ${ }^{2}$. ${ }^{1}$ Denver Health and Hospital Authority, Denver, CO; ${ }^{2}$ University of Colorado Denver, Denver, CO; ${ }^{3}$ Centers for Disease Control and Prevention, Atlanta, GA; ${ }^{4}$ University of Utah, Salt Lake City, UT. (Control ID \#1337689)

BACKGROUND: Eleven vaccines are recommended for routine use in adults, including catch-up vaccines. Immunization information systems (IIS) are confidential, computerized, population-based systems that collect and consolidate vaccination data from multiple providers. IIS are available in 49 out of 50 states. While use of IISs has primarily been encouraged for pediatric vaccines, $82 \%$ of IIS nationwide have the capacity to track vaccination of persons of all ages. The objectives of this study were to assess and compare among general internists (GIM) and family medicine physicians (FM) routinely recommended vaccines stocked in the practice and awareness and use of IISs.

METHODS: Between September 2011 and January 2012, we administered an Internet and mail survey to a national network of 428 GIM physicians representative of the American College of Physicians membership and 420 FM physicians representative of the American Academy of Family Physicians.

RESULTS: Response rates were 58\% (247/428) for GIM and $61 \%$ $(255 / 420)$ for FM. Over $95 \%$ of GIM and FM reported providing vaccines to adults. The percent who reported stocking the various vaccines by specialty are presented in Table $1(n=470)$. FM were more likely to stock a greater number of adult vaccines than GIM. GIM stocked an average of 3 (range 1-4) vaccines and FM an average of 6 (range 1-7) $(\mathrm{p}<0.001)$. FM were more likely than GIM to stock vaccines commonly required for catch-up including HPV, MMR, varicella and meningococcal vaccines. Regarding IIS, $70 \%$ of GIM and $36 \%$ of FM were unsure if their region or state had one, $21 \%$ of GIM and $60 \%$ of FM reported that their state or region did have an IIS, and $9 \%$ of GIM and $4 \%$ of FM reported that their state or region did not $(\mathrm{p}<.001)$. Of the physicians who reported that their state or region had an IIS, $55 \%$ of GIM and $82 \%$ of FM participate in it $(\mathrm{p}<.001)$.

CONCLUSIONS: Many adult physicians, GIM more than FM, are not currently providing all routinely recommended adult vaccines, particularly catch-up vaccines, creating a situation where patients might need to be vaccinated outside of the medical home. Increasing awareness and use of IISs, particularly among GIM physicians, would allow for centralized information collection for patients receiving immunizations at multiple locations and would allow physicians to more readily track immunization status, potentially reducing missed opportunities for vaccination. 
Table 1

\begin{tabular}{llll}
\hline \hline & \multicolumn{2}{l}{$\begin{array}{l}\text { Percent reported } \\
\text { stocking vaccine }\end{array}$} & p-value* \\
& GIM & FM & \\
Hepatitis A & 65 & 75 & 0.02 \\
Hepatitis B & 74 & 84 & 0.007 \\
HPV & 54 & 79 & $<.0001$ \\
High dose influenza & 42 & 50 & 0.07 \\
Injectable influenza & 94 & 97 & 0.09 \\
Intranasal influenza & 22 & 38 & 0.0003 \\
Meningococcal & 46 & 81 & $<.0001$ \\
MMR & 51 & 79 & $<.0001$ \\
Pneumococcal & 98 & 98 & 0.95 \\
Td & 87 & 88 & 0.99 \\
Tdap & 93 & 94 & 0.58 \\
Varicella & 33 & 75 & $<.0001$ \\
Zoster & 52 & 54 & 0.69 \\
\hline
\end{tabular}

Abbreviations: GIM, general internists; FM, family medicine physicians; HPV, human papillomavirus vaccine; MMR, measles-mumps-rubella vaccine; Td, tetanus-diphtheria vaccine; Tdap, tetanus-diphtheria-acellular pertussis vaccine. * $\chi 2$ test for comparison of GIM and FM

PRIMARY CARE WORKLOAD AND BLOOD PRESSURE MANAGEMENT DID NOT WORSEN AT AN URBAN SAFETY-NET HOSPITAL IN MASSACHUSETTS FOLLOWING HEALTH REFORM Karen E. Lasser ${ }^{1,3}$; Steven D. Pizer ${ }^{2,3}$; Aaron Legler ${ }^{1}$; Meredith D'Amore $^{\top}$; Arlene S. Ash ${ }^{4}$; Jeroan Allison ${ }^{4}$; Christopher W. Shanahan ${ }^{1}$; William G. Adams ${ }^{1}$; Dan Berlowitz ${ }^{5,3}$; Nancy R. Kressin ${ }^{2,1}$. ${ }^{1}$ Boston Medical Center/Boston University School of Medicine, Boston, MA; ${ }^{2} \mathrm{VA}$ Boston Healthcare System, Boston, MA; ${ }^{3}$ Boston University School of Public Health, Boston, MA; ${ }^{4}$ University of Massachusetts Medical School, Boston, MA; ${ }^{5}$ Bedford VA Medical Center, Bedford, MA. (Control ID \#1317106)

BACKGROUND: Following Massachusetts (MA) health reform in 2006, 500,000 previously uninsured individuals gained health insurance, which may have burdened primary care capacity to treat such individuals and ensure high quality care. Prior studies have not examined the effect of health reform on clinical outcomes such as blood pressure (BP) control. We hypothesized that an influx of newly insured patients with a fixed supply of primary care providers (PCPs) would increase PCP workload, which in turn would lead to greater delays in BP normalization after an episode of uncontrolled BP.

METHODS: We analyzed 2005-2009 data from general internal medicine visits at one urban safety-net hospital in MA. We included all adult (age> 18) visits with a diagnosis of essential hypertension (based on ICD-9 codes), defining the start of a hypertensive episode as the date of a second consecutive primary care visit with uncontrolled BP (SBP $>140$ OR DBP $>$ 90) within a 90-day period. For patients with multiple episodes, we included one randomly selected hypertensive episode in the analysis. Our primary outcome was time to BP normalization (BP $<140 / 90$ at any visit [primary care or subspecialty]); our secondary outcome was time to next appointment. We followed patients for up to 12 months, censoring observations at 12 months or after the last BP in the observation window, whichever occurred earlier. We measured primary care workload monthly, using the median number of patient visits per PCP-month, and classified PCPs into quartiles of workload. We performed a multivariate discrete time to event analysis to determine whether primary care workload was an independent predictor of time to BP normalization in the years prior to and following MA health reform, controlling for month and year effects.

RESULTS: We studied 17,421 hypertensive episodes. Median time to BP normalization was 70 days in 2005, 67 in 2006, 64 in 2007, 66 in 2008, and 64 in 2009; median days to next appointment over the same 5 years was $44,42,41,42$, and 39 , while primary care workload (visits per PCPmonth) fluctuated, at $66,62,55,57$, and 59 . Over the entire study period, relative to hypertensive episodes treated by PCPs in the lowest workload quartile, hypertensive episodes treated by PCPs in the highest workload quartile were: similarly likely to have a follow-up appointment (odds ratio [OR] 1.0; 95\% confidence interval [CI], 0.9-1.1, $\mathrm{p}=0.7)$ and slightly more likely to have normalized (OR 1.1; $95 \%$ CI, (1.0-1.2).

CONCLUSIONS: Following MA health reform, BP control did not worsen in primary care. Time to next appointment following an episode of uncontrolled BP did not increase, nor did primary care workload. Thus, it appears that newly insured patients did not overwhelm the primary care capacity at this single safety-net institution. Future analyses should examine whether emergency department use for uncontrolled BP increased following health reform.

PRIMARY CARE FOR ADULTS WITH DOWN SYNDROME: ADHERENCE TO PREVENTIVE HEALTH CARE RECOMMENDATIONS Kristin M. Jensen ${ }^{1}$; Laura C. Taylor ${ }^{2}$; Matthew M. Davis ${ }^{2,3}$. ${ }^{1}$ University of Colorado School of Medicine, Aurora, CO; ${ }^{2}$ University of Michigan, Ann Arbor, MI; ${ }^{3}$ University of Michigan, Ann Arbor, MI. (Control ID \#1314995)

BACKGROUND: Due to significant medical improvements, persons with Down syndrome now live well into adulthood. Consequently, primary care for adults with Down syndrome needs to incorporate routine care with screening for condition-specific comorbidities. This study seeks to evaluate the adherence of primary care physicians to age- , gender- and Down syndrome-specific preventive care in a cohort of adults with Down syndrome.

METHODS: In this retrospective observational cohort study, preventive screening was evaluated in patients with Down syndrome ages 18-45 years who received primary care in an academic medical center from 2000-2008. Comparisons were made based on the field of patients' primary care providers (Family Medicine or Internal Medicine).

RESULTS: This cohort included 62 patients, median index age $=33$ years. $40 \%$ of patients received primary care by Family Physicians, with $60 \%$ seen by Internal Medicine practices. Patient demographics, comorbidities, and overall screening patterns were similar between provider groups. Despite near universal screening for obesity and hypothyroidism $(>90 \%$ in both domains), adherence to preventive care recommendations was otherwise inconsistent. Screening was "moderate" $(50-80 \%)$ for cardiac anomalies, reproductive health, dentition, and behavior, psychological, or memory abnormalities. The only areas of discrepancy observed between provider groups were in reproductive health care (adherence=Family Medicine $72 \%$, Internal Medicine $43 \%, \mathrm{p}=0.003$ ) and in screening for cardiac anomalies (adherence $=$ Internal Medicine $81 \%$, Family Medicine $48 \%, \mathrm{p}=0.006)$. Both provider groups demonstrated low adherence $(<50 \%)$ in screening for obstructive sleep apnea, atlanto-axial instability, hearing loss, and vision loss.

CONCLUSIONS: We observed inconsistent preventive care in adults with Down syndrome over this 8.5 year study. This is concerning, given that the long-term consequences of many of these conditions can be prevented if discovered in a timely fashion. Further studies must evaluate the implications of screening practices and more timely identification of comorbidities on clinical outcomes.

PRIMARY CARE PROVIDERS' RESPONSE TO THE USPSTF DRAFT RECOMMENDATIONS ON SCREENING FOR PROSTATE CANCER Craig E. Pollack ${ }^{1}$; Elizabeth A. Platz ${ }^{3}$; Gary Noronha ${ }^{2}$; Gene E. Green $^{2}$; Nrupen A. Bhavsar ${ }^{3}$; H. B. Carter ${ }^{1} .{ }^{1}$ Johns Hopkins University School of Medicine, Baltimore, MD; ${ }^{2}$ Johns Hopkins Community Physicians, Baltimore, MD; ${ }^{3}$ Johns Hopkins Bloomberg School of Public Health, Baltimore, MD. (Control ID \#1331321)

BACKGROUND: The US Preventive Services Task Force issued draft recommendations against routine PSA-based screening for prostate cancer in October 2011. Primary care providers' views on the draft guidelines and 
their willingness to change clinical practice patterns in response remain unknown.

METHODS: We performed a self-administered survey of 141 primary care practitioners from a university-affiliated practice network in November 2011. The network includes primary care physicians, family practice physicians, internal medicine/pediatric-trained physicians, and nurse practitioners working in 26 practice settings.

RESULTS: The response rate was $88.7 \%$ (125 out of 141). Nearly half $(49.1 \%)$ agreed or strongly agreed with the recommendations while $36.0 \%$ disagreed or strongly disagreed. Few providers $(1.8 \%)$ said that they would no longer order routine PSA testing and $21.9 \%$ would be much less likely to do so. Both agreement with the recommendations and expectations as to how the recommendations would change practice did not significantly vary by years since residency graduation, gender, or race/ethnicity. Even among those clinicians who agreed with the draft recommendations, less than half $(41.1 \%)$ stated that they would either no longer order routine PSA screening or be much less likely to do so. Providers who were most likely to screen at baseline were least likely to believe the recommendations would affect their practices $(45.8 \%$ of providers who typically recommend PSA screening did not think the draft recommendations would change their screening behavior compared to $28.3 \%$ of those who typically let the patient decide, $\mathrm{p}<0.001$ ). Providers identified multiple barriers to stopping routine PSA screening including patient expectations, lack of time to explain changes, fear of malpractice litigation, and discomfort with uncertainty associated with stopping screening.

CONCLUSIONS: If finalized, the USPSTF recommendations may encounter significant barriers to adoption, even among those primary care providers who agree with the recommendations. To the extent that PSA screening should be reduced, it may be necessary to address these barriers.

PRIOR BUPRENORPHINE EXPERIENCE IS ASSOCIATED WITH OFFICE-BASED BUPRENORPHINE TREATMENT OUTCOMES Chinazo Cunningham ${ }^{1}$; Robert Roose ${ }^{1}$; Joanna L. Starrels ${ }^{1}$; Angela G. Giovanniello'; Nancy Sohler ${ }^{2}$. ${ }^{1}$ Albert Einstein College of Medicine \& Montefiore Medical Center, Bronx, NY; ${ }^{2}$ Sophie Davis School of Biomedical Education, City College of the City University of New York, New York, NY. (Control ID \#1323667)

BACKGROUND: As buprenorphine treatment is increasing and illicit buprenorphine use is also increasing, many patients seeking buprenorphine treatment will have prior experience with buprenorphine. Although these two types of prior buprenorphine use (prescribed and illicit) may reflect differences in patient populations and may be associated with different treatment outcomes, both types of buprenorphine use provide individuals with an experience taking buprenorphine, a partial opioid agonist with unique pharmacologic properties and challenges. Thus, examining how any prior experience with buprenorphine may be associated with treatment outcomes is warranted. Little evidence is available to guide optimal treatment strategies for patients with prior buprenorphine experience.

METHODS: To examine whether prior buprenorphine experience is associated with buprenorphine treatment outcomes, we conducted an analysis of a longitudinal cohort study of 87 individuals who initiated buprenorphine treatment at an urban health center. Participants were interviewed at baseline, 1 , 3 , and 6 months, and medical records were extracted at 6 months. Dependent variables were 6-month treatment retention (measured via visit and prescription data from medical records) and self-reported opioid use (measured via interviews). The main independent variable was self-reported prior prescribed or illicit buprenorphine experience. We examined associations between prior buprenorphine experience and 6-month treatment retention using logistic regression models, and prior buprenorphine experience and opioid use using non-linear mixed models.

RESULTS: Participants' mean age was 43.5 years, and most were men (73.6\%), Hispanic (73.2\%), and unemployed (69.0\%). At baseline, $67.8 \%$ reported using heroin, $52.9 \%$ methadone, and $26.4 \%$ opioid analgesics. Most $(57.4 \%)$ reported prior buprenorphine experience; of these, $40 \%$ used prescribed buprenorphine, and $60 \%$ used illicit buprenorphine. Compared to buprenorphine-naïve participants, those with prior buprenorphine experience had better treatment retention $(\mathrm{AOR}=2.69,95 \% \mathrm{CI}=1.01-7.17)$, but no difference in opioid use $(\mathrm{AOR}=1.27,95 \% \mathrm{CI}=0.37-4.40)$. When we explored type of buprenorphine experience (prescribe buprenorphine vs. buprenorphine-naïve; illicit buprenorphine vs. buprenorphine-naïve), we found similar findings for treatment retention, but qualitatively different findings for opioid use, though none reached statistical significance (opioid use with prior prescribed buprenorphine use vs. buprenorphine-naïve, $\mathrm{AOR}=2.00,95 \% \mathrm{CI}=$ 0.53-7.50; opioid use with prior illicit buprenorphine use vs. buprenorphinenaïve, $\mathrm{AOR}=0.42,95 \% \mathrm{CI}=0.06-3.09$ ).

CONCLUSIONS: Among individuals seeking buprenorphine treatment at our health center, prior buprenorphine experience was common. We found better treatment retention in those with prior buprenorphine experience (prescribed or illicit buprenorphine use) than those who were buprenorphine-naïve. As buprenorphine treatment continues to expand, and illicit buprenorphine use appears to be increasing, prior experience with buprenorphine is likely to be more common among individuals presenting for buprenorphine treatment. Understanding how prior buprenorphine experience affects treatment outcomes has important clinical and public health implications.

PROFESSIONALISM IN SOCIAL MEDIA - DO EXISTING GUIDELINES FAIL A REALITY CHECK? Neil Mehta ${ }^{2,1}$; Ehsan $\mathrm{H}$. Balagamwala $^{1}$; Bryan A. Sisk ${ }^{1}$; Ilka Decker ${ }^{1}$; Jason Ho ${ }^{1}$; J. H. Isaacson ${ }^{2,1}$; Amy S. Nowacki ${ }^{1}$. ${ }^{1}$ Cleveland Clinic, Cleveland, $\mathrm{OH} ;{ }^{2} \mathrm{Cleveland}$ Clinic, Cleveland, OH. (Control ID \#1336563)

BACKGROUND: The increasing popularity of social media (SoMe) has raised many controversies regarding professionalism. Multiple guidelines have been authored to help us address professionalism in the digital age. Professionalism in SoMe is a highly complex and controversial area. We designed and implemented a workshop for third year students at the Cleveland Clinic Lerner College of Medicine to initiate discussion. Our aim was to study a) whether students could reach consensus regarding these areas with the help of existing guidelines and $b$ ) the impact of a discussion exposing them to various different points of view on this topic.

METHODS: The 2 hour workshop introduced students to various SoMe applications (such as Facebook, Twitter and blogs) with a demonstration of practical applications of SoMe for learning and building a professional identity. We used a real life twitter post that had led to a lively debate in the online community regarding appropriate behavior by medical professionals in SoMe. Students were subsequently divided into 4 small groups, with each group being assigned one of the controversies (see Table) to guide their discussion. The workshop concluded with a large group discussion and a conference call with one of the physicians involved in the controversy. Students completed an online questionnaire before and after the workshop to report their usage of and attitudes towards social media. The questionnaire was anonymous and approved by the Institutional Review Board. Each questionnaire had 4 Likert-type questions. The pre and post workshop surveys were not linked by student and results were analyzed using Fisher's exact test.

RESULTS: All 32 third year students participated in the workshop, and 32 and 28 completed the pre- and post-workshop questionnaires, respectively. Many students utilized SoMe applications such as Facebook (82\%), Twitter $(24 \%)$ and blogged more than one time per month $(6 \%)$. At baseline, majority of the students felt that doctors should not post about their patients in SoMe ( $85 \%)$ and all students felt that humor could be misunderstood in SoMe. After the workshop, students were more polarized in their opinions regarding these two questions $(\mathrm{p}=0.002$ and 0.005 , respectively). As a result of the workshop students also developed a stronger opinion on each side of the issue regarding posting anonymously in SoMe $(\mathrm{p}<0.001)$. Notably, at baseline and after the workshop a majority of the student's felt that "Doctors' professionalism should not be judged by their posts in SoMe".

CONCLUSIONS: When exposed to real life situations, and after discussions with peers, several students changed their beliefs about professionalism in SoMe. In general, there was a decrease in consensus on some of these issues after attending the workshop. This suggests that professionalism in SoMe is a complex, multifaceted issue 
that is not adequately handled by existing guidelines thus requiring further study. Workshops that allow students to review and discuss real cases of use of SoMe will help increase awareness of these complexities.

\begin{tabular}{|c|c|c|c|c|c|c|c|}
\hline \multirow{2}{*}{$\begin{array}{l}\text { Question } \\
\text { It is not professional to post about } \\
\text { your patients. }\end{array}$} & & Strongly Agree & Agree & Neutral & Disagree & Strongly Disagree & $\overline{\text { p-value }}$ \\
\hline & Pre-seminar & $65.6 \%(21)$ & $18.8 \%(6)$ & $6.3 \%(2)$ & $9.4 \%$ (3) & $0 \%(0)$ & 0.002 \\
\hline \multirow{3}{*}{$\begin{array}{l}\text { Doctors should not post } \\
\text { anonymously. }\end{array}$} & Post-s & 21.4 & $\%(15)$ & $17.9 \%(5)$ & $7.1 \%(2)$ & $0 \%(0)$ & \\
\hline & Pre-seminar & $9.1 \%(3)$ & $9.1 \%(3)$ & $69.7 \%(23)$ & $0 \%(0)$ & $12.1 \%(4)$ & $<0.001$ \\
\hline & Post-seminar & $3.6 \%(1)$ & $32.1 \%(9)$ & $39.3 \%(11)$ & $25 \%(7)$ & $0 \%(0)$ & \\
\hline \multirow[t]{2}{*}{$\begin{array}{l}\text { Doctors' professionalism should } \\
\text { not be judged by their posts } \\
\text { in social media. }\end{array}$} & Pre-seminar & $12.5 \%(4)$ & $12.5 \%(4)$ & $15.6 \%(5)$ & $43.8 \%(14)$ & $15.6 \%(5)$ & 0.76 \\
\hline & Post-seminar & $7.1 \%(2)$ & $14.3 \%(4)$ & $10.7 \%(3)$ & $39.3 \%$ & $28.6 \%(8)$ & \\
\hline \multirow{2}{*}{$\begin{array}{l}\text { Humor can be misunderstood } \\
\text { in social media. }\end{array}$} & Pre-seminar & $59.4 \%(19)$ & $40.6 \%(13)$ & $0 \%(0)$ & $0 \%(0)$ & $0 \%(0)$ & 0.005 \\
\hline & Post-seminar & $85.7 \%(24)$ & $7.1 \%(2)$ & $0 \%(0)$ & $3.6 \%(1)$ & $3.6 \%(1)$ & \\
\hline
\end{tabular}

PROGRESSION OF SHARED DECISION MAKING IN THE LAST 15 YEARS IN THE HIGHEST IMPACT FACTOR JOURNALS IN GENERAL INTERNAL MEDICINE Xavier Blanc ${ }^{1}$; Tinh-Hai Collet ${ }^{1}$; Reto Auer ${ }^{2}$; Roland Fischer ${ }^{1}$; Isabella Locatelli ${ }^{1}$; Jacques Cornuz ${ }^{1}$. ${ }^{1}$ University of Lausanne, Lausanne, Switzerland; ${ }^{2}$ University of California, San Francisco, CA. (Control ID \#1340290)

BACKGROUND: Shared Decision Making (SDM) is increasingly advocated as a model for decision making in the medical encounter. A previous report showed an exponential growth of publications on SDM in the overall medical literature since the mid-1990s. Despite this growth, there is still low use of SDM in clinical practice. High impact factor (IF) journals might represent an efficient way for promoting SDM. We aimed to determine the dissemination of SDM among major journals in general internal medicine (GIM).

METHODS: We conducted a full text search of the phrase "shared decision making" using advanced search on the websites of the 15 journals with the highest 2010 5-year IF in the GIM category that published at least original articles, letters and editorials. We included publications released between 1996 and 2010. We used a multivariate longitudinal analysis to predict the percentage of publications addressing SDM, according to the year of publication as a continuous variable and the IF as a dichotomous variable, based on the median value (5.6).

RESULTS: Over the last 15 years, the numbers of publications per journal addressing SDM ranged from 2 ( $0.1 \%$ of the number of annual publications) to $181(12.1 \%)$. We found a significant increase in both absolute and relative mean numbers of publications per year, from $1.1(0.1 \%)$ in 1996 to $3.3(0.5 \%)$ publications in 2010 (both $\mathrm{p}<0.001$ ). The increase was greater among journals with $\mathrm{IF}<5.6(0.2 \%$ to $1.3 \%$ vs. $0.4 \%$ to $0.7 \%$, p of the difference $=0.03)$. The two highest IF journals had the lowest proportion of publications addressing SDM over the last 15 years $(0.10 \%$ and $0.08 \%)$.

CONCLUSIONS: We found an increase in both absolute and relative numbers of publications addressing SDM in the highest IF journals in GIM within the 15 last years. However, we observed a wide variation of the dissemination among the studied journals. Notably, the proportion of publications addressing SDM was low in the two highest IF journals. This significant but heterogeneous increase in major journals suggests a path for implementing SDM among the GIM community. Future research focusing on the way SDM is addressed in high IF journals might help to better understand the spread of SDM over recent years.

PROSPECTIVE COMPARISON OF CLINICAL PROGNOSTIC SCORES FOR MAJOR BLEEDING IN ELDERLY PATIENTS WITH VENOUS THROMBOEMBOLISM Nathalie Scherz'; Marie Méan $^{1}$; Andreas Limacher ${ }^{2}$; Marc Righini ${ }^{3}$; Kurt Jäger ${ }^{4}$; Markus Aschwanden ${ }^{4}$; Hans-Jürg Beer ${ }^{5}$; Beat Frauchiger ${ }^{6}$; Joseph Osterwalder ${ }^{7}$. Christian M. Matter ${ }^{9}$; Nils Kucher ${ }^{8}$; Martin Banyai ${ }^{10}$; Anne AngelilloScherrer ${ }^{11}$; Bernhard Lämmle ${ }^{12}$; Michael Egloff ${ }^{5}$; Henri Bounameaux ${ }^{3}$; Jacques Cornuz ${ }^{13}$; Nicolas Rodondi ${ }^{1}$; Drahomir Aujesky ${ }^{1} .{ }^{1}$ Bern University Hospital, Bern, Switzerland; ${ }^{2}$ University of Bern, Bern, Switzerland;
${ }^{3}$ Geneva University Hospital, Geneva, Switzerland; ${ }^{4}$ Basel University Hospitall, Basel, Switzerland; ${ }^{5}$ Cantonal Hospital of Baden, Baden, Switzerland; ${ }^{6}$ Cantonal Hospital of Frauenfeld, Frauenfeld, Switzerland; ${ }^{7}$ Cantonal Hospital of St. Gallen, St. Gallen, Switzerland; ${ }^{8}$ Bern University Hospital, Bern, Switzerland; ${ }^{9}$ University of Zurich, Zurich, Switzerland; ${ }^{10}$ Cantonal Hospital of Lucerne, Lucerne, Switzerland; ${ }^{11}$ Centre Hospitalier Universitaire Vaudois, Lausanne, Switzerland; ${ }^{12}$ Bern University Hospital, Bern, Switzerland; ${ }^{13}$ University of Lausanne, Lausanne, Switzerland. (Control ID \#1323496)

BACKGROUND: The Outpatient Bleeding Risk Index (OBRI) and the Kuijer, RIETE, and Kearon scores are clinical prognostic scores for bleeding in patients (pts) receiving anticoagulants for venous thromboembolism (VTE) and other diseases. To date, the prognostic performance of these scores was never examined in elderly pts with VTE. We prospectively compared the performance of these scores in predicting the risk of major bleeding and in identifying pts at high-risk of bleeding in a multicenter cohort of elderly pts with VTE.

METHODS: We studied 634 in- and out-pts aged $\geq 65$ years with acute symptomatic, objectively confirmed VTE diagnosed at 9 Swiss hospitals (11/ 2009-03/2011). The outcome was major bleeding (defined as fatal bleeding, symptomatic bleeding in a critical organ, bleeding with a reduction of hemoglobin $\geq 20 \mathrm{~g} / \mathrm{L}$, or leading to the transfusion $\geq 2$ units of packed red blood cells) within 3 months after the index VTE. We classified pts into 3 categories of bleeding risk (low, moderate, and high) according to each score. We dichotomized pts as high vs. low or moderate risk in all 4 scores. We calculated the area under the receiver operating characteristic (ROC) curve, positive predictive value (PPV), and positive likelihood ratio (pLHR) for each score. RESULTS: Overall, 28 of 634 pts $(4.4 \%, 95 \%$ confidence interval $[\mathrm{CI}]$ : 3.0-6.3\%) developed a major bleeding within 3 months. The rate of major bleeding varied from $1.9 \%$ to $2.2 \%$ among low-risk and from $3.8 \%$ to $8.0 \%$ among high-risk pts. The discriminative power of the scores to predict major bleeding was poor to moderate, with areas under the ROC curve ranging from 0.50 to 0.61 (p-value for equality: 0.19 ; Table). PPVs and pLHRs to predict major bleeding were generally low.

CONCLUSIONS: Existing bleeding risk scores do not have sufficient power to discriminate between elderly pts with VTE who are at high-risk of major bleeding and those who are not. Novel, more accurate risk stratification methods must be developed to predict bleeding risk in elderly pts receiving anticoagulants for VTE.

Table

\begin{tabular}{llll}
\hline Clinical score & Area under ROC & PPV, \% (95\% CI) & pLHR (95\% CI)
\end{tabular}

OBRI curve $(95 \% \mathrm{CI})$

$0.54(0.50-0.58)$

Kuijer score $\quad 0.50(0.46-0.54)$

RIETE score $0.61(0.57-0.65)$

$4.7(1.3-15.5)$

$1.06(0.27-4.15)$

Kearon score

$0.60(0.56-0.64)$

$5.8(2.7-12.1)$

$1.34(0.64-2.78)$ 
PROSPECTIVE COMPARISON OF CLINICAL PROGNOSTIC SCORES IN ELDERLY PATIENTS WITH PULMONARY EMBOLISM Daniela Zwierzina ${ }^{1}$; Marie Méan ${ }^{1}$; Andreas Limacher ${ }^{2}$; Marc Righini $^{3}$; Kurt Jäger ${ }^{4}$; Hans-Jürg Beer ${ }^{5}$; Beat Frauchiger ${ }^{6}$; Joseph Osterwalder ${ }^{7}$; Nils Kucher ${ }^{8}$; Christian M. Matter'; Martin Banyai ${ }^{10}$; Anne Angelillo-Scherrer ${ }^{11}$; Bernhard Lämmle ${ }^{12}$; Michael Egloff ${ }^{5}$; Markus Aschwanden ${ }^{4}$; Henri Bounameaux ${ }^{3}$; Jacques Cornuz $^{13}$; Nicolas Rodondi ${ }^{1}$; Drahomir Aujesky ${ }^{1} .{ }^{1}$ Bern University Hospital, Bern, Switzerland; ${ }^{2}$ University of Bern, Bern, Switzerland; ${ }^{3}$ Geneva University Hospital, Geneva, Switzerland; ${ }^{4}$ Basel University Hospital, Basel, Switzerland; ${ }^{5}$ Cantonal Hospital of Baden, Baden, Switzerland; ${ }^{6}$ Cantonal Hospital of Frauenfeld, Frauenfeld, Switzerland; ${ }^{7}$ Cantonal Hospital of St. Gallen, St. Gallen, Switzerland; ${ }^{8}$ Bern University Hospital, Bern, Switzerland; ${ }^{9}$ Zurich Center for Integrative Human Physiology, University of Zurich, Zürich, Switzerland; ${ }^{10}$ Cantonal Hospital of Lucerne, Lucerne, Switzerland; ${ }^{11}$ Centre Hospitalier Universitaire Vaudois, Lausanne, Switzerland; ${ }^{12}$ Bern University Hospital, Bern, Switzerland; ${ }^{13}$ University of Lausanne, Lausanne, Switzerland. (Control ID \#1316460)

BACKGROUND: The Geneva Prognostic Score (GPS), the Pulmonary Embolism Severity Index (PESI), and its simplified version (SPESI) are validated clinical prognostic scores for pulmonary embolism (PE). To our knowledge, the prognostic performance of these scores has never been examined in elderly patients (pts) with PE who have a particularly high risk of adverse outcomes. We prospectively compared the accuracy of these scores in predicting short-term mortality and in identifying pts with PE at low risk of mortality in a multicenter cohort of elderly pts with venous thromboembolism

METHODS: We studied 449 consecutive in- and out-pts aged 65 or older with objectively diagnosed, symptomatic PE from 9 Swiss hospitals (11/ 09-03/2011). The outcome was 30-day overall mortality. We dichotomized pts as low- vs. higher-risk in all 3 scores using the following thresholds: GPS scores $\leq 2$ vs. $>2$, PESI risk classes I-II vs. III-V, and sPESI scores 0 vs. $\geq 1$. Based on these cutoff-points, we calculated sensitivity, specificity, negative predictive value (NPV), and negative likelihood ratio (nLHR) for each score. We also compared the areas under the receiver operating characteristic curve (ROC).

RESULTS: Overall, 17 pts $(3.8 \%, 95 \%$ CI: $2.2-6.0 \%)$ died at 30 days. Although the GPS classified substantially more pts as low-risk, the PESI and the sPESI more accurately identified low-risk pts with PE than the GPS (see table). The areas under the ROC curves did not significantly differ $(\mathrm{p}=0.47)$.

CONCLUSIONS: Overall, the PESI and SPESI are more accurate than the GPS in predicting 30-day mortality in elderly pts with PE. The PESI and sPESI are also more accurate in identifying low-risk pts, and as such, are more useful in safely identifying the most appropriate pts for out-pt treatment or an abbreviated hospital stay.

\begin{tabular}{|c|c|c|c|}
\hline & $\begin{array}{l}\text { GPS }(95 \% \\
\text { CI })\end{array}$ & $\begin{array}{l}\text { PESI }(95 \% \\
\mathrm{CI})\end{array}$ & $\begin{array}{l}\text { sPESI }(95 \% \\
\mathrm{CI})\end{array}$ \\
\hline Low-risk pts, \% & $92(89-94)$ & $36(32-41)$ & $40(35-44)$ \\
\hline $\begin{array}{l}\text { Mortality in low-risk pts, } \\
\%\end{array}$ & $3.4(1.9-5.6)$ & $0.6(0-3.4)$ & $0(0-2.1)$ \\
\hline $\begin{array}{l}\text { Mortality in higher-risk } \\
\text { pts, \% }\end{array}$ & $8.3(1.8-22.5)$ & $5.6(3.2-8.9)$ & $6.3(3.7-9.9)$ \\
\hline Area under ROC & $\begin{array}{l}0.71(0.66- \\
0.75)\end{array}$ & $\begin{array}{l}0.76(0.72- \\
0.80)\end{array}$ & $\begin{array}{l}0.77(0.72- \\
0.81)\end{array}$ \\
\hline Sensitivity, $\%$ & $18(6-41)$ & 94 (73-99) & $100(82-100)$ \\
\hline Specificity, \% & $92(90-95)$ & $38(33-42)$ & $41(37-46)$ \\
\hline NPV, \% & 97 (94-98) & 99 (97-100) & $100(98-100)$ \\
\hline nLHR & $\begin{array}{l}0.89(0.71- \\
1.11)\end{array}$ & $\begin{array}{l}0.16(0.02- \\
1.05)\end{array}$ & $\begin{array}{l}0.00(0.00- \\
1.04)\end{array}$ \\
\hline
\end{tabular}

PUTTING PATIENTS FIRST: USING THE PATIENT PERSPECTIVE TO ENGINEER PATIENT-ORIENTED CLINIC HANDOFFS (EPOCH) Wei Wei Lee; Amber Pincavage; Kimberly Beiting; Vineet Arora. University of Chicago, Chicago, IL. (Control ID \#1328265)
BACKGROUND: Although the year-end resident clinic handoff affects millions of patients annually and has implications for patient safety and satisfaction, little research to date has described the patient experiences as they transition to a new resident primary care physician (PCP). The aim of our study is to identify patients' perceptions of risks associated with the resident clinic handoff and elicit their suggestions to inform development a new patient-oriented handoff process.

METHODS: Graduating internal medicine residents at a single academic institution identified their highest risk patients during the year-end clinic handoff. Three months after transfer to the new resident PCP, a trained research assistant conducted telephone interviews with patients using critical incident technique and appreciative inquiry to elicit both positive and negative experiences associated with the transfer to a new PCP. The interviews were audio recorded and transcribed to ensure accuracy. Using constant comparative method, two investigators independently reviewed ten transcripts to develop an initial coding classification, which was then applied to the remainder of the transcripts using ATLAS.ti software.

RESULTS: Of 323 high-risk patients that departing residents identified, 37 were excluded due to incorrect contact information, change of insurance, transfer of care to other clinics, inability to consent, or death. Of the 286 eligible patients, 103 telephone interviews have been completed. The interviews revealed patient barriers that were categorized into four overarching themes: (1) breakdowns in transition process (i.e. patient unaware transition occurred); (2) clinic logistics (i.e. difficulty rescheduling appointments); (3) doctor-patient relationships (i.e. difficulty building rapport with new PCP); (4) patient safety issues (i.e. missed lab results). Specifically, they reported seeking acute care visits (ER or urgent care) due to delayed care, missed test results or running out of medications during the transition period. For example, one patient reported: "I have to hold everything in until I see [my new doctor]...my pressure was so high until...they sent me to emergency." Patients valued early notification and preparation for the transition. They also appreciated 'telephone visits' with their new PCP prior to their first clinic visit and reported that 'personal sharing' from the new PCP helped build rapport. For example, one patient reported: "Dr. X was...getting ready for her marriage... when I talked to her on the phone...I was like...you're not supposed to be worried about your patients, go get married, we'll talk when you get back." Patients who were aware of their role in the training mission were more understanding of the process: "You hate to see them go but they have to, they have no choice because they are residents."

CONCLUSIONS: By interviewing patients about their experiences with the resident PCP transition, unique insights into the challenges and solutions to this problem can be developed. This data can be used to develop and implement a patient-centered clinic handoff for medicine residents. Such solutions could include a patient 'transition packet' to facilitate early notification of the handoff and introduction to their new PCP, a systematic rescheduling process for missed appointments, formally recognizing and thanking patients for their role in the resident training mission, and implementing "telephone visits" prior to the first visit with the new PCP.

QUALITATIVE APPROACH TO UNDERSTANDING MEDICATION CHALLENGES AMONG OLDER ADULTS AFTER HOSPITAL DISCHARGE Dandan $\mathrm{Liu}^{1,2}$; Stephanie Rennke ${ }^{2}$; Han-Lin $\mathrm{Chi}^{2}$; Michael Steinman ${ }^{1,2}$. $\mathrm{SF}$ VA Medical Center, San Francisco, CA; ${ }^{2}$ UCSF, San Francisco, CA. (Control ID \#1310468)

BACKGROUND: Patients age 65 years and older have a $19 \%$ risk of 30 day readmission after hospital discharge, and medication safety plays an important role in reducing this risk. We piloted a telephone-based qualitative study to better understand the needs older adults have with medications after recent hospital discharge.

METHODS: The study population included patients 2-4 weeks after discharge from the general medicine service at an academic teaching hospital. Inclusion criteria were age $\geq 65$ years, English-speaking, and discharged home with $\geq 5$ medications. We used semi-structured, openended questions assessing problems with medication since discharge, resources patients have, and what additional help they need. Transcriptions were analyzed from a grounded theory approach. For participants who answered "no problems," we used common scenarios based on the medical 
literature as prompts. Concurrent with our study, hospital discharge nurses attempted to reach all participants with a standard post-discharge telephone call that included 2 medication questions (Did you fill your prescriptions? Did you understand your medication instructions?).

RESULTS: We have conducted 9 interviews. All interviewees endorsed some medication-related problem including adherence, titration, side effects, and financial difficulty. Three initially denied any problems, but in response to prompts did endorse at least one. Six of the participants received the standard post-discharge phone call, but none of these revealed any medication problems. Participants identified needs at discharge along themes of lack of communication ("I don't think anyone has any time to discuss with me before I left") and inclusion of family in discharge planning. Four participants denied needing any additional help at discharge with reasons being family support or confidence in self ability. The latter group, however, qualified their response by saying they did "not yet" need assistance.

CONCLUSIONS: Our study suggests that the brief post-discharge phone call after discharge fails to identify common medication problems among older patients. The themes of family and communication suggest focal points for future study and design of discharge programs. Even participants who denied needing assistance still qualified their response with a "yet," suggesting needs are a moving target.

RACIAL DIFFERENCES IN GLUCOSE CONTROL: A SURVEY OF PATIENTS WITH TYPE 2 DIABETES ON COPING, DIET TEMPTATIONS, AND TRUST IN PHYSICIANS Kathleen O. Degnan $^{1}$; Renee M. Betancourt ${ }^{2}$; Judith A. Long ${ }^{3}$. ${ }^{1}$ Perelman School of Medicine at the University of Pennsylvania, Philadelphia, PA; ${ }^{2}$ University of California San Francisco, San Francisco, CA; ${ }^{3}$ University of Pennsylvania, Philadelphia, PA. (Control ID \#1332558)

BACKGROUND: Blacks have a higher prevalence of diabetes and worse clinical outcomes compared to whites. A qualitative study found that compared to well-controlled blacks and whites, poorly-controlled black diabetics were more likely to note barriers to self-care and psychosocial factors as affecting their diabetes control and were less likely to note positive health care experiences. This study quantifies the effects of psychosocial factors on glucose control in an attempt to explain racial disparities in diabetes control.

METHODS: We enrolled adult Type 2 diabetics with providers in the University of Pennsylvania Health System (UPHS) and a recent Hemoglobin A1c (HbAlc). Patients were called within one month of their most recent $\mathrm{HbA} 1 \mathrm{c}$ and a telephone survey was given to patients wishing to participate. The survey contained demographic and clinical questions and several standardized scales including the Jalowiec Coping Scale (JCS), the Dieter's Inventory of Eating Temptations-SE (DIET-SE), and the Trust in Physician scale (TIP).

RESULTS: 332 patients ( 230 blacks, 71 whites, 31 other race) completed the survey. Race significantly correlated with glucose control $(\mathrm{Hbalc}<$ $8 \%$ ), $33.0 \%$ of blacks vs $16.9 \%$ of whites were poorly controlled $(\mathrm{p}=$ 0.023 ). Analysis of JCS responses showed that a confrontive coping style correlated with poor control in whites $(\mathrm{p}<0.001)$ but was not associated with control in blacks; emotive and evasive coping styles were not associated with control. From the DIET-SE, confidence in resisting social and internal dietary temptations correlated with good glucose control in both blacks and whites $(p=0.037$ and $p=0.082$ respectively); high caloric and negative emotion temptations were not associated with control. Scores on the TIP scale did not correlate with glucose control in diabetics of either race. These findings persisted in multivariate models adjusting for age, socio-economic status (education, income, employment, and insurance), duration of diabetes, diabetic medications, complications from diabetes, an interaction term for race and confrontive coping style and possible mediators including self-reported diet and medication adherence.

CONCLUSIONS: Findings confirm significant racial differences in glucose control; however, as measured coping styles, response to dietary temptations and trust in physicians did not account for this racial disparity. At this time there is no evidence that interventions to address these issues would reduce disparities in glucose control.
RACIAL DISPARITIES IN INTERMEDIATE OUTCOMES OF DIABETES CARE IN A PATIENT-CENTERED MEDICAL HOME Joseph A. Simonetti ${ }^{1}$; Michael J. Fine ${ }^{1,2}$; Deborah M. Simak ${ }^{1}$; Yi-Fan $\mathrm{Chen}^{3}$; Rachel Hess ${ }^{1}{ }^{1}$ University of Pittsburgh Medical Center, Pittsburgh, PA; ${ }^{2}$ VA Pittsburgh Healthcare System, Pittsburgh, PA; ${ }^{3}$ University of Pittsburgh Graduate School of Public Health, Pittsburgh, PA. (Control ID \#1318208)

BACKGROUND: Racial disparities in the processes and outcomes of care for patients with diabetes (DM) have been well-documented using administrative data. Such disparities are not well-described in primary care practices organized as patient-centered medical homes (PCMH). Our aim was to assess racial disparities in DM care, controlling for potential patient and provider level confounders.

METHODS: We conducted a retrospective cohort study of patients receiving primary care (July 2009-August 2010) in a level 3, National Committee for Quality Assurance PCMH-designated, university-affiliated, internal medicine practice. We included adults $($ age $>17)$ with $>1$ ICD-9 code for DM or use of DM medications during both the study period and preceding year. We excluded patients with unknown race (n $=2)$ and race other than non-Hispanic white or black $(n=151)$ due to small numbers. We used data routinely collected in the electronic medical record including patient demographics (age, race, gender, medical insurance, educational and marital status), SF-36 mental and physical health scores, DM complications, social support, and primary care provider type (attending vs. resident). Our key independent variable was race (non-Hispanic white vs. black). DM processes of care were the percent of patients who had A1C and low-density lipoprotein cholesterol (LDL-C) testing, and foot and dilated retinal examinations during the study period; intermediate outcomes were percent of patients with an $\mathrm{A} 1 \mathrm{C}<7.0 \%$ (good control), $\mathrm{A} 1 \mathrm{C}>9.0 \%$ (poor control), LDL-C $<100 \mathrm{mg} / \mathrm{dL}$, and $\mathrm{BP}<140 /<90 \mathrm{mmHg}$ as their last measured value during the study period. We used logistic regressions to assess the independent associations between race and processes of care and intermediate outcomes, controlling for patient characteristics and provider type.

RESULTS: Of 1,457 eligible patients with DM, 589 (40.4\%) were black and $868(59.6 \%)$ were non-Hispanic white. They saw one of 89 providers [41 (46.1\%) attending and $48(53.9 \%)$ resident]. At baseline, compared to white patients, black patients were significantly younger (median age 57.0 vs. 61.0 ), less often college attendees $(42.5 \%$ vs. $71.1 \%)$, more often single $(53.0 \%$ vs. $25.3 \%$ ), more often Medicaid-insured ( $25.5 \%$ vs. $6.2 \%$ ), less often treated by an attending (67.6\% vs. $93.7 \%)$, and reported lower median physical (36.0 vs. $43.0)$ and mental health scores $(41.0$ vs. 46.0$)(\mathrm{p}<0.001$, all comparisons). In unadjusted analyses, black patients were less likely than white patients to have had an eye exam $(60.6 \%$ vs. $66.1 \%, p=0.03)$; there were no significant differences in foot exam (75.7\% vs. $74.8 \%)$, LDL-C screening $(79.8 \%$ vs. $83.4 \%$ ) and A1C testing (91.9\% vs. $93.4 \%$ ). Black patients were significantly less likely to have $\mathrm{BP}<140 /<90 \mathrm{mmHg}(64.9 \%$ vs. $75.8 \%)$, LDL-C $<100 \mathrm{mg}$ / dL $(57.5 \%$ vs. $67.0 \%)$, and $\mathrm{A} 1 \mathrm{C}<7.0 \%(45.2 \%$ vs. $51.4 \%)$, and more likely to have $\mathrm{A} 1 \mathrm{C}>9.0 \%(21.0 \%$ vs. $11.8 \%)(\mathrm{p}<0.001$, all comparisons $)$. After covariate adjustment, no racial differences were observed for the four DM processes of care. Black patients continued to have significantly lower odds of achieving $\mathrm{BP}<140 /<90 \mathrm{mmHg}(\mathrm{OR}=0.56 ; 95 \% \mathrm{CI} 0.41-0.75)$ and LDL-C $<100 \mathrm{mg} / \mathrm{dL}(\mathrm{OR}=0.68 ; 95 \% \mathrm{CI} 0.50-0.93)$ and significantly higher odds of having $\mathrm{A} 1 \mathrm{C}>9.0 \%(\mathrm{OR}=1.52 ; 95 \% \mathrm{CI} 1.05-2.17)$.

CONCLUSIONS: In a level 3 PCMH providing primary care to over 1,400 patients with DM, despite high levels of performance with racial equity for all processes of care, we observed clinically and statistically significant racial disparities in intermediate outcomes.

RACIAL DIFFERENCES IN MEN WITH BREAST CANCER Tezo karedan $^{1}$; Siva K. Talluri ${ }^{1}$; Radhika Kakarala ${ }^{1}$; Madhuri Kakarala ${ }^{2}$. ${ }^{\mathrm{T}}$ McLaren Regional Medical center, Flint, MI; ${ }^{2}$ University of Michigan, Ann Arbor, MI. (Control ID \#1310568)

BACKGROUND: Breast cancer in men is rare and constitutes less than 1\% of all cancers in males. The objective of our study is to compare the presenting 
characteristics, and survival rates in Caucasian and African-American men with breast cancer.

METHODS: We analyzed a retrospective cohort of breast cancer patients included in National Cancer Institute's Surveillance, Epidemiology, and End Results (SEER) Registry from 1990 to 2007. Seventeen registries are enrolled in the SEER database and include approximately $26 \%$ of the US population. Racial differences in demographic and tumor characteristics at the time of diagnosis were compared using chi-square test. Breast cancer and racial specific survival was compared using Kaplan-Meier method. Cox proportional hazards regression model was used to determine the independent effect of race on survival after adjusting for age of patient, stage, grade and receptor status of tumor.

RESULTS: We included 2348 men with breast cancer in our study; they were Caucasians (2075), and African-American (273). A greater proportion of African American men presenting with breast cancer were younger than 60 years old as compared to Caucasians ( $46 \%$ vs. $31 \%)$ p $<$ 0.001 . They also more often presented with advanced disease $(12 \%$ vs. $6 \%$ ), higher grade lesions ( $34 \%$ vs. $29 \%$ ), tumor size greater than $2 \mathrm{~cm}$ $(48 \%$ vs. $37 \%)$ and lymph node involvement (35\% vs. $32 \%)$ compared to Caucasians. Tumors were more likely to be progesterone receptor negative $(21 \%$ vs. $11 \%)$ and estrogen receptor negative $(8 \%$ vs. $4 \%)$. All differences were statistically significant $(\mathrm{p}<0.05)$. The mean survival was lower among African Americans (12.6 yrs.) compared to Caucasians (14.3 yrs.) $\mathrm{p}<0.001$ (Log-rank test). After adjusting for age of the patient, stage, grade, and receptor status of the tumor, African -Americans had shorter survival time (Hazard ratio 1.59, 95\% CI: $1.11-2.29)$

CONCLUSIONS: African American men present with more advanced disease and have shorter survival from breast cancer compared to Caucasian men. While male breast cancer is rare, education to raise awareness of this issue, particularly among African Americans, may help promote earlier diagnosis.

RACIAL DIFFERENCES IN SELF-EFFICACY AMONG AFRICANAMERICANS AND WHITES WITH HEART FAILURE Raegan W. Durant $^{1,2}$; Nicole Redmond ${ }^{1,3}$; Zhiying You ${ }^{1}$; Sandral Hullett ${ }^{3}$; A. Robert Sheppard ${ }^{4}$; Isabel Scarinci ${ }^{1}$. ${ }^{1}$ University of Alabama at Birmingham, Birmingham, AL; ${ }^{2}$ Birmingham Veterans Affairs Medical Center, Birmingham, $\mathrm{AL} ;{ }^{3}$ Cooper Green Mercy Hospital, Birmingham, AL; ${ }^{4} \mathrm{DCH}$ Regional Medical Center, Tuscaloosa, AL. (Control ID \#1324371)

BACKGROUND: African Americans with heart failure are hospitalized more frequently than whites. Though chronic disease management programs have been designed to decrease hospitalization rates by enhancing patients' self-efficacy for heart failure self-care, little is known about potential racial differences in self-efficacy. Therefore, we examined the relationship between race and self-efficacy.

METHODS: 734 African-American and white heart failure patients, receiving care at 3 hospitals in Alabama, completed a telephone or inperson survey. The primary outcome was a self-efficacy subscale comprised of two items from the Kansas City Cardiomyopathy Questionnaire: "How sure are you that you know what to do, or whom to call, if your heart failure gets worse?" and "How well do you understand what things you are able to do to keep your heart failure symptoms from getting worse? (for example, weighing yourself, eating a low salt diet, etc)". Combined response data from the two items was scored on a continuous scale $(0-100$, higher score=higher self-efficacy). Continuous scale scores were dichotomized into categories of low (bottom quartile of scale scores) and high self-efficacy (top 3 quartiles of scale scores). The primary independent variable was self-reported race. Other independent variables were categorized into 4 domains: 1) sociodemographics (age, gender, household income, education, marital status) 2) clinical status (hospital site, number of years since heart failure diagnosis, severity of heart failure, type of doctor [primary care vs. cardiologist] managing heart failure), 3 ) social support 4) other psychosocial factors (knowledge of heart failure, depression, trust in physicians). Sequential model-building was used to assess the impact of the successive addition of domain factors to a multivariable logistic regression model.
RESULTS: The study population was $65 \%$ African-American and $54 \%$ female. The mean age was $63(+/-12)$ years. African Americans were more likely to report low heart failure self-efficacy compared to whites $(21 \%$ vs. $10 \%, \mathrm{p}=0.0002)$. The relationship between African American race and low self-efficacy was attenuated slightly when other sociodemographics were added to the model, but the relationship remained statistically significant despite further multivariable adjustment via sequential model-building (see table). The mode of survey administration did not alter the relationship between race and selfefficacy when added to the models.

CONCLUSIONS: Multifaceted chronic disease management programs, focused on improving patients' clinical status, heart failure knowledge, and social support, may still be insufficient to eliminate potential racial disparities in heart failure self-efficacy. The exploration of additional novel facets of chronic disease management may be needed to address the disparities in heart failure self-efficacy.

Unadjusted and adjusted ORs (95\% CI) of low self-efficacy according to race

\begin{tabular}{llllll}
\hline \hline Characteristic & Model 1 & Model 2 & Model 3 & Model 4 & Model 5 \\
Race African & 2.4 & 1.9 & $1.8(1.1$, & $1.8(1.1$, & 1.9 \\
American White & $(1.5,3.8)$ & $(1.2,3.3)$ & $3.1)$ & $3.0)$ & $(1.1,3.2)$ \\
(ref) & & & & & \\
\hline
\end{tabular}

Model $1($ Base model $)=$ race alone Model $2=$ Model $1+$ sociodemographics (age, gender, education, household income, marital status) Model $3=$ Model $2+$ clinical factors (severity of heart failure, time since heart failure diagnosis, type of doctor [primary care vs. cardiologist] managing heart failure) Model 4=Model 3+social support Model 5=Model 4+other psychosocial factors (knowledge of heart failure, trust in physicians, depression)

RARE PRESENTATION OF COCCIDIOIDOMYCOSIS WITH SPONTANEOUS PNEUMOTHORAX Saira Hussain ${ }^{1}$; Natalya Goldshytn ${ }^{1}$; Richard Lazzaro ${ }^{2}$. 'new york methodist hospital, Brooklyn, NY; ${ }^{2}$ new york methodist hospital, Brooklyn, NY. (Control ID \#1319743)

BACKGROUND: $60 \%$ of Coccidioidomycosis cases are completely asymptomatic. The other $40 \%$ of cases are symptomatic with presentation ranging from influenza-like symptoms to a wide spread disseminated infection. Hence, it was to our surprise when our patient presented initially with a spontaneous pneumothorax, and was later diagnosed with Coccidioidomycosis.

METHODS: A 20 year old female with past medical history of diabetes mellitus type 1 since 7 years of age and secondary left lower extremity neuropathy, presented to the emergency room with sudden onset of, fever, dyspnea and right sided chest and shoulder pain for 1 day. Patient was born in Texas but had resided in Arizona for the past 10 years and had recently moved to NY. She did not have a primary care physician and hence was self-managing her diabetes. On arrival, patient was found to have uncontrolled diabetes. Her physical examination was significant for markedly decreased breath sounds on the entire right lung field. Chest $\mathrm{x}-$ ray showed right sided pneumothorax with complete collapse of the lung with left shift of the mediastinum. Emergent left sided pig tail pleural drainage catheter was inserted causing a decrease in the size of the pneumothorax.

RESULTS: Patient then underwent video assisted decortication and wedge ressection. Pathology of the specimen showed a broncho-pleural fistula that was positive for Coccidiomyces immitis/posadasil. The patient was started on itraconazole $200 \mathrm{mg}$ bid with plans to treat for approximately 6 months. CONCLUSIONS: Of the $40 \%$ of Coccidioidiomycosis infections that are symptomatic, approximately, $35 \%$ develop symptomatic pneumonia, $5 \%$ erythema nodosum and $5 \%$ a disseminated disease. One study found that the incidence of pyopneumothorax, and or bronchopulmonary fistula is quite small. Upon the review of 274 cases, it was found that only $2.6 \%$ had a pneumothorax as a complication of the infection. When a spontaneous pneumothorax is discovered, endemic fungal infections should be considered, especially if the patient has a history of being from an endemic 
area. This identifies the importance of gathering an epidemiologic history and expanding the differential diagnosis. This patient presented in NY, but because of her history of residing in Arizona, Coccidioidomycosis was suspected. Also, the importance of submitting the tissue specimen for specific testing cannot be overestimated. In this case, fungal cultures were ordered in anticipation of possible endemic fungal infection, which was confirmed.

REAL WORLD CLINICAL AND PRESCRIBING EXPERIENCE MANAGING PATIENTS WITH ALZHEIMER'S DISEASE: A HEALTH CARE PROVIDERS PERSPECTIVE Russell L. Knoth ${ }^{3}$; Ann Hake; ${ }^{1}$ Malaz Boustani ${ }^{2}$; Sonali N. Shah ${ }^{4}$; Jana Oresayna ${ }^{5}$; Kathy Wyrwich ${ }^{5}$. ${ }^{1}$ Indiana University School of Medicine, Indianapolis, IN; ${ }^{2}$ Regenstrief Institute, Indianapolis, IN; ${ }^{3}$ Eisai, Inc., Woodcliff Lake, NJ; ${ }^{4}$ Pfizer, New York, NY; ${ }^{5}$ United BioSource Corporation, Bethesda, MD. (Control ID \#1337693)

BACKGROUND: The acetylcholinesterase inhibitors (AchEI)s have long been used in the treatment mild, moderate and severe Alzheimer's disease (AD). Donepezil, the most commonly used AchEI, was recently approved by the FDA in a $23 \mathrm{mg} /$ day formulation for patients diagnosed with moderate to severe $\mathrm{AD}$ who had been maintained on a dose of $10 \mathrm{mg}$ daily for at least 3 months. Although most physicians have experience prescribing the lower dosages of donepezil, few have experience with the higher dosage. The study surveyed health care providers (HCPs) who had prescribed the new dosage of donepezil to gain insight into how they identified appropriate patients for treatment, sources of information used in this evaluation, and assessment of outcomes.

METHODS: A survey was used to assess clinical practice in $\mathrm{AD}$ and included a web-based Harris Interactive poll of clinicians' assessment and treatment of $\mathrm{AD}$, followed by an in-depth interactive telephone interview. Appropriate HCPs were identified through market research data as having prescribed donepezil $23 \mathrm{mg}$ to at least two patients. Identified HCPs were contacted via email and provided with a link to the web-based survey administered by Harris Interactive. The survey consisted of 28 questions and took approximately 15 minutes to complete. Participation in the survey was not paid, but those who completed the survey were provided the opportunity to select 1 of 4 charitable organizations to receive a donation of $\$ 20$. Subsequently, a subset of HCPs participating in the web study was invited to participate in the in-depth telephone survey. One-on-one interviews were conducted and HCPs received $\$ 200$ as monetary compensation for their participation.

RESULTS: A total of 1,871 individuals were contacted by email and invited to participate in the web based survey. A total of $40 \mathrm{HCPs}$ met the inclusion criteria and completed the survey. Average age of the respondents was 49.8 years, $85 \%$ were male, and average time in practice was 20 years For medical practice, $95 \%$ were physicians, the most common medical specialties were neurology $(38 \%)$ and geriatrics $(25 \%)$, and the majority $(53 \%)$ worked in a group practice. In the diagnosis of $\mathrm{AD}$, the most common patient was classified as having mild to moderate symptoms (48\%), and initial diagnostic testing included caregiver history (95\%), neurological exam (93\%), and brief neurological screen (93\%). Imaging studies such as CT or MRI were also common $(83 \%)$. In the treatment of $\mathrm{AD}$, for mild disease the most common medication prescribed was donepezil alone (27\%) or an AchEI and memantine combination (27\%). For moderate and severe AD, combination therapy was most common treatment, $30 \%$ and $33 \%$ of the time, respectively. Other medications prescribed included antidepressants $(54 \%)$, anxiolytics (24\%), and antipsychotics (22\%). The most common reasons given for escalating the dosage of donepezil from $10 \mathrm{mg}$ to $23 \mathrm{mg}$ was cognitive decline $(70 \%)$, the ability to tolerate the lower doses of donepezil $(62 \%)$, decline in daily functioning (62\%), and caregiver burden (60\%). Effectiveness of the $23 \mathrm{mg}$ dosage was primarily evaluated by input from caregivers $(85 \%)$ and the patient $(70 \%)$.

CONCLUSIONS: The results of this study give insight into the diagnosis and treatment of $\mathrm{AD}$ in the typical outpatient medical practice. The results also speak to the importance of caregiver input to the physician in the evaluation and treatment of $\mathrm{AD}$.
REAL-WORLD PRACTICE PATTERNS, CLINICAL AND ECONOMIC OUTCOMES AMONG US PATIENTS WITH TYPE 2 DIABETES INITIATING ONCE-DAILY INJECTABLE PEN THERAPY WITH INSULIN GLARGINE OR LIRAGLUTIDE Swetha Raparla'; Wenhui Wei $^{2}$; Michael Grabner'; Robert M. Cuddihy ${ }^{2}$; Wenli $\mathrm{Hu}^{2}$; Ralph Quimbo ${ }^{1}$. ${ }^{1}$ HealthCore, Inc., Wilmington, DE; ${ }^{2}$ sanofi-aventis U.S., Bridgewater, NJ. (Control ID \#1340378)

BACKGROUND: Initiation of injectable therapy is a major event for patients with type 2 diabetes mellitus (T2DM), and to conduct real-world comparative effectiveness studies of such therapies one needs to first understand patient characteristics and the associated treatment patterns and outcomes. This study aims to examine real-world practice patterns and outcomes among T2DM patients initiating once-daily injectable pen therapy, with insulin glargine disposable pen (GLA-P) or liraglutide pen (LIRA), a glucagon-like peptide-1 analog.

METHODS: This is a retrospective study of a large US commercial health plan using administrative claims data from the HealthCore Integrated Research Database (HIRD[SM]). Included were adult T2DM patients who were previously treated with oral antidiabetic drugs (OADs) only and initiated GLA-P or LIRA between January and June 2010, with the index date representing start of injectable therapy for T2DM. Patients were required to have continuous health plan coverage for $\geq 6$ months before (baseline period) and $\geq 1$ year after (follow-up period) the index date. Differences in baseline characteristics between the 2 cohorts were tested. One-year follow-up measures were assessed descriptively, including treatment persistence, hypoglycemia-related events, healthcare utilization, and cost.

RESULTS: A total of 1,709 unmatched T2DM patients (GLA-P $\mathrm{n}=1,188$; LIRA $\mathrm{n}=521$ ) were included. At baseline, significant differences (all $\mathrm{P}<$ 0.001 ) existed between the 2 cohorts. GLA-P initiators, compared with LIRA initiators, were: older (mean age 56.9 vs. 53.2 years); male (57.7 vs. $47.8 \%$ ); less obese $(9.2$ vs. $18.8 \%$ ); less often on metformin (74.8 vs. $84.6 \%$ ) but more often on sulfonylureas (63.1 vs. $42.8 \%$ ); had higher comorbidity (Quan-Charlson comorbidity index $1.05 \pm 1.67$ vs. $0.73 \pm 1.28$ ); had higher A1C among those with data available (GLA-P $\mathrm{n}=283$ vs. LIRA $\mathrm{n}=113$; mean $9.1 \pm 1.90$ vs. $7.7 \pm 1.38 \% ; 11.3 \%$ in GLA-P had $\mathrm{A} 1 \mathrm{C}<7 \%$ vs. $33.6 \%$ in LIRA, and 47.7 vs. $16.8 \%$ had $\mathrm{A} 1 \mathrm{C} \geq 9 \%$ ); and were more likely to have hospitalizations ( 11.3 vs. $5.0 \%)$, but less likely to have endocrinologist visits (22.1 vs. 34.2\%). During follow-up, treatment persistence was $60.2 \%$ in GLA-P and $50.9 \%$ in LIRA (mean persistence days: GLA-P: 221.4; LIRA: 165.0). Average daily drug usage was 28 units in GLA-P and $1.44 \mathrm{mg}$ in LIRA. Overall hypoglycemic event rates were 25.3 (GLA-P) and 5.6 (LIRA) per 100 patients/year, with hospital- or emergency room-related hypoglycemic event rates being low in both cohorts ( 3 and 1 per 100 patients/year). Hospitalization rate was $18.4 \%$ in GLA-P and $10.6 \%$ in LIRA. Mean 1-year total all-cause healthcare costs were $\$ 16,466$ in GLA-P (7.0\% from study drug) and $\$ 14,579$ in LIRA (18.9\% from study drug), and pharmacy costs were $\$ 6,184$ in GLA-P (18.7\% from study drug) and $\$ 7,549$ in LIRA (36.5\% from study drug). CONCLUSIONS: This real-world study shows significant baseline differences between T2DM patients initiating GLA-P vs. LIRA as their first injectable therapy. Patients initiating on GLA-P were older and sicker, with more comorbidities and significantly different baseline A1C levels. About $1 / 3$ of LIRA initiators had A1C $<7.0 \%$ at baseline, suggesting that a substantial number of patients might not have initiated LIRA primarily for glycemic control. The data showed that patients initiating LIRA may not be directly comparable to patients initiating GLA-P, implying challenges in conducting related comparative effectiveness research.

REASONS FOR READMISSIONS IN A HIGH-RISK POPULATION Theodore Long; Leora I. Horwitz. Yale Dept of Internal Medicine, New Haven, CT. (Control ID \#1339048)

BACKGROUND: Hospital readmissions represent a significant cost to the healthcare system and are a burden to patients. There is a paucity of qualitative data regarding the perspectives of patients with multiple readmissions. 
Furthermore, very little is known about the factors contributing to readmissions in the urban underserved population, who comprise a disproportionate share of readmissions for many academic medical centers. We gathered qualitative data to elucidate the reasons for readmissions in a high-risk population of underserved patients at Yale-New Haven Hospital.

METHODS: We conducted semi-structured qualitative interviews of patients receiving primary care services from the Yale Primary Care Center (a low-income health center serving New Haven) who had four or more readmissions in the previous six months and were currently readmitted to the hospital. All interviews were transcribed by an independent company. Two investigators independently generated codes from the primary data and developed a final code list using the constant comparative method. These codes were organized into 11 main themes.

RESULTS: To date eight interviews have been completed. We identified three major themes: "self-triage," primary care discontinuity, and adequacy of formal services. Patients in the study typically went directly to the Emergency Department when they experienced a change in health status without consulting with their primary provider. Prevalent reasons for this "self triage" included poor telephone access to the Primary Care Center, poor access to urgent visit appointments, and the belief that the Primary Care Center could not treat acute illness. Another contributor to readmission was that patients either could not name their primary provider or stated that they did not have a primary provider. Conversely, every patient reported being able to obtain medications without undue financial burden, and every patient reported receiving adequate formal home services such as visiting nurse services, home health aides, or transportation assistance.

CONCLUSIONS: Our results suggest that there may be factors contributing to readmissions in this underserved high-risk population that are not addressed by most current interventions, which are targeted at access to medications and formal home and nursing services. In particular, patients consistently reported using "self triage" stemming from inadequate communication with providers when they had a change in health status. As future interventions are developed for prevention of readmissions, improvement of continuity and communication with outpatient providers should be considered.

REASONS FOR DELAYED RECEIPT OF HORMONAL THERAPY FOR BREAST CANCER AT AN URBAN HOSPITAL Meaghan M. Crowley ${ }^{1}$; Molly E. McCoy ${ }^{2}$; Sharon $\mathrm{Bak}^{2}$; Sarah E. Caron ${ }^{2}$; $\overline{\text { Faber Alvis }}{ }^{5}$; Lisa A. Kachnic ${ }^{4}$; Tracy Battaglia ${ }^{2}$. ${ }^{1}$ Boston University School of Medicine, Boston, MA; ${ }^{2}$ Boston University School of Medicine, Boston, MA; ${ }^{3}$ Boston Medical Center, Boston, MA; ${ }^{4}$ Boston Medical Center, Boston, MA. (Control ID \#1338277)

BACKGROUND: Significant racial and economic disparities in breast cancer mortality may be partially explained by underutilization or delay of proven treatments in vulnerable populations. Accordingly, quality standards for timely treatment have been established. However, the underlying reasons for not meeting quality standards of treatment are poorly understood in these populations. Our objective was to identify characteristics of patients who fail to receive quality breast cancer treatment as defined by the American College of Surgeons (ACOS) Cancer Program Practice Profile Reports.

METHODS: This retrospective observational study was conducted at Boston Medical Center, an ACOS Commission on Cancer accredited center. We included women diagnosed with non-metastatic Estrogen Receptor or Progesterone Receptor positive breast cancer at an inner-city safety net hospital from 2006 to 2008 . Our outcome had three levels: 1) timely hormone therapy (within 365 days of diagnosis) which met compliance with ACOS practice reports, 2) delayed hormone therapy (>365 days) and 3) no hormone therapy. Demographic and clinical data were collected from the institution's cancer registry and a chart review of the electronic medical record was conducted for all noncompliant cases (receipt of hormone therapy $<365$ days of diagnosis) to identify causes of delay. Bivariable analysis was performed to compare socioeconomic variables as well as tumor and treatment characteristics between the compliant and noncompliant cases. Descriptive analysis assessed how patient, provider, and system level variables factored into noncompliance. RESULTS: Among 113 eligible cases, mean age was 57 years; majority were of racial or ethnic minority (56\%), Stage II (53\%), not married (60\%), and had public or no health insurance $(71 \%)$. Among all cases, $74(65 \%)$ were timely or compliant, $37(33 \%)$ were delayed, and $2(2 \%)$ did not receive hormone therapy. Compared to noncompliant cases, compliant cases were more likely to be Hispanic $(41 \%$ v. $59 \%, p=.02)$, and stage III at diagnosis $(40 \%$ v. $60 \%, \mathrm{p}=.001)$, and less likely to be white $(84 \% \mathrm{v}$. $16 \%, \mathrm{p}=.003)$. A more detailed review of the noncompliant cases found: $39 \%$ spoke a primary language other than English and 13\% lived at a primary residence outside of the US. Median days from diagnosis to hormone therapy for the delayed group was 460 days, range 370 to 1208 . Two or more primary surgeries were required in $75 \%$ of these patients. The most common reasons for noncompliance were: active medical comorbidity or treatment complication (49\%); socio-cultural barriers $(33 \%)$, such as patient or family requests for delay in treatment, anxiety/poor understanding of cancer diagnosis, or patient not returning to clinic; completion of herceptin treatment was recommended prior to starting hormone therapy $(21 \%)$; diagnosis occurred at outside hospital prior to entering our system (18\%); financial barriers $(8 \%)$; and transferring care $(8 \%)$. Among the two cases who never received hormonal therapy, one moved back to her home country and the other declined therapy.

CONCLUSIONS: Our results demonstrate that in an underserved diverse breast cancer population, reasons for noncompliance with recommended hormonal therapy are complex and range from complex medical co-morbidity and multi-agent treatment regimens to diverse socio-cultural factors. These findings suggest multiple areas must be targeted in order to reduce disparities in receipt of breast cancer treatment.

REASONS FOR NOT PRESCRIBING GUIDELINE-RECOMMENDED MEDICATIONS TO VETERANS WITH HEART FAILURE Michael A. Steinman $^{1,2}$; Liezel Dimaano ${ }^{2} .{ }^{1} \mathrm{SF}$ VA Medical Center, San Francisco, $\overline{\mathrm{CA} ;{ }^{2} \mathrm{UCSF}}$, San Francisco, CA. (Control ID \#1335207)

BACKGROUND: Performance measures for heart failure implicitly assume that higher rates of guideline-recommended treatment are better. However, little is known about whether patients not receiving guidelineconcordant care have legitimate reasons for foregoing care, or about the distribution of reasons for non-treatment.

METHODS: We conducted comprehensive chart reviews of veterans age 50 years and older in 4 VA health care systems who had systolic heart failure and were not prescribed a beta blocker and/or an ACE inhibitor or angiotensin receptor blocker (ARB). Reasons for non-treatment with these medications were identified from clinic notes based on explicit or strongly implicit statements by the treating clinician and were coded using an established taxonomy. We also interviewed subjects' primary care clinicians about their reasons for non-prescribing to patients identified in the chart review.

RESULTS: Among 2846 patients screened, 301 met inclusion criteria, of whom $70 \%$ were not prescribed an ACE inhibitor or ARB, $18 \%$ were not prescribed a beta blocker, and $11 \%$ were not prescribed both drug classes. Mean age was $75(+/-10)$ years, and $85 \%$ had seen a primary care clinician in the past 6 months. Chart review identified 235 reasons for non-prescribing in 195 patients $(65 \%)$. The most common reason for non-prescribing was clinical contraindications to drug treatment $(58 \%)$. In addition, $18 \%$ of patients had at least one nonbiomedical reason for non-prescribing, including reasons related to patient attitudes, adherence, or drug misuse (10\%) and comanagement with other clinicians $(6 \%)$. We compared these results with interviews of 61 clinicians whose patients were the subject of chart review. The interviews identified nearly twice as many reasons for non-prescribing as the chart (mean 1.6 vs. 0.9 reasons per patient, $\mathrm{P}<.001$ ). While clinical contraindications were often cited $(70 \%$ of patients), one or more other reasons for non-prescribing were described for $66 \%$ of patients. These other reasons included patient attitudes, adherence, or 
misuse; comanagement with other clinicians; and believing that the drug is not indicated $(21-27 \%$ of patients each).

CONCLUSIONS: Two-thirds of veterans with heart failure who do not receive guideline-recommended medications have a reason for nonprescribing documented in the chart, mostly involving clinical contraindications. However, clinicians report that non-biomedical reasons for not prescribing are equally common. Guidelines and performance measures should better account for non-biomedical reasons for not providing guideline-recommended care.

REDUCING ALERT FATIGUE IN ELECTRONIC MEDICAL RECORDS Shobha Phansalkar ${ }^{1,2}$; David W. Bates ${ }^{1,2}$; Douglas S. Bell ${ }^{3}$; Blackford Middleton ${ }^{1,2}$; Amrita Desai ${ }^{1} .{ }^{1}$ Partners Healthcare, Wellesley gateway, MA; ${ }^{2}$ Brigham and Women's Hospital, Boston, MA; ${ }^{3}$ RAND Corporation, Santa monica, CA. (Control ID \#1340482)

BACKGROUND: Alert override rates in electronic health records (EHRs) are between $49-96 \%$. This essentially translates to the fact that despite the benefits of making clinical decision support available in EHRs, most providers end up ignoring these alerts. The Office of the National coordinator (ONC) issued a task order to study how alert fatigue could be reduced in EHRs. To a large extent alert fatigue exists because spurious interactions that do not merit interrupting a provider's workflow are included in medication knowledge bases. In this study, we will describe the findings of an international expert panel to identify such interactions which could be safely suppressed from interruptive alerting.

METHODS: We convened a panel of experts with the goal of identifying those DDIs which were frequently presented in EHRs but were overridden $>96 \%$ of the times. Over 4000 DDI pairs $(n=4077)$ were assessed, these were responsible for a total of 158,794 alerts being fired in the 6-month time period. These interacting pairs were normalized to represent a final list of 1339 drug-class \& class-class DDIs. From the top $0.2 \%$ DDI alerts we discussed the top 50 interactions with the panel. Panelists included medication knowledge base vendors, EHR vendors, in-house knowledge base developers from academic medical centers, and both federal and private agencies involved in the regulation of medication use. The panel evaluated these based on the consequence of the interaction, severity levels assigned to them across various medication knowledge bases, availability of therapeutic alternatives, monitoring/management options, predisposing factors, and the probability of the interaction based on the strength of evidence available in the literature.

RESULTS: Of the 50 interactions that were assessed, the panel voted for 16 interactions to be interruptive in nature while the remaining 24 could safely be used to generate non-interruptive alerts. These 34 class based interactions account for $36 \%$ of the alerts. Thus, suppressing these alerts to be informational rather than interruptive could possibly reduce alert fatigue by about a third.

CONCLUSIONS: We identified a set of clinically insignificant drug interactions that can safely be suppressed from generating interruptive alerts in EHRs and thus make a large difference in the alert fatigue experienced by providers today.

REDUCING THE RATES OF PERSISTENTLY UNCONTROLLED DIABETES IN AMBULATORY PRACTICE THROUGH ACTIVE SURVEILLANCE AND MONTHLY INTERVENTION Muhammad A. Zafar; Jennifer Neville; Sana F. Khan; Sana Waheed; Wajeeha Yousaf; Colin Carracher; Ali Raza; Benni Hensley; Tiffiny Diers; Eric J. Warm. University of Cincinnati, Cincinnati, OH. (Control ID \#1312268)

BACKGROUND: Diabetes and its related complications are an enormous health care burden and its prevalence is expected to double by 2030 Chronic hyperglycemia is the primary risk factor for most of the related complications. The majority of health benefits in terms of cost reduction and prevention of complications can be achieved by targeting the relatively small proportion of patients with uncontrolled diabetes. Objective: To reduce the rate of persistently uncontrolled diabetes at 3 monthly followups in a resident-run primary care clinic.
METHODS: The study was conducted in an internal medicine resident clinic at University of Cincinnati over a period of six months. Intervention group (IG) and Control group (CG) comprised of patients being followed by six and nine residents respectively. Uncontrolled Diabetes (UD) was defined as $\mathrm{HbA} 1 \mathrm{C}>9$. A 3-step-intervention was performed on a monthly basis in the IG comprising of: 1) Active surveillance of Electronic Medical Record by the clinic nurse for patients with $\mathrm{HbA} 1 \mathrm{c}>9$ followed by each resident. ii) PCP-directed individualized intervention for each patient with UD, if needed. iii) Monthly contact with UD patients by at least one clinic member, either PCP, clinic nurse, pharmaco-therapist or diabetic educator. This contact could be in a form of a clinic encounter, a phone call or a letter. Data were collected at baseline, at 3 months and at 6 months follow up.

RESULTS: At baseline, the mean numbers of patients with UD per resident in IG and CG were 10.16 and $10.33(\mathrm{p}=0.93)$ respectively. At 3 months, the percentages of patients with persistent UD in relation to baseline were $44 \%$ in IG and $72 \%$ in CG $\quad(p=0.002)$. At 6 months, the percentages of patients with persistent UD in relation to 3 month follow up were $54 \%$ in IG and $86 \%$ in CG (p=0.002). At 6 months, the mean numbers of patients with UD per resident were 6.33 in IG and 8.88 in CG $(p=0.06)$ showing a reduction in prevalence of $37.7 \%$ in IG and $14 \%$ in $\mathrm{CG}$, compared to baseline.

CONCLUSIONS: Our model of 'active surveillance and monthly contact along with intervention as needed' is highly efficacious in reducing the prevalence and rates of persistently uncontrolled diabetes in the ambulatory setting. This model is easy to adapt and distributes the burden of care over different members of the clinic staff.

REFERRALS FOR OUTPATIENT PHYSICIAN AND SOCIAL SERVICES FOLLOW-UP AMONG PATIENTS DISCHARGED FROM US EMERGENCY DEPARTMENTS AFTER TREATMENT FOR VIOLENT INJURY: A NATIONAL PERSPECTIVE. Paulvalery Roulette $^{1}$; Eric Fleegler ${ }^{3,1}$; Danny McCormick ${ }^{2,1}$. ${ }^{1}$ Harvard Medical School, Boston, MA; ${ }^{2}$ Cambridge Health Alliance, Cambridge, MA; ${ }^{3}$ Children's Hospital, Boston, MA. (Control ID \#1337034)

BACKGROUND: For those who experience violent injury and survive, physical and psychological sequelae are common, including depression, anxiety, PTSD and an increased risk for both experiencing another or committing a violent injury. Many violent injuries are first treated in emergency departments (ED) and studies suggest that referral to a physician or social services from the ED can mitigate these sequelae. However, few data exist regarding national patterns of referral for follow-up care from the ED for such patients, particularly among racial and ethnic groups at high risk for assault.

METHODS: We used data from the National Hospital Ambulatory Medical Care Survey (NHAMCS) from 1999-2008 to study a nationally representative sample of ED visits for patients of all ages to examine ED referrals for follow-up physician care and social services for patients discharged after treatment for violent injury. We excluded patients who were admitted to the hospital, transferred to another medical facility, left against medical advice, were dead on arrival or who died in the ED. We calculated the percentage and annual number of patients discharged with: 1 . referral to a physician; 2 . referral to social services; or 3. had no planned follow-up. We used multivariate logistic regression to identify patient and hospital characteristics associated with receiving referral to social services at ED discharge (compared with no social service referral). In addition, using data from the U.S. Census Bureau from the years 1999-2008 we calculated US population rates of ED visits for violent injury and ED discharge with no follow-up, according to race/ethnicity.

RESULTS: Among the 3,735 ED visits (representing 12.3 million nationally) for violent injury that we studied, at discharge $68.7 \%$ [95\% CI 66.2\%-71.2\%] were referred to a physician $(845,554$ annually), $4.5 \%$ [95\% CI 3.7\%-5.4\%] were referred to social services (55, 902 annually) and 26.7\% [CI 95\% 24.2\%$29.3 \%$ ] were discharged with no follow-up (328,615 annually). Female gender $(\mathrm{OR}=2.5[95 \% \mathrm{CI}, 1.6-3.7]$ Age $65+$ or $<10(\mathrm{OR}=10.2$ [95\% CI, 3.7-28] and $\mathrm{OR}=14.2$ [95\% CI, 6.8-29.9] respectively, compared with age 20-29), drug/ alcohol use $(\mathrm{OR}=3.5$ [95\% CI, 2.0 - 6.1] and rape (compared with unarmed fight) as the type of assault (OR=7.4 [95\% CI, 3.6-15.1]) were positively 
associated with referral to social service in multivariate analyses; race/ ethnicity, co-existing psychiatric diagnosis, insurance type and region of the country were not. ED visit rates (per 10,000 US population) according to race were: 100.6 for African Americans, 40.6 for Hispanics and 33.0 for Whites. US rates (per 10,000 US population) for being discharged from an ED visit for violent injury with no follow-up were nearly 3-fold higher for African Americans (27.6) than for Hispanics (9.6) and Whites (9.4).

CONCLUSIONS: For violently assaulted patients, discharge with social service follow-up is rare and discharge with no follow-up is common in US EDs. While rates of referral from the ED do not vary by race/ethnicity, because African Americans are substantially more likely to be assaulted, high rates of discharge with no planned follow-up disproportionately affects African Americans.

\section{RELATIONSHIP BETWEEN PATIENT EXPERIENCE OF CARE} AND PHYSICIAN PRODUCTIVITY MEASURES Charlotte E. Ward; Jeffrey M. Ashburner; Yuchiao Chang; Steven J. Atlas. Massachusetts General Hospital, Boston, MA. (Control ID \#1338232)

BACKGROUND: Improving patients experience when interacting with the healthcare system has become an important quality of care measure. At the same time, greater emphasis is being placed on increasing primary care physician's (PCPs) productivity and efficiency of care. Previous studies suggest that efforts to improve productivity may adversely affect patient experience of care. Our goal was to examine the relationship between patient experience of care, using the Consumer Assessment of Healthcare Providers and Systems (CAHPS) survey, and measures of provider productivity among PCPs within one, large, academic practice network.

METHODS: We studied 14,857 patients cared for by 156 PCPs in 13 primary care practices within the Massachusetts General Hospital practicebased research network with completed CAHPS surveys $(n=18,424)$ between August 2008 and June 2011. For each survey, we calculated CAHPS composite measures for the access ( 5 items) and communication (6 items) domains by taking the average of each items 'top box' score which was calculated as the proportion of items where "Always" was chosen . For each PCP, productivity measures adjusted for clinical full-time equivalents (FTEs) for fiscal year 2010 included visit-based measures (total work relative value units [WRVUs] and total visits) and patient panel size. To examine the relationship between PCP productivity measures and CAHPS composite measures or individual items, we used linear regression models adjusting for patient characteristics including patient age, gender, insurance status, race, language, education, and Charlson score, accounting for clustering by PCP.

RESULTS: Productivity measures varied considerably among PCPs: median 40.4 WRVUs (interquartile range [IQR] 36.4-45.7), median 31.8 visits per FTE (IQR 27.9-35.8), median 2.3 panel per FTE (IQR 1.7-2.9). Patient characteristics also differed among PCP panels including gender (range 1.3$81.8 \%$ male), ethnicity (range 5.6- 95.5\% non-Hispanic white), mean age (range 38.9-82.7 years), insurance (0-37.9\% Medicaid/uninsured), Charlson score (9.2-73.3\% Charlson score $\geq 2)$, education (9.0-81.8\% college or above), and language (15.6-100\% English speaking). There was no significant relationship between productivity measures and access composite scores or individual items. Similarly, WRVUs and visits per FTE were not associated with the communication composite. However, increasing panel size was associated with better performance on the communication composite (beta 0.023, $\mathrm{p}=0.001$ ). Communication composite scores were higher among PCPs in the top tertile of panel size compared to the bottom tertile (adjusted mean $86.9 \%$ vs. $83.4 \%, p<0.0001$ ). These findings were consistent among items within the communication composite, except for time spent with the patient. For this item, spending more time with the patient was inversely associated with WRVUs and visits per FTE ( $<<0.05$ for each), but not associated with panel size $(\mathrm{p}=0.43)$.

CONCLUSIONS: Among PCPs practicing within the same primary care network, visit-based productivity measures were not associated with patient perceptions about access to care and quality of provider communication. Somewhat unexpectedly, PCP panel size was not associated with access to care, but was associated with better communication. As new payment models emphasize managing populations of patients, more research will be needed to assess how measures of provider panel size impact patient experience of care.
RELATIONSHIP BETWEEN THE PROGNOSTIC EXPECTATIONS OF DIALYSIS PATIENTS AND THEIR NEPHROLOGISTS Melissa W. Wachterman $^{1}$; Edward R. Marcantonio ${ }^{1}$; Roger B. Davis ${ }^{1}$; Robert A. $\overline{\text { Cohen }}^{2}$; Sushrut S. Waikar ${ }^{3}$; Russell Phillips ${ }^{1}$; Ellen P. McCarthy ${ }^{1} .{ }^{1}$ Beth Israel Deaconess Medical Center, Boston, MA; ${ }^{2}$ Beth Israel Deaconess Medical Center, Boston, MA; ${ }^{3}$ Brigham and Women's Hospital, Boston, MA. (Control ID \#1322478)

BACKGROUND: Selected patients undergoing hemodialysis (HD) have an annual mortality rate that exceeds $20 \%$. Perceptions of prognosis and expectations for transplant may influence goals of care. We compared HD patients' and their nephrologists' perceptions of prognosis and transplant candidacy, and explored the relationship between patients' expectations and goals of care.

METHODS: We conducted in-person interviews with seriously ill HD patients from 2 urban $\mathrm{HD}$ units and their nephrologists. We used 2 published prognostic models to identify HD patients with $\geq 20 \%$ probability of dying in the next year. Of the 207 patients treated at these HD units between October 2010 and April 2011, 151 (73\%) had $\geq 20 \%$ mortality risk. We excluded 70 of these patients because of cognitive impairment or limited English proficiency. Of the remaining 81 eligible patients, 63 participated (78\% response rate). All nephrologists $(n=13)$ for these patients were interviewed. We assessed prognostic expectations by asking patients and their nephrologists what they thought the chances were that the patient would be alive in one year $(\geq 90 \%, 61-89 \%, 40-60 \%, 11-39 \%, \leq$ $10 \%$ ). We asked whether they thought kidney transplant was a possibility (yes, no). We then asked patients about their goals of care (prefer a course of care focused on life-extension vs. symptom-directed care). We defined "transplant discordance" as present when patients reported they were transplant candidates but their nephrologist reported they were not. We examined whether patients' and nephrologists' beliefs about prognosis and transplant candidacy differed using McNemar's test for paired data. We then explored the association between goals of care and prognostic expectations and transplant discordance, respectively using Fisher's exact tests.

RESULTS: Of 63 patients interviewed, $57 \%$ were female, $51 \%$ were African American, and the mean age was $68 \pm 10$ years. Patients were significantly more likely than their nephrologists to report a $\geq 90 \%$ chance of being alive at 1 year $(82 \%$ vs. $25 \%, p<0.0001)$. Overall, in $60 \%$ of patient-nephrologist pairs, patients were more optimistic than their physician, whereas physicians were more optimistic in $10 \%$ of pairs. Patients were also significantly more likely than their nephrologists to report they were transplant candidates ( $66 \%$ vs. $46 \%, \mathrm{p}<0.01)$. Of the $82 \%$ of patients reporting $\geq 90 \%$ chance of being alive at 1 year, $49 \%$ preferred care focused on extending life even if it meant more pain and discomfort, compared to $11 \%$ among patients reporting a lower probability of survival $(\mathrm{p}=0.04) .67 \%$ of patients whose beliefs about transplant candidacy were optimistically discordant with their nephrologists wanted care focused on life extension, compared to $32 \%$ of those whose transplant expectations aligned with their nephrologist $(\mathrm{p}=0.03)$

CONCLUSIONS: Patients undergoing HD appear to be more optimistic about their prognosis and transplant candidacy than their nephrologists, and patients' unrealistic expectations for positive outcomes may influence their treatment choices.

RELATIONSHIP OF TIMING AND QUALITY OF DISCHARGE SUMMARIES TO CLINICAL EXPERIENCE Leora I. Horwitz; Christine Chen ${ }^{2}$; Grace Y. Jenq ${ }^{1}$; Sandhya V. Kanade ${ }^{2}$; Katy Araujo ${ }^{1}$; Peter Van Ness' ${ }^{1}$; Boback Ziaeian ${ }^{2}$; John Moriarty ${ }^{1} .{ }^{1}$ Yale University, New Haven, CT; ${ }^{2}$ Yale New Haven Hospital, New Haven, CT. (Control ID $\# 1338260)$

BACKGROUND: Discharge summaries are an essential component of a safe transition from hospital to home, yet timeliness and content are known to be suboptimal. Whether clinical experience affects the quality of the discharge summary is unknown.

METHODS: A prospective cohort of patients admitted to the medical service with pneumonia, heart failure or acute myocardial infarction and discharged home was enrolled. Discharge summaries were reviewed by trained nurse abstractors for timeliness of summary dictation and the presence of individual content, including data related to hospitalization and data related to transitional 
care. Two summary scores were created, one for six content elements recommended by the Joint Commission (reason for hospitalization, significant findings, procedures and treatment provided, patient's discharge condition, patient and family instructions and attending physician's signature), and one for seven elements recommended by a multispecialty group (principal diagnosis and problem list, medication list, transferring physician name and contact information, cognitive status of the patient, test results, and pending test results). Timeliness and content were compared by training level (hospitalist, second or third year house staff, and cardiology advanced practice RNs [APRNs]).

RESULTS: The study cohort included 377 patients. Included patients had a mean age of 77 years; $54 \%$ were male. A total of $195(52 \%)$ had acute coronary syndrome, $146(39 \%)$ had heart failure, and $91(24 \%)$ had pneumonia. Discharge summaries were completed for $376 / 377$ patients. A total of 114 $(30 \%)$ had discharge summaries dictated by hospitalist attendings, $123(33 \%)$ had discharge summaries dictated by house staff, and 140 (37\%) had discharge summaries dictated by APRNs. The median days to dictation was 1 day after discharge (IQR 0-7, range 0-95 days); 255 (67.4\%) were dictated within 48 hours of discharge. Summaries dictated by hospitalists were most likely to be done within 48 hours of discharge $(81.6 \%$ hospitalists, $65.9 \%$ house staff, $57.6 \%$ APRNs, Chi-square test, $2 \mathrm{df}, \mathrm{p}<0.001)$. Diagnosis, hospital course and tests/procedures performed during admission were included in $>97 \%$ of discharge summaries. However, data related to post-discharge care such as social support (39.3\%), functional capacity $(26.3 \%)$, physical exam at discharge $(16.5 \%)$ and whether any test results were pending $(13.0 \%)$ were rarely included. Only $6.1 \%$ of summaries included a call-back number for the inpatient physician; only $4 \%$ of summaries for patients with heart failure included the discharge weight. On average, summaries included 5.6 of the 6 elements required by the Joint Commission and 4.0 of the 7 elements recommended by a multispecialty group. Hospitalists, APRNs and house staff included the same average number of Joint Commission elements (5.6), but hospitalists on average included slightly more multispecialty elements (4.3) than did house staff (4.0) or APRNs (3.8), Kruskal-Wallis test, 2df, $\mathrm{p}<0.001$.

CONCLUSIONS: Discharge summaries lack key information relevant to post-discharge care and are hospitalization-focused rather than transitionfocused. Hospitalists produce more timely summaries that include slightly more key content than house staff or APRNs; however, no group produced consistently timely or high quality summaries. Medical training should specifically include discharge summary skills focused on transition rather than documentation.

RELATIVE INFLUENCE OF PHYSICIANS VERSUS PATIENTS IN MEASURES OF DIABETES CONTROL Calie Santana; Joseph Deluca; Elisabeth Ihler; Marta Rico; William Southern; Yuming Ning. Montefiore Medical Center, Bronx, NY. (Control ID \#1336118)

BACKGROUND: While quality improvement (QI) interventions are often delivered to providers and staff, their evaluation is based on patient-level outcomes. One such intervention, performance reports, often meets resistance from providers who do not consider themselves responsible for the patientlevel outcomes included in the reports. Although patient-level factors are known to account for the majority of differences in outcomes among patients, we sought to perform an analysis to measure the contribution of individual primary care physicians (PCPs) on measures of diabetes control.

METHODS: We used data from a randomized study of resident PCPs at our three teaching clinics in Bronx, NY. The experimental group received quarterly performance reports documenting appropriate testing and level of control for their patients with diabetes. In addition, they completed worksheets by ordering necessary testing and titrating medications as appropriate for the 5 patients with the highest $\mathrm{HbAlc}$. The control group received performance reports only. Our outcomes were $\mathrm{HbAl} \mathrm{c} \leq 8 \%, \mathrm{LDL} \leq 100$, microalbumin $\leq 30$, $\mathrm{BP} \leq 130 / 80$ or $\leq 140 / 90$ for the most recent value in the past 12 months. Our analysis at 6 months showed improved blood pressure control in the experimental arm compared to the control arm but these differences were present at baseline. To measure the relative contribution of individual PCPs, we measured the percent of variance explained by PCP in a hierarchical model that included two levels or sources of variance (patient-level repeated measures and PCP). The model was adjusted for baseline performance, clinic at which each patient received care, the PGY level of the PCP, and patient age, sex, race/ ethnicity and insurance. Our model considered PCP as a random effect since it defines the hierarchical level that we are interested in. The intra-correlation effect of repeated measures of each patient was taken into account using generalized estimating equation (GEE) method in our hierarchical model.

RESULTS: Our study included 2,091 patients of 141 residents. The individual PCP significantly influenced the variance of all 5 outcomes measured. The percent of the variance in the outcomes between arms explained by the PCP was $11.2 \%$ for $\mathrm{HbA} 1 \mathrm{c} \leq 8 \%$ ( $\mathrm{p}=0.002), 15.8 \%$ for $\mathrm{LDL} \leq 100(\mathrm{p}<0.001), 45.2 \%$ for microalbumin $\leq 30(\mathrm{p}<0.001), 10.1 \%$ for $\mathrm{BP} \leq 130 / 80(\mathrm{p}=0.002)$, and $16.4 \%$ for $\mathrm{BP} \leq 140 / 90(\mathrm{p}<0.001)$, with the rest of the variance being explained by patient-level factors. For example, in the $\mathrm{HbAlc}$ outcome, we observed a 13 -fold difference in the odds of meeting the control outcome cutoff $(\leq 8 \%)$ for some resident PCPs versus others.

CONCLUSIONS: In our teaching sites, diabetes control is significantly associated with the individual resident PCP assigned to each patient even after adjustment for site of care, patient characteristics, and PGY year of the PCP. Our study highlights that providers play an important role in disease control for patient with diabetes and that measuring patient-level outcomes is a valid way to measure differences in performance among PCPs even in a medically and socially complex patient population. As health systems transform into PatientCentered Medical Homes and strive to become Accountable Care Organizations, we must identify and foster those panel management practices and processes followed by trainees associated with improved diabetes control, and organize systems of care that support those best practices.

RESIDENT PHYSICIANS IDENTIFY NEED FOR MORE POLICY EDUCATION AND HEALTHCARE IMPROVEMENT Julia Skapik ${ }^{1}$; Elan D. Cohen ${ }^{1}$; Hillary Lum ${ }^{2}$; Peggy Hasley ${ }^{1} .{ }^{1}$ University of Pittsburgh Medical Center, Pittsburgh, PA; ${ }^{2}$ University of Colorado, Denver, CO. (Control ID \#1339161)

BACKGROUND: Virtually no research has assessed both knowledge and attitudes regarding health policy among graduate medical residents, but prior surveys in physicians and students indicate a knowledge gap in health policy. Ongoing health care reform efforts have led to increased interest in and need for education in health policy among physicians.

METHODS: The aim of this study was to assess current knowledge and attitudes among resident physicians. We electronically administered a survey of residents PGY1-3 in varying residency programs at a large university-affiliated medical center and two community hospitals that was designed using current data on healthcare systems, prior questions about health policy attitudes, and general questions about health policy education and experience. Responses were elicited via email link over a period of 4 months using the program SurveyMonkey and participation was rewarded with a small gift card to Starbucks. Descriptive analyses were generated and factor analyses, regresssions, and t-tests were performed using SAS.

RESULTS: All PGY1-3 residents from internal medicine, family practice, obstetrics and gynecology, psychiatry, pediatrics, general and subspecialty surgery, radiology, emergency medicine, pathology, and neurology were invited to participate in a survey on health policy; 273/635 (43.0\%) responded (ages 24-41). On the knowledge section, residents performed poorly, answering only $25.3 \%$ of questions about health systems correctly and were not more likely to perform well even if they indicated experience with or interest in health policy. Most residents reported they were somewhat $(45.2 \%)$ or very $(13.5 \%)$ interested in health policy. Of residents surveyed, $85 \%$ felt that more healthcare leaders should be physicians, and felt that the amount of health policy education they received was very $(43.7 \%)$ or somewhat $(38.1 \%)$ inadequate. Only one-quarter $(27.9 \%)$ somewhat or strongly agreed they had a "basic understanding of the structure and function of the U.S. healthcare system." Interestingly, residents who reported they were planning to enter a primary care specialty (general internal medicine, family medicine, pediatrics) were significantly more likely to report that healthcare was a human right, that they would accept patients with public insurance despite being reimbursed at a lower rate, that mental health should be covered at an equal level to other medical coverage, and that they supported the provisions of the 2010 Affordable Care Act. Just over half (50.4\%) reported they would be hesitant to recommend a career in medicine to their friends or children given the current state of the healthcare system. Only $27.5 \%$ of 
residents described the quality and safety of healthcare in the US as "adequate."

CONCLUSIONS: Overall, residents both anticipated and demonstrated poor knowledge of health policy and healthcare systems. The majority of resident physicians reported a need for more health policy physician-leaders, more health policy education, and improvements in healthcare quality and safety. Residents planning to enter primary care careers were more likely to support the Affordable Care Act, mental health parity, and a universal right to healthcare. Although the response rate and location of this study may limit the generalizability of results, they strongly suggest resident physicians have an interest and would benefit from more health policy education and leadership opportunities.

\section{RESIDENT SATISFACTION AND PREPAREDNESS AT A LEVEL THREE PATIENT-CENTERED MEDICAL HOME Shana Ratner;} Kristen Amann; Paul Chelminski; Brooke B. McGuirt. UNC Hospital, Chapel Hill, NC. (Control ID \#1333749)

BACKGROUND: Prior studies have shown internal medicine residency graduates are dissatisfied with continuity clinics, often citing shortcomings related to under-resourced, disorganized, and inefficient practice environments. However, it is unknown whether the level of the practice performance influences resident satisfaction; that is, whether a highly functioning practice environment leads to improved resident satisfaction with the continuity experience. Our aim was to assess resident satisfaction and preparedness at a level three Patient-Centered Medical Home (PCMH).

METHODS: In December 2011, we asked residents to complete an anonymous clinic satisfaction survey. Residents were asked to rate their satisfaction with elements of continuity clinic using a 5-point Likert scale ranging from 1 (not at all satisfied) to 5 (very satisfied). Additionally, residents rated their preparedness to treat common ambulatory conditions, such as diabetes and headache, on a 5-point Likert scale ranging from 1 (not at all prepared) to 5 (very prepared). Data was analyzed using descriptive statistics as well as a qualitative analysis of free text comments. RESULTS: Survey response rate was 85\% (29/34) with 11 PGY-1, 7 PGY-2, and 11 PGY-3. Of the residents completing the survey, 52\% (15/29) had considered a career in primary care. Overall satisfaction with continuity clinic was $48 \%$. Residents rated satisfaction with three chronic disease management programs highly, with most residents satisfied or highly satisfied with depression $(83 \%)$, diabetes $(93 \%)$, and chronic pain $(72 \%)$. When asked for free text comments regarding what residents liked the most about clinic, $45 \%$ $(9 / 20)$ listed the chronic disease management programs and visit planners. Although patient care in these disease management programs is provided almost exclusively by mid-level providers, residents who responded rated their preparedness to take care of these conditions as high, with most feeling prepared or very prepared to manage depression $(100 \%, 27 / 27)$, diabetes $(100 \%, 27 / 27)$, and chronic pain $(78 \%, 21 / 27)$.

CONCLUSIONS: Residents with continuity clinic at level three PCMH reported high satisfaction with chronic disease management programs specializing in care management and patient self-support, as well as patient tracking and registry functions embedded in our clinic's visit planner. Additionally, residents independently noted PCMH criteria to be the best aspects of their clinic experience. Participating in a comprehensive multidisciplinary disease management program does not detract from resident perceptions of their preparedness to treat these chronic illnesses. Future work should focus on determining if these $\mathrm{PCMH}$ criteria improve resident education and performance.

RESIDENT SATISFACTION WITH CLINIC AND CHOOSING A CAREER IN GENERAL INTERNAL MEDICINE Sean Tackett ${ }^{1}$; Lauren Peccoralo ${ }^{2}$; Lawrence Ward ${ }^{3}$; Ira Helenius ${ }^{4}$; Alex Federman ${ }^{2}$; Colleen Christmas ${ }^{1}$; David C. Thomas ${ }^{2} .{ }^{1}$ Johns Hopkins Bayview Medical Center, Baltimore, MD; ${ }^{2}$ Mount Sinai School of Medicine, New York City, NY; ${ }^{3}$ Temple University School of Medicine, Philadelphia, PA; ${ }^{4}$ University of Virginia School of Medicine, Charlottesville, VA. (Control ID \#1320776)

BACKGROUND: Amid America's primary care crisis, the percentage of internal medicine trainees entering general internal medicine (GIM) has been declining. Many believe that improving resident continuity clinic could increase interest in GIM. This study assessed which elements of resident clinic may influence pursuing employment in GIM.

METHODS: Surveys were administered at the end of the academic year to all 250 internal medicine residents with continuity clinic at 3 programs: Temple University School of Medicine, Mount Sinai School of Medicine, and Johns Hopkins Bayview Medical Center. The survey was a modified and re-validated version of the VA Learners Perception Survey, assessing, across a 4-point Likert scale, satisfaction with 32 clinic elements, grouped into 6 domains: (1) clinical preceptors, (2) educational environment, (3) ancillary staff, (4) time management, (5) clinic records and space, and (6) personal experience. Each respondent was also asked to rate the likelihood that they would "consider a future employment opportunity in GIM" (1) before their continuity clinic experience and (2) as a result of their continuity clinic experience. Bivariate analyses were performed between each clinic element and whether the respondent was likely vs. unlikely to pursue GIM as a result of continuity clinic. A generalized estimating equation was used for multivariate analysis.

RESULTS: 225 (90\%) residents, evenly distributed across training year and gender, completed surveys. $48 \%$ were likely to enter GIM before continuity clinic; $38 \%$ as a result of continuity clinic. On average, $83 \%$ were satisfied or very satisfied with the 32 clinic elements, with highest ratings for the clinical preceptor domain ( $96 \%$ satisfied or very satisfied) and lowest for the time management domain $(66 \%$ satisfied or very satisfied). Bivariate analyses showed significant differences $(p<0.002)$ between those likely vs. unlikely to enter GIM as a result of clinic, in the percentage of those "very satisfied" with 6 of 32 clinical elements: faculty mentorship ( $76 \%$ vs. $53 \%)$, time for patients ( $29 \%$ vs. $11 \%)$, number of patients $(76 \%$ vs. $34 \%)$, personal reward from work $(52 \%$ vs. $23 \%)$, relationship with patients (64\% vs. $42 \%)$, and continuity with patients (57\% vs. $32 \%$ ). Being likely to enter GIM before clinic (OR 29.2, CI $20.8-$ 41.2 ) and being "very satisfied" with the continuity of relationships with patients (OR 3.04, CI 1.79-5.15) were the strongest independent predictors of being likely to enter GIM as a result of clinic in the multivariate analysis.

CONCLUSIONS: Across 3 internal medicine training programs, resident satisfaction with most aspects of continuity clinic was high; yet, continuity clinic appeared to have an overall negative influence on residents' attitudes towards GIM careers. While it is likely that increasing interest in GIM before trainees begin residency would have the greatest impact on increasing the number of trainees who choose GIM careers, there is potential to increase interest in GIM by improving residents' continuity with patients and time management skills in resident continuity clinic.

RESIDENT AND FACULTY PERCEPTION OF PHYSICAL EXAMINATION LEARNING DURING RESIDENCY John Ragsdale; Kevin Kraemer; Melissa McNeil. University of Pittsburgh, Pittsburgh, PA. (Control ID \#1338697)

BACKGROUND: Physical exam (PE) skills are systematically taught in medical school with the expectation that they will continue to improve during residency through routine patient care. The available data indicate that these skills do not continue to improve; residents often perform no better than medical students. Promoting skill development in residency is difficult because not enough is known about how PE is learned in residency. We conducted this study to examine the perceived impact of various barriers, to evaluate whether residents value the skills, and to evaluate the effectiveness of various teaching methods. We surveyed residents and faculty to compare their perceptions.

METHODS: We conducted a cross-sectional survey of internal medicine residents and faculty. The survey was developed through extensive literature review and structured interviews. The survey included three principal domains: (1) barriers to learning PE, (2) self-reported value of PE and perceived value of PE to the other group, and (3) effectiveness of PE teaching methods. The scope of the survey was limited to the inpatient general medicine service. The survey was administered on-line anonymously. We used summary statistics to describe the residents and faculty. Ratings of barriers and teaching methods did not consistently fit a normal distribution so the Wilcoxon Rank Sum test was used for comparisons between the two groups. For assessment of 
value, a Wilcoxon Signed Rank test was used to analyze paired responses within groups.

RESULTS: The survey was sent to 190 residents with 140 responses (74\%) and to 39 faculty with 26 responses (67\%). Residents ranged from PGY-1 to PGY-4 and faculty had been in practice from $<5$ years to $>20$ years. Residents rated competing demands as the barrier which most hindered their PE skill development, whereas faculty rated lack of accountability as the most important barrier. Faculty perceived 3 of the barriers as more significant than the residents did: lack of feedback, lack of accountability, and lack of teaching. When respondents were asked to rate the importance of PE compared to history, labs, and imaging, both groups rated PE high (approximately 5 on a scale of 1-7) but lower than the other 3 factors. There was no significant difference between the groups. For residents, when the ratings of selfimportance were compared to perceived faculty importance, there was no difference for PE. For faculty, when the ratings of self-importance were compared to perceived resident importance, the faculty rated PE as less important to residents than to themselves $(\mathrm{p}=0.007)$. Both residents and faculty rated demonstration of skills by an attending as the most helpful teaching method.

CONCLUSIONS: Faculty appear to believe that certain barriers are more significant than residents do. These data could be used to prioritize system changes and to promote improved communication regarding barriers. Lack of interest in PE by the residents is frequently cited in the literature as a major teaching barrier, an opinion faculty at our institution appear to hold. However, it appears that residents and faculty place similar value on PE. Helping faculty to appreciate that residents value and want to learn PE skills may encourage faculty to teach PE skills. Finally, both groups strongly favor learning PE skills through demonstration by an attending, highlighting the importance of bedside teaching for PE.

RESIDENT PERCEPTIONS OF FACTORS CONTRIBUTING TO ADVERSE EVENTS AND NEAR MISSES Anu R. Lamba; Kathlyn E. Fletcher. Milwaukee VAMC/Medical College of Wisconsin, Milwaukee, WI. (Control ID \#1339508)

BACKGROUND: In the last 10 years, the concepts of patient safety and medical errors have gained attention. Patient safety is becoming a more prominent part of the medicine culture, and both hospitals and residency programs have started to increase patient safety education. Current ways to detect adverse events include chart auditing and automated data mining of laboratory and pharmacy data. These methods are useful; however, they may only be able to capture adverse events and not catch "near misses." In our study, we sought to find out what factors house staff perceive are contributors to near misses and adverse events.

METHODS: A survey was developed by the study team after a review of the literature. It was reviewed by the chief residents and program directors for content and clarity. The survey was anonymous and contained demographic questions and 5 additional questions about adverse events/ near misses and perceived contributors to them. Adverse events, near misses and categories of potential contributors were defined in the survey. The study was approved by our institutional review board. Participants were internal medicine interns and residents at a single Midwestern internal medicine residency program. The survey was distributed at a regularly scheduled educational meeting. Participants were asked to state if they could recall either an adverse event or near miss occurring within the last 12 months. Participants were then asked to state which of the following factors the adverse event or near miss was related to 1) Patient factors, 2) Task and technology factors, 3) Staff/Individual factors, 4) Work environmental factors, 5) Organizational and management factors, 6) Institutional context factors.

RESULTS: Participation rate was $91 \%$. The survey was completed by 30 interns and 39 residents (PGY2 and above). Twenty-nine (42\%) were male. Mean age was 28.6 (SD 2.5). Most (79\%) were able to recall an adverse event within the last 12 months. All but one intern (97\%) could recall an event, while $65 \%$ of residents could recall an event $(\mathrm{p}<0.01$ for the comparison between interns and residents). Participants identified a mean of 1.8 (SD 0.8) contributing factors per event. Staff/individual factors were cited most commonly as contributors to adverse events/near misses, reported by $74 \%$ of those who reported an event. This was noted equally commonly by interns and residents. Task and technology factors were reported by $38 \%$, organization/management factors by $32 \%$, patient factors by $19 \%$, environmental factors by $13 \%$ and institutional context by $6 \%$.

CONCLUSIONS: Our study shows that the majority of interns and residents have experienced an adverse event and/or near miss within the last 12 months. A larger percentage of interns as compared to residents recalled an adverse event or near miss. Staff/individual factors were cited by both interns and residents as the most common type of contributing factor. This suggests that our current culture of medicine still focuses on individual blame rather than systems issues as the source of errors.

RESIDENTS' PERSPECTIVES ON DISCHARGE DIDACTICS Jennifer Carnahan ${ }^{2}$; Kathlyn E. Fletcher ${ }^{1,2}$. ${ }^{1}$ Milwaukee VAMC, Milwaukee, WI; ${ }^{2}$ Medical College of Wisconsin, Milwaukee, WI. (Control ID \#1334955)

BACKGROUND: Patients discharged from the hospital without adequate discharge planning are at risk of serious post-hospitalization complications. Therefore, teaching trainees safe discharge practices is extremely important. Few internal medicine programs have a discharge planning curriculum in place, instead leaving this critical education to the informal curriculum. We conducted the following study to gain a more detailed understanding of internal medicine house staff perspectives on various aspects of the discharge planning process.

METHODS: We conducted an anonymous survey of internal medicine house staff at a Midwestern residency program. The 37-question survey was developed after an extensive literature. We asked about respondent demographics, confidence in performing various aspects of discharge planning, general approach to discharge planning and education received about discharge planning. Preliminary and categorical interns and residents participated. The survey was distributed at a regularly scheduled house staff meeting during a 10-15 minute allotted time period. We report descriptive statistics for the group.

RESULTS: The response rate was $100 \%(n=62)$. Most house staff $(92 \%)$ agreed that they were confident in their discharge plan when discharging patients from the hospital. However, respondents were less confident about specific aspects of the discharge process. On a scale of not at all confident to completely confident, only $40 \%$ were completely confident about ordering dietary restrictions, $34 \%$ were completely confident about fluid restrictions, 24\% about activity restrictions, 53\% about discharge medications and $42 \%$ about educating patients about danger signs requiring return to the hospital. When questioned on their experience with formal education in nine key areas of the discharge process, $63 \%$ of residents reported no formal instruction in any of the nine areas. Nearly all (60/62) respondents reported that the discharge plan was reviewed with them by a resident, fellow or attending at least once during internship, with $98 \%$ reporting that occurring at least half the time. However, $39 \%$ of subjects reported that a resident, fellow or attending physician never reviewed a discharge summary with them. Most $(93 \%)$ of residents said that they believed that the way that primary care physicians learned about their patients' hospitalization was from the discharge summary, yet less than $75 \%$ of residents agreed that primary care physicians would have access to their patients' discharge summaries by the time they see them in clinic post-hospitalization.

CONCLUSIONS: Despite a reportedly high level of confidence in general with discharging patients, when asked about specific aspects of the discharge process many more residents reported perceived inadequacies. Our respondents reported little formal education about discharge planning, and the informal education is inconsistent and non-standardized. In addition to specific content gaps, we also identified a need for education on how to effectively transmit discharge summaries to primary care providers. This is concerning because discharge summaries are one of the mainstays of communication between inpatient and outpatient providers. We believe that these issues are likely to exist at other institutions as well. The next step for this work is to develop a formal curriculum on discharge planning that can be disseminated to other institutions. 
RESIDENTS' EXPERIENCES WITH HOME VISITS: A MIXEDMETHODS STUDY Jared W. Klein; Jill M. Watanabe. University of Washington, Seattle, WA. (Control ID \#1311697)

BACKGROUND: Home visits have long been an enjoyable and meaningful part of many physicians' practices. Unfortunately, most internal medicine residency programs do not have formal home visit curricula. This study explores internal medicine residents' experiences with home visits and how these experiences inform residents' practice of medicine.

METHODS: Participants were internal medicine residents whose continuity clinic was located at a single academic general internal medicine clinic. As part of the curriculum, residents performed home visits to at least one patient on their continuity clinic panel. The week of their scheduled home visit, residents were invited to complete a brief, anonymous online survey. In the spring, after the completion of most home visits, semistructured focus groups lasting 30 minutes were conducted with small groups of residents. Sessions were audio-recorded, transcribed and deidentified. Using the principles of grounded theory, the authors created a coding scheme and independently analyzed the transcripts to identify key themes. Discrepancies were resolved by discussion until consensus was achieved.

RESULTS: Eighty-two percent (28/34) of residents completed an online survey, of whom $79 \%(22 / 28)$ successfully completing a scheduled home visit. $96 \%$ of residents felt home visits were valuable and $78 \%$ would like to perform two or more home visits per year. $76 \%(26 / 34)$ of residents participated in at least one of nine focus groups, providing over four hours of audio recordings. Among many compelling stories, our analysis identified several primary content domains: 1) "There's more than the 20 minutes that you see." Providing residents with context for their patients' lives: Uncovering physical obstacles (small spaces, clutter, mobility issues), revealing lack of resources (poverty, social isolation, absence of family support), allowing for direct observation of health behaviors (medication administration, dietary intake), exposing unique patient attributes (previously unknown interests, backgrounds, idiosyncrasies) and re-conceptualizing the patient as a caregiver. 2) "I just feel that relationship is stronger." Developing the patient-resident relationship: Rapport-building and socializing, permitting time and space for questions, earning patient trust, fostering patient pride by hosting a visit. 3) "I went to go see him before he passed away." Doctoring: Exhibiting empathy, displaying compassion, developing listening skills, enhancing residents' sense of responsibility for their patients, providing a service or convenience for patients. Residents largely felt that home visits enhanced their clinical care by facilitating communication, improving follow-up, reprioritizing goals of care, enlisting new resources (obtaining medisets, placing referrals), intervening early to ameliorate potential problems and raising awareness of the social complexity of many patients' lives. Residents identified several challenges with their participation in home visits including busy schedules, limited experience with the practice and difficulty getting some patients to agree to a visit.

CONCLUSIONS: Residents found home visits to be useful and memorable experiences that offer unique opportunities to learn about their patients' lives and provide the best, most appropriate care. Efforts should be made to encourage internal medicine residents' participation in home visits and reduce barriers to incorporating home visits into the training curriculum.

RESISTANT HYPERTENSION AT US PHYSICIANS' OFFICES: CAN WE DO BETTER? Valy Fontil ${ }^{1}$; Mark J. Pletcher ${ }^{2,3}$; David Guzman $^{1}$; Raman R. Khanna ${ }^{5}$; Ronald G. Victor ${ }^{4}$; Kirsten BibbinsDomingo ${ }^{1}$. ${ }^{1}$ University of California San Francisco, San Francisco, CA; ${ }^{2}$ University of California San Francisco, San Francisco, CA; ${ }^{3}$ University of California San Francisco, San Francisco, CA; ${ }^{4}$ Cedars-Sinai Medical Center, Los Angeles, CA. (Control ID \#1340129)

BACKGROUND: Many patients' blood pressure remains elevated despite being prescribed multiple antihypertensive medications. Guidelines for resistant hypertension include optimizing diuretic therapy by using chlorthalidone and adding an aldosterone antagonist if blood pressure remains elevated. The extent to which U.S physicians follow these guidelines has not been fully examined. This analysis describes the prevalence of resistant hypertension among visits to US physicians' offices and the antihypertensive use recorded during these visits.

METHODS: We analyzed the National Ambulatory Medical Care Survey 2006-2009 and included all visits of non-pregnant adult patients with a history of hypertension, at least one anti-hypertensive medication, and a recorded blood pressure. Resistant hypertension was defined as the concurrent use of four or more antihypertensive medications or an elevated blood pressure despite the use of three or more medications. We calculated the prevalent use of recommended antihypertensive agents in patients taking multiple antihypertensive medications. We also compared visits of patients taking diuretics (loop or thiazide) versus those who were not, based on patient socio -demographic characteristics and type of physician.

RESULTS: From 2006-2009, 13,232 of the office visits to US ambulatory sites were available for this analysis; of these, 9.1 percent met criteria for resistant hypertension. Among visits of patients with resistant hypertension, $80 \%$ reported use of either a thiazide or loop diuretic; this rate dropped to $49 \%$ among those with only 2 antihypertensive medications whose blood pressure was poorly controlled. Only $0.99 \%$ of those with resistant hypertension were taking chlorthalidone, and $8 \%$ were using an aldosterone antagonist. The most frequently used classes of anti-hypertensive medications among patients with resistant hypertension were beta blockers (79\%), calcium channel blockers (64\%), ACE inhibitors (57\%), and thiazide diuretics $(50 \%)$. The use of diuretics was not associated with patient characteristics or type of physician.

CONCLUSIONS: Visits of patients with resistant hypertension account for nine percent of all outpatient visits in the US among hypertensive patients. Increased use of diuretics, particularly chlorthalidone and aldosterone antagonist, is warranted.

RESULTS OF AN ENHANCED CLINIC HANDOFF ON RESIDENT PROFESSIONAL RESPONSIBILITY AND PATIENT SAFETY Marcus Dahlstrom ${ }^{1}$; Megan Prochaska ${ }^{1}$; Kimberly Beiting ${ }^{1}$; Shana Ratner $^{2}$; Julie L. Oyler ${ }^{1}$; Lisa M. Vinci ${ }^{1}$; Vineet Arora ${ }^{1}$; Amber Pincavage ${ }^{1}$. ${ }^{1}$ University of Chicago, Chicago, IL; ${ }^{2}$ University of North Carolina - Chapel Hill, Chapel Hill, NC. (Control ID \#1334592)

BACKGROUND: Year-End Internal Medicine resident clinic handoffs are a vulnerable time when patients may fall through the cracks. Despite this high risk, few interventions to improve this process have been described. Our study aims to evaluate the effectiveness of an enhanced handoff protocol on this transition.

METHODS: Our needs assessment in 2010 demonstrated that although clinic scheduling was effective, patients were lost to follow-up 6 months after the handoff due to missed visits. Patients who missed visits were at higher risk for a poor handoff as they were more likely to see the wrong PCP, have pending studies missed, be lost to follow-up and have an acute visit in the ED or hospital after the handoff. Furthermore, residents reported they did not take responsibility for a patient until they have been seen in the clinic. Using this data, we formalized a 2011 handoff protocol consisting of resident education, greater scheduling coordination, automatic rescheduling for missed visits, and protected time for PGY2s to call new clinic patients to establish care during telephone visits. In both years, graduating residents listed patients they perceived to be at "high risk" during the clinic handoff on a structured template which they used to handoff their patients during a designated meeting. PGY2 residents assuming care were surveyed regarding their beliefs of the clinic handoff process. Signouts and charts were reviewed to determine if and when patients were scheduled, if they saw the correct PCP, and acute care visits (ED visits or hospitalizations). Descriptive statistics, including Fisher exact and chi squared tests, were used for analysis.

RESULTS: In 2011, 27 graduating residents signed-out 323 high risk patients to 27 PGY2s. This was similar in 2010 (30 graduating residents, 258 high risk patients and 20 PGY2s). Most PGY2s completed surveys ( $92.5 \%$ vs. $95 \%$, p $=$ 0.11). Compared with 2010 (baseline), 2011 residents reported longer handoffs ( $>20$ minutes, $52 \%$ vs. $6 \%$, p $<0.001)$, more verbal handoffs ( $80 \%$ vs. $38 \%$, p $=$ 
0.003), seeing more transfer patients 3 months post-handoff ( $>20$ patients, $52 \%$ vs. $5 \%, p=0.001)$, and more patients who were aware of the handoff $(100 \%$ vs. $74 \%, p=0.01)$. Many residents from $2011(76 \%)$ reported using a telephone visit to establish care, and $44 \%$ reported discovering a missed test at that time. Fewer 2011 residents felt uncomfortable with paperwork for new clinic patients not yet seen ( $40 \%$ vs. $74 \%, \mathrm{p}=0.03)$, and reported taking ownership of handoff patients before the first clinic visit ( $56 \%$ vs. $26 \%, p=0.05)$. Nearly all patients [98\% (317/323) vs 97\% (250/258), $\mathrm{p}=0.48]$ were scheduled for a follow-up appointment in both years. However, significantly more patients saw their correct PCP in 2011 ( $82 \%$ vs $44 \%, p<0.001)$ with a trend for patients to be seen in clinic during the month that their physician intended $(40 \%$ vs $33 \%$, $p=$ 0.056). Finally, a trend towards a decreased number of patients with acute care visits (ED and hospital stays) 3 months post-handoff was observed in the 2011 patient panel $(20 \%$ vs $26 \%, \mathrm{p}=0.06)$.

CONCLUSIONS: Our intervention successfully improved the handoff process between residents, and was associated with an increased likelihood that patients saw the correct PCP in a timely manner and a trend towards reduced acute care visits 3 months after the handoff. Internal medicine residency clinics and their patients may benefit from adopting similar interventions targeting this handoff.

RISK ADJUSTMENT AND RISK SELECTION IN MEDICARE ADVANTAGE J. Michael McWilliams. ${ }^{1}$ Harvard Medical School, Boston, MA; ${ }^{2}$ Brigham and Women's Hospital, Boston, MA. (Control ID \#1319873)

BACKGROUND: To address favorable selection in Medicare Advantage (MA), adjustment of plan payments for clinical diagnoses by the Centers for Medicare and Medicaid Services Hierarchical Condition Categories (CMSHCC) model and an enrollment lock-in were implemented from 2004 to 2007. The CMS-HCC model will also serve as the basis for risk adjustment of spending targets for accountable care organizations (ACOs) and plan revenues in state health insurance exchanges. The potential benefits of exchanges and ACOs could be undermined if plans and provider organizations avoid chronically ill and costly patients for whom these reforms were intended to improve coverage and care. The performance of the CMS-HCC risk adjustment system may therefore be an important determinant of success for key programs established by the Affordable Care Act, but its effectiveness in reducing favorable risk selection in MA remains unclear.

METHODS: Using survey and Medicare enrollment data from the nationally representative and longitudinal Medicare Current Beneficiary Survey, we estimated changes from 2001-2003 to 2006-2007 in differences in selfreported medical utilization and health between beneficiaries who switched into or out of MA and other beneficiaries in traditional Medicare (TM) or MA. We also compared differences in utilization and health between all MA and TM beneficiaries over this period.

RESULTS: Compared with TM beneficiaries in 2001-2003, new MA enrollees reported $33 \%$ lower total utilization $(\mathrm{P}=0.002), 45 \%$ fewer hospitalizations $(\mathrm{P}=$ $0.02), 18 \%$ fewer prescription drug fills $(\mathrm{P}=0.02)$, and better health (difference in general health score: $-0.19 ; \mathrm{P}=0.04$ ), consistent with favorable selection into MA. By 2006-2007, however, these differences were significantly narrowed or reversed to $8 \%$ lower $(\mathrm{P}=0.10), 3 \%$ fewer $(\mathrm{P}=0.81), 13 \%$ higher $(\mathrm{P}=0.007)$, and $-0.01(\mathrm{P}=0.82)$, respectively. Differences between new and incumbent MA enrollees were similarly reduced. Annual rates of disenrollment from MA decreased from $10 \%$ in $2001-2003$ to $3 \%$ in 2006-2007. Those disenrolling became significantly more costly and less healthy from 2001-2003 to 2006-2007 relative to other beneficiaries, but comparisons of all MA enrollees to all TM beneficiaries indicated net reductions in favorable selection.

CONCLUSIONS: Adjustment of capitated payments for clinical diagnoses and an enrollment lock-in were associated with reduced selection of less costly and healthier beneficiaries into MA. Similar risk adjustment of prospective or global payments may help mitigate incentives for ACOs and plans competing in health insurance exchanges to select patients with favorable clinical risks.

RISK FACTOR MODEL TO PREDICT A MISSED CLINIC APPOINTMENT IN AN URBAN, ACADEMIC AND UNDERSERVED SETTING. Orlando L. Torres; Michael Rothberg; Owolabi Ogunneye. Baystate Health, Springfield, MA, MA. (Control ID \#1334709)
BACKGROUND: In the chronic care model setting, a missed clinic appointment (or no-show) decreases continuity, adversely affects scheduling efficiency and can harm quality of care. The aim of this study is to identify the predictors of a missed clinic appointment and to develop a model to effectively predict an individual's likelihood of missing an appointment.

METHODS: We performed a retrospective study in an urban, academic and underserved outpatient Internal Medicine clinic from January 2008 to June 2011. All patients scheduled with an attending, resident or advanced practitioners were included. The primary endpoint was whether the patient attended or missed the appointment. A missed appointment was defined as a no-show or cancellation within 24 hours of the appointment time. Each patient was included only once, using the most recent appointment. Patient variables included age, gender, marital status, race, zip code, English language and number of previous visits. The visit variables included provider type, day of the week, time of the day, season and time from booking to actual appointment. The patient population was divided into two randomly selected samples, a test sample $(70 \%)$ and a validation sample (30\%). The test sample was used to generate a logistic model. The validation sample was used to validate the model using the c-statistic and the Hosmer-Lemeshow goodness of fit test.

RESULTS: During the course of 3.5 years, 11,546 patients generated 163,554 encounters. The test population missed an appointment $45 \%$ of the time. In the multivariate model, male gender (OR 1.14, 95\% CI 1.02,1.26), provider type (OR resident vs. attending $1.43,95 \%$ CI 1.26,1.63) and season (OR fall vs. winter $1.25,95 \%$ CI $1.03,1.51$ ) were all positively associated with a missed appointment. The strongest predictors were percentage of previously missed appointments (OR per $1 \%$ increase 1.02 , $95 \%$ CI 1.02,1.02), time from booking to actual appointment (OR per day $1.02,95 \%$ CI 1.02,1.02) and younger age (OR Age Group 55-65 vs. 18-30 $0.45,95 \%$ CI $0.37,0.54$ ). Non-English Proficiency (OR 0.84, 95\% CI 0.74, 0.95 ) and Day of the week (OR Thursday vs. Monday 0.82, 95\% CI $0.70,0.97)$ were all negatively associated with a missed appointment. In the validation set, the multivariable model produced deciles of mean predicted risk from $16 \%$ to $74 \%$, while the mean observed risk over the same deciles ranged from $14 \%$ to $71 \%$ (Figure 1). The predicted and observed rates of a missed appointment were $41 \%$. The model showed no gross lack of fit $(\mathrm{p}=0.63)$, and the $\mathrm{c}$-statistic was 0.71 .

CONCLUSIONS: A simple risk factor model can assist in predicting the likelihood that an individual patient will miss an appointment.

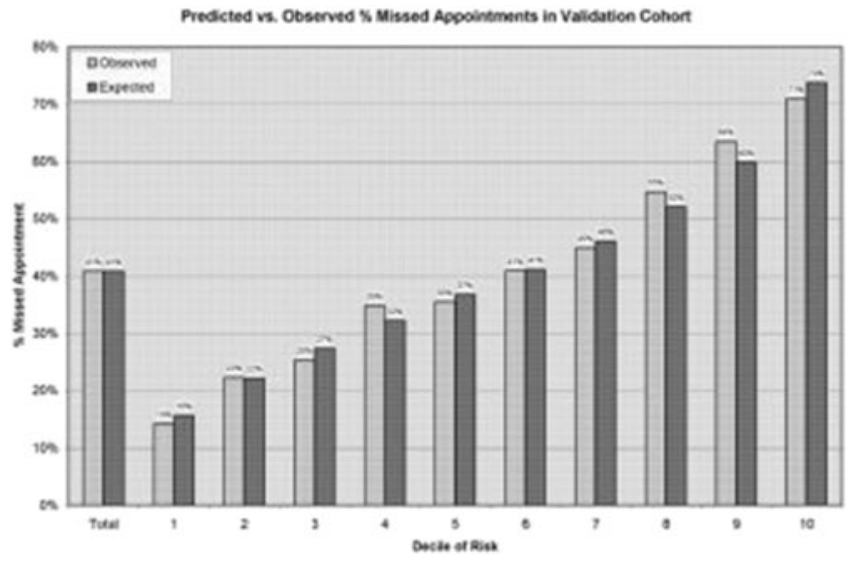

RISK FACTORS OF FALL AMONG COMMUNITY-DWELLING OLDER ADULTS IN TAIWAN Chi Hua Yen. ${ }^{1}$ Chung Shan Medical University Hospital, Taichung, Taiwan; ${ }^{2}$ Chung Shan Medical University, Taichung, Taiwan. (Control ID \#1336621)

BACKGROUND: Falling constitutes a significant hazard to the health and well-being of seniors. Preventing fall in the elderly is one of the important public health issues. The purpose of this research is to identify the risk factors for fall among the elderly population in Taiwan. 
METHODS: Data were drawn from the "Survey of Health and Living Status of the Elderly in Taiwan", a national longitudinal study. A total of 2310 seniors, aged 70 years and older were recruited in 1999. 1645 complete respondents were followed up in 2003, the lost cases were due to death and incomplete data. The independent variables collected in 1999 as risk factors to predict the occurrence of falls within 2003. A fall as dependent variable was defined according to self-report. A Chisquare test was used for univariate analyses first. Secondly, the significant independent variables were entered into the logistic regression analyses by three models including age, gender, education, life style factors and medication use, chronic diseases, nutrition status and general health, physical impairments, psycho-social status, activity and mobility.

RESULTS: The prevalence rate of fall at the 4-year follow-up point was $24 \%(n=395): 11.6 \%$ reported one fall, $12.2 \%(n=395)$ reported two falls or more. By logistic regression analysis, risk factors of fall existed among persons with prior fall history $(\mathrm{OR}=1.7,95 \% \mathrm{CI}=1.3-2.3)$, incontinence $(\mathrm{OR}=1.6,95 \% \mathrm{CI}=1.1-2.4)$, low education level $(\mathrm{OR}=1.5,95 \% \mathrm{CI}=1.1$ $2.0)$ and diabetes history $(\mathrm{OR}=1.4,95 \% \mathrm{CI}=1.0-2.0)$.

CONCLUSIONS: We conclude that prior fall history, incontinence, low education level and diabetes history are independent risk factors for fall. Those who are at high risk of falls should require more detailed assessment and active management to prevent falling.

RISK OF FALLS AND MAJOR BLEEDS IN PATIENTS ON ORAL ANTICOAGULATION THERAPY Jacques Donze ${ }^{1,4}$; Carole Clair ${ }^{2}$; Balthasar L. Hug ${ }^{3}$; Nicolas Rodondi ${ }^{5}$; Gerard Waeber ${ }^{4}$; Jacques Cornuz $^{2}$; Drahomir Aujesky ${ }^{5}$. ${ }^{1}$ Brigham and Women's Hospital, Boston, MA; ${ }^{2}$ University of Lausanne, Lausanne, Switzerland; ${ }^{3}$ University hospital of Basel, Basel, Switzerland; ${ }^{4}$ University hospital of Lausanne, Lausanne, Switzerland; ${ }^{5}$ University hospital of Bern, Bern, Switzerland. (Control ID \#1316112)

BACKGROUND: The risk of falls is the most commonly cited reason for not providing oral anticoagulation (OAC), although data on the risk of bleeding associated with falls on $\mathrm{OAC}$ remain conflicting. We aimed to evaluate whether patients on OAC with high fall risk have an increased risk of major bleeding

METHODS: We prospectively studied all consecutive adult patients who were discharged on OAC between January 1, 2008 and March 31, 2009 from the department of medicine at a Swiss university hospital. The outcome was the time to a first major bleeding within a 12-month follow-up period. Major bleeding was defined as a fatal bleeding, a symptomatic bleeding in a critical organ or a bleeding causing a fall in hemoglobin level $\geq 20 \mathrm{~g} / \mathrm{L}$ or leading to a transfusion $\geq 2$ units of packed red cells. We assessed the risk of falls using two validated screening questions: 1) Did you fall during the last year? If not 2) Did you notice any problem with gait, balance, or mobility? Patients who answered yes to $\geq 1$ screening question were considered at high risk of falls. All other patients were considered to be at low risk. To examine the association between fall risk and major bleeding, we used a Cox proportional hazards model, adjusted for age, gender, alcohol abuse, number of drugs, concomitant treatment with antiplatelet agents, and history of stroke or transient ischemic attack.

RESULTS: Among 515 enrolled patients, 35 had a first major bleeding during follow-up (incidence: 7.5 per 100 patient-years). Overall, 308 patients (59.8\%) were at high risk of falls, and these patients had a non-significantly higher crude incidence of major bleeding than patients at low risk of falls ( 8.0 vs. 6.8 per 100 patientyears, $\mathrm{P}=0.64)$. In multivariate analysis, a high fall risk was not associated with major bleeding (hazard ratio [HR] 1.09, 95\% confidence interval [CI] 0.54-2.21). Only the number of medications (HR 1.15 per additional drug taken, 95\% CI 1.04-1.26) and female gender (HR 2.19, 95\% CI 1.00-4.80) were significantly associated with major bleeding (Table). Overall, only $3 / 35$ major bleedings were directly related to a fall (incidence: 0.6 per 100 patient-years).

CONCLUSIONS: In this prospective cohort, patients on OAC at high risk of falls did not have a significantly increased risk of major bleeds. These findings suggest that being at risk of falls is not a valid reason to avoid OAC in medical patients.

Table. Risk of major bleeding in Cox multivariable-adjusted analyses $(\mathrm{n}=515)$

\begin{tabular}{lll}
\hline \hline Variable & HR & $95 \%$ CI \\
High risk of falls & 1.09 & $(0.54-2.21)$ \\
Number of medication(s), per additional drug taken & 1.15 & $(1.04-1.26) *$ \\
Female & 2.19 & $(1.00-4.80)$
\end{tabular}

$* \mathrm{P}<0.05$

RISK-BENEFIT DISCUSSION PRIOR TO COMPUTED TOMOGRAPHY IMAGING - INFREQUENT AND INEFFECTIVE? Tanner Caverly ${ }^{1,2}$; Daniel Richlie ${ }^{2}$; Allan V. Prochazka ${ }^{2,1}$; Margaret K. Cook-Shimanek $^{3}$; Mary Pawlak ${ }^{3}$; Jennifer Woodward ${ }^{3}$; Chad Stickrath ${ }^{2,1}$. ${ }^{1}$ University of Colorado Denver, Denver, CO; ${ }^{2}$ Denver Veterans Affairs Medical Center, Denver, CO; ${ }^{3}$ University of Colorado Denver, Denver, CO. (Control ID \#1339382)

BACKGROUND: There have been calls for mandatory informed consent prior to ordering computed tomography (CT) imaging given best estimates of the risk of developing cancer after a single scan (about 1 case of cancer out of 300-2000 people scanned). The frequency and quality of shared decision making prior to ordering CT imaging is not well studied. We evaluated the frequency and impact of risk-benefit discussions for patients getting outpatient, non-emergent scans.

METHODS: This was a cross-sectional, single-center survey at the Denver Veteran Affairs Medical Center. Consecutive patients in the outpatient CT scan waiting room were invited to complete a short, selfadministered survey. The survey contained items assessing participant demographics, knowledge of radiation risks, presence of risk-benefit discussion, and preference for more information (Table 1). Using a 0-10 Likert scale $(0=$ no radiation; $10=$ high radiation $)$, participants gave a subjective value to the amount of radiation associated with $1 \mathrm{CT}$ scan, living 1 year in Colorado, chest x-ray, and MRI. Pearson's Chi-squared test of association was used to determine general associations.

RESULTS: Of 286 patients invited, 271 responded representing a $94.8 \%$ response rate. $85.5 \%$ of respondents were over 50 years old, $92.1 \%$ were male, and $26.2 \%$ had a high school education or less. Only $17.4 \%(n=46)$ reported all of the following: having a shared final decision, discussing the risks, and discussing the benefits with their provider. To assess the effect that risk-benefit discussion had on knowledge, we used knowledge that a CT scan has a higher level of radiation than a chest x-ray. Overall, $40.7 \%(n=101)$ of respondents ranked the radiation exposure from a CT scan higher than a chest $\mathrm{x}$-ray. This knowledge was no different between participants based on the presence of a risk-benefit discussion; $38.3 \%$ answering correctly of those who reported having a risk-benefit discussion versus $41.6 \%$ of those not reporting a riskbenefit discussion (p-value 0.617). Likewise, knowledge was no different between participants who reported a shared final decision and those who did not ( $47.1 \%$ versus $36.5 \%$; p-value 0.103$)$. When participants recalled discussing both risks and benefits with their provider they were much less likely to feel they needed more information; $5.7 \%$ feeling the need for more information of those recalling a risk-benefit discussion versus $31.3 \%$ of those who did not recall a risk-benefit discussion ( $\mathrm{p}$-value $<0.00001$ ). Similarly, when patients reported a shared final decision they were less likely to feel they needed more information (15.1\% versus $26.9 \%$; -value 0.028$)$.

CONCLUSIONS: Few patients recall discussing both risks and benefits prior to undergoing CT imaging. Although patients who did report a riskbenefit discussion did not feel they needed more information, these discussions did not seem to improve even basic knowledge of the relative radiation dose. This calls into question the quality of the few discussions 
taking place. Future studies should evaluate which tools and practices can more effectively inform patients.

Risk-benefit discussion and preference for more information

\begin{tabular}{|c|c|}
\hline $\begin{array}{l}\text { Who made the final decision to have a CT scan done? ( } \mathrm{n}= \\
\text { 267) }\end{array}$ & $\begin{array}{l}\text { Frequency } \\
(\%)\end{array}$ \\
\hline The doctor and I made the decision together. & $34.8 \%$ \\
\hline $\begin{array}{l}\text { Did your provider discuss the potential benefits of the test } \\
\text { with you }(n=267) \text { ? YES }\end{array}$ & $67.4 \%$ \\
\hline $\begin{array}{l}\text { Do you feel you need more information about the benefits } \\
(\mathrm{n}=266) \text { ? YES }\end{array}$ & $25.9 \%$ \\
\hline $\begin{array}{l}\text { Did your provider discuss the potential risks of the test } \\
\text { with } \\
\text { you }(n=266) \text { ? YES }\end{array}$ & $35.3 \%$ \\
\hline $\begin{array}{l}\text { Do you feel you need more information about the risks } \\
(\mathrm{n}=264) \text { ? YES }\end{array}$ & $43.6 \%$ \\
\hline
\end{tabular}

ROFLUMILAST TREATMENT WITH CONCOMITANT TIOTROPIUM: EFFECT ON LUNG FUNCTION IN SEVERE COPD PATIENTS Leonardo M. Fabbri ${ }^{1}$; Fernando J. Martinez ${ }^{2}$; Udo-Michael Goehring ${ }^{3}$; Manja Brose ${ }^{3}$; Hassan Lakkis ${ }^{4}$; Paul Rowe ${ }^{4}$. ${ }^{1}$ University of Modena \& Reggio Emilia, Modena, Italy; ${ }^{2}$ University of Michigan Medical Center, Ann Arbor, MI; ${ }^{3}$ Nycomed GmbH: A Takeda Company, Konstanz, Germany; ${ }^{4}$ Forest Research Institute, Jersey City, NJ. (Control ID \#1336196)

BACKGROUND: Chronic obstructive pulmonary disease (COPD) is characterized by chronic respiratory symptoms and airflow limitation; bronchodilators are central to symptom management in COPD. In the pivotal studies, roflumilast, a treatment to reduce COPD exacerbation rates, also significantly improved lung function. In the M2-128 study, roflumilast- and placebo-treated patients received concomitant tiotropium, a bronchodilator, to evaluate the add-on effects of roflumilast on lung function (1). The present analysis examined a subset of severe COPD patients (defined by FEV1 between $30 \%-50 \%$ of a patient's predicted value), a cohort that is likely to be seen by clinicians and that is similar to the approved treatment population for roflumilast.

METHODS: Data were analyzed from this 24-week study examining the effect of roflumilast in moderate to severe COPD patients randomized to roflumilast $500 \mu \mathrm{g} \mathrm{QD}+$ tiotropium $18 \mu \mathrm{g} \mathrm{QD}(\mathrm{ROF}+\mathrm{TIO})$ or placebo+ tiotropium $18 \mu \mathrm{g}$ QD ( $\mathrm{PBO}+\mathrm{TIO})$. Patients were required to have chronic cough or sputum production and frequent short-acting beta-2 agonist use. These prespecified analyses assessed lung function (mean changes from baseline during treatment in pre- and postbronchodilator FEV1) in the overall population (1) and a subset of patients with severe COPD.

RESULTS: A total of 743 patients were randomized to ROF+TIO ( $\mathrm{n}=$ 371) or PBO+TIO $(n=372)$. Of these, 244 patients had severe COPD $(\mathrm{ROF}+\mathrm{TIO}, \mathrm{n}=125 ; \mathrm{PBO}+\mathrm{TIO}, \mathrm{n}=119)$. Demographics were similar between treatment groups in the overall population and severe patient subset. Among the severe COPD subset patients, treatment with ROF+TIO resulted in significant improvements in pre- and postbronchodilator FEV1 at all time points versus $\mathrm{PBO}+\mathrm{TIO}$ (both $\mathrm{P}<0.05$ ). Lung function with ROF+TIO treatment was also improved from baseline during treatment over $\mathrm{PBO}+\mathrm{TIO}$ treatment, with differences of $68 \mathrm{~mL}$ and $73 \mathrm{~mL}$ ( $\mathrm{p}=0.015$ and 0.008 ) for pre- and postbronchodilator FEV1, respectively.

CONCLUSIONS: Bronchodilators are central to COPD management, although a subgroup of patients experience recurrent exacerbations even with bronchodilator treatment. Roflumilast reduces the risk of exacerbations in patients with severe COPD associated with chronic bronchitis and history of exacerbations. Here, effects on lung function with concomitant roflumilast and tiotropium treatment were examined. Among severe COPD patients, roflumilast treatment with concomitant tiotropium significantly improved lung function versus tiotropium use alone. These results suggest that in patients with severe COPD, chronic cough and sputum production, roflumilast in combination with tiotropium may provide further improvements in lung function. 1) Fabbri LM, Calverley PM, Izquierdo-Alonso JL, Bundschuh DS, Brose M, Martinez FJ, Rabe KF; M2-127 and M2-128 study groups. Roflumilast in moderate-to-severe chronic obstructive pulmonary disease treated with longacting bronchodilators: two randomised clinical trials. Lancet. 2009 Aug 29;374(9691):695-703.

ROLE OF SELF-EFFICACY IN THE MANAGEMENT OF TYPE 2 DIABETES MELLITUS - A SYSTEMATIC REVIEW Apoorva K. Chandar $^{1}$; Corinna Falck-Ytter ${ }^{2}$; Adam T. Perzynski ${ }^{2}$; Alida M. Gertz ; Donna Windish ${ }^{5}$; Carl V. Tyler ${ }^{3}$; Paulette A. Sage ${ }^{1}$; Shari Bolen ${ }^{2} .{ }^{1}$ Case Western Reserve University, Cleveland, OH; ${ }^{2}$ MetroHealth Medical Center, Cleveland, OH; ${ }^{3}$ Cleveland Clinic, Cleveland, OH; ${ }^{4}$ The Johns Hopkins Hospital, Baltimore, $\mathrm{OH} ;{ }^{5}$ Yale School of Medicine, New Haven, CT. (Control ID \#1339881)

BACKGROUND: Theories of health behavior change (including The Theory of Reasoned Action and The Transtheoretical Model) posit that a person's self-efficacy has a strong positive influence on health behavior change. Little is known about the extent to which self-efficacy improves across behavior change interventions and the extent to which improvements in self-efficacy are associated with improvements in intermediate diabetes outcomes like $\mathrm{HbAlc}$. Therefore, we conducted a comprehensive review of published literature to assess the role of self-efficacy in selfmanagement interventions of adults with Type 2 Diabetes Mellitus (DM). METHODS: We searched MEDLINE, EMBASE, CINAHL and Cochrane databases from inception for original English Language articles combining the search terms for Type 2 DM, Randomized Controlled Trials, and selfmanagement interventions. Using standardized protocols, reviewers extracted data from each article that assessed the impact of self-efficacy and also measured an intermediate outcome (A1c, SBP, weight, lipids), a long term outcome (i.e. complications or mortality), or a safety outcome. Quantitative and qualitative analysis of the data was conducted to determine the effect of self-efficacy in mediating health behavior change in Type 2 DM patients.

RESULTS: Of the 10,219 citations available, twenty-seven articles discussed self-efficacy out of 100 articles from a larger systematic review evaluating the effectiveness and safety of self management interventions for adults with Type $2 \mathrm{DM}$. We were unable to combine the data in a meta-analysis due to heterogeneity in study populations, interventions, and measurement scales. More than half $(\mathrm{n}=14)$ of the studies were based in the US. The mean sample size was 240 (Range $=57$ to 824 ) with a mean study duration of 10 months. Twenty-two percent of studies discussed self efficacy as a theoretical construct or mentioned it qualitatively but did not report a quantitative outcome. Of the 21 studies that quantitatively measured self-efficacy, most (71\%) used fully validated scales such as the "DMSES" (Diabetes Management Self-Efficacy Scale). Among these, significant improvement in self-efficacy of treatment over control groups was seen in $57 \%(n=12)$. Fifty-six percent of the articles that reported a significant improvement in self-efficacy also reported a significant improvement in HbAlc (5 of 9 studies), while only $1 \%$ of the articles with no significant improvements in self-efficacy showed significant improvements in HbAlc (1 of 7 articles). Two studies reported that selfefficacy levels returned to baseline values or lower despite having shown initial improvement. Too few studies $(n=3)$ reported mediation effects of selfefficacy levels on other positive health behaviors such as self-management, goal setting and problem solving to draw any firm conclusions.

CONCLUSIONS: Improving self-efficacy is associated with improvements in important physiological outcomes such as HbAlc. However, improving self efficacy alone may not be sufficient, and we should continue to investigate other mechanisms to help promote positive health behavior change.

ROUNDING PRACTICES AT AN ACADEMIC MEDICAL CENTER Marwa Shoeb; Raman R. Khanna; Daniel Westerdahl; Bradley Monash. UCSF, San Francisco, CA. (Control ID \#1339310)

BACKGROUND: Patient centered rounds (PCR), where the presentation of the history and physical and the discussion of the care plan occur in the presence of the patient, have historically served as the primary means for inpatient attending physicians to teach trainees, to learn about their patients, and to model humanistic behavior. With changing resident work hour regulations, increased reliance on information technology, and the 
advent of hospital medicine, rounds have shifted away from this patient centered, bedside model. Our study aimed to determine current and optimal rounding practices at our institution.

METHODS: We conducted a survey of inpatient attending physicians at a large tertiary care urban academic medical center. Surveys were distributed through our teaching attending listserv. We outlined three models for attending rounds based on our discussion with several inpatient clinician educators and associate program directors: 1) patient-centered rounds (PCR), defined above; 2) hallway rounds (HR), where the discussion of the patient and care plan occurs partially outside the patient's room and partially at the patient's bedside in the presence of the patient and team; and 3) card-flipping rounds (CFR), where the discussion of the patient and care plan occurs entirely outside of the patient's room and the team does not see the patient together. We ascertained demographic information and the frequency with which different models are used. We also assessed the perceived value of each model for promoting ACGME core competencies, the primary goals of rounding, and the perceived barriers to conducting PCR

RESULTS: Of the 39 attending physicians who responded, 25 completed the survey (64\%). 52\% were women and $54 \%$ had at least 3 years of experience attending on inpatient teaching services. HR proved to be the model used most frequently for new and established patients (56\% and $45 \%$ respectively), followed by CFR ( $38 \%$ and $45 \%$ ). Of the attending physicians surveyed, most agreed or strongly agreed that compared with CFR, PCR and HR promoted the following ACGME competencies: patient care $(32 \%, 76 \%$ and $96 \%$ respectively, $\mathrm{p}<0.001)$, systems-based practice $(60 \%, 60 \%$ and $76 \%, \mathrm{p}=$ $0.34)$, professionalism $(24 \%, 84 \%$ and $80 \%, \mathrm{p}=0.02)$, and interpersonal and communication skills $(16 \%, 96 \%$ and $92 \%, \mathrm{p}=0.04)$. As opposed to CFR, PCR and HR also scored well in achieving the following goals of rounds: role modeling $(17 \%, 88 \%$ and $79 \%, p<0.001)$, patient understanding of care plan $(4 \%, 68 \%$ and $84 \%, \mathrm{p}=0.003)$, and patient involvement in care $(4 \%, 88 \%$ and $72 \%, p=0.001)$. CFR functioned well in promoting medical knowledge and team communication, compared with PCR and HR $(88 \%, 44 \%$ and $84 \%$, p= $0.007)$. Despite its perceived virtues, $68 \%$ of surveyed attending physicians said they never used PCR. Time constraints and patient psychosocial complexities were frequently cited as barriers to performing PCR $(84 \%$ and $52 \%)$

CONCLUSIONS: PCR and HR are perceived to be equally valuable for promoting the ACGME competencies of patient care, systems-based practice, professionalism, and communication and interpersonal skills, but PCR is considered superior for role modeling and HR for team communication. Approximately two thirds of inpatient attending physicians never use PCR, with time constraints and patient psychosocial complexities most frequently perceived as significant barriers. Despite being considered mostly inferior to PCR or HR, CFR remains frequently employed.

\section{SMAD4 MUTATION INDICATIVE OF JUVENILE POLYPOSIS IN A LARGE FAMILY INITIALLY DIAGNOSED WITH MENETRIER DISEASE Steven H. Yale ${ }^{1,3}$; Deanna S. Cross $^{2}$; James K. Burmester ${ }^{2}$. ${ }^{1}$ Marshfield Clinic, Marshfield, WI; ${ }^{2}$ Marshfield Clinic Research Foundation, Marshfield, WI; ${ }^{3}$ Marshfield Clinic Research Foundation, Marshfield, WI. (Control ID \#1337741)}

BACKGROUND: Menetrier disease (MD) is a rare disease characterized by hypertrophic folds within the fundus and body of the stomach with antral sparing accompanied by increased proliferation of mucous cells within the gastric glands. Clinical presentation includes hypochlorhydria, hypoproteinemia and enhanced mucous production. Current treatment involves blocking epidermal growth factor receptor (EGFR) with neutralizing antibodies or surgical gastrectomy. Understanding the role of genes which regulate TGF $\alpha$, EGFR, PdX1, SMAD4 and BMPR1A in Menetrier disease is critical toward gaining insight into disease pathogenesis, which may translate into more efficient, safe, and cost-effective treatments. The goal of this study is to sequence regulatory regions of the genes encoding TGF $\alpha$ and PdX1 known to be overexpressed in a family of 50 individuals, some of whom have Menetrier disease, in order to determine if overexpression is caused by a mutation in the promoter region. We sequenced SMAD4 and BMPR1A in an attempt to determine if genes involved in Juvenile Polyposis Syndrome, a condition closely related to Menetrier disease, are also involved in this disease.
METHODS: A family of more than 100 individuals across 4 generations was identified. This family had 5 cases of MD, 1 case of MD and juvenile polyposis (JP) and 2 cases of JP. Subjects provided blood for DNA extraction and completed a questionnaire regarding gastric polyps, gastritis, gastric cancer, gastroesophogeal reflux, gastrointestinal bleeding, helicobacter pylori infection, hemorrhagic telangiectasia, epistasis, juvenile polyposis, diabetes and other diseases that may be involved in the development of MD. After diagraming the family pedigree, selected affected and non-affected family members were DNA sequenced at the coding regions of the SMAD4 and BMPR1A genes and the regulatory regions of TGF- $\alpha$ and Pdx 1 genes.

RESULTS: No mutations were identified in the sequenced regions of BMPR1A, TGF- $\alpha$ or Pdx1. A dominant 1244_1247delACAG mutation of SMAD4 was identified in each of the subjects with JP as well as each of the subjects with MD. Although this mutation segregated through the family in association with disease there were also unaffected/undiagnosed carriers of the mutation.

CONCLUSIONS: The 1244 1247delACAG mutation of SMAD4 is the cause of JP and the likely cause for MD in a large family initially diagnosed with MD. This study provided the first evidence of a genetic link shared by JP and MD. Gene polymorphisms identified as associated with this disease will thus serve as markers for determining risk of acquiring Menetrier disease in unaffected family members. All family members were advised to meet with their physician and discuss genetic testing for this mutation since there are specific screening recommendation for JP and hereditary hemorrhagic telangiectasia for carriers of the SMAD4 mutation.

SUBSTITUTION TREATMENT FOR ALL DEPENDENT OPIOID USERS IS POSSIBLE IN JAIL: A CASE STUDY OF SWITZERLAND Hans Wolff; Thierry Favrod-Coune; Mariem Baroudi; Jean-Pierre Rieder; Laurent Gétaz; Javier Barro; Jean-Michel T. Gaspoz; Barbara Broers. Geneva University Hospitals and University of Geneva, Geneva, Switzerland. (Control ID \#1321592)

BACKGROUND: Detainees are a vulnerable population with limited access to healthcare previous to incarceration due to educational, social and economic disadvantage. They have a high burden of disease, particularly blood borne infections and addiction. The prevalence of illicit drug use in correctional facilities is estimated 50 to $80 \%$ in most European countries and the US, mainly due to cannabis, cocaine and heroin. Opioid substitution treatment (OST) prevents transmission of blood borne infections and overdose. In contradiction with international rules and recommendations OST is not available in every prison. In Switzerland largest pre-trial prison OST is provided by trained primary care physicians and available since 20 years for all opioid dependent prisoners. The aim of this study was to provide a detailed description of OST in Switzerland's largest remand prison, to establish if it respects the international recommendations and if OST is feasible in a pre-trial prison setting.

METHODS: This retrospective longitudinal study included all standardized health records of opioid using detainees entering Switzerland's largest remand prison during 2007. OST dependence was determined by history of current opioid use with 1 . a positive urine test, 2. participation in an OST program (written confirmation by direct contact with the treating physician) or 3. objective opioid withdrawal signs. Detailed information was reported concerning: sociodemographic characteristics, diagnosis and characteristics of substance use, type of opioid substitution treatment, complications during OST or death. Analyzes were descriptive.

RESULTS: A total of 2566 health records were reviewed. Mean age was 29.6 years (SD 7.1); $95.4 \%$ were male and $92.8 \%$ were of foreign origin. Main regions of origin were: Western Europe (28.9\%), followed by North Africa, Middle East (27.5\%) and Sub-Saharan Africa (20.1\%). Length of stay was short, as $27 \%$ stayed less than one week and $78 \%$ less than three months. Two hundred forty-one $(9.4 \%)$ used opioids. Of them, $68.9 \%$ were opioid dependent and $31.1 \%$ had occasional use. History of intravenous use was identified in $40.4 \%$ among dependent users. Other routes of use were intranasal (61\%), smoked (34\%) and unknown for 4.6\%. OST was proposed to all dependent users. A quarter $(27.1 \%)$ had established OST in the community and received OST. The majority $(72.3 \%)$ of all entering opioid users had no current OST. They received a first dose of methadone of max. $40 \mathrm{mg}$ with progressive adaptation according to withdrawal signs; no refusal was observed. The prescribed OST was methadone in $95.8 \%$ with a mean dose of $41.7 \mathrm{mg}$ (SD 29.1). No death by overdose or serious side effect were 
observed. Follow-up was organized for $81.2 \%$ of dependent and for $18.2 \%$ of occasional opioid users.

CONCLUSIONS: Prescription of OST for all opioid dependent detainees, by trained physicians, is feasible in a pre-trial setting. Treatment was overall in accordance with international guidelines, although methadone dose was low. Better implementation of OST is needed in all correctional facilities -worldwide- and helps to improve access to community OST facilities after detention.

\section{SAFETY OF ONCE-DAILY GABAPENTIN FOR THE TREATMENT} OF POSTHERPETIC NEURALGIA IN PATIENTS WITH A CREATININE CLEARANCE $<\mathbf{8 0}$ ML/MIN John T. Mathis ${ }^{1}$; Daniel Kantor $^{2}$; Richard L. Rauck ${ }^{3}$; Gordon Irving ${ }^{4}$; Michael Sweeney ${ }^{\text {; }}$ Geertrui F. Vanhove ${ }^{1} .{ }^{1}$ Depomed Inc, Menlo Park, CA; ${ }^{2}$ Neurologique Foundation, Ponte Vedra, FL; ${ }^{3}$ Carolinas Pain Institute, Winston-Salem, NC; ${ }^{4}$ Swedish Pain Center, Seattle, WA. (Control ID \#1331882)

BACKGROUND: Gabapentin is a first-line treatment for postherpetic neuralgia $(\mathrm{PHN})$. However, due to gabapentin's short elimination half-life and its absorption by a saturable transporter with limited distribution in the proximal small bowel, gabapentin needs to be dosed TID. Gabapentin TID is associated with a high incidence of somnolence and dizziness that may prevent efficacious dosages from being reached. Recently, a once-daily gastroretentive formulation of gabapentin (G-QD) was approved for the treatment of PHN. Upon contact with gastric fluid, G-QD tablets swell to a size that promotes retention in the fed stomach. This prolonged retention (approximately 8 to 10 hours) allows gabapentin to be gradually released to the site of absorption, minimizing the chance of saturating transporter uptake and resulting in greater absorption. This permits once-daily dosing and may result in less somnolence and dizziness, G-QD is renally excreted; dosing adjustment is required in patients with a creatinine clearance $(\mathrm{CrCL})$ between 30 and $60 \mathrm{~mL} / \mathrm{min}$. G-QD should not be administered in patients with a $\mathrm{CrCL}<30 \mathrm{~mL} / \mathrm{min}$ or in patients undergoing hemodialysis.

METHODS: An 11-week, Phase 3, double-blind, randomized, placebocontrolled study evaluated the safety and efficacy of G-QD (1800 mg, qd) in patients with PHN. The primary endpoint was the change in "average pain for the past 24 hours" (ADP) as assessed by the Numeric Pain Rating Scale (NPRS), with scores recorded every morning from Baseline to Week 10 . Safety was evaluated by periodic assessments of adverse events (AEs), baseline and end of study vital signs, and routine hematology and blood chemistry. Patients with an estimated $\mathrm{CrCL}<50 \mathrm{~mL} / \mathrm{min}$ as calculated by the Cockcroft and Gault equation, were excluded from the study. AE subgroup analyses were performed on patients with an estimated CrCL $<80 \mathrm{~mL} / \mathrm{min}$.

RESULTS: 452 patients (mean age, 65.6 years) were randomized; 377 patients completed the study (84\%, G-QD; $83 \%$, placebo). G-QD was generally well tolerated and AEs were reported by $118(53 \%)$ patients in the G-QD arm and 92 (40\%) patients in the placebo arm; dizziness (11\% G-QD; $2 \%$, placebo), somnolence ( $5 \%$, G-QD; $3 \%$, placebo), and peripheral edema ( $3 \%$ G-QD; $<1 \%$, placebo) were some of the more common AEs, with $9 \%$ of G-QD patients and $4 \%$ of placebo patients withdrawing due to AEs. Of the patients randomized, 205 patients (100, G-QD; 105, placebo) had an estimated $\mathrm{CrCL}$ of $<80 \mathrm{ml} / \mathrm{min}$. In this subgroup, a total of $56(56 \%) \mathrm{G}-\mathrm{QD}$ patients and $46(44 \%)$ placebo patients reported at least one AE. The incidence of dizziness $(13 \%)$, somnolence $(4 \%)$ and peripheral edema $(2 \%)$ in patients treated with G-QD in this subpopulation were comparable to the overall safety population. CONCLUSIONS: G-QD was generally safe and well tolerated and had a similar AE profile in the subgroup with a $\mathrm{CrCL}<80 \mathrm{~mL} / \mathrm{min}$ compared to the overall safety population. G-QD can be an effective, well-tolerated treatment option for $\mathrm{PHN}$ even in patients with a $\mathrm{CrCL}<80 \mathrm{~mL} / \mathrm{min}$.

SCHWARTZ CENTER CONNECTIONS: QUALITY WITH COMPASSION James M. Richter ${ }^{2}$; Beth Lown ${ }^{1}$; Karen Gareis ${ }^{3}$; William Kormos $^{2}$; Gila Kriegel ${ }^{4}$; Daniel A. Leffler ${ }^{4}$; Colleen Manning ${ }^{3}$; Gregg Meyer $^{2}$; Ann Louise Puopolo ${ }^{5}$; Kenneth Sands ${ }^{4}$; Amy Ship ${ }^{4}$; Eric M. Weil ${ }^{2}$. ${ }^{1}$ Schwartz Center for Compassionate Healthcare, Boston, MA; ${ }^{2}$ Massachusetts General Hospital, Boston, MA; ${ }^{3}$ Goodman Research Group, Inc., Cambridge, MA; ${ }^{4}$ Beth Israel Deaconess Medical Center, Boston, MA; ${ }^{5}$ CRICO/RMF, Boston, MA. (Control ID \#1319446)
BACKGROUND: Effective communication between clinicians, support staff, and patients is essential for efficient diagnosis and follow-up. Communication lapses compromise clinical care quality and patient safety resulting in poor health outcomes and increased malpractice risk. Communication between disciplines can be especially challenging given their "siloed" departmental structures. We implemented Schwartz Center Connections to discuss causes of communication lapses in outpatient settings and to build relationships across disciplines.

METHODS: We invited primary care and gastroenterology clinicians and support staff at two academic medical centers to attend five pilot program sessions conducted over nine months. Sessions were facilitated, case-based discussions of communication lapses involving patients shared by both disciplines, derived from closed malpractice claims. We used a pre-post program-comparison group design to evaluate the program. The evaluation sample included 112 clinicians; 56 attended two or more sessions and 56 non-attendees comprised the comparison group. Data collection included online surveys and individual and focus group interviews with participants and stakeholders. ANOVAs were conducted to examine the relationship between program participation and changes in the intended outcomes.

RESULTS: Ten survey items with high internal consistency were included in a composite "Communication Lapse Prevention Scale." Scale items included knowledge about systems that support information sharing, roles of team members within and across departments to ensure communication about diagnostic findings, strategies to clarify responsibility for communicating about abnormal findings and for patient follow-up, and for discussing bad news and errors with patients, strategies to help patients assume responsibility for communicating and following up with providers, and for resolving differences in judgment among providers. The effect size of the program on this scale was large $\left(d^{\prime}=0.79\right)$. The program had a significant impact $(p<0.01)$ on the knowledge of communication strategies and self-reported communication behaviors for the treatment group at both pilot sites relative to the comparison group. At program conclusion, $91 \%$ of attendees reported increased likelihood of recognizing communication lapses; 93\% reported increased likelihood of intervening to avert lapses. In addition, participants affirmed that the program fostered relationships and promoted communication across participating disciplines and energized existing quality initiatives at both hospitals.

CONCLUSIONS: The Schwartz Center Connections program resulted in significant changes in knowledge about communication lapses, and in reported behaviors that prevent lapses which contribute to delay in diagnosis and inadequate follow-up of screening and diagnostic tests. Combining discussion sessions with working group sessions to implement suggestions for policy and/or system changes may enhance the effect of the program on patient care. The program's curriculum design, educational methods and assessment processes can be easily adapted for use across other disciplines and settings within institutions, and across health care systems. Adoption of this program may facilitate improvement in interprovider and patient-provider communication necessary to reduce the occurrence of adverse events, poor patient health outcomes, and subsequent malpractice claims.

SECONDHAND SMOKE EXPOSURE AMONG YOUNG ADULTS ATTENDING BARS Sara Kalkhoran; Pamela M. Ling. University of California, San Francisco, San Francisco, CA. (Control ID \#1337813)

BACKGROUND: Both active tobacco use and exposure to secondhand smoke (SHS) have serious health consequences. Young adults are increasingly at risk for smoking initiation, and cessation before age 30 eliminates most negative health effects of smoking. Young adults have high rates of SHS exposure, but little is known about how this relates to smoking behavior. Since bar attendance is associated with smoking, we aimed to describe sources of SHS exposure among young adults attending bars and clubs and to assess associations between SHS exposure and smoking cessation and initiation.

METHODS: Randomized time-location samples of bar-going young adults aged $18-26$ in San Diego, CA $(\mathrm{N}=1305)$ and Oklahoma City $(\mathrm{OKC})(\mathrm{N}=1264)$ and Tulsa $(\mathrm{N}=1250)$, OK completed cross-sectional surveys from Sept 2010 to July 2011. Respondents reported exposure to 
SHS in the past seven days in various locations (work, home, car, bar). Multivariate logistic regression was used to evaluate associations between SHS exposure and intention to quit in current smokers, and between SHS exposure and susceptibility to start smoking in nonsmokers, controlling for age, race, gender, and education.

RESULTS: Over $90 \%$ of respondents at both the California and Oklahoma study sites reported any exposure to SHS, and over $70 \%$ reported exposure in a bar (figure 1); the lowest exposure rates were in indoor workplaces. Respondents in San Diego reported SHS exposure at all locations significantly less frequently. Exposure to SHS at home was negatively associated with attempts to quit in daily smokers $(\mathrm{OR}=0.7095 \%$ CI $[0.51,0.96])$. Among nondaily smokers, exposure to SHS in a car was positively associated with attempts to quit $(\mathrm{OR}=1.48[1.16,1.90])$. Among nonsmokers, any SHS exposure was associated with susceptibility to start smoking $(\mathrm{OR}=1.66[1.12,2.47])$, as was reporting SHS exposure in a car $(\mathrm{OR}=1.75[1.39,2.21])$.

CONCLUSIONS: Young adults attending bars continue to report very high SHS exposure rates, particularly in bars. Though rates were significantly lower in California than Oklahoma, overall rates were higher than reported in statewide data $(80 \%$ of young adults were exposed in CA in 2008). Daily smokers without smokefree homes were less likely to have tried to quit smoking, and nonsmokers exposed to any SHS were more susceptible to start smoking. Promoting smokefree environments may enhance cessation in established smokers or prevent initiation in nonsmoking young adults. Intense SHS exposure (such as in a car) may motivate quit attempts in nondaily smokers, perhaps because they are more bothered by this exposure. Clinicians seeing young adult patients should screen for and counsel against SHS exposure, and encourage them to make their homes and cars smokefree.

\section{Secondhand smoke exposure}

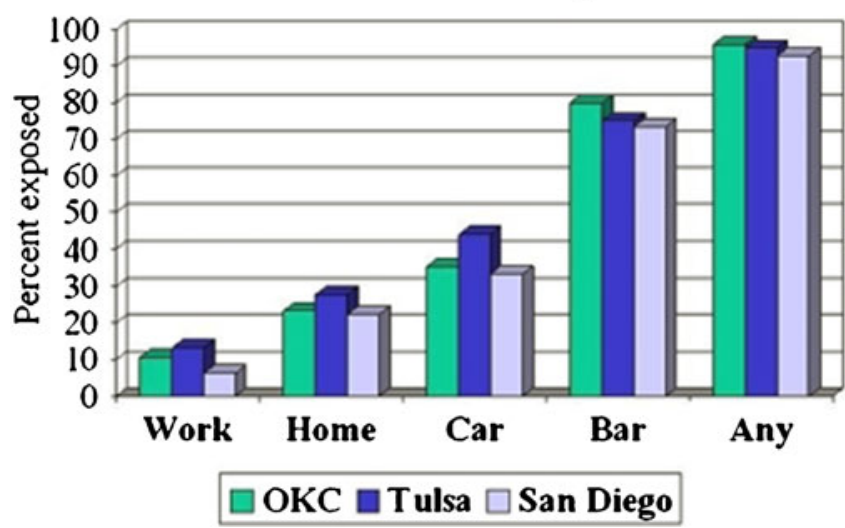

SELF-ADMINISTERED SUBSTANCE USE SCREENING AND ASSESSMENT IN PRIMARY CARE SETTINGS: TEST-RETEST RELIABILITY OF AN AUDIO COMPUTERIZED-ASSISTED SELFINTERVIEW (ACASI) VERSION OF THE ASSIST Jennifer McNeely ${ }^{1}$; Shiela Strauss ${ }^{2}$; Rubina Khan ${ }^{1}$; Shana Wright ${ }^{1,3}$; John Rotrosen $^{3}$; Marc N. Gourevitch ${ }^{1} .{ }^{1}$ NYU School of Medicine, New York, NY; ${ }^{2}$ NYU College of Nursing, New York, NY; ${ }^{3}$ NYU School of Medicine, New York, NY. (Control ID \#1319841)

BACKGROUND: To maximize the use of limited clinical time, medical providers are increasingly turning to patient self-administered health assessments. This approach is particularly relevant for behavioral health conditions such as substance use, which have high prevalence but frequently go undetected, and often require detailed assessments to guide clinical interventions. The WHO Alcohol and Substance Involvement Screening Test (ASSIST) is a comprehensive substance use screening and assessment instrument that has been validated in interview format, but not as a self-administered measure.
We therefore studied the test-retest reliability of an audio guided computer-assisted self-interview (ACASI) version of the ASSIST in identifying unhealthy use of tobacco, alcohol and illicit drugs, as a first step in establishing its feasibility in primary care settings.

METHODS: We adapted the ASSIST to ACASI format and administered it on touch-screen tablet computers. Patients were approached consecutively in the waiting area of a large urban safety-net primary care clinic and screened for eligibility. Basic eligibility criteria were: current clinic patient, English or Spanish speaking, age 18-65. We oversampled patients anticipated to have greater difficulty using the ACASI instrument (less than high school education, 50-65 years old, primary language Spanish). Participants completed the ACASI ASSIST at the initial visit, and were asked to return 1-3 weeks later to repeat it. Agreement between ASSIST results at the first and second administration was evaluated with intraclass correlation coefficients (ICC) and McNemar's tests.

RESULTS: The 87 participants were $55 \%$ female, with mean age 44 years (range 19-63 years, $\mathrm{SD}=9$ ). The majority $(59 \%)$ was foreign born, and 63\% were Hispanic, 30\% African American. 44 (51\%) had less than high school education/GED. 46 participants completed the ASSIST in English, 41 in Spanish. Mean time to complete the ACASI ASSIST on the first administration was 5.4 minutes (range 1.4$14.8 \mathrm{~min}, \mathrm{SD}=2.4)$. $22(25 \%)$ participants screened positive for current (past 3 months) use of tobacco, 48 (55\%) for alcohol, and $27(30 \%)$ for illicit drugs, including nonmedical use of prescription opioids or stimulants. Based on ASSIST scores, $39(45 \%)$ had moderate or high risk use of alcohol and/or illicit drugs (4 alcohol, 23 illicit drugs, 12 both). The $48(55 \%)$ participants who returned for the second visit had similar demographics, but slightly higher levels of illicit drug use, than those who did not. We found excellent correlation between global ACASI ASSIST scores on the first and second administration $(\mathrm{ICC}=0.77, \mathrm{P}<0.001)$. Substance specific risk scores, which distinguish between low, moderate and high risk use, had excellent correlation for tobacco and alcohol (tobacco ICC $=0.80$ and alcohol ICC $=0.83, \mathrm{P}<0.001$ ) and good correlation for illicit drugs $(\mathrm{ICC}=0.64, \mathrm{P}<0.001)$. There were no significant differences between test administrations in detecting moderate to high risk use, based on McNemar's tests, for tobacco $(\mathrm{P}=0.63)$, alcohol $(\mathrm{P}=$ $1.00)$, or for any illicit drug $(\mathrm{P}=0.22)$.

CONCLUSIONS: The self-administered ACASI ASSIST detected high levels of substance use among primary care patients, and had good testretest reliability. Further research will evaluate the validity of ACASI ASSIST responses, as well as its feasibility in primary care practice and acceptability to patients.

SELF-PERCEPTION OF CARDIOVASCULAR RISK AMONG NICOTINE-DEPENDENT SMOKERS Benoit Desgraz ${ }^{1}$; Tinh-Hai Collet $^{1}$; Nicolas Rodondi ${ }^{2,1}$; Jacques Cornuz ${ }^{1}$. ${ }^{1}$ University of Lausanne, Lausanne, Switzerland; ${ }^{2}$ University Hospital of Bern, Bern, Switzerland. (Control ID \#1334352)

BACKGROUND: Previous studies suggest that smokers have a misperception of their 10-year cardiovascular risk. We aimed to compare 10-year cardiovascular risk self-perception among smokers with 10-year cardiovascular risk calculated by Framingham score, and the determinants of this possible misperception.

METHODS: We collected data on cardiovascular risk factors (RF) and demographics of 514 participants recruited for a randomized controlled trial on smoking cessation. We defined cardiovascular RF as follows: Hypertension as $\geq 140$ systolic $\mathrm{mmHg}$ and/or 90 diastolic $\mathrm{mmHg}$, except for participants with diabetes mellitus $\geq 130$ and/or $80 \mathrm{mmHg}$; Dyslipidemia, according to ATP-III guidelines, as LDL-cholesterol $\geq 2.6 \mathrm{mmol} / \mathrm{L}, \geq$ $3.4 \mathrm{mmol} / \mathrm{L}, \geq 4.1 \mathrm{mmol} / \mathrm{L}$ for high $(>20 \%)$, moderate $(10-20 \%)$ and low $(<10 \%)$ risk subjects, respectively; Diabetes mellitus as fasting blood glucose $\geq 7.0 \mathrm{mmol} / \mathrm{L}$. Participants were asked to estimate their 10 -year cardiovascular risk using a 3-item scale corresponding to high, moderate and low risk categories. We determined and compared the characteristics of participants according to their self-reported risk categories and their 
Framingham score categories. We used multi-variate logistic regression models to determine characteristics of participants who underestimate their risk vs. those who correctly estimate it.

RESULTS: Participants (mean age 51.1 years, range $40-70$ years; $46 \%$ women) smoked an average of 24 cigarettes per day with a median duration of 32 years. 214 smokers $(42 \%)$ correctly estimated their 10 -year cardiovascular risk compared to their calculated 10-year cardiovascular risk. 39\% (200) overestimated their cardiovascular risk while 19\% (100) underestimated it. An underestimated 10-year cardiovascular risk was associated with male gender (OR 6.33 ; CI 2.90-13.84), increasing age (OR per 5 years $=1.36$; CI 1.02-1.81), dyslipidemia (OR 2.23 ; CI 1.16-4.26) and diabetes mellitus (OR 6.27; CI 1.94-20.27). Socioeconomic status was not associated with inappropriate risk perception.

CONCLUSIONS: In our study of nicotine-dependent smokers, $58 \%$ of participants had a misperception of their 10-year cardiovascular risk. Male gender, older age, the presence of diabetes mellitus and dyslipidemia were associated with cardiovascular risk underestimation. These findings may help physicians emphasize on cardiovascular risk perception of patients with such characteristics to improve their health behaviors and adherence to risk-reduction therapy.

SELF-REPORTED MEDICATION ADHERENCE AND GLYCEMIC CONTROL AMONG A COHORT OF LATINO DIABETICS Bhavana Pendurthi ${ }^{1}$; Hua $\mathrm{Li}^{2}$; Olveen Carrasquillo ${ }^{1}$. ${ }^{1}$ University of MIami, Miami, FL; ${ }^{2}$ University of Miami, Miami, FL. (Control ID \#1339236)

BACKGROUND: Patient adherence to pharmacotherapy is a complex but important factor in achieving positive clinical outcomes in different chronic diseases including diabetes mellitus; a disease which disproportionately affects the Latino population. However, the association between self-reported medication adherence and diabetes intermediate outcomes has not been well explored among Latinos. In this abstract, we present preliminary data on the relationship between self-reported medication adherence and glycemic control among Latinos collected as part of an ongoing prospective study.

METHODS: The Miami Healthy Heart Initiative is an ongoing randomized trial testing the impact of a community health worker intervention on diabetes intermediate outcomes (A1C, LDL, SBP) among poorly controlled Latino diabetics (inclusion $\mathrm{A} 1 \mathrm{C} \geq 8.0$ ). To date, we have randomized 164 of our target 300 patients. As part of a comprehensive baseline assessment, we measured self-reported medication adherence using the Morisky Medication Adherence Scale (MMAS). The MMAS was originally developed to measure medication adherence for blood pressure medications but has also been validated for use in a range of chronic diseases and among various other racial and non-Latino ethnic populations. For Spanish speakers ( $95 \%$ of our sample), we translated the seven item MMAS into Spanish and then back translated the questionnaire to ensure comprehension. Patients with scores of 0-4 were considered as having low adherence (32\%), 4-6 intermediate (46\%) and 7 as high adherence $(21 \%)$. We used ANOVA to test for statistically significant differences in A1C among the 3 groups.

RESULTS: Approximately $30 \%$ of our sample is Cuban, the rest the study subjects comprise a variety of other Latino ethnicities with no other group representing $>15 \%$ of the sample. The mean AIC of the sample was $(8.9 \%$ $\pm 1.7 \%$ ). AIC level was correlated with adherence as follows; low adherence $(9.2 \pm 1.8)$, intermediate (8.9 \pm 1.4$)$, and high $(8.7 \pm 1.7)$. However, our current sample size limits the statistical power of our comparison (at present, we are only powered to detect larger differences of AIC $>1.0$ at $\beta$ of .80 ). Interestingly, although our questions ask about adherence to diabetes medications, we also saw similar patterns for LDL; $105 \mathrm{mg} / \mathrm{dl} \pm 46$ for low adherence, $101 \mathrm{mg} / \mathrm{dl} \pm 38$ for intermediate, and $87 \mathrm{mg} / \mathrm{dl} \pm 40$ for high, $p=0.05$. Such trends were not observed for systolic blood pressure. CONCLUSIONS: Our preliminary analysis suggests self-reported medication adherence as measured by the MMAS may be correlated with glycemic control and LDL in this study population. Thus, the MMAS may be a reasonable marker of adherence to include in studies examining diabetes control among Latinos. By May 2012, we expect to have enrolled another fifty subjects into the MHHI and thus will have greater statistical power for our comparisons.
SEXUAL ACTIVITY IS RELATED TO VAGINAL MUCOSAL MATURITY IN POSTMENOPAUSAL WOMEN Rachel Hess'; Sam Poloyak $^{1}$; Stacey Dillon ${ }^{1}$; M. B. Minnigh ${ }^{1}$; Shenay Jeffrey ${ }^{1}$; Genevieve Neal-Perry ${ }^{2}$. ${ }^{1}$ University of Pittsburgh, Pittsburgh, PA; ${ }^{2}$ Albert Einstein College of Medicine, Bronx, NY. (Control ID \#1339722)

BACKGROUND: The menopause is characterized by decreased systemic levels of estrogens; a hormonal milieu that heralds the onset of menopausal symptoms (hot flashes and vaginal atrophy) which frequently negatively impact women's quality of life. While hot flashes often resolve over time, vaginal changes persist and become more prominent with advancement of the menopause. The goal of this pilot study was to examine the associations among sexual activity in postmenopausal women, sex steroids levels, and vaginal maturation indices.

METHODS: We enrolled 40 sexually active (participated in vaginally penetrative sexual activities with a partner at least 2 times a week) or sexually inactive (had not participated in partnered sexual activities in the last 6 months) postmenopausal women. We excluded women using any local or systemic hormone therapy. Women self-collected vaginal swabs for calculation of the vaginal maturation index (VMI), an objective assessment of vaginal hormone response as well as overall hormonal environment. Higher values represent more mature (better) vaginal mucosa. Blood was collected and assayed for estradiol, estrone, androstendione, and testosterone using liquid chromatography tandem mass spectrometry. Women completed demographic and sexual functioning questionnaires. We compared VMI and sex-steroid hormone levels between sexually active and inactive women using Student's t-test. We then examined the correlation between VMI and sex steroid hormone levels using Pearson's correlations coefficients. Finally, we examined the association among VMI (outcome) and sexual activity and sex steroid hormone levels (independent variables) using multiple linear regression.

RESULTS: Of the 40 women enrolled, 17 were sexually active and 23 were sexually inactive. Sexually active women were younger [age (standard deviation (SD) 53 (4.9) vs. 58 (7.1) years, $\mathrm{p}=.02$ ] and had more mature vaginal indices [VMI (SD); 38 (17) vs. 28 (8), p=.02]. Sex-steroid hormone levels did not differ between sexually active and inactive menopausal women (Table). The correlations between VMI and sex steroid hormone levels were small, ranging from 0.02 for testosterone to 0.3 for estrone. In models that included individual sex steroid hormones, being sexually active was consistently associated with more mature VMI regardless of sex steroid hormone level. The relationship between sexual activity and an improved VMI remained after adjusting for age.

CONCLUSIONS: In this pilot study, participation in partnered sexual activities, but not sex-steroid hormone levels, was related to more mature vaginal mucosa in postmenopausal women. While confirmation is needed in a larger, more diverse sample, behavior modification should be considered as a method to limit the development of vaginal atrophy and improve sexual functioning and quality of life in postmenopausal women.

Table. t-test sexually active vs. inactive women for VMI* and each sex steroid hormone

\begin{tabular}{llll}
\hline \hline & $\begin{array}{l}\text { Sexually } \\
\text { active } \\
(\mathrm{n}=17)\end{array}$ & $\begin{array}{l}\text { Sexually } \\
\text { inactive } \\
(\mathrm{n}=23)\end{array}$ & $\begin{array}{l}\mathrm{p}- \\
\text { value }\end{array}$ \\
VMI (mean (sd)) & $38(17)$ & $28(8)$ & 0.02 \\
Estradiol pg/mL (mean (sd)) & $27(59)$ & $12(29)$ & 0.29 \\
Estrone $\mathrm{pg} / \mathrm{mL}($ mean (sd)) & $42(44)$ & $27(14)$ & 0.14 \\
Testosteronepg/mL (mean (sd)) & $204(112)$ & $166(109)$ & 0.29 \\
$\begin{array}{l}\text { Androstendione } \mathrm{pg} / \mathrm{mL}(\mathrm{mean} \\
\text { (sd)) }\end{array}$ & $496(294)$ & $477(270)$ & 0.84 \\
\hline
\end{tabular}

*Abbreviations: VMI: vaginal maturation index, sd: standard deviation, pg: picograms, ml: milliliter; $\mathrm{P}<0.05$ statistically significant.

SIGN-OUT; WHAT REALLY GOES ON? Lauren Shapiro ${ }^{1,2}$; Natalie Zelta $^{1}$; Kevin Hauck ${ }^{1}$; Joseph Deluca ${ }^{1,2}$; Hillary Kunins ${ }^{1,2} .{ }^{1}$ Montefiore Medical Center, Bronx, NY; ${ }^{2}$ Albert Einstein College of Medicine, Bronx, NY. (Control ID \#1339351) 
BACKGROUND: Verbal and written sign-outs facilitate information transfer during clinical handoffs. Recent restrictions on resident duty hours have increased the number of handoffs and highlight the need for high quality sign-outs. In this observational study, we investigated the quality of sign-outs among residents on an inpatient medicine rotation and compared sign-out quality between covering and primary residents.

METHODS: For four days, all oral sign-outs between the day and night residents on the internal medicine teaching service at Montefiore Medical Center were audiotaped and written components collected. Day residents were either interns who were the primary caretakers of the patient or covering residents for interns in clinic or post-call. Night residents were interns assigned to cover a group of patients overnight. No formal instruction on sign-out was provided at the time of the study.

RESULTS: 235 sign-outs were evaluated; ten sign-outs had missing data and could not be coded. 183 sign-outs contained both written and verbal elements; 33 were written only. The table shows the number and proportion of sign-outs containing each of the six components and globally rated as adequate.

CONCLUSIONS: Key to safe clinical handoffs are sign-outs which include the clinical condition of the patient, anticipatory guidance, and reason and plan for assigned tasks. We found that a majority of sign-outs during the four-day evaluation period included these components and were rated as globally adequate. The most common omissions were clinical condition, anticipatory guidance and plan and rationale for tasks assigned. Sign-outs by primary residents were more likely to include code status, give anticipatory guidance, and plan and rationale for tasks. These differences likely result from covering residents' lack of familiarity with the patient and could portend poorer patient outcomes. Based on these findings, we developed a curriculum in clinical handoffs, and plan to assess whether it leads to improved sign-out quality and reductions in adverse patient outcomes.

\begin{tabular}{|c|c|c|c|c|}
\hline Sign-out Component & $\begin{array}{l}\text { Total N } \\
(\%)\end{array}$ & $\begin{array}{l}\text { Signouts } \\
\text { by Primary } \\
\text { Resident N } \\
(\%)\end{array}$ & $\begin{array}{l}\text { Signouts } \\
\text { by Covering } \\
\text { Resident N } \\
(\%)\end{array}$ & $\begin{array}{l}\mathrm{p} \\
\text { value* }\end{array}$ \\
\hline Clinical Condition & $185(82)$ & $121(92)$ & $64(67)$ & $<0.001$ \\
\hline Code Status & $210(93)$ & $127(97)$ & $83(87)$ & 0.006 \\
\hline $\begin{array}{l}\text { Recent/Scheduled } \\
\text { Events }\end{array}$ & $214(95)$ & 127 (97) & $87(91)$ & 0.08 \\
\hline $\begin{array}{l}\text { Anticipatory } \\
\text { Guidance }\end{array}$ & $139(62)$ & $95(73)$ & $44(47)$ & $<0.001$ \\
\hline $\begin{array}{l}\text { Tasks to be } \\
\text { Completed }\end{array}$ & $150(66)$ & $75(57)$ & 75 (79) & 0.01 \\
\hline Plan for Task & $73(49)$ & $40(55)$ & $33(45)$ & 0.29 \\
\hline Rationale for Task & $79(53)$ & $43(54)$ & $36(46)$ & 0.29 \\
\hline Globally Adequate & $205(91)$ & $121(92)$ & $83(88)$ & 0.30 \\
\hline
\end{tabular}

${ }^{*}$ Chi-Square Test

SIMPLIFYING A SCORING TOOL TO SELECT THE BEST CLINICAL VIGNETTES Ryan Kraemer; Jeremiah Newsom; Carlos Estrada; Lisa L. Willett; Jason L. Morris. University of Alabama at Birmingham, Birmingham, AL. (Control ID \#1322023)

BACKGROUND: Academic organizations use clinical vignette presentations to foster interest in internal medicine. The best clinical vignettes are chosen for presentation at academic meetings through standardized scoring tools. In past work, we simplified a scoring tool allowing scorers to save time without sacrificing quality. In this study, we aimed to to validate the simplified scoring tool in a multi-institutional setting and to explore whether the scoring tool could be further simplified to a single item in order to select the best clinical vignettes for presentation at academic meetings.

METHODS: In a prospective study, 70 clinician educators reviewed 211 vignette abstracts submitted to the Southern Society of General Internal Medicine meeting (2011). Reviewers independently rated abstracts on 5 items: a) clarity of presentation, b) relevance to clinical practice, c) relevance to general internal medicine, d) teaching value, and e) overall assessment (Likert scale; $1=$ low, $7=$ high). We examined internal consistency with Cronbach's alpha and factor analysis; and also, calculated Kappa agreement for accepted oral presentations using 5-items compared to 3 -items (relevance to general medicine, teaching value, and overall assessment) and to a single item (overall assessment).

RESULTS: A total of 1,215 ratings were available; each reviewer rated a mean of 17.4 (SD 0.7) abstracts; each abstract was rated by a mean of 5.8 reviewers (SD 0.7); 19\% (40/211) top-rated abstracts were accepted for oral presentation. The internal consistency was almost perfect (Cronbach's alpha= 0.93 ); and it remained excellent (Cronbach's alpha $=0.91$ ) after simplifying to three items (relevance to general medicine, teaching value, and overall assessment) using factor analysis and inter-item correlations. The agreement between the number accepted for oral presentation using the 5-item average score with the number that would have been accepted using the 3-item average score was almost perfect with 36 of the previously accepted 40 vignettes being the same (Kappa $=0.88 ; 95 \%$ confidence interval $[\mathrm{CI}], 0.79$ to 0.96 ). The agreement remained high comparing the 5 -item tool with a single item, overall assessment, with 34 of the previously accepted 40 vignettes being the same (Kappa $=0.83,95 \%$ CI, 0.73 to 0.93 ).

CONCLUSIONS: A 5-item scoring tool to select the best clinical vignettes for presentation at academic meetings could be further simplified to a single item. Hence, a prospective validation of a single item scoring tool is warranted.

\section{SMOKING BEHAVIORS IN A COMMUNITY-BASED COHORT} OF HIV-INFECTED UNSTABLY HOUSED AND HOMELESS ADULTS Maya Vijayaraghavan ${ }^{1}$; Joanne Penko ${ }^{2}$; David Guzman ${ }^{2}$; Christine Miaskowski ${ }^{2}$; Margot Kushel ${ }^{2}$. ${ }^{1}$ University of California, San Diego, San Diego, CA; ${ }^{2}$ University of California, San Francisco, San Francisco, CA. (Control ID \#1320912)

BACKGROUND: The prevalence of smoking is high among homeless adults, however limited data exists on patterns of tobacco use and cessation. In a longitudinal study of a community-based cohort of HIV-infected unstably housed and homeless adults, we examined smoking behaviors, factors associated with initiating a quit attempt, and successful quitting.

METHODS: At a community-based field site, we interviewed participants every three months over a two-year interval about demographics, illicit substance use, alcohol use, and smoking. At baseline, we determined eversmoking rates (smoked at least 100 cigarettes), frequency of smoking, intention to quit, and the quit ratio among ever smokers (former/ever smoker). At follow-up, we asked whether participants had smoked in the past 30 days, the number of days smoked and the number of cigarettes smoked. Among current smokers, we defined a quit attempt as having no cigarette consumption during a follow-up visit, and successful quitting as having no consumption over two consecutive follow-up visits. Using multivariable logistic regression (clustered on participant and accounting for repeated measurements), we determined factors associated with initiating a quit attempt. We used Poisson regression to determine factors associated with successful quitting accounting for interval-censored data.

RESULTS: Among the 296 participants, $41.2 \%$ were African American, $82.1 \%$ had a lifetime history of homelessness, and $34.8 \%$ had used illicit substances in the past 90 days. Of the 296 participants, 251 (84.8\%) were ever smokers. The prevalence of current smoking among ever smokers was $86.6 \%$ and the quit ratio $13.2 \%$. Among current smokers, $20.5 \%$ had an intention to quit in the next one to six months, $54.9 \%$ had an intention but not in the next six months, and $24.7 \%$ had no intention to quit at baseline. Of the 218 current smokers, 45 (20.6\%) initiated a quit attempt. Of the 45 participants who made a quit attempt, 24 (53.3\%) successfully quit. Persons with a history of illicit drug use (Adjusted odds ratio (AOR) $0.2,95 \%$ CI 0.1-0.6), and alcohol abuse or dependence (AOR $0.2,95 \%$ CI $0.1-0.8$ ) were less likely to initiate a quit attempt, whereas those who intended to quit within one to six months at baseline were more likely to do so (AOR 4.4, 95\% CI 1.3-15.5). In Poisson regression, illicit drug use was associated with a decreased likelihood of successfully quitting (AOR 0.1, 95\% 0.03-0.5), whereas having more than a high school education (AOR 4.1, 95\% CI 1.1-15.0) and an intention to quit within one to six months at baseline (AOR 2.6, $95 \%$ CI 1.0-6.7) was associated with an increased likelihood.

CONCLUSIONS: In this high-risk cohort, current smoking prevalence was 4.5 times higher than the $19 \%$ national smoking rate, and the quit 
ratio 4 times lower than the $50 \%$ national average for quit ratios. Less than one-fourth made a quit attempt during the study interval, and half of those successfully quit. Smoking cessation should be prioritized among this population. Interventions should focus on assisting persons with an intention to quit to increase their likelihood of success. Substance abuse treatment programs should motivate people to move through the stages of quitting.

SOCIAL DETERMINANTS OF PRIMARY-CARE PATIENTS FORGOING HEALTH CARE IN THE SWISS COST-SHARING SYSTEM Patrick Bodenmann ${ }^{1}$; Thomas Bischoff ${ }^{2}$; Hans Wolff ${ }^{3}$; Francesco Panese $^{4}$; Lilli Herzig ${ }^{2}$; Paul Vaucher ${ }^{1,3} .{ }^{1}$ University of Lausanne, Lausanne, Switzerland; ${ }^{2}$ University of Lausanne, Lausanne, Switzerland; ${ }^{3}$ University of Geneva, Geneva, Switzerland; ${ }^{4}$ University of Lausanne, Lausanne, Switzerland. (Control ID \#1317342)

BACKGROUND: Consequences of out-of-pocket expenses causing health disparities can be addressed by primary-care physicians in complement to upstream reforms focused on social determinants of health. By considering social conditions in clinical decision-making, primary-care physicians can address mismatches between patients' health care needs and financial abilities. The aim of this study is to identify and model social determinants explaining patients' decisions to forgo health care for economic reasons, and investigate whether questioning patients' subjective perception of their social situation is more relevant than recording objective socio-economic status (SES).

METHODS: This Swiss primary-care practice cross-sectional survey questioned a random sample of 2,025 patients over 16 years of age attending one of 47 private primary-care practices in western Switzerland between September 2010 and February 2011. Patients concerned by health care renunciation were those who reported a household member not to have sought treatment for economic reasons during the previous 12 months. For subjective social determinants, patients were questioned on their state of deprivation (DiPCare-Q), their subjective social status (subjective SES ladder), and state of health (EQ-5D). We also collected objective socioeconomical determinants (age, gender, nationality, education level, daily income, and household's source of income). Using regression analysis and coefficients of determination $\left(\mathrm{R}^{2}\right)$, we compared the load of self-perceived subjective social determinants to the load of objective socio-economic determinants in explaining the decision to forgo health care. Likelihood ratio test was used to assess significant level of observed differences.

RESULTS: During the 2,945 monitored random consultations, physicians saw 2,811 different patients of which 2,025 were included in the analysis (response rate $72 \%$ ). Among them, $10.7 \%$ (CI95\% 9.4 to 12.1 ) were concerned by restricted health care during the 12 previous months. Forgoing health care was independently explained by level of material deprivation, social deprivation, subjective social status, health status, daily income, and source of income (related to age). Questioning patients on their subjective perceived state of material deprivation was more relevant in explaining their decision than collecting objective socio-economical status $\left(\mathrm{R}^{2}=0.226\right.$ vs. $\left.0.097 ; \mathrm{P}<0.0001\right)$, which nevertheless remains better than having physicians estimate their patient's subjective social status $\left(\mathrm{R}^{2}=\right.$ 0.029; $\mathrm{P}<0.0001$ )

CONCLUSIONS: Firstly, financial difficulties, social isolation, chronic conditions, mental disorders, and younger age favor forgoing health care. This model is however limited to households for which at least one member attends a primary care physician, and does not explore other, non-economic, reasons for not accessing healthcare. Secondly, during social history, physicians are recommended to favor questioning their patients on subjective perceived social conditions over common socioeconomic determinants to detect underlying social risks of restricting access to health care. This seems particularly important in developed countries given the current increase in the number of patients concerned by rapid changes in their socio-economic situation.

SOME TALK, NOT MUCH WALK: DISCORDANCE OF PHYSICIANORDERED AND NURSING-RECORDED ACTIVITY AMONG HOSPITALIZED ELDERS Hilary Mosher. University of Iowa Hospitals and Clinics, Iowa City, IA. (Control ID \#1336326)
BACKGROUND: Functional decline related to low mobility during hospitalization of acutely ill elders is well-recognized. Interventions have shown variable results in relating increased ambulation with improvements in clinically meaningful outcomes including functional ability, hospital length of stay (LOS), and discharges home. Despite the acknowledged importance of maintaining functional abilities of hospitalized elders, a culture of increased activity has not broadly taken hold. The objective of this study was to assess the association between physician-ordered activity and excess LOS (defined as the difference between actual and expected LOS). A secondary objective was to compare physician-ordered activity with nursing-recorded activity level to determine if physician orders were reflected in patient activity.

METHODS: A retrospective chart review was done in a convenience sample of all patients aged 65 years or older admitted to a general medicine ward of an academic medical center over a 3-month period in 2010. Data on age, LOS, discharge disposition, physician-ordered activity, nursing-recorded activity level, and physical therapy evaluation were abstracted from the electronic health record. Data analysis, including frequency statistics and ANOVA, was performed using SAS. RESULTS: A total of 329 patients accounting for 365 admissions were included in the study. Physician activity orders were for bed rest in $34(9 \%)$ admissions, ambulate 4 times daily in $83(23 \%)$, up ad lib in $161(44 \%)$ and not ordered in $87(24 \%)$ admissions. Ambulate 4 times daily orders were associated with the greatest excess days beyond expected LOS ( 2.84 days, SE $0.48, \mathrm{P}<.0001$ ), no orders were associated with 2.12 excess days (SE 0.87, $\mathrm{P}=.015$ ), and up ad lib orders were associated with 1.14 excess days (SE $0.34, \mathrm{P}=.001$ ). Bed rest orders were not significantly associated with excess LOS. Physician-ordered activity and nursingrecorded activity level were discordant during approximately 50\% percent of admissions, with bed rest recorded at greater frequency $(72 \%$ of admissions) in the nursing records. Physical therapy assessments were performed in approximately $75 \%$ of admissions. Physical therapists often documented ability to stand or walk in patients with a nursing-recorded activity level of bed rest. The electronic health record at the study institution lacked a convenient provision by which nursing staff could communicate number of times per day a patient ambulated.

CONCLUSIONS: Despite the growing recognition of the importance of maintaining mobility and function among hospitalized elders, numerous barriers to safe and appropriate mobilization remain. This descriptive study suggests that physicians, nurses, and physical therapists have a high level of discordance regarding intended and actual patient activity levels. This discordance may well account for the significant association found between ambulate 4 times daily orders and excess LOS: patients with a recognized but unmet need for increased mobilization may be at particular risk for prolonged hospitalization. Moreover, physicians failed to prescribe activity level in nearly 1 out of 4 admissions, indicating that mobility remains a frequently disregarded component of hospital care.

\section{STATIN USE ON INCIDENT IMMUNE-MEDIATED AND INFECTIOUS CONDITIONS IN THE VETERANS ADMINISTRATION HEALTH SYSTEM Dominic J. Cirillo ${ }^{1,2}$; Robert B. Wallace ${ }^{2,1} .{ }^{1}$ University of Iowa, Iowa City, IA; ${ }^{2}$ University of Iowa, Iowa City, IA. (Control ID \#1337695)}

BACKGROUND: Statins are commonly used cholesterol-lowering medications with immunologic properties. The main study hypothesis was that statin use may be associated with alterations in immune cell function, potentially impacting subsequent diagnosis of immune-mediated conditions, including rheumatology diseases and infections.

METHODS: A modified case-cohort study was formed using administrative databases from the Midwest Veterans Administration (VA) region. To be eligible for inclusion in the study, subjects needed at least one year of medical claims and at least one pharmacy claim. Cases were identified by inpatient or outpatient medical claims using International Classification of Disease, Ninth Revision (ICD-9) codes between FY 2003-2004. The incident cases $(n=28,642)$ included non-mutually exclusive groups of immune-mediated $(n=2,327)$, infectious $(n=8,221)$, and non-immunologic $(n=10,730)$ comparison diagnoses. The referent sub-cohort was formed by randomly sampling 10,000 subjects with medical and pharmacy benefits 
during fiscal year (FY) 2002. Demographic and medical variables were obtained from FY 2001-2004, and pharmacologic data from FY 2002-2004. Cox proportional hazards regression modeling was used to estimate hazard ratios for the current statin use (within the last 180 days) and former statin use, compared to non-users, including time-dependent variables for demographic factors, comorbidity as measured by Elixhauser and Chronic Disease Score variables, medications, and visit rates after initiating statins. Sensitivity analyses were conducted using variations of drug exposure definition, variations of case definition, new-user analysis, and propensity scores.

RESULTS: Results for statin use and immune-mediated conditions are listed in Table 1. The results for incident infection and statin use are included in Table 2. Statin use was not associated with multiple sclerosis, thyroiditis, sarcoidosis, temporal arteritis, influenza, shingles, histoplasmosis, or pyelonephritis.

CONCLUSIONS: Although current statin use appeared protective for some study conditions, selection bias, misclassification, healthy user effects, adherence bias, confounding by indication, and surveillance bias were considered as possible explanations of the study findings. The associations are intriguing for possible positive preventive interventions using statins if causal links are confirmed.

Summary of Major Findings for Immune-mediated Conditions

\begin{tabular}{llllll}
\hline \hline Disease Outcome & \multirow{2}{*}{$\mathrm{N}$} & \multicolumn{2}{c}{ Current Users } & \multicolumn{3}{c}{ Former Users } \\
& & HR & $95 \% \mathrm{CI}$ & HR & $95 \% \mathrm{CI}$ \\
Psoriasis & 674 & 0.63 & $(0.52,0.77)$ & 1.09 & $(0.59,2.02)$ \\
Rheumatoid arthritis & 662 & 0.68 & $(0.56,0.83)$ & 1.22 & $(0.71,2.12)$ \\
IBD & 307 & 0.60 & $(0.45,0.81)$ & 0.69 & $(0.22,2.18)$ \\
Polymyalgia Rheumatica & 251 & 1.08 & $(0.81,1.43)$ & 2.11 & $(1.06,4.23)$ \\
Spondyloarthropathy & 197 & 0.76 & $(0.53,1.09)$ & 0.91 & $(0.28,2.95)$ \\
DCTD & 123 & 0.66 & $(0.41,1.05)$ & 1.72 & $(0.61,4.89)$ \\
SLE & 59 & 0.52 & $(0.26,1.01)$ & 1.46 & $(0.33,6.40)$ \\
AIHA & 30 & 0.49 & $(0.18,1.33)$ & 9.19 & $(2.47,34.19)$ \\
Pneumonia (Bacterial) & 2,986 & 0.77 & $(0.70,0.84)$ & 1.04 & $(0.83,1.29)$ \\
Urinary Tract Infections & 2,820 & 0.73 & $(0.67,0.80)$ & 1.13 & $(0.88,1.44)$ \\
Cellulitis & 2,184 & 0.75 & $(0.68,0.84)$ & 1.11 & $(0.84,1.47)$ \\
Sepsis & 843 & 0.75 & $(0.51,0.72)$ & 1.44 & $(1.01,2.05)$ \\
Candidiasis & 565 & 0.72 & $(0.58,0.90)$ & 1.45 & $(0.91,2.29)$ \\
Osteomyelitis & 454 & 0.75 & $(0.59,0.94)$ & 1.88 & $(1.18,2.99)$ \\
\hline
\end{tabular}

$\mathrm{N}=$ Number of cases; $\mathrm{HR}=$ Hazard ratio; $\mathrm{CI}=$ Confidence Interval; $\mathrm{IBD}=$ Inflammatory bowel disease; $\mathrm{DCTD}=$ Diffuse connective tissue disease; $\mathrm{SLE}=$ Systemic lupus erythematosus; $\mathrm{AIHA}=$ Autoimmune hemolytic anemia

STILL PAINFUL AFTER ALL THESE YEARS? EFFECTS OF A CURRICULUM ON RESIDENT KNOWLEDGE AND ATTITUDES REGARDING CHRONIC PAIN Tracey G. Simon; Edward Feller; Donnah Mathews; Mark J. Fagan. Warren Alpert Medical School, Brown University, Providence, RI. (Control ID \#1338962)

BACKGROUND: Despite studies documenting internal medicine (IM) residents' unease when managing patients with chronic non-malignant pain (CNMP), few have evaluated the impact of educational interventions designed to improve resident experience with CNMP. The purpose of this study was to assess and compare IM residents' attitudes and experiences with CNMP, before and after the implementation of a longitudinal, multidimensional curriculum focused on CNMP care.

METHODS: We anonymously surveyed all residents within the IM program at Lifespan/Brown University, first in 2004 (124 residents), and again in 2011 (117 residents). In 2005, we implemented a required, longitudinal CNMP curriculum, including 5 hours of didactic training as well as case-based discussion modules. The survey instrument included Likert-style questions assessing frequency of exposure to CNMP, perceived level of self-confidence when managing CNMP, resident experience with CNMP compared with the management of diabetes $(1=$ much easier; $5=$ much more difficult $)$, and the impact that interactions with CNMP patients had on residents' overall clinic experience $(1=$ very negative, $5=$ very positive $)$. We compared mean responses across gender, residency year of training, and residency track using ANOVA and chi-square analyses. The results from pre-intervention respondents were then compared to those of the post-intervention group by chi-square analysis.

RESULTS: The survey response rate was $88.7 \%(110 / 124)$ in 2004 , and $82.9 \%(97 / 117)$ in 2011. Compared to pre-intervention respondents, fewer post-intervention respondents rated their experience with CNMP as "poor" or "very poor" ( $86 \%$ vs. $76 \%, \mathrm{p}<0.002)$. Self-rated experience improved with increasing level of training: the mean Likert response at the PGY3 level was 3.4, compared to only 2.1 and 2.3 at the PGY1 and PGY2 levels, respectively $(p<0.001)$. There were no statistically significant differences between the pre- and post-intervention groups, with regard to self-rated level of preparation or confidence in managing CNMP. However, significantly more post-intervention respondents reported $>5$ cumulative hours of dedicated CNMP instruction ( $45 \%$ in 2011 vs. $29 \%$ in 2004; $\mathrm{p}=$ 0.004). Additionally, significantly fewer post-intervention respondents reported a "large" or "very large" uncertainty about opioid pharmacology and side effects $(44 \%$ in 2004 vs. $7 \%$ in $2011 ;$ p $<0.001)$. Only $37 \%$ of post-intervention General Internal Medicine (GIM) residents reported that managing CNMP patients had "negative" or "very negative" impact on their view of primary care as a career, compared to $73 \%$ of pre-intervention GIM respondents $(\mathrm{p}=0.021)$. There was no statistically significant difference between pre- and post-intervention categorical residents, with regard to the impact of CNMP management on their view of primary care.

CONCLUSIONS: After the introduction of a multi-dimensional curriculum dedicated to the management of CNMP, residents at this institution reported less-negative overall experiences with CNMP, as well as increased knowledge of CNMP pharmacology and side effects. Increasing year of training and participation in the GIM residency track were both associated with more positive attitudes. Nevertheless, respondents continued to find CNMP management difficult, lacked confidence in managing patients with CNMP, and did not gain confidence over the course of training. These results highlight continued barriers to care, as well as future opportunities to improve residents' experience with CNMP.

STROKE-PREVENTION BELIEFS PREDICT BLOOD PRESSURE CONTROL IN STROKE AND TIA SURVIVORS L. Alison Phillips ${ }^{1}$; Ian Kronish ${ }^{2}$; Carol Horowitz ${ }^{3}{ }^{1}$ The George Washington University, Washington, DC; ${ }^{2}$ Columbia University Medical Center, New York, NY; ${ }^{3}$ The Mount Sinai Hospital, New York, NY. (Control ID \#1340813)

BACKGROUND: Blood pressure (BP) control is essential for preventing recurrent stroke. In general hypertensive populations, patients who endorse a "stress model" of hypertension (i.e., that controlling stress will control BP) have been shown to be less adherent to medications and to have worse control of their BP than those who endorse a "medical model" of hypertension (i.e., that medications, diet, and exercise are required for controlling BP) (Hekler et al., 2008). Stroke survivors have the highest risk for stroke, and BP control is the most effective strategy to prevent recurrent stroke. Yet, little is known about the health belief factors that influence whether stroke-survivors will have controlled BP as compared with a general hypertensive population. The purpose of the current study was to determine if stress-model endorsement in a post-stroke population is associated with their adherence to medication and their BP control.

METHODS: Participants $(\mathrm{n}=510)$ participated in a stroke-prevention intervention targeted at stroke survivors in underserved communities in New York City (inclusion criteria: $\geq 40$ years, had at least one stroke or transient ischemic attack (TIA) in the past 5 years). Prior to starting the intervention, medication adherence was measured via survey using the 8item Morisky scale; patients' BP was measured three times and considered to be high if systolic BP was $\geq 140$ and/or if diastolic BP was $\geq 90$. Patients were asked to name the 3 most important things they would do to lower their risk of stroke; patients were considered to be "stress-modelendorsers" if they stated that reduction of stress is important for preventing stroke and "medical-model-endorsers" if they stated that taking medication (BP and/or stroke) was important for preventing stroke. Logistic regression 
(for BP control) and ANOVA (for adherence) were used to assess the relationships of interest.

RESULTS: Fifty-three patients (10\%) endorsed both the stress model and the medical model, 227 patients (45\%) endorsed neither, 127 patients (25\%) endorsed only the stress model, and 103 patients $(20 \%)$ endorsed only the medical model. There was no significant difference in adherence between those who endorsed a stress model (and rejected the medical model) vs those who did not endorse a stress model, endorsing only a medical model (mean difference in adherence $=.19(\mathrm{SD}=.21), \mathrm{p}=.81$, $95 \% \mathrm{CI}$ for difference $=-.72, .35$ ). However, the latter group had significantly higher BP than the former group (OR of stress-model-only endorsers having high BP compared to medical-model-only endorsers $=.48$, Wald $=6.03$, $\mathrm{p}=.01$ ). There were no differences in adherence or BP control for those who adopted a combination of a stress and medical model or neither model.

CONCLUSIONS: Stress-model endorsement alone should not necessarily cause clinicians concern regarding their post-stroke patients' BP control However, those patients who endorsed a stress-model and rejected the notion that taking medications will control their BP did have poorer BP control compared to patients who endorsed only a medical model of BP control/stroke prevention. This is despite no differences in their selfreported adherence. Clinicians may consider eliciting beliefs about BP control in this high-risk group and devising/testing strategies to educate them about medical management of hypertension.

SUBCLINICAL THYROID DYSFUNCTION AND THE RISK OF HEART FAILURE EVENTS: AN INDIVIDUAL PARTICIPANT DATA ANALYSIS FROM SIX PROSPECTIVE COHORTS. Baris Gencer $^{1}$; Tinh-Hai Collet ${ }^{1}$; Douglas Bauer ${ }^{2,3}$; Jacobijn Gussekloo ${ }^{4}$; Anne R.Cappola ${ }^{5}$; David Nanchen ${ }^{1}$; Wendy P. den Elzen ${ }^{4}$; Philippe Balmer ${ }^{1}$; Robert N. Luben ${ }^{6}$; Vincenzo Triggiani ${ }^{7}$; Jacques Cornuz ${ }^{1}$; Anne B. Newman $^{8}$; Wouter Jukema ${ }^{9}$; Massimo Iacoviello ${ }^{10}$; Kay-Tee Khaw ${ }^{6}$; Rudi G. Westendorp ${ }^{11}$; Drahomir Aujesky ${ }^{12}$; Nicolas Rodondi ${ }^{12}$. ${ }^{1}$ University of Lausanne, Lausanne, Switzerland; ${ }^{2}$ University of California, San Francisco, CA; ${ }^{3}$ University of California, San Francisco, CA; ${ }^{4}$ University of Leiden, Leiden, Netherlands; ${ }^{5}$ University of Pennsylvania, Philadelphia, PA; ${ }^{6}$ University of Cambridge, Cambridge, United Kingdom; ${ }^{7}$ University of Bari, Bari, Italy; ${ }^{8}$ University of Pittsburgh, Pittsburgh, PA; ${ }^{9}$ Leiden University Medical Center, Leiden, Netherlands; ${ }^{10}$ University of Bari, Bari, Italy; ${ }^{11}$ University of Leiden, Leiden, Netherlands; ${ }^{12}$ University of Bern, Bern, Switzerland. (Control ID \#1306405)

BACKGROUND: Heart failure (HF) is among the most frequent cause of hospitalization in persons older than 65 years. Subclinical thyroid dysfunction is common in older individuals and has been associated with systolic and diastolic dysfunction. However, few prospective data exist regarding the association between subclinical thyroid dysfunction and heart failure (HF) events. Currently controversy persists as to whether screening and treatment of subclinical hypothyroidism is warranted, as evidence about its risks is limited.

METHODS: After a literature search we performed a pooled analysis of individual participant data using all available prospective cohorts with thyroid function measurements and subsequent follow-up of HF events. Individual data on 25,738 participants with 216,668 person-years of follow-up were supplied from 6 prospective cohorts in the United States and Europe. Euthyroidism was defined as a TSH 0.45-4.49 mIU/L, subclinical hypothyroidism as a TSH between 4.5-19.9 mIU/L and subclinical hyperthyroidism as a $\mathrm{TSH}<0.45 \mathrm{mIU} / \mathrm{L}$ both with normal free thyroxine levels. HF events were defined as acute HF events, hospitalization or death related to $\mathrm{HF}$ events.

RESULTS: Among 25,378 participants, 2,065 had subclinical hypothyroidism (8.1\%), 648 subclinical hyperthyroidism $(2.6 \%)$ and 22,665 were euthyroid. During follow-up, 2,069 participants had HF events. In age- and gender-adjusted analyses, the risk of HF events among adults with subclinical hypothyroidism increased with higher TSH levels: hazard ratio (HR) was 1.02 (95\% confidence interval [CI], 0.82-1.27) for a TSH level of 4.5-6.9 mIU/L, 1.59 (CI 0.82-3.08) for a TSH level of 7.0-9.9 mIU/L, and 2.13 (CI 1.29-3.51) for a TSH level of 10.0-19.9 mIUL/L ( $\mathrm{p}$ for trend=0.001). Results were similar after further adjustment for traditional cardiovascular risk factors. Among participants with TSH 10-19.9 $\mathrm{mIU} / \mathrm{L}$, the association remained significant after excluding those with preexisting HF (HR 1.76 , CI 1.10-2.81). In age- and gender- adjusted analyses, the risk of HF events among participants with subclinical hyperthyroidism was 1.31 (CI $0.88-1.95$ ) for a TSH of $0.1-0.44 \mathrm{mIU} / \mathrm{L}$ and $1.87(0.98-3.57)$ for a TSH $<0.10 \mathrm{mIU} / \mathrm{L}$ ( $\mathrm{p}$ for trend=0.058).

CONCLUSIONS: This first pooled analysis of large cohorts examining the association between subclinical thyroid dysfunction and HF events shows that subclinical hypothyroidism is associated with an increased risk of HF events, particularly among those with a TSH level $\geq 10 \mathrm{mIU} / \mathrm{L}$. The findings of our study might be useful to better define the TSH thresholds for thyroxine replacement. Given the high prevalence of both subclinical hypothyroidism and HF in the elderly, the impact of thyroxine substitution on HF events will be investigated over the next years with an appropriately powered randomized controlled trial with other clinical outcomes (TRUST study) among 3000 older adults.

Figure 1. Hazard ratios for Heart Failure Events According to Elevated Thyroid Stimulating Hormone (TSH)

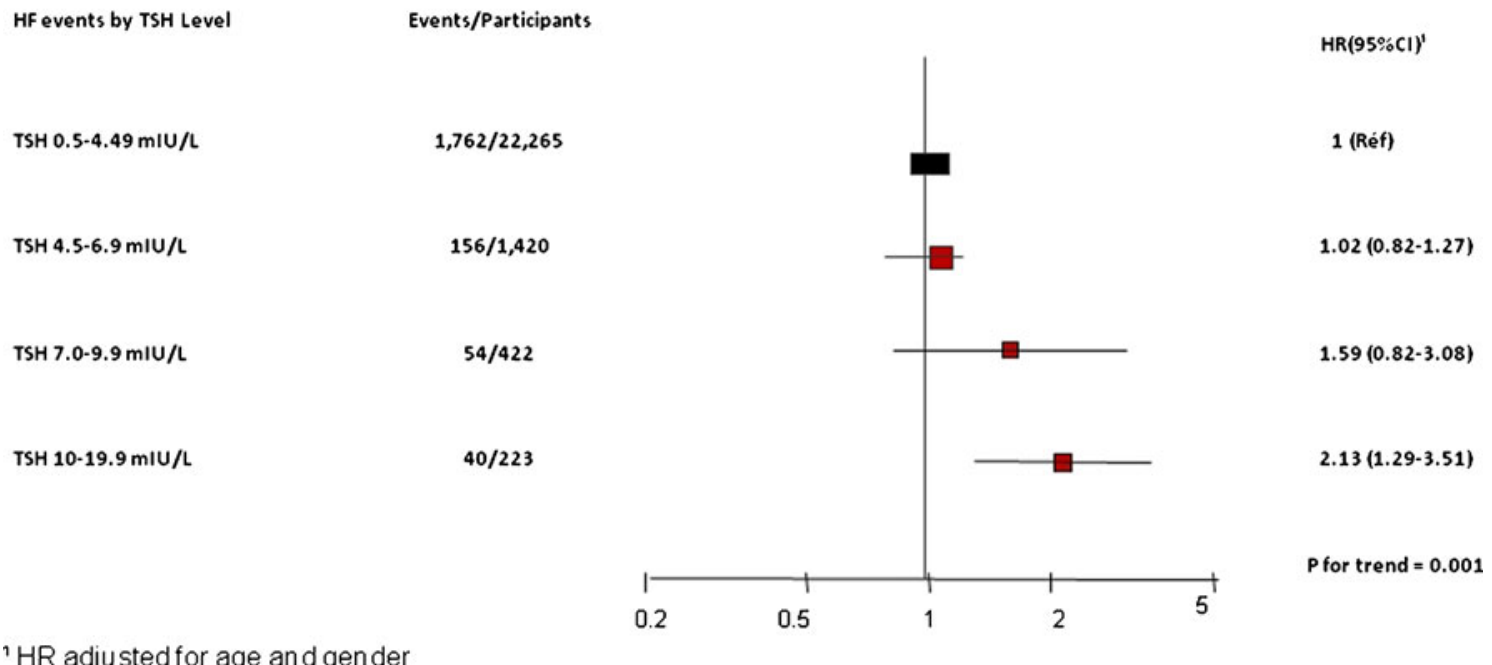

'HR adjusted for age and gender 
SUBJECTIVE AND OBJECTIVE SOCIOECONOMIC STATUS AND CONTROL OF HYPERTENSION AND DIABETES JOSE Delgado $^{1}$; Alicia Fernandez ${ }^{2}$; Nancy E. Adler ${ }^{3}$; Keegan Korthauer ${ }^{4}$; Elizabeth Jacobs ${ }^{5}$. ${ }^{1}$ Georgetown University Hospital, Washington, DC; ${ }^{2}$ University of California, San Francisco, San Francisco, CA; ${ }^{3}$ University of California, San Francisco, San Francisco, CA; ${ }^{4}$ University of Wisconsin School of Medicine and Public Health, Madison, WI; ${ }^{5}$ University of Wisconsin - Madison, Madison, WI. (Control ID \#1336272)

BACKGROUND: Poor control of diabetes and hypertension is common among minority and low-income populations compared to higher income and white populations. Objective measures of social status, such as income and education, have been shown to account for some of this disparity. Subjective social status (SSS) defined as an individual's perception of where they stand in society, has been related to general health status and the presence of diabetes and hypertension. It is not clear if SSS might also be related to disparities in diabetic and hypertensive control. Our objective was to investigate whether SSS was significantly related to control of these chronic illnesses in a diverse, vulnerable population.

METHODS: Data was obtained from the Immigration, Culture and Health Care Study (ICHC), a large survey and data abstraction study of a convenience sample of patients with diabetes seeking care for diabetes in safety-net clinics in Chicago and the San Francisco Bay Area. Subjective social status was measured by showing participants a drawing of two ladders. Participants were told one represents their community and the other represents the overall US society. In each ladder participants were asked to choose the step of the ladder that better represents their current position within the ladders' respective group. The most recent blood pressure measurement and hemoglobin A1c values were obtained from the participant's electronic medical record. We defined hypertensive control as systolic blood pressure bellow $130 \mathrm{mmHg}$ and a diastolic blood pressure bellow $80 \mathrm{mmHg}$. Diabetes control was defined as having a hemoglobin A1c lower than $8 \%$. Univariate chi-square analyses were used to determine racial/ ethnic differences in SSS while sequential multivariate logistic regression models were used to assess the association of SSS with diabetes and blood pressure control. Additionally we examined the interaction between each measure of subjective social status and race/ethnicity a final multivariate model. Multivariate models were adjusted for age, gender, income, education, marital status and medication adherence.

RESULTS: Participants included 107 whites, 200 black and 404 Mexican Americans. Hypertension was controlled in $46.5 \%$ of participants, while $52 \%$ met our criterion for glycemic control. Neither measure of SSS nor race/ethnicity was significantly associated with blood pressure or diabetes control in the overall population. However, in the final model for glycemic control, there was a significant interaction between SSS and race/ethnicity when participants compared themselves with the overall US society indicating that it did matter in some racial/ethnic groups compared to others: the odds ratio for diabetes control of Mexican Americans was 2.07 $(p>0.05)$ and African Americans $1.10(p>0.05)$ for each unit increase in SSS when compared with whites, while the odds for diabetes control among African Americans was $0.50(\mathrm{p}=0.02)$ compared to Mexican Americans.

CONCLUSIONS: In this population of diabetes patients who seek care in safety net clinics, we found that one measure of SSS was significantly related to difference in diabetic control across racial ethnic groups but that neither measure of SSS was associated with hypertensive control. This suggests that measures of SSS may contribute to disparities above and beyond objective social status.

SURVEY RESPONSES AND MEDICATION ADHERENCE AMONG DIABETIC PATIENTS Chuan-Fen Liu ${ }^{1,2}$; Mark Perkins ${ }^{1}$; Edwin Wong ${ }^{1}$; Christopher L. Bryson ${ }^{1,2}$. ${ }^{1}$ VA Puget Sound Health Care System, Seattle, Seattle, WA; ${ }^{2}$ University of Washington, Seattle, WA, WA. (Control ID \#1274896)

BACKGROUND: Medication adherence is generally described using large administrative databases or smaller, survey based data. Both methods have strengths and weaknesses. Combining patient surveys and administrative data could provide more information. However, response bias may significantly hinder generalizability compared to administrative methods. This study examines the relationship between survey response and medication adherence to oral hypoglycemic agents (OHAs) by diabetic patients.

METHODS: This study includes all diabetic patients on an OHA seen in a VA primary care clinic from FY2006-07 (504,778 patients), identified from administrative datasets. We use pharmacy refill data to calculate a medication possession ratio and classify patients as adherent if they had $\geq 80 \%$ of their OHA regimen during the first quarter of 2007. Of the study population, 52,725 were given the Primary Care Satisfaction Survey in 2006, and 63.9\% responded. We classified our sample into three categories: non-surveyed patients $(452,053)$, survey respondents $(33,710)$, survey non-respondents $(19,015)$. We used a logit regression to adjust for patient characteristics.

RESULTS: The proportions of adherence were significantly different among the three groups $(\mathrm{p}<0.0001)$ with the highest from the complete survey respondents $(0.74)$, followed by non-surveyed patients $(0.70)$, and incomplete patients respondents (0.66). Compared to non-surveyed patients, after adjusting for patient characteristics, survey respondents were more likely to be adherent to OHAs (odds ratio $=1.13, \mathrm{p}<0.0001$ ), while survey non-respondents were less likely to be adherent (odds ratio $=0.87$, $\mathrm{p}<0.0001$ ). Survey non-respondents were more likely to be younger, unmarried, and to have mental health conditions, including depression, drug abuse, and PTSD, which were accounted for in the analyses.

CONCLUSIONS: After adjusting for available characteristics, survey nonrespondents had the lowest medication adherence compared to survey respondents and non-surveyed patients. For medication adherence, non-response bias may be a serious threat to the generalizability of survey data or interventions based on surveys to the general clinic population.

TAILORING SUSTAINABLE PATIENT AND FAMILY INTERVENTIONS TO REDUCE RACE DISPARITIES IN HYPERTENSION AMONG URBAN AFRICAN AMERICANS Sarah J. Flynn ${ }^{1}$; Patti Ephraim $^{3}$; LaPricia Lewis-Boyer ${ }^{1}$; Jeffrey M. Barbers ${ }^{1}$; Jessica Ameling ${ }^{1}$; Lee Bone ${ }^{1,3}$; David Levine ${ }^{1}$; Felicia Hill-Briggs ${ }^{1,2}$; Deborah Roter ${ }^{3}$; Jennifer L. Wolff; ; Leon Purnell ${ }^{4}$; Annette Fisher ${ }^{4}$; L. Ebony Boulware,1, ${ }^{1}$ Johns Hopkins University School of Medicine, Baltimore, MD; ${ }^{2}$ Johns Hopkins University, Baltimore, MD; ${ }^{3}$ Johns Hopkins Bloomberg School of Public Health, Baltimore, MD; ${ }^{4}$ Johns Hopkins Center to Eliminate Cardiovascular Health Disparities, Baltimore, MD. (Control ID \#1335656)

BACKGROUND: High rates of uncontrolled hypertension among urban African Americans persist despite the existence of efficacious interventions to improve hypertension self-management. Studies identifying patients' and their families' perceived barriers to improving hypertension control could inform efforts to enhance existing interventions' effectiveness and sustainability in this population.

METHODS: Using the Community-Based Participatory Research (CBPR) framework, we performed focus groups to inform the design of behavioral self-management interventions intended to improve hypertension control among urban African Americans. We conducted 90-minute focus groups of patients with controlled (1 group) and uncontrolled (1 group) hypertension receiving care in an urban community health center as well as their family members (2 groups). Trained community members moderated all groups to assess participants' perceived barriers to improving their (a) self-management skills; (b) understanding of hypertension care; (c) knowledge of community resources; and (d) patient, family, and physician communication about hypertension. Moderators also assessed participants' views regarding the contributions of family members and the community health center itself in helping or hindering patients' self-management. The sessions were audio-recorded, transcribed, and independently coded by three investigators to distinguish themes.

RESULTS: Thirty members participated in 4 separate focus groups: patients with controlled hypertension $(n=8$, mean age $=61$, mean hypertension duration $=19$ years $)$, their family members $(n=5$, mean age $=50$, $40 \%$ with hypertension), patients with uncontrolled hypertension $(n=10$, mean age $=60$, mean hypertension duration $=19$ years), and their family members $(n=7$, mean age $=43,71 \%$ with hypertension). Patients from both groups described difficulties with taking medication, quitting smoking, reducing sodium intake, and having few educational and community resources as barriers to their hypertension self-care. Of those patients who 
had previously worked with community outreach workers, uncontrolled hypertensive patients discussed difficulties maintaining longterm relationships with them, "I cancelled out...she and I were knocking heads." Although both groups identified the long waiting room time as a barrier to care, uncontrolled hypertensive patients reported leaving the clinic without seeing their doctor if they had been waiting too long. Patients desired more education about nutrition and co-morbidities. Family members perceived patients to have problems with personal motivation and difficulties "[doing] the right thing." While some family members supported the patients by taking an active role in doctors' visits, others didn't attend visits because of privacy concerns. Family members of patients with uncontrolled hypertension requested more education for themselves.

CONCLUSIONS: Themes reflected a desire among urban African American participants for interventions addressing numerous behavioral challenges and educational needs related to their hypertension selfmanagement. Family members recognized patients' difficulties with selfmanagement and wanted their own interventions to support patients. Community clinic operations may also influence patients' engagement in hypertension care. Tailoring interventions to directly address concerns raised by urban African Americans with hypertension may improve interventions' effectiveness and sustainability.

TARGETING SPECIFIC PHYSICIAN GROUPS TO IMPROVE ADHERENCE TO ESTABLISHED DIAGNOSTIC GUIDELINES IN THE EVALUATION OF SYNCOPE. David Graham; Heidel E. Robert; Mark Rasnake. University of Tennessee Knoxville, Knoxville, TN. (Control ID \#1340163)

BACKGROUND: The 2009 European Society of Cardiology Task Force for the Diagnosis and Management of Syncope states that CT or MRI in uncomplicated syncope should be avoided unless indicated by neurological evaluation. It further states that for all practical purposes, a TIA concerns a focal deficit without loss of consciousness and syncope the opposite. The 2006 American Heart Association Statement on the Evaluation of Syncope recommends that neurological causes of syncope should only be pursued if suggested by the history or physical. Syncope is defined as a transient loss of consciousness due to transient global hypoperfusion characterized by a rapid onset, short duration, and spontaneous complete recovery. Studies have looked at educating Internal medicine residents on the workup of syncope in attempts to avoid prodigal usage of diagnostic tests with no change in clinical practice. Our study intends to identify if our physicians' ordering tendencies in the evaluation of syncope are according to established guidelines and to categorize the physician groups ordering these tests to identify those whose ordering tendencies should be targeted for intervention.

METHODS: All patients admitted to our hospital from 9/30/09 to 9/30/11 with the primary diagnosis of syncope (ICD-9 code 780.2) were included. Of 166 patients, 26 patients were excluded for having neurologic deficits or prolonged seizure activity documented by history or exam. Of the 140 patients meeting criteria, frequency statistics were utilized to calculate the prevalence of a) CT Head (CT) ordered for patients with and without head trauma, b) CT, MRI, and Carotid Ultrasound (US) tests ordered for patients with and without neurological deficits or prolonged seizures indicated by history or physical exam, b) US ordered for patients with and without a carotid bruit c) significant test results, d) costs per significant test result, and e) specialty of physician ordering CT, MRI, and US tests. All analyses were conducted using SPSS Version 19. A significant test result is a diagnostic test result that contributed to, confirmed, or established a diagnosis or management decision including follow up for further evaluation.

RESULTS: Of the 140 patients meeting inclusion criteria, only $29(20.7 \%)$ had documented head trauma, yet 98 people received CT and only 2/98 $(2.0 \%)$ yielded significant results. ED attendings ordered $71.4 \%$ of these. Only 5 patients had carotid bruits, yet 73/140 (52.1\%) received US Hospitalists, Internal Medicine (IM) residents, and Family practice (FP) residents accounted for $75.3 \%$ of the US's ordering physicians. MRI brain and stem was ordered for $41 / 140(29.2 \%)$ of the patients, of which hospitalists and IM residents accounted for $68.3 \%$ of the ordering physicians. Interestingly, $\$ 142,238$ was spent on the MRI studies of which none were significant. Notably, $\$ 53,949$ and $\$ 28,175$ were spent per significant result for CT and US respectively.

CONCLUSIONS: To improve cost effective utilization and adherence to established guidelines in the diagnostic evaluation of syncope, ED physicians should be targeted for intervention and training in guiding the selective usage of CT; IM residents and Hospitalists targeted for guiding the usage of MRI; and FP residents, IM residents and Hospitalists targeted for guiding the usage of US.

TELEMONITORING IN PATIENTS WITH HEART FAILURE: A SINGLE-CENTER EXPERIENCE Raid Abu-awwad; Yaser Alkhatib; Aymen Bukannan; Ghassan Bandak; Mazen El Atrache; Jacqueline Pflaum; Mohammad Zaidan; Kimberly Baker-Genaw. Henry Ford Hospital, Detroit, MI. (Control ID \#1285999)

BACKGROUND: Despite advances in medical treatment for heart failure (HF), rates of death and readmission after hospitalization remain high and impact the entire health system resources. Recently, two large randomized controlled studies showed that telemonitoring does not intrinsically carry a significant benefit in terms of improving HF outcomes. This contradicts with the findings of previous systematic reviews and meta-analyses of small scale studies which showed benefits of such strategy. The aim of our study is to assess the benefits of adopting such strategy in a focused, patient centered team care clinic as opposed to the large multicenter studies.

METHODS: The study was a retrospective chart review and analysis of 212 patients who were enrolled in the "The Heart Failure Tele-Assurance Program" at a tertiary medical center between the years 2007 and 2011. Only patients with at least 6 months of adherence to the program after enrollment were included in the study. Analysis included data collected over a time period of 6 months prior to enrollment through 6 months after enrollment. The primary end-points were number of hospitalizations, emergency department (ED) visits and clinic visits, for any reason. Secondary end-points included hospitalization for HF, admission to the intensive care unit (ICU) and number of days in the hospital. Comparison of pre-enrollment data to post-enrollment data was done using Wilcoxon signed-rank tests.

RESULTS: Of the 212 patients that were included in the study, mean age was $71,51 \%$ were female, and $78 \%$ were African-American. Mean ejection fraction (EF) was $41 \%$. There were significantly fewer overall hospitalizations, ED visits and clinic visits 6 months post-enrollment [decrease of $38 \%$ ( $<<$ $0.0001), 28 \%(\mathrm{p}=0.0002)$ and $24 \%(\mathrm{p}<0.0001)$ respectively]. There were also fewer hospitalizations for $\mathrm{HF}$ and total number of days in the hospital [decrease of $44 \%(p<0.0001)$ and $47 \%(p<0.0001)$ respectively]. Reduction in the admissions to the intensive care unit was marginally significant $(\mathrm{p}=0.0507)$

CONCLUSIONS: Despite the conflicting data in regards to the benefits of telemonitoring in heart failure patients, our study showed a significant benefit in terms of improving HF outcomes. This effect can be attributed in part to the triage of patients by a well-trained telemonitoring case manager at the initial signs of clinical deterioration, and the intervention using a prespecified protocol. Also our patients represent a population at a higher risk for readmission as compared to previous studies; older patients with lower mean EF and multiple comorbidities. We believe that identification of such patients by well trained medical professionals following a unified protocol in the proper setting can produce promising results for the health care systems adopting such strategy.

TEN-YEAR TRENDS IN HYPERTENSION, HYPERTENSION UNAWARENESS, UNTREATED HYPERTENSION AND UNCONTROLLED HYPERTENSION AMONG ADULTS IN GENEVA, SWITZERLAND Idris Guessous ${ }^{1,2}$; Murielle Bochud ${ }^{2}$; Jean-Marc Theler ${ }^{1}$; Jean-Michel T. Gaspoz ${ }^{1}$; Antoinette Pechere ${ }^{1} .{ }^{1}$ Geneva University Hospitals, Geneva 14, Switzerland; ${ }^{2}$ Univesity Institute of Social and Preventive Medicine, Lausanne, Switzerland. (Control ID \#1336629)

BACKGROUND: Hypertension is one of the leading causes of disease burden worldwide. Guidelines recommand the screening, treatement and 
control of high blood pressure. In Switzerland, there are no recent time trends in hypertension, hypertension unawareness, untreated hypertension and uncontrolled hypertension. The objectives of this study were to analyze these trends and to determine the associated factors.

METHODS: A population-based study conducted in the Canton of Geneva, Switzerland, which collects information on cardiovascular risk factors, diet and physical activity using a stratified random sampling. Hypertension was defined as mean systolic/diastolic BP $\geqq 140$ / $90 \mathrm{mmHg}$ or self-reported hypertension or presence of anti-hypertensive medication. Hypertension unawareness, untreated and uncontrolled hypertension were determined by questionnaires and BP measures. Non parametric trend tests were used to assess trends of prevalence rates across survey year. Multiple logistic regressions were used to estimate adjusted odds ratios (OR).

RESULTS: A total of 9,215 participants over a 10-year period were included. The hypertension prevalence rate increased between 1999 and 2009 from $31.1 \%$ to $36.5 \%$ ( $\mathrm{p}=0.04$ ). A significant increase was found in men $(35.6 \%$ to $43.5 \%, \mathrm{p}=0.04)$ but not in women $(26.2 .0 \%$ to $30.1 \%, \mathrm{p}=$ 0.45). During the same period, the prevalence rates of hypertension unawareness decreased from $37.6 \%$ to $16.7 \%$ ( $\mathrm{P}<0.001$ ), untreated hypertension increased from $43.8 \%$ to $48.9 \%(\mathrm{P}=0.09)$, and uncontrolled hypertension decreased from $69.1 \%$ to $50.0 \%(\mathrm{P}<0.001)$. Prevalence rates of untreated and uncontrolled hypertension were about $50 \%$ in 2009 . Factors associated with hypertension unawareness were current smoking $(\mathrm{OR}=1.27,95 \% \mathrm{CI}, 1.04-1.52)$, male gender $(\mathrm{OR}=1.47,1.23-1.75)$, hypercholesterolemia $(\mathrm{OR}=1.47,1.23-1.75)$, older age (OR 65-74y vs $35-49 y=1.36,1.10-1.69$ ), and alcohol consumption (OR upper vs lower tertile $=1.20,1.06-1.36)$. High level of education was associated with untreated hypertension $(\mathrm{OR}=1.39,1.14-1.17)$. Male gender $(\mathrm{OR}=1.49$, 1.12-1.96), sedentarity ( $\mathrm{OR}=0.79,0.64-0.99)$, and older age (OR 65-74y vs $35-49 y=1.58,1.02-2.43)$ were associated with an increased risk of uncontrolled hypertension. The magnitude of these associations decreased between the 1999-2002 and the 2003-2009 periods, with the exception of the association of smoking status with uncontrolled hypertension, which increased. The prevalence rate of hypertension linearly decreased with monthly household income (from $50.0 \%$ for $<3$ ' $000 \mathrm{CHF}$ to $23.4 \%$ for $>13^{\prime} 000 \mathrm{CHF} ; \mathrm{p}<0.001$ ), and the prevalence rates of untreated hypertension linearly increased with income (from $30.0 \%$ for $<3$ '000CHF to $50.7 \%$ for $>13^{\prime} 000 \mathrm{CHF} ; \mathrm{p}=0.03$ ). Hypertension and untreated hypertension prevalence rates differed by job position. Hypertension prevalence rate was the highest among the Retired, jobless, or disability insurance category $(59.5 \%)$, which however had the lowest prevalence rates of untreated hypertension $(26.8 \%)$

CONCLUSIONS: In a representative sample of Switzerland, the prevalence rate of hypertension increased between 1999 and 2009. While favourable trends in hypertension unawareness, untreated and uncontrolled hypertension occurred during this period, about half of hypertensive subjects were not treated or had uncontrolled high BP in 2009. This study identified determinants that should guide interventions aimed to improving hypertension treatement and control.

THE 12-MONTH COST-EFFECTIVENESS OF TELEPHONEDELIVERED COLLABORATIVE CARE FOR POST-CABG DEPRESSION Bruce L. Rollman; Julie M. Donohue; Bea Herbeck Belnap; Aiju Men; Fanyin He; Mark S. Roberts. University of Pittsburgh, Pittsburgh, PA. (Control ID \#1312829)

BACKGROUND: Depressive symptoms commonly follow coronary artery bypass graft $(\mathrm{CABG})$ surgery and are associated with poorer clinical outcomes. We demonstrated that telephone-delivered collaborative care $(\mathrm{CC})$ for post-CABG depression provided in concert with patients' PCPs reduces mood symptoms, and improves health-related quality of life (HRQoL) and physical functioning more than usual care (UC) at 8-months follow-up (Rollman BL, et al. JAMA 2009). We now report the cost-effectiveness of our intervention so as to guide clinicians, employers, insurers, and health systems on whether to adopt similar treatment strategies for treating depression following an acute cardiac event.
METHODS: From 3/04-9/07 we enrolled 302 post-CABG patients who screened positive for depression prior to hospital discharge; had at least a moderate level of mood symptoms two weeks later (PHQ-9 $\geq 10$ ); met all eligibility criteria; and were randomized to either our 8-month intervention or to UC. Later, we obtained insurance claims data for $189(63 \%)$ patients with $\geq$ 12 months continuous enrollment from Medicare and the two largest health insurers in our region. We applied 2007 Medicare prices to inpatient and outpatient claims and approximated intervention costs (e.g., care manager time, pharmacotherapy) to estimate incremental costs between UC and CC from the payor's perspective, and used generalized linear models with gamma distribution to correct for skewness in cost data. Then we converted SF-36 MCS scores collected at baseline, 2-, 4-, 8-, and 12-months follow-up to preference-based utilities, and calculated the incremental cost per qualityadjusted life year (QALY) gained for CC relative to UC.

RESULTS: At baseline, the 189 patients with continuous 12-month claims data were similar by: (a) sociodemographic and clinical characteristics to the 113 excluded from our analyses due to incomplete claims data; and (b) randomization status ( $90 \mathrm{CC}$ and $99 \mathrm{UC}$; mean age: 67 years, $61 \%$ male). At 12 -months, CC patients had $\$ 449$ lower mean total costs than UC $(\$ 18,172$ vs. $\$ 18,621)$, at an incremental cost effectiveness ratio of $-\$ 9,889$ per QALY vs. UC $(95 \%$ CI: $-\$ 11,940$ to $-\$ 7,838)$.

CONCLUSIONS: Telephone-delivered collaborative care for post-CABG depression is both more effective and less costly than PCPs' usual care and compares very favorably to other medical interventions at improving HRQoL. Future studies should examine: (1) how Accountable Care Organizations can provide similar treatment strategies for treating post-CABG depression in routine care; and (2) whether our treatment strategy is as effective and costeffective at treating depression in patients with other cardiovascular disorders.

\section{THE ASSOCIATION BETWEEN HOSPITALIST PHYSICIAN} WORKLOAD AND HOSPITAL LENGTH OF STAY Daniel J. Elliott ${ }^{1}$; Robert Young ${ }^{2}$; Paul Kolm ${ }^{1}$; Joanne C. Brice ${ }^{1} .{ }^{1}$ Christiana Care Health System, Newark, DE; ${ }^{2}$ Northwestern University, Chicago, IL. (Control ID \#1339782)

BACKGROUND: Hospitalist physicians are in increasingly high demand, largely because of perceived improvements in the efficiency of care. However, little data exist to determine the association between hospitalist workload and overall efficiency as measured by length of stay (LOS). The objective of this study is to determine the association between daily hospitalist physician workload and overall LOS.

METHODS: We conducted a retrospective cohort study of inpatients over 18 admitted to a large, private hospital medicine service between February 1, 2008 and January 31, 2011. We excluded patients who were admitted directly to an intensive care unit, were not discharged prior to the end of the study period, or had a hospital length of stay (LOS) $<0.5$ or $>100$ days. The exposure was the average physician workload on the day of a billed visit for each physician who billed a visit during the patient's hospitalization. Daily workload was calculated for each physician as the total Relative Value Units (RVU) generated each day. We standardized RVU values to 2011 CMS guidelines. The primary outcome was overall hospital LOS. Key covariates included patient demographics, the fragmentation of care index of physician continuity, and hospital characteristics including occupancy. We included all diagnoses and used severity-adjustment algorithms from Thomson-Reuters. We used linear mixed-effects models clustered by patient to determine the association of daily workload and LOS. LOS was log-transformed for all analyses to account for skewed data.

RESULTS: Overall, 20,406 hospitalizations met study criterion. Mean daily workload was 32.5 RVU. Mean LOS was 5.4 days. The figure shows the results of the adjusted analyses. LOS was shortest when workload was in the lowest quintile $(\mathrm{p}<0.001)$. LOS increased significantly as workload increased, with a maximum LOS between 31 to 37 RVUs per physician per day. The average LOS decreased for patients in the highest quintile of workload (>37 RVUs/day).

CONCLUSIONS: Hospitalist Physician workload is strongly associated with LOS, even after adjusting for patient and hospital factors. Importantly, LOS decreases at the highest levels of physician workload, which may reflect changes in practice that occur at the extremes of workload. Further studies to 
determine the impact of these LOS changes on quality and safety should be conducted, with particular attention to potential cost shifting that may occur with LOS reductions at the highest levels of physician workload

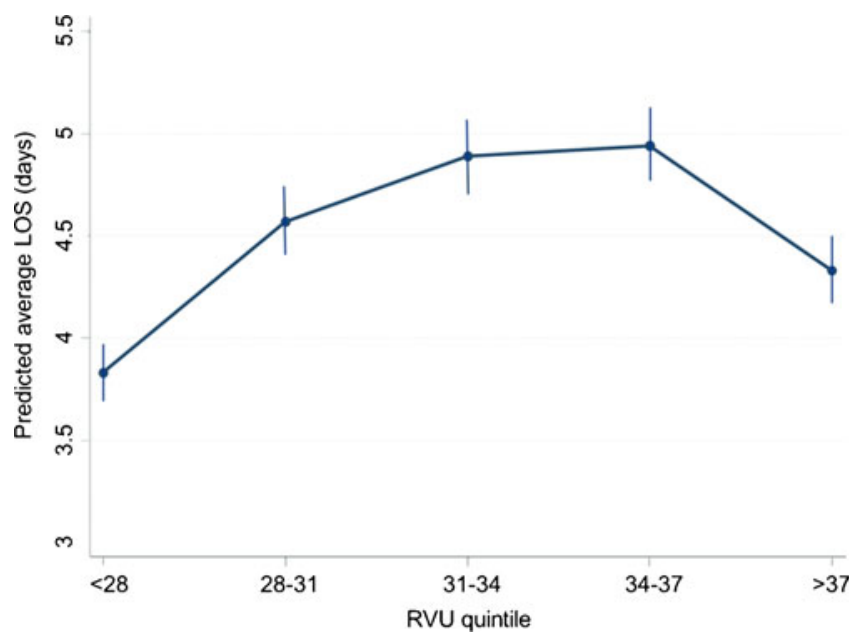

THE ASSOCIATION BETWEEN MEDICAL HOME READINESS, QUALITY OF CARE, AND RESOURCE USE Lena M. Chen ${ }^{1,2}$. Joseph Sakshaug ${ }^{2}$; David C. Miller ${ }^{2}$; Michael $\mathrm{Wu}^{2}$; John M. Hollingsworth ${ }^{2}$. ${ }^{1}$ VA Ann Arbor Healthcare System, Ann Arbor, MI; ${ }^{2}$ University of Michigan, Ann Arbor, MI. (Control ID \#1315619)

BACKGROUND: Hopes are high that reorganizing primary care practices into medical homes will improve the value of chronic disease care. Some data suggest that medical home model implementation is associated with modest quality gains, but less is known about the association between medical home readiness and resource use. Therefore, we sought to describe whether visits to medical home ready practices are associated with both higher quality care and less resource use.

METHODS: We conducted a retrospective analysis of visits by adults 18 years or older to a nationally representative sample of office-based primary care physicians in the United States. Our primary predictor was the medical home readiness of the practice in which each physician worked. We determined this by mapping physician responses from the 2007 and 2008 National Ambulatory Medical Care Survey (NAMCS) to the 2011 National Committee on Quality Assurance's (NCQA's) medical home standards. We created two groups: physicians who worked in practices that met $\geq 35 \%$ of NCQA points for medical home readiness ("medical home ready") vs. all other physicians. Our primary quality outcome was performance on each of nine previously validated outpatient quality indicators. Our outcomes for resource use were: ordering of $\geq 1$ lab test or $\geq 1$ radiology test, referral to a specialist, and admission to the hospital or emergency department. For each of the two NCQA groups, we estimated the proportion of visits meeting quality standards, and then used t-tests to test for group differences. Multivariate linear regression was used to assess the relationship between medical home readiness and resource use while adjusting for other factors, including age, gender, race/ethnicity, severity of illness, and type of visit (general medical exam or not).

RESULTS: Our cohort included 14,144 visits to general practitioners and 5,245 visits to general internists in 2007 and $2008.73 \%$ of all visits occurred at practices that were medical home ready. Patients with chronic conditions (e.g., $\mathrm{CHF}, \mathrm{CAD}, \mathrm{COPD}, \mathrm{CKD}$, or $\mathrm{DM}$ ) were as likely to visit medical home ready practices, as patients without these conditions. For all quality indicators, performance at medical home ready practices was higher, but the differences were only significant $(\mathrm{p}<0.05)$ for four indicators: beta blocker for $\mathrm{CHF}$, diuretic or beta-blocker for HTN, and diet and exercise counseling. Visits at which at least one lab or radiology test, or specialist referral was ordered, had a higher odds of occurring at a medical home ready practice (OR: 1.43, 1.36, 1.47 respectively, all $\mathrm{p}<0.05$ ). Outpatient visits which resulted in emergency department or hospital visits were equally likely to occur at practices that were or were not medical home ready.

CONCLUSIONS: There was a trend towards improved quality, but more frequent ordering of tests and consults, at visits to practices that were medical home ready. Efforts to increase medical home readiness may result in modestly higher quality of care, but greater resource use at the time of the outpatient visit.

THE ASSOCIATION BETWEEN REGIONAL VARIATIONS IN MEDICARE SPENDING AND PATIENTS' OUT-OF-POCKET MEDICAL EXPENDITURES Lena M. Chen ${ }^{1,2}$; Sidney $\mathrm{Le}^{3}$; Kenneth $\mathrm{M}$. Langa $^{1,2}$; Arnold M. Epstein ${ }^{3}$. VA Ann Arbor Healthcare System, Ann Arbor, MI; ${ }^{2}$ University of Michigan, Ann Arbor, MI; ${ }^{3}$ Harvard School of Public Health, Boston, MA. (Control ID \#1315603)

BACKGROUND: Wide regional variations in the costs of US medical care exist. Some policymakers hope that reducing utilization in high cost areas will decrease spending and increase efficiency. However, broad-based public support for this approach has been slow to materialize. To date, no one has examined how much regional variations in costs of care directly impact the finances of patients. We examined three questions. Compared to residents of low cost regions, do residents of high cost regions: 1) have higher out-ofpocket medical expenditures (OOPEs), 2) perceive themselves to be at higher risk for future catastrophic medical expenditures, and 3) more frequently purchase supplemental insurance coverage to protect themselves against future risk?

METHODS: We used the Health and Retirement Study (HRS) linked to Medicare data to identify a nationally representative cohort of noninstitutionalized, fee-for-service elderly Medicare beneficiaries who were interviewed in 2006 and 2008. To create our primary predictor spending quintile by hospital referral region (HRR) - we used data on total per capita standardized 2009 Medicare payments for fee-forservice Medicare beneficiaries 65 years of age or older. Our primary outcome was a self-reported, validated measure of OOPEs in the two years prior to the 2008 interview, and included OOPEs for hospital and outpatient visits, outpatient surgery, home health care, dental care, and medications. Secondary outcomes included: 2006-2007 Medicare payments for our cohort, self-reported estimates of the likelihood that medical expenses would eliminate household savings, and supplemental insurance rates.

RESULTS: Our cohort included 5,590 Medicare beneficiaries in 248 HRRs. Compared to residents of the lowest cost quintile, residents of the highest cost quintile were more likely to be poor, sick, and non-White (all $\mathrm{p}<0.05$ ). Predictors of higher OOPEs included higher net worth and White race (all $\mathrm{p}<$ $0.05)$. Median Medicare payments were $\$ 6,574(\$ 3,780)$ in the highest (lowest) cost quintile (ratio $=1.74, \mathrm{p}<0.001)$. Median OOPEs were $\$ 1,440$ $(\$ 1,448)$ in the highest (lowest) cost quintile (ratio $=0.99)$. Mean OOPEs were $\$ 2,637(\$ 2,330)$ in the highest (lowest) cost quintile (ratio $=1.13, p=0.006)$. Estimates of the likelihood of future catastrophic medical spending, and rates of supplemental insurance coverage did not differ between the highest and lowest cost quintiles (all p-values $>0.05$ )

CONCLUSIONS: Wide regional variations in costs of care do not translate into similarly large regional variations in OOPEs or differences in patients' concerns about future medical expenses. The protection that Medicare provides against wide regional variations in out-of-pocket medical expenditures may limit public support for commonly proposed strategies to reduce geographic variations in health care utilization and costs.

THE ASSOCIATION OF BMI AND NEIGHBORHOOD SOCIAL CAPITAL AMONG ADULTS IN LOS ANGELES COUNTY Adebowale Odulana $^{1}$; Eliana Perrin ${ }^{1}$; Tamera Coyne-Beasely ${ }^{1}$; Crystal Cene ${ }^{1}$; Feng-Chang Lin ${ }^{1}$; Arleen Brown ${ }^{2}$; Giselle Corbie-Smith ${ }^{1} .{ }^{1} \mathrm{UNC}$ at Chapel Hill School of Medicine, Chapel Hill, NC; ${ }^{2}$ Univeristy of California at Los Angeles, Los Angeles, CA. (Control ID \#1339394) 
BACKGROUND: Environmental factors have increasingly been recognized as important contributors to health. One environmental factor, neighborhood social capital, is associated with improved health behaviors and outcomes independent of other social indicators. Social capital defined as availability of, and accessibility to, group-level resources as a result of membership within a social group-has been shown to be an important determinant of health behaviors and therefore could affect body mass index (BMI). However, the relationship between neighborhood social capital and adult BMI is not clearly understood. In order to better understand how social capital may influence BMI, we examined the association between BMI and domains of neighborhood social capital in adults living in Los Angeles County.

METHODS: We used multi-level data from a cross sectional study of adults $(n=1986)$ in the Los Angeles Family and Neighborhood Survey (2006-2008). Neighborhood social capital was measured by the following accepted subdomains: social cohesion, social support, informal social control, social leverage, and neighborhood organization participation. We constructed multivariate models of the association between the outcome variable, BMI, and neighborhood social capital, initially adjusting for individual characteristics (age, gender, race/ethnicity, physical activity, income), and then adjusting for the subdomains of neighborhood social capital. We used a random-effects model to estimate the association and account for clustering in neighborhoods. RESULTS: Of the adults (34\% male, 60\% Hispanic, $19 \%$ White, $8 \%$ African American, 5\% Asian, mean age 39 yrs), mean BMI was 29.5, mean income was $\$ 31,360$, and mean self-reported physical activity was 1.8 times/7 days. Most (91\%) neighborhoods had moderate social cohesion; $52 \%$ had high social support; $50 \%$ had high informal social control; $58 \%$ had moderate social leverage; and $90 \%$ had low organization participation. Adjusting solely for individual characteristics, females $(p=0.001)$, decreased physical activity $(0.007)$, increased age $(p=0.001)$, Hispanics $(p=$ 0.001), and African Americans ( $\mathrm{p}=0.001$ ) had higher BMIs. Adjusting for individual characteristics and subdomains of neighborhood social capital, adults in neighborhoods with low informal social control had higher BMIs than those with moderate informal social control $(\mathrm{p}=0.046)$, and, similarly, adults in neighborhoods with low social support had higher BMIs than those with high social support $(\mathrm{p}=0.034)$.

CONCLUSIONS: Low informal social control and low social support seem to be important domains in the relationship of neighborhood social capital and higher BMIs. This relationship provides insight into how environmental factors can lead to adverse health outcomes. These findings can be leveraged in the design of interventions that take into account and prompt both individual and group level behavioral change.

THE ASSOCIATION OF RACE CONSCIOUSNESS WITH THE PATIENT-PHYSICIAN RELATIONSHIP, MEDICATION ADHERENCE, AND BLOOD PRESSURE IN URBAN PRIMARY CARE PATIENTS LaPrincess Brewer ${ }^{1,4}$; Kathryn A. Carson ${ }^{2}$; Camara P. Jones ${ }^{3}$; Lisa A. Cooper $^{1,2}$. Johns Hopkins University School of Medicine, Baltimore, MD; ${ }^{2}$ Johns Hopkins Bloomberg School of Public Health, Baltimore, MD; ${ }^{3}$ Centers for Disease Control, Atlanta, GA; ${ }^{4}$ Johns Hopkins Bayview Medical Center, Baltimore, MD. (Control ID \#1334803)

BACKGROUND: Discrimination has been linked to poorer adherence and impaired patient-physician relationships among African Americans (AAs). Previous studies also suggest associations between discrimination and blood pressure (BP) among ethnic minorities; however, patterns of association remain unclear. Internalized racism is one mechanism by which discrimination may negatively impact BP. We hypothesized that race consciousness, a measure of internalized racism, would be associated with poorer ratings of the patient-physician relationship, lower medication adherence, and higher BP, among AA, but not among White patients with hypertension

METHODS: This cross-sectional analysis includes 266 patients with hypertension seeing 41 physicians in 14 urban community-based primary care clinics serving predominantly minority or low income populations in Baltimore, MD. The predictor variable, race consciousness, was dichotomized as ever think about one's race and never think about one's race. Continuous outcomes assessed were systolic (SBP) and diastolic (DBP) blood pressures. Dichotomous outcomes were perceived respect from the physician, likelihood of recommending the physician to a friend and adherence on the Morisky medication adherence scale. Stratifying by patient race, generalized estimating equations (GEE) were used to control for patients being nested within physicians. Models were adjusted for patient's age and race concordance with the physician, but race concordance was then excluded because it did not significantly change the estimates for race consciousness.

RESULTS: Mean age was 61.3 years, $62 \%$ were AA and $65 \%$ were women. AA patients were more likely to ever think about race than white patients $(49 \%$ vs. $21 \%, \mathrm{P}<0.001)$. There were no significant associations between race consciousness and ratings of the physician or adherence among AAs. Race-conscious AA patients had significantly higher DBP (79.3 vs. $74.6 \mathrm{mmHg}, \mathrm{P}<0.05)$ and somewhat higher $\mathrm{SBP}(138.7$ vs. $134.8 \mathrm{mmHg}, \mathrm{P}=$ 0.12 ) than those who were not race-conscious. Race-conscious whites were more likely to perceive respect from $(57.3 \%$ vs. $27.8 \%, \mathrm{P}=0.008)$ and recommend their physician to a friend $(51.8 \%$ vs. $20.9 \%, \mathrm{P}=0.008)$, but had significantly lower medication adherence $(62.8 \%$ versus $83.2 \%, \mathrm{P}<0.05)$ than those who were not race-conscious. There was no association between race consciousness and BP among whites.

CONCLUSIONS: Race consciousness is associated with higher DBP, and may be associated with higher SBP among AA, but not among white patients with hypertension. In contrast, race consciousness is associated with ratings of the patient-physician relationship and adherence among whites, but not AAs. Future work should explore disparities in race consciousness and its impact on health and healthcare.

THE CHOLESTEROL, HYPERTENSION, AND GLUCOSE EDUCATION (CHANGE) STUDY: RESULTS FROM A RANDOMIZED CONTROLLED TRIAL IN AFRICAN AMERICANS WITH DIABETES Benjamin Powers ${ }^{1,2}$; Janet M. Grubber' ${ }^{2}$ Maren Olsen ${ }^{2}$; Matthew $\frac{{ }_{\text {Crowley }}{ }^{1,2} \text {; Hayden bOSWORTH }}{1,2}$. ${ }^{1}$ Duke University Medical Center, Durham, NC; ${ }^{2}$ Durham VA Medical Center, Durham, NC. (Control ID \#1340768)

BACKGROUND: Cardiovascular disease (CVD) and diabetes account for over one third of the mortality difference between African Americans and white patients. Health services interventions focusing on patient selfmanagement and physician medication management have been proposed as means of improving chronic disease control, and some studies suggest a particular benefit from these interventions among African Americans. We sought to evaluate the effect of a nurse telephone intervention targeting CVD risk reduction in African Americans with diabetes.

METHODS: We randomized 359 African Americans receiving primary care for type 2 diabetes from one of the two participating clinics to receive either usual care or a tailored nurse telephone intervention. The intervention provided monthly self-management support by phone tailored to patient needs and quarterly facilitation of medication management through electronic communication with the physician providers. Patients were surveyed about health beliefs, health behaviors, and knowledge at baseline and 12 months. The three co-primary outcomes were change in clinic-measured systolic blood pressure, $\mathrm{Hb}$ A1c, and LDL cholesterol over the 12 months of intervention delivery. Data were analyzed using linear mixed models assuming a linear trend over time and adjustment for the site stratification variable.

RESULTS: Of the 359 patients included, $70 \%$ were female, $69 \%$ reported graduating from high school, $46 \%$ read below a 9 th grade level, and $38 \%$ reported annual income under $\$ 10,000$. The overall model-estimated mean systolic blood pressure, LDL cholesterol, and $\mathrm{Hb}$ Alc at baseline was $136.7 \mathrm{mmHg}, 99.3 \mathrm{mg} / \mathrm{dl}$, and $8.0 \%$, respectively. The intervention patients received a mean of $9.9(\mathrm{sd}+3.0)$ intervention sessions from the nurses. In the intervention arm, the nurses contacted the physician providers at 3,6 , and 9 months; providers replied to $76 \%$ of these encounters, and $18 \%$ of these contacts resulted in physician recommendations for medication changes. There was no significant between group difference at 12 months for SBP $(2.4 \mathrm{mmHg}$ higher in intervention arm; 95\% CI: $-1.2,6.0 ; \mathrm{p}=0.18$ ), LDL cholesterol $(0.9 \mathrm{mg} / \mathrm{dl}$ higher in intervention group; $95 \% \mathrm{CI}-6.6,8.4 ; \mathrm{p}=0.81)$ or $\mathrm{Hb} \mathrm{Alc}$ $(0.07 \%$ lower in intervention group; $95 \% \mathrm{CI}:-0.39,0.25 ; \mathrm{p}=0.66)$.

CONCLUSIONS: In spite of prior studies suggesting benefit of nurse telephone self-management interventions in African Americans and patients 
with limited literacy, we found no significant difference in any of the three outcomes for this high risk population with limited resources. While patient participation in the phone calls was high, provider medication changes were low with this model of care. Consistent, effective, and sustainable interventions for high risk African Americans with diabetes remain elusive.

THE DIABETES LITERACY AND NUMERACY EDUCATIONAL TOOLKIT LATINO (DLNET-LATINO): A PRE-POST FEASIBILITY STUDY OF AN EDUCATIONAL INTERVENTION FOR LATINO ADULTS WITH DIABETES. Richard O. White ${ }^{1}$; Tebeb Gebretsadik ${ }^{3}$; Sunil Kripalani ${ }^{2}$; Russell L. Rothman ${ }^{2}{ }^{\text {I }}$ Meharry Medical College, Nashville, TN; ${ }^{2}$ Vanderbilt University Medical Center, Nashville, TN; ${ }^{3}$ Vanderbilt University Medical Center, Nashville, TN. (Control ID \#1340073)

BACKGROUND: Latinos are now the most populous minority group in the United States and bear a disproportionate burden of type 2 diabetes and its related complications. Deficits in health literacy and numeracy have been documented in Latinos and likely contribute to disparities in diabetes care. The aim of this study was to evaluate the feasibility of a diabetes educational intervention for adult Latinos with limited literacy and numeracy skills and to explore its potential for impacting knowledge, self-efficacy, self-care activities, and glycemic control.

METHODS: A convenience sample of adult Latino patients with diabetes was recruited from a community-based academic medical center and a federally-qualified health clinic which serve a growing immigrant population with many socio-demographic challenges in Nashville, TN. We designed a novel curriculum, the DLNET-Latino, a culturally-tailored, literacy/numeracy sensitive educational intervention with 6 modules that address general diabetes knowledge, diet, exercise, medication/insulin management, stress, and the medical visit. Participants received 4 weekly diabetes educational sessions led by a bilingual nutritionist trained in the content and delivery of the DLNET-Latino. Baseline data collection included demographics, psychosocial factors, behaviors, and clinical variables (A1c, lipids). Measures were repeated immediately post intervention and during a follow-up visit that ranged from 3-6 months later. Our primary outcome was the feasibility of intervention delivery. We also explored changes in knowledge, self-efficacy, self-care activities, and glycemic control from baseline to follow-up using Wilcoxon signed-rank tests. Results were also stratified by literacy status and compared using Wilcoxon rank-sum tests. RESULTS: 36 adults were enrolled with 22 completing all intervention sessions, and 16 providing complete follow-up data. Median age and time in the U.S. were 49 and 12 years respectively. Participants were mostly women, uninsured, had low levels of acculturation, and were of Mexican nationality. $91 \%$ earned $<20 \mathrm{~K}$ annually, $92 \%$ had $<$ HS education, and $42 \%$ had limited health literacy. In unadjusted bivariate analyses of baseline, post-intervention, and follow-up measures, significant improvements were observed immediately post-intervention in self-reported dietary behavior $(\mathrm{p}$ $=0.003)$ and medication adherence $(p=0.08)$. Participants with higher literacy reported significantly improved exercise behavior immediately post-intervention $(p=0.029)$; however, none of these findings were sustained at follow-up. No improvements were seen in knowledge, glycemic control or other self-care activities.

CONCLUSIONS: The DLNET-Latino intervention was accepted by a sample of adult Latinos with diabetes. We experienced a number of challenges during the post-intervention period of our program with moderate retention at 3-6 months follow-up. This was due largely in part to the many socio-demographic challenges faced by this vulnerable population. Nonetheless, our intervention shows promise for impacting self-care behaviors among adult Latinos with limited health literacy and diabetes and warrants further evaluation.

THE ECONOMICS OF IN-PATIENT STRESS TESTING AND ITS PERCEIVED BENEFIT TO LOW RISK PATIENTS Mukesh Gopalakrishnan ${ }^{1}$; Rojina Pant ${ }^{1}$; Jeffrey Cook $^{2}$; Lloyd W. Klein ${ }^{2} .{ }^{1}$ Advocate Illinois Masonic Medical Center, Chicago, IL; ${ }^{2}$ Advocate Illinois Masonic Medical Center, Chiacgo, IL. (Control ID \#1315852)

BACKGROUND: "Acute Coronary Syndrome Rule Out" accounts for 37\% of telemetry admissions. Our aim was to determine the financial burden of such patients without known coronary artery disease (CAD), evaluate the effect of stress test on their hospital course and assess outpatient testing as a safe alternative for low risk patients.

METHODS: We conducted a retrospective observational study of 265 consecutive patients who underwent myocardial perfusion imaging. Patients were categorized into low, intermediate and high pretest probability with Diamond Forrester Risk Score (DFR) and Framingham Risk Score (FRS) for CAD. Incidence of abnormal stress tests, change in management prior to discharge with modification of cardiovascular medication regimen, interventions or consultations, and final discharge diagnosis were compared in each group. Cost analysis was done based on 24-hour hospital stay in a telemetry unit, services rendered and subsequent benefit to patients.

RESULTS: Inpatient management did not change for $93.7 \%$ of those in the low-risk FRS group, $(\mathrm{p}<0.001)$ and $92.9 \%$ in the low-risk DFR group, $(p=0.002)$ based on stress tests. There were $82 \%$ with significant change in medical management that belonged to intermediate risk group. Comparing costs for inpatient and outpatient stress testing demonstrated nearly double the expense for inpatient testing to obtain an abnormal stress test $\mathbf{\$ 7 5 , 9 6 7}$ versus $\$ 32,886$ ). Table 1 explains cost to obtain an abnormal stress test, cost for CAD diagnosis, and cost to provide benefit in terms of change in management to one patient under the three risk categories. For every patient in the low-risk group with a meaningful change in management, 2 and 5 patients in the intermediate- and high-risk groups, respectively, would have benefited from utilization of equivalent resources. $45 \%$ of physicians surveyed felt defensive medicine played a role in the decision to order stress testing.

CONCLUSIONS: It is ideal to restrict inpatient management and further stress testing within intermediate- and high-risk groups, while relegating outpatient stress testing to low-risk patients. Based on the 2008 U.S. National Hospital Ambulatory Care Survey, this would translate to billions of dollars, yet provide a similar patient safety profile.

Table 1

\begin{tabular}{llll}
\hline \hline & $\begin{array}{l}\text { Cost to Abnormal } \\
\text { Stress Test }\end{array}$ & $\begin{array}{l}\text { Cost to CAD } \\
\text { Diagnosis }\end{array}$ & $\begin{array}{l}\text { Cost to Benefit } \\
\text { One Patient }\end{array}$ \\
Low Risk & $\$ 30,625$ & $\$ 576,450$ & $\$ 108,525$ \\
Intermediate & $\$ 17,500$ & $\$ 134,226$ & $\$ 50,645$ \\
Risk & $\$ 14,000$ & $\$ 42,940$ & $\$ 21,705$ \\
High Risk & $\$$ & & \\
\hline
\end{tabular}

THE EFFECT OF A STANDARDIZED CHEST PAIN PROTOCOL ON TROPONIN AND STRESS TEST UTILIZATION AND ITS IMPACT ON LENGTH OF STAY Aziz Ansari ${ }^{1}$; Mark Speyer ${ }^{1}$; Micky Simwenyi ${ }^{2}$; Elizabeth Schulwolf ${ }^{1}$. ${ }^{1}$ Loyola University Medical Center, Lisle, IL; ${ }^{2}$ Loyola University Medical Center, Lisle, IL. (Control ID \#1339833)

BACKGROUND: Observation units are increasingly common and one of the more frequent admission diagnoses is low risk chest pain (CP). Variation in practice exists regarding number of serum troponins and type of stress test ordered which may impact length of stay (LOS). Factors such as time intervals between each troponin and differences in duration of the stress test and time to test results can all influence length of stay. In April 2011 we implemented a CP protocol in an effort to standardize care for patients with low risk $\mathrm{CP}$ among our hospitalist group, which included ordering two troponins 6 hours apart, as well as guidelines for ordering stress tests.

METHODS: We analyzed administrative data of patients admitted to an observation unit managed by hospitalists only in an urban, academic medical center for evaluation of low risk $\mathrm{CP}$ from a 3-month period prior to initiation of the CP protocol (October 1, 2010 through December 31, 2010) and a 3-month period after (April 1,2011 through June 30, 2011). Variables included number of troponins and type of stress test. LOS was determined from date and time of admission to date and time of discharge. Continuous variables were compared using the Student's t-test and dichotomous variables compared using the chi-square test. 
RESULTS: A total of 525 patients were identified: 251 (47.8\%) preprotocol and 274 (52.2\%) post-protocol. A total of 191 (36.4\%) patients underwent a stress test: $91(47.6 \%)$ treadmill stress echo, $70(36.6 \%)$ nuclear and $29(15.2 \%)$ dobutamine stress echo. A total of $255(48.6 \%)$ had more than 2 troponins drawn. Mean LOS (pre 21.2 vs. post 22.2 hours) and mean total charges (pre $\$ 8014$ vs. post $\$ 8282$ ) were not significantly different. The proportion of patients receiving more than 2 troponins was significantly higher before the protocol when compared to after implementation $(183(72.9 \%)$ vs. $72(26.2 \%), p<0.001)$. The LOS was significantly longer in patients with more than 2 troponins but did not undergo a stress test $(22.0$ vs. 17.6 hours, $\mathrm{p}<0.001)$. Similarly, patients who underwent a stress test and had more than 2 troponins had a significantly longer LOS (27.7 vs. 23.1 hours, $\mathrm{p}=0.002)$. In patients who underwent stress tests, LOS by stress test showed significant differences in treadmill stress echo and nuclear stress test. Patients undergoing treadmill stress tests had a shorter $\operatorname{LOS}[\mathrm{n}=91,23.0$ vs. 26.9 hours $(\mathrm{p}=0.009)]$ and patients undergoing nuclear medicine tests had a longer LOS $[n=70,27.3$ vs 23.8 hours $(\mathrm{P}=0.02)]$. There was no difference in LOS in the dobutamine stress echo group.

CONCLUSIONS: The number of troponins and type of stress test ordered can impact LOS. The differences in LOS by stress test suggest that evaluation of the patient flow for those tests is warranted. Additionally, since 2 troponins obtained at least 6 hours apart is sufficient to rule out acute coronary syndrome in patients with low risk chest pain, establishing protocols to standardize evaluation of patients with this diagnosis could improve care efficiency and allow for improved flow of patients in an observation unit.

THE EFFECT OF DISTANCE TO PRIMARY CARE ON RESPONSE TO A HYPERTENSION TELEMEDICINE INTERVENTION AND CHANGE IN SYSTOLIC BLOOD PRESSURE Michael E. Bowen ${ }^{1,2}$; Hayden Bosworth ${ }^{3,4}$; Christianne Roumie ${ }^{1,2}$. ${ }^{1}$ VA Tennessee Valley Healthcare System, Nashville, TN; ${ }^{2}$ Vanderbilt University Medical Center, Nashville, TN; ${ }^{3}$ Durham VA Medical Center, Durham, NC; ${ }^{4}$ Duke University, Durham, NC. (Control ID \#1336158)

BACKGROUND: Distance to primary care is a significant barrier to healthcare access. Telemedicine clinical encounters that do not rely on face-to-face visits have been shown to improve blood pressure control; however, less is known about the relationship between distance to care and the response to telemedicine interventions. The objective of this study was to examine the interaction between distance to primary care and exposure to a hypertension telemedicine intervention on systolic blood pressure (SBP) within the Veterans Health Administration (VHA). We hypothesized that greater distance to care would be associated with a greater improvement in SBP among telemedicine intervention participants.

METHODS: We conducted a cross-sectional analysis of 503 veterans enrolled in the Hypertension Intervention Nurse Telemedicine study. Eligible veterans had a diagnosis of hypertension, took antihypertensive medication(s), had a primary care provider at the Durham VA Medical Center, and had an 18-month SBP measurement. Veterans were stratified by distance to primary care ( $<30$ miles vs. $\geq 30$ miles $)$ and subsequently grouped into usual care $(\mathrm{N}=147)$ and intervention $(\mathrm{N}=444)$ groups. The interaction between distance and the telemedicine intervention on 18 month SBP was examined using multivariable linear regression adjusting for baseline SBP, age, race, and marital status. Important subgroups were also examined including those with uncontrolled baseline SBP or a diagnosis of diabetes.

RESULTS: The median distance to primary care was 41 miles (IQR 1859). Veterans living $\geq 30$ miles from care were younger (63 years vs. 65 years; $p=0.006$ ) than veterans living $<30$ miles from care. Patients were $92 \%$ male, $48 \%$ were white, and $42 \%$ had diabetes. Mean (SD) baseline SBP was 129 (19) $\mathrm{mmHg}$ for patients living $\geq 30$ miles from care versus 131 (21) $\mathrm{mmHg}$ for those $<30$ miles $(\mathrm{p}=0.25)$. There was also no difference in baseline SBP by exposure to intervention $(p=0.36)$. In the adjusted regression model, the interaction between distance and intervention on 18 month SBP was non-significant (beta $0.13 ; \mathrm{p}=0.97$ ). For an average, 65 year old, single, white, veteran receiving usual care and living $<30$ miles to care, the SBP was 128 (95\% CI 122-134). No difference in the effect of distance $\geq 30$ miles on SBP in usual care (beta $0.13 ; p=0.97$ ) and intervention groups (beta $-0.80 ; \mathrm{p}=0.79$ ) was observed. Subgroup analyses of veterans with diabetes or uncontrolled SBP at study entry were also non-significant. Veterans had a median of 5 completed primary care visits during the study time period.

CONCLUSIONS: We did not observe an interaction between distance to primary care and the telemedicine intervention on SBP. Veterans had frequent clinical encounters and well controlled baseline SBP regardless of distance to care, possibly related to the high prevalence of diabetes and travel pay incentives provided by VHA. Although telemedicine can improve hypertension control, further work examining the relationship between distance to care and response to telemedicine interventions is needed.

THE EFFECT OF IMPROVEMENTS TO RACE/ETHNICITY DATA COLLECTION PRACTICES ON THE RACIAL/ETHNIC DISTRIBUTION OF HOSPITALIZED PATIENTS Rosette Chakkalakal ${ }^{1}$; Jeremy C. Green ${ }^{3}$; Harlan M. Krumholz'; Brahmajee K. Nallamothu ${ }^{2}$. ${ }^{1}$ Robert Wood Johnson Foundation Clinical Scholars Program, Yale University School of Medicine, New Haven, CT; ${ }^{2}$ University of Michigan, Ann Arbor, MI; ${ }^{3}$ Yale University, New Haven, CT. (Control ID \#1340440)

BACKGROUND: Hospitals collect and report data on patient race/ ethnicity that researchers and policymakers commonly use to identify and track healthcare disparities. However, substantial variation in the categories and procedures used by individual hospitals to obtain this information may make these data unreliable, particularly for smaller minority groups. In 2007, New Jersey hospitals adopted "Guidelines for Systematic Collection of Data on Patient Race and Ethnicity" throughout the state. This program sought to improve the process of collecting race/ethnicity data by: (1) requiring all New Jersey hospitals to use uniform categories for race/ethnicity and (2) training healthcare workers to solicit self-identified race/ethnicity data from patients using standard procedures. The purpose of our project was to determine if and how the racial/ethnic distribution of patients hospitalized for congestive heart failure (CHF), pneumonia, and acute myocardial infarction (AMI) in New Jersey changed as a result of this program.

METHODS: We used the Healthcare Utilization Project (HCUP) State Inpatient Databases (SID) to compare the racial/ethnic distribution of patients 18 years and older hospitalized with CHF, pneumonia, and AMI in New Jersey before (2005-2006) and after (2008-2009) implementation of the data collection changes relative to New York, a state with a similar racial/ethnic distribution of patients that has not implemented changes to data collection. Multinomial logistic regression was used to fit a difference-in-differences (DD) model where race/ethnicity was estimated as a function of the state in which the patient was hospitalized, the time period, the interaction of state and time period, and patient age and gender. Coefficients from the fitted model were used to predict racial/ethnic distributions for each diagnosis before and after implementation of the New Jersey program for 5 categories: non-Hispanic white, non-Hispanic black, Hispanic, Asian/Pacific Islander, and other. The percent change was calculated as the DD divided by the 2005-2006 proportions for New Jersey. Statistical significance was tested using bootstrap standard errors.

RESULTS: See Table 1 .

CONCLUSIONS: The racial/ethnic distribution of patients hospitalized for CHF, pneumonia, and AMI in New Jersey changed significantly as a result of a statewide effort to improve hospital data collection practices. Changes were most evident in the increased proportion of patients identified as Asian/Pacific Islander and decreased proportion of patients identified as other for all 3 diagnoses. More widespread adoption of standardized data collection strategies within hospitals could substantially improve our understanding of healthcare needs in smaller minority groups, including Asians and Pacific Islanders, by improving the identification of these groups within healthcare data. 
Table 1. Percent Change in Proportion of Individuals Identified in each Racial/Ethnic Group by Diagnosis

\begin{tabular}{|c|c|c|c|}
\hline & $\begin{array}{l}406,326) \\
\text { Change }\end{array}$ & $\begin{array}{l}\text { Pneumonia } \\
(\mathrm{n}=341,891) \\
\text { Percent Change } \\
(95 \% \mathrm{CI})\end{array}$ & $\begin{array}{l}\text { AMI }(\mathrm{n}=227,487) \\
\text { Percent Change } \\
(95 \% \text { CI })\end{array}$ \\
\hline spanic & $\begin{array}{l}3.0 \\
(2.2\end{array}$ & $\begin{array}{l}1.20 * * \\
(0.28,2.12)\end{array}$ & $0.98 * *(0.04,1$. \\
\hline $\begin{array}{l}\text { Non-Hispanic } \\
\text { Black }\end{array}$ & $-1.75)$ & $2.71(-0.55,5.98)$ & $\begin{array}{l}5.60 * * \\
(0.08,11.12)\end{array}$ \\
\hline Hispanic & $\begin{array}{l}-7.71^{*} \\
(-11.4\end{array}$ & $*$ & $-1.25(-8$ \\
\hline acific & $\begin{array}{l}16.50^{* *} \\
(1.38,31.62)\end{array}$ & $\begin{array}{l}35.61 * * * \\
(23.71,47.5)\end{array}$ & $\begin{array}{l}31.53^{* * *} \\
(14.72,48.34)\end{array}$ \\
\hline & $\begin{array}{l}-45.83 * * * \\
\quad(-57.09,-34.56)\end{array}$ & $\begin{array}{l}-45.74 * * * \\
\quad(-56.83,-34.64)\end{array}$ & $\begin{array}{l}-55.67 * * * \\
(-65.7,-45.64)\end{array}$ \\
\hline
\end{tabular}

Analysis restricted to adults age 18 and over. ${ }^{* * *} \mathrm{p}<0.01,{ }^{* *} \mathrm{p}<0.05$

THE EFFECT OF SHIFT DURATION ON THE EFFICACY AND TOLERABILITY OF ARMODAFINIL IN PATIENTS WITH EXCESSIVE SLEEPINESS ASSOCIATED WITH SHIFT WORK DISORDER John Harsh ${ }^{1}$; Steven G. Hull ${ }^{2}$; Ronghua Yang ${ }^{3} .{ }^{1}$ The University of Southern Mississippi, Hattiesburg, MS; ${ }^{2}$ Vince and Associates Clinical Research, Overland Park, KS; ${ }^{3}$ Teva Pharmaceuticals Ltd., Frazer, PA. (Control ID \#1337964)

BACKGROUND: Shift work disorder (SWD) is a circadian rhythm sleep disorder characterized by excessive sleepiness and/or insomnia in individuals working shifts. The wakefulness-promoting agent armodafinil has been shown to significantly improve clinical condition and wakefulness during the last 4 hours of the night shift, as well as overall functioning in patients with SWD. This post-hoc analysis examined whether the length of the night shift worked ( $\leq 9 \mathrm{hrs}$ vs. $>9 \mathrm{hrs}$ ) affected the efficacy and tolerability of armodafinil in patients with SWD.

METHODS: Patients in this study were diagnosed with SWD (DSM-IV and ICSD-2 criteria), worked at least five 6- to 12-hour night shifts per month, had mean Karolinska Sleepiness Scale (KSS) score $>6$, and Global Assessment of Functioning (GAF) score $<70$. Following randomization, patients received $150 \mathrm{mg}$ armodafinil or placebo on nights worked for 6 weeks. For the current analysis, patients were divided into 2 groups: those working $\leq 9$-hour shifts and those working $>9$-hour shifts. Efficacy assessments included change in Clinical Global Impression-Change (CGI-C) score related to excessive sleepiness late in the shift (including the commute home [4:00 AM to 8:00 AM]), GAF, late-in-shift KSS, and modified Sheehan Disability Scale (SDS-M) from baseline to final visit. The SDS-M was modified to capture the effect of shift work on work, family, and social life. Final visit data included last observation carried forward.

RESULTS: Of the 383 patients enrolled in the study, 279 (73\%) worked shifts $\leq 9 \mathrm{hrs}$ ( $\mathrm{n}=132$ armodafinil; $\mathrm{n}=147$ placebo) and $104(27 \%)$ worked shifts $>9$ hrs $(\mathrm{n}=61$ armodafinil; $\mathrm{n}=43$ placebo). At final visit, a significantly greater proportion of armodafinil patients demonstrated an improvement in late-in-shift CGI-C score from baseline compared to placebo regardless of shift duration $(\leq 9 \mathrm{hrs:} 78 \%$ vs. $60 \%$ [p=0.0017]; $>9$ hrs: $77 \%$ vs. $46 \%$ [p=0.002]). Significantly greater improvements with armodafinil were also observed in both shift duration groups for the GAF ( $\leq 9$ hrs: +9.5 vs. $+5.4[\mathrm{p}<0.0001] ;>9$ hrs: +9.6 vs. $+4.3[\mathrm{p}=0.0019])$ and late-in-shift KSS scores ( $\leq 9$ hrs: -2.9 vs. -1.9 [p=0.0002]; $>9$ hrs: -2.8 vs. $-1.6[p=0.0028])$ at final visit. However, armodafinil treatment led to significantly greater improvement in composite SDS-M scores at final visit only in those patients working $>9 \mathrm{hr}$ shifts $(\leq 9 \mathrm{hrs}$ : -6.8 vs. -5.0 $[\mathrm{p}=0.0536] ;>9$ hrs: -6.8 vs. $-2.7[\mathrm{p}=0.0086])$. The most common adverse events were nausea and headache and a greater proportion of patients working $>9 \mathrm{hrs}$ had at least one adverse event compared with those working $\leq 9 \mathrm{hrs}$.

CONCLUSIONS: These findings indicate that shift duration did not affect the improvement with armodafinil over placebo in terms of late-in-shift clinical condition and wakefulness and overall functioning in patients with SWD. Only those patients working $>9 \mathrm{hrs}$ demonstrated improvements over placebo in disability following armodafinil treatment. A greater proportion of patients working $>9 \mathrm{hrs}$ reported adverse events associated with armodafinil, although the types of events reported were similar between shift duration groups. This study was funded by Cephalon, Inc, now a part of Teva Pharmaceuticals Ltd.

\section{THE EFFECT OF STATE MEDICAID EXPANSIONS ON MORTALITY} AND INSURANCE COVERAGE AMONG NON-ELDERLY ADULTS Benjamin D. Sommers; Katherine Baicker; Arnold M. Epstein. Harvard School of Public Health, Boston, MA. (Control ID \#1317587)

BACKGROUND: Several states significantly expanded Medicaid eligibility for adults in the past decade, and the Affordable Care Act will expand Medicaid dramatically in 2014. Yet the effect of acquiring Medicaid on health outcomes among adults remains unclear. Our objective was to examine whether Medicaid expansions resulted in any changes in all-cause mortality, as well as coverage, access to care, and self-reported health status.

METHODS: Our study used a differences-in-differences quasi-experimental design. The intervention group included three states significantly expanding adult Medicaid eligibility since 2000 (New York, Maine, and Arizona). The control group included neighboring states without expansions. The sample contained all adults aged 20-64, observed 5 years before and 5 years after expansions. Data sources were the CDC's 1997-2007 Compressed Mortality File for county-level mortality $(\mathrm{N}=68,012)$; the Current Population Survey for insurance coverage and health status $(\mathrm{N}=169,124)$; the Behavioral Risk Factor Surveillance System for barriers to care $(\mathrm{N}=192,148)$; and the Area Resource file for county-year covariates. Multivariable regression models adjusted for demographics, county of residence, and local economic conditions. Prespecified subgroup analyses examined differential effects by age, race, and county poverty rates. Our primary outcome was all-cause mortality rate per 100,000 adults. Secondary outcomes were insurance coverage, deferring needed care due to costs, and self-reported health status.

RESULTS: Medicaid expansions were associated with a significant reduction in all-cause mortality ( -19.6 deaths per $100,000, p=0.001$; from a baseline of 320 deaths per 100,000$)$. Mortality reductions were greater for older adults, non-whites, and residents of poorer counties. Expansions increased the percentage of adults with Medicaid coverage $(+2.2$ percentage points, $\mathrm{p}=$ $0.014)$; decreased the proportion uninsured $(-3.2, \mathrm{p}<0.001)$; decreased the percentage deferring care due to costs $(-2.9, \mathrm{p}=0.002)$; and increased the percentage reporting "excellent" or "very good" health $(+2.2, \mathrm{p}=0.045)$.

CONCLUSIONS: State Medicaid expansions to low-income adults were associated with significant reductions in all-cause mortality over a five-year follow-up period. Our analyses of secondary outcomes provide a plausible causal chain for these mortality RESULTS: Medicaid eligibility expansions were associated with higher Medicaid coverage rates, lower rates of being uninsured, reduced financial barriers to care, and improved self-reported health. The Affordable Care Act expands Medicaid eligibility starting in 2014 to a similar group as these state expansions - primarily low-income childless adults. In contrast, budget pressures have led several states to consider cutting eligibility from previous expansions. Policymakers should be aware that major changes in Medicaid - either expansions or reductions in coverage - may have significant effects on the health of vulnerable populations.

THE EFFECT OF A SELF-AFFIRMATION WRITING EXERCISE ON RACE-DISCORDANT PATIENT-PROVIDER COMMUNICATION Rebecca Hanratty ${ }^{1,2}$; Edward P. Havranek ${ }^{1,2}$; Channing Tate ${ }^{1}$; L Miriam Dickinson $^{2}$; John F. Steiner ${ }^{3}$; Geoffrey Cohen ${ }^{4}$; Irene V. Blair ${ }^{5}$. ${ }^{1}$ Denver Health Medical Center, Denver, CO; ${ }^{2}$ University of Colorado School of Medicine, Aurora, CO; ${ }^{3}$ Kaiser Permanente of Colorado, Denver, CO; ${ }^{4}$ Stanford University, Palo Alto, CA; ${ }^{5}$ University of Colorado-Boulder, Boulder, CO. (Control ID \#1340110)

BACKGROUND: Although provider bias is thought to play a role in the genesis of health disparities by race, documentation of this has generally been lacking. Fear of stereotyping on the part of patients might be affecting race- 
discordant patient-provider encounters. The process of self-affirmation has been shown to lessen the impact of stereotype threat.

METHODS: Within a single community health center, we randomized 99 African American patients with hypertension to perform either a selfaffirmation exercise or a control exercise prior to a visit with their primary care provider and to have the visit audiotaped. We compared the patientprovider communication for the two groups quantitatively using the Roter Interaction Analysis System (RIAS), and evaluated visit satisfaction, trust, stress, and mood after the visit by questionnaire.

RESULTS: Patients were generally middle aged (self affirmation group mean $53.6 \pm 9.1$ years, control group mean $57.3 \pm 10.5$ years), predominantly female $(69.1 \%$ and $65.9 \%)$ and had a high school education or less (76.4\% and 63.6\%). Patients in the intervention group requested and received more information about their medical condition (number of statements $66.3 \pm 6.8$ in the self-affirmation group, $48.1 \pm 5.9$ in the control group, $\mathrm{p}=0.02$ ). Patient communication in the intervention group was rated more interested, friendly, responsive, interactive, and respectful $(\mathrm{p}=0.03)$ and less depressed and distressed $(\mathrm{p}=0.02)$. Questionnaires did not detect differences in visit satisfaction, trust, stress, or mood.

CONCLUSIONS: A self-affirmation exercise improved some aspects of patient-provider communication in race-discordant primary care visits.

THE EFFECT OF CIGARETTE SMOKING ON DIABETIC PERIPHERAL NEUROPATHY: A SYSTEMATIC REVIEW AND META-ANALYSIS Carole Clair ${ }^{1,2}$; Marya J. Cohen ${ }^{3}$; Florian S. Eichler ${ }^{4}$; Nancy A. Rigotti ${ }^{2}$. 'Lausanne University, Lausanne, Switzerland; ${ }^{2}$ Massachusetts General Hospital, Boston, MA; ${ }^{3}$ Massachusetts General Hospital, Boston, MA; ${ }^{4}$ Massachusetts General Hospital, Boston, MA. (Control ID \#1335435)

BACKGROUND: Diabetic peripheral neuropathy (DPN) is a common and incapacitating syndrome affecting almost one-third of people with diabetes. About one-fifth of people with diabetes also smoke. Tobacco use is a known risk factor for development of cardiovascular complications in people with diabetes and studies suggest that it might also be a risk factor for development of microvascular complications such as DPN. The objective of the systematic review and meta-analysis is to assess the relationship between smoking and peripheral neuropathy in people with type 1 or type 2 diabetes.

METHODS: A systematic review of MEDLINE, EMBASE, and Cochrane Clinical Trials databases was conducted from 1966 to August 2011. Each study was reviewed for eligibility independently by 2 authors. Studies were included if they reported DPN as an outcome and smoking status as an exposure in a population with type 1 or type 2 diabetes. Data from the included studies were extracted and quality assessed. We performed separate analysis for prospective cohort studies and cross-sectional studies. All pooled analyses were based on random effects models and heterogeneity was assessed using I2 statistics. We used Review Manager to perform the statistical analyses.

RESULTS: From 1,644 abstracts, 24 studies ( 9 prospective cohorts and 15 cross sectional) met the inclusion criteria and agreement between reviewers was good (Kappa 0.72). The prospective cohort studies included 5,477 participants who did not have DPN at baseline. In 5 studies participants had type 1 diabetes, in 3 studies they had type 2 diabetes and in one study they had both. During a follow up ranging from 2 to 9 years, 1,539 cases of DPN occurred. Most studies reported an increased risk of DPN associated with smoking but 3 studies reported a decreased risk. Odds ratios (ORs) for DPN associated with smoking ranged from 0.22 to 2.20. The pooled OR of developing DPN associated with smoking using a random effect model was 1.16 (95\% Confidence Interval (CI) 0.76-1.78). There was significant heterogeneity between studies. In sub-analysis restricted to studies where smoking status was well-defined and ORs were adjusted for at least $\mathrm{HbA} 1 \mathrm{c}$ and diabetes duration, the pooled OR was 1.62 (95\% CI 1.30-2.01) and there was no evidence of heterogeneity. There was no evidence of publication bias. The cross sectional studies included 5,627 participants, 11 with type 2 diabetes, 2 with type 1 diabetes and 2 with both. Only 7 studies had analysis adjusted for the main confounders and ORs ranged from 0.68 to 3.32 . The pooled OR of DPN associated with smoking was 1.68 (95\% $1.30-2.17)$ using a random effect model. There was no evidence of publication bias.
CONCLUSIONS: People with diabetes who smoke have an increased risk of having or developing neuropathy compared with non smokers. Targeting smoking cessation is a key factor for improvement of diabetic complications such as DPN.

THE EPIDEMIOLOGY OF WEIGHT COUNSELING IN THE UNITED STATES: A CASE OF POSITIVE DEVIANCE Christopher Sciamanna ${ }^{1}$; Jennifer Kraschnewski ${ }^{1}$; Kathyrn Pollak ${ }^{2}$; Heather Stuckey ${ }^{1}$; Nancy Sherwood ${ }^{3} .{ }^{1}$ PennState Hershey, Hershey, PA; ${ }^{2}$ Duke University, Durham, NC; ${ }^{3}$ University of Minnesota, Minneapolis, MN. (Control ID \#1311395)

BACKGROUND: Primary care providers (PCPs) rarely counsel about weight, despite NIH guideline recommendations. To improve the rate of weight counseling, a full understanding of the epidemiology of weight counseling in primary care is needed. Although many have examined the rate of weight counseling using visit-based data from the National Ambulatory Medical Care Survey (NAMCS), no study has described the frequency of weight counseling at the level of the provider. The objective of this study was to measure the frequency of weight counseling at the level of the PCP and to identify the characteristics of patients who are seen by "positive deviant" PCPs who engage in higher rates of weight counseling.

METHODS: Data were examined from the 2007 to 2008 NAMCS, a national survey designed to provide reliable data about the provision of ambulatory medical care services in the U.S. We performed a cross-sectional study of 21,220 U.S. adult (age >17) outpatient primary care visits with 954 PCPs (general/family practitioner and general internal medicine). Rate of counseling was determined per PCP by dividing the number of visits with weight counseling by the total number of visits per PCP. Positive Deviance is an approach that studies successful individuals and compares them to those who are less successful. Positive deviant (PD) physicians were those who: 1) performed higher levels of weight counseling and 2) as a group, provided half of all weight counseling by PCPs in NAMCS. Visit and patient characteristics seen by $\mathrm{PD}$ and non-PD physicians were then compared to understand whether the higher rates of weight counseling among PD physicians were due primarily to previously reported patient characteristics (e.g., age, ethnicity, insurance). Sample weights were applied to account for the complex sampling design and to allow extrapolation of national estimates. Chi-squared and analysis of variance were used to compare visits to PD and non-PD physicians. Logistic regression was used to describe the association between visits to PD and nonPD physicians and receipt of weight counseling, adjusting for covariates.

RESULTS: Most (58\%) PCPs performed no weight counseling during any patient visits. Eighty-five (8.9\%) PCPs provided $52 \%$ of all weight counseling and were categorized as PD physicians. Patients seeing PD physicians were older, more likely to be male and to have hypertension, diabetes, and obesity. After adjusting for patient characteristics, including patient age, gender, race, ethnicity, insurance status, hypertension, diabetes and obesity, strengthened status was strongly associated with receipt of weight counseling during visits [adjusted $\mathrm{OR}=13.2$ (95\% CI; 11.5-15.7)].

CONCLUSIONS: In conclusion, a minority of PCPs (Positive Deviants) provide the majority of primary care weight counseling in the US. Studies of these PCPs may help to identify practical methods to increase weight counseling in primary care settings.

THE FAST PATH PROGRAM: ENGAGEMENT AND DEPENDENCE OUTCOMES IN A PRIMARY CARE-BASED ADDICTION TREATMENT PROGRAM Alexander Y. Walley ${ }^{1}$; Joseph Palmisano ${ }^{2}$; Amy SorensenAlawad ${ }^{1}$; Kaylyn Duerfeldt ${ }^{1}$; Anita Raj ${ }^{3,1}$; Donald Allensworth-Davies ${ }^{2}$; Jeffrey H. Samet ${ }^{1,2}$; Mari-Lynn Drainoni ${ }^{1,2}$. ${ }^{1}$ Boston University School of Medicine, Boston, MA; ${ }^{2}$ Boston University School of Public Health, Boston, MA; ${ }^{3}$ University of California, San Diego School of Medicine, San Diego, CA. (Control ID \#1333471)

BACKGROUND: Addiction and medical outcomes can improve when treatment for these conditions are coordinated. Yet, it is not clear what factors are associated with engagement and reduction in substance 
dependence over time within coordinated programs. To understand better how to implement coordinated care, we explored factors associated with engagement in treatment and substance dependence over time in a primary care-based addiction treatment program at an urban medical center.

METHODS: After establishing a primary care-based addiction treatment program for patients with alcohol or drug dependence and HIV or risk for HIV, we conducted a cohort study of $81 \%(178 / 220)$ of the patients enrolled between February 1, 2008 and December 31, 2010 who completed both baseline and 6-month follow-up interviews. The main outcomes were engagement, defined using standard criteria ( 2 addiction treatment encounters within the first 14 days and 2 more within the next 30 days), and persistent alcohol or drug dependence, measured at 6-months with the Composite International Diagnostic Interview -Short Form (CIDI-SF). Using logistic regression we examined the following potential factors associated with study outcomes: HIV status, self-reported serious depression previous 30 days, housing status, polysubstance use at baseline, and receipt of buprenorphine treatment during follow-up. Adjusted models included these potential factors and the following covariates: age, gender, race/ethnicity, and education.

RESULTS: Patient characteristics at baseline were 61\% HIV-infected, $74 \%$ serious depression, $21 \%$ homeless, $51 \%$ polysubstance use; at $6-$ month $63 \%$ had been treated with buprenorphine. Engagement occurred in $66 \%$. Persistent alcohol or drug dependence was present in $48 \%$. In adjusted analyses, receipt of buprenorphine treatment was associated with engagement (Odds Ratio (OR) 5.8 95\% CI: 2.75-12.4). Baseline depression was associated with persistent substance dependence (OR 4.58 95\% CI: 2.02-10.4).

CONCLUSIONS: Buprenorphine treatment was a major driver of treatment engagement within a primary care-based addiction treatment program. Engaging patients not receiving or eligible for maintenance medication presents a challenge. With a substantial burden of self-reported depression at the beginning of treatment, including mental health treatment as part of integrated care may improve addiction treatment outcomes.

Factors associated with engagement and persistent substance dependence in a primary care clinic-based substance abuse treatment program

\begin{tabular}{|c|c|c|c|}
\hline & All $\mathrm{N}=178$ & $\begin{array}{l}\text { Engagement: } \\
\text { Adjusted } \\
\text { Odds Ratio } \\
(95 \% \mathrm{CI})\end{array}$ & $\begin{array}{l}\text { Substance } \\
\text { Dependence: } \\
\text { Adjusted } \\
\text { Odds Ratio } \\
(95 \% \text { CI })\end{array}$ \\
\hline $\begin{array}{l}\text { Age in years, Mean } \\
\text { (Std Dev) }\end{array}$ & $44(9)$ & $1.05(1.00-1.09)$ & $0.95(0.91-1.00)$ \\
\hline Female & $33 \%$ & $0.71(0.32-1.55)$ & $0.70(0.33-1.47)$ \\
\hline White, Non-Hispanic & $37 \%$ & Ref & Ref \\
\hline Hispanic & $31 \%$ & $1.04(0.39-2.78)$ & $0.63(0.25-1.52)$ \\
\hline $\begin{array}{l}\text { Black/African American, } \\
\text { Non-Hispanic }\end{array}$ & $24 \%$ & $0.45(0.15-1.29)$ & $0.60(0.22-1.63)$ \\
\hline Other, Non-Hispanic & $7.3 \%$ & $0.35(0.09-1.46)$ & $0.23(0.06-0.95)$ \\
\hline Completed 12th grade & $67 \%$ & $0.92(0.41-2.08)$ & $0.87(0.41-1.85)$ \\
\hline HIV infected & $61 \%$ & $1.76(0.79-3.92)$ & $0.94(0.45-1.96)$ \\
\hline $\begin{array}{l}\text { Self-reported } \\
\text { serious depression }\end{array}$ & $74 \%$ & $0.95(0.42-2.13)$ & $4.58(2.02-10.4)$ \\
\hline Homeless & $21 \%$ & $0.74(0.30-1.81)$ & $1.11(0.47-2.63)$ \\
\hline Polysubstance Us & $51 \%$ & $1.33(0.64-2.79)$ & $1.91(0.97-3.80)$ \\
\hline Buprenorphine treatment & $63 \%$ & $5.82(2.75-12.4)$ & $0.82(0.40-1.68)$ \\
\hline
\end{tabular}

Multiple logistic regression: Odds Ratios adjusted for all variables in the table. *Defined as use of $>1$ substance, past 30 days, at baseline: alcohol, heroin, cocaine, or marijuana

THE FEASIBILITY OF INTEGRATING DIABETES EDUCATION WITH COMMUNITY PARTNERSHIPS Tonya Roberson ${ }^{1}$; Anna Goddu $^{1}$; Abigail E. Wilkes ${ }^{1}$; Marla C. Solomon ${ }^{2}$; Shelley Scott ${ }^{3}$; Sheila Harmon $^{3}$; Marshall Chin ${ }^{1,2}$; Monica Peek ${ }^{1,2}$. ${ }^{1}$ University of Chicago, Chicago, IL; ${ }^{2}$ University of Chicago, Chicago, IL; ${ }^{3}$ Access Community Health Network, Chicago, IL. (Control ID \#1338185)
BACKGROUND: Identifying effective interventions to improve diabetes self-management and outcomes among African-Americans is a national priority. Tailoring diabetes self-management interventions to the culture and practical needs of urban African-Americans has been shown to be effective. Nevertheless, few diabetes education interventions incorporate local community resources. We piloted diabetes education integrated with community partnerships. Our aim was to develop effective partnerships with local food organizations to support newly educated and empowered diabetes patients. We sought to assess the feasibility and preliminary effectiveness of this integrated approach to classroom and communitybased education.

METHODS: Patients participated in 10 weeks of culturally-tailored diabetes education at a clinic on Chicago's South Side. Participants learned diabetes self-management, patient activation communication skills, and tips on healthful eating. Community-based grocery store tours taught hands-on skills identifying healthy choices. Partnerships with food organizations linked participants to affordable healthy food: gift cards were donated by a local food retailer in tandem with a store tour; participants were directed to a farmer's market where LINK dollars were doubled; free fresh produce was given to participants at a food pantry, along with healthy recipes, nutrition advice, and free exercise lessons. Changes in clinical outcomes, diabetes selfmanagement and self-efficacy were measured pre, post, and three months following the class (six months post-intervention data is currently being collected).

RESULTS: All patients were African-American; the mean age was 66 years. The majority was female (88\%), and the annual household income was $<\$ 25 \mathrm{k}$. All participants attended at least half the classes; $86 \%$ attended at least $70 \%$ of the classes. All patients "strongly agreed" that they were satisfied with the program. Participants experienced improvements in clinical outcomes, diabetes self-efficacy and self-management, including reported nutrition patterns. The mean $\mathrm{HbA} 1 \mathrm{c}$ of participants improved from $8.2 \%$ preintervention to $7.3 \%$ three months post $(\mathrm{p}=0.021)$. Self-efficacy improved in all patients, including confidence in diabetes management $(\mathrm{p}=0.047)$, feeling capable of handling their diabetes $(\mathrm{p}=0.011)$, and feeling capable of doing routine care $(\mathrm{p}=0.002)$. Improvements were observed in the mean number of days/week participants ate $5+$ servings of fruits and vegetables ( 4.3 vs. $4.9, \mathrm{p}=$ $0.041)$, inspected their shoes ( 2.8 vs. $5.6 ; \mathrm{p}=0.003)$, and monitored their glucose ( 4.6 vs. $5.6 ; \mathrm{p}=0.047$ ). Participants anecdotally expressed appreciation for the resources offered by the community partnerships.

CONCLUSIONS: We aimed to assess the feasibility of integrating community partnerships into diabetes education, and the effectiveness of a program thus tailored to the culture and pragmatic needs of urban AfricanAmericans. The partnerships proved feasible, and the education effective. Educated participants are ready and willing to eat healthily, but they need support to do so. Incorporating community partnerships that offer discounted/ free fresh produce and/or guidance in healthy food choices anchors new nutrition information and relates it to the everyday logistical, financial, and personal realities of patients. Integrating diabetes education with community partnerships has great potential to support patients as they work to sustain healthy behavior change.

\section{THE HIDDEN CURRICULUM, PATIENT SAFETY, AND ETHICAL EROSION: EXPOSURE TO ROLE MODELING AND RESIDENTS' DISCLOSURE OF MEDICAL ERRORS William Martinez; Lisa Lehmann. Brigham \& Women's Hospital, Boston, MA. (Control ID \#1326375)}

BACKGROUND: Prior research suggests that role models for responding to medical errors are important to trainees and may constitute part of a "hidden curriculum" that may impact attitudes toward disclosure. We measured residents' exposure to negative and positive role models for responding to medical errors and examined the association between exposure to these role models and residents' own attitudes and behaviors regarding error disclosure.

METHODS: We conducted a multicenter, cross-sectional survey of residents' attitudes and experiences regarding errors and their exposure to role modeling. We administered an anonymous, electronic questionnaire to 436 residents across surgical and non-surgical residency programs at two, 
large academic medical centers. The questionnaire asked respondents about: (1) Personal experience with medical errors; (2) Disclosure training; (3) Unprofessional behaviors related to disclosure; (4) Frequency of exposure to role modeling related to disclosure, which included a negative role modeling scale ( 2 items, score range: $2-8^{*}$, Cronbach $\alpha=.76$ ) and a positive role modeling scale (3 items, score range: $3-12 *$, Cronbach $\alpha$ $=.92$ ); (5) Attitudes regarding disclosure, which included a disclosure attitudes scale ( 9 items, score range: $9-36^{*}$, Cronbach $\alpha=.77$ ); and (6) Demographics. (*Higher scores represent more frequent exposure and more positive attitudes, respectively.) Univariate statistics were used to describe the frequency of exposure to negative and positive role models. Multivariate linear regression was used to assess independent predictors of attitudes regarding disclosure. Multivariate logistic regression was used to assess independent predictors of unprofessional behavior related to disclosure, which was a composite outcome of respondents who reported not disclosing a harmful medical error to the patient, not disclosing a harmful medical error to more senior team members, or attempting to evade responsibility for a medical error. Our primary predictors were disclosure training and exposure to role modeling.

RESULTS: The overall response rate was $59 \%(259 / 436)$. More than $80 \%$ of residents reported exposure to positive role modeling related to disclosure; while more than $50 \%$ of residents reported exposure to negative role modeling. Independent predictors of attitudes regarding disclosure included, training, which had the largest positive effect on attitudes and, negative role modeling, which had the largest negative effect on attitudes (standardized effect estimate, $0.33, \mathrm{P}<.001$ v. $-0.29, \mathrm{P}<.001)$. Positive role modeling had a smaller, positive effect on attitudes (standardized effect estimate, 0.21, $\mathrm{P}<.001$ ). Only two variables were independently associated with unprofessional behavior related to disclosure. More frequent exposure to negative role modeling was associated with an increased likelihood of unprofessional behavior (OR 1.31, 95\% CI 1.02-1.70; $\mathrm{P}=.03$ ); while more positive attitudes toward disclosure was associated with a decreased likelihood of unprofessional behavior (OR $0.83,95 \%$ CI $0.73-0.94 ; \mathrm{P}=.03$ ).

CONCLUSIONS: Reducing exposure to negative role models may increase residents' likelihood of meeting their ethical obligation to disclose harmful errors to patients. Training residents on how to respond to medical errors is important, but may be insufficient to ensure professional conduct in response to errors. Attention should be paid to identifying and remediating faculty who act as negative role models.

THE IMPACT OF 2011 ACGME DUTY HOUR REFORM: BASELINE DATA OF A SURVEY OF RESIDENT AND ATTENDING PERCEPTIONS OF RESIDENT TRAINING, WELL-BEING, AND PATIENT SAFETY Caroline Tse; Francois Rollin; Christina E. Payne. Emory University DOM, Atlanta, GA. (Control ID \#1340239)

BACKGROUND: In an effort to improve resident quality of life and reduce patient safety errors, the latest Accreditation Council for Graduate Medical Education (ACMGE) resident duty hour reform, effective July 1, 2011, decreased the intern shift length from 30 to 16 hours, while continuing previous standards of an 80-hour weekly limit. The impact of these new restrictions on patient safety, handoffs, and resident well-being remains unclear. The purpose of this study is to examine resident and attending perceptions of the quality of resident training, resident self-efficacy, and the perceived effects on patient care, before and after the 2011 ACGME work hour restrictions.

METHODS: 105 categorical post-graduate-year (PGY) 1 and PGY-2 residents and 81 attendings on medical wards were surveyed in May and June 2011, before the resident work hour restriction changes. The electronically administered survey was voluntary, anonymous, and results were obtained through aggregate totals. Responses were weighted on a 5-point scale.

RESULTS: Baseline data: 40 residents $(\mathrm{n}=105 ; 38 \%$ response rate) responded to the survey, with 28 PGY-1 responses. 19 attendings $(n=81$; $23 \%$ response rate) responded to the survey, with a median number of postresidency experience of 4-6 years. Resident Quality of Life: Average shift length was reported as 20.6 hours, with 61-70 median duty hours weekly. The median amount of sleep reported was 6 hours. The majority of residents felt depressed (51\%) and overwhelmed with work or patient care $(81 \%)$ some or most days in the week. $65 \%$ of residents believed personal relationships were negatively affected during an inpatient wards rotation. Patient Care: Medical Errors and Handoffs Residents perceived the majority of medical errors to occur during cross-cover, with medical errors being secondary to inadequate time (weighted scale: 2.7 out of 5), excessive workload (2.7), and fatigue (2.5). Attendings perceived resident medical errors to be related to inadequate knowledge base (2.7) and felt that adherence to duty hours is a barrier to patient care (3.7). Both residents and attendings felt there are too many patient handoffs $(3.6 ; 3.8)$; however, the majority of interns felt their patients saw them as the primary caregiver (3.9) and felt personally responsible for their patients (4.3). Overall, residents felt their ability to learn was hindered by fatigue (3.9). Despite this, residents did not feel that duty hour compliance affected their attendings' ability to teach on the wards and the majority felt prepared to advance to the next level of training (4.1).

CONCLUSIONS: This study collected baseline data on resident and attending perceptions of patient care and resident well-being prior to the most recent ACGME duty hour changes. Preliminary data show that the majority of residents surveyed on medical wards feel overwhelmed; with fatigue, excessive workload, and inadequate time as the most commonly perceived causes of medical errors. While the majority of residents note there are too many patient handoffs, residents still feel they are the primary caregiver for their patients and agree that they are prepared for their responsibilities in the next year of training. Future studies will further examine the impact of the ACMGE resident duty hour reform.

THE IMPACT OF BUPRENORPHINE/NALOXONE TREATMENT ON HIV RISK BEHAVIORS AMONG HIV-INFECTED, OPIOIDDEPENDENT PATIENTS: RESULTS FROM THE BHIVES COLLABORATION E. J. Edelman ${ }^{1,2}$; Tongtan Chantarat ${ }^{3}$; Sarah Caffrey ${ }^{4}$; Amina A. Chaudhry ${ }^{5}$; Linda Weiss ${ }^{3}$; David A. Fiellin ${ }^{4}$; Lynn E. Fiellin ${ }^{4} .{ }^{1}$ Yale University School of Medicine, New Haven, CT; ${ }^{2}$ VA Connecticut Healthcare System, West Haven, CT; ${ }^{3}$ New York Academy of Medicine, New York, NY; ${ }^{4}$ Yale University School of Medicine, New Haven, CT; ${ }^{5}$ Johns Hopkins University, Baltimore, MD. (Control ID \#1340021)

BACKGROUND: Needle-sharing and sexual risk behaviors are prevalent among opioid-dependent patients. This finding is particularly concerning for individual and public health when considering patients who are HIVinfected. Opioid agonist treatment is associated with decreased needlesharing practices among opioid-dependent injection drug users. The impact of buprenorphine/naloxone treatment on needle-sharing and sexual risk behaviors among HIV-infected patients over time is unknown. Therefore, we sought to assess the changes over time in needle-sharing and sexual risk behaviors among HIV-infected, opioid-dependent patients receiving buprenorphine/naloxone treatment.

METHODS: We analyzed data from the Buprenorphine-HIV Evaluation and Support Demonstration Project (BHIVES), a prospective study of HIVinfected, opioid-dependent patients initiating treatment with buprenorphine/ naloxone at 9 U.S. sites between July 2005 and December 2007. Patients completed assessments every 3 months. Outcomes of interest included selfreported needle-sharing and sexual risk behaviors, defined as non-condom use with vaginal or anal sex. Descriptive statistics were used to determine the characteristics of the population and prevalence of HIV risk behaviors over time. RESULTS: The study sample $(\mathrm{n}=303)$ had a mean age of 45 years, was $68 \%$ male, 23\% White, $51 \%$ Black, 22\% Hispanic, $81 \%$ heterosexual, and $50 \%$ unmarried. Sixty-one percent of patients were on combination antiretroviral therapy, with the most recent CD4 count less than 199 in $67 \%$ and HIV-1 RNA viral load greater than 400 copies in $58 \%$ of patients. Needle-sharing decreased over time ( $9 \%$ at baseline vs. $3 \%$ at 1 year, $\mathrm{p}=0.05$ ), though non-condom use did not change ( $24 \%$ vs. $21 \%, \mathrm{p}=0.82$ ) (Figure 1 ).

CONCLUSIONS: Buprenoprhine/naloxone treatment is associated with decreased needle-sharing, but not decreased sexual risk behaviors, in HIVinfected, opioid-dependent patients. Developing interventions that specifically address sexual risk behaviors in this patient population receiving buprenorphine/naloxone may increase the public health impact of this treatment. 
THE IMPACT OF PRIMARY CARE WEIGHT ADVICE Christopher Sciamanna $^{1}$; Andrew Pool ${ }^{1}$; Jennifer Kraschnewski ${ }^{1}$; Heather Stuckey ${ }^{1}$; Kevin Hwang ${ }^{2}$; Kathryn Pollak ${ }^{3}$; Deborah F. Tate ${ }^{4}$; Erik Lehman ${ }^{1} .{ }^{1}$ Penn State Hershey, Hershey, PA; ${ }^{2}$ University of Texas, Houston, TX; ${ }^{3}$ Duke University, Durham, NC; ${ }^{4}$ University of North Carolina, Chapel Hill, NC. (Control ID \#1311422)

BACKGROUND: Individuals in the United States continue to be greatly affected by the epidemic of overweight and obesity. Additionally, physicians struggle with identifying and providing effective weight counseling to their patients. Thus, there is a need for simple and effective interventions for physicians to help their overweight and obese patients to lose weight. Physician acknowledgement of a patient's weight has recently been shown to have a significant effect on patients' perceptions of their own weight, in addition to their desire and attempts to lose weight. The objective of this study is to examine the association of a doctor's acknowledgement of a patient's weight status with reported weight loss by comparing 2005 to 2008 data from the National Health and Nutrition Examination Survey (NHANES).

METHODS: We analyzed data from the 2005 to 2008 National Health and Nutrition Examination Survey to examine the association of a doctor's acknowledgement of patient weight status and patient reported weight loss. We included nonpregnant overweight and obese (body mass index $[\mathrm{BMI}] \geq 25$ ) participants between the ages of 20 and 64 years who had responded to the question, "Has a doctor or other health professional ever told you that you were overweight?" $(\mathrm{n}=5054)$. The main outcome measure was the proportion of participants who lost at least $5 \%$ of their body weight in the past year. Bivariate relationships by BMI category were evaluated using chi square tests between participants who had and had not been told by a doctor that they were overweight, demographic characteristics and weight loss. To determine the odds of losing at least $5 \%$ weight, a logistic regression was performed, controlling for the following variables: age, sex, education, marital status, poverty to income ratio (PIR), ethnicity, place of routine care, number of physician visits in the past year, and doctor's acknowledgement of weight status.

RESULTS: Overweight participants were significantly more likely to report a $5 \%$ loss of weight in the past year if their doctor told them they were overweight (adjusted OR 2.08; 95\% CI 1.62-2.66). Obese participants reported similar results (adjusted OR 1.81; 95\% CI 1.34-2.44). Also, overweight and obese patients, respectively, were also more likely to report a $10 \%$ loss of body weight in the past year if their doctor told them they were overweight (adjusted OR 2.81; 95\% CI 1.93-4.06 for overweight; adjusted OR 2.27; 95\% CI 1.31-3.94 for obese).

CONCLUSIONS: Physicians' recognition of their patients' weight status is associated with significant patient weight loss. Specifically, overweight and obese individuals have almost two times the odds of reporting a 5\% loss of weight in the past year - a weight loss amount that has been found to significantly improve the comorbidities associated with being overweight or obese. In addition, overweight and obese individuals were more than twice as likely to report a $10 \%$ weight loss in the past year if their doctor had told them that they were overweight. This suggests that physician acknowledgement of weight status may have a measurable impact on weight.

THE IMPACT OF REAL TIME LABORATORY COST DISPLAY ON PRIMARY CARE PHYSICIAN ORDERING PATTERNS Daniel M. Horn ${ }^{1,2}$; Kate E. Koplan ${ }^{1}$; Margaret D. Senese ${ }^{1}$; Endel J. $\overline{\text { Orav }^{2} \text {; }}$ Thomas D. Sequist ${ }^{1,2}$. ${ }^{1}$ Harvard Vanguard Medical Associates and Atrius Health, Newton, MA; ${ }^{2}$ Brigham and Women's Hospital, Boston, MA. (Control ID \#1338560)

BACKGROUND: Physicians are under increased pressure to help control rising health care costs, though they may lack information regarding the relative cost implications of their patient care decisions. We evaluated whether real time display of costs with an electronic health record impacted primary care physician ordering of common laboratory tests in the outpatient setting.

METHODS: We conducted this study within a physician group practice in Massachusetts utilizing an integrated electronic health record. Beginning in April 2011, the average Medicare reimbursement rate for 22 commonly overused laboratory tests with either high annual volume $(n=15)$ or relatively high individual cost $(n=7)$ was displayed to primary care physicians at the time of electronic ordering. High annual volume tests included creatinine, BUN, glucose, ALT, electrolyte panel, basic metabolic panel, comprehensive metabolic panel, hemoglobin A1c, iron binding profile, lipid profile, sedimentation rate, thyroid stimulating hormone, prostate specific antigen, ferritin, and hemogram. High-cost tests included antimitochondrial antibody, brain natriuretic peptide, parathyroid hormone, testosterone, tissue transglutaminase antibody, vitamin $\mathrm{D}$, and alpha fetoprotein. We used electronic health record data to measure test orders for 12 months prior to the cost display intervention and 6 months following the intervention start. Order rates (orders/ monthly patient visits) were analyzed for 157 "intervention" primary care physicians receiving the cost information and 61 "control" primary care physicians not receiving the cost information within the group practice. We assessed the longitudinal impact of the intervention on laboratory ordering using an interrupted time series analysis with a parallel control group. For each laboratory test, multivariable hierarchical binomial regression was used to compare the change-in-slope of the ordering rate in intervention physicians to the change-in-slope in control physicians. The model used the number of laboratory orders per month, per physician, as the dependent variable and accounted for both the number of patient visits per month and within-physician correlation.

RESULTS: The average number of monthly patient visits was 192 among intervention physicians and 261 among control physicians. Compared to the control group, the display of laboratory cost information among intervention physicians was associated with a directional decrease in physician ordering rates for 12 of the 15 high volume laboratory tests, with $6(40 \%)$ achieving statistical significance $(\mathrm{p}<0.05)$. These statistically significant decreases in ordering high volume tests included lipid profile (mean decrease in intervention compared to control group -2.40 orders/100 visits), creatinine (2.40 orders $/ 100$ visits), glucose ( -2.36 orders $/ 100$ visits $)$, ALT ( -1.96 orders/ 100 visits), electrolyte panel ( -0.35 orders/ 100 visits), and BUN (-0.03 orders/ 100 visits). For high cost laboratory tests, the intervention was associated with a directional decrease in physician ordering for only 2 of the 7 tests, and neither reached statistical significance.

CONCLUSIONS: Real time display of cost information can reduce ordering of high volume laboratory tests, and has no effect on physician ordering of lower volume, high cost tests. Future work should focus on understanding physician perceptions of cost information provision and how to optimize the value of laboratory test utilization in the primary care setting.

THE IMPACT OF RESIDENT PHYSICIAN BURNOUT ON THE QUALITY OF CARE OF HOSPITALIZED PATIENTS Rebecca A. Mazurkiewicz; Kristofer L. Smith; Deborah Korenstein; Jonathan Ripp. Mount Sinai School of Medicine, New York, NY. (Control ID \#1321067)

BACKGROUND: While the impact of burnout on resident physicians has been described, few studies have explored the repercussions of trainee burnout for their patients. We sought to examine the relationship between burnout in internal medicine (IM) resident physicians early in training and the quality outcomes of hospitalized patients under their care. We hypothesized that burnout among resident physicians would be associated with poorer outcomes for their hospitalized patients.

METHODS: We surveyed members of the Mount Sinai Hospital IM residency graduating classes of 2011 and 2012 during their post-graduate year 1 (PGY1) orientation, collecting demographic information and administering the Maslach Burnout Inventory (MBI), a validated instrument measuring the 3 domains of burnout (emotional exhaustion, depersonalization, and impaired sense of personal accomplishment). In keeping with the most commonly used definition in the literature, participants were considered to be burnt out if they had high scores on either the emotional exhaustion $(\geq 27)$ or depersonalization $(\geq 10)$ subscales of the MBI. Subsequently, we collected data from the medical records of hospitalized patients under the care of each participant (matched by author of the discharge summary) for the following year including demographics, level of illness, co-morbidities, length of stay index (the ratio of observed length of stay to expected length of stay controlled for age, sex, race, socioeconomic status, admit source and status, severity of 
illness, and co-morbidities), mortality index (calculated similarly to length of stay index), and 30-day readmissions, as well as each participant's rate of discharge summary completion within 48 hours and patient satisfaction scores as measured by the Hospital Consumer Assessment of Healthcare Providers and Systems (HCAHPS) survey. Generalized estimating equations were used to fit linear or logistic regression models, as appropriate, to examine associations between resident physician burnout and patient outcomes.

RESULTS: Of the 104 eligible resident physicians, 91 (88\%) participated in the study and were linked to the care of 7941 hospitalized patients. Thirty-three $(36 \%)$ study subjects met criteria for burnout at the start of training. When controlling for characteristics of patient encounters and resident physician demographics, there was a trend toward an association between resident physician burnout and an increased length of stay index ( $\beta=0.05, p=0.06)$. There was no association between burnout and mortality, readmissions, discharge summary completion, or patient satisfaction.

CONCLUSIONS: Our study demonstrates a trend towards an association between burnout among physicians beginning IM residency and a higher length of stay. By nature of the index, the impact of this finding will vary from patient to patient; however, the prolonged length of stay doesn't appear to affect patient care in terms of the other quality markers we measured. IM residents, burnt out at the start of training, may be able to provide quality care and ensure patient safety, perhaps due to support from other members of the treatment team, the residency program, or other resources of the hospital.

THE IMPACT OF SOCIOECONOMIC STATUS ON THE PRESCRIBING OF OPIATES IN THE EMERGENCY SETTING IN THE UNITED STATES Michael Joynt; Meghan Train; Brett Robbins; Jill Halterman; Enrico Caiola; Robert J. Fortuna. University of Rochester, Rochester, NY. (Control ID \#1332919)

BACKGROUND: Racial and ethnic disparities in opioid prescribing in the emergency setting are well described, yet the influence of socioeconomic status on the prescribing of opioids remains unclear. The objectives of this study were to: 1) evaluate the effect of both poverty and race on the prescribing patterns of opioids in the emergency setting in adult patients suffering moderate to severe pain, and 2) determine whether differences by poverty explain the racial differences in prescribing previously described.

METHODS: We used cross-sectional data from the National Hospital Ambulatory Medical Care Survey between 2006-2009 to examine the prescribing of opioids to patients, age 18 and over, presenting to the emergency department with either moderate or severe pain. We used logistic regression models to evaluate the independent association between poverty, race, and the prescribing of opioids. Models were adjusted for pain level, age, ethnicity, injury status, frequency of emergency visits in the past year, type of hospital (nonprofit, government, proprietary), region of the country, and location within urban or rural areas. Poverty was determined based on the percent poverty within a patient's zip code and analyzed by quartiles.

RESULTS: We examined 183.9 million weighted visits from adults with moderate to severe pain. Opiates were prescribed more frequently to patients of the highest socioeconomic status, including percent poverty ( $49.0 \%$ vs. $39.4 \%$, $\mathrm{P}<0.001$ ), household income ( $47.3 \%$ vs. $40.6 \%, \mathrm{P}<0.001$ ), and educational level ( $46.3 \%$ vs. $42.5 \%, \mathrm{P}=0.01)$, compared to patients in the lowest quartile. White patients were consistently prescribed opiates more frequently than black patients across all quartiles of poverty, including poverty $<5.00 \%(50.0 \%$ vs. $42.4 \%, \mathrm{P}=0.02)$, poverty $5.00-9.99 \%$ ( $49.0 \%$ vs. $40.2 \%, \mathrm{P}<0.001), 10.00$ $19.99(44.3 \%$ vs. $40.1 \%, \mathrm{P}=0.02)$, and poverty $>20.00 \%(42.2 \%$ vs. $36.5 \%$, $\mathrm{P}<0.001$ ). In adjusted models, both black patients (AOR 0.73; 95\% CI $0.67-$ 0.80 ) and patients from poorer areas (AOR $0.76 ; 95 \%$ CI $0.68-0.84$ ) were significantly less likely to receive opioids after accounting for pain level, age, injury status, and other covariates.

CONCLUSIONS: Patients presenting to emergency departments from poorer areas were less likely to receive opioids for equivalent levels of pain than those from more affluent areas. Black patients were also less likely than whites to receive opioids for equivalent levels of pain. Poverty did not explain racial differences in prescribing of opioids in the emergency setting.
THE MEDICARE PART D LOW-INCOME COST SUBSIDY (LICS) AND ADHERENCE TO MEDICATIONS FOR SECONDARY PREVENTION OF CARDIOVASCULAR DISEASE O. Kenrik Duru; Sarah Edgington; Carol Mangione; Norman Turk; Chi-hong Tseng; Lindsay Kimbro; Susan Ettner. University of California, Los Angeles, Los Angeles, CA. (Control ID \#1320374)

BACKGROUND: Out-of-pocket costs are an important barrier to medication adherence. High copayments can be a major obstacle for patients who have already experienced a cardiovascular event, and are taking medications which are quite expensive but also critical for secondary prevention of recurrent events. Medicare Part D includes a low-income cost subsidy (LICS) for eligible low-income patients that reduces the out-of-pocket costs for chronic maintenance medications. To date, there have been few studies examining the association between LICS enrollment and medication adherence among Medicare beneficiaries.

METHODS: We used 2006 and 2007 administrative data from diabetic patients within Medicare Advantage Prescription Drug (MAPD) plans offered by a large, national Part D insurer. The analytic sample was limited to patients with ICD-9 and/or CPT codes for a myocardial infarction (MI), coronary stent, or coronary artery bypass graft (CABG) in 2006, who had at least one postevent fill for a statin (MI, CABG), or clopidogrel (stent). We classified patients who were enrolled in LICS at any point during the 12-months post-event as the sample of interest, and we used nearest neighbor propensity score matching to identify a comparison group of non-LICS patients for each of the three events. We matched on age, gender, institutionalization, comorbidities, days' supply of the last medication fill in the study window, and median poverty level in the provider billing zip code. We then constructed three multivariate logistic regression models (MI, stent, $\mathrm{CABG}$ ) with these covariates to examine the associations between LICS status and the likelihood of good adherence over the 12-month window, as defined by a Proportion of Days Covered (PDC) of $>80 \%$. In a separate multivariate regression model, we evaluated the association between LICS status and premature discontinuation of clopidogrel after coronary stent placement, defined as the absence of clopidogrel fills for 120 days between the end of the previous days' supply and the end of the 12 month window. We expressed results as differences in predicted probabilities.

RESULTS: Our sample after propensity score matching ( $\mathrm{n}=5,332)$ included 2,405 LICS patients and 2,208 non-LICS patients with an MI, stent, and/or CABG. Among post-MI patients $(n=2,764)$, LICS patients had a higher likelihood of good adherence to statins compared to non-LICS patients $(35.8 \%$ vs. $28.3 \%, \mathrm{p}<0.001)$. Among post-stent patients $(\mathrm{n}=$ 1,971), LICS patients had a higher likelihood of good adherence to clopidogrel $(54.2 \%$ vs. $45.2 \%, \mathrm{p}<0.001)$ and a lower likelihood of premature clopidogrel discontinuation $(15.9 \%$ vs. $23.5 \%, \mathrm{p}<0.001)$, compared to non-LICS patients. We did not find a significant association between LICS status and statin adherence among post-CABG patients.

CONCLUSIONS: We found that among Medicare patients with diabetes, the LICS benefit is associated with a higher likelihood of good adherence to medications for secondary prevention after an MI or stent. This differential in adherence may translate into lower rates of recurrent MIs, less stent thrombosis, and fewer readmissions among LICS patients. Although clopidogrel and most statin medications are now off-patent, there are other very expensive drugs for which good adherence is important to prevent adverse cardiovascular outcomes. Our work underscores the need for ongoing efforts to identify and enroll eligible Medicare beneficiaries with diabetes and cardiovascular disease in the LICS benefit.

THE NATURAL HISTORY OF DIABETES COMPLICATIONS IN OLDER ADULTS: THE DIABETES AND AGING STUDY Priya John'; Andrew J. Karter ${ }^{2}$; Jennifer Y. Liu ${ }^{2}$; Howard H. Moffet ${ }^{2}$; Neda Laiteerapong ${ }^{1}$; Elbert Huang ${ }^{1} .{ }^{1}$ University of Chicago, Chicago, IL; ${ }^{2}$ Kaiser Permanente of Northern California, Oakland, CA. (Control ID \#1323964)

BACKGROUND: Understanding the modern natural history of diabetes in older adults has implications for setting current diabetes treatment priorities. Patients over the age of 65 represent a large (40\%) and growing 
segment of the diabetes population. Despite their prevelance, most of our understanding of the natural history of diabetes complications in older people is based on epidemiological data from the 1980s and 1990s. There are reasons to believe that the modern history of diabetes complications in older people is now different due to secular changes in treatment and diagnosis and needs to be updated. Using a contemporary cohort, we sought to describe the natural history of diabetes in older adults by age and duration.

METHODS: We conducted a longitudinal cohort study of patients with type 2 diabetes, with no prior history of major diabetes complications, aged 60 years and older in the Kaiser Permanente Northern California Diabetes Registry. Incidence densities (events per 1000 patient years) were calculated for various age categories and duration of diabetes (0-9 year's duration vs. $10+$ years). Outcomes of interest included incidence of diabetes related microvascular complications (lower extremity amputation, eye disease and peripheral vascular disease), macrovascular complications (coronary artery disease, cerebrovascular disease and congestive heart failure), and all-cause mortality. RESULTS: Mean age of subjects was 71, and a mean hemoglobin A1c of 7 (SD). For 60-69 years olds, incidence of macrovascular complications was higher than mortality and microvascular complications (e.g $\geq 10$ years duration: 30.8 (Macrovascular) vs. 28.2 (Mortality) vs. 23.4 (Microvascular)). For 70-79 year olds, mortality had the highest incidence followed by macrovascular complications (e.g. $\geq 10$ years duration: 55.3(Mortality) vs. 41.6 (Macrovascular) vs. 22.7 (Microvascular)). The incidence of mortality greatly exceeded the incidence of complications in patients $\geq 80$ years (e.g. $\geq 10$ years duration: 114.1 (Mortality) vs. 61.9 (Macrovascular) vs. 21.1 (Microvascular)). Across all ages, longer duration ( $\geq 10$ years) was associated with higher incidence of complications and mortality. For those with long durations of diabetes, age did not have a significant assocation on the incidence of microvascular complications.

CONCLUSIONS: There is a relatively greater incidence of macrovascular versus microvascular complications in patients 60 years and older with diabetes, and this pattern increases dramatically with age and duration of diabetes. This look at the modern natural history study will have implications for the future of health care utilization and costs in the aging diabetes population.

Incidence of Complication and Mortality per 1000 patient years by Age and Durations (95\% CI)

\begin{tabular}{|c|c|c|c|c|c|c|}
\hline & $<10$ years duration & & & $>=10$ years Duration & & \\
\hline Age & Macrovascular* & Microvascular* & Mortality* & Macrovascular* & Microvascular & Mortality* \\
\hline $60-69$ & $16.41(15.25,17.66)$ & $5.33(4.72,6.03)$ & $10.99(0.08,11.98)$ & $25.93(23.50,28.62)$ & $20.28(17.95,22.91)$ & $\begin{array}{l}13.41 \\
(11.76,15.30)\end{array}$ \\
\hline $70-79$ & $25.55(23.83,27.4)$ & $6.46(5.68,7.34)$ & $23.76(2.09,25.56)$ & $37.68(34.48,41,17)$ & $17.95(15.78,20.43)$ & $\begin{array}{l}30.61 \\
(27.77,33.74)\end{array}$ \\
\hline $80+$ & $43.05(39.37,47.08)$ & $8.97(7.45,10.81)$ & $62.20(7.57,67.21)$ & $58.42(52.28,65.27)$ & $17.75(14.66,21.5)$ & $\begin{array}{l}75.34 \\
(68.17,83.26)\end{array}$ \\
\hline
\end{tabular}

* $\mathrm{P}$ value for trend significant at $\mathrm{p}<0.01$ across age categories

THE OUTCOME OF CARDIOPULMONARY ARREST IN CRITICALLY ILL CANCER PATIENTS Tashfeen Mahmood ${ }^{1}$; Matt Chua $^{1}$; Megan Slaton ${ }^{1}$; Mohammad Abu-Zaid ${ }^{2}$; Mahmoud Kamel ${ }^{2}$; Faisal Khasawneh ${ }^{1} .{ }^{1}$ texas tech university health sciences center, Amarillo, TX; ${ }^{2}$ University of Arizona, Tucson, AZ. (Control ID \#1327168)

BACKGROUND: The success of cardiopulmonary resuscitation is variable and depends on the patient's age, the presence of comorbidities, and the promptness of appropriate medical interventions. Survival among cancer patients who suffer in-hospital cardiopulmonary arrest (CPA) is poor. Higher survival rates were reported in patients cared for at dedicated cancer centers. The predictors of successful cardiopulmonary resuscitation in cancer patients are poorly characterized. Objectives: 1. To characterize the outcome of CPA in critically ill cancer patients admitted to a closed intensive care unit (ICU) in a comprehensive cancer center. 2. To identify resuscitation outcome predictors in critically ill CPA cancer patients.

METHODS: A retrospective chart review of all CPA cases in cancer patients admitted to a comprehensive cancer center ICU between 1/2008 and 12/2009. Patients' demographics, type of malignancy, extent of disease, presence of organ failure, ICU length of stay (LOS), hospital LOS, the presence of multidrug resistant bacteria colonization and outcome data were collected. Descriptive statistics were used to summarize the data and describe the patients. Comparisons between different variables and CPA outcome were conducted using Fisher's exact test.

RESULTS: During the study period, 128 cardiopulmonary arrests occurred in 104 patients ( 65 males and 39 females). The mean age of the cohort was 49.7 years with an average APACHE II score of 23.8. Twenty seven percent of the patients had leukemia, $13 \%$ had lymphoma, $13 \%$ had lung cancer, $9 \%$ had colon cancer and $34 \%$ had different other cancers. Forty seven percent of the patients had a progressing or a relapsed cancer. Seventy four percent of the patients were on vasopressors, $82.7 \%$ were on mechanical ventilation, $27.1 \%$ had acute kidney injury and $38.5 \%$ were colonized by multidrug resistant bacteria. The mean time from ICU admission to CPA was 4.7 days. The most common initial rhythm was pulseless electrical activity/asystole. Acute respiratory failure and refractory shock were the most common causes of CPA. The mean duration of cardiopulmonary resuscitation was 17.6 minutes. Thirty four percent of cardiopulmonary arrests were resuscitated successfully but only 9 patients $(8.7 \%)$ left the ICU alive and 6 patients (5.7\%) left the hospital alive. The average ICU and hospital LOS were 5.8 days 31.6 days, respectively.

CONCLUSIONS: The outcome of CPA in critically ill cancer patients is poor. Once cancer patients suffer a CPA in the ICU, acute kidney injury ( $p$ 0.008 ), mechanical ventilation (p 0.003), refractory shock (p 0.001), multidrug resistant bacteria colonization (p 0.001) and CPA duration of more than 10 minutes ( $\mathrm{p}$ 0.001) predicts failed cardiopulmonary resuscitation efforts.

THE PATIENT-CENTERED MEDICAL HOME, A SYSTEMATIC REVIEW Ranee Chatterjee; George Jackson; Janet Bettger; Benjamin Powers; Alex Kemper; Rowena Dolor; John Williams. Duke University, Durham, NC. (Control ID \#1328180)

BACKGROUND: As part of the Agency for Healthcare Research and Quality's (AHRQ's) "Closing the Quality Gap" series, this systematic review sought to identify completed and ongoing evaluations of the comprehensive patient-centered medical home $(\mathrm{PCMH})$, summarize current evidence for this model, and identify gaps in the evidence.

METHODS: We searched PubMedR, CINAHLR, and the CDSR for published English language studies, and a wide variety of databases and web resources to identify ongoing or recently completed studies. Two investigators per study screened abstracts and full-text articles for inclusion, abstracted data, and performed quality ratings and evidence grading. Our functional definition of PCMH was based on the definition used by AHRQ. We included studies that explicitly claimed to be 
evaluating PCMH and those that did not but which met our functional definition.

RESULTS: Seventeen studies with comparison groups evaluated the effects of PCMH. Older adults in the United States were the most commonly studied population ( 8 of 17 studies). PCMH interventions had a small positive impact on patient experiences, and small to moderate positive effects on preventive care services (moderate strength of evidence [SOE]). Staff experiences were also improved by a small to moderate degree (low SOE). There was little to no evidence of improved clinical outcomes or reduction in utilization or total costs. Twenty of 26 studies reported approaches that addressed all seven major PCMH components, including team based-care, sustained partnership, reorganized or structural changes to care, enhanced access, coordinated care, comprehensive care, and a systems-based approach to quality. A total of 51 related strategies were abstracted, with great variability across studies. Twenty-one of 26 studies reported information on financial systems used to implement PCMH, implementation strategies, and/or organizational learning strategies for implementing PCMH. The 30 studies included in the horizon scan of ongoing PCMH studies were broadly representative of the U.S. health care system, both in geography and in the complexity of private and public health care payers and delivery networks.

CONCLUSIONS: Published studies of PCMH interventions often have similar broad elements, but precise components of care varied widely. The PCMH holds promise for improving the experiences of patients and staff, and potentially for improving care processes. However, there is little to no evidence of improved patient clinical outcomes or reduced economic burden. Ongoing studies identified through the horizon scan have potential to greatly expand the evidence base relating to $\mathrm{PCMH}$.

THE PLACEBO EFFECT IN TRIALS OF HEADACHES Elizabeth Cogbill; Jeffrey L. Jackson. Zablocki VAMC, Milwaukee, WI. (Control ID \#1337950)

BACKGROUND: Headaches are common. Since other symptom-based syndromes have been shown to have a significant placebo effect, our purpose was to examine the placebo effect among treatment trials of headaches.

METHODS: We have recently conducted three systematic reviews examing the efficacy of prophylactic medications in 1) migraine headaches and 2) tension headaches and a third evaluating the efficacy of botulinum injections for both headache types. All three studies searched MEDLINE, EMBASE and the Cochrane Register through Fall 2011 (exact search terms and last search date varied). In all three reviews, data was abstracted and quality rated independently by reviewers with good inter-rater reliability. In all three the effect of treatment for both the active and placebo drugs were abstracted at all time-points reported. In this paper, we analyzed the placebo effect at each time point by calculating the standardized effect $(\mathrm{mean} / \mathrm{SD})$ for subjects receiving placebo. We pooled these at each time point using random effects methods and examined for a time-effect of placebo using random effects meta-regression with adjustment for clustering by study.

RESULTS: Our search strategies produced 8703 total articles (drugmigraine: 3943, drug-tension: 4511, botulinum: 249). From these we included 30 placebo-controlled trials of migraine headaches, 12 placebocontrolled trials of tension headaches and 27 placebo-controlled trials of botulinum injections for various headache types. Study durations ranged from 4-90 weeks. In total, there were 3,647 patients who received placebo for periods ranging from 4-90 weeks (average: 14). The pooled headache severity at time zero was $4.8(95 \%$ CI: $6.1-29.3, \mathrm{Q}=8404, \mathrm{df}=121, \mathrm{I} 2=$ 98.6). By 4 weeks this had declined to a standardized mean of 3.68 (95\% CI: 2.3-5.0, $\mathrm{Q}=716, \mathrm{df}=54, \mathrm{i} 2=92.5)$ and by 12 weeks, headaches had decreased to $2.84(95 \% \mathrm{CI}: 1.34-4.33, \mathrm{Q}=617, \mathrm{df}=45, \mathrm{I} 2=97.2)$. There was linear improvement over study durations reported $(\beta=-0.01,95 \% \mathrm{CI}$ : -0.007 to -0.02), meaning that for every week patients were given placebo, they experienced a decrease in their headaches of 0.01 standard deviations. Dropout rates varied widely, with longer trials having greater dropout rates.
CONCLUSIONS: There is a strong placebo effect in randomized trials of headache treatment. Case series or other methods that examine the efficacy of headache treatment will overstate benefit. The placebo effect appeared to increase over time, though enthusiasm for this time-effect observation should be tempered by the relatively high drop out rates seen in many headache trials.

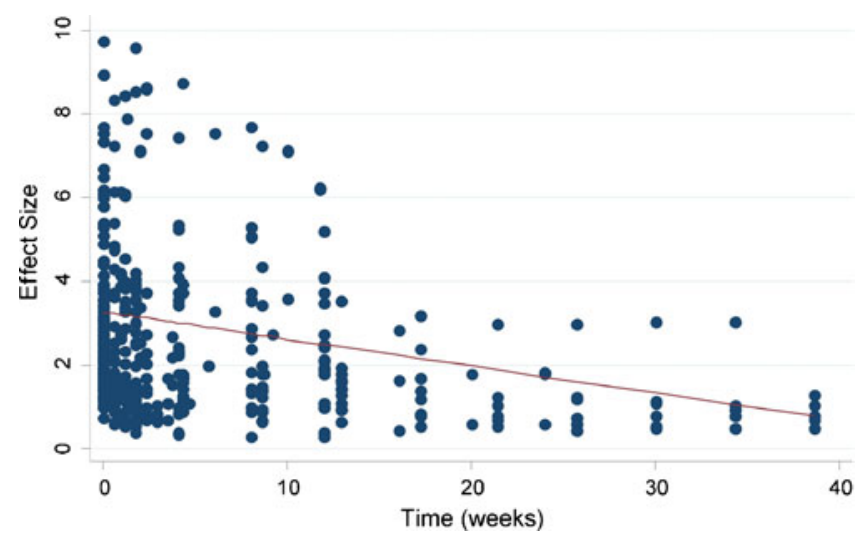

THE PREVALENCE OF MENTAL HEALTH DISORDERS IN FREQUENTLY HOSPITALIZED HEART FAILURE PATIENTS ENROLLED IN A VA HOME BASED PRIMARY CARE PROGRAM SALLY NAMBOODIRI, JEFFREY COONEY, BROOK WATTS Sally Namboodiri ${ }^{1}$; Jeffrey M. Cooney ${ }^{2}$; Brook Watts ${ }^{1} .{ }^{1}$ Cleveland VA Medical Center, Cleveland, OH; ${ }^{2}$ Cleveland VA Medical Center, Cleveland, $\mathrm{OH}$. (Control ID \#1317614)

BACKGROUND: Heart failure patients with frequent hospital readmissions may benefit from home-based care programs targeted to improve self-management skills and medication adherence. However, the benefits of these programs may not be fully realized if patients suffer from concomitant mental health disorders such as post-traumatic stress disorder (PTSD), depression and neurocognitive impairment (NCI). In order to enhance the quality of such home care services, we sought to characterize the prevalence of PTSD, depression and NCI in a population of heart failure patients with frequent admissions who were also enrolled in a VA home-based care program.

METHODS: We retrospectively reviewed complete medical records of all patients with diastolic or systolic heart failure enrolled in an established VA Home Based Primary Care (HBPC) program and who had 3 or more inpatient admissions (including at least one for heart failure) from 11/1/09 to 11/30/10. Chart reviews were completed by an interdisciplinary team and included examination of standardized social work screenings for mental health disorders using validated instruments (i.e. PTSD 4Q for post-traumatic stress disorder, Depression PHQ-2 for depression, and the St. Louis University Mental Status Exam (SLUMS), a 30-point screening questionnaire that tests for orientation, memory, attention, and executive functions).

RESULTS: 39 patients meeting criteria were identified and all records were reviewed. Mean age of patients was 72 (range 55-96), with $95 \%(\mathrm{~N}=37)$ male. Forty-one percent $(n=16)$ of patients had a preexisting diagnosis of PTSD, depression,or both. An additional $10 \%(n=4)$ had a positive screen for PTSD and/or depression or exhibited signs of depression. Overall, $51 \%$ of patients $(n=20)$ had evidence of PTSD and/or depression as determined by prior mental health evaluation or HBPC evaluation. Neurocognitive screening (NCS) was offered to all patients $(n=39)$ of which 32 patients received screening (6 patients refused and 1 patient was not screened due to a preexisting dementia diagnosis). Of the 32 patients screened, $28 \%$ of patients $(n=9)$ had screens suggestive of significant NCI (SLUMS score 1-20). Fifty percent of patients $(n=16)$ had screens suggestive of mild NCI (SLUMS score 21-26). Overall, 78\% $(n=25)$ of the patients screened had results suggesting some degree of NCI.

CONCLUSIONS: This retrospective evaluation of frequently hospitalized heart failure patients who were enrolled in a VA Home Based Primary Care 
program found a high prevalence of PTSD, depression and neurocognitive impairment. These concomitant conditions may serve as barriers to the success of HBPC programs in preventing readmissions. This pilot data provides an important foundation for further studies that examine the role of co-treatment of mental health issues in HBPC programs, as well as the use of PTSD, depression and cognitive screenings to identify vulnerable patients who may benefit from more intense support initiatives focused on in-home chronic disease management.

THE RELIABILITY AND VALIDITY OF READMISSION RATES AS A HOSPITAL QUALITY MEASURE: IMPLICATIONS FOR CURRENT POLICIES Matthew J. Press ${ }^{1,3}$; Dennis P. Scanlon ${ }^{4}$; Jingsan $\mathrm{Zhu}^{3}$; Andrew Ryan ${ }^{1}$; Amol Navathe ${ }^{5,5}$; Jessica N. Mittler ${ }^{4}$; Kevin G. Volpp ${ }^{2,3}$. ${ }^{1}$ Weill Cornell Medical College, New York, NY; ${ }^{2}$ Philadelphia VAMC, Philadelphia, PA; ${ }^{3}$ University of Pennsylvania, Philadelphia, PA; ${ }^{4}$ The Pennsylvania State University, State College, PA; ${ }^{5}$ Harvard Medical School, Boston, MA. (Control ID \#1339736)

BACKGROUND: Recent national policies intended to encourage hospitals to reduce readmissions utilize risk-standardized readmission rates to measure hospital quality. Our objective was to assess the reliability and validity of readmission rates as a measure of hospital quality. We hypothesized that if readmission rates change year-to-year on account of statistical variance rather than true changes in quality of care, then reliability would be low, making it difficult to identify the best- and worst-performing hospitals with a high degree of certainty. Further, if readmission rates are unrelated to other measures of hospital quality, then validity would be low, challenging the premise that hospitals are the appropriate target for policies to reduce readmissions.

METHODS: We conducted a retrospective analysis of hospital data published through the Hospital Compare website in 2009 and 2011 for acute myocardial infarction (AMI), congestive heart failure (CHF), and pneumonia. To assess the reliability of readmission rates, we compared hospital rankings based on risk-standardized readmission rates in 2009 and in 2011. To assess the validity of readmission rates, we estimated the correlation between risk-standardized readmission rates and widely accepted indicators of hospital quality, including risk-standardized mortality rates, volume, teaching status, and process measure performance.

RESULTS: For each clinical condition, approximately $45 \%$ of hospitals in the highest and lowest readmission rate quartiles in 2009 moved into other quartiles in 2011. Hospitals with higher readmission rates in 2009 tended to improve by 2011, and hospitals with lower readmission rates in 2009 tended to worsen by 2011 (R-squared values for the relationship between readmission rate in 2009 and percent change in readmission rate from 2009-2011 were $0.18,0.21$, and 0.27 for AMI, CHF, and pneumonia, respectively). This pattern of longitudinal change is suggestive of regression to the mean. Hospital readmission rates had minimal correlation with mortality rates. For AMI, CHF, and pneumonia, respectively, changes in readmission rates from 2009-2011 explained $0.1 \%, 0.3 \%$, and $0.8 \%$ of the variance in changes in mortality rates. In addition, $18 \%$ (AMI), 22\% (CHF), and 10\% (pneumonia) of hospitals designated in Hospital Compare as "worse than the U.S. national rate" for readmission were designated as "better than the U.S. national rate" for mortality. Readmission rates had no consistent correlation with hospital volume or process measure performance and were higher in teaching hospitals than in non-teaching hospitals, which is contrary to the expectation of better outcomes in teaching hospitals

CONCLUSIONS: The degree and pattern of change in hospital rankings based on risk-standardized readmission rates, in addition to the poor correlation between readmission rates and other indicators of hospital quality, raise concerns about the reliability and validity of readmission rates as a measure of hospital quality. Low reliability and validity of readmission rates could undermine the impact of current policies, including payment reform, that use readmission rates to identify low quality hospitals and create incentives to encourage these hospitals to reduce readmissions.

THE ROLE OF CARE COORDINATORS IN IMPROVING CARE COORDINATION: THE PATIENT'S PERSPECTIVE Michelle M. Doty; Ashley-Kay Fryer; Anne-Marie J. Audet. The Commonwealth Fund, $\overline{\text { New }}$ York, NY. (Control ID \#1311682)
BACKGROUND: Evidence suggests that care coordination can improve provider and health system quality and efficiency. To date, little is known about the impact of various approaches to improving care coordination from the patient's perspective. This paper focuses on the experiences of patients who received care from multiple doctors and explores whether patients who say they have a care coordinator, better access to primary care, and strong provider-patient communication are less likely to experience care coordination problems.

METHODS: Data come from a telephone survey conducted in 2010 among a nationally representative sample of 19,738 adults in eleven countries. We examine self-reported coordination gaps related to medical records or tests, communication failures between providers including specialists, and failure to provide information about care received during a hospitalization and/or ER visit post discharge. We use multivariate logistic regression models to examine whether having a care coordinator, accessible care, and a strong patient-provider relationship reduces the risk of coordination gaps after controlling for cofactors.

RESULTS: Transitions between different care settings are an especially vulnerable period for patients because of the potential for incomplete or inaccurate information transfer and lack of appropriate follow-up care. Having someone that helps coordinate or arrange the care patients receive from other doctors reduces the risk of coordination problems. Patients with a care coordinator were less likely to report that their test results or medical records were not available at their scheduled appointment $(10 \%$ versus $23 \%, \mathrm{p}<.001)$, they received conflicting information from different doctors $(21 \%$ versus $34 \%, \mathrm{p}<.001)$, or someone failed to follow-up about their test results $(14 \%$ versus $34 \%, \mathrm{p}<.001)$. Patients who saw specialists and had a care coordinator were also more likely to report that their regular doctor and specialist were sharing information about their care. Among hospitalized patients, those lacking a care coordinator were more likely than those with a coordinator to report that that no one made arrangements for a follow-up visit with a doctor post-discharge $(35 \%$ versus $22 \%$, $\mathrm{p}<.001)$ and that their regular doctor was not informed about the care they received while they were hospitalized $(32 \%$, versus $13 \%$ versus $\mathrm{p}<.001)$ and in the emergency room ( $46 \%$ versus $27 \%$ versus, $\mathrm{p}<.001)$. Having a care coordinator reduces the risk for all coordination problems, even after controlling for cofactors, including whether a patient has accessible care or a strong patient-doctor relationship.

CONCLUSIONS: Physicians care for a high percentage of patients with multiple chronic conditions who may be co-managed by numerous physicians and health care professionals in various settings. Not surprising, patients who receive care from multiple physicians can experience fragmented and uncoordinated care. Our findings indicate that patients who report having a care coordinator as part of their care team and who also report having a positive patient-provider relationship and easy access and communication with their practice are significantly less likely to experience care coordination problems. Results suggest that including care coordinators in care teams can be a promising strategy for improving the care of patients with complex healthcare needs.

\section{THE SYMPTOM BURDEN OF PATIENTS CONSULTED ON BY} PALLIATIVE CARE IS UNDERESTIMATED BY THE PRIMARY INPATIENT CARE TEAM Nicole M. LaRue ${ }^{1}$; Linda Pang ${ }^{1}$; Michael K. Paasche-Orlow ${ }^{1}$; Angelo E. Volandes ${ }^{2}$. ${ }^{1}$ Boston University Medical Center, Boston, MA; ${ }^{2}$ Massachusetts General Hospital, Boston, MA. (Control ID \#1334505)

BACKGROUND: The delivery of high quality end-of-life care relies upon the accurate assessment of patients' symptoms. Prior work focusing mainly on patients with advanced cancer, has demonstrated poor agreement between patients and health care providers regarding health related quality of life. We are unaware of prior studies evaluating concordance in ratings of symptom intensity using the validated Edmonton Symptom Assessment Scale (ESAS) for providers and their patients consulted on by an inpatient palliative care service.

METHODS: Data was gathered through interviews with patients and/or proxies who were consulted on by an inpatient palliative care service and enrolled in a study of a video decision support tool to supplement goals-of- 
care discussions. Following the palliative care consultation, symptoms were measured across the nine different domains of pain, tiredness, nausea, depression, anxiety, drowsiness, well-being, appetite and shortness of breath using the validated ESAS instrument. Each symptom was rated on a 10 -point scale with zero meaning the symptom was absent and 10 indicating the worst possible intensity. Subsequent to the interview with the patient and/or proxy, the ESAS was administered to the attending of record, resident physicians and nursing staff of the primary team caring for the subject. The level of agreement was analyzed across each patientprovider dyad. For each symptom domain, a difference score was calculated as the patient's score minus that of the provider. The proportion of cases in which providers under- and over-estimated patients' symptom burden was compared across all nine domains.

RESULTS: This study included 62 patient-provider dyads. The prevalence of patient reported symptoms rated as five or greater ranged from $26 \%$ to $55 \%$ across the domains of pain, nausea, depression and appetite versus $71 \%$ to $90 \%$ across the domains of tiredness, drowsiness, anxiety, well-being and shortness of breath. Providers underestimated patients' symptoms in near to or greater than half of cases in the domains of drowsiness (73\%), tiredness $(58 \%)$, shortness of breath (58\%), pain (56\%), well-being (54\%) and anxiety $(49 \%)$. The prevalence with which providers underestimated a symptom by five or more points in these domains ranged from $16 \%$ to $29 \%$, with the exception of the domain of well-being in which symptoms were underestimated by five or more points in only $4 \%$ of cases. Symptom assessments showed greater concordance between patients and providers in the domains of depression, nausea and appetite, with symptoms underestimated in only $26 \%$ to $33 \%$ of cases. The prevalence with which providers overestimated patients' symptoms by five or more points ranged from $2 \%$ to $10 \%$ across all domains with the exception of depression and appetite, in which the prevalence of overestimation by five or more points was $20 \%$ and $18 \%$ respectively.

CONCLUSIONS: Patients who are consulted on by an inpatient palliative care service have a significant symptom burden that is frequently underestimated by the primary healthcare team. A failure to accurately assess the symptoms of patients with advanced disease may delay appropriate palliative care referrals and lead to unmet physical, emotional and psychosocial needs of patients and their loved ones.

THE USE OF CLAIMS DATA ALGORITHMS TO RECRUIT ELIGIBLE PARTICIPANTS INTO CLINICAL TRIALS Leonardo Tamariz; Ana M. Palacio; Ivonne H. Schulman; Gabriel Contreras. University of Miami, Miami, FL. (Control ID \#1336618)

BACKGROUND: International classifications of diseases (ICD-9) codes are frequently used to identify potential clinical research participants. However, this recruitment strategy usually focuses on a single ICD-9 code and does not include exclusion criteria. The purpose of this study is to validate a claims based algorithm to identify, from VA administrative data, eligible participants to be recruited into the Systolic Blood Pressure Intervention Trial (SPRINT).

METHODS: We created an algorithm to recruit elderly hypertensive subjects without diabetes and stroke into the SPRINT trial. Subjects were labeled as eligible if they were older than 75 years of age, had a hypertension ICD-9 code (401.x-405.x, 437.2) and did not have a diabetes (250.xx) or stroke ICD-9 codes (430.x-436.x, 437.1, 437.9, 438.x). We compared the eligible subjects with the medical record, which was considered the gold standard. In the medical record we defined hypertensionif hypertension was listed as a problem in the assessment and plan of at least oneprimary care clinical note and if the subject was taking blood pressure medication. Subjects were classified as diabetics if they met the 1997 American Diabetes Association definition of diabetes and stroke if they had documentation of stroke in the clinical notes. We calculated the positive predictive value (PPV) as the outcome.

RESULTS: The algorithm identified 3591 elderly Veterans with hypertension with no diabetes or stroke and we reviewed the medical records of 75 randomly selected patients identified using the algorithm. In the sample of medical record review the mean age was $83.3 \pm 5.4,44 \%$ had coronary artery disease, $23 \%$ had chronic kidney disease and $5 \%$ were Black. The mean systolic blood pressure was
134.4 \pm 15.7 . The most commonly used ICD-9 code to identify hypertension was 401.9 in $95 \%$ of the population. When compared to the medical record the PPV, for any hypertension code was $94 \%(95 \%$ CI: $87-97)$ and for the entire algorithm including older than 75 years and absence of both diabetes and stroke the PPV was $90 \%$ (95\% CI: 81-95)

CONCLUSIONS: The use of any ICD-9 code for hypertension is useful to identify elderly patients with hypertension. The algorithm to identify elderly patients with hypertension and without diabetes or stroke is a useful tool to identify eligible patients for clinical trial participation.

THE USE OF DIRECT OBSERVATION AND FEEDBACK TO ENHANCE THE TEACHING SKILLS OF RESIDENTS: A MULTIDISCIPLINARY RANDOMIZED CONTROL TRIAL AT TWO INSTITUTIONS Laura K. Snydman ${ }^{1}$; Lori Lyn Price ${ }^{2}$; Daniel Chandler ${ }^{1}$; Kimberly Dowdel1 ${ }^{1}$; Jessica K. Paulus ${ }^{2}$; H. Barrett Fromme ${ }^{3}$; Joseph Rencic ${ }^{1}{ }^{1}$ Tufts Medical Center, Boston, MA; ${ }^{2}$ Tufts Medical Center, Boston, MA; ${ }^{3}$ University of Chicago Medical Center, Chicago, IL. (Control ID \#1339288)

BACKGROUND: Little is known about the type and quality of resident teaching in the setting of work rounds, which is one of the main venues for residents to teach and lead autonomously. Yet students feel that a significant amount of their education derives from these interactions. Previous data have demonstrated improvement in attending teaching after direct observation and feedback, but no study has evaluated whether feedback in real-time clinical practice actually changes the teaching behaviors of residents. The objective of this study was to determine the effects of direct observation and feedback on ratings of inpatient teaching by pediatric and internal medicine residents as measured by resident selfratings, learner ratings, and trained faculty observer ratings.

METHODS: Pediatric and internal medicine residents at two academic medical centers participated in a randomized controlled trial involving direct observation of work rounds. Residents were randomized to a feedback group or control group. Each resident was observed on two occasions 3 days apart. After the first observation, residents in the feedback group received verbal feedback from a trained attending observer using a standardized feedback process. Residents in the control group did not receive feedback after the first observation. All residents received feedback from a trained attending observer after the second observation. The faculty observers assigned to the second observations were blinded as to whether the residents were in the feedback group or the control group. After the observation, the attending observer, the interns, and the students completed a validated tool (the Clinical Teaching Effectiveness Instrument) to assess the type and quality of teaching that occurred. The residents who were observed also completed a similar tool. Faculty observers were trained via video clips of teaching during work rounds. The rater pairs used at both sites had discussion and built consensus to achieve $>80 \%$ concordance.

RESULTS: Twenty-five internal medicine residents and 11 pediatric residents participated. A total of 380 completed forms were collected with an additional 8 forms having missing information. The majority of residents $(97.22 \%)$ had 6 or more CTEI evaluation forms. The results did not differ statistically between control group and feedback group for either institution alone or combined. One interesting finding was that residents who received feedback noted less improvement retrospectively than their peers in the control group (mean 2.19 vs. 2.94). Of the residents who received feedback, the competencies with the greatest improvement were as follows: organizing time, giving feedback, and asking questions. Teaching cost-appropriate care consistently ranked the lowest in terms of resident self-assessed competence and had the least improvement compared to the other competencies.

CONCLUSIONS: Our data showed no significant difference in learner, attending observer, and resident self-assessment between groups. However our data does demonstrate a difference in self-assessed improvement, with residents in the feedback group noting less improvement. This finding suggests that attending feedback may serve as a "reality check" which then manifests as the residents having a higher standard for themselves. 
THE UTILITY OF REPEAT BONE DENSITOMETRY IN WOMEN ON TREATMENT FOR LOW BONE MINERAL DENSITY Brandon Combs; Tanner Caverly; Stephen Ross; Wendolyn Gozansky; Daniel Matlock. University of Colorado, Denver, CO. (Control ID \#1339624)

BACKGROUND: It has been established that approximately $98 \%$ of patients initiated on bisphosphonate therapy have an increase in bone mineral density (BMD) and routine monitoring may not be necessary. Also, once treatment is started changes in BMD may be misleading as they have been shown to vary widely between individual dual energy $\mathrm{x}$-ray absorptiometry (DXA) scans and weakly correlate with fracture risk. This study explored the utility of repeat DXA in an average risk population on treatment for low BMD with the hypothesis that repeat testing will infrequently lead to changes in therapy.

METHODS: We identified 1782 unique patients with $>1$ DXA between Jan 1, 2003 and August 1, 2011 who had been seen within the past 18 months at one of five primary care clinics at the University of Colorado Hospital. Men $(n=120)$ and patients on medications or with conditions known to cause secondary osteoporosis $(n=580)$ were excluded. Of 1082 patients remaining, 552 were on therapy for low BMD (Vitamin D and calcium supplementation were not considered treatment). We conducted a chart review of a random sample of 25 women on therapy. Charts of each patient were reviewed to calculate the patients' 10-year fracture risk using the FRAX ${ }^{\circledR}$ score and to determine if repeat DXA led to changes in drug therapy within six months of DXA or next clinic visit. Considered scenarios that would require data from DXA were: change in drug class or medication change within class in the setting of significant decrease in $\mathrm{BMD}$, drug holiday after 5 years of treatment in the setting of stable or increased BMD, or drug stoppage in an osteopenic patient whose FRAX ${ }^{\circledR}$ score would not merit treatment (10-year probability of hip or combined major osteoporotic fracture of $<3.0$ or $<20$ percent). We considered changes as being due to factors other than repeat DXA if: changes occurred due to drug side-effects or payment reasons, if there was a medication change in the setting of stable or increased BMD, or if a drug holiday occurred in the setting of significantly decreased BMD.

RESULTS: Mean age of the sample was 68 years. Mean calculated 10year probability of hip or combined major osteoporotic fracture was $3.25 \%$ and $13.89 \%$, respectively. There were 58 repeat DXA scans performed during the follow up period and changes following repeat DXA are reported in Table 1. Treatment at the time of repeat DXA was with bisphosphonate in 53 of 58 instances (91.4\%). Fifty (86.2\%) DXA scans led to no change in therapy. Two (3.4\%) repeat DXA scans led to change in drug class, while one $(1.7 \%)$ led to change within class. Five repeat DXA scans were followed by stoppage of treatment though two of these did not meet drug holiday criteria - one was after four years of treatment and another was after DXA showed significant decrease in BMD. One repeat DXA leading to stoppage revealed a FRAX score too low to merit treatment, while two others met drug holiday criteria.

CONCLUSIONS: In this small single center study, repeat DXA scans infrequently led to changes in drug management. The current study highlights a need for additional studies assessing the utility of routine repeat DXA in patients on treatment before this practice can be justified.

Table 1. Changes in Therapy Due to Repeat DXA

\begin{tabular}{ll}
\hline \hline Type of change & Number of repeat DXAs $(\%)(\mathrm{n}=58)$ \\
No change & $50(86.2)$ \\
Change drug class & $2(3.4)$ \\
Change drug within class & $1(1.7)$ \\
Stop drug & $3(5.2)$ \\
\hline
\end{tabular}

THE VALUE OF BEDSIDE ROUNDS - A QUALITATIVE ANALYSIS Jed Gonzalo ${ }^{1}$; Brian S. Heist ${ }^{1}$; Briar Duffy ${ }^{2}$; Michael Elnicki ${ }^{1}{ }^{1} \mathrm{U}$. of Pittsburgh, Pittsburgh, PA; ${ }^{2}$ U. of Minnesota, Minneapolis, MN. (Control ID \#1334200)

BACKGROUND: Bedside rounds have traditionally been integral to teaching services, but their frequency has decreased. Medical educators have expressed concern about displacement of the patient from the forefront of education and lost opportunities to learn clinical skills, such as history-taking, physical-examination, communication, and professionalism. The transition away from the bedside has also weakened patientphysician relationships. No work has formally investigated bedside teachers' motivation for performing bedside rounds. To assist in promoting bedside rounds as an educational tool, we sought to elucidate physicians' motivations and reasons for performing bedside rounds on teaching services.

METHODS: Using purposeful sampling, we identified 10 academic institutions and a site principal investigator at each location to identify physicians who perform "bedside rounds" according to a pre-determined definition and serve as inpatient teaching attending. Two investigators conducted digitally-recorded, semi-structured telephone interviews, consisting of closed- and open-ended questions pertaining to motivations and reasons for performing bedside rounds. Interview transcripts were coded using an inductive thematic analysis to identify emerging themes. Quality control was maintained with 2 researchers independently coding the data and a 3 rd-analyst serving as a cross check. The Institutional Review Board at all institutions approved the study.

RESULTS: All 10 institutions identified at least 3 participants for inclusion. We completed 34 interviews, the largest subset with assistant professors (44\%). Participants averaged 13.7 years of academic experience and 18 weeks on the teaching services with housestaff over the previous 2 years. In response to questions pertaining to motivations for performing bedside rounds, 6 categories emerged: 1. Skill development for all learners - "[Learners] will never learn how to gleam physical findings from the patient or even history without somebody showing them.," 2. Observation and feedback - "Unless you see patients at the bedside with learners, there is no way you can assess their skills or give them feedback on [them].," 3 . Role-modeling - "[During conference room rounds], you can model what a good discussion should be, but you can't show them how physicians should interact with patients, how to be human, how to focus on patients.," 4. Team-Building - "[Bedside rounding] builds trust between all of us because if you establish the team dynamics right, we are all learning from each other and the patients trust us more.," 5. Improved Patient-Care Delivery - "You can hone in on information quickly and correct misinformation as the [learner] is telling you the story. It actually saves [us] a lot of time.," and 6. The Culture of Medicine as Patient-Centered Care - "[Bedside rounds] discourage stereotyping of patients. It keeps everybody professional and focused on the patient, rather than on test results."

CONCLUSIONS: In an era of advanced technology and duty hours, our participants believe bedside rounds enable skill development, observation, feedback, role-modeling, and team-building, all essential in fostering high quality, patient-centered care. In the context of competency-based education, raised concerns about the inadequacies of learners' clinical skills, and recent efforts to promote team-building skills, bedside rounds may offer an ideal venue for the development of these skills and improved patient care delivery.

THE ABILITY OF SINGLE SCREENING QUESTIONS FOR UNHEALTHY ALCOHOL AND OTHER DRUG USE TO IDENTIFY SUBSTANCE DEPENDENCE IN PRIMARY CARE Richard Saitz ${ }^{1,2}$; Debbie M. Cheng ${ }^{1,2}$; Donald Allensworth-Davies ${ }^{2}$; Michael Winter ${ }^{2}$; Peter C. Smith ${ }^{1,2}$. ${ }^{1}$ Boston Medical Center, Boston, MA; ${ }^{2}$ Boston University, Boston, MA. (Control ID \#1333544)

BACKGROUND: Single Screening Questions (SSQs) are recommended to identify unhealthy alcohol and other drug use (spectrum of risky use through dependence). But SSQs may also provide information on severity necessary to inform brief intervention thought to be obtainable only from longer questionnaires. We assessed SSQ accuracy for identifying patients with dependence.

METHODS: In a cross sectional study in an urban primary care practice, subjects were administered the SSQs asking about heavy drinking and drug use ["How many times in the past year have you had 5 (4 for women) or more drinks in a day?" \& "How many times in the past year have you used an illegal drug or used a prescription medication for nonmedical reasons?"], the Alcohol Use Disorders Identification Test-Consumption 
items (AUDIT-C), the Drug Abuse Screening Test (DAST), \& the Composite International Diagnostic Interview reference standard for current dependence. All possible cutoffs were evaluated by receiver operating characteristic (ROC) curve. Sensitivity (Ss), specificity (Sp), positive predictive value (PPV) and likelihood ratios positive and negative (LR+, LR- along with 95\% confidence intervals [CIs]) were assessed at cut points maximizing the sum of $\mathrm{Ss}$ and $\mathrm{Sp}$ (alcohol screening tests for alcohol dependence (AD), drug screening tests for drug dependence (DD)). RESULTS: Of 286 patients, 9\% had AD and 12\% DD; 31\% reported heavy drinking $\geq 3$ times, $22 \% \geq 8$ times in the past year; $30 \%$ reported drug use $\geq 3$ times, $22 \% \geq 8$ times in the past year. The area under the ROC curve (AUC), the probability of distinguishing those with and without dependence, was high for all tests. The optimal cut points were $\geq 8$ times for the alcohol SSQ, score $\geq 3$ for the 3 -item AUDIT-C, $\geq 3$ times for the drug SSQ, and $\geq 4$ for the 10-item DAST. Test characteristics appear in the table.

CONCLUSIONS: Single screening question (SSQ) results appear consistent with moderate to large changes in pre- to post-test probability of alcohol and other drug dependence (LR+5-10 and LR- 0.1-0.2 generate moderate changes in pre-to post-test probability (approximately $+/-30$ $45 \%$ ); LRs $>10$ or $<0.1$ may generate larger, clinically important changes). SSQs can identify alcohol and other drug dependence, with test characteristics similar to, or in the case of alcohol, possibly better than longer screening tools (based on positive likelihood ratio CIs). If confirmed in other studies SSQs may be useful for both screening and for severity assessment (to identify substance dependence), providing information needed and overcoming a barrier (lengthy questionnaires) to dissemination of screening and brief intervention in primary care settings. Financial Support: NIAAA (R01 AA10870), NIDA (R01s DA10019, DA025068)

\begin{tabular}{lllllll}
\hline \hline Test & AUC & Ss & Sp & PPV & LR+(CI) & LR-(CI) \\
SSQ Alcohol & 0.88 & $88 \%$ & $84 \%$ & $35 \%$ & $5.6(4.1,7.7)$ & $0.1(0.05,0.4)$ \\
AUDIT-C & 0.87 & $92 \%$ & $71 \%$ & $23 \%$ & $3.2(2.5,3.9)$ & $0.1(0.02,0.4)$ \\
SSQ Drug & 0.93 & $97 \%$ & $79 \%$ & $38 \%$ & $4.6(3.6,5.9)$ & $0.04(0.01,0.2)$ \\
DAST & 0.96 & $100 \%$ & $84 \%$ & $46 \%$ & $6.3(4.7,8.3)$ & 0 \\
\hline
\end{tabular}

THE ASSOCIATION BETWEEN BLOOD GLUCOSE AND OUTCOMES IN ADULTS HOSPITALIZED WITH ACUTE CHRONIC OBSTRUCTIVE PULMONARY DISEASE EXACERBATION Melissa Simpson $^{1}$; Yusuf Kasirye ${ }^{1}$; Narendranath Epperla ${ }^{1}$; Liang Hong ${ }^{2}$; Steven H. Yale ${ }^{1}{ }^{1}$ Marshfield Clininc Research Foundation, Marshfield, WI; ${ }^{2}$ Marshfield Clinic Research Center, Marshfield, WI. (Control ID \#1337039)

BACKGROUND: Hyperglycemia has been shown to be associated with adverse health outcomes. Recent evidence suggests that chronic obstructive pulmonary disease (COPD) complications are more common among patients with metabolic syndrome. However, little evidence exists concerning hyperglycemia specifically and outcomes associated with acute COPD exacerbations (AECOPD). The purpose of this study is to examine blood glucose (BG) during AECOPD hospitalization and clinical outcomes. We hypothesized that increased BG is associated with worse clinical outcomes. METHODS: We retrospectively studied a cohort of 209 hospitalized patients (40-80 years of age) with a physician-validated AECOPD diagnosis from $1 / 1 / 2004$ to $12 / 31 / 2008$. Inclusion requirements for this study were: meeting the Global Initiative for Chronic Obstructive Lung Disease criteria for COPD, AECOPD diagnosis at admission and discharge, first BG ascertained within 6 hours of admission, and $\geq 2$ BG measurements during hospitalization. Regression analyses accounting for repeated BG during hospitalization were performed to estimate the odds ratio (OR) for daily mean $\mathrm{BG}$ (per $100 \mathrm{ng} / \mathrm{ml}$ ) and length of hospitalization, 30 day mortality and hospital readmission, and 90 day all-cause mortality. Recognizing that the pathophysiologic process may be different in people with and without diabetes, we tested this association stratified by diabetes status.

RESULTS: Mean length of hospitalization was 3 days. Adjusting for age, and diabetes status, decreased BG was associated with longer length of hospitalization (OR: $0.72,95 \%$ CI: $0.54-0.96$, p-value: 0.03 ). Stratified analysis adjusting for age showed no association (diabetes: OR: $0.81,95 \%$ CI: $0.56-1.15, \mathrm{p}=0.24$, no diabetes: $0.64,95 \% \mathrm{CI}: 0.38-1.09, \mathrm{p}=0.10$ ).
Forty one patients $(19 \%)$ were readmitted to the hospital within 30 days of discharge from their index hospitalization. Adjusting for previous covariates and length of hospitalization, BG was not associated with 30 day hospital readmission (OR: $0.82,95 \% \mathrm{CI}$ : 0.54-1.22, p-value: 0.32), nor was it associated after stratification (diabetes: OR: $0.64,95 \%$ CI: $0.33-1.25, p=$ 0.19 , no diabetes: $1.01,95 \%$ CI: $0.33-1.81, \mathrm{p}=0.96)$. Nine patients $(4 \%)$ died within 90 days of their index hospitalization. Adjusting for previous covariates and readmission, decreased BG was associated with increased odds of 90 day all cause mortality (OR: $0.30,95 \%$ CI: $0.11-0.86$, p-value: 0.02 ) and the association is among people with diabetes (diabetes: OR: $0.22,95 \%$ CI: $0.07-$ $0.74, \mathrm{p}=0.01$, no diabetes: $0.72,95 \%$ CI: $0.14-3.61, \mathrm{p}=0.69$ ).

CONCLUSIONS: We found that among patients with diabetes, decreased blood glucose is associated with increased odds of 90 day all-cause mortality. Perhaps BG response (or lack thereof) in light of hyperglycemic agents is a proxy for a patient's overall physiological status, especially given that $96 \%$ of this population received corticosteroids during hospitalization. The physiology of glycemic control in this setting is extremely complex; in order to better understand the real time response to medical interventions and their association with outcomes, future studies may want to apply continuous glucose monitoring to this group.

THE ASSOCIATION BETWEEN BODY IMAGE DISSATISFACTION AND WEIGHT LOSS IN THE SMALL CHANGES AND LASTING EFFECTS (SCALE) PILOT STUDY Ginger J. Winston; Erica Phillips; Jessica Hippolyte; Mary Charlson. Weill Cornell Medical College, New York, NY. (Control ID \#1319284)

BACKGROUND: Obesity is a major health problem in the United States disproportionately affecting black and Hispanic adults. Perception of current and ideal body size may play a role in weight loss patterns, however, this relationship has not been well established among overweight/ obese adults.

METHODS: The SCALE pilot study was a 12-week behavioral weight loss intervention of eligible black and Hispanic adults with a BMI $\geq 25 \mathrm{~kg} / \mathrm{m}^{2}$ recruited at two clinical and two church sites in Harlem and the South Bronx, New York. Current and ideal body size were measured using the Gardner scale, a 13 figure scale of human silhouettes of increasing body size. Body image dissatisfaction was calculated as the difference between the participant's estimate of their ideal and current body size using the Gardner scale. T-tests were used to analyze the difference in mean values of linear variables. Multivariable regression models were used to assess the relationship between body image dissatisfaction and weight change with adjustment for age, gender, race/ethnicity, insurance and education. Interaction terms were used to assess for effect modification by race/ethnicity and gender.

RESULTS: 61 participants completed the 12 week pilot study $(74 \%$ women, $26 \%$ men, $43 \%$ black, $57 \%$ Hispanic). Mean weight loss at study close out was $2.5 \mathrm{lbs}$ and did not differ significantly by gender or race/ ethnic group (women $1.9 \mathrm{lbs}$ vs. men $4.2 \mathrm{lbs}, \mathrm{p}=0.2$; black $1.4 \mathrm{lbs}$ vs. Hispanic $3.4 \mathrm{lbs}, \mathrm{n}=0.3$ ). Body image dissatisfaction was higher among Hispanics compared to blacks $(p=0.001)$. There was no significant association between body image dissatisfaction and weight loss in the study sample, however, among black participants there was a trend towards increased weight loss as body image dissatisfaction increased.

CONCLUSIONS: In the SCALE pilot study, there was no significant association between body image dissatisfaction and weight loss. There was a trend towards increased weight loss among black participants as body image dissatisfaction increased despite greater body image dissatisfaction among Hispanics.

THE ASSOCIATION BETWEEN FREE PSA VELOCITY AND PROSTATE CANCER DIAGNOSIS BY BIOPSY Shoshana Weiner; Jianbo Li; Ahmed El-Shafei; J. S. Jones. Cleveland Clinic, Cleveland, $\mathrm{OH}$. (Control ID \#1314652)

BACKGROUND: Screening for prostate cancer with total serum prostate specific antigen (PSA) has inconsistently found a mortality benefit for prostate cancer patients. This has led to a search for new biomarkers. PSA 
velocity has been widely studied, but free PSA (fPSA) velocity, the change in the concentration unbound serum PSA over time, has not been previously considered.

METHODS: All patients who presented to Cleveland Clinic between 2000 and 2009 who had at least two prostate biopsies, with one initial negative biopsy, were considered for the study. A case-control study was conducted to compare free PSA velocity between patients diagnosed with prostate cancer after a previously negative biopsy, and controls, who are patients with two sequential negative biopsies. Univariate analyses were conducted to compare absolute differences and velocities of PPSA and PSA between cases and controls. Age, race, prostate volume, positive digital rectal exam and the pathological features of prostatic intraepithelial neoplasia (PIN), atypia and inflammation were compared between cases and controls for each biopsy. An iterative logistic regression model was constructed in order to compare fPSA velocity between cases and controls. All statistics were considered significant at a p-value of 0.05 . Bonferroni correction was used in the univariate analysis.

RESULTS: Forty-nine cases and 128 controls had the necessary fPSA values, which were restricted to being within two months prior to each biopsy. In addition, all biopsies required at least 10 cores and needed to be at a minimum one year apart from the previous biopsy. Our population was mainly Caucasian (86\%). The median ages for the first and second biopsies were 62 (IQR: 56, 67) and 64 (IQR: 58,69), respectively. $9(18 \%)$ of the positive biopsies had a Gleason score of 7 or greater, and most were Gleason score $6(78 \%)$. There was no significant difference in the fPSA velocity between cases (-0.21, IQR: -2.32 , $1.06)$ and controls $(0$, IQR: $-1.73,0.81)$. For the second biopsy, samples from cases had significantly more PIN ( $49 \%$ versus $26 \%$,p-value $=0.003$ ) than controls, and controls had significantly more inflammation (69\% versus $43 \%$, $\mathrm{p}$-value $=0.002)$. fPSA velocity was not significantly associated with having a positive prostate cancer biopsy in the logistic regression model (Odd ratio: $0.92 ; 95 \%$ CI $0.75-1.12$ ), which adjusted fPSA for PSA velocity and absolute change in PSA

CONCLUSIONS: fPSA velocity was not associated with a positive prostate biopsy for cancer in this population. This may be due to confounding pathological findings on biopsy such as inflammation and/ or the need for a larger sample-size.

THE CONSEQUENCES OF GOOD INTENTIONS: HIV/AIDS MEDIA DIRECTED TOWARDS AFRICAN-BORN PERSONS IN THE UNITED STATES Demetri Blanas ${ }^{1,2}$; Kim Nichols ${ }^{2}$; Carol Horowitz ${ }^{1} .{ }^{1}$ Mount Sinai School of Medicine, New York, NY; ${ }^{2}$ African Services Committee, New York, NY. (Control ID \#1340450)

BACKGROUND: The number of African-born persons in the US increased by $750 \%$ between 1980 and 2009, a period concurrent to the epidemic expansion of HIV/AIDS in sub-Saharan Africa. In spite of this, there is a dearth of research addressing this population's HIV screening needs, and despite a number of culturally tailored screening programs conducted by a local community-based organization, screening rates remain low.

METHODS: In order to identify effective strategies to increase HIV testing and referral rates, researchers and community partners conducted focus groups to uncover barriers and potential facilitators of screening. We recruited 39 African-born persons ( $46 \%$ women) residing in New York City, representing a wide range of African immigrants, including taxi drivers, hair braiders, streetvendors, students, mosque and church attendees. Four focus groups were held at a community-based organization and were audiotaped, transcribed, and translated from French and Wolof to English.

RESULTS: Grounded theory analysis using ATLAS.ti ${ }^{\circledR}$ revealed four previously described themes: fear of deportation; fatalistic attitudes; misinformation about HIV treatment options; and HIV stigma. Unexpectedly, we also identified two novel themes: 1) negative responses to public health messaging directed exclusively to Africans as it was viewed as inappropriately associating HIV/AIDS with Africans; and 2) preference for non-African providers so as to decrease the risk of a breach of confidentiality.

CONCLUSIONS: We found that current efforts to offer culturally-tailored HIV screening that exclusively targets African immigrants and provision of ethnically similar providers can paradoxically lead to concerns about being stereotyped and loss of privacy. Solutions proposed in the focus groups include public health messaging that portrays both African and non-African individuals, and cultural training for non-African counselors and screeners and anonymous translation services (translation phones) in testing centers.

THE EFFECT OF A COMMUNITY-BASED DIABETES SELFMANAGEMENT EMPOWERMENT PROGRAM ON MENTAL WELL-BEING: A CAUSAL MEDIATION ANALYSIS OF A RANDOMIZED CONTROLLED TRIAL. Takehiro Sugiyama ${ }^{1,2}$; Neil Steers $^{1}$; Neil Wenger ${ }^{1}$; O. Kenrik Duru ${ }^{1}$; Carol Mangione ${ }^{1}{ }^{1}$ University of California, Los Angeles, Los Angeles, CA; ${ }^{2}$ University of Tokyo, Tokyo, Japan. (Control ID \#1328528)

BACKGROUND: Diabetes impairs both physical and mental healthrelated quality of life (HRQL). Programs grounded in empowerment theory improve glycemic control, but their effects on mental HRQL are unknown. We investigated the effect of a community-based group diabetes selfmanagement program on mental well-being and explored whether the effect is direct or mediated by improved glycemic control and other concurrent physiological factors.

METHODS: We performed a secondary data analysis of the Diabetes Self-Care Study, a randomized community-based intervention that improved glycemic control. Study participants $(n=516)$ were African Americans and Latinos 55 years or older with poorly controlled diabetes $(\mathrm{HgbA} 1 \mathrm{c} \geq 8)$ recruited from senior centers and churches in Los Angeles. Participants were randomly assigned to intervention and control groups. Control group participants received 6 lectures on geriatric topics. Intervention group participants received 6 small-group self-care sessions based on empowerment theory. For these analyses, our primary outcome was change in Mental Component Summary score from the SF-12 (MCS12) between baseline and 6-month follow-up. HgbA1c, body mass index (BMI), low-density lipoprotein cholesterol (LDL), and systolic blood pressure (SBP) also were measured at baseline and 6-month follow-up. For these analyses, changes in those clinical variables were used as mediators. First, we compared baseline to 6 month change in MCS-12 between study groups using Student's t-test. Second, we performed a causal mediation analysis with $\mathrm{HgbA} 1 \mathrm{c}$ as a mediator in order to determine whether there was a direct effect of the diabetes empowerment program on mental well-being apart from the indirect effect mediated by HgbAlc change. Causal mediation analyses were repeated by inserting BMI, LDL and SBP as the potential mediator. In addition, we performed sensitivity analyses to assess the robustness of the results against the violation of no-confounder (between the mediator and the outcome) assumption. We performed causal mediation analysis and sensitivity analysis using the "mediation" package of software R. RESULTS: The 258 participants in each group had no significant differences in baseline characteristics and there was not a significant difference in follow-up between groups (follow-up data available for $79 \%$ intervention v $76 \%$ control participants). MCS-12 increased by a mean of 1.4 points in the intervention group and decreased by a mean of 0.2 points in the control group, for a difference in change of 1.6 points (95\% CI: 0.1 to 3.1). In causal mediation analysis, the empowerment intervention was shown to have a direct effect on MCS-12 improvement (1.7, 95\% CI: 0.2 to 3.2$)$ separate from the indirect effect mediated via HgbAlc change (-0.1, $95 \% \mathrm{CI}-0.4$ to 0.1$)$. We found no significant indirect effects of the other mediators. In sensitivity analyses, the causal mediation analyses were robust.

CONCLUSIONS: This Diabetes Self Care Study empowerment intervention had a positive impact on mental well-being not mediated by improvement in glycemic control or other physiological factors. The MCS-12 change of 1.6 points was equivalent to the difference of MCS- 12 between people with and without type 2 diabetes found in prior studies. This favorable effect of DSME on mental well-being may confer a clinical advantage that is separate from benefits achieved by pharmacotherapy.

THE EFFECT OF AGE-SPECIFIC GUIDELINES ON SCREENING OLDER WOMEN FOR CANCER Adam Licurse ${ }^{1,2}$; Pamela Soulos ${ }^{3}$; Jennifer L. Quon ${ }^{3}$; Cary Gross ${ }^{3}$. ${ }^{1}$ Brigham and Women's Hospital, Boston, MA; ${ }^{2}$ Harvard Medical School, Boston, MA; ${ }^{3}$ Yale School of Medicine, New Haven, CT. (Control ID \#1339917) 
BACKGROUND: Because the benefits of cancer screening diminish with increasing age, some guidelines recommend against screening for cervical and colorectal cancer in older women. However, it is unclear whether these guidelines have affected patterns of screening in the Medicare population. METHODS: We created a sample of women from Medicare's 5\% random sample of beneficiaries from 2000 through 2009, and reviewed major agespecific screening recommendations released during this period. For Pap smear, we included the 2002 American Cancer Society cut-off age of 70, and the 2003 United States Preventive Services Task Force cut-off age of 65. For colonoscopy, we included the 2008 USPSTF recommended cut-off age of 75. For each year, our sample included women 67-94 who did not have a cancer diagnosis or history of cancer in the prior two years (per ICD9), and were continuously enrolled in Medicare during that year and two years prior. Patients were further excluded from the colorectal or cervical screening samples if they had claims for similar procedures in the preceding 9 months, and were thus ineligible for routine screening during the index year. Our primary outcome was receipt of Pap smear, colonoscopy, and mammography each year. Mammography was used as a secular comparison, as no age-specific guidelines for breast cancer screening were released during the study period. For years when a major age-specific discontinuation recommendation was announced, we com- pared screening rates during the 2 years prior to the recommendation with the year after the recommendation using a Chi-square test. For each screening test, we assessed the trend for the entire period using a Poisson test.

RESULTS: From 2000 through 2009 (N $\approx 120,000$ per year), the percent of women age $\geq 67$ years undergoing mammography remained unchanged at approximately $30 \%$ ( $\mathrm{p}$-value for trend $=0.15$ ). Over the same period in the same age group, there was a significant decline in the use of Pap smear ((rate ratio (RR) for each year: $0.97 ; \mathrm{p}<0.001)$ ). About $13.3 \%$ of women in 2000 and 2001 received a pap smear (pre-guideline period), compared to $12.2 \%$ after the guidelines in $2004(\mathrm{p}<0.001)$. Conversely, there was an increase in the use of screening colonoscopy from 2000 up until the USPSTF guidelines were released after 2007 ( $R R$ for each year: $1.07 ; \mathrm{p}=0.02$ ) for women $\geq 75$ years of age. In the same age group, $1.5 \%$ of women in 2006 and 2007 (pre-guideline period) received colonoscopy, compared to $1.0 \%$ after the guidelines in $2009(\mathrm{p}<0.001)$.

CONCLUSIONS: National screening practices for colorectal and cervical cancer screening among Medicare beneficiaries were sensitive to guideline changes, and were associated with a decrease in use of screening among women with a lower likelihood of benefit. More attention should be paid to generating and disseminating guidelines applicable to older patients.

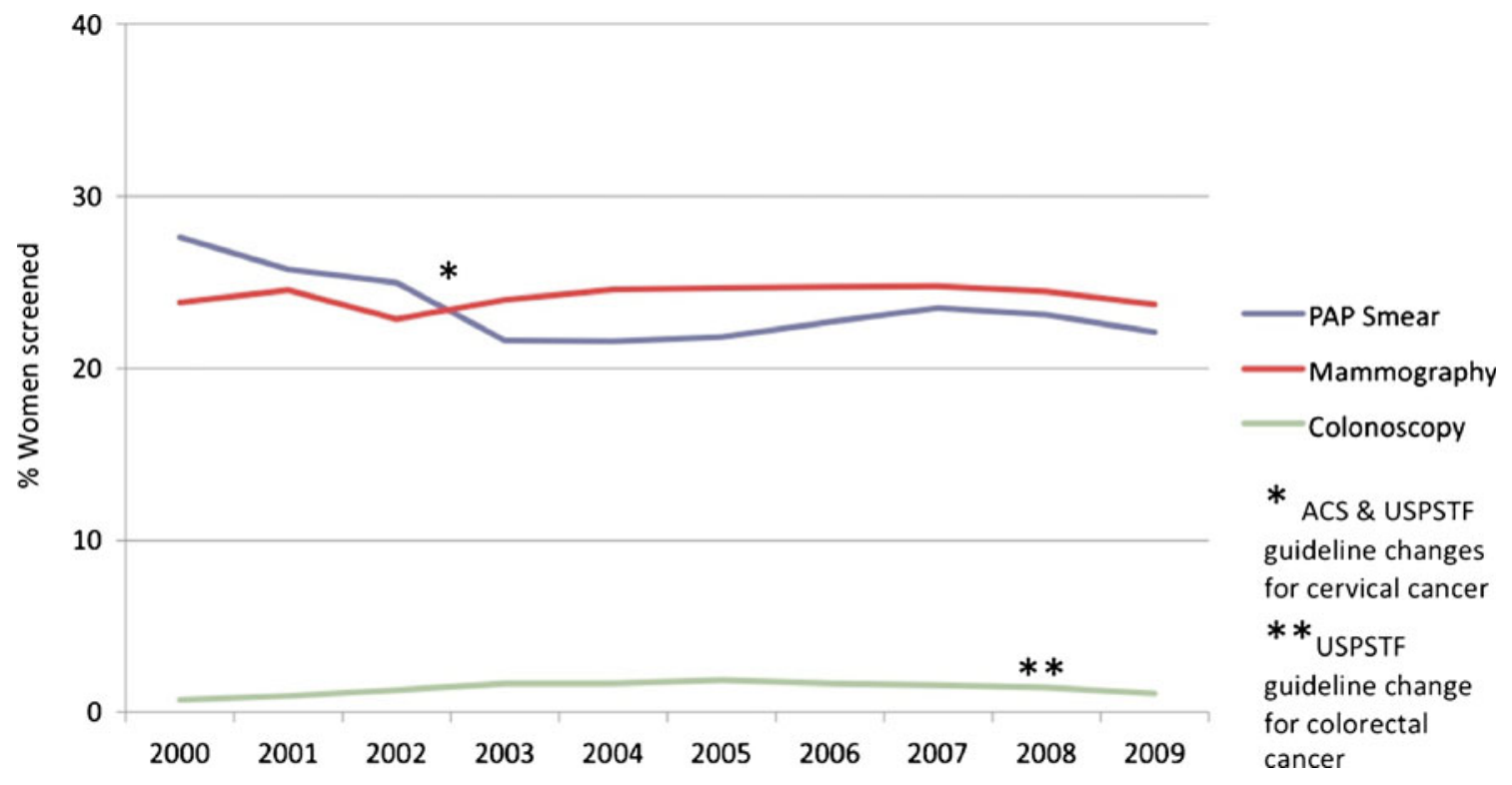

THE GOOD, THE BAD, AND THE TIME-CONSUMING: ATTITUDES TOWARD AND USE OF A COMMON AMBULATORY EMR Anil Makam $^{1}$; Holly Lanham ${ }^{2,3}$; Kim Batchelor ${ }^{4}$; Lipika Samal ${ }^{5}$; Lynne Kirk ${ }^{4}$; $\overline{\text { Brett }}$ $\overline{\text { Moran }}^{4,6}$; Manjula Cherukuri ${ }^{4}$; Temple Howell-Stampley ${ }^{4}$; Noel Santini ${ }^{6}$; Luci Leykum ${ }^{2,3}$; Ethan Halm ${ }^{4}$. ${ }^{1}$ University of California San Francisco, San Francisco, CA; ${ }^{2}$ South Texas Veterans Health Care System, San Antonio, TX; ${ }^{3}$ University of Texas Health Science Center at San Antonio, San Antonio, TX; ${ }^{4}$ University of Texas Southwestern, Dallas, TX; ${ }^{5}$ Brigham and Women's Hospital, Boston, MA; ${ }^{6}$ Parkland Health \& Hospital System, Dallas, TX. (Control ID \#1319842)

BACKGROUND: Despite intense interest in "meaningful use" of electronic medical records (EMR), realizing their potential benefits to improve quality has been difficult. Most positive studies come from earlyadopter institutions with 'home-grown' EMRs. Less is known about the use of commercial EMRs especially in non-HMO, ambulatory settings. METHODS: We surveyed 210 adult primary care providers (PCPs) in 11 general internal medicine (GIM) and family medicine (FM) practices affiliated with 3 academic health systems in TX. The survey focused on providers' attitudes, satisfaction, and use of the Epic EMR system, a common commercial EMR. Most practices had $>5$ yrs of experience with Epic. Items on early adoption of technology, use of EMR features, and impact of EMRs on quality were based on prior surveys. Most ratings were 5 -point Likert scales. Responses were dichotomized and multivariable regression examined factors associated with time using the EMR and attitudes of early adopters.

RESULTS: 144 PCPs completed the survey (69\% response rate)-64\% were attendings, $26 \%$ residents, and $10 \% \mathrm{NP} / \mathrm{PAs} ; 75 \%$ were GIM and $25 \%$ FM providers, $62 \%$ were female, and $61 \%$ were in practice for $\geq 10$ yrs. Over half (55\%) had $\geq 3$ yrs of experience using an EMR, and $64 \%$ were classified as early adopters. Most PCPs agreed the EMR was easy to use $(78 \%)$, easy to learn $(62 \%)$, improved clinical workflow $(69 \%)$, and helped them be more thorough in their work $(78 \%)$. However, $69 \%$ said the EMR made it difficult to maintain eye contact with a patient $(\mathrm{Pt})$ and $51 \%$ said it interfered with clinician-Pt communication. Most PCPs reported positive effects of the EMR on communication with other MDs (88\%), prescription refills (94\%), and timely access to medical records $(94 \%)$. PCPs were more likely to agree that future versions of the EMR would have positive effects on quality compared to the current version of the EMR on: preventing adverse drug interactions $(89 \%$ agreed future EMR 
would help this v. $78 \%$ current EMR), preventing medication errors $(85 \% \mathrm{v}$. $75 \%$ ), delivering preventive care that meets guidelines ( $88 \%$ v. $61 \%)$, delivering chronic disease care that meets guidelines ( $84 \%$ v. $51 \%)$, delivering high quality of care $(87 \%$ v. $71 \%)$, and preventing things from falling through the cracks $(86 \%$ v. $61 \%)$ [ $p<.01$ for all]. Documentation practices varied with about half of PCPs using free text for the history $(51 \%)$, note templates for the physical exam (57\%), and free text for the A \& P (54\%). Two-thirds used more sophisticated features of the EMR like smart phrases ( $\geq$ some of the time) and $47 \%$ used electronic communication with Pts and staff. For each half day clinic session, $43 \%$ reported spending $\geq 1 \mathrm{hr}$ beyond their session to complete EMR documentation and $29 \%$ reported spending $\geq 1 \mathrm{hr}$ to respond to messages in the EMR from Pts, staff and MDs. In multivariable regression, documentation time was not influenced by being an early adopter, EMR experience, yrs in practice, or other characteristics. Early adopters were more likely to say the EMR: was easy to learn, improves clinical workflow, helps them be more thorough, and discover new ways to use it $(\mathrm{p}<.05$ for all).

CONCLUSIONS: PCPs using a common commercial EMR had positive attitudes towards the EMR, perceived it to negatively affect patient communication and were hopeful future versions of the EMR would have a greater impact on quality than the current one. EMR documentation is time-consuming, even for PCPs who are early adopters of technology and have years of experience using it.

THE IMPACT OF HEPATITIS C VIRUS ON HIV RISK BEHAVIOR DURING METHADONE TREATMENT Karran A. Phillips; David H. Epstein; Kenzie L. Preston. NIDA/NIH, Baltimore, MD. (Control ID \#1340372)

BACKGROUND: Drug users have high rates of hepatitis $\mathrm{C}$ virus (HCV) infection, and they are important contributors in HIV, HCV, and sexually transmitted infection (STI) transmission. Better information about the specific risk behaviors in sub-populations of drug users may allow for more targeted $\mathrm{HIV} / \mathrm{HCV} / \mathrm{STI}$ risk reduction efforts.

METHODS: In this 40-week study, 140 methadone-maintained, heroinand cocaine-abusing individuals completed the HIV Risk-Taking Behaviour Scale (HRBS) every 2 weeks. In addition, to the 11 standard questions asked, a 12 th question on condom use during anal sex was added. This resulted in a 6 question drug-related risk subscale and 6 question sex-related risk subscale. Information on drug use and social/legal history and characteristics was obtained at baseline via the Addiction Severity Index (ASI) and HCV status was determined on admission. Longitudinal data was analyzed accounting for repeated measures and fixed and random effects using a multilevel mixed effects model in Stata 10.

RESULTS: Forty three percent of participants were HCV+. HCV+individuals had significantly higher scores on the drug-related risk subscale than HCVindividuals $(\mathrm{z}=11.5, \mathrm{p}<0.001)$. This finding appeared largely due to route of administration. $\mathrm{HCV}+$ individuals had significantly lower sex-related risk scores than $\mathrm{HCV}$ - individuals $(\mathrm{z}=-2.73, \mathrm{p}=0.006)$. The total HRBS was higher in $\mathrm{HCV}+$ individuals compared to HCV- individuals $(\mathrm{z}=4.96, \mathrm{p}<0.001)$ and this seemed largely driven by their higher drug-related risk subscale scores. Regardless of HCV status, HIV risk decreased with increasing time in methadone treatment.

CONCLUSIONS: When compared to HCV-individuals, $\mathrm{HCV}+$ individuals had higher drug-related and lower sex-related HIV risk behavior. Methadone treatment appeared to be an effective tool in reducing HIV risk. The data reported here argue for tailored prevention and treatment interventions aimed at changing drug use and HIV risk behavior and accounting for HCV status.

THE IMPACT OF INSURANCE INSTABILITY ON CARE AFTER ABNORMAL CANCER SCREENING Karen Freund ${ }^{1}$; Amresh Hanchate $^{1}$; Alexis P. Isabelle ${ }^{1}$; Richard Kalish $\overline{{ }^{2} \text {; Alok Kapoor }}{ }^{1}$; Sharon Bak $^{1}$; Marc C. Flore $^{1}$; Rebecca Grochow ${ }^{3}$; Swati Shroff ${ }^{3}$; Tracy Battaglia ${ }^{1}$. ${ }^{1}$ Boston University School of Medicine, Boston, MA; ${ }^{2}$ South Boston Community Health Center, Boston, MA; ${ }^{3}$ Boston Medical Center, Boston, MA. (Control ID \#1317085)

BACKGROUND: The intent of health insurance reform is to improve care through the expansion of access to care for minority, vulnerable, and underinsured populations. In 2006 the Massachusetts Health Reform Legislation sought to improve access to care by increasing insurance coverage. We sought to assess the impact of insurance instability on vulnerable women with an abnormal screening event, looking at whether they achieved a diagnostic resolution, comparing pre and post insurance reform cohorts. We studied women at 6 Community Health Centers, as they care for a disproportionate group of women with unstable insurance coverage.

METHODS: We analyzed billing data for all women following an abnormal breast or cervical cancer screening exam at 6 Community Health Centers in two groups: 2004 - 2005 (pre reform) and 2007 - 2008 (post reform). We observed insurance claims for eighteen months before and after the abnormal screening exam, and recorded insurance coverage and frequency of health insurance switches. We categorized switches into five levels of favorability from the most favorable representing women who were always privately insured to the least favorable representing women who were always uninsured. The outcome of interest is the time it takes to reach diagnostic resolution, dichotomized to those who resolved within 365 days of abnormal screening and those who did not. We conducted Cox proportional hazards regression analyses to observe if insurance instability changed the proportion of women with diagnostic resolution between the pre and post reform periods. We also generated Kaplan-Meier survival curves of the percent resolved by time.

RESULTS: We examined 1946 women, 434 women in the pre reform period and 1512 women in the post reform period. Subjects had an average age of $43( \pm 15)$ years and were $35 \%$ white, $32 \%$ black, $28 \%$ Hispanic, and $4 \%$ other, primarily Asian. Women in the sample received care at their Community Health Center for an average of 25 months and during that time had an average of 17 visits. At the time of the abnormal cancer screening in the pre reform period, $39 \%$ of women were uninsured, $30 \%$ had public insurance, and $31 \%$ had private insurance. We placed women into 5 categories of insurance instability: $22 \%$ were always privately insured, $16 \%$ were always publically insured, $21 \%$ had at least one switch but were never uninsured, $22 \%$ had at least one switch to an uninsured state, and $18 \%$ were consistently without insurance. The proportion always uninsured dropped from $25 \%$ to $16 \%$, and the proportion always privately insured increasing from $18 \%$ to $23 \%$ in the pre- compared to postinsurance reform period, X2 27.8, $\mathrm{p}<.0001$. We did not find that insurance stability was associated with women reaching diagnostic resolution within one year, comparing pre and post periods, Mantel-Haenszel X2 (df 4) 5.03, $\mathrm{p}=0.28$.

CONCLUSIONS: Limitations of the study include the inability to assess the length of non coverage between switches or to identify switches which occurred between health care visits. Insurance reform significantly improved coverage, with fewer women consistently uninsured. Our results did not show an association between changes in insurance stability and delays in time to diagnostic resolution.

THE INTERACTION BETWEEN GENDER AND AMOUNT OF SMOKING IN THE PREDICTION OF ONE-YEAR WEIGHT GAIN AFTER SMOKING CESSATION Isabella Locatelli ${ }^{1}$; Carole Clair ${ }^{1}$; Tinh-Hai Collet ${ }^{1}$; Nicolas Rodondi ${ }^{2}$; Jacques Cornuz ${ }^{1}$. ${ }^{1}$ University of Lausanne, Lausanne, Switzerland; ${ }^{2}$ Inselspital, University of Bern, Bern, Switzerland. (Control ID \#1314857)

BACKGROUND: Smoking cessation is associated with weight gain. We aimed to determine the amount of weight gain and its predictors among primary care smokers after 1 year of smoking cessation.

METHODS: We merged two randomized controlled studies (RCTs) assessing new interventions helping smokers to quit. The first RCT ( $\mathrm{n}=$ 477) evaluated a program of moderate intensity physical activity; the second $(n=536)$ assessed the effect of carotid plaque screening by ultrasound. Both RCTs studied the intervention effect on smoking cessation rates after one year compared to a control program of intensive counseling and nicotine replacement therapies. The similarities between both RCTs and the identical control programs allowed us to merge the two studies, regardless of the individual assignment to the RCT-specific control/intervention group. The analysis included all subjects that made at least one quit attempt during the study $(\mathrm{n}=810)$. We used a multivariable linear regression model to test the effect on the mean weight gain at one year for the following potential predictors and all their interactions: age 
(dichotomized at the median, 47 years), sex, education level, number of cigarettes at baseline and duration of smoking abstinence. We applied a backward procedure to select the best model $(\mathrm{p}<0.05)$.

RESULTS: At baseline, subjects (mean age 46.9 years, $43.7 \%$ women) smoked a median of 22 cigarettes per day, ranging from 8 to 70 , and median smoking abstinence was 18 weeks during follow-up. After 1 year, the mean weight gain was 2.6 kilograms $(\mathrm{kg}, 5.7 \mathrm{lbs})(3.6 \%$ of the initial weight). Among smokers that were abstinent more than 18 weeks, the mean weight gain was $3.8 \mathrm{~kg}$ (5.3\% of the initial weight). Age, sex and number of cigarettes at baseline were significantly associated with weight gain at one year. Older subjects ( $>47$ years) gained $0.7 \mathrm{~kg}$ less than younger $(p=0.001)$. We found an interaction between sex and the number of cigarettes smoked at baseline $(\mathrm{p}=0.003)$ : men gained more weight than women when smoking $<30$ cigarettes per day; the reverse was observed for heavy smokers (Figure). According to this model, a woman younger than 47 and smoking more than 30 cigarettes/day could gain up to $5.5 \mathrm{~kg}$ on average one year after smoking cessation ( $9 \%$ of her initial weight). A man of the same age group and smoking the same amount would expect a mean weight gain of $4.5 \mathrm{~kg}$ ( $6 \%$ of his initial weight).

CONCLUSIONS: Age, sex and number of cigarettes at baseline predicted 1-year weight gain among smokers who quit, and a significant interaction between sex and number of cigarettes smoked was found. Young women smoking heavily at baseline were those with the highest risk of weight gain after quitting and should be followed closely by their physicians during abstinence periods.

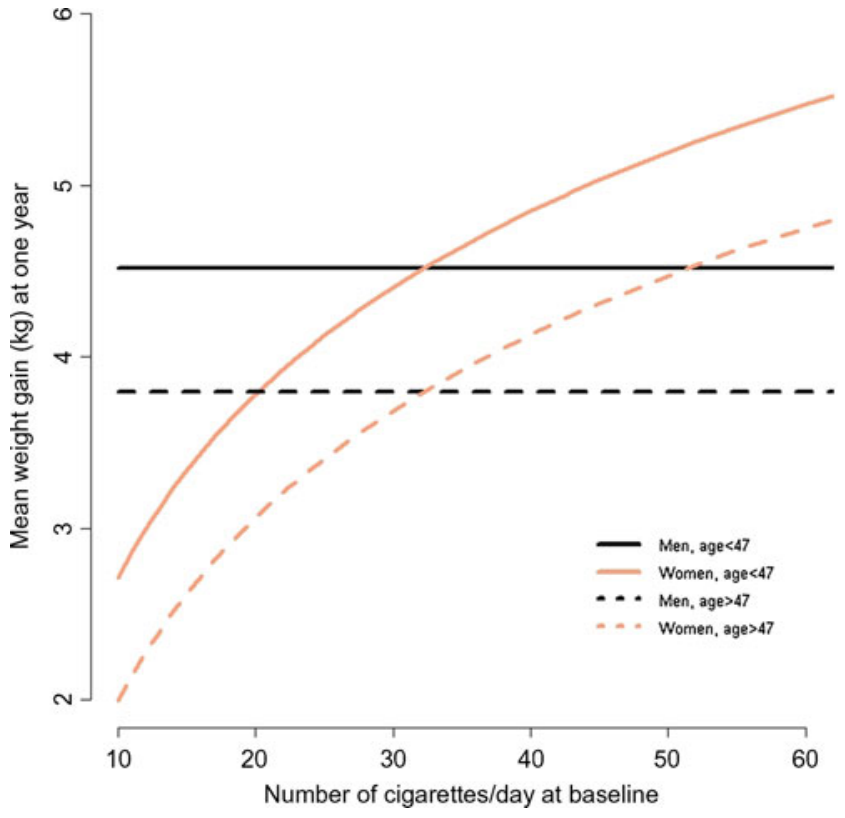

THE LEVEL OF PATIENT SATISFACTION WITH INFORMED CONSENT IS HEIGHTENED WITH THE CHANGE OF THE SYSTEM OF OUTPATIENT CLINIC OF GENERAL MEDICINE FROM TREATING ALL OUTPATIENTS TO ONLY NEW OUTPATIENTS. Hitoshi Eguchi ${ }^{1}$; Midori Nishii ${ }^{1}$; Yoshinori Tokushima ${ }^{2}$; Naoko Eguchi ${ }^{1}$; Motoshi Fujiwara ${ }^{1}$; Masaki Tago ${ }^{1}$; Yuta Sakanishi ${ }^{2}$; Motosuke Tomonaga $^{1}$; Tsuneaki Yoshioka ${ }^{1}$; Masaki Hyakutake ${ }^{1}$; Yuichiro Eguchi $^{1}$; Sei Emura ${ }^{1}$; Takashi Sugioka ${ }^{2}$; Shu-ichi Yamashita ${ }^{1}{ }^{1}$ Saga University Hospital, Saga, Japan; ${ }^{2}$ Saga University Hospital, Saga, Japan. (Control ID \#1335128)

BACKGROUND: Doctors of outpatient clinic in the department of general medicine take charge of almost all patients including patients on the long-term follow-up and new patients in Japan. Doctors working in outpatient clinic of the department of general medicine, Saga University Hospital also had taken charge of all patients since 1978, however, we changed our system of outpatient clinic treating all patients to the new system treating only new ones in October 2011. Recently, there is a tendency that hospitals survey the patient satisfaction to improve hospital functions and quality. Although previous studies have indicated the correlation between patient satisfaction and age, elapsed times, doctor's attitude and car parking space, correlation with the system of outpatient clinic remains to be elucidated. This study investigated the change of patient satisfaction with the change of the system of outpatient clinic. METHODS: 680 patients who visited to outpatient clinic of the department of general medicine, Saga University Hospital for the first time were enrolled in from August to November 2011. System of outpatient clinic changed from treating all patients including patients on the long-term follow-up and new outpatients to treating only new ones in October 2011. We investigated age, elapsed time, doctors in charge, presence or absence of medical referral letter, involvement of students and a motivational condition to visit out clinic. The level of patients' satisfactions with time, doctors, nurses, other health care providers, co-medical, informed consent, response to patient's expectation, were evaluated by the questionnaire which scaled one-to-five. Total score was also evaluated.

RESULTS: A total of 680 patients were evaluated (male 305, 44.9\%, female $375,55.1 \%)$. There was significant sexual difference about total score $(\mathrm{p}<$ 0.05 , male $3.86 \pm 0.70$, female $3.75 \pm 0.76$ ). There were 374 (55\%) patients who visited before the system-change (Pre) in August and September 2011, and there were $306(45 \%)$ patients who visited after the system-change (Post) in October and November 2011. There was higher level of satisfaction with informed consent in Post than Pre with significant difference $(p<0.05$, Pre $3.95 \pm 0.68$, Post $4.05 \pm 0.70$ ). Although there were tendency that the level of patients' satisfactions with time score, doctors, nurses, total score was increased, there were no significant differences. There were also no significant differences in elapsed time and response to patients' expectation.

CONCLUSIONS: Our study indicated that it is important in order to satisfy new outpatients especially with informed consent, to change system of outpatient clinic from treating all patients including patients on the longterm follow-up and new outpatients to treating only new ones in outpatient clinic of general medicine.

Difference of the level of patients satisfaction between treating all patients and treating only new patients.

\begin{tabular}{llll}
\hline \hline & $\begin{array}{l}\text { All patients } \\
(374,55 \%)\end{array}$ & $\begin{array}{l}\text { Only new patient } \\
(306,45 \%)\end{array}$ & $\mathrm{p}$ value \\
Age (y.o) & $55.66 \pm 17.0$ & $54.5 \pm 20.0$ & 0.425 \\
Time (hour) & $2.13 \pm 1.15$ & $2.17 \pm 1.13$ & 0.659 \\
Time-score & $2.59 \pm 0.83$ & $2.67 \pm 0.81$ & 0.181 \\
Dr-score & $4.09 \pm 0.79$ & $4.15 \pm 0.73$ & 0.269 \\
Ns-score & $3.99 \pm 0.69$ & $4.06 \pm 0.7$ & 0.225 \\
Co medical-score & $3.91 \pm 0.76$ & $3.96 \pm 0.74$ & 0.409 \\
Informed Consent & $3.95 \pm 0.68$ & $4.05 \pm 0.70$ & $0.046^{*}$ \\
response to patient's & $3.82 \pm 0.71$ & $3.87 \pm 0.80$ & 0.444 \\
expectation & & & \\
Total-score & $3.77 \pm 0.73$ & $3.83 \pm 0.75$ & 0.255 \\
\hline
\end{tabular}

THE LOWER QUALITY OF PRIMARY CARE AMONG ASYLUM SEEKERS IN A COUNTRY WITH UNIVERSAL HEALTHCARE COVERAGE Yonas Martin ${ }^{1}$; Tinh-Hai Collet $^{1}$; Lukas Zimmerli ${ }^{2}$; Edouard Battegay ${ }^{2}$; Jean-Michel T. Gaspoz ${ }^{3}$; Jacques Cornuz ${ }^{1}$; Nicolas Rodondi ${ }^{4,1}$. ${ }^{1}$ University of Lausanne, Lausanne, Switzerland; ${ }^{2}$ University Hospital of Zurich, Zurich, Switzerland; ${ }^{3}$ University Hospitals of Geneva, Geneva, Switzerland; ${ }^{4}$ Inselspital, University of Bern, Bern, Switzerland. (Control ID \#1334067)

BACKGROUND: Differences of quality of preventive care among different population subgroups have been demonstrated in the US. In Switzerland, no study has examined the factors associated with the quality of preventive care. We assessed the association between patient and physician factors, and the quality of preventive care and chronic care of cardiovascular (CV) risk factors in Swiss university primary care settings. METHODS: Our study was based on a retrospective cohort of a random sample of 1,002 patients aged 50-80 years followed for 2 years in 4 Swiss university primary care settings. Indicators derived from RAND's Quality 
Assessment Tools were used. We constructed aggregate scores for preventive care and chronic care of $\mathrm{CV}$ risk factors. Percentages of recommended preventive and chronic care of $\mathrm{CV}$ risk factors offered to eligible patients of the different socio-demographic subgroups were calculated.

RESULTS: Overall, patients received $69 \%$ of recommended preventive care. Men had higher scores than women $(72.9 \%$ vs. $65.3 \%$; $<<0.001)$ and prevention rates declined with age $(50-59$ years: $71.2 \%$; $60-69$ years: $69.8 \%$; $70-80$ years: $66.8 \%$; $p$ for trend $<0.03$ ). Prevention indicators were more likely to be met among Swiss patients than asylum seekers $(71.9 \%$ vs. $61.2 \% ; p=0.001)$, mostly because of lower rates of cancer screening and influenza immunization. The overall score for chronic care of CV risk factors was $83 \%$. The elderly had significantly lower scores $(70-80$ years: $79.9 \%$ ) than the youngest patients $(50-59$ years: $84.0 \%$; p for trend 0.033 ). Rates of chronic care of CV risk factors did not differ by gender and legal status. Female physicians provided significantly more preventive care than male physicians $(\mathrm{p}=0.02)$ for female patients $(66.6 \%$ vs. $63.9 \%)$, and male patients (74.0\% vs. $71.7 \%)$. Chronic care of $\mathrm{CV}$ risk factors did not differ according to patient gender or physician gender.

CONCLUSIONS: Despite universal healthcare coverage, adults in Swiss university primary care settings received less preventive care according to their gender, age and legal status. Greater attention should be paid to vulnerable populations, particularly for cancer screening and influenza immunization. Even though quality of preventive care varies between subgroups of the population, the differences are much smaller than the gap separating provided from recommended preventive care.

THE RELATIONSHIP BETWEEN EXPERIENCE AND OUTCOMES: ANOTHER LOOK AT THE JULY EFFECT Jennifer Carnahan ${ }^{1}$; Alexis M. Visotcky ${ }^{2}$; Kathlyn E. Fletcher ${ }^{3,1}$. ${ }^{1}$ Medical College of Wisconsin, Milwaukee, WI; ${ }^{2}$ Medical College of Wisconsin, Milwaukee, WI; ${ }^{3}$ Milwaukee VAMC, Milwaukee, WI. (Control ID \#1335009)

BACKGROUND: The "July effect" is an oft cited, occasionally proven, and pervasively feared phenomenon that refers to the supposed ill-effect of the July influx of inexperienced house staff on patient outcomes. Various studies have found evidence for and against the July effect on patient outcomes. The aim of the current study is to explore the association between the inexperience of residents earlier in the academic year and selected patient outcomes.

METHODS: This project was part of a larger study of discontinuity in hospitalized general medicine patients. The data for this project comes from retrospective chart review. Patient charts from 3 sites were randomly chosen and evenly distributed over a one-year period between March 2009March 2010. The 3 sites included a VAMC, an academic tertiary care medical center and a community teaching hospital. To be included in the study, patients were either assigned to a house staff team or a hospitalist team. Patients were excluded if their hospital stay was $<48$ hours. Trained nurse abstractors did the chart review which included demographics, comorbidity data, adverse events, readmission within 30 days, and ER visit within 30 days of discharge. We used Wilcoxon ranked sum tests and chisquared analyses to compare the readmission, ER visits post-discharge and adverse events in patients by quarter of the year. We used the patients admitted to hospitalist teams as "controls" in order to evaluate for evidence of different outcomes in the first quarter of the academic year ("July-September" phenomenon). All tests were 2 -tailed, with significance at $\mathrm{p}<0.05$.

RESULTS: The sample contained 1180 patients. Mean age was 61 years (SD 18). $41 \%$ of the sample was female. Racial breakdown included $51 \%$ Caucasian, 43\% African-American, and 6\% other. Mean Charlson score was 2.3 (SD 2.1). Mean length of stay was 5.2 (SD 4.1) days. Overall readmission rate was $22 \%$. There was no difference in readmission rate between quarters for either the house staff or the hospitalist patients. Neither were there differences in readmission rates when house staff and hospitalist teams were compared to each other quarter by quarter. However, when differences in adverse events by quarter were tested between house staff and hospitalist teams, we found 2 differences. First, mean adverse events per patient in the last quarter of the academic year were significantly higher in the house staff patients (1.14) when compared to the hospitalist patients $(0.80), \mathrm{p}<0.05)$. Also in the last quarter, more house staff patients $(47 \%)$ than hospitalist patients $(34 \%)$ had at least one adverse event, $p<0.05$ No other quarters showed an adverse event disparity. In the third quarter of the academic year, we found that hospitalist patients had a significantly shorter length of stay (4.2 days, SD 3.1) when compared to the house staff patients (6.1 days, SD 5.4), $\mathrm{p}<0.05$. There were no significant differences in length of stay noted in other quarters.

CONCLUSIONS: Prior evidence is variable for the existence of a July effect, and our study failed to confirm its existence. Interestingly, just prior to the end of an academic year we did find a significant difference in adverse events when hospitalist and house staff teams were compared. This could be related to resident burn-out or a decrease in supervision. Therefore, although the myth of the July effect persists in the literature and in residency culture, the reality of the "June effect" warrants further attention.

THEY DON'T KNOW WHAT THEY DON'T KNOW: INTERNAL MEDICINE RESIDENTS' KNOWLEDGE AND CONFIDENCE IN URINE DRUG TEST INTERPRETATION FOR PATIENTS WITH CHRONIC PAIN Joanna L. Starrels; Aaron Fox; Hillary Kunins; Chinazo Cunningham. Albert Einstein College of Medicine \& Montefiore Medical Center, Bronx, NY. (Control ID \#1328158)

BACKGROUND: Urine drug testing (UDT) can help to identify misuse or diversion of opioid medications among patients with chronic pain. However, misinterpreting results can lead to false reassurance or erroneous conclusions about drug use. Misinterpretation might occur when physicians who lack knowledge about UDT interpretation are overly confident in their ability to interpret UDT results. Previous studies have not evaluated the association between physicians' knowledge about UDT interpretation and confidence in their ability to interpret UDT results.

METHODS: As part of a broader needs assessment, we conducted a cross-sectional study of internal medicine residents to explore the relationship between residents' knowledge and confidence in interpreting UDT results. All 148 internal medicine residents in a university-based health system in the Bronx were invited to complete a 53-item questionnaire, via email or distribution at educational sessions, from October 2010 to July 2011. Residents' knowledge of UDT interpretation was assessed using an 8-item scale ("UDT knowledge score"). Residents" confidence in UDT interpretation was assessed using a single statement ("I feel confident in my ability to interpret the results of urine drug testing"), to which participants rated their level of agreement on a 5-point Likert Scale (strongly disagree through strongly agree). Responses were dichotomized according to confident (agree or strongly agree) or not confident. We conducted chi-square tests, t-tests, and logistic regression to determine the association between knowledge and confidence, and to examine whether resident characteristics such as gender (male, female), training level (PGY-1, PGY-2 or -3), or UDT use (routine, rare) moderated the relationship between knowledge and confidence.

RESULTS: 109 residents participated in the study (74\% response rate) and 10 were excluded from the current analysis because they did not provide data on knowledge or confidence in UDT interpretation. Among 99 residents, the mean UDT knowledge score was 3.2 out of 8 (SD 1.4). Though $55(56 \%)$ of residents felt confident in their ability to interpret UDT results, $38(69 \%)$ of confident residents had a knowledge score of 3 or lower. Knowledge score was not associated with confidence among the full sample or when stratified by training level or UDT use. The association between knowledge and confidence differed significantly by gender (interaction term $\mathrm{p}<0.01$ ). Adjusting for training level and UDT use, knowledge was positively associated with confidence among females (AOR 1.51 for each additional knowledge item answered correctly, $95 \%$ CI: $0.98,2.32$ ), and negatively associated with confidence among males (AOR $0.57,95 \%$ CI: $0.30,1.05$ ). While neither association was statistically significant, these findings suggest that among females, greater knowledge was associated with feeling confident, and among males, lower knowledge was associated with feeling confident.

CONCLUSIONS: Despite poor knowledge about UDT interpretation, most resident physicians felt confident in their ability to interpret UDT results. Gender differences warrant further exploration, but even confident physicians who use UDT should evaluate their proficiency in interpreting UDT results. Educational initiatives to improve monitoring for patients prescribed opioids for chronic pain should emphasize the complexities of UDT interpretation. 
THOUGHT PROCESS DURING MULTIPLE-CHOICE EXAMINATIONS Brian S. Heist ${ }^{1}$; Jed Gonzalo ${ }^{1}$; Steven Durning ${ }^{2}$; Dario M. Torre ${ }^{3}$; Michael

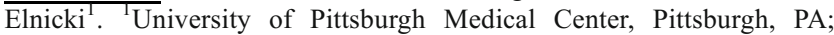
${ }^{2}$ Uniformed Services University of the Health Sciences, Bethesda, MD; ${ }^{3}$ Drexel University College of Medicine, Philadelphia, PA. (Control ID \#1324048)

BACKGROUND: Since the 1970s extensive research has been conducted on thought processes of physician trainees when approaching clinical scenarios in an attempt to enhance the teaching of clinical reasoning. However, little is known about the thought processes of physician trainees when approaching clinical vignette multiple-choice questions (MCQs), the standardized testing method commonly assumed to evaluate clinical reasoning. This study attempted to characterize the different thought processes used by resident physicians when solving clinical vignette MCQs, and to correlate processes with those used in clinical work and with scores on high stakes MCQ exams.

METHODS: We selected 6 clinical vignette MCQs representing 6 different organ systems from a question bank. Toward the end of their PGY-1 year, all residents training in internal medicine (transitional-year, categorical internal medicine, and med-peds residents) at our hospital were solicited for participation. During individual sessions, each subject completed the MCQs using a think-aloud protocol. Qualitative analysis of data transcriptions was performed independently by 2 investigators using a constant-comparative approach. Codes were re-evaluated until consensus was reached on activities used by the subjects. The subjects' application of the activities was then correlated with scores on the USMLE Step2CK, the high stakes exam most similar to the question bank.

RESULTS: Of 27 eligible residents, 10 participated. Subjects were allotted one hour but all completed the 6 questions in $\leq 25$ minutes. Transcript process coding found activities were consistent for a given subject, rather than for a given question. The following activities were identified: 1 . reading question prior to stem, 2 . reading stem with frequent summarization, 3. querying the test writer's objective, 4. summarizing key features of stem after reading alternatives, 5 . using inductive reasoning, 6 . considering alternatives systematically, 7. admitting knowledge deficits, 8. reaching closure prematurely, 9. reaching closure with difficulty, 10. negative suggestion effects (application of incorrect knowledge or concept without feedback), 11. generating own answer prior to reading alternatives (limited to $5 / 60$ questions total performed by $4 / 10$ subjects, a far lower rate than for most other activities). Subjects' USMLE Step 2CK scores ranged from 189 - 260. Subjects with the two lowest Step 2CK scores $(189,199)$ did not systematically consider answers or admit knowledge deficits, demonstrated premature closure in $\geq 2$ questions, and experienced the most negative suggestion effects ( $3 / 6$ and $4 / 6$ questions respectively). Subjects with the two highest Step 2 CK scores $(251,260)$ systematically considered answers, admitted knowledge deficits, did not generate their own answers prior to reading alternatives, did not experience premature or difficulty with closure, and experienced one negative suggestion effect collectively.

CONCLUSIONS: Though limited by the small number of study subjects and questions, the data suggest different patterns of thought process activities used by higher performers and lower performers during high stakes clinical vignette style MCQs. They also suggest generating an answer prior to reading alternatives, an activity important in clinical practice, is not valued by MCQs. Implications include the potential to assist lower test performers whose activities may be maladaptive, and to reconsider question format to value one's own generation of the answer.

THROMBOPROPHYLAXIS - WHEN IT FAILS Asif A. Ansari ${ }^{1}$; Purnachander R. Vangala ${ }^{1}$; Harneet Pahwa ${ }^{1}$; Kristy Pahl ${ }^{3}$; Alan T. Davis 1 ; Thomas Gribbin $2 .{ }^{1}$ GRMEP/MSU, Grand Rapids, MI; ${ }^{2}$ Cancer and Hematology centers of west michigan, Grand Rapids, MI; ${ }^{3}$ Univeristy of Rochester, Rochester, NY. (Control ID \#1320400)

BACKGROUND: Venous thromboembolism (VTE) is a major cause of morbidity and mortality that affects more than one million patients every year. A number of global registries have documented poor compliance to thrombophylaxis guidelines. Our hospital has adopted a systematic electronic admission policy to ensure compliance to guidelines. There still remains a subset of patients who develop VTE in hospital despite appropriate prophylaxis. To our knowledge, there has been no study that details this high risk group failing prophylaxis. Our aim was to characterize the population failing thromboprophylaxis and scrutinize our practice.

METHODS: This was a retrospective cohort study reviewing charts beginning from 2007 to 2010, of all patients who developed VTE after 3 days of hospitalization. We analyzed the incidence of thromboembolic events, characterized the risk factor profiles of these high risk patients in addition to the choice of prophylaxis, treatments used and their outcomes.

RESULTS: The incidence of VTE in patients despite prophylaxis was $0.15 \%$ (95\% CI: $0.12 \%-0.19 \%$ ). Seventy-two patients developed VTE during this period, of which 58 had received appropriate prophylaxis. Enoxaparin was used for prophylaxis in 35 patients, while heparin was used in 23 patients. Of the 14 patients who did not receive heparin based prophylaxis, 13 had a relative or absolute contraindication for the use of heparin products. The mean age of patients was 65 years; and $57 \%$ of them were male. Thirty patients had a DVT, 17 had a PE while 11 were diagnosed with both. The average time for the development of a DVT or PE was 7 days. There was no significant difference between the patients in the heparin and enoxaparin groups. Patients with cancer, post orthopedic or surgical intervention, or sepsis/severe inflammatory response syndrome were at a high risk to develop VTE with an incidence of $88.9 \%(95 \% \mathrm{CI}: 79.3 \%-95.1 \%$, p value $<0.000001)$. The incidence of treatment failure was $0.59 \%$ (95\% CI: $0.45 \%-0.76 \%)$. Implementation of electronic records improved the rate of documentation of VTE prophylaxis from $55 \%$ to $80 \%(\mathrm{p}=0.11)$ and risk stratification from $20 \%$ to $72 \%$ ( $\mathrm{p}=0.0008)$.

CONCLUSIONS: Our study details characteristics of this high risk patients failing prophylaxis. VTE during hospitalization is likely to worsen outcomes and thus must be prevented. Further studies are needed to determine the optimal dose of thromboprophylaxis in this high risk group, perhaps higher doses of heparin or enoxaparin. Electronic record keeping is likely to improve compliance to local VTE guidelines.

TIME SERIES ANALYSES OF THE EFFECT OF FDA WARNINGS ON PRESCRIBING OF WEIGHT LOSS MEDICATIONS Jason P. Block $^{1,2}$; Niteesh K. Choudhry ${ }^{3}$; Angela Tong ${ }^{3}$; William Shrank ${ }^{4,3}$. ${ }^{1}$ Harvard Pilgrim Health Care Institute/Harvard Medical School, Boston, MA; ${ }^{2}$ Brigham and Women's Hospital, Boston, MA; ${ }^{3}$ Harvard Medical School, Boston, MA; ${ }^{4}$ Centers for Medicare and Medicaid Services, Baltimore, MD. (Control ID \#1339856)

BACKGROUND: The Food and Drug Administration has released multiple recent warnings regarding the use of prescription weight loss medications. Final warnings were released in January 2010 for sibutramine (Meridia) regarding cardiovascular risks and in May 2010 for orlistat (Xenical) regarding case reports of severe hepatic injury. How these warnings have impacted prescribing rates of weight loss medications is unclear.

METHODS: We conducted a time series analysis of pharmacy claims data from the national pharmacy benefits manager, CVS Caremark, to examine the impact of the FDA warnings on prescribing rates of orlistat, sibutramine, and phentermine, the three medications FDA-approved for the treatment of obesity. We used data from August 2007 through December 2010 to ensure adequate data prior to and following warnings. The sample included patients continuously enrolled in CVS Caremark for six months prior to their first prescription for a weight loss medication and for at least one year after the first prescription, isolating new users with stable enrollment. We examined the impact of warnings on 1) the rates of discontinuation of each prescription medication and 2) substitution to an alternate weight loss medication in the three month period following discontinuation (e.g., switching from sibutramine to phentermine or orlistat after the warning for sibutramine). Patients were identified as discontinuers if they did not refill their medication 90 days after the previous supply was scheduled to run out. We used segmented linear regression models to evaluate level changes and trend changes associated temporally with the release of warnings.

RESULTS: From August 2007 through December 2010, 11,915 subjects received prescriptions for orlistat, 18,676 for sibutramine, and 108,295 for phentermine from among over 117 million Caremark enrollees. The overall prevalence of use of any of these medications was low: $0.06 \%$ of all Caremark enrollees used any of these medications in August 2007 and $0.02 \%$ used them 
in December 2010. Most subjects receiving these prescriptions were women (76.6\% to $83.5 \%$ ). Subjects receiving orlistat were older and had more comorbidities, defined according to the number of additional medications taken $(38.2 \%$ of orlistat users received 5 or more other medications compared to $26.5 \%$ for sibutramine and $16.5 \%$ for phentermine). In the time series analyses, we found that rates of discontinuation of each of the medications were similar before and after warnings $(\mathrm{P}$ value for level change of nonpersistence rate post-warning was 0.11 for orlistat, 0.85 for sibutramine, and 0.55 for phentermine). The trend change of non-persistence post-warning also was not significant. Subjects who discontinued sibutramine had an increased trend post-warning to start phentermine than before the warning $(\mathrm{P}=0.01)$.

CONCLUSIONS: We found no evidence for an effect of FDA warnings on discontinuation of prescription weight loss medications. Subjects who discontinued use of sibutramine were more likely to switch to phentermine postwarning than prior, a concerning finding because of ongoing safety concerns about phentermine. These findings raise concern about the effectiveness of communication strategies when FDA warnings are issued.

TIMELINESS OF FOLLOW-UP AFTER ABNORMAL SCREENING MAMMOGRAPHY AT FACILITIES SERVING VULNERABLE WOMEN L. E. Goldman ${ }^{1}$; Rod Walker'; Rebecca Hubbard ${ }^{2}$; Karla Kerlikowske'. 'University of California San Francisco, San Francisco, CA; ${ }^{2}$ Group Health Research Institute, Seattle, WA. (Control ID \#1337750)

BACKGROUND: Delays in breast cancer diagnoses contribute to higher mortality rates that disproportionately affect women with low income, limited education, racial or ethnic minorities, and those who live in rural areas. It is unknown whether the timeliness of evaluation following abnormal mammography at facilities serving vulnerable women compared to other facilities contributes to these disparities.

METHODS: We examined mammography that received a recommendation for subsequent imaging (herein 'recall') and mammography that received a recommendation for biopsy (herein 'biopsy recommendation') performed from 1998 to 2006 on Medicare recipients age 65 or older at 4 sites participating in the Breast Cancer Surveillance Consortium (BCSC). BCSC data were linked to Medicare claims to obtain dates of subsequent imaging and biopsy procedures. We estimated the association between timeliness of subsequent imaging and biopsy and vulnerability status of the facility. Facilities were characterized as serving vulnerable women based on the proportion of mammograms performed on racial/ethnic minorities, women with lower educational attainment or income, and those living in rural areas. Times to follow-up imaging and biopsy were estimated based on Kaplan-Meier curves, and adjusted hazard ratios (HRs) for these events were estimated using Cox regression, accounting for clustering at the level of the facility and adjusting for mammography indication, registry site, age, and prior screening mammography.

RESULTS: We analyzed 35,764 mammograms recalled for additional imaging at 142 facilities and 12,443 mammograms with biopsy recommendations at 128 facilities. Following recall, women at facilities serving smaller proportions of women with limited education and smaller proportions of racial/ ethnic minorities received follow-up imaging sooner than women at facilities serving more vulnerable women on the basis of these characteristics [education: median time to imaging 11 vs. 14 days; adjusted hazard ratio (HR) 1.44 (95\% confidence intervals (CI) 1.22, 1.71)] [race/ethnicity: 11 vs. 12 days; adjusted HR $1.31(1.00,1.73)]$. Following biopsy recommendation, women returned for biopsy sooner at facilities serving smaller proportions of rural women (median time to biopsy 18 vs. 23 days; adjusted HR $1.35(1.16,1.57))$ and smaller proportions of limited income women (19 vs. 24 days; adjusted HR 1.35 (1.20, 1.51)). Overall, at facilities serving vulnerable women were less likely to return for biopsy within 3 months after a biopsy recommendation: facilities that serve women with limited education vs. those that did not $(76.7 \%$ vs. $82.0 \%$ : $\mathrm{p}<$ $0.01)$, facilities that serve racial/ethnic minorities vs. those that did not $(75.8 \%$ vs. $81.8 \%$ : $\mathrm{p}<0.01$ ), facilities that serve rural residents vs. those that did not ( $79.5 \%$ vs. $82.5 \%$ : $\mathrm{p}<0.01)$, facilities that serve women with limited income vs. those that did not $(78.9 \%$ vs. $82.3 \%$ : $\mathrm{p}<0.01)$.

CONCLUSIONS: While women at facilities serving non-vulnerable women receive follow-up imaging sooner, the differences in time to follow-up do not seem clinically significant. However, the greater likelihood that women at facilities serving vulnerable women do not return for biopsy within 3 months after biopsy recommendation may be an indicator of quality of care and an important target for interventions to decrease diagnostic delays in breast cancer diagnoses among vulnerable women.

TOTAL HOURS MATTER: REDESIGNING RESIDENCY SCHEDULES TO MINIMIZE FATIGUE Lauren Block'; Sanjay Desai $^{2}$; Leonard S. Feldman ${ }^{1}$; Albert W. Wu ${ }^{1} .{ }^{1}$ Johns Hopkins University School of Medicine, Baltimore, MD; ${ }^{2}$ Johns Hopkins University School of Medicine, Baltimore, MD. (Control ID \#1321094)

BACKGROUND: The 2011 ACGME residency work hour mandate reaffirms the need to design residency schedules in a way that optimizes patient safety and minimizes resident fatigue. Changes to residency schedules should be informed by an understanding of the relationship between these factors. We sought to determine whether scheduling factors were related to sleepiness, burnout, and poor patient care outcomes.

METHODS: A cross-sectional survey of medicine interns at a large academic medical center ( 29 interns), a smaller academic hospital (15 interns), and a community hospital (11 interns) was conducted in June 2011. The survey assessed scheduling characteristics including hours worked and adherence to the relevant ACGME mandates, such as duty hours and the 80-hour work week. Fatigue and sleepiness were measured using a modified Maslach Burnout Index and Eppworth Sleepiness scale. Other outcome measures included resident assessments of quality of life, education, handoffs, and quality of patient care, as well as attitudes towards safety (measured by an abbreviated Safety Attitudes Questionnaire), and medical errors (measured by an errors index). Reponses were analyzed using two-tailed t-tests and multivariate linear and logistic regression. RESULTS: Response rate was $82 \%(\mathrm{~N}=55)$. Average hours worked in the past week was 76 (range 50-107). 45\% of residents surveyed met criteria for high burnout (Maslach score $\geq 17$ ) and 53\% for sleepiness (Eppworth score $\geq 10$ ). Total hours worked, sleepiness, residency program, and traditional overnight call rotation were independently associated with burnout. Residency program and traditional overnight call rotation were independently associated with sleepiness. In a multivariate model adjusting for age, sex, and sleepiness; total hours worked, overnight rotation, and residency program were associated with burnout, but number of days off, leaving on time post-call, and adherence to the 80-hour work week were not. Interns with high burnout were more likely than interns without high burnout to report making errors due to fatigue $(84 \%$ vs. $60 \%, \mathrm{p}=0.03)$ and to excessive workload $(70 \%$ vs. $90 \%, \mathrm{p}=0.04)$, and fewer reported positive attitudes towards safety $(74 \%$ vs. $97 \%, p=0.01)$. Those with high burnout were less likely to report satisfaction with quality of patient care provided ( $52 \%$ vs. $97 \%, \mathrm{p}<0.001)$, with work life ( $35 \%$ vs. $83 \%, \mathrm{p}=.001)$, and with personal life ( $35 \%$ vs. $63 \%$, $\mathrm{p}=0.03)$, and fewer felt prepared to be a PGY2 resident $(58 \%$ vs. $96 \%, p=0.001)$. Interns with high sleepiness scores were less likely to report spending at least the median time on handoffs $(47 \%$ vs. $74 \%, \mathrm{p}=.03)$, and less likely to report receiving high quality handoffs $(74 \%$ vs. $96 \%, p=0.05$ ) than interns without high sleepiness scores.

CONCLUSIONS: Sleepiness and burnout were prevalent among interns in this study and were independently associated with poor quality of life and undesirable patient care outcomes. While total hours worked and a traditional overnight call rotation were associated with resident burnout and sleepiness, other scheduling factors, including adherence to the 80-hour work week, leaving on time after on-call shifts, and number of days off, were not. In designing residency schedules, efforts to reduce total number of hours worked and using day and night team scheduling may be effective in improving fatigue, burnout, and patient safety.

TRANSITIONS OF CARE INTERNAL MEDICINE PGY 1 AMBULATORY EDUCATION: PILOT YEAR 1 Rachel K. Miller ${ }^{1}$; Karen M. Goldstein ${ }^{2}$; Jennifer Lapin ${ }^{1}$; Jerry Johnson ${ }^{1}$. 'University of Pennsylvania, Philadelphia, PA; ${ }^{2}$ Durham VA Medical Center, Durham, NC. (Control ID \#1339811)

BACKGROUND: In one out of every five hospital discharges, there is an adverse event which can lead to hospital readmission or an ER visit. In July 2007, the American College of Physicians, Society of Hospital Medicine, and Society of General Internal Medicine came together to address quality issues and to develop consensus standards for transitions of care between inpatient and outpatient settings. While the learning of care transitions is a hidden curriculum at many medical institutions, trainees are still expected to master 
this challenging skill set by the end of residency. We proposed that introducing proper transitions of care strategeies via small group, interactive didactics and a piloted post-discharge hospital home visit in the PGY1 internal medicine curriculum would increase confidence in the implementation of safer discharges for hospitalized patients.

METHODS: In pilot year 1 at the University of Pennsylvania, the first week of PGY1 internal medicine ambulatory blocks from July through December included an hour long didactic session on "Transitions of Care". This interactive, small group session discussed pertinent topics such as: identifying vulnerable patients, working with multidisciplinary team members, the basics of home services and skilled nursing facilities, medication reconciliation, discharge summaries/instructions, and patient communication. During the next 3 weeks of the block, half of Internal Medicine interns were randomized to go on a post-discharge home visit. Home visits were piloted in 2009 with the University of Pennsylvania Transitions of Care Nursing Team led by Mary Naylor. On the last didactic session of the month, we reviewed discharge summaries/instructions. There was also a debriefing session for those who attended a post- discharge home visit.

RESULTS: An IRB approved pre- intervention survey was given immediately before the first and last session to all the interns and 27 paired evaluations were reviewed. Overall, the interns showed an increased degree of confidence in: identifying potential threats to a well executed transition between sites of care $(p<0.001)$; anticipating the consequences of a poorly executed care transitions $(\mathrm{p}<0.001)$, and knowledge of the community resources available to patients with chronic illness $(\mathrm{p}<0.001)$. In addition, they showed increased knowledge in the roles of physical therapists $(\mathrm{p}<0.001)$, occupational therapists $(p<0.001)$, nursing $(p<0.010)$, and social work $(p<0.030)$.

CONCLUSIONS: This pilot transitions of care education initiative for internal medicine interns showed increased confidence in high risk discharge issues and increased knowledge of community resources and the role of multidisciplinary team members in safe transitions of care. Future directions include having all interns participate in post hospital discharge home visits and further evaluation of the long term impact of a transitions of care education program.

TRAUMATIC BRAIN INJURY IN HOMELESS PATIENTS Carol A. Waldmann. Frederick Memorial Hospital, Federick, MD. (Control ID \#1339873)

BACKGROUND: Traumatic brain injury (TBI) Is the leading cause of death and disability among children and young adults in the United States (US). These disabilities include neuropsychological dysfunction and behavioral problems which can interfere with a person's ability to maintain stable housing, employment and relationships. Previous studies have found high rates of cognitive impairment among homeless individuals. A recent study in Toronto, Canada, found that in a representative sample of homeless individuals, $58 \%$ of men and $42 \%$ of women had a history of TBI. No published study has examined the issue of TBI among homeless individuals in the US.

METHODS: English speaking patients at each of the top ten attended Boston Healthcare for the Homeless (BHCHP) clinical sites over the age of 17 were consecutively recruited to answer a 97 item questionnaire. 227 interviews occurred from $12 / 2006$ though $8 / 2007.190$ of these patients were technically homeless The recruitment rate was $74 \%$. To assure adequate distribution by clinic site when $3 \%$ of the annual patient load was reached at any site no more surveys were there. . Responses by patients reporting TBI were compared with those without TBI. Chi square and T-test analysis were used to determine statistically significant differences between the two groups. Prevalence and scope of TBI including severity, cause, and sequelae were studied. Questions about co-morbidities, risk factors, history of homelessness, and demographic information were also asked and examined.

RESULTS: Of the 190 homeless patients surveyed 129 (68\%) reported having at least one TBI. Of these subjects: $69 \%$ reported having had more than 1 TBI, $1.3 \%$ reported losing consciousness, $6 \%$ reported being hospitalized, $5.7 \%$ reported using alcohol or drugs when the TBI occurred. The average age at first TBI was 19 , over $25 \%$ of first TBI occurred before the age of 10 suggesting that TBI may have preceded homelessness in many patients. Assault was the most common cause of TBI $(29 \%)$ followed by motor vehicle accident $(27 \%)$ and other accidents (25\%) Patients with TBI had multiple statistically significant differences in co-morbidities and possible sequelae ot TBI including higher rates of alcohol and narcotic abuse, mental illness including depression, criminal conviction and symptoms of irritability and anger and symptoms associated with headache including nausea, photophobia, visual changes, and aura. They were more likely to be veterans and were more likely to have problems with word finding.

CONCLUSIONS: TBI is common in homeless patients seeking care at BHCHP medical services. Patients who have had a TBI exhibit associated symptoms and conditions that may impair their ability to maintain housing, employment and adequate self care. This suggests that all clinicians caring for homeless individuals should consider screening for a history of TBI. Care plans of homeless patients particularly those with a discovered history of TBI should include consideration of patients' possible impairments due to TBI including poorer emotional regulation, cognition and health status. An understanding of the impact of TBI on homeless person's health creates an opportunity to develop interventions that can improve the quality and efficiency of care to this complicated patient population.

TREATING CHRONIC NON CANCER PAIN WITH NARCOTICSDOES IT WORK? Asif A. Ansari ${ }^{1}$; Angela Embree ${ }^{1}$; Michael Bouthillier ${ }^{2}$; Jeevarathna Subramanian ${ }^{1}$. ${ }^{1}$ GRMEP/MSU, Grand Rapids, MI; ${ }^{2}$ Ferris State University, Grand Rapids, MI. (Control ID \#1334573)

BACKGROUND: Opioid analgesics are widely used by physicians to treat chronic non cancer pain (CNCP). Several professional societies and organizations have endorsed guidelines for judicial and rational use of opioids to treat CNCP. The aim of the study was to scrutinize our practice of opioid prescription to treat CNCP. In addition, we describe patient profiles and behavior patterns, role of vitamin D supplementation and association with depression.

METHODS: This was a prospective study from July 2010 of all patients at our resident out-patient clinic on long term narcotics for managing CNCP. Patients were followed on a monthly basis for 18 months, and a survey on patient perceptions was conducted by patient interview at the end of study. Patients with vitamin D deficiency were treated with supplementation for 1 year. Performance was compared to a study completed in the same setting in 2008 ( $n=31$ ). A modified oswentry scoring tool was used to assess disability. RESULTS: 50 patients were identified to be treated with long term opioids for $\mathrm{CNCP}$ in our practice. 28 of these patients were female. Back pain was the most common site of pain, and was present in more than half of the patients. $64 \%$ of patients claimed disability due to pain. $50(100 \%)$ patients had a signed narcotic agreement, compared to $65 \%$ in $2008(\mathrm{p}<0.0001)$. There was reduction in number of patients seeking narcotics from an alternative facility from $64 \%$ in 2008 to $24 \%$ during the study period ( $\mathrm{p}=0.0004$ ). All 50 patients has a random drug screen performed as per guidelines, treatment goals established and functional assessment performed at regular intervals, compared to 9, 20 and 3 patients retrospectively in $2008(p<0.0001)$. Half of the patients in our study had vitamin D deficiency and a similar number had depression. Patients felt pain was better controlled with treated depression. There was no significant statistical improvement in pain control or reduction in opioid requirement after vitamin D supplementation. Although, 84 percent of patients felt improved function subjectively in activities of daily living with long term opioids, there was no significant difference objectively in pain relief score or modified oswentry score for disability at the end of study.

CONCLUSIONS: The results showcase an improved performance in delivering quality care to patients on long term opioids to treat CNCP. This has been largely possible with recruitment of nurse specialist with special interest in chronic pain and formal education of resident physicians. Centers managing patients with chronic pain should follow standardized guidelines, formalize patient relationship with a narcotic agreement, and audit their performance. Patients with CNCP are likely to have depression and vitamin D deficiency; and treating these conditions may improve their quality of life. Until larger controlled studies are done, it may be prudent to screen and treat patients with these underlying comorbities.

TREATMENT OF POSITIVE URINE CULTURES IN HOSPITALIZED PATIENTS: A DRIVER OF ANTIMICROBIAL MISUSE Sarah Hartley'; Staci Valley ${ }^{1}$; Latoya $\mathrm{Kuhn}^{2}$; Tejal N. Gandhi ${ }^{1}$; Laraine Washer $^{1}$; Carol Chenoweth ${ }^{1}$; Jennifer Meddings ${ }^{1}$; Anurag Malani ${ }^{3,1}$; Sanjay Saint ${ }^{2,1}$; Scott Flanders ${ }^{1} .{ }^{1}$ University of Michigan, Ann Arbor, MI; ${ }^{2}$ Ann Arbor VAMC, Ann Arbor, MI; ${ }^{3}$ St. Joseph Mercy Hospital, Ann Arbor, MI. (Control ID \#1310198) 
BACKGROUND: Obtaining a urine culture in hospitalized patients is extremely common. Positive cultures frequently trigger antimicrobial therapy, but the appropriateness of this treatment remains unclear. We sought to describe physician management of positive urine cultures and the extent to which treatment contributes to antimicrobial overuse.

METHODS: We randomly selected adult patients admitted to a large academic center between February 2008 and February 2009 who had positive urine cultures. Patients were excluded if they were admitted to intensive care, had a major urinary procedure (e.g., renal transplant), were actively being treated for a urinary tract infection (UTI) at the time of admission or $>48$ hours prior to urine collection. Two hospitalists performed retrospective medical record review to determine the presence of signs or symptoms of UTI, urinary catheter presence, antimicrobial therapy, reason for and duration of antimicrobials. Appropriateness criteria for diagnostic testing and antimicrobial treatment was defined using national and professional society guidelines (i.e., Centers for Disease Control and Prevention and Infectious Diseases Society of America) and final determination of appropriateness was adjudicated by the hospitalist reviewers and 2 infectious disease physicians.

RESULTS: Of 153 patients, 73 (48\%) had an appropriate reason documented to obtain a urine culture. The most common reasons for obtaining a culture were fever $(27 \%)$, altered mental status $(16 \%)$ and change in character of urine $(15 \%)$. A total of $94(61 \%)$ had asymptomatic bacteriuria, including 39 patients $(41 \%)$ on antimicrobials at the time the urine culture was sent. Despite the lack of signs or symptoms of UTI, $60(64 \%)$ were treated for a UTI within 72 hours of the urine culture, including $59(98 \%)$ who were newly started on antimicrobials for treatment. Patients with asymptomatic bacteriuria received a mean of 6.6 days of antimicrobials. Of 59 patients (39\%) who met criteria for UTI, 15 (25\%) had a catheter associated UTI. Fifty-five (93\%) were started on initial antibiotics that were consistent with guidelines. The duration of therapy was incorrect for 16 patients (27\%) including 4 patients treated an average 6 days beyond the recommended course and 12 patients with an insufficient duration of treatment. The most common reason for truncated therapy was inappropriate categorization of a patient as having an uncomplicated UTI.

CONCLUSIONS: In hospitalized patients, systemic symptoms were the most common drivers of orders for urinary culture. Over half of patients with positive urine cultures had asymptomatic bacteriuria. A majority of these patients inappropriately received antimicrobials. In patients meeting criteria for UTI, antimicrobial use was inappropriate in over one quarter. Strategies to promote appropriate diagnostic testing and treatment for possible UTI in hospitalized patients are urgently needed and will likely reduce antibiotic misuse. Hospitalists can help lead these improvement efforts.

TRENDS IN ETHICS CONSULTATION PRACTICES IN A LARGE HEALTH SYSTEM David Alfandre; Kenneth Berkowitz; Ellen Fox. VA National Center for Ethics in Health Care, New York, NY. (Control ID \#1336394)

BACKGROUND: The discipline of health care ethics consultation (EC) has been limited by the lack of both high quality data and quality standards. To promote high quality ethics consultation practices, staff at the National Center for Ethics in Health Care within the Veterans Health Administration (VHA) developed 2 specific EC tools, ECWeb and the EC Feedback Tool. ECWeb is a web-based database tool that promotes process standards consistent with "CASES," VA's systematic approach to ethics consultation. The EC Feedback Tool, which links to ECWeb records, enables consultation participants to rate their experience on various aspects of EC. This paper describes the ethics consultation requests, processes, and evaluations from all facilities in our system.

METHODS: We analyzed data from completed ethics consultations from ECWeb records initiated between October 2008 and September 2011. For each consultation record, users documented in ECWeb the data related to utilization of the EC service (e.g., type of consultation request, requester role (i.e., physician, nurse, patient). Additionally, ECWeb users documented, as applicable, various processes performed during the ethics consultation (e.g., capacity assessment $(\mathrm{y} / \mathrm{n})$, a face-to-face patient visit $(\mathrm{y} / \mathrm{n})$, and if the consult was identified as being symptomatic of underlying issues that are best addressed at the systems level). The EC Feedback Tool asked respondents to rate the ethics consultant(s) on 12 specific ethics knowledge and skill areas as well as their overall experience with ethics consultation, both on a 5 point Likert scale.
RESULTS: We analyzed ECWeb data for 4628 completed consults from 140 facilities across the VHA health system . Median consultation volume per facility was 7 in 2009 (mean=9.6, range $=0-60$ ), 8 in 2010 (mean $=12.4$, range $=0-106$ ), and 8 in 2011 (mean $=12.1$, range $=0-119$ ). The majority of consultations were classified by the consultants as related to shared decision making (73\%). Most consultations $(63 \%)$ related to patients in the inpatient setting, $25 \%$ in the outpatient setting, and $10 \%$ in long term care settings. EC processes showed minimal change over the time period studied with the exception of an increase in underlying systems issues recorded from $37 \%$ in 2009 to $42 \%$ in 2011. Of all completed ethics consultations, $32 \%$ had at least one evaluation recorded. From 2009 to 2011, participant ratings improved for the overall experience, as well as for all 12 specific knowledge and skills. In particular, ratings of the consultant on "providing a helpful service" and "clarifying decisions that had to be made" rose from $85 \%$ to $89 \%$ and $79 \%$ to $85 \%$ respectively over the time period studied. CONCLUSIONS: Data from over 4,500 ethics consultations highlight the current trends in ethics consultation requests, processes, and evaluations in our integrated health system. Developing and implementing these EC tools has set EC standards for VHA, helped to promote a quality improvement approach to EC practices, and in the case of participant satisfaction, demonstrated improved ethics quality. Further work is needed to establish relationships between these data elements and other measures of EC quality such as desirable outcomes and overall content quality. Wider adoption of EC standards outside of VA is recommended to better understand EC practices and improve EC quality as well as to establish its accountability.

TRENDS IN HEART FAILURE ASSOCIATED HOSPITALIZATIONS IN THE UNITED STATES, 2001-2009 Saul Blecker; Margaret Paul; Gbenga Ogedegbe; Glen Taksler; Stuart Katz. NYU School of Medicine, New York, NY. (Control ID \#1339520)

BACKGROUND: Heart failure is among the most common reasons for hospitalizations in the United States. Recent data from Medicare suggest that the number of hospitalizations with a primary diagnosis of heart failure has declined over the past decade. However, heart failure may increase hospitalization rates for related comorbidities and individuals with heart failure are commonly admitted for other reasons. Using a nationally representative sample of hospital admissions, we studied trends in hospitalizations with both a primary and a secondary diagnosis of heart failure.

METHODS: We evaluated trends in heart failure hospitalizations from 2001 to 2009 using the Nationwide Inpatient Sample (NIS), the largest all-payer inpatient database in the United States. We included hospitalizations with an International Classification of Diseases, Ninth Revision discharge diagnosis codes of 402.X1, 404.X1, 404.X3, 428.XX in any position; these codes in the primary position are used by The Centers for Medicare \& Medicaid Services for reporting heart failure quality measures. Admissions were categorized as either primary heart failure hospitalization, if heart failure was the primary discharge code, or heart failure associated hospitalization, if heart failure was listed as a secondary diagnosis. National estimates of heart failure hospitalizations were calculated using the sampling weights and stratified sample design of the NIS. Yearly hospitalization rates were determined by dividing the number of hospitalizations by the United States population in a given year. Population estimates were obtained from the United States Census Bureau.

RESULTS: The total number of heart failure hospitalizations in the United States increased from 3,900,305 in 2001 to 4,398,376 in 2006 and then decreased to $4,253,937$ in 2009 . The number of primary heart failure admissions decreased from $1,139,607$ in 2001 to $1,087,913$ in 2009, while the number of heart failure associated hospitalizations increased from $2,760,698$ to $3,166,024$ over the same period. Primary heart failure hospitalization rates also decreased from 2001 to 2009, whereas heart failure associated hospitalization rates increased from 2001 and 2006 and then slightly decreased (figure).

CONCLUSIONS: Although primary heart failure hospitalizations declined nationally over the past decade, heart failure associated hospitalizations increased during the same period. These divergent trends may reflect improved treatment for heart failure but not for related comorbidities, resulting in increased admissions for causes which may be associated with, but not directly due to, heart failure. Reduced coding of heart failure as the primary diagnosis due to public reporting of heart failure quality measures and readmissions may have also contributed to these trends. 


\section{Rates of Hospitalization for Heart Failure, 2001-2009}

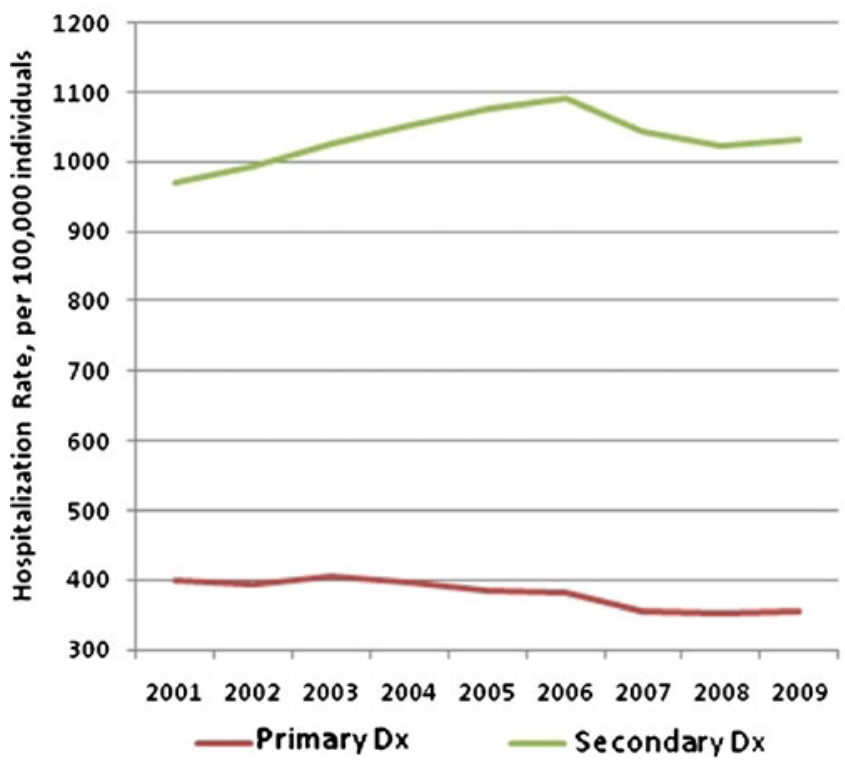

TRENDS IN US PHYSICIAN WORK HOURS AND CAREER SATISFACTION Andrea S. Christopher ${ }^{1,2}$; Andrew P. Wilper ${ }^{1,2}$; Rick Tivis ${ }^{3}$. ${ }^{1}$ University of Washington, Seattle, WA; ${ }^{2}$ Boise Veterans Affairs Medical Center, Boise, ID; ${ }^{3}$ Idaho State University Meridian Health Science Center, Meridian, ID. (Control ID \#1325066)

BACKGROUND: U.S. physician work hours declined in recent decades. We evaluated the association between physician work hours and career satisfaction overall with a focus on trends over time for primary care and subspecialty physicians.

METHODS: We analyzed the Community Tracking Survey (CTS), a publicly available data set generated from telephone surveys of a nationally representative sample of physicians. We reviewed data from the four survey cycles: 1996-97, 1998-99, 2000-01, and 2004-05. We quantified hours spent in all medically relevant work as well as the distribution of work into direct patient care and charity care responsibilities. We tested for changes in demographics using chi-square tests and repeated measure ANOVA, testing trends over time. Career satisfaction was quantified on a scale of one to five in the survey instrument. First we examined the relationship of hours in medicine with career satisfaction by dividing hours in medicine into quartiles. Next, we analyzed the proportion of physicians reporting moderate to extreme career satisfaction per quartile using chi square tests. Finally, we developed a multivariate logistic regression to examine the relationship between US physician career satisfaction and work hours. We accounted for the CTS complex survey design and used SAS 9.2 for this analysis. Weights were used to derive national estimates. The Puget Sound VA IRB approved this study.

RESULTS: Mean physician age increased from 48.5 (95\% confidence interval $(\mathrm{CI})=48.3$, to 48.7$)$ years in $1996-97$ to 50 years $(95 \% \mathrm{CI}=49.6$, $50.3)$ in 2004-05 $(\mathrm{p}<0.001)$. The proportion of female physicians increased from $17.6 \%(95 \% \mathrm{CI}=16.8,18.4)$ in $1996-1997$ to $25.0 \%(95 \% \mathrm{CI}=23.2$, $26.8)$ in 2004-05 $(\mathrm{p}<0.0001)$. We did not detect any other meaningful trends $(p>.01)$ in other population characteristics. The mean total number of weeks per year spent in practice decreased significantly among both primary care and non primary care providers $(-0.2$ weeks each, $(\mathrm{p}<.01))$. The mean hours per week spent in all medically related activities (HMRA) decreased significantly across both groups $(\mathrm{p}<.0001)$. For primary care providers, HMRA decreased from $54.4(95 \% \mathrm{CI}=53.9,54.8)$ in 1997 to $51.8(95 \% \mathrm{CI}=50.9,52.7)$ in 2005 (5\% decrease). For non-primary care providers, HMRA decreased from $57.4(95 \% \mathrm{CI}=56.9,57.9)$ in 1997 to $54.9(95 \% \mathrm{CI}=54.0,55.8)$ in 2005 (4\% decrease). The hours per week spent performing charity work also decreased significantly amongst primary care and subspecialty physicians (down $6 \%$ and $17 \%$ per week, respectively). The percentage of physicians reporting career satisfaction increased from $80.82 \%$ in $1996-97$ to $84.26 \%$ in $2004-08$ ( $p<0.001)$. Examining changes in career satisfaction at different levels of HMRA over time reveled a significant difference in proportion reporting satisfaction $(\mathrm{p}<.0001)$ with those at the lower quartile on HMRA showing the greatest increase in proportion satisfied (from $82 \%$ to $90 \%$ ) over time. Lastly, in our multivariate logistic regression analysis, weeks worked and HMRA were significant positively $(p=.0002)$ related to with the degree of career satisfaction, while controlling for age, gender, US education, primary care, type of practice, region and several others.

CONCLUSIONS: Our study demonstrates trends towards decreased work hours, regardless of physician subtype. This is associated with a concomitant increase in physician career satisfaction that correlates with the decrease in work hours.

TRUST YET VERIFY: PHYSICIANS AS TRUSTED SOURCES OF HEALTH INFORMATION ON HPV FOR BLACK WOMEN IN UNDERSERVED COMMUNITIES Cheryl R. Clark ${ }^{1,2}$; Nashira Baril ${ }^{3}$; Erline Achille ${ }^{3}$; Shauntell Foster ${ }^{3}$; Kalahn Taylor-Clark ${ }^{4}$; Natacha Johnson ${ }^{2}$; Joshua Gagne $^{6}$; Oluwakemi Olukoya ${ }^{3}$; Carrie Huisingh ${ }^{2}$; Mark Ommerborn ${ }^{2}$; Vish Viswanath ${ }^{5,7}$. ${ }^{1}$ Brigham and Women's Hospital, Boston, MA; ${ }^{2}$ Brigham and Women's Hospital, Boston, MA; ${ }^{3}$ Boston Public Health Commission, Boston, MA; ${ }^{4}$ National Partnership for Women and Families, Washington D. C., DC; ${ }^{5}$ Dana Farber Cancer Institute, Boston, MA; ${ }^{6}$ Dana Farber Cancer Institute, Boston, MA; ${ }^{7}$ Harvard School of Public Health, Boston, MA. (Control ID \#1331496)

BACKGROUND: Human Papilloma Virus (HPV) infection in the US is highest among Black women and women of low socioeconomic position. However, these groups are also least likely to have information about HPV or strategies for preventing its transmission. The principal channels through which Black women of low socioeconomic position obtain information regarding HPV and HPV vaccination are not fully known. Our study sought to understand key information channels for delivering health information regarding HPV and the HPV vaccine to Black women of low socioeconomic position in Boston, Massachusetts.

METHODS: We conducted a qualitative analysis using focus groups to explore constructs in the theory of communication inequality. We conducted five focus groups among 25 women in underserved neighborhoods in Boston, Massachusetts. We hypothesized that Black women of low socioeconomic position would prefer information from trusted and accessible sources including friends, family, and community agencies.

RESULTS: Contrary to our hypothesis, we found that women in all of the focus groups preferred to receive health information in general, and specific health information regarding HPV, from a physician or health care center. Focus group participants indicated that they preferred to receive information in print form from clinical sources so that they could check the veracity of the information from providers with external sources. Participants stated their actual sources of information regarding HPV were chiefly via television commercials. Participants perceived that time pressure during the clinical encounter limited physicians' accessibility as a source of general health education.

CONCLUSIONS: Our study suggests that physicians are trusted and preferred sources of information on HPV and vaccination for Black women of low socioeconomic position in Boston. Our focus group participants preferred to receive print materials from physicians and health care institutions, and to triangulate this information with data from other sources, including information received in community settings. Our data suggest the need for intervention research to design effective strategies for physicians to provide appropriate print materials and community education on HPV to Black women of low socioeconomic position.

UNDERSTANDING OBESITY: IMPACT OF SOCIO ECONOMIC STATUS ON OBESITY RATES. Rajesh Krishnamoorthi; Arun K. Muthusamy; Palaniappan Manickam; Theresa E. Vettese. Wayne State University, Detroit, MI. (Control ID \#1336826)

BACKGROUND: In underdeveloped and developing countries, lower socio economic status(SES) is associated with poor nutrition and lower BMI. However, this is not true in developed countries where fast foods have become 
a source of nutrition. The less expensive fast foods have higher calorie content which can potentially increase the risk of obesity in population belonging to low socio economic status. There is limited data directly supporting this hypothesis and we wanted to perform a study analyzing it. Our objective was to study the impact of socio economic status on prevalence of obesity. We also investigated if demographic factors like race and ethnicity influence obesity rates.

METHODS: We performed a retrospective analysis of the limited access dataset from the National Ambulatory Medical Care Survey from 2007 to 2009. All patients less than 65 years of age were included in the study and categorized into lower, lower- middle, upper-middle and upper SES and obesity rates (BMI $>30$ ) were calculated for each SES. The study population was further categorized based on race and ethnicity into Caucasians, African Americans, Hispanics and NonHispanics. Multivariate logistic regression was used to study the impact of socio economic status, race and ethnicity on obesity rates. All statistical analysis was performed using SAS

RESULTS: 66014 patients with complete details on socio economic status were included in the study. The prevalence of obesity in lower, lowermiddle and upper-middle SES was significantly higher than upper SES (Table 1). On sub-group analysis based on race and ethnicity, the results were the same in Caucasian and Non- Hispanic populations. However in African American and Hispanic populations, obesity rates were not statistically different in different SES (Table $2 \& 3$ ).

CONCLUSIONS: Our results confirm the hypothesis that socio economic status has an inverse relationship with obesity rates. Also, race and ethnicity are independent risk factors for obesity. Obesity rates were the same across different SES in African Americans and Hispanics unlike Caucasians and Non-Hispanics, suggesting that these populations have other confounding risk factors contributing to obesity in addition to SES. Further studies are needed to identify these risk factors to understand obesity better.

Impact of Socio Economic Status on Obesity Rate.

Table.1- Total Study Population

Socio Economic Status

Lower SES

Lower Middle SES

Total (n)

14344

15028

16838

19804

Upper SES

Population

Socio Economic Status Total (n)

Lower SES

Lower Middle SES

3455

1775

1536 1080

Upper SES

Table.3- Hispanic Population

Socio Economic Status

Lower SES

Lower Middle SES

Total (n)

2645

2146

2182

1747
Obese (n)

2236

2210

2320

2078

Obese (n)

726

372

271

181

Obese (n)

380

284

310

246
Odds Ratio $95 \% \mathrm{Cl}$

1.23 (1.03 to 1.46$)$

$1.29(1.10$ to 1.51$)$

1.31 (1.15 to 1.50$)$

1 (Reference)

Odds Ratio 95\% CI

$1.04(0.77$ to 1.41$)$

$1.29(0.88$ to 1.89$)$

$1.13(0.77$ to 1.66$)$

1 (Reference)

Odds Ratio $95 \% \mathrm{CI}$ $1.21(0.83$ to 1.76$)$ $1.21(0.84$ to 1.74$)$ 1.23 ( 0.92 to 1.65$)$ 1 (Reference)

There is an inverse relationship between socio economic status and obesity rates.
UNDERSTANDING AND DECISION MAKING ABOUT SCREENING COLONOSCOPY FOR OLDER PERSONS WITH MULTIMORBIDITY Carmen L. Lewis ${ }^{1}$; Terri Fried ${ }^{2}$; Joseph S. Ross' ${ }^{2}$; Jenerius Aminawung $^{2}$; Lisa Werner ${ }^{1}$; Christine Kistler ${ }^{1}$; Mary Tinetti ${ }^{2}$; Katherine McKenzie $^{2}$; Inginia Genao ${ }^{2}$; Cary Gross ${ }^{2} .{ }^{1}$ University of North Carolina, Chapel Hill, NC; ${ }^{2}$ Yale University School of Medicine, New Haven, CT. (Control ID \#1326001)

BACKGROUND: Although current guidelines recommend against colorectal cancer screening for older persons with multi-morbidity and limited life expectancy, evidence suggests that many older adults who may not benefit undergo screening colonoscopy. The purpose of this study was to assess whether an understanding of the risks and benefits of screening colonoscopy with increasing age and multi-morbidity would influence patient decision making about whether to undergo screening. METHODS: We recruited a convenience sample of participants ages 70 and older with at least two comorbidities and taking 5 or more medications in 2 geographical areas. Participants reviewed a paper teaching aid (TA), which included information and graphics describing the diminishing benefits and increasing risks associated with screening colonoscopy in the setting of increasing age and multi-morbidity. We used an open ended question to assess understanding of the information. Chi -squared tests were used to compare the associations between understanding the information in the teaching aid and the decision to undergo screening colonoscopy. Logistic regression was used to estimate the odds of a decision to screen.

RESULTS: Among 101 participants, mean age was 78, 76\% were white, $71 \%$ were women, $45 \%$ had a college degree or higher, and $96 \%$ reported having previously been screened for colon cancer. After viewing the teaching aid, $55(55 \%)$ participants understood either the diminishing benefits or the increasing risks of screening colonoscopy. When asked whether participants would choose to undergo screening colonoscopy if they were in poor health, $9(17 \%)$ of participants who understood the information presented in the teaching aid stated that they would choose to do so compared to $25(57 \%)$ of the participants who did not understand the information $(p<0.001)$. When asked whether participants would choose to undergo screening in their current health, results were similar for the two groups $(43(80 \%)$ who understood vs. $38(83 \%)$ who did not understand; $\mathrm{p}=0.38)$. Thirty-nine $(39 \%)$ of participants classified themselves as having serious medical problems. After adjusting for perceived health status, understanding was strongly associated with a decreased odds of choosing to be screened in poor health (OR 0.15 ; $95 \% \mathrm{CI}, 0.06$ to 0.39 ), but not in the current state of health (OR 0.8 ; $95 \%$ CI, 0.28 to 2.15 ). Results stratified by self-reported health status are shown in the table.

CONCLUSIONS: Participants who understood the diminishing benefits or increasing risks of screening colonoscopy in the setting of increasing age or multi-morbidity appeared to have decreased interest in screening colonoscopy in the context of poor health. However, a majority of participants indicated that they would undergo screening colonoscopy in their current state of health, regardless of their self-perceived health or their level of understanding. An understanding of the risks and benefits of screening colonoscopy may not be sufficient to discourage screening in older persons who may not benefit from it.

\begin{tabular}{|c|c|c|c|c|c|c|}
\hline \multirow[b]{2}{*}{ Decision to Screen } & \multicolumn{2}{|c|}{ With Serious Medical Problems } & \multirow[t]{2}{*}{$\overline{P \text { value }}$} & \multicolumn{2}{|c|}{ Without Serious Medical Problems } & \multirow[t]{2}{*}{$\overline{\overline{\text { P value }}}$} \\
\hline & $\begin{array}{l}\text { Understood* } \\
\text { information in } \\
\text { TA } n=23\end{array}$ & $\begin{array}{l}\text { Did not understand } \\
\text { information in TA } \\
n=16\end{array}$ & & $\begin{array}{l}\text { Understood* } \\
\text { information } \\
\text { in TA } n=30\end{array}$ & $\begin{array}{l}\text { Did not understand } \\
\text { information in TA } \\
\mathrm{n}=28\end{array}$ & \\
\hline $\begin{array}{l}\text { Would undergo } \\
\text { Screening } \\
\text { in poor health }\end{array}$ & $6(26 \%)$ & $10(63 \%)$ & 0.02 & $3(10 \%)$ & $15(54 \%)$ & $<0.001$ \\
\hline $\begin{array}{l}\text { Would undergo } \\
\text { screening } \\
\text { in current health }\end{array}$ & $15(65 \%)$ & $13(81 \%)$ & 0.27 & $27(90 \%)$ & $25(83 \%)$ & 0.45 \\
\hline
\end{tabular}

Participant demonstrated understanding of decreased benefits or increased risks 
UNDERSTANDING THE CARE GAP AND MISSED OPPORTUNITIES FOR HEPATITIS C CONFIRMATORY VIRAL TESTING Yang $\mathrm{Liu}^{3}$; Renee H. Lawrence ${ }^{1}$; Brook Watts ${ }^{1,3}$; Yngve Falck-Ytter ${ }^{1,3}$; Amy Hirsch ${ }^{2,3}$. ${ }^{1}$ Department of Medicine, Louis Stokes Cleveland Department of Veterans Affairs Medical Center, Cleveland, OH; ${ }^{2}$ Pharmacy Service, Louis Stokes Cleveland Department of Veterans Affairs Medical Center, Cleveland, $\mathrm{OH} ;{ }^{3} \mathrm{Case}$ Western Reserve University, School of Medicine, Cleveland, OH. (Control ID \#1323493)

BACKGROUND: Clinical practice guidelines state that diagnosis of chronic Hepatitis $\mathrm{C}(\mathrm{HCV})$ in high risk patients or those suspected of having HCV requires screening with the HCV antibody (HCVab) test followed by confirmatory viral testing. Published physician performance measure sets include rates of $\mathrm{HCV}$ confirmatory testing as a measure. Failure to obtain confirmatory testing may result in missed opportunities for treatment, or unnecessary emotional stress or medical care if an $\mathrm{HCV}$ diagnosis is inappropriately given or referenced in future documentation. To date there are no published studies that summarize information about patients in this care gap.

METHODS: Using an established HCV patient registry, we conducted a retrospective chart review of 419 patients at a large veterans affairs medical center who had history of at least one positive $\mathrm{HCV}$ antibody result but lacked confirmatory viral testing. For each patient, we collected demographic data (e.g., age, race, gender, etc). For each subject's first instance of a positive HCVab, we determined who, PCP or non-PCP (e.g., provider from mental health, inpatient medicine, etc.) ordered the HCVab, whether the positive HCVab was acknowledged, and by whom. If acknowledged, we then determined what the interpreting provider did in response to the result (e.g., entering $\mathrm{HCV}$ into the patient problem list, placing an $\mathrm{HCV}$ clinic consult, etc.). We also determined whether the term "Hepatitis C" was documented by a future medical provider who was not the ordering or acknowledging provider.

RESULTS: The average age of subjects was 60 years, and $97 \%$ were male. $47 \%$ of subjects were black, $45 \%$ white, and subjects had received health care through the VA center for an average of 8.0 years. Thirty-one percent of the positive HCVab tests were unacknowledged by any provider. Of the labs that were acknowledged, a non-PCP ordering and acknowledging the lab was more likely $(\mathrm{p}<.05)$ than a PCP to inappropriately enter $\mathrm{HCV}$ into the problem list in the absence of confirmatory viral testing, without taking further action ( $6 \%$ and $3 \%$, respectively). Furthermore, nonPCPs were more likely than PCPs to inappropriately document $\mathrm{HCV}$ in the progress note only $(17 \%$ and $6 \%)$. However, the majority of the time, both PCPs and non-PCPs took HCV follow-up actions (HCV education, HCV consult, etc), with PCPs more likely to do so $(65 \%$ and $57 \%)$. Of those who took other actions, a PCP ordering and acknowledging the test was associated with an HCV clinic consult being placed $(p<.001)$ more than non-PCPs (34\% versus 6\%). Overall very few consults were placed (38 out of 251). Entering HCV into the problem list was associated with evidence of future medical providers referencing the diagnosis $(p<.001)$ in their documentation, which occurred in $86 \%$ of the 145 instances.

CONCLUSIONS: In this care gap, one-third of the positive HCVab tests were unacknowledged. When a positive HCVab was acknowledged, both PCPs and non-PCPs had an inadequate understanding of the diagnosis of $\mathrm{HCV}$, with subsequent actions that suggested that a diagnosis was made in the absence of confirmatory testing. Furthermore, while the majority of providers provided some level of HCV follow-up, this rarely resulted in a desired specialty HCV clinic referral. Entering HCV into a patient problem list was significantly associated with other providers acknowledging the diagnosis

USE OF ADVANCE DIRECTIVES IN AN ELDERLY HOME-BOUND POPULATION Laura Montague; Barbara A. Boland; Charmaine Wright. University of Pennsylvania, Philadelphia, PA. (Control ID \#1306443)

BACKGROUND: Advance directives, including living wills and appointment of health care proxies, are valuable tools for preserving patient autonomy at the end of life, particularly in populations that interact frequently with health systems. There are 3.6 million home-bound adults in the United States over the age of 65 . These individuals suffer from a wide range of medical and psychiatric co-morbidities, and are repeatedly hospitalized. It is important for providers to engage these patients in discussions of advance directives and end of life care, as physicians and family members are not able to consistently predict patient preferences. Despite their importance, few patients complete advance directives. Furthermore, the use of advance directives in the high-risk home bound population has not been previously examined. This study aims to determine the prevalence of advance directive completion in a cohort of elderly home-bound patients residing in West Philadelphia. It also seeks to identify avenues for future interventions that may increase advance directive completion in this population.

METHODS: We performed a cross sectional survey of a population of elders $(n=16)$ enrolled in a home-based primary care program at an urban academic medical center. An 11-question survey on advance directives was administered to subjects over the telephone or in person. If subjects were unable to participate as a result of dementia or intellectual disability, family members were asked to complete the survey. Demographic information was collected from the electronic medical record.

RESULTS: Mean (SD) age was 81.3 (12.9) years, with $56 \%$ female, and $100 \%$ black. The average (SD) number of prescribed medications was 9.3 (5.3). All of the participants had at least 2 diagnoses; $25 \%$ of the participants carried a diagnosis of dementia or intellectual disability, and had family involvement in survey completion. Although $87.5 \%$ of participants were familiar with living wills (LW), only $25 \%$ had created a written LW. In addition, 94\% reported that they understood the role of a power of attorney (POA) for healthcare, but only $44 \%$ of participants had an officially appointed POA. Of those that had a POA, only $43 \%$ of participants had discussed their wishes for end of life care and resuscitation with the POA. Slightly less than half $(43 \%)$ of those surveyed were interested in more information on advance directives; most of these individuals $(86 \%)$ desired to receive information directly from their physician, while $29 \%$ asked for written educational materials. Two-thirds (67\%) identified not being asked by their physician as the primary reason that they had not yet created a LW or named a POA.

CONCLUSIONS: Elderly home bound patients understand the meaning of the terms "living will" and "power of attorney," but very few of these patients have established advance directives. Those that had named a POA had not communicated their thoughts or wishes regarding future medical care to that individual. Most home-bound patients wish to learn more about advance directives from their physician. Future interventions should be directed at both patients and their physicians to improve communication and patient education about the importance of the LW and POA in order to facilitate increased use of advance directives.

USE OF INTERPRETERS BY PHYSICIANS FOR HOSPITALIZED LIMITED ENGLISH PROFICIENCY PATIENTS AND ITS IMPACT ON PATIENT OUTCOMES Lenny Lopez ${ }^{1,2}$; Jane R. Soukup ${ }^{2}$; LeRoi Hicks ${ }^{3}$. ${ }^{1}$ Massachusetts General Hospital, Boston, MA; ${ }^{2}$ Brigham and Women's Hospital, Boston, MA; ${ }^{3}$ University of Massachusetts Medical Center, Worcester, MA. (Control ID \#1337842)

BACKGROUND: Use of professional medical interpreters for limited English-proficiency (LEP) patients is associated with increased patient satisfaction and improved disease-specific process measures however data related to clinical outcomes are less well elucidated. More specifically, whether the use of interpreters by physicians in the hospital setting impacts hospitalized patients' clinical outcomes is not clear. We hypothesized that the use of interpreters is associated with decreased length of stay (LOS), 30-day post discharge emergency department (ED) visits and 30- day hospital readmission rates for LEP patients.

METHODS: We reviewed hospital administrative and interpreter services data for all hospitalized patients in 2009 admitted to the general medicine service at a large tertiary academic center $(n=4224)$. For patients self-reported as LEP in administrative data, we collected data regarding use of interpreters during each episode of hospitalization from the hospital interpreter service database and categorized as: (1) interpreter used by non-MD (i.e., nurse); (2) interpreter used by a non-Hospitalist MD; (3) interpreter used by Hospitalist; and (4) no interpreter used during hospitalization. We examined the association of English proficiency and interpreter use (English-speaking vs. each LEP category) on outcomes utilizing poisson models with log transformed LOS and 
logistic regression for the 30-day ED visits and readmission outcomes with adjustment for patient clustering at the physician level. Each model adjusted for patients' age, gender, race/ethnicity, insurance, discharge diagnosis, Charlson comorbidity score, type of admitting attending physician (Hospitalist/non-Hospitalist), and the number of a patient's hospital admissions in the previous year. Similar stratified analyses were conducted among only LEP patients

RESULTS: Of 4224 patients, 564 (13\%) were LEP. Of these LEP patients, $65.8 \%$ never had a documented interpreter visit, $16.8 \%$ utilized an interpreter with a non-MD, $12.6 \%$ utilized an interpreter with a nonHospitalist MD and $4.8 \%$ utilized an interpreter with a hospitalist present. In adjusted models, compared to English speakers, LEP patients with no interpreters (OR 0.83 [CI: 0.76-0.90]) and those who had interpreter use with a non-MD (OR 0.83 [CI: 0.78-0.89]) had significantly shorter LOS Among LEP patients compared to those with no interpreter use, those who had a physician present with interpreter use (both hospitalist [OR 1.32 [CI: 1.09-1.11] and non-hospitalist [OR 1.28 [CI: 1.10-1.53]) had a longer LOS. There were no differences in unadjusted and adjusted readmission rates (17\% vs. $18.3 \%, p=0.47)$ and ED utilization (both $19.9 \%, p=0.97)$ between LEP and non-LEP patients.

CONCLUSIONS: Academic hospital use of interpreters remains highly variable. After accounting for patients' demographic characteristics and severity of disease, LEP patients who had a physician present with interpreter use had longer LOS compared to LEP patients without an interpreter. There were no differences in 30-day post discharge ED visits or readmission rates for LEP patients compared to English speaking patients. Our findings suggest that physicians may selectively be using interpreters for the sickest patients and that use of interpreters may be associated with use of other unmeasured inpatient services that may appropriately increase LOS. Interventions are needed to increase the consistent use of interpreters for all hospitalized LEP patients.

USE OF INTERPRETERS FOR LIMITED ENGLISH PROFICIENT PATIENTS WITH CANCER Lisa C. Diamond; Lalanthica Yogendran; Jennifer Leng; Abraham Aragones; Julia Ramirez; Javier Gonzalez; Francesca Gany. Memorial Sloan-Kettering Cancer Center, New York, NY. (Control ID \#1341117)

BACKGROUND: Underserved minority groups have worse cancer outcomes. Having limited English proficiency (LEP) adds to these disparities. LEP patients with cancer are less accurate in their knowledge of diagnosis. The use of professional interpreters has been shown to improve outcomes for LEP patients but ad-hoc interpreters, such as a patient's family member, may exacerbate misunderstandings. This study identifies which interpreter modalities were used at various types of outpatient visits for LEP oncology patients and what factors influence knowledge of cancer diagnosis.

METHODS: Patients were recruited at an urban hospital-based cancer clinic in New York City between September 2008 and December 2010. Bilingual staff administered a survey of demographic and self-reported clinical information to $n=60$ oncology patients. A retrospective chart review verified the diagnosis. Bivariate analyses were conducted using X2, Fishers Exact test and T-tests to assess factors associated with knowledge of cancer diagnosis. Generalized linear mixed models were used to account for clustering and compare interpreter modality and visit type.

RESULTS: All patients in the sample were LEP with $85 \%$ born in a Latin American country. The majority had been in the US more than 10 years. More than $80 \%$ had no primary care physician and had not seen a social worker. Over a third were uninsured and $19 \%$ had less than a 6th grade education. Eighteen percent failed to correctly identify their cancer diagnosis. LEP patients with insurance were more likely to know their cancer diagnosis than those without $(\mathrm{p}<.0001)$. Professional interpreters less likely to be used in visits for chemotherapy $(\mathrm{p}<.0001)$ and more likely to be used in social work visits $(\mathrm{p}=.01)$ compared to other visit types. Ad-hoc interpreters were more likely to be used in surgery and oncology clinic visits $(p=.01, p=.0004)$. Despite an identified need for an interpreter, none was used in chemotherapy visits $(p=.0002)$ more commonly and in oncology clinic visits $(\mathrm{p}=.04)$ compared to other visit types. There were no significant differences in visit types when clinicians use their own non-English language skills to communicate directly with LEP patients.
CONCLUSIONS: Many LEP patients with cancer at an urban medical center did not know their cancer diagnosis. Having insurance may help improve knowledge of cancer diagnosis due to increased access to the healthcare system. Using professional interpreters at all types of visits may improve knowledge of cancer diagnosis.

USE OF VIGNETTES TO STIMULATE REFLECTION ON PROFESSIONAL DILEMMAS Elizabeth C. Bernabeo ${ }^{1}$; Eric Holmboe ${ }^{1}$; Shiphra Ginsburg ${ }^{2} .{ }^{1}$ American Board of Internal Medicine, Philadelphia, PA; ${ }^{2}$ Mount Sinai Hospital, Toronto, ON, Canada. (Control ID \#1326726)

BACKGROUND: Many believe that the growth and renewal of professional values can serve as a stable foundation for physicians to draw upon amidst challenging and unpredictable times in health care. However, while most physicians embrace professional values, many experience challenges to exhibiting consistent behaviors in practice. Reflecting on their response to professional dilemmas may help practicing physicians identify both internal and external factors contributing to (un) professional behavior.

METHODS: We developed a set of vignettes designed to stimulate physicians' reflection on professional dilemmas. The vignettes are grounded in theory and extant professionalism literature, and emphasize domains such as physician- patient relationships, self- regulation, allocation of resources, conflict of interest, and confidentiality. Five focus groups were conducted during which physicians responded to the vignettes. Groups $(\mathrm{N}=40)$ were comprised of outpatient specialists, outpatient generalists, inpatient specialists, inpatient generalists, and one mixed group. Data were analyzed using a constructivist grounded theory approach.

RESULTS: Each scenario was effective in stimulating discussion, resulting in an average of 50 codes per scenario (range 36-93). The scenarios were perceived as authentic and familiar to participants, who endorsed facing similar dilemmas on a regular basis. Debates surrounding areas of professional ambiguity, where there was a perceived lack of understanding of a "right" or "wrong" response to the vignette, were salient, and these cases in particular generated a broad range of responses. Two main sets of factors appeared to underpin participants' responses to the dilemmas: guiding principles and modifiers. Guiding principles $(\mathrm{N}=14)$ included patient welfare and satisfaction, efficiency, confidentiality, availability, transparency, evidence-based medicine, reimbursement and other financial considerations, and legal concerns such as a fear of being sued. Each principle arose in all five FG discussions to some degree. We also identified several $(\mathrm{N}=6)$ modifiers, factors which influenced how participants interpreted and acted upon the principles. These included the relationship with the patient or colleague, risk of danger or harm, familiarity with the request, and the type of patient or nature of the illness. Importantly, these two categories are not mutually exclusive, and are often activated together and interact with each other in response to a given scenario. Our findings therefore suggest that physicians respond to professional dilemmas in a highly individualized manner, taking into consideration their resources, environment, and setting on a case by case basis.

CONCLUSIONS: Providing an opportunity for physicians to reflect on professional behavior in an open and safe forum may be a practical way to guide physicians to assess themselves on professional behavior and engage with the complexities of their work. The finding that the focus groups led to reflection at a group level suggests that reflection on professional behavior may require a socially interactive process. Our finding that physicians rely on a number of principles and modifiers that interact in complex and often unpredictable ways may help explain the discordance between professional values and behaviors. Emphasizing both the behaviors and the context in which they occur can therefore be seen to be critically important for understanding professionalism in practicing physicians.

USE OF A CLINICAL DECISION SUPPORT TOOL TO PROMOTE JUDICIOUS USE OF ANTIBIOTICS IN PRIMARY CARE Cara Litvin; Andrea Wessell; Paul Nietert; Ruth Jenkins; Lynne Nemeth; Steven M. Ornstein. Medical University of South Carolina, Charleston, SC. (Control ID \#1339061)

BACKGROUND: Antibiotics are often inappropriately prescribed for acute respiratory infections (ARIs). Clinical decision support tools (CDS) 
may be able to target multiple factors affecting a provider's decision to prescribe antibiotics for ARIs. The purpose of this report is to present the results of a pilot project assessing the impact of a CDS on antibiotic prescribing for ARIs in PPRNet, a national primary care practice based research whose members use a common EHR (McKesson Practice Partner ${ }^{\mathbb{R}}$ ) and pool data for quality improvement and research.

METHODS: Thirty-nine providers in nine PPRNet practices in nine states participated in this 15 month study between January 1, 2010 and March 31, 2011. A CDS was designed as an electronic health record (EHR) progress note template, presenting diagnosis and treatment guidelines based on a patient's symptoms along with relevant patient education. Each practice received two site visits for academic detailing and CDS training, sent representatives to two network meetings for "best-practice" dissemination, and received quarterly performance reports on antibiotic prescribing for ARIs. Data on CDS use, ARI diagnoses, and antibiotic prescriptions within three days of an ARI encounter were obtained from the PPRNet database. Study measures included prescription of antibiotics for ARI conditions for which antibiotics are rarely appropriate (non-specific upper respiratory infection (URI), acute bronchitis, acute non-strep pharyngitis) and use of broad spectrum antibiotics to treat these conditions.

RESULTS: During the study, the CDS was used 12,664 times for ARI encounters in adults over 18 years age. Median practice use of the CDS for ARIs was $60.8 \%$ (range $41.1 \%$ to $77.6 \%$ ) of all ARI encounters. At these encounters, there were 3260 diagnoses of URI, 1602 diagnoses of acute bronchitis, 712 of non-strep pharyngitis and 1395 encounters with more than one of these diagnoses. Median practice antibiotic prescribing for these conditions was $29.6 \%$ (range $24.1 \%$ to $72.1 \%$ ); lower than a comparable national average in 7 of 9 practices. Antibiotics were prescribed for $14.3 \%$ of encounters for URI, $53.5 \%$ for acute bronchitis, $36.8 \%$ for non-strep pharyngitis and $69.4 \%$ for multiple diagnoses. Median practice use of broad spectrum antibiotics when antibiotics were prescribed was $53.7 \%$ for these conditions; lower than a comparable national average in 5 of 9 practices. Broad spectrum antibiotics comprised $43.9 \%$ of antibiotic prescriptions for URIs, $68.7 \%$ for acute bronchitis, $28.9 \%$ for acute pharyngitis and $54.9 \%$ for multiple rarely appropriate diagnoses.

CONCLUSIONS: Although there was great variability in antibiotic prescribing for ARIs by both practice and by ARI diagnosis, practices using this CDS prescribed antibiotics more appropriately for these ARI conditions compared to national prescribing rates. A CDS, implemented within the context of academic detailing, user training and performance review, shows promise for promoting judicious antibiotic use for ARIs.

USE OF RETICULOCYTE PRODUCTION INDEX IN ANEMIC INPATIENTS Ariel Katz ${ }^{1,2}$; Chioma A. Ekeh ${ }^{1}$; Magdalena A. Danch ${ }^{1}$; Emad U. Hakemi' ${ }^{1}$; German E. Giese ${ }^{1}$; Franklin Njoku ${ }^{1}$; Maryam Sanati ${ }^{1,2}$; Brian P. Lucas ${ }^{1,2}$. ${ }^{1}$ Stroger Hospital of Cook County, Chicago, IL; ${ }^{2}$ Rush University, Chicago, IL. (Control ID \#1314451)

BACKGROUND: The reticulocyte production index (RPI) is an early branch point in most anemia algorithms: an RPI greater than 2.0 indicates an adequate bone marrow response. In our hospital, however, we have noticed that many patients with anemia never have an RPI drawn. Moreover, among those who do, it seems that values are often low (less than 2.0) except in patients with established causes of anemia where RPIs are used to monitor and not diagnose them (e.g., rule out an aplastic crises in a patient with sickle cell disease). Among a cohort of anemic inpatients, therefore, we hypothesized that few have an RPI drawn, and for those who did, the diagnostic yield would be low. METHODS: Our cohort was drawn from 13667 hospitalizations to the general medicine service of a public teaching hospital from July 2009 through June 2010. The inclusion criterion was a hospitalization with anemia defined as any hemoglobin $<13 \mathrm{gm} / \mathrm{dL}$ for men and hemoglobin $<12 \mathrm{gm} / \mathrm{dL}$ for women (World Health Organization criteria). We calculated the RPI as the reticulocyte percentage multiplied by the most recent hemoglobin divided by 15 and multiplied by the inverse of the maturation factor: 1 for hemoglobin $>=$ $13.3 \mathrm{gm} / \mathrm{dL} ; 1.5$ for hemoglobin $<13.3 \mathrm{gm} / \mathrm{dL}$ and $>=10 \mathrm{gm} / \mathrm{dL} ; 2$ for hemoglobin $<10 \mathrm{gm} / \mathrm{dL}$ and $>=6.67 \mathrm{gm} / \mathrm{dL}$; and 2.5 for hemoglobin $<6.67$ $\mathrm{gm} / \mathrm{dL}$. Hospitalizations of anemic patients with RPIs greater than 2.0 were reviewed by 2 physician investigators and verified by a third. Elevated RPIs were considered useful when they contributed to a diagnosis that was not already established or otherwise immediately obvious.

RESULTS: 13459 hospitalizations had at least one hemoglobin level. During two thirds (8602 of 13459) of these hospitalizations, patients were anemic. Of these 8602 hospitalizations, 979 RPIs were checked on 905 unique patients. Of these 905 patients, only 5\% (47 of 905) had an RPI greater than 2.0. The causes of the elevated RPIs were sickle cell disease $(n=30)$, autoimmune hemolytic anemia $(n=8)$, acute blood loss $(n=4)$, other hemolytic anemia $(n=4)$, and unknown $(n=1)$. In less than $1 \%$ (7 of 905) the elevated RPI contributed to a diagnosis that was neither already established nor otherwise immediately obvious. These diagnoses included acute blood loss $(\mathrm{n}=3)$, autoimmune hemolytic anemia $(n=2)$, sickle cell trait $(n=1)$, and spherocytosis $(n=1)$.

CONCLUSIONS: Despite well-known diagnostic algorithms for anemia, RPIs are checked infrequently among anemic inpatients. This may be justified, however, because less than 1 in 100 RPIs are both elevated and meaningfully contribute to an anemia diagnosis. Whereas the RPI is useful in some clinical settings, it may not be useful in routinely diagnosing anemia in general medicine inpatients.

\section{USING DIFFERENT ROUNDING TECHNIQUES TO EVALUATE} PATIENT DATA RETENTION BY MEDICAL INTERNS IN THE ICU Vikram Chabra; Farzin Rahmanou; Edison Gavilanes; Stephen Karbowtiz. New York Hospital Queens, Flushing, NY. (Control ID \#1334513)

BACKGROUND: Bedside rounds are conducted daily in the ICU. Interns present new cases and provide interim medical histories on already established ICU patients. They are responsible for understanding these histories, reason for ICU admission, and plan of care for the day. This study's goal is to evaluate intern retention and understanding of these basic information items for each of 12 patients in our unit. Two different modes of rounding were used and compared.

METHODS: The ICU team consists of three interns. Senior team members include a second year medical resident, a pulmonary-critical care fellow, and one intensivist attending. Interns were on 24-hour call every fourth day. To accomplish this call schedule, an intern "float" was utilized once every fourth day to cover. Every intern is assigned between 3-6 patients each day in our 12-bed unit. Using a questionnaire we studied three consecutive months from March-2010 to May-2010 to determine if changing the rounding technique would improve intern's basic knowledge of each patient. The questionnaire was comprised of three items: 1)What is the patient's diagnosis? 2)Why is the patient in the ICU? 3)What is the plan for the day? The answers were scored to allow up to a maximum of nine points per patient. A point was awarded for every aspect of the question he/ she got correct. Interns could also score maximal points for knowing their patients with lower acuity who had less than 3 aspects per question to answer. The Fellow administered the questionnaire after rounds and each question was awarded a percent score of $0 \%, 33 \%, 50 \%, 66 \%$ or $100 \%$. For the first two weeks (Technique \#1) of the month, patients were presented by their own assigned intern. These mornings the team would hear presentations from at least 2 but usually 3 interns. Patients admitted overnight were presented by the overnight on call intern. For the second two weeks (Technique \#2) of the month, all patients were presented by the overnight on call intern. Each weekday after rounds the ICU Fellow would quiz the intern on call using the questionnaire. If the intern on call that day was a float intern a random intern was chosen for the questionnaire.

RESULTS: A statistically significant correlation was achieved showing that regardless of which rounding technique was employed, interns showed a better understanding of the 3-6 patient they are assigned compared to patients they are not assigned. There were no overall statistical differences between rounding techniques 1 and 2 when it came to intern's recollection of patient diagnosis, reason in ICU, or plan of care except for one isolated month which showed technique 1 was superior. Although not statistically significant there when the data broken down by intern category; technique 2 was better for the preliminary interns.

CONCLUSIONS: Despite our efforts to invoke a sense of responsibility on the part of each individual 1st year medical, preliminary, and emergency room rotator, and to suggest that they share all the patients in the unit 
equally, it appears that 1 st year providers show a bias of information retention favoring those patients that are directly assigned to them. This occurs even when they are not the intern responsible for morning presentations. Whether or not this is a function of experience or dependant on other unknown factors, we should continue to seek out better methods of rounding and education to improve upon our current models.

USING PHOTOVOICE TO IDENTIFY INTERVENTIONS TO IMPROVE MENTAL HEALTH AMONG RECENT LATINA IMMIGRANTS Sarah E. Paraghamian ${ }^{1}$; Melissa Green ${ }^{2}$; Laura C. Braswell ${ }^{2}$; Rachel Page $^{2}$; Georgina Perez ${ }^{3}$; Anh N. Tran ${ }^{3}$; Connie Blumenthal ${ }^{2}$; Michelle J. Lyn ${ }^{3}$; Giselle Corbie-Smith ${ }^{4} .{ }^{1}$ The University of North Carolina at Chapel Hill, Chapel Hill, NC; ${ }^{2}$ The University of North Carolina at Chapel Hill, Chapel Hill, NC; ${ }^{3}$ Duke University Medical Center, Durham, NC; ${ }^{4}$ The University of North Carolina at Chapel Hill, Chapel Hill, NC. (Control ID \#1277321)

BACKGROUND: Latino immigrants face numerous social, economic, and political challenges as they navigate transition to life in the United States. Disparities in mental health outcomes and quality of life compared to non-Latinos are well documented, yet access to health care and mental health services is limited. We used Photovoice to elicit the perspectives of Latina mental health promotoras on barriers to well-being among their peers and to identify possible interventions to improve mental health.

METHODS: Amigas Latinas Motivando el Alma (ALMA) is an academic community partnership to improve mental health among immigrating Latinas. Promotoras completing the ALMA stress reduction training served as partners using Photovoice. Through photography and guided discussion, promotoras recorded and reflected on community strengths and concerns regarding mental health. Discussions were audio recorded, transcribed, and coded using content analysis to identify salient themes. Promotoras reviewed codes for verification (member checking) and development of themes that were presented by the promotoras in a community forum. RESULTS: Nine Promotoras aged 30 to 43 participated in Photovoice. The women have lived in the US for an average of 10 years (range 3 to 17) and most are from Mexico (78\%). Two-thirds are currently employed and $44 \%$ have a college degree while $22 \%$ did not attend high school. The promotoras identified three interrelated themes that impact the mental health of newly immigrating Latinos. 1) Intergenerational tension that challenges communication between parents and children. Tension stems from concern about loss of values and acculturation, the division between foreign-born parents and their US-born children, and time constraints. 2) Limited education and employment opportunities. While many came to the US for work, participants voiced concerns about factors such as low educational attainment, limited English proficiency, lack of recognition of degrees obtained outside of the US and immigration status that limited work opportunities. 3) Language barriers and cultural isolation. In this emerging and rapidly changing immigrant community, exposure to only Spanish-language social networks and media sources was felt to limit knowledge of and access to resources. Physical barriers such as lack of transportation (complicated by immigration status) and distance from family and friends, as well as racial tension among other minorities were also discussed as factors increasing isolation. Over 70 stakeholders attended the community forum and proposed the following in response to the themes that were presented: increasing awareness of mental health resources through partnerships with Spanish-language media, churches, and businesses; school programs that foster involvement of Latino parents; and workshops to train community leaders and more promotoras to address the stigma around mental illness in order to increase utilization of existing mental health services.

CONCLUSIONS: Photovoice is an effective tool to give Latina women with limited English proficiency and other vulnerable populations a platform to inform interventions designed to improve community health.

USING SOCIAL MEDIA TO ENHANCE CONTINUING MEDICAL EDUCATION: A SURVEY OF INTERNAL MEDICINE CME COURSE PARTICIPANTS Amy T. Wang; Nicole P. Sandhu; Christopher M. Wittich; Jayawant N. Mandrekar; Thomas J. Beckman. Mayo Clinic College of Medicine, Rochester, MN. (Control ID \#1338638)
BACKGROUND: Social media (SM) is widely used by millions of physicians. According to recent surveys, over half of all students, residents and practicing doctors use some form of SM. Several studies have focused on the professionalism implications of SM; however, there has been little research on the utility of SM for enhancing medical learning and we are unaware of any studies on the use of SM in continuing medical education (CME). Therefore, we conducted a cross-sectional survey of U.S. and Canadian physicians attending a Mayo Clinic Internal Medicine CME course to determine their use of SM, to evaluate their attitudes regarding the value of SM for enhancing CME education, and to explore potential associations between CME participants' characteristics and attitudes towards SM.

METHODS: This was a cross-sectional survey and validation study that included all 539 U.S. and Canadian participants at a Mayo Clinic Internal Medicine CME course in 2011.The Social Media Use and Perception Instrument (SMUPI) consists of 10 items (5-point Likert scales) along with categorical response options for demographic variables. SMUPI content was based on existing literature and input from experts in scale design and CME assessment. Factor analysis was performed on the Likert-scaled survey items. Internal consistency reliability was calculated using Cronbach alpha. Associations between SMUPI item scores and participants' characteristics were determined using the Kruskal-Wallis test. The threshold for statistical significance was set at $\mathrm{p}<0.05$.

RESULTS: A total of 327 of 539 CME participants (response rate $=61 \%$ ) completed the survey. $291(89 \%)$ of participants reported using social media, with YouTube (189; 58\%), Facebook (163; 50\%), and Skype (142; $43 \%$ ) being the most common. Factor analysis revealed a two-dimensional assessment of CME course participants' attitudes, with Factor 1 representing the value of SM to course participants (items 1-5), and Factor 2 representing the value of SM to CME course directors (items 6-9). One item was eliminated due to an ambiguous item loading. Internal consistency reliability (Cronbach alpha) was excellent for Factor $1(0.94)$, Factor 2 (0.89) and overall (0.95). CME course participants' favorable attitudes towards SM were associated with characteristics (mean scores; p-value) of being younger in years $(20-29=3.13 ; 30-39=3.40 ; 40-49=3.39$; $50-59=3.18 ; 60-69=2.93 ;>70=2.92 ; \mathrm{p}=0.02)$, using SM frequently (never $=2.49$; $<$ once monthly $=2.75$; once monthly $=3.21$; weekly $=3.31$; daily $=3.81 ; \mathrm{p}<0.0001)$, and professional degree $(\mathrm{PhD}=3.00 ; \mathrm{MD}=3.05$; $\mathrm{DO}=3.35 ; \mathrm{PA}=3.42 ; \mathrm{NP}=3.50 ; \mathrm{p}=0.01$ ).

CONCLUSIONS: We describe the first validated measure of Internal Medicine CME participants' attitudes regarding personal use of SM and the value of SM for learning CME. The association between positive attitudes on using SM in CME with younger age and increased frequency of SM use, suggests that CME course directors might want to direct SM learning strategies towards more youthful, technology-savvy CME physicians, and that the utilization of SM in CME will become increasingly worthwhile as junior physicians enter the profession. There remains a need for more research on developing effective methods for enhancing CME learning with SM.

USING PHOTO-ELICITATION TO IDENTIFY SOURCES OF WASTE IN AN ACADEMIC MEDICAL CENTER Sarah L. Goff ${ }^{1}$, Reva Kleppel ${ }^{2}$; Michael Rothberg ${ }^{2}$. ${ }^{1}$ Baystate Medical Center/Tufts University School of Medicine, Springfield, MA; ${ }^{2}$ Baystate Medical Center/Tufts University School of Medicine, Springfield, MA. (Control ID \#1307623)

BACKGROUND: Rising medical costs in the U.S. have made health care unaffordable for many patients and force payers to make difficult coverage decisions. Identifying and reducing sources of waste in the medical system is an attractive alternative to limiting necessary health care coverage. Using photo-elicitation, an innovative approach to qualitative study, we sought to identify sources of waste in a large tertiary care academic medical center. METHODS: Participants were recruited from a broad range of departments throughout the hospital via word of mouth and were invited to take up to 10 photos of examples of waste they encountered during their workday. Participants then described the waste they captured in their photos during a semi-structured interview with a research staff member. A standardized interview guide was used; interviews were audio-taped and professionally transcribed. Transcriptions were reviewed independently 
and iteratively by two study team members and a code book was developed from the transcripts. The code book was revised with each successive interview and organized into pertinent themes. Interviews were conducted until theoretical saturation was reached. Agreement in code assignment between reviewers was assessed.

RESULTS: Eighteen individuals participated in this study and 140 photos were taken; all were included in the study. Participants represented a range of health professionals, including nurses, attending physicians from a number of disciplines, respiratory therapists, administrators and administrative support personnel. Agreement between transcript reviewers reached $85 \%$. Major types of waste described included time and tangible resources such as food, paper and energy. Factors identified in creation of waste included poorly designed or inefficient systems, medical education, poor communication and false economies. Barriers to reducing waste included inertia and regulatory systems. Consequences of waste included suboptimal patient care and satisfaction as well as physician disengagement. Although some recommendations for waste reductions appeared simple to implement, many were complex.

CONCLUSIONS: Individuals working within the health care system can offer unique insights into sources of waste they encounter in their daily routine. Although reducing waste may prove challenging, the results of this photo-elicitation study serve to generate a broad range of testable hypotheses regarding sources of waste existing in a large tertiary care hospital.

USING THE AUDIT-PC TO PREDICT ALCOHOL WITHDRAWAL IN HOSPITALIZED PATIENTS Edward Ewen ${ }^{1}$; Anna Pecoraro ${ }^{2}$; Terry Horton $^{1}$; Paul Kolm ${ }^{3}$; Ruth A. Mooney ${ }^{4}$; Patty McGraw ${ }^{1}$; George E. Woody ${ }^{2}{ }^{1}$ Christiana Care Health System, Newark, DE; ${ }^{2}$ University of Pennsylvania, Philadelphia, PA; ${ }^{3}$ Christiana Care Health System, Newark, DE; ${ }^{4}$ Christiana Care Health System, Newark, DE. (Control ID \#1333538)

BACKGROUND: Alcohol dependent hospitalized patients are at risk for alcohol withdrawal syndrome (AWS); however there are currently no measures to predict the risk of AWS in these patients. Early identification of those at risk for AWS could be used to alert clinicians and lead to more timely initiation of appropriate pharmacotherapy to prevent or treat withdrawal. The Alcohol Use Disorders Identification Test-PC (AUDIT-PC), a short 5 question survey instrument, is well validated to detect problem drinking in the primary care setting, however it has never been assessed in the hospital setting or used to predict AWS. This study examines the discriminating ability overall, and by age and gender, of the AUDIT-PC score in predicting AWS in hospitalized patients.

METHODS: All medical-surgical hospitalizations from 10/2009 to 10/ 2010 in a large single health system were examined and a retrospective case-control study was conducted. Beginning in 10/2009, AUDIT-PC scores were obtained routinely during initial nursing assessment on all adult admissions. We randomly selected 300 patients with a primary or secondary discharge diagnosis of AWS and matched them 1:1 to randomly selected controls by age, sex, and race. AUDIT-PC scores were identified by manual chart review. A hierarchical (hospital unit), case-control, weighted logistic regression was performed and area under the ROC curve (aROC) was calculated. Models included AUDIT-PC score by age $(\geq 65)$ and sex (male) interaction terms. Descriptive statistics were compared using the Chi-square and Mann-Whitney $\mathrm{U}$ tests.

RESULTS: We identified 589 (1.4\%) patients with AWS out of 40,908 hospitalizations. Overall, those with AWS were younger (age 52 [SD 13] vs. 62 [SD 18], $\mathrm{p}<0.001)$ more frequently male $(76.7 \%$ vs. $47.0 \%, \mathrm{p}<$ $0.001)$, and less frequently African-American (17.8\% vs. $21.3 \%, \mathrm{p}=0.039)$. Of the 300 case-control pairs, 97 were missing one or both AUDIT-PC scores and the analysis was conducted on the remaining 203 pairs. There were no significant differences in age $(\mathrm{p}=1.0)$, sex $(\mathrm{p}=0.48)$, or race $(\mathrm{p}=$ 0.18 ) between those excluded and those included in this analysis. The sensitivity was $88 \%$ and specificity $90 \%$ at a cutoff value of 5 for the AUDIT-PC score (see figure). The aROC was 0.94 (95\% CI, $0.92-0.97)$. aROCs were not significantly different for age $(\mathrm{p}=0.68$, or $\operatorname{sex}(\mathrm{p}=0.12)$, but, there were statistically significant AUDIT-PC by age and by sex interactions $(\mathrm{p}=0.003$ and $<0.001)$.

CONCLUSIONS: The admission AUDIT-PC score is an excellent discriminator of hospitalized patients developing AWS and could be an important contributing factor in the development of future clinical prediction rules. Further study including calibration and validation on a large prospective cohort is needed.

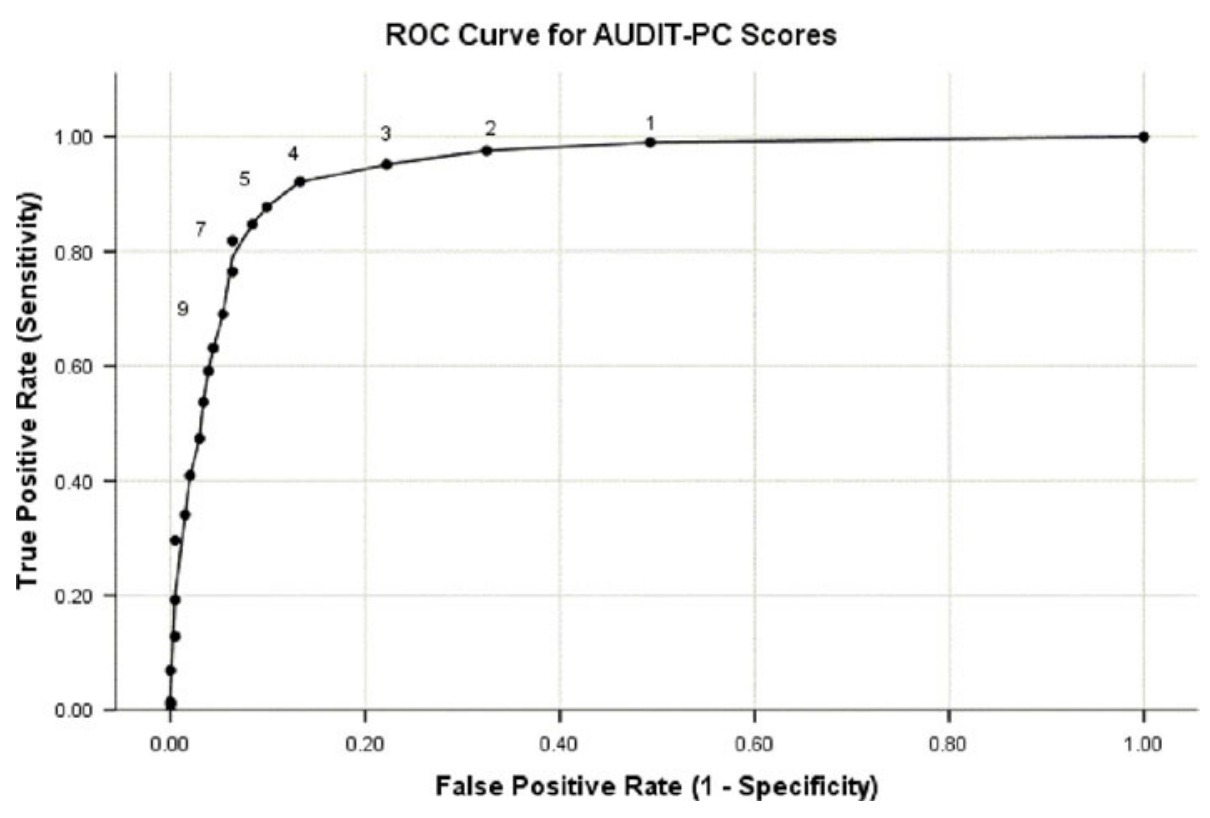

USING THE PARIHS FRAMEWORK TO INFORM THE IMPLEMENTATION OF THE VA STEPPED CARE MODEL FOR PAIN MANAGEMENT IN A MULTI-SITE COMMUNITY HEALTH CENTER Daren Anderson. Community Health Center, Inc., Middletown, CT. (Control ID \#1282928)
BACKGROUND: Most patients suffering from chronic pain receive pain care from a primary care provider. However, primary care providers express low confidence in their ability to effectively manage pain, and studies suggest wide variation in adherence to best practice guidelines for pain care. Strategies are needed to improve pain care in primary care. The 
Veterans Health Administration has developed an effective model for managing chronic pain, the Stepped Care Model for Pain Management (SCM-PM). The objective of this study was to use the PARIHS framework to conduct a formative assessment of pain care practice across a multisite, statewide Community Health Center to inform the process of adapting and implementing the SCM-PM.

METHODS: We selected a broad range of measures to assess the PARIHS domains of Evidence and Context. Data was collected from the electronic health record, chart reviews, and surveys of health center staff. Patients with pain were identified using pain scores and prescription records to identify chronic opioid use. Aggregate data was evaluated for two cohorts: adults with two or more pain scores $>=4$ separated by 90 days or more and adults receiving prescription opioid medications for $>=90$ days. Charts of patients with chronic pain were chosen at random and reviewed by a trained research assistant. Surveys on knowledge, attitudes, and beliefs about pain care were administered to primary care providers. All primary care staff completed an organizational change readiness assessment.

RESULTS: There were 6746 patients with two or more pain scores $>4$, and 1013 patients receiving opioids for $>90$ days. Patients with pain and chronic opioid use had an average of 15 visits per year as compared to the agency average of six visits per year. Few were referred to pain-related specialties, and only $25 \%$ were co-managed by onsite behavioral health providers. Approximately two thirds of patients on chronic opioids had signed an opioid agreement and had a urine toxicology screen in the past year. There were large gaps in recommended documentation standards. There was wide provider variability in the prescription of opioids to treat pain. Surveys found substantial variance in pain care knowledge, a lack of confidence in ability to manage pain, and dissatisfaction with the resources available to support chronic pain care. The change readiness assessment revealed higher scores on mandate for change, quality of communication, and leadership, and lower scores on peer input and on confidence that adequate resources would be allocated.

CONCLUSIONS: The PARIHS framework provided a useful construct to prepare for successful project implementation. High primary care utilization and low referrals and behavioral health co-management suggest the need for multidisciplinary collaboration and better coordination of care. Low confidence and variable knowledge scores indicates a need for more training in pain care. Variation in charting, follow up, and adherence to guidelines for opioid prescribing and monitoring suggests a need for stricter policies and systems to monitor and provide feedback to providers. Based on these findings we have developed a multi-faceted intervention aimed at increasing options for behavioral health and complementary medicine support, increasing access to specialty consultation, providing pain-specific CME for providers, and improving documentation of pain care in the electronic health record.

UTILIZATION OF ANTIDEPRESSANT MEDICATIONS AMONG PATIENTS WITH DEPRESSION: COMPARISON BETWEEN USUAL CARE AND COLLABORATIVE CARE USING CARE MANAGERS. Ramona S. DeJesus; Kurt Angstman; Mark Williams. Mayo Clinic, Rochester, MN. (Control ID \#1284940)

BACKGROUND: Depression is responsible for an estimated economic cost of more than 40 billion annually, and has a large impact on quality of life and productivity. Yet it remained under diagnosed and inadequately treated. The Sequenced Treatment Alternatives to Relieve Depression (STAR*D) trial confirmed that several sequential treatment steps are often needed to obtain remission. Only $28 \%$ of patients remitted after 12 weeks of adequate trial on a single agent; switching to another agent or augmentation with a second agent was often necessary. The collaborative care model, using care managers, has been consistently showed in numerous studies to be an effective way to manage depression and achieve sustained outcomes compared to usual care. In March 2008, the DIAMOND (Depression Improvement Across Minnesota Offering a New Direction) project, a collaborative model using care managers coordinated by the Institute for Clinical Systems Improvement, was implemented at Mayo Family Clinics Northwest in Rochester, Minnesota. The model was subsequently rolled out to the remaining primary care sites. We hypothesize that utilization of antidepressant medications among patients with depression managed under the collaborative care management (CCM) model would be different from usual care.
METHODS: Data was abstracted from medical records of patients who received a diagnosis of depression from March of 2008 defined as a screening score of 10 or greater on the Patient Health Questionnaire- 9 (PHQ-9), had given permission to have their records reviewed and had at least a 6 month follow-up. Pattern of antidepressant medications utilization were compared between patients with depression enrolled in the CCM model and those under usual care. Demographic data was also obtained. Data was analyzed using Fisher exact test. Sub-analysis was done on geriatric patients defined as those aged 65 and older.

RESULTS: There were three hundred thirty three $(\mathrm{N}=333)$ patients who met study criteria; two hundred forty two $(\mathrm{N}=242)$ were enrolled under $\mathrm{CCM}$ and ninety one $(\mathrm{N}=91)$ were in usual care. There was no statistical difference in demographics (age, gender, race, ethnicity, marital status, initial PHQ-9 score) between the two groups. At six months, the mean PHQ-9 score of those enrolled in CCM was statistically lower compared to those in usual care (4.44 vs. 7.13 ; p value: 0.002$)$. Likewise the mean difference in PHQ-9 score from baseline was also greater among those in CCM compared to usual care. Those patients who were followed under CCM had significantly greater utilization of anti-depressant medications at the end of one year ( $p$ value: $<0.001$ ). They likewise had more change in the number of medications from March, 2008 to March, 2009.

CONCLUSIONS: The collaborative care model for depression management is associated with greater anti-depression medication utilization compared to usual care. It is also statistically significantly associated with greater reduction in PHQ-9 scores at 6 months and remission when compared to usual care. These findings have significant implications in depression management particularly among primary care settings.

VA ELECTRONIC PATIENT PORTAL: INCREASING ACCESS FOR VETERANS RECEIVING HOME-BASED PRIMARY CARE Rebecca Grochow ${ }^{1,2}$; Max D. Stewart ${ }^{1,7}$; Gemmae Fix ${ }^{4,3}$; Keith McInnes $^{4,5}$; Judith B. Boardman ${ }^{5,6}$; Steven R. Simon ${ }^{1,7}$. ${ }^{1}$ VA Boston Healthcare System, Boston, MA; ${ }^{2}$ Boston University School of Medicine, Boston, MA; ${ }^{3}$ Boston University School of Public Health, Boston, MA; ${ }^{4}$ VA Medical Center ENRM, Bedford, MA; ${ }^{5}$ VA Boston Healthcare System, Boston, MA; ${ }^{6}$ Salem State University, Salem, MA; ${ }^{7}$ Brigham and Women's Hospital, Boston, MA. (Control ID \#1336036)

BACKGROUND: My HealtheVet (MHV), the VA's web-based patient portal, allows patients to access health information and communicate via secure messaging with their healthcare team. This electronic tool has the potential to improve the health of all Veterans, especially those with physical and/or psychological limitations that impede their ability to travel to a VA site for care. To date, adoption of MHV has been slow among all Veterans, including among those Veterans who receive their care through the Home Based Primary Care (HBPC) program. Eight percent of all HBPC Veterans have registered for MHV and only $1 \%$ have completed in-person authentication (IPA) to gain access to key MHV features, such as their personal medical records and secure messaging with providers. Little is known about how these Veterans perceive the potential value of MHV and the barriers to its use. We undertook a qualitative study to characterize the perspectives of Veterans and healthcare providers in preparation for developing an intervention to increase access to MHV for this vulnerable patient population.

METHODS: Using a literature-based semi-structured interview guide, we conducted in-depth interviews with HBPC-enrolled Veterans. We also interviewed HBPC providers to further inform the development of an intervention to improve enrollment. A multi-disciplinary team used content analysis to identify key themes.

RESULTS: We have interviewed 8 Veterans and 3 providers to date. All Veterans had experience using computers and the majority $(5 / 8)$ had access to a computer and the internet in their homes. Four of the Veterans had heard of MHV, but only 2 had accessed it, and only 1 had completed IPA. Both MHV-enrolled Veterans had heard about MHV via an HBPC provider. Others had received flyers by mail but had not pursued access, noting that the flyer did not sufficiently explain the function and benefit of the patient portal. All Veterans expressed interest in learning about MHV from HBPC providers. HBPC providers suggested that a social worker, during the initial in-home assessment, could assess Veterans' ability to use MHV and provide enrollment information. HBPC providers noted that they 
would need training and expressed willingness to learn how to complete the IPA process to obviate the Veterans' need to visit the Medical Center in-person. All Veterans and providers identified the ability to refill medications and to communicate electronically with healthcare providers as key features the Veterans would find useful. Many Veterans expressed frustration with the VA telephone system when trying to reach a provider or clinic. They identified the patient portal as a potentially more direct method of communication.

CONCLUSIONS: HBPC-enrolled Veterans are vulnerable patients given their limited physical access to the VA and their high need for services. Barriers to accessing the patient portal included lack of information as well as computer access for some. Veterans expressed enthusiasm for the portal features that could provide them with health information and greater access to healthcare providers. With appropriate training, the clinical team may be able to promote adoption of MHV among these Veterans, thereby enhancing a patient-centered care model.

VACCINE TRACKING AMONG GENERAL INTERNISTS: CURRENT PRACTICES AND HOW A 2-D BARCODING SYSTEM MIGHT HELP Laura Hurley $^{1,2}$; Lori Crane $^{2}$; Erin D. Kennedy ${ }^{3}$; Sean T. O'Leary ${ }^{2}$; $\overline{B r e n d a ~ B e a t y}^{2}$; Shannon Stokley ${ }^{3}$; Mandy A. Allison ${ }^{4,2}$; Michaela Brtnikova $^{2}$; Andrea Clinger ${ }^{2}$; Allison Kempe ${ }^{2} .{ }^{1}$ Denver Health and Hospital Authority, Denver, CO; ${ }^{2}$ University of Colorado Denver, Denver, CO; ${ }^{3}$ Centers for Disease Control and Prevention, Atlanta, GA; ${ }^{4}$ University of Utah, Salt Lake City, UT. (Control ID \#1338472)

BACKGROUND: In August 2011, the Food and Drug Administration announced it would consider requests from vaccine manufacturers to permit alternative identification methods, including two-dimensional (2D) barcodes, on vaccines that would include product identifier, lot number and expiration date. The objectives of this study were to assess among general internists (GIM) 1) current systems for tracking vaccine supplies and recording vaccinations 2 ) attitudes about a 2-D vaccine barcoding system (barcode scanner/associated interface with computer) 3) barriers to using a vaccine barcoding system and 4) functions needed for the practice to adopt a barcoding system.

METHODS: Between September 2011 and January 2012, we administered an Internet and mail survey to a national network of 428 GIM physicians representative of the American College of Physicians membership.

RESULTS: Response rate was $58 \%(247 / 428)$. Over $90 \%$ of respondents stock some of the recommended adult vaccines. When asked about how their practices monitor vaccine inventory, most physicians reported they simply order vaccine when stock looks low $(65 \%)$, when they know demand is about to pick up (57\%), or use a paper-based inventory system $(34 \%)$. Few physicians used an Internet-based system $(7 \%)$ or an inventory software system (4\%). Although $74 \%$ were satisfied with their current method for monitoring vaccine inventory, $40 \%$ reported it was time consuming to keep track of inventory and 39\% reported their practices frequently run out of vaccine doses. The most common methods for recording vaccine product, lot number and expiration date in the medical record were to enter information directly into the patient's electronic $(50 \%)$ or paper-based $(31 \%)$ record. Forty-three percent reported recording this information in two or more places. Fifty-nine percent noted that recording vaccine information was time consuming and $28 \%$ reported problems with accurately maintaining vaccine records. Physicians agreed that barcodes could facilitate tracking of vaccine inventory $(90 \%)$, would be more reliable and accurate than current recording systems $(90 \%)$, would improve patient safety $(92 \%)$ and could improve the efficiency of vaccine administration (79\%). The only major barriers reported to the use of a barcode inventory system included the need for software (22\%), the need for information technology support (14\%), lacking an electronic medical record $(14 \%)$, and the need for computer equipment (12\%). Functions needed for the practice to adopt a barcoding system included: 1) recording dose information in an electronic medical record $(86 \%), 2)$ identifying patients who had received a recalled lot of vaccine (89\%) 3) maintaining vaccine inventory records $(71 \%), 4)$ recording that a Vaccine Information Statement was given to a patient $(69 \%)$ and 5) billing for vaccine doses $(70 \%)$. A majority of physicians $(70 \%)$ would definitely or probably adopt a barcoding system if the total costs were less than $\$ 1000$.
CONCLUSIONS: Most physicians report using inefficient systems for tracking vaccine doses and inventory and realize multiple potential benefits of incorporating a vaccine barcoding system. Costs will need to be contained and technological barriers will need to be addressed to facilitate adoption of vaccine barcoding systems.

VALIDATING THE PRESENT-ON-ADMISSION INDICATOR FOR HOSPITAL-ACQUIRED VENOUS THROMBOEMBOLISM IN ADMINISTRATIVE DISCHARGE DATA Sharon B. Kim ${ }^{1}$; Raman R. Khanna ${ }^{1}$; Ian Jenkins ${ }^{2}$; Robert El-Kareh ${ }^{2}$; Nasim Afsarmanesh ${ }^{3}$; Alpesh $\mathrm{Amin}^{4}$; Heather Sand ${ }^{4}$; Andrew Auerbach ${ }^{1}$; Catherine Chia ${ }^{5}$; Gregory Maynard $^{2}$; Richard H. White ${ }^{5}{ }^{1}$ University of California, San Francisco, San Francisco, CA; ${ }^{2}$ University of California, San Diego, San Diego, CA; ${ }^{3}$ University of California, Los Angeles, Los Angeles, CA; ${ }^{4}$ University of California, Irvine, Irvine, CA; ${ }^{5}$ University of California, Davis, Davis, CA. (Control ID \#1339388)

BACKGROUND: Since 2007, the Centers for Medicare \& Medicaid Services (CMS) has required hospitals to flag all medical diagnoses using a present-on-admission (POA) indicator, which it uses to differentiate preexisting/admission-coincident medical conditions from hospital-acquired ones. CMS currently considers venous thromboembolism (VTE) after total knee or hip replacement a preventable hospital-acquired condition and thus does not reimburse hospitals for VTE events not POA. CMS may eventually use the POA indicator to decide reimbursement for all hospital-acquired VTE, including among medical patients; however, the accuracy of the POA indicator for hospital-acquired VTE events in a medical population is unknown. The aim of our study was to determine the positive predictive value (PPV) of the POA indicator in an inpatient medical population.

METHODS: We analyzed administrative discharge data from October 1, 2008 to September 30, 2010 from five University of California academic medical centers. We included hospitalizations for a medical illness (where the MS-DRG indicated a medical condition) with a secondary medical international classification of disease (ICD-9-CM) code for VTE. We excluded hospitalizations that were principally for VTE. Using a weighted sampling strategy we then selected approximately 50 hospitalizations with a POA indicator of "yes" $(\mathrm{POA}=\mathrm{Y})$ and 50 hospitalizations with a POA indicator of "no" $(\mathrm{POA}=\mathrm{N})$ at each medical center $(\mathrm{N}=504$ total). Using a standardized instrument, trained abstractors blinded to the POA indicator then reviewed the medical record for each hospitalization and categorized each VTE event as a pre-existing/admission-coincident condition, hospital-acquired condition, or timing indeterminate condition, with the latter two counted together in keeping with CMS reimbursement rules. The abstraction classification was then compared to the POA indicator. Abstractors also ascertained the date of VTE diagnosis, tests used to confirm the diagnosis, type of VTE, location, clinical presentation, and association with an indwelling vascular catheter.

RESULTS: During the time period analyzed there were a total of 1,941 secondary VTE events flagged $\mathrm{POA}=\mathrm{Y}, 339$ events flagged $\mathrm{POA}=\mathrm{N}$, and 5 indeterminate events out of 2,072 hospitalizations. (Seven percent of hospitalizations had more than one VTE event.) Of the $254 \mathrm{POA}=\mathrm{N}$ cases abstracted, $62 \%$ were classified as hospital-acquired and $20 \%$ were clinically indeterminate. The positive predictive value of the $P O A=N$ flag for hospital-acquired VTE was thus $82 \%(95 \%$ confidence interval $[\mathrm{CI}], 76 \%-86 \%$ ). The positive predictive value (PPV) of the $\mathrm{POA}=\mathrm{Y}$ flag for a pre-existing/admission-coincident VTE was $78 \%(95 \% \mathrm{CI}$, 74\%-84\%). Both PPV and NPV varied considerably across institutions, across VTE anatomic location, and by hospital day of diagnosis (TABLE). Extrapolating from our weighted random sample to the hospital population, we estimate there were actually 654 hospital acquired VTE events (almost twice as many as $\mathrm{POA}=\mathrm{N}$ events); of these, $60 \%$ of were incorrectly flagged $\mathrm{POA}=\mathrm{Y}$.

CONCLUSIONS: Our data suggest that despite a moderately high positive predictive value for both $\mathrm{POA}=\mathrm{Y}$ and $\mathrm{POA}=\mathrm{N}$, the $\mathrm{POA}$ indicator falsely classifies many pre-existing/admission-coincident VTE events as hospital-acquired while actually missing most hospital-acquired VTE events. 
VALIDATION OF A 10-YEAR MORTALITY INDEX FOR COMMUNITY-DWELLING OLDER ADULTS Marisa $\mathrm{Cruz}^{1}$; Irena Stijacic Cenzer ${ }^{2}$; Sei Lee ${ }^{2} .{ }^{1}$ UCSF, San Francisco, $\mathrm{CA} ;{ }^{2}$ San Francisco Veterans Affairs Medical Center, San Francisco, CA. (Control ID \#1335073)

BACKGROUND: Studies suggest that the benefits of both breast and colorectal cancer screenings typically are not seen for up to 10 years after screening, leading guidelines to recommend targeting cancer screening to patients with an extended life expectancy. Although a 10- year mortality index could help identify patients most likely to benefit from cancer screening, few mortality indexes have been validated beyond 5 years. Thus, we validated a previously published 4-year mortality index for 10year mortality.

METHODS: The original prognostic index was developed and validated for 4-year mortality in 19710 older adults enrolled in the 1998 Health and Retirement Study (HRS), a nationally representative cohort of communitydwelling US residents. The twelve independent risk factors comprising the index were demographic factors (age and gender), comorbidities (diabetes, heart disease, lung disease and cancer), behavioral factors (body mass index and smoking) and functional limitations (bathing, managing finances, walking and pushing large objects). Cox regression was performed with these predictors, using survival through 2008 (10 year mortality) as the outcome. Model calibration was determined by comparing observed 10-year mortality rates and index point scores for the original development (Eastern, Midwestern and Western US) and validation (Southeastern US) cohorts. Model discrimination was determined using the Harrell's c-statistic.

RESULTS: Our index was highly predictive of 10 -year mortality in both the development and validation cohorts. The $34 \%$ of respondents in the validation cohort with risk scores of $0-3$ had an observed 10 -year mortality risk of $7 \%$, while the $11 \%$ of respondents with scores of 8-9 points had an observed risk of $55 \%$, and the $5 \%$ with scores of 13 or more points had an observed risk of $92 \%$. The quartiles of risk within this cohort similarly ranged from $5.8 \% 10$-year mortality risk for the lowest quartile to $70.5 \%$ risk for the highest quartile. The 10-year index demonstrated excellent discrimination with Harrell's c-statistic of 0.784 .

CONCLUSIONS: Our index, using self-reported demographic, behavioral, functional and comorbidity factors, accurately predicts 10 -year mortality as well as 4-year mortality for community-dwelling adults over 50 years of age. Use of this and similar prognostic indices may help clinicians identify patients at increased mortality risk, who are unlikely to benefit from further preventive interventions such as cancer screening.

VARIABILITY IN PROVIDER PRACTICE IN PATIENTS ADMITTED WITH LOW RISK CHEST PAIN AND EFFECTS ON PRACTICE AFTER IMPLEMENTATION OF A STANDARDIZED CARE PROTOCOL Elizabeth Schulwolf ${ }^{1}$; Mark Speyer ${ }^{1}$; Micky Simwenyi ${ }^{2}$;

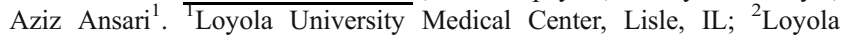
University Medical Center, Lisle, IL. (Control ID \#1339918)

BACKGROUND: Observation units are increasingly common and one of the more frequent admission diagnoses is low risk chest pain (CP). Guidelines indicate that ordering 2 troponins in patients with low risk $\mathrm{CP}$ to rule out acute coronary syndrome is sufficient. In April 2011 we implemented a $\mathrm{CP}$ protocol in an effort to standardize care for patients with low risk CP among our hospitalist group. The protocol recommends 2 troponins 6 hours apart and if needed a stress test, with treadmill stress echo the preferred modality unless clinical factors indicated an alternative stress test. Variation in practice likely exists regarding care including number of troponins obtained and type of stress test ordered.

METHODS: We analyzed administrative data of patients admitted to an observation unit managed by hospitalists in an urban, academic medical center for evaluation of low risk CP from a 3-month period prior to initiation of the CP protocol (October 1, 2010 through December 31, 2010) and a 3-month period after (April 1,2011 through June 30,2011). Variables included number of troponins and type of stress test. Dichotomous variables were compared using the chi-square test.
RESULTS: A total of 525 patients were identified: 254 (48.4\%) preprotocol and 271 (51.6\%) post-protocol. A total of 191 (36.4\%) patients underwent a stress test: $91(47.6 \%)$ treadmill stress echo, $70(36.6 \%)$ nuclear and 29 (15.2\%) dobutamine stress echo. During our study period, 16 hospitalists independently cared for patients in the observation unit with the range of patient numbers per provider being 2 to 24 . Three providers tended to select particular stress tests: 1 ordered nuclear stress tests more often (total stress tests ordered $=17,64.7 \%$ nuclear, $\mathrm{p}=0.01$ ) and 2 other providers ordered treadmill stress echos more often (provider 1: total stress tests ordered $=21,61.9 \%$ treadmill, $\mathrm{p}=0.06$; provider 2 : total stress tests ordered $=24,75 \%$ treadmill, $\mathrm{p}=0.01$ ). No other providers demonstrated a trend in stress test selection. Among providers, 2 tended to order more than 2 troponins (provider 1: $71.45 \%, \mathrm{P}=0.007$; provider $2: 75.0 \%, \mathrm{P}=0.03$ ) and 2 providers tended to order 2 or fewer troponins (provider 1: $75.0 \%$, $\mathrm{P}=0.04$; provider $2: 81.0 \%, \mathrm{P}=0.03$ ). Within providers, following the initiation of the protocol, 5 providers significantly changed their practice from ordering 3 or more troponins more often to 2 or fewer troponins. CONCLUSIONS: These results suggest that there is provider variability in selecting stress tests and the number of troponins ordered and further evaluation is needed to understand this practice variability. It is also evident that the protocol affected individual provider practice where some providers reduced the number of troponins ordered after the protocol was implemented. Our study is limited by the small numbers of patients seen by each provider individually and evaluating the data over a longer time period should be considered.

VARIATION IN PATIENT AND PHYSICIAN EMOTIONAL INTENSITY DURING DISCUSSIONS ABOUT PAIN IN PRIMARY CARE VISITS Stephen G. Henry ${ }^{1,2}$; Susan Eggly ${ }^{3} .{ }^{1}$ VA Ann Arbor Healthcare System, Ann Arbor, MI; ${ }^{2}$ University of Michigan, Ann Arbor, MI; ${ }^{3}$ Wayne State University, Detroit, MI. (Control ID \#1334228)

BACKGROUND: Patients and primary care physicians report that discussions about pain are often emotionally difficult, but questions remain about whether patients and physicians actually display more negative emotions when discussing pain compared to other topics. Understanding the emotional dimension of discussions about pain may be useful for improving communication and pain management in primary care. We used a "thin slice" approach to compare patient and physician affect (i.e. displayed emotion) during discussions about pain to patient and physician affect during discussions about non-pain topics.

METHODS: We observed and analyzed an existing archive of videorecorded primary care visits collected at a clinic serving predominantly low-income, black patients. We developed and applied a structured coding system to identify the video segments when patients and physicians discussed pain during each visit. We systematically selected 30 -second video segments (i.e. "thin slices") from the beginning, middle, and end of all visits that included discussions about pain $(n=85)$. For each visit, we selected 1-2 segments that included discussions about pain and 1-2 segments that included discussions about non-pain topics for a total of 132 pain-related and 121 non pain-related. Four trained raters independently observed all video segments and rated patient and physician affect (in separate steps) during each segment on six affective dimensions. We calculated standardized mean ratings across raters for each segment and performed factor analysis to identify groupings among affect variables. We used linear regression to compare mean patient and physician affect ratings for pain-related vs. non pain-related video segments within each visit. We used a 2-level hierarchical model with visit as a fixed effect to control for segments clustered within visits.

RESULTS: Estimated reliability for mean ratings was 0.70 for nearly all variables. Based on factor analysis results, we constructed four composite variables for our primary analysis: patient and physician "positive affect" (warm/friendly+engaged/attentive) and patient and physician "negative affect" (upset/distressed+tense/anxious for patients; hesitant/uncomfortable+ tense/anxious for physicians). Within visits, patients were rated as having both significantly greater negative affect (beta-coefficient 0.36 [95\% CI 0.17 to 0.47 ] $\mathrm{p}<0.001$ ) and significantly greater positive affect (beta-coefficient 0.21 [95\%CI 0.06 to 0.35$], \mathrm{p}=0.005$ ) during pain-related segments compared to non pain-related segments. For physicians, ratings for pain-related vs. non 
pain-related segments did not differ significantly for either negative affect (beta-coefficient $-0.11,[95 \% \mathrm{CI}-0.25$ to 0.03$], \mathrm{p}=0.09$ ) or positive affect (beta-coefficient 0.02, [ $95 \% \mathrm{CI}-0.13$ to 0.16$], \mathrm{p}=0.84$ ).

CONCLUSIONS: The "thin slice" approach is a reliable method for measuring variation in patient and physician affect during video-recorded clinical visits. Findings showed patients, but not physicians, displayed both significantly greater positive and negative affect when discussing pain compared to non-pain topics. Findings suggest that for this patient population, discussions about pain are associated with greater overall emotional intensity than discussions about other topics. Additional research is needed to better understand whether patients' emotional intensity influences patients' perceptions of physicians, adherence, or physicians' treatment recommendations.

VIDEO DECISION SUPPORT TOOL TO SUPPLEMENT GOALSOF-CARE DISCUSSIONS WITH HOSPITALIZED PATIENTS WITH ADVANCED ILLNESSES: PRELIMINARY FINDINGS Nicole M. LaRue ${ }^{1}$; Michael K. Paasche-Orlow ${ }^{1}$; Angelo E. Volandes ${ }^{2} .{ }^{1} \overline{\text { Boston }}$ University Medical Center, Boston, MA; ${ }^{2}$ Massachusetts General Hospital, Boston, MA. (Control ID \#1334457)

BACKGROUND: End-of-life discussions are often inadequate, leading to care that is inconsistent with patients' and families' wishes. The use of video to reinforce verbal descriptions of treatment options may better inform patients and families engaged in end-of-life decision making. We are unaware of prior studies to assess the effect of a video decision support tool on preferences for end-of-life care in patients and surrogate decision makers consulted on by an inpatient palliative care service.

METHODS: We analyzed the preliminary findings of a temporal intervention study, to be conducted until 25 subjects are enrolled each into the observational and intervention phases. Eligible subjects included adult patients consulted on by an inpatient palliative care team (PCT) who were appropriate for a goals-of-care discussion. Subjects in the observational phase received the standard of care provided by an inpatient PCT. During the intervention phase, a video illustrating specific treatments for three levels of care (life-prolonging, basic, and comfort oriented) was integrated into the standard palliative care consultation. Following the consultation, all subjects were surveyed regarding preferences for care near the end of life, the level of certainty regarding their decision, and pain and symptom burden. The primary study outcome was the difference in proportions of subjects in each group who preferred comfort oriented care. The secondary outcomes included the level of uncertainty regarding treatment preferences (score range, 0-100; higher score indicating greater uncertainty), concordance between patient reported treatment preferences and documentation in the electronic medical record (EMR), and satisfaction with pain control and symptom management, as measured by the modified Palliative Care Outcome Scale (score range, 0-36; higher score indicating worse symptom control) and the Edmonton Symptom Assessment Scale (total symptom distress score range, 0-90; higher score indicating greater symptom burden).

RESULTS: Of 25 subjects in the observational phase, 6 preferred lifeprolonging care, 6 preferred limited care, 11 preferred comfort care and 2 were unsure. Of the 9 subjects enrolled to date in the intervention phase, 2 preferred life-prolonging care, 2 preferred limited care, 4 preferred comfort care and 1 was unsure. The mean uncertainty score was 21.9 and 22.9 in the observational and intervention phases respectively. Patient reported preferences were discordant with the EMR in $28 \%$ of encounters in the observational phase and $22 \%$ of encounters in the intervention phase. The mean score for subjects on the Palliative Care Outcome scale was 14.7 (SD 6.4) and 15.2 (SD 7.1), in the observational and intervention phases respectively. On the Edmonton Symptom Assessment scale, subjects in the observational phase had a mean score of 46.5 (SD 14.3) compared to 43.0 (SD 19.2) during the intervention phase.

CONCLUSIONS: A video decision support tool illustrating treatment options is a feasible and acceptable method to supplement goals-of-care discussions with patients and families consulted on by an inpatient palliative care team. The use of video images may provide an easily reproducible method for more accurately eliciting preferences and ultimately improve end-of-life care.
WEB-BASED PTSD EDUCATION FOR MILITARY FAMILY MEMBERS Michael J. Roy ${ }^{1}$; Patricia Taylor ${ }^{1}$; Will Runge ${ }^{1}$; Evonne Grigsby $^{2}$; Tonya Torgeson ${ }^{2}$. Uniformed Services University, Bethesda, MD; ${ }^{2}$ DefenseWeb Technologies, Inc., San Diego, CA. (Control ID $\# 1340441)$

BACKGROUND: Posttraumatic stress disorder (PTSD) is common after military service in Iraq or Afghanistan, but its presentation is frequently delayed until months after return from deployment. This is believed to be largely attributable to a "honeymoon effect" resulting from the fact that service members find themselves out of harm's way and reunited with loved ones. Failure to promptly recognize stress-related symptoms in service members may lead to deleterious behaviors, such as abuse or dependence upon alcohol or illicit drugs, social withdrawal, and expressions of anger towards others, and even suicide. PTSD impairs service members' functional status in multiple domains, including at home with family members. Since service members typically have several weeks of vacation upon their return from deployment, their family members are ideally positioned to identify warning signals, but may lack the knowledge to recognize such signals and then respond in an effective manner. We hypothesized that an educational website developed expressly for military family members could provide them with the knowledge to improve outcomes.

METHODS: This is a three-phase study to try to significantly improve the knowledge of military family members iregarding PTSD, and further, to determine whether this knowledge translated into behavioral changes. First, focus groups were conducted with military family members across the US. and their feedback was incorporated into an educational website to improve family members' knowledge of PTSD. A pilot study then tested the initial iteration of the site and a 25-item knowldege questionnaire. The site and questionnaire were modified based on the results of the pilot, and then PTSD-related knowledge was assessed in 497 military family members before and after their use of the website.

RESULTS: Use of an educational website improved military family member PTSD-related knowledge on a 25 -item test, with an increase from a mean 13.9 correct responses beforehand to 18.7 after website use $(p<.001$; effect size 1.2). For 23 of the 25 items on the PTSD Knowledge questionnaire, the proportion of correct responses significantly increased between pre and post administration $(\mathrm{p}<.002)$. In addition, 217 family members returned to the site $>10$ days after their initial visit; $57 \%$ had taken actions such as discussing the service member's symptoms with them, or persuading them to get medical attention. Seventy-four percent of those who had a discussion with the service member about their symptoms thought it was helpful, as did $82 \%$ of those who persuaded the service member to see a primary care provider only, and $95 \%$ of those who persuaded them to see a mental health provider with our without primary care. The knowledge acquired with the initial use of the site was retained at the time of return to the site, manifest by similar scores on the knowledge questionnaire.

CONCLUSIONS: A web-based intervention can both improve PTSD-related knowledge and foster behavioral changes in military family members.

WEB-BASED WALKING TO WELLNESS: ADOPTION AND IMPACT OF AN INSURANCE-INCENTIVIZED INTERNETMEDIATED WALKING PROGRAM FOR OBESE ADULTS Donna M. Zulman ${ }^{1,2}$; Ryan G. Smith ${ }^{4}$; Paul Resnick ${ }^{5}$; Erin Krupka ${ }^{5}$; Laura Damschroder ${ }^{3}$; Caroline Richardson ${ }^{6,3}$. ${ }^{1}$ Stanford University, Stanford, CA $;{ }^{2}$ VA Palo Alto Health Care System, Menlo Park, CA; ${ }^{3}$ Ann Arbor VA, Ann Arbor, MI; ${ }^{4}$ Thomas Jefferson University, Philadelphia, PA; ${ }^{5}$ University of Michigan, Ann Arbor, MI; ${ }^{6}$ University of Michigan, Ann Arbor, MI. (Control ID \#1336486)

BACKGROUND: Rising obesity rates and associated chronic conditions and costs are driving demand for effective and inexpensive weight loss interventions. While Internet-mediated programs have been shown to increase physical activity levels in randomized controlled trials, it is unclear whether such programs can affect population-level behavior change. The objective of this study was to evaluate adoption and acceptance of a novel, insurance-incentivized Internet-mediated walking 
program, and to assess the program's population-level impact on physical activity among obese adults.

METHODS: We conducted a formative mixed-methods evaluation of the adoption, acceptance, and impact of an Internet-mediated walking program implemented by a large Midwest insurance company. Beginning in 2010 , individuals with a BMI $>30$ who were insured by this company could receive enhanced benefits if they participated in an Internet-mediated walking program or a traditional Weight Watchers program. Individuals who enrolled in the walking intervention received a free pedometer and could upload and monitor their daily step-counts (number of steps per day) on a personalized, interactive website. Participants who uploaded their step-count data at least once a month and achieved a goal of 5,000 average daily steps over three months were eligible for lower co-payments and deductibles-equivalent to $20 \%$ lower out-of-pocket expenses. We assessed program adoption and participation rates, and explored program impact among a subset of adherent participants, using a paired t-test to examine changes in step-counts over a four-month period. We complemented these findings with quantitative and qualitative analyses of participant feedback to a web-based survey.

RESULTS: Among the 15,387 individuals who met eligibility criteria for the incentivized weight management program over the first nine months, $6,546(43 \%)$ enrolled in the Internet-mediated walking program, and 5,045 $(33 \%)$ enrolled in Weight Watchers. Participants in the walking program documented an average of 6,963 steps per day (SD 2,626 steps). Over 75\% of walking program participants were classified as adherent based on uploads of valid pedometer data for at least 3 of 4 enrolled days. In a preliminary analysis of 1,818 adherent participants who had valid pedometer data during the first and last two weeks of the program's first four months, there was an average increase of 585 steps per day (SEM 53 days, paired $\mathrm{t}$-test $\mathrm{t}=11.05, \mathrm{p}<.0001$ ). In a web-based survey (response rate $12 \%), 51 \%$ of respondents reported that they appreciated the value of the program for improving their health and decreasing their health care costs. Another $17 \%$ of respondents initially joined the program for the financial incentive and were unhappy with the program, but ultimately appreciated the program benefits.

CONCLUSIONS: In this formative study of a novel insurance-incentivized Internet-mediated walking program for obese adults, we found high rates of adoption, adherence, and satisfaction. Among adherent participants, physical activity rates increased significantly over a four-month period, suggesting that such programs - when implemented in the population - have the potential to affect widespread change in physical activity levels. Additional evaluations should explore the long-term impact of these programs on individual and population-level health, utilization, and costs.

WEIGHT CHANGES IN THE TRANSITION TO ADULTHOOD: RESULTS FROM NHANES Arlene E. Chung ${ }^{1,2}$; Asheley C. Skinner ${ }^{2}$. ${ }^{1}$ University of North Carolina at Chapel Hill School of Medicine, Chapel Hill, NC; ${ }^{2}$ University of North Carolina at Chapel Hill School of Medicine, Chapel Hill, NC. (Control ID \#1334638)

BACKGROUND: Although adolescents who are obese are more likely to remain so into adulthood, little is known about the amount of weight loss and gain by BMI status in adolescents during the years of transition from adolescence to young adulthood. Our objective was to examine weight loss and gain by BMI status and sex from adolescence to young adulthood in a nationally representative sample of older US adolescents.

METHODS: We examined 16-21 year olds from the National Health and Nutrition Examination Survey from 1999-2008 ( $\mathrm{n}=5,827)$. We calculated weight loss or gain based on self-reported current weight and weight from one year ago. Measured height and weight was used to categorize individuals as overweight/obese, healthy weight, or underweight, using CDC's age-for-sex BMI percentile for those aged 16-20 and BMI in $\mathrm{kg} / \mathrm{m} 2$ for those 21+. We used chi-square tests and t-tests to examine differences in any weight loss or gain and average amount of weight change by weight status, stratified by sex.

RESULTS: $61 \%$ of the participants were Caucasian, $15 \%$ African American, $18 \%$ Hispanic, and $6 \%$ other race/ethnicity. $27 \%$ had family income below the federal poverty level. The sample had $45 \%$ that were overweight/obese, $51 \%$ healthy weight, and $4 \%$ underweight. $21 \%$ of participants reported having any weight loss in the previous year $(44 \%$ male); $56 \%$ reported weight gain over the past year (54\% male). The average loss was 16 pounds (lbs) and average gain was $14 \mathrm{lbs}$. Overall, females were significantly more likely to report weight loss $(\mathrm{p}<0.01)$ and less likely to report weight gain $(\mathrm{p}<0.01)$, compared to males. Among overweight/obese females, $25 \%$ reported weight loss in the past year vs. $21 \%$ of healthy weight females $(\mathrm{p}<0.05)$; any weight gain did not differ by BMI. Among overweight/obese males, $24 \%$ reported weight loss in the last year vs. $13 \%$ of healthy weight males $(\mathrm{p}<0.001)$. However, $54 \%$ of overweight/obese males reported gain in the last year vs. $67 \%$ of healthy weight males $(\mathrm{p}<0.001)$. Among those who lost any weight, overweight/ obese females lost more than healthy weight females (19 vs. $10 \mathrm{lbs}, \mathrm{p}<$ $0.001)$, with a similar finding for males ( $22 \mathrm{vs} .13 \mathrm{lbs}, \mathrm{p}<0.001)$. However, when there was any weight gain, obese females gained more than healthy weight females ( 20 vs. $8 \mathrm{lbs}, \mathrm{p}<0.001)$. This was also similar for males $(19$ vs. $13 \mathrm{lbs}, \mathrm{p}<0.001$ )

CONCLUSIONS: Although some fluctuation of weight in adolescence could be normal, the greater gain among those who are overweight or obese is concerning. Significant changes in weight occur during the period of transition from adolescence to adulthood with more than half reporting weight gain in the past year, and greater gain among those who are obese or overweight. Primary care physicians should counsel their patients about healthy lifestyles to prevent weight gain among both healthy and overweight/obese patients during the transition to adulthood.

WEIGHT GAIN AMONG FORMER WORLD TRADE CENTER RESCUE AND RECOVERY WORKERS AND VOLUNTEERS Camille Napier ${ }^{1,2}$; Rafael E. de la Hoz ${ }^{1,2}$; Ositadinma L. Mbadugha ${ }^{1}$. ${ }^{1}$ Mount Sinai School of Medicine, New York, NY; ${ }^{2}$ Mount Sinai School of Medicine, New York, NY. (Control ID \#1339090)

BACKGROUND: Despite a growing national awareness, obesity continues to rise in prevalence in the United States. Studies among former World Trade Center (WTC) workers and volunteers described a high prevalence of overweight and obesity, and identified obesity markers, and weight gain during surveillance as risk factors for accelerated expiratory flow decline. We conducted a descriptive survey to identify risk factors for obesity in this patient population, including occupational WTC related exposures, employment and disability status, as well as dietary and exercise-related risk factors.

METHODS: 223 patients from the Mount Sinai WTC Treatment Program were sequentially invited to participate in a 10-minute survey to assess their occupational WTC exposures, employment and disability status, medical comorbidities, dietary and exercise habits, as well as height and weight. Body mass index was utilized to determine the obesity (BMI $\geq$ $30 \mathrm{~kg} / \mathrm{m} 2$ ) status of the subjects, and univariate and bivariate analyses ( $\mathrm{t}$ and Chi square tests, as appropriate) were used to explore its association with risk factors.

RESULTS: As expected from WTC worker cohorts, the population showed a substantial predominance of the male sex, with an average age of 51 years (SD 9.4 years), and obesity was highly prevalent (Table 1). We did not detect an association between obesity and WTC exposure variables (early arrival or prolonged stay at the WTC disaster site), disability status, or with self-reported depression/ anxiety. Employed individuals (whether full- or part-time, 99/223) were more likely to be obese $(51.9 \%$ vs. $37.4 \%, p=0.03)$. Obese individuals were more likely to report less weekly exercise (3.75 vs. 4.5 times/week, $\mathrm{p}=0.03$ ), and drinking more glasses of soda daily ( $0.84 \mathrm{vs}$. $0.55, \mathrm{p}=0.04$ ).

CONCLUSIONS: Obesity is highly prevalent among former WTC workers and volunteers, and we detected associations of obesity with active employment, decreased weekly exercise, and increased daily consumption of soda drinks. These associations deserve further exploration, and may guide future interventions to prevent obesity related comorbidities, including accelerated lung function decline in this patient population. 
Characteristics of study population

\begin{tabular}{lll}
\hline \hline $\begin{array}{l}\text { Male sex } \\
\text { Ethnicity }\end{array}$ & 152 & $68.2 \%$ \\
Latinos & 116 & \\
Caucasian & 81 & $52.0 \%$ \\
African American & 23 & $36.3 \%$ \\
Asian & 3 & $10.3 \%$ \\
WTC exposures & & $1.3 \%$ \\
Arrival 48 hr & 130 & \\
Exposure $\geq 60$ d & 163 & $58.3 \%$ \\
WTC occupation & & $73.1 \%$ \\
Law enforcement & 35 & \\
Laborer & 113 & $58.3 \%$ \\
Construction & 16 & $50.7 \%$ \\
Other city employee & 14 & $7.2 \%$ \\
Firefighter & 5 & $6.3 \%$ \\
Ironworker & 5 & $2.2 \%$ \\
Volunteers & 15 & $2.2 \%$ \\
Presently employed & 99 & $6.7 \%$ \\
Overweight & 80 & $44.4 \%$ \\
Obesity & 108 & $35.9 \%$ \\
\hline
\end{tabular}

WHAT CAN I DO? RECOMMENDATIONS FOR RESPONDING TO ISSUES IDENTIFIED BY PATIENT-REPORTED OUTCOMES ASSESSMENTS USED IN CLINICAL PRACTICE Claire F. Snyder ${ }^{4}$; Elizabeth F. Hughes ${ }^{1}$; Albert W. $\mathrm{Wu}^{2}$; Michael A. Carducci ${ }^{3}$. ${ }^{1}$ Johns Hopkins School of Nursing, Baltimore, MD; ${ }^{2}$ Johns Hopkins Bloomberg School of Public Health, Baltimore, MD; ${ }^{3}$ Sidney Kimmel Comprehensive Cancer Center at Johns Hopkins, Baltimore, MD; ${ }^{4}$ Johns Hopkins School of Medicine, Baltimore, MD. (Control ID \#1315114)

BACKGROUND: There is growing interest in using patient-reported outcome (PRO) assessments in clinical practice to improve patient management. The use of PROs in clinical practice involves patients completing questionnaires and feeding the individual's results to the patient's clinician(s) to help them monitor the patient's progress and manage issues in functioning and well-being. We have developed the PatientViewpoint website (www.patientviewpoint.org) which allows clinicians to assign, and patients to complete, PRO questionnaires at regular intervals. The results are available to the clinicians through the PatientViewpoint website and are linked to the electronic medical record. Based on previous research and preliminary testing of PatientViewpoint in breast and prostate cancer patients, providing clinicians guidance on how to address issues identified by the PRO questionnaires can facilitate the intervention's effectiveness. In this project, we conducted a multi-stage multi-disciplinary process to develop consensus suggestions for responding to potential problems identified by PRO questionnaires.

METHODS: We investigated 20 domains commonly covered in PRO questionnaires: anxiety, constipation, appetite loss, depressed mood, diarrhea, dyspnea, fatigue, nausea and vomiting, pain, insomnia, cognitive function, emotional function, financial problems, physical function, role function, social function, sexual function, overall quality of life, patient care \& support needs, and health system \& information needs. We first searched the MEDLINE database and key palliative care textbooks to summarize recommendations for addressing each of the 20 issues. We then held one-on-one interviews with experts from a variety of disciplines: internal medicine, palliative care, cancer outcomes research, medical oncology (breast and prostate), radiation oncology (breast and prostate), social work, psychiatric liaison nursing, triage nursing, clergy, and patient advocates (breast and prostate cancer). The results from the one-on-one interviews were combined with the literature recommendations to develop draft consensus statements for each issue. Finally, we held a panel meeting attended by all the experts where the draft consensus statements were reviewed and modified to develop the final consensus recommendations. RESULTS: Consensus statements were developed for each of the 20 domains. Both the literature review and the expert input supported the importance of first determining the nature of the issue, so all recommendations begin with an assessment and evaluation of the problem (e.g., its history, acute versus chronic nature, impact on patient quality-of-life). The consensus recommendations also included a range of suggestions that incorporated the expert panel members' various perspectives, ranging from medication adjustments (e.g., prescribing anti-emetics) to lifestyle modifications (e.g., addressing sleep hygiene) to referrals to other disciplines (e.g., social work).

CONCLUSIONS: Clinicians presented with their patients' PRO assessments may fail to act on them because they are uncertain about the most effective action to take. We developed consensus guidelines clinicians can access by clicking on a link "What can I do?" when reviewing patients' PRO results using our PatientViewpoint web system. While the initial pilot-testing has been done in cancer patients, the intervention is designed to be applicable across settings.

WHAT DIABETES EDUCATION IS DISCUSSED IN PRIMARY CARE VISITS? Kavitha Srighanthan ${ }^{1}$; Paulette A. Sage ${ }^{2}$; Adam T. Perzynski ${ }^{1}$; Kurt Stange ${ }^{3}$; Denise Kaiser ${ }^{1}$; Shari Bolen ${ }^{1,4} .{ }^{1}$ MetroHealth Medical Center/Case Western Reserve University, Cleveland, $\mathrm{OH} ;{ }^{2} \mathrm{Case}$ Western Reserve University, Cleveland, $\mathrm{OH} ;{ }^{3}$ Case Western Reserve University, Cleveland, $\mathrm{OH} ;{ }^{4}$ Case Western Reserve University, Cleveland, OH. (Control ID \#1335620)

BACKGROUND: Although diabetes education improves clinical outcomes, less is known regarding what diabetes education is given by primary care providers during patient visits with under-served populations and whether the education is consistent with diabetes education standards. As new health care delivery models are created, this knowledge will help health care providers incorporate diabetes education within primary care practices for under-served groups.

METHODS: We observed, audio-taped, and transcribed doctor-patient visits for 10 adults with type 2 diabetes from four different primary care physicians with high quality of care scores for diabetic patients at an underserved clinic. We conducted a qualitative analysis, purposefully coding for the presence of educational messages. The messages were coded and categorized into specific groups based on the American Diabetes Association's National Standards for Diabetes Self-Management Education. The groups are as follows: description of the diabetes disease process and treatment options; incorporating nutritional management into lifestyle; incorporating physical activity into lifestyle; using medication safely and effectively; monitoring blood glucose; preventing, detecting and treating acute and chronic complications; developing personal strategies to address psychosocial issues and concerns as well as promote health and behavioral change. The educational messages were compared with 'Type 2 Diabetes Basics', a textbook used widely as the basis for teaching by diabetic educators in group sessions. We also assessed whether the physician or patient brought up the education and whether or not a follow up plan was developed.

RESULTS: In a mainly African American (90\%) population with fair control of intermediate outcomes (HbA1c $7.5 \%$, BP $134 / 81 \mathrm{mmHg}$, and LDL $100 \mathrm{mg} / \mathrm{dl}$ ), the mean total education issues discussed in a scheduled 20 minute primary care visit was 8 (range 4 to 17). The majority of the 79 education issues covered diabetes-related topics $(77 \%)$, and most educational points were brought up by the physician $(72 \%)$. Educational topics covered the full range of ADA recommended national educational standards. The two diabetes education topics brought up most frequently by providers were preventing, detecting and treating chronic complications $(27 \%)$ and monitoring blood glucose (22\%). Similarly, the two educational topics most frequently brought up by patients were preventing, detecting, and treating chronic complications (58\%) and using medication safely and for maximum therapeutic effectiveness (33\%). Fifteen percent of educational messages were not in the diabetes handbook used for group sessions due to the individualized message, three percent were inconsistent with the diabetes handbook, and the remaining $83 \%$ were either consistent with the handbook or not applicable. The majority of educational topics (57\%) had follow up plans.

CONCLUSIONS: Diabetes education is common and individualized at primary care visits with diabetic patients. While doctors and patients in our sample routinely brought up a wide range of educational issues, the issues most often discussed were those in areas closest to provider expertise and 
training. Continuing integration of diabetes educators in primary care would further support provider education on a wide range of diabetes issues, and would enhance the education on self management topics that are covered less frequently at the primary care visit.

\section{WHAT DOES MY MAMMOGRAM RESULT MEAN? DEVELOPMENT} OF A TOOL TO IMPROVE BLACK WOMEN'S KNOWLEDGE ABOUT SCREENING MAMMOGRAM OUTCOMES. Erin N. Marcus. ${ }^{1}$ University of Miami Miller School of Medicine, Miami, FL; ${ }^{2}$ University of Miami Miller School of Medicine, Miami, FL. (Control ID \#1330386)

BACKGROUND: Effective communication of results is an important component of efforts to improve follow up after an abnormal mammogram. Federal law requires centers send every woman a letter explaining her results, but recent analyses suggest these letters may be written at a level too difficult for much of the U.S. population to understand. Research has found that many women incorrectly state their mammogram results when surveyed. New tools are needed to improve women's understanding of their results and follow up plan. The objectives of this project are to develop, refine, and pilot test a culturally targeted, literacy appropriate informational brochure explaining what happens after a routine screening mammogram, in order to improve knowledge of how common it is to have an abnormal test and why it is important to keep a follow-up appointment. We targeted our intervention to urban black English-speaking women because they are at increased risk of presenting with advanced stage breast cancer at time of diagnosis.

METHODS: Using principles of community-based participatory research, we conducted a qualitative study of black women recruited from the community in inner city Miami. 6 focus groups were convened and the discussion was audiorecorded and transcribed. The initial focus groups have been described previously and discussed the women's preferences regarding how mammogram centers communicate results while eliciting advice regarding the development of materials to improve this communication. The final 2 focus groups discussed women's preferences regarding the refinement of these materials. The investigators analyzed the transcriptions using an immersion and crystallization approach. A pilot study to evaluate the brochure's efficacy in improving followup and understanding of results among low income black, English-speaking women with a BIRADS 0 (incomplete, requiring additional follow up) mammogram center is now being conducted.

RESULTS: Focus group members stated that the brochure's incorporation of a 1800 informational number and its messages "don't be afraid to come back" and "you are not alone" were its most important features. They found icon arrays and pie charts confusing and preferred risk be explained through the use of absolute numbers stated simply. They were receptive to the incorporation of testimonials as well as photographs of smiling realistic-appearing minority women of all age groups . They were unenthusiastic about photos of women who appeared too "corporate."

CONCLUSIONS: Women in our groups were receptive to culturally targeted photographs and testimonials and clear, uncomplicated messages regarding breast cancer risk and the importance of follow up after an abnormal mammogram. Including clear "next-step" information and telephone numbers to call is important when creating educational materials about mammogram outcomes. Additional study is needed to assess the effectiveness of these materials in improving mammogram follow-up among vulnerable populations.

WHAT IS ON THE HORIZON?: RESIDENTS FORECAST THE CONSEQUENCES OF THE LATEST DUTY HOUR REFORM Elizabeth A. Paesch ${ }^{1}$; Furman S. McDonald ${ }^{2}$; Lisa L. Willett ${ }^{3}$; Saima Chaudhry $^{4}$; Karen Chacko ${ }^{5}$; Andrew J. Halvorsen ${ }^{6}$; Vineet Arora ${ }^{1} .{ }^{1}$ University of Chicago, Chicago, IL; ${ }^{2}$ Mayo Clinic, Rochester, MN; ${ }^{3}$ University of Alabama at Birmingham, Birmingham, AL; ${ }^{4}$ North Shore-Long Island Jewish Health System, Manhasset, NY; ${ }^{5}$ University of Colorado Denver, Aurora, CO; ${ }^{6}$ Mayo Clinic, Rochester, MN. (Control ID \#1340453)

BACKGROUND: Recent single-center and small multi-center studies have shown that residents believe duty hour reform brings positive and negative consequences. Interestingly, junior residents have been less concerned than senior residents about possible negative consequences of duty hour restrictions. However, it is not known if the differences of opinion between residents at different training levels seen in these studies hold true for all U.S. internal medicine residents. Nor is it known if differences of opinion exist among residents who are not alike in other respects such as IMG status.

METHODS: Data from the Internal Medicine In-Training Examination 2010 Residents Questionnaire was analyzed. The exam and questionnaire were administered to approximately $75 \%$ of the categorical and primary-care internal medicine residents in the United States in 2010-2011. PGY levels, IMG status, and attitudes regarding the new duty hour regulations were extracted from the database. For example, residents were asked "What do you think the consequences of the new regulations will be on resident ownership of patients?" Their response options were "uncertain, decrease, stay the same, and increase." Descriptive and inferential statistics including the chi-squared test were used to describe the data and look for inter-group differences.

RESULTS: 16,205 residents were included in the study of whom $36 \%$ were PGY-2 s, 33\% were PGY-3 s, 52\% were USMGs, and $45 \%$ were female. Residents were concerned duty hour restrictions would worsen resident ownership of patients ( $41 \%$ thought it would worsen vs. $10 \%$ thought it would improve), autonomy ( $24 \%$ vs. $10 \%)$, clinical experience ( $40 \%$ vs. $13 \%$ ), quality of educational experience ( $27 \%$ vs. $25 \%)$, quality of handoffs ( $29 \%$ vs. $23 \%)$, errors due to handoffs ( $34 \%$ vs. $24 \%$ ), and faculty morale $(20 \%$ vs. $15 \%)$. Residents were hopeful duty hour restrictions would improve resident fatigue ( $58 \%$ thought it would improve vs. $6 \%$ thought it would worsen), work intensity ( $41 \%$ vs. $16 \%)$, time for education ( $31 \%$ vs. $25 \%)$, time for electives ( $33 \%$ vs. $20 \%$ ), time for ambulatory rotations ( $21 \%$ vs. $15 \%)$, patient safety ( $36 \%$ vs. $15 \%)$, errors due to fatigue ( $56 \%$ vs. $4 \%$ ), errors due to workload ( $47 \%$ vs. $7 \%$ ), and resident morale ( $34 \%$ vs. $12 \%$ ). PGY- 2 s and PGY-3 s were more worried than PGY-1 s about the possible negative consequences of the new duty hour restrictions (PGY-2 ORs=1.38-1.68, PGY-3 ORs=1.38-2.02, all $\mathrm{p}<.0001)$. As well, USMGs were more concerned than IMGs about potential consequences of duty hour reform (USMG ORs $=2.85-3.68$, all $\mathrm{p}<.0001$ ).

CONCLUSIONS: This observational study demonstrates that residents anticipate both positive and negative consequences from the new duty hour regulations. Interns are less worried than junior and senior residents about the negative consequences new duty hour reform might bring; and IMGs are less concerned than USMGs. Residents may be more worried than interns because they have some experience in the system, so they may either fear change or have more insight into the possible consequences of change. IMGs may be less concerned than USMGs because they have foreign clinical experience which shapes their perception of the consequences of the changes, and they strongly desire to fit into the U.S. system. Future research should examine these and similar hypotheses as well as track the opinion of residents via-à-vis duty hour restrictions after more have trained only under the new requirements.

WHAT'S COST GOT TO DO WITH IT? CHARACTERISTICS OF 'HIGH COST' USERS OF HEALTH CARE AND THE OVERLAP BETWEEN COSTS, HOSPITAL ADMISSIONS, AND EMERGENCY DEPARTMENT VISITS Oanh K. Nguyen; Ning Tang; John M. Hillman; Ralph Gonzales. University of California San Francisco, San Francisco, CA. (Control ID \#1340176)

BACKGROUND: Care management programs targeted at 'high cost' individuals, or those deemed at risk for becoming high cost - those with frequent hospital admissions or readmissions, emergency department (ED) visits or outpatient visits - are increasingly common among health systems seeking to reduce costs. Among 'high cost' individuals, it is unclear what proportion of costs are potentially responsive to care management and to what extent 'high cost' individuals are also frequent users of the hospital and ED. We examined a population of 'high cost' patients within a university-based primary care population to explore the extent of overlap between cost, admissions, readmissions and ED visits.

METHODS: We analyzed hospital cost and use metrics from July 12010 to June 302011 for all adults $\geq 18$ years old with a primary care provider (PCP) at the University of California San Francisco (UCSF) with at least one hospital admission at UCSF $(n=2,727)$. We defined cutoffs for the top $10 \%$ of individuals by hospital costs $(>\$ 63,239, n=273)$ and hospital admissions $(\geq 3$, $\mathrm{n}=251$ ). Demographic characteristics between groups were compared using McNemar's chi-square and two-way analysis of variance. Hospital costs (excluding physician fees), admissions, emergency department visits and 
medical diagnosis groups as coded by Centers for Medicare \& Medicaid Services Diagnosis Related Groups (CMS-DRGs) were obtained from administrative billing data. To identify admissions for the top 3 CMS core conditions, we used CMS-DRGs 089, 090, 121, 122, 123 and 127 to identify admissions for pneumonia, myocardial infarction and heart failure.

RESULTS: 'High cost' individuals represented $10 \%$ of the study population but accounted for $46 \%$ of hospital costs and $22 \%$ of hospital admissions. Compared to those who were not 'high cost', they were more likely to be male $(49 \%$ vs. $36 \%$, p $<0.001)$, African-American $(24 \%$ vs. $14 \%$, p $<0.001)$ and have Medicare or Medicaid as a primary payor $(77 \%$ vs. $57 \%, \mathrm{p}<0.001)$. Fewer than half (48\%) of high cost individuals were also within the top $10 \%$ by admissions. Those who met cost criteria alone had an increased length of hospital stay ( $15.6 \pm 17.3$ vs. $8.35 \pm 6.0$ days, $\mathrm{p}<0.001)$; greater average costs per admission $(\$ 72,666 \pm 64,942$ vs. $\$ 30,183 \pm 36,432)$; and a smaller proportion of readmissions ( $16 \%$ vs. $47 \%, \mathrm{p}<0.001)$ compared to those who met both cost and admissions criteria. Additionally, those who met cost criteria alone had a greater proportion of admissions for surgical procedures $(56 \%$ vs. $28 \%, \mathrm{p}<0.001)$ and had a lower proportion of admissions for a CMS core condition $(3 \%$ vs. $6 \%, \mathrm{p}=0.07)$ compared to those who met both cost and admissions criteria. Fewer than half $(43 \%)$ of high cost individuals had 1 or more ED visits within the study period. A significantly smaller proportion of those meeting only cost criteria had $\geq 1$ ED visit (35\%) compared to those who met both cost and admissions criteria $(52 \%, \mathrm{p}=0.005)$.

CONCLUSIONS: Defining 'high use' by costs alone may not adequately identify the population with expenditures potentially amenable to intervention, while defining 'high use' by volume of service (admissions and ED visits) alone may inadequately capture costly patients. A multi-faceted definition of high users incorporating both cost and service volume data may more effectively identify high users with preventable costs and use and help to inform more effective strategies to improve the quality of care for these patients.

WHEN DO SUPERVISING PHYSICIANS DECIDE TO ENTRUST RESIDENTS WITH UNSUPERVISED TASKS? A QUALITATIVE STUDY Kevin $\mathrm{Choo}^{1}$; Vineet Arora ${ }^{2,1}$; Paul Barach ${ }^{3}$; Julie K. Johnson ${ }^{4}$; Jeanne M. Farnan ${ }^{2,1}$. ${ }^{1}$ University of Chicago, Chicago, IL; ${ }^{2}$ University of Chicago, Chicago, IL; ${ }^{3}$ University Medical Centre Utrecht, Utrecht, Netherlands; ${ }^{4}$ University of New South Wales, New South Wales, NSW, Australia. (Control ID \#1326394)

BACKGROUND: Attending physicians are often challenged by the decision of when to allow trainees autonomy in procedural tasks and clinical decision-making. Medical educators have struggled to find ways to evaluate trainees and assist faculty in determining trainees' preparedness to independently perform tasks. The aim of this study was to create a conceptual framework to identify factors determining attending and resident perceptions of trust in clinical decision-making.

METHODS: Internal medicine residents and attending physicians were interviewed between January and November 2006, at the conclusion of their Internal Medicine rotation. Participants at a single academic medical center were asked, using the Critical Incident Technique, to describe important entrustment decisions made during their rotation and final call night. Audiotaped interviews lasted on average 45 minutes. All personal and patient data were de-identified during transcription. Interview transcripts were reviewed and analyzed using a deductive approach and the Entrustable Professional Activities (EPA) framework. Data were coded to construct themes of trust, and to identify the factors that promoted, undermined, or otherwise described trust. Two investigators (JMF, KJC) independently reviewed representative portions of the transcripts until consensus was achieved. The inter-rater reliability was calculated using a generalized kappa-statistic $(\mathrm{K})$. The coding scheme was then applied to the entire set of transcripts.

RESULTS: Eighty four percent (42/50) of residents and $80 \%(40 / 50)$ of attending physicians were interviewed. The analysis yielded 535 discrete mentions of trusting factors, which were coded into 35 sub-themes. The inter-rater Kappa for coding between the two raters was high at 0.84 . Four major domains of trust were described, each with specific subthemes: trainee factors (confidence, recognition of limitations, area of specialty/career plans); supervisor factors (approachability, area of clinical expertise, perception of clinical obligations); task factors (urgency/ severity of illness, transitions, level of difficulty, situational characteristics); and, systems factors (workload, duty hours, training philosophy). Supervisors frequently base entrusting decisions on direct observation of trainee performance. Situational factors such as adequacy of support staff and team dynamics were noted to influence the entrustment decisions. Relational factors such as personality characteristics and prior work experience were frequently mentioned. Attendings noted that the career plan or sub-specialty choice influenced their provision of resident autonomy, with those pursuing a subspecialty perceived as more competent and worthy of trust.

CONCLUSIONS: The development of trust is multi-factorial and comprises factors driven by the supervisor, trainee, task and environment. Trust is often driven, despite objective metrics, by subjective conclusions drawn from direct trainee observation. Supervising physicians base decisions on personal characteristics, including honesty, disposition, and self-confidence, which may not correlate with trainee competency. It is important to recognize bias toward sub-specialty bound residents, which may hinder the growth of those planning generalist careers. Future studies should address drivers behind these decisions, correlations with patient outcomes, and tools to enable faculty to justify their entrustment decisions and assess readiness of residents to proceed without supervision.

WHICH WOMEN VETERANS ARE MOST LIKELY TO LEAVE VHA? Sarah A. Friedman ${ }^{1}$; Ciaran S. Phibbs ${ }^{2,1}$; Alison Hamilton ${ }^{3}$; Donna L. Washington ${ }^{3}$; Elizabeth Yano $^{3}$; Patricia M. Hayes ${ }^{4}$; Sally G. Haskell ${ }^{4,5}$; Susan M. Frayne ${ }^{1}{ }^{1}$ VA Palo Alto HSR\&D Center of Excellence, Menlo Park, CA; ${ }^{2}$ VA Palo Alto Health Care System, Menlo Park, CA; ${ }^{3}$ VA Greater Los Angeles HSR\&D Center of Excellence, Sepulveda, CA; ${ }^{4}$ VA Central Office, Washington DC, DC; ${ }^{5}$ VA Connecticut Healthcare System, West Haven, CT. (Control ID \#1339187)

BACKGROUND: Nationally, $30 \%$ of women Veterans new to VHA do not return within 3 years. In a research/clinical partnership, we investigated whether attrition is higher among new women patients, controlling for other factors, and explored factors contributing to their attrition.

METHODS: Data came from VHA enrollment and utilization databases. We examined predictors of attrition (alive but did not return for VHA outpatient care in 2nd-3 rd years after first FY06 visit) using two logistic regression models: Model 1 tests whether, among women Veteran with an FY06 face-toface visit, new users (no outpatient services in three years before first FY06 visit) are as likely to leave VHA as return users, controlling for age, serviceconnected status, receipt of fee-basis care, receipt of inpatient care, and outpatient visit use (overall, primary care, mental health). Model 2 tests whether, among the subset of women new to VHA, these control variables predict attrition. Both models allow for non-linearity in age and visit counts. Only adjusted odds ratios (AORs) significant at $\mathrm{p}<.05$ are reported.

RESULTS: Of the full cohort $(\mathrm{n}=232,491), 11 \%$ had attrition. New women were more likely than returning women to leave VHA ( $30 \%$ vs. $8 \%$ ), even when controlling for other factors (Model 1 new user AOR: 2.8). Model 2 found that among the $13 \%$ who were new, odds of attrition were lower in those 45+ years old (versus 18-44 year olds) (45-64 AOR: 0.72; 65+ AOR: 0.88), and in those with service-connected status (AOR: 0.92), fee-basis use (AOR: 0.84 ), or any primary care visit (AOR: 0.65 ), and higher in those with inpatient use (AOR: 1.26). Also in new users, frequent use in their first year of care was associated with especially low attrition (9+ visits [versus 1 visit] AOR: 0.10 ). CONCLUSIONS: Attrition is higher in new women patients, even after controlling for other factors. Among new women, odds of attrition vary by age: those older than 45 years were most likely to stay, even among Medicare eligible women. Attrition was lower in new women who used primary care during the first year of care. To reduce the loss of new women patients, strategies may include age-relevant services for the youngest new VHA patients, and continued aggressive efforts to assure access to primary care for patients entering the system.

WHO LEAVES AGAINST MEDICAL ADVICE (AMA): PREVALENCE AND PREDICTORS OF LEAVING AMA IN UNITED STATES Siva K. Talluri $^{1}$; Pramod K. Kalagara ${ }^{1}$; Steven J. Pierce ${ }^{2}$; Siddesh $\overline{\text { Besur }^{1} \text {; }}$ Jyosthna Talluri ${ }^{1}$; Anne Dohrenwend ${ }^{1} .{ }^{1}$ McLaren Regional Medical Center, Flint, MI; ${ }^{2}$ Michigan State University, East Lansing, MI. (Control ID \#1336490) 
BACKGROUND: Health care providers are often frustrated when patients decide to self-discharge against medical advice (AMA). Such patients are frequently readmitted within weeks resulting in substantial utilization of financial resources. Our goal was to estimate AMA discharge prevalence and its predictive patient characteristics.

METHODS: We conducted a secondary data analysis of a retrospective patient cohort in the 2008 National Hospital Discharge Survey (NHDS). NHDS is a publicly available, nationally representative sample survey with discharge information from 208 U.S., non-federal, acute care hospitals. All adult patients ( $>19$ years) discharged routinely and those who left AMA were included. We excluded deaths and discharges to short and long term rehabilitation facilities. Categorical demographic characteristics were compared using chi- square tests and the continuous variables with Wilcoxon rank-sum tests. We identified significant $(\mathrm{P}<0.05)$ patient variables by univariate analysis and used logistic regression analysis to identify independent risk predictors.

RESULTS: The NHDS survey included 153,055 patients. Total study patients were 106,714 and included those routinely discharged $(105,004)$ and those leaving AMA $(1,710)$. We excluded 46,341. The prevalence of patients leaving AMA was $1.3 \%$.Patients who leave AMA as compared to those routinely discharged were more likely to be younger $(47+16$ years vs. $53+20$ years), male ( $58 \%$ vs. $38 \%)$, African-American ( $23 \%$ vs. $13 \%)$, not currently married ( $36 \%$ vs. $28 \%$ ) and admitted through the emergency room $(75 \%$ vs. $47 \%)$. They predominantly were either self-pay ( $16 \%$ vs. $6 \%$ ) or had Medicaid insurance ( $25 \%$ vs. $16 \%$ ); had shorter length of stay ( 2.7 vs. 4.1 days) and left AMA within 24 hours of admission. The oddsratios (ORs) and corresponding 95\% confidence intervals (CIs) for patient characteristics that independently predicted leaving AMA are: age less than 60 years $(\mathrm{OR}=3.38, \mathrm{CI}=2.51-4.57)$; male sex $(\mathrm{OR}=2.31, \mathrm{CI}=1.91$ 2.80); African-American race $(\mathrm{OR}=1.33, \mathrm{CI}=1.06-1.66)$; not currently married $(\mathrm{OR}=1.39, \mathrm{CI}=1.05-1.85)$; non-private payment sources of Medicare $(\mathrm{OR}=3.11, \mathrm{CI}=2.31-4.18)$, Medicaid $(\mathrm{OR}=2.82, \mathrm{CI}=2.18-3.65)$, other $(\mathrm{OR}=2.44, \mathrm{CI}=1.59-3.74)$ and self-pay $(\mathrm{OR}=3.82, \mathrm{CI}=2.87-5.09)$. The diseases that were listed as first diagnosis in those who left AMA in order of frequency included alcohol withdrawal, viral meningitis, alcohol dependence, acute pancreatitis and congestive heart failure. CONCLUSIONS: We found that the prevalence of those leaving AMA is low. Young men, African-Americans and those without insurance were more likely to leave AMA and frequently had an alcohol-related diagnosis.

“A LOT OF MEDICINE, OKAY?": GAPS IN COMMUNICATION IN OUTPATIENT CARDIOLOGY CLINIC ENCOUNTERS Anna Neumeier; Eliseo Perez-Stable; Andrea Lopez; Kirsten Bibbins-Domingo; Urmimala Sarkar. University of California, San Francisco, San Francisco, CA. (Control ID \#1338892)
BACKGROUND: Effective patient-physician information exchange is necessary for ambulatory care. Although evidence of sub-optimal communication in primary care abounds, less is known about communication for outpatient sub-specialty visits. Thus, we sought to examine the adequacy of communication needed for self-management in outpatient visits to a cardiology clinic within a safety-net health care system.

METHODS: We reviewed 10 clinical encounters obtained as part of a larger observational communication study of patients cared for in an outpatient county-based cardiology clinic. Data analysis is ongoing. The encounters were audiotaped, transcribed, and de-identified (English, $n=12$; Spanish, $n=14$ ). One investigator read and listened to all recordings and created a coding scheme which was collaboratively revised by the entire team. Then 2 investigators reviewed and applied codes to the transcripts. Differences were resolved by discussion. Thematic saturation was obtained with 10 interviews. All analyses were done using Atlas.ti software. For this analysis, we report on themes relating to medication use, diagnosis, and symptom assessment, which are core aspects of ambulatory chronic disease care. We calculated the Medication Communication Index (MCI), a validated measure of medication communication adequacy, for each visit. MCI ranges from $0-5$, with higher scores indicating better communication.

RESULTS: 10 interviews have been analyzed to date. Nearly half of the interview content was medication-related $(118 / 256)$. The majority of these themes related to medication reconciliation (87/118). There was usually an approach to obtain a comprehensive patient medication list (7/10) with frequent discussion of the medication names (33/87) but often not the directions (13/87), dose $(9 / 87)$ or medication indication $(9 / 87)$. When new medications were introduced, name, directions and indications were mentioned (5/5). Anticipated duration and side effects were omitted. The average MCI score was $2 / 5$. Symptom assessment was variable, with an average of 2 symptoms per visit, but ranged from no symptom discussion $(2 / 10)$ to 11 symptoms (3/10) discussed. Most commonly discussed symptoms included palpitations $(5 / 10)$ and shortness of breath $(5 / 10)$. In 4/10 interviews an assessment of the patient's functional status/New York Heart Association class was obtained. During an encounter, 1-4 patient diagnoses were discussed either explicitly or implied. "the problem is you have a weak heart now due to the heart attack." Most common diagnoses were hypertension and heart failure. Investigators were unable to deduce the diagnosis from the transcript in 4 cases.

CONCLUSIONS: In the outpatient cardiology setting, discussion of medication-related topics occupies the majority of the clinical encounter, yet falls short of recommended communications standards. Symptom assessment and determination of functional status, although critical for provision of anticipatory guidance, is variable and at times absent. Similarly, communication about specific diagnoses was less frequent than expected and may impact patients' understanding of their illness. Overall, our findings imply that communication in outpatient visits does not adequately support patient self-management for cardiac diseases.

\author{
MD: A lot of medicine, okay? I'll write it down ... enalapril, 2.5 and I should say two times. Carvedilol, 12.5, two times. Isordil, 20 milligrams, two times. \\ Hydralazine, 10 milligrams two times a day.

Figure: Medication reconciliation exchange

"I'M TALKING ABOUT PAIN". SICKLE CELL DISEASE PATIENTS WITH EXTREMELY HIGH HOSPITAL USE Danie Weisberg ${ }^{1}$; Gabriela Balf-Soran ${ }^{3}$; William Becker ${ }^{2}$; William Sledge ${ }^{3}$. ${ }^{\text {TY }}$ Yale University School of Medicine, New Haven, CT; ${ }^{2}$ Yale University School of Medicine, New Haven, CT; ${ }^{3}$ Yale University School of Medicine, New Haven, CT. (Control ID \#1339405)

BACKGROUND: Sickle cell disease (SCD) is marked by painful vaso-occlusive crises that may precipitate inpatient admission. Most individuals with SCD manage their pain at home, with sporadic admissions occurring on average 1.5 times per patient year. A small minority of patients with SCD accounts for the great majority of inpatient hospital stays. Admitted as often as several times a month, over successive years, this cohort of patients has not been studied in depth despite their disproportionate contribution to inpatient hospital costs in SCD. The objective of this study is to characterize the subjective experience of extremely high hospital use in patients with $\mathrm{SCD}$, and generate hypotheses about the precipitants and consequences 
of this phenomenon with the ultimate goal of aiding the design of interventions that will improve quality of care.

METHODS: This is a qualitative study involving in-depth, open-ended interviews using a standardized interview guide. Participants consisted of eight individuals of varying age and gender identified through hospital claims data as among the highest hospital use patients with SCD over a three-year period at a single urban academic medical center. The design, interview process, and subsequent analysis were based on the principles of grounded theory for qualitative research. A qualitative review was performed by four members of the research team. The analysis involved creating narrative summaries according to an established process in phenomenological research. These summaries were used to organize emergent themes into an analytic paradigm, and facilitate a return to the interview transcripts in order to catalog data.

RESULTS: A common narrative emerged from the interview transcripts. Participants were exposed to the hospital environment and intravenous opioids at a young age, and this exposure was associated with extremely high hospital use in adulthood evident in descriptions of multiple dimensions of their lives: their upbringing, their history of hospitalization and opioid use, and their personal relationships with peers, caregivers, and family members. Opioid use, due to its reinforcing effects and side effects of therapy, as well as hospitalization itself perpetuated developmental failures that hindered participants' ability to manage SCD-related pain apart from the hospital-based healthcare system and resulted in further hospitalization, lost social and vocational opportunities, and isolation from support structures. Escalating opioid use appeared central to the failure to fulfill social roles, a hallmark of addiction. That this escalation was propagated by clinicians complicates the patient-caregiver relationship.

CONCLUSIONS: Our results suggest a systematic process of alienation from mainstream society, support structures, and caregivers based on increasing hospitalization, growing dependency on opioid medications, as well as missed developmental milestones. Breaking this spiraling cycle may be possible with early interventions, and alternative strategies in pain management, and renewed effort to delineate the pathologies of acute pain, chronic pain, and addiction. Further study should be geared towards formally defining extremely high hospital use in SCD, and quantifying its prevalence. Resources and research focused on this group will likely benefit SCD care at large.

"SHE MAKES ME FEEL LIKE AN ALL-STAR": PATIENTS' EXPERIENCES WITH SELF-MANAGEMENT EDUCATION IN AN INTERVENTION FOR CHRONIC MUSCULOSKELETAL PAIN Marianne S. Matthias ${ }^{1,2}$; Laura J. Myers ${ }^{1,3}$; Edward J. Miech ${ }^{1,2}$; Christy Sargent ${ }^{1}$; Matthew J. Bair ${ }^{1,2}$. ${ }^{1}$ Roudebush VAMC, Indianapolis, IN; ${ }^{2}$ Regenstrief Institute, Inc., Indianapolis, IN; ${ }^{3}$ Indiana University, Indianapolis, IN. (Control ID \#1322172)

BACKGROUND: Chronic pain is prevalent, costly, and exerts a significant burden on both primary care providers (PCPs) and patients. In this study, we elicited patients' experiences following completion of a stepped-care intervention for chronic musculoskeletal pain.

METHODS: We conducted qualitative interviews with patients who participated in the intervention arm of a randomized controlled trial for chronic pain management at a VA Medical Center. Step 1 of the intervention consisted of analgesic treatment coupled with pain self-management strategies, followed by brief cognitive behavioral therapy in Step 2. A nurse care manager delivered all elements of the intervention via telephone. At the end of this trial, we asked patients open-ended questions about their experiences in the RCT. Interviews were audio-taped, transcribed, and checked for accuracy. Sampling continued until theoretical saturation was reached. We used grounded theory and constant comparative methods to analyze the data.

RESULTS: Patients $(\mathrm{N}=26)$ were 24 to 62 years old; four were women; all had moderate to severe chronic musculoskeletal pain. While patients varied in their descriptions of the stepped-care intervention and the selfmanagement education received in the study, they all spoke of the important role played by the nurse care manager. Three themes emerged related to the nurse care manager's role in pain self-management. Theme 1, Finding What Works: Patients appreciated having someone they knew to talk to about different options pain self-management: "The best part is having somebody there to talk to, to go over ideas you have about what works and what doesn't, and get feedback on your progress." (Participant 7) Theme 2, Being Held Accountable: Patients felt accountable to the nurse. They knew she would call them, and they wanted to be able to tell her (truthfully) that they were using their self-management strategies. "It kept me accountable... Usually nobody asks me, 'Are you walking? Stretching?' I don't want to lie to her, so I do it, where normally I'd just do nothing." (Participant 19) Theme 3, Motivation/Emotional Support: For some, the nurse's phone calls provided motivation to continue with their selfmanagement strategies. For others, emotional support was more critical: "When I got off the phone, I felt better. I was more relaxed and I felt that somebody's helping me. You're in a bad spot and somebody cares enough to lend a hand. It was a big deal. It's comforting." (Participant 25) One veteran simply valued the personalized attention: "She makes me feel like I'm an allstar." (Participant 11)

CONCLUSIONS: This study highlights the important role played by a nurse care manager in helping patients self-manage their chronic pain. Specifically, the nurse helped patients find different self-management options, held patients accountable in self-management goals, and provided emotional support and motivation to patients. Incorporating nurses into pain management in primary care may potentially alleviate some of the burden on PCPs caring for patients with chronic pain.

\section{CLINICAL VIGNETTES}

"A PAINFUL JOURNEY" Adam Abraham; Alison R. Landrey; Rachel Swigris. UCH-Denver, Denver, CO. (Control ID \#1339830)

LEARNING OBJECTIVE 1: Identify potential solutions to help minimize inappropriate opiate prescriptions for chronic non-malignant pain (CNMP) in resident clinic.

CASE: From 1996-2011, a 59-year-old female with a history Crohn's disease, polysubstance abuse and chronic abdominal pain presented to our resident clinic 90 times, often because of pain. She was treated with opiates in progressively increasing doses. There was ample evidence that her Crohn's was inactive and not her pain source. There was extensive documentation of both opiate seeking behavior and untoward side effects. Chart review revealed over 26 patient calls for early opiate renewals, 7 reported falls, 4 reports of stolen opiates, and an episode of walking into oncoming traffic. Clinicians did not document clear functional goals, a discussion about stopping opiates or referring her to a treatment program until 2011. In 2011, a review of the Colorado Prescription Drug Monitoring Program database indicated continued outpatient opiate refills during a hospitalization and receipt of opiates from non-University providers. Her clinician stopped prescribing her opiates and offered a methadone clinic referral.

DISCUSSION: This case highlights three system shortcomings which we feel contribute to inappropriate prescribing patterns in resident clinics: (1) lack of care continuity, (2) limited provider knowledge regarding CNMP treatment guidelines and (3) inability to quickly identify red flags during clinic visits. This patient saw over 22 resident and 24 attending physicians, leading to repeated instances where different, relatively inexperienced providers had to decide whether to refill opiates or modify a prior treatment plan. Nearly every time, they continued opiates despite evidence of potential harms. One mitigating strategy would be to have an attending physician, not just supervise, but also share ownership of each patient with CNMP. This would enhance continuity when the resident is out of clinic and upon graduation. The American Academy of Pain Medicine recommends documenting pain intensity, level of functioning, progress toward achieving functional goals and presence of adverse events when prescribing opiates. Tapering patients off opiates who engage in repeated aberrant drug-related behaviors is also advised. In our case, these steps were not taken. We suspect most providers are unfamiliar with these guidelines and believe that enhanced training in them is crucial. Our electronic medical record (EMR) had no easy way for providers to rapidly detect red flags and identify previous opiate misuse. On 5 occasions, clinicians documented that the patient had no evidence of aberrant seeking behavior, despite contradictory evidence, scattered throughout the chart. EMRs should be customized so red flags are present on a single page and accessible with one mouse click. Prescription painkiller overdoses killed more than 15,000 people in 2008 , a rise of over $300 \%$ from 1999 . We 
believe that implementation of the proposed tactics can help training clinics meet our primary obligation, "First, do no harm."

"CRYO"-ING OUT OVER RENAL FAILURE Aarti Ravikumar; Michal Gross; Sheira Schlair. Montefiore Medical Center, New York, NY. (Control ID \#1338994)

LEARNING OBJECTIVE 1: Recognize the types and clinical manifestations of cryoglobulinemia

LEARNING OBJECTIVE 2: Recognize causes of renal failure in a patient with chronic lymphocytic leukemia

CASE: 47 year-old male presented with lower extremity edema, progressive dyspnea and decreased urinary output for two weeks. The patient had a history of extranodal marginal B cell lymphoma and chronic lymphocytic leukemia (CLL) on bone marrow biopsy, diagnosed in 2009, for which he was followed regularly by an oncologist and had not received any treatment. Physical Exam: Patient was tachypneic and breathing 25 breaths per minute. His lungs and heart were clear. Patient had bilateral lower and unilateral upper extremity edema. He had petechiae on his legs. He had no hepatosplenomegaly or lymphadenopathy. An echocardiogram was normal. Initial lab tests revealed acute renal failure (BUN 99, Cr 2.2) along with progressive oliguria that eventually required hemodialysis. Urinalysis revealed $100 \mathrm{mg} / \mathrm{dl}$ protein and large blood. He was found to a white blood cell count of 13,700 (88\% granulocytes and 6\% lymphocytes). An autoimmune panel was sent, which was positive for low C3 (63) and C4 (3); all other tests were normal. Cryoglobulin screen was positive and $\mathrm{HCV}$ was negative. A bone marrow biopsy was done which was unchanged from the prior done in 2009. Renal biopsy showed membranoproliferative glomerulonephritis (MPGN) type I with IgG-IgM codeposits, consistent with mixed cryoglobulinemia and interstitial deposition of previously diagnosed CLL.

DISCUSSION: Renal failure in CLL is a problem that should be recognized by the internist and trigger a broad differential diagnosis that includes cryoglobulinemia, lymphangitic spread of CLL, and transformation to acute leukemic crises with tumor lysis. Cryoglobulins are immunoglobulins that precipitate out of blood at temperatures lower than $37^{\circ} \mathrm{C}$. This precipitation causes a systemic inflammatory syndrome. Type I is a pure monoclonal IgG or IgM, often associated with an underlying lymphoproliferative disorder. Symptomatic Type 1 causes hyperviscosity symptoms and/or thrombosis. Type II and III are both "mixed cryoglobulinemia." Type II involves polyclonal IgG and monoclonal IgM, and type III involves polyclonal IgG and IgM. Mixed cryoglobulinemia (MC) tends to be associated with various infectious (especially $\mathrm{HCV}$ ), immunological and neoplastic disorders. There are no universal diagnostic criteria, but the triad of circulating cryoglobulins, low $\mathrm{C} 4$ and skin purpura are the hallmarks of MC, and biopsy of suspected tissue is the best diagnostic method. The typical pathological finding of $\mathrm{MC}$ is a leukocytoclastic vasculitis of the small and medium vessels. Mean survival is $50-60 \%$ at 10 years after diagnosis, but those with renal involvement have a worse prognosis. Nearly $10-27 \%$ of cases of MC have renal involvement. The most common renal manifestation of MC is type I membranoproliferative glomerulonephritis (MPGN), which accounts for $80 \%$ of cases. In cases of MPGN type I secondary to malignancy, the treatment consists typically of immunosuppression with steroids and cytotoxic agents, with or without plasmapheresis. Renal failure is a common finding, and for those with an underlying malignancy, cryoglobulinemia should be considered in the differential even if the malignancy does not appear to be active.

"PACEMAKERS ARE FOR LIVING - NOT FOR FIDDLING!" Tatyana Der; Venkataramanan Gangadharan; Vijay Ramu. ETSU, Quillen College of Medicine, Johnson City, TN. (Control ID \#1275983)

LEARNING OBJECTIVE 1: - Recognise Twiddler syndrome and its importance

LEARNING OBJECTIVE 2: - Management of Twiddler syndrome

CASE: Mrs. X is a functional 94 year old woman admitted with complaints of chest pain, few days in duration. She had a past medical history significant for complete heart block with permanent pacemaker placement 7 years ago, hypertension, gout and osteoporosis. Physical exam revealed an elderly woman with moderate build with normal controlled vital signs. Patient was worked up for possible acute coronary syndrome by obtaining cardiac isoenzymes, troponin $\mathrm{T}$, an $\mathrm{EKG}$ and routine chest $\mathrm{x}$-ray. Lab work was essentially benign as was her EKG revealing a ventricular paced rhythm. Routine chest $\mathrm{x}$-ray, however, revealed significant twisting and descent of her pacemaker device since its initial implantation. Patient herself denied any manipulation of the device either voluntarily or involuntarily. The device had no specific abnormalities on interrogation. Since the patient was pacemaker dependent, elective revision was performed and the device was re-implanted by creating a new pocket superior and lateral to the previous pocket, and device was sutured to the fascia with more secure knotting. The leads weren't extracted but moved and reattached to the new generator. Follow up chest $\mathrm{x}$-ray and device interrogation revealed the corrected position and normal function.

DISCUSSION: Twiddler's syndrome is rare but a potentially dangerous complication of device therapy for arrhythmias. It is important for primary care physicians and cardiologists to be aware of this syndrome since it can be easily prevented. Twiddler's syndrome refers to the intentional or unintentional twisting of the generator within the pacemaker pocket by the patient resulting in either lead dislodgement or fracture without damage to the generator itself. Patients at risk for this condition are usually elderly, obese and often female presumably due to their relaxed subcutaneous tissue that facilitates the rotation. Patients with psychiatric illnesses present an understandable challenge too. Diagnosis is often incidental but could also present as problems on device interrogation such as loss of capture, diaphragmatic stimulation, stimulation of the pectoral muscle. Clinically it can manifest as twitching of the pectoral or abdominal muscles, chest pain, dysrhythmias, syncope or pre-syncope. Treatment proposals include limiting the pocket size or changing its location, use of a Parsonnet pouch or a Dacron patch for the patients at risk, sturdier tethering of the device to the underlying fascia or using abdominal rectus muscle for device implantation. With this case we also hope to advocate that careful patient selection with appropriate counseling on the dangers of generator manipulation is essential before any form of device implantation.

\section{"PANCORONARITIS SYNDROME"; UNCOMMON PRESENTATION} OF A COMMON DISEASE PROCESS Abhishek Biswas ${ }^{1}$; Anisha $\mathrm{Shah}^{2}$; Patricio A. Sanchez Cueva ${ }^{3} .{ }^{1}$ UPMC Mercy, Pittsburgh, PA; ${ }^{2} \mathrm{UPMC}$ Mercy, Pittsburgh, PA; ${ }^{3}$ University of Pittsburgh, Pittsburgh, PA. (Control ID \#1309906)

LEARNING OBJECTIVE 1: Recognize an important cause,clinical setting and pathophysiology of sudden cardiac death.

LEARNING OBJECTIVE 2: Manage all cases of v fib cardiac arrest appropriately with cardiac catheterization even in the absence of classic EKG and clinical findings suggestive of acute occlusive coronary artery disease.

CASE: A 84 year old diabetic lady was recovering after laparoscopic cholecystectomy when she developed chest pain and shortness of breath. She went into ventricular fibrillation, was resuscitated per ACLS protocol and transferred to the $\mathrm{CCU}$ for $\mathrm{TH}$ protocol. EKG on arrival showed normal sinus rhythm with evidence of inferior $\mathrm{Q}$ waves. Troponin was 2.67 at arrival and went up to 552.34 within eight hours. She was immediately cooled to a target of 33 degrees Celsius. Coronary angiogram showed $100 \%$ acute occlusion of the mid LAD and RCA, both of which were stented. An echo showed non dilated LV with akinesis of the septum, lateral and inferior walls with apical clot and an EF of $25-30 \%$. She subsequently developed a pneumothorax, required increasing doses of inotropes and subsequently was made CMO by her family. She passed away after 24 hours.

DISCUSSION: Atherosclerosis is regarded as an inflammatory state. In light of that view, plaque instability should not be regarded as a random "vascular accident", but rather a result of a "pan-coronary" inflammatory process. The phenomenon of simultaneously developing multiple coronary thromboses, although infrequently observed by cardiologists, has been known to pathologists for years. It seems likely that multiple coronary thrombosis often has a rapid and fatal course which may explain its rarity in the clinical setting. A remarkable postmortem study on patients with SCD showed that $38 \%$ of such patients had more than one discontinuous 
segment with thrombosis (multiple coronary thrombi).It is suggested that this pancoronary process of inflammation leads to simultaneous multiple plaque instability and thrombi generation when provoked by other stressors such as post operative states. This case also illustrates the fact that acute multivessel thrombosis is quite common in the post operative state. Unfortunately electrocardiograms are unable to predict acute coronary occlusions in a significant proportion of cardiac arrests.Hence, immediate coronary angiography in all such patients may be feasible in view of the poor predictive value of clinical findings and electrocardiograms in identifying acute coronary occlusion. Multiple simultaneously occurring coronary occlusion is clinically rare,hard to diagnose on the EKG and has a significant mortality rate,hence we must have a high index of suspicion for it in the appropriate clinical setting. TH: therapeutic hypothermia V fib: Ventricular fibrillation SCD: Sudden cardiac death CMO: Comfort measures only

'ROID RAGE Anwar Rizvi; Joshua Pinner; Sander Koyfman. New York Methodist Hospital, Brooklyn, NY. (Control ID \#1320524)

LEARNING OBJECTIVE 1: Corticosteroids are widely used to treat many inflammatory conditions. Along with their numerous beneficial effects, however, come many adverse side effects. One such side effect is corticosteroid-induced psychosis.

CASE: 61 year old male with a history of hepatitis B and hypertension, but with no known psychiatric or substance abuse history, was admitted for shortness of breath. He received methylprednisolone $60 \mathrm{mg}$ every 8 hours for presumed idiopathic pulmonary fibrosis (IPF) with improvement of symptoms. He was discharged on tapering doses of prednisone over 5 days. At home the patient became manic and agitated and was re-admitted. Patient was given haloperidol with good effect and discharged. Patient continued to fluctuate as to mania and required eight readmissions over five months. Lorazepam was added for acute mania and lead to moderate symptom resolution alongside with haloperidol up to $5 \mathrm{mg}$ daily. Patient never fully returned to premorbid cognitive functioning but was sufficiently improved to be placed in the community. Extensive work-up found no independent causes of altered mental status. CT and MRI of the brain were negative for vasculitits and intracranial hemorrhage; arterial blood gas revealed no hypoxia; blood and urine cultures were negative; serum vitamin B12, ceruloplasmin, antibodies for autoimmune disorders or vasculitis, urine toxicology for drug abuse, alcoholrelated studies, and lumbar puncture were all negative or normal. Biopsy showed a "respiratory bronchiolitis-like pattern." Corticosteroids were presumed to be the likely precipitant for the observed mental status changes.

DISCUSSION: Corticosteroids often cause a sense of euphoria; however, many patients develop disturbing psychiatric symptoms. The incidence of steroid induced psychosis in patients treated with prednisone doses $<40 \mathrm{mg} /$ day is $1 / 3 \%$, but is $4.6 \%$ in patients treated with doses of 41 $80 \mathrm{mg} /$ day, and $18.4 \%$ in patients receiving doses above $80 \mathrm{mg} /$ day. Some of these side effects can occur quickly, within days of initiating corticosteroids. Psychosis can occur at any dose, but almost exclusively at doses above $20 \mathrm{mg} /$ day. Approximately $10 \%$ of patients have persistent symptoms despite reduction of corticosteroids. Before the availability of antipsychotic drugs, psychiatric symptoms usually remitted between 14 and 210 days after discontinuing corticosteroids, with up to $80 \%$ of cases remitting by the sixth week. Now, with the use of antipsychotics, symptoms remit in 1-150 days, with a mean recovery at 22 days. Of note, $40 \%$ of patients respond to antipsychotic drugs within 7 days, $55 \%$ recover fully within 14 days, and $>90 \%$ recover within 42 days. However, our patient's psychiatric symptoms took approximately 5 months to remit requiring re-admissions, despite corticosteroid discontinuation and adequate antipsychotics. Corticosteroid-induced psychosis should be one of the earlier adverse effects picked up by physicians today. Although steroidinduced psychosis can easily be treated by discontinuing the corticosteroid and beginning antipsychotic therapy, our patient's course was unusually long. Greater selectiveness when prescribing corticosteroids is advisable because steroid psychosis affects $3-6 \%$ of patients treated with corticosteroids for medical illnesses.
26 YEAR-OLD WOMAN WITH ABDOMINAL PAIN AND XANTHOMAS Rishi Jain; Jason A. Korcak. Montefiore Medical Center, Bronx, NY. (Control ID \#1339922)

LEARNING OBJECTIVE 1: Recognize the pathophysiology and clinical features of hypertriglyceridemia.

LEARNING OBJECTIVE 2: Diagnose and manage hypertriglyceridemiainduced pancreatitis.

CASE: A 26 year-old woman presented with worsening epigastric pain for 2 days. The pain radiated to the back and was associated with nausea, vomiting, and anorexia. The patient had a history of diabetes mellitus type 1 and hypertriglyceridemia. She reported taking long-acting insulin daily, but was not compliant with a low-fat diet and other medications, including simvastatin, fenofibrate, and mealtime insulin. She also recently began an estrogen and progesterone vaginal ring for contraception. Her exam was notable for tachycardia, diffuse abdominal tenderness to palpation, eruptive xanthomas over the elbows and knees, and yellowish discoloration of the palmar creases. Initial laboratory values demonstrated serum sodium $122 \mathrm{meq} / \mathrm{L}$, measured serum osmolality $295 \mathrm{mosm} / \mathrm{kg}$, total cholesterol $967 \mathrm{mg} / \mathrm{dL}$, triglycerides $5456 \mathrm{mg} / \mathrm{dL}$, and lipase $248 \mathrm{U} / \mathrm{L}$. Extensive peripancreatic edema consistent with pancreatitis was present on abdominal CT scan. Further evaluation revealed a VLDL to triglyceride ratio of 0.36 , supporting a diagnosis of familial dysbetalipoproteinemia.

DISCUSSION: While acute pancreatitis is a problem commonly encountered by internists, hypertriglyceridemia-induced pancreatitis presents unique diagnostic and management challenges. Although the exact mechanism is unknown, hypertriglyceridemia-induced pancreatitis likely begins with capillary obstruction by chylomicrons and VLDL. The resultant ischemia promotes pancreatic enzyme release and exposure of triglycerides to lipase, creating proinflammatory free fatty acids and damaging free radicals. There is no threshold triglyceride value for the development of pancreatitis, but levels are typically greater than $1000 \mathrm{mg} /$ $\mathrm{dL}$. It is not uncommon for individuals with hypertriglyceridemia-induced pancreatitis to have an underlying disorder of lipid metabolism. In familial dysbetalipoproteinemia, a deficiency in apolipoprotein E prevents clearance of VLDL and chylomicrons by the liver. Additional factors such as poorly controlled diabetes, high-fat diet, or changes in estrogen, provide a "second hit" to lipid metabolism, raising triglycerides to dangerous levels. Patients with severe hypertriglyceridemia may develop various types of xanthomas due to lipid leakage from the vasculature into surrounding tissues. For instance, lipid deposition in the palmar creases, striae palmaris, is pathognomonic for familial dysbetalipoproteinemia. Patients with hypertriglyceridemia-induced pancreatitis may also present with pseudohyponatremia, as volume displacement by extreme hyperlipidemia causes artificially low measurement of serum sodium. In addition to the typical treatment modalities for acute pancreatitis, a continuous insulin infusion helps to lower triglycerides by increasing lipoprotein catabolism and decreasing lipolysis. Severe, refractory hyperlipidemia may also warrant the use of plasmapheresis to lower triglyceride levels. Moreover, antioxidant therapy, such as vitamin E, may help prevent recurrent pancreatitis in hyperlipidemic patients by reducing free radical activity. Through an understanding of the pathophysiology and clinical features of hypertriglyceridemia, internists can more effectively identify and treat hypertriglyceridemia-induced pancreatitis.

ALCALIGENES XYLOSOXIDANS AS A CAUSE OF PNEUMONIA IN PATIENTS WITH IMPAIRED COUGH REFLEX Sagar S. Mungekar; Daniel Eiras; Benjamin Wertheimer. NYUSoM, New York,

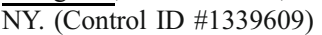

LEARNING OBJECTIVE 1: Recognize the clinical manifestations of $A$. xylosoxidans infections

LEARNING OBJECTIVE 2: Manage patients with complicated pneumonia who are unable to clear secretions

CASE: An 81-year-old woman presented with four days of fever, shortness of breath, and cough. She has a history of cervical and transverse myelitis due to progressive multiple sclerosis resulting in triplegia and dysphagia; she is on steroid maintenance therapy of prednisone 10 milligrams daily. Upon presentation to the emergency room, the patient's oxygen saturation 
was $79 \%$ on room air, which resolved with suctioning and supplemental oxygen. A pulmonary examination was notable for rhonchi heard throughout bilateral lung fields that did not clear with cough. The patient was initially treated for presumed community-acquired pneumonia with azithromycin and ceftriaxone, and was also given metronidazole for anaerobic bacteria coverage given her high risk for aspiration. The patient continued to have daily fevers on this regimen and serial chest roentgenograms demonstrated evolving bibasilar and retrocardiac consolidations. Blood cultures had not shown any growth; cultures of sputum from expectoration were reported as growing normal pharyngeal flora. Videofluoroscopic assessment of swallowing demonstrated significant amounts of penetration into the laryngeal vestibule with thin liquids and an inconsistently elicited cough reflex in response to misdirected material. Culture of a sputum sample retrieved by deep tracheal aspiration revealed innumerable Alcaligenes xylosoxidans subspecies denitrificans resistant to multiple antibiotics including ceftriaxone but sensitive to piperacillin/tazobactam in vitro. Infusion of this antibiotic was begun. The patient continued to have an oxygen requirement and developed hypotension that responded to fluid resuscitation. The patient underwent a therapeutic thoracentesis to improve her respiratory status.

DISCUSSION: A. xylosoxidans is an opportunistic, aerobic, Gram-negative bacillus that causes infections including pneumonia, bacteremia, and meningitis. It has been found to colonize the human gut. Cases of $A$. xylosoxidans infections have been reported often in neutropenic and cystic fibrosis (CF) patients, often nosocomial or as a result of instrumentation. This case of $A$. xylosoxidans pneumonia is unique in that it occurred in a patient on low-dose steroids but who is otherwise immunocompetent and not recently hospitalized. Airway inflammation and chronic pulmonary disease is found in CF patients presumably due to their inability to clear secretions adequately. This patient's bed-bound state and impaired gag and cough reflexes functionally mimic this phenomenon. This case illustrates a severe infection by a rare organism that may be a normal colonizer, but can be pathogenic and is difficult to treat given its resistance to multiple drugs. This patient's risk factors of microaspiration and impaired cough reflex predisposed her to a rapid deterioration. Patients with multiple risk factors for infections and limited ability to clear secretions should be identified early and treated with broad-spectrum antibiotics. In patients with impaired cough reflex, sputum retrieved from deep tracheal aspirates may yield more microbiological culture data than those retrieved from expectoration. Although A.xylosoxidans may be a component of normal oropharyngeal flora, it should be considered pathogenic when recovered from deep tracheal aspirates in a patient with pneumonia.

A 24 YEAR OLD MALE WITH A MYOCARDIAL INFARCTION AND CARDIAC ARREST SECONDARY TO AN UNCOMMON ETIOLOGY Daniel Gutteridge; Kavitha Kesari. McLaren Regional Medical Center, Flint, MI. (Control ID \#1333681)

LEARNING OBJECTIVE 1: Recognize a rare familial cause of myocardial infarction in a non-traditional population.

CASE: A 25-year-old male with no previous medical history presented to emergency medical services after developing typical left sided chest pain radiating to the left arm at rest. The patient had a normal body habitus, no family history of early coronary artery disease and no illicit drug use. The only traditional risk factor present was smoking. Initial electrocardiogram done by emergency medical services showed ventricular tachycardia that led to ventricular fibrillation and cardiac arrest. Upon arrival at the emergency department resuscitation was continued and was converted to atrial fibrillation with ST elevation in anterior lateral leads and depression in inferior leads. Emergent cardiac catheterization showed complete occlusion of the left anterior descending coronary artery requiring intravascular thrombolysis and placement of 2 bare metal stents and temporary intraaortic ballon pump. A hypercoagulable workup was done, showing a positive result for homozygous factor $\mathrm{V}$ Leiden. He recovered and was discharged with life long anticoagulation and genetic counseling. DISCUSSION: Factor V Leiden (Factor V G1691A) mutation is the most common hereditary blood coagulation disorder in the United States. The prevalence of Factor V Leiden for heterozygous carriers is 3-5\% and 1\% of those total carriers are homozygous. Association of resistance to activated protein $\mathrm{C}$ with deep venous thrombosis is well established, where as arterial thrombosis is unknown. The frequency of MI among patients aged less than 45 is rare and is typically associated with atheromatous coronary artery disease, non-atheromatous coronary artery disease, hypercoagulable states and MIs related to substance abuse. There have been case-control studies showing an increased correlation with myocardial infarction at an early age in female patients, 18-44 years that have Factor V Leiden and concomitant tobacco use. Research up to this point has been based on heterozygous mutations due to the rarity of homozygous cases. Individuals with homozygous factor $\mathrm{V}$ Leiden mutation who smoke may be at a significantly increased risk for MI as young adults. In particular homozygous Factor V Leiden individuals are at risk for both arterial and venous embolism, even unprovoked, and will require lifelong anticoagulation when identified. The effect of homozygous mutation on arterial thrombus, especially with concomitant risk factors like smoking, needs to be studied further.

A 26 YEAR-OLD MAN WITH METASTATIC PARAGANGLIOMA. Emily E. Hurstak ${ }^{1}$; Adrienne Green ${ }^{1}$; Aaron B. Neinstein ${ }^{2} .{ }^{1}$ UCSF, San Francisco, CA; ${ }^{2}$ UCSF, San Francisco, CA. (Control ID \#1340075)

LEARNING OBJECTIVE 1: Diagnose catecholamine excess in a patient with a neuroendocrine tumor.

LEARNING OBJECTIVE 2: Management of catecholamine crisis in a critically ill patient

CASE: A 26 year-old man with metastastic paraganglioma presented with shortness of breath. In 2005 he was diagnosed with a spermatic cord tumor, which was resected. In 2009, he presented with low back pain, lower extremity weakness, and facial numbness. An MRI demonstrated vertebral lesions with cord compression and a skull base mass consistent with paraganglioma. $\mathrm{He}$ received radiation therapy, but the disease progressed causing diplopia from a sixth nerve palsy. At the time of presentation, he complained of two months of palpitations, diaphoresis, weight loss, and one week of progressive shortness of breath. He was afebrile, hypertensive, and tachycardic. He had a II/VI systolic murmur and coarse breath sounds. Initially alert and oriented, he rapidly became confused with increased work of breathing and hypoxia requiring intubation. Initial labs included a white count of 17, platelets of 59, fibrinogen 180, anion gap 17, lactate 6, and a troponin 1.2. His EKG demonstrated sinus tachycardia. Chest CTA demonstrated bilateral ground glass opacities with a dense lower lobe consolidation and bilateral pleural effusions. He had diffuse lytic sclerotic rib metastases, a T6 vertebral body compression fracture, and a soft tissue mass within the spinal canal and surrounding the T5-T7 vertebra. A TTE showed moderate depression of left ventricular function with increased LV wall thickness, and global hypokinesis. The patient was felt to have mixed septic and cardiogenic shock. He was started on broad-spectrum antibiotics, placed on the ARDS net protocol, and admitted to the ICU. By the third day of hospitalization the patient was persistently febrile, tachycardic, and hypertensive. Cultures of blood, urine, and respiratory sources had no growth. The patient was started on phenoxybenzamine. On hospital day 6 , he became pulseless and expired after multiple rounds of CPR. The following labs resulted post-mortem: serum free metanephrine $97(\mathrm{nl}<57)$, normetanephrines 58,279 (ULN 412), a chromogranin A level $2600(\mathrm{nl}<15)$ and total metanephrines 58,629 (nl<604). The family declined a post-mortem autopsy.

DISCUSSION: This patient with metastatic paraganglioma died from a catecholamine induced cardiomyopathy and subsequent malignant arrhythmia. Paraganglioma are tumors arising in neuroendocrine tissues. Parasympathetic paragangliomas are typically located in the head and neck and $95 \%$ are non-secretory, while sympathetic paragangliomas originate in the thorax, abdomen and pelvis and are typically secretory. The initial treatment of secretory neuroendocrine tumors involves alpha blockade with secondary addition of low doses of beta blockers for adjunctive blood pressure control. The definitive treatment is surgical. A diagnosis of hereditary paraganglioma syndrome was likely in this case given the clinical history and genetic testing. The nuclear genes SDHD, SDHC, and SDHB encode subunits of the mitochondrial enzyme succinate dehydrogenase; mutations are inherited in an autosomal dominant manner. Detection of these genetic mutations is helpful in guiding surveillance and early detection of neuroendocrine tumors. Catecholamine crisis is rarely encountered on the medical service; rapid diagnosis and treatment are essential for good clinical outcomes. 
A 30 YEAR-OLD MAN WITH HEADACHE AND FEVER Sarah A. Weiss. Montefiore Medical Center, Bronx, NY. (Control ID \#1318368)

LEARNING OBJECTIVE 1: Diagnose tuberculous meningitis. LEARNING OBJECTIVE 2: Recognize cerebrospinal fluid (CSF) characteristics of tuberculous meningitis.

CASE: A 30 year-old Puerto Rican man presented with 5 days of severe headache associated with vomiting, photophobia, neck stiffness, and fevers. He denied head trauma, sick contacts, or upper respiratory symptoms. He worked in construction, recently travelled to the Dominican Republic, and denied toxic habits. Physical exam revealed a temperature of 100.9 F, photophobia, clear lungs, and no focal neurologic deficits. Lumbar puncture (LP) showed: glucose $61 \mathrm{mg} / \mathrm{dl}$, protein $98 \mathrm{mg} / \mathrm{dL}$, and cell count 336 leukocytes $/ \mathrm{mL}$ with $87 \%$ lymphocytes. Empiric treatment with Ceftriaxone, Vancomycin, and Acyclovir was begun and discontinued after bacterial CSF cultures and HSV PCR were negative. Laboratory testing was negative for HSV, VZV, and enterovirus CSF PCR, CSF Cryptococcal antigen, RPR, and HIV. Repeat LP showed a lymphocytic pleocytosis and elevated protein, but now low glucose $(35 \mathrm{mg} / \mathrm{dl})$. Tuberculin skin test resulted in a $10 \mathrm{~mm}$ induration. Empiric treatment for tuberculous meningitis (TBM) was immediately initiated. Initial CSF gram stain and cultures for acid fast bacilli (AFB) were negative. A third LP after starting treatment showed a cell count of 713 leukocytes $/ \mathrm{ml}$ with $55 \%$ neutrophils. MRI Brain revealed basilar meningeal enhancement. Clinical course was significant for waxing and waning mental status and persistent headaches, but no neurologic deficits. The patient was discharged on directly-observed anti-tuberculosis therapy. Mycobacterium tuberculosis was isolated on AFB culture 3 months later.

DISCUSSION: TBM occurs in $1 \%$ of all tuberculosis cases in the U.S. Prompt clinical suspicion of TBM is imperative because of high mortality rates $(20-50 \%)$ and lasting neurologic deficits (20-30\%). Risk factors include young age, HIV-infection, malnutrition, alcoholism, malignancy, and use of immunosuppressive drugs. TBM is caused by Mycobacterium tuberculosis that seeds the meninges and forms tubercles that rupture into the subarachnoid space. Diagnosis of TBM is challenging because of variable presentation and CSF characteristics that may mimic subacute meningitis, cryptococcosis, neurosyphilis, parameningeal infections, and herpes encephalitis. A 1-2 week prodrome of fever, headache, and irritability can progress to cranial nerve palsies, seizures, and eventually coma and death. CSF usually demonstrates a lymphocytic pleocytosis (100-500 leukocytes/mL), low glucose $(<45 \mathrm{mg} / \mathrm{dL})$, and high protein $(100-500 \mathrm{mg} / \mathrm{dL})$. Once treatment is begun, a paradoxical response occurs in which the CSF cell count becomes neutrophilic, due to a hypersensitivity reaction caused by tubercular protein release. Gold standard for diagnosis is identification of AFB in CSF gram stain and culture. AFB are seen on gram stain in only $10-20 \%$ of cases and cultures are slow growing with low sensitivity $(25-70 \%)$. Sensitivity can be increased by examining at least 6 $10 \mathrm{~mL}$ of CSF for 30 minutes by a skilled technician. CT and MRI may show tuberculomas, basilar meningeal exudates, and hydrocephalus, all nonspecific findings. Treatment is 4 drug therapy (rifampin, isoniazid, pyrazinamide, and ethambutol) for 2 months followed by 7-10 months of isoniazid and rifampin. Corticosteroid use remains controversial. Clinical suspicion of TBM requires immediate treatment, even in the absence of definitive diagnostics, given its high morbidity and mortality.

A 47-YEAR OLD WOMAN WITH BILATERAL ANKLE ARTHRITIS Michelle Fox; Brigid M. Dolan. Brigham and Women's Hospital, Boston, MA. (Control ID \#1334642)

LEARNING OBJECTIVE 1: Devise a differential diagnosis for bilateral ankle arthritis

LEARNING OBJECTIVE 2: Describe the classic presentation and diagnosis of Lofgren's syndrome

CASE: A 47-year old African-American female presented to urgent care with 2 weeks of bilateral ankle pain, limiting her ability to walk or wear shoes, and diffuse muscle aches. A trial of non-steroidal anti-inflammatory medication afforded minimal relief. She denied associated fevers, chills, cough, rash, or conjunctivitis. Exam revealed tender, swollen ankles bilaterally without other joint involvement or rashes. Chest x-ray revealed bilateral hilar adenopathy. A diagnosis of Lofgren's sarcoidosis was made, although the patient declined mediastinoscopy for tissue biopsy. The patient was treated with prednisone with significant improvement in her symptoms.

DISCUSSION: Here we present a case of a 47-year old woman with bilateral ankle arthritis and hilar adenopathy, consistent with Lofgren's Sarcoidosis. The CDC estimates that nearly 50 million Americans have arthritis, and as a many $25 \%$ of primary care visits are related to musculoskeletal complaints [1]. Bilateral ankle arthritis is an example of an uncommon presentation and its presence should prompt a chest xray to evaluate for the presence of hilar adenopathy. The presence of bilateral ankle arthritis and hilar adenopathy is consistent with the diagnosis of Lofgren's sarcoidosis[2]. Lofgren's syndrome, first described by Sven Lofgren in 1953, is a variant of sarcoidosis classically described as the constellation of hilar adenopathy, erythema nodusm, and other joint symptoms [3, 4]. More recently, bilateral ankle arthritis has been recognized as a hallmark of this syndrome as well [5]. Some argue that these classic findings are sufficient to seal the diagnosis, even in the absence of tissue biopsy[6]. It is important for clinicians to distinguish Lofgren's sarcoidosis from other forms of sarcoidosis, as Lofgren's has a better prognosis, higher rates of remission, and significantly lower relapse rates [7]. 1. CDC, Arthritis: Meeting the Challenge, in At A Glance, N.C.f.C.D.P.a.H. Promotion, Editor. 2011. 2. Figueira-Coelho, J. and P. Mendonca, Male presenting with bilateral ankle arthritis? Request a chest X-ray! Mod Rheumatol. 20(6): p. 640-2. 3. Lofgren, S., Primary pulmonary sarcoidosis. II. Clinical course and prognosis. Acta Med Scand, 1953. 145(6): p. 465-74. 4. Lofgren, S., Primary pulmonary sarcoidosis. I. Early signs and symptoms. Acta Med Scand, 1953. 145(6): p. 424-31. 5. Mana, J., et al., Periarticular ankle sarcoidosis: a variant of Lofgren's syndrome. J Rheumatol, 1996. 23(5): p. 874-7. 6. Reich, J.M., et al., Mediastinoscopy in patients with presumptive stage I sarcoidosis: a risk/benefit, cost/benefit analysis. Chest, 1998. 113(1): p. 147-53. 7. Mana, J., et al., Lofgren's syndrome revisited: a study of 186 patients. Am J Med, 1999. 107(3): p. 240-5.

A BONNET FULL OF SNAKES Mrunal L. Shah; Zahraa Hajiiri; John Eiser; Chetan Mittal; Raymond Hobbs. Henry Ford Health System, Detroit, MI. (Control ID \#1314346)

LEARNING OBJECTIVE 1: Recognize that visual hallucinations may occur in otherwise normal individuals with extremely poor vision.

LEARNING OBJECTIVE 2: Diagnose the Charles Bonnet Syndrome and realize that failure to make the correct diagnosis can lead to mislabeling the patient as psychotic and result in unnecessary institutionalization.

CASE: A 76 year-old nearly blind woman complained of seeing "snakes and lizards" three days after receiving intravitreal, bevacizumab injections for wet age related macular degeneration. She knew the visions were unreal and could make them disappear by closing her eyes. There was no history of psychiatric illness, alcoholism or drug abuse. Laboratory testing, CT scans and an EEG were normal. She had the Charles Bonnet syndrome, an obscure condition that is unknown to most physicians. Such patients can be erroneously diagnosed with dementia, delirium, psychosis, etc and inappropriately institutionalized. DISCUSSION: The Swiss naturalist, Charles Bonnet, first described the syndrome that bears his name in 1760 when he reported complex visual hallucinations in his 87 year old grandfather who suffered from cataracts. It was relatively unknown in the English literature until 1982. The syndrome occurs in patients with loss of visual acuity or visual field loss. Although $11-15 \%$ of geriatric patients with impaired vision have visual hallucinations many will not report them for fear of being labeled with a psychiatric problem. Current theory suggests that visual sensory deafferentation leads to disinhibition of the visual cortex which then fires spontaneously. The characteristic that makes them different from typical hallucinations is that the patient realizes they are not real. Our patient received monthly bevacizumab injections to help her vision. After each round of therapy she had hallucinations but this time they were more distressing and persisting longer. Her hallucinations paradoxically may have been made worse as her visual acuity improved since the hallucinations may stop when blindness occurs. She was treated with reassurance and distraction therapy. Low dose atypical antipsychotics can be useful as well. Perhaps the most important intervention was explaining that she did not have a psychiatric problem and giving her a card with the diagnosis to present to future doctors who may not be aware of the condition. 
A BUG ON THE BACK: A UNIQUE CASE OF SALMONELLA OSTEOMYELITIS SACHIN MOHAN MD, PHD AND SATYAJEET ROY MD, FACP DEPARTMENT OF INTERNAL MEDICINE, COOPER UNIVERSITY HOSPITAL, CAMDEN, NEW JERSEY 08103, USA Sachin Mohan; Satyajeet Roy. Cooper University Hospital, Camden, NJ. (Control ID \#1334723)

LEARNING OBJECTIVE 1: To recognize that metastatic lesions in Salmonellosis can cause spinal osteomyelitis, presenting as non-specific back/abdominal pain.

LEARNING OBJECTIVE 2: To diagnose and treat osteomyelitis of spine associated with Salmonella

CASE: A 62 year-old male with history of recent overseas travel, presented with fever, fatigue, dry cough, decreased appetite, weight loss, mild pain and increased sensitivity over left upper abdomen for one month. On further questioning, the patient reported an episode of self-limited diarrhea lasting about four days during his stay in India. Labs revealed no leukocytosis or leucopenia. Liver function tests, urine analyses, and tuberculin skin testing were unremarkable. An Ultrasound-Abdomen and a CT-Abdomen/Pelvis with contrast did not reveal any major pathology. Surprisingly, a CT-Chest showed T8-T11 osteomyelitis with para-vertebral extension, and mild-moderate cord compression. Interestingly, both the stool and the spinal-biopsy identified Salmonella typhi that was ampicillin-sensitive but levofloxacin-resistant. Per-sensitivity, empiric coverage with levofloxacin was switched to ceftriaxone and ampicillin, and thoracic spinal abscess drainage, laminectomy, and fusion were done. The patient recovered well and was able to resume all of his activities of daily living.

DISCUSSION: Salmonella are principally motile, Gram-negative, rodshaped Enterobacteria that cause diseases like typhoid fever, paratyphoid fever, and food-borne illness. Salmonella typhi is specific to humans and spreads by contaminated food and water or via a chronic carrier. Though extraintestinal dissemination is widely seen, spinal involvement occurs in only 0.8 $\%$ of Salmonella infections. As a disease entity, Salmonella osteomyelitis comprises only $0.45 \%$ of all types of osteomyelitis. Other than S.typhi, osteomyelitis has also been reported with S.typhimurium, S.panama and S.enteritidis. Though Salmonella osteomyelitis is classically described in patients with sickle cell disease, pre-existing bone disease, and hemoglobinopathies; typhoid osteomyelitis is more commonly seen in patients with liver disease, diabetes, lymphoma, prior surgery, prolonged steroid use and at extremes of age. Usual sites of infection are diaphysial regions of long bones like femur and humerus, with other important sites including lumbar vertebrae, radius, ulna and tibia. While single bone involvement is usual, multiple bone involvement has also been seen. Remarkably, the symptom-free period between the initial infection and osteomyelitis can extend from months to decades. Definitive diagnosis is by culture of blood, urine, stool or marrow along with serology, while anemia, leukocytosis/leucopenia, along with raised ESR in the appropriate context should raise early clinical suspicion. Bone lesions usually show destruction of bone with erosion of diaphysis. In wake of ampicillin and bactrim resistance, ceftriaxone combined with debridement is recommended for severe systemic illness, while ciprofloxacin is used empirically. Here, we report a rare presentation of vertebral typhoid osteomyelitis in an otherwise healthy individual that was successfully treated by surgery and antibiotics. Given the significant morbidity associated, early suspicion for typhoid osteomyelitis in the setting of travel, prolonged continuous fever and diarrhea is critical in successful identification and management of the disease.

A CASE REPORT OF IGA NEPHROPATHY IN ANTI-GLOMERULAR BASEMENT MEMBRANE GLOMERULONEPHRITIS HIroshi Yamaguchi $^{1}$; Hideki Takizawa ${ }^{1}$; Yayoi Ogawa ${ }^{3}$; Christine Kwan ${ }^{2}$; Tamaki Takada ${ }^{1}$; Izumi Yamaji ${ }^{1}$; Nobuyuki Ura ${ }^{2}{ }^{1}$ Teine keijinkai Medical Center, Sapporo, Japan; ${ }^{2}$ Teine Keijinkai Medical Center, Sapporo, Japan; ${ }^{3}$ Hokkaido Renal Pathology Center, Sapporo, Japan. (Control ID \#1340344)

LEARNING OBJECTIVE 1: Recognize that anti-glomerular basement membrane (GBM) glomerulonephritis (GN) can coexist with IgA nephropathy (NP)

CASE: A 46 year-old Japanese man with significant past medical history of well-controlled hypertension, diabetes mellitus, hyperlipidemia, and microscopic hematuria with intermittent proteinuria for 3 years presents with 1 week's history of nausea, vomiting, and lower abdominal pain. $\mathrm{He}$ reports preceding fevers, chills, and malaise while denying dyspnea, hemoptysis, arthralgias, myalgias, and bowel movement changes. $\mathrm{He}$ smokes 5 cigarettes /day for 26 years; his family history is unremarkable. Physical examination shows blood pressure of $134 / 83 \mathrm{mmHg}$, heart rate of 84 beats/minute, normal respiratory rate range, and body temperature of $37.5^{\circ} \mathrm{C}$. No skin lesions, joint tenderness, effusions, or edema has been found; the rest of the examination is unremarkable. Significant laboratory data include hemoglobin of $11.9 \mathrm{~g} / \mathrm{dL}$, white blood cell count of $17,350 /$ $\mathrm{mm} 3$, C-reactive protein of $16.7 \mathrm{mg} / \mathrm{dL}$, blood urea nitrogen of $64.9 \mathrm{mg} /$ $\mathrm{dL}$, creatinine of $5.2 \mathrm{mg} / \mathrm{dL}$ (baseline $0.7 \mathrm{mg} / \mathrm{dL}$ ), IgA level of $640 \mathrm{mg} / \mathrm{dL}$, and anti-GBM antibody level of $214 \mathrm{EU}$. Urinalysis shows proteinuria $(2.98 \mathrm{~g} / \mathrm{gCr})$ and $>100 \mathrm{red}$ blood cells/high power field. Serology for human immunodeficiency virus types 1 and 2, hepatitis B and C, PR3ANCA, MPO-ANCA, cryoglobulin, C3, C4, and CH50 are within the normal range. Computed tomography shows normal sized kidneys and no pulmonary abnormalities. With rising creatinine levels to $6.6 \mathrm{mg} / \mathrm{dL}$, renal biopsy reveals 1 of 16 glomeruli with global sclerosis while the remainder shows cellular crescents. Light microscopy shows fragmented basement membranes; no mesangial proliferation has been observed. The diffuse tubular and interstitial infiltrates are composed of monocytes and neutrophils. In immunofluorescence (IF) microscopy, linear staining for IgG along the GBM is accompanied by granular staining of IgA in the mesangial region. Electron microscopy shows glomeruli's exhibiting wrinkling and irregularity of the GBM. No electron-dense deposits, even in the mesangium, has been observed. The diagnosis, therefore, of antiGBM GN and IgA NP has been established; his renal function, however, does not recover so he undergoes hemodialysis.

DISCUSSION: Rapidly progressive GN (RPGN) is characterized clinically by a rapid progression to end-stage renal disease and histologically by profuse epithelial proliferation often with epithelial crescents. RPGN can be classified into three categories: pauci-immune, anti-GBM, and immune complex. Among them, antiGBM GN can occur in all ages and is pathologically and clinically the severest form of GN. While its coexistence with another disease having an additional distinctive pattern of glomerular IF staining does not occur frequently, concomittent processes of ANCA disease and membranous glomerulopathy have been observed. This case, however, illustrates an even rarer occurrence of having coexisting anti-GBM GN and IgA NP. In crescentic anti-GBM GN, the average creatinine level at the time of diagnosis is higher than any other type of glomerular disease. Given its severity and rapidity, the number of glomeruli free from destruction is very limited. For this reason, the pathological feature of IgA NP may not be observed, even when IgA NP precedes it, thereby possibly causing a lack of case reports describing the combination of anti-GBM GN and IgA NP.

A CASE OF ACUTE COPPER TOXICITY Punal Patel; K. Andhavarapu; P. Shah; N. Menon; J. Feldman. SJHMC, Phoenix, AZ. (Control ID \#1323769)

LEARNING OBJECTIVE 1: Wilson disease, also known as hepatolenticular degeneration, is due to an autosomal recessive defect in copper transportation. Ceruloplasmin represents circulating copper in the plasma. The hepatic production of apoceruloplasmin, ceruloplasmin without copper, causes the low level of serum ceruloplasmin found in patients with Wilson disease. Furthermore, the inappropriate function of the Wilson disease protein, ATP7B, leads to an impairment in biliary copper excretion, which allows copper to accumulate and deposit in the hepatic, neurologic, renal, and ophthalmic systems. Important clinical features of the disease include cirrhosis, acute liver failure, movement disorders, psychosis, and premature osteoporosis.

CASE: 25 year old male with a history of panhypopituitarism s/p removal of a suprasellar mass secondary to langerhan's histiocytosis presented with altered mental status, generalized weakness, and lethargy. Of note, he had discontinued use of his DDAVP for several weeks. On admission he was found to have a serum sodium level of 172 . He was immediately started on pituitary hormone replacement therapy which included, desmopressin, solucortef, and synthroid. Furthermore, he was found to have new onset elevated liver function tests, ammonia, and INR. Initially, his altered mentation was presumed to be a combination of hypernatremia and hepatic 
dysfunction. As a result, he received lactulose and rifaximin. His sodium level improved, however, he remained encephalopathic. His acute liver failure was further evaluated by gastroenterology and hepatology. Diagnostic laboratory tests revealed low ceruloplasmin, high serum copper, and an elevated urinary copper level. He subsequently underwent a liver biopsy which demonstrated portal fibrosis and steatosis stage III-IV, and portal and parenchymal infiltrates stage II-IV, both were consistent with Wilson disease. He was started on pencillamine which led to immediate improvement in his mental status. The ATP7B gene was pending on discharge. He will undergo a slit lamp exam on an outpatient basis. He was discharged home with close gastroenterology and hepatology follow up.

DISCUSSION: Wilson disease should be considered in any individual between ages 3 and 55 with liver abnormalities of uncertain cause, and must be excluded in any patient with unexplained liver disease accompanied by a rapid neurological deterioration. Diagnosis of Wilson dsease is made by a slit lamp examination to identify Kayser-Fleischer rings, which represent deposition of copper in decemet's membrane of the cornea. Biochemical tests demonstrate elevated liver function tests, low levels of ceruloplasmin, and high levels of serum and urinary copper excretion. Increased hepatic parenchymal copper content provides critical diagnostic information and should be obtained in cases where the diagnosis is not straightforward. Histological abnormalities found on liver biopsy include steatosis, hepatocellular necrosis, fibrosis, and cirrhosis. If the diagnosis remains elusive, molecular genetic studies such as the ATP7B mutation can be obtained. Treatment options include D-penicillamine, a chelator that promotes urinary copper excretion, trientine (also a chelator) which is indicated in patients intolerant of pencillamine, and zinc which interferes with uptake of copper from the gastrointestinal tract.

A CASE OF AVASCULAR NECROSIS OF HIP JOINTS Emran Rouf; Maybelline Lezama; Lisa Forrester. Scott and White Healthcare, Temple, TX. (Control ID \#1303096)

LEARNING OBJECTIVE 1: 1) Diagnose avascular necrosis (AVN) of hip when typical risk factors are absent

CASE: A 33-year-old white male presented to our clinic with progressively worsening low back and hip pain for about 6 months. At the onset, he noticed spontaneous low back pain with radicular symptoms, which was then followed by moderate-to-severe bilateral hip pain, left worse than right. He denied any leg weakness, bowel or bladder incontinence. He is a welder and denied any major trauma to his back or hip regions. He had no history of hip dysplasia as a child, nor did he report any surgeries to his back or hip. His medical history is notable for peptic ulcer disease which is treated with a PPI. Although he was not on any chronic steroid therapy, he reported at least two episodes of intermittent systemic steroid therapy in the preceding 12 months, once for a bout of interstitial pneumonitis, and the other for an acute treatment of his low back pain. Primary care physician, neurologist and a neurosurgeon evaluated his pain. MRI of his lumbosacral spine was notable for mild degenerative joint disease, and he was managed conservatively. Two months prior to presentation, he developed progressive worsening of his bilateral hip pain, and at that point he sought medical care at our institution. His physical examination was only remarkable for significantly reduced range of motion at both hip joints, left worse than the right, along with an antalgic gait. X-ray of bilateral hip joints revealed significant avascular necrosis of both hips, although he had more disease on left. An urgent referral to Orthopedics was done. He underwent an uncomplicated left total hip replacement and is doing well since then.

DISCUSSION: Avascular necrosis of bone is a devastating, yet treatable condition that requires prompt diagnosis and often surgical management. Although a definitive pathogenesis is unknown, critical ischemia is thought to be the final etiologic mechanism. Hip joints are affected in most patients, although shoulder, knee and ankle joints can be involved as well. For symptomatic patients, as described in our vignette, a plain X-ray of the affected joint usually yields the right diagnosis. Our case highlights two major learning points: first, when hip pain accompanies lumbosacral radiculopathy, it can be attributed to the lumbosacral pathology as a referred pain, and this type of reasoning may miss a concurrent, potentially severe diagnosis. In such a scenario, physician should be meticulous to determine if hip pain is exceedingly more severe than what a referred pain would suggest. In our case, hip pain was much more severe and disabling which alerted us to think about a possible alternate pathology. Second, in the absence of established risk factors for osteonecrosis, such as chronic steroid use and sickle cell disease, one should consider any past treatment of systemic steroid as a potential risk factor. Case reports of avascular necrosis have been reported after a single corticosteroid injection. Our vignette serves as an example of how careful historytaking and diagnostic reasoning help physicians in clinical problemsolving.

A CASE OF CAPNOCYTOPHAGUS AND THE FUTURE OF IDENTIFYING INFECTIOUS DISEASE PATHOGENS. William J. Rust $^{1}$; Danny Spinuzzi ${ }^{1}$; Robert Volosky ${ }^{1,2} .{ }^{1}$ Allegheny General Hospital, Pittsburgh, PA; ${ }^{2}$ Infectious Disease Associates of Western Pennsylvania, Pittsburgh, PA. (Control ID \#1283611)

LEARNING OBJECTIVE 1: Recognize Capnocytophagus as a potential source of sepsis in those at risk.

LEARNING OBJECTIVE 2: Recognize Ibis T5000 as a game-changing tool in rapidly isolating and identifying pathogens from a broad range of samples.

CASE: A 67-year-old man presented to an outside hospital with altered mental status and acute respiratory failure, with a past history of asplenia secondary to a gunshot wound. The patient's wife reported acute onset of "confusion" on the evening prior, along with several episodes of vomiting, a temperature of 104 degrees Fahrenheit and a non-productive cough. The patient became increasingly short of breath and was evaluated at the outside hospital ER. The patient was febrile at 105.2 and had a white blood count of 16.6. He was pan-cultured and started on vancomycin, ceftriaxone, acyclovir, as well as steroids for presumed meningitis. A lumbar puncture was unable to be performed because of a TENS unit. The patient was intubated and transferred to our MICU. Upon arrival, the patient had cold extremities with multiple petechiae. The tip of his nose was also involved and cultures were redrawn. When checking a blood count, the lab noted "rod-shaped bacteria intra/extra-cellularly." Infectious disease and critical care staff assessment included sepsis (encapsulated organism versus Listeria versus other potential pathogen), as well as DIC, lactic acidosis, and acute kidney injury. On hospital day one, the wife asked if a "dog bite" could be responsible, as the family dog "nipped" at the patient's nose and caused a minor break in the skin on the day prior to evaluation. The differential now included Capnocytophaga Canimorsus, a gram-negative bacillus in the canine flora that requires an oxygen rich environment. The organism is very difficult to isolate in culture. The patient's DIC persisted and his extremity/nose necrosis continued to worsen. His somnolence continued despite sedation discontinuation. The family requested withdrawal. Multiple cultures from our facility were negative, as well as samples sent to outside facilities. The initial blood culture from the outside hospital was later reported as likely Capnocytophagus species. Post-mortem, six of the blood samples were analyzed using state-of-the-art Ibis T5000, which is a universal biosensor that allows one to identify a broad range of pathogens without requiring an anticipated organism. All six of our patient's samples were grossly positive with Capnocytophaga Canimorsus.

DISCUSSION: This vignette highlights the importance of taking a thorough history and identifying Capnocytophagus as a potential source of sepsis in those at risk. Our patient was asplenic and the initial concern was for typical encapsulated organisms, however Capnocytophagus can cause devastating DIC and sepsis in asplenics. Capnocytophagus is very difficult to isolate, requiring an oxygen-rich environment. We were unable to grow the organism from multiple samples. This case introduces Ibis T5000 technology as a potential game-changer in the world of infectious disease and overall patient care. The system incorporates mass spectrometry and PCR technology to identify a broad range of pathogens, including bacteria, fungi, viruses, and protozoa, without requiring the individual to state what they are anticipating. The system can identify pathogens, including their resistance and virulence factors, in less than eight hours. The technology allowed our staff to identify an organism that we were unable to grow in culture. 
A CASE OF DENGUE IN THE COLORADO ROCKY MOUNTAINS Ingrid Lobo. University of Colorado, Denver School of Medicine, Aurora, CO. (Control ID \#1333772)

LEARNING OBJECTIVE 1: Recognize and diagnose Dengue in a non endemic area.

LEARNING OBJECTIVE 2: Manage and treat Dengue.

CASE: 33 year old female of Thai origin presented from an urgent care with 1 day history of bloody diarrhea, heavy menstrual bleeding, and nosebleeds. Preceding this, she complained of a 3 day history of fevers, nausea, vomiting, and headaches. Patient lives in Colorado but had recently traveled to Thailand. At the urgent care, patient was found to be thrombocytopenic and sent to the ER. Her review of systems revealed blurry vision, bruising, and myalgias. She denied confusion, congestion, cough, breathing issues, chest pain or hematuria. She had no past medical history or known bleeding issues. She denied any family history for hematologic disorders. In Thailand, they prescribed her an unknown antiemetic and antibiotic for gastroenteritis 2 weeks prior. She had not taken any other remedies or drugs. She was mainly in Bangkok but did travel to the coast. She was slender in no distress with normal vitals. She was oriented with no focal findings. She had dried blood on her nose. Her exam was otherwise normal. Negatives included no petichiae, rash, murmurs, crackles, hepatosplenomegaly or lymphadenopathy. Pertinent laboratory findings included thrombocytopenia [7 (150-400)] with a normal white count and hemoglobin, abnormal liver function tests (AST $228 \mathrm{U} / \mathrm{L}$, ALT $120 \mathrm{U} / \mathrm{L}$, albumin 3.2, with normal alkaline phosphatase and bilirubin), elevated d-dimer (2210 FEU), elevated PT (26.5 seconds), elevated PTT (41.9 seconds), elevated LDH (636 U/L), and a normal creatinine and fibrinogen. A peripheral smear showed no schistocytes. The patient was transfused platelets and Hematology was consulted. The differential included an autoimmune etiology, infection, TTP, HUS, or a medication reaction. The patient was thought to have low grade DIC. Hematology suggested an Infectious disease consult and prompted us to start steroids for an immune mediated process. Infectious disease thought the presentation was most suggestive of Dengue, but asked us to test for Salmonella, blood borne pathogens, HIV and malaria as well. Dengue IgM and IgG antibodies were elevated. The patient improved with fluids, a 1 week prednisone taper, and no further transfusions.

DISCUSSION: Dengue virus is a mosquito-borne infection that affects millions worldwide. The World Health Organization (WHO) defines Dengue Fever as an acute febrile illness with 2 of the following: headache, retro-orbital pain, myalgia, arthralgia, rash, hemorrhagic manifestations, or leukopenia. Dengue hemorrhagic fever, a clinically worse scenario, must have the following: fever, active bleeding, thrombocytopenia, and evidence of vascular permeability. Dengue Shock Syndrome is when the disease progresses to circulatory failure. On literature review, diagnosis can be made clinically, but confirmed with antibody titers and PCR. It can be difficult to differentiate Dengue from other febrile illness in endemic areas, but the above clinical signs are suggestive. Upper respiratory symptoms, sore throat, congestion and cough are less likely. Treatment is largely supportive and steroids have not been found to be helpful. Our patient met WHO criteria for Dengue hemorrhagic fever. This case illustrates that clinicians must remember that a good history and a broad differential is key in diagnosis as even a tropical illness can present itself in a non tropical mountain region.

A CASE OF DYNAMIC DESATURATION Carolyn D. Sy ${ }^{1}$; Jennifer Hsieh $^{2}$; Yelena Averbukh ${ }^{2} .{ }^{1}$ Albert Einstein College of Medicine, Bronx, NY; ${ }^{2}$ Montefiore Medical Center, Bronx, NY. (Control ID \#1288451)

LEARNING OBJECTIVE 1: Consider intrapulmonary shunts in patients with platypnea

LEARNING OBJECTIVE 2: Identify diagnostic modalities for intrapulmonary shunts

CASE: An 89 year-old woman with no smoking history or respiratory disease presented with worsening dyspnea on exertion. This was her fourth admission for the same complaint in five months. On prior admissions, she received nebulizer treatments with limited effect. The patient was a frail woman in no distress. Her respiratory exam was clear to auscultation bilaterally. She had a regular rate and rhythm without murmurs; the JVP was not elevated. She had no lower extremity edema or tenderness. On room air, the patient's respiratory rate was 18 breaths per minute and her oxygen saturation was $98 \%$. After walking, she dynamically desaturated to $90 \%$. Her saturation returned to $98 \%$ when supine and without supplemental oxygen. $\mathrm{CBC}$, electrolytes and liver function tests were within normal limits. Chest XRay revealed no pulmonary consolidation, effusion or fluid overload. Non-contrast chest CT revealed mild pulmonary emphysema. Transthoracic echocardiogram revealed normal left ventricular wall motion and an ejection fraction of $60 \%$. Following injection of agitated saline augmented with the valsalva maneuver, late bubbles entered the left atrium from the pulmonary veins four heart beats after their appearance in the right heart; indicating an intrapulmonary shunt.

DISCUSSION: Dyspnea on exertion causing hypoxemia is a common clinical complaint. Most cases can be explained by acute insults like pneumonia and MI, or chronic cardiac or pulmonary conditions like CHF, COPD, and interstitial fibrosis. However, when the etiology is not readily apparent, further investigation is required. This patient exhibited signs of platypnea-orthodeoxia; wherein dyspnea and desaturation are exacerbated in the upright position and relieved when supine. Platypnea requires two components to develop: a shunt and a functional component that promotes shunting. In this patient, exertion acted to promote shunting through the intrapulmonary shunt revealed by the bubble study. While they are commonly congenital abnormalities or secondary to cirrhosis, evidence suggests large intrapulmonary shunts exist in otherwise healthy individuals and can lead to significant impairment of gas exchange. Several modalities are available for visualizing intrapulmonary shunts. Contrast-enhanced echocardiography using agitated saline has a sensitivity approaching $100 \%$ in detecting them. Due to the possibility of revealing clinically insignificant shunts, further anatomic evaluation is warranted. Spiral CT with contrast is the preferred modality for confirming intrapulmonary shunts. However, this technique may not depict small shunts and may make visualization of large shunts challenging due to the breath holding requirement. Pulmonary angiography is the gold standard for defining the anatomy of intrapulmonary shunts due to its high specificity and treatment potential. However, its invasive nature makes other imaging modalities preferred. In patients with dyspnea on exertion without a clear cause, consideration should be given to other causes for hypoxemia, like intrapulmonary shunts. Non-invasive diagnostic modalities like contrast-enhanced echocardiography or CT have high sensitivity and can lead to better patient care.

A CASE OF MULTIFOCAL OSTEONECROSIS AND ACQUIRED IMMUNODEFICIENCY SYNDROME. Tierney Sparks; Jennifer Adams. University of Colorado School of Medicine, Aurora, CO. (Control ID \#1333787)

LEARNING OBJECTIVE 1: Recognize risk factors for developing osteonecrosis in patients with HIV.

LEARNING OBJECTIVE 2: Diagnose osteonecrosis in patients with HIV.

CASE: 47-year-old male with PMH of AIDS, Hepatitis C and alcohol and tobacco abuse presented to his PCP in 2009 with bilateral hip and low back pain. Exam revealed diminished range of motion and significant pain in his right hip. An $\mathrm{x}$-ray was consistent with bilateral osteonecrosis of his hips, and an MRI demonstrated stage IV disease on the right and stage II disease on the left. Several months later, he underwent right hip arthroplasty. Six months after surgery, the patient presented again to his PCP with a new complaint of right shoulder pain. An x-ray demonstrated osteonecrosis. Prior to evaluation by orthopedics, the patient returned complaining of left shoulder pain and osteonecrosis was evident on $\mathrm{x}$-ray of this joint. The patient was diagnosed with multifocal osteonecrosis of bilateral hips and shoulders. Prior to the diagnosis, the patient had been treated intermittently for three years with prednisone for idiopathic thrombocytopenia and had multiple admissions for acute pancreatitis secondary to alcohol abuse. The patient had been on antiretroviral therapy (ART) for three years. At this time, the patient is scheduled for a hip arthroplasty on the left and is awaiting orthopedic evaluation for his bilateral shoulder disease. 
DISCUSSION: Osteonecrosis is characterized by the in situ death of bone leading to joint destruction. The hip is the most frequently affected site, although the shoulder, knee, wrist, and ankle may also be affected. Osteonecrosis is insidious in onset, affects adults between the ages of 20 and 50 , and is more common in males. The pain is periarticular and triggered by weight bearing or moving the affected limb. The physical exam may reveal decreased range of motion or joint pain. Among HIVinfected patients, the incidence of mono-articular osteonecrosis is less than $0.1 \%$. Few cases of multifocal osteonecrosis in patients with HIV have been reported. Risk factors for osteonecrosis in patients with HIV include the use of systemic corticosteroids, hypercoagulable states, alcohol abuse, inflammatory states (i.e. pancreatitis), hyperlipidemia, osteopenia or osteoporosis, tobacco use, and ART. Glucocorticoids are the most commonly implicated risk factor for osteonecrosis. Steroids are thought to alter lipid metabolism leading to fatty infiltration of the bone marrow and obstruction of blood flow. Steroid- and alcohol-induced hyperlipidemia promotes fat embolization within the bone vasculature. ART has direct effects on bone metabolism thought to contribute to the development of osteonecrosis and, in addition, is associated with independent risk factors for osteonecrosis including hyperlipidemia, pancreatitis, and osteopenia. An HIV-infected patient presenting with periarticular pain and risk factors for osteonecrosis should undergo evaluation for osteonecrosis. Initial work up should include Xray of the affected joint, with a sensitivity of $40 \%$. If the X-ray is nondiagnostic, MRI should be pursued and has sensitivity over $90 \%$. A bone scan may be considered if clinical suspicion remains high and MRI is not diagnostic. MRI is used for staging. Conservative management, including decreasing weight bearing is recommend for limited involvement of the bone $(<15 \%$ of the bony head affected). For advanced disease, core decompression, bone grafting, and joint arthroplasty may be considered.

A CONSCIENTIOUS WORK-UP OF REGIONAL LYMPHADENOPATHY Jonathan S. Lee; Hollis Day; Peggy Hasley. University of Pittsburgh Medical Center, Pittsburgh, PA. (Control ID \#1339348)

LEARNING OBJECTIVE 1: Evaluate and manage regional lymphadenopathy

LEARNING OBJECTIVE 2: Diagnose and treat cat scratch disease

CASE: A 40 year old man with no significant PMH presented with one month of worsening neck pain and swelling. Following upper respiratory symptoms, the patient had developed two painful marble sized masses in the left side of his neck. He was initially treated with a two week course of oral clindamycin without improvement. He also noted occasional night sweats but denied fevers, chills or weight loss. The patient had a history of incarceration with previous negative PPDs. He had not traveled outside of the United States. He reported having a cat at home with a recent flea infestation. The patient's exam was remarkable for multiple firm, tender neck masses with minimal overlying erythema. A CT scan showed multiple necrotic cervical lymph nodes. Fine needle aspirate showed focal acute inflammation and granulomatous reaction favoring an infectious process. Gram stain, culture and AFB were negative. Studies for HIV, streptococcus, tuberculosis, mononucleosis and toxoplasma were negative. The patient was treated with broad spectrum antibiotics. Serology later returned positive for Bartonella henselae IgM with an IgG titer of $1: 256$.

DISCUSSION: Regional lymphadenopathy is a common presenting complaint. The differential diagnosis is primarily comprised of infectious versus malignant etiologies. Infectious causes include mononucleosis (EBV or CMV), bacterial infection (commonly strep or staph), toxoplasma, scrofula from tuberculosis, and cat scratch disease. Regional malignant causes focus on metastatic head and neck cancers. While malignancy is always a concern, only 1.1 percent of patients presenting to their PCP with lymphadenopathy are estimated to have malignancy. Age is the most important risk factor with those 40 years or older having an estimated tenfold increase in risk ( 4 vs 0.4 percent). Evaluation begins with a thorough history including onset, duration and progression of swelling, associated symptoms (fever, weight loss, night sweats, fatigue, URI), and travel and exposure history (insects, animals, sun, tobacco, alcohol, radiation). Physical exam should focus on the size, consistency, tenderness and location of the nodes. The thyroid, salivary glands, skin and oropharynx should also be carefully examined. Disease specific laboratory testing and treatment is performed based on the initial evaluation. Patients with suspected malignancy and those who do not improve after 1 month of empiric antibiotics or observation should undergo further testing with contrast-enhanced CT scanning and fine-needle aspiration or biopsy. Cat scratch disease is caused by Bartonella henselae, a gram-negative bacterium that is believed to be transmitted to humans through cat scratches and bites. Tender regional lymphadenopathy is the hallmark of cat scratch disease but disseminated disease can also occur. Diagnosis is typically based on serology because of difficulty culturing and visualizing Bartonella from tissue. A positive IgM or an IgG titer $>1: 256$ strongly supports a diagnosis of cat scratch disease. Most cases of cat scratch disease manifest solely as regional lymphadenopathy and are self-limited. However, given that disease can disseminate to the liver, spleen, eye and central nervous system, some experts advocate treating with antibiotics, though clinical evidence studying the benefits of treatment is sparse.

A CURIOUS CASE OF FORGETFULNESS Salman J. Bandeali ${ }^{1}$; Fawad Aslam ${ }^{2}$; Anna Kolpakchi ${ }^{1}$; Lee Lu ${ }^{1} .{ }^{1}$ Baylor College of Medicine, Houston, TX; ${ }^{2}$ University of Arkansas for Medical Sciences, Little Rock, AR. (Control ID \#1339533)

LEARNING OBJECTIVE 1: Recognize limbic encephalitis (LE) as a cause of forgetfulness and mental status change.

LEARNING OBJECTIVE 2: Review idiopathic LE

CASE: A 40-year-old previously healthy female presented with a three week history of increased forgetfulness and intermittent dull headache. She denied fever, nausea, vomiting and photophobia. The family reported paranoid behavioral changes, episodes of lip smacking, and staring spells lasting one minute. There were no jerky movements, bowel or bladder incontinence. On exam, she was oriented to person but not to time and place. Frequent lip smacking was observed. The rest of her neurological exam was normal. A MRI of the brain showed hyperintense signal on T2weighted images in bilateral hippocampi. A lumbar puncture revealed lymphocytic pleocytosis, no RBCs with normal glucose and protein. Cultures were negative for bacteria, HSV, VZV, CMV, Borrelia Burgdorferi and VDRL. ANA was positive with 1:320 titers; Anti-Smith, Rheumatoid factor, and Anti-dsDNA were negative. Other laboratory studies included normal TSH, normal complement levels, negative EBV antibody, HIV, RPR, hepatitis panel and HHV-6 PCR. The patient was treated empirically with acyclovir without clinical response. An electroencephalogram showed epileptiform activity in the bilateral temporal lobes. She was started on Levetiracetam, and the lip smacking and staring spells resolved. Based on the clinical presentation, the lack of response to anti-viral therapy, and MRI findings with a negative infectious work up, she was diagnosed with limbic encephalitis (LE). The search for an occult neoplasm was initiated. Workup was negative for Anti-Hu, Anti-Yo, Anti-Ri, Anti-GAD65, Anti-CV2 and Anti-Ma2 antibodies. A mammogram, PAP smear, transvaginal ultrasound, CT scan of the thorax and MRI of abdomen and pelvis were normal. Hence, a final diagnosis of idiopathic LE was made. She was started on prednisone $60 \mathrm{mg}$ daily which was tapered over six months with clinical improvement. A year later and off steroids, her mental status remains at normal baseline.

DISCUSSION: Limbic encephalitis is typically associated with $0.01 \%$ of all cancers. Recently, $70 \%$ of LE has been reported without an associated malignancy, known as idiopathic LE. The incidence is not known. Newonset anterograde amnesia and psychiatric symptoms are the hallmark features. Diagnosis is based on the clinical presentation, CSF and MRI findings. Eighty percent of patients have CSF lymphocytic pleocytosis, normal glucose and elevated protein, and 70 to $80 \%$ of the MRI findings show hyperintense signal in the medial aspect of the temporal lobes on T2 images. LE, as a paraneoplastic syndrome, is usually associated with lung cancer $(50 \%)$, testicular germ cell malignancy $(20 \%)$, and breast cancer $(8 \%)$. Therefore, the diagnosis of idiopathic LE can only be made if there is no identifiable malignancy. However, surveillance for malignancy with full body imaging is recommended for at least 2 years after the diagnosis. Treatment options for idiopathic LE include immune modulation using steroids, azathioprine, rituximab, intravenous immunoglobulins, and plasmapheresis. Patients typically respond well with full recovery. Thus, idiopathic LE should be recognized as an entity causing anterograde amnesia and psychosis, especially in patients with a negative infectious and malignancy work up. 
A CURIOUS CASE OF LOWER URINARY TRACT SYMPTOMS CLINICAL SIGN IDENTIFYING ILIOPSOAS MUSCLE STRAIN AS ETIOLOGY OF "CHRONIC PROSTATITIS" Ayodeji O. Shedu'; Bruce Karlin ${ }^{1}$; Mike Roberts Roberts ${ }^{2} .{ }^{1}$ St Vincent Hospital, Worcester, MA; ${ }^{2}$ Central Massachusetts Physical Therapy, Worcester, MA. (Control ID \#1313038)

LEARNING OBJECTIVE 1: Consider Iliopsoas strain in the differentials of lower urinary tract symptoms

LEARNING OBJECTIVE 2: Use appropriate clinical sign to elicit iliopsoas muscle strain in setting of chronic prostatitis

CASE: A 50yo otherwise healthy male, presented to the outpatient clinic with urinary urgency and frequency of 7 days duration, and nocturia up to 3 times nightly. He had mild lower back pain which he attributed to multiple repetitions of abdominal crunches he recently added to his vigorous daily workout. He denied fever, urethral discharge, or change in sexual habits. He had no medical history and family history was not contributory. He had seasonal allergies and worked as an engineer. Vital signs were stable and physical examination revealed no focal back tenderness. DRE elicited mild tenderness of prostate. Despite negative UA, UTI was presumed and treated with ciprofloxacin. 3 days later, patient returned to clinic with worsening symptoms. He was referred to a Urologist who made a diagnosis of inflammatory prostatitis and started a 2 weeks course of Tamsulosin. For the next 15 months, various urologists prescribed multiple courses of antimuscarinic drugs and antibiotics for "Chronic Prostatitis". His symptoms improved after 6 months treatment with Solifenacin and it was tapered. Two months later, his urinary symptoms returned along with increasing back pain. IPSS was 23 , back exam was negative, and PVR was negligible. A cystoscopy was negative. An MRI was initially read as L2-3 root impingement but neurosurgery dissented. A physical therapy assessment revealed tenderness to palpation along the iliopsoas muscle belly. Palpation of the proximal portion of the iliopsoas was done by palpating at the midpoint of the umbilicus and anterior superior iliac spine at the lateral border of the rectus abdominis muscle with patient supine and lower limb extended. The patients head was resting on a pillow to relax the abdominal musculature. A diagnosis of iliopsoas strain was made and patient was treated with physical therapy intervention consisting of manual myofascial massage, neuromuscular reeducation and stretching. His symptoms completely resolved by the end of the 5 th session. He remains asymptomatic.

DISCUSSION: Symptoms suggestive of "chronic prostatitis" has a prevalence of about $8 \%$, accounting for nearly 2 million ambulatory care encounters annually. The diagnosis of "Chronic Prostatitis" currently encompasses many conditions unrelated to the prostate. Abdominal and/ or pelvic floor muscle tenderness have been associated with this condition. The patient is an avid rower and was preparing for a competition by doing up to 500 crunches at a time immediately prior to symptom onset. His lower urinary tract symptoms probably developed secondary to Iliopsoas strain as a result of his vigorous exercises. Elicitation of exquisite iliopsoas muscle tenderness correctly identified myofascial strain as a contributor to his symptoms. Routine clinical assessment of muscular involvement in prostate symptoms can easily identify patients who may respond to physical therapy intervention. This will engender significant reduction in patient's suffering and health care cost.

A DEVIATED TONGUE Alice Jacob; Kurt J. Pfeifer; Ehab Atallah. Medical College of Wisconsin, Milwaukee, WI. (Control ID \#1314701)

LEARNING OBJECTIVE 1: Describe the presentation of extramedullary leukemia

LEARNING OBJECTIVE 2: Review the treatment of chronic myelogenous leukemia and extramedullary leukemia.

CASE: A 43-year-old man with no significant medical history presented with slurred speech and tongue deviation for 2 days. He also had night sweats, migratory arthralgias, 30-pound weight loss over 2 months and intermittent headache. On admission he had an isolated left cranial nerve XII palsy. Other pertinent exam findings included a right scapular mass and bilateral testicular enlargement. Laboratory studies were notable for hemoglobin of $8.7 \mathrm{~g} / \mathrm{dl}$, platelet count $429,000 / \mathrm{cu} \mathrm{mm}$ and white blood cell count of $18,100 / \mathrm{cu}$ mm with an absolute neutrophil count of $12,800 / \mathrm{cu}$ $\mathrm{mm}$. Infectious etiologies, including HIV and tuberculosis, were ruled out. AFP and Beta-HCG were within normal limits. Brain MRI revealed a clivus mass impinging on the left hypoglossal nerve. Scrotal ultrasound was suggestive of an infectious or lymphoproliferative disorder. A positron emission tomography scan showed patchy infiltrates in both lungs, hypoattenuating areas within the muscle surrounding the right scapula and abnormal left kidney uptake. Bone marrow biopsy demonstrated a myeloid neoplasm - Philadelphia chromosome-positive $(\mathrm{Ph}+)$ chronic myelogenous leukemia (CML) with $1.6 \%$ blasts, $90 \%$ of which were myeloid. Scapular mass biopsy was consistent with an extramedullary lymphoid blast crisis of $\mathrm{Ph}$ + CML. Given that the brain and testes are not well targeted by chemotherapy, he received brain and testicular irradiation. His chemotherapy regimen consisted of dasatinib and HyperCVAD (cyclophosphamide, vincristine, doxorubicin and dexamethasone) followed by methotrexate and cytarabine. On completion of radiation therapy and his first few cycles of chemotherapy, he had resolution of his tongue deviation and testicular enlargement.

DISCUSSION: Chronic myelogenous leukemia (CML) is a clonal malignancy characterized by the chromosomal translocation of the breakpoint cluster region gene (BCR) from chromosome 22 fusing with the $A B L$ gene on chromosome 9, commonly known as the Philadelphia chromosome. Granulocytic sarcomas (GS) or extramedullary tumors occur most frequently in acute myelogenous leukemia (AML) but also in CML and other myeloproliferative disorders. Their occurrence in AML is seen in 2.5$9.1 \%$ of patients but is five times less frequent in CML patients. Patients with GS are frequently asymptomatic, and $50 \%$ of cases are diagnosed only at autopsy. These tumors can involve any part of the body, including bone, spine, brain, and other visceral organs, and multiple sites simultaneously. CML has three phases: chronic, accelerated and blastic. Blast crisis is the terminal phase of CML and behaves like acute leukemia. The BCR-ABL fusion gene product is a tyrosine kinase.Treatment for patients with myeloid blast crisis, lymphoid blast crisis and $\mathrm{Ph}+\mathrm{ALL}$ includes tyrosine kinase inhibitors such as imatinib, dasatinib, and nilotinib. Recent studies show that although the BCR-ABL tyrosine kinase inhibitor imatinib is effective in $\mathrm{Ph}+$ leukemias, relapse does occur. In such cases, dasatinib induces hematologic and cytogenetic responses in patients with CML or Ph+ALL who cannot tolerate or are resistant to imatinib.

A DIAGNOSIS THAT IS HARD TO SWALLOW: COMPLEXITIES OF MEDICAL MANAGEMENT Andrew Ayers. Creighton University Medical Center, Omaha, NE. (Control ID \#1334785)

LEARNING OBJECTIVE 1: Recognize clinical features of Amyotrophic Lateral Sclerosis (ALS) as part of the differential diagnosis when working up progressive dysphagia.

LEARNING OBJECTIVE 2: Manage multiple general and medical subspecialty resources to diagnose and treat ALS.

CASE: A 78 year old male with a past medical history of CAD with five coronary stent placements, hypertension, hyperlipidemia, and diabetes mellitus presented as a new patient to internal medicine clinic with a complaint of difficulty swallowing solids for several months. Prior to this clinic visit, he had seen multiple primary care providers and had undergone esophageal dilatation twice, with trace improvement in symptoms. Upon presentation, the patient noted that in addition to dysphagia, his voice would become hoarse after talking for 10 to 15 minutes, and after a brief period of rest, his ability to speak would recover. He also relayed that he felt like he could not breathe while lying flat but had no symptoms when lying on his side. He was admitted 3 months prior for chest pain for which catheterization revealed a $70 \%$ RCA lesion not requiring intervention. $\mathrm{He}$ was discharged with plans to undergo a cardiac stress test but was lost to follow up. At the time of clinic evaluation, the patient had a hoarse voice but no other pertinent physical exam signs. He was referred to gastroenterology and otolaryngology and was set up for EGD with plans for esophageal dilation if needed. Prior to the procedure, he required clearance with cardiac stress testing which demonstrated acute ischemia requiring admission. At that time, his dysphagia had progressed. He had developed a slow gait, and his head was continuously in a flexed position. CT and MRI brain were pertinent for only mild brain atrophy. Cardiac catheterization was performed, and a bare metal stent was placed in the $\mathrm{RCA}$ lesion to reduce the time needed for anticoagulation, given the 
patient's impending EGD and progressing symptoms. Subsequently, neurology was consulted for suspected visual changes. Patient admitted that visual changes were chronic in nature but, newly performed neurological examination revealed hyperactive reflexes in the lower extremities and fasciculations over the deltoid muscles on hammer strike. $\mathrm{He}$ underwent immediate EMG which revealed active denervation of multiple muscle groups consistent with ALS, bulbar onset type. EGD was cancelled, and the patient was started on riluzole.

DISCUSSION: ALS presents as asymmetric limb weakness in approximately $80 \%$ of patients with a prevalence of $1.7 / 100,000$ among the general population. Bulbar onset, as described above, may occur in up to $20 \%$ of patients presenting with ALS. Clinical suspicion for neurological disorders with this presentation should be higher in patients without a significant smoking history or traditional symptoms of gastroesophageal reflux. This patient's post-hospital management included chronic disease management and preventive care by internal medicine, regular follow up and treatment with neurology, gastrostomy tube placement by gastroenterology, and assistance by palliative care for end of life issues. Recognizing the presentation of ALS in this patient with active cardiac disease and history of multiple esophageal procedures was only possible once this patient established care with a permanent primary medical provider. This also allowed for the implementation of a coherent treatment plan among multiple medical specialties.

A DIAGNOSTIC ENIGMA: ANCA POSITIVE PNEUMONIA Halis K. Akturk; Priyanka Vashisht; Daniel Wehrmann; Gretchen Butler; Andrea Giomi; Againdra K. Bewtra. Creighton University Medical Center, Omaha, NE. (Control ID \#1279064)

LEARNING OBJECTIVE 1: Identify the different causes of refractory cough and dyspnea.

LEARNING OBJECTIVE 2: Understand the clinical presentation of cryptogenic organizing pneumonia (COP). Comprehend the differential diagnosis of positive serological markers like cytoplasmic antineutrophil cytoplasmic antibody (c-ANCA).

CASE: A previously healthy 62-year-old African American male presented with six week history of dry cough, shortness of breath and seven pound weight loss. The patient had dyspnea with his routine activities and often had prolonged coughing spells. He had a 40-pack year smoking history. He had no history of allergies, animal exposure, recent travel or occupational risk factors. There was no personal or family history of pulmonary disease. He was prescribed a 10-day course of moxifloxacin with no relief. He was treated with piperacillin/tazobactam and levofloxacin for 1 week without improvement and had 4 liters oxygen requirement. The patient presented to the emergency, as his symptoms were progressive worsening without any relief. The patient was afebrile, tachycardic and tachypneic. Respiratory exam revealed coarse rhonchi bilaterally with scattered wheezing. Rest of the physical exam was unremarkable. Lab investigation revealed leukocytosis $(12.000 \mathrm{WBC} / \mathrm{mm} 3)$ with eosinophilia $(12 \%)$ and hypoalbuminemia $(2 \mathrm{~g} / \mathrm{dl})$. Erythrocyte sedimentation rate was 101 , c-ANCA was positive (1:320), and antibodies to myeloperoxidase and Proteinase-3 were negative. An initial chest x-ray showed bilateral interstitial shadows. Computed tomography revealed bilateral patchy ground glass opacities with areas of honeycombing. Pulmonary function test showed mild airflow obstruction with severely reduced diffusion capacity. Bronchoscopy revealed moderate lymphocytic inflammation throughout the tracheobronchial tree. Bronchoscopic biopsies were inconclusive so video-assisted biopsy of lung was obtained. It showed fibroblastic proliferation and granulation tissue filling terminal bronchioles and alveolar spaces consisted with Cryptogenic Organizing Pneumonia (COP). High dose of intravenous steroids were started and followed by oral taper. His pulmonary function tests improved dramatically with the steroid therapy.

DISCUSSION: It is important to recognize the presentation of refractory cough and shortness of breath. COP previously termed Bronchiolitis Obliterans-Organizing Pneumonia (BOOP) is a clinical, radiological and pathological diagnosis, when no definite etiology such as infection or connective tissue disease is found. The onset of illness is usually acute and presents as a flu like illness. Histological sampling is imperative because corticosteroids and cytotoxics are contraindicated in many differential diagnoses. Transbronchial biopsies usually do not provide a sufficient sample and an open/video-assisted lung biopsy is recommended to confirm the diagnosis. It is critical to understand the relevance of immunological test and the diagnosis should not be made solely on their basis. Although C-ANCA has a specificity of 88-100 \% for Wegener's Granulomatosis, it should be reviewed in the context of the overall picture. An inappropriate diagnosis based solely on the results of ANCA testing could have serious consequences.

A DIZZYING DIFFERENTIAL DIAGNOSIS Tara Shankar. UPMC, Pittsburgh, PA. (Control ID \#1339409)

LEARNING OBJECTIVE 1: Differentiate between peripheral and central vertigo and state initial diagnostic evaluation

LEARNING OBJECTIVE 2: Recognize the clinical features of pontine infarct

CASE: A 48 year old nonsmoking female with history of hypertension and family history of migraines presented with two day history of sudden onset dizziness with sensation of movement, right-sided tinnitus, nausea and vomiting. Patient additionally reported intermittent symptoms of diplopia, bitemporal headache, and transient right upper extremity weakness. Physical exam was notable for bilateral miosis, left horizontal nystagmus that did not suppress with ocular fixation, and mild dysphagia. Rinne and Weber testing was normal bilaterally and Dix-Hallpike maneuver was negative. MRI brain was performed which revealed a small infarct within the left paramedian pons, interpreted to be lacunar stroke in setting of known hypertension. Hypercoagulabilty workup was negative, as was evaluation for arrhythmia or patent foramen ovale. Antiplatelet therapy was initiated and patient referred for acute stroke rehabilitation.

DISCUSSION: Dizziness and vertigo rank among the most common complaints in medicine and account for approximately 7.5 million primary care visits annually. Vertigo may pose a diagnostic challenge for physicians as it represents a large range of diagnoses from benign to immediately life threatening; therefore, distinguishing between its peripheral and central etiologies is essential. Peripheral etiologies, which frequently present with pronounced nausea and vomiting, auditory symptoms such as hearing loss, tinnitus or pain, and nystagmus suppressed with visual fixation, tend to resolve in hours to days. Conversely, central etiologies present with severe imbalance but less prominent movement illusion and nausea, vertical or pure rotary nystagmus, and other neurologic signs such as motor and sensory deficits that tend to resolve in days to weeks. As blood supply to the inner ear and vestibulocochlear nerve arise from the basilar artery, infarction in the pontine distribution may present as vertigo of either peripheral or central origin. More commonly in pontine infarction, vertigo is accompanied by motor findings such as transient or persistent weakness and incoordination as well as oculomotor findings of nystagmus, miosis and horizontal gaze palsy. In particular, weakness of the bulbar muscles, commonly manifested by symptoms of dysphagia and dysarthria, is an important cause of morbidity in these patients. Therefore, the initial diagnostic evaluation of a patient with vertigo should utilize a combination of focused history taking, physical exam maneuvers, and diagnostic tests to evaluate the most common and most serious etiologies. History of stroke risk factors, migraines, positional symptoms and temporal relationship may help direct exam. Physical exam should include hearing and neurologic testing, as well as maneuvers such as the Head Impulse Test, which has a high specificity for peripheral vertigo syndromes. Neuroimaging is indicated if there are neurologic signs on physical examination, if there is a new headache, and if the physical exam and history are not entirely consistent with a peripheral lesion. In this scenario, MRI and MRA of the brain, which has a specificity and sensitivity greater than $95 \%$ in detecting occlusion of posterior circulation is the test of choice.

A FIRE BEING KINDLED Holly Peek; Chayan Chakraborti. Tulane University Health Sciences Center, New Orleans, LA. (Control ID \#1311833)

LEARNING OBJECTIVE 1: Recognize the clinical significance of the kindling phenomenon in patients with multiple alcohol withdrawal episodes. 
LEARNING OBJECTIVE 2: Identify alternative treatments to benzodiazepines for patients experiencing multiple withdrawal episodes. CASE: A 55-year-old woman with an extensive history of alcohol dependence presented to the emergency department with progressive generalized weakness over the previous several months. Her past medical history included hypertension, cirrhosis, and heavy alcohol use with several withdrawal episodes. Her heart rate was $108 \mathrm{bpm}$, but the other vital signs were normal. She was ill-appearing, pale, icteric, jaundiced, and moderately confused and oriented to person and place. She had stigmata of chronic liver disease, but had no asterixis. Her laboratory evaluation revealed anemia, thrombocytopenia, hyponatremia, hyperkalemia, hypocarbia, elevated total bilirubin, elevated ALT, and elevated alkaline phosphatase. Her urinalysis was indicative of a urinary tract infection; the toxicology screen was normal. Despite resolution of the urinary tract infection with antibiotics, adequate electrolyte replacement, and improved hyper-ammonemia with lactulose therapy, her mental status remained poor. She was not oriented to person, place, or time, requiring one-to-one observation for severe agitation and occasional treatment with risperidone and haloperidol. A brain MRI/MRA was normal and no seizure activity was noted. The medical team treated the patient with benzodiazipines for alcohol withdrawal. The mental status initially improved but worsened when benzodiazipines were tapered. The patient's condition was ultimately determined to be prolonged alcohol withdrawal associated with the kindling phenomenon.

DISCUSSION: Alcohol withdrawal is a common problem facing general internists. The kindling phenomenon occurs after several prior episodes of alcohol withdrawal building up to result in a more prolonged episode and may manifest as persistent mental decline and psychomotor agitation. Animal studies have demonstrated permanent imbalance in excitatory and inhibitory neurotransmission in animals exposed to multiple cycles of alcohol exposure, abstinence, withdrawal, and re-exposure. This is clinically supported in both human and animal studies that have demonstrated worsening withdrawal symptoms with each successive withdrawal cycle. History of multiple withdrawal episodes in these studies increased susceptibility to more severe future withdrawals. In our patient, due to the difficulty in tapering benzodiazepine treatment and her declining mental status, she was given a 5-day taper of oral carbamazepine. Studies have shown carbamazepine to be equal in efficacy to benzodiazepine for alcohol withdrawal treatment and may be superior in treating those with multiple withdrawal episodes. Carbamazepine prevents alcohol withdrawal seizures and improves symptoms common in withdrawal states such as sleep disturbances, anxiety, and mood instability. Carbamazepine has lower abuse potential and sedating effects compared to benzodiazepines. Most promising, patients treated with carbamazepine compared to lorazepam were found to drink significantly less and take longer to return to drinking if they relapsed. Over the ensuing five days of carbamazepine treatment, the patient's mental status and orientation markedly improved, no longer requiring medications for agitation. She was discharged to a nursing home and one month later due to complications of cirrhosis.

A HEART CRUSHING DIAGNOSIS OF ABDOMINAL PAIN Camila

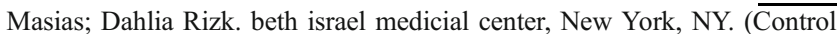
$\overline{\mathrm{ID} \# 13} 40380)$

LEARNING OBJECTIVE 1: Recognize the broad differential of abdominal pain, including rare causes

LEARNING OBJECTIVE 2: Diagnose Morgagni hernia as a cause of abdominal pain

CASE: 36 year old hispanic male, no remarkable past medical history, presented to the emergency department with a 6 month history of intermittent abdominal pain that became severe 3 days prior to admission. Pain was localized in the epigastrium and radiated to both upper quadrants. He had been taking omeprazole $40 \mathrm{mg}$, ranitidine $150 \mathrm{mg}$, and aluminum hydroxide liquid without relief. He reported nausea, but denied vomiting, diarrhea, melena, hematochezia or constipation. He also reported intermittent abdominal distention associated with increase pain. The patient was seen at two other institutions with the same symptoms. Chest x-ray, abdominal x-ray and ultrasound were normal. Esophagogastroduodenoscopy showed chronic gastritis. Abdominal computerized tomography (CT) scan showed an anterior left diaphragmatic hernia, which contained transverse colon abutting the heart. Despite findings, symptoms improved, and patient was discharged home with esomeprazole $40 \mathrm{mg}$ daily. On presentation 3 months later to our institution, patient reported intermittent bloating, distention, and was in distress secondary to pain. On exam, there was no distention, however the abdomen was tender in the upper quadrants and epigastric region. There was no guarding and no masses were palpated. Laboratory tests were normal, including liver panel, amylase and lipase. Chest CT scan again showed Morgagni hernia in the anterior chest containing a loop of transverse colon and omentum with mass effect on the heart. Laparoscopic repair of the hernia was done successfully by retracting transverse colon from the mediastinal cavity. The patient was discharged 3 days after the surgery, and was followed as an outpatient with resolution of symptoms.

DISCUSSION: While evaluating a patient with abdominal pain, it is important to have a broad differential of common causes, however, rare causes must also be considered. Morgagni's hernias are secondary to congenital defects in the anterior diaphragm, that later stretch secondary to the rapid rise in intraperitoneal pressure. For this reason pregnancy, trauma, obesity, constipation, and chronic chough have been found as common predisposing conditions. Morgagni's hernias seem to be more common in women, and present mostly with pain or pressure in the chest or abdomen. Occasionally, due to compression on the heart wall, dyspnea or arrhythmia may be present. Some cases are asymptomatic. Delays in diagnosis, however, may lead to serious complications such as obstruction or strangulation. Although there has been a rise in the number of cases reported, due to low clinical suspicion, this diagnosis may be present more frequently than the literature suggests. To confirm diagnosis, a plain chest radiography may be adequate, however, since the appearance differs depending on the contents of the hernia, diagnosis may be missed. CT scan is considered a more accurate method for diagnosis. The treatment is surgical, even in asymptomatic patients, as this may prevent future complications. Clinicians must have an increased awareness of rare causes of recurrent epigastric pain, chest pain or dyspnea. Morgagni's hernia is one such case that may have serious consequences if gone undiagnosed or untreated.

\section{A HIGH PRESSURE SYSTEM: WHEN CHEMO GOES TERRIBLY} WRONG S. M. Soni; Allison DeKosky. University of California, San Francisco, San Francisco, CA. (Control ID \#1338710)

LEARNING OBJECTIVE 1: Learn about a rare cause of meningitis LEARNING OBJECTIVE 2: Recognize the signs of increased intracranial pressure

CASE: A 51 year-old woman presents with severe headache for two days. She has a history of advanced non-small cell lung cancer with metastases in her cerebrospinal fluid. She had undergone left upper lobe segmental resection, Ommaya reservoir placement and six cycles of intrathecal methotrexate as well as systemic chemotherapy. Three days prior to admission, she received her first dose of intrathecal liposomal cytarabine. On initial exam, she was inattentive with waxing and waning mental status, but oriented to self, place and date and answering questions appropriately. She had bilateral proptosis, pupils were reactive 5 to $2 \mathrm{~mm}$ bilaterally, extraocular movements were intact. She had no photophobia. Her cranial nerves 2-12 were intact and neck was supple. She had 5/5 strength in upper and lower extremities with intact sensation to light touch throughout. Five hours later she was found face down on her bed unresponsive. Her repeat exam was notable for heart rate ranging from 55-67 beats per minute and blood pressures of 160-220/98-110. Her pupils were sluggish, she had an absent gag reflex, meningismus and was leaking CSF from her Ommaya port. She was no longer following commands, repeating "yes" to all questions. She had 5-10 beats of clonus of the right foot that was not present on the left. CT scan of brain demonstrated mild interval increase in ventricle size concerning for early hydrocephalus compared to past imaging with Ommaya catheter tip in appropriate position. CSF analysis of fluid drawn off Ommaya port had an opening pressure of $>55 \mathrm{mmHg}$ with $78 \mathrm{WBC}, 850 \mathrm{RBC}, 69 \mathrm{PMNs}$, glucose of 81 and protein of 53 (serum glucose of 130 and serum total protein of 6.2). On further questioning of the patient's family, it was revealed that patient had not taken any of her post-chemotherapy doses of dexamethasone. They also recalled that she had previous hospitalizations for intracranial hypertension. 
DISCUSSION: Although headaches are commonly seen in patients receiving chemotherapy and managed in the outpatient and inpatient setting, quick identification of red flags in patient presentations and implementation of emergent management are critical skills for internists. In this patient there were several concerning features, including the patient's history of malignancy with recent intrathecal chemotherapy and altered mental status. Her clinical status quickly deteriorated as her intracranial pressure increased,leading to CSF leakage through her Ommaya port. Further, she developed a profound Cushing's response (bradycardia and hypertension), a finding that requires emergent intervention. Other markers of increased intracranial pressure include headache, emesis, papilledema, and cranial nerve palsies. Once these findings are present, neurosurgical consultation is mandated. Our patient underwent placement of an external ventricular drainage device. Within hours, she was alert and oriented without any residual neurologic deficits. Although this patient had an excellent recovery, fatal chemical ventriculomeningitis after intrathecal cytarabine has been reported (Butto A, et al. 2011. Fulminant chemical ventriculomeningitis following intrathecal liposomal cytarabine administration. Journal of Clinical Neuroscience 18(10):1417-1418). This episode appears to be triggered by her medication noncompliance with dexamethasone and perhaps her predisposition to hydrocephalus.

A LYTIC LESION BY ANY OTHER NAME, IS STILL A LYTIC LESION Ramsey Al-Hakim; Adam Rodman. Tulane University Health Sciences Center, New Orleans, LA. (Control ID \#1311870)

LEARNING OBJECTIVE 1: 1. Identify the differential diagnosis of a lytic bone lesion 2. Understand the diagnostic approach to aneurysmal bone cysts 3 . Identify the risks associated with a percutaneous transpedicular vertebral biopsy

CASE: A 50 year-old woman presented with a three-day history of acute onset low back pain. The lumbar pain came on suddenly after she leaned over to pick up a light load of laundry. The pain radiated to her legs bilaterally, and was associated with leg numbness. Her symptoms improved with standing, and worsened with sitting. She denied lower extremity weakness, incontinence, saddle anesthesia, or fevers. Her past history was significant only for hypertension, diabetes, and hyperlipidemia. She was afebrile, and her vital signs were normal. There was exquisite tenderness to palpation of the fifth lumbar vertebrate. Lumbar flexion and gait were limited secondary to pain. Straight leg testing was positive bilaterally. Reflexes were normal, and her sensation and motor strength were intact. An MRI revealed an expansile lesion in the L5 vertebral body and a pathological fracture of the superior and inferior endplates; there was significant loss of vertebral height. A CT of the T/L/C-spine, abdomen/ pelvis, and thorax revealed only the L5 lytic lesion. A bone scan revealed increased uptake in L5. A percutaneous transpedicular biopsy of the lytic lesion was performed, revealing blood clots on histology and scant clusters of atypical spindle cells on cytology. Age appropriate cancer screening was performed, as was a bone marrow biopsy and immunoelectrophoresis; all diagnostic tests were normal. A repeat biopsy of the lytic lesion six months after initial presentation confirmed the aneurysmal bone cyst.

DISCUSSION: Back pain and lytic bone lesions are commonly encountered by the general internist. It is important that the general internist has a disciplined approach to the evaluation of an expansile lytic lesion, recognizing that not all lytic lesions are indicative of metastatic disease. Once metastatic disease has been excluded with age-appropriate cancer screening, a primary bone disease should be considered for Lodwick grade II or III lesions. A percutaneous transpedicular vertebral biopsy with good approach can reliably detect plasmacytomas and chondrosarcomas. Repeat imaging of a lytic lesion without demonstration of significant expansile growth can effectively exclude a giant cell tumor, particularly in adults. While percutaneous transpedicular biopsy is regarded as safe with a high diagnostic rate, the risk of further collapse and worsening of symptoms increases in severely diseased vertebrae. For the general internist, it is important to understand the diagnostic approach to a lytic bone lesion. Once primary and secondary malignant processes have been excluded with a successful biopsy and appropriate imaging/studies, aneurysmal bone cyst can reliably be diagnosed without exposing a patient to further risks associated with repeat transpedicular biopsies in a severely diseased bone. Further, knowledge of this disease can avert untoward patient stress and anxiety by prematurely jumping to the conclusion that a cancer is present.

A MOVING STORY: FAHR DISEASE John Humphrey. Tulane University Health Sciences Center, New Orleans, LA. (Control ID \#1311725)

LEARNING OBJECTIVE 1: Recognize the clinical and radiographic presentation of Fahr disease.

LEARNING OBJECTIVE 2: Distinguish between the classifications of movement disorders as they relate to the function of the basal ganglia.

CASE: A 38 year-old man experienced three months of progressive gait instability and declining functional capacity. Prior to the onset of these symptoms, he had had no medical history. He described no signs and symptoms suggestive of endocarditis or thromboembolic disease, but did note that members of his extended family had experienced similar symptoms during the fourth and fifth decade of life. His vital signs were normal. His cardiac, pulmonary, abdominal and skin examinations were norma. He was severely dysarthric but able to follow commands. Movement of the extremities was symmetric but clumsy, bradykinetic, and rigid. His gait was unstable. A computed tomography scan of the brain revealed extensive symmetric mineralization of the caudate nucleus, thalami, putamen, subcortical region, and brain stem. Subsequent laboratory testing revealed normal serum calcium, phosphorous, lead, copper, and ferritin levels. The HIV ELIZA and ANA tests were negative, and a clinical and radiographic diagnosis of familial idiopathic basal ganglia calcification (Fahr disease) was made.

DISCUSSION: Over time, general internists have assumed more and more of the primary diagnosis and management of neurologic disease. While movement disorders are not as common as stroke, it is important that the general internist have a disciplined approach to the diagnosis of new-onset movement disorders. Familial idiopathic basal ganglia calcification, also known as Fahr disease, is a disorder of movement characterized by symmetric calcification of the basal ganglia in the setting of progressive neurologic dysfunction. Diagnosis is confirmed by brain imaging after excluding other metabolic, infectious, toxic, or traumatic causes. Autosomal inheritance patterns have been observed, though the cause of the disorder remains unknown. Hence, a family history of the disorder raises the pretest probability of the disease. Once the diagnosis is made, immediate family members should be offered neurologic and neuropsychiatric evaluation. This should include genetic counseling and screening neuroimaging regardless of whether one is exhibiting symptoms of the disease. As there is no cure for Fahr disease, management is focused on pharmacologic treatment of the neurologic and psychiatric manifestations. These therapies may include anxiolytics, antipressants, antispasmodic, or antiepileptics. The general internist's ability to distinguishing between the clinical presentation of the most common movement disorders, as they relate to the function of the basal ganglia, is important in identifying and managing these diseases.

A MYSTERIOUS CASE OF ESOPHAGEAL VARICES David W. Walsh. Medical University of South Carolina, Charleston, SC. (Control ID \#1338514)

LEARNING OBJECTIVE 1: Overview the initial work up of patients with esophageal varices and negative liver biopsies

LEARNING OBJECTIVE 2: Describe the natural history of Idiopathic Portal Hypertension

CASE: Patient is a 69 year old male with a PMH of DM, aspirin use who presented with new onset massive hematemesis requiring intubation, blood products, and vasopressor pressure support. Urgent EGD was preformed showing 3 medium sized column varices in the esophagus and clips were placed. Initial laboratory values were significant for thrombocytopenia and normal liver function tests. The patient subsequently stabilized and was able to be extubated. A liver biopsy at the time of discharge showed no evidence of cirrhosis or non-cirrhotic portal hypertension. The patient was discharged on Nadolol. He subsequently underwent MR and hypercoagulable work up to evaluate for portal occlusive disease, however the 
portal system was widely patent. Out of concern for sampling error, the patient underwent a repeat liver biopsy. It again was negative cirrhosis, however it did show sinusoidal dilation and Stage I portal fibrosis consistent with idiopathic portal hypertension.

DISCUSSION: Idiopathic portal hypertension is a rare, poorly understood condition that leads to clinical features of portal hypertension (i.e.- varices, spleenomegaly, etc.) with no evidence of cirrhosis or clear etiology of portal occlusive disease. Terms such as hepatoportal sclerosis, obliterative portal venopathy of the liver, and phlebosclerosis have been applied to the disease, but likely represent the same process. Clinically, it appears to act similar in nature to other patients with variceal disease and respond well to appropriate therapy. In contrast, though, these patients do not share other similar features to cirrhotic patients (recurrent infection, encephalopathy, etc). IPH is a diagnosis of exclusion; therefore it is important to rule other possible etiologies of portal hypertension including cirrhosis, venoocclusive disease, and hypercoagulable states. Once a negative liver biopsy is obtained, a reasonable next step is to perform MR to evaluate the patency of the portal system. IPH carries a better prognosis than it's cirrhotic counterpart and the main cause of death is related to esophageal variceal bleeding. Patients seem to do well with beta blockade therapy and subsequent reduction in portal pressures. The 10 year survival approaches $80 \%$

A MYSTERIOUS CAUSE FOR A LUNG MASS Maria Sobolevi; Andrew Lee ${ }^{2}$; Robert Ashton ${ }^{3}$; Lewis A. Eisen ${ }^{4}$; Ariel L. Shiloh ${ }^{4}$. ${ }^{1}$ Montefiore Medical Center, Albert Einstein College of Medicine, Bronx, NY; ${ }^{2}$ Montefiore Medical Center, Albert Einstein College of Medicine, Bronx, NY; ${ }^{3}$ Montefiore Medical Center, Albert Einstein College of Medicine, Bronx, NY; ${ }^{4}$ Montefiore Medical Center, Albert Einstein College of Medicine, Bronx, NY. (Control ID \#1337481)

LEARNING OBJECTIVE 1: Recognize foreign body aspiration as a cause for recurrent or prolonged lung disease.

CASE: A 49 year-old man with mild-intermittent asthma, a 90-pack-year smoking history, and multiple admissions for pneumonia was admitted for increasing cough, right sided chest pain, dyspnea, and hemoptysis over a three year course. There was no fever, chills, night sweats, or weight loss. Physical exam revealed right base rales. Chest X-ray demonstrated a right lower lobe infiltrate. Laboratory testing was significant for a leukocytosis (WBC $12.8 \mathrm{~K} / \mathrm{uL}$ ). Urine legionella, fungal cultures, PPD, and AFB testing were negative. Chest $\mathrm{CT}$ revealed a $4 \mathrm{~cm} \mathrm{x} 4 \mathrm{~cm}$ right lower lobe mass with an endobronchial component and ipsilateral, mediastinal, and hilar lymph nodes measuring up to $1 \mathrm{~cm}$ in size, consistent with malignancy. PET CT displayed a hypermetabolic mass in the same location without evidence of metastatic disease. Bronchoscopy identified an endobronchial mass surrounded by necrotic tissue almost completely occluding the right lower lobe. Cultures grew E. coli and the patient was treated with antibiotics. The transbronchial biopsy, washings, and cytology were nondiagnostic. The patient underwent right lobectomy via video-assisted transthoracic surgery. Final pathology revealed a spherical object, grossly resembling a peanut and microscopically consistent with vegetable matter, within the bronchus. There were changes suggestive of a post-obstructive pneumonia, without evidence of malignancy in the specimen or lymph nodes. On further questioning, the patient could not recall an aspiration. DISCUSSION: Among the recognized cases of FBAs, $80 \%$ occur in children. Most cases in adults, occur in patients who are elderly, have a neurological disorder, abuse alcohol or use sedatives. Adult FBAs are less likely to be fatal. Presentation of aspiration may be immediate or delayed for months to years, in a rare case described, for more than 20 years. Patients often don't recall choking and the material retrieved from the airways is not readily recognized, causing a diagnosis to be overlooked. Aspirated foreign bodies commonly lodge in the right main stem bronchus, with the right lower lobe being the most common site of impaction, presumably because of the vertical orientation of the bronchus. Complications associated with long-standing FBAs, including recurrent pneumonias, lung abscesses, bronchial stenosis, empyemas, and bronchocutaneous fistulas, make it more difficult to detect FBA radiographically. Diagnosis and occasionally treatment can be made by flexible bronchoscopy, but often rigid bronchoscopy is required. There are procedural challenges related to the location, or the foreign body itself, which can be surrounded by granulation tissue posing difficulty in removing the foreign body. Moreover, if the diagnosis of FBA is not known a priori, visualization on bronchoscopy is less likely, especially with organic material, which within hours of impaction tends to be surrounded by mucosal inflammation and granulation tissue, requiring histological examination. In our case, as there was no history of aspiration, radiographic studies and direct visualization led to misdiagnosis of bronchogenic carcinoma with surgical lobectomy leading to the correct diagnosis. FBA is often not suspected in healthy adults and may present a diagnostic challenge. Aspiration should be included in the differential diagnosis of recurrent or prolonged lung disease.

A MYSTERIOUS SLOW DECLINE: HTLV ASSOCIATED MYELOPATHY Md J. Ahmed; Michael Ryan; Nazrul Chowdhury; Roger D. Smalligan. Texas Tech University Health Sciences Center, Amarillo, TX. (Control ID \#1334585)

LEARNING OBJECTIVE 1: Recognize an uncommon cause of a slowly progressive lower extremity weakness in an adult.

LEARNING OBJECTIVE 2: Diagnose HTLV Associated Myelopathy in adults.

CASE: A 58yo African American man presented with slowly progressive weakness in his lower extremities (LE) over an 8 year period. On walking short distances he had severe fatigue in his legs and would frequently fall. He had tingling in his feet, dizziness on arising, urinary urgency and nocturia. The patient denied headaches, visual or hearing changes, neck and back pain. He had no history of trauma or stroke and denied foreign travel. PMH was significant for hepatitis $\mathrm{C}$ and $\mathrm{HTN}$. FH was negative for neurologic diseases. P/S history: positive for smoking; denied alcohol and current drug use. PE: orthostatic changes in blood pressure and pulse but otherwise normal heart, lung and abd exams. Neuro: cranial nerves intact, $4 / 5$ strength in LEs, DTRs brisk except decreased at the ankles. Vibratory sense was absent at the toes and decreased at the ankles. Pinprick was diminished to the midfoot bilaterally and toes were upgoing. Gait was halting and unsteady. Lab: normal $\mathrm{CBC}$, chemistries, coagulation panel, HbA1c, CPK, vit B12, copper and urinalysis. AST/ALT were mildly elevated. HIV, RPR, influenza were negative. CSF: WBC 11, $(98 \%$ lymphs, $2 \%$ monos), normal gluc/protein and gram stain. Brain MRI showed enhancing white matter lesions on T2/FLAIR in the frontal/parietal lobes, thalamus and pons. MRI of the spine showed diffuse atrophy of the cord. HTLV I/II titers in serum and CSF were positive. NCV/EMG showed neuropathy without myopathy in LEs. The patient was treated with a trial of steroids and had some increased strength and less falls.

DISCUSSION: This patient's history, exam, lab and imaging studies meet WHO criteria for the diagnosis of HTLV-Associated-Myelopathy (HAM). HTLV-I is known to be the causative agent of several diseases including adult T cell leukemia, HAM (also referred to as Tropical Spastic Paraparesis (TSP)) and distinct forms of uveitis, dermatitis and myositis. The virus is endemic in Japan, the Caribbean and parts of Africa. Transmission is by breastfeeding, blood products and intravenous drug use. It is believed that 20-30 million people are infected worldwide; $1-3 \%$ of infected individuals develop HAM/ TSP. The time from infection to onset of symptoms ranges from years to decades and between 35 to 45 years of age. The clinical picture is much like our patient's: slowly progressive, symmetric LE weakness with some spasticity, hyper-reflexia and Babinski signs; upper extremities are spared. Urinary and bowel dysfunction are common as are parasthesias, although motor complaints usually predominate. HAM can mimick primary progressive multiple sclerosis except that HAM does not have a relapsing-remitting course. Diagnosis is suggested by the history and physical and confirmed with images and labs including positive IgG titers to HTLV I/II in serum and CSF. MRI can be normal but $50 \%$ of patients have white matter lesions as in our patient. Spinal cord MRI may show atrophy or degeneration. Small treatment trials have shown anti-virals to be ineffective and mixed results from steroids. Progression to wheelchair bound status occurs on average at 21 years from diagnosis but the disease is rarely fatal. Further research is needed to identify a more effective treatment regimen. Internists need to suspect HTLV associated myelopathy in any middle-aged patient with progressive weakness combined with spasticity. 
A POEMS THAT DOES NOT RHYME: AN UNUSUAL CASE OF POLYNEUROPATHY Patrick Green ${ }^{1,2}$; Lara Paraskos ${ }^{1,2}$; Yoel Brito ${ }^{1,2}$; Erin N. Marcus ${ }^{1}$. ${ }^{1}$ University of Miami Miller School of Medicine, Miami, FL; ${ }^{2}$ Jackson Memorial Hospital, Miami, FL. (Control ID \#1339320)

LEARNING OBJECTIVE 1: Recognize the diagnostic criteria for POEMS Syndrome.

LEARNING OBJECTIVE 2: Understand the limitation of serum protein electrophoresis for the diagnosis of monoclonal plasma cell disorders.

CASE: A 59 year old male with no past medical history presented to his primary care physician with generalized abdominal pain, lower extremity numbness and tingling pain, fatigue, and erectile dysfunction. Initial studies revealed hypothyroidism, low testosterone, low Vitamin B12, thrombocytosis, and hepatosplenomegaly. Treatment was initiated with levothyroxine, testosterone replacement, a PDE-5 inhibitor, gabapentin, and B12 injections but his symptoms worsened. A fat pad biopsy was negative for amyloidosis. Four months after his initial presentation he went to an emergency department for increased abdominal pain and girth. Paracentesis showed spontaneous bacterial peritonitis. Physical exam was remarkable for ascites, hepatosplenomegaly, static mechanical allodynia and edema of the lower extremities, facial telangectasias, and a nonblanching rash on the dorsum of his feet. He was admitted and started on Cefotaxime and albumin. Laboratories showed normal liver function tests and $\mathrm{CBC}$ with a creatinine of 1.2 , which continued to worsen. Ultrasound showed hepatosplenomegaly and patent hepatic vasculature. Hepatitis B, Hepatitis C, HIV, anti-smooth muscle antibody, anti-mitochondrial antibody, and a 24-hour urine copper were normal. Transhepatic biopsy showed congestion with negative congo red and rhodamine stains. Electromyography showed diffusely slowed nerve velocities and lack of motor responses consistent with a demyelinating process such as chronic inflammatory demyelinating polyneuropathy. The medical team entertained a diagnosis of POEMS syndrome. SPEP revealed an IgA isotype with elevations of both lambda and kappa light chains with a normal kappa/ lambda ratio interpreted as no monoclonal spike. He had an elevated serum VEGF level. Two weeks after admission he acutely developed respiratory arrest, and died. Autopsy showed an incidental low grade follicular lymphoma in the mesenteric lymph nodes.

DISCUSSION: POEMS is a clinical syndrome characterized by polyneuropathy, organomegaly, endocrinopathies, monoclonal plasma cell proliferative disorder, and skin changes. Diagnosis requires polyneuropathy and a monoclonal plasma cell disorder plus one major criteria (sclerotic bone lesions, Castleman's disease, or elevated VEGF level) and one minor criteria (organomegaly, endocrinopathy - but not isolated diabetes or hypothyroidism, skin changes, papilledema, extravascular volume overload, thrombocytosis, or polycythemia). The majority $(90 \%)$ have elevated lambda chains. Our patient had all of the characteristic findings except the monoclonal spike. Review of the literature revealed a series of 50 patients in which there was a correlation between renal failure and elevated lambda and kappa levels resulting in a normal ratio and lack of an M-spike on SPEP. Reports of the sensitivity vary with most studies citing sensitivities around $70 \%-98 \%$ based on methodology and the disease for which it is being used to screen. In cases with no monoclonal spike on SPEP, determination of a clonal plasma cell disorder can be made via bone marrow biopsy, flow cytometry, serum protein immunofixation. In this case, examination of the bone marrow on autopsy may have revealed a monoclonal plasma cell disorder.

A PAIN IN THE NECK: VERTEBRAL ARTERY DISSECTION AFTER CERVICAL MANIPULATION William A. Hammond. Dartmouth-Hitchcock Medical Center, Lebanon, NH. (Control ID \#1339528)

LEARNING OBJECTIVE 1: Recognize, assess, and expeditiously treat vertebral artery dissections and associated posterior circulation cerebrovascular accidents.

LEARNING OBJECTIVE 2: Appropriately counsel patients about potential risks of chiropractic cervical manipulation

CASE: A 33 year old right-handed man with history of mild hypertension was well until about a month prior to presentation to the Emergency
Department when he experienced pneumonia with severe cough. He recalled a prolonged coughing bout, after which his neck began to ache. This persisted, so he sought chiropractic treatment. On his second visit, immediately following cervical spine manipulation, he noted light-headedness, tingling in the right hand and foot, and became diaphoretic. He attempted to stand but noted dizziness and listing to the right. When symptoms did not rapidly resolve, he was transported to the ED and was found to be hypertensive with systolic blood pressure greater than $200 \mathrm{mmHg}$. Exam revealed mild right ptosis, right beating nystagmus, and anisocoria with a miotic right pupil. He was diaphoretic, but mental state was intact. He exhibited mild right arm ataxia and a muted right plantar reflex, however the remainder of his neurologic exam, including strength and sensation, was unremarkable. Computed tomography of his head and neck revealed an extensive right vertebral artery dissection, and subsequent magnetic resonance imaging of the brain revealed right sided cerebellar and medullary infarcts consistent with the clinical presentation of lateral medullary infarction, or Wallenberg syndrome. He was started on a heparin infusion and transitioned to full anticoagulation with warfarin for a planned course of six months of therapy

DISCUSSION: Vertebral artery dissection is a rare complication of chiropractic manipulation, however, may contribute to more injuries than clinically recognized. A body of literature exists of multiple case reports indicating that dissection may be quite common. The arterial injury may be preceded by an incident causing an intimal tear, perhaps accompanied by cervicalgia. This tear allows propagation of an arterial intramural dissection when shearing forces from cervical manipulation of the vertebral bodies apply unnatural pressure to the vertebral arteries as they course through the transverse foramina. Given the anatomical predisposition to this sort of injury, patients should be warned about this potential complication of chiropractic therapy. This is increasingly pertinent as the use of complementary therapeutics becomes more ubiquitous. The most common presenting symptom of dissection is dizziness, indicating vestibulocerebellar involvement, but can also include findings similar to the aforementioned case including nystagmus, ipsilateral limb ataxia, and ipsilateral Horner's syndrome. These findings are related to the infarction territory in the lateral medulla with vestibular nuclei and the descending sympathetic nervous system. The abnormal sweating and labile blood pressure is explained by lesions involving the dorsal motor nucleus of the vagus nerve. This syndrome should be recognized early so that therapy can be initiated with rapid imaging and subsequent full anticoagulation, if no contraindications exist.

A PANCREATIC PSEUdOCYST GOING ROGUE Alice Williamson; Jamie Nguyen; Joseph Luka; Peter S. Reynaud. Tulane $\overline{\text { University Health }}$ Sciences Center, New Orleans, LA. (Control ID \#1340486)

LEARNING OBJECTIVE 1: 1. Recognize the clinical presentation of extra-abdominal pancreatic pseudocyst. 2 . Understand the pathophysiology of pancreatic pseudocyst formation.

CASE: A 49 year-old woman with chronic pancreatitis presented with new-onset fever and epigastric tenderness. She noted diffuse abdominal tenderness without distention, or changes in her bowel habits. There was associated intermittent pleuritic chest pain. She had a history of rheumatoid arthritis, COPD, HIV, and multiple recurrent pneumonias over the previous two years. She had a forty-pack year history of smoking and alcohol use, but had stopped drinking three years earlier. Her vital signs were normal. Her cardiac, pulmonary and neurologic examinations were normal. She had diffuse tenderness to abdominal palpation, but no guarding or rebound tenderness; her liver size was small. The amylase and lipase levels were normal, as was her $\mathrm{CBC}$ and INR. The chest X-ray revealed left lower lobe airspace disease. Given her history of recurrent pneumonia and chronic pancreatitis, a CT scan of her chest, abdomen, and pelvis was obtained, revealing a peripherally-enhancing, air-containing, complex fluid collection within the thoracic cavity. The fluid collection extended from the pancreas through the esophageal hiatus into the posterior pleural space bilaterally with two main components measuring $6.0 \times 4.0 \mathrm{~cm}$ on the left and $3.0 \times 1.0 \mathrm{~cm}$ on the right. A CT-guided drainage and contrast injection revealed contrast flowing through the medial right and left pleural spaces, along the 
medial and inferior aspects of the stomach, then into the pancreatic duct - a pancreatic pseudocyst. Of note, the fluid culture grew VRE for which she was appropriately treated.

DISCUSSION: Pancreatitis is a commonly encountered diagnosis by the general internist. Past establishing the diagnosis, the internist must also be adept at recognizing the complications of this disease. Pancreatic pseudocysts are cystic cavities bound to the pancreas by inflammatory tissue. They are infrequent complications of pancreatitis and more commonly occur in alcohol-induced pancreatitis. Most pseudocysts develop in the peripancreatic area; however, they rarely extend elsewhere, preferring the paths of least resistance and presenting with symptoms of irritation to the local areas. In this case, the pathway was through the esophageal hiatus and into the posterior pleural space bilaterally. Given the complex nature of this pseudocyst and the patient's recurrent illness, she needed surgical excision. She underwent exploratory laparotomy, extensive lysis of adhesions, distal pancreatectomy, and splenectomy with pleural fistula excision. Unfortunately, she passed away five days after the surgery.

A QUESTIONABLE CLOT Samuel E. Cohen; Manuela Calvo; David de Gijsel. Montefiore Medical Center, Bronx, NY. (Control ID \#1324666)

LEARNING OBJECTIVE 1: 1) Discuss a case where the diagnosis of pulmonary embolism was investigated.

LEARNING OBJECTIVE 2: 2) Highlight the importance of clinical epidemiology in medical decision-making.

CASE: A 52 year-old woman, with a history of scimitar syndrome (a right pulmonary vein which empties directly to the inferior vena cava), presented with shortness of breath and chest pain. The shortness of breath started two years ago and had been gradually worsening since then. It was now present at rest and there was associated three-pillow orthopnea. The chest pain was pleuritic, left-sided and radiated to the back. She was tachycardiac to 104 beats per minute. A V/Q scan was performed and revealed a segmental perfusion defect in the right lower base, which is considered to be high-probability for pulmonary embolism. Anticoagulation was initiated. Upon further examination, it was discovered that the pain was reproducible on palpation and the d-dimer was $1.0 \mathrm{ug} / \mathrm{dl}$ (low-probability). The V/Q scan was thought to be a false positive. The patient was discharged with the diagnosis of costochrondritis. Two weeks later, the patient presented to the Emergency Department with the same symptoms. At that time, an echocardiogram was done and revealed severe global left ventricular dysfunction, with an ejection fraction of $20 \%$. Due to uncertainty regarding the interpretation of the V/Q scan from the previous admission, a CT of the thorax with contrast was performed. It revealed no embolus, but showed "bronchial-arterial dissociation" in the right inferior lung i.e. the bronchial tree and arterial tree did not share the same course.

DISCUSSION: While a high probability V/Q scan in a patient with chest pain and shortness of breath would appear to make a strong argument in favor of the diagnosis of pulmonary embolism, a closer look at the clinical context of the patient's presentation cautions a shrewder approach. According to the PIOPED study, a high probability $\mathrm{V} / \mathrm{Q}$ scan in the setting of a low clinical suspicion as judged by a physician was positive for $\mathrm{PE}$ in 5 out of $9(56 \%)$ patients. In the setting of intermediate suspicion, a high probability V/Q scan was positive for a PE in 70 out of $80(88 \%)$ patients. As judged by the attending physician at the time of the patient's first hospital admission, a pulmonary embolism was not the most likely diagnosis based on the history and physical examination. Additionally, the Modified Wells Score was 1 and the Simplified Geneva Score was 2, both considered low probability. This low risk provides a framework by which to interpret the subsequent lab tests and radiological studies. Unique to this case, the patient's anomalous pulmonary vein clued in the medical team to a plausible, alternative explanation for mismatched ventilation and perfusion. Congenital heart defects, as well as heart failure, COPD, and pneumonia may cause positive V/Q scans. Consequently, the gold standard, CT angiography, corroborated and accounted for the falsepositive $\mathrm{V} / \mathrm{Q}$ scan. This raises an interesting point regarding the paradoxical nature of false-positive results and management of the subsequent uncertainty: when can a presumed false positive be disregarded and when is additional confirmatory testing required?
A RARE ASSOCIATION OF MONOCLONAL GAMMOPATHY OF UNDETERMINED SIGNIFICANCE. Girish Singhania; Dipaben Patel; Soujanya Sodavarapu; Swapna Chalasani. OSF St Francis Medical Center, Peoria, IL. (Control ID \#1311950)

LEARNING OBJECTIVE 1: Monoclonal Gammopathy of Undetermined Significance (MGUS) can present as protein losing enteropathy by association with localized Gastrointestinal (GI) amyloidosis.

CASE: An 85-yr-old Hispanic female with history of hypertension presented with abdominal pain, diarrhea and increased abdominal distension for 4 days. She also had increased fatigue, dysphagia and edematous legs for 4 months. She denied shortness of breath, chest pain, fever or dysuria. On examination, she was alert and oriented but fatigued. There was no jaundice or macroglossia. She had decreased bibasilar breath sounds, normal heart sounds, distended abdomen without hepatosplenomegaly and +2 lower extremity edema. Initial labs showed $\mathrm{CBC}$, electrolytes, creatinine, lipase, bilirubin and lactate within normal limits. Her albumin was found to be $1.5 \mathrm{gm} / \mathrm{dL}$. Stool was negative for helminthic ova, giardia and clostridium difficile antigen. Chest X-ray showed small pleural effusions. CT abdomen revealed diffuse fluid-filled dilation of the small bowel. Upper GI series showed moderate presbyesophagus and spasm at the esophagogastric junction. Esophagogastroduodenoscopy showed multiple soft nodules in the gastric antrum with normal esophagus and duodenum. Esophageal and duodenal biopsy showed amorphous eosinophilic material which demonstrated apple-green birefringence with Congo red stain under polarized light suggestive of Amyloidosis. Alcian Blue, Periodic acid-Schiff, Warthin-Starry stain were negative for GI metaplasia, Whipple's disease and Helicobactor pylori respectively. Etiological work-up for amyloidosis included ESR $4 \mathrm{~mm} / \mathrm{hr}$, C-reactive protein $1.3 \mathrm{mg} / \mathrm{dL}$, Calcium $8.6 \mathrm{mg} / \mathrm{dL}$, urine protein $384 \mathrm{mg} / 24 \mathrm{hr}$ and negative Rheumatoid factor and Antinuclear antibodies. Serum protein electrophoresis detected a paraprotein spike at $1.44 \mathrm{gm} / \mathrm{dL}$. Urine protein electrophoresis showed a faint gamma band. Immunofixation revealed increased $\mathrm{IgG} /$ Lamba monoclonal immunoglobulin suggestive of MGUS. Immunohistochemistry and flow cytometry from peripheral smear were negative for clonal plasma cells or malignant B-cells. Echocardiography showed no infiltrative cardiomyopathy. The patient was started on tube feeds because of dysphagia. Her clinical status continued to deteriorate with worsening dyspnea and severe malnutrition secondary to protein losing enteropathy. Percutaneous gastrostomy tube was suggested. However, after extensive discussions, the family refused and decided to withdraw all life support measures and the patient died.

DISCUSSION: MGUS is defined by the presence of a serum monoclonal protein concentration less than $3 \mathrm{gm} / \mathrm{dL}$. It has rarely been shown to be complicated by amyloidosis. Our case describes a rare association of localized GI amyloidosis with MGUS. No clinical, laboratory and imaging findings were consistent with cardiac, renal and liver involvement ruling out systemic amyloidosis. As MGUS can be complicated by amyloidosis and multiple myeloma transformations, a follow-up of these patients and further research is needed to establish guidelines for better management.

A RARE CASE OF COMPLETE HEART BLOCK Ehtesham U1 Haq; Abdul Haseeb; Subhraleena Das; Amit S. Dhamoon. SUNY Upstate Medical University, Syracuse, NY. (Control ID \#1334129)

LEARNING OBJECTIVE 1: Recognize cardiac manifestations of Rabies

LEARNING OBJECTIVE 2: Learn to monitor cardiac function in Rabies patients

CASE: A 24 year old male with no significant past medical history was bitten by a stray dog on thenar eminence of right hand. According to the patient, the dog was captured, killed and his brain was biopsied which did not show any infection with Rabies virus. The patient received a vaccine at that time but he did not remember the details of treatment. Subsequently, the patient presented seven months later with severe numbness and tingling of right hand. He also had intractable nausea, vomiting and throat tightness when drinking fluids. Physical exam was within normal limits at the time of admission. He started becoming anxious and agitated on 2 nd day of hospitalization. CT scan of the head was normal. The patient became very 
agitated and required intravenous Ativan, intramuscular Zipresidone and eventually required endotracheal intubation for airway protection. A provisional diagnosis of Rabies was made. Subsequently nuchal skin biopsy, salivary sample and CSF analysis were all consistent with Rabies encephalitis. After confirmation of the diagnosis, the patient was put on the Milwaukee protocol for Rabies encephalitis and was treated with Ketamine, Ribavarin, Amantadine and Fentanyl. Patient became bradycardiac with a heart rate in the 30s and went into Mobitz type II and third degree heart block. He was given atropine intravenously. Cardiology was consulted urgently and a transvenous pacemaker was placed immediately at the bedside. Cardiac injury panel remained normal in this patient. Pacemaker was rechecked multiple times during the hospital stay and it was found to have excellent capturing. Due to the complexity of ongoing medical issues, permanent pacemaker was not inserted. Hospital course was complicated by Diabetes Insipidus, ARDS requiring extracorporeal membrane oxygenation and intracranial hemorrhage. Intracranial bleed with large midline shift was not amenable to any surgical intervention. Patient was made comfort care at this point and he died.

DISCUSSION: Rabies is a preventable zoonotic disease that has the highest case fatality rate of any infectious disease. Viral replication occurs in the CNS and then virus spreads along neural pathways to Heart, Skin and other organs. In Heart, Rabies virus can cause cardiac arrhythmias and myocarditis. Myocarditis may reflect both hyperadrenergic state and direct viral infection. Cardiac injury panel should be checked periodically and patients should be kept on telemetry to monitor for myocarditis and high degree AV blocks respectively. There have been very few case reports in literature mentioning high degree heart block requiring pacemaker in Rabies patients. Our patient went into high degree AV block from Rabies requiring pacemaker. Heart block was managed appropriately with urgent pacemaker placement but multi organ failure led to his death.

A RARE CASE OF SHIGELLA BACTEREMIA Darshan Kothari; Alexis Z. Tumolo; Rebecca Hutchinson. BIDMC, Boston, MA. (Control ID \#1340418)

LEARNING OBJECTIVE 1: Review causes of acute onset bloody diarrhea, recognizing Shigella as a less common cause of enteritis and bacteremia.

LEARNING OBJECTIVE 2: Recognize the importance of blood culture surveillance and immediate therapy in immunocompromised hosts.

CASE: A 55 year-old male with past medical history of HIV (CD4 121, viral load 4,160), hepatitis $C$, and gastric lymphoma presented with a four day history of hematochezia. The patient reported 5-10 bloody bowel movements per day, without abdominal or rectal pain. He also denied fevers, chills, nausea, vomiting, melena, sick contacts, recent travel, dietary changes, prior episodes of bloody diarrhea, recent sexual contact, or drug use. On the day of admission, he was found to be lethargic and with altered mental status. In the emergency department, the patient had $500 \mathrm{cc}$ of rectal bleeding with an associated four-point hematocrit drop. Labs were notable for a leukocytosis to 17,200 with $9 \%$ bands and an anion gap with a lactate of 1.9. An abdominal CT demonstrated thickened mucosa in the terminal ileum and diffusely throughout the colon. Given these findings, intravenous ciprofloxacin and metronidazole were initiated for empiric therapy of colitis. Blood cultures were drawn on admission and within 48 hours grew Shigella flexneri, sensitive to penicillins, cephalosporins, and fluoroquinolones. Stool cultures were initially negative however ultimately grew $S$. flexneri. Antibiotics were tailored to ciprofloxacin. Diarrhea and mental status improved over his hospital course. As his mental status improved, he complained of severe rectal pain concerning for perirectal abscess. However, a pelvic MRI was remarkable only for findings consistent with colitis. Pain was ultimately attributed to a rectal tube placed in the intensive care unit. The patient was discharged to complete two weeks of ciprofloxacin. At two-week follow up with his PCP, his symptoms had resolved.

DISCUSSION: Dysentery is an inflammatory state of the intestine caused by an enteroinvasive pathogen resulting in bloody diarrhea. The common bacterial pathogens causing bloody diarrhea include E. coli O157:H7, Shigella, Campylobacter, and Salmonella. Bacteremia is a rare complication of dysentery but has been reported with Shigella infections. This is more frequently reported in the pediatric population, however also occurs in immunocompromised adults. In 1993, Huebner et al. reviewed twelve reported cases in Shigella bacteremia in HIV-positive patients [1]. A review article in the Archives of Internal Medicine from 1987 reported higher mortality rates in cases with isolated bacteremia than in patients with Shigella cultured from blood and stool in patients with HIV [2]. Thus, it is imperative that blood cultures be obtained from patients on presentation before initiating antibiotic treatment, especially in immunocompromised hosts. Positive blood cultures should then define the antibiotic course, with gram-negative-rod bacteremia usually requiring two weeks of treatment. References: 1. Huebner J, Czerwenka W, Gruner E, von Graeventiz A. Shigellemia in AIDS patients: care report and review of the literature. Infection. 1993 Mar-Apr;21(2):122-4. 2. Morduchowicz G, Huminer D, Siegman-Igra Y, Drucker M, Block Cs, Pitlik SD. Shigella bacteremia in adults. A report of five cases and a review of the literature.Arch Intern Med. 1987 Nov; 127(11):2034-7.

A RARE CASE OF HEPATOSPLENIC LYMPHOMA: THE DIAGNOSTIC CHALLENGE OF INFECTION VS MALIGNANCY Elizabeth Selden; Andrew A. Chang. NYU, New York, NY. (Control ID \# $\# \overline{1323721)}$

LEARNING OBJECTIVE 1: Recognize hepatosplenic lymphoma as a rare subtype of T-cell lymphoma

LEARNING OBJECTIVE 2: Recognize the dangers of early diagnostic closure

CASE: A 30 year-old Guinean man with no past medical history presented with cough, fever, night sweats and weight loss for two months. The patient immigrated to the US 6 years ago. Exam showed cachexia, HR 122, T 101.3, BP 105/72, RR 22, O2 96\% on RA, splenomegaly, normal lung exam and no appreciable lymphadenopathy. Labs were significant for a WBC 3.0 with $73 \% \mathrm{~N}$, Hbg of 7 and a negative rapid HIV. CXR was unremarkable. Patient was isolated for possible tuberculosis. CT revealed splenomegaly, multiple scattered low-density lesions in the liver, prominent mediastinal, hilar, axillary and retroperitoneal lymphadeopthy, with the largest node measuring $3.7 \times 2.8 \mathrm{~cm}$. CT-guided biopsy of the retroperitoneal lymph node revealed fibrocollagenous tissue with necrosis. AFB, fungal stains, and cultures were negative. High fevers persisted to Tmax 105.7 F. Despite three negative AFB sputums, patient was started on a liver-sparing TB regimen given clinical suspicion for extra-pulmonary tuberculosis and worsening liver function. A bone marrow biopsy was performed revealing normal bone marrow with slight megakaryocyte hyperplasia but no evidence of lymphoma. Repeat core biopsy of the retroperitoneal lymph node showed necrotic tissue with few $\mathrm{CD} 3+\mathrm{T}$ cells; AFB and cultures again negative. The course was further complicated by acute hepatic failure and transfusion dependent pancytopenia. On day 20, as the risks of invasive surgical testing increased, decision was made to perform excisional biopsy of a small superficial inguinal lymph node seen on CT. Preliminary pathology showed T-cell lymphoma with cytotoxic features. TB treatment was stopped and chemotherapy with a modified $\mathrm{EPOCH}$ regimen was initiated. After biopsy, flank pain, severe agitation from hepatic encephalopathy and DIC developed. This was concerning for retroperitoneal bleed and led to intubation and sedation. The patient's clinical picture rapidly declined and multi-organ system failure ensued. The patient expired one month after hospital admission. Final pathology of lymph node revealed hepatosplenic T-cell lymphoma of the alpha/beta subtype.

DISCUSSION: Hepatosplenic T-cell lymphoma (HSTCL) is a rapidly progressive variant of peripheral T-cell lymphoma, in which mature T cells infiltrate the spleen, liver and bone marrow. These T-cells are usually of the gamma/delta subtype; however in rare cases such as this one, they express the alpha/beta T-cell receptor. These lymphomas account for less than one percent of non-hodgkin's lymphomas, and typically affect men in their thirties. Median survival is less than 2 years. This case of HSTCL was unusual in that there was significant diffuse lymphadenopathy, the T-cells were alpha/beta subtype rather than delta/gamma, and hepatomegaly was not present. Given the aggressive nature of HSTCL, expedient diagnosis is critical. The patient's presenting symptoms and emigration from an area endemic for tuberculosis rightly raised concern for TB; however, it is essential to consider other diagnostic pursuits and not have early closure on diagnosis. In retrospect, an excisional biopsy should have been performed earlier in his hospital course given the inconclusive core biopsy of the 
retroperitoneal lymph node. It is unclear whether this would have ultimately changed the outcome of the case, but it may have lead to earlier diagnosis and treatment.

A RARE CASE OF RECURRENT COCAINE INDUCED PSEUDOVASCULITIS Ayodeji O. Shedu ${ }^{1}$; Yongli $\mathrm{Ji}^{1}$; Gregory Williams ${ }^{2}$; Alice Williams ${ }^{2} .{ }^{1}$ St vincent Hospital, Worcester, MA; ${ }^{2}$ Reliant Medical Group, Worcester, MA. (Control ID \#1304923)

LEARNING OBJECTIVE 1: Consider cocaine as etiology of vasculitis during work up

CASE: A 43 year old woman was admitted for a two day history of rash, swelling and pain in both hands. She also reported chills and sore throat. Further review of systems was negative except for bilateral knee pain that had resolved by admission. Her past history includes depression, heavy use of alcohol, and osteomyelitis of the right index finger secondary to a cat bite about 6 weeks before the present illness which required partial amputation. Her home medications included sublingual buprenorphine and oral clindamycin. The patient had presented with similar symptoms two years ago after an episode of cocaine consumption. C-ANCA and cardiolipin IgM at the time were positive. The lesions resolved after she had abstained from cocaine. On exam she had a low grade fever of $100.1^{\circ}$. A purpuric rash was found on the earlobes, hands, gluteal and suprapubic regions, and both lower extremities. Some of the affected areas had bullous changes with necrosis. Severe tenderness was noted. Laboratory studies were remarkable for a positive ANA, IgM of 24, and p-ANCA 1:640. PR-3 and MPO specificity were negative. Cryoglobulin was negative and C3, C4 and $\mathrm{CH} 50$ were normal. Toxicology screening was positive for cocaine and alcohol. PT/INR/PTT were normal. Fibrinogen was elevated at 427 but fibrin split products were negative. Blood cultures and RPR were negative. The patient was advised to abstain from cocaine and was managed symptomatically, with complete resolution of vasculitis in six weeks.

DISCUSSION: Pseudovasculitis is often clinically difficult to differentiate from vasculitis. Cocaine use is a known but rare cause of pseudovasculitis. This patient had repeated episodes of small vessel cutaneous vasculitis which were associated with cocaine use. Her laboratory results were inconclusive for any of the primary vasculitides, and there were no identifiable triggers aside from cocaine use. The lesions resolved after she stopped snorting cocaine. This case illustrates the importance of considering cocaine as the etiology of vasculitis during the work up of patients. Identification of the etiology of pseudovasculitis and subsequent removal of the stimulus prevents unnecessary, often dangerous and expensive treatments.

A RARE CASE OF SPONTANEOUS PNEUMOTHORAX IN A YOUNG WOMAN Lindsay A. Lucas ${ }^{1}$; Tariq Cheema ${ }^{2,1}$. ${ }^{1}$ West Penn Allegheny General Health System, Pittsburgh, PA; ${ }^{2}$ Allegheny General Hospital, Pittsburgh, PA. (Control ID \#1322870)

LEARNING OBJECTIVE 1: Recognize the clinical features of thoracic endometriosis.

LEARNING OBJECTIVE 2: Describe the hypothesized pathophysiology behind catamenial pneumothorax.

CASE: A 20-year-old woman presented with shortness of breath and stabbing left-sided chest pain that started at a concert that night. Pain was constant, worse with inspiration, and associated with anxiety. She is a nonsmoker, and denied trauma or recent illness. History was notable for congenital hypothyroidism and three spontaneous pneumothoraces, the first of which occurred at age 16. Family history was significant for endometriosis in her mother. In the last 18 months, she was hospitalized twice for pneumothoraces requiring chest tube decompression and had been to the emergency department several times for panic attacks with similar pain. Recently she left college and returned home due to missed classes and anxiety. Her only medication was levothyroxine. Physical exam disclosed a tall, anxious-appearing, thin young woman, BMI 18. She was mildly hypoxemic on room air. Increased work of breathing and decreased breath sounds over the left apex were noted. Chest radiograph revealed a large pneumothorax with collapse of the left upper lobe. A chest tube was placed in the ER, and she underwent video-assisted thoracic surgery the next morning for pleurodesis, wedge resection of the bleb, and pleural biopsy. Alpha-1 antitrypsin, CBC, CMP, and thyroid studies were normal. Pathology revealed nonspecific fibrosis and chronic inflammation. Further history revealed she was menstruating prior to the onset of symptoms. She was referred to gynecology and started on oral contraceptives for catamenial pneumothorax. At six month follow up she has had no further pneumothoraces, anxiety has improved, and she doing well in community college.

DISCUSSION: Catamenial pneumothorax (CP) and catamenial hemothorax are uncommon clinical entities related to thoracic endometriosis, characterized by recurrent pneumo- or hemothorax within 72 hours of menstruation. Once thought to be rare, new data suggest thoracic endometriosis is under recognized as a cause of secondary pneumothorax. In three large studies of recurrent pneumothorax in women of reproductive age, prevalence of $\mathrm{CP}$ ranged from less than $1 \%$ to $24.6 \%$. Little is known about the exact mechanism by which CP occurs. Histological evidence of endometrial tissue is identified in less than $13 \%$ of biopsied blebs, suggesting the mechanism of injury is not necessarily related to sloughing of endometrial implants on \visceral pleura, but may also include indirect effects of hormones or prostaglandins on existing blebs, and progressive destruction of alveoli from circulating endometrial cells that enter the thoracic cavity through congenital diaphragmatic defects. $\mathrm{CP}$ should be considered a clinical rather than histopathological diagnosis. Initial treatment includes rest for mild cases and thoracostomy for larger lesions. Long-term management is not well defined due to limited identification of the condition. Invasive strategies include pleurectomy, pleurodesis, diaphragmatic defect repair, or ablation of endometrial implants. Medical therapies are aimed at hormonal management including oral contraceptives and GnRH agonists. Thoracic endometriosis is a rare but potentially treatable cause of recurrent hemothorax/pneumothorax in women of reproductive age, and a source of significant stress on those affected. Identification and continued research will be the keys to identifying optimal treatment in the future.

A RARE HEPATIC PARASITIC INFECTION IN CENTRAL PENNSYLVANIA: THE STORY OF SHEEP AND DOGS Abdulla Damluji ${ }^{1}$; Tareq Yasin ${ }^{2}$; Thomas J. McGarrity ${ }^{2}$; Nicole Swallow'. 'Penn State University, Hershey, PA; ${ }^{2}$ Penn State University, Hershey, PA. (Control ID \#1340065)

LEARNING OBJECTIVE 1: Hydatid disease is a zoonotic infection that is caused by the larval form of tapeworms of the genus Echinococcus. It is endemic in Australia, Latin America, Eastern Europe, the Middle East, and Africa (1). In central Pennsylvania the disease is very rare. We report a case of Ecchinococcus cystic disease of the liver that presented to the Penn State MS Hershey Medical Center.

CASE: An 85-year-old male with a past medical history of hypertension, dyslipidemia, atrial fibrillation on systemic anticoagulation, peripheral vascular disease and chronic obstructive pulmonary disease presents with few days of fevers and generalized fatigue. The fevers are low grade and continuous in nature. He denies any respiratory symptoms or severe pruritis. His review of system was significant for abdominal fullness, early satiety, and weight loss over a month time. He has no history of anaphylaxis, hepatitis, jaundice, or known liver disease. His past surgical history is significant for multiple inguinal and abdominal hernia repairs. $\mathrm{He}$ lived in central Pennsylvania all his life. His temperature was 38.6, HR 65, RR 20, BP 102/54, Sp O2 93\% on room air. His physical examination showed no scleral icterus. His Abdomen was soft, non-tender, and his bowel sounds were normal. There was no evidence of hepatomegaly or ascitis. Laboratory values revealed his white cell count was 9.4, neutrophils 93.1\%, and eosinophils were normal. LFT showed AST 400, ALT 133, T Bilirubin 2.3, ALP 202, and INR 3.8. Lipase and TSH were normal. Abdominal ultrasound showed numerous bilateral hepatic cysts. A follow up abdominal MR scan showed multiple benign hepatic cysts involving both liver lobes, the largest of which measures $2.4 \mathrm{~cm}$. No cysts were found in his lungs or brain. A follow up AFP was normal. His Echinococcal antibody ( $\operatorname{IgG})$ tested highly positive. His blood cultures grew $\mathrm{E}$ Coli that was thought to be biliary in origin. He was started on cefazolin for his E Coli Bacteremia and Albendazole $400 \mathrm{mg}$ by mouth twice daily for his hydatid disease. He was scheduled for preoperative evaluation as an outpatient prior to surgical resection of his cysts. 
DISCUSSION: Hydatid disease is rare in the United States. Most cases are due to E. Granulosus. It is prevalent where livestock is raised in association with dogs, such as in central Pennsylvania. In primary Echinococcal disease any organ can be affected with liver being most common site. Cysts can become symptomatic if they exert pressure on adjacent tissues. Cyst rupture causes spillage of viable protoscolices, which can cause secondary disease. Surgery can be curative (2). High level of suspicion is needed in rural areas with low prevalence of the disease for prompt diagnosis and management. 1. Ammann RW, Eckert J. Cestodes. Echinococcus. Gastroenterol Clin North Am 1996;25(3):655-689. 2. Guidelines for treatment of cystic and alveolar echinococcosis in humans. WHO Informal Working Group on Echinococcosis. Bull World Health Organ 1996;74(3):231-242.

A RASH: OH M-EYE! Kate Hust; Christian Fauria-Robinson. Tulane University Health Sciences Center, New Orleans, LA. (Control ID \#1340478)

LEARNING OBJECTIVE 1: 1. Recognize clinical signs of zoster ophthalmicus. 2. Understand implications of zoster \& appropriate clinical assessment. 3. Identify appropriate treatment regimen.

CASE: A 64 year-old man presented with four days of a rash over the left side of his face. He had a painful cyst on his nose four days earlier; he subsequently developed painless, red patches over his temple that enlarged and coalesced. He had no past medical history, including no immunecompromising conditions. He was taking no medications and he had no drug allergies. His vital signs were normal, as were his cardiac, pulmonary and abdominal examinations. There were maculopapular lesions extending from the left temple superiorly to the hairline. The lesions were hyperesthesetic, but there were no abnormalities to cranial nerve function. The remainder of his skin examination was normal. The left conjunctiva is mildly injected; a slit-lamp and retinal examination were normal. The $\mathrm{CBC}$ and electrolyte panels were normal.

DISCUSSION: The general internist is frequently confronted with first presentations of dermatologic abnormalities. While most abnormalities are benign and self-limiting, it is important for the general internist to recognize dermatologic emergencies. In an aging population, herpes zoster infection becomes more prevalent, and early recognition of herpes zoster infection is critical for decreasing immediate and long-term complications. Pain followed by the appearance of maculopapular or vesicular lesions is the classic presentation of zoster infection. The most common locations are the chest and abdomen as the varicella zoster virus is reactivated from the thoraco-lumbar dorsal root ganglia; however, lesions of the face due to trigeminal or facial nerve involvement are not uncommon. Hutchinson's sign, the appearance of a vesicle over the tip of the nose, is a signal of nasociliary nerve involvement. The nasociliary nerve, from the ophthalmic branch $(\mathrm{V} 1)$ of the trigeminal nerve, innervates both the nose and the cornea. Recognizing nasociliary involvement is critical such that a complete ophthalmic examination can be undertaken to assess for corneal and other ocular involvement. The persistent or recurrent pain of postherpetic neuralgia after classic zoster may occur with zoster ophthalmicus. More gravely, however, if ocular manifestations of zoster ophthalmicus are missed and left untreated, progressive vision loss and blindness may result. Treatment of zoster begins with antiviral agents to decrease duration of symptoms and risk of long-term complications. Acyclovir is the preferred agent for zoster ophthalmicus though valacyclovir or famciclovir have also been shown to be effective and have simpler dosing. Further treatment includes pain control and corticosteroids to decrease symptoms and accelerate return to baseline. The specific regimen for ocular involvement includes topical lubricants and steroids, and should be guided by an ophthalmologist. Topical antibiotics should be added for zoster conjunctivitis because of the risk of secondary infection with gram-positive organisms.

A RECURRING HAZE Naree Whang; Henry J. Hefler. Tulane University Health Sciences Center, New Orleans, LA. (Control ID \#1311820)

LEARNING OBJECTIVE 1: 1. Identify the clinical manifestations of neurosyphilis 2 . Recognize how the diagnostic approach to neurosyphilis is altered in patients with HIV. 3. Identify HIV as a risk factor for relapsing neurosyphilis.

CASE: A 62-year-old man with AIDS presented with a progressive history of photophobia and blurry vision in his left eye. He denied any pain, discharge, redness or floaters. There was no associated change in mental status, fever, headache, neck stiffness, or nausea. Eighteen months ago he had blurry vision in both eyes and was diagnosed with anterior uveitis secondary to neurosyphilis, for which he completed a full course of treatment. Since that time, he reported compliance with his HAART regimen and abstinence from sexual activity. The vital signs were normal. His pupils were reactive to light and accommodation. Slit lamp examination revealed cells and flare in the anterior chamber of the left eye, and visual acuity was 20/60; the right eye was normal. The remainder of his exam was normal. Laboratory studies revealed a serum RPR of greater than 1:128. The lumbar puncture revealed a VDRL titer of $1: 4$, a protein of $68 \mathrm{mg} / \mathrm{dL}$, and a WBC of 42 cells $/ \mu \mathrm{L}$ with $94 \%$ lymphocytes.

DISCUSSION: With improvements in retroviral therapy, patients with HIV are living longer lives, and general internists are increasingly providing care for HIV patients at an older age. Recognizing the common co-existent complications of HIV, including syphilis, is important fro the general internist's management of patients with HIV. Syphilis, an infection caused by the spirochete Treponema pallidum, results in systemic, chronic inflammation. Neurosyphilis can occur during any of the four stages of syphilis. Common symptoms include tabes dorsalis, seizure, stroke, meningitis, or altered mental status. Ocular manifestations, as seen in this case, include uveitis, neuroretinitis, or optic neuritis. While a reactive CSF-VDRL is diagnostic for neurosyphilis, a nonreactive test does not exclude it. In non-HIV infected patients with suspected neurosyphilis who do not have a reactive test, a CSF lymphocyte count $>5$ cells $/ \mu \mathrm{L}$ or a protein concentration $>45 \mathrm{mg} / \mathrm{dL}$ is consistent with the diagnosis. Because the CSF leukocyte count usually is elevated in patients with $\mathrm{HIV}$, using a higher cutoff $(>20$ cells $/ \mu \mathrm{L})$ improves the specificity of the diagnosis. Additional evaluation using FTA-ABS testing should be considered with a nonreactive CSF-VDRL; neurosyphilis is highly unlikely with a negative CSF FTA-ABS test. The treatment of choice for neurosyphilis is aqueous crystalline Penicillin G 3-4 million units IV q4 hours or continuous infusion for 10-14 days. In this case, the patient experienced recurrence of anterior uveitis, raising concern for relapsing neurosyphilis, which has a higher prevalence in patients who are co-infected with HIV. Of the 143 patients in one study, $65 \%$ were co-infected with HIV. It is important for the general internist to recognize that patients co-infected with HIV can have relapse of syphilis despite treatment, and that these patients should be carefully monitored in the outpatient setting.

A REPORT OF NEWLY DIAGNOSED HIV/AIDS IN AN ELDERLY MAN Toru Naganuma ${ }^{1}$; Christine Kwan ${ }^{1}$; Simi Padival ${ }^{2}$; Hitoshi Honda ${ }^{3}$. ${ }^{1}$ Teine Keijinkai Medical Center, Sapporo, Japan; ${ }^{2}$ Beth Israel Deaconess Medical Center, Boston, MA; ${ }^{3}$ Teine Keijinkai Medical Center, Sapporo, Japan. (Control ID \#1323653)

LEARNING OBJECTIVE 1: Recognize that the elderly population is still vulnerable to human immunodeficiency virus (HIV) infection

LEARNING OBJECTIVE 2: Recognize that no age threshold should exist for obtaining sexual history and testing for HIV

CASE: A 74 years old Japanese male with a past medical history of chronic obstructive pulmonary disease presents with one month's history of progressive fever and dyspnea despite taking an antimicrobial agent for 3 weeks. He also reports a cough productive of yellow sputum, diarrhea, and 5-kg weight loss for 6 months. He has used 2 packs/day of tobacco for 40 years but quit 15 years ago. He denies recent travel history and sick contacts. He works as an accountant and lives with his wife and children, but he does not have pets. His physical examination includes significant findings of a fever of 39.6 degrees Celsius, oxygen saturation of $90 \%$ on room air, and fine crackles throughout the bilateral lungs. Laboratory examination at presentation reveals white blood cell of 6,820 counts $/ \mu \mathrm{L}$ with lymphocytopenia $(550$ counts $/ \mu \mathrm{L})$. Blood and sputum cultures are all negative. Although chest X-ray does not show any significant infiltrates, a 
computed tomographic (CT) scan shows diffuse emphysema and interstitial infiltration of the bilateral lower lobes. On further investigation, he has been found to have a history of bacterial pneumonia, trichophytosis, seborrheic dermatitis, oral-esophageal candidiasis, and amebic colitis in the past 9 months. Moreover, he discloses that he has had sexual intercourse with men for the past 20 years since his mid-fifties; he continues to be sexually active at the time of presentation with multiple male partners. Testing for HIV is subsequently positive with CD4 count of 124 counts $/ \mu \mathrm{L}$ and HIV viral load of 230,000 copies $/ \mathrm{mL}$. Despite negative sputum Pneumocystis jiroveci polymerase chain reaction, his clinical presentation, advanced HIV status, and CT scan are consistent with Pneumocystis jiroveci pneumonia (PCP) for which trimethoprim/sulfamethoxazole and prednisolone are instituted. Antiretroviral (ARV) therapy is started once the genotype result becomes available. He has had overall improvement in his symptoms and has been discharged with continuing ARV as an outpatient. DISCUSSION: Although the prevalence of HIV infection and acquired immune deficiency syndrome (AIDS) in Japan is still low compared to western countries, the incidence rate of HIV/AIDS continues to increase in Japan, especially in the younger population of men who have sex with men. As a sexually transmitted disease (STD), newly diagnosed HIV usually occurs in the younger population. Sexual history in the elderly tends to be overlooked although STD Treatment Guidelines, 2010 from the Centers for Disease Control and Prevention recommends all healthcare providers routinely obtain sexual history from all adult patients. Even though this patient has been diagnosed with several opportunistic infections and followed by a pulmonologist, gastroenterologist, and dermatologist, none of them has obtained a sexual history from him or suspected HIV/AIDS until he has developed PCP. This case, therefore, highlights the fact that the elderly population is still vulnerable to HIV infection and the importance of obtaining sexual history from all patients despite their age, especially in those suspected of having an infection.

A RIDDLE WRAPPED IN AN ENIGMA, BUT PERHAPS THERE IS A KEY! Sarita K. Sapkota; Greg Sutton; Bhumin Patel; Lisa Staton; Mukta Panda. University of Tennessee, Chattanooga, TN. (Control ID \#1319987)

LEARNING OBJECTIVE 1: Discuss the differential diagnosis of cirrhosis in a young adult

LEARNING OBJECTIVE 2: Discuss significance of work-up for Wilson's with diagnostic procedures, the importance of a strong clinical suspicion, and of continuity of care and communication among healthcare providers.

CASE: A 26-year-old female with a past medical history of cryptogenic cirrhosis, diagnosed at age 22, presents in December 2011 with a 2-week history of abdominal pain and abdominal swelling. With the loss of insurance about 10 months prior, she was unable to maintain proper dosage of lactulose and lasix and became dependent on the many subsequent ER visits for acute care. The pain characteristics were: sharp, squeezing, pressure sensation, located diffusely over the entire abdomen, and has a severity as high as 10/10. She complained of some nausea and vomiting, fever, and episodes of altered mental status. On exam she was afebrile, normotensive, and in no acute distress. She had significant scleral icterus, and had no apparent changes of mental status. Abdomen was firm and distended, tender to palpation with a positive fluid wave and mild splenomegaly. Abnormal labs: hemoglobin 10.7, hematocrit 32.1, negative hepatitis panel, alkaline phosphatase 532, AST 132, total bilirubin 24.8 (direct 13.6; indirect 11.2), MELD score 26 and her Child Pugh score of 10 , PTT of 36, PT 29.7, and INR of 1.88. Review of her previous records from 2010 revealed a normal serum ceruloplasmin level, 24 hour copper excretion of 34 and a positive liver biopsy for copper but without any quantification. The antimitochondrial antibody, ANA, and hemochromatosis gene mutation were negative. She had been treated for depression and substance abuse $(\mathrm{EtOH})$, which was the reason she had not qualified for liver transplant. However, upon further questioning we found out that she only recently used alcohol for coping after she lost her insurance. Our work-up yielded a faint Kayser-Fleischer ring, ceruloplasmin level of 20.1, elevated 24 hour copper at 86 and cooper/creatinine ratio of 78 . At this point we concluded that she met the diagnostic criteria for Wilson disease and initiated treatment with D-penicillamine.
DISCUSSION: Wilson's disease is an autosomal recessive disorder (gene ATP7B) of copper transport with a prevalence of approximately $1 / 30000$. Typical presentation includes neurological and hepatic dysfunction. Diagnosis usually requires a high index of clinical suspicion due to overlapping symptoms with other disorders. Delayed and misdiagnosis is not uncommon like in our case especially where there is a possible substance abuse history and when lack of insurance becomes a limiting factor for proper follow up and care. Therefore the importance of continuity of care and communication between the physicians cannot be over emphasized. Diagnostic criteria include: presence of Kayser-Fleishcer rings on slit-lamp, ceruloplasmin of $<0.20 \mathrm{~g} / \mathrm{l}, 24 \mathrm{~h}$ urinary free-copper excretion, penicillamine challenge test, liver copper measurement, and the presence of abnormal gene mutation. Mainstays of therapy include penicillamine, trientine, and zinc. For advanced disease, as in our patient, the long-term treatment is liver transplant.

A STEALTHY INFILTRATOR Ethan Kuperman. Penn State College of Medicine, Hershey, PA. (Control ID \#1320884)

\section{LEARNING OBJECTIVE 1: Recognize when further evaluation is indicated in syncope.}

LEARNING OBJECTIVE 2: Identify sarcoidosis as a potential cause of infiltrative cardiomyopathy in a young adult.

CASE: A 34-year-old man presented with two episodes of syncope within six hours. The episodes were preceded by several seconds of dimming vision and light-headedness and lasted less than one minute before rapid recovery. He denied chest pain or abnormal movement and review of systems was negative. His past medical history was otherwise significant for a single episode of syncope four years previous and controlled hypertension. The patient was employed in a warehouse, which required heavy lifting. His only medication was lisinopril. On exam, he was afebrile, pulse 88 beats per minute and regular, and blood pressure 128/ $88 \mathrm{mmHg}$. Body mass index was $37 \mathrm{~kg} / \mathrm{m} 2$. The patient was alert, oriented, and appeared well. There was no palpable adenopathy. His heart sounds were regular, without murmurs, rubs, or gallops. There was no jugular venous distention and point of maximal impulse was difficult to palpate due to obesity. Lungs were clear to auscultation and neurologic exam was nonfocal. There was no peripheral edema. There were no skin lesions. Laboratory studies included a normal complete blood count, thyroid stimulating hormone, and serum troponin. His electrocardiogram showed new bifasicular block. Chest x-ray was normal. An echocardiogram revealed a globally hypokinetic left ventricle with an ejection fraction of $30 \%$. Cardiac MRI was positive for a dilated cardiomyopathy with delayed hyperenhancement and identified mediastinal lymph nodes not visualized on chest $\mathrm{x}$-ray. Bronchoscopy with biopsy was not diagnostic, but mediastinoscopy was positive for sarcoidosis. The patient proceeded to AICD implantation and was started on immunosuppression. He had no subsequent syncopal episodes and maintained an excellent functional status.

DISCUSSION: General internists routinely evaluate syncopal patients, rarely finding a specific diagnosis. A complete history and physical with an electrocardiogram is typically recommended to decide if further evaluation is appropriate. This patient had recurrent episodes and an abnormal ECG; red flags which justified further workup. The combination of depressed ejection fraction and conduction disorder suggests an infiltrative process. Cardiac MRI is an increasingly available modality to evaluate disorders of the myocardium. The differential diagnosis of a dilated infiltrative cardiomyopathy includes sarcoidosis, Wegener's granulomatosis, and hemochromatosis. The typical MRI appearance of sarcoidosis includes patchy late gadolinium enhancement of the basal and inferolateral walls. Cardiac sarcoidosis can have subtle presentation and requires a high index of suspicion. It is frequently undiagnosed, particularly in the young population. Cardiac involvement is found in $40 \%$ of patients with sarcoidosis. Diagnosis can be confirmed by tissue diagnosis of affected skin or bronchoscopy when lesions are accessible. This patient had no other manifestations of sarcoidosis and plain film imaging was negative for adenopathy, necessitating invasive diagnostic procedures. Involvement may be limited to the heart in two-thirds of patients with cardiac sarcoidosis. Steroids remain the foundation of treatment, but fiveyear mortality may be as high as $40 \%$. Early diagnosis is necessary due to the risk of sudden cardiac death. 
A SWEET DIAGNOSIS Alexis C. Ferguson ${ }^{1}$; Rajan Kapoor ${ }^{2}$; Anu Batra $^{3}$; Ritu Gothwal ${ }^{4}$; Prasanta Basak ${ }^{1}$; Stephen Jesmajian ${ }^{1} .{ }^{1}$ Sound Shore Medical Center of Westchester and New York Medical College, New Rochelle, NY; ${ }^{2}$ Westchester Medical Center, Valhalla, NY; ${ }^{3}$ University of Arkansas Medical Center, Little Rock, AR; ${ }^{4}$ Women's Correctional Institute, Muncy, PA. (Control ID \#1313135)

LEARNING OBJECTIVE 1: Sweet syndrome is a rare dermatosis, frequently associated with hematological malignancies and solid tumors including malignancies of the breast, gastrointestinal and genitourinary tracts. We present a rare association of Sweet syndrome with myelodysplastic syndrome (MDS)

CASE: An 80 year old female with hypertension, was admitted with progressive generalized weakness and decreased appetite for 3 weeks. She denied any chest pain, shortness of breath, abdominal pain, diarrhea, gastrointestinal bleed, or any symptoms suggestive of upper respiratory tract infection. She was tachycardic (110-120/min), had conjunctival pallor. Laboratory data revealed pancytopenia (Hemoglobin: $4.3 \mathrm{mg} / \mathrm{dl}$, Hematocrit: $12.4 \%$, MCV: 112 fL, WBC: 4,200/mm3 and platelets of $38,000 /$ $\mathrm{mm} 3$ ). Blood smear and bone marrow biopsy were consistent with MDS. She was symptomatically treated with red cell and platelet transfusions. On hospital day 4, she developed recurrent febrile episodes and painful erythematous plaques and nodules on her left forehead and right arm. She subsequently developed renal and respiratory failure and had to be intubated. Extensive work up for sepsis, including sputum and blood cultures, urine analysis, CSF exam, CT scan and Chest X-ray were noncontributory. Biopsy of a forehead skin lesion revealed diffuse neutrophilic infiltrate in the upper dermis consistent with Sweet syndrome. The patient was promptly started on intravenous steroids resulting in complete resolution of skin lesions, improvement in renal function and was successfully extubated 3 days later.

DISCUSSION: Acute febrile neutrophilic dermatosis or Sweet syndrome was first described by Robert Douglas Sweet in 1964. It is presumed to be a hypersensitivity reaction and is characterized by fever, neutrophilia, tender, erythematous skin lesions (papules, nodules and plaques) and a diffuse infiltrate consisting predominantly of mature neutrophils that are typically located in the upper dermis. It has been postulated that inflammatory cytokines may have an etiologic role in the development of the dermatosis. Twenty percent of Sweet syndrome cases are associated with MDS or other hematological malignancies. Skin lesions of Sweet syndrome may precede the diagnosis of MDS by a median of 3.5 years. Sweet syndrome occurring in patients with MDS could be a marker of advancing MDS. A skin biopsy is necessary to clinch the diagnosis of this interesting syndrome.

A TREATMENT APPROACH TO METHICILLIN-RESISTANT STAPHYLOCOCCUS AUREUS (MRSA) SEPTIC PULMONARY EMBOLI AND BACTEREMIA WITHOUT EVIDENCE OF ENDOCARDITIS Vinitha Reddy; Abdulla Damluji. Penn State Milton S. Hershey Medical Center, Hershey, PA. (Control ID \#1340458)

LEARNING OBJECTIVE 1: Daptomycin is a bacteriocidal lipopeptide antibacterial with a spectrum of activity limited to gram-positive organisms including highly resistant species of (MRSA, VISA, VRSA, and VRE). Daptomycin has demonstrated treatment failure in lung MRSA infections (1) and success with right-sided endocarditis and septic pulmonary emboli (2). No treatment algorithm was recommended by the Infectious Disease Society of America (IDSA) guidelines for patient with MRSA septic pulmonary emboli in the absence right-sided endocarditis. For that subgroup of patients, we report a successful eradication of MRSA bacteremia with intravenous daptomycin and linezolid in a veteran presenting to the Lebanon VA Medical Center.

CASE: An 84-year-old man with a history of hypertension and benign prostatic hyperplasia presents with increasing lethargy and confusion one day prior to hospital admission. He was febrile, tachycardic, and hypotensive. He had an elevated white cell count with a left shift. The patient was fluid resuscitation and empiric antibiotics including Vancomycin were started. His initial peripheral blood cultures showed MRSA susceptible to Vancomycin. The patient's fever and leukocytosis continued despite appropriate trough levels that were kept in the range of 15 to 20 . A CT scan of the chest was obtained to search for possible abscess. It revealed bilateral pulmonary cavitary lesions suspected to be septic emboli that were necrotic in nature. Transesophageal echocardiogram did not reveal any vegetations. CT scan of the pelvis revealed presacral fluid collections, which was subsequently drained under radiologic guidance. Due to treatment failure, Vancomycin was discontinued and Linezolid and high-dose Daptomycin were started. Subsequently, the patient was afebrile, his white cell count normalized, and blood cultures remained negative. He was continued on antibiotic therapy for six weeks after hospital discharge.

DISCUSSION: High failure rates in the treatment of severe MRSA pneumonia were observed with Vancomycin due to poor penetration into pulmonary tissue and lung epithelial lining fluid (3). Failure rates with high-dose Daptomycin were also reported and thought to be due to intactivation by lung surfactant (1). However, high-dose Daptomycin is FDA-approved for treatment of complicated MRSA bacteremia and rightsided endocarditis. Adjunctive therapy with protein synthesis inhibitor (i.e. clindamycin or linezolid) was proposed by IDSA as a "consideration" in cases of necrotizing pneumonia or severe sepsis. We report a successful treatment of cavitary MRSA pneumonia without evidence of endocarditis using a combination of high-dose Daptomycin and Linezolid. This regimen could be considered in this subgroup of severe MRSA pneumonia. References: 1. Koplowicz et al. Clin Infect Dis 2009;49(8):1286-1287. 2. Rehm et al. J Antimicrob Chemother 2008;62(6):1413-1421. 3. Cruciani et al. J Antimicrob Chemother 1996;38(5):865-869.

A UNIQUE CASE OF COEXISTING ABPA AND SEMI-INVASIVE ASPERGILLOSIS IN AN IMMUNOCOMPETENT PATIENT Oluwakemi Fagbami; Alec B. Platt. The Reading Hospital and Medical Center, West Reading, PA. (Control ID \#1310713)

LEARNING OBJECTIVE 1: Semi-invasive aspergillosis is an infection usually seen in immunosuppressed individuals. Rarely, semi-invasive aspergillosis can overlap with allergic bronchopulmonary aspergillosis (ABPA)in an immunocompetent patient.

CASE: A 61 year-old previously healthy male with mild asthma and seasonal allergies was evaluated two months post hospitalization for community acquired pneumonia and methicillin sensitive Staphylococcus aureus bacteremia. Transesophageal echocardiogram (TEE) showed no vegetation and repeat blood cultures during four weeks of treatment and surveillance were negative. A CT scan showed mild bronchiectasis; resolution of bilateral lower lobe and progressive enlargement and cavitation of multiple $1 \mathrm{~cm}$ bilateral pulmonary nodules. There were no prior hospitalizations for asthma, childhood history of pneumonia or other chronic infections. He had only rare episodes of sinusitis. He had not been on any steroids or immune suppressive medications. The patient, a nonsmoker, had a history of occupational exposure to mold in an office setting. Physical examination including lung exam was normal. Total IgE was $6155 \mathrm{IU} / \mathrm{ml}$; IgA, IgG and IgM were normal. Aspergillus fumigatus $\operatorname{IgG}(121 \mathrm{IU} / \mathrm{ml})$ and peripheral blood eosinophils $(9.8 \%)$ were elevated. ESR, ANA, rheumatoid factor, ANCA, HIV, PPD, cystic fibrosis screens were negative. Pulmonary function tests were normal. Fiber optic bronchoscopy washings of a left upper lobe area of cavitation were negative for AFB but grew Aspergillus fumigatus and methicillin resistant staph aureus. Transbronchial biopsies were negative. Repeat surveillance blood cultures were negative. The patient was treated first with oral Linezolid for four weeks and then Voriconazole for six weeks. The cavitary lesions progressed on serial CT scans and patient subsequently developed Methicillin resistant Staphylococcus aureus endocarditis and pneumonia. A video assisted thoracoscopic wedge resection of a left lower lobe cavitary lesion demonstrated Aspergillus fumigatus organisms with limited surrounding tissue necrosis classified by outside expert histologic review as semi-invasive aspergillosis. The patient received six weeks of Vancomycin and began a six month course of Prednisone and Voriconazole. With the exception of mild intermittent asthma, he remains symptom free on follow-up. Repeat CT scan imaging three months into treatment showed partial resolution of the cavitary lesions and a decrease in IgE to $695 \mathrm{IU} / \mathrm{ml}$.

DISCUSSION: Pulmonary spergillosis infection can be subdivided into five categories: simple aspergilloma, allergic bronchopulmonary aspergil- 
losis (ABPA), chronic necrotizing aspergillosis (also known as semiinvasive aspergillosis), airway-invasive aspergillosis and angioinvasive aspergillosis. ABPA is seen in patients with long-standing bronchial asthma while aspergilloma is seen in patients with underlying obstructive or fibrotic lung disease. Chronic necrotizing aspergillosis is rarely seen in immune competent hosts and has not been well reported in association with ABPA. This case illustrates the need to be aware of the different forms of aspergillosis which may coexist rarely in immunocompetent patients.

\section{A CASE OF HEMOPHAGOCYTIC LYMPHOHISTIOCYTOSIS} AFTER EPSTEIN-BARR VIRUS INFECTION SIMULATING SEVERE SEPSIS Eduardo J. Bazan; Claudia P. Taramona Espinoza; Ravneet Thind; Javier Diaz-Mendoza. Henry Ford Hospital, Detroit, MI. (Control ID \#1335024)

LEARNING OBJECTIVE 1: To recognize the clinical features of Hemophagocytic Lymphohistiocytosis (HLH) in a patient admitted for severe sepsis in a Medical Intensive Care Unit (MICU).

LEARNING OBJECTIVE 2: To discuss the importance of early diagnosis of HLH for treatment success.

CASE: A 27 year-old previously health female presented with a 2-week history of fever. Her boyfriend had been febrile one week prior to the beginning of her symptoms. She was initially prescribed amoxicillinclavulanate and cephalexin with no improvement. She developed jaundice and a diffuse rash which prompted her admission to the hospital. The patient developed fever, tachycardia, hypotension and hypoxemia requiring admission to the MICU for possible sepsis. Physical exam revealed cervical lymphadenopathy and hepatomegaly. Laboratory workup showed elevated AST, ALT, total bilirubin, INR, D-dimer, ferritin, triglycerides as well as low fibrinogen, anemia, thrombocytopenia and a brief period of neutropenia. IV fluids were given for hypotension with good clinical response as well as IV antibiotics. Work up for hepatitis, HIV, CMV and autoimmune disease was negative. IgG for Parvovirus and serology for Epstein- Barr virus (EBV) were positive. Pancultures were negative. Abdominal ultrasound showed hepatomegaly with mild bilateral pleural effusions. Secondary HLH was considered in the differential and a bone marrow biopsy suggested this diagnosis. IV antibiotics were discontinued and oral steroids were started. She improved clinically and was discharged home on tapering doses of steroids which were later on discontinued. She is now following only with her PCP.

DISCUSSION: HLH is a rare disease with high mortality. It is classified as primary (a familial form) and secondary. The latter one is most commonly related to infections, being EBV the most frequent etiology. Secondary HLH varies widely from inflammation that resolves spontaneously to progressive disease with hematopoietic cell transplantation as final treatment. The diagnosis is based on clinical, laboratory and histopathological findings. Patients with HLH suffer from prolonged fever, cytopenias and diseminated intravascular coagulation-like presentation who are unresponsive to antibiotics. This clinical course has to prompt us to look for a complete HLH evaluation. Based on the diagnostic guidelines proposed by the Histiocytic Society in 1991 and updated in 2004 , it is not necessary to wait for completion of all criteria before initiating therapy. In this case, the patient had 5 out of 8 of the following diagnostic criteria: fever, cytopenia, hyperferritinaemia, hypertriglyceridemia and haemophagocytosis in the bone marrow. The patient did not have splenomegaly on physical examination. NK-cell activity or $\mathrm{sCD} 25$, the two left criteria, were not checked. The therapy has to be adjusted depending on the grade of disease severity. This patient experienced a mild disease as she was clinically stable, requiring only conservative therapy with oral dexamethasone and supportive therapy in MICU. High clinical suspicion is critical. Understanding of the pathophysiology of HLH by the clinicians is crucial for early diagnosis and treatment to prevent poor outcome including death.

A CASE OF KETOSIS-PRONE TYPE 2 DIABETES MELLITUS IN A NIGERIAN MALE Rebecca Braunstein ${ }^{1}$; Vafa Tabatabaie ${ }^{2}$; Cristina M. Gonzalez ${ }^{1,2}$. ${ }^{1}$ Albert Einstein College of Medicine, Bronx, NY; ${ }^{2}$ Montefiore Medical Center, Bronx, NY. (Control ID \#1339517)
LEARNING OBJECTIVE 1: To recognize the diagnosis of KetosisProne Type 2 Diabetes Mellitus (DM) in a patient presenting with severe hyperglycemia or diabetic ketoacidosis (DKA).

LEARNING OBJECTIVE 2: To describe the natural disease course of this subtype of DM.

CASE: A 54 year old Nigerian male with no significant medical history presented with fatigue, polyuria, polydipsia, polyphagia, and weight loss. He endorsed dry mouth, and denied fevers, dysuria, cough, chest pain, shortness of breath, nausea, vomiting, abdominal pain, blurry vision, or headache. He denied toxic habits. Family history was positive for type 2 DM. Initial vitals were T $97.5^{\circ} \mathrm{F}, \mathrm{P} 106 \mathrm{BPM}$, BP 138/93 mmHg, RR 19 RPM, FSG $592 \mathrm{mg} / \mathrm{dL}$. Pertinent exam findings included dry oral mucosa, lungs clear to auscultation, tachycardia, benign gastrointestinal exam, and no neurological deficits. Labs were significant for WBC $8.2 \times 10^{3} / \mu \mathrm{L}$, an anion gap metabolic acidosis (AG 19), positive serum ketones, $\mathrm{pH} 7.395$, and troponin- $\mathrm{T}<0.01 \mathrm{ng} / \mathrm{mL}$. Urinalysis was negative for infection. The patient was treated with aggressive fluid resuscitation, intravenous insulin drip, and electrolyte repletion. $\mathrm{HbAlc}$ was $12.7 \%$. One month later he was doing well on oral hypoglycemics with a HbA1c of $8.4 \%$.

DISCUSSION: DM is one of the most frequently encountered diagnoses by the internist. Ketosis-Prone Type 2 DM, also known as "Flatbush Diabetes," is a subtype of DM typically seen in African American or Hispanic patients. It has unique pathophysiologic and prognostic features. This subtype of DM presents similarly to type 1 DM with severe hyperglycemia or DKA, but has many characteristics of type 2 DM. Specifically, patients do not have islet cell autoantibodies and typically display insulin resistance. Following initial treatment, studies have shown that these patients are often able to discontinue insulin therapy and maintain glycemic control with oral agents or diet alone. The prevalence of this subtype of DM in the United States is thought to be between $20-50 \%$ of African American and Hispanic patients with new-onset DM that present in DKA. The typical patient is an obese, middle-aged (mean age of 40) male (3:1 predominance) with a strong family history of type $2 \mathrm{DM}$, who presents acutely with DKA or severe hyperglycemia in the absence of common triggers such as infection or myocardial infarction. Physiologically, the initial presentation is due to acute $\beta$-cell failure, resulting in transient insulinopenia. There is concurrent defective insulin function and insulin resistance. $\beta$-cell dysfunction often resolves after treatment. Studies have revealed that patients have significant pancreatic insulin reserve, and that 42 $76 \%$ of patients achieve near-normoglycemic remission (defined as $\mathrm{HbAlc}$ of $<6.3 \%$ and a fasting plasma glucose of $<120 \mathrm{mg} / \mathrm{dL}$ ) 3 months after therapy with all pharmacologic agents discontinued. In patients who achieve remission, $\beta$-cell function, as measured by serum levels of $\mathrm{C}$-peptide (basal and glucagon stimulated), is improved by $80 \%$ shortly after resolution of DKA or hyperglycemia. C-pepide levels and presence of autoantibodies have been used as markers to predict likelihood of near-normoglycemic remission. Positive autoimmune markers suggests slow-onset type $1 \mathrm{DM}$ or latent autoimmune DM, making future remission less likely. In conclusion, KetosisProne Type $2 \mathrm{DM}$ is important to recognize and understand in order to properly manage and anticipate outcomes in patients with this diagnosis.

A CASE OF REVERSIBLE POSTERIOR ENCEPHALOPATHY SYNDROME IN OVARIAN HYPERSTIMULATION SYNDROME. Cara J. Tsoi. California Pacific Medical Center, San Francisco, CA. (Control ID \#1310555)

LEARNING OBJECTIVE 1: To recognize that Posterior Reversible Encephalopathy Syndrome may arise in the context of Ovarian Hyperstimulation Syndrome.

LEARNING OBJECTIVE 2: To manage neurologic symptoms in the setting of Ovarian Hyperstimulation Syndrome.

CASE: A 38 yr-old Indian woman, G2 P1, at 4 weeks gestation, presented with visual changes and headache. The patient complained of an intermittent right parietal headache for 3 days. This was associated with episodic visual changes resembling "shattered glass" in her peripheral vision bilaterally, as well as left-sided colorful shapes, and right visual field loss. The patient had been undergoing experimental fertility treatments with a three drug regimen consisting of a GnRH antagonist, HCG, and recombinant FSH dosed either orally or as a long-acting weekly injection. As the patient 
was enrolled in a phase III trial, it is unknown whether the patient received the oral or the injectable form. All of these drugs had been discontinued 2 weeks prior to arrival. It was unknown whether the patient had received the experimental drug or not. Of significance, she had recently been diagnosed with OHSS, with complaints of ascites and shortness of breath, and had undergone culdoparesis (transvaginal removal of ascitic fluid) with removal of $2 \mathrm{~L}$ of fluid prior to presentation. The patient's exam was notable only for mild distention of the abdomen without tenderness, and normal cranial nerve and visual field as well as cerebellar exams. A funduscopic exam was normal. MRI revealed findings significant for numerous foci of abnormal signal involving the high left parasagittal pariental lobe as well as parietooccipital pole. There was suggestion of potential associated cytotoxic change. These findings were consistent with PRES. The patient's symptoms improved in-house and she was sent home on hospital day 3.

DISCUSSION: Both cerebral autoregulation and endothelial dysfunction have been implicated in the pathophysiology of PRES, as well as hypoperfusion secondary to vasospasm. Specifically, excessive autoregulation is thought to lead to dilation of cerebral arterioles and increase in cerebral blood flow which causes hyperperfusion. This hyperperfusion may lead to the breakdown of the blood brain barrier, allowing extravasation of fluid and blood products into the brain parenchyma and resulting in the clinical features of PRES. OHSS has been associated with release of VEGF with resulting increased capillary permeability. VEGF has been shown to be a potent vascular permeability factor in the brain as well. Therefore, we hypothesize that this patient's OHSS caused excessive VEGF which then may have been related to development of PRES. Thus far there has been no research linking VEGF to the pathogenesis of PRES, however it may present a future topic.

A CASE OF PULSE STEROID INDUCED BRADYARRHYTHMIA IN A PATIENT WITH SLE Tushar A. Tuliani; Hemanckur Makker; Kashish Goel; Maithili Shenoy; Diane L. Levine. Wayne State University/ Detroit Medical Center, Detroit, MI. (Control ID \#1336296)

LEARNING OBJECTIVE 1: Recognizing a rare side effect of pulse steroids in a patient with SLE.

CASE: A 20 year old woman with Systemic Lupus Erythematosus diagnosed at 14 and stage 3 lupus nephritis (FSGS) was admitted for fever and symptoms of upper respiratory tract infection. Her medications included: hydrochloroquine, acetaminophen, hydrocodone, mesna, trimethorpim-sulfamethoxazole, sildenafil, pantoprazole, ondansetron, mycophenolate mofetil, methylprednison, leuprolide, folic acid, diphenhydramine, cyclophosphamide, and calcium carbonate. Vital signs revealed a temperature of $39.1 \mathrm{C}$, blood pressure $114 / 77$, heart rate 89 , respiratory rate 18 . On examination the patient had a dry discoid rash present over her face, arms and posterior neck. The remainder of her exam was normal without signs of infection. Initial WBC was 3.7 (neutrophils of 2.5 and lymphocytes of 0.7 ). Serum electrolytes were normal. Estimated GFR was $36 \mathrm{~mL} / \mathrm{min} / 1.73 \mathrm{~m} 2$. Urinalysis showed $3+$ blood, $2+$ protein with 2-5 granular casts per low power field. The initial differential diagnosis was infection versus a lupus flare. Treatment was initiated for health care associated pneumonia in the Emergency Depratment. The patient was started on pulsed methylprednisone at $500 \mathrm{mg}$ IVPB BID for a total of three days for lupus flare. The patient developed asymptomatic bradycardia while she was on steroids. EKG revealed sinus bradycardia 37/ min with normal intervals (PR $0.154 \mathrm{~s}$, QRS $0.096 \mathrm{~s}$, QTc $0.412 \mathrm{~s}$ ). Previous EKGs showed normal sinus rhythm. Troponins were normal. Thyroid function was normal. Bradycardia could not be attributed to her medications as the patient had not been on anti hypertensive agents for more than a week. The patient's heart rate ranged from 55-38/min during her course of steroid treatment. Five hours after her last dose her heart rate had risen and varied between 51-68/min. No abnormalities were noted upon cardiology follow up.

DISCUSSION: The major cardiovascular adverse effects of pulse steroid therapy include myocardial infarction, asystole, supraventricular arrhythmias, atrial flutter and fibrillation, ventricular tachycardia and cardiac arrest. However high dose pulse steroid induced bradycardia has been reported in the past in the pediatric population. In our case, underlying involvement of the cardiac conduction system secondary to SLE is unlikely. The patient had normal EKGs both before and after completion of pulse steroids. The patient had no alternative cause of bradycardia. Overt hypothyroidism was ruled out by a low free T4 and normal TSH (euthyroid sick syndrome). She was not on any medications known to cause bradycardia and her anti hypertensive agents had been discontinued for more than a week. An Echocardiogram revealed pulmonary hypertension and elevated right ventricular systolic pressure of $60-65 \mathrm{~mm}$ of $\mathrm{Hg}$ but no other abnormalities. Chronotropic competence was intact during her hospital stay with her heart rate increasing to more than $60 / \mathrm{min}$ on walking. Practitioners should be aware of the potential for bradycardia with high-dose pulse steroids in both children and young adults.

A COMMON DISEASE AS A HARBINGER OF CANCER Yogita Segon; Ankur Segon; Kurt J. Pfeifer. Medical College of Wisconsin, Milwaukee, WI. (Control ID \#1311761)

LEARNING OBJECTIVE 1: Recognize that new onset diabetes mellitus can be the initial presentation of pancreatic cancer, especially in older patients

CASE: An 82-year-old woman with a past medical history of hypertension, hyperlipidemia and stroke presented with fatigue, nausea and poor oral intake over 3-4 weeks. She was referred for hospital admission by her primary care physician when she found the patient's blood glucose to be $600 \mathrm{mg} / \mathrm{dL}$ and her HbA1C $11.5 \%$. She was treated as new-onset type 2 diabetes mellitus and was discharged home after a few days on long-acting insulin and pioglitazone. She returned to the emergency room 5 days after discharge with complaints of persistent nausea and vomiting. She also reported vague, epigastric abdominal pain. Her physical examination was significant for diffuse abdominal distension and epigastric tenderness. Her hepatic function panel was normal with a slightly elevated amylase and normal lipase. Upper endoscopy suggested retention of food in the stomach and an increased amount of fluid in the second portion of the duodenum. Subsequent small bowel follow-through suggested poor gastric motility with narrowing of the third part of the duodenum. Pancreas protocol abdominal CT showed a $6 \times 3 \mathrm{~cm}$ pancreatic tail lesion causing significant compression of distal duodenum. Also noted were numerous lesions in both hepatic lobes, very likely liver metastases in the setting of primary pancreatic malignancy. Ultrasound-guided liver biopsy was subsequently declined by the family, and the patient was discharged home on hospice. She passed away 1 month later.

DISCUSSION: Age is the greatest risk factor for pancreatic cancer and incidence rises with each decade after age 50 years. The most common location is the head of the gland with the most common symptoms being epigastric pain radiating to the back and painless jaundice due to common bile duct obstruction. Laboratory findings can be nonspecific with increases in amylase, lipase, hepatic enzymes and/or CA 19-9. A pancreas protocol abdominal $\mathrm{CT}$ is the most important diagnostic and staging tool for pancreatic cancer, with a sensitivity of greater than $90 \%$. Endoscopic ultrasonography is as accurate as CT scan for detecting tumors and may be more sensitive for tumors smaller than $2 \mathrm{~cm}$ in diameter. Patients with unresectable, locally advanced disease usually survive for 10 months or less; patients with metastatic disease for 6 months or less. More than $75 \%$ of patients with pancreatic cancer are hyperglycemic or diabetic, but it is unclear if diabetes is a risk factor or complication of pancreatic cancer. Although type $2 \mathrm{DM}$ is common in the same population at risk for pancreatic malignancy, new-onset diabetes may also be the initial presentation of pancreatic cancer especially when the tumor is present in the body or tail of the pancreas. While evidence and guidelines for pancreatic cancer screening are lacking, this etiology should be considered in any elderly patient who presents with new-onset diabetes.

A COUGH UNFIT FOR A LADY Jonathan Kirsch; Brian Bramson. University of North Carolina, Chapel Hill, NC. (Control ID \#1336304)

LEARNING OBJECTIVE 1: Recognize the clinical presentation of chronic pulmonary mycobacterial avium complex (MAC) in an elderly patient.

LEARNING OBJECTIVE 2: Identify pulmonary MAC with the use of imaging, sputum cultures and AFB staining. 
CASE: An 80 year-old woman presented with an acute on chronic productive cough with yellow sputum. Over the previous 12 months, she had developed worsening a dry cough, dyspnea on exertion, anorexia, diarrhea, and experienced a $13 \mathrm{~kg}$ weight loss. She denied fevers, chills or hemoptysis. She had been treated by her primary care physician (PCP) with a course of azithromycin followed by levofloxacin without improvement. She was told by her cardiologist that her heart failure was stable. She was seen again by her PCP for a productive cough. A chest radiograph revealed multifocal infiltrates and she was referred for hospital admission. She was afebrile, respiratory rate was 21 breaths per minute and oxygen saturation was $96 \%$ on room air. Her weight was $51 \mathrm{~kg}$ and body mass index was 16 . She was pleasant with mild memory loss. She was cachectic and her skin was dry. She had scattered rhonchi and bilateral rales, more in the right middle lung field. She has a history of diastolic heart failure, hyperlipidemia and paroxysmal atrial fibrillation. She drinks four glasses of wine nightly and quit smoking cigarettes 50 years ago. She lives alone. Her complete blood count and differential were normal. Her sputum gram stain was negative. Computed tomography scan of her chest revealed diffuse ground glass nodular opacities with bronchiectasis and mucus plugging. She was treated for community acquired pneumonia without improvement. Repeat sputum specimens were obtained and sent for acidfast bacillus (AFB) staining and culture. Sputum was positive for acid-fast organisms with PCR testing negative for M. tuberculosis species. She was diagnosed with pulmonary mycobacterium avium complex (MAC) and cultures were later confirmed. She was discharged on ethambutol, clarithromycin, and rifampicin. Months later, she was seen for follow-up and her symptoms had improved. She was gaining weight and continued her antibiotic treatment.

DISCUSSION: Indolent cough with chest radiograph abnormalities is commonly encountered by general internists and hospitalists. Pulmonary MAC is being recognized with increasing frequency as an indolent infection in middle-aged and elderly women with no pre-existing lung disease, known as "Lady Windermere Syndrome". It was once thought to occur in women that suppressed cough to appear more "lady-like" and is named after a Victorian character in an Oscar Wilde play. Pulmonary MAC infection should be considered in a patient with a chronic pulmonary infiltrate, especially in a patient not responding to standard therapies as this organism will not respond to commonly prescribed empiric antibiotics. The diagnosis may be missed without proper imaging and sputum cultures. Diagnosis in the right setting requires abnormalities on $\mathrm{CT}$ or chest $\mathrm{X}$-ray plus one of the following: 1) acidfast bacilli, granulomas, or positive culture from a tissue specimen; 2) 3 positive sputum cultures without positive smears; 3 ) 2 positive sputum cultures with 1 positive smear; or 4 ) a bronchial wash culture with a $2+$ or greater smear or $2+$ or greater growth in culture. Treatment is difficult, requiring combination therapy for at least 12 months.

\section{A PRESENTATION OF ACUTE COMPARTMENT SYNDROME DUE TO SEVERE BLEEDING LEADS TO A DIAGNOSIS OF MULTIPLE MYELOMA AND AN ACQUIRED COAGULOPATHY Sahar Soleymani; Peter Y. Chung. UCLA-Olive View Medical Center, Sylmar, CA. (Control ID \#1334409)}

LEARNING OBJECTIVE 1: Recognize that multiple myeloma may present with acute severe bleeding due to an acquired coagulopathy. LEARNING OBJECTIVE 2: Diagnose and manage bleeding diatheses due to an acquired coagulopathy in a patient with multiple myeloma. CASE: A 43 year-old male presented with sudden, spontaneous onset of left leg swelling and pain. He was found to have acute compartment syndrome secondary to hemorrhage and underwent multiple fasciotomies with subsequent amputation. During outpatient recovery, he experienced a second episode of spontaneous severe bleeding in his left arm necessitating fasciotomy for acute compartment syndrome. Physical exam demonstrated tense edema, induration of the left arm and thigh, oozing of blood and clots from surgical wounds, cachexia and kyphosis. Laboratory and imaging demonstrated WBC 5.8 (P82\% L7.5\% M8.9\%), hgb $8.6 \mathrm{~g} / \mathrm{dl}$, platelets 93, total protein $8.0 \mathrm{~g} / \mathrm{dL}$, albumin $2.8 \mathrm{~g} / \mathrm{dL}$, serum electrophoresis M-protein $3.1 \mathrm{~g} / \mathrm{dL}$, serum immunofixation IgA-Kappa, PT and PTT of 26.2 and $74.4 \mathrm{sec}$. without correction on 1:1 mixing, fibrinogen $226 \mathrm{mg} / \mathrm{dL}$, prolonged thrombin time (TT) $32 \mathrm{sec}$, normal reptilase time (RT) $19 \mathrm{sec}$.
VWF antigen, factor II, V, VII, VIII, IX, X activity levels were normal. CT Scans revealed large bilateral gluteal hematomas arising from the pelvic bone, numerous skeletal lytic lesions and compression fractures. Marrow examination of the resected left femur revealed sheets of plasma cells.

DISCUSSION: Multiple myeloma, a clonal proliferation of malignant plasma cells that secrete a monoclonal serum immunoglobulin and light chain, often presents with abnormalities of screening coagulation tests; however, severe bleeding due to acquired coagulopathy is rare. The underlying mechanism in most case reports is attributed to interference of fibrin monomer polymerization by the M-protein, resulting in an unstable clot more susceptible to fibrinolysis. Other mechanisms, such as direct autoantibody inhibition of coagulation factors (i.e. VIII, XIII, thrombin) or the detection of circulating heparin-like glycoaminoglycans, have been rarely reported. Diagnostic evaluation is clinically challenging and little data exists to guide therapeutic management. Our patient's abnormal PT and PTT mixing times suggested the presence of a defect in the common factor pathway. The prolonged TT and normal RT implied that the abnormal PT and PTT were more likely due to an acquired thrombin inhibitor, rather than a dysfibrinogemia or fibrin polymerization disorder. Despite initiating a bortezomib-cyclophosphamide chemotherapy regimen, our patient continued to have recurrent, painful limb bleeding refractory to factor replacement. Plasmapheresis was employed and resulted in a cessation of bleeding with a reduction of M protein, correction of PT, PTT and TT. However, bleeding at the femoral vein catheter site on day 5 of pheresis necessitated catheter removal and discontinuation of pheresis. Shortly thereafter, the patient had recurrent limb bleeding with an increase in M-protein, prolongation of PT, PTT, and TT, due to refractoriness to bortezomib-based chemotherapy. Attempts to neutralize the suspected thrombin inhibitor with IVIG and to bypass it with recombinant factor VIIa were ineffective. Fortunately, treatment with melphalan-thalidomide chemotherapy eventually resulted in the remission of bleeding, reduction of M-protein and correction of coagulation tests.

A RARE CAUSE OF COMPlete heART BLOCK; A CASE REPORT Wasim A. Hamarneh; Subhraleena Das; Sujith Cherian; David Landsberg. SUNY Upstate Medical University, Syracuse, NY. (Control ID \#1339978)

LEARNING OBJECTIVE 1: 1. Recognise a rare cardiac complication of hydromorphone administration

CASE: A 75 -year- old female with history of ischemic cardiomyopathy presented to our institute with right upper quadrant pain, nausea and vomiting. The patient after appropriate workup was found to have acute cholecystitis and was admitted for cholecystectomy the next day. For severe abdominal pain patient received $2 \mathrm{mg}$ of IV hydromorphone. 15 mins later patient was found unresponsive and severely bradycardic. She was diagnosed to be in complete heart block (CHB), with ventricular escape at 20-25 bpm. Patient was transcutaneously paced following which patient again became responsive. During this time patient was also given $2 \mathrm{mg}$ of IV naloxone. 5 mins after administration of naloxone, patient reverted back to normal sinus rhythm and remained so making requirement for pacing redundant. Acute coronary syndromes were ruled out. Given no other precipitating factor, the direct temporal association with hydromorphone administration and reversal with naloxone, hydromorphone was determined to be the cause of CHB.

DISCUSSION: Review of the medical literature revealed a few isolated reported cases implicating opioids to cause varying degrees of heart block: 1 . Christensen et al described complete heart block with morphine in a 40 year old female. 2. Heaney et al described LBBB with first degree heart block in a 20 year old male with propoxyphene. 3 . Stein et al demonstrated cardiodepressant actions of morphine including bradycardia and atrioventricular block in murine models. Multiple theories have been proposed: 1. Increased parasympathetic activity from stimulation of the dorsal nucleus of vagus nerve by opioids. The fact atropine fails to reverse, refutes this to some extent. 2 . Secondly, varying degrees of verapamil like calcium channel blockade by opioids. Consistently, in all the reported cases and as in our case, naloxone was found to reverse the cardio-depressant action completely. To conclude, our case reiterates the adverse cardiac outcomes possible with opioid usage apart from being the first reported case of CHB secondary to hydromorphone in medical literature, to the best of our knowledge. 
A RARE DISEASE WITH A NOVEL TREATMENT; A CASE REPORT Wasim A. Hamarneh; Subhraleena Das; Sujith Cherian. SUNY Upstate Medical University, Syracuse, NY. (Control ID \#1339934)

LEARNING OBJECTIVE 1: 1. Recognize the diagnostic challenge and the broad differential for acute symmetric polyarthritis.

LEARNING OBJECTIVE 2: 2. Diagnose and manage parvovirus arthropathy

CASE: A 35-year-old- female post splenectomy for Beta thalassemia major was admitted with rapid onset of hand pain and swelling of four days. She had morning stiffness lasting more than an hour. Review of systems was unremarkable. Examination revealed synovitis in a symmetrical distribution, involving metacarpophalangeal (MCP), proximal interphalangeal (PIP), wrist and ankle joints with restriction of movement. Radiographs showed no erosive changes. Rheumatoid factor and anti- CCP (anti- citrullinated cyclic peptide) were negative but antinuclear antibody was transiently positive. She was started on steroids with a presumptive diagnosis of seronegative RA (rheumatoid arthritis) but her joint symptoms soon relapsed. Secondary hemosiderosis was considered, given her history of multiple transfusions; iron chelation therapy was contemplated. Serum levels of Parvovirus B19 antibodies were sent which returned strongly positive with an IgM level at $11.3(<0.9)$. Intravenous immunoglobulin (IVIG) infusions were started for parvovirus arthritis with good clinical response. Follow up after 3 months showed substantial reductions in the IgM level and elevated $\operatorname{IgG}$ levels.

DISCUSSION: Parvovirus B19 is known to cause arthropathy in adults. It presents with a symmetrical polyarthritis (RA-like) and usually resolves without joint erosions. Patients with immunosuppression and shortened red blood cell lifespan are at risk for persistent infection. Proposed mechanisms for B19- associated arthritis include immune-complex deposition, phospholipase-A2 like activity of viral capsomer protein (VP1) and molecular mimicry (VP-2 protein and articular collagen) with auto-antibody production. Viral persistence in synoviocytes may be a crucial factor in the development of arthropathy. Parvoviral infection has been linked to RA; causality has never been established. Higher viral seroprevalence and increased synovial fluid viral DNA persistence in RA cases serve as arguments for the association and merit further study. Most cases of parvoviral arthritis do not require specific therapy. Commercial IVIG (Intravenous immunoglobulin) is a good source of neutralizing anti-parvo antibodies. IVIG may also suppress TNFa (tumour necrosis factor a) and increase IL-2 (interleukin-2) production. Parvovirus arthropathy often responds to a 5-day course of IVIG. In patients at risk, it is prudent to consider parvoviral infection as a cause of persistent arthropathy. IVIG can be considered as a therapeutic option if arthropathy persists in spite of conventional treatment.

A RARE PRESENTATION OF TAKOTSUBO CARDIOMYOPATHY Waleed T. Kayani ${ }^{1}$; Salman J. Bandeali ${ }^{1}$; Anam Khan ${ }^{2}$; Ali Hashmi ${ }^{1}$; Himabindu Kadiyala ${ }^{1} .{ }^{1}$ Baylor College of Medicine, Houston, TX; ${ }^{2}$ Aga Khan University, Karachi, Pakistan. (Control ID \#1339973)

LEARNING OBJECTIVE 1: Recognize thyroid storm as a rare cause of Takotsubo cardiomyopathy

CASE: A 50 year old female with no prior diagnosed illnesses who presented with three days of nausea, low grade fevers, ongoing right upper quadrant (RUQ) abdominal pain and one episode of non specific chest pain. Admission examination revealed a febrile lady with sinus tachycardia, RUQ guarding and tenderness. Findings of leucocytosis on blood tests and positive Murphy's sign on abdominal sonogram revealed a picture consistent with acute cholecystitis. A screening electrocardiogram showed diffuse precordial STE elevations and T wave inversions. Troponin levels returned mildly elevated $(0.07 \mathrm{ng} / \mathrm{mL}$. An emergent cardiac catheterization was undertaken which did not show any evidence of obstructive coronary artery disease or plaque rupture. A transthoracic echocardiogram was done which showed moderately depressed ejection fraction $(30-34 \%)$ with severe akinesis of mid- distal left ventricular segments. Her presentation was consistent with stress induced cardiomyopathy precipitated by acute cholecystitis. She underwent open cholecystectomy on day 2 of admission. Her post operative course was complicated by lethargy, persistent sinus tachycardia to $150 /$ min and unremitting fever up to $103 \circ \mathrm{F}$. All infectious workup returned negative. As no apparent cause of persistent tachycardia and fever was evident, thyroid function studies were sent which revealed TSH $<0.01 \mathrm{uIU} / \mathrm{ml}$, free T4 $7.42 \mathrm{pg} / \mathrm{ml}$, free T3 $8.61 \mathrm{pg} / \mathrm{ml}$ consistent with severe hyperthyroidism. Her overall picture was consistent with thyroid storm (Burch Wartofsky score of 55). She was started on metoprolol, methimizole and hydrocortisone with resolution of tachycardia and fever in next 2 days. Her thyroid stimulating antibodies were elevated at 491\% (normal 0 - 139\%). A transthoracic echocardiogram done in 2 weeks showed return of ejection fraction to normal and resolution of impaired wall motion. Final diagnosis of stress induced cardiomyopathy from thyroid storm (which in turn was precipitated by acute cholecystitis) was made. Patient continues to do well on antithyroid therapy as an outpatient.

DISCUSSION: Takotsubo cardiomyopathy is a reversible form of left ventricular dysfunction which is typically preceded by an episode of intense physical or emotional stress. The presentation mimics that of a myocardial infarction with chest pain, elevated cardiac enzymes and ST segment elevation (usually anterior); however, coronary angiography does not reveal significant coronary stenosis or evidence of plaque rupture. There is increasing evidence that the disorder may be caused by diffuse catecholamine induced microvascular spasm or dysfunction resulting in myocardial stunning or by direct catecholamine-associated myocardial toxicity. Given increased concentrations of adrenoreceptors at cardiac apex, apical ballooning may reflect focal damage from circulating catecholamines, however, it remains a hypothesis at this point. Thyroid hormones are known to increase myocardial susceptibility to catecholamines and this may explain occurrence of Takotsubo's in patients with thyroid storm. Review of literature reveals only four cases of Takotsubo's cardiomyopathy induced by thyroid storm. Keeping in mind the high mortality associated with thyroid storm, physicians should keep hyperthythyroidism a consideration while working up such patients.

A RARE PULMONARY COMPLICATION OF SYSTEMIC LUPUS ERYTHEMATOSUS; A CASE REPORT Sujith Cherian; Subhraleena Das; Wasim A. Hamarneh. SUNY Upstate Medical University, Syracuse, NY. (Control ID \#1339789)

LEARNING OBJECTIVE 1: 1. Recognizing and diagnosing shrinking lung syndrome (SLS), an extremely rare pulmonary complication associated with systemic lupus erythematosus (SLE).

CASE: A 27-year-old female with known history of active SLE was referred to the pulmonary clinic by her rheumatologist for workup of exertional dyspnea and pleuritic chest pain. The patient had two recent admissions for the same in the past few months where radiological investigations revealed only low lung volumes and no other acute processes. CT scan of the chest was negative for any evidence of embolism and $2 \mathrm{D}$ echo was normal. Laboratory investigations confirmed the presence of active lupus with high ESR, elevated anti ds DNA titers and anti Ro/ SSA titers. Pulmonary function tests suggested severe restrictive ventilatory defect which revealed a FEV1 of $1.5 \mathrm{~L}, \mathrm{FVC}$ of $1.21 \mathrm{~L}$ and DLCO of $11.8 \mathrm{ml} / \mathrm{mmHg} / \mathrm{min}(37 \%, 39 \%$ and $43 \%$ of predicted respectively). A diagnosis of SLS was considered and the patient was put on an increased prednisone dosage at $1 \mathrm{mg} / \mathrm{kg} /$ day with symptomatic improvement and no further admissions, but was found to have no objective evidence of improvement on follow up.

DISCUSSION: SLE is a systemic autoimmune disease with pleuropulmonary involvement in 60-80\% of patients. SLS is a rare progressive pulmonary complication associated with SLE, characterized by dyspnea, pleuritic chest pain, reduced lung volumes and restrictive ventilatory defects without parenchymal abnormalities. The mechanism responsible is unclear although diaphragmatic dysfunction has been suggested. It generally presents in the third decade of life with a female predominance $(9: 1)$ and has a reported association with anti Ro/ SSA antibody positivity. There is no definitive therapy, but based on anecdotal evidence, increasing systemic glucocorticoid or increasing immunosuppressive agents as second line approach has been found to be useful. Therefore, in a patient with SLE, a strong index of clinical suspicion needs to be maintained for SLS, especially in patients presenting repeatedly with a constellation of dyspnea and pleuritic chest pain and no objective findings. 
A STRANGE PRESENTATION IN THE OFFICE - "MY HOUSE IS ON FIRE!” Tulsi Sharma; Pankaj Mehta. SUNY Upstate Medical University, Liverpool, NY. (Control ID \#1311834)

LEARNING OBJECTIVE 1: Organic brain lesions may present with atypical neurological presentations including psychiatric symptoms.

LEARNING OBJECTIVE 2: Brain imaging should be considered for patients with atypical symptoms or a sudden change in clinical presentation of psychiatric symptoms.

CASE: Introduction: Patients with organic brain lesions in neurologically silent brain areas might present only with psychiatric symptoms. Case: We present the case of a 21-year-old lady who was transferred from an outside facility with a possible diagnosis of encephalitis. She woke up one day with the sensation of her house burning down. This sensation was shortlasting but later in the day she developed mild headache with nausea. She was evaluated at the office of her primary care and sent for CT head, which revealed some signal abnormalities in the temporal area. An MRI was done the same day which suggested possible encephalitis. Lumbar puncture was attempted by a neurologist but was unsuccessful, so it was abandoned. EEG was negative for any focal discharges and she was empirically started on acyclovir. Her headache improved and she was discharged home on oral acyclovir. Over the next few days she felt depressed and emotionally labile. She said that she had been crying and this is not her usual self. She is actually a very happy person with no past medical history. She went back to her primary physician and repeat head imaging was unchanged. Her emotional lability persisted but she did not have any neurological signs. She was transferred to University hospital for an opinion and a repeat CT again revealed similar findings. Lumbar puncture done was normal and PCR for HSV was negative. MR spectroscopic examination showed very high choline peak, markedly diminished N-acetylaspartate (NAA) and decreased creatine. This metabolic profile on MR spectroscopy was suggestive of a probable tumor rather than infection or inflammatory process. Our suspicion was confirmed on the biopsy which revealed a glioblastoma. She has undergone surgery and is currently on chemo-radiation.

DISCUSSION: We present this case to highlight the fact that patients with organic brain lesions may present with a variety of psychiatric symptoms. This is especially important in the outpatient primary care setting as the symptoms may include depression, personality changes, cognitive deterioration, and anorexia nervosa. Diagnosis is often delayed and needs a high clinical suspicion in the office setting. 1. Studies have reported that $1 / 1000$ of hospitalized psychiatric patients have brain tumors. This rate is 20-times higher than in general population. 2 . Visual hallucinations have been reported but olfactory hallucinations are a rare presenting symptom for brain tumors. 3. Brain imaging should be considered not only for psychiatric patients with neurologic symptoms and signs, but for all psychiatric patients with atypical symptoms or a sudden change in clinical presentation of psychiatric symptoms. Delay to perform brain imaging might have a direct negative effect on treatment options and quality of life of such patients. 4. This case highlights the role of MR Spectroscopy in the early differentiation of infectious, metabolic and malignant pathologies of the brain using metabolic indicators. MR spectroscopy helped in the early diagnosis of this patient and hopefully a favorable outcome.

\section{A SYMPTOMATIC CASE OF R. PARKERI INFECTION DETECTED BY POLYMERASE CHAIN REACTION AMPLIFICA- TION FROM BLOOD AND ESCHAR SWAB SPECIMENS: A NOVEL DIAGNOSTIC APPROACH FOR A RARE CONDITION Patrick Daly ${ }^{1}$; Jason Maguire ${ }^{2}$; Tahaniyat Lalani ${ }^{2} .{ }^{1}$ Naval Medical Center Portsmouth, Portsmouth, VA; ${ }^{2}$ Naval Medical Center Ports- mouth, Portsmouth, VA. (Control ID \#1314593)}

LEARNING OBJECTIVE 1: Introduce novel approach to diagnosis of "R. parkeri" through cell culture isolate or PCR of eschar swab.

LEARNING OBJECTIVE 2: Demonstrate that "R. parkeri" should be considered in all patients with suspected rickettsial infection as "R. parkeri" prevalence is likely higher than previously reported due to cross-reactivity of the existing rickettsial serologic assays.
CASE: The zoonotic, vector-borne rickettsia are clinically important worldwide and considered an emerging risk for humans. Several spotted fever group (SFG) rickettsia cause human disease such as Rickettsiae rickettsii, $\mathrm{R}$. felis and R. akari. R. rickettsii, the cause of Rocky Mountain spotted fever (RMSF) is the most commonly recognized rickettsial disease in the U.S. with approximately 500-2000 cases reported annually and a case fatality rate in untreated patients as high as $25 \%$. Within the past seven years, a previously unrecognized cause of human disease, R. parkeri, has been reported as a confirmed cause of illness on six occasions, and was recovered in cell culture from an eschar. A 43 year-old gentleman presented to his primary care clinic in May 2011 after developing an eschar on the lateral aspect of his left knee, where he had removed an embedded tick several days prior. He was prescribed topical antibiotics, though subsequently developed fevers to $104 \mathrm{~F}$ accompanied by chills and a diffuse rash. He returned to the primary care clinic during the following week and was prescribed a two-week course of doxycycline. Within three to four days of starting the doxycycline, the patient reported resolution of his symptoms. Approximately three weeks later, the patient noted onset of asymmetric arthralgias including bilateral knee pain along with intermittent left wrist and ankle pain without swelling. Polymerase chain reaction (PCR) performed on the patient's blood and a swab of the eschar obtained at the time of his acute illness prior to antibiotic administration for $\mathrm{R}$. rickettsii and $\mathrm{R}$. parkeri and revealed $\mathrm{R}$ parkeri.

DISCUSSION: While R. rickettsii is the predominant pathogen involved in human rickettsial disease, the actual prevalence of $\mathrm{R}$. parkeri is unknown because most commercial serological assays do not distinguish between SFG species. Serum specimens from previously diagnosed RMSF patients react with protein isolates from R. parkeri. Seroprevalence studies have also demonstrated higher than expected R. rickettsii sero-positivity patients without history of RMS, which suggests that they may have been infected by less pathogenic SFG rickettsii such as R. parkeri. This pathogen has military relevance as the fourth reported case of R. parkeri infection in humans with three of the four cases involving US service members in the Tidewater region of Southeastern Virginia. We also report the first instance of diagnosing acute R. parkeri infection through non-invasive means. R. parkeri should be considered in the presence of single or multiple eschars in a patient with suspected rickettsial infection. In the current absence of serologic testing, cell culture isolate or PCR can be performed on eschar biopsy specimens and possibly eschar swabs. The clinical presentation of reported cases in contrast to RMSF will be reviewed in addition to the novel diagnostic approach employed in this case.

A WOMAN WHO PASSES OUT ON EATING Ojas Bansal ${ }^{1}$; Janardhana Gorthi ${ }^{1}$; Aryan Mooss ${ }^{1,2}$. ${ }^{1}$ Creighton University Medical Center, Omaha, NE; ${ }^{2}$ Creighton University Medical Center, Omaha, NE. (Control ID \#1335116)

LEARNING OBJECTIVE 1: Learn the presentation and diagnosis of Swallow Syncope

LEARNING OBJECTIVE 2: Discuss various management options for Swallow Syncope

CASE: 84 year old female presented to the hospital after she had recurrent episodes of syncope. First episode occurred two days ago while she was eating lunch at a restaurant when she felt nauseous, lightheaded and lost consciousness. She regained consciousness spontaneously in about a minute. She had another episode the following morning while eating breakfast at which point she decided to seek medical attention. On presentation her Blood pressure was $176 / 89$, heart rate $85 /$ minute, Cardiac exam was normal with S1 S2 regular rhythm without any murmurs. During her hospitalization she had a similar episode of syncope while eating a big piece of sandwich. Telemonitoring captured Sinus bradycardia with heart rates in 40's and blood pressure drop to $70 / 40 \mathrm{~mm} \mathrm{Hg}$. Her past medical history included hypertension for which she was on Metoprolol succinate $200 \mathrm{mg}$, Amlodipine $10 \mathrm{mg}$ and Lisinopril $20 \mathrm{mg}$ daily. We stopped her beta blocker and lisinopril with marked improvement in symptoms. She subsequently underwent esophagogastroduodenoscopy which showed a schatzki ring in the distal esophagus which was then dilated. She was started on soft diet which she tolerated well and was advised to eat small bites slowly. She did not have any further symptoms. 
DISCUSSION: Swallow (or Deglutition) syncope is a relatively rare type of Situational syncope. Manifestations vary from dizziness and presyncope to complete temporary loss of consciousness associated with swallowing. There have been about 80 reported cases of swallow syncope in the literature and only 2 of them were associated with Schatzki ring. It's a hypersensitive vagotonic reflex. Activation of the mechanoreceptors on esophageal distension leads to stimulation of the $\mathrm{SA}$ and $\mathrm{AV}$ node via the vagus nerve resulting in paroxysmal bradyarrhythmias and heart blocks. Others have observed that swallow syncope can occur even in the absence of bradycardia, as increased afferent vagal stimulation may lead to syncope via sympathetic withdrawal, resulting in peripheral vasodilation and hypotension, as seen in our patient. The diagnosis of swallow syncope requires careful elicitation of the temporal relationship between swallowing and syncope. Provocative testing with various types of liquid and solid foods should be attempted. As esophageal disorders may be associated with a majority of cases of swallow syncope, further work-up to exclude possible structural or functional esophageal pathology has been suggested. Echocardiogram and resting and ambulatory EKG should be performed to rule out any underlying cardiac pathology. Management of swallow syncope involves withdrawal of all medications causing delay in cardiac conduction and vasodepression. Avoidance of carbonated fluids or other agents associated with symptoms, as well as behavioral modification to change eating habits may be successful in patients with infrequent episodes of syncope. In patients with significant bradyarrythmias and AV blocks, placement of a pacemaker with a rate drop feature has been effective in controlling the symptoms. While syncope is a common presenting complaint, swallow syncope is a rare condition that may only be ascertained through thorough history, proper diagnostic work up and high index of clinical suspicion.

A "FORGOTTEN" CAUSE OF SEPSIS AND PNEUMONIA IN A 30 YEAR OLD MALE Neeraj N. Shah; Valay Parikh; Neville Mobarakai. Staten Island University Hospital, Staten Island, NY. (Control ID \#1312108)

LEARNING OBJECTIVE 1: Recognize Lemierre syndrome as an important cause of $\mathrm{F}$. necrophorum sepsis.

LEARNING OBJECTIVE 2: Diagnose Lemierre syndrome in absence of classical clinical features.

CASE: A 30 year old male presented to Emergency Department with complaints of pleuritic chest pain, abdominal pain, nausea, vomiting \& diarrhea. Review of systems revealed fever, chills, malaise, myalgias, generalized weakness \& decreased appetite since the past week. He was a smoker (7.5 pack-years). He appeared pale, diaphoretic \& dehydrated. Vitals showed tachycardia \& hypotension. Respiratory system examination revealed bilateral lower lobe ronchi. White blood count was $30,000 / \mu \mathrm{L}$ with neutrophilia. Other laboratory tests were normal except for platelet count of $53,000 / \mu \mathrm{L}$, BUN of $24 \mathrm{mg} / \mathrm{dl} \&$ mild conjugated hyperbilirubinemia. CT-chest showed consolidated airspace opacity in the left lower lobe along with multiple nodular opacities with cavitation in the right base. Blood cultures were sent \& empiric broad spectrum antibiotics were started. A 2D echo was negative for vegetations, ruling out infective endocarditis. Two days later, gram negative rods started growing in the blood cultures, \& antibiotics were switched to linezolid, ertapenem \& moxifloxacin. A week later, the blood culture report showed Fusobacterium necrophorum. Upon suspicion of Lemierre syndrome, a duplex ultrasound of neck vessels was done, which showed a partially occlusive thrombus in the right internal jugular vein. A diagnosis of Lemierre syndrome was established \& antibiotics were switched to penicillin \& clindamycin. The patient denied any sore throat or neck pain or swelling in the recent past \& examination of the head \& neck was normal, except for mild periodontitis. The patient was started on tinzaparin therapy for IJV thrombus. There was gradual clinical improvement in the patient's condition \& repeat ultrasound showed resolution of the thrombus 20 days later. After 3 weeks of intravenous antibiotics, the patient was discharged on oral clindamycin for 10 days and oral warfarin (target INR of 2-3) for 6 months.

DISCUSSION: Lemierre syndrome is an anaerobic suppurative thrombophlebitis of internal jugular vein (IJV) occurring following tonsillitis, pharyngitis, otitis media, sinusitis or dental infections. The most common pathogen is Fusobacterium necrophorum. It is more common in adolescents and young adults. Septic emboli to lungs, systemic sepsis \& metastatic abscesses can occur. In the preantibiotic era, it was common and fulminant, with a mortality of $90 \%$. However, it is now so rare (incidence 0.8 cases per million general population) that many clinicians are unaware of its existence, thus leading to the term "forgotten disease". With timely antibiotic treatment, the mortality maybe quite low (4.6\%). The classic presentation of Lemierre syndrome is an episode of fever \& sore throat followed by neck pain \& swelling. In our case, however, the patient presented with abdominal pain, vomiting, diarrhea, sepsis and chest pain, which can have a broad etiology. Few cases in the literature have reported sepsis \&/or cavitating lung lesions, without any evidence of pharyngitis, as the presenting features of Lemierre syndrome. This case highlights that in patients with positive blood cultures for F. necrophorum, in absence of classic features of Lemierre syndrome, it is important to perform radiologic testing for thrombosis of IJV in order to diagnose this syndrome. Without emergent antibiotic treatment, this disease can be rapidly fatal.

ACCELERATED ACUTE TUBULAR NECROSIS DUE TO VANCOMYCIN Sunay Shah; Papia Nasiri; Jose Vazquez. Henry Ford Hospital, Detroit, MI. (Control ID \#1339723)

LEARNING OBJECTIVE 1: Review the risk factors of vancomycininduced acute tubular necrosis.

CASE: A 31-year-old African American male with a history of a chronic left plantar foot ulcer, type 2 non-insulin dependent diabetes mellitus, and morbid obesity was admitted with the diagnosis of acute bacterial cellulitis on the plantar surface of his left foot with an associated ulceration. The physical examination revealed that the patient was afebrile and the second digit of the left lower extremity was markedly swollen with a linear ulceration on the lateral aspect, as well as a $3 \times 3 \mathrm{~cm}$ stage 3 plantar ulcer. Radiographs of the left foot demonstrated gas within the soft tissues and an area of bone destruction in the proximal phalanx of the second digit. The patient was empirically started on Vancomycin (VAN) 2 gm IV every 6 hrs, cefepime, and metronidazole. After $52 \mathrm{hrs}$ of hospitalization, the patient had received a cumulative dose of 16 gm of VAN. On the morning of hospital day three, the patient's labs revealed a serum creatinine of $5.3 \mathrm{mg} / \mathrm{dL}$, an increase of $4.5 \mathrm{mg} / \mathrm{dL}$ from the initial measurement [Table 1]. The first VAN trough level was found to be $76 \mu \mathrm{g} / \mathrm{mL}$, measured $44 \mathrm{hrs}$ after the first VAN dose and after a total of $12 \mathrm{gm}$. A renal biopsy demonstrated abnormalities consistent with ATN with extensive tubular epithelial cytoplasmic microvacuolization. Despite withholding VAN, the patient's serum creatinine continued to rise and eventually peaked on hospital day six at $12.1 \mathrm{mg} / \mathrm{dL}$. On hospital days seven and eight, he underwent two sessions of hemodialysis secondary to volume overload. Over the next few weeks, the patient's serum creatinine gradually improved and eventually stabilized at $1.4 \mathrm{mg} / \mathrm{dL}$. The patient completed antimicrobial treatment with a six-week course of ceftaroline for a severe diabetic foot infection and underlying osteomyelitis.

DISCUSSION: Vancomycin is one of the most commonly used antibiotics for the empiric and definitive treatment of MRSA and many other gram positive infections. Recently, several risk factors have been described that have been associated with increased VAN-induced nephrotoxicity. These include VAN serum trough concentrations $\geq 15 \mu \mathrm{g} / \mathrm{mL}$, prolonged duration of therapy $\geq 14$ days, obese patients weighing $>101 \mathrm{~kg}$, and VAN dosing regimens of $>4$ gm per day. Several recent studies suggest that AKI occurs more commonly in patients who reside in the intensive care units, have had a prior episode of AKI, or have received a vasopressor and/or other nephrotoxins, such as loop diuretics or aminoglycosides. This case report is unique for several reasons. It is only the second report documenting VAN as the sole etiologic cause of ATN. Moreover, this case also demonstrates the rapidity in the development of VAN-nephrotoxicity (case patient within $72 \mathrm{hrs}$ of admission). The primary risk factors associated with VAN-induced nephrotoxicity are directly related to the total amount of VAN being administered. Special considerations for calculating VAN doses and intervals should be considered in the diabetic and morbidly obese populations, since we frequently overestimate the patients' actual creatinine clearance. Given the information discussed herein, should we consider the use of alternative, less toxic antimicrobials in these high-risk patient populations? 
ACQUIRED THROMBOTIC THROMBOCYTOPENIC PURPURA IN AN 83 YEAR OLD WOMAN Allison E. Jordan; Merrideth A. Morris; Brad A. Keith. Medical University of South Carolina, Charleston, SC. (Control ID \#1335727)

LEARNING OBJECTIVE 1: Recognize the clinical features of thrombotic thrombocytopenic purpura in a geriatric patient.

LEARNING OBJECTIVE 2: Treat with standard therapy of plasmapheresis and incorporate rituximab for refractory cases of thrombotic thrombocytopenic purpura.

CASE: The patient is an 83 year old woman with a past medical history of hypertension, nicotine dependence, and monoclonal gammopathy of unknown significance who was transferred from an outside hospital with a chief complaint of abdominal pain, nausea, and bilious vomiting for several days. During initial evaluation at the outside hospital, she was noted to have platelets of 83,000 and hemoglobin of 8.3 . Due to her gastrointestinal problems, an EGD was performed which found esophageal candidiasis. Upon transfer, the patient also complained of a productive cough which had persisted for two weeks and sputum cultures had heavy growth of Pseudomonas aeruginosa. On admission to our hospital, her platelets were 70,000 and hemoglobin was 9.4. Her LDH was 661, Ddimer 4.37, haptoglobin 50 and creatinine 1.7. Her peripheral smear showed numerous schistocytes and direct Coomb's test was negative. Hematology and nephrology were consulted and the patient was emergently started on plasmapheresis for presumed thrombotic thrombocytopenic purpura. An ADAMTS13 activity returned $<5 \%$ and ADAMTS13 inhibitor was $<0.4$. Despite plasmapheresis for over two weeks, the patient continued to have thrombocytopenia. The patient was started on rituximab and received two doses while hospitalized. The patient was also treated with a two week course of fluconazole for the esophageal candidiasis and cefepime for Pseudomonas pneumonia. The patient's platelet count was 103, LDH 400, D-dimer 1.59, haptoglobin 21 and creatinine 1.7 at the time of discharge. The patient was discharged to a rehabilitation facility and followed up with hematology to receive her remaining two doses of rituximab as an outpatient. Her platelet count has ranged from 240 to 330 several weeks after her final treatment and she is considered to be in remisssion.

DISCUSSION: Acquired thrombotic thrombocytopenic purpura (TTP) is a rare condition that is a medical emergency. It is caused by a deficiency of a von-Willebrand factor-cleaving protease that has autoantibodies directed against it. The etiology for most cases of acquired TTP is idiopathic. Patients present with neurological abnormalities, renal failure, hemolytic anemia and thrombocytopenia. The diagnosis can be confirmed with testing for ADAMTS13 activity however it is recommended that treatment be initiated even if there is uncertainty about the diagnosis if the patient has thrombocytopenia and microangiopathic hemolytic anemia. Treatment involves plasmapheresis which removes the patient's circulating antibody to ADAMTS13 and von-Willebrand factor multimers. Patients are also infused with normal plasma that contains ADAMTS13. For patients with refractory cases or neurologic abnormalities, rituximab has been used in a small number of patients. After four once-weekly doses, most patients go into remission. Our patient was treated with two doses of rituximab while an inpatient due to continued thrombocytopenia despite plamapheresis and her platelets improved with this therapy. After completing the four doses her platelet count has normalized. More investigation is needed to determine if rituximab should be used as part of the initial treatment of TTP with plasmapheresis.

ACQUIRED HIGH-GRADE PAROXYSMAL ATRIOVENTRICULAR BLOCK DURING PREGNANCY Patrick Daly ${ }^{1}$; Eric Schwartzman ${ }^{2}$. ${ }^{1}$ Naval Medical Center Portsmouth, Portsmouth, VA; ${ }^{2}$ Naval Medical Center Portsmouth, Portsmouth, VA. (Control ID \#1314592)

LEARNING OBJECTIVE 1: Improve comprehension of mechanisms of $\mathrm{AV}$ block arising in pregnancy.

LEARNING OBJECTIVE 2: Demonstrate safe application of pacemaker placement during pregnancy.

CASE: High-grade atrioventricular block (AVB) arising in pregnancy is an exceptionally rare occurrence with only a few reported cases in the literature. In a review of admissions to a high-volume obstetric service, there were less than 2/100,000 cases of high-grade AVB with one attributed to congenital heart disease. Due to the limited number of reported cases in the literature, the underlying physiology and outcome of patients with acquired heart block during pregnancy is not well understood. We present the unusual case of a pregnant woman with multiple syncopal events and high-grade AVB on holter monitor. A 28 year-old female (G3P1) with history of depression and attention deficit hyperactivity disorder presented to the Emergency Department (ED) at approximately 10 weeks of pregnancy reporting dizziness, fatigue, and exertional dyspnea. She was discharged from the ED with an event monitor that documented variable degrees of AVB. She had no previous history of AVB or other cardiac conditions and had never experienced similar symptoms. Her first pregnancy was complicated by intrauterine fetal demise at 24 weeks gestational age, while she delivered a healthy infant at term during her second pregnancy. Patient remained active during this pregnancy and was not limited by her symptoms. Within several weeks of initial presentation, she had one syncopal event at rest after eating as well as a pre-syncopal episode while walking in her house. The remainder of her pregnancy was remarkable for pre-syncopal and syncopal episodes with electrocardiographic documentation on outpatient event monitors of high-grade AVB. A transthoracic echocardiogram was normal. A transvenous pacer was placed prior to the patient developing active labor. She delivered a healthy infant during an uncomplicated vaginal delivery, after which the transvenous pacer was removed. She continued to suffer from presyncopal episodes several times per week postpartum. Five months following delivery a dual-chamber pacemaker placed with subsequent resolution of her symptoms.

DISCUSSION: The physiology of AVB onset during pregnancy has not been established. There are several possible mechanisms with the most likely being a mechanical phenomenon involving increased vagal tone due to uterine compression of abdominal and pelvic vasculature. The hormonal changes occurring during pregnancy may alter the refractory period of the $\mathrm{AV}$ node or His-Purkinje system. Alternatively, autoantibodies to elements of the cardiac conduction system that arise during pregnancy could be the cause of the variable AVB. Certainly more research is needed to determine the etiology of new-onset AVB in pregnancy. Given the rarity of pregnancy-induced AVB, optimal therapy has not been well-established. These patients must be monitored closely with the knowledge that the AVB will likely be progressive in severity. Both temporary and permanent pacemaker placement during pregnancy has been performed without maternal or known fetal complications.

ACUTE HIV-1 INFECTION MISDIAGNOSED AS STREPTOCOCCAL PHARYNGITIS Aalok D. Patel ${ }^{1}$; Girish L. Kalra ${ }^{2} .{ }^{1}$ Emory University, Atlanta, GA; ${ }^{2}$ Emory, Atlanta, GA. (Control ID \#1322694)

LEARNING OBJECTIVE 1: Recognize the clinical features of acute HIV1 infection.

CASE: Case A 33-year-old African American man presented to the emergency department with several days of fever, sore throat, chest discomfort, vague difficulty swallowing, and occasional nausea/vomiting. Documented exam was notable for pharyngeal erythema and cervical lymphadenopathy. Rapid strep antigen screen returned positive. He was treated for streptococcal pharyngitis with intramuscular penicillin and discharged. He returned two weeks later for persistent symptoms and anorexia. Vital signs and exam were unremarkable. We did not appreciate cervical lymphadenopathy or pharyngeal erythema. Laboratories revealed a mildly elevated serum creatinine of $1.4 \mathrm{mg} / \mathrm{dL}, \mathrm{CPK}$ of $2294 \mathrm{U} / \mathrm{L}$, and AST of $108 \mathrm{U} / \mathrm{L}$. Upon detailed questioning, he endorsed a history of routine unprotected sex with male and female partners, intermittent homelessness, and a remote history of treated syphilis. An investigational point-of-care HIV screening test was negative. He was admitted for dehydration due to swallowing difficulty. He was treated conservatively with fluids, analgesics, and a proton pump inhibitor. He experienced intermittent fevers. Endoscopy revealed three superficial ulcerations in the mid-esophagus; biopsy later revealed severe inflammation without evidence of infection. Given persistent symptoms and a history of high-risk behaviors, the possibility of acute HIV infection was entertained, and formal HIV antibody and RNA testing were ordered. Initial ELISA screen 
was positive, but confirmatory western blot returned negative. The diagnosis of acute HIV was confirmed when his RNA viral load was found to be $>10$ million copies $/ \mathrm{mL}$. CD4 count was $326 / \mathrm{mcL}(39 \%)$. His swallowing gradually improved, and he was discharged with a plan for close follow-up with an infectious diseases specialist.

DISCUSSION: In acute human immunodeficiency virus (HIV)-1 infection, patients often present with a constellation of varied signs and symptoms, including fever, fatigue, rash, myalgias, headache, pharyngitis, gastrointestinal disturbance, and lymphadenopathy, beginning 1-4 weeks posttransmission. Painful mucocutaneous ulcerations, such as those found in our patient, are also commonly seen. The acute illness lasts days to weeks and is commonly misdiagnosed because of its nonspecific nature. Diagnosis is particularly challenging because HIV antibody tests are typically negative or indeterminate for several weeks following transmission. P24 antigen testing enhances sensitivity, but still has considerable diagnostic lag time. The most valuable test for early detection is the HIV RNA viral load, though it is not routinely ordered during screening. Thus, high clinical suspicion is necessary in order to diagnose acute HIV-1 infection. This case highlights the importance of asking patients about uncomfortable topics such as sexual history, homelessness, and drug use. In our patient, the diagnosis was delayed partly due to a positive rapid strep antigen screen; it is unclear whether this represents rare coincidence, colonization, or lab error. Nonetheless, detailed history-taking and recognition of the clinical syndrome at the time of follow-up led to the correct diagnosis.

ACUTE RESPIRATORY DISTRESS SYNDROME RESULTING FROM PRE-EXCLAMPSIA IN THE POST-PARTUM SETTING, A RARE BUT POTENTIALLY DEADLY COMPLICATION Daniel Gutteridge; Bhavana Siddegowda Bangalore. McLaren Regional Medical Center, Flint, MI. (Control ID \#1333654)

LEARNING OBJECTIVE 1: Recognizing risk factors for acute respiratory distress syndrome in the post-partum setting.

CASE: A 24-year-old G1P1 38 week pregnant female was delivered by Csection after failed induction for pre-eclampsia. Postoperatively, she was transferred to the intensive care unit due to progressive hypoxia. Findings suggestive of evolving ARDS on x-ray with impending respiratory failure led to intubation and mechanical ventilation. She was noted to be febrile with urine cultures showing pseudomonas and sputum cultures positive for staphylococcus aureus. Broad spectrum antibiotics were initiated. Hypoxia persisted and continued to worsen; ultimately she was transferred to a university hospital for rapid oscillator ventilation and evaluation for extracorporeal membrane oxygenation. After 9 days of treatment and subsequent stabilization she was transferred back to the referring hospital's intensive care unit for continued care.

DISCUSSION: Acute respiratory distress syndrome (ARDS) is a severe lung injury with rapid decline in pulmonary oxygenation status and subsequent respiratory failure requiring mechanical ventilation. There is severe hypoxemia with acute alveolar-capillary injury leading to non-hydrostatic pulmonary edema. As of 2005 there were approximately 200,000 cases in the published literature with in-hospital mortality rates ranging from $25 \%$ to $60 \%$, depending on patient age. Typical pathologies include severe sepsis, trauma, drug overdose and pancreatitis. ARDS is a rare and lethal complication in the postpartum setting with an incidence 1 in 10,113 deliveries. Prolonged ventilator support is typical and justified in this setting in order to decrease the otherwise high mortality rate. The majority of ARDS cases in the postpartum setting stem from infection and pre-eclampsia. In our case the patient presented with both, potentially increasing the risk. With identifiable causes of ARDS in the setting of pregnancy, prevention is the mainstay of treatment. Once ARDS presents, supportive care with mechanical ventilation with low tidal volumes and treatment of underlying cause is critical.

ACUTE CARDIOMYOPATHY SECONDARY TO NUTRITIONAL DEFICIENCY Ming Yeong Lim. Mayo Clinic, Rochester, MN. (Control ID \#1279476)

LEARNING OBJECTIVE 1: Recognize the importance of screening patients at risk of malnourishment for selenium deficiency
LEARNING OBJECTIVE 2: Recognize that selenium deficiency can lead to acute cardiomyopathy, which can mimic acute coronary syndrome (ACS)

CASE: A 69-year old male presented with a 1-day history of generalized weakness and dyspnea. Two months prior, he underwent a right hemicolectomy for a carcinoid tumor in the terminal ileum. Post-operatively, he developed atrial flutter, which resolved spontaneously. Echocardiogram then showed normal left ventricular size; ejection fraction (EF) $61 \%$. Serial cardiac biomarkers were negative. Post-surgery, he developed 5-6 watery bowel movements daily. To limit the frequency of diarrhea, he reduced his oral intake substantially. On examination, he was cachetic, dehydrated, tachycardic and hypotensive $(92 / 59 \mathrm{mmHg})$. Lung auscultation was clear bilaterally. Abdominal examination showed a healed incision scar. Electrocardiogram showed sinus tachycardia with non-specific ST and Twave changes. Initial troponin- $\mathrm{T}$ was $1.58 \mathrm{ng} / \mathrm{mL}(\mathrm{N}<0.01)$. Bedside echocardiogram demonstrated left ventricular enlargement with global hypokinesis;EF 35\%. He was transferred to the Coronary Care Unit due to concern for ACS. Serial troponin-T levels were $1.84 \mathrm{ng} / \mathrm{mL}$ and $1.99 \mathrm{ng} /$ $\mathrm{mL}$ at 3 and 6 hours respectively. He declined invasive diagnostic intervention with cardiac catheterization. He underwent $\mathrm{CT}$ angiogram of his coronary arteries, which revealed normal coronary arteries, thus ruling out ACS. With his cachexia, he was tested for nutritional deficiencies and found to be selenium deficient at $55 \mathrm{ng} / \mathrm{mL}(95-165 \mathrm{ng} / \mathrm{mL})$. He was diagnosed with selenium-deficient cardiomyopathy and started on oral selenium $(80 \mathrm{ug} /$ day $)$. On dismissal, he received medical therapy for heart failure (ACE-I and $\beta$-blockers). Clinical improvement was noted after selenium supplementation for 2 weeks, which correlated with echocardiogram findings of normalization of his left ventricular size; EF 43\%.

DISCUSSION: Selenium-deficient cardiomyopathy was first described in 1935 in Keshan, China, where the soil was poor in selenium, and was reversed with selenium supplementation. As selenium is absorbed in the small intestine, patients with malabsorption states, on chronic total parenteral nutrition, with small intestine surgery or disease are at risk for selenium-deficient cardiomyopathy. Selenium is an integral part of the enzyme glutathione peroxidase, which catalyzes active oxygen species, thus protecting cells from free radical damage. Loss of this protective mechanism with accumulation of free radicals has been proposed as the pathophysiology of selenium-deficient cardiomyopathy. Despite its importance, the daily requirement remains unclear due to the wide range of reference values for selenium and the variability in expression of symptomatic selenium deficiency. Also, selenium presents a nutritional conundrum as it is both essential and highly toxic. The World Health Organization suggests an intake of 30-40ug/day to meet healthy adult requirements. However, treatment of symptomatic selenium-deficiency patients requires intakes of $80 \mathrm{ug} /$ day. This case highlights the importance of selenium awareness in patients with malabsorptive or malnutrition states. Screening these patients for selenium deficiency is recommended. Early detection allows for prompt correction, thus avoiding potentially lifethreatening complications, such as in our patient.

ACUTE CHOLECYSTITIS AND PRIMARY EPSTEIN-BARR VIRUS INFECTION: A RARE COMPLICATION Alexander Zider; Magdalena Ptaszny. University of California, Los Angeles, Los Angeles, CA. (Control ID \#1340235)

LEARNING OBJECTIVE 1: Recognize cholecystitis as a rare complication of primary Epstein Barr virus (EBV) infection

CASE: A healthy, obese 32 year old female presented to the emergency room with five days of progressive headaches and three days of photophobia. In addition to these symptoms, she also had fevers (recorded as $100^{\circ} \mathrm{F}$ at home), night sweats, sore throat, anorexia with five pounds of weight loss, diarrhea, and generalized myalgias. Lastly, she had non-focal abdominal pain present on deep inspiration. Laboratory workup revealed a lymphocytosis with atypical lymphocytes (peak absolute lymphocyte count of 8700 on HD\#8), thrombocytopenia (nadir of 117 on HD\#4), and a transaminitis (peak aspartate and alanine aminotransferase (AST and ALT) of 266 and $286 \mathrm{IU} / \mathrm{L}$, respectively, on HD\#5). Her initial heterophile antibody and EBV-specific serologies were negative; these became positive 15 days after initial presentation, with positive heterophile and EBV-VCA (viral capsid antigen) IgM antibodies. Of note, on hospital day \#4, the 
patient's abdominal pain worsened and localized to the right upper quadrant (RUQ). Her total bilirubin had elevated slightly from $0.4 \mathrm{mg} / \mathrm{dL}$ on admission to $1.2 \mathrm{mg} / \mathrm{dL}$ with a conjugated bilirubin of $0.3 \mathrm{mg} / \mathrm{dL}$ (from $0.1 \mathrm{mg} / \mathrm{dL}$ ). A RUQ ultrasound had a positive sonographic Murphy's sign and demonstrated gallbladder wall thickening, pericholecystic fluid, and cholelithiasis. The patient underwent cholecystectomy on HD\#5, which confirmed calculous cholecystitis grossly and on histological examination (Figure). She denied any prior symptoms of biliary colic.

DISCUSSION: EBV is a ubiquitous virus, with carriage by over $90 \%$ of adults world-wide (1). Primary EBV infection, known as "infectious mononucleosis" or "glandular fever," afflicts about 500 people per 100,000 per year (1). Classic signs and symptoms of infectious mononucleosis include fatigue, fevers, pharyngitis, lymphadenopathy, hepatitis and splenomegaly. Acalculous cholecystitis in the setting of acute EBV infection is a rarely reported complication. There may be a female predominance, in contrast to the slight male predominance usually seen in acalculous cholecystitis (2). Concomitant Gilbert's syndrome has been seen in some patients (3). There have been several hypotheses regarding the mechanisms of EBV infection leading to cholecystitis. It may be akin to acalculous cholecystitis seen in critically ill patients (4). Alternatively, EBV hepatitis has been shown to cause cholestasis (5), which may lead to acute cholecystitis (4). Lastly, some have proposed direct invasion of the gallbladder by EBV (6). This type of "viral cholecystitis" has been seen with hepatitis A, where viral antigen in the gallbladder was identified on histology (7). EBV-DNA has been observed in surgical cholecystitis specimens: a case series noted $70 \%$ of cholecystitis patients had EBV-DNA detectable by PCR on cholecystectomy specimens. Of note, all of these patients were EBV-VCA IgM (-) and IgG (+), suggesting latent EBV infection (8). This appears to be the first reported case of calculous cholecystitis in the setting of primary EBV infection, at least in the United States. Possibly, her cholelithiasis simply may have been an unrelated concurrent process. The gallstones may have exacerbated the inflammation already present from cholestasis or EBV invasion. Most likely, the combination of her EBV hepatitis, cholelithiasis, dehydration and severity of infection all contributed to the development of her cholecystitis.

\section{ACUTE VISUAL LOSS AND HALLUCINATIONS IN A GERIATRIC PATIENT: GRAVE'S OPHTHALMOPATHY AND THE CHARLES BONNET SYNDROME. Cecily K. Peterson; Wenjing Liu. Duke Univer-} sity Health System, Durham, NC. (Control ID \#1338676)

LEARNING OBJECTIVE 1: Diagnose and manage Grave's ophthalmopathy

LEARNING OBJECTIVE 2: Recognize partial sightedness as a risk for visual release hallucinations: Charles Bonnet Syndrome

CASE: An 88-year-old woman with a history of diabetes, coronary disease, and macular degeneration presented with double vision and vision loss in her left eye. The patient reported occasional horizontal and vertical binocular diplopia during the preceding nine months. She was found to have thyrotoxicosis and began treatment with methimazole. During the three weeks after initiation of therapy, she noticed a decline in the vision of her left eye. She also noted right frontal headaches occurring two to three times per week but denied jaw claudication. Visual acuity was 20/70 in the right eye and 20/400 in the left eye. She had bilateral proptosis, limited elevation and abduction of both eyes, and a left afferent pupillary defect. The erythrocyte sedimentation rate was 61 seconds. Thyroid stimulating hormone was $0.05 \mathrm{microIU} / \mathrm{mL}$ (low), free thyroxine $1.21 \mathrm{ng} / \mathrm{dL}$ (normal), and free triiodothyronine $2.34 \mathrm{pg} / \mathrm{mL}$ (normal). The patient began intravenous methylprednisolone for 5 days and a temporal artery biopsy revealed no arteritis. Orbital MRI showed bilaterally enlarged extraocular muscles. Grave's ophthalmopathy was diagnosed. Her methimazole dose was increased and a subTenon injection of triamcinolone resulted in no visual improvement. During her hospital stay, she reported exquisitely detailed and increasingly frequent non-threatening visual hallucinations that she knew were not real. The images she reported were in far more detail than her visual acuity could distinguish and she and her family were puzzled and distressed by this. Due to cardiovascular risk factors, the patient chose radiation therapy to the orbits. Visual acuity improved to 20/ 50 in the right eye and 20/60 in the left eye.
DISCUSSION: The clinical presentation and course of Grave's ophthalmopathy (GO) is varied. Presenting symptoms may include eyelid retraction, exophthalmos, extraocular muscle dysfunction, ocular pain, and lacrimation. A minority of patients with Graves' disease can also develop sight-threatening optic neuropathy or corneal breakdown. Initiation of anti-thyroid therapy has been associated with worsening ophthalmopathy. Differential considerations include temporal arteritis, ischemic optic neuropathy, myasthenia gravis, orbital myositis, statininduced orbital myopathy and orbital tumors. Once a diagnosis of GO is established, treatment options include glucocorticoids, immunomodulators, surgical decompression, and radiation therapy. In patients with visual loss or visual field loss, it is important to recognize Charles Bonnet syndrome (CBS) as a cause of visual release hallucinations. Any vision loss can be associated with CBS but is commonly due to age-related macular degeneration, diabetic retinopathy, cataract, multiple sclerosis, or occipital infarction. Symptoms often go unreported for fear that psychiatric illness is responsible, but survey data suggests upwards of $15 \%$ of older patients with visual impairment have hallucinations. The natural history of visual release hallucinations varies with the rapidity of onset, underlying cause for visual impairment, and further change in vision over time. Early recognition with education and reassurance is helpful for many patients.

ADENOMAS AND ANTIBODIES: A STICKY SITUATION Kristy Bojazi; Varsha Somasekharan; Domnica Fotino. Tulane University Health Sciences Center, New Orleans, LA. (Control ID \#1337248)

LEARNING OBJECTIVE 1: 1 . Recognize the clinical manifestations of anti-phospholipid antibody syndrome 2 . Identify the differential diagnosis of anti-phospholipid antibody syndrome 3 . Understand the pathophysiology and treatment of anti-phospholipid antibody syndrome

CASE: A 44 year-old diabetic man presented with the sudden onset of severe headaches, blurry vision, and photophobia. The pain was frontal, radiating down his right neck. On the day of admission, he had fallen, striking his head. Subsequently, he noted left upper and lower extremity weakness. He denied neck stiffness, fevers, or chills. His temperature was $100.7 \mathrm{~F}$; remaining vital signs were normal. He had bi-temporal hemianopsia. His motor strength was $5 / 5$, and his upper and lower extremity reflexes were $2+$ and $1+$. His white blood cell count was 8,100 cells $/ \mathrm{mm} 3$; his electrolyes were normal. The total protein was 8.8 and the albumin was 4.1 . The hemoglobin $\mathrm{A} 1 \mathrm{C}$ was $11.9 \%$. A bilateral carotid artery ultrasound, transthoracic echocardiogram, and CT angiography of the head and neck were normal. An MRI of the brain revealed multiple two-centimeter acute and sub-acute infarcts in the right cerebral hemisphere. A three-centimeter pituitary macroadenoma was compressing and displacing the suprasellar anatomical structures; cavernous sinus involvement was indeterminate. The prolactin level was low at 0.8 ; a cortisol stimulation test was normal. The antiphospholipid antibody level was elevated at 11.6, and the lupus anticoagulant was elevated at 55. The patient was diagnosed with ischemic stroke caused by a hyper-coagulable state secondary to antiphospholipid syndrome, in turn due to the pituitary macroadenoma.

DISCUSSION: Although atherosclerosis is the most common cause of stroke, the general internist must entertain other etiologies when confronted by a young patient with headache and unilateral weakness. This differential diagnosis should include hemorrhagic stroke, a cerebral mass lesion, atypical seizures, septic emboli, and complicated migraine. One uncommon etiology of ischemic stroke is a hyper-coaguable state secondary to anti-phospholipid syndrome (APS). APS should be tested for in patients who present with signs and symptoms of multiple infarcts. APS is marked by venous and arterial thromboses in the presence of serum antibodies directed against phospholipidprotein complexes. Our patient met the criteria for APS with both an elevated antiphospholipid antibody and lupus anticoagulant. His pituitary mass's slight displacement of the intracranial vessels was considered clinically insignificant in his multiple infarcts. The pituitary infarcts were likely secondary to APS. Low-dose aspirin is commonly used for ischemic stroke, but its effectiveness in APS has not been proven. Patients with severe APS may require corticosteroids, immunosuppression, intravenous immunoglobulin and/or 
plasma exchange. In our patient, long-term oral anticoagulation with warfarin was the treatment of choice.

\section{ADULT OSTEOPETROSIS AND MARKED EXTRAMEDULLARY HEMATOPOESIS : A CAUSE OF RESPIRATORY FAILURE Surabhi Thakar; Nikhil Kalva. University of Illinois College of Medicine

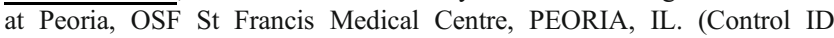 \#1311964)}

LEARNING OBJECTIVE 1: Osteopetrosis or Albers Schonberg's disease is a rare heterogeneous inherited metabolic bone disorder caused by impaired osteoclastic activity resulting in impaired bone resorption. It results in striking generalized osteosclerosis (Figure 1) with characteristic $\mathrm{X}$-ray findings of "bone within bone" appearance. Due to impaired osteoclastic function, Osteopetrosis presents with recurrent jaw osteomyelitis, fractures with poor healing and rarely marrow failure with extramedullary hematopoiesis involving spleen and liver. Here we describe a case of Osteopetrosis with marked extramedullary hematopoiesis involving paravertebral region (Figure 2) resulting in progressive respiratory failure and death

CASE: A 65 yr old male was admitted for evaluation of increasing progressive dyspnea, decreased appetite and weight loss over 1 year. He had chronic dyspnea from COPD, a result of long standing smoking. He reported no symptoms of chest pain, orthopnea or PND. He had noticed a marked reduction in his activity of daily living with symptom onset on minimal exertion. He appeared cachectic, BMI 19, tachypneic, using accessory muscles and supplemental oxygen to maintain saturations along with wheezing and diminished breath sounds. His admission hemogram showed markedly decreased reticulocyte count (0.5), macrocytosis (MCV 102) with normal folate and vitamin B12 levels, and thrombocytopenia $(73,000)$ which is below his usual baseline. His admission Chest $\mathrm{X}$ ray was remarkable for striking generalized osteosclerosis and increasing paraspinal densities. A CT chest, abdomen and pelvis revealed paravertebral soft tissue densities in the thorax, retroperitoneal and parasacral region with features suggestive of extramedullary hematopoiesis. His respiratory status continued to deteriorate and required endotracheal intubation complicated by failure to wean off the mechanical ventilator needing tracheostomy. $\mathrm{He}$ eventually died from respiratory complications despite aggressive attempts to liberate from the mechanical ventilator. The cause of death is likely multifactorial from COPD along with paravertebral extramedullary hematopoiesis contributing to respiratory failure.

DISCUSSION: Extramedullary hematopoiesis usually presents in the setting of primary marrow failure syndromes such as Primary Myelofibrosis or rarely with "Myelophthisic Process" from secondary marrow encroachment like in this patient. In certain primary marrow failure syndromes, cytoreductive therapy such as hydroxyurea has been shown to control respiratory symptoms. Alternatively low dose thoracic radiation can also be attempted to control the symptoms. Both thoracic radiation and hydroxyurea were not considered in this patient due to concern of worsening respiratory and marrow failure respectively.

AEROCOCCUS URINAE CAUSES AN INFECTIOUS ENDOCARDITIS Muhammed Sherid; Salih Samo; Samian Sulaiman; Meenu Singh. University of Illinois at Chicago, St.Francis Hospital, Evanston, IL. (Control ID \#1314374)

LEARNING OBJECTIVE 1: To suspect infectious endocarditis in a new onset stroke and fever

LEARNING OBJECTIVE 2: To understand that Aerococcus urinae is highly implicated as a causative agent of endocarditis in the presence of bacteremia

CASE: A 65 year old male presented with a twelve hour history of right-sided weakness with slurred speech and several days history of fever. There was no history of recent travel or sick contacts. He denied nausea, vomiting, diarrhea, abdominal pain or urinary symptoms. There was no history of cough, shortness of breath, headache or loss of consciousness. Past medical history was significant for left sided stroke two years ago with no residual defects. He did not have any history of substance abuse. He was married and was monogamous with his wife. On physical examination, blood pressure was157/79, pulse 121 , temperature $101.6 \mathrm{~F}$, oxygen saturation $96 \%$. He was pale and anicteric. There was no jugular venous distension or lymphadenopathy. Chest was clear to auscultation. There was a 2/6 systolic murmur in the base of the heart without any radiation. The abdomen was soft, non tender and did not reveal any organomegaly. Neurological exam was significant for right sided hemiparesis. Cranial nerves were intact. He did not have any pedal edema or skin rash. Laboratory studies showed an elevated white blood cell count at 14.2, platelet of $560, \mathrm{Hb} 9.6$ and Hct 28.5. Comprehensive metabolic panel was within normal limits. A CT scan of the head was unremarkable following which an MRI of the brain was done which showed acute ischemic changes along the medial cortex of the left frontal lobe. A chest $\mathrm{x}$-ray was unremarkable. Urinalysis showed pyuria. He was started on broad spectrum antibiotics. Urine culture grew mixed gram positive bacteria indicative of possible contamination. His blood culture grew Aerococcus urinae. A transesophageal echocardiogram was done which showed large vegetations noted on the mitral valve accompanied by severe mitral regurgitation and a smaller vegetation on the tricuspid valve. The antibiotics were changed to ampicillin and gentamycin after the organism was identified. However, despite therapy his condition deteriorated and the patient expired approximately two weeks after admission.

DISCUSSION: Aerococcus urinae is a gram positive alpha hemolytic coccus that grows in pairs and clusters. This organism is commonly misidentified as streptococcus or enterococcus. It commonly causes UTI, but has also been frequently incriminated in cases of endocarditis. This organism is associated with a high mortality when isolated from patients with endocarditis. A retrospective study of endocarditis caused by A.urinae showed that 9 out of 13 patients died. Review of literature shows that A.urinae is usually treated with penicillins or vancomycin with gentamycin.

ALCOHOL WITHDRAWAL: A RISK FOR DEVELOPING TAKOTSUBO CARDIOMYOPATHY Carmen Campbell; Shobhina Chheda. UW Madison, Madison, WI. (Control ID \#1338970)

LEARNING OBJECTIVE 1: Recognize the diagnostic criteria of takotsubo cardiomyopathy (TM).

LEARNING OBJECTIVE 2: Consider patients undergoing alcohol withdrawal at risk for development TM.

CASE: A 49-year-old male with a history of chronic pancreatitis complicated by pseudocyst and alcohol abuse presented with tremor, anxiety, and hypertension. He reported drinking approximately one liter of hard alcohol daily for the past week; his last drink was the day of presentation. His blood pressure was $145 / 95$, pulse 108 , temperature 36.7 Celsius, and respiratory rate 18 . Physical exam was notable for diffuse abdominal tenderness, tremor, and orientation only to person and place. Laboratory studies were notable for elevated liver function tests and negative blood alcohol level. EKG demonstrated sinus tachycardia without any ST or $\mathrm{T}$ wave changes. Patient was admitted to the ICU for management of severe alcohol withdrawal. On day 3, new T-wave inversion and ST depression were noted on EKG. Patient was delirious, but endorsed a history of exertional chest pressure. Serial troponins were followed and peaked at $0.23 \mathrm{ng} / \mathrm{ml}$. Patient was treated with aspirin and beta blockade. Cardiology thought that the EKG changes and troponin leak were secondary to demand ischemia in the setting of tachycardia. A transthoracic echocardiogram was obtained which demonstrated global wall motion abnormalities involving the anterolateral, apical, and inferoapical segments and an LVEF of $40 \%$. Patient underwent cardiac catheterization, which revealed no significant fixed coronary artery lesions. Patient was diagnosed with TM. Patient was started on carvedilol and continued on lisinopril. Two months later, a transthoracic echocardiogram demonstrated normalization of left ventricular function and resolution of wall motion abnormalities.

DISCUSSION: General internists in the hospital setting commonly encounter alcohol withdrawal. Case studies have been published in which alcohol withdrawal was identified as the stressor that precipitated TM among male patients. TM should be included in the differential diagnosis 
of acute coronary syndrome (ACS). Two decades ago, several Japanese patients presented with abnormal left ventricles similar in appearance to the eponymous Japanese octopus trap (narrow necks, wide bases). TM accounts for at least $2 \%$ of patients who present with ACS. TM most often affects women ( $>90 \%$ cases) who are middle-aged; however, cases have been documented in all age groups. Patients typically present with chest pain/pressure (70-90\%); other symptoms include: dyspnea $(20 \%)$, ventricular arrhythmias, cardiogenic shock, and cardiac arrest. Often an emotional stressor, such as loss of a loved one, preceded the diagnosis. The proposed diagnostic criteria for TM include: 1) Transient hypokinesis/ dyskinesis of left ventricular mid segments that extends beyond a single coronary bed, 2) No obstructive coronary disease or evidence of acute plaque rupture, 3) New electrocardiographic changes (ST elevation, T-wave inversion) or mild troponin elevation, and 4) Absence of pheochromocytoma or myocarditis. The pathophysiology of TM remains unknown, but is thought to be in part secondary to catecholamine surge. Treatment of TM acutely is similar to that of patients with ACS. Sub-acutely and chronically, patients are often treated with an ACE inhibitor and beta-blocker. Complete recovery of left ventricular systolic function occurs in the vast majority of patients, over a period of days to weeks. Recurrence occurs in up to $10 \%$ of patients.

\section{AN 85 YEAR OLD WOMAN PRESENTING WITH WHIPPLE'S} TRIAD FOR HYPOGLYCEMIA Yasuko Nagasaka, ${ }^{1,2}$; Annie A. Lee ${ }^{1,3}$; Junsung Rho ${ }^{4}$; Kristin A. Cox ${ }^{1,4}$. Newton-Wellesley Hospital, Boston, MA; ${ }^{2}$ Massachusetts General Hospital, Boston, MA; ${ }^{3}$ Massachusetts General Hospital, Boston, MA; ${ }^{4}$ Tufts University, School of Medicine, Boston, MA. (Control ID \#1334135)

LEARNING OBJECTIVE 1: Recognize the causes of hypoglycemia. LEARNING OBJECTIVE 2: Recognize the clinical presentation of an IGF-2 secreting tumor.

CASE: AH is an 85 year old woman with PAF, CHF, HTN, hypothyroidism and liver mesenchymal sarcoma $\mathrm{s} / \mathrm{p}$ chemoembolization who presented with hypoglycemia to 40 checked on her home glucose monitor. She reported increasing frequency of hypoglycemia over two months and often awoke at night feeling sweaty and lightheaded. These symptoms resolved with eating snacks. Her medications were notable for prednisone $5 \mathrm{mg}$ daily, metoprolol, furosemide, spironolactone, levothyroxine, and coumadin. VS: T 98.1, HR 91, BP 118/72, RR 20, 94\% on RA. PE was notable for an elderly, overweight woman with coarse facial features and a large tongue, systolic ejection heart murmur, clear lung exam, and distended abdomen with palpable, enlarged, firm liver, and trace lower extremity edema. Admission labs were significant for glucose of $97 \mathrm{mg} / \mathrm{dl}$, WBC of $9.4 \times 103 / \mu \mathrm{L}$, AST of $14 \mathrm{IU} / \mathrm{L}$, ALT of $19 \mathrm{IU} / \mathrm{L}$. During the patient's hospitalization, she had several episodes of blood sugars ranging from 40-45 in spite of a D10W infusion. When fasting, patient became hypoglycemic to 44 within four hours. While fasting and hypoglycemic, labs revealed a low insulin level $(0.1 \mu \mathrm{IU} / \mathrm{mL})$, low c-peptide level $(0.3 \mathrm{ng} / \mathrm{mL})$, low proinsulin level $(2.9$ $\mathrm{pmol} / \mathrm{L})$, low beta-hydroxybutyrate level $(0.1 \mathrm{mmol} / \mathrm{L})$ and negative sulfonylurea screen. Her hypoglycemia rapidly improved from 44 to 95 with intravenous glucagon. Insulin-like growth factor 2 (IGF-2) was elevated $(818 \mathrm{ng} / \mathrm{ml})$ whereas IGF-1 was normal. Due to frequent and severe episodes of nocturnal hypoglycemia, the patient was started on diazoxide which failed to adequately treat her hypogyclemia and was complicated by hyponatremia. Subsequently, octreotide was administered concomitantly with prednisone $20 \mathrm{mg}$ daily, which resulted in resolution of her hypoglycemia.

DISCUSSION: This patient presented with Whipple's triad: symptoms of hypoglycemia, a low glucose level, and resolution of these symptoms with treatment of hypoglycemia. The differential diagnosis of hypoglycemia includes surreptitious insulin use, insulinoma, sulfonylurea use, depletion of liver glycogen stores, and hypoglycemia mediated by an insulin-like factor. In this case, exogenous insulin use, insulinoma, and sulfonylurea use was excluded by low levels of insulin and c-peptide, and a negative sulfonylurea screen. Correction of hypoglycemia with glucagon suggested that she had sufficient liver glycogen stores to produce glucose. The elevated IGF-2 level confirmed the diagnosis of hypoglycemia mediated by IFG-2 which was likely produced by her liver tumor. Management of hypoglycemia from an IGF-2 secreting tumor involves treatment of the tumor or medications. This patient's hypoglycemia did not respond to three chemoembolizations and she was felt not to be a candidate for tumor resection given invasion of nearly the entire liver. Diazoxide, which inhibits insulin secretion from the pancreas, can be used to treat hypoglycemia but can cause worsening congestive heart failure. Prednisone can be used to treat hypoglycemia but has significant side effects. Octreotide also reduces insulin secretion and although typically ineffective in treatment of hypoglycemia mediated by IGF-2 was effective in this patient when used in combination with prednisone.

AN ACUTE PRESENTATION OF POLYMYALGIA RHEUMATICA (PMR) OR SOMETHING DIFFERENT? Shiva Taasoobshirazi; Bhavin Adhyaru. Emory University, Atlanta, GA. (Control ID \#1334998)

LEARNING OBJECTIVE 1: Understand the clinical presentation of the RS3PE syndrome

LEARNING OBJECTIVE 2: Recognize the differences between the RS3PE syndrome and PMR

CASE: A 62 year old African-American male presented to the ED with altered mental status after being found down in his apartment in his urine and feces by his landlord. In the ED, the patient was minimally responsive, would not open his eyes, and was unable to move his extremities. Friends of the patient noted he was in his usual state of health the previous week, but did notice he had some difficulty with stepping into a car. Friends also noted that the patient had been complaining of shoulder pain, weakness, and difficulty completing tasks like dressing and bathing over the past couple months. Of note, the patient had presented to the ED the previous month with a rash and fever and was discharged the same day. On exam, the patient was oriented only to person and was agitated and diffusely tender to touch. He had dry oral mucosa, decreased skin turgor, and ulcerated lesions on his lips and tongue. He was unable to lift both his upper and lower extremities, had decreased range of motion in all extremities, and had generalized muscle atrophy more proximally than distally. He also had a diffuse scaly, macular rash. In addition, he had swelling of the wrists bilaterally with pitting edema. Laboratory data showed an elevated ESR and CRP. Aspiration of the wrist joint was consistent with inflammatory arthritis. The differential included PMR but given the patient's presentation, he was diagnosed with Remitting Seronegative Symmetrical Synovitis with Pitting edema (RS3PE) syndrome and it was thought that his altered mental status was secondary to dehydration as he was bed-bound from pain and weakness. He was started on a low dose of prednisone (5 mg BID) and had dramatic improvement in his strength. Within a week he was able to walk using a walker.

DISCUSSION: The RS3PE syndrome is classified as an autoimmune disease similar to polymyalgia rheumatic (PMR) and in the same spectrum as Seronegative Rheumatoid Arthritis. It was first described in 1985 in a case series. Since its discovery there has been some controversy on whether this and PMR are the same syndrome but they share different HLA haplotypes. The criteria for diagnosis include the following: bilateral pitting edema of both hands, sudden onset of polyarthritis, age $>50 \mathrm{y} / \mathrm{o}$, and negative rheumatoid factor. The syndrome is generally more prevalent in males, responds well to very low dose steroids, and has good long-term prognosis. Some recent case series show that RS3PE syndrome may be a paraneoplastic syndrome with underlying malignancy. Our patient presents with a classic presentation of the RS3PE syndrome and the patient did have a diagnosis of prostate cancer that was treated in the past. The presentation of AMS is likely a manifestation of dehydration from decreased oral intake from the profound weakness and pain this syndrome had on the patient. This case illustrates that RS3PE syndrome should be considered in patients presenting with acute polyarthritis and pitting edema in the hands or feet and if found, they should have appropriate work-up for underlying malignancy.

AN ALTERNATIVE TREATMENT OPTION FOR COLLAGENOUS SPRUE WITH LYMPHOCYTIC GASTRITIS Sandhya Bogi; Sushma P. Banda. St.Joseph Mercy Hospital, Ypsilanti, MI. (Control ID \#1339583)

LEARNING OBJECTIVE 1: To learn treatment options for rare gastrintestinal disease. 
CASE: A 60-year-old woman with insulin resistence presented with a twomonth history of severe watery diarrhea, nausea and intractable vomiting associated with a 40-pound weight loss. Previous endoscopy revealed Helicobacter pylori associated gastritis, which had been treated. Physical examination revealed an ill appearing woman. Laboratory studies were remarkable only for hypoalbuminemia. Work up of her diarrhea included normal serum gastrin, vasoactive intestinal peptide (VIP) and 24 -hour urine for 5- hydroxyindole acetic acid (5-HIAA). Celiac serologies and stool studies were negative. Stool electrolytes were not suggestive of secretory diarrhea. Computerized tomography (CT) scan of the abdomen was normal. Colonoscopy with random biopsies was non diagnostic. Repeat endoscopy was unremarkable but duodenal biopsies revealed villous blunting and intraepithelial lymphocytosis with increased sub-epithelial collagen deposition. These findings were consistent with collagenous sprue. Antral biopsies revealed lymphocytic gastritis, which has been reported rarely in association with this disease.

DISCUSSION: There are seventy six reported cases of collagenous sprue in the literature since 1970; only four other cases have been associated with lymphocytic gastritis. Historically, collagenous sprue has been treated with immune-suppressants such as prednisone and azthiopine. Rare reports of treatment with Budesonide exist. Our case is the first example of a positive response with the use of Budesonide in a case of collagenous sprue with lymphocytic gastritis. It is yet to be determined whether this agent will be effective in maintaining this response and in preventing the severe complications associated with this disease.

AN ANAESTHESIA INTRICACY: METHEMOGLOBINEMIA. Jasleen Kaur ${ }^{1}$; Alaeddin Maeza ${ }^{1}$; Cosmin Dascalu ${ }^{1}$; Palaniappan Manickam ${ }^{1}$; Iuliana Niculescu $2 .{ }^{1}$ WSU/Crittenton Hospital Medical Center, Rochester, MI; ${ }^{2}$ William Beaumont Hospital, Royal Oak, MI. (Control ID \#1334365)

LEARNING OBJECTIVE 1: Methemoglobin is a form of hemoglobin which cannot bind oxygen due to Iron oxidation from ferrous state to ferric state. Methemoglobinemia can be Congenital or Acquired. Acquired Methemoglobinemia is more common and life threatening particularly due to exposure to certain drugs or toxins.

LEARNING OBJECTIVE 2: Diagnose methemoglobinemia with early treatment.

CASE: A 81 y/o female,known hypertensive with past h/o TIA ,presented with sudden onset of headache,nausea and vomiting for 2 hours. Associated symptoms include lightheadedness,generalised weakness, disorientation and confusion. On examination, she was hypertensive with systolic BP ranging $160-170 \mathrm{~mm} \mathrm{Hg}$ and bradycardic with 50 beats per min. Rest of the examination was unremarkable. CT scan and MRI indicated an acute ischemic change in the right temporal lobe and right occipital lobe adjacent to thalamus, compatible with involvement of cortical branches of right posterior cerebral artery (PCA). Echocardiography and U/S Carotids findings were insignificant. To rule out cardiac source of emboli in the setting of PCA Stroke, patient was taken for Transesophageal Echocardiography. During the procedure, local Hurricane $20 \%$ was given to back of her throat in the form of 2 sprays. Within $10 \mathrm{~min}$,she developed blue discoloration on her face and extremities notably on hands, dizziness and altered mental status. She was short of breath dropping her sats to $68 \%$ on $100 \%$ rebreather mask.The first set of $\mathrm{ABG}$ revealed 'chocolate colored' blood with MethHb level of 61.0. She immediately received Methylene Blue $6 \mathrm{mg}$ over $5 \mathrm{~min}$. She recovered significantly after $2 \mathrm{hrs}$ without any respiratory compromise.

DISCUSSION: Methemoglobinemia occurs when RBC's contain $>1.5 \%$ of methemoglobin. It is clinically characterised by cyanosis, low pulse oximetric readings and chocolate brown blood on ABG sampling with normal arterial Po2 values. This condition requires prompt diagnosis and treatment with Methylene Blue given in a dose of 1-2 $\mathrm{mg} / \mathrm{Kg}$ over 3-5 min and repeated every $30 \mathrm{~min}$ as necessary. Although the association between Benzocaine and Methemoglobinemia remains unclear but patients with co-morbidities such as anemia, cardiovascular disease, lung disease, sepsis, or presence of other abnormal hemoglobin species (e.g. carboxyhemoglobin or sickle hemoglobin) may experience moderate to severe symptoms at much lower levels (as low as 5-8\%). Acquired Toxic Methemoglobinemia can be life-threatening. Multiple cases have been reported in the literature including 2 large studies,especially with the use of Benzocaine. Since the warnings issued by FDA Public Health
Advisory in 2006, 72 new cases have been reported including 3 deaths. FDA continues to warn against the use of Benzocaine and recently issued guidelines for the proper doses and concentrations to be used in various settings. Healthcare professionals should be more aware of this serious reaction in order to make early diagnosis and prompt institution of treatment to prevent complications.

AN ATYPICAL CASE OF AUTO-IMMUNE HEPATITIS: CAN WEIGHT-LOSS AND HERBAL SUPPLEMENTS ACT AS TOXININDUCED PRECIPITATORS IN A SUSCEPTIBLE HOST? Madeline Sterling ${ }^{1,2}$; Sarang $\mathrm{Kim}^{1} .{ }^{1}$ UMDNJ-RWJMS, New Brunswick, NJ; ${ }^{2}$ UMDNJ-SPH, New Brunswick, NJ. (Control ID \#1310831)

LEARNING OBJECTIVE 1: Recognize an atypical case of Autoimmune Hepatitis (AIH)

LEARNING OBJECTIVE 2: Consider toxin-induced acute hepatic necrosis and evaluate sources of injury.

CASE: A 29 year-old Hispanic female presented with a 3 week history of abdominal pain, decreased appetite and jaundice. She reported gray-white colored bowel movements and darker colored urine for the past two weeks. She denied any fevers, chills, pruritus, rashes or bleeding. She denied recent infections, sick exposures, previous hepatitis, blood transfusions, or ingestion of medications or mushrooms. She was 'trying to lose weight' with herbal and dietary supplements hydroxycut and herbalife, which she took for 1 month 3 months prior. Her past medical history was significant for 2 spontaneous abortions. She does not smoke, drink alcohol, or use drugs. She saw her PMD 2 days prior to arrival because of her jaundiced skin; initial labwork revealed AST of 2409, ALT of 2000, TBili of 15.1 and AlkPhos of 154 , and she was advised to go to the emergency room. On physical exam she had scleral icterus, jaundice and mild mid-epigastric tenderness without hepato-splenomegaly, asterexis or mental status changes. Diagnostic evaluation was negative for hepatitis A, B, C, CMV and EBV. Autoimmune panels revealed an elevated ANA at 1:1280, A-SMA 1:10, normal AMA and LKM1 titers. Ceruloplasmin, Ferrittin, TSH and lipid panels were WNL. An USguided liver biopsy showed findings consistent with both acute hepatocyte necrosis secondary to toxin exposure and ongoing AIH. During her hospitalization, her hyperbilirubinemia and jaundice resolved and her ALT and AST improved without intervention to 1375 and 1149 respectively. At outpatient follow-up, she was found to have persistent transaminitis and was started on prednisone and AZT for AIH. She has been counseled on the dangers of herbal supplements and has agreed to discontinue this means of weight loss.

DISCUSSION: Dietary supplements are common causes of liver toxicity and hepatic injury. Case reports have shown Hydoxycut and Herbalife to be two of the most commonly used supplements, both of which contain components likely to inflict hepatocellular injury. This case demonstrates a temporal relationship between dietary supplement use and subsequent liver injury, though hepatic necrosis was not the only culprit. Her clinical and laboratory presentation, along with her liver biopsy results, indicate that an autoimmune process was present as well. Several drugs have been implicated in the onset of $\mathrm{AIH}$, including minocycline, nitrofurantoin, methyldopa, NSAIDS, and hydralazine. In this patient, weight loss medication may have precipitated an autoimmune hepatitis or caused hepatic necrosis which subsequently unmasked subclinical AIH. As a result of the obesity epidemic, supplements advertising weight loss continue to flourish. This case highlights the need for healthcare providers to be aware of such products and their dangers, especially for patients with underlying chronic or autoimmune diseases. More clinical and epidemiologic research is needed to ascertain the mechanism by which they cause damage and the prevalence and effects of their use among susceptible populations.

AN IGNORED IATROGEN Adegboyega O. Olayode; Lindsay C. Northam; Temple Brannan. Creighton University Medical Center, Omaha, NE. (Control ID \#1323226)

LEARNING OBJECTIVE 1: - Recognize the clinical features of serotonin syndrome 


\section{LEARNING OBJECTIVE 2: - Manage serotonin syndrome}

CASE: A 53 year old male who had been previously seen at two medical facilities was transferred due to findings of fever, tachycardia, elevated white blood cell count (WBC) with bandemia and elevated creatine phosphokinase (CPK). Preliminary diagnoses from the referring facilities included sepsis and rhabdomyolysis. Prior to transfer he was started on IV fluids and given a $2 \mathrm{mg}$ dose of IV lorazepam for agitation. History revealed anxiety, agitation, restlessness, diaphoresis and a mild nonproductive cough. On careful review of his outpatient medications, it was observed that he was simultaneously taking three serotonergic agents: bupropion, citalopram and tramadol. On initial assessment of vital signs he was febrile, tachycardic and had a blood pressure of 104/68. Physical examination revealed resting tremors and hyperreflexia predominant in the lower extremities. Initial lab data revealed a WBC of 16.7 with $19 \%$ bands and a CPK of 1496. Investigative procedures revealed no demonstrable foci of infection. Urine drug screen was negative. Serotonergic agents were held at the time of admission and IV fluids were continued. The patient was placed on continuous cardiac monitoring and treated symptomatically with IV lorazepam for tremor and agitation. Symptom resolution, including cessation of tremor and agitation, occurred quickly in less than 24 hours and was accompanied by normalization of white blood cell count and significant decrease in CPK level.

DISCUSSION: It has been reported that over $85 \%$ of physicians are unaware of serotonin syndrome as a clinical diagnosis. This case illustrates how easily it may be misdiagnosed and highlights the importance of taking a detailed history including the use of prescription drugs, over-the-counter medications and illicit substances along with performing a thorough physical examination. Serotonin syndrome commonly presents as a triad of mental status changes (agitation, restlessness, delirium, disorientation), autonomic dysfunction (diaphoresis, tachycardia, hyperthermia, BP instability, vomitting, diarrhea) and neurologic abnormalities (tremor, hyperreflexia, myoclonus, muscle rigidity, bilateral Babinski sign). The diagnosis of serotonin syndrome is a clinical diagnosis. Serum serotonin concentrations don't correlate with clinical findings and no lab tests confirm the diagnosis. Nevertheless it can be associated with some non-specific lab findings namely: leukocytosis, elevated CPK, transaminitis, metabolic acidosis and these can be used to monitor for potential complications. The Hunter Toxicity Criteria Decision Rules represent the most accurate diagnostic criteria for it. To fulfill the Hunter Criteria a patient must have taken a serotonergic agent and have ONE of the following: spontaneous clonus, inducible clonus plus agitation or diaphoresis, ocular clonus plus agitation or diaphoresis, tremor and hyperreflexia, hypertonia, temperature above $38^{\circ} \mathrm{C}$ plus ocular clonus or inducible clonus. In mild cases, discontinuation of inciting medications, supportive care, and sedation with benzodiazepines is generally sufficient. Moderately ill patients require more aggressive treatment of autonomic instability and possibly treatment with a serotonin antagonist (cyproheptadine). Hyperthermic patients are critically ill and often require paralysis and endotracheal intubation.

\section{AN INCIDENTAL AORTIC ULCER IN A PATIENT DIAGNOSED WITH COLON CANCER: HICKAM'S DICTUM VERSUS OCCAM'S RAZOR Christiane N. Mbianda; Jennifer Yacub; Peter-Trung Phan; Siddhartha Singh. Medical College of Wisconsin, Milwaukee, WI. (Control ID \#1287468)}

LEARNING OBJECTIVE 1: To recognize clostridium septicum aortitis and its strong association with colon cancer

LEARNING OBJECTIVE 2: To highlight the diagnostic dilemma faced in a patient with Clostridium Septicum Infection.

CASE: A 73-year- old male was hospitalized for evaluation of a four-week history of diarrhea. On admission, he was comfortable and notably afebrile. The initial evaluation revealed peripheral leukocytosis $(\mathrm{WBC}=16,000 / \mu \mathrm{l})$; elevated inflammatory markers $(\mathrm{ESR}=88 \mathrm{~mm} / \mathrm{Hr})$ and an abdominal $\mathrm{CT}$ showed colonic thickening around the hepatic flexure. Blood cultures were drawn on admission. A colonoscopy revealed an ulcerating, necrotic mass in the cecum which was confirmed to be an adenocarcinoma on pathological exam. Awaiting further workup, the patient had an episode of atrial flutter and underwent a contrast enhanced chest CT as an evaluation for suspected pulmonary embolism (PE). The chest CT showed no PE but incidentally revealed an aortic ulcer in the distal aortic arch. This was initially considered to be an atherosclerotic ulceration. A PET scan requested as a staging work up for the colon cancer showed no metastatic disease, but marked uptake in the region of the aortic ulcer, suggesting inflammation. A literature search revealed an association between infectious aortitis and colon cancer but blood cultures drawn on admission remained negative, the patient had no chest pain and remained afebrile. Subsequently, the patient was noted to have a hoarse voice, which prompted repeat imaging that revealed marked progression of the aortic ulcer and a $3.3 \mathrm{~cm}$ pseudo aneurysm. The patient underwent emergent surgery and the resected necrotic aortic arch grew Clostridium septicum. The patient initially made a remarkable recovery, but 3 days prior to the planned resection of his colon cancer - he suffered a cardiac arrest and died.

DISCUSSION: This case is notable for two reasons. Firstly, Clostridium septicum is an uncommon cause of aortitis and has a striking association with colon cancer. To the best of our knowledge, we are reporting the 27th case of C. septicum aortitis and the 21 st case associated with colon cancer. Secondly, the diagnostic journey in our case is intriguing. The incidental discovery of an aortic ulcer in a patient with newly diagnosed colon cancer set into play an age old conflict between Occam's razor and Hickam's dictum. Occam's razor, which states "plurality must not be posited without necessity" is the classic heuristic of medical diagnosticians but with our aging patient population suffering an epidemic of chronic diseases, Hickam's dictum, which states "a patient can have as many diagnoses as he darn well pleases" is holding true more often. In our case, as blood cultures were negative and the patient remained afebrile, Hickam's dictum dominated initially - prompting the hypothesis of an atherosclerotic aortic ulcer and an unrelated diagnosis of colon cancer. This changed with the development of hoarseness of voice (involvement of the recurrent laryngeal nerve) - which signaled the progression of the aortic arch pathology. This prompted repeat imaging, surgery and subsequent pathological exam which revealed a parsimonious explanation for the patient's clinical conditions and the ultimate triumph of Occam's razor: Proliferation of $\mathrm{C}$. septicum in the anaerobic, necrotic areas of the colon cancer with eventual occult C. septicum bacteremia and resulting aortitis.

\section{AN INTERESTING CUTANEOUS FINDING IN A YOUNG ADULT WITH DISSEMINATED COCCIDIOMYCOSIS Lisa Ngo. SJHMC,} Phoenix, AZ. (Control ID \#1336472)

LEARNING OBJECTIVE 1: Coccidiomycosis is caused by Coccidioides immitis, a dimorphic soil fungus native to the San Joaquin Valley of California, southern portions of Arizona, northern portions of Mexico, and scattered areas in Central America and South America. The disease is usually mild, with flu-like symptoms and rash. Serious complications include severe pneumonia, lung nodules, and disseminated disease. The disseminated form of "valley fever" can devastate the body, causing skin ulcers, abscesses, bone lesions, severe joint pain, pericarditis, urinary tract infection, meningitis, and often death. The skin is the most common site of dissemination. Involvement ranges from superficial maculopapules, keratotic nodules, and verrucous ulcers to subcutaneous fluctuant abscesses CASE: 25 year old African American male without significant past medical history presented with complaint of left scapular and shoulder pain for five months. Pt also initially complained of a small nodule medial to his left scapula which progressively increased in size. Pt noted night sweats for one month, but denied fever, chills, weight loss, cough, or dyspnea. Pt was initially treated with rest, narcotics, and physical therapy with minimal improvement. Pt was eventually sent for imaging studies including CT of neck and chest, and MRI of the cervical and thoracic spine, which shown a heterogeneous soft tissue mass involving the left posterior chest wall as well as the cervicothoracic spine region. Pt underwent a CT-guided biopsy of the mass, pathology revealed granulomatous inflammation with rare spherules consistent with coccidioides. Cocci serology was positive for IgM and IgG with a titer of $>1: 256$. Pt was treated with long term treatment with fluconazole. Pre- and post-treatment imaging demonstrated near resolution of his subcutaneous abscess.

DISCUSSION: Fewer than $1 \%$ of infected individuals develop disseminated coccidiomycosis. The clinical manifestations of disseminated coccidiomycosis range from a fulminant illess that is fatal within a few weeks if left untreated, to an indolent chronic disease that persists for 
months or years. One or more sites may e involved, but the skin, soft tissue, bones, joints, and meninges are most commonly affected. Cutaneous and subcutaneous lesions are among the most common manifestations of disseminated cocidiomycosis. Cutaneous lesions may be single or multiple and can persist for long periods. They may appear as verrucous papules, erythematous plaques, and nodules. The large, cold abscess that may develop in soft tissue with $\mathrm{C}$. immitis can be mistaken for tuberculosis, sebaceous cyst, or lipoma. This case was remarkable because of the size of his subcutaneous abscess and also how he was misdiagnosed for a prolonged period of time because of his atypical presentation. Disseminated coccidiomycosis has a broad range of clinical presentations. This case emphasizes the importance of including coccidiomycosis as a differential diagnosis in unexplained skin, soft tissue, bone and joint manifestations for patients in areas with a high prevalence for coccidiomycosis.

\section{AN INTERESTING CUTANEOUS FINDING IN A YOUNG ADULT WITH DISSEMINATED COCCIDIOMYCOSIS Lisa Ngo. SJHMC,} Phoenix, AZ. (Control ID \#1336472)

LEARNING OBJECTIVE 1: Coccidiomycosis is caused by Coccidioides immitis, a dimorphic soil fungus native to the San Joaquin Valley of California, southern portions of Arizona, northern portions of Mexico, and scattered areas in Central America and South America. The disease is usually mild, with flu-like symptoms and rash. Serious complications include severe pneumonia, lung nodules, and disseminated disease. The disseminated form of "valley fever" can devastate the body, causing skin ulcers, abscesses, bone lesions, severe joint pain, pericarditis, urinary tract infection, meningitis, and often death. The skin is the most common site of dissemination. Involvement ranges from superficial maculopapules, keratotic nodules, and verrucous ulcers to subcutaneous fluctuant abscesses

CASE: 25 year old African American male without significant past medical history presented with complaint of left scapular and shoulder pain for five months. Pt also initially complained of a small nodule medial to his left scapula which progressively increased in size. Pt noted night sweats for one month, but denied fever, chills, weight loss, cough, or dyspnea. Pt was initially treated with rest, narcotics, and physical therapy with minimal improvement. Pt was eventually sent for imaging studies including CT of neck and chest, and MRI of the cervical and thoracic spine, which shown a heterogeneous soft tissue mass involving the left posterior chest wall as well as the cervicothoracic spine region. Pt underwent a CT-guided biopsy of the mass, pathology revealed granulomatous inflammation with rare spherules consistent with coccidioides. Cocci serology was positive for $\operatorname{IgM}$ and IgG with a titer of $>1: 256$. Pt was treated with long term treatment with fluconazole. Pre- and post-treatment imaging demonstrated near resolution of his subcutaneous abscess.

DISCUSSION: Fewer than $1 \%$ of infected individuals develop disseminated coccidiomycosis. The clinical manifestations of disseminated coccidiomycosis range from a fulminant illess that is fatal within a few weeks if left untreated, to an indolent chronic disease that persists for months or years. One or more sites may e involved, but the skin, soft tissue, bones, joints, and meninges are most commonly affected. Cutaneous and subcutaneous lesions are among the most common manifestations of disseminated cocidiomycosis. Cutaneous lesions may be single or multiple and can persist for long periods. They may appear as verrucous papules, erythematous plaques, and nodules. The large, cold abscess that may develop in soft tissue with C. immitis can be mistaken for tuberculosis, sebaceous cyst, or lipoma. This case was remarkable because of the size of his subcutaneous abscess and also how he was misdiagnosed for a prolonged period of time because of his atypical presentation. Disseminated coccidiomycosis has a broad range of clinical presentations. This case emphasizes the importance of including coccidiomycosis as a differential diagnosis in unexplained skin, soft tissue, bone and joint manifestations for patients in areas with a high prevalence for coccidiomycosis.

AN UNCOMMON CAUSE OF INFLAMMATION May Tun Saung; Luciana Catanese; Priya Joshi. Boston Medical Center, Boston, MA. (Control ID \#1340212)
LEARNING OBJECTIVE 1: Recognize clinical features of hemophagocytic lymphohistiocytosis (HLH)

LEARNING OBJECTIVE 2: Treat HLH

CASE: A 53 year-old woman presented to an outside hospital with complaints of 1 week of fevers, myalgias, URI symptoms, and 1 episode of "fainting." She was hypotensive, but responded to fluid resuscitation. Marked hematologic derangements were seen, including leukopenia of 1.3 with $15 \%$ bands, transaminitis AST 397/ALT 239, ferritinemia 65,000 and LDH 2,000. A chest radiograph showed a possible infiltrate, leading to the diagnosis of presumed severe sepsis secondary to community-acquired pneumonia, but pancytopenia and transaminitis worsened despite broadspectrum antibiotics, prompting the transfer to our hospital. Additional labs at our hospital showed neutropenia of 0.5 , hypertriglyceridemia 379 , and a positive DIC panel. Flow cytometry and a peripheral smear did not indicate malignancy. All infectious and rheumatologic studies were negative except for a positive EBV DNA PCR. A bone marrow biopsy showed hemophagocytosis with numerous nucleated erythroid precursors engulfed by histiocytes, indicative of HLH.

DISCUSSION: There are two types of HLH which present similarly. Primary HLH is due to an autosomal recessive trait and is predominantly seen in children. Secondary HLH is seen more often in adults, and is caused by the body's reaction to another condition such as infections (EBV is the most commonly associated infection), autoimmune disease, malignancies, immune deficiencies or suppression, and post-transplantation. The central pathophysiology of HLH is an excessive immune response. A cytokine storm leads multi-organ dysfunction via infiltration of activated T-lymphocytes, natural killer (NK) cells and histiocytes. Autoantibodies may also contribute to the cytopenia. The key diagnostic finding is hemophagocytosis of blood cells in the bone marrow, spleen and lymph nodes. HLH can typically be diagnosed if at least five of the following criteria are fulfilled: fever, splenomegaly, cytopenia, hypertriglyceridemia or hypofibrinogemia, hemophagocytosis, hepatitis, low or absent NK cell activity, marked ferritinemia and elevated IL-2 receptor. Though HLH is a rare condition, incidence may be higher given the need for complex diagnostic workup and is commonly misdiagnosed as severe sepsis (as in this patient's case), FUO, hepatitis, renal failure, ARDS, and various CNS diseases to name a few. Prompt diagnosis is crucial, since without treatment, median survival is 1 to 2 months. To address this, the first international treatment protocol, HLH-94, was developed and studies have shown a significant improvement in survival rates. The HLH-2004 protocol (Grade 1B) is the current standard of care: immediate immunochemotherapy with dexamethasone and etoposide, aggressive supportive therapy and for selected patients, hematopoietic cell transplantation (HCT). Whether cyclosporine should be administered upfront as proposed by HLH-94 or later is controversial. This patient was treated with dexamethasone and intravenous immunoglobulin since the latter is an appropriate adjunct for most viral infections. The patient improved following 2 days of therapy and supportive care, and etoposide was never started. EBVassociated HLH can range from spontaneously resolving inflammation to persisting illness requiring $\mathrm{HCT}$, and the patient's presentation is closer to the former. During her outpatient follow-up visit, her laboratory values normalized except for a slight down-trending ferritinemia.

AN UNLIKELY FOE: IATROGENIC MULTI-SYSTEM ORGAN DYSFUNCTION Aliza Norwood; Allison DeKosky; Manisha Israni. UCSF Medical Center, San Francisco, CA. (Control ID \#1340146)

LEARNING OBJECTIVE 1: Recognize the unanticipated adverse effects of prolonged courses of broad-spectrum antibiotics

LEARNING OBJECTIVE 2: Recognize an unusual cause of altered mental status in hospitalized patients.

CASE: A 59 year-old man with diabetes, Stage I congestive heart failure, and crytogenic organizing pneumonia on chronic steroids was admitted for surgical treatment of recurrent retrocalcaneal abscesses. He received two weeks of vancomycin and piperacillin-tazobactam before a flap repair. Intra-operative wound cultures grew pseudomonas and the patient was therefore switched to cefepime. Two weeks post-operatively, repeat deep wound cultures grew resistant enteroccoccus and empiric vancomycin (restarted three days prior) was changed to linezolid; cefepime remained on 
medication list throughout. Four days later, he was found to be somnolent and confused. Computed tomography of his brain was normal. Over the next twelve hours, his mental status worsened and he developed facial edema and a macular, confluent erythematous rash that spread from his torso to his face, ears, and upper extremities with no mucosal involvement. He became hemodynamically unstable with fever, tachycardia, hypotension and anuria. His creatinine had increased over the previous two days from 0.7 to 3 , while maintaining a normal BUN. He developed leukocytosis with 3.5\% eosinophils. His urinalysis revealed pyuria with no nitrite, bacteria or casts, consistent with acute interstitial nephritis. He ultimately required vasopressor support in the ICU and continuous veno-venous hemodialysis. Cefepime was then stopped and meropenem was initiated. Within a few days, his mental status and renal function improved. No organisms grew on multiple repeat blood and wound cultures. High-dose steroids were initiated for a concern of DRESS syndrome (Drug Rash, Eosinophilia and Systemic Symptoms). A biopsy of the rash revealed perivascular infiltrates containing eosinophils.

DISCUSSION: Cefepime hydrochloride, a fourth-generation cephalosporin, is a common tool in physicians' arsenals for treating severe infections, especially Pseudomonal infections. Prolonged use of cefepime increases the risk of complications, which are compounded when renal function deteriorates. Our patient presented with rapid progression of altered mental status and acute renal failure in the setting of long term antibiotic treatment with vancomycin and cefepime for recurrent abscesses. Cefepime has been associated with encephalopathy, myoclonus, and seizures that usually resolve within $24-48 \mathrm{hr}$ of discontinuation of the drug. The drug is removed easily by hemodialysis, which accounts for the rapid improvement in mental status seen in patients with cefepime neurotoxicity treated with dialysis. This patient had multiple cefepime-induced toxicities including severe neurotoxicity resulting in profound encephalopathy, more likely to be seen with high doses of cefepime; interstitial nephritis, and the less known DRESS syndrome. This drug hypersensitivity syndrome typically develops two to six weeks after the responsible drug is begun, consistent with this patient's time course. Fever and erythroderma are common characteristics, while the facial edema and ear involvement are hallmark features. DRESS syndrome's constellation of symptoms can be mistaken for sepsis-induced multi-organ failure or toxic shock syndrome. Internists should be aware of the unusual but critical complications associated with commonly used drugs like cefepime.

AN UNUSUAL CASE OF MENINGITIS Jordan B. Strom; Cynthia M. Cooper. Massachusetts General Hospital, Boston, MA. (Control ID \#1311012)

LEARNING OBJECTIVE 1: Diagnose and distinguish medicationrelated etiologies of community acquired aseptic meningitis.

LEARNING OBJECTIVE 2: Recognize the role of taking a thorough medication history in the assessment of a patient with suspected meningitis.

CASE: A 58-year-old woman presented with a severe headache with onset three hours after taking prophylactic amoxicillin for a routine dental cleaning. She described a constant bifrontal headache with mild photophobia, multiple episodes of emesis, diarrhea, fevers, chills, and myalgias. She denied any phonophobia, visual changes, neck stiffness, or other neurologic symptoms. She described similar symptoms five months prior, 6 hours after amoxicillin use for a dental cleaning, which resolved spontaneously in less than 24 hours without medical intervention. Past medical history was remarkable for a right total hip replacement one year ago after a traumatic fracture and anaphylaxis to soy, nuts, peanuts, beans and legumes. She was married, a non-smoker, with no history of alcohol or illicit drug abuse and employed as a health care lawyer. Her only medications were amoxicillin and epinephrine 1:1000 pen. On exam, she was febrile to 103 degrees Fahrenheit, but otherwise non-toxic. Physical exam and detailed neurologic exam were normal. Cerebrospinal fluid was obtained by lumbar puncture. There were 611 nucleated cells in tube 1 (92\% neutrophils, 370 red blood cells) and 624 nucleated cells in tube 4 ( $90 \%$ neutrophils, 17 red blood cells). Total protein was $228 \mathrm{mg} / \mathrm{dL}$ and glucose $67 \mathrm{mg} / \mathrm{dL}$. The patient received meningitic dosing of vancomycin, ceftriaxone, and acyclovir. Culture, gram stain, and herpes simplex virus DNA amplification of her cerebral spinal fluid were all negative. Her symptoms rapidly resolved within 12 hours. An allergy consultation was obtained. Given the recurrent pattern of symptoms with amoxicillin exposure, she was felt to have amoxicillin-induced aseptic meningitis. She was discharged home with instruction to avoid all penicillin-based products.

DISCUSSION: Drug-induced aseptic meningitis (DIAM) is an uncommon cause of community-acquired aseptic meningitis. Its true incidence is unknown. DIAM has been associated with use of nonsteroidal antiinflammatory drugs, Cox-2 inhibitors, antibiotics, anticonvulsants, and immunomodulation therapies, e.g, IVIG and OKT3 antibodies. It typically presents with a neutrophilic pleocytosis, and can be mistaken for infectious meningitis. DIAM appears to be more common in patients with autoimmune disease. There are only eight other case reports of amoxicillin-induced meningitis in the literature. The mechanism of DIAM is unknown, but hypersensitivity and immune complex formation have been postulated. Resolution occurs within days of antibiotic cessation. This report adds to the evidence-base and emphasizes the importance of taking a thorough medication history in individuals with suspected meningitis.

AN UNUSUAL CASE OF SEPTIC ARTHRITIS DUE TO STREPTOCOCCUS PNEUMONIAE Tina Constantin; Karen Schmitz; Girish L. Kalra. Emory University School of Medicine, Atlanta, GA. (Control ID \#1334313)

LEARNING OBJECTIVE 1: Diagnose septic arthritis in a patient with inflamed joints and fever - even when the fluid analysis fails to impress. LEARNING OBJECTIVE 2: Recognize features of pneumococcal septic arthritis, an uncommon but important entity.

CASE: A 51-year-old African American woman with type 2 diabetes mellitus presented to the emergency department with one week of progressively worsening pain and swelling involving her right knee, left ankle, and proximal right thumb. She also complained of severe fatigue, myalgias, and subjective fevers. She denied any precipitating injury, but recalled feeling ill one day before the onset, after a strenuous day at work as a housekeeper. 12 days before the onset of her illness, she saw a podiatrist for chronic ankle pain and was treated with bilateral intraarticular steroid injections. On admission, she was tachycardic to $135 \mathrm{bpm}$ and febrile to 38.9 C. Exam revealed warm, tender effusions and painfully restricted motion in the right knee and both ankles. She had frank synovitis on her dorsal right thumb over the metacarpophalangeal (MCP) joint. Labs revealed white blood cell (WBC) count of $20.6 \mathrm{~K} / \mathrm{mcL}$; this later rose to $32.8 \mathrm{~K} / \mathrm{mcL}$. Joint aspirate from her right knee showed only $4,150 \mathrm{WBC} /$ mcL with $80 \%$ neutrophils and negative gram stain. She received one dose of vancomycin and was transitioned to ceftriaxone to cover possible gonococcal arthritis. Cultures from joint aspirate and blood later grew Streptococcus pneumoniae. Chest $\mathrm{x}$-ray and subsequent transthoracic echocardiogram failed to identify other sources for her bacteremia. Repeat arthrocentesis of her right knee the following day revealed pus and 20,100 $\mathrm{WBC} / \mathrm{mcL}$. She underwent surgical lavage of her right knee and thumb and slowly improved on ceftriaxone therapy, though she remained weak and largely immobile. On hospital day 12, she was discharged to a skilled nursing and rehabilitation facility to complete 6 weeks of IV ceftriaxone. DISCUSSION: Streptococcus pneumoniae is an uncommon cause of septic arthritis, comprising only $3-5 \%$ of cases. As this case illustrates, polyarticular involvement is common and seen in $36 \%$ of adults, while bacteremia is particularly characteristic and seen in $72 \%$; rates of both findings are lower with other causes of nongonococcal bacterial arthritis. The most commonly affected joint is the knee, followed by the shoulder and elbow. Patients are typically systemically ill, with fever and leukocytosis present in more than half. Synovial fluid is purulent and mean WBC is 127,000 . The most commonly identified extra-articular source of pneumococcal infection is pneumonia; others include meningitis and endocarditis. However, as is seen here, in half of patients with pneumococcal septic arthritis, no extra-articular focus of infection is found. This case highlights the importance of maintaining a high clinical suspicion for septic arthritis in any patient with an unexplained inflammatory arthritis, particularly if fever is present. Indeed, the American College of Rheumatology recommends synovial fluid analysis even in those with a flare of an established cause of arthritis (eg, rheumatoid arthritis) if febrile. 
Moreover, though synovial fluid leukocytosis exceeding $50,000 / \mathrm{mcL}$ is present in most patients with septic arthritis and values below 25,000 modestly reduce the likelihood, a mildly inflammatory cell count alone is insufficient to exclude the diagnosis if clinical suspicion is high, as is illustrated here. In these cases, it is imperative to await culture results.

AN UNUSUAL CAUSE FOR DYSPNEA? Lauren M. Maragh. New York- Presbyterian, New York, NY. (Control ID \#1339305)

LEARNING OBJECTIVE 1: 1.) Recognize the importance of sexual education in regards to prophylactic practice in those at low risk for sexually transmitted infections.

LEARNING OBJECTIVE 2: 2.) Recognize clinical manifestations of tertiary syphilis and potential complications if left untreated.

CASE: A 63 year-old woman presented to her primary care provider concerns for dyspnea on exertion. She had been treated in the past for hypertension, hyperlipidemia, and GERD. She reported that she had been compliant with her medications. Within the last 6 months she reported decreased exercise tolerance due to dyspnea. She had no other complaints. A physical exam in the office was notable for a new late peaking systolic murmur and several ulcerations on her nose and upper lip. Initial labs and chest $\mathrm{x}$-ray are unremarkable. Echocardiography showed mild aortic regurgitation. She additionally underwent nuclear stress tests which were interpreted as normal. She had been seen by several specialists and undergone a skin biopsy which showed the lesions on her face to be consistent with granulomatous disease. She is about to start a course of oral prednisone for possible sarcoidosis when she reported to a local emergency room with concerns for a transient ischemic attack. The patient was discharged and instructed to follow-up with her primary care provider. There she underwent a secondary evaluation for her neurologic symptoms which included serologic immunofluorescence studies that revealed the presence of Treponema pallidum antigen. The patient has become sexually active in the last 2 years. She reported that both she and her ex-husband had been tested for sexually transmitted infections over 20 years ago. She does not regularly use prophylactics during sexual intercourse because she believes this was more for prevention of pregnancy. She recently completed a 10 day course of intramuscular penicillin $\mathrm{G}$ with oral probenecid without complications and continues to receive serologic monitoring.

DISCUSSION: Syphilis is a disease caused by Treponema pallidum and is commonly spread through sexual intercourse (vaginal, anal or oral) with an individual who has syphilis chancre. Tertiary syphilis manifestations may, but classically include the cardiovascular system (syphilitic aortitis), central nervous system, and visceral cutaneous manifestations (gummas). Biopsied gummas often appear as granulomas and can be mistaken for other granulomatous type diseases such as sarcoidosis. The manifestation of gummas is uncommon given ease in detection and treatment in this era; however, there are still individuals who have not been treated for primary disease. The Centers for Disease Control reported from 2001-2009 the annual rate of primary and secondary syphilis increased by $4.1 \%$ from the previous decade which saw record lows. The number of cases of latent syphilis has increased $4.3 \%$. The groups with the highest incidence of infection were men who have sex with men and those in the age group of 20 to 39 . Those 45 years or older still are at risk and may be diagnosed later than their younger counterparts due to misconceptions regarding sexuality in older individuals and condom use. Syphilis itself is an independent risk factor for HIV and persons aged 50 and older account for $15 \%$ of new HIV/AIDS diagnosis and $24 \%$ of individuals living with HIV/AIDS. Lower risk does not assume lack of risk. Therefore, the importance of educating this group on how to prevent sexually transmitted infections is important.

AN UNUSUAL CAUSE OF GI BLEEDING Salih Samo; Muhammed Sherid; Vinod Kharti; Samian Sulaiman; Samaneh Dowlatshahi; Shahriar Dadkhah; Harvey Friedman. Saint Francis Hospital/University of Illinois at Chicago, Evanston, IL. (Control ID \#1275998)

LEARNING OBJECTIVE 1: To recognize that metastatic melanoma of the colon may occur many years after the primary lesion, but rarely.

LEARNING OBJECTIVE 2: It may even present as gastrointestinal bleeding.
CASE: An 81 year old male was admitted to the hospital to undergo coronary angiogram after he had chest pain during stress test. He denied chest pain,breathlessness, cough, abdominal pain, or change in bowel movements at time of admission. His past medical history was significant for scalp melanoma which was excised along with right neck dissection 6 years ago (stage 3 ) and bladder cancer. On physical examination patient was awake and alert. Vital signs were unremarkable. Neck was remarkable for surgical scar in right neck. Heart exam revealed an ejection systolic murmur at left sternal border radiates to both carotids. Lung exam was unremarkable. Abdomen was soft, non-tender, and non-distended. Bowel sounds were present. Rectal exam was unremarkable but FOBT was positive. Laboratory studies revealed anemia with hemoglobin of 8.3 , hematocrit of 25.7, MCV of 94.5, and $\mathrm{MCH}$ of 30.5. During hospitalization he underwent colonoscopy which revealed an ulcerated, hemorrhagic, fungating mass in the descending-sigmoid colon junction area. Histopathology showed diffuse sheets of large malignant cells with pleomorphic nuclei with large nucleoli. Histochemistry staining of HMB-45 as well as S100 was positive which confirms the diagnosis of melanoma. CT-chest and abdomen showed a mass measuring $3.5 \times 2.5 \times 2.5$ in the lingual of upper lobe of the left lung. Biopsy from lung mass was consistent with melanoma. Subsequently, patient underwent laparoscopic limited sigmoid resection. Readmission: Three weeks later he presented with melena and $\mathrm{Hgb}$ that dropped from 10 to $8 \mathrm{mg} / \mathrm{dl}$. Colonoscopy revealed normal colon with normal anastomosis without any bleeding. A re-review of the abdominal CT scan showed thickening in the proximal jejunum. The small bowel study showed a filling defect in the ileoceal area. Upper endoscopy revealed a mass in the proximal jejunum. The patient underwent laparatomy and found to have a mass approximately $15 \mathrm{~cm}$ beyond ligament of Treitz and another lesion approximately one foot distal to the first one. Both lesions were resected. Biopsy from both lesions revealed findings consistent with melanoma. Patient was discharged home after surgery uneventfully.

DISCUSSION: Metastatic melanoma of gastrointestinal (GI) tract is well documented. The small bowel is the most common site followed by stomach. Colonic metastasis is distinctly rare and on review of literature, none of them have presented with GI Bleeding. In our case GI bleeding was probably occurring from multiple sites. The interval time between diagnosis of the primary malignant melanoma and metastatic disease to the colon is long; more than 7 years according to the literature.

AN UNUSUAL CAUSE OF 'BLACK' URINE AND ACUTE KIDNEY INJURY Kunal V. Domakonda; Ashish Verma; Golla Venkata; Matthew Trainor; Konstantin Abramov. University of Massachusetts Medical School, Shrewsbury, MA. (Control ID \#1327657)

LEARNING OBJECTIVE 1: Manage parental injection of bleach leading to acute kidney injury.

LEARNING OBJECTIVE 2: Recognize the importance of early hemodialysis in preventing nephrotoxicity in the parental injection of bleach.

CASE: An 18-year-old white female presented with black urine following self-infusion of $100-\mathrm{mL}$ of domestic bleach through a Port-A-Cath meant for administration of antibiotics for chronic Lyme disease. Past history was significant for depression, attempted suicide, anxiety, chronic Lyme disease, Babesiosis and Bartonellosis. On admission, patient was awake, confused and had tachycardia. Blood pressure, oxygen saturation and systemic physical exam were normal. A tunneled internal jugular catheter was noted on the anterior chest wall. Admission labs and several sets of labs thereafter were hemolyzed. First Creatinine was $0.63 \mathrm{mg} / \mathrm{dL}$ on a handheld blood analyzer. It rose rapidly thereafter despite adequate volume resuscitation and continued to worsen. Hemolysis was evident on serial labs. Urine remained black for several days and sediment only showed monomorphic RBCs. A renal biopsy was performed. Light microscopy showed extensive loss of proximal tubular epithelium. Many tubular lumens contained fragmented, strongly eosinophilic, anucleate cellular debris, likely representing hemolyzed red blood cells. Interstitium contained focal acute inflammatory infiltrates surrounding necrotic tubules with no significant chronic parenchymal changes. Hemodialysis was initiated the next day for removal of toxin as patient remained oliguric 
and uremic. She required six dialysis sessions before renal function improved. Patient was hemodialysed for removal of toxin and a week later, she had complete renal recovery with resolution of hemolysis. Port-a-cath was removed as infectious disease evaluation showed no evidence of ongoing Lyme disease.

DISCUSSION: $\mathrm{NaClO}$ has been used as disinfectant for syringes, dialysis machines and in dental procedures. Large volume injection of IV bleach causes severe AKI which is usually reversible with early initiation of dialysis. Nephrotoxicity is due to direct tubular injury and hemolysis from rapid protein degradation in the presence of a strongly alkaline and hypertonic solution. No antidote is known but forced diuresis, hydration and sodium thiosulfate have been used with unclear benefits. $\mathrm{NaClO}$ molecule is easily dialyzable due to low molecular weight and small volume of distribution. Given that early hemodialysis may prevent toxic injury, as clinicians, bleach toxicity should be on the radar in the setting of AKI and 'black urine'. Cresol poisoning, hemoglobinuria, myoglobinuria, alkaptonuria, tyrosinuria, porphyrinuria and melaninuria (in malignant melanoma) are the other causes of 'black' urine.

AN UNUSUAL PRESENTATION OF ATRIAL MYXOMA Fritzie S. Albarillo; Azhar Kothawala; Ahmet A. Oktay; Tanmayee Bichile. St. Francis Hospital, Evanston, IL. (Control ID \#1339792)

LEARNING OBJECTIVE 1: List the differential diagnoses of dysphagia. LEARNING OBJECTIVE 2: Manage a patient with atrial myxoma CASE: A 68 year-old male presented to the ER after a mechanical fall 3 days prior. He also complained of a $90-\mathrm{lb}$ weight loss for the past 3 months secondary to progressive dysphagia for solid foods as well as no urine output for the past 4 days. He is single, lives with his sister and has no other significant social history.His vital signs were within normal limits. Physical examination revealed a disheveled, cachectic male with a grade 4 systolic murmur best heard at the apex and left sternal border, and a palpable mass in the hypogastric area. There were no focal neurologic deficits.Laboratory tests showed hemoglobin of 9.5, hematocrit of 28, WBC of 12.4 , BUN of 204, creatinine of 12.40 and phosphorus of 9.1. CT scan of the head without contrast was unremarkable. CT scan of the abdomen and pelvis revealed a distended bladder.Foley catheter was inserted draining approximately $2700 \mathrm{ml}$ of dark brown urine. Patient was started on liquid diet and a swallow evaluation was done which showed frequent regurgitation of pureed liquid.Esophagogastroduodenoscopy did not reveal any pathology. A transthoracic echocardiogram showed severe aortic stenosis and a $7.3 \times 2.8 \mathrm{~cm}$ lobulated mass of soft tissue consistency with a ball valve effect attached to the anterior mitral valve leaflet. This was later confirmed by a transesophageal echocardiogram as most consistent with an atrial myxoma.MRI of the brain reported a $3 \mathrm{~mm}$ punctuate area of acute ischemia in the left frontal lobe.Patient underwent resection of the left atrial mass as well as aortic valve replacement on hospital day 5. Biopsy of the specimen confirmed atrial myxoma.Patient recovered from the surgery well and reported improvement of dysphagia. DISCUSSION: Primary cardiac tumors are very uncommon. Metastatic cardiac tumors are 20 times more common. Atrial myxoma is the most common benign primary tumor of the heart which accounts for $40-50 \%$. $90 \%$ are solitary and pedunculated and $75-80 \%$ occur in the left atrium. Its presentation varies from being asymptomatic to sudden cardiac death. The cases may present with dyspnea, fatigue, dizziness, syncope or symptoms related to embolization. The most common cause of dysphagia is usually a pathology within the esophagus itself, e.g, neoplasm, achalasia, strictures and webs. However, there are a few cases where dysphagia can be caused by an abnormality in adjacent structures that can compress the esophagus. In our case the patient's dysphagia could either have been caused by direct compression of the esophagus by the left atrial mass or an embolization causing a stroke. However, the prompt improvement of dysphagia after resection of the myxoma points more towards the former. Furthermore, the findings of an acute ischemia in the left frontal lobe by MRI do not correlate with dysphagia. Surgical resection is the treatment of choice. Long-term prognosis is excellent with a $1-5 \%$ recurrence rate. But recurrence after 4 years is rare.
AN UNUSUAL CAUSE OF MULTIPLE PULMONARY NODULES Tulsi Sharma; Pankaj Mehta; Roberto Izquierdo. SUNY Upstate Medical University, Liverpool, NY. (Control ID \#1311838)

LEARNING OBJECTIVE 1: Pulmonary nodules that exhibit lack of growth for more than two years are generally considered benign. But there are a few exceptions to this rule, especially in the presence of multiple nodules.

LEARNING OBJECTIVE 2: Papillary thyroid carcinoma is the most common malignant thyroid cancer with the best prognosis but may present with atypical presentations especially in the younger patients.

CASE: Introduction: Many diseases can present with a miliary lung pattern, especially in immunocompromised patients. What can be the differential in a young asymptomatic and immunocompetent individual? Case: We present the case of a 21-year-old lady who had been in her usual state of health until October 2008 when she had a motor vehicle accident. A CT-thorax obtained at an outside facility did not reveal any traumatic injury, however, it revealed a diffuse reticulonodular pulmonary process. Subsequent sputum for AFB and PPD were negative and she was asymptomatic. She did not return for her follow-up appointments and returned to her PCP in November 2009 for a regular yearly evaluation. Repeat CT-thorax revealed a stable nodular pattern. She was then referred to Upstate Medical University with possible diagnosis of sarcoidosis. At the time of referral in early 2010 she still denied any constitutional symptoms. Clinical exam was normal. Repeat CT-thorax revealed persistence of the miliary pattern. Blood work, ACE-level and PPD were negative. What would cause these numerous nodules which have been stable for 2 years and without any clinical manifestations? After discussion of the risks and benefits of bronchoscopy, the patient agreed to the procedure. Bronchoscopy with transbronchial biopsy revealed metastatic well-differentiated papillary thyroid carcinoma (PTC)! Sonography of the thyroid revealed a small solid nodule in the left thyroid lobe with internal and peripheral vascular flow on doppler interrogation of the nodule. The patient underwent a near-total thyroidectomy and a limited central compartment neck dissection. The patient has since undergone radioactive iodine therapy and is on thyroid hormone replacement therapy. Her follow up hypothyroid I-131 whole body scan showed a significant decrease in uptake in the lungs.

DISCUSSION: Papillary thyroid carcinoma is the most common malignant thyroid cancer, and accounts for approximately $80 \%$ of thyroid cancers. Involvement of regional lymph nodes is seen in $20-80 \%$ of patients with PTC at the time of initial surgery. Diffuse lung metastatic disease from an occult thyroid cancer is however extremely rare. Diagnosis based on a transbronchial biopsy in an asymptomatic patient with incidental nodules makes it even more intriguing. The growth rate of pulmonary nodules is often used to help differentiate benign from malignant disease. Pulmonary nodules that exhibit lack of growth for more than two years are generally considered benign. However, nodular lung metastases from papillary thyroid carcinoma are an exception and may demonstrate lack of significant growth over years. The arrest of metastatic growth in PTC is more common in the younger patients and is thought to be related to the immune response to the cancer. The exact cause of this growth arrest is however not known. PTC has the best prognosis of the thyroid malignancies with a 90\% 10-year survival. Even in the presence of metastatic spread, survival periods may exceed 20 years, especially in the young. Hopefully the accident was actually a blessing in disguise!

AN INTERESTING CASE OF “CRAZY PAVING” Sourabh Prabhakar; Susan Mathews; Kameron Ashker; Rohit Varghese; Marvin Balaan. Allegheny General Hospital, Pittsburgh, PA. (Control ID \#1340481)

LEARNING OBJECTIVE 1: Recognize a relatively rare but now treatable cause of progressive dyspnea

LEARNING OBJECTIVE 2: Treatment of Pulmonary alveolar proteinosis using Veno-Venous ECMO (Extracorporeal Membrane Oxygenation) and whole lung lavage

CASE: A Young female presenting with progressive shortness of breath and $35 \mathrm{lbs}$ weight loss over past 4 months. 34 year old Caucasian female with history of asthma presented with flu-like symptoms and a 4 month 
history of "feeling unwell". This included episodes of dry cough, chest congestion, progressive dyspnea and a $35 \mathrm{lbs}$ weight loss. She had only mild response to intermittent courses of antibiotics and steroid tapers. In addition, she had no travel or sick contact history. Finally, the patient progressively became worse and presented to the ER were she was found to have oxygen saturations in 70 's. Her chest radiography revealed diffuse alveolar infiltrates, EKG was normal, and CT chest showed diffuse bilateral ground glass opacities throughout lungs with septal thickening and yields a "crazy paving" appearance. Her ABG showed pH 7.4; Pco2 33 mm Hg; pO2 $34 \mathrm{~mm} \mathrm{Hg}$ on $4 \mathrm{~L}$ via nasal cannula. All her bloodwork was normal except for LDH value of 494. Bronchial aveolar lavage was positive for PAS stain consistent with Pulmonary Alveolar Proteinosis (PAP) and was had not growth on gram stain and negative cultures. Unfortunately she was persistently hypoxic and was became difficult to oxygenate therefore required intubation. She then underwent Veno-venous Extracorporeal Membrane Oxygenation (V-V ECMO) and subsequently bilateral sequential whole lung lavage(WLL). Another important level revealed was the level of Anti-Granulocyte Macrophage CSF 1,537.4 pg/mL (Normal $<0.4 \mathrm{pg} / \mathrm{ml}$ ). After these critical interventions, she was extubated and was discharged without need for supplemental oxygen.

DISCUSSION: PAP is a rare pulmonary disorder characterized by accumulation of lipo-proteinaceous material within the alveolar spaces. It presents in three forms: congenital, secondary and most commonly, acquired. Symptoms are nonspecific and include dyspnea, dry cough or fever. Physical exam can exhibit rales, clubbing or cyanosis. An elevated LDH is frequently seen. High-resolution CT reveals the "crazy paving" appearance shown by a reticular, nodular airspace disease with intra/interlobular thickening. There is little or no lung inflammation, and the underlying lung architecture is preserved. The milky BAL fluid under light microscopy exhibits large foamy macrophages with increased lymphocytes and minimal inflammatory cells which stains PAS positive. As in this case, a whole lung lavage utilizes a large quantity of saline, up to 50 liters, divided into small aliquots. The procedure is terminated when the lavage effluent becomes clear. There have very few cases (less than 10) where bilateral sequential WLL was performed under V-V ECMO. Until recently, the pathogenesis has eluded investigators. Experimental trials with human recombinant GM-CSF, have shown that it activates alveolar macrophages, improving surfactant clearance, leading to clinical, physiological and radiographic improvements. With an accurate diagnosis and treatment, progression of PAP can be halted, improving the clinical course and outcome.

AN INTERESTING DIFFERENTIAL FOR UNILATERAL PLEURAL EFFUSION Ojas Bansal ${ }^{1}$; Swapna Kanuri ${ }^{1}$; Nachiket Patel ${ }^{1}$; Dan Schuller ${ }^{2,3} .{ }^{1}$ Creighton University Medical Center, Omaha, NE; ${ }^{2}$ Creighton University Medical Center, Omaha, NE; ${ }^{3}$ VA hospital Omaha, Omaha, NE. (Control ID \#1335079)

LEARNING OBJECTIVE 1: Describe clinical presentation of pancreaticopleural fistula

LEARNING OBJECTIVE 2: Management strategies for pancreaticopleural fistula

CASE: 53 year old Caucasian male was admitted with severe left sided chest pain radiating to his left shoulder and fever. He had similar presentation without fever four weeks ago and a CT scan at that time showed moderate left pleural effusion and a $10 \mathrm{~cm}$ splenic cyst. He underwent thoracentesis and cyst aspiration that were inconclusive for any specific etiology from the limited labs performed on the fluid. Past history is significant for hypertension, diabetes and smoking. He has a history of alcohol abuse but quit drinking 25 years ago. He was never hospitalized for any complications of alcoholism namely severe withdrawal symptoms or acute pancreatitis. On examination Vitals- Temperature- 101, BP120/59, HR$128, \mathrm{RR}-30, \mathrm{SaO} 2-95 \%$ on RA. He appeared dehydrated with normal heart and lung examination. Abdomen was diffusely tender with no guarding or rebound. Relevant labs include WBC count- 30.86 with $4 \%$ bands, amylase374 and lipase-694. Liver function tests were normal and cardiac enzymes negative. EKG revealed sinus tachycardia. A CT chest and abdomen redemonstrated a moderate sized left pleural effusion, compressive atelectasis, splenic cyst $(9.9 \times 6.2 \mathrm{~cm})$ and multiple pancreatic calcifications. He was started on empiric antibiotics vancomycin, zosyn and ciprofloxacin with aggressive rehydration and pain management. Thoracentesis revealed a turbid exudative effusion with PH- 6.9, WBC count 20000, Amylase and lipase levels in the pleural fluid were $>20000$ and $>6000$ respectively, Cytology and pleural fluid cultures were negative. After a tube thoracostomy, he underwent a magnetic resonance cholangiopancreatography (MRCP) showing multiple fluid collections consistent with pseudocysts, proximal duct dilatation with suspected pancratolith and pancreatic calcifications. Endoscopic retrograde cholangiopancreatography (ERCP) confirmed a pancreatic duct fistula at the junction of the body and tail. A stent was placed after a pancreatolith was removed at the neck. Amylase and lipase levels in splenic cyst aspirate were very high confirming a pseudocyst.

DISCUSSION: Pancreatico-pleural fistula is an extremely rare complication of pancreatitis occurring only in $1 \%$ of the cases. These patients usually have a history of excessive alcohol intake or recurrent episodes of pancreatitis, predominantly present with chest symptoms. The underlying mechanism is usually a leak of incompletely formed or ruptured pseudocyst or direct pancreatic duct leakage. CT scan is often the initial imaging modality. MRCP is non invasive and is superior to CT scan in identifying the fistula. ERCP is critical in confirming the diagnosis and aids in therapeutic intervention. Medical management with synthetic somatostatin analogs significantly reduces output and hastens the closure of fistula. Endoscopic pancreatic stenting is an effective therapeutic option, creates a least pathway of resistance allowing the fistula to heal. Pancreatic duct strictures and stones downstream can be treated with balloon dilation, basket extraction and stent placement. Patients with large cysts, multiple strictures and complete duct disruption are managed with surgery usually a distal pancreatectomy followed by pancreatico jejunostomy. Following appropriate management the prognosis is good.

AN UNCOMMON CAUSE OF TONGUE LESIONS Mili Shum. Albert Einstein College of Medicine, Montefiore Medical Center, Bronx, NY. (Control ID \#1339119)

LEARNING OBJECTIVE 1: Recognize the variable presentation of tongue amyloidosis as a cause of oral lesions in multiple myeloma patients. LEARNING OBJECTIVE 2: Recognize that tongue amyloidosis is a debilitating disease that may severely impact the patient's quality of life and is associated with poor outcomes in multiple myeloma patients.

CASE: A 65 year-old woman presented with two years of progressively worsening tongue pain. She has a history of light chain multiple myeloma (MM) diagnosed four years ago and was treated with multiple courses of chemotherapy including melphalan and other cytotoxic agents. She complained of ulceration and soreness mainly involving the anterior region of her tongue and was experiencing difficulty with speech and eating. This subsequently led to fatigue and weight loss. Antifungal treatment was ineffective at treating her tongue lesions and mucositis cocktails provided little relief. Head and neck examination revealed a diffusely stiff tongue that was enlarged with firm nodules and ulcers on the anterior $1 / 3$ of tongue and whitish flat plaque on the posterior $1 / 3$ of tongue. Serum cytomegalovirus and herpes simplex virus testing revealed no evidence of infection. A biopsy of the left lateral tongue did not demonstrate features of candida, herpes simplex virus, or cytomegalovirus infection. It revealed benign squamous mucosa with subepithelial amyloid deposition. A positive Congo Red stain confirmed the diagnosis of amyloid. She received mucositis cocktails and topical lidocaine for symptomatic treatment of her tongue lesions. She concurrently received chemotherapy for multiple myeloma. Her tongue symptoms persisted resulting in inadequate oral intake and deterioration in functional status. As a result, she was no longer suitable for further chemotherapy due to her functional status. She died seven months after the diagnosis of tongue amyloid in association with MM.

DISCUSSION: Amyloidosis is caused by extracellular deposition of insoluble fibrils. In the setting of multiple myeloma, the insoluble fibrils are composed of excess immunoglobulin light chains from the abnormal proliferation of plasma cells. MM-associated amyloidosis portends a poor prognosis with a mean survival of 5 to 15 months. This case demonstrates that oral amyloidosis can have rapid and debilitating consequences. Amyloidosis affects $10-20 \%$ of patients with MM. Up to one-third of patients with amyloid have oral manifestations. Macroglossia with tooth 
indentations along the lateral border and mucosal pain are the most common presentation. However, mucosal nodules are considered to be more specific of oral amyloid. Tongue ulceration is an infrequent presentation. This patient had diverse tongue lesions that included white plaques, ulcerations and nodules highlighting the heterogenous presentation of oral amyloid. Tissue biopsy and Red Congo staining is considered a gold standard and should be obtained for the diagnosis of amyloidosis. Clinicians should be aware of the variable ways in which oral amyloid may present within the oral cavity and undergo a thorough investigation of oral discomfort or lesions in a patient with multiple myeloma. This patient had symptoms for 2 years before diagnosis was made. Screening for oral symptoms may allow for earlier diagnosis and management and possibly result in reduced morbidity associated with the disease.

AN UNFORTUNATE COMPLICATION OF ANTI-PLATELET THERAPY: A CASE REPORT Subhraleena Das; Sujith Cherian; Wasim A. Hamarneh; Ehtesham Ul Haq; David Landsberg. SUNY Upstate Medical University, Syracuse, NY. (Control ID \#1339683)

LEARNING OBJECTIVE 1: Recognize the rare but potentially fatal complication of diffuse alveolar hemorrhage (DAH) with clopidogrel. LEARNING OBJECTIVE 2: Manage clopidogrel induced DAH. CASE: A 72-year-old man was transferred to the ICU with hemoptysis and dyspnea. The patient had been admitted for an elective carotid endarterectomy a day prior and had been given $150 \mathrm{mg}$ of clopidogrel before the procedure and was continued on aspirin and clopidogrel post procedure. Examination revealed tachypnea and bilateral rales. A Chest X-ray and CT scan of thorax revealed bilateral alveolar infiltrates. Both aspirin and clopidogrel were held, with improvement in respiratory status. Aspirin was restarted without complications. However, an attempt to restart clopidogrel resulted in sudden respiratory deterioration. The patient was started on pulse dose steroids with no improvement and was subsequently intubated. 2 D echo revealed diastolic dysfunction, and vasculitis workup was negative for ANA, ANCA, anti- GBM antibody with normal complement levels. Flexible bronchoscopy revealed blood throughout the broncho-alveolar tree. Broncho-alveolar lavage was negative for infectious agents and cytology negative for malignant cells. Clopidogrel was again held with marked improvement, thus confirming the diagnosis of clopidogrel induced DAH.

DISCUSSION: Clopidogrel is a thienopyridine derivative used to inhibit platelet aggregation by irreversible binding of adenosine diphosphate to the low affinity type 2 platelet receptors. It is commonly associated with increased incidences of gastrointestinal and intracerebral hemorrhage, and very rarely with DAH. DAH is most likely, an idiosyncratic hypersensitive response to clopidogrel, with a potential fatal outcome. Diagnosis is established by demonstrating a temporal relationship with the drug and ruling out other infectious, vasculitic and neoplastic processes by bronchoscopy. Due to the long half life (277- 433 hours) of the drug, even after stopping the drug, DAH may worsen. Therefore, supportive treatment with mechanical ventilation, if needed, is key. Of note, platelet infusions are futile as donor platelets are also irreversibly inhibited by clopidogrel in the system. Thus, our case highlights the importance of identifying DAH secondary to clopidogrel, which if not diagnosed in time may prove to be fatal. We reiterate the need for maintaining a high index of suspicion in these patients as it may easily be overlooked as pulmonary edema in the setting of acute coronary syndrome, where the drug is frequently used.

\section{AN UNUSUAL CASE OF AUTOIMMUNE PANCREATITIS} PRESENTING AS PANCREATIC MASS AND OBSTRUCTIVE JAUNDICE Betre Workie. SJHMC, Phoenix, AZ. (Control ID \#1324256)

LEARNING OBJECTIVE 1: There are two types of autoimmune pancreatitis (AIP) - Type 1 AIP and Type 2 AIP. Type 1 AIP (the classical form) is associated with elevated serum IgG4 levels and tissue infiltration by IgG4+ plasma cells.

CASE: A 53-year-old female presented with three weeks history of abdominal pain, nausea, vomiting and diarrhea. She was unable to tolerate any oral intake because she vomited after every meal. She reported being afraid to eat anything and had consequent weight loss which she could not quantify. She also reported dark urine. On the day of presentation, patient continued to have dull, aching abdominal pain in bilateral upper quadrants, nonradiating, with $8 / 10$ severity. She described her diarrhea as brownish, semi-solid stool with no blood. She denied any hematemesis, fever, chills, itchiness or any such episode in the past. She reported drinking alcohol socially, denied any tobacco or illicit drug use and she was not on any medication. On physical exam, patient was afebrile, hypertensive at 220/106 and the rest of her vitals were within normal limit. She had scleral icterus and her abdomen was soft, tender to palpation on bilateral upper quadrants with no rebound tenderness and bowel sounds were normal. Laboratory studies showed normal CBC, AST 339, ALT 423, total bilirubin 7.9, direct bilirubin 5.5, serum alkaline phosphatase 539, amylase 29, lipase less than 4. CT scan showed diffuse mild enlargement of the pancreas with "sausagelike" appearance, dilated intrahepatic bile ducts, hydropic gallbladder and prominent common bile duct measuring $1.3 \mathrm{~cm}$. Serum immunoglobline measurement showed elevated total $\mathrm{IgG}$ at 1625 with $\mathrm{IgG} 4$ subclass at 284. ERCP showed a $2 \mathrm{~cm}$ stricture in CBD which was stented and biliary bushing was obtained which was found to be nonmalignant. Endoscopic ultrasound showed $33 \mathrm{~mm}$ mass at head of pancreas which appeared to be inflammatory in nature. FNA of the pancreatic mass was non-diagnostic. Given the radiologic, laboratory and extra-pancreatic findings, patient was diagnosed with auto-immune pancreatitis type 1 and was initially treated with IV steroids and later transitioned to oral steroid. Her liver enzymes rapidly improved after stenting of biliary stricture. A follow up MRI showed resolution of biliary ductal dilatation and improved pancreatic edema. On day of discharged, all labs and vitals were within normal limit and patient was tolerating oral intake.

DISCUSSION: There are two types of autoimmune pancreatitis (AIP) Type 1 AIP and Type 2 AIP. Type 1 AIP (the classical form) is associated with elevated serum IgG4 levels and tissue infiltration by IgG4+ plasma cells. Main symptoms include pancreatic mass, cholestatic jaundice, chronic diarrhea and abdominal pain. Extra-pancreatic lesions include bile duct strictures, retroperitoneal fibrosis, inflammatory bowel disease, and Sjogren's syndrome. Elevated IgG4 (>135 mg/dL) is the most sensitive $(95 \%)$ and specific $(97 \%)$ for AIP type 1. Imaging features include irregular narrowing of the pancreatic duct, a peri-pancreatic capsule-like rim, and enhancement at the late phase of contrast-enhanced images. A dramatic response to steroids is another characteristic. Biopsy would show lymphoplasmocytic sclerosing pancreatitis. Corticosteroids are the standard treatment for type 1 AIP. Other therapies which can be considered for refractory cases include immunosuppresion with azathioprine or 6mercaptopurine, and rituximab administration.

\section{AN UNUSUAL CAUSE OF CHEST PAIN - SYMPTOMATIC GIANT} CORONARY ARTERY ANEURYSM. Jeffrey S. Wilkinson ${ }^{1}$; Ahmad Moustapha ${ }^{2}$; Paul Schulte'; Payam Dehghani ${ }^{2} .{ }^{~}$ Queen's University, Kingston, ON, Canada; ${ }^{2}$ University of Saskatchewan, Regina, SK, Canada. (Control ID \#1310886)

LEARNING OBJECTIVE 1: Review the differential diagnosis, diagnostic approach, and important features of coronary aneurysms.

LEARNING OBJECTIVE 2: Discuss the utility of CT angiography for assessing coronary artery anatomy.

CASE: A 58 year-old man presented with severe retrosternal chest pain radiating to his jaw and left arm. He was obese, had a 40 pack-year history of smoking, and was on statin therapy for hypercholesterolemia. He did not report any significant personal or family history of coronary artery disease, vasculitis, connective tissue disorders, or history of Kawasaki's as a child. $\mathrm{He}$ had non-specific ECG findings and a mild enzyme rise. Cardiac angiography showed a large aneurysm of the left main artery (LM). It involved the ostial left anterior descending artery (LAD) - with a double density contour at the aneurysm segment. It was difficult to determine if the haziness in the aneurysmal segment represented artery dissection, plaque rupture, or de novo thrombus formation secondary to stasis. A cardiac CT angiogram was ordered urgently to help delineate this ambiguous lesion. The aneurysm of the LAD had dimensions of $3.0 \mathrm{x}$ 
$1.8 \times 1.6 \mathrm{~cm}$ and the hypodense filling defect was in fact clot, representing impending embolization. An emergent consultation with cardiovascular surgery was requested. The patient was taken for surgery due to the ongoing ischemic chest pain and suggestion of impending embolization of clot into the mid-segment of the LAD. The surgeon performed an aneurysmorrhaphy, bypass grafting, and removed the clot en bloc. Finger-like projections forming a cast of septal perforators could be seen. The post-operative period was uncomplicated. He was discharged on coumadin, aspirin, an ACE inhibitor, and a beta-blocker. The surgical pathology report showed benign fibromuscular tissue representing true aneurysmal dilatation.

DISCUSSION: Coronary artery aneurysm (CAA) is defined as the abnormal dilation of a coronary artery greater than $50 \%$ of its normal size. The incidence of CAA has been estimated to be $4.9 \%$ in one series of 978 patients who underwent coronary angiography. CAA's are most commonly related to atherosclerosis, but have also been known to occur in Kawasaki's disease, connective tissue disorders such as Ehlers-Danlos, sepsis leading to mycotic aneurysm, trauma, cocaine use, and in various vasculitides including polyarteritis nodosa and Takayasu arteritis. When symptoms occur as the primary presentation they have been documented to cause angina, mimic an acute coronary event, arrythmia, coronary rupture, and obstruction resulting in superior vena cava syndrome. Definitive diagnosis with invasive angiography may be difficult because dissection, plaque wall rupture, and acute thrombus may mimic a true aneurysm. In our case, CT angiography provided a better assessment of the coronary anatomy due to its inherent capability of investigating the vessel wall, the filling defect, the embolization potential of the clot, and identification of extra-vascular structures for surgical planning. As CT angiography continues to become more cost-effective, improve performance and image quality and decrease radiation dosage, this tool will have increasing utility in the management of patients with coronary artery disease. This case highlights the importance of individualized assessment and treatment, which requires a broad assessment with a number of specialties including internal medicine, cardiology, rheumatology, radiology, and surgery.

AN UNUSUAL COMPLICATION OF CRACK ABUSE Melissa Stein. ${ }^{1}$ Albert Einstein College of Medicine, Bronx, NY; ${ }^{2}$ Montefiore Medical Center, Bronx, NY. (Control ID \#1318722)

LEARNING OBJECTIVE 1: Identify complications of crack cocaine abuse with attention to technique and paraphernalia used to smoke crack cocaine.

LEARNING OBJECTIVE 2: Recognize severity of compulsion to smoke crack cocaine.

CASE: A 50 year-old woman presented to the Emergency Department (ED) complaining of dyspnea for two hours. She had a history of laryngeal cancer and tracheostomy several years prior but because of longstanding discomfort at the tracheostomy site, she did not have a tracheostomy tube in place. She had a history of polysubstance abuse and was enrolled in a drug treatment program where she received methadone and psychosocial support. Urine toxicology reports at the program were intermittently positive for cocaine. In the ED she reported that an object had fallen into her tracheostomy. On physical examination the patient was tachypneic and agitated. Her lungs were clear to auscultation. Her oxygen saturation was $78 \%$ on room air. Chest radiography revealed a tubular radiodensity overlying the region of the carina and right mainstem bronchus. Bronchoscopy was performed urgently and a glass tube $3.2 \mathrm{~cm}$ in length was removed from the right mainstem bronchus. The tube was the size and shape of a pipe used to smoke crack cocaine. Copious secretions had accumulated behind the obstruction, but no injury to the airways was observed. The patient recovered uneventfully and continued intermittent cocaine abuse until just prior to her death three years later. She died due to a recurrence of her laryngeal cancer.

DISCUSSION: Cocaine, including crack, is the second most commonly abused non-prescription illicit drug with 1.9 million Americans reporting use in the past month. Cocaine is associated with more emergency department visits than any other illicit drug. Cocaine abuse can lead to dysfunction of multiple organ systems. For example, injecting cocaine can lead to skin and soft tissue infections as well as transmission of viral hepatitis and HIV. Smoking crack increases risk of pulmonary infections and chronic pulmonary disease. By any route, cocaine can cause myocardial infarction, cardiac arrhythmia and seizure. Cocaine is highly addictive. Few psychosocial interventions have proved effective in treating cocaine abuse. Though multiple medications have been tried, none have proved effective in treating cocaine abuse in randomized controlled trials. Cocaine vaccine is currently being investigated as a tool to prevent relapse among prior cocaine users. Amongst poor urban populations, cocaine is most frequently smoked in the form of crack, the least expensive form of cocaine. Crack is commonly smoked from a glass pipe ("stem") with a steel wool filter. Oral injuries such as burns and lacerations are common among crack abusers and can be routes of viral infection when smoking paraphernalia is shared. Tracheal and esophageal aspiration of smoking paraphernalia are rare sequelae of crack abuse. Published reports include cases of aspiration of bagged cocaine by a "body packer", aspiration and ingestion of a steel wool filter and ingestion of a crack pipe. This unusual case graphically illustrates the tremendous compulsion to smoke crack, even in the face of extreme risk. Given the morbidity associated with cocaine abuse, efforts to prevent cocaine abuse as well as identify and treat patients who abuse cocaine are warranted.

AN UNUSUAL PRESENTATION OF WEGENER'S GRANULOMATOSIS OF GENITALIA Waqas Qureshi; Marilyn Karam; Salman Siddiqui; Sean Drake. Henry Ford Health Systems, Detroit, MI. (Control ID \#1311917)

LEARNING OBJECTIVE 1: 1. To understand that autoimmune diseases of skin might present with superimposed infectious process.

LEARNING OBJECTIVE 2: 2 . To elucidate the work up of destructive skin disease also includes autoimmune work up, if the biopsy does not reveal malignancy and symptoms are not improving with broad coverage antibiotics.

CASE: A 52 year old Caucasian gentleman developed a non-pruritic and non-tender papular erythematous well demarcated rash on the shaft of his penis associated with a $4 \mathrm{~cm}$ growth. This rash extended on the dorsal surface of the penis over the course of 2 weeks. Since, it was suspicious of a malignancy, an initial biopsy was done, but the biopsy showed chronic inflammation. Even though the biopsy did not reveal malignancy, due to a a high suspicion of malignancy, the patient was referred to a urologist for deep penile biopsy and circumsicion after a month of initial symptoms. Over the next 1 week, his rash progressed quickly involving most of the skin of penis. Patient was admitted to the hospital with fever, chills and pyuria. He was treated for complicated urinary tract infection (UTI) and another biopsy was done showing methicillin resistant Staphylococcus aureus (MRSA). Antibiotics were continued. Meanwhile, the rash continued to progress slowly. After a week of treatment, the rash started healing which had progressed to the lower part of the abdomen but the penile lesion still looked the same. Now, the patient started developing palpable purpura on the lower extremities. Autoimmune work up was done and biopsy of the purpura was also carried out. Penilectomy was also performed and the specimen was sent for biopsy. A diversion urethrostomy was also carried out after the penilectomy. The biopsy from the lower extremities revealed leukocytoclastic vasculitis and autoimmune work up showed strongly positive c-ANCA. There were necrotizing vasculitic granulomas present on the biopsy of the penis. A CT scan with contrast was done of the chest to find any pulmonary lesions thinking that if this is wegener's granulomatosis, then there might be pulmonary involvement. The CT scan showed granulomas of the lungs. Patient was started on high dose pulse steroids, with improvement in the lesions.

DISCUSSION: This is a rare case of limited Wegener's granulomatosis, which was not diagnosed until 2 months after the development of skin disease. Only few similar case reports exist. Our case was unique because the initial lesion that presented was a growth, rather than an ulcer which was the presentation in other case reports. Late diagnosis led our patient to undergo penilectomy. There have been other cases of penile Wegener's granulomatosis and a vasculitis should be considered in the differential diagnosis after ruling out malignancy as it is a treatable condition and can prevent the patient from developing significant morbidity. 
ANGIOIMMUNOBLASTIC T-CELL LYMPHOMA: A DIAGNOSIS IN DISGUISE Brittany K. Ragon; Matthew Semler. Vanderbilt University Medical Center, Nashville, TN. (Control ID \#1334410)

LEARNING OBJECTIVE 1: Distinguish the unique presentation of Angioimmunoblastic T-Cell Lymphoma from the rheumatologic, infectious, and malignant diagnoses raised by the condition's clinical and laboratory findings.

LEARNING OBJECTIVE 2: Recognize the importance of clinical judgment in interpreting diagnostic testing when the results do not fit the clinical picture.

CASE: A 58-year-old Caucasian male with coronary artery disease and type 2 diabetes mellitus presented to the emergency department with 6 weeks of joint pain and rash. Initially, he had experienced the gradual onset of dull, aching pain and swelling in his ankles which progressed over several days to involve his knees and then hands. Four days after the onset of symptoms, he noticed a painless rash resembling tiny "pinpricks" appearing intermittently on his lower extremities. His abdomen and chest developed diffuse pruritic erythema, and he began to experience fever, chills, fatigue, intermittent diarrhea, and a 20-lb weight loss. Seen by his primary care physician, he received doxycycline out of concern for Rocky Mountain Spotted Fever. He did not improve and was referred to dermatology where a biopsy was read as "leukocytoclastic vasculitis" and attributed to his metformin and trazadone. At this time a c-ANCA was positive. Despite discontinuation of both medications, the patient continued to worsen and was admitted to the hospital for further evaluation. Physical exam at admission showed a well-appearing man in no distress with normal vital signs. Further examination showed diffuse, large, and firm cervical lymphadenopathy. Heart and lung exam were normal. Abdominal exam did not show hepatosplenomegaly. A faint, reticular rash diffusely covered his trunk, and resolving purpura were visible on both shins. Laboratory studies showed a mild eosinophilia, elevated ESR and CRP, low complement, increased total protein, negative ANA, and positive c-ANCA. An ELISA for HIV came back positive, and CD4 count, PCR, and Western Blot were sent. A radiograph of the chest showed 2-3 cm bilateral hilar adenopathy. Fine needle aspiration of a cervical lymph node showed lymphoid infiltrate, negative for malignancy. Finally, an excisional biopsy of an inguinal lymph node was performed and revealed effacement of the nodal architecture with an infiltrate of lymphocytes admixed with eosinophils and histiocytes with CD4 and CD10 positive staining confirming a diagnosis of Angioimmunoblastic T-Cell Lymphoma (AITL). Review of his prior skin biopsy showed evidence of AITL, and his repeat cANCA and HIV Western Blot and PCR returned negative.

DISCUSSION: AITL is an aggressive form of nodal, mature T-cell neoplasm that presents with fever, generalized lymphadenopathy, pruritic or vasculitic rashes, arthritis, and a unique set of confounding laboratory abnormalities including eosinophilia, polyclonal hypergammaglobulinemia, and rarely, a falsely positive HIV ELISA (1). Because AITL is characterized by diffuse symptoms resembling infectious or rheumatologic conditions and falsely positive antibody tests for these conditions, recognizing AITL requires a high index of clinical suspicion. This case demonstrates both a classic presentation of AITL and the importance of employing clinical judgment when the results of diagnostic testing do not fit the clinical picture. 1. Seiji et al. False-positive HIV antibody test and autoimmune hemolytic anemia in a patient with AITL. Intern Med 50:2383-2387, 2011

ANOGENITAL PYODERMA GANGRENOSUM IN INFLAMMATORY BOWEL DISEASE Daniel Lin; Jason A. Korcak. Montefiore Medical Center, Bronx, NY. (Control ID \#1339186)

LEARNING OBJECTIVE 1: Recognize the clinical presentation of pyoderma gangrenosum

LEARNING OBJECTIVE 2: Identify the relationship between pyoderma gangrenosum and inflammatory bowel disease.

CASE: A 24 year-old man presented with perianal and genital lesions for three months. He described the lesions as red bumps, which gradually grew into larger, non-draining ulcers. The patient also reported intermittent episodes of watery brown stool with small amounts of blood and occasional crampy abdominal pain in his left lower quadrant over the prior six months.
He had chills, night sweats, and a ten-pound weight loss during this time. The patient had a temperature of $100.8 \mathrm{~F}$. His abdomen was soft, but tender to palpation in the left lower quadrant. He had painful ulcerations with irregular borders in the perianal area and at the base of the penis. There were no oral ulcers, other skin lesions, or palpable lymph nodes. The white blood cell count was $16.0 \mathrm{~K} / \mu \mathrm{L}$. Stool culture and assays for Clostridium difficile, ova and parasites, and fecal fat were negative. HIV, Hepatitis B and C, gonorrhea, chlamydia, and RPR tests were also negative. Pancolitis was present on abdominal CT scan, and colonoscopy revealed an erythematous mucosa with deep ulcerations and severe active colitis. A biopsy of the perianal skin showed neutrophil rich dermatitis without evidence of infection. The patient was diagnosed with inflammatory bowel disease (IBD) and associated pyoderma gangrenosum (PG).

DISCUSSION: Rare skin lesions, such as PG, present a diagnostic challenge for the general internist. PG is a non-infectious neutrophilic dermatosis likely caused by dysregulated cellular immunity and abnormal neutrophil function. PG begins with tender papules or pustules that rapidly progress to painful ulcers with erythematous, violaceous borders. Lesions primarily present on the lower extremities and are seldom described in genital or perianal regions, as in our patient. The lesions may endure for months to years and heal with an atrophic cribriform scar. Histopathology is non-specific, with neutrophilic infiltration of the dermis, as well as hemorrhage and necrosis of the epidermis. PG most often occurs in adults 25-54 years of age and is found equally in men and women. Although PG may present as an idiopathic condition, at least half of PG cases are associated with systemic diseases, including autoimmune disorders, such as lupus erythematosus, hematologic disorders, such as acute myeloid leukemia, and most commonly, IBD. The prevalence of PG is estimated at $1-10 \%$ in ulcerative colitis and 0.5 $20 \%$ in Crohn's disease. The onset of PG may coincide with active colitis, but can also occur before or after the clinical development of IBD. Like all forms of PG, lesions may arise at sites of trauma, such as after colostomy placement. While PG remains a diagnosis of exclusion, a better understanding of the clinical presentation of PG and its association with IBD will help internists recognize this rare skin disorder.

ANOTHER GREAT MASQUERADER Rachel R. Johnson. Medical College of Wisconsin, New Berlin, WI. (Control ID \#1334874)

LEARNING OBJECTIVE 1: Describe an interesting presentation to an increasingly prevalent disease.

LEARNING OBJECTIVE 2: Highlight the importance of early diagnosis to prevent spread of infection and to begin treatment promptly.

CASE: A 19-year-old gentleman with a past medical history of chronic constipation and seasonal allergies presented with complaints of rectal pain for 1 week along with fever, chills and malaise. Prior to presentation, he had been evaluated at an outside hospital and underwent a rectal exam under anesthesia with no identification of abnormalities. On presentation at our institution, physical exam was significant for a low grade fever, maculopapular rash on his torso, and perianal mucosal ulcerations. Initial lab evaluation was significant for elevation of hepatic transaminases and thrombocytopenia. He was admitted for further treatment and evaluation, and ceftriaxone was initiated to cover for Gram-positive bacterial infections, including sexually transmitted diseases. Rectal swabs were negative for herpes simplex virus (HSV) and bacteria, including group A streptococcus and $\mathrm{H}$. ducyeri. Further laboratory work-up was negative for viral hepatitis, syphilis, gonorrhea, and chlamydia. Initial HIV antibody assay was also negative, but serum HSV serologies (IgG and IgM) were positive. When informed of his HSV infection, the patient admitted to having 13 male sexual partners in the last year, which prompted concern for an acute retroviral infection. HIV RNA PCR was ordered and revealed an HIV viral load of over 700,000 . The patient was discharged home with outpatient infectious disease clinic follow-up. As an outpatient, antiretroviral medications were started, and the patient's HIV viral load eventually became undetectable after 6 months of therapy.

DISCUSSION: Acute retroviral syndrome (ARS) describes the clinical signs and symptoms of primary HIV infection which includes the period from initial HIV infection to HIV seroconversion. The initial presenting signs and symptoms of primary HIV infection are often nonspecific. The most common presentation is an acute mononucleosis-like illness characterized by fever, 
sore throat and lymphadenopathy. Other symptoms include lethargy, malaise, myalgias, weight loss, headache and a diffuse maculopapular rash. Acute retroviral syndrome can also involve multiple organ systems. Mucosal ulcerations of the oropharynx, esophagus and the genitalia have been reported to occur in $28 \%, 17 \%$, and $6 \%$ of patient's respectively. Involvement of the gastrointestinal system can include vomiting and diarrhea along with pancreatitis, colitis and epiglottitis. Hepatitis is a common presentation that often resolves as the host's immune system gains control over the initial viral replication. Other laboratory abnormalities include anemia, leukopenia and thrombocytopenia. Neurologic involvement most commonly includes headaches but can also include aseptic meningitis or encephalitis. The signs and symptoms of ARS usually begin within 24 weeks of initial HIV infection and last approximately 2-3 weeks. During the primary HIV infection, the viral load is very high and the patient is highly infectious. Early identification is important, not only for initiation of antiretroviral therapy to preserve the host's immune responses, but also to decrease transmission through patient education and therapy. Clinicians should have a high index of suspicion of ARS in patients with generalized mononucleosis-like symptoms and risk factors for HIV infection.

\section{ANTI-COAGULANT/ANTI-PLATELET MEDIATED SAPHENOUS VEIN GRAFT RECANALIZATION Suchet Kaur ${ }^{1}$; Hemendra Sarda ${ }^{2}$;} Dexter Dexter ${ }^{3}$; Sulaiman Rathore ${ }^{3}$; Timothy Ball ${ }^{4}$. ${ }^{1}$ carilion clinic, Roanoke, VA; ${ }^{2}$ Carilion Clinic, Roanoke, VA; ${ }^{3}$ Carilion Clinic, Roanoke, VA; ${ }^{4}$ Carilion Clinic, Roanoke, VA. (Control ID \#1310613)

LEARNING OBJECTIVE 1: Systemic anticoagulation with dual antiplatelet therapy may provide a treatment option for the rare patient presenting with an SVG with heavy thrombus burden not amenable to PCI. CASE: A 51 year old man with history of hyperlipidemia and tobacco use presented with ST segment elevation myocardial infarction (STEMI). Emergent angiography revealed multi-vessel coronary artery disease and he was referred for emergent CABG with conduit composed of the right internal mammary artery, radial artery and saphenous vein. On post-op day 18 the patient presented with chest pain and was diagnosed with a nonSTEMI. Coronary angiography revealed heavy thrombus burden within the SVG to the ramus intermedius. The native ramus was stented with four drug-eluting stents. Post-procedure, the patient developed heparin induced thrombocytopenia and thrombosis (HITT); he was treated with lepirudin, bridged to warfarin and discharged on aspirin, clopidogrel and warfarin for 6 months. Over the ensuing months the patient developed progressive exertional angina, stress testing was positive for antero-apical ischemia and repeat coronary angiography demonstrated complete recanalization of the thrombosed SVG to the ramus and patent stents in the ramus. Three months after discontinuation of warfarin, the patient presented again with chest pain and he was again diagnosed with a non-STEMI. Coronary angiography revealed complete mid-graft occlusion of the SVG to the ramus with widely patent ramus stents.

DISCUSSION: Aorto-coronary grafting is a common method for surgical myocardial revascularization, which is often complicated by early and late graft thrombosis. An in depth review of the literature identified three reports of thrombus resolution with recanalization of the SVG without Percutaneous Coronary Intervention (PCI). We report a case of thrombus regression in a thrombus laden SVG in a patient placed on systemic anticoagulation. The authors believe that systemic anticoagulation therapy for the treatment of HITT resulted in resorption of the thrombus within the SVG possibly protection from rethrombosis owing to the fact that rethrombosis of the SVG occurred within 3 months of the discontinuation of warfarin therapy. As with venous thrombosis, the treatment goal of systemic anticoagulation is to prevent clot propagation and recurrence while allowing normal thrombus resorption and vascular recanalization. We propose that systemic anticoagulation with dual anti-platelet therapy may provide a treatment option for the rare patient presenting with an SVG with heavy thrombus burden not amenable to PCI.

ANTIPHOSPHOSPHOLIPID SYNDROME WITH MULTI-SYSTEM ORGAN FAILURE Maciej Walczyszyn; Raghu Thirumala; Larry DiFabrizio. Lenox Hill Hospital, New York, NY. (Control ID \#1339631)
LEARNING OBJECTIVE 1: To recognize early signs of a life threatening autoimmune disease.

CASE: A 65 year old, previously healthy, female presented with weakness and progressive exertional dyspnea for 10 days. She complained of orthopnea, palpitations, chills, and loss of appetite; yet, denied chest pain and fevers. Her past history was significant for hysterectomy, bilateral mastectomy, and epilepsy controlled on daily carbamazepine. On physical exam, the patient was in respiratory distress with a respiratory rate of 30 / min, temperature of $97.1 \mathrm{~F}$, heart rate of $110 / \mathrm{min}$ and blood pressure of $180 / 90 \mathrm{mmHg}$. Cardiopulmonary exam was notable for bilateral rales and a $2 / 6$ systolic murmur in the aortic region. The remainder of the exam was normal. Labs were significant for hemoglobin of $7.3 \mathrm{gm} / \mathrm{dl}$, serum creatinine of $2.37 \mathrm{mg} / \mathrm{dl}$, peak troponin of $2.5 \mathrm{ng} / \mathrm{ml}$, and BNP of $147,060 \mathrm{pg} / \mathrm{ml}$. EKG revealed sinus tachycardia. Chest X-ray showed cardiomegaly with pulmonary edema. Cardiac echo revealed moderate aortic regurgitation and stenosis, elevated pulmonary artery systolic pressure $(65 \mathrm{mmHg})$, moderate left ventricular hypertrophy, dilated left atrium, akinetic apex and generalized hypokinesis with an estimated ejection fraction of $25 \%$. The patient was treated for acute systolic congestive heart failure with i.v. nitroglycerin and diuretics as well as transfused packed RBCs; which resulted in improvement of hemodynamics and renal function. While awaiting cardiac catheterization, the patient had an acute change in mental status. MRI of the brain revealed multiple infarcts of bilateral cerebral hemispheres, cerebellum, basal ganglia and thalami, consistent with emboli. Subsequently, a transesophageal echo found a large, ill-defined mass attached to the aortic valve. A lung perfusion scan showed a wedge shaped defect within the right upper lobe consistent with pulmonary embolism. The patient was started on anticoagulation along with antibiotics to cover for presumed infective endocarditis. Blood cultures remained negative and a gallium scan did not identify inflammatory uptake; thus, antibiotics were stopped. Serologic studies were strongly positive for antinuclear, anti-double standed DNA, antiphospholipid (aPL), and anti- $\beta 2$-glycoprotein I antibodies; consistent with Systemic Lupus Erythematosus (SLE) and Libman-Sacks endocarditis. Multiple thromboemboli to brain and lung, and multiorgan dysfunction were consistent with catastrophic antiphospholipid syndrome (APS), which was treated with high dose steroids and anticoagulation. Steroids were subsequently tapered with the addition of mycophenolate mofetil. The patient improved and is undergoing physical therapy.

DISCUSSION: Catastrophic APS is a rare cause of acute multi-system organ failure due to wide spread thrombosis. The presence of aPL antibodies in SLE patients increases the risk of valvular heart disease and thromboembolic complications. Diagnosis requires: 1) evidence of involvement of at least 3 organs; 2) development of manifestations within 1 week; 3) histopathology confirming small vessel occlusion; and 4) laboratory confirmation of the presence of an aPL antibody. In conclusion, maintaining a broad differential and a high index of suspicion is necessary to diagnose non-infective endocarditis in the setting of a life threatening immunologic disorder, to appropriately initiate early treatment with anticoagulation and immunosuppresion.

AORTIC THROMBUS IN GIANT CELL ARTERITIS Catherine A. Barry; Mala Joneja; Phillip Wattam. Queen's University, Kingston, ON, Canada. (Control ID \#1319961)

LEARNING OBJECTIVE 1: Assess the causes of an aortic thrombus and aortitis

LEARNING OBJECTIVE 2: Recognizing the complications of large vessel vasculitis

CASE: An 80 year female with a one year history of giant cell arteritis and a recent history of unilateral leg deep vein thrombosis presented to her Rheumatologist with a few day history of increasing dyspnea. Her past medical history included transient ischemic attacks, hypertension and dyslipidemia. She was taking a tapering course of prednisone for her vasculitis and anticoagulation for the DVT. Two weeks prior to presentation, she was admitted to hospital for syncope. At that time her INR was found to be supratherapeutic and her ongoing anticoagulation was held. Due to a miscommunication at follow up, it was never restarted. At this presentation she was tachypneic and hypoxemic. She was treated empirically in the 
emergency department with heparin for possible pulmonary embolus. Her CT pulmonary angiogram showed small segmental pulmonary emboli, and more remarkably a $6.5 \mathrm{~cm}$ thrombus in her thoracic aorta causing $75 \%$ narrowing of the aortic lumen without evidence of aortitis, dissection, aneurysm, or artherosclerotic disease. There was no evidence of peripheral or end-organ ischemia clinically or on investigation. In consultation with Rheumatology it was suggested that the thrombus developed as a complication of aortitis from her giant cell arteritis and the patient was started on higher doses of prednisone. She remained stable through the next few days and was sent home with low molecular weight heparin treatment for her aortic thrombus. Investigations were also sent for antiphospholipid antibody syndrome.

DISCUSSION: Aortic thrombus, most often caused by artherosclerotic disease, has been described in aortitis and rarely in antiphospholipid antibody syndrome. Aortitis is a known complication of large-vessel vasculitis such as giant cell arteritis and Takayasu's arteritis. However it is rare for an aortic thrombus to develop from aortitis in the absence of an aneurysm. It is also unusual for thrombus to develop in the thoracic aorta. There have been a few case reports linking anti-phospholipid syndrome and aortitis/aortic thrombosis. The quandary in this patient's case is that imaging of the aorta did not show a clear cause of thrombus formation, and it's etiology remains unknown. Although this patient has risk factors for atherosclerosis, sub-radiographic aortitis from giant cell arteritis in association with an undiagnosed thrombophilia could have resulted in this patient's thoracic aortic thrombus.

APPROACHING THE GRAVE \$4 AT A TIME Rudy Kimmerling. UC Denver, Aurora, CO. (Control ID \#1312882)

LEARNING OBJECTIVE 1: Recognize the risks inherent in outpatient transitions of care.

LEARNING OBJECTIVE 2: Assess adverse drug effects based on history and exam.

CASE: A 60 year-old female presented to clinic for a regular follow up exam. One month prior, her home health agency had finished their period of care and stopped weekly medication and lab monitoring of electrolytes and INR. Since then, the patient endorsed increasing weakness to the point of being unable to ambulate for the past week. She decided to wait until her scheduled appointment to be seen because of cost concerns, having recently become a Medicare and Medicaid beneficiary. Review of systems revealed easy bleeding though the patient denied dark stools. She had lost $4 \mathrm{lbs}$ over 6 weeks. Medications had previously been prescribed predominantly from the " $\$ 4$ list." They included spironolactone, levothyroxine, lisinopril, warfarin, amiodarone and furosemide, all new medications prescribed upon discharge from a prior hospitalization. Thorough medication reconciliation revealed the patient had been accidentally taking a double dose of furosemide, she was taking the other medications as previously prescribed. Vital signs obtained by medical assistants showed normal temperature and heart rate though there was difficulty obtaining a blood pressure. Examination revealed a slightly disoriented tremulous woman in no acute distress. Mucous membranes were dry with dried blood on her lips. Lungs were clear; heart rhythm was irregular with a new $2 / 6$ systolic ejection murmur heard diffusely throughout the chest. Neurologic exam showed diffuse hyperreflexia. The vitals were rechecked and the patient was found to have a heart rate of 80 and a manual blood pressure of $66 / 40$. The patient was placed in a wheelchair and taken to the emergency department by the physician. EKG showed atrial fibrillation with wide QRS complexes and tall peaked $\mathrm{T}$ waves. Laboratory abnormalities included a potassium of $8.0, \mathrm{CO} 2$ of $14, \mathrm{BUN}$ of 276 , creatinine of 16.2 , and INR >10.4. Other electrolytes, LFTs and TSH were normal. Her hematocrit had dropped from $42.5 \%$ to $25.6 \%$. The patient was admitted for urgent dialysis and blood product transfusion. No evidence of active GI bleeding was found. She recovered rapidly and was discharged home 4 days later on a reduced drug regimen, all symptoms apparently caused by adverse medication effects exacerbated by iatrogenic hypotension and occult GI bleeding.

DISCUSSION: Transitions of care have increasingly become "danger points" for patients as they move through our fragmented health care system. In our current system with multiple providers involved in patient care, medication and laboratory monitoring can fall through the cracks. According to the Commonwealth Fund, 11 to 15 of every 1,000 Americans visit a health care provider because of adverse drug effects in a given year. Of these, more than one quarter are considered ameliorable with better communication between physicians and patients. In this patient, poor communication between providers left a critical gap in monitoring of common but potentially dangerous medications. By establishing better tools and incentives for coordination and communication within care delivery systems, potentially fatal adverse events like the one above can be reduced.

ARE SURGICAL COMPLICATIONS WITHIN THE PURVIEW OF THE INTERNIST? Shuchi Gulati; Jorge Scheirer. The Reading Hospital and Medical Center, West Reading, PA. (Control ID \#1339080)

LEARNING OBJECTIVE 1: Recognize complications of ablative techniques used to treat renal cell carcinoma

CASE: A 78 year old male was noted to have a heterogeneous mass arising from the posterior pole of the right kidney, measuring $13 \mathrm{~mm} \mathrm{X}$ $15 \mathrm{~mm}$ on an abdominal CT scan obtained during evaluation for a bowel obstruction. On serial follow up, the SRM increased to $22 \mathrm{~mm}$. The urologist elected to treat the SRM with open exploration and cryoablation therapy. 2 weeks after hospital discharge, the patient presented to his primary care internist with complains of a twenty-pound weight gain, fatigue and weakness. On examination he was pale and orthostatic. The abdominal exam revealed a tender palpable mass in the right upper quadrant. The $\mathrm{CBC}$ revealed hemoglobin of $11.3 \mathrm{~g} / \mathrm{dL}$ (compared to $9.8 \mathrm{~g} / \mathrm{dL}$ at the time of discharge) and the BUN and creatinine were $28 \mathrm{mg} / \mathrm{dL}$ and $1.22 \mathrm{mg} / \mathrm{dL}$, respectively. CT scan of the abdomen revealed a $13 \times 12 \times 23 \mathrm{~cm}$ fluid collection adjacent to the surgical site of the right kidney. Ultrasound guided drainage of the collection yielded 1.8 liters of blood-tinged urine; a culture of this urine was negative. Definitive drainage was achieved via a short-term percutaneous catheter. Follow up CT images over the next several months confirmed resolution of the urinoma.

DISCUSSION: Renal cell carcinoma (RCC) is the $7^{\text {th }}$ most common cause of cancer and the $10^{\text {th }}$ most common cause of cancer death in the United States. Treatment has long been surgical excision by radical nephrectomy. For small renal masses (SRM's, defined as a mass $<4 \mathrm{~cm}$ ), ablative therapies such as cryoablation, radiofrequency ablation are being used with increasing frequency. Advantages to ablative approach include lower morbidity, shorter lengths of hospital stay with short term and intermediate oncologic outcomes similar to nephrectomy. Though deemed to be safer than nephrectomy, ablative techniques used to treat SRM's are associated with a $4.9 \%$ complication rate. Common complications associated with ablative techniques include hemorrhage requiring transfusion, loss of renal function, and injury to adjacent organs. A meta-analysis of 46 series including data on 1,055 patients undergoing renal ablation revealed that urinary leaks or urinoma formation occurred in less than $0.1 \%$ of the cases. If left untreated, large urinomas can lead to complications like abscess formation, sepsis, hydronephrosis, paralytic ileus, and electrolyte abnormalities. Given the propensity to discharge patients undergoing minimally invasive surgery within 48 hours, it is likely that patients with complications of renal cryoablation will present to their family physician. As cryoablation becomes commonplace, ambulatory internists will need to be familiar with complications of this procedure.

ARTERIOVENOUS FISTULA: A RARE PRECIPITANT OF CONGESTIVE HEART FAILURE IN HEMODIALYSIS PATIENTS Prajit Arora ${ }^{1}$; Ambreen $\mathrm{Gul}^{2}$; Susie Stokley ${ }^{2} .{ }^{1}$ University of New Mexico, Albuquerque, NM; ${ }^{2}$ University of New Mexico, Albuquerque, NM. (Control ID \#1308421)

LEARNING OBJECTIVE 1: To recognize high output cardiac failure as a potential etiology of decompensated heart failure in patients getting hemodialysis (HD) through an Arteriovenous fistula (AVF) 
LEARNING OBJECTIVE 2: Diagnosis and treatment options available for high output cardiac failure secondary to AV fistula in Hemodialysis patients

CASE: 55 year old female with End stage Renal Disease (ESRD) on thrice weekly Hemodialysis (HD) secondary to Diabetes Mellitus, Hypertension admitted with complaints of shortness of breath, cough, subjective fevers and generalized fatigue. The patient had HD one day prior to admission and had been compliant with her dialysis regimen. On exam, patient was afebrile, slightly tachypneic and hypoxic with oxygen saturations of 70 percent on room air. She had jugular venous distension, bilateral basilar crepitations and trace bilateral lower extremity pitting edema. The patient also had an AV fistula noted at Left Upper extremity with bruit/thrill and aneurysmal dilatation with an additional aneurysm noted at Left Upper Extremity vein proximal to the fistula. Laboratory data was significant for leukocyte count of 4100 cells $/ \mu \mathrm{L}$, hemoglobin $11.0 \mathrm{gm} / \mathrm{dL}$, platelets 124,000 cells $/ \mu \mathrm{L}$, Sodium $137 \mathrm{mEq} / \mathrm{L}$, potassium $3.5 \mathrm{mEq} / \mathrm{L}$, BUN $10 \mathrm{mg} / \mathrm{dL}$, creatinine $2.7 \mathrm{mg} / \mathrm{dL}$.Troponin was normal, BNP $98,996 \mathrm{pg} / \mathrm{ml}$ and Lactate was $0.7 \mathrm{mEq} / \mathrm{L}$. The patient had a chest $\mathrm{x}$-ray done which showed findings of congestive heart failure and pulmonary edema with possibility of pneumonia not excluded. Transthoracic Echocardiogram showed Normal Ejection Fraction with additional findings of restrictive filling pattern and Right Ventricle Pressure/Volume Overload. The patient did have a fistulogram done for evaluation of Arteriovenous fistula as concern present for increasing dilatation at site, which did show aneurysmal dilatation of Left Upper extremity cephalic vein and patent arteriovenous anatomizes, with tortuosity and kinking noted in the vein in the lower upper portion of the upper arm. Flow velocities were obtained which showed increased flow rate greater than 2 liters/minutes. The patient was started on treatment for Health care associated pneumonia and received daily Hemodialysis with Decrease on EDW from 56 to 49.4 $\mathrm{kgs}$. The patient noted improvement in symptoms with decreased oxygen requirements. Vascular Surgery was consulted and the patient is planned for surgical revision of her AV fistula as an outpatient.

DISCUSSION: High Output Cardiac failure can result from a number of etiologies such as systemic AV fistulas, Chronic Anemia, Thyrotoxicosis, beri beri and psoriasis. Physical exam may reveal findings such as tachycardia, central venous hum, particularly over the Deep Internal Jugular vein and bounding pulse with wide pulse pressure. These conditions are associated with increased Cardiac index and low Systemic vascular resistance. The degree of increase in cardiac output secondary to the AV fistula depends on the size of the shunt. There is a shunting of blood from high pressure Artery to low pressure vein with decrease in Systemic Vascular Resistance and compensatory changes such as an increase in Stroke Volume, Heart rate and total plasma volume as well. Treatment involves surgical revision/banding of the site. Angiotensin receptor blockers and beta blockers may worsen the condition secondary to further decrease in Systemic vascular resistance. Upper arm AV fistulas and male gender pose an increased risk and this condition is generally associated with flow rate greater than 2 liters $/ \mathrm{min}$.

ARTHRITIS DUE TO AN UNUSUAL HUMAN PATHOGEN Angela H. Shin; Amy Sargious; Ramin Motarjemi. university of california, San Diego(UCSD), San Diego, CA. (Control ID \#1324350)

LEARNING OBJECTIVE 1: Familirize Physicians with Streptococcus equi infection in humans.

LEARNING OBJECTIVE 2: While a rare cause for infection in humans, should be considered in patients who have systematic or local signs and symptoms and are in contact with horses.

CASE: Case Presentation: This is a 65 year old male with diabetes and hypertension who presented to the emergency room with a complaint of right shoulder pain. He was found to be febrile with a tender,erythematous and swollen shoulder. A shoulder arthrocentesis was attempted but was unsuccessful in aspirating synovial fluid. Blood cultures were drawn and the patient left the emergency room against medical advice. The blood cultures grew positive for gram positive cocci and the patient was called to return to the emergency room. Further questioning revealed that he had a subjective fever for 3-4 weeks, mild fatigue, but otherwise no localizing signs or symptoms of infection other than his shoulder pain. He also noted that he lived on a ranch with horses, with one particular horse that had been sick with "distemper" who had to be isolated from the rest of the animals. The patient was initially started on vancomycin and ceftriaxone until speciation of the blood culture revealed streptococcus equi. The patient was continued on ceftriaxone alone. His AC joint fluid analysis revealed WBC 3490, PMN 95\%, and grew strep equi. The glenohumoral joint aspiration was unsuccessful in aspirating fluid after 3 separate attempts. The patient's fever and leukocytosis resolved by day 2 of antibiotics, CRP decreased from 38.2 to 8.94 , and a TTE revealed no vegetations. The patient was discharged with a 4 week course of IV ceftriaxone.

DISCUSSION: DISCUSSION: Streptococcus equi from group C streptococci is the etiologic agent for the upper respiratory disease in horses commonly referred to as strangles or distemper. The clinical signs in horses are fever, usually Lymphadenopathy, Mucopurulent nasal discharge, Pharyngitis and upper airway stridor. Subsequently, there is swelling of cervical nodes, which can drain purulent material. Transmission is direct horse-to-horse contact. Human infections are rare, but have been described in case reports. The clinical presentations in humans include pharyngitis, septicemia, meningitis, purulent arthritis and endocarditis. Poststreptococcal glomerulonephritis has also been described in connection with human infections. The source of human infection is often traced back to contact with horses. Our case lived in a ranch, and bred horses. There were 3 cases of strangles in his horses, which they all had pustules with draining pus. Patient later commented that he did not use gloves in several occasions when he was cleaning the pustules. We believe that he got infected through direct contact, and the bacteremia caused the arthritis. He was treated successfully, and repeat cultures were negative.

ASEPTIC MENINGITIS ASSOCIATED WITH INTRAVENOUS IMMUNOGLOBULIN THERAPY IN A CASE OF MILLER-FISHER SYNDROME. Sean Raj; Elena Katz; Mario Romagnoli. Lenox Hill Hospital, New York, NY. (Control ID \#1318238)

LEARNING OBJECTIVE 1: Recognize the clinical features of aseptic meningitis associated with IVIG in a case of Miller-Fisher Syndrome. CASE: Intravenous immunoglobulin (IVIG) therapy is widely utilized in the treatment of Guillain-Barre Syndrome (GBS). Although generally considered to be safe, IVIG has been associated with rare cases of aseptic meningitis. We report a case of aseptic meningitis associated with high dose IVIG in the treatment of Miller-Fisher Syndrome, a variant of Guillain-Barre. A 42-year-old male triathlete presented to the ER with acute-onset double vision worsening over five days prior to admission. The patient also reported new upper and lower extremity numbness and tingling for three days prior to admission that progressed from his hands and feet proximally to his elbows and knees, respectively. At the initial onset of blurry vision, the patient was seen in the ER of an outside facility, where a MRI/MRA of the head and neck was unrevealing. An outpatient neurologist performed EMG studies, which were unremarkable, but advised the patient to return to the ER for further work-up of possible Guillain-Barre syndrome if his symptoms did not improve. Ten days prior to developing these symptoms, the patient suffered a bout of bloody diarrhea with positive stool cultures for Campylobacter jejuni. Of note, the patient competed in the Ironman triathlon, during which he swam in the Hudson River, recently polluted with a sewage spill just two weeks earlier. On presentation to the ER, the patient had lateral gaze palsy in his right eye, decreased patellar reflexes, and decreased sensations to light touch in both his lower and upper extremities, but strength that remained intact throughout. A lumbar puncture (LP) on admission, revealed a normal cerebrospinal fluid analysis (CSF) with a WBC $0 / \mu \mathrm{L}$. Treatment with IVIG daily (high-dose of $0.4 \mathrm{~g} / \mathrm{kg} /$ day) for presumptive diagnosis of Miller-Fisher Syndrome and ciprofloxacin for Campylobacter jejuni infection was initiated - GQ1B-IgG Antibody positivity later confirmed GBS. After completion of IVIG, on admission day five, the patient developed an acuteonset severe "migraine-type" headache, photophobia, neck-stiffness, and was febrile to $100.5 \mathrm{~F}$. LP demonstrated a CSF with normal protein and glucose levels, CSF culture with no growth, but a WBC of $296 / \mu \mathrm{L}$, suggestive of aseptic meningitis. The patient's meningeal signs resolved over the subsequent three days after discontinuation of IVIG therapy and 
supportive care. On discharge, mild residual lateral gaze palsy and blurry vision remained, with a near-normal return of sensations throughout, normal VC and NIF. He was discharged with neurology follow-up as an outpatient. DISCUSSION: In patients receiving high-dose intravenous immunoglobulin therapy, clinicians should include aseptic meningitis in their differential for the constellation of symptoms of fever and a headache in the 6-48 hour period following the completion of an IVIG infusion.

\section{ASYMPTOMATIC ISOLATED LEFT VENTRICULAR NONCOM-} PACTION (LVNC) IN SIBLINGS- A THERAPEUTIC DILEMMA. Robin Singh ${ }^{1}$; James D. Holloway ${ }^{2} .{ }^{1}$ University of Louisville, Louisville, KY; ${ }^{2}$ Baptist Health Heart Institute, Little Rock, AR. (Control ID \#1327631)

LEARNING OBJECTIVE 1: Recognition of isolated LVNC as a specific form of genetic cardiomyopathy and its characteristic echocardiographic features.

LEARNING OBJECTIVE 2: Understanding the variable prognosis and the therapeutic challenges of this disease.

CASE: A 33 year old male (Case A) was referred for cardiac evaluation because of his elder brother's abnormal echocardiogram aged 37 (Case B) and a history of sudden cardiac death (SCD) in the father at age 38 . The patient was totally asymptomatic and had unremarkable past medical and social histories. Physical examination, EKG and Chest Xray were normal. Echocardiogram revealed normal left ventricular (LV) internal dimension and $\mathrm{EF}>55 \%$ with normal cardiac valves. Deep trabeculations were noted in the LV myocardium with intertrabecular recesses involving a large part of the LV myocardium. This gave the appearance of a spongy myocardium and LV hypertrophy. Color flow Doppler demonstrated communication between LV and intertrabecular recesses. The characteristic echocardiogrpahic findings led to the diagnosis of LVNC. The ratio of the noncompacted to the compacted myocardium was 3. RV morphology and function was normal. The echocardiogram report of case $\mathrm{B}$, done at another facility, was obtained and was reported to have possible apical hypertrophic cardiomyopathy or prominent trabeculations. Echocardiogram was repeated and demonstrated identical features of isolated LVNC as in case A with normal LV EF. Case B was also asymptomatic and had normal physical examination, EKG and Chest X-ray. Due to the rarity of the condition, not much is known about the prognosis except in patients with severely reduced LV EF or documented episodes of ventricular tachycardia (VT). Based on the limited observational data, both the patients were advised to have implantable cardiac defibrillators (ICD) to reduce the risk of SCD because of the history of SCD in the father at age 38. Case A declined to have the ICD implant at this time. Case B opted to have ICD implant but had no inducible sustained VT during EP study prior to ICD implant.

DISCUSSION: Isolated LVNC is a rare disorder and is classified as a primary genetic cardiomyopathy with varying genetic heterogeneity. It can be either sporadic or familial. Autosomal dominant inheritance is more common than recessive or X-linked inheritance. It is characterized by an altered myocardial wall with prominent trabeculae and deep intertrabecular recesses resulting in a thickened myocardium with two layers consisting of compacted and noncompacted myocardium. Echocardiogram is very helpful in making the diagnosis and can distinguish it from dilated and hypertrophic cardioymypathy. Cardiac MRI and CT may be helpful. Common clinical presentations include heart failure, arrhythmias and embolic events. ICD implant in patients with reduced LVEF of $<35 \%$, sustained VT, and history of SCD are considered acceptable indications. The prognosis of LVNC in asymptomatic patients with normal LVEF is not well known but the family history of sudden cardiac death prompted advice for ICD implants in our patients.

ATRIAL SEPTAL DEFECTS IN THE ADULT PATIENT: A CASE REPORT AND LITERATURE REVIEW Christopher Sankey. Yale School of Medicine, New Haven, CT. (Control ID \#1316191)

LEARNING OBJECTIVE 1: Appreciate the prevalence and potential consequences of atrial septal defects in the adult patient.
LEARNING OBJECTIVE 2: Recognize the clinical presentation of atrial septal defects and identify the options and recommendations for defect closure.

CASE: A 26-year-old Hispanic female without a significant medical history presented with new-onset diabetic ketoacidosis. She was admitted, and blood sugars and metabolic derangements quickly normalized with insulin and intravenous fluids. Physical examination on repeat assessment was notable for a systolic ejection murmur at the left upper sternal border with a prominent split of the second heart sound. She had no exertional symptoms, and denied any family history of congenital heart disease. Chest radiograph revealed mild enlargement of the right atrium and pulmonary arteries. Electrocardiogram demonstrated sinus tachycardia, with $\mathrm{P}$ wave morphology suggestive of right atrial enlargement. Transthoracic echocardiogram subsequently revealed an enlarged right atrium and right ventricle with diastolic interventricular flattening, consistent with volume overload. Injection of agitated saline confirmed the presence of a left-to-right intracardiac shunt, suggestive of an atrial septal defect (ASD). Transesophageal echocardiogram further demonstrated left-to-right shunting across a large secundum ASD. The patient was referred for outpatient percutaneous device closure.

DISCUSSION: ASD is an important diagnostic entity in the adult patient evaluated by General Internists and Hospitalists, with considerable morbidity and mortality if left unrepaired. ASDs account for approximately one third of all adult congenital cases. Three quarters of ASDs are secundum defects, which are more common in women than men. Most ASDs represent spontaneous genetic mutations, though some are associated with heritable chromosomal abnormalities. Symptoms at presentation are nonspecific and most often include fatigue and exertional dyspnea. Long-term complications of uncorrected ASDs include atrial arrhythmias, right ventricular dilatation and failure, pulmonary hypertension, recurrent pulmonary infection, paradoxical embolism and cerebrovascular accident. Atrial fibrillation is rare in patients younger than 40 , but there is an age-related increase in prevalence after age 40 which is independent of subsequent repair. Percutaneous device closure is standard of care for secundum ASDs in tertiary medical centers worldwide. Procedure efficacy has been demonstrated with experienced providers and appropriately selected defects. Compared with surgery, percutaneous closure is associated with a significantly lower risk of complication and has reduced hospital lengths of stay. Device closure of ASDs also affords significant improvement in exercise capacity, left ventricular filling, and cardiac output. Surgery remains first-line therapy in patients with specified defect complexities. Symptoms and evidence of structural right heart abnormalities are predominant indicators for immediate intervention. In conclusion, ASD is a frequent congenital cardiac abnormality, the physiologic subtlety of which often results in the delay of diagnosis well into adulthood. The significant agerelated morbidity and mortality associated with ASDs can be mitigated with timely defect closure, and is recommended in most patients regardless of age.

ATRIO-ESOPHAGEAL FISTULA; A RARE BUT DEADLY COMPLICATION IN THE TREATMENT OF ATRIAL FIBRILLATION Lindsay A. Lucas ${ }^{1}$; Michael Errico ${ }^{2}$; Anthony Zikos ${ }^{3}$. ${ }^{1}$ West Penn Allegheny General Health System, Pittsburgh, PA; ${ }^{2}$ West Penn Allegheny Health System, Pittsburgh, PA; ${ }^{3}$ Allegheny General Hospital, Pittsburgh, PA. (Control ID \#1339485)

LEARNING OBJECTIVE 1: Recognize atrio-esophageal fistula as a potential risk of pulmonary vein isolation in the treatment of atrial fibrillation.

LEARNING OBJECTIVE 2: Identify the signs and symptoms of atrioesophageal fistula.

CASE: A 57-year-old man with a history of chronic atrial fibrillation on anticoagulation and previous right parietal ischemic stroke was admitted with acute-onset fevers, vomiting, and disorientation. His surgical history was notable for mitral valve replacement, PFO closure five years prior, and radiofrequency pulmonary vein isolation for restoration of sinus rhythm 30 days prior to admission. On presentation he was hypotensive, tachycardic, febrile, and complaining of a headache. He was volume resuscitated, pancultured, and empirically started on intravenous antibiotics for meningitis. Physical exam revealed equal and reactive pupils with mild periorbital edema and pale conjunctiva, bibasilar rales, and no focal neurologic deficit or abnormal cardiovascular findings. Laboratory studies 
revealed an INR of 1.5 and lactic acid of 3 , but otherwise unremarkable complete blood count and metabolic panel. CSF was unrevealing. CT head showed a subacute left frontal infarct and an old right parietal infarct. Cultures and chest plain film were negative. Initially his symptoms improved with antibiotics, but overnight he developed dark blood per rectum, vertigo, weakness, and shock, requiring intubation and fluid resuscitation. His clinical signs strongly indicated a left atrio-esophageal fistula formation with septic embolic infarct. A CT chest confirmed a fistulous connection between the esophagus and left atrium. MRI brain revealed innumerable, acute, bilateral cerebral and cerebellar embolic infarcts. The patient was immediately taken to the operating room and underwent successful closure of the left atrium and esophageal tear. Despite early surgical intervention, the patient's mental status failed to recover and his family withdrew care.

DISCUSSION: Left atrial catheter ablation to encircle the pulmonary veins is becoming increasingly recognized as an effective treatment for atrial fibrillation in cases where the arrhythmic activity originates in the muscle sleeves of the pulmonary vasculature. Less recognized is the small but potentially lethal risk of esophageal injury caused by creating a radiofrequency-induced deep tissue injury to the left atrial posterior wall. While left atrio-esophageal fistula formation occurs in fewer than $1 \%$ of cases, it should be considered in any patient presenting with symptoms of meningitis, endocarditis, stroke, or gastrointestinal bleed with a history of pulmonary vein catheter ablation within the last 30 days. Mortality is greater than $50 \%$ and those who survive are usually recognized early and have few co-morbidities. Treatment is immediate surgical correction of the defect in the tissues walls, antibiotics, and supportive care. Research in risk reduction is limited, due to the relative infrequency of the condition. Low frequency radiation and continuous monitoring of esophageal temperatures is thought to reduce risk of injury, however, these have not been shown to significantly decrease mortality. Left atrio-esophageal fistula is a rare but often fatal complication of pulmonary vein isolation in the treatment of atrial fibrillation and should be considered in any patient presenting subacutely with sepsis, stroke, or GI bleed.

ATYPICAL STRESS INDUCED CARDIOMYOPATHY Aiman M. Smer; Manu Kaushik; Dennis Esterbrooks. Creighton University Medical Center, Omaha, NE. (Control ID \#1293250)

LEARNING OBJECTIVE 1: Recognize the clinical features and types of stress induced cardiomyopathy

LEARNING OBJECTIVE 2: Diagnose stress induced cardiomyopathy using the Mayo Clinic Criteria

CASE: A 58-year-old woman presented to a tertiary center with new onset chest pain associated with shortness of breath and diaphoresis. This started a few minutes after hearing news of her husband's demise. Her initial ECG revealed normal sinus rhythm with T-wave inversions in leads I and aVL. Troponin was elevated at 2.7. Patient was initially treated as ACS with medical therapy with anti-platelets and anticoagulants. Cardiac catheterization showed normal coronary arteries. Left ventriculography revealed akinesis of the midventricular segments predominantly inferiorly. The remaining ventricular segments were hyperdynamic. Serial echocardiography demonstrated improving LV function. A diagnosis of atypical stress induced cardiomyopathy was made based on the transient LV systolic dysfunction at the mid-ventricular segments, ECG changes, and normal coronaries. The patient was medically managed and discharged in stable condition.

DISCUSSION: Stress induced cardiomyopathy is a rapidly reversible cardiomyopathy characterized by transient systolic dysfunction in absence of obstructive coronary disease. The typical form of SIC first described in Japan in 1990 by Sato et al is characterized by hypokinesis of the apex with hyperkinesis of the basal ventricular segments. SIC usually occurs in postmenopausal women and is triggered by psychological or physical stress. Although, the pathogenesis of SIC is not well understood, it has been suggested that SIC maybe caused by catecholamine excess causing coronary spasm and myocardial stunning. Initial presentation typically mimics ACS with ST segment elevation and T-wave inversions in the precordial leads being the most common ECG finding. Recently, four diagnostic criteria were proposed to diagnosis SIC. 1.Transient hypokinesis, akinesis or dyskinesis of the left ventricular mid segments with or without apical involvement
2.Absence of obstructive coronary disease 3.New ECG changes (either STsegment elevation and/or T wave inversion) or modest elevation in cardiac troponin 4.Absence of pheochromocytoma or myocarditis. Acute complications of SIC include acute heart failure, arrhythmias, cardiogenic shock, and transient left ventricular outlet obstruction. Patients who survive the acute episode typically recover normal left ventricular function within one to four weeks. This case suggests that atypical forms of SIC may have partial and noncircumferential mid-ventricular involvement, and that no region of ventricular myocardium is spared from possible involvement SIC.

AXONAL PERIPHERAL NEUROPATHY; AN UNCOMMON SEQUELA OF ALCOHOL ABUSE. Mira Kaga; Aprajita Mattoo. Montefiore, Bronx, NY. (Control ID \#1339122)

LEARNING OBJECTIVE 1: Recognize the clinical presentation of alcoholic polyneuropathy.

LEARNING OBJECTIVE 2: Identify the differential diagnosis of peripheral neuropathy.

CASE: A 60 year-old female with no significant past medical history presented with three months of progressively worsening weakness, numbness, and tingling in her upper and lower extremities. She also had occasional sharp, lancinating pain throughout her legs over this same period of time.These symptoms were of insidious onset, first appearing several years prior to presentation and then precipitously worsening over the last three months. Her weakness, numbness and tingling was present in a stocking-and-glove distribution. There was evidence of muscle wasting in the medial and lateral aspects of her thigh and calf muscles bilaterally and in the thenar and interossei muscles of her hands. Though her sensory exam was intact, both her vibrioception and propioception were decreased and she had a scissored, ataxic gait with a positive rhomberg sign. A basic metabolic panel revealed a sodium of 132, a potassium of 2.8 and a magnesium of 1.0. The mean corpuscle volume was found to be elevated to 114 with a folate deficiency of 3.8. Total vitamin $\mathrm{D} 2+\mathrm{D} 3$ was deficient to 5.8 and a recent hemoglobin $\mathrm{A} 1 \mathrm{c}$ was 4.8. Electromyography of the lower extremities demonstrated sensorimotor axonal peripheral neuropathy without evidence of demyelination. These findings were consistent with nutritional deficiencies secondary to chronic alcohol abuse. On further questioning, the patient revealed a history of one to three glasses of white wine per day for approximately thirty years with an increase in consumption over the past three months to 6-10 glasses per day. Her alcohol history was unknown to her primary care physician.

DISCUSSION: Weakness and parethesia are problems commonly encountered by the internist. A methodical approach to peripheral neuropathy must include less common causes of the disease state such as chronic alcohol use. Like diabetic neuropathy, alcoholic neuropathy is a primary axononal sensorimotor peripheral polyneuropathy that manifests distally first. Symptoms are typically symmetric and follow a stocking-and-glove distribution, involving the hands and arms once lower extremity involvement progresses past the ankle. Sensory symptoms such as numbness, dysesthesias, paresthesias, and loss of position and vibration sense are commonly seen and usually manifest prior to motor symptoms such as weakness and muscle wasting. Gait ataxia may be caused by cerebellar degeneration, sensory ataxia, or simply distal muscle weakness. As with the case above, patients who have alcoholic neuropathy often have nutritional deficiencies as well. Electrolyte abnormalities such as those demonstrated in our vignette and vitamin deficiencies, particularly thiamine, B12, and folate, are therefore supportive of the diagnosis. The pathophysiology behind the disease state is still an area of debate but it is believed that the segmental thinning and loss of myelin that is seen in alcoholic neuropathy is a combination of both nutritional deficiencies and the direct toxic effects of ethanol on neural tissue. Identification of patients who abuse alcohol is an important aspect of primary care that is often not addressed during patient visits. Treatment includes improving nutrition and the cessation of drinking. Although complete recovery from severe neuropathy is uncommon, low doses of tricyclic antidepressants, or gabapentin can be used to control dysesthesias.

AZATHIOPRINE HYPERSENSITIVITY MIMICKING SEPSIS IN A PATIENT WITH CROHN'S DISEASE. Oxana Haflund; Asif Iqbal. Aurora Sinai Medical Center, Milwaukee, WI. (Control ID \#1313477) 
LEARNING OBJECTIVE 1: Recognize a case of Azathioprine hypersensitivity mimicking sepsis in a patient with Crohn's disease.

CASE: A 68 years old man with past medical history of atrial fibrillation and recent diagnosis of Crohn's disease presented with two days history of fevers as high as $102.8 \mathrm{~F}$, chills, mild nasal congestion and mild cough with production of small amount of whitish sputum. He did not complain of any abdominal pain or diarrhea. He did not have any chest pant, joint pain, rash or urticaria. Patient was recently diagnosed with Crohn's disease after having multiple episodes of bloody diarrhea. Diagnosis was confirmed by colonic biopsy. Treatment was started with Azathioprine and Prednisone. On our examination patient looked ill and was having severe rigors and high grade fevers. Abdominal exam showed mild tenderness of the left lower quadrant on deep palpation without guarding or rebound tenderness and normal bowel sounds. Laboratory date showed normal white count and venous lactate level. Chest X-ray, urine analysis, and blood culture results were unremarkable. CT scan of the abdomen and pelvis with contrast showed mild intrahepatic biliary dilation with normal common biliary duct and gallbladder and diverticulosis without abscess or any other pathology. Suspecting an intra-abdominal abscess, treatment with Cefoxitin was started. Patient continued to have fevers and rigors after starting antibiotics. Azathioprine was then stopped suspecting a hypersensitivity reaction. Fever and rigors promptly disappeared after discontinuation of Azathioprine. Patient clinically improved and did not have any more fevers and rigors after Azathioprine was discontinued.

DISCUSSION: Systemic hypersensitivity is a rare side effect of azathioprine. The common side effects include gastrointestinal disturbances, granulocytopenia and hepatocellular injury. The majority of reactions occur in the first four weeks of initiation of the treatment. Hypersensitivity should be suspected and included in the differential diagnosis if a patient experiences fever, malaise, hypotension and renal failure. The above mentioned symptoms could relate to a true adverse drug reaction in some versus exacerbation of underlying disease or sepsis in others. We suspected hypersensitivity to azathioprine in this patient due to: timing of initiation of azathioprine, presence of above mentioned symptoms and improvement after discontinuation of azathioprine. A rechallenge test may be used to confirm the diagnosis of azathioprine hypersensitivity. Optimally rechallenge should be done under careful supervision. Rechallenge was not conducted in this case because it is dangerous and can lead to life-threatening reoccurrence of symptoms.

B CELL LYMPHOMA MASQUERADING AS GROSS HEMATURIA Lindsay A. Mazotti ${ }^{2}$; Jaclyn Watkins ${ }^{1}$; Hugh Benedict ${ }^{2}$; Thomas N. Hackford $^{2}$; Stacy Shoshan ${ }^{2}$; Benjamin L. Maring ${ }^{2}$. ${ }^{1}$ UC San Francisco, School of Medicine, San Francisco, CA; ${ }^{2}$ Kaiser Permanente: Oakland Medical Center, Oakland, CA. (Control ID \#1340083)

LEARNING OBJECTIVE 1: Recognize gross hematuria as a possible presentation of B cell lymphoma

LEARNING OBJECTIVE 2: Recognize uric acid nephropathy in the setting of spontaneous tumor lysis syndrome

CASE: A 72 year old man with a history of diabetes mellitus type 2, hypertension, asthma, and GERD presented to the emergency department with two episodes of painless gross hematuria, recent night sweats, and two weeks of intermittent non-bloody diarrhea. Physical examination revealed bilateral petechiae on the legs and mild bilateral CVA tenderness. Urinalysis was notable for $2+$ uric acid crystals. Abdominal CT revealed bilateral obstructing stones without marked hydronephrosis. The patient was found to be in anuric acute renal failure with a creatinine of 4.84 compared to baseline of 1.01. Further evaluation revealed new anemia and thrombocytopenia. Though TTP-HUS was entertained given recent diarrhea, serial blood smears did not reveal schistocytes. Lab work on hospital day two revealed a serum uric acid of $22.4 \mathrm{mg} / \mathrm{dL}$ and markedly elevated LDH at $44977 \mathrm{U} / \mathrm{L}$, raising the suspicion of spontaneous tumor lysis syndrome with acute uric acid nephropathy. After bilateral ureteral stent placement, the patient was emergently hemodialyzed and started on allopurinol. He remained anuric for three days. Renal function gradually improved with alternating IVF and IV furosemide. A bone marrow biopsy obtained on hospital day two initially suggested leukemia. However, further staining of the sample revealed high-grade B cell lymphoma with extensive bone marrow involvement. The patient was started on R-CHOP. At discharge, creatinine was 0.96 . Several weeks after presentation, the patient developed multiple cranial nerve palsies secondary to CNS lymphoma. Intrathecal chemotherapy failed to resolve the palsies, and the patient entered Hospice care.

DISCUSSION: Tumor lysis syndrome is defined by several metabolic complications that may arise in the setting of rapidly proliferating tumors, a large tumor burden, or during treatment of a drug-sensitive neoplasm. Metabolic abnormalities associated with TLS include serum uric acid $>=8 \mathrm{mg} / \mathrm{dL}$ or a 25 percent increase from baseline, serum potassium $>=6.0 \mathrm{mmol} / \mathrm{L}$ or a 25 percent increase from baseline, serum phosphate $>=6.5 \mathrm{mg} / \mathrm{dL}$ or a 25 percent increase from baseline, and serum calcium $<=7 \mathrm{mg} / \mathrm{dL}$ or a 25 percent decrease from baseline. The symptoms of TLS include those associated with the metabolic abnormalities (eg nausea, vomiting, arrhythmia, seizure, tetany, syncope, sudden death). Additionally, if uric acid or calcium precipitates in the urinary tract leading to nephrolithiasis, flank pain may result. Uric acid precipitation within the renal tubules with resulting oliguric or anuric renal failure is known as acute uric acid nephropathy (UAN). UAN typically results from a combination of overproduction and overexcretion of uric acid as seen in patients with lymphoma and leukemia. UAN should be considered when there is acute kidney injury in the context of marked hyperuricemia $(>15 \mathrm{mg} / \mathrm{dL})$. UAN secondary to spontaneous TLS may be treated with allopurinol, rasburicase, and hemodialysis. Gross hematuria is not a typical presentation of UAN. However, it has been described, mostly in case reports, as a presentation of leukemia or urinary tract non-Hodgkin's lymphoma. Based on CT, our patient did not have evidence of urinary tract tumor burden. However, biopsy of the urinary tract was not performed.

B-CELL LYMPHOMA MANIFESTING AS A HEPATIC GRANULOMATOSIS Kerry-Anne Machado. Allegheny General Hospital, Pittsburgh, PA. (Control ID \#1339946)

LEARNING OBJECTIVE 1: Demonstrate a unique etiology of hepatic granulomas.

LEARNING OBJECTIVE 2: Illustrate the clinical and laboratory features of granulomatous hepatitis in an adult.

CASE: This is a 42-year-old Caucasian female who was admitted for intermittent fevers for 3 months. She complained of weakness, diaphoresis, weight loss, and right upper quadrant abdominal pain with associated nausea and vomiting. She denied jaundice, confusion, pruritus, dark urine, and diarrhea, melena or hematochezia. She underwent a cholecystectomy one year ago. She denied sick contacts or recent travel. She is a nonsmoker and denied alcohol use. Family history was significant for cirrhosis that her father had developed as a complication of NASH. Her vital signs demonstrated a temperature of $102^{\circ} \mathrm{F}$, the pulse rate $115 / \mathrm{min}$, blood pressure $110 / 60 \mathrm{mmHg}$ and respiration rate $12 / \mathrm{min}$. On physical examination sclera was nonicteric and there were no palpable lymph nodes. Her abdomen revealed moderate tenderness in the epigastrium and right upper quadrant without rebound, guarding, organomegaly or masses. The heart and lung exams were normal Initial lab work was notable for an elevated alkaline phosphatase of $143 \mathrm{U} / \mathrm{L}$ with normal synthetic function. There were mild intermittent rises in AST and ALT, though chronic liver disease panel was unremarkable including anti-mitochondrial antibody, anti-smooth muscle antibody, viral hepatitis serologies, ANA, anti-LKM antibody, HSV, EBV and CMV serologies. Infectious workup including blood and urine cultures as well as further imaging including chest $\mathrm{CT}$ and transthoracic echocardiogram were unremarkable. An enhanced abdominal CT scan demonstrated hepatomegaly with multiple ill-defined, low-density liver lesions up to $1.3 \mathrm{~cm} \times 2.5 \mathrm{~cm}$ in size. Subsequently, a liver biopsy was done which showed non-caseating epithelioid granulomas and mild steatosis. An extensive workup was done for her granulomatous disease including studies for bacterial and fungal infection, malignancy, and autoimmune mediated causes. In addition a bone marrow biopsy, bronchoscopy with broncheoalveolar lavage, and transbronchial biopsies were done, all of which were unrevealing. The etiology of her granulomatous disease was unclear and suspicions leaned towards autoimmune 
mediated disease. The patient was treated with NSAIDs, prednisone and ursodiol, with mild improvement in her constitutional symptoms, however she did not defervesce. The patient became progressively ill and sought additional care at a tertiary care center. A PET scan demonstrated numerous hypermetabolic foci throughout the liver with involvement in the spleen. Subsequently a repeat biopsy of the liver supported the diagnosis of EBV-positive B-cell lymphoma. Large atypical cells were strongly positive for CD20, PAX-5, CD30, MUM-1. In situ hybridization recognized EBV encoded RNA, further solidifying the diagnosis. Given these findings the patient was referred to her local oncologist to initiate therapy for her lymphoma.

DISCUSSION: Hepatic granulomas are not uncommon, seen in 2-10\% of patients who undergo routine liver biopsy and may be caused by a variety of conditions. However, the presence of noncaseating epithelioid granulomas in association with B-cell lymphoma is both distinctive and rare. Very little has been published to demonstrate the diversity of etiologic factors in granulomatous liver diseases. Therefore, this case serves to emphasize a less common histopathologic diagnosis.

BABESIOSIS IN A NEW ENGLANDER-FROM PRIMARY CARE TO INTENSIVE CARE Asa Z. Oxner; Jeffrey William. Beth Israel Deaconess Medical Center, Boston, MA. (Control ID \#1334637)

LEARNING OBJECTIVE 1: Distinguish patients at risk for Babesia infection over other co-infections

LEARNING OBJECTIVE 2: Recognize laboratory values and evidence of organ damage that accompany severe babesiosis

CASE: A 63 year-old gentleman with history of Non-Hodgkin lymphoma (NHL) presented to his community hospital with two weeks of neck stiffness, body/joint aches, and daily fevers to $102-105^{\circ} \mathrm{F}$ with rigors. He is an air quality scientist who frequently hikes in the backwoods of New England. He recalled removing two ticks engorged with blood prior to presentation. His NHL was treated with chemotherapy and in remission since 2010 , confirmed by CT imaging. The community hospital started doxycycline due to concern for Lyme disease and discharged him home. A few days later he returned with persistent fevers and worsening thrombocytopenia and transaminitis. He was hypotensive despite six liters of volume resuscitation and required vasopressor initiation. Azithromycin and atovaquone were added to doxycycline and he was transferred to our intensive care unit. Within hours of arrival, volume resuscitation was continued and his vasopressor requirement decreased. Physical exam was notable for mild jaundice but absence of erythema multiforme or petechiae. His laboratory studies were significant for: platelet count of $36 \mathrm{~K} / \mu \mathrm{L}$, hematocrit of 33.4, creatinine $1.2 \mathrm{mg} / \mathrm{dl}$, AST/ALT 838/777 IU/L, LDH $2399 \mathrm{IU} / \mathrm{L}$, total and direct bilirubin of 3.9 and $2.8 \mathrm{mg} / \mathrm{dl}$. Urinalysis showed large blood with only 7 RBCs. A thin parasite smear showed many red cells with single ring forms with an estimated parasitemia of $10 \%$ consistent with severe babesiosis. Antibiotics were changed to quinine and clindamycin, remaining on doxycycline until Lyme, Anaplasma, and Ehrlichia serologies returned negative. He underwent exchange transfusion pheresis with resolution of parasitemia to $2 \%$. He returned home to complete a 10-day course of atovaquone and azithromycin.

DISCUSSION: Babesiosis is an uncommon human illness caused by a parasite called Babesia microti, transmitted by the Ixodes scapularis tick, most prevalent in the mid-Atlantic and New England regions of North America. Most people infected with the Babesia parasite are asymptomatic, but severe infections can present with symptoms and signs similar to malaria: high fevers, rigors, and hemolytic anemia. Immunosuppressed patients are at increased risk for clinically significant babesiosis, including those with functional asplenia, HIV/AIDS infection, malignancy, age> 50 years, or on chronic immunosuppressive medications. Severe babesiosis is characterized by parasitemia $>10 \%$ on peripheral blood smear, hemolysis, respiratory distress, disseminated intravascular coagulation, renal/hepatic failure, or death with a mortality rate of $5-10 \%$. No early clinical markers foretell a severe Babesiosis nor distinguish it from Lyme. However, labs showing early organ dysfunction should hint that more than Lyme is at play. Given the risk of co-infections carried by the Ixodes tick, doxycycline $100 \mathrm{mg}$ PO BID is an important therapy to initiate until serologies return negative. Mild infection should be treated with atovaquone $750 \mathrm{mg}$ PO BID and azithromycin $250 \mathrm{mg}$ PO daily and severe infection with clindamycin $600 \mathrm{mg}$ IV Q6H and quinine $650 \mathrm{mg} \mathrm{PO}$ $\mathrm{Q} 8 \mathrm{H}$; both courses are 7-10 days. In rare cases with parasitemia $>10 \%$ or evidence of organ damage, exchange transfusion is a life-saving therapy to remove the parasites and hemolysis byproducts.

BEFORE THE CURTAIN FALLS Cady Blackey. Tulane University Health Sciences Center, New Orleans, LA. (Control ID \#1311862)

LEARNING OBJECTIVE 1: 1. Recognize the clinical presentation of temporal arteritis 2 . Identify the most useful symptoms, physical examination findings, and laboratory data for diagnosing temporal arteritis 3. Identify how to obtain accurate temporal artery biopsy results CASE: A 63 year-old woman presented with three months of a progressive headache. She reported episodes of stabbing pain in her left forehead lasting seconds to minutes, occurring multiple times a day and increasing in frequency and severity. She denied associated photophobia, phonophobia, or nausea. She reported pain in her forehead and jaw after eating. She denied fever or a stiff neck. She had two episodes of vision loss in her left eye that she described as "a curtain coming down over my eye." She complained of fatigue, in addition to stiffness and soreness in her wrists each morning that resolved after 2-3 hours. The physical exam revealed a thickened and tender left temporal artery; the retina were normal bilaterally. Her neurologic examination was normal. The c-reactive protein was less than 0.5 , and the sedimentation rate was was 30 . An oral prednisone regimen was initiated, and she was immediately sent for a temporal artery biopsy. The biopsy revealed mild lymphocytic inflammation in arterial adventitia and mild intimal hyperplasia. Her symptoms improved with prednisone, without further episodes of visual disturbance.

DISCUSSION: Headaches are one of the most commonly encountered chief complaints by the general internist. Maintaining appropriate suspicion for temporal arteritis is imperative, as the diagnosis is largely established on historical information, and timely diagnosis is imperative: blindness can develop rapidly if it is not promptly treated. Signs and symptoms that should raise suspicion for temporal arteritis include headache, particularly in an older patient, jaw claudication, visual symptoms, scalp tenderness, fever, malaise, arthralgias and tender, beaded, engorged or pulseless temporal arteries. However, the presentation of this disease can vary greatly depending on which symptoms predominate. Temporal artery biopsy is the gold standard for diagnosis, but immediate biopsy is often logistically impractical. Knowing how the presence or absence of given signs and symptoms impacts the likelihood of having the disease is essential in determining which patients to treat. Jaw claudication (+LR 4.3), diplopia (+LR 3.5) and temporal artery abnormalities on exam (+LR 4.6) have been shown to be the most useful in predicting positive biopsy results; normal ESR levels (-LR 0.2) and young age are valuable in excluding the disease. Treatment should begin promptly if temporal arteritis is considered likely. Steroids are the mainstay of therapy, but dosing, route and duration vary depending on the clinical presentation. Steroids do not impact biopsy results if suspicion of temporal arteritis is high, so treatment should not be delayed. However, inadequate biopsy specimen length and greater duration of time between steroid initiation and biopsy have both been shown to decrease the number of positive biopsies. Thus, a biopsy should be performed by an experienced specialist within two weeks of steroid initiation. With an aging population in the US, physicians should be adept at diagnosing this cause of headache from which significant morbidity is possible.

BING NEEL SYNDROME- A RARE COMPLICATION OF WALDENSTROM'S MACROGLOBULINEMIA Akshiv Malhotra; Toni Pacioles; Teresa Gentile. SUNY Upstate Medical University, Syracuse, NY. (Control ID \#1339584)

LEARNING OBJECTIVE 1: Recognize the neurological complications of Waldenstrom's macroglobulinemia.

LEARNING OBJECTIVE 2: Diagnose Bing Neel syndrome based on the MRI and CSF findings.

CASE: 70 year old female presented to the Emergency Department with worsening left upper extremity weakness, dysarthria and confusion. Her past 
medical history is significant for Waldenstrom's macroglobulinemia (WM) diagnosed 1 year ago, breast cancer diagnosed 11 years ago, status post lumpectomy, radiation and Tamoxifen. About a year ago, she was noted to have anemia and paraprotein in her blood and underwent a bone marrow biopsy which showed a lymphoplasmacytic infiltrate. She then underwent CT scans to look for enlarged lymph nodes. She was found to have a mass of the left kidney which on biopsy showed Lymphoplasmacytic lymphoma with an IgM monoclonal protein (WM). She was treated with 4 cycles of Rituximab to which she responded well. However, she started having intermittent confusion and balance problems and now, presented to the ER with left upper extremity weakness, dysarthria and confusion. The patient was admitted to the neurology service. The symptoms resolved within 6 hours of onset. She underwent $\mathrm{CT}$ of the head without contrast which showed no evidence of acute bleed/ infarct. MRI of the brain showed dural enhancement. Lumbar puncture was performed which showed Protein of $768 \mathrm{mg} / \mathrm{dl}$ and White count of $108 / \mathrm{cu} \mathrm{mm}$ with $100 \%$ monocytes. The cytopathology report and flow- cytometry came back consistent with previously diagnosed B cell lymphoma ( Lymphoplasmacytic lymphoma). Decadron $4 \mathrm{mg}$ every 6 hours was started. An intraventricular reservoir was placed and Intrathecal methotrexate was started twice a week for 8 cycles. CSF cleared up with no evidence of malignancy. She was then treated with craniospinal irradiation and has remained in remission for 4 months.

DISCUSSION: Bing Neel (BN) syndrome is a very rare complication of Waldenstrom's Macroglobulinemia (WM) that should be considered in patients with neurologic symptoms and a history of WM. Neurologic complications occur in $25 \%$ cases of WM, but are most commonly due to serum hyperviscosity syndrome and immune related neuropathy. Direct malignant lymphoid infiltration of the CNS, the so called Bing Neel syndrome is extremely rare. BN syndrome is defined as WM with perivascular infiltration of small lymphocytes, lymphoplasmacytoid cells, and plasma cells in the CNS. BN syndrome refers specifically to the involvement of perivascular infiltrates rather than stroke or hemorrhage due to hyperviscosity. Symptoms may include seizures, confusion, cognitive decline, headache, blurry vision, pain, numbness, paresthesias, hearing loss, or weakness. Serum laboratory tests show macroglobulinemia, normocytic anemia, IgM kappa or lambda light chain restriction. CSF analysis shows lymphocytic pleocytosis of $100-500 \mathrm{cells} / \mathrm{cu} \mathrm{mm}$, elevated protein 40-2000 $\mathrm{mg} / \mathrm{dl}$ and CD20+ lymphoplasmacytoid cells in flow cytometry and cytology. MRI shows T2/FLAIR hyperintensity and gadolinium enhancement. Remission in BN syndrome has been reported with the use of intrathecal Methotrexate alone, or with radiation therapy.

BLISTER IN THE SUN: A CASE OF PORPHYRIA CUTANEA TARDA Judith Griffin; Regina Lee. Montefiore Medical Center, New York, NY. (Control ID \#1334532)

LEARNING OBJECTIVE 1: Discuss the presentation and diagnosis of porphyria cutanea tarda

LEARNING OBJECTIVE 2: Review the relationship between PCT and hepatitis $\mathrm{C}$

CASE: A 67 year-old man with a history of hypertension and end stage renal disease on hemodialysis presented with a blistering skin rash on his hands and face. Two weeks prior, the patient noticed blisters on his right and left hands; a blister on his lower lip developed and burst. The lesions were painful but not pruritic. On exam the patient's blood pressure was elevated, but he was afebrile and vital signs were otherwise normal. His non-dermatologic exam was normal. The skin exam revealed a swollen, hypopigmented lower lip, and multiple fleshtoned plaques and ulcers on palmar and dorsal hands. No vesicles were intact, and there were no lesions on his trunk, lower extremities, or genitals. An HIV ELISA was negative, but the hepatitis C (HCV) viral load $>100,000 / \mathrm{mL}$. Fractionated plasma porphyrins were elevated with uroporphyrin $262.4 \mathrm{mcg} / \mathrm{L}(<0.2 \mathrm{mcg} / \mathrm{L})$, heptaporphyrin $214.4 \mathrm{mcg} / \mathrm{L}$ $(<0.2 \mathrm{mcg} / \mathrm{L})$, hexaporphyrin $7.8 \mathrm{mcg} / \mathrm{L}(<0.3 \mathrm{mcg} / \mathrm{L})$, pentaporphyrin $81.5 \mathrm{mcg} / \mathrm{L}(<0.4 \mathrm{mcg} / \mathrm{L})$ and coproporphyrin $12.8 \mathrm{mcg} / \mathrm{L}(<0.8 \mathrm{mcg} / \mathrm{L})$. Skin biopsy of the right hand was consistent with porphyria cutanea tarda (PCT). Based on the presence of elevated plasma porphyrins and characteristic skin biopsy, the patient was diagnosed with PCT.
DISCUSSION: PCT is a metabolic condition resulting from the deficiency of hepatic uroporphyrinogen decarboxylase, the fifth enzyme in the heme synthetic pathway. The deficiency leading to PCT can be hereditary or acquired and the incidence of PCT is one in 25,000 , typically occurring in patients over thirty. PCT has been described in patients on chronic hemodialysis, with the pathogenesis likely due to the lack of clearance of porphyrin precursors and their accumulation in the serum. Multiple studies have also revealed a strong association between $\mathrm{PCT}$ and $\mathrm{HCV}$, with significant geographic variation between regions depending on $\mathrm{HCV}$ prevalence. In North America, prevalence of $\mathrm{HCV}$ in patients with PCT may be as high as 66 percent. The mechanism linking the two is not known, but several have been proposed including release of iron from hepatocytes due to damage from HCV leading to oxidative stress. PCT is associated with accumulation of porphyrins in the liver, which typically result in mild elevations in serum alanine aminotransferase and aspartate aminotransferase. Porphyrins also accumulate in the skin and exposure to ultraviolet or visible light creates oxidized substances that activate collagenases, leading to disruption of cell components in the subepidermis and the formation of bullae on sun-exposed areas. Other dermatological findings include hypo- and hyperpigmentation and scarring also on sun-exposed areas. PCT should be suspected in any patient who presents with blistering on sun-exposed areas, especially if risk factors are present. The diagnosis is confirmed by demonstration of markedly elevated porphyrins in the plasma or urine. Patients diagnosed with PCT should also undergo testing for $\mathrm{HCV}, \mathrm{HIV}$ and iron overload. In patients with or without iron overload, the treatment of PCT is repeated phlebotomy. Low-dose hydroxychloroquine is an alternative therapy if phlebotomy is not tolerated. Supportive care, such as analgesia, avoiding sun exposure, and wearing protective clothing, is also recommended during therapy.

BLOOD TRANSFUSIONS IN PATIENTS WITH SICKLE CELL DISEASE: A DOUBLE EDGED SWORD Jonathan Kirsch; Pahresah Roomiany. University of North Carolina, Chapel Hill, NC. (Control ID \#1318358)

LEARNING OBJECTIVE 1: Recognize the clinical presentation of delayed transfusion reaction.

LEARNING OBJECTIVE 2: Differentiate delayed transfusion reaction from sickle cell pain crisis

CASE: A 21 year-old man with sickle cell anemia (SS disease) presented with acute lower extremity pain. He noted fatigue and subjective low-grade fevers. His symptoms began on the day of presentation and were constant. He did not have trauma to the legs, Wells score was zero and he did not have sputum production or cough. He had a history of acute chest syndrome and delayed hemolytic transfusion reactions. He was transfused with packed red blood cells for acute chest syndrome 1 week prior to admission. At that time hemoglobin rose from 3.8 to 6 grams per deciliter and hemoglobin A was low but present at $38.2 \%$ with a hemoglobin S of $55 \%$. A three out of six systolic murmur was heard loudest at the left lower sternal border. His lower extremities were globally tender to palpation. No rash, effusion or erythema was present. Chest $\mathrm{X}$ ray was normal. White blood cell count was $16 \times 10^{\wedge} 9$ per liter. Hemoglobin was 4 grams per deciliter and dropped to 3 grams per deciliter within two days. LD was 1600 units per liter and total bilirubin was $5.5 \mathrm{mg}$ per deciliter. Lactate dehydrogenase was 1500 units per liter and Coombs test was negative. Reticulocyte count was $10 \%$ and haptoglobin was less than 20 milligrams per deciliters. Hemoglobin A was no longer present and hemoglobin $\mathrm{S}$ was $92 \%$. He had developed a delayed transfusion reaction.

DISCUSSION: Sickle cell disease is commonly encountered by internists, particularly in the hospital setting. Recognizing complications of blood transfusions in this population is important for treating sickle cell disease in both the short and long-term. Delayed hemolytic transfusion reactions can be a life-threatening complication in patients with sickle cell disease. Exposure to red blood cell transfusion increases the likelihood of occurrence as antibodies to red blood cell antigens develop. Findings associated with delayed hemolytic transfusion reaction include pain, anemia and evidence of hemolysis. Delayed hemolytic transfusion reactions should remain on the differential when patients with sickle cell 
disease present with pain crisis. Inquiring about transfusion history and recent hospitalization is important. Diagnosis of hemolytic transfusion reaction is based on history, labs consistent with hemolysis and absence of hemoglobin A on electrophoresis. Once the diagnosis has been made, further red blood cell transfusions should be withheld unless critical. Treatment is aimed at improving anemia and suppressing the immune response to blood cells. As patients respond to therapy, their anemia will improve and reticulocyte count will increase appropriately.

\section{BOERHAAVE SYNDROME REVISITED: PROXIMAL ESOPHAGEAL} RUPTURE MANAGED CONSERVATIVELY Kalpana Nagarkar; Marta Herrera; Sundip Kaur; Ronald Warren; Joseph DeAntonio; Daniel Goldsmith. Capital Health Regional Medical Center, Trenton, NJ. (Control ID \#1319929)

LEARNING OBJECTIVE 1: Recognition of unexpected physical findings in Boerhaave Syndrome can lead to early diagnosis and management.

LEARNING OBJECTIVE 2: A subset of proximal esophageal ruptures is appropriate for conservative management and can present in unusual ways.

CASE: A 56 year old African American male patient presented to the ED with acute difficulty breathing and swallowing for a few hours. He reported that he woke up with sudden onset of breathlessness, and had difficulty swallowing and hence had to spit out repeatedly. He denied any history of chest pain or cough. He further reported vomiting multiple times the prior day associated with nausea and retching. Past medical history was pertinent for essential hypertension and alcohol abuse. Physical examination revealed that the patient preferred a sitting position and was constantly spitting. He was able to speak but the voice was muffled. Oral examination revealed swelling and a large bubble-like lesion of the right pharyngeal wall. Chest radiography was normal apart from mild elevation of the right hemidiaphragm. CT scan of the neck and chest revealed extensive free air tracking through the fascial planes of the neck, extensive mediastinal air tracking along the esophagus greatest in the oral pharyngeal regions surrounding the epiglottis and aryepiglottic folds suggestive of a proximal esophageal tear. The Gastrograffin esophagram showed focal out-pouching of contrast from the cervical esophagus. After conservative management, subsequent $\mathrm{CT}$ of the neck showed resolution of the air and repeat esophogram showed that the leak was contained and the patient recovered without further intervention.

DISCUSSION: Unexpected physical findings may be the key to the correct diagnosis. Proximal esophageal rupture with para-esophageal air tracking may be difficult to appreciate on physical examination, until the air reaches the oropharyngeal cavity and can be seen on oral inspection. Further, the patient's general appearance of preferring upright position and constant spitting suggests a proximal swallowing obstruction. Despite the dramatic and worrisome nature of the diagnosis, proximal esophageal ruptures are managed differently from distal, and can frequently be successfully treated with conservative observation.

BOWEL BEYOND BORDERS: A MASSIVE PARAESOPHAGEAL HERNIA MIMICKING A PULMONARY EMBOLISM Michael Farbaniec; James Lamberg; Ethan Kuperman. Penn State University Hershey Medical Center, Hershey, PA. (Control ID \#1339107)

LEARNING OBJECTIVE 1: Distinguish between the different types of hiatal/paraesophageal hernias and recognize less common presenting symptoms.

LEARNING OBJECTIVE 2: Understand the management of paraesophageal hernias.

CASE: An 87-year-old woman with gastroesophageal reflux disease but no history of cardiopulmonary disease presented with a fracture of her right femoral neck after a mechanical fall. The night of her admission, she became acutely short of breath, tachycardic, and developed increased oxygen requirements. Chest auscultation revealed decreased breath sounds along the left lung field but occasional bowel sounds could be heard in the bases and midlung fields bilaterally. With initial suspicion for pulmonary embolism, computed tomography of her chest was performed. No pulmonary embolism was identified but a $7 \times 10 \mathrm{~cm}$ type IV paraesophageal hernia was revealed, which included the entirety of her stomach and pancreas, portions of the duodenum, the splenic flexure of the colon, and associated vasculature. She remained stable after conservative treatment and was taken to surgery the following day for a right hip hemiarthroplasty. The procedure was uncomplicated and she was extubated successfully. After extubation, her oxygen requirements had diminished. Cardiothoracic surgery was consulted for management of her large paraesophageal hernia. The patient decided to decline any further surgical intervention at this time and remained asymptomatic upon discharge.

DISCUSSION: Hiatal hernias are classified into four different types. Type I classifies sliding hernias and accounts for $95 \%$ of hiatal hernias while II, III, and IV are collectively known as paraesophageal hernias. Type II classifies herniation of the stomach through the hiatus while the gastroesophageal junction remains below the diaphragm. Type III is classified by the gastroesophageal junction herniating through the diaphragm. Type IV is classified by any other intraadbominal organs besides the stomach herniating through the diaphragm. Most common symptoms include reflux and vomiting but about 3\% may develop dyspnea. Fewer than $8 \%$ of paraesophageal hernias involve viscera beyond the stomach. The small bowel and colon are often involved, but only five previous cases have reported pancreatic involvement. This patient's unique hernia is the only documented paraesophageal hernia to contain the entirety of the pancreas as well as being one of the largest currently documented in the literature. Management of these hernias has been controversial. A majority of past publications suggest correction of all paraesophageal hernias regardless of symptomatology. However, large database studies show a mortality rate of $1.4 \%$ in elective repair but only $1.1 \%$ developed acute symptoms requiring emergent surgery. Indications for elective surgery include failed medical management of reflux or persistent vomiting. Indications for emergent surgery include volvulus, obstruction, bleeding, and respiratory failure. In this case, the patient's dyspnea resolved after her surgery and she remained asymptomatic. This did not make her a candidate for elective surgical repair.

BROKEN-HEARTED? NON-ACS CAUSES OF ELEVATED TROPONINS Cady Blackey. Tulane University Health Sciences Center, New Orleans, LA. (Control ID \#1311854)

LEARNING OBJECTIVE 1: 1. Identify etiologies of elevated troponins 2. Determine the most likely cause of an elevated troponin level 3. Identify the utility and limitations of troponin as a diagnostic tool

CASE: A 44 year-old woman presented with severe, sub-sternal chest pain that radiated to her left arm. The pain was associated with diaphoresis and relieved with nitroglycerin. She denied prior episodes of chest pain, and she reported no recent stress. She was a nonsmoker, but her mother had died of a myocardial infarction at the age of 53. The patient had been recently diagnosed and treated for right thigh myosarcoma Her vital signs were normal. She was an overweight, slightly anxious woman. Her cardiovascular exam was normal, and her lungs were clear; she had no jugular venous distension or peripheral edema. Her chest pain was not reproducible and not affected by position. The EKG revealed a normal sinus rhythm without ST changes or Q waves, but her troponin was 6.75. She was treated for a non-ST elevation myocardial infarction; her troponin levels peaked at 12 . A left heart catheterization and coronary angiography revealed patent coronary arteries and normal pressures with focal hypokinesis of the mid-anterolateral wall. The echocardiogram was normal.

DISCUSSION: Coronary artery disease (CAD) affects 17.6 million Americans, making it one of the most common diagnoses encountered by the general internist. Highly sensitive troponin assays have revolutionized the diagnosis of acute coronary syndrome (ACS); however, this test is not a sufficient substitute for sound clinical reasoning. The physician must suspect other etiologies when the clinical picture is inconsistent with ACS. General internists should be equipped with a differential diagnosis for common non-ACS etiologies of elevated troponins. Causes include pulmonary embolus, congestive heart failure, sepsis, cardiac arrest, cardiac 
ablation/cardioversion, stroke, renal failure, cardiac contusion, pericarditis and myocarditis. The presence of risk factors for atherosclerosis, typical chest pain, and ischemic changes on echocardiography or electrocardiogram increase the probability of cardiac ischemia. In the absence of these signs and symptoms, a thorough history and physical exam can identify most non-ACS causes of elevated troponin. Securing the proper diagnosis prevents unnecessary intervention and allows for appropriate treatment. Very rarely, a thorough history and exam fail to reveal an alternative source for elevated troponin. Both tropnin isomers, $\mathrm{cTnI}$ and $\mathrm{cTnT}$, are specific for cardiac muscle; however, earlier generation assays have detected elevated troponin levels in circumstances when skeletal muscle is damaged and regenerating. Purely analytical error can also cause false positive troponin results in as many as 1/2000 assays, due to cross reactivity between cTnI assay components and a product in the patient's blood (rheumatoid factor, heterophile antibodies, macroglobulin, fibrin clots, bilirubin). In a patient with muscle disease, it is important to consider the possibility of cross-reactivity of abnormal skeletal muscle with the troponin assay. Such was the case in our patient who had recently underwent surgical resection and chemotherapy for a sarcoma. Though troponin levels are helpful in ruling out cardiac ischemia, elevations in troponin are not specific for ACS. Physicians cannot allow themselves to be lured towards a convenient conclusion without first developing a differential diagnosis.

BRONCHOMEDIASTINAL FISTULA PRESENTING AS PURULENT PERICARDITIS IN A HEALTHY 32 YEAR-OLD MAN Aiman M. Smer; Jamil Abuzetun; Mahmoud A. Abu Hazeem; Alok Saurav; Aryan Mooss. Creighton University Medical Center, Omaha, NE. (Control ID \#1293075)

LEARNING OBJECTIVE 1: Recognize the clinical features and complications of bronchomediastinal fistula

CASE: A previously healthy 32-year-old Hispanic man presented with acute onset retrosternal chest pain associated with subjective fever, dry cough, and dyspnea on exertion. Physical examination was remarkable for pericardial friction rub. Laboratory studies showed leukocytosis with left shift and elevated ESR. The electrocardiogram revealed sinus tachycardia with diffuse ST segment elevation, and PR segment depression consistent with the diagnosis of acute pericarditis. Patient was treated with Ibuprofen. On day 2 of admission, the patient's clinical condition deteriorated and became more dyspnic and tachypnic. Transthoracic echocardiogram showed moderate pericardial effusion with no evidence of tamponade (Figure 1). Computed tomographic scan of the chest revealed left bronchomediastinal fistula with mediastinal adenophathy, along with moderate pericardial and bilateral pleural effusions (Figure 2). A rigid bronchoscopy confirmed the presence of $1 \mathrm{~cm}$ in size left bronchomediastinal fistula (Figure 3). Thoracocentesis was performed and $130 \mathrm{ml}$ of pus was drained. Both Blood culture and pleural fluid culture grew eikenella corrodens. The patient was started on intravenous ampicillin after sensitivity and susceptibility testing. There was no obvious cause for the bronchomediastinal fistula, especially that the patient never had symptoms of respiratory or oropharyngeal disease or previous trauma. Barium swallow study and esophagogastroduodenoscopy were normal. The patient underwent operative drainage of the pericardial sac, debridement and decortications of the left lung, and placement of silicone stent at the left main bronchus (Figure 4). He completed four weeks of intravenous ampicillin. The patient's clinical condition continues to improve and he was discharged home three weeks after the surgery. The bronchial stent was removed six weeks later during follow up visit.

DISCUSSION: Acute purulent pericarditis is rarely caused by anaerobic bacteria and is almost always a complication of another disease. In our case mediastinitis due to spontaneous bronchomediastinal fistula was found to be the primary source of infection. Esophagomediastinal fistula, odontogenic, or pleuropulmonary infections have been reported to be the primary source of purulent pericarditis. To our knowledge this is the fourth case of eikenella corrodens pericarditis reported in the literature. This case illustrates the importance of a diligent search for the primary source of infection in order to diagnose and treat these patients properly. Treatment of purulent pericarditis hinge on pericardial fluid drainage and appropriate antibiotic therapy
CAN BUPRENORPHINE MAKE YOUR LIVER ANGRY? Houman

Sharifi; Thomas Daniel; Nazrul Chowdhury; Matt Chua. Texas Tech University of Health Sciences, Amarillo, TX. (Control ID \#1324418)

LEARNING OBJECTIVE 1: Recognize clinical features and laboratory results of Buprenorphine induced hepatitis in patients on therapeutic dose of this medication.

LEARNING OBJECTIVE 2: Recognize rare side effects of Buprenorphine and its mechanism to cause these side effects since its wide spread use to control opiate withdrawal symptoms.

CASE: 48 year old white female admitted to psychiatric department for opiate withdrawal symptoms. She has past medical history of bipolar disease, hepatitis C, GERD and prescribed opiate dependency for years. She was on multiple medications including trazodone, clonazepam, duloxetine and hydrocodone/acetaminophen. She was abusing hydrocodone/acetaminophen and her last use was 3 days prior to admission. Her basic labs and liver function test was normal prior to admission. She was started on buprenorphine $0.3 \mathrm{mg}$ IM every 8 hours. She developed tremor, mild nausea and right upper quadrant pain on the second day of treatment. It was the first time she had buprenorphine in a treatment program. On physical exam her temperature was 98.4 , blood pressure of $112 / 71 \mathrm{mmHg}$, heart rate of $78 \mathrm{bit} / \mathrm{min}$ and respiratory rate of 18 . She had unintentional tremor but no asterixis and right upper quadrant tenderness without hepatomegaly. Laboratory data showed AST of $759 \mathrm{U} / \mathrm{L}$ and ALT of $1524 \mathrm{U} / \mathrm{L}$, Alkaline phosphatase of $150 \mathrm{U} / \mathrm{L}$. She had normal Bilirubin Total and Amylase/Lipase. Her acetaminophen level was within normal limits. Her right upper quadrant ultrasound was normal. Buprenorphine induced acute hepatitis was suspected and the drug was discontinued and supportive care initiated, her abdominal pain and extremity tremor subsequently improved and liver function test on next day showed trending down of enzymes. She was discharged home after 6 days and later follow up care after 2 weeks showed her liver function test back to normal.

DISCUSSION: Buprenorphine is a semi-synthetic opioid derived from thebaine which is an alkaloid of opium. It has partial agonist antagonist effect on mu receptor and some effects on kappa receptor. It has been approved for opiate dependency detoxification and maintenance therapy by FDA in 2002 with or without Naloxone in sublingual form but using IM form is also common in psychiatric wards. Common side effects of buprenorphine include: nausea and vomiting, dry mouth, constipation, insomnia, drowsiness, dizziness, orthostatic hypotension and sweating. Among less common but more serious side effects are acute hepatitis and renal failure, respiratory depression, bronco-spasm, angioneurotic edema and anaphylactic shock. Buprenorphine is mainly metabolized in liver by cytochrome P450 (CYP) 3A4 and less than 10\% is excreted by the kidney. Studies showed that buprenorphine concentrates within mitochondria and it uncouples and inhibit mitochondrial respiration and also inhibits fatty acid beta-oxidation in higher serum levels causing liver toxicity. Although liver toxicity is a rare adverse effect of buprenorphine but with widespread use of this medication, it is strongly recommended that patients with risk factors like chronic viral hepatitis, concurrent use of alcohol or $\mathrm{CYP} 3 \mathrm{~A} /$ mitochondrial competitor drugs should undergo liver function test within the first week of treatment of Buprenorphine.

CAN'T DIAGNOSE HEPATIC FAILURE? BETTER HODG(KINS) YOUR BETS Ahmed Mohiuddin; Casey Dunn. Tulane University Health Sciences Center, New Orleans, LA. (Control ID \#1339245)

LEARNING OBJECTIVE 1: 1 . Recognize the clinical signs and symptoms of Hodgkin's lymphoma-related idiopathic cholestasis. 2. Understand the implications of cholestasis on prognosis of Hodgkin's lymphoma 3. Identify treatment options

CASE: A 19 year-old woman presented with two weeks of fever, nausea, bloody vomitus and worsening jaundice. She was not taking any medications and denied any recent drug or alcohol use. She has a history of anemia diagnosed at childbirth, but no other past medical history. She has a heart rate of 112 beats $/ \mathrm{min}$; the remaining vital signs were normal. Her cardiac and pulmonary examinations were normal. She had diffuse jaundice, including scleral and sublingual icterus. The abdominal examination was normal with no tenderness or hepatomegaly. She had a diffuse macular 
rash extending from her abdomen to her lower back. Her total bilirubin was 9.0, the direct bilirubin was 6.0, and her AST and ALT were 540 and 805; the alkaline phosphatase was 1375 . Acetaminophen and salicylate levels were normal; her INR was 2.0. A viral hepatitis panel was normal. An ultrasound of her abdomen was revealed a contracted gallbladder, but no focal dilatation. An MRCP and EGD were without any abnormalities. A CT of the abdomen revealed multiple anterior mediastinal soft tissue masses, the largest one measuring $8 \times 7 \times 5 \mathrm{~cm}$. A subsequent biopsy confirmed Hodgkin's lymphoma of the nodular sclerosing subtype.

DISCUSSION: The general internist is commonly confronted with acute hepatic failure, often without a clear etiology on presentation. Hodgkin's lymphoma-related idiopathic cholestasis is a rare but often lethal cause of hepatic failure that must be recognized quickly for appropriate treatment. Hodgkin's lymphoma-related idiopathic cholestasis often presents as hepatic failure of unknown etiology. The patient is often jaundiced and has other signs of hepatic failure including nausea and vomiting. The diagnosis should be considered after the more common etiologies of viral and toxic hepatic failure have been excluded. Importantly, the general internist should recognize that the cholestasis due to lymphoma-induced liver failure can be deadly. Although a third of patients with this syndrome survive with reversible hepatic injury, most will die due to fulminant liver failure or sepsis. Early recognition and treatment of the lymphoma with a hepatic sparing chemotherapy regimen is the most aggressive and effective treatment for this condition. It is imperative to identify early and treat Hodgkin's lymphoma-related idiopathic cholestasis as this rare disease is often fatal but can be reversible with appropriate treatment.

CAN'T HEAR, CAN'T SEE, AND MY NOSE BLEEDS. NOT YOUR USUAL PRESENTATION OF MULTIPLE MYELOMA. Phyllis Kim; Jeffrey Miller. UCLA-Olive View Medical Center, Sylmar, $\overline{\text { CA. (Control }}$ ID \#1339599)

LEARNING OBJECTIVE 1: Recognize hyperviscosity syndrome as a presentation of multiple myeloma.

CASE: A 56 year old male presents with 4 months of dizziness, fatigue, recurrent epistaxis, bilateral vision loss, and unilateral hearing loss. Initial workup revealed anemia (Hgb $7.9 \mathrm{~g} / \mathrm{dL}$ ), mild thrombocytopenia, coagulopathy, hypoalbuminemia, elevated total protein $(10.8 \mathrm{~g} / \mathrm{dL})$, and a large $\mathrm{M}$ spike on SPEP $(3.2 \mathrm{~g} / \mathrm{dL})$. Fundoscopic exam revealed bilateral central retinal vein occlusion with significant macular edema. The constellation of symptoms was concerning for hyperviscosity syndrome. The patient was immediately started on plasmapheresis and had a marked clinical and laboratory response. A serum viscosity level was later confirmed to be 24.1 $\mathrm{cp}$ (normal 1.4-1.8 cp). Despite nondiagnostic bone marrow biopsies, the patient had compelling evidence for a diagnosis of Multiple Myeloma (MM) by virtue of a monoclonal protein (IgG kappa monoclonal band), hyperviscosity syndrome, and myeloma related end organ damage (anemia, severe osteoporosis). A full body CT scan excluded extramedullary involvement or lymphoma. Peripheral blood smear revealed rouleaux formation. Based on both the Durie-Salmon and International Staging System, the patient has symptomatic Stage III IgG kappa Multiple Myeloma. Total protein levels were noted to rise while not receiving plasmapheresis and induction chemotherapy was begun.

DISCUSSION: Multiple Myeloma classically presents with the CRAB symptoms (hypercalcemia, renal insufficiency, anemia, bone lesions), however up to $20 \%$ of patients with MM present with a variety of other non-CRAB manifestations. These include hyperviscosity syndrome, neurologic disease, extramedullary plasmacytomas, hemorrhage/coagulopathy, generalized weakness, weight loss, and infection. This case demonstrates hyperviscosity as an uncommon presentation of myeloma. Hyperviscosity is more frequently associated with monoclonal hypergammaglobulinemia as seen in the plasma cell dyscrasias Waldenström's macroglobulinemia and MM. However, the incidence of symptomatic hyperviscosity is significantly higher in Waldenström's macroglobulinemia $(10-30 \%)$ as compared to myeloma (2-6\%). Symptoms usually develop with serum viscosity levels exceeding $4-5 \mathrm{cp}$. Clinical features include constitutional symptoms, bleeding, as well as ocular, neurologic, and cardiovascular disease. Bleeding occurs due to paraprotein coating of platelets and clotting factor, especially Factor X. Ocular symptoms include blurred vision or diplopia. Uncommonly, central retinal vein occlusion and retinal detachment can occur. Impairment of the CNS microcirculation due to hyperviscosity results in headache, dizziness, impaired hearing, seizure, stroke, and coma. High output heart failure may develop as a result of expanded plasma volume. In symptomatically hyperviscous patients, immediate therapy with plasmapheresis is directed at reducing paraprotein levels. As viscosity is logarithmically related to protein levels, even a modest reduction in proteins results in dramatic symptom relief. Transfusions should be avoided in anemic patients until after plasmapheresis as this may aggravate hyperviscosity and trigger lethal complications. Long term management is aimed at treating the underlying disorder and reducing paraprotein disease burden.

CARDIAC TAMPONADE FROM CHYLOPERICARDIUM Syed Zaidi; Jose Cuellar-Silva; Christopher Pallas; Vincent Robinson; Deepak Kapoor. Georgia Health Sciences University, Augusta, GA. (Control ID $\# 1315588)$

LEARNING OBJECTIVE 1: Diagnose chylopericardium, a rare cause of pericardial effusion in patients with end stage renal disease.

LEARNING OBJECTIVE 2: Recognize unsual complication of catheter induced thrombosis of central veins.

CASE: 32 year old black female with end stage renal disease on hemodialysis presented to emergency department with dyspnea for 1 week. She had a left brachiocephalic vein thrombosis 5 months ago as a complication from tunneled catheter in left Internal Jugular vein and was treated with removal of catheter and mechanical thrombectomy. She has no history of trauma or cardiac surgery. She was afebrile, tachycardiac and hypotensive. She had elevated jugular venous distention, distant heart sounds and bibasilar crackles. An echocardiogram showed large pericardial effusion with tamponade physiology. She underwent pericardiocentesis and $500 \mathrm{ml}$ of fluid was drained. Fluid analysis showed milky colored fluid, elevated triglycerides $(2160 \mathrm{mg} / \mathrm{dl})$, cholesterol $(101 \mathrm{mg} / \mathrm{dl})$, elevated lymphocytes (98\% of white blood cells) with negative cultures. Lymphoscintigraphy didn't reveal lymphatic drainage into the pericardial space. She continued to drain fluid for 4 days through pericardial catheter. A repeat echocardiogram showed small pericardial effusion with no hemodynamic compromise. Unfortunately, patient's pericardial drain came out and she insisted to leave without undergoing further evaluation. She was sent home on a low fat diet enriched with medium-chain triglycerides.

DISCUSSION: Chylopericardium is composed of chyle, the normal content of lacteals and thoracic duct Chylopericardium may be idiopathic or most commonly results from obstruction or injury to the thoracic duct. The diagnosis is confirmed by analysis of the pericardial fluid, which is milky and opaquea with a triglyceride level greater than $500 \mathrm{mg} / \mathrm{dL}$, cholesterol/triglyceride ratio of less than 1 , negative cultures and lymphocyte predominance. Catheter induced thrombosis was the probable cause of chylopericardium in this patient. The hypothesized mechanism of chylopericardium formation is obstruction of the thoracic duct ostium with presumed reflux of chyle back into the pericardium. Lymphoscintigraphy has poor sensitivity to show a macroscopic connection between the pericardium and thoracic duct. Substantial leakage of chyle causes serious nutritional, metabolic, and immunologic abnormalities. In addition, cardiac complications can occur including cardiac tamponade, acute pericarditis or chronic constriction. Chylopericardium without treatment is associated with a high mortality rate. Treatment varies with the etiology and includes adherence to low fat diet enriched with medium-chain triglycerides, ligation of thoracic duct and pericardiotomy or pericardiectomy. In cases of thrombosis-associated chylopericardium treatment is similar. Additional treatment includes removal of the indwelling catheter if present, anticoagulation, thrombolytics, mechanical thrombectomy as well as stent placement within a thrombosed vessel. Chylopericardium, although rare, should be included among the serious sequelae of catheter induced thrombosis of central veins.

CARDIOMYOPATHY: A RARE BUT SERIOUS COMPLICATION OF SCLERODERMA Damanpreet K. Grewal; Sara Taherkhani; Brent Hardman; Arpit Bhargava. Allegheny General Hospital, Pittsburgh, PA. (Control ID \#1340056) 
LEARNING OBJECTIVE 1: Importance of recognizing scleroderma as a multi-organ condition.

LEARNING OBJECTIVE 2: Early treatment of Cardiomyopathy due to scleroderma can reduce morbidity and mortality associated with this condition.

CASE: A 43 year-old Caucasian female with a 3-year history of untreated systemic sclerosis presented with 2 weeks of shortness of breath associated with chest heaviness, nausea and decreasing exercise tolerance. Upon further review, patient revealed multiple visits to local ER over the last 8 months for chest pressure and palpitations with elevated cardiac enzymes and BNP at each visit. An Echo and PET CT scan done 2 months after initiation of symptoms were normal. A prior cardiac catherization from late 2007 was also normal. These visits were attributed to anxiety and panic attacks. Initial work-up, including a CT Angiogram, was negative for a PE, but significant for pleural effusions, dilated esophagus, cardiomegaly, mediastinal adenopathy and pericardial effusion. Again, the patient was noted to have elevated cardiac enzymes, with an echocardiography significant for an EF of $5-10 \%$. A cardiac MRI revealed an LVEF of $8 \%$ and RVEF of $28 \%$ with a non-coronary distribution pattern and abnormal late-gadolinium enhancement, including prominent lateral and inferior epicardial enhancement, typical of a connective tissue inflammatory disorder. Rheumatology was consulted and patient was started on $1 \mathrm{~g}$ solumedrol daily for a total of 3 days. Subsequently, she underwent a cardiac catherization, significant for moderately elevated right atrial and pulmonary artery wedge pressures, and a normal cardiac output. A biopsy of the heart tissue was inconclusive and showed occasional hypertrophic myocytes. At this point, IV Cytoxan was initiated with significant improvement in patient's symptoms. A repeat echocardiogram prior to discharge showed an EF of $15-20 \%$ and the patient was discharged with a life-vest. DISCUSSION: Systemic sclerosis is a rare condition characterized by immune activation, vascular damage and buildup of collagen in the extracellular matrix. The peak onset occurs at 30-40 years of age with an annual incidence of 200 per 100,000. It affects women more than men, with a female-to-male ratio of 3-6:1. The condition typically involves the skin, but can frequently occur in other organs. Although rare, clinically significant cardiomyopathy associated with this condition is rare, but early diagnosis using frequent evaluation of cardiac enzymes, BNP and echocardiography can help with timely initiation of aggressive treatment and improved outcomes. This case demonstrates the need for close surveillance of patients with scleroderma given the possibility of internal organ involvement and significant morbidity and mortality benefits.

CATHECHOLAMINe CATASTROPHE Jodie Bryk; Peggy Hasley; Hollis Day. University of Pittsburgh, Pittsburgh, PA, PA. (Control ID \#1317155)

LEARNING OBJECTIVE 1: 1 . To list the "5 Ps" of pheochromocytoma 2. To describe tests for pheochromocytoma in low and high risk patients 3 . To summarize the pre-operative management of pheochromocytomas CASE: A 34 year old presented for establishment of primary care. At her initial appointment she complained of panic attacks for the past two years. During these episodes, she experienced palpitations, headaches, chest tightness, perspiration, and tremulousness. She had been previously treated with multiple benzodiazepines and antidepressants for presumed anxiety and depression without relief of her symptoms. Her blood pressure was found to be elevated to $180 /$ $120 \mathrm{mmHg}$ and her fasting blood glucose was $158 \mathrm{mg} / \mathrm{dL}$. Her plasma free metanephrines were elevated to $2132 \mathrm{mcg}(\mathrm{Nl}<57)$. A 24 -h urinary fractionated metanephrines and catecholamines were significantly elevated. MRI of the abdomen and pelvis showed a $5.7 \times 5.3 \times 5.7 \mathrm{~cm}$ spherical well-circumscribed left adrenal mass. MIBG scan showed no additional extraadrenal involvement. She underwent laparoscopic removal of the left adrenal gland mass after pre-operative management with phenoxybenzamine and then propranolol. Pathology showed an encapsulated pheochromocytoma. Postresection, her plasma free metanephrines decreased to $25 \mathrm{mcg}$. Her mood significantly improved and she was able to discontinue all psychiatric medications. She underwent complete sequencing of the Von Hippel Lindau, RET, neurofibromatosis 1, and familial paraganglioma genes whereupon an inherited predisposition to pheochromocytoma was ruled out.
DISCUSSION: Pheochromocytomas present clinically with the "five Ps" of pressure, pain, palpitations, perspiration, and pallor. If a patient is suspected to be high risk of having a pheochromocytoma, plasma free metanephrines is the test of choice given its $99 \%$ sensitivity. However, if a patient is suspected to be at low risk for pheochromocytoma, 24-h urinary fractionated metanephrines and catecholamines is the preferred test given its higher specificity. It is important to note that acute illness, tricyclic antidepressants, and labetalol may interfere and cause false positives for both tests. Treatment of pheochromocytoma involves first alpha blockade with phenoxybenzamine followed by beta-blockade with propranolol immediately prior to laparoscopic removal. Plasma free metanephrines and chromogranin A levels are monitored post-operatively. All patients with diagnosed pheochromocytoma should be referred for genetic counseling for an extensive evaluation for hereditary causes of pheochromocytoma including MEN syndromes, von Hippel-Lindau, neurofibromatosis, and succinate dehydrogenase abnormalities.

CAUGHT IN THE ACT: THROMBUS IN TRANSIT WITH PARADOXICAL EMBOLISM Rajan Garg; Daniel Goldsmith; Bipinpreet Nagra. Capital Health Regional Medical Center, Trenton, NJ. (Control ID \#1336951)

LEARNING OBJECTIVE 1: Significance of early diagnostic intervention using Echocardiogram in patients presenting with symptoms of Stroke LEARNING OBJECTIVE 2: Treatment modalities for Paradoxical Embolism

CASE: A 72 year old male with recent hospitalization for TIA presented with left hemiparesis. Workup revealed an acute large right MCA stroke with small hemorrhagic transformation in the right frontal lobe. A right lower extremity DVT was also found. Transthoracic echocardiogram (TTE) revealed long mobile thrombi in both left and right atria extending into both ventricles. TEE done subsequently the same day revealed no thrombus in the ventricles, but emboli were seen in the pulmonary artery and multiple large mobile emboli in the descending aorta along with a large PFO. The patient could not be treated with IPA secondary to the hemorrhagic conversion of stroke. Subsequent CT scan of chest revealed bilateral pulmonary emboli for which an IVC filter was placed. The patient also developed acute left leg ischemia from aortic emboli and underwent urgent thrombus retrieval. Because of multiple comorbidities and hemodynamic instability, the patient's family chose comfort measures and the patient expired on hospital day three.

DISCUSSION: Diagnosis of a paradoxical embolus usually is inferred from the presence of DVT, embolic phenomena and a PFO. The capture of a thrombus in transit by echocardiogram demonstrating movement of the thrombi from venous to arterial circulation confirms that our inferred pathophysiology is likely correct. Given the high mortality associated with paradoxical embolism, urgent anticoagulation, thrombolysis and/or embolectomy is required, if no contraindications.

CAUSES AND DANGERS OF NON-HEPATIC ACUTE HYPERAMMONEMIA Brita Roy. University of Alabama at Birmingham, Birmingham, AL. (Control ID \#1339767)

LEARNING OBJECTIVE 1: Recognize that acutely elevated serum ammonia levels can cause cerebral herniation and understand how to prevent/treat.

LEARNING OBJECTIVE 2: Emphasize non-hepatic causes of hyperammoniemia, and that some inborn errors of metabolism may present in adulthood.

CASE: A 41-year-old white woman with a history of bipolar disorder presented initially to an outside hospital with nausea, vomiting diarrhea and encephalopathy for 3 days following exposure to a sick contact. She then became progressively somnolent and was intubated on day 2 for airway protection and vasopressors were initiated for hypotension. Her husband denied a history of ingestions, suicidal ideation or alcohol abuse. Her family history was positive only for coronary disease and diabetes mellitus. She was transferred to our hospital on day 3 , and at that time vital signs were temperature $100 \mathrm{~F}$, heart rate 114 , respiratory rate 37 , blood 
pressure 124/63 (on vasopressors), and oxygen saturation $98 \%$ on $40 \%$ FiO2. Examination revealed crackles in the right middle lung field; abdomen was benign without organomegaly; neurologic exam revealed positive corneal reflex, $3+$ reflexes throughout, downgoing toes. Laboratory evaluation was significant for a serum $\mathrm{pH} 7.29, \mathrm{pCO} 2$ 22, pO2 208, pHCO3 10.6, anion gap 26, lactic acid 3.1, creatinine 1.7 , white blood cell count 6.18 with $92 \%$ neutrophils, ALT 75, AST 255, alkaline phosphatase 49, creatine kinase 9805, amylase 733, lipase 15, and ammonia of 547. Chest roentenogram revealed a right middle lobe infiltrate and broad spectrum antibiotics were initiated for septic shock. Due to the severity of hyperammonemia, dextrose infusion, lactulose, rifaximin, sodium benzoate-sodium phenylacetate and arginine were administered to prevent intracranial hypertension. Urine and plasma amino acid levels were examined for evidence of an inborn error of metabolism which were consistent with isovaleric acidemia.

DISCUSSION: Independent of the cause, acute elevation of serum ammonia (NH4) levels above $200 \mu \mathrm{mol} / \mathrm{L}$ can precipitate intracranial hypertension and cerebral herniation. Thus, agents to peripherally convert ammonia such as sodium benzoate-sodium phenylacetate should be rapidly administered in addition to lactulose and rifaximin to reduce serum ammonia levels quickly. If these measures are ineffective, hemodialysis may be initiated. Additionally, administration of mannitol and/or hypothermia may be considered to reduce intracranial pressure. Administration of intravenous dextrose to prevent muscle breakdown and subsequent elevation of $\mathrm{NH} 4$ is also important. Of note, unlike acute hyperammonemia, chronic elevation of NH4 has much lower risk of cerebral edema. If hyperammonemia is thought not to be due to acute hepatic failure, workup for alternate causes should be initiated. Non-hepatic causes of elevated NH4 include infection with herpes or urease-producing bacteria, muscle breakdown, starvation, gastrointestinal hemorrhage, and malignancies like multiple myeloma. Additionally, drugs such as HMG CoA reductase inhibitors, non-steroidal anti-inflammatory agents, antibiotics, antifungals, antiretrovirals, antiepiliptics, antidepressants and total parenteral nutrition can cause hyperammonemia. Finally, inborn errors of metabolism such as urea cyle disorders, organic academias (ie, isovaleric acidema diagnosed in our patient) and fatty acid oxidation defects may present with hyperammonemia in adults during times of metabolic stress.

CEREBRAL IMMUNE RECONSTITUTION INFLAMATORY SYNDROME SECONDARY TO NEUROSYPHILIS Zouyan $\mathrm{Lu}^{1}$; Jane N. Wainaina ${ }^{2} .{ }^{1}$ Medical College of Wisconsin, Milwaukee, WI; ${ }^{2}$ Medical College of Wisconsin, Milwaukee, WI. (Control ID \#1304683)

LEARNING OBJECTIVE 1: Describe the clinical presentation of immune reconstitution inflammatory syndrome

LEARNING OBJECTIVE 2: Review risk factors and treatment for development of immune reconstitution inflammatory syndrome

CASE: A 45 year-old gentleman with history of HIV presented with acute onset aphasia approximately 1 year after self-discontinuing his HAART. CT of the head showed acute ischemic stroke and potential vasculitis. Cerebrospinal fluid (CSF) analysis was consistent with meningitis, and serum free treponemal antibody was reactive. He was treated for meningovascular syphilis with 2 weeks of intravenous penicillin and restarted on his previous HAART of efavirenz and lamivudine/zidovudine. One day after completing treatment he returned with complaints of fever, neck pain and acute onset headache. CT scan showed intraventricular hemorrhage, new right parietal infarcts and findings consistent with vasculitis. He was started on empiric treatment with IV vancomycin, ceftriaxone, and acyclovir. Within 24 hours of admission, he had acute neurological decline, repeat imaging showed new infarcts. CSF was significant for elevated red blood cell count, low glucose and neutrophilic pleocytosis. Gram stain and culture were negative. CSF cryptococcal antigen, HSV PCR, and VDRL were also negative. His HIV viral load, when compared to values obtained 3 weeks prior, showed a $3 \log$ decrease and his CD4 increased from 30 to 52. Brain MRI showing more new infarcts and leptominingeal enhancement. Given his rapid decline in viral load as well as signs of continued vasculitis, there was concern for immune reconstitution inflammatory syndrome (IRIS). Empiric antibiotics were stopped and high-dose steroids were started with initial response. But the patient later developed step-wise decline in neurologic status when steroids were tapered down. The patient eventually became minimally responsive with extensor posturing. After discussion with his family, he was transferred to home hospice.

DISCUSSION: IRIS often presents with a paradoxical decline in a patient's clinical condition after initiation of HAART. IRIS is though to be the result of a pathologic response of the host's reconstituted immune system to antigens of opportunistic pathogens, including mycobacterial, fungal and viral infections. Some investigators have reported an incidence as high as $23 \%$ in patients starting HAART. The risk for developing IRIS appears to increase with the burden of opportunistic infections. IRIS should be suspected when patients present with unusual symptoms or disease course with a temporal relation to immunological recovery following initiation of HAART. It is most commonly described in association with mycobacterial, fungal, and viral infections. While, any organ system can be involved, reports of CNS IRIS are less common. There are no published trials for the treatment of IRIS, but case studies suggest appropriate treatment of the underlying opportunistic infection with or without the addition of corticosteroids.

CERVICAL LYMPHADENOPATHY IN A YOUNG, HEALTHY FEMALE: AN UNCOMMON DIAGNOSIS Amy Iwamaye; Stephen Gluckman. University of Pennsylvania, Philadelphia, PA. (Control ID \#1339505)

LEARNING OBJECTIVE 1: Recognize and diagnose other benign causes of persistent lymphadenopathy

CASE: A 29 year old female, with no significant past medical history, presented to her primary care physician complaining of 4 weeks of left-sided, non-tender, posterior cervical lymphadenopathy. She denied any fevers, chills, or preceding illness. She had ten pounds of intentional weight loss. On physical exam, she had multiple, enlarged lymph nodes, which were firm, rubbery, and mobile along the L posterior cervical chain. She had no other adenopathy. The remainder of her exam was within normal limits. Her laboratory tests including $\mathrm{CBC}$ and BMP were unremarkable. A fine-needle aspiration was performed and flow cytometry was negative for a clonal population. An excisional biopsy was performed which showed histiocytic necrosis with numerous foamy macrophages and occasional crescentic nuclei, consistent with necrotizing lymphadenitis. No Reed Sternberg cells were found. T-cell rearrangement studies excluded a T-cell lymphoma. Ultimately, the patient was given a diagnosis of Kikuchi's Disease. Her symptoms resolved within 1 month of her diagnosis without treatment.

DISCUSSION: Kikuchi's Disease is an uncommon, benign cause of cervical lymphadenopathy. It was first described in Japan and is often associated with young Asian women, but has been described in both sexes, with a slight female predominance, and has been found in various racial and ethnic groups. The etiology is unknown. There is histologic suggestion for infectious etiology, including HHV-6, EBV, and parainfluenza, although studies have failed to confirm this. Clinical symptoms usually include persistent cervical lymphadenopathy, but can be systemic, as well as fever. Most common lab abnormalities include leukopenia, anemia, and elevated ESR. The condition is generally self-limited and benign, and symptoms usually resolve in 1-4 months. Steroids have been used for treatment in symptomatic cases. It should be noted that pathologically this condition can be misdiagnosed for SLE and malignant lymphoma, leading to unnecessary treatment including, in some cases, chemotherapy. This case report serves to illustrate that Kikuchi's disease, an uncommon benign condition, should be included in the differential diagnosis of unexplained persistent localized lymphadenopathy.

CHEST PAIN IN A 21-YEAR OLD WITH SLE - WHEN TRADITIONAL MODELS BREAK DOWN Satish Misra; Grant Chow; Steven Schulman. Johns Hopkins, Baltimore, MD. (Control ID \#1311095)

LEARNING OBJECTIVE 1: Recognize SLE as an independent risk factor of coronary artery disease and acute coronary syndrome. 
LEARNING OBJECTIVE 2: Management of coronary artery disease in SLE patients.

CASE: A 21-year-old female with a history of systemic lupus erythematosus (SLE), treatment for which was self-discontinued three years prior, presented to the emergency room with 12 hours of pleuritic, sharp, left sided chest pain that worsened with laying supine. When an electrocardiogram revealed mild ST-elevations in the inferior leads and PR depression in the inferior and lateral leads, she was initially diagnosed with pericarditis. However, her pain worsened and a bedside echo revealed mild inferior and inferolateral wall hypokinesis. Coronary angiography revealed a $100 \%$ left circumflex artery occlusion, with diffuse disease noted in the left anterior descending and right coronary arteries. She underwent percutaneous coronary intervention (PCI) with thrombectomy and placement of two sequential drug-eluting stents in the left circumflex artery. She was subsequently treated with aspirin $325 \mathrm{mg}$, clopidogrel, and a low-dose beta blocker. An ACE-inhibitor could not be started due to low blood pressure, and statin therapy was discontinued due to rising liver function tests (LFT). Further evaluation revealed antiphospholipid syndrome, for which she was started on warfarin. Due to advanced atherosclerosis and newly diagnosed lupus nephritis, she was started on high-dose steroids, hydroxychloroquine, and mycophenolate mofetil. As an outpatient, she was initiated on atorvastatin that was rapidly uptitrated to maximum dose with frequent LFT monitoring. She was additionally started on lisinopril $2.5 \mathrm{mg}$ that has been slowly increased. Finally, she has received frequent dietary counseling and was initiated in a cardiac rehabilitation program.

DISCUSSION: There is a well-documented association between SLE and accelerated atherosclerosis, with the relative risk for patients with SLE to have nonfatal myocardial infarction or coronary artery disease-related death estimated to be 10 -fold and 17 -fold greater than what would be predicted by traditional risk factors. The etiology is thought to be related to the proinflammatory state of SLE, with proposed mechanisms including generation of pro-inflammatory high density lipoprotein (HDL) molecules and generation of LDL-containing antibody complexes. Recognition of SLE as an independent risk factor for coronary artery disease is critical for diagnosis of acute coronary syndrome (ACS) in patients who would otherwise be deemed 'low-risk' based on traditional scoring methods. Regarding management, aggressive risk factor modification per standard guidelines for coronary artery disease is critical. It is also imperative that any associated SLE complications, such as anti-phospholipid antibody syndrome (APS) or lupus nephritis, be aggressively treated due to further increased risk of coronary and vascular disease. There may be a cardioprotective effect of hydroxychloroquine in SLE patients, with conflicting data on steroids and azathioprine. In summary, this case of an atypical presentation of ACS in a young woman with SLE highlights a critical risk factor for patients who would otherwise be considered low-risk. Patients with SLE who present with chest pain should be managed with a high index of suspicion for cardiovascular disease.

CHEST PAIN WITH ST SEGMENT ELEVATION-BRUGADA SYNDROME-AN IMPORTANT NON-ISCHEMIC CAUSE IN WOMEN Manju Bengaluru Jayanna; Syed M. Mohiuddin; Janardhana Gorthi. Creighton University Medical Center, Omaha, NE. (Control ID \#1340523)

LEARNING OBJECTIVE 1: Recognize Brugada Syndrome as a differential in diagnosis of Chest pain with ST elevation.

LEARNING OBJECTIVE 2: Recognize Hypokalemia unmasks asymptomatic Brugada Syndrome.

CASE: 55 year Old Caucasian female with hypertension, hypothyroidism, recent laparoscopic cholecystectomy and pyelonephritis presented with two day symptoms of progressive exertional dyspnea, retrosternal chest pain radiating to her shoulder blades, subjective fever and fatigue .Examination was unremarkable except for a temperature of $99.3 \mathrm{~F}$ and few bilateral scattered wheezes. Lab data showed leukocytosis of 13,000; elevated D dimer of 4.98, hypokalemia of 2.7 and normal serum troponin. Urinalysis showed infection. EKG revealed new coved type ST segment elevation with $\mathrm{T}$ wave inversion in leads V1-V3 compared to her previous EKG. Echocardiogram was unremarkable with normal LV systolic function and no regional wall motion abnormalities.Emergent CT scan of chest was found to be normal.She was treated with IV potassium chloride and antibiotics for Urinary tract infection. Serial EKGs revealed complete resolution of ST segment elevation of the over the next 12 hours and her symptoms of chest pain resolved over the next 24 hours.CT abdomen revealed malposition of previously placed left double $\mathrm{J}$ stent and left renal stone and was treated surgically Since she had asymptomatic Brugada syndrome with no symptoms of syncope, no documented ventricular fibrillation, ventricular tachycardia and no family history of sudden cardiac death she managed with close follow up.

DISCUSSION: The patient's EKG changes were consistent with Brugada syndrome(BS) Type 1. This syndrome typically occurs in male patients in their twenties or thirties, and is characterized by distinct ECG changes in the right precordial leads (V1-V3) and susceptibility to ventricular fibrillation in the absence of structural heart disease. The Brugada pattern is much more common in men than in women, being as much as nine times higher in one analysis.BS is usually diagnosed in adulthood. The average patient at diagnosis is 41 years. It's much more common in Asian populations and prevalence in United states vary anywhere between 0.4 and 0.012 percent.EKG changes in BS are transient. Various provoking factors include pacing, vagal maneuvers, increased alpha-adrenergic drive, beta blockers, tricyclic antidepressants, lithium, local anesthetics, fever, hypokalemia, hyperkalemia, hypercalcemia, and alcohol and cocaine toxicity. Characteristic ECG abnormalities may be exposed by a sodium channel blocker, such as flecainide, thereby identifying those at risk. Possible provoking factor in our patient was severe hypokalemia. She had additional factors, including mild fever and increased sympathetic drive secondary to her proinflammatory state. The exact mechanisms by which Brugada pattern is uncovered by all the various provoking factors is yet be determined. There have been reports of Chest pain and ST elevation associated with fever in patients with asymptomatic BS.In conclusion it's important to consider BS in a patient who presents with chest pain and characteristic EKG pattern and avoid unnecessary invasive interventions, but rather treat underlying provoking factors emergently because ST elevation in BS may be a harbinger of malignant cardiac rhythms including ventricular fibrillation and sudden cardiac death.

CHOLESTASIS NOT DUE TO STASIS Stephanie Haimowitz ${ }^{1}$; Yelena Averbukh $^{2,1}$; Jennifer Hsieh ${ }^{2} .{ }^{1}$ Albert Einstein College of Medicine, Bronx, NY; ${ }^{2}$ Montefiore Medical Center, Bronx, NY. (Control ID \#1311805)

LEARNING OBJECTIVE 1: To consider Hydroxycut use as an inciting factor for hepatic failure.

LEARNING OBJECTIVE 2: To recognize a clinical syndrome consistent with Porphyria syndromes in the adult population as a cause for cholestatic liver failure.

CASE: A 23 year-old man with no significant past medical history except a few months of upper extremity photosensitive rash presented with nausea, vomiting and abdominal pain for four days. Remaining review of systems was negative, but the patient did admit to using the OTC weight loss remedy, Hydroxycut, for the past few months. Physical exam was significant for a toxic appearing man with temperature of $102 \mathrm{~F}$, heart rate of 125, marked jaundice, and right upper quadrant abdominal tenderness. Notable labs included WBC 40.3 , total bilirubin of $24.4 \mathrm{mg} / \mathrm{dL}$, conjugated bilirubin of $17.5 \mathrm{mg} / \mathrm{dL}$, alkaline phosphatase of 91, AST 194, ALT 92 and INR 2. Abdominal ultrasound showed no cholelithiasis or biliary duct dilatation. CT scan of the abdomen and pelvis was unremarkable. Extensive infectious disease work up, including bacterial, mycobacterial, viral, and parasitic etiologies, was non-diagnostic. Persistent leukocytosis and fevers led to work-ups for hematologic malignancies which were also non-diagnostic. Because the patient continued to deteriorate despite supportive care and discontinuation of Hydroxycut, other etiologies of liver failure were explored. Given his history of a photosensitive rash along with his cholestatic liver failure, it was hypothesized that he may have porphyria. Measurement of plasma porphyrin showed a total porphyrin level of $100.2 \mathrm{mcg} / \mathrm{L}$, (20 times the upper limit of normal), and a coproporphyrin level of $93.6 \mathrm{mcg} / \mathrm{L}$ (normal $<0.9 \mathrm{mcg} / \mathrm{L}$ ). Genetic testing identified a mutation in the cpox gene, which is suggestive of hereditary 
coproporphyria. Thus, the patient was diagnosed with porphyria, treatment with hemin was started, and the patient improved.

DISCUSSION: Garcinia, a component of Hydroxycut, has been associated with hepatotoxicity in 23 case reports, perhaps due to increased oxidative stress. Upon cessation of the drug, liver function is expected to improve within 7 weeks, normalizing by week 14 . However, because our patient did not improve as expected, we considered rarer etiologies as the cause of his liver failure. Porphyrias are a heterogenous group of metabolic syndromes that result from abnormal enzymatic activity in the heme biosynthetic pathway. In hepatic porphyrias like hereditary coproporphyria, neurovisceral symptoms, such as abdominal pain, motor neuropathy, hyponatremia, seizures and cutaneous manifestations such as photosensitivity, result from abnormal heme synthesis which is needed for the production of hepatic CYP enzymes. In these patients, an oxidative insult to the liver from the use of hepatotoxic medications like Hydroxycut, can rapidly induce acute porphyria symptoms despite discontinuation of the precipitating agent. However, an acute attack is reversible if treated promptly with intravenous hemin. Work-up includes testing urine, serum, or feces for elevated porphyrins. Definitive diagnosis is made via genetic testing and mutation analysis for specific abnormalities. Cholestatic liver failure is commonly seen by primary care physicians. Once common etiologies are ruled out, rarer causes should be pursued. While porphyrias account for less than $1 \%$ of all patients presenting with cholestatic liver failure, a timely diagnosis and treatment can be lifesaving.

CLINICAL CLUES TO RECOGNIZE A RARE CAUSE OF ACUTE PANCREATITIS Mansumeet Singh. Creighton University, Omaha, NE. (Control ID \#1311960)

LEARNING OBJECTIVE 1: Recognize clinical clues to diagnose Intraductal Papillary Mucinous Neoplasm (IPMN), a rare cause of Acute Pancreatitis (AP)

CASE: A 78 year old Caucasian male with history of Ischemic Cardiomyopathy and Diabetes was admitted with chest pain and dyspeptic symptoms. Initial work up for cardiac causes of chest pain was negative. Further investigations revealed elevated levels of Amylase (193 U/L) and Lipase $(698 \mathrm{U} / \mathrm{L})$, and normal liver function tests. A Computerized Tomography scan of the abdomen revealed an atrophic appearing pancreas and a dilated pancreatic duct. No history of alcohol use was elicited and the patient had undergone a cholecystectomy 20 years prior to presentation. There was no history of recent change of medications, or use of over the counter products, or herbal agents. Endoscopic ultrasonographic (EUS) exam of the pancreas was later undertaken as the etiology of pancreatitis was not clear. Findings of EUS and fine needle aspiration (EUS-FNA) were consistent with a diagnosis of main duct IPMN of the pancreas. The patient was referred for a surgical consultation; however he passed away within a few weeks due to cardiac related mortality.

DISCUSSION: 1) Main duct IPMN should be recognized as a rare etiology of AP once the common causes of acute pancreatitis have been ruled out. 2) Clinical clues for IPMN as the etiology of AP include initial presentation of AP in the elderly, history of diabetes, and presence of pancreatic duct dilation on transabdominal imaging studies. 3) EUS is a relatively noninvasive modality for the evaluation of AP with an unclear etiology.

CLOSTRIDIUM PERFRINGENS BACTEREMIA IN A PATIENT WITH PROSTHETIC AORTIC VALVE: ENDOCARDITIS OR COLON CANCER - THAT IS THE QUESTION? Amaninderapal S. Ghotra $^{1}$; Tanmay S. Panchabhai ${ }^{1}$; Raul Nakamatsu ${ }^{2}$. ${ }^{1}$ University of Louisville, Louisville, KY; ${ }^{2}$ University of Louisville, Louisville, KY. (Control ID \#1339824)

LEARNING OBJECTIVE 1: Discuss the differential diagnosis of gram positive bacilli in blood.

LEARNING OBJECTIVE 2: Review the association of colon cancer with bacteremia caused by bacteria from genus Clostridium.

CASE: A 66 year old male presented with symptoms of nausea, vomiting and fever since 3-4 days. The emesis was non-projectile, non-bloody and was associated with diarrhea with constipation, on and off for the past one month. The patient's past medical history was significant for a prosthetic aortic valve (for aortic stenosis), uncontrolled diabetes, chronic kidney disease and a negative screening colonoscopy one year prior to admission. The patient was febrile $\left(101.7^{\circ} \mathrm{F}\right)$ and physical examination revealed a $4 / 6$ ejection systolic murmur best heard in the aortic area, a distended abdomen with intact bowel sounds and negative rectal examination. An abdominal radiograph revealed partial abdominal obstruction. Initial laboratory data was unrevealing except left shift ( $85 \%$ neutrophils). Nasogastric suction with bowel rest was initiated and blood cultures were drawn in view of the new onset fever and prosthetic aortic valve. Preliminary gram stain revealed gram positive bacilli. With a working diagnosis of infective endocarditis with accompanying bowel obstruction, possible organisms being considered were Clostridium, Bacillus, Corynebacterium, Listeria, Rhodococcus and Nocardia. Intravenous Ampicilin-Sulbactam was initiated and a CT scan of the Abdomen/pelvis with oral contrast revealed a $10 \mathrm{~cm}$ circumferential rectal thickening surrounded by induration and stranding. Four blood cultures grew Clostridium perfringens and antibiotics were changed to high dose penicillin with clindamycin (for decreasing toxin production). A Transesophageal echocardiogram (TEE) did not reveal vegetations or abscess formation suggestive of endocarditis. In view of the negative colonoscopy done a year ago, infective and inflammatory colitis causing persistent bacteremia was higher on our differential. The patient was continued on antibiotics and had two negative blood cultures. A flexible sigmoidoscopy showed no grossly ulcerative/obstructive lesions, however, multiple cold forceps biopsies from the area of thickening seen on CT scan were positive for adenocarcinoma of the rectum. Bacteremia resolved with high dose penicillin which was continued for 14 days and the colon cancer was staged at IIIB. Being a poor operative candidate, platinum based chemotherapy was initiated.

DISCUSSION: Our case clearly defines the need for high suspicion in patients with gram positive bacilli causing persistent bacteremia as very few organisms like Clostridium, Bacillus, Corynebacterium, Listeria, Rhodococcus and Nocardia fall in to this category. Our patient presented with persistent bacteremia from Clostridium perfringens with a prosthetic aortic valve and rectal thickening increasing the diagnostic challenge as one case of clostridial endocarditis has been previously reported in literature. With Clostridium bacteremia, a detailed physical examination to look for any obvious source where the anaerobic organism can grow, especially feet, oro-genital areas and any infected wounds is warranted to formulate the initial approach for management. Genus Clostridium, especially the septicum species have been associated with colon cancer, the mechanisms being tumor necrosis, ulceration and bacterial translocation. Other clostridium species however have been implicated as well which needs to be kept in mind.

CLUBBING IN THE DIGITAL ERA Lynn Sauers; Thomas Jaeger. Mayo School of Graduate Medical Education, Rochester, MN. (Control ID \#1296845)

LEARNING OBJECTIVE 1: Recognize the clinical manifestations of hypertrophic osteoarthropathy

LEARNING OBJECTIVE 2: Identify underlying disease states associated with hypertrophic osteoarthropathy

CASE: A 59 year-old male with no significant past medical history presented to the outpatient clinic for evaluation of bilateral knee pain of four months' duration. He had been evaluated by an orthopedist at another institution, and suspicion was for degenerative joint disease, though MRI revealed relatively mild degenerative changes. The patient noted no significant benefit from multiple intra-articular steroid injections during that time. He reported that his pain was now interfering with his ability to work, which required him to stand on a hard surface for up to ten hours at a time. Social inquiry was significant for a 40 pack-year smoking history as well as history of alcoholism, now sober for over 10 years. On physical examination, mild tenderness over the medial joint space and patella was noted bilaterally, but knee exam was otherwise unremarkable. Digital clubbing was noted, however, prompting chest imaging in addition to knee. 
Knee X-ray demonstrated diffuse cortical thickening of the femoral, tibial, and fibular shafts, consistent with hypertrophic osteoarthropathy. Chest Xray revealed a $7 \mathrm{~cm}$ lobulated right apical lung mass. The patient ultimately proceeded to a right upper lobectomy. Pathology revealed stage IIIB adenocarcinoma (grade 3 of 4), with invasion of the visceral pleura and chest wall. On follow-up one month after surgery, he noted complete resolution of his knee pain.

DISCUSSION: Hypertrophic osteoarthropathy (HOA) is characterized by digital clubbing and periostosis of tubular bones. It is most often secondary to processes involving a possible arteriovenous shunt, including non-small cell lung cancer, cyanotic congenital heart disease, and cystic fibrosis. It has been estimated to occur in up to $30 \%$ of non-small cell lung cancers, most commonly adenocarcinoma and squamous cell types. The finding has been described for over a century, but pathophysiology remains poorly understood. Recent hypotheses have suggested possible mechanisms involving vascular endothelial growth factor, platelet-derived growth factor, or prostaglandin E2. The finding of digital clubbing should prompt investigation into intrathoracic pathology. Bone scan is the best modality for identifying affected bones. The pain of HOA can be severe and is frequently refractory to typical analgesics, but treatment of the primary cause frequently leads to regression of signs and symptoms.

\section{COCCIDIOIDES ENDOPHTHALMITIS IN AN IMMUNOCOMPETENT} HOST. Michael Cheng; Edward Ha. University of California, Los Angeles, Los Angeles, CA. (Control ID \#1334560)

LEARNING OBJECTIVE 1: Recognize endophthalmitis as an unusual presentation of Coccidioides infection.

LEARNING OBJECTIVE 2: Manage medical workup and treatment of fungal endophthalmitis.

CASE: A 55 year-old white male from Santa Clarita, CA developed a severe pneumonia four months prior to admission. With his symptoms almost resolved, he reported scratching his left eye with his eyeglasses, and thereafter developed increasing injection and pain with progressive loss of vision from 20/10 without correction to hand motion only. The patient underwent vitrectomy with intravitreal injection of empiric vancomycin, ceftazidime, voriconazole, and dexamethasone. Aqueous fluid culture returned positive for mold. He was admitted for systemic anti-fungal therapy. The patient had no significant past medical history or risk factors for HIV. Social history was notable for avid mountain biking in the Central Valley, and prior handling of birds as a falconer. Physical exam was notable for left visual acuity limited to hand motion only, limited extra-ocular movement, conjunctival injection, and intravitreal "fluff balls." WBC was elevated at 15,700 . HIV testing was negative. CT chest demonstrated micro-nodules in the right upper lobe suspicious for prior pulmonary coccidioidal infection. The patient began IV voriconazole at $4 \mathrm{mg} / \mathrm{kg}$ IV Q12H. Aqueous fluid cultures grew Coccidioides, and serum IgG coccidioidal antibody was elevated. CT brain, lumbar puncture, and bone scan were normal. The patient was discharged on oral voriconazole $350 \mathrm{mg}$ twice daily. He was transitioned to oral fluconazole $800 \mathrm{mg}$ daily one month later. The patient subsequently underwent 13 subsequent intravitreal injections of amphotericin and voriconazole, as well as two vitrectomies. As of eight months following discharge, the patient's best-corrected visual acuity was 20/25.

DISCUSSION: Coccidioidomycosis is endemic in the southwest United States. Symptoms vary, from subclinical infection to acute pneumonia to disseminated disease. Disseminated disease occurs more commonly in immunosuppressed individuals, typically involving the skin, meninges and bone. Intraocular coccidiomycosis is being recognized with greater frequency, but remains very rare [1]. A high index of suspicion should be retained in endemic regions, as early diagnosis and treatment may mitigate visual loss and extension of disease. Evaluation for other foci of disease should include careful history and physical examination, bone scan, CT chest, imaging of the brain, and lumbar puncture. Evaluation for immunosuppression, including HIV status, should be pursued. Diagnosis of fungal endophthalmitis can be difficult, and empiric therapy often must be started before the etiologic agent is known. While fluconazole has good CNS and ocular penetration, it has a limited spectrum and lacks activity against many of the common etiologic agents of fungal endophthalmitis, notably Aspergillus, Fusarium, and Paecilomyces. Voriconazole is a second-generation derivative of fluconazole with an expanded spectrum [2]. Future research may be directed to evaluate whether voriconazole or fluconazole is superior after Coccidioides has been identified, when broad coverage can safely be narrowed, and optimal treatment duration. 1. Cutler JE, et al. Metastatic coccidioidal endophthalmitis. Arch Ophthalmol 1978;96:689-91. 2. Marangon FB, et al. In vitro investigation of voriconazole susceptibility for keratitis and endophthalmitis fungal pathogens. Am J Ophthalmol 2004; 137:820-825.

COINFECTION OF CLOSTRIDIUM BUTYRICUM AND CLOSTRIDIUM DIFFICILE IN AN ADULT PATIENT Jennifer Hsieh; Sharon Leung. Montefiore Medical Center, Bronx, NY. (Control ID \#1339943)

LEARNING OBJECTIVE 1: Recognize that Clostridium butyricum is an uncommon cause of bacteremia.

LEARNING OBJECTIVE 2: Review the risk factors, prevalence, and management of $\mathrm{C}$. butyricum bacteremia.

CASE: A 63 year-old woman with a history of peripheral vascular disease with a left below the knee amputation and a recent right above the knee amputation (AKA) presented from nursing home with altered mental status and lethargy for one day. Upon arrival, BP was 108/54, pulse 132 , respiration rate 42 , temperature $100.4 \mathrm{~F}$ and oxygen saturation $88 \%$ on room air. The patient was unresponsive and breathing was shallow. Physical exam was notable for diffuse abdominal tenderness, right AKA stump with foul smelling pus, and multiple stage IV sacral and right gluteal decubitus ulcers along with copious amounts of loose stool. Pertinent labs included WBC 25, Na 150, K 5.5, HCO3 12, BUN 122, Cr 2.9, pH 7.20, lactate 8.4 and an anion gap of 24. CT imaging revealed left lower lobe pneumonia, bowel thickening in sigmoid colon and rectum as well as soft tissue emphysema in the left ischiorectal fossa extending into the perineum, but no soft tissue emphysema along the fascial planes of the muscle to suggest necrotizing fasciitis. The patient was resuscitated with 6 liters of fluid and empirical antibiotic coverage with intravenous vancomycin, piperacillin/tazobactam, clindamycin and metronidazole was initiated. The blood culture grew Clostridium butyricum, the respiratory culture grew multidrug resistant Klebsiella pneumoniae, and the wound culture grew Pseudomonas aeruginosa. Clostridium difficile toxin was also positive. The patient received extensive debridement of necrotic tissue from the sacral and gluteal region, and her antibiotic regimen was changed to imipenem/cilastatin and $\mathrm{PO}$ vancomycin in order to cover the multidrug resistant Klebsiella and C. difficile infection, respectively. She subsequently improved.

DISCUSSION: C. butyricum has been cultured from the stool of healthy children and adults, and is commonly found in soured milk and cheeses. It is an anaerobic endospore-forming Gram-positive rod that is uncommonly reported as a human pathogen. To date, it has only been reported as a causative pathogen in an adult with history of intravenous drug use, and in pediatric patients with underlying gastrointestinal infections, or abdominal, rectal, and oropharyngeal abscesses. Similarly, in adults, Clostridia infections often arise from a gastrointestinal source. Risk factors include patients who are elderly, have underlying conditions such as diabetes, or malignancy who are receiving chemotherapy and/or radiation therapy. Other extremely uncommon clostridium species, such as C. novyi, have also been reported to cause extensive and progressive wound infections, resulting in septic shock. In general, Clostridia are generally susceptible to antibiotics used in the treatment of anaerobic infections. Studies have shown that the susceptibility of C. butyricum isolated from intra-abdominal infections has a reasonable minimum inhibitory concentration (MIC) with metronidazole (MIC of $90 \%$ isolates was 2). Because Clostridium infections are associated with high mortality, a high index of suspicion, early administration of antibiotics and aggressive surgical debridement of the affected tissue is crucial to better ensure a successful outcome. To our knowledge, this is first reported coinfection with two Clostridium species in an adult, causing extensive C. difficile colitis and widespread cellulitis from C. butyricum.

COMMON THINGS BEING COMMON Marissa Shams; Domnica Fotino. Tulane University Health Sciences Center, New Orleans, LA. (Control ID \#1311733) 
LEARNING OBJECTIVE 1: 1. Recognize the clinical presentation of primary headache in HIV/AIDS population. 2. Identify the treatment of headache in patients with HIV. 3. Utilize cost-efficient diagnostic strategies in the diagnosis of headache

CASE: A 27 year-old woman presented with three-weeks of a progressive frontal headache. The headache was associated with dizziness and blurry vision. She had taken no medications for the headache, and could not recall any inciting event. She had a history of HIV with a CD4 count of 240 cells/ $\mathrm{mm} 3$. Her vital signs were normal; she was afebrile. Her cardiac, pulmonary, abdominal and skin examinations were normal. The neurologic examination was also normal; there were no sensory or motor defects, and her cranial nerves were intact. Her BMI was 33. She had a history of positive cryptococcal antigen titer 1:64, with prior negative CSF fungal culture. A head CT was negative for intracranial mass or acute lesion. An MRI/MRV of the head was negative for cranial thrombosis. CSF studies from revealed normal glucose, protein and cell counts.

DISCUSSION: In patients with HIV, headache is the most common chief complaint encountered by general internists. Despite the fear of intracranial infections or malignancies, it is important that the general internist recognize that more than two thirds of HIV patients with headache are diagnosed with primary headaches, including migraine, tension and cluster headaches. Despite the high prevalence, many patients remain undiagnosed after their initial presentation and suffer from headache-associated morbidity, even after AIDS-defining neurologic complications have been excluded. Primary headache in AIDS patients is usually associated with depression, anxiety or insomnia. Like patients without HIV, these patients often have a positive family history or a personal history of headache prior to seroconversion. Clinical prediction rules based on symptoms and CD4 counts have been developed to aid in the recognition of patients that require prompt diagnostic imaging or invasive testing for headache management. The algorithms are utilized to decrease the risks associated with invasive procedures and imaging while helping control rising medical costs. Patients are stratified as low, intermediate and high risk for intracranial mass/lesion based on the presence of focal neurologic symptoms, altered mental status, past history of seizure, and CD4 count. Low-risk patients are treated clinically. Both intermediate and high-risk patients deserve imaging and invasive testing for a complete workup. This patient's headache was relieved with a regimen of ketorolac, divalproex sodium and promethazine. She was discharged on a daily regimen of topiramate. Primary headache is a clinical entity that occurs in a many HIV/AIDS patients. Prompt recognition and appropriate treatment can restore a patient's quality of life and decrease both treatment and diseaseassociated morbidity.

COMMON VARIABLE IMMUNODEFICIENCY IN A PATIENT WITH RECURRENT BRONCHITIS PRESENTING WITH PNEUMOCOCCAL SEPSIS Natasha Parekh ${ }^{1}$; Farhan Zaidi ${ }^{1}$; Jo-Anne Suffoletto ${ }^{1,2}$. ${ }^{1}$ University of Pittsburgh Medical Center, PIttsburgh, PA; ${ }^{2}$ VA Pittsburgh Health System, Pittsburgh, PA. (Control ID \#1321195)

LEARNING OBJECTIVE 1: Consider immunodeficiency in a patient with a history of recurrent infections

LEARNING OBJECTIVE 2: Distinguish common variable immunodeficiency (CVID) from other immunodeficiencies using history and laboratory testing

CASE: A 29 year-old male presented with a 2-day history of fever, dyspnea, and pleuritic chest pain. On admission, he was febrile, diaphoretic, and tachycardic; CBC showed leukocytosis (WBC 33,000/ $\mu \mathrm{L}$ ) and chest X-ray showed left lower lobe opacity. Blood cultures were positive for Streptococcus pneumonia. Labs revealed he was in acute kidney injury (AKI) with a creatinine of $1.6 \mathrm{mg} / \mathrm{dL}$ (baseline 0.7 ); his urinalysis showed proteinuria and hematuria. He denied cough, meningismus, or gastrointestinal and urinary symptoms. Further questioning revealed a 16-year history of 1-2 bronchitis episodes a year. He clinically improved and blood cultures cleared within 48 hours of hydration and treatment with intravenous (IV) antibiotics. Given his history of recurrent bronchitis, current sepsis, and AKI with proteinuria and hematuria, an extensive lab work-up was performed, including HIV, Hepatitis B and C, ANA, p-ANCA, c-ANCA, anti-GBM, complements, and immunoglobu- lins. This was remarkable for an IgA level of $<6.67 \mathrm{mg} / \mathrm{dL}$ (normal 53 334 ), IgG of $36.1 \mathrm{mg} / \mathrm{dL}$ (normal 53-334), and $\mathrm{IgM}<4.17 \mathrm{mg} / \mathrm{dL}$ (normal 53-334). Titers for diphtheria, pertussis, tetanus, hemophilus influenza B, and streptococcus pneumonia showed absent response. Based on his markedly reduced immunoglobulin levels, deficient response to immunization, and absence of any other identifiable immunodeficiency, a tentative diagnosis of CVID was made. Repeat immunoglobulin titers showed persistently low $\operatorname{IgA}$ and $\operatorname{IgM}$ but an increase in $\operatorname{IgG}$ from 31.6 to 519 . Because of the IgG increase, he was given Tdap and pneumococcal vaccines and post-vaccination titers will be sent shortly to gauge response and confirm diagnosis. If the diagnosis is confirmed, he will receive his first dose of IVIG.

DISCUSSION: CVID is a rare entity, affecting 1 in 50,000 patients. It is a primary antibody deficiency that can lead to a host of pathologies, including recurrent infections, chronic lung disease, gastrointestinal issues, autoimmune disorders, and malignancy. It is defined as low serum levels of IgG, IgA, and IgM with poor or absent antibody production and exclusion of other causes of immunoglobulin deficiency. Sinopulmonary infections (like otitis media, sinusitis, bronchitis, and pneumonia) are the most common presentations of patients with CVID, occurring in up to $73 \%$ of patients. Pneumonia in CVID can be severe and frequently requires IV antibiotics. Complications of pneumonia include sepsis, pleurisy, bronchiectasis, and empyema. When considering immunodeficiency, it is vital to distinguish between CVID and other immunodeficiencies. Age of onset is important as patients over 15 years rarely present with X-linked (Bruton's) agammaglobulinemia (characterized as decreased serum immunoglobulins and absent B cells) or X-linked hyper-IgM syndrome. Serum immunoglobulin levels are essential to distinguish CVID from hyper-IgM syndromes and selective IgA deficiency. Medication history is important as certain drugs can induce hypogammaglobulinemia such as anti-malarials, steroids, and sulfasalazine. This case illustrates a severe complication as the presentation of a rare medical problem. It is essential to consider a broader differential in a patient with recurrent infections, and to differentiate CVID from other immunodeficiency syndromes.

\section{COMPETENT ENOUGH FOR CRYPTOCOCCUS: CRYPTOCOCCUS} GATTII MENINGOENCEPHALITIS Starr Steinhilber ${ }^{1}$; Victoria A. Johnson ${ }^{2}$. ${ }^{1}$ University of Alabama at Birmingham, Birmingham, AL; ${ }^{2}$ University of Alabama at Birmingham, Birmingham, AL. (Control ID \#1334651)

LEARNING OBJECTIVE 1: Emphasize Cryptococcus gattii as an emerging pathogen causing meningoencephalitis in immunocompetent hosts.

LEARNING OBJECTIVE 2: Recognize that C. gattii is more difficult to treat and clear than $\mathrm{C}$. neoformans.

CASE: A previously healthy 49-year-old African American male presented with weeks of fever, headache, back pain, and vomiting unresolved after multiple ER visits and empiric treatment with doxycycline for a tick bite. His headache persisted with new blurry vision. Examination revealed an oriented male with meningismus and bilateral nystagmus upon left lateral gaze. There was no rash, fever, seizures, history of trauma, or travel outside of Alabama in over twenty years. Immunologic work up including HIV antibody, CD4 lymphocyte assay, serum complement and immunoglobulin levels were negative or normal. Lumbar puncture (LP) revealed opening pressure of $23 \mathrm{mmHg}$, cerebrospinal fluid (CSF) protein of $129 \mathrm{mg} / \mathrm{dl}$, and CSF glucose of $29 \mathrm{mg} / \mathrm{dl}$. India ink was positive for encapsulated yeast. CSF cryptococcal antigen was positive (1:2048). Amphotericin lipid complex $5 \mathrm{mg} / \mathrm{kg} / \mathrm{d}$ and flucytosine $100 \mathrm{mg} / \mathrm{kg} / \mathrm{d}$ were administered. CSF cultures were positive for Cryptococcus species and remained positive for fourteen days despite combination therapy. His headache and blurry vision resolved with serial therapeutic LPs. Opening pressures were as high as $46 \mathrm{mmHG}$, and a ventriculoperitoneal shunt was placed on day fourteen of hospitalization. MRI revealed cryptococcomas in the cerebrum and spinal cord. Interferongamma was initiated on day twenty for refractory infection. The Cryptococcus species was identified as C. gattii, and he received seventy-five days of amphotericin and was discharged on long-term fluconazole.

DISCUSSION: Meningoencephalitis is one of the most serious consequences of cryptococcosis, and is a well-known pathogen among immunocompromised 
patients. Primary infection occurs through the respiratory tract, but it has a propensity for the central nervous system. Cryptococcus is a basidiomycetous yeast and is further classified into two species with important differences, C. neoformans and C. gattii. C. neoformans affects primarily immunocompromised, whereas C. gattii affects immunocompetent hosts. A case series from Australia showed that all cases of C. gattii were in immunocompetent hosts, and most cases presented insidiously with evolving neurologic symptoms, similar to our patient. Though $\mathrm{C}$. neoformans infection has a higher mortality rate, C. gattii causes a greater number of cryptococcomas, leading to more neurological sequelae and slower response to treatment. C. gattii has been cultured from trees in Australia and was originally seen in places where those trees were sent. However, since 1999, over 200 immunocompetent cases of $\mathrm{C}$. gattii infection have been reported in British Columbia and the northwestern United States with rare cases are now being reported in other areas of the US. C. gattii is known to develop resistance to antifungal agents and treatment with Amphotericin and flucytosine is often required for longer than a month. Interferon-gamma was added as adjunct therapy due to its suggested benefit in decreasing time to clearance of resistant $\mathrm{C}$. neoformans in immunocompromised patients. C. gattii is still rare in most of the US but is emerging in immunocompetent hosts. Education is needed as it can present more indolently than other more common causes of meningoencephalitis and often requires lengthy and more complicated treatment regimens for successful outcomes.

COMPLICATED GASTRO-ESOPHAGEAL REFLUX DISEASE FOLLOWING LAPAROSCOPIC ADJUSTABLE GASTRIC BANDING. Adetokunbo F. Oluwasanjo; Richard Alweis. The Reading Hospital and Medical Center, West Reading, PA. (Control ID \#1333825)

LEARNING OBJECTIVE 1: Recognize and manage new-onset gastroesophageal reflux (GERD) disease as a complication of laparoscopic adjustable gastric banding (LAGB).

LEARNING OBJECTIVE 2: Identify patients at risk for esophageal complications following LAGB.

CASE: A 68 year old man presented with a one month history of cough, heartburn and episodes of nocturnal regurgitation. He had no history of gastro-esophageal reflux disease (GERD) and had undergone laparoscopic adjustable gastric banding (LAGB) 8 months prior to presentation. His past medical history was notable for chronic obstructive pulmonary disease (COPD), obstructive sleep apnea, type II diabetes, hypertension and hyperlipidemia; and a review of his preoperative records showed radiographic evidence of a small hiatal hernia. Omeprazole $20 \mathrm{mg}$ twice daily was initiated and an upper gastrointestinal series demonstrated a more dilated hiatal hernia, significant reflux and probable stricture formation. His band was loosened, but his symptoms persisted. Over the next 15 months, he underwent three surgical repairs for recurrent hiatal hernias and was hospitalized for left lower lobe pneumonia. His symptoms progressively worsened with nasal and oral regurgitation, but he had achieved significant weight loss, with a reduction in his body mass index (BMI) from 41 to 27 ; and was reluctant to have his band completely deflated. One month after being discharged from the hospital, he presented with a productive cough, worsening dyspnea, anorexia and fatigue. His chest radiograph indicated persistent left lower lobe infiltrate and a chest computed tomography (CT) scan revealed bilateral pulmonary nodules that were too numerous to count. The diagnosis of exclusion was metastatic disease. After 7 days of levofloxacin and prednisone, he underwent bronchoscopy with evacuation of copious amounts of vegetable matter and mucus plugs. His lung biopsy was consistent with organizing aspiration pneumonia and follow-up chest CTs at one and three months showed continued resolution of the nodules, strongly supporting an inflammatory etiology. With persistent symptoms resulting in poor quality of life and radiographic evidence of esophageal dysmotility, the decision was made to completely deflate the gastric band. Over the next 4 months, his symptoms completely resolved.

DISCUSSION: Laparoscopic adjustable gastric banding (LAGB) is gaining popularity as a relatively safe and effective bariatric procedure with the advantages of being minimally invasive and reversible. There are however long-term esophageal complications of this procedure, including dilatation, dysmotility and GERD. The reported incidences are conflicting, suggesting a sub-set of patients may be at a higher risk of developing these complications. While there is no established tool to help risk-stratify patients, a review of literature suggests that abnormal preoperative $\mathrm{pH}$ monitoring and an esophageal caliber $>35 \mathrm{~mm}$ are risk factors, while abnormal preoperative esophageal manometry and a preceding history of GERD or hiatal hernias are inconsistent predictors. In most patients, removal or partial deflation of the band leads to symptom resolution in most patients; however, there are reports of irreversible complications. Prior to LAGB, preoperative risk-stratification should be performed and patients educated on the potential long-term complications.

CONFUSED? TAKE A [2ND] LOOK AT THE SMEAR Edward Mannina; Daniel Salerno. Tulane University Health Sciences Center, New Orleans, LA. (Control ID \#1338507)

LEARNING OBJECTIVE 1: 1. Recognize the presentation of malaria and complicated malaria. 2. Understand the diagnosis and treatment of Plasmodium infection. 3. Appreciate the importance of a detailed social history.

CASE: A 53 year-old man presented with altered mental status following a motor vehicle accident. He had no medical, surgical, family, allergy, prescription or recreational drug history. He worked as a helicopter pilot in Nigeria. He complained of diarrhea, dark urine and dry mouth. On admission, his temperature was $104 \mathrm{~F}$; his heart rate was 130 beats/min., the blood pressure was $142 / 77 \mathrm{mmHg}$, and his respiratory rate was 24 breaths $/ \mathrm{min}$. He was diaphoretic and confused with no focal deficits. His cardiac, pulmonary and abdominal examination was normal. A CBC revealed a WBC of 2,800 cells $/ \mathrm{mm} 3$, a hemoglobin of $10.1 \mathrm{~g} / \mathrm{dl}$, and a platelet count of 26,000 cells $/ \mathrm{mm} 3$. He had a bilirubin of 1.5 , a lactate 4.5 and an LDH of 527. A peripheral blood smear showed normocytic red blood cells with thin ring forms, consistent with Plasmodium falciparum infection denoted as "severe" or "complicated" by cerebral malaria. Intravenous quinidine was unavailable, so intravenous artesunate was initiated. Since his fever paroxysms were more frequent than expected, a second look at the peripheral smear revealed rare enlarged/ovoid red blood cells with larger ring forms, suggesting concurrent Plasmodium ovale infection. In addition to the artesunate, oral atovaquone/proguanil was initiated. With smears negative for four days, and after glucose-6-phosphate dehydrogenase deficiency was excluded, he was discharged for an additional forteen days of oral primaquine.

DISCUSSION: As the global economy continues to expand, general internists are likely to encounter more and more patients who work abroad as part of their primary employment. Recognizing diseases acquired abroad, even in patients presenting within the continental United States, will become increasingly important for the general internist. Plasmodium infection produces nonspecific findings of fevers, tachycardia, tachypnea, headache, and myalgias. Laboratory findings are also nonspecific: low cell counts, hyper-bilirubinemia, and an elevated BUN and creatinine. The social, travel and employment history is therefore the key to establishing the pre-test probability requisite for pursing the diagnosis. Peripheral smears remain the diagnostic gold standard and are used to quantify disease burden as percent parasitemia. Infection of more than $5 \%$ of the red blood cells/hpf denotes "severe malaria," an advanced condition involving the kidney, liver, lungs or brain with hemolysis, intravascular coagulopathies and circulatory collapse described. Due to chloroquine resistance, oral artemisinin-combination therapies are recommended while parenteral artesunate or quinidine is used for complicated disease. Infection with Plasmodium ovale or P. vivax requires an agent such as primaquine to kill liver hypnozoites preventing relapse.

CONFUSION AND UPPER EXTREMITY WEAKNESS IN AN ELDERLY WOMAN; CASE OF LYME ENCEPHALOPATHY AND RADICULNEURITIS Naba R. Mainali; Madan R. Aryal; Madan Badal; Anup Subedee. The Reading Hospital and Medical Center, West Reading, PA. (Control ID \#1310520) 
LEARNING OBJECTIVE 1: Recognize that lyme disease may present with peripheral neuropathy involving non- cranial nerves.

LEARNING OBJECTIVE 2: Diagnose and treat neurological involvement of Lyme disease.

CASE: Lyme disease is a tick borne, multi system disease caused by the spirochete, Borrelia burgdorferi. Early symptoms include fever, headache, fatigue, and a characteristic rash called erythema migrans. Lyme encephalitis and radiculoneuritis are rare entities among seropositive individuals which can present months to years after initial infection. Patients with lyme encephalitis usually present with mild confusion, language difficulties and sleep disturbances. Here we present an interesting case of elderly female who presented with confusion and weakness 6 weeks after the tick bite which was finally diagnosed as lyme encephalopathy and radiculoneuritis. A 78 year old female with history of Lyme disease 2 years ago, treated with doxycycline was again bitten by a bug 6 weeks back without characteristic rash. Subsequently, she started noticing mild confusion, slurred speech, and more recent onset of weakness and mild pain in bilateral upper extremities. Neurological exam revealed intact cranial nerves. Extra ocular movements were full without nystagmus or diplopia. Muscle strength on bilateral upper extremities across all the joints was $3 / 5$. However muscle strength in lower extremity were full across all joints. There was decreased sensation in glove and stocking distribution bilaterally on upper and lower extremities. MRI of the brain showed mild periventricular and subcortical white matter hyperdensities consistent with multiple sclerosis or Lyme disease. CT of the cervical spine was normal. Lumbar puncture revealed glucose of 54, total protein 274, white blood cells 296 with $92 \%$ lymphocytes. Lyme ELISA in the blood was positive. Western blot IgG and IgM for Lyme were positive. Culture of stool for Campylobacter, EHEC and Giardia were negative. CSF West Nile virus serology, Ehrlichia and anaplasma serologies were negative. CSF IgM for Lyme was positive. However, Lyme by PCR was negative. EEG was normal. Patient was started on ceftriaxone $2 \mathrm{~g}$ daily after which the patient had remarkable improvement.

DISCUSSION: Clues to the diagnosis of Lyme encephalopathy and radiculoneuritis include history of tick exposure and confusion, elevated CSF protein and lymphocytic pleocytosis, positive serologies and abnormal brain imaging. Although cranial neuropathy is the most common focal nervous system abnormality, presentations of early dissiminated Lyme disease may include mononeuritis multiplex or radiculoneuritis elsewhere. Symptoms as well as MRI scan can sometimes be confused with multiple sclerosis. Confirmation of CNS infection requires either presence of intrathecal antibodies or positive PCR for DNA. The prominent feature of Lyme radiculoneuritis is pain and resembles mechanical radiculopathy like sciatica. The neurological abnormality is usually treated with ceftriaxone or cefotaxime for 2 to 4 weeks.

\section{CRYPTOCOCCAL MENINGITIS MASQUERADING AS NORMAL PRESSURE HYDROCEPHALUS IN AN IMMUNOCOMPETENT} INDIVIDUAL. Vijai Bhola; Ekta Bansal; Jean Smith. Carilion Clinic, Roanoke, VA. (Control ID \#1340206)

LEARNING OBJECTIVE 1: Cryptococcal meningitis is a rare cause of meningitis in immunocompetent patients, and often presents atypically. Diagnosis is thus often delayed leading to poor outcomes. We present a unique case of cryptococcal meningitis to highlight its atypical presentation and discuss the management options and possible pitfalls.

CASE: A $54 \mathrm{yr}$ old male with no impressive past medical history presented with a one-month history of nausea, vomiting, urinary incontinence, falls and unsteady gait. He denied fever, chills, sick contacts, recent travel. On examination, the patient was afebrile, vital signs within normal range. Unsteady gait and positive Romberg's sign were noted. Labs revealed normal white cell count, hemoglobin, creatinine, thyroid function, vitamin B12, and folate. Negative urine drug screen and HIV. With a glycosylated Hemoglobin of 8.5, he was diagnosed with Diabetes Mellitus. Magnetic Resonance Imaging of the brain showed moderate enlargement of the ventricular system and he was diagnosed with Normal Pressure Hydrocephalus (NPH). Lumbar Puncture (LP) revealed normal opening pressure, White Cell Count 107/cmm, Lymphocytes $71 \%$, Protein $319 \mathrm{mg} / \mathrm{dl}$, Glucose $59 \mathrm{mg} / \mathrm{dl}$. $50 \mathrm{cc}$ of CSF was withdrawn and patient had some improvement in gait. CSF Cryptococcal Antigen returned positive. Blood and Cerebrospinal (CSF) cultures were negative. Amphotericin $\mathrm{B}$ and Flucytosine were started on Day 8 and the patient had slow clinical improvement to baseline by discharge.

DISCUSSION: Cryptococcal meningitis presents in immunocompetent individuals with features of a more indolent neurological disease, with symptoms such as headache, anorexia and neurologic abnormalities such as ataxia. It can therefore be misdiagnosed with diseases such as migraine, tension headache or NPH. One small series described median time lag between symptom onset and diagnosis to be 44 days, diagnosis typically made only after multiple physician encounters and time delay. CSF antigen testing (sensitivity, $93-100 \%$; specificity, $93-98 \%$ ) is useful pending culture results. Cryptococcus Gatti is a Cryptococcal variant, identifiable by molecular speciation. First seen in the United States in 2007, this variant is associated with increased incidence of hydrocephalus and cryptococcomas. Treatment recommendations are a combination of amphotericin B and flucytosine for a 4-6 week induction phase, followed by a consolidation phase of fluconazole $800 \mathrm{mg}$ daily for 8 weeks, then maintenance, at $200 \mathrm{mg}$ daily for 6-12 months. Amphotericin B can be substituted with liposomal preparation if renal failure develops. An Immune Reconstitution Syndrome (IRIS) can be seen during treatment, and needs to be distinguished from treatment failure, as steroids plus acetazolamide can be indicated. Repeat LP or drainage procedures are indicated for refractory elevations of CSF pressures. An increased index of suspicion for cryptococcal meningitis may lead to earlier diagnosis and initiation of appropriate treatment for this disease entity, decreasing morbidity and mortality.

CUT TO THE CASE Taimur Khan; Marissa Karpoff; John Scopetta; Marcia Glass. Tulane University Health Sciences Center, New Orleans, LA. (Control ID \#1338576)

LEARNING OBJECTIVE 1: 1. Understand the importance of early microbial diagnosis when initiating antimicrobial therapy in patients with multiple comorbidities.

LEARNING OBJECTIVE 2: 2. Recognize the clinical presentation of histoplasmosis

CASE: A 35-year-old woman with a history of systemic lupus erythematosus and polymyositis, previously on leflunomide, presented with four weeks of progressively worsening cellulitis refractory to multiple antibiotics. On initial presentation, she had cutaneous lesions on her inner left thigh. She was afebrile with negative blood and urine cultures. An outside facility initiated broad-spectrum antibiotics, including linezolid, pipericillin/tazobactam and fluconazole. MRI of the thigh revealed focal edema and fluid of the lateral soft tissues near the periosteum with no suggestion of deep abscess or osteomyelitis. Wound cultures were positive for Pseudomonas. Ceftazadime was empirically initiated. The patient continued to worsen clinically, with increased wound purulence and spiking fevers. Fluconazole was initiated for fungal coverage. Fungal stains of punch biopsy returned as Histoplasma. We then adjusted her therapy to itraconazole for the remainder of the hospitalization. The patient went home on itraconazole but soon returned with weakness and jaundice. She eventually expired during this readmission. DISCUSSION: The early diagnosis of infectious etiologies is paramount is avoiding unnecessary side effects. By empirically treating these patients, unintentional harm may be caused. Physicians should be particularly conservative when approaching patients with multiple comorbidities, such as autoimmune processes, immunosuppression secondary to treatment, and chronic renal insufficiency. Without a final diagnosis, delaying broad-based treatment may be preferable to avoid detrimental side effects. With this particular patient, an earlier diagnosis would have helped us provide a focused and successful antifungal regimen and, likely, a much better outcome by sparing her multiple, toxic antimicrobials. This is especially important as this patient adversely responded to most of the medications she received. She presented with signs of heparin-induced thrombocytopenia, with elevated liver enzymes levels, and with pancytopenia - each problem likely a drug side effect. Perhaps, treating her symptomatically until we received the biopsy results could have spared her renal function and the other complications. Whereas most immunocompromised patients 
may require more aggressive, empiric treatment in order to halt infection as early as possible, a highly sensitive patient may require a less aggressive approach despite also being immunocompromised. Furthermore, since this patient was iatrogenically immunocompromised, she may have benefited in the interim by inducing her immune system more aggressively. With the average patient, a reflexive reaction to empirical antimicrobials is often the safest option. However, in patients who are physiologically fragile and are presenting unusually, deferring empiric treatment may help patients avoid unfavorable side effects. It is this high level of attention to such details that will make the difference in the healthcare provided to these unique patients.

CUTTING OUT THE NEUTROPHILS: LEVAMISOLE TOXICITY IN CHRONIC COCAINE USERS Robert Li; Amit Kothari. UT Southwestern Medical School, Dallas, TX. (Control ID \#1339977)

LEARNING OBJECTIVE 1: Recognize the clinical presentation of levamisole toxicity

LEARNING OBJECTIVE 2: Manage complications of adulterated cocaine abuse

CASE: A 51 year-old woman presents with one week of swelling, blistering, and tenderness of the left ring finger. She has a history of neutropenia, cocaine dependence, and has had multiple finger amputations secondary to dry gangrene and osteomyelitis. On admission, the patient's temperature was $39.1^{\circ} \mathrm{C}$ with a heart rate of $91 \mathrm{bpm}$. On exam, tender purpura were seen on both cheeks and extensive morbilliform rashes were noted on the abdomen, legs, and toes. Examination of the left ring finger showed circumferential swelling of the middle and distal phalanges and a $1 \mathrm{~cm}$ long dorsal lesion near the PIP joint. The affected finger was tender to palpation with mild erythema but there was no warmth, purulence, or signs of crepitance. CBC revealed a WBC count of 1200 cells $/ \mathrm{mm} 3$ and an ANC of 120 cells $/ \mathrm{mm} 3$; hemoglobin was $9.2 \mathrm{mmol} / \mathrm{L}$ and the platelet count was 171,000 per mm3. X-ray of the left hand showed diffuse soft tissue swelling of the fourth digit. Rheumatologic workup revealed a negative cANCA, positive p-ANCA, positive proteinase-3, positive rheumatoid factor, and equivocal meyloperoxidase. The patient was given appropriate wound care, ticaracillin/clavulanate, vancomycin, and G-CSF which successfully resolved her fever, modestly improved her ANC, and preserved the finger.

DISCUSSION: Given her recurrent presentations with digit gangrene and significant neutropenia, systemic etiolgies to the patient's illness including vasculitis, arteritis, malignancy, infection, and autoimmune etiologies were considered and worked up. In a chronic cocaine user, especially one with dermatologic findings, the differential diagnosis for neutropenia should also include levamisole toxicity. Levamisole is an anti-helminthic drug used as a cutting agent to adulterate up to $70 \%$ of street cocaine. Previous use of levamisole as an immunomodulator for rheumatoid arthritis and for adjuvant therapy in colorectal cancer revealed agranulocytosis as a side effect of therapy. Recently, the CDC has linked levamisole to 21 cases of agranulocytosis associated with cocaine use. Levamisole toxicity is also associated with pruritic rashes, ulcers, lichenoid eruptions, fixed drug eruptions, necrotizing thrombotic vasculitis, and the generation of autoimmune antibodies. Detection of levamisole is difficult as the plasma half-life is 5.6 hours and relatively little $(\sim 12 \%)$ is recovered in the urine. A gas chromatograph/mass spectrometer (GC-MS) is required for confirmation of the presence of levamisole in serum or urine samples. No commercial tests are available and therefore, a high clinical suspicion and positive urine toxicology for cocaine remain the most viable route for diagnosis. Treatment of levamisole toxicity includes G-CSF administration, steroids, and avoiding further exposure. A complete dermatological and serological recovery, even without G-CSF, usually occurs within weeks to months. However, it remains critical to suspect levamisole toxicity in a cocaine user as a reversible cause of agranulocytosis given the high morbidity and mortality associated with complications such as bacterial sepsis.

CYTOMEGALOVIRUS AND EPSTEIN BARR VIRUS COINFECTION IN AN AIDS PATIENT Mamatha Racheruvu; Kumar Sanam; Jyoti S. Samant. UAB Montgomery, Montgomery, AL. (Control ID \#1339805)
LEARNING OBJECTIVE 1: Distinguish infection versus neoplastic process causing neurologic complications in a HIV patient.

CASE: 42 year old white female with a history of HIV infection for $14 \mathrm{yrs}$ presented with right-sided facial weakness, double vision, vertigo and gait instability. She began having tinnitus and fullness in her right ear two months back, which continued to worsen, causing bilateral hearing loss. She also complained of productive cough. She was not receiving highly active antiretroviral therapy (HAART). Physical exam revealed complete paralysis of the right side of the face, bilateral hearing loss, bilateral ophtalmoplegia and nystagmus, remaining exam was normal. Initial differential was stroke or Central Nervous System (CNS) infection. MRI of brain revealed dorsal pontine abnormality, with enhancement in the ependymal/subependymal region adjacent to the lateral ventricles suspecting infectious process. Lumbar puncture (LP) was done.Opening pressure was $16 \mathrm{cmH} 2 \mathrm{O}$. Cerebrospinal fluid (CSF) was clear, colorless with glucose of $30 \mathrm{mg} / \mathrm{dl}$, protein of $99.4 \mathrm{mg} / \mathrm{dl}$, W.B.C of $23 / \mathrm{mm} 3$, with differential of $94 \%$ monocytes. CSF was negative for toxoplasma, mycobacteria, cryptococcus, or syphilis. CD4 count was $27 \mathrm{cells} / \mathrm{mm} 3$ with a viral load of $33,126 \mathrm{c} / \mathrm{mL}$. She was started on HAART but her neurological symptoms worsened.There was concern for Immune Reconstitution Inflammatory Syndrome, so HAART was stopped and was started on steroids. She continued to worsen so MRI and LP were repeated. CSF PCR was positive for Cytomegalovirus (CMV) and Epstein Barr Virus (EBV) with viral load of $2914 \mathrm{c} / \mathrm{ml}$ and $14,900 \mathrm{c} / \mathrm{ml}$ respectively. MRI revealed progression of pontine lesion with extension into right cerebellar hemisphere periventricular tissues and worsening of subependymal enhancement. Ganciclovir and foscarnet were started and HAART was resumed. A chest $\mathrm{x}$-ray done for pneumonia showed ill-defined mass in left lung, CT of the chest confirmed the mass along with multiple nodular densities in both lungs. Lung biopsy was done.Pathology revealed Lymphomatoid granulomatosis with focal progression to diffuse large B-cell lymphoma and EBV positive lymphocytes. Lesions in brain were difficult to biopsy, so flow cytometry of CSF was done which revealed B-cell lymphoma. As she was a poor candidate for chemotherapy, and patient wished for comfort care, so supportive care was provided. She died after one month of initial presentation.

DISCUSSION: This case illustrates an unusual neurologic viral coinfection in an AIDS patient. Our patient developed EBV related primary CNS lymphoma (PCNSL) and CMV encephalitis. We describe the first case of CMV and EBV coinfection in an AIDS patient leading to rapid neurologic decline. Neurologic manifestations of CMV disease in AIDS patients are encephalitis, ventriculoencephalitis, cerebral mass lesions, myelitis, polyradiculitis. Occurrence of CMV disease in AIDS is associated with poor prognosis and higher mortality. PCNSL is an important alternative condition in the differential diagnosis of intracranial mass lesions in toxoplasma seronegative AIDS patients. Lesions can be single or multiple and can produce edema and mass effect. CSF EBV PCR is helpful in confirmation of diagnosis. Open brain biopsy is required for tissue confirmation before cranial irradiation for PCNSL. We hope our case raises the awareness of CMV as an important differential in intracranial mass lesions in AIDS patients which can lead to rapid neurologic decline and increased mortality.

CYTOMEGALOVIRUS GASTRITIS IN AN IMMUNOCOMPROMISED PATIENT: CHALLENGES IN DIAGNOSIS Pranjal Sharma; Savio Reddymasu; Pallavi Bellamkonda. Creighton University, Omaha, NE. (Control ID \#1339708)

LEARNING OBJECTIVE 1: Recognize the clinical presentation of cytomegalovirus (CMV) gastritis in an immunocompromised patient. LEARNING OBJECTIVE 2: Identify that CMV infection is a known cause of mortality and morbidity in patients with solid organ transplantation and understand that even if the serum PCR is negative, gastric biopsies and cultures should be done to further confirm the diagnosis.

CASE: A 61 year-old man with history of idiopathic pulmonary fibrosis and status post bilateral lung transplantation and taking immunosuppressive medications for the past 4 years, presented with epigastric pain associated with nausea, weight loss and dyspepsia. Pertinent positives in physical examination were mild distress secondary to pain and epigastric tenderness. Complete blood count revealed pancytopenia with absolute neutrophil count of 400 . Comprehensive metabolic panel and abdominal 
X-rays were within normal limits. Cytomegalovirus Polymerized Chain Reaction (PCR) serology was negative. Gastroenterology was consulted and Esophagoduodenoscopy (EGD) with biopsies was done; EGD showed small ulcerations and biopsy results were positive for cytoplasmic CMV inclusions in gastric epithelial cells. Patient was treated with Ganciclovir and later showed clinical improvement.

DISCUSSION: The complaints of epigastric pain and dyspepsia are commonly encountered in the hospital setting and should be taken seriously, especially in immunosuppressed patients. Complete history and physical examination are critical in arriving at an accurate diagnosis. CMV infection is a known cause of mortality and morbidity in patients with solid organ transplantation and CMV gastritis can present with symptoms of epigastric pain, dyspepsia and also GI bleeding (hemorrhagic gastritis). Diagnosis is with DNA PCR and also Endoscopy with biopsies should be done. Gastric biopsies would show cytomegalic "owl-eye" cells. There should be a high level of suspicion of CMV infection in immunocompromised patients who present with acute to sub-acute gastrointestinal symptoms and even if the DNA PCR is negative, these patients warrant further investigation i.e., Endoscopy with gastric biopsies.

DRESSED AS IRIS Jorien G. Breur ${ }^{1}$; Yelena Averbukh ${ }^{2}$; Hanna Lee ${ }^{2}$ ${ }^{1}$ Albert Einstein College of Medicine, New York, NY; ${ }^{2}$ Montefiore Medical Center, New York, NY. (Control ID \#1332568)

LEARNING OBJECTIVE 1: Consider IRIS in AIDS patients presenting with fever and rash after recent initiation of HAART

LEARNING OBJECTIVE 2: Recognize DRESS syndrome in patients presenting with rash and fever after exposure to new medications CASE: A 31-year-old woman with AIDS and history of cryptococcal meningitis, disseminated Mycobacterium avium complex (MAC), hepatitis B (HBV), P. jirovecii pneumonia, and multiple drug allergies presents with highgrade fevers and a diffuse rash in the setting of recent initiation of HAART and MAC therapy. On physical exam the patient exhibited a diffuse, macular, blanching, pruritic rash, diffuse lymphadenopathy, and was febrile to $102.5^{\circ} \mathrm{F}$. Laboratory findings were significant for $7 \%$ eosinophilia, IgE levels elevated to $21839(\mathrm{nl}<180 \mathrm{IU} / \mathrm{mL})$ and elevated aspartate transaminase to 444 , alanine transaminase to 208, and alkaline phosphatase to 933. Infectious disease workup was significant for positive MAC blood culture and elevated HBV viral load. Since initiation of HAART with Raltegravir, Emtriatabine, and Tenofovir two weeks prior, the patient's viral load decreased from 1.7 million to 816 , and her CD4 count increased from 3 to 14 . Allergic drug reaction was suspected and as the patient's medications were stopped the rash, fever, and liver laboratory abnormalities began to improve. The patient's MAC regimen was restarted without any adverse reaction and the patient was scheduled for outpatient drug allergy testing prior to initiation of HAART to evaluate a causal relationship.

DISCUSSION: Given the similarities in presentation between immune reconstitution inflammatory syndrome (IRIS) and Drug Rash with Eosinophilia and Systemic Symptoms (DRESS) syndrome, potential allergic drug reactions should be considered in AIDS patients presenting with fever and rash in the setting of recent HAART initiation. Initiation of HAART in the profoundly immune-deficient patient can be associated with a paradoxical clinical worsening due to opportunistic infection (OI) as a direct result of immune restitution. The exuberant inflammatory reaction seen in IRIS typically presents from weeks to months of initiating HAART and is manifested clinically with fever, lymphadenopathy, and multi-organ dysfunction depending on the underlying OI. Allergic drug reaction, which occurs at a much higher frequency in $\mathrm{HIV}+$ patients, must be ruled out whenever a patient treated with a new drug develops an exacerbation or a new medical problem. DRESS syndrome reflects a serious hypersensitivity reaction to drugs which usually begins several weeks after exposure to the offending drug. Clinical features include fever, lymphadenopathy, rash, hypereosinophilia $>1,500 / \mu \mathrm{L}$, and multi-organ dysfunction. HAART agents Raltegravir, Emtriatabine, and Tenofovir have all been implicated in DRESS syndrome. While the prognosis of IRIS is relatively benign, the overall mortality of DRESS syndrome is $10 \%$. IRIS treatment is supportive and HAART therapy is continued, while DRESS syndrome necessitates that all potentially offending agents be stopped immediately. Our patient was at risk for both IRIS and DRESS syndrome due to her severely immuno-deficient state, presence of OIs, and recent HAART initiation.
Based on the presence of hypereosinophilia and elevated IgE levels, elevated liver transaminases, and cutaneous involvement a clinical diagnosis of DRESS syndrome was made. Clinicians should be aware of DRESS syndrome when prescribing $\mathrm{s}$ new drug due to the potential mortality if drug therapy is not discontinued.

DABIGATRAN TOXICITY IN A 65 YEAR OLD MALE WHO FAILED OTHER ANTICOAGULATION METHODS Rachel Harold ${ }^{2}$; Matthew Tuck ${ }^{1,2}$. ${ }^{1}$ Department of Veterans Affairs Medical Center, Washington, DC; ${ }^{2}$ George Washington University, Washington, DC. (Control ID \#1329853)

LEARNING OBJECTIVE 1: Recognize the interaction between dabigatran and P-glycoprotein (PGP) inhibitors and inducers

CASE: When dabigatran was introduced to the US market in 2010 as an oral anticoagulant, it was heralded as a welcome alternative to warfarin. Among its reputed benefits, dabigatran does not require monitoring of levels. This case report describes a 65 year old male who developed dabigatran toxicity on the manufacturer's recommended dosage. The patient had a history of chronic atrial fibrillation maintained on amiodarone, pulmonary embolism, ischemic cardiomyopathy with ejection fraction $25 \%$, mitral and tricuspid regurgitation and chronic kidney disease with a creatinine clearance $(\mathrm{CrCl})$ of $52.8 \mathrm{~mL} / \mathrm{min}$, who was admitted for a heart failure exacerbation. Prior to admission, he reported inconsistent adherence to medications, including dabigatran $150 \mathrm{mg}$ twice a day, which was started months earlier after failing to reach therapeutic levels with warfarin and developing heparin induced thrombocytopenia on dalteparin. On hospital day four, he had an episode of melena. Labs showed a markedly elevated PTT of 113.3, INR 3.4 and PT 33.7. Dabigatran was held. Amiodarone was also held out of concern that the interaction with dabigatran led to toxic levels. Fortunately, the patient had no further episodes of melena. Endoscopy demonstrated gastric erosions without active bleeding. As expected, based on the elimination half-life of dabigatran of 12-17 hours, the coagulation studies improved by the following day: PTT 66.7, INR 1.8 and PT 20.5. With these anticoagulation challenges, electrical cardioversion was planned to address the atrial fibrillation, but the procedure was aborted when a thrombus was discovered in the left atrium. In consultation with hematology, warfarin was restarted so that levels could be monitored.

DISCUSSION: The patient likely developed dabigatran toxicity on the recommended manufacturer's dose due to concomitant amiodarone use. As a result of a number of post-marketing surveillance reports, the manufacturer recently changed the prescribing information to include warnings of interactions with PGP inhibitors, such as amiodarone. Specifically, the package insert recommends lowering the dose to $75 \mathrm{mg}$ twice a day when co-administered with dronedarone or ketoconazole as well as a contraindication in patients with a $\mathrm{CrCl}<30 \mathrm{~mL} / \mathrm{min}$ when coadministered with a PGP inhibitor. The recommended dose of dabigatran $150 \mathrm{mg}$ twice a day may present added risk without dramatic added benefit over a lower dose for patients with atrial fibrillation. The Randomized Evaluation of Long-Term Anticoagulant Therapy trial showed the $150 \mathrm{mg}$ dose of dabigatran was superior to both warfarin and the $110 \mathrm{mg}$ dose of dabigatran in preventing stroke and systemic emboli. Importantly, the $110 \mathrm{mg}$ dose was noninferior to warfarin, but with a decreased risk of bleeding when compared to the $150 \mathrm{mg}$ dose among all subgroups. The FDA approved the higher dose because it wagered the added benefit of preventing systemic emboli outweighed the reduced risk of bleeding afforded by the lower dose. For patients with a $\mathrm{CrCl}$ of $15-30 \mathrm{~mL} / \mathrm{min}$, the suggested dose is $75 \mathrm{mg}$ twice daily. No other dosing options are available and there is no antidote for dabigatran. When prescribing dabigatran for a patient taking PGP inhibitors or inducers with concomitant chronic kidney disease, one should use alternate dosing and consider monitoring coagulation studies or drug levels.

DANGER LURKING IN THE DARK: THE INCIDENTAL FINDING OF A MASSIVE SUPRARENAL TUMOR. Javier Perez-Rodriguez ${ }^{1,2}$; Arooj Hyat ${ }^{2} .{ }^{1}$ Beth Israel Deaconess Medical Center, Boston, MA; ${ }^{2}$ Mount Auburn Hospital, Cambridge, MA. (Control ID \#1296242) 
LEARNING OBJECTIVE 1: Outline the endocrinology work up after detection of a suprarenal mass.

LEARNING OBJECTIVE 2: Describe differential diagnosis and management of suprarenal "incidentalomas."

CASE: A 20 year-old African-American woman G1P1 presented with persistent postpartum bleeding 6 weeks after an uneventful vaginal delivery. The patient was nursing her healthy infant, had no significant $\mathrm{PMH}$ and review of systems was positive only for right shoulder pain worsened by lying supine. A transvaginal ultrasound was performed and revealed a large right-sided suprarenal mass. Abdominal MRI with contrast showed a $13 \times 11.4 \times 6.9 \mathrm{~cm}$ retroperitoneal mass overlying the superior pole of the right kidney and displacing the vena cava. Extensive lab work up included CBC, BMP, aldosterone, renin, adrenal androgens (DHEAS, androstenedione, testosterone, 17-OH progesterone), cortisol stimulation test and plasma/urine levels of metanephrines and catecholamines. These tests were within normal limits. Surgical consult was obtained and recommended immediate excision given the likelihood of adrenocortical carcinoma by radiologic criteria. However, due to patient's preference, a CT guided needle biopsy was performed. Preliminary diagnosis of ganglioneuroma was made after which the patient consented to surgery. The procedure posed serious technical difficulties as the tumor abutted the aorta medially, the IVC laterally, the right renal vein inferiorly and the porta hepatis anteriorly. The capsule was adherent to adjacent vessels and the adrenal gland (which had to be partially excised), but there was no tissue invasion. The pathological analysis of the specimen confirmed the diagnosis of ganglioneuroma.

DISCUSSION: Our patient presented with an incidentaloma that topographically was suspicious for an adrenal mass. In this anatomic location differential diagnoses of adrenal origin include adenoma, pheochromocytoma or adrenocortical carcinoma. Potential non-adrenal etiologies of retroperitoneal tumors include benign soft tissue growths, soft tissue sarcoma, lymphoma, extragonadal germ-cell tumors, peripheral nerve tumors and metastatic disease. After such finding, a full adrenal work up is indicated given that treatment and/or preoperative preparation depend on activity or lack thereof of the tumor. In the case of adrenal incidentaloma, criteria for excision are functionality of the mass or imaging criteria suspicious for malignancy. Biopsy is not indicated in most cases as it cannot differentiate adenoma from carcinoma, a hypertensive crisis could be precipitated if the neoplasm is a pheochromocytoma and needle track seeding might occur in case of malignancy. However, this approach might be appropriate after pheochromocytoma has been ruled out. In this case a biopsy yielded the diagnosis of ganglioneuroma which was later confirmed by analysis of the surgical specimen. Ganglioneuromas are benign, encapsulated, slow growing nerve sheath tumors of the sympathetic chain more commonly found in female patients before the age of 20 . These tumors are asymptomatic until they start exerting a local mass effect and are usually discovered after their diameter exceeds $7 \mathrm{~cm}$. Although benign, complete excision is sometimes impossible due to adherence of the tumor capsule to important anatomic structures. Postoperative autonomic dysfunction is uncommon.

DEEP VEIN THROMBOSIS IN KLINEFELTER'S SYNDROME Abhishek Seth ${ }^{1,2}$; Robert L. Penn ${ }^{2,1}$. ${ }^{1}$ LSU Health Sciences Center, Shreveport, LA; ${ }^{2}$ Overton Brooks VA Medical Center, Shreveport, LA. (Control ID \#1339753)

LEARNING OBJECTIVE 1: Association of deep vein thrombosis with Klinefelter's syndrome.

LEARNING OBJECTIVE 2: Bring attention to association between genetic mutations and predisposition to thrombosis in Klinefelter's syndrome patients.

CASE: We report a 40 year old white male with Klinefelter's syndrome ( 47XXY karyotype), on testosterone replacement therapy, who presented with a month long history of left lower extremity pain, warmth and swelling . Venous doppler ultrasonography of the extremities revealed thrombosis in the proximal, mid and distal superficial femoral vein, popliteal vein and the peroneal vein. His past medical history has been significant for osteopororsis, hypertension, dyslipidemia, seizure disorder and major depression. Family history was positive for deep vein thrombosis in his mother. The patient was admitted to the inpatient medicine service for anti coagulation and appropriate workup. Coagulation studies showed a PT of 34 seconds, PTT of 14.3 seconds, and an INR of 1.1. Tests for various genetic causes of hypercoagulability states were found to be normal, including the protein $\mathrm{C}$, protein $\mathrm{S}$, homocysteine and anti thrombin III levels. Mutation in the prothrombin gene and Factor V Leiden were absent. Other risk factors for thrombosis such as prolonged immobility, malignancy, recent surgery and major trauma also weren't present in our patient. Varicose veins, stasis dermatitis and other signs of venous insufficiency were absent as well. However, our patient was found positive for MTHFR-C677t and MTHFRA1298 heterozygous mutations, increased Factor VIIIc activity and increased Ig M anti-cardiolipin antibody (repeat testing is pending). Anticoagulation was initiated with enoxaparin and warfarin, and he was discharged home to continue oral warfarin therapy.

DISCUSSION: Klinefelter's syndrome is the most common cause of primary testicular failure. It is characterized by small and firm testes, azoospermia, gynecomastia and a variable degree of eunuchoid features. An association between Klinefelter's syndrome and venous and arterial thrombo embolic disease has been found in a case series and several case reports. The exact causes for this association are unknown, but hypoandrogenism leading to hypofibrinolysis has been postulated as the most likely underlying mechanism. Most of the reported Klinefelter's patients with thromboembolic disease were found to have some congenital or acquired conditions which predisposed them to thrombosis. Previous reports of Klinefelter's patients with thrombosis have found heterozygous mutations of the MTHFR-677 C $>\mathrm{T}$ and 1298A $>\mathrm{C}$ genes, factor $\mathrm{V}$ Leiden with and without the G20210A prothrombin mutation, and hyperhomocysteinemia. The exact role of MTHFR mutations in thrombosis is poorly understood. Our patient is the first case reported with Klinefelter's syndrome and deep vein thrombosis, who was found to have both MTHFR mutations and elevated factor VIIIc levels. The results of the pending repeat anti-cardiolipin assay will clarify the importance of the elevated titer found during the acute presentation. Optimum duration of anticoagulation for deep vein thrombosis in patients with Klinefelter's syndrome is unknown and needs more clarification.

DEMENTIA AS THE INITIAL PRESENTATION OF HIV/AIDS Christopher Goodman; Kristina L. Lundberg. emory, Atlanta, GA. (Control ID \#1337851)

LEARNING OBJECTIVE 1: Recognize HIV as a cause of progressive dementia

CASE: We present the case of a 58yom with history of CKD, HTN, and previous CVA (without lasting deficit) who was brought by friends to the hospital for progressive decline in functional and mental status over two weeks prior with prominent apathy and memory difficulty such that the patient now required help with all iADLs. The patient was a poor historian and seemed oblivious to the deterioration described by his friends denying there were any problems. He did agree to $15 \mathrm{lb}$ wt loss over the last few months, and his only complaint was feeling short of breath despite appearing comfortable. He denied changes in speech or vision, focal weakness, and sensory changes. He denied fever, chills, and chest pain. T: $36.9^{\circ} \mathrm{C}$ P: 93 R: 20 BP: 138/83 Pox: 95\%, RA Pertinent physical exam demonstrated a thin, adult male who appeared older than stated aged and malodorous. He was AAOx2, not oriented to time. He had poor dentition. Lung bases had coarse crackles. Neuro exam revealed symmetric hyperreflexia (3+), 4/5 strength to LUE and LLE with 5/5 to RUE/RLE, negative Romberg, absent Babinski but sustained ankle clonus b/l. MMSE: 19. Work-up initially focused on his prominent neurological symptoms. Routine lab studies including TSH, B12, RPR were all normal. CT head demonstrated microvascular changes and possible subacute stroke suggestive of vascular dementia. On day three of admission the patient became progressively more hypoxic with $\mathrm{O} 2$ sats in low 80 s while on $4 \mathrm{~L} \mathrm{nc}$. While awaiting bronchoscopy and CT imaging which would eventually confirm the diagnosis of PCP, the initial HIV screen came back positive CD4 returned at $2 / 1 \%$. When the patient was more stable, an MRI head confirmed the vascular findings of the CT head as well as white matter changes consistent with HIV dementia. LP studies did not yield an alternate diagnosis. 
DISCUSSION: HIV-associated dementia (HAD) has been a recognized clinical entity (although it has undergone changes in definition and nomenclature) since AIDs was first recognized in the 1980s with a wideranging reported prevalence of $15 \%$ to $60 \%$ likely reflecting the difficulty in both making and defining diagnoses of neurocognitive impairment. Classically, it is considered a subcortical dementia with decline occurring over weeks to months; faster progression may be seen with greater immunosuppression. Dementia as the initial presentation of AIDS is fairly uncommon. From surveillance data by the CDC this was the presenting diagnosis in about $3 \%$ of AIDS patients. However, this was data from the mid-1990s and the incidence of HAD has been steadily declining since the introduction of HAART. For our patient, it would have been even easier to miss given the presence of significant vascular disease on imaging suggesting vascular dementia as the cause of his symptoms. Furthermore, HIV/AIDs is becoming increasingly common in patients over age 50 and not just because more people are living with the disease. In 2005, based on data from the CDC, individuals over the age of 50 accounted for $15 \%$ of new HIV/AIDs diagnoses. This case provides a good reminder of the importance of regular HIV screening of older individuals, especially those with evidence of dementia. If the diagnosis had been delayed or not considered it could possibly have left him at greater risk of mortality from untreated OIs such as the PCP that became apparent during his admission.

DIABETIC MUSCLE INFARCTION: A RARE BUT SERIOUS COMPLICATION OF DIABETES MELLITUS Jessica Clima; Sara L. Swenson. California Pacific Medical Center, San Francisco, CA. (Control ID \#1334735)

LEARNING OBJECTIVE 1: Recognize diabetic muscle infarction as a rare but serious complication of long-standing diabetes

LEARNING OBJECTIVE 2: Distinguish diabetic muscle infarction from other causes of thigh pain in diabetics

CASE: A 48 year-old African American female presented with a 2-month history of progressive left thigh pain and swelling. She had long-standing type 2 diabetes mellitus complicated by end stage kidney disease requiring hemodialysis, severe peripheral neuropathy, retinopathy, and hypertension. Two months prior to admission, the patient reported left medial thigh pain. One month later, she reported persistent left medial thigh pain that worsened with movement and hemodialysis. The pain was constant, accompanied by intermittent swelling, and she noted a masslike sensation. She denied parasthesias, fevers, or joint symptoms. Interval work-up included doppler ultrasounds that were negative for deep vein thrombosis and the recommendation of physical therapy. In the week prior to admission, her pain intensified to the point that she could not walk so she presented to the emergency department. On examination, her left anteriomedial thigh was swollen and exquisitely tender to palpation without palpable masses, erythema, crepitus, or inguinal adenopathy. Pertinent laboratory studies included a WBC of $7.1 \mathrm{~K} / \mathrm{uL}, \mathrm{CPK}$ of 447 $\mathrm{U} / \mathrm{L}$ and negative blood cultures. Given her severe pain, we obtained an MRI. It revealed diffuse intramuscular and perifascial edema of the left vastus medialis and vastus intermedius without focal fluid collections. Similar, but less severe findings were seen in the right thigh adductors and vastus medialis. We managed the patient conservatively with rest and pain control, and her symptoms gradually improved.

DISCUSSION: Diabetic muscle infarction (DMI) is a rare vascular complication of poorly controlled, long-standing diabetes mellitus. It results from ischemic necrosis of skeletal muscle with local inflammation. It is slightly more common in women(59\%) and type 1 diabetics(62\%). As in our case, patients typically have microvascular complications, such as nephropathy $(71 \%)$, retinopathy $(57 \%)$ and neuropathy(55\%). Common symptoms include pain $(80 \%)$, swelling $(76 \%)$, mass(34\%), and fever(10$50 \%$ ). DMI usually occurs in the thigh, most commonly the vastus lateralis and medialis muscles. Bilateral involvement occurs in $8-30 \%$ of cases. Laboratory data is non-specific but can include leukocytosis and an elevated creatinine kinase or erythrocyte sedimentation rate. MRI shows a hyperintense signal on T2-weighted images. Muscle biopsy showing microvascular disease with muscle necrosis and inflammation can confirm the diagnosis. As with our patient, diagnosis is often delayed due to the rarity of the disease and misdiagnosis of more common etiologies such as infections or neuropathies. The differential diagnosis includes focal myositis, polymyositis, pyomyositis, deep vein thrombosis, nectrotizing fasciitis and calciphylaxis. Our patient's case highlights features that suggest DMI, including her characteristic pain and swelling associated with typical MRI findings. MRI findings are generally confined to a single muscle or muscle group and lack the focal, well-demarcated intramuscular fluid or gas collections of pyomyositis or necrotizing fasciitis. Our patient also lacks the rapid progression of severe systemic symptoms that herald necrotizing fasciitis or pyomyositis. Other helpful diagnostic features include the absence of dermal and subcutaneous fat necrosis seen in calciphylaxis.

DIAGNOSING VITAMIN B12 DEFICIENCY - WHEN NUMBERS LIE! Nataliya Mar; Anita Pudusseri; Robert E. Graham. Lenox Hill Hospital, New York, NY. (Control ID \#1320149)

LEARNING OBJECTIVE 1: Recognize the limitations of serum cobalamin levels in diagnosing vitamin B12 deficiency and be aware of alternative tests available.

LEARNING OBJECTIVE 2: Know when to institute empiric B12 replacement therapy.

CASE: A 75-year old male presented with generalized weakness and dizziness for 3 weeks. His medical history included colon adinocarcinoma treated with resection 13 years ago, hypertension, and mild thrombocytopenia of unclear etiology. He denied fevers, dyspnea, chest pain, bleeding, or diarrhea. Vital signs were notable for a blood pressure of 106/82 with positive orthostatics. Physical exam was unremarkable while rectal exam showed heme-negative stool. Laboratory analysis was as follows: white cell count, $5,900 / \mu \mathrm{L}$; hemoglobin, $8.9 \mathrm{~g} / \mathrm{dL}$ (from $13.4 \mathrm{~g} / \mathrm{dL} 4$ months ago); mean corpuscular volume, $121.4 \mathrm{fL}$; platelets, $79,000 / \mu \mathrm{L}$ (from $105,000 / \mu \mathrm{L} 4$ months ago); lactate dehydrogenase, $527 \mathrm{U} / \mathrm{L}$; total bilirubin, $1.8 \mathrm{mg} / \mathrm{dl}$; direct bilirubin, $0.4 \mathrm{mg} / \mathrm{dl}$; haptoglobin, $<8 \mathrm{mg} / \mathrm{dl}$; negative direct antiglobulin test; negative urine hemosiderin; reticulocyte count, $1.6 \%$; and thyroid stimulating hormone, $1.13 \mathrm{mclU} / \mathrm{ml}$. Peripheral smear showed $3+$ schistocytes, moderate spherocytes and ovalocytes, and hypersegmented neutrophils. The patient received 1 unit of packed red cells and vitamin B12/folate deficiency was suspected. However, folate level was $12.3 \mathrm{ng} / \mathrm{ml}$ [normal, $3.1-17.5 \mathrm{ng} / \mathrm{ml}$ ] and vitamin B12 level was $489 \mathrm{pg} / \mathrm{ml}$ [normal, $254-1320 \mathrm{pg} / \mathrm{ml}$ ]. Repeated vitamin B12 level was $321 \mathrm{pg} / \mathrm{ml}$. Bone marrow biopsy showed erythroid hyperplasia, megaloblastic changes, hypersegmented neutrophils, increased iron stores, and normal flow cytometry/cytogenetics. Empiric daily cyanocobalamin (1 gram intramuscularly) was initiated with good hematologic response. With time, the following tests were resulted: methylmalonic acid, $28 \mathrm{nmol} / \mathrm{ml}$ [normal, $<0.4 \mathrm{nmol} / \mathrm{ml}$ ]; homocysteine, $72.4 \mathrm{mcmol} / \mathrm{L}$ [normal, $3.2-10.7$ $\mathrm{mcmol} / \mathrm{L}]$; and markedly increased anti-parietal cell and intrinsic factor antibody titers. A formal diagnosis of vitamin B12 deficiency due to pernicious anemia was made.

DISCUSSION: This case highlights the difficulty in establishing a diagnosis of vitamin B12 deficiency due to repeatedly false-negative serum cobalamin $(\mathrm{sCbl})$ assay results. In clinical practice, $\mathrm{sCbl}$ levels are the diagnostic test of choice due to being easily-accessible and costeffective [2]. However, they lack sensitivity and specificity, missing many patients with tissue B12 deficiency [1,3]. Several reports also commented on false-negative $\mathrm{sCbl}$ results due to intra-assay variability when B12 deficiency was clinically evident $[4,5]$. As such, measurements of serum methylmalonic acid (MMA) and homocysteine (Hcy) levels, which are elevated in $\mathrm{B} 12$ deficiency, become useful when sCbl levels are equivocal but clinical suspicion remains high [2]. Short-term intra-individual variations in MMA and Hcy values are also common, leading to false normal results [6]. Accumulating evidence indicates that serum holotranscobalamin (holoTC), an earlier marker that decreases before total sCbl, may be superior to other assays and has been proposed for use as a firstline diagnostic test $[2,3,7]$. A major challenge arises when deciding on whether to institute empiric B12 therapy when laboratory results contradict the clinical picture or additional tests are pending. In this setting, it is prudent to start replacement therapy and continue treatment provided a clinical improvement is evident or until a final diagnosis can be made $[2,6]$. 
DIAGNOSIS OF DISSEMINATED HISTOPLASMOSIS WITH A PERIPHERAL SMEAR Richard A. Lau. UCLA - Olive View, Sylmar, CA. (Control ID \#1330160)

LEARNING OBJECTIVE 1: Recognize that the different diagnostic modalities available for the confirmatory diagnosis of systemic histomplamosis infection can take days to weeks to result, with the exception of the examination of the buffy coat on a peripheral smear which can be performed and completed within hours.

LEARNING OBJECTIVE 2: Recognize the presentation of disseminated histoplasmosis.

CASE: Pt is a 38 year old Guatamalan male, recently moved to California, presenting with 1 month history of fevers, fatigue, abdominal pain, and diarrhea. The patient initially experienced symptoms of subjective fevers and fatigue. Several days later, he began experiencing unremitting abdominal pain. He localized the pain as diffusely over his abdomen and characterized the pain as a "hot" and "gurgling" sensation. Then several days prior to presentation, the patient began having profuse, non-bloody, watery diarrhea. Initial vital signs were T 35, HR 122, BP 114/67, RR 20 and O2 sat 99\% on RA. Physical exam was significant for leukoplakia, hepatosplenomegaly, minimal diffuse abdominal tenderness to deep palpation, and mildly enlarged bilateral inguinal LNs. Labs notable for a CBC diff showed WBC $1.9(79 \%$ neutrophils, 13\%lymphs,6\%mono) Hb/Hct 8.6/26 PLT 25. CT scan of the c/a/p revealed enlarged mediastinal $\mathrm{LAD}$, bilateral nodular opacities in the lungs, HSM, multiple enlarged retroperitoneal LNs extending from the level of the kidneys to the common iliac arteries. The patient's clinical presentation at this point was concerning for HIV. Subsequently, HIV lab tests were sent, which came back positive, with HIV VL of 683,395 copies $/ \mathrm{mL}$ and a CD4 count of $1 /$ cubic mm. After confirmation of HIV, additional tests were done throughout the hospital course to assess for disseminated histoplasmosis, including urine histoplasma antigen, fungal blood cxs, bone marrow bx, and a retroperitoneal $\mathrm{LN} \mathrm{bx}$ and cx. All such tests were consistent for disseminated histoplasmosis. However, of all the tests mentioned, the peripheral smear confirmed the diagnosis days to weeks prior to all the other tests. After confirmation with peripheral smear, pt was started on liposomal amphoteracin with good response to therapy.

DISCUSSION: In the patient described in the case, his clinical course was consistent with disseminated histoplasmosis. Numerous modalities are available for the diagnosis of disseminated histoplasmosis. The most common of which is the histoplasma antigen of the urine, serum or csf. Other diagnostic tests include fungal cxs of blood or tissue specimens, histoplasma serologies, and histologic examination of biopsy specimens. However, all such tests are limited by time, up to days to weeks, either because of processing or procurement. Examination of the buffy coat on a peripheral smear is an old and forgotten, but fast and inexpensive test for the diagnosis of certain disseminated infections. The detection of small yeasts in circulating mononuclear cells is very specific for histoplasmosis, as no other fungi which causes human disease can have such an appearance within white blood cells. However, because of the lack of data on the use of buffy coats to diagnose disseminated histoplasmosis, the exact sensitivity is unknown. Therefore, although a negative buffy coat examination does not rule out disseminated histoplasmosis, a positive result is very specific and can expeditiously guide initial therapy, even before the results of the other diagnostic modalities (serologies, cultures, biopsies) return days to weeks later.

DiAGNOSTIC DELAY IN ACHALASIA Ahmet A. Oktay. Saint Francis Hospital, Evanston, IL. (Control ID \#1338687)

LEARNING OBJECTIVE 1: Considering achalasia within the differential diagnosis of vomiting and dysphagia

LEARNING OBJECTIVE 2: Timely use of barium swallow evaluation CASE: 26 year-old African American female with no significant past medical history presented to the emergency department with history of vomiting and dysphagia which was described as inability to swallow solids or liquids. Patient's symptoms began one week after returning from a trip to Nigeria. She was initially experiencing epigastric burning pain accompanied by difficulty swallowing solids without vomiting. Her symptoms progressively worsened within weeks and for the past 4 months she had had bouts of emesis typically within 60 seconds after any oral intake. She had lost 32 pounds over this time period. She denied any intentional component of dysphagia. She reported being evaluated for her symptoms during 5 different visits to the emergency departments in Chicago-land area. She denied any hospital admissions. She was given treatment with proton pump inhibitors and antibiotics for possible gastroesophageal reflux disease and Helicobacter Pylori. But she hadn't had any relief of her symptoms. Her physical examination revealed body mass index at $17.7 \mathrm{Kg} / \mathrm{m} 2$, blood pressure at $96 / 67 \mathrm{mmHg}$, heart rate at 82 . Physical exam was otherwise unremarkable. Her laboratory tests were not significant except albumin $3.2 \mathrm{mg} / \mathrm{dL}$, lipase $12 \mathrm{IU} / \mathrm{L}$, hemoglobin $10.7 \mathrm{mg} / \mathrm{dL}$, bicarbonate $16 \mathrm{mmol} / \mathrm{L}$. Obstructive series in ED demonstrated nonobstructive bowel gas pattern. Patient underwent esophagogastroduodenoscopy procedure, which revealed food retention in distal esophagus. Lower esophageal sphincter was passed with modest pressure on endoscope. Barium swallow evaluation of the esophagus demonstrated sharp tapering of the distal esophagus characteristically known as a "birds beak" sign. There was subsequent dilatation of the distal half of the esophagus. Loss of peristalsis was noted within the distal esophagus and a fluid-fluid level was noted indicative of esophageal dysmotility. Those findings were suggestive of achalasia. Patient underwent motility studies at another medical center, which confirmed the diagnosis (verbal report).

DISCUSSION: This case illustrates the importance of considering achalasia within the differential diagnosis of vomiting and dysphagia. Achalasia findings can sometimes misinterpreted as GERD or H. Pylori infection. Timely use of barium swallow evaluation would have established the correct diagnosis earlier in this case. And with the use of pneumatic dilatation or surgical myotomy procedures, the severe gastrointestinal discomfort for 4 months and the significant weight loss would have been prevented.

DIET PILL SHOCK Sean Condon ${ }^{1}$; Eric Green ${ }^{2}$; Manzoor Rather ${ }^{2}$ ${ }^{1}$ Drexel University College of Medicine, Philadelphia, PA; ${ }^{2}$ Mercy Fitzgerald Hospital, Darby, PA. (Control ID \#1339083)

LEARNING OBJECTIVE 1: Recognize potential side effects of over the counter (OTC) diet pills

LEARNING OBJECTIVE 2: Recognize caffeine induced anaphylaxis CASE: A 24-year-old African American female presents with 12 hours of diaphoresis and severe stabbing abdominal pain, which awoke her from sleep. She also notes the appearance of a red pruritic maculopapular rash covering her body including palms and soles, periorbital edema, lip swelling, and shortness of breath. She began an over the counter (OTC) diet pill, D4 Thermal Shock, seven days ago. She had a similar, yet less severe reaction to another diet pill called Oxyproelite. Medical, social, and family histories are non-contributory, and dietary history is notable for very rare ingestion of caffeinated beverages. She has no known allergies. Physical exam was remarkable for a temperature of 103.9 degrees F, tachypnea, diffuse expiratory wheezes bilaterally, a diffuse blanching maculopapular rash and 2+ lower extremity edema. Labs including EKG, chest X-ray, electrolytes, complete blood count, and a urine drug screen were normal. She was treated initially with epinephrine, then admitted and treated with antihistamine and steroid therapy. Her symptoms abated within 36 hours and she was discharged home with instructions to avoid OTC weight loss pills. DISCUSSION: This patient's history strongly suggests anaphylaxis. Her lack of other dietary or medication triggers, past history of an adverse reaction from diet pills, and her recent use suggests the OTC diet pill was the culprit. A large number of OTC products have been marketed for weight loss, and $15.2 \%$ of adults have used a weight loss supplement. However, the adverse effects of OTC diet pills have been poorly described. In part this is likely because almost all of these agents are marked as dietary supplements and therefore are not subject to FDA oversight for safety and efficacy, nor are they subject to after-market collection of adverse effects. Anaphylaxis has never previously been reported as a side effect from OTC diet pills. On review of the ingredient list for both diet pills, the only common ingredient was caffeine. Thus, this patient may be allergic to caffeine or one its metabolites. Although rare, there have been 5 documented cases of caffeine causing hypersensitivities including urticaria, rash, anaphylaxis and even death. A 21 year-old boy presented very similarly with pruritus of the soles and palms, generalized urticaria, cough, wheezing 
and shortness of breath after drinking a cup of coffee. Cases were confirmed with skin prick tests and oral tolerance. In all cases, caffeine induced urticaria/ anaphylaxis was dose dependent. It remains to be seen whether caffeineinduced urticaria represents IgE-mediated type I allergy or a nonallergic hypersensitivity. Further studies are needed to determine the exact pathophysiology. Regardless, due to the prevalence of caffeine and its use, a caffeine allergy may be the underlying cause of many cases inappropriately termed idiopathic anaphylaxis.

DIFFICULTY GETTING UP IN THE MORNING: A CASE OF THYROTOXIC PERIODIC PARALYSIS Cliff W. Hampton ${ }^{1}$; Joseph R. Sweigart ${ }^{1}$; Susan M. Nikels ${ }^{1}$; Michael Hanley ${ }^{2}$. ${ }^{1}$ University of Colorado Denver, Aurora, CO; ${ }^{2}$ University of Colorado Denver, Aurora, CO. (Control ID \#1327979)

LEARNING OBJECTIVE 1: Recognize thyrotoxic periodic paralysis as an uncommon complication of thyrotoxicosis

LEARNING OBJECTIVE 2: Treat thyrotoxic periodic paralysis with nonselective beta blockade and cautious potassium supplementation CASE: A 47 year old man of Eastern European descent was brought to the emergency department after awakening with profound muscle weakness. He got out of bed at 6:00 am, but was so weak he fell to the floor and was unable to get up. After some time, he pulled his phone to the floor and called emergency medical services. He had noticed some mild myalgias in his right thigh the previous night, but had no other recent symptoms. On exam, he was afebrile, heart rate was 90 beats per minute and blood pressure 160/94 $\mathrm{mmHg}$. He had flaccid proximal quadriparesis and diffuse mildly-diminished reflexes. Mental status, sensation, speech, and cranial nerves were normal. He denied recent exertion, high carbohydrate intake, or stimulant use. However, he did admit to being under significant stress related to personal life events. Labs revealed severe hypokalemia of $1.7 \mathrm{mmol} / \mathrm{L}$ and subsequent ECG showed associated changes. Further investigation revealed an undetectable thyroid stimulating hormone and a moderately elevated free thyroxine value of $2.2 \mathrm{ng} / \mathrm{dL}$. Other labs were unremarkable. He did not present with obvious symptoms of hyperthyroidism, but in retrospect did recall recent episodes of loose stool, fine hand tremors, and palpitations over the preceding months. Thyrotoxic periodic paralysis was diagnosed and the patient was admitted overnight for close monitoring. After receiving propranolol and gentle potassium repletion the hypokalemia resolved, the muscle weakness improved dramatically, and the ECG normalized. Prior to discharge, he was started on methimazole and beta blockade. As an outpatient, he was later diagnosed with Grave's disease.

DISCUSSION: Thyrotoxic periodic paralysis (TPP) is a well-described complication of hyperthyroidism. It is relatively common in patients of Asian lineage. However, it remains quite rare in other races. Patients tend to be young adult men presenting with flaccid paralysis that progresses from the lower to the upper extremities. Sensation is unaffected. Patients most often present early in the morning after an overnight onset. The event is often preceded by high carbohydrate intake, alcohol intake, or strenuous exercise. This patient's attack seems instead to have been precipitated by personal life stress, suggesting a common pathway involving glucocorticoid and insulin excess. It is thought that the excess thyroxine simultaneously increases the adrenergic response, the activity of the $\mathrm{Na}+/ \mathrm{K}+$-ATPase pumps and insulin release, all of which shift potassium ions intracellularly. The functional hypokalemia causes hyperpolarization of the motor neurons leading to paralysis. Neither the severity, duration, nor the etiology of the hyperthyroidism are correlated with TPP. When treating TPP, total body stores of potassium are not decreased and overaggressive potassium repletion can be fatal. Potassium repletion is, however, important in prevention of fatal hypokalemic arrhythmias in the acute setting. Ongoing supplementation for prophylaxis against attacks is not helpful. Administration of a non-selective beta blocker may be as useful as potassium chloride administration and is without the risk of rebound hyperkalemia. These should be taken until the patient reaches a euthyroid state and definitive treatment for hyperthyroidism is completed.

DIFFUSE LARGE B-CELL LYMPHOMA OF THE NECK COMPLICATED BY TRACHEAL PERFORATION Janine Adamczyk Montefiore Medical Center, Bronx, NY. (Control ID \#1340131)
LEARNING OBJECTIVE 1: Recognize Non-Hodgkin lymphomas as highly invasive and rapidly enlarging cancers which can lead to unusual complications such as tracheal perforation

LEARNING OBJECTIVE 2: Identify the critical role of a good physical exam in clinical decision-making

CASE: An 80 year old female with a history of asthma presented with shortness of breath and painless swelling of her neck. An intravenous contrast enhanced CT scan showed a $7.3 \times 5.8 \mathrm{~cm}$ central neck mass with necrotic areas and surrounding adenopathy. A lymph node biopsy established the diagnosis of a diffuse large B cell lymphoma which was classified as stage $2 \mathrm{~B}$. Increasing respiratory distress necessitated endotracheal intubation of the patient. Chemotherapy according to the R-CVP protocol (Rituximab, Cyclophosphamide, Vincristine, Prednisolone) was initiated. A repeat CT neck showed a significant reduction in tumor mass after the first cycle. However, the patient failed multiple attempts to wean her from the ventilator. Two days into the second therapy-cycle she developed a sudden inspiratory stridor. A CT neck revealed a tracheal perforation at the thoracic inlet with the endotracheal tube extending through the defect. Bronchoscopy confirmed a tracheal perforation between the 2nd and 7th tracheal ring with extensive tissue necrosis. The patient was managed conservatively by bridging the defect with an endotracheal tube and by starting antibiotics. One week later, she was successfully extubated and remained stable, a repeat bronchoscopy after two weeks showed beginning granulation of the lesion.

DISCUSSION: Respiratory distress is a common complaint encountered by internal medicine physicians. Malignancy inside or outside of the thorax is an important differential diagnosis. With an incidence of 7 cases per 100 000 person years, diffuse large B-cell lymphoma represents the most common histologic subtype of Non-Hodgkin lymphoma (approximately $25 \%$ ). NHL can be highly invasive and rapidly enlarging. The disease most commonly arises from lymph nodes in the neck and abdomen but can virtually affect any tissue. Depending on the stage, 5-year survival rates between 21 and 83 percent can be achieved after treatment with systemic chemotherapy with or without involved-field radiotherapy. Perforation of the gastrointestinal tract in patients with gastrointestinal lymphoma undergoing chemotherapy has been well described. Only a few case reports link lymphoma with tracheal perforation. Tracheal rupture itself is a rare condition most commonly caused by head and neck trauma or by intubation, tracheostomy, bronchoscopy, radiotherapy or infection. In this case, rapid tissue necrosis due to chemotherapy-induced vascular compromise caused the patients' tracheal perforation. Previously described risk factors for tracheal perforation are mainly anatomic in nature, e.g. tracheal distortion by lymph node collections or smoking-related COPD. The management includes surgical closure, stent placement or conservative therapy. Complications such as mediastinitis and tracheal stenosis have been described. In our patient, an interventional closure was not possible due to the inability to anchor a tracheostomy for ventilation below the defect. Overall, the detection of inspiratory stridor in physical exam led to rapid diagnosis of this potentially lethal complication and a positive outcome in this case.

DIFFUSE PULMONARY CAVITARY LESIONS AS A RARE PRESENTATION OF ACUTE SARCOIDOSIS. Bassam Yaghmour; George Yaghmour; Mustafa Abas; Michael Eichenhorn. HFHS, Detroit, MI. (Control ID \#1340088)

LEARNING OBJECTIVE 1: Recognize pulmonary cavitary sarcoidosis as a rare form of acute sarcoidosis.

LEARNING OBJECTIVE 2: Review of Pulmonary cavitary sarcoidosis including pathogenesis, characteristics, natural history and treatment. CASE: A 27-year-old African-American female presented to the hospital with complaints of acute upper respiratory tract infection symptoms and blurred vision of five days duration. These symptoms were overlying a one month history of progressive dyspnea and non-productive cough associated with constitutional symptoms including fatigue, subjective fever, chills, night sweats and a $15 \mathrm{lb}$ weight loss. There was no history of sick contacts, travel outside of Michigan, occupational exposure, or TB exposure. Chest x-ray showed multifocal cystic changes, patchy opacity, and hilar lymphadenopathy. CT scan of the chest showed multiple, bilateral cavitations as well as 
hilar and mediastinal lymphadenopathy. Laboratory data showed a high level of serum angiotensin converting enzyme 172. The patient was placed in isolation and TB was ruled out. Histological findings of specimens obtained by transbronchial lung biopsy and by transbronchial fine-needle aspiration of mediastinal lymph nodes demonstrated noncaseating granulomatous inflammation consistent with sarcoidosis. Infection, autoimmune, and malignancy evaluations were negative. Since the patient was symptomatic with dyspnea and blurry vision, she was started on prednisone $40 \mathrm{mg}$ daily. As the patient began to improve clinically, she decided to forgo an ophthalmologic exam to evaluate for uveitis and further radiological images.

DISCUSSION: Pulmonary cavitary lesions are a rare and often confusing manifestation of sarcoidosis. Clinicians' unfamiliarity with this atypical presentation is compounded by the relative scarcity of reported cases in the United State as compared with other parts of the world. A retrospective series performed in France estimated the prevalence of cavitary sarcoidosis with no pulmonary comorbidities to be $2.2 \%$. Cavitations do not appear to be specific to radiologic stage of disease as they have been reported in stages II through IV. Regardless, most cases were reported in patients with severe and active sarcoidosis including a majority associated with extrapulmonary sarcoidosis. Additionally, the serum angiotensin-converting enzyme was more than 2 times the upper limit of normal range in most of the reported cases indicating highly active disease. The pathogenesis of cavitation in the context of sarcoidosis is unclear as there have been several mechanisms hypothesized to contribute, however, it is accepted that cavitary lesions are related to ischemic necrosis with extrusion of hyaline material from conglomerate sarcoid granulomas. The evolution of the cavitations is unpredictable as a literature review of case reports has shown inconsistent improvement versus developments of complications when the patient is treated with steroids for extrapulmonary manifestations. Despite the observation that some patients' cavitations completely resolve, complications frequently arise in patients with cavitary sarcoidosis. These include aspergilloma, hemoptysis, secondary infection, and pneumothorax. Selected case reports in which there was a reduction in cavitary lesions suggests that systemic steroids are effective in the treatment of cavitary sarcoidosis.

DISSEMINATED INTRA-VENTRICULAR ENDOCARDITIS (DIVE) SYNDROME Sara Taherkhani; Damanpreet Grewal; Robert Biederman; Triston Smith. Allegheny General Hospital, Pittsburgh, PA. (Control ID \#1335078)

LEARNING OBJECTIVE 1: Recognize the embolic manifestations of endocarditis in a timely fashion and utilize the best method for accurate diagnosis of intra-ventricular endocarditis.

CASE: A 30 year old male IVDU was initially found unresponsive at home and though alert in ED, became progressively lethargic, tachypneic and hypotensive requiring intubation and MICU admission. CBC showed leukocytosis of 25,000 with a left shift. Physical exam was remarkable for bilateral subconjunctival hemorrhages, diffuse rhonci, 2/6 apical systolic murmur, needle tracks on upper extremities and Janeway lesions on several fingers and toes. Empiric broad spectrum antibiotics were initiated after blood cultures were drawn, given the concern for endocarditis, which subsequently grew MSSA. CT showed bilateral, scattered, subcentimeter pulmonary nodules, multiple splenic infarcts and various small foci of intraparenchymal hemorrhages in the brain, all suggestive of septic emboli. Initial TTE was normal but subsequent TEE with $3 \mathrm{D}$ reconstructed images showed multiple highly mobile densities extensively throughout the left ventricle and the outflow tract attached to the surface, as well as a large mobile mass attached to the anterior infindibulum of the right ventricle with resulting outflow tract obstruction. No valvular involvement was present, nor were there any structural abnormalities. Unfortunately the patient presented too late in the course of his illness and was no longer thought to benefit from surgical intervention. Despite aggressive antibiotic therapy, he succumbed to intracranial hemorrhage.

DISCUSSION: Although Staphylococcus aureus is capable of infecting normal heart valves, infection is usually preceded by injury to the endocardium by various turbulent hemodynamic mechanisms. In an IVDU without known preexisting cardiac disease or immunosuppression, infection is presumed to be due to trauma to heart valves by contamination with debris and bacteria during injection. Though rare, mural endocarditis has been reported in the past but our case is the first to diffusely involve both ventricles with diagnosis by TEE alone. Although TTE is considered the best method for detecting masses in the LV apex, TEE proved to be superior in our patient. Hence, when suspicion for endocarditis is high, it is advised to proceed directly to TEE as its sensitivity and specificity are greater than TTE. Embolization, with its high mortality, is the most feared complication of mural endocarditis, which warrants attention and awareness as early diagnosis with surgical intervention, when possible, is of paramount importance in the patient's outcome.

DISSEMINATED HISTOPLASMOSIS IN A PATIENT WITH CROHN'S DISEASE WHO IS MAINTAINED ON INFLIXIMAB, PREDNISONE AND AZATHIOPRINE Abdallah Abou Zahr'; Pete Yunyongying ${ }^{1,2}$. ${ }^{1}$ university of texas south western medical center, Dallas, TX; ${ }^{2}$ Dallas VA medical center, dallas, TX. (Control ID \#1333846)

LEARNING OBJECTIVE 1: Diagnose histoplasma infection in patients with crohn's disease who are maintained on immunosuppressive regimen.

CASE: The patient is 43 yo man with Crohn's Disease who is maintained on azathioprine, prednisone and infliximab. He presented to his private gastroenterologist with worsening odynophagia. Esophagogastroduodenoscopy (EGD) was performed and showed inflammatory changes in the oropharynx including the epiglottis. He was managed as Crohn's flare so his immunosuppressive regimen was increased. However the patient's symptoms worsened. So additional work up was done including laryngoscopy and biopsy of the epiglottis was taken. The result showed histoplasma epiglotittis. The patient's immunosuppressive regimen was tapered down and he was started on amphotericin initially and then itraconazole with rapid improvement in his symptoms.

DISCUSSION: Crohn's disease is associated with significant infectious complications, both treatment and nontreatment related. A broad spectrum of treatment related infectious complications has been reported and these include viral, bacterial, protozoal and fungal infections. Among these infections disseminated histoplasma is interesting because its presentation can mimic crohn's flares. Data regarding fungal infections in IBD patients who are maintained on immunosuppressive regimens including infliximab is limited to case reports and case series. 240 cases of histoplasmosis associated with use of tnf alfa inhibitors has been reported to FDA AERS till September 2008. Clinically 70 to $90 \%$ of patients with disseminated histoplasmosis have gastrointestinal involvement. The GI symptoms include nausea, vomiting, diarrhea, bleeding, abdominal pain, weight loss, obstruction, and perforation. Many of these GI symptoms overlap with Crohn's flare. Also there is significant overlap among the endoscopic findings that include plaques, small polyps, mucosal edema, ulcerations, strictures, and masses. So the differentiation between histoplasma infection and crohn's flare is not easy given the significant overlap in clinical and endoscopic features. However it is very important to be able to recognize histoplasma infection in these patients because the treatment of Crohn's disease with immunosuppressive regimens can worsen histoplasma infection. In this case there are several clinical clues that hint at infectious reasons for patient presentation. First the patient did not have symptoms of Crohn's flare elsewhere as he usually has. Another important clue is that symptoms became worse after the escalation of the immunosuppressive regimen which is unusual for Crohn's flare. A high index of suspicion is needed in patients with worsening symptoms despite being on multiple immunosuppressive regimen. A diagnostic work up should be initiated and it includes fungal sputum and blood cultures, histoplasma antibodies and antigen in blood and urine, as well as histopathological testing. However histopathology is only $50 \%$ sensitive. This case presents a diagnostic challenge because of significant overlap between some of treatment related infectious complications of Crohn's disease and Crohn's flare. A high index of suspicion is needed because missing the diagnosis of disseminated histoplasmosis can result in deleterious consequences. 
DOC, WHY DOES MY KNEE HURT? GOUT, THE FALL, THIS TICK OR NONE OF THE ABOVE? Jonathan Kirsch; Jack Kuritzky; Mukhtar Adem. University of North Carolina, Chapel Hill, NC. (Control ID \#1339600)

LEARNING OBJECTIVE 1: Understand the approach to a patient with acute inflammatory joint pain through history and synovial fluid analysis. LEARNING OBJECTIVE 2: Recognize the clinical presentation and three pathogenic mechanisms of primary meningococcal arthritis

CASE: A 66 year-old man presented with left knee swelling, pain, fever malaise and a rash. He had been treated with doxycycline for 3 days for a presumed tick-borne illness after finding a tick on his arm. His knee pain and swelling progressed and he presented to the hospital when he could no longer ambulate. $\mathrm{He}$ was also involved in a motorcycle accident one month prior to admission, but his knee pain resolved quickly. He had a history of gout, affecting his toes and knees, but hadn't had any recent flares. He was afebrile. His neurological exam was unremarkable. He had a petechial rash on his lower extremities. He had normal passive range of movement in both of his knees without reproduction of pain. His left knee was tender to palpation with a moderate-sized effusion. His serum white blood cell count was $16,900 \mathrm{x}$ $10^{\wedge} 9 / \mathrm{L}$ with a normal serum chemistry profile. Arthrocentesis of his left knee effusion yielded monosodium urate crystals and 5800 white blood cells per high powered field with $90 \%$ neutrophils. There were no organisms on gram stain. Joint fluid was sent for culture. The patient was started on prednisone for presumed gout and continued on doxycycline for possible tick-borne illness. His knee pain and swelling didn't improve. On day 2 of his hospitalization, the culture from his arthrocentesis yielded gram-negative diplococci and ceftriaxone was initiated. On day 4, this organism was speciated as Neisseria meningitidis. Blood cultures remained negative. He was diagnosed with primary meningococcal arthritis.

DISCUSSION: Knee pain with effusion is commonly encountered by the internist. An approach to determine the etiology of knee pain should include an analysis of synovial fluid. While gram stain, culture, and polarized light microscopy may yield a specific diagnosis, the leukocyte count helps to categorize the effusion. Non-inflammatory conditions, such as osteoarthritis, tend to have a synovial leukocyte count $<2,000$ per $\mathrm{m}^{\wedge} 3$. Counts between $2,000-100,000$ leukocytes per $\mathrm{mm}^{\wedge} 3$ suggest inflammatory etiologies such as gout, rheumatoid arthritis, and other rheumatologic conditions. Finally, septic arthritis is associated with at least 15,000 leukocytes per $\mathrm{mm}^{\wedge} 3$ in the synovium and it often exceeds 100,000 per $\mathrm{mm}^{\wedge} 3$. This patient had a knee infection with Neisseria meningitides and no other signs of neurologic involvement, consistent with a diagnosis of primary meningococcal arthritis. Infections with Neisseria meningitides present with rheumatologic manifestations in 2$10 \%$ of cases. Patients with arthritic complications tend to be less than 60 years old and present with monoarticular symptoms affecting the knees and ankles. Approximately $30 \%$ of patients also experience an erythematous, maculopapular rash. The development of meningococcal arthritis can be via one of the following mechanisms: direct hematologic spread, immunologic mechanism with meningococcal antigen-antibody complexes and disseminated intravascular coagulation causing hemarthrosis in fulminant meningococcemia.

DOCTOR'S DILEMMA: DIFFERENTIATING BETWEEN REALITY AND DELUSION IN A PSYCHOTIC PHYSICIAN Molly A. Fisher ${ }^{4}$; Jessica Campbell ${ }^{1,2}$; Lilia Cervantes ${ }^{1,2}$; Aaron Scott ${ }^{2}$; Kilmberly Breidenback ${ }^{3}$. ${ }^{1}$ Denver Health, Denver, CO; ${ }^{2}$ University of Colorado, Denver, CO; ${ }^{3}$ University of Colorado, Denver, CO; ${ }^{4}$ University of Colorado, Denver, Denver, CO. (Control ID \#1335872)

LEARNING OBJECTIVE 1: Highlight the challenges faced when providing medical care to physicians with psychiatric illness

LEARNING OBJECTIVE 2: Differentiate between different forms of delusions of parasitosis

CASE: : BP is a 55-year-old retired physician who was admitted to our medicine service for observation after suffering a scalp laceration and small subdural hematoma related to a fall outside of a tavern. As the patient sobered, he informed the team that he was a family physician with a rare case of disseminated dermatophytosis from tinea capitis. He believed the tinea was growing in the sutures of his skull and causing a host of neurological symptoms. Due to the patient's vast medical knowledge, he created convincing explanations to validate his bizarre beliefs. His explanations were medically sound and created considerable debate among the team as to what extent to entertain the validity of his ideas and work-up his complaints. During his hospitalization the team recognized that whenever a test or study disproved his medical logic he created an alternative explanation and his certainty about his diagnosis never wavered. BP became outraged when the team denied his requests for specific studies and quickly discredited doctors who disagreed with him. His frustration led to angry outbursts and agitation. The situation was complicated by possible delirium secondary to the subdural hematoma, and the patient's use of prescribed amphetamines. Ultimately, he was admitted to the inpatient psychiatry unit.

DISCUSSION: Delusions of parasitosis is a rare condition in which a person believes that he or she is infested by parasites. It is categorized by DSMIV as a somatic-subtype delusional disorder. Rarely delusions of parasitosis involve tinea, a fungal infection limited to the dermis. There are no previously documented cases in the medical literature of a physician with delusions of parasitosis. The greatest challenge in treating this case stemmed from BP's medical training. It was unsettling to watch as his distorted expertise wreaked havoc on his emotional state; we could imagine ourselves in his place. Our clinical decision-making was muddled with our emotional reaction to the situation making it difficult to decide how far to indulge his delusions. At times we hoped to find disseminated dermatophytosis to legitimize him and calm our own fears. This unusual case demonstrates the importance of acknowledging our reaction to patients, the difficulty of treating a physician and the challenge of effectively managing delusions.

DON'T GET TRAPPED: ASSESSING PATIENTS WITH ULNAR NEUROPATHY Meena Raj; Jillian S. Catalanotti. George Washington University, Washington, DC. (Control ID \#1334655)

LEARNING OBJECTIVE 1: Develop a differential diagnosis of intrinsic hand muscle atrophy

LEARNING OBJECTIVE 2: Recognize indications for surgical involvement of ulnar nerve entrapment

CASE: A 60 year old woman with a history of diabetes mellitus presented to primary care clinic with right hand pain. The patient is a legal secretary and right hand dominant. Her symptoms started after a blood draw was taken from the ulnar aspect of her right wrist six months prior to her visit. Since then, she noted severe pain with associated weakness and diminished dexterity of her fingers. On physical exam, vital signs were normal and she was well-appearing. Her neck revealed full range of motion and a non-tender cervical spine. She had $2+$ radial and ulnar pulses bilaterally and her wrist exam showed no deformity, full range of motion, and no strength deficits. She had severe atrophy of the first dorsal interosseous muscle as well as the hypothenar and loss of abduction of her fifth digit (Waternberg's sign). Tinel's sign was positive distal to Guyon's canal but negative over the ulnar nerve at the elbow. There was mildly reduced sensation and prolonged two-point discrimination along the fifth digit. These clinical findings were consistent with ulnar nerve palsy. Work-up was initiated with EMG, which confirmed a severe distal ulnar neuropathy. MRI demonstrated an abnormal growth distal and anterior to the styloid process of the ulna, near the course of the ulnar nerve. She was referred to orthopaedic surgery and underwent surgical decompression in Guyon's canal. Pathology of the mass revealed mature adipose tissue. The patient was disappointed postoperatively, with no improvement in her significant atrophy as well as ongoing pain and decreased motor control of the fifth digit.

DISCUSSION: Muscle atrophy generally occurs where there is complete, chronic muscle denervation. Diagnosing intrinsic hand muscle atrophy includes both elucidating an etiology and discerning the location of the lesion. Systemic conditions which affect the hand muscles often present bilaterally and include Pancoast syndrome, Hansen's disease, rheumatoid arthritis, amyotrophic lateral sclerosis and poliomyelitis. Unilateral findings may be due to chronic repetitive trauma, fractures of the wrist bones, 
thrombosis, aneurysms, or masses such as lipomas, ganglia, or synovial cysts. Sites of possible nerve involvement include the anterior horn cells (i.e. ALS), nerve roots (i.e. cervical spondylosis), brachial plexus, peripheral nerves (median or ulnar nerves), or the muscle itself (i.e. congenital myopathies). In our patient, severe ulnar neuropathy was secondary to entrapment of the nerve at the wrist by what appeared to be a lipoma and was likely exacerbated by the chronic repetitive movements from her long career as a legal secretary. Indications for surgery in ulnar nerve entrapment are lack of improvement in symptoms 2-3 months after attempts at conservative therapy, progressive palsy/paralysis or clinical evidence of a long-standing lesion such as demonstrated in our patient by muscle wasting. Post-operative prognosis is affected by duration of nerve entrapment and severity of weakness and paresthesias. In chronic cases, improvement may be limited but further progression of damage can be halted.

DON'T OVERLOOK THE WARNING SIGN. Thandar Aung ${ }^{1}$; Harvey Friedman $^{2}$; Glynn . Elliott ${ }^{1}$; Karoon Nititham ${ }^{3}$. 'Saint Francis Hospital, Evanston, IL; ${ }^{2}$ Saint francis hospital, evanston, IL; ${ }^{3}$ North Suburban Cardiology Group, Evanston, IL. (Control ID \#1311848)

LEARNING OBJECTIVE 1: Transient ischemic attack(TIA) is a serious warning sign for potential stroke, cardiovascular events and death Therefore, finding consistent with TIA deserves urgent workup due to risk of significant morbidity.

LEARNING OBJECTIVE 2: Complex aortic arch plaque has taken its place along with Atrial Fibrillation(AF) and Carotid Atherosclerosis as an important cause of cerebral ischemia and peripheral embolism. Aortic arch plaque is also an independent predictor of Myocardial Infarction, Cerebral ischemia and vascular death.

CASE: 84 y/o Male, heavy smoker, with past medical history of hypertension,COPD and type 2 Diabetes came to ER because he experienced four episodes of TIA within one month. During each episode, he suddenly lost consciousness for few minutes followed by facial droop, slurring of speech and weakness of the extremities and completely resolved within an hour. In each episode, only one side of the body was affected but both sides had shown symptoms across multiple episodes. He also had 2 similar episodes last one and a half years ago. No significant family history. Physical examination, MRI of the brain, Transthoracic echocardiogram and Electrocardiogram were unremarkable. He was discharged on the same day with Aspirin and Statin. Seven months later, he was admitted to the hospital for new onset AF and heart failure due to non ST elevation Myocardial Infarction(NSTEMI). In addition to AF, physical examination revealed he had tachypnea, diffuse wheezing, decreased breath sound in bilateral lower lung field and bilateral lower extremities edema. He underwent CT chest with contrast showed no pulmonary embolism, bilateral pleural effusion, enlarged heart and there were two approximately $1 \mathrm{~cm}$ in thickness non calcified ulcerated linear plaque in each ascending and descending portion of aortic arch. Thoracocentesis with fluid study showed Transudate pleural effusion. He was medically treated for heart failure, COPD exacerbation, AF and NSTEMI . TEE was done for ruling out thrombus in the heart. TEE showed $1 \mathrm{~cm}$ thickness ulcerated plaque with protruding thrombus and a finger like mobile thrombus $\left(1^{*} 0.6 \mathrm{~cm}\right)$ in ascending portion of aortic arch . Later, he underwent angiogram, stent placement. Cardioversion was done for AF but his heart rhythm was controlled for only 2 days. He was discharged with Aspirin ,Plavix and Statin. After one month, his heart failure was controlled but heart rthym was still uncontrollable.

DISCUSSION: Complex aortic plaque is the plaque that contains mobile thrombi or ulceration or is $4 \mathrm{~mm}$ or greater in thickness. Particularly Plaques $4 \mathrm{~mm}$ or more in thickness proximal to origin of left subclavian artery are associated with cerebral ischemia and constitute one third of patient with cryptogenic stroke. Ulcerated, Thrombotic and Non calcified plaques are more likely to have complication and are high risk of recurrence vascular event. TEE best evaluates large vessel/ aortic disease . Patient with cryptogenic stroke, TEE revealed a potential embolic source more than TTE. There is still no firm evidence-based algorithm of treatment for atherosclerotic plaque and thrombus. A retrospective study indicates a likely benefit from statin drugs. The result for an ongoing randomized trial of warfarin vs antiplatelet therapy(Aspirin and plavix) are still pending. There is insufficient evidence to recommend prophylactic endarterectomy or aortic arch stenting for purposes of stroke prevention.

DON'T FORGET YOUR SOCIAL HISTORY! TOBACCO SMOKING ASSOCIATED ACUTE EOSINOPHILIC PNEUMONIA IN A 20-YEAR-OLD FEMALE Robert Freed; Benjamin Lloyd. The Reading Hospital and Medical Center, West Reading, PA. (Control ID \#1339926)

LEARNING OBJECTIVE 1: Recognize tobacco smoking and other toxic inhalants as a trigger for Acute Eosinophilic Pneumonia

LEARNING OBJECTIVE 2: Distinguish Acute Eosinophilic Pneumonia from Acute Respiratory Distress Syndrome (ARDS) based on bronchoalveolar lavage findings

CASE: A 20-year-old previously healthy female presented to the emergency department with fever, myalgias, dyspnea and productive cough. She was on no medications, family history was unremarkable and patient had no allergies. Review of social history showed no recent travel, or exposure to toxins or chemicals, but she did admit to smoking cigarettes for the first time, one pack-per-day, during the two weeks leading up to her initial presentation. Her temperature was $38.0 \mathrm{~F}$, heart rate 130 , respiration rate 28 , blood pressure $98 / 63$ and oxygen saturation of $92 \%$ on nonrebreather at 10 liters/min. Her examination was significant for diffuse rhonchi, but was otherwise normal. Lab work showed leukocytosis of $28.4 \mathrm{~K} / \mu \mathrm{L}$ with neutrophilia and CXR exhibited diffuse alveolar infiltrates. She was started on broad spectrum antibiotics for presumed atypical pneumonia. Her respiratory failure progressed rapidly, requiring intubation and mechanical ventilation three days after onset of her symptoms. Serial chest $\mathrm{x}$-rays and a chest $\mathrm{CT}$ scan showed progressively worsening dense alveolar infiltrates bilaterally. Extensive infectious workup, including bacterial, viral and fungal entities was negative. TEE showed no evidence of heart failure or pulmonary hypertension. Serial labs were significant for rising serum eosinophilia, reaching a peak of $14 \%$. Bronchoalveolar lavage was obtained, which showed marked eosinophilia of $43 \%$. Antibiotics were discontinued and intravenous corticosteroids were initiated leading to a dramatic improvement in her respiratory failure and extubation within 48 hours.

DISCUSSION: First described in 1989, roughly 150 cases of acute eosinophilic pneumonia (AEP) have been reported worldwide. Common to all reported episodes is an extrinsic inflammatory insult, most commonly cigarette smoking, but other toxin exposures have been also described including tear gas, gasoline, inhalation from fireworks and cleaning supplies, acute cocaine usage and one reported post-September 11th rescuer. Symptoms and imaging are poorly specific and patients are often initially treated for presumed infectious causes. Much like acute respiratory distress syndrome (ARDS), AEP requires the exclusion of cardiogenic pulmonary edema and pulmonary infection, but the presence of eosinophilia is a key criteria of AEP. Eosinophil count of $25 \%$ or greater in bronchoalveolar lavage fluid is required for the diagnosis of AEP, but concomitant rise in serum eosinophil count to above $10 \%$ may also be present. Despite being uncommon, with early diagnosis and appropriate steroid treatment, the prognosis of affected AEP patients remains excellent with survival exceeding $95 \%$ based on two retrospective studies of 55 patients. In summary, despite being exceedingly uncommon, AEP should be considered in the differential diagnosis of ARDS, in patients with suspected toxin exposure for whom antimicrobial treatments have failed.

DON'T TAKE HYPERCALCEMIA TOO LIGHTLY Rachel Anquez; Robin Klein. Emory University, Atlanta, GA. (Control ID \#1339551)

LEARNING OBJECTIVE 1: Identify classic clinical and laboratory features of light chain myeloma

LEARNING OBJECTIVE 2: Review the diagnostic serum and urine testing for multiple myeloma

CASE: A 76 year old female presents with back pain for six months. She reports progressively worsening back pain along with fatigue, weight loss, and dizziness. Two months prior, she was found to have hypercalcemia with elevated PTH levels. She underwent parathyroidectomy 
for hyperparathyroidism. However, her calcium remained elevated following her operation. Exam was notable for point tenderness over the thoracic spine. Laboratories revealed a creatinine of $1.35 \mathrm{mg} / \mathrm{dl}$, calcium $10.9 \mathrm{mg} / \mathrm{dl}$, albumin of $4.3 \mathrm{gm} / \mathrm{dl}$, and total protein of 7.5 $\mathrm{gm} / \mathrm{dl}$. WBC was $5,900 / \mathrm{mcl}$ and hemoglobin was $11.5 \mathrm{~g} / \mathrm{dl}$. No protein was detected on urinalysis. PTH was $82 \mathrm{pg} / \mathrm{ml}$. MRI of the spine revealed multiple spinal masses causing spinal canal stenosis and pathologic compression fractures. CT scan to evaluate for primary lesions found no suspicious masses but showing extensive lytic lesions throughout the osseous skeleton including multiple ribs and vertebrae, scapula, clavicles, and pelvis. Serum protein electrophoresis (SPEP) showed no monoclonal elevation in gamma globulins. Serum immunofixation revealed increased beta-2 microglobulin (386 mg/l). Serum free light chain (SFLC) analysis showed elevated kappa protein at $6209 \mathrm{mg} / \mathrm{dl}$. Urine protein electrophoresis (UPEP) showed 1.2 grams of protein with $78 \%$ kappa light chain paraprotein, and was confirmed with urine immunofixation. Bone marrow biopsy revealed a plasma cell dyscrasia consistent with myeloma.

DISCUSSION: Multiple myeloma (MM) is a plasma cell malignancy leading to excessive and abnormal antibody production. Myeloma can produce all classes of immunoglobulins as well as kappa light chains, lambda light chains, or any of the five types of heavy chains. Isolated light chain myeloma comprises $16-20 \%$ of myeloma cases. Patients typically present with anemia, renal failure, proteinuria, hypercalcemia, and a globulin gap. In our case, the presentation with hypercalcemia, bone pain, anemia and renal insufficiency was suspicious for myeloma. However, the diagnosis of MM was not pursued due to the elevated PTH, lack of proteinuria and absence of globulin gap. These values were falsely reassuring. As light chains are small proteins, they are usually not detected by urine dipstick and do not lead to the textbook globulin gap that is considered synonymous with MM. As such, proteinuria and globulin gaps are not prominent features of light chain myeloma. Furthermore, in our case, initial serum testing with SPEP was unremarkable. However, SPEP alone will not capture the diagnosis of MM in every patient and addition of serum immunofixation and UPEP increases the sensitivity significantly. $82 \%$ of MM patients will have a monoclonal band evident on SPEP. Addition of serum immunofixation increases the sensitivity of serum testing to $93 \%$, and addition of UPEP and urine immunofixation increases the sensitivity of detection to $97 \%$. In patients with suspected MM, testing with SPEP in combination with immunofixation, and confirmatory urine studies are necessary so as to not miss light chain disease. Conclusion The diagnosis of light chain myeloma is challenging due to the absence of classic findings such as proteinuria and globulin gap. Understanding the utility and limitations of serum and urine diagnostic testing is necessary to avoid missing the diagnosis.

DOUBLE THE DOSE OF DéJéRINE: A RARE CASE OF BILATERAL MEDIAL MEDULLARY INFARCT Lindsey Wooliscroft; Aiman Shokr; Nazrul Chowdhury; Matt Chua. Texas Tech University Health Sciences Center, Amarillo, TX. (Control ID \#1311077)

LEARNING OBJECTIVE 1: To recognize the heterogeneous clinical features of acute bilateral medial medullary infarction.

LEARNING OBJECTIVE 2: Appreciate the prognosis of this rare infarct.

CASE: A 59-year-old male with history of CAD, HTN and CABG 18 months prior, presented with right-sided weakness in the upper and lower extremities. On admission he was afebrile, BP 191/109 mmHg, HR $68 / \mathrm{min}$, RR $16 / \mathrm{min}$, and O2 sat of $97 \%$ on room air. At that time, he was alert and oriented and exam revealed motor strength was $5 / 5$ in the left side and $1 / 5$ on the right side, no sensory deficit, and cranial nerves were grossly intact. CT of the brain neither showed obvious infarct nor hemorrhage. However, within 36 hours, he developed weakness of the left upper and lower extremities with motor strength of $1 / 5$. There was decreased tone noted in all four extremities. Bilateral Babinski sign and hyperreflexia were also noted. The patient also began to exhibit mild to moderate dysarthria with dysphagia, confirmed by a swallow evaluation. The patient continued to have no sensory deficits. The MRI of the brain showed bilateral medullary pyramid infarctions, with increased signal intensity on diffusion weight as well as $\mathrm{T} 2$ and flare. His transthoracic echocardiogram and carotid Doppler were unremarkable. MRA revealed only mild atherosclerotic change in the vertebrobasilar system without suggestion of significant stenosis. There was no evidence for myelitis, or cervical compression myelopathy. He was monitored closely and stroke management was instituted, unfortunately there was no significant neurologic recovery during his hospital stay. He was subsequently transferred to a rehab hospital for further physical therapy.

DISCUSSION: Even unilaterally, medial medullary infarction is rareaccounting for approximately $0.5 \%$ of all acute brain infarctions. Bilaterally, this condition becomes even more uncommon with less than 20 reported cases by 2010 . Medial medullary infarction is characterized by Déjérine's syndrome, with consequent dysfunctions of hypoglossal nerve fibers (lingual palsy or dysarthria), medullary pyramids (contralateral hemiparesis), and the medial lemniscus (sensory loss). However, the symptoms are heterogeneous depending on the extent of the infarct, with motor pareses being the most common clinical manifestation in documented cases. Most of these infarcts result from infarction of the vertebral or anterior spinal artery or their small branches. Prognosis for these patients is guarded, with respiratory infections or respiratory failure being the most common cause of death. Therefore, it is imperative to continue to monitor respiratory function and watch for infection.

DRUG INDUCED ASEPTIC MENINGITIS ASSOCIATED WITH INTRATHECAL TRASTUZUMAB IN THE TREATMENT OF LEPTOMENINGEAL CARCINOMATOSIS FROM HER2/NEU POSITIVE METASTATIC BREAST CANCER. George Yaghmour; Doyle Thomas. henry ford health system, Detroit, MI. (Control ID \#1340231)

LEARNING OBJECTIVE 1: Recognize the significant adverse event associated with use of intrathecal trastuzumab as a first published report. CASE: 43 year old African American female who presented with a history of frontal headache, anorexia, weakness, and ataxia for the last 7 days. No focal neurologic deficits were noted. Her past medical history was significant for stage IV metastatic inflammatory breast ductal carcinoma, strongly positive $(+3)$ for the HER-2/neu oncogenic protein, ER positive / PR negative with metastasis to the liver and lungs. Following her diagnosis, she received neoadjuvant chemotherapy followed by a modified radical mastectomy, radiation, and adjuvant chemotherapy. Despite several chemotherapy agents, her disease continued to progress. In the emergency department the patient received MRI of her brain as well as a LP. An Abnormal enhancement of the leptomeningeal surfaces was found; suspicious for metastatic disease.CSF analysis was significant for elevated protein, low glucose, and 58 atypical cells which were determined as adenocarcinoma cells. Infectious etiologies were excluded. She was diagnosed with Leptomeningeal carcinomatosis. Upon admission the patient was a suitable candidate for intrathecal chemotherapy with trastuzumab. The patient was started on dexamethasone which did yield some improvement in aphasic symptoms. Twelve days after her admission, she received her first dose of $30 \mathrm{mg}$ of trastuzumab intrathecally via Ommaya reservoir. Within 2 hours of receiving trastuzumab, she had sudden mental status change with nausea and vomiting episodes as well as marked obtundation, severe dizziness, and urinary incontinence. She received a subsequent $\mathrm{CT}$ scan of her head which ruled out intracranial hemorrhage or acute ischemia. The Ommaya reservoir was not noted to be misplaced on imaging. An EEG suggested moderate encephalopathy but no seizure activity. Five days after administration, a repeat CSF analysis was positive for $9 \%$ malignant adenocarcinoma cells, slightly low glucose levels $(37 \mathrm{mg} / \mathrm{dL})$, elevated protein $(127 \mathrm{mg} / \mathrm{dL}), 3310$ red blood cells, and 50 white blood cells, $21 \%$ of which were neutrophils. With no infection etiology. She continued to have slurred speech, delayed reaction time, and difficulty following commands. Her case deteriorated without improvement.

DISCUSSION: Leptomeningeal carcinomatosis refers to the seeding of the leptomeningeal membrane of the CNS by malignant cells originating from solid tumors. LC is associated with significant morbidity and mortality and is diagnosed in 5\% of patients with breast cancer. Common presenting symptoms include headaches, mental status changes, cranial nerve palsies, hemiparesis, diplopia, and motor weakness. In general, 
median survival is less than 3 months from diagnosis, however if left untreated death typically occurs within 6 weeks. Intrathecal administration of trastuzumab has been described as a treatment for LC secondary to HER-2 positive breast cancer with positive results in overall survival and a lack of significant adverse effects. The use of intrathecal trastuzumab has been described in 10 published case reports using doses up to $100 \mathrm{mg}$ all without any significant adverse effects. This is the first published report of any significant adverse event associated with use of intrathecal trastuzumab. Clinicians must be aware of this potential adverse effect to a therapy with which limited experience exists in treating a rare but serious complication to metastatic Breast cancer.

DRUG INDUCED ASEPTIC MENINGITIS IN A HEALTHY YOUNG FEMALE Pinky Jha; Mallory Cohen. Medical College Of Wisconsin, Milwaukee, WI. (Control ID \#1310501)

LEARNING OBJECTIVE 1: To recognize Trimethoprim-Sulfamethoxazole (TMP-SMX) as a cause of aseptic meningitis in a healthy young female.

CASE: A healthy, immunocompetent 26 year old female with no significant past medical history presented to the ED with a severe headache and fever. The pain was located at the top of her head radiating to the neck. On exam she was in severe distress due to headache and had fever of 101.1 F. She had nuchal rigidity and positive kernig's sigh. She underwent a lumbar puncture and was started on empiric iv antibiotic for possible meningitis based on the history and exam. Cerebrospinal Fluid (CSF) analysis showed Glucose 51, CSF Protein 110, CSF WBC Count 152, CSF RBC Count 2, CSF Monocytes 16, CSF Polysegmented Neutrophils 28. The CSF gram stains showed no PMNS, few mononuclear cells, and no bacteria. CSF culture and viral PCR was negative. Infectious disease was consulted. On further questioning she gave a history of using TMP-SMX for a UTI prior to admission. After completing the three day course of TMP-SMX she had developed back pain followed by this severe headache and fever. Given her presentation and recent use of TMP-SMX a diagnosis of aseptic meningitis was made. IV antibiotics were stopped as per ID as the infectious disease workup was negative. She was discharged to home in stable condition after three days. She was advised to avoid trimethoprimsulfamethoxazole in the future.

DISCUSSION: : Aseptic meningitis is the cause of meningitis in a patient who has clinical and laboratory evidence of meningitis without a positive bacterial culture or viral PCR. Drugs, viruses, and malignancies are known causes of aseptic meningitis. Drug-induced aseptic meningitis is an uncommon and mysterious adverse reaction to some commonly used medications. This condition can mimic the signs and symptoms of true infectious meningitis. The most common drugs to cause aseptic meningitis are NSAIDs and antibiotics. The first documented case of drug-induced aseptic meningitis was reported in 1963 in a patient who had taken 2 tablets of sulfamethizole. Drug-induced aseptic meningitis is a rare but important and often-challenging diagnosis . Trimethoprim-Sulfamethoxazole has been shown in case reports to cause aseptic meningitis in immunocompromised and older patients. This is rarely shown in young, healthy females as in this patient.

DUODENAL DIVERTICULA AND ACUTE PANCREATITIS Sandipani Sandilya ${ }^{1}$; Andrea Porrovecchio ${ }^{2} .{ }^{1}$ Montefiore Medical Center, Bronx, NY; ${ }^{2}$ Montefiore Medical Center, Bronx, NY. (Control ID \#1334444)

LEARNING OBJECTIVE 1: Recognize duodenal diverticula as a possible etiology for acute pancreatitis

LEARNING OBJECTIVE 2: Understand the pathophysiology of duodenal diverticula and how they can play a role the etiology of acute pancreatitis

CASE: A 67 year old woman presented with sharp abdominal pain radiating to the back, nausea, and vomiting for one day. She had a history of cholelithiasis and was status post-laparoscopic cholecystectomy. She was taking celecoxib, pregabalin, and cyclobenzaprine, and denied any recent changes in medication. She was a smoker and had a 50 pack year history, but denied drugs or alcohol. She was afebrile and hemodynamically stable, and had epigastric tenderness and guarding on examination. Significant laboratory values were amylase of 1,829 and lipase of 8,125. An ultrasound revealed mild dilatation of the pancreatic and common bile ducts with no gallstones and a hypoechoic structure posterior to the head of the pancreas. Triglycerides were normal. The CT was consistent with pancreatitis and revealed a large juxtapapillary duodenal diverticula (JPDD) compressing the pancreatic head, pancreatic duct and common bile duct. The patient was treated with bowel rest, intravenous hydration, and pain control, and made an uneventful recovery.

DISCUSSION: Acute pancreatitis is extremely common cause of hospital admission, with an incidence of 79.8 cases per 100,000 in the United States, frequently encountered by the internist. The majority of cases are caused by alcohol and cholelithiasis. Rarer causes include hypertriglyceridemia, trauma (including iatrogenic), medications, infections, and autoimmune processes. Cases in which no etiology is found $(8-44 \%)$ are labeled "idiopathic" pancreatitis. The patient had an unexpected finding of JPDD on imaging which was an etiological factor for her acute pancreatitis. The incidence of JPDD increases with age - retrospective analyses of ERCPs have revealed a $6 \%$ prevalence among patients below 50,22\% among patients between 60 and 69 , and $30 \%$ among patients above 70 . While a congenital factor may be involved, the major etiology is thought to be due to an increase in intraduodenal pressure and a weakening of duodenal smooth muscles. The majority of diverticula are asymptomatic, but they have been associated with serious complications like hemorrhage and perforation, which are surgical emergencies. Compression of the pancreatic duct from distension of the diverticulum is implicated in the etiology of pancreatitis. A retrospective analysis on 433 patients who underwent ERCP over a 2 year period observed that the prevalence of "idiopathic" pancreatitis was $14 \%$ in patients with JPDD and $2 \%$ in patients without JPDD. The recommendation of the study was that JPDD be excluded before making a diagnosis of idiopathic pancreatitis, especially in older patients. There are no established guidelines on the management of duodenal diverticula. The main surgical option is diverticulectomy, which is not recommended for asymptomatic diverticula. In summary, idiopathic pancreatitis is a common diagnosis, and a number of studies support a pathophysiological role for JPDD. While there are no guidelines on the management of diverticula, it is important for the internist to be aware of the increasing prevalence of these anatomical abnormalities and their relationship to pancreatitis in an increasingly aging population.

ECTHYMA GANGRONOSUM SECONDARY TO METHICILLINSENSITIVE STAPHYLOCOCCUS AUREUS SEPTICEMIA IN A PATIENT WITH DRUG - INDUCED AGRANULOCYTOSIS. Shahrzad Shidfar; Jay Jahanmir. umassmemorial, Worcester, MA. (Control ID \#1311941)

LEARNING OBJECTIVE 1: Recognize Staphyloccous Aureus as a etiology of ecthyma gangronosum.

LEARNING OBJECTIVE 2: Identify predisposing factors to ecthyma gangronosum.

CASE: A 47 year-old man presented with five days of rash. The rash began on his fingers as red firm bumps or blisters and then spread to chest, arms, back, neck and face and roof of his mouth and a large red tender swelling in right axilla over a period of a week. These lesions became more painful and developed black centers over the next few days. He also had subjective fever, chills, sweats and nausea two days prior to presentation. He developed shortness of breath and right lower chest wall pain on the day of presentation. Eight days prior to his presentation, he had returned from a 10-day trip to Brazil where he he spent all his time in urban areas. He denied any sick contact or contact with sheep, sheepskins or animal skins while in Brazil. He had contact with domesticated parrots and had a few mosquito bites. He had history of hypertension and diabetes controlled with diet and exercise. He lived at home with his dog. He was afebrile with heart rate of hundred beats/minutes, respiratory rate of eighteen and normal oxygen saturation. He had several discrete red nodular plaques with black necrotic centers on right nasal ala, left inferior jaw, chest and abdomen. He had two pustules on the hard palate. There was approximately an eight $\mathrm{cm}$ firm, red and 
tender swelling in right axilla that was well demarcated with surrounding erythema and central black plaque. He had pleural friction rub at right lung base. There were no heart murmurs, hepatosplenomegaly, joint edema or peripheral stigmata of endocarditis. CBC revealed WBC $400 \mathrm{th} / \mathrm{mm} 3$ with $12 \%$ segmented cells, $75 \%$ lymphocytes, $13 \%$ monocytes, $\mathrm{Hb} 13 \mathrm{gr} / \mathrm{dl}$, Platelet $111000 \mathrm{th} / \mathrm{mm} 3$. HIV serology was negative. Cat scan of chest revealed multiple foci of ground glass opacities of varying sizes with bilateral axillary and mediastinal adenopathy and right lower lobe pneumonia. There were no valvular vegetations on the transthoracic echocardiogram; left ventricular ejection fraction was mildly depressed. Skin Punch biopsies revealed epidermal necrosis with prominent collection of gram positive cocci consistent with ecthyma gangronosum. Blood culture grew Methicillin sensitive Staphyloccous Aureus. On further questioning the patient said he took 5 doses of an over the counter medication called Dipyrone for isolated headaches in Brazil.

DISCUSSION: Ecthyma gangrenosum is an skin lesion commonly associated with Pseudomona aeruginosa septicemia in immunocompromised patients. Microorganisms invade the media and adventitia of vessels, causing ischemic necrosis. The most common predisposing factors include leukemia, Hodgkins' disease, chemotherapy and immunsuppression and lymphoma. Less commonly ecthyma gangronosum presents in patients with a history of burns, pneumonia, tuberculosis or urinary tract infection. Our case is unique in that the patient had none of the usual predisposing factors and skin lesions, which preceeded chest pain and shortness of breath by five days, were associated with methicillin -sensitive staphylococcus aureus septicemia. Our possible explanation for his Neutropenia is agranulocytosis as a side effect of Dipyrone. Dipyrone is an over the counter powerful analgesic that carries a small risk of causing agranulocytosis and widely used in Brazil. He was started on Naficillin and his Absolute Neutrophil Count recuperated to normal levels as the infection cleared.

EMBOLIC STROKE, A DELAYED SEQUELA OF BLUNT CHEST TRAUMA. Sonali Arora ${ }^{1}$; Auras R. Atreya ${ }^{1}$; Taraka V. Gadiraju ${ }^{1}$; Srikanth Penumetsa ${ }^{1,2}$; William L. Hiser ${ }^{1,2} .{ }^{1}$ Baystate Medical Center/Tufts University School of Medicine, Springfield, MA; ${ }^{2}$ Baystate Medical Center/Tufts University School of Medicine, Springfield, MA. (Control ID \#1339845)

LEARNING OBJECTIVE 1: Recognize coronary artery dissection as a potential complication of blunt chest trauma.

CASE: A 30 year old healthy man presented to an outside facility with word finding difficulty and right sided neglect lasting for few minutes during a wrestling match. An MRI brain showed 3 punctate lesions involving the left frontal and parietal hemisphere, suggestive of embolic etiology. A transthoracic echocardiogram (TTE) showed a $1.5 \times 1.5 \mathrm{~cm}$ mass present in the left ventricular (LV) apex. The patient was transferred to our institution for further evaluation by cardiac surgery and possible removal of mass. On detailed questioning, he reported an incident of blunt chest trauma during a martial arts exhibition fight that took place 2 years back. He had experienced acute chest pain radiating to both arms, diaphoresis and dyspnea following the episode. $\mathrm{He}$ was taken to an ER but was sent home after a period of observation. Reportedly, no diagnosis was made. Given this history, a pre-operative cardiac catheterization was done to exclude coronary artery dissection. It showed $30 \%$ stenosis in mid-LAD without any other significant obstructive lesion. A transesophageal echocardiogram showed akinesis of the LV apex and confirmed TTE finding of a mass, the appearance of which was consistent with an apical thrombus. The decision to operate was deferred and patient was started on anticoagulation. A cardiac MRI done 2 weeks later showed akinesis of the apical segment. Patient's remote history of blunt trauma was consistent with possible left anterior descending artery (LAD) dissection causing apical infarction and akinesis predisposing to formation of LV thrombus.

DISCUSSION: An embolic stroke as a sequela of remote blunt chest trauma is a rare clinical presentation. Blunt chest trauma can cause various acute cardiac complications like arrhythmias, myocardial rupture, injury to coronary arteries resulting in dissection and myocardial infarction. However, delayed consequences such as left ventricular thrombus resulting in thromboembolic phenomena are reported infrequently. LAD is the most commonly affected coronary vessel by blunt traumatic injuries, likely due to its vulnerable anatomical position on the anterior aspect of the heart. A variety of mechanisms including intimal tear, rupture and spasm have been implicated in the pathogenesis of myocardial infarction after blunt chest trauma. A subacute development of an intimal tear might escape early diagnosis such as this case. A history of trauma should be sought in otherwise healthy patients with only a single vessel disease. Patients who present with stable dissection without occlusive thrombus on angiography can be managed conservatively. However, in the event of significant thrombus burden, angioplasty and stenting might be necessary. In summary, although coronary artery dissection leading to myocardial infarction in the setting of blunt chest trauma is rare, it should remain a consideration.

EMPLOYMENT RELATED BACK PAIN Kenneth Cerreta. Tulane University Health Sciences Center, New Orleans, LA. (Control ID \#1311864)

LEARNING OBJECTIVE 1: 1. Recognize the complications of vertebral osteomyelitis 2 . Identify the differential diagnosis of neurologic dysfunction in the setting of back pain 3. Understand the pathophysology of spinal epidural abscess

CASE: A 54 year-old man presented with one month of progressively worsening back pain and bilateral leg numbness and weakness, progressing to paralysis one week prior to admission. He noted urinary incontinence for the past three days, but no fevers or night sweats. Vital signs were normal. Cranial nerves were normal, and the upper extremities had full strength and equal sensation. He had decreased sensation to light touch and cold stimuli inferior to the nipples; lower extremities had $0 / 5$ strength, flaccid tone, upgoing toes, and five beats of clonus. Rectal tone was flaccid. There was point tenderness over the T1-3 vertebral processes. MRI revealed a large mass destroying the T2 vertebral body and extending into T1 and T3 vertebral bodies, the paraspinal soft tissue, and the epidural compartment from T1-4, compressing the thecal sac and deforming the spinal cord. Biopsy of the mass demonstrated filamentous fungi consistent with coccidiomycosis. On further questioning, the patient reported that he had been working as a landscaper in western Texas prior to the onset of his weakness. A detailed evaluation demonstrated no evidence of immunologic dysfunction.

DISCUSSION: Spinal cord dysfunction is a common problem encountered by the general internist. Unlike other diagnostic dilemmas, there is no one diagnosis that stands out as being more prevalent than the others. As a result, it is important that the internist have a systematic method for approaching spinal cord dysfunction to systematically evaluate all potential etiologies. Also unlike other diagnostic dilemmas, the physical examination is particularly important to the evaluation of spinal cord dysfunction. One diagnostic method is to trace the nerves starting from the cerebral cortex caudally. The level where neurologic dysfunction begins is typically $1-2$ levels caudal to the site of the lesion. In this case, cranial nerve function was not impaired and upper extremities had full strength and sensation with dysfunction beginning at T4. The location of pathology, therefore was likely to be two levels rostral to T4. An MRI was subsequently obtained, confirming the destructive lesion located at T2. The epidural space of the spine, unlike the that of the skull, is a space between the ligamentum flavum/posterior longitudinal ligament and the dura. This space consists of loose fatty tissue, lymphatics, small arteries, and the epidural venous plexus. Microorganisms can gain access to this space through hematogenous spread, direct extension from infected vertebral bodies, or direct inoculation during instrumentation. While in the space, organisms can grow rapidly, especially in the thoracolumbar region where this space is larger and contains more infection prone fatty tissue. The neurologic sequela are directly related to degree of spinal cord impingement. While coccidiomycosis is a rare pathogen for the epidural space, especially in an immunocompetent patient, it is not unheard of. Coccidiomycosis, unlike other fungal infections, can infect the immunocompetent host, and the general internist must be aware of this potential pathogen affecting the central nervous system. As in the case, the history, particularly employment and travel, is instrumental in raising pre-test probability of the diagnosis.

ENCEPHALOPATHY DUE TO HYPERAMMONEMIA IN A PATIENT WITH HEREDITARY HEMORRHAGIC TELAGECTASIA: A CASE REPORT Alvin Wycoco; Shebene Chacko; Jose Concepcion; Jitendra Patel. Kingsbrook Jewish Medical Center, Brooklyn, NY. (Control ID \#1335746) 
LEARNING OBJECTIVE 1: Recognize symptoms of hereditary hemorrhagic telangectasia.

CASE: A 70-year-old female with a history of recurrent nose bleeds presented to the emergency room with an acute change in mental status, persistent epistaxis, coffee ground emesis and rectal bleeding. She was brought to the hospital after becoming severely agitated and confused. Past medical history was significant for a brain aneurysm with surgical clipping. Patient was hemodynamically stable and had a significant flapping tremor on presentation but was otherwise neurologically intact. Blood drawn on admission revealed Hemoglobin $9.3 \mathrm{~g} / \mathrm{dl}$ and a hematocrit $28 \%$. The rest of the $\mathrm{CBC}$ and metabolic panel were within normal limits. Ammonia was also elevated at $1.08 \mathrm{ug} / \mathrm{ml}$ and stool guaiac was positive. CT of the head on admission was normal except for the surgical clips. Contrast abdominal MRA revealed hypertrophied common hepatic artery and collateral branches from the superior mesenteric artery extending to the hepatic parenchyma with extrahepatic vascular anomalies suggestive of arteriovenous malformation. Small telangectasias were suspected in the spleen. The patient was subsequently diagnosed with OslerWeber-Rendu Syndrome and transferred to the ICU for persistent bleeding. The patient required intubation with worsening mentation and progressively increasing ammonia levels. She was started on intravenous fluids, blood transfusions, intravenous PPI, octerotide drip, lactulose and decompressed via gastric suctioning. Nasal bleeding and GI bleeding spontaneously resolved later in the course with marked improvement of ammonia levels. Further vascular imaging MRI studies showed multiple telangectasias in the abdomen and aorta. The patient was extubated uneventfully and returned to baseline mental status prior to discharge with complete resolution of bleeding.

DISCUSSION: Hereditary Hemorrhagic Telangectasia also known as OslerWeber-Rendu Syndrome, is a genetic disorder inherited in an autosomal dominant pattern. This disorder manifests by mucocutaneous telangiectases and arteriovenous malformations and typically presents with epistaxis. This disorder shows lesions in the nasopharynx, central nervous system, lungs, liver, spleen as well as the urinary and GI tracts. In the United States frequency has been estimated at 1 case per 16,500 persons in a Vermont study, Guttmacher AE et al. HHT can be identified with diagnostic imaging studies such as contrast enhanced MRI or CT scans particularly in the vasculature of affected organs. Endoscopy may reveal telangiectasias in patients with GI bleeding. Hyperammonemia may occur as a complication of HHT as patients develop hepatic arteriovenous shunts, in turn causing hepatic encephalopathy. Ammonia entry to the brain is a primary cause of neurologic disorder. Treatment of HHT includes multi-system therapy. Controlling bleeding especially those in the brain and GI tract must have emergent intervention such as intracranial clipping of aneurysms and GI endoscopy to identify varices and AVM's. Protein intake should be limited, if not stopped, to help prevent encephalopathy with hyperammonemia. Lactulose aids in acidifying colonic contents to inhibit diffusion of ammonia back into the blood. Genetic therapy has not yet been successful in treatment of HHT, however awareness and detection of HHT may aid in improvement of outcome before significant clinical complications occur.

EOSINOPHILIA SECONDARY TO TOXOCARA CANIS Laila Shiekh Sroujieh; Mona Hassan; Mayur Ramesh. Henry Ford Hospital, Detroit, MI. (Control ID \#1334281)

LEARNING OBJECTIVE 1: Approach to a patient with severe Eosinophilia

LEARNING OBJECTIVE 2: Diagnostic Approach to Toxocara canis CASE: A 25-year-old male patient with history of autism and end stage renal disease presented to the ER with fever and chills associated with abdominal pain. Basic blood work showed evidence of severe eosinophilia [54\%; $9.99 \mathrm{~K} / \mathrm{uL}])$. The patient was admitted for further diagnostic evaluation and to rule out hypereosinophilic syndrome. Hematology was consulted and a bone marrow biopsy was performed and showed reactive eosinophilia without evidence of hypereosinophilic syndrome. The patient denied any history of allergy. Infectious work up including stool for ova and parasites and fungal panel was unremarkable. CT scan of the abdomen, chest and pelvis revealed focal hypodensities in the liver and soft tissue nodules with ground glass halos within the lungs. LFTs were within normal limits. Clinically, there was no evidence of specific organ involvement. Autoimmune work up including RF, ANA, ESR and CRP were within normal limits. Immunoglobulin panel revealed mildly elevated IgG. The patient's medications were reviewed and none of his medications were known to cause hypereosinophilia. After a thorough history was taken, it was noted the patient spent much time with his pet dog. Given his history of autism and exposure to a dog, it was likely patient may have contracted a parasite from the dog's saliva. It was decided to test the patient for Toxacara Canis antibodies. The antibodies returned positive and the patient was started on albendazole. The patient's eosinophilia improved with treatment.

DISCUSSION: Eosinophilia is not an uncommon finding in clinical practice and when it is associated with additional signs and symptoms, it can guide physicians to eventually establish a certain diagnosis. Secondary or reactive eosinophilia can develop in response to parasitic helminthic infections or allergens as well as in the setting of solid tumors or lymphomas. Rarely, eosinophilia is clonal in nature resulting from hematopoietic stem cell mutation. Eosinophilia is defined as a blood eosinophil count exceeding 500 cells/uL. Eosinophilia can further be differentiated into mild (500 to 1500 cells/uL), moderate (1500 to 5000 cells/uL), and severe (> 5000 cells/ uL). The level of blood eosinophilia is an incorrect assessment for the potential of eosinophil-mediated tissue damage. However, an eosinophilic cell count of $>1500$ cells/uL is classically considered the level above which organ damage is more likely to occur. Helminthic parasite associated eosinophilia can be constant or fluctuate over time and can be observed at any level of severity. Toxocariasis can be contracted after ingestion of soil/vegetables contaminated by excrement from infected dogs and is often relatively asymptomatic. Although stool examination for ova and parasites are warranted in patients with eosinophilia, these examinations are insensitive and therefore serologic testing is recommended in all eosinophilic patients in the appropriate clinical suspicion.

EOSINOPHILIA WORK UP IN PRIMARY CARE-THINKING BEYOND THE USUAL CAUSES Leena Jalota; Madan R. Aryal; Richard Alweis. Reading Hospital and Medical Center, West Reading, PA. (Control ID \#1335685)

LEARNING OBJECTIVE 1: Recognize how to diagnose and treat Non Allergic Rhinitis with Eosinophilia Syndrome (NARES) in a patient presenting with eosinophilia

CASE: Non-allergic rhinitis with eosinophilia syndrome (NARES) is a relatively rare condition with an estimated prevalence of $13-33 \%$ in patients with non-allergic rhinitis. It presents with symptoms similar to allergic rhinitis, but is characterized by absence of atopy and more than $20 \%$ eosinophils in the nasal cytology. We present a case in which a prolonged work-up for eosinophilia was finally diagnosed as NARES. A 40 year old female with past medical history of Multiple Sclerosis, stable on glatiramer acetate, and obstructive sleep apnea, presented to the outpatient clinic with profuse watery rhinorrhea, nasal pruritus, and nasal obstruction for one month. She also complained of occasional shortness of breath and loss of smell, which she specifically noticed while cooking. Review of systems was negative except as noted. Family history was non contributory. Physical examination was negative except for occasional crackles noted in the bibasilar lung fields. She received one week of amoxicillin for possible bronchitis, but her clinical condition did not improve. Blood work was performed, and the differential of the compete blood count revealed an eosinophlilia of $13.3 \%$. On further questioning for possible causes of eosinophilia, she denied weight loss or a loss of appetite, joint pain, eczema, rashes, tick exposure or recent history of travel outside the United States. Further workup for eosinophila was carried out, including: chest radiograph, pulmonary function tests with methacholine challenge, TSH and HIV antibody, all of which were negative. She was ultimately referred for allergen skin testing, which was also negative. Serum IgE and total immunoglobulin were normal. While total immunoglobulin and total IgE levels in nasal secretions were normal, nasal cytology revealed more than $25 \%$ eosinophils. Serum IgE antibody to specific allergen was absent. She was treated with intranasal beclomethasone. There was significant improvement in her symptoms over the next few weeks, although she continues to have recurrences several times per year

DISCUSSION: NARES usually presents with sneezing, watery rhinorrhea, nasal obstruction, anosmia and nasal itching, precipitated by nonspecific irritants and weather changes. It is more common in females, with some cases having a positive family history. The pathophysiology of NARES is poorly 
understood, but a key component involves a self-perpetuating, chronic eosinophilic nasal inflammation with development of nasal micropolyposis and polyposis. Anosmia is a prominent feature, which can differentiate it from allergic rhinitis. It can be associated with sleep apnea and aspirin sensitivity. Diagnose is made by nasal eosinophilia, in the absence of allergy in patient's history, negative skin tests and normal IgE. NARES responds well to nasal steroids and there may also be benefit from treatment with oral antihistamines.

\section{EOSINOPHILIC PNEUMONIA AFTER INFLUENZA VACCINATION} Mouhamad Mansour ${ }^{1}$; Daniel A. King ${ }^{2}$; Rishi Sharma ${ }^{1}{ }^{1}$ Henry Ford Hospital, Detroit, MI; ${ }^{2}$ Wayne State University, Detroit, MI. (Control ID \#1336498)

LEARNING OBJECTIVE 1: To review the clinical manifestations of chronic eosinophilic pneumonia

LEARNING OBJECTIVE 2: To report an association between influenza vaccine and the development of chronic eosinophilic pneumonia

CASE: A 74 year old non-smoker male with history of asthma and allergic rhinitis presented with a 3-week history of malaise, low-grade fever, nonproductive cough and shortness of breath. He denied recent travel, sick contacts, pet exposure, inhalation of chemicals, tobacco, or starting new medications. He had received his yearly influenza vaccine a week before the onset of his symptoms. On exam, the patient was in no distress. Pulse oximetry measured $95 \%$ on room air. Auscultation revealed fine crepitations in lung bases bilaterally. Laboratory exam was remarkable for leukocytosis $(30.6 \mathrm{k} / \mathrm{uL})$ with $71 \%$ eosinophils. Chest radiography showed slight atelectasis and effusion at the right lung base, and high-resolution chest computed tomography demonstrated bilateral lower lobe airspace disease with ground glass opacities and septal thickening, compatible with eosinophilic pneumonia. Further testing showed increased $\mathrm{IgE}(918 \mathrm{IU} / \mathrm{mL})$ but testing for Aspergillus-specific IgE was negative, as were p-ANCA, and cANCA. Sputum culture was negative. The patient was started on treatment with oral prednisone, with remarkable improvement of respiratory symptoms, radiographic appearance and resolution of peripheral eosinophilia. A spirogram, done 2 weeks after the initiation of steroids, was normal.

DISCUSSION: Chronic eosinophilic pneumonia (CEP) was first described in 1969 as a potentially life-threatening illness with fever, night sweats, weight loss and severe dyspnea. Important characteristics include association with asthma, "photo-negative" of pulmonary edema x-ray apperance, and rapid response to corticosteroid treatment. Approximately $50 \%$ of patients have history of asthma with a mean onset in the fourth decade of life, with a female predominance. The etiology of the CEP is idiopathic, and is diagnosed after considering other differentials of pulmonary eosinophilia. Those typically include helminthic and other infections, allergic bronchopulmonary aspergillosis, churg-strauss syndrome, malignancy, medications, toxins, and vaccines. Vaccination with inactivated respiratory syncytial virus reportedly results in pulmonary eosinophilia with substantial inflammation on re-infection, but we did find reports of association with influenza vaccine. Given that our patient had no clearly identifiable triggers, administration of influenza vaccine may have triggered eosinophilic pneumonitis in this case.

EXPAND YOUR MIND (AND BRAIN) WITH KNOWLEDGE OF C. NEOFORMANS Bobbie Jo Dodson; Jason Halperin. Tulane University Health Sciences Center, New Orleans, LA. (Control ID \#1338783)

LEARNING OBJECTIVE 1: 1. Recognize the manifestations of Cryptococcus neoformans infection 2. Identify the differential diagnosis of headache in an HIV-positive patient 3. Understand the role of serum cryptococcal antigen in the diagnosis and monitoring of extra-pulmonary disease.

CASE: A 38 year-old man presented with two weeks of headaches and a tender enlarging head mass. Over the past week, he had noted a large fluctuant mass over the right parietal region. He had no fevers, altered mental status or nuchal rigidity. He was HIV positive, with a CD4 count of 560 and no history of opportunistic infections. His vital signs were normal, as were his cardiac, pulmonary, abdominal and neurologic examinations. CSF analysis yielded clear fluid with a normal opening pressure; there were 4 WBCs, 1 RBC, 69.5 protein, 85 glucose, VDRL negative, and negative cryptococcal antigen. An MRI of the brain demonstrated an epidural subgaleal soft tissue mass eroding through the cranial bone into the sub-pericranial space enhancing with contrast and consistent with an abscess. Laboratory studies were unrevealing with the exception of a positive serum cryptococcal antigen(Titer 1:32). Cranial bone biopsy grew encapsulated yeast, positive for Cryptococcus neoformans. Neurosurgery debrided the abscess with craniectomy and dissection confirmed an intact dura with cryptococcal epidural abscess and overlying osteomyelitis. He was started on intravenous liposomal amphotericin B and oral flucytosine for a six-week course.

DISCUSSION: The general internist commonly encounters headache in an HIV-infected patient as a diagnostic dilemma. The differential diagnosis is broad, including including HIV-associated aseptic meningitis, cryptococcal meningitis, HSV meningoencephalitis, CNS toxoplasmosis, primary central nervous system lymphoma, and infective intra-cranial abscesses. Timely contrast-enhanced imaging and CNS analysis are necessary for evaluation and prompt diagnosis. Unrecognized infectious or malignant causes of headache in an HIV positive patient can be fatal. CNS infections represent the most common extrapulmonary site of Cryptoccocal neoformans infection; manifestations include meningitis, cryptococcomas, and rarely epidural abscesses. Those most at risk for extra-pulmonary infection include patients with HIV, Hodgkin disease, long-term corticosteroid therapy, transplant recipients, TNF-inhibitor therapy or patients undergoing chemotherapy.. $95 \%$ of HIV-infected patients have a positive serum cryptococcus antigen in the setting of cryptococcal meningitis. $83 \%$ of patients with extrapulmonary disease have a positive serum crpytococcal antigen, which most likely demonstrates disseminated disease. Persistently elevated serum cryptococcal antigen in HIV-infected patients carries a poor prognosis and likely indicates ongoing production of fungal spores. In the majority of patients the serum cryptococcal antigen titers decrease over time, yet it has not been shown to be useful in the monitoring of invasive disease. Headache in an immunocompromised patient can alert the general internist to primary CNS involvement or a disseminated disease process. Extra-meningeal Cryptococcus is a rare presentation of headache but must be considered for immediate surgical intervention and prompt initiation of antifungal therapy.

FROM EMPTYNESS TO A TOTAL ECLIPSE OF THE HEART Jerson Munoz Mendoza; Ricardo Correa; Willy Marcos Valencia Rodrigo; Veronica Pinto; Kargi Atil. University of Miami-Jackson Memorial Hospital, Coral Gables, FL. (Control ID \#1339793)

LEARNING OBJECTIVE 1: Recognize panhypopituitarism as a cause of cardiac tamponade

LEARNING OBJECTIVE 2: Identify the clinical presentation of empty sella syndrome

CASE: A 39-year-old Hispanic female presented to the emergency room with progressively worsening shortness of breath, pleuritic chest pain and fatigue. The information was provided by her family, who described a $30-$ lb-weight lost over 6 month, denied fever, cough or sick contacts. The patient had hypothyroidism and familial hypokalemic periodic paralysis, with four female relatives affected by the latter. She was in acute respiratory distress. Her skin was dry and cold skin, without lesions or hyperpigmentation, absent pubic and axillary hair. Her pulse was $110 \mathrm{bpm}$ and blood pressure was $50 \mathrm{mmHg}$. She had a $7-\mathrm{cm}$ jugular vein distention. The thyroid was not palpable. The lungs were clear to auscultation bilaterally. The heart sounds were distant without murmurs, rub or gallops. She had a $1+$ bilateral pitting edema below the knees. Her blood tests showed normocytic, normochromic anemia, leukocytosis and normal platelet count, hypokalemia, non-anion gap metabolic acidosis with increased lactic acid, and elevated creatinine. Liver tests were within normal limits. The TSH was normal $(0.84 \mu \mathrm{IU} / \mathrm{mL})$. The EKG showed low voltage QRS complexes. A chest x-ray showed interstitial pulmonary edema. A transthoracic echocardiogram revealed pericardial effusion, signs of diastolic collapse of the right atrium, and normal ventricular function. Emergent pericardiocentesis removed $100 \mathrm{~mL}$ of straw-colored fluid, yet the patient remained hemodinamically unstable, requiring increasing doses 
of vasopressors. Infectious and rheumatologic causes were ruled out. A stimulation test with $250 \mu \mathrm{g}$ of cosyntropin showed serum cortisol of 1.0 and $2.8 \mu \mathrm{g} / \mathrm{dL}$, and IV steroids were started. The pressors were decreased but could not be weaned off. A hormonal panel was obtained, with low levels of prolactin, corticotropin, gonadotropins and estradiol, while free $\mathrm{T} 4(0.15 \mathrm{ng} / \mathrm{ml})$, free T3 $(0.5 \mathrm{pg} / \mathrm{ml})$ and reverse $\mathrm{T} 3(12 \mathrm{ng} / \mathrm{ml})$ were also low. Levothyroxine and then liothyronine were added, and slow clinical improvement followed. A MRI of the brain found an empty sella turcica with residual pituitary tissue. After thirty days, the patient was discharged with a regimen of levothyroxine and dexamethasone daily, follow up instructions and bracelet alert for adrenal insufficiency and hypothyroidism.

DISCUSSION: Most cases of empty sella syndrome are asymptomatic For those who develop hormonal defficiency, partial or total panhypopituitarism may occur. Hypothyroidism is considered a rare cause of cardiac tamponade, much less frequent is the association with central etiology. Two isolated reports suggest that adrenal insufficiency may play a role in the pathogenesis of pericardial effusion as well. Our literatture review revealed five reported cases of pericardial effusion secondary to panhypopituitarism, three due to a pituitary mass and two due to Sheehan syndrome. To our knowledge, we report the first case of empty sella syndrome manifesting with panhypopituitarism leading to cardiac tamponade. Prompt recognition of this association, pericardiocentesis and proper hormonal replacement are paramount to effective therapy of this life-threatening condition.

FAT LEAKING FROM A BROKEN TIBIA: NEAR DEATH AND BACK AGAIN IN TWO WEEKS Brian S. Heist. University of Pittsburgh Medical Center, Pittsburgh, PA. (Control ID \#1333161)

LEARNING OBJECTIVE 1: Diagnose fat embolism syndrome from its clinical, imaging, and laboratory manifestations.

CASE: A 31 year-old physically fit Caucasian man with unremarkable medical history experienced closed fracture of his left tibia and fibula while snowboarding in rural Hokkaido, Japan. He was evaluated promptly at a local hospital where he was admitted, with surgery scheduled for a week later. Two days after admission, he experienced fever (39 degrees C) and dyspnea, and imipenem-cilastatin was commenced for suspected pneumonia. Over the following 2 days, he developed intermittent confusion, visual changes, left eye ptosis, and then several hours of progressive stupor and respiratory distress (SpO2 60\% on room air), prompting emergent air flight to our hospital $50 \mathrm{~km}$ away. On arrival, the patient experienced hemoptysis. Respiratory rate was 30 breaths per minute with $\mathrm{SpO} 297 \%$ on $6 \mathrm{~L} \mathrm{O} 2$ facemask. Fever persisted. Vitals were otherwise normal. In addition to the ptosis, anisocoria was present. Lungs were clear to auscultation. Petechiae were observed in the conjunctiva and diffusely over the neck, chest, and abdomen. Based on the history and physical exam, the diagnosis of fat emboli syndrome (FES) was made. Blood tests were remarkable for normal WBC $(8300$ cells $/ \mu \mathrm{L})$ and hematocrit $(36.0 \mathrm{mg} / \mathrm{dL})$, but also thrombocytopenia $(124 \mathrm{~K} / \mu \mathrm{L})$ and elevated PTT ( $29.3 \mathrm{sec})$, INR (1.44), and FDP $(12 \mu \mathrm{g} / \mathrm{ml})$ worrisome for impending DIC. Fibrinogen was elevated $(552 \mathrm{mg} / \mathrm{dL})$ and CRP was markedly elevated $(12.95 \mathrm{mg} / \mathrm{dL})$. Emergent intubation and open reduction external fixation of the tibial fracture were performed without complication. Chest CT showed diffuse peripheral infiltrates and ground glass opacification concerning for alveolar hemorrhage and ARDS. Over the next 36 hours, the hemoptysis and respiratory status worsened, as did the DIC serum markers. Ventilator support was provided per ARDSnet protocol (FiO2 0.8 and PEEP 10 required on post-op day \#2). Six units of pRBCs AND 14 units of FFP were administered. Subsequently, however, the patient's blood tests as well as respiratory and mental status steadily and rapidly improved. Brain MRI was completely normal. On post-op day $\# 5$ he was extubated to room air. Neurologic exam was normal. He was transferred to the orthopedic ward and one week later, discharged with a flight home to the U.S. the next day. Ten weeks later, he reported complete recovery from his fractures.

DISCUSSION: This case exemplifies the rapidly fatal consequences of fat emboli syndrome and the consequent need to monitor for its presentation in any patient with a long bone fracture. Trauma-associated FES is identified with pelvic and femoral fractures, but cases secondary to tibial fractures have also been reported. While the mechanisms underlying the diverse manifestation of FES have not been completely clarified, they are understood to involve mechanical injury from fat droplet embolization, biochemical conversion of embolized fat into toxic metabolites, and activation of the complement system and coagulation cascade by tissue thromboplastin released from the marrow. In turn, FES typically presents 12-72 hours after fracture, with progression from respiratory to neurologic, and then hematologic manifestations as observed here. This case also demonstrates the dramatic recovery that may occur with expeditious repair of the fracture and supportive care, at least in an otherwise healthy young individual such as this patient. At present, no other measure has proven helpful.

FATIGUE AND APATHY: DEPRESSION OR KLINEFELTER SYNDROME Jasleen K. Pannu; Vamsi K. Emani; Sriranjini C. Ramaswamy; Aron Blecher. Western Reserve Care System/NEOUCOM, Youngstown, OH. (Control ID \#1340261)

LEARNING OBJECTIVE 1: Recognize Klinefelter syndrome as an important differential in a man with chronic, unrelenting depression and fatigue even with normal sexual function and advanced age.

CASE: A 56 year old Caucasian man with history of hyperlipidemia, depression, recurrent falls and irritable bowel syndrome came for a regular office visit with complaints of progressive fatigue since few weeks and difficulty climbing stairs. He did not report any shortness of breath at rest, weight loss or loss of appetite. He was a heterosexual and reported having a normal sexual life. He reported having morning erections and normal libido. He was seen in the office intermittently over a period of 10 years and had long history of recurrent falls, apathy and depression. At baseline he was always socially withdrawn and had lagged behind his peers most of his life. Review of systems was negative. On physical exam at baseline he was pale with height of 5' 10", weight $178 \mathrm{lbs}$ and with scant hair on his body. Genital exam showed presence of testicles on lower side of normal in terms of size and normal in consistency. Pubic, axillary and facial hairs were scant but present. No gynaecomastia was seen. No change from his baseline on physical examination was seen. Testosterone level was $66 \mathrm{ng} / \mathrm{dl}$ and $22 \mathrm{ng} / \mathrm{dl}$ on repeated testing. Free testosterone levels low $(3 \mathrm{pg} / \mathrm{ml})$. Luteinizing hormone levels was high $(32 \mathrm{mIU} / \mathrm{ml})$. Levels of sex hormone binding globulin and cortisol were normal. Free T4 level was $0.82 \mathrm{pmol} / \mathrm{L}$ and TSH was $0.6 \mathrm{mIU} / \mathrm{L}$. Subsequently, karyotyping revealed XXY pattern and diagnosis of Klinefelters syndrome was established. Testosterone therapy followed and marked improvement was noticed in his physical and psychiatric symptoms over the next several weeks. Periodic assessment over 3 year period revealed resolution of fatigue and depression along with dramatic and sustained enhancement of his physical abilities and psychiatric symptoms.

DISCUSSION: Sporadic case reports in the past have attributed depression and psychosocial problems to an organic brain syndrome secondary to Klinefelters syndrome. Dramatic improvement following testosterone therapy in our patient further validates this hypothesis. Further studies to reveal pathogenesis of such occurrence are warranted. Also, it is imperative to keep Klinefelters syndrome as an important differential while treating males with chronic depressive psychosocial disorder and disabling fatigue.

FEVER OF UNKNOWN ORIGIN; FINDING THE NEEDLE IN A HAYSTACK Sujith Cherian; Subhraleena Das; Wasim A. Hamarneh. SUNY Upstate Medical University, Syracuse, NY. (Control ID \#1339897)

LEARNING OBJECTIVE 1: Diagnosing the cause when faced with fever of unknown origin

LEARNING OBJECTIVE 2: Distinguishing and recognizing the rare intravascular variant of large B cell lymphoma

CASE: A 65-year-old Caucasian male was referred to our institution by his primary care physician for evaluation of fever of 3 weeks duration. Review of the systems was positive for the presence of decreased appetite, a weight loss of around 15 pounds and increasing fatigue over the previous 4 weeks. His past medical history was significant for diabetes mellitus and hypertension, both of which were well controlled. He had no history of recent travel or any exposure to pets. He was sexually active with one partner for the last 30 years. Physical examination did not reveal any 
abnormalities with no evidence of any masses or organ enlargement. Laboratory data revealed normal leucocyte count with monocytosis (18\%) on differential, calcium level of $11.4 \mathrm{mg} / \mathrm{dL}$, and lactate dehyrdogenase (LDH) level of $1276 \mathrm{IU} / \mathrm{L}$. Rheumatology workup blood, urine cultures, serum /urine protein electrophoresis were negative. Other electrolytes and hepatic function panel was within normal limits. After admission, radiological investigations done were significant only for mild splenomegaly. Workup for fungal etiology and endocarditis remained negative. Ultimately, the patient underwent a bone marrow biopsy which revealed large atypical CD20 positive lymphoid cells in clusters intravascularly, with markedly irregular nuclei and multiple nucleoli, clinching the diagnosis of intravascular large B cell lymphoma. The patient was subsequently started on chemotherapy with R-CHOP (Rituximab, Cyclophophamide, Adriamycin, Vincristine and Prednisone). Following four cycles of R-CHOP and intra-thecal methotrexate the patient has been afebrile and doing well after 4 months of disease diagnosis.

DISCUSSION: Intravascular large B cell lymphoma (IVLBCL) is a rare and often fatal variant of extranodal diffuse large B cell lymphoma with only around 300 cases reported so far. Median age of diagnosis is in the sixth to seventh decade with no sex predilection. It is characterized by lymphoma cells that are confined to lumina of small vessels, hence they do not present with masses or lymphadenopathy. Clinical presentation appears to differ by country of origin with involvement of the nervous system and skin tending to be more common in the western countries, whereas involvement of the bone marrow, liver and spleen being more common in the asian countries. Fever of unknown origin may be the presenting feature in $60 \%$ of cases. Most common laboratory abnormalities include elevated lactate dehydrogenase and beta 2-microglobulin (80-90\%). Diagnosis needs a high index of suspicion and is established by demonstrating the presence of large lymphoma cells within small to medium sized blood vessels. Treatment is generally with systemic chemotherapy, i.e- anthracycline based chemotherapy (R-CHOP) and central nervous system directed therapy with methotrexate and radiation. If a timely diagnosis is made, remission with $60 \%$ response rate to chemotherapy and $30 \%$ three year survival is seen. Our case highlights the importance of a thorough workup when faced with the problem of fever of unknown origin and sheds light on one of the rarer causes.

FIRST SIGNS OF METASTATIC CANCER: AFTER VIRCHOW'S NODE, NOW THROMBOSED NECK VEINS. Jasleen K. Pannu; Sriranjini C. Ramaswamy; Ritha Kartan. Western Reserve Care System NEOUCOM, Youngstown, OH. (Control ID \#1340274)

LEARNING OBJECTIVE 1: Recognize that local or distant malignancy (Trousseau's syndrome) is an unusual but significant cause of spontaneous thrombosis of internal jugular veins and calls for an extensive work up.

CASE: A 61 year old Caucasian male with COPD, hypertension, hyperlipidemia and gastroesophageal reflux disease presented to our hospital with swelling and mild pain on right side of his neck for three days. He had no complaints of dyspnea, dysphagia, odynophagia, chest pain, syncope, weight loss, fever or chills. Patient reported resection of right sided Warthin's tumor 10 years prior without any post operative complications. On examination, his vital signs were stable. There was mild edema and tenderness on the right side of the neck. Cardiorespiratory and abdominal exam was normal. Laboratory studies were normal except hemoglobin level of $9.2 \mathrm{gm} / \mathrm{dl}$. CT scan of neck with contrast revealed a long segment of thrombus within the right jugular vein without evidence of collateral circulation. Mild subcutaneous inflammatory change without evidence of abscess and mild right sided deep cervical lymphadenopathy was also seen. Patient was started on intravenous heparin therapy and warfarin was added subsequently. The workup for hypercoagulability was negative. By third day of anticoagulation patient's hemoglobin dropped to $7.2 \mathrm{gm} / \mathrm{dl}$. Fecal hemoccult test was positive.He never had a colonoscopy before and previous fecal hemoccult tests were negative. Patient refused colonoscopy and opted to continue anticoagulation. His hemoglobin stabilized after blood transfusion and he was discharged home on warfarin. $\mathrm{He}$ was readmitted 6 weeks later with critically high INR, a near syncopal episode and hemoglobin of $4.2 \mathrm{gm} / \mathrm{dl}$. Alkaline phosphatase levels were
$664 \mathrm{U} / \mathrm{L}$ with SGOT of $41 \mathrm{U} / \mathrm{L}$ and SGPT of 59U/L. Esophagogastroduodenoscopy and ERCP with biopsy revealed poorly differentiated gastric adenocarcinoma causing biliary and gastric outlet obstruction. Metastatic lymph nodes were found in mediastinum and retroperitoneum. Also multiple venous thrombosis developed in left jugular, axillary and subclavian veins. Patient was subsequently discharged on chemotherapy after duodenal and bile duct stenting

DISCUSSION: Thrombosis of neck veins as the first sign of occult malignancy has been sparsely reported. Adenocarcinomas are notorious for causing hypercoagulability and thromboembolic phenomena. Hence, in the setting of spontaneous jugular and mediastinal vein thrombosis with unknown precipitating cause, extensive diagnostic work up to rule out an occult malignancy is warranted. Also, benign appearing skin manifestations as seen in our patient should be viewed with high suspicion for underlying thrombosis even in the absence of other signs of malignancy.

\section{FIRST DO NO HARM. WHAT YOU SHOULD CONSIDER PRIOR} TO TREATING SJOGREN'S SYNDROME Angel Brown; Michael Brit. UT Chattanooga College of Medicine, Chattanooga, TN. (Control ID \#1313962)

LEARNING OBJECTIVE 1: To recognize extra-hepatic manifestations of hepatitis $\mathrm{C}(\mathrm{HCV})$ and what impact these manifestations have on the treatment of $\mathrm{HCV}$.

LEARNING OBJECTIVE 2: To become familiar with differences between primary Sjogren's syndrome(pSS), secondary Sjogren's syndrome(sSS) and Sjogren's-like syndrome.

CASE: A 64 year-old male presented to a primary care provider with right eye pain. He was referred to an ophthalmologist. His CBC and BMP were normal. ANA, SSA and SSB were negative. Rheumatoid factor(RF) and total protein were elevated. Albumin was decreased. Non-contrasted head CT showed bilateral enlargement of the lacrimal and parotid glands with a diffuse miliary pattern of abnormal enhancement. The otolaryngologist who evaluated him noted xerostomia, firmness and enlargement of submandibular, lacrimal and parotid glands. Biopsy of minor salivary and parotid glands was performed. The pathologic diagnosis was chronic sialadenitis. Histology revealed a fibrotic stroma with diffuse lymphocyte and plasma cell infiltration. Diagnosis of pSS was suggested. He was started on prednisone therapy. His symptoms failed to improve. He was referred to a rheumatologist. Review of systems at the rheumatologist's office was positive for dry eyes and physical exam revealed enlarged, hardened parotid glands. Further testing revealed negative ANCA, HIV, RPR and hepatitis B. ESR and IgG were elevated. Hepatitis C antibody was detected. The patient was referred to a hepatologist.

DISCUSSION: Sjogren's syndrome is a chronic autoimmune inflammatory disease. It targets exocrine glands. It is characterized by sialadenitis, keratoconjunctivitis sicca and xerostomia. Symptoms are due to lymphocytic infiltration of salivary and lacrimal glands which leads to gland failure. Primary Sjögren's syndrome occurs without underlying rheumatic disease, whereas SSS is associated with underlying rheumatic disease. Sjögren's affects $0.1-4 \%$ of the world's population. It is more prevalent in females. Sjögren's-like syndrome describes individuals with sicca symptoms and chronic HCV. HCV impacts individuals worldwide. An estimated 3 million people are infected with HCV yearly. 130-170 million people are chronically infected with HCV. Chronic HCV infections can be asymptomatic or have extra-hepatic manifestations which include arthalgia, neuropathy and sicca syndrome. There is a risk of misdiagnosing patients with $\mathrm{HCV}$ and sicca symptoms as pSS. Patients in both groups can have positive RF. Histology of exocrine glands will usually show lymphocytic infiltration. However, patients with $\mathrm{HCV}$ are more likely to have abnormal liver function, whereas patients with pSS usually test positive for ANA, SSA and SSB. Different patterns of immunological response have been described for these two groups. The lymphocytes in pSS are predominantly CD4+T-cells. In HCV CD8+ T-cells predominate. Treatment for Sjogren's involves symptom relief, DMARDs and immunosuppressive agents. Immunosuppressive therapy and DMARDs can increase hepatitis $\mathrm{C}$ viral replication and worsen hepatic function. There is data that sicca symptoms improve after treatment of HCV. In conclusion, the above case illustrates the importance of considerating HCV infection in individuals with decreased salivary and lacrimal gland secretion 
and enlargement. Confusion exists in nosology of sicca syndrome. As such a diagnosis of sSS should not be applied for cases of HCV, or the definition of sSS should not be limited to rheumatic disorders.

FIRST DO NO HARM: A CASE OF DRESS Anna Corey. University of Wisconsin Hospital and Clinics, Madison, WI. (Control ID \#1340087)

LEARNING OBJECTIVE 1: Recognize the clinical features of DRESS syndrome and identify common medication culprits.

LEARNING OBJECTIVE 2: Verify reported medical diagnoses in new patients prior to continuing or starting medications.

CASE: A 42-year-old woman with a history of atypical absence seizures presented with three weeks of fevers, lymphadenopathy and rash. She first developed tender axillary and inguinal adenopathy, followed by fevers and a painful papular erruption on her shins that progressed to involve her entire body. The rash was pruritic, severely painful and burning in quality. Her medication regimen included levetiracetam and gabapentin with the recent addition of lamotrigine one month earlier. Her vital signs were normal. Skin examination revealed tender, erythematous papules on the lower extremities and edematous, erythematous macules and thin plaques on the upper extremities, abdomen and chest, which coalesced into erythrodermic plaques on the back. Tender, mobile adenopathy was noted in the cervical, axillary and inguinal areas. Laboratory evaluation revealed leukocytosis with eosinophilia and mild hepatitis. Out of concern for drug reaction, possible culprits lamotrigine and levetiracetam were discontinued. Prednisone was initiated at a daily dose of $1 \mathrm{mg} / \mathrm{kg}$. Dermatopathology revealed focal spongiosis and superficial mixed dermal inflammation consistent with a medication reaction. Lamotrigine-triggered DRESS syndrome was thought to best fit the clinical and laboratory data. Over the next two weeks, her rash improved, laboratory parameters normalized and prednisone was slowly tapered. Unfortunately, it was discovered that the patient's diagnosis of epilepsy was questionable. Her staring spells, which were her usual absence seizures, were captured on video EEG monitoring and not associated with EEG changes. She was evaluated by neurology and evidence for a seizure disorder was not found. Her frequent requests for additional medication prompted a thorough chart review which revealed that several historical diagnoses stated by the patient, a former nurse, had never been confirmed.

DISCUSSION: The Drug Reaction with Eosinophilia and Systemic Symptoms or DRESS syndrome is a life-threatening drug reaction with hallmark features of severe skin rash, fever, eosinophilia, atypical lymphocytes, lymphadenopathy and internal organ involvement, most commonly hepatitis, pancreatitis, interstitial pneumonitis or nephritis. DRESS usually develops 2-6 weeks after initiation of the drug and can persist or worsen despite discontinuation. The most frequent offenders are allopurinol, sulfonamides, and anticonvulsants such as phenytoin, phenobarbital, carbamazepine and lamotrigine although over fifty drugs have been implicated. The pathogenesis of DRESS is incompletely understood but thought to include detoxification defects leading to reactive metabolite formation and subsequent immunological reactions and reactivation of EBV and HHV-4,6 and 7. Treatment involves removal of the offending agent and initiation of corticosteroids. Prompt diagnosis is paramount as the mortality rate is up to $10 \%$. This case exemplifies a serious complication arising from a medication prescribed for a condition that the patient did not likely have. Physicians must verify diagnoses prior to prescribing and ensure that the potential benefits of a medication do indeed outweigh the risks.

FIRST IS THE WORST Dandan $\mathrm{Liu}^{2,1}$; Alvin Rajkomar'; Sumant Ranji ${ }^{2}$; Sumana Kesh ${ }^{2}$. ${ }^{1}$ SFVA Medical Center, San Francisco, CA; ${ }^{2}$ UCSF, San Francisco, CA. (Control ID \#1304372)

LEARNING OBJECTIVE 1: Recognize limits of laboratory data in diagnosing acute pancreatitis

LEARNING OBJECTIVE 2: Review treatment of hypertriglyceridemic pancreatitis (HTGP)

CASE: A 23 year old man with no significant medical history presented to the emergency department with sudden onset abdominal pain and 6 episodes of nonbloody emesis along with new polyuria and polydipsia. $\mathrm{He}$ had abstained from alcohol for two years and denied any ingestions. On initial exam, he had a soft abdomen that was nontender. Labs revealed glucose of $321 \mathrm{mg} / \mathrm{dL}$, anion gap of 29, normal lactate, and lipase of 59 units/L. He was treated with IV fluids and insulin drip for presumed new onset diabetic ketoacidosis (DKA) as well as pain control. Overnight, patient was noted to have increasing pain medication requirements, and his abdomen was increasingly distended with reproducible tenderness in the epigastric region. An abdominal CT with contrast confirmed diagnosis of pancreatitis with no evidence of gallstones. The patient's blood was noted to be lipemic. Morning labs showed elevated triglyceride $7470 \mathrm{mg} / \mathrm{dL}$ and elevated lipase 143 units/L. He was continued on an insulin drip for management of hypertriglyceridemia induced acute pancreatitis (HTGP) and concomitant DKA. By hospital day 3, his triglycerides decreased to $720 \mathrm{mg} / \mathrm{dL}$, and he was transitioned to subcutaneous insulin. His GAD and islet cell antibodies were negative. He was discharged on hospital day 6 with minimal abdominal pain, tolerating food, with plans for close outpatient follow-up for his diabetes and hypertriglyceridemia.

DISCUSSION: Acute pancreatitis is commonly precipitated by gallstones or alcohol, but HTGP, with triglycerides $>1000 \mathrm{mg} / \mathrm{dl}$, accounts for $2-10 \%$ of cases. Serum lipase is a specific test for pancreatitis ( $\operatorname{spec} 82-97 \%$, sens $67-85 \%$ ). While there is not much literature on the reliability of lipase in lipemic samples, serum amlyase levels are known to be spuriously low in lipemic blood. DKA itself can conversely cause nonspecific elevations in amylase and lipase apart from pancreatitis in approximately $10 \%$ of cases. When the history and lab findings are incongruent with an evolving exam, the diagnostic "gold standard" should be ordered: a contrast enhanced CT. The mechanism of HTGP is not clearly defined, but there is likely secondary hydrolysis of triglycerides by lipase in pancreatic arteries, leading to release of free-fatty acids that are toxic to the capillaries or acinar cells. A cascade of capillary ischemia then promotes activation of trypsinogen and thus pancreatitis. Management is fairly uniform despite etiology. Most commonly, insulin infusion is used, thought to induce lipoprotein lipase activity. Heparin infusion can also be used, although it ultimately leads to a decrease in lipolytic activity. If refractory, plasmapharesis, lipid pheresis, and extra-corporeal lipid elimination are possible, although there are no direct comparisons between these treatment methods, and they are not readily available. Long-term treatment of hypertriglyceridemia is aimed to reduce levels $<1,000 \mathrm{mg} / \mathrm{dL}$ to decrease the likelihood of pancreatitis. Dietary restriction with $<20 \%$ of calories from fat is the first step to reduce chlomicron-mediated contribution to elevated triglycerides. Pharmaceuticals including niacin, fibrates, and fish oil can also be employed, although each carries its own set of side effects and costs. This case, with close outpatient follow-up and patient adherence to dietary restrictions should illustrate the adage "first is worst."

FOOLED TWICE AND MAYBE MORE: AN INSIDIOUS CASE OF BRUCELLOSIS Susanna Tan; Alissa Detz; Paul Aronowitz; David Busch. California Pacific Medical Center, San Francisco, CA. (Control ID \#1334990)

LEARNING OBJECTIVE 1: Recognize cognitive biases that lead to diagnostic error.

CASE: A 77-year old Spanish-speaking woman with history of arthritis presented with fever and fatigue for one week. She received antibiotic treatment for a urinary tract infection but returned with worsening arthralgias, abdominal pain, and decreased appetite. On admission, she was found to be hypercalcemic $(12.3 \mathrm{mg} / \mathrm{dl})$ and was diagnosed with primary hyperparathyroidism (PTH $224 \mathrm{pg} / \mathrm{ml}$ and 25-OH Vitamin D $13 \mathrm{ng} / \mathrm{ml}$ ). Fever (38.1 C) was attributed to a lower extremity deep vein thrombosis. Six weeks later, she presented with persistent malaise and fevers. She was again hypercalcemic and treated for urinary tract infection. She was discharged but was readmitted when gram-positive cocci grew in two blood cultures. The organism was too fastidious for routine identification. A transesophageal echocardiogram was negative. When repeat blood cultures were negative, the initial blood cultures were considered a contaminant, and she was discharged. Five days later, the second set of blood cultures grew a Gram positive organism that again defied identification. She was then admitted for her third hospitalization in two months with continued complaints of fevers, malaise, 
arthralgias, and worsening back pain. MRI of the spine showed T9-10 diskitis with epidural abscess. Blood cultures and a culture from a bone biopsy grew an organism that was finally determined to be Gram-negative and was identified as Brucella melitensis. She was started on antibiotics with slow symptom improvement; she eventually required debridement of the epidural abscess. A more thorough history later revealed travel to central Mexico three months prior to symptom onset. In Mexico, she had consumed milk from local ranches.

DISCUSSION: This case highlights the importance of thorough history taking and exemplifies several cognitive biases that led to delays in care. The patient's diagnosis of primary hyperparathyroidism likely dissuaded physicians from considering alternative diagnoses, an example of anchoring bias. The case also highlights the importance of clinical correlation in pursuing identification of positive blood cultures. Fastidious organisms may not grow rapidly in culture, and Gram stain interpretation may be difficult. This is an example of expectation bias, the tendency to only believe and pursue data that agree with one's expectations. At the time of diagnosis, the patient had been seen by more than seventy physicians at multiple hospitals. Lack of continuity of care and the patient's non-English speaking status also contributed to her delayed diagnosis. As physicians, we must be aware of cognitive biases that may influence our thinking and result in delay of care to our patients.

FOR WHOM THE BELL TOLLS Ryan Brown. Tulane University Health Sciences Center, New Orleans, LA. (Control ID \#1339195)

LEARNING OBJECTIVE 1: 1. Identify the clinical presentation of conversion disorder-induced paralysis. 2. Recognize the clinical presentations of Bell's Palsy. 3. Identify the treatment of acute Bell's Palsy.

CASE: A 19 year-old man presented following the sudden onset of leftsided upper and lower extremity paralysis while lying in bed. $\mathrm{He}$ experienced no pain or loss of consciousness. His initial symptom began the previous night, when his lips felt numb at dinner. Later, when he went to bed, he was unable to close his right eye. These symptoms persisted until late the following morning, when the paralysis occurred. He had no past medical history, and no risk factors for stroke or embolic disease. He reported two years of anhedonia and decreased energy for the past two years, citing unemployment and a friend's death as the causes. At the time of his presentation, his paralysis had resolved. His cranial nerves were intact and he had 5/5 strength and $2+$ reflexes throughout; his gait and cerebellar examination were normal. He was again able to close his right eye, but his lips remained numb and now his left cheek was numb. He had a flat affect, low, mumbled speech, and poor eye contact. A head CT revealed no abnormalities. Thyroid function testing was normal, and periodic paralysis was excluded on clinical grounds. He was admitted for observation and subsequent testing. Overnight, he developed a right facial droop including forehead paralysis. To exclude multiple sclerosis, a noncontrast MRI of the brain was performed, revealing enhancement of the right facial nerve, consistent with Bell's Palsy. He was discharged on a prednisone taper with outpatient psychiatric follow-up.

DISCUSSION: The general internist is frequently the first to encounter physical manifestations of psychiatric disease. Conversion disorder is the unconscious manifestation of a physical CNS symptom secondary to psychiatric conflict (usually anxiety or depression). Conversion disorder, unlike somatization disorder, manifests as a single neurologic symptom, often abruptly. The treatment of conversion disorder focuses on treating the underlying psychiatric disorder with antidepressants, and psychotherapy, gently illustrating to the patient the connection between their psychiatric illness and their physical symptom. Hospital admission with inpatient treatment (namely, physical therapy), validation of symptoms, and allowance of slow, face-saving improvement are methods that have proven effective. Bell's Palsy is the most common cause of unilateral facial paralysis. Normally, it is an acute, lower motor neuron paralysis of the facial nerve that resolves in $80-90 \%$ of cases. Etiology varies, but a viral source, particularly reactivation of Herpes Simplex 1 and Herpes Zoster, is the most consistent and validated cause. Presentation may include unilateral facial droop, inability to close eye, hyperacusis, and ageusia. Presence of forehead paralysis suggests the lesion is peripheral, not central. Meta-analyses suggest that oral glucocorticoid treatment within three days of onset of symptoms is beneficial. Using an adjunctive antiviral, such as acyclovir, is helpful in severe cases, but remains controversial in routine cases. Beyond three days, medications do not affect the outcome.

FORAGING FOR LIVER FAILURE Alvin Htut; Lauren Shapiro. Montefiore Medical Center, BRONX, NY. (Control ID \#1334207)

LEARNING OBJECTIVE 1: Recognize the clinical manifestations and treatment options of acute mushroom toxicity.

LEARNING OBJECTIVE 2: Remind clinicians of the time course of Amatoxin poisoning.

CASE: A healthy 44 year-old woman presented with crampy abdominal pain, vomiting and watery diarrhea for one day with subjective fevers and myalgias. Her symptoms began 10 hours after eating an omelet made with mushrooms found in her backyard. Her initial vital signs were normal. On exam the patient was tender to palpation in the left lower quadrant of her abdomen without guarding or rebound. Initial laboratory and imaging evaluations were also normal. Repeat laboratory studies 24 hours later revealed aspartate and alanine aminotransferases of $360 / 349 \mathrm{u} / \mathrm{L}$ respectively, an INR of 1.5 , bicarbonate of $15 \mathrm{mEq} / \mathrm{L}$ and lactic acid of 3.8. $\mathrm{mmol} / \mathrm{L}$. Her transaminases peaked at 10,556/9,302 u/L and her INR was 2.4 after another 12 hours. Total bilirubin peaked at $60 \mathrm{mg} / \mathrm{dL}$ several days later. The patient underwent rapid transplant evaluation for acute liver failure, received IV Penicillin G and N-Acetylcysteine and was transferred to the ICU. Acetaminophen levels were normal as were markers of viral hepatitis. Her course was complicated by recurrent watery diarrhea and lower gastrointestinal bleeding. Although the patient was listed urgently for a liver transplant, she made a full recovery with supportive care. The mushrooms the patient ingested were confirmed to be from the Amatoxin producing Gallerina genus.

DISCUSSION: Acute liver failure is a serious clinical entity encountered by general practitioners. The differential diagnosis often includes Acetaminophen toxicity, acute viral hepatitis, ischemic injury and other toxic exposures such as wild mushrooms. Amatoxin mushrooms accounts for 90\% of mushroom related fatalities, with Amanita Phalloides being the most potent species. Once ingested, the toxins enter the portal circulation and are actively transported into hepatocytes, stopping protein synthesis and inducing apoptosis. Approximately 6-24 hours following ingestion the patient develops gastroenteritis with abdominal pain, diarrhea and vomiting with normal liver tests. 24-36 hours following ingestion, mild elevations in transaminases are detected. Two to four days afterwards, hepatocyte death, disruption of hepatic venous and biliary flow occurs, with significant elevations in transaminases and hyperbilirubinemia. Coagulopathy and encephalopathy follow, which can progress to multi-organ failure and death. Treatment is supportive but measures can be taken to decrease toxicity. IV Penicillin blocks uptake of toxin to hepatocytes and NAcetylcysteine limits damage from oxidative stress. Assessing the need for liver transplantation is critical because delays can lead to death as these patients can progress rapidly. Retrospective analysis of previous cases indicate that there are some tests that may help guide the clinician towards transplantation, including an elevated serum creatinine $(>1.2 \mathrm{mg} / \mathrm{dL})$ with a decreased prothrombin index $(<10 \%) 3-4$ days after ingestion. Both appear to be indicators of a poor outcome; neither criterion was met by our patient. Initial evaluation of patients with suspected mushroom toxicity may not reveal any abnormalities. The delayed onset of symptoms following ingestion and the rapid progression of hepatotoxicity are telltale signs of Amatoxin ingestion, a rare but often fatal cause of acute liver failure.

FOREIGN BODY INGESTION AS A CAUSE OF ACUTE DYSPHAGIA IN THE ELDERLY Shuchi Gulati; Priyanka Vyas; Richard Alweis. The Reading Hospital and Medical Center, West Reading, PA. (Control ID \#1339130)

LEARNING OBJECTIVE 1: Recognize foreign body ingestion as a cause of dysphagia in elderly. LEARNING OBJECTIVE 2: .

CASE: A 73 year old man called his primary care physician with complaint of sudden onset of difficulty in swallowing solids and liquids for one day with regurgitation of undigested food. Past medical history was 
significant for GERD on proton pump inhibitors and chronic atrial fibrillation for which he was on warfarin. Colonoscopy done one year earlier was normal. The patient did not have a history of smoking or alcohol abuse. Physician recommended urgent esophageal barium swallow which showed a $3 \mathrm{~cm}$ disc-like foreign body lodged within the distal esophagus. Patient was advised to go to the emergency department (ED) for endoscopic removal of the foreign body. The foreign body was thought to be a coin. Emergent endoscopy was planned despite therapeutic INR as patient was symptomatic for almost 48 hours. When the foreign body was removed endoscopically, it was found to actually be a circular $20 \mathrm{~mm}$ energizer 2032 lithium button battery. Endoscopy also showed focal severe esophagitis at the site of impaction. Later on, patient admitted to the accidental ingestion of the lithium battery.

DISCUSSION: Button battery ingestion is an uncommon scenario, with an incidence of 10-15 cases per million population per year. Lithium batteries are commonly used as a household battery, hence fatal outcomes associated with lithium battery ingestion have been increasing over the last 10 years. Potential complications include severe local esophagitis, perforation, tracheoesohageal fistula, esophageal stricture, vocal cord palsy, mediastinitis, aspiration pneumonia and cardio pulmonary arrest. Although systemic absorption is possible after ingestion of lithium button battery, systemic toxicity has not been reported. Lithium batteries produce local corrosive effect by producing electrolytic current which hydrolyzes tissue fluids and produces hydroxide at negative pole. The outcome of lithium button battery ingestion depends upon anatomical position, diameter, chemistry and age of the battery. Injury-free outcome can be achieved by removing battery in less than 2 hours. This case illustrates that foreign body ingestion should be included in the differential diagnosis of acute dysphagia because early endoscopic removal can prevent fatal complications. This case further illustrates the need to expand beyond a coin to more toxic ingestions when one is considering foreign body ingestion as a likely etiology for acute dysphagia, and expedite evaluation.

FRESH WATER PERIL Claire Zeigler. Oregon Health Science University, Portland, OR. (Control ID \#1295560)

LEARNING OBJECTIVE 1: Diagnosis and epidemiology of leptospirosis LEARNING OBJECTIVE 2: Role of convalescent antibody testing in confirming diagnosis

CASE: Leptospirosis is a spirochetal infection associated with freshwater exposure. Its primary manifestations of fevers and flulike symptoms with or without multiorgan failure are non-specific, making it a diagnostic challenge. A 47 year old previously healthy man was transferred from a referring hospital for fevers, acute renal failure, and thrombocytopenia concerning for TTP. He initially presented to the referring ED one day prior to admission with fevers of 101-102; labs at that time were significant for a normal creatinine, platelets of 139 , hematocrit of 38.4 , and negative urinanalysis and blood cultures. He was sent home, but returned the following day with recurrent fevers and rigors. At this time, he reported developing myalgias, abdominal pain and watery, non-bloody diarrhea five days prior. As a competitive wind surfer, he had recently competed on the Oregon coast and in Hood River. Travel history was also notable for wintering in Mexico three months out of the year. He denied weight loss, night sweats, lymphadenopathy, and rash. On presentation, physical exam was significant for fever, tachycardia, and slight conjunctival pallor. Labs were significant for a creatinine of 5.4, BUN 40, hematocrit 36.6, platelets 90,000 , and mild transaminitis. In order to rule out TTP, an LDH was ordered but was normal. CXR, blood cultures, echocardiogram, anti-streptolysin, ANCA, peripheral smear, leptospirosis serology were ordered. He was started on empiric broad-spectrum antibiotics including doxycycline. Urine sediment revealed muddy brown and granular casts, consistent with acute tubular necrosis. Over the course of several days, his fevers remitted, his renal function began to improve and his platelets rebounded. Initial leptospirosis antibody was negative, but treatment with doxycyline was continued for 10 days because of strong suspicion for this diagnosis. Convalescent leptospirosis titers, done through the state health department, resulted as strongly positive after discharge. He was seen in Infectious Disease clinic several weeks later where he reported to be doing well.
DISCUSSION: Leptospirosis is a surprisingly common yet under diagnosed infection. It should be considered in patients with abrupt onset of fever, abdominal pain, renal or hepatic failure, and exposure history (in this case, recent fresh water wind surfing). Physical examination is usually not helpful in diagnosis, but occasionally, conjunctival injection, hepatosplenomegaly and edema are observed. In severe cases, complications include organ dysfunction, specifically ARDS, renal failure, myocarditis, uveitis and rhabdomyolysis. Diagnosis is usually by serologic testing, though blood and urine cultures are positive in approximately $50 \%$ of the time. Microscopic agglutinin test is considered gold standard, but because it is not widely available, IgM antibody and PCR are more commonly used. As in this case, convalescent antibodies should be sent several weeks after onset of symptoms for serologic confirmation.

FULMINANT NEUROGENIC PULMONARY EDEMA FROM SUBARACHNOID HEMORRHAGE Madan R. Aryal; Leena Jalota; Naba Mainlai; Madan Badal; Anthony Donato. The Reading Hospital and Medical Center, West Reading, PA. (Control ID \#1329267)

LEARNING OBJECTIVE 1: Recognize neurogenic pulmonary edema in the setting of acute CNS lesion.

LEARNING OBJECTIVE 2: Describe the management of neurogenic pulmonary edema.

CASE: The fulminant form of neurogenic pulmonary edema (NPE) is a rare, life threatening complication in patients with subarachnoid hemorrhage. It may develop very quickly within minutes and may be the initial presentation of an acute CNS event. We report a fatal case of NPE that was associated with subarachnoid hemorrhage. A previously healthy 47 year old female was brought to the ED by an EMS after having intense headache followed by loss of consciousness and jerking movements of the hands and legs. On examination, patient was dyspneic with respiratory rate $30 / \mathrm{min}$, blood pressure $152 / 80 \mathrm{~mm}$ $\mathrm{Hg}$, HR $140 / \mathrm{min}$ and oxygen saturation $88 \%$ on room air. She was intubated in the ED for airway protection and required $80 \% \mathrm{FIO} 2$ to maintain saturation. Following intubation she was noted to have copious pinkish frothy discharge from the endotracheal tube. She was then transferred to the ICU. She rapidly developed hypotension that was refractory to infusion of $6 \mathrm{~L}$ of normal saline and $2 \mathrm{~L}$ of albumin and maximum doses of norepinephrine and vasopressin. In lung exam, she had fine crackles in the lung bases. Heart examination was unremarkable. Chest $\mathrm{X}$ ray showed diffuse bilateral pulmonary edema pattern, EKG showed normal sinus rhythm with nonspecific ST, T wave changes. Cardiac enzymes were normal. BNP was $720 \mathrm{pg} / \mathrm{ml}$. Bedside echocardiogram done immediately in the ED was normal. Echocardiogram done after 3 hours showed left ventricular ejection fraction of $43 \%$ and decreased wall motion of the left ventricle. Pulmonary capillary wedge pressure was $19 \mathrm{~mm} \mathrm{Hg}$. In the subsequent 5 hours the neurological examination did not demonstrate any findings consistent with brainstem, higher cerebral and cranial nerve functioning. The $\mathrm{CT}$ scan and $\mathrm{CT}$ angiogram showed diffuse subarachnoid hemorrhage from rupture of aneurysm of posterior inferior cerebellar artery (PICA) with hydrocephalus. Patient died after 6.5 hours of arrival in ED and 7 hours of symptom onset.

DISCUSSION: Fulminant NPE usually occurs within minutes to hours from a severe CNS insult with acute onset of dyspnea. Higher grades of CNS insult radiologically as well as PICA aneurysmal bleeding are important factors for the development of NPE. NPE shows broad clinical spectrum ranging from asymptomatic patients to the rapid development of respiratory failure. Differential diagnosis for NPE includes aspiration pneumonitis, ventilator associated pneumonia and ventilation induced lung injury. Chest radiograph classically shows bilateral alveolar infiltrates. Initial studies of cardiac function including EKG, echocardiogram, CVP and PCWP are usually unremarkable; however serial monitoring of cardiac function may demonstrate reduced left ventricular function and regional wall motion abnormalities. PCWP may increase and approach high levels. Increase permeability of the vascular bed as well as stunned myocardium are proposed mechanisms of this condition. The treatment is supportive with the primary aim of decreasing the intracranial pressure. Prognosis depends on the underlying neurological problem unless significant respiratory complications develop. 
GOO! WHERE TO START? Edward Mannina; Ken Harang; Danielle King. Tulane University Health Sciences Center, New Orleans, LA. (Control ID \#1338656)

LEARNING OBJECTIVE 1: 1. Learn to manage the symptomatology of gastric outlet obstruction. 2. Recognize the acid-base disturbances that can result from prolonged gastric outlet obstruction. 3. Implement a treatment strategy for starvation and define refeeding syndrome.

CASE: A 54 year-old man presented with four months of nausea and nonbloody vomiting. He reported a forty-pound weigh loss and decreased appetite; he had not had a bowel movement for over a week. He had a past medical history of hypertension. His vital signs were normal with the exception of a blood pressure of 94/43 $\mathrm{mmHg}$. He had hypoactive bowel sounds and tenderness to palpation in the epigastrum; there was no evidence of hepatosplenomegaly. Serum chemistries included a potassium of 3.2, a choloride of 69 , and a bicarbonate of 45 ; the BUN was 58 and the creatinine was 4.2. Liver enzyme and function tests were normal. An arterial blood gas revealed a $\mathrm{pH}$ of 7.57, a pCO2 of 60 , and a pO2 of 151 . Plain films of the abdomen and chest were normal. He was started on intravenous pantoprazole, ondansetron and compazine. A nasogastric tube was placed and set to suction, returning $2 \mathrm{~L}$ of brownish fluid. Aggressive fluid resuscitation was initiated. The hypotension resolved, and the acute kidney injury began to correct. A diagnosis of metabolic alkalosis secondary to prolonged vomiting was established. His anion gap starvation ketoacidosis was treated with fluid resuscitation followed by peripheral parenteral nutrition. Esophagogastroduodenoscopy revealed gastric outlet stricture by a sub-mucosal mass. Dilation was performed for symptom alleviation. Electrolytes were monitored for evidence of re-feeding syndrome; potassium and phosphate were repleted as needed. Following biopsy results, the patient was referred to hematology-oncology for treatment of a gastric adenocarcinoma.

DISCUSSION: The general internist is frequently confronted with the symptoms of nausea, vomiting and weight loss. While most cases are due to self-limited diseases, it is important for the internist to recognize the signs and symptoms that suggest more serious etiologies. Months of nausea and vomiting with decreased oral intake and significant weight loss should prompt an investigation of the upper gastrointestinal tract to exclude obstruction. In addition to visualization of the gastrointestinal tract, is equally important that the internist suspect a mixed acidbase disorder and electrolyte abnormalities secondary to vomiting and starvation. The treatment should aim to alleviate symptoms of gastric outlet obstruction while aggressively fluid resuscitating and supplementing nutrition. As nutrition restoration prompts insulin release and anabolism, serum electrolytes can be depleted - a condition called refeeding syndrome. Abnormal serum values of potassium and phosphate can cause muscular dysfunction, including the myocardium. Electrolyte repletion and telemetric monitoring are thus advised.

GRAM NEGATIVE SEPSIS IN A WOMAN WITH ORIENTAL CHOLANGIOHEPATITIS. Joshua C. Obuch; Amy R. Weinstein. Beth Israel Deaconess Medical Center, Boston, MA. (Control ID \#1339601)

LEARNING OBJECTIVE 1: Characterize Oriental Cholangiohepatitis $(\mathrm{OCH})$ and review its epidemiology

LEARNING OBJECTIVE 2: Understand the pathophysiology of $\mathrm{OCH}$ cholangitis, anatomical assessment, and treatment options

CASE: 74 year old Vietnamese female with $\mathrm{OCH}$ and prior cholecystectomy and Roux-en-Y hepaticojejunostomy for repeated biliary stones presented to her hepatology visit with fevers for several weeks after returning from a month in Vietnam. She also had epigastric pain, anorexia, 6lbs weight loss, and fatigue. She denied other GI issues, jaundice, night sweats, pulmonary or urinary symptoms. In Vietnam, she received antibiotics for a presumed UTI. She takes omeprazole and risendronate. She does not smoke or drink. History is notable for being a fishing industry supplier in Vietnam. On presentation she had fevers to $102 \mathrm{~F}$, epigastric tenderness, alkaline phosphatase of 818 and WBC of 12.5 without bandemia. IV ampicillin/sulbactam was started, but switched to ceftriaxone and metronidazole for continued fevers. MRCP showed old biliary strictures and dilation, caudate lobe inflammation, necrosis, abscess posterior to the IVC, and areas of ongoing cholangitis. ERCP was deferred, but PTC showed contrast flow from the left duct into the jejunum, but moderate degree of stenosis, stricturing, and stasis in the central left and right duct. Stricture biopsies and bile cultures were done, and a drainage catheter was placed. Meropenem (MPM) was started due to continued fevers. Bile cultures showed eggs concerning for trematode infection. Stool and urine were sent for ova and parasites. Blood cultures grew highly resistant E.Coli sensitive to MPM. She remained afebrile with clinical improvement on MPM and stayed in house with pending biopsies and trematode serologies.

DISCUSSION: Oriental cholangiohepatitis $(\mathrm{OCH})$ entails recurrent bouts of cholangitis with strictures and dilation of biliary ducts along with pigment stone formation within the ducts. It typically affects $20-40$ year olds, and is endemic to southeast Asia. Pathogenesis is thought to be from chronic biliary infestation with parasites, such as Clonorchis sinensis and Ascaris lumbricoides, causing dilation, inflammation, stricture, sludging, and stone formation. Parasitic eggs and sloughed epithelial cells can block ducts causing stasis and bacterial proliferation. This cyclical process leads to repeated stricture formation and episodes of cholangitis. Patients can present with RUQ pain, fever, and jaundice, with evidence of obstructive cholangiopathy, hepatic injury, and infection on labs. Workup includes US, CT, and MRCP. US can show extra and intrahepatic duct dilatation and narrowing of smaller intrahepatic branches, along with presence of stones. CT can provide biliary detail and evidence of chronic disease with abscess and necrosis. MRCP provides additional detail of proximal ducts and possible intraductal mass lesions. Mild symptoms can be treated with oral fluoroquinolones. Moderate to severe cholangitis needs broader GNR and enterococci coverage. If improvement fails, coverage changes to ESBL GNR's and VRE. It is unclear if fluke treatment changes the chronic disease course. ERCP can stent and dilate strictures and remove stones. If ERCP cannot be done, PTC can accomplish duct drainage, stenting, and biliary samples. Localized disease may be surgically resected with hepatic lobectomy or duct resection via Roux-en-y hepaticojejunostomy. In refractory disease, orthotopic liver transplant should be considered.

HIV AND MALIGNANCY IN THE POST-HAART ERA- A STORY OF BURKITT'S LYMPHOMA Reshma Gupta; Santiago Neme; Shireesha Dhanireddy. University of Seattle, Washington, Seattle, WA. (Control ID \#1324075)

LEARNING OBJECTIVE 1: Assess differences in disease outcomes between AIDS-related Burkitt's lymphoma and other AIDS defining malignancies

LEARNING OBJECTIVE 2: Distinguish the correlation between virologic suppression, CD4 recovery, and progression of AIDS-related Burkitt's lymphoma

CASE: 28 year old HIV-positive man with history of Burkitt's lymphoma presented with severe upper back pain and vomiting. He was diagnosed with HIV four years ago (CD4 count 300) and highly active antiretroviral therapy (HAART) was initiated. One year ago, he presented with a right axillary mass and small bowel obstruction. He was diagnosed with stage IV Burkitt's lymphoma, negative for bone marrow and CNS involvement. He received E-POCH with methotrexate, RDHAP, and auto-stem cell transplantation. At baseline, he was highly functional with a CD4 count 192 and undetectable viral load on efavirenz-tenofovir-emtricitabine. Upon admission, he was afebrile, ambulating well with no alarming laboratory findings. At 9 am the next day, he had worsened back pain and difficulty ambulating. By noon, he was unable to move his lower extremities and had reduced lower extremity strength, T4-T11 point tenderness, T3 sensory level, and urinary retention. MRI lumbothoracic spine showed a dorsal epidural mass lesion from T2-T11 with compression of the thecal sac. Dexamethasone was started, and he emergently underwent a T3-T6 laminectomy and decompression. Inflammatory gelatinous vascular tissue compressing the spinal cord was found in the epidural space. Post-operatively, he regained sensation but not strength. Cytology of intraoperative tissue showed high-grade Burkitt's lymphoma. He started radiation therapy, however, given CNS involvement and relapse after 
transplantation, his prognosis was poor and no further therapy was recommended.

DISCUSSION: Burkitt's lymphoma is an aggressive B-cell lymphoma and one of the most common initial AIDS-defining illnesses. With the advent of HAART, many AIDS-related malignancies have decreased in incidence. However, in the post-HAART era, survival for AIDSrelated Burkitt's lymphoma has not improved significantly compared to other AIDS-related malignancies (38.1 months for Kaposi's sarcoma vs 5.1 months for Burkitt's lymphoma, $\mathrm{p}<0.005)$. People with HIV are still at increased risk of mortality to AIDS-related Burkitt's lymphoma independent of the degree of immunodeficiency or antiretroviral therapy. This patient remained physically active and adherent to HAART with an undetectable viral load; however, he still developed a devastating CNS lymphoma. Progression of AIDS-related Burkitt's lymphoma involves unique mechanisms that require targeted interventions plus HAART. HIV causes loss of T-cell function reducing immunoregulation of Epstein-Barr virus-infected $\mathrm{B}$ cells and chronic B-cell hyperactivation that increases genetic mutations. These are targets for new therapies for AIDS-related Burkitt's lymhoma, a treatment challenge in the post-HAART era.

HAEMOPHILIA IS NOT ONLY MEN'S DISEASE Nayan K. Desai; Vijay Rajput. Cooper university Hospital, UMDNJ, Camden, NJ. (Control ID \#1319349)

LEARNING OBJECTIVE 1: Approach to a patient with isolated elevation of partial thromboplastin time.

LEARNING OBJECTIVE 2: Recognise the hematological causes of bleeding in postpartum females.

CASE: BACKGROUND: Haemophilia A is a hereditary disorder of young men. Acquired Haemophilia A (AHA) is rare but critical diagnosis in young women with postpartum bleeding, caused by an autoantibody to factor VIII. It must be distinguished from congenital hemophilia and other acquired bleeding disorders for appropriate and timely treatment in young women with postpartum bleeding. Case report: A 24 year Asian -Indian women presented with recurrent, spontaneous bruising over her lower limbs and continuous noncyclical vaginal bleeding over four months after normal delivery. On examination she had pallor, ecchymotic patches on lower limb with swelling and tenderness of right calf. Her haematological parameters were consistent with iron deficiency anaemia and platelet count was within range. Coagulation tests revealed elevated activated partial thromboplastin time (aPTT) and normal prothrombin time. aPTT on mixing studies did not decrease by $50 \%$. Factor VIII levels were less than $1 \%$, consistent with severe hemophilia. Inhibitors to factor VIII were positive with levels of 5 Bethesda units $/ \mathrm{ml}$. Lupus Anticoagulant was negative. Ristocetin and ADP aggregation test were normal. Diagnosis of Postpartum AHA was made based on clinical and laboratory presentation. She responded to oral corticosteroids.

DISCUSSION: Von Willebrand disease, platelet function defects and acquired factor inhibitors are rare but important haematological conditions in women with postpartum bleeding. AHA is an acquired autoimmune disease caused by polyclonal autoantibody to factor VIII, which leads to FVIII deficiency, which results in insufficient generation of thrombin through the intrinsic pathway. The incidence is estimated to be $0.2-1.0$ case per 1 million persons per year. More than two third women with postpartum bleeding have autoantibody against factor VIII. Hepatitis B and C; solid and haematological malignancies; drugs are other causes of AHA. Mortality ranges from $8-22 \%$ if not diagnose early in first week of bleeding. Deep soft tissue, mucosal and subcutaneous bruising is common, but haemarthoses is relatively uncommon compared to hereditary hemophilia A. The diagnosis is confirmed by the finding of low FVIII and raised inhibitor titer on Bethesda assay. The principles of treatment are to control of bleeding with recombinant Factor VIIa; eradication of inhibitor by immunosuppressive agents; removal of inhibitors with plasmapheresis or immunoabsorption. Corticosteroids are first line therapy for inhibitor eradication either alone or in combination with cyclophosphamide. Rituximab is second line agent for refractory cases or failure of first line therapy. Most patients spontaneously clear inhibitors after a median period of 30 months and less likely to recur with subsequent pregnancies. Conclusion: Isolated elevation of aPTT with postpartum bleeding in primiparous female is a key towards diagnosis of AHA. Early recognition and treatment can avoid the life threatening bleeding and its complications.

HAH, WHAT ARE YOU SAYING? BY: SARA HANNA, MD, PETER SIDAROUS, MD, PRAMIL CHERIYATH, MD Sara Hanna; Peter Sidarous; Pramil Cheriyath. Pinnaclehealth, Harrisburg, PA. (Control ID \#1340422)

LEARNING OBJECTIVE 1: To recognize the features of cryptococcal meninigitis in healthy subjects

CASE: Introduction: Cryptococcus is the most common fungal infection of the CNS and meningitis is the most frequent CNS involvement. It mostly infects immunocompromised individuals but can happen in healthy subjects. Other presentations are also seen such as pneumonia, osteomyelitis and skin infections. This is a case presentation of Cryptococcal meningitis in an apparently healthy patient who presents with bilateral hearing impairment as well as deteriorating mental functions. Case Presentation: This is a 58 years old African American male with past medical history of Diabetes mellitus, hypertension, Hepatitis $\mathrm{C}$ virus, Genital herpes who presented with history of weakness and multiple falls for two month. According to his family, he has not been acting himself lately. His main other complaint was migraine that are not improving on any medication. On presentation, his examination was benign except for impairment in hearing which is sensorineural in nature. His initial laboratory finding was only significant for hyperglycemia of $350 \mathrm{mg} / \mathrm{dl}$ and hyponatremia of $129 \mathrm{mmol} / \mathrm{L}$ after correction. CT brain was done due to history of falls which did not reveal any hemorrhage or acute infarct. Overnight, he became more confused. All labs including ammonia, TSH, vitamin B12, folic acid were within normal limits. A lumbar puncture was done that revealed glucose of $4 \mathrm{mg} / \mathrm{dl}$, protein of $61 \mathrm{mg} / \mathrm{dl}$ and nucleated cells of $183 \mathrm{cell} / \mathrm{mm}$ cube with lymphocytic predominance. India ink was positive and Cryptococcus neoformans was cultured. Blood cultures were also done that showed disseminated disease. HIV testing was negative twice as well as the viral load. CD4 count was 87 with an absolute percent of $56 \%$ that was attributed to sepsis. Patient was started on Amphotericin and Flucytosine followed with treatment with Fluconazole. MRI was done that excluded cryptococcoma. Repeat of lumbar puncture after 2 weeks revealed Cryptococcus which did not grow on cultures. Patient recovered gradually and his hearing impairment improved but functional and mental status never returned to normal.

DISCUSSION: Cryptococcal meningitis was a fatal disease until Amphotericin and Flucytosine were used in the treatment. Clinical presentation is variable and usually extends for several months before the diagnosis is made. Headache, lethargy, coma, personality changes and memory loss are some of the typical symptoms. Deafness is seen in up to $30 \%$ of cases and is characterized by being mostly bilateral and senisorineural through direct effect of the auditory nerve is either by infection or compression. Other cranial nerve involvement was also described, most commonly the optic nerve. Treatment consists of antifungal treatment in the form of Amphotericin B with Flucytosine for 2-4 weeks followed by Fluconazole for consolidation therapy. Treatment should also include management of increased intracranial pressure and improving immunologic status. Prognostic factors indicating treatment failure include low CSF glucose, high CSF lactate, high CSF cryptococcal antigen titer, level of consciousness, seizure and hydrocephalus. Early diagnosis, initiation of treatment and correction of electrolyte abnormalities are important in successful management.

HARD TO FIGURE OUT DARLINGS Allison Heinen ${ }^{1,2}$; Lee $\mathrm{Lu}^{1,2}$, Anna Kolpakchi ${ }^{1,2}$. ${ }^{1}$ Baylor College of Medicine, Houston, TX; ${ }^{2}$ Michael E. Debakey Veteran's Affairs Medical Center, Houston, TX. (Control ID \#1316275)

LEARNING OBJECTIVE 1: Histoplasmosis can frequently mimic sarcoidosis

LEARNING OBJECTIVE 2: To avoid fatal outcome, histoplasmosis should be considered before treatment of sarcoidosis with steroid is initiated. 
CASE: A 44 year old Caucasian man with psoriatic arthritis has been receiving infliximab for one year. He presented initially to his private doctor with shortness of breath and fatigue for one week. He denied fever, chills, cough, and weight loss. Physical exam showed no lymphadenopathy and clear lungs. Chest radiography showed mediastinal lymphadenopathy only. A lymph node biopsy revealed non-caseating granulomas. The patient was diagnosed with sarcoidosis and treated with prednisone with improvement in his symptoms. He presented one month later with worsened shortness of breath, fever to $1030 \mathrm{~F}$, fatigue, and $10 \mathrm{lb}$ weight loss. Physical exam showed hypoxia with $\mathrm{O} 2$ saturation of $70 \%$ on room air, fever of $102.30 \mathrm{~F}, 2 \mathrm{~cm}$ ulceration over the lower lip, and diffuse rhonchi. Chest radiography showed diffuse bilateral interstitial infiltrates. Abnormal laboratory studies included: sodium 127, alkaline phosphatase 268, ALT 199, AST 593, ESR 14, CRP 4.59, and LDH 9378. Urine and serum histoplasma antigen were elevated at $>39 \mathrm{ng} / \mathrm{ml}$ (normal $<0.6 \mathrm{ng} / \mathrm{ml}$ ). The patient was diagnosed with disseminated histoplasmosis, treated with amphotericin B and itraconazole with full recovery.

DISCUSSION: Sarcoidosis can frequently mimic lymphoma or diseases characterized by a mononuclear cell granulomatous inflammatory process, such as the mycobacterial and histoplasma infections. Sarcoidosis and histoplasmosis (a.k.a Darling's disease, Cave disease, Ohio valley disease, Reticuloendotheliosis, Spelunker's Lung, and Caver's disease) can present with similar clinical and laboratory features and distinguishing the two can be difficult at times. Erythema nodosum, uveitis, retinitis, splenomegaly, skin or mucosal ulcers, and mediastinal lymphadenopathy are frequent physical findings in both. Elevated ESR, LDH, alkaline phosphatase, angiotensin converting enzyme, hypercalcemia, and non-caseating granulomas are also common in both conditions. The added challenge to diagnose histoplasmosis, is the fact that sputum and blood cultures take many weeks to grow histoplasma and they can be negative. Sensitivity for cultures can be as low as $15 \%$ in acute pulmonary histoplasmosis, between $50-85 \%$ in chronic pulmonary histoplasmosis, and less than $25 \%$ in mediastinal histoplasmosis. Histopathology is often unreliable. Histoplasma antibodies are often falsely negative especially in patients with sarcoidosis. Urine and serum histoplasma antigen tests have the highest sensitivity and spesificity. In patients with disseminated histoplasmosis (excluding patients with AIDS), 92\% have antigenuria and 50\% antigenemia. As shown in this case, diagnosing histoplasmosis can be a challenge. But if it is misdiagnosed as sarcoidosis and treated with steroid, histoplasmosis can become disseminated which can lead to fatal outcome.

HARLEQUIN SYNDROME: EXERCISE-INDUCED FLUSHING IN ZUMBA CLASS Charlotte M. Carlson. UCSF, San Francisco, CA. (Control ID \#1333752)

LEARNING OBJECTIVE 1: 1) Recognize the diagnostic approach to unilateral facial flushing

LEARNING OBJECTIVE 2: 2) Distinguish Harlequin syndrome from other neurologic disorders

CASE: A 46 year-old premenopausal Brazilian female presented to primary care with a 2 month history of unilateral facial flushing after joining a Zumba exercise class. During intense exertion, the right side of her face showed excessive flushing and sweating, while the contralateral side remained pale and dry. She denied any associated visual changes, dizziness, neck pain, or headache. Her past medical history was significant for well-controlled hyperlipidemia on simvastatin. She was a nonsmoker and denied any alcohol or drug use. Neck, cardiac, and respiratory examination were normal. Neurological examination was normal. Formal ophthalmologic testing revealed no ptosis, miosis, or anhidrosis. Basic laboratory tests were normal. Chest X-ray and MRI thoracic spine were normal.

DISCUSSION: Unilateral facial flushing is typically caused by injury or activation of the autonomic nervous system on one side of the body. When the sympathetic nervous system is involved, lesions can occur in the hypothalamus, descending sympathetic tract in the brainstem, spinal cord, or in the cervical sympathetic ganglia, which then have fibers that ascend around the internal carotid artery. A unilateral ptosis and miosis syndrome is often concurrently found. While most cases of facial flushing are of benign nature, imaging of the chest to exclude a Pancoast's tumor, the carotid arteries to exclude a carotid dissection, and often imaging of the brainstem and thoracic spinal cord are indicated. If workup is otherwise negative or no clear evidence of other sympathetic dysfunction is found (e.g. ptosis, miosis), a diagnosis of an idiopathic autonomic disorder, known as Harlequin syndrome, can be entertained. Harlequin syndrome is considered to be an idiopathic, benign condition, resulting from compromise of vasomotor sympathetic nerve supply to one side of the face with compensatory overreaction of corresponding fibers on the intact side. It is an uncommon disease with onset is typically in childhood, and if it presents later in life, a structural lesion should be excluded.

HEART BLOCK AS A PRESENTING SIGN IN A PATIENT WITH WEGENER'S GRANULOMATOSIS Sandra Zaeh ${ }^{1}$; Stephen K. Lau ${ }^{2}$; Dustin T. Smith ${ }^{1,3}$. ${ }^{1}$ Emory University School of Medicine, Atlanta, GA; ${ }^{2}$ Atlanta Veterans Affairs Medical Center, Decatur, GA; ${ }^{3}$ Atlanta Veterans Affairs Medical Center, Decatur, GA. (Control ID \#1310105)

LEARNING OBJECTIVE 1: Recognize both typical and atypical presentations of Wegener's Granulomatosis

LEARNING OBJECTIVE 2: Understand organ systems commonly affected by Wegener's Granulomatosis

CASE: A 62 year-old male with no past medical history was placed on hemodialysis after being found in renal failure of unknown etiology. The patient reported dyspnea as well as fevers, night sweats, weight loss, and swelling. His vital signs were normal except for decreased oxygen saturation. Physical exam revealed uveitis, regular heartbeat without murmur, bibasilar lung crackles, and an ulcerated skin lesion on his ankle. Laboratory investigation showed a leukocytosis, elevated BUN and creatinine, but normal electrolytes. Urinalysis revealed hematuria and proteinuria. Renal ultrasound had findings of medical renal disease. Chest imaging demonstrated a cavitary lung lesion. Electrocardiogram revealed complete heart block. Investigation for infection was negative including bacterial cultures and serologies for viral hepatitides, HIV, and other infectious etiologies. Serum complement levels were normal. Antiproteinase-3 antibody levels were elevated. The patient was admitted to the intensive care unit for monitoring where he experienced variable degrees of atrioventricular (AV) block. Echocardiography showed normal ejection fraction. The patient spontaneously converted to first-degree AV block and his shortness of breath improved. He remained stable from a cardiovascular standpoint during his hospitalization. Renal biopsy revealed necrotizing crescentic glomerulonephritis. The patient was diagnosed with Wegener's granulomatosis based on his biopsy results and clinical findings. He was treated with prednisone, cyclophosphamide, plasma exchange, and hemodialysis.

DISCUSSION: Wegener's granulomatosis (WG) is a rare cause of kidney failure that is typically associated with upper and lower respiratory tract signs and symptoms. It has been reported that up to $44 \%$ of Wegener's cases have cardiac findings, including supraventricular tachycardia, pericarditis, coronary artery vasculitis, and aortic valve disease. There have been only a few case reports of reversible heart block associated with WG. The etiology of heart block in WG is unknown but may be due to disease involvement of the coronary arteries or involvement of the conduction system secondary to small vessel vasculitis. Granuloma formation is another possibility. The cytoplasmic pattern on immunofluorescence for anti-proteinase-3 antibodies has high sensitivity and specificity in the diagnosis of WG. Renal biopsy typically demonstrates segmental necrotizing glomerulonephritis with multiple crescents and supports the diagnosis. Granulomas can also be found in tissue specimens. Treatment of WG is directed to prevent end-organ damage. Cyclophosphamide and prednisone can induce temporary remission in most patients. Plasma exchange has been shown in trials to decrease mortality and improve renal function in patients who present acutely with severe renal disease. This case recognizes the clinical manifestations of Wegener's granulomatosis and reports the rare finding of heart block associated with this disease.

HEMOGLOBIN CHICAGO, A RARE VARIANT OF ALPHA THALASSEMIA. Namratha R. Vontela ${ }^{1}$; Pranitha Naini ${ }^{2}$; Alva B. Weir ${ }^{2}$. ${ }^{1}$ University of Tennessee, memphis, TN; ${ }^{2}$ University of Tennessee, memphis, TN. (Control ID \#1309280) 
LEARNING OBJECTIVE 1: Recognize coexistent thalassemias in patients with iron deficiency anemia.

CASE: Patient is a 43 year old African American female with history of hypertension and diabetes who presented to the clinic with fatigue. She reported a long standing history of heavy menstrual bleeding and denied any hematuria or rectal bleeding. Her last colonoscopy an year ago showed benign polyps. Physical examination was normal. Laboratory data at presentation demonstrated microcytic, hypochromic anemia with $\mathrm{Hb}$ of $9.6 \mathrm{~g} / \mathrm{dL}$ and very low MCV of $69.3 \mathrm{fL}$. RDW was 19.6. Ferritin level was $2.9 \mathrm{ng} / \mathrm{mL}$ and transferrin saturation was $2 \%$ leading to the impression of iron deficiency anemia. However her RBC count was $4.4 \mathrm{M} / \mathrm{uL}$ which was in the normal range in spite of the anemia. This lead to the suspicion of an additional factor contributing to the low MCV apart from her established iron deficiency anemia. Therefore hemoglobin electrophoresis was performed which demonstrated "Hemoglobin Chicago", a rare variant of alpha thalassemia. Patient was started on oral iron and repeat labs in 3 weeks showed RBC $-4.8 \mathrm{M} / \mathrm{uL}$, $\mathrm{Hb}-10.3 \mathrm{~g} / \mathrm{dL}$. Her MCV - $69.2 \mathrm{fL}$ remained the same. Her ferritin improved to $5.1 \mathrm{ng} / \mathrm{mL}$ and transferrin saturation increased to $3 \%$.

DISCUSSION: We report a rare case of "Hemoglobin Chicago" in a woman who presented with iron deficiency anemia. Hemoglobin Chicago is a rare variant of alpha thalassemia in which the leucine residue at position 136 of alpha chain is substituted by methionine. This rare variant was first described in 1986 in a black newborn girl and her father and has since had few reported cases. This mutation is either carried alone or in combination with sickle cell trait or sickle cell disease. In our patient, as Hemoglobin Chicago was coexistent with iron deficiency anemia the diagnosis had been missed for several years. Hence physicians should consider ruling out thalassemia in cases of iron deficiency with normal $\mathrm{RBC}$ count and microcytosis out of proportion to the degree of anemia, and especially in cases where serum ferritin corrects with iron therapy but the MCV remains low. This approach may protect patients with coexistent thalassemias from iron overload caused by long term iron therapy in an attempt to correct hemoglobin and MCV rather than true iron deficiency.

HEMOLYTIC ANEMIA IN SYSTEMIC LUPUS ERYTHEMATOSUS Sumana Nagireddy ${ }^{1}$; Robert Avery ${ }^{2} .{ }^{1}$ UAB Montgomery, Montgomery, AL; ${ }^{2} \mathrm{UAB}$ Montgomery, Montgomery, AL. (Control ID \#1339614)

LEARNING OBJECTIVE 1: Recognize various types of anemia in SLE and diagnose SLE presenting as auto immune hemolytic anemia (AIHA) LEARNING OBJECTIVE 2: Differentiate autoimmune hemolytic anemia from micorangiopathic hemolytic anemia

CASE: A 27 year old female with history of discoid rash, hair loss and hypertension for one year was hospitalized for generalized weakness and fatigue. On admission vitals were stable other than temperature which was elevated at $101.9 \mathrm{o}$ F. She was alert but showed limited communication. Examination showed discoid lesions on all extremities. Her blood cultures were negative with no source of infection noted. Lab studies showed hemoglobin of $3.4 \mathrm{~g} / \mathrm{dl}$, MCV of $112.7 \mathrm{fl}$, Platelets $150,000 \mathrm{k} /$ cumm, creatinine $2.34 \mathrm{mg} / \mathrm{dl}$ and total bilirubin $1.5 \mathrm{mg} / \mathrm{dl}$. Serum folate and B12 levels were normal. Hemolytic anemia was suspected so, hemolytic indices were tested which showed LDH of $1749 \mathrm{IU} / \mathrm{L}$ (normal 98-192 IU/L), and haptoglobin $<14 \mathrm{mg} / \mathrm{dl}$ (normal 30-200 mg/dl). Hemoglobin electrophoresis was normal. . Peripheral smear showed nucleated red cells, spherocytes but no schistocytes thus ruling out microangiopathic anemia. .Direct antiglobulin test was positive confirming the diagnosis of AIHA. ANA was positive with titer of 1:160 (normal $<1: 40$ ), dsDNA was elevated to $67 \mathrm{IU} / \mathrm{ml}$ (>10 positive), complement $\mathrm{C} 3$ and $\mathrm{C} 4$ were low, $44 \mathrm{mg} / \mathrm{dl}$ (Normal 75$175 \mathrm{mg} / \mathrm{dl})$ and $7 \mathrm{mg} / \mathrm{dl}(14-40 \mathrm{mg} / \mathrm{dl})$ respectively. She met diagnostic criteria for lupus ( $>4$ out of 11) and her initial presentation was with AIHA, alopecia, discoid rash, nephritis and cerebritis. She was started on pulse dose steroids for AIHA and her clinical status and anemia improved over the time and she was discharged for outpatient follow up.

DISCUSSION: Anemia in lupus may be caused by multiple reasons including chronic inflammation, renal insufficiency and hemolysis. Hemolytic anemia in patients with lupus frequently presents with considerable diagnostic and therapeutic difficulties to clinician as its incidence is low. In lupus patients hemolysis may be because of auto immune hemolytic anemia (AIHA) or thrombotic microangiopathic hemolytic anemia (MAHA). AIHA is a hematologic diagnosis that is based on the presence of anemia, signs of hemolysis, and detection of red cell-reactive antibodies (positive coombs test). AIHA may be a primary (idiopathic) or a secondary disease. Secondary AIHA may be from lymphomas and systemic autoimmune disorders, and less frequently from organ transplantation, infections, or solid tumors. For AIHA first line of treatment is glucocorticoids and second line of treatment is splenectomy. In refractory cases rituximab (anti-CD20) and mycophenolate mofetil are effective. If MAHA associated with fever, neurological symptoms, kidney involvement HUS should be ruled out as it needs urgent management. In lupus patients less severe MAHA can be treated with high dose steroids but more severe MAHA involving major organs needs treatment with plasmapheresis as in TTP-HUS. This case illustrates the importance to recognize various types of anemia in SLE diagnose AIHA as a initial presentation of SLE and differentiate AIHA with MAHA as prognosis and treatment are different.

HEPATITIS B REACTIVATION WITH FULMINANT HEPATITIS AFTER RITUXIMAB CHEMOTHERAPY IN A PATIENT WITH FOLLICULAR LYMPHOMA Aditi Kumar ${ }^{1}$; Ioannis Politikos ${ }^{1}$; Chirag Acharya ${ }^{2}$. ${ }^{1}$ Mount Auburn Hospital, Cambridge, MA; ${ }^{2}$ Dana Farber Cancer Institute, Boston, MA. (Control ID \#1333887)

LEARNING OBJECTIVE 1: To recognize Hepatitis B reactivation as a complication of rituximab chemotherapy.

LEARNING OBJECTIVE 2: To recognize the importance of checking Hepatitis B serologies before institution of rituximab chemotherapy.

CASE: 83 year old male with follicular B cell lymphoma who received rituximab chemotherapy presented to our hospital with nausea and fatigue. $\mathrm{He}$ had received 6 cycles of rituximab, cytoxan, vincristine and prednisone followed by one dose of rituximab two months after the last cycle as part of maintenance therapy. After two months of the last rituximab dose, the patient came to the hospital and had rapidly rising aminotransferase and bilirubin levels. He had positive serology for $\mathrm{HBsAg}, \mathrm{HBeAg}$ and $\mathrm{HBsAb}$ and negative for HBcAb. HBV load detected by PCR was elevated to $467000 \mathrm{IU} / \mathrm{ml}$. His Hepatitis B status prior to start of chemotherapy was unknown. His presentation was considered to be secondary to rituximab induced Hepatitis $\mathrm{B}$ reactivation. The patient was started on telbivudine but died of fulminant hepatic failure despite anti viral therapy.

DISCUSSION: This case illustrates late reactivation of hepatitis B virus after immunosuppressive and cytotoxic therapies such as rituximab. Full Hepatitis B serologies must be obtained in all patients prior to administering intensive immunosuppressive therapies. For patients who are HBsAg positive, a baseline HBV DNA should be performed and preemptive lamivudine therapy should be given and continued for at least 3 months after cessation of chemotherapy. Patients with high baseline HBV DNA levels may need prolonged lamivudine therapy (12 months or more). $\mathrm{HBeAg}$ positive patients should continue therapy till $\mathrm{HBeAg}$ seroconversion occurs. However, HBV reactivation may still occur while patients are on lamivudine due to development of drug resistant HBV variants with YMDD mutations . The development of mutants is more commonly associated with prolonged lamivudine administration and is accompanied by a surge in HBV DNA levels. It is therefore necessary to monitor patients on lamivudine with HBV DNA and liver transaminase levels. Newer nucleoside analogues such as entacavir, adefovir, telbivudine and tenofovir are available for treatment of lamivudine resistant Hepatitis B infection. Early use of these agents may be justifiable when prolonged anti HBV therapy is necessary for patients requiring prolonged immunosuppression. Another concern with lamivudine use is the occurrence of withdrawal hepatic flares upon cessation of antiviral therapy and this is usually associated with high prechemotherapy viral load. Therefore close monitoring of serum HBV DNA and aminotransferase levels for evidence of withdrawal flares is recommended after withdrawal of lamivudine therapy. Despite lamivudine, HBV associated mortality has been reported in upto $20 \% \mathrm{HBsAg}$ positive patients treated due to delay in administration of antiviral at a time when severe hepatic impairment has already occurred. The best strategy in managing patients with occult HBV infection is less clear. These patients are HBsAg negative and $\mathrm{HBcAb}$ positive. Among these patients, the HBsAb negative patients have higher likelihood of HBV 
reactivation. Serial monitoring of HBV DNA and transaminase levels for early diagnosis of $\mathrm{HBV}$ reactivation and beginning antiviral therapy when HBV DNA is detected in the serum is a reasonable strategy though the optimal frequency and duration of such HBV DNA monitoring is not clear.

\section{HEROIN INDUCED RHABDOMYOLYSIS AND COMPARTMENT SYNDROME: EARLY RECOGNITION TO PREVENT MITIGATION} OF SERIOUS MORBIDITY AND MORTALITY Jordan Brodsky; Elizabeth Gilbert. Beth Israel Medical Center, New York, NY. (Control ID \#1277337)

LEARNING OBJECTIVE 1: The need for clinicians to possess a high level of suspicion for rhabdomyolysis and compartment syndrome in heroin users presenting with non specific pain.

LEARNING OBJECTIVE 2: The mitigation of serious morbidity and mortality is dependent on early diagnosis and adequate therapy of rhabdomyolysis.

CASE: A 26 year old man with a history of intravenous heroin presented to the Emergency Department with right lower extremity pain and swelling for three days. History revealed that he had traveled on a plane to Mexico ten days prior to arrival in the Emergency Department and used heroin intravenously in the right antecubital fossa three days prior. One day after intravenously heroin use he experienced increased pain and swelling in his right lower extremity calf. He denied any history of overdosing, seizures, unconsciousness, trauma, recent exercise or extensive walking. In the Emergency Department physical exam revealed a right swollen red leg below the knee that was firm and painful to touch on the medial calf along the anterior lateral leg. Strength on right foot was $3 / 5$, pulses $2+$ diffuse and patient was unable to dorsiflex his right foot; there was decreased pin prick sensation on the right foot. Labs revealed a CPK 112,747 IU/L, BUN $9 \mathrm{mg} / \mathrm{dL}$, creatinine $2.77 \mathrm{mg} / \mathrm{dL}$, Calcium $8.8 \mathrm{mg} / \mathrm{dL}$, Phosphate $5.2 \mathrm{mg} / \mathrm{dL}$, potassium $4.8 \mathrm{mEq} / \mathrm{L}$, AST $1389 \mathrm{U} / \mathrm{L}$, ALT $450 \mathrm{U} / \mathrm{L}$, urine analysis showed large blood with only 3 RBC. The patient was diagnosed with rhabdomyolysis and acute renal failure. He was started on half normal saline with bicarbonate $1.5 \mathrm{amps}$ at $200 \mathrm{ml} / \mathrm{hr}$. X-ray of tibia/fibula showed no evidence of fracture. Ultrasound was done and showed no signs of a deep vein thrombosis. Compartment pressure was measured at $10 \mathrm{mmHg}$. Right anterior compartment fasciotomy was performed as he was diagnosed with right anterior compartment syndrome after his pain and neurological deficit increased. Post surgery his numbness subsided, sensation and movement returned to his right lower extremity. His creatinine trended down and fluids were decreased to $150 \mathrm{ml} / \mathrm{hr}$ and subsequently stopped. He clinically improved and CPK trended down to $273 \mathrm{IU} / \mathrm{L}$. The patient underwent split thickness skin graft and wound closure. He was subsequently discharged home with visiting nurse service for wound care.

DISCUSSION: In recent years, Emergency Departments have reported an increased number of cases of non traumatic rhabdomyolysis associated with heroin addiction. Even if the last time heroin was used was day's prior, early diagnosis and treatment of rhabdomyolysis after intravenous heroin, is imperative in preventing complication such as electrolyte abnormalities, renal failure and compartment syndrome. While the causes of rhabdomyolysis vary, compartment syndrome is a serious complication that must be recognized early. The diagnosis of compartment syndrome can be missed or delayed in the setting of rhabdomyolysis. Inexperience with non traumatic rhabdomyolysis leading to compartment syndrome may delay necessary surgical treatment and lead to permanent disability. Physicians must take into account that rhabdomyolysis and the development of compartment syndrome can occur days after the administration of a drug. A high index of suspicion for rhabdomyolysis and compartment syndrome is essential in drug abusers presenting to the Emergency Department with non specific pain as the prognosis and treatment is imperative to prevent morbidity and mortality.

HICKAM'S DICTUM ILLUSTRATED: THE SIMULTANEOUS PRESENTATION OF TWO METASTATIC CANCERS Brandon Verdoorn; Jeremy Larsen; Elise C. Carey. College of Medicine - Mayo Clinic, Rochester, MN. (Control ID \#1312636)
LEARNING OBJECTIVE 1: Recognize the typical pattern of metastasis of differentiated thyroid cancer.

LEARNING OBJECTIVE 2: Use pattern of metastasis to help predict primary tumor site(s) in a patient with newly suspected metastatic cancer on imaging.

CASE: A 56 year-old male with remote 18 pack-year smoking history was admitted to the hospital with cough and low back pain. Ten days prior to admission he was evaluated for a one-month history of cough. He was found to have cervical lymphadenopathy and right middle lobe consolidation on chest $\mathrm{x}$-ray. He was treated with azithromycin, but returned two days prior to admission with persistent cough and new, severe low back pain. Computed tomography (CT) scan of the neck and chest revealed a large, cystic right thyroid mass, cervical and mediastinal lymphadenopathy, innumerable pulmonary nodules, lytic lesions throughout the spine, bilateral adrenal masses, and right lower lung consolidation. The patient was admitted to the hospital with suspected widely metastatic cancer. Magnetic resonance imaging of the head and spine demonstrated multiple ring-enhancing cerebral and cerebellar lesions with surrounding vasogenic edema and confirmed extensive lytic disease throughout the spine. The patient underwent fine needle aspiration of an enlarged cervical lymph node and the right thyroid mass. Pathology showed papillary thyroid carcinoma (PTC). Positron emission tomography scan revealed uptake in the brain, axial skeleton, pulmonary nodules, right lower lung consolidation, mediastinal lymph nodes, bilateral adrenal glands, right supraclavicular fossa, and a right cervical lymph node. The thyroid gland had no uptake. The aggressive nature of his disease was felt inconsistent with typical PTC and he subsequently underwent CT-guided biopsy of an L2 vertebral lesion. Pathology showed adenocarcinoma of unknown primary origin, most consistent with lung or upper gastrointestinal tract malignancy. The patient was discharged from the hospital, and underwent palliative radiation therapy to the brain and spine. A course of carboplatin and Taxol was offered for the adenocarcinoma, but the patient elected to pursue hospice care and passed away shortly thereafter.

DISCUSSION: The simultaneous presentation of multiple metastatic malignancies is rare. Pattern of organ involvement on staging work-up is an important clue to synchronous occurrence of tumors originating from multiple primary sites. Papillary and follicular carcinomas of the thyroid gland, often referred together as differentiated thyroid cancer (DTC), are metastatic at presentation in only $1-9 \%$ of cases. When this does occur, solitary lung or bone metastases are most common. Only rarely are multiple metastatic sites involved at presentation. In a patient with newly diagnosed DTC and imaging evidence of widely metastatic disease, evaluation for a second primary tumor may be warranted. Synchronous occurrence of DTC and other primary cancers has been reported. To our knowledge, simultaneous diagnosis of metastatic DTC and adenocarcinoma of unknown primary origin has not been previously reported in the literature.

HIDDEN WHIRLPOOL Tashfeen Mahmood; Roger D. Smalligan; James ". Walker. Texas Tech University Health Sciences Center, Amarillo, TX. (Control ID \#1327392)

LEARNING OBJECTIVE 1: Recognize and diagnose an unusual cause of abdominal pain in adults.

LEARNING OBJECTIVE 2: Manage midgut volvulus with early surgical intervention to reduce morbidity and mortality.

CASE: A 38yo man with uncontrolled type 1 diabetes presented with nausea, slightly bloody vomitus, abdominal pain, myalgias and subjective fever for 7 days. He also complained of some blood in his stools recently. Surgeries - none. Personal/Social - methamphetamine and cocaine use. Incarcerated for several months. Denied alcohol and tobacco use. Physical exam: Alert \& oriented in mild distress, T 98.6, P137, BP 75/60, RR 20 O2 sat $96 \%$ on $2 \mathrm{~L} \mathrm{O}$. Dry mouth, clear lungs, abdomen with mild distention, mild tenderness in the RLQ, BS present, no rebound. Lab: WBC $7.8 \mathrm{k}$ with $38 \%$ bands, Hgb 15.9, PLT 67 k, Na 123, BUN 76, Cr 1.92, glucose 481, CO2 20, lactate 5.3, albumin 1.5, INR 1.6. Imaging: abdominal CT showed a "whirlpool sign" with clockwise swirling of the superior mesenteric artery (SMA) and vein twisted around each other suggesting intestinal volvulus. The patient was resuscitated with IV fluids and taken for laparotomy where 
volvulus of the midgut and necrosis of the jejunum were found and resected. End to end anastomosis was performed and the patient recovered well. DISCUSSION: Although well known among neonates, midgut volvulus is a rare entity in adults. A search of Pubmed found less than 40 cases in the literature of volvulus leading to malrotation in adults. Review of these cases shows that some adult patients present acutely with symptoms of bowel obstruction and ischemia while others, especially those with a midgut or cecal volvulus, present more insidiously with chronic, vague abdominal pain. Diagnosis is typically made with ultrasonography (US), computed tomography (CT), angiography or emergent exploratory laparotomy depending on the presentation. The "clockwise whirlpool sign" on color Doppler US or CT has been described as essentially a pathognomonic finding in midgut volvulus. It consists of wrapping of the SMV and the mesentery around the SMA in a clockwise pattern as the SMA descends into the midgut region. In malrotation the mesenteric root, which normally forms a diagonal line from the ligament of Treitz to the cecum, is shortened. This shorter mesenteric attachment of the small bowel permits its rotation around the axis of the SMA resulting in midgut volvulus. Clinical findings depend on the severity of the twist around the SMA and range from mild abdominal pain with protein losing enteropathy, if only the venous and lymphatic structures are involved, to more severe abdominal pain, bilious vomiting, and bloody stools if a complete twist is involved. The most severe cases present as an acute abdomen due to severe bowel ischemia and necrosis of the entire jejunum and ileum. Surgical treatment remains the Ladd procedure as described in our case. This case reminds internists to maintain a high index of suspicion of volvulus in patients with signs of obstruction or chronic abdominal pain in order to make a prompt diagnosis and save as much viable bowel as possible with definitive surgical treatment.

HIDDEN IN THE TUNNEL! Muhammed Sherid; Samian Sulaiman; Salih Samo; Meenu Singh. University of Illinois at Chicago, St.Francis Hospital, Evanston, IL. (Control ID \#1314389)

LEARNING OBJECTIVE 1: To consider Trichinella between the differential diagnosis for any muscle lesion.

LEARNING OBJECTIVE 2: To recognize that cases of Trichinella still can be found in the US even it is rare.

CASE: A 48 year female underwent surgical release of median nerve for the left hand carpal tunnel syndrome. She had have numbness and tingling in the first three fingers in the left hand for a year which developed after fracture in the same wrist a year prior. She had local steroid injection twice without any relief of symptoms. Finally, the decision was made to go for surgery after the failure of all conservative measures. . During surgery, in addition of release of the median nerve, her surgeon took a biopsy from her left thenar because he felt a small lump in that area. Her past medical history was consistent with asthma and depression which were controlled by advair and sertraline respectively. Her social history; she was nonsmoker non alcoholic, was living in Chicago. Surprisingly, the biopsy from the thenar showed one encapsulated larvae between muscle cells (see figure1). Once the biopsy result came back, a detailed history was taken regarding her exposure to any kind of parasite. She did not have any pets and denied exposure to alive animals. As for her consumption of raw or undercooked meat, she stated that she was in Hawaii 6 months prior. She ate once a meat from pork cooked on the ground. The source of animal was not clear. She stated it could be a wild boar. Also, she recalled that after 2 weeks from the consumption of that meat she developed flu-like symptoms and had worsening asthma symptoms which required systemic steroids for 2 weeks. Also both, her son and husband, developed similar symptoms around that time, and her husband had conjunctivitis and periorbital edema. She recalled also one of her friends, from the same group went to Hawaii, had 1 week history of nausea, vomiting diarrhea and abdominal pain. Her laboratory studies showed negative Toxocara antibodies, but Trichinella Ig G was positive. The diagnosis of trichinellosis was made. Her family members and friends checked for trichinella, but all of them were negative.

DISCUSSION: Trichinella is parasitic nematode that infects primarily pigs, but it can infect horses, wild animals such as foxes, wolves, bears, skunk, raccoons, rats, and other small mammals. Humans can get infected when they consume raw or undercooked meat even tasting very small pieces of undercooked meat during preparation. There are still 11 cases reports to CDC every year, most of the same are not related to pork meat. Trichinellosis has 2 phases, first is gastrointestinal symptoms such as nausea, vomiting and diarrhea which occur during the period ingested larvae release and mature in the intestine. Second phase is muscular symptoms such as myalgia, fever, periorbital edema and conjunctivitis which occur when newborn larvae penetrate to vessels and spread throughout the body until reaching their final location, mostly muscles. The symptoms vary up to the burden of larvae which could minimal and goes without diagnosis. The diagnosis is made either by muscle biopsy or positive anti-trichinella antibody in appropriate clinical scenario. The treatment is albendazole or mebendazole for 10-15 days. Steroids should be given when muscular symptoms are very severe to decrease the body reaction and inflammation.

HILAR MASS IN YOUNG FEMALE - TB??/ LYMPHOMA??/ SARCOID?? THINK AGAIN!! Sashank Kolli; Chanunya Srihawan; Thomas Liao. Advocate Illinois Masonic Medical Center, Chicago, IL. (Control ID \#1339209)

LEARNING OBJECTIVE 1: Introduction: Hilar/ mediastinal masses have a broad differential including malignancy, infectious and connective tissue disorders. In young people tuberculosis (TB), lymphoma or sarcoid are prime considerations. The patient may present with respiratory or constitutional symptoms, or a mass may be discovered incidentally on routine chest $\mathrm{x}$-ray. Further evaluation is usually undertaken; options include CT chest, bronchoscopy and thoracic biopsy by thoracic surgery. CASE: Our patient is a 27 year old female with a past medical history significant for pulmonary embolism, who initially presented to her PCP for persistent cough. She had no systemic symptoms. Chest x-ray showed a left hilar mass, which was new compared to xray one year ago. CT scan confirmed a left hilar mass which also was causing mass effect on pulmonary arteries and left lower lobe bronchus. Additional mediastinal lymphadenopathy was also observed. Bronchoscopy showed narrowing of lingula and bronchus but lavage and biopsies were inconclusive. After evaluation by thoracic surgery she underwent thoracotomy, which showed a friable mass with adherence to pulmonary arteries. Biopsy was done and was most consistent with fibrosing mediastinitis, negative for malignancy and infections. Additional work up revealed positive Histoplasma titres and Quantiferon TB Gold was indeterminate.

DISCUSSION: Fibrosing mediastinitis is a rare disorder, with extensive fibrotic reaction in the mediastinum. Most commonly identified cause of this reaction is infection with Histoplasma capsulatum although it may be drug related (methysergide) or idiopathic. It can be a progressive disease and diagnosed when complications occur secondary to mass effect on mediastinal structures such as airways, great vessels, heart and the esophagus. Prognosis is variable with no proven treatment benefits with either antifungals or steroids. Surgery is considered when life threatening complications arise from mass effect such as obstruction of the superior vena cava, pulmonary artery or major airway. Ours is a very rare case of fibrosing mediastinits which is consistent with literature for being secondary to Histoplasma infection. Not all hilar lymphadenopathy is $\mathrm{TB}$, lymphoma or sarcoid.

HISTO FOR HISTO Zouyan Lu; Karrie L. Martin; Adam Meyers. Medical College of Wisconsin, Milwaukee, WI. (Control ID \#1289805)

LEARNING OBJECTIVE 1: Review available diagnostic strategies for histoplasmosis.

LEARNING OBJECTIVE 2: Recognize patient factors which impact selection of the optimal pharmacologic treatment of histoplasmosis.

CASE: A 73-year-old woman on long-term infliximab for Crohn's disease presented with a two-week history of recurrent high fevers. She also complained of headache, sore throat, intermittent non-productive cough, malaise, and occasional night-sweats. Her additional medical history included monoclonal gammopathy of undetermined significance, hypothyroidism, and short-gut syndrome requiring chronic total parenteral nutrition 
(TPN). Following unremarkable initial laboratory testing, she was admitted to the hospital, and a chest CT revealed multiple, new pulmonary nodules, and hilar and mediastinal lymphadenopathy. Blood cultures were negative, a transthoracic echocardiogram was unremarkable, and urine antigens for Histoplasma and Blastomyces were negative. Bronchoalveolar lavage (BAL) was performed, and transbronchial biopsy of the right upper lobe revealed nonnecrotizing granulomas with negative GMS and AFB stains. The patient continued to have intermittent fevers, but without a definitive source of infection, treatment was not initiated. In order to obtain a diagnosis, biopsy of an enlarged pre-tracheal lymph node was performed and revealed multiple epithelioid cell granulomas with central necrosis and GMS stain positive for multiple $\mathrm{H}$. capsulatum organisms. Later, culture of BAL fluid also grew histoplasma organisms, and the patient was started on oral itraconazole. Though she became afebrile, she did not achieve therapeutic levels of itraconazole, and follow-up CT showed disease progression. Therapy was changed to high-dose oral fluconazole, and after three weeks of treatment, repeat scans showed radiographic response.

DISCUSSION: Histoplasma capsulatum is endemic to the Ohio and Mississippi River valleys. Severity of infection is related to level of exposure and host immune status. Histoplasmosis can range from mild, self-limited disease to life-threatening, disseminated infection. Pulmonary histoplasmosis often presents with fever, chills, headache, chest pain, and cough. It should be considered in patients with mediastinal or hilar lymphadenopathy and pulmonary nodules. The gold standard for diagnosis is fungal culture; however, results may take weeks. More rapid diagnosis is often made by antigen or antibody detection in urine, blood, or BAL fluid, or by histopathologic examination of fluid or tissue. Urine antigen testing may be negative in pulmonary disease, and serologic testing is less useful in immunosuppressed patients due to a lack of antibody response. Itraconazole is the drug of choice for treatment of mild-moderate histoplasmosis, but bioavailability is variable when taken by mouth. Despite evidence that fluconazole is less effective, it has $90 \%$ bioavailability, and as in our patient, may be more successful in achieving therapeutic levels in patients with short-gut syndrome.

HORNER SYNDROME-NOT TO BE SNEEZED AT! Krishna Khatri; Vinod Khatri; Tanmayee Bichile; Harvey Friedman. St. Francis Hospital, Evanston, IL. (Control ID \#1339140)

LEARNING OBJECTIVE 1: Dissection of cervical internal carotid artery can occur without any apparent trauma.

LEARNING OBJECTIVE 2: Horner's syndrome may be the only sign on presentation.

CASE: 62 yrs old Caucasian male was admitted with 7 history of headache which started after an upper respiratory tract infection. Headache was dull aching, left sided, mild to moderate in intensity and was only relieved for few hours with analgesics. He had no significant past medical history and was in good health. On examination his vital signs were stable, he had no carotid bruit. The cardiac and respiratory exams were essentially normal. On CNS exam he had mild ptosis on the left side and his left pupil was smaller than the right. Rest of the neurological exam was non-focal. On evaluation his routine labs were normal. An MRI/MRA head revealed decrease in the signal involving the left distal internal carotid artery in the cervical portion. The lumen of the cervical left internal carotid artery was narrowed over approximately $2.5 \mathrm{~cm}$ length which appeared to be from internal carotid artery dissection that started several $\mathrm{cm}$ after the carotid bulb and internal carotid artery origin. There was eccentric hyper-attenuation within the wall consistent with an intramural hematoma suggestive of a sub-acute nature. $\mathrm{He}$ was started on anticoagulation and was discharged home and advised regular follow up.

DISCUSSION: Internal carotid artery dissection is a potentially lifethreatening condition and carries a substantial risk of disabling stroke. Carotid dissection is under-recognized as a cause of Horner syndrome and can be missed. Spontaneous dissection of the cervical internal carotid artery causes, in more than $90 \%$ of patients, carotid territory ischemia, local signs and symptoms on the side of dissection, or both, whereas the remaining are clinically asymptomatic. Local signs and symptoms include head, facial, or neck pain, Horner syndrome, pulsatile tinnitus, and cranial nerve palsy. Horner syndrome consisting essentially of miosis and ptosis is detected in about one third. Most of cases of Horner syndrome due to internal carotid artery dissection are painful. It is important to diagnose dissection because anticoagulation can prevent carotid thrombosis and embolism. The investigation of choice is magnetic resonance imaging and angiography scan of the head and neck. The treatment advocated for dissection is anticoagulation for 3-6 months.

HUMORAL HYPERCALCEMIC CRISIS AND ADENOCARCINOMA OF THE GALL BLADDER Kalpana Nagarkar; Tania Calzada; Emily Chen; Shodhan Patel. Capital Health Regional Medical Center, Trenton, NJ. (Control ID \#1319938)

LEARNING OBJECTIVE 1: Rare presentation of humoral hypercalcemic crisis secondary to adenocarcinoma of the gall bladder.

LEARNING OBJECTIVE 2: The presentation of high serum calcium and PTHrP early in the course of disease appears to correlate to a poor response to treatment and rapid disease progression

CASE: A 54-year-old African American female presented with persistent nausea and vomiting along with abdominal pain. CT scan of the abdomen and pelvis revealed large metastases in the right lobe and medial left lobe of the liver. There were also gallstones in the neck of the gallbladder, focal gallbladder wall thickening and a soft tissue density in the medial wall of the gallbladder. Serum calcium was $11.9 \mathrm{mg} / \mathrm{dL}$ at presentation. Elevated PTHrP $(109 \mathrm{pg} / \mathrm{ml})$ and low PTH $(8 \mathrm{pg} / \mathrm{ml})$ suggested humoral origin of the hypercalcemia due to malignancy. She underwent liver biopsy and was diagnosed with adenocarcinoma arising from the gallbladder with liver metastases. A bone scan showed no evidence of bony metastatic disease. The patient was managed with intravenous fluids and bisphosphonate therapy without resolution of the hypercalcemia and she was referred to oncology for chemotherapy.

DISCUSSION: Parathyroid hormone-related peptide (PTHrP) is a tumorderived circulating factor that has been associated with hypercalcemia of malignancy and is generally seen in squamous cell malignancy. The role of PTHrP as a prognostic indicator remains unclear. Studies suggest that it may function as a growth factor; and, recently, the ability of PTHrP to induce cytokine expression has been described. It is postulated that PTHrP is associated with systemic inflammation and adverse nutritional status. We expect the hypercalcemia to improve with definitive treatment of the underlying malignancy and reduction of the PTHrP level. Teaching points: There are very few cases in the literature that describe hypercalcemia associated with adenocarcinoma of the gall bladder. The elevated PTHrP level, sub-baseline PTH level, and absence of bony metastases confirmed that the hypercalcemia was humoral, mediated by the adenocarcinoma of the gallbladder. The presentation of high serum calcium and PTHrP early in the course of disease appears to correlate to a poor response to treatment and rapid disease progression. Though it can be postulated that presence of PTHrp itself with or without hypercalcemia predicts prognosis, more such studies are needed.

HYDRONEPHROSIS IN A HEALTHY 40 YEAR OLD: WHAT'S THE ROLE OF ROUTINE SCREENING? Christian Suarez-Fuentes; Ashwin Sridharan; Ari Kriegsman; Lauren Shapiro. Montefiore Medical Center, Bronx, NY. (Control ID \#1338336)

LEARNING OBJECTIVE 1: To make internists aware of hydronephrosis and renal failure secondary to cervical cancer, an infrequent occurrence in the developed world because of routine screening

LEARNING OBJECTIVE 2: To draw attention to health disparities in cervical cancer rates and mortality

CASE: A 40 year old Hispanic female with no past medical history was brought into the emergency room for altered mental status. As per her family, she had a three month history of lower abdominal pain radiating to her back exacerbated by her periods. Her menses were increasingly irregular and heavy and her urine output was less. One week prior to admission her previously unlimited exercise tolerance was severely limited secondary to fatigue and shortness of breath. In the ED her temperature was $94.5 \mathrm{~F}$, her pulse was 105 , her blood pressure was 189/91, and her respiratory rate was 22 . On exam, she 
appeared pale and was breathing rapidly. A friction rub and rales were noted. She was oriented only to person with no other neurological deficits. Laboratory evaluation showed a serum creatinine of 15.3 , BUN of 155 , bicarbonate of 7 , potassium of 9.2, and a hemoglobin of 5.1. She was intubated, dialyzed, transfused blood, and transferred to the MICU. CT scan of the abdomen and pelvis showed bilateral hydronephrosis with relative atrophy of the left kidney and lower right retroperitoneal lymphadenopathy. She was extubated on hospital day 2. As her mental status improved, the patient recalled a Papanicolaou smear showing a high grade squamous intrapeithelial lesion five years ago. She had not followed-up with doctors for this since she had lost her insurance. Cervical biopsy showed cervical intraepithelial neoplasia III, consistent with cervical cancer stage IIIB. Bilateral nephrostomy tubes were placed with improvement in renal function. She was started on radiation therapy and discharged with follow-up.

DISCUSSION: This case demonstrates advanced sequelae of cervical cancer infrequently seen in the United States because of comprehensive preventative health care services. This patient knew about her abnormal screening test five years before but was unable to seek appropriate medical care. Cervical cancer can cause hydronephrosis through several etiologies, including adhesion to the pelvic sidewall, direct bladder outflow obstruction, and obstruction secondary to diffuse bulky lymphadenopathy. Cervical cancer was the third most commonly diagnosed cancer in women and the fourth leading cause of cancer death in women worldwide in 2008. It disproportionally affects women in the developing world, where screening is less readily available. In the United States, cervical cancer disproportionately affects underprivileged minorities. In 2007, there were 7.9 cases per 100,000 women across all races, with 11.5 and 10.2 cases per 100,000 among black and hispanic women, respectively. These women were also more likely to die from cervical cancer than whites and asians. A causative role for the human papillomavirus has been established, with a vaccine now available against the four most common serotypes; however, another $30 \%$ of cervical cancers are caused by other strains so routine screening must be continued. In summary, hydronephrosis due to cervical cancer is infrequent but preventable. Our case highlights the importance of routine papanicolaou smears for screening and appropriate gynecologic treatment for positive results, especially in poor minority populations.

HYPERBARIC OXYGEN AS A TREATMENT OPTION FOR DIABETIC MYONECROSIS :A CASE REPORT Tanvi Tiwari; Malvika Varma. saint francis hospital, Evanston, IL. (Control ID \#1309929)

LEARNING OBJECTIVE 1: Diagnose diabetic myonecrosis, an under recognised complication of longstanding diabetes mellitus. Illustrate a rare occurance of this condition in a patient without advanced diabetic end organ damage.

LEARNING OBJECTIVE 2: Investigate role of Hyperbaric oxygen as a potential treatment option.

CASE: 31 year old female with type 1 diabetes mellitus presented with pain and swelling of the right medial thigh since two weeks. There was no history of fever, trauma, joint pain, joint stiffness or sensory loss. She had a similar episode two years ago involving both calf muscles treated with rest and analgesics and took several months to resolve. Past medical history was significant for type 1 diabetes, Grave's disease, malignant neoplasm of thyroid $\mathrm{s} / \mathrm{p}$ thyroidectomy, post surgical hypothyroidism. She was non compliant with her medications which included levothyroxine, NPH insulin and Lisinopril. Clinical examination was significant for low grade fever and sinus tachycardia. Right lower extremity was edematous and extremely tender without erythema or crepitus. The medial right thigh was most tender without any fluctuant mass. There was no swelling or stiffness of the knee and distal neurovascular function was intact. Muscle strength was difficult to assess proximally due to severe pain but was normal distally. Sensations were intact to monofilament, light touch, pin prick and joint position. Labs were remarkable for a mildly elevated white count, microcytic iron deficiency anemia and a normal renal function. Thyroid stimulating hormone was 105.6 and free $\mathrm{T} 4$ was 0.675 . Total creatine kinase was 309 . Glycated hemoglobin was $10.8 \%$. Urine microalbumin to creatinine ratio was 330.7 suggestive of occult diabetic nephropathy. Hypercoaguable workup was positive for lupus anticoagulant. ANA panel was negative. CT scan showed massive intramuscular, subcutaneous and fascial edema of vastus musculature specially vastus medialis. Arterial and venous dopplers of the lower extremities were negative for deep vein thrombosis and arterial occlusion. She was initially treated with intravenous antibiotics, opioid analgesics. MRI done 48 hours later confirmed the CT findings and now showed edema extending proximally into the pelvic muscles and distally into the calf muscles. Needle biopsy of vastus medialis revealed acute inflammation and hemorrhagic necrosis of the skeletal muscle consistent with diabetic muscle infarction(DMI) and she was started on NSAIDs and aspirin. A trial of daily hyperbaric oxygen treatment (HBOT) at 2.5 atmospheres absolute (ATA) was started after informed consent. By the fourth treatment her symptoms were reduced by $50 \%$ and she was discharged on day ten after 5 HBOTs, with remaining treatments scheduled as outpatient. Patient's recovery was gauged by symptomatic improvement.

DISCUSSION: Diabetic muscle infarction is a rare and under recognized complication of diabetes mellitus, characterized by localized acutely painful inflammation and necrosis of muscles involving the extremities. It is typically associated with long standing poorly controlled diabetes type1. Pathogenesis of the condition remains unclear but largely involves muscle ischemia secondary to diabetic vasculopathy. Since the underlying process in diabetic myonecrosis involves tissue ischemia, HBOT could possibly play a crucial role in its treatment.

HYPERKALEMIC ASCENDING PARALYSIS Abdur Baig; Hector Castro; Giselle Guerra; Oliver Lenz. University of Miami, Miller School of Medicine, Miami, FL. (Control ID \#1339565)

LEARNING OBJECTIVE 1: Recognize the differential diagnosis of ascending paralysis.

LEARNING OBJECTIVE 2: Importance of measuring the serum potassium in acute ascending paralysis.

CASE: A 79-year-old man with a history of stage IIIA follicular lymphoma, recently transformed to diffuse large B-cell lymphoma, presented with acute onset of ascending paralysis. Before this presentation he was complaining of generalized weakness, malaise, loss of appetite, and weight loss of about 5 pounds. On the day of admission he tripped and fell, and was unable to move any extremities. Medications included amlodipine, aspirin, levothyroxine, lovastatin, and nadolol. Physical examination showed a blood pressure of 136/ $76 \mathrm{mmHg}$, pulse 68 beats/min, temperature $36.6 \mathrm{C}$ and respirations 18/min. He was alert, awake, oriented, and had flaccid paralysis with absent reflexes in both upper and lower extremities. No extrapyramidal signs were noted. Sensation was present, but disturbed. Laboratory data included sodium of $120 \mathrm{mEq} / \mathrm{L}$, potassium $9.3 \mathrm{mEq} / \mathrm{L}$, blood urea nitrogen $81 \mathrm{mg} / \mathrm{dl}$, creatinine $9.9 \mathrm{mg} / \mathrm{dl}$, phosphorus $5.7 \mathrm{mg} / \mathrm{dl}$, calcium $8.5 \mathrm{mg} / \mathrm{dl}$ and uric acid $9.6 \mathrm{mg} / \mathrm{dl}$. Electrocardiogram showed heart rate of 70 per minute with widened QRS complexes with disappearance of $\mathrm{p}$-waves, but $\mathrm{T}$-waves were not peaked. $\mathrm{He}$ was immediately treated with intravenous calcium gluconate, regular insulin, dextrose $50 \%$, albuterol nebulizations, and multiple doses of oral sodium polystyrene sulfonate. He also received aggressive intravenous fluids. Potassium decreased to $7.2 \mathrm{mEq} / \mathrm{L}$ within four hours, quadriparesis improved significantly. QRS widening also disappeared with appearance of $\mathrm{P}$ waves as potassium level decreased. Within 24 hours potassium became $4.3 \mathrm{mEq} / \mathrm{L}$, renal function progressively improved and later normalized. He received first cycle of chemotherapy.

DISCUSSION: Ascending paralysis is an uncommon complication of hyperkalemia that generally presents with flaccid motor paralysis with intact sphincter tone. Sensory and cranial nerve examinations are normal. Secondary respiratory failure has also been reported. Richardson et al. first described this clinical entity in 1953, which may mimic the Guillain-Barré syndrome. Other differential diagnosis includes traumatic spinal cord injury, botulism, drug-induced weakness, and a variety of metabolic abnormalities. By decreasing the intracellular to extracellular potassium ratio, hyperkalemia adversely affect the function of excitable tissues, and cardiac muscle affected before the skeletal muscle. Our patient developed hyperkalemia as part of tumor lysis syndrome (TLS). He also had hyperphosphatemia, hyperuricemia, and renal failure. In 1997 Evers S et al. reviewed all the published cases of secondary hyperkalaemic paralysis and found only 17 cases. The most probable cause was intake of spironolactone or amiloride. In majority of the cases (12) chronic renal failure was the underlying mechanism of hyperkalemia. Six 
patients required dialysis. Two patients died of cardiac arrest or convulsions due to hyperkalemia and one of pulmonary embolism. None was reported having TLS. Management includes immediate intravenous infusion of calcium, close monitoring of the vital signs, and in case of respiratory compromise mechanical ventilation. If digoxin toxicity is suspected magnesium sulfate should be used instead of calcium. This is followed by infusion of insulin and glucose, sodium polystyrene sulfonate resin, furosemide or dialysis. Prompt diagnosis and treatment is crucial to prevent fatal outcome.

HYPOCALCEMIA; A RARE CAUSE OF ST ELEVATION MYOCARDIAL INFARCTION !! Ehtesham Ul Haq; Abdul Haseeb; Subhraleena Das; Amit S. Dhamoon. SUNY Upstate Medical University, Syracuse, NY. (Control ID \#1334005)

LEARNING OBJECTIVE 1: Learn to diagnose one of the rarest causes of STEMI

LEARNING OBJECTIVE 2: Learn the relationship between hypocalcemia and coronary vasospasm

CASE: ST elevation Myocardial Infarction is a life threatening emergency mostly caused by cardiac ischemia. There have been very few case reports in the literature mentioning hypocalcemia as one of the rarest causes of STEMI. A 53 year old male was diagnosed with a cancerous thyroid nodule and underwent thyroidectomy which was complicated by an accidental parathyroidectomy three weeks prior to this admission. He was brought to the hospital because of numbness and tingling in both hands and face for 1-2 days duration. On admission, he denied any other complaints. His physical exam was only significant for a positive Chvostek's sign. His labs showed total Calcium of $5.4 \mathrm{mg} / \mathrm{dl}$, Ionized Calcium $0.08 \mathrm{mmol} / \mathrm{L}$, Albumin $3.8 \mathrm{gm} / \mathrm{dl}$, Magnesium $2.3 \mathrm{mEq} / \mathrm{L}$, CPK $145 \mathrm{U} / \mathrm{L}$, CKMB $2.5 \mathrm{ng} / \mathrm{ml}$ and Troponin I of $2.4 \mathrm{ng} / \mathrm{ml}$. Rest of the labs were within normal limits. EKG was normal. Stat echocardiogram showed EF of 55-60 percent with no wall motion abnormalities. He received intravenous and oral Calcium replacement during his hospitalization. On his 2nd hospital day, he developed left sided chest discomfort with no associated symptoms. Repeat EKG showed ST segment elevation $>1 \mathrm{~mm}$ in inferior leads. Repeat set of cardiac enzymes showed CPK of $1573 \mathrm{U} / \mathrm{L}$, CKMB $173 \mathrm{ng} / \mathrm{ml}$, Troponin I of $62.6 \mathrm{ng} / \mathrm{ml}$. Emergent cardiac catheterization showed normal coronary vasculature and normal LV function. Chest pain improved with sublingual nitroglycerin and intravenous morphine. Meanwhile, he was kept on Calcium replacement. After about $72 \mathrm{hrs}$ of admission, ionized Calcium level went up to $1.15 \mathrm{mmol} / \mathrm{L}$ and troponin I level dropped down to $6.4 \mathrm{ng} / \mathrm{ml}$. ST elevations on EKG also normalized with Calcium supplementation. At the time of discharge, the patient was chest pain free and was sent home on oral Calcium supplements. DISCUSSION: Calcium is essential for cell function, cell membrane stability, neuronal transmission, blood homeostasis and cell signaling. Calcium has central role in myocardial contraction coupling and hypocalcemia reduces myocardial function. Coronary vasospasm must be considered as the most likely cause of myocardial infarction in the setting of hypocalcemia. Hypocalcemia has been reported as reversible cause of cardiac failure; due to its rarity, it is largely ignored in clinical teaching and standard textbooks. Hypocalcemia can result in several cardiac manifestations including QT prolongation on EKG and more rarely congestive heart failure and Cardiomyopathy that is reversible once the hypocalcemia is corrected. In this case, hypocalcemia was the most likely cause of coronary vasospasm which improved after treatment with nitrates.

HYPOTHERMIA IN SEPTIC SHOCK: PROTECTIVE OR HARMFUL? Jilalu A. Kelbe; Harvey Friedman. Saint Francis Hospital of Evanston, Evanston, IL. (Control ID \#1338821)

LEARNING OBJECTIVE 1: Describe the effects of hypothermia on blood lactate level.

LEARNING OBJECTIVE 2: Recognize the potential protective effect of hypothermia in septic shock with multiple organ failure.

CASE: A 36 yr-old man presented with altered mental status of 1 day duration. The patient was unable to provide further history. His past medical history is remarkable for advanced multiple sclerosis and the patient is wheel chair bound because of it. On examination, he was comatose. Vital signs were: Heart rate 48/min, BP 79/44 mmHg, T 84 F (rectally). He had a scalp wound with purulent drainage. Chest was clear and no murmur on cardiac examination. He was in deep coma with minimal grimace to painful stimuli. Lab tests revealed WBC $11,600 / \mathrm{mm} 3$ with $28 \%$ bands; platelets $28,000 / \mathrm{mm} 3$. The metabolic panel showed BUN 63, creatinine 0.82, CO2 31; AST 217, ALT 139. Lactic acid was 2.2, PT $16.3 \mathrm{sec}$; PTT $40 \mathrm{sec}$. Scalp wound, urine, and blood cultures grew MRSA. The patient was treated with IV fluids, norepinephrine and antibiotics for septic shock. He was also treated with warm normal saline infusion for hypothermia. His renal function worsened in the first 2 days but afterwards he progressively improved by all parameters. His lactate level remained in the normal range. DISCUSSION: The patient presented with septic shock and multiple organ failure as evidenced by altered mental status, abnormal liver function tests, lab tests consistent with DIC and the subsequent development of azotemia. However, his lactate level remained normal. One possible explanation for the normal lactate level despite the severe septic shock could be the severe hypothermia the patient had at the time of presentation. There was no apparent cause for hypothermia other than sepsis. Although hypothermia is one of the manifestations of sepsis, could it be protective of damage from tissue hypoperfusion in the setting of septic shock? In a study of 81 patients with TBI, mild hypothermia therapy was shown to reduce blood lactate levels. The role of hypothermia protocols in improving neurologic outcomes in post-cardiac arrest patients is well established but its role in septic shock has not been reported. This observation will hopefully stimulate further study to understand the role of hypothermia in the setting of septic shock and how aggressive reversal of hypothermia should be.

ISONIAZID, A RARE CAUSE OF ACUTE PANCREATITIS Johny S. Kuttab; David Veltre; Paul Yi; Vikrum Rangan; Lisa E. Norton. Boston University Medical Center, Boston, MA. (Control ID \#1338726)

LEARNING OBJECTIVE 1: Recognize INH as a potential culprit in the differential diagnosis of acute pancreatitis.

LEARNING OBJECTIVE 2: Early recognition, and withdrawal of INH results in excellent prognosis

CASE: A 74 year old female, with a history of reflux disease, Iron Deficiency anemia, hyperlipidemia, and latent TB infection ( LTBI) on INH monotherpay ( $300 \mathrm{mg}$ daily) inititated 3 months prior to presentation presented with one day of gradually worsening 6/10 RUQ pain, dull, constant, non radiating, associated with multiple episodes of non-bloody, non-bilious emesis, exacerbated by oral intake of solids and liquids, and not relieved by any measures. There were no associated fevers or chills, no change in bowel habits, no jaundice, hematochezia, melena, dysuria or hematuria. Physical exam was remarkable for normal vital signs, RUQ/ RLQ tenderness, slight guarding and hypo-active bowel sounds. The rest of the exam was normal. Labs showed: WBC $17.7 \mathrm{~K} / \mathrm{UL}$, HCT 41 (baseline of $35 \%$ ), glucose $137 \mathrm{mg} / \mathrm{dl}$, normal chemistry panel, normal LFTs, with slightly elevated Lipase to $167 \mathrm{U} / \mathrm{L}$, in the setting of a normal amylase. Abdominal Ultrasound showed a normal GB, without any stones. An abdominal CT with contrast was significant for inflammatory changes around the pancreatic head, uncinate process, and $2 \mathrm{nd} / 3 \mathrm{rd}$ part of the duodenum consistent with groove pancreatitis. Antiemetics were given for nausea and she was admitted to the geriatric service for further management. Gallstone and alcohol induced pancreatitis were both ruled out based on imaging and history. Given that she was on INH for 3 months at that point, INH induced pancreatitis was the leading diagnosis. INH was discontinued immediately. The patient was managed conservatively with bowel rest, IV fluids, and pain medications with complete resolution of her symptoms on the 3 rd day. On the 4 th day she was discharged home, tolerating a full diet.

DISCUSSION: Medications are an uncommon cause of acute pancreatitis. Based on reporting of adverse events in German and Swiss centers, the incidence of drug induced-pancreatitis is believed to be between $0.3-2 \%$. Drug-induced pancreatitis has no distinguishing features. Therefore a high index of suspicion and a careful drug history are important in the diagnosis. Onset could vary from weeks to months depending on the offending agent. The pathogenesis is poorly understood, but may be due to an idiosyncratic reaction or direct toxic effects. Other proposed theories include alteration 
in the zymogen granule formation, stability, migration and release. INH is considered a Category I medication in the causation of acute pancreatitis. i.e. At least one case report with a positive recurrence after drug rechallenge. Most of the reported cases of INH induced pancreatitis had an onset within 3 weeks of drug initiation, however in our case the onset was delayed to 3 months, which may suggest an alternative mechanism of injury, or earlier onset pancreatitis which was misdiagnosed. (Our patient was diagnosed with food poisoning a few months earlier) Regardless of the offending agent, the prognosis of drug-induced pancreatitis is generally excellent. In one report of 22 cases, 19 were associated with interstitial pancreatitis and none were associated with necrosis on CT and none died. In our case, the patient had evidence of necrosis, however the area that was involved was small ( groove pancreatitis). There were no reported deaths in this case series suggesting a low overall mortality rate.

IDIOPATHIC RETROPERITONEAL FIBROSIS: AN UNCOMMON CAUSE OF BACK PAIN Sadia Moinuddin. SJHMC, Phoenix, AZ. (Control ID \#1339030)

LEARNING OBJECTIVE 1: Low back pain is one of the most common chief complaints in primary care setting. We present a case of idiopathic retroperitoneal fibrosis diagnosed in the evaluation of back pain

CASE: A 50 year-old male presented to his PCP with low back pain at rest, dull, accompanied by muscle spasms, $8 / 10$, with radiation to abdomen and groin area. There was no preceding trigger event and pain was unrelieved by anti-inflammatory medications, muscle relaxants or opiates. Patient denied having fever, chills, weight loss, and recent travel. An Xray showed enlarged aorta and CT showed peri-aortitis with diffuse circumferential encroachment of the aorta and vena cava, characteristic of retroperitoneal fibrosis. Patient denied use of beta-blockers, ergot derivatives, and no infections were found during work-up. CTA demonstrated thickened aortic wall without aneurysm. Laboratory data showed elevated CRP, ESR, and serum IgG4. Patient was treated with corticosteroids with clinical improvement. Repeat MRI one month later showed decreased peri-aortic soft tissue swelling. After tapering and discontinuing steroids patient had recurrence of back pain and imaging showed interval increase of the ring of soft tissue surrounding the aorta as well as fibrotic process surrounding both ureters at the level of the aortic bifurcation resulting in mild bilateral hydronephrosis.

DISCUSSION: Idiopathic retroperitoneal fibrosis (IPRF) should be considered in the presence of chronic peri-aortitis. It is characterized by fibro-inflammatory tissue surrounding the abdominal aorta and iliac arteries. The inflammatory tissue is composed of lymphoplasmacytic inflammation and fibrosis with large number of the plasma cells expressing IgG4. Pathogenesis is unclear, however, leading theories propose IPRF is either an enhanced inflammatory reaction in response to aortic atherosclerosis by oxidized LDL or a systemic manifestation of autoimmune disease, the latter being supported by the presence of constitutional symptoms, elevated acute phase reactants, and auto-antibodies. Other pathogenetic processes may also include the presence of IgG4 producing plasma cells. Symptoms include a non-specific dull low back pain in early stages and obstructive uropathy in advanced disease. Most common cause is idiopathic and secondary causes being medications, infections, malignancies, trauma, surgery, radiation. Diagnosis should start by excluding possible primary causes of IPRF, such as drugs, infections, malignancies, radiotherapy, trauma, or previous surgery. IPRF is diagnosed by MRI which provides a more exact anatomic definition than $\mathrm{CT}$, because of its multi plane capabilities; CT is usually the initial test. CT reveals a periaortic soft tissue mass that may extend laterally to entrap the tumors. When the mass presents atypically, biopsy is recommended. Management of IRPF often requires surgical, urological, or medical intervention to address anatomic complications for example ureteral obstruction or ureterolysis. Medical treatment can be directed to treat the acute inflammation and suppress the chronic immunologic process with subsequent fibrosis. Glucocorticoids are generally considered the mainstay of therapy for IPRF due to the non-specific inflammatory nature of the disease but other steroid sparing agents such as tamoxifen, azathioprine, mycophenolate mofetil also may have a role. Overall IPRF responds to corticosteroid therapy initially but often recurs without treatment as is the case with our patient.
IDIOPATHIC RETROPERITONEAL FIBROSIS: AN UNCOMMON CAUSE OF BACK PAIN Sadia Moinuddin. SJHMC, Phoenix, AZ. (Control ID \#1339030)

LEARNING OBJECTIVE 1: Low back pain is one of the most common chief complaints in primary care setting. We present a case of idiopathic retroperitoneal fibrosis diagnosed in the evaluation of back pain.

CASE: A 50 year-old male presented to his PCP with low back pain at rest, dull, accompanied by muscle spasms, $8 / 10$, with radiation to abdomen and groin area. There was no preceding trigger event and pain was unrelieved by anti-inflammatory medications, muscle relaxants or opiates. Patient denied having fever, chills, weight loss, and recent travel. An Xray showed enlarged aorta and CT showed peri-aortitis with diffuse circumferential encroachment of the aorta and vena cava, characteristic of retroperitoneal fibrosis. Patient denied use of beta-blockers, ergot derivatives, and no infections were found during work-up. CTA demonstrated thickened aortic wall without aneurysm. Laboratory data showed elevated CRP, ESR, and serum IgG4. Patient was treated with corticosteroids with clinical improvement. Repeat MRI one month later showed decreased periaortic soft tissue swelling. After tapering and discontinuing steroids patient had recurrence of back pain and imaging showed interval increase of the ring of soft tissue surrounding the aorta as well as fibrotic process surrounding both ureters at the level of the aortic bifurcation resulting in mild bilateral hydronephrosis.

DISCUSSION: Idiopathic retroperitoneal fibrosis (IPRF) should be considered in the presence of chronic peri-aortitis. It is characterized by fibro-inflammatory tissue surrounding the abdominal aorta and iliac arteries. The inflammatory tissue is composed of lymphoplasmacytic inflammation and fibrosis with large number of the plasma cells expressing IgG4. Pathogenesis is unclear, however, leading theories propose IPRF is either an enhanced inflammatory reaction in response to aortic atherosclerosis by oxidized LDL or a systemic manifestation of autoimmune disease, the latter being supported by the presence of constitutional symptoms, elevated acute phase reactants, and autoantibodies. Other pathogenetic processes may also include the presence of IgG4 producing plasma cells. Symptoms include a non-specific dull low back pain in early stages and obstructive uropathy in advanced disease. Most common cause is idiopathic and secondary causes being medications, infections, malignancies, trauma, surgery, radiation. Diagnosis should start by excluding possible primary causes of IPRF, such as drugs, infections, malignancies, radiotherapy, trauma, or previous surgery. IPRF is diagnosed by MRI which provides a more exact anatomic definition than CT, because of its multi plane capabilities; CT is usually the initial test. CT reveals a peri-aortic soft tissue mass that may extend laterally to entrap the tumors. When the mass presents atypically, biopsy is recommended. Management of IRPF often requires surgical, urological, or medical intervention to address anatomic complications for example ureteral obstruction or ureterolysis. Medical treatment can be directed to treat the acute inflammation and suppress the chronic immunologic process with subsequent fibrosis. Glucocorticoids are generally considered the mainstay of therapy for IPRF due to the nonspecific inflammatory nature of the disease but other steroid sparing agents such as tamoxifen, azathioprine, mycophenolate mofetil also may have a role. Overall IPRF responds to corticosteroid therapy initially but often recurs without treatment as is the case with our patient.

IMPRESSIVE LASTING EFFECTS OF A "REVERSIBLE" CONDITION Alice Williamson; Erin Boswell; William B. Rothwell; Peter S. Reynaud. Tulane University Health Sciences Center, New Orleans, LA. (Control ID \#1338834)

LEARNING OBJECTIVE 1: 1. Recognize the clinical presentation of Posterior Reversible Encephalopathy Syndrome (PRES). 2. Identify the hypothesized pathophysiology of PRES.

CASE: A 29-year old woman with HIV presented with one day of intractable seizures. She had as history of end-stage renal disease, hypertension and Posterior Reversible Encephalopathy Syndrome (PRES), for which she had received supportive care. She had never had seizures prior to her initial episode of PRES nor had she seized prior to this 
admission. On presentation, her blood pressure was 251/210 $\mathrm{mmHg}$ and her heart rate was 138 beats/min; her remaining vital signs were normal. She was lethargic; her cardiac, pulmonary and abdominal examinations were normal. There were no neurologic deficits aside from the frequent seizures. Her white blood cell count was 10,800 cells $/ \mathrm{mm} 3$. Blood cultures grew Staph epidermidis and Staph haemolyticus; she refused a lumbar puncture. A head CT revealed no findings of PRES. After her blood pressure was controlled, she had no further seizures. The bacteremia was treated with vancomycin.

DISCUSSION: PRES, Posterior Reversible Encephalopathy Syndrome, is a constellation of symptoms seen in immunocompromised patients, including altered mental status, hypertension, visual changes, and seizures with specific radiologic evidence of cerebral edema. This patient presented as she had with her prior episodes of PRES; however, this third presentation of seizures was without radiographic evidence of PRES. PRES is a typically a reversible cause of seizures; however, it is important that the general internist recognizes that it can be a recurrent disease. This patient with hypertension, AIDS, and end-stage renal disease on hemodialysis had the rare combination of recurrent PRES with resulting epilepsy. This patient likely had permanent cerebral dis-autoregulation resulting from the recurrent episodes of brain edema, requiring indefinite anticonvulsant therapy.

INCURABLE BUT TREATABLE PLASMA CELL CANCER Darshan Patel; Rachana Kanaujia; Jiwon Lee; William Hauger. Conemaugh Valley Memorial Hospital, Johnstown, PA. (Control ID \#1338938)

LEARNING OBJECTIVE 1: Waldenstrom's Macroglobulinemia, also known as Lymphoplasmacytic lymphoma is a very rare type of B-cell nonHodgkin's lymphoma with an incidence rate of 3 cases per million per year that is characterized by an increased proliferation of immunoglobulin (Ig) M paraprotein.

CASE: A 55 year old African American male contractor, with no past medical history presented to the ER with new onset mild pain and fullness of left upper quadrant of the abdomen that had increased in severity over the past 4 weeks. Over the course of time patient lost sixteen pounds, complained of early satiety, and occasional night sweats. He mentioned having an unknown blood disorder in a first degree relative. Physical examination did not reveal frank distention but he had massive splenomegaly that extended beyond the pelvic brim and was quite firm and tender with no palpable lymphadenopathy. Initial laboratory data revealed leukopenia, anemia and prolonged PT and PTT. CT scan of the abdomen confirmed massive splenomegaly with mass effect compressing the stomach, displacing the left kidney inferiorly, loops of bowel to the right and inferiorly. A lymphoplastic disorder was suspected. SPEP revealed IgM level of 2107. Urine protein electrophoresis showed small monoclonal protein typing as free kappa light chain. On the second day, bone marrow biopsy revealed lymphocytic infiltrate of monoclonal B-cells. Immunohistochemistry showed monoclonal B cell population positive for CD 19, CD 20, CD 23, CD 38 plasmacytoid cells with kappa light chain restriction, while negative for CD5 and CD10 with kappa chain restriction. Diagnosis of Waldenstrom's Macroglobulinemia was established. Following that a serum viscosity of 2.2 was noted. Plasma exchange was done prior to beginning treatment in anticipation of disease flare post treatment. Patient was started on Velcade, Rituxan and Dexamethasone. Meanwhile, patient's abdominal pain had resolved by 4th day at the hospital, although labartory values remained unchanged, notably the elevated PT and PTT.

DISCUSSION: Waldenstroms Macroglobulinemia can manifest with pancytopenia due to bone marrow infiltration and direct affect of IgM monoclonal gammapathy in the blood leading to spontaneous bleeding, peripheral neuropathy, hyperviscosity syndrome, lymphadenopathy, and organomegaly. Risks of developing this condition include genetic factors, environmental exposure not limited to but including wood dust, organic solvents as could be the case in this patient. It is important to recognize that high levels of paraprotein can lead to alterations in platelet function by inhibition of fibrin polymerization and coaguloapathy leading to spontaneous bleeding. Although in our case, the patient did not exhibit severe signs of hyperviscosity syndrome, plasmapheresis may be necessary to prevent adverse effects related to paraprotein. Due to rarity of this condition, Waldenstroms macroglobulinemia can often be overlooked and misdiagnosed. Consequence can lead to life threatening complications. However, with appropriate treatment patients may have years of symptom free remission and live active lifestyles.

INITIAL EMPIRIC THERAPY FOR STAPHYLOCOCCUS AUREUS BACTEREMIA: IS VANCOMYCIN MONOTHERAPY ENOUGH? Renuka Tipirneni; Ana Weil; Xiaosong Zhang; Daniel P. Hunt. Massachusetts General Hospital, Boston, MA. (Control ID \#1334986)

LEARNING OBJECTIVE 1: Recognize the limitations of vancomycin monotherapy for empiric treatment of $S$. aureus bacteremia.

LEARNING OBJECTIVE 2: Assess risk factors for community-acquired methicillin-resistant $S$. aureus (MRSA).

CASE: A 53-year-old woman with a recent diagnosis of breast cancer presented with fever and low back pain. She had undergone an uncomplicated mastectomy with reconstruction three months prior to admission, and had not received chemotherapy or radiation. Two weeks prior to admission she developed fever, shaking chills and oral ulcers that resolved with ibuprofen. Four days prior to admission she developed recurrence of fever and chills, and also experienced myalgias and progressive severe low back pain. On initial exam, her temperature was 98.2. She was hemodynamically stable and ill appearing. Exquisite tenderness was apparent upon palpation over the L5 vertebra. She had no murmurs, intravascular catheters, prostheses, or rashes. MRI of the lumbar spine showed L5/S1 osteoarthritic disc changes without epidural collection or osteomyelitis. On arrival to the medical floor, blood cultures resulted with growth of gram-positive cocci in clusters. Given high suspicion of $S$. aureus infection, vancomycin was given. Later that night her temperature rose to 104.6. Nafcillin was added to her antibiotic regimen. Four of four blood cultures later grew $S$. aureus, susceptible to penicillin, methicillin and vancomycin (minimum inhibitory concentration, or MIC, $=2 \mathrm{mcg} / \mathrm{mL}$ ). Her antibiotic regimen was narrowed to penicillin and surveillance blood cultures were negative. Subsequent imaging revealed L5 osteomyelitis and abscess formation at the L5/S1 vertebral space.

DISCUSSION: Is vancomycin monotherapy adequate empiric treatment for $S$. aureus bacteremia? Since its introduction in the 1950's, vancomycin has become the standard initial treatment for resistant $S$. aureus infections. In an era of increasing incidence of MRSA, including community acquired MRSA (CA-MRSA), vancomycin is frequently initiated for gram-positive cocci bacteremia, pending further susceptibility data. However, vancomycin monotherapy has limitations. First, it is less bactericidal than betalactams, which may result in longer periods of bacteremia. Second, it has reduced penetration into some tissues, including bone, lung and CSF. Third, with high inocula drug efficacy is reduced. Fourth, resistance is emerging, with rising MICs and the breakpoint MIC is now considered 2 $\mathrm{mcg} / \mathrm{mL}$. In light of these limitations, it is not surprising that in cases of methicillin-sensitive $S$. aureus (MSSA) bacteremia, patients treated with vancomycin have higher rates of relapse, reinfection and treatment failure, when compared with beta-lactam antibiotics such as nafcillin. In our patient, the addition of nafcillin may have led to increased bactericidal activity, improved penetration of bone, and increased efficacy in the setting of high grade bacteremia. She had some traditional risk factors for MRSA infection (recent hospitalization and chronic illness). However, she did not use injection drugs or have recent antibiotic exposure, and lacked additional risk factors for CA-MRSA, which include skin trauma (her surgical incision was well healed), contact sports, crowded or unsanitary living conditions, and men who have sex with men. Given that our patient was ill, with risk factors for vancomycin failure, and low probability of CA-MRSA, the addition of a beta-lactam while awaiting susceptibilities was a reasonable choice over vancomycin alone.

INTESTINAL PURPURA: AN ATYPICAL PRESENTATION OF ADULT-ONSET HENOCH-SCHöNLEIN PURPURA Melissa Y. Wei; Tanvi A. Dhere. Emory University, Atlanta, GA. (Control ID \#1339263)

LEARNING OBJECTIVE 1: Recognize atypical features of adult-onset Henoch-Schönlein purpura (HSP)

LEARNING OBJECTIVE 2: Appreciate HSP without palpable purpura as a syndrome of $\operatorname{IgA}$ enteropathy subset of HSP or alternatively as a novel 
entity of IgA-mediated systemic vasculitis under which HSP encompasses individuals with cutaneous involvement

CASE: A 23 yo G1P0101 African American woman with immunoglobulin A (IgA) nephropathy presented with abdominal pain and watery diarrhea for 4 days. She was diagnosed with biopsy-proven IgA nephropathy and treated with high-dose prednisone and two doses of Cytoxan until she became pregnant. During her third trimester she developed preeclampsia and underwent a Caesarean-section. She delivered a healthy girl and was discharged with prednisone for persistent renal failure. Two days after discharge she developed severe, diffuse abdominal pain with multiple episodes of non-bloody watery diarrhea. On admission she was afebrile with blood pressure 150/101. Her abdomen was guarded with epigastric tenderness. No palpable purpura, joint tenderness or synovitis were appreciated. Her labs revealed creatinine $4.5 \mathrm{mg} / \mathrm{dL}$ (baseline $2.2 \mathrm{mg} / \mathrm{dL}$ ), WBC $17.2 \times 10^{\wedge} 3 / \mu \mathrm{L}$ and albumin $2 \mathrm{~g} / \mathrm{dL}$. Her urinalysis had massive proteinuria, hematuria and granular casts. Her ANA panel, complement, ANCA, anti-dsDNA, HIV and hepatitis panel were negative. Stool lactoferrin was positive but other stool studies were negative. An abdominal MRI/MRA showed small bowel loop dilation with wall thickening and edema but no vasculitis. A repeat renal biopsy showed IgA nephropathy with $>8 \%$ scarring. Due to continued abdominal pain and diarrhea, a small bowel enteroscopy was performed that showed multiple ulcerated, purpuric lesions in the jejunum. Biopsies of the lesions showed leukocytoclastic vasculitis with intravascular thrombi and vascular wall staining with IgA consistent with gastrointestinal involvement by HSP. With methylprednisolone her abdominal pain and diarrhea resolved, and her creatinine decreased to a new baseline of $3.5 \mathrm{mg} / \mathrm{dL}$. She was discharged home on prednisone.

DISCUSSION: HSP is an IgA immune complex-mediated systemic leukocytoclastic vasculitis that primarily affects children with the classic tetrad of palpable purpura, polyarthralgia, acute abdominal pain and nephropathy. The prevalence of gastrointestinal symptoms is up to $85 \%$, and petechiae referred to as "intestinal purpura" may occur throughout the GI tract (Saito 2011), particularly the small bowel. Adult onset HSP is rare but often more severe, requiring aggressive therapy with high dose steroids and possibly cytotoxic immunosuppressive agents. Diagnosis in adults is challenging due to an atypical clinical presentation, particularly absence or delayed onset of pathognomonic purpura. Our patient presented with renal and GI manifestations of HSP. While palpable purpura was absent, her jejunum revealed multiple purpuric lesions and a large hemosiderin-laden ulcer with histopathologic analysis consistent with HSP vasculitis. HSP without palpable purpura is rare but has been reported in an adult (Nakamura 2010) and case series of children (Kato 2004, Murayama 2007). These individuals may be classified under a proposed syndrome of IgA enteropathy subset of HSP, or alternatively under a novel entity of IgA-mediated systemic vasculitis under which HSP encompasses individuals with cutaneous involvement. While the exact diagnostic category may be debated, aggressive therapy should be initiated early to prevent irreversible disease progression.

INVASIVE TONGUE ASPERGILLOSIS: A RAPIDLY PROGRESSIVE INFECTION Shuang Guo; Jason A. Korcak. Montefiore Medical Center, Bronx, NY. (Control ID \#1339418)

LEARNING OBJECTIVE 1: Recognize the clinical presentation of invasive tongue aspergillosis.

LEARNING OBJECTIVE 2: Develop a differential diagnosis of necrotic tongue lesions.

CASE: An 86 year-old woman presented with three days of generalized weakness and diarrhea. She had a past medical history of hypertension and atrial fibrillation, as well as a recent admission for lower extremity cellulitis. On presentation, the patient became hemodynamically unstable and was intubated. The oropharynx was unremarkable prior to intubation. The patient was started on broad-spectrum antibiotics and high dose intravenous steroids with clinical improvement and was extubated on hospital day four. Three days later, the patient complained of difficulty swallowing secondary to tongue pain. A gray-green plaque without bleeding or ulceration was present on the anterior tongue. Metronidazole was discontinued given suspicion for a drug reaction. The tongue lesion rapidly increased in size with loss of normal architecture and necrotic changes. Fungal elements were present on tongue biopsy. The patient was given fluconazole and oral nystatin without improvement. Further fungal speciation revealed Aspergillus flavus and antibiotics were changed to intravenous voriconazole. The tongue lesion failed to improve, and the patient expired several days later from overwhelming sepsis of unclear etiology.

DISCUSSION: The broad differential diagnosis of necrotic tongue lesions presents a challenge for the general internist. The initial appearance of our patient's lesion led to a consideration of oral candidiasis, lichen planus, medication side effect, or trauma from intubation as possible causes. The rapid onset of tongue necrosis expanded the differential to include invasive fungal, bacterial, and viral infections, malignancy, and vasculitis. Although not present in our case, other types of vascular compromise, such as disseminated intravascular coagulation, and chemical or radiation exposure can lead to a similar presentation. Invasive aspergillosis is a rapidly progressive and often life-threatening infection that is characterized by invasion of blood vessels resulting in ischemia and necrosis. Direct oral mucosal invasion is uncommon. There are 27 reported cases of primary oral aspergillosis, most commonly affecting the gingiva, and only two reported cases involving the tongue. Risk factors for invasive aspergillosis include severe and prolonged neutropenia and other drugs or conditions that chronically impair cellular immune responses. In all reported cases of oral aspergillosis, the patients were immunosuppressed from a primary malignancy or chemotherapy. Several factors may have increased our patient's susceptibility to an invasive fungal infection, including immunosuppression from high dose corticosteroids, alteration in normal oral flora from broad-spectrum antibiotics, and inoculation during traumatic endotracheal tube placement. Although invasive aspergillosis is rare, the development of an oral lesion that rapidly becomes necrotic should raise suspicion for the diagnosis, especially in immunocompromised patients. This case highlights the need for internists to form a broad differential diagnosis when encountering necrotic tongue lesions.

\section{ISOLATED 6TH NERVE PALSY- AN UNCOMMON PRESENTATION} OF LYME'S DISEASE Leena Jalota; Robert Freed; Shashank Jain. The Reading Hospital and Medical Center, West Reading, PA. (Control ID \#1340434)

LEARNING OBJECTIVE 1: Recognize common signs and symptoms of isolated sixth nerve palsy

LEARNING OBJECTIVE 2: Appreciate the differential diagnosis including rare causes such as Lyme's disease

CASE: Introduction: The clinical manifestations of Lyme disease can generally be divided into 3 phases: early localized, early disseminated and late disease. However patients may present in a later stage without a history of prior signs or symptoms of early disease. In particular, early disseminated cases can present subacutely after initial inoculation with neurological findings which, although rare, may include isolated nerve palsies. CASE: A 55-year-old male with hypertension and diabetes, both controlled with oral medication, presented to the emergency room with complains of new-onset double vision of one-day duration. He initially deemed it trivial, even driving long-distance to a sporting event, but later presented to the hospital with progressive binocular diplopia. Specifically his vision was worsened with distance and he denied eye pain at rest or with movement. He denied associated trauma or similar episodes in the past and denied other neurological deficits. Patient was a truck driver and prior smoker who drank alcohol occasionally. Family history was pertinent for stroke and cardiovascular disease in his father and brother. The patient denied foreign travel, exposure to sick individuals and specifically denied bug and tick bites. Review of systems was positive for horizontal diplopia only. On exam: He was afebrile with elevated blood pressure of $172 / 86$, vitals were otherwise normal. Patient was awake, alert and oriented X 3 . Neck was supple, sclera anicteric and pupils of $4 \mathrm{~mm}$ bilaterally, equally round, and reactive to light. On right lateral gaze, the patient had a convergent strabismus, consistent with a sixth nerve palsy. Extra-ocular muscles otherwise intact and remainder of examination was entirely unremarkable. Laboratory: $\mathrm{CBC}$, electrolytes and coagulation panel were normal. Both TSH and Free T4 were also normal. CVA work-up including non-contrast CT, MRI, echocardiogram and carotid duplex ultrasonography 
were normal. Other diagnosis entertained included temporal arteritis, HIV and syphilis all of which were deemed less likely with normal ESR, negative HIV ELISA and negative RPR, respectively. Initial Lyme ELISA, however returned positive with subsequent confirmatory Western blot testing. Patient was begun on Doxycycline $100 \mathrm{mg}$ twice daily for 28 days with appropriate follow-up in place. Upon follow up, patient had mild to moderate improvemet in symptoms.

DISCUSSION: Patients with isolated 6th or abducens nerve palsy often complain of diplopia which should be binocular and purely horizontal. It is typically worse when looking at objects at a distance and in lateral gaze towards the affected side, although some patients may also complain of visual deficit in primary position. New onset cranial nerve palsies in adults most often result from myasthenia gravis, diabetes melitus, meningitis, microvascular disease or giant cell arteritis. Other less likely causes include Lyme disease, CNS tumors, metastatic lesions, autoimmune disorders and vitamin deficiencies. After ruling out common causes appropriate workup should ensure to rule out rare and reversible causes. Lyme disease, although a rare cause, can present with isolated cranial nerve palsies and should be ruled out even in diabetic patients, regardless of overt tick exposure.

ISOLATED PULMONARY ANTI-GBM DISEASE Yaser Alkhatib ${ }^{1}$; Ghassan Bandak ${ }^{1}$; Waqas Qureshi ${ }^{1}$; James E. Novak ${ }^{2}$. ${ }^{1}$ Henry Ford Hospital, Detroit, MI; ${ }^{2}$ Henry Ford Hospital, Detroit, MI. (Control ID \#1328098)

LEARNING OBJECTIVE 1: Although Anti-GBM disease usually involves the kidneys, it might very rarely presents exclusively in lungs LEARNING OBJECTIVE 2: Early recognition and treatment of Anti-GBM disease might delay or prevent renal involvement

CASE: A 30-year-old Caucasian lady presented to the hospital with severe dyspnea that started one week after exposure to smoke from burning leaves. The dyspnea progressively worsened until she became symptomatic at rest prior to presentation. It was associated with non-productive cough but not chest pain, paroxysmal nocturnal dyspnea, orthopnea, hemoptysis, wheezing, or fever. Her past medical history was negative for lung disease. She was not taking any medications. Social history was significant for 2 packs per day cigarette smoking. In the emergency room, she quickly progressed to hypoxemic respiratory failure requiring intubation and mechanical ventilation. Initial chest X-ray revealed alveolar infiltrates and she was diagnosed with acute pulmonary edema. She was treated with diuretics but became hypotensive and oliguric. She was also started on empirical antibiotics for possible pneumonia. The lack of response in the next 72 hours prompted discontinuation of antibiotics, and flexible bronchoscopy suggested alveolar hemorrhage. At this point, the patient developed renal failure requiring hemodialysis. Infectious and rheumatological work up was negative and anti-glomerular basement membrane (anti-GBM) antibodies were found to be strongly positive. There was a concern that renal failure might be secondary to anti-GBM disease. The patient was started on high dose steroids on day 5 . Since the patient was too unstable to undergo kidney biopsy, plasmapheresis was given empirically for 14 days. Oxygenation improved, and the patient was extubated on day 9. Subsequently, kidney biopsy was performed that revealed acute tubular necrosis but not anti-GBM disease. The patient resumed urinating after three weeks, and creatinine improved to almost normal prior to discharge.

DISCUSSION: Anti-GBM disease is a rare autoimmune disease with an annual incidence of 0.5 cases per million in the general population. The disease is secondary to anti-GBM antibodies against the noncollagenous 1 domain of the alpha 3 chain of type IV collagen found in the glomerular basement membrane. The disease manifests in type IV collagen-rich organs such as kidney (anti-GBM glomerulonephritis) and lungs (Goodpasture's disease). Very rarely, it may only present in the lungs, as in our case. Isolated pulmonary anti-GBM disease has been reported in the literature. Renal involvement might be absent, subtle, or eventually develop months to years from initial presentation. The inciting event is usually environmental exposure to inhalational toxins, representing an environmental trigger in a genetically predisposed individual. It was hypothesized that early recognition and treatment prevented overt renal involvement in our patient. Alveolar hemorrhage is an important differential diagnosis of alveolar infiltrates on chest X-ray and should prompt a physician to check for anti-GBM antibodies in a patient without a clear etiology for ARDS, pneumonia, or pulmonary edema. Timely diagnosis and treatment could be life-saving. Follow up is essential in patients with isolated pulmonary antiGBM disease. Recurrence is common and can occur months to years after the initial presentation as an isolated pulmonary disease or with frank pulmonary-renal syndrome.

ISOLATED RIGHT VENTRICULAR PULSUS ALTERNANS IN SEVERE PULMONARY HYPERTENSION ASSOCIATED WITH HIV INFECTION. Robin Singh ${ }^{1}$; Hanan Makhoul ${ }^{2}$. ${ }^{1}$ University of Louisville, Louisville, KY; ${ }^{2}$ Little Rock Diagnostic Clinic, Little Rock, AR. (Control ID \#1327677)

LEARNING OBJECTIVE 1: HIV associated pulmonary arterial hypertension (PAH) needs an early diagnosis and treatment, as 2D Echo and Doppler should be utilized early in the evaluation of dyspnea in HIV patients.

LEARNING OBJECTIVE 2: Isolated right ventricular (RV) and pulmonary arterial (PA) pulsus alternans in HIV associated PAH signifies severe right ventricular dysfunction.

CASE: A 31 year old African American female with a 9 year history of HIV presented with progressively exertional dyspnea of 1 year duration. She had mild leg edema but no history of fever, productive cough, chest pain, palpitation, syncope, orthopnea or PND. She had mild hypertension. She had no history of tobacco, alcohol or drug abuse, and family history was noncontributory. Medications included antiretroviral drugs and Furosemide. Physical Examination: No acute distress, BP 150/90, 80/min/regular, elevated JVP, loud P2, 3/6 holosystolic murmur in the left parasternal area without respiratory variation. Lungs clear to auscultation bilaterally and mild leg edema. CD4 count 128/cmm with HIV PCR count of 1910 copies/ml. EKG: sinus rhythm, right axis, RBBB. Chest x-ray: moderate cardiomegaly, no pulmonary edema or infiltrates. Echocardiogram: markedly dilated RV and RA. Ventricular septum was flattened indicating increased RV pressure and volume. LV EF was $50 \%$. No valvular disease. Doppler showed moderate tricuspid regurgitation and severe PAH with an estimated PA systolic pressure of $115 \mathrm{mmHg}$. Moderate pericardial effusion was also noted. Right Heart Catheterization confirmed severe PAH (PA pressure $100 / 40$, mean 60 , wedge pressure of 10 , RV diastolic pressure of $15 \mathrm{mmHg}$ ). Cardiac output $2.0 \mathrm{~L} / \mathrm{min}$. PA saturation: $17 \%$. Intermittent PA and RV pulsus alternans during regular sinus rhythm. Radial arterial saturation: 95\%. Radial pressure wave form did not show alternans. Pulmonary scan: low probability for PE. Diagnosis of HIV associated severe PAH with congestive heart failure was made. Patient was treated with Bosentan and Warfarin

DISCUSSION: Noninfectious cardiovascular complications of HIV such as PAH remain poorly understood. HIV associated PAH occurs at all stages of the disease and does not seem to be related to the degree of immune deficiency. HIV infected patients have a 2500 fold increased risk of developing PAH compared to the general population (1-2 per million). Echocardiogram has an important role in the early evaluation of HIV associated cardiovascular diseases. This case illustrates the association of severe PAH with HIV.- the interesting hemodynamic finding of isolated right ventricular and pulmonary artery pulsus alternans as noted in this patient has been very rarely reported in PAH in contrast to systemic arterial pulsus alternans in patients with severe LV dysfunction. The exact mechanism of alternans is not known but may be secondary to alternation of loading conditions or contractility or alteration of intracellular calcium. Presence of RV and PA alternans represents significant RV systolic dysfunction and carries a poor prognosis. The severe right ventricular dysfunction explains the lack of respiratory variation of the Tricuspid regurgitation murmur.

IT'S TIME TO QUIT SMOKING - A CASE OF PULMONARY LANGERHANS CELL HISTIOCYTOSIS Sarat Chandra Ayyagari; Randhir Jesudoss; Sandeep Kapur; Rajan Prakash. The Christ Hospital, Cincinnati, OH. (Control ID \#1303510) 
LEARNING OBJECTIVE 1: * Consider Pulmonary Langerhans Cell Histiocytosis in the differential diagnosis of Interstitial lung disease in smokers.

LEARNING OBJECTIVE 2: * Early diagnosis is important because the clinical course of the disease may be dramatically altered by a single intervention i.e. smoking cessation.

CASE: A 50 year old Caucasian female with no prior respiratory ailments presented with worsening non productive cough and exertional dyspnea for four months. She denied fevers, weight loss or lower extremity swelling. She was prescribed multiple courses of antibiotics, oral steroids and bronchodilators with no subjective improvement. In fact, her cough worsened to a point where she sustained bilateral rib fractures after a prolonged coughing spell. She reported an 18 pack year history of ongoing smoking. She denied any exposure to metallic dusts, recent travel or any unusual pets. Her physical exam was unremarkable. Chest CT revealed lower rib fractures, mild hyperinflation and bilateral pulmonary nodules measuring up to $5 \mathrm{~mm}$ predominantly in the upper lobes. A comprehensive autoimmune, allergic and infectious workup that included bronchoscopy failed to identify an etiology of her interstitial lung disease .Differential diagnosis at this point included sarcoidosis, lymphangiomatosis, eosinophilic pneumonia and diffuse metastases. She subsequently underwent an open lung biopsy, which showed multiple stellate nodules that were S-100 protein-positive and CD-68-positive diagnostic of Langerhans Cell Histiocytosis. At this point, she was strongly recommended to quit smoking. On a follow up visit at six months, she denies any cough and dyspnea markedly improved.

DISCUSSION: Pulmonary Langerhans Cell Histiocytosis (PLCH) is a rare interstitial lung disease occurring exclusively in young cigarette smokers. The extremely low incidence and non-specific presentation often poses a diagnostic challenge to clinicians. Pulmonary LCH belongs to a spectrum of diseases characterized by the proliferation of specific histiocytes, known as Langerhans cells, and their infiltration of organs. An ongoing debate exists over whether $\mathrm{LCH}$ is a reactive or neoplastic process. Its association with smoking has been well described and it is now classified under smoking related interstitial lung diseases. Patients typically present with chronic non productive cough, dyspnea or spontaneous pneumothorax. Extra pulmonary manifestations including cystic bone lesions and diabetes insipidus have been described. In our patient, it is unclear whether the rib fractures represent a purely mechanical complication versus extra pulmonary involvement. Radiological appearance is typical with nodular opacities with upper lobe predominance and interstitial fibrosis on a HRCT; however, most patients require a surgical biopsy for confirmation. Smoking cessation is the cornerstone of management. No other intervention consistently results in clinical stabilization. In fact, cases of complete radiological resolution following smoking cessation have been reported. Steroids and chemotherapy have been tried in refractory cases. Long term complications include pulmonary hypertension and respiratory failure. Physicians should consider PLCH in the differential of interstitial lung disease in young smokers. Early diagnosis is important because the clinical course of the disease may be dramatically altered by a single intervention i.e. smoking cessation.

KAPOSI'S SARCOMA PRESENTING AS ATYPICAL CONJUNCTIVITIS Schuyler D. Livingston; Valeria Cantos; Minh Nguyen. Emory University SOM, Atlanta, GA. (Control ID \#1339822)

LEARNING OBJECTIVE 1: Recognize ocular Kaposi's sarcoma (KS) LEARNING OBJECTIVE 2: Identify indications for systemic chemotherapy in KS

CASE: A 35 year-old African-American male presented to clinic with a 2week history of progressive right eye redness with associated nasal congestion. Redness had spread to the left eye several days before. The patient had been diagnosed with AIDS five months prior, with an initial CD4 count of 4, and viral load of 140,000. Physical exam was notable for bilateral conjunctival erythema, with thickening of the right lacrimal caruncle. Moderate nasal mucosal edema was noted. Further exam did not reveal additional mucosal or cutaneous lesions. On laboratory evaluation, viral load was now undetectable on boosted darunavir and fixed-dose tenofovir/emtricitabine. The patient returned to clinic 1 month later with worsening bilateral eye redness and facial edema. Physical exam revealed a beefy-red, crescent-shaped right conjunctival mass with mildly dilated surrounding vasculature. Violaceous nodules were present in the posterior oropharynx and left superior gingiva. There were two small $(\sim 1 \mathrm{~cm})$ hyperpigmented plaques on the right thigh and upper abdomen. Marked facial edema and narrowing of the nasal passages had developed. Though no respiratory or constitutional symptoms were present, chest X-ray revealed diffuse nodular infiltrate, with perihilar predominance. A clinical diagnosis of Kaposi's sarcoma (KS) related to immune reconstitution was made, with pulmonary involvement. Due to a shortage of doxorubicin, chemotherapy consisting of paclitaxel was administered the following day. The patient reported rapid improvement in symptoms over the next few days.

DISCUSSION: KS is a well-known complication of AIDS, associated with human herpesvirus 8 infection. Visceral involvement is common, with predilection for the oral cavity, GI tract, and lungs. Ocular disease is seen in approximately $20 \%$ of cases, affecting the conjunctiva, eyelids, and lacrimal sacs. Conjunctival lesions are usually bright red or violaceous in color, slightly raised, and may bleed. The inferior fornix is the most common site of involvement. Early lesions may appear similar to subconjunctival hemorrhage. Eyelid KS is more common, typically presenting as a raised purple lesion, similar to an ecchymosis. Associated lymphedema is common with $\mathrm{KS}$ and may be severe, possibly explaining the facial edema and nasal congestion seen in this case. Treatment with systemic chemotherapy is indicated for $\mathrm{KS}$ in the following settings: extensive cutaneous disease, lymphedema, symptomatic visceral disease, and immune reconstitution inflammatory syndrome (IRIS). In this patient, KS manifested after 6 weeks of antiretroviral therapy, supporting a diagnosis of IRIS. IRIS refers to unmasking or worsening of an opportunistic infection with response to combined antiretroviral therapy (cART). It occurs in about $10 \%$ of patients with AIDS starting cART. Affected patients usually have low baseline CD4 counts and high baseline viral loads. KS is among the most common underlying diseases in IRIS, presenting as early as 3 weeks to as late as 1 year following initiation of therapy. In one case series, IRIS occurred in 12/41 (29\%) cases of pre-existing Kaposi's sarcoma. Visceral KS occurring in IRIS tends to be aggressive, with potential for chronic lymphedema, and mortality estimated at 50\%. Systemic chemotherapy with doxorubicin or paclitaxel is potentially life-saving in such patients.

LABOR: NOT YOUR TYPICAL PAIN IN THE NECK Lauren Shapiro; Naeema Ginwala. Montefiore Medical Center, Bronx, NY. (Control ID \#1320839)

LEARNING OBJECTIVE 1: Learning objective: To make internists aware of bilateral carotid dissection as a rare, but serious, complication of childbirth.

CASE: 34 year-old female with no significant past medical history, presented to the emergency room with complaints of right hand weakness and numbness on the right side of her face. Two weeks prior, a few days after a normal spontaneous vaginal delivery, the patient reported feeling a left-sided neck pain. Ten days later, the patient noted numbness of the right side of her face, and subsequently weakness of her right arm and hand that limited her ability to grasp and pick up objects. When she presented to the hospital she was slightly hypertensive at $151 / 98$ with a right hand grip $3 / 5$ and finger extension 2/5. Evaluation with a CT scan of the head was negative for an intracranial bleed, however an MRI of the brain showed several foci of acute left frontal and parietal infarctions, suggestive of arterial emboli. She subsequently underwent an MRA of the neck, which showed bilateral proximal internal carotid artery narrowing suggestive of bilateral carotid artery dissections. The patient was treated conservatively with anticoagulation. Her neurological deficits gradually improved and were completely resolved approximately two months following the event.

DISCUSSION: The case above demonstrates the rare, but dangerous, labor complication of carotid artery dissection leading to embolic stroke. There have been few case reports of women who have experienced postpartum internal carotid artery dissections (ICAD), and none that describe bilateral dissections related to labor. Ipsilateral head and face pain, with or without neck pain, is the most common sign for dissection. ICAD have been described in patients with predisposing conditions such as 
fibromuscular dysplasia, cystic medial necrosis, Marfan's, and Ehlers Danlos syndromes. However, several case reports have documented women with no previous risk factors to dissection as having experienced this postpartum. There are several physiological reasons why parturition may cause or exacerbate ICAD. In pregnancy, physiologic vascular wall remodeling enhances arterial diameter and compliance, increasing vulnerability to dissection. Elevated blood flow and cardiac output during pregnancy may intensify shear forces and subsequently increase the risk of intimal tear. In addition, the hypercoagulable state of pregnancy helps in the development of thrombi at the site of dissection, allowing for subsequent embolization. Lastly, expulsive forces have been proposed as precipitating factors to ICAD for women reaching the second stage of labor. Studies show that pregnancy increases the risk of stroke as much as 13-fold, and that peri- and postpartum periods are the most at-risk times. The incidence of ICAD, however, as the cause of puerperium stroke is rare, and bilateral dissections even more so. Physiologic changes to women's vasculature and hypercoagulability make pregnancy a high-risk state for ICAD. Strain during labor has often been overlooked as a form of trauma that may leave the internal carotids arteries vulnerable to injury. ICAD is an important complication of pregnancy and cause of stroke that internists should be aware of in their patients.

\section{LAMBL'S EXCRESCENCES AND CEREBROVASCULAR ACCIDENT} Leon I. Igel. New York Presbyterian/Weill Cornell Medical Center, New York, NY. (Control ID \#1316208)

LEARNING OBJECTIVE 1: To review the potential etiologies of recurrent embolic stroke in a young patient

LEARNING OBJECTIVE 2: To compare Lambl's Excrescences and Papillary Fibroelastomas

CASE: An athletic 25-year-old man with no known past medical history presented with sudden onset of slurred speech and right-sided body weakness. Emergent head CT and follow-up MRI brain revealed the presence of an acute stroke as well as evidence of a subacute embolic stroke of unclear age. The patient subsequently received tPA with complete neurologic recovery. Stroke and hypercoagulability workups were completed, with the only abnormality encountered being a $13 \mathrm{~mm}$ mass seen on the cusp of the patient's aortic valve on both transthoracic and transesophageal echocardiograms. Given that no additional risk factor or alternative etiology for the recurrent emboli could be found, the patient underwent open heart surgery for resection of the aortic valve mass. Histopathological studies performed on the excrescence revealed fibrous tissue with myxoid change and the mass was termed a large Lambl's Excrescence. The patient has remained without further incident since the time of his procedure and follow-up transthoracic and transesophageal echocardiograms have demonstrated absence of new valvular mass development

DISCUSSION: There are many plausible causes for recurrent embolic stroke. However, in a young, healthy patient without known risk factors (i.e. hypertension, diabetes mellitus, dyslipidemia, known hypercoagulable state, tobacco use, known cardiac arrhythmias including atrial fibrillation) the possible etiologies are substantially narrowed, with the most likely cause being a cardioembolic source. It is estimated that a cardioembolic source is responsible for approximately $25 \%$ of all ischemic strokes in the general population. Potential cardiac sources for emboli include masses (i.e. thrombi, atherosclerotic plaque, valvular calcification, vegetations, and tumors) and aberrant passageway for embolism (including patent foramen ovale and atrial septal defect). Lambl Excrescences are fibrinous strands most often found at the contact margins of the aortic valve. There is a great deal of controversy as to whether these valvular excrescences are etiologically associated with embolic events, but the predominant sentiment is that they are rarely, if ever, associated with cardioembolic stroke. Giant Lambl's Excrescences have been documented, and these larger filamentous masses have been causally associated with embolic stroke in case reports. However, at the size described in this case $(13 \mathrm{~mm})$, it would be important to consider a small Papillary Fibroelastoma as part of the differential diagnosis. Fibroelastomas are benign cardiac tumors that are most frequently located on the aortic $(36 \%)$ or mitral (29\%) valves. They often have frond-like arms emanating from a stalked central core (similar in appearance to large Lambl's Excrescences), with a mean size of $9 \mathrm{~mm}$. They can be a source of systemic embolization either from migration of thrombus from the tumor surface, or secondary to tumor embolization. Surgical excision of fibroelastomas is frequently recommended in patients who experience embolic events and in patients who have large $(>10 \mathrm{~mm})$ tumors

LEFT BLACK AND BLUE BY A VACCINE Kokila Bindiganavile Nagendran; Harvey Friedman; Salih Samo. Saint Francis Hospital, Evanston, IL. (Control ID \#1339700)

LEARNING OBJECTIVE 1: Immune thrombocytopenia can be a potential life threatening complication of Hepatitis B Vaccination in adults. LEARNING OBJECTIVE 2: The hepatitis B vaccine has a good level of efficiency and its rare adverse effects, including ITP, must not put its use in question.

CASE: We present the case of a $57 \mathrm{y} / \mathrm{o}$ male with ESRD on hemodialysis who was transferred from the nursing home due to an abnormally low platelet count $(3000 / \mathrm{mm} 3)$ found on a blood test. The patient had noticed bruising around his left eye, hemorrhagic blebs on his tongue, hematuria, tarry stools and diffuse ecchymosis over his extremities. He also reported a mild frontal headache. History was significant for receiving the recombinant hepatitis B vaccine 2 days prior to admission. He had received the vaccine previously with no documented adverse reaction. Past history revealed splenectomy at age eleven after a motor vehicle accident. Physical examination revealed ecchymosis, conjunctival haemorrhage and mucosal bleeding in the mouth. A CT head showed no intra cranial haemorrhage. On admission, the results of analytical tests were: white blood count of $11,200 / \mathrm{mm} 3$, haemoglobin of $12.8 \mathrm{~g} / \mathrm{dL}$, platelet count of $3000 / \mathrm{mm} 3$, and peripheral smear showed no schistocytes, clumping and normal platelet morphology; prothrombin time and active partial thromboplastin time were normal as were assays for heparin-induced antibodies, heparin induced platelet aggregation, antinuclear antibodies. Serological assays for hepatitis $\mathrm{B}$ virus, hepatitis $\mathrm{C}$ virus and Human immunodeficiency virus were negative. He was initially transfused single donor platelets with no improvement in the platelet count. A diagnosis of immune thrombocytopenic purpura was made and he was started on IV solumedrol, intravenous immunoglobulin and aminocaproic acid. By the end of the fifth day, the platelet count was $102,000 / \mathrm{mm} 3$ and was $259,000 / \mathrm{mm} 3$ at discharge. He was sent to the nursing home on tapering oral steroids for a month. Platelet counts remained normal on a six month follow -up.

DISCUSSION: Universal vaccination with Hepatitis B vaccine was instituted since 1991 .The vaccine is usually well tolerated, with few side effects; but several cases of thrombocytopenia after recombinant vaccine have been described in literature since 1994. ITP is thought to be caused by the presence of autoantibodies to glycoprotein IIb/IIIa molecules present in the platelet membrane. Repeated doses of vaccine may function as a booster to autoantibody formation. Previously recognized cases had a latency period of three to four weeks in patients who received their second or third dose. Compared to that, our patient had rapid onset of thrombocytopenia. Diagnosis remains one of exclusion. Most cases resolve spontaneously and do not experience recurrence even after the same vaccination months later. Steroids may be used as a first line agent with prompt increase in platelet count.

LEMIERRE'S SYNDROME: RECALLING THE 'FORGOTTEN DISEASE" Ramon Jacobs; Bora Toklu. NYU School of Medicine, New York, NY. (Control ID \#1319359)

LEARNING OBJECTIVE 1: Distinguish clinical clues to assess for Lemierre's Syndrome.

LEARNING OBJECTIVE 2: Manage Lemierre's Syndrome when appropriate anaerobic antibiotics are not sufficient.

CASE: A 24-year-old healthy female was admitted after presenting with fever, sore throat, neck and pleuritic chest pain that started 3 days prior to admission. On physical examination, the patient appeared in mild respiratory distress requiring supplemental oxygen, and noted to have swelling and tenderness along the left sternocleidomastoid muscle with associated left tonsillar exudate and bibasilar pulmonary rales. Her initial 
complete blood count revealed isolated mild thrombocytopenia and a bandemia of $79 \%$. A rapid strep test returned negative and a rapid influenza A/B RNA test also was negative. Following admission, a CT scan of the neck with contrast showed left peritonsillar abscess extending into the hypopharyngeal region with extensive lymphadenopathy. The patient was subsequently started on piperacillin-tazobactam and metronidazole after her admission blood culture began growing gram-negative anaerobes, which later speciated as Fusobacterium necrophorum. As the patient continued to spike high grade fevers, metronidazole was switched to intravenous sulbactam-ampicillin. On appropriate antibiotic coverage, the patient continued to complain of worsening dyspnea requiring increased supplemental oxygen by nasal cannula. A CT scan of the chest then showed multifocal pneumonia and multiple lung nodules concerning for septic emboli. In search for the source of her septic emboli, an echocardiogram showed normal findings, while a repeat CT of the neck with contrast revealed new findings of multiple necrotic lymph nodes and left internal jugular vein thrombus leading to a diagnosis of Lemierre's Syndrome. Subsequently, given clinical deterioration, patient was initiated on intravenous anticoagulation and the left internal jugular vein was resected. Over the ensuing several days, the patient's clinical picture had improved significantly on the same antibiotic coverage. She was eventually discharged on oral antibiotics to complete a 4-week course.

DISCUSSION: Lemierre's Syndrome is a potentially fatal complication of oropharyngeal bacterial infections, most commonly of anaerobic Fusobacterium species, leading to ipsilateral jugular vein suppurative thrombophlebitis and subsequent septic emboli. It is a rare disease, but incidence is reportedly increasing, postulated to be due to more conservative patterns of antibiotic prescription for oropharyngeal infections. Any oropharyngeal infection involving adjacent pharyngeal tissue with an anaerobic septicemia and/or respiratory distress should prompt clinicians to evaluate for Lemierre's Syndrome by a simple cervical venous duplex scan or a more sensitive CT scan of the neck with contrast. Although potentially fatal, it can be treated easily if diagnosed in a timely fashion. A high degree of clinical suspicion and prompt initiation of anaerobic antibiotic therapy are of paramount importance to reduce the associated risk of mortality. Although the role of anticoagulation and jugular vein excision is controversial, we believe these measures should be considered if the clinical picture deteriorates on anaerobic antibiotic therapy alone.

LITHIUM TOXICITY IN THE SETTING OF NON STEROIDAL ANTI INFLAMMATORY MEDICATIONS Zahraa Hajjiri ${ }^{1}$; Sandeep K. Walia ${ }^{1}$; Syed Hassan ${ }^{1}$; Sandeep Soman ${ }^{2}$. ${ }^{1}$ Henry Ford Health System, detroit, MI; ${ }^{2}$ Henry ford Hospital, Detroit, MI. (Control ID \#1335286)

LEARNING OBJECTIVE 1: Lithium toxicity is known to affect multiple organ systems, including the central nervous system. Lithium levels have been used in the diagnosis of toxicity and assessing response to management. There is evidence that non steroidal anti inflammatory medications (NSAID) can increase lithium levels and decrease renal lithium clearance. We present a case of lithium toxicity, which demonstrates this effect and also highlights the fact that lithium levels do not correlate with clinical improvement, especially the neurological deficit.

CASE: The patient is a 51 year old AA male with a history of schizophrenia and bipolar disorder who was chronically taking lithium, valproate, quetiapine, and risperidone. He presented to the emergency department with progressively worsening mental status that was preceded by dysarthria, abnormal gait, and diarrhea. Prior to presentation, he was taking ibuprofen (an NSAID) for pain control in the setting of a recent dental procedure and had poor oral intake. He was found to have an elevated lithium level $(3 \mathrm{Mmol} / \mathrm{L})$ with mild renal failure (serum creatinine $1.6 \mathrm{mg} / \mathrm{dl}$ ) and was treated with intravenous hydration and supportive care. Hemodialysis (HD) was not initiated as renal function along with his lithium levels improved rapidly. His serum lithium levels normalized without improvement in his mental status. Subsequently, he required intubation with infectious/metabolic workup being negative, an unremarkable CT scan of the head, and an electroencephalography revealing metabolic encephalopathy. With a few days of supportive care, the patient was extubated and his mental status returned to baseline.

DISCUSSION: We report a case of lithium toxicity in the setting of NSAID use, where a patient had normalized serum lithium levels with delayed improvement in mental status. It is recommended that lithium levels be checked every 4-5 days after starting an NSAID to assess for toxicity. We emphasize that lithium levels may be helpful in the primary diagnosis of toxicity; however, serial levels should not be used to assess response to treatment. In cases of acute toxicity, lithium is mainly an extracellular water soluble ion rapidly cleared by intravenous hydration or HD. However, in cases of toxicity following chronic lithium ingestion, intracellular and intracerebral concentrations are high. When the serum lithium level normalizes, intracellular concentrations remain elevated and further clinical decompensation is possible. This occurs because lithium equilibrates slowly between both compartments, requiring multiple prolonged HD treatment sessions. Reviewing this case retrospectively reinforces the current guidelines for the management of lithium toxicity. Any patient who comes in with altered mental status associated with toxic lithium levels (greater than 2.5 $\mathrm{Mmol} / \mathrm{L}$ ) should undergo multiple prolonged HD sessions to adequately deplete intracellular lithium.

LOST ART OF PHYSICAL EXAM Nikhil Mukhi; Tushar Shah; Tauseef Ahmed. Westchester Medical Center, Valhalla, NY. (Control ID \#1327566)

LEARNING OBJECTIVE 1: A good physical exam should be used to guide the diagnostic workup of a patient.

LEARNING OBJECTIVE 2: A good physical exam helps avoid delay in diagnosis and unnecessary testing.

CASE: A 29 years-old Hispanic construction worker admitted for evaluation of an abdominal mass and weakness. Patient noted a painless mass in his abdomen 1 year ago which he stated was initially the size of a lemon and had gradually progressed in size. For 3 months he was experiencing progressive dysphagia to solid foods and markedly diminished appetite with approximately 25 pound weight loss. One month ago he started having episodic projectile nausea and vomiting occurring immediately after food intake. The vomitus was bilious, contained food but no blood and was associated with burning chest pain. He denied abdominal pain or change in bowel movements. He developed weakness one week prior to admission which prompted him to seek medical attention. He had subjective high grade fevers not associated with chills or night sweats for one month. He is a non smoker, occasionally drinks a beer and doesn't use any recreational drug. The physical examination was entirely normal other than mild tachycardia and a $15.0 \times 15.0 \mathrm{~cm}$ firm, mildly tender, non-pulsatile mass with smooth borders occupying the area between symphysis pubus to umbilicus. No hepatosplenomegaly or adenopathy was appreciated. Initial labs revealed WBC count of $4.4 \mathrm{k} / \mathrm{mm} 3$ (Diff - 68\% Neutrophils, 14\%Lymphocytes and $15 \%$ monocytes), $\mathrm{Hb}$ of $11 \mathrm{gm} / \mathrm{dl}$ (MCV 83.6 and RDW 15.6) and platelet count of $193 \mathrm{k} / \mathrm{mm} 3$. Chemistry revealed BUN: $34 \mathrm{mg} / \mathrm{dl}, \mathrm{Cr}: 2.54 \mathrm{mg} / \mathrm{dl}, \mathrm{K}: 3.3 \mathrm{meq} / \mathrm{l}, \mathrm{LDH}$ : 786U/1, Alkaline Phosphate: 453U/l, Calcium: $14.6 \mathrm{mg} / \mathrm{dl}$, Uric acid: $10.3 \mathrm{mg} / \mathrm{dl}$. In the setting of monocytosis, hypercalcemia, hyperuricemia and abdominal mass, a preliminary diagnosis of lymphoma was made and patient was transferred to our facility. Bone marrow biopsy revealed a normal trilineage and the cytogram analysis showed no evidence of lymphoma/leukemia. CT Chest/Abdomen/Pelvis revealed a large $(8.9 \mathrm{x}$ $10.5 \mathrm{~cm}$ ) homogeneous posterior mediastinal mass extending inferiorly into the retroperitoneum and two additional soft tissue masses $(7.8 \times 17.4 \times 14.4 \mathrm{~cm}$ and $10 \times 15.7 \times 14 \mathrm{~cm}$ ) within the intraperitoneal space which contained areas of necrosis and calcification. On repeat exam, patient had a normal left testis and an absent right testis. Ultrasound of the scrotum confirmed the findings. Patient underwent a CT guided biopsy of the intraperitoneal mass which showed a classic type seminoma associated with non-necrotizing granulomas. Patient's Serum AFP was $1.6 \mathrm{ng} / \mathrm{ml}$ and $\beta-\mathrm{hCG}$ was $42.3 \mathrm{mIU} / \mathrm{ml}$. Patient was diagnosed with Stage 3 Seminoma, has received 4 cycles of BEP regimen [bleomycin, etoposide, cisplatin] and is currently awaiting postchemotherapy evaluation.

DISCUSSION: Testicular cancer is the most common solid malignancy affecting males between the ages of 15 and 35, although it accounts for only 1 percent of cancers in men. With a five year survival rate of over $95 \%$ even in advanced germ cell tumors, it is one of the most readily treatable cancers. Varghese et al described how the electronic medical record and advanced imaging technology have led doctors away from the bedside but also devalued the importance of a good bedside physical exam. 
$\mathrm{He}$ also stated that clinicians who are skilled at the bedside examination make better use of diagnostic tests and order fewer unnecessary tests. Our case illustrates that physical exam should be used to guide the diagnostic tests.

LOST IN TRANSLATION? HOT FLASHES IN A MAN Jaseena Elikkottil; Tseganesh Selameab. HCMC, Minneapolis, MN. (Control ID \#1308485)

LEARNING OBJECTIVE 1: Review diagnosis and treatment of prostatic disease in resource poor settings

LEARNING OBJECTIVE 2: Recognize cultural differences in patientprovider communication

CASE: A 76-year-old Amharic speaking male from Ethiopia presented with chief complaint of "hot flashes". He had arrived in the United States less than one month prior. When interviewed with a professional interpreter, he reported being healthy till one year prior when he had surgery for removal of his "seeds" to relieve urinary obstructive symptoms. Patient denied ever having a history of malignancy and denied having any other treatments, such as injections or radiation. Since that surgery the patient reported feeling alternately "hot and cold", and often waking up at night drenched in sweat. On his initial physical exam, the patient was afebrile but diaphoretic and was noted to have a supra-pubic horizontal scar. The genital exam was remarkable for absence of testes bilaterally. Hemogram, TSH and comprehensive metabolic panel were unremarkable. Further evaluation revealed an elevated PSA and a very low testosterone level. After the initial visit, a family member revealed to the care team that the patient had been diagnosed with prostate cancer in Ethiopia and had undergone bilateral orchiectomy and prostatectomy. The diagnosis of prostate cancer had been kept from the patient by both the family and the medical team caring for him in Ethiopia.

DISCUSSION: Bilateral orchiectomy is commonly used as the first line treatment for prostate cancer in many parts of Africa and other resource poor areas. According to one study from Africa, $89 \%$ of the prostate cancer cases presented as locally advanced or metastatic disease. Diagnosis is commonly made by a digital rectal exam due to the expenses associated with lab testing. A histological diagnosis was obtained prior to surgery in only $39 \%$ of cases. Bilateral orchiectomy was done in $71 \%$ of the cases, and $28 \%$ of those received additional therapy. Additionally, there are practice differences amongst cultures regarding revealing bad news, specifically a diagnosis of cancer, to the patient. In many Eastern cultures, the diagnosis is disclosed to a close family member first and their input may be sought on how and when to break the news to the patient. Our patient was not aware of his diagnosis of prostate cancer made in Ethiopia. These possibilities should be kept in mind when working with a culturally diverse population. Obtaining collateral history from family becomes even more important in such a scenario.

LUMPS, BUMPS, AND SLE Stephanie J. Davis; Shana Ratner. University of North Carolina Hospitals, Chapel Hill, NC. (Control ID \#1339487)

LEARNING OBJECTIVE 1: Recognize lymphadenopathy as a potential presenting symptom of Systemic Lupus Erythematosus

LEARNING OBJECTIVE 2: Learn about Familial Multiple Angiolipomatosis, a rare yet benign inherited condition

CASE: A 49 year old adopted female presented to the General Medicine clinic to establish care. She complained of the sudden appearance of greater than twenty lumps beneath the skin that seemed to bruise easily. She denied fevers, chills, or unexplained weight loss but did note night sweats for many years which she attributed to menopause. Her past medical history included squamous cell carcinoma of the eyelid treated with excision, and menorrhagia requiring blood transfusion, resolved since uterine ablation. She had not been in contact with her birth parents, and thus did not know her family history. Review of systems revealed a photosensitive rash on the cheeks that she had been told was "atypical rosacea." On exam she was found to have nontender, bilateral, rubbery, $1 \mathrm{~cm}$ epitrochlear nodes, as well as nontender, scattered, rubbery soft tissue nodules on the inner aspects of her arms and anterior and posterior thighs that appeared bruised. No cervical, supraclavicular, axillary, or inguinal lymphadenopathy was found on exam. Labs were notable for mild leukopenia, positive ANA with a titer of 1:640, and a positive RNP. Other rheumatologic serologies, HIV, RPR, and urinalysis were unremarkable. Based on concern for lymphoma versus systemic lupus erythematosus (SLE) presenting with lymphadenopathy, the patient was sent for excisional biopsy. Biopsy of a presumed epitrochlear node and a nodule on the anterior thigh both revealed angiolipoma on pathology. Due to the abundance of these angiolipomas, the patient may have familial multiple angiolipomatosis; however, due to her adoption status, she is unaware of her family history. During the workup of this benign condition, the patient was also found to have SLE, which was likely an unrelated, yet interesting, finding.

DISCUSSION: Familial multiple angiolipomatosis is a rare, benign entity that is inheritied in an autosomal-dominant fashion and may be a subtype of Familial Multiple Lipomatosis (FML), a condition that presents in the third to fifth decade of life, characterized by numerous, encapsulated lipomas on the trunk and extremities. Treatment of the angiolipomas for cosmetic purposes (as the lipomas are benign) typically consists of surgical excision or liposuction. In this case, the presence of bilateral nodules in the epitrochlear region was concerning for lymphadenopathy, prompting a workup for malignancy, autoimmune condition, or systemic inflammatory disease. While the angiolipomas in this patient were found to be benign by pathologic evaluation, oddly enough, systemic lupus erythematosus happened to also be present and, thus far, undiagnosed in this patient. Although only occurring in approximately $5 \%$ of SLE patients, generalized lymphadenopathy can be a presenting symptom of SLE.

LYMPHOMA PRESENTING AS HYPERCALCEMIA AND NORMALIZATION OF PARATHORMONE IN A PATIENT WITH UNDERLYING SECONDARY HYPERPARATHYROIDISM Abhishek Singla $^{1,2}$; Vrinda Agrawal ${ }^{1,2}$; James Fulton ${ }^{1,2}$; Mary Tadros ${ }^{2,1}$. ${ }^{\mathrm{T}}$ Creighton University Medical Center, Omaha, NE; ${ }^{2}$ VA Omaha, Omaha, NE. (Control ID \#1333798)

LEARNING OBJECTIVE 1: To emphasize the need to evaluate every case of severe hypercalcemia for underlying malignancy.

LEARNING OBJECTIVE 2: To review the management of severe hypercalcemia caused by lymphoma and identify the role of early steroid and chemotherapy.

CASE: A 86 year old caucasian male with past medical history of chronic kidney disease (Stage III) with secondary hyperparathyroidism, dilated cardiomyopathy with poor EF, chronic obstructive lung disease; presented with severe fatigue, anorexia, weight loss and back pain. On examination, spleen was palpable. A complete laboratory work up was done with the following RESULTS: Calcium $14.8 \mathrm{mg} / \mathrm{dL}$ (normal 8.2-10.2), Phosphorus $3.5 \mathrm{mg} / \mathrm{dL}$ (2.6-4.9), alkaline phosphatase $50 \mathrm{IU} / \mathrm{L}$ (38-126), BUN $55 \mathrm{mg} / \mathrm{dL}$ (9-20), creatinine $3.2 \mathrm{mg} / \mathrm{dL}$ (0.7- 1.5), TSH $1.18 \mathrm{mIU} / \mathrm{L}(0.47-6.20)$, PTH $17 \mathrm{pg} / \mathrm{ml}$ (12-88), Vitamin D $91 \mathrm{pg} / \mathrm{mL}$ (18-72), PTH-rP $27 \mathrm{pg} / \mathrm{mL}$ (14 -27). Review of labs done four months prior showed calcium $10.4 \mathrm{mg} / \mathrm{dL}$ and PTH $180 \mathrm{pg} / \mathrm{ml}$. Urine electrophoresis was positive for monoclonal protein noted in the beta-gamma region and free kappa light chains identified by immunofixation electrophoresis but no evidence of monoclonal protein on serum electrophoresis. CT scan of abdomen showed large left upper quadrant mass $(13 \mathrm{~cm} \times 13 \mathrm{~cm} \times 15 \mathrm{~cm})$ resulting in anterior displacement of the abdominal viscera with multiple large lymph node in the left para-aortic region. Biopsy of mass showed diffuse infiltrate of intermediate to large atypical B-cells positive for CD45, CD20; suggestive of high-grade B-cell lymphoma. Molecular cytogenetic studies were positive for 3q27 (BCL6) and 18q21 (BCL2) and negative for $\mathrm{t}(14 ; 18)(\mathrm{q} 32 ; \mathrm{q} 21)$; which is most consistent with diffuse large B-cell lymphoma. Bone marrow examination was normal. Patient was treated with IV fluids, palmidronate, calcitonin. After confirmation of type of lymphoma patient was started on vincristine and prednisone. Patient's calcium normalized; however, treatment was soon complicated by tumor lysis syndrome.

DISCUSSION: Patients presenting with severe hypercalcemia should be evaluated for malignancy irrespective of past history. Lymphomas can cause severe hypercalcemia most commonly by overproduction of calcitriol, which results in decreased parathormone production. Furthermore, hypercalcemia is 
usually associated with aggressive lymphomas, and early treatment with steroids and chemotherapy becomes paramount.

MMM: MYSTERIOUS MEDICATION MANIA Shawn Brickner; Carl Fichtenbaum. University of Cincinnati College of Medicine, Cincinnati, OH. (Control ID \#1309443)

LEARNING OBJECTIVE 1: Describe common manifestations of baclofen withdrawal particularly acute psychosis.

LEARNING OBJECTIVE 2: Understand and be able to explain to patients that baclofen should never be stopped abruptly.

CASE: Introduction: Baclofen is commonly prescribed to alleviate pain in patients with muscle spasms. Abrupt withdrawal of baclofen can lead to frank psychosis. Case Presentation: A 60-year-old male with hypertension, diabetes mellitus and chronic lumbago presented with an episode of fatigue, shaking, abnormal speech and altered mental status. The evening prior to admission, the patient had been exhausted from performing physical labor at his home and fell asleep without taking his medications. He awoke the next morning still tired and very shaky, to the point that he could not hold a glass of water without spilling it. The day progressed and his wife noticed his abnormal speech. He developed memory problems. EMS found him to be hypotensive at 73/40 but normoglycemic with a blood glucose of 102 . Admitting physicians did not want medications to complicate his altered mental status and held his home medications of gabapentin, zolpidem, citalopram and baclofen. An extensive work-up for metabolic, cardiac, and neurologic causes of altered mental status revealed only an elevated CK of 2022. By hospital day 3 , his mental status had deteriorated to the point that three separate episodes of psychiatric codes were called on him. He was found to be screaming, tremulous, rigid, hyper-religious and having visual hallucinations. His acute psychosis caused concern for a toxidrome and poison control was called. After discussing the case with poison control, his baclofen was restarted. Within a few hours, he returned to his normal self.

DISCUSSION: Baclofen has a short half-life of 3-4 hours. Symptoms of withdrawal can develop within 12-72 hours of cessation. Patients can present with symptoms of tachycardia, autonomic instability, seizures, hyperthermia and spasticity. Spasticity can lead to rhabdomyolysis. If baclofen is not restarted, patients can develop frank psychosis or even die from the withdrawal. The exact cause is unknown, but thought to be due to baclofen causing continuous inhibition of monoamine neurotransmitters. The neurotransmitter receptors become highly sensitive to monoamines and once baclofen is stopped and norepinephrine and dopamine reach the receptors, it leads to autonomic arousal and delirium. Readministration of baclofen leads to rapid resolution of delirium. When baclofen is prescribed, patients should be advised to never stop it abruptly. If baclofen needs to be discontinued, it should be tapered over 1-2 weeks to prevent withdrawal. Finally, it is important to remember to continue baclofen when patients are hospitalized, even for those with altered mental status.

MEDIASTINAL CLUTTER CAN MAKE YOUR HEART FLUTTER! Vinod Khatri; Krishna Khatri; Harvey Friedman. St. Francis Hospital, Evanston, IL. (Control ID \#1339066)

LEARNING OBJECTIVE 1: Large mediastinal masses can cause pressure symptoms on the heart.

LEARNING OBJECTIVE 2: This elevated mediastinal pressure may lead to atrial flutter/fibrillation.

CASE: CASE: 173 yrs old gentle man with past medical history significant for indolent lymphoma presented to hospital with history of progressive dysphagia, exertional dyspnea. He denied any constitutional symptoms. On examinations his vital signs were WNL. His neck exam revealed a mobile, rubbery about $2.5 \mathrm{~cm}$ lymph node in ant cervical group on left side. Chest was clear to auscultation, Cardiac exam revealed regular rate \& rhythm, normal S1S2, with no murmurs. On evaluation his CT scan of chest showed extensive bulky mediastinal lymphadenopathy which was causing mass effect on right side of heart. He underwent mediastinoscopy and lymph node biopsy, which was consistent with marginal zone lymphoma. While in hospital, patient developed an episode of atrial flutter with 2:1 block which converted to sinus rhythm after amiodarone infusion.
Pt had another episode of atrial fibrillation with rapid ventricular response during the course of hospitalization which was treated successfully with amiodarone again. CASE: 268 yrs old female with past medical history of HTN and dyslipidemia was admitted with shortness of breath. She denied any chest pain, fever or cough. On examination her vital signs revealed T97.2, P-130/min, BP-114/70, and RR-20/min. Chest exam revealed focal rales in the right infraclavicular \& mammary regions. On cardiac exam she had an irregularly irregular rhythm and had no murmur. On evaluation her routine labs were WNL. EKG revealed Afib with RVR, so patient was anticoagulated and started on Multaq and digoxin. On radiological evaluation patient's chest $\mathrm{CT}$ showed a large $(7.8 \times 5.8 \times 10 \mathrm{~cm})$ anterior mediastinal mass located in the right hemithorax abutting major vascular structures. A CT guided biopsy of the mass was consistent with thymoma. About a month later when patient underwent resection of tumor, the mass was noted to encircle the innominate vein extending up to its junction with SVC. As a result of extensive adhesions the innominate vein had to be resected along with tumor. Final biopsy confirmed the diagnosis of widely invasive thymoma.

DISCUSSION: Both of above cases illustrate that large mediastinal mass lesions can cause pressure symptoms on heart, and may present with atrial fibrillation/flutter. On review of literature there are few case reports of mediastinal tumors causing atrial arrhythmias, many of which were a result of chemotherapy. Lymphomas and thymomas have also been reported to invade the pericardium and cause cardiac tamponade as a result of mass effect, but till date no case has been reported to cause atrial flutter/ fibrillation as a result of mass effect on right side of heart or invasion of innominate vein or SVC.

METHADONE MAINTENANCE THERAPY- A POTENTIAL TWISTER! Krishna Khatri; Vinod Khatri; Muhammad Shahreyar; Harvey Friedman. St. Francis Hospital, Evanston, IL. (Control ID \#1340205)

LEARNING OBJECTIVE 1: Methadone maintenance therapy for opioid dependence has been associated with prolonged QT interval in EKG in several patients.

LEARNING OBJECTIVE 2: These patient are also predisposed to torsade de pointes.

CASE: 26 yr old female was admitted with 2 episodes of syncope on day of admission. Each episode lasted for less than a minute. There was no history of shaking, tongue bite or urinary incontinence. She reported abusing cocaine and clonidine few hours prior to presentation. She denied having chest pain, shortness of breath or palpitations. Her past medical history was significant for anxiety and heroine abuse for which she was on methadone maintenance program for past three years. On admission she was alert, awake and oriented X 3; vital signs were stable and had no evidence of orthostatic hypotension. Cardiovascular revealed no JVD normal S1, S2 and no murmurs, neurologic exam was essentially non focal. On evaluation her routine labs including were found to be normal. Her urine toxicology screen was positive for cocaine and benzodiazepines. Her EKG revealed sinus bradycardia with prolonged QT interval $(626 \mathrm{~ms})$ which was at $454 \mathrm{~ms}$ a year back. During hospital course had multiple ventricular arrhythmias including premature ventricular contractions, bigeminy, runs of ventricular tachycardia and multiple episodes of torsade de pointes. Most of these were self limited and one methadone was tapered and stopped patient's QT interval reduced back to $444 \mathrm{~ms}$ and patient had no more ventricular arrhythmias.

DISCUSSION: Methadone is associated with prolongation of the QT interval (QT) as well as torsade de pointes. It has recently been suggested that methadone promoted sudden cardiac death based on the absence of structural heart disease in an autopsy cohort. Abnormalities in voltage-gated potassium channels have been shown to lead to prolonged action potentials that are expressed as long QT intervals, and methadone has been found to interact with the voltage-gated potassium channels of the myocardium. Because not every patient experiences QT interval prolongation with methadone, recent research has elucidated risk factors that predispose patients to this adverse effect, including female sex, hypokalemia, high-dose methadone, drug interactions, underlying cardiac conditions, unrecognized congenital long QT interval syndrome, and predisposing DNA polymorphisms. A baseline electrocardiogram (ECG), personal and family history of syncope, and a complete 
medication history should be obtained before a patient begins treatment with methadone. Buprenorphine, a partial micro-opiate-receptor agonist and a kappa-opiate-receptor antagonist does not cause QTP or torsade de pointes. It is a useful and effective alternative to methadone in a select group of patients, including those with documented ventricular arrhythmias on methadone. Pacemakers or defibrillators should be reserved for patients who have failed buprenorphine or a reduced methadone dose.

METHADONE MAINTENANCE THERAPY- A POTENTIAL TWISTER! Krishna Khatri; Vinod Khatri; Muhammad Shahreyar; Harvey Friedman. St. Francis Hospital, Evanston, IL. (Control ID \#1340205)

LEARNING OBJECTIVE 1: Methadone maintenance therapy for opioid dependence has been associated with prolonged QT interval in EKG in several patients.

LEARNING OBJECTIVE 2: These patient are also predisposed to torsade de pointes.

CASE: 26 yr old female was admitted with 2 episodes of syncope on day of admission. Each episode lasted for less than a minute. There was no history of shaking, tongue bite or urinary incontinence. She reported abusing cocaine and clonidine few hours prior to presentation. She denied having chest pain, shortness of breath or palpitations. Her past medical history was significant for anxiety and heroine abuse for which she was on methadone maintenance program for past three years. On admission she was alert, awake and oriented X 3; vital signs were stable and had no evidence of orthostatic hypotension. Cardiovascular revealed no JVD, normal S1, S2 and no murmurs, neurologic exam was essentially non focal. On evaluation her routine labs including were found to be normal. Her urine toxicology screen was positive for cocaine and benzodiazepines. Her EKG revealed sinus bradycardia with prolonged QT interval $(626 \mathrm{~ms})$ which was at $454 \mathrm{~ms}$ a year back. During hospital course had multiple ventricular arrhythmias including premature ventricular contractions, bigeminy, runs of ventricular tachycardia and multiple episodes of torsade de pointes. Most of these were self limited and one methadone was tapered and stopped patient's QT interval reduced back to $444 \mathrm{~ms}$ and patient had no more ventricular arrhythmias.

DISCUSSION: Methadone is associated with prolongation of the QT interval (QT) as well as torsade de pointes. It has recently been suggested that methadone promoted sudden cardiac death based on the absence of structural heart disease in an autopsy cohort. Abnormalities in voltagegated potassium channels have been shown to lead to prolonged action potentials that are expressed as long QT intervals, and methadone has been found to interact with the voltage-gated potassium channels of the myocardium. Because not every patient experiences QT interval prolongation with methadone, recent research has elucidated risk factors that predispose patients to this adverse effect, including female sex, hypokalemia, high-dose methadone, drug interactions, underlying cardiac conditions, unrecognized congenital long QT interval syndrome, and predisposing DNA polymorphisms. A baseline electrocardiogram (ECG), personal and family history of syncope, and a complete medication history should be obtained before a patient begins treatment with methadone. Buprenorphine, a partial micro-opiate-receptor agonist and a kappa-opiatereceptor antagonist does not cause QTP or torsade de pointes. It is a useful and effective alternative to methadone in a select group of patients, including those with documented ventricular arrhythmias on methadone. Pacemakers or defibrillators should be reserved for patients who have failed buprenorphine or a reduced methadone dose.

MICROANGIOPATHIC HEMOLYTIC ANEMIA IN A PATIENT WITH HEMOGLOBIN C TRAIT: TTP OR B 12 DEFICIENCY - THAT IS THE QUESTION? Tanmay S. Panchabhai ${ }^{1}$; Alireza Abdolmohammadi ${ }^{2}$; Elizabeth C. Riley ${ }^{2}$; Charlene K. Mitchell ${ }^{1}$. 'University of Louisville School of Medicine, Louisville, KY; ${ }^{2}$ University of Louisville School of Medicine, Louisville, KY. (Control ID \#1321283)

LEARNING OBJECTIVE 1: Discuss the similarities and differences of the microangiopathic hemolytic anemia picture in TTP and that due to vitamin $\mathrm{B}_{12}$ deficiency
LEARNING OBJECTIVE 2: Recognize the role of hyperhomocysteinemia in the causation of microangiopathic hemolytic anemia from vitamin $\mathrm{B}_{12}$ deficiency

CASE: A 42 year-old AAM with type II diabetes mellitus presented with pre-syncope and diabetic ketoacidosis. History revealed a relatively normal dietary pattern, polyuria, and a daughter with "sickle cell disease." Initial laboratory data showed glucose of $466 \mathrm{mg} / \mathrm{dl}$; anion gap of 19; urine ketones (+); and toxicology screen (-). He had pancytopenia with $\mathrm{Hgb}$ $8.1 \mathrm{~g} / \mathrm{dl}$ (baseline 14.5) with an MCV of $107.1 \mathrm{fl}$, WBC $4,100 / \mathrm{mm}^{3}$, and platelets $39,000 / \mathrm{mm}^{3}$. The peripheral smear had macrocytes, schistocytes (1+), occasional teardrop cells, and hypersegmented neutrophils. Serum ferritin was $102 \mathrm{ng} / \mathrm{ml}$ with iron of $174 \mathrm{mcg} / \mathrm{dl}$, saturation of $87.4 \%$, and TIBC of $199 \mathrm{mcg} / \mathrm{dl}$. The workup for infectious causes was negative. DKA resolved with an insulin drip and he was transitioned to home insulin. An evaluation for hemolytic processes revealed: serum LDH $5368 \mathrm{U} / \mathrm{L}$, haptoglobin $<5.83 \mathrm{mg} / \mathrm{dl}$ with normal fibrin-split products and fibrinogen; hemoglobin electrophoresis showed $50.8 \% \mathrm{HbA}$ and $49.2 \% \mathrm{HbC}$ diagnostic for Hemoglobin C trait. As TTP was an initial concern with its high associated mortality, plasmapheresis and parenteral steroid therapy was initiated, but there was no improvement in blood counts. Subsequently, a profound Vitamin $B_{12}$ deficiency $\left(B_{12}<159 \mathrm{pg} / \mathrm{ml}\right)$ was identified with markedly elevated homocysteine $(88.3 \mathrm{Umol} / \mathrm{dl})$, and normal serum folate $(17.9 \mathrm{pg} / \mathrm{ml})$ levels. Parenteral $\mathrm{B}_{12}$ and oral folate were initiated. MTFR (methylene tetrahydrofolate reductase) mutation analyses to evaluate hyperhomocysteinemia and an ADAMTS 13 mutation analysis for TTP were both negative. Marked clinical response was observed with B12 replacement (platelets 39,000 to 111,000 , MCV 107.1 to 97.6, LDH 5368 to 642 , homocysteine 88.3 to 14.5 ). Plasmapheresis was discontinued prior to normalization of platelets because of anaphylactoid reactions. An intrinsic factor antibody was positive and the patient was discharged with $\mathrm{B}_{12}$ and folate supplementation.

DISCUSSION: Our patient's megaloblastic anemia, high iron saturation, and absent reticulocytosis could not be explained by TTP. However, hemolytic anemia complicates severe $\mathrm{B}_{12}$ deficiency secondary to ineffective erythropoiesis in the marrow. Recent reports have fueled interest in hyperhomocysteinemia in $\mathrm{B}_{12}$ deficiency causing a pseudothrombotic angiopathy by causing microvascular thrombi. Homocysteine, a reactive thiol, is metalolized by remethylation (requiring MFFR, $B_{12}$ and folate). $B_{12}$ deficiency when coupled with MTFR mutations have been shown to produce marked elevations in homocysteine causing microangiopathic hemolysis in addition to marrow hemolysis. Our patient tested negative for MTFR mutations but the presence of $\mathrm{HbC}$ trait may have lowered the threshold for peripheral microangiopathic hemolysis triggered by the hyperhomocysteinemia. Normalization of laboratory parameters in our patient with $\mathrm{B}_{12}$ replacement supports this point.

MILKY MALIGNANCY Kate Hust. Tulane University Health Sciences Center, New Orleans, LA. (Control ID \#1339127)

LEARNING OBJECTIVE 1: 1. Recognize pleural effusion and its causes. 2. Understand Light's Criteria for exudative effusions. 3. Identify chylous effusion and its causes. 4. Understand appropriate treatment for chylothorax.

CASE: A 44 year-old woman with stage IV lung adenocarcinoma was admitted for dyspnea secondary to malignant ascites and malignant pleural effusion. Her breath sounds were decreased at both bases and throughout the right lung fields, where there was also dullness to percussion. An initial chest x-ray confirmed extensive right-sided and small left-sided pleural effusions. Symptoms initially improved after therapeutic removal of strawcolored fluid from the peritoneal and right-sided pleural cavities, but patient subsequently developed respiratory distress. After right-sided pleurodesis for recurrent effusion, dyspnea again improved but never completely resolved. Concurrently, chest x-rays showed worsening left-sided effusion, resulting in near-complete opacification of left lung field. Two liters of milky fluid were removed from left pleural cavity, and fluid analysis revealed LDH of 220 units/L and triglycerides of $1392 \mathrm{mg} / \mathrm{dL}$.

DISCUSSION: Pleural effusions often contribute to dyspnea, a complaint commonly faced by general internists. These occur when the absorption of pleural fluid is overwhelmed by the formation of fluid. It may be a disorder 
of excess pleural fluid creation or of decreased absorption. Pleural effusions may be a consequence of both systemic and local processes. Transudative effusions result from systemic diseases such as heart failure, cirrhosis, and nephrotic syndrome. Exudative effusions are caused by local processes including malignancy, infection, and collagen-vascular diseases. Less commonly gastrointestinal disease, medications, sarcoidosis, and chylothorax may lead to exudative effusion. To find the etiology of pleural effusion, it must first be classified as transudate or exudate. Light's Criteria indicate an effusion is exudative if one of the following are met: (1) ratio of pleural fluid protein to serum protein greater than 0.5 , (2) ratio of pleural fluid LDH to serum LDH greater than 0.6, or (3) pleural fluid LDH greater than two-thirds the upper limit of normal for serum LDH. When an exudate is identified, further studies to elucidate etiology should include glucose and amylase levels, cell count and differential, microbiology, and cytology. In the case of milky fluid, chylous effusion should be suspected and fluid analyzed for triglycerides; diagnosis is made with triglycerides greater than $110 \mathrm{mg} / \mathrm{dL}$. The first step in planning treatment of chylothorax is identifying the underlying cause. Chylothorax is often secondary to trauma when damage to the thoracic duct results in leakage of chyle into the pleural space. Non-traumatic chylothorax results from obstruction of the thoracic duct. Lymphoma is the primary non-traumatic cause of chylothorax though malignancy, mediastinal radiation, and congenital abnormalities may also contribute. Most important in treating chylothorax is treating its cause. Decompression with placement of chest tube alleviates symptoms but should not be used as long-term therapy because recurring chylous effusions can lead to immunologic incompetence and malnutrition. A low fat diet and bowel-rest with parenteral nutrition may also be used. Pleural effusions are commonly encountered by the hospitalist and understanding their large differential diagnosis is important in guiding the most efficient treatment.

MOTOR COMPLICATIONS OF HERPES ZOSTER Tania Calzada; Damian Casadesus; Manuel Vergara; Sarah Sordo. Capital Health Regional Medical Center, Trenton, NJ. (Control ID \#1327746)

LEARNING OBJECTIVE 1: Physicians must be aware that segmental motor paresis is a debilitating complication of herpes zoster even in immunocompetent patients.

LEARNING OBJECTIVE 2: A high level of suspicion should be maintained because prompt diagnosis and treatment are critical to minimize morbidity.

CASE: A 63 year-old male presented to the outpatient office complaining of a vesicular rash in the left groin. He had a history of diabetes mellitus, hyperlipidemia, hypertension and a resected squamous cell carcinoma of the skin. The rash was followed by severe low back pain and few days later by the extension of the rash on the left thigh and leg associated with weakness of that extremity. The pain was described as sharp and burning with hypersensitivity to touch in the back, thigh, groin, leg and gluteal regions. At physical examination, there was an extensive vesicular rash to the left groin, inner part of the proximal thigh, and the antero-medial part of the leg. Neurological examination showed weakness of the left lower extremity: hip flexion $2 / 5$, hip adduction $3 / 5$, knee extension $0 / 5$, with other muscle groups spared. All the deep tendon reflexes were absent in the left leg. There was hypersensitivity to touch in the anterior thigh, and decreased pinprick and temperature to the inner aspect of the thigh and left foot was noted. He needed support to ambulate and limped on the left side. He received treatment with acyclovir, pregabalin and physical rehabilitation, but the weakness has only improved slightly.

DISCUSSION: Segmental motor paresis develops in approximately $3 \%$ of patients with herpes zoster. Peripheral motor weakness is felt to result from spread of Varicella-Zoster virus from the dorsal root to the anterior horn. The onset of motor paresis is coincident with the development of pain and cutaneous eruption in a dermatomal distribution. Varicella-Zoster becomes latent in the cranial nerve and dorsal root ganglia and frequently reactivates decades later to produce a skin rash with the dermatome distribution of the affected nerve. Segmental motor paresis is a rare complication resulting in muscle atrophy; however, more than $75 \%$ of patients experience gradual recovery of motor strength with early diagnosis and treatment.
MULTIPLE MYELOMA, BUT NO MONOCLONAL PROTEIN ERI Jou; Radha Raghupathy. Montefiore Medical Center, Bronx, NY. (Control $\overline{\mathrm{ID}} \# 1334857)$

LEARNING OBJECTIVE 1: Diagnose multiple myeloma in the absence of monoclonal protein in the serum and urine.

LEARNING OBJECTIVE 2: Recognize the characteristics of nonsecretory myeloma.

CASE: A healthy 57 year old male presented with a four month history of right lateral chest wall pain that had worsened over one week. The pain was exacerbated by movement and was minimally responsive to ibuprofen. He also noted a 10 pound weight loss over 6 months but denied fevers or night sweats. Exam was significant for diffuse fullness with tenderness in the right lateral chest wall over the 4 th to 6 th ribs. Laboratory data on presentation was remarkable for hemoglobin of $10.9 \mathrm{gm} / \mathrm{dL}$ and creatinine of $1.6 \mathrm{mg} / \mathrm{dL}$. Calcium was $10.4 \mathrm{mg} / \mathrm{dL}$, total protein $7.3 \mathrm{~g} / \mathrm{dL}$ and albumin $5.1 \mathrm{~g} / \mathrm{dL}$. Chest radiography showed a large right extrapleural chest wall mass with destruction of the underlying 4 th rib and multiple bilateral rib fractures. Skeletal survey revealed multiple lytic lesions throughout all visualized bony structures. Monoclonal protein (M-protein) was not detected in the serum or urine by electrophoresis or immunofixation. Serum free light chains showed normal kappa at $5.9 \mathrm{mg} / \mathrm{L}$ and slightly decreased lambda at $3.5 \mathrm{mg} / \mathrm{L}$. Quantitative $\mathrm{IgG}$, IgA and $\mathrm{IgM}$ were significantly decreased at 413,16 and $8 \mathrm{mg} / \mathrm{dL}$ respectively. Bone marrow biopsy was performed and showed a hypercellular marrow diffusely replaced by sheets of atypical plasma cells. In the absence of M-protein or clonal light chains and the presence of clonal marrow plasmacytosis, a diagnosis of nonsecretory myeloma was made.

DISCUSSION: Multiple myeloma (MM) is often discovered by internists through routine bloodwork. It has an incidence of $4-5$ per 100,000 in the US and constitutes $1 \%$ of all cancers. MM is characterized by a proliferation of malignant plasma cells producing an overabundance of monoclonal immunoglobulin. The detection of this M-protein in the serum or urine is a major criterion for diagnosis. Patients present with signs and symptoms related to infiltration of plasma cells into the bone or other organs, or to kidney damage from excess light chains; common clinical features include anemia, bone pain, renal failure, and hypercalcemia. Nonsecretory myeloma is a rare variant of MM and accounts for $1-5 \%$ of all myelomas. It is characterized by the absence of a detectable M-protein or clonal excess of free light chains in the serum and urine. Defective immunoglobulin synthesis, increased intracellular proteolysis of synthesized immunoglobulins, defective immunoglobulin efflux mechanisms or rapid extracellular degradation of secreted paraproteins may contribute to the nonsecretory state. Clinical manifestations are similar to classic MM but renal failure is less common since there are no detectable light chains. Hypogammaglobulinemia is frequently seen due to failure of malignant plasma cells to synthesize normal immunoglobulins. Diagnosis is often delayed due to the absence of serum markers. Induction therapy and response rates for nonsecretory myeloma are similar to classic MM and consolidation with high dose melphalan and autologous stem cell transplant is offered to eligible patients. Assessment of response to therapy remains a challenge and reduction in marrow plasmacytosis should be demonstrated to confirm a symptomatic response. Our case is a rare presentation of nonsecretory myeloma with a chest wall plasmacytoma and mild renal failure, and emphasizes that the diagnosis of myeloma should not be overlooked in the absence of M-protein.

\section{MY CALCIUM IS DRIVING ME CRAZY! TREATMENT OF HYPERCALCEMIC ENCEPHALOPATHY IN A PATIENT WITH DECOMPENSATED HEART FAILURE Raquel Villavicencio. Indiana University Medical Group, Indianapolis, IN. (Control ID \#1311955)}

LEARNING OBJECTIVE 1: Managing hypercalcemic crisis in a patient with decompensated cardiomyopathy, fluid restriction, and vitamin D deficiency in today's dynamic healthcare setting.

CASE: Ms. $\mathrm{X}$ is a 26 year-old black woman with history of neuroblastoma status post irradiation and doxorubicin, who presented to the ED in heart failure. Echocardiogram revealed left ventricular ejection fraction of $22 \%$. Aggressive diuresis was initiated with IV furosemide leading to improved 
dyspnea and tachycardia. However, it was not long before her new, euvolemic state unveiled evidence of hypercalcemia, peaking at $12.9 \mathrm{mg} / \mathrm{dL}(8.5-10.5)$. Other lab values were as follows (normal reference ranges in parentheses): parathyroid hormone (PTH) $183 \mathrm{pg} / \mathrm{mL}$ (14-72); $25-\mathrm{OH}$ vitamin D $7 \mathrm{ng} / \mathrm{mL}$ (30-80); magnesium $0.9 \mathrm{mg} / \mathrm{dL}$ (1.3-2.7); phosphorus $2.9 \mathrm{mg} / \mathrm{dL}$ (2.4-5.1); and $\mathrm{Cr} 0.9 \mathrm{mg} / \mathrm{dL}(0.5-1.1)$. As her calcium rose, her mental status plummeted causing increased somnolence and a decline in speech and cognition. Treatment of Ms. X's hypercalcemia in the face of heart failure, fluid restriction, and low vitamin $\mathrm{D}$ posed a true management dilemma.

DISCUSSION: Labs were consistent with primary hyperparathyroidism as PTH was inappropriately elevated given her hypercalcemia. This was later confirmed by neck ultrasound showing a $2.1 \mathrm{~cm}$ hypoechoic structure posterior to the right thyroid consistent with parathyroid adenoma. Definitive treatment would be parathyroidectomy, however controlling her serum calcium in the short-term was of optimal concern. First line treatment of hypercalcemic crisis consists of administration of normal saline, with consideration of addition of furosemide only after any volume deficit has been corrected. Given her recently diagnosed decompensated cardiomyopathy, addition of fluids was contraindicated and any further furosemide would push her into a negative fluid balance and actually increase serum calcium instead of decreasing it. Bisphosphonates decrease calcium levels by inhibiting osteoclast activity and reducing bone resorption. This would be the next step in treatment; however, given the degree of vitamin D deficiency, there was concern for precipitation of hypocalcemia on a bisphosphonate. Nonetheless, the benefit outweighed the risk and the patient received one dose of pamidronate $30 \mathrm{mg} I V$ with subsequent decline of calcium to a level of $10.4 \mathrm{mg} / \mathrm{dL}$ coinciding with an improvement in cognition. She was also started on gentle vitamin D repletion with 2,000 units of cholecalciferol per day and scheduled for weekly calcium monitoring as an outpatient to detect potential hypocalcemia. In summary, severely hypercalcemic patients with low cardiac output and vitamin D deficiency should be considered for bisphosphonate therapy and followed closely thereafter for possible hypocalcemia. This type of management and follow-up requires good communication amongst physicians. As our health systems grow, so do the potential for errors. This complicated patient was cared for by multiple providers which could have resulted in confusion, delays in care, and catastrophic outcomes. However, through the collaboration of her inpatient team, consultants, surgeon, and primary care physician she was treated successfully and had a smooth transition to the outpatient setting.

MY TEETH WON'T LET ME EAT Ravi J. Patel; James M. Sosman. University of Wisconsin Hospitals and Clinics, Madison, WI. (Control ID \#1334808)

LEARNING OBJECTIVE 1: To recognize the differential diagnosis for a patient presenting with trismus.

LEARNING OBJECTIVE 2: To distinguish disease manifestations and review management of a patient with Tetanus.

CASE: A 78 year-old female presented to the ED by EMS after being found on the floor of her home by neighbors. She was found unresponsive with possible seizure-like activity. An oral airway was initially placed, but removed after improvement in her mental status. The patient lived in unsanitary conditions with a dirt floor and each room filled with hoarded items. In the ED, the patient complained "my teeth won't let me eat." She was hungry but stopped eating due to fear of choking. She also complained of weakness and sacral pain due to a fall. Her VS: BP 138/48, HR 84, RR 22, T 37.8 C. Her exam revealed episodes of interrupted speech due to clenching her jaw which lasted only a few seconds. She had no other neurologic deficits. She had no visible wounds, however her feet and toe-nails were caked with dirt. Her labs revealed WBC $12.0 \mathrm{~K} / \mathrm{uL}, \mathrm{CK} 2369$ (N 0-175), troponin 0.29 (N 0-0.05), Ca 9.1, BUN 60, Cr 1.6. EKG showed anterolateral T wave flattening and the CXR had RML infiltrates. Initial therapy included a heparin drip for possible NSTEMI and IV Ceftriaxone for aspiration pneumonia. The differential diagnosis included Tetanus, so she was begun on IV Metronidazole and Tetanus IG which required several hours to obtain sufficient doses from other health care facilities. Subsequent tests revealed a normal TTE and EEG negative for seizure-like activity. The patient's symptoms quickly progressed with frequent episodes of trismus along with apnea and hypoxemia. She also developed spastic contractions of her upper extremities, muscle rigidity, and 1 st and 2nd degree AV block with 5 second pauses. The patient was given muscle relaxants, and 3000 units of IM Tetanus IG. She refused intubation or CPR. She developed progressive apnea and bradycardia despite atropine and died within 48 hours. Her blood cultures revealed no growth.

DISCUSSION: Tetanus is a disorder caused by the toxin producing anaerobe Clostridium tetani. Now rare in the developed world, the CDC estimates an annual incidence of 1 per 10 million people in the US, and 2.3 per 10 million in those ages $>65$. The bacterium is ubiquitous in soil and remains a threat to all unvaccinated people. Infection occurs through penetrating injury with a foreign body, or within devitalized tissue. In $10 \%$ of cases, no cause is identified. C. tetani spores produce tetanospasmin that irreversibly binds to receptors of anterior horn cells. This results in autonomic instability, increased muscle tone, and severe spasms. Tetanus is a clinical diagnosis with $50 \%$ of cases presenting with trismus, a forceful spasm of the masseter muscle. Differential diagnosis includes Strychnine poisoning, drug-induced dystonias, neuroleptic malignant syndrome, and Stiff-person Syndrome. Treatment includes wound care and antibiotics to halt toxin production, neutralizing unbound toxin with Tetanus IG, sedatives to control spasms, and intubation with paralysis for weeks. Though the majority of patients survive with optimal management, mortality is as high as $50 \%$ in those who are treated conservatively. Unfortunately, our patient had not received health care or vaccinations for over two decades. This case underscores the importance of continued tetanus vaccination even during a time of advanced medical care.

MY EYES ARE DROOPING! Waleed T. Kayani ${ }^{1}$; Salman J. Bandeali ${ }^{1}$; Anam Khan ${ }^{2}$; Ali Hashmi ${ }^{1}$; Radha M. Rao ${ }^{1}$; Himabindu Kadiyala ${ }^{1}$. ${ }^{1}$ Baylor College of Medicine, Houston, TX; ${ }^{2}$ Aga Khan University, Karachi, Pakistan. (Control ID \#1339849)

LEARNING OBJECTIVE 1: Recognize the most common cause of acute Horner's Syndrome

LEARNING OBJECTIVE 2: Review internal carotid artery (ICA) dissection

CASE: A 53 year old Caucasian male with history of aortic valve replacement, hypertension, DM and hyperlipidemia who presented with complaints of drooping of the right eyelid for 2 days. He also noted some redness, tearing, and a deep pressure behind the right eye. Patient denied any history of trauma or falls. On examination, significant findings included conjunctival injection, miotic right pupil, $1 \mathrm{~mm}$ anisocoria in light and $2 \mathrm{~mm}$ anisocoria in dark. Mild ptosis of the right eye was noted which resolved after instillation of phenylephrine eye drops. Extraocular movements were intact bilaterally and no anhydrosis was observed. There were no carotid bruits appreciated. An emergent CT Angiogram was ordered which revealed dissection of right ICA as it entered the skull base. The patient was started on unfractionated heparin infusion. Any invasive intervention was deferred by neuroradiology given the location of his dissection and risk of extension. He continued to improve as an inpatient and was discharged 2 days later. His only complaint on discharge was mild eye pressure, and only exam finding, residual conjunctival injection. He was advised to avoid any heavy lifting and observe strict control of hypertension and diabetes. He was bridged to warfarin. His eye symptoms gradually resolved and the patient continues to do well.

DISCUSSION: Horner's syndrome, also called oculosympathetic paresis is characterized by miosis, anhydrosis and ptosis. Causes range from benign to serious, requiring a methodological approach to diagnostic evaluation. Acute Horner's syndrome with neck or facial pain should be presumed to be caused by carotid dissection until proven otherwise. Around 50 percent of patients with internal carotid artery dissections present with an isolated painful partial Horner's syndrome. Partial Horner syndrome is used because anhidrosis is absent. The sympathetic fibers innervating the facial sweat glands are anatomically located on the external rather than internal carotid artery; thus, anhidrosis is not a finding in the setting of internal carotid dissection. Patients often have an antecedent history of neck trauma, but this can be subtle, and a number of carotid dissections are spontaneous events. Patients with acute carotid dissection are at a high risk for cerebral infarction, which usually occurs within days or few weeks after onset of the Horner's syndrome. An axial MRI of the neck with T1-weighted, fat-suppressed sequences and magnetic resonance 
angiography (MRA) or CT scan with CT angiogram will detect most internal carotid artery dissections. However, conventional angiography remains the gold standard. Patients with ICA dissection, who have neurologic symptoms, should be treated with anticoagulation, using unfractionated heparin followed by warfarin for six months. At that time, as long as symptoms are not recurrent and the arterial lesion is thrombosed or healed, warfarin can be stopped and long-term antiplatelet therapy initiated. Transcranial Doppler, duplex Doppler, CT angiography, and MR angiography can help decide the status of the arterial system prior to discontinuing therapy.

NAIL PATELLA SYNDROME (NPS): AN INTERNIST'S CHALLENGE Bhavana Siddegowda Bangalore; Parul Sud. Mclaren Regional Medical Center, Flint, TN. (Control ID \#1339960)

LEARNING OBJECTIVE 1: Recognize the diagnostic features of NPS, a rare congenital syndrome.

LEARNING OBJECTIVE 2: Recognize the co-morbidities associated with NPS.

CASE: A 46 year old Caucasian female with a diagnosis of NPS presented with chronic generalized pain and intermittent numbness of the left arm. Past medical history included club feet at birth, multiple corrective joint surgeries and irritable bowel syndrome (IBS). Family history was significant for NPS in father and sons. She denied smoking or drinking. Patient was thinly built with a high forehead and fine hair. Her gait and posture were normal. She had bilateral dystrophic thumb nails, triangular lunules in finger nails, absence of dorsal skin crease on index fingers, hypoplastic patellae, iliac horns, club feet and orthopedic surgical scars. Elbows had limited extension. She exhibited 18/18 painful trigger points. Pertinent lab results included albumin to creatinine ratio of 21, osteoporosis on bone mineral density study and left ulnar neuropathy on electromyography/nerve conduction studies. Pain persisted despite multiple medications including narcotics, muscle relaxants, steroid injections at trigger points, physical therapy and counseling. Extreme emotional stress resulted from her conviction that she would be wheelchair bound

DISCUSSION: NPS consists of a clinical tetrad of hypoplastic or absent patellae, dystrophic nails, dysplastic radial heads, and iliac horns. Prevalence is estimated at one in 50,000. It is a rare autosomal dominant disorder with mutations of LMX1B gene of the distal end of the long arm of chromosome 9 . This gene is necessary for dorso-ventral patterning of limbs. This explains the pattern of decreasing severity of dystrophic changes in nails marching from lateral to medial. This is purely a clinical diagnosis. Nail features are present in $98 \%$ of patients, primarily a symmetric pattern of dystrophic or absent nails. Triangular lunules in finger nails are considered diagnostic. Loss of dorsal distal digital crease is a sensitive $(96 \%)$ sign. About $75 \%$ of patients have absent or hypoplastic patellae. Dislocation or subluxation of knee joints is common. Iliac horns, bilateral conical bony processes at the anterior-superior iliac crest most commonly noted on $\mathrm{x}$-ray, is sine qua non of NPS. Fibromyalgia (FM) and decreased bone density are reported co-morbidities. Open angle glaucoma, IBS, entrapment neuropathies and depression may also coexist. Renal involvement occurs in up to $25 \%$ of patients, is sporadic and may result in renal failure, which is associated with a poor prognosis. Renal transplant, however, carries a favorable outcome. Management recommendations include annual screening for proteinuria, hypertension, and monitoring for glaucoma. Genetic counseling should be offered to all patients. The management challenge in our patient was pain control. Patients with NPS experience joint and ligamentous pains, however, our patient's pattern of pain met clinical criteria for FM. Anchoring heuristics of the patient and lack of physician experience led to an overemphasis on NPS, thus delaying identification and treatment of FM. Our patient eventually accepted her dual diagnosis of NPS and FM, resulting in improved functionality and pain control. Management barriers in NPS result from its rarity, compounded by physicians' lack of knowledge and experience. Patients and providers should be aware of anchoring heuristics which may cloud clinical judgment especially when dealing with rare disorders.

NECROTIZING PULMONARY SARCOIDOSIS MASQUERADING AS SUPERFICIAL THROMBOPHLEBITIS John Matulis. DartmouthHitchcock Medical Center, Lebanon, NH. (Control ID \#1334933)
LEARNING OBJECTIVE 1: Recognize and manage superficial migratory thrombophlebitis

LEARNING OBJECTIVE 2: Identify a rare extra-pulmonary manifestation of sarcoidosis

CASE: Sarcoidosis is a multi-system inflammatory disease of unknown etiology which predominantly affects the lungs and intra-thoracic lymph nodes; it's hallmark is the presence of non-caseating granulomas in affected tissue. Sarcoidosis has been documented to initially present with a variety of symptoms and is often a challenging diagnosis. Here I document an unusual presentation of pulmonary sarcoidosis. A 72 y/o caucasian female presented with non painful, slightly pruritic raised lesions on her bilateral lower extremities. She was referred to dermatology and a biopsy was taken which showed superficial venous thrombosis consistent with a migratory thrombophlebitis. Labarotory testing at that time revealed normal blood counts, chemistries, ANA, thrombosis panel, ENA, ANCAS, SPEP, ACE level, CA 19-9 and CA-125. Her CRP was 24.1 and her ESR was 32. A CT of the chest and abdomen revealed circumferential thickening of the ascending colon, scattered enlarged mesenteric lymph nodes and non-specific prominent right paratracheal lymph nodes. A mammogram and colonoscopy were unrevealing and a BAL was nondiagnostic. Endobronchial ultrasound guided mediastinal lymph node biopsy revealed dense, lymphoid tissue without evidence of malignancy or connective tissue disease. The patient's symptoms remained stable and further workup was deferred. A few months later repeat CT scans showed no progression of the lymphadenopathy, and she began treatment for her thrombophlebitis with ibuprofen, colchicine and plaquenil with partial response. The patient remained symptomatically well for an additional 6 months before presenting with several weeks of progressing dyspnea; she was found to have an oxygen saturation of $85 \%$ and new bi-basilar crackles. Repeat $\mathrm{Ct}$ scan showed extensive pulmonary nodules throughout her lung fields with her pre-tracheal adenopathy remaining stable. Open lung biopsy and VATs procedure was performed and tissue histology was diagnostic of necrotizing sarcoidosis. She was started on high dose steroids and seen in follow-up with improvement, but not resolution of her symptoms. Her superficial thrombophlebitis is largely resolved since initiation of steroid therapy.

DISCUSSION: This case illustrates the presentation and workup of superficial migratory thrombophlebitis as well as an unusual presentation of occult pulmonary sarcoidosis. The key teaching point in this case is the workup of superficial migratory thrombophlebitis. Recent reviews have shown that isolated, superficial thrombophlebitis is much less commonly a presentation of occult malignancy than previous thought (between 2 and $5 \%$ ). It can generally be managed in a more conservative manner and will most often resolve without further adverse sequelae. In this case, Sarcoidosis remained on the differential diagnosis throughout her evaluation, but the unusual presentation made her case perplexing. It is unclear whether sampling other sites of inflammation or lyphadenopathy would have made for a more timely diagnosis, but awareness of unusual presentations of connective tissue diseases can certainly be helpful in determining sequence of diagnostic testing. In this case, Rheumatological laboratory values were also difficult to interpret, illustrating their limited utility in making a diagnosis.

\section{NEUROINVASIVE WEST NILE VIRUS: RAPIDLY INCREASING} IN PREVALENCE BUT RARELY PART OF THE DIFFERENTIAL Daniel Gutteridge; Bhavana Siddegowda Bangalore. McLaren Regional Medical Center, Flint, MI. (Control ID \#1333634)

LEARNING OBJECTIVE 1: Recognize the increasing prevalence of neuroinvasive West Nile virus.

LEARNING OBJECTIVE 2: Diagnostic evaluation in the suspicion of neuroinvasive West Nile virus.

CASE: A 58 year old Caucasian female with no significant past medical history was found lying in her bathroom by EMS and was brought to the emergency room. In the ER patient was alert, oriented and cachectic. Patient apparently had flu-like symptoms three days before presentation and was suspected to have been unresponsive for more than 14 hours. There was multi-organ failure at presentation with rhabdomyolysis (CPK: 14000), acute kidney failure, leukocytosis, elevated troponins and elevated liver enzymes. She was intubated later for respiratory distress but chest $\mathrm{x}$-ray 
was negative for infiltrates. Initially treatment was directed at septic inflammatory response syndrome. Post-extubation on third day patient was found to have profound proximal and distal muscle weakness, greater in the lower limbs, areflexia and mildly reduced vibratory sense in the lower limbs. Computed tomography of the head and MRI of the brain and spinal cord demonstrated no acute findings. Lumbar puncture was positive for IgG and IgM antibodies for West Nile virus. Patient progressively improved on supportive treatment and was transferred to a long-term inpatient rehabilitation unit.

DISCUSSION: In the past decade a new infectious disease with serious consequences has rapidly spread across the United States. West Nile virus (WNV) started raising some red flags in 2000 when 19 cases of neuroinvasive disease were reported in New York. Over 11,000 confirmed cases have been identified in 48 states since then. Neuroinvasive WNV cases represent $41 \%$ of all documented WNV disease and both are believed to be strongly under-reported. The challenge has been the non-specific presentation with symptoms ranging from flu-like symptoms to flaccid paralysis and altered levels of consciousness. Diagnosis is suspected based upon thorough history and physical exam, then confirmed by finding IgM antibody in cerebrospinal fluid and radiologic findings of signal intensity abnormalities on magnetic resonance imaging (MRI). Initial presentation of a patient in this manner forces a physician to explore a broad differential diagnosis. Difficulty lies in the fact that it takes time to obtain immunologic testing results for a growing, but still uncommon, disease. The lack of typical radiologic findings can further delay treatment. When working up a patient with new onset severe neurologic deficits and no clear diagnosis, WNV should be in the differential diagnosis due to its epidemic prevalence over the past 10 years.

NEUROSARCOIDOSIS: MORE THAN MEETS THE EYE Lauren Chan; Priscilla Yee. CPMC, San Francisco, CA. (Control ID \#1340126)

LEARNING OBJECTIVE 1: Cranial neuropathy is the most common presentation of neurosarcoidosis given that it is primarily a clinical and pathologic diagnosis.

LEARNING OBJECTIVE 2: MRI plays a supportive role in the diagnosis of neurosarcoidosis and should be used as an adjunctive diagnostic technique. It may be helpful in following regression of disease with steroid treatment.

CASE: 41 year old African American male presents with one month of headache and several weeks of diplopia, L ptosis, L periorbital/maxillary "stinging" pain, diaphoresis of the forehead, with worsening of these symptoms one week prior to presentation. He also noted an unintentional twenty pound weight loss over 2 months. On exam the patient had stable vitals, normal fundoscopic exam without lymphadenopathy or papilledema. His exam was notable for $\mathrm{L}$ ptosis, inability to adduct the $\mathrm{L}$ eye medially or gaze upward, dilated L pupil, paresthesia and decreased sensation over $\mathrm{L}$ forehead and cheek. Basic labs, Quantiferon, induced sputum cultures for AFB and fungal, HIV, RPR, myasthenia gravis antibodies were all negative. ACE was elevated at 96 and LP revealed a lymphocytic predominance with elevated protein and no malignant cells or organisms. CT chest/abdomen/pelvis showed mediastinal and pretracheal lymphadenopathy and bilateral upper lobe interstitial infiltrates. MRI brain showed a mass in L Meckel's cave with extension to the $\mathrm{L}$ cavernous sinus. Nasal endoscopy ruled out infection of sinuses. Brain biopsy was deferred in favor of transbronchial lung biopsy which demonstrated non-necrotizing micro-granulomas highly suggestive of sarcoidosis. The patient was started on Decadron with resolution of symptoms within a couple days of treatment.

DISCUSSION: Neurosarcoidosis is primarily a clinical and pathologic diagnosis and appears in about 5\% of patients with sarcoidosis. This diagnosis relies heavily upon the clinical findings especially when brain biopsy presents significant risk. Clinicians should recognize common presentations of neurosarcoidosis such as cranial neuropathies which occur in up to $50 \%$ of patients with neurosarcoidosis. Facial nerve palsy is the most common. Other presentations include encephalopathy, peripheral neuropathy, meningitis, seizure, spinal cord dysfunction, and myopathy. In this case, the patient presented with findings consistent with $\mathrm{CN}$ III and $\mathrm{V} 1 / \mathrm{V} 2$ palsies. A lymphocytic predominance with protein elevation in the CSF was suggestive for neurosarcoidosis but not specific. The findings of mediastinal lymphadenopathy and bilateral upper lobe infiltrates, elevated $\mathrm{ACE}$, congruent $\mathrm{CSF}$ profile, lung biopsy showing non-necrotizing microgranulomas, and rapid resolution of neurological findings with steroid treatment strongly favored the diagnosis of neurosarcoidosis. While imaging defined a cavernous sinus mass and identified the mediastinal lymphadenopathy and bilateral infiltrates suggestive of sarcoid, the MRI was not the definitive diagnostic technique. The literature suggests there is poor correlation between imaging findings and clinical symptoms. About $40 \%$ of symptomatic cranial nerve deficits are not visible on imaging and around $40 \%$ of cranial nerve lesions seen on imaging are asymptomatic. Depending upon the location of the lesion, MRI may demonstrate resolution of enhancement with resolution of symptoms. Clinical and imaging improvements after steroid treatment are most likely to correlate in cranial nerve and spinal lesions, and less likely in dural and parenchymal lesions.

NON-OPERATIVE MANAGEMENT OF STREPTOCOCCAL HEPATIC ABSCESS Elizabeth Selden; Andrew A. Chang. New York University, New York, NY. (Control ID \#1321104)

LEARNING OBJECTIVE 1: Identify indications for surgical intervention in the case of hepatic abscesses

LEARNING OBJECTIVE 2: Review appropriate management of new pleural effusions

CASE: A 56 year-old woman presented with weight loss, malaise and polyuria for two months. On admission, HR 135, BP 107/80, RR 22, T 101, O2 $94 \%$ on RA. Exam revealed cachexia, bilateral lower extremity edema, decreased breath sounds in lower half of right lung with decreased tactile fremitis and coarse crackles superiorly. Labs were notable for WBC 33 and glucose 559. EKG showed sinus tachycardia. CXR revealed a right pleural effusion. Patient was pan-cultured and empirically started on vancomycin, piperacillin/tazobatam and azithromycin for presumed pneumonia. Despite insulin and IV fluids, she remained tachycardic. CT angiogram revealed a large pulmonary embolus in the left basal pulmonary artery and a large loculated right pleural effusion with no pleural enhancement. Abdominal CT revealed $8 \mathrm{~cm}$ septated hepatic mass, hepatic vein thrombus extending into the IVC, and a small amount of air in the wall of the gallbladder. Heparin drip was started and patient was taken for percutaneous drainage of hepatic mass and thoracentesis. Only small samples were obtained given loculation. Pleural fluid was exudative, gram stain showed white cells but no organisms, $\mathrm{pH} 7.22$, glucose 169. Chest tube was placed. Strep viridans grew from pleural and hepatic fluid; blood cultures grew strep anginosus and c. albicans. Antibiotics were narrowed to ceftriaxone and caspofungin. Given pneumobilia, hepatic abscess was presumed to be from GI source with empyema formation from local spread of infection and hepatic vein thrombus from local inflammation. EGD/colonoscopy failed to find communicating track between bowel and biliary systems. Surgical intervention was deemed risky given large clot burden and patient was managed with antibiotics alone. Repeat imaging two months later at discharge showed significant resolution of hepatic and pulmonary collections. She completed five weeks of ceftriaxone followed by four weeks of oral ciprofloxacin and a total of six weeks of fluconazole. She continues to do well without recurrence of sepsis.

DISCUSSION: Strep anginosus, a subgroup of strep viridans, lives in the normal flora of the GI tract. It often presents in polymicrobial infections and produces a cytolytic toxin that is a virulence factor for deep-seated infections which are most commonly seen in the head, neck and abdomen. Traditionally, solitary liver abscesses have been managed with surgical or percutaneous drainage. Arenas-Jimenez et al. demonstrated that antibiotics alone are safe in stable patients with hepatic abscesses, but those with signs of sepsis should be managed with drainage. This case represents an example of successful management of pyogenic liver abscess with antibiotics alone despite sepsis. On admission, CT revealed pleural effusion, which, per radiology, was not suggestive of empyema given lack of pleural enhancement. However, thoracentesis done nearly 24 hours later revealed a complicated parapneumonic effusion bordering on empyema. This raises the question of whether CT can exclude empyema. Studies show that although there are typical findings on CT that suggest empyema - pleural thickening and extrapleural fat enhancement lack of these findings cannot rule out empyema. Any new pleural effusion with fever should prompt clinicians to perform immediate thoracentesis to rule out empyema regardless of radiologic findings. 
NOT ALL IT'S CRACKED UP TO BE: A NEW PUBLIC HEALTH CAMPAIGN Noelle Northcutt ${ }^{2}$; Ramya Mishra ${ }^{2}$; Colleen Barry ${ }^{2}$; Katarzyna Mastalerz ${ }^{1}$; Mary Maher ${ }^{1}$; Jessica Campbel1 ${ }^{1} .{ }^{1}$ Denver Health, Denver, CO; ${ }^{2}$ University of Colorado Anschutz Medial Campus, Aurora, CO. (Control ID $\# 1340113$ )

LEARNING OBJECTIVE 1: Diagnose the clinical manifestations of levamisole-associated thrombotic vasculitis.

LEARNING OBJECTIVE 2: Raise awareness about a growing substanceabuse related public health topic

CASE: A 41-year-old female with a history of substance abuse presented to the Emergency Department with one month of bilateral lower extremity rash and severe pain. On arrival patient was noted to have altered mental status and met SIRS criteria of fever and tachycardia. She was also found to be leukopenic with an ANC of 1600. Initially, the rash was purpuric with stellate lesions and associated erythematous borders. A few of the lesions were bullous and necrotic. A poorly demarcated erythematous macule was noted on the right ear. Workup yielded normal renal function, computed tomography negative for acute thrombotic stroke, no sign of infection, and urinalysis with trace protein with no evidence of hematuria. P-ANCA was noted to be strongly positive at a titer of 1:2650 and polyreactive to MPO Ab and PR3. Vancomycin was empirically started for suspicion of MRSA super-infection. Supportive care was provided with volume resuscitation, anti-pyretics, and wound care. Thigh skin biopsy histopathology taken during a similar presentation to an outside hospital three months prior revealed cocaine microangiopathy, or levamisoleassociated thrombotic vasculitis. On this admission, her urine drug screen was positive for cocaine metabolites and patient admitted to using the day prior to presentation

DISCUSSION: This is the 9th confirmed case of levamisole-associated thrombotic vasculitis at our hospital. Levamisole was historically used as an immunomodulator in rheumatoid arthritis and colon cancer until its withdrawal from the US market in 2000 due to agranulocytosis. The synthetic imidazothiazole derivative continues to be used today as an effective antihelminthic in veterinary medicine, and outside the US for childhood nephrotic syndrome. In 2010, it was found as a contaminant in $78 \%$ of cocaine at this safety net hospital. The clinical presentation of levamisole-associated thrombotic vasculitis includes characteristic retiform purpura with propensity for pinna involvement, high-titer ANCAS reactive to multiple target antigens, and neutropenia is common. Treatment for this condition is not well established and remains largely supportive. Unless targeted efforts to eradicate levamisole laced cocaine are undertaken, cases like this may become a common presentation of cocaine abuse. Epidemiologic data for the Midwest and Western US indicates increasing prevalence of levamisole-adulterated cocaine. Unfortunately, cocaine and crack use is the second-most common illicit drug-associated cause of ED visits in the Denver metro area. There is need for a robust public health campaign targeted at cocaine users regarding this life-threatening and potentially deforming drug related complication

NOT CRYING WOLF: AN ATYPICAL MANIFESTATION OF SYSTEMIC LUPUS ERYTHEMATOSUS Alexis Eastman; Khin Mae Hla. University of WI Hospitals and Clinics, Madison, WI. (Control ID \#1339586)

LEARNING OBJECTIVE 1: Identify an uncommon cutaneous complication of systemic lupus erythematosus (SLE)

LEARNING OBJECTIVE 2: Diagnose and treat lupus erythematosus profundus

CASE: A 56 year-old African-American female with well-controlled SLE on hydroxychloroquine and low dose prednisone presented to clinic with a new right volar forearm mass. Initially, the mass was $1.5 \mathrm{~cm}$ in diameter, mildly tender, without fluctuance or skin changes. She denied fevers, chills, night sweats, weight loss, or trauma. An ultrasound was ordered which showed non-specific findings of subcutaneous edema and fat globules, with a broad differential concerning for infection, post-traumatic ossification, or malignancy. A week later, the mass had increased to $4 \mathrm{~cm}$ with tenderness, a small fluctuant area, erythema and accompanying daily subjective low-grade fevers. An MRI was obtained and was suggestive of an infectious or inflammatory process with no drainable fluid collection. She was started on empiric antibiotics, and surgical biopsy was obtained. Pathology showed a benign dense lymphoplasmacytic infiltrate involving the dermis and subcutis, rare perivascular fibrinoid necrosis, consistent with lupus erythematosus profundus. In the interim, her fevers had resolved and the mass had become non-tender. Her hydroxychloroquine was continued, antibiotics were stopped, and the lesion subsequently resolved.

DISCUSSION: Cutaneous manifestations of systemic lupus erythematosus (SLE) are common, occurring in $75 \%$ of patients with SLE. The majority of these are discoid lupus, of which lupus erythematosus profundus (LEP) is a variant. LEP is usually found prior to or independent of SLE (10-42\% of cases); only $2-5 \%$ of patients with pre-existing SLE will develop LEP. It is more prevalent in women, with a 2-9:1 female:male ratio. It presents asymmetrically on the face, proximal extremities and trunk as a tender subcutaneous nodule that resolves with subsequent lipoatrophy and skin depression. Presentation on the distal extremities is unusual. The differential includes infection and malignancy. Patients with SLE are at increased risk of infection from underlying leukopenia and immunosuppressive medications. Historically, infection is one of the leading causes of death in SLE, especially in patients with early SLE (less than 2 years). Given this, infectious processes must always be ruled out. Surgical biopsy is the standard diagnostic tool. Histologically, LEP shows a mostly lobular dense infiltrate of lymphocytes and morphologically unremarkable plasma cells, dense perivascular and periappendigeal lymphocytic infiltrate in the dermis. Subcutaneous panniculitis-like T-cell lymphoma can also closely mimic LEP, and can be difficult to differentiate histologically: recent case series found significant overlapping findings. Thus, if patients present atypically with subcutaneous masses, it is important for primary care givers to be aware of LEP and the possibility that the findings are actually an evolving lymphoma. The treatment of choice for LEP is hydroxychloroquine for both acute and maintenance therapy. Intralesional corticosteroids may worsen the eventual lipoatrophy, and should be avoided.

NOT ALL BREAST MALIGNANCIES ARE BREAST CANCER: SMALL CELL LUNG CARCINOMA METASTASIS TO THE BREAST. Laura E. Paletta; Kimberly Pedram. VCU, Richmond, VA. (Control ID \#1337439)

LEARNING OBJECTIVE 1: 1. Recognize when a chronic cough warrants further work-up

LEARNING OBJECTIVE 2: 2. Understand that extra-mammary breast metastases are rare cause of breast masses.

CASE: A 48 year-old female smoker with no significant history presented with a chronic productive cough for 2 months and a mass in her left breast. She was initially treated with cough suppressants and an antibiotic but her cough persisted. She denied a history of fevers, chills, night sweats or hemoptysis. She reported that her breast mass was without tenderness or nipple discharge. Her physical exam was notable only for diffusely coarse breath sounds and a 2centimeter, firm mass in the upper outer quadrant of her left breast. Laboratory evaluation was unremarkable. An x-ray and later chest CT revealed a large right hilar mass and mediastinal nodal extension highly suspicious for malignancy with evidence of a post-obstructive pneumonia as well as left breast mass. Biopsies from the paratracheal and hilar mass obtained with endobronchial ultrasound revealed small cell carcinoma. Needle biopsy of the breast mass also demonstrated small cell carcinoma.

DISCUSSION: Chronic cough is a common complaint encountered by a general internist. A cough that persists for longer than eight weeks without known cause needs to be worked up further with imaging. While it is a concern, lung cancer is the cause of cough in less than $2 \%$ of patients presenting with a chronic cough. In this patient, the laryngeal nerve irritation caused by mediastinal small cell lung carcinoma was the cause of her presenting symptom. Metastatic lesions of the breast are also rare, accounting for $0.4-6.6 \%$ of all breast malignancies. The most common cancers to metastasize to breast are malignant melanoma and leukemia/lymphoma. Others that have been reported include carcinoma of the kidney, stomach and lung. These patients usually present with a small, painless lump in the upper outer quadrant in contrast to the pain, tenderness and nipple discharge commonly seen with a primary breast malignancy. In addition, metastases to 
breast will often spare the adjacent skin architecture and often do not show characteristic calcification or spiculation on mammogram. The majority of these patients have a previously diagnosed non-mammary malignancy; in this patient the lung mass and breast mass were discovered concomitantly. The prognosis for patients with cancer metastasized to the breast is poor; it is reported that $80 \%$ of these patients will die within one year. It is not uncommon for general internists to make the first diagnosis of breast malignancy. In this patient, it was fortuitous that she presented with a chronic cough that prompted an X-ray and CT. Otherwise, her primary malignancy may have been missed.

\section{NOT ALWAYS MOANS, GROANS AND BONES: RECOGNIZING} THE DIFFERENT FACES OF HYPERCALCEMIA. Maliha I. Jumani; Ioannis Politikos; Arooj Hyat. Mount Auburn Hospital, $\overline{\text { Cambridge, MA. }}$ (Control ID \#1309745)

LEARNING OBJECTIVE 1: Emphasize the importance of a thorough history while evaluating patients with common presentations.

LEARNING OBJECTIVE 2: Recognize the resurgence of milk alkali syndrome as a cause of hypercalcemia.

CASE: A 53 year old male with past medical history of obesity, type 2 diabetes, hypertension, atrial fibrillation and chronic kidney disease presented to clinic with three days of vomiting and decreased oral intake. He was noted to be slightly hypotensive and was sent to the ED. On arrival at the ED, patient's vital signs were notable for blood pressure 93/60. His physical exam revealed clear lungs, normal heart sounds and abdominal exam showed no tenderness, guarding or rigidity. The initial diagnosis was gastroenteritis and patient was started on IV hydration, zofran and pantoprazole. His labs were notable for creatinine of 2.6 and calcium of 15.4. Upon further questioning, patient revealed that he had eaten at a fast food restaurant 5 days ago and developed significant heartburn; subsequently he had started taking calcium carbonate tablets six to seven times a day for relief of his symptoms. Two days after this he developed intractable vomiting and anorexia. In light of this information, the patient was diagnosed with hypercalcemia secondary to milk alkali syndrome and acute on chronic renal failure. PTH level was also checked during hospitalization and was noted to be 4.2, further supporting the diagnosis. The patient was given IV fluids and his calcium level was monitored. Upon discharge his calcium level had normalized at 10.2 and creatinine returned to his baseline of 1.4.

DISCUSSION: Our patient had a common clinical presentation of persistent vomiting and resultant hypotension. These symptoms were initially perceived to be due to gastroenteritis. It was only after a careful history was taken with reference to the chronology of events and lab work became available that it became apparent the cause of his symptoms was hypercalcemia due to milk alkali syndrome. This demonstrates the importance of a thorough history especially in the evaluation of common clinical presentations. Although in recent times patients with milk alkali syndrome are noted to be asymptomatic and findings of hypercalcemia and acute renal failure are incidental, our patient presented with the classic symptoms of the acute form of this syndrome including nausea and vomiting. The milk alkali syndrome was originally seen in association with the use of milk and sodium bicarbonate for treatment of peptic ulcer disease. Its incidence dropped significantly with the initiation of modern day treatment for peptic ulcer disease. The syndrome re-emerged in the 1990s with the increased use of over-the-counter antacids and is now the third leading cause of hypercalcemia after primary hyperparathyroidism and malignancy. The modern presentation differs from the classical presentation in that the patients are often asymptomatic, hypophosphatemia is common (due to lack of the phosphate load caused by milk drinking and the phosphate binding properties of calcium carbonate) and hypocalcemia may develop as a result of therapy.

NOT EVERY FECAL INCONTINENCE AND ABDOMINAL PAIN IS FROM GI TRACT SOURCE: A CASE OF GIANT LAMINATED BLADDER STONE Muhammed Sherid; Salih Samo; Samian Sulaiman; Meenu Singh. University of Illinois at Chicago, St.Francis Hospital, Evanston, IL. (Control ID \#1314361)

LEARNING OBJECTIVE 1: To understand the pathogenesis and clinical manifestations of bladder stones
LEARNING OBJECTIVE 2: To recognize this uncommon disorder in the appropriate clinical settings

CASE: A 75 year old man presented with a two week history of fecal incontinence while urinating, associated with severe suprapubic abdominal pain. The fecal incontinence occurred while urinating in both the sitting and standing positions. The patient had a history of urinary frequency, urgency and suprapubic abdominal discomfort for duration of one month. He denied any history of diarrhea, constipation, nausea, vomiting or fever. He was treated with two courses of antibiotics without improvement. Past medical history is significant for removal of bladder stones two times, thirteen and ten years ago (the size of stone was more than $5 \mathrm{~cm}$ both the times). He was diagnosed with benign prostatic hypertrophy three years ago. Social history: $\mathrm{He}$ is from Belize in Central America and works as a teacher. No family history of kidney or bladder stones. On physical examination, he was afebrile with normal vital signs. Cardiopulmonary examination was unremarkable. Abdomen was soft. A firm mass was palpated in the suprapubic area associated with mild tenderness. He also had tenderness in the right costovertebral angle. Rectal exam revealed normal sphincter tone; prostate was enlarged with no nodules or masses. An attempt at urethral catheterization failed. Laboratory studies showed hematuria and pyuria with positive nitrite and leukocyte esterase. Urine culture grew Escherichia Coli. Complete blood count and comprehensive metabolic panel were unremarkable except for mild elevation of creatinine. CT scan of the abdomen and pelvis showed a large radioopaque laminated bladder stone measuring $8 \times 7 \times 6 \mathrm{~cm}$. Bilateral hydronephrosis was noted as well. Patient underwent an open cystolithotomy with removal of the bladder stone which weighed one kilogram. The analysis showed uric acid $80 \%$ and calcium oxalate $20 \%$. After surgery, his symptoms, bilateral hydronephrosis, and acute renal failure all resolved. The patient had an uneventful hospital course and was advised to increase his oral fluid intake and get six monthly ultrasound examinations.

DISCUSSION: Bladder stones occur in adult men in the vast majority of cases. They are usually secondary to bladder outlet obstruction. However, in some patients they originate from the upper urinary tract and migrate into the bladder where they grow after additional deposition of crystals. The incidence of bladder stones has been declining in developed countries while it still remains common in developing countries. The majority of these stones are composed of uric acid. Calcium oxalate, phosphate and struvite stones are next in frequency.

NOT EVERY GRANULOMA IS SARCOID: PULMONARY TALCOSIS CAUSED BY BABY POWDER IN ADULT. Chanunya Srihawan; Sashank Kolli; Soamsiri Niwattisaiwong; Thomas Liao. Advocate Illinois Masonic Medical Center, Chicago, IL. (Control ID \#1335063)

LEARNING OBJECTIVE 1: Importance of history taking for the diagnosis of pulmonary talcosis.

CASE: A 63 year-old-male, nonsmoker, was referred to the pulmonary consult service for evaluation of multiple bilateral lung nodules incidentally found on a computed tomography (CT) scan of the abdomen during a workup for abdominal pain. The patient had no respiratory symptoms. Both lungs were clear to auscultation. The rest of physical examination was unremarkable. Subsequent CT scan of the chest revealed ill-defined spiculated nodules scattered throughout both lungs, most pronounced in upper lobes. The largest nodule measured 1.5 to 2 centimeters. Sarcoidosis was considered and bronchoscopy discussed, but patient opted for observation. The patient was followed with serial CT scans and after four years of follow-up, patient still had no respiratory symptoms. However, the follow-up CT scan of the chest eventually showed a subtle increase in size of confluent nodules associated with architectural distortion and bronchiectasis. Pulmonary function testing revealed a moderate obstructive pattern, slightly responsive to bronchodilators. On bronchoscopy, bronchoalveolar lavage was negative for mycobacterium, fungus and other infectious organisms. Transbronchial biopsies revealed non-caseating granulomas with refractile particles, which raised our suspicion for non-infectious causes of non-caseating granulomas. A detailed history revealed the use of baby talcum powder in a small, non-ventilated room everyday for at least 10 years. The diagnosis of pulmonary talcosis was made based on the history, radiological, and histological findings. The patient was advised to stop using baby powder to prevent further progression of disease. 
DISCUSSION: Pulmonary talcosis should be in the differential diagnosis of generalized lung nodules. This condition is usually either underdiagnosed or misdiagnosed as more common granulomatous lung diseases because of unrecognized talc exposure history and non-specific radiologic findings. Modifying patient's behavior by discontinuation of talc exposure will prevent disease progression to an irreversible obstructive or restrictive pulmonary disease.

NOT FOR HUMAN CONSUMPTION Eric Edwards. University of North Carolina School of Medicine, Chapel Hill, NC. (Control ID \#1291853)

LEARNING OBJECTIVE 1: Recognize that the abuse of "bath salts" is a rapidly growing problem.

LEARNING OBJECTIVE 2: Recognize the signs and symptoms of bath salts intoxication.

CASE: A 49 year-old man presented with a one day history of chest pain as well as visual and auditory hallucinations. He denied past medical history and medication use. He admitted to binge drinking and smoking marijuana. He was mildly tachypneic, but physical examination was otherwise unremarkable. His white blood cell count was $18,000 / \mu \mathrm{L}$, blood alcohol level was $114 \mathrm{mg} / \mathrm{dL}$, and creatine kinase level was 5280 units/L. Troponin I was undetectable. Urine toxicology screen was positive for cannabinoids. On EKG, T waves were inverted in leads II, III, V5, and V6. He was treated with intravenous fluids and lorazepam for agitation. His visual and auditory hallucinations resolved over the next 24 hours. Serial creatine kinase values trended downward. On further questioning, the man revealed that he had been snorting "bath salts" that he had purchased from a tobacco store.

DISCUSSION: Drug induced hallucinations are commonly encountered in the emergency department. A rapidly growing cause for this type of presentation is a group of synthetic drugs commonly referred to as "bath salts." These drugs are sold in powder or crystal form under a variety of names such as Bliss, Purple Wave, Ivory Wave, and Vanilla Sky. Bath salts contain stimulant compounds such as 3,4-methylenedioxypyrovalerone (MDPV), mephedrone, and methylone. The use of bath salts has risen rapidly in the United States during the past year, particularly among teenagers and young adults. In 2010, less than 300 calls in reference to bath salts were placed to poison control centers nationwide; in 2011, over 5600 calls were placed as of October 31st. Bath salts are commonly sold at "head shops," convenience stores, and over the internet. Packaging is often labeled with the phrase "not for human consumption." Until recently, the use of bath salts was still legal in many states. However, in October of 2011, the United States Drug Enforcement Administration (DEA) exercised emergency scheduling authority to control MDPV, mephedrone, and methylone, effectively making the sale and possession of bath salts illegal. Bath salts may be taken orally, intranasally, intravenously, or rectally. Signs and symptoms of bath salts intoxication include agitation, tachycardia, delusions, hallucinations, tremors, seizures, and rhabdomyolysis. Of note, routine urine toxicology screens do not detect the active ingredients of bath salts. Treatment of bath salts intoxication is supportive, including benzodiazepines for agitation and intravenous fluids for rhabdomyolysis. With the abuse of these synthetic drugs on the rise, physicians need to be familiar with the existence of bath salts and the clinical presentation and management of bath salts intoxication.

NOT JUST ANOTHER CASE OF "RULE OUT MI" Vijaya Rao; Vibhav Rangarajan; Lisa Dunning; Karen Orjuela; Elizabeth Schulwolf. Loyola University Medical Center, Maywood, IL. (Control ID \#1338844)

LEARNING OBJECTIVE 1: Recognize spontaneous coronary artery dissection (SCAD) as a rare cause of angina in middle aged male patients LEARNING OBJECTIVE 2: Managing SCAD in non-pregnant patients without other obvious risk factors for developing SCAD

CASE: The patient is a 55 year old male with past medical history significant for hypertension, hyperlipidemia, and GERD who presented with a one month history of progressive dyspnea and exertional angina. His electrocardiogram on admission was notable for prior inferior and anterior wall infarct. Serum troponin was negative. Patient also underwent stress echocardiogram that showed inferior and apical septal hypokinesis with localized thrombus in the apical segment; transthoracic echocardiogram revealed an ejection fraction of $45 \%$, left ventricular hypertrophy, mild hypokinesis of the left ventricle, akinesis of the apical septal segment, and again left ventricular apical thrombus. The patient ultimately underwent coronary angiography which revealed a healed spontaneous coronary artery dissection of the mid-distal left anterior descending artery (LAD). The patient denied any history of blunt trauma to the chest or connective tissue disorders, though his mother died of scleroderma and patient had recently been concerned that he is developing the disease. He was ultimately ruled out for scleroderma (Anti-Scl 70, ANA, and ENA negative). He was discharged with a medication regimen to optimize his heart failure (carvedilol, aspirin, lisinopril, simvastatin) and warfarin $5 \mathrm{mg}$ for anticoagulation for the LV thrombus.

DISCUSSION: Spontaneous coronary artery dissection (SCAD) is a rare phenomenon, estimated to comprise approximately $0.2-0.3 \%$ of cases acute coronary syndromes in the general population. Reported cases have been typically associated with the peri or post-partum period in young women, intense exercise, blunt trauma to the chest, cocaine use, connective tissue diseases such as Ehlers-Danlos or Marfan's syndrome, fibromuscular dysplasia, use of oral contraceptives or drugs such as cyclosporine, as well as history of atherosclerosis. Still, the underlying pathophysiology remains elusive. SCAD encompasses a broad spectrum of clinical presentations, ranging from asymptomatic to both stable and unstable angina, acute myocardial infarction, arrhythmias, and sudden cardiac death. However, one study reports that $77 \%$ of all SCAD reported in the literature presented with chest pain, with reportedly $65 \%$ revealing ST-segment elevation on electrocardiogram. The diagnosis is typically confirmed with cardiac computed tomography or coronary angiography; approximately half of the lesions found are in the LAD. In regards to an appropriate treatment plan, the results are mixed. Some studies have shown that conservative management may simply lead to delay of an inevitable procedure given that approximately $60 \%$ of lesions managed without intervention remained the same or worsened on repeat angiography. Others state that recurrent dissection is generally rare. The extent of the ischemia, amount of preserved coronary flow, and location of the dissection should ultimately dictate the possibility of intervention. In the case of our patient, coronary angiography revealed a well-healed mid-distal dissection with preservation of the remainder of his coronary vasculature. Given that the dissection was not acute, was in the mid-distal LAD, and coronary flow was preserved, conservative medical therapy with close follow up was chosen as a reasonable approach.

NOT SO BENIGN BACITRACIN Jessica Prange; Kurt J. PfeiferMedical College of Wisconsin, New Berlin, WI. (Control ID \#1337438)

LEARNING OBJECTIVE 1: Identify Patients at Increased risk for bacitracin induced anaphylaxis

LEARNING OBJECTIVE 2: Manage anaphylacitic shock

CASE: A 94 year old female presents for a routine pacemaker generator change. The original dual chamber device was un-eventfully placed for sick sinus syndrome seven years ago. Prior to the procedure the patient received fentanyl, vancomycin, and xylocaine. The genrator was replaced, and the pocket was irrigated with a dilute bacitracin solution. Within minutes of the irrigation, the patient experienced acute pruritis, shortness of breath, hypotension, facial and toungue edema. She required emergent nasopharyngeal intubation, IV fluids, and IM epinephrine. She was stablilized and then transferred to the cardiovascular ICU. On exam she was afebrile, normotensive, and found to have severe facial and toungue edema without rash. Laboratory and imaging studies were unremarkable. Clinical course included 5 days of steroid therapy, diphenhydramine, and famotidine. Allergy/Immunology was consulted, and it was felt that the bacitracin used to irrigate the pacemaker pocket was the most likely cause of her anaphylactic shock. On hospital day 5 she was extubated, and ultimately was discharged to a subacute rehab facility. DISCUSSION: Bacitracin, although thought to be a benign drug, is known to be a potent sensitizer for allergic reactions. Therefore, patients at the highest risk of reaction are those that have had prior exposure to the drug. Reactions to topical bacitracin have been well described, but anaphylaxis due to intraoperative bacitration irrigation solution is extremely rare with less than 10 cases reported in the literature. There is good evidence to support the use of prophylactic IV antibiotic administration prior to cardiac 
device placement to reduce post-procedure infections. Antibiotic irrigation during cardiac device placement on the other hand, has little data to support its use. Careful assessment of the risks versus benefits of using bacitracin irrigation should be considered for all patients undergoing cardiac device placement, especially in those patients with prior exposure to the drug.

NOTHING IN ISOLATION Joseph A. Avalos. Tulane University Health Sciences Center, New Orleans, LA. (Control ID \#1311875)

LEARNING OBJECTIVE 1: 1. Identify the differential diagnosis of acute kidney injury. 2. Recognize the clinical presentation of multiple myeloma 3. Identify the appropriate imaging in the setting of acute renal failure.

CASE: A 48 year-old man with history of well-controlled hypertension presented with one week of lethargy, lightheadness, and a ten-pound weight gain. He noted decreased appetite and oral intake, as well as a recent decline in urine output. The abdominal examination was normal; there were no masses, the bladder was not distended, and there were no spinal, paraspinal, or CVA tenderness. His lungs were clear, the JVP was normal and he had no edema. The BUN was 98 , the creatinine was 17 , and the potassium was $6 \mathrm{mmol} / \mathrm{L}$. His calcium was 10.8 ; the total protein was 6.7 , the albumin was 4.2 , and his hemoglobin was $10.6 \mathrm{~g} / \mathrm{dl}$. The urinalysis revealed a protein $30 \mathrm{mg} / \mathrm{dL}$. A renal ultrasound was obtained, revealing mildly enlarged bilateral kidneys without hydronephrosis. There were multiple lytic lesions to the spine and pelvis on CT of abdomen and pelvis. An immuno-electrophoresis and subsequent bone marrow biopsy confirmed the diagnosis of multiple myeloma with a predominance of kappa light-chains.

DISCUSSION: Acute kidney insufficiency is commonly encountered by the general internist. While the most common causes include urinary obstruction and inadequate perfusion of the kidneys, the internist must have a disciplined diagnostic approach to identify the less common causes of acute renal insufficiency. Once pre-renal and post-renal causes are excluded, the general internist should have a disciplined approach to evaluating potential intrinsic renal damage. Intrinsic insults can occur due to prolonged hypo-perfusion or obstruction, or exposure to either exogenous or endogenous nephrotoxic agents. Common exogenous toxins are aminoglycosides and contrast; common endogenous nephrotoxins included heme-containing products, uric acid, or Bence-Jones proteins. Based upon the age of our patient, and the normal physical examination, post-renal obstruction was quickly excluded. His decreased oral intake increased the pre-test probability of pre-renal azotemia, as was suggested by the initial laboratory findings of an elevated BUN and creatinine. This case illustrates, however, that it is important that the general internist not succumb to premature closure in the diagnosis of pre-renal azotemia based upon only a few laboratory findings in isoloation. When interpreted in the context of the anemia and hyper-calcemia, the elevated BUN and creatinine suggested an intrinsic renal toxicity. Multiple Myeloma (MM) is characterized by renal failure, skeletal involvement, anemia, and hypercalcemia; common presenting symptoms include bone pain, impaired renal function, anemia, hypercalcemia, recurrent infections, and hyperviscosity. Although the median age at presentation of myeloma is seventy years, fifteen percent are younger than sixty years of age at diagnosis. In our patient, the context of acute renal insufficiency, anemia, hyper-calcemia and lethargy prompted the clinical suspicion for myeloma. In such cases, a skeletal survey is the best initial imaging modality, as the lesions of myeloma are purely lytic, and will thus not be revealed by a bone scan. The role of CT scanning is reserved for clarifying indistinct lesions, or to better evaluate difficult regions such as the ribs, sternum, and scapula. The role of MRI is limited to assessing suspected cord compression

NUMB CHIN SYNDROME: A HARBINGER FOR METASTATIC PROSTATE CANCER. Raji Shameem; Robert E. Graham. Lenox Hill Hospital, New York, NY. (Control ID \#1339942)

LEARNING OBJECTIVE 1: Recognize Numb Chin Syndrome in the setting of systemic malignancy.

LEARNING OBJECTIVE 2: Identify Numb Chin Syndrome as a precursor for Metastatic Prostate Cancer.
CASE: A 59-year-old male presented to the hospital with a one-month history of severe and diffuse bone pain most prominent in the spine. Of note, three months prior to the onset of diffuse bone pain the patient had noticed persistent decreased sensation and pain over his jaw. The patient did not consider the symptom to be of any significance and did not go see a physician for evaluation. Eventually severe bone pain brought him to the hospital. On clinical examination there was hypothesia over the chin. In addition, on rectal examination the prostate was enlarged with a nodular consistency. Prostate specific antigen was substantially elevated at 1,920. CT imaging revealed widespread osteoblastic bony metastases, including the vertebrae, ribs, and mandible. These findings were consistent with metastatic prostate cancer.

DISCUSSION: Numb Chin Syndrome is an uncommon condition. It is characterized to be a neuropathy of the mental nerve, a branch of the trigeminal nerve. Affected individuals describe decreased sensation and/or pain over the sensory distribution of the mental nerve. The sensory distribution involves the skin of the chin, lower lip, and the gingiva. A wide variety of conditions are associated with Numb Chin Syndrome. The extensive list includes multiple sclerosis, dental infection, sickle cell anemia, and systemic malignancy. Numb Chin Syndrome has been shown to be a symptom in the setting of systemic malignancy. Examples include breast, ovarian, lung, and prostate cancer. The fact that the majority of these malignancies are widespread with severe diffuse involvement emphasizes the importance of clinical recognition of Numb Chin Syndrome. Prostate cancer rarely has been shown to be a culprit behind Numb Chin Syndrome. Previous case reports that have linked Numb Chin Syndrome with prostate cancer have been when the cancer had metastasized. However, in this case, Numb Chin Syndrome was an apparent initial presenting factor for prostate cancer months before the onset of generalized bone pain. Given the temporal relationship of initial symptoms and the subsequent development of diffuse bone pain we argue that Numb Chin Syndrome appears prior to the metastasis of prostate cancer. Clinicians must be cautious of Numb Chin Syndrome with a high index of suspicion because of its ominous association with malignancies such as prostate cancer. If this is done malignancy will be detected earlier prior to the onset of metastasis.

OMENTAL INFARCT: RARE CAUSE OF UNEXPLAINED ABDOMINAL PAIN Jochebed A. Pink; Robin Klein. Emory University School of Medicine, Atlanta, GA. (Control ID \#1339693)

LEARNING OBJECTIVE 1: - Recognize the clinical presentation and risk factors of omental infarction

LEARNING OBJECTIVE 2: - Review the treatment and management of omental infarct

CASE: A 48-year-old male with a history of obesity, diabetes mellitus, and hypertension presented with chest and abdominal pain. Three days prior, he reported the sudden onset of left sided chest pain over his lower ribs. The pain was sharp and constant, independent of exertion, deep breathing, or position. The following day, the pain spread to his left upper quadrant. The pain was unrelated to eating, gas, or bowel movements. He denied shortness of breath, nausea, vomiting, fever, chills, and changes in weight. On exam, his abdomen was soft, nontender, and nondistended without guarding. Bowel sounds were normal and no masses were palapable. Laboratories revealed white cell count 9.2 × $109 \mathrm{~K} / \mathrm{mcL}$. Lipase was elevated at $77 \mathrm{U} / \mathrm{L}$ and lactic acid was $1.3 \mathrm{mmol} / \mathrm{L}$. CT scan of the abdomen and pelvis revealed stranding of the fat tissue in the upper abdominal mesentery with a ring of hyperattenuation measuring $1.2 \mathrm{x}$ $5.0 \mathrm{~cm}$ consistent with omental infarction with epiploic appendigitis. $\mathrm{He}$ was diagnosed with omental infarction. He was initially treated with intravenous levofloxacin, metronidazole, and heparin, which were discontinued after consulting general surgery who confirmed that omental infarction is a benign condition. The patient's pain was well controlled with hydrocodone/acetaminophen and he was discharged home.

DISCUSSION: Omental infarction occurs when blood supply to the abdominal omentum is compromised. This can occur in the setting of spontaneous venous thrombosis, torsion of the omentum around its vascular pedicle, vasculitis of the omental vessels, or venous outflow obstruction. Risk factors include obesity, strenuous activity, congestive heart failure, recent abdominal surgery, and abdominal trauma. Typically, patients present with acute pain that can arise in any abdominal quadrant. The presentation may 
mimic other more common causes of abdominal pain such as cholecystitis, diverticulitis, appendicitis, perforated peptic ulcer, or ruptured ovarian cyst. Given its nonspecific presentation, accurate diagnosis relies upon on imaging. Both abdominal CT and ultrasonography can demonstrate inflammatory changes in the fat consistent with infarction. Fortunately, omental infarction is a benign, self-limiting condition. Pain typically resolves in 1 week with conservative treatment with analgesics. However, the imaging findings may persist for up to 6 months. While benign, accurate and timely recognition of omental infarction is key. Clinically, it is indistinguishable from more ominous causes of pain such as an acute abdomen. Misdiagnosis may lead to unnecessary treatment with antibiotics and even surgical intervention. While rare, physicians need to consider omental infarction in patients presenting with abdominal pain given the potential morbidity of misdiagnosis.

ONE MORE WAY THAT SMOKING IS BAD FOR YOUR HEALTH Jeffrey DellaVolpe. Tulane University Health Sciences Center, New Orleans, LA. (Control ID \#1338803)

LEARNING OBJECTIVE 1: Recognize acute pulmonary eosinophilia as a cause of fever, dyspnea, and cough especially in the setting of a recent airborne exposure

LEARNING OBJECTIVE 2: Understand the differences in treatment of acute pulmonary eosinophilia versus the treatment of infectious pneumonia CASE: A 22 year-old man on military deployment presented to a remote clinic in Central Africa. He had experienced two days of worsening dyspnea on exertion, fever, and fatigue; he denied a cough. His symptoms worsened despite treatment with azithromycin by the field medic. He was a nonsmoker, however, over the last two weeks, he had been smoking local cigarettes to help him stay awake during night patrols. On physical examination, his temperature was $103.9 \mathrm{oF}$, his heart rate was 120 beats/ minute, and his respiratory rate was 32 times/minute; his oxygen saturation was $80 \%$. He had decreased air movement bilaterally, in addition to bilateral inspiratory crackles best heard at the bases of the lungs. There was a tactile fremitus on the right side. Laboratory capabilities were limited, but rapid malaria and rapid influenza tests were negative. His chest X-ray demonstrated diffuse alveolar infiltrates and homogenous opacification of the right hemithorax. He was placed on continuous oxygen by ventimask and started on intravenous ceftriaxone and vancomycin. He was given normal saline, which had a modest effect on his heart rate. Attempts to wean his oxygen consumption were accompanied by an immediate desaturation to $80 \%$. Because of the limited supplies and minimal improvement, he was transported to a facility with greater capabilities. His CBC revealed a leukocytosis with no eosinophils. He underwent bronchoalveolar lavage (BAL) which showed 30\% eosinophils. A diagnosis of acute eosinophilic pneumonia was established, and he was started on prednisone. $\mathrm{He}$ experienced a rapid resolution of symptoms and was completely weaned off oxygen two days later. The following week he was released from the hospital and able to return home to his unit.

DISCUSSION: Acute eosinophilic pneumonia (AEP) is part of a heterogenous group of disorders known as the eosinophilic lung syndromes. The syndrome typically consists of an acute febrile illness, severe hypoxia, pulmonary infiltrates, increased eosinophils on BAL, and an absence of infection or other cause. The most commonly documented presenting signs and symptoms are dyspnea, fever, cough, and crackles on inspiration. The diagnosis is established based on pulmonary eosinophilia and exclusion of chronic causes of eosinophilic pulmonary disease. It is important to recognize, as was the case in our patient, that pulmonary eosinophilia can exist in the absence of a peripheral eosinophilia due to a pulmonary eosinophil sequestration. Although idiopathic causes have been described, patients usually develop the syndrome following an airborne toxin exposure. One key exposure that has been well described is new onset smoking, which was seen with our patient. Typically, patients are in their mid-20s and develop symptoms consistent with AEP within one month of initiation of smoking. The treatment of AEP is steroids, typically IV methylprednisolone. Dosages vary, but the consensus is $60-125 \mathrm{mg}$ of methylprednisolone every six hours followed by an oral prednisone taper. Relapses of AEP have not been described in the literature, and the prognosis is typically excellent if identified rapidly and treated appropriately.
PRES AS UNCOMMON CAUSE FOR SUDDEN BLINDNESS Khaled

M. Abouelezz; Abhilash Akinapelli; Pranjal Sharma; Mahmoud A. Abu Hazeem; Bruce L. Houghton. Creighton university medical center, Omaha, NE. (Control ID \#1339044)

LEARNING OBJECTIVE 1: Recognize Posterior Reversible Encephalopathy Syndrome (PRES) as a possible cause of sudden blindness.

LEARNING OBJECTIVE 2: Recognize possible causes that can lead to PRES.

CASE: 66yo Caucasian female admitted with paraspinal abscess and MSSA bacteremia, hospitalized for drainage, laminectomy and IV nafcillin. Patient developed Acute Kidney Injury and required blood transfusion for her low hemoglobin during hospital course. Blood pressure was normal since admission but started to elevate on days 9, was not treated aggressively due to risk of hypotension. On day 10, patient started to have an acute onset of dizziness, frontal headache radiating to the base of the skull, decreasing visual acuity and color vision. 7 hours later she developed complete blindness in both eyes. On examination blood pressure was $171 / 86$, baseline 120/71, pulse 91 , RR 22, T 96.9, she was conscious but confused. There was no light perception in both eyes. She had normal fundoscopic examination, otherwise normal neurological exam and normal electrolytes. Urgent MRI w/o contrast was done showed increased diffusion and T2 signal in the bilateral occipital and posterior partial regions most consistent with posterior reversible encephalopathy syndrome. Patient was transferred to ICU for tight control of her blood pressure and close monitoring. Follow up MRI after 5 days from the first one showed picture consistent with resolving PRES. On day 11, patient appreciated hand motion vision. Her vision gradually improved to near normal on day 20 . DISCUSSION: DISCUSSION: PRES is a clinical radiographic syndrome characterized by unique pattern of brain vasogenic edema of heterogeneous etiologies that are grouped together because of similar findings on neuroimaging studies, first described by Hinchy et al. Frequently PRES was described in association with hypertensive encephalopathy, eclampsia, and the use of cytotoxic and immunosuppressants. PRES can also be seen in infection specially gram positive bacteria, acute or chronic renal diseases, blood transfusion, chemotherapy, TTP, HUS , vasculitis, Porphyria, hypercalcemia, hypomagnesemia, contrast media exposure (cerebral, coronary angiography). In our patient PRES may be multifactorial due to transfusion, renal insufficiency but mainly due to infection with GR+ve cocci and moderate elevation of blood pressure. In one study it is found that PRES associated with Infection/sepsis gram-positive organisms predominate $(84 \%)$, and in $40 \%$ of patients, blood pressure is normal or only minimally increased. Clinical manifestations include insidious onset of headache, confusion, visual changes, and seizures. In our patient it is manifested with acute onset of bilateral complete cortical blindness. DD: Other neurologic conditions can be manifested with similar presentation such as stroke, venous thrombosis, toxic, metabolic encephalopathy, demyelinating disorders, vasculitis, or encephalitis. Management: usually treating the cause but hypertension is a feature in the majority of PRES patients, regardless of etiology. With blood pressure lowering, patients will often improve dramatically. Prognosis: Most patients recover within two weeks. MRI findings can be helpful in identifying patients with worse prognosis. CONCLUSION: PRES may be considered as an important differential diagnosis in hospitalized patients with sudden onset of visual changes when infection, hypertension, blood transfusion or kidney injury is present.

PTU: SUPPRESSING MORE THAN THYROID HORMONE Thomas Jensen ${ }^{1}$; Jerald Marifke ${ }^{2} .{ }^{1} \mathrm{MCW}$, Milwaukee, WI; ${ }^{2} \mathrm{MCW}$, Milwaukee, WI. (Control ID \#1328071)

LEARNING OBJECTIVE 1: To describe a rare case of PTU-induced agranulocytosis in a patient with a relapse of Graves' hypothyroidism, who previously tolerated the medication.

CASE: A 56 year-old woman with a past medical history of Graves' disease presented with a chief complaint of fever, chills, nonproductive cough, and general malaise for 4 weeks. Her symptoms began immediately after initiation of propylthiouracil (PTU) for recurrence of hyperthyroidism, though she failed to report this until a day before admission. She was diagnosed with Graves' hypothyroidism in 1996 and initially treated with 
methimazole. However, she did not tolerate this and was switched to PTU. She received this therapy for two years, at which time it was stopped due to remission of her disease. At this presentation the patient had a temperature of $100.3 \mathrm{~F}$, rigors, exudative tonsillar lesions, and an erythematous pharynx. She had a normocytic anemia (hgb of $9.2 \mathrm{~g} / \mathrm{dL}$ ), and thrombocytopenia $(123 \mathrm{e} 3 / \mathrm{uL})$, but more substantially her white cell count (WBC) was $1.3 \times 10^{\wedge} 9 / \mathrm{L}$, and an absolute neutrophil count of 0 . Her TSH was $0.015 \mu \mathrm{U} / \mathrm{mL}$. PTU was held and hematology concurred a diagnosis of PTU-induced agranulocytosis. Granulocyte colony stimulating factor (GCSF) was not started since evidence was inconclusive as to it being a benefit in PTU-induced agranulocytosis. Her neutrophil count recovered on hospital day 10 to $1.8 \times 10^{\wedge} 9$ and remained above $1.5 \times 10^{\wedge} 9 / \mathrm{L}$ during the remainder of the hospital stay. She had a WBC of $4.8 \times 10^{\wedge} 9 / \mathrm{L}$, hemoglobin $11.8 \mathrm{~g} / \mathrm{dL}$, and platelet count of $265 \mathrm{e} 3 / \mathrm{uL}$ on day of discharge. She underwent radioactive iodine treatment for definitive treatment.

DISCUSSION: Agranulocytosis is a rare, serious complication of thionamides occurring in $0.3 \%$ of patients. Of interest, there are very few case reports of PTU-induced agranulocytosis on second time exposure. Therefore, physicians and patients must be aware of symptoms of agranulocytosis even if they previously tolerated the medication, discontinue the thionamide, and perform further investigation immediately. Routine monitoring is not recommended since agranulocytosis develops suddenly, though typically within the first three months of initiation of therapy. The median time to resolution of agranulocytosis is 10-14 days. Conflicting data exists from retrospective and small randomized control studies as to whether GCSF improves recovery time. Management includes discontinuation of the thionamide and supportive treatment with antibiotics for neutropenic fever is recommended. Either RAI or thyroidectomy should then be pursued for definitive treatment of Graves' disease. Agranulocytosis is a serious complication of thionamides that can occur even after prior tolerance to the medication.

PACING THE BLOCK Kate Hust; Chayan Chakraborti. Tulane University Health Sciences Center, New Orleans, LA. (Control ID \#1339148)

LEARNING OBJECTIVE 1: 1. Recognize atrioventricular block. 2. Identify etiologies of atrioventricular block. 3. Understand pathophysiology and natural history of atrioventricular block. 4. Identify appropriate treatment for atrioventricular block.

CASE: A 58 year-old man presented with three weeks of chest pain, shortness of breath, and weakness. He reported past episodes upon exertion, but now had resting symptoms. He had no past medical history, and he was taking no medications. His admission vital signs and physical examination were normal. The troponin was $0.07 \mathrm{ng} / \mathrm{mL}$, the BNP was less than $20 \mathrm{pg} / \mathrm{mL}$; his urine toxicology was positive for cocaine. An initial EKG showed J-point elevation in the anterior leads, but no other abnormalities. He was admitted for observation. He remained asymptomatic overnight. His troponin level normalized, but an EKG revealed a first-degree atrioventricular (AV) block. Telemetry recorded intermittent episodes of bradycardia, second-degree AV block, and pauses up to four-seconds. An echocardiogram and stress test were normal, and he was discharged with a cardiac event monitor. He re-presented two days later with recurrent symptoms and pre-syncope. Interrogation of his event monitor showed corresponding bradycardia and Mobitz II seconddegree AV block.

DISCUSSION: Cardiac arrhythmias are frequently encountered in hospitalized patients, and recognizing heart block is important for guiding appropriate medical care. Natural conduction of a cardiac impulse is from sinoatrial node to atrioventricular (AV) node and then along the Bundle of His, bundle branches, and Purkinje fibers. Prolongation of the PR-interval indicates slowed conduction, generally within the AV node, and is diagnostic of first-degree AV block. Second-degree blocks are evidenced by P-waves without corresponding QRS complexes. They are subdivided into Mobitz I (Wenckebach), with progressively prolonged PR-interval leading to a dropped QRS complex, and Mobitz II, with dropped QRS complexes lacking the progressive PR-prolongation. Complete dissociation of the P-waves and QRS complexes is diagnostic for third-degree or complete AV block. These advanced degrees of AV block often occur distal to the AV node in the Bundle of His. Conduction abnormalities in AV block are caused by chronic infiltrative and inflammatory diseases leading to permanent fibrous changes. Fibrosis may also occur after infections such as syphilis or Lyme disease. Other irreversible heart blocks may be caused by diseases affecting cardiac structure - congenital heart disease, ischemia, trauma, or neoplasm. Common, reversible etiologies for AV block include metabolic derangements, medication side effects, and vasovagal or autonomic stimulation. When treating AV block, a reversible cause must first be ruled out. Providing heart rate support is the mainstay of therapy. Medications such as atropine may be used in the short-term, but definitive treatment is pacing. Permanent pacemakers are indicated in symptomatic bradycardia or irreversible second- and third-degree block. Waking heart rate below forty, left ventricular dysfunction, wide-complex QRS in second-degree block, and cardiomegaly with acquired third-degree block may be indications for pacemaker placement in asymptomatic individuals. AV blocks are not uncommonly seen by hospitalists, and recognizing these bradyarrhythmias is important to optimize care.

PANCREATIC ASCITES, A CASE OF TWO PSEUDOCYSTS Mona Hassan; Laila Shiekh Sroujieh. Henry Ford Hospital, Detroit, MI. (Control ID \#1334661)

LEARNING OBJECTIVE 1: An approach to pancreatic ascites and pancreatic pseudocyst causing it.

LEARNING OBJECTIVE 2: ERCP transpapilary stent placement as a treatment option for pancreatic ascites secondary to a pancreatic fistula. CASE: A 47 year old female with cirrhosis and chronic pancreatitis secondary to alcohol abuse, presented with epigastric abdominal pain radiating to the back of 3 days duration following an episode of binge drinking. Serum amylase and lipase were elevated and $\mathrm{CT}$ of the abdomen showed inflammatory changes consistent with acute pancreatitis, pancreatic body small pseudocyst $(2.0 \times 1.9 \mathrm{~cm})$, large pancreatic tail perisplenic pseudocyst $(7.3 \times 8.7 \mathrm{~cm})$ and ascites. Ultrasound guided diagnostic paracentesis was performed and showed findings consistent with pancreatic ascites (serum ascitic albumin ratio of $<1.1$, ascitic fluid protein $>3 \mathrm{~g} / \mathrm{dl}$, ascitic fluid amylase was $15954 \mathrm{IU} / \mathrm{L}$ and the ascitic fluid to serum amylase (741) ratio was $>6.0$ ). An ERCP was performed due to suspected drainage from the pancreatic pseudocyst into the peritoneal cavity. ERCP revealed a large pseudocyst at the tail of the pancrease with complete disruption of the end of the pancreatic duct with free flow of fluid from the pseudocyst into the peritoneum. A $5 \mathrm{fR}, 12 \mathrm{~cm}$ pancreatic stent was inserted that ended inside the pseudocyst. There was a good flow of pancreatic juice through the stent into the duodenum. The pancreatic enzymes continued to trend down after stent placement and the ascites clinically decreased.

DISCUSSION: Pancreatic ascites accounts for $1 \%$ of all cases of ascites. It occurs in $3.5 \%$ of patients with chronic pancreatitis and 6$14 \%$ of patients with pancreatic pseudocyst. ERCP with stent placement is a treatment option for pancreatic ascites as surgical management is associated with $15-25 \%$ chance of morbidity and mortality. A case of pancreatic ascites secondary to a pancreatic pseudocyst draining into the peritoneal cavity is presented her. ERCP with stent placement is presented as a treatment option. Either conservative management with prolonged NPO for pancreatic rest or surgery are management options for pancreatic fistulae. An alternative treatment for pancreatic fistula with evidence of disruption of the pancreatic duct and causing ascites is endoscopic placement of a transpapillary stent. The presence of pancreatic ascites should raise the suspicion of a pseudocyst draining into the peritoneal cavity. This case encourages us to consider ERCP with stent placement as a treatment option in pancreatic ascites not responding to conservative medical therapy and provides a safer alternative to surgery.

PANCREATICOPLEURAL FISTULA (PPF) Madan Badal; Madan R. Aryal; Naba R. Mainali. Reading Hospital, Reading, PA. (Control ID $\# 1340452$ )

LEARNING OBJECTIVE 1: When to suspect the case of Pancreaticopleural fistula (PPF)

LEARNING OBJECTIVE 2: How to manage the case of Pancreaticopleural fistula (PPF) 
CASE: A 45 years old Caucasian woman with a history of chronic pancreatitis secondary to alcoholism presented with a 2 week history of progressive shortness of breath, dry cough and right sided pleuritic chest pain. On physical examination, she was found to have decreased breath sound at right lung base, stony dull percussion note on the area with positive vocal fremitus on auscultation. As per patient, she visited acute care center and got pleural fluid aspiration done twice over last two months for increased shortness of breath. Chest $\mathrm{x}$ - ray on admission confirmed the clinical finding of large right sided pleural effusion. Thoracentesis was performed yielding 2.5 liter serosanguinous pleural fluid with an exudative pattern. The findings were: $\mathrm{pH} 7.37$, lactate dehydrogenase (LDH) $493 \mathrm{U} / \mathrm{L}$ (serum LDH $144 \mathrm{U} / \mathrm{L}$ ), total protein $1.4 \mathrm{~g} / \mathrm{dl}$ (serum total protein $6.5 \mathrm{~g} / \mathrm{dl}$ ), lipase $60778 \mathrm{U} / \mathrm{L}$ (Serum lipase $63 \mathrm{U} / \mathrm{L}$ ) and amylase 45,500 U/L (Serum amylase $165 \mathrm{U} / \mathrm{L}$ ). Liver function tests were within normal limit. Contrast CT scan of thorax/ abdomen revealed massive right pleural effusion and a pseudo cyst adjacent to the pancreatic head which was tracking superiorly and entering the right pleural space. The patient was managed conservatively with bowel rest and started on intravenous octeotride. ERCP with possible endoscopic stenting of pancreatic duct was planned for the following week.

DISCUSSION: Pancreaticopleural fistula (PPF) is rare but a known serious complication of chronic pancreatitis or pancreatic trauma. It is estimated to occur in $0.5 \%$ of patients presenting with pancreatitis. Unlike the small pleural effusions that can usually be seen in acute pancreatitis, PPF can produce large and recurrent pleural effusions. PPF requires a high index of suspicion in patients presenting with chest symptoms or pleural effusion with history of pancreatitis or alcoholism. Extremely high amylase and lipase in pleural fluid aspirate are common findings. Early suspicion and recognition of a PPF is crucial, as delay in definitive treatment is associated with a higher rate of complication, with mortality up to $5 \%$. A ductal disruption on the anterior surface of the pancreas usually leads to pancreatic ascites, whereas the posterior ductal leakage usually results in thoracic fluid collections. The pancreatic juice spreads retroperitoneally through the paths of least resistance, commonly through the aortic or esophageal hiatus. The management options in PPF include conservative treatment and endoscopic or surgical intervention. Medical therapy of PPF fails in $59 \%-69 \%$ of cases. Treatment of obstruction of a main pancreatic duct proximal to the fistula is to decompress the obstructed duct endoscopically and for cases refractory to endoscopic management, surgery is recommended.

PARASPINAL COMPARTMENT SYNDROME IN THE SETTING OF RHABDOMYOLYSIS Garrett M. Chinn; Kathleen Finn. Massachusetts General Hospital, Boston, MA. (Control ID \#1286064)

LEARNING OBJECTIVE 1: Recognize when to consider paraspinal compartment syndrome as a cause of back pain.

LEARNING OBJECTIVE 2: Understand who is at risk for paraspinal compartment syndrome.

CASE: A 34 year old man with history notable for myocardial infarction at age 29 , polysubstance abuse, hepatitis $\mathrm{C}$, and lumbar disc disease presented with debilitating back pain and extremity weakness. The patient is a mixed martial arts fighter who had been restricting fluids while training to make a lower weight class for a fight. During the match he suffered repeated blows to the back. Afterward he ingested an unknown amount of ibuprofen and snorted cocaine. He then slept for 1 hour but awoke with severe back pain and could not move his arms or legs. He stated his strength returned but noted lower back numbness and "tightness" without radiation. Physical exam revealed hard left paraspinal muscles and tenderness to palpation over this area, but no swelling. Pain was out of proportion to exam. Sensation was intact and he had normal bilateral lower extremity motor strength. Initial laboratory measurements were as follows: serum creatinine, $3.5 \mathrm{mg} / \mathrm{dL}$; aspartate aminotransferase, $1106 \mathrm{U} / \mathrm{L}$; alanine aminotransferase, $289 \mathrm{U} / \mathrm{L}$; creatinine kinase (CK), 91,790. Lumbar xrays showed a loss of lordosis and a non-contrast computed tomography (CT) demonstrated mild degenerative changes of the spine without fracture or paraspinal muscle abnormality. Compartment pressure measurements were obtained. Left and right proximal paraspinal muscle pressures were 20 and $38 \mathrm{mmHg}$ respectively. Fasciotomy was not performed at this time because the differences between compartment and diastolic pressures $(83-86 \mathrm{mmHg})$ were greater than 40 . The following day his CK had improved to 63,310. However, persistent severe back pain prompted repeat pressure measurements showing left and right sided pressures of 110 and $115 \mathrm{mmHg}$ respectively. He was taken for an emergent fasciotomy.

DISCUSSION: Acute compartment syndrome occurs when increased pressure within fascial spaces results in neurovascular compromise and ischemia. While rare, paraspinal compartment syndrome occurs in both traumatic and non-traumatic cases. Risk factors include strenuous physical exertion, trauma, or impaired circulation during aortic bypass surgery. It is most commonly reported in young men who weight lift or ski. All but one were admitted for rhabdomyolysis and reported significant back pain. Paraspinal compartment syndrome can share findings with other common causes of back pain. However, physical exam findings include tender paraspinal muscles, loss of lumbar lordosis, absent bowel sounds, and localized sensory loss. Pain is out of proportion to exam and is exacerbated by valsalva maneuvers, passive and active spinal flexion, or straight leg maneuvers. CT imaging can show swelling of the musculature, but may be normal. Magnetic resonance imaging demonstrates increased T2-weighted signal intensity. Although compartment pressure measurement remains the gold standard for diagnosis, there is no consensus regarding an absolute pressure threshold for fasciotomy. Some use $30 \mathrm{mmHg}$ as a cut off, while others utilize the difference between diastolic and compartment pressures (delta pressure). Definitive management includes surgical decompression. Early recognition of paraspinal compartment syndrome in a patient with severe back pain and rhabdomyolysis is crucial to prevent permanent muscle and nerve damage.

PEDIATRIC SYNDROMES FOR INTERNISTS: OPSOCLONUS MYOCLONUS ATAXIA IN AN ADULT Kalpana Nagarkar; Tsao-Wei Liang; Manuel Vergara; Rajeshree Anandakrishnan. Capital Health Regional Medical Center, Trenton, NJ. (Control ID \#1319784)

LEARNING OBJECTIVE 1: Opsoclonus myoclonus ataxia can be mistaken for anxiety, status epilepticus, cerebellar ataxia, Sydenham's chorea and others.

LEARNING OBJECTIVE 2: Internists need to be aware that though this is mostly a pediatric syndrome, it can present in adults and unlike in children, the patient can show better response to IVIG than to ACTH treatment.

CASE: A 19 year old African American female presented with acute onset of unstable gait, chaotic eye movements and tremulousness of the whole body associated with nausea and vomiting. She denied fever or recent flulike symptoms. Vital signs were normal including no fever. She was alert and oriented with normal mental status. Cranial nerve examination showed conjugate, arrhythmic, chaotic, large amplitude eye movements, predominantly in horizontal direction. Voluntary ocular movements were full but tended to exacerbate the opsoclonus. She had severe truncal and appendicular ataxia, along with truncal and limb myoclonus. Her myoclonic movements markedly increased on attempted movement. The deep tendon reflexes were normal and no paresis or sensory deficit could be found. Remaining neurological and systemic examination was normal. EEG was negative for epileptiform activity. LP revealed $19 \mathrm{WBC} / \mathrm{hpf} 96 \%$ lymphocytes and normal protein and glucose. CSF was negative for HSV, Lyme, VDRL and enterovirus. MRI of the brain was normal. She underwent intensive negative evaluation to rule out malignancy. She was diagnosed with opsoclonus myoclonus ataxia (OMA), and initially received high dose corticosteroids and ACTH therapy without any response. Later she received IVIG treatment with improvement.

DISCUSSION: OMA affects mainly young children with a mean age of 1.5 to 2 years. Non-paraneoplastic OMA has been reported in adults in association with various infections, including Lyme disease, enterovirus, West Nile virus, Epstein Barr virus, HIV, salmonella, cytomegalovirus, after anti-Rubella vaccination, and post-streptococcal infection. Many cases of non-paraneoplastic OMA are idiopathic and often assumed to be parainfectious in origin. Our case was presumed to be a post viral syndrome as other causes were ruled out, CSF pleocytosis was present, and symptoms responded to IVIG treatment. Teaching Points: This infrequent syndrome can be mistaken for anxiety, status epilepticus, cerebellar ataxia, Sydenham's chorea and others. Unlike in children, our patient showed better response to IVIG than to ACTH treatment. In order to develop novel and effective therapeutic strategies, further studies on the immunopathogenesis and pathophysiology of OMA are required. 
PERSISTENT HEADACHE: A FUSS OVER CRYPTOCOCCUS Leila Zuo; Amit Kothari. UT Southwestern Medical School, Dallas, TX. (Control ID \#1339930)

LEARNING OBJECTIVE 1: Identify the clinical presentation of cryptococcal meningitis.

LEARNING OBJECTIVE 2: Recognize the utility of corticosteroids and surgical intervention as adjunctive therapy for cryptococcoma.

CASE: A 36 year-old man presented with a 3-week history of headache. $\mathrm{He}$ also noted neck stiffness, photophobia, persistent nausea, and intermittent vomiting during this time. Ibuprofen, acetaminophen, and marijuana did not provide relief. He denied high risk sexual behavior and was not known to be HIV positive. Past medical history is only notable for psoriasis without previous immunosuppressive therapy. Lumbar puncture revealed elevated protein \& low glucose. Vancomycin and ceftriaxone were started as empiric therapy for presumed bacterial meningitis. Subsequent CSF culture revealed budding yeast identified as Cryptococcus neoformans. Vancomycin and ceftriaxone were discontinued, and a 6-week course of ambisome and flucytosine was started. A right temporal ring-enhancing $3 \mathrm{~cm}$ mass with central necrosis was seen on initial MRI, consistent with cryptococcoma. His hospital course was complicated by partial seizures for which he was treated with phenytoin. After ventriculo-peritoneal shunt placement and 5-weeks of antifungal treatment, the patient's headaches persisted. Repeat MRI/CT scan revealed stable size of the lesion but with worsening cerebritis and more prominent vasogenic edema. Due to ongoing headache and increased edema, dexamethasone was added to his treatment regimen. Four days of treatment with corticosteroids did not improve his headaches, and he underwent resection of the cryptococcoma.

DISCUSSION: General internists should recognize the triad of headache, photophobia, and nuchal rigidity as possible meningitis. Internists should understand how to work-up this patient, as his subacute presentation points to a differential of a wide variety of infectious, inflammatory, and malignant etiologies. To determine the cause of subacute meningitis, patients should undergo a complex workup that includes serologic assays, imaging tests, and repeated lumbar puncture. Though more common in immunocompromised patients, cryptococcal meningitis should be considered in any immunocompetent individual presenting with subacute to chronic meningitis and antigen testing of the CSF for C. neoformans should therefore be performed. Cerebral cryptococcomas can cause significant neurologic morbidity and are difficult to treat, often requiring prolonged antifungal treatment with multiple rounds of induction therapy. As there are no prospective studies of therapy for cerebral cryptococcomas, current treatment recommendations are based on case reports and retrospective analyses. Corticosteroids and surgical resection should be considered for cases refractory to antifungal therapy. Although unusual, cases of immunocompetent individuals with cryptococcoma successfully treated with antifungal drugs, corticosteroids, and surgical resection have been reported. In this patient's case, corticosteroids were initiated to decrease intracranial edema and for symptom management. Future MRI results and post-operative evaluation of his condition could potentially add to the sparse scientific literature regarding treatment strategy for cryptococcoma.

PHEOCHROMOCYTOMA PRESENTING AS CARDIOGENIC SHOCK: AN ATYPICAL CASE. Danny Spinuzzi; William J. Rust. Allegheny General Hospital, Pittsburgh, PA. (Control ID \#1283926)

LEARNING OBJECTIVE 1: To recognize pheochromocytoma as a potential cause in patients who present with shock of unknown etiology.

LEARNING OBJECTIVE 2: To identify the various manifestations of pheochromocytoma, as well as tools to screen and treat.

CASE: A 51 year old white female with a past medical history of hypertension and diabetes mellitus (DM) presented to the emergency department complaining of chest pain, shortness of breath and several episodes of emesis over the preceding 2 days. Vital signs include a blood pressure (BP) of $60 / 30 \mathrm{~mm} \mathrm{Hg}$, heart rate (HR) of 130 beats per minute (bpm) and respiratory rate (RR) of $34 / \mathrm{min}$. Physical exam revealed bilateral crackles and cool extremities. Sinus tachycardia with diffuse ST segment and $\mathrm{T}$ wave changes were seen on the initial electrocardiogram (ECG). Initial cardiac enzymes were elevated. Other pertinent presenting laboratory values included a leukocyte count of $19.7 \mathrm{k} / \mathrm{ml}$, BUN of
$27 \mathrm{mg} / \mathrm{dL}$, and a creatinine of $2.5 \mathrm{mg} / \mathrm{dL}$. An arterial blood gas revealed a $\mathrm{pH}$ of 6.99 and $\mathrm{HCO} 3$ of 6.6. A chest X-Ray revealed bilateral pulmonary edema. She was emergently intubated, and started on dopamine and dobutamine. An emergent cardiac catheterization found normal coronary arteries and severe left ventricular (LV) dysfunction with an ejection (EF) of $10 \%$. An intra-aortic balloon pump was placed for hemodynamic support and the patient was evaluated for possible left ventricular assist device. In the CCU, systolic blood pressure ranged from $90-220 \mathrm{~mm} \mathrm{Hg}$ and $\mathrm{HR}$ ranged from $120-280 \mathrm{bpm}$. She had urine metanephrine of 3195(Normal 30-180 ug/24 hr), total metanephrine 4838(Normal 164-588 ug/24 hr) and normetanephrine of 1643(Normal 124$484 \mathrm{ug} / 24 \mathrm{hr}$ ). Anti-hypertensive medical therapy included phentolamine, phenoxybenzamine and labetolol. Computed Tomography (CT) revealed a left adrenal mass highly suspicious for pheochromocytoma. Pathology from an abdominal tumor resection confirmed the diagnosis. The hospital course was complicated by acute renal failure and respiratory failure, but she gradually improved post-operatively and a repeat echocardiogram revealed an EF of $65 \%$. She was discharged to home without anti-hypertensive therapy.

DISCUSSION: Pheochromocytoma is a rare neuroendocrine tumor derived from enterochromaffin cells and is found in less than $0.3 \%$ of hypertensive individuals. $0.01 \%$ present with shock. Symptoms typically include a triad of headache, palpitations and diaphoresis. Less common cardiac manifestations include acute myocardial infarction, arrhythmias, dilated cardiomyopathy, and in some extreme cases, acute heart failure. This case illustrates a near fatal presentation of Pheochromocytoma. Sudden release of catecholamines may precipitate hemodynamic compromise and multisystem organ failure. Catecholamine induced focal myocardial necrosis with inflammatory infiltration can cause pulmonary edema and LV dysfunction as seen in this patient and appears to be reversible with both medical management and subsequent removal of the tumor. Although rare, the diagnosis of pheochromocytoma should be considered in patients that present with cardiogenic shock of unknown etiology. This case illustrates the significant morbidity and mortality associated with pheochromocytoma and the importance of recognizing its various clinical manifestations.

PITYRIASIS LICHENOIDES ET VARIOLIFORMIS ACUTA (PLEVA): A CASE REPORT Peter T. Georges; Menhel Kinno. Umassmemorial health care, Worcester, MA. (Control ID \#1312840)

LEARNING OBJECTIVE 1: The purpose of reporting this case is to increase awareness of this rare disease, and promote research of its pathophysiology, prognosis, and therapy.

CASE: This is a 29 year old Caucasian male who presented with a rash and systemic symptoms for 1 month. Initially, he developed painful papules on the trunk which spread to the extremities and head, with palmar and plantar sparing. Additionally, he had fever, bodyaches, and sore throat. His serology was positive for varicella and he was treated with 1 week of valacyclovir. He presented to the hospital for therapy failure. He had tender papules with central hemorrhagic crust distributed over trunk, extremities, genitalia, and head, with sparing of mucosa, palms, and soles. He was febrile and tachycardic. He denied any past medical history. He smokes 1.5 ppd of cigarettes, denies IVDA, and is homosexual with 1 partner. Laboratory results showed leukocytosis, negative HIV test, and positive VZV and HSV serology. The infectious disease team evaluated the patient and treated him with IV acyclovir for 10 days. His symptoms did not resolve and dermatology was consulted. They performed a skin biopsy of a lesion which showed features consistent with PLEVA and plasma cells, which is atypical for this disease. A syphilis test was performed prior to discharge. Patient was discharged on Azithromycin for 10 days for treatment of PLEVA. The syphilis test was positive and the patient's primary care physician was notified and he received treatment with Penicillin as an outpatient. He followed up with the dermatology clinic and had mild improvement in his symptoms following treatment for syphilis.

DISCUSSION: PLEVA, or Mucha-Habermann disease, is a cutaneous disorder with erythematous macules and papules, usually on the trunk and extremities. The lesions present at different stages with either a central punctum or evolve into vesicopustules with hemorrhagic necrosis or crust. It is seen in an acute or chronic form. It occurs most frequently in the second or third decades of life and is more frequent in men. Its etiology is unclear but it is suggested to be an inflammatory reaction triggered by 
infectious agents or an immune complex-mediated hypersensitivity. EBV, adenovirus, toxoplasma gondii, parvovirus B19, s. aureus, and s. pyogenes are pathogens thought to cause PLEVA. Upper respiratory tract infections, chickenpox, and streptococal pharyngitis may precede the onset of PLEVA. PLEVA is diagnosed by skin biopsy and histopathology shows perivascular and diffuse lymphocytic and histiocytic infiltration that obscures the dermoepidermal junction. There is a febrile ulcerative Mucha-Habermann disease (FUMHD), which is a potentially lethal variant. Treatment includes tetracycline, macrolides, or phototherapy. Treatment of FUMHD is more challenging and includes steroids, methotrexate, and dapsone.

PLASMAPHORESIS IN THYROID STORM WITH MULTI-ORGAN FAILURE Deepa Rani Nandiwada; Andrew A. Chang. NYU Langone Medical Center, New York, NY. (Control ID \#1339438)

LEARNING OBJECTIVE 1: Diagnose thyroid storm causing multiorgan failure

LEARNING OBJECTIVE 2: Assess the use of plasmaphoresis in the treatment of thyroid storm

CASE: A 31 year-old man presented with palpitations and worsening shortness of breath for two months associated with worsening orthopnea and decreased exercise tolerance. Over the past two weeks he noted worsening chest pain, yellowing of his skin and eyes, darkening of his urine, and twentypound weight loss. He denied fever, chills, cough, chills, diarrhea or abdominal pain. On exam, he appeared cachectic in moderate respiratory distress with $\mathrm{T}$ 101.7 F, HR 165 , RR 20 O2 sat $95 \%$ on room air. Scleral icterus, exophthalmos, jugular venous distension, decreased breath sounds at bilateral bases crackles, and hepatomegaly were appreciated. On laboratory exam the patient had a white blood cell count of 9,800 and hemoglobin of 10.3,AST 56 , ALT 22, ALP 179, direct bilirubin 4.8, INR 2.8 TSH $<0.04$, free T4 5.4. Chest $\mathrm{X}$-Ray demonstrated bilateral pleural effusions and increased vascular congestion. EKG revealed atrial flutter with 2:1 conduction and a ventricular rate of 165 . An echocardiogram revealed an ejection fraction of $25 \%$. He was admitted to the Intensive Care Unit and started on propylthiouracil, potassium iodide, and hydrocortisone. A beta-blocker drip was started, but was ineffective in controlling his heart rate. Eventually he became hypotensive requiring multiple pressors. Vancomycin and Pipercillian-tazoactam were started empirically as the patient was hypotensive and febrile, although initial cultures were negative. He eventually required intubation and continuous venous-venous hemofiltration. Fulminant hepatic failure ensued presumably due to shock liver on top of his thyrotoxic liver injuries. Plasmaphoresis was trialed while the patient was being evaluated for possible liver transplant. This resulted in temporary improvement in mental status and hormone levels. As the patient was undocumented, he did not qualify for transplant. A multidisciplinary conference was held and the patient's prognosis was deemed futile without a liver transplant. The patient ultimately expired.

DISCUSSION: In-hospital mortality from thyroid storm is high, reaching up to $30 \%$, with multi-organ failure seen in less than $10 \%$ of these cases. Diagnosing and treating thyroid storm early can reduce mortality and prevent multi-organ failure. Based on the Burch-Wartofsky scale, the patient met criteria for high suspicion of thyroid storm. Hepatotoxicity is reported in up to $30 \%$ of patients with thyrotoxicosis, however fulminant hepatic failure is only reported in 4 case reports and is associated with $80 \%$ mortality without transplant. Vulnerable populations can be even more challenging due to lack of access to primary care for early disease management and diagnosis. As seen in our patient transplant was not an option due to his undocumented status. The mainstays of thyroid storm treatment include medications that control increased adrenergic tone and medications that control the release and conversion of T3 and T4 including thionamide, iodine solution, iodinated radiocontrast, and glucocorticoids. Plasmaphoresis in thyroid storm has been attempted in only a few case reports, often with positive outcomes. Indications for plasmaphoresis include treatment failure, thyrotoxicosis induced cardiotoxicity, neurotoxicity, coma, and rapid clinical decompensation.

POST TRANSFUSION PURPURA VERSUS HEPARIN INDUCED THROMBOCYTOPENIA: A DIAGNOSTIC DILEMMA Subhraleena Das; Sujith Cherian; Wasim A. Hamarneh; Ehtesham Ul Haq. SUNY Upstate Medical University, Syracuse, NY. (Control ID \#1334621)
LEARNING OBJECTIVE 1: 1. Recognize the complexity of sudden onset thrombocytopenia in an inpatient setting.

LEARNING OBJECTIVE 2: 2. Distinguish between post transfusion purpura (PTP) and heparin induced thrombocytopenia (HIT) when faced with the dilemma.

CASE: A 59 year- old- African American man with history of hypertension and severe peripheral vascular disease was admitted for evaluation of fever The patient was just recently discharged 7 days prior to the admission, during which he underwent a left below knee amputation, which was complicated with severe bleeding, requiring 2 units of packed red cells (PRBC) transfusion. During the present admission, review of systems was negative. Physical examination was benign except for a temperature of 101.5 degrees F. Labs revealed anemia with a hematocrit of 25 , for which 2 units of $\mathrm{PRBC}$ was given. Over the next day, platelet counts plummeted from 160,000 to 4,000.All heparin products were discontinued empirically. However, HIT antibody was negative. PTP was considered, given the previous history of blood transfusion, and he was started on intravenous immunoglobulin (IVIG). A positive human platelet alloantigen (HPA) antibody confirmed the diagnosis. Platelet counts subsequently improved. After a week, the patient developed right upper extremity pain and was a Doppler ultrasound confirmed the diagnosis of basilic vein thrombosis, and blood counts at the time also revealed a rapid drop in platelet count raising a suspicion for HIT. HIT antibody was positive and the diagnosis of HIT was confirmed with serotonin release assay (SRA). Patient was started on argatroban infusion thereafter and discharged subsequently on warfarin.

DISCUSSION: PTP and HIT are two immune syndromes causing extreme thrombocytopenia, but with marked differences in pathogenesis and treatment. PTP is characterized by marked thrombocytopenia $<15 \mathrm{Gpt} / \mathrm{L}$, with platelet alloantibodies (most commonly anti HPA-1a antibody) following human platelet alloantigen incompatible blood product transfusion which may be complicated by severe hemorrhagic complications. Treatment options include mainly IVIG therapy. HIT is associated with platelet nadir between 20 to $150 \mathrm{Gpt} / \mathrm{L}$, usually with generation of antibodies to heparin and platelet factor 4 (PF4) antigen complexes, and thrombotic complications. Treatment is discontinuation of all heparin products with initiation of danaparoid products or recombinant hirudin. Delayed onset HIT can occur as late as even 3 months after heparin products are discontinued. Our case is unique with the presence of both HPA 1a antibody and HIT antibodies, which has been reported only once before, emphasizing the importance of making the right diagnosis due to the difference in treatment of both the conditions.

POSTINFECTIOUS GLOMERULONEPHRITIS IN AN ELDERLY PATIENT-A CASE REPORT AND REVIEW OF THE LITERATURE Mamatha Racheruvu'; Zipporah Krishnasamy ${ }^{2} .{ }^{1} \mathrm{UAB}$ Montgomery, Montgomery, AL; ${ }^{2}$ University of Alabama at Birmingham, Birmingham, AL. (Control ID \#1334206)

LEARNING OBJECTIVE 1: Recognize the cause of rapidly progressive renal failure in an elderly patient

CASE: 65 yr old white male with history of Diabetes, Hypertension, Congestive Heart Failure, Aortic Stenosis status post aortic valve replacement six weeks earlier was admitted with Methicillin Resistant Staphylococcus Aureus (MRSA) infection at surgical site. On admission Creatinine $(\mathrm{Cr})$ was 1.5 with baseline $\mathrm{Cr}$ of 1.1-1.3. Cr started to increase slowly to 5.5 over a period of one month. During the hospital stay patient was on vancomycin for MRSA infection. On physical exam patient had petechial rash on both lower limbs, 2+pitting edema at sacrum and auscultation of lungs revealed bilateral basal crepitations. Remaining exam was normal. Chemistries showed sodium of $138 \mathrm{mEq} / \mathrm{L}$, potassium of $4.2 \mathrm{mEq} / \mathrm{L}$, chloride of $99 \mathrm{mEq} / \mathrm{L}$, bicarbonate of $30 \mathrm{mEq} / \mathrm{L}$, blood urea nitrogen of $48 \mathrm{mg} / \mathrm{dL}$, and creatinine of $5.5 \mathrm{mg} / \mathrm{dL}$. On Hematology, white blood cell count was 11,000 with differential of $76 \%$ neutrophils, and $6 \%$ eosinophils (Eos). Urine analysis showed yellow, cloudy urine with $\mathrm{PH}$ of $5.0,3+$ protein, $2+$ blood and $1+$ Eos. Random urine sodium was $64 \mathrm{mEq} / \mathrm{L}$, with Fractional excretion (Fe) of sodium of $4 \%$, FeUrea of $67 \%$. 24 hour urine protein was $3.3 \mathrm{~g} / \mathrm{dL}$. Serum Antinuclear Antibody titer was less than 1:80, Rheumatoid factor was negative, complement $\mathrm{C} 3$ and $\mathrm{C} 4$ levels were normal, Anti-neutrophilic cytoplasmic antibodies (ANCA)-C and ANCA-P 
were negative and Hepatitis B was negative. Renal Ultrasound was benign with both kidneys of normal size of $12 \mathrm{~cm}$ each. Biopsy of the petechial rash revealed leukocytoclastic vasculitis due to moxifloxacin, which was started four weeks earlier. Initially the Renal failure was attributed to Allergic Interstitial Nephritis and moxifloxacin was stopped, but $\mathrm{Cr}$ subsequently worsened and then it was thought due to cardio-renal syndrome or Vancomycin toxicity as Vancomycin trough levels were $25 \mathrm{mcg} / \mathrm{ml}(15-20 \mathrm{mcg} / \mathrm{ml})$. Since therapeutic approach for management of these possible etiologies differs, and with worsening renal function it was thought he would benefit from kidney biopsy. Renal Biopsy showed Post Infectious Glomerulonephritis with diffuse mesangial staining for $\operatorname{IgA}(2+)$, C3 (3+) along with one large hump type subepithelial deposit.

DISCUSSION: This case illustrates IgA-dominant acute postinfectious glomerulonephritis (APIGN).The presence of diabetes with recent MRSA infection at the surgical site were the risk factors predisposing to APIGN. So far 109 cases of APIGN were reported. The average age at diagnosis was 60 years. Most patients were Caucasian (53\%) and Asian (26\%), with few African Americans, Hispanics and Native Americans. Underlying diabetes mellitus was present in 27 patients (55\%), others include malignancy, IV drug use, alcoholism, HIV infection and atopic dermatitis. The most common site of infection was skin, infectious agent identified was Staphylococcus in $76 \%$. The latent period between infection and onset of renal disease was typically 1 to 6 weeks. Clinical presentation included renal failure, hematuria, proteinuria and hypertension. The pathogenetic mechanism likely involves specific host responses to the inciting pathogen. On pathology, stronger immunofluorescence staining for $\mathrm{C} 3$ than $\operatorname{IgA}$, and the presence of subepithelial humps on electron microscopy are characteristic of APIGN Despite therapy directed to the infection, prognosis is guarded with less than a fifth of patients fully recovering renal function.

POTASSIUM WASTING IN AN HIV PATIENT ON HIGHLY ACTIVE ANTIRETROVIRAL THERAPY Genevieve Lozier; Stephen Harder. University of Texas - Southwestern, Dallas, TX. (Control ID \#1338825)

LEARNING OBJECTIVE 1: Recognize the potential for proximal tubular toxicity in HIV patients on tenofovir.

LEARNING OBJECTIVE 2: Assess risk factors for the onset of tenofovir-induced Fanconi syndrome.

CASE: A 48 year-old man with HIV treated with Atripla (efavirenz/ emtricitabine/tenofovir) presented with incidental findings of hypokalemia and hyponatremia. Since being diagnosed with HIV he had experienced gradual weight loss, but described no other recent changes in medication or general health and had no diarrhea or vomiting. On physical exam, he was cachetic with dry mucous membranes. Initial laboratory studies demonstrated potassium of $2.7 \mathrm{meq} / \mathrm{L}$, sodium $131 \mathrm{meq} / \mathrm{L}$, chloride $99 \mathrm{meq} / \mathrm{L}$, bicarbonate $19 \mathrm{meq} / \mathrm{L}$, albumin $3.7 \mathrm{~g} / \mathrm{dL}$, and anion gap $13 \mathrm{meq} / \mathrm{L}$, indicative of a nonanion gap metabolic acidosis. A proximal renal tubular acidosis was suggested by a urine sodium of $38 \mathrm{meq} / \mathrm{L}$, potassium $28 \mathrm{meq} / \mathrm{L}$, chloride $35 \mathrm{meq} / \mathrm{L}$, osmolality $285 \mathrm{mOsm} / \mathrm{kg}$, pH 7.0, and urine anion gap $31 \mathrm{meq} / \mathrm{L}$. With a transtubular potassium gradient of 7.6, renal potassium wasting was present. Twenty-four hour urine studies showed additional wasting of phosphate and protein, confirming the diagnosis of Fanconi syndrome.

DISCUSSION: Fanconi Syndrome is characterized by proximal renal tubular dysfunction causing proteinuria, amino aciduria, phosphaturia, glycosuria, and bicarbonate wasting. Fanconi syndrome is most often caused by monoclonal gammopathies and heavy metals, but may be drug-induced as with tenofovir, a first-line component of highly active anti-retroviral therapy (HAART). Tenofovir is excreted by both renal filtration and secretion from proximal tubular cells, which are injured by intracellular tenofovir accumulation. In this case, weight loss was the inciting factor leading to a higher dose relative to the patient's weight. Other risk factors for tenofovir-induced toxicity include advancing age, decreasing renal function, and the addition of nephrotoxic agents. While tenofovir-induced Fanconi syndrome is rare, there is evidence that subclinical renal tubular toxicity is common, and monitoring is warranted. Physicians investigating a renal tubular acidosis in an HIV patient on HAART should keep tenofovir-induced proximal renal tubule dysfunction in their differential diagnosis.
POTASSIUM FOR A BROKEN HEART Sumaira Shaikh'; Suhail Shaikh ${ }^{2}$; James M. Sosman ${ }^{1}$. ${ }^{1}$ University of Wisconsin Hospitals and clinics, Madison, WI; ${ }^{2}$ Sea Mar CHC, Olympia, WA. (Control ID \#1334458)

LEARNING OBJECTIVE 1: Recognize the clinical features of Tako-Tsubo cardiomyopathy and the difficulty in differentiating between Tako-Tsubo cardiomyopathy and Ischemic cardiomyopathy based on history, physical exam, EKG, cardiac markers and echocardiogram

LEARNING OBJECTIVE 2: Recognize that hypokalemia can affect the musculature of the heart along with the electrical system, leading to Tako-Tsubo Cardiomyopathy

CASE: : A 62 yr old lady presented with progressive weakness over the past 6 months requiring her to use a walker to ambulate. She reported extreme anorexia and a $50 \mathrm{lb}$ weight loss. She had not seen a physician in 38 years since the birth of her daughter. PE revealed HR 88, BP 100/60, dry skin, clear chest, cardiac exam with palpitations but no murmurs, and non-focal neuro exam with generalized $4 / 5$ muscle strength. Lab tests revealed $\mathrm{K} 1.2, \mathrm{Mg} \mathrm{0.8}$, Ca 5.7, $\mathrm{Na} 136, \mathrm{Cl}$ 90, $\mathrm{HCO} 3$ 35, BUN 12 Cr 1.36 Glu 95, TSH 35, Hgb 9.9 and elevated Troponin 0.65. EKG showed prolonged PR and QT with LBBB. Her electrolytes were replaced and her PR and QT interval normalized and LBBB resolved. Her weakness was considered secondary to low K . A TTE revealed EjFx 40\% and anterior and anteroseptal wall motion abnormality $\mathrm{c} / \mathrm{w}$ infarction. She was started on metoprolol but had persistently low BP $(90 \mathrm{~s} / 50 \mathrm{~s})$. That same night, the patient reported dyspnea. Exam revealed elevated JVP and bilateral chest crackles. CXR showed bilateral pulmonary edema. Repeat EKG showed anterior infarct along with poor $\mathrm{R}$ wave progression. Troponin increased to 2.33. She was taken for cardiac catheterization next morning, which revealed minimal $\mathrm{CAD}$ with maximum stenosis $<25 \%$ but ventricular apical ballooning with EjFx 25\%. She was thought to have Tako-Tsubo cardiomyopathy, as a result of severe hypokalemia. The hypokalemia may have been from GI losses secondary to laxative abuse or renal losses secondary to diuretic abuse, vomiting, Barter's or Gitelman's syndrome. She was continued on metoprolol tartrate, low dose levothyroxine, potassium replacement and discharged home with outpatient physical therapy.

DISCUSSION: Transient left ventricular (LV) apical ballooning syndrome or Tako-Tsubo cardiomyopathy (TTC) is a syndrome characterized by transient LV dysfunction, ECG changes that mimic acute MI with myocardial enzyme release in the absence of obstructive CAD. TTC was first described in 1991 in Japan and named Takotsubo due to its similar echo appearance to a Japanese pot with a round bottom and narrow neck used for trapping octopuses (LV apical ballooning). This case illustrates how difficult it is to differentiate between TTC and ischemic cardiomyopathy. TTC has been increasingly recognized mostly in post-menopausal women $(90 \%)$, who present with dyspnea and chest pain from a presumed acute MI (prevalence $2.5 \%$ ). TTC is often preceded by emotional or physical stress. Hypokalemia has known effects on the electrical system of the heart which were noted on the patient's admission EKG. Severe hypokalemia may have been the triggering factor for Takotsubo cardiomyopathy in this patient. The patient was seen in cardiology clinic 4 weeks after discharge. She reported feeling better with improved energy and no return of chest pain or dyspnea. Most patients with TTC have complete resolution of cardiomyopathy, within days to weeks after the event.

POTENTIALLY FATAL MUSCLE SPASMS Sana F. Khan; LeAnn Coberly. University of Cincinnati Academic Health Center, Cincinnati, OH. (Control ID \#1310136)

LEARNING OBJECTIVE 1: Recognize the clinical presentation of Tetanus, as timely diagnosis and treatment can prevent severe morbidity. CASE: A healthy 56 year old Caucasian male presented with a 16 hour history of facial, neck and upper extremity twitching and spasms. The symptoms initially started with bilateral, symmetric, painless spasms occurring over the lower face, lasting for a few seconds and resolving spontaneously. The patient was unable to talk during spasms, and occasionally felt a choking sensation. The spasms worsened by talking or activity, and were associated with five episodes of locked jaw earlier in the 
day. The physical exam was otherwise unremarkable. There was no history of recent wounds, sutures, soil or animal exposures. The patient was admitted to the ICU anticipating the need for respiratory support and close monitoring of vital signs. Treatment was initiated with IM tetanus immunoglobulin, IM tetanus toxoid, IV metronidazole and IV diazepam. A dark quiet environment was provided and supportive IV fluids initiated. Supportive care resulted in resolution of symptoms and the patient was transitioned to oral diazepam and metronidazole, and was subsequently discharged home.

DISCUSSION: Tetanus is a potentially fatal disease, with case fatality rates in developed countries as high as $20 \%$. With the advent of successful immunization strategies, only 31 cases per year have been reported from 2002 to 2007. Generalized tetanus is the most commonly recognized type of tetanus and diagnosis is mainly on clinical findings. Though most cases are associated with contaminated wounds, $7 \%$ to $21 \%$ of cases are cryptogenic. Symptoms usually start with episodes of muscle spasms and trismus, followed by stiffness of neck, shoulder and back muscles. Sustained contraction of facial and back muscles produce characteristic risus sardonicus and opisthotonus respectively. Spasms are spontaneous and provoked by slight stimulation. Laryngeal along with respiratory muscle spasms results in respiratory failure which is the leading cause of death. Additional complications include autonomic instability, spinal fractures and rhabdomyolysis with renal failure. Other conditions mimicking generalized tetanus include seizures, dystonic reactions, meningitis/encephalitis, hypocalcemia, and strychnine poisoning. Treatment is aimed at wound debridement, antimicrobial therapy and supportive care. Tetanus immunoglobulin is used to neutralize unbound toxin, whereas metronidazole is currently the preferred antimicrobial. Ventilatory support and an environment without tactile and auditory stimuli are essential. Benzodiazepines are commonly used to control rigidity and spasms. Specific therapies for autonomic instability have not been defined, but labetalol, magnesium sulfate and morphine have been shown to be effective. Besides immunoglobulin, primary immunization with tetanus toxoid is also initiated at time of diagnosis.

PRIMARY EFFUSION LYMPHOMA IN AN HIV-NEGATIVE PATIENT WITH ABDOMINAL DISTENSION Jessica Taff; Nicole Adler. New York University Langone Medical Center, New York, NY. (Control ID \#1334581)

LEARNING OBJECTIVE 1: Primary effusion lymphoma can present atypically with complaints of abdominal distension and ascites.

LEARNING OBJECTIVE 2: Although most common in HIV positive patients or those with immunocompromise, primary effusion lymphoma may occur in HIV negative and otherwise immunocompetent patients. CASE: A 76 year-old Filipino man presented with two weeks of generalized weakness and decreased exercise tolerance. He also endorsed worsening polyuria, polydipsia, and diffuse abdominal swelling that prompted admission. On physical exam, the patient was alert and oriented to self and place. He had jugular venous distension to the angle of the mandible and bibasilar crackles. His abdomen was distended with shifting dullness. He had pitting edema to the thighs bilaterally. Labs were notable for a hemoglobin of $9.2 \mathrm{~g} / \mathrm{dL}$, platelets of $106,000 / \mathrm{mm} 3$, sodium of $109 \mathrm{mmol} / \mathrm{L}$, and chloride of $81 \mathrm{mmol} / \mathrm{L}$. Aspartate aminotransferase was $66 \mathrm{U} / \mathrm{L}$ and alkaline phosphatase was $81 \mathrm{U} / \mathrm{L}$. B-type natriuretic peptide was $883.5 \mathrm{pg} / \mathrm{ml}$ and lactate dehydrogenase was 391U/L. A diagnostic paracentesis revealed 5650 red blood cells $/ \mathrm{mm} 3$ and 930 white blood cells/ $\mathrm{mm} 3$, with $69 \%$ lymphocytes. Abdominal CAT scan revealed a large amount of abdominal and pelvic ascites with ill-defined stranding and nodularity of the peritoneal surface. Liver and spleen were normal. A peripheral blood smear revealed large neoplastic lymphoid cells. Hematoxylin and eosin staining showed pleomorphic large atypical cells with nuclear stippling. The cells were CD38 positive on flow cytometry and positive for CD 138 and Human Herpesvirus - 8 (HHV-8) on immunohistochemistry. The patient was diagnosed with primary effusion lymphoma and elected against treatment. He was transferred to hospice where he died several weeks later.

DISCUSSION: Primary effusion lymphoma (PEL) occurs when there is infection of tumor-clone cells by HHV-8 with subsequent liquid phase growth in serous cavities in the absence of tumor mass. It was originally classified as a distinct type of B cell Non-Hodgin's Lymphoma in AIDS patients who were also positive for HHV-8. The malignant cells are recognized as pre-terminally differentiated B cells. The cells traditionally express a common gene profile distinct from other Non-Hodgkin's lymphoma, with the tumor cell corresponding to a stage of B-cell development intermediate between that of immunoblasts and plasma cells. HHV-8 is thought to be a requirement for pathogenesis by promoting tumor growth. Approximately $70 \%$ of PEL patients are also co-infected with Epstein-Barr virus (EBV), as was our patient, although this relationship is less well clarified. Although patients with PEL most often present with symptomatic pleural effusions, this patient's main complaint was abdominal distension with ascites. His fluid had characteristic findings of PEL, with ascites plasmablastic cellular morphology and positivity for CD 138, HHV-8, and EBV without B-cell markers. Also unique about this case is that the patient was HIV negative. Although there are case reports of PEL in HIV negative patients, these people usually have an associated degree of immunocompromise. Other than older age, this patient did not have any known risk factors for an immunocompromised state. In conclusion, this case presents an unusual diagnosis of primary effusion lymphoma in an HIV negative patient without additional evidence for an immunocompromised state. While rare, his presentation had many of the typical features of this uncommon disease.

PRINZMETAL'S ANGINA: AN UNDER RECOGNIZED CAUSE OF RECURRENT ST ELEVATION MI Pratik Choksy; Rebecca Napier; Gyanendra Sharma. Georgia Health Sciences University, Augusta, GA. (Control ID \#1339356)

LEARNING OBJECTIVE 1: Prinzmetal's angina is a well documented but under recognized etiology of recurrent ST elevation MI, which should be considered in patients with recurrent angina with both typical and atypical cardiac risk factors.

LEARNING OBJECTIVE 2: Medical management with nondihydropyridine calcium channel blockers and nitrates is the key for treatment of Prinzmetal's Angina and helps in preventing recurrent cardiac catheterization.

CASE: A 67 year old male with past medical history significant for hypertension, hyperlipidemia, and tobacco use presented to his primary care physician with complaint of intermittent chest discomfort during the previous 2-3 weeks. The pain was described as a substernal pressure which was nonradiating and occurred daily lasting 5-15 minutes per episode. The pain occurred spontaneously and there was no identifiable association with rest or exertion. EKG was obtained at presentation and consistent with ST elevation in the inferior leads. Patient subsequently underwent urgent cardiac catheterization notable for $90 \%$ proximal and $90 \%$ distal RCA (right coronary artery) lesions for which he underwent uncomplicated balloon angioplasty without stenting. Off note patient had a peak troponin of 0.02 and ejection fraction (EF) of $65 \%$ with mild inferior wall hypokinesis by left ventriculography. At two week follow up, the patient continued to complain of recurrent chest discomfort often occurring upon awakening in the mornings. Patient developed chest pain during his office visit. EKG showed $3 \mathrm{~mm}$ ST elevation in leads II, III and aVF and reciprocal ST depression in leads I and aVL. Patient was given nitroglycerin and repeat EKG showed gradual decrease in ST elevations with symptomatic improvement in the chest discomfort. Given concern for reocclusion of RCA, the patient again underwent urgent cardiac catheterization showing subtotal occlusion of the proximal RCA. During the procedure, intracoronary nitroglycerin was administered with visible resolution of the occlusion via angiography consistent with coronary vasospasm. Patient was therefore initiated on therapy with amlodipine and isosorbide mononitrate and remained symptom free at subsequent follow up visits.

DISCUSSION: Prinzmetal's or variant angina is characterized by a transient reduction in luminal diameter of a coronary artery resulting in spontaneous and often recurrent episodes of angina with EKG findings consistent with ST elevation. Such findings can often result in repeat invasive studies such as cardiac catheterization. Key findings for diagnosis of coronary vasospasm include simultaneous resolution of ST segment changes and anginal symptoms spontaneously or with nitroglycerin, or reversibility of coronary occlusions upon intraluminal injections of nitroglycerin. Though the underlying pathogenesis of variant angina is unclear, there appears to be some component 
of autonomic and endothelial dysfunction resulting in spasm of vascular smooth muscle. Those with variant angina are typically younger, often female, and lack traditional cardiovascular risk factors, though vasospasm may occur in association with atherosclerotic plaques. Symptoms often occur in the early morning hours awakening patients from sleep and may occur in association with exercise, hyperventilation, or substance abuse. Medical therapy is aimed at cardiovascular risk factor reduction and pharmacological management through the vasodilatory effects of non dihydropiridine calcium channel blockers and nitrates.

\section{PROPOFOL USE IN A PATIENT WITH NEUROLEPTIC} MALIGNANT SYNDROME INDUCING HYPERTHERMIA Somy S. George; Swati Choudhary; Narendra Khanchandani; Mark Villeneuve. St. Mary Mercy Hospital, Livonia, MI. (Control ID \#1311432)

LEARNING OBJECTIVE 1: Neuroleptic malignant syndrome (NMS) is a life threatening neurologic emergency associated with the use of neuroleptic agents and characterized by a distinctive clinical syndrome of mental status change, rigidity, hyperthermia and dysautonomia. Central dopamine receptor blockade in the hypothalamus may cause hyperthermia and other signs of dysautonomia. Propofol has been commonly used as a sedative in the intensive care unit and is generally considered safe. Case reports have shown that Propofol can cause hyperthermia in patients who have predisposing factors such as neuroleptic malignant syndrome.

CASE: Patient was a 41 year old caucasian female with past medical history significant for Diabetes Mellitus Type II, Bipolar Disorder and Hypertension was admitted with acute respiratory failure, altered mental status, and a temperature of 104. She was intubated and broad spectrum antibiotics were given for suspicion of pneumonia. Pan-culture was done which showed no bacterial growth. Her home medications included Benztropine, Chlomipramine, Clonazepam, Lamotrigine, Lithium and Ziprasidone. All her medications were stopped on the day of admission secondary to the possibility of Serotonin Syndrome versus Neuroleptic Malignant Syndrome (NMS). She was started on Propofol drip due to agitation and was weaned off slowly within 3 days. Fever resolved within 2 days of admission. Patient was extubated on day 4 but then was re-intubated the following day secondary to continuing respiratory failure. During second intubation, jaw clenching was noticed and one dose of succinylcholine was given for rapid sequence intubation and Propofol was restarted. Patient's temperature slowly started to rise with a rate of approximately 1 degree F/day to a maximum of 107 F. Propofol was then discontinued and patient was given Dantrolene and Bromocriptine for possibility of malignant hyperthermia. Her temperature started decreasing to normothermia within 2 days after discontinuation of Propofol. During the time of her hospital stay, the patient developed multi-organ failure. Due to patient's poor prognosis the family made her hospice and she passed 19 days after admission. Multiple factors were responsible for hyperthermia, including NMS, use of succinylcholine, and propofol. But due to the duration of onset of hyperthermia, propofol was the most likely culprit.

DISCUSSION: Propofol should be used cautiously in patients who have mulitple risk factors to hyperthermia. Alternative sedative agents should be used. Further studies need to be done to understand the exact mechanism of hyperthermia caused by propofol in these high risk patients.

PROTON-PUMP-INHIBITOR INDUCED HEPATITIS Jennifer K. Lue; Daniel Eiras; Nicole Adler. New York University School of Medicine, New York, NY. (Control ID \#1334557)

LEARNING OBJECTIVE 1: Recognize and distinguish the clinical features of drug-induced hepatitis from other etiologies of hepatitis LEARNING OBJECTIVE 2: Manage acute drug-incuded hepatitis with concomitant hepatitis $\mathrm{C}$ infection

CASE: TA 61 year-old Hispanic female presented with jaundice, dark urine and progressive fatigue of three weeks duration. TThe patient presented to her primary care physician three weeks prior to admission with bloating and early satiety, and was started on rabeprazole $20 \mathrm{mg}$ daily. After one week, blood tests were drawn which were notable for an AST/ ALT of 1680/1123 u/L, alkaline phosphatase of $283 \mathrm{u} / \mathrm{L}$, and total bilirubin of $1.7 \mathrm{mg} / \mathrm{dL}$. TThe patient subsequently noted worsening abdominal discomfort, fatigue and jaundice. She was referred for an endoscopy that demonstrated severe gastritis. The patient was then prescribed omeprazole $40 \mathrm{mg}$ daily and ranitidine $20 \mathrm{mg}$ daily, with discontinuation of rabeprazole. IDue to progression of her symptoms the patient presented to our hospital where she was found to have an AST/ALT of 3423/1620 u/L, alkaline phosphatase of $269 \mathrm{u} / \mathrm{L}$, total bilirubin $10 \mathrm{mg} / \mathrm{dL}$, and a direct bilirubin $6 \mathrm{mg} / \mathrm{dL}$. Physical exam was significant for jaundice, icteric sclera, and mild right upper quadrant pain. A CAT scan of the abdomen and pelvis demonstrated several small hepatic cysts, but was otherwise normal. TThe patient's medications including omeprazole were held, and her hepatic enzymes subsequently improved, which was suggestive of a diagnosis of drug-induced hepatitis. ๆIn addition, workup of the patient's liver function abnormalities revealed a positive hepatitis $C$ antibody and viral load of $7 \times 10^{6}$ copies $/ \mathrm{mL}$. qDue to suspicion of acute hepatitis $\mathrm{C}$ versus drug-induced hepatitis, a liver biopsy was performed which was significant for acute drug induced hepatitis intermixed with elements of chronic hepatitis $\mathrm{C}$.

DISCUSSION: ๆIn cases of acute hepatitis of unclear etiology, medications should always be considered as possible culprits and reviewed thoroughly. Although Proton-Pump-Inhibitors (PPIs) are generally recognized as low-risk medications, they should be taken into consideration during the evaluation of hepatotoxicity of unknown origin. \The more common side effects of PPIs are diarrhea, nausea, vomiting, and abdominal pain. Generally, one can see a minimal elevation in liver enzymes secondary to PPI use ${ }^{1}$. However, case reports of acute hepatitis in subjects without previous liver disease have been reported for omeprazole, lansoprazole, and recently pantoprazole ${ }^{2-5}$, but to our knowledge, there are no published cases involving rabeprazole. Our patient had a similar course to those described in the literature, where the development of acute hepatitis resolved spontaneously when the PPI was held. "This patient's acute presentation was likely complicated by her underlying hepatitis $\mathrm{C}$ infection, and highlights the important point that underlying hepatic dysfunction has been shown to alter PPI pharmacokinetics, and may exacerbate the acute presentation of drug-induced hepatitis, although the impairment was less significant in patients with hepatitis compared to cirrhotics ${ }^{6}$. In addition, patients with non-alcoholic fatty liver disease were more likely than patients with hepatitis $\mathrm{C}$ to develop drug-induced hepatitis ${ }^{7}$. It is likely that baseline hepatic dysfunction made our patient more susceptible to liver injury, and this report may be the first known case of drug-induced hepatitis in a patient with chronic hepatitis $\mathrm{C}$.

PULMONARY AMYLOIDOMA: A RARE CAUSE OF DYSPNEA Qura Tul Ain Rashid; Robin Klein; Dominique Cosco. Emory University School of Medicine, Atlanta, GA. (Control ID \#1337547)

LEARNING OBJECTIVE 1: 1. Review the pathophysiology of amyloidosis. 2. Recognize the presentation of nodular pulmonary amyloidosis. 3. Consider pulmonary amyloidosis in the differential diagnosis in patients with calcified nodules on imaging.

CASE: 65 y/o caucasian female presented with new onset dyspnea on exertion for three months. Past medical history was significant for hypertension and pulmonary embolism. Patient denied chest pain, fever or weight loss. Physical exam was unremarkable. Echocardiogram showed normal ejection fraction and mild left ventricular hypertrophy. Right heart catheterization was negative for pulmonary hypertension. Calcified lesions were noted on the chest $\mathrm{x}$-ray. CT chest showed multiple irregular soft tissue masses abutting pleural surface bilaterally. Bronchoscopy revealed normal airway. Biopsy of lung mass showed amorphous eosinophilic material with in lung parenchyma, consistent with amyloidosis. Follow up diagnostic studies including serum and urine protein electrophoresis, free light chain analysis, abdominal fat pad aspiration and bone marrow biopsy were negative for systemic amyloidosis. Patient was diagnosed with nodular form of pulmonary amyloidosis. Pulmonary function test showed mild obstructive disease. Patient is currently being followed by pulmonary service as an out patient for symptom surveillance.

DISCUSSION: Amyloidosis is characterized by the deposition of insoluble amyloid fibrils in extracellular tissue. Classically, these fibrils demonstrate apple-green birefringence under polarized microscopy confirming the presence of amyloid proteins. Protein deposition may occur in association with autoimmune, hereditary, neoplastic or inflammatory conditions. It may involve 
multiple organs systems or localize to a single site. The type of precursor protein, the tissue distribution, and the amount of amyloid deposition largely determine the clinical manifestations. Sites of clinically important amyloid deposition are the kidneys, heart, and liver. Localized pulmonary amyloidosis is defined as amyloid deposition isolated to respiratory tract without systemic deposition. Typically, nodular pulmonary amyloidosis presents in older individuals as asymptomatic nodules identified on chest $\mathrm{x}$-ray. Symptomatic presentations are uncommon. Histology reveals well circumscribed, consolidated masses of amyloid with inflammatory cell infiltrate. Calcification of these lesions is common. We present a case of nodular pulmonary amyloidosis. While often asymptomatic, our patient had subacute dyspnea due to her underlying disease process. The combination of symptoms and calcified nodules on imaging raised suspicion for malignancy. However, demonstration of classic histologic findings confirmed the diagnosis of amyloidosis. Physicians should consider pulmonary amyloidosis in the differential diagnosis of calcified pulmonary nodules.

PULMONARY AND OROPHARYNGEAL KAPOSI SARCOMA IN A HIV-INFECTED MALE Sabyasachi Roy. SJHMC, Phoenix, AZ. (Control ID \#1336671)

LEARNING OBJECTIVE 1: Epidemic or AIDS associated Kaposi Sarcoma (EKS) is a vascular tumor associated with Human Herpes Virus 8 (HHV8) and is an AIDS defining illness. EKS is seen primarily among men who have sex with men as compared to other HIV-infected groups (intravenous drug users, women, transfusion recipients). Research from nine population-based cancer registries reporting to the Surveillance, Epidemiology, and End Results (SEER) program of the National Cancer Institute, demonstrated a significant decline in KS. Rates among white men had risen from 0.5/100,000 people/year in 1973, to between 31.3 and 33.3 from 1987 through 1991, and then declined to 2.8 in 1998. With the introduction of highly active antiretroviral therapy (HAART), EKS is an uncommon finding in HIV-infected persons.

CASE: We report the case of a 44 year old homosexual male patient diagnosed with HIV seven years ago who now presented with new chest $\mathrm{X}$ ray findings of bilateral perihilar opacities and violaceous discoloration of the inside of his mouth. He did not have any skin lesions, cough or fevers. He had a history of alcohol abuse and was non-compliant with his HAART medications. We proceeded with a palatal biopsy which was definitively positive for Kaposi Sarcoma (KS), and a bronchoscopy, which showed multiple violaceous lesions in the tracheobronchial tree. Transbronchial biopsy was negative for KS. Bronchoalveolar lavage (BAL) studies for bacteria, viruses, PCP were also negative. A presumptive diagnosis of pulmonary KS and a definitive diagnosis of oropharyngeal KS was made and the patient was started on Taxol (Paclitaxel) and HAART therapy. DISCUSSION: The prevalence and aggressiveness of EKS has dramatically decreased since the introduction of HAART. Cutaneous lesions are still the most common presentation. Visceral manifestations are rarer and, in the absence of any skin findings can be a challenging diagnosis. Common extra-cutaneous sites involved are the oral cavity, respiratory system and GI tract. Bronchoscopy, upper GI endoscopy and colonoscopy are required for definitive diagnosis. Skin lesions vary in color, are often elliptical, along skin tension lines and may be papular, plaque like or fungating in appearance. Oral lesions commonly affect the palate and gingival mucosa. GI lesions occur as isolated or confluent hemorrhagic nodules in any part of the GI tract, and they may be asymptomatic or they may cause pain, nausea, bleeding, malabsorption or obstruction. These are present in about $40 \%$ of patients at time of diagnosis in the absence of HAART. Respiratory symptoms include chest pain, cough, dyspnea, fever, hemoptysis or asymptomatic with variable finding on chest radiographs. Bronchoscopic lesions typically appear as raised cherry-red spots. Widespread systemic involvement has also been described in the heart, pancreas, liver, testes, bone marrow and even skeletal muscle. Biopsy is the mainstay of definitive diagnosis, but a presumptive diagnosis can be made, as in the above case with characteristic lesions in the trachea and negative BAL studies for other pathogens. Physicians and dentists should maintain a high degree of suspicion in identifying EKS lesions even in the absence of classical skin findings, and proceed with invasive evaluations if deemed necessary.
RASH AND ARTHRITIS Qura Tul Ain Rashid; Robin Klein; Kristina L. Lundberg. Emory University School of Medicine, Atlanta, GA. (Control ID \#1337107)

LEARNING OBJECTIVE 1: Recognize parvovirus infection as a cause of poly arthritis in immunocompetent adult.

LEARNING OBJECTIVE 2: Recognize the difference between clinical features of parvovirus infection in children and adults.

CASE: 30 year old African American male presents with diffuse joint pains and rash for four days. The nonpruritic rash started on his wrist, then spread to abdomen, chest and lower extremities, sparing palms and soles. He reported pain and swelling in his wrists and knees. He had no recent travel and denied fever, chills or weight loss. He denied sick contacts but did have small children at home. He did report unprotected sex a week before onset of symptoms. He denied a history of arthritis or autoimmune disease. Skin exam revealed blanching violaceous macules and papules on his abdomen, anterior chest, and extremities. Right wrist, hand and knee joint were mildly swollen and tender. Admission labs revealed an ESR of $87 \mathrm{~mm} / \mathrm{h}$, AST of $98 \mathrm{u} / \mathrm{L}$, and ALT of $189 \mathrm{u} / \mathrm{L}$. Patient was treated empirically for disseminated gonococcal infection. HIV viral load, RPR, and hepatitis panel were negative. Urine gonorrhea test was negative and antibiotics were discontinued. Rheumatoid factor and ANA were negative; complement levels were normal. Parvovirus antibody was elevated with $\operatorname{IgM}$ of $2.2 \mathrm{mg} / \mathrm{dL}$ and $\mathrm{IgG} 4.8 \mathrm{mg} / \mathrm{dL}$. He was diagnosed with parvovirus infection, was treated with analgesics, and symptoms improved over weeks.

DISCUSSION: Parvovirus B19 is a member of the Parvoviridae family of DNA viruses that affects humans. In children and adolescents, parvovirus infection presents with the classic 'slapped check' rash, a lacy rash on the trunk and extremities, or papules in a stocking glove distribution. In adults, the rash is less prominent and infection typically manifests as arthritis in the hands, wrists, and knees. Symptomatic infections are more common in females than males. Typically, symptoms last 2 to 3 weeks but in up to $20 \%$ of patients, arthritis can last weeks to months. Parvovirus is highly infectious and easily spreads between individuals by respiratory droplets. About half of household contacts will be infected following exposure to an infected family member. Since $50 \%$ of adults were exposed as children, many will not manifest symptoms due to prior antibody production. It is most contagious during active viral replication which occurs 5 to 10 days after initial exposure. Rash and arthralgia signify B19 specific antibody production and patients are no longer contagious once these symptoms appear. Given the nonspecific symptoms, parvovirus infection is often confused with autoimmune diseases such as rheumatoid arthritis, lupus, seronegative arthropathies, or other infections. In our patient, the initial suspicion for acute HIV or gonococcal disease was high due to his sexual history. Parvovirus was considered only after he reported contact with young children given its highly infectious nature. As this case illustrates, parvovirus infection should be considered in adults with arthritis despite the absence of the classic features that are common in children.

RASH AND BODY ACHES IN A RETURNING TRAVELER James Y. Wang; Glenn Mathisen; Michael Rotblatt. Olive View - UCLA Medical Center, Sylmar, CA. (Control ID \#1308400)

LEARNING OBJECTIVE 1: Recognize the initial clinical presentation of Dengue fever in patients with recent travel

LEARNING OBJECTIVE 2: Utilize the CDC website to obtain information on epidemics in various countries

CASE: A 54-year-old healthy woman developed fever, an itchy leg rash, mild nausea, and epigastric pain immediately upon return from a trip to El Salvador. She presented to the ED one week later with new-onset severe "leg and joint pain." She denied sick contacts during her travel. Upon presentation to the ED, the patient was afebrile and the exam was significant for epigastric tenderness and scattered oropharyngeal and bilateral lower extremity petechiae. The leukocyte count and coagulation studies were normal; however, the patient had thrombocytopenia $(19,000$ cells $/ \mathrm{mm} 3)$ and abnormal liver tests (AST;ALT were 5-10 times above normal). The patient was admitted and treated with IV fluids. Studies including peripheral smear (for malaria), urinalysis, blood/urine cultures and assays for HIV, ANA, CMV, 
EBV and leptospirosis were ordered and found to be negative. During her hospital stay, the patient remained afebrile without hemodynamic instability. No new petechiae were noted and platelet counts trended upward rapidly; the liver tests improved and trended back to baseline. At discharge, the patient was clinically improved and her platelet count had increased to 80,000 cells $/ \mathrm{mm} 3$. One week later, specific serologies drawn at the time of admission (ELISA IgM) confirmed dengue as the cause of the patient's clinical syndrome.

DISCUSSION: Dengue fever should be high on the differential diagnosis in patients with fever and rash who have recently traveled to an endemic region. Since serological confirmation of dengue viral infection may take up to one week, diagnosis and therapy must be initially based on clinical suspicion. The presence of abrupt onset of fever, severe myalgias/ arthralgias, and severe headache are common. The presence of a blanching macular rash is also an early clue to the diagnosis. In addition, there is a biphasic pattern to the disease with subsequent recurrence of fever, often accompanied by a generalized maculopapular rash. Patients may develop petechiae in association with thrombocytopenia and/or capillary fragility. In dengue hemorrhagic fever, the most severe form of dengue, widespread hemorrhage may occur with associated hypotension. Our patient presented relatively late during the recovery period - she had a history of previous rash; however, on our exam the only finding was petechiae due to thrombocytopenia. The abnormal liver tests suggested a concomitant hepatitis, a common finding in dengue. Fortunately, our patient improved rapidly and did not have evidence of hemorrhage. Diagnosis of dengue depends on the typical clinical presentation with subsequent serological confirmation. Treatment is primarily supportive with transfusions and IV fluids for those with hypotension or extensive bleeding. In the returning traveler with fever, the clinician needs a high index of suspicion and should look for the presence of clinical clues including the typical rash, severe myalgias or arthralgias, relative bradycardia, and the common clinical/ laboratory findings associated with dengue. In our case, going to the CDC website after her serologies returned revealed that, at the time of the patient's presentation, El Salvador was in the midst of a dengue fever epidemic. Checking this website on initial presentation would have helped us make an earlier presumptive diagnosis.

REACTIVATION OF TROPICAL DISEASE AS A PRESENTING DIAGNOSIS OF AIDS Alison B. Rapoport ${ }^{1,2}$; Genevieve Bergeron ${ }^{1,2}$; Linda Shipton ${ }^{1,2} .{ }^{1}$ Cambridge Health Alliance, Cambridge, MA; ${ }^{2}$ Harvard University, Cambridge, MA. (Control ID \#1324749)

LEARNING OBJECTIVE 1: Recognize the clinical features of visceral leishmaniasis and its occurrence with HIV co-infection.

CASE: A 32 year old man from El Salvador with no significant past medical history presented in the summer to a Boston-area hospital with subjective fevers, chills, diaphoresis, myalgias, abdominal bloating and cough. He immigrated to the U.S. in 2005, lives in an apartment with his brother and works in a restaurant preparing food. He is sexually active with females only and admits to recent encounters with sex workers. He denied recent travel, history of intravenous drug use or alcoholism. Vital signs: temperature 103.8 , heart rate 112 , BP $101 / 59$, respiratory rate 16 . Physical exam was significant for a distended, non-tender abdomen with a palpable spleen tip and no hepatomegaly. Cardiopulmonary, musculoskeletal, neurologic and skin exams were normal. Significant laboratory abnormalities included leukopenia (WBC $2.5 \mathrm{cells} / \mathrm{ml}$ ), anemia (Hemoglobin $8.7 \mathrm{~g} / \mathrm{dl}$, normal MCV), thrombocytopenia (platelets $50,000 \mathrm{TH} / \mathrm{uL}$ ) and elevated $\mathrm{LDH}$ (421 IU/L). Peripheral smear confirmed thrombocytopenia but was otherwise normal. Blood chemistry, liver function tests, CXR and head CT were unremarkable. On hospital day 1, testing was done for HIV $1 / 2$ antibodies and viral load, Parvovirus, Epstein Barr Virus, Cytomegalovirus, Hepatitis B and C, Borrelia Burgdorferi, Ehrlichia and Babesia. Blood cultures were done and a PPD was placed. Abdominal ultrasound revealed splenomegaly of $17 \mathrm{~cm}$ without hepatomegaly, portal hypertension or ascites. CT of the chest, abdomen, and pelvis revealed splenomegaly and nonspecific paraaortic and retroperitoneal lymphadenopathy. On hospital day 2, the patient's HIV antibody returned positive with a CD4 count of 15 cell/uL and viral load $>500,000$ copies $/ \mathrm{ml}$. EGD and CSF evaluations were normal. He was started on prophylaxis for Mycobacterium Avium Complex and Pneumocystis
Jiroveci. Several days into hospitalization he remained febrile. All other studies returned negative, prompting the decision to pursue bone marrow biopsy to evaluate for infectious and malignant processes. Biopsy revealed "macrophages containing numerous amastigotes with a few well visualized kinetoplasts". Samples were sent to the CDC for PCR analysis, confirming the diagnosis of Leishmania Donovani Chagasi. Of interest, serum indirect florescent antibody testing was negative for $\mathrm{L}$. Donovani. The patient was started on antiretrovirals and Liposomal Amphotericin, therapies he continues to date, now five months after initial presentation. He has returned to work and his HIV viral load is undetectable. Despite these gains, he remains pancytopenic (WBC $1.8 \mathrm{TH} / \mathrm{uL}, \mathrm{Hgb} 9.3 \mathrm{~g} / \mathrm{dl}$, Plt $67 \mathrm{TH} / \mathrm{uL}$ ) with a CD4 count of 16 cells/uL, worsening splenomegaly (now $>20 \mathrm{~cm}$ ) and the need for indefinite Liposomal Amphotericin therapy pending CD4 reconstitution.

DISCUSSION: HIV and Visceral Leishmaniasis (VL) co-infection can lead to highly variable clinical presentations. Serologic testing for VL exists (IFA, ELISA, DAT), however poor sensitivity in co-infection often requires PCR testing or visualization of amastigotes (typically in bone marrow or splenic specimens) for diagnosis. In the U.S., VL is treated with Liposomal Amphotericin given its favorable safety and efficacy profile, but cost limits its use in the developing world. Treating VL in HIV-infected patients is especially challenging due to diminished drug efficacy, long treatment courses and high rates of relapse and death despite treatment.

RED AND SWOLLEN: A CASE OF POLYARTICULAR GOUT ARTHRITIS. Bassel Obaid; Jennifer Dooley. UT, Chattanooga, Chattanooga, TN. (Control ID \#1339925)

LEARNING OBJECTIVE 1: To highlight a case of polyarticular gout arthritis as first manifestation of gout in a patient with multiple risk factors of gout.

CASE: 57 year-old Caucasian male presented to ED complaining of painful swollen knees and ankles started 3 days ago, and gradually got worse that he became chair-bound for 24 hours prior to admission. He denies fever chills, skin rash, any recent trauma or over use of joints or bug bites. He had similar episode about a year ago that lasted few days before gradually subsided, he did not seek any medical help then. Past medical history includes diabetes mellitus type 2 , hypertension, coronary artery disease status post arteriography and angioplasty, chronic kidney disease stage 3 and tobacco abuse. No history of sexually transmitted disease before. He is unemployed and lives with girlfriend with whom he has monogamous relationship for the past three years. He denies alcohol or drug abuse. His medications include insulin, carvidelol, furosemide, aspirin, digoxin and lisinopril. Vital signs were: oral temperature 98.2, blood pressure 142/74, Heart rate 100, RR 17. On exam he appears uncomfortable due to pain but otherwise nontoxic, he was awake and fully oriented. Musculoskeletal exam reveals swelling tenderness, warmness and mild erythema over knees and ankles bilaterally with limited range of motion in both knee joints due to pain, no evidence of trauma, bites, rash or tophaceous deposits on skin exam. Other joints appear within normal limits. Rest of physical exam was unremarkable. Initial laboratory studies showed WBC of 14.9, $\mathrm{Hb}$ 13.4, Sodium 132, Urea 38 and creatinine 2.1(baseline 1.2), glucose 99, uric acid was $12.5, \mathrm{CRP}>16 \mathrm{mg} / \mathrm{dl}$, negative RPR and HIV test and normal liver function test. Patient had left knee arthrocentesis and joint fluid analysis showed WBC $>90,000$ with $90 \%$ PMNs, protein 5.2 grams and glucose of 53 . Microscopic exam reveals uric acid crystals whereas gram stain and culture were negative. Patient was admitted to the hospital and started on oral prednisone with good response.

DISCUSSION: Gout is a common inflammatory arthritis caused by articular precipitation of monosodium urate crystals. It usually affects the first metatarsophalangeal joint of the foot and less commonly other joints, such as wrists, elbows, knees and ankles. It is characterized by recurrent episodes of inflammatory arthritis, tophaceous soft tissue deposits of monosodium urate crystals, uric acid renal calculi and chronic nephropathy. Polyarticular gouty arthritis is the initial manifestation in less than 20 percent of patients with gout, but occurs with increasing frequency in later flares. Polyarticular symptoms are particularly common late in the course of untreated gout, when multiple recurrences, short or absent symptom-free intervals, and palpable tophaceous deposits are common. Treatment options of acute gout arthritis include NSAIDS, Colchicine, or corticosteroids 
(systemic or intraarticular). Use of interlukine-1 inhibitors (kanacinomab) is still under investigation. For patients with polyarticular involvement, systemic glucocorticoid is preferred. In our case, we chose to treat with $20 \mathrm{mg}$ of oral prednisone that was gradually tapered over 7 days.

\section{RENAL INVOLVEMENT IN LEVAMISOLE CONTAMINATED} COCAINE VASCULITIS. Adetokunbo F. Oluwasanjo; Richard Alweis. The Reading Hospital and Medical Center, West Reading, PA. (Control ID \#1333855)

LEARNING OBJECTIVE 1: Recognise renal complications of levamisolecontaminated cocaine vasculitis

CASE: A 46 year old Hispanic male with a history of cocaine abuse presented initially with recurrent painful necrotic purpuric plaques involving his earlobes, trunk and extremities associated with diffuse arthralgias and hematuria. Significant laboratory findings revealed leukopenia, acute kidney injury, urine toxicology positive for cocaine and blood toxicology positive for levamisole. A presumptive diagnosis of levamisole-induced vasculitis was made and he was started on $30 \mathrm{mg}$ of Prednisone twice a day. On day 4 of admission, the patient was discharged with significant resolution of his skin lesions, normal white cell count and resolving renal failure. His work up was positive for $\mathrm{p}$ ANCA and lupus anticoagulant, but negative for anti-cardiolipin antibodies. Over the next 9 months, the patient demonstrated non-compliance to medications and poor follow-up with medical appointments. He presented twice to the emergency department during this time with diffuse arthralgias and was noted on both occasions to have worsening renal function. He claimed he had abstained from cocaine usage over the last 5 months, which was corroborated by the absence of cutaneous lesions as well as negative urine screens for cocaine on both occasions. Due to his worsening renal function (peak creatinine of $5.2 \mathrm{mg} / \mathrm{dL}$ ), a renal biopsy was done that revealed a pauciimmune, segmental, necrotizing, concentric and sclerosing glomerulonephritis. This overall picture was consistent with microscopic polyangiitis and he was started on high dose corticosteroids and rituximab. His renal function slowly improved, with his creatinine decreasing to $2.2 \mathrm{mg} / \mathrm{dL}$ prior to discharge DISCUSSION: Levamisole is a veterinary anti-helmintic that has been used as a human anti-neoplastic immunomodulatory agent. It is illegally used to adulterate cocaine presumably because it is cheap and adds bulk, and also because it enhances the dopaminergic effects of cocaine. Recently, there have been a number of case reports describing a classic ANCA-associated cutaneous vasculopathy secondary to the use of levamisole-contaminated cocaine. Skin biopsies of cutaneous lesions in this disease are also reportedly consistent with microscopic polyangiitis. The incidence appears to be increasing and is gaining public health significance as the amount of cocaine entering the US containing levamisole is reportedly as high as $82 \%$. The progression and pathology of levamisole-induced vasculitis is not fully understood and there are rising concerns about the systemic complications of levamisole as more becomes known about this agent. In this case report, the patient had resolution of the cutaneous manifestations of the vasculitis but persistent worsening of his renal function in spite of reported abstinence and laboratory findings confirming this. Clinicians need to be aware of the fact that while abstinence improves the skin manifestations of the disease, it appears that systemic involvement may continue to progress and require long term therapy and follow-up.

RETAINED RISK Julie Kim; Brian Brinkerhoff; Kurt J. Pfeifer. Medical College of Wisconsin Affiliated Hospitals, Milwaukee, WI. (Control ID \#1333782)

LEARNING OBJECTIVE 1: Recognize epithelioid angiosarcomas as a rare complication of chronically retained synthetic materials

LEARNING OBJECTIVE 2: Identify patients at risk for development of epithelioid angiosarcomas

CASE: A 70-year old man with a history of end-stage renal disease secondary to hypertension treated with deceased donor kidney transplantation presented with a four-month history of recurrent coagulase-negative Staphylococcus softtissue infection directly over his right upper arm polytetrafluoroethylene (PTFE) hemodialysis graft site. He reported no complications with the graft while undergoing hemodialysis for approximately three years prior to receiving his donated kidney. After his transplant, the patient inquired about removing the graft but was advised against elective surgery to avoid unnecessary complications. The graft remained in place for an additional seven years to the time of his recurrent infections. Wound cultures were positive for coagulasenegative Staphylococcus infection, and the patient failed multiple oral and intravenous antibiotic therapies. Due to the patient's immunocompromised status and failure to respond to antibiotics, magnetic resonance imaging (MRI) of his right upper arm graft site was performed, which showed an $8.2 \times 13.6 \mathrm{~cm}$ vertical mass concerning for ulcerated malignancy. The mass was resected, and histopathological analysis revealed diffuse skin infiltration with a high grade malignant vascular neoplasm which stained positive for endothelial cell marker CD31, consistent with a diagnosis of epithelioid angiosarcoma. Two weeks later, a $2.5 \times 2.0 \mathrm{~cm}$ right thigh mass was found, excised, and confirmed to be an epithelioid angiosarcoma metastasis. He was referred to oncology for recommendations regarding chemotherapy treatment.

DISCUSSION: Epithelioid angiosarcomas are extremely rare and aggressive neoplasms comprising $<1 \%$ of all soft tissue tumors. They typically arise de novo in superficial or deep soft tissues but have been known to occur in the setting of retained synthetic materials, particularly Dacron(C) grafts. Epithelioid angiosarcomas often present as large hemorrhagic masses and are easily confused with hematomas or local infections. These tumors readily metastasize to local lymph nodes, lung, bone, and remote soft tissue and carry a median survival of 7 months. Negative prognostic factors include older age, histologic grade, size $(>5 \mathrm{~cm})$, and retroperitoneal location. Very few cases of angiosarcoma arising in arteriovenous (AV) fistulae of renal transplant patients have been documented in the literature. It is well established that renal transplant patients have an increased risk of malignancy overall due to immunosuppression with common malignancies including basal cell carcinoma, Kaposi's sarcoma, and lymphoma. Complicating the diagnosis of epithelioid angiosarcomas in the setting of an $\mathrm{AV}$ graft site is their association with hematoma and infection. Given the dismal prognosis of epithelioid angiosarcomas, prompt clinical evaluation and diagnosis is critical.

RETURN OF "THE GREEN DEMON": ABSINTHE AS A POTENTIAL FACTOR IN EXACERBATING EXERTIONAL RHABDOMYOLYSIS Jill Jin; Elijah Wasson. Olive View-UCLA Medical Center, Sylmar, CA. (Control ID \#1338795)

LEARNING OBJECTIVE 1: Recognize eccentric exercise such as the P90x Extreme Home Fitness System as a risk factor for exertional rhabdomyolysis even in baseline physically active individuals.

LEARNING OBJECTIVE 2: Recognize consumption of Absinthe alcohol as a potential factor in exacerbating exertional rhabdomyolysis. CASE: A 21-year-old previously healthy and physically active male presented to the Emergency Department for two days of progressive thigh and buttocks pain as well as dark urine following a one-hour P90x workout involving eccentric strength-training exercises. Patient was physically active at baseline with both aerobic and nonaerobic exercise, though never with P90x. On exam, he was markedly tender in the bilateral thighs/ buttocks but otherwise neurovascularly intact. Initial creatine kinase (CK) level was $>45,100$ (maximum reported value) and urinalysis showed large blood without red blood cells. Patient was diagnosed with exertional rhabdomyolysis and treated supportively with intravenous fluids. On the 5th hospital day however, patient's CK level remained $>45,100$ despite continuous intravenous hydration, which raised concern for a secondary process exacerbating muscle injury. Patient had no prior history of rhabdomyolysis, even with much more prolonged and intensive exercise. Patient denied any drug use including cocaine, but further questioning revealed that he had consumed six shots of Absinthe alcohol following his workout, which he had previously never done. Patient's CK level finally decreased to $<5000$ on the 8 th hospital day, at which time he was discharged with subsequent full recovery. Patient's kidney function remained normal throughout admission.

DISCUSSION: This case of exertional rhabdomyolysis is unusual in the severity of muscle injury and length of recovery time out-of-proportion to level of exertion. The patient's presentation was complicated by two factors: 1) eccentric nature of exercise, and 2) consumption of significant amount of Absinthe alcohol following exercise. Though the development 
of exertional rhabdomyolysis can be unpredictable in relation to type and amount of exercise, and is certainly more often seen with eccentric exercise, generally CK levels peak 1-3 days following exertion and decline rapidly within 3-5 days. There are case reports of exertional rhabdomyolysis where CK levels take over one week to resolve, but these often involve a secondary muscle insult such as medications (e.g. statins), genetic predisposition (e.g. sickle cell trait, metabolic myopathy), or extreme hot weather/hyperthermia, none of which were present in this case. Although alcohol in itself can also cause rhabdomyolysis, this is usually in the context of prolonged immobilization/coma secondary to severe intoxication, also not present here. Thus, in this case where no clear secondary insult could be identified, it is possible that Absinthe consumption resulted in some level of direct muscle toxicity leading to the severe presentation. Absinthe is a highly alcoholic (45-74\% alcohol by volume) spirit made from the Wormwood plant historically associated with hallucinations and seizures, causing it to be banned in the U.S. from 1915 until 2007 when the FDA instituted new regulations on the maximum content of thujone in Absinthe. The mechanism of action of thujone is unclear. There are currently no case reports on Absinthe alone causing rhabdomyolysis, though it may be an interesting area of further case reporting, especially with the recent legalization of Absinthe in the U.S.

RHABDOMYOLYSIS INDUCED BY A MILD CASE OF SALMONELLA GASTROENTERITIS Anjali Singla; Anunta Virapongse. Lenox Hill Hospital, New York, NY. (Control ID \#1338951)

LEARNING OBJECTIVE 1: Recognize that rhabdomyolysis can be a rare complication of infectious diarrhea

CASE: We report a case of a 19 -year-old male with no past medical history who presented with two days of abdominal pain, chills, diarrhea, and dark colored urine. Upon presentation, he described dull left lower quadrant abdominal pain and cramping, as well as 10 bouts of diarrhea with occasional blood over 24 hours. The patient denied subjective fevers, previous similar episodes, sick contacts, recent travel or alcohol use. On examination, he was afebrile with stable vital signs, dry mucous membranes, and with mild abdominal tenderness in the epigastric area and left lower quadrant without guarding or rebound tenderness. Labs were significant for aspartate aminotransferase $1602 \mathrm{U} / \mathrm{L}$, alanine aminotransferase $452 \mathrm{U} / \mathrm{L}$, alkaline phosphatase $59 \mathrm{U} / \mathrm{L}$, and white blood cells (WBC) $6.2 \times 103 / \mathrm{uL}$. Serum electrolytes, calcium and phosphate were within normal limits. Urinanalysis revealed 5-10 WBC/HPF, 5-10 RBC/HPF, and large blood and was negative for nitrites and leukocyte esterase. A computerized tomography scan of the abdomen showed wall thickening and pericolonic inflammatory changes consistent with infectious or inflammatory colitis. The patient was started on empiric antibiotics and stool studies were sent to the lab for evaluation for likely gastroenteritis. Given the degree of transaminitis and large blood in the urine, a serum creatinine kinase $(\mathrm{CK})$ was obtained and found to be $112,360 \mathrm{U} / \mathrm{L}$. Erythrocyte sedimentation rate was normal at $11 \mathrm{~mm} / \mathrm{hr}$. Upon further questioning, the patient revealed that four days prior to admission he had exerted himself with strenuous running and weight-lifting. Antibiotics were discontinued and intravenous hydration with normal saline was increased to $300 \mathrm{cc} /$ hour to treat rhabdomyolysis. We closely monitored the patient's clinical status, CK level, renal function, electrolytes (calcium, phosphorous, potassium), and fluid balance to ensure the patient was recovering from both the gastroenteritis and rhabdomyolysis. Symptoms and labs improved within the next 24 hours. Stool studies later revealed Salmonella species (further speciation pending).

DISCUSSION: Rhabdomyolysis is a rare complication of Salmonella infection, and generally presents with other severe complications, such as acute renal failure, hepatitis, cholecystitis, pancreatitis, and septicemia. The pathophysiology behind Salmonella induced rhabdomyolysis is poorly understood, and is likely multifactorial. Current literature suggests involvement of increased intracellular calcium concentrations, along with factors such as tissue hypoxia, bacterial toxin release, direct bacterial muscle invasion, and alteration of enzyme activity. To our knowledge, this is the first case of rhabdomyolysis seen in Salmonella gastroenteritis without concomitant sepsis and multi-organ system failure. In this case, the patient's Salmonella-induced rhabdomyolysis may have been exacerbated by recent exercise, thereby explaining the degree of CK elevation in the setting of relatively mild gastroenteritis. Clinicians are urged to monitor patients with even mild Salmonella gastroenteritis closely, as rhabdomyolysis, infectious involvement of other organs, sepsis, and multi-organ failure are all possible complications that can be avoided with early recognition and awareness.

RUNNING ON EMPTY Anjali Masand. Montefiore Medical Center, Bronx, NY. (Control ID \#1334950)

LEARNING OBJECTIVE 1: Recognize anemia as an important cause of heart failure.

LEARNING OBJECTIVE 2: Understand the pathophysiology of anemia-induced high output heart failure.

CASE: A 43 year-old woman with a history of menorrhagia presented with three weeks of progressively worsening shortness of breath and lower extremity swelling. She had a heart rate of 120 beats per minute and conjunctival pallor. Her jugular venous pressure was elevated, and she had an enlarged, laterally displaced PMI. There was lower extremity pitting edema extending up to the thighs bilaterally. There was four chamber dilatation by echocardiography, with an ejection fraction of $60 \%$. The initial hemoglobin level was $2.5 \mathrm{~g} / \mathrm{dL}$, and the mean corpuscular volume was $47.2 \mathrm{fL}$. Results of iron studies revealed severe iron-deficiency anemia, with an iron level of $13 \mathrm{ng} / \mathrm{mL}$, total iron-binding capacity of $410 \mathrm{ng} / \mathrm{mL}$, percent iron saturation of $3 \%$, and ferritin of $5 \mathrm{ng} / \mathrm{mL}$. After 2 units of packed red blood cells and several days of intravenous Furosemide, she had improvement of her symptoms.

DISCUSSION: Heart failure is among the most common conditions that an internist treats, but the diagnosis is not always secondary to structural heart disease. Typically, patients with heart failure have either systolic or diastolic dysfunction with a low or normal cardiac output, respectively. However, in some cases the resting cardiac index is elevated beyond the normal range of 2.5 to $4.0 \mathrm{~L} / \mathrm{min}$ per $\mathrm{m} 2$. A number of disorders can lead to a rise in cardiac output resulting in high output heart failure, and iron deficiency anemia is an important cause. Other conditions that can lead to high output heart failure include sepsis, systemic arteriovenous fistulas, thyrotoxicosis, beriberi, multiple myeloma, obesity, pregnancy, and carcinoid syndrome. Several characteristic findings are usually seen on physical examination in patients with high output heart failure. The pulse pressure is typically wide, and the pulse is usually bounding with a quick upstroke. Pistol-shot sounds auscultated over the femoral arteries and a systolic bruit heard over the carotid arteries are both highly suggestive of elevated left ventricular stroke volume due to a hyperdynamic state. In high output heart failure, patients typically have warm rather than cold extremities due to low systemic vascular resistance and peripheral vasodilation. Patients with chronic high output may develop signs of pulmonary and systemic congestion associated with low output heart failure, including raised jugular venous pressure, pulmonary rales, and peripheral edema. Only in cases of severe anemia (hemoglobin less than $5 \mathrm{~g} / \mathrm{dL}$ ) does heart failure develop in the absence of underlying heart disease. Although the mechanism is not completely understood, it is postulated that anemia can cause peripheral vasodilation through increased renal and vascular nitric oxide production. This, in turn, causes low systemic vascular resistance. Severe anemia also results in reduced serum viscosity. Ineffective blood pressure and volume leads to chronic activation of the sympathetic nervous system, renin-angiotensinaldosterone axis, and increased serum vasopressin concentrations. Over time, chronic volume overload and increased stroke volume gradually cause ventricular enlargement, remodeling, and heart failure. Clinicians should be able to recognize the signs and symptoms of high output heart failure, as it is often associated with a potentially reversible etiology.

ST SEGMENT ELEVATION IN AVR HERALDING LEFT MAIN CORONARY ARTERY (LMCA) OCCLUSION IN FATAL MYOCARDIAL INFARCTION Chinyelu Ofodile. Virginia Tech Carilion School of Medicine, Roanoke, VA. (Control ID \#1340296)

LEARNING OBJECTIVE 1: The importance of lead aVR to localizing and predicting Left main coronary artery obstruction

LEARNING OBJECTIVE 2: evaluation of studies showing the imprtance of lead aVR 
CASE: A 41 year old female, known hypertensive smoker, presented to Emergency Department (ED) with a two hour history of severe, substernal chest pain. Electrocardiogram (EKG) in ED was significant for $4 \mathrm{~mm} \mathrm{ST-}$ elevation in AVR, $2 \mathrm{~mm}$ ST elevation on Lead V1, and diffuse ST depression in all other leads. Initial Troponin I was $0.31 \mathrm{ng} / \mathrm{ml}$. She was taken emergently for cardiac catheterization, which revealed $99 \%$ ostial stenosis of the LMCA, patent Right Coronary Artery (RCA). Emergent Coronary Artery Bypass Grafting (CABG) was considered, however patient went into cardiac arrest and expired.

DISCUSSION: Lead aVR is the only ECG lead that records electrical activity from the basal interventricular septum. This portion receives its blood supply either from the proximal septal branches of the left anterior descending artery or from the posterior descending branch of the RCA in those with prior Left Coronary Artery (LCA) occlusion. Patients with aVR ST-segment elevation may have severe proximal LCA disease, usually LMCA disease. High ST-elevation in lead aVR, compared to V1 was a useful indicator for predicting acute LMCA obstruction, mandating immediate aggressive treatment. aVR ST-elevation has been associated with higher 30-day mortality independent of concomitant ST-segment changes in the other ECG leads. This association was strong for patients with Anterior AMI with an ST-level cut point $>=1.5 \mathrm{~mm}$, and for patients with an inferior AMI with an ST level cut point of $>=1 \mathrm{~mm}$. When STelevation was present, 30-day mortality was high and similar for both Anterior and inferior AMI. ST-elevation in aVR also has an important role in exercise stress testing where it has an overall predictive accuracy of $80 \%$ for LMCA or ostial LAD stenosis. In some cases it can be used as an adjunct to cardiac scintigraphy, conferring an increased sensitivity to detecting subendocardial ischemia. ST-segment depression in aVR also has prognostic significance. In patients with acute Anterior MI, the concomitant presence of aVR ST-depression was also associated with higher 30day mortality. Resolution in ST-depression in aVR in patients undergoing fibrinolytic therapy was associated with lower mortality. The utility of aVR extends out of the scope of ischemic heart disease. Prominent PR segment elevation can be indicative of acute pericarditis and a prominent $\mathrm{R}$ wave can indicate significant tricyclic antidepressant poisoning. Lead aVR is often neglected in routine clinical practice and in education of medical students and residents, perhaps because it is directionally non-adjacent to any other ECG lead. More attention should be paid to this important but largely ignored lead in the screening for, diagnosis and prognosis of cardiac ischemia, as well as other non ischemic pathologies. Physicians should maybe simply think of AVR as "A-cute V-essel R-evascularization" needed.

STAY OFF THE METRO(NIDAZOLE) WITH THE DARK URINE Brian Zwecker; Anna Kolpakchi; Lee Lu. Baylor College of Medicine, Houston, TX. (Control ID \#1334605)

LEARNING OBJECTIVE 1: To realize that commonly used medications can precipitate an acute intermittent porphyria (AIP) attack.

LEARNING OBJECTIVE 2: To review the clinical presentation of an AIP attack.

CASE: A 23-year-old Hispanic woman with past medical history of AIP presented with a three-day history of generalized abdominal pain. Physical exam was significant for cervical motion tenderness and the presence of a minimal white vaginal discharge. The patient was diagnosed with pelvic inflammatory disease (PID) and treated with ceftriaxone IM and fourteen days of oral doxycycline and metronidazole. Over the subsequent three days, she developed nausea and had three episodes of non-bloody non-bilious emesis, darker than usual urine, and worsening of abdominal pain. On admission, she was afebrile with blood pressure of 122/81 and heart rate of 93; physical exam was remarkable for moderate diffuse abdominal tenderness without rebound or guarding. There was no organomegaly. Complete blood count and comprehensive metabolic panel were within normal limits. UA showed 3+ leukoesterase, 9 WBCs, and a few bacteria. However, urine studies revealed elevation in urinary porphobilinogen of 95.6 (normal $0-2.0$ ). She was diagnosed with an AIP attack due to metronidazole. She was treated with intravenous hemin and $10 \%$ dextrose with complete resolution of nausea and vomiting and discharged home with some residual abdominal pain well controlled on oral pain medication.
DISCUSSION: AIP is an autosomal dominant disorder caused by mutations in the porphobilinogen deaminase (PBGD) gene, leading to heme precursor build up with an estimated prevalence of 1-5 per 100,000 in the U.S. Most patients present with severe abdominal pain. Additional symptoms may include a prodrome of anxiety or restlessness, nausea with or without emesis, constipation, red or dark-colored urine, and such manifestations of increased sympathetic activity as tachycardia, hypertension, and excessive sweating. AIP attacks may be precipitated by hormone fluctuations, fasting, infections, or drugs. Commonly used medications which can precipitate acute attacks include oral contraceptives, sex hormones, simvastatin, pravastatin, tetracylines, nitrofurantoin, and metronidazole. Some of these medications are believed to be porphyrogenic through their actions on hepatic P450 enzymes, whereas others, such as sex hormones, have been shown to directly stimulate heme biosynthesis. The mechanism through which metronidazole may induce AIP attacks is unclear, but metronidazole is listed by several international porphyria societies as an inducer of AIP attacks as compiled by the Norwegian Porphyria Centre. The incidence is unknown. Our case illustrates the importance of recognizing that commonly prescribed medications such as metronidazole should be avoided in patients with a history of AIP to prevent attacks.

SCURVY FROM "TOO MUCH COCA-COLA" IN A MALNOURISHED AND BIPOLAR WOMAN. Payal Jhawar; Richard Steingart; Jackcy Jacob. Baystate Medical Center, Springfield, MA. (Control ID \#1309707)

LEARNING OBJECTIVE 1: To recognize the specific populations affected by scurvy in the industrialized world.

LEARNING OBJECTIVE 2: To identify the clinical signs and symptoms of vitamin $\mathrm{C}$ deficiency.

CASE: A 54-year-old malnourished female with poorly treated bipolar disorder was sent to the emergency room by a caseworker for concerning behavior including two weeks of food refusal, not bathing and placing bags of feces around her room. On arrival to the ER, she was severely anemic with a hemoglobin of 5.9 and hematocrit of 17.4. She denied any hematuria, melena, hematochezia, bleeding gums, menorrhagia, trauma or sexual assault. She also denied use of NSAIDs or Aspirin and had no history of a colonoscopy. She attributed bruising on her skin to excessive Coca-Cola ingestion, and instead started drinking "Sprite with no lemon lime because it is pure." On examination, her blood pressure was $76 / 48$, but she was asymptomatic. Her skin and conjunctiva were pale, and she had poor dentition and gingival swelling. She had significant lower extremity pitting edema and palpable purpura with perifollicular hemorrhages and coiled hairs in addition to ecchymoses on her left thigh. We were initially concerned for sepsis and DIC given her hypotension and ecchymotic thigh; however, her diet history had stimulated a nutritional panel, which revealed a vitamin $\mathrm{C}$ level of zero. With prompt vitamin $\mathrm{C}$ treatment, her clinical findings of scurvy disappeared within 1 week.

DISCUSSION: Scurvy is a disease caused by a deficiency of vitamin C, which is necessary for the formation of mature collagen and blood vessel integrity. It is prevalent in approximately $13 \%$ of the US population according to the NHANES III, and serum concentrations of $<11.4 \mu \mathrm{mol} / \mathrm{L}$ are indicative of substantial vitamin $\mathrm{C}$ deficiency. ${ }^{1}$ The earliest symptom of fatigue occurs after weeks of being deficient. ${ }^{2}$ Other clinical findings include hypotension, ecchymoses, anemia, cutaneous follicular hyperkeratosis, perifollicular hemorrhages, palpable purpura, poor wound healing, leg edema, coiled body hairs, and gingival swelling. ${ }^{3}$ Administration of vitamin $\mathrm{C}$ leads to dramatic improvement in clinical findings within a few days to weeks. Scurvy is not just a disease of the sailors! In patients with nutritional deficiency, it is an important diagnosis to consider because if left untreated, it can be fatal. With supplementation, manifestations of scurvy improve dramatically, making the prognosis excellent. References: 1. Schleicher, RL et al. Serum vitamin $\mathrm{C}$ and the prevalence of vitamin c deficiency in the United States: 2003-2004 National Health and Nutrition Examination Survey (NHANES). Am J Clin Nutr 2009;90:1252-63. 2. Hirschmann, JV et al. Adult Scurvy. J Am Acad Dermatol 1999;41:895-906. 3. Velandia, B et al. Scurvy is Still Present in Developed Countries. J Gen Intern Med 23(8):1281-4. 
SCURVY IN AN AIDS PATIENT Torrey R. Schmidt; Richard Alweis; Jorge Scheirer. Reading Hospital and Medical Center, West Reading, PA. (Control ID \#1304419)

LEARNING OBJECTIVE 1: Scurvy is very rare in modern America because of enriched food products.

LEARNING OBJECTIVE 2: Scurvy should be considered in the chronically malnourished patient population.

CASE: A 54 year old male with advanced HIV/AIDS (CD4 142) on HAART complicated by Mycobacteria xenopi osteomyelitis of the spine, and resistant candida esophagitis, presented with fatigue, lower extremity edema, sore gums with pustular discharge, and easy bruising on his arms. His appetite was poor and he did not eat citrus fruits or green vegetables. His total caloric intake was estimated to be $1,000 \mathrm{kcal}$ per day. He did not take vitamin supplements, aspirin or non-steroidal anti-inflammatory drugs. Over the preceding 3 months, his weight decreased from $71 \mathrm{~kg}$ (BMI 21.4) to $57 \mathrm{~kg}$ (BMI 17.2). Physical examination revealed cachexia with erythematous and edematous gingiva, upper extremitiy ecchymoses, and lower extremity edema. The platelet count was 189,000 cells per cubic mm (CMM, normal 130-400,000 CMM), a PT and PTT were normal. Ascorbic acid level was $0.1 \mathrm{mg} / \mathrm{dL}$ (normal range 0.6 to $2.0 \mathrm{mg} / \mathrm{dL}$ ). He began to take ascorbic acid $500 \mathrm{mg}$ daily by mouth with subsequent improvement in his gingival symptoms. A repeat ascorbic acid level after 3 weeks of treatment was $2.0 \mathrm{mg} / \mathrm{dL}$.

DISCUSSION: Water soluble vitamin deficiencies are uncommon in the supplement crazed modern world, but should be suspected in patients with poor nutritional status, including advanced HIV/AIDS. Ascorbic acid deficiency or scurvy typically appears 2 to 3 months after consuming a diet lacking Ascorbic acid. Symptoms include, fatigue, easy bruising, petechiae, gingival bleeding, and extremity edema. The pathogenic mechanism underlying these signs is increased vascular fragility owing to defective collagen synthesis. The dermatologic findings in scurvy can easily be misdiagnosed as vasculitis. Physicians should be cognizant of the signs and symptoms of all vitamin deficiencies including ascorbic acid, and monitor for them in their chronically malnourished patient population, as well as alcohol and drug abusers, and those living in poverty without access to vitamin supplements or nutritious foods.

SCURVY: NOT GONE, BUT OFTEN FORGOTTEN Juan A. Pena ${ }^{1}$; Erin Wilmer ${ }^{1}$; Dean Abtahi ${ }^{1}$; Rebecca Beyth ${ }^{2,1}$. ${ }^{1}$ University of Florida College of Medicine, Gainesville, FL; ${ }^{2}$ North Florida South Georgia GRECC, Gainesville, FL. (Control ID \#1336105)

LEARNING OBJECTIVE 1: Recognize populations at risk for Vitamin C deficiency

LEARNING OBJECTIVE 2: Identify clinical signs and symptoms suggestive of scurvy

CASE: A debilitated 72 year old man with multiple comorbidities including rheumatoid arthritis, alcohol and tobacco abuse presented with malaise, generalized weakness, myalgias, joint pain, non-pruritic rash, coffee-ground emesis, renal insufficiency and transaminitis. He had a similar episode 4 months prior that resolved after a prolonged hospitalization; all lab studies and skin biopsy were non-diagnostic. He was afebrile, HR 97 and BP 100/53. On exam he had multiple regions of petechiea, hemorrhagic papules with overlying necrosis, areas of coalesced ecchymoses and purpura on his extremities, torso and face, periungal hemorrhages and diffusely tender joints. Stools were guiaic positive and his hemoglobin progressively declined. Differential diagnosis for the rash included septic emboli, rheumatoid vasculitis, coagulopathy and vitamin $\mathrm{C}$ deficiency. Infectious causes were unrevealing with multiple negative cultures and transthoracic echo without valvular vegetations. Hepatitis, vasculitis and coagulopathy panels were negative. Vitamin $\mathrm{C}$ level was $<0.12 \mathrm{mg} / \mathrm{dL}(0.2-1.7 \mathrm{mg} / \mathrm{dL})$. Interestingly, the patient was craving extra fruit with meals. He was treated with oral Vitamin $\mathrm{C}$ and the rash improved within days.

DISCUSSION: Acute vitamin C deficiency leads to scurvy, a renowned disease of 15th-16th century sailors, but it is an often forgotten diagnosis in the modern era; $7 \%$ of the US population is Vitamin C deficient. High risk groups include alcoholics, smokers, anorexics, patients with psychiatric illness, those with low socioeconomic status and restricted diets (1-2).
Smokers and rheumatoid arthritis patients have much lower levels of Vitamin C compared to healthy controls (3). Scurvy is primarily a clinical diagnosis supported by a history of inadequate vitamin $\mathrm{C}$ intake. Clinical manifestations vary and relate to the diverse biological roles of Vitamin $\mathrm{C}$ including energy metabolism via carnitine production and collagen biosynthesis, which is required for vascular integrity. Deficiency results in symptoms of weakness, malaise and myalgia and blood vessel fragility (petechiae, purpura, ecchymoses, perifollicular hemorrhages, GI bleeding, and hemarthroses) (5). Thus, it is easily mistaken for a systemic vasculitis. Decreased levels of Vitamin C in the setting of oxidative stress (i.e., sepsis, injury, inflammatory disease) may contribute to microvascular dysfunction and organ failure (6). In this patient with multiple risk factors, weakness, myalgia, petechiae, mucosal bleeding and GI bleeding were harbingers of an underlying vitamin C deficiency. Scurvy is easily treated with oral vitamin C, but the results can be devastating if the diagnosis is missed. 1.Schleicher RL, Carroll $\mathrm{MD}$, et al. Serum vitamin $\mathrm{C}$ and the prevalence of vitamin $\mathrm{C}$ deficiency in the US: 2003-2004 NHANES. Am J Clin Nutr. 2009 Nov;90(5):1252-63. 2.Velandia B, Centor RM, et al. Scurvy is still present in developed countries.JGIM 2008; 23:1281-4. 3.Jaswal S, Mehta HC, et al.Antioxidant status in rheumatoid arthritis and role of antioxidant therapy.Clin Chim Acta. 2003 Dec;338(1-2):123-9. 4.Rebouche CJ.Ascorbic acid and carnitine biosynthesis. Am J Clin Nutr.1991 Dec;54(6 Suppl):1147S-1152S. 5.De Luna RH, Colley BJ 3 rd, et al.Scurvy: an often forgotten cause of bleeding. Am J Hematol.2003 Sep;74(1):85-7. 6.Wilson JX, Wu F.Vitamin C in sepsis.Subcell Biochem.2012;56:67-83.

SEEING THE FOREST THROUGH THE TREES: THE NEED FOR CLINICAL AWARENESS IN A COMPLICATED AIDS CASE Lee Chang; Bradley Monash. UCSF, San Francisco, CA. (Control ID \#1340042)

LEARNING OBJECTIVE 1: Recognize the clinical features of Multicentric Castleman's Disease

LEARNING OBJECTIVE 2: Recognize the anchoring heuristic, including its pitfalls and how to overcome them

CASE: A 46-year-old man presented with recurrent fevers, diffuse lymphadenopathy, and worsening Kaposi sarcoma lesions on his feet. $\mathrm{He}$ was in his usual state of health until five months prior to admission when he presented with a syphilitic rash. Two months prior to admission, he developed painful "bruising" on the arches of his feet. His CD4 count and HIV viral load were 99 and 1 million, respectively. Skin biopsy revealed Kaposi's sarcoma (KS). He began a regimen of truvada, atazanavir, and ritonavir, but his functional status deteriorated and he was hospitalized multiple times for severe foot pain, fungal esophagitis, and suicidal ideation. On admission, his feet were so painful he could no longer walk. He complained of xerostomia, confusion and fatigue. He had generalized, bulky lymphadenopathy. Violaceous plaques were scattered on his arms and covered the plantar surface of both feet; none were detected on his oral mucosa. Serial cognitive assessments revealed attention and memory deficits. He remained despondent throughout his hospitalization. Multiple transfusions were required for Coombs-positive anemia. Persistent hyponatremia remained stable with fluid restriction. A repeat CD4 count and viral load were 332 and 4500, respectively. He had intermittent fevers, with a negative infectious workup. His ongoing symptoms were attributed to immune reconstitution inflammatory syndrome (IRIS). Chemotherapy was offered to treat his KS in the setting of IRIS, but he opted instead for irradiation of his plantar lesions, which proved unsuccessful. While awaiting placement for physical rehabilitation, further consideration of his signs and symptoms led to an excisional lymph node biopsy, which was diagnostic for hyaline vascular multicentric Castleman's disease. After starting rituximab, his fevers resolved and his lymphadenopathy and altered mental status improved. He reported less pain and regained his ability to walk. However, a new lesion suspicious for KS was detected on his palate and he developed hematochezia, suggestive of visceral involvement, hastening plans to initiate chemotherapy.

DISCUSSION: Despite public health efforts at early diagnosis, many patients with HIV continue to present with AIDS-defining illnesses. For AIDS patients who present with KS, the differential should include Castleman's disease given its strong association with human herpesvirus 
8 and rising incidence. In these patients, the presence of unexplained fever, lymphadenopathy, pulmonary symptoms, splenomegaly, central nervous system findings, xerostomia, or anemia is an indication for lymph node biopsy. Moreover, this case illustrates the danger of prematurely anchoring to a diagnosis and failing to adjust the differential in the presence of new or seemingly disparate data. For our patient presenting with recurrent fevers and worsening $\mathrm{KS}$, we were sold on the first logical correlation presented: immune reconstitution inflammatory syndrome. However, fixating on IRIS, and its nebulous time to resolution, clouded our capacity to extend our differential to account for his other problems. This delayed our diagnosing of multicentric Castleman's disease. Although anchoring is instinctive and sometimes efficient, patients are ultimately best served by constant reevaluation of all available information and diagnostic possibilities.

SENSE AND SENSITIVITIES Erin L. Reigh; Adam F. Binder; Anita Vanka. Beth Israel Deaconess Medical Center, Boston, MA. (Control ID \#1340032)

LEARNING OBJECTIVE 1: List the signs and symptoms of ovarian vein thrombosis.

LEARNING OBJECTIVE 2: Contrast the treatment of malignancyassociated ovarian vein thrombosis with postpartum ovarian vein thrombosis. CASE: This is an 81 year old female with a history of bladder cancer status post trans-urethral resection and ureteral stent placement who presented with urinary frequency and malodorous urine for two days. She also reported a temperature of 100.3 , chills, sweats, and left flank pain. Initial lab data was remarkable for a white blood cell count of $11.4 \mathrm{~K} / \mathrm{uL}$ and an elevated BUN and $\mathrm{Cr}$ of 49 and $2.3 \mathrm{mg} / \mathrm{dL}$, respectively (baseline creatinine $0.7 \mathrm{mg} / \mathrm{dL}$ ). Urinalysis was positive for leukocyte esterase and 182 white blood cells. A CT abdomen/pelvis was obtained without contrast. It showed malposition of the ureteral stent, uretero-hydronephrosis, and extensive bladder cancer metastases. A percutaneous nephrostomy tube was placed and she was started on vancomycin and cefepime for pyelonephritis. Urine culture revealed E. coli sensitive to cefepime. Despite appropriate antibiotic therapy, the patient continued to have fevers. A repeat CT abdomen/ pelvis was obtained with contrast. It demonstrated resolution of the hydronephrosis and no abscess, but revealed a new finding of left ovarian vein thrombosis. She was started on enoxaparin $1 \mathrm{mg} / \mathrm{kg}$ BID for treatment of malignancy-related venous thromboembolism (VTE). She defervesced and was discharged in stable condition.

DISCUSSION: Ovarian vein thrombosis (OVT) is rare and most commonly related to pregnancy. In these cases, it involves the right ovarian vein $90 \%$ of the time due to physiologic changes that occur in pregnancy. Over $50 \%$ of cases are associated with infection. Symptoms include flank/pelvic pain, fever, and a palpable cord in the abdomen. The diagnosis is often made on CT or MRI imaging when a febrile postpartum patient does not respond to antibiotics. Complications include PE (13\%), ureteral obstruction, renal vein thrombosis, abscess, ovarian infarction, and death. This case is unusual as the patient was not postpartum and likely developed an OVT secondary to her active malignancy. Malignancy-associated OVT is rare; there are only a few dozen cases reported. Her concomitant pyelonephritis may have also played a role, given the association between OVT and infection. For treatment of OVT, observational studies in postpartum patients report varying lengths of anticoagulation, from 10 days to 6 months. There is little data on OVT treatment in malignancy, but studies on VTE in this population demonstrate a mortality benefit when treated with low-molecular-weight heparin (LMWH), which reduces recurrence of VTE without increasing bleeding risk compared to coumadin (NEJM 2003; 349). Dosing is $1.5 \mathrm{mg} / \mathrm{kg}$ daily or $1 \mathrm{mg} / \mathrm{kg}$ twice daily. Some studies indicate twice daily dosing is more efficacious, but further study is needed (J Oncol Pharm Pract 2011; Sep). Duration of treatment is an ongoing debate, but continued anticoagulation in patients with residual malignancy is considered reasonable. Some data even indicate a survival benefit in patients who are maintained on LMWH indefinitely (Pol Arch Med Wewn 2008; 118 (4)). In summary, OVT is a rare complication of malignancy and should be considered in a patient with flank pain, intra-abdominal pathology, and fevers unresponsive to appropriate antibiotics. Treatment data is limited, so it is reasonable to manage OVT in a fashion similar to VTE, with LMWH as first-line therapy.
SEVERE HYPONATREMIA AND SEIZURES FOLLOWING COLONOSCOPY PREPARATION Quang V. Ton; Mark Nader; Shaheryar Siddiqui; Susan M. Clifford. Englewood Hospital Medical Center, Englewood, NJ. (Control ID \#1340487)

LEARNING OBJECTIVE 1: Recognize the clinical risk factors for hyponatremia.

LEARNING OBJECTIVE 2: Manage the adverse effects of bowel preparations.

CASE: An 80-year old female without previous neurological disease presented with unresponsiveness and generalized seizure. Three days prior, patient began bowel preparation for routine colonoscopy consisting of clear liquids with low sodium and protein intake followed by bisacodyl. On night of admission patient was scheduled for polyethylene glycol. It was never taken as patient was found by family unresponsive in bathroom. At baseline, she has normal cognitive function and is independent of all activities of daily living. Past medical history included mild depression on venlafaxine and hyperlipidemia on atorvastatin. On examination, she was afebrile with stable vitals. Neurological examination found the patient not responsive to voice but to noxious stimuli, GCS of 6/15. Pupils were reactive, toes up-going, bite marks on tongue noted. Remaining physical exam was unremarkable. During examination, patient began to have generalized tonic clonic seizure abated by lorazepam $2 \mathrm{mg}$ intravenous. She was then was post-ictal. Significant laboratory results drawn before seizure revealed serum sodium $112 \mathrm{mmol} / \mathrm{L}$, potassium $3.7 \mathrm{mmol} / \mathrm{L}$, chloride $75 \mathrm{mmol} / \mathrm{L}$, bicarbonate $25 \mathrm{mmol} / \mathrm{L}$, calcium $8.3 \mathrm{mg} / \mathrm{dl}$, phosphorus $1.8 \mathrm{mg} / \mathrm{dl}$, blood urea nitrogen $12 \mathrm{mg} / \mathrm{dl}$, creatinine $0.5 \mathrm{mg}$ / $\mathrm{dl}$, creatinine kinase $463 \mathrm{u} / \mathrm{L}$, serum osmolality 237 , urine osmolality 446 , urine sodium $112 \mathrm{mmol} / \mathrm{L}$, and urine potassium $31 \mathrm{mmol} / \mathrm{L}$. CT scan of the brain showed no acute abnormality. Patient was intubated, admitted to intensive care unit, and prescribed $3 \%$ hypertonic saline at a rate of $30 \mathrm{ml} /$ hr. Venlafaxine was stopped. Hypertonic saline was stopped when serum sodium was $121 \mathrm{mml} / \mathrm{L}$. Serum sodium rose to $128 \mathrm{mmol} / \mathrm{L}$ in 24 hours Desmopressin and D5W were given to slow the rate of sodium correction. Patient was extubated and returned to baseline mentation. Sodium was stable at $138 \mathrm{mmol} / \mathrm{L}$ prior to discharge.

DISCUSSION: Bisacodyl is a diphenylmethane stimulant laxative that is used in the treatment of constipation and part of bowel preparation before colonoscopy. Electrolyte and fluid imbalances are well recognized adverse effects of all bowel preparations but rarely of significance. There have been only five reports of hyponatremic seizures with non-phosphate bowel preparation and eight reports with oral sodium phosphate-containing purgatives. For the patient described in our report, her advanced age, gender and SNRI use may have led to SIADH. The clear liquid diet and stimulant laxative were contributing factors resulting in severe hyponatremia leading to seizures. Thus, clinicians should use caution when prescribing bowel preparation regimens in elderly patients. Patients should be encouraged to keep hydrated with electrolyte containing solutions.

SHIGELLA FLEXNERI: AN UNRECOGNIZED SEXUALLY TRANSMITTED INFECTION Jason Halperin. Tulane University, New Orleans, LA. (Control ID \#1313018)

LEARNING OBJECTIVE 1: Recognize Shigella Flexneri as a serious cause of enterocolitis in the HIV positive population.

LEARNING OBJECTIVE 2: Recognize that shigellosis can be sexually transmitted and therefore a thorough social history is imperative to identify risk factors

CASE: A 21-year-old HIV positive man (CD4 count 270 at 24\%) presented with a three-day history of abdominal cramping, diarrhea and vomiting. He described frequent stooling with non-bloody bowel movements. He denied anorexia and hematemesis. His pain was relieved with defecation. He denied sick contacts, change in diet, or previous episodes. $\mathrm{He}$ denied exposure to unpasteurized foods, daycare facilities, nursing homes or recent antibiotic use. He had not traveled recently nor had any animal contacts. He was diagnosed with HIV five years ago during routine screening. He recently stopped taking his anti-retroviral medications (HAART) because "he was feeling so good." He described frequent sexual contact with both men and women, engaging in both insertive and 
receptive sex as well as direct oral-anal contact. He reported condom use and did not know of any sexual contacts with similar symptoms. His physical exam demonstrated a mildly distressed man, alert and oriented with normal build. He was febrile to 102.6, tachycardic, normotensive and positive for orthostatics. His mucous membranes were dry and his oropharynx was clear. His skin exam showed decreased skin turgor. His abdominal exam demonstrated tenderness in all four quadrants without rigidity, rebound tenderness and negative for murphy's, roevsing's and psoas sign. Diagnostic testing revealed acute renal failure. Abdominal CT scan showed severe inflammation in the sigmoid colon with a normal gallbladder and appendix. Testing was negative for acute hepatitis. Stool studies were positive for fecal leukocytes, and negative for clostridium difficile toxin assay, giardia antigen, and cryptosporidium antigen. His stool culture grew Shigella Flexneri, sensitive to ciprofloxacin. He was discharged with resolved acute kidney injury and a 10-day course of antibiotics. He was referred for re-initiation of HAART and counseled on safe sexual practices.

DISCUSSION: In the industrialized world, shigella species are typically transmitted by direct or indirect fecal-oral contact and frequently associated with daycare facilities, nursing homes and contaminated food or water. Yet, HIV infection has been recognized as an important risk factor for shigellosis. The Center for Disease Control (CDC) demonstrated a 30 times greater incidence of infection in the HIV positive population compared to HIV negative. Furthermore, HIV positive individuals are four times more likely to require hospitalization. Since the mid-1970s, outbreaks of shigella infections have been recognized in the MSM population. Efficient sexual transmission has been suggested due to direct fecal-oral transmission and the very small inoculums of shigella-as low as 10 organisms-able to cause disease. In addition, concurrent HIV infection has been suggested to have several effects on shigella transmission including extended carriage of shigella species as well as increased asymptomatic shedding. A recent population-based study of shigella cases in San Francisco demonstrated independent associations for MSM, HIV and specifically direct oral-anal sexual contact with an odds ratio of 8 for each risk factor.

SHOT TO THE HEART Kate Hust. Tulane University Health Sciences Center, New Orleans, LA. ( $\overline{\text { Control ID } \# 1340501)}$

LEARNING OBJECTIVE 1: 1. Identify signs and symptoms of heart failure. 2. Recognize non-ischemic etiologies of dilated cardiomyopathy. 3. Identify medications and lifestyle modifications for treatment of alcoholic cardiomyopathy. 4. Integrate an understanding of the disease course of alcoholic cardiomyopathy for patient counseling.

CASE: A 64 year-old man presented with three days of bilateral lower extremity swelling. He had had no trauma to the lower extremities, and no immobilization. He noted associated orthopnea and a recently decreased exercise tolerance from twenty to five blocks. He had no known past medical history. Further history revealed chronic alcohol use, characterized as one-half pint of whisky several times weekly. His vital signs were normal. The heart sounds were distant but regular; there were no murmurs. Pulmonary crackles were absent, though his JVP was elevated. He had pitting edema extending to the knees bilaterally. A trans-thoracic echocardiography showed an ejection fraction of less than $20 \%$, grade III/IV diastolic dysfunction, and a pulmonary artery pressure of 50$69 \mathrm{mmHg}$. Subsequent right- and left-heart catheterizations showed unobstructed coronary arteries. After six-months of treating the heart failure and abstinence from alcohol, his ejection fraction improved to $35 \%$, the diastolic dysfunction reduced to a grade I/IV, and the pulmonary artery pressure normalized.

DISCUSSION: Both heart failure and alcohol abuse are frequently addressed issues by the general internist. Understanding their interaction is important for appropriate treatment and counseling. Though ischemic disease is the most common cause of dilated cardiomyopathy, the general internist must keep an open mind to the additional causes of cardiomyopathy. Genetic variations may result in familial forms of dilated cardiomyopathy. Often myocardial damage is secondary to infection, toxic agents, or metabolic end-products. Glycogen storage diseases, connective tissue disorders, and muscular dystrophies can all lead to dilated cardiomyopathy.
Common toxic etiologies include the anthracycline derivatives, cocaine, and alcohol. Alcoholic cardiomyopathy may occur from several mechanisms including direct toxic effects of alcohol or additives to alcoholic beverages, concurrent thiamine deficiency leading to beriberi, or hypertensive changes. Ensuring the appropriate treatment for alcoholic cardiomyopathy is essential as it is a reversible cardiomyopathy. As in heart failure of any etiology, the mainstays of therapy are angiotensin-converting enzyme inhibitors, for their impact on cardiac remodeling, and beta-blockers, for their impact on remodeling as well as anti-arrhythmic effects. Though without mortality benefit, diuretics should be added for relief of fluid overload symptoms. Additionally, alcohol cessation is critical. Cessation will not only prevent further damage but also allow for reversal of current damage. The reversibility of alcoholic cardiomyopathy should be stressed to patients upon diagnosis and continually reinforced during follow-up as encouragement for continued abstinence. Alcoholism and heart failure are two conditions for which the general internist commonly offers care, and it is important to recognize their intersection to offer appropriate counseling and treatment.

SIMULTANEOUS DETECTION OF HEPATITIS E AND EPSTEIN-BARR VIRUSES ASSOCIATED WITH ACUTE HEPATITIS Damian Casadesus; Syed Hassan; Tania Calzada; Izabella Zathureczky; Joseph DeAntonio; Daniel Goldsmith. Capital Health Regional Medical Center, Trenton, NJ. (Control ID \#1278529)

LEARNING OBJECTIVE 1: Practitioners need to be aware of the presence of Hepatitis E in the United States

LEARNING OBJECTIVE 2: Positive results of EBV antibody testing can be potentially a false positive in the setting of acute hepatitis.

CASE: A 39-year-old Indian male presented to the emergency department with recent onset of anorexia, 5 pounds of weight loss, and one week of jaundice. $\mathrm{He}$ recently traveled to India three months before admission. There was no significant past medical history, except an evaluation for infertility that was negative. At presentation, physical examination showed jaundice, itching and dark urine. Relevant clinical laboratory data showed AST 1734 U/L, ALT 2125 $\mathrm{U} / \mathrm{L}$, alkaline phosphatase $215 \mathrm{U} / \mathrm{L}$, total bilirubin $14.9 \mathrm{mg} / \mathrm{dl}$, total protein $7.5 \mathrm{~g} / \mathrm{dl}$, albumin $3.7 \mathrm{~g} / \mathrm{dl}$, prothrombin time $13.7 \mathrm{~s}$, Hepatitis A IgM negative, all Hepatitis B studies were negative, and Hepatitis C antibody and RNA titer were negative. By ultrasound, the liver was normal in size and heterogeneous in appearance suggesting focal fatty infiltration. Liver biopsy showed moderate to severe hepatitis of uncertain chronicity and etiology, moderate steatosis and mixed hepatocellular and reticuloendothelial siderosis. Further laboratory data showed Hepatitis E (HEV) IgM positive, Epstein-Barr virus (EBV) IgM and IgG positive and Cytomegalovirus IgM negative. Overall, a diagnosis of acute hepatitis $\mathrm{E}$ was made and we hypothesize that the patient was probably infected by fecal-oral transmission during his trip to India.

DISCUSSION: We describe a case with positive serology for two viral agents which may be implicated in the etiology of acute hepatitis. Three different possibilities can explain the simultaneous detection of EBV and HEV: i) an acute infection by HEV with false positivity for EBV, ii) an acute infection by EBV with false positivity for anti-HEV IgM, iii) a double viral infection. To our knowledge, this is the third case described with positive serology findings for both HEV and EBV, but the explanation of which virus produced a false positive result remains uncertain. Previous studies support the theory that hepatitis viruses can induce false positive results for phylogenetically similar viruses. In our patient, the detection of EBV IgG suggests a distant infection, and his recent travel to an endemic area of hepatitis $E$ and the presence of HEV IgM makes the diagnosis of hepatitis $\mathrm{E}$ with false positive reactivity for EBV most persuasive. Further, the acute illness of EBV infection is not typically associated with his pattern of liver function abnormalities. This case illustrates the need for practitioners to be aware of the presence of Hepatitis $E$ in the United States, such that positive results of EBV antibody testing are recognized as being potentially a false positive in the setting of a clinical syndrome consistent with acute hepatitis.

SIN, SEX AND HEARTACHE: AN UNUSUAL PRESENTATION, EVEN FOR "THE GREAT IMITATOR" Maria G. Frank ${ }^{1,2}$; Barbara Statland ${ }^{1,2}$; Jessica Campbell ${ }^{1,2}$. ${ }^{1}$ Denver Health Hospital Authority, Denver, CO; ${ }^{2}$ University of Colorado, School of Medicine, Aurora, CO. (Control ID \#1310287) 
LEARNING OBJECTIVE 1: Diagnose atypical presentations of early syphilis.

LEARNING OBJECTIVE 2: Recognize the importance of obtaining a detailed sexual history and review of systems.

CASE: A 21 year-old male with no significant past medical history presents with two episodes of acute pericarditis in a one month period. The first episode was complicated by cardiac tamponade requiring pericardial drain. Work up during initial presentation revealed a large pericardial effusion with tamponade physiology and serologic studies that were unrevealing as to the underlying etiology. Patient remained asymptomatic for 4 weeks after his discharge, until his chest pain recurred and was readmitted with diagnosis of recurrent acute pericarditis. Review of symptoms obtained during this admission was notable for a resolved $0.5 \mathrm{~cm}$ non-tender penile ulcer of two months duration. Exam revealed bilateral inguinal lymphadenopathy. Patient was found to have a positive RPR as well as a reactive T.pallidum IgG. Hence, he was treated with Penicillin for early syphilis with no further relapses of pericarditis.

DISCUSSION: We present a case of a patient with recurrent pericarditis attributed to early syphilis. Syphilis has re-arisen worldwide. Cases of early syphilis detected in Colorado have increased almost 300\% since 2007. Although known to affect almost every organ system, including the heart in tertiary syphilis, pericarditis is not a frequently recognized manifestation of the disease. We found only 3 case reports of syphilis pericarditis in the literature. In this case, the temporal relationship of symptoms and the resolution of them after treatment point to syphilis as the cause of the pericarditis. This case highlights the importance of a careful sexual history and review of symptoms in the diagnosis of syphilis, especially as new cases of syphilis are growing. Even today syphilis remains "The Great Imitator"; Osler's words ring true still, "the physician who knows syphilis knows medicine"

SKIN, LIGHT, AND MEDS: LOVE OR HATE? Shant Ayanian; Jillian S. Catalanotti. George Washington University Medical Center, Arlington, VA. (Control ID \#1335561)

LEARNING OBJECTIVE 1: Diagnose and treat photosensitivity reactions

LEARNING OBJECTIVE 2: Identify common medications that may cause photosensitivity

CASE: WF is a 67 year old African American gentleman with diabetes, hypertension, dyslipidemia and prostate cancer who presented to the general internal medicine clinic with a rash over his face and neck of three months duration. He described his skin as darkening, scaling and thickening since August. Each morning he used a razor blade to remove scaling skin from his cheeks and neck. He denied using any new cosmetic or topical products, any new ingestions, use of new soaps or detergents, changes to his medications, or any previous rashes. The patient reported regular sun exposure of the face during twice monthly golf games. $\mathrm{He}$ denied personal or family history of lupus or other autoimmune or photosensitive diseases. His medications included atenolol, nifedipine, hydrochlorothiazide, lisinopril, aspirin and simvastatin. He had no known drug allergies. Physical exam was remarkable only for violaceous, scaly hyperkeratotic plaques over the face and neck with notable sparing of the post-auricular and peri-orbital areas as well as directly under the chin. Punch biopsy was performed and revealed hyperkeratosis, parakeratosis, irregular epidermal hyperplasia, spongiosis and perivascualr inflammation predominantly of lymphocytes and eosinophils consistent with either spongiotic (eczematous) dermatitis or hypersensitivity dermatitis. He was referred to a dermatologist, who diagnosed photoallergy, most likely due to hydrochlorothiazide. He was treated with desonide $0.5 \%$ cream twice daily for three weeks and advised to use mild soap and sunblock with SPF 30 or higher with good effect. Hydrochlorothiazide was discontinued. The rash responded well to treatment.

DISCUSSION: Photosensitivity consists of phototoxicity and photoallergy. It can be caused by either topical or systemic agents. Phototoxicity is an immediate reaction that presents after sufficient UVA exposure and manifests hours later as an exaggerated sunburn. Histology shows an acute inflammatory pattern. The exact mechanism of phototoxicity depends on the inciting molecule, but a common trend is the radicalization of an elemental chlorine atom. In contrast, photoallergy is a far less common, type IV delayed, immune-mediated hypersensitivity reaction that that seems to be Tcell mediated. Photoallergy usually presents as a papulovesicular or eczematous rash that typically appears on sun exposed areas days to weeks after sun exposure. Biopsy reveals a spongiotic dermatitis pattern. Both phototoxicity and photoallergy are caused by exogenous substances that interact with one or multiple specific wavelengths of light to induce a biochemical change in the tissues; if caused by an administered medication they are termed an adverse drug reaction. The most commonly associated medications are diuretics, nonsteroidals, antimicrobials, and antipsychotics. The true incidence of photosensitivity is difficult to assess but is thought to be in the range of $1.4-12 \%$. The investigation of photosensitivity reactions should include a detailed exposure history, skin biopsy and photopatch testing. Treatment includes withdrawal of the inciting agent, regular and consistent use of sunblock with SPF of at least 30, and consideration of short-term topical steroids.

SMALL LEADS TO BIG: THE PERILS OF THROMBOCYTOSIS IGNORED Siyang Leng ${ }^{1}$; Leonard J. Appleman ${ }^{2}$; Gregory M. Bump ${ }^{1}$. ${ }^{1}$ University of Pittsburgh Medical Center, Pittsburgh, PA; ${ }^{2}$ University of Pittsburgh Medical Center, Pittsburgh, PA. (Control ID \#1314555)

LEARNING OBJECTIVE 1: Recognize that the myeloproliferative disorders are associated with thrombocytosis and thrombosis.

LEARNING OBJECTIVE 2: Distinguish between reactive and clonal thrombocytosis.

CASE: A 44 year old man who has no medical problems presented with severe, substernal, burning chest pain that woke him up one hour prior. $\mathrm{He}$ has had similar pain for the past one month that was not exertional and lasted for hours to days each time. There is no family history of coronary artery disease, and the patient does not smoke, consume alcohol, or use recreational drugs. On exam, he was in significant distress, clutching his chest. Cardiac auscultation revealed regular rate and rhythm with no murmurs, extra heart sounds, jugular venous distention, or lower extremity edema, and the rest of his exam was similarly unremarkable. An EKG found ST elevations in leads V2-6, I, and aVL. The initial troponin was $0.08 \mathrm{ng} / \mathrm{mL}$. The patient underwent left cardiac catheterization, which found a $100 \%$ occlusion in the proximal left anterior descending artery, which was then stented. No significant coronary artery disease was found. The troponin peaked at $127 \mathrm{ng} / \mathrm{mL}$. Lipid panel and hemoglobin A1c were unremarkable. However, it was noted on the admission CBC that the patient had a platelet count of $771,000 / \mathrm{mL}^{\wedge} 3$. The patient remembered being informed over 10 years ago while donating blood that he had elevated platelet counts in the $500-600,000 / \mathrm{mL}^{\wedge} 3$ range. A JAK2 mutation was checked, and was positive. Bone marrow biopsy found an increased number of megakaryocytes, some of which were dysplastic. Trilineage hypercellularity and reticulin fibrosis were also noted. The pathological diagnosis was myelofibrosis. The patient was started on aspirin and hydroxyurea and discharged home.

DISCUSSION: This patient developed a myocardial infarction secondary to his myeloproliferative neoplasm. He did not have significant atherosclerosis on coronary angiography, suggesting that hypercoagulability rather than coronary artery disease was the predisposing factor for his myocardial infarction. The myeloproliferative neoplasms significantly increase the risk of thrombosis, with arterial thromboses being more frequent than venous. Paradoxically, they also increase the risk of hemorrhage, although this is less frequent and severe than thrombosis. Multiple mechanisms are thought to account for these bleeding diatheses hyperviscosity, platelet aggregation abnormalities, leukocytosis, and downstream effects of the JAK2 mutation that is commonly seen in these disorders. In addition to thrombosis, the myeloproliferative neoplasms also predispose to thrombocytosis. While we initially thought that our patient's thrombocytosis was secondary, or reactive, further history taking suggested that it in fact was clonal, or myeloproliferative, in nature. Reactive etiologies are most commonly infection, rebound thrombocytosis, tissue damage, inflammation, and malignancy. Distinguishing between reactive and clonal thrombocytosis can be difficult. Findings that suggest clonal processes include pruritus (often after showering), erythromelalgia, splenomegaly, dysplastic megakaryocytes or myelofibrosis on bone marrow exam, and positive JAK2 mutation testing. The JAK2 mutation is prevalent in the 
myeloproliferative neoplasms, and should be checked when a patient has persistent thrombocytosis without an apparent reactive cause.

SMOOTH TRANSITIONS? COMPLICATIONS OF POOR TRANSITION OF CARE IN SICKLE CELL PATIENTS Matthew Calzetta $^{3}$; Dina E. Adimora-Nweke ${ }^{3}$; Princess Dennar ${ }^{3}$; Michael D. Landry ${ }^{1}$. ${ }^{1}$ Tulane University, New Orleans, LA; ${ }^{2}$ Southeast Louisiana Veterans Healthcare system, New Orleans, LA; ${ }^{3}$ Tulane University, New Orleans, LA. (Control ID \#1332854)

LEARNING OBJECTIVE 1: Recognize common medical and psychosocial complications arising from poor care transitions from pediatric to adult healthcare systems in sickle-cell patients.

LEARNING OBJECTIVE 2: Identify essential concepts for appropriate care transitions including key advancements from Patient Centered Medical Homes

CASE: A 32 year-old man with Hemoglobin SS sickle-cell disease presented to Med-Peds clinic with a two year history of untreated, nonprogressive, constant $8 / 10$ shoulder pain. He described his shoulder pain as swollen, numb, and typical of his many sickle cell pain crises. He denied taking any medication including analgesics, hydroxyurea, folic acid, or penicillin. His immunizations were not current. Laboratory studies revealed a hemoglobin of $7.8 \mathrm{~g} / \mathrm{dL}$, a reticulocyte count: $17 \%$, and albumin $2.3 \mathrm{~g} / \mathrm{dL}$. Medical records show that he transitioned from a pediatric to an adult hematologist at 18 . Since then, he had multiple primary care physicians, a disproportionate number of emergency room visits and duplicate work-ups at multiple sites. He showed poor compliance with his care plan and primary care appointments. He had numerous x-rays showing cardiomegaly and an echocardiogram showing right heart dilatation with mild pulmonary hypertension, but was never seen by Cardiology. The patient dropped out of college at 21 and applied for disability. He was never formally evaluated for depression. He started his individualized medical care plan including pain management, preventive care, specialty referrals and imaging studies.

DISCUSSION: This patient demonstrates the result of poor transition of care from pediatric to adult healthcare systems in chronically ill patients. His disease progressed without the attenuating effects of proven sickle-cell treatment. He suffers daily symptoms, a prognostic sign for early mortality in sickle-cell disease. He lacks the benefits of reduced pain crises and decreased mortality seen with increased fetal hemoglobin levels from hydroxyurea treatment. Overutilization of the healthcare system, with multiple emergency room visits and varied primary care physicians, places financial burdens on the healthcare system. The Society for Adolescent Medicine defines successful transitions of care as a purposeful and planned process with continuity of care amongst providers, patients and families encompassing medical, psychosocial and educational patient specificneeds. Ideal transition programs account for developmental and temporal differences in individuals. The transition process begins at diagnosis with education of the entire family with careful attention to patient-specific medical and psychosocial needs. As an adolescent, formal transition programming must prepare patients for the culture of adult medicine, promote self-advocacy in seeking assistance from schools and employers, and address funding for future health care services. Through each stage, patients at risk for poor outcomes require further patient-specific interventions to ensure proper transition. Our case highlights the negative impact of poor transitional care: worsening disease burden, diminished quality of life, psychosocial and educational strain, and poor utilization of the healthcare system. This case demonstrates the essential need for established protocols in the transition from pediatric to adult healthcare systems in chronic medical illness. Advancement of key components of the Patient Centered Medical Home may provide structural frameworks that will improve transitions of care.

SO, YOU ARE TELLING ME I JUST HAVE TO DEAL WITH MY P.M.S.?" Aaron Kline; Gary Malakoff; Jacob Noe; Mukta Panda. University of Tennessee, Chattanooga, TN. (Control ID \#1319984)

LEARNING OBJECTIVE 1: Recognize the indications for head imaging in the context of new onset seizure
LEARNING OBJECTIVE 2: Review the presentation initial management and imaging of cerebral venous thrombosis

CASE: A 45-year-old euthyroid white female with a history of bilateral carpel tunnel syndrome, depression and oral contraceptive hormone use for perimenopausal complaints presented with her first witnessed generalized tonic-clonic seizure. Review of systems revealed a headache for the past two weeks requiring increasing ibuprofen and acetaminophen doses. The patient had no family history of clotting disorders. Initial laboratory studies showed no significant abnormalities. Computerized Tomography (CT) imaging of her head revealed likely sagital sinus thrombosis. She was placed on heparin drip and antiepileptic medication. Re-imaging with Magnetic Resonance Imaging (MRI), Magnetic Resonance Arteriography (MRA) and Magnetic Resonance Venography (MRV) of her head confirmed the suspected sagital sinus thrombosis. Hormonal therapy was discontinued. The patient was monitored for hemorrhagic conversion of thrombosis for 72 hours. She was transitioned to warfarin. The patient begrudgingly agreed to abstain from her hormonal therapy at discharge

DISCUSSION: For adult patients presenting with an unprovoked first seizure, head imaging should be performed (1). In the emergency department, a CT is often ordered to rule out an intracranial bleed. The preferred brain imaging modality in the setting of seizure is MRI. Cerebral venous thrombosis is relatively uncommon with an annual incidence of less than $1 / 100,000$. Women are three times more commonly affected than men. Risk factors include hypercoaguable conditions, such as oral contraceptive hormone use, intracerebral lesions and trauma. Headache, of varying duration, is a predominate symptom in women. Other symptoms include focal neurological symptoms, encephalopathy or seizure. In the context of cerebral venous thrombosis, it is not uncommon for a patient to have a normal brain CT. Initial management is aimed at reversing the cause of the hypercoaguable state and starting anticoagulation therapy. Monitoring for thrombosis hemorrhagic conversion is prudent, especially at the initiation of anticoagulation therapy. Over half of patients presenting with cerebral venous thrombosis are on oral contraceptive hormones (2). This case illustrates the importance of maintaining a high index of suspicion for rare entities in the context of risk factors. References: 1. Adams SM and Knowles PD. Evaluation of a first seizure. Am Fam Physician. 2007 May 1;75(9):1342-7. 2. Saposnik G, et al. Diagnosis and management of cerebral venous thrombosis: a statement for healthcare professionals from the American Heart Association/American Stroke Association. Stroke 2011, 42:1158-1192

SOME LIKE IT HOT: A CASE OF CANNABINOID HYPEREMESIS SYNDROME Jeffrey D. Welder. University of Iowa, Iowa City, IA. (Control ID \#1326703)

LEARNING OBJECTIVE 1: Recognize the clinical features of cannabinoid hyperemesis syndrome

LEARNING OBJECTIVE 2: Manage cannabinoid hyperemesis syndrome CASE: A 29 year-old male with possible fructose-intolerance presented with acute abdominal pain and vomiting. He had a 10 -year history of recurrent pain and vomiting for which he had been hospitalized multiple times, most recently 2 months prior, improving with 1-2 days of hydration and pain medication. An extensive workup including labs, lactose breath test, multiple CT scans and ultrasounds had always been negative. Fructose tolerance testing performed at the onset of symptoms was inconclusive. Yet, the patient attributed his symptoms to this diagnosis. Physical exam revealed a diaphoretic and moderately distressed individual curled in the fetal position and retching. He was afebrile and tachycardic. Mild periumbilical tenderness was present but the abdomen was soft. Labs were notable for phosphorus of $0.7 \mathrm{mg} / \mathrm{dL}$ and $2+$ urine ketones. The patient was given IV fluids, morphine and phosphorus. Over the next 2 days the patient had resolution of symptoms. On every attempt to interview the patient, however, he was found in the shower. Nursing documented that the patient was spending hours each day taking hot showers and refused to come out for meals or receive medications. Upon further questioning, he admitted that he had been spending hours almost every day for the past 6 months in the shower, which relieved his nausea and pain. He began this practice around 4 years ago. Cannabinoid hyperemesis syndrome was suspected and at this point the patient admitted to daily marijuana use starting at age 16 . He held a medical marijuana 
card for nausea and smoked daily. He had never divulged this information to past providers because he didn't find it relevant. The patient was counseled on the association between daily marijuana use and vomiting and agreed to a trial of abstinence. The patient was contacted 4 weeks after discharge and reported complete resolution of pain and vomiting during this period.

DISCUSSION: Cannabinoid hyperemesis syndrome (CHS) is a condition of cyclic vomiting and compulsive bathing to relieve symptoms in patients with chronic daily marijuana use. It is more common in males, and typically presents after 10 years of daily marijuana use, with most patients beginning use as teenagers. These patients spend an average of 5 hours daily in hot baths when symptomatic. In a large review of cases it was found that the diagnosis was made an average of 4.5 years after onset of symptoms, with patients receiving repeated radiologic imaging and invasive procedures with no diagnosis. Significant clinical improvement is seen within 12-24 hours with cannabis cessation. In all cases, complete resolution of CHS is found with abstinence, which is the only treatment. The etiology remains unclear, but is likely related to chronic stimulation of cannabinoid-like 1 (CB1) receptors in the enteric plexus by Delta-9tetrahydrocannabinol (THC), which slows gut motility. While these receptors usually mediate an anti-emetic effect, a paradoxical effect is thought to induce emesis in CHS. As for the hot baths, it is thought that warm water counteracts the effects of $\mathrm{THC}$ on $\mathrm{CB} 1$ receptors in the hypothalamus, which controls temperature. CHS should be suspected in patients with recurrent abdominal pain and vomiting. Recognition of this of this under-diagnosed syndrome will decrease unnecessary testing and provide a solution for these patients.

SOMETIMES RED SOMETIMES BLUE; I WONDER WHY MY FEET CHANGE THEIR HUE! Fatima Khalid; Syed Hassan; Waqas Qureshi; Kelly Caverzagie. Henry ford Hospital, Detroit, MI. (Control ID \#1311895)

LEARNING OBJECTIVE 1: To identify the clinical features of Erythromelalgia.

LEARNING OBJECTIVE 2: To recognize the role of aspirin in the treatment of Erythromelalgia.

CASE: We present a case of a 64 years old female who presented to the Emergency Department with bilateral foot pain, redness and warmth, more prominent in the right foot. The pain was excruciating, 10/10, burning in quality and worsened with ambulation as well as being placed in a dependent position. Her medical history was significant for hypertension, myeloproliferative disorder in remission and stroke for which she was started on aspirin two years ago. One week prior to presentation she was found to have right iliac artery thrombosis and anticoagulation with Coumadin was initiated with discontinuation of aspirin. Other medications included gabapentin, anagrelide, omeprazole and atenolol. On examination she was hemodynamically stable and afebrile, distal pulses were intact and there was blanchable erythema in the bilateral lower extremities. Laboratory studies showed normal WBC and platelet count with an elevated INR. Initial differential diagnosis included cellulitis, arterial or venous insufficiency which were systematically excluded. A presumptive diagnosis of erythromelalgia was made and aspirin $325 \mathrm{mg}$ was initiated with symptomatic improvement within 24 hours.

DISCUSSION: Erythromelalgia is a rare disease characterized by redness, warmth and severe burning pain of extremities aggravated by temperature changes. It usually involves the lower extremities and is more prevalent in women. Diagnosis is usually based on history and physical examination. Primary erythromelalgia is classified into idiopathic, familial (autosomal dominant) and sporadic subtypes. Secondary erythromelalgia is associated with myeloproliferative disorders, lupus, multiple sclerosis, diabetes and the use of some medications including verapamil, nifedipine and bromocriptine. Etiopathogenesis is not clear, however, a prevalent theory is the vascular hypothesis suggesting improper distribution of nerves to skin vasculature causing endothelial edema, hypoxia and activation and aggregation of platelets that release prostaglandins causing pain and erythema. The familial variety has been attributed to mutations in the $\mathrm{Na}$ (v)1.7 sodium channel, expressed in the dorsal root ganglia cells and sympathetic neurons, causing perturbation in pain sensation. There is no definite medical management Aspirin, misoprostol, sodium nitroprusside and gabapetin have been found to be helpful but with limited success. Prior case descriptions of erythromelalgia describe this entity in the setting of significantly elevated platelet counts. Our case is unique as the platelet count was normal at the time of presentation because the patient was on anagrelide. Another unique feature is that the symptoms were precipitated by withdrawal of aspirin in a patient who did not have a known diagnosis of erythromelalgia.

SPINAL TUBERCULOSIS: A BIG PAIN IN THE BACK. Stephan Hanses; Jillian S. Catalanotti. George Washington University, Washington,

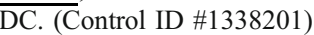

LEARNING OBJECTIVE 1: Recognize spinal tuberculosis as a cause of intractable back pain.

CASE: A 24 year old otherwise healthy man presented to clinic with new onset, severe $9 / 10$ back pain in the spinal and para-spinal lumbar region, which radiated into the right buttock, and right posterior thigh. It was aching in character, and lasted up to several hours a day. Movement, in particular, back extension and hip flexion, exacerbated the pain. Leaning forward while ambulating alleviated the pain. It improved with rest, but would not resolve completely, and at times the pain awakened him from sleep. He denied injury, radicular symptoms, or neurologic deficits. $\mathrm{He}$ took no medications, had no allergies, and no family history of illness. Social history was significant for immigrating to the US from India at the age of 6 . Review of systems was otherwise negative. Vital signs were unremarkable. Physical exam revealed tenderness to palpation along the lumbar paraspinal muscles bilaterally, and spinal tenderness at several points distal to L2. Strength, sensation and reflexes were normal. He was treated for a presumed back strain with ibuprofen and cyclobenzaprine. This provided relief, however, over the course of a year, he continued to suffer bouts of back pain. He presented to the emergency room, where a spinal MRI showed multiple, noncontiguous abscesses in his thoracic and lumbar vertebrae. There was also an $8 \mathrm{~cm}$ lesion in his right psoas muscle. PPD and interferon gold tests were positive, and aspiration of the psoas abscess showed acid fast bacilli. Culture grew mycobacterium tuberculosis

DISCUSSION: Lumbar muscle strain is the most common etiology of back pain in a young adult, and accounts for more than $90 \%$ of cases. More serious causes of spondylar disease should be considered for intractable pain that fails to respond to conservative treatment, pain that occurs at night while resting, accompanied by systemic symptoms or neurologic deficits, or if there is spinal tenderness on exam. 11,182 TB cases of active tuberculosis were reported in the United States in 2010.Tuberculous spondylitis (Pott's Disease) is an uncommon form of active tuberculosis and occurs in about $1 \%$ cases. It is usually seen in older children and young adults from TB endemic countries. Spinal tuberculosis is the result of either hematogenous spread, lymphatic spread, or extension of contiguous disease from an extraspinal source.Lesions are most commonly seen in the lower thoracic and upper lumbar spine, and can spread to the intervertebral disc, adjacent vertebrae, distant vertebrae, epidural space, psoas muscle, and the posterior iliac crest. Physical exam often shows spinal tenderness. Advanced disease can lead to muscle weakness and paralysis. Systemic tuberculosis symptoms are often absent, and Xrays can miss early lesions. MRI is the most sensitive in identifying early disease and is the standard for evaluating disk-space infection, osteomyelitis of the spine, and extension of disease into soft tissues. Tuberculomas on MRI show thin, smooth enhancement of the abscess wall, whereas pyogenic spondylitis is characterized by thick and irregular enhancement of the abscess wall. The diagnosis of spinal TB is confirmed with an AFB positive aspirate. Treatment is with antimycobacterial agents.

\section{SPONTANEOUS BACTERIAL PERITONITIS (SBP) IN A WOMAN} WITH METASTATIC GASTRIC CANCER WITHOUT LIVER INVOLVEMENT Jamie Osman; Tanping Wong. NYU, New York, NY. (Control ID \#1309954)

LEARNING OBJECTIVE 1: Recognize a case of malignant ascites complicated by SBP with a high serum-ascites albumin gradient (SAAG) despite absence of primary liver disease. 
LEARNING OBJECTIVE 2: Broaden a diagnostic differential for peritoneal infection in the setting of peritoneal carcinomatosis.

CASE: A previously healthy 41yo Ecuadorian woman presented with 2 months of intermittent sharp epigastric pain that improved with eating, and worsening abdominal distention accompanied by a $15 \mathrm{lb}$ weight loss, dysphagia, and dyspnea; days before admission, she developed nausea, nonbilious/non-bloody emesis, constipation and fevers. She denied sick contacts or recent travel, was taking no medications besides herbals for gastritis, and denied all toxic habits. Vitals: T 102 F, BP 115/81, HR 130 s, R 22, O2 94\% on RA. She was cachectic with bitemporal wasting, anicteric sclera, shallow breathing and decreased bibasilar breath sounds. Her abdomen was massively distended with fluid wave, dull to percussion, diffusely tender, with no hepatosplenomegaly. Extremities were cool, moist, non-edematous, with normal pulses. Labs showed serum Na 121; AST 43, ALT 16, Alk phos 78, albumin 3.4; WBC $19.7(92 \% \mathrm{~N}), \mathrm{Hb} 11.3$, MCV 69, RDW 30; guaiac negative. Abdominal CT showed massive ascites with jejunal and gastric fundus thickening, peritoneal carciomatosis, normal liver and spleen. Paracentesis yielded 4.5 L of cloudy yellow fluid, RBC 1500, WBC $4890(81 \%$ seg), protein 4.3, albumin 2.3 (SAAG 1.1), negative gram stain, cytology with inflammatory but no malignant cells, and culture with gram negative rods (Klebsiella oxytoca). The patient's family later revealed her recent diagnosis at an outside hospital with stage IV gastric cancer, confirmed by biopsy report showing high grade invasive signet ring cell type gastric adenocarcinoma. Her course was complicated by multiple bilateral pulmonary emboli, bilateral pleural effusions, electrolyte disturbances, and labored breathing requiring nighttime bipap. On day 24 , she was discharged to hospice.

DISCUSSION: This was a case of a 41yo woman with gastric adenocarcinoma and peritoneal carcinomatosis with $\mathrm{K}$. oxytoca SBP and SAAG of 1.1. SBP was diagnosed using standard criteria: ascitic fluid PMNs $>=250$ cells mm3 (3961), positive culture without secondary causes, and monomicrobial infection. Portal HTN is an instigator of SBP via suppression of the hepatic reticuloendothelial system (RES) function, intestinal bacterial overgrowth due to intestinal hypomotility, and venous status causing increased permeability to enteric bacteria. Although our patient had a SAAG of 1.1, and SAAG $>=1.1$ is 97\% specific for ascites due to portal hypertension, she had a non-dilated IVC, no parenchymal liver disease or sequelae of hepatic failure. Isner at al. reported a case of strep pneumonia SBP in a patient with metastatic gastric adenocarcinoma, without portal hypertension, proposing that massive metastases led to SBP via loss of the hepatic RES. Makharia et al. incriminated chemotherapy-induced immunosuppression and active severe GI bleed or perforation with increased mucosal permeability due to bowel ischemia as additional causes of SBP. However, our case did not involve these established prerequisites for malignancy-related SBP (liver metastasis, portal hypertension, bowel hemorrhage/perforation). Furthermore, the high ascitic protein of 4.3 and infection with common gut flora defied the standard SBP milieu, adding evidence for altered gut permeability secondary to cancer-related physiologic changes.

SPONTANEOUS PNEUMOTHORAX: THE GATEWAY TO A UNIQUE CLINICAL ENTITY Rosana Ayoub; Christine Yu; Michael Rotblatt. Olive-View UCLA Medical Center, Sylmar, CA. (Control ID \#1339902)

LEARNING OBJECTIVE 1: Recognize the numerous clinical and radiologic manifestations of tuberous sclerosis

LEARNING OBJECTIVE 2: Recognize that Sirolimus is a new groundbreaking treatment for certain manifestations of tuberous sclerosis

CASE: A 27 year old woman with PMH of a seizure disorder presented to the ED complaining of sudden onset chest pain and DOE. The physical exam was significant for decreased breath sounds over the left lung, multiple facial lesions over her bilateral cheeks and nasal bridge, hypopigmentation of her arms, and pitting of her nails. The CXR revealed a left sided pneumothorax, and the CT demonstrated mixed solid and fat containing lesions suspicious for pulmonary lymphangioleiomyomatosis and large renal angiomyolipomas. Taken together, the history, exam and radiologic findings were suspicious for tuberous sclerosis, and a brain MRI further revealed multiple cortical and subcortical tubers, numerous subependymal nodules, and a white matter hamartoma. To provide a complete assessment of this condition, the patient was referred for Ophthalmic examination, which identified bilateral retinal hamartomas. This constitution of clinical findings, along with her history of seizure disorder and facial adenomas, reinforced the diagnosis of tuberous sclerosis. A chest tube was placed to treat the pneumothorax, but treatment of the renal angiomyolipomas and lymphangioleiomyomatosis proved more difficult. A decision was made to begin treatment with sirolimus, which has been documented in a recent RCT to be effective in reducing the size of renal angiomyolipomas and improving spirometric measurements in patients with lymphangioleiomyomatosis from tuberous sclerosis.

DISCUSSION: Tuberous sclerosis is a disease of widespread hamartomas, commonly in the CNS, kidneys, and skin, commonly identified clinically with a triad (Vogt's triad) of seizures, mental retardation, and facial angiofibromas. However, these hamartomas can occur in almost any organ system, including pulmonary lymphangioleiomyomatosis, renal angiomyolipomas, cardiac rhabdomyoma, retinal nodular hamartomas, and CNS cortical tubers and supendymal nodules. Enlargement of renal angiomyolipomas pose a significant threat of pain and enlargement with possibility of hemorrhage, and renal cell carcinoma can rarely occur in less that two percent of patients. Trials involving use of sirolimus as a suppressor of mTOR (mammalian target of rapamycin) signaling have found a significant reduction in the size of renal angiomyolipomas during the duration of administration. The major pulmonary manifestation in women with tuberous sclerosis is lymphangioleiomyomatosis, which is characterized by formation of parenchymal cysts and the infiltration of smooth muscle cells. With sirolimus treatment of tuberous sclerosis patients, gas trapping and airflow can improve significantly. However, the most common cause of death is neurologic disease, such as status epilepticus and subependymal giant cell tumors, which are not affected by sirolimus treatment. The manifestations of tuberous sclerosis are numerous and, as in our patient, become more obvious with more complications. It remains crucial for clinicians to identify the constellation of findings in order to diagnose the disease as early as possible, and consider innovative treatments such as sirolimus.

SPURRING CONVERSATIONS OF PATIENT CARE: SPUR CELL ANEMIA IN LIVER CIRRHOSIS PATIENTS Mary J. Dennison; William Niehaus; Ankur Segon. Medical college of Wisconsin, Milwaukee, WI. (Control ID \#1297231)

LEARNING OBJECTIVE 1: Recognize the association between end stage liver disease and spur cell anemia

LEARNING OBJECTIVE 2: Recognize spur cell anemia as an ominous prognostic marker in end stage liver disease

CASE: A 57-year-old Caucasian male with child class $\mathrm{C}$ alcoholic liver cirrhosis (current drinker), DM-2, and chronic kidney disease presented to the ED from a skilled nursing facility due to altered mental status and a hemoglobin of $4.8 \mathrm{mg} / \mathrm{dL}$ (baseline $7-8 \mathrm{mg} / \mathrm{dL}$ ). Upon arrival, he was lethargic but arousable, icteric, and oriented only to person. Workup showed stable vital signs, clear chest X-ray, hemolytic anemia, and elevated creatinine. He was transferred to the MICU where he received 6 units of RBCs, had a paracentesis that was negative for bacteria, and had an NG tube placed. He was transferred to the floor after 6 days but continued to respond poorly to treatment with lactulose, rifaximin, and zinc. While on the floor, he regularly needed blood transfusions. His encephalopathy worsened despite treatment and his creatinine began to rise. A family meeting was held to determine goals of care for the patient. It was communicated that he was not a liver transplant candidate and he would likely have a poor prognosis, but the family decided to continue life saving care. On review of peripheral smear, it was discovered that the patient had Spur Cell Hemolytic Anemia. The fact that presence of spur cells confers a particularly poor prognosis in end stage liver disease was communicated to the family in the next family meeting. Based in part upon this information, the family decided to withdraw current support and focus solely on comfort care, with death as the expected outcome. The patient was discharged 16 days after admission in stable and terminal condition to a hospice facility close to his family.

DISCUSSION: $5 \%$ of patients with end stage liver disease also have spur cell anemia. Alcohol related liver disease is most commonly associated with spur cell anemia. The presence of spur cells on peripheral smear in a 
patient with advanced hepatocellular disease is an ominous marker and portends a survival of a few weeks. Liver transplant can be curative in this situation: however, such patients might not be candidates for transplantation due to multiple other reasons. In our case, we found this objective finding to be a useful facilitator of goals of care discussion with family members.

STREPTOCOCCUS BOVIS BACTEREMIA ASSOCIATED WITH COMPOSITE LYMPHOMA Ahmet A. Oktay ${ }^{1}$; Fritzie S. Albarillo ${ }^{1}$; Janis Atkinson ${ }^{2} .{ }^{1}$ Saint Francis Hospital, Evanston, IL; ${ }^{2}$ Saint Francis Hospital, Evanston, IL. (Control ID \#1338539)

LEARNING OBJECTIVE 1: Recognizing the importance of work-up to rule out underlying malignancy in patients with Streptococcus bovis (S. bovis) bacteremia.

LEARNING OBJECTIVE 2: Learning the general features of a very rare form of lymphoma; composite lymphoma.

CASE: An 86-year-old male patient with past medical history of mild dementia, deep vein thrombosis, hypertension, and type-2 diabetes mellitus was admitted for generalized weakness and asymptomatic hypoglycemia. Family members reported that the patient had been having generalized weakness, poor appetite and had fallen multiple times recently without loss of consciousness. His medications had included warfarin, metformin and glyburide. When he presented to the ED his vital signs were within normal limits. On examination he was found to be lethargic, diaphoretic and oriented only to person and place. Initial laboratory tests were unremarkable except for serum glucose of $69 \mathrm{mg} / \mathrm{dL}$. Initial urinalysis showed hematuria without any sign of urinary tract infection. Blood cultures and urine culture from the day of admission were negative. On the third day of admission he was found to have severe sepsis. His blood cultures on that day reported S. bovis and Enterococcus faecalis. The latter also grew in the urine culture. The patient responded well to intravenous Vancomycin and fluid replacement. An extensive work-up was done to identify the source of S. bovis bacteremia. Trans-esophageal echocardiography showed no evidence of thrombus or vegetation. His colonoscopy showed a single sessile polyp with no evidence of malignancy. CT of the chest, abdomen and pelvis showed a $4.2 \times 5.6 \mathrm{~cm}$ intra-abdominal mass adjacent to the liver and diffuse thoracic, abdominal and pelvic lymphadenopathy. An incisional biopsy of deep cervical/scalene lymph node and immunophenotypic analysis revealed composite lymphoma: classical Hodgkin's lymphoma and CD5 positive B-cell lymphoproliferative disorder.

DISCUSSION: Streptococcus bovis (S. bovis) infections in humans are usually associated with bacteremia and infective endocarditis (IE). Gastrointestinal tract is the main portal of entry for S. bovis. It has been well known that S. bovis bacteremia, with or without IE, is associated with underlying malignancy of the colon as well as extra-colonic malignancy or liver disease. Every patient with S. bovis bacteremia should undergo evaluation for IE and gastrointestinal malignancy. S. bovis bacteremia with underlying lymphoma, mostly gastric lymphoma, was reported in very few case reports. To our knowledge this is the first $\mathrm{S}$. bovis bacteremia case reported to be associated with composite lymphoma which is a rare disease and defined by the presence of two or more distinct lymphoma types in a single lymph note. The underlying mechanism for the association between S. bovis infection and malignancy remains elusive. We believe that, in our case, the liver involvement has provided a portal of entry from the hepatobiliary tree for $\mathrm{S}$. bovis. This case supports the fact that $\mathrm{S}$. bovis bacteremia cases need extensive workup to rule out underlying malignancy.

STROKE IN YOUNG ADULT: THINK OF CAROTID ARTERY DISSECTION Mohamed El khashab. Creighton University Medical Center, Omaha, NE. (Control ID \#1336098)

LEARNING OBJECTIVE 1: Carotid Artery Dissection is a common and easily missed cause of stroke in young adults.

LEARNING OBJECTIVE 2: Presentation of Carotid Artery Dissection might be similar to complicated Migraine headache

CASE: A 28-years old female presented with right arm and leg weakness/ numbness for two hours. Associated symptoms included frontal headache and expressive aphasia. The patient had complete resolution of her neurological symptoms in 2 hours. Physical exam was unremarkable at time of presentation as the symptoms had resolved. CT of the brain was unremarkable. However, within few hours of admission the patient started to have upper limb weakness. MRI/MRA showed diffusion restriction at the level of left the insula consistent with acute ischemia involving distal L MCA. MRA of the neck showed absence of flow within the left internal carotid artery, findings was suggestive of carotid artery dissection and secondary progressive thrombo-embolic events. The patient was started on Heparin and aspirin. However, she experienced worsening of neurological status to include right leg. Interventional cardiology performed carotid angiogram which showed long linear dissection of L ICA, and a $4 \mathrm{~mm}$ bare metal stent was placed in $\mathrm{L}$ ICA with good results and distal flow was considered excellent. The patient immediately experienced increased strength in the right leg but continued to have right arm weakness and aphasia.

DISCUSSION: Carotid Artery Dissection accounts for 5-20\% of CVA in young adults. It is usually related to trauma and connective tissue disease causing arteriopathies. Spontaneous Carotid Artery Dissection is not uncommon in clinical practice. This might be associated with intramural thrombus formation. Focal neurological deficit and frank stroke with fronto-temporal and hemi-cranial headache are the frequent presentations of carotid artery dissection. Some of these presentations could be misinterpreted as complicated migraine headache. Carotid bruit may be heard over the carotid artery and ecchymosis would be seen in cases of trauma. Carotid angiogram is the gold standard diagnostic tool. MRA is considered an alternative to angiography for evaluation of carotid dissection. Angiography would be considered if there was significant discrepancy between the findings of MRA and Dupplex ultrasonography that would impact on intervention planning. Clinical judgment with prompt investigations and early treatment can prevent devastating neurological consequences in these young adults. At that time the benefit of anticoagulation, intervention with stenting and less commonly surgical intervention would improve the clinical outcome.

SUBMUCOSAL GASTRIC LIPOMA PRESENTING WITH ABDOMINAL PAIN AND SYMPTOMS OF OBSTRUCTION Sterling K. Hansen. SJHMC, Phoenix, AZ. (Control ID \#1339006)

LEARNING OBJECTIVE 1: Although gastric lipomas (GL) are benign, they can be large, causing abdominal pain, symptoms of obstruction, and/or GI bleeding. Abdominal CT is the imaging modality of choice, but a definitive diagnosis requires tissue either from upper GI endoscopy or postresection biopsies. Surgical resection is the mainstay of treatment for symptomatic GL.

CASE: An 80-year-old, Hispanic female, with past medical history of type II diabetes mellitus, remote cholecystectomy and partial hysterectomy, presented with a 3-day history of sharp, intermittent RUQ abdominal pain, nausea, and vomiting. She had had no recent diarrhea or constipation. She noted a $20-1 \mathrm{~b}$ unintentional weight loss over the preceding 2-3 months. On physical exam, she was hemodynamically stable. Abdomen was soft and non-distended, with mild diffuse abdominal tenderness to palpation. No rebound or guarding was elicited, and no abdominal masses were palpated. Upon admission, abdominal CT showed gastric outlet obstruction likely secondary to a $5.6 \times 4.6 \mathrm{~cm}$ mass, consistent with a GL, at the gastroduodenal junction. A NGT was placed, and the patient was placed on NPO status with some relief of pain. Upper GI endoscopy with biopsies performed on hospital day 2 confirmed a diagnosis of submucosal GL. General surgery was consulted, and after medical clearance, an exploratory laparotomy with partial gastrectomy and excision of GL was performed on day 11. Patient recovered well post-operatively, was able to tolerate a regular diet, and was discharged home on hospital day 13.

DISCUSSION: GL are rare, representing 2-3\% of benign gastric tumors and less than $1 \%$ of all gastric tumors 1 . Tumor growth is usually indolent with the peak incidence in the 7th decade of life2. GL are mostly asymptomatic when smaller than $4 \mathrm{~cm}$ but can become symptomatic with various presentations when larger1. A PubMed search using the terms 'gastric lipoma' returned multiple case reports describing the more common presentation of GI hemorrhage secondary to ulceration of the overlying mucosa. Only 8 cases 
were found with presentations of abdominal pain and/or obstruction, as seen in this case. Dyspepsia has also been reported as a presenting symptom1. Abdominal CT is a highly-specific imaging modality in the detection of GL, with radiological findings generally showing a homogenous, non-invasive structure with negative Hounsfield units, indicative of a fat density3. MRI and high-resolution ultrasound can be useful in the detection of GL1. Upper GI endoscopy with biopsy also aids in the diagnosis of GL but often does not reach the submucosal layer, where $95 \%$ of GL originate 3 . As a result, a definitive diagnosis of GL is often made from biopsies obtained post-resection. The treatment of asymptomatic GL is controversial, but symptomatic GL are treated with surgical resection. Laparoscopic resection is advised for tumors less than $6 \mathrm{~cm}$ in diameter, though larger tumors have been removed laparascopically.

\section{SUCCESSFUL TREATMENT OF ACQUIRED ANGIOEDEMA} USING RITUXIMAB Chang $\mathrm{Na}^{1}$; David Podell ${ }^{1}$; David Dreyfus ${ }^{2}$; Christopher Randolph ${ }^{2}$; Denise M. Kearney ${ }^{2} .{ }^{1}$ Yale Primary Care Program, Waterbury, CT; ${ }^{2}$ Allergy, Asthma \& Immunology Group, Waterbury, CT. (Control ID \#1279059)

LEARNING OBJECTIVE 1: Learn about the immunobiology underlying acquired angioedema (AAE)

LEARNING OBJECTIVE 2: Make treatment decisions based on mechanism of disease and the latest literature data

CASE: A 41 year old woman with a past medical history of myasthenia gravis and anti-phospholipid syndrome, presented with recurrent diffuse abdominal pain. She had low C1q, C2, C3, and C4 levels and $\mathrm{CT}$ abdomen revealed thickened ascending colon consistent with bowel edema. She was diagnosed with AAE based on her low complement levels, recurrent angioedema symptoms and the presence of $\operatorname{IgG}$ antibodies $(\mathrm{Ab})$ against $\mathrm{C} 1$ inhibitor $(\mathrm{C} 1-\mathrm{INH})$. Despite the established association between $\mathrm{AAE}$ and lymphoproliferative disorders (LPD), our patient had a subsequent normal CT chest/abdomen/ pelvis, normal SPEP, and flow cytometry that showed normal lymphoid phenotype. She continued to have recurrent episodes of angioedema involving the colon and the larynx requiring intubations. She was treated with various medications including loratadine, cetirizine, and ranitidine for prevention and methylprednisolone without benefit. She also did not appear to benefit from ecallantide, a novel kallekrein inhibitory peptide that is used in acute exacerbations of hereditary angioedema. The patient was eventually treated with rituximab (RTX) with significant improvement in severity of her latest angioedema episode. After she finished the course of RTX, a decrease of anti-C1-INH Ab titer was observed and she remained without further angioedema episodes.

DISCUSSION: AAE is a rare condition that is characterized by acquired deficiency of $\mathrm{C} 1-\mathrm{INH}$, hyperactivation of the classical complement pathway and recurrent angioedema symptoms. C1-INH is a protease inhibitor that regulates the activation of kallikrein and the classical complement pathway. C1-INH deficiency, which can be hereditary or acquired due to anti-C1-INH $\mathrm{Ab}$, can lead to unregulated activation of the complement system and increased generation of bradykinin via kallikrein activation. Clinically, it is thought that uncontrolled bradykinin production results in angioedema. In hereditary angioedema, there is either a lack of synthesis of C1-INH or synthesis of a nonfunctional protein. In AAE, there is either an excessive consumption of $\mathrm{C} 1$-INH or formation of a circulating anti$\mathrm{C} 1-\mathrm{INH} \mathrm{Ab}$ that cleaves $\mathrm{C} 1-\mathrm{INH}$ into a nonfunctional form. Acquired C1-INH deficiency has been associated with LPD and autoimmune disorders. Treatment includes emergent therapies for potentially fatal episodes, such as laryngeal edema, and therapies to curb recurrences by treating the underlying disease and using antifibrinolytics and immunosuppresants. RTX has been shown to be effective as adjunctive treatment of LPD, in particular B-cell lymphomas, and there have been recent case reports of RTX effectively treating AAE secondary to LPD. It is postulated that RTX, a monoclonal $\mathrm{Ab}$ directed against glycoprotein $\mathrm{CD} 20$ expressed on B cells, works by depleting $\mathrm{B}$ cells and subsequent auto-Ab production, thereby making it an effective treatment of AAE mediated by high circulating auto-Ab. This case is unique in that RTX was successfully used to treat AAE in a patient without LPD. RTX was chosen in this patient since she had other underlying autoimmune disorders that are associated with auto- $\mathrm{Ab}$ production by $\mathrm{B}$ cells, which may also underlie the $\mathrm{C} 1 \mathrm{INH}$ auto- $\mathrm{Ab}$ production and subsequent AAE. Our case highlights that RTX can be an effective treatment for acquired C1-INH deficiency that is not related to LPD.

SURGICAL CURE FOR AUTOIMMUNE HEMOLYSIS-NOT THE SPLEEN Yue Sha ${ }^{1}$; Cheng-jin Huang ${ }^{1}$; Gurpreet Dhaliwal ${ }^{2}$; Wei-gang Fang ${ }^{1}$; Xin-yan Liu ${ }^{3}$; Xin-yu Ren ${ }^{4}$; Hui Zhang ${ }^{5}$; Xue-jun Zeng ${ }^{1} .{ }^{1}$ Division of General Internal Medicine, Department of Medicine, Peking Union Medical College Hospital, Beijing, China, Beijing, China; ${ }^{2}$ Department of Medicine, University of California San Francisco and San Francisco VA Medical Center, San Francisco, CA; ${ }^{3}$ Department of Obstetrics and Gynecology, Peking Union Medical College Hospital, Beijing, China; ${ }^{4}$ Department of Pathology, Peking Union Medical, Beijing, China; ${ }^{5}$ Department of Emergency, Peking Union Medical, Beijing, China. (Control ID \#1310384)

\section{LEARNING OBJECTIVE 1: Recognize Ovarian Teratoma Induced} AIHA

LEARNING OBJECTIVE 2: Treat Ovarian Teratoma Induced AIHA

CASE: A 39-year-old woman was evaluated for palpitations, fatigue, and dizziness for one week. She had lost consciousness for minutes when standing up several times before admission. She denied abdominal pain or dark urine. She was previously healthy other than a large right ovarian mass $(9 \times 9 \times 9 \mathrm{~cm})$ that was detected 3 months earlier by ultrasound ordered to evaluate irregular menstruation. The mass was suspected to be an ovarian teratoma. She took no medications or illegal drugs and did not smoke or alcohol. She was afebrile. Her heart rate was 100 beats per minute and her supine blood pressure was $125 / 75 \mathrm{mmHg}$ without orthostatic changes. Her conjunctivae were pale and scleral icterus was present. Heart, lung, and abdominal exam were normal. No hepatosplenomegaly. The hemoglobin was $4 \bullet 0 \mathrm{mg} / \mathrm{dL}$ with a reticulocyte count of $23 \%$ and MCV $105 \mathrm{fl}$. Ferritin $342 \mathrm{ng} / \mathrm{ml}$ (normal 14-336 ng/ml), serum iron $135 \mathrm{ug} / \mathrm{dl}$ (normal 50-150 ug/dl), total iron-binding capacity $315 \mathrm{ug} / \mathrm{dl}$ (normal 300-430 ug/dl), transferrin saturation $42 \%$ (normal $25-50 \%$ ), vitamin B12 $1500 \mathrm{ng} / \mathrm{mL}$ (normal 180-914 ng/mL), folic acid $20 \mathrm{ng} / \mathrm{L}$ (normal $>3 \mathrm{ng} / \mathrm{L}$ ). The serum level of total bilirubin was $2 \bullet 8 \mathrm{mg} / \mathrm{dL}$, conjugated billirubin $1 \bullet 0 \mathrm{mg} / \mathrm{dL}$, lactate dehydrogenase 743U/L (normal 97-270U/L), anti-IgG direct antiglobulin test was positive (4+). Anti-nuclear antibody (ANA) and anti-double stranded DNA antibody were negative. She received oral prednisolone ( $1 \mathrm{mg} / \mathrm{kg} /$ day), intravenous cyclophosphamide, and intermittent blood transfusion for one month for idiopathic autoimmune hemolytic anemia. After the initiation of immunosuppression and several transfusions $(800 \mathrm{ml}$ of RBC in total), her hemoglobin gradually increased to $9 \bullet 4 \mathrm{mg} / \mathrm{dL}$, but then returned to a stable level of $7 \bullet 6 \mathrm{mg} / \mathrm{dL}$ with a simultaneous increase in the reticulocytes from $10 \%$ to $25 \%$. A decision was made to remove the teratoma. One week after the operation her hemoglobin rose to $11 \bullet 0 \mathrm{mg} / \mathrm{dL}$ with the reticulocyte count dropping to $6 \%$. Cyclophosphamide was stopped and corticosteroids were gradually tapered over 2 months. During 2 years of follow up her hemoglobin, reticulocyte count, and Coombs test have remained normal. DISCUSSION: AIHA can be induced by autoimmune diseases, lymphoproliferative disorders, infections, neoplasms, and drugs. Ovarian teratoma is a rare cause of AIHA. We identified less than 30 cases of hemolytic anemia associated with ovarian teratoma or dermoid cyst in PubMed from 1966 through 2011. All patients had hemolytic anemia refractory to steroids, other immunosuppressants, plasmapheresis, or splenectomy. All responded to resection of the teratoma or dermoid cyst. The mechanism of autoimmune hemolysis induced by ovarian teratoma is unknown.Ovarian teratoma has been associated with other autoimmune phenomena, including Graves' disease, encephalitis, and the palmar fasciitis and arthritis syndrome. The relationship has typically been recognized after unanticipated reversal of the autoimmune symptoms following removal of the tumor. In the recently described cases of ovarian teratoma with encephalitis, anti-NMDA (N-methyl-D-aspartate) receptor antibodies were found to be pathogenic. This case reminds clinicians to consider alternative antigenic sources such as teratoma when cases of unexplained or refractory autoimmune hemolytic anemia are encountered, similar to the approach that is increasingly adopted for young patients with unexplained encephalitis. 
TB OR NOT TB, THAT IS THE QUESTION Lucius Howell; Michael Queen; Michael D. Landry. Tulane University Health Sciences Center, New Orleans, LA. (Control ID \#1339185)

LEARNING OBJECTIVE 1: 1. Identify hemoptysis and weight loss as symptoms in chronic mycobacterial infections 2 . Understand the epidemiology and treatment of non-tubercular mycobacterium infections 3. Recognize the critical role of transitions of care in preventing re-hospitalization

CASE: An 83 year-old man with myelodysplastic syndrome presented with one month of hemoptysis and a fifty-pound weight loss. He noted increasing fatigue, anorexia and dysguesia. Three months earlier, he was diagnosed with reactivation pulmonary tuberculosis based on a positive Purified Protein Derivative (PPD), an abnormal chest X-ray and sputum smear positive for acid fast bacilli (AFB). He was treated with standard rifampin, isoniazid, pyrazinamide, ethambutol (RIPE) therapy without symptomatic improvement. On presentation, he had bilateral temporal and extremity wasting with coarse breath sounds bilaterally. His labs revealed pancytopenia. A chest X-ray revealed a large opacification in the right upper field. A follow-up CT with contrast revealed scattered nodular opacities with a right apical cavitary lesion. An AFB smear and culture was positive for Mycobacterium abscessus. A review of his medical record revealed his prior positive culture for Mycobacterium abscessus. Appropriate inpatient treatment was initiated.

DISCUSSION: Unintentional weight loss, hemoptysis, upper lobe cavitary lesion, and a positive PPD is the classic presentation of Mycobacterium tuberculosis (TB). While TB is the most common of the mycobacterial infections, the general internist must recognize that it is not the only infection to present in this way. While patients suspected of having TB are commonly discharged on antibiotics, it is important that the internist recognize the importance of following-up on cultures and sensitivities to confirm the diagnosis. Prior to instituting long-term treatment, it is important to confirm that the etiology by following up on culture results, antibiotic sensitivities and ensuring patient follow up. Mycobacterium abscessus is a non-tubercular mycobacterium that exists in water, sewerage, and soil. It is endemic to the Southeastern United States, but is reported throughout North America. Risk factors for M. Abscessus include immunosuppression, chronic lung diseases and prior tuberculosis. Among patients with hematological malignancies associated with pancytopenia, there is a higher prevalence of non-tuberculous mycobacteria infections. Mycobacterium avium-intracellulare is the most commonly isolated species, followed by $\mathrm{M}$. abscessus, M. fortuitum, and M. kansasii. The Initial evaluation of symptomatic patients includes excluding Mycobacterium tuberculosis and underlying malignancy followed by sputum culture. The treatment of non-tubercular pulmonary infections is based on active symptoms and the overall risk versus benefit of treatment. The treatment typically includes six months of an oral macrolide plus intravenous amikacin, tigecycline or cefoxitin. Antibiotics may only prevent dissemination and may not be curative secondary to the bacteria's relative resistance to antibiotics. Continual treatment may be chosen if the patient remains symptomatic with cough, hemoptysis or "B" symptoms. Surgery is the only definitive treatment.

TMP/SMX-INDUCED SEVERE THROMBOCYTOPENIA Ramon Jacobs; Bora Toklu. NYU School of Medicine, New York, NY. ( $\overline{\text { Control }}$ ID \#1323997)

LEARNING OBJECTIVE 1: Recognize that TMP/SMX can induce a severe, potentially life-threatening, isolated thrombocytopenia.

CASE: A 50-year-old healthy female without significant medical history presented with a day history of non-pruritic red rash on her torso. The patient was in her usual state of good health until three days prior to admission when she starting trimethoprim/sulfamethoxazole (TMP/SMX) for a possible dental infection. Except TMP/SMX, she was not taking any other prescribed or over the counter medication. Two days after starting taking TMP/SMX, she noticed the rash and presented to the hospital. On initial physical examination, the patient noted to have scattered nonblanching red petechial rash over her torso extending down to the bilateral lower extremities. The rest of her physical exam and review of systems were unremarkable. Her initial complete blood count $(\mathrm{CBC})$ revealed an isolated thrombocytopenia with a platelet count of $4.000 / \mathrm{mm} 3$. The rest of her blood work including chemistry, coagulation, liver function and hemolysis panels were all within normal range. A subsequent peripheral smear confirmed thrombocytopenia with large platelets, but otherwise was normal. Bactrim was held off as a possible causative agent, and the patient was being evaluated for possible idiopathic thrombocytopenic purpura (ITP). Patient's platelet count responded poorly to the first unit of single donor platelets (SDP) transfusion, while a second unit of SDP tranfusion led to appropriate increase in platelet count. Within 36 hours of her hospital stay, the platelet count recovered to a normal range without any further transfusion requirement or glucocorticoids for initially presumed ITP. This led to a diagnosis of TMP/SMX-induced severe thrombocytopenia. Her presenting petechial rash also gradually resolved over hospital course. Patient was discharged on hospital day 3 with a platelet count of 202.000/ $\mathrm{mm} 3$. A week after discharge, her repeat platelet count was $425.000 / \mathrm{mm} 3$. DISCUSSION: Based on the Naranjo probability scale, TMP/SMX is a probable cause of thrombocytopenia in our patient. Hematologic adverse effects associated with TMP/SMX, although uncommon, can involve any of the three cell lines. TMP/SMX-induced severe isolated thrombocytopenia is a very rare finding, and only a few case reports of this exists in literature. Although very rare, this is a potentially life threatening adverse effect. We would like to remind clinicians that serious and potentially fatal side effects exist even with frequently prescribed medications, such as in our case.

TAKING SYPHILIS TO HEART April Evans. Tulane University Health Sciences Center, New Orleans, LA. (Control ID \#1311712)

LEARNING OBJECTIVE 1: Identify the differential diagnosis for isolated aortic insufficiency.

LEARNING OBJECTIVE 2: Identify the diagnostic tests necessary for staging syphilis infection.

CASE: A 70 year-old man presented with three days of sudden-onset severe unilateral knee pain and swelling. He reported progressive fatigue over the past three months. He noted no fever, dyspnea; there was no inciting trauma to the knee. His vital signs were normal with the exception of a wide pulse pressure; the blood pressure was $158 / 65$ mmHG. The pulmonary, abdominal and neurologic examinations were normal. He had isolated left knee swelling which was exquisitely tender to touch and movement. He had a four-out-of-four blowing diastolic murmur located at the cardiac base that increased in intensity when he leaned forward and held his breath. The murmur also increased in intensity when in the Trendelenberg position. There was also a two-out-of-four mid-diastolic murmur at the cardiac apex; the PMI was displaced down and to the left. De Musset's sign and Becker's sign were present. The pulse was bounding and arterial pulsations were seen extending down into the arms bilaterally. Joint aspiration of the left knee showed monosodium urate monohydrate crystals and 34,000 white blood cells $/ \mathrm{mm} 3$. Severe, isolated aortic insufficiency was revealed on echocardiogram; the left ventricle was dilated. An eight centimeter dilated aortic root without calcification was revealed on CT scan. The RPR was reactive and TP-MHA test was positive. CSF studies revealed a protein of 58 and a negative TP-MHA.

DISCUSSION: Syphilitic aortitis is an uncommon finding after the advent of penicillin, but it is substantially more common in patients who have not had access to penicillin-based drugs for incidental infections via primary care. As such, the general internist providing care to underserved patient populations must still be vigilent for the signs and symptoms suggestive of a systemic syphilitic infection. Recognizing the physical manifestations of aortic insufficiency is one such clinical presentation of which the general internist must be aware. The finding of an isolated aortic regurgitation, especially in the younger patient without signs and symptoms of endocarditis, merits screening for syphilis. Primary, secondary, latent, tertiary and neurosyphilis are treated with different penicillin-based regimens and appropriate identification is important to define the duration of treatment. Cardiovascular manifestations automatically stage the syphilis infection as tertiary. Importantly, the general internist should recognize that at the time of aortic insufficiency, it is likely that the patient has nonatherosclerotic coronary artery disease caused by obliterative endarteritis at the coronary ostia. In patients with late latent syphilis or tertiary syphilis that is not considered to be neurosyphilis, the recommended treatment is intramus- 
cular benzathine penicillin $\mathrm{G}$, in a dosage of 2.4 million $\mathrm{U}$ once weekly for three weeks.

TELLING SIGNS OF TROUBLE: A SWOLLEN NECK AND TIGHT RINGS Shanthini Kasturi; Leonor Fernandez. Beth Israel Deaconess Medical Center, Boston, MA. (Control ID \#1334598)

LEARNING OBJECTIVE 1: Recognize the physical exam findings suggestive of superior vena cava (SVC) syndrome

LEARNING OBJECTIVE 2: Manage emergent and non-emergent SVC syndrome

CASE: A 56 year old woman presented to her primary care physician complaining of three days of "swollen neck veins." She had been seen one week earlier when she was started on amlodipine for uncontrolled hypertension and wondered if this was a side effect of the medication. She reported a cough and congestion of five days, but denied dyspnea, chest pain, hoarseness, stridor, or dysphagia. She had not noticed increased swelling of her extremities, but on questioning noted that her rings felt tighter on her fingers. She denied recent fevers, nightsweats, or weight loss. Her past medical and social history was notable for hypertension and a sixty-pack year smoking history. Her family history was remarkable for throat cancer in her father. On examination, vital signs were within normal limits. The patient had visibly engorged external jugular veins without significant facial swelling or flushing. There was no cervical or supraclavicular lymphadenopathy. Lungs were clear to auscultation and cardiac exam was without murmurs or gallops. There was slight swelling of the fingers, but no lower extremity edema. A chest X-ray was obtained which showed a mediastinal mass at the right tracheobronchial angle. The patient was sent for urgent computed tomography of the chest, which revealed a $5.8 \times 6 \mathrm{~cm}$ superior right mediastinal mass obliterating the superior vena cava and narrowing the right mainstem bronchus. She was admitted for expedited work up with flexible bronchoscopy and needle aspiration of the mass yielding a diagnosis of small cell lung cancer. Given rapid progression of facial and upper extremity edema, a stent was placed in her SVC to relieve obstruction prior to the initiation of chemotherapy.

DISCUSSION: This case highlights how jugular venous distention, a familiar physical exam sign most typically associated with heart failure, can herald a less common diagnosis. The appearance of engorged neck veins with subtle signs of upper body edema in the absence of generalized volume overload is an early sign of SVC syndrome. SVC syndrome is characterized by edema of the head, neck, and arms, often with associated facial plethora. Urgent chest imaging is indicated to distinguish extrinsic compression versus internal obstruction of the SVC. The differential diagnosis of SVC syndrome is divided into malignant $(65 \%)$ and nonmalignant etiologies, the latter including thrombosis, often associated with intravascular devices, or rarely aortic aneurysms or fibrosing mediastinitis. A smoker who presents with upper body swelling without history of intravascular manipulation, has a high likelihood of an underlying diagnosis of pulmonary neoplasm. Management of SVC syndrome involves relieving symptoms of obstruction, typically by treating the underlying malignancy. Supportive care with head elevation and diuretics are commonly employed though there is no definitive data documenting their efficacy. Ideally a tissue diagnosis is obtained prior to initiating definitive treatment with chemo or radiation therapy. If symptoms are severe- marked by stridor, respiratory compromise, or depressed central nervous system function- SVC stent placement, localized thrombolysis, and/or emergent radiation therapy may be indicated.

TESTING FOR STDS, DISCLOSURE OF RESULTS AND PARTNER NOTIFICATION - AN ETHICALLY SLIPPERY SLOPE? Eleanor Weinstein; Lisa Rucker. Jacobi Medical Center Albert Einstein College of Medicine, Bronx, NY. (Control ID \#1341486)

LEARNING OBJECTIVE 1: To Increase awareness of the ethical issues surrounding testing for STDs and disclosure of results to patients and contacts.

LEARNING OBJECTIVE 2: To become knowledgeable about the role of EPT - its potential advantages and ethical considerations.
CASE: AJ, a 35 yo man, was diagnosed and treated for chlamydia The patient asked his provider to treat his spouse, witholding information as to the specifics of the infection. The provider disclosed that she was being treated for a "urinary tract infection".

DISCUSSION: Telling the truth in a clinical context is an ethical obligation but determining just what constitutes "the truth" remains a clinical judgment. Truth telling must be seen in association with other ethical principles of beneficence, nonmaleficence, and protection of the community. Is there a place in clinical care to disclose less than the full "truth" or to deceive? There are well described situations where it appears to be justified to withhold information. At times, patients themselves may request not to be given full disclosure of information. This practice may not ordinarily violate major ethical principles of veracity, autonomy and beneficence. However, in some cases respecting the patient's request not to know may create a danger to the patient themselves or to others. In this case, the patient's request may not be respected as it would violate core principles of beneficence and nonmaleficence. Additionally, in certain cultures it may be more acceptable to see the individual patient as a part of a family unit or extended family, rather that as an autonomous entity. What about the moral obligation of the physician to properly inform the partner of her husband's transgressions, her risk for future exposure to infection and her possible risk for other STDs and HIV? Participating in the deception prevents this full disclosure and thus prevents any meaningful education or opportunity to counsel the partner. The deception however results in a desired outcome - treatment of chlamydia. In our case, the physician would have the right to disclose the wife's test results without the index patient's permission and to discuss the implications - since she now had consented to her own treatment and had entered into an independent relationship with the physician. Considering the many factors involved, it is not surprising that partners of index patients infected with STDs may not receive treatment despite the high likelihood that they are also infected. Available data suggests that about $50 \%$ of all partners of persons with gonorrhea or chlamydia infection receive appropriate treatment. Expedited partner therapy (EPT) is the practice of treating the sex partners of persons with STDs without an intervening medical evaluation or professional counseling. This is usually achieved through patient delivered partner therapy (PDPT). Recent evidence suggests that EPT is a useful option to facilitate partner management among heterosexual men and women with gonorrhea and chlamydia and the Center for Disease Control advocates that EPT be available to clinicians in addition to the current standard practices such as patient referral or provider assisted referral. Despite concerns regarding missed opportunities for medical evaluation, screening for other STDs, and professional counseling, as well as the concern for adverse effects from medications dispensed, EPT appears to be an ethically acceptable and practical option in states where it is legal and available.

THE "EYES" HAVE IT: AN UNUSUAL CASE OF OPHTHALMOPLEGIA Matthew Lander. University of Pittsburgh Medical Center, Pittsburgh, PA. (Control ID \#1339854)

LEARNING OBJECTIVE 1: Recognize the proper approach to evaluation of ophthalmoplegia.

LEARNING OBJECTIVE 2: Identify the various malignancies that can present in the ocular orbit.

CASE: A 70 year old woman presented with eight months of restricted right eye movement. Her PMHx included hypertension, a previous TIA, and basal cell carcinoma of the right upper eyelid. Symptoms were reported as right eye pain, headache, and diplopia associated with a forty pound loss of weight over six months. She denied any fevers, chest or abdominal pain, numbness, or weakness. Physical examination revealed that she was afebrile and her right eye was notable for injection, decreased visual acuity, and severely restricted gaze in all directions. There was also some milky discharge from the epicanthus. An MRI revealed diffuse abnormal enhancement of the right orbital tissues and extra-ocular muscles. A biopsy of a mass in the right inferior fornix initially was interpreted as undifferentiated carcinoma. Cytology of ascites fluid showed malignant cells with staining suggestive of metastatic breast carcinoma. Mammogram of her right breast showed 5 lesions in total, all less than $1 \mathrm{~cm}$ in diameter. Pathology revealed an invasive breast carcinoma. Re-staining of the 
previous orbital biopsy revealed similar pathology confirming the diagnosis of metastatic breast carcinoma to the orbit.

DISCUSSION: Impairment of extra-ocular movements is commonly seen by general internists. The differential diagnosis for this condition is broad and requires knowledge of the involved patho-physiology and neurologic pathways. Basic understanding of the six extra-ocular muscles and the three cranial nerves that supply them is vital. A lesion, impingement, or injury anywhere along the tracts, such as the muscle itself, the cavernous sinus, or brainstem, can result in abnormal extra-ocular movements. Pertinent information for discovery of a cause includes time of onset, unilateral versus bilateral, and associated symptoms such as eye pain, numbness, or weakness. Execution of a thorough physical examination can elucidate a diagnosis. This would include full ocular movements, but also evaluation for proptosis, conjugate gaze palsy, nystagmus, and Argyll Robertson pupils. The ability of a physician to accurately determine the cause of ophthalmoplegia can lead to the discovery of important and life-threatening diagnoses, which may otherwise go unseen Breast carcinoma is one of several malignancies that has been found to metastasize to the orbit. Excluding the eye itself still leaves numerous structures that may be affected. Primary malignancies of the lacrimal gland, optic nerve, optic sheath, bone, and connective tissue are all possibilities. One review has stated only $2-7 \%$ of all orbital tumors are from solid tumor metastasis. In this rare subset of patients, lung and breast carcinomas are by far the most common primary tumors, followed by skin, genitourinary, and gastrointestinal tract. A retrospective analysis of a small patient population showed $88 \%$ of patients that underwent fine needle aspiration had identification of their primary malignancy. This has important implications for the internist who is faced with such a rare presentation for a common malignancy.

THE ALLURE OF A FIRST DIAGNOSIS Vincent Wong. Baylor, Houston, TX. (Control ID \#1333906)

LEARNING OBJECTIVE 1: Expand the differential diagnosis of elevated transaminases in young alcoholic patients.

LEARNING OBJECTIVE 2: Review risk factors and diagnostic challenge of cholangiocarcinoma (CCA).

CASE: A 29-year-old male with a history of heavy alcohol abuse presented with epigastric and left upper abdominal pain associated with nausea, pruritis, dark urine, clay stools, and jaundice for several days. $\mathrm{He}$ denied fever, weight loss, recent illness, travel, IVDA, and medication use. He reported drinking 80-90 cans of beer weekly. Exam was significant for jaundice and epigastric and left upper quadrant pain without rebound and guarding. Laboratory studies revealed ALT $804 \mathrm{U} / \mathrm{L}$, AST $487 \mathrm{U} / \mathrm{L}$, ALP $507 \mathrm{U} / \mathrm{L}$, total bilirubin $9.6 \mathrm{mg} / \mathrm{dL}$, direct bilirubin $7.7 \mathrm{mg} / \mathrm{dL}$, and GGT $2254 \mathrm{U} / \mathrm{L}$. Total protein was $8 \mathrm{~g} / \mathrm{dL}$, albumin was $4.4 \mathrm{~g} / \mathrm{L}$, and platelets were normal. Hepatitis panel, EBV IgM, ANA, alphal anti-trypsin, antismooth muscle antibody, cerulopasmin, and iron saturation were negative/ normal. An ultrasound showed increased echogenicity of liver and a contracted gallbladder but no masses and no ductal dilation. He was diagnosed with alcoholic hepatitis and was discharged home with recommendation of alcohol cessation. Patient continued to drink ETOH and returned one week later with severe abdominal pain. His liver panel worsened. A MRCP revealed a beaded hepatic ductal pattern consistent with primary sclerosing cholangitis (PSC) and an exophytic hepatic mass. CA19-9 was $66.78 \mathrm{U} / \mathrm{ml}(0-35 \mathrm{U} / \mathrm{ml})$. Core biopsy and pathology showed invasive cholangiocarcinoma, Klatskins tumor. The mass was deemed non-resectable, and he was offered chemotherapy and possible liver transplant.

DISCUSSION: Cholangiocarcinoma occurs in patients 50 to 70 years of age but can present two decades earlier with PSC. In patients with PSC, there is an annual incidence of approximately $1 \%$ of developing CCA, with half being detected at the same time or within 1 year of diagnosis. The other known risk factors include parasitic infections, hepatolothiasis, and biliary duct cysts. Other less established risks are IBD, hepatitis B and C, obesity, smoking, and alcohol abuse. Altaee et al, in a retrospective study, reported that heavy alcohol consumption was present in nearly half of 112 patients with CCA, but subsequent studies did not find this cause-effect relationship. Thus, whether excessive ETOH abuse is a risk factor for this patient remains controversial. The pathogenesis is unclear but possibly reflects a step wise accumulation of defects in oncogenes (eg K-ras, EGFR) and tumor suppressor genes (eg p53). Symptoms include jaundice, weight loss, and abdominal pain. Diagnosing CCA is challenging. CA 19-9 has a sensitivity and specificity of 79 and $98 \%$, respectively. Ultrasound, CT and MRI have positive predictive values of $48 \%, 38 \%$, and $40 \%$ in identifying CCA. ERCP and MRCP have lesser positive predictive values. Brush cytology has $100 \%$ specificity but possesses low sensitivity $(18-40 \%)$. There is no established standard therapy, but 5-FU, gemcitabine, cisplatin and oxaliplatin have activity. Survival after 2 years is extremely rare. Liver transplantation following neoadjuvant therapy is recommended for early stage ( $<3 \mathrm{~cm}$ in diameter without metastases) not amenable to resection. This case illustrates the importance of broadening the differential diagnoses of elevated transaminases in young alcoholic patients.

THE ANSWER IS IN THE HISTORY: AN UNCOMMON CAUSE OF PALPITATIONS Elizabeth Sandman. Beth Israel Deaconess Medical Center, Boston, MA. (Control ID \#1339763)

LEARNING OBJECTIVE 1: Explain the evaluation and diagnosis of palpitations.

LEARNING OBJECTIVE 2: Describe the clinical presentation, diagnosis and treatment of pheochromocytoma.

CASE: A 40 year old Caucasian woman with no past medical history presented to clinic with several months of palpitations. Medical work up including TSH and toxicology screen were negative. She was diagnosed with generalized anxiety disorder and started on as needed clonazepam. One year later, the patient returned to clinic with report of an intermittent tremor worsened during times of stress. She was diagnosed with essential tremor and declined medical therapy. Two years later, she presented to an outside emergency room with severe abdominal pain. She was found to be hypertensive to 204/100 with a pulse of 138 . She underwent CT scan for evaluation of abdominal pain but developed hypoxia and delirium and was emergently intubated before the scan was completed. EKG demonstrated ST elevations in V2-V4. She was transferred to our facility for cardiac catheterization, which showed no flow limiting disease. Echocardiography showed an ejection fraction of $20 \%$ with apical sparing. Subsequently she developed hypotension ultimately requiring three pressor support. Upon questioning of her husband, he reported the patient recently complained of increased diaphoresis and diarrhea for the last month. Bedside renal ultrasound was performed given high clinical suspicion for pheochromocytoma and a $5 \mathrm{~cm}$ hypervascular mass was seen superior to the right kidney. After hemodynamic stability was achieved, she underwent right adrenalectomy and pathology returned as pheochromocytoma. Following surgery she recovered completely. After a brief stay in a rehabilitation facility she was discharged home.

DISCUSSION: Palpitations are a common complaint in the primary care setting. The most common cause is anxiety disorder. Older age at presentation may suggest a non-psychiatric cause. The most common non-psychiatric, noncardiac causes of palpitations include thyroid disease, anemia, alcohol, and stimulants. As most patients presenting with palpitations are without symptoms at time of presentation, initial work up should include a careful history to elucidate underlying cause. A 12-lead EKG is the first diagnostic test that can be performed in the office. If suspicion is high for a cardiac arrythmia as the cause of palpitations and work up for systemic disease is negative, ambulatory cardiac monitoring can be done. Pheochromocytoma is a rare malignancy that classically presents with headache, tachycardia and diaphoresis though the majority of patients do not demonstrate this triad. It should be considered in patients presenting with hyperadrenergic episodes. Cardiomyopathy from catecholamine excess is a rare presentation of pheochromocytoma but may demonstrate a 'reverse Takosubo' pattern on echo with apical sparing. Treatment for pheochromocytoma is initially medical with alpha-adrenergic blockade followed by surgical resection. In rare cases where there is family history of other neuroendocrine tumors, a neoplastic syndrome such as multiple endocrine neoplasia 2a should be considered and genetic testing performed. Pheochromocytoma is a rare cause of palpitations that should be considered in patients with other signs and symptoms of a hyperadrenergic state.

THE CASE OF THE MISSED PHYSICAL EXAM Yasmeen Kabir; Jeffrey Miller; Michael Rotblatt. UCLA-Olive VIew Program, Sylmar, CA. (Control ID \#1334506) 
LEARNING OBJECTIVE 1: Recognize metastatic testicular carcinoma as part of the differential diagnosis in young men who present with GI bleeding.

LEARNING OBJECTIVE 2: Recognize the importance of the comprehensive admission physical exam, especially the GU exam that is often neglected.

CASE: A 30-year-old Hispanic male with a history of heavy NSAID use presented with complaints of melena and severe abdominal pain for approximately 3 weeks. He had presented to the Emergency Department several times previously over the past 4-5 months for similar, but milder symptoms. The patient was severely anemic with $\mathrm{Hg} 7.8$ and MCV 78 on admission. A "complete" physical exam revealed no pertinent findings. Endoscopy revealed a small ulcer with erosions in the gastric antrum, and a large ulcer with heaped up edges and overlying clot in the second portion of the duodenum. These findings were concerning for malignancy vs. NSAID induced ulcer. The patient only then endorsed a right swollen testicle, which had been missed on all previous exams, and a non-tender enlarged right testicle was then found on GU examination. Testicular UTZ revealed a $3.5 \times 2.0 \times 2.0 \mathrm{~cm}$ mass. Tumor markers were elevated: HCG 50, AFP 1990, LDH 534. CT demonstrated a $16 \times 11 \mathrm{~cm}$ enhancing mass of the right psoas, right hydronephrosis due to encasement of the right ureter, and invasion of the duodenum. The initial EGD Biopsy revealed malignant germ cell tumor (GCT), consistent with embryonal carcinoma. Due to extensive tumor involvement, the patient was treated with systemic chemotherapy and a right orchiectomy with good response.

DISCUSSION: Testicular carcinoma most commonly presents as a painless testicular mass. The presenting manifestations of testicular cancer are attributable to metastatic disease in approximately $10 \%$ of patients. Symptoms vary with the sites of metastases. Back pain can occur with skeletal metastases or retroperitoneal disease involving the psoas muscle. Pulmonary symptoms such as shortness of breath, chest pain, and hemoptysis can occur in patients with advanced pulmonary disease or primary mediastinal GCT. Approximately $5 \%$ of patients with GCT of the testis present with GI metastasis. GI metastasis is associated with nonseminomatous germ cell tumors of which the duodenum is the most commonly affected part of the small intestine. Testicular metastasis to the duodenum occurs as a result of lymphatic drainage of the testes to the interaortocaval lymph nodes and para-aortic nodes. Direct spread from tumor masses can also invade the duodenum and cause ulceration, as in our patient. The most common presenting symptom among patients with duodenal metastasis is GI hemorrhage with melena, hematemesis, and abdominal pain, most of which occurred in our patient. GI bleeding is an important presenting finding in a small percentage of cases of germ cell tumors. When men (especially young men) present with symptoms suggestive of GI bleeding it is essential to recognize metastatic testicular carcinoma as part of the differential diagnosis. As with all hospital admissions, a complete comprehensive physical exam is required, including the frequently neglected GU examination.

THE COCCI IN CLUSTERS CONUNDRUM John Humphrey. Tulane University Health Sciences Center, New Orleans, LA. (Control ID \#1339036)

LEARNING OBJECTIVE 1: 1. Identify the risk factors and clinical features of spinal epidural abscess. 2. Understand the diagnostic approach to confirming a spinal epidural abscess 3 . Recognize the treatment of spinal epidural abscess

CASE: A 57 year-old man presented with three days of back pain, fever and confusion. He had a history of Crohn's disease and was taking prednisone and infliximab. He additionally reported injuring his back while playing basketball one week prior to presentation, and acknowledged receiving intravenous analgesics at home during the days leading up to presentation. His vital signs were normal. Kernig and Brudzinski signs were positive, and there was a grade $3 / 6$ holosystolic murmur at the apex. The white-cell count (WBC) was $20,000 / \mathrm{mm} 3$ with $88 \%$ neutrophils. The lumbar puncture yielded cloudy fluid containing WBC 29,028 cells $/ \mu 1$. A Gram stain revealed gram-positive cocci in clusters, later identified as methicillin-sensitive Staphylococcus aureus. He was initially given ceftriaxone for presumed meningitis. However, the history of back pain prompted concern for a spinal epidural abscess. Contrast-enhanced MRI confirmed a dorsal epidural abscess at level L1-4 that was not compressing the spinal cord. This was managed non-operatively with antibiotics. An echocardiogram visualized a two-centimeter mitral valve vegetation causing severe mitral regurgitation. This required mitral valve repair; he recovered without residual neurologic or cardiac dysfunction.

DISCUSSION: The general internist must maintain a high index of suspicion for spinal epidural abscess, as prompt diagnosis has been cited as the most important factor in patient outcome. In this case, the presence of back pain, fever, and elevated inflammatory markers provided important clues to the diagnosis. Lumbar puncture is not indicated to diagnose spinal epidural abscess as instrumentation may seed the cerebral spinal fluid to induce meningitis. It is likely that the treating physician inadvertently sampled fluid from the lumbar epidural abscess rather than the subarachnoid space for two reasons: first, the typical CSF WBC count in bacterial meningitis is $1,000-5,000$ cells $/ \mathrm{mm} 3$, far below that obtained in our patient; second, $\mathrm{S}$. aureus is responsible for up to $70 \%$ of cases of SEA, compared to $1-3 \%$ of bacterial meningitis cases. In this patient, intravenous drug use while taking immunosuppressant medications likely led to endocarditis, which by hematogenous dissemination, served as a source for infection of the epidural space. The history of a recent back injury in this patient may serve to connect the two by the principle of locus minoris resistentiae, in which bacteria preferentially seeds an area of recent trauma. Physicians must be cognizant about the possibility of spinal epidural abscess given the importance of early diagnosis in disease outcome.

THE CURIOUS CASE OF EXTREMITY WEAKNESS AND AMBULATORY DIFFICULTY: AN UNUSUAL PRESENTATION OF POLYMYALGIA RHEUMATICA (PMR) Anuradha L. Mookerjee; Bert M. Bieler; Sachin Mohan. Cooper University Hospital, Camden, NJ. (Control ID \#1334504)

LEARNING OBJECTIVE 1: Recognize an unusual presentation of Polymyalgia Rheumatica (PMR) in an elderly man

LEARNING OBJECTIVE 2: Treat the symptoms of PMR early to enable effective regression of symptoms

CASE: A 63 year-old Caucasian male presented with two days of lower extremity weakness and difficulty in ambulation. Two weeks prior, the patient had bilateral shoulder pain and marked stiffness. He continued to experience a burning sensation which migrated from his upper arms to his fingertips and grew in intensity. This was associated with weakness in the upper girdle and an inability to raise his arms above his shoulders. Review of systems was positive for subjective fevers and diarrhea five days prior to admission, but had resolved at time of admission. Family history was negative for any rheumatologic disorders. Medications included metoprolol, lisinopril, atorvastatin, and aspirin for his known hypertension, hyperlipedimia and coronary artery disease. On physical exam, the patient had normal vital signs and the neurologic exam revealed decreased tone and power in motor function of both his upper and lower extremities, with normal sensations and reflexes. The cranial nerves, the cerebellar exam and gait were all within normal limits. The laboratory data for chemistry, liver function tests, $\mathrm{CK}$, TSH and aldolase were all within the normal ranges. The RF, ANA and Lyme antibodies were negative. The patient did show a significant rise in his ESR at 64 and in CRP which was 10. Based on the clinical presentation and elevated inflammatory markers, the patient was diagnosed with PMR and started on steroids. He showed remarkable improvement in all of his symptoms. The lower extremity pain and weakness along with the burning sensation in the arms and fingers was completely resolved within twenty four hours and he was discharged home. A three week follow-up confirmed that the patient continued to do well on a very slow taper of steroids.

DISCUSSION: This case of PMR reminds us that uncommon disorders may present uncommonly. Classically PMR occurs in Caucasians over 50 years, with a 3:1 female to male ratio. It is associated with shoulder and hip girdle pain and an elevated ESR, usually above 40 . This case was unusual in that the patient presented with weakness and ambulatory dysfunction, potentially delaying the diagnosis. The differential diagnosis included inflammatory myopathy, statin-induced myositis, systemic vasculitis, radiculopathy, Guillain-Barre, myasthenia gravis, Lambert-Eaton syndrome, ALS, severe hypothyroidism, RA, and fibromyalgia. Key to 
solving the puzzle was a good history. Initially, with the patient's main complaints, one might have been steered towards a neurological disorder or myositis as the diagnosis. When further questioned, the symptoms included shoulder girdle pain of a waxing and waning nature. With an elevated ESR and CRP and an otherwise normal exam and laboratory studies, PMR became the leading diagnosis. Rapid response to steroid therapy clinched the diagnosis.

THE FLEXNERI REPORT Jason Halperin; Obinna Nnedu. Tulane University Health Sciences Center, New Orleans, LA. (Control ID \#1311857)

LEARNING OBJECTIVE 1: Identify Shigella flexneri as a serious cause of enterocolitis in the HIV positive population.

LEARNING OBJECTIVE 2: Recognize that shigellosis can be sexually transmitted and therefore a thorough social history is imperative to identify risk factors.

CASE: A 21-year-old an with HIV (CD4=270 cells/mm3) presented with a three-day history of abdominal cramping, diarrhea and vomiting. $\mathrm{He}$ described frequent stooling with non-bloody bowel movements. He denied anorexia and hematemesis. His pain was relieved with defecation. $\mathrm{He}$ denied sick contacts, change in diet, or previous episodes. He denied exposure to unpasteurized foods, daycare facilities, nursing homes or recent antibiotic use. He had not traveled recently nor had any animal contacts. He was diagnosed with HIV five years earlier during routine screening. He recently stopped taking his anti-retroviral medications because "he was feeling so good." He described frequent sexual contact with both men and women, engaging in both insertive and receptive sex as well as direct oral-anal contact. He did not know of any sexual contacts who had experienced similar symptoms. He was febrile to $102.6^{\circ} \mathrm{F}$, tachycardic, normotensive and orthostatics. His cardiac, pulmonary, neurologic and skin examinations were normal. His abdominal exam demonstrated tenderness in all four quadrants without rigidity; Murphy's, Roevsing's and the psoas sign were absent. Diagnostic testing revealed acute renal failure. An abdominal CT scan revealed severe inflammation in the sigmoid colon with a normal gallbladder and appendix. He was negative for viral hepatitis. Stool studies were positive for fecal leukocytes, and negative for Clostridium difficile toxin assay, giardia antigen, and cryptosporidium antigen. On the second hospital day, his stool culture was positive for Shigella flexneri, sensitive to ciprofloxacin. He was discharged once the acute kidney insufficiency had resolved, and he was treated with a ten-day course of antibiotics. He was referred for re-initiation of anti-retroviral therapy and counseled on safe sexual practices

DISCUSSION: In the industrialized world, shigella species are typically transmitted by direct or indirect fecal-oral contact and frequently associated with daycare facilities, nursing homes and contaminated food or water. It is important, however, that the general internist recognize HIV-infected patients as being at higher risk for shigella infection, especially when unsafe sexual practices are a part of the history. The Center for Disease Control (CDC) demonstrated a 30 times greater incidence of infection in the HIV positive population compared to HIV negative. Furthermore, HIV positive individuals are four times more likely to require hospitalization. Efficient sexual transmission has been demonstrated by direct fecal-oral transmission; very small inoculums of shigella, as low as 10 organisms, are able to initiate disease. Concurrent HIV infection is associated with extended carriage of shigella species, as well as increased asymptomatic shedding. A recent populationbased study of shigella cases in San Francisco demonstrated independent associations of HIV and men who have sex with men, specifically direct oralanal sexual contact, with an odds ratio of 8 for each risk factor.

THE G.I.S.T. OF NEUROFIBROMATOSIS Akshiv Malhotra; Jonathan Wright; Ajeet Gajra. SUNY Upstate Medical University, Syracuse, NY. (Control ID \#1338977)

LEARNING OBJECTIVE 1: Recognize the increased incidence of gastrointestinal stromal tumors in patients with neurofibromatosis.

CASE: A 64-year-old man with known Neurofibromatosis type 1 ( NF1) during preoperative workup for a CABG was incidently noted to have a large inhomogeneous pelvic mass with dimensions of $9 \mathrm{~cm} \times 10 \mathrm{~cm} \times$ $7.8 \mathrm{~cm}$ on a CT abdomen/pelvis. Biopsy of the mass showed palisaded appearing long spindle cells. On immunohistochemical testing, S-100 was negative (ruling out schwannoma) and there was strong and diffuse positivity for CD117( KIT) and CD34 indicating a gastrointestinal stromal tumor (GIST). The tumor was considered to be marginally resectable, so neoadjuvant treatment with Imatinib $400 \mathrm{mg}$ daily was started to decrease the tumor size preoperatively. After three months on the repeat CT abdomen/pelvis multiple foci of air were seen in the mass, suggestive of necrosis though the size remained stable at $11 \times 9.7 \times 7.7 \mathrm{~cm}$. His tumor was then surgically resected en-bloc. Large cavity was noted within the tumor along with fistula formation necessitating partial excision of small intestine. After the surgery he was restarted on Imatinib $400 \mathrm{mg}$ daily for 36 months. DISCUSSION: Neurofibromatosis type 1 (NF1), also known as VonRecklinghausen's disease is amongst the most commonly transmitted hereditary autosomal dominant diseases, with an estimated birth incidence of 1:3,000. Loss of NF1 tumor suppressor gene on chromosome 17 leads to the development of benign and malignant tumors in NF1. In addition to cutaneous, soft tissue, and visceral (plexiform) neurofibromas, this syndrome is associated with several types of gastrointestinal (GI) and abdominal tumors. Here we present the case of a patient with NF1 who was incidentally found to have an extraintestinal Gastro-Intestinal Stromal Tumor (GIST). Extra-intestinal GIST is a very rare entity, accounting for $<$ $5 \%$ of cases of GIST. There is a known correlation between NF1 and GIST. GIST develops in $7 \%$ of patients with NF1, although the incidence of GIST in the general population is $1.5 / 100,000 /$ year. NF1 patients tend to develop GIST at a younger age (median 49 years) than sporadic GIST (56 years). This brings forward a need to formulate guidelines to screen for GIST in patients with NF1 at an earlier age. GIST may be associated with gastrointestinal bleed, perforation or obstruction as demonstrated by pathological findings in our case. Imatinib has greatly enhanced outcomes in patients with GIST.

THE GREAT MASQUERADER Haleh Moazen ${ }^{1}$; Amir Ansari-Ezabadi ${ }^{2}$. ${ }^{1}$ Montefiore Medical Center, Bronx, NY; ${ }^{2}$ Montefiore Medical Center, Bronx, NY. (Control ID \#1310573)

LEARNING OBJECTIVE 1: Recognize amyloid as a cause of rapidly progressive congestive heart failure.

LEARNING OBJECTIVE 2: Describe the clinical, echocardiogram and cardiac magnetic resonance imaging features of cardiac amyloid.

CASE: A 54 year old woman was admitted for the evaluation of shortness of breath on exertion and paroxysmal nocturnal dyspnea for three months. History elicited a recent diagnosis of eosinophilic gastroenteritis after a colon biopsy revealed eosinophilic infiltration. The patient also had a history of hypertension. Physical exam was remarkable for rales.Labs revealed eosinophilia. Radiograph demonstrated heart failure. Echocardiogram revealed moderate pericardial effusion and a normal ejection fraction .Workup for eosinophilia was initiated and revealed a positive Strongyloides IGg titer. Empiric treatment for Strongyloides and heart failure were started. Symptoms initially improved but two months later the patient returned with recurrent heart failure and worsening pericardial effusion. Echocardiogram again revealed moderate to large pericardial effusion and a normal ejection fraction. The patient received a pericardial window. A pericardial biopsy revealed chronic inflammation with occasional granulomas but no acid fast bacilli was noted. Treatment for presumed tuberculosis pericarditis was initiated. Two months later the patient returns with rapidly progressive heart failure despite appropriate treatment for heart failure and tuberculosis pericarditis. On computerized tomography of the chest, the pericardium was noted to be nodular with a tubular configuration of the heart suggesting constriction. Cardiac magnetic resonance imaging demonstrated pericardial nodularity and straightening of the interventricular septum suggestive of constrictive pericarditis. Cardiac catheterization revealed both constrictive and restrictive patterns. The biopsies of the pericardium and the gastrointestinal tract done prior were restained with Congo red stain and demonstrated applegreen birefringence under polarized light microscopy consistent with $\mathrm{AL}$ amyloidosis. A bone marrow biopsy revealed multiple myeloma.

DISCUSSION: Systemic AL amyloidosis is a multiorgan systemic disease Fifty percent of patients with AL amyloidosis initially present with clinical 
features of heart failure. Cardiac disease in AL amyloidosis is an important cause of progressively worsening heart failure and conduction system abnormalities. Clinically, amyloid heart disease may mimic constrictive pericarditis, coronary artery disease, valvular heart disease, and idiopathic hypertrophic or congestive cardiomyopathy. No single noninvasive test nor abnormality is classic for cardiac amyloid. The diagnosis of cardiac amyloid is considered to be more likely if there is evidence of reduced electrocardiographic voltages in combination with the following echocardiographic findings; thickened left ventricular wall,biatrial dilation, increase echogenicity and less commonly thickened valves and a small pericardial effusion. Cardiac magnetic resonance imaging findings occur late in the disease and usually reveal global subendocardial late gadolinium enhancement of the myocardium. Amyloid is a very difficult disease to diagnosis and has been known as the great masquerader. This case illustrates that an infiltrative myocardial cardiomyopathy such as amyloid should be included early in the differential diagnosis of patients presenting with rapidly progressive heart failure.

THE HEART BRAIN CONNECTION Robert L. Glover; Robert E. Graham; Ranjit Suri. Lenox Hill Hospital, New York, NY. (Control ID \#1333931)

LEARNING OBJECTIVE 1: Recognize an uncommon underlying cause of seizures

CASE: The patient is a 73 year old male with 3 year history of a seizure disorder, hyperlipidemia, left bundle branch block and mitral regurgitation who presented to an outside hospital after experiencing seizures. The patient's wife reports that the patient experienced a seizure event with generalized shaking and incontinence while asleep lasting 2-3 minutes. Shortly after, the patient suffered an additional event of similar quality for roughly eight minutes. Emergency medical services were called and the patient seized again while in transit to the hospital. $1 \mathrm{mg}$ of ativan and $100 \mathrm{mg}$ of keppra were given intravenously with successful resolution. The patient was placed on telemetry and had paroxysmal complete heart block with an unstable escape rhythm resulting in pauses up to 5.5 seconds. The patient did not seize during this event and there were no significant metabolic abnormalities, changes in cardiac enzymes or notable findings on intracranial imaging. Transfer was arranged from the outside hospital to our tertiary cardiac center for emergent permanent pacemaker placement. The following day a permanent pacemaker was placed without complication. Concurrently during hospitalization an EEG was performed that did not demonstrate any seizure activity.

DISCUSSION: Details regarding the patient's history of seizure disorder revealed that the patient had first started experiencing seizures at the age of 70 . These seizures generally occurred in three to six month intervals and lasted approximately 2-3 minutes with incontinence and generalized shaking. The patient was referred to a neurologist who performed a CT, MRI and EEG scan of the brain which did not reveal any overt cause of seizures. Despite pharmacological treatment with keppra, the patient continued to have intermittent seizures of cryptogenic origin. This case highlights the importance of an expanded differential diagnosis in the event that an underlying neuro structural origin for seizures cannot be found, especially in a patient with known cardiac conduction disease. It is hypothesized that this patient's seizure disorder was triggered by transient hypoxia secondary to episodes of third degree heart block. This condition is called Stokes-Adams syndrome. StokesAdams syndrome is more broadly defined as loss of consciousness with or without seizures caused by a sudden decrease in cardiac output. Seizures in Stokes-Adams syndrome are notable for lacking an aura and starting and ending abruptly. Witnesses may also describe the patient has suddenly becoming very pale just prior to seizure onset. The patient has been asymptomatic since pacemaker implantation - suggesting that the original diagnosis of "seizure" disorder is likely incorrect.

THE IMPORTANCE OF DONATING BLOOD Anna Postolova; Jason Halperin. Tulane University Health Sciences Center, New Orleans, LA. (Control ID \#1339076)

LEARNING OBJECTIVE 1: 1. Identify the differential diagnosis of hemoptysis. 2. Recognize the clinical presentation of Eisenmenger syndrome. 3. Understand the pathophysiology of Eisenmenger syndrome.
CASE: A 44 year-old Honduran man presented with four hours of hemoptysis, muscle weakness, dyspnea and fatigue. Each episode had produced approximately three tablespoons of blood. On physical examination a IV/VI holosystolic murmur was heard best at the upper left sternal border. Significant jugular venous distention was present with an associated hepatojugular reflux. There was wheezing in the lower lung fields, bilaterally. The abdomen was distended, soft, and mildly tender without hepatosplenomegaly. He had fingernail clubbing, 2+ lower extremity edema, and no cyanosis. Laboratory studies revealed a hemoglobin of 18.6 , a hematocrit of 60.4 , and a brain naturetic peptide of 1,911 . The cardiac silhouette was enlarged by chest X-ray. Echocardiography demonstrated a patent ductus arteriosous, a pulmonary artery pressure of $160 \mathrm{~mm} \mathrm{Hg}$ and Eisenmenger physiology.

DISCUSSION: Hemoptysis is a symptom commonly encountered by the general internist. While the differential diagnosis is broad, the physical examination can be instrumental in the general internist's attempts to narrow the differential diagnosis. In our patient, the clinical presentation of shortness of breath, an elevated JVD, edema and clubbing were indicative of heart failure. The presence of hepatojugular reflux suggested right ventricular impairment, an important clue in considering the diagnosis of Eisenmenger syndrome, which was later confirmed by echocardiography. Eisenmenger syndrome is an advanced form of pulmonary arterial hypertension secondary to a congenital heart defect. The development of Eisenmenger syndrome begins with left-to-right shunting of blood flow generating increased pulmonary blood flow, endothelial dysfunction and vascular remodeling. The ensuing increased pulmonary vascular resistance leads to pulmonary infarction or rupture of dilated pulmonary arterioles and hemoptysis. As the shunt reverses, due to the equalization of pressures in the right and left ventricle, circulating deoxygenated blood does not meet physiologic demand resulting in erythrocytosis, polycythemia, and hyperviscosity. Symptoms of hyperviscosity include headache, muscle weakness, fatigue and hemoptysis. The treatment for symptomatic hyperviscosity includes lowering the red blood cell mass via phlebotomy. This must be performed conservatively and asymptomatic patients, regardless of their hematocrit, should not be treated nor should treatment be targeted to a predetermined hematocrit. Treatment should be targeted to symptoms; excessively aggressive treatments can lead to iron deficiency, further exacerbating the inadequate delivery of oxygenated blood. Hemoptysis is frequently encountered by the internist. Eisenmenger physiology though is rare and often due to inadequately treated congenital heart disease, uncommon in the United States. Yet, with dynamic migratory patterns in our increasingly globalized world, the primary care physician will need to be prepared to consider rare causes of common presentations such as hemoptysis due to an untreated patent ductous arteriousus.

THE USE OF TRANSHEPATIC CATHETERS IN URGENT DIALYSIS Jennifer Yehl; Munazza Anis; Sujeev S. Bains. Medical University of South Carolina, Charleston, SC. (Control ID \#1339088)

LEARNING OBJECTIVE 1: Indications for urgent dialysis LEARNING OBJECTIVE 2: Alternative access sites for hemodialysis and complications

CASE: CB is a 33 year old African American male with a past medical history of end stage renal disease (ESRD) secondary to posterior urethral valves, chronic hypocalcemia secondary to parathyroidectomy, recurrent symptomatic hypotension secondary to diastolic heart failure, history of DVT, and anemia who presented to an outside hospital with significant metabolic abnormalities $(\mathrm{K}+=7.6, \mathrm{Ca} 2+=5.5, \mathrm{Phos}=13.3, \mathrm{Cr}=22)$ indicating the need for urgent dialysis. CB has a history of difficult vascular access requiring peritoneal dialysis along with medical non-compliance. Consequent to his poor compliance, his peritoneal site became infected with gram positive cocci. Unable to gain alternative access for dialysis, CB was subsequently transferred to the MUSC MICU for placement of a transhepatic catheter to serve as his access site. This is achieved by advancing a needle at the right midaxillary line at the level of the twelfth rib and contrast is infused as the needle is withdrawn to the point when the hepatic vein is opacified. A wire is then advanced centrally into the right atrium and a tunnel is created in the right abdominal wall. Following this, the dialysis catheter is then placed over the guidewire into the right atrium under fluoroscopic guidance. Real-time 
fluoroscopy was used to visualize catheter placement and confirm appropriate placement using radiographic films. $\mathrm{CB}$ was able to undergo dialysis to correct his electrolyte abnormalities and improved enough to be transferred to the general medicine team. He was discharged the subsequent day with follow-up for continued dialysis and monitoring.

DISCUSSION: The National Kidney Foundation recommends initiation of dialysis for chronic kidney disease (CKD) for patients with eGFR < $15 \mathrm{~mL} / \mathrm{min} / 1.73 \mathrm{~m} 2$ or stage 5 CKD (1). While CKD may take years to advance to stage 5 , some conditions indicate the need for more immediate dialysis. Urgent indications include acidemia, drug intoxication, electrolyte imbalance, fluid overload, and uremia. Dialysis access sites include arteriovenous grafts, peritoneal dialysis, and transhepatic catheters. While AV grafts are commonly used, the use of transhepatic catheter access is still rare. Currently, the literature has very limited data regarding the ease of access, longitudinal efficacy, and possible complications of transhepatic catheter dialysis. One study describes six complications that may arise during catheter placement (2). Of these complications, bleeding accounted for four of the six difficulties patients experienced. The various bleeding sites included an access site bleed, a liver capsule tear, a catheter tract bleed, and a massive intraperitoneal hemorrhage which proved to be fatal. One patient experienced a kink in his catheter, while another patient died from a previously diagnosed subdural hematoma following the procedure. The remaining patients in the study showed successful results. The catheters lasted an average of 138 days (ranging from 0 to 599 days), and each patient received a range of 1 to 6 catheter exchanges. While this type of access is not often implemented in emergent hospital settings, it is a viable option for patients with multiple failed attempts and difficult vascular access (2).

THE WAY TO A MAN'S HEART IS THROUGH HIS STOMACH Nachiket Patel ${ }^{1}$; Kyle G. Ulveling ${ }^{2}$; Tom $\mathrm{Hee}^{2} .{ }^{1}$ Creighton University Medical Center, Omaha, NE; ${ }^{2}$ Creighton University Medical Center, Omaha, NE. (Control ID \#1340409)

LEARNING OBJECTIVE 1: Recognize deglutition syncope as a unique cause of loss of consciousness.

CASE: The patient was a 64-year-old white male with a PMH significant for HTN who was transferred from an OSH for evaluation of three episodes of loss of consciousness (LOC) over a 6-hour period. He presented to the OSH ED after LOC while driving. For three days prior to his presentation, he had epigastric discomfort, which he described as "gas bloating," which was relieved with antacids. On day of admission, the pain was more intense and lasted longer. He denied any associated N/V/D, melena, or hematochezia. Pt bought food at a local fast food restaurant on his drive home. While driving on country roads, he experienced symptoms of nausea and tunnel vision lasting $<10$ seconds, followed by LOC. Pt woke up in a cornfield. There was no trauma to himself, or damage to his vehicle. Pt called his family who drove him to the local ED. Pt's vitals signs were within normal limits. His $\mathrm{CBC}$ and electrolytes were unremarkable. Troponins, amylase, and lipase were negative. Physical exam was significant for epigastric abdominal tenderness. Heart and lung exams were unremarkable. ECG showed NSR with a HR of $80 \mathrm{bpm}$. In the ED, pt had 2 witnessed episodes of LOC, lasting 5-10 seconds each. Telemetry at the time demonstrated high-degree AV block with rates in the 30s. Pt was transferred to Creighton for further management. On arrival pt had a CT chest/ abdomen which showed an intrathoracic stomach due to a large hiatal hernia. Surgical repair of the pt's hiatal hernia was scheduled. Pt was started on a clear liquid diet. Immediately after he ingested $\sim 20 \mathrm{cc}$ 's of broth, his HR slowed from a baseline of $75 \mathrm{bpm}$ to a bradycardic $30-40 \mathrm{bpm}$, to sinus arrest with junctional escape beats, at which time pt lost consciousness. A nurse who was in the room started chest compressions. After the second compression, pt regained consciousness, and his rhythm reverted back to sinus. Pt was kept NPO and underwent laproscopic take down of his stomach and diaphragmatic repair. Following the surgery, pt was able to tolerate food without any symptoms.

DISCUSSION: Deglutition syncope, also known as swallow syncope, is an uncommon cause of LOC, and is neurally mediated. The afferent path of the reflex is thought to be in the vagus nerve (but also documented in the glossophyrngeal nerve). The intense afferent stimulation results in sympathetic inhibition. It also results in an efferent signal returning down the vagus nerve resulting in a decrease in HR, and peripheral vasodilitation, resulting in hypotension. Swallow syncope was first described in "Classical Descriptions of Disease" by Spens in 1793. It was more thoroughly described in 1972 by Levin \& Posner in the journal Neurology. The description of the event typically includes dizziness, light-headedness, confusion, and/or fainting during swallowing of food or liquids. Numerous disorders have been reported with deglutition syncope including diffuse esophageal spasm, hiatal hernia, esophageal diverticulum, esophageal cancer and achalasia, resulting in CHB, SVT, and atrial fibrillation. Treatment includes avoidance of the inciting food/beverage and anticholenergic medications. In our pt, the intrathoracic pressure from the hiatal hernia, precipitated by swallowing, likely resulted in intense vagal nerve stimulation causing sinus node slowing followed by sinus arrest resulting in the transient syncopal episodes.

\section{THE ENDANGERED PANDA (SIGN): THE DIAGNOSTIC DILEMMA OF PULMONARY NECROTIZING GRANULOMAS Efrain Irizarry.} New York Methodist Hospital, Brooklyn, NY. (Control ID \#1315664)

LEARNING OBJECTIVE 1: Necrotizing granulomas of the lung are usually observed in the setting of infection, most commonly mycobacterium. In contrast, non-necrotizing granulomas in pulmonary tissue are considered the hallmark of pulmonary sarcoidosis.

CASE: A 27 year old African American man was admitted with cough productive of green sputum, progressive dyspnea on exertion, and intermittent fevers for one month. The patient denied weight loss, night sweats, hemoptysis, exposure to tuberculosis, occupational exposures, and family history of autoimmune disease. He smoked $1 / 2$ pack-per-day of cigarettes for 14 years and quit four months ago. Chest $\mathrm{x}$-ray demonstrated hilar lymphadenopathy. Computerized tomography of the chest showed mediastinal adenopathy and scattered sub-centimeter pulmonary nodules. Laboratory studies were as follows: Quantiferon Gold - negative, erythrocyte sedimentation rate of $68 \mathrm{~mm} / \mathrm{H}$ (elevated), c-reactive protein of $75 \mathrm{u} / \mathrm{L}$ (normal), human immunodeficiency virus-negative, anti-nuclear antibody-negative, and rheumatoid factor-negative. Tuberculin skin test was non-reactive. Daily sputum stains over a five-day period for acid-fast bacilli were negative. Fiberoptic bronchoscopy with biopsy was unrevealing. Mediastinoscopy with sampling of station $4 \mathrm{R}$ paratracheal nodes revealed "caseating" granuloma; stains for acid-fast bacilli and fungal elements were negative. The patient was started on four-drug antituberculosis therapy (rifampin, isoniazid, pyrazinamide, and ethambutol). While on therapy, fevers continued despite a normal leukocyte count. Due to the inconsistent clinical and laboratory findings, as well as elevated liver enzymes, anti-TB therapy was stopped and an alternate diagnosis was considered. The patient underwent a total body gallium 67-citrate scan which showed intense uptake in the parotid glands (Panda sign) and bilateral lungs (Lambda sign) consistent with pulmonary sarcoidosis. Prednisone therapy was initiated and anti-tuberculous (TB) treatment was restarted following improvement in liver function test. He demonstrated quick clinical improvement and was discharged from the hospital on this regimen. On follow-up, cultures and nucleic acid amplification (NAA) were negative for Mycobacterium tuberculosis (99\% specificity and sensitivity of $75 \%$ to $90 \%$ ). With these results in hand, anti-tuberculosis medications were discontinued.

DISCUSSION: Sarcoidosis is a multisystemic inflammatory condition with pulmonary manifestations in over $90 \%$ of cases; distinction from pulmonary Mycobacterium tuberculosis is at times challenging. In general, sarcoidosis is confirmed with non-caseating granulomas on histopathology; however, a recent rigorous study of 500 lung biopsies containing granulomas found necrotizing granulomas in $4 \%$ of sarcoidosis specimens. In our patient, initial treatment was influenced by the findings of necrotizing granulomas on pathologic specimens suggesting pulmonary tuberculosis. Utilizing both old and new technologies, we successfully continued the patient on appropriate therapy with steroids, and discontinued anti-tuberculous medications. There exists no definitive diagnostic tool for sarcoidosis at this time, and part of the initial evaluation requires dismissing other causes, specifically Mycobacterium tuberculosis infection. 
Therefore, one of the first diagnostic tests that should be instituted should be the NAA for tuberculosis from sputum, cultures or biopsies.

\section{THE EVALUATION OF EOSINOPHILIA IN THE OUTPATIENT SETTING Caroline DeFilippo; Athanasia Vasiliadis. Mount SInai Medical Center, New York, NY. (Control ID \#1314879)}

LEARNING OBJECTIVE 1: Identify an approach to eosinophilia in the ambulatory setting

LEARNING OBJECTIVE 2: Recognize the clinical manifestations of Strongyloides in an immunocompetent host

CASE: A 33-year old Peruvian male with a past medical history of congenital deafness presented to an outpatient clinic with ten years of intermittent right lower quadrant abdominal pain. He does not recall when he first noted the pain but describes it as dull and with no identifiable exacerbating or relieving factors. He denies pain elsewhere in his abdomen and denies any change in the pain with eating or fasting. He has no dyspepsia, nausea, vomiting, diarrhea or constipation. He does endorse occasional right testicular fullness. His weight has remained stable during this time and he denies fevers or chills. He has no family history of any abdominal illnesses or malignancies. He takes no medications. He is a non smoker with no drug abuse history and travels to Peru annually. He is sexually active with one partner. On exam, the patient was afebrile, normotensive and well appearing. His abdominal exam was notable from mild pain in the right lower quadrant with no guarding, normoactive bowel sounds and a soft abdomen. He had no flank tenderness. A testicular exam was within normal limits. The remainder of his head, neck, cardiovascular, pulmonary and skin exam were within normal limits. A complete blood count was significant for a white count of $7400 / \mu \mathrm{L}$ initially with $19.1 \%$ eosinophilia. Over the course of four visits, the patient was noted to have a normal renal function panel, liver function tests, negative hepatitis $\mathrm{b}$ and $\mathrm{c}$ serologies, negative HIV panel, negative urine gonorrhea and Chlamydia tests, a normal urinalysis and negative stool ova and parasite exam. A testicular ultrasound was within normal limits and a serum Strongyloides $\operatorname{IgG}$ was strongly positive. The patient was treated with a one-time weight based dose of Ivermectin.

DISCUSSION: This case highlights the importance of evaluating eosinophilia detected on complete blood counts. The differential of eosinophilia can be remembered through the pneumonic NAACP: neoplasms, atopy/allergic disease, adrenal insufficiency, collagen vascular disease and parasites. The patient was screened for testicular cancer with an ultrasound given his symptoms of fullness and age. He had no history of atopy or allergies. He had no history or symptoms to suggest an immune disorder and his vital signs and lab values were not consistent with adrenal insufficiency. Finally he had no signs of collagen vascular disease. Strongyloides is a nematode endemic to tropical regions worldwide. There is no gold standard for diagnosis. Stool studies have poor sensitivity. Serum antibody testing, as was performed in this patient, can be helpful but does not distinguish between old and active infections. Strongyloides has a broad range of clinical manifestations depending on the host's immune status. In immunocompetent hosts, such as this patient, they are often asymptomatic or have chronic intestinal infections. These can manifest as nonspecific abdominal complaints. Often patients will have respiratory complaints with cough and shortness of breath that mimic asthma or COPD. Ivermectin is the preferred treatment for Strongyloides and can be given in one or two doses with cure rates up to $100 \%$

THE IMMUNOCOMPROMISED HOST: WHAT LIES BENEATH THE ITCHY RASH? Shuchi Gulati; Gaurav Gulati; Elizabeth Dohan; Richard Alweis. The Reading Hospital and Medical Center, West Reading, PA. (Control ID \#1339018)

LEARNING OBJECTIVE 1: Recognize that immunosuppressed patients are at a high risk of developing atypical infections

LEARNING OBJECTIVE 2: Earlier recognition of skin infections is critical, especially when confounded by underlying rheumatologic/ dermatologic conditions

CASE: A 51 year old female with history of dermatomyositis and uncontrolled diabetes was admitted to the hospital in an obtunded state secondary to severe diabetic ketoacidosis and sepsis. Over the last few months, her dose of prednisone for dermatomyositis had been increased to as high as $80 \mathrm{mg}$ a day due to worsening symptoms. In addition, she was receiving methotrexate $20 \mathrm{mg}$ weekly and hydroxychloroquine $600 \mathrm{mg}$ daily. On physical exam, she was found to be febrile with a temperature of $39.5^{\circ}$ Celcius and was obtunded. On skin exam, she had diffuse erythematosus and hyperkeratotic patches covered with thick scales. Lesions were especially prominent in interdigital spaces of her hands, scalp, breasts and upper arms. Diffuse excoriation marks were noted. She had twice seen her PCP with this rash in the preceding 2 weeks, and her prednisone dosage has been increased as the rash was attributed to dermatomyositis. Initial laboratory evaluation revealed: normal $\mathrm{CBC}$ with differential; chemistries significant for blood glucose of over $1000 \mathrm{mg} / \mathrm{dL}, 3+$ serum ketones, serum sodium of $151 \mathrm{mEq} / \mathrm{L}$ (normal $135-153 \mathrm{mEq} / \mathrm{L}$ ), and potassium $7.1 \mathrm{mEq} / \mathrm{L}$ (normal 3.5-5.3 mEq/L); and an elevated anion gap metabolic acidosis with $\mathrm{pH}$ of 7.16 and gap of $21 \mathrm{mEq} / \mathrm{L}$. Serum osmolality was $378 \mathrm{Mosm} / \mathrm{K}$ (normal 280-290 Mosm/K). CSF analysis was normal. Urine culture indicated a Klebsiella pneumoniae urinary tract infection. The patient's metabolic derangements were corrected with subsequent return of consciousness over 2 days. Upon awakening, she revealed that she had been living at an assisted living facility where there had been an outbreak of scabies. This prompted a direct microscopic examination of skin scrapings with demonstration of numerous mites and eggs. A diagnosis of crusted or Norwegian scabies was made. Treatment was initiated with permethrin $5 \%$ cream for 3 days and repeated after 1 week. Because of the generalized manifestations, additional oral ivermectin at a dose of $0.2 \mathrm{mg} / \mathrm{kg}$, to be repeated at weekly intervals for 4 weeks was administered. There was rapid clinical response with almost complete resolution of skin lesions at the time of discharge

DISCUSSION: Atypical clinical infections are more prevalent in institutionalized or debilitated patients, or those who are immunosuppressed from underlying disease or drug therapy. Norwegian or crusted scabies is one such infection. It is a fulminant and highly infectious form of scabies which results from a failure of the host immune response to control the proliferation of the scabies mite in the skin, thus causing hyperinfestation. Clinically, the hyperkeratotic skin lesions with crusting and scaling can resemble rheumatologic conditions like psoriasis. Though documented as "rare" in previously published papers, with the recent surge in the use of immunosuppressant agents for treating rheumatologic conditions and cancers, Norwegian scabies will become more prevalent. A high index of suspicion is required to enable early recognition and treatment, to prevent associated morbidity and spread.

THE MANY FACES OF ARTEMIS: A PECULIAR CASE OF POLYARTERITIS NODOSA Anuradha L. Mookerjee; Sachin Mohan; Bert M. Bieler. Cooper University Hospital, Camden, NJ. (Control ID \#1334538)

LEARNING OBJECTIVE 1: Recognize radiological findings of beading and aneurysms of abdominal arteries as an acceptable alternative to establish the diagnosis of PAN (Polyarteritis Nodosa).

LEARNING OBJECTIVE 2: Diagnose and treat PAN in the acute phase to prevent further complications.

CASE: A 55 year old Caucasian female presented with one week history of diffuse abdominal pain, symptomatic anemia and syncope. She also reported intermittent skin irritation, dryness of eyes and mouth, nasal sores, hair loss, weight loss of 50 pounds in less than one year, repeated infections, fevers, weakness and numbness in her left leg, and muscle and joint pains in her knees and feet. Her past medical history was significant for myocardial infarction, stroke and Thrombotic Thrombocytopenic Purpura leading to acute renal injury about four years ago. Physical exam revealed a cachectic female with marked abdominal tenderness in all quadrants and mild guarding. Her laboratory tests revealed elevated inflammatory markers and marked anemia. ANCA was negative. HIV and hepatitis panel were also negative. CT scan of abdomen showed acute intra peritoneal hematoma. The distal Superior Mesentric Artery was noted to have irregular beaded appearance suggestive of microaneurysms and vasculitis. A biopsy of the artery could not be performed due to increased risk of bleeding but she underwent successful evacuation of her hematoma. With a radiologically convincing diagnosis of PAN, the patient was started 
on steroids. The patient responded dramatically over the next week with marked improvement of all her symptoms.

DISCUSSION: PAN is a multisystem vasculitis involving medium and small sized arteries of the viscera and sparing the pulmonary arteries. PAN represents a spectrum of disease rather than a single entity. Deposition of circulating antigen antibody complexes has been proposed as a possible mechanism of narrowing of the arteries. The diagnosis of PAN remains a challenge as most patients present with vague signs and symptoms like fever, weight loss, abdominal pain, malaise and myalgias. There is no specific laboratory diagnostic test with usual findings being elevation in leukocyte count, anemia, raised ESR and hypergammaglobulinemia. Although biopsy of the involved vessels with demonstration of characteristic vasculitic changes is diagnostic; radiological findings with aneurysmal changes in the involved arteries is an acceptable alternative to establish diagnosis. Overall prognosis of untreated patients remains poor with five year survival rate of less than twenty percent. In conclusion, we present a case of Polyarteritis Nodosa with abdominal hematoma and bleeding, diagnosed radiographically with its beaded appearance of the superior mesenteric artery, which responded effectively to steroids.

THIS ADMISSION IS A PAIN Morgan Katz; Michelle M. Guidry. Tulane University Health Sciences Center, New Orleans, LA. (Control ID \#1340471)

LEARNING OBJECTIVE 1: 1. Recognize the clinical presentation of pyomyositis 2 . Identify an approach to diffuse pain in the hospitalized patient

CASE: A 25 year-old man with no past medical history presented with three days of a severe bilateral "gnawing" pain in his calves and forearms. The pain began in his right calf and spread to the opposite calf and both forearms; in both cases, the pain was preceded by overlying erythema. He recalled several recent episodes of profuse sweating and chills but denied other complaints. He also denied recent muscle trauma, vigorous exercise, alcohol or drug use and was taking no medications. He had been stationed in Haiti and Iraq within the past eighteen months while serving in the army. The vital signs were normal. Both calves and forearms were exquisitely tender to palpation and the skin overlying the right forearm was erythematous and warm. Laboratory studies were significant for leukocytosis, an elevated erythrocyte sedimentation rate and creatine phosphokinase; blood cultures were negative. Serology for HIV and antinuclear antibody were negative. A lower extremity MRI revealed multifocal microabscesses in both gastrocnemius muscles consistent with pyomyositis. The diagnosis was confirmed by muscle biopsy which showed perimysial and endomysial inflammation with myophagocytosis and myofiber necrosis. Empiric antibiotic therapy was initiated and within two days the patient was asymptomatic.

DISCUSSION: The general internist is frequently tasked with uncovering the cause of diffuse pain. In many cases diffuse pain is often a constitutional symptom referable to a primary diagnosis. Nonetheless, it is important for the hospitalist to have a defined method in assessing pain and formulating a cogent differential diagnosis. Pyomyositis is a purulent infection of skeletal muscle occurring predominantly in tropical areas, often in otherwise healthy individuals. In contrast, cases in temperate climates have been associated with previous trauma, infection or immunocompromised state. Muscle groups most commonly affected include quadriceps, gluteal, iliopsoas and abdominal. Multisite involvement is noted in up to twenty percent of cases but to our knowledge bilateral, multifocal involvement has been reported only once previously in immunocompetent residents of temperate areas. Laboratory findings are typically nonspecific but bacteremia is observed in $10 \%$ and $35 \%$ of tropical and temperate cases, respectively. MRI is the gold standard for diagnosis as other imaging modalities may reveal only nonspecific changes. Treatment includes surgical drainage of abscesses and at least 24 weeks of antibiotics which cover Staphylococcal aureus, responsible for up to $90 \%$ of cases with an identifiable pathogen. Mortality is reported to be less than $1 \%$ when treatment is initiated early but increases to $15 \%$ when delayed; overwhelming sepsis is the most common cause of death. The differential diagnosis of myalgias includes a variety of conditions that cause symptoms similar to pyomyositis. In the absence of positive blood cultures, laboratory findings are nonspecific. Our patient highlights the importance of obtaining a travel history and an early MRI in the evaluation of unexplained myalgias and severe muscle tenderness. The prompt initiation of antibiotics may be curative in this unfamiliar but potentially fatal disease.

THOSE WHO SEEK WILL FIND - UNRELENTING MSSA BACTEREMIA AND VERTEBRAL OSTEOMYELITIS Moises Auron $^{1}$; Kai Wang ${ }^{2}$; Sravani Avula ${ }^{1}$; Lucileia Johnson ${ }^{3} .{ }^{1}$ Cleveland Clinic, Cleveland, $\mathrm{OH} ;{ }^{2}$ Cleveland Clinic, Cleveland, $\mathrm{OH} ;{ }^{3} \mathrm{Cleveland}$ Clinic, Cleveland, OH. (Control ID \#1338856)

LEARNING OBJECTIVE 1: Recognize MSSA bacteremia as a life threatening condition which requires a persistent diagnostic approach. LEARNING OBJECTIVE 2: Diagnose vertebral osteomyelitis in the setting of an initial negative MRI of the spine.

CASE: A 77 year old African American man with HTN, AFib and osteoarthritis with chronic low back and neck pain was admitted after one week of worsening back pain and intermittent fever. The onset of pain was one day after discharge from other hospital where was admitted for Afib; at that admission he developed right hand superficial phlebitis at an IV site with wound culture positive for MSSA treated with topical mupirocin. Examination revealed fever $38.4 \mathrm{oC}$, and cervical and lumbar spine tenderness with no neurological deficits. Laboratory showed WBC 22,460/ $\mu \mathrm{L}$, CRP $40.8 \mathrm{mg} / \mathrm{L}$, ESR $115 \mathrm{~mm}$, Procalcitonin $5.71 \mathrm{pg} / \mathrm{mL}$, BUN $66 \mathrm{mg} / \mathrm{dl}$, creatinine $2.12 \mathrm{mg} / \mathrm{dl}$, Potassium $3.9 \mathrm{mEq} / \mathrm{L}$ and Bicarbonate $20 \mathrm{mEq} / \mathrm{L}$. A cervico-thoraco-lumbar spine MRI without contrast showed advanced degenerative spondylosis with multilevel moderate to severe central foraminal stenosis and disk edema, predominantly at L1-L2. Empiric iv antibiotics (vancomycin and piperacillin/tazobactam) and vigorous iv hydration were started; blood culture grew MSSA. Vancomycin was switched for i.v. oxacillin. Patient continued to have back pain and daily fever up to $38.9 \mathrm{oC}$ and all daily blood cultures from day 1- day 9 grew MSSA. Renal function normalized by day 8 Tagged WBC scan showed increased L1-L5 uptake concerning for osteomyelitis. Echocardiogram and transesophageal echocardiogram showed normal cardiac function and no vegetations. Due to persistent fever and worsening lumbar pain a repear lumbar MRI with contrast was done on day 8 showing disk space infection and osteomyelitis of L1-L2 with new bilateral psoas abscess and extensive epidural abscess from L1-S1. On day 9, emergency L1-L2 laminectomy and drainage of pockets of epidural abscesses was done. Oxacillin dose was increased to $2 \mathrm{~g}$ i.v every $6 \mathrm{~h}$. Fever remitted and blood cultures converted to negative on day 10. Abscess cultures were negative. A repeat MRI one week post-op showed interval improvement of epidural and psoas collections. CRP dropped to $15 \mathrm{mg} / \mathrm{L}$ prior to discharge and patient was discharged on day 17 to LTAC to complete 6 weeks of IV oxacillin.

DISCUSSION: Pyogenic vertebral osteomyelitis is a severe infection with an incidence of 2.4/100,000 and results most often from hematogenous spread, being Staphylococcus aureus and E. coli the most commonly implicated microorganisms. Clinicians are most fearful of resistant bacteria; however even when MSSA is the etiology it should be treated aggressively. Magnetic resonance imaging (MRI) is the imaging study of choice and although it has a specificity of $90 \%$ for diagnosing spinal osteomyelitis, a diagnostic challenge occurs in erosive osteochondrosis whose features may mimic those of vertebral osteomyelitis. Direct bone biopsy/aspiration with culture is the gold standard for diagnosis. In our case initial MRI without contrast did not show evidence of osteomyelitis, which was evident on a repeat MRI with contrast 8 days afterwards. A tagged WBC scan was suggestive of osteomyelitis on day 5 of admission suggesting that in cases with equivocal results in initial imaging, alternative persistent diagnostic approaches should be undertaken.

THROMBUS IN TRANSIT Armaan Shaikh; Kurt J. Pfeifer. Medical College of Wisconsin Affiliated Hospitals, Milwaukee, WI. (Control ID \#1339757)

LEARNING OBJECTIVE 1: Recognize patent foramen ovale (PFO) and paradoxical embolus as causes of systemic embolization

LEARNING OBJECTIVE 2: Describe treatment options for PFO-associated thrombus 
CASE: A 35-year-old woman presented with a 24-hour history of fatigue, chest tightness and dyspnea at rest. Additionally, she developed nausea and lightheadedness with episodic near-syncope on the morning of admission. Her past medical history was remarkable for Brugada syndrome and ventricular tachycardia found 4 months prior which lead to placement of an implantable cardiac defibrillator (ICD). Initial evaluation included a chest radiograph showing an enlarged cardiac silhouette and an echocardiogram showing a large pericardial effusion with tamponade physiology. She underwent pericardiocentesis with removal of $300 \mathrm{cc}$ of sanguineous pericardial fluid. Despite these interventions, the patient's dyspnea persisted, and she developed episodic paresthesias. Repeat echocardiogram showed a significant decrease in the pericardial effusion but also noted a mobile, left atrial mass. Transesophageal echocardiogram revealed a large, filamentous, mobile mass attached to the septum and passing through a large patent foramen ovale (PFO). A small mobile mass was also noted at the tip of the right atrial lead. Therapeutic intravenous heparin was immediately initiated, and the patient was taken for minimally invasive surgical PFO closure and thrombus evacuation. The patient did well post-operatively and was discharged home 4 days later on long-term anticoagulation.

DISCUSSION: A paradoxical embolus originates in the venous system and enters the arterial circulation through an anomalous connection between the two systems, such as a PFO. While there is an increased incidence of PFO in patients with cryptogenic stroke, the detection of a PFO in a patient with embolic stroke does not prove a cause-and-effect association. Therefore the diagnosis of a paradoxical embolus is often one of exclusion, unless imaging reveals a thrombus in transit. When a thrombus in transit is identified, treatment is aimed at inhibiting growth of the thrombus through anticoagulation with eventual clot dissolution. In severe cases, surgical evacuation of the thrombus may be necessary.

\section{TOXOPLASMA ENCEPHALITIS IN HIV: IS A CD4+ COUNT} GREATER THAN 200/ $\mu$ L REASSURING? Auras R. Atreya ${ }^{1}$; Sonali

Arora ${ }^{1}$; Taraka V. Gadiraju ${ }^{1}$; José Martagón-Villamil ${ }^{1,2}$. ${ }^{1}$ Baystate Medical Center/Tufts University School of Medicine, Springfield, MA; ${ }^{2}$ Baystate Medical Center/Tufts University School of Medicine, Springfield, MA. (Control ID \#1340366)

LEARNING OBJECTIVE 1: Recognize the possibility of Toxoplasma Encephalitis (TE) in an HIV positive patient with CD4+ count $>200 / \mu \mathrm{L}$. CASE: Ms. XYZ is a 55-year-old female with a history of HIV/AIDS, well controlled on HAART, CD4+ count in the $300 / \mu \mathrm{L}$ range for $>1$ year, as well as stage II (T3 N0) adenocarcinoma of the appendix , status post appendectomy, who presented with the acute onset of headache, nausea and vomiting. A brain CT scan revealed lesions suspicious for metastases. Subsequent brain MRI was consistent with multiple ring-enhancing supratentorial and infra-tentorial parenchymal lesions, which further pushed the working diagnosis towards metastatic disease, especially given history of adenocarcinoma. The possibility of TE and primary CNS lymphoma were considered but deemed unlikely given her robust maintenance of $\mathrm{CD} 4+$ count near $300 / \mu \mathrm{L}$ for over a year. She had been on her current HAART consisting of raltegravir, co-formulated emtricitabine/tenofovir and etravirine for 3 years and had been off Pneumocytis primary prophylaxis for 10 months (trimethoprimsulfamethoxazole also acts as prophylaxis for TE). Her Toxoplasma IgG titer was $>250 \mathrm{IU} / \mathrm{mL}$, but given the high seroprevalence in the community, this was not considered diagnostic of an active TE. She had no other evidence of metastatic disease in her body. Since her primary appendiceal adenocarcinoma had been low grade, resected over a year ago with no evidence of metastasis to the liver, all medical and surgical teams were in favor of obtaining a tissue biopsy due to her unusual presentation. It was felt that an attempt to characterize the lesions histologically would be prudent. The brain biopsy obtained via craniotomy demonstrated Toxoplasma tachyzoites within necrotic tissue. Immunohistochemistry and in-situ hybridization studies showed no evidence of lymphoma or carcinoma. Subsequently, treatment was started with sulfadiazine, pyrimethamine and leucovorin with good clinical response.

DISCUSSION: The primary differential of multifocal enhancing lesions in the CNS in HIV-positive patients may include TE, primary CNS lymphoma, metastatic lesions, Nocardia, brain abscesses, among others. Although TE is a common occurrence in HIV-positive patients, it is almost always seen with CD4+ counts less than $100 / \mu \mathrm{L}$. Indeed, discontinuation of prophylaxis for TE is recommended when CD4+ counts are above $200 / \mu \mathrm{L}$ for 3-6 months, as close follow-up in observational and randomized studies demonstrated no cases of TE. A likely explanation for this unusal presentation could be a defect in CD4+ cells. The first line treatment of TE is sulfadiazine, pyrimethamine and leucovorin for 6 weeks followed by lower dose as secondary prophylaxis. In circumstances such as this, there are no recommendations about cessation of secondary prophylaxis. This case illustrates the gray zone between work-up of multifocal lesions in HIV-positive patients with CD4 + count $<200 / \mu \mathrm{L}$ and those who are immunocompetent. Although one would not expect the development of Toxoplasmosis in this patient's CD4+ range to be common, it can occur and remains a challenge to address this unususal presentation.

TRANSFUSION - TRANSMITTED BABESIA Chiti Parikh; Erica Phillips. New York Presbytarian Hospital, New York, NY. (Control ID \#1331734)

LEARNING OBJECTIVE 1: To recognize Babesiosis as a possible cause of unexplained post transfusion fever and hemolytic anemia, especially in asplenic patients.

CASE: A 47 year old female with transfusion dependent beta thalassemia intermedia status post spleenectomy, presented to her primary hematologist with 1 week of right upper quadrant pain and fever upto $102 \mathrm{~F}$. Examination in the providers office revealed epigastric tenderness. Labs were ordered including complete blood count, complete metabolic panel, blood and urine cultures, which were significant for hemoglobin of 5.7, down from 6.9 the day before. She was transfused two units of packed red blood cells. Abdominal ultrasound and MRCP were also ordered, which were negative. She continued to experience abdominal pain with fevers and presented to the emergency room two days later for further work up. Her history is notable for bimonthly blood transfusion due to thalassemia since age two and hepatitis $\mathrm{C}$ with undetectable viral load. She has been on iron chelation therapy for over 20 years. On presentation to the emergency room, she was febrile to $101.8 \mathrm{~F}$, physical exam was significant for right upper quadrant tenderness. Labs showed hemoglobin of 7.7, total bilirubin 2.7, indirect bilirubin 2.3, elevated reticulocyte count with normal LDH and haptoglobin. Blood smear was positive for B. microti with $0.59 \%$ parasitemia, later confirmed with PCR. Her blood cultures, urine cultures, EBV, CMV, parvovirus PCR were negative. CT scan of abdomen and pelvis was negative for any acute changes. She was started on Atovaquone and Azithromycin for a six week course and iron chelation therapy was held. Her symptoms improved over three days after which she was discharged. Her parasitemia continued to decrease while on treatment and was cleared after four weeks.

DISCUSSION: There has been a recent increase in cases of transfusion transmitted Babesia. Based on recent CDC data, 159 B. microti transfusion-associated cases were documented between 1979 - 2009. 77\% of those cases occurred during 2000-2009 and most of them were in the 7 main B. microti-endemic states in the northeast and upper Midwest. This case illustrates the potential for B. microti to cause post transfusion infections which can often be life threatening in a population that is chronically ill and requires frequent blood transfusions. This includes patients with malignancy, hemoglobinopathies, and liver failure. Most of these patients often have functional asplenia which increases their risk of complications from B. microti. As our blood donor pool becomes more diverse, many of them are likely to have been exposed to more exotic microbes. Since B. microti is an intra-erythrocytic microbe, leukoreduced blood products do not reduce risk of transmission. Currently, there is no way to reduces transmission by screening blood besides donor deferral. Thus our differential for post transfusion hemolytic anemia and fever should include B. microti, especially in asplenic patients, since early identification and treatment can be life saving.

TRUE - TRUE - RELATED: AN UNUSUAL CAUSE OF BOWEL OBSTRUCTION Marwa Shoeb; Susan Wlodarczyk; Somnath Mookherjee. UCSF, San Francisco, CA. (Control ID \#1339220)

LEARNING OBJECTIVE 1: Recognize that catastrophic sequela of blunt abdominal trauma can occur many weeks after the event. 
LEARNING OBJECTIVE 2: Recognize radiological mimickers of intussusception and small bowel obstruction.

CASE: A previously healthy 38 year-old man presented with abdominal distention, nausea, and vomiting. Two weeks prior, he was admitted to an outside hospital with the same symptoms. At the time, computerized tomography (CT) scan showed small bowel obstruction (SBO) with transition point in the left mid-abdomen and terminal ileum wall thickening. He was treated with nasogastric tube decompression, but left against medical advice without a diagnosis when his symptoms improved. He presented to our emergency department with recurrentf abdominal distention and new feculent emesis. He denied fevers, chills, blood in his stool, melena, or history of abnormal bowel movements. He reported that three weeks prior to the first hospitalization, he had suffered a bicycle collision in which the handlebars struck his abdomen, resulting in severe abdominal pain. There had been no open wound or blood per rectum after the accident. On physical exam at the time of admission, he had normal vital signs and mild abdominal distention and tenderness. Laboratories showed normal complete blood count, electrolytes, aminotransferases, bilirubin, and lipase. CT scan of the abdomen and pelvis with intravenous contrast showed wall thickening of the distal jejunum and proximal ileum, probable ileal enteroenteric fistula, two segments of jejunojejunal intussusception with upstream bowel dilation, and diffuse lymphadenopathy. Taken together, the findings were thought to be most consistent with inflammatory bowel disease (IBD). Unfortunately, before a diagnostic colonoscopy was done, the patient ate a large meal. Overnight, he developed severe abdominal pain, went into shock and was taken emergently to the operating room. He was found to have obstruction of the terminal ileum due to an adhesion and upstream perforation with food in the peritoneal cavity. $\mathrm{He}$ underwent resection of 140 centimeters of terminal ileum and end-ileostomy. Final pathology showed a mixed acute and chronic inflammatory reaction within the serosa associated with small vessel proliferation, organization, and foreign material; there was no evidence of IBD or cancer. The diagnosis was felt to be intussusception and SBO as a result of intestinal trauma from his bicycle accident.

DISCUSSION: Inpatient providers often care for patients with undifferentiated SBO. Blunt abdominal trauma can cause injury to the mesentery, hemorrhagic mucosal infarction, and subclinical bowel perforation. Subsequent healing with fibrosis and stricture can lead to intussusception and SBO with a delayed onset of symptoms. Intussusception and SBO after abdominal trauma are rare; there are 22 reports representing 91 cases in the literature. Patients typically present with intermittent abdominal pain and vomiting within 4 to 8 weeks of the trauma, but symptoms can be delayed by years. Diagnostic criteria include (1) prior blunt abdominal trauma, (2) absence of intestinal disease, (3) new intestinal symptoms, (4) radiographic confirmation of stenosis, and (5) lack of inflammatory or neoplastic changes in resected segment of bowel. In managing patients with abdominal pain or SBO, inpatient providers should consider prior blunt abdominal trauma in the differential and appreciate the variable timing in symptom onset.

TWO BLOWS, BUT NO HIT Patrick Richard; Bassam Maalouf. Tulane University Health Sciences Center, New Orleans, LA. (Control ID \#1311742)

LEARNING OBJECTIVE 1: 1. Identify the differential for thrombocytopenia. 2. Identify the etiologies, pathophysiology, and clinical manifestations of HIV-associated immune thrombocytopenia (ITP). 3. Recognize the association between secondary ITP and solid malignancy. 4. Recognize the treatment for HIV-ITP and when to initiate.

CASE: A 64 year-old man with HIV and metastatic lung cancer was admitted from clinic after it was discovered that his platelet count was 3,000 cells $/ \mathrm{mm} 3$; his baseline platelet count had been 150,000 cells $/ \mathrm{mm} 3$. He denied any bleeding symptoms except mild epistaxis. He had been hospitalized one week earlier, and had been started on heparin for DVT prophylaxis at that time. His vital signs were normal. He had ecchymoses on his hands and petechiae on his ankles and palate. His fibrinogen was $727 \mathrm{IU}$, and the d-dimer $3.7 \mathrm{IU}$. His INR was 0.9; the PT and PTT were 10.9 and 24.7. His peripheral smear did not reveal schistocytes. The heparin-induced thrombocytopenia antibody was negative. Despite being transfused twelve platelet transfusions, his platelet count dropped to 2,000 cells/mm3. He was diagnosed with malignancy and HIV-associated secondary ITP and was initiated on prednisone $1 \mathrm{mg} / \mathrm{kg}$ oral daily. His platelets increased to 13,000 cells $/ \mathrm{mm} 3$ at discharge; in clinic, two days later, his platelets had increased to 108,000 .

DISCUSSION: Thrombocytopenia is a common condition encountered by the general internist. Further, as anti-retroviral therapy has prolonged the life expectance of patients with HIV, general internists are increasingly encountering long-term complications of HIV, as well as age-expected comorbid diseases independent of HIV. Because ITP is a diagnosis of exclusion, it is important that the general internist utilize a systematic approach to diagnosing the cause of the thrombocytopenia. Our patient had HIVassociated ITP; however, other causes such as HIT, DIC, and TTP were first excluded prior to settling on the diagnosis of ITP. HIV-ITP has a complex pathophysiology including reduced platelet survival and decrease production of megakaryocyte progenitors. Patients with HIV-ITP are at increased risk of thrombocytopenia from other causes, as was the case in our patient, whose lung cancer contributed to the paraneoplastic thrombocytopenia. General internists should be aware of the recent American Society of Hematology guidelines, which no longer recommend a bone marrow biopsy for patients older than 60 years of age who have ITP without evidence of depression of other cell lines. Treatment should be based on symptoms, and not the absolute platelet count. If HIV-associated ITP is confirmed, zidovudine should be initiated. In patients developing ITP who are already on anti-retroviral therapy, as was the case with our patient, prednisone at $1 \mathrm{mg} / \mathrm{kg}$ for 21 days is indicated. For contraindications or cases refractory to steroids, IVIG or splenectomy may be pursued. Using a systematic approach to thrombocytopenia, the internists will efficiently diagnose secondary ITP. An understanding of the treatment options and timing for HIV-associated ITP will prevent unnecessary, ineffective treatments and improve outcomes.

TWO CASES OF SPINAL CORD INFARCTION - A COMPARISON Muhammed Sherid; Samian Sulaiman; Salih Samo; Meenu Singh. University of Illinois at Chicago, St.Francis Hospital, Evanston, IL. (Control ID \#1314386)

LEARNING OBJECTIVE 1: To suspect spinal cord infarction in nonaortic surgical settings

LEARNING OBJECTIVE 2: To understand the etiology of spinal cord infarction.

CASE: Case 1: A 64 year old Romanian man presented to the ED with a two hour history of severe, sharp circumferential lower chest and back pain, associated with left leg weakness and numbness. These symptoms started when he was at work at his auto-body shop. He did not have headache, loss of consciousness, urinary or fecal incontinence. Past medical history was significant for diabetes, dyslipidemia, hypertension, and infrarenal abdominal aortic aneurysm. His vital signs and general examination were unremarkable. Neurological examination revealed normal level of consciousness. Cranial nerves were intact. Motor examination revealed a power of $0 / 5$ in all muscle groups of the left lower extremity. Sensations to light touch, pain, vibration and position sensations were normal. He was unable to distinguish between warm and cold temperature sensations from the level of the nipples down to the mid thigh bilaterally. Deep tendon reflexes were absent in the left knee and ankle. Babinski's sign was positive on the left and neutral on the right. Rectal tone was decreased but he was able to squeeze the examiner's finger. Laboratory studies and electrocardiogram were unremarkable. CT scan showed marked atherosclerotic changes with a protruding thrombus, plaque and penetrating ulcers with subintimal hematoma in the descending thoracic aorta to the upper abdominal aorta. An infrarenal abdominal aneurysm measuring $3.7 \times 3.8 \mathrm{~cm}$ unchanged from the previous study was identified. MRI brain and spinal cord were unremarkable. A diagnosis of anterior spinal cord infarction was made. His symptoms resolved within twenty four hours without residual deficits. Case 2: A 51 year old African American man presented to the ED with sudden onset paralysis of both the upper and lower extremities associated with neck pain. There was no headache, loss of consciousness or bowel and bladder disturbances. Past medical history was significant for diabetes mellitus, atrial fibrillation, and hypertension. On examination; his blood pressure was 180/ 97 with irregular heart rate of 114 . On neurological examination he was alert, 
awake and oriented to time, place and person. Cranial nerves were intact. Motor examination revealed a power of $0 / 5$ in bilateral upper and lower extremities. Sensations to touch and pain were intact. Deep tendon reflexes were absent in all four extremities and Babinski's sign was positive bilaterally. Laboratory studies were unremarkable. MRI revealed infarction in the cervical spinal cord from C2-4. MRA showed occlusion in left vertebral artery. The patient was intubated initially due to respiratory distress. His symptoms improved over the next twenty four hours and extubated. He was discharged with mild left sided weakness.

DISCUSSION: Spinal cord infarction is a rare disorder. Aortic surgery is the most common etiology; however, other etiologies include atherosclerosis, vasculitis, infection, embolic and thrombotic occlusion, and severe hypotension. The clinical presentation is acute onset of paraparesis or quadriparesis. A therapeutic algorithm exists regarding this condition in the setting of aortic surgery, but no definitive therapy has been shown to be of benefit in other settings.

TYPE B LACTIC ACIDOSIS IN ESOPHAGEAL CARCINOMA: AN INTERESTING CASE. Sandesh Murali; Shabeta Sahore; Harsha Ramchandani. St. Mary Mercy Hospital, Livonia, MI. (Control ID \#1311164)

LEARNING OBJECTIVE 1: Identification of different types of lactic acidosis

LEARNING OBJECTIVE 2: Pathogenesis of Lactic Acidosis in Malignancy

CASE: A 53 year old Caucasian male was admitted to general medical floor for sepsis. His past medical history was significant for Diabetes Mellitus, Hypertension, Hyperlipidemia, Stage 4 esophageal adenocarcinoma with metastasis to lung, spine and liver. Esophageal cancer was diagnosed two months prior to current admission. He had received 2 cycles of Taxol/carboplatinum. His last chemotherapy was 3 weeks before admission. On day 1 , positive findings in physical exam included hypotension, tachycardia, crackles in right lung base and stage 2 noninfected decubitus ulcer on the sacrum; his significant blood labs were WBC- 15.7, lactic acid-5.7, AST- 83, ALT- 49. He was treated with antibiotics vancomycin and piperacillin-tazobactum to cover organisms causing health care associated pneumonia. On day 6 of admission, his lung exam was clear bilaterally, vital signs were stable and blood labs wereWBC 10.8, lactic acid 6.5, AST- 41 ALT -51. Despite pneumonia resolution, and improving sepsis, his lactic acid levels increased from 5.7 on admission to 12.1 on day 10 . He was not on any medications known to cause lactic acidosis. Lactic acidosis was worsening despite resolution of hypotension, hypoperfusion and sepsis. Due to poor prognosis, patient changed his code status to DNR and requested discharge to hospice without further workup. Patient passed away at hospice the day after discharge.

DISCUSSION: Lactic acidosis is the most common cause of metabolic acidosis in hospitalized patients. There are three different types of lactic acidosis type A, B and D. Type A lactic acidosis is due to tissue hypoperfusion and acute severe hypoxaemia. Type B lactic acidosis could be due to hereditary metabolic diseases, drugs/ toxins (Biguanides, salicylates, nucleoside reverse transcriptase inhibitors and methanol), systemic disorders or malignancies. Type D Lactic acidosis can occur in patients with short bowel syndrome or other forms of malabsorption. In tumors, Type B lactic acidosis pathogenesis may involve liver dysfunction due to massive liver metastasis leading to lactate underutilization and subsequently lactic acidosis. During the last decade evidence has emerged supporting the role of overproduction of lactic acid due to ischemia in the neoplastic tissue bed and cancer cells having an aberrant energy production. Lactic acidosis in malignancy was first described in patients with acute leukemia by Field et al. in 1963. Since then, it has been observed often in hematological malignancies, but rarely in solid non-hematological tumors such as small cell lung cancer, cholangiocarcinoma, breast cancer, gynecological cancers, hepatoma and metastasis from unknown primary carcinoma. Our case illustrates an uncommon cause of lactic acidosis. Treatment of lactic acidosis due to malignancy includes chemotherapy to decrease tumor burden, bicarbonate infusion and hemodialysis. Lactic acidosis in the background of malignancy is an indicator of poor prognosis.
UNFOLDING THE ENDOCRINOPATHY SOUP James W. Ragins; Patrick Siler; Mukta Panda. University of TN, College of Medicine, Chattanooga, Chattanooga, TN. (Control ID \#1289489)

LEARNING OBJECTIVE 1: Review the differentiating features between primary and secondary adrenal insufficiency

LEARNING OBJECTIVE 2: Discuss the key features of Polyglandular Autoimmune Syndrome (PAS) Type I and II

CASE: A 46-year-old Caucasian female presented initially in November 2002 after a syncopal episode and was found to have a $14 \times 18 \mathrm{~mm}$ pituitary adenoma. She had low levels of TSH, FSH, LH and random cortisol. The patient treated for panhypopituitarism with prednisone and synthroid and subsequently underwent transforaminal hypophysectomy for lymphocytic hypophysitis. In July 2011, the patient presented to the emergency department with nausea, vomiting and diarrhea for three days associated with cramping and tingling in her upper and lower extremities. Her home medications included synthroid $112 \mathrm{mcg}$ daily, prednisone $10 \mathrm{mg}$ daily and over the counter Tylenol. On presentation her blood pressure of $108 / 73 \mathrm{mmHg}$, pulse of $100 /$ min, respiratory rate of $20 / \mathrm{min}$, and temperature of 98.9 F. Physical examination was significant for mild left upper quadrant tenderness and multiple hypopigmented areas on bilateral upper extremities consistent with vitiligo. Laboratory data showed hyponatremia ( $\mathrm{Na}$ 127), hyperkalemia (K 6.2), leukocytosis (WBC 16.5), TSH 0.114 and free T4 0.85. Urinalysis revealed a large amount of leukocyte esterase, no nitrites and WBC 47, low vitamin B12(233) and increased methyl melonic acid.Primary adrenal insufficiency and pernicious anemia were considered. She had positive adrenal and anti parietal antibodies.She was admitted and treated for acute adrenal crisis and urinary tract infection.

DISCUSSION: PAS should be considered when a patient presents with two or more endocrinopathies. There are two main classifications of PAS: type I and type II. Type I PAS is usually seen in childhood and characterized by deficiency in thyroid and adrenal function along with mucocutaneous candidiasis. Type II PAS or Schmidt syndrome, is seen more frequently in adult women and is characterized by adrenal insufficiency, thyroiditis or type 1 dibetes mellitus. Other associated conditions include celiac disease, pernicious anemia, Grave's disease, hypophysitis and vitiligo. Type II PAS has an autosomal dominant inheritance pattern that is not manifested in all persons of a family who posses the affected gene. Autoantibodies seen in PAS type II can include antibodies against the thyroid gland, adrenals and pancreas. Because the clinical manifestations of adrenal insufficiency may be difficult to detect and fatal if not diagnosed,it is important to screen patients at risk for PAS routinely with the cosyntropin stimulation test. The key differences between primary and secondary disease lies with the ACTH and aldosterone level. Primary disease have a high ACTH level which likely result in hyperpigmentation of skin due to elevated melanocyte-stimulating hormone and a low aldosterone level because the rennin-angiotensin system is dysfunctional resulting hyperkalemia. Secondary disorder will not have the hyperpigmentation or hyperkalemia. Screening studies for adrenal insufficiency should be done routinely every 1-2 years up to about the age of 50 in families with PAS type II and until about the age of 40 in patients with type I PAS. Treatment includes managing each individualized endocrine dysfunction, usually with hormonal replacement. Special attention should be towards the recognition and prevention of acute adrenal crisis.

UNLEASHING THE WOLFF: A CASE OF LIFE THREATENING COCAINE INDUCED TACHYCARDIA Jonathan S. Lee; Hollis Day; Peggy Hasley. University of Pittsburgh Medical Center, Pittsburgh, PA. (Control ID \#1339264)

LEARNING OBJECTIVE 1: Describe the ECG characteristics, acute manifestations and management of Wolff-Parkinson-White syndrome

LEARNING OBJECTIVE 2: Recognize that cocaine can induce life threatening reentrant tachycardias in patients with WPW

CASE: A 50 year old female with no cardiac history presented from home following the acute onset of palpitations, weakness, nausea and vomiting after imbibing only 3 drinks of alcohol. EMS was called to evaluate the patient and found her pre-syncopal with narrow complex tachycardia at a rate of 280 beats per minute. She was DC cardioverted into normal sinus rhythm and immediately felt better. On presentation to the hospital the 
patient appeared very anxious but her exam was otherwise unremarkable. Urine was positive for cocaine and tetrahydrocannabinol. An ECG revealed normal sinus rhythm at a rate of 90 beats per minute with a PR interval of 90 milliseconds and slurring of the QRS upstroke suggesting the diagnosis of Wolff-Parkinson-White syndrome. After discussion of treatment options, the patient underwent catheter-based radiofrequency ablation. She was also advised to abstain from cocaine.

DISCUSSION: The ECG pattern in Wolff-Parkinson-White is a result of conduction from the atria simultaneously through the AV node and through an accessory tract bypassing the AV node. The bypass tract leads to faster initial ventricular depolarization compared to the AV node but slower conduction within the ventricular myocardium compared to the HisPurkinje system. This results in ventricular pre-excitation and the characteristic findings of slurring of the QRS upstroke - the delta wave - and a short PR interval less than 120 milliseconds. The prevalence of the WPW ECG pattern has been estimated as high as 1 in 400 people in the general population. Diagnosis is complicated by the fact that the ECG pattern can be intermittent and can disappear over time. In addition, not all patients develop tachyarrhythmias. In one study only 1.8 percent of patients with the WPW ECG pattern had episodes of tachyarrhythmias, and the risk of sudden cardiac death, the most feared complication, was estimated to be very low at 0 to 0.4 percent. Treatment of WPW is generally reserved for symptomatic patients with episodes of AVRT or atrial fibrillation. They should be referred to electrophysiology for ablation which is potentially curative. Acute pharmacologic therapy generally involves intravenous antiarrhythmics such as procainamide. It is important to recognize that in patients with wide-complex tachycardia or atrial fibrillation and a known history of WPW, AV nodal blockers such as beta-blockers, calcium-channel blockers, adenosine and digoxin are contraindicated as they can potentially precipitate ventricular fibrillation and sudden cardiac death. Cardioversion is still first-line therapy in an unstable patient. Cocaine inhibits the reuptake of norepinephrine and dopamine at nerve terminals. This increase in sympathetic tone along with direct cardiotoxic effects is hypothesized as the cause for the wide range of arrhythmias with which cocaine has been associated. In addition, at high doses cocaine acts as a sodium channel blocker with class I antiarrhythmic effects, which can theoretically promote reentrant arrhythmia. While cannabis has not been associated with arrhythmias, it may increase plasma levels of cocaine, thereby increasing arrhythmogenic potential. In this patient, it is possible that all of these factors played a role.

UNMASKING OF UNDIAGNOSED PRE-EXISTING CENTRAL DIABETES INSIPIDUS AFTER RENAL TRANSPLANTATION Jerson Munoz-Mendoza; Veronica Pinto. University of Miami - Jackson Memorial Hospital, Miami, FL. (Control ID \#1335028)

LEARNING OBJECTIVE 1: Recognize that central diabetes insipidus may be masked by chronic kidney disease and disclosed by renal transplantation

LEARNING OBJECTIVE 2: Identify empty sella syndrome as a cause of central diabetes insipidus

CASE: A 59-year-old Hispanic woman with end stage renal disease (ESRD) of unknown etiology on dialysis was admitted to the hospital to undergo deceased donor renal transplantation. The pre-operative history and physical examination, along with blood work and other investigations were unremarkable. Diuresis started within the first hour and rapidly exceeded $600 \mathrm{ml} / \mathrm{h}$ with a total urinary output of 10 liters during the first 24 hours after the surgery. In the early postoperative course the patient developed increased urinary frequency, thirst and water intake. Over the coming days, despite treatment with free water, her urine output remained 10 liters per day and serum sodium level and serum osmolality increased to $161 \mathrm{mmol} / \mathrm{L}$ and $324 \mathrm{mOsm} / \mathrm{Kg} \mathrm{H} 2 \mathrm{O}$ respectively. Since urine osmolality was persistently low, diabetes insipidus was suspected and intravenous desmopressin $4 \mu \mathrm{g}$ was initiated on the sixth hospital day. Two and half hours later the urine osmolality rose from 67 to $219 \mathrm{mOsm} / \mathrm{kg}$, and the plasma sodium level and serum osmolality dropped from 159 to $146 \mathrm{mmol} / \mathrm{L}$ and from 317 to $297 \mathrm{mOsm} / \mathrm{Kg} \mathrm{H} 20$ respectively, which is consistent with the diagnosis of CDI. Maintenance therapy with intranasal desmopressin $10 \mu \mathrm{g}$ was instituted with subsequent improvement of the symptoms including a decrease urine output. MRI revealed empty sella. The anterior pituitary hormonal panel was within normal limits. The patient was discharged on the twelfth hospital day with diagnosis of CDI and empty sella syndrome, with serum creatinine of $0.9 \mathrm{mg} / \mathrm{dL}$, sodium level of $141 \mathrm{mmol} / \mathrm{L}$, and daily urinary output of approximately 3.5 liters per day.

DISCUSSION: Central Diabetes Insipidus (CDI) is a rare condition characterized by vasopressin deficiency, manifested as polydipsia and polyuria. In the setting of dialysis-dependent chronic kidney disease, newonset CDI is largely asymptomatic and may therefore go unrecognized. We describe a case of CDI in a renal transplant recipient, in whom restoration of renal function unmasked preexisting CDI, leading to severe polyuria and electrolyte abnormalities. CDI rarely complicates the perioperative management of kidney transplantation, there are only 5 reported cases in the literature worldwide. CDI symptoms usually become apparent within 24 hours after renal transplantation and should be suspected in the setting of persistent polyuria. Treatment with desmopressin facilitated the fluid and electrolyte management without impairment of the allograft function. In our patient, CDI was most likely secondary to empty sella syndrome (ESS), which is an uncommon cause of CDI. This clinical presentation of ESS, characterized by an isolated vasopressin deficiency with intact anterior pituitary function, is unique as well.

UNUSUAL PRESENTATION OF BREAST CANCER Laura Lourdes; Umna Ashfaq. University of Florida, Gainesville, FL. (Control ID \#1338227)

LEARNING OBJECTIVE 1: Learn the unusual presentation of breast cancer.

CASE: 55-year-old post-menopausal female who with a significant past medical history of severe head trauma complicated by subarachnoid hemorrhage requiring insertion of ventriculo-peritoneal shunt (VPS) for hydrocephalus, and previous gastric bypass surgery presents to her primary care practitioner with a lump on her abdomen just below her right breast 3 years after insertion of the VPS close to the shunt site. She was reassured initially that this was scar tissue related to the shunt surgery. 2 months later she informs the neurosurgeon that this lump was getting larger and painful. An ultrasound is performed and reveals a $2 \times 1.4 \mathrm{~cm}$ mass in the inferio-medial quadrant of the right breast overriding the shunt catheter and a smaller confluent mass tracking inferiorly along the shunt. Biopsy of the mass revealed invasive papillary carcinoma ER/PR positive, HER-2 negative and TFT negative. Pathological interpretation was that this was most likely breast or ovarian tumor. CT chest, abdomen and pelvis revealed lesions within the spleen measuring up to $2.5 \mathrm{~cm}$. There were areas of abnormal density were noted within the peritoneum on both the left and right measuring from 1 to $2.5 \mathrm{~cm}$, not including a $2.9-\mathrm{cm}$ soft tissue mass at the peritoneum in the region of the umbilicus. Nodularities surrounding the shunt through the right breast with right anterior wall were also seen. On PET scan there was increased FDG activity uptake of these same lesions. No obvious breast or ovarian lesion was identified. MRI brain showed no evidence of metastasis. An initial trial of hormonal therapy with letrozole failed and since then she has been treated with multiple lines of chemotherapy and her disease remains controlled 3 years from diagnosis. A PUBMED search done with the following words 'breast cancer' AND 'VP Shunt' revealed no results. However there has been a case report of ovarian carcinoma with leptomeningeal metastasis via VPS, pancreatic carcinoma seeding along a VPS as well as intracranial malignancies metastasizing to the peritoneum via VPS. Hence this is the first reported care of breast cancer with peritoneal metastasis that presented with subcutaneous nodules tracking along a VPS

DISCUSSION: This case highlights that peritoneal involvement of carcinoma, no matter the origin can metastasize along a VP shunt. As primary care practitioners, we need to be aware and have a low threshold to biopsy any masses that present as such.

UNUSUAL CAUSE OF NAUSEA, VOMITING AND ABDOMINAL PAIN Shaghayegh Khayambashi. SJHMC, Phoenix, AZ. (Control ID \#1324040)

LEARNING OBJECTIVE 1: Cannabis is the third most commonly used drug after tobacco and alcohol and is the most commonly abused illicit 
drug in the United States. This drug is often used as anti-emetic and appetite-stimulating agent in cancer patients and in those with acquired immunodeficiency syndrome. As such, cannabinoid hyperemesis is often an unrecognized side effect of chronic marijuana use in patients presenting with abdominal pain, nausea and cyclic vomiting unresponsive to antiemetics. Given the increasing recreational and medicinal use of Marijuana in the United States, physicians need to be more aware of this paradoxical side effect.

CASE: A 23-year-old female presented with abdominal pain, nausea, and refractory bilious vomiting for the past 4 days. She had recurrent episodes of these symptoms over the past 4 years and the symptoms started approximately 2 years after she began using Marijuana regularly. She received conservative treatment in the emergency room on multiple occasions and was never admitted as all lab results were within normal limits. She described a non-radiating, sharp, right upper quadrant abdominal pain with $9 / 10$ severity. Patient's father described his daughter taking multiple long hot baths throughout the day to help reduce episodes of nausea and vomiting. On physical exam, the patient appeared dehydrated but was afebrile and vital signs were stable. Laboratory studies showed mild leukocytosis with white blood cell count of 12.7 which decreased to 10.4 the next day, increase in AST of 148 and ALT of 116, both of which decreased to 29 and 46 respectively. Laboratory results including pregnancy test, hepatitis panel, amylase, lipase and basic metabolic panel were normal. $\mathrm{CT}$ of abdomen and pelvis was normal except for hypodensities in the liver described as vascular shunt in the subsequent MRI study. Patient's symptoms resolved in 48 hours with conservative treatment and abstinence from Marijuana. She was discharged

DISCUSSION: Cannabinoid hyperemesis was first described by JH Allen in Australia. He noted this condition in a group of nineteen patients with chronic cannabis abuse and cyclic vomiting who habitually bathed in warm showers or baths to uniquely find temporary relief of their symptoms. Many subsequent cases described the association between cannabinoid use and cyclical vomiting and abdominal pain. Although the centrally mediated anti-emetic effect of cannabinoids has been described, the mechanism for its emetic action is not precisely known. With the recent legalization of Marijuana in many states, physicians will encounter cannabinoid hyperemesis more frequently. Unnecessary expensive and invasive diagnostic, medical and surgical treatment can be avoided with the knowledge of this phenomenon.

USING THE LOUISIANA SIDS RISK REDUCTION PROGRAM AS A MODEL FOR EVALUATION OF PROGRAMS THAT AIM TO IMPROVE THE ACCURACY OF VITAL RECORDS Arta Lahiji. UCLA Olive View Medical Center, Redondo Beach, CA. (Control ID \#1341363)

LEARNING OBJECTIVE 1: A public health program that aims to decrease national and local rates of SIDS/SUID should have a system of accurately documenting the incidence of SIDS/SUID

CASE: The Louisiana Office of Public Health (LAOPH) has worked to reduce the rate of Sudden Infant Death Syndrome (SIDS) and sudden unexplained infant deaths (SUID) by improving the accuracy and consistency of SIDS/SUID diagnoses. Nationally, collecting complete and accurate records on SIDS/SUID deaths has been hindered by a lack of standardized training, diagnostic criteria, and system for reclassification. The LAOPH aims to develop a more consistent system for reporting SIDS/ SUID, to both improve accuracy and potentially decrease the overall rate of SIDS/SUID. The Sudden Infant Death Syndrome Risk Reduction Program (SIDSRRP) aims to improve the accuracy of SIDS/SUID reporting through standardized coroner and death scene investigator training, incentive programs for data collection, and legislation compliance. This work was done as part of the Centers for Disease Control Program Evaluation Training for Harvard School of Public Health students. A mixed-method approach using both quantitative and qualitative data was utilized. Four key informant interviews (SIDSRRP program coordinator, Child Death Review (CDR) Medical Director, facilitator, and state-wide coordinator) were conducted. Three investigative forms (death scene investigation (DSI) form, coroner's invoice, and SUID home visit form), two pieces of legislation (Coroner's Law, Child Death Investigation statute), and the Title
Five Maternal and Child Health Block Grant were reviewed. This was used to design a programmatic logic model and an evaluation method for assessing the arm of the SIDSRRP that aims to improve the accuracy of SIDS/SUID reporting. A time-series evaluation design and suggested measurable process indicators : 1- Number/percent of complete SIDS records 2- Number/percent of reclassified records are proposed. As coroners and death scene investigators receive standardized training on SIDS/SUID classification and comply with legislation requiring that they submit full autopsies, DSIs and SUIDI forms, the expected number/percent of complete SIDS/SUID records will increase. This is a measurable indicator of the program's process. It is assumed that as the number/percent of complete SIDS/SUID records increase, the amount of information coroners have to make an accurate initial diagnosis will also increase. Furthermore, the number/percent of reclassified records can serve as an indicator of the ultimate impact goal of improved accuracy of the SIDS/ SUID reported rate. We can expect with standardized training and compliance with regulations, forms, and procedures, there will be an improved initial accuracy of SIDS/SUID diagnosis and decreased reclassification rate over time by the CDR.

DISCUSSION: By improving the accuracy of reporting, SIDS rate in Louisiana may be decreased, in itself fulfilling the program aims. The proposed evaluation of Louisiana's SIDSRRP aim of improving accurate SIDS/SUID reporting can be beneficial for their program planning and resource allocation. The proposed evaluation of Louisiana's SIDSRRP can be used as a model for other state programs that aim to improve the accuracy of SIDS/SUID reporting in vital records. A public health program that aims to decrease national and local rates of SIDS/SUID should have a system of accurately documenting the incidence of SIDS/SUID.

VARICEAL BLEEDING WITHOUT CIRRHOSIS? Victor Estacio; Zeeshan Qureshi; Lee Lu. Baylor College of Medicine, Houston, TX. (Control ID \#1321258)

LEARNING OBJECTIVE 1: Review the etiology and pathophysiology of Left-sided Portal Hypertension (LSPH)

LEARNING OBJECTIVE 2: Discuss diagnosis and management options of LSPH.

CASE: A 37-year-old Caucasian male presented with two weeks of melena followed by four days of light-headedness, weakness, and shortness of breath. He denied abdominal pain, nausea, vomiting, hematemesis, early satiety, or NSAID use. He was monogamous with his wife, rarely drank alcohol, and denied drug use. Exam showed pallor without icterus or jaundice. There were no stigmata of chronic liver disease, no hepatosplenomegaly, and no ascites. The rest of the exam was unremarkable. Admission $\mathrm{Hgb}$ was $5.9 \mathrm{~g} / \mathrm{dL}$, platelets were $88,000 / \mathrm{uL}$, and iron panel showed severe deficiency. Liver function tests were within normal limits. Ultrasound did not reveal cirrhosis or splenomegaly. He was transfused 4 units pRBC, and endoscopy showed large grape-like fundal varices with a sentinel clot, without esophageal varices. To further investigate the underlying etiology, an MRI of the abdomen revealed a $5.3 \times 7.2 \mathrm{~cm}$ splenic hilar mass encasing the pancreatic tail and a $2.8 \times 3 \mathrm{~cm}$ periaortic retroperitoneal adenopathy. The pathology of the fine needle biopsy of the periaortic lymph node was consistent with a non-small cell carcinoma with squamoid features. AFP, CEA, CA19-9, and CA-125 were negative. Staging CT chest, repeat EGD/ colonoscopy, and testicular ultrasound could not identify possible primaries or metastases. He thus underwent distal pancreatectomy and splenectomy and was diagnosed with squamous neoplasm of the pancreatic tail with extension to the spleen and involvement of the retroperitoneal lymph nodes. The gastric varices were mainly due to LSPH secondary to splenic vein obstruction by the mass.

DISCUSSION: LSPH is an uncommon clinical entity caused by splenic vein obstruction (SVO), leading to venous hypertension distally, and predisposing to increased left-sided venous collateral circulation flow. It accounts for less than $0.5 \%$ of all causes of portal hypertension. Most LSPH originate from pancreatic disorders. These include pancreatitis, pancreatic cancer, or pancreatic expansion as in pseudocysts. Other causes include abdominal surgical procedures, metastatic cancers, hypercoagulability, and retroperitoneal fibrosis. LSPH leads most commonly to isolated gastric or combined gastroesophageal varices, although LSPH-associated isolated esophageal or colonic varices have been described. While most cases are asymptomatic, patients can present with 
abdominal pain, jaundice, fatigue, gastrointestinal bleeding, or weight loss. Splenomegaly is a common finding. Work up should include an MRI or MRV to look for SVO. Treatment involves splenectomy in those with known variceal bleeding. However, primary prophylactic splenectomy is discouraged in patients without history of bleeding due to low bleeding rates. Prognosis is intimately related to the primary pathology causing the LSPH. Those with pancreatic cancers were found to have 2-12 months survival with deaths not related to bleeding. In benign processes, prognosis remains good once bleeding is controlled, and further rebleeding is uncommon at $0-3 \%$. Hence, in patients with varices or variceal bleeding without evidence of liver disease or portal vein thrombosis, LSPH should be considered and further work up is warranted.

VARICELLA ZOSTER VIRUS (VZV) MENINGOENCEPHALITIS IN AN IMMUNOCOMPETENT HOST Brandon Verdoorn; Ryan Hurt. College of Medicine - Mayo Clinic, Rochester, MN. (Control ID \#1312623)

LEARNING OBJECTIVE 1: Recognize the typical presentation of VZV meningoencephalitis in an immunocompetent host.

LEARNING OBJECTIVE 2: Diagnose VZV meningoencephalitis using cerebrospinal fluid (CSF) analysis.

CASE: A 72-year old immunocompetent male with past medical history of hypertension, hyperlipidemia, and obesity presented to his local Emergency Department with 2-day history of fever, confusion, generalized weakness, and painful erythematous vesicular rash affecting his left torso. He was transferred to a tertiary care center following an unremarkable head CT. On initial presentation to our facility, he was noted to be febrile $\left(38.5^{\circ}\right.$ C) with otherwise normal vital signs. On examination, he was fully oriented with a Glasgow Coma Scale (GCS) of 15. There were no focal neurologic signs or findings of meningeal irritation. A unilateral vesicular rash was noted on the left chest wall, in approximately a T4 dermatomal distribution. Examination was otherwise unremarkable. Laboratory studies were unremarkable aside from a mild normocytic anemia (Hemoglobin $12.8 \mathrm{gm} / \mathrm{dl}$ ), mild thrombocytopenia (Platelets $114 \times 10^{\wedge} 9 / \mathrm{L}$ ), and mild monocytosis (Monocytes 1,100/mm^3; 17.3\%) with normal leukocyte count. Blood cultures were obtained, and he was initiated empirically on intravenous (IV) acyclovir given suspicion for disseminated herpes zoster infection. He was admitted to the hospital and noted to have waxing and waning confusion. Lumbar puncture was performed, revealing clear cerebrospinal fluid with elevated total protein $(85 \mathrm{mg} / \mathrm{dl})$ and elevated cell count $(159 / \mathrm{uL})$ with $56 \%$ lymphocytes, $42 \%$ monocytes, and $1 \%$ neutrophils. PCR for VZV DNA was positive, while bacterial culture, Gram's stain, and PCR for HSV DNA were negative. He was diagnosed with VZV meningoencephalitis, and continued on a course of IV acyclovir for a total of fourteen days. Human immunodeficiency virus (HIV) screening test was negative. His confusion progressively improved and eventually resolved, and he was dismissed on hospital day seven.

DISCUSSION: Varicella zoster virus (VZV) infection in adults is typically due to reactivation of latent virus, which lies dormant in sensory ganglia following primary infection (varicella, or chickenpox). Reactivation manifests as herpes zoster (shingles), characterized by a painful vesicular rash that is usually unilateral and restricted to the distribution of the dermatome supplied by the involved sensory ganglion. The rash may be preceded by pain ("preherpetic neuralgia"), and pain may persist chronically following resolution of the rash ("postherpetic neuralgia"). Treatment with antiviral agents may be effective in shortening duration of symptoms and reducing risk of postherpetic neuralgia when started early in the course of the illness. Systemic dissemination of reactivated VZV is rare. Varicella zoster virus (VZV) meningoencephalitis is an uncommon clinical entity, typically affecting immunocompromised hosts, particularly those with human immunodeficiency virus (HIV) infection. Cases in immunocompetent hosts have been reported, however, and it is important to consider VZV meningoencephalitis in the differential of undifferentiated acute neurologic disease, particularly in patients with skin findings suggestive of zoster infection. With prompt intravenous (IV) antiviral treatment, most patients recover to their baseline level of neurologic function.
VARICELLA ZOSTER VIRUS VASCULITIS IN AN HIV-POSITIVE MALE PRESENTING WITH STROKE Molly Kraus. SJHMC, Phoenix, AZ. (Control ID \#1339132)

LEARNING OBJECTIVE 1: The approach to an HIV-infected patient that presents with an abnormal neurologic exam can be challenging. In patients with CD4 cell count $<200 /$ microL, the differential diagnosis is broad. Varicella Zoster Virus (VZV) vasculitis is important to consider in this population. Findings on imaging and CSF fluid analysis can lead you to this diagnosis.

CASE: A 35 year-old hispanic male with HIV presented for a 3 day history of headache, right sided numbness and weakness, and difficulty ambulating for 3 days. He had been diagnosed with HIV/AIDS 2 months prior to this admission and was started on HAART therapy. Past medical history was relevant for syphilis treated with penicillin 3 years ago. His last CD4+ count was 8 . On exam, he was an ill-appearing, frail male, who was alert and oriented, tachycardic at 106. Cranial nerves were intact; there was mild nuchal rigidity, and multiple skin lesions in lower extremities. Strength was decreased on right lower extremity to $3 / 5$, right upper and left lower extremities $4 / 5,2+$ reflexes throughout. Laboratory was significant for normocytic anemia with hemoglobin of 8.6. A lumbar puncture was performed which was positive for VZV by PCR, RPR was positive. CTangiagraphy showed infarct in the posterior limb on the left with suggestion of vasculitis. An MRI of the brain revealed an acute infarct involving the left thalamus with moderate chronic small vessel ischemic changes. A CT-angiogram was performed and beaded appearance of vasculature was noted. The patient was started on IV acyclovir and steroids for the treatment of VZV vasculitis leading to the presentation of stroke. DISCUSSION: Strokes caused by VZV are most often ischemic strokes. Vasculitis can be caused by either a primary infection or with viral reactivation, affects large and small arteries and this complication can occur without the characteristic zoster rash. The classic clinical presentation of VZV vasculopathy in adults is opthalmic distribution zoster followed by acute contralateral hemiplegia. Clinical presentations vary widely and include mental status changes, aphasia, ataxia, hemi-sensory loss and visual changes. Two-thirds of patients will have a history of rash within the previous several months. Prior to antiretroviral therapy, VZV CNS infections were detectable in 1.5 to 4.4 percent of HIV-positive patients at autopsy. Most cases occur in patients with severely low CD4 count. VZV causes stroke by the direct infection of the cerebral arteries leading to inflammatory and noninflammatory processes such as necrosis, dissection, and aneurysm formation. The proposed transaxonal spread is through afferent fibers from trigeminal and dorsal root ganglia to both intracranial and extracranial blood vessels. Differential diagnosis includes a broad number of infections and diagnosis including: HIV vasculitis, CMV infection, intracranial lymphoma, cerebral toxoplasmosis among others. MRI typically shows superficial and deep lesions in both the gray and white matter, particularly at the gray-white matter junctions. Multifocal lesions are common. Typical angiographic changes are segmental constriction with post-stenotic dilatation, or beading. In about two-thirds of patients with CSF analysis show modest pleocytosis, usually fewer than 100 cells $/ \mathrm{mm} 3$, monocytes predominance. CSF protein is also typically elevated, with oligoclonal bands. Intrathecal production of anti-VZV antibodies or VZV DNA in the CSF.

WAITING TO EXHALE Sharifa Llemit; Ravi S. Hira. Baylor College of Medicine, Houston, TX. (Control ID \#1284932)

LEARNING OBJECTIVE 1: Recognize septic pulmonary embolism (SPE) as a complication from a peripheral intravenous line.

LEARNING OBJECTIVE 2: Review septic pulmonary embolism.

CASE: A 47-year-old white male presented with blurring of vision, progressive dyspnea on exertion, and hemoptysis. He was diagnosed with relapsing remitting multiple sclerosis 4 months prior and was treated 1 week ago with IV Steroids for a relapse. Two days prior to presentation, he noted blurry vision in his left eye, increasing dyspnea on exertion, and later had an episode of hemoptysis. He denied fevers or chills but did complain of bilateral pleuritic chest pain. On exam, he was afebrile and tachypneic, lung exam was clear with decreased breath sounds and vocal resonance in the right base and oxygen saturation of $95 \%$ on $2 \mathrm{~L}$ by nasal 
canula. Additionally, he was noted to be tachycardic without any murmurs or rubs and skin exam revealed no abnormal lesions. CXR showed multiple vague densities suggestive of metastatic disease. CT Chest with contrast revealed bilateral lung nodules, some of which had cavitation suggestive of septic pulmonary emboli. On ophthalmological exam, an intra-vitreal abscess was found in his left eye which was subsequently incised and drained. Blood cultures were positive for MRSA. Transthoracic and transesophageal echocardiograms did not reveal valvular vegetation. The patient later developed pain, swelling, and tenderness in his right cubital fossa at the site of his previous peripheral IV access and was found to have septic thrombophlebitis of his cephalic vein, requiring vein resection. The culture from the vein also grew MRSA. Patient was diagnosed with SPE caused by a peripheral IV line and treated with IV Vancomycin for 6 weeks.

DISCUSSION: Superficial thrombophlebitis is a common complication from peripheral IV lines with an incidence of 25 to $35 \%$ and suppurative thrombophlebitis occurring in 0.2 to $2 \%$. SPE is one of the rare but serious sequelae with an infected thrombus embolizing to the lungs. Risk factors include age over 50 years, underlying immunocompromised status and intravenous drug abuse (IVDA). Our patient had been on steroid therapy. The common organisms identified are coagulase-positive Staphylococcus aureus and group A Streptococcus. Historically, IVDA has been a major risk factor as reported in a case series in 1978 describing 60 patients with SPE over a 5 -year period with $78 \%$ of their cases caused by IVDA. However, with heightened awareness of needle hygiene over the years, SPE is becoming a rare complication of IVDA. A recent case series of 14 patients with SPE in 2005 found only 1 patient to be an IVDA. The others were Lemierre's syndrome, central line infections, prosthetic valves, infected pacemaker leads and abscesses. All 3 patients with central line infections were immunocompromised. Symptoms of SPE include fever, cough, dyspnea and hemoptysis. Positive blood cultures may aid in diagnosis. CXR is often the initial study but is usually non-diagnostic. The use of CT has increased the sensitivity and specificity in diagnosing SPE. If SPE originates from suppurative thrombophlebitis, in addition to antibiotics, surgical resection of the involved vein is the treatment of choice. Hence, in the current medical era where peripheral IV lines are often used, physicians must be cognizant of SPE as a potential complication, especially in immunocompromised patients.

WALKS LIKE SEPSIS, TALKS LIKE SEPSIS, BUT STILL NOT SEPSIS: HEMATOPHAGOCYTIC LYMPHOHISTIOCYTOSIS Asma Khaliq ${ }^{1}$; Marc Zumberg ${ }^{2}$; Ge Xiong ${ }^{1}$; Margaret C. Lo ${ }^{1} .{ }^{1}$ University of Florida, Gainesville, FL; '2University of Florida, Gainesville, FL. (Control ID \#1321277)

LEARNING OBJECTIVE 1: Distinguish between the clinical and diagnostic features of sepsis versus hematophagocytic lymphohistiocytosis (HLH)

LEARNING OBJECTIVE 2: Recognize the diagnostic need for serial bone marrow assessments (BMA) and close clinicopathologic collaboration in $\mathrm{HLH}$

CASE: A 65 year-old, African-American female with rheumatoid arthritis presented as a hospital-to-hospital transfer for fever of unknown origin after 2-weeks of recurrent fever spikes, anorexia, confusion, and hypotension. She failed to respond to vasopressors, broad-spectrum antibiotics, and neupogen for presumed septic shock with neutropenic fever. Addition of micofungin for subsequent candidemia ( 1 of 4 bottles) provided no clinical benefit. Full body CT revealed no infections. BMA revealed hypercellular marrow, but was otherwise nondiagnostic. Upon transfer, physical examination revealed a cachectic, confused, febrile $(103 \mathrm{~F})$ female with severe ulnar deviation of fingers and massive hepatosplenomegaly. Labs demonstrated trilinage pancytopenia (WBC 1,300/mm3, Platelets 10,000/mm3, Hgb $6.9 \mathrm{~g} / \mathrm{dL}$ ), LFT abnormalities (AST 370U/L, ALT $1400 \mathrm{U} / \mathrm{L}$, total bilirubin $10 \mathrm{mg} / \mathrm{dL}$, direct bilirubin $7 \mathrm{mg} / \mathrm{dL}$, alkaline phospatase $35 \mathrm{mg} / \mathrm{dL}$ ), and DIC with hypofibrinogenemia $(35 \mathrm{mg} / \mathrm{dL})$. Evaluation for infectious etiologies was negative i.e. pancultures, viral hepatitis, EBV, CMV, HSV, Parvovirus, HIV. Rheumatoid serologies and ceruloplasmin were normal. Ferritin was significantly elevated $(71,000 \mathrm{ng} / \mathrm{ml})$. Repeat BMA revealed erythroid-predominant trilineage hypercellular marrow, without increased blasts or plasma cells, but noted extensive hemophagocytosis. Patient remained refractory to broad-spectrum antibiotics, antifungals, and multiple blood product transfusions. Our differential diagnosis was Felty's syndrome with severe sepsis, large granular lymphocytic leukemia or secondary HLH. Diagnosis of HLH secondary to rheumatoid arthritis was made upon meeting 5 of the 8 diagnostic criteria (fever; splenomegaly; cytopenia $\geq 2$ cell lines; hypertriglyceridemia; hypofibrinogenemia; ferritin $\geq 500 \mu \mathrm{g} / \mathrm{l} ; \mathrm{sCD} 25 \geq 2400 \mathrm{U} / \mathrm{ml}$; decreased/absent NK-cell activity; hemophagocytosis in bone marrow, CSF or lymph nodes). Patient did not respond to IVIG therapy and became critically ill from severe lactic acidosis secondary to bowel ischemia from celiac arterial thrombosis. Family chose palliative care; patient died shortly thereafter.

DISCUSSION: This case demonstrates that severe systemic inflammatory response syndrome (SIRS) does not always represent sepsis and can result from noninfectious processes such as HLH. Literature reports many cases of HLH mistakened for sepsis. HLH is a severe immune dysfunction with hypercytokinemia and impaired NK and cytotoxic T-cell hyperfunction causing life-threating tissue damage. Sensitivity of such diagnostic criteria as fever, hypofibrinogenemia, and splenomegaly is $<50 \%$. Literature promotes BMA as the optimal test in HLH recognition. Yet, initial BMA maybe false-negative and serial BMA are needed for positive results. Close clinicopathologic collaboration is recommended for diagnostic accuracy. Although many parallels exist in the clinical and pathophysiologic features of HLH and sepsis, the exaggerated immune response of HLH requires the prompt use of immunosuppressants such as IVIG, etoposide, or steroids, per Histiocyte Society's 2004 HLH protocol. Early recognition of the differences between sepsis and HLH is important for optimal patient outcome given the rapidly progressive and drastically different therapy of HLH.

WATCH OUT FOR THE STORM! Bhavana Siddegowda Bangalore; Daniel Gutteridge; Radhika Kakarala; Raj Ethiraj. Mclaren Regional Medical Center, Flint, TN. (Control ID \#1339909)

LEARNING OBJECTIVE 1: Recognize the increasing prevalence of electrical storm in the automatic implantable cardioverter-defibrillator (AICD) era.

LEARNING OBJECTIVE 2: Recognize management dilemmas that surround an electrical storm.

CASE: A 49 year old Caucasian female with multiple co-morbidities, arrived with a chief complaint of her AICD firing. It was accompanied by a sudden onset of transient dizziness and mild shortness of breath just before the firing. She had two episodes of appropriate firing in the ER. Pertinent medical history is significant for dialated ischemic cardiomyopathy with systolic heart failure (ejection fraction 20\%) status post AICD in 2010, COPD, PAD, CAD with multiple stents. AICD interrogation showed 10 episodes of shocks delivered within 48 hours which were appropriate. Despite antiarrhythmic therapy with intravenous (IV) amiodarone, subsequently combined with procainamide, repeated AICD firing were triggered by episodes of sustained ventricular tachycardia (VT). Coronary angiogram was done which showed patent stents. She was transferred to a university hospital for ablation therapy evaluation due to persistent episodes of sustained VT. However ablation was contra-indicated secondary to left ventricular thrombus and she was ultimately discharged. She was re-admitted due to repeated AICD firing, 23 shocks in 24 hrs, which took place as an outpatient. After two days of persistent arrhythmias and shocks in spite of IV antiarrthymics the patient opted to turn off the AICD and eventually passed away.

DISCUSSION: Electrical storm or defibrillator storm is an emerging phenomenon in the defibrillator era representing a highly lethal cardiac state of recurrent ventricular fibrillation or hemodynamically unstable ventricular tachycardia. In people with defibrillators it is defined as 3 or more appropriate shocks delivered for ventricular arrthymias. Electrical storm has multiple risk factors including acute myocardial ischemia and new or worsening heart failure with a low ejection fraction. It occurs at a rate of 10 to 28 percent during the initial 3 years post defibrillator implantation based upon the definition and population of interest. There are contradictory views about the prognostic value of electrical storm although the AVID and MADIT II trials demonstrate it to be a poor prognostic 
factor. There is emerging evidence demonstrating cluster VTs to be a poor prognostic factor. Intracellular calcium overload secondary to recurrent VF and recurrent shocks may be responsible for ineffective defibrillation serving as a trigger for recurrent VF. Treatment options include IV amiodarone $+/$ - procainamide or catheter ablation. Although it has a high incidence and hospitalization rate, awareness among internists remains low. There is a need for increased knowledge about this phenomenon. Whether rapid deterioration of myocardial function due to recurrent shocks actually decreases survival needs to be further studied. The role of an active AICD in patients with a poor prognosis as well as ethical considerations of switching off AICDs in these patients should be considered. The question that remains unanswered is "Should we switch off AICDs in patients with electrical storm who have a poor prognosis and end stage heart disease?"

WHAT A COUPLE OF JERKS: LANCE-ADAMS SYNDROME Brita Roy. University of Alabama at Birmingham, Birmingham, AL. (Control ID $\overline{\# 1339638)}$

LEARNING OBJECTIVE 1: Recognize an important complication of cerebral anoxia resulting in significant, prolonged morbidity.

LEARNING OBJECTIVE 2: Distinguish the difference between myoclonic status epilepticus and Lance-Adams syndrome.

CASE: A 47-year-old white woman presented from home complaining of new onset falls that began two days ago. She had been hospitalized the previous week due to a cardiac arrest secondary to status asthmaticus. Upon discharge from the hospital three days prior, she was walking normally without assistance. However, the following day she described that she would suddenly lose control of her legs when ambulating, resulting in a fall. She had multiple episodes like this daily, and symptoms worsened over the course of two days preceding presentation. She denied loss of consciousness, seizure-like activity, post-ictal state, headache, chest pain, and dyspnea. Past medical history was significant for asthma, congestive heart failure, and history of non-ST elevation myocardial infarction. She denied family history of movement disorders. Home medications included aspirin, moxifloxacin, prednisone, enalapril, and pravastatin. Vital signs, including orthostatics, were normal. Neurologic examination revealed fully intact cranial nerves, myoclonic jerks in all four extremities which limited testing for dysmetria, hyperreflexia throughout with clonus, and no fasciculations. Laboratory evaluation including electrolytes, thyroid panel and cardiac enzymes were normal except for a white blood cell count of 16,000. Electrocardiogram revealed T-wave inversions in lateral leads that were unchanged from previous. CT head showed no abnormalities and recent echocardiogram was normal. She was diagnosed with Lance-Adams syndrome, or post-anoxic myoclonus, and treated with low-dose clonazepam with rapid improvement in symptoms.

DISCUSSION: Lance-Adams syndrome (LAS) is a rare disorder, first described in the 1960s, that presents in survivors of profound hypoxic episodes, similar to our patient. The incidence of this disorder is unknown as the diagnosis is underrecognized, but over 100 cases have been reported. The pathophysiology of LAS is not well understood, but one hypothesis is that anoxia leads to death of Purkinje cells at the fastigial nucleus of the cerebellum, and the subsequent loss of GABA-inhibition of the motor thalamus results in myoclonus. Alternate hypotheses include depression of the serotonergic system. LAS can be difficult to differentiate from myoclonic status epilepticus, but consciousness is undisturbed and electroencephalogram may show spike discharges preceding myclonic jerks by 7-32 milliseconds in patients with LAS. LAS results in significant disability, with uncontrollable myoclonus upon purposeful movement that typically develops days to weeks post anoxic injury, and thus may present during post-hospital discharge clinic visits. LAS is not curable but may be treatable. Clonazepam seems to be the most effective benzodiazepine used to treat LAS. Anti-epileptic agents such as valproic acid, leviteracetam and zonisamide may also be used to control symptoms. If these agents are ineffective, continuous globus pallidus internus stimulation was recently found to be successful.

WHAT IS HIDDEN BEHIND SUDDEN-ONSET ABDOMINAL PAIN? Chiaki Terashima; Michinori Mayama; Naoki Misumida; Naomi Otowa; Mitsunori Iwase. Toyota Memorial Hospital, Toyota, Japan. (Control ID \#1323425)
LEARNING OBJECTIVE 1: Identify isolated dissection of the superior mesenteric artery as a possible cause of sudden-onset abdominal pain.

LEARNING OBJECTIVE 2: Recognize the clinical features of isolated dissection of the superior mesenteric artery.

CASE: A 46-year-old Japanese-Brazilian man presented to the emergency department complaining of severe epigastric pain. The pain had begun two hours prior to presentation and he could recall the exact time of its onset. The pain was persistent and radiated to the left upper quadrant as well as his back. He denied having nausea, vomiting, or diarrhea. His past history was significant for hypertension, which had been medicated with telmisartan. He did not smoke, had no family history of cardiovascular diseases. His vital signs were normal except for a slightly elevated blood pressure of 140/ $96 \mathrm{mmHg}$ and no differences were noted between the left and the right extremities. On physical examination, his bowel sounds were normal and his abdomen was soft to palpation. He complained of slight epigastric tenderness, but there was no bruit on auscultation and no guarding or rebound tenderness on palpation of the same area. His laboratory tests were unremarkable except for a subtle elevation in white cell count; $10,800 / \mathrm{mcL}$. Other tests, including chest X-ray, EKG, and plain abdominal CT were unremarkable. The sudden-onset nature of his abdominal pain, however, warranted further investigation and contrast-enhanced abdominal CT was ordered. It revealed an intramural hematoma of the superior mesenteric artery (SMA) and no aortic lesions were found. He was diagnosed as having an isolated dissection of the SMA. There was no sign of ischemia so conservative management with hypotensive drugs was initiated and the patient's blood pressure was well controlled. His pain subsided by day three of admission and the clinical course was uneventful. He was discharged from the hospital on day seven without any symptoms.

DISCUSSION: Although isolated dissection of the SMA is uncommon, in recent years, more cases have been reported because of the development of diagnostic imaging technology. Most patients with isolated dissection of the SMA present with sudden-onset abdominal pain in the epigastrium. It is critical to diagnose this condition at an early phase for intestinal ischemia may develop. Mortality rate rises when the patient has intestinal ischemia so it is prudent to consider isolated dissection of the SMA as a possible cause when seeing a patient with sudden-onset abdominal pain. The sudden-onset nature may be the only clue to reach the diagnosis as demonstrated in this case. Fibromuscular dysplasia, atherosclerosis, smoking, and hypertension are some reported risk factors. Typical CT findings include intramural hematoma, intimal flap, and/or enlarged diameter of the SMA. No standard treatment protocol exists for this condition but surgery, stents, and conservative management are currently available options. When the patient presents with signs and symptoms of intestinal ischemia, emergency surgical treatment is essential. Patients put on conservative management usually follow a good clinical course, though clinicians should watch, with extreme caution, for the development of intestinal ischemia.

WHAT? I CAN'T HEAR YOU! GENETIC SYNDROME PRESENTING WITH UNILATERAL HEARING LOSS Kathleen M. Buchheit; Katherine T. Johnston. Beth Israel Deaconess Medical Center, Boston, MA. (Control ID \#1339479)

LEARNING OBJECTIVE 1: Explain the primary care evaluation of unilateral hearing loss

LEARNING OBJECTIVE 2: Recognize the clinical features of neurofibromatosis type 2

CASE: Ms. B is a 30 year-old female without significant past medical history who presents for her annual exam. She reports unilateral hearing impairment for one year. When in loud environments, she cannot hear well in her right ear; sounds are "muffled." She denies tinnitus, head trauma, headaches, or visual changes. No vertigo or gait imbalance. As a child she had multiple ear infections requiring tympanostomy tubes bilaterally. She takes an oral contraceptive daily. Remaining history is non-contributory. Complete physical examination was normal. Normal tympanic membranes with tympanostomy tube scar on left. Neurologic exam with hearing intact to whisper and finger rub bilaterally. Weber test non-lateralizing. Rinne test with air conduction greater than bone conduction bilaterally. No plaques or nodules present on skin exam. She was referred for audiogram, which demonstrated right sensorineural hearing loss. MRI head revealed a large 
mass extending from right internal auditory canal, most consistent with large vestibular schwannoma and numerous $(>10)$ extra-axial masses, most consistent with meningiomas. A diagnosis of neurofibromatosis type 2 (NF2) was made due to the vestibular schwannoma and multiple meningiomas present. She was referred to neurosurgery for removal of the vestibular schwannoma. Genetic testing for the patient and screening for siblings was advised.

DISCUSSION: Sensorineural hearing loss results from pathologic changes to inner ear structures and may be genetic or acquired from exposure to loud noise, inner ear infections, toxic exposures, or systemic diseases. Patients with sensorineural hearing loss typically have difficulty filtering background noises. The first screening test in primary care practice involves asking the patient about hearing impairment. The whispered voice test is also a useful screening test and patients with positive results should be referred for formal testing. Rinne and Weber tests are not useful as screening tools, but help to distinguish conductive from sensorineural hearing loss, which helps direct further evaluation. Formal audiograms are required for a complete evaluation of hearing loss and should always be performed in patients with unilateral hearing loss. NF2 is caused by an autosomal dominant or de novo mutation predisposing people to nervous system tumor development; thus, family history may not be present. The average age of onset of NF2 is $18-24$ years. Individuals with NF2 also develop schwannomas of other cranial and peripheral nerves, meningiomas, and ependymomas. Patients may have visual impairment from cataracts and optic nerve meningiomas. Cutaneous manifestations include hyperpigmented plaque-like lesions and subcutaneous nodules. Following suspected or clinical diagnosis of NF2, the extent of the disease should be evaluated by a head MRI, hearing evaluation, ophthalmologic evaluation, and cutaneous exam. Treatment of vestibular schwannoma is primarily surgical. Treatment for hearing loss from vestibular schwannoma includes hearing aids or cochlear or brainstem implants. All affected individuals need coordinated care among their primary care providers, neurologists, neurosurgeons, and genetic counselors. Family members should also be referred for evaluation.

WHAT'S THE ITIS? UNRESOLVING GASTROINTESTINAL SYMPTOMS IN A YOUNG ADULT Hector R. Perez ${ }^{1}$; Andrea Porrovecchio $^{2}$. ${ }^{1}$ Montefiore Medical Center, Bronx, NY; ${ }^{2}$ Montefiore Medical Center, Bronx, NY. (Control ID \#1338540)

LEARNING OBJECTIVE 1: Recognize the clinical presentation of eosinophilic gastroenteritis.

LEARNING OBJECTIVE 2: Recognize the differential diagnosis of gastroenteritis with a peripheral eosinophilia.

CASE: A 25 year-old woman with an allergy to milk presented with three weeks of severe post-prandial epigastric pain, severe nausea and vomiting, and diarrhea. She had originally presented twice with similar symptoms to an outside hospital in the two weeks prior and was admitted with the inability to tolerate oral intake. An endoscopy was performed before her discharge. Her gastrointestinal symptoms persisted. During the current presentation, her vital signs were normal. She had minimal epigastric tenderness, and her stool guaiac was negative. Her white blood count was 15,000 and her eosinophil count was 4100. A CT scan revealed extensive intraabdominal ascites along with thickening and distention of the small bowel. A paracentesis revealed a cloudy yellow fluid containing 8500 white blood cells with a 94\% eosinophilic predominance. Stronglyloides serologies and stool testing for ova and parasites were negative. Serum IgE was elevated to 861 (normal is less than 180). Biopsy results of the endoscopy performed at the outside hospital revealed chronic gastritis, duodenitis, and jejunitis with numerous eosinophils consistent with eosinophilic gastroenteritis. She was started on prednisone and improved clinically.

DISCUSSION: Abdominal pain is a common problem encountered by the internist. A stepwise approach to diagnosing abdominal pain is important, particularly when symptoms fail to resolve after empiric treatment. Eosinophilic gastroenteritis (EG), a rare disease in which eosinophils infiltrate the walls of the gastrointestinal tract, can present with a wide variety of gastrointestinal symptoms depending on the extent of the involvement. Most commonly, it is associated with post-prandial abdominal pain, nausea, vomiting, and diarrhea. Over $50 \%$ of patients have histories of allergic disorders. The major laboratory abnormality is a significant peripheral eosinophilia; ascites fluid, if present, also demonstrates significant eosinophilia. In patients with abdominal symptoms and peripheral eosinophilia, the differential includes intestinal parasites and gastrointestinal malignancies. Stool testing and serologies for Stronglyoides and other parasites should be performed. Endoscopy with biopsy is necessary to make the diagnosis of EG, as a normal appearing mucosa can still harbor significant eosinophilic infiltration. A thorough allergy history and workup should follow once a diagnosis is settled, as food hypersensitivity plays an important but undetermined role in EG pathogenesis. Treatment has not been evaluated in a randomized clinical trial and is limited to case reports. Clinical and histologic remission can occur with a six-week elimination diet. Based on the clinical severity, systemic steroids are often used and have also been shown to lead to remission. Long term data on the natural history of EG is lacking, but most experts consider EG to be a chronic disorder characterized by relapses and remissions. While EG is rare, making the diagnosis requires a high index of suspicion. A timely diagnosis along with treatment can have lasting effects on a patient's morbidity and quality of life.

WHEN ANCHOVY PASTE IS UNSAVORY: AMEBIC LIVER ABSCESS IN A YOUNG MAN Mahmuda Islam; Roger D. Smalligan; Md J. Ahmed; Nazrul Chowdhury; James ". Walker. Texas Tech University Health Sciences Center, Amarillo, TX. (Control ID \#1332505)

LEARNING OBJECTIVE 1: Diagnose amebic liver abscess. LEARNING OBJECTIVE 2: Recognize the epidemiology of extraintestinal manifestations of Entamoeba histolytica.

CASE: A 20-year-old male Burmese refugee presented with diffuse, intermittent abdominal pain for two weeks. The pain made him unable to eat solids and he had been drinking only liquids for several days and was losing weight. He denied fever, chills, nausea, vomiting and diarrhea but was having sweats. Past medical history and family history were negative. He smoked 1 pack-per-day of cigarettes and denied alcohol and drug use. Physical exam: BP 106/82, P 110, RR 30, T 98.5, O2 sats $100 \%$ on RA. Lungs and heart were normal. Abdomen was tender in the right upper and lower quadrants and there was mild guarding and rigidity. Bowel sounds were normal. Labs: WBC $27.8 \mathrm{k}$; neutrophils $94.8 \%$; bands $42 \%$; platelets 645,000; INR 1.64, AST 18, ALT 16, alk phos 238; bilirubin 1.2; albumin 2.5; total protein 7.7. Abdominal CT showed an $8 \times 8 \mathrm{~cm}$ hypodense mass in the right lobe of the liver. Aspirate of the abscess showed an anchovy paste like substance with negative Gram, AFB and fungal stains as were bacterial cultures. IgG for Entamoeba histolytica later returned strongly positive with negative antibodies for Echinococcus. Hospital course: Patient was treated with metronidazole and albendazole and he responded well.

DISCUSSION: Many cities in the USA participate in the US Refugee Program that facilitates the immigration and resettlement of people from developing or war-torn countries each year (over 50,000 were received in 2011). This influx of people requires internists who treat these patients to broaden their differential diagnoses to include tropical diseases not frequently encountered in this country. Extra-intestinal manifestations of E. histolytica are not common but when they do occur, amebic liver abscess is most common followed by pleuropulmonary, cardiac and brain involvement. Amebic liver abscess can occur after travel exposure as short as four days but the condition is predominantly seen in immigrants from endemic countries. Approximately 3,000 cases are seen annually in the USA with about 10 deaths. For travelers returning from an endemic area, presentation usually occurs within 8 to 20 weeks, although a longer lag time (sometimes years) has been reported. For unclear reasons, amebic liver abscess is 10 times more common in men than women and is rare in children. Clinically, patients generally present with slowly progressive RUQ pain and fever and may have malaise and weight loss like our patient. Hepatomegaly is common (over 50\%) and recent diarrhea may be reported in $30 \%$ of patients. Leukocytosis and elevated alkaline phosphatase are common though jaundice is uncommon. Diagnosis can be made by ultrasound, CT or MRI. Aspiration can be performed which will yield the anchovy paste type substance with negative stains, in contrast to a pyogenic liver abscess which would show bacteria and neutrophils. Antibodies to E. histolytica are present in over $95 \%$ of patients. Treatment is usually successful with metronidazole for 7-14 days, often followed by paramomycin to eliminate intraluminal cysts. Internists must include amebic liver abscess in their differential of RUQ abdominal pain in immigrant patients or travelers to endemic areas. 
WHEN HALLUCINATIONS ARE NOT DUE TO DELIRIUM OR PSYCHIATRIC DISEASE: CHARLES BONNET SYNDROME Taraka V. Gadiraju $^{1}$; Auras R. Atreya ${ }^{1}$; Sonali Arora $^{1}$; Maura J. Brennan ${ }^{1,2}$. Baystate Medical Center/Tufts University School of Medicine, Springfield, MA; ${ }^{2}$ Baystate Medical Center/Tufts University School of Medicine, Springfield, MA. (Control ID \#1334597)

LEARNING OBJECTIVE 1: Distinguish atypical hallucinations in Charles Bonnet Syndrome from those occurring in delirium and psychiatric illnesses.

CASE: A 92 year old man with hypertension had a subacute on chronic worsening of vision. (He had acquired ocular toxoplasmosis 10 yrs prior). $\mathrm{He}$ was a retired French teacher with no personal or family history of neuropsychiatric illness. He was well overall but had 3 days of intermittent, complex visual hallucinations which first occurred as he awoke. He saw "pink snow" that later became red and blue finally turning into smoke. At other times he saw children, a witch, and huge trees. He retained insight and knew these visions were "not real." The hallucinations lasted minutes to hours and occasionally disappeared with an attempt to touch them or by closing his eyes. He did not drink alcohol, had no head trauma and family did not report any cognitive change (except for some difficulty finding his way due to worsening vision). He was attentive and organized in his thought processes. He did not appear ill in any way and denied depression. Cranial nerves were intact (except for poor vision) with no other neurological deficits. His score on the MiniMental State Examination (barring those items which require intact eyesight) was perfect. An electroencephalogram and MRI head were unremarkable. In short, he showed no signs of delirium, dementia or depression. A diagnosis of Charles-Bonnet Syndrome (CBS) was made given his classic presentation of advanced age, progressive visual loss and isolated vivid visual hallucinations in the absence of mental illness. He was reassured, his symptoms eased over time and his anxiety about the meaning of the hallucinations abated.

DISCUSSION: The classic triad of CBS consists of visual hallucinations, visual impairment of any etiology and intact cognition. Also called visual release hallucinations, CBS was first described by Charles Bonnet in 1769. A decline in eyesight precedes the onset; patients maintain intact reality recognizing the hallucinations are not real. CBS is quite prevalent; approximately $50-60 \%$ of those with severe visual loss experience hallucinations. However, it remains under-reported by patients who may fear being labeled as psychiatrically impaired. It is also under-diagnosed by clinicians many of whom are unaware of its existence. CBS is thought to result from disordered neuronal activity between the thalamus and the visual cortex. The broad differential for bizarre hallucinations includes atypical seizures, Lewy Body Dementia, drug withdrawal, delirium, narcolepsy, peduncular hallucinations, psychiatric illness, etc. This patient had an unremarkable EEG, normal brain imaging and no evidence of dementia, depression, delirium or sleep disorders. CBS is usually self-limited; there are no clear treatment recommendations, although various medications, behavioral strategies, and ophthalmologic interventions can be used if symptoms are troubling. Reassurance remains the mainstay of treatment. Physicians must be able to recognize and diagnose CBS. This allows both appropriate reassurance of patients and avoidance of iatrogenic injury from unneeded procedures, hospitalizations and investigations especially in elders. This will also conserve scarce healthcare resources.

WHEN LATENT MAY BECOME FATAL Jonathan Katz ${ }^{1}$; Jordan Brodsky $^{1}$; Dahlia Rizk ${ }^{1}$; Ya Ju Chang ${ }^{1}$; David Beyda ${ }^{2}$; Jose A. Cortes ${ }^{1}$. ${ }^{1}$ Beth Israel Medical Center, Manhattan Campus of the Albert Einstein College of Medicine, New York, NY; ${ }^{2}$ University Hospital of Brooklyn at Long Island College Hospital, Brooklyn, NY. (Control ID \#1296876)

LEARNING OBJECTIVE 1: Recognize risk factors, signs, symptoms, and potential complications of Strongyloides stercoralis.

LEARNING OBJECTIVE 2: Recognize the importance of diagnosis and treatment of latent as well as active strongyloidiasis in light of the rise in incidence.

CASE: CASE PRESENTATIONS Case 1: 69 yo F Chinese immigrant with rheumatoid arthritis on prednisone and methotrexate presented with weakness, abdominal discomfort, cough, fever and chills. She developed sepsis with bilateral lung opacities. Endoscopic gastric biopsy revealed Strongyloides stercoralis. Case 2: 72 yo M Cuban immigrant with chronic obstructive pulmonary disease, lumbar plexopathy, and recent exposure to steroids was admitted for increasing dyspnea. He developed sepsis with bilateral interstitial infiltrates and respiratory failure. Bronchoalveolar lavage revealed S. stercoralis. Case 3: 70 yo F Puerto Rican with lupus nephritis and recent initiation of prednisone presented with weakness, anorexia, and rash over the chest and abdomen. The hospital course was complicated by respiratory failure and sepsis. Skin biopsy showed S. stercoralis larvae. Case 4: 45 yo $\mathrm{F}$ from China recently diagnosed with lupus nephritis on prednisone and mycophenolate mofetil presented with generalized weakness and progressive dysphagia. She developed sepsis and respiratory failure. Computer tomography (CT) showed bilateral pneumothoraces, pneumomediastinum, pneumoperitoneum, subcutaneous emphysema, and ground-glass opacities. Bronchoscopy revealed diffuse alveolar hemorrhages and multiple filariform larvae consistent with $\mathrm{S}$. stercoralis. Case 5: 56 yo M from Dominican Republic with multiple myeloma treated with dexamethasone, melphalan, and thalidomide presented with weakness of the lower extremities. After radiation therapy for bone lesions, he developed gastrointestinal complaints and sepsis. CT chest showed extensive ground-glass opacities. Bronchoalveolar lavage showed filariform larvae consistent with S. stercoralis. We describe five cases of patients with hyperinfection originally from endemic regions that were all secondary to immunosupressive agents. All patients were treated with antihelmintics. 3 of the 5 patients did not survive.

DISCUSSION: Strongyloides stercoralis, an intestinal nematode, affects 30-100 million people worldwide. While latent or asymptomatic infection is most common, hyperinfection, a rare complication, has a mortality rate reported as high as $85 \%$. The incidence of strongyloidiasis may be rising in developed countries due to globalization, increased use of immunosuppressive therapies, and the prevalence of immunocompromised patients. The presence of sepsis or fever with any level eosinophil count, chronic anorexia, bloating, weakness, skin rash or wheezing, in a patient from an endemic area or undergoing immunosuppression, should prompt testing for S. stercoralis. Practitioners planning on prescribing medicines that will affect immune status must be aware of this potential complication and consider screening prior to starting treatment. In cost effectiveness studies for the treatment of intestinal parasites in immigrants, it was concluded that presumptive administration of albendazole to all immigrants at risk for parasitosis would save lives and money, while screening-based treatment would be less cost effective. Although ivermectin is more effective than albendazole, it is more costly. Consideration should be given to creating guidelines for screening and prophylaxis in high-risk populations.

WHEN A POSITIVE TURNS NEGATIVE: CASE REPORT OF A FALSE POSITIVE CLUSTER IN RAPID HIV TESTING Rachel Zhuk; David C. Thomas; Yasmin S. Meah. Mount Sinai School of Medicine, New York, NY. (Control ID \#1317405)

LEARNING OBJECTIVE 1: To report a cluster of false positive oral fluid HIV tests in a primary care clinic

LEARNING OBJECTIVE 2: To understand the reliability of rapid HIV testing in light of post-market surveillance

CASE: In 2008, rapid oral fluid HIV testing was introduced at the studentrun, attending-directed free clinic of the Mount Sinai School of Medicine. Screening was performed with the OraQuick ADVANCE ${ }^{\circledR}$ Rapid HIV-1/2 Antibody Test, a qualitative immunoassay compatible with oral fluid, whole blood or plasma. A cluster of false positive results, however, raised concerns about the validity of the oral test. Patient 1 was a 58 -year-old woman with multiple past sexual partners and recent unprotected sexual activity with an abusive partner. Informed consent was obtained and a rapid oral fluid test performed. One student and two attendings perceived a faint pink line in the 'test zone,' considered a preliminary positive. The patient was distressed by the result and considered confronting her recent partner. When blood was drawn for confirmatory testing, two ELISA tests were negative. The patient was notified immediately and noted relief, describing the experience as a 'wake-up call'. Within one week, a second rapid test was positive. Patient 2 was a 50 -year-old obese man in a monogamous 5 year relationship with a woman. The test was reactive according to both student and attending. In the following week, the patient 
reported significant depression and anxiety. Again, ELISA testing showed the patient to be HIV-negative.

DISCUSSION: Though clusters of false positives have been noted, the specificity of Oraquick oral fluid testing is well above the FDA-required $98 \%$, and close to $99.8 \%$ premarket specificity (Orasure, 2007). Data from NYC STD Clinics and several large, multi-center prospective studies showed $99.6 \%-99.8 \%$ specificity, with the $95 \%$ CI above $98 \%$. Whole blood testing was marginally more specific. (CDC, 2008; Delaney, 2006; Wesolowski, 2006; Zelin, 2008). However, six documented false positive clusters nationally have distressed both patients and providers. While specificity within these clusters generally remained near $98 \%$, it fell to $95.9 \%$ in one study, with a positive predictive value (PPV) of just $28 \%$. Investigation revealed no connection to test lots, operators, or patient characteristics. Retraining produced no change in false positive rate (CDC, 2008; Delaney, 2006; Wesolowski, 2006; Zelin, 2008; Walensky, 2008; Brown, 2007). A test's PPV significantly impacts the experience of patients and providers. It is difficult to trust a test when, even temporarily, nearly $3 / 4$ of patients with positive results do not have HIV. Given the potential for psychological trauma, as well as the risk of compromising trust between patient and provider, our clinic emulated NYC STD Clinics and discontinued oral fluid testing. Instead, whole blood testing from fingersticks was adopted for its higher specificity. Our clinic remains committed to rapid testing which increases both testing rate and percentage of patients who receive their results (Hutchinson 2006). Test completion rates were similar between fingerstick and oral fluid testing in a randomized study, and in our clinic, patient refusal rates have declined since implementing fingerstick testing (Spielberg, 2005; Stephens 2010). This simple change in testing protocol has allowed us to continue providing HIV testing to our uninsured population while diminishing test-related anxiety.

WHEN THE END OF LIFE COMES TOO CLOSE TO THE BEGINNING: CARE OF A TERMINALLY ILL YOUNG ADULT PATIENT Aaron D. Losey ${ }^{1}$; Gene R. Quinn'; James A. Thomas ${ }^{2}$. ${ }^{1}$ University of California, San Francisco, San Francisco, CA; ${ }^{2}$ University of California, San Francisco, San Francisco, CA. (Control ID \#1335106)

LEARNING OBJECTIVE 1: Describe the clinical presentation, prognosis, and treatment options for anaplastic thyroid carcinoma.

LEARNING OBJECTIVE 2: Recognize the unique challenges of end-oflife care in critically ill young adults.

CASE: A 25-year-old single mother with anaplastic thyroid cancer metastatic to the lungs and mediastinum presented with two weeks of progressively worsening shortness of breath, orthopnea, and pleuritic chest pain. On exam she was noted to have an oxygen saturation of $80 \%$ on room air, bilateral severely decreased lung sounds, and was in respiratory distress. A CT scan revealed the bilateral lungs largely replaced with tumor, compressing the bronchi. She was placed on high flow oxygen and admitted to the intensive care unit for respiratory care. The patient had been in good health until a year and half prior to admission when she presented with a rapidly enlarging neck mass. After pathology showed anaplastic carcinoma, she underwent total thyroidectomy and subsequent extensive neck dissection. Multiple cycles of radiation and chemotherapy were attempted, all with continued progression of her disease. She was enrolling in a phase I clinical trial at the time of admission. Despite aggressive respiratory therapy, the patient continued to have increasing oxygen requirements and work of breathing. After consultation with multiple subspecialty services, no further treatment options could be identified. The patient expressed her wishes not to be intubated if there was no foreseeable possibility of extubation. Child life services were contacted and worked with the patient's three-year-old daughter to say goodbye. Our social worker helped the patient document her wishes for her daughter's guardianship. In consultation with palliative care medicine, comfort care measures were initiated for her dyspnea. She passed away peacefully on hospital day four with her family present. The patient's final wish was for others to learn from her case.

DISCUSSION: Anaplastic thyroid carcinoma (ATC) is a rare, rapidly progressive tumor with a median survival of less than 6 months. Patients typically present with a rapidly enlarging neck mass and receive the diagnosis after pathology reveals poorly differentiated cells. Metastases are present in $50 \%$ of patients at the time of diagnosis. Surgery in combination with radiation and chemotherapy is the preferred treatment for resectable tumors. For metastatic disease, the treatment is palliative. Caring for young patients at the end of life presents unique challenges for providers. Young adults are more likely to receive aggressive care with the goal of cure at the end-of-life and die in the intensive care unit than their older counterparts. Physicians must learn when to shift focus to ensure proper palliative care and ease suffering. Physicians must initiate and facilitate difficult conversations with young patients and their families. For parents with young children, the overriding concern at the end-of-life is usually about the impact of their death on their children. A multidisciplinary team including social workers, psychologists, child life specialists and chaplains is vital. Provider reflection and formal debriefing is also important for ensuring physician well-being. Strategies for shifting focus to palliation, ensuring physician well-being during tragedy, and comprehensive end-of-life care for young patients will be presented.

WHEN THE PHYSICAL EXAM TELLS MORE THAN THE LABORATORY RESULTS. Maggie K. Benson; Peggy Hasley. University of Pittsburgh Medical Center, Pittsburgh, PA. (Control ID \#1338317)

LEARNING OBJECTIVE 1: Recognize the importance of the cardiovascular exam during an evaluation of abdominal pain.

LEARNING OBJECTIVE 2: Discuss the advantages and disadvantages of treatment options for malignant pericardial effusion.

CASE: A 57 year old man with a history of tobacco abuse presented to the emergency department with one day of progressive dyspnea on exertion and vague right upper quadrant abdominal pain. He denied experiencing fevers, weight loss, cough or chest pain. His AST and ALT were mildly elevated, alkaline phosphatase was normal and abdominal ultrasound showed pericholecystic fluid and thickening of the gallbladder wall. He underwent emergent cholecystectomy during which his liver appeared "congested." The next morning, he had acute liver injury with AST and ALT in the 3000 's, prompting his transfer to a tertiary care center. On arrival, his exam revealed tachycardia, an elevated jugular venous pressure, $>10 \mathrm{mmHg}$ drop in blood pressure with inspiration, and a hepatojugular reflex. A CT scan of the chest revealed a moderate pericardial effusion, a $1.9 \times 2.7 \mathrm{~cm}$ spiculated right upper lobe lung mass and heterogenous liver enhancement. An echocardiogram confirmed the presence of a pericardial effusion with diastolic collapse of the right ventricular free wall, consistent with cardiac tamponade. An emergent pericardiocentesis was performed with drainage of $900 \mathrm{~mL}$ of fluid. Cytology was positive for malignant cells, non small cell lung cancer type. He had continued drainage from his pericardiocentesis catheter, so pericardial sclerosis with doxycycline was attempted, but unsuccessful. He ultimately required placement of a pericardial window. His liver function gradually returned to normal, and he was discharged with plans for systemic chemotherapy.

DISCUSSION: Pericardial effusions are present in up to $20 \%$ of patients with malignancy. Although malignant pericardial effusions (MPE) more commonly present with gradual accumulation of fluid and slowly progressive dyspnea, the presentation can be more dramatic with acute tamponade physiology. Approximately $80 \%$ of MPE's occur in the setting of lung cancer, breast cancer or hematologic malignancy. Malignant cells invade the pericardium through either direct or hematogenous spread. The presence of malignant cells in the pericardial fluid is a poor prognostic indicator. A careful history and focused physical examination are key in the diagnosis of cardiac tamponade. The sensitivity of dyspnea, tachycardia, elevated jugular venous pressure and pulsus paradoxus $>10 \mathrm{mmHg}$ are all greater than $75 \%$ and should increase suspicion for tamponade. Mild transaminase elevation and right upper quadrant abdominal pain in the setting of other signs of heart failure may be an indication of congestive hepatopathy. The hepatojugular reflex has a specificity of $93-96 \%$ and thereby helps to rule in right heart dysfunction. The management of MPE presents a challenge for internists. Pericardiocentesis can be performed emergently, with low complication rate, and provide immediate relief of symptoms, however recurrence rates are high. Various sclerosing agents instilled into the pericardium have been effective in preventing effusion reaccumulation, but have variable side effect profiles. Finally, surgical intervention with subxiphoid pericardial window, thoracoscopy with pericardiopleural window or thoracot- 
omy with pericardiectomy are effective, although more invasive and must take into consideration overall prognosis and goals of care.

WHEN THERE'S MORE THAN MEETS THE EYE: NOT YOUR TYPICAL FUNGAL INFECTION. Nadia N. Amin; Hilit F. Mechaber. University of Miami, Miami, FL. (Control ID \#1339691)

LEARNING OBJECTIVE 1: Differentiate between malignant and benign dermatologic lesions of the genital area in elderly patients LEARNING OBJECTIVE 2: Recognize Extramammary Paget's disease as a marker of malignancy

CASE: 64 y/o Nicaraguan male with a history of rheumatoid arthritis, HTN, $\mathrm{BPH}$, osteoporosis presents to the clinic with an erythematous, pruitic rash of the genital area. He notes that it has been worsening over the past few days, which he attributed to the hot humid weather. He has not tried any over the counter medication and is concerned with potential interactions with his current RA therapy. On physical exam the intertrigenious folds were erythematous with some flaking of the skin that extended onto the upper thigh. Pt was treated for a case of presumptive tinea cruris with clotrimazole cream but there was incomplete resolution, so he was referred to dermatology for further evaluation. Pt was diagnosed with lichen simplex chronicus and started on clobetasol and ketoconazole. Pt used the clobetasol and ketoconazole for approximately one month with minimal resolution of symptoms, so a shave biopsy was done and patient was tentatively treated with zinc oxide for possible irritation dermatitis. The biopsy results showed extramammary Paget's disease and the patient underwent a full evaluation for possible malignancy

DISCUSSION: Extramammary Paget's disease (EMPD) is a rare, cutaneous adenocarcinoma that affects mainly elderly, Caucasian females. EMPD usually presents with an exquisitely pruitic lesion in the vulva, perianal, perineal, scrotal or penile region but it can appear in any apocrine gland containing site. Approximately $25 \%$ of cases of EMPD are associated with neoplastic disease such as colorectal cancer, bladder carcinoma, prostate carcinoma, and various uterine malignancies. EMPD is often misdiagnosed due to it's nonspecific findings, with an average time to biopsy of approximately 1 year. Of note, the site of the EMPD can give an indication to the type of malignancy as scrotal lesions had a greater incidence of genitourinary malignancies and perianal lesions had a greater incidence of gastrointestinal malignancies. EMPD is particularly important for the primary care provider to identify as it may be a marker for malignancy, and its diagnosis is often delayed. After an underlying malignancy has been fully investigated, primary EMPD limited to the dermis can be treated with an immunomodulator, Imiquimod, for 12 weeks. Mohs surgery and radiation therapy have also been used.

WHO IS THE FIRST VICTIM - THE LUNG OR THE KIDNEY? Kurt J. Pfeifer; Sana S. Gafoor. Medical College of Wisconsin, Wauwatosa, WI. (Control ID \#1338417)

LEARNING OBJECTIVE 1: Recognize microscopic polyangitis (MPA) as a key differential diagnosis in patients presenting with pulmonary-renal syndrome.

LEARNING OBJECTIVE 2: Diagnose and treat MPA early to reduce remission rates and improve overall mortality.

CASE: A 65-year-old woman with a history of nonspecific interstitial pneumonitis (NSIP), diabetes mellitus, and hypertension presented with the chief complaint of fatigue. The patient was on chronic steroid therapy for the past five years for her NSIP, but her steroid therapy was stopped three months prior to the start of her symptoms due to stabilization of her disease. She was transferred from an outside facility after her initial evaluation revealed new laboratory findings of a microcytic anemia (hemoglobin $6.0 \mathrm{~g} / \mathrm{dl}$ ) and azotemia with a creatinine of $7.23 \mathrm{mg} / \mathrm{dl}$. Her associated symptoms included lower extremity myalgias, shortness of breath, and a dry cough over the last four weeks. Significant physical exam findings included bibasilar lung crackles and a 2/6 ejection murmur in the aortic area. She had no clear signs of uremia, rashes, or musculoskeletal findings. Urinalysis revealed $2+$ blood and protein in addition to numerous granular casts, waxy casts, and dysmorphic red blood cells. Renal biopsy revealed a myeloperoxidase antibody-positive (p-ANCA) necrotizing crescenteric glomerulonephritis. The patient was given IV methylprednisolone for 3 days and then transitioned to oral prednisone. Given the histological findings, absence of granulomas, and p-ANCA positivity, a working diagnosis of MPA was given. The patient was continued on immunosuppressive therapy with oral prednisone and monthly cyclophosphamide. By time of discharge, the patient's shortness of breath had improved and her renal function had stabilized.

DISCUSSION: MPA typically presents as a systemic necrotizing vasculitis with few or no immune complexes that mainly affects small vessels. The affected vessel caliber and absence of granulomatous inflammation are key differentiating features from polyarteritis nodosa and Granulomatosis with Polyangiitis respectively. As in the discussed patient, renal manifestations are the most predominant feature of MPA. In our patient, it is not clear if the steroid responsive NSIP was the initial presentation of the disease process or if the chronic steroid therapy prevented progression of an undiagnosed MPA. Further research of the patient's records revealed that she had been found to have a positive $\mathrm{p}$ ANCA serology in the past. Combined therapy with high-dose steroids and cyclophosphamide is the first-line treatment of MPA. Earlier diagnosis may help prevent further progression of renal disease and decrease the potential for requiring renal replacement therapy. MPA should be a consideration in the differential diagnosis of any pulmonary-renal syndrome since tailored therapy has been shown to decrease disease relapse rates and mortality.

WHOLE LOTTA SHAKIN' GOING ON Aimee Aysenne. Tulane University Health Sciences Center, New Orleans, LA. (Control ID \#1340495)

LEARNING OBJECTIVE 1: 1. Recognize neurological symptoms associated with Acute Human Immunodefiency Virus (HIV) infections. 2. Identify the association of acute HIV meningitis and myoclonus. 3. Understand how to interpret the results of cerebral spinal fluid (CSF) in HIV patients.

CASE: A 33 year-old woman presented with five hours of abnormal and uncontrollable movements of her legs. The movements began with spontaneously with jerking, then facial twitching and grunting began. Each episode lasted about one minute, occurring every fifteen minutes; she was asymptomatic between episodes. She had been to another hospital prior to this admission, at which time a head CT was found to be normal; she was sent home on diazepam and ibuprofen. On this admission, she was found to have a flaccid paresis of the lower extremities and hyperreflexia. During one of the episodes, she was tachycardic with symmetric non-rhythmic movements of her legs and increased tone in both arms. Also noted was twitching of the face symmetrically and loud grunting; she was unable to follow verbal commands. A lactic acid level was $3.9 \mathrm{mmol} / \mathrm{L}$, and a creatine kinase was $8,000 \mathrm{IU} / \mathrm{L}$. The remainder of her laboratory values were normal. She developed a fever and acute kidney injury. The episodes worsened: occurring more frequently, involving the face, arms and legs, increasing heart rate to 200 beats per minute during the episodes and including hypoxic which required positive pressure ventilation. Brain imaging remained normal. The CSF had 26 leukocytes per $\mu \mathrm{L}$ with $92 \%$ lymphocytes and a protein level of $76.8 \mathrm{mg} / \mathrm{dl}$. All cultures were negative. An HIV test was reactive with a viral load of 1,500,000-copies/ $\mathrm{mL}$ and a CD4 count of 408 cells per $\mu \mathrm{L}$. The diagnosis of acute HIV syndrome associated with myoclonus was made. She treated with levetiracetam, clonazepam and anti-retroviral therapy, with subsequent improvement of the symptoms.

DISCUSSION: It is important for the general internist to recognize the neurologic manifestations of acute HIV syndrome. Common symptoms include meningitis, encephalitis, polyradiculoneuritis, and peripheral mononeuritis. Primary symptomatic infection (PSI) with HIV occurs two to six weeks after infection and lasts one to two weeks. The initial phase of viral replication occurs systemically, meningeal or both. CSF pleocytosis with lymphocytic predominance and elevated levels of several soluble immunological markers can be found in acute HIV. Patients are typically asymptomatic. Generally, CSF HIV infection responds well to anti-retroviral therapy. The relative rates of viral decay in the serum and CSF may differ in some patients, with HIV viral concentrations falling more slowly in the CSF than in serum. Mild mononuclear pleocytosis is common in untreated, 
asymptomatic HIV infected individuals with blood CD4 counts greater than 50 cells per $\mu \mathrm{L}$. Meningitis should be suspected and investigated when CSF cell counts are greater than 20 cells per $\mu \mathrm{L}$ in untreated HIV patients, CSF cell counts are greater than 5 cells per $\mu \mathrm{L}$ in patients with blood CD4 counts less than 50 cells per $\mu \mathrm{L}$, or CSF cell counts are greater than 5 cells per $\mu \mathrm{L}$ in patients taking anti-retroviral therapy regardless of serum HIV viral load and CD4 count. While this is an unusual case, the general internist should be aware of neurologic presentations of acute HIV syndrome and be able to interpret the CSF analysis in HIV infected patients.

WHOOPS! PERTUSSIS AFTER VACCINATION FAILURE Torrey R. Schmidt; Richard Alweis; Gaurav Gulati. Reading Hospital and Medical Center, West Reading, PA. (Control ID \#1304413)

LEARNING OBJECTIVE 1: Vaccinations vary in efficacy rate, with pertussis being $92 \%$ effective

LEARNING OBJECTIVE 2: Pertussis should be considered in a coughing case lasting longer than 5 days, despite the lack of the classic "whoop."

CASE: A 43 year old otherwise healthy male presented to the Emergency Department (ED) with continued shortness of breath and a two week history of low grade fever and non-productive cough without whoop. The patient had received Tdap vaccination a year prior. He had previously been seen twice in his primary care provider's office with these complaints and an initial sore throat. After his first visit, he was thought to have a viral URI and given supportive care. On day 7, he was placed on prednisone and albuterol for putative bronchospastic complications manifesting as occasional wheezing. His symptoms failed to improve requiring admission for further management. He complained of dyspnea at rest and on exertion but denied hemoptysis or chest pain. His exam was notable for hypoxia $(85 \%$ on Room Air) with dry rales and end-expiratory wheezing, without respiratory distress. Routine labwork was unremarkable; chest radiograph and CT scan did not demonstrate signs of infection. Because of the lack of specific findings, prolonged symptoms and significant hypoxia, atypical infections were considered. Serology for mycoplasma, chlamydia and Bordetella were sent. Bordetella IgM and IgG titers were significantly elevated, indicating a vaccine failure. He was successfully treated with azithromycin.

DISCUSSION: Despite advancements in vaccination, cases of pertussis are still seen sporadically in the adult population. This has led to the original recommendation for a Tdap booster between the ages of 19 to 64 and now also after age 65. The Acellular Pertussis Vaccine Trial (APERT) was a multi-center, double blinded, controlled study that demonstrated Tdap efficacy to be $92 \%$, although many providers assume this number to be higher. In this case, despite a recent well-publicized outbreak less than 20 miles away, providers did not consider a vaccination failure due to this assumption, resulting in significant morbidity requiring hospitalization. Therefore, although rare, pertussis vaccination failure does occur, and should be considered in the workup for a coughing illness lasting more than 5 days, even without the classic "whoop".

WILSON'S DISEASE WITH NORMAL LIVER FUNCTION AND LOW COPPER LEVELS: AN UNCOMMON PRESENTATION Gaurav Gulati; Shuchi Gulati; Hai P. Nguyen. The Reading Hospital and Medical Center, West Reading, PA. (Control ID \#1338991)

LEARNING OBJECTIVE 1: Diagnose Wilson's disease in a patient with normal liver function and low serum copper.

LEARNING OBJECTIVE 2: Recognize Wilson's disease in young patients presenting with Parkinsonian features.

CASE: A 24 year old previously healthy female was hospitalized with recurrent numbness and headaches accompanied by Parkinsonian symptoms (tremors, slurred speech and drooling with difficulty swallowing). She had a significant family history of psychiatric illnesses in her mother and sister. Physical exam was notable only for mild dysarthria and subjective weakness on the left side. Initial laboratory data including a $\mathrm{CBC}$, basic metabolic panel, liver function tests, thyroid function, ESR, CRP, Lyme PCR, HIV and
RPR/VDRL were all negative. She had a normal CSF exam with negative paraneoplastic panel, IgG index, viral studies and cryptococcal antigen. Urinalysis was unremarkable and urine drug screen unrevealing. Autoimmune workup including ANA and rheumatoid factor was negative. A CT scan of the head revealed abnormal, symmetric, diffuse hypo-densities of bilateral basal ganglia. An MRI to further evaluate the soft tissue abnormalities showed increased T2 signal within the right and left lenticular nucleus. A heavy metal screen was ordered. Arsenic, mercury, lead, cadmium and copper levels were all low. Given the high index of suspicion a ceruloplasmin level was ordered which was extremely low at $3.1 \mathrm{mg} / \mathrm{dL}$ (normal: $14-78 \mathrm{mg} / \mathrm{dL}$ ). A liver biopsy showed mild chronic active hepatitis grade 2, septal fibrosis stage 3 and retained copper within hepatocytes, confirming a diagnosis of Wilson's disease. An ophthalmologic exam under slit lamp showed clear Kayser-Fleischer (KF) rings. She was started on trientine and zinc, and over subsequent follow up her KF rings disappeared and neurological symptoms showed modest improvement.

DISCUSSION: Hepatolenticular degeneration (Wilson's disease) is an autosomal recessive defect in copper export with a prevalence rate of one in 30,000 live births. It is caused by reduced biliary excretion of Copper leading to its buildup in the liver, subsequently progressing to other organs. Diagnosis is based on clinical presentation combined with physical, biochemical and histologic findings. Neurologic disease (present in 35 percent patients) includes Parkinsonian tremor, rigidity and clumsiness of gait, speech problems and drooling. Ten percent patients exhibit psychiatric symptoms varying from subtle personality changes to overt depression, catatonia and paranoia. Serum aminotransferases are usually elevated, but their severity does not correlate with histologic hepatic injury. Total body copper levels are elevated, but serum levels may be low in patients with low ceruloplasmin levels. In the presence of only neurological symptoms with normal liver function and low copper levels, a slit lamp exam to look for KF rings should be considered. If positive, it strongly suggests Wilson's disease. A liver biopsy may be required for further confirmation of the diagnosis.

WITHOUT A TARGET: UNDERSTANDING ATYPICAL PRESENTATIONS OF LYME DISEASE Bethany J. Hyduke; Robert Krippendorf; Kurt J. Pfeifer. Medical College of Wisconsin, Milwaukee, WI. (Control ID \#1337133)

LEARNING OBJECTIVE 1: Recognize nonspecific array of symptoms as manifestations of disseminated Lyme disease

LEARNING OBJECTIVE 2: Treatment of Lyme disease once it reaches the CNS

CASE: A 75 year old male presented with a 3-week history of weakness, low back pain, upper extremity tremors and double vision. As a result of these symptoms, he reported having difficulty writing, dialing phone numbers and reading. His daughter also observed word-finding difficulty and a weight loss of 20 pounds over several weeks. The patient attributed this to decreased appetite, dysphagia and odynophagia. Prior to the onset of these symptoms, the patient was very active, including working as a substitute teacher. He denied possible ingestions of toxins, inappropriate use of medications, recent travel and insect or tick bites. The patient's neurologic exam was significant for decreased reflexes throughout his extremities but normal muscle tone, bulk and power. He had a positive Romberg test, intention tremor with finger-to-nose testing, delayed rapid alternating hand movements on his left side and a wide-based gait. He also had vertical diplopia with leftward gaze and scored only 13 out of 30 on Montreal cognitive assessment. Brain MRI was significant for slightly prominent ventriculomegaly and global volume loss. He underwent lumbar puncture, and cerebrospinal fluid (CSF) analysis revealed elevated protein, elevated leukocyte count, normal glucose, negative cytology, and negative gram stain and culture. Subsequent MRI of his lumbar spine revealed diffuse leptomeningeal enhancement along the visualized lower cord, conus and cauda equina. Eventually, CSF Lyme serologies returned positive for IgG and were confirmed by western blot. The patient was treated with a 2-week course of ceftriaxone and was improved at the end of his therapy. He was still experiencing achy low back pain and parethesias in his upper extremeties. However, his cognitive function was improved on his follow up mental status exam. 
DISCUSSION: Lyme disease is the most common tick-borne disease in the United States and Europe. The most common clinical manifestation is the classic target lesion of erythema migrans, which develops 7 to 14 days after tick detachment. Patients also present without erythema migrans with nonspecific complaints such as headache, arthralgias, fatigue, cognitive slowing and memory difficulty. The more serious clinical sequelae of Lyme disease develop as a consequence of the hematogenous spread of the spirochete. Approximately 10 percent of patients with erythema migrans who go untreated will have a neurologic manifestation, such as trigeminal neuralgia, facial nerve palsy, meningitis or encephalopathy. Lyme encephalomyelitis is a rare observation that occurs when inflammatory appearing parenchymal abnormalities appear in the brain or spinal cord. Randomized trials have shown that doxycycline, amoxicillin and cefuroxime are effective oral treatments for Lyme disease. Those patients who have evidence of disseminated infection, including neurologic manifestations or Lyme carditis, might be considered for parenteral antibiotics. Prevention of tick bites by using repellants and keeping skin covered as much as possible are the most effective ways of avoiding transmission.

WORKING UP RIGIDITY: KEEPING THE DIAGNOSIS FLEXIBLE Prameela Rao; Coral Parikh; Andrea Porrovecchio. Montefiore Medical Center, Bronx, NY. (Control ID \#1336499)

LEARNING OBJECTIVE 1: Review the natural history and differential diagnosis of Parkinsonism

CASE: An 81 year old female presented with three months of progressive bilateral lower extremity weakness and stiffness. She was fully functional prior to the onset of a slow gait and neck stiffness. Two weeks prior to admission, her walking was characterized as 'locking.' One week prior, her upper extremities, neck, and shoulder girdle were involved. She was bradyphrenic with a masked face, but with normal mental status.Strength was $3 / 5$. She had severe rigidity of all extremities. There was increased tone in all muscle groups, but no tremors or clonus. Her sensory exam was normal. She was unable to stand. TFTs and CPK were normal and an LP was negative. Since extensive brain imaging was normal, a paraneoplastic process was on the differential, but a malignancy workup was negative. EMG showed continuous motor unit activity which is seen in Stiff Person Syndrome(SPS). Anti-glutamic acid decarboxylase (GAD) antibodies were not elevated. An empiric trial of high dose steroids was initiated, followed by IVIG therapy resulting in some increased mobility.

DISCUSSION: Rigidity is a common ailment amongst the elderly Neurologic conditions can present with stiffness and result in gait abnormalities therefore internists should be cognizant of the natural history of these conditions. Due to the patient's age and symptoms, Parkinson's disease, a movement disorder occurring in 2.5 percent of patients over the age of 80 , was initially considered. Parkinson's disease comprises $75 \%$ of all cases of Parkinsonism, which is diagnosed clinically in those with signs of bradykinesia, rigidity, resting tremor, shuffling gait, and postural instability. The rapid progression and symmetric nature of her presentation makes Parkinson's disease far less likely. Primary neurological disorders such as progressive supranuclear palsy (PSP) or Lewy body dementia can also present with Parkinsonism. PSP is characterized by gait impairment, early falls, bulbar abnormalities, and anti-NMDA antibodies, which were negative in our patient. Lewy body dementia is characterized by visual hallucinations and onset of dementia within one year of Parkinsonism. The acuity of her symptoms and intact cognition argue against these diseases. Secondary Parkinsonism can be seen in paraneoplastic syndromes,; associated malignancies include: small cell lung cancer, breast, ovarian, testicular, thymoma and Hodgkin's lymphoma. SPS is characterized by progressive muscle stiffness and muscle rigidity. Presentation of SPS is usually between the third and fifth decades of life, and equal amongst the sexes. Symmetric rigidity and stiffness begin in the axial muscle groups and progress proximally. Sixty percent of patients are found to have anti-GAD antibodies in the CSF and serum. These antibodies are found to inhibit GABA-ergic nerve terminals in the CNS, a similar effect produced by the tetanus toxin. Anti-GAD antibodies are familiar to the internist due to their new role in identifying type 1 diabetics. The presence of these antibodies in both SPS and type 1 diabetes supports the hypothesis that SPS is an autoimmune phenomenon. Most diseases associated with Parkinsonism are diagnosed clinically, therefore variations from the expected time course should alert physicians to be more flexible in their diagnoses.

YET ANOTHER PATIENT WITH DYSPNEA! ANCA NEGATIVE PAUCI IMMUNE GLOMERULONEPHRITIS - A CASE REPORT Chitra Srinivasan. Saint Francis Hospital, Evanston, IL. (Control ID \#1340476)

LEARNING OBJECTIVE 1: Pauci immune crescentic glomerulonephritis is a common cause of rapidly progressive glomerulonephritis. In a majority of patients, it is a manifestation of ANCA associated vasculitis. However, ANCA can be absent in a subset of patients.

CASE: 83 y/o male presented to the emergency room with a 3 day history of shortness of breath. He also complained of fatigue and loss of appetite for the last 2 weeks. Past medical history was significant for hypertension and sick sinus syndrome. Physical exam revealed bilateral crackles at the lung bases and trace pedal edema. Laboratory examination showed BUN 71, Creatinine - 4.8 ( 0.8 one month ago), Potassium - 6 and Hemoglobin 8.9. Chest $\mathrm{X}$ ray revealed bilateral pulmonary congestion. Patient was administered intravenous furosemide and kayexalate with some improvement in symptoms. Urinanalysis was positive for proteins, blood and eosinophils. A renal ultrasound showed normal sized kidneys with increased corticomedullary differentiation. Serological tests showed a positive ANA (SS/B in a titer of 2.5). ANCA was negative. C 3 levels were slightly decreased and C4 levels were normal. SPEP and hepatitis panel were negative. Hemodialysis was initiated due to worsening renal function. A renal biopsy showed focal segmental necrotizing crescentic glomerulonephritis. There was severe interstitial fibrosis and tubular atrophy. Acute interstitial nephritis with eosinophilic infiltration was also seen involving $25 \%$ of the cortex. Immunoglobulin deposits were absent. A diagnosis of ANCA negative pauci immune crescentic glomerulonephritis was made. He was started on immunosuppressive therapy with prednisone and cyclophosphamide

DISCUSSION: ANCA negative pauci immune glomerulonephritis accounts for less than $5 \%$ of cases of rapidly progressive glomerulonephritis. Patients can present with acute onset of macroscopic hematuria, decreased urine output and edema. Alternatively, onset can be insidious with initial symptoms being just fatigue or edema. Early and accurate diagnosis with serologic testing and renal biopsy is essential to initiate appropriate therapy and prevent progression to irreversible renal failure. Renal biopsy typically shows a focal necrotizing and crescentic glomerulonephritis with little or no glomerular staining for immunoglobulins by immunofluorescence microscopy. As with ANCA positive vasculitis, treatment involves induction therapy with intravenous methylprednisone and cyclophosphamide followed by oral prednisone and cyclophosphamide for six to nine months. Azathioprine is used to maintain remission.

ZOSTER RASH MANIFESTING WITH ENCEPHALITIS AND PARESIS Jennifer C. Fuller ${ }^{1}$; Vinhfield X. Ta ${ }^{2}$; Suzanne Donovan ${ }^{2}$. ${ }^{1}$ David Geffen School of Medicine at UCLA, Los Angeles, CA; ${ }^{2}$ Olive View-UCLA Medical Center, Sylmar, CA. (Control ID \#1339929)

LEARNING OBJECTIVE 1: Recognize that encephalitis and segmental motor paresis are rare but serious complications of varicella zoster virus (VZV) in an immunocompetent adult with typical zoster rash.

CASE: A 63-year-old diabetic man presented with a subacute onset of right lower extremity weakness over four days and one day of confusion and wordfinding difficulty. He had a mild headache, but denied fever, neck stiffness, dizziness, loss of consciousness, back pain, or incontinence. He also complained of tingling pain on his right lateral leg. History was negative for recent trauma, animal or insect bites, travel, and tobacco, alcohol or other drugs. The patient was found to be febrile to $38.8 \mathrm{C}$, tachycardic and mildly hypertensive. He had no meningismus or lymphadenopathy. On neurological exam, he was alert and oriented with fluent speech, but slowed mentation, inability to follow 3 -step commands, $0 / 3$ delayed recall, instability on standing, and a right foot drop that worsened from $4 / 5$ to $1 / 5$ during hospitalization. A few small red papules were initially noted on the dorsum of the right foot, which evolved over a day to form many scattered non-tender, 
non-pruritic, blanching erythematous macules with 1-2 mm pustules in the L5 distribution. An initial workup for infection and stroke was unrevealing, with the absence of peripheral leukocytosis, blood or urine culture growth, and brain CT or MRI findings. However, lumbar puncture revealed elevated cerebral spinal fluid (CSF) protein, normal glucose, monocytic pleocytosis, and negative Gram and India ink stains. While awaiting further studies, acyclovir, ceftriaxone, and fluconazole were empirically started. Two days later, VZV polymerase chain reaction (PCR) of the CSF and VZV total antibody returned positive. Skin biopsy of the right leg rash was also consistent with VZV. The patient completed a 14-day course of IV acyclovir. Over this time, his mental status quickly returned to baseline, the pustular rash crusted over, and the right foot drop persisted, although the patient was able to walk with physical therapy.

DISCUSSION: This case illustrates rare but important complications of herpes zoster reactivation. Our patient presented with altered mental status secondary to encephalitis and focal motor weakness prior to the typical cutaneous and sensory signs. Segmental motor paresis, seen in 3-5\% of cases, occurs in the same distribution as the cutaneous eruption due to spread of VZV from the dorsal root ganglion to the anterior root or horn, most commonly affecting C5-C7 or L1-L4, within 2 weeks of skin findings. Rarely, as in this case with L4-5 motor and L5 cutaneous involvement, weakness may precede the rash. Importantly, complete or partial motor recovery is seen in $75 \%$ but may take up to $1-2$ years. Another uncommon complication in our patient was VZV encephalitis. This is believed to result from a vasculopathy and most commonly occurs in immunocompromised patients, but can also affect immunocompetent adults. Most cases are seen with cranial nerve or cervical involvement, making this case unique. As in this case, $80 \%$ of cases have normal neuroimaging and typical CSF findings include moderate pleocytosis $(52 \%$ lymphocyte- and $26 \%$ monocyte-predominant), elevated protein, and normal glucose. CSF PCR is a rapid and sensitive means of diagnosis. Mortality is $5-10 \%$ and $10-20 \%$ have long-term sequelae. Vaccination can prevent shingles and its potential complications, and is currently recommended for adults aged 60 and over.

'RAPIDLY PROGRESSIVE DEMENTIA: SOMETIMES IT IS A ZEBRA' Jennifer L. Neville; Carl Fichtenbaum. University of Cincinnati, Cincinnati, OH. (Control ID \#1312807)

LEARNING OBJECTIVE 1: To describe the salient features and differential diagnosis of encephalitis.

LEARNING OBJECTIVE 2: To define clinical evaluation for encephalopathy and Creutzfeldt-Jakob Disease.

CASE: A 54 year-old white male with diabetes mellitus and hyperlipidemia presented with right-sided weakness, dizziness, and shortness of breath. On the morning of admission he developed numbness and weakness on his right side with a foot drop that resolved prior to presentation. The history obtained from the family revealed a functional status decline over the past several months. They described increasing confusion, memory loss, ataxia, tremors, occasional double vision, tinnitus, decreased appetite, dysphagia, choking on food, frequent throat clearing, sixty pound weight loss over the past three to four months, insomnia, sleep talking, and odd jerking movements while asleep. Prior to the onset of symptoms, the patient was highly functional. He had a history of significant alcohol intake for 30 years (six beers/day) but had quit 4 months prior to presentation. Prior ambulatory evaluation by a neurologist suggested a possible diagnosis of Frontal Lobe Parkinson's and treated with rivastigmine. A family medical history was significant for a maternal grandfather with 'early' onset Alzheimer's disease and his paternal grandmother was diagnosed with Alzheimer's disease in her seventies.

DISCUSSION: The hallmark presentation for CJD is a rapidly progressive dementia with myoclonus. The incidence in the United States is one case per million person-years. Mental decline presents as dementia with behavioral abnormalities, and deficits involving higher cortical function. Mood changes such as apathy and depression are more common where as emotional lability and anxiety are less frequent. Extrapyramidal signs, cerebellar manifestations, and corticospinal tract involvement can develop. Brain biopsy is the gold standard test for CJD. The WHO criteria for the diagnosis of CJD include MRI and/or EEG abnormalities consistent with CJD, CSF protein studies, and ruling out other causes. Clinicians should be aware of CJD and consider this in the differential diagnosis of persons presenting with dementia particularly those with a rapidly progressive declining clinical status. “A DANGEROUS WOLF IN SHEEP'S CLOTHING:” A CASE
REPORT OF DESTRUCTIVE ENDOCARDITIS CAUSED BY
STAPHYLOCOCCUS LUGDUNENSIS Daiki Morikawa. Teine Keijinkai
Medical Center, Sapporo, Japan. (Control ID \#1335636)

LEARNING OBJECTIVE 1: Recognize that a positive blood culture of coagulase-negative staphylococci may not always be a contaminant LEARNING OBJECTIVE 2: Recognize the clinical signs and symptoms of endocarditis caused by Staphylococcus lugdunensis

CASE: A 70 year-old Japanese woman with a past medical history of cerebral palsy presents with 1-month's history of decrease in appetite and weight. She also reports generalized body weakness while denying fevers, chills, chest pain, palpitations, shortness of breath, cough, urinary changes, and rashes. Her significant physical findings include temperature of $35.3^{\circ} \mathrm{Celsius}$, blood pressure of $85 / 48 \mathrm{mmHg}$, heart rate of 87 beats/minute, respiratory rate of 30 breaths/minute, and oxygen saturation of $100 \%$ on 10 liters of oxygen. She is lethargic and disoriented to time, place, and person; she also has poor oral hygiene with saprodontia. She has no heart murmurs, costovertebral angle tenderness, back pain, or skin lesions. The remainder of her examination is unremarkable. In addition, significant laboratory findings consist of white blood cell count (WBC) of $23,240 / \mu \mathrm{L}$ ( $4 \%$ bands, $93 \%$ segmented neutrophils), hemoglobin of $7.9 \mathrm{~g} / \mathrm{dL}$, platelets of $65,000 /$ $\mu \mathrm{L}$, blood urea nitrogen of $68 \mathrm{~g} / \mathrm{dL}$, and creatinine of $1.2 \mathrm{mg} / \mathrm{dL}$. Urinalysis shows $2+$ protein , $3+$ heme, $>100$ red blood cells/high power field (HPF), $>100 \mathrm{WBC} / \mathrm{HPF}$, and $3+$ bacteria. A computed tomographic scan of the chest, abdomen, and pelvis reveals bilateral pleural effusions and bladder masses with hydronephrosis. Given her septic shock from presumed urinary tract infection, she has been empirically started on meropenem. Her encephalopathy, however, does not improve, and she even develops right-sided hemiparesis; a magnetic resonance imaging of the brain shows multiple areas of embolic cerebral infarcts. She also develops a renal infarction, splenic abscess, and iliopsoas abscess for which their drainage shows no bacteria. Her antimicrobial regimen has thus been changed to ceftriaxone and vancomycin for suspicion of infectious endocarditis; a transesophageal echocardiogram shows a vegetation on the mitral valve with regurgitation. During this time, all 3 sets of blood cultures have grown coagulase-negative staphylococcus (CNS), Staphylococcus lugdunensis in particular. Despite aggressive medical management and scheduled mitral valve replacement, the patient dies after 18 days of admission.

DISCUSSION: As part of the normal skin flora, CNS has often been identified as a blood culture contaminant but has become increasingly recognized as a clinically significant pathogen even in cases of native valve endocarditis (NVE). In particular, S. lugdunensis can cause severe infections frequently attributable to Staphylococcus aureus. While both of these species produce a bound coagulase, S. lugdunensis, however, does not produce a free coagulase. Patients with immunosuppression and foreign bodies such as prosthetic devices and intravascular catheters are at a higher risk of infection with S. lugdunensis. The clinical presentation of NVE caused by CNS is indistinguishable from Streptococcus viridans and includes fever, generalized weakness, weight loss, and anorexia; about $25-33 \%$ of patients exhibit Osler's nodes, Janeway lesions, and splinter hemorrhages. While this case may have clinical signs and symptoms similar to $\mathrm{S}$. viridans, one must not discount blood culture results initially showing CNS as just a blood culture contaminant.

"BATH SALTS": A NEW HIGH, NOT FOUND IN THE HYGIENE AISLE William A. Hammond. Dartmouth-Hitchcock Medical Center, Lebanon, NH. (Control ID \#1333709)

LEARNING OBJECTIVE 1: Recognize "bath salts" as new designer drugs of abuse undetectable by current readily available screening techniques 
LEARNING OBJECTIVE 2: Recognize signs of intoxication and adverse effects of bath salt use and appropriately advise at risk patient populations of these adverse effects.

CASE: A 32 year old man with chronic low back pain (LBP) and a remote history of opioid and other substance abuse presented to an Emergency Department (ED) with new onset LBP after lifting a washing machine. Despite being febrile to 38.8 degrees Celsius, he was diagnosed with mechanical injury and discharged with opioids, benzodiazepine, and prednisone. Over the ensuing 24 hours, he experienced worsening fever, systemic symptoms, and new onset left-sided facial droop. His fiancée returned him to the ED for reevaluation. He quickly became septic with decreased mental state and was transferred to a tertiary care facility after tracheal intubation. Just prior to his dramatic decline, he admitted to recently injecting "bath salts", a legal synthetic amphetamine. Exam on transfer revealed temperature of 38 degrees Celsius, hyperdynamic pulses, multiple skin pustules, bilateral wrist track marks, and involuntary neck flexion with flexion of the knees. Lumbar puncture returned frank pus, and subsequent magnetic resonance imaging of the spine and brain revealed a large psoas abscess with extension into the epidural space, ventriculomegaly with ventriculitis, and bilateral pontine infarcts. He was treated with intravenous antibiotics, as well as rapid extraventricular drain placement to remove purulent fluid and reduce intracranial pressure. Cultures returned with methacillin sensitive Staphylococcus aureus. After multiple surgical drainages, he made a surprising recovery to discharge to a local rehabilitation facility.

DISCUSSION: "Bath salts" are new synthetic amphetamine-like substances that have been legally sold, prior to October 2011, in smoke shops with the intent of abuse. These products were sold legally with the label "not for human consumption" under clever labels such as "bath salts", "plant food", or "insect repellant". Manufacturers circumvent the law by creating substances with slightly altered structure or side chain so that the substances do not require Drug Enforcement Agency monitoring. The most common substances are synthetic cathinones, most commonly methylenedioxypyrovalerone (MDPV), however numerous other substances have been produced and sold similarly. The substances are typically consumed orally or via inhalation, however they can also be taken intravenously, such as the patient in the clinical presentation. The effects are similar to those of amphetamines, producing euphoria, hyperactivity, and hypersexuality, however negative effects including hallucinations, paranoia, and seizures. Public media reports of emotional lability with injury to self and others, as well as suicidality among teenage and young adult users have been increasing. Currently, there are no readily available screening tests for bath salts, so the diagnosis must be made on clinical suspicion along with elimination of other substances as the causative agent in an intoxicated patient.

"CRACK" THE CASE: REPORT OF COCAINE/LEVAMISOLEINDUCED CUTANEOUS VASCULITIS Cory Walker; Andre C. Eaddy; Cathryn Caton. Medical University of South Carolina, Charleston, SC. (Control ID \#1340456)

LEARNING OBJECTIVE 1: Recognize the characteristic clinical presentation and serologic findings of cocaine/levamisole-induced vasculitis to facilitate rapid diagosis.

LEARNING OBJECTIVE 2: Review the proposed role of levamisole (an antihelminthic used as a cocaine cutting agent) in cocaine-induced vasculitis. CASE: 40 year old female with a 1 year history of lower extremity cutaneous vasculitis with limited response to systemic steroids treated at an outside hospital presents with 1 month worsening of rash and polyarthralgias. Her past medical/surgical and family history are unremarkable. On physical exam, she had diffuse necrotic appearing palpable purpuric and bullous lesions extensively on bilateral lower extremities, on arms bilaterally, and smaller lesions on the vertex of the head and on the abdomen. Although the patient denied cocaine use, lesions were suspicious for cocaine-induced vasculitis. Skin biopsy revealed leukocytoclastic vasculitis. Urine drug screen was positive for cocaine. MPO and RF were positive and ANCA was positive with a perinuclear pattern. This patient was newly diagnosed with Hepatitis $\mathrm{C}$. Levamisole, a cocaine cutting agent associated with cocaine-induced vasculitis, was measured in serum by HPLC. These findings are all consistent with case reports of cocaine/ levamisole-induced vasculitis. Although other causes/contributors to cutaneous vasculitis such as ANCA-, SLE-, Hepatitis C- associated vasculitis cannot be definitively ruled out, this patient's condition improved with abstinence from cocaine use and supportive care. The patient was discharged in improved and stable condition and was provided resources to pursue rehabilitation.

DISCUSSION: Levamisole, an antihelminthic agent used in veterinary medicine, is present in $30-40 \%$ of U.S. "crack" cocaine and is used as a cutting agent because of its physical similarities to crack cocaine and its reported hallucinatory effects. Levamisole-contaminated cocaine has been associated with thrombotic vasculopathy and a leukocytoclastic vasculitis in several case reports and is becoming increasingly more prevalent. Unfortunately, neither the mechanism nor threshold of toxicity has been elucidated. Interestingly, cocaine/levamisole-induced vasculitis has been associated strongly with ANCA positive findings and moderately associated with ANA, Hepatitis C, anti-phospholipid, dsDNA, RNP and/or MPO antibody positive patients in the setting of detectable levamisole in either urine or serum. This cutaneous vasculitis, usually occurring in middle aged women, has a characteristic physical presentation involving the extremities and/or earlobes and these patients have numerous serological findings. There are patients who also require debridement and reconstructive surgery, compounding the sequelae of exposure to this agent. We encourage clinicians to recognize and quickly diagnose cocaine/levamisole-induced vasculitis in patients at high risk for drug abuse and with vasculitis of unknown etiology.

"DOCTOR, I CAN NOT STOP VOMITING!" Salman J. Bandeali ${ }^{1}$; Jolie Britt'; Anna Kolpakchi ${ }^{2}$; Lee Lu ${ }^{1} .{ }^{1}$ Baylor College of Medicine, Houston, TX; ${ }^{2}$ Baylor College of Medicine, Houston, TX. (Control ID \#1317605)

LEARNING OBJECTIVE 1: Recognize that common gastrointestinal (GI) symptoms of nausea and vomiting could be the initial presentation of immunoglobin light-chain GI amyloidosis.

LEARNING OBJECTIVE 2: Review GI amyloidosis.

CASE: A 47-year-old African American male with poorly controlled hypertension presented with nausea, vomiting, and early satiety with a 30 pound unintentional weight loss. He denied dysphagia, odynophagia, fever, abdominal pain, diarrhea or constipation. He had no sick contacts or recent travel. The patient's symptoms started 2 months prior at which time he was admitted with dehydration and acute kidney injury with a creatinine $(\mathrm{Cr})$ of $3.4 \mathrm{mg} / \mathrm{dL}$ from a normal baseline Cr. Urinalysis showed granular casts and proteinuria. He was treated with supportive care, and his $\mathrm{Cr}$ improved to $2.0 \mathrm{mg} / \mathrm{dL}$ but failed to normalize completely. He was presumed to have developed chronic kidney disease due to long-standing hypertension as evidenced by medical renal disease on ultrasound. His nausea and vomiting persisted with intermittent flares requiring multiple emergency room visits. One month later, he was admitted again for the same complaints. An abdominal CT scan showed an incidental supraumbilical hernia along with a ventral hernia, but no evidence of incarceration. The ventral hernia was thought to be the source of his intractable nausea and vomiting; thus, a laparoscopic hernia repair was performed. Few weeks later, his symptoms recurred. Physical exam at that time was significant for hyperactive bowel sounds and a liver span of $14 \mathrm{~cm}$. Laboratory studies showed a $\mathrm{Cr}$ of $2.9 \mathrm{mg} / \mathrm{dL}$ and $3(+)$ proteinuria. Esophagogram was normal, and gastric emptying study showed accelerated gastric emptying. An esophagogastroduodenoscopy revealed antral erythema, and the biopsy of duodenal mucosa was positive for Congo red staining. Serum protein electrophoresis identified IgG kappa and lambda M spikes. Bone marrow biopsy showed $5 \%$ plasma cells. A diagnosis of "AL" amyloidosis with gastrointestinal involvement was made. The patient received bortezomib and dexamethasone treatment with resolution of his symptoms.

DISCUSSION: Nausea and vomiting are common GI complaints, and the long differentials usually do not include GI amyloidosis as an etiology. Gastric and duodenal involvement in "AL" amyloidosis occurs in $8 \%$ of patients by biopsy, with only $1 \%$ cases being symptomatic. As an initial presentation of "AL" GI amyloidosis, nausea and vomiting are extremely rare. Patients typically present with epigastric pain, diarrhea, constipation, or bleeding. Due to the non-specific symptoms and endoscopic findings, diagnosing GI 
amyloidosis in patients without a prior history of amyloidosis can be challenging and delayed. The endoscopic findings vary from polypoid protrusions to mucosal friability and erosions. Most common site of involvement is small intestine. Biopsy of the intestinal mucosa with positive Congo red staining confirms the diagnosis. Treatment usually is with supportive care for the specific symptoms, and the chemotherapy used in multiple myeloma may be administered to reduce the abundance of the amyloidogenic precursor protein to improve organ dysfunction. Weight loss is noted to be an independent poor predictor of survival. Hence, although rare, GI amyloidosis can present with intractable nausea and vomiting and should be considered in the differential diagnosis.

\section{"I'VE LIVED A GOOD LIFE"-ETHICAL DILEMMAS IN THE TREATMENT OF GERIATRIC PATIENTS WITH HIGH RISK SURGICAL CONDITIONS AND COGNITIVE IMPAIRMENT Jennifer Carnahan; Kurt J. Pfeifer. Medical College of Wisconsin, Milwaukee, WI. (Control ID \#1334863)}

LEARNING OBJECTIVE 1: Recognize appropriate circumstances to recommend aortic valve replacement.

LEARNING OBJECTIVE 2: Manage ethical dilemmas of surgical referral of geriatric patients with cognitive impairment

CASE: An 87-year-old male presented from home complaining of intermittent, exertional chest pain, associated with an increase in exertional dyspnea. He had known aortic stenosis, atrial fibrillation, diabetes, and mild cognitive impairment and had been hospitalized recently after sustaining several falls. He lived in his own home with a caretaker-friend who received free room and board from him in exchange for help with his activities of daily living. On physical exam, he had a loud systolic murmur at the left upper sternal border that radiated to the neck. He was delirious at admission and during much of his hospital course. An echocardiogram showed a normal left ventricular ejection fraction with an aortic valve area of 0.4$0.5 \mathrm{~cm} 2$ and a mean valve gradient of $29 \mathrm{mmHg}$. This represented a significant progression of his aortic stenosis. Cardiac catheterization showed three-vessel disease not amenable to stenting. When the possibility of coronary artery bypass graft $(\mathrm{CABG})$ and aortic valve replacement was introduced to this patient he responded, "I've lived a good life," and expressed a desire to not have surgery. He was assessed by the geriatrics consult team and deemed decisional. A family meeting was held and after much discussion with his family and caretaker, he reversed his decision and elected to have the aortic valve replacement and CABG. Two weeks after the surgery he died of disseminated intravascular coagulation.

DISCUSSION: Indicators for aortic valve replacement in severe aortic stenosis include heart failure, chest pain, and syncope. Without surgical intervention the average five-year survival rate for patients with severe symptomatic aortic stenosis is less than $50 \%$. Geriatric and surgical literature is rife with discussion on whether or not advanced age can or should be used as a criterion for or against surgery. However, pre-operative mental status is also an important consideration. On the one hand, our patient's mental status called into question his decisionality regarding the surgery. Assuming he was decisional, the acceptability of family influence or coercion is also a major concern. Our patient clearly had indications for surgery; however, whether that was the best decision for him is debatable. As generalists, we are often at the front line of decision-making for geriatric surgical candidates. Patients and their families solicit our opinions regarding surgery. We must take a patient's full medical picture into account when we make recommendations. More importantly, we must take into account more elusive considerations such as the patient's quality of life and assumed wishes. With geriatric patients we must also negotiate with patients' family members to arrive at a decision. Such complex issues are encountered daily but cannot be solved in a day.

"POLIOMYELITIS": BUT ...NOT DUE TO POLIO Irem Nasir greenwich hospital, Greenwich, CT. (Control ID \#1333244)

LEARNING OBJECTIVE 1: To recognize acute flaccid paralysis (AFP) as a symptom of West Nile Virus (WNV) neuroinvasive disease.

LEARNING OBJECTIVE 2: To name interferon A (INF A) as possible therapy for WNV neuroinvasive disease.
CASE: A 63 year old male, residing in Greenwich, CT, presented in late summer, with 1 week of low grade fevers, nonproductive cough, myalgias, frontal headache without photophobia, neck and low back pain. He had acute RLE weakness $\mathrm{x} 1$ day, that progressed to flaccid paralysis.He denied any bowel/bladder incontinence, saddle anesthesia, or sensory deficits. There was no dysphagia, gastrointestinal symptoms, or dysuria.He denied sick contacts. There was no history of IVDA or risky sexual behavior. He traveled to Canada 2 months prior.He did not recall any tick or mosquito bites. On exam, he was febrile at 101.4 F. He was fully conversant, nontoxic. His lung exam was normal. Cranial nerves 2-12 were intact with no nystagmus. His RLE was flaccid at $0 / 5$ strength. DTRs were absent in the R ankle and knee.Sensation was intact. Sphincter tone was normal. Labs revealed a WBC $7.8(82 \% \mathrm{PMN})$ and ESR 2. CXR was normal. CT and MRI brain were negative. Urgent MRI Cervicothoracolumbar spine was negative for abscess or abnormal enhancement. Spinal fluid had WBC 271, (71\% PMN, 24\% Lymphs), glucose 64 , protein 63 . CSF gram stain revealed many polys, no bacteria. Ceftriaxone and vancomycin were empirically started. CSF HSV 1 , 2, varicella, enterovirus PCR, and cryptococcal Ag were all negative. CMV, adenoviral, HSV, and varicella rectal cultures were negative. Blood and urine cultures were negative.Given the above symptoms, there was a high index of suspicion for WNV meningomyelitis. INF A therapy at 3 milliunits daily for 5 days was initiated. WNV IgM in CSF and serum resulted positive on day 5 of admission. He was discharged to rehab. After 4 months, he had persistent RLE paralysis.

DISCUSSION: WNV, a mosquito-borne RNA flavivirus, has the highest incidence in summer months. While $80 \%$ of WNV infections are subclinical, $20 \%$ have a self-limited febrile illness $\mathrm{w} /$ headache, fatigue, and rash (WN Fever), $<1 \%$ develop neuroinvasive disease (encephalitis, meningitis, and $\mathrm{AFP}$ ). AFP is the least common and results when there is direct spinal cord anterior horn (SCAH) cell destruction, resulting in an irreversible asymmetric "poliomyelitis-like syndrome" with severe long-term morbidity. On CSF initially, there can be a left shift pleocytosis, which then converts to lymphocytosis. Guillain-Barre, a postviral peripheral demyelinating syndrome, has been included in the differential, but this is symmetric. In a patient with the above symptoms, WN IgM in the CSF is diagnostic of acute neuroinvasive disease, indicating intrathecal synthesis. WNV PCR is highly specific, but not sensitive, and is used largely in the surveillance of birds and mosquitoes. MRI signal enhancement of SCAH has been noted in the literature, but the absence of such changes, as in our patient, does not preclude "poliomyelitis". Treatment for WNV neuroinvasive disease is largely supportive, and control of mosquito vectors may reduce incidence of human infections. Benefits for INF A have been seen only in case reports and are difficult to interpret due to a highly variable clinical course for WNV neuroinvasive disease. In endemic areas, clinicians must maintain a high index of suspicion that assymetric AFP without sensory deficits can be of spinal origin due to WNV. This will help prevent unnecessary diagnostics and therapy.

"PROSTATE CANCER AFFECTING THE LUNG WITHOUT BONY METASTASIS" VIKRAM CHABRA DO, EDISON GAVILANES MD, FARZIN RAHMANOU DO, KENNETH SHA MD NEW YORK HOSPITAL QUEENS, FLUSHING NY Vikram Chabra; Edison Gavilanes; Farzin Rahmanou; Kenneth Sha. New York Hospital Queens, Flushing, NY. (Control ID \#1334465)

LEARNING OBJECTIVE 1: Prostate adenocarcinoma is generally described as a slow growing malignancy that can metastasize in predictable order to other areas of the body. Bone and lymph nodes are commonly affected. Prostate cancer screening remains a debatable issue and is responsible for the detection of some cancers in asymptomatic patients. It is also among the differential for patients presenting with lower back pain, other bone pain, difficulty in urinating, and erectile dysfunction.

CASE: The patient is a 73 year old male with a history of Benign Prostatic Hyperplasia who presented with dry cough for three weeks associated with hoarseness of voice and anorexia. His other medical history included hypothyroidism, osteoarthritis, and type two diabetes mellitus. Prior to his admission he was evaluated by ear nose and throat and was placed on montelukast without relief. On physical exam he had a hoarse voice and clear lung fields. The balance of his history and exam were unremarkable with the 
exception that he had a family history of lung cancer in mother and sister, and gastric cancer in father. A chest CT showed innumerable bilateral pulmonary nodules some of which formed cavities. A CT of the abdomen/pelvis revealed an enlarged heterogeneous prostate measuring $6 \mathrm{~cm}$ in diameter which was correlated with a total PSA of 244 to make the presumptive diagnosis of metastatic prostate cancer. A subsequent nuclear medicine whole body bone scan however failed to reveal any evidence of metastatic bone disease. The patient underwent bronchoscopy followed by CT guided biopsy of the left lung. These specimens were CK7, CK 20, TTF-1, CDX-2 negative, and PSA positive. A diagnosis of metastatic adenocarcinoma consistent with prostate primary was made. The patient was started on bicalutamide for the treatment of his prostate cancer and was discharged home to follow up with his oncologist.

DISCUSSION: We report on the rare case presentation of metastatic prostate cancer with extensive lung pathology not involving the musculoskeletal system. Prostate cancer with metastasis to lung without metastasis to bone is rare. Although metastatic pathways in prostate cancer are not entirely understood, patterns of progression in prostate cancer are highly predictable. Metastasis to bone is the most common primary site and usually precedes metastasis to lung or any other organ.

“THERE WILL BE BLOOD” Shubhum Misra; Dhruti Patel; Halis Sonmez; Armen Simonion. Capital Health, Trenton, NJ. (Control ID \#1335764)

LEARNING OBJECTIVE 1: LEARNING OBJECTIVES: Symptomatic paraesophageal hernias account for less than $5 \%$ of all subtypes of hiatal hernias encountered during endoscopic evaluation of the upper GI tract. The major risk factor for developing a symptomatic paraesophageal hernia is morbid obesity due to increased intra-abdominal pressure. The major complications are severe anemia and strangulation of the esophagus, which is a surgical emergency.

CASE: CASE DESCRIPTION: This is a case of a 52-year-old African American male that presents with complaints of fatigue and progressively worsening dyspnea over 18 months. He denies melena, hematochezia, change in bowel habits, weight loss, dyspepsia, diarrhea, constipation, or hematemesis. The patient originally noticed easy fatigability, while working construction, but did not seek medical attention. Eventually the patient was laid off from work due to poor productivity and sought help from social services for public disability and unemployment benefits. The patient was referred to a PMD for routine lab work, which revealed severe anemia. The patient was immediately referred to our emergency department for acute care. At that time the $\mathrm{CBC}$ showed hemoglobin of 4.4, hematocrit of $16.8, \mathrm{MCV}$ of 60.3 , WBC of 4000, and platelets of 340,000. Iron studies were performed and reveled severe iron deficiency anemia. In addition, his reticulocyte count was $2.2 \%$, yielding a reticulocyte index of 0.33 . The initial physical exam of the patient was grossly normal except for BMI of 45 , grade $2 / 6$ systolic ejection murmur at the apex, mucosal pallor and weakly positive Heme-occult test. Subsequent fecal occult blood tests were negative three times, but a colonoscopy was performed to rule out an occult lower GI bleed and was normal. Next an esophogastroduodenoscopy was performed which showed a sliding type hiatal hernia with linear streaking pattern associated with submucosal aggregation of blood vessels and occult blood loss. It was decided that the hiatal hernia required further investigation with upper GI series with barium swallow, and this revealed a large type II sliding/paraesophageal hernia which was non-strangulating. The patient was transfused 6 units packed red blood cells and was discharged with follow-up for elective hernia repair and iron supplements.

DISCUSSION: DISCUSSION: Morbidly obese patients should have thorough examination of any hernias encountered during endoscopic evaluation of the upper gastro-intestinal tract in order to rule-out a potentially lethal complication such as paraesophageal hernia.

\section{CLINICAL PRACTICE INNOVATIONS}

A CLINICAL DECISION AID FOR HOSPITALISTS: SAFE DISCHARGE ASSESSMENT FOR LOW RISK CHEST PAIN ADMISSIONS Andrew McWilliams ${ }^{1}$; Christopher Caulfield ${ }^{1}$; Jonathan Weeks ${ }^{2}$; E. Allen Liles ${ }^{1}$; Jonathan Kirsch ${ }^{1}{ }^{1}$ University of North Carolina, Chapel Hill, NC; ${ }^{2}$ North Carolina State University, Raleigh, NC. (Control ID \#1340410)
STATEMENT OF PROBLEM OR QUESTION (ONE SENTENCE): There is a paucity of data to help guide hospitalists in risk stratifying patients who are low risk for Acute Coronary Syndrome (ACS) but are referred for admission with chest pain.

OBJECTIVES OF PROGRAM/INTERVENTION (NO MORE THAN THREE OBJECTIVES): This study aims to: further characterize a cohort of low-risk chest pain patients referred for admission to our hospitalist group; demonstrate current outcomes for this cohort, and to develop an efficient clinical decision aid. The primary outcome is 30 and 90-day cardiac event rate (MI, NSTEMI, unstable angina, revascularization, sudden unexplained death, or death from a cardiac cause). Secondary outcomes are length of stay, 30 day readmission and Emergency Department (ED) visit rates, and rates of significant non-ACS conditions at discharge.

DESCRIPTION OF PROGRAM/INTERVENTION, INCLUDING ORGANIZATIONAL CONTEXT (E.G. INPATIENT VS. OUTPATIENT, PRACTICE OR COMMUNITY CHARACTERISTICS): Chest pain accounted for 5.5 million ED visits in the United States during 2007-2008. While $13.0 \%$ of these patients had acute coronary syndrome (ACS), the majority did not. Correctly identifying patients without ACS, who can be expeditiously discharged home, continues to be a challenge. At our academic, suburban, tertiary care hospital, the hospitalist group is called upon to admit the majority of low risk chest pain patients. The hospitalists perform a secondary assessment (following that of the ED staff) of ACS risk. The opportunity to perform a secondary assessment of ACS risk prior to admission may uniquely position the hospitalist to identify a subset of patients that can be safely discharged. We retrospectively reviewed the charts of all patients referred for admission to the hospitalist service from the ED with chest pain as a chief complaint. Patients were excluded if they had a history of coronary artery disease, previous myocardial infarction, or age $>70$ years.

MEASURES OF SUCCESS (DISCUSS QUALITATIVE AND/OR QUANTITATIVE METRICS WHICH WILL BE USED TO EVALUATE PROGRAM/INTERVENTION): Our database (including patients' demographic data, presenting symptoms, laboratory and clinical data, discharge data, and 30 and 90 day outcomes data) will allow for outcomes analysis on the cohort as well as multivariate analysis of risk factors and prediction modeling. Our study is designed with $80 \%$ power to show a $0.1 \%$ adverse event rate (goal $n=300$ patients).

FINDINGS TO DATE (IT IS NOT SUFFICIENT TO STATE "FINDINGS WILL BE DISCUSSED"): Data collection is currently in process. The average age of our preliminary cohort (current $n=65$ patients) was 53 years, $49.2 \%$ had hypertension, $75.4 \%$ had diabetes, $78.5 \%$ had hyperlipidemia, $52.3 \%$ were smokers and $51.0 \%$ had a family history of coronary artery disease. Of these, $78.5 \%$ had chest pain not relieved by rest or nitroglycerin and $69 \%$ had chest pain not worsened by exertion. The average length of stay for our cohort was 47.9 hours. Thirty and ninety day readmission/ED visit rates were $20 \%$ and $23.1 \%$ respectively. Cardiac event rates will be further verified with follow-up phone call interviews.

KEY LESSONS FOR DISSEMINATION (WHAT CAN OTHERS TAKE AWAY FOR IMPLEMENTATION TO THEIR PRACTICE OR COMMUNITY?): Outcomes data and prediction rules are key resources to help guide physicians making a bedside determination of the need for hospital admission. Our research will help inform the decision making process for hospitalists performing secondary assessments of patients with low-risk chest pain.

A COLLABORATIVE EFFORT BETWEEN A PATIENT CENTERED MEDICAL HOME AND A PATIENT ADVISORY GROUP TO CREATE A PATIENT GUIDE TO SERVICES Scott Joy; Henry Wooten; Lynn Daugherty; Marilyn Hartman; Ron Witt; George Snowden; Elizabeth Domingoes. Duke University, Durham, NC. (Control ID \#1327085)

STATEMENT OF PROBLEM OR QUESTION (ONE SENTENCE): TO take advantage of all the services offered by a Patient Centered Medical Home (PCMH), patients must first be aware of current services offered, understand how to best access those services, and be given the opportunity to provide input into the development and description of the services offered. 
OBJECTIVES OF PROGRAM/INTERVENTION (NO MORE THAN THREE OBJECTIVES): 1. Identify key services and processes offered by the PCMH 2. Create a Patient Advisory Board (PAB) where patient advisors would discuss relevant topics with PCMH leadership 3. Create a document resulting from this collaborative communication that empowers patients to optimize clinical services

DESCRIPTION OF PROGRAM/INTERVENTION, INCLUDING ORGANIZATIONAL CONTEXT (E.G. INPATIENT VS. OUTPATIENT, PRACTICE OR COMMUNITY CHARACTERISTICS): Patients need to be aware of services offered by a PCMH, and PCMH leadership need patient's perspectives regarding their understanding of these services, if the processes to access these services need to be improved, or if new services need to be offered. Clinical services and processes in need of review were identified, including after- hours care, follow-up after ER, urgent care visit, hospitalization, contacting a care manager, scheduling an appointments, receiving test results, using an online patient portal, managing medication refills, requesting copies of medical records and completion of forms, and obtaining referrals . A PAB was created by asking each provider in the practice to nominate a patient who they felt would be an active contributor in a group setting and had an online patient portal account and e-mail address. Nominated patients were contacted by the practice medical director, and those who agreed to participate were invited to meet every 2 months for 2 hours at the practice site. Patient advisors, practice medical director, health center administrator, nurse manager, and clerical manager attended the meetings. Creating a patient guide to services was determined to be the best starting project, as it created a framework for discussion, all participants had experience with the services and processes being discussed, and the effort would result in a tangible product (printed brochure).

MEASURES OF SUCCESS (DISCUSS QUALITATIVE AND/OR QUANTITATIVE METRICS WHICH WILL BE USED TO EVALUATE PROGRAM/INTERVENTION): 1 . Establish a PAB and create an ongoing forum for collaboration and discussion between patient advisors and PCMH leadership 2. Create a patient-centered brochure that outlines the services offered by a PCMH and describes the optimal processes that will empower patients to access these services

FINDINGS TO DATE (IT IS NOT SUFFICIENT TO STATE "FINDINGS WILL BE DISCUSSED"): 1. A total of 6 hours (3 meetings) were required for 8 patient advisors and PCMH leadership to discuss, revise, edit, and create the patient guide to services 2. A brochure was created that listed the practice address, phone/fax numbers and e-mail address, and described the optimal processes for requesting medication refills, lab/x-ray results, and copies of medical records. 3. Methods for appropriate use of telephone triage and care management, accessing afterhours care, and follow-up after ER, urgent care and inpatient hospitalization were also described. 4 . Initial printing was 10,000 brochures, and the brochures are widely available in the practice waiting room and exam rooms for distribution to all patients.

KEY LESSONS FOR DISSEMINATION (WHAT CAN OTHERS TAKE AWAY FOR IMPLEMENTATION TO THEIR PRACTICE OR COMMUNITY?): Creating a patient guide to services is an excellent initial opportunity for engaging a Patient Advisory Board and promotes a sense of communication, collaboration, empowerment, and accomplishment for all parties involved.

A COMPARISON OF REFERRAL MODALITIES FOR DISEASE MANAGEMENT PROGRAMS IN A PRIMARY CARE SETTING Scott Joy ${ }^{1}$; Patrick J. Maheney ${ }^{2}$; William Schiff ${ }^{2}$; Peter D. Jacobi ${ }^{2}$; Christina L. Crosby ${ }^{2}{ }^{1}$ Duke University, Durham, NC; ${ }^{2}$ Duke University, Durham, NC. (Control ID \#1327124)

STATEMENT OF PROBLEM OR QUESTION (ONE SENTENCE): What is the most effective referral process in primary care (PC) to enroll patients in an external disease management (DM) program?

OBJECTIVES OF PROGRAM/INTERVENTION (NO MORE THAN THREE OBJECTIVES): 1.Develop and offer multiple methods for referral from $\mathrm{PC}$ to an external DM program, including telephone referral, written prescription, or electronic referral 2.Evaluate the effectiveness of different options by determining number of referrals given using each modality 3.Compare referral rates/enrollment into DM program after 1 year of implementation

DESCRIPTION OF PROGRAM/INTERVENTION, INCLUDING ORGANIZATIONAL CONTEXT (E.G. INPATIENT VS. OUTPATIENT, PRACTICE OR COMMUNITY CHARACTERISTICS): As part of a basic benefit package, DM services are often offered to patients, but may be underutilized. Many DM programs are centrally based, and receive referrals from multiple practices in an area. This often results in a disconnect between patient, provider, practice and DM service. Improving the referral process into DM programs is thus paramount to optimizing use of these programs. Our DM program is available to 39,566 covered lives as an employee benefit. The DM services are marketed to participating PC practices in multiple ways, including quarterly practice visits, all provider meetings, and staff/provider in-services. However, sustaining referrals has been a challenge. With collaborative input from health plan and practice administrators, physicians, and practice and DM nurses, a menu of options for referrals was developed. 1.Telephone Referral: PC practice staff would call the DM team with referrals, and the DM team would then contact the patient to schedule. 2.Written Prescription: Prescription pads were given to each PC practice to be used by providers at the point of care for patients deemed interested or suitable for DM. The patient's name would be written on the prescription that included a contact phone number for the DM program that the patient could call to enroll. A carbon copy of each prescription was also generated and was placed in a box for members of the DM team to collect who would also then proactively contact the patient by telephone to schedule. 3.Electronic Referral: An electronic process was developed within the electronic health record (EHR, McKesson HAC) that allowed a PC provider/nurse to electronically send a DM referral to the DM team. Upon receiving the referral, DM care managers would contact the patient, and sent an electronic message back to the referring $\mathrm{PC}$ provider.

MEASURES OF SUCCESS (DISCUSS QUALITATIVE AND/OR QUANTITATIVE METRICS WHICH WILL BE USED TO EVALUATE PROGRAM/INTERVENTION): 1 . Evaluate uptake of different methods for referrals into DM program 2. Increase in overall number of referrals to DM from previous year

FINDINGS TO DATE (IT IS NOT SUFFICIENT TO STATE "FINDINGS WILL BE DISCUSSED"): Type of referral and number of each referral submitted for 2010 and 2011: Telephone: 1 in 2010, 11 in 2011: Written Prescription: 31 in 2010, 41 in 2011: Electronic: 0 in 2010, 267 in 2011: Total: 32 in 2010, 319 in 2011 Telephone referrals decreased dramatically over the year as electronic referrals were implemented 7 patients were eligible for and enrolled in high level DM programs in 2010, 126 patients were eligible and enrolled in 2011

KEY LESSONS FOR DISSEMINATION (WHAT CAN OTHERS TAKE AWAY FOR IMPLEMENTATION TO THEIR PRACTICE OR COMMUNITY?): 1.Multiple referral processes can improve engagement and enrollment in DM programs 2.Electronic referrals were shown to be the most common method for referral into the DM program, suggests that integrating DM care managers into the electronic workflow of providers and practices is an effective method to refer patients to DM programs.

A HUB AND SATELLITE MODEL TO IMPROVE THE QUALITY OF CARE FOR PATIENTS WITH CONGESTIVE HEART FAILURE Michael E. Bowen ${ }^{1,3}$; Christianne Roumie ${ }^{1,3}$; Ann Minnick ${ }^{4}$; Beth Donaghey ${ }^{4}$; Amy S. Wilson ${ }^{5}$; Cynthia A. Fink ${ }^{2}$; Henry Ooi ${ }^{2} .{ }^{1}$ VA Tennessee Valley Healthcare System, Nashville, TN; ${ }^{2}$ VA Tennessee Valley Healthcare System and Vanderbilt University Medical Center, Nashville, TN; ${ }^{3}$ Vanderbilt University Medical Center, Nashville, TN; ${ }^{4}$ Vanderbilt University School of Nursing, Nashville, TN; ${ }^{5}$ VA Tennessee Valley Healthcare System, Nashville, TN. (Control ID \#1336642)

STATEMENT OF PROBLEM OR QUESTION (ONE SENTENCE): Evidence-based treatments for congestive heart failure (CHF) can modify disease progression and reduce admissions; however, a quality gap between current and desired practice exists.

OBJECTIVES OF PROGRAM/INTERVENTION (NO MORE THAN THREE OBJECTIVES): 1 . To provide CHF management support to community primary care providers (PCPs) from a regional CHF team 2 . To determine model impact on CHF performance metrics 3. To determine model acceptability and facilitators/barriers to implementation 
DESCRIPTION OF PROGRAM/INTERVENTION, INCLUDING ORGANIZATIONAL CONTEXT (E.G. INPATIENT VS. OUTPATIENT, PRACTICE OR COMMUNITY CHARACTERISTICS): The model consisted of a multidisciplinary CHF team (heart failure physician, nurse practitioner, and pharmacist) at a regional "hub" center that provided clinical support to 6 PCPs in 3 community "satellite" clinics within the Veterans' Health Administration. The model consisted of 4 components: 1) A 3 day CHF management course; 2) Availability of a clinical pharmacist to assist in medication titration; 3) Open-access communication with the CHF "hub" team; 4) Quarterly performance feedback to PCPs. CHF patients with an ejection fraction $<40 \%$ were included in a before-after analysis. CHF patients followed by non-study providers in the same clinics formed a concurrent control group.

MEASURES OF SUCCESS (DISCUSS QUALITATIVE AND/OR QUANTITATIVE METRICS WHICH WILL BE USED TO EVALUATE PROGRAM/INTERVENTION): Nine CHF quality metrics were assessed by chart review at baseline and 12 months after the intervention. Metrics included: weight, activity and volume assessment, use of ACE-inhibitor/ $\mathrm{ARB}$ and beta blockers, and the percent target dose achieved. Coumadin use in atrial fibrillation and use of evidence based beta blockers were also measured. Structured interviews were conducted with study providers prior to training and at study conclusion.

FINDINGS TO DATE (IT IS NOT SUFFICIENT TO STATE "FINDINGS WILL BE DISCUSSED"): Two hundred fifty-eight patients (129 intervention; 129 control) followed by 32 PCPs (6 intervention; 26 control) were followed from May 2010-May 2011. Patient characteristics and performance on quality metrics were similar between groups at baseline and after intervention. Performance exceeded $80 \%$ on 5 metrics at baseline including: weight, volume, use of $\mathrm{ACE} / \mathrm{ARB}$, beta blockers, and evidence based beta blockers. The subgroup referred for pharmacist-led medication management improved performance on evidence based betablocker use and beta blocker target dose; however, less than $20 \%$ of eligible patients were referred. Intervention PCPs saw CHF patients only twice during the study and contacted the CHF team a total of 3 times. More than $85 \%$ of study patients were followed by a cardiologist, and PCPs cited perceptions that patients were already "in the system" as a key barrier to utilizing the program. Interviews found high satisfaction with the educational training and performance feedback. PCPs reported that the program increased their confidence and ability to manage complex CHF patients.

KEY LESSONS FOR DISSEMINATION (WHAT CAN OTHERS TAKE AWAY FOR IMPLEMENTATION TO THEIR PRACTICE OR COMMUNITY?): The model enhanced PCP confidence in CHF management, and PCPs were receptive to education and performance feedback. High performance on quality metrics at baseline and infrequent PCP visits may limit opportunities for improvement. Contamination of the control group by the intervention may have limited the ability to detect a difference. Improved implementation strategies are needed to increase provider engagement. Inclusion of pharmacists in CHF management teams may improve performance on medication measures. Greater understanding of roles and responsibilities among members of the CHF care team may enhance outcomes of disease management programs.

A SUCCESSFUL PDSA: IMPROVING COMPLIANCE WITH PLATELET TRANSFUSION GUIDELINES. Elena Katz; Malgorzata Klek; Randy Levine; Robert E. Graham. Lenoxhill Hospital, New York, NY. (Control ID \#1318228)

STATEMENT OF PROBLEM OR QUESTION (ONE SENTENCE): How can physician compliance with up-to-date platelet transfusion guidelines be improved?

OBJECTIVES OF PROGRAM/INTERVENTION (NO MORE THAN THREE OBJECTIVES): To improve physician compliance with up-to-date platelet transfusion guidelines through an educational intervention. DESCRIPTION OF PROGRAM/INTERVENTION, INCLUDING ORGANIZATIONAL CONTEXT (E.G. INPATIENT VS. OUTPATIENT, PRACTICE OR COMMUNITY CHARACTERISTICS): All patients receiving platelet transfusions over a five-month period from Jan-Feb, and April-June 2010, admitted to the medical, critical care and cardiac services were reviewed. The medical record clinical indication was then evaluated against the ASH 2007 "Evidence-Based Platelet Transfusion Guidelines" (Slichter SJ. Hematology 2007): bleeding and platelets $\leq 50,000 / \mu \mathrm{L}$, preinvasive procedure and platelets $\leq 50,000 / \mu \mathrm{L}$, prophylactic transfusion for platelets $\leq 10,000 / \mu \mathrm{L}$ and $\mathrm{WHO}$ bleeding grade $\geq 2$. We also assessed how the patients' clinical indication met the previous prophylactic threshold for platelet transfusion of $\leq 20,000 / \mu \mathrm{L}$. Following initial data collection, we implemented an educational intervention by giving a lecture, reviewing all indications for platelet transfusions, and distributing a pocket card to the house-staff on the medical wards in August, 2010. We subsequently gathered post-intervention data following the methods described above for four consecutive months from Sept-December, 2011.

MEASURES OF SUCCESS (DISCUSS QUALITATIVE AND/OR QUANTITATIVE METRICS WHICH WILL BE USED TO EVALUATE PROGRAM/INTERVENTION): Descriptive statistics were collected on the pre and post intervention groups and data analysis was conducted using a Z-test with $\alpha=0.05$ and $Z$ critical value $=1.96$.

FINDINGS TO DATE (IT IS NOT SUFFICIENT TO STATE "FINDINGS WILL BE DISCUSSED"): Eighty-six patients on the selected units received a total of 241 platelet transfusions pre-intervention and 81 patients received a total of 237 platelet transfusions postintervention. The patient's clinical indication met the old guidelines in $63 \%$ of cases pre-intervention and in $81 \%$ of cases post-intervention with a significant $\mathrm{p}$-value of $<0.05$. The patient's clinical indication met the currently accepted 2007 ASH guidelines in $41 \%$ of cases pre-intervention and in $53 \%$ of cases post-intervention with a significant p-value of $<0.05$. KEY LESSONS FOR DISSEMINATION (WHAT CAN OTHERS TAKE AWAY FOR IMPLEMENTATION TO THEIR PRACTICE OR COMMUNITY?): Based on the significant difference observed between the pre- and post-educational intervention groups, updating physicians on current evidence-based guidelines for platelet transfusions is a simple and effective means to improve transfusion practices. Periodic educational interventions and their assessment may prove to even further enhance physician compliance with up-to-date guidelines.

\section{A COMPREHENSIVE DISCHARGE TRANSITION INTERVENTION} REDUCES READMISSIONS IN A GENERAL MEDICINE POPULATION Christopher Wong ${ }^{1}$; Andrew White ${ }^{1}$; Susan Merel ${ }^{1}$; Carol Charles ${ }^{2}$. ${ }^{1}$ University of Washington, Seattle, WA; ${ }^{2}$ University of Washington, Seattle, WA. (Control ID \#1332748)

STATEMENT OF PROBLEM OR QUESTION (ONE SENTENCE): We present the results of a quality improvement initiative to reduce readmissions after discharge from the general medicine service at an academic hospital.

OBJECTIVES OF PROGRAM/INTERVENTION (NO MORE THAN THREE OBJECTIVES): 1 . Reduce the 30 day readmission rate of the general medicine service. 2. Improve intermediate outcomes pertaining to optimal transitions of care, including discharge summary completion rates by housestaff.

DESCRIPTION OF PROGRAM/INTERVENTION, INCLUDING ORGANIZATIONAL CONTEXT (E.G. INPATIENT VS. OUTPATIENT, PRACTICE OR COMMUNITY CHARACTERISTICS): We applied the following multi-modality intervention to a general medicine service at an urban academic hospital: 1. Enhanced primary care provider (PCP) communication via a team assistant. 2. Housestaff education through a monthly teaching session pertaining to transitions of care, daily interaction with a nurse care coordinator to discuss discharge planning, and a discharge (DC) summary timeliness intervention utilizing provider-level real-time data. 3. Enhanced follow up via a team assistant arranging appointments and nursing follow up phone calls through a parallel hospital initiative. 4. Medication reconciliation and an improved "patient-friendly" medication list on discharge. 5. Nurse care coordination, including care plans for frequently readmitted patients.

MEASURES OF SUCCESS (DISCUSS QUALITATIVE AND/OR QUANTITATIVE METRICS WHICH WILL BE USED TO EVALUATE PROGRAM/INTERVENTION): 1.30 day readmission rate for the General Medicine Service. 2. Intermediate measures: \% DC summaries complete within 48 hours of discharge, percentage of PCPs identified and 
contacted on admission, percentage of DC Summaries sent to PCPs, \% follow up appointments completed within 21 days of discharge, \% medication reconciliation on admission and on discharge, use of Patient friendly medication lists, provider feedback regarding nurse care coordination.

FINDINGS TO DATE (IT IS NOT SUFFICIENT TO STATE "FINDINGS WILL BE DISCUSSED"): The baseline 30 day readmission rate for the General Medicine Service was $18.3-18.7 \%$ for the preceding 2 years. Post intervention, the 30 day readmission rate decreased to $14.5 \%$, consisting of 3 consecutive months of readmission rates lower than the pre-intervention baseline. Secondary outcome measures showed improvements in PCP identification (up to $100 \%$ of eligible patients), discharge summaries sent to PCPs (up to 96\%), discharge summary timeliness (98$100 \%$, most showing improvement pre and post receipt of individual performance data), follow up appointment completion ( $90 \%$ at 21 days), and follow up phone calls made (up to $83 \%$ ). Medication reconciliation remained at a high baseline (96-100\%).

KEY LESSONS FOR DISSEMINATION (WHAT CAN OTHERS TAKE AWAY FOR IMPLEMENTATION TO THEIR PRACTICE OR COMMUNITY?): A multi-modality intervention focused on improving care transitions lowered 30 day readmission rates in a general medical population cared for predominantly by medical residents. Behavior change among physicians was able to be achieved by providing the rationale for the promoted behavior (teaching key concepts of transitions of care) combined with an accurate and timely feedback mechanism (report of discharge summary timeliness). Quality metrics for PCP communication and follow up showed high levels of success but less reliabilitydiminished intermediate outcomes during team assistant absences revealed a gap in the improvement intervention. Future directions include diagnosisspecific interventions, improving patient education via additional teachback training, improving written patient discharge materials, development of patient coaching, improving rates of patient follow up calls, and increasing primary care clinic involvement. Behavior change interventions will continue to require adequate training and feedback mechanisms.

A SUCCESSFUL ROLE FOR A "PAIN PHARMACIST" IN A LARGE ACADEMIC TEACHING PRACTICE Lori Tishler ${ }^{1}$; Michele Matthews ${ }^{3,2}$; Edgar Ross ${ }^{2}$; Robert Jamison ${ }^{2} .{ }^{1}$ Brigham and Women's Hospital, Boston, MA; ${ }^{2}$ Brigham and Women's Hospital, Boston, MA; ${ }^{3}$ Mass College of Pharmacy and Health Sciences, Boston, MA. (Control ID \#1310763)

STATEMENT OF PROBLEM OR QUESTION (ONE SENTENCE): How can teaching practices help residents and staff learn the best practices for caring for patients with chronic pain?

OBJECTIVES OF PROGRAM/INTERVENTION (NO MORE THAN THREE OBJECTIVES): 1 . To help patients with chronic pain in a familiar setting. 2. To help residents and faculty learn best practices for monitoring patients on opioids. 3. To facilitate team-based care.

DESCRIPTION OF PROGRAM/INTERVENTION, INCLUDING ORGANIZATIONAL CONTEXT (E.G. INPATIENT VS. OUTPATIENT, PRACTICE OR COMMUNITY CHARACTERISTICS): Our practice is a large academic teaching practice. Like many teaching practices, we are challenged by patients with chronic pain. Our desire to help treat their pain is balanced by our concerns about addiction, aberrant behavior, and drug diversion. While we have a wonderful consultative pain service, the care of these patients is often best done in the practice. Yet, particularly for newer physicians, at both the resident and faculty level, this care can be quite challenging. Working together with our pain service, we discussed ways that we might improve patient care. We have had success with a clinical pharmacist for co-management of chronic diseases and ultimately, the Jen Center and the pain service decided to try embedding a pharmacist with particular interest in chronic pain into our practice. Our "pain pharmacist" comes to the practice one afternoon/week, where she is free to meet with patients as well as consult with residents and staff. In her downtime in our practice, she is reviewing our log of patients on chronic opioids and helping to make suggestions for better processes and protocols. When she is not in the practice, she easily and quickly consults with residents, faculty, and staff via phone or e-mail. She has integrated well and it's made a huge impact on our practice and resident experience.
MEASURES OF SUCCESS (DISCUSS QUALITATIVE AND/OR QUANTITATIVE METRICS WHICH WILL BE USED TO EVALUATE PROGRAM/INTERVENTION): We will use the following measures to help us determine success of the program. These questions speak to both the need for improved knowledge and process. 1. Treatment of chronic pain is an issue in my practice (pretest response was 53/59 respondents agreed) 2. I am confident in my ability to manage chronic pain (pretest: 22/ 59 respondents agreed) 3. I always follow a defined protocol when I prescribe opioids. (pretest:14/59 respondents agreed) 4 . I am afraid that my patients will become addicted if I prescribe chronic opioids (38/59 respondents agreed)

FINDINGS TO DATE (IT IS NOT SUFFICIENT TO STATE "FINDINGS WILL BE DISCUSSED"): While our post intervention study has not yet been completed, we have found that the embedded pain pharmacist has been quite successful in the following areas: 1. The availability of a real-time consultant has been extremely helpful in guiding us towards best practice. We see, for example, more appropriate use of urine screens and pain agreements in the practice. 2. As we move toward a PCMH model, the embedded pharmacist has been an excellent example of collaborative, team-based care. 3. An embedded pain pharmacist in primary care has helped enhance communications between our practice and the pain service.

KEY LESSONS FOR DISSEMINATION (WHAT CAN OTHERS TAKE AWAY FOR IMPLEMENTATION TO THEIR PRACTICE OR COMMUNITY?): Key lessons from our project to date include: 1 . There is tremendous value in working collaboratively with specialty services, but keeping care based within a primary care practice. 2 . Without adding FTE resources, we can provide improved care for patients with chronic pain who use opioids. 3. There is tremendous need for improved care and communication to allow primary care physicians to manage opioids independently and competently.

ADDRESSING POOR DIABETIC CONTROL IN AN UNDERSERVED POPULATION WITHIN THE FRAMEWORK OF A PATIENTCENTERED MEDICAL HOME Sarah Richards; Thomas G. Tape; Rachel Bonnema; Andrew Vasey. University of Nebraska Medical Center, Omaha, NE. (Control ID \#1340426)

STATEMENT OF PROBLEM OR QUESTION (ONE SENTENCE): There are several barriers to care that inhibit adequate diabetes management in low-income patients.

OBJECTIVES OF PROGRAM/INTERVENTION (NO MORE THAN THREE OBJECTIVES): 1. Improve access to care 2. Improve patient education 3. Improve overall diabetes management DESCRIPTION OF PROGRAM/INTERVENTION, INCLUDING ORGANIZATIONAL CONTEXT (E.G. INPATIENT VS. OUTPATIENT, PRACTICE OR COMMUNITY CHARACTERISTICS): Midtown Clinic is a resident-run Internal Medicine clinic that functions as a PatientCentered Medical Home. Over $32 \%$ of patients serviced are self-pay or Medicaid. The clinic board of directors has developed several innovative programs within the context of the PCMH model to help our low-income diabetic patients. 1. Improved Access to Care: Internal Medicine residents raise several thousand dollars per year via a department-wide raffle. Our full-time social worker uses a portion of these funds to ensure that patients have transportation to and from their clinic appointments by providing free bus tickets or paying for their taxi fare. The clinic is also involved in a collaborative project with the College of Nursing in which nursing students do post-hospital discharge home visits. The nursing students ensure our patients know about their follow-up appointment and the transportation options available. 2. Improved Patient Education: Our clinic employs a full-time on-site Diabetes Educator who provides same-day diabetes selfmanagement counseling. Additionally, we offer free, group Diabetes Education classes. We look forward to offering a free Diabetes Cooking Class in January 2012 as part of a collaborative project with the dietetic students. The aforementioned classes are made possible completely via donations and volunteers. 3. Improve Overall Diabetes Management: We provide several generic medications to patients free of charge. Specifically, the clinic appropriates donations and fundraising dollars to buy metformin, glipizide and lisinopril as well as meters in bulk at a reduced cost. Our social 
worker also helps our patients with the paperwork necessary to obtain insulin via patient assistance programs.

MEASURES OF SUCCESS (DISCUSS QUALITATIVE AND/OR QUANTITATIVE METRICS WHICH WILL BE USED TO EVALUATE PROGRAM/INTERVENTION): 1 . Failed appointment rates are tracked on a monthly basis. 2. Surveys are sent to patients after they attend our diabetes education classes. 3. Hgb A1C, LDL and systolic blood pressure readings of diabetic patients have been tracked over the last three years.

FINDINGS TO DATE (IT IS NOT SUFFICIENT TO STATE "FINDINGS WILL BE DISCUSSED"): 1. Failed appointment rates have dropped from $15.3 \%$ prior to July 2011 to a current rate of $12.7 \%$. 2 . Patients have reported a high level of satisfaction after attending education classes. 3. Among our diabetic patients, the percentage of patients with a $\mathrm{Hgb} \mathrm{A} 1 \mathrm{C}$ level greater than $9 \%$ has dropped from $20.4 \%$ to $20.1 \%$ in the last three years. The percentage of systolic blood pressure readings greater than 140 has dropped from $30.7 \%$ to $28.6 \%$. Further, average LDL levels have dropped from $94.6 \mathrm{mg} / \mathrm{dl}$ to $87.6 \mathrm{mg} / \mathrm{dl}$ over the last three years. We are awaiting new data from the last 6 months and expect an even greater improvement in these measures.

KEY LESSONS FOR DISSEMINATION (WHAT CAN OTHERS TAKE AWAY FOR IMPLEMENTATION TO THEIR PRACTICE OR COMMUNITY?): 1. Identify barriers to care among low-income diabetic patients 2 . Consider fundraising as a means to provide patients with free or reduced cost medications and transportation. 3. Invest in collaborative and multidisciplinary projects

AN INNOVATIVE AND TRANSPARENT ELECTRONIC HAND-OFF MODEL TO IMPROVE PATIENT SAFETY Mukesh Gopalakrishnan; Federico Silva-Palacios; Mohammed Samee. Advocate Illinois Masonic Medical Center, Chicago, IL. (Control ID \#1317418)

STATEMENT OF PROBLEM OR QUESTION (ONE SENTENCE): Recent ACGME resident duty hour guidelines increased frequency of transfers in patient care thus imposing the need to create an efficient, safe and standardized hand-off process.

OBJECTIVES OF PROGRAM/INTERVENTION (NO MORE THAN THREE OBJECTIVES): 1. Improve Patient Safety - The clinical handover is one of five key areas identified under the World Health Organization Alliance for Patient Safety High $5 \mathrm{~s}$ Project. 2. Improve efficiency - Verbal handovers are associated with loss of all data, written handovers lose $31 \%$ of data, and a combination of written and verbal handover results in minimal loss of data. The medical model of handover stresses on standardization and a minimal dataset.

DESCRIPTION OF PROGRAM/INTERVENTION, INCLUDING ORGANIZATIONAL CONTEXT (E.G. INPATIENT VS. OUTPATIENT, PRACTICE OR COMMUNITY CHARACTERISTICS): Ours is a 440bed community based teaching hospital with resident teams following patients from admission to discharge. A cross-cover or night float team takes care of overnight events, with communication between the teams established via this electronic tool. Our focus was to create an electronic standardized handover tool that avoids redundancy and provides consistent, easy access to data while improving patient safety and decreasing errors during transitions of care. The goal was to transfer information on patient presentation, current status, and anticipatory guidance. This custom-built HIPAA-compliant online module was created within Microsoft SharePoint ${ }^{\mathbb{R}}$. Built-in automation pulls patient demographics and allows for simultaneous viewing and editing capability by all care providers including physicians, nursing, and pharmacy staff. During the identification of handover fields to be included in the module, key areas common to different departments were identified. This led to the creation of a collaborative electronic handover to augment verbal transaction.

MEASURES OF SUCCESS (DISCUSS QUALITATIVE AND/OR QUANTITATIVE METRICS WHICH WILL BE USED TO EVALUATE PROGRAM/INTERVENTION): Adverse patient safety events were monitored as a measure of success with initial goal at implementation being to maintain patient safety at the same level as before implementation. User feedback was taken into consideration to assess the user friendly nature of this module and make updates.
FINDINGS TO DATE (IT IS NOT SUFFICIENT TO STATE "FINDINGS WILL BE DISCUSSED"): Allowing for a team-based approach streamlined care and provided a complete picture of the patient. The electronic clinical handover successfully identified unstable patients and clinical concerns, forwarded pending tasks, and provided anticipatory guidance. Furthermore, psychological and social needs could be addressed. Patient safety events did not show any deterioration after implementation and are being monitored to ensure further improvement. Feedback from users showed increasing familiarity with the module improved efficiency with less time being spent on preparing a hand off.

KEY LESSONS FOR DISSEMINATION (WHAT CAN OTHERS TAKE AWAY FOR IMPLEMENTATION TO THEIR PRACTICE OR COMMUNITY?): Our solution provides a standardized and a streamlined dataset, collaboration within the patient care team, and an electronic platform that is HIPAA-compliant. This defines and establishes a separation between the handover and the clinical content in electronic medical records. This provides a consistent handover process, yet affords flexibility for mobile solutions. Technology allows for advanced capabilities, such as instant messaging, web-based group meetings, and audio and video uploads, making this method truly distinctive. Our tool lays the foundation for a reliable patient-centric handover. This unique implementation of teambased approach is innovative in the realm of patient safety. Our copyrighted application will be expanded to other institutions within our network.

AN INNOVATIVE MODEL FOR HIGH QUALITY, LOW COST, END OF LIFE CARE Kristy Deep. Univ of Kentucky, Lexington, KY. (Control ID \#1340432)

STATEMENT OF PROBLEM OR QUESTION (ONE SENTENCE): A large proportion of Americans die in acute care hospitals where care is expensive, fragmented, and provides inadequate pain and symptom management.

OBJECTIVES OF PROGRAM/INTERVENTION (NO MORE THAN THREE OBJECTIVES): Provide higher quality, lower cost care for dying adult patients and their families using a hospice model.

DESCRIPTION OF PROGRAM/INTERVENTION, INCLUDING ORGANIZATIONAL CONTEXT (E.G. INPATIENT VS. OUTPATIENT, PRACTICE OR COMMUNITY CHARACTERISTICS): Our 473 bed academic medical center partnered with a free-standing community hospice to develop an inpatient hospice service to offer expert level care for actively dying patients. Inpatient hospice services are provided throughout several hospital units. The hospice team includes 0.5 FTE palliative physician (who becomes attending of record) and 1 FTE nurse, social worker, and chaplain. These team members are employed by the hospice and work alongside hospital staff. Patients actively dying or terminally ill with uncontrolled symptoms are referred for hospice admission by their primary admitting service. If their care meets the requirements for inpatient hospice under Part A Medicare Hospice Benefit (MHB), they are administratively discharged from acute care and admitted with a new encounter to inpatient hospice while remaining in the same facility. Under the MHB, a per diem rate $(\$ 623)$ is paid to the hospice with a portion (67\%) passed through to the hospital. A similar structure applies for Medicaid and commercial insurers.

MEASURES OF SUCCESS (DISCUSS QUALITATIVE AND/OR QUANTITATIVE METRICS WHICH WILL BE USED TO EVALUATE PROGRAM/INTERVENTION): Administrative measures include average daily census (ADC) and average length of stay (ALOS). We calculate a capture rate defined as the proportion of expected adult deaths admitted to inpatient hospice when documentation indicated that the patient was receiving symptom focused care (code v66.7). Quality measures include symptom management ratings and family and referring provider satisfaction. Financial impact is assessed by cost avoidance compared to non-hospice comfort care deaths.

FINDINGS TO DATE (IT IS NOT SUFFICIENT TO STATE "FINDINGS WILL BE DISCUSSED"): The mean hospice admissions per month is 24 (range 19-35). ADC is 4 patients with ALOS of 5.74 days. Overall, $88 \%$ of hospice inpatients die with the remainder discharged home or to long term care with hospice. The monthly capture rate of comfort care deaths exceeds $40 \%$. Inpatient hospice deaths do not affect a hospital's 
mortality rate which has led to a statistically significant drop in our observed/expected mortality index $(\mathrm{YTD}=0.61)$. Surveys of bereaved family members show $91 \%$ rate their inpatient hospice care as excellent with $94 \%$ stating the patient received adequate pain management. The majority of referring physicians $(80 \%)$ rate ease of referral as excellent and $92 \%$ find the service helpful for their patients. The most common barrier identified was bed availability as only some units offer hospice. Financial analysis reveals substantially lower costs for hospice deaths compared to usual care expected deaths. We demonstrated a 54\% reduction in total costs for the patients' last 3 days of life (cost avoidance of $\$ 1204.45 /$ day). The greatest cost avoidance was seen in direct variable costs. This signifies the hospice team's decreased utilization of lab, pharmacy, imaging and other diagnostic or therapeutic services that were non-essential for supporting the patient's comfort.

KEY LESSONS FOR DISSEMINATION (WHAT CAN OTHERS TAKE AWAY FOR IMPLEMENTATION TO THEIR PRACTICE OR COMMUNITY?): A collaborative initiative between an acute care hospital and a community hospice led to highly rated patient and family centered care at a lower cost to both the hospital and society. This model requires little start-up investment and is highly generalizable.

AN INTERACTIVE, POINT-OF-CARE COMPUTER PROGRAM TO FACILITATE DIAGNOSIS AND TREATMENT OF FEMALE URINARY INCONTINENCE IN PRIMARY CARE Alison Huang; Ralph Gonzales; Anne Chang; Jeanette S. Brown. UCSF, San Francisco, CA. (Control ID \#1317557)

STATEMENT OF PROBLEM OR QUESTION (ONE SENTENCE): Urinary incontinence affects up to a quarter of adult women, but is underrecognized and under-treated in primary care. Patients may avoid seeking attention for incontinence because they are embarrassed or uncomfortable, while providers may lack time during clinic visits or may be unfamiliar with evidence-based diagnosis and treatment.

OBJECTIVES OF PROGRAM/INTERVENTION (NO MORE THAN THREE OBJECTIVES): 1) To develop a brief, interactive computer program designed for touch-screen computers in primary care waiting rooms to facilitate diagnosis and treatment for incontinence in women; 2) To evaluate the feasibility of integrating this program into two types of primary care practices (general medicine and general gynecology).

DESCRIPTION OF PROGRAM/INTERVENTION, INCLUDING ORGANIZATIONAL CONTEXT (E.G. INPATIENT VS. OUTPATIENT, PRACTICE OR COMMUNITY CHARACTERISTICS): A brief $(<10$ minute), point-of-care computer module was developed to guide female patients in self-diagnosing and self-classifying incontinence, assess their interest in treatment, and identify co-morbid problems that could complicate care. For women with weekly stress, urgency, or equallymixed incontinence and no co-morbidities requiring specialist referral (e.g., major neurologic condition, pelvic cancer or surgery), the module was designed to print out the results of the evaluation for their providers, along with recommendations for evaluation and treatment. After pretesting, the module was field-tested in the waiting rooms of an academic internal medicine practice and general gynecology practice, using a touch-screen tablet computer. Female patients (regardless of age) were invited to try the computer while waiting to see their providers; those who reported weekly, classifiable incontinence in the absence of major co-morbidities, expressed interested in treatment, and received provider printouts were invited to participate in a follow-up telephone call.

MEASURES OF SUCCESS (DISCUSS QUALITATIVE AND/OR QUANTITATIVE METRICS WHICH WILL BE USED TO EVALUATE PROGRAM/INTERVENTION): We tracked the numbers of patients trying the computer program who: 1) successfully completed the module in the waiting room; 2) self-identified as having any or weekly incontinence; and 3) self-diagnosed as having stress-, urgency-, and mixed-incontinence in the absence of co-morbidities requiring referral. Of women completing follow-up calls, we examined rates of patient-provider discussion about incontinence and patient perceptions of the module.

FINDINGS TO DATE (IT IS NOT SUFFICIENT TO STATE "FINDINGS WILL BE DISCUSSED"): During 60 half-days of fieldtesting, 371 women tried the computer program, and 206 (56\%) completed the program in the waiting room. Of these, $131(50 \%)$ reported any incontinence, and $61(30 \%)$ reported weekly incontinence in the past 3 months. Twenty-six (45\%) self-classified as having stress, $13(22 \%)$ as having urgency, and $12(21 \%)$ as having mixed incontinence. Of the women completing follow-up phone calls, $100 \%$ reported showing their printouts to their providers, and $80 \%$ reported discussing their incontinence at that visit. No women were referred to see a specialist for incontinence. Over $80 \%$ felt that the module had had a positive effect, and none felt that it had a negative effect on their visit.

KEY LESSONS FOR DISSEMINATION (WHAT CAN OTHERS TAKE AWAY FOR IMPLEMENTATION TO THEIR PRACTICE OR COMMUNITY?): An interactive, point-of-care computer program has the potential to overcome barriers to diagnosing and treating female incontinence in primary care, although systematic administration may be necessary to consistently identify women with symptoms. Assisting physicians and their patients in discussing incontinence may have important implications for women's quality of life.

AN OUTPATIENT INTRAVENOUS DIURESIS CLINIC AS AN INNOVATIVE APPROACH TO THE MANAGEMENT OF HEART FAILURE Sunal Makadia ${ }^{1}$; Ugonna Nwosu ${ }^{1}$; Tanya Simmons ${ }^{1,2}$; Nowreen $\mathrm{Haq}^{1}$; Kapil Parakh ${ }^{1,2}$. ${ }^{1}$ Johns Hopkins Bayview Medical Center, Baltimore, MD; ${ }^{2}$ Johns Hopkins Medical Institutes, Baltimore, MD. (Control ID \#1332888)

STATEMENT OF PROBLEM OR QUESTION (ONE SENTENCE): In this IRB approved study, we present pilot data from a recently launched, institution-based intravenous (IV) diuresis clinic as a novel approach to provide treatment and education for patients with heart failure.

OBJECTIVES OF PROGRAM/INTERVENTION (NO MORE THAN THREE OBJECTIVES): (1) To establish an institution-based outpatient IV diuresis clinic as a novel treatment strategy in heart failure (2) To utilize the outpatient IV diuresis clinic as a novel venue for heart failure education (3) To reduce costs associated with heart failure by reducing heart failure hospitalizations

DESCRIPTION OF PROGRAM/INTERVENTION, INCLUDING ORGANIZATIONAL CONTEXT (E.G. INPATIENT VS. OUTPATIENT, PRACTICE OR COMMUNITY CHARACTERISTICS): A clinic was set up at an academic community hospital to provide IV diuretics to outpatients. Protocol: On arrival, vitals are checked, and labs and EKG are obtained for every patient. A cardiologist evaluates all patients and assesses suitability for IV diuretic therapy. Where appropriate, IV furosemide is administered at a pre-determined dose with electrolyte supplementation as needed. Patients are monitored in clinic for at least 3 hours for adverse events. Urine output is recorded as well as weight on arrival and discharge. During diuresis, patients receive education from a dedicated HF nurse. Clinically unstable patients are stabilized and referred directly to the hospital. Patients are re-assessed by the cardiologist prior to discharge. Arrangements are made for subsequent sessions as appropriate. Patients are followed up by telephone call within 30 days to evaluate symptoms, quality of life, and number of readmissions to the hospital. All data reported in this study is routinely collected as part of clinical services provided.

MEASURES OF SUCCESS (DISCUSS QUALITATIVE AND/OR QUANTITATIVE METRICS WHICH WILL BE USED TO EVALUATE PROGRAM/INTERVENTION): (1) Rate of admission to the hospital within 30 days (2) Quality of life within 30 days (3)Heart failure knowledge

FINDINGS TO DATE (IT IS NOT SUFFICIENT TO STATE "FINDINGS WILL BE DISCUSSED"): To date, we have data from 66 total IV diuresis sessions from 16 patients. No patients required admission to the hospital after outpatient IV diuresis. One patient experienced dizziness and orthostatic hypotension the day after diuresis but these symptoms resolved. The cardiologist determined to not administer IV diuretics on 10 sessions based on clinical data (hypokalemia $n=2$, elevated creatinine, $n=5$, orthostatic hypotension $n=3$ ). One patient left clinic prior to receiving diuresis. Two patients were determined to require emergent care on arrival to the diuresis clinic and were referred directly to the hospital. Average length of visit was 3 to 4 hours and mean fluid loss was 
$1160 \pm 520 \mathrm{ml}$ per session. To date, two of our patients have required inpatient admission for decompensated heart failure within 30 days of treatment. One of these patients died after admission. All patients treated have voiced significant satisfaction with IV therapy in clinic and report better quality of life. Patients report significant satisfaction with $\mathrm{HF}$ education and report better understanding of their illness and disease management. Providers and referring physicians expressed much satisfaction with the service.

KEY LESSONS FOR DISSEMINATION (WHAT CAN OTHERS TAKE AWAY FOR IMPLEMENTATION TO THEIR PRACTICE OR COMMUNITY?): Pilot data from the Johns Hopkins Bayview Diuresis Clinic suggest that outpatient IV diuresis appears to be a promising strategy to provide treatment and education for heart failure patients. As the cost of a diuresis clinic visit is significantly less than the cost of hospitalization, there is substantial potential for cost reduction. Further studies are needed to establish the safety, cost-effectiveness, efficacy, and impact on readmissions.

ANALYZING READMISSIONS AT A COMMUNITY HOSPITAL Lee Park ${ }^{1,3}$; Danielle Andrade ${ }^{1}$; Andrew Mastey ${ }^{1}$; LeRoi Hicks ${ }^{2} .{ }^{1}$ Newton Wellesley Hospital, Newton, MA; ${ }^{2}$ University of Massachusetts Medical Center, Worchester, MA; ${ }^{3}$ Harvard School of Public Health, Boston, MA. (Control ID \#1338518)

STATEMENT OF PROBLEM OR QUESTION (ONE SENTENCE): Despite high readmission rates, our institution did not have a process for systematically evaluating medical discharges that led to readmissions. OBJECTIVES OF PROGRAM/INTERVENTION (NO MORE THAN THREE OBJECTIVES): 1 . Evaluating whether or not hospitalists were aware of readmissions. 2. To determine institutional-specific risks factors for readmission by reviewing processes for readmitted patients compared to those without readmission.

DESCRIPTION OF PROGRAM/INTERVENTION, INCLUDING ORGANIZATIONAL CONTEXT (E.G. INPATIENT VS. OUTPATIENT, PRACTICE OR COMMUNITY CHARACTERISTICS): Our institution is a community hospital with a hospitalist group that cares for most medical admissions. Readmission was defined as rehospitalization within 30 days of discharge. We first surveyed 21 hospitalists in May 2010 about 34 recently discharged patients to evaluate whether or not they were aware of their readmissions and to ascertain their opinions on the cause for readmission. We then conducted a review of 100 discharges with diagnoses of either congestive heart failure (CHF) or pneumonia (PNA) utilizing data from the Transitions Systems Incorporated database and data from the electronic medical record as well as from the hospitalist group records to compare characteristics between cases that were and were not readmitted. We then collected data from all inpatient medical discharges $(n=4012)$ from January 1, 2009 until December 31, 2010 with diagnoses of CHF, PNA and/or chronic obstructive pulmonary disease (COPD) excluding patients who were transferred to another acute care hospital or expired during the admission.

MEASURES OF SUCCESS (DISCUSS QUALITATIVE AND/OR QUANTITATIVE METRICS WHICH WILL BE USED TO EVALUATE PROGRAM/INTERVENTION): We are currently evaluating the larger dataset and have preliminarily found that readmissions at our institution have similar characteristics to those documented in the literature. We will further analyze these data to examine our hospital-specific factors identified from the survey and limited chart review.

FINDINGS TO DATE (IT IS NOT SUFFICIENT TO STATE "FINDINGS WILL BE DISCUSSED"): Of our surveyed hospitalists, $13(62 \%)$ responded regarding 21 patients. Of these, $9(42 \%)$ were aware of the readmissions with 4 of these patients $(44.4 \%)$ readmitted to the same hospitalist. In the smaller analysis of 100 charts, we examined factors that reflected processes at our institution including hospitalist hours and census on the day of discharge, census of the floor and floor of discharge, service (housestaff vs. non-housestaff), day of discharge (hospitalist and housestaff switch days vs. non-switch days). We found a significant trend between readmissions and switch day when compared with a chi-square test (OR 2.5 [0.99-2.26]). Of the 4,012 discharges from the medical service, 697 (17.3\%) were readmitted in 30 days. In preliminary analyses, we found discharges leading to readmissions are more likely to be older (mean age $77.5 \mathrm{yrs}$ vs.
75.6 yrs), have an admission in the previous calendar year ( $22.8 \%$ vs. $14.8 \%)$, have a longer length of stay (mean 5 days vs. 4 days), have a higher Elixhauser sum score (mean 4.3 vs. 3.9), and were more likely to have initially been discharged to a facility $(42.6 \%$ vs. $34.0 \%)$. We have also found that readmissions are more likely to come from discharges from the housestaff service ( $85 \%$ vs. $80.4 \%)$.

KEY LESSONS FOR DISSEMINATION (WHAT CAN OTHERS TAKE AWAY FOR IMPLEMENTATION TO THEIR PRACTICE OR COMMUNITY?): We found that hospitalists are not aware of their readmissions the majority of the time and that it is possible at a community hospital to find ways to link readmissions back to the previous discharge and discharging hospitalist to systemically analyze readmissions.

AUTOMATED COMPUTER ALERTS TO IMPROVE WARFARIN PRESCRIBING AMONG HOSPITALIZED INPATIENTS Michele Fang; Todd Burstain; Peter Cram. University of Iowa Carver College of Medicine, Iowa City, IA. (Control ID \#1331740)

STATEMENT OF PROBLEM OR QUESTION (ONE SENTENCE): Anticoagulant drugs are a common cause of preventable adverse drug events in hospitalized patients.

OBJECTIVES OF PROGRAM/INTERVENTION (NO MORE THAN THREE OBJECTIVES): We developed and implemented computer alerts into our electronic medical record (Epic Systems Madison, WI) based upon the Joint Commission National Patient Safety Goal for Anticoagulants. The alerts consisted of an automated warning, a link directing providers to correct the deficiency, and a brief explanation of the rationale for the alert. For example, a computer alert would be triggered reminding the provider to check an INR level if a patient receiving warfarin did not have an INR ordered within the last 72 hours. Initiation of the reminder alerts was supplemented by an educational session for residents on proper monitoring of anticoagulants and use of the warning alerts. Our goals were to use the alerts to improve compliance with National Patient Safety Goals for anticoagulants and decrease the proportion of supratherapeutic INRs and bleeding complications.

DESCRIPTION OF PROGRAM/INTERVENTION, INCLUDING ORGANIZATIONAL CONTEXT (E.G. INPATIENT VS. OUTPATIENT, PRACTICE OR COMMUNITY CHARACTERISTICS): We conducted a retrospective before-after study of the effectiveness of the computer alerts and resident education session. The analysis included all adult patients admitted to a tertiary referral center between September 1, 2010 through February 19, 2011 during 3 different time periods: (phase 1: alerts fired silently without notification to providers, phase 2 : alerts fired with notification of providers, phase 3 . alerts notifying providers with resident education).

MEASURES OF SUCCESS (DISCUSS QUALITATIVE AND/OR QUANTITATIVE METRICS WHICH WILL BE USED TO EVALUATE PROGRAM/INTERVENTION): 1) proportion of INR $>4$; 2) the percentage of patients experiencing bleeding complications; 3) the number of alerts relative to anticoagulation orders; 4) the number of alerts that resulted in an appropriate response.

FINDINGS TO DATE (IT IS NOT SUFFICIENT TO STATE "FINDINGS WILL BE DISCUSSED"): We observed a 55\% decrease in the number the proportion of patients with INR $>4$ when comparing Phase 3 to Phase 1 (143 out of 10699 in the post-alert period, 201 out of 6751 in the pre-alert period $)(\mathrm{P}<.001)$ We did not see a significant decline in the number of bleeding episodes (1.7\% in Phase $1,2.1 \%$ in Phase 2$)$. There were signs that the computer alerts changed physician behavior. There was on average a $55 \%$ decrease in the firing of warfarin computer alerts in Phases 2 and 3 when compared to Phase 1 (one-tailed proportion test $\mathrm{p}<0.001$ ) even as the number of warfarin prescriptions remained stable. Approximately $33 \%$ of computer alerts resulted in the recommended response by the clinician during Phase 2. After completion of resident education (Phase 3), approximately $47 \%$ rate of alerts resulted in the appropriate response.

KEY LESSONS FOR DISSEMINATION (WHAT CAN OTHERS TAKE AWAY FOR IMPLEMENTATION TO THEIR PRACTICE OR COMMUNITY?): Use of computer alerts embedded in the electronic medical record with mandatory stops is an inexpensive method to improve 
compliance with anticoagulant monitoring and decrease the rate of supratherapeutic INRs. A single session of resident education seemed to enhance provider response to the alerts.

BAYVIEW PATIENT CONNECTION (BPC): AN AMBULATORY INTENSIVE CARE EXPERIENCE FOR HIGH-UTILIZING MEDICAID PATIENTS IN AN INTERNAL MEDICINE RESIDENCY CONTINUITY CLINIC Marc Larochelle; Melissa Dattalo; Huy Do; Sean Tackett; Justin Elfrey; Ryan E. Childers; Lauren Graham; Lana Elpert; Wendy L. Bennett; Laura Hanyok. Johns Hopkins Bayview Medical Center, Baltimore, MD. (Control ID \#1334968)

STATEMENT OF PROBLEM OR QUESTION (ONE SENTENCE): Delivering high quality care to high-utilizing medically and psychosocially complex patients in residency continuity clinics is challenging, and this experience may discourage residents from pursuing careers in general internal medicine (GIM).

OBJECTIVES OF PROGRAM/INTERVENTION (NO MORE THAN THREE OBJECTIVES): 1 . Improve care for high-utilizing Medicaid patients through translation of evidence-based interventions. 2. Reduce unnecessary inpatient admissions and emergency department (ED) visits. 3 . Improve resident skill and satisfaction with caring for challenging patients.

DESCRIPTION OF PROGRAM/INTERVENTION, INCLUDING ORGANIZATIONAL CONTEXT (E.G. INPATIENT VS. OUTPATIENT, PRACTICE OR COMMUNITY CHARACTERISTICS): High-utilizing patients from a Medicaid HMO who receive primary care at the Johns Hopkins Bayview GIM Clinic were identified. 15 new July 2011 interns were assigned a high-utilizing patient whose PCP was an outgoing PGY3 resident. A multifaceted intervention was designed and implemented with the intern-patient pairs. Intervention patients were offered a home visit with their new PCP, with the goal of identifying psychosocial factors influencing health behaviors. Interns received training in shared decision making and motivational interviewing, and an Action Plan document was created to record and track goals. Every two weeks, interns met as a group with a faculty mentor and multidisciplinary team members to share experiences and problem solve. Intervention patients were scheduled office visits every six weeks with their assigned intern. Each intervention patient was assigned a case manager from the Medicaid HMO. 23 high-utilizing patients with rising PGY2 and PGY3 resident PCPs served as a usual care cohort for comparison.

MEASURES OF SUCCESS (DISCUSS QUALITATIVE AND/OR QUANTITATIVE METRICS WHICH WILL BE USED TO EVALUATE PROGRAM/INTERVENTION): Administrative data from the Medicaid HMO will be used to compare the number of inpatient admissions and ED visits. Patient-perceived quality of care, quality of the patient-provider relationship, and satisfaction with the outpatient experience for patients and residents will be compared using validated surveys. Resident specific outcomes include their attitude toward challenging patients and selfreported adequacy of training in Systems-Based Practice. Number of visits, phone notes, and Action Plans from the clinic EMR will be tracked to assess the degree of intervention implementation.

FINDINGS TO DATE (IT IS NOT SUFFICIENT TO STATE "FINDINGS WILL BE DISCUSSED"): Of the 165 Medicaid HMO patients who regularly attend the Bayview GIM clinic, 49 (30\%) accounted for $82 \%$ of hospital utilization with a mean of 8.0 inpatient admissions and ED visits for the 12 months ending February 28, 2011. During the program's first 6 months, intervention patients attended, on average, a similar number of office visits (3.0 visits, $53 \%$ with PCP) compared to usual care patients (2.9 visits, $60 \%$ with $\mathrm{PCP}$ ). Intervention patients had more contact with their PCPs outside of clinic visits. Ten $(67 \%)$ interns visited their intervention patients at home, while no usual care patients received home visits. Intervention patients had phone contact with their PCP 2.0 times on average compared with 0.6 times for usual care patients. KEY LESSONS FOR DISSEMINATION (WHAT CAN OTHERS TAKE AWAY FOR IMPLEMENTATION TO THEIR PRACTICE OR COMMUNITY?): This intervention has the potential to transform resident frustration with challenging outpatients into a learning opportunity that may increase self-efficacy and inspire more trainees to pursue GIM careers. If the model is successful, dissemination to other academic practices has the potential to add tremendous value with minimal resources.
BUILDING AN ACADEMIC PRIMARY CARE-BASED CENTER FOR INTEGRATIVE MEDICINE: THE BETH ISRAEL DEACONESS MEDICAL CENTER (BIDMC) EXPERIENCE Kim D. Ariyabuddhiphongs. Beth Israel Deaconess Medical Center, Boston, MA. (Control ID \#1342331)

STATEMENT OF PROBLEM OR OUESTION (ONE SENTENCE): Surveys have shown that nearly $40 \%$ of Americans use complementary and alternative medicine (CAM) annually and there is emerging evidence on the utilization, safety, and efficacy of CAM for common medical conditions including musculoskeletal pain, mood disorders, insomnia, hyperlipidemia, hypertension, and diabetes.

OBJECTIVES OF PROGRAM/INTERVENTION (NO MORE THAN THREE OBJECTIVES): Integrative Medicine (IM) embodies many aspects of exceptional primary care: patient-centered care that attends to the whole person, features healing partnerships between providers and patients, and creates a culture of self-care and wellness. Integrative medicine also combines both conventional treatments and CAM practices. Our academic IM center at BIDMC aims to promote healthy behavior and self-care by providing IM services to support our patients in attaining and maintaining optimal health.

DESCRIPTION OF PROGRAM/INTERVENTION, INCLUDING ORGANIZATIONAL CONTEXT (E.G. INPATIENT VS. OUTPATIENT, PRACTICE OR COMMUNITY CHARACTERISTICS): Our center is one of the first of its kind in Boston: an innovative primary-care based model where patients can engage in integrative therapies alongside and under the supervision of their primary care physician (PCP). The development of our program has required significant planning- obtaining support from hospital leadership, designing a plan for sustainability, recruiting a team of administrators, and IM-trained physicians, and selecting a PCP to champion the program. The center will be located within our primary care practice serving more than 40,000 patients. We will provide IM services with trained providers in healing traditions (acupuncture, Tai Chi, yoga), massage therapy, and behavioral and lifestyle therapies (nutrition, exercise, health coaching). Our center will also offer consultations on integrative nutrition, cardiovascular health, and mind-body medicine, provided by physicians with IM fellowship training. All providers will collaborate in monthly team meetings with case-based discussion.

MEASURES OF SUCCESS (DISCUSS QUALITATIVE AND/OR QUANTITATIVE METRICS WHICH WILL BE USED TO EVALUATE PROGRAM/INTERVENTION): In addition to clinical services, our program will educate physicians and the community on IM and healthy behaviors through continuing medical education programs, resident rotations, and public programs on topics such as exercise counseling. We will develop tailored IM programs to specific disease populations, such as Tai Chi for patients with congestive heart failure, yoga for patients with asthma, and demonstrations on healthy cooking for patients with diabetes. Successful dissemination of our program will involve demonstrating referral to the Center for Integrative Care by greater than $90 \%$ of PCPs.

FINDINGS TO DATE (IT IS NOT SUFFICIENT TO STATE "FINDINGS WILL BE DISCUSSED"): Because a comprehensive center of this scope had not previously existed at BIDMC, we have undertaken a process facilitated by a start-up fund from philanthropists. An IM Advisory Group composed of key division leaders, IM fellowship-trained physicians, and donors, has been central to the Center's development. Discussions on efficacy, safety and legal aspects have been instrumental for hospital approval. In addition, attention has been paid to physician supervision, communication, and documentation. Future steps will involve recruitment, hiring, and credentialing of IM providers. KEY LESSONS FOR DISSEMINATION (WHAT CAN OTHERS TAKE AWAY FOR IMPLEMENTATION TO THEIR PRACTICE OR COMMUNITY?): Our clinical practice innovation details initial steps necessary for the creation of our Center for Integrative Care including: hospital executive, legal, patient safety, and credentialing approval, team member recruitment, programmatic development, and development of a physician consult in Integrative Care.

CONTINUED BENEFIT OF A DIABETES GROUP CLINIC (DGC) IN AN URBAN CLINIC POPULATION Corinna Falck-Ytter; Guptha Baskaran; Christina Sanders; Lucinda Newshutz; Douglas Einstadter. MetroHealth Medical Center at Case Western Reserve University, Cleveland, OH. (Control ID \#1339328) 
STATEMENT OF PROBLEM OR QUESTION (ONE SENTENCE): We examined the effects of the DGC on Hemoglobin A1c (HBA1c) for all patients and for those with a comorbid mental health disorder.

OBJECTIVES OF PROGRAM/INTERVENTION (NO MORE THAN THREE OBJECTIVES): Improve Hemoglobin A1c and selfmanagement skills.

DESCRIPTION OF PROGRAM/INTERVENTION, INCLUDING ORGANIZATIONAL CONTEXT (E.G. INPATIENT VS. OUTPATIENT, PRACTICE OR COMMUNITY CHARACTERISTICS): The DGC offers group visits for up to 13 patients at a time. Initially the patients are assessed individually based on information from a diabetes summary that is available through our electronic medical record. A group discussion on topics chosen by the attendees and led by a diabetes educator follows. Topics include diet, exercise, medications and cost of medications, and adjustment to illness and self-management. Each patient is then seen individually by the MD or NP and therapy is adjusted, referrals made and exams performed as needed. When necessary, additional individual sessions to teach skills such as insulin injection or self-monitoring are completed. Patients are referred to the DGC by their primary care physician (PCP) when eligible (type 2 diabetes, Hemoglobin A1c (HBA1c) $>7 \%$, English speaking, no gestational diabetes). Patients follow up once a month until their target HBA1c is achieved and continue to see their PCP as needed. DGC sessions are conducted by an RN and CDE (scheduling, assessment, group education, individual teaching), MD and NP (exam, medication adjustment, referrals) and a nurse who manages the diabetes registry for the Medical Home and can refer patients directly to the DGC. The individual care plans that are part of the Medical Home are given to the patients at the end of the visit and contain general information about their diabetes and individualized goals and commitments until their next visit.

MEASURES OF SUCCESS (DISCUSS QUALITATIVE AND/OR QUANTITATIVE METRICS WHICH WILL BE USED TO EVALUATE PROGRAM/INTERVENTION): Before and after HbAlc for DGC attendees compared with non-attendees.

FINDINGS TO DATE (IT IS NOT SUFFICIENT TO STATE "FINDINGS WILL BE DISCUSSED"): Between October 2009 and August 2011, 166 patients were referred, $85(51 \%)$ of whom attended at least one visit to the DGC. The mean age for patients attending DGC was 51.3 years, $44 \%$ were women, $51 \%$ African American, $33 \%$ uninsured and $60 \%$ had a documented MHD. The mean baseline HbAlc was $10.4 \%$ for the attendees and $10.2 \%$ for non-attendees. Those who attended experienced a $1 \%$ decrease in their $\mathrm{HbA} 1 \mathrm{c}$ over the subsequent 6 months. During the same time period the $\mathrm{HbA} 1 \mathrm{c}$ for non-attendees decreased by $0.1 \%$. In the attendee group the HbA1c decreased by $0.5 \%$ for those with a MHD and decreased by $1.3 \%$ for those without a MHD. In the non-attendee group the HbAlc increased by $0.82 \%$ for those with a MHD and decreased by $0.5 \%$ for those without.

KEY LESSONS FOR DISSEMINATION (WHAT CAN OTHERS TAKE AWAY FOR IMPLEMENTATION TO THEIR PRACTICE OR COMMUNITY?): At our institution, the Diabetes Group Clinic has been associated with improved outcomes for patients with poorly controlled diabetes in an urban resident clinic, including those with coexisting MHD. In our experience it is important to incorporate the data available through the electronic medical record to educate patients about their diabetes and to formulate clear goals between visits. For patients with diabetes and MHD, we are exploring the establishment of joint group visits co-led by both mental health and internal medicine providers

CONTINUITY OF CARE IN AN INTERNAL MEDICINE RESIDENCY AMBULATORY CLINIC:FACT OR FICTION Maria Palomata; Delaram Moazami. Capital Health, Trenton, NJ. (Control ID \#1339635)

STATEMENT OF PROBLEM OR QUESTION (ONE SENTENCE): Is high rate of continuity of care attainable in an Internal Medicine Residency Ambulatory Clinic or is it an idealistic goal?

OBJECTIVES OF PROGRAM/INTERVENTION (NO MORE THAN THREE OBJECTIVES): To evaluate the effectiveness of systems based changes in continuity of care in an Internal Medicine Residency Ambulatory Clinic serving an ethnically diverse urban population.
DESCRIPTION OF PROGRAM/INTERVENTION, INCLUDING ORGANIZATIONAL CONTEXT (E.G. INPATIENT VS. OUTPATIENT, PRACTICE OR COMMUNITY CHARACTERISTICS): Continuity of care powerfully affects patient-focused outcomes and has shown to benefit patients with chronic medical illness. It is also associated with patient and provider satisfaction, high quality of care and decreased cost. There is a scarcity of published reports specifically for Internal Medicine Residency Ambulatory Clinics regarding continuity. The ACGME and Internal Medicine RRC support continuity in resident clinics and require residents to attend a minimum of 130 longitudinal continuity clinic sessions during their training. Every effort is made to maintain continuity. However, rotation requirements limit residents' availability. Recent changes in healthcare delivery promote team-based care. From July 2010, systems based changes, including 1) establishing a distinct resident/patient practice team 2) chart color coding to identify patients' practice team and 3) staff education and close monitoring of appointment assignment, were implemented

MEASURES OF SUCCESS (DISCUSS QUALITATIVE AND/OR QUANTITATIVE METRICS WHICH WILL BE USED TO EVALUATE PROGRAM/INTERVENTION): Residents' patient panels were identified based on consecutive clinic registration from July 2010-June 2011. A retrospective analysis of data from registration and medical record review was completed for July 2009-June 2011. We included patients seen at least 3 times each academic year. We collected the total number of patient visits per academic year, visits with primary resident, visits with primary resident's team, or other providers. Three indices were used to measure continuity: Usual Provider Continuity Index (UPC-visits with usual provider/total visits), Team Provider Continuity Index (TPC-visits with team provider/total visits) our modification of UPC to measure care provided by the primary provider's team and Modified, Modified Continuity Index (MMCI-a measure that considers the number of providers seen). Data from one academic year before and one year after implementation of systems based changes were measured and compared using independent sample T-test.

FINDINGS TO DATE (IT IS NOT SUFFICIENT TO STATE "FINDINGS WILL BE DISCUSSED"): Data from 112 eligible patients were included in the analysis after review of 773 patients' registration record and 148 charts. 19 residents were identified as usual providers for these eligible patients and were grouped into 5 teams. Mean number of visits for each eligible patient was 5.11 before systems based changes and 4.76 after. Initial mean UPC, TPC and MMCI were $0.73,0.89$ and 0.69 respectively. After systems based changes, these improved to $0.81,0.94$ and 0.76 ( $\mathrm{p}$ value $<.01,<.01,<.05)$.

KEY LESSONS FOR DISSEMINATION (WHAT CAN OTHERS TAKE AWAY FOR IMPLEMENTATION TO THEIR PRACTICE OR COMMUNITY?): Data validated that for patients seen at least 3 times per year, continuity of care can be maintained to a high degree in an Internal Medicine Residency Ambulatory Clinic. Systems based efforts improved continuity. Analysis confirms that we outperform the average continuity indices in published literature for academic settings (UPC 0.43 0.68; MMCI 0.44-0.64). Having a team based approach and a new index to measure adherence (Team Provider Continuity Index/TPC) allowed scheduling flexibility for better patient access if the primary resident is not available, yet it limited the number of providers a patient can see.

CREATING MODELS FOR GLOBAL HEALTH AND PRIMARY CARE: A TEAM-BASED APPROACH FOR MANAGING GLOBAL HEALTH RESIDENT PATIENT PANELS Charlotte A. $\mathrm{Wu}^{1}$; Rose $\mathrm{M}$. Kakoza $^{1}$; Joy E. O'Brien ${ }^{1}$; Charles A. Morris ${ }^{1}$; Lori Tishler ${ }^{1}$; Joseph J. Rhatigan'; Andrew L. Ellner ${ }^{1,2}$. 'Brigham and Women's Hospital, Boston, MA; ${ }^{2}$ Harvard Medical School, Boston, MA. (Control ID \#1334436)

STATEMENT OF PROBLEM OR QUESTION (ONE SENTENCE): Despite shared missions within primary care and global health to serve vulnerable populations and develop innovative health care delivery approaches, balancing global health training with resident continuity clinic is challenging and few models exist for practicing physicians to pursue global health and stay in primary care

OBJECTIVES OF PROGRAM/INTERVENTION (NO MORE THAN THREE OBJECTIVES): The Global Health Equity Resident 
Primary Care (GHE-PC) team in The Jen Center for Primary Care (PJC) was established in order to: -Create a robust system for team-based care delivery to support GHE residents while away -Improve continuity of care and communication -Increase patient, staff and provider satisfaction

DESCRIPTION OF PROGRAM/INTERVENTION, INCLUDING ORGANIZATIONAL CONTEXT (E.G. INPATIENT VS. OUTPATIENT, PRACTICE OR COMMUNITY CHARACTERISTICS): Residents in the Global Health Equity (GHE) residency at Brigham \& Women's Hospital (BWH) complete 12 months of global health training over 4 years. The PJC, a faculty/resident clinic at BWH with $\sim 40,000$ patient visits/yr, is the training site for nearly 80 residents including the majority of GHE residents. The GHE-PC team embedded in the PJC includes: -A team of GHE residents with a dedicated faculty preceptor and a NP -New care plans for high-risk patients with clarified hand-off, communication, and coverage models There are 2 implementation phases: Phase 1: establishment of a dedicated joint preceptor for GHE residents for co-management, pilot-testing integrated support package; Phase 2: addition of NP

MEASURES OF SUCCESS (DISCUSS QUALITATIVE AND/OR QUANTITATIVE METRICS WHICH WILL BE USED TO EVALUATE PROGRAM/INTERVENTION): Quantitative and qualitative evaluations of pre/post implementation metrics for a sample of GHE resident patients: -Continuty of care: Percentage of out-of-team urgent care visits/contact -Patient utilization of hospital/ambulatory resources: ED utilization rates, 30day hospital re-admission rates -Patient, resident, and staff experiences: Qualitative data obtained through patient focus groups and surveys

FINDINGS TO DATE (IT IS NOT SUFFICIENT TO STATE "FINDINGS WILL BE DISCUSSED"): Preliminary data after Phase 1 Patient Outcomes: -Decreased care fragmentation for high risk patients and urgent/ post-discharge visits Patient Experience: -Increased comfort with seeing the team preceptor in transitional care -Increased satisfaction and feeling of security by being seen more often -Decreased sense of abandonment when PCPs are away Team Member Experience: -Increased resident satisfaction in their primary care experience and delivery of care to medically and psychosocially complex patients -Substantial decrease in staff complaints about GHE resident absences and impact on patient care -Improved communication with clear sign-outs when GHE residents go abroad

KEY LESSONS FOR DISSEMINATION (WHAT CAN OTHERS TAKE AWAY FOR IMPLEMENTATION TO THEIR PRACTICE OR COMMUNITY?): This model addresses the significant issue of fragmented care created by balancing global health and primary care training. Preliminary data shows that our model may significantly improve service utilization and patient, staff, and provider experience Lessons learned can inform: -Other practice settings with providers with interest in global health, creating career paths for combining primary care and global health, and fostering active exchange of care delivery strategies -Team-based ambulatory training models for all residents to support discontinuity in outpatient care -New measures of continuity of care In the movement towards team-based primary care, we implemented a model for building capacity among non-physician staff to support vulnerable populations within a fragmented care system

DECREASING OPERATING ROOM DELAYS WITH COMPREHENSIVE PREOPERATIVE CARE Barbara Slawski; Kurt J. Pfeifer. Medical College of Wisconsin, Milwaukee, WI. (Control ID \#1338267)

STATEMENT OF PROBLEM OR QUESTION (ONE SENTENCE): Operating room (OR) delays cause significant patient and physician dissatisfaction and have financial impact on medical facilities, who often set goals to decrease OR delays.

OBJECTIVES OF PROGRAM/INTERVENTION (NO MORE THAN THREE OBJECTIVES): 1 . Standardize preoperative medical evaluations and testing. 2. Centralize administrative functions related to preoperative care, such as chart preparation. 3. Improve OR efficiency by utilizing a standardized, interdisciplinary program.

DESCRIPTION OF PROGRAM/INTERVENTION, INCLUDING ORGANIZATIONAL CONTEXT (E.G. INPATIENT VS. OUTPATIENT, PRACTICE OR COMMUNITY CHARACTERISTICS): The Pre Admission Testing Clinic (PAT) is a multidisciplinary preoperative clinic at an academic center performing over 15,000 OR cases annually. Causes of OR delays are numerous and include lack of chart completion and unaddressed medical problems. The goal of PAT is to provide safe, consistent and reliable preoperative care in a comprehensive manner to surgical patients. Key services provided in PAT include standardized preoperative evaluations performed by Anesthesiology, Internal Medicine, or non-physician providers; diagnostic testing completed in a protocolized and streamlined manner; medication reconciliation by a pharmacist; patient education; and completion of required preoperative RN evaluations and paperwork. Referral of preoperative patients to PAT is elective at the discretion of the surgeon. Currently, about 8,000 patients are seen in the clinic annually.

MEASURES OF SUCCESS (DISCUSS QUALITATIVE AND/OR QUANTITATIVE METRICS WHICH WILL BE USED TO EVALUATE PROGRAM/INTERVENTION): Scheduled Procedural Start to Wheels In and Scheduled Procedural Start to Actual Procedural Start are dashboard metrics routinely measured by surgical services. "Wheels In" is the actual time the patient enters the OR. These outcomes were compared for surgical patients in August and September 2011 who were seen and not seen in PAT Clinic.

FINDINGS TO DATE (IT IS NOT SUFFICIENT TO STATE "FINDINGS WILL BE DISCUSSED"): Patients seen in PAT had shorter surgical delays than patients not seen in PAT. The Scheduled Procedural Start to Wheels In was on average 20.0 minutes for patients who were seen and 35.1 minutes for patients who were not seen in PAT (95\% CI 15.9-24.0 and 29.8-40.4 respectively, $\mathrm{p}=0.0004$ ). The Scheduled Procedural Start to Actual Procedural Start was an average of 14.2 minutes less for patients who were seen in PAT $(95 \% \mathrm{CI}$ 50.6-59.2 and 63.874.4 min respectively, $\mathrm{p}=0.001$ ). OR availability may have allowed early surgical starts, but when negative start times were adjusted to zero, data remained significant with an average difference in surgical delays of 19.4 and 15.9 minutes, respectively ( $<<0.0001$ for both).

KEY LESSONS FOR DISSEMINATION (WHAT CAN OTHERS TAKE AWAY FOR IMPLEMENTATION TO THEIR PRACTICE OR COMMUNITY?): Perioperative medicine is a growing clinical area within Internal Medicine. There is not a single accepted model for preoperative care. This preoperative clinic focuses not only on delivery of standardized clinical care, but also on the administrative processes required to prepare patients and associated documentation for surgery. Use of the preoperative clinic decreases the number of minutes patients are delayed in the OR, improving efficiency, and having the possibility of improving staff and patient satisfaction. Establishing and expanding a sustainable preoperative clinic is challenging, as these clinics are often viewed as non-revenue producing. Creation of a sustainable perioperative program requires identification and demonstration of improved measures that are aligned with institutional goals. OR costs are often estimated at over $\$ 20$ a minute. Linking improvement in OR efficiency to a preoperative clinic can establish significant financial benefit of a preoperative clinic to the institution.

DESIGN AND DEVELOPMENT OF A CHURCH-BASED, PHOTOVOICE INTERVENTION FOR LATINOS WITH DIABETES Arshiya A. Baig ${ }^{1}$; Cara A. Locklin ${ }^{2}$; Michael T. Quinn ${ }^{1}$; Marla C. Solomon $^{3}$; Lisa Sanchez-Johnsen ${ }^{4}$; Deborah L. Burnet ${ }^{1}$; Marshall Chin ${ }^{1}$. ${ }^{1}$ University of Chicago, Chicago, IL; ${ }^{2}$ University of Illinois at Chicago, Chicago, IL; ${ }^{3}$ University of Illinois at Chicago, Chicago, IL; ${ }^{4}$ University of Illinois at Chicago, Chicago, IL. (Control ID \#1326842)

STATEMENT OF PROBLEM OR QUESTION (ONE SENTENCE): Churches provide a novel setting for chronic disease self-management programs; however, data from Latino communities are lacking.

OBJECTIVES OF PROGRAM/INTERVENTION (NO MORE THAN THREE OBJECTIVES): To assess the effect of a church-based diabetes self-management intervention on diabetes outcomes among Latinos

DESCRIPTION OF PROGRAM/INTERVENTION, INCLUDING ORGANIZATIONAL CONTEXT (E.G. INPATIENT VS. OUTPATIENT, PRACTICE OR COMMUNITY CHARACTERISTICS): We partnered with two Latino churches in an urban, low-income community. In Phase I, we conducted focus groups with 37 Latino adults with diabetes and their family members to assess preferences for church-based diabetes interventions. In Phase II, we formed a community advisory board (CAB) of 
key stakeholders to guide the development of the intervention. We used input from the $\mathrm{CAB}$ and the data from the focus groups to design a churchbased diabetes intervention for Latinos with diabetes, Picture Good Health (PGH). PGH is an 8-week church-based diabetes intervention that consists of weekly diabetes self-management classes led by trained lay leaders, a photovoice component, an exercise program and patient navigation services. For the photovoice, participants receive disposable cameras to take photos of their lives with diabetes. The photos are used in the class to facilitate discussion around diabetes self-empowerment. For the exercise component, participants engage in weekly church-based aerobics and exercises classes. To enhance linkages to the healthcare system, patient navigators assisted participants in finding primary care physicians. In Phase III, we are now recruiting English and Spanish-speaking adults with diabetes to enroll in a community-based, randomized control study to assess the impact of the intervention on diabetes outcomes. Participants in the control group are offered a one-hour diabetes education class and diabetes educational materials.

MEASURES OF SUCCESS (DISCUSS QUALITATIVE AND/OR QUANTITATIVE METRICS WHICH WILL BE USED TO EVALUATE PROGRAM/INTERVENTION): A sample size of 100 participants provide $80 \%$ power to detect mean differences between groups of $1.26 \%$ in $\mathrm{HBalc}$ at any two time points. The primary outcome is change in $\mathrm{HbA} 1 \mathrm{c}$ from baseline to follow-up at 3 and 6 months. Secondary outcomes include changes in systolic blood pressure, weight, lipids, diabetes selfmanagement, self-efficacy, self-empowerment, knowledge and medication adherence. We are also conducting a process evaluation to understand the feasibility and challenges of implementing the intervention.

FINDINGS TO DATE (IT IS NOT SUFFICIENT TO STATE "FINDINGS WILL BE DISCUSSED"): To date, we have recruited 72 Latino adults with diabetes. Three intervention classes have been given and the control group has received two diabetes lectures. We have 3 month follow-up data on 20 participants (10 control and 10 intervention). Participants in the intervention arm have had a $0.98 \pm 0.23 \%$ drop in $\mathrm{HbA1c}$ versus a $0.51 \pm 0.4 \%$ drop in the control arm $(\mathrm{p}=0.20)$. In preliminary theme analyses, we found participants took photographs of food, the neighborhood, their homes, family and friends, medication, and diabetic supplies.

KEY LESSONS FOR DISSEMINATION (WHAT CAN OTHERS TAKE AWAY FOR IMPLEMENTATION TO THEIR PRACTICE OR COMMUNITY?): A multi-component, church-based diabetes intervention shows an early trend towards improved HbAlc among Latinos with diabetes.

DEVELOPING A PRACTICAL, SELF-SUSTAINING, CLINIC-BASED YOGA PROGRAM FOR PATIENTS WITH CHRONIC LOW BACK PAIN AT AN URBAN ACADEMIC HEALTH CENTER Brian C. Hilgeman; Steve Hillson; Anna Cox; Nissa Valdez; Sheri Thorson; Adrian Sotro. Hennepin County Medical Center, Minneapolis, MN. (Control ID $\# 1327420)$

STATEMENT OF PROBLEM OR QUESTION (ONE SENTENCE): Can an Internal Medicine clinic located in a large county hospital successfully implement and sustain a Yoga program for patients with chronic low back pain?

OBJECTIVES OF PROGRAM/INTERVENTION (NO MORE THAN THREE OBJECTIVES): - Implement and sustain weekly Yoga classes for our patients with chronic low back pain - Objectively and subjectively assess for improvements in pain and disability among our patients - Measure the financial feasibility of such a program

DESCRIPTION OF PROGRAM/INTERVENTION, INCLUDING ORGANIZATIONAL CONTEXT (E.G. INPATIENT VS. OUTPATIENT, PRACTICE OR COMMUNITY CHARACTERISTICS): Therapeutic yoga is a form of exercise involving stretching, breath work, and meditation that has strong evidence supporting its efficacy in treating chronic mechanical low back pain. However, its utility has not been proven to be practical outside of the research realm. Further, practical limitations (such as availability, cost, transportation and scheduling) and patients' concerns about community-based programs made it difficult to engage our patients in off-site yoga programs. We sought to develop an on-campus, low- or no-cost therapeutic yoga program for these patients. Our intervention was conducted at Hennepin County Medical Center in Minneapolis, MN, a large community-based county health system. Our internal medicine clinic serves a total of 10,328 patients with multiple staff and resident providers. Our patients are $55 \%$ male, $69 \%$ minority with $48 \%$ African American, and the majority of our patients rely on public health insurance. We recruited patients with chronic, mechanical low back pain via provider referral. We were able to procure 43 referrals and enroll 14 patients in our first session. Our intervention consisted of weekly 75 minute classes for a total of 12 weeks held in the Chapel of our hospital. Each patient had a brief staff or resident physician visit in the group setting, vital signs were taken and medical concerns were addressed. Typically, a level 1 or 2 provider encounter was billed to insurance. The classes were led by a professional Yoga instructor implementing therapeutic and restorative Yoga postures specifically focusing on relieving chronic low back pain. We provided our patients with Yoga mats, blankets, bolsters, and blocks to use throughout the class. In attendance, we had a range of 3-8 patients with an average age of 50 (42-72), 33\% men, all enrolled in public insurance (4 MA, 6 Medicare), and 7/10 of African-American or African-born heritage. All of our patients had no exposure to Yoga prior to our intervention and came with significant physical limitations.

MEASURES OF SUCCESS (DISCUSS QUALITATIVE AND/OR QUANTITATIVE METRICS WHICH WILL BE USED TO EVALUATE PROGRAM/INTERVENTION): We plan to analyze average weekly pain scores, Oswestry Diability Scores (from start of intervention to completion of intervention), and subjective reports of pain improvement to measure the success of our program.

FINDINGS TO DATE (IT IS NOT SUFFICIENT TO STATE "FINDINGS WILL BE DISCUSSED"): We have thus far analyzed basic demographics as outlined above. Analysis of average weekly pain scores and disability scores will be available by the time of poster presentation. We also hope to have data about the financial feasibility of this intervention.

KEY LESSONS FOR DISSEMINATION (WHAT CAN OTHERS TAKE AWAY FOR IMPLEMENTATION TO THEIR PRACTICE OR COMMUNITY?): - There is strong evidence to support the use of Yoga to treat chronic low back pain - Implementing a Yoga program for patients with chronic low back pain is practically and financially feasible (and professionally satisfying) in a community based clinic, specifically in a county academic health center that serves a largely under-served, minority, and impoverished population.

DEVELOPING A TOOLKIT TO ENHANCE PATIENT CENTERED MEDICAL HOME IMPLEMENTATION: IMPROVING HYPERTENSION AND SMOKING OUTCOMES THROUGH PANEL MANAGEMENT Mark D. Schwartz ${ }^{1,2}$; Jaclyn Fox ${ }^{1,2}$; Stella Savarimuthu ${ }^{1,2}$; Katelyn Bennett ${ }^{1,2}$; Karolina Pekala $^{1,2}$; Joseph Leung ${ }^{1,2}$; Anne Dembitzer ${ }^{1,2}$; Scott Sherman ${ }^{1,2}$; Colleen Gillespie ${ }^{1,2}$; Alfredo Axtmayer ${ }^{1,2}$. ${ }^{1}$ Veteran Affairs Hospital, New York, NY; ${ }^{2}$ NYU School of Medicine, New York, NY. (Control ID \#1337677)

STATEMENT OF PROBLEM OR QUESTION (ONE SENTENCE): To determine how adding a non-clinical member to primary care teams can improve hypertension and smoking cessation outcomes in Veteran Affairs New York Harbor Healthcare System's (VA NYHHS) implementation of the VA's Patient Centered Medical Home (PCMH) model, known as Patient Aligned Care Teams (PACT).

OBJECTIVES OF PROGRAM/INTERVENTION (NO MORE THAN THREE OBJECTIVES): As part of the Program for Research on Outcomes of VA Education (PROVE) study, we sought to define a toolkit of panel management strategies that Panel Management Assistants (PMAs) will use to improve outcomes in smoking cessation and hypertension across patient panels.

DESCRIPTION OF PROGRAM/INTERVENTION, INCLUDING ORGANIZATIONAL CONTEXT (E.G. INPATIENT VS. OUTPATIENT, PRACTICE OR COMMUNITY CHARACTERISTICS): Coincident with the nation-wide implementation of PACT across the VA system, PROVE explores the incremental impact of panel management and clinical microsystem education on hypertension and smoking outcomes. Two-thirds of randomly selected PACT teams in ambulatory care clinics 
at the Brooklyn and Manhattan campuses of the VA NYHHS had a PMA added to the team. Based on literature review and qualitative interviews of clinicians and key stakeholders at VA NYHHS, we developed a core toolkit of strategies utilizing clinical databases to target subsets of smokers and hypertensive patients that could benefit from specialized panel management interventions outside of the patient visit, such as identifying smokers who have not recently received tobacco cessation medications.

MEASURES OF SUCCESS (DISCUSS QUALITATIVE AND/OR QUANTITATIVE METRICS WHICH WILL BE USED TO EVALUATE PROGRAM/INTERVENTION): Prior to PROVE's intervention, we determined baseline rates of hypertension (uncontrolled and controlled) and smoking for all PACT panels. To assess PROVE's effectiveness of integrating panel management strategies by PACT teams, we will survey providers and nurses at baseline, 6 and 12 months to measure the teams' changing views and patients at baseline, 6 and 12 months. We will also measure the change in number of smoking quit attempts, quit rate, rates of controlled versus uncontrolled hypertension, increased medication adherence, medication possession ratio and no-show rate. Additionally, PMAs will keep activity trackers to monitor intervention attempts and team cohesion to identify the most successful strategies for improving panel wide outcomes.

FINDINGS TO DATE (IT IS NOT SUFFICIENT TO STATE "FINDINGS WILL BE DISCUSSED"): Researchers conducted semistructured interviews with 12 primary care providers and 5 administrators and staff to identify care gaps amenable to PMA intervention. These 9 care gaps became the basis for the strategy toolkit and are addressed by 10 specific PMA strategies. The gaps were grouped into 5 domains - access to care, patient centeredness, patient education, and hypertension- and smoking cessation-specific strategies. Toolkit examples include: 1) Domain: Access to care; Gap: Increasing outreach to vulnerable patients; Strategy: Perform database queries and follow-up for uncontrolled hypertensive patients not seen in 1 year. 2) Domain: Smoking cessationspecific strategies; Gap: Addressing barriers to quitting; Strategy: Followup phone call two weeks after patient prescription of nicotine replacement. KEY LESSONS FOR DISSEMINATION (WHAT CAN OTHERS TAKE AWAY FOR IMPLEMENTATION TO THEIR PRACTICE OR COMMUNITY?): PROVE will provide a foundation for implementing panel management in primary care clinics. We will determine which PMA toolkit activities are most successful in improving health outcomes and patient and provider satisfaction, and thus facilitate adaptation of panel management to various settings and locations.

DEVELOPMENT AND EFFECTIVENESS OF GROUP WEIGHT LOSS VISITS IN A FEDERALLY QUALIFIED HEALTH CENTER Adam G. Tsai, ${ }^{1,2}$; Elizabeth Raube ${ }^{1}$; Judith Conrad ${ }^{1}$; Jeanne M. Rozwadowski ${ }^{1,2}$. ${ }^{1}$ Denver Health, Denver, CO; ${ }^{2}$ University of Colorado, Aurora, CO. (Control ID \#1309589)

STATEMENT OF PROBLEM OR QUESTION (ONE SENTENCE): Obesity and its co-morbidities are extremely common in primary care settings, but the brief primary care visit is not well suited to an in-depth discussion of weight management.

OBJECTIVES OF PROGRAM/INTERVENTION (NO MORE THAN THREE OBJECTIVES): 1) Establish group visits for weight loss in our federally qualified health center 2) Motivate patients to attend weekly classes for 5 weeks and to keep records of food and physical activity during that time 3 ) Induce weight losses of 1-2 pounds per week during the period of the group visits

DESCRIPTION OF PROGRAM/INTERVENTION, INCLUDING ORGANIZATIONAL CONTEXT (E.G. INPATIENT VS. OUTPATIENT, PRACTICE OR COMMUNITY CHARACTERISTICS): We offered a 90 minute group visit, once weekly for five weeks. The group visit is offered quarterly and is open to all patients from our practice and from 7 other primary care practices that make up the outpatient department of Denver Health (the city hospital for Denver, Colorado). Materials used were from the National Institutes of Health and the American Dietetic Association. These materials were supplemented with visual aids to teach portion size and calorie counting. Classes are co-led by a registered nurse and either a nurse practitioner or a primary care physician. We seek to have patients keep records of food intake and physical activity and to lose approximately 1-2 pounds per week. Each class consists of a "check-in" for patients to report calorie intake and physical activity, time for didactic teaching, time for questions, and time at the end of each class for patients to set goals. We are able to bill for these visits using a primary diagnosis code for obesity, and by individualizing patients' goals.

MEASURES OF SUCCESS (DISCUSS QUALITATIVE AND/OR QUANTITATIVE METRICS WHICH WILL BE USED TO EVALUATE PROGRAM/INTERVENTION): We have tracked referrals, enrollments, attendance, and weight loss over a period of 7 quarters (21 months). FINDINGS TO DATE (IT IS NOT SUFFICIENT TO STATE "FINDINGS WILL BE DISCUSSED"): During the first 21 months, a total of 180 patients were referred to the groups. Of these 180 , only 58 patients attended a first class. Of the 58 that attended a first class, 47 finished the 5 weeks of the group visits. These 47 individuals lost 2.9 pounds between week 1 and week 5 of the groups and regained 1.7 pounds at 22 weeks after the end of the group visits (weights abstracted from the medical record). We informally surveyed 30 patients that registered but did not attend a group. most of these individuals reported that they wanted to attend the groups but had other more pressing commitments (child or family care, seeking work).

KEY LESSONS FOR DISSEMINATION (WHAT CAN OTHERS TAKE AWAY FOR IMPLEMENTATION TO THEIR PRACTICE OR COMMUNITY?): 1) Weight losses of nearly 1 pound per week can be achieved in a low income patient population with a high percentage of ethnic minorities. 2) Weight regain was observed after the end of the 5 week group visit. This is common in behavioral interventions and shows that longer/more intensive intervention may be needed. 3) The no show rate for the group visits was very high, illustrating the challenges of conducting behavioral interventions in a low income patient population. We are attempting to overcome this by registering a larger number of patients for each group.

DISCHARGE PROCESS IMPROVEMENT ON A GENERAL INTERNAL MEDICINE INPATIENT TEAM Gwen Crevensten; Maria Winne; Kathleen Buckley; Sara Macchiano; Theresa Mills; Jennifer Harris. Massachusetts General Hospital, Boston, MA. (Control ID \#1340508)

STATEMENT OF PROBLEM OR QUESTION (ONE SENTENCE): The hospital discharge process coordinated by inpatient general medicine teams can be hurried, inefficient, and disorganized, resulting in patient and staff dissatisfaction, discharge delays, and potentially errors in patient care. OBJECTIVES OF PROGRAM/INTERVENTION (NO MORE THAN THREE OBJECTIVES): Our team sought to improve the organization and fluidity of patient discharge as measured by predictability of discharge timing and staff perception of the ease of discharge.

DESCRIPTION OF PROGRAM/INTERVENTION, INCLUDING ORGANIZATIONAL CONTEXT (E.G. INPATIENT VS. OUTPATIENT, PRACTICE OR COMMUNITY CHARACTERISTICS): The discharge process improvement pilot took place on the Academic Hospitalist Service, an inpatient general medicine team at Massachusetts General Hospital with an average census of 10-13 patients. This service encompasses portions of two general medicine inpatient floors, and includes patients cared for by hospitalists as well as MGH-affiliated primary care providers in conjunction with nurse practitioners. Patients included in the pilot were discharged to home, skilled nursing facilities, acute rehab, and long-term acute care. The pilot was coordinated by a multidisciplinary team comprised of case management, MD's, NP's, floor nurses, social work, and physical therapy. Prior to the intervention, a survey was conducted to collect background data to better define the causes for inefficient, delayed, or hurried discharges. Interventions were developed targeting the most frequent causes for delay or disorganization identified in this survey. The team's interventions focused on a multidisciplinary approach to predicting the estimated date of discharge, and a structured communication of this estimated date to the patients, their families, and the extended care team. The interventions were iterated weekly in response to feedback gathered during a brief huddle with the clinical team. 
MEASURES OF SUCCESS (DISCUSS QUALITATIVE AND/OR QUANTITATIVE METRICS WHICH WILL BE USED TO EVALUATE PROGRAM/INTERVENTION): Measures of success for this pilot were to both improve the staff's perception of the organization of the discharge process, and to improve the team's ability to accurately predict the date of discharge.

FINDINGS TO DATE (IT IS NOT SUFFICIENT TO STATE "FINDINGS WILL BE DISCUSSED"): Over a 3-month period in which the pilot was implemented and iterated, the perception of an organized and well-coordinated discharge rose from 46 to $80 \%$. The ability of the team to predict an accurate discharge date increased from $30 \%$ to $48 \%$.

KEY LESSONS FOR DISSEMINATION (WHAT CAN OTHERS TAKE AWAY FOR IMPLEMENTATION TO THEIR PRACTICE OR COMMUNITY?): Coordination of the discharge process is a critical component in the care of hospitalized patients. Clinical decisions and arrangements for follow-up made at the time of discharge have the potential to significantly impact the patient's health and safety, as well as chance of readmission. Improved management of the discharge process also creates more time to spend with patients and their families to discuss their hospitalization and plans for follow-up, and to communicate with outpatient providers. Depending on patient volume, acuity, and level of involvement of residents, inpatient medicine teams in many other settings likely experience disorganized, rushed, or delayed discharges. The interventions developed here, while suited to this particular general medicine inpatient team, highlight two approaches that may be useful to teams in diverse settings: drawing on multi-disciplinary input to estimate the date of discharge, and implementing a structured method to communicate and disseminate this date.

DRIVE TO BEAT SHINGLES EXCEEDS EXPECTATIONS Radha M. Rao; Himabindu Kadiyala; Charles Wright; Nicholas Masozera. Michael E Debakey, Houston, TX. (Control ID \#1338848)

STATEMENT OF PROBLEM OR QUESTION (ONE SENTENCE): Herpes Zoster vaccination rates have traditionally been low in our outpatient primary care clinics at the Michael E Debakey center after it was approved for patients 60 and older in 2006

OBJECTIVES OF PROGRAM/INTERVENTION (NO MORE THAN THREE OBJECTIVES): To see if vaccination rates for Zoster can be improved in a tertiary VA medical center through educating nurses and providers and increasing supply

DESCRIPTION OF PROGRAM/INTERVENTION, INCLUDING ORGANIZATIONAL CONTEXT (E.G. INPATIENT VS. OUTPATIENT, PRACTICE OR COMMUNITY CHARACTERISTICS): Zoster vaccine was approved for adults 60 and older in 2006 . The most recent federal survey reports state as of 2009 only 10 percent of adults 60 and older were vaccinated against shingles Obstacles like intermittent shortages that last months have kept Merck from consistently marketing the vaccine and have forestalled public health campaigns that could have built awareness of the need for it. With Zostavax again in short supply, a C.D.C. advisory committee in June declined to vote on whether to recommend the vaccine for people in their $50 \mathrm{~s}$, even though the F.D.A. had already approved it for that group in March 2011, Recent attention on this vaccine prompted us to review our electronic medical records and we found that only 272 veterans had received the zoster vaccine out of a total of 18,656 eligible patients from 2006 to 0 ctober 2011.700 vials of Zoster vaccine were purchased from Merck in bulk in October 2011.All the Primary care providers were notified that there was fairly large supply of vaccines available. Veterans aged 60 and above both males and females, coming into the primary care clinics at the Houston VA for routine visit were identified by the epidemiologist in the department. Nurses received education from the epidemiologist and the pharmacy representative. Nurses and providers were given a list of eligible patients that had no contraindications at the beginning of the week between October 24 th 2011 and Dec 9th 2011. These patients were offered the zoster vaccine

MEASURES OF SUCCESS (DISCUSS QUALITATIVE AND/OR QUANTITATIVE METRICS WHICH WILL BE USED TO EVALUATE PROGRAM/INTERVENTION): Data regarding zoster vaccination was entered by the nurses in the electronic medical records. This data was later reviewed for accuracy and collected by the epidemiologist on site.It was determined that about 1000 patients above the age of 60 visited the primary care clinics in those 9 weeks between October 24th and December 9th 2011 and of those 700 got vaccinated

FINDINGS TO DATE (IT IS NOT SUFFICIENT TO STATE "FINDINGS WILL BE DISCUSSED"): A record number of vets, approximately 700 of them were vaccinated between the months of Oct 24th 2011 and December 9th 2011

KEY LESSONS FOR DISSEMINATION (WHAT CAN OTHERS TAKE AWAY FOR IMPLEMENTATION TO THEIR PRACTICE OR COMMUNITY?): Raising awareness through education and reminders among providers and nurses and increasing vaccine supply on site can dramaticaly improve vaccination rates

ECHO: AN INNOVATIVE CAMPUS-COMMUNITY PARTNERSHIP FOR MANAGING RESISTANT HYPERTENSION IN AN URBAN UNDERSERVED AREA Christopher Masi ${ }^{1}$; Tamara Hamlish ${ }^{2}$; Andrew M. Davis ${ }^{1}$; Kristine Bordenave ${ }^{3}$; Stephen Brown ${ }^{4}$; Brenda Perea ${ }^{5}$; Glen Aduana $^{6}$; Marcus B. Wolfe ${ }^{7}$; George Bakris ${ }^{1}$; Daniel Johnson ${ }^{2} .{ }^{1}$ University of Chicago, Chicago, IL; ${ }^{2}$ University of Chicago, Chicago, IL; ${ }^{3}$ Humana Inc., Chicago, IL; ${ }^{4}$ University of Illinois, Chicago, IL; ${ }^{5}$ Advocate Healthcare Systems, Chicago, IL; ${ }^{6}$ Chicago Family Health Center, Chicago, IL; ${ }^{7}$ University of Chicago, Chicago, IL. (Control ID \#1291887)

STATEMENT OF PROBLEM OR QUESTION (ONE SENTENCE): Providers at community health centers often have limited access to specialists for assistance in managing complex, chronic diseases, including resistant hypertension.

OBJECTIVES OF PROGRAM/INTERVENTION (NO MORE THAN THREE OBJECTIVES): The objectives of this intervention were to increase 1) hypertension management knowledge and 2) hypertension management self-efficacy among primary care providers (PCP's) at six Federally Qualified Health Centers (FQHC's) on Chicago's South Side.

DESCRIPTION OF PROGRAM/INTERVENTION, INCLUDING ORGANIZATIONAL CONTEXT (E.G. INPATIENT VS. OUTPATIENT, PRACTICE OR COMMUNITY CHARACTERISTICS): We created an interactive videoconference network in collaboration with six urban FQHC's to support Project ECHO (Extension for Community Healthcare Outcomes), a 12-session educational program designed to teach state-of-the-art management of resistant hypertension. Each one-hour session began with a 20-minute lecture by a university-based hypertension specialist. PCP's then presented cases of patients with resistant hypertension. After each case, the hypertension specialist led an interactive discussion across the participating sites regarding management of the case. Learning occurred through the hypertension lectures, as well as through the case discussions. We hypothesized that this 'mini-fellowship,' case-based approach would enhance hypertension management knowledge and self-efficacy in the intervention group but not among controls.

MEASURES OF SUCCESS (DISCUSS QUALITATIVE AND/OR QUANTITATIVE METRICS WHICH WILL BE USED TO EVALUATE PROGRAM/INTERVENTION): Carter's 26-item hypertension management questionnaire was used to measure PCP knowledge at baseline and immediately following the intervention. We adapted Arora's disease management self-efficacy scale $(1=$ no skill at all, $7=$ expert $)$ to measure PCP confidence in managing hypertension at baseline and post-intervention. Twelve PCP's (9 intervention and 3 controls) participated in the study.

FINDINGS TO DATE (IT IS NOT SUFFICIENT TO STATE "FINDINGS WILL BE DISCUSSED"): Demographic characteristics of the 9 intervention participants included a mean age of 33 years $(\mathrm{SD}=4.3)$, mean duration of practice of 6.8 years $(\mathrm{SD}=10.49), 7$ female, 7 physicians, and 2 physician assistants; 3 were Caucasian, 1 African-American, 1 Latino, 2 Asian/Pacific Islander, and 2 East Indian. The mean number of correct answers on the 26-item hypertension knowledge test increased significantly in the intervention group (13.11 $(\mathrm{SD}=3.06)$ to $17.44(\mathrm{SD}=1.59), \mathrm{p}<.01)$ but not in the control group (14.33 $(\mathrm{SD}=3.21)$ to $13.00(\mathrm{SD}=3.46), \mathrm{p}=.06)$. Similarly, the mean score on the 7-item hypertension management selfefficacy scale increased in the intervention group $(4.68(\mathrm{SD}=.75)$ to 5.41 $(\mathrm{SD}=.55), \mathrm{p}<.01)$ but not in the control group $(5.29(\mathrm{SD}=.49)$ to 5.62 
$(\mathrm{SD}=.62), \mathrm{p}=.11)$. Upon completion of the follow-up surveys, the PCP's requested additional curricula in diabetes, congestive heart failure, obesity, rheumatology, and dermatology. They also suggested that the sessions be archived for future use

KEY LESSONS FOR DISSEMINATION (WHAT CAN OTHERS TAKE AWAY FOR IMPLEMENTATION TO THEIR PRACTICE OR COMMUNITY?): By creating a community of learners using videoconference technology, the ECHO model increased hypertension management knowledge and self-efficacy among PCP's in an urban underserved area. The case-based, interactive discussions created learning opportunities for all participants, not just for those who presented cases. Videoconferencing is a convenient way to enhance interaction between community health center providers and university-based specialists, thereby increasing the likelihood that uninsured and underinsured patients will receive state-of-the-art care for complex, chronic diseases.

ELECTRONICALLY REPORTED INFORMATION FOR PATIENTCENTERED PRIMARY CARE Arlene E. Chung ${ }^{1,2}$; Matthew Waters ${ }^{1}$; Shaun McDonald ${ }^{1}$; Carmen L. Lewis ${ }^{1}$. ${ }^{1}$ University of North Carolina at Chapel Hill School of Medicine, Chapel Hill, NC; ${ }^{2}$ University of North Carolina at Chapel Hill, Chapel Hill, NC. (Control ID \#1318391)

STATEMENT OF PROBLEM OR QUESTION (ONE SENTENCE): Patient-reported information is difficult to obtain in time for patient visits, so innovative approaches to obtain this information are needed to improve patient-centered care.

OBJECTIVES OF PROGRAM/INTERVENTION (NO MORE THAN THREE OBJECTIVES): 1.To leverage health IT to facilitate patient-centered care through a web-based tool to collect electronically reported patient information. 2.To integrate this electronic information into the electronic health record (EHR) for review prior to visits.

DESCRIPTION OF PROGRAM/INTERVENTION, INCLUDING ORGANIZATIONAL CONTEXT (E.G. INPATIENT VS. OUTPATIENT, PRACTICE OR COMMUNITY CHARACTERISTICS): Our innovative program was implemented in a large, academic general medicine clinic. A web-based survey tool was created to improve patient-centered care by enabling collection of electronic information. The tool allows patients to enter 3 top health issues (agenda setting) that he/she want to discuss at their upcoming primary care provider (PMD) visit and collect other patient information. The tool interfaced to link the data securely into our EHR, which sent PMDs a note with agenda items for review before the visit. Inclusion criteria were all patients who had PMD visits and an email address on file. The tool has other functions, which will be added over the coming months including review of systems, decision support tools, etc. MEASURES OF SUCCESS (DISCUSS QUALITATIVE AND/OR QUANTITATIVE METRICS WHICH WILL BE USED TO EVALUATE PROGRAM/INTERVENTION): Completion/non-completion of the survey tool were assessed. Patient's opinions about the survey tool were collected and categorized as positive, neutral, or negative. Patient satisfaction including questions which were rated on a 5 point scale (1-strongly agree, 5 -strongly disagree): "I liked being able to tell my doctor about issues I wanted to address before my clinic appointment;" "I liked being contacted by email to prepare for my appointment and share my needs in advance," "The website was easy to use;" and "This is an important new service that Internal Medicine should continue to offer." For non-completers, we attempted to contact patients to determine reasons for non-completion. Patient's comments about the survey were elicited and recorded verbatim by a research assistant and were coded by 2 investigators for themes on barriers/facilitators for completion and attitudes about the survey tool.

FINDINGS TO DATE (IT IS NOT SUFFICIENT TO STATE "FINDINGS WILL BE DISCUSSED"): 168 patients (pts) were eligible and sent an email web-link to the survey tool over 4 months. $26 \%$ (43 pts) completed the tool and $74 \%$ did not. 9 of 25 , who did not complete the survey, logged on. For those who completed the tool, average satisfaction (see above) ranged from 1.72-1.95 (1=strongly agree, 5=strongly disagree). 25 of 43 pts completed the satisfaction survey. Over $80 \%$ had positive opinions about the survey tool vs. $58 \%$ among non-completers. 8 pts had negative opinions with most citing "security" concerns. Comments elicited were grouped into themes: liked the tool, felt it helped prepare for their visit, felt it helped providers prepare, security concerns, felt it was unnecessary/too time-consuming, or preferred other communication. 54 of 87 pts were contacted and reached in post-survey follow-up for non-completers. The reasons for non-completion were mainly not receiving the email link or the patient forgetting to complete the survey (64\%).

KEY LESSONS FOR DISSEMINATION (WHAT CAN OTHERS TAKE AWAY FOR IMPLEMENTATION TO THEIR PRACTICE OR COMMUNITY?): 1.Validate email addresses by sending test messages prior to full implementation. 2.Consider providing information about data security since patients cited security concerns. 3.To ensure timely delivery of information to PMDs and to sustain the program, it must be integrated into existing EHR systems.

EVIDENCED-BASED DECISION SUPPORT TO ENHANCE STRESS TEST ORDERING: A QI PROJECT John Mafi ${ }^{1}$; Diane Brockmeyer $^{1}$; Ryan Nall ${ }^{1}$; Kristin A. Cox ${ }^{2}$; Kenneth Mukamal ${ }^{1}$. ${ }^{1}$ Beth Israel Deaconess Medical Center, Brookline, MA; ${ }^{2}$ Newton-Wellesley Hospital, Newton, MA. (Control ID \#1338808)

STATEMENT OF PROBLEM OR QUESTION (ONE SENTENCE): Despite the publication of multiple evidenced-based guidelines, cardiac stress imaging remains an expensive and increasingly over-utilized diagnostic modality.

OBJECTIVES OF PROGRAM/INTERVENTION (NO MORE THAN THREE OBJECTIVES): A guideline-based electronic decision support tool to enhance stress test ordering will significantly reduce cost, radiation exposure, unnecessary downstream procedures, and potentially improve cardiac catheterization diagnostic yield. The intervention will provide non-inferior quality of care among primary care patients with stable chest pain syndromes.

DESCRIPTION OF PROGRAM/INTERVENTION, INCLUDING ORGANIZATIONAL CONTEXT (E.G. INPATIENT VS. OUTPATIENT, PRACTICE OR COMMUNITY CHARACTERISTICS): We have designed an evidenced-based electronic decision support tool that calculates the pretest probability of coronary ischemia based on age, gender, and anginal sub-type. The provider answers two brief multiplechoice questions, and the program uses those answers along with the electronic record to prompt to the appropriate stress test to order. For example, for an ambulatory patient who has a normal ECG and a low pretest probability of ischemia, the tool will prompt to a non-imaging exercise treadmill stress test. The use of imaging would require free-text justification. The study will then prospectively analyze primary care providers who use the electronic decision support tool to order stress tests versus usual care. The intervention groups will consist of 2 out of 4 academic primary care clinics with 2 comparable control clinics.

MEASURES OF SUCCESS (DISCUSS QUALITATIVE AND/OR QUANTITATIVE METRICS WHICH WILL BE USED TO EVALUATE PROGRAM/INTERVENTION): Outcomes of interest include (1) number of stress tests and stress test type ordered (including order attempts/diversions) over a 1 year period among intervention versus controls (including associated costs and radiation exposure), (2) ratio of positive to negative tests in both groups, (3) cardiac catheterization diagnostic yield (those with stenosis $>50 \%$ ), (4) provider satisfaction with the decision support tool, and (5) rates of MI and all-cause mortality between the two groups.

FINDINGS TO DATE (IT IS NOT SUFFICIENT TO STATE "FINDINGS WILL BE DISCUSSED"): Findings to date reveal the following: of the 51 cardiac stress tests ordered in February, 2011, 32 or 59\% used imaging. After retrospectively applying the guideline-based electronic decision support tool, 15 or $47 \%$ of providers would have been prompted to order a non-imaging exercise treadmill stress test (all 15 were ambulatory and had normal ECGs). While imaging was usually not inappropriate, it was not necessary to use imaging in any of these 15 cases. This could significantly reduce healthcare costs and prevent 10 milliSieverts ( $=200$ chest x-rays) of radiation exposure per nuclear study avoided.

KEY LESSONS FOR DISSEMINATION (WHAT CAN OTHERS TAKE AWAY FOR IMPLEMENTATION TO THEIR PRACTICE OR COMMUNITY?): In the new era of Accountable Care Organizations, rational decision support tools for expensive diagnostics will need to play a central role in providing cost-effective and high quality care. Changing 
clinician behavior remains one of the most vexing challenges in modern medicine. Education is necessary but not sufficient in implementing real change, as evidence reveals its effects alone are temporary at best. Seamless and intelligent decision support can bring guidelines to the point of care, improving the cost-effectiveness and quality of healthcare.

EVOLUTION AND REFINEMENT OF A DEPRESSION DISEASE MANAGEMENT PROGRAM IN AN ACADEMIC INTERNAL MEDICINE PRACTICE Kristen Amann; Paul Chelminski. University of North Carolina at Chapel HIll, Chapel HIIl, NC. (Control ID \#1312415)

\section{STATEMENT OF PROBLEM OR QUESTION (ONE SENTENCE)} Depression is common in general practice and complicates the management of chronic disease.

OBJECTIVES OF PROGRAM/INTERVENTION (NO MORE THAN THREE OBJECTIVES): We developed and refined a depression disease management program in an academic internal medicine practice with both resident and faculty providers. The objectives of this quality improvement project were: 1 . Apply a validated screening and treatment metric clinic-wide for depression, the Patient Health Questionnaire 9 (PHQ-9). 2. Implement a depression algorithm coupled with an electronic registry to optimize population-based management. 3. Integrate a mid-level provider with expertise in problem-solving therapy into a multi-disciplinary team.

DESCRIPTION OF PROGRAM/INTERVENTION, INCLUDING ORGANIZATIONAL CONTEXT (E.G. INPATIENT VS. OUTPATIENT, PRACTICE OR COMMUNITY CHARACTERISTICS): Our intervention unfolded over several years with multiple PDSA cycles spearheaded by residents. The major cycles were: 1 . A first investigator described the burden of depression in clinic patients with diabetes (2009). 2. An evidence-based screening and treatment algorithm was incorporated into the clinic's visit planner and refined over five PDSA cycles (2009-2010). 3. The clinic hired a social worker experienced in problem-solving therapy (2010). 4. Three interim analyses of algorithm uptake led to refinement of depression care (2010-2011). MEASURES OF SUCCESS (DISCUSS QUALITATIVE AND/OR QUANTITATIVE METRICS WHICH WILL BE USED TO EVALUATE PROGRAM/INTERVENTION): Measures of treatment algorithm adherence varied according to PDSA cycle. These measures included: 1 . Percentage of patient visits where depression was addressed and managed according to the algorithm. 2. Change in patient PHQ-9 scores.

FINDINGS TO DATE (IT IS NOT SUFFICIENT TO STATE "FINDINGS WILL BE DISCUSSED"): A needs assessment (2009) indicated under-diagnosis and under-treatment of depression. This was especially true in patients with a high burden of non-psychiatric co-morbidities. We found that $24 \%$ of diabetic patients had affective or anxiety disorders and that non-intervention was common. For example, only $39 \%$ of patients with mental illness had a documented mood assessment. This led to the implementation of PHQ-9 screening for diabetic patients. High levels of depression were found, but non-intervention persisted. Subsequent PDSA cycles investigated barriers to provider intervention. Some barriers were systems based; others, provider based. To improve intervention, we next developed a depression algorithm and integrated a provider trained in problem-solving therapy. An interim analysis (July 2011) of 897 patients screened over a two-month period showed that providers adhered to the algorithm in $69 \%$ and $85 \%$ of patients with moderate and severe depression, respectively. After further process refinements, algorithm adherence rates were assessed on 150 patients. This analysis showed an increase in adherence from $69 \%$ to $92 \%$ in patients with moderate depression and from $85 \%$ to $93 \%$ in patients with severe depression. KEY LESSONS FOR DISSEMINATION (WHAT CAN OTHERS TAKE AWAY FOR IMPLEMENTATION TO THEIR PRACTICE OR COMMUNITY?): Over three years, multiple PDSA cycles led to substantive improvements in process measures of depression care linked to better outcomes. Resident physicians spearheaded these cycles. Quality improvement is a powerful educational tool that benefits patients.

FITNESS NOW: A PHYSICIAN-RUN EXERCISE PROGRAM Charmaine S. Wright; Sara Slattery; Edernst Noncent; Cindy Armstrong. University of Pennsylvania School of Medicine, Philadelphia, PA. (Control ID \#1339465)
STATEMENT OF PROBLEM OR QUESTION (ONE SENTENCE): In an urban academic Internal Medicine practice in Philadelphia, $42 \%$ of all patients have BMI (body mass index) $>30 \mathrm{~kg} / \mathrm{m} 2$ and $15 \%$ have diabetes mellitus type II. This population demonstrates several previouslystudied barriers to fitness including low self efficacy, low health literacy, and limited disposable income to engage in structured exercise and nutrition instruction.

OBJECTIVES OF PROGRAM/INTERVENTION (NO MORE THAN THREE OBJECTIVES): The goal of Fitness Now is to create a supervised low cost exercise program in the waiting room of the clinical practice, centered on partnership between the patient and practice physicians, emphasizing safety.

MEASURES OF SUCCESS (DISCUSS QUALITATIVE AND/OR QUANTITATIVE METRICS WHICH WILL BE USED TO EVALUATE PROGRAM/INTERVENTION): Components of the first pilot included 32 sessions of live exercise class with fitness lesson moderated by a personal trainer and practice physician, pedometer usage, financial incentive, and social support provided by phone and text messages. The majority of patients were referred by their health care provider $(n=38)$, but a few self-referred after seeing posted fliers in the waiting room $(n=10) .41$ participants were women, 40 designated their race as black, 18 were diabetic, and all had BMI $>28 \mathrm{~kg} / \mathrm{m} 2$.

MEASURES OF SUCCESS (DISCUSS QUALITATIVE AND/OR QUANTITATIVE METRICS WHICH WILL BE USED TO EVALUATE PROGRAM/INTERVENTION): Of those followed for 16 weeks with post program evaluation, 12 were regular class participants receiving supportive calls and texts, and 18 received calls and texts alone and never participated in a class. There was no difference between these two groups in the following measures taken at the start of the program: mean health rating (3.1 on a 5 point Likert scale), confidence that they would follow through with the class (4.5), or self-efficacy for exercise (29 out of 50 on a 10 -question validated scale). However, weight loss was larger among those who came to class $(-7.3$ (3.0) pounds versus 0.5 (5.5) pounds, $\mathrm{p}=0.001)$ and self-efficacy for exercise in post program evaluation was higher among those who came to class (1.2 (4.0) versus $-11.2(8.3), \mathrm{p}=0.003)$.

FINDINGS TO DATE (IT IS NOT SUFFICIENT TO STATE "FINDINGS WILL BE DISCUSSED"): This pilot identified several barriers to exercise and healthy eating in an urban patient population namely compliance, but shows significant short-term weight loss and increased selfefficacy for exercise when virtual support was coupled with live classes.

KEY LESSONS FOR DISSEMINATION (WHAT CAN OTHERS TAKE AWAY FOR IMPLEMENTATION TO THEIR PRACTICE OR COMMUNITY?): We demonstrate the importance of motivating healthy behavior change in high-risk patients in places both real and virtual, outside of the regular office visit. Planned further piloting of the program and a randomized controlled trial is needed to test this intervention on a larger scale with long-term follow-up.

IDENTIFYING OPERATIONAL FACTORS AFFECTING PATIENT'S UNDERSTANDING OF TREATMENT Jessica J. Chen; John Fontanesi. University of California at San Diego, La Jolla, CA. (Control ID \#1341290)

STATEMENT OF PROBLEM OR QUESTION (ONE SENTENCE): Can clinic operation condition affect pt's understanding of treatment? OBJECTIVES OF PROGRAM/INTERVENTION (NO MORE THAN THREE OBJECTIVES): confirming operational factors do affect patient's understanding of treatment identifying operational factors which affect patient's understanding of treatment

DESCRIPTION OF PROGRAM/INTERVENTION, INCLUDING ORGANIZATIONAL CONTEXT (E.G. INPATIENT VS. OUTPATIENT, PRACTICE OR COMMUNITY CHARACTERISTICS): 75 clinic encounter workflow observations were conducted at two primary care clinics in a single academic center. Observations were encoded using Observational Checklist of Patient Encounter (OCPE), which document time requirement and operation conditions occurring at the time of service. MEASURES OF SUCCESS (DISCUSS QUALITATIVE AND/OR QUANTITATIVE METRICS WHICH WILL BE USED TO EVALUATE PROGRAM/INTERVENTION): At conclusion of each observed clinic visit, patients were asked to select from a checklist of specific treatment 
recommendations and/or discussion topics for specific test results. Checklist items included 'your doctor discussed laboratory results, your doctor ordered laboratory studies, your doctor reviewed your medications, your doctor changed your medications etc. Patient recollection was then compared against provider documentation and scored for congruence.

FINDINGS TO DATE (IT IS NOT SUFFICIENT TO STATE "FINDINGS WILL BE DISCUSSED"): patient's ability in hearing provider recommendation are strongly correlate with the amount of wait time before seeing the provider the "wait-time" was strongly correlated with provider to staff ratio and provider to exam room ratio

KEY LESSONS FOR DISSEMINATION (WHAT CAN OTHERS TAKE AWAY FOR IMPLEMENTATION TO THEIR PRACTICE OR COMMUNITY?): recognizing that patient's understanding of treatment plan is not solely dependant on the quality of physician-patient direct interaction excessive patient wait-time could diminish quality of physician-patient interaction and understanding of treatment plan excessive wait-time are closely linked to provider to staff ratio and provider to exam ratio clinic operation factors can affect the quality of patient care

IMPLEMENTATION AND EVALUATION OF AN INNOVATIVE CHILDHOOD CANCER SURVIVOR FOLLOW-UP CLINIC: TACTIC Linda Overholser ${ }^{1}$; Brian Greffe ${ }^{3}$; Timothy Garrington ${ }^{3}$; Kristin Leonardi-Warren ${ }^{4}$; Kristin Kilbourn ${ }^{2}$; Traci Yamashita ${ }^{1}$. ${ }^{1}$ University of Colorado Denver, Denver, CO; ${ }^{2}$ University of Colorado Denver, Denver, $\mathrm{CO} ;{ }^{3}$ Childrens Hospital Colorado, Denver, CO $;{ }^{4}$ University of Colorado Hospital, Denver, CO. (Control ID \#1334953)

STATEMENT OF PROBLEM OR QUESTION (ONE SENTENCE): A mechanism to facilitate the transition of care for childhood cancer survivors into adulthood must include coordination between pediatric oncologists and adult primary care due to the significantly increased risk for late and long term effects in this population.

OBJECTIVES OF PROGRAM/INTERVENTION (NO MORE THAN THREE OBJECTIVES): 1) Implement a primary care based clinic for survivors of childhood cancer; 2) Evaluate patient satisfaction with a primary care based survivorship clinic; 3) Evaluate the effect of a survivorship clinic intervention on self reported health behaviors, knowledge and confidence regarding managing health as a cancer survivor.

DESCRIPTION OF PROGRAM/INTERVENTION, INCLUDING ORGANIZATIONAL CONTEXT (E.G. INPATIENT VS. OUTPATIENT, PRACTICE OR COMMUNITY CHARACTERISTICS): The TACTIC (Thriving After Cancer Treatment is Complete) Clinic at the University of Colorado Denver was started in 2008 and is housed in the Division of General Internal Medicine adult outpatient clinic. The clinic serves adults over the age of 18 who have been treated for any childhood cancer, are at least 5 years from diagnosis and are at least 2 years from completion of therapy. After consultation with a general internist, pediatric oncologist, health psychologist and oncology nurse educator, patients receive a detailed treatment summary and risk-based survivorship care plan.

MEASURES OF SUCCESS (DISCUSS QUALITATIVE AND/OR QUANTITATIVE METRICS WHICH WILL BE USED TO EVALUATE PROGRAM/INTERVENTION): Surveys are conducted at baseline and 2 and 8 weeks following receipt of survivorship care plans; domains include patient satisfaction, health habits, self-reported health, knowledge and confidence about managing one's health. Analysis includes frequency distributions and use of Likert scales. Non-parametric methods are used to evaluate changes in knowledge and self efficacy from baseline to 8 weeks. FINDINGS TO DATE (IT IS NOT SUFFICIENT TO STATE "FINDINGS WILL BE DISCUSSED”): Since its inception 63 patients have been seen; sixteen (16) have consented to be part of the study since IRB approval was received in 2010. Median age of consenting survivors is 28 , with an average of 17.9 years since childhood cancer diagnosis. From baseline to 8 weeks after receipt of their survivorship care plan respondents $(n=7$ completing both baseline and 8 week follow up surveys) demonstrated significant improvements in ability to identify cancer survivorship resources, knowing who to talk to about specific medical issues, and identifying unique health risks associated with their cancer experience $(\mathrm{p}=.03)$. Trends towards significant improvements in ability to identify late and long-term effects of their cancer treatment were identified $(\mathrm{p}=.06)$. Patients have expressed satisfaction with the format and content of survivorship care plans. Most have preferred the adult healthcare setting (4 on a scale of 1-5) versus pediatric oncology.

KEY LESSONS FOR DISSEMINATION (WHAT CAN OTHERS TAKE AWAY FOR IMPLEMENTATION TO THEIR PRACTICE OR COMMUNITY?): The TACTIC clinic model represents a feasible, acceptable and effective way to provide risk-based care to pediatric cancer survivors and emphasizes the important role that primary care has in survivorship care. Challenges include supporting the significant resources required to prepare the treatment summaries and care plans.

IMPLEMENTATION OF FORMAL TEACHBACK TRAINING FOR NURSES TO IMPROVE POST-DISCHARGE MEDICATION ADHERENCE Joshua Metlay ${ }^{2}$; Deepshikha Charan ${ }^{1}$; Kathryn Green ${ }^{3}$; Lisa Fidyk ${ }^{4}$; Emmanuel King ${ }^{2}$. ${ }^{1}$ Perelman School of Medicine at the University of Pennsylvania, Philadelphia, PA; ${ }^{2}$ Perelman School of Medicine at the University of Pennsylvania, Philadelphia, PA; ${ }^{3}$ Hospital of the University of Pennsylvania, Philadelphia, PA; ${ }^{4}$ Penn Medicine, Philadelphia, PA. (Control ID \#1337436)

STATEMENT OF PROBLEM OR QUESTION (ONE SENTENCE): Reducing unplanned hospital readmissions is a national quality and patient safety priority and there is evidence that patient misunderstanding of medications is an important cause of avoidable readmissions.

OBJECTIVES OF PROGRAM/INTERVENTION (NO MORE THAN THREE OBJECTIVES): To determine if training nurses in the innovative teachback method would have an impact on medication related post-discharge outcomes.

DESCRIPTION OF PROGRAM/INTERVENTION, INCLUDING ORGANIZATIONAL CONTEXT (E.G. INPATIENT VS. OUTPATIENT, PRACTICE OR COMMUNITY CHARACTERISTICS): A team of nurses and physicians developed a formal training course on teachback for nurses on a general medicine, non-housestaff hospitalist unit. Teachback utilizes serial patient teaching sessions, followed by a thorough assessment of patient understanding. The first stage involved developing educational booklets which were used at a 4 hour teachback retreat with didactics on adult learning theory and health literacy. Role-playing allowed nurses to use teachback in such challenging situations as educating a diabetic patient new to insulin. Every nurse rotated as a role-player or observer, leading to self-reflection and external feedback. The session concluded with a multidisciplinary panel on transitions of care, including physicians, home care and clinic nurses, and a patient who shared his experience about the discharge transition. All 44 nurses on the unit have participated in the program, with plans for expansion to other units starting April 2012.

MEASURES OF SUCCESS (DISCUSS QUALITATIVE AND/OR QUANTITATIVE METRICS WHICH WILL BE USED TO EVALUATE PROGRAM/INTERVENTION): We studied patients discharged between April and October 2011 who were enrolled in home care nursing. Home care nurses administered a 24 question survey on medication related issues in the immediate post-discharge period. We compared responses between patients with and without teachback education, using chi-square statistics with $\mathrm{p}>.05$ indicating statistical significance.

FINDINGS TO DATE (IT IS NOT SUFFICIENT TO STATE "FINDINGS WILL BE DISCUSSED"): Of 99 patients in the study, 38 (38.4\%) had been taught by teachback trained nurses. Most $(n=55,59.1 \%)$ were on 8 or more medications, and most were on at least one "high-risk" medication such as warfarin or insulin $(\mathrm{n}=58,58.6 \%)$. Few appeared to be in the hospital directly due to medication issues $(\mathrm{n}=4,4.7 \%)$. Comparing patients who received teachback to those who did not, a similar percentage reported that they had been taught about their medications pre-discharge $(81.5 \%$ vs. $88.6 \%, p=0.40$ ). Likewise, a similar percentage were felt by the home care nurse to have a good understanding of their medications $(74.3 \%$ vs. $86.2 \%, \mathrm{p}=0.15$ ). There was no significant difference in the frequency of medication discrepancies between patients with and without teachback education $(21.6 \%$ vs. $8.6 \%, p=0.07)$. There were only two areas where we observed a significant difference. Patients who had teachback more frequently reported multiple past hospitalizations due to medications $(14.7 \%$ vs. $1.8 \%$, $\mathrm{p}=0.016$ ), and were more likely to have had multiple medication changes prior to discharge ( $50 \%$ vs. $24.5 \%, \mathrm{p}=0.01)$. 
KEY LESSONS FOR DISSEMINATION (WHAT CAN OTHERS TAKE AWAY FOR IMPLEMENTATION TO THEIR PRACTICE OR COMMUNITY?): At this preliminary stage, teachback training does not appear to have an impact on medication-related patient outcomes in the post-discharge setting. The only significant correlations appear to be in the numbers of patients who had more frequent medication-related past hospitalizations or multiple medication changes prior to discharge. This suggests that higher risk patients may have been unintentionally assigned to teachbacktrained nurses. We will follow more outcomes, including readmission rates, to further study the value of teachback training.

IMPLEMENTING A SYSTEM OF INTEGRATED POST DEPLOYMENT CARE FOR RETURNING COMBAT VETERANS Lucile Burgo-Black ${ }^{1,2}$; Stephen C. Hunt ${ }^{3,4} .{ }^{1}$ VA Connecticut Healthcare System, West Haven, CT; ${ }^{2}$ Yale University, New Haven, CT; ${ }^{3}$ VA Puget Sound Healthcare System, Seattle, WA; ${ }^{4}$ University of Washington, Seattle, WA. (Control ID \#1340184)

STATEMENT OF PROBLEM OR QUESTION (ONE SENTENCE): This presentation will describe the health impacts of combat on military personnel returning from Iraq and Afghanistan and the comprehensive, interdisciplinary system of post-deployment integrated care that has been implemented nation-wide in VHA through the collaborative efforts of primary care, mental health, social work and rehabilitation services.

OBJECTIVES OF PROGRAM/INTERVENTION (NO MORE THAN THREE OBJECTIVES): Describe the most common health concerns of Veterans returning from Iraq and Afghanistan Describe effective approaches for addressing co-morbid health concerns in this population Review data from a study of system wide implementation of the program

DESCRIPTION OF PROGRAM/INTERVENTION, INCLUDING ORGANIZATIONAL CONTEXT (E.G. INPATIENT VS. OUTPATIENT, PRACTICE OR COMMUNITY CHARACTERISTICS): The conflicts in Iraq and Afghanistan have exposed combat Veterans to risks of physical injury (including traumatic brain injury from blast wave exposure), psychological trauma, environmental agent exposure and numerous psychosocial stressors potentially impacting financial, social, and family life. A broad spectrum of mental health conditions and psychosocial difficulties are common among these Veterans; clinical presentations involve complex combinations of physical and mental health symptoms and conditions and a variety of psychosocial issues that vary widely from Veteran to Veteran. A VA-wide education and training campaign collaboratively created and implemented by all of the programs and disciplines involved in post-combat care (primary care, mental health, social work, rehabilitation services, addictions services and pain services) was created and implemented to educate and train VA staff nationally. The symptom overlap and frequency of co-occurring PTSD, chronic pain, mild TBI and substance abuse have highlighted the need for inter-disciplinary, integrated care and proactive case management/social work support. This session will describe the impacts of combat on the lives and health of Veterans returning from Iraq and Afghanistan and the model of post-deployment integrated care that has been implemented in VA to address these health issues.

MEASURES OF SUCCESS (DISCUSS QUALITATIVE AND/OR QUANTITATIVE METRICS WHICH WILL BE USED TO EVALUATE PROGRAM/INTERVENTION): Initial evaluation by Mental Health, Social Work and Primary Care on same day Continuity of care with same clinicians over time Team based care plan Integrated team meetings Team function supported by shared notes, instant messaging, warm handoffs, curbside consults. Completion of relevant clinical reminders Psychosocial screening rates Enrollment in VA secure messaging

FINDINGS TO DATE (IT IS NOT SUFFICIENT TO STATE "FINDINGS WILL BE DISCUSSED"): From the inception of the Post-Deployment Integrated Care Initiative in 2008 until end of 2010: 84\% of VA Centers have dedicated resources to specialized primary care arrangements for post-combat care. 54\% of VA Centers link their primary care, mental health and social work initial evaluations on the same day to reduce the number of visits the Veteran must make to the center. In $65 \%$ of facilities regular integrated team meetings are held, primarily between PC, $\mathrm{MH}$, and SW; $54 \%$ rated the meetings as highly useful; other providers attending these meetings include polytrauma/rehab medicine staff, suicide risk reduction staff, Pain staff, SUDs staff and Women's Health clinicians.
KEY LESSONS FOR DISSEMINATION (WHAT CAN OTHERS TAKE AWAY FOR IMPLEMENTATION TO THEIR PRACTICE OR COMMUNITY?): Train the trainer implementation nationally Importance of outreach, transition support, intake, interdisciplinary assessment and ongoing case/care management Central role of OEF/OIF/OND Program managers and case management Necessity of educating teams on military culture and unique concerns of returning service members Network of champions

IMPROVING ADHERENCE TO BEST PRACTICES FOR OPIOID PRESCRIBING IN NON-CANCER CHRONIC PAIN PATIENTS IN A PRIMARY CARE PRACTICE JoAnne Gottridge ${ }^{1}$; Mark Stokes ${ }^{1}$; Pauline Leong ${ }^{1}$; Janet R. Zolli ${ }^{1}$; Sandy Balwan ${ }^{1}$; Nissa Mazzola ${ }^{2,1}$. ${ }^{1}$ Hofstra North Shore LIJ School of Medicine, Great Neck, NY; ${ }^{2}$ St. John's University, Queens, NY. (Control ID \#1336281)

STATEMENT OF PROBLEM OR QUESTION (ONE SENTENCE): Opioid prescribing for non-cancer chronic pain has increased and been associated with large increases in abuse and overdose of prescription opioids, despite publication of best practices that can reduce these risks.

OBJECTIVES OF PROGRAM/INTERVENTION (NO MORE THAN THREE OBJECTIVES): 1. Implement current best practices for opioid prescribing in a primary care practice; 2. provide education, guidelines and decision support for best practices, including at the point of care; 3 . measure the adherence to best practices

DESCRIPTION OF PROGRAM/INTERVENTION, INCLUDING ORGANIZATIONAL CONTEXT (E.G. INPATIENT VS. OUTPATIENT, PRACTICE OR COMMUNITY CHARACTERISTICS): A large General Internal Medicine practice certified by the NCQA as a Patient Centered Medical Home, using an Electronic Medical Record (EMR) as the only patient record, and serving as a site for Internal Medicine Resident Education, cares for approximately 1000 individuals receiving chronic narcotics. To standardize and improve the care of these patients a series of interventions were developed and implemented in late, 2011. Firstly, a practice Policy and Procedure Document for narcotic prescribing was developed, based on recently published clinical guidelines and expert opinion. This Policy and Procedure document was discussed and reviewed with all providers. Secondly, a "chronic opioid" templated note for the EMR, that allows for easy documentation (point and click) of mandatory elements from the Policy and Procedure document, was provided. This templated note was developed to promote adherence to policies and procedures at the point of care, and to improve documentation of the care that was provided. Thirdly, a templated Opioid Flow Sheet in the EMR, populated by elements in the templated opioid note, was implemented, to serve as a quick review of adherence to guidelines. Lastly, a practice Narcotics Review Committee, with participation by a Clinical Pharmacist, was developed to review and make decisions about any chronic opioid patient care challenges in a team setting

MEASURES OF SUCCESS (DISCUSS QUALITATIVE AND/OR QUANTITATIVE METRICS WHICH WILL BE USED TO EVALUATE PROGRAM/INTERVENTION): Reports will be generated from the EMR to determiine adherence to policies and procedures post-intervention, including: completion of the tool for assessment of risk of opioid abuse, periodic urine drug testing, presence of narcotics agreement in the medical record, and adherence to refill protocols. The number of patient cases referred for discussion at Narcotics Review Committee will also be tracked.

FINDINGS TO DATE (IT IS NOT SUFFICIENT TO STATE "FINDINGS WILL BE DISCUSSED"): Preliminary indications are that provider acceptance and adherence to the established policies and procedures for narcotic prescribing is high. It is too soon after the intervention to determine any significant metrics.

KEY LESSONS FOR DISSEMINATION (WHAT CAN OTHERS TAKE AWAY FOR IMPLEMENTATION TO THEIR PRACTICE OR COMMUNITY?): If these described interventions are successful in improving adherence to best practices for opioid prescribing, they may serve as an example for implementation in other primary care practices that prescribe a significant amount of opioid medications. 
IMPROVING ALLERGY RECONCILIATION PROCESS IN THE AMBULATORY SETTING: FIVE MINUTES THAT CAN SAVE A LIFE AND LOTS OF MONEY! Stephen B. Erban ${ }^{1,2}$; Trudy Manchester ${ }^{1,2}$; Aimon C. Miranda ${ }^{3}$; Michelle E. Conroy ${ }^{1,2}$; Marisela Navarro ${ }^{1}{ }^{1}$ UMass Medical School, Worcester, MA; ${ }^{2}$ UMass Memorial Medical Center, Worcester, MA; ${ }^{3}$ University of South Florida, Tampa, FL. (Control ID \#1321079)

STATEMENT OF PROBLEM OR QUESTION (ONE SENTENCE): In the era of electronic health records (EHRs), information interfaces, "copy \& paste" notes, and meaningful use (MU), medication allergies and adverse drug reactions tend to accumulate in the EHR and are rarely critically reviewed or reconciled; this project highlighted the importance of allergy reconciliation along with providing educational interventions for clinicians to improve allergy reconciliation.

OBJECTIVES OF PROGRAM/INTERVENTION (NO MORE THAN THREE OBJECTIVES): 1.Review the impact of inaccurate allergy data on the cost and quality of care. 2. Describe the intervention and impact. 3. Future directions for work.

DESCRIPTION OF PROGRAM/INTERVENTION, INCLUDING ORGANIZATIONAL CONTEXT (E.G. INPATIENT VS. OUTPATIENT, PRACTICE OR COMMUNITY CHARACTERISTICS): UmassMemorial Healthcare in is an integrated delivery system in Central Massachusetts that utilizes the Allscripts Enterprise EHR in most ambulatory sites. There are approximately 400,000 active patient records in the EHR. Approximately 36,200 (9\%) of these records have "penicillin allergy" and 22,000 (5.5\%) have some version of "sulfa allergy". Prior work suggests that most these labels are not valid when more detailed history or skin testing is utilized. The intervention was made in a hospital-based, adult general internal medicine practice with 31 attending physicians and 4 nurse practitioners. Residents were excluded (low patient volumes). A multifaceted intervention was designed to improve the accuracy of the allergy data in the EHR. Components included: 1) Patient education and patient self-review of their allergy data; 2) Face-to-face didactic education for physicians and NPs on allergy reconciliation; 3) email distribution of educational materials to clinicians after the didactic presentation.

MEASURES OF SUCCESS (DISCUSS QUALITATIVE AND/OR QUANTITATIVE METRICS WHICH WILL BE USED TO EVALUATE PROGRAM/INTERVENTION): The Allscripts EHR tracks allergy data activity to allow queries for how often data was viewed (allergy tab clicked on) or changed (created, deleted or edited). Baseline rates for viewing and changing allergy data were measured for all clinicians. Post-intervention rates were reassessed about 10 weeks after the presentation and about 2 weeks after the emailed distribution of information.

FINDINGS TO DATE (IT IS NOT SUFFICIENT TO STATE "FINDINGS WILL BE DISCUSSED"): Two of 4 NPs and 15 of 31 physicians attended a single $30^{\prime}$ presentation on this topic. It is unclear how many clinicians viewed the emailed intervention. Follow-up rates of review and modification of allergy data in the EHR was measured. Baseline rate for physician viewing and changing allergy data was $52.3 \%$ and $12.6 \%$, respectively. Post-intervention rates were $49.8 \%(-2.5 \%)$ and $12.4 \%(-0.2 \%)$, respectively. Baseline rates for NPs viewing and changing were $75.8 \%$ and $10.6 \%$ respectively. The post-intervention rates were $90 \%$ $(+14.3 \%)$ and $10 \%(-0.6 \%)$.

KEY LESSONS FOR DISSEMINATION (WHAT CAN OTHERS TAKE AWAY FOR IMPLEMENTATION TO THEIR PRACTICE OR COMMUNITY?): 1 . There is no "gold standard" as to how often allergy data should by viewed, edited, and/or reconciled - or by whom and in what setting. 2. Individual provider behavior with respect to viewing, editing, and reconciling allergy data varies widely. It appears that allergy reconciliation is often ignored, which may lead to lower quality and higher cost of care. 3. Improving performance will likely require more than a multifaceted educational approach involving patients, providers and staff. 4. Nurse practitioners in our setting responded positively to this intervention, whereas there was no significant change in physician behavior. Strategies to incorporate nursing support staff and/or pharmacists may be more successful and help to leverage scarce resources to do this work.
IMPROVING CANCER SCREENING USING A NOVEL PATIENTCENTRIC, POPULATION-BASED HEALTH INFORMATION TECHNOLOGY SYSTEM Jeffrey M. Ashburner; Adrian Zai; Richard W. Grant; Sanja Percac-Lima; Steven K. Wong; Charlotte E. Ward; Steven J. Atlas. Massachusetts General Hospital, Boston, MA. (Control ID \#1336967)

STATEMENT OF PROBLEM OR QUESTION (ONE SENTENCE): Evidence suggests health information technology (HIT) can improve quality of care, but it remains uncertain how to use HIT systems to most effectively and efficiently deliver care.

OBJECTIVES OF PROGRAM/INTERVENTION (NO MORE THAN THREE OBJECTIVES): To implement a novel HIT system that uses a visit-independent, patient-centric approach to screen a primary care population for preventive cancer screening. We hypothesize that involving primary care providers (PCPs) by using their unique knowledge about her/his patient panel to direct outreach will result in more effective and efficient care compared to an automated HIT screening system.

DESCRIPTION OF PROGRAM/INTERVENTION, INCLUDING ORGANIZATIONAL CONTEXT (E.G. INPATIENT VS. OUTPATIENT, PRACTICE OR COMMUNITY CHARACTERISTICS): We designed the TopCare system (Technology for Optimizing Population Care in A Resource-limited Environment) to identify all patients seen in primary care practices affiliated within an academic hospital-based network who are eligible and overdue for breast, cervical, and/or colorectal cancer screening. Practices were randomly assigned to intervention or control groups. In intervention practices, PCPs screen their panel of overdue patients and decide whether and by which method to contact a patient. Providers may choose to 1 ) have a reminder letter sent to the patient followed by tracking and outreach by a scheduling delegate, 2) directly send the patient to a delegate for prioritized outreach, or 3) directly send a limited number of patients to a health navigator for more intensive outreach. In control practices, this process is sequentially automated: Overdue patients first receive a reminder letter and are transferred to a scheduling delegate list. Patients unscheduled after 4 months are referred to a health navigator if risk-based decision support identifies them as likely to not undergo screening. Patients in intervention practices default to the automated outreach if their PCP does not take action.

MEASURES OF SUCCESS (DISCUSS QUALITATIVE AND/OR QUANTITATIVE METRICS WHICH WILL BE USED TO EVALUATE PROGRAM/INTERVENTION): Primary outcomes include average cancer screening test completion over 1-year of follow-up for each eligible patient in all eligible cancers, and for each individual cancer. Secondary outcomes include measures of system usage, cancer detection rates, provider satisfaction, and cost-effectiveness analyses.

FINDINGS TO DATE (IT IS NOT SUFFICIENT TO STATE "FINDINGS WILL BE DISCUSSED"): 96,491 patients were eligible for breast, cervical, and/or colorectal cancer screening as of the trial start date of June 15, 2011. As of October 31, 2011, 73 of 94 (78\%) intervention providers have used the TopCare tool and reviewed 6969 patients overdue for at least one screening test. Among these patients, 5141 were selected to receive a reminder letter, 334 were sent directly to a scheduling delegate for follow-up, and 26 were sent to a health navigator. Another 6600 patients received letters through an automated mechanism after no provider action was taken. Additionally, providers removed 1468 patients from their roster by deferring screening $(n=$ 1113), indicating the patients receive care from a different provider and/or practice $(n=140)$, permanently excluding screening $(n=120)$, or adding updated out of network screening data $(n=95)$. In control practices, 14,196 letters were mailed to overdue patients without provider review.

KEY LESSONS FOR DISSEMINATION (WHAT CAN OTHERS TAKE AWAY FOR IMPLEMENTATION TO THEIR PRACTICE OR COMMUNITY?): Population-based HIT systems can be used to identify and contact patients regarding care outside of routine office visits, but require providers and staff input to redesign care processes and integrate such systems into existing visit-based approaches. 
IMPROVING CARE AT A SAFETY-NET RESIDENT CLINIC Michael E. Hochman ${ }^{1}$; Arek Jibilian ${ }^{2}$; David Goldstein ${ }^{2}$; Steven Asch $^{3}$; Carol Mangione ${ }^{4}$. ${ }^{1}$ robert Wood Johnson Clinical Scholars Program at UCLA and the VA, Los Angeles, CA, Los Angeles, CA; ${ }^{2}$ Keck School of Medicine, Los Angeles, CA; ${ }^{3}$ Veterans Administration of Palo Alto Healthcare System, Palo Alto, CA; ${ }^{4}$ David Geffen School of Medicine at UCLA, Los Angeles, CA. (Control ID \#1322293)

STATEMENT OF PROBLEM OR QUESTION (ONE SENTENCE): National health care reform will provide new opportunities to promote patient-centric primary care. However, implementing such changes will be challenging, particularly in safety-net settings and in resident teaching clinics where the residents are present only intermittently.

OBJECTIVES OF PROGRAM/INTERVENTION (NO MORE THAN THREE OBJECTIVES): In collaboration with the Los Angeles Department of Health Services, and with grant support, we have implemented a pilot program to expand access to care and to improve care management at a safety-net primary care internal medicine clinic operated by resident physicians.

DESCRIPTION OF PROGRAM/INTERVENTION, INCLUDING ORGANIZATIONAL CONTEXT (E.G. INPATIENT VS. OUTPATIENT, PRACTICE OR COMMUNITY CHARACTERISTICS): The program offers the following new services for patients: 1) Enhanced telephone services including telephone triage by resident physicians and after-hours access to an on-call resident physician for urgent questions; 2) medication renewals by telephone; 3) urgent care appointment availability; 4) expanded care management; and 5) outreach to patients who visit the hospital and emergency room.

MEASURES OF SUCCESS (DISCUSS QUALITATIVE AND/OR QUANTITATIVE METRICS WHICH WILL BE USED TO EVALUATE PROGRAM/INTERVENTION): Here, we describe a process evaluation of the program in its first four months following implementation. We are planning a formal, controlled evaluation that will also assess the program's impact on: 1) patient satisfaction; 2) resident physician satisfaction; 3) emergency room and hospital utilization; and 4) hemoglobin A1C and LDL control among patients with diabetes. Two other resident clinics located at the same medical center will serve as controls.

FINDINGS TO DATE (IT IS NOT SUFFICIENT TO STATE "FINDINGS WILL BE DISCUSSED"): During the first four months, $2,752(64 \%)$ of the approximately 4,300 clinic patients visited the clinic and were exposed to the new services. During month four, the call center received an average of 35.3 (SD 9.2) calls per weekday, of which 11.5 (SD 3.9) were distinct patients requiring telephone advice from a resident physician. Each week, 15.6 medications were prescribed by telephone (mostly medication renewals, but occasionally new medications). Each day, patients used 3.2 (SD 1.3) urgent care appointment slots. The care coordinators completed an average of 3.8 (SD 2.5) care management tasks per weekday and outreach to 28.3 clinic patients per week who visited the emergency room or hospital. Feedback surveys completed by clinic staff and resident physicians indicate high satisfaction with the program with scores of $\geq 4.0$ on a scale of 1 (low satisfaction) - 5 (high satisfaction) for all questions. Preliminary results from the controlled evaluation show that emergency room and hospital utilization has remained stable since program implementation: At baseline, there was an average of 79 emergency room and hospital visits per 1,000 patients per month in both the intervention and control clinics. In the first two months since program implementation, there has been an average of 78 emergency room and hospital visits per 1,000 patients in the intervention clinic vs. 80 in the control clinics $(\mathrm{P}=0.81)$.

KEY LESSONS FOR DISSEMINATION (WHAT CAN OTHERS TAKE AWAY FOR IMPLEMENTATION TO THEIR PRACTICE OR COMMUNITY?): We have implemented a program providing expanded access to care and enhanced care management at a resident safety-net primary care clinic. Thus far, feedback from residents and clinic staff has been positive. In contrast to some similar programs, we have not seen an increase in emergency room and hospitalization rates as access to care has been expanded. We believe this program, if it continues to be successful, could serve as a model for resident safety-net primary care clinics in Los Angeles County and perhaps elsewhere.
IMPROVING COORDINATION OF MEDICAL AND MENTAL HEALTH CARE FOR CO-MANAGED PREGNANT VETERANS Neha Pathak. ${ }^{1}$ Providence VA Medical Center, Providence, RI; ${ }^{2}$ Alpert Medical School Brown University, Providence, RI. (Control ID \#1316263)

STATEMENT OF PROBLEM OR QUESTION (ONE SENTENCE): We anticipate that demand for pregnancy-related care will increase among veterans and it is unlikely that prenatal care can be delivered through the VHA alone; so it is imperative that a clearly defined procedure exists between VA and non-VA providers to offer coordinated, comprehensive care as new evidence suggests an increased risk of medical and mental health problems among veterans during pregnancy.

OBJECTIVES OF PROGRAM/INTERVENTION (NO MORE THAN THREE OBJECTIVES): Our goal is to use the VA Patient Align Care Team (PACT) Model (known as the patient centered medical home in the community) to improve coordination of care between VA and non-VA providers by: 1) monitoring the number of requests for prenatal care 2) monitoring for and enhancing the management of developing mental health disorders during pregnancy within the VA 3) monitoring outcomes of pregnancy in our veterans

DESCRIPTION OF PROGRAM/INTERVENTION, INCLUDING ORGANIZATIONAL CONTEXT (E.G. INPATIENT VS. OUTPATIENT, PRACTICE OR COMMUNITY CHARACTERISTICS): We are developing a templated system utilizing the EMR and PACT within the Providence VA Women's Health Outpatient Clinic to aid our pregnant veterans in navigating a fragmented system of care. 1) Provider to complete Templated Pregnancy Note and request creation of Pregnancy Flag in EMR a) Templated Pregnancy Note to trigger: i) Review of patient history and medications ii) Referral to Clinic Nurse for authorization for release of information iii) Referral to Social Work for benefits information iv) Referral to Behavioral Health (BH) b) Copy of templated note sent to non-VA provider at initial visit c) Pregnancy Outcomes TRACKED by clinic nurse 2) BH Provider to evaluate veteran at time of pregnancy diagnosis, then 3, 6, 8 months, and post-partum with PHQ-9 (a standardized tool used to monitor for development of mental health issues in all veterans). a) Screen for post-partum depression with the Edinburgh Postnatal Depression Scale (EPDS) at 8 months and postpartum. b) Patient to be seen more frequently or referred to psychiatric services if necessary. c) Development of new behavioral health issues TRACKED by BH provider.

MEASURES OF SUCCESS (DISCUSS QUALITATIVE AND/OR QUANTITATIVE METRICS WHICH WILL BE USED TO EVALUATE PROGRAM/INTERVENTION): 1) Creation of pregnancy template note/ flag within EMR 2) Creation of standard operating procedures for each member of PACT 3) Creation of Patient Brochures to aid Pregnant Veterans in navigating the co-managed system 4) Monitoring: requests for prenatal care, completion of pregnancy template note/flag, regular VA BH follow up, documentation of new mental health diagnosis, outside documentation of pregnancy care and outcomes 5) Assessment of patient satisfaction via survey instrument

FINDINGS TO DATE (IT IS NOT SUFFICIENT TO STATE "FINDINGS WILL BE DISCUSSED"): Data prior to implementing our intervention show: 1) An annual increase in the request for prenatal care over the past 3 years. Over the past year, 17 veterans, between the ages of 21-43, requested prenatal care. 2) All veterans received prenatal care outside of the VA and did not return for medical or mental health care until after pregnancy. 3) VA providers did not formally assess for new mental health issues at any time during pregnancy, though data suggest that veterans are at higher risk for mental health disorders during pregnancy. 4) No documentation of pregnancy care or outcome from non-VA providers was scanned within EMR.

KEY LESSONS FOR DISSEMINATION (WHAT CAN OTHERS TAKE AWAY FOR IMPLEMENTATION TO THEIR PRACTICE OR COMMUNITY?): By realigning the already existing PACT resources and utilizing the EMR, we can create a sustainable, efficient protocol that can easily be implemented in other VA Medical Centers to coordinate co-managed care for an increasing numbers of pregnant veterans.

IMPROVING OSTEOPOROSIS SCREENING IN A RESIDENT-RUN PRIMARY CARE URBAN CLINIC Zahi Mitri; Anthony Gamboa; Rinku Chatterjee; Nurcan Ilksoy. Emory University, Atlanta, GA. (Control ID \#1332420) 
STATEMENT OF PROBLEM OR QUESTION (ONE SENTENCE):

The U.S preventive services task force recommends routine osteoporosis screening in all women aged 65 and older, as well as younger women whose fracture risk is equal to or greater than that of a 65 year-old white woman who has no additional risk factors.

OBJECTIVES OF PROGRAM/INTERVENTION (NO MORE THAN THREE OBJECTIVES): Bone density measurements accurately predict the risk of fractures in this patient population. Unfortunately, many of these women, despite clinic attendance, do not undergo timely screening for osteoporosis. They thus go untreated and are at increased risk of fractures. This project aimed at improving the prevalence of bone density screening through DEXA scanning to $80 \%$ in women 65 years and older in a primary care clinic.

DESCRIPTION OF PROGRAM/INTERVENTION, INCLUDING ORGANIZATIONAL CONTEXT (E.G. INPATIENT VS. OUTPATIENT, PRACTICE OR COMMUNITY CHARACTERISTICS): This quality improvement project was conducted as part of the Emory Internal Medicine Residency Performance Improvement Curriculum. It was held in the resident continuity clinic at a community teaching hospital. Team members included Emory Internal Medicine residents, the nursing and ancillary staff from Grady Memorial Hospital. A retrospective chart review was done initially to determine a baseline screening percentage, and identify potential barriers to screening. These fell into four categories: provider, patient, system, and nursing/ancillary staff related. The first test of change was implemented in 1/2011, and consisted of verbal reminders between providers. The second test of change was implemented on 2/2011, and consisted of adding a written reminder for DEXA screening to the electronic medical record clinic template. The project spanned from 11/1/2010 until 5/1/2011.

MEASURES OF SUCCESS (DISCUSS QUALITATIVE AND/OR QUANTITATIVE METRICS WHICH WILL BE USED TO EVALUATE PROGRAM/INTERVENTION): The results consisted of monthly chart reviews to tabulate the percentage of eligible patients screened for osteoporosis. The barriers to screening were divided into the aforementioned four categories. The goal was to reach $80 \%$ screening rates by the end of the project. The results were calculated using basic statistical tools. FINDINGS TO DATE (IT IS NOT SUFFICIENT TO STATE "FINDINGS WILL BE DISCUSSED"): The baseline screening rate based on data from $11 / 1 / 2010-1 / 1 / 2011$ was $50 \%$. This was similar to that found in the previous year in the same clinic. The rate of screening did not improve after the first test of change, remaining at $50 \%$. The rate however increased to $73 \%$ after the second test of change. The main barrier to screening was identified as being provider related, accounting for $100 \%$ of cases at baseline, $67 \%$ of cases after the first test of change, and $50 \%$ after the second test of change. A follow up analysis after the end of the project was done, spanning the timeline from 5/1/2011 to 7/1/2011. The rates of screening dropped down to $65 \%$ in $5 / 2011$ and $55 \%$ in $6 / 2011$. The main barrier to screening was provider related, accounting for $91 \%$ of cases.

KEY LESSONS FOR DISSEMINATION (WHAT CAN OTHERS TAKE AWAY FOR IMPLEMENTATION TO THEIR PRACTICE OR COMMUNITY?): The key lessons learned through this project were first that a large fraction of eligible patients were not undergoing routine screening for osteoporosis, the main barrier to that being provider related factors. In addition to that, it was noted that verbal reminders were ineffective in changing clinical practices. On the other hand, written reminders were successful in improving screening rates, as well as decreasing the barrier proportion that is provider related. Finally, the drop in screening rates after the end of the project may be an indicator for the need for additional tools, in addition to written reminders, to maintain awareness for appropriate screening for patients in a primary clinic setting.

INPATIENT MANAGEMENT OF THE END-STAGE RENAL DISEASE PATIENT: COMPARING GENERALISTS TO NEPHROLOGISTS AS PRIMARY CAREGIVERS Vipulkumar Rana; Julie L. Mitchell; Siddhartha Singh; David S. Marks; Hariprasad Trivedi; Sundaram Hariharan; Kory Koerner; Kavita Naik; Pinky Jha; Molly Robischon. Medical Collegel of Wisconsin, Milwaukee, WI. (Control ID \#1339395)
STATEMENT OF PROBLEM OR QUESTION (ONE SENTENCE): Is primary coordination of inpatient care for patients with End-Stage Renal Disease (ESRD) achieved more efficiently with nephrologists or generalists? OBJECTIVES OF PROGRAM/INTERVENTION (NO MORE THAN THREE OBJECTIVES): 1. Maintain or Improve Mortality and Length of Stay (LOS) Index for ESRD patients 2. Maintain or Reduce Direct and Total Costs per case for ESRD patients 3. Maintain or Improve inpatient capacity despite reduced resident work hours

DESCRIPTION OF PROGRAM/INTERVENTION, INCLUDING ORGANIZATIONAL CONTEXT (E.G. INPATIENT VS. OUTPATIENT, PRACTICE OR COMMUNITY CHARACTERISTICS): Traditionally, we have admitted ESRD patients under the care of Nephrologists to our hospital. Starting July 1st 2011, we admitted ESRD patients to general medical teams staffed by hospitalists or general internists with a Nephrology consultation for specialty care. This now allowed nephrologists to concentrate on specialty care, nephrology procedures, and outpatient visits. We hired 2.4 FTE hospitalists and 1 FTE physician assistant to staff a new Hospitalist-Physician assistant team and eliminated a resident based Nephrology team. To facilitate this transition, the nephrology service provided generalists education on the care of the ESRD patient and created systems to increase responsiveness of their consultation services.

MEASURES OF SUCCESS (DISCUSS QUALITATIVE AND/OR QUANTITATIVE METRICS WHICH WILL BE USED TO EVALUATE PROGRAM/INTERVENTION): We used University Healthsystem Consortium (UHC) methodology to calculate Mortality Index, the ratio of observed to expected deaths, and LOS Index, the ratio of observed to expected inpatient LOS. We also calculated total and direct costs per case. Direct Cost includes such services as radiological and laboratory studies. We compared data for ESRD patients discharged 12 months prior and 4 months after the intervention date ( 5 months for cost data).

FINDINGS TO DATE (IT IS NOT SUFFICIENT TO STATE "FINDINGS WILL BE DISCUSSED"): We analyzed LOS and mortality data on 1783 discharges. There were 111 patients per month before and after the intervention. The LOS index decreased from 1.16 to $0.92(\mathrm{p}<0.01)$. The mortality index increased from 0.8 to $1.1(\mathrm{p}=0.16)$. 30-day readmission rate did not change. The total and direct costs per case decreased: respectively, from $\$ 27 \mathrm{k}$ to $\$ 24 \mathrm{k}(\mathrm{p}=0.11)$, and, from $\$ 17 \mathrm{k}$ to $\$ 15 \mathrm{k}(\mathrm{p}=0.23)$. Administrative data demonstrated no loss of wRVU production by providers in either the hospitalist or nephrology services.

KEY LESSONS FOR DISSEMINATION (WHAT CAN OTHERS TAKE AWAY FOR IMPLEMENTATION TO THEIR PRACTICE OR COMMUNITY?): We found generalists were able to lower LOS in ESRD patients compared to nephrologists in the inpatient setting. The intervention may have lowered hospital costs and increased physician wRVUs. We were able to maintain inpatient capacity in the context of decreasing resident work hours. While our intervention had the possibility of worsening efficiency of care via the decreased coordination of dialysis services by generalists and relative decrease in clinical expertise of generalists, our preliminary analysis has shown the opposite to be true. We hypothesize that the decrease in LOS is the result of a responsive nephrology consultation service, preparatory education for generalists and the system expertise of generalists. We plan on building on our findings by exploring a wider variety of clinical and operational outcomes as well as studying patient and provider experience over a longer follow up period. In conclusion, our preliminary findings have merit and relevance for the planning of inpatient services and show that a transition to generalist inpatient care of ESRD patients may reduce LOS and cost.

LESSONS LEARNED IN ESTABLISHING A MEDICAL NEIGHBORHOOD BETWEEN A PRIMARY CARE MEDICAL HOME AND DERMATOLOGY SPECIALTY SERVICES. Kathleen Waite ${ }^{1}$; Scott Joy ${ }^{1}$; Linda Dorman ${ }^{2}$; Russell P. Hall ${ }^{2} .{ }^{1}$ Duke University, Durmam, NC; ${ }^{2}$ Duke University, Durham, NC. (Control ID \#1333579)

STATEMENT OF PROBLEM OR QUESTION (ONE SENTENCE): The process by which to integrate a subspecialty "neighbor" into a Patient Centered Medical Home (PCMH) to improve patient access to, and care coordination with, specialty services (SS), has not been optimally defined. 
OBJECTIVES OF PROGRAM/INTERVENTION (NO MORE THAN THREE OBJECTIVES): 1 . Create an electronic form to refer patients from a PCMH to SS (Dermatology) that defines the reason and urgency of the referral and establishes ongoing care coordination for patients. 2.Evaluate improvement in access to dermatology with the new process.

DESCRIPTION OF PROGRAM/INTERVENTION, INCLUDING ORGANIZATIONAL CONTEXT (E.G. INPATIENT VS. OUTPATIENT, PRACTICE OR COMMUNITY CHARACTERISTICS): Duke Primary Care at Pickett Road is a General Internal Medicine, Tier 3 NCQA certified PCMH in Durham, NC. The Department of Dermatology at Duke University entered into an agreement with this PCMH to serve as a medical neighbor with the intent to improve access to dermatology services for patients. Using the McKesson HAC electronic health record, a web-based electronic form was created that served multiple purposes: 1. placing a referral, 2. documenting reason for the referral, 3 . documenting the urgency of the referral (urgent - within 1 week, priority within 2 weeks, routine - within 1 month), and 4 . setting the expectation for care coordination (pre-consultation exchange to expedite care, formal consult to address discrete question, co-management with shared management of disease, co management with principal care limited time, transfer of patient to specialist for entirety of care). During the trial period, two physicians within the PCMH used the electronic form/ process to directly communicate with a triage nurse in dermatology who reviewed, processed, and scheduled the patient's referral and visit. The remaining 6 physicians used the standard referral process, creating an electronic referral sent to an in-practice referral coordinator who then contacted the dermatology scheduling hub. No comment was required regarding urgency of the referral or expectations for follow-up care.

MEASURES OF SUCCESS (DISCUSS QUALITATIVE AND/OR QUANTITATIVE METRICS WHICH WILL BE USED TO EVALUATE PROGRAM/INTERVENTION): 1 . Compare the number of days between the date of the referral and the date of the dermatology appointment between the two different referral processes. 2.Identify the most common referral priority level. 3.Observe if there was a difference in wait time depending on the referral priority level.

FINDINGS TO DATE (IT IS NOT SUFFICIENT TO STATE "FINDINGS WILL BE DISCUSSED"): From January 2011 through September 2011, 28 referrals were generated ( 23 routine, 4 priority, 1 urgent). Of the routine referrals, 1 patient declined an appointment when contacted and 4 referrals were not completed for unclear reasons. $93 \%$ of the referrals were formal consult to address a specific question and/or for procedure. There was improvement in dermatology access with the median time from date of routine referral to appointment being 36.5 calendar days (mean 38 days). This compared to the wait time for referrals done via the standard referral process during this same time varying between 150 and 180 days. Priority referrals were seen with a median of 13.5 calendar days (mean 12.5 days)

KEY LESSONS FOR DISSEMINATION (WHAT CAN OTHERS TAKE AWAY FOR IMPLEMENTATION TO THEIR PRACTICE OR COMMUNITY?): 1 . Creating an electronic process for dermatology referrals and a medical neighbor relationship improved patient access to dermatology evaluation and care. 2. Having a level of priority attached to a referral allowed patients with more clinically urgent issues to be seen before routine referral. 3. Reason for referral and expectations for follow-up care plans can be established at time of referral using a standard template.

LESSONS LEARNED WITH PATIENTS COMPLETING AN ONLINE HRA VIA A PATIENT PORTAL PRIOR TO A PRIMARY CARE OFFICE VISIT Scott Joy; Geoff Ginsburg; Jeffrey Saville; Pete L'Engle; Boyd Carlson. Duke University, Durham, NC. (Control ID \#1327053)

STATEMENT OF PROBLEM OR QUESTION (ONE SENTENCE): Health Risk Assessments (HRA's) can help providers better understand a patient's family, social, and exercise history and personal health goals, and thus it is important to develop new methods to efficiently collect this type of data before the visit and display in the electronic health record (EHR) prior to patient contact.

OBJECTIVES OF PROGRAM/INTERVENTION (NO MORE THAN THREE OBJECTIVES): 1. Select key questions from a current, paper-based HRA, convert into electronic format, integrate into an online patient portal, and push to patients to complete online prior to their annual visit 2. Include this document in the EHR for providers to review before or during the office visit 3 . Measure the time required by patients to complete the HRA, evaluate the number of patients completing each section, and identify most common personal health goals

DESCRIPTION OF PROGRAM/INTERVENTION, INCLUDING ORGANIZATIONAL CONTEXT (E.G. INPATIENT VS. OUTPATIENT, PRACTICE OR COMMUNITY CHARACTERISTICS): HRA's are often obtained outside of the office visit, are not integrated into clinical work flow, and patient responses to HRA questions are often not documented in the EHR. A HRA (Insight) has commonly been used by our HR department as part of disease management/wellness program offered by the health plan. This HRA is completed on paper or by telephone interview, and individual patient information from the HRA has not been integrated into the clinical or electronic work flow of the primary care practices. As a result, individual practices often have patients, staff or providers wastefully duplicate this effort during the office visit. We chose key questions (family history, alcohol, tobacco use, exercise patterns, personal health goals) from the existing HRA and created an original electronic document that was integrated into our patient portal and could be used to meet meaningful use criteria. Patients received an e-mail prior to their appointment that encouraged them to check in online. Patients choosing to check-in online received a message from their provider requesting that they follow a link to complete the HRA questions online prior to their visit. Patients were able to print off a summary of their responses after completing the HRA questions. The completed HRA was then stored as a document in the EHR and was able to be reviewed by the providers prior or during the patient's annual visit. A six week trial period in March-April of 2011 was chosen to analyze the operation feasibility and the data received.

MEASURES OF SUCCESS (DISCUSS QUALITATIVE AND/OR QUANTITATIVE METRICS WHICH WILL BE USED TO EVALUATE PROGRAM/INTERVENTION): Successful integration of the HRA into the patient portal and the EHR

FINDINGS TO DATE (IT IS NOT SUFFICIENT TO STATE "FINDINGS WILL BE DISCUSSED"): 107 patients completed an HRA online (estimated $15 \%$ of those receiving e-mail); Average time to complete HRA online: 4 minutes, 25 seconds (range 54 seconds to 1 hour 1 minute, 41 seconds); $100 \%$ completed exercise, smoking, and alcohol use history; $4 \%$ reported interest in reducing smoking; $8 \%$ reported interest in reducing alcohol consumption; 100\% reported interest in increasing frequency of exercise; $72 \%$ completed family history; $84 \%$ completed personal health goals; Most common health goals were reach a healthy weight and get the right amount of exercise

KEY LESSONS FOR DISSEMINATION (WHAT CAN OTHERS TAKE AWAY FOR IMPLEMENTATION TO THEIR PRACTICE OR COMMUNITY?): 1. A paper HRA can be converted into electronic form that meets meaningful use criteria, pushed to patients via an online patient portal, and a summary of the patients responses can be posted in the EHR 2. All patients completed categories for smoking, alcohol and exercise histories, fewer patients responded in categories related to family history and personal health goals

MAINTAINING CONNECTIONS BETWEEN HOSPITALIZED PATIENTS AND THE PRIMARY CARE PRACTICE AT A TEACHING COMMUNITY HEALTH CENTER James L. Wofford; Claudia Campos; Kirsten Feieriesel; Carolyn R. Pedley; Ramon Velez; Robert E. Jones. Wake Forest University, Winston-Salem, NC. (Control ID \#1320823)

STATEMENT OF PROBLEM OR QUESTION (ONE SENTENCE): When patients are hospitalized, they often lose their connections to their primary care provider and practice; as a result, follow-up appointments are haphazard, uncoordinated, confusing both patients and clinicians. OBJECTIVES OF PROGRAM/INTERVENTION (NO MORE THAN THREE OBJECTIVES): (1) Improve availability of hospital follow-up visits for established patients in the practice. (2) Improve awareness/involvement of the practice during the patient's hospitalization. 
(3) Assess changes in continuity and co-management that occur with hospitalization.

DESCRIPTION OF PROGRAM/INTERVENTION, INCLUDING ORGANIZATIONAL CONTEXT (E.G. INPATIENT VS. OUTPATIENT, PRACTICE OR COMMUNITY CHARACTERISTICS): In the hopes of making safer post-hospital transitions, we tracked all patients from a singlesite community health center clinic practice who were hospitalized at the parent hospital during a two-week period (November 2011). Newly hospitalized patients were identified through a daily computerized query of the practice registry, a spreadsheet maintained separately from the EMR. Through daily huddles, multiple rapid learning cycles, and staff meetings, we made incremental improvements in tracking hospitalized patients who belong to the practice and to an individual $\mathrm{PCP}$, and developing stronger practice ownership of the post-hospital transition period with specific timelines and policies for hospital follow-up visits (add-on to PCP's continuity schedules within two weeks of discharge)

MEASURES OF SUCCESS (DISCUSS QUALITATIVE AND/OR QUANTITATIVE METRICS WHICH WILL BE USED TO EVALUATE PROGRAM/INTERVENTION): (1) Proportion of patients who are scheduled to see their PCP within 2 weeks of hospital discharge. (2) Proportion of patents who actually attend their hospital follow-up visit. FINDINGS TO DATE (IT IS NOT SUFFICIENT TO STATE "FINDINGS WILL BE DISCUSSED"): 46 adult patients from the practice were hospitalized during the two-week pilot period (29 on general medicine/hospitalist services, 17 on speciality services). Three patients were hospitalized for symptoms suggestive of pneumonia, 10 for chest pain, and 1 for heart failure. Length of stay averaged 2.6 days $(+1.3)$, with 6 patients $(12 \%, 6 / 46)$ still in the hospital at the end of the two-week pilot. At the time of hospitalization, 3 patients had already transferred to another continuity relationship (1 patient to the VA system, one to a nursing home, and 1 dialysis patient who did not clearly belong to the practice). One patient expired during the hospitalization. Of the remaining 36 patients, 27 $(75 \%, 27 / 36)$ were given follow-up appointments in the practice by a hospitalist team with an average number of days of 7.6 days $(+3.5)$ until the hospital follow-up visit. The majority of follow-up appointments were scheduled within one week $(59 \%, 16 / 27)$ and $89 \%(24 / 27)$ within 2 weeks. Although 70\% (19/27) of patients had PCPs with available clinic schedules during the follow-up period, only $30 \%(9 / 27)$ of patients were scheduled to see their PCP. 9 patients had PCPs who were not available (maternity leave), or had resident providers who had left the practice and had not yet been reconnected with a new resident physician.

KEY LESSONS FOR DISSEMINATION (WHAT CAN OTHERS TAKE AWAY FOR IMPLEMENTATION TO THEIR PRACTICE OR COMMUNITY?): Initial identification of practice patients who are hospitalized can best come through electronic practice registry query and notification, and should not depend on personal communication from hospitalists. Follow-up visit strategy can best come from the practice, not the hospital, in order to maintain continuity and optimize patient safety. Reengineering of follow-up strategies requires vigilance on the part of the primary care practice to manage post-hospital transitions in a fragmented healthcare system.

MULTIDISCIPLINARY ENGAGEMENT TO IMPROVE MEDICATION RECONCILIATION IN AN ACADEMIC OUTPATIENT PRACTICE USING LEAN METHODOLOGY Zhou Zhang ${ }^{1,3}$; Jason $\mathrm{Fish}^{2,3}$; Edward $\mathrm{Hui}^{3}$; Brandon Koretz ${ }^{3}$; Katherine Steinberg ${ }^{3}$; Wendy Senelick ${ }^{3}$; Katherine Serrano ${ }^{3}$; Krisan Soriano ${ }^{3} .{ }^{1}$ Kasier Permanente, Woodland Hills, CA; ${ }^{2}$ University of Texas Southwestern, Dallas, TX; ${ }^{3}$ University of California, Los Angeles, Los Angeles, CA. (Control ID \#1311376)

STATEMENT OF PROBLEM OR QUESTION (ONE SENTENCE): Medication errors are the third leading cost of healthcare costs behind cardiac problems and cancer (Schumock 2000), a large percentage of medication errors can be attributed to suboptimal medication reconciliation process.

OBJECTIVES OF PROGRAM/INTERVENTION (NO MORE THAN THREE OBJECTIVES): 1 . To improve the safety of medication usage for patients during and between office visits through the development and implementation of an appropriate medication reconciliation program based on the Joint Commission's National Patient Safety Goal. 2. To rework the medication reconciliation process in a multidisciplinary manner to ensure sustainability.

DESCRIPTION OF PROGRAM/INTERVENTION, INCLUDING ORGANIZATIONAL CONTEXT (E.G. INPATIENT VS. OUTPATIENT, PRACTICE OR COMMUNITY CHARACTERISTICS): A committee composed of physicians, medical students, nurses, patient services representatives, administrative leaders, and performance improvement staff was organized to better understand and improve the medication reconciliation process in an outpatient general internal medicine office. The key measurement was Joint Commission recommended documentation with listing of drug name, route, dose and frequency. Using LEAN methodology, an "A3" value stream map identified the scope of the problem and mapped out the current processes and stakeholders across multiple disciplines. Baseline data was collected based on current work-flow, which identified that only $8 \%$ of clinic visits and $0 \%$ between clinic visits had successful medication reconciliation. A future state map was subsequently created that included the use of an electronic medication prescribing program with a reworking of clinic flow to generate and update the medication list, and to perform electronic prescribing.

MEASURES OF SUCCESS (DISCUSS QUALITATIVE AND/OR QUANTITATIVE METRICS WHICH WILL BE USED TO EVALUATE PROGRAM/INTERVENTION): Percentage of clinic visits that maintain a complete list of drug names, route, dose, and frequency; percentage of patient charts with updated medication changes during and in between visits; and percentage of patient charts with medication list readily available to physician and patients.

FINDINGS TO DATE (IT IS NOT SUFFICIENT TO STATE "FINDINGS WILL BE DISCUSSED"): Three months were spent generating appropriate protocols, with full implementation of the pilot across four physician practices beginning in month four. After three months of full implementation, a random sample of 50 patient visits were reviewed, as well as 25 random sample of patient visits from physician practices not in the pilot but practicing in the same clinic (control group). In the intervention group, $60 \%$ of the patient visits satisfied the Joint Commission National Patient Safety Goal, up from $8 \%$, whereas only $4 \%$ of the visits from the control group satisfied the Goal. When examining between-visit medication reconciliation in a random sample of 20 visits for the pilot group and 20 visits for the control group, $72 \%$ of the intervention group visits had medication reconciliation completed compared to $0 \%$ in the control group.

KEY LESSONS FOR DISSEMINATION (WHAT CAN OTHERS TAKE AWAY FOR IMPLEMENTATION TO THEIR PRACTICE OR COMMUNITY?): Using LEAN methodology, individuals across multiple disciplines participated in improving the medication reconciliation process with adherence to the Joint Commission National Patient Safety goals and little disruption of clinic productivity. We were able to demonstrate that the electronic and multi-disciplinary medication reconciliation process significantly increased the accuracy of patients' medication lists, although more rapid cycling is needed to improve this process further.

ONLINE PHYSICIAN PEER REVIEW PORTAL: REINVENTING A TRADITION FOR CHANGING TIMES Sandhya K. Rao. Massachusetts General Hospital, Boston, MA. (Control ID \#1334406)

STATEMENT OF PROBLEM OR QUESTION (ONE SENTENCE): Is it feasible to develop an online peer review portal that facilitates efficient review of clinical documents, supports continuing education, meets physician performance evaluation requirements of hospitals, and results in measurable improvements in documentation in a way that satisfies physicians?

OBJECTIVES OF PROGRAM/INTERVENTION (NO MORE THAN THREE OBJECTIVES): 1. Re-engage physicians in the tradition of peer review as both a continuing education initiative and quality improvement activity 2. Transform paper-based chart audit focused on quality assurance into an automated, efficient experience focused on measurable quality improvement 3. Develop a tool to support efforts to meet JACHOs Ongoing Physician Performance Evaluation requirement 
DESCRIPTION OF PROGRAM/INTERVENTION, INCLUDING ORGANIZATIONAL CONTEXT (E.G. INPATIENT VS. OUTPATIENT, PRACTICE OR COMMUNITY CHARACTERISTICS): Clinical leaders develop a clinical documentation scoring instrument, which is programmed into the web-based tool. The tool pairs participating physicians, presents a sample of ambulatory notes based on an automated selection algorithm, and enables note review and data collection in one view. The physician receives a report of completeness, clarity, relevance and overall scores. Participants address an improvement area and complete a second review to receive $\mathrm{CME}$ credits in accordance with the AMA PI CME model. Three departments within our academic medical center have participated in this pilot.

MEASURES OF SUCCESS (DISCUSS QUALITATIVE AND/OR QUANTITATIVE METRICS WHICH WILL BE USED TO EVALUATE PROGRAM/INTERVENTION): 1. Quality of Notes: To date, we have calculated the change in scores produced by the review. We will conduct an independent review of notes from before and after the intervention using blinded non-participants and will use the average of individuals' change in scores as outcome measures. 2. Physician Satisfaction: A survey of participants addressed if physicians felt there were opportunities to improve documentation, if the exercise led to identification of improvement opportunities, and if it was worth the time spent.

FINDINGS TO DATE (IT IS NOT SUFFICIENT TO STATE "FINDINGS WILL BE DISCUSSED"): The average change in overall scores reported by participating physicians in one group that has completed two reviews was a modest $.88 \%$. More interestingly, the average change in completeness was $-2 \%$. In the absence of blinded review by non participants, it is impossible to know if there was an actual decline in performance or if there was change in participants' standards for completeness after participating in the intervention. This finding raises important concerns about the reliability of peer review data for performance appraisal. Forty-five percent of physicians in one department identified an area for improvement in documentation and $94 \%$ reported that they will change how they perform clinical documenation. However, $50 \%$ of physicians did not find the review questions clear and almost $40 \%$ would not recommend the tool to another practice.

KEY LESSONS FOR DISSEMINATION (WHAT CAN OTHERS TAKE AWAY FOR IMPLEMENTATION TO THEIR PRACTICE OR COMMUNITY?): Despite advances in performance measurement based on adminstrative and clinical data, physicians still value peer feedback and may make changes in practice based on it. It is feasible to engage physicians in peer review using an online tool that reduces the burden of paper based review and enables improvement activities through which they can earn CME credit. In one practice, peer-reported completeness scores were lower in the follow up review. This may be the result of decline in completeness after physicians received feedback, a shift in scoring as participants progress through the intervention, or lack of clarity of the questions. Objective review by non-participants is a critical component of evaluation of such programs.

OPTIMIZING THE USE OF BEDSIDE PATIENT SAFETY ASSISTANTS TO IMPROVE PRODUCTIVITY, DECREASE COST, AND IMPROVE OUTCOMES Christine Andre ${ }^{1}$; Michelle Ryerson ${ }^{2}$; David Paul ${ }^{2} .{ }^{1}$ University of Texas Health Science Center San Antonio, San Antonio, TX; ${ }^{2}$ University Health System, San Antonio, TX. (Control ID \#1322056)

STATEMENT OF PROBLEM OR QUESTION (ONE SENTENCE): We sought to determine whether applying a standardized protocol for when to use patient safety assistants (PSAs) and other safety measures would improve staffing productivity without compromising patient safety as monitored by rates of falls, injuries and elopement.

OBJECTIVES OF PROGRAM/INTERVENTION (NO MORE THAN THREE OBJECTIVES): 1 . Improve productivity with better utilization of limited staffing resources. 2. Decrease costs to University Hospital. 3. Show that adherence to the protocol does not compromise patient safety.

DESCRIPTION OF PROGRAM/INTERVENTION, INCLUDING ORGANIZATIONAL CONTEXT (E.G. INPATIENT VS. OUTPATIENT, PRACTICE OR COMMUNITY CHARACTERISTICS): We used quality tools such as fishbone diagrams and flowcharts to determine the baseline utilization patterns of PSAs at University Hospital in San Antonio. After performing a literature review of best practices, we selected a process algorithm to guide appropriate PSA utilization. The tool was implemented on a single inpatient general medicine ward on July 5, 2011. Data was measured for 60 days and we ran control charts on PSA utilization before and after our intervention as well as on rates of patient falls and elopements.

MEASURES OF SUCCESS (DISCUSS QUALITATIVE AND/OR QUANTITATIVE METRICS WHICH WILL BE USED TO EVALUATE PROGRAM/INTERVENTION): Decrease in total number of PSAs used per day. Decrease in total hours of PSAs used per month. Decrease in overtime hours. No increase from baseline in the volume of falls, falls with injury, and elopements.

FINDINGS TO DATE (IT IS NOT SUFFICIENT TO STATE "FINDINGS WILL BE DISCUSSED"): The total hours of Patient Safety Assistant utilization decreased by $69 \%$ (average of 3832 hours per month from January 1 through June 30, 2011 decreased to 1188 hours per month by the end of August). The average number of PSAs used decreased by $60 \%$. The average number of patients requiring a PSA decreased from 5 to 2 per shift. The total amount spent on overtime for PSA staffing decreased for the entire hospital by $41 \%$ (from an average of 627.5 hours biweekly over 9 pay periods to an average of 370.5 hours biweekly over 4 pay periods). Overall, this project decreased costs by $\$ 49,003$ with an annual estimated hard savings of $\$ 576,000$ and a return on investment of $295 \%$. There was an increase in total falls in July to 13 , one with moderate injury, one with minor but during August, this rate stabilized and returned to the pre-pilot baseline of 6 total falls. None were with injury. There was 1 elopement in July and 2 in August but data was insufficient to show a trend. At baseline there are 1 to 3 elopements per month.

KEY LESSONS FOR DISSEMINATION (WHAT CAN OTHERS TAKE AWAY FOR IMPLEMENTATION TO THEIR PRACTICE OR COMMUNITY?): Implementing an algorithm for appropriate utilization of patient safety assistants has led to improved use of hospital employee resources with a substantial cost savings without an increase in falls or elopements.

PREDICTING AND DECREASING LOW-ACUITY ED VISITS FOR AN ACADEMIC PRIMARY CARE CLINIC WITH AVAILABLE URGENT CARE Neil Wagle; Dave Chokshi; Laura Gandy; Dorothy Goulart; Monica Hynes-Payack; Ravi Kavasery; Adam Licurse; Morgan Maglich; Mary Montgomery; Rita Nguyen; Audrey Provenzano; Sonja Rakowski; Daniel Solomon; Charles A. Morris. Brigham and Women's Hospital, Boston, MA. (Control ID \#1340514)

STATEMENT OF PROBLEM OR QUESTION (ONE SENTENCE): Decreasing utilization of the emergency department (ED) by patients with low-acuity complaints is a goal for primary care practices that aim to provide better continuity of care at lower cost, but this population is heterogeneous and factors contributing to this utilization are unclear. OBJECTIVES OF PROGRAM/INTERVENTION (NO MORE THAN THREE OBJECTIVES): To measure and analyze demographic factors that predispose patients to utilize the ED for low-acuity visits; To understand the motivations behind low-acuity ED utilization; To decrease low-acuity ED utilization in favor of in-clinic Urgent Care utilization

DESCRIPTION OF PROGRAM/INTERVENTION, INCLUDING ORGANIZATIONAL CONTEXT (E.G. INPATIENT VS. OUTPATIENT, PRACTICE OR COMMUNITY CHARACTERISTICS): Primary care residents at Brigham and Women's Hospital received formal training in Lean process improvement methods. Using this framework, we addressed the prevalence of patients from a single clinic, The Phyllis Jen Center for Primary Care (PJC) using the Emergency Room for low acuity problems. Residents analyzed patients' demographic data and worked with the clinic staff to perform chart reviews and structured patient interviews. A new automated data extraction tool allowed for implementation of post-ED visit follow up wherein a nurse performed phone call outreach both for continuity of care and to understand the motivation behind utilizing the ED. This helped to create a structured visit closure process including business cards with bilingual information for patients regarding PJC Urgent Care. 
MEASURES OF SUCCESS (DISCUSS QUALITATIVE AND/OR QUANTITATIVE METRICS WHICH WILL BE USED TO EVALUATE PROGRAM/INTERVENTION): Awareness of Urgent Care among providers and patients Utilization of visit closure resources (cards). Call volume to Urgent Care ED utilization for low-acuity issues FINDINGS TO DATE (IT IS NOT SUFFICIENT TO STATE "FINDINGS WILL BE DISCUSSED"): We followed 18,814 PJC patients and tracked their ED utilization over 9 months. There were a total of 3,616 ED visits, 1,010 of which were classified by the pre-existing ED triage system as "low-acuity". Black and Hispanic patients were at much greater risk of a low-acuity ED visit compared to White patients (Relative Risk: Black=3.86, Hispanic $=3.64$ ). Younger patient were also at greater risk compared to patients aged 65-80 (Relative Risk: age $<35=2.38$, age $35-50=2.18$, age $50-65=1.44$, age $>80=1.48$ ). Patients with Spanish as primary language were not at significantly greater risk of a low-acuity ED visit compared to English-speaking patients (Relative Risk: 1.09). Patients of residents were 2.9 times more likely to have a low-acuity ED visit than patients of faculty members, though this may be partially explained by payor mix. Full multivariate analysis is pending. The post-ED visit follow-up demonstrated increased patient satisfaction, possibly improved continuity of care, and provided qualitative information regarding why patients utilized the ED. Patients using the ED for low-acuity visits were often unaware of the PJC's capacity to accommodate urgent care. This led to a new visit closure process, the effect of which is still pending.

KEY LESSONS FOR DISSEMINATION (WHAT CAN OTHERS TAKE AWAY FOR IMPLEMENTATION TO THEIR PRACTICE OR COMMUNITY?): Use of a process improvement framework revealed key opportunities for intervention in our ambulatory clinic and previously underappreciated disparities in care. Once identified, the barriers to urgent care utilization can be successfully addressed within a rapid-cycle improvement framework. This work permitted identification of populations at particularly high risk for ED utilization, which will allow for more targeted interventions in the future.

PRIMARY CARE PROVIDERS DRIVE GENERIC PRESCRIPTION RATES OF A MULTI-SPECIALTY PRACTICE Julie L. Mitchell; Karen Fickel; Robert Acker; Nandita Nanchal; Christopher Spahr; Siddhartha Singh. Medical College of Wisconsin, Milwaukee, WI. (Control ID \#1327341)

STATEMENT OF PROBLEM OR QUESTION (ONE SENTENCE): In the climate of value-based medicine, how can we increase our use of lower-cost prescriptions?

OBJECTIVES OF PROGRAM/INTERVENTION (NO MORE THAN THREE OBJECTIVES): 1.Increase the rate of lower-cost medications in a large multi-specialty practice 2 .Respond to a pay-for-performance ( $\mathrm{P} 4 \mathrm{P})$ initiative

DESCRIPTION OF PROGRAM/INTERVENTION, INCLUDING ORGANIZATIONAL CONTEXT (E.G. INPATIENT VS. OUTPATIENT, PRACTICE OR COMMUNITY CHARACTERISTICS): Over one year, we sent letters to all providers, met with clinical leaders, set benchmarks including the national overall and specialty-specific average generic rates for our state, developed a website with patient- and provider-oriented information about generics and links to formularies, and changed our EHR's e-prescribing defaults so generic prescriptions are prompted first. We targeted commonly prescribed non-preferred medications with low-cost alternatives.

MEASURES OF SUCCESS (DISCUSS QUALITATIVE AND/OR QUANTITATIVE METRICS WHICH WILL BE USED TO EVALUATE PROGRAM/INTERVENTION): We used pharmacy claims data from our two largest commercial payors. Payor \#1 designates a tiered formulary where the lowest tier ("preferred medications") includes nearly all generics plus other selected medications. Payor \#2's lowest tier is essentially generics and generics alone. We compared our historical preferred prescription rates (averaged over 12-months) to our post-intervention rates (averaged over 5-months).

FINDINGS TO DATE (IT IS NOT SUFFICIENT TO STATE "FINDINGS WILL BE DISCUSSED"): Our multi-specialty adult and pediatric group included an average of about 900 physicians over the study period. For Payor $\# 1$, our preferred prescription rate increased from $65 \%$ to $71 \%(\mathrm{p}<0.01) ; 129,808$ prescriptions were dispensed post-intervention. While primary care providers (PCPs) in internal medicine, family medicine and pediatrics represented $18 \%$ of the group's providers, they were responsible for $54 \%$ of the rate increase, largely because PCPs prescribed $47 \%$ of the prescriptions. General internists represented $9 \%$ of all providers but prescribed $27 \%$ of the prescriptions and were responsible for $34 \%$ of our rate improvement. Their rate increased from $68 \%$ to $76 \%(p=0.03)$. For Payor \#2, the generic rate increased from $67 \%$ to $72 \%$ ( $\mathrm{p}=0.16$ ); 23,666 prescriptions were dispensed post-intervention. The most frequently prescribed non-preferred medications were the same for both payor data sets. While PCPs wrote $43 \%$ of the prescriptions, they were responsible for only $22 \%$ of the payor's medication costs.

KEY LESSONS FOR DISSEMINATION (WHAT CAN OTHERS TAKE AWAY FOR IMPLEMENTATION TO THEIR PRACTICE OR COMMUNITY?): We were able to increase the rate of lower-cost prescriptions about 5 percentage points, meeting our P4P goals. We found payors were interested in hearing about our strategies. We encountered resistance from physicians. A higher generic rate reduces costs and likely improves patient adherence to chronic medications. Our initiative, part of a $\mathrm{P} 4 \mathrm{P}$ program for commercial payors, may have been perceived to be aimed at lowering costs rather than improving patient care, and thus may be less of a motivator for physicians. Physicians had trouble negotiating the payor's formularies. Although our practice subscribes to a point-ofprescribing service linking a patient's individual pharmacy benefits to our EHR, we found several limitations to this service, such as ease of use and untimely formulary updates. Primary care handles the bulk of prescriptions in a large multi-specialty practice. Any intervention to lower the cost of prescriptions must involve primary care to succeed. Future strategies will include making a stronger case for improving patient care with use of lower-cost of medications and a systematic approach to providing individual physician feedback on their prescribing practices.

PRIMARY CARE-BASED CARE MANAGEMENT FOR HIGH RISK PATIENTS: IMPLEMENTING EVIDENCE-BASED PRACTICE IN A REAL-WORLD CLINIC Elizabeth Davis ${ }^{1}$; Julia Janssen ${ }^{1}$; Anneliese Johnson'; Fern Ebeling'; Claire Horton ${ }^{1}$ ' San Francisco General Hospital, University of California San Franisco, San Francisco, CA; ${ }^{2}$ San Francisco General Hospital, San Francisco, CA. (Control ID \#1340196)

STATEMENT OF PROBLEM OR QUESTION (ONE SENTENCE): In the General Medicine Clinic (GMC) at San Francisco General Hospital, there is a small group of primary care patients that account for a disproportionate number of GMC patient hospitalizations, reflecting both the poor health of these patients and the high cost of their care.

OBJECTIVES OF PROGRAM/INTERVENTION (NO MORE THAN THREE OBJECTIVES): For patients in the GMC Complex Care Management Program (CCMP), we seek to: (1) Reduce ambulatory care sensitive admissions, (2) Reduce cost of care, and (3) Improve patient satisfaction and functional status.

DESCRIPTION OF PROGRAM/INTERVENTION, INCLUDING ORGANIZATIONAL CONTEXT (E.G. INPATIENT VS. OUTPATIENT, PRACTICE OR COMMUNITY CHARACTERISTICS): The design of CCMP is based on a literature review of care management for high risk patients in geriatric primary care clinics, Medicaid health plans, and homeless populations, all of which show improved utilization, improved health, and reduced cost of care. Our program is unique in that it adapts lessons from research to a real-world primary care clinic, but instead of solely focusing on geriatric or homeless patients, we are including all high utilizing patients in our clinic. The CCMP team, composed of an RN, behavioral health staff, and health workers, works closely with primary care providers to achieve program objectives. The process begins with an in-home patient-centered assessment focusing on self-management, function, social issues, behavioral health, and safety. From this assessment, the CCMP team develops a care plan together with the patient and primary care provider. Care managers proactively work with patients toward care plan goals and patients have direct access to care managers by phone. There are three tiers of care management intensity of contact, ranging on from weekly patient contact initiated by the care team to solely patient-initiated 
contact. Patients can move up or down tiers depending on their stability. Patients move to the highest tier when they have new events or transitions of care. The CCMP team has direct access to GMC's electronic medical record, where they document care plans and medication reconciliation. CCMP uses an electronic registry to track patients. The activities of care managers are tracked using a program on their mobile phones.

MEASURES OF SUCCESS (DISCUSS QUALITATIVE AND/OR QUANTITATIVE METRICS WHICH WILL BE USED TO EVALUATE PROGRAM/INTERVENTION): Hospitalizations, HbAlc, BP, and adherence to guidelines for diabetes, COPD, and CHF will be measured quarterly. Patient experience will be measured using patient satisfaction surveys and a quality of life measure. Health care costs will be measured quarterly.

FINDINGS TO DATE (IT IS NOT SUFFICIENT TO STATE "FINDINGS WILL BE DISCUSSED"): As our program is beginning now, we do not yet have evaluation data, but we do have data about our target population. In GMC, only $2.7 \%$ of patients account for $35 \%$ of all hospital admissions. For this group, the top three admission diagnoses- CHF, angina, and pneumonia - are all ambulatory care sensitive and accounted for $26.7 \%$ of primary admission diagnoses. Diabetes and COPD, also ambulatory care sensitive conditions, were in the top 12 primary admission diagnoses.

KEY LESSONS FOR DISSEMINATION (WHAT CAN OTHERS TAKE AWAY FOR IMPLEMENTATION TO THEIR PRACTICE OR COMMUNITY?): CCMP is a patient-centered model that other primary care clinics can learn from as they try to improve care for their highest risk patients. This model combines lessons learned from research on care management and adapts them to a real-world primary care clinic. Our use of information technology demonstrates how a primary care clinic can use its EMR and existing resources to meet program needs.

QUALITY IMPROVEMENT IN THE ADMISSIONS PROCESS: A RESIDENT-LED ANALYSIS OF THE EMERGENCY DEPARTMENT DOOR-TO-FLOOR TIME Krishan Soni; Gene Quinn; Elizabeth Le; Gabrielle Berger; Seth Berkowitz; Stacy Brenner; Jennifer T. Chang; Nathaniel Gleason; Yinchong E. Mak; Erika Moseson; Jorge Tello; Arpana Vidyarthi; Read Pierce. University of California, San Francisco, San Francisco, CA. (Control ID \#1339974)

STATEMENT OF PROBLEM OR QUESTION (ONE SENTENCE): Despite significant efforts, emergency room wait times and admission times at our institution continue to be prolonged.

OBJECTIVES OF PROGRAM/INTERVENTION (NO MORE THAN THREE OBJECTIVES): For academic medical centers, the involvement and input of house-staff are critical to achieving meaningful results in decreasing the "ED Door-to-Floor time" (ED D2F). We describe here a resident-led analysis of the ED D2F process in an attempt to reduce wait times. We set out to conduct a comprehensive resident-led analysis of the ED D2F time and present recommendations to the medical center leadership for improvement using novel strategies, particularly with regards to (1) interdepartmental teamwork and communication, (2) effective resource utilization and (3) improved care transitions.

DESCRIPTION OF PROGRAM/INTERVENTION, INCLUDING ORGANIZATIONAL CONTEXT (E.G. INPATIENT VS. OUTPATIENT, PRACTICE OR COMMUNITY CHARACTERISTICS): The analysis of the Emergency Department Door-to-Floor process for a 600 bed academic medical center was undertaken over several months in the spring of 2011. Residents embarked on a comprehensive literature review, interviewed key staff across multiple departments, engaged in discussion with thought leaders and representatives at peer institutions and carried out process mapping and failure modes effects analysis to better understand the scope and details of the ED D2F time problem. Additionally, the residents designed and piloted a unique staff position, called the "Triage Hospitalist," comprised of an Internal Medicine Hospitalist charged with facilitating appropriate patient admissions while in the ED, to try and decrease the door-to-floor time while safely triaging admitted patients.

MEASURES OF SUCCESS (DISCUSS QUALITATIVE AND/OR QUANTITATIVE METRICS WHICH WILL BE USED TO EVALUATE PROGRAM/INTERVENTION): The medical center is continuing to track ED Door to Floor times.
FINDINGS TO DATE (IT IS NOT SUFFICIENT TO STATE "FINDINGS WILL BE DISCUSSED"): Overall results of our analysis focused on the need for simultaneous attention to workflow, culture, and incentives to achieve change in a complex system. With regards to workflow, an early emphasis on destination, reducing redundancy, and unbundling of specialized monitoring services was emphasized. Changes in the culture of the admitting process emphasized training house-staff to make early triage decisions, shifting traditional ED tasks upstairs and the creation of a safe and appropriate environment for expedited care on the medicine floors. Lastly, attention was paid to identifying stakeholders and their incentives, avoiding the creation of unintended incentives, and the role of continuous feedback in modifying behavior. Strong positive feedback during presentation to the medical center leadership has resulted in the inclusion of residents on the Patient Flow Committee, streamlining of the admissions process from the house-staff perspective and increased collaboration between the Emergency Department and admitting services. Since presentation of our analysis to the medical center, median ED D2F times have fallen by approximately 20 minutes.

KEY LESSONS FOR DISSEMINATION (WHAT CAN OTHERS TAKE AWAY FOR IMPLEMENTATION TO THEIR PRACTICE OR COMMUNITY?): Prolonged wait times in the Emergency Department have a strong negative impact on both patient satisfaction and inpatient health outcomes. A resident-led analysis to shorten admission times highlights the importance of simultaneously addressing workflow, culture, and incentives to create sustainable and appreciable change.

REDUCING CONGESTIVE HEART FAILURE READMISSIONS: A SUCCESSFUL PROGRAM BETWEEN AN ACUTE HOSPITAL AND SKILLED NURSE FACILITY. Diego F. Martinez-Vasquez ${ }^{1}$; Jan Lear ${ }^{1}$; Marsha J. Butler ${ }^{2}$; Suzanne Proctor ${ }^{3}$. ${ }^{1}$ Franklin Square Hospital Center, Baltimore, MD; ${ }^{2}$ Genesis Health Care, Towson, MD; ${ }^{3}$ Medstar Health Visiting Nurse Association, Calverton, MD. (Control ID \#1313452)

\section{STATEMENT OF PROBLEM OR QUESTION (ONE SENTENCE):} Congestive Heart Failure (CHF) is among the leading cause for hospital readmissions. Discharge to Skilled Nurse Facility (SNF) occurs in approximately 1 in 5 Medicare beneficiaries after hospitalization for CHF. Among these patients $23.5 \%$ are readmitted to acute hospitals within 30 days. CHF readmissions worsens patient outcomes are very costly and can be preventable in approximately $40 \%$ of the cases.

OBJECTIVES OF PROGRAM/INTERVENTION (NO MORE THAN THREE OBJECTIVES): Develop a program to decrease CHF readmissions from patients discharged to a SNF.

DESCRIPTION OF PROGRAM/INTERVENTION, INCLUDING ORGANIZATIONAL CONTEXT (E.G. INPATIENT VS. OUTPATIENT, PRACTICE OR COMMUNITY CHARACTERISTICS): The program started in February, 2011 and focused on CHF admissions that came to the hospital and were further discharged to a SNF. One specific SNF was chosen for this program. Upon admission to the hospital a readmission tool was administered to early detect the risk for a future readmission. A multidisciplinary team including the attending and nurse carrying for the patient, pharmacist, social worker, cardiologist and a SNF and VNA liaison performed medical rounds. After the acute hospitalization, patients who met criteria for SNF placement were sent to the specific SNF chosen for the program. This SNF has also developed a multidisciplinary team similar to the hospital one. After discharge from the SNF patients went home with or without VNA services.

MEASURES OF SUCCESS (DISCUSS QUALITATIVE AND/OR QUANTITATIVE METRICS WHICH WILL BE USED TO EVALUATE PROGRAM/INTERVENTION): Readmission tool Multidiciplinary team metrics and goals for each patient Appropriate coordination of care checklist between acute hospital and Skilled Nurse facility including discharge summaries, medication reconciliation and consults $\mathrm{P}$ value One way anova

FINDINGS TO DATE (IT IS NOT SUFFICIENT TO STATE "FINDINGS WILL BE DISCUSSED"): The data analyzed is until November 30th 2011.The readmission tool helped the multidisciplinary team focused on high yield reasons for potential readmission. During this period the hospital sent 68 patients with a Dx of CHF to the SNF vs. 20 same 
period previous year. Out of the patients sent by the hospital to the SNF, the hospital had 17 readmissions (25\%) within 30 days in comparison with 13 readmission $(65 \%) \mathrm{P}<0.05$. from previous year. Among the readmissions $10(59 \%)$ came from the SNF and $7(41 \%)$ from home. 8 readmissions $(47 \%)$ had a diagnosis of CHF. Among the readmissions $15(88 \%)$ did not have a VNA service set up when they went home from the SNF compared with only $2(12 \%)$ who had a VNA service. KEY LESSONS FOR DISSEMINATION (WHAT CAN OTHERS TAKE AWAY FOR IMPLEMENTATION TO THEIR PRACTICE OR COMMUNITY?): The program decreased readmissions from patients sent to SNF by $40 \%$. VNA services should be a part of a readmission program. Building programs of care between acute hospitals and SNF are fundamental to achieve reduction in CHF readmissions.

REDUCING HOSPITAL READMISSIONS: DEVELOPING A TRANSITIONS-OF-CARE PROGRAM AT UCSF'S GENERAL INTERNAL MEDICINE CLINIC Ning Tang. UCSF, San Francisco, CA. (Control ID \#1340044)

STATEMENT OF PROBLEM OR QUESTION (ONE SENTENCE): How can general internal medicine (GIM) clinics develop a comprehensive program to reduce hospital readmissions?

OBJECTIVES OF PROGRAM/INTERVENTION (NO MORE THAN THREE OBJECTIVES): 1 . Track and understand trends in allcause 30-day readmission rates in a GIM clinic 2. Test interventions to reduce readmission rates 3 . Improve communication and collaboration between inpatient services and the GIM clinic around transitions-of-care (TOC)

DESCRIPTION OF PROGRAM/INTERVENTION, INCLUDING ORGANIZATIONAL CONTEXT (E.G. INPATIENT VS. OUTPATIENT, PRACTICE OR COMMUNITY CHARACTERISTICS): Starting in June 2010, UCSF's GIM clinic has been tracking 30-day readmission rates for its panel of 21,000 patients. Targeting services where the majority of our patients were hospitalized, we collaborated with inpatient physician leaders towards the common goal of reducing readmissions. The initial focus of our program was to ensure patients were offered outpatient follow-up appointments within 14 days of discharge. Our clinic nurses called patients who refused, cancelled, or did not arrive for the appointment. In the second phase of our program, we improved communication between inpatient and outpatient physicians at the time of discharge and developed standardized processes for the post-hospitalization appointment, including reserving appointment slots to ensure patient access, longer appointment times with a nurse visit prior to the physician visit, completing full medication reconciliation, and notifying the primary care physician $(\mathrm{PCP})$ about the outcome of the follow-up appointment. A final component of our TOC program has been reviewing cases of patients who are repeatedly hospitalized to identify modifiable causes of readmission and identifying a list of "high-risk" patients for active surveillance and case management. MEASURES OF SUCCESS (DISCUSS QUALITATIVE AND/OR QUANTITATIVE METRICS WHICH WILL BE USED TO EVALUATE PROGRAM/INTERVENTION): The primary outcome measure is allcause 30-day readmission rate. Secondary outcome measures include percentage of patients with post-hospitalization follow-up appointments within 14 days of discharge and PCP satisfaction with the TOC program. FINDINGS TO DATE (IT IS NOT SUFFICIENT TO STATE "FINDINGS WILL BE DISCUSSED"): During our intervention months, we offered over $90 \%$ of our patients follow-up appointments within 14 days of discharge, or had a nurse call patients who refused, cancelled, or did not arrive for their appointment. Reducing 30-day readmission rates has been more difficult to sustain. Prior to the intervention, all-cause 30day readmission rates averaged $15.3 \%$. In the 4 months following the intervention, the readmission rate averaged $12.8 \%$. However, the monthly readmission rate has fluctuated, ranging from $10.2 \%$ to $22.2 \%$. In informal discussions, PCPs showed appreciation for communication about hospital discharges.

KEY LESSONS FOR DISSEMINATION (WHAT CAN OTHERS TAKE AWAY FOR IMPLEMENTATION TO THEIR PRACTICE OR COMMUNITY?): - Tracking patients on admission, at discharge, and on the appointment day is very labor intensive. Automated computer systems could streamline the process. - Patients may refuse follow-up appointments if they are already seeing a specialist. Patients may refuse follow-up appointments with an acute provider if they have an appointment with their PCP at a later date. - Physician access is variable. Proactive steps should be taken to reserve appointment slots for physicians with high hospital discharge volume. - There may be an increased role for nurse phone call after discharge, especially in patients who refuse the 14-day follow-up appointment or where physician access is limited. - Post-hospitalization follow-up appointments frequently require more time (up to 1 hour). It can be difficult to schedule adequate time for these appointments.

REDUCING EMERGENCY ROOM VISITS IN A HIGH RISK POPULATION IN A TEACHING COMMUNITY HEALTH CENTER Carolyn F. Pedley; James L. Wofford; Mary M. Smoak; David Mount; Miriam Baird. Wake Forest, Winston-Salem, NC. (Control ID \#1339780)

STATEMENT OF PROBLEM OR QUESTION (ONE SENTENCE): The Medicaid population has a high use of the emergency room for nonemergent reasons which greatly increases the cost of caring for this population.

OBJECTIVES OF PROGRAM/INTERVENTION (NO MORE THAN THREE OBJECTIVES): 1 To identify high users of the emergency room in the Medicaid population assigned to a community clinic 2 . To devise interventions based in the clinic to reduce emergency room use by these individuals 3 Improve use of the community health clinic by these individuals DESCRIPTION OF PROGRAM/INTERVENTION, INCLUDING ORGANIZATIONAL CONTEXT (E.G. INPATIENT VS. OUTPATIENT, PRACTICE OR COMMUNITY CHARACTERISTICS): Medicaid patients assigned to a teaching community health clinic were screened for emergency room use by utilizing an alert system and the electronic medical record. Individuals going to the emergency room three or more times in the previous year were enrolled in interventions based in the clinic. Patients were contacted by telephone, mailings and were given information about the clinic's desire to have them access the clinic rather than going to the emergency room They were given the telephone numbers of two health navigators and were made aware of extended clinic hours and weekend clinc hours Patients also received proactive calls from the Navigators who arranged for them to be seen in the clinic when they felt the need to be seen urgently The patients were also invited to participate in support groups run by a Psychologist. The Navigators also assisted in arranging transportation and access to medications.

MEASURES OF SUCCESS (DISCUSS QUALITATIVE AND/OR QUANTITATIVE METRICS WHICH WILL BE USED TO EVALUATE PROGRAM/INTERVENTION): The number of emergency room visits were counted one year before and at four months and eight months following enrollment in the interventions.

FINDINGS TO DATE (IT IS NOT SUFFICIENT TO STATE "FINDINGS WILL BE DISCUSSED"): Fifty five patients were identified as having gone to the emergency room three or more times in the previous year, were part of the practice and were willing to participate in the interventions One year prior to enrollment these patients went to the emergency room an average of six times or $.5 \mathrm{ED}$ visits per patient per month. Four months after enrollment ED visits by these 55 were reduced to .45 visits per patient per month and after eight months the ED visits were reduced to .36 visits per patient per month. The 12 month figures are now being gathered. At the eight month rate more than 90 emergency room visits would be avoided in a year.

KEY LESSONS FOR DISSEMINATION (WHAT CAN OTHERS TAKE AWAY FOR IMPLEMENTATION TO THEIR PRACTICE OR COMMUNITY?): A program to identify frequent emergency room users and to intervene by proactive strategies can result in significant reductions in emergency room use by these individuals. At the same time these patients can be more actively engaged in their home clinic. These reductions in emergency room use will result in cost savings and enhanced engagement in the clinic will result in better continuity of health care

REDUCING INPATIENT HYPOGLYCEMIA: DIABETES- A TEAM APPROACH Sunil Asnani; Lyudmila Shvets; Christian Kaunzinger. Jersey Shore University Medical Center, Neptune, NJ. (Control ID \#1340189) 
STATEMENT OF PROBLEM OR QUESTION (ONE SENTENCE):

Hypoglycemia in an inpatient setting is associated with an increased length of stay, higher health care costs and an increased risk of inpatient mortality. OBJECTIVES OF PROGRAM/INTERVENTION (NO MORE THAN THREE OBJECTIVES): 1 . To identify etiology of inpatient hypoglycemia. 2. To reduce inpatient hypoglycemia 3. To establish protocols and pathways in the hospital to minimize the risk of inpatient hypoglycmia

DESCRIPTION OF PROGRAM/INTERVENTION, INCLUDING ORGANIZATIONAL CONTEXT (E.G. INPATIENT VS. OUTPATIENT, PRACTICE OR COMMUNITY CHARACTERISTICS): Retrospective chart review of patients with severe hypoglycemia $(<40 \mathrm{mg} / \mathrm{dl})$ was conducted over a continuous 6 months period from January-June 2007. After an intervention period of 4 years, a similar chart review was conducted from May-November 2011. The planning phase was extensive and multidisciplinary including administration, medical staff, nursing, dietary, radiology and surgical services. Intervention phase was over 2 years and is ongoing.

MEASURES OF SUCCESS (DISCUSS QUALITATIVE AND/OR QUANTITATIVE METRICS WHICH WILL BE USED TO EVALUATE PROGRAM/INTERVENTION): Severe hypoglycemia $(<40 \mathrm{mg} / \mathrm{dl})$ rates over a 6 month period will be compared pre and post intervention.

FINDINGS TO DATE (IT IS NOT SUFFICIENT TO STATE "FINDINGS WILL BE DISCUSSED"): PRE-INTERVENTION: 474 episodes of severe hypoglycemia were confirmed over 6 months; $54 \%$ occurred in ICU; $20 \%$ in type 1 diabetes, $30 \%$ between $12-6$ AM; over $50 \%$ had altered nutritional status (NPO/TPN/tube feeds/perioperative): $35 \%$ had only 'sliding scale insulin' ordered; $20 \%$ were on steroid taper. INTERVENTION- Strategies were developed to tackle each high risk group. Nursing, dietary and patient education regarding insulin-meal match was undertaken and continues on an ongoing basis. Pre-meal fingerstick blood glucose and insulin administration and consistent meal delivery from kitchen is now a goal at each nursing unit. The insulin infusion protocol at the ICU is now 'trended' and hypoglycemia rates are down by over $90 \%$ in the ICU. The medical staff of the hospital is constantly being urged to adopt basalbolus insulin when ordering instead of the 'sliding scale' culture; order sets are being developed to assist the physicians to guide them down this pathway. Diabetes management team consults are being encouraged in patients with type 1 diabetes, steroid taper, and those with known history of severe hypoglycemia. POST-INTERVENTION: After 4 years of intervention, education and ongoing culture changes, the data collection was reattempted. 190 episodes of severe hypoglycemia were noted over 6 months; ICU rates were down $90 \%$. Majority of episodes still occurred in type 1 diabetes, steroid taper and altered nutritional status. Use of basal insulin has increased but 'sliding scales' remain visible.

KEY LESSONS FOR DISSEMINATION (WHAT CAN OTHERS TAKE AWAY FOR IMPLEMENTATION TO THEIR PRACTICE OR COMMUNITY?): Inpatient hypoglycemia can be largely preventable in a majority of patients ( $>60 \%)$. It is, however, a collective effort. Developing strategies to prevent or to reduce hypoglycemic events should include identifying high risk patients, recognizing precipitating factors, use of appropriate scheduled insulin and appropriate nutrition support for hospitalized patients. Nursing should be constantly alert and physician orders for anti hyperglycemic therapy must be frequently revised in high risk patients to avoid hypoglycemia. Diabetes management team consults may be helpful in certain high risk patient populations. Constant physician education is necessary to break the grip of 'sliding scales'. A hospital-wide policy should be established for the appropriate response to triggering events. Optimal diabetes care and improving outcomes in an inpatient setting needs a team approach.

REVIEW OF INDIVIDUAL READMISSIONS BY HOSPITALISTS: PRELIMINARY FINDINGS OF A QUALITY IMPROVEMENT PROJECT. Sumanta Chaudhuri; Vipulkumar Rana; Kartik Reddy; Ritesh Panwar; Adil Jadoon; Bipin Thapa; Kathleen Idstein; Binod Dhakal; Conti Mary; Siddhartha Singh. Medical College of Wisconsin, Milwaukee, WI. (Control ID \#1339814)

STATEMENT OF PROBLEM OR QUESTION (ONE SENTENCE): Readmissions are considered a costly and avoidable waste in the healthcare system yet reasons for readmissions are poorly understood and reducing readmissions has been challenging.

OBJECTIVES OF PROGRAM/INTERVENTION (NO MORE THAN THREE OBJECTIVES): We undertook a quality improvement project where each faculty and physician assistant in our hospitalist section reviewed charts of patients discharged by them who were readmitted within 30 days. Its objectives were: 1 . To allow hospitalists to learn from reviewing readmission cases and change their individual practice 2 . To discuss important lessons learned and translate them into systems based changes 3. To reduce readmission rates to our hospitalist section

DESCRIPTION OF PROGRAM/INTERVENTION, INCLUDING ORGANIZATIONAL CONTEXT (E.G. INPATIENT VS. OUTPATIENT, PRACTICE OR COMMUNITY CHARACTERISTICS): Starting January 2011, we provided all hospitalists a weekly list of patients discharged by them who were readmitted within 30 days. We asked them to write a short report on each case with an emphasis on strategies to prevent that readmission. Starting March 2010, we asked the hospitalists to use an online Microsoft Access based tool to abstract data from each case reviewed to allow easier collation of data. The fields in this tool are partly populated through the discharge abstract database and partly filled in manually following the review of the chart. It is housed on a shred but secure password protected drive. We also conduct monthly meetings where individual data on readmission rates are shared, strategies to reduce readmissions are discussed and a few exemplary cases are reviewed in detail in a group setting.

MEASURES OF SUCCESS (DISCUSS QUALITATIVE AND/OR QUANTITATIVE METRICS WHICH WILL BE USED TO EVALUATE PROGRAM/INTERVENTION): During the period March 2011 till May 2011 we have asked the hospitalist group to review a total of a 152 cases and a review has been completed on 79 (52\%). Rich data has been collected about factors contributing to readmissions such as discovering that in approximately $10 \%$ of readmissions were related to medication changes made during index admission; only $22 \%$ of patients readmitted saw their primary care provider after discharge and in $18 \%$ each of cases social and psychiatric issues contributed to the readmission. In addition the monthly meetings have increased the collective awareness of the group to the problem of readmissions and we have brainstormed a number of issues relevant to reducing readmissions such as improvements in discharge planning, in the care of the non-compliant patient, and better input with consulting services at discharge.

FINDINGS TO DATE (IT IS NOT SUFFICIENT TO STATE "FINDINGS WILL BE DISCUSSED"): The readmission rates for our hospitalist group for the period while we have reviewed readmissions (January to April 2011) of $15.9 \%$ has decreased significantly compared to a similar period last year (January to April 2010; 19.9\%; $\mathrm{P}=0.005$ ) and also when compared to the immediate 4 months prior to our intervention (September to December, 2010; 18.7\%; P=0.019).

KEY LESSONS FOR DISSEMINATION (WHAT CAN OTHERS TAKE AWAY FOR IMPLEMENTATION TO THEIR PRACTICE OR COMMUNITY?): Hospitalists reviewing their own individual readmissions is feasible and is a valuable learning tool to determine factors contributing to readmissions. It also has the potential to act as an intervention to reduce readmissions.

SPREAD: AN EFFECTIVENESS TRIAL COMPARING A COMMUNITY HEALTH WORKER DISCHARGE INTERVENTION TO STANDARD CARE IN POST-MYOCARDIAL INFARCTION PATIENTS Tanvir Hussain ${ }^{1}$; Denis Xavier ${ }^{2}$; Rajeev Gupta ${ }^{3}$; Philip Devereaux ${ }^{4}$; $\overline{\text { Alben } \text { Sigmani }^{2}}$; A. Soumya ${ }^{2}$; Salim Yusuf ${ }^{4} .{ }^{1}$ Brigham \& Women's Hospital, Boston, MA; ${ }^{2}$ St. John's Medical College, Bangalore, India; ${ }^{3}$ Fortis Escorts Hospital, Jaipur, India; ${ }^{4}$ McMaster University, Hamilton, ON, Canada. (Control ID \#1340484)

STATEMENT OF PROBLEM OR QUESTION (ONE SENTENCE): While mortality is highest in the first year after a myocardial infarction (MI), there is inadequate infrastructure to support patient adherence to medical recommendations post-discharge, particularly in communities with poor health literacy. 
OBJECTIVES OF PROGRAM/INTERVENTION (NO MORE THAN THREE OBJECTIVES): 1 . To test the feasibility of training community health workers (CHWs) in post-MI care in resource-poor settings. 2. To study the effectiveness of lay health workers in improving lifestyle and medication adherence post-MI.

DESCRIPTION OF PROGRAM/INTERVENTION, INCLUDING ORGANIZATIONAL CONTEXT (E.G. INPATIENT VS. OUTPATIENT, PRACTICE OR COMMUNITY CHARACTERISTICS): This effectiveness trial will randomize patients diagnosed with MI from fourteen hospitals across India for follow-up care at the time of discharge: 400 to standard care and 400 to the CHW intervention. CHWs will conduct patient visits at discharge, months $1,3,5,7$, and 9 of follow-up, and finally at 12 months. CHWs will identify "red flag" symptoms; increase health literacy; conduct medicine reconciliation; encourage lifestyle and medication adherence through motivational interviewing, personalizing solutions to overcome barriers, goal setting and action planning, and identifying social supports. One CHW has been identified per hospital; selection criteria included: age $>18$ years, no more than secondary education, and fluency (written and spoken) in the local language. A CHW training manual and workbook were developed by the above authors. Content includes cardiovascular physiology, pathophysiology of ischemic heart disease, lifestyle recommendations and medications for secondary prevention. Skills-training covers rapport building, vital signs measurement, and behavioral change techniques. CHWs were evaluated during a week-long capstone training using simulated patient encounters.

MEASURES OF SUCCESS (DISCUSS QUALITATIVE AND/OR QUANTITATIVE METRICS WHICH WILL BE USED TO EVALUATE PROGRAM/INTERVENTION): This mixed-methods study is powered to detect a $10 \%$ difference between groups in adherence to post-MI medications at one year. Secondary quantitative outcomes include cardiovascular events, adherence to healthy lifestyle, and patient knowledge of and attitudes toward cardiovascular disease prevention. Qualitative assessment will include structured interviews of CHWs and of a selection of trial participants. Also, patient-encounter reports completed by CHWs will be reviewed.

FINDINGS TO DATE (IT IS NOT SUFFICIENT TO STATE "FINDINGS WILL BE DISCUSSED"): CHWs are currently conducting follow-up visits for enrolled patients. Effectiveness data are not yet reportable; however, it is also critical to evaluate the CHW training process for this clinical innovation. Defining the CHW role was informed by multi-stakeholder focus groups, which revealed consensus that physicians should not task-shift clinical duties to CHWs; rather, CHWs should provide patient and community support (with adherence and health care navigation). Decentralized CHW training, led by study site officers using a standard training manual, provided logistical ease and individualized pacing without jeopardizing quality. After didactic instruction, CHWs still felt unprepared to facilitate behavior change around adherence, so an additional training component was included: CHWs shadowed and participated in fifty physician-led patient counseling sessions. Of the fourteen $\mathrm{CHW}$ selected, two were replaced: one for failure to demonstrate required competencies; another due to personal circumstances.

KEY LESSONS FOR DISSEMINATION (WHAT CAN OTHERS TAKE AWAY FOR IMPLEMENTATION TO THEIR PRACTICE OR COMMUNITY?): CHWs may add clinical value and increase social capital in communities with poor health literacy.

STREAMLINING FOLLOW-UP: A NEW TECHNOLOGY FOR PATIENT-CENTERED CARE. Sabrina Felson ${ }^{1}$; Lauri Calkins ${ }^{2}$; Mae Callanan ${ }^{3}$. ${ }^{1}$ Veterans Health Administration New York Harbor Healthcare, New York, NY; ${ }^{2}$ Veterans Health Administration East Orange, New Jersey, $\mathrm{NJ} ;{ }^{3}$ Veterans Health Administration New York Harbor Healthcare, New York, NY. (Control ID \#1317885)

STATEMENT OF PROBLEM OR QUESTION (ONE SENTENCE): Primary care providers need a way to formalize and simplify complicated follow-up instructions so patients can successfully navigate the health-care system in between visits with their doctor.

OBJECTIVES OF PROGRAM/INTERVENTION (NO MORE THAN THREE OBJECTIVES): The individualized Electronic Primary Care Follow-up Plan is designed to maximize the efficiency and utility of face-to-face visits; encourage patient participation; and facilitate communication between the patient and health care team.
DESCRIPTION OF PROGRAM/INTERVENTION, INCLUDING ORGANIZATIONAL CONTEXT (E.G. INPATIENT VS. OUTPATIENT, PRACTICE OR COMMUNITY CHARACTERISTICS): Using the Veterans Affairs (VA) electronic medical record we designed a Follow-up Plan that translates physician follow-up orders into a single page, individualized instruction sheet for the patient to take home at the end of each primary-care visit. This templated note not only supplies the patient with an instruction tool; it also provides the physician with the convenience of ordering all referrals from one place. Physicians view a checklist of all possible relevant follow-up orders, expandable when prompted to offer additional, more specific options. For instance, if a physician orders imaging, a menu box of options appears with different imaging modalities; if a referral is required, a list of specialty clinics appears. The selected follow-up then automatically links to electronic orders. The final chart note pulls in only patient-relevant data. The patient leaves the primary care visit with a one-page personalized instruction sheet explaining how, when and why to accomplish each follow-up task. Checkout lines are minimized as patients no longer wait in line to schedule appointments. The note, which documents that patient communication took place with an electronic signature, remains in the medical record and can be consulted and reprinted at any time between visits by any member of Patient Aligned Care Team (PACT). The follow-up plan shows how the healthcare system can harness technology to improve patient care. MEASURES OF SUCCESS (DISCUSS QUALITATIVE AND/OR QUANTITATIVE METRICS WHICH WILL BE USED TO EVALUATE PROGRAM/INTERVENTION): A primary measure of the Plan's success thus far is the excitement that it has generated among providers and the administration. Most primary care providers in NY Harbor, including residents, use the note. As part of a leadership fellowship, Dr. Laurie Calkins imported the Follow-Up Plan note, which she renamed E-FUN, to primary care at the VA NJ. We plan to roll out the note to all facilities in the NY/NJ Veterans Integrated Service Network, which includes 9 large primary care facilities. Next month we will introduce it to a group of national VA primary care leaders for possible adoption on a larger scale. FINDINGS TO DATE (IT IS NOT SUFFICIENT TO STATE "FINDINGS WILL BE DISCUSSED"): Dr. Calkins developed a chart review comparing 60 patients with primary care visits in VA NJ and found that $94 \%$ of patients with E-FUN compared with $81 \%$ of patients without it completed recommended follow-up tasks. A phone survey of the 30 patients who received E-FUN revealed that $37 \%$ found it "very helpful and "60\% found it "helpful." We plan a similar study with the more urban and indigent population at NY Harbor.

KEY LESSONS FOR DISSEMINATION (WHAT CAN OTHERS TAKE AWAY FOR IMPLEMENTATION TO THEIR PRACTICE OR COMMUNITY?): We recommend the presence of a local champion, preferably a physician, who collaborates directly with an IT expert in constructing a note that enhances workflow for the busy provider and facilitates follow-up by the patient. We have prepared a detailed instruction guide for exporting E-FUN to other VA sites for possible inclusion in the national PACT "toolkit." We are also working to offer a patient version of instructions in Spanish.

SUCCESSFULLY ACHIEVING THE TRIPLE AIM FOR THE HIGH-RISK HIGH-COST MEDICARE POPULATION: LESSONS LEARNED FROM AN INTENSIVE PRIMARY CARE-BASED CARE MANAGEMENT PROGRAM Norifumi Kamo. Massachusetts General Hospital, Boston, MA. (Control ID \#1340255)

STATEMENT OF PROBLEM OR QUESTION (ONE SENTENCE): High-risk Medicare patients account for a disproportionate amount of spending, while interventions to improve quality of care and reduce costs have had mixed results.

OBJECTIVES OF PROGRAM/INTERVENTION (NO MORE THAN THREE OBJECTIVES): Improve health outcomes and achieve cost savings for high-risk Medicare beneficiaries

DESCRIPTION OF PROGRAM/INTERVENTION, INCLUDING ORGANIZATIONAL CONTEXT (E.G. INPATIENT VS. OUTPATIENT, PRACTICE OR COMMUNITY CHARACTERISTICS): The MassGeneral Care Management Program (CMP) is a Medicare demonstration project that utilizes an intensive primary care-based care management 
program for high-risk, high-cost Medicare beneficiaries. The program is based at 19 hospital and community-based primary care practices associated with a large academic medical center. The CMP program assigned primary carebased nurse care managers with extensive medical-surgical experience to 2600 program participants. 800 additional participants were added after one year. In close collaboration with primary care physicians, nurse case managers provide customized interventions to improve self-management of chronic conditions, facilitate access to resources, assist with care transitions, and address changes in participants' clinical conditions. CMP also includes other specialized services for program participants (e.g. a mental health team). The program is supported by an integrated health IT system and data analytics to evaluate trends in hospitalizations and costs

MEASURES OF SUCCESS (DISCUSS QUALITATIVE AND/OR QUANTITATIVE METRICS WHICH WILL BE USED TO EVALUATE PROGRAM/INTERVENTION): Quantitative metrics were assessed by Research Triangle International, Inc., which conducted regression models comparing costs, hospitalization rates, emergency room visits, 90-day readmission rates, mortality rates between the intervention cohort and a comparison cohort. Qualitative analysis of the program's success factors were evaluated by a focus group attended by nurse care managers and other CMP staff, site visits, semi-structured interviews with program staff and primary care physicians.

FINDINGS TO DATE (IT IS NOT SUFFICIENT TO STATE "FINDINGS WILL BE DISCUSSED"): Over a three-year period, the program demonstrated cost savings, reduced number of hospitalizations and emergency department visits, decreased mortality rate, and improved beneficiary quality of life relative to a comparison cohort. CMP did not reduce 90 day readmission rates. Success factors included: - Appropriately targeting patients where benefit from intensive outpatient care management would outweigh costs - Leveraging organizational capabilities of the institution (e.g. strong primary care network, integrated health IT system) Involving stakeholders early in the design of the program and obtaining institutional support - Tightly integrating the CMP program with existing primary care practices - Hiring and training clinically experienced nurse care managers who anticipate and promptly respond to changes in patients' clinical status - Actively modifying the program based on real-time data and promoting a culture dedicated to continuous improvement

KEY LESSONS FOR DISSEMINATION (WHAT CAN OTHERS TAKE AWAY FOR IMPLEMENTATION TO THEIR PRACTICE OR COMMUNITY?): The MassGeneral CMP demonstrated that a comprehensive primary care-based care management program is one approach to improving health care outcomes while producing savings for high-cost highrisk Medicare patients. Similar programs will likely be successful in the context of tight integration with primary care practices within integrated health organizations. Given the complexity of the high-cost high-risk Medicare population and the highly customized nature of care management interventions, similar programs will need continuous modifications guided by strong program leadership.

TELEPHONE FOLLOW-UP AFTER HOSPITAL DISCHARGE Amy R. Schwartz ${ }^{1,2}$; Gail Barrows ${ }^{1}$; Carl M. Dillon ${ }^{1}$; Jill Edwards ${ }^{1}$; Johanna $\overline{\text { Giovanniello }}^{1}$; Rosemary Lettiero ${ }^{1}$; Catherine Lobner ${ }^{1}$; Esterina Messeder ${ }^{1}$; Maureen Roche ${ }^{1}$; Christopher Ruser ${ }^{1,2}$; Gloria Satti-Langlois ${ }^{1}$; Monique Simmons ${ }^{1}$; Lashonda Sullivan ${ }^{1}$; Donna C. Vogel ${ }^{1}{ }^{1}$ VA Connecticut Healthcare System, West Haven, CT; ${ }^{2}$ Yale University, New Haven, CT. (Control ID \#1339766)

STATEMENT OF PROBLEM OR QUESTION (ONE SENTENCE): Transition from the hospital to home is difficult; patients often have medication and other clinical questions or concerns and are at risk for readmission and other adverse events

OBJECTIVES OF PROGRAM/INTERVENTION (NO MORE THAN THREE OBJECTIVES): 1 . Call patients within 2 business days of discharge from the medical, surgical, or psychiatric inpatient services at VA Connecticut. 2. Identify medication, appointment, home care, and clinical concerns and intervene. 3. Decrease hospital readmission.

DESCRIPTION OF PROGRAM/INTERVENTION, INCLUDING ORGANIZATIONAL CONTEXT (E.G. INPATIENT VS. OUTPATIENT, PRACTICE OR COMMUNITY CHARACTERISTICS): Patients are called within 2 business days after discharge by their team RN (patients discharged to home) or RN or SW case manager (patients discharged to a facility). A templated note is used to enquire regarding medical reconciliation, appointment needs, and clinical concerns. The intervention has been successfully spread to all primary care sites in the VA Connecticut Healthcare System.

MEASURES OF SUCCESS (DISCUSS QUALITATIVE AND/OR QUANTITATIVE METRICS WHICH WILL BE USED TO EVALUATE PROGRAM/INTERVENTION): 1 . Successful telephone call completion. 2. Identification and resolution of medication, appointment, home care, and clinical concerns. 3. Readmission rates.

FINDINGS TO DATE (IT IS NOT SUFFICIENT TO STATE "FINDINGS WILL BE DISCUSSED"): Telephone calls following discharge identify medication concerns in about $20 \%$ of patients, appointment issues in about $26 \%$, case management needs in $5 \%$, and other clinical issues in $11 \%$. Our pilot teams found that patients were often confused about their medications at discharge, and this information was used to redesign the discharge instruction and medication education process on the inpatient services. We were able to spread the process throughout all VA sites in our state. Data regarding readmission is being collected.

KEY LESSONS FOR DISSEMINATION (WHAT CAN OTHERS TAKE AWAY FOR IMPLEMENTATION TO THEIR PRACTICE OR COMMUNITY?): Discharge follow-up calls identify clinical problems and are well-received by patients. When developing the process for discharge follow-up calls it is helpful to have an interdisciplinary workgroup, create a clear playbook, employ team-to-team training, and periodically track contact rates and reasons for inability to contact patients.

TEXT MESSAGING TO ENGAGE PARENTS OF CHILDREN PARTICIPATING IN A COMMUNITY-BASED AFTERSCHOOL NUTRITION AND PHYSICAL ACTIVITY PROGRAM Michael T. Quinn $^{1}$; Althera Steenes ${ }^{1}$; Marla C. Solomon ${ }^{3}$; Lori McClinton-Powell ${ }^{2} ;$ F. Kweku Embil ${ }^{2}$; Amanda Gawin ${ }^{1}$; Shantanu Nundy ${ }^{1}$; Deborah L. Burnet ${ }^{1}$. ${ }^{1}$ University of Chicago, Chicago, IL; ${ }^{2}$ Woodlawn Community School, Chicago, IL; ${ }^{3}$ University of Illinois at Chicago, Chicago, IL. (Control ID \#1339524)

STATEMENT OF PROBLEM OR QUESTION (ONE SENTENCE): While the afterschool setting offers an ideal venue for promoting healthy nutrition and activity among children, engaging parents regarding the knowledge and skills their children are acquiring in the afterschool program can be challenging. Cellphone texting represents a promising approach to engage parents of children in an after-school program focused on healthy nutrition and exercise.

OBJECTIVES OF PROGRAM/INTERVENTION (NO MORE THAN THREE OBJECTIVES): This pilot test of a text messaging system for parents is being conducted as part of a larger initiative designed to promote healthy nutrition and activity among children in grades 3-5 who are attending an afterschool program in an urban community. The objectives of the text messaging component are: 1) develop a system of brief text messages designed to communicate with parents and engage them in the nutrition and physical activity lessons their children receive in the afterschool program, and 2) evaluate the feasibility and acceptability of the text messaging system with a sample of parents whose children attend the afterschool program

DESCRIPTION OF PROGRAM/INTERVENTION, INCLUDING ORGANIZATIONAL CONTEXT (E.G. INPATIENT VS. OUTPATIENT, PRACTICE OR COMMUNITY CHARACTERISTICS): A total of 25 children in the $3 \mathrm{rd}$, 4th, and 5th grades at one urban public elementary school are currently enrolled in the Power Up program, a 20-week afterschool educational program designed to promote healthy nutrition and physical activity. The program is delivered by regular classroom teachers. Effectively engaging parents within the afterschool time frame has been challenging, as opportunity for face-to-face interaction is limited to the brief time when parents pick up their children and rush home to prepare dinner. A promising adjunct to this face-to-face interaction is the use of brief text messages via cell-phone. We developed a system of brief text messages for parents that are aligned with the afterschool curriculum and designed to raise awareness of content covered in class (e.g., "Today, your child learned about 
the Nutrition Fact Label on packaged foods"), support the value of that information (e.g., "Knowing how much sugar is in a food can help your child make healthy food choices."), suggest behavioral application of that information (e.g., "At the grocery store, ask your child to compare the nutrition facts of a granola bar and a cereal."), and ask a related question (e.g., "Ask your child to show you a portion size for rice or pasta."). We are currently recruiting a convenience sample of 10-15 parents to participate in this onemonth pilot study of the feasibility of this brief text messaging system.

MEASURES OF SUCCESS (DISCUSS QUALITATIVE AND/OR QUANTITATIVE METRICS WHICH WILL BE USED TO EVALUATE PROGRAM/INTERVENTION): We are using participant surveys and semi-structured interviews to assess acceptability and ease of use of the text messaging system, recall of content, clarity of messages, impact on confidence in changing dietary and physical activity behaviors, and effectiveness in prompting actual behavior changes.

FINDINGS TO DATE (IT IS NOT SUFFICIENT TO STATE "FINDINGS WILL BE DISCUSSED"): Teachers and parents expressed interest and enthusiasm about participating in the text messaging pilot; data will be collected and analyzed this winter.

KEY LESSONS FOR DISSEMINATION (WHAT CAN OTHERS TAKE AWAY FOR IMPLEMENTATION TO THEIR PRACTICE OR COMMUNITY?): Text messaging is widely used by parents, and inexpensive technologies for creating and sending automated text messages are readily available. This technology affords an innovative opportunity to engage parents in participating in and reinforcing their child's learning and to promote their family's healthy lifestyle changes.

THE MGH COMPLEX CARE SERVICE: A CONTINUITY-FOCUSED CARE MODEL FOR MEDICALLY-COMPLEX INPATIENTS Ryan Thompson; Deirdre Sweeney; David Finn; Steve Levisohn. Massachusetts General Hospital, Boston, MA. (Control ID \#1340054)

STATEMENT OF PROBLEM OR QUESTION (ONE SENTENCE): The most complex medical inpatients often involve long length-of-stay, multiple medical team changes, and many consulting physicians; therefore maintaining inpatient continuity of care can be difficult.

OBJECTIVES OF PROGRAM/INTERVENTION (NO MORE THAN THREE OBJECTIVES): 1) Provide team-based continuity of care to medically-complex, long length-of-stay inpatients. 2) Improve patient and family satisfaction with inpatient care through higher levels of care coordination. 3) Provide enhanced coordination of services with nursing, allied health professionals, and consulting teams through the Complex Care nurse practitioner.

DESCRIPTION OF PROGRAM/INTERVENTION, INCLUDING ORGANIZATIONAL CONTEXT (E.G. INPATIENT VS. OUTPATIENT, PRACTICE OR COMMUNITY CHARACTERISTICS): The Complex Care Service at Massachusetts General Hospital (MGH) began in 2008 to address the needs of the most medically-complex inpatients at $\mathrm{MGH}$. Patients referred to the service have had several common themes, including multiple consulting teams, expected long length-of-stay, and extended time spent in an intensive care unit. The service consists of three outpatientbased general internists skilled in inpatient care, and one inpatient nurse practitioner. The physicians do not rotate on- and off-service, and thus provide continuity of care throughout their patients' referral admission, and on subsequent admissions if re-admitted. Referrals to Complex Care come from case management, housestaff, hospitalists, and specialist physicians. The service can follow up to six inpatients at a time, with weekend coverage provided by an affiliated primary care practice. Salary for the nurse practitioner is paid for by the hospital. Physicians receive an augmented inpatient fee and weekly management fee to compensate for the additional time required to care for these patients.

MEASURES OF SUCCESS (DISCUSS QUALITATIVE AND/OR QUANTITATIVE METRICS WHICH WILL BE USED TO EVALUATE PROGRAM/INTERVENTION): Evaluating the operational value of the service (e.g. cost savings, patient outcomes) compared to a control group is difficult given the "outlier" status of these patients. However, we plan to assess patient, family, and nursing satisfaction with the service through validated survey tools that assess continuity of care. We also plan to develop formal acceptance criteria for the service that will help characterize the referral base, and allow for more systematic identification of patients who will derive greatest benefit from the service.

FINDINGS TO DATE (IT IS NOT SUFFICIENT TO STATE "FINDINGS WILL BE DISCUSSED"): Since 2008, the Complex Care Service has accepted 88 unique patients onto the service, spanning 193 admissions. Referral to the service was made, on average, 26.4 days into the patient's admission (median 21 days). Most referrals came from case management $(59 \%)$ or other physicians $(36 \%)$, both medical and surgical. The average number of consulting services per patient at the time of referral was 5.5. The average length-of-stay on the Complex Care Service for the referral admission was 21.8 days. The range of primary diagnoses for Complex Care patients has been varied, with common themes being rare complicated diagnoses (e.g. catastrophic antiphospholipid syndrome, calciphylaxis), complicated systemic infections, and inpatient medical complications. Other themes include tertiary or quaternary referrals, complicated discharge dispositions, and chronically-ill adolescent patients transitioning to adult care. KEY LESSONS FOR DISSEMINATION (WHAT CAN OTHERS TAKE AWAY FOR IMPLEMENTATION TO THEIR PRACTICE OR COMMUNITY?): Outpatient general internists with skill and interest in inpatient care can partner with an inpatient nurse practitioner to provide continuity of inpatient care and enhanced care coordination to the most medically-complex, long length-of-stay inpatients.

THE USE OF A PERSONALIZED GENE EXPRESSION TEST TO IMPROVE DECISION MAKING IN THE EVALUATION OF PATIENTS WITH SUSPECTED CORONARY ARTERY DISEASE Michael F. Conlin ${ }^{1}$; Mark Mouton ${ }^{3}$; Lee E. Herman ${ }^{1}$; May Yau ${ }^{4}$; Mark Monane $^{4}$; John McPherson ${ }^{5}$; Michael Elashoff ${ }^{4}$; Larry Wilson ${ }^{2} .{ }^{1}$ Johns Creek Primary Care, Suwanee, GA; ${ }^{2}$ Wake Forest Family Medicine, Wake Forest, NC; ${ }^{3}$ Mark Mouton, Baker, LA; ${ }^{4}$ CardioDx, Palo Alto, CA; ${ }^{5}$ Vanderbilt Heart and Vascular Institute, Nashville, TN. (Control ID \#1338662)

STATEMENT OF PROBLEM OR QUESTION (ONE SENTENCE): The evaluation of patients (pts) with suspected coronary artery disease (CAD) is highly variable and is associated with test overutilization, high costs, and iatrogenic complications.

OBJECTIVES OF PROGRAM/INTERVENTION (NO MORE THAN THREE OBJECTIVES): The main objective of the program is to assess the clinical utility of a personalized gene expression score (GES) among pts with suspected CAD in medical decision making by primary care physicians around referral to a cardiologist. We also assessed downstream use of cardiac noninvasive and invasive testing in these referred and non-referred pts.

DESCRIPTION OF PROGRAM/INTERVENTION, INCLUDING ORGANIZATIONAL CONTEXT (E.G. INPATIENT VS. OUTPATIENT, PRACTICE OR COMMUNITY CHARACTERISTICS): The GES is a validated quantitative diagnostic test for non-diabetic pts, measuring expression levels of 23 genes from peripheral blood to determine the likelihood of a pt having at least one vessel with $\geq 50 \%$ coronary artery stenosis. The GES has a negative predictive value of $96 \%$ in a recent study evaluating pts referred for myocardial perfusion imaging. We selected three community-based primary care practices for evaluation: these sites underwent education and training in the use and interpretation of the GES. Stable pts with chest pain with suspected $\mathrm{CAD}$ had a peripheral blood sample drawn, which was sent to a central reference lab: this lab reported the GES to the physician within 3-4 days. A total of 184 pts presented to these practices with chest pain and underwent gene expression testing from January to September 2011. We extracted information on GES, patient demographics, and chest pain symptoms as well as diagnostic tests and cardiology referrals ordered. All pts are being followed for late major adverse cardiac events.

MEASURES OF SUCCESS (DISCUSS QUALITATIVE AND/OR QUANTITATIVE METRICS WHICH WILL BE USED TO EVALUATE PROGRAM/INTERVENTION): We measured percentage of low GES $(\leq 15)$ pts, rate of cardiology referrals, rate of noninvasive and invasive cardiac testing, and diagnostic yield at cardiac catheterization.

FINDINGS TO DATE (IT IS NOT SUFFICIENT TO STATE "FINDINGS WILL BE DISCUSSED"): The median age was 56 years old, and $49 \%$ were female. The cohort included 184 pts with typical and 
atypical symptoms $(112,61 \%)$, asymptomatic pts with $\geq 3$ risk factors (64, $35 \%)$ and asymptomatic pts with $<3$ risk factors for CAD $(8,4 \%)$. There were $88(48 \%)$ pts with low GES (mean=9) and $96(52 \%)$ with non-low GES $($ mean $=25)$. The primary analysis was the proportion of referrals to a cardiologist among low and non-low GES pts. A total of $47(25 \%)$ pts were referred to cardiology: $6 \%(n=5)$ of low GES and $44 \%(n=42)$ of non-low GES. The odds ratio by logistic regression for referral among low GES pts was $0.17(\mathrm{p}=0.011)$, controlling for age, gender, type of symptoms, and practice site. Additional cardiac testing was noted in $6 \%(\mathrm{n}=5)$ of low GES pts and $36 \%(n=35)$ of non-low GES pts. Of note, there were 7 angiograms performed in the low $(n=1)$ and non-low $(n=6)$ GES pts: $2 / 7$ pts (all nonlow GES scores)were found to have clinically significant obstructive CAD KEY LESSONS FOR DISSEMINATION (WHAT CAN OTHERS TAKE AWAY FOR IMPLEMENTATION TO THEIR PRACTICE OR COMMUNITY?): The use of the personalized gene expression test was efficient in separating pts into low (48\%) and non-low (52\%) GES groups. Pts in the low GES were $\sim 6$ times less likely to be referred to a cardiologist. The low GES was associated with lower downstream cardiac testing effects as well. The findings suggest that this clinical practice innovation involving personalized gene expression scores may be used by primary care physicians to rule out low risk pts as well as identify appropriate pts needing further testing.

UNIVERSAL OPT-IN HIV SCREENING AT MASSACHUSETTS GENERAL HOSPITAL CHELSEA URGENT CARE CLINIC Jordan Lane $^{1}$; Rachel Bender Ignacio ${ }^{1,2}$; Jacqueline $\mathrm{Chu}^{2}$; Jeffrey Collins ${ }^{2}$; Valerie E. Stone ${ }^{1,2}$. ${ }^{1}$ Harvard Medical School, Boston, MA; ${ }^{2}$ Massachusetts General Hospital, Boston, MA. (Control ID \#1339840)

STATEMENT OF PROBLEM OR QUESTION (ONE SENTENCE): Despite CDC guidelines that recommend routine screening of all patients 13-64 years old in all health care settings, many urgent care clinics and primary care providers do not offer universal HIV screening.

OBJECTIVES OF PROGRAM/INTERVENTION (NO MORE THAN THREE OBJECTIVES): 1) Determine the feasibility of opt-in HIV screening at the Massachusetts General Hospital Chelsea Urgent Care Clinic in a 4 month pilot. 2) Assess the correlation between having an MGH Primary Care Physician and prior HIV screening to determine the need for screening in our urgent care clinic and to improve screening in the primary care setting. 3) Determine the acceptability of screening to patients and providers as well as the rate and demographics of undiagnosed HIV among screened patients.

DESCRIPTION OF PROGRAM/INTERVENTION, INCLUDING ORGANIZATIONAL CONTEXT (E.G. INPATIENT VS. OUTPATIENT, PRACTICE OR COMMUNITY CHARACTERISTICS): As of the 2010 census, $62.1 \%$ of the population of Chelsea, MA, a densely populated city within the urban area of Boston, self-identified as Hispanic, $38 \%$ of the population was foreign born, and the HIV prevalence was one of the highest in the state. From Oct. 2011 to Jan. 2012, all patients 18-65 years old presenting to the MGH Chelsea Urgent Care Clinic were offered HIV screening regardless of risk or reason for presentation. Patients who consented (required by MA law) were tested using our hospital's standard Enzyme Immune Assay, confirmed by Western Blot, if positive. Initially, all negative results were given by phone. An interim review necessitated having patients pick up negative results due to burden on work-flow. HIV positive individuals were notified in person with followup arranged with an HIV team.

MEASURES OF SUCCESS (DISCUSS QUALITATIVE AND/OR QUANTITATIVE METRICS WHICH WILL BE USED TO EVALUATE PROGRAM/INTERVENTION): We will determine acceptance of the program through consent and testing rates. Staff perceptions will be obtained in an anonymous post-pilot survey. Prior HIV screening and MGH primary care will be determined by an electronic medical records review. The key measure of success will be integration of this HIV screening protocol into standard of practice upon the pilot's end.

FINDINGS TO DATE (IT IS NOT SUFFICIENT TO STATE "FINDINGS WILL BE DISCUSSED"): Of the 1243 patient encounters reviewed to date, $297(23.9 \%)$ were screened. One HIV+result was encountered, although this led to the discovery of undiagnosed HIV in their partner as well. This is a prevalence of $0.34 \%$ undiagnosed HIV. A prevalence $\geq 0.1 \%$ has previously been shown cost effective. Of the 874 patients with an active MGH Primary Care Physician, 368(42.1\%) had no record of prior screening. Of those patients not previously tested, 75 (20.4\%) underwent testing when offered.

KEY LESSONS FOR DISSEMINATION (WHAT CAN OTHERS TAKE AWAY FOR IMPLEMENTATION TO THEIR PRACTICE OR COMMUNITY?): $22 \%$ of patients seen did not have a primary care physician. These patients often have less health literacy, non-legal immigrant status, and less social stability. Many of these characteristics not only increase the risk of acquiring HIV, but also decrease the likelihood of receiving preventive care. This pilot demonstrates it is possible to routinely screen for HIV in our urgent care setting with a reasonable rate of uptake and minimal expenditure of clinical time and resources. The rate of undiagnosed HIV indicates that screening is warranted. Also, screening rates among patients receiving their primary care within the MGH system indicate that having a primary care physician is not predictive of having received screening. This data will promote better screening in our primary care and underscores the importance of screening at a health center/hospital-affiliated urgent care clinic despite theoretical availability of testing through primary care.

USE OF A RESIDENT-DRIVEN LONGITUDINAL APPROACH TO ACCOMPLISH A PRACTICE IMPROVEMENT PROJECT Dana M. Carne; Antonio L. Perez; Shiri B. Feingold; Oluseyi Ojeifo; Daniel J. Friedman; Onyinye I. Iweala; Kerri L. Palamara; Blair W. Fosburgh. The Massachusetts General Hospital, Boston, MA. (Control ID \#1316177)

STATEMENT OF PROBLEM OR QUESTION (ONE SENTENCE): Due to complicated resident work schedules, many trainee-driven practice improvement projects are difficult to execute.

OBJECTIVES OF PROGRAM/INTERVENTION (NO MORE THAN THREE OBJECTIVES): 1. Design and execute a longitudinal resident-driven practice improvement project involving all residents that are part of one large primary care practice. 2 . Increase the number of patients over age 65 in the resident outpatient panels who have had an informed discussion regarding advance directives by April 2012. Document this discussion in the longitudinal electronic medical record (EMR)

DESCRIPTION OF PROGRAM/INTERVENTION, INCLUDING ORGANIZATIONAL CONTEXT (E.G. INPATIENT VS. OUTPATIENT, PRACTICE OR COMMUNITY CHARACTERISTICS): All residents in one primary care practice mutually selected to undertake a practice improvement project to increase the number of patients who have a documented code status discussion. Every two weeks, a set of project priorities was established and accomplished by residents who were rotating on an ambulatory block. At the end of each block, work-inprogress, results, and next steps were communicated to the incoming ambulatory residents. To obtain code status baseline data, we selected three patients over age 65 from the panel of each practice resident who were seen in clinic in the last three months. The patient's EMR was searched for documentation of a code status discussion. In the intervention stage, patients over age 65 who were seen in clinic during January 2011 through March 2012 were sent a letter prior to their PCP visit. This letter conferred the importance of thinking about code status and discussing this decision with family. At the office visit, the PCP discussed the issue further and offered educational materials as needed. The patient was then brought back for a second visit where the PCP reviewed the patient's decision regarding code status and documented this decision in the EMR.

MEASURES OF SUCCESS (DISCUSS QUALITATIVE AND/OR QUANTITATIVE METRICS WHICH WILL BE USED TO EVALUATE PROGRAM/INTERVENTION): At the end of the study period, a second sample of medical records of patients over 65 seen by residents in the last three months was searched for code status discussions. This rate will be compared to our baseline rate detailed below to determine if the intervention and study design were successful.

FINDINGS TO DATE (IT IS NOT SUFFICIENT TO STATE "FINDINGS WILL BE DISCUSSED"): Sixty-seven patients were included in the first sample, of which $16(24 \%)$ patients had documented 
discussions regarding code status with providers. Data for the postintervention sample will be presented in full at the meeting.

KEY LESSONS FOR DISSEMINATION (WHAT CAN OTHERS TAKE AWAY FOR IMPLEMENTATION TO THEIR PRACTICE OR COMMUNITY?): Outpatient quality improvement projects are often difficult for residents to execute due to complicated resident work schedules and short nature of individual ambulatory blocks. Our group-based, resident-driven improvement project showed that trainees can achieve meaningful accomplishments by sharing longitudinal projects over time. This process ensures that residents can work on these efforts when they have dedicated time and can pass-off project responsibilities to their colleagues as they rotate off ambulatory rotations. Attending guidance, practice support and strong resident-toresident project pass-offs are key factors necessary for success in this approach. The majority of our patients did not have a documented code discussion. This intervention will afford patients the opportunity to discuss their wishes, while healthy, for code status with their families and healthcare proxies.

USE OF THE "HAWTHORNE EFFECT" TO INCREASE COMPLIANCE WITH HOSPITAL ADMISSION MEDICATION RECONCILIATION BY INTERNAL MEDICINE RESIDENTS Alfred Burger ${ }^{1,2}$; Amy Esposito $^{1}$; Georges Ephrem ${ }^{1}$; Andrew S. Korman ${ }^{1}$; Jonathan Mazurek ${ }^{1}$; Yuichi Shimada $^{1}$; Jose A. Cortes ${ }^{1,2}$; Daniel I. Steinberg ${ }^{1,2}$. ${ }^{1}$ Beth Israel Medical Center, New York, NY; ${ }^{2}$ Albert Einsctein College of Medicine, Bronx, NY. (Control ID \#1330120)

STATEMENT OF PROBLEM OR QUESTION (ONE SENTENCE): Medication reconciliation at hospital admission and discharge is critical to patient safety; many institutions have difficulty ensuring consistent provider compliance.

OBJECTIVES OF PROGRAM/INTERVENTION (NO MORE THAN THREE OBJECTIVES): 1 . Obtain a high physician compliance rate with admission medication reconciliation after launch of a new, computer order entry (CPOE) based system. 2. Sustain this high rate of compliance.

DESCRIPTION OF PROGRAM/INTERVENTION, INCLUDING ORGANIZATIONAL CONTEXT (E.G. INPATIENT VS. OUTPATIENT, PRACTICE OR COMMUNITY CHARACTERISTICS): In spring of 2011 our institution transitioned from a paper-based medication reconciliation process to one embedded in our CPOE. Initial house staff compliance after launch, which included education and groupbased orientation/training, was zero percent. Further educational efforts such as noon conferences by the residency program did not improve compliance. We then started twice weekly audits of our 14 inpatient medicine teams. A minimum of 10 patient charts per resident team were randomly selected for audit via computer. Results were made public to entire house staff via group email. Accountability for results was assigned to the PGY-2/3 level residents. Those who achieved high compliance rates were praised in the program wide email by name from the residency program leadership $(>90 \%$ compliance warranted strong praise, $100 \%$ compliance resulted in highest praise). Lower performing residents were not singled out by name in the email but were approached and counseled individually by a program director or chief resident.

MEASURES OF SUCCESS (DISCUSS QUALITATIVE AND/OR QUANTITATIVE METRICS WHICH WILL BE USED TO EVALUATE PROGRAM/INTERVENTION): Success was determined by the percentage of patient charts for which the medication reconciliation form was completed within 24 hours of admission. An overall goal of $>95 \%$ compliance was set.

FINDINGS TO DATE (IT IS NOT SUFFICIENT TO STATE "FINDINGS WILL BE DISCUSSED"): After implementation of our feedback and reporting program overall compliance rose steadily, and at 6 months compliance was sustained at over 95\%. A Joint Commission visit occurred during this time, in which medication reconciliation was noted by the inspectors to be an institutional strength. After a two month break in the auditing process, a follow up audit to check for sustained effect revealed that average compliance had fallen to $69.5 \%$.
KEY LESSONS FOR DISSEMINATION (WHAT CAN OTHERS TAKE AWAY FOR IMPLEMENTATION TO THEIR PRACTICE OR COMMUNITY?): The "Hawthorne Effect", first described by psychologists in the 1930's studying workers at the Hawthorne Works factory, is generally accepted as a method for improving compliance. It states that individual behaviors will improve in the setting of known monitoring. Physicians are increasingly being held individually accountable for quality and patient safety outcomes, often with data reported in a public way. The ACGME requires that residents be involved in patient safety and quality improvement initiatives, and that they receive data on the outcomes of patients specifically under their care. Our intervention combined all 3 of these principles and found that an email based "public reporting" system improved and sustained compliance with a new admission medication reconciliation process. Withdrawal of this system likely led to a drop off in compliance. This approach was effective but may need to be done on a permanent or more long-term basis to effect durable change. In addition, such an approach might be effective in increasing housestaff compliance with other behaviors (for example, completion of medical records, evaluations, etc).

USING PILLBOX INTERVENTION FOR IMPROVING PATIENT SAFETY UPON DISCHARGE FROM HOSPITAL Nowreen Haq; Chakan Smith. Johns Hopkins School of Medicine, Baltimore, MD. (Control ID \#1340904)

STATEMENT OF PROBLEM OR QUESTION (ONE SENTENCE): Elderly patients on home self-care often face the challenge of poly pharmacy and lack of knowledge for their home medications-therefore, often get under dosed or overdosed.

OBJECTIVES OF PROGRAM/INTERVENTION (NO MORE THAN THREE OBJECTIVES): 1 . To see if pillboxes can reduce the error of inappropriate home medication dosage 2. To enhance confidence among the elderly patients and to create ownership of their health by improving their knowledge base on the use of medications 3. To augment patient safety and to reduce the error of inappropriate home medication dosage

DESCRIPTION OF PROGRAM/INTERVENTION, INCLUDING ORGANIZATIONAL CONTEXT (E.G. INPATIENT VS. OUTPATIENT, PRACTICE OR COMMUNITY CHARACTERISTICS): All elderly patients aged $>60$ years, admitted to the Johns Hopkins Bayview Medical Center with intact cognition levels getting discharged on $>3$ medications to home self-care is our target group. On the day of discharge, the discharging physician, nursing staff or clinical pharmacists will be asking the target group patients 6 pre-intervention survey questions focusing on their home medication organization and level of knowledge on the indication, dosage of the home medications. If patients are not using pillboxes at home, the clinical staff will obtain patients' permission to teach them about using the pillboxes to organize medications. The patients then will demonstrate the staff on using the pillbox and should be comfortable using this before the planned discharge. Any issue regarding the pillbox use will be addressed at this time along with teaching on the clinical indication of the home medications and the typical side-effects of the prescribed home medication. The target group patients will then be supplied with pillboxes and the clinical staff will be contacting the patients within 3-7 days of discharge to ask the post-intervention survey questions.

MEASURES OF SUCCESS (DISCUSS QUALITATIVE AND/OR QUANTITATIVE METRICS WHICH WILL BE USED TO EVALUATE PROGRAM/INTERVENTION): We will evaluate the target group patients for any improvement on their level of knowledge about the clinical use, side effects of their home medications and organization of home medications in pillboxes by asking them 6 questions that will focus on the relevant areas upon 3-7 days of their hospital discharge to home. Basically, each patient will serve as their own control by answering the same survey questions before and after the pillbox intervention.

FINDINGS TO DATE (IT IS NOT SUFFICIENT TO STATE "FINDINGS WILL BE DISCUSSED"): A pilot survey was constructed among 10 patients who met the above mentioned criteria and the survey report showed that there was a significant improvement with answers from likert scale $0-1$ to level 3. For example, 2 elderly patients have answered 
the question which asked "How comfortable you are to understand why your home medications are prescribed". Before intervention, the answer was $0=$ not at all. After intervention, the response to the same question was 3 =Extremely comfortable. (other choices, $1=$ somewhat comfortable, $2=$ mostly comfortable). By March 2012, we will get the survey data and we plan to summarize the report with displays of findings in tables, graphical chart and pie charts.

KEY LESSONS FOR DISSEMINATION (WHAT CAN OTHERS TAKE AWAY FOR IMPLEMENTATION TO THEIR PRACTICE OR COMMUNITY?): 1 . To enhance patient safety level among elderly home self-care patients on poly pharmacy by reducing the fatal error of over dosage or under dosage of medications by pillbox intervention and teaching on clinical indications/side-effects of medications while in hospital by clinical staff. 2 . To disseminate this safety practice among community patients by adapting this approach for outpatient practice as well. 3 . To boost up the independence and confidence among elderly home self-care patients to encourage ownership of their own health

USING A PATIENT PORTAL TO COMMUNICATE LABORATORY TEST RESULTS IN COMMUNITY PRACTICES Caitlin A. Colling ${ }^{1}$, Lynn A. Volk ${ }^{2}$; Chelsea Jenter ${ }^{1}$; Marti Dembowitz ${ }^{2}$; David W. Bates ${ }^{1,2}$; Steven R. Simon ${ }^{3,1}$. ${ }^{1}$ Brigham and Women's Hospital, Boston, MA; ${ }^{2}$ Partners HealthCare, Boston, MA; ${ }^{3}$ VA Boston Healthcare System, Boston, MA. (Control ID \#1337102)

STATEMENT OF PROBLEM OR QUESTION (ONE SENTENCE): Timely notification of laboratory test results is an element of CMS's meaningful use criteria and a JCAHO Patient Safety Goal. However, little is known about the use of web-based patient portals to communicate test results in community practices.

OBJECTIVES OF PROGRAM/INTERVENTION (NO MORE THAN THREE OBJECTIVES): Objective 1: Evaluate timeliness of test result notification using a patient portal of an electronic health record (EHR) in community-based primary care practices. Objective 2: Examine how physicians' and patients' portal use for laboratory test result notification changes after the initial implementation.

DESCRIPTION OF PROGRAM/INTERVENTION, INCLUDING ORGANIZATIONAL CONTEXT (E.G. INPATIENT VS. OUTPATIENT, PRACTICE OR COMMUNITY CHARACTERISTICS): Following the adoption of EHR-based patient portals by 3 community practices, we studied the time and method of test result notification. After results are provided to the EHR, the physician must review results, click a box that marks the results as reviewed, and indicate the results should be released to the patient portal. At the same time, the EHR generates a HIPAAcompliant e-mail message notifying the patient of new portal activity. For analysis, we extracted automated data related to laboratory test RESULTS: date result delivered to the EHR; date result reviewed by physician; whether result was posted to the portal; date of posting; and patient log-in date. We also extracted variables to indicate patient notification of test results via other routes (e.g., telephone encounter or letter). For each practice, we defined 3 time periods: 1) 6 months immediately prior to portal adoption ("pre-adoption"); 2) 6 months beginning four months after portal activation ("early post-adoption"); and 3) 6 months from $6 / 1 / 10$ to 12/1/11 ("late post-adoption")

MEASURES OF SUCCESS (DISCUSS QUALITATIVE AND/OR QUANTITATIVE METRICS WHICH WILL BE USED TO EVALUATE PROGRAM/INTERVENTION): The primary outcome measure was the time to patient notification of a test result after physician review. Secondary measures included 1) whether a patient was notified of an abnormal result within the assigned time frame and 2) whether clinician follow-up was performed within an appropriate time frame.

FINDINGS TO DATE (IT IS NOT SUFFICIENT TO STATE "FINDINGS WILL BE DISCUSSED"): In a single pediatric practice, 1840 results were reviewed in the early post-adoption period. To minimize autocorrelation, we included one randomly selected laboratory test per patient per period. A total of $56 \%$ of laboratory tests reviewed were sent to the portal. For test results posted to the portal, $40 \%$ of patients logged into the portal within 30 days of the posting, with an average of 7.6 days between posting and patient log-in. In the late post-adoption period, the percent of results posted to portal dropped to $50 \%$, with fewer patients (31\%) logging into the portal within 30 days of posting. For those patients who logged in following result posting, the average time between posting and log-in dropped to 3.0 days.

KEY LESSONS FOR DISSEMINATION (WHAT CAN OTHERS TAKE AWAY FOR IMPLEMENTATION TO THEIR PRACTICE OR COMMUNITY?): Understanding patient preferences for the use of a patient portal for test notification will help to improve timely results management. The data from a single practice, to be confirmed in the two other practices, suggest that, over time, patients using a portal log in more quickly following the posting of a test result. However, given the reduction in both the percentage of results posted to the portal and the proportion of patients with tests posted who logged in, this route may not be preferred by the majority of patients. Thus, when implementing a portal, physicians need to assess patient preferences to make informed decisions on the optimal use of the portal for test result notification.

VIDEOCONFERENCING FOR REMOTE SPANISH INTERPRETATION IN A COMMUNITY HEALTH CENTER: LESSONS LEARNED USING THE TABLET COMPUTER Claudia Campos; Dominic Johnson; Nancy M. Denizard-Thompson; Monica T. Brown; James L. Wofford. Wake Forest University, Winston-Salem, NC. (Control ID \#1321932)

STATEMENT OF PROBLEM OR QUESTION (ONE SENTENCE): The advent of more mobile, more reliable, and more affordable videoconferencing technology finally makes it realistic to offer remote foreign language interpretation in the office setting; still, such technology deserve proof of acceptability to clinicians and patients before there is widespread acceptance and routine use.

OBJECTIVES OF PROGRAM/INTERVENTION (NO MORE THAN THREE OBJECTIVES): We sought to examine (1) the audio and video technical fidelity of $\mathrm{iPad} /$ Facetime (TM) software. (2) the acceptability of videoconferencing to patients and clinicians.

DESCRIPTION OF PROGRAM/INTERVENTION, INCLUDING ORGANIZATIONAL CONTEXT (E.G. INPATIENT VS. OUTPATIENT, PRACTICE OR COMMUNITY CHARACTERISTICS): The convenience sample included Spanish-speaking adult patients presenting to a community health care medicine clinic in August-November 2011. Videoconferencing was accomplished using Facetime ${ }^{\mathrm{TM}}$ software on 2 Apple iPads ${ }^{\mathrm{TM}}$, a swiveling computer stand in the exam room, and a wireless network of 2.0 Mps bandwidth. As clinicians and interpreters gained comfort with the technology, the introduction/orientation to the patient by the interpreter took place over the videoconferencing device rather than in-person. The clinician was oriented to the device (rationale, computer positioning) by an investigator with no other special instructions. During the clinical encounter, the interpreter was seated in a separate, quiet office elsewhere in the building. The interpreter spoke with the patient in-person after the clinical encounter to administer the survey and debrief.

MEASURES OF SUCCESS (DISCUSS QUALITATIVE AND/OR QUANTITATIVE METRICS WHICH WILL BE USED TO EVALUATE PROGRAM/INTERVENTION): A five-item survey was used to solicit opinions from patients and clinicians on overall quality of the videoconferencing device, audio/video integrity/fidelity, perception of encounter duration, and attitude toward future use.

FINDINGS TO DATE (IT IS NOT SUFFICIENT TO STATE "FINDINGS WILL BE DISCUSSED"): Participants were most often female (20 $25,80 \%$ ), and of mean age $42.3(+5.1) .3$ patients refused to use the device. 18 clinicians and 5 interpreters participated in the project. Most patients rated overall quality of videoconferencing as good/excellent (24/25), with only 1 "'fair" rating. The quality of audio/video was rated as good/excellent by $72 \%$ (18/25). 11 patients rated the amount of time as no longer than in-person, and 9 reported it as shorter than in-person. Most patients 94\% (24/25) favored using videoconferencing during future visits. For the 18 clinicians, the results were similar. 94\% (17/18) of clinicians found the overall experience good/excellent and the audio/video fidelity acceptable, with only 1 "fair" rating. 12 clinicians rated the amount of time as no longer than in-person, and 4 of shorter duration than in-person. 14/18 clinicians favored using the device again in the future. Challenges included concerns over ambient noise and multiple conversations 
during the clinical encounter, and the absence of a dedicated room for the interpreter.

KEY LESSONS FOR DISSEMINATION (WHAT CAN OTHERS TAKE AWAY FOR IMPLEMENTATION TO THEIR PRACTICE OR COMMUNITY?): Based on our experience at a single-site community health center, the audio/video fidelity of the videoconferencing technology appeared to be flawless, and both patients and clinicians were satisfied. Implementation still required attention to quality of the wireless network, orientation and device positioning strategies, and psychological effects on interpreters. Expansion of videoconferencing to other off-site healthcare professionals( i.e., sign language interpreters, diabetic educators, mental health counselors) should be considered in the search for more cost-effective healthcare.

WHO NEEDS DETOX? AN EVIDENCE-BASED PROTOCOL FOR SYSTEMATIC EVALUATION OF PATIENTS WITH ALCOHOL DEPENDENCE AND WITHDRAWAL. John Stephens; Ria Dancel; Jonathan Kirsch; Edmund A. Liles; Michael Gilchrist; Kelly Stepanek. UNC Hospitals, Chapel Hill, NC. (Control ID \#1308493)

STATEMENT OF PROBLEM OR QUESTION (ONE SENTENCE): Our tertiary hospital medicine practice noted a high number of admissions and readmissions to the internal medicine service for acute detoxification from alcohol, and also noted a lack of a clear, evidence-based system for determining which patients would benefit from inpatient detoxification versus those who might be safely treated as an outpatient.

OBJECTIVES OF PROGRAM/INTERVENTION (NO MORE THAN THREE OBJECTIVES): 1) Develop an evidence-based protocol to evaluate alcohol dependent patients presenting to the emergency department requesting inpatient alcohol detoxification, specifically addressing safety of outpatient detoxification and identifying risk factors for complicated alcohol withdrawal 2) Improve co-ordination with community resources for alcohol dependence treatment 3) Measure specific metrics before and after implementation of the protocol to see if protocol would impact utilization of inpatient services

DESCRIPTION OF PROGRAM/INTERVENTION, INCLUDING ORGANIZATIONAL CONTEXT (E.G. INPATIENT VS. OUTPATIENT, PRACTICE OR COMMUNITY CHARACTERISTICS): We formed a task force to develop the protocol for alcohol detoxification. The task force reviewed best available evidence on acute alcohol dependence treatment. Data are limited on this specific topic, with majority of literature focusing on predictors of delirium tremens, and these risk factors informing expert opinion on need for inpatient versus outpatient treatment for alcohol detoxification. Accordingly, we reviewed evidence on predictors of delirium tremens in developing our protocol. Briefly, the developed protocol calls for careful assessment of risk factors for complicated withdrawal coupled with Clinical Institute Withdrawal Assessment (CIWA) scores, in the context of medical and psychological history. We met with representatives from the largest community alcohol treatment facility, and the protocol incorporates a process for referring patients for alcohol dependence treatment. The protocol was reviewed with emergency room physicians and the hospital medicine group. After a period for commentary and modification was provided, the protocol was implemented July $1,2011$.

MEASURES OF SUCCESS (DISCUSS QUALITATIVE AND/OR QUANTITATIVE METRICS WHICH WILL BE USED TO EVALUATE PROGRAM/INTERVENTION): The metrics to be followed will include number of admissions to inpatient service for alcohol detoxification, 30day readmission rate, and length of stay index before and after implementation of protocol. Other metrics may be added as data are further analyzed, including number of referrals to community alcohol treatment.

FINDINGS TO DATE (IT IS NOT SUFFICIENT TO STATE "FINDINGS WILL BE DISCUSSED"): Preliminary data have yielded the following RESULTS: For the 6 months preceding initiation of protocol there was an average of 18.33 admissions/month for alcohol detoxification, with a $20 \% 30$-day readmission rate and length of stay index of 0.61 . For the 2 months following initiation of protocol there was 11.5 admissions/ month with a $17.5 \%$ readmission rate and a length of stay index of 0.92 .
KEY LESSONS FOR DISSEMINATION (WHAT CAN OTHERS TAKE AWAY FOR IMPLEMENTATION TO THEIR PRACTICE OR COMMUNITY?): With use of a standardized protocol, patients with alcohol dependence presenting to emergency department for substance abuse can be systematically evaluated for need for inpatient versus outpatient treatment. Utilization of the protocol appears to lower admissions to the hospital and 30day readmission rates for alcohol detoxification.

\section{INNOVATIONS IN MEDICAL EDUCATION (IME)}

"BOLUS" AND "DRIP" QUALITY IMPROVEMENT CURRICULA FOR INTERNAL MEDICINE RESIDENTS Amanda Carmel; Laura Fanucchi; Jennifer Lee; Lia S. Logio. Weill Cornell Medical College, New York, NY. (Control ID \#1339311)

NEEDS AND OBJECTIVES: Familiarity and experience with QI is essential for preparing residents for future careers and is an area highlighted in two of the six core competencies defined by the Accreditation Council for Graduate Medical Education. Given the many educational goals and work hour requirements, incorporating formal QI education into training programs is a challenge. At New York-Presbyterian Hospital/Weill Cornell (NYPH/ WC), we developed two QI curricula: an intensive 2-week curriculum for senior Internal Medicine residents (bolus), and a 6-month longitudinal curriculum for junior residents (drip). The goals of each were to explain the fundamentals of QI and how they impact medical practice and to mentor residents in the design of a QI project using the Model for Improvement (Plan-Do-Study-Act (PDSA)).

SETTING AND PARTICIPANTS: Junior and senior internal medicine residents at NYPH/WC.

DESCRIPTION: Both "bolus" and "drip" curricula occur during the ambulatory care block. The senior curriculum is an intensive 2-week experience where residents spend 3 afternoons a week (18 hrs) dedicated to learning and applying QI methodology. The junior residents spend 2 mornings per week during 3 two-week blocks spaced at 6 week intervals ( $36 \mathrm{hrs}$ ). The main focus of both is to design and implement QI projects using the PDSA cycle. Didactic sessions on measurement strategies, data analysis, and other QI tools are provided. At the end of these educational units, the resident teams present their projects to an audience of key stakeholders and peers and submit a summary poster. Residents complete the validated Quality Improvement Knowledge Assessment Test (QIKAT) before and after each course.

EVALUATION: Over 8 weeks in July and August 2011, all 46 senior internal medicine residents participated in the senior curriculum. From July 2011 to December 2011, 24 junior residents participated in the junior curriculum. A total of 16 posters were submitted by resident teams (12 from PGY3s and 4 from PGY2s). The average QIKAT score before the "bolus" curriculum was 8.7 (out of 15 possible points) and rose to 13.5 at the end (junior resident data pending). Both courses were rated highly among the residents with a mean evaluation score of 4.1 (Likert scale 1-5). The spectrum of projects included introducing rapid administration neostigmine injections to the Cardiac Care Unit and revising smoking cessation online resources and resident education materials. DISCUSSION / REFLECTION / LESSONS LEARNED: Both intensive (bolus) and longitudinal (drip) curricula provide in depth instruction on various components of QI and allow residents to conduct their own QI initiatives using the PDSA cycle. The senior residents' understanding of QI concepts improved after the curriculum as measured by the QIKAT, and we anticipate similar improvements for the junior residents. Residents rate the experience highly in follow up evaluations. Internal Medicine residents are interested in and motivated by learning about QI. Our work is unique in that it provides two effective models for QI education, "bolus" and "drip." We anticipate that given the two schedule options, our curriculum models can be applied to other residency programs and thus will allow better integration of QI into resident teaching, mentorship, and clinical practice.

3+1: A NEW MODEL FOR RESIDENT TRAINING Teresa Cheng ${ }^{1}$; Maria C. DeOliveira ${ }^{2}$; Subha Ramani ${ }^{1}$; Rajlakshmi Krishnamurthy ${ }^{1}$; Angela Jackson ${ }^{1} .{ }^{1}$ Boston University, Boston, MA; ${ }^{2}$ Beth Israel Deaconess Medical Center, Boston, MA. (Control ID \#1334456) 
NEEDS AND OBJECTIVES: In 2009, the ACGME increased the number of required continuity clinics from 108 to 130 over 36 months of medicine residency training. At Boston University/ Boston Medical Center, the Medicine Residency Program believed that the previous model of residency training could not achieve the need for increased continuity clinics, high quality ambulatory clinical and educational experiences and compliance with duty hour regulations. The Program designed a new " $3+1$ " model to: 1 . Meet the number of mandatory resident continuity clinics 2 . Decrease tension between the inpatient and ambulatory commitments 3 . Develop meaningful and broad-based subspecialty ambulatory experiences 4 . Decrease resident stress and fatigue

SETTING AND PARTICIPANTS: The $3+1$ model was implemented in July 2010 for categorical and primary care residents at the Boston University Medical Residency Program.

DESCRIPTION: Residents alternate between 3 week blocks of wards, units, Emergency Department or elective rotations and 1 week of ambulatory experiences. These 10 half days are scheduled with 4 primary care continuity clinic sessions, one session of unscheduled patient management time, 4 sessions of a single specialty clinic that rotates every 3 months and one session for dedicated seminar style intensive education, which complements an enhanced pre-clinic and midday conference curriculum.

EVALUATION: All residents will successfully meet the new ACGME clinic requirements. Residents were surveyed confidentially before and 8 months after implementation of the $3+1$ model. The survey queried satisfaction with clinic experience, preceptors, learning and work environment and included a 2 item scale from the Maslach Burnout Inventory and the Quality of Life (QOL) Analog Scales. The post-survey also added a question on their $3+1$ experience. 57 respondents $(40 \%)$ answered the June 2010 survey and 52 (34\%) answered the February 2011 survey. Overall satisfaction with continuity clinic increased from $46 \%$ to $64 \%$. Satisfaction with ability to focus in clinic without interruption was 33\% pre change and $67 \%$ post change. Measures of burnout and overall QOL did not appear to vary but our QOL measure of fatigue improved, from 5 to 7 , where 10 is defined "as good as it gets". Qualitative analysis of comments revealed key themes categorized as work environment, ambulatory clinic environment, educational environment, and preceptors. Burn-out was mentioned often pre $3+1$. Following the change, several residents expressed relief that an ambulatory week broke up the inpatient rotations. Residents appreciated the ability to focus on their inpatient and outpatient experiences separately and the ambulatory curriculum was well received. Clinic preceptors were viewed positively pre and post implementation. There was initial apprehension about the $3+1$ model, but residents seem satisfied overall 8 months later.

DISCUSSION / REFLECTION / LESSONS LEARNED: We successfully implemented a new $3+1$ model for residency training which easily met the target goal of 130 continuity clinics over 3 years. Separation of inpatient and outpatient experiences resulted in enhanced ambulatory clinical and educational experiences in continuity clinic and specialty clinics and a decrease in resident stress and fatigue. Major challenges include extensive coordination across multiple clinic sites with varying abilities to accommodate schedule changes and major changes in faculty schedules.

\section{A SPACED EDUCATION CURRICULUM TO IMPROVE BONE} HEALTH CARE BY INTERNAL MEDICINE RESIDENTS Brigid M. Dolan $^{1}$; Graham T. McMahon ${ }^{2}$; Maria Yialamas ${ }^{2} .{ }^{1}$ Brigham and Women's Hospital, Boston, MA; ${ }^{2}$ Brigham and Women's Hospital, Boston, MA. (Control ID \#1337189)

NEEDS AND OBJECTIVES: The increasingly compressed schedule of residents creates a need for educational interventions to effectively and efficiently generate behavior change. We have hypothesized that a spaced education intervention will be acceptable to residents and generate measurable improvements in clinical practice. We chose osteoporosis as the topic for this study, as osteoporosis is a common condition that is frequently undertreated. A sample chart review of residents in our clinic revealed that only $70 \%$ of eligible patients had received bone density screening and only $11 \%$ of osteopenic patients had a FRAX score calculated. We have designed, implemented and will evaluate a novel curriculum to help residents improve the quality of their osteoporosis care using online spaced education. We sought to examine if this innovation would meaningfully change ambulatory practice performance by internal medicine residents.

SETTING AND PARTICIPANTS: A convenience sample of junior and senior residents was chosen from an academic primary care practice in our institution. Fifty residents have been randomized.

DESCRIPTION: The intervention curriculum delivers content by email using spaced education, a method that sends short questions and explanations over time, with incorrectly answered items recycled with an increased frequency. This method has been shown to improve knowledge retention and change faculty behaviors, however, to our knowledge this has not been applied to resident practice behaviors in the primary care setting. Residents in the intervention arm received an email every 3 days containing 2 multiplechoice questions. The question set was validated prior to delivery. The curriculum adapted to learner competence resulting in a duration that varied by user, lasting between 2.5 and 6 months. The standard curriculum consisted of a one-hour lecture; these residents also received one-time access to the multiplechoice questions and answers used in the intervention arm.

EVALUATION: Of the 25 residents randomized to the intervention arm, 22 residents enrolled in the curriculum. $56 \%$ of residents responded to at least $75 \%$ of the curriculum. $28 \%$ of residents completed the entire curriculum, needing an average of 64 responses total to correctly answer each question twice. Initial reports indicate that email fatigue is emerging as a challenge. In Spring 2012, residents will complete a knowledge assessment to determine retention of osteoporosis knowledge. A chart review will assess resident patient care outcomes in osteoporosis screening and treatment.

DISCUSSION / REFLECTION / LESSONS LEARNED: Our initial evaluation of the acceptability of the intervention indicates that the majority of residents voluntarily participated in this online longitudinal educational format. Residents did endorse "email fatigue" towards the end of the course. Using an interface that would limit emails or lengthening the delivery time of the course may be necessary if this tool is used outside of pilot intervention, particularly if content is extended to reflect the broader curricular needs of internal medicine residents. The knowledge retention tool and chart review portion of this evaluation will demonstrate if there is sufficient behavior change benefit to merit using this tool to create a comprehensive, longitudinal primary care curriculum for internal medicine residents.

A COMPARATIVE TEACHING STRATEGY FOR INTERPROFESSIONAL COMMUNICATION Hollis Day ${ }^{1}$; Susan Meyer ${ }^{2} .{ }^{1}$ University of Pittsburgh School of Medicine, Pittsburgh, PA; ${ }^{2}$ University of Pittsburgh School of Pharmacy, Pittsburgh, PA. (Control ID \#1312206)

NEEDS AND OBJECTIVES: Interprofessional communication has long been recognized by the Institute of Medicine as a critical element to patient care. While such communication has been a key topic in all health professional schools, a national conference in 2011 specifically outlined the core competencies in interprofessional care and education that have been adopted by the major accrediting bodies of the health professions. This study compared two methods of teaching pharmacy students how to communicate with physicians in challenging scenarios: standardized colleagues (adaptation of standardized patients) and video triggers/group discussions. Objectives were 1) Design scenarios representing authentic interprofessional interactions and communication challenges to facilitate the development of interprofessional communication skills; 2) Evaluate the effectiveness of standardized colleagues as a strategy to facilitate the development of interprofessional communication skills compared to a traditional strategy of using video triggers and role play.

SETTING AND PARTICIPANTS: 104 second year pharmacy students in the context of their Profession of Pharmacy class.

DESCRIPTION: All students attended a one hour preparatory lecture for communication skills in difficult conversations. Fifty-seven students were randomized to interact with medical faculty as standardized colleagues portraying particular professional roles, attitudes, and communication styles. Cases were based on real-life situations representing the seven crucial conversations identified in Silence Kills, a seminal work on poor 
healthcare communication. Faculty provided feedback on demonstrated behaviors impacting communication effectiveness. Forty-seven students were randomized to view videos demonstrating interprofessional interactions and participate in facilitated discussions of the demonstrated interprofessional communication skills. A self-evaluation of comfort and confidence in communication skills adapted from a validated instrument was administered at baseline, three, and six months. Students completed an evaluation of the perceived helpfulness of the activity.

EVALUATION: Data from students with scores on all three time points were used in the analysis $(\mathrm{n}=92)$. Results of the repeated measures ANOVA demonstrated an increase in comfort and confidence over time $(\mathrm{F}=42.508, \mathrm{df}=$ $2, \mathrm{p}<.001)$. Paired samples t-tests showed a significant increase between baseline and three months $(\mathrm{t}=-7.615, \mathrm{df}=99, \mathrm{p}<.001)$. An independent samples t-test revealed a significant difference in helpfulness, confidence, and comfort between the video and standardized colleagues methods $(\mathrm{t}=-2.396, \mathrm{df}=82.69, \mathrm{p}=.019)$.

DISCUSSION / REFLECTION / LESSONS LEARNED: Using standardized colleagues can enhance students' confidence and comfort in their abilities to communicate effectively in challenging situations. This methodology provides a safe way for students to practice difficult conversations that affect patient care. Case scenarios can be adapted to multiple health professions schools. Limitations include cost, ability to recruit medical professionals to portray physician roles, and the potential for reinforcing negative stereotypes of physicians. Other health professionas may be more eager to adopt this methodology than medicine.

A CURRICULUM FOR ELECTRONIC PATIENT-DOCTOR COMMUNICATION Bradley H. Crotty; Arash Mostaghimi. Beth Israel Deaconess Medical Center, Boston, MA. (Control ID \#1334944)

NEEDS AND OBJECTIVES: Despite the rise in patient-doctor electronic communication via email and patient portals, no formal training exists for online communication. In many practices, physicians are expected to use e-mail or secure messaging with patients outside of visits. In our internal medicine training program, we created a curriculum and assessment tool for patient-centered electronic communication using PatientSite, a secure patient portal at Beth Israel Deaconess Medical Center (BIDMC).

SETTING AND PARTICIPANTS: The curriculum was developed in the Internal Medicine Residency Training Program at BIDMC. Residents from all three years practicing on-site were enrolled in PatientSite $(n=116)$. Those practicing off-site $(n=32)$ received portions of the curriculum and assessment but did not participate in the portal.

DESCRIPTION: Our curriculum consists of a one-hour lecture, a one-hour case-based small group session, and experiential learning through PatientSite. We organized the curriculum around four domains: patients, the patient experience, the physician experience, and systems-based practice. The cases include themes of unequal access to online resources, health literacy and patient-centered communication, patient empowerment, and choosing the appropriate medium of communication for a given concern. We gave a preintervention questionnaire to assess baseline attitudes toward patient portals and to assess self-perceptions of communication skills. We also asked respondents to rank appropriateness of different communication media in each of 4 scenarios. We created simulated patient e-mail exchanges to assess response time, comprehensibility, clarity, and empathy. At the end of the intervention a post-test will be administered.

EVALUATION: Residents completed pre-intervention surveys. Most residents felt that the portal would increase their work $(\sim 57 \%)$, but would be better for patients. Nearly $90 \%$ of residents expressed concern about medical liability from missed or inappropriate messaging. Three months into the educational intervention, 104 patients have been enrolled.

DISCUSSION / REFLECTION / LESSONS LEARNED: Residents must learn evidence-based skills for electronic communication to be ready for ever-evolving practice models. While we are currently piloting this curriculum, housestaff have had robust discussions during the small group sessions with heterogeneous perspectives. The combination of didactics and experiential learning is promising for preparing physicians for electronic communication with patients.

ONLINE RESOURCE URL (OPTIONAL): http://themetrc.org/ a-curriculum-for-patient-doctor-electronic-communication/
A DEBATABle APPROACH TO EVIDENCE BASED MEDICINE Harry Burke; Brian Cohee; Scott Hopkins. Walter Reed National Military Medical Center, Bethesda, MD. (Control ID \#1324181)

NEEDS AND OBJECTIVES: There are many ways to teach residents how to use the empirical medical literature to assist them when they are making real clinical decisions in real time with real patients. One popular approach is a "mini-journal club" format, where residents are given a clinical question and they must find and present an article relevant to the question. One problem with this approach is that the article rather than the patient is the main focus of the discussion. We created a "debate" format for evidence-based medicine.

SETTING AND PARTICIPANTS: The residents who had participated in the mini-journal club format were surveyed. We changed to the debate format and asked the residents who participated in the debate format the same survey questions. We assessed our six ward teams.

DESCRIPTION: For the debate format, every month, over three days, two teams at a time debated a clinical question. The question was announced when they arrived for the debate. They were assigned to argue either the affirmative or the negative. The team was allowed 30 minutes to research the medical literature and create their argument, 8 minutes to argue their side, 4 minutes for rebuttal, and 3 minutes for summation. After the debate, an attending, two chief residents, and a third year resident discussed how the evidence was obtained, its quality, and the strengths and weakness of each side. A winning team was chosen each month.

EVALUATION: Twenty-two residents answered the mini-journal club survey and 43 residents answered the debate format. Seven questions were asked, five of which were not significantly different between the journal club and debate formats. The two key questions were: "This exercise was a valuable use of my time." and "For the amount of time I put into the exercise, I received a commensurate increase in learning." The residents felt that the value was greater for the debate format (lower scores were better, journal club, mean 2.0; debate, mean $1.67 ; \mathrm{p}=0.035$, two-sided) and they felt that the debate was a good use of their time (lower is better, journal club, mean 2.0; debate, mean 1.60; $\mathrm{p}=0.048$, two-sided).

DISCUSSION / REFLECTION / LESSONS LEARNED: Residents felt that the debate-style format was more valuable and a better use of their time than the mini-journal club format. Out goal is to create a format that requires the residents to obtain, synthesize, and use evidence-based clinical information in real time so that they can use this skill both in the hospital and in outpatient clinic. We believe that out debate format provides a realistic approach for achieving our goal that the residents find valuable and a good use of their time.

A LONGITUDINAL SIMULATION BASED MEDICAL EDUCATION CURRICULUM IN AN INTERNAL MEDICINE RESIDENCY PROGRAM Raquel K. Belforti; Ngina Muigai; Mihaela Stefan. Baystate Medical Center, Springfield, MA. (Control ID \#1335687)

NEEDS AND OBJECTIVES: At teaching hospitals across the country, medical residents of varying knowledge and skill levels are responsible for providing all aspects of patient care. Traditional medical education accepted the "see one, do one, teach one" mentality for learning, in which residents practice and master skills on their patients. Currently, due to society's change in expectations of medical care there has been a tremendous emphasis to ensure patient safety. This creates a struggle for residency programs on how to provide physicians-in-training with the opportunities to practice, attain competence, and master skills, as well as protect patients from the errors that coincide with learning. The answer to this great ethical dilemma may be in simulation based medical education (SBME). SBME can be defined as any educational activity which uses simulative aids to replicate clinical scenarios. Human Patient Simulation (HPS) provides learners with the opportunities to repeatedly practice clinical skills, and more importantly learn from errors without any harm to patients. The Accreditation Council of Graduate Medical Education has recognized the need for alternative teaching tools and has stated that the role of simulation in formative and summative competency evaluation is growing. Often the barrier to implementing a simulation curriculum to meet a program's needs is the unfamiliarity with the plethora of ways simulation can be used within residency training programs and how to get started. 
SETTING AND PARTICIPANTS: The Baystate Medical Center Internal Medicine Residency Program has integrated SBME using HPS into their residency curriculum that provides longitudinal experiential learning to all levels of training.

DESCRIPTION: We have three main simulation programs. Intern Boot Camp is a 2-week intensive program for July interns to learn how to manage acutely ill hospitalized patients. Teaching Team Simulation which occurs weekly during medicine ward rotations for interns to manage patients with complicated medical conditions and for senior residents to practice debriefing and teaching skills. ACLS Leadership Training which provides weekly simulation sessions for second year residents to learn and practice crisis resource management skills integral for effective ACLS implementation.

EVALUATION: Our current state of evaluation of the effectiveness of our simulation curriculum is based on debriefing and direct feedback from residents. Each curriculum focuses on teaching and providing immediate feedback on the core competencies. After each simulation session a debriefing is held to illicit peer feedback as well as provide immediate verbal evaluation. We are currently in the process of developing and validating an evaluation tool to be used to assess our interns' performance in the simulation lab caring for unstable hospitalized patients. The goal is for this evaluation tool to be used in conjunction with other evaluations of interns' performance by the clinical competency committee to determine progression onto second year of residency

DISCUSSION / REFLECTION / LESSONS LEARNED: Overall, Baystate Medical Center Internal Medicine Residency Program has a well established simulation curriculum for all levels of learners. We are currently in the process of transitioning from using our simulation curriculum for formative to summative competency evaluation.

A MULTI-STRATEGY APPROACH TO IMPROVE MEDICAL RESIDENT'S CONFIDENCE IN DISCUSSING END-OF-LIFE CARE William P. Moran; Patty J. Iverson; Patrick D. Mauldin; Paul Rousseau. MUSC, Charleston, SC. (Control ID \#1337730)

NEEDS AND OBJECTIVES: Elderly patients want to discuss advanced care directives but residents are not adequately trained to confidently have such discussions with their patients, and may avoid the topic. This paper describes an innovative approach to (1) increase patient opportunity to discuss end of life care wishes with their physician and (2) improve resident's confidence to lead discussions about ACDs. Objectives: (1) Create an environment where residents are cued to ask patients about advanced care directives (ACD). (2) Educate residents about ACDs and provide a tool to facilitate good communication skills in discussing advanced care directives with elderly patients. (3) Increase resident's confidence in leading end of life care discussions through multiple learning strategies.

SETTING AND PARTICIPANTS: This program, conducted in an outpatient internal medicine resident clinic at a southeastern United States medical university, included nearly 100 residents and 600 elderly patients. DESCRIPTION: Education: Over a 3 month period, internal medicine residents were offered multiple learning strategies about advanced care products and discussed and practiced ways to improve their confidence in talking with their patients about their wishes. Residents were offered lecture, academic detailing by faculty in continuity clinic, faculty observation of patient discussions, and small group simulations. System change: Clinic Patient Care Technicians gave elderly patients the booklet, "Isn't it Time We Talked" and asked if they had ACDs. The patient's response $(\mathrm{Y}$ or $\mathrm{N})$ was noted on the chart, prompting the resident to address the following during the visit: (1) If Yes, the resident asked about their wishes and checked for a copy in the medical record; or (2) If No, the resident asked the patient if he/she wanted to discuss advanced directives now or schedule a follow-up appointment for discussion. The resident was prompted to document responses in the patient's electronic medical record (EMR).

EVALUATION: On a pre- post-test, residents were asked: "On a scale of 1-4 with 1 being no confidence and 4 being very confident, rate your confidence in your ability to discuss advanced care planning with an older adult patient." Resident participation in academic detailing, small group simulations, and faculty observation were tracked and cross referenced with the resident's reported change in confidence.

DISCUSSION / REFLECTION / LESSONS LEARNED: We found elderly patients do want to discuss their end-of-life care wishes with their physician and residents need training to improve their confidence in leading such discussions. When provided information and opportunity, the majority of resident continuity clinic patients expressed interest in talking with their physician about their wishes. $77 \%$ of patients asked reported no $\mathrm{ACD}$ and $74 \%$ of those patients wanted to talk about it during their visit or at a follow-up appointment. After education and prompting, IM resident physicians reported they were more confident having these discussions with their patients. Of the 58 residents $(60 \%$ of program) who completed the pre- and post-tests, $24 / 58(41 \%)$ reported improved confidence; $27 / 58$ $(46 \%)$ the same; and $7 / 58(12 \%)$ less confidence. Some medical universities offer communication training in medical school however few offer on-going opportunities for residents to sharpen their skills during residency when they actually have direct patient-doctor conversations.

ONLINE RESOURCE URL (OPTIONAL): http://mcintranet.musc.edu/ agingq3/endoflifecareacove

\section{A PRIMARY CARE RESIDENCY'S CORE DNA INSERTED AT} PROGRAM OUTSET TO BLOOM INTO A TIGHT SPIRAL CURRICULUM Richard E. Greene; Jennifer Adams; Sondra Zabar; Rob Caldwell; Les Chuang; Carrie Mahowald; Negar Aliabadi; Kathleen Hanley; Andrew A. Chang; Julianne Cameron; Mack Lipkin. NYU School of Medicine, New York, NY. (Control ID \#1339602)

NEEDS AND OBJECTIVES: Our annual residency retreat brainstorms innovations to meet needs. In 2010 needs were: to introduce foundation concepts and enable primary care (PC) residents to feel/be competent in clinic earlier; to spiral learning of core concepts, skills and attitudes from the start; and to have residents and faculty connect from the outset. We aim to equip PC clinicians to deliver bio-psychosocial, comprehensive, best evidence-based systems savvy care and to become change agents, leaders, and scholars. To meet these aims we designed a learner centered, team oriented, skills-based Essentials for PC Clinicians (EPIC) curriculum utilizing an initial, rigorous 4 week block with spiral reinforcement through 3 years. The innovation is a comprehensive, reproducible, effective method to ensure residents' progress on paths of clinical, humanistic, and intellectual excellence consistent with the generalist paradigm.

SETTING AND PARTICIPANTS: EPIC is part of the NYU Internal Medicine PC Residency. Residents attend public hospital and community continuity clinics. 8 interns take the EPIC block and 24 residents spiral through the curriculum.

DESCRIPTION: EPIC begins with a 4 week intern block dedicated to core topics in PC; is reinforced in precepting and subsequent blocks; and has a weekly EPIC conference where these topics are deepened and extended. EPIC Block: The overarching themes throughout the 4 weeks focus on understanding and practice of core skills: workshops/precepting on time management, efficient use of EHR, obtaining best practices, consultation, how one learns best, practice in the medical home and engaging community resources. Week 1 focuses on diabetes, and introduces the pillars: psychosocial medicine, evidence-based practice, and systems-based policy awareness and skill. The second week focuses on hypertension. The last 2 weeks introduce 7 common, high-risk high gain conditions from smoking to hepatitis B. Teaching methods combine group learning and reflective written exercises; clinical experiences; core lectures; and a block-long problem-based group learning case set to integrate epidemiology, communications, data (physical, lab, imaging), evidencebased management, and systems quality. Spiral curriculum: All preceptors are trained in the pillar approaches and which is integrated within precepting and 10 subsequent PC blocks. EPIC conference: Weekly, in 1 hour a resident presents on a core PC topic generated by that resident's practices. This allows residents \& program leaders to monitor learners' needs based on their patients in a learner-centered way, modeling selfeducation/discovery.

EVALUATION: The EPIC Block and the subsequent curriculum are evaluated at block's end by a 2-hour 4 station OSCE that reviews and assesses learning and skills, through evaluations and formative debriefing. 
An annual 10 station OSCE is a broad assessment of skills for all 3 years of residents. Unannounced standardized patients appear in residents' practices on core topics.

DISCUSSION / REFLECTION / LESSONS LEARNED: Lessons learned include that EPIC is feasible, well received, meets the initial goals, and sets the interns up for positive future experiences. Implementing early in residency quickly sets expectations/standards, engages learners, immunizes beginners against bad habits, and jump starts core PC skills. The planning effort is major but eye opening for the faculty involved.

ONLINE RESOURCE URL (OPTIONAL): http://medicine.med.nyu.edu/ EDUCATION/ABOUT-PROGRAM/PROGRAM/PRIMARY-CARE/ PC-CURRICULUM

A PRIMARY CARE-BASED TEAMWORK SKILLS CURRICULUM FOR RESIDENTS AND NURSE PRACTITIONER STUDENTS Rebecca L. Shunk ${ }^{1,2}$; Calvin L. Chou ${ }^{1,2}$; Maya H. Dulay ${ }^{1,2}$; Susan L. Janson $^{3,1}$; Shalini Patel ${ }^{1}$; Timothy Carmody ${ }^{1}$; Stephen Rao ${ }^{1}$; Patricia A. Cornett ${ }^{1,2}$; Bridget OBrien ${ }^{2,1}$. ${ }^{1}$ San Francisco VA Medical Center, San Francisco, CA; ${ }^{2}$ Univ of California, San Francisco, San Francisco, CA; ${ }^{3}$ University of California, San Francisco, San Francisco, CA. (Control ID \#1340050)

NEEDS AND OBJECTIVES: Primary care clinics at the San Francisco VA (SFVA) have transitioned to a VA-mandated interprofessional teambased model of patient care called Patient Aligned Care Teams (PACTs). Trainees in these clinics are part of the team-based model and must work more closely with clinic staff than in traditional outpatient clinics. Highfunctioning teams require members to learn and practice skills together, and to know each other both professionally and personally. To facilitate development of these interprofessional skills, we created a curriculum and formative evaluation process.

SETTING AND PARTICIPANTS: In July 2011, we added trainee squads ( 2 second-year internal medicine residents and 1 second-year nurse practitioner student) to each of 8 pre-existing "teamlets" ( 1 registered nurse, 1 clinical associate, 1 clerical associate) in three SFVA primary care clinics. Each trainee acts as primary provider for a panel of patients. When primary providers are unavailable, trainee squad members provide backup coverage. Teamlets collaborate with trainee squads to deliver care to their patients.

DESCRIPTION: We created a multifaceted curriculum to address core competencies associated with effective interpersonal communication. Interactive small group sessions addressed elements of the TeamSTEPPS model, including handoff communication, debriefing, huddling, feedback, and conflict resolution. In addition, we held a daylong retreat attended by all trainees and teamlet members to enhance team building and to practice key communication skills. Preceptors reinforce skills and provide ongoing feedback during teamlet huddles. To assess overall team effectiveness, in September 2011, members of all 8 teams were asked to complete the Team Development Measure (TDM), created by Peace Health.

EVALUATION: Trainees rated the overall quality of the training sessions (mean 3.6 to 4.1 on a 5 point scale, $1=$ poor $5=$ excellent) and of the retreat (mean 4.4 out of 5). On the TDM, response rates for each team ranged from $67 \%$ to $86 \%$. Individual ratings were combined to obtain aggregate scores for each team, which ranged from 55 to 68 out of maximum score of 100. Each team discussed TDM results and made plans for improvement during a 1 hour session with a faculty facilitator. The TDM will be completed again in February 2012, and teams will have another opportunity to discuss and plan for improvement based on the results.

DISCUSSION / REFLECTION / LESSONS LEARNED: Teamwork and communication skills may seem intuitive, but they require training and practice. TDM results showed that all teams had substantial room for further development and improvement. After three months, most teams were cohesive but were still establishing role clarity and working towards consistent effective communication. We found it important to teach skills in an authentic way, reinforcing them in daily practice. Time for teams to collectively reflect upon and discuss team process is critical; facilitators are helpful to guide the process and help the team generate a plan for improvement.
A RESIDENT-LED MANDATORY INTERN MEDICINE SIMULATION CURRICULUM: THE MASSACHUSETTS GENERAL HOSPITAL EXPERIENCE Fernando M. Contreras ${ }^{1}$; Susan K. Mathai ${ }^{1}$; Eli M. Miloslavsky ${ }^{1}$; Emily Hayden ${ }^{2,3}$; James A. Gordon ${ }^{2,3}$; Paul F. Currier ${ }^{1}$. ${ }^{1}$ Massachusetts General Hospital, Boston, MA; ${ }^{2}$ Massachusetts General Hospital, Boston, MA; ${ }^{3}$ Massachusetts General Hospital, Boston, MA. (Control ID \#1312101)

NEEDS AND OBJECTIVES: Mannequin-based simulation for residents across specialties has gained widespread use in recent years, but has not been expanded as a core training element on the general medicine wards. Possible explanations may include logistical challenges and resource investment. We report here on the Massachusetts General Hospital (MGH) Department of Medicine's experience with a mandatory, residentled mannequin-based simulation curriculum. The goal of the program was to introduce clinical decision-making training using medical simulation into the residency program.

SETTING AND PARTICIPANTS: Mandatory simulation sessions for interns were offered during required ambulatory blocks over the first four months of the 2011-2012 academic year and held four days per week, accommodating up to six interns at a time. All categorical and primary care interns ( 54 total) attended three to four sessions and completed six to eight cases. Junior and senior residents, 30 in total, participated as facilitators supervised by fellows and faculty.

DESCRIPTION: The curriculum was composed of eight simulated acute clinical situations (e.g., hypertensive emergency, rapid atrial fibrillation). Each session was sixty minutes long and covered two cases. Interns worked in groups of two to four on each mannequin. Each case began with 10 minutes of mannequin-based patient management where learners managed the clinical scenario in groups of two to three, followed by a 15-minute debriefing session led by residents. Online surveys designed to assess interns' satisfaction with the program were administered.

EVALUATION: Fifty interns completed the online survey $(92.6 \%$ response rate). Seventy-six percent of the interns rated the sessions as "excellent" ( 5 on a 5 point scale) and the remainder as "good" ( 4 on a 5 point scale). One hundred percent of the interns reported that the sessions significantly $(52 \%)$ or moderately improved $(48 \%)$ their differential diagnosis skills. Eighty-eight percent of interns responded that the simulations should be mandatory. Comments regarding program strength focused on the opportunity to debrief with residents about practical management of acute clinical scenarios, emphasizing the "insider knowledge" that residents brought to the cases. Almost all interns requested that there be more frequent resident-led simulation sessions as part of their residency training, and they did not believe these sessions conflicted with other residency activities.

DISCUSSION / REFLECTION / LESSONS LEARNED: Our program utilized several techniques to overcome barriers to implementation of a simulation curriculum in a large residency. Utilizing residents as facilitators significantly decreased the amount faculty hours required to run the program and was well received by the residents. Scheduling the sessions during ambulatory rotations allowed interns to participate without violating ACGME work hour rules. The initial MGH experience has been met with widespread enthusiasm by the participants. Most interns believed that this type of program should be mandatory, specifically noting that senior residents' leading the debriefings was one of the highlights of the program. Notably, they felt that the scheduling of the sessions did not interfere with their clinical duties or other learning activities. Based on this experience, the Department of Medicine will continue to run a mandatory simulation program for interns and to develop new resident-led sessions for internal medicine residents.

A SMALL REWARD CAN CHANGE THE WARDS: HOW MENTORED DELIBERATE PRACTICE TRANSFORMS ACADEMIC MEDICINE Robert K. Hudon ${ }^{3}$; Jessica Campbell ${ }^{1,3}$; Maria G. Frank ${ }^{1,3}$; Caroline Leclair'; Mark Reid ${ }^{1,3}$; Lilia Cervantes ${ }^{1,3}$. ${ }^{1}$ Denver Health, Denver, $\mathrm{CO} ;{ }^{2}$ University of Colorado, Denver, Denver, CO; ${ }^{3}$ University of Colorado, Denver, Denver, CO. (Control ID \#1339758) 
NEEDS AND OBJECTIVES: To test an innovative approach for creating expert academic hospitalists and trainees by: 1) promoting engagement in academic works with award and recognition 2) establishing crossgenerational mentorship within medical teams on the wards 3 ) increasing participation in conferences 4) encouraging collaboration across departments and institutions.

SETTING AND PARTICIPANTS: Denver Health is an academic teaching hospital with 38 academic hospitalists on staff. Each year approximately 300 trainees rotate through on their internal medicine clerkship.

DESCRIPTION: Expertise and advancement in academic medicine are closely tied to academic productivity, yet the rate of publication among new attendings is consistently low. For example, a recent multi-institutional survey revealed that half of academic hospitalists have not produced an abstract, poster or first-author paper. Deliberate practice, mentorship, and reward have proven to build expertise and engagement but have not found consistent applications in medicine. The Rick Albert Prize is awarded to the medical team (ie medical student, resident and attending) with the best clinical vignette of 2011-2012. Each team is matched with an academically successful hospitalist who mentors the way from abstract to poster, to presentation at local and national conferences and ultimately publication. The winning team is given a cash prize, recognized on a plaque, and celebrated at an awards ceremony. Throughout the year, cases are disseminated through online publications and multiple presentation opportunities. Students and faculty are surveyed at the end of the academic year to determine effectiveness of this intervention and impact on job satisfaction and academic preparedness

EVALUATION: In less than four months we have seen a dramatic $800 \%$ increase in abstract, poster, and conference submissions. Attending noon conference attendance has gone from $<10 \%$ to $>80 \%$ and interdepartmental collaboration is blossoming.

DISCUSSION / REFLECTION / LESSONS LEARNED: The Rick Albert prize is the first studied intervention to promote academic achievement among academic hospitalists. This successful program engages physicians at all levels of training, facilitates a new culture of mentorship and learning, and provides physicians and students with an encouraging environment that promotes involvement in patient outcomes and their own pursuit of expertise. Since the inception of this team based academic mentorship program in deliberate practice, we have seen dramatic increases in scholarly activity on the wards at Denver Health.

ONLINE RESOURCE URL (OPTIONAL): http://www.denverhealth org/Services/HospitalMedicine/TheRickAlbertPrize.aspx

A WEB-BASED STUDENT-LED JOURNAL CLUB FOR MEDICAL STUDENTS Jane J. Lee ${ }^{1}$; Maura M. Manion ${ }^{1}$; Megan Mcnamara ${ }^{2,1}$. ${ }^{1}$ Case Western Reserve University School of Medicine, CLEVELAND, OH; ${ }^{2}$ Louis Stokes Cleveland Veterans Affairs Medical Center, Cleveland, OH. (Control ID \#1317331)

NEEDS AND OBJECTIVES: Journal clubs are a valuable tool for introducing students to primary research literature. In contrast to traditional formats which are often faculty led with little student input, a studentdriven electronic journal club allows students to choose articles that specifically interest them and learn at their own pace without pressure or self-consciousness. However, only a handful of student-led journal clubs exist in US medical schools, and student-led electronic web-based journal clubs have not been described to date. The primary objective of this Electronic-journal club (E-journal club) is to increase exposure of recent publications to medical students through an informal web-based platform. Secondary objectives are to help students understand the importance of evidence based medicine and to foster skills in critiquing primary literature. SETTING AND PARTICIPANTS: Medical students in their first 3 years at Case Western Reserve University School of Medicine (512 total students) were provided access to the E-journal club.

DESCRIPTION: The E-journal club is a weekly web blog featuring recent articles selected by fourth-year medical students. Articles are selected from high-impact medical journals (JAMA, NEJM, Annals of Internal Medicine, The Lancet), journal review platforms (Journal Watch, Johns Hopkins Medicine Podcast), and news outlets (The New York
Times, Science Daily). Articles are screened for relevance to medical students, innovativeness, and likelihood to impact clinical practice. Each reviewed article is preceded by a relevant clinical scenario, and the study design is described using the PICO format (patient population, intervention, comparison, outcome). The comments section includes study strengths and weaknesses, analysis of findings in the context of current practice, and commentary from faculty. A short quiz at the end of each issue is used to test the highlighted concepts.

EVALUATION: Student participation was gauged using an online statistics service from weeks 4 to 8 . Also, an online satisfaction survey was conducted to assess the level of interest in the E-journal club and its effect on students' article reading habits. There were 135 unique IP addresses logged. Excluding the 2 weeks of holiday season, there was an average of 37.7 visitors to the site per week. 64 of 512 students responded to the survey $(12.5 \%) .89 .5 \%$ of students were satisfied with the amount of information covered in the E-Journal club. There was a statistically nonsignificant increase in the volume of article reading after the E-Journal Club was initiated - a $4.7 \%$ increase in the number of students reading 1-2 articles/month, $7.9 \%$ for $3-5$ articles/month, and $4.8 \%$ for $>5$ articles a month. The majority of students felt that their critical appraisal skills have not changed after participating in the E-journal club.

DISCUSSION / REFLECTION / LESSONS LEARNED: We have presented a model for introducing primary literature to medical students using a student-organized web-based journal club. Despite the absence of any external incentives, over $1 / 4$ of the students participated in the Ejournal club. Although not statistically significant, there was a trend toward increased volume of article reading, which was encouraging. However, there is much room for improvement in developing students' critical appraisal skills. To further enhance the effectiveness of the E-journal club, we propose the use of an online comments function to encourage discussion about strengths and weakness of study.

ONLINE RESOURCE URL (OPTIONAL): http://casemed-ejournalclub. tumblr.com

ADDRESSING HEALTH DISPARITIES: EXPLORING NEW AND NONTRADITIONAL PATHS TO THE HEALTH PROFESSIONS Katy Hicks ${ }^{1}$; Akua Brown ${ }^{2}$; Rachel True ${ }^{3} .{ }^{1}$ Alameda County Medical Center, Oakland, CA; ${ }^{2}$ Latin American Medical School, Havana, Cuba; ${ }^{3}$ MEDICC, Oakland, CA. (Control ID \#1324216)

NEEDS AND OBJECTIVES: The 2004 Sullivan Report (Missing Persons: Minorities in the Health Professions, 2004) and Institute of Medicine's landmark study, Unequal Treatment: Confronting Racial and Ethnic Disparities in Health Care (Smedley, B, Stith, A, Nelson, A, eds., 2003), both recommend increasing the number of minority health professionals as a key strategy to eliminating health disparities. Yet as of 2010 , these groups still constitute less than $13 \%$ of the health care workforce (AAMC, Diversity in the Physician Workforce: Facts \& Figures 2010). Since 2001 Cuba has been providing full scholarships for students from other countries to study medicine at the Latin American Medical School (ELAM). The US students at ELAM represent ethnic and socioeconomic demographics in proportions rarely seen in US medical schools yet face unique challenges in gaining access to clinical rotations and hence residencies in the US.

SETTING AND PARTICIPANTS: Through their participation in a unique community health project, several physicians and the CEO of the Alameda County Medical Center (ACMC) in Oakland, California traveled to Cuba in 2009. While there they observed US students in their academic setting at ELAM. Recognizing their potential as a pool of strong, diverse residency candidates, ACMC, in collaboration with MEDICC (Medical Education Cooperation with Cuba, a non-profit organization also based in Oakland), developed a new clinical clerkship program. The 4-week program provides six US ELAM students per year with practical experience serving a complex mix of poor and underserved patients.

DESCRIPTION: The program was piloted at ACMC in 2010 with two tracks, (1) an outpatient clerkship for medical students in their first through third years of ELAM training and, (2) an inpatient medicine ward clerkship for students who have completed core rotations and passed Step 1. Several challenges were identified and addressed during the first two years. First, 
because most students have limited financial resources, funding for stipends had to be secured. Second, individual liability insurance policies had to be identified and processed (ELAM does not provide insurance coverage). Third, schedules and orientations had to be arranged with very little communication due to limited internet access in Cuba and the brief period students are present in the US. Fourth, since US and ELAM medical school curricula differ, the expectations for each of the clerkships needed to be carefully evaluated by faculty and students.

EVALUATION: Students and faculty completed online evaluations which the faculty used to improve the program. Faculty were impressed with the medical knowledge, clinical skills, professionalism and practice improvement of the ELAM students. A majority of the students reported that clinical expectations and the adjustment to the US systems were complex but manageable. One hundred percent of the students felt the program was a valuable addition to their training.

DISCUSSION / REFLECTION / LESSONS LEARNED: In an era of demographic shifts toward an increasingly diverse population, demand for culturally competent, bilingual and bicultural doctors is intensifying. ACMC and MEDICC's experience developing and implementing a clerkship program for US ELAM students indicates that these graduates may be a viable source of residents and physicians in the near future. It is hoped that other academic institutions will adopt this model and create similar rotations for US ELAM students in other parts of the country.

AMBULATORY HANDOFFS: A CURRICULUM AND TOOL TO FACILITATE CONTINUITY OF CARE IN A TEAM-BASED PRACTICE MODEL Melissa Bachhuber ${ }^{1,2}$; Shalini Patel ${ }^{1,2}$; Nicholas Moy ${ }^{1,2}$; Bridget O'Brien ${ }^{1}{ }^{1}$ University of California, San Francisco, San Francisco, CA; ${ }^{2}$ San Francisco VA Medical Center, San Francisco, CA. (Control ID \#1324692)

NEEDS AND OBJECTIVES: Team-based models of care and reduced duty hours increase the need for safe and effective patient handoffs among trainees. Few guidelines or training materials exist for handoffs in outpatient trainee continuity clinics. Our aim was to conduct a needs assessment on current and desirable handoff practices for ambulatory patients and use this information, along with literature on handoffs in other settings, to create an ambulatory handoff tool and curriculum.

SETTING AND PARTICIPANTS: In the San Francisco VA Medical Center (SFVAMC) primary care clinics, Internal Medicine residents work as practice partners in which one partner spends a two month block performing inpatient duties while his/her partner performs ambulatory duties. In July 2011 one NP student joined each partner team. Team members interchangeably see their partners' patients during any absence greater than two weeks.

DESCRIPTION: In our needs assessment survey completed by 29 residents, $66 \%$ indicated concern that there are times when patients should be handed off to a practice partner but are not and 33\% reported that a patient sustained a preventable adverse event or near miss due to a suboptimal handoff. Residents reported the main barriers to completing handoffs were lack of time and lack of a structured system. We designed an innovation to address this patient safety issue which included an outpatient handoff curriculum, an ambulatory handoff template tool, and protected time for handoff completion. The curriculum included an overview of handoff literature and a one hour case-based session on a complex diabetic patient requiring handoff to a practice partner. The case prompted discussion of criteria to identify patients at "high risk" for adverse events and served as an example of how to use the ambulatory handoff template. The template contains the most desired information identified by residents on the needs assessment survey: Active Clinical Problems, Issues Requiring Immediate Follow Up, and Recommendations for Further Management. The completed handoffs are stored on a secure server accessible to trainees and clinic preceptors. Protected time is provided for trainees to complete handoffs before going off clinic service and practice partners are expected to review handoffs while covering their partners' patients. Quarterly check-in sessions are held to obtain feedback on the tool.

EVALUATION: Twenty-three trainees (16 R2s and $7 \mathrm{NP}$ students) participated in our curriculum. Fifteen trainees $(65 \%)$ have used the template to date, entering between 1 and 4 patients per handoff. Examples of handoffs entered included patients with substance abuse and depression, and weight loss undergoing active work up. Prior to participation in the curriculum, trainees completed a survey. When asked how prepared they feel to see their partners' complicated patients, the mean response was 3.8 (SD 1.2) on a 6-point scale from strongly disagree(1) to strongly agree(6). The mean response to confidence and satisfaction with handoffs was 4.6 (SD 0.7) and 4.3 (SD 1.1) respectively. We will resurvey trainees at the end of the academic year to evaluate effectiveness of the innovation.

DISCUSSION / REFLECTION / LESSONS LEARNED: Creating a structured system and curriculum for ambulatory handoffs is feasible in a team-based practice model and could be adapted to other outpatient settings.Trainee input into system improvement is critical to adoption and sustainability of new processes. Future work is needed to evaluate the impact of ambulatory handoff systems on patient safety.

AMBULATORY PROCEDURE CADAVER CLINIC Kurt J. Pfeifer; Cynthia Kay; Theodore MacKinney. Medical College of Wisconsin, Milwaukee, WI. (Control ID \#1306139)

NEEDS AND OBJECTIVES: 1. Increase resident training in common clinic-related procedures. 2. Provide risk-free but realistic practice performing arthrocentesis and dermatologic procedures. 3. Improve residents' confidence in performing ambulatory procedures.

SETTING AND PARTICIPANTS: The Internal Medicine (IM) Residency Program at our institution is comprised of 99 residents rotating between 3 hospitals and engaging in a variety of inpatient and ambulatory electives and mandatory rotations. During these experiences, residents can perform bedside procedures as opportunities arise during their patient care duties. First-year residents also participate in a one-day workshop utilizing simulators and models to teach common procedures. Our program, like most others, requires competency in performance of only the limited procedures mandated by the American Board of Internal Medicine (ABIM). For the approximately $30 \%$ of our residents who eventually practice outpatient general internal medicine, these requirements and educational experiences may be insufficient for preparing them to perform common, clinic-based procedures.

DESCRIPTION: Five 2-hour sessions were conducted in the gross anatomy laboratory of the Medical College of Wisconsin. Fourteen internal medicine residents of all post-graduate levels and two faculty attendings took part in these sessions. No more than 5 participants were assigned to a single day, and all but one participant attended just one session. Each workshop was run by a faculty attending with over twenty years of experience performing arthrocentesis of various joints, punch biopsies and skin excisions. Prior to the start of the workshop, participants completed a short survey rating their experience with knee, shoulder and wrist injections and skin punch and wedge biopsies, as well as their comfort level for each procedure. Each workshop was conducted in a similar manner, with the faculty director first demonstrating each procedure. Demonstrations of external anatomy and bony landmarks were done on participants and on cadavers. Then, each participant had time to practice the procedure on the available cadavers, until they felt comfortable. For arthrocentesis, some cadavers were available with joints already dissected so that joint anatomy could be easily reviewed. Faculty worked with each resident until they could reliably do knee and shoulder arthrocentesis and skin punch and wedge biopsies. Approximately 4 weeks following the workshop, participants again completed a survey rating their experience and comfort level with the procedures.

EVALUATION: Participants rated their confidence in performing the procedures on a scale from 1 to 5 , with 1 corresponding to "have no experience or knowledge" and 5 correlating to "confident to perform, teach and evaluate." Data from the surveys revealed that participants' confidence rating increased by an average of one point for all procedures. Most notable were skin wedge biopsy that increased from 1.625 to 2.625 and shoulder arthrocentesis, which increased from 1.937 to 2.937 .

DISCUSSION / REFLECTION / LESSONS LEARNED: Though there were a small number of participants in this first cadaver-based procedure 
workshop, the data supports the idea that any practice improves confidence, even after a single workshop. In addition, the use of cadavers provides a more realistic experience when compared to manikins. This workshop could serve as a valuable supplement to traditional clinical experience, which typically relies upon chance and preceptors' own procedural confidence.

AN 8-MODULE INTERN NIGHT FLOAT CURRICULUM: ENHANCING THE EDUCATIONAL VALUE OF INTERN "NIGHT FLOAT" AND THE TEACHING SKILLS OF SENIOR RESIDENTS Erin J. Goss; Danit Arad. Montefiore Medical Center, Bronx, NY. (Control $\overline{\mathrm{ID} \# 1339810 \text { ) }}$

NEEDS AND OBJECTIVES: In order to adhere to increased intern work hour restrictions, many internal medicine residency programs have increased the amount of time interns spend on overnight rotations like night float. Night float systems have been associated with reduced medical errors, but they have also been associated with dissatisfaction with the learning environment, and do not improve standardized testing scores. Overnight residents are well situated to be teachers for night floats. In addition, recent LCME guidelines suggest that residency programs formalize "resident as teacher" training. To enhance the educational experience of interns on night float and teaching skills of senior residents, we developed an interactive night float curriculum delivered by senior residents to night float interns.

SETTING AND PARTICIPANTS: Internal Medicine interns at MMC complete one to two, 2-week blocks of night float at either of two university hospital locations in the Bronx, NY. Four senior residents rotate through a medicine consult rotation monthly at the Moses campus. Senior residents deliver the pilot curriculum to interns at the Moses campus; interns on night float at Weiler campus receive their regular orientation.

DESCRIPTION: Senior and chief residents collaborated to design eight 30-minute evidence-based and interactive powerpoint modules along with example signouts. Modules simulate relevant clinical scenarios and focus on acute management of high yield topics such as chest pain, hypotension, respiratory distress, delirium, alcohol withdrawal, electrolyte abnormalities, DKA and pain management. In the first few days of the rotation, residents on medicine consult rotation receive an educational session on "Resident as Teacher" where they brainstorm and role-play teaching to adult learners and receive peer and faculty feedback on their teaching performance. The session, developed for this curriculum, is part of a larger "Resident as Teacher" series. Each senior resident is then responsible for presenting one module per week to each pair of night floats, so that night float interns receive all 8 didactic sessions.

EVALUATION: To determine whether modules enhance intern's medical knowledge, the change in scores between pre- and post-testing on a 16 item medical knowledge exam will be calculated and compared between intervention and control sites using paired student $t$ tests. Interns will also report their confidence in managing acute changes in patients' medical conditions on a Likert scale. To assess the impact on resident's skill in teaching, interns will rate residents on the quality of their teaching. Finally, senior residents are asked to rate their confidence on a Likert scale in teaching interns and medical students at the beginning and end of the rotation.

DISCUSSION / REFLECTION / LESSONS LEARNED: Currently being piloted with the first group of night floats and residents, this curriculum is innovative in its ability to formalize the overnight clinical teaching while building teaching skill capacity of senior residents. We hypothesize that this multi-layered curriculum will increase intern's knowledge of acute care management of overnight issues and will increase their confidence in their role as night-float. In addition, we hypothesize that this series of activities will enhance residents' teaching skills and build their confidence as teachers. Downstream effects that should be measured going forward include impact on resident teaching outside the modular curriculum, patient outcomes and frequency of medical errors.

ONLINE RESOURCE URL (OPTIONAL): www.night-float.org
AN EXPERIENTIAL, LONGITUDINAL INTERPROFESSIONAL QI CURRICULUM FOR HOSPITALIST RESIDENTS Darlene Tad-y; Lisa Price; Dimitriy Levin; Jeffrey Glasheen. University of Colorado Denver School of Medicine, Aurora, CO. (Control ID $\# 1332650)$

NEEDS AND OBJECTIVES: Formal training in quality improvement (QI) has become increasingly important. Both the Accreditation Council for Graduate Medical Education and the American Board of Internal Medicine have created competencies in the areas of practice-based learning improvement (PBLI), systems-based practice (SBP) and communication that must be met. Most QI curricula for internal medicine residents are implemented in the outpatient setting and are often limited to resident learners. As part of an inpatient training program for internal medicine (IM) residents, we developed a curriculum to improve residents' knowledge, attitudes, and skills in QI, and to meet competency requirements set by the ACGME in the realms of PBLI, SBP, and communication. SETTING AND PARTICIPANTS: The Quality Improvement Program (QuIP) Curriculum was developed within the Hospitalist Training Program (HTP) at the University of Colorado, which started in 2004. The HTP is a training program for 2 nd and 3 rd year residents in our IM Residency Training program pursuing a career in hospital medicine. Learners, which included hospitalist residents, medical students and pharmacy residents, worked in faculty-mentored teams (QuIP Teams) to design, implement, and measure a QI project in conjunction with the relevant hospital committees as part of the curriculum.

DESCRIPTION: The QuIP curriculum is a 2-year program that incorporates didactic teaching, targeted coaching sessions, and an experiential project embedded in hospital-led initiatives. Didactic components were delivered in 2 settings: monthly educational sessions and a 1month dedicated QI rotation. Learners also completed online QI educational content provided by the Institute for Healthcare Improvement. In the pilot year, 4 projects were undertaken by QuIP Teams: 1 . Improving the time to TPA for Stroke Alerts, 2. Improvement of the Quality and Timeliness of Discharge Summaries, 3. A Physician-Centered Initiative to Decrease Inpatient Falls, 4.Reducing Informal Restraints for the Frail Elderly Patient. Faculty mentors and QuIP Team members attended educational and coaching sessions together to facilitate team learning. EVALUATION: Educational, scholarly, and clinical outcomes were evaluated throughout the program. Results demonstrated that learners had increased confidence in key QI knowledge and skill sets. Additionally, all learners earned the Institute for Healthcare Improvement's Advanced certificate in QI and presented a scholarly abstract at a regional Hospital Medicine meeting. Clinical process outcomes were promising,including decreased time to TPA, implementation of EMR fall risk notification, and improved timeliness of discharge summaries. Measures of sustainability and wide dissemination are yet to be determined.

DISCUSSION / REFLECTION / LESSONS LEARNED: A longitudinal QI curriculum can successfully be implemented primarily in the inpatient setting. Interprofessional learners can master QI knowledge and skills and collaborate on projects that produce meaningful change. Internal medicine residents who participate in an inpatient QI curriculum can be better prepared to design and implement QI initiatives upon graduation from residency.

ATTENDING EVALUATION OF INTERNAL MEDICINE RESIDENTS' CLINICAL ADMISSION PERFORMANCE DURING THEIR NIGHT FLOAT ROTATION Maryann T. Ally; Harry Burke; Moromoke Odina. Walter Reed National Military Medical Center, Bethesda, MD. (Control ID \#1327333)

NEEDS AND OBJECTIVES: We have instituted a night float resident rotation but it is not known how to best evaluate residents' clinical performance. We developed a structured attending evaluation form to assess internal medicine residents' night float clinical admission performance.

SETTING AND PARTICIPANTS: Over one month, hospitalist attendings evaluated internal medicine residents during their night float rotation during a weekly night float medicine conference. 
DESCRIPTION: We created a two-page, structured evaluation form. A hospitalist attending selected one night float admission per resident to review. The first page of the evaluation form was filled out by the resident, who recalled information provided in the patient's history and physical. The second page was filled out by the attending, who performed a face-toface and written review of the resident's workup, differential diagnoses, and treatment plan. The attending scored each area and provided a summary score for each section. This evaluative process took fifteen to twenty minutes.

EVALUATION: Hospitalist attendings evaluated fourteen internal medicine residents over one month in terms of above average, average, and below average performance on six clinical measures. Of these fourteen residents, 5 were PGY-1 and 9 were PGY-2 or PGY-3. The six measures and the results, as assessed by the attending, were: What was the chief complaint or night float issue? $64.2 \%$ above, $35.7 \%$ average, and $0 \%$ below. What were your differential diagnoses and how did you work it up? $35.7 \%$ above, $64.2 \%$ average, and $0 \%$ below. What results did you obtain and what conclusions did you come to? $57.1 \%$ above, $42.8 \%$ average, and $0 \%$ below. Recalling that night and the events of subsequent days, would you say that you would have done something differently in terms of the first three questions? If so, what and why? $57.1 \%$ above, $42.8 \%$ average, and $0 \%$ below. Was there clear documentation in the chart of the house staff's thought process and plan of action? $57.1 \%$ above, $35.7 \%$ average, and $7.1 \%$ below.

DISCUSSION / REFLECTION / LESSONS LEARNED: Using a structured evaluation form, we assessed the internal medicine residents' clinical performance during their night float rotation. This evaluative process identified areas for resident improvement including developing more robust differential diagnoses and more comprehensive documentation. We will address these needs by working with residents on an individual basis and by implementing system-based improvement initiatives.

BULIDING A CURRICUUM IN GLOBAL AND DOMESTIC HEALTH DISPARITIES THROUGH A LONGITUDINAL MENTORED LEADERSHIP TRAINING PROGRAM Brent C. Williams; Julie S. Perry; Andrew J. Haig; Patricia Mullan; Joy Williams. University of Michigan, Ann Arbor, MI. (Control ID \#1318841)

NEEDS AND OBJECTIVES: A substantial number of UM medical school applicants and enrolled students have expressed interest in, and independently pursue, learning experiences related to underserved populations. The Dean's office charged five faculty members to develop a structured opportunity for motivated students to pursue training in the care of underserved populations in the U. S. and abroad - termed the Global Health and Disparities Path of Excellence (GHD PoE). Following review of existing disparities curricula, competencies and objectives were identified to anchor the program, within the overall mission to "integrate foundational, investigative and experiential learning that will prepare the medical students to be agents of sustainable change to reduce domestic and global health disparities." Competency domains include: 1) Social Determinants of Health Disparities, 2) Tools and Strategies to Promote Sustainable Change, 3) Health Care Systems and Policy, and 4) Professional and Leadership Development. Foundational goals of the GHD-PoE are to create a flexible, mentored, self-regulated environment to shape student learning.

SETTING AND PARTICIPANTS: Working groups focusing on learning methods, assessment and leadership designed the program with substantial student involvement. To date, the GHD PoE overall framework has been developed, endorsed by the school's curriculum policy committee, and initial components implemented. 3 faculty mentors have been recruited and supported at $10 \%$ each to implement the program. The GHD PoE is being implemented beginning with the entering class of 2011 (M1s), 35 of whom have expressed interest in the Path.

DESCRIPTION: Key planned features of the GHD PoE include: a) curriculum-based lecture series open to all M1s, b) regular small group meetings with faculty mentors throughout students' four years, c) required capstone field projects, and d) use of electronic portfolios to document and measure progress towards goals, communicate with fellow students and mentors, and network with GHD alumni to promote project and career development.

EVALUATION: Learner assessment will be multimodal and include: a) structured review of capstone field projects, b) review of written assignments applying readings to case studies emphasizing core curricular elements, and c) reflective essays applying principles of leadership to group field projects.

DISCUSSION / REFLECTION / LESSONS LEARNED: Compared to scholarly co-curricula at other medical schools, distinctive features of the GHD PoE include: a) focus on both domestic and international health disparities, b) central role of, and substantive investment in, faculty mentors, c) emphasis on leadership skills to promote sustained change in health care in underserved settings, d) focus on career development and professional networking, and e) multidimensional use of portfolios by students for planning, implementing, documenting, and assessing learning experiences. As the program is developed, anticipated challenges are: a) accommodating students at widely varying levels of experience and skills, b) teaching and measuring competency in leadership skills, and c) providing ongoing faculty development support for longitudinal and project mentors.

CANCER SURVIVORSHIP: A NEEDS ASSESSMENT FOR CURRICULUM DEVELOPMENT IN RESIDENT EDUCATION Chi Kim; April Barbour; Gabriel Rivera; Jessica Logan. George Washington University, Washington, DC. (Control ID \#1319793)

NEEDS AND OBJECTIVES: There are nearly 12 million cancer survivors in the United States. With ongoing innovations in cancer treatment, that population will grow to approximately 20 million by 2020. As the surviving population ages, who should oversee medical care for cancer survivors - internists or oncologists? Based upon prior surveys, oncologists feel comfortable with survivorship care, but lack the time and resources to provide long-term survivorship care to millions of patients; general internists, however, feel that they lack adequate knowledge and confidence in providing survivorship care. To date, internal medicine residents have not been assessed regarding their attitudes towards cancer survivorship care. Our unique survey was intended to assess the knowledge and attitudes of internal medicine residents towards survivorship care to prepare for the implementation of a survivorship residency curriculum. This longitudinal curriculum is aimed at increasing internal medicine residents' awareness of long term effects and their confidence in treating cancer survivors.

SETTING AND PARTICIPANTS: All internal medicine residents at George Washington were asked to complete an IRB approved survey during a town hall meeting in July 2011.

DESCRIPTION: A 10-item questionnaire was provided to assess the knowledge and attitudes of internal medicine residents on survivorship care. This survey included demographic information (PGY level, residency tract) as well as likert scale questions designed to assess the interest and confidence in providing survivorship care to patients in their practice.

EVALUATION: Seventy-two $(70 \%)$ of the internal medicine residents completed the survey. Overwhelmingly primary care (100\%) and nonprimary care bound $(76 \%)$ residents felt cancer survivors should receive their long term care from both hematologist/oncologist and internists. Of those surveyed, $99 \%$ stated they were interested in learning how to provide survivorship care to their patients; however, only $61 \%$ indicated they would feel comfortable monitoring for late and long-term effects in cancer survivors. In addition, $50 \%$ of respondents stated they were familiar with late effects of cancer treatment. Finally, $37 \%$ stated they were familiar with resources or support services for cancer survivors.

DISCUSSION / REFLECTION / LESSONS LEARNED: There is a growing need to provide long-term cancer survivorship care to millions of survivors. Based on our questionnaire results, internal medicine residents are motivated to participate in the care of cancer survivors; however, they appear to lack the knowledge and confidence to provide this care. Our results are consistent with the survey results of practicing internists who also feel they lack adequate knowledge and confidence in survivorship care. Background studies establish the need for cancer survivorship education. Our results suggest that a collaborative effort between internists and oncologists would be the preferred model for resident education. In 
sum, our study shows that a cancer survivorship curriculum should be part of resident education to train future internists who will be providing longterm care to our rapidly growing population of cancer survivors in the United States.

ONLINE RESOURCE URL (OPTIONAL): http://www.gwmed. $\mathrm{com} /$ joomla/index.php?0ption $=$ com_content $\&$ view $=$ article $\&$ id $=128 \&$ Itemid $=234$

COMPETENCY-BASED TEST OF INPATIENT GERIATRIC MANAGEMENT SKILLS Ethan U. Cumbler ${ }^{1}$; Jeannette Guerrasio ${ }^{1}$; Jean Youngwerth $^{1}$; Judy T. Zerzan ${ }^{1}$; Sonja Rosen ${ }^{2}$; Suzanne Brandenburg ${ }^{1}$; Eva M. Aagaard ${ }^{1}$; Heidi Wald ${ }^{1}{ }^{1}$ University of Colorado School of Medicine, Aurora, CO; ${ }^{2}$ University of California Los Angeles, Los Angeles, CA. (Control ID \#1310407)

NEEDS AND OBJECTIVES: Mulitple choice or short answer examinations fail to assess important aspects of clinical behavior. Increasing emphasis is being placed on competency-based assessments in geriatric medicine. Practical, inexpensive, structured clinical examinations which assess applied knowledge and clinical skills measured across discrete competencies in the inpatient geriatric setting are needed. ObjectiveDerivation and validation of a novel examination designed to evaluate application of medical knowledge to clinical care in the inpatient setting using Core Competencies from Geriatric and Hospital Medicine. Hypotheses- 1. Competency-based examination will distinguish clinical skills based on level of experience and training 2. Performance on competencybased examination should improve after a dedicated educational experience designed to teach these clinical skills

SETTING AND PARTICIPANTS: Cohorts- Construct validity examined in a cohort of 10 third year medical students (MSIII), 10 interns (R1) without prior inpatient geriatrics training, and 6 experienced third year residents (R3-post) who had completed an ACE rotation. Hypothesis 2 was tested in an independent cohort of 11 second year residents both before (R2-pre) and after (R2-post) an inpatient ACE rotation.

DESCRIPTION: A standardized history and physical of a geriatric admission was designed. It describes an elderly woman with increased falls after surgery and anticholinergic medication initiation. The acute trigger for hospitalization was increased weakness induced by infection. Decreased oral intake caused acute on chronic renal failure with drug toxicity from impaired clearance. The final event bringing her to medical attention was inability to rise after a fall. The test evaluates ten selected competencies of geriatric and hospital medicine through analysis of the actions ordered by the learner in free-written admission orders. For external validity the case was reviewed by 20 expert educators in geriatrics and hospital medicine at 6 academic medical centers nationally. The scoring system applied relative weighting for action on each competency using average judgment of the 20 experts standardized to a 100 point scale.

EVALUATION: Average test performance was: MSIII 49.4\% (IQR 34\%63\%), R1 62\% (IQR 56\%-68\%), R2-pre 70\% (IQR 65\%-76\%), R2-post 92\% (IQR 87\%-100\%), R3-post 86\% (IQR 80\%-88\%). Statistical analysis revealed significant relationship between level of trainee and score on the pre-tests $(p=0.02)$. There was also a significant difference between the R2 scores pre and post exposure to inpatient geriatric rotations $(\mathrm{p}<0.0001)$. No difference was seen between R2 and R3 scores after the inpatient geriatric rotation.

DISCUSSION / REFLECTION / LESSONS LEARNED: The competency-based admission order practical exam is a new tool to evaluate clinical practice behavior across a range of learner experience. As predicted by hypothesis 1 , it distinguishes superior clinical management based on level of training. Consistent with hypothesis 2 that proficiency in these competencies can be accelerated through focused training, the test scores improved significantly in the cohort of second year residents after inpatient geriatric training and competency was indistinguishable between second and third year residents after completion of an inpatient geriatrics rotation. Methodology used in the development of this examination can be applied to the creation of other competency-based practical examinations.

ONLINE RESOURCE URL (OPTIONAL): Examination and Scoring System at: http://www.pogoe.org/node/2455
CREATION AND IMPLEMENTATION OF A WOMEN'S LEADERSHIP CURRICULUM FOR INTERNAL MEDICINE RESIDENTS Brigid M. Dolan ${ }^{1}$; Joel T. Katz ${ }^{2}$; Maria Yialamas ${ }^{2} .{ }^{1}$ Brigham and Women's Hospital, Boston, MA; ${ }^{2}$ Brigham and Women's Hospital, Boston, MA. (Control ID \#1339141)

NEEDS AND OBJECTIVES: While women comprise approximately half of the physician community, women hold far fewer academic leadership positions. Prior studies indicate that women who participate in leadership development curricula are more likely to attain deanships, department chairmanships, or full professorship. Few women's leadership training programs for residents have been described. Female residents at our institution express perceived deficiencies in leadership skills and career development training for women. We created and implemented a Women's Leadership Curriculum with the following objectives. Residents will identify personal leadership strengths and compare these to those measured by an inventory tool; examine the contrasting careers of prominent women faculty, using these models to inform their own career development plan; evaluate strategies for effective communication and demonstrate ability to use skills in small groups; and evaluate different negotiation strategies and apply negotiation skills to daily activities

SETTING AND PARTICIPANTS: Interns, junior residents and senior resident groups participate in separate half-day seminars each year. Clinical duties are covered by others for these sessions to allow all women in a given class to participate. Most sessions rely on faculty leaders, minimizing program expense. Senior residents facilitate selected sessions with more junior attendees to encourage peer-mentoring.

DESCRIPTION: The session objectives are aligned with the learners' career development stage. The intern session focuses on identifying leadership strengths and learning strategies to resolve conflict and lead teams. The junior session addresses career planning and communication; methods include a workshop on how to be effectively mentored, a faculty panel on career transitions, and an interactive communication session. The senior session provides residents with basic negotiation skills.

EVALUATION: Senior residents felt that the objectives of the session were important to career development (93\% strongly agreed, $7 \%$ agreed) and that they are now better able to recognize negotiation opportunities (62\% strongly agreed, $38 \%$ agreed). Participants have indicated in followup surveys that they have used newly developed negotiation skills in daily work activities (31\%). Qualitative responses indicate that the emphasis on negotiation skill development is considered especially valuable.

DISCUSSION / REFLECTION / LESSONS LEARNED: Our preliminary findings indicate that women residents appreciate a dedicated curriculum in leadership skills. With departmental support, launching the program was feasible. Our residents prioritized content in negotiation, communication and self-evaluation. In this first year of implementation, the curriculum is highly valued by residents who express belief that topics are important and useful. As the program continues, we hope to develop a strategy for assessing these skills in the inpatient and outpatient settings via direct observation on rounds and resident self-review of videotaped sessions.

CURRICULUM DEVELOPMENT FOR CLINICAL OFFICER INTERNS IN NAIROBI, KENYA Kimberly M. Ganster ${ }^{1}$; Hillary Dunlevy ${ }^{1,3}$; Carole Okoth ${ }^{4}$; Jennifer Cohn ${ }^{2}$. ${ }^{1}$ University of Pennsylvania, Philadelphia, PA; ${ }^{2}$ University of Pennsylvania, Philadelphia, PA; ${ }^{3}$ Children's Hospital of Philadelphia, Philadelphia, PA; ${ }^{4}$ Mbgathi District Hospital, Nairobi, Kenya. (Control ID \#1333542)

NEEDS AND OBJECTIVES: Clinical officers (COs) serve a valuable role in providing health care in Sub-Saharan Africa in areas where there are few doctors. A large proportion of medical care in inpatient, outpatient, rural and urban settings is through these providers. After high school and graduation from a three year clinical officer training program, COs complete a one year internship including rotations in medicine, surgery, pediatrics and obstetrics. In Kenya, $\mathrm{CO}$ interns must pass a national qualifying test after internship, however, there is little standardized or structured curriculum during this training, and the education received 
during the medicine clerkship varies significantly by hospital. We aimed to evaluate a self-contained, replicable curriculum including a paper curriculum, power point lectures, and pocket cards on the medicine training of clinical officer interns in a hospital in Nairobi, Kenya.

SETTING AND PARTICIPANTS: The evaluation took place from October to November 2011 in Mbgathi District Hospital (MDH), a public training hospital in Nairobi. Thirteen CO interns completing their three month clerkship in internal medicine participated

DESCRIPTION: The curriculum was developed through collaborative efforts between the University of Pennsylvania Global Health Equities Residency Track and the education coordinator at MDH. It was based on core internal medicine principles, Kenya's National Guidelines, and basic $\mathrm{CO}$ training topics provided by the Ministry of Health. At the start of the internal medicine rotation, the $\mathrm{CO}$ interns were given a 30 question pretest, covering topics such as the management of hypertension, ascites, and tuberculosis. Each intern was given a 35 page inclusive paper curriculum. Weekly for six weeks the interns attended lunchtime power point lectures given by a visiting resident from the University of Pennsylvania and were given a pocket card related to that topic. The power points were designed to be self-contained and easily administered by a medical provider without specific training on the curriculum. After completion of the curriculum, the $\mathrm{CO}$ interns were given the same 30 question post-test.

EVALUATION: Thirteen $\mathrm{CO}$ interns completed the pre-test and eleven completed the post-test. The average pre-test score was 59\% (17.8/30) with a range of 43 to $73 \%$. The average post test score was $68 \%$ (20.5/30) with a range of 53 to $87 \%$.

DISCUSSION / REFLECTION / LESSONS LEARNED: This evaluation serves as an important first step in creating a medicine curriculum for clinical officer interns, a significant accomplishment as clinical officers provide a considerable portion of the health care in sub-Saharan Africa and their training is vital to the well being of the population. Test scores improved after the curriculum and the curriculum was well received by the trainees. There were certain limitations to this evaluation. Although designed to be easily delivered without training, during this evaluation the lecture series was given by one of the internal medicine residents who helped design it. The pre-test and post-tests were not validated nor were they compared to a control group. Future directions include evaluating the tests in a control group who does not receive the curriculum, assessing the process when given by a local medical provider, and studying patient care outcomes. With the continued evaluation and success of this curriculum there is the potential for reproduction and replication in other training hospitals in Kenya as well as other parts of sub-Saharan Africa.

DESIGNING A RESIDENT CURRICULUM TO INTEGRATE LOCAL AND GLOBAL PRIMARY CARE TRAINING Renuka Tipirneni; Joseph Joyner; David Munson; Sarah Wakeman; Elizabeth Cunningham; Patrick T. Lee. Massachusetts General Hospital, Boston, MA. (Control ID \#1334594)

NEEDS AND OBJECTIVES: Residency training has historically separated curricula for residents interested in promoting health equity into two tracks: advocacy for underserved populations in the U.S., and health delivery in resource-poor international settings. However, common lessons may be shared by integrating global health and primary care training. Our goal was to design a curriculum that allows residents to explore the full arc of health care delivery for vulnerable populations, from policy and planning to implementation and community engagement in order to develop future leaders in health equity in the U.S. and across the globe.

SETTING AND PARTICIPANTS: Learners were 13 internal medicine interns at Massachusetts General Hospital on an ambulatory care block. Primary care interns were required to participate in the Global Primary Care curriculum, while categorical interns requested to join the block. The 4-week learning block included 14 half-day sessions of didactic and casebased teaching, and 7 experiential sessions at community organizations. Instructors included 4 senior residents involved in the curriculum design, and various faculty and leaders in community and international organizations.
DESCRIPTION: The curriculum focused on teaching general principles of health reform and community-oriented primary care through case-based examples. Teaching modalities included assigned readings, didactic lectures, interactive case discussion, and team-based discussion and projects. The class-based sessions included: examining health reform in the U.S., Mexico and Liberia; discussing community-oriented primary care and local community health center initiatives; discussing the role of community health workers and health promoters in the U.S., Haiti and Bangladesh; evaluating primary care/public health efforts in Uganda; and a session on critical reflection. The experiential sessions included visits to: a refugee health assessment program, an HIV health promoters program, a homeless health program, a health care improvement organization, and two community health centers. Through individual and team discussions, learners evaluated various health system reform and community health initiatives, articulated lessons learned, and generated proposals for improvements in primary health care planning and delivery.

EVALUATION: Two types of assessments were performed before and after the curriculum to examine the impact on learners' preparedness to apply comparative health systems thinking to the care of vulnerable populations. Quantitative knowledge assessments and Likert questions on preparedness and practice intentions were performed. Qualitative interviews were also conducted to assess the depth, breadth, and complexity of learners' responses to health policy and delivery scenarios. Evaluation is currently in the analysis phase.

DISCUSSION / REFLECTION / LESSONS LEARNED: An innovative curriculum linking the traditionally separate domains of training in primary care and global health is achievable. With minimal preparation, interns were able to explore policy and implementation issues and stimulate in-depth discussion on a variety of topics. Going forward, we believe it is important to strengthen the learning environment by: 1) streamlining the scheduling process in order to maximize participation; and 2) refining and providing further training for learners/facilitators on the case-based method.

ONLINE RESOURCE URL (OPTIONAL): https:/hub.partners.org/ globalprimarycare/Programs/Curriculum

DEVELOPING A LONGITUDINAL INTERDISCIPLINARY CLERKSHIP (LIC) AT AN URBAN TERTIARY MEDICAL CENTER: THE MOUNT SINAI INTERACT PROGRAM Yasmin S. Meah; Allison Gault; Neloofar R. Naderi; Rainier P. Soriano; Valerie Parkas. Mount Sinai School of Medicine, New York, NY. (Control ID \#1319855)

NEEDS AND OBJECTIVES: 1) To describe the inception and implementation of a nascent longitudinal interdisciplinary clerkship (LIC) and 2) To discuss challenges and highlight successes of this innovative program.

SETTING AND PARTICIPANTS: Rising third year medical students $(n=8-11)$ were selected through a competitive application process to participate in an 8-11 week LIC. Selected students demonstrated interest in longitudinally caring for vulnerable patients.

DESCRIPTION: Longitudinal integrated clerkships are gaining momentum nationally as innovative platforms to educate students in the care of patients over time. To date, only UCSF had previously implemented an LIC at a tertiary medical center. In 2010, Mount Sinai School of Medicine launched an LIC, uniquely centered in a tertiary care setting with a homebound medical program and a student-run free clinic at its primary care core. The Interclerkship Ambulatory Care Track (InterACT) is more interspersed throughout the traditional inpatient curriculum than the exclusively longitudinal model of parent programs. Created for 8-12 students, foundational ambulatory care venues of the standard curriculum traditionally taught singularly during the block clerkships are transformed into a multidisciplinary integrated longitudinal experience. During a typical InterACT week, students see patients with dedicated mentors in pediatrics, general medicine, geriatrics, homebound medicine, surgery and a studentrun free clinic. InterACT didactics focus on themes central to primary care and are heavily derived from students' patient encounters. Though the model significantly differs from those of more established programs, the central concepts and missions are similar: to augment integrated rather than 
siloed clinical reasoning; to inculcate strong patient ownership by nurturing students' understanding of the vicissitudes of illness and students' advocacy for patients with chronic illnesses over time; to foster longitudinal mentorship, learning and evaluation.

EVALUATION: Individual and programmatic evaluations are on going and evolving. In our pilot year, InterACT students performed as well or better on all NBME subject shelf examinations. Overall mentor and student evaluations of the clerkship were positive stressing the most valuable aspects of the clerkship as longitudinal mentorship and assessing and shaping the growth of students' skills over time. Future evaluations will focus on program impact on career choice, advocacy skills, patientcentered care and empathy compared to traditional clerkships.

DISCUSSION / REFLECTION / LESSONS LEARNED: To date, InterACT is the only LIC to incorporate homebound care and care within a student-run free clinic (SRFC) as core to the structure of this program. These have proven the most successful venues to promote student ownership and advocacy for vulnerable populations. Program challenges include recruiting dedicated quality mentors; finding additional venues that promote student ownership and stress the care of vulnerable populations; establishing a grading system that balances the LIC student evaluations with the non-longitudinal core clerkship student evaluations. Lessons learned: faculty buy-in from the top down is essential to recruiting the right mentors; working with clerkship directors to mold the clerkship and disseminate the mission is key; regular student input in shaping the clerkship, designing the didactic curriculum, recruiting and retaining the right mentors and incorporating ideal clinical venues is fundamental to the growth and success of an LIC.

DEVELOPING AN IMMERSION ELECTIVE FOR RESIDENTS COMMITTED TO CARE OF UNDERSERVED COMMUNITIES Marya J. Cohen; Jessica Zeidman; Patrick T. Lee; Kristian Olson. MGH, Boston, MA. (Control ID \#1336580)

NEEDS AND OBJECTIVES: Internal medicine residents interested in primary care for underserved urban communities have few opportunities to provide clinical care while gaining educational expertise in these settings during training. Our objective is to design an elective that provides an opportunity to provide care to vulnerable populations in Boston, in addition to honing educational expertise through participation in a novel curriculum that incorporates teaching, literature review, and presentation development.

SETTING AND PARTICIPANTS: Internal Medicine Residents at Massachusetts General Hospital complete two week rotations at the Crimson Care Chelsea Clinic, a student-faculty collaborative clinic run by physicians of Massachusetts General Hospital (MGH) Chelsea Health Center and Harvard Medical School students. MGH Chelsea is a federally-qualified health center with 40 years of service to a multilingual, ethnically diverse urban area in Massachusetts. The Crimson Care Chelsea Clinic is an innovative primary care clinic that seeks to improve access to the most vulnerable members of this community, particularly recently incarcerated individuals and immigrants without a usual source of care

DESCRIPTION: To provide a foundation to inform their involvement in the clinic, residents review literature in three areas with the clinic director: (1) health of immigrant communities in the US, (2) health of postincarceration populations, and (3) the role of student-faculty partnerships as safety-net institutions. Residents have an opportunity to gain an understanding of community resources through introduction to health center initiatives and exposure to innovative primary care programs there. During the rotation, residents teach clinical skills to first to fourth year medical students, and lead a weekly case-based discussion. Based on their review of the literature and experience in the clinic, the resident's culminating project is an hour-long lecture to medical students on a topic relevant to care of these communities; for example, health disparities, health policy, a clinical case of interest, or an educational module for use in the medical student curriculum.

EVALUATION: Participants fill out online anonymous evaluations of their elective experience.
DISCUSSION / REFLECTION / LESSONS LEARNED: Involvement in the elective has provided a foundation for deeper learning for residents interested in care of vulnerable populations; there has been significant interest in the elective and in volunteering to lead casebased discussions. Residents have been incorporated successfully into the operational and educational structure of this collaborative clinic. Embedding this experience within a student-faculty collaborative enhances the educational value of the elective by providing hands-on opportunities for instruction and mentorship of medical students. Running case - based discussions and providing training on basic clinical skills has enriched the medical students' experience and offers an opportunity for residents to reflect on the role of service learning in providing care for vulnerable communities. Future directions include expanding the clinical role of the resident through leading clinical skills workshops as well as mentoring quality improvement and research initiatives.

DEVELOPING AND IMPLEMENTING A PATIENT SAFETY ROTATION Abby Spencer ${ }^{1}$; Anasastasios Kapetanos ${ }^{1}$; Andrew Sahud ${ }^{2}$; Kathy Hayes-Light' ${ }^{2}$ Diane Frndak ${ }^{2} .{ }^{1}$ Allegheny General Hospital, Pittsburgh, PA; ${ }^{2}$ Allegheny General Hospital, Pittsburgh, PA. (Control ID \#1342262)

NEEDS AND OBJECTIVES: New guidelines highlight the need to expand patient safety education for residents via formal curricula, experiential activities, and integration of residents into safety programs at their hospitals. The goal of our curriculum is to provide didactic and experiential education that promotes a culture of patient safety, systems-thinking, error analysis, and systems-level improvement.

SETTING AND PARTICIPANTS: Mandatory 2-week PGY3 patient safety rotation.

DESCRIPTION: Residents learn the fundamentals of patient safety from a series of didactics, online modules, video discussions, and experiential activities including participation in RCAs, participation on hospital committees, and conducting a safety investigation project. Residents have bi-weekly mentoring sessions with a safety officer to reflect on their observations \& make recommendations for systems-improvements. They interview safety officers \& leaders throughout our system to better understand their various roles. They also attend patient safety meetings to observe how the hospital system manages safety/quality. Residents visit hospital "hot spots" where they observe practices and make recommendations for error reduction. Residents are required to investigate a medical error and develop a set of recommendations for improvement. For example, residents may work with pharmacy, nursing, and IT to improve the system by which heparin is safely delivered to patients or will work with a similar team to respond to a medication error on the floor. The patient safety resident plays an important role in departmental M\&M conferences, supplementing clinical education with quality/safety education and plans for process improvement.

EVALUATION: We evaluate resident learning and curricular effectiveness. In addition to post-test knowledge/attitude questions, residents write a reflection piece and an apology letter on the first and last day of the rotation. Changes in essays show more in-depth understanding of no-blame and just culture, of process issues, of individual accountability for safe care, and empowerment to address patient safety issues. The large majority felt that after the rotation they were more likely to report errors/near-miss and that they would change the way they care for patients. Activities they found beneficial were attending RCAs, preparing safety analyses of M\&M conferences, learning about initiatives to prevent hospital acquired infections, and observing hand hygiene practices of staff. Residents unanimously felt they were empowered to address safety issues, one resident stated "I believe every physician should have exposure to this field." Residents felt the rotation taught them to think logically about errors $\&$ enact change. They felt it will impact their patient care, documentation, and encouraged them to increase the involvement of patients and their families in decision making.

DISCUSSION / REFLECTION / LESSONS LEARNED: Our new rotation improved resident knowledge, changed attitudes, and most 
importantly, changed actions. After the rotation, residents said they were more likely to recognize, report, and prevent errors. Developing a two-week patient safety rotation is feasible, effective, and provides a forum for medical educators to incorporate safety curricula into their training programs and to integrate residents into the safety operations of hospitals. Our rotation can serve as a useful model for program directors struggling to meet the challenges of meeting new ACGME requirements.

DISCHARGE VISITS IN THE MEDICINE CLERKSHIP: PILOT DATA Deborah Jones ${ }^{1}$; James Noble ${ }^{2}$; Katherine Nickerson ${ }^{1} .{ }^{1}$ Columbia University College of Physicians \& Surgeons, New York, NY; ${ }^{2}$ Columbia University College of Physicians \& Surgeons, New York, NY. (Control ID \#1322100)

NEEDS AND OBJECTIVES: A number of trends in healthcare threaten the smooth transition of care between hospital and home as the acuity of inpatients rises: the rarity of doctors who work both inand outpatient environments; pressure to limit time in the hospital; and minimal contact between inpatient and outpatient providers. Pressure is also mounting to lower costs and reduce high rates of readmissions. In this environment it is essential that medical students acquire explicit knowledge, skills and attitudes to orchestrate successful transitions in care. However, nationally students report poor preparation to take on this role. Presently, students who graduate from our medical school without a Medicine Clerkship curriculum on transitions in care report inadequate instruction on continuity of care more frequently than at other schools ( $31 \%$ vs. $18 \%)$. The Medicine Clerkship is a natural locus to teach principles of transitions in care due to the high prevalence of chronic illness. Several medical student programs have demonstrated success with post-discharge home visits, but it is unknown if postdischarge office visits can improve student confidence and knowledge about discharges and transitions in care.

SETTING AND PARTICIPANTS: Commencing with the Major Clinical Year in January 2012, all members of the class of 2014 will discharge a patient in the initial weeks of their 6 week Medicine Clerkship. Two groups on neurology early in 2012 will serve as a controls. In the five-week Neurology Clerkship students will discharge adult patients but not attend a specifically planned follow up visit.

DESCRIPTION: Students will be provided information on discharge planning in the form of a discharge planning information card at clerkship orientation. The student will be required to attend a post-discharge outpatient follow up visit, if the visit will be with an attending physician affiliated with the home institution or is medical school faculty. The student will write up that visit and submit a report to their preceptor, identifying the issues at that visit. Students will use a template provided by the clerkship director.

EVALUATION: Evaluation of the education program will have a quantitative and qualitative component. At the beginning and conclusion of medicine and neurology clerkships students will respond to a 9-item anonymous, electronic, 5-point Likert scale survey regarding their confidence on topics related to transitions in care. All responses will be compared at the group level with a non-paired t-test. Pilot data from approximately 70 students are anticipated by May, 2012. The student write ups will be qualitatively analyzed for their thematic and reflective content with an analytic tool currently under development.

DISCUSSION / REFLECTION / LESSONS LEARNED: Opportunity to improve student knowledge and confidence in transitions in care, while helping outpatient doctors and patients with transitions, makes a discharge visit an appealing addition to the Medicine Clerkship. Many attempts have been made to teach students about continuity of care such as longitudinal curricula, and visits to homes and long term care facilities. An office based, post-discharge visit incorporated into a Medicine Clerkship is a feasible addition for all medical schools. Linking outpatient and inpatient care within the Medicine Clerkship improves student knowledge of chronic disease management, medication reconcilation and adherence, discharge planning, fosters professionalism and should ultimately improve patient outcomes.
DISEASE OF THE MONTH: A MONTH-BY-MONTH MULTIDISCIPLINARY CURRICULUM FOR AMBULATORY BLOCK OR PRIMARY CARE CLINIC H. Keels S. Jorn. Mayo Clinic, Jacksonville, FL. (Control ID \#1339800)

NEEDS AND OBJECTIVES: Educators and learners in internal medicine resident clinics have a wealth of information available to them but it can be difficult to tailor the available information to needs of learners in a given clinic setting. In Disease-of-the-Month Curriculum, the material presented incorporates recognized resources for medical knowledge and patient care with multidisciplinary providers, emphasizing systems based practices of the medical center in which it is shared. In contrast to many educational resources that focus on hospital management of acute exacerbations of chronic disease, this curriculum attempts to guide clinicians in the management of patients for "the 51 weeks of the year that they aren't in the hospital", with emphasis on local resources. Basics of quality of care and practice-based learning and improvement for each resident's continuity panel in context of the Disease-of-the-Month

SETTING AND PARTICIPANTS: 4-5 Once-weekly 1-2 h conferences during Ambulatory Block for PGY1's and PGY2's. Occasional "field trips" to relevant areas of clinic, such as to physical therapy department to learn about PT for back pain. Presenters vary with the topic, but could be internal medicine physicians, home health providers, pharmacists, diabetes educators, and others. Curriculum could be adapted to a pre-clinic conference for continuity clinic.

DESCRIPTION: Common ambulatory conditions (diabetes, COPD, HTN, mental illness, etc) are selected as "Disease-of-the-Month". The month is structured. Week 1: Medical overview of the condition, discussion of quality indicators for that condition, resident-selected quality measures for self-audit of their continuity patients. Week 2: PharmD lectures on pharmacology relevant to the DOM. Week 3 or 4: Nonphysician provider contribution to chronic or outpatient management. Week 4 or 5: review of results of resident self-audit. Diabetes (example) Week 1: Medical overview, selection of quality measures. Week 2: Pharmacology review of medications in diabetes, emphasis on oral agents to treat diabetes and medications that can aggravate diabetes. Week 3: Diabetic nurse educator brings injection practice kits, teaches residents to use insulin pens vs. syringes. Week 4: Review of findings from self-audit and action plans for next 6 months Back Pain (example) Week 1: Medical overview, differential diagnosis, "red flag" features, and selection of quality measures for self-audit. Week 2: Pharmacology review, emphasis on non-narcotic medications used in back pain Week 3: "field trip" to physical therapy department to see, touch, experience equipment for back pain Week 4-5: chiropractor discusses non-allopathic management of back pain, review of quality measures Other examples will be available if/when presented.

EVALUATION: End of month evaluations, resident reflection on level of interest and comfort in managing chronic conditions in clinic, re-audit to verify quality items addressed

DISCUSSION / REFLECTION / LESSONS LEARNED: Having a structure for the monthly cycle of conferences helps to assure that nonphysician resources not immediately recognized in the practice are made more familiar to learners. Physician seen as member of treatment team is clarified. Educators from other institutions could use the "skeleton" of this conference as basis for their own, site specific resources. One downfall is that only the residents on the rotation experience that "Disease-of-theMonth". Additional effectiveness evaluation methods are needed. Curriculum in use for 6 years.

ESTABLISHING KLIC- THE UCSF-KAISER PERMANENTE LONGITUDINAL INTEGRATED CLERKSHIP Lindsay A. Mazotti ${ }^{1,2}$; Juan Guerra ${ }^{1}$; Stanton $\mathrm{Siu}^{1}$; Leslea Brickner ${ }^{1}$; Wendy Smith $^{1}$; Ann Poncelet ${ }^{2}$. ${ }^{1}$ Kaiser Permanente, Oakland, CA; ${ }^{2}$ University of California San Francisco, San Francisco, CA. (Control ID \#1328731)

NEEDS AND OBJECTIVES: Medical education is in an exciting period of transformation as medical schools work to incorporate modern learning principles into their structure and align forces impacting delivery of care 
with educational priorities. Guiding principles of longitudinal integrated clerkships (LICs) emphasize continuity with preceptors, patients and site. LIC benefits include enhanced patient-centeredness, moral development, observation and feedback on clinical skills, identity formation, and countering of the hidden curriculum. Creating LICs, however, can be both faculty and resource intensive. This educational transformation may require symbiotic partnerships with integrated community health systems such as Kaiser Permanente (KP), with potential benefits for both institutions. Our objective was to create a one year LIC at KP that emphasized: longitudinal relationships between patients, students, teachers, and healthcare systems, the course of chronic illness and the patient's experience of disease, the development of clinical reasoning skills through early introduction to patients with undiagnosed illness, and the skills and integrated knowledge needed for compassionate, effective patient-centered care.

SETTING AND PARTICIPANTS: KLIC [Kaiser Permanente (KP)University of California San Francisco (UCSF) Longitudinal Integrated Clerkship (LIC)], a one year LIC for 8 UCSF third year medical students at KP Oakland, CA.

DESCRIPTION: Academic leaders in established LICs at UCSF began discussions with Northern California Regional KP in fall of 2009. In 201112, KP Oakland was chosen to pilot 8 students in an LIC, mirroring the PISCES program at UCSF. Students complete all 8 core clinical clerkships over one year with supervision from one preceptor for each clerkship. Students establish a patient "cohort" panel of 50-75 patients for whom they will provide longitudinal care over the course of one year. Unique aspects of the KLIC program include a novel leadership, health systems and quality improvement curriculum "PULSE."

EVALUATION: Students are evaluated with formative and summative evaluations including Brief Structured Clinical Observations and faculty quarterly R-I-M-E evaluation sessions. Each clerkship is evaluated by the students at midpoint and end of year (currently pending), as well as the overall course. Didactics and PULSE leadership sessions are also evaluated. Program evaluation is semi-annual and ongoing.

DISCUSSION / REFLECTION / LESSONS LEARNED: As medical education continues to transform, it will be important for university settings to collaborate further with community training sites. Benefits to UCSF may include additional resources available at the community site, a sustainable model of LIC training, opportunity for innovation across the continuum of UME and GME at KP, and a primary care focused educational site. Benefits to KP may include faculty development and faculty appointments at the university for staff, participation in an educational program aligned with the structure of KP's healthcare system, opportunity to train physician workforce who will be competent in systems-based practice and practicebased learning and to recruit future residents and staff, as well as train future doctors who can appreciate and disseminate KP values. This symbiotic LIC clerkship site is expected to be replicated at between UCSF and other local KP medical centers, but can also be exported to other university-community alliances.

FOSTERING TRANSFORMATIVE LEARNING THROUGH ASSIGNED SELF-REFLECTION AND ACTIVE DISCOURSE FOR THE FIRST YEAR MEDICAL STUDENTS. Yelena Averbukh. Montefiore Medical Center, Bronx, NY. (Control ID \#1333662)

NEEDS AND OBJECTIVES: Doctors in training have minimal guidance on how to process and learn from emotions during and following clinical encounters. The Transformational Learning Theory holds that "the way adult learners interpret and reinterpret their sense experience is, central to making meaning and hence learning" (Mezirow, 1991). Our objective was to assess how a first-year medical student curriculum emphasizing selfreflection, paired reflection and active discourse in a small group format enhances trainees' communication and professionalism during introductory clinical encounters.

SETTING AND PARTICIPANTS: Introduction to Clinical Medicine course at Albert Einstein College of Medicine in Bronx, NY. Series of fourteen 2.5-hour long precepted clinical encounters in inpatient setting at affiliated tertiary hospital.
DESCRIPTION: Students are divided in 2 groups. The students in the control group have weekly clinical encounters under an individual supervision of a preceptor with focus on standard medical interview technique and skills. The intervention group students are paired and assigned to one preceptor per pair. In addition to standard medical interview course assignments, they complete weekly written free style self-reflection assignments regarding emotions that they experienced during clinical encounters, which are then discussed during following session with their paired partner under preceptor supervision. Preceptor's role is to foster transformative learning by assisting learners in becoming aware and critical of their own emotions, assumptions, beliefs, points of view as well as assumptions of others. Small group discussions enable participants to engage in active discourse while becoming better at recognizing frames of references and alternate perspectives. This experience may facilitate transformational processing of information and actions on behalf of the participants.

EVALUATION: By the end of the 14 sessions students in both groups are given anonymous surveys with 1 to 5 Likert scale assessing their comfort level in communicating with certain challenging patients (angry, depressed, psychotic, demented and disruptive) and in certain challenging situations (patient in physical discomfort, unable to provide good history). Students are then asked to read 5 clinical vignettes and choose an answer from a set of multiple-choice questions hypothetically assessing an impact of the intervention on their future professional behavior.

DISCUSSION / REFLECTION / LESSONS LEARNED: The preliminary data in this pilot project indicates a trend for enhanced comfort level in communication and professionalism in the intervention group. It is known that health care providers may fail to connect to patients in physical or emotional distress for a wide variety of reasons. Structured selfreflection and small group discussion of clinical encounters in the preclinical years may not only enhance self-assessment capabilities (regarding counter-transference) but may also have an impact on trainee satisfaction, and enhance patient satisfaction. Certainly, formal written processing of trainee emotions may ensure acknowledgement and prevent denial and/or avoidance. Deepening ability to reflect through structured paired discussions may help to identify emotion experienced and enable productive utilization of the current experience while validating the expression of and reflection on emotions experienced in clinical encounters. Finding effective tools and appropriate settings for transformational learning in trainees is of interest to many clinician educators.

IMPACT OF MILESTONES-BASED EVALUATIONS ON AN INTERNAL MEDICINE RESIDENCY PROGRAM Jaya Raj. St. Joseph's Hospital and Medical Center, Phoenix, AZ. (Control ID \#1338725)

NEEDS AND OBJECTIVES: The fair and accurate evaluation of residents presents a big challenge to program directors and faculty. The subjectivity and unreliability of global evaluations and the tendency of faculty to inflate residents' ratings limits their value in providing useful feedback to residents. Objectives: 1. To create an evaluation tool which accurately assesses residents' achievement of specific, observable milestones 2. To design an evaluation form that empowers faculty to be more specific and candid in their evaluations

SETTING AND PARTICIPANTS: Thiry-five residents and nine key clinical faculty in the St. Joseph's Internal Medicine Residency Program participated. The innovation spanned both the inpatient and outpatient setting, but outcomes were measured in the inpatient setting only.

DESCRIPTION: In 2008-2009, we revised all our program's goals and objectives and our promotion criteria to correspond to "milestones," which we developed shortly before the release of the draft milestones document by the joint ACGME/ABIM task force. The milestones were used to design new faculty, peer, nursing/ancillary staff, and self evaluations, which were implemented in July 2009. We compared the mean and the range of summative scores from faculty evaluations in 2008-2009 versus 2010 in each competency. We also measured the correlation between residents' summative scores in medical knowledge and their performance on the ABIM certification exam. In addition, the faculty were asked to 
anonymously rate each graduating senior on their "ability to provide autonomous care" and the likelihood they would "engage in lifelong learning." We then measured the correlation between the anonymous faculty ratings and the ratings that the same residents received in their evaluations.

EVALUATION: In 2010, compared to 2008-2009, faculty assigned a wider range of scores to residents in all ACGME competencies except Professionalism. This effect was most pronounced for Medical Knowledge (range of 4 in 2010-2011 versus 0 in 2009). There was no statistically significant difference in mean scores. The residents' summative scores for Medical Knowledge correlated better with their scores on the ABIM certification exam in $2010(\mathrm{r}=.861, \mathrm{p}=.027)$ compared to $2008-2009(\mathrm{r}=$ $0.579, \mathrm{p}=0.038$ ) but did not predict pass/fail. The anonymous ratings of "ability to provide autonomous care" correlated with residents" global evaluation scores in Medical Knowledge (Pearson correlation .536, $\mathrm{p}<.05$ ) but not in Patient Care or Overall Competence. The anonymous ratings of "lifelong learning" correlated with residents' scores in Medical Knowledge (Pearson correlation .695, $\mathrm{p}<.01$ ), Problem Based Learning \& Improvement $(.625, \mathrm{p}<.01)$, and Overall Competence $(.521, \mathrm{p}<.05)$.

DISCUSSION / REFLECTION / LESSONS LEARNED: The new milestones-based evaluations have resulted in small but observable changes in faculty behavior. Faculty are more likely to assign residents a range of scores in each competency, rather than the same score across the board. However, faculty's global evaluations do not necessarily reflect their actual perceptions of residents' competence or predict their performance on examinations. The milestones may be useful for programs in designing evaluation tools based on entrustable professional activities (EPA's), which encompass the milestones but describe more fully the key activities that physicians must be able to perform. Despite its limitations, the milestones concept is a useful step in moving residency programs toward true competency-based progression.

IMPLEMENTING A LONGITUDINAL INTEGRATED CLERKSHIP (LIC) FOR THE THIRD YEAR AT THE COMMONWEALTH MEDIAL COLLEGE Christian Adonizio; Valerie D. Weber; Janet Townsend; Susan M. Perlis; Maurice Clifton. The Commonwealth Medical College, Scranton, PA. (Control ID \#1311299)

NEEDS AND OBJECTIVES: The Commonwealth Medical College (TCMC) is a new (2009) community based, allopathic medical school which provides a distributed model of education with three regional campus "hubs" over a 16 county region in northeastern and north central Pennsylvania. TCMC sought to design a third year curriculum which would offer opportunities for meaningful longitudinal relationships with patients and faculty and avoid the fragmentation of educational experiences seen in traditional third year hospital based clerkships.

SETTING AND PARTICIPANTS: All third year medical students (63) at The Commonwealth Medical College, a community-based allopathic medical school with three regional campuses, located in both small urban and rural communities.

DESCRIPTION: The longitudinal integrated clerkship (LIC) is a model of third year education where students: 1)participate in the comprehensive care of patients over time; 2) experience continuous learning relationships with faculty; and 3) meet the core clinical competencies across multiple disciplines simultaneously. Students are assigned to six preceptors, one in each of the six core disciplines. Students spend one half day weekly with each preceptor in office-based settings. Three half days of 'white space' allow students to follow patients longitudinally into other care settings (hospital, OR, specialty appointments, etc) and a half day of weekly structured educational sessions round out the curricular experience. In addition, six one week 'bursts' in inpatient Surgery and Medicine allow for concentrated experiences in the hospital setting. Cross-campus comparability is ensured through central oversight and a strong regional educational leadership structure. The success of the model is enhanced by robust faculty development.

EVALUATION: Students log all clinical encounters into an e-portfolio. These are reviewed regularly by the educational leadership team to ensure the educational objectives are being met. All students complete OSCEs and take NBME content exams in each of the six disciplines. In addition, faculty evaluations at 30 days, 6 months and end of year are completed along with measures of professionalism such as cultural competency and empathy.
DISCUSSION / REFLECTION / LESSONS LEARNED: Recent reports from the Macy and Carnegie Foundation support the expansion of the longitudinal integrated clerkship as a model that supports longitudinal exposure to patients and faculty, enhances patient-centered attitudes and the maintenance of empathy, while demonstrating equivalent or better outcomes on measures such as written examinations and OSCEs compared with traditional inpatient block clerkships. Yet, no other US medical schools have as of yet expanded this curricular model broadly. TCMC offers a model for expansion of LICs that is generalizable to other US medical schools including best practices for ensuring comparability, monitoring of educational outcomes, and faculty development to support the model.

IMPROVEMENT IN RESIDENT KNOWLEDGE AND CLINICAL SKILL IN KNEE AND SHOULDER EXAMS AFTER LARGE GROUP TRAINING IN THE CONTINUITY CLINIC SETTING. Frances Norlock $^{1,2}$; Laura Sadowski ${ }^{1,2}$; Steve Clar ${ }^{1,2}$; Ernest Fontecha ${ }^{1,2}$. ${ }^{1}$ Stroger Hospital of Cook County, Chicago, IL; ${ }^{2}$ Rush University, Chicago, IL. (Control ID \#1335011)

NEEDS AND OBJECTIVES: Training and proficiency in musculoskeletal examination skills is variable and frequently inadequate during medical school. Residents may be hesitant to diagnose and manage common musculoskeletal problems and refer patients unnecessarily resulting in delay of care. At our institution General Internal Medicine faculty collaborated with physiatrists to develop, implement and evaluate a workshop to internal medicine residents aimed at building their knowledge and clinical skill in the examination of the shoulder and knee joints.

SETTING AND PARTICIPANTS: Residents who were not on vacation, an away rotation or in the intensive care units attended the workshop during their ambulatory clinic session (one-half day). We implemented a pilot workshop to residents who had clinic on Monday $(n=19)$. After minor improvements, we implemented the workshop for residents during four clinic sessions, Tuesday through Friday $(n=83)$. The approach in training residents in this material in a large group setting has not been described in the literature before.

DESCRIPTION: The workshop was composed of: 1) two large group interactive didactic sessions including observing the knee and shoulder exam of live model; 2) two small group hands-on sessions for the knee and shoulder exam with live model (4-5 learners per instructor); and 3) small group session to practice aspiration using knee joint models .

EVALUATION: Residents completed a 13 item written exam, before and after the workshop to assess their knowledge of the diagnosis and management of common knee and shoulder disorders. Apriori we developed criteria for a passing score (9 of 13 correct), as well as our goal to improve knowledge scores accounting for baseline scores. All PGY1s and PGY3s passed the written exam after the workshop; 5 of the 24 PGYs $(79 \%)$ passed $(p=.005)$. The knowledge improvement goal was reached in the majority of PGY1s and PGY3s ( $82 \%$ and $80 \%$ respectively). Barely half of the PGY2s met our expectations for improvement of knowledge (54\%). Improvement in knowledge scores differed by residency levels $(\mathrm{PGY} 1=3.1$; PGY2 $=1.6$; PGY3 $=2.3, \mathrm{p}=0.058)$. Observed structured clinical exams (OSCE) were used to assess the skill of each resident in performing a knee exam at the end of the workshop. General Medicine faculty preceptors observed each resident performing a knee exam on a human model in a patient exam room, and evaluated their technique on 11 skills. The performance score of each skill ranged from 0 to $2(0=$ did not perform, $1=$ partially completed, $2=$ adequately performed). Apriori we set a passing score to be 11 points out of a maximum of 22 points. The mean OSCE score was 15.5 (range 15.0 (PGY3s) to 15.9 (PGY1s) with no statistical significance between groups $(p=0.74)$. The mean OSCE score did not differ by self-reported previous training in the knee exam; 34 residents reported prior training and had a mean OSCE score of 15.1. PGY1s were most likely to pass the OSCE (95\%) followed by PGY2s (88\%) and lastly PGY3s (83\%).

DISCUSSION / REFLECTION / LESSONS LEARNED: Our workshop succeeded in improving the knowledge and skills of residents. The improvement in knowledge was greatest in the PGY1s and PGY3s. The performance of PGY2s was largely influenced by a few outliers who performed very poorly ( $>2$ standard deviations below the mean). The 
mean OSCE score for the knee exam was of 15.5 (out of 22) and did not differ by residency level or prior training, suggesting that all residents may benefit from this type of workshop training.

IMPROVING WRITTEN SIGNOUTS THROUGH EDUCATION \& STRUCTURED AUDIT: THE UPDATED APPROACH Allison DeKosky ${ }^{1}$; Ananya Gangopadhyaya ${ }^{2}$; Bobby Chan ${ }^{3}$; Vineet Arora ${ }^{4} .{ }^{1} \mathrm{UCSF}$ Medical Center, San Francisco, CA; ' University of Illinois at Chicago Medical Center, Chicago, IL; ${ }^{3}$ MacNeal Hospital, Berwyn, IL; ${ }^{4}$ University of Chicago Medical Center, Chicago, IL. (Control ID \#1339970)

NEEDS AND OBJECTIVES: The July 2011 ACGME guidelines mandate that residency programs monitor and ensure resident competency in patient handoffs. The guidelines' concomitant restrictions on resident duty hours are likely to increase both the volume and complexity of handoffs. The new duty hours will also likely change resident teams' structures in ways that will necessitate handoffs to and by "bridge" or "float" residents with no prior knowledge or ownership of the patients. This has potential for dangerous errors. In this setting, structured handoffs often become the only continuity for several hours a day. Ensuring proper creation and vigilant maintenance of the written signout is critical in today's environment. Prior curricula to teach adequate signout communication have focused on verbal handoffs, and tools to facilitate teaching, monitoring, and evaluation of written signouts are especially lacking. The aim of this educational innovation was to develop, implement and evaluate an interactive curriculum for use in teaching and evaluating written signouts through evidence-based audits.

SETTING AND PARTICIPANTS: Workshops were held at three Chicago-area teaching hospitals, with 130 4th year medical students and residents using the UPDATED mnemonic to teach signout strategies.

DESCRIPTION: The UPDATED approach emphasizes the importance of the following: Updated administrative data, Prioritized problem list by importance, Diagnoses in the one-liner, Anticipated problems clear, preventing Too much information, Error-prone medications highlighted, and Directions that are clear. The UPDATED mnemonic was also transformed into an audit tool used during the workshop to facilitate peer or self-assessment of the written signouts

EVALUATION: Almost all participants (95\%) felt more confident in creating a written signout, and reported the process of auditing a written signout helpful (93\%). The practice behaviors with most commitment to change were to include the diagnosis in the one-line summary $(98 \%)$, to provide clear and specific directions with anticipated guidance and to-do items $(95 \%)$, to prioritize the problem list $(95 \%)$, and to prevent too much information $(94 \%)$. To preliminarily assess whether this education translated into changes in practice, a random selection of actual signouts from one of the institutions was evaluated by the investigators before and after the workshop. The proportion of signouts rated as "Good" rose from $13 \%$ to $73 \%\left[\chi^{2}=35.7\right.$, $\mathrm{P}<0.0001]$

DISCUSSION / REFLECTION / LESSONS LEARNED: As residency training programs are now required to ensure competency in the process of handoffs, development of tools with structured teaching and monitoring of signouts has become crucial, particularly given the rapid proliferation of electronic health records combined with multiple daily handoffs. The UPDATED approach is effective in addressing trainees' competency in written signout, with an emphasis on the daily synthesis and integration of large amounts of data critical to safe patient care.

INTEGRATION OF MENTAL HEALTH AND PRIMARY CARE SERVICES IN A STUDENT-FACULTY COLLABORATIVE PRIMARY CARE CLINIC: AN APPROACH FOR PSYCHIATRY EDUCATION Chuan-Mei Lee; John Heintz; Janine Knudsen; Oriana Vesga-Lopez; Lazaor Zayas; Mary Lyons Hunter; Derri Shtasel; Marya J. Cohen. MGH, Boston, MA. (Control ID \#1334734)

NEEDS AND OBJECTIVES: To demonstrate a model of integrating mental health and primary care services as an innovative approach for psychiatric education.
SETTING AND PARTICIPANTS: The Crimson Care Collaborative (CCC) at Chelsea is a Harvard Medical School student-resident-faculty collaborative clinic located at the Massachusetts General Hospital (MGH) Chelsea community health center. The clinic features co-located, integrative mental health and primary care services and serves two main patient populations: 1) recently incarcerated individuals and 2) patients who have been unable to establish routine primary care and are high utilizers of urgent care services. Harvard medical students are involved with all aspects of the clinic, from clinical care and social services to day-to-day administrative work.

DESCRIPTION: The authors will describe the operational structure of the clinic. Medical student participants will be asked to describe how the colocation of psychiatric and primary care services informed their approach to patient care.

EVALUATION: The CCC Chelsea clinic offers several learning opportunities in psychiatry with an emphasis on interdisciplinary teamwork: 1) student participation in psychiatric patient care, 2) a case conference co-led by residents from psychiatry and internal medicine, 3) a student-led casemanagement system that helps patients navigate social service resources available in the community, and 4) student-initiated discussions between psychiatric and primary care teams. Descriptive results of medical students' recognition of the effect of co-located psychiatric care will be presented.

DISCUSSION / REFLECTION / LESSONS LEARNED: A studentresident-faculty collaborative clinic provides a unique learning opportunity for exposure to psychiatry in the context of an interdisciplinary care model.

INTERDISCIPLINARY TEACHING SAFE TRANSITIONS CARE BASED SESSION Rachel K. Miller; Eric Goren; Christina R. Whitehouse; Anne Norris; Jennifer S. Myers. University of Pennsylvania, Philadelphia, PA. (Control ID \#1339869)

NEEDS AND OBJECTIVES: Transitions of Care, the coordination of discharge care outside the hospital, has been identified as a key element of patient care and critical component of health professions education by nationwide by medical institutions, Joint Commission, and Medical Education Accreditation Commitees. In addition, the AAMC has newly identified Core Competencies for Interprofessional Collaborative Practice. We believed that the introduction of safe care transitions and the interprofessional team model would be ideal on internal medicine intern orientation day. At the end of the session, participants will be able to: 1 . Identify the importance of a safe discharge from the hospital 2. Identify the complexities of creating a safe transition of care out of hospital to home or facility. 3. Identify high risk discharge issues 4 . Discuss entry criteria and scope of care provided by the potential sites of care for hospital discharge. 5. Identify the health professionals involved in facilitating a safe discharge and their respective roles in the process. 6 . Describe the interdisciplinary discharge process and value its role in facilitating a safe discharge 7 . Describe the importance of communication with patient and family members in care transitions

SETTING AND PARTICIPANTS: During intern orientation day, small group session lasting 40 minutes. Each group had an internal medicine physician-moderator, social worker, clinical pharmacist, and a visiting nurse.

DESCRIPTION: First, during the intern orientation lecture section, a fifteen minute overview of transitions of care was given. This included definition, key concepts, brief introduction to upcoming small group session, and overview of future transitions of care education initiatives during their residency. In the later part of the afternoon, there was a small group session lasting 40 minutes Each group had an internal medicine physician-moderator, social worker, clinical pharmacist, and a visiting nurse. Each group reviewed 2 cases which focused safe discharge from the hospital to home or skilled nursing facility. Each case highlighted the role of the interprofessionals and each discipline was given ample opportunity to provide information for the case, highlight their role, and emphasize key high risk discharge issues.

EVALUATION: An IRB approved survey was given immediately following the session to all the interns using a 5 point Likert scale and 
56 evaluations were collected. 24 (42.8\%) agreed and 31 (55.3\%) strongly agreed that the session enhanced their ability to identify threats to a safe hospital discharge. $33(58.9 \%)$ agreed and $23(41 \%)$ strongly agreed that after attending the session, they feel more confident in their understanding of the roles of other health care professionals who participate in the discharge process. $8(14.2 \%)$ agreed and $48(85.7 \%)$ strongly agreed that they believe that the use of other healthcare professionals (social worker, nurse, etc) to teach this topic was effective.

DISCUSSION / REFLECTION / LESSONS LEARNED: This interprofessional case-based discussion on teaching safe transitions demonstrated an overall increased confidence in knowledge of discharge transitions, ability to identify threats to a safe hospital discharge, roles of other healthcare professionals in discharge process, and the role of physician in discharge transition. Future directions include expanding this session and further enhancing transitions of care curriculum throughout the entirety of internal medicine residency.

INTERPROFESSIONAL TEAM-BASED LEARNING AMONG PRIMARY CARE TRAINEES Bennett Lee; Brigitte L. Sicat; Bruce Rybarczyk; Benjamin D. Lord. Virginia Commonwealth University, Richmond, VA. (Control ID \#1326921)

NEEDS AND OBJECTIVES: Internal medicine residents, pharmacy residents, and psychology doctoral trainees routinely co-manage patients in our academic medical center primary care clinic. The management provided, however, is multidisciplinary versus interdisciplinary. Last year, the authors developed three Interprofessional Education (IPE) Team-Based Learning (TBL) modules whereby learners could learn with, from, and about each other. Objectives: 1. to educate primary care trainees from different professions about the expertise of each discipline 2. To foster interdiscplinary collaboration among primary care trainees 3 . To educate trainees on topics commonly seen in a primary care

SETTING AND PARTICIPANTS: Academic Medical Center 2 hour conference sessions Participants: Psychology doctoral students Pharmacy residents Internal medicine residents Pastoral care trainees one session DESCRIPTION: Three 2-hour IPE TBL modules were designed to promote a belief in the importance and effectiveness of interdisciplinary team work as well as application of content knowledge in the following areas: (1) Introduction to the Professions/Motivational Interviewing, (2) Depression, and (3) Non-Cancer Pain. The modules (learning objectives, readiness assessment tests, cases, and case discussion points) were developed collaboratively by an interdisciplinary faculty team. During each 2-hour learning session, learners were placed in teams of 7-9 members with each discipline represented on the teams. Interdisciplinary faculty team served as facilitators. Surveys were administered to participants after each TBL module to evaluate their perceptions of achievement of learning objectives and of learning gains from participating in the module. The Attitudes Toward Health Care Teams Scale was administered to evaluate their attitudes toward health care teams at baseline and after the second and third TBL modules.

EVALUATION: Each module had 70 attendees and an average of 51 participants completed the survey each session $(63 \%$ medical residents, $17 \%$ clinical psychology doctoral trainees, $15 \%$ pharmacy residents, and $4 \%$ chaplains; $35 \%$ male, $65 \%$ female). In an anonymous survey of the trainees in attendance, there was a uniform report of moderate gain to good gain ( 2 and 3 on a 5 -point scale ranging from $0=$ no gain to $4=$ great gain) on the learning objectives. Disciplines were generally similar in their ratings. The TBL components were also rated in terms of how much help they provided in meeting the learning objectives. On a $0-4$ scale $(0=$ no help, $1=$ a little help, $2=$ moderate help, $3=$ much help, and $4=$ great help) the results were as follows: individual readiness assurance test $=2.4$, team readiness assurance test $=2.7$, case-based discussion within the team $=3.3$, and case-based discussion with the full group=3.2. Additionally, the Attitudes Toward Health Care Teams Scale showed that there was significant positive change toward viewing teams as more efficient after participating in the first two TBL modules. This change was evident in all three main participant groups (medical residents, pharmacy residents, and psychology doctoral trainees)
DISCUSSION / REFLECTION / LESSONS LEARNED: The IPE TBL sessions appear to be an effective format for teaching interdisciplinary communication as well as a belief in the importance and effectiveness of interdisciplinary team work. Additionally, it appears to serve as an effective tool for applying current clinical knowledge in areas where interdisciplinary work is beneficial in primary care.

INTERPROFESSIONAL PHYSICIAN-NURSE TEAM TRAINING USING A STANDARDIZED CLINICAL ENCOUNTER INVOLVING THEATER STUDENTS. Tabassum Salam ${ }^{1}$; Robert Dressler ${ }^{1}$; Ann Marie Baker $^{4}$; Susan C. Zern ${ }^{5}$; Michelle Collins ${ }^{4}$; Amy Cowperthwait ${ }^{3}$; Allan Carlsen ${ }^{2} .{ }^{1}$ Christiana Care Health System, Newark, DE; ${ }^{2}$ University of Delaware, Newark, DE; ${ }^{3}$ University of Delaware, Newark, DE; ${ }^{4}$ Christiana Care Health System, Newark, DE; ${ }^{5}$ Christiana Care Health System, Newark, DE. (Control ID \#1331618)

NEEDS AND OBJECTIVES: In order to facilitate the delivery of excellent patient care, it is imperative that physician-nurse teams work in a collaborative manner. We wanted to create a forum for the joint training of physician-nurse teams in order to allow them to use their training and skills in a complementary and collaborative fashion. This educational program was designed to allow resident physicians and novice nurses to work together to care for a simulated inpatient in crisis. It also allowed the physician-nurse team to work with a 'live' standardized patient, so that they could also practice collaborative verbal and non-verbal communication with the patient.

SETTING AND PARTICIPANTS: The venue was the Virtual Education and Simulation Center at Christiana Care Health System, in a facsimile of a full equipped inpatient hospital room, [including equipment to assess vital signs, a sham electronic medical record, computerized order entry, medication cart]. Undergraduate students from the Theater Department of the University of Delaware were trained to portray inpatients in active alcohol withdrawal. Each learner team was comprised of an Internal Medicine resident physician and a novice nurse from the nurse training program. A pair of nursing and physician educators jointly facilitated debriefing sessions with the pair of learners at the end of the bedside encounter.

DESCRIPTION: The nurse learner was given verbal signout on the patient, and initiated the encounter with a bedside assessment of the patient. Thereafter, she could page the physician learner to the patient's bedside. The physiciannurse team was expected to communicate verbally and nonverbally with the patient, communicate and collaborate with each other, recognize the symptoms of alcohol withdrawal, and formulate a treatment plan. After the 10 minute bedside scenario, physician and nursing educators facilitated a joint debriefing session for each pair of physician-nurse learners.

EVALUATION: 3 variables were measured. Improvements were seen across all variables after the adaptive clinical encounter. These were: Confidence in ability to identify alcohol withdrawal [44\% of participants pre-encounter to $94 \%$ of participants post-encounter], Comfort level with the CIWA protocol $[41 \%$ of participants pre-encounter to $72 \%$ of participants post-encounter], Ability to communicate with team members [ $55 \%$ of participants pre-encounter to $81 \%$ of participants post-encounter]. DISCUSSION / REFLECTION / LESSONS LEARNED: Resident physicians and novice nurses are often thrown into stressful situations with complex inpatients. This training method will help them develop the skills needed to communicate with their clinical partner, collaborate with each other and come up with a workable treatment plan. Today's resident physicians get a lot of exposure to learning through simulation. However, our residents commented that their experiences in team-training in the past had involved a mannequin, and not a live patient. They felt that the ability to practice verbal and non-verbal communication with the patient, in collaboration with their nurse partner, was a novel experience. Overwhelmingly, the learners felt the experience was exceptionally valuable for its authenticity. The education plan was implemented in fall of 2011 as a pilot, with the intent to expand the program in 2012. Other clinical scenarios under development include the management of an inpatient with delirium, communicating bad news to patients, and educating patients about their medications at time of discharge. 
LACHMAN'S AND JOBE'S A YEAR LATER: A MUSCULOSKELETAL WORKSHOP FOR INTERNAL MEDICINE RESIDENTS AIMED AT RETAINING CONFIDENCE, KNOWLEDGE, AND SKILLS Darlene LeFrancois; Sharon Leung; Cindy Sadikot. Montefiore Medical Center and Albert Einstein College of Medicine, Bronx, NY. (Control ID \#1315199)

NEEDS AND OBJECTIVES: Musculoskeletal complaints are some of the most common reasons for primary care visits, yet most Internal Medicine (IM) residents do not feel confident or skilled in their ability to diagnose and treat these complaints. A primary care provider's ability to examine and diagnose musculoskeletal complaints can promote cost effective care and contribute to patient and provider satisfaction. The aim of our project is to institute and assess the immediate and 1 year effectiveness of a musculoskeletal workshop on IM residents' confidence, knowledge, and skills in the examination and diagnosis of common shoulder and knee complaints.

SETTING AND PARTICIPANTS: Montefiore Medical Center's categorical residency program in IM is located in the Bronx, New York City amongst a large underserved poor urban population. The musculoskeletal workshop was delivered to our PGY2 residents during their ambulatory care rotation (ACR)

DESCRIPTION: The one hour workshop consisted of a didactic powerpoint presentation of shoulder and knee anatomy, pathology, and examination techniques followed by demonstration of exams and specific maneuvers. Housestaff then paired and practiced supervised exams until they were able to complete them correctly. Before, immediately after, and 1 year post workshop the residents completed a 4 item Likert confidence scale from 1 (strongly disagree) to 5 (strongly agree) and 8 multiple-choice case-based knowledge questions on the evaluation of common knee and shoulder complaints. A standardized case-based shoulder ( 8 items) and knee (9 items) examination checklist was used to measure resident observed performance at 1 year. The checklist was based on the American College of Physicians Musculoskeleletal Examination Clinical Skills Series and each item was scored as "not completed", "partially completed", or "completed correctly".

EVALUATION: All 45 housestaff $(100 \%)$ who rotated on the 7 ACR months from June 2010 to June 2011 attended the workshop (range 4 to 8 subjects/month). After the workshop confidence was significantly improved in diagnosing and examining common shoulder and knee complaints $(p<0.001)$. For the knowledge score, the median percent answered correctly for the shoulder and knee questions pre- and post-workshop was $48 \%$ and $70 \%$, and $52 \%$ and $81 \%$ respectively $(p<0.001)$. Of the 9 residents who have currently met the 1 year post workshop mark knowledge scores did not decline from the immediate post workshop scores but only $18 \%$ and 19\% of all shoulder and knee checklist items respectively were "completed correctly". The remaining approximate $80 \%$ of musculoskeletal exam checklist items were either "not completed" or only "partially completed".

DISCUSSION / REFLECTION / LESSONS LEARNED: While both confidence and knowledge improved as a result of an intensive musculoskeletal workshop intervention, observed resident examination skills at 1 year were poor in this pilot study. As such, a current study is underway that randomizes half the ACR residents to receive subspecialty ambulatory clinic training in both orthopedic and rehabilitative medicine in addition to the musculoskeletal workshop intervention that all residents will continue to receive. Confidence, knowledge, and musculoskeletal exam performance will be assessed by blinded observers against the standardized checklist at baseline, immediate post intervention, and on 1 year follow up. Retention of housestaff musculoskeletal examination skills is preferable to isolated confidence and knowledge improvements as an outcome measure of teaching effectiveness.

LEARNING SYSTEMS-BASED PRACTICE IN STUDENT-RUN CLINICS Cindy $\mathrm{Lai}^{3}$; Leslie Sheu ${ }^{1}$; Bridget $\mathrm{O}^{\prime} \mathrm{Brien}^{2,3}$; Patricia S. O'Sullivan ${ }^{2,3}$; Austin Kwong ${ }^{4}{ }^{1}$ University of California, San Francisco, San Francisco, CA; ${ }^{2}$ University of California, San Francisco, San Francisco, CA; ${ }^{3}$ University of California, San Francisco, San Francisco, CA ${ }^{4}$ University of California, Berkeley, Berkeley, CA. (Control ID \#1333952)

NEEDS AND OBJECTIVES: In undergraduate medical education (UGME), the competency of systems-based practice (SBP) is often considered the most difficult to teach and assess, particularly in preclerkship years. Student-run clinics (SRCs), most of which provide care for the underserved and have limited operating budgets, exist at many medical schools and offer potential opportunities for pre-clerkship students to gain meaningful exposure to SBP. To explore ways of linking SRCs with our school's formal curriculum and competency-based assessment system, we identified domains of SBP in students' SRC experiences and mapped them to specific SBP milestones.

SETTING AND PARTICIPANTS: Four SRCs exist at our institution Each SRC provides a variety of services to distinct underserved communities in San Francisco. Over $70 \%$ of first-year medical students voluntarily participate in one or more SRCs, and approximately $20 \%$ of second years volunteer as SRC coordinators.

DESCRIPTION: We interviewed a convenience sample of current and past student coordinators and faculty advisors for our initial evaluation. Interviewees responded to a semi-structured set of questions on noncoordinator and coordinator roles, clinic operations, and personal reflections. Through an iterative consensus building process, we generated a list of themes on student engagement in SBP.

EVALUATION: Thirteen student coordinators and 4 faculty advisors participated. Preliminary thematic analysis revealed five major domains of SBP common to all coordinators' SRC experiences: interdisciplinary patient care (roles and collaboration), resource acquisition and allocation, navigation of the healthcare system (access to care, community partnerships, insurance, referrals, follow-up processes), clinic organization (leadership and faculty roles, training of volunteers, clinic flow), and quality improvement (patient care, clinic operations, volunteer satisfaction). When asked whether SBP experiences in SRCs differed from SBP education in the formal curriculum, students and faculty unanimously agreed that engaging in SBP in SRCs significantly enhanced what they learned in the formal curriculum. When asked about their depth of understanding of SBP compared to non-coordinator volunteers, most coordinators and faculty felt that they had a deeper understanding of SBP, but expressed mixed opinions as to whether this was because of specific coordinator roles, or increased frequency of volunteering which allowed greater exposure to systems issues.

DISCUSSION / REFLECTION / LESSONS LEARNED: SRC coordinators at our institution have substantial exposure to and participate actively in SBP. Domains that emerged from our analysis paralleled those postulated in a previous article, with two key differences: 1. "clinic operations' was not outlined previously, and 2. 'quality improvement' better captures our participants' experiences than 'quality care,' which focuses primarily on individual patient outcomes. Our findings provide insight into developmentally appropriate SBP milestones for UGME, and will help guide assessment plans for student achievement of these milestones. One limitation of our preliminary analysis is that student interviewees were coordinators, and thus may not reflect experiences of non-coordinator volunteers. The next phase will assess whether intensity of participation impacts the degree to which students learn about and engage in SBP through interviews with non-coordinator volunteers.

MAKING THE MOST OF 1/3 AMBULATORY Kerri L. Palamara; Hasan Bazari; Eva Chittenden; Tanya Milosh; Beverly M. Biller Massachusetts General Hospital, Boston, MA. (Control ID \#1310405)

NEEDS AND OBJECTIVES: : In exploring three core ACGME requirements (1) that all residents must have exposure to and demonstrate the ability to manage patients in each of the medical subspecialties, (2) the program must identify a Subspecialty Education Coordinator (SEC) in each subspecialty, (3) there must be a minimum of $1 / 3$ ambulatory time, the program sought to use this opportunity to improve outpatient subspecialty experiences. Through program evaluation and resident feedback, we identified variability in our existing subspecialty ambulatory experiences and a perceived need to increase the academic rigor of these rotations to ensure that residents achieved the appropriate level of competence related to each subspecialty by the end of their training. Therefore, the residency program set a goal of creating subspecialty rotations with uniform standards and expectations that promoted a high level of rigor and learning. A secondary aim was to create a community 
of subspecialty educators who could share best practices, problem solve around challenges, and be academically productive as a group.

SETTING AND PARTICIPANTS: The setting is the MGH Department of Medicine and its subspecialty divisions. Participants include Residency Program Administration, SECs, Subspecialty Faculty and MGH Housestaff.

DESCRIPTION: MGH restructured its ambulatory training paradigm with the specific aim of making outpatient rotations as rigorous as inpatient rotations. The elements of a rigorous rotation were defined and a Subspecialty Education Task Force formed to develop goals, strategies and an infrastructure to support high quality education in the ambulatory subspecialty setting. SECs were appointed, funded, and assumed responsibility for the development and coordination of 2-week ambulatory rotations, overseeing the overall curriculum in their subspecialty, and for mentoring residents interested in their field. SECs met monthly and agreed on mandatory rotation components. Rotation goals and objectives, schedules, curricula and readings are posted in standardized format on the residency intranet site. The SEC committee collaborated to design standardized rotation evaluation forms, patient logs, and resident evaluation forms to be completed by faculty. At the end of the first year, each SEC met individually with the co-chairs of the SEC Committee to review resident feedback and to plan improvements based on the feedback.

EVALUATION: Based on end of year and rotation evaluations, resident satisfaction with their ambulatory experiences increased with the above described innovation. Resident attendance during ambulatory rotations significantly improved with clarification of rotation objectives and expectations and with increased involvement and presence of the SECs. The secondary goal of the Subspecialty Education Task Force to develop a faculty community was also achieved through monthly meetings to collaborate and share common challenges.

DISCUSSION / REFLECTION / LESSONS LEARNED: The model of a community of SECs working closely together with the Program Director to design, implement and evaluate rigorous outpatient rotations is likely of interest to many medicine programs struggling to create meaningful outpatient experiences. This educational model is improving the quality of outpatient education and can be applied to community and university institutions alike. Next steps will include developing or adopting online curriculum modules and offering faculty development opportunities for the ambulatory subspecialty faculty.

\section{MEDICINE, POLICY AND PUBLIC LEADERSHIP: THE NEXT PARADIGM FOR PRIMARY CARE EDUCATION? Ali Khan ${ }^{2,1}$; Theodore Long ${ }^{2,1}$; Rebecca Brienza ${ }^{1,2}$. ${ }^{1}$ VA Connecticut Healthcare System, West Haven, CT; ${ }^{2}$ Yale University School of Medicine, New Haven, CT. (Control ID \#1338487)}

NEEDS AND OBJECTIVES: Increased attention has been placed in recent years on the need for greater integration of health policy and teambased improvement in medical education. Inclusion of training in health policy and leadership in medical education, however, remains infrequent. Existing curricula overwhelmingly emphasize either the international or private sector, thereby neglecting trainees' potential for leadership in public service. As part of the VACHS Center of Excellence $(\mathrm{CoE})$ in Primary Care Education, we have developed an innovative curriculum focusing on health policy and public leadership. Our curriculum emphasizes grounding in translational health policy - with a focus on the skill set necessary to succeed as agents of change. Objectives: 1) to teach trainees the core concepts, history of and current trends in domestic health policy, health economics, health care delivery systems and health law; 2) to teach trainees the core skills, principles and applications of public leadership, organizational strategy, political analysis, media relations, advocacy and community organizing while promoting application of these concepts in everyday practice; 3) to determine if longitudinal retention of content and skills can be enhanced through a mix of didactic and experiential components.

SETTING AND PARTICIPANTS: Internal medicine residents and nurse practitioner fellows $(n=9)$ participating in the VACHS Center of Excellence in Primary Care Education.
DESCRIPTION: Our curriculum, (MedLEAD: Medical Leadership, Economics And Domestic Policy) is learner-designed and taught by senior internal medicine residents. The program focuses on domestic health policy, leadership and public sector engagement. It is structured around three primary domains: Diagnosis, Prescription for Change, and Public Leadership. The MedLEAD curriculum is based on four guiding principles: scalability, portability, return on investment maximization and value creation. The curriculum minimizes diversion of faculty FTEs, integrates didactics, case-based learning, simulation, audience response and other adult learning techniques.

EVALUATION: To date, the model has been piloted on the first group of trainees $(n=9)$ participating in the VACHS CoE in Primary Care Education. We are evaluating the impact of MedLEAD training on trainee knowledge and attitudes. Preliminary data indicate that trainees: 1) have greater knowledge of core health policy and leadership concepts; 2) feel more comfortable with application of those concepts in clinical practice; 3) feel the learner-driven model offers greater engagement with course material; 4) have greater interest in effecting cultural, structural or policy change.

DISCUSSION / REFLECTION / LESSONS LEARNED: We believe MedLEAD's public sector focus, learner-driven design and implementation and inter-professional application represents an innovative curricular model in medical education. The survey instrument includes assessment of confidence in newly acquired knowledge - and, uniquely, the application of those concepts in everyday practice. Given that existing health policy/ leadership curricula overwhelmingly emphasize international and/or private sector action, MedLEAD's focus on domestic public sector engagement represents a portable and scalable model for training in medicine and public service. Future work will explore how best to 1) measure application of core policy and leadership content; 2) how best to ensure curricular sustainability in a learner-driven model.

MUTUAL LEARNING THROUGH COMMUNITY COLLABORATION Joslyn Fisher; Cara Foldes; Achilia Morrow. Baylor College of Medicine, Houston, TX. (Control ID \#1340114)

NEEDS AND OBJECTIVES: Although physicians often are called upon to participate in community activities such as health education presentations to local community organizations, most medical school and residency curricula do not emphasize the skills needed to facilitate responsible community involvement for our future physician leaders. Upon completion of the Internal Medicine Women's Health Elective, internal medicine residents will (1) demonstrate the ability to design and implement a health presentation for a lay community audience and (2) develop increased confidence in speaking to the public (community). A separate, yet related objective for the curriculum anticipates that community members will find the presentations to be beneficial.

SETTING AND PARTICIPANTS: As a component of a women's health elective, one internal medicine resident each month gives an oral presentation to women living in a shelter for survivors of domestic violence.

DESCRIPTION: Each month, one senior level internal medicine resident rotates on the women's health elective, during which, the resident visits various relevant clinics at our public hospital. The residents have time built in the month-long schedule to develop a short 20-30 minute oral (non-electronic) presentation on a women's health topic of their choice. The presentation outline is reviewed for content, accuracy, and interactivity by the elective faculty prior to actual implementation. The resident presents his or her discussion at the end of the month at the women's shelter and allows time for the shelter clients to ask questions.

EVALUATION: Quantitative and qualitative assessments are obtained from medicine residents. Verbal feedback is sought from shelter clients via the shelter director. Approximately 15 medicine residents have completed the rotation since the initiation of the collaboration. Between five and twenty different shelter clients attend each month's resident-led health presentation. Evaluations indicate that (1) shelter clients' and residents' enthusiasm for the program is high and (2) medicine residents gain confidence in their ability to present publicly. While over half of the 
residents responding to course evaluation are not planning on practicing outpatient primary care (nor will they specialize in women's health), they all felt it was important or very important for physicians to give health presentations to the community. All residents (37\%) who had never given a previous presentation to a lay audience gained confidence in their ability to do a presentation in public. Consistently, the medicine residents noted that prior to the presentation, they felt "nervous" and uncertain of what to expect. After the presentation, the residents noted positive feelings and made comments about the experience, such as "really rewarding" and felt "it really made a difference." Shelter clients found the presentations to be informative

DISCUSSION / REFLECTION / LESSONS LEARNED: To ensure the sustainability of this community collaboration, the presentation sessions must be built into the residents' schedule. The medicine residents recommend making the presentations simple, involving the audience, and focusing on practical aspects of health and healthcare. This community-oriented program is a model for resident advocacy that can be replicated at relatively low cost and high yield at other medical schools and residency programs.

NEW LIFESTYLE: A WEB-BASED TEACHING AND LEARNING PROGRAM FOR NUTRITION, EXERCISE, AND WEIGHT MANAGEMENT David P. Miller ${ }^{1}$; Sonia Crandall ${ }^{2}$; Mara Z. Vitolins ${ }^{3}$; Stephen Davis ${ }^{2}$; Donna Kronner ${ }^{4}$; Edward $\mathrm{Ip}^{4}$; Karen Vaden ${ }^{2}$; John Spangler ${ }^{2} .{ }^{1}$ Wake Forest School of Medicine, Winston-Salem, NC; ${ }^{2}$ Wake Forest School of Medicine, Winston-Salem, NC; ${ }^{3}$ Wake Forest School of Medicine, Winston-Salem, NC; ${ }^{4}$ Wake Forest School of Medicine, WinstonSalem, NC. (Control ID \#1337442)

NEEDS AND OBJECTIVES: Obesity is the second leading cause of preventable death in the US. Guidelines from the Association of American Medical Colleges urge medical schools to develop curricula on obesity, including its epidemiology, harms, and treatment.

SETTING AND PARTICIPANTS: A team of interdisciplinary faculty at Wake Forest School of Medicine (WFSM) developed NEW Lifestyle (Nutrition, Exercise and Weight management), a comprehensive web-based program to educate all medical students about weight management issues. More detailed learning objectives may be viewed at the curriculum's website (listed below) which is available for use online at no cost.

DESCRIPTION: Funded by grants from the National Cancer Institute, NEW Lifestyle consists of 8 self-contained web-based educational modules, all of which have pre- and post-quizzes. The modules cover obesity related topics including: 1) Epidemiology, 2) Cancer Risk, 3) Adverse Health Effects, 4) Energy Balance, 5) Factors Affecting BMI, 6) Counseling Skills, 7) Bias and Stigmatization, and 8) Obesity Treatment Guidelines. Unique to this program are its emphasis on confronting anti-obesity bias among providers, training medical students in culturally sensitive weight management counseling, and emphasizing the connection between obesity, inactivity, and cancer risk. Each module requires 10-15 minutes to complete. The web site resides in the public domain, allowing learners to complete the modules anytime. At WFSM, the modules were embedded into the basic science and clinical clerkship core curricula beginning with the Class of 2012 .

EVALUATION: The WFSM Class of $2012(n=116)$ was the first class to receive the full curriculum. Across all modules, average pre-quiz scores ranged from $47 \%-80 \%$. Overall, the percent correct increased on the post-quizzes by a mean of $28 \%$ (range $5.4 \%-43 \%, \mathrm{p}<0.01$ for all comparisons). After the curriculum was completed, all students were evaluated on an encounter with an obese standardized patient (SP) who complained of inability to lose weight. Students were not aware they were being evaluated on their obesity management skills. We compared the performance on the SP encounter for the Class of 2012 (full curriculum) to the Class of 2011 (no curriculum). Students who received the curriculum more often took a dieting history ( $85 \%$ vs. $53 \%, p<0.0001$ ), asked about normal eating habits ( $87 \%$ vs. $78 \%$, $\mathrm{p}=0.08)$, suggested an exercise plan ( $91 \%$ vs. $83 \%, \mathrm{p}=0.08)$, advised patients not to skip meals $(84 \%$ vs. $70 \%, p=0.01)$, and expressed empathy about weight struggles $(89 \%$ vs. $80 \%, p=0.07)$. In contrast, students who did not receive the curriculum were more likely to investigate medical issues, e.g., hypothyroidism ( $45 \%$ vs. $14 \%, \mathrm{p}<0.0001)$, sleep disturbances or snoring $(15 \%$ vs. $3 \%, p<0.01)$, and family history of weight disorders (35\% vs $16 \%$, $\mathrm{p}<0.001)$. Non-curriculum students also were more likely to stress the need for medical follow-up ( $56 \%$ vs. $18 \%, \mathrm{p}<0.0001)$, and obesity's negative health effects $(62 \%$ vs. $21 \%, \mathrm{p}<0.0001)$.

DISCUSSION / REFLECTION / LESSONS LEARNED: Focus groups with students provided essential input for designing the web modules to match students' learning styles. Courses that offered extra credit for module completion had higher participation rates $(80 \%)$ than those in which the modules were less emphasized (43\%). Therefore, we suggest requiring students to email their post-test scores to course directors. Overall, the webbased program enhanced students' obesity management skills, making NEW Lifestyle a valuable addition to medical school's curricula.

ONLINE RESOURCE URL (OPTIONAL): www.NEWLifestyle.org

PILOT OF A RESIDENT CURRICULUM IN TEAM-BASED CARE AND PANEL MANAGEMENT Nivedita Ghosh ${ }^{2}$; Jessica L. O'Brien ${ }^{1}$; Charles A. Morris ${ }^{2}$; Lori Tishler ${ }^{2}$; Rebecca J. Cunningham ${ }^{2} .{ }^{1}$ Harvard Medical School, Boston, MA; ${ }^{2}$ Brigham and Women's Hospital, Boston, MA. (Control ID \#1334880)

NEEDS AND OBJECTIVES: Increasingly, primary care practices are turning to team-based models of care, such as the patient centered medical home (PCMH), to meet patient needs and performance targets. Most medical resident clinics, however, function in more traditional models of primary care, leaving trainees ill-prepared for modern practice. To address this training gap, we piloted a curriculum that aimed to: 1. Expose trainees to new and evolving care delivery models. 2 . Increase trainees' knowledge of the skills and scope of practice of ambulatory care team members. 3 . Teach trainees social work, nutrition, and clinical pharmacy pearls to use in their own practices. 4. Introduce the concept of panel management and provide each resident with personal panel data to encourage populationlevel thinking and problem-solving.

SETTING AND PARTICIPANTS: Junior internal medicine residents $(\mathrm{n}=18)$ rotated through a two-week ambulatory block, spending time at Brigham and Women's Advanced Primary Care Associates, a new PCMH practice.

DESCRIPTION: Through group workshops and direct patient care experiences, key non-physician health professionals educated residents about their scope of practice, role in the care team, and unique skills. In addition to group didactics and take-home exercises, residents spent one half-day each with a clinical social worker, clinical pharmacist, and nutritionist, who taught and modeled skills that residents can implement in their own practices. Finally, residents were provided diabetic and hypertension quality metrics from their own patient panels and were expected to devise a plan to systematically assist patients not meeting treatment goals. Development of this plan necessitated that residents learn how to better utilize resources within their own clinics and begin to conceptualize patient care beyond the individual. Rotation feedback was regularly elicited through quantitative and qualitative surveys. Overall, residents felt that the group workshops and individual sessions with nonphysician health care workers were valuable. The most common suggestions for curriculum improvement were to schedule more patient visits during the half-day sessions and include more panel data.

EVALUATION: To measure the educational impact of the pilot rotation, a 26-item survey was developed to assess changes in residents' self-reported knowledge and skills on aspects of primary care. Questions used a 5-point Likert scale to indicate the extent to which residents agreed or disagreed with statements. Comparing responses to the pre- and post-rotation survey, there were statistically significant increases in residents' self-reported knowledge of new models of care, panel management concepts, and the roles and skills of social workers, pharmacists, and nutritionists $(\mathrm{p}<.001)$. Of note, all differences were greater than 1.3 points on the scale. A similar increase was reported for confidence in responding to domestic violence and using diabetic equipment $(\mathrm{p}<.02)$; there was also an increase in comfort in dietary counseling and engaging patients in their own healthcare. However, residents reported no significant difference in their confidence in implementing systems-based solutions $(p=.07)$.

DISCUSSION / REFLECTION / LESSONS LEARNED: A brief, easily adaptable curriculum had a significant impact on resident knowledge 
and attitudes towards interdisciplinary care and shows promise for higher utilization of health care teams. Having multiple clinic sites remains a challenge to providing uniform panel data and information on local resources.

PRIMARY CARE REDESIGN - A NOVEL RESIDENCY ELECTIVE Carol K. Bates; Kelly Graham; Bruce E. Landon. Beth Israel Deaconess Medical Center, Boston, MA. (Control ID \#1334522)

NEEDS AND OBJECTIVES: Most resident continuity practices are not patient-centered medical homes. We developed a two-week elective rotation to immerse residents into PCMH concepts with the goal of encouraging residents to consider primary care careers by exposing them to opportunities in primary care innovation and leadership.

SETTING AND PARTICIPANTS: The elective was developed for Internal Medicine Residents at Beth Israel Deaconess Medical Center, in Boston, MA. Participants were selected based on expressed interest in an annual survey used by the residency program to assign elective rotations. Three groups of 4-6 residents have completed the elective during academic years 2010-2011 and 2011-2012, 12 of 16 were in the Primary Care Track. Residents visited multiple innovative practice settings in the Boston metropolitan area, and completed an innovation project at HealthCare Associates, our hospital-based primary care practice site.

DESCRIPTION: The course includes background reading, meetings with local leaders in primary care redesign, and exposure to a group visit. In addition, each group of residents completes a practice redesign project focused upon improving a specific aspect of care delivery or the resident experience in Healthcare Associates (HCA), our hospital-based primary care practice, and presents their plan to practice and hospital leadership as the culminating activity of the elective. Resident schedules are weighted with visits and meetings in week one and are relatively unscheduled in week two to allow for group project work. All residents visited core practice sites; individual groups met with additional resources based upon their projects. The three projects to date included a feasibility analysis of providing servies on Saturdays, a work plan for a new administrative support position for residents, and an analysis of the highest cost patients covered under a risk contract.

EVALUATION: Feedback has been obtained through an anonymous webbased survey. Ten of 16 residents were probably or definitely planning careers in primary care both before and after the elective, though $87 \%$ did feel that the elective experience increased their interest in primary care practice. Approximately $2 / 3$ of residents thought they would have a role in practice management in their futures both before and after the elective. The degree to which residents agreed that new approaches to care delivery would improve physician work life did not change significantly. After the elective, residents felt better prepared for practicing cost effective medicine, participating in quality improvement activities, population management, and practicing in managed care settings. Residents reported their knowledge as improved in the realms of financial management, physician compensation, productivity measures, customer service, national healthcare organization, and management of our practice. $80 \%$ would definitely recommend this elective to others.

DISCUSSION / REFLECTION / LESSONS LEARNED: Feedback has been obtained through an anonymous web-based survey. Ten of 16 residents were probably or definitely planning careers in primary care both before and after the elective, though $87 \%$ did feel that the elective experience increased their interest in primary care practice. Approximately $2 / 3$ of residents thought they would have a role in practice management in their futures both before and after the elective. The degree to which residents agreed that new approaches to care delivery would improve physician work life did not change significantly. After the elective, residents felt better prepared for practicing cost effective medicine, participating in quality improvement activities, population management, and practicing in managed care settings. Residents reported their knowledge as improved in the realms of financial management, physician compensation, productivity measures, customer service, national healthcare organization, and management of our practice. $80 \%$ would definitely recommend this elective to others.
SENIOR PRECEPTORSHIP IN CLINICAL TEACHING: A LONGITUDINAL EXPERIENTIAL AND DIDACTIC TEACHING IMPROVEMENT PROGRAM FOR MEDICAL STUDENTS William Kormos $^{1,3}$; Pamela Vohra-Khullar ${ }^{2,3}$. ${ }^{1}$ Massachusetts General Hospital, Boston, MA; ${ }^{2}$ Beth Israel Deaconess Medical Center, Boston, MA; ${ }^{3}$ Harvard Medical School, Boston, MA. (Control ID \#1339985)

NEEDS AND OBJECTIVES: Many training programs recognize the importance of training residents to be more effective teachers. However, fourth-year students are eager to learn these skills in anticipation of residency, and possess the knowledge and skills to assist more junior students. Although fourth year students are often used as teaching assistants in the basic science curriculum, and electives may be offered in clinical teaching skills, few medical schools have courses that combine the pedagogy of clinical teaching with a longitudinal experience in clinical teaching. The objectives of this course are: 1) To develop an approach to clinical teaching 2) To improve physical examination skills by teaching others 3) To understand future teaching roles in medicine.

SETTING AND PARTICIPANTS: This program is an elective rotation for fourth-year medical students, who serve as peer tutors to second year students over a six month period. Tutors are assigned to a hospital site affiliated with the second year physical examination course, and meet with second year students during afternoon sessions. This experiential learning is combined with a nine session curriculum in clinical teaching skills.

DESCRIPTION: The Senior Preceptorship in Clinical Teaching enrolls 40-60 4th year students (tutors) each year. The longitudinal design of the course allows tutors to gain experience in clinical teaching and reflect on their own performance. Nine two-hour workshops are provided throughout the course. The initial sessions focus on clinical teaching basics: adult learning theory, feedback, use of questions, and methods for teaching psychomotor skills. The middle sessions focus on physical exam skills, and the final sessions provide opportunities to apply lessons learned to teaching during residency and beyond. These sessions rely on active learning through role playing, small group teaching, and peer feedback. The experiential part of the course allows students to gain personal teaching experience and to observe faculty preceptors. Tutors provide direct observation and feedback to 2nd year students, participate in small group teaching, and serve as OSCE faculty.

EVALUATION: Pre- and post-course surveys were electronically administered to tutors. Assessment data is provided with the most recent two years available $(\mathrm{n}=69)$. Tutors valued the course highly $(4.51$ on a 5 point scale), and $92 \%$ agreed that they would take the course again if offered the opportunity. $87 \%$ of 4 th year students reported the course increased their comfort as teachers. Students reported a significant increase $(p<0.01)$ in confidence in giving feedback ( 3.83 vs. 4.19$)$ and bedside teaching(3.31 vs. 4.04). Tutors valued the opportunity to mentor second year students and to review the physical exam. They identified scheduling conflicts with residency interviews and other rotations as the top barrier to effectively integrating into the course.

DISCUSSION / REFLECTION / LESSONS LEARNED: Our course provides 4th year medical students with a meaningful experience in clinical teaching coupled with didactic instruction. Students are eager to learn about effective teaching strategies and have a wealth of learning experience on which to reflect. Fourth year students are an invaluable part of our physical examination course, and provide a role that complements the teaching faculty. Challenges focus on time constraints due to clinical responsibilities of fourth year students. In addition, the large number of hospital sites and locations used by the tutors makes direct observation of their teaching difficult.

SHOW ME THE MONEY: IMPLEMENTATION OF A UNIQUE COST AWARENESS CURRICULUM FOR MEDICAL RESIDENTS Christopher Moriates; Krishan Soni; Andrew Lai. University of California, San Francisco (UCSF), San Francisco, CA. (Control ID \#1318294)

NEEDS AND OBJECTIVES: Approximately \$700B of annual healthcare spending is wasted, with physicians directly influencing $87 \%$ of this expenditure. Medical training has emphasized quality improvement but 
few programs are addressing the ACGME requirement that physicians-intraining now "incorporate considerations of cost awareness" into practice. Our objective was to develop an inpatient curriculum to promote cost awareness, highlight current clinical guidelines, improve physician attitudes towards cost control, and cultivate more cost-effective physician ordering behaviors.

SETTING AND PARTICIPANTS: Medical students, internal medicine residents, and attending physicians at a university-based academic medical center.

DESCRIPTION: We first reviewed the literature on strategies addressing discrepancies between physicians' desired and actual knowledge of health care costs. The most valued educational strategy for residents has been case-based conferences. We selected 12 "core topics" of commonly encountered internal medicine clinical scenarios with frequent practice and resource-utilization variability, including chest pain, syncope, pulmonary embolism, and low back pain. We created a longitudinal curriculum involving all PGY1 residents, as well as a monthly case-based conference for on-service medical students, residents, and attendings. Each month five PGY1 residents participated in a one-hour introductory cost awareness session during a quality improvement rotation. We provided them with background reading and an anonymous, itemized hospital statement for one of the "core" diagnoses. The learners were divided into two groups: one reviewed evidence-based guidelines and the other evaluated common practices and relevant charges. We reconvened these groups for a facilitated session to present and integrate their findings. This portion of the curriculum involved two one-hour sessions and less than five hours of independent work for the PGY1 residents. We then prepared a casebased noon conference from these collective lessons. During this conference, we reviewed a specific case and underscored appropriate, evidence-based, cost-effective care.

EVALUATION: Our early experience with this curriculum, during the 2011-12 academic year, has been highly positive. We received 116 evaluations from six conferences involving medical students $(n=38)$, residents $(n=65)$ and attendings $(n=13)$. Respondents reported that the conferences were highly relevant to their clinical practices (mean of 4.47 $+/-0.65$ on a 5-point Likert scale) and that they were likely to change their ordering behaviors based on the conferences (mean of $4.19+/-0.72$ ).

DISCUSSION / REFLECTION / LESSONS LEARNED: A residentled educational innovation involving a monthly PGY1 curriculum, with active preparation of a facilitated, case-based conference emphasizing evidence-based and cost-effective medical practices, can be well-received, highly relevant, and likely to change ordering behaviors of a diverse internal medicine audience. The time and resources required to implement this curriculum are relatively minimal, making this paradigm sustainable and adaptable to other institutions.

SPEED DATING AS AN INNOVATIVE METHOD FOR HELPING MEDICAL STUDENTS LEARN ABOUT INTERNAL MEDICINE TRAINING AND CAREERS Jennifer Adams; Nina Yeboah; Kathleen Hanley; Sondra Zabar; Jennifer Gillman; Kathryn Jors; Ross Mccormack; Z-Hye Lee; Colleen Gillespie. NYU School of Medicine, New York, NY. (Control ID \#1339777)

NEEDS AND OBJECTIVES: Despite an increasing need for physicians trained in Internal Medicine (IM), the number of medical students entering residencies in IM has declined. Misconceptions about careers in IM, pay differentials between disciplines, student debt and work hours are thought to contribute to this decline. We developed an "IM Speed Dating Event" to increase first year medical student's awareness of the breadth and richness of IM training and careers.

SETTING AND PARTICIPANTS: Faculty members from each Division within the Department of Medicine at our institution were asked to participate to emphasize the diversity of careers paths after IM training. Medical Students were recruited via email, flyers and word-of-mouth. Over 3 years of the event (2009-2011), 51 medical students participated (14-19/year)

DESCRIPTION: This "speed dating" event was structured so that students rotated, in timed, five-minute blocks, speaking to a total of 10 faculty. Faculty members were organized to optimize diversity of disciplines to which students were exposed. Students asked questions about faculty members' career and training paths, current roles/responsibilities, work life, and work/life balance. The event was very informal, easy to set up and organize, and the speed dating format encouraged friendly, compelling and direct, but brief, discussions.

EVALUATION: All 51 participants $(n=18$ in 2009, 19 in 2010, and 14 in 2011), completed a pre-event anonymous assessment of their attitudes toward and understanding of IM residency and career pathways and practices as well as their specialty and career intentions. After the event, 47 completed an evaluation of the "Speed Dating" event including listing 3 things they learned and the degree to which the event led them to become more interested in exploring IM. Pre-event assessment results suggest that medical students are quite unsure about IM careers (e.g., 45\% reported being not sure whether faculty within IM Departments have all done IM residencies and $58 \%$ reported being not confident at their specialty choice) After the Speed Dating Event, 85\% strongly agreed that they would recommend this event to future students. $64 \%$ strongly and 30\% somewhat agreed that this event made them more interested in exploring IM. Students' reports of what they learned fell into 5 broad themes: the breadth of IM; the diversity of career pathways; the work lives associated with different specialties/physician roles; program requirements; and a better sense of the multiple roles physicians fulfill.

DISCUSSION / REFLECTION / LESSONS LEARNED: With the ever decreasing supply of internists, it is imperative that medical schools expose their students to the field of IM early in their education. This innovative approach provided a fun, educational event that exposed students to a variety of IM faculty members. It is clear from our survey that students have minimal understanding of IM training and early exposure to the field is necessary for them to understand the breadth and richness of the field. Future events can better target specific segments of IM and be provided later in training to give further guidance to students considering a career in IM. Additional research should investigate whether events such as this actually influence students' career choice.

STRUCTURED PEER OBSERVATION AND FEEDBACK TO OPTIMIZE ATTENDING TEACHING Somnath Mookherjee; Bradley Monash; Bradley A. Sharpe. UCSF, San Francisco, CA. (Control ID \#1338881)

NEEDS AND OBJECTIVES: Much of the clinical teaching in medical education occurs in the inpatient setting, yet ward attendings rarely receive structured feedback on their teaching. Attending feedback is generally limited to end-rotation performance evaluation by learners, rather than formative assessment of teaching effectiveness. Therefore, we sought to develop an educational program that is based on peer observation and feedback (allowing both observers and teachers to benefit); uses structured feedback anchored in validated and observable measures; and includes evaluation of actual teaching practices with longitudinal reassessment.

SETTING AND PARTICIPANTS: All internal medicine ward attendings at a single institution were eligible to participate if they ward attended at least twice during the academic year. Learners present during teaching rounds were asked to participate

DESCRIPTION: We derived a Structured Peer Feedback Tool (SPFT-10) from the validated Stanford Faculty Development Program (SFDP) framework. From the list of 25 effective teaching behaviors in the SFDP teaching assessment tool, we selected the 10 items felt to be most easily observable and salient for effective attending teaching rounds. In a 2-hour session, participants watched videos of teaching, learned to identify the 10 selected behaviors, developed constructive and reinforcing comments, and practiced giving peer feedback. For actual teaching observation, participants were paired into feedback "dyads" in which each observes the teaching rounds of the other twice over the year. Learners present at the teaching are asked to rate overall teaching effectiveness on a scale of 1 to 5 ( $1=$ very poor, $5=$ excellent). We hypothesize that attending teaching performance will improve as measured by improvement in SPFT-10 scores, learner ratings, and retrospective self-assessment. At year-end, we 
will also assess attending reaction to the program, attitudes towards peer observation, and knowledge of key teaching behaviors.

EVALUATION: Of 37 eligible ward attendings, 22 enrolled (59\%), averaging 2.2 years $(+/-2.1$ years SD) experience. Half-way through the academic year, 16 hospitalists $(73 \%$ of enrollees) have participated in a total 13 observations. The average SPFT-10 score is $7.69(+/-2.0 \mathrm{SD})$ out of 10 . The 3 items least likely to be present are, "motivated learners to learn on their own" (46\%), "called attention to time" (54\%) and "stated goals clearly and concisely" (69\%). Representative written constructive comments included encouragement to speak more clearly, use the names of learners, make session goals clear, and to pay attention to time. Twentynine learners have evaluated speakers, with an average overall teaching efficacy score of $4.76(+/-0.44 \mathrm{SD})$.

DISCUSSION / REFLECTION / LESSONS LEARNED: We have demonstrated the feasibility of a peer observation and feedback program to improve attending teaching. Attendings can successfully observe for behaviors known to contribute to effective teaching, and provide structured feedback to each other. Common deficiencies have been identified; these behaviors will be addressed in a mid-year "refresher" didactic session. While scores from peers and learners are high, there is room for improvement. Further assessment at year-end will evaluate the efficacy of the program in improving attending teaching, overall reaction to the program, and attitudes about peer observation.

SWIMMING IN THE MURKY WATERS OF SOCIAL MEDIA - DON'T LET YOUR WHITE COAT GET DIRTY: A WORKSHOP FOR MEDICAL STUDENTS. J. H. Isaacson ${ }^{2,1}$; Bryan A. Sisk ${ }^{1}$; Ehsan H. Balagamwala $^{1}$; Ilka Decker ${ }^{1}$ Jason $\mathrm{Ho}^{1}$; A my S. Nowacki ${ }^{1}$; Neil Mehta ${ }^{2,1}$. ${ }^{1}$ Cleveland Clinic, Cleveland, $\mathrm{OH} ;{ }^{2}$ Cleveland Clinic, Cleveland, $\mathrm{OH}$ (Control ID \#1339475)

NEEDS AND OBJECTIVES: Increasing popularity of social media (SoMe) has raised many controversies regarding professionalism. Lapses in online professionalism can denigrate the physician/patient relationship and mar the image of the profession. Professionalism in SoMe is highly complex and current guidelines leave many uncertainties. We identified four especially controversial areas regarding the use of SoMe by medical professionals. Our aims were a) to introduce students to this vital discussion through the lens of a recent controversy, and b) to determine whether students could reach a consensus regarding these controversial areas.

SETTING AND PARTICIPANTS: We designed and implemented a workshop for third year students at the Cleveland Clinic Lerner College of Medicine to initiate a discussion about SoMe.

DESCRIPTION: This two-hour workshop introduced students to various SoMe applications (e.g. Facebook, Twitter and blogs) with a demonstration of practical applications of SoMe for learning and building a professional identity. We used an actual Twitter post that had led to a lively debate in the online community. The specific case involved one physician publicly rebuking another physician for an anonymous Tweet of patient information in an inappropriate context. Students were subsequently divided into small groups, with each group discussing one of four questions. The workshop concluded with a large group discussion and a conference call with the rebuking physician involved in the controversy.

EVALUATION: Students were organized in small groups with each group discussing one the controversial areas. The discussion of each group was recorded and summarized and presented to the large group for further discussion. 1) Should physicians ever post about patients in SoMe? This group demonstrated a spectrum of opinions; however, students agreed that appropriateness is influenced by the purpose, venue, content and context of the posting. 2) Is it okay to post anonymously in SoMe? The consensus was that "anonymous" posting is a fallacy; it is not possible to truly remain anonymous on today's internet. Thus, authors should not post anything anonymously that they would be ashamed of posting non-anonymously. A subset of students described a sense of security when writing anonymously about personal issues; they maintained that posting anonymously could still be carried out in a responsible manner. 3) Does concern for professionalism extend beyond HIPAA compliance? Students concluded that the level of professionalism in SoMe should exceed that which is expected in other forms of communication, due to the ease with which information can spread once posted on the internet. 4) Did the rebuking physician in this specific controversy do the right thing? Students appreciated the rebuking physician's role in highlighting the controversy, but they regretted the negative tone that the online discussion took.

DISCUSSION / REFLECTION / LESSONS LEARNED: After completion of this workshop, students were unable to reach consensus on various professionalism and SoMe issues. Although professional organizations have published guidelines for the use of SoMe, our workshop with a real-life scenario showed that current guidelines insufficiently address the complexities of SoMe. A number of "gray areas" remain, which need further analysis. Workshops that allow students to review and discuss real cases of professionalism and SoMe will help to raise awareness of these complexities, thus serving to further the dialogue of how the medical profession should adapt to SoMe.

TEACHING QUALITY IMPROVEMENT TO RESIDENTS USING ABIM PRACTICE IMPROVEMENT MODULES: THE YALE PRIMARY CARE EXPERIENCE Daniel G. Tobin. Yale Primary Care Internal Medicine Residency Program, Waterbury, CT. (Control ID \#1336687)

NEEDS AND OBJECTIVES: The Accreditation Council for Graduate Medical Education (ACGME) requires residents to systematically analyze their practice using quality improvement methods, and implement changes with the goal of practice improvement. This curriculum intended to teach fundamental principles of quality improvement using lectures and validated audit tools developed by the American Board of Internal Medicine (ABIM). Secondarily, the project intended to expose residents to the ABIM's Maintenance of Certification Program.

SETTING AND PARTICIPANTS: The project was headquartered at the Chase Center where Yale Primary Care Medicine Residents have their continuity clinic practices. Participants included 27 second and third-year residents. 9 faculty and the clinical staff at the practice site also participated. Residents participated during their 3-month Ambulatory Block rotation and the project was implemented in phases over a 2 year period. Uniquely, residents were divided into longitudinal quality improvement teams, each responsible for a specific phase of the project as well as their own patient assessments.

DESCRIPTION: Participants were enrolled in the ABIM's Hypertension Practice Improvement Module (PIM). Hypertension was chosen as a common and easily measurable condition with data driven treatment recommendations. Residents participated during their 3-month Ambulatory rotations. Each Ambulatory Block team worked collaboratively to advance project design and implementation and each block began with a didactic series about medical error and the quality improvement process. During the first year, team 1 educated colleagues and patients about the project. Team 2 standardized and implemented the chart audit and patient survey process. Team 3 reevaluated progress to date and modified processes. Team 4 managed data entry and submitted aggregate data to the ABIM. Participants conducted chart audits and collected patient surveys for their own hypertensive patients; personal results were reviewed with their preceptor. In the second year, team 1 analyzed results and picked an improvement target (DASH diet counseling). Team 2 developed educational materials and an implementation protocol. Team 3 executed the DASH diet counseling plan, and team 4 performed a targeted re-measurement to see if counseling rates improved. At the conclusion of the project, the data was submitted to the ABIM and faculty earned both $\mathrm{CME}$ and MOC points for their participation.

EVALUATION: The project achieved it's primary and secondary objectives. Residents reported satisfaction and a deeper understanding quality improvement principles including rapid cycles of change and the importance of a reflective practice style. DASH diet counseling increased $235 \%$ over baseline. Time constraints, language barriers, and patient willingness to complete surveys were significant barriers.

DISCUSSION / REFLECTION / LESSONS LEARNED: Implementing an outpatient quality improvement curriculum using tools employed by the ABIM MOC program is feasible within a busy residency training program. 
Participants worked enthusiastically as teams and coordinated their efforts longitudinally. Data collection was hindered by time constraints, language barriers, and lack of patient willingness to complete surveys; response rates may be improved by offering patient incentives. Aggregate data analysis supplemented individual reflection. DASH diet counseling was grossly underutilized. Residents were completely unfamiliar with the ABIM's MOC process prior to completing the project.

TEACHING SELF-DIRECTED LEARNING: CAN THIS BE DONE? Maureen D. Willcox ${ }^{1}$; Michael F. O'Connor ${ }^{2}$; Jennifer Glick ${ }^{3}$; Patrick D. $\mathrm{O}^{\prime}$ Connor $^{4}$; Susan Glick ${ }^{5}$. ${ }^{1}$ Pritzker School of Medicine, Chicago, IL; ${ }^{2}$ University of Chicago, Chicago, IL; ${ }^{3}$ Washington University, St. Louis, MO; ${ }^{4}$ New Trier High School, Chicago, IL; ${ }^{5}$ University of Chicago, Chicago, IL. (Control ID \#1335087)

NEEDS AND OBJECTIVES: Self-directed learning is a requisite for lifelong learning. Third-year students are expected to be self-directed learners, selecting which content to study and the appropriate resources. Unfortunately, for most students, the educational experience in both college and the preclinical years is overwhelmingly teacher-directed, and thus students are often ill prepared for the transition to self-directed learning. How best to prepare medical students to become self-directed learners is unknown. The Foundations in Clinical Medicine (FICM) course is an immersive, 7-day experience intended to prepare rising third-year students for the clinical years. One aim of the course is to develop students' self-directed learning skills in preparation for the third-year clerkships and beyond.

SETTING AND PARTICIPANTS: 47 rising third-year students participated in this classroom-based course

DESCRIPTION: The FICM course consists of 7 distinct content areas, 3 of which focus on self-directed learning. For 2 of these content areas (Data Interpretation and Hypothesis-Driven History and Physical Examination), we created a series of structured paper and pencil exercises that required self-directed learning. Students were provided relevant resources (textbooks, original articles, Up-to-Date) and were encouraged to seek others. Faculty were present to answer questions but instructed not to initiate or lead discussion. For the other content area (FICM Laboratory), we created an unstructured setting for students to work individually or in groups to revisit content they had not yet mastered and to extend their understanding of the material. Faculty responded to students' questions, but did not initiate or lead discussion.

EVALUATION: In order to determine the effectiveness of our teaching methodology, self-directed learning was assessed on the first and last days of the course using a single instrument that combined two validated measures of self-directed learning: Abd-El-Fattah's Self-Directed Learning Aptitude Scale (SDLAS) and Lee's Self-Assessed Self-Directed Learning Ability (SASDLA). This project received IRB exemption. The response rate was $100 \%(n=47)$. Scores were calculated by assigning a point value to each answer $(5=$ strongly agree to $1=$ strongly disagree $)$, and then dividing the total number of points by the total number of questions answered. Use of the mean score instead of total score was necessary to correct for unanswered items. Student's t-test was utilized to compare the change in self-management, motivation, self-monitoring and the total score (Abd-El-Fattah's SDLAS) as well as the total score (Lee's SASDLA) before and after the course. Following the course, there was statistically significant improvement in the score for each subscale and for both total scores. For Abd-El-Fattah's SDLAS, scored on a 4-point Likert scale, the mean improvement in the total score was 0.127 (95\% CI 0.064-0.189, $\mathrm{p}<$ $0.001)$. The mean improvement in the self-management subscale was 0.126 (95\% CI $0.0201-0.232, \mathrm{p}=0.021)$, the motivation subscale $0.119(95 \% \mathrm{Cl}$ $0.0542-0.183, \mathrm{p}<0.001)$, and the self-monitoring subscale $0.139(0.0526$ $0.226, \mathrm{p}<0.001)$. For Lee's SASDLA, scored on a 5-point Likert scale, the mean improvement was 0.486 (95\% CI 0.310-0.663, p <0.001)

DISCUSSION / REFLECTION / LESSONS LEARNED: We cultivated self-directed learning in our students by immersing them in time-pressured problem-solving situations and providing them access to appropriate resource materials and faculty to keep them on-track. Self-directed learning can be taught to medical students.
TEACHING TEAMWORK: ENHANCING RESIDENT CONTINUITY TRAINING IN AN URBAN ACADEMIC CLINIC Reena Gupta; Ryan Laponis; Neda Ratanawongsa; Elizabeth Davis; Claire Horton. San Francisco General Hospital, University of California, San Francisco, San Francisco, CA. (Control ID \#1340466)

NEEDS AND OBJECTIVES: As primary care moves toward medical home models of care, residents increasingly need to be trained to deliver effective patient-centered care in health care teams. Team-based care requires skills beyond traditional patient-provider relationships and clinical knowledge. Educational initiatives that emphasize skills in multidisciplinary teamwork and team-based population management are needed. We redesigned resident continuity experience and implemented a new curriculum to teach residents skills in multidisciplinary team care and team-based panel management. Our secondary objective was to enhance resident satisfaction with continuity clinic experience through strengthened team-based care.

SETTING AND PARTICIPANTS: The General Medicine Clinic (GMC) at San Francisco General Hospital (SFGH) is an urban safety net practice site for 50 internal medicine residents with a patient population of over 6500 ethnically diverse, low-income patients with complex disease.

DESCRIPTION: The GMC Enhanced Medical Service (GEMS) curriculum was launched in January, 2011. The curriculum focuses on building multidisciplinary teams and teaching residents skills in panel management. Resident clinic sessions are blocked for the first hour every other week for the curriculum and team care activities. Each GEMS session includes a didactic component and 30 minutes for team huddles and panel management. Didactic sessions cover skills in team communication and huddles; optimizing teamwork with medical assistant, nurse practitioner, and clerical team members; panel management; and review of team quality metrics. We evaluated a 6 month pilot using an anonymous survey and resident focus groups.

EVALUATION: 32 of 50 residents (64\%) completed the survey at the end of the GEMS curriculum. $89 \%$ felt the GEMS curriculum enhanced their understanding of clinic teams and helped them work more effectively with staff team members. $76 \%$ of residents reported "huddling" with their medical assistant teams during clinic. $82 \%$ responded that the GEMS curriculum helped them understand and implement panel management. 93\% of residents felt GEMS made clinic less stressful. One resident reflected, "I was often in clinic for over 4 hours after my clinic day. It [made] primary care feel like an incredible burden on top of busy inpatient responsibilities. Panel management time alleviates some of this feeling and helps us get feedback on our performance and outcomes."

DISCUSSION / REFLECTION / LESSONS LEARNED: As more primary care practices become medical homes, resident ambulatory education increasingly requires training to build resident skills in multidisciplinary teamwork and population management. The GEMS curriculum helped residents function in clinic teams and learn skills of panel management. Further efforts are needed to increase team efficacy and assess residents' competency in providing comprehensive primary care in team-based systems.

TEACHING INFORMATION LITERACY WITH MEDICAL RESOURCE APPS AND OPTIMIZED MOBILE WEBSITES. Sarang Kim; Kerry O'Rourke. UMDNJ-Robert Wood Johnson Med School, New Brunswick, NJ. (Control ID \#1311920)

NEEDS AND OBJECTIVES: Information literacy is the ability to access information resources to make informed patient care decisions. Effective teaching of information literacy requires hands-on interaction, which can be challenging with a large group of students, and actual use of resources is limited without easy access. With widespread use of Smartphones, apps and mobile optimized web sites improve access to medical resources, and may enhance the teaching of information literacy. The objective of this educational innovation was to explore the use of apps and mobile optimized web sites in teaching information literacy to medical students.

SETTING AND PARTICIPANTS: 3 rd year students at a US medical school 
DESCRIPTION: We conducted a 90 minute workshop for 120 third year medical students on efficient use of medical resources. Students were asked to bring their Smartphone and were provided instruction prior to the session on downloading select medical resource apps and optimized mobile websites. At the workshop, students were first introduced to 5 medical resources (DynaMed, Essential Evidence Plus, First Consult, MD Consult, Access Medicine), then given instructions about how to use those resources with their Smartphones to answer clinical questions provided. After students navigated through the resources on their own, they were challenged to answer new clinical questions using the EBR apps in less than 4 minutes as a practical exercise to test out how efficiently the resources may be used in real clinical settings. Pre and post tests were administered to identify students' utilization of medical resources.

EVALUATION: Eighty-nine students completed the pre-test and 83 completed the post-test. On pre-test, the most commonly used resources to answer clinical questions was Google ( $88 \%$ of students) and textbooks $(67 \%)$. A majority of students indicated that they were unfamiliar with or never used the resources discussed during the session. On posttest, a majority of students reported being comfortable with the resources discussed (54-66\%). When asked which resource they planned to use to answer clinical questions, DynaMed ranked highest, and the most commonly reported reason was its up to date content. Overall, $86 \%$ of students rated the workshop content as important to their professional education, and $88 \%$ reported that they planned to use the mobile resources in clinical care.

DISCUSSION / REFLECTION / LESSONS LEARNED: Hands-on learning utilizing students' mobile devices is an effective means of teaching information literacy to a large group of students. Instructing students to download the apps prior to the session ensured access to the resources during the session and in their clerkships. We plan to survey the students at the end of their academic year to determine actual use of these resources in their clinical clerkships.

THE CLINICIAN EDUCATOR PATHWAY: MEETING THE NEEDS OF TOMORROW'S MASTER TEACHERS Maryann K. Overland ${ }^{1}$; Lauren R. Thronson ${ }^{2} .{ }^{1}$ University of Washington, Seattle, WA; ${ }^{2}$ Harborview Medical Center, Seattle, WA. (Control ID \#1310895)

NEEDS AND OBJECTIVES: During an era of decreased resident work hours and increasing clinical obligations, we must identify new and innovative educational curricula to better prepare our internal medicine trainees who wish to pursue a career as a Clinician Educator. Objectives: 1. Provide residents with a toolbox of best practices for teaching in a variety of settings 2. Facilitate and encourage educational scholarship 3. Create a framework for construction of a Teaching Portfolio

SETTING AND PARTICIPANTS: University of Washington Internal Medicine Residency has selected four second year residents to participate in a pilot Clinician Educator Pathway. Pathway development involved focus group feedback from residents and Clinician Teacher faculty. The pathway was created using data gained from these feedback sessions as well as reviews of the literature and evaluation of similar programs around the country.

DESCRIPTION: The pathway has three main interventions. First, the participants are paired with mentors who are working within their desired field. The goal of these partnerships is to facilitate ongoing educational scholarship in a variety of forms, including original research and curriculum development. A secondary goal is to provide mentorship and advice about career goals and the teaching portfolio. Second, the participants will engage in a 4-week educational immersion block in January of 2012. During their immersion experience, they will participate in a variety of teaching activities and small-group didactic sessions. The didactics include precepting in the outpatient setting, bedside teaching, constructing a teaching portfolio, providing feedback, and formal group teaching. They will also have the opportunity to teach in unique settings. They will facilitate problem-based learning sessions (PBL) with the preclinical medical students, an experience that will extend for eight weeks into their next clinical rotation. They will precept 4th-year medical students in a primary care setting. As proctors for observed standardized clinical examination (OSCE's), they will formally evaluate and give immediate feedback to students under the guidance of experienced educators. They will develop and present a lecture for their resident peers. Additionally, they will have individual sessions with the University of Washington's most highly regarded Master Teachers. Finally, the pathway participants will build a Teaching Portfolio, including a personal teaching philosophy statement and examples of their teaching and educational scholarship, to be a living document by the time they complete their residency.

EVALUATION: Although this program is in its infancy, the pathway is garnering great enthusiasm among residents and faculty. However, based on our focus group discussions, it is clear that residents' understanding of educational scholarship and the academic promotion process is quite limited. Evaluation will include pre- and post-surveys for the participating residents.

DISCUSSION / REFLECTION / LESSONS LEARNED: The inaugural immersion block will be completed February 3 rd, 2012, and will therefore have reflections on the successes and challenges of that experience at the time of the SGIM meeting in May, 2012. Additionally, we will have suggestions on pitfalls to avoid and key must-haves for a successful education immersion experience.

THE CRIMSON CARE COLLABORATIVE CHELSEA CLINIC: INTEGRATING MEDICAL, MENTAL, AND SOCIAL HEALTHCARE FOR POST-INCARCERATION AND URGENT CARE PATIENTS IN A STUDENT-FACULTY CLINIC Divya Mallampati ${ }^{1,2}$; Janine Knudsen ${ }^{1,2}$; Marya J. Cohen ${ }^{2,1}$; Jonathan Cunningham ${ }^{1,2}$; Chuan-Mei Lee ${ }^{1,2}$; Luis Ticona ${ }^{1,2}$; Rachel Bender Ignacio ${ }^{3,2}$; Brennan Bollman ${ }^{1,2}$. ${ }^{1}$ Harvard Medical School, Cambridge, MA; ${ }^{2}$ Massachusetts General Hospital, Boston, MA; ${ }^{3}$ Massachusetts General Hospital, Boston, MA. (Control ID \#1334975)

NEEDS AND OBJECTIVES: Social determinants of health influence outcomes both in exam rooms and in the community, yet medical students receive little exposure to social medicine in practice. In addition, students are rarely able to explore innovative models of primary care. A new student-resident-faculty collaborative clinic at Massachusetts General Hospital's Chelsea Community Health Center (MGH-Chelsea) and Harvard Medical School (HMS) therefore seeks to educate medical students by enabling them to design comprehensive primary care strategies that deliver effective care to two underserved populations: people returning to Chelsea post-incarceration and frequent urgent care users without a usual provider.

SETTING AND PARTICIPANTS: In October 2011 HMS expanded its student-faculty clinic model, the Crimson Care Collaborative (CCC), to a new site at an MGH-Chelsea. This new clinic provides students with a valuable opportunity to care for vulnerable populations, explore social medicine in practice, and understand how medical teams implement new strategies to enhance patient-centered care. Our integrative model trains students to address the socioeconomic barriers to their patients' health and the unique health care needs of two underserved populations.

DESCRIPTION: We have developed a unique clinic structure to address the strong need for coordinated primary care, mental health care, and social services in our populations. Patients first meet with a social services navigator, a student who screens the patient for non-clinical issues that might impact their overall health, such as food security, employment, and legal issues. The patient next meets with student and faculty clinicians to establish a longitudinal primary care relationship. Finally, the social navigator returns to provide referrals to relevant social services organizations and establish a follow-up plan. Patients requiring additional mental health care are referred to our co-located mental health team of two students and a psychiatry resident for further assessment and management. In the future, our team will also implement a patient education program for topics including substance abuse and chronic disease.

EVALUATION: The clinic team works together closely to ensure that our socially complex patient populations have access to appropriate, highquality services that meet both provider- and patient-identified needs. To achieve this goal, our student roles extend beyond the medical clinic. Students actively follow up with their patients for medical and social 
services and are encouraged to develop strong relationships with them. In addition, a student outreach contingent has conducted structured interviews with non-profits in Chelsea and compiled an online database of more than 20 local social service assistance programs for use in our clinic.

DISCUSSION / REFLECTION / LESSONS LEARNED: Since the clinic's establishment in October 2011 we have provided care to more than 50 patients, one quarter of whom are transitioning into the community from incarceration. Around $90 \%$ of our patients have received at least 1 social services referral to a local organization, and $40 \%$ have been referred to and seen by our mental health teams. Finally, more than 40 medical students and 5 residents have participated in the clinic's design and operations, and our volunteer numbers continue to rise. Through our innovative, comprehensive clinic model, we will continue to educate a new generation of socially-minded, creative physicians familiar with new models of care while providing care to two underserved populations.

THE CROSSOVER CURRICULUM: PREPARING INTERNAL MEDICINE RESIDENTS TO CARE FOR PATIENTS WITH CHILDHOOD-ONSET CHRONIC DISEASES Zadok Sacks; Anna Volerman; Niraj Sharma. Brigham and Women's Hospital/Children's Hospital Boston, Boston, MA. (Control ID \#1334351)

NEEDS AND OBJECTIVES: Research has demonstrated that internal medicine residents (IMRs) are less comfortable caring for adults with childhood-onset chronic diseases (COCDs) than their pediatric counterparts. Other work has shown that this may negatively impact the quality of care these patients receive after transitioning to adult providers. A survey conducted among IMRs at our institution suggested significant interest in a curriculum focusing on the management of these patients. For each of ten COCDs identified, over $75 \%$ of respondents $(\mathrm{N}=53)$ felt that formal teaching of those topics would improve their care of these patients. To address this need, the Crossover Curriculum (CC) initiative was developed by our Medicine-Pediatrics (Med-Peds) residency program. Program objectives include increasing IMRs' confidence in managing adult patients with COCDs; creating a unique educational niche for the Med-Peds residency program; and giving IMRs the opportunity to learn from master pediatric educators.

SETTING AND PARTICIPANTS: Our survey revealed that $84 \%$ of IMR respondents had no prior training related to the transitioning of young adults with COCDs. When surveyed regarding the preferred teaching format, approximately $90 \%$ of IMRs indicated interest in case-based morning reports or noontime lectures. Accordingly, five morning report sessions and two lectures to IMRs have been completed.

DESCRIPTION: Individual CC morning report sessions have focused on anorexia, severe asthma, cystic fibrosis (CF), inflammatory bowel disease (IBD), and adult survivorship of pediatric cancer, while lectures have focused on sickle cell disease and hyperglycemic crises. All sessions have involved guest discussants from our affiliated pediatric institution. Discussions typically focus on management issues and highlight the challenges involved in transitioning patients with COCDs.

EVALUATION: Feedback was obtained from IMRs both before and after this initial set of sessions. Over $75 \%$ of respondents who attended at least one session $(\mathrm{N}=70)$ felt that morning reports and noontime lectures were effective vehicles for learning about the management of adult patients with COCDs, while nearly all attendees felt that hearing from pediatric subspecialists enhanced their learning. Qualitative feedback has been similarly favorable. A strong majority of respondents felt that the $\mathrm{CC}$ adds to their education overall, with many commenting that the sessions have changed their practice. Specifically, individuals commented that they "feel more knowledgeable about IBD and CF...[and] more comfortable when taking care of adult patients with those diseases." Other IMRs described that they will be "less aggressive with IV fluids in diabetic ketoacidosis" and "trust sickle cell patients [about] what dose of pain medication works for them in a pain crisis."

DISCUSSION / REFLECTION / LESSONS LEARNED: The CC is an innovative program in which there has been a great deal of interest from IMRs at our institution, who view it as a high-yield educational experience with the potential to impact management of patients with COCDs. Based on the feedback obtained to date, we plan to add additional topics to the CC (e.g. adult congenital heart disease, cerebral palsy, intellectual disability, and genetic disorders) and continue the $\mathrm{CC}$ in the future. The $\mathrm{CC}$ can be generalized to any Med-Peds residency program and its affiliated IM residency program, and fills an important educational niche; as one IMR commented, "Children with these diseases are living longer now, and internists have to know how to care for them."

\section{THE INTERNAL MEDICINE SIMULATED CASE SCENARIO} PROJECT Bridget B. Stiegler. Banner Good Samaritan Medical Center, Phoenix, AZ. (Control ID \#1319891)

NEEDS AND OBJECTIVES: The restriction of resident work hours has prompted the need for creation of a supplementary educational forum wherein we evaluate resident's knowledge and ability to manage basic ward diagnoses. In response to this need the Banner Good Samaritan Academic Medical Service (AMS) has created a program utilizing high fidelity mannequins in a standardized patient hybrid simulation experience. In addition to creating high yield teaching time between our residents, their clinical attendings and specialists, this program also increases the annual number of direct observation faculty evaluations.

SETTING AND PARTICIPANTS: This exercise involves second year residents on elective rotations, which occur every other month, as the residents are on a call/non call rotating schedule. Cases run monthly, prior to the start of Academic Half Day lectures, thus each resident runs approximately six cases per year. In the context of this project the resident obtains a thorough history and physical examination on his or her patient (mannequin), the voice of which is provided by an internal medicine teaching attending. After completing an exam the resident is then asked to discuss admission orders, and receives immediate feedback from his attending regarding performance and the depth of understanding of the case topic.

DESCRIPTION: Prior to test day, residents are provided with literature relevant to the medical case in question. Residents also recieve a quiz to complete prior to the simulation to ensure their preparation for the exercise. Quiz questions are management/critical thinking based, and are drawn from the assigned reading as well as from the weighted priority items as determined by the AMS attendings. Literary references and articles are chosen by the members of the SIMS Center Project committee. The SIMS Center Project host reviews the quiz answers and revisits major learning points for the case prior to the resident leaving the SIMET Center.

EVALUATION: All mannequin runners have the same case outline and patient background information to reduce inter-rater variability. The weight of each item in the history and physical examination is pre-determined by the AMS group as "vital" or "non-vital," in the achievement of an "acceptable" or "not acceptable" grade. Residents have a comprehensive grade comprised of three elements; score on pre-test quiz, weighted case items, and "Attending Evaluation". The evaluation specifically addresses learning milestones for second year residents. Deficiency in any one of these elements leads to a score of "Not Acceptable", warranting close monitoring of the resident's academic and clinical progress by the program director and the resident's advisor.

DISCUSSION / REFLECTION / LESSONS LEARNED: Outcomes to this point have been learning based. We created 52 additional direct observation experiences during the pilot year for this project. Residents have communicated appreciation for the opportunity to practice patient management, and for one-on-one feedback that they receive from their attendings. The faculty members have been pleased with the opportunity to choose and distribute literary references they feel are vital for clinical management. Currently we are evaluating the Simulated Case Scenario Project as a teaching tool, by studying long-term retention of information covered in the simulated cases. A research proposal to evaluate the effectiveness of this teaching model using knowledge retention as endpoint is currently under review by our Institutional Review Board. 
THE VA PRIMARY CARE CLINIC: AN IDEAL SETTING TO TRAIN INTERNAL MEDICINE RESIDENTS TO WORK WITHIN THE PATIENT-CENTERED MEDICAL HOME Neha Pathak ${ }^{1,2}$; Karen Woolfall-Quinn ${ }^{1,2}$. ${ }^{1}$ Providence VA Medical Center, Providence, RI; ${ }^{2}$ Alpert School of Medicine Brown University, Providence, RI. (Control ID \#1318386)

NEEDS AND OBJECTIVES: Research suggests that transforming primary care delivery to the Patient-Centered Medical Home (PCMH) model could reduce health care costs AND improve the quality of acute and chronic disease care. There is an increasing trend toward creating outpatient practices that fit the PCMH model, emphasizing: 1) ongoing relationship with a physician, 2) providing continuous and comprehensive care within teams, 3) patient self-management, 4) care facilitated by information technology, 5) care provided according to patient need, such as the use of asynchronous communication, expanded hours and open-access scheduling, and 6) use of evidence-based medicine and clinical decision support tools. Internal medicine residency ambulatory education must be redesigned to produce physicians capable of effectively practicing within the PCMH. We have developed a rigorous, patient-centered, team-based curriculum in the ambulatory setting at the Providence VA Medical Center utilizing both didactic and experiential components. Our objective is to train internal medicine residents to provide comprehensive, coordinated, and continuous care for a panel of patients within the Patient Aligned Care Team (PACT) structure in the primary care clinic.

SETTING AND PARTICIPANTS: The Department of Veterans Affairs is currently funding a $\$ 250$ million effort to adopt the PCMH model nationwide (called PACT within the VA) and this provides an ideal setting to train internal medicine residents within a PCMH. The Providence VA is an ambulatory care continuity clinic site for 18 Brown University Internal Medicine Residents paired with 1 of 5 dedicated faculty preceptors. Each resident is expected to provide care for a panel of patients utilizing the PACT. DESCRIPTION: Curriculum A. Didactics a) Pre-clinic Seminars on PACT topics: 1) Structure of PACT: meeting team members and understanding roles 2) Alternative modes of communication: ie telephone visits, secure messaging 3) Interdisciplinary VA resources: Nutrition, Pharmacy, Mental Health, Social Work b) Pre-clinic Outpatient Practice Improvement Seminars: Monthly review of patient panel data: 1) "out-of range" diabetes, lipid, hypertension metrics 2) Review of ED utilization and hospitalizations B. Clinical Experience with a Patient Panel a) Ambulatory Clinic appointments: routine follow up and open access for "sick visits" b) Telephone appointments c) Panel management using PACT to improve metrics d) Brief Team meetings with Primary Preceptor, Clinic nurse, resident colleagues, health techs

EVALUATION: A. Resident Evaluation a) Preceptor evaluation of resident in the following categories: 1) outpatient practice improvement 2) knowledge of and ability to work within PACT TEAM $b$ ) Patient evaluation of resident $B$. Curriculum Evaluation a) Resident to complete PACT Recognition Survey- a tool to ascertain knowledge of the principles of PACT

DISCUSSION / REFLECTION / LESSONS LEARNED: The Providence VA presents an ideal site for resident education within the structure of a patient-centered medical home as this model of care has been steadily developed within our VA for many years, most recently by a reorganization of our clinical teams. In addition, our size is an asset. Our medical center is small, allowing for geographic proximity of the interdisciplinary teams, creating an environment of easy access for consultation with team members, Mental Health providers, pharmacy, etc; this further instills the importance of the "warm hand-off" and providing patient-centered care in a timely, efficient manner.

THE IMPACT OF INTERDISCIPLINARY CODE SIMULATION ON PERCEPTIONS OF COLLABORATION AND TEAM PERFORMANCE AMONG INTERNAL MEDICINE RESIDENTS AND NURSING STUDENTS Cynthia J. Herrick ${ }^{1}$; Michael Nasiak ${ }^{1}$; Aditi Singh ${ }^{1}$; Sandhya Wahi-Gururaj ${ }^{1}$; Jessica Doolen ${ }^{2}$; Kevin Gulliver ${ }^{2}$; Carolyn S. Witt ${ }^{2}$. ${ }^{1}$ University of Nevada School of Medicine, Las Vegas, NV; ${ }^{2}$ University of Nevada-Las Vegas, Las Vegas, NV. (Control ID \#1339653)

NEEDS AND OBJECTIVES: Crisis resource management (CRM) encompasses communication, leadership, situation awareness, and deci- sion-making. These skills are critical to patient safety and quality improvement in healthcare settings. Simulation provides an opportunity for training in interdisciplinary teams facing situations comparable to those encountered in the hospital. Internal medicine residents receive ACLS training prior to starting residency, but may not have an opportunity to hone CRM skills prior to leading a code team. Similarly, nursing students have limited direct exposure to physicians during their education.

SETTING AND PARTICIPANTS: Twenty-seven second year Internal Medicine residents from the University of Nevada School of Medicine and one hundred and twenty three second and fourth semester nursing students from the UNLV School of Nursing participated in high fidelity simulation at the Clinical Simulation Center of Las Vegas as part of their training during Summer 2010 and 2011.

DESCRIPTION: Residents and students participated in two collaborative emergency codes (Ventricular Fibrillation \& Pulseless Electrical Activity algorithms). Nursing students acted as the nursing resuscitation team and residents acted as the on-call staff physician and team leader. Each scenario was designed to run approximately twenty minutes including initial assessment, patient decompensation, pulseless arrest, and recovery. After completing the exercise, nursing and medicine faculty facilitated video guided debriefing of the team, focusing on CRM fundamentals.

EVALUATION: After each scenario, participants completed the Jefferson Scale of Attitudes toward Physician-Nurse Collaboration (JSA) and the Mayo High Performance Teamwork Scale (MHPTS). Each instrument was completed before and after each debriefing. The JSA assesses general attitudes about collaborative education and the MHPTS evaluates perceptions of team performance. Statistically significant differences were seen in attitudes about collaborative education and perceptions of team performance. On the JSA, RM ANOVA for pretest 1 to post-test 1 revealed $\mathrm{F}(1,158)=25.69(\mathrm{p}=0.005)$ and pretest 2 to post-test 2 showed $F(1,164)=15.32(p=0.0005)$. On the MHPTS, statistically significant differences were only observed between pretest 2 and post-test $2(\mathrm{~F}(1,161)=19.24,(\mathrm{p}=0.0005)$.

DISCUSSION / REFLECTION / LESSONS LEARNED: Participants reported higher mean scores for both measures on the second scenario and for the JSA on the first scenario. This suggests that the educational intervention improved general attitudes regarding collaborative education. Perceptions of team performance were also enhanced, but this occurred more slowly than changes in attitudes about collaboration. Interdisciplinary code simulation and debriefing focusing on CRM skills can be an important tool in fostering physician-nurse teamwork. Future evaluation of data from this intervention will focus on objective evaluation of team performance and its correlation with ACLS algorithm adherence.

TRAINING IN CROSS-CULTURAL COMMUNICATION FOR IMG PHYSICIANS Eric Green ${ }^{1,2}$; E. Amy Janke ${ }^{3}$; Arnold Eiser ${ }^{1,2}$. ${ }^{1}$ Mercy Catholic Medical Center, Darby, PA; ${ }^{2}$ Drexel Univiersty College of Medicine, Philadelphia, PA; ${ }^{3}$ University of the Sciences of Philadelphia of, Philadelphia, PA. (Control ID \#1310829)

NEEDS AND OBJECTIVES: Most models of cross-cultural communication in medicine presume the teacher and student share a common health belief system that differs from the patient. At least one third of all U.S. graduate medical trainees are international medical graduates (IMGs) whose culturally-related health beliefs may differ significantly from that of their American-bred faculty. We hypothesized that training IMGs in communication skills and U.S. health beliefs would increase their ability to conduct appropriate cross-cultural communication during clinical interactions. We created a multi-modal crosscultural training program to meet this need.

SETTING AND PARTICIPANTS: Our intervention is set in a university-affiliated community teaching hospital based residency based in urban and suburban Philadelphia where approximately $75 \%$ of residents are IMGs. Residents were drawn from all years

DESCRIPTION: Our multi-modal intervention is designed to increase an IMG's understanding of U.S. health beliefs and stimulate improved doctorpatient communication between IMGs and patients. We focused on African-American patient perspectives with particular reference to the 
management of non-malignant pain and shared medical decision making. The intervention included two simulated patient (SP) encounters that highlighted areas of cross-cultural differences among U.S. born teachers, U.S. born patients, and internationally educated residents; an online module; assigned readings; and an hour-long interactive didactic session. Our residents were observed during their SP encounters by a clinical psychologist who provided immediate, in vivo feedback using a behaviorally-anchored rating scale. Residents completed a self-report assessment derived from Like's Clinical Cultural Competency Questionnaire which uses a 5-point Likert scale ranging from 1 ("not at all") to 5 ("very") to collect pre- and post-training data on resident's knowledge, skill, and attitudes in cross-cultural care. We analyzed survey results using descriptive techniques and student T-test.

EVALUATION: 15 residents completed the training program. The residents had a mean age of 32 , and $40 \%$ were men. $53 \%$ were from India, with remainder from Africa or elsewhere on the Indian subcontinent $20 \%$ were PGY 1, 47\% were PGY2, and 33\% PGY3. 67\% reported some previous training in cultural diversity. Self-ratings on 8 items measuring cross-cultural knowledge improved from a mean of 2.94 pre-training to 3.93 post-training $(p<.001)$, while self-reported culturally-competent skills improved from 3.26 to $4.27(\mathrm{p}<.001)$. Residents reported improvement in their ability to interpret non-verbal clues $(2.87$ to $3.92, p=.003)$ and interpreting cultural expressions for pain $(2.73$ to $3.92, \mathrm{p}<.001)$. The residents universally enjoyed the training as whole (mean $4.83 \pm .39$ on 5 point scale), and rated each component favorably (all rated $>4$ on a 5-point scale). We were able create the training for approximately $\$ 3000$, and the cost per resident is approximately $\$ 100$.

DISCUSSION / REFLECTION / LESSONS LEARNED: A multimodal training program designed to enhance cultural competence for IMGs successfully improved residents' self reported knowledge and skills in areas of cultural competency. This preliminary study also demonstrates that such a program can be developed and maintained in a cost-effective way with minimal additional time burden for training staff or residents. It appears to be a promising approach to improving communication skills and enhancing cultural competence for IMG physicians.

TRANSFORMING HEALTHCARE THROUGH A UNIT-BASED CLINICAL LEADERSHIP MODEL Robert Dressler; Virginia U. Collier; Michael Eppehimer; Barbara A. Monegan; Janet Cunningham; Diane Talarek; Vernon L. Alders; Sharon L. Anderson. Christiana Care Health System, Newark, DE. (Control ID \#1339521)

NEEDS AND OBJECTIVES: The Christiana Care Department of Medicine has an attending staff of approximately 439 physicians and oversees 500 of the 1,150 beds in Christiana Care hospitals. Multiple departments, including Medicine, Nursing, Quality and Safety, Operational Excellence, and System Learning, collaborated to expand the existing unitbased medical director role. The new model's goal further develops our medical director-nurse manager leadership teams to effectively lead quality improvement activities on their units and advance the value of care provided to patients.

SETTING AND PARTICIPANTS: The unit-based clinical leadership model concentrates on three specific areas; increased time commitment on the floor by the medical director, a structured training program to educate the medical director and nurse manager in leadership skills and improvement methods, and embedded support by process improvement and quality resources. The training program began April 2011 for eight inpatient medicine patient care units.

DESCRIPTION: The Unit-Based Clinical Leadership Teams (15 nurse managers and medical directors; one director assigned to two units) completed an initial 18 hours of training and development over 5 learning sessions offered between April and June 2011. Key elements of the program included: Team Kickoff - Led by Senior Leadership, introduction to Christiana Care's Annual Operating Plan, Quality and Patient Safety goals and the expectations for Unit-Based Clinical Leaders. Team Building - Individual thinking preference profiles for individuals and teams, structured discussion about leadership team expectations and working styles. Leading Through Vision - Business case studies on the importance of a guiding vision, creation of specific unit vision for each team. Performance Improvement, Part I - Introduction to Lean / PDCA improvement tools and methodology, creation of "current state" process map. Team Dynamics \& Change Management - Expected life cycle of teams leading change efforts, team communication and problem identification. Performance Improvement, Part II - Continued focus on Lean / PDCA tools, root cause analysis, problem identification and prioritization and future state mapping. Each nurse-physician team identified one initial performance improvement project focused on improving patient care on their units. From June through September 2011, the skills were applied to structured performance improvement efforts. Monthly meetings occurred to review progress toward goals and to prepare the teams for a 90 day report out to senior leadership.

EVALUATION: All eight unit based teams were able to apply the acquired skills and successfully completed improvements with measurable results within 90 days. Additional measures included session evaluations, project outcomes and impact, and team function status at 2 month milestone and post initial project completion (baseline data completed; post intervention data collection scheduled for end of year 2011).

DISCUSSION / REFLECTION / LESSONS LEARNED: The UnitBased Clinical Leader model fosters interaction and close collaboration between the medical and nursing staff. Building core competencies in improvement and leadership supports organizational capability in delivering value based healthcare to patients. We are leveraging this program to further refine the Medicine Value Process (a standardized approach to quality and patient safety) in our faculty development efforts.

ULTRASOUND FOR INTERNAL MEDICINE PHYSICIANS: THE FUTURE OF PHYSICAL EXAM Megan M. Dulohery; John Eaton; Tanya Tajouri; Anjali Bhagra. Mayo Clinic, Rochester, MN. (Control ID \#1336178)

NEEDS AND OBJECTIVES: Internal medicine physicians frequently perform bedside invasive procedures such as thoracentesis, paracentesis, and central venous catheter placement. With the advent of handheld ultrasound devices, it is now easier for these physicians to enhance their physical exam and procedural skills through the use of bedside ultrasound. Literature suggests that ultrasound guidance can also enhance patient safety during invasive procedures. Despite this new tool and data, few of these physicians have formal ultrasound training. The objective of this study sought to understand the physician's baseline knowledge and skill, provide education in ultrasound principles and use, and demonstrate that internal medicine physicians can learn this skill in a timely manner.

SETTING AND PARTICIPANTS: This project was completed at the Mayo Clinic, Rochester, Minnesota, USA, in June 2010 and 2011. The participants included junior internal medicine house staff in the first three years of training. The workshop took place in a multidisciplinary simulation centre. The workshop was led by subspecialty fellows, staff, and senior medical residents.

DESCRIPTION: The project involved a novel teaching intervention including didactics and hands-on ultrasound experience in human and cadaver models in a multidisciplinary simulation centre. Our study was a multidisciplinary educational intervention which included a pre- and postassessment of knowledge of ultrasound and skill of image acquisition. EVALUATION: A total of 136 physicians completed the workshop. One hundred thirty-three participated in the pre-survey, and all participants completed the post-survey. Forty-four (33\%) participants were able to identify air in the pre-test compared to $132(97 \%)$ on the post-test. Ninetysix $(72 \%)$ were able to identify fluid on the pre-test compared to 136 $(100 \%)$ on the post-test. Image acquisition was tested by assessment of the internal jugular (IJ) vein. One hundred thirty-five participants had adequate data to be included in this assessment. Eighty-five (63\%) were able to demonstrate the IJ in the pre-test compared to $126(93 \%)$ in the post-test. Eighty-three (61\%) could demonstrate compressibility of the IJ in the pretest compared to $126(93 \%)$ in the post-test. Fifty-one (38\%) were able to demonstrate appropriate gain in the pre-test compared to $116(86 \%)$ on the post-test. Thirty-nine $(29 \%)$ were able to demonstrate appropriate depth 
compared to $113(84 \%)$ on the post-test. Time to image acquisition improved by an average of 30 seconds (median 35 seconds).

DISCUSSION / REFLECTION / LESSONS LEARNED: A multidisciplinary educational intervention resulted in improvement in ultrasound knowledge and its use for adequate image acquisition by internal medicine physicians in the first three years of practice. Recent trends show that ultrasound is emerging as a clinical stethoscope and is likely to become an essential element of the physicians' tool box for providing timely, safe, and quality care. Our findings are very encouraging and demonstrate that this skill can be learned in a timely manner to improve the skill set of the physicians.

USING ART TO ENHANCE REFLECTION ON PROFESSIONAL ATTRIBUTES Lynn Byars ${ }^{1}$; Gerald D. Denton ${ }^{2} .{ }^{1}$ Walter Reed National Military Medical Center, Bethesda, MD; ${ }^{2}$ Uniformed Services University of the Health Sciences, Bethesda, MD. (Control ID \#1322296)

NEEDS AND OBJECTIVES: Professional behavior of doctors is increasingly discussed in the popular press and medical literature. Methods to actively teach appropriate professional behavior (beyond implicit methods like role modeling) in undergraduate medical education are beginning to emerge. We created a novel curriculum for our Internal Medicine Clerkship, which actively teaches professional attributes utilizing art as a catalyst for reflection.

SETTING AND PARTICIPANTS: This curriculum was designed for 3 rd year medical students on the Internal Medicine Clerkship.

DESCRIPTION: Recognizing that modeling appropriate behavior, the historically cited way to teach professionalism to medical students, is a necessary but not sufficient method, we designed a curriculum to transition the learning of professional behavior from a subconscious process to a conscious one. To accomplish this, we developed a self-paced online educational module that students began during week one of our twelveweek internal medicine clerkship. The attributes laid out in Dr. Herbert Swick's June 2000 article on medical professionalism were introduced. Students were instructed to pay attention to the behaviors modeled around them in the clinics and on the wards, as well as their own behavior. Next, the students selected one of 10 different paintings available in the online module and gave a short interpretation of the artwork, followed by a discussion of the attribute of professionalism they felt was represented in the artwork. These tasks were intended to enhance the student's ability to reflect upon professional behavior by allowing the artwork to serve as a surrogate for self and a memory prompt to recall a critical incident they observed. During the sixth week of the clerkship, the students wrote a brief reflective essay outlining the critical incident, applying their chosen professionalism attribute, describing their impressions and discussing how this experience might change their future practice behavior. These essays were discussed in the preceptor small group sessions, allowing fellow students to gain insight from the events witnessed by their colleagues and allowing the preceptor to provide feedback on the event and the student's response to it.

EVALUATION: The curriculum was implemented at the start of this academic year as an optional project and has been well received by students and faculty. 34 of 82 students have opted to complete the project. The faculty reviews have been enthusiastically positive, with one attempt by faculty to publish a project with the student and two attempts by faculty to give feedback to other faculty regarding egregious professional behavior observed by the student. Based on initial lessons learned, a more robust assessment instrument was developed and implemented.

DISCUSSION / REFLECTION / LESSONS LEARNED: This project is an effective and palatable format to incorporate explicit professionalism teaching into the clerkship curriculum. This exercise has educational implications beyond the individual student, as small group discussions generalize the concepts learned. Further, de-identified essays were presented to house staff to promote reflection and we plan to do the same with faculty, calling attention to behaviors occurring in our own program and witnessed by students. Additionally, the curriculum could easily be adopted by other clerkships or for house staff.
USING RESEARCH TO DEMONSTRATE AND IMPROVE QUALITY OF CARE IN STUDENT-LED CLINICS Janine Knudsen ${ }^{1,2}$; Laura Huppert ${ }^{1,2}$; Kyle E. Dempsey ${ }^{1,2}$; Karen Donelan ${ }^{2,1}$; Rebecca Berman ${ }^{2,1} .{ }^{1}$ Harvard Medical School, Cambridge, MA; ${ }^{2}$ Massachusetts General Hospital, Boston, MA. (Control ID \#1334974)

NEEDS AND OBJECTIVES: Student-run clinics fill crucial health care gaps for our nations' underserved citizens and provide valuable medical education opportunities. However, these $100+$ clinics remain underrecognized and are often rumored to provide sub-optimal care, in part due to the sparse literature evaluating their success. Student-run clinics therefore have a special imperative to demonstrate that they provide high quality, patient-centered care.

SETTING AND PARTICIPANTS: At Harvard's Crimson Care Collaborative (CCC), a consortium of student-faculty clinics, we aim to provide health care to those who lack access, to reduce unnecessary hospitalizations, and to expose medical students to primary care. Since the clinic's start in 2009, we have used research to better understand our patient population and to enhance the care that we provide. Here, we describe our research operations, patient population, and data-driven quality improvement efforts.

DESCRIPTION: Student researchers collect data through patient intake surveys, medical chart reviews, and clinic flow mapping to better understand patient demographics and medical-social needs, assess care quality, and measure clinic outcomes. We have also adapted the Consumer Assessment of Healthcare Providers and Systems (CAPHS) survey to evaluate patient satisfaction. Our research efforts over the last year have demonstrated positive results and also exposed areas for clinic improvement.

EVALUATION: Our original clinic at the Massachusetts General Hospital's Internal Medicine Associates has served 263 patients, including 182 urgent care patients and 81 "Bridge to Care" (BTC) patients, who receive up to 1 year of primary care before transferring to a regular PCP. Our BTC patients are typically low-income (56.2\%), not college educated (68.2\%), underemployed (78.2\%), insured through MassHealth or Mass Safety Net (59.2\%), and request social services (50.9\%). The experience of all of our patients reflects the need for primary care access: $49.2 \%$ of our patients visited the ED multiple times in the past year and $42.7 \%$ attributed this to the lack of physician availability. Our patient satisfaction data suggests that most of our patients would recommend the clinic to their friends and family members $(95 \%, \mathrm{n}=41)$. Although many did not know prior to the visit that both medical students and physicians would see them $(41.5 \%)$, they still rate our students and faculty members highly (8.9 and 9.1 , respectively, out of 10 ).

DISCUSSION / REFLECTION / LESSONS LEARNED: By gathering data about the patients we serve, our clinic has more effectively met our patients' needs and expectations. To improve patient understanding of the student-faculty collaborative model, we have revised our brochures and educated the office staff to better explain the role of the medical students in their care. Our student-run resource center uses our demographic information to help patients secure affordable medications, health insurance, food stamps, and other needs. Additionally, for the $58.5 \%$ of our patients who live with chronic disease, we have piloted an education program that utilizes motivational interviewing to help them with diet, exercise, and medication management. By addressing these patients' wide range of medical and social needs, our student volunteers are exposed to the breadth of primary care. This evidenced-based, patient-centered research approach provides a model for future data-driven research and operations at other student-run clinics.

UTILIZATION OF GERIATRIC ASSESSMENT TOOLS IN AMBULATORY CARE SETTING FOR IMPROVING THE QUALITY OF PATIENT CARE PROVIDED BY THE RESIDENT TRAINEES (SPONSORED BY REYNOLDS SAGE GRANT) Sujata Bhushan. Dallas VA Medical Center, Dallas, TX. (Control ID \#1334 $\overline{4634)}$

NEEDS AND OBJECTIVES: As the US population ages, primary care clinicians are encountering more patients with geriatric syndromes, such as 
urinary incontinence,dementia,gait instability, polypharmacy. Current evidence suggests that care of these conditions does not meet expected standards and that residents in training would benefit from tools objectively evaluating these specific conditions. We developed condition specific templates within the electronic medical record, both to improve care of patients with geriatric problems, and to provide the internal medicine residents with tools to learn and practice the essence of comprehensive assessment of the geriatric outpatient in an interdisciplinary setting.

SETTING AND PARTICIPANTS: We incorporated four validated geriatric assessment tools,namely, MINI COG TEST, Get Up and Go Test, urinary incontinence algorithm and polypharmacy, in the initial outpatient evaluation of patients aged 70 years and above, formulating a treatment plan using available resources/consults. The project was implemented in the residents' primary care continuity clinic at the VA. The residents received educational material in the form of formal lectures and periodic one on one/ group discussions addressing the unique aspects of care of the elderly, in particular addressing the Competency based Objectives, including medical knowledge,practice based learning,communication and interpersonal skills,system based practice and patient care. The residents were guided by the clinic attending.

DESCRIPTION: Seventy one resident trainees, with continuity clinic at the Dallas VA were participants in the project. The tools were incorporated as computerized templates into the EMR ( CPRS) at the VA after close collaboration with the CPRS team. A periodic chart review was conducted by the research team to ensure compliance and proper usage of the assessment tools . Outcome measures were improved clinical skills and level of comfort of the resident trainees in geriatric care and patient satisfaction regarding the quality of comprehensive care. The residents were tested at the beginning and at the end of the year by means of a knowledge and attitude/perception quiz. Any improvement in patient satisfaction was assessed by a questionnaire after completion of the project. EVALUATION: A total of 361 patients enrolled in the study. Of those 361,137 completed the one year follow up survey. There were significant differences between the pre and post assessment responses pertaining to discussion of memory problems with the physician $(45.3 \%$ vs $26.6 \%$, $\mathrm{p}<.001$ ). Also, there was a $5 \%$ decrease in the incidence of falls on the post assessment surveys. There was no significant difference in the continuous survey items regarding patient perception of care received. 82 residents completed the pre study geriatric test (including some residents who did not have continuity clinic at the VA). Only 27 residents completed the follow up quiz. Only 9 residents completed both a baseline and follow up quiz, therefore our analyses were substantially underpowered.There was a $10 \%$ improvement knowledge-wise from pre to post assessment. The resident survey items assessing attitudes regarding geriatric practice revealed no statistically significant differences, but revealed noteworthy improvements in knowledge,attitudes and understanding of issues pertaining to geriatric care.

DISCUSSION / REFLECTION / LESSONS LEARNED: Because of the small numbers, our pilot study was substantially underpowered, but the results are promising, encouraging us to continue using the tools in our clinic

WELCOME TO THE NEIGHBORHOOD: TEACHING THE SOCIAL DETERMINANTS OF HEALTH Jada Bussey-Jones ${ }^{1}$; Maura George $^{1}$; Carmen Mohan ${ }^{1}$; Minesh Shah ${ }^{2}$; Stacy Higgins ${ }^{1}$; Michael Saenger $^{1}$; Rinku Chatterjee ${ }^{1}$; Jason Schneider ${ }^{1}$; Mehul Tejani ${ }^{1}$; Schuyler Livingston ${ }^{1}$. ${ }^{1}$ Emory University, Atlanta, GA; ${ }^{2}$ University of Illinois, Chicago, IL. (Control ID \#1338530)

NEEDS AND OBJECTIVES: Physicians entering the workforce require broad medical knowledge and practiced procedural skills. Traditional training would suffice in decreasing disease burden if medical care were the only factor relevant to patient health outcomes. Research suggests, however, that multiple complex social determinants (e.g. neighborhood characteristics, social policies, available resources) play a significant role in producing or mitigating health outcomes. Further, studies suggest students' knowledge about health access and attitudes toward the underserved actually become more negative throughout medical school. Most current educational environments do not prepare learners for engagement with broader health issues. We designed a curricular intervention with the following objectives: 1) To teach learners the complexity and impact of community and other social determinants of health and 2)To demonstrate ways to connect public service and advocacy with their clinical and academic work through a combination of didactic, experiential, and direct service activities. SETTING AND PARTICIPANTS: Multi-disciplinary Emory University month long elective

DESCRIPTION: 3 core activities are used. 1. SCHOLARSHIP - Includes lectures and readings on relevant topics (advocacy, literacy, community engagement, etc). Also includes a required scholarly activity (oral presentation or submission to scientific meeting or peer-reviewed publication). 2. EXPERIENCES - (1) Observational: observing and engaging community partners as appropriate for a given module. (e.g. neighborhood assessment of available resources such as green space, fresh vegetables, pharmacy supply, safety, billboards). Learners also participate in simulation experiences and are asked to navigate public hospital as a patient (e.g. request financial assistance, get prescription filled, go to clinic appointment - assessing wait times, barriers, etc). (2) Direct Service: Participants engage one of our community partners' sites using an asset model approach to collaborate and participate in public service interventions. Our learners visit and engage several essential community resources that provide context for the health of many including, for example, homeless shelters, nursing homes, and prison medical facilities. Finally, they are trained and asked to complete advocacy tasks such as meeting with legislators or writing position papers. (3) Clinical experiences: Learners provide services (when appropriate) at designated community partner sites. 3 . REFLECTION - Includes group discussions with faculty and community facilitator, journaling, photo journaling.

EVALUATION: 1 . Assessment the effect of the Social Medicine Elective on learners' attitudes compared to matched controls through validated survey instruments (MSATU, AREA) administered at 0, 1 and 12 months. 2. Semistructured interviews at the conclusion of the elective to characterize the kinds of learners who choose Social Medicine and identify components of the elective that are best received. 3. Electronic database to facilitate longitudinal analysis of participants in the social medicine elective.

DISCUSSION / REFLECTION / LESSONS LEARNED: Our pilot month was by all accounts a success. Our core faculty work group participated in the curriculum alongside the students, attending lectures, meeting legislators, and reflecting on the determinants of health. Most found the learning activities to be interesting, innovative, and applicable. Given the complexity of the schedule, a coordinator would be a valuable asset.

WOMEN'S HEALTH (WH) CURRICULUM FOR INTERNAL MEDICINE (IM) RESIDENTS: IMPLEMENTATION BASED ON IDENTIFICATION OF KNOWLEDGE AND COMFORT LEVEL DEFICITS IN RESIDENTS Stephanie Hartman ${ }^{1}$; JoAnn Porter ${ }^{1}$; Rachel Bonnema ${ }^{2}$. ${ }^{1}$ Creighton University Medical Center, Omaha, NE; ${ }^{2}$ University of Nebraska Medical Center, Omaha, NE. (Control ID \#1339346)

NEEDS AND OBJECTIVES: The American Board of Internal Medicine and Federated Council for Internal Medicine published WH competencies include medical knowledge and counseling skills, WH procedures, and inclusion of WH content on the American College of Physicians (ACP) in-training exam and the ABIM Board exam. Recent studies suggest gaps still exist for knowledge and comfort in $\mathrm{WH}$ for both residents and practicing physicians. Objective: Assess IM resident knowledge and comfort with regard to medical practices specific to women or areas of medicine with important gender differences and create a dynamic curriculum to include lecture-based, web-based, and small group problem-based learning.

SETTING AND PARTICIPANTS: IM residents in a University-based program.

DESCRIPTION: Residents completed a 27-question pre-test assessment of knowledge, an 11-question demographic and prior training related to WH survey, and a 34-question Likert questionnaire on their comfort level discussing specific topics with patients, evaluation and treatment, and procedural skills. 
EVALUATION: $52 / 72$ residents completed the initial assessment tools, 51.9\% female, 38.5\% PGY1, 36.5\% PGY2, 25\% PGY3, 36.5\% American medical graduates. Previous exposure to $\mathrm{WH}$ included: completion of an ambulatory medicine rotation $(65.4 \%)$, simulated pelvic exam with model (76.9\%), or gynecologic teaching aide (GTA) $(88.5 \%)$, simulated breast exam with model $(69.2 \%)$ or GTA $(61.5 \%)$. Comfort analysis of procedures using 6-point Likert score revealed least comfort with performing and reviewing vaginal wet mount (mean 2.2) and most comfort with breast exam (mean 4.1). Residents were most comfortable discussing safe sexual practices (mean 3.9) and least comfortable discussing infertility (mean 2.9). Regarding evaluation and treatment, residents were most comfortable with cardiovascular disease (mean 3.8) and least comfortable with medications safe in pregnancy (mean 2.7). Two-way ANOVA was used to evaluate the impact of training level on comfort and knowledge. Comfort level with procedures was significantly different based on training level; PGY1 $2.96 \pm 0.99$, PGY2 3.09 \pm 0.65 , PGY3 3.86 \pm 1.16 , $(p=0.024)$.

There was no difference in comfort with discussion with patients or evaluation and treatment based on training level. In multivariate analysis increased exposure to simulation experience and foreign medical graduate status was associated with increased total comfort score $(\mathrm{p}=0.034$ and $\mathrm{p}=0.004$ respectively). Pre-test knowledge differed by training level with total score PGY1 15.1 \pm 2.8 , PGY2 17.7 \pm 2.8 , PGY3 17.1 $\pm 2.2(\mathrm{p}=0.010)$. No difference in knowledge scores was present between genders, medical school location, future employment plans or location of continuity clinic. DISCUSSION / REFLECTION / LESSONS LEARNED: Overall, IM residents lack confidence in multiple areas of WH procedures, treatment and evaluation. As training level increases WH knowledge is improved, although comfort was not significantly improved. Comfort was improved with increased exposure to simulation experience, which indicates it is an important curriculum tool and should be included as a part of a comprehensive WH curriculum. The next step: A lecture-based, webbased, and small group problem-based learning series will be developed based on the areas of least knowledge and perceived least comfort. At the completion of the series all residents will be asked to complete a post-test assessment including both knowledge and comfort assessments to assess improvements in knowledge and comfort level related to WH topics. 